\title{
Hanford Atmospheric Dispersion Data: 1960 Through June 1967
}

Prepared by P. W. Nickola, J. V. Ramsdell, C. S. Glantz, R. E. Kerns

Pacific Northwest Laboratory

Operated by

Battelle Memorial Institute

- Prepared for

U.S. Nuclear Regulatory

Commission 
This report was prepared as an account of work sponsored by an agency of the United States Government. Neither the United States Government nor any agency thereof, or any of their employees, makes any warranty, expressed or implied, or assumes any legal liability of responsibility for any third party's use, or the results of such use, of any information, apparatus, product or process disclosed in this report, or represents that its use by such third party would not infringe privately owned rights.

\section{Availability of Reference Materials Cited in NRC Publications}

Most documents cited in NRC publications will be available from one of the following sources:

1. The NRC Public Document Room, 1717 H Street, N.W. Washington, DC 20555

2. The NRC/GPO Sales Program, U.S. Nuclear Regulatory Commission, Washington, DC 20555

3. The National Technical Information Service, Springfield, VA 22161

Although the listing that follows represents the majority of documents cited in NRC publications, it is not intended to be exhaustive.

Referenced documents available for inspection and copying for a fee from the NRC Public Document Room include NRC correspondence and iriternal NRC memoranda; NRC Office of Inspection and Enforcement bulletins, circulars, information notices, inspection and invertigation notices; Licensee Event Reports; vendor reports and correspondence; Commission papers; and applicant and licensee documents and correspondence.

The following documents in the NUREG series are available for purchase from the NRC/GPO Sales Program: formal NRC staff and contractor reports, NRC-sponsored conference proceedings, and NRC booklets and brochures. Also available are Regulatory Guides, NRC regulations in the Code of Federal Regulations, and Nuclear Regulatory Commission Issuances.

Documents available from the National Technical Information Service include NUREG series reports and technical reports prepared by other federal agencies and reports prepared by the Atomic Energy Commission, forerunner agency to the Nuclear Regulatory Commission.

Documents available from public and special technical libraries includgenll open literature items, such as books, journal and periodical articles, and transactions. Federary state legislation, and congressional reports can usually be obtained fromk

Documents such as theses, dissertations, foreign reports and translations, and non- 1 conference proceedings are available for purchase from the organization sponsoring the publicat,

Single copies of NRC draft reports are available free upon written request to the Df fion of Tech. nical Information and Document Controi, U.S. Nuclear Regulatory Commission, W. Hityn, DC
20555 .

Copies of industry codes and standards used in a substantive manner in the NRC regulatory process are maintained at the NAC Library, 7920 Norfolk Avenue, Bethesda, Maryland, and are availablethere for reference use by the public. Codes and standards are usually copyrighted and may be purchased from the originating organization or, if they are American National Standards, from the ' American National Standards fr. Tute, 1430 Broadway, New York, NY 10018. 
NUREG/CR-3456

PNL-4814

RB

\section{Hanford Atmospheric Dispersion Data: 1960 Through June 1967}

Manuscript Completed: October 1983

Date Published: November 1983

Prepared by

P. W. Nickola, J. V. Ramsdell, C. S. Glantz, R. E. Kerns

Pacific Northwest Laboratory

Richland, WA 99352

\section{Prepared for}

Division of Health, Siting and Waste Management

Office of Nuclear Regulatory Research

U.S. Nuclear Regulatory Commission

Washington, D.C. 20555

NRC FIN B2384 


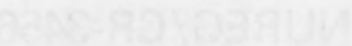

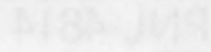

and
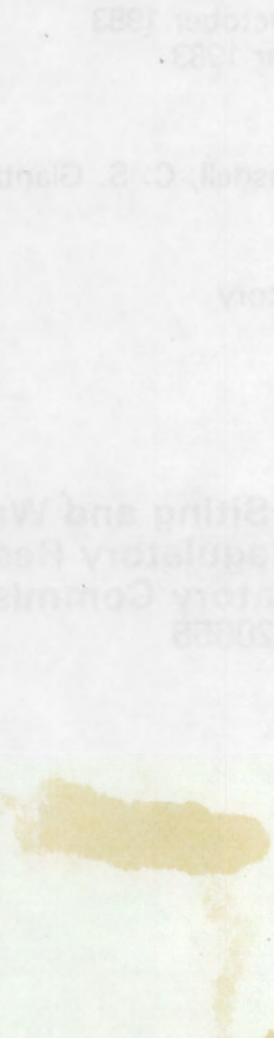

(2)

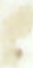




\section{PREFACE}

Our ability to prepare this data volume is due almost entirely to the efforts of the senior author, Paul W. Nickola. He was involved in the development of the experimental methods that made it possible to collect and assay the large number of samples affordably; he was involved in the field work during the experiments and in the assay of the samples; he collected and preserved the original data logs that were used in compilation of this volume, and he worked to have the data published so that others could benefit from them. Unfortunately, Paul isn't here to receive the credit that is due him.

Work on this data volume was begun before Paul's death in August 1982 . He had organized the original data records and collected supporting documentation. To these, he had added comments that reflected his personal recollections on and insight to the experiments. Finally, he had begun to prepare the data for publication. This data volume follows the example he set in the Hanford 67-Series data volume (Nickola 1977).

We, the remaining authors, hope that our efforts in completing the data volume have been adequate in comparison with his. We have included all of the data that he assembled along with his annotations. In doing this, we may have included data that he would have excluded, and certainly, we have not been able to make further annotations as he would have. 



\section{ACKNOWLEDGEMENTS}

The data reported in this volume were collected in research programs funded by the U.S. Atomic Energy Commission from 1960 through 1967. The authors wish to thank the U.S. Department of Energy Office of Health and Environmental Research for making the data available for publication, and the U.S. Nuclear Regulatory Commission for making the publication possible. The NRC technical contract monitor, Dr. R. F. Abbey of the Earth Sciences Branch of the Division of Health, Siting and Waste Management, was instrumental in the identification of the potential application of these data to the NRC's needs in estimating diffusion and transport of nuclear power plant effluents under normal operating conditions and in the event of an emergency. His contribution is gratefully acknowledged. 



\section{ABSTRACT}

This volume presents dispersion and supporting meteorological data from experiments conducted over relatively flat terrain at Hanford, Washington from January 1960 through June 1967. The nature of the experiments, the sampling grids, and the tracer techniques used are described in the narrative portion of the document. Appendices contain the time-integrated concentrations for samplers within the plumes, summaries of the concentration distributions across the plumes, and wind and temperature profile data for each release period. 



\section{EXECUTIVE SUMMARY}

A large number of diffusion experiments were conducted on the Hanford diffusion grid from 1959 through 1974. These experiments involved surface and elevated releases of one or more tracers. Samples were collected near ground-level $(1.5 \mathrm{~m})$ at distances between 100 and $25,600 \mathrm{~m}$ and on towers to distances of $3200 \mathrm{~m}$. This report contains the diffusion and supporting meteorological data for 134 releases made from 1960 through June 1967. It is anticipated that these data are pertinent to many current areas of research, despite their age. Data for most of the other Hanford experiments have been published in previous data volumes. With the publication of this volume, essentially all of the conventional diffusion data obtained in Hanford experiments through 1974 have been made available. 



\section{CONTENTS}

PREFACE $\ldots \ldots \ldots \ldots \ldots \ldots$

ACKNOWLEDGEMENTS. . . . . . . . . . . . . . .

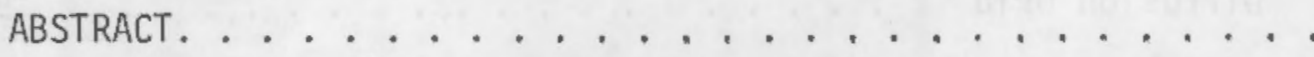

EXECUT IVE SUMMARY

INTRODUCT ION.

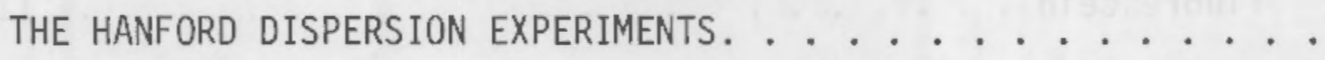

THE FIELD GRID.

ZINC SULFIDE FLUORESCENT PARTICLES

FLUORESCEIN

XENON .

DESCRIPTION OF THE DATA

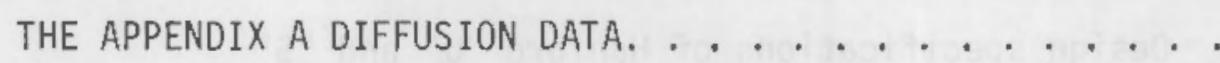

THE APPENDIX B DIFFUSION SUMMARIES . . . . . . . .

THE APPENDIX C METEOROLOGICAL DATA .........

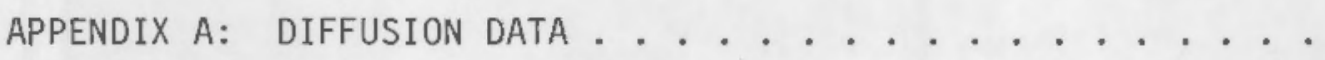

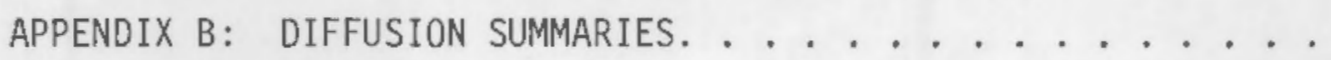




\section{FIGURES}

1 Diffusion test chronology ........... 6

2 View looking south along arc $1600 \mathrm{~m}$ from S-source ... 8

3 Aircraft view of near-source arcs on Hanford Diffusion Grid ............... 9

4 Aircraft view showing sampling arcs to distances of $1600 \mathrm{~m}$ from $\mathrm{U}$ - and $\mathrm{S}$-sources . . . . . . . . 10

5 Insecticide sprayers used in dispensing $\mathrm{ZnS}$ and fluorescein ................ 11

6 The diffusion grids superimposed on a contour map . . . . 12

7 Assembled Rankin counter (right) and disassembled Rankin counter (left) with several field filter assemblies in place for assay . . . . . . . . 18

\section{TABLES}

1 Distribution of tracer releases by time-of-day and release height, number of ZnS releases/ number of fluorescein and rhodamine releases ..... 5

2 Design specifications of Hanford " $U$ " and "S" diffusion grids ................ 14

3 General grid, source, release and meteorological information ............. 25 


\section{INTRODUCT ION}

A large number of diffusion experiments were conducted on the Hanford diffusion grid from 1959 through 1974. These experiments involved surface and elevated releases of one or more tracers. Samples were collected near ground-level $(1.5 \mathrm{~m})$ at distances between 100 and $25,600 \mathrm{~m}$ and on towers to distances of $3200 \mathrm{~m}$. This report contains the diffusion and supporting meteorological data for 134 releases made from 1960 through June 1967. It is anticipated that these data are pertinent to many current areas of research, despite their age. Data for most of the other Hanford experiments have been published in previous data volumes. With the publication of this volume, essentially all of the conventional diffusion data obtained in Hanford experiments through 1974 have been made available.

The data volume is organized as follows: A narrative section briefly describes the Hanford experiments, the sampling grid, the tracers, tracer dispersal techniques, and sample collection and assay. The narrative section is followed by three appendices that contain the diffusion and supporting meteorological data. Appendix A contains the diffusion data for each of the experiments. The information presented includes: a description of the experiment by release height, time-of-day, tracer, amount released and wind speed; the normalized time-integrated concentrations (exposures) for each sampling position, and the crosswind integrated exposures for the ground-level samplers at each distance. Appendix B contains diffusion estimates derived from the data. These estimates include the mean, standard deviation and coefficients of skewness and kurtosis of the crosswind concentration distributions, the three highest exposures at each distance, and the crosswind integrated exposures for each distance. Finally, Appendix C contains the supporting meteorological data for the experiments. These data include vertical profiles of temperature, wind speed, wind direction and standard deviation of the wind direction for each release period. 



\section{THE HANFORD DIFFUSION EXPERIMENTS}

The Hanford diffusion experiments have been assigned identifying names or codes. The names are associated with groups of experiments having similar objectives and experimental designs.

The first, and best known group of experiments, was Green Glow. These experiments were conducted during the summer of 1959 and consisted of 28 releases of fluorescent pigment (ZnS) near ground-level during nighttime, stable atmospheric conditions. The data for the Green Glow experiments have been published by Barad and Fuquay (1962a and 1962b).

The Hanford 30 series experiments from 1960 through 1962 followed the Green Glow design except that they were conducted during neutral and unstable atmospheric conditions. There were 42 releases in the 30 Series, giving a total of 70 ground-level releases in the combination of the Green Glow and 30 Series. Fuquay, Simpson and Hinds (1964) presented a summary of the data from the best of the Green Glow and 30 Series experiments. This volume contains the available 30 series data. Data for 8 of the tests are missing.

The $U$ Series experiments were started in 1960 and continued through 1969. These experiments departed from the Green Glow design by using elevated release points. This is the largest group of Hanford experiments. Of the 92 experiments in the U Series, the first 53 were conducted under neutral and unstable atmospheric conditions, and the last 37 were conducted under stable conditions. The number of tracer releases in the $U$ Series was larger than the number of experiments because, starting at test $U 40$, each experiment involved the simultaneous release of $\mathrm{ZnS}$ and fluorescein at different heights. Nickola (1977) presents the data for experiments U-56 through U-92; the data for experiments $\mathrm{U}-2$ and $\mathrm{U}-5$ through $\mathrm{U}-55$ are contained in this volume.

Prior to changing the U Series emphasis from neutral and unstable atmospheric conditions to stable atmospheric conditions, two sets of preliminary experiments were conducted. These were the ' 63 and '64 Series experiments. In a11, there were 33 experiments in these two series. This volume contains the data for the ' 63 and ' 64 Series experiments with the exception of '63-2 and '64-3.

The last two major experimental series at Hanford were krypton-85 releases that occurred between 1967 and 1974 and the $V$ Series experiments in 1972 and 1973. The krypton-85 experiments consisted of 19 puff and continuous releases near ground-level. They provide data on the temporal variations of concentration as well as time-integrated concentrations. During the early experiments in this series, the sampling was conducted along the 200 and $800 \mathrm{~m}$ sampling arcs used initially in the Green Glow experiments. During the later krypton 
experiments, special sampling grids were used when low wind diffusion measurements were being made. Data for the initial krypton-85 experiments are published in Nickola, Ramsdell and Ludwick (1970), and timeintegrated data for these experiments are also contained in Nickola (1977).

The eight $V$ Series experiments were conducted to evaluate the depletion of particulate plumes by deposition. The experiments consisted of multiple tracer releases from a height of $26 \mathrm{~m}$. The tracers used were $\mathrm{ZnS}$, rhodamine dye, and krypton-85. Concentration measurements were made on towers and at ground-level. The data for these experiments are contained in Nickola (1977).

In addition to the seven experimental series just mentioned, there were many other releases on the Hanford diffusion grid and at other locations on the Hanford site. Some of these releases have been combined into seven secondary series. These secondary experimental series were generally related to development of new tracer techniques. Where appropriate, previously unpublished data for these secondary series have been included in this volume.

The MT Series of 4 releases from 1962 through 1964 was related to the development of multiple tracer techniques, and four D Series releases in 1967 carried the multiple tracer technique development further by providing data for evaluation of simultaneous releases from a common source. Data for the D Series experiments were published by Nickola (1977). Data for the last three MT experiments are included in this volume. This volume also contains the data from the single $Z$ experiment conducted in 1962.

Four $X$ Series releases in 1964 were made to evaluate trichloroethane as a carrier for use in dispersing ZnS particles in place of water. The data for two of these releases are included in this data volume.

Two series of tests have been given an A designator. The first A Series occurred in 1964 and 1965, and the second in 1966 and 1967 . Both sets of releases were made to test a real-time sampler for $\mathrm{ZnS}$ and to evaluate use of the sampler in moving vehicles and aircraft. Groundlevel fixed samplers were activated for some of these releases. Available data from the fixed samplers for these experiments have been included in this data volume.

In 1965, there were five multiple tracer experiments in which xenon-133 was one of the tracers. These experiments, known collectively as the Xenon Series, were to evaluate xenon for use as a non-depositing tracer in studying the dry deposition of ZnS and fluorescein. The other tracers in these experiments were $\mathrm{ZnS}$ and fluorescein. Data for all of these experiments are included in this data volume. 
The last secondary experimental series of consequence is the PECON Series. This series consisted of $11 \mathrm{ZnS}$ releases made near the Hanford diffusion grid from 1965 through 1967. Seven of the releases were made at the upwind corner of a small building having a rectangular crosssection, and the remaining releases were made under similar meteorological conditions over flat terrain without any obstructions. Samples were collected to a distance of $100 \mathrm{~m}$ using both fixed samplers and a real-time sampler. Hinds (1967 and 1969) describe the experiments in detail and present analyses of the PECON data.

The chronological relationship among the sets of experiments is summarized in Figure 1. Table 1 shows the distribution of the releases of $\mathrm{ZnS}$, fluorescein and rhodamine by release height and time-of-day. Time-of-day is roughly equivalent to atmospheric stability.

Table 1. Distribution of Tracer Releases by Time-of-Day and Release Height, Number of ZnS Releases/Number of Fluorescein and Rhodamine Releases

\begin{tabular}{lccccc}
\hline $\begin{array}{l}\text { Time- } \\
\text { of-Day }\end{array}$ & \multicolumn{5}{c}{ Release Height (m) } \\
& 1 & 7 & 26 & 56 & 111 \\
\hline $0000-0159$ & $6 / 5$ & & $17 / 0$ & $6 / 19$ & $9 / 0$ \\
$0200-0359$ & $10 / 12$ & $11 / 0$ & $8 / 0$ & $6 / 2$ & $3 / 0$ \\
$0400-0559$ & $24 / 4$ & $7 / 0$ & $3 / 5$ & & \\
$0600-0759$ & $2 / 1$ & $1 / 0$ & & & \\
$0800-0959$ & $5 / 0$ & $1 / 0$ & $2 / 2$ & $5 / 0$ & \\
$1000-1159$ & $8 / 4$ & & $9 / 3$ & $15 / 0$ & $1 / 0$ \\
$1200-1359$ & $2 / 2$ & & $4 / 1$ & $10 / 0$ & \\
$1400-1559$ & $2 / 2$ & & $2 / 0$ & $8 / 0$ & \\
$1600-1759$ & $1 / 1$ & & $1 / 0$ & & \\
$1800-1959$ & $17 / 0$ & & & & \\
$2000-2159$ & $17 / 0$ & & & \\
$2200-2359$ & $15 / 1$ & & & & \\
& & & & & \\
\hline
\end{tabular}




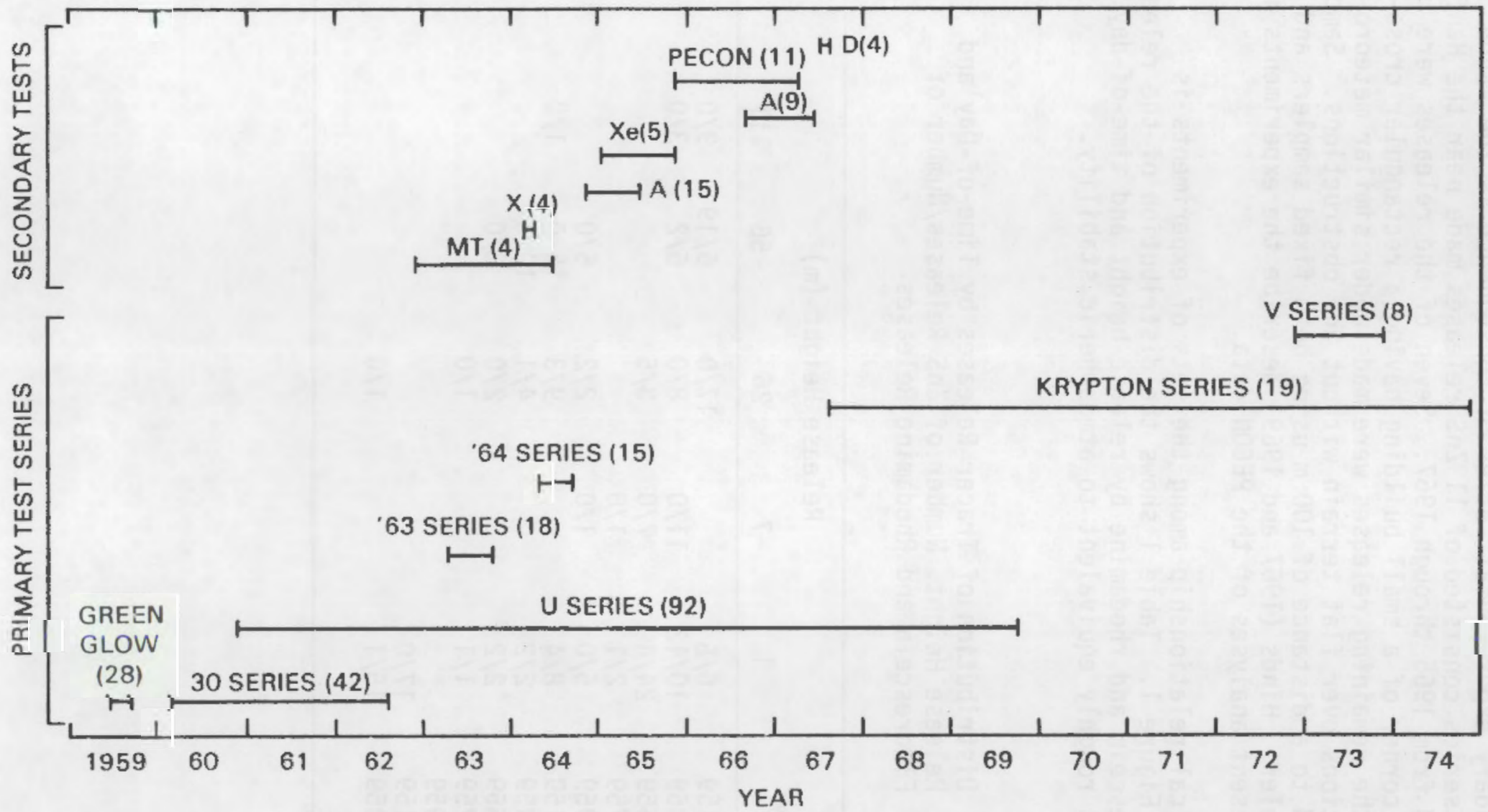

FIGURE 1. Diffusion test chronology. 


\section{THE FIELD GRID}

Hanford is located in a semi-arid region in the southeast corner of the State of Washington. The natural vegetation in the area is sagebrush, 1 to $2 \mathrm{~m}$ in height, interspersed with steppe grasses. Figures 2 through 5 give some idea of the nature and density of the vegetation. Boundary layer wind measurements indicate that a roughness length of $0.03 \mathrm{~m}$ is generaliy appropriate for Hanford.

The Hanford diffusion grids are located near the center of an approximately $40 \mathrm{~km}$ diameter basin. The center of the basin is about $200 \mathrm{~m}$ above mean sea level, and the hills and bluffs that nearly surround the basin reach as high as $1000 \mathrm{~m} \mathrm{~ms}$. Figure 6 shows the diffusion sampling grids superimposed on a contour map. The bulk of the sampling arcs are located in a relatively flat area where the extremes in elevation range from $200 \mathrm{~m}$ to $230 \mathrm{mms}$. The elevation of the most distant tracer sampling arc shown, $12.8 \mathrm{~km}$ from the source, is about $35 \mathrm{~m}$ lower than the nearer-source sampling arcs. A more distant sampling $\operatorname{arc}(25.6 \mathrm{~km})$ was used during the Green Glow experiments, but was not used during any of the experiments reported on in this data volume.

The approximately 1000 primary or "ground-level" samplers on the Hanford diffusion grids are located at a height of $1.5 \mathrm{~m}$, a height that approximates the breathing height of man. Samples are collected by drawing air through filters using vacuum pumps. Power for the field vacuum pumps is supplied by gasoline or propane fueled internal combustion engines. A single engine/pump assembly draws vacuum for 1 to 9 sampling stations depending on the flow rate required at the stations. The flow at each sample station is controlled by a critical flow orifice in the vacuum line just downstream of the filter-filter holder assembly. Flow through each sampler is constant as long as the pressure drop across the control orifice is greater than one-half atmosphere. The pressure drop for each engine/pump assembly is monitored by a vacuum gauge in the vacuum line immediately downstream of the orifice at the most remote sampler serviced by that assembly.

Ground-level sampling stations are supplemented by 365 tower-mounted sampling stations. The towers as tall as $62 \mathrm{~m}$ and as far removed as $3.2 \mathrm{~km}$ from the tracer release point, are discussed in more detail later in this section. Vacuum and flow control for the tower samplers are accomplished in the same manner as for the ground-level samplers.

The sampling grid(s) used at Hanford evolved from grids laid out in 1959 and 1960. The grids were designated the "U" and the "S" grids because their original uses were restricted to thermally unstable (U) and thermally stable (S) atmospheres, respectively. The U-grid is laid out as a series of circular arcs, concentric about a $122 \mathrm{~m}$ tower. This configuration can be seen in Figures 3 and 4 . In these figures, several 


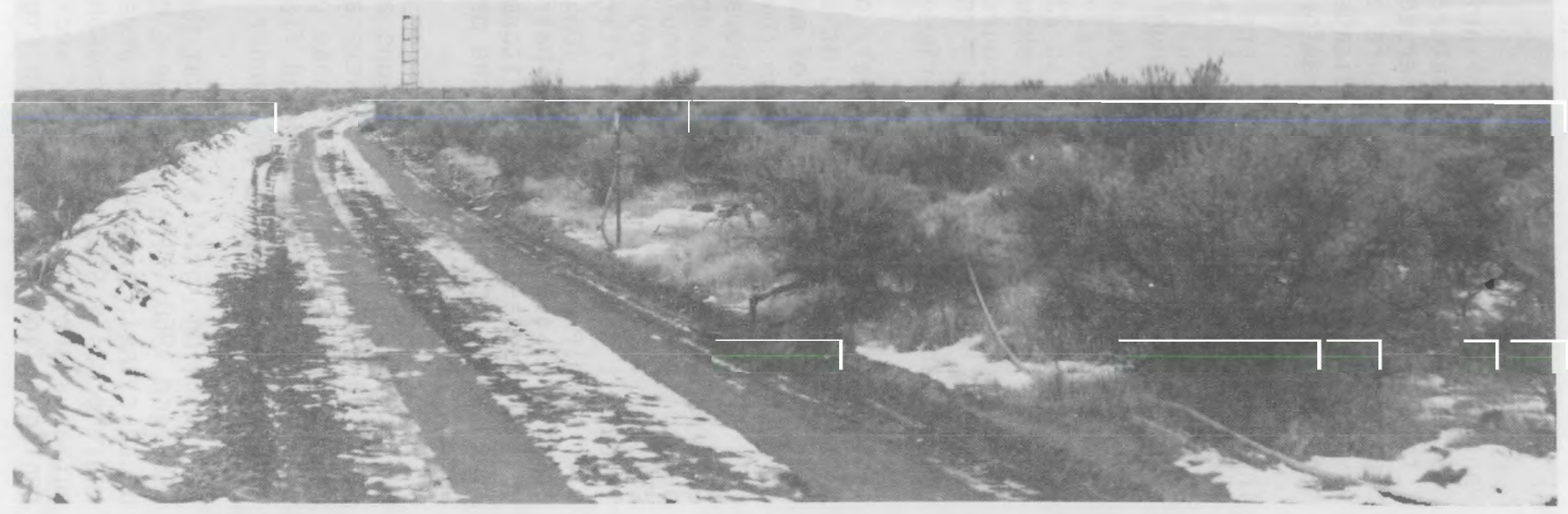

Figure 2. View looking south along arc $1600 \mathrm{~m}$ from S-source. 


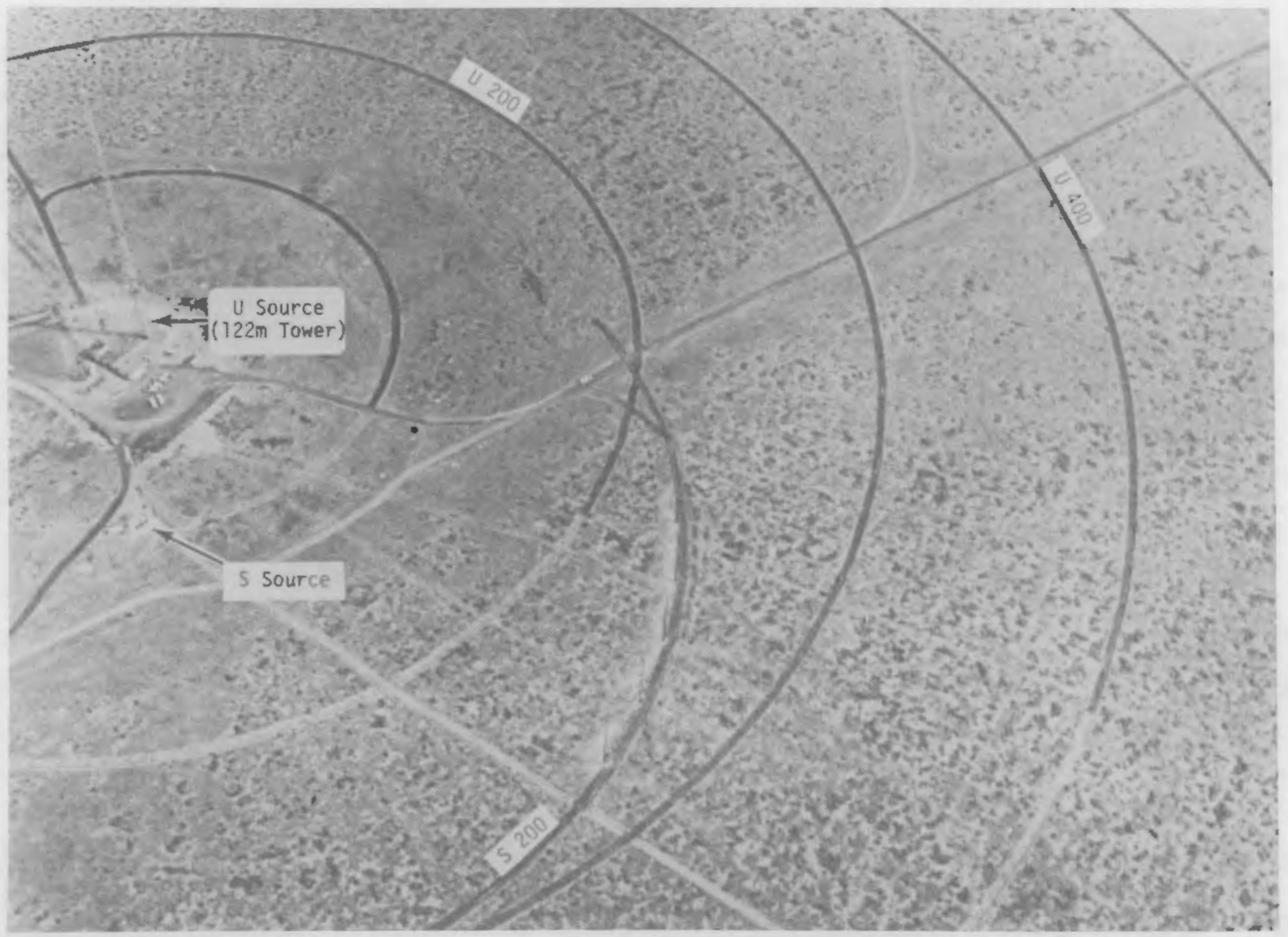

FIGURE 3. Aircraft View of Near-Source Arcs on Hanford Diffusion Grid 


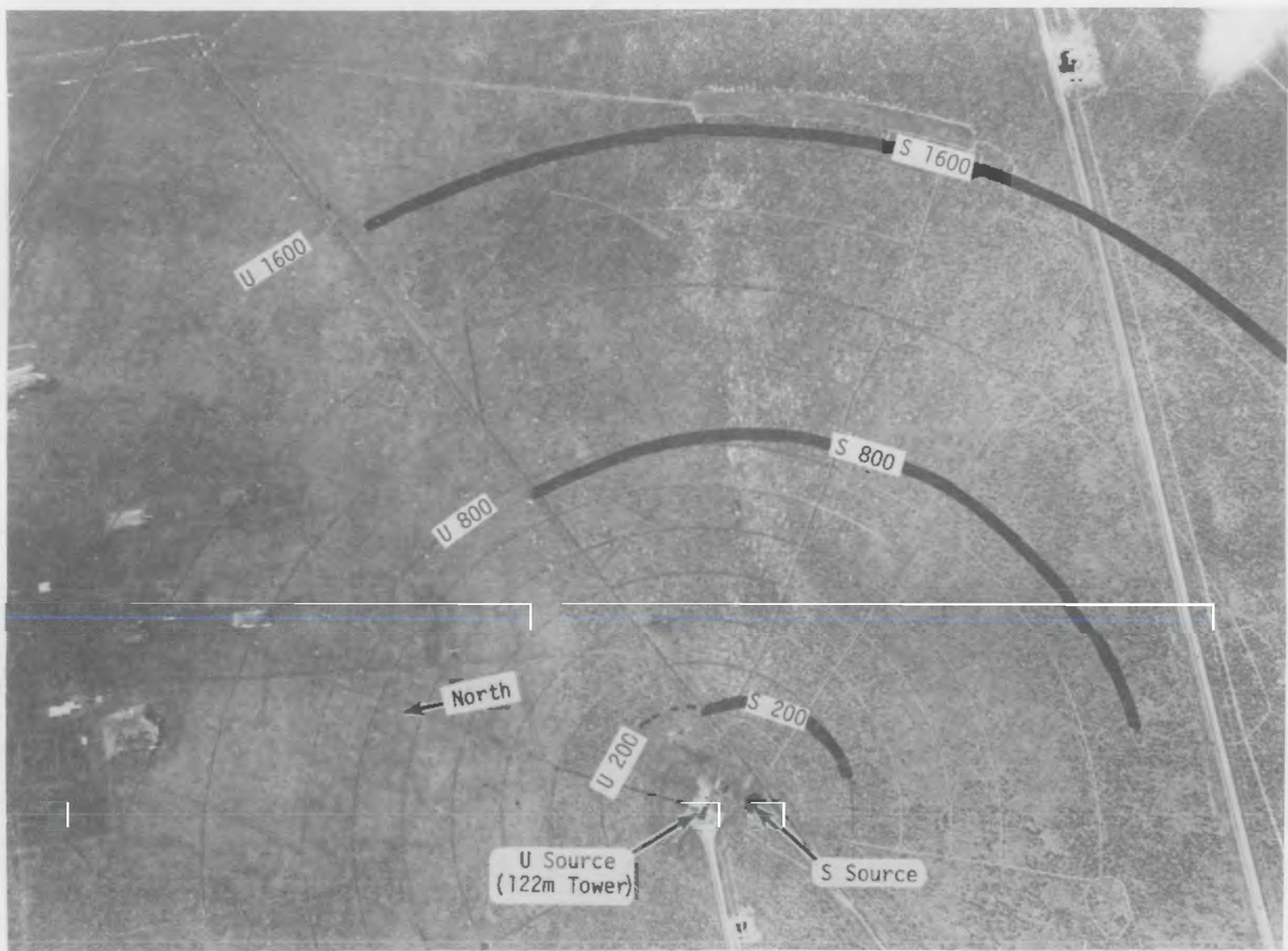

FIGURE 4. Aircarft View Showing Sampling Arcs to Distances of $1600 \mathrm{~m}$ from $\mathrm{U}$ - and S-sources 


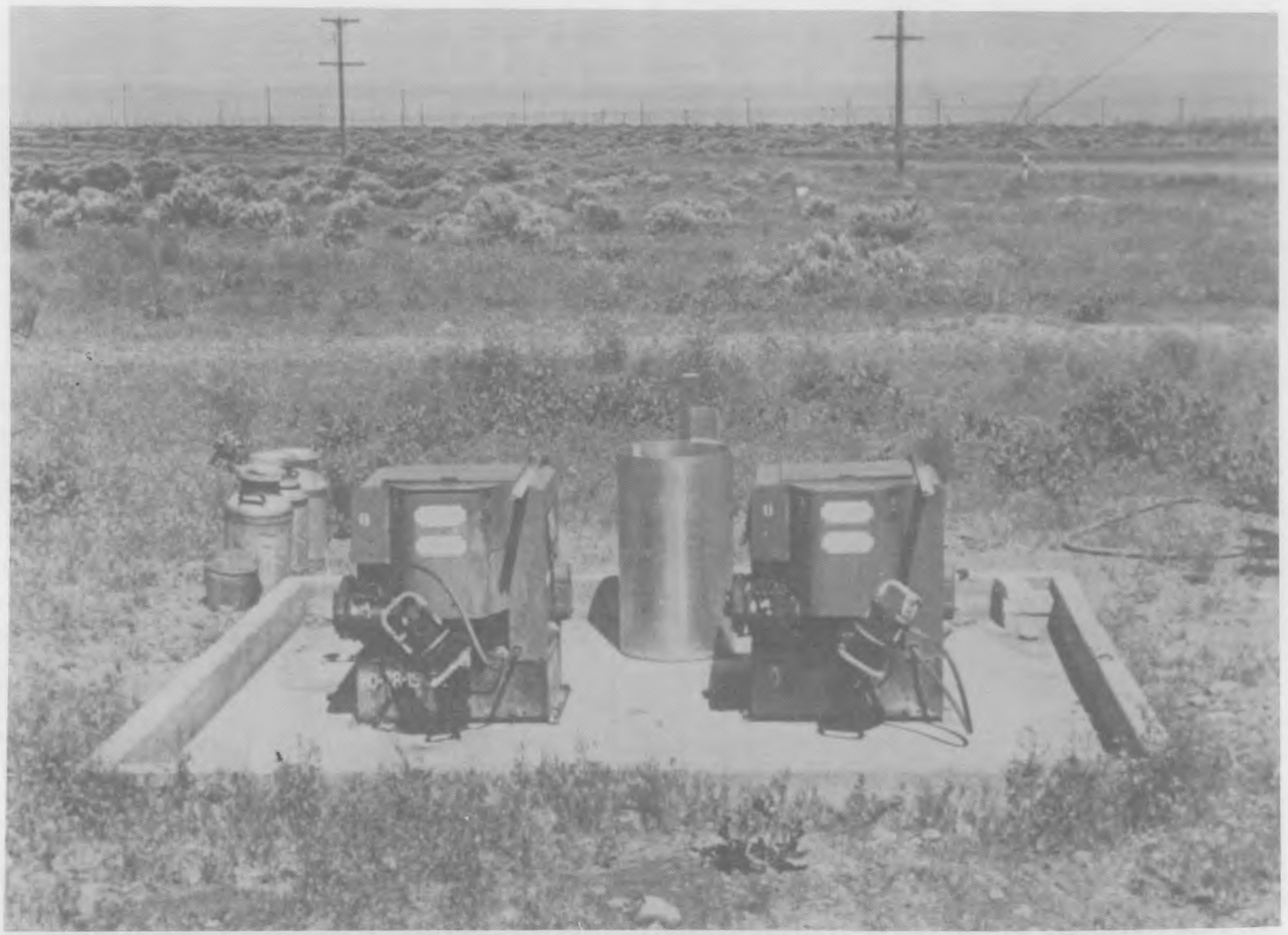

FIGURE 5. Insecticide sprayers used in dispensing $\mathrm{ZnS}$ and fluorescein 


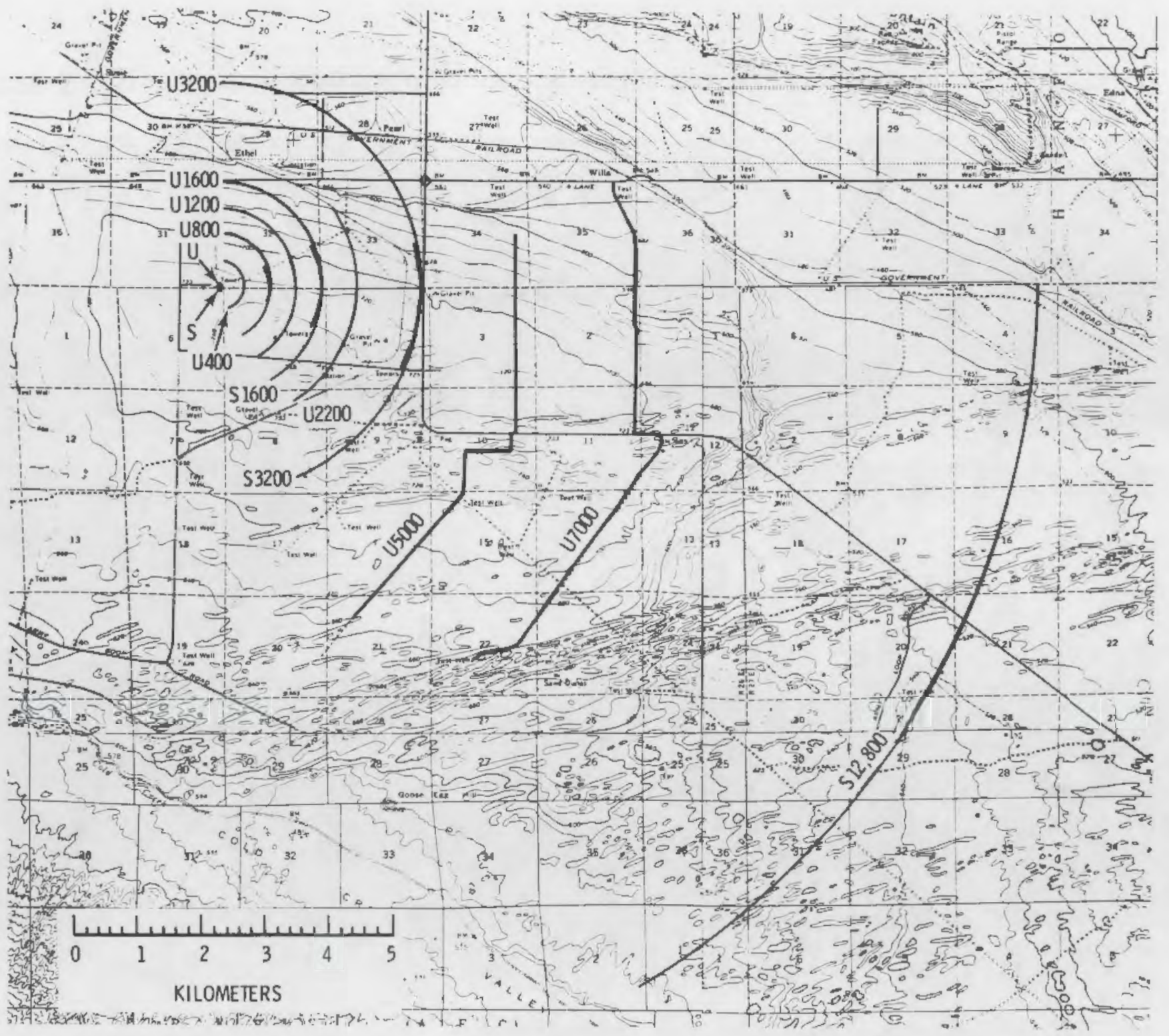

FIGURE 6. The diffusion grids superimposed on a contour map 
of the arcs on this grid are labeled with the letter "U" followed by the radial distance in meters from the source to the arc. The design specifications for the U-grid, including the arc distances and the crosswind extent of the arcs, are given in Table 2. However, not all of the arcs, nor even all of the samplers on any selected arc, were used during a given field experiment. Experimental objectives, meteorological conditions, and the manpower available were factored into decisions as to which samplers should be activated.

Two more "arcs" of opportunity were laid out with azimuths related to the U-source. These so-called arcs were not arcs of circles, but were merely tracer sampling stations set out along existing roads or trails. They were dubbed the U5000 and U7000 arcs in correspondence with the approximate source-to-sampler distances involved. Figure 6 shows the configuration of the $\mathrm{U} 5000$ and U7000 arcs.

The S-grid source, used with only near ground-level tracer releases, is located $100 \mathrm{~m}$ due south of the U-grid source. This location was selected to minimize the wake effect of buildings at the base of the $122 \mathrm{~m}$ tower. Figure 5 is a view looking "upwind" from the S-source. The S-source is also indicated in Figures 3 and 4 . There are fewer concentric arcs on the S-grid than on the U-grid. The three arcs closest to the S-source are darkened and labeled in Figure 4. S-grid arcs used for the experiments documented here were $\$ 200, S 800, \$ 1600$, $\mathrm{S} 3200$ and $\mathrm{S} 12800$. The number designation, as with the U-arcs, gives the radial distance in meters from $S$-source sampling arc. Further detail on the S-grid is given in Table 2 .

There are twenty towers, instrumented for tracer sampling, on the Sgrid. These towers are 1ocated at azimuths of 98, 106, 114, 122 and 130 of the $\$ 200$, $\$ 800$, $\$ 1600$ and $\$ 3200$ arcs. The tower heights are $27,42,62$ and $62 \mathrm{~m}$ on the $\$ 200, \$ 800$, $\$ 1600$ and $\$ 3200$ arcs, respectively. 
Table 2. Design Specifications of Hanford "U" and "S" Diffusion Grids

\begin{tabular}{|c|c|c|c|c|c|c|}
\hline \multirow{2}{*}{$\begin{array}{l}\text { Grid Source and } \\
\text { Distance from } \\
\text { Source (m) }\end{array}$} & \multirow{2}{*}{$\begin{array}{c}\text { Range }{ }^{2} \text { of } \\
\text { 1. } 5 \text { m Sampling } \\
\text { Azimuths }\end{array}$} & \multirow[b]{2}{*}{$\begin{array}{l}\text { Sampler } \\
\text { Spacing }\end{array}$} & \multirow{2}{*}{$\begin{array}{c}\text { Sampler Flow } \\
\text { Rate } \\
\left(x 10^{-4} \mathrm{~m}^{3} / \mathrm{sec}\right)\end{array}$} & \multicolumn{3}{|c|}{ Towers } \\
\hline & & & & $\begin{array}{l}\text { Samples/ } \\
\text { Tower }\end{array}$ & Height (m) & \\
\hline U100 & $340^{\circ}$ to $122^{\circ}$ & $4^{\circ}$ & 5.07 & 17 & 33 & $102^{\circ}, 110^{\circ}, 118^{\circ}$, \\
\hline U200 & $342^{\circ}$ to $160^{\circ}$ & $4^{\circ}$ & 5.07 & & & $126^{\circ}, 134^{\circ}$ \\
\hline U300 & $340^{\circ}$ to $120^{\circ}$ & $4^{\circ}$ & 5.07 & & & \\
\hline $\mathrm{U} 400$ & $342^{\circ}$ to $154^{\circ}$ & $4^{\circ}$ & 5.07 & & & \\
\hline U 500 & $340^{\circ}$ to $120^{\circ}$ & $4^{\circ}$ & 5.07 & & $=$ & \\
\hline U600 & $342^{\circ}$ to $122^{\circ}$ & $4^{\circ}$ & 5.07 & & & 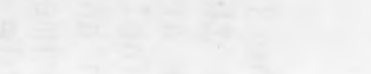 \\
\hline U700 & $340^{\circ}$ to $120^{\circ}$ & $4^{\circ}$ & 5.07 & & & \\
\hline U 800 & $340^{\circ}$ to $121^{\circ}$ & $3^{\circ}$ & 5.07 & & & \\
\hline U1000 & $001^{\circ}$ to $121^{\circ}$ & $2^{\circ}$ & 9.09 & & & \\
\hline U1200 & $360^{\circ}$ to $156^{\circ}$ & $2^{\circ}$ & 9.09 & & & \\
\hline U1600 & $360^{\circ}$ to $122^{\circ}$ & $2^{\circ}$ & 9.09 & & & \\
\hline U3200 & $360^{\circ}$ to $114^{\circ}$ & $2^{\circ}$ & 17.5 & & & \\
\hline $\mathrm{U} 7000^{3}$ & $76^{\circ}$ to $146^{\circ}$ & $2^{\circ}$ & 17.5 & & & \\
\hline $\mathrm{S} 200$ & $68^{\circ}$ to $158^{\circ}$ & $2^{\circ}$ & 1.48 & 17 & 27 & $\begin{array}{l}98^{\circ}, 106^{\circ}, 114^{\circ}, \\
122^{\circ}, 130^{\circ}\end{array}$ \\
\hline 5800 & $70^{\circ}$ to $160^{\circ}$ & $2^{\circ}$ & 1.53 & 18 & 42 & $\begin{array}{l}98^{\circ}, 106^{\circ}, 114^{\circ}, \\
122^{\circ}, 130^{\circ}\end{array}$ \\
\hline $\mathrm{S} 1600$ & $70^{\circ}$ to $154^{\circ}$ & $1^{\circ}$ & 2.32 & 19 & 62 & $\begin{array}{l}98^{\circ}, 106^{\circ}, 114^{\circ}, \\
122^{\circ}, 130^{\circ}\end{array}$ \\
\hline S3200 & $72^{\circ}$ to $157^{\circ}$ & $1^{\circ}$ & 5.13 & 19 & 62 & $\begin{array}{l}98^{\circ}, 106^{\circ}, 114^{\circ}, \\
122^{\circ}, 130^{\circ}\end{array}$ \\
\hline $\mathrm{S} 12800$ & $91^{\circ}$ to $151^{\circ}$ & $1^{\circ}$ & 17.5 & & & \\
\hline
\end{tabular}




\section{TRACER TECHNIQUES}

Data are presented in this volume for three different tracers released in the Hanford experiments of the early and mid-1960's. The tracers were: a zinc sulfide fluorescent powder (ZnS or FP), fluorescein, and xenon-133, a radioactive inert gas. A third fluorescent tracer, rhodamine, and another radioactive inert gas, krypton-85 were used in some of the experiments in the late 1960's and 1970's, which are reported in the Hanford 1967 Series Data Volume (Nickola 1977). The particulate tracers were collected on membrane filters, and concentrations were determined using laboratory procedures that made use of the fluorescent properties of the tracers. Assay techniques were able to discriminate among the tracers to the extent that collection of $\mathrm{ZnS}$ and fluorescein on a common filter did not result in an analytical problem.

\section{ZINC SULFIDE FLUORESCENT PARTICLES}

Use of fluorescent paint pigment (FP) as an atmospheric tracer was initiated in 1946 and documented in 1955 at Stanford University under the auspices of the U.S. Army Chemical Corps (Leighton 1955). The Hanford meteorology group began investigation of the use of such fluorescent pigments in 1952, and made use of FP field techniques on a relatively modest scale through 1958. An optical-electronic device, called a Rankin counter, developed in 1958 (Rankin 1958; Barad and Fuquay 1962a) increased the speed of the 1aboratory assay of FP samples by eliminating a tedious "man-and-microscope" sample assay procedure. This development made possible the analys is of the large number of samples collected during the Green Glow and subsequent Hanford field diffusion programs.

The FP selected for use in the Hanford techniques was Helecon Fluorescent Pigment 2210 manufactured by U.S. Radium Corp., Morristown, New Jersey. It is ZnS with an activator placed interstitially in its crystalline structure. These particles have a specific gravity of 4.1 . Based on optical microscope sizings at 1000X magnification, the number median (geometric mean) diameter of FP 2210 is about $2.1 \mu \mathrm{m}$. Assuming that Stokes' law for spheres applies, the terminal fall velocities should be between $1.9 \mathrm{~m} / \mathrm{hr}$ and $7.6 \mathrm{~m} / \mathrm{hr}$.

The ZnS tracer was dispensed through a commercial insecticide sprayer. Two of these devices are shown in Figure 5. A measured quantity of the tracer (generally 1 to $4 \mathrm{~kg}$ ) was added to a known volume of liquid carrier in an approximately 200 \& cylindrical tank (Figure 5) and maintained in suspension by a heavy-duty industrial propeller. The tracercarrier suspension was drawn directly from the cylindrical tank by the commercial sprayer unit and pumped to a nozzle assembiy. There it was atomized by mixing with a jet of heated air as it was dispensed. The mass of tracer dispensed in an experiment was determined by measuring liquid level in the tank before and after the release. 
The rate at which the tracer is released with this technique has been demonstrated to be constant. Further details of the rate determination --and of the wet dispensing technique--are given in Chapter $V$ of the Green Glow documentation (Barad and Fuquay 1962a).

The possibility that the wet dispensing technique could result in a significant agglomoration of individual tracer particles has been considered. However, in the years preceding development of the Rankin counter, a great number of filters were examined and particles were visually counted with the aid of a microscope and ultra-violet illumination. Very few agglomorates were observed during these microscopic examinations, even though the wet dispensing technique was in use at that time.

The temperature of the air used in the atomization was approximately $400^{\circ} \mathrm{C}$. Evaporation of the liquid carrier aided in the dissipation of heat so that the effluent from the generator felt dry and themally comfortable to the hand within 1 to $2 \mathrm{~m}$ of the nozzle.

In the early and mid 1960's, the liquid carrier used was water. Some concern developed over the possibility that, under high humidity conditions, the evaporation of the water carrier in the nozzle spray might take place so slowly that there would be qravitational settling. Therefore, a more volatile liquid, trichloroethane $\left(\mathrm{CH}_{3} \mathrm{CC}_{3}\right)$, was used as the carrier for ZnS for most of the experiments conducted after the summer of 1963. Although it is difficult to assess any differences in the data that might be due to the change in carrier, qualitatively at Hanford there was no obvious effect attributable to the carrier.

One problem that was associated with use of trichloroethane was that it did not act as a lubricant for the insecticide sprayer, as water apparently did. Many more mechanical difficulties or failures of the tracer dispensing equipment occurred when trichloroethane was used.

A comment should be made regarding the high temperature to which the ZnS fluorescent particles were subjected during the dispersal process. There was concern that the $400^{\circ} \mathrm{C}$ temperature might alter the fluorescent properties of the tracer even though the high temperature was experienced for only a fraction of a second. In 1963, Nickola (Nickola and Scoggins 1964) subjected samples of FP 2210 to temperatures of $1000^{\circ} \mathrm{C}$ for periods up to $20 \mathrm{sec}$. There were no discernible changes between the pre-heated and post-heated masses indicated when the samples were assayed using the Rankin counter.

It was also demonstrated by Nickola and Scoggins (1964) that FP 2210 was not affected by exposure to bright sunlight -- as has been observed for other fluorescent powders (Eggleton and Thompson 1961). Filters were selected from a field experiment in which the release, sampling and filter retrieval all occurred during hours of darkness. The filters 
were stored in an opaque box until they were assayed for FP 2210. After the initial assay, the filters were exposed to bright sunlight for more than 7 hours and then reexamined. (The Rankin counter does not destroy or alter the samples.) There was no reduction in the measured mass of FP on the filters.

Three different filters have been used to collect particulate samples at Hanford. AM-1 filters were used prior to 1962; GM-1 filters were used from 1962 through mid-1965, and VM-1 filters have been used since mid-1965. The filters are $47 \mathrm{~mm}$ in diameter and offer a compromise between the opposing requirements of minimal restriction to flow through the filter and a relatively hard, flat surface upon which the $\mathrm{ZnS}$ can be retained. The filter is inserted into a polyethylene filter holder assembly that leaves a $41 \mathrm{~mm}$ diameter circular area exposed for tracer collection.

Figure 7 shows several of these assemblies in place in the turntables of the Rankin counter. Between the counters, two of the filter assemblies are shown turned face down to display the ribbed nozzle which can be inserted in an neoprene grommet at each field sampling location. A dust cap (as on the assembly marked "1-122" on Figure 7) is placed over the filter-filter holder assembly during handling and storage. To minimize tracer contamination from experiment to experiment, filterfilter holder assemblies are used only once.

An assembled Rankin counter is shown at the right in Figure 7 , and one with top removed to expose a turntable is shown at the left. Filters are assayed by removing the dust cap and inserting the remaining filterfilter holder assembly into a circular cavity in the turntable. Several filters can be seen in these cavities in Figure 7 . The turntable is rotated until the filter to be assayed lies directly below a multiplier phototube. Here a 200-microcurie plutonium source, in the shape of an annulus about the face of the phototube, bombards the face of the filter with alpha particles. If there is any FP on the filter face, it is excited to fluorescence by the alpha bombardment. The resulting scintillations are monitored by the phototube, amplified and counted by a scaler. By retaining the bulk of the FP on its surface rather than allowing deeper penetration, the filter enhances the probability of the short-range alpha particles reaching the FP and of the resulting scintillations being seen by the photomultiplier.

Design of the Rankin counter permits the operator to insert or remove filters from the turntable at the same time another filter is being counted under the phototube. This procedure is a significant time saver in view of the large number of filters assayed for each experiment.

The relationship between the count from the Rankin counter and the mass of $\mathrm{ZnS}$ on the filter is essentially linear over a wide range. The linearity has been determined by comparing Rankin counter and X-ray 


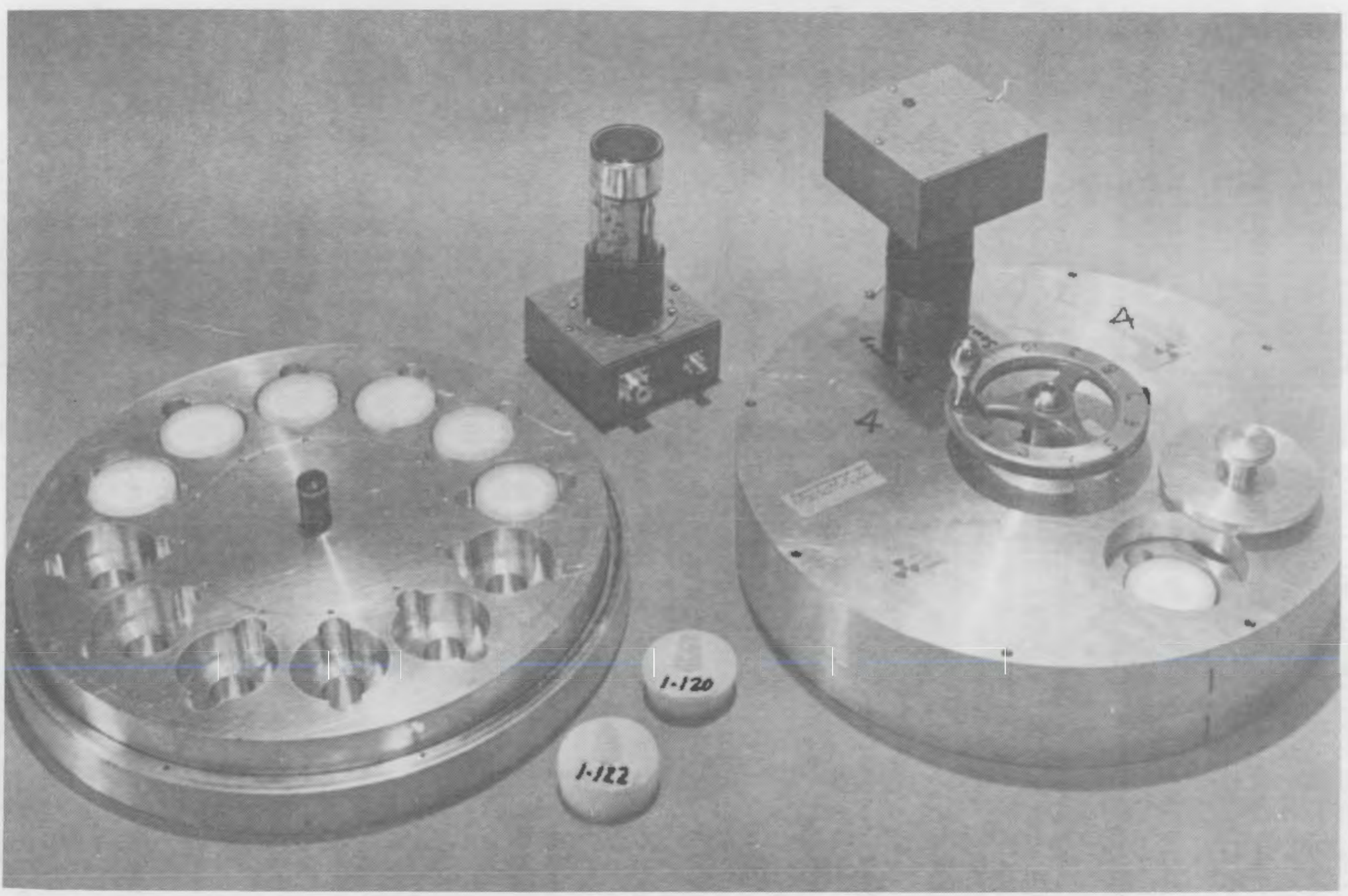

FIGURE 7. Assembled Rankin counter (right) and disassembled Rankin counter (left) with several field filter assemblies in place for assay 
fluorescence evaluation of many filters. Perhaps as a result of differences in filter surface characteristics, the Rankin counter calibration is a function of filter type.

The Rankin counter underwent primary calibrations against a series of filters of well-established mass several times during the experimental series. The calibration for the AM-1 and GM-1 filters used in most of the experiments reported here is

$$
M=5.23 \times 10^{-10} \mathrm{C}
$$

where $M$ is mass of ZnS FP 2270 in grams and $C$ is Rankin counts/min. The calibration for the VM- ] filters used for the experiments reported in the Hanford 67-Series data volume and a few of the more recent experiments in this data volume is

$$
M=2.06 \times 10^{-10} \mathrm{C}
$$

The level of detection with confidence is considered to be 20 counts $/ \mathrm{min}$ (about 3 times the maximum field background), and the corresponding mass is about $4 \times 10^{-9}$ grams. Count rate reproducibility is good with the Rankin counter, particularly at the higher count rates. The ratios of count rate standard deviation to mean count rates for $100,1000,10,000$ and 100,000 counts/min are $0.16,0.048,0.038$ and 0.012 , respectively.

The Rankin counter is checked, and tuned electronically, if necessary, against a standard filter that is left in one of the twelve turntable cavities. Inasmuch as the standard filter is counted each time it passes beneath the photomultiplier, a secondary calibration occurs once for each 10 field filters assayed. A background filter occupies the twelfth turntable cavity.

The normal counting time used with the Rankin counter is one minute. Unexposed filters generally have zero counts, and field exposed background filters have counts from 0 to about 6 due, presumably, to foreign fluorescent material in the ambient atmosphere. Despite all precautions there is strong evidence of contamination of a smali number of exposed filters. This contamination is generally associated with tower samples where it was more difficult to minimize the handling of filter assemblies during field deployment and collection.

Atmospheric dust -- or carbon from the internat combustion engines associated with the fjeld vacuum system -- can collect on the filter face and reduce the number of scintillations seen by the photomultiplier tube. To evaluate this effect, a series of previously assayed filters 
that had a clean appearance were intentionally subjected to tracer-free but dust-laden air. The filters were then ranked subjectively from 1 (clean) to 10 (extremely heavy dust load) according to their post-dust visual appearance and re-assayed. It was found that the count rates of filters with dust ratings of 4 or less were essentially unaffected by the dust. With successively higher dust ratings, increasingly greater count rate degradation was observed. Therefore, dust ratings are indicated in data in Appendix A for filters graded 5 or greater.

A liquid scintillation counting technique (Barad and Fuquay 1962a; Ludwick and Perkins 1961) is available that, to a large extent, overcomes the ZnS assay problems caused by dust-laden filters. However, the laboratory procedure is a much more tedious "wet" procedure than the simple Rankin counting approach. This fact, plus the relatively few dusty filters encountered during the experimental series, led to a decision not to employ the more elaborate "wet" assay technique.

In order for a filter to properly sample particles carried in air, the air velocity through the filter face should equal the ambient air velocity. In this isokinetic flow situation, the streamlines of the flow neither diverge nor converge at the filter. Therefore, particles carried with the fluid are sampled properly. However, if the velocity through the filter is substantially greater or less than the ambient air velocity, the particles will not (by virtue of their greater density than air) faithfully follow the streamines in the vicinity of the filter.

Sehme? (1966) investigated non-isokinetic sampling effects in a wind tunnel study using ZnS FP 2210 and the standard Hanford field filter. He derived corrections for non-isokinetic flow which are functions of wind speed and filter flow rate. On the Hanford grids, the filter face velocities are essentially always less than ambient wind speed, resulting in sub-isokinetic sampling. As a result, Sehmel's corrections have been applied to all the ZnS data presented in this report.

\section{FLUORESCEIN}

An atmospheric tracer technique using uranine dye, the sodium salt of fluorescein, was reported by Robinson, Macleod and Lapple (1959). The possibility of using this dye as a tracer to complement the existing $\mathrm{ZnS}$ FP 2210 technique was first investigated at Hanford in 1961, with early field results reported by Ludwick (1961), Ludwick and Nickola (1963) and Nickola (1965). The term fluorescein, rather than the specific salt, uranine, has been applied to the dye in Hanford nomenclature. This dye is available through industrial chemical suppliers. 
Fluorescein has a specific gravity of 1.53. Based on microscope sizing at 1000x magnification, the specific batch of fluorescein used had a number median diameter of $1.4 \mu \mathrm{m}$ and a mass median diameter of 18.6 $\mu \mathrm{m}$. The number and mass median diameters translate to terminal fall veiocities of about 0.4 and $64 \mathrm{~m} / \mathrm{hr}$ if Stokes' law is applied.

As with the ZnS, fluorescein was dispensed using a cormercial insecticidal sprayer. The liquid carrier used with fluorescein in the experiments reported here was water. In later experiments (Nickola 1977), trichloroethane was used as the carrier. The technique used to dispense the fluorescein was as described for the $\mathrm{ZnS}$ releases.

The same filters were used to collect fluorescein and ZnS. In the cases where both ZnS and fluorescein tracers were used simultaneously, the ZnS assay of the dry filters was carried out first. The filters were then placed in individual glass vials. Distilled water was added to dissolve the fluorescein particles, leaving the insoluble $\mathrm{ZnS}$ on the filter. The fluorescein solution was then assayed using a calibrated spectrophotofluorometer with excitation and emission wave lengths tuned for optimum perfomance. The analytical technique is explained in greater detail in Ludwick and Nickola (1963).

Although less than $1 \times 10^{-11}$ gram of fluorescein is detectable under laboratory conditions, atmospheric pollutants and filter-to-filter background variation make $5 \times 10^{-9}$ grams a more realistic estimate of the detection Timit for actual samples. The variation in background made definition of the tails of crosswind or vertical tracer distributions more difficult with fluorescein than with ZnS. In contrast with the deleterious effect of dust on the ZnS assay, the assay of fluorescein in solution was minimally affected by dust.

Although no laboratory tests were made to directly investigate the possible effects of the subjection of fluorescein to high temperatures during dispersai, Nickola (1965) found that ZnS and fluorescein released from the same location gave compatible downwind field concentrations. Reduction of fluorescence due to mixture with air at $400^{\circ} \mathrm{C}$ caused no obvious problems in these 1964 field tests. No corrections have been made for non-isokinetic sampling of fluorescein.

XENON

In 1965 five releases of xenon-133 were made to evaluate this inert gas for use in studies of the deposition of $\mathrm{ZnS}$ and fluorescein. Xenon-133 is a moderately short-lived radioisotope (5.3 day half-life) that decays with a weak beta enission $(0.35 \mathrm{MeV})$.

The technique used to collect the xenon samples involved trapping the gas on charcoal filters. Air was drawn through the membrane filters used to coliect the ZnS and fluorescein samples. It was then dried, carbon dioxide was removed, and the remaining gases were cooled by passing 
through glass bead traps containing a dry ice/acetone mixture. Finally, the gases were passed through a charcoal trap about $0.50 \mathrm{~m}$ in length. The charcoal traps were maintained at $-80^{\circ} \mathrm{C}$ while in the field and being returned to the laboratory, where they were stored at $-195^{\circ} \mathrm{C}$.

The samples were prepared for assay by desorption of the gases off the charcoal, and purification and dissolution of the xenon-133 into a liquid scintillator for 1iquid scintillation counting. This process took several hours and involved slowly warming the samples from $-195^{\circ} \mathrm{C}$ to $175^{\circ} \mathrm{C}$. The samples were assayed by counting for one hour, waiting about 5 days, then counting again for one hour.

Ludwick (1965) describes the sample collection, preparation and assay procedure in detail.

Compared with the other tracer technique in use at Hanford in 1965, the collection and analysis of xenon-133 data was extremely time consuming and costly. It also involved significantly more elaborate field equipment. As a result, the number of samples collected during each experiment was limited. It appears from the records available that fewer than 20 xenon samples were collected in each of the tests. There are not enough xenon samples to estimate diffusion coefficients, but the normalized xenon time-integrated concentrations can be compared with the time-integrated concentrations of $\mathrm{ZnS}$ and fluorescein collected at the same locations. 
The experiments that produced the data presented in this data volume were conducted on 128 days during the eight and one-half year period from January 1960 through June 1967. The data were collected in nine experimental series. Of these series, four are primary series, i.e., the 30 Series, the $U$ Series, the ' 63 Series and the ' 64 Series. These series contribute data for 117 experiments. The remaining five series are secondary and contribute data for 17 experiments. ZnS was released in all of the experiments, and most (115) of the experiments were limited to the release of a single tracer. Fluorescein data have been included for 17 of the experiments, and xenon data for four.

Tracer sampling equipment was activated before the tracer release began and continued in operation for a period deemed long enough for the bulk of the tracer to pass. The delay in shutting off the samplers was generaily 2-?/2 times as long as necessary for the uninhibited transport to the sampler, assuming the $15 \mathrm{~m}$ wind speed.

The cataloging of the diffusion and meteorological data in this volume is chronological within each experimental series. The data from the primary series are presented first, in the order in which they were begun, then data from the secondary series are presented. Again, the sequence of presentation of the series is the order in which they were begun. Table 3 lists the experiments in the order in which the data are presented. It lists the meteorological conditions during release, the tracer release points, the type of tracers, the release elevations, and the extent of the sampling. The meteorological conditions for each experiment are characterized by the wind speed at $2 \mathrm{~m}$ and a bulk Richardson number for the layer between 2 and $30 \mathrm{~m}$. The bulk Richardson number is defined as:

$$
R i_{b}=\frac{g}{T_{2}} \frac{\left(T_{30}-T_{2}\right)}{U_{15}{ }^{2}} \Delta Z
$$

where $g$ is the gravitational acceleration, $T_{2}$ and $T_{30}$ are the potential temperatures at 2 and $30 \mathrm{~m}$, respectively, $U_{15}$ is the wind speed at $15 \mathrm{~m}$ and $\Delta Z$ is the layer thickness $(28 \mathrm{~m})$. Data from the $122 \mathrm{~m}$ tower in Appendix $C$ were used for determination of the butk Richardson number.

Although the ideal diffusion experiment would entail a point source release into an undisturbed atmosphere, such releases are impossible. A11 of the elevated releases were, undoubtedly, affected to some degree by the $122 \mathrm{~m}$ tower from which the releases took place. This tower is a rather sturdy structure triangular in cross section and about $3.6 \mathrm{~m}$ on a side. An idea of the appearance of the tower can be gained from Figure 3 . 
Near ground-level releases from the U-source were subjected to the worst wake situation. First, the tracer dispensing equipment (to be seen in Figure 5) was mounted on a small trailer; second, the wake of the tower a few meters away added to the turbulence, and third, a small building stood near the base of the tower.

\section{THE APPENDIX A DIFFUSION DATA}

Appendix A gives the individual field diffusion measurements. These data form the bulk of the data volume. The experiments are presented in the order given in Table 3. For multiple tracer experiments the ZnS data are presented first. They are followed by fluorescein data. The data for the xenon samples is presented following the data for the ZnS and fluorescein samples. General comments pertinent to each experiment precede the listing of the data for the experiment. The date and time of release, tracer release elevation and wind speed at the release height are included in the heading for the data from each arc or tower.

The first column in the body of the Appendix A tables gives the azimuth with respect to the source, and the distance from the source is given in the last column.

The second column in the Appendix A tables lists exposures (timeintegrated concentrations). For towers, a column specifying sampling height precedes the exposure column. The exposure data for particulate tracers results from division of the mass of tracer on the filter by the flow rate through the filter. In the exposure column, the magnitudes of the individual numbers are directly related to the mass of the tracer collected on the filters, and are therefore relatec to the confidence that can be placed in the samples.

The exposure column has been left in digital format, as opposed to the scientific notation used in the following two columns, to give an arialog appearance to the concentration distributions across an arc or up a tower. In most cases the shape of the distribution is relatively obvious with a glance at the column.

In the column headed $E / G$, exposures have been normalized by dividing by the total mass emitted (or curies released in the case of xenon).

$E U / Q$ is the exposure normalized to both unit emission and unit wind speed. The mean wind speed (U) used in this normalization is the speed listed in the heading immediately preceding the data for the arc or tower. It is the mean wind speed at the release height during the period of release. In cases where there were multiple tracer releases but the release periods differed, the wind speed used is the average for the longer release period. 
TABLE 3. General Grid, Source, Release and Meteorological Information

\begin{tabular}{|c|c|c|c|c|c|c|c|c|c|}
\hline Test & $\begin{array}{c}\text { Grid } \\
\text { Source }\end{array}$ & $\begin{array}{l}\text { 'ind Speed } \\
\qquad \frac{12.0 \mathrm{~m})^{*}}{(\mathrm{~m} / \mathrm{s})^{\prime}}\end{array}$ & $\frac{\text { Stabjity }}{\mathrm{Ri}_{b}}$ & $\begin{array}{l}\text { Release } \\
\frac{\text { Height }}{(m)}\end{array}$ & $\begin{array}{c}\text { Type } \\
\text { of } \\
\text { Tracer }\end{array}$ & $\begin{array}{c}\text { Duration } \\
\text { of } \\
\text { Release } \\
\text { (min) }\end{array}$ & $\begin{array}{l}\text { Samplin } \\
\text { from } 5 \\
\text { Nearest }\end{array}$ & $\begin{array}{l}\text { Discance } \\
\text { urce, m } \\
\text { Farthest }\end{array}$ & $\begin{array}{l}\text { Total } \\
\text { Towers } \\
\text { Active }\end{array}$ \\
\hline$F T=30$ & s & $2.5^{\star}$ & -.063 & 2 & $F P$ & 20 & 200 & 3200 & 20 \\
\hline$F \bar{i}-31$ & 5 & $3.4 *$ & -.044 & 2 & $F ?$ & 30 & 200 & 3200 & 20 \\
\hline $\mathrm{FT}-40$ & S & 2.5 & -.040 & 2 & $\mathrm{FP}$ & 60 & 200 & 3200 & 20 \\
\hline FT -41 & s & $6.7^{*}$ & -.005 & 2 & FP & 30 & 200 & 3200 & 20 \\
\hline $\mathrm{Fi}-42$ & 5 & 3.2 & .100 & 2 & FP & 60 & 200 & 3200 & 20 \\
\hline FT -43 & 5 & $2.3^{*}$ & .069 & 2 & $\mathrm{FP}$ & 30 & 200 & 3200 & 20 \\
\hline FT-44 & 5 & $2.1 *$ & .076 & 2 & $F P$ & 50 & 200 & 3200 & 20 \\
\hline fT -45 & 5 & $6.3^{*}$ & -.024 & 2 & $\mathrm{FP}$ & 29 & 200 & 3200 & 20 \\
\hline$\Gamma T-46$ & 5 & $1.6^{*}$ & .178 & 2 & $F P$ & 51 & 200 & 3200 & None \\
\hline CT -47 & 5 & 3.6 & -.043 & 2 & FP & 39 & 200 & 3200 & 20 \\
\hline FT- 48 & $\mathrm{~s}$ & $2.9 *$ & -.068 & 2 & FP & 30 & 200 & 3200 & 20 \\
\hline FT- 49 & s & $5.7 *$ & -.048 & 2 & $=p$ & 30 & 200 & 3200 & 20 \\
\hline FT-50 & s & $4.7^{\star}$ & .015 & 2 & $F P$ & 30 & 200 & 3200 & 20 \\
\hline FT-51 & S & $4.8^{\star}$ & -.345 & 2 & $F p$ & 30 & 200 & 3200 & 20 \\
\hline$F F-52$ & $\grave{s}$ & $3.8^{\star}$ & - .015 & 2 & $F p$ & 30 & 200 & 3200 & 20 \\
\hline FT -53 & $\mathrm{~s}$ & $3.2^{*}$ & -.013 & 2 & $\mathrm{FP}$ & 21 & 200 & 12800 & 20 \\
\hline FT- 54 & $s$ & $1.9 *$ & .153 & 2 & FP & 30 & 200 & 3200 & 20 \\
\hline FT-55 & s & $2.5^{\star}$ & .050 & 2 & FP & 30 & 200 & 3200 & 20 \\
\hline$F \div-56$ & $\mathrm{~s}$ & $0.8^{*}$ & .186 & 2 & $\mathrm{FP}$ & 30 & 200 & 3200 & 20 \\
\hline $\mathrm{FT}-57$ & $\mathrm{~s}$ & $2.6 *$ & .052 & 2 & $F p$ & 30 & 200 & 3200 & 20 \\
\hline FT-58 & 5 & $1.0^{\star}$ & 683 & 2 & Fo & 15 & 200 & 3200 & 20 \\
\hline FT-59 & $\dot{s}$ & $2.8 *$ & -.057 & 2 & $F p$ & 30 & 200 & 3200 & 20 \\
\hline$F T-60$ & 5 & $3.0^{\star}$ & -.021 & 2 & FP & 21 & 200 & 3200 & 20 \\
\hline$F T-6 i$ & 5 & $4.2^{\star}$ & -.025 & 2 & $F P$ & 31 & 200 & 3200 & 20 \\
\hline$F T-62$ & $s$ & 0.7 & .406 & 2 & $F P$ & 30 & 200 & 3200 & 20 \\
\hline FT- 63 & 5 & $1.3^{\star}$ & .247 & 2 & FP & 196 & 200 & 12800 & 10 \\
\hline $\mathrm{FT}-64$ & $\mathrm{~s}$ & $3.7^{\star}$ & .235 & $\overline{2}$ & $\mathrm{FP}$ & 35 & 200 & 3200 & 10 \\
\hline$F T-55$ & $\mathrm{~s}$ & $4.9 *$ & .010 & 2 & $\mathrm{FP}$ & 15 & 200 & $: 2800$ & 10 \\
\hline$=T-66$ & $\mathrm{~s}$ & $3.3 *$ & .029 & 2 & Fp & 60 & 200 & 12800 & 10 \\
\hline$F i-67$ & s & $1.7^{\star}$ & .147 & 2 & $F P$ & 45 & 200 & 3200 & 10 \\
\hline FT- 68 & 5 & $3.8^{\star}$ & .021 & 2 & 5P & 45 & 200 & 3200 & 10 \\
\hline ГT- 59 & 5 & $4.5 *$ & .013 & 2 & $\mathrm{FP}$ & 60 & 200 & 12800 & 10 \\
\hline FT-70 & 5 & $2.9 *$ & .019 & 2 & $c p$ & 135 & 200 & 12800 & 10 \\
\hline FT-7I & $s$ & 3. $9 *$ & .022 & 2 & FP & 51 & 200 & 3200 & 10 \\
\hline$!-2$ & iJ & 5.7 & -.006 & 56 & FP & 30 & 100 & 1600 & Sone \\
\hline $3-5$ & ! & 4.6 & -.018 & 56 & $\mathrm{FP}$ & 60 & 100 & 1600 & None \\
\hline J -6 & iv & 6.8 & -.013 & 56 & $\mathrm{FP}$ & 60 & 100 & 1600 & None \\
\hline$U-7$ & ป & $6.5^{\star}$ & -.027 & 56 & $F P$ & 60 & 100 & 1600 & None \\
\hline $11-8$ & U & $7.5^{*}$ & -.003 & 56 & FP & 35 & 100 & 1600 & Yone \\
\hline$U-9$ & u & $5.0^{\star}$ & -.015 & 56 & $\mathrm{FP}$ & 60 & 100 & 7600 & None \\
\hline$U-10$ & is & $2.7 *$ & -.112 & 56 & $\mathrm{FP}$ & 25 & 100 & 1600 & Nane \\
\hline U-11 & U & $1.8^{*}$ & -.153 & 56 & $F P$ & 60 & 100 & 1600 & None \\
\hline U- 12 & u & $2.0^{*}$ & -119 & 56 & FP & 60 & 100 & 1600 & None \\
\hline $\mathrm{U}-13$ & U & $7.2^{\star}$ & -010 & 56 & $F P$ & 60 & 100 & 1600 & None \\
\hline$U-14$ & is & $4.2^{\star}$ & -.012 & 56 & $\mathrm{FP}$ & 60 & 100 & 1600 & None \\
\hline U-15 & U & $5.5 *$ & -.004 & 56 & FP & 60 & 100 & 1600 & None \\
\hline ป 16 & U & $4.7 *$ & -.002 & 56 & $F P$ & 60 & 100 & 1600 & None \\
\hline $0-17$ & iv & $5.6^{\star}$ & -.011 & 56 & $\mathrm{FP}$ & 17 & 100 & 1600 & None \\
\hline U-18 & U & $5.6 \star$ & -.011 & 56 & FP & 50 & 100 & 1600 & None \\
\hline$u-19$ & is & $3.1 *$ & ו ו & 56 & $f p$ & 60 & 100 & 3200 & Nore \\
\hline$y-20$ & u & $7.0^{*}$ & -.306 & 56 & Fp & 50 & 100 & 1600 & None \\
\hline$!-21$ & is & $5.2 *$ & - ots & 56 & FP & 60 & 100 & 1600 & None \\
\hline $13-22$ & u & $3.5^{\star}$ & -.071 & 56 & EP & 57 & 100 & 1600 & None \\
\hline$!-23$ & ij & $3.1 *$ & -.041 & 56 & $\mathrm{FP}$ & 46 & 100 & 1600 & None \\
\hline $13-24$ & iJ & $5.4^{*}$ & -.052 & 56 & FP & 60 & 100 & 1600 & None \\
\hline$U-25$ & !」 & $1.9 *$ & -.078 & 56 & $\mathrm{FP}$ & 24 & 100 & 1600 & None \\
\hline $15-26$ & 3 & $3.5 *$ & -.083 & 56 & FP & 60 & 100 & 1600 & None \\
\hline $\mathrm{U}-27$ & U & $2.2^{\star}$ & -.192 & 56 & $F P$ & 60 & 100 & 1600 & None \\
\hline J -28 & U & $5.5 *$ & -.025 & 56 & FP & 60 & 100 & 1600 & None \\
\hline$U-29$ & U & $1.7^{\star}$ & -.177 & 56 & $F$ & 60 & 100 & 1600 & Yone \\
\hline$U-30$ & U & $6.0^{*}$ & -.010 & 56 & FP & 60 & 100 & 3200 & None \\
\hline $\mid 3-31$ & j & $3.9 *$ & .010 & 56 & FP & 38 & 100 & 1600 & None \\
\hline$\sqrt{3}-32$ & IJ & $4.5 \star$ & .002 & 56 & FD & 50 & 100 & 1600 & None \\
\hline v-33 & 3 & $6.0^{*}$ & -.030 & 56 & FP & 58 & 100 & $? 600$ & fione \\
\hline
\end{tabular}


TABLE 3. (Cont'd)

\begin{tabular}{|c|c|c|c|c|c|c|c|c|c|}
\hline Test & $\begin{array}{c}\text { Grid } \\
\text { Source } \\
\end{array}$ & $\begin{array}{l}\text { Wind Speed } \\
\frac{1.5 \mathrm{~m}(2.0 \mathrm{~m})}{(\mathrm{m} / \mathrm{s})}\end{array}$ & $\frac{\text { Stability }}{R_{0}}$ & $\begin{array}{l}\text { Release } \\
\frac{\text { Height }}{(m)}\end{array}$ & $\begin{array}{c}\begin{array}{c}\text { Type } \\
\text { of } \\
\text { iracer }\end{array} \\
\end{array}$ & $\begin{array}{c}\text { Duration } \\
\text { of } \\
\text { Release } \\
\frac{(\text { min) }}{}\end{array}$ & $\begin{array}{c}\text { Sampl in } \\
\text { from } \\
\text { kearest } \\
\end{array}$ & $\begin{array}{l}\text { Distance } \\
\text { urce, m } \\
\text { Farthest }\end{array}$ & $\begin{array}{l}\text { Total } \\
\text { Towers } \\
\text { Active } \\
\end{array}$ \\
\hline iJ -34 & $u$ & $5.0^{\star}$ & -.041 & 56 & $F P$ & 37 & 100 & 3200 & Кone \\
\hline-35 & u & $5.6^{\star}$ & -.044 & 56 & $F P$ & 26 & 100 & 1600 & None \\
\hline $\mathrm{U}-36$ & v & 2.9 & -.152 & 26 & FP & 25 & 100 & 1600 & None \\
\hline !J -37 & U & 2.7 & -.043 & 111 & $F p$ & 61 & 300 & 3200 & None \\
\hline$j-38$ & u & 3.4 & -.076 & 26 & $\mathrm{FP}$ & 17,5 & 100 & 1600 & None \\
\hline L-39 & U & 6.6 & -.019 & 56 & $\mathrm{FP}$ & 52 & 300 & 1600 & None \\
\hline $11-40$ & ij & 5.9 & -011 & 56 & $F ?$ & 49 & 100 & 3200 & None \\
\hline IJ-41 & ij & $6.2^{\star}$ & -.027 & 26 & FP & 38 & 100 & 1600 & None \\
\hline$v-42$ & U & 5.1 & -.014 & 56 & FP & 55 & 400 & 3200 & None \\
\hline$U-43$ & U & 2.8 & .046 & 26 & Fo & 40 & 100 & 1600 & None \\
\hline$i-44$ & 1! & 1.9 & -.187 & 26 & $F P$ & 30 & 100 & 1600 & Mone \\
\hline U- 45 & 0 & 6.0 & -.001 & 1.5 & $F P$ & 30 & 200 & 1600 & None \\
\hline & & & & 26 & $\mathrm{FL}$ & 30 & 200 & 1600 & None \\
\hline$J-46$ & U & 6.0 & -.015 & 1.5 & $\mathrm{FL}$ & 30 & 200 & 1600 & None \\
\hline & & & & 26 & FP & 30 & 200 & 1600 & Vone \\
\hline$\Downarrow-47$ & v & $4.6^{\star}$ & -.015 & 3.5 & $\mathrm{FL}$ & 30 & 100 & 1600 & None \\
\hline$v-48$ & & & & 26 & $\mathrm{FP}$ & 30 & 100 & 1600 & None \\
\hline$v-48$ & J & $1.5^{*}$ & .102 & $\begin{array}{r}1,5 \\
26\end{array}$ & $\begin{array}{l}\text { FL } \\
\text { cọ }\end{array}$ & $\begin{array}{l}30 \\
30\end{array}$ & $\begin{array}{l}100 \\
100\end{array}$ & $\begin{array}{l}1600 \\
1600\end{array}$ & $\begin{array}{l}\text { None } \\
\text { None }\end{array}$ \\
\hline$U-49$ & U & 1.0 & -.090 & 1.5 & $\mathrm{FL}$ & 30 & 100 & 1600 & None \\
\hline & & & & 26 & $F P$ & 30 & 100 & 1600 & None \\
\hline JJ-50 & !] & 5.7 & -.009 & 1.5 & $\mathrm{FL}$ & 30 & 300 & 1600 & None \\
\hline & & & & 26 & FP & 30 & 100 & 1600 & None \\
\hline$U-51$ & U & $4.8^{*}$ & -.030 & $i .5$ & $\overline{F L}$ & 30 & 100 & 1600 & None \\
\hline & & & & 26 & $F P$ & 30 & 100 & 1600 & None \\
\hline$J-52$ & ! & $6.8^{*}$ & -.024 & 1.5 & $\mathrm{Fl}$ & 47 & 100 & 1600 & None \\
\hline & & & & 26 & Fp & 47 & 100 & 3600 & None \\
\hline $1 J-53$ & U & 8.9 & .000 & 3.5 & $\mathrm{FL}$ & 60 & 100 & 1600 & None \\
\hline & & & & 26 & $F \vec{P}$ & 60 & 100 & 1600 & None \\
\hline$!-54$ & $j$ & $3.3^{*}$ & .037 & 1.5 & $\mathrm{Fl}$ & 45 & 100 & 1600 & None \\
\hline & & & & 26 & $F P$ & 45 & 100 & 1600 & Hone \\
\hline$U-55$ & U & 3.0 & -.079 & $\begin{array}{r}1.5 \\
26\end{array}$ & $\begin{array}{l}F L \\
F P\end{array}$ & $\begin{array}{l}59 \\
59\end{array}$ & $\begin{array}{l}100 \\
100\end{array}$ & $\begin{array}{l}1500 \\
1600\end{array}$ & $\begin{array}{l}\text { None } \\
\text { None }\end{array}$ \\
\hline$F i-1-63$ & $s$ & $0.6 *$ & .439 & 7 & $\mathrm{FP}$ & 45 & 200 & 3200 & 20 \\
\hline FT -3.63 & 5 & $3.4 \star$ & 14.8 & 7 & $\mathrm{fp}$ & 30 & 200 & 3200 & 20 \\
\hline$F T-4-63$ & $s$ & $3.4^{*}$ & .039 & 7 & $F P$ & 30 & 200 & 3200 & 20 \\
\hline$F T-5-63$ & 5 & $2.7^{\star}$ & .081 & 7 & $\mathrm{FP}$ & jo & 200 & 3200 & 20 \\
\hline $8 T-6-63$ & 5 & $1.3 *$ & .112 & 7 & $\mathrm{FP}$ & 23 & 200 & 3200 & 20 \\
\hline $\mathrm{FT}-7-63$ & 5 & $2.7^{\star}$ & .025 & 7 & $F P$ & 43 & 200 & 3200 & 20 \\
\hline $\mathrm{FT}-8-63$ & 5 & $1.2^{*}$ & .040 & 7 & FP & 40 & 200 & 3200 & 20 \\
\hline$F T-9-63$ & $s$ & $2.2^{\star}$ & .077 & 7 & $\mathrm{FP}$ & 60 & 200 & 3200 & 20 \\
\hline$F T-10-63$ & $\mathrm{~S}$ & $1.7 *$ & .153 & 7 & FP & 39 & 200 & 3200 & 20 \\
\hline$F T-11-6 \hat{3}$ & 5 & i. $6^{*}$ & .149 & 7 & FP & 41 & 200 & 3200 & 20 \\
\hline FT-12-63 & $\mathrm{s}$ & $1.7 *$ & .265 & 7 & FP & 30 & 200 & 3200 & 20 \\
\hline$=T-13-53$ & 5 & $0.5^{\star}$ & .597 & 7 & $\mathrm{FP}$ & 26 & 200 & 3200 & 20 \\
\hline$F[-14-63$ & 5 & $2.0^{*}$ & .056 & 7 & FP & 43 & 200 & 3200 & 20 \\
\hline$F-15-6 \hat{3}$ & 5 & $0.8 \star$ & .236 & 7 & $F p$ & 37 & 200 & 3200 & 20 \\
\hline CT-16-63 & 5 & $0.4^{\star}$ & .777 & 7 & $F P$ & 42 & 200 & 3200 & 20 \\
\hline$F T=17-63$ & 5 & $0.8 \star$ & .283 & 7 & $F P$ & 30 & 200 & 3200 & 20 \\
\hline$F T-18-63$ & s & $3.3^{*}$ & -.066 & 7 & FP & 79 & 200 & 3200 & 20 \\
\hline$S-64-1$ & 5 & $1.3^{*}$ & .066 & 56 & $F P$ & 38 & 200 & 12800 & 20 \\
\hline $5-64-2$ & s & $1.3 *$ & .182 & 56 & FP & 26 & 200 & 12800 & 20 \\
\hline $5-64-4$ & 5 & $1.0^{*}$ & .250 & 56 & $\mathrm{FP}$ & 26 & 200 & 12800 & 20 \\
\hline $5-64-5$ & 5 & i. $9 *$ & .770 & 56 & $\tilde{r} p$ & 32 & 1600 & 12800 & 10 \\
\hline$S-64-6$ & 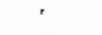 & $1.0 *$ & & 56 & $5 p$ & 20 & 1600 & 12800 & To \\
\hline $5-64-7$ & 5 & $2.8^{\star}$ & .070 & 56 & $F P$ & 30 & 200 & 3200 & 20 \\
\hline $5-64-8$ & $s$ & $0.8^{*}$ & .146 & 56 & $p$ & 50 & 1500 & 12800 & 10 \\
\hline$\leqslant-64-9$ & $s$ & $1.4 *$ & .241 & 56 & $F P$ & 60 & 1600 & 12800 & 10 \\
\hline$S-64-10$ & 5 & 2.4 & .079 & 56 & $F P$ & 60 & 1600 & 12800 & 10 \\
\hline $5-64-17$ & 5 & 1.2 & .578 & 56 & Fo & 21 & $\$ 600$ & 12800 & 10 \\
\hline $5-64-12$ & $s$ & ?. . 5* & .232 & 56 & Ep & 57 & 1600 & 12800 & 10 \\
\hline $5-64-13$ & 5 & $1.1 *$ & .348 & 111 & $\vec{r} p$ & 72 & 1500 & 12800 & 10 \\
\hline $5-54-14$ & $\$$ & $2.2 *$ & .053 & 111 & FP & 40 & 1000 & $128 \mathrm{CO}$ & None \\
\hline $3-64-15$ & 5 & $\therefore 2$ & .302 & 111 & $=0$ & 15 & 16100 & 12800 & 10 \\
\hline
\end{tabular}


TABLE 3. (Cont'd)

\begin{tabular}{|c|c|c|c|c|c|c|c|c|c|}
\hline Test & $\begin{array}{c}\text { Grid } \\
\text { source } \\
\end{array}$ & $\begin{array}{l}\text { Wind Speed } \\
\frac{1.5 m(2.0 m) *}{(m / s)}\end{array}$ & $\frac{\text { stability }}{R i_{b}}$ & $\begin{array}{l}\begin{array}{l}\text { Release } \\
\text { Height }\end{array} \\
(\mathrm{m})\end{array}$ & $\begin{array}{r}\text { Type } \\
\text { of } \\
\text { rracer } \\
\end{array}$ & $\begin{array}{l}\text { Duration } \\
\text { of } \\
\frac{\text { zelease }}{(\min )}\end{array}$ & $\begin{array}{l}\text { Sampi in } \\
\text { from So } \\
\text { Vearest }\end{array}$ & $\begin{array}{l}\text { Distance } \\
\text { rce, m } \\
\text { Farthest }\end{array}$ & $\begin{array}{l}\text { Total } \\
\text { Towers } \\
\text { Active }\end{array}$ \\
\hline $2-1$ & 5 & $2.5^{*}$ & .052 & 26 & $\mathrm{FP}$ & 39 & 200 & 3200 & 20 \\
\hline $\begin{array}{l}\text { MT }-2 \\
M T-3 \\
M T-4\end{array}$ & $\begin{array}{l}S \\
S \\
S\end{array}$ & $\begin{array}{l}3.8^{*} \\
1.1^{*} \\
2.2^{*}\end{array}$ & $\begin{array}{l}.016 \\
.222 \\
.039\end{array}$ & $\begin{array}{l}1.5 \\
1.5 \\
1.5\end{array}$ & $\begin{array}{l}E P, F L \\
F P, F L \\
F P, F L\end{array}$ & $\begin{array}{l}33 \\
21 \\
28\end{array}$ & $\begin{array}{l}200 \\
200 \\
200\end{array}$ & $\begin{array}{l}3200 \\
3200 \\
3200\end{array}$ & $\begin{array}{l}20 \\
20 \\
20\end{array}$ \\
\hline $\begin{array}{l}x-2 \\
x-4\end{array}$ & $\begin{array}{l}S \\
5\end{array}$ & $\begin{array}{l}1.2^{*} \\
2.1^{*}\end{array}$ & $\begin{array}{l}.187 \\
.106\end{array}$ & $\begin{array}{l}7 \\
7\end{array}$ & $\begin{array}{l}F P \\
F P\end{array}$ & $\begin{array}{l}28 \\
26\end{array}$ & $\begin{array}{l}800 \\
200\end{array}$ & $\begin{array}{l}3200 \\
3200\end{array}$ & $\begin{array}{l}! 5 \\
20\end{array}$ \\
\hline $\begin{array}{l}x_{e}-1 \\
x_{e}-2 \\
x_{e}-3 \\
x_{e}-4 \\
y_{e}-5\end{array}$ & $\begin{array}{l}5 \\
5 \\
5 \\
5 \\
5\end{array}$ & $\begin{array}{l}1.5 \\
5.3 \\
. .7 \\
2.2 \\
3.2\end{array}$ & $\begin{array}{l}.534 \\
.021 \\
.096 \\
.096 \\
.073\end{array}$ & $\begin{array}{l}1.5 \\
1.5 \\
1.5 \\
1.5 \\
1.5\end{array}$ & $\begin{array}{l}F P, X e \\
F P, X e \\
F P, F L, X e \\
F P, F L, X e \\
F P, F L, X e\end{array}$ & $\begin{array}{r}30 \\
30 \\
4 \\
7.5 \\
6\end{array}$ & $\begin{array}{l}200 \\
200 \\
200 \\
200 \\
200\end{array}$ & $\begin{array}{l}3200 \\
3200 \\
1600 \\
1600 \\
1600\end{array}$ & $\begin{array}{r}20 \\
\text { None } \\
15 \\
15 \\
15\end{array}$ \\
\hline $\begin{array}{l}\dot{A}-2 \\
A-5 \\
A-0 \\
1-7 \\
A-5 \\
A-9\end{array}$ & $\begin{array}{l}5 \\
5 \\
S \\
5 \\
5 \\
5\end{array}$ & $\begin{array}{l}5.6^{\star} \\
2.7 \\
. .4 \\
2.6 \\
1.9 \\
1.2\end{array}$ & $\begin{array}{l}.010 \\
.029 \\
.096 \\
.026 \\
.133 \\
.445\end{array}$ & $\begin{array}{l}1.5 \\
\vdots .5 \\
1.5 \\
1.5 \\
1.5 \\
1.5\end{array}$ & $\begin{array}{l}F P \\
F P \\
F P \\
F P \\
F P \\
F D\end{array}$ & $\begin{array}{l}61 \\
60 \\
50 \\
55 \\
55 \\
54\end{array}$ & $\begin{array}{l}200 \\
800 \\
800 \\
800 \\
800 \\
800\end{array}$ & $\begin{array}{l}3200 \\
3200 \\
3200 \\
3200 \\
3200 \\
3200\end{array}$ & $\begin{array}{l}5 \\
5 \\
5 \\
5 \\
5 \\
5\end{array}$ \\
\hline
\end{tabular}

$F P=$ Fracer Flourescent Pigment 2210

$F \mathrm{~L}=$ Fluorescein

te $=$ xenon 
In many cases, a one-character symbol precedes the azimuth column in Appendix A. These symbols indicate that something less than ideal was associated with the sample. A detailed explanation of the alphabetical symbols is given at the beginning of the appendix. The numerical symbols used in Appendix A were mentioned earlier; they are associated with dust loadings on the filters. Values of 5 or more indicate that the apparent ZnS mass may be less than the true mass because of dust on the filter. Ratings of less than 5 can be ignored.

Since each $1.5 \mathrm{~m}$ sample was used in the computation of crosswind integrated concentrations and the statistics presented in Appendix $B$, it was deemed wiser to substitute a reasonable estimate for an obviously erroneous sample than to have an erroneous number go into the computations. These estimates are frequently merely interpolations or extrapolations of data collected on the same arc. At times it was necessary to plot the distributions of the tracer and interpolate concentrations between arcs.

In some instances, the amount or quality of data on an arc is so low that no estimations were attempted. Also, since no vertical moments or vertical crosswind sums were computed, the incentive to supply estimates for poor tower samples was not as great as it was for poor $1.5 \mathrm{~m}$ samples. Thus, the poorer tower measurements are frequent7y reported directly with an accompanying remark symbol other than "E" for "estimate".

Following Nickola (1977), data for measurements at backaround levels have been omitted. Also omitted are data for measurements where there was a total failure of a sampler.

APPENDIX B DIFFUSION SUMMARIES

Appendix B summarizes the ground-level data for each of the releases. The summary for each release starts by repeating the specific experiment remarks given in Appendix $A$. The heading then identifies the specific tracer and gives pertinent release information.

The first column of the tabular data indicates the distance from the source. The next four columns give statistics relating to the crosswind distribution. The first of these columns gives the location of the mean of the crosswind distribution. The standard deviation $\left(\sigma_{y}\right)$ and the coefficients of skewness and kurtosis for the distribution follow. The next three columns give the azimuth and the magnitude of the three largest exposures measured on each arc. Three values are given to reduce the chance of considering a "sport" (erroneous measurement) as representative of the plume centerline. The last two columns give crosswind integrated exposure values. 
The symbol "?" following the tabulated data indicates that there is some uncertainty in the data. The uncertainty may involve estimates in sample values given in Appendix A, or there may be some question about the performance of the laboratory assay equipment. The symbol is intended to alert a user to the fact that something not completely routine went into the generation of the associated number. The remarks preceding the table or the appropriate data field in Appendix A should indicate the reason for the symbol. Where estimates of missing data were on the tails of a distribution, or where a broad distribution reduced the consequences of a single estimate near the center of a distribution, a "?" may not have been appended in the Appendix $B$ tables. The decisions to append the symbol or not are subjective. Therefore, a second review of the data would be unlikely to result in precisely the same tagging.

Appending of the symbol " $x$ " to Appendix B data symbolizes a much more serious difficulty with the data. The data are either incomplete or invalid. For instance, the computations of mean, standard deviation, skewness, kurtosis and crosswind integrated exposure from a badly truncated distribution Tack significance. An " $X$ " is then appended. However, if the truncated distribution is such that there is little doubt that the plume center] ine was observed, no " $X$ " is appended in the maximum exposure columns.

THE APPENDIX C METEOROLOGICAL DATA

Vertical profiles of temperature, wind speed and wind direction were measured on a $122 \mathrm{~m}$ tower at the $\mathrm{U}$-source. In addition, wind speed and direction were measured on a $25 \mathrm{~m}$ tower -- also generally near the tracer release point.

Temperature measurements on the $122 \mathrm{~m}$ tower were made by Foxboro thermohms. The aspirated thermohms were exposed at elevations of 0.9 , $15.2,30.5,45.7,61.0,76.2,91.4$, and $122 \mathrm{~m}(3,50,100,150,200$, 250,300 and $400 \mathrm{ft}$ ). Recording was a series of points on a strip chart. About 3-1/2 minutes were required to cycle through all temperature sensors.

Wind speed and direction on the $122 \mathrm{~m}$ tower were measured by seven Aerovane anemometers mounted at elevations of $2.1,15.2,30.5,45.7$, $61.0,91.4$ and $122 \mathrm{~m}(7,50,100,150,200,300$ and $400 \mathrm{ft})$. The starting speed for Aerovane propellers is approximately $1 \mathrm{~m} / \mathrm{sec}$. The Aerovane is a rather large stream 1 ined vane/anemometer assemb1y about $80 \mathrm{~cm}$ in length. The assembly has a distance constant of about $4.5 \mathrm{~m}$ for speed and about $10 \mathrm{~m}$ for direction.

Wind direction transducers employed on the $25 \mathrm{~m}$ tower were Beckman and Whitley Model M1565 vanes. These vanes respond to winds of less than $0.4 \mathrm{~m} / \mathrm{sec}$ and have a distance constant of about $1.5 \mathrm{~m}$. The output signals from these iightweight vanes were smoothed by a filter having 
a 5-sec time constant. The filter output was sampled for 60 msec once each $5 \mathrm{sec}$, digitized and recorded. The digitized data were used for computing mean directions and standard deviations of direction.

The Beckman and Whitley cups and vanes were mounted at elevations of $0.76,1.5,3.0,6.1,12.2$ and $24.4 \mathrm{~m}(2.5,5,10,20,40$ and $80 \mathrm{ft})$ during most of the field experiments. Thus, the spacing between wind sensors on the shorter tower was closer than was the spacing between Aerovanes on the $122 \mathrm{~m}$ tower. Wind speeds indicated by the Beckman and Whitley cups frequently averaged slightly higher than those computed from the Aerovanes, although the shapes of the profiles in the area of common measurements were in excellent agreement.

Two sets of wind direction and wind direction standard deviation $\left(\sigma_{\theta}\right)$ data were deveioped. One set is based on the Aerovane measurements, and the other is based on the Beckman and Whitley vane measurements. With a few exceptions for light wind cases, these data show that agreement between the Aerovane and Beckman and Whitley data is reasonably good. The agreement in mean direction profiles is rather expected. However, in view of the difference in physical characteristics of the large Aerovane and the relatively lightweight Beckman and Whitley vane, and the difference in the method of digitizing direction, the agreement in $\sigma_{\theta}$ profiles is more surprising.

The data presented in Appendix $\mathrm{C}$ are based on measurements made during the period of tracer release. When two or more tracers were released during the same experiment and the periods of release were not the same, the meteorological data apply to the longer tracer release period. 


\section{REFERENCES}

Barad, M. L. and J. J. Fuquay (Eds.). 1962a. The Green Glow Diffusion Program. Geophysical Research Papers No. 73, Vo1. I, AFCRL-62-251 (I), Air Force Cambridge Research Laboratories, Bedford, Massachusetts.

Barad, M. L. and J. J. Fuquay (Eds.). 1962b. The Green Glow Diffusion Program. Geophysical Research Papers No. 73, Vol. II, AFCRL-62-251 (II, Air Force Cambridge Research Laboratories, Bedford, Massachusetts.

Eggleton, A. E. J. and N. Thompson. 1967. "Loss of Fluorescent Particles in Atmospheric Diffusion Experiments by Comparison with Radio-xenon Tracer." Nature 192:935-936.

Fuquay, J. J., C. L. Simpson and W. T. Hinds. 1964. "Prediction of Environmental Exposures from Sources Near the Ground Based on Hanford Experimental Data." J.App1. Meteorol. 3(6):761-770.

Hinds, W. T. 1967. On the Variance of Concentration in Plumes and Wakes. BNWL-SA-1435, Pacific Northwest Laboratory, Richland, Washington.

Hinds, W. T. 1969. "Peak-to-Mean Concentration Ratios from GroundLeve1 Sources in Building Wakes." Atmospheric Environ. 3:145-156.

Leighton, P. A. 1955. The Stanford Fluorescent-Particle Tracer Techniques. (Defense Documentation Center, AD 91-94), Dept. of Chemistry, Stanford University, Palo Alto, California.

Ludwick, J. D. 1961, Dual Atmospheric Tracer Techniques for Diffusion Studies Using Phosphorescence - Fluorescence AnaTysis. HW-70892, Hanford Atomic Products Dperation, General Electric Co., Richland, Washington.

Ludwick, J. D. and R. W. Perkins. 1961. "Liquid Scintillation Techniques Applied to Counting Phosphorescence Emission." Anal. Chem. $33(9): 1230-1235$.

Ludwick, J. D. and P. W. Nickola. 1963. "A Fluorescein Analysis Technique for Use in Atmospheric Diffusion Studies." in Hanford Radiologica? Sciences Research and Development Report for 7962 , HW-77609, Hanford Atom ic Products Operation, General Electric Co., Richland, Washington.

Ludwick, J. D, 1965. "Investigation of Inert Atmospheric Tracers; Xe133." in Hanford Radiological Sciences Research and Development Annual Report for 1964, BNwL-36, Pacific Northwest Laboratory, Richland, Washington. 
Nickola, P. W. and M. T. Scoggins. 1964. "Atomspheric Tracer Technology." in Hanford Radiological Sciences Research and Development Annual Report for 1963, HW-81746L 1.43 - 1.51, Hanford Atomic Products Operation, General Electric Co., Richland, Washington.

Nickola, P. W. 1965. Field Testing of a Fluorescein - Zinc Sulfide Dual Atmospheric Tracer Techiniques. BNWL-103, Battelle, Pacific Northwest Laboratories, Richland, Washington.

Nickola, P. W., J. V. Ramsde11, Jr. and J. D. Ludwick. 1970.

Detailed Time-Histories of Concentrations Resulting from Puff and Short-Period ReTeases of an Inert Radioactive Gas: A VoTume of Atmospheric Diffusion Data. BNWL-1272, Battel7e, Pacific Northwest Laboratories, Richland, Washington.

Nickola, P. W. 1977. The Hanford 67-Series: A Volume of Atmospheric Field Diffusion Measurements. PNL-2433, Pacific Northwest Laboratory, Richland, Washington.

Rankin, M. 0. 1958. A Zinc Sulfide Particle Detector. HW-55917, Hanford Laboratories, GeneraT ETectric Co., Richland, Washington.

Robinson, E., J. A. Macleod and C. E. Lapple. 1959. "A Meteorological Tracer Technique Using Uranine Dye." J. Meteorol. 16:63-67.

Sehmel, G. A. 1966. Subjsokinetic Sampling of Particles in an Air Stream. BNWL-217, Battelle, Pacific Northwest Laboratories, Richland, Washington. 
APPENDIX A

DIFFUSION DATA

A-1 

MEANING OF REMARK SYMBOLS THAT PRECEDE

AZIMUTH COLUMN IN APPENDIX A TABLES

Symbol

Significance

$E$

H

$W$

C

P

$x$

Q

I

L

M

D

$S$

$T$

F

$\mathrm{R}$

EST IMATED values.

DOA (dead on arrival), HOT. At time of filter pickup, vacuum supply to filter was found to have fajled due to dead engine. Engine hot, however, indicating vacuum missing only a short time and hence likely a valid sample.

DOA, WARM. Same as $H$, except engine only warm to touch. Possibly valid sample, but more doubt than with $H$.

DOA, COLD. Same as $H$, except engine cold to touch, and no fuel in tank. Sampling occurred as long as fuel lasted, but ended considerably before filter pickup.

DOA, cold, PARTIAL tank of gasoline. Same as $C$ except sampling was

likely of shorter duration since engine failed before burning all the fuel.

DOE, cold, full tank. Engine failed shortly after starting. Sample is essentially due to impaction on filter rather than to flow through filter.

DOA, other. Engine DOA, but no other field note given.

IMPACTION sample. Engine for vacuum supply was never started. Sample is due to impaction on fijter rather than flow through filter.

Filter LOST or, in the case of krypton, Geiger-Muller tube not operating.

Filter was MISSING from nolder assembly. The supporting crepe paper roll was often assayed in the case of the ZnS tracer, but it is a much less efficient sampler than the membrane filter.

Filter assembly DROPPED. Effect unknown.

Filter SLIGHTLY cracked. Effect likely minimal.

Fi]ter TORN badly. Effect likely significant.

Apparent FINGERPRINT on filter. Time of placement and effect, if any, unknown.

For Rankin counting of ZnS, a RECOUNT of a filter with an unexpected mass assessment, but with essentially unchanged mass indicated on the repeat assessment. 
GEIGER-MULLER tube noisey or questionable

LOW VOLTAGE on Geiger-Muller tube. Likeiy low exposures indicated.

B

Tracer likely extends BEYOND this last active sampler. (The symbol was not always appended to top vertical samplers even though tracer obviously extended higher.)

$N$

Samplers operated, but N0 tracer observed on the arc (tower).

possibly no tracer passed samplers or possibly concentration below detection limit. 
Z Any other field or assessment remark. (A check of field notes-or laboratory assay notes would be needed to elaborate on each Z remark).

$\left.\begin{array}{l}1 \\ 2 \\ 3 \\ 4\end{array}\right\}$

\begin{tabular}{l|}
5 \\
6 \\
7 \\
8 \\
9 \\
0
\end{tabular}

Increasing

significance

of

visible

dust on

filters.*

*The numerical remark symbols are qualitative ranking of visible dust on field filters. Larger numbers indicate darker filters -- except "0" indicates the most. severe loading. Ins assay is degraded most severiy by dust, although a nomenclature of Tess than 5 can be considered of minimal importance. 

30-SERIES EXPEPINENT: 30 (FT-30)

THIS EXPERIIENT IS THE FIRST EXPERIIIENT OF THE 3O-SERIES.

GROU:ID LEVEL AND TOHEP SAHPL ING 200-3200 HARCS

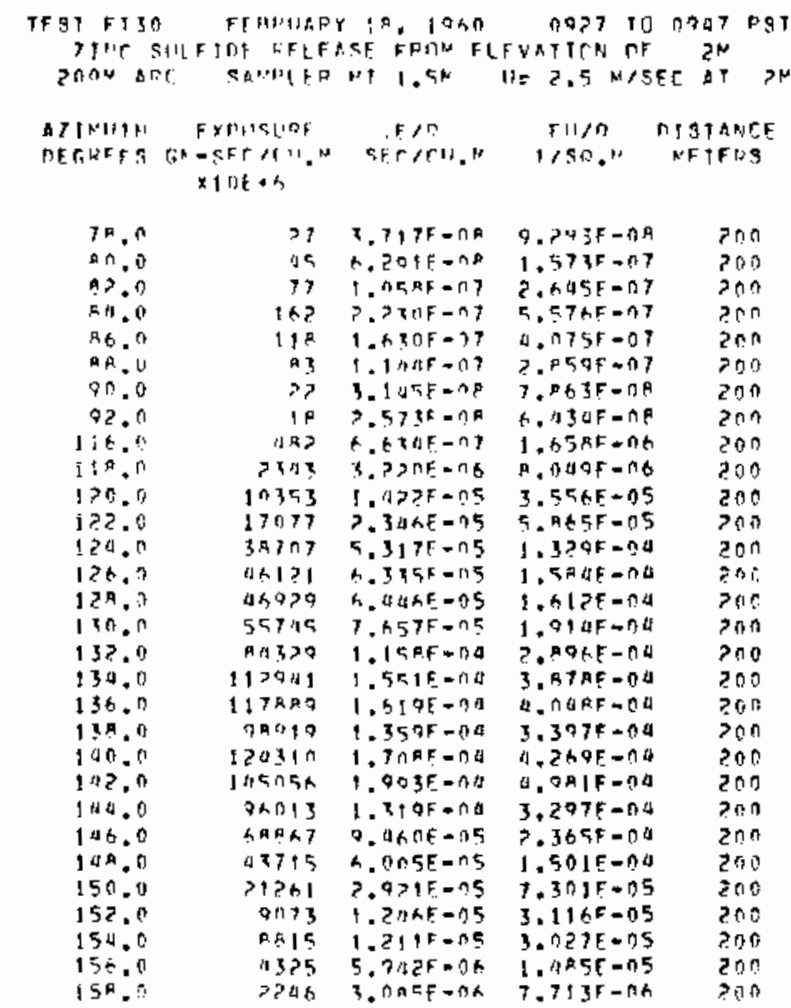

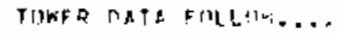

IFST FT3 5.

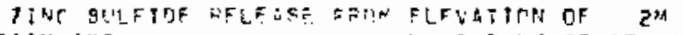

ZOGN $\triangle \mathrm{KE}$ WIE P.5 N/SEC AT $2 \mathrm{M}$

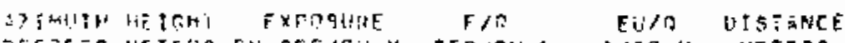

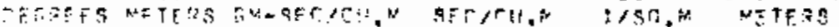
$x+\infty=+h$

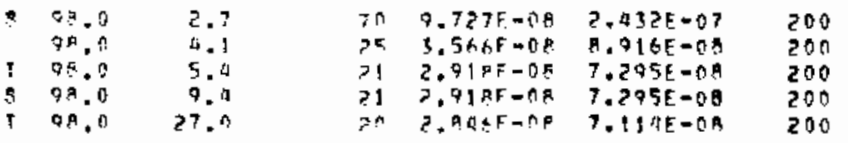

DIIMUTH HEIGHT EXPOSURE F/O EU/O DIETANEE

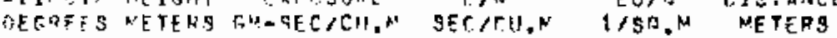
$x \rightarrow A+\infty$

\begin{tabular}{|c|c|c|c|c|c|}
\hline $\begin{array}{l}106.0 \\
106.0 \\
106.01\end{array}$ & $\begin{array}{l}2.7 \\
4.2 \\
6\end{array}$ & $\begin{array}{l}49 \\
23 \\
319\end{array}$ & $\begin{array}{l}3.309 E-04 \\
3.245 F=04 \\
4.21=F=08\end{array}$ & $\begin{array}{l}1.702 E=07 \\
9106 E=02 \\
1.050 E=07\end{array}$ & $\begin{array}{l}200 \\
240 \\
200\end{array}$ \\
\hline
\end{tabular}

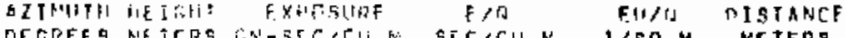

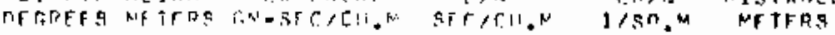

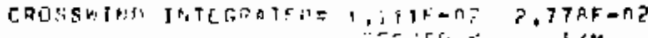

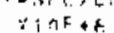

\begin{tabular}{|c|c|c|c|c|c|}
\hline 119.0 & 0.7 & 19 & $2.647 \mathrm{~F}=\mathrm{n}$ 月 & $6.619 E-0 n$ & 200 \\
\hline 114.0 & 1.3 & 16 & $2.276,008$ & $5.67 \mathrm{HE}=08$ & 200 \\
\hline $1+4.0$ & 7.7 & 1,7 & $1.7515=07$ & $4.377 E=07$ & 200 \\
\hline i 14.5 & 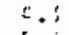 & $4=$ & $\therefore$ व व & i. $702 E=0 ?$ & $2 n n$ \\
\hline 914.8 & $5 . \therefore$ & $\mathrm{G}=$ & $3 . \therefore x_{1}=0 \mathrm{~A}$ & $5.54 \cap E=87$ & 200 \\
\hline $3: 4.0$ & $n \cdot a$ & $P 5$ & $3,50 h F=0 A$ & $A .9 ; A E=O A$ & 200 \\
\hline 194.0 & R.! & ah & 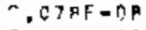 & 2.27 DE $=07$ & 200 \\
\hline $114 . ?$ & 0.4 & $4=$ & $5.85+5-08$ & $1.459 \mathrm{E}-07$ & 200 \\
\hline 114.0 & $10 .{ }^{\circ}$ & $\mathrm{c}^{\mathrm{c}}$ & $3.56 \mathrm{fE}-0 \mathrm{~g}$ & 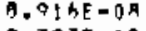 & 200 \\
\hline 114.0 & 13.5 & $>\mathrm{A}$ & $F . R Q ; E-O R$ & $9.727 \mathrm{E}-0 \mathrm{~A}$ & 200 \\
\hline $1+40$ & to. & ק & $\dagger .135 \mathrm{G}=07$ & ?.A37F-0? & 200 \\
\hline
\end{tabular}




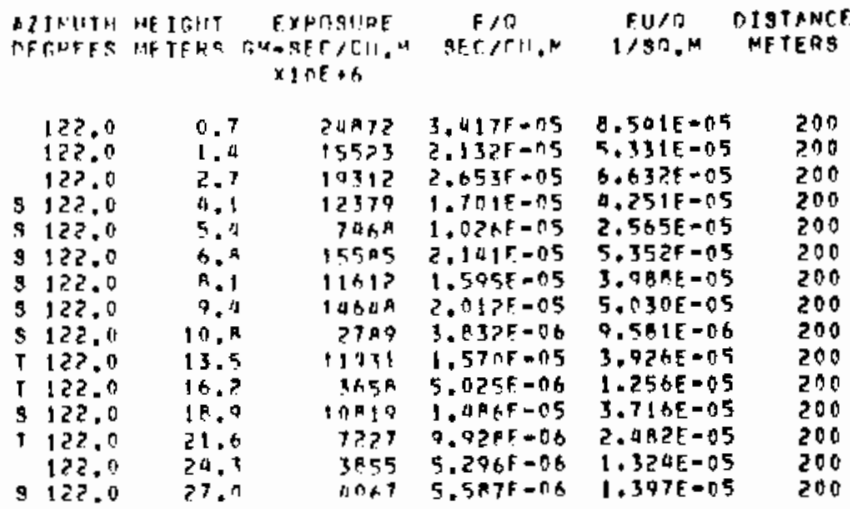

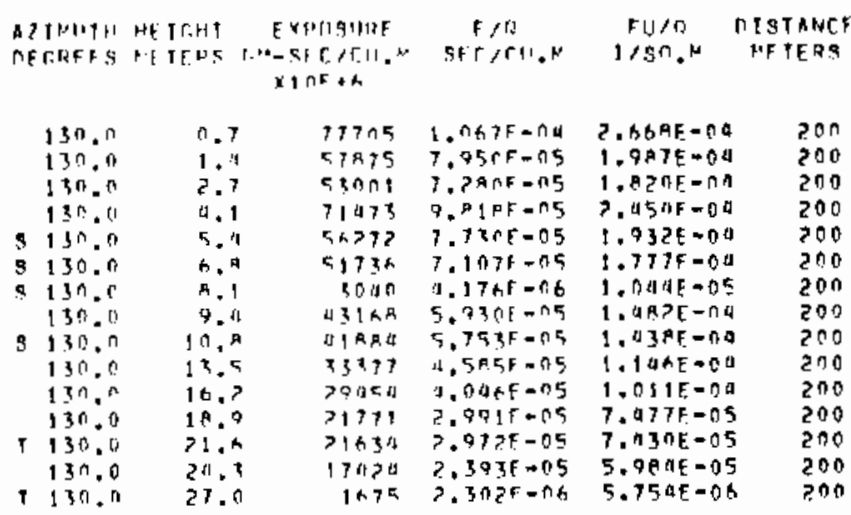

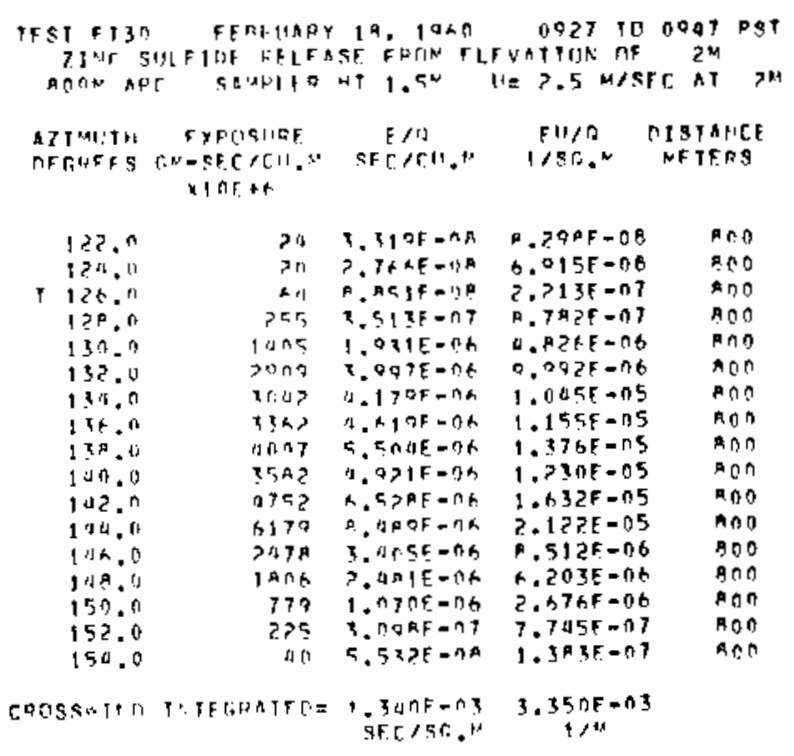


TOAFA DATA FOLLU,

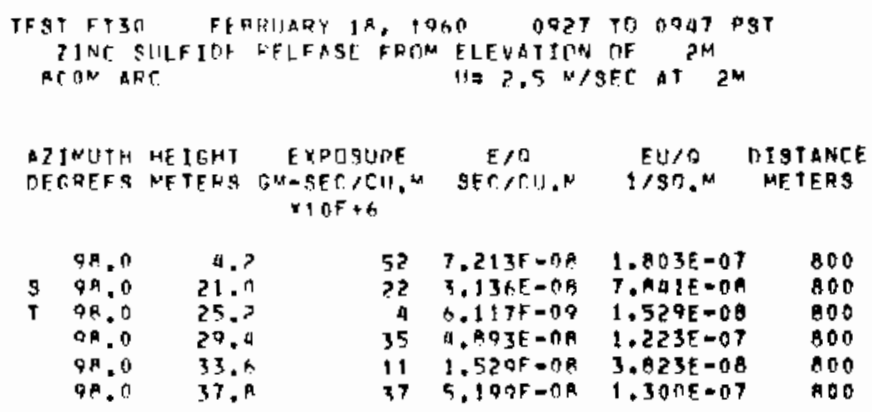

\begin{tabular}{|c|c|c|c|c|c|}
\hline $\begin{array}{l}\text { AZJNUTH } \\
\text { MEGREES }\end{array}$ & $\begin{array}{l}\text { HF ITHE } \\
\text { ME TFRS }\end{array}$ & $\begin{array}{c}\text { EXPOSUPE } \\
G M-9 E C / C ! 1 . * \\
\times 1 O F+6\end{array}$ & $\begin{array}{c}E / t \\
s e c / C \theta . m\end{array}$ & $\begin{array}{l}E \cup / 0 \\
1 / 9 n . M\end{array}$ & $\begin{array}{l}\text { OI TT ANCE } \\
\text { METERS }\end{array}$ \\
\hline $\begin{array}{l}106.0 \\
106.0 \\
106.0\end{array}$ & $\begin{array}{l}10.5 \\
12.6 \\
14.7\end{array}$ & $\begin{array}{l}30 \\
43 \\
19\end{array}$ & $\begin{array}{l}4.700 E=0 A \\
5.950 F=0 A \\
2.500 F=0 A\end{array}$ & $\begin{array}{l}1.176 E-07 \\
1.900 E=07 \\
6.273 E=08\end{array}$ & $\begin{array}{l}800 \\
800 \\
800\end{array}$ \\
\hline
\end{tabular}

A2TVUTH HEIGHT FXPISLIOF F/U FHIC DISTANCF

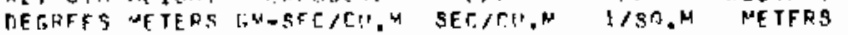
$x \perp \cap+6$

\begin{tabular}{|c|c|c|c|c|c|c|}
\hline & 1.9 .0 & 7.1 & 30 & $2 . A 2 \pi \mathrm{F}=0 \mathrm{~B}$ & $7.057 \mathrm{E}=0 \mathrm{~A}$ & an \\
\hline$T$ & 1.9. 0 & 4,2 & 13 & $1.8 R 2 F-C B$ & $4.7045=0$. & AOn \\
\hline & $1: 4.0$ & 0.3 & $3^{A}$ & $5,332 F=08$ & $1.335 E=07$ & AOA \\
\hline & $1: 0.0$ & 9. 4 & $>0$ & $535=08$ & $57 E-0$ & 200 \\
\hline 3 & $1: 4.0$ & 10.5 & 25 & 1. $45 \cap F=0 A$ & $4.625 F=08$ & 800 \\
\hline & $1 ! 4.0$ & 14.7 & 31. & $a .391 F=n B$ & $1.09 B E=07$ & 900 \\
\hline 1 & 114,0 & $16, n$ & 63 & $A, 7 A+E=0 B$ & $2.195 E-07$ & 010 \\
\hline & $1: 4.0$ & $2 !, 0$ & 20 & $2.825 F-O R$ & $7.057 E-0 A$ & 800 \\
\hline & $1+0.0$ & $25 . ?$ & ad & $6.117 E-18$ & $1.520 E-07$ & $A O D$ \\
\hline & 114.0 & 29.7 & $?$ & D5 AE - C9 & $646 E \cdot 09$ & 800 \\
\hline 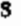 & 110.0 & 33.5 & 20 & $.753 F-0.8$ & A $A$ B!E $\approx A$ & 800 \\
\hline
\end{tabular}

\begin{tabular}{|c|c|c|c|c|c|}
\hline $\begin{array}{l}\text { AZINUTH } \\
\text { PETEEFS }\end{array}$ & $\begin{array}{l}\text { HEIGHT } \\
\text { MFTENS }\end{array}$ & 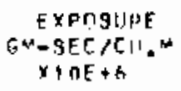 & $\begin{array}{c}F / 0 \\
\text { SEC }\end{array}$ & $\begin{array}{l}E(1 / B \\
1 / 5 n . M\end{array}$ & $\begin{array}{l}\text { DISTANCE } \\
\text { METEAS }\end{array}$ \\
\hline $\begin{array}{l}122,0 \\
3422.0 \\
9 \\
122.0\end{array}$ & $\begin{array}{l}12.6 \\
29.4 \\
33.4\end{array}$ & $\begin{array}{l}22 \\
17 \\
13\end{array}$ & $\begin{array}{l}3.136 F=0 A \\
3.44 Y E=08 \\
1.935 E=0 A\end{array}$ & $\begin{array}{l}\text { T. A A IE }=0 B \\
6.11 \text { TE }=00 \\
4.5 A B E=0\end{array}$ & $\begin{array}{l}100 \\
000 \\
000\end{array}$ \\
\hline
\end{tabular}

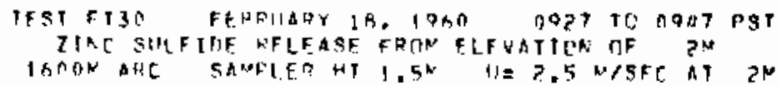

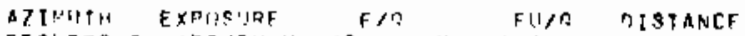

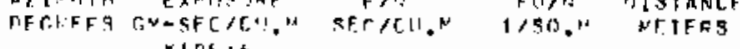
$\times \operatorname{lnt}+\infty$

\begin{tabular}{|c|c|c|c|c|}
\hline 131.0 & 133 & $>$ 3APF-07 & $5.055 \mathrm{~F}-07$ & 1600 \\
\hline 132.0 & 141 & $1.91,0 F=07$ & $A .873 E-07$ & 1600 \\
\hline 133.0 & $3 n 3$ & $4.7>1 E-07$ & 1. IRAF $=0 B$ & $160 n$ \\
\hline 134.0 & $5 \mathrm{AA}$ & A. A7AF-197 & $? .019 F=06$ & $\operatorname{ssn} 0$ \\
\hline 135.0 & $3 \times 7$ & $1.6345-n 7$ & $1.150 \mathrm{~F}=\mathrm{ns}$ & 1600 \\
\hline 136,0 & |३। & $6.757 F=07$ & $1.6 R Q E=06$ & 1600 \\
\hline $137 . n$ & +19 & A. $5: 1 F=07$ & $2.178 F=06$ & 1500 \\
\hline 5932.0 & 432 & A. $5 A$ AL -07 & $2.171 E=16$ & 1sos \\
\hline 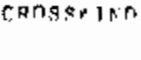 & TEFI:ATER= & $\begin{array}{r}1.2775=74 \\
9 F F>00 . N\end{array}$ & $\begin{array}{c}3.1975=014 \\
1 / N\end{array}$ & \\
\hline
\end{tabular}

TOWFP MATA FOLLTE....

TERT FT30 FEAHHARY 19 , 1900 DO TE OQAT PST

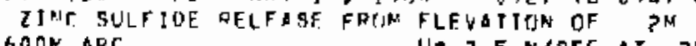
$1600 \mathrm{~N}$ ARC II T.5 NSBFC AT $? N$

AIINITH HE JGH EXPTSUDE EOD EU/O DISTANCE

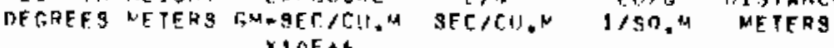
$x \in E+h$

\begin{tabular}{|c|c|c|c|c|c|}
\hline $\begin{array}{l}130.0 \\
7130.0 \\
5130.0 \\
1130.0 \\
130.0 \\
130.0 \\
130.0 \\
130.0 \\
130.0\end{array}$ & $\begin{array}{l}19.5 \\
21.7 \\
29.8 \\
31.0 \\
37.2 \\
13.4 \\
09.5 \\
55.8 \\
62.0\end{array}$ & $\begin{array}{l}11 \\
17 \\
18 \\
25 \\
72 \\
1 \\
1 \\
17 \\
16\end{array}$ & $\begin{array}{l}1.32 A F=08 \\
2.410 F=08 \\
2.595 F=08 \\
3.538 F=08 \\
3.067 F=08 \\
2.359 F=09 \\
2.1665=09 \\
2.599 F=08 \\
4.984 E=08\end{array}$ & $\begin{array}{l}4.020 E=0 B \\
6.025 E=01 \\
6.087 E-08 \\
0.446 E=09 \\
7.666 E=08 \\
5.097 E=09 \\
5.414 E=09 \\
6.497 F=08 \\
1.245 E=07\end{array}$ & $\begin{array}{l}1600 \\
1600 \\
1600 \\
1600 \\
1600 \\
1000 \\
1600 \\
1600 \\
1600\end{array}$ \\
\hline
\end{tabular}

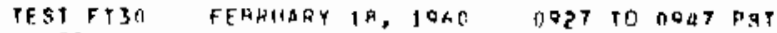

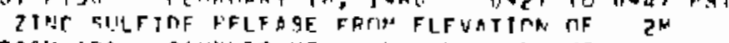

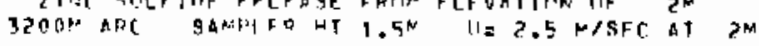

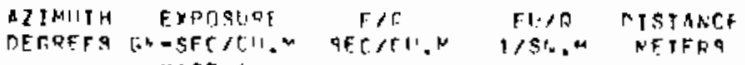
$\times 10$. +6

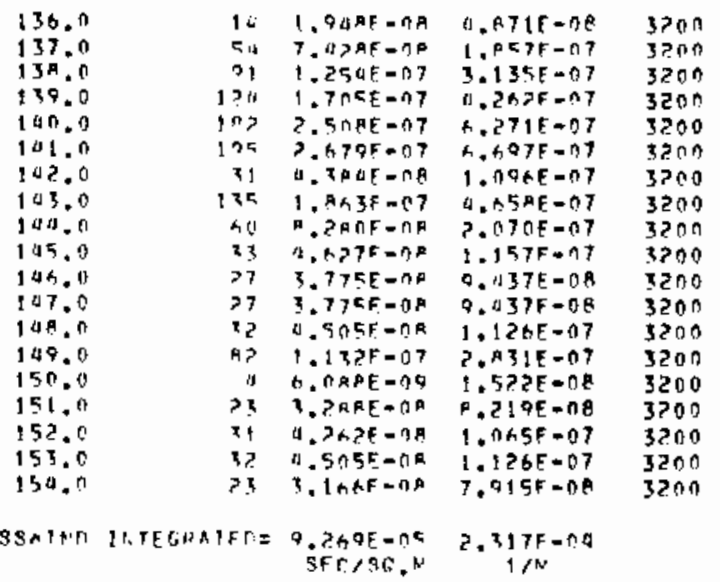


30-SERIES EXPERIMENT: 31 ( FY-31

GROUHD LEVEL AND TOWER SAMP! ING 200-3200 H APSS

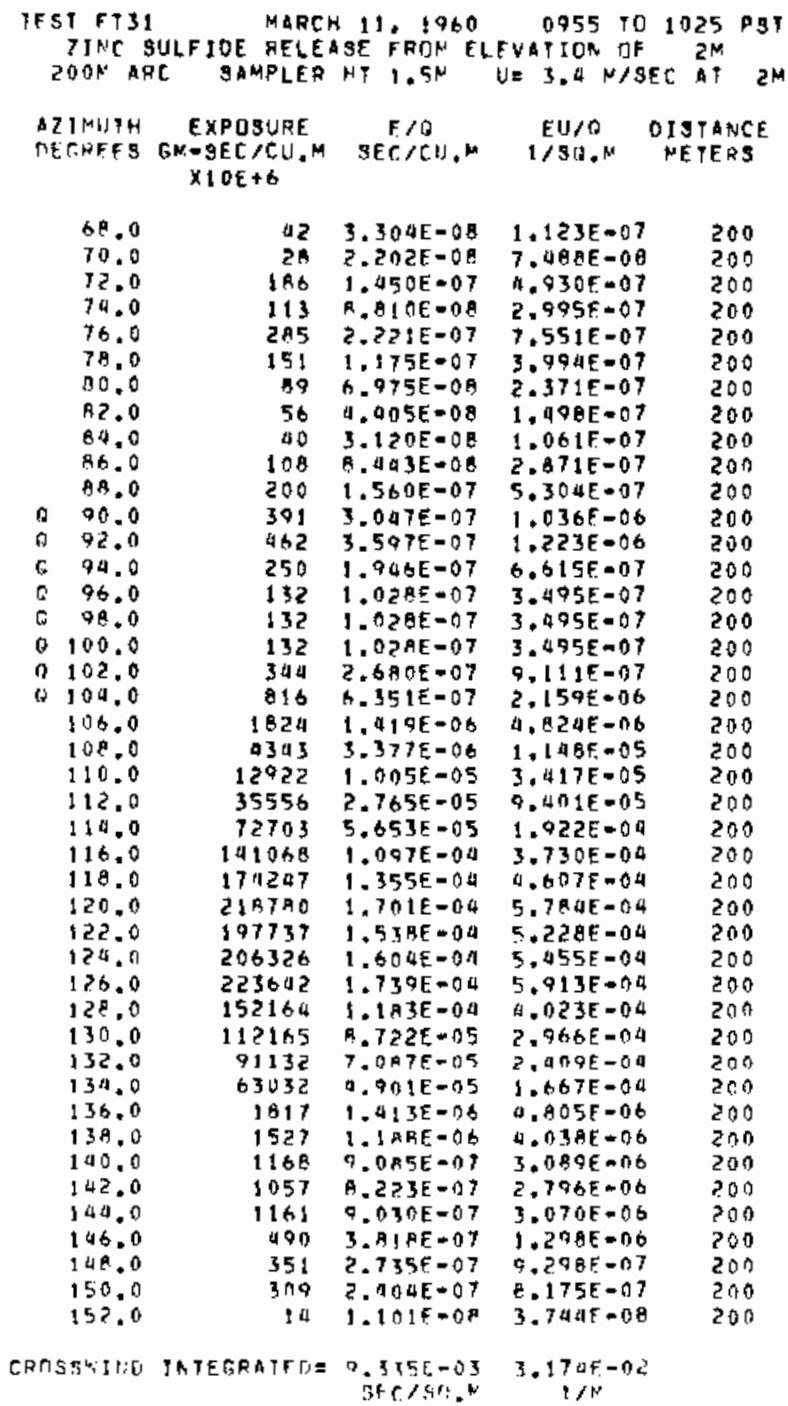

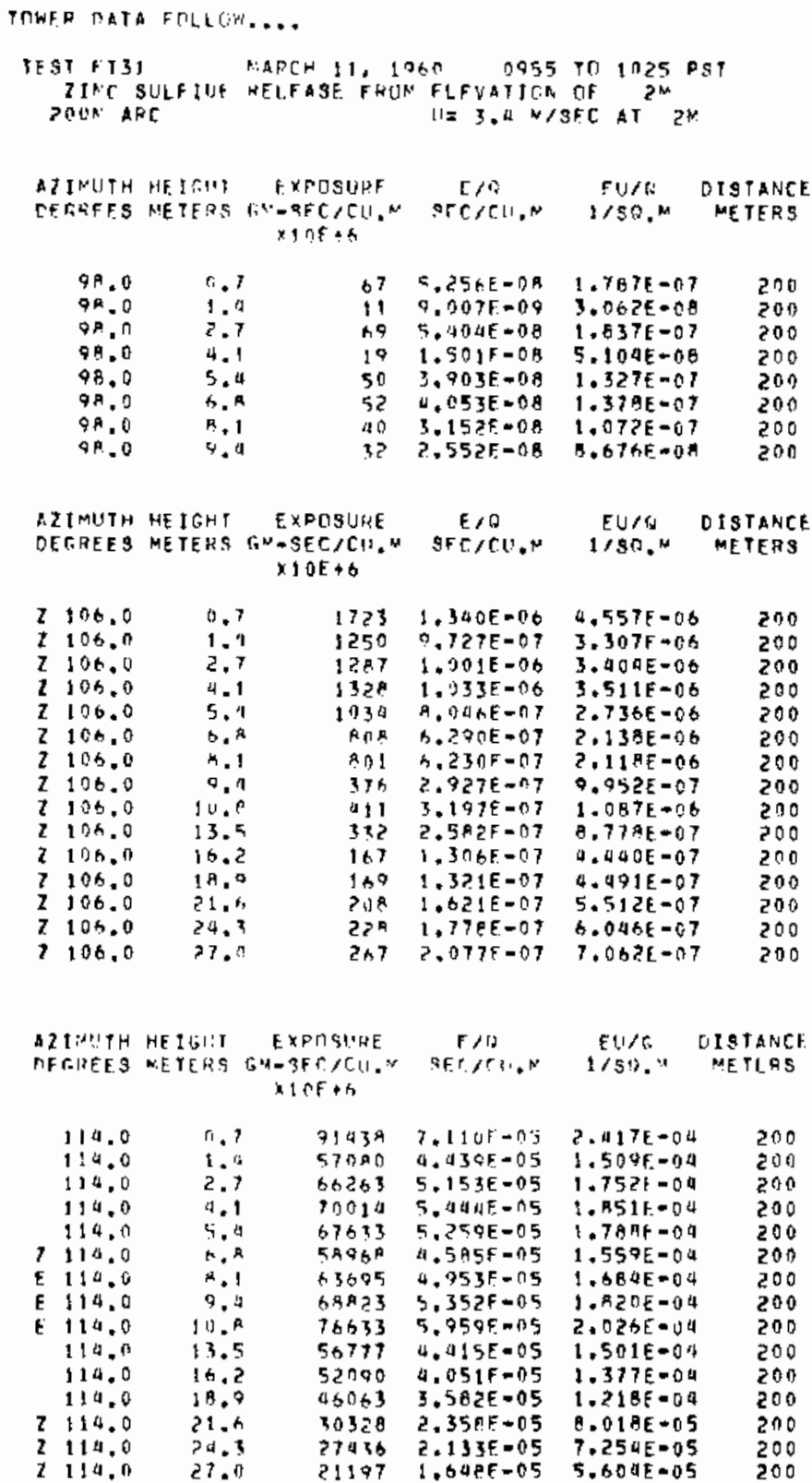




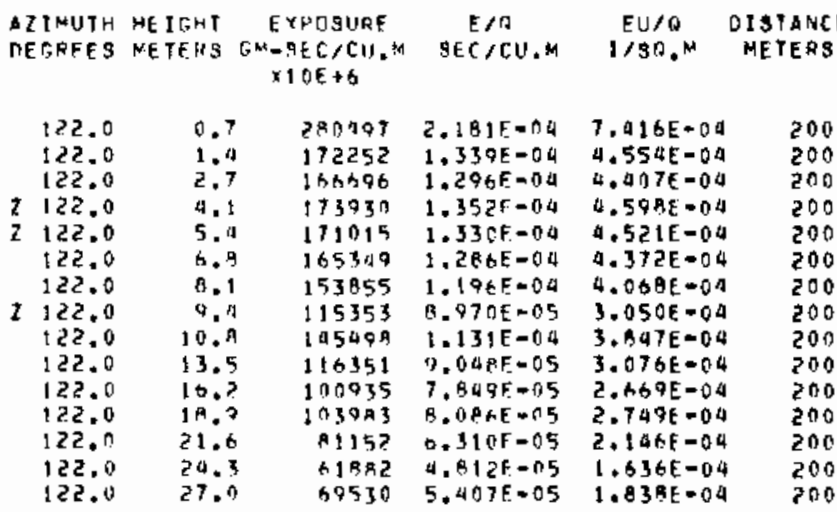

AZJMHTH HEILHT EXPRBLAE EID DISTANCE

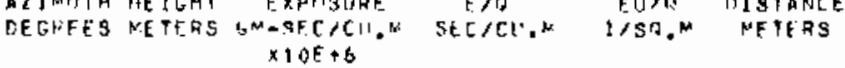

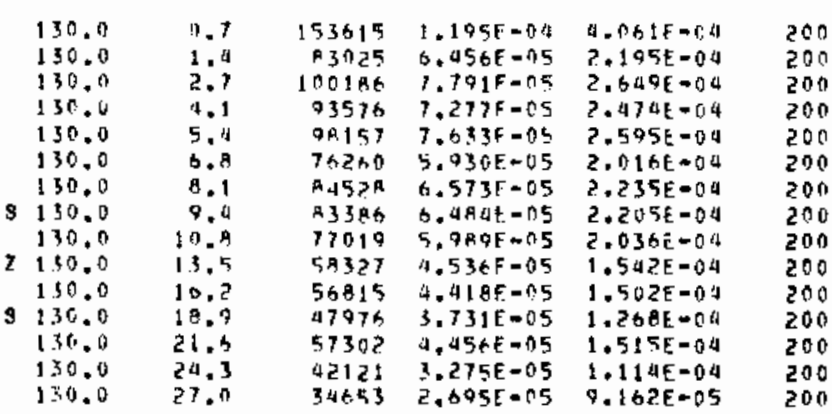

TE IT FI31 MARCH 11, 1960 O955 TO 1025 PST

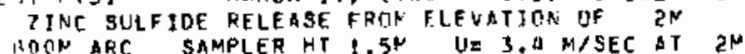

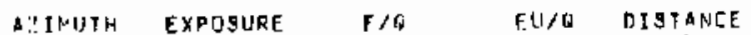
DITRFES GN-SECICU.M SECICU,N T/SD.M RETEHS $X \perp 0 E+b$ 00 00 20 200 200 200 200 200 200 200

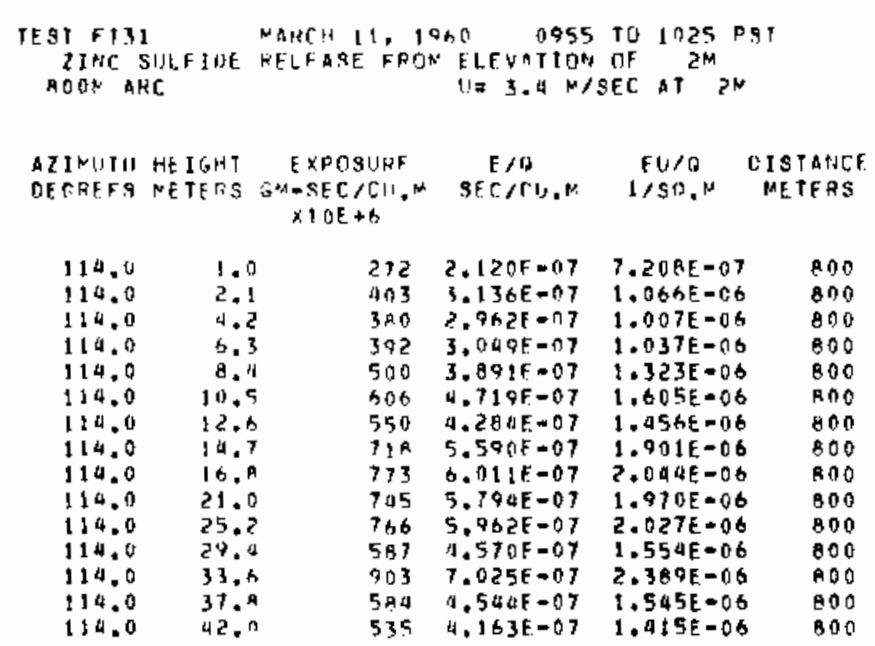

\begin{tabular}{|c|c|c|c|c|c|}
\hline $\begin{array}{l}\text { AZTMUTH } \\
\text { DEGREES }\end{array}$ & $\begin{array}{l}\text { HE IGHT } \\
\text { METERS }\end{array}$ & $\begin{array}{c}\text { EXPOSUEE } \\
\text { GM-SEC/CI! M } \\
\times 1 U E+6\end{array}$ & $\begin{array}{c}E / a \\
s e c / C l 1 . N\end{array}$ & $\begin{array}{c}E \cup / 0 \\
1>B S . M\end{array}$ & $\begin{array}{l}\text { D ISTANCE } \\
\text { ME TERS }\end{array}$ \\
\hline $\begin{array}{l}122.0 \\
122.0 \\
122.0 \\
122.0 \\
12200 \\
122.0 \\
122.0 \\
122.0 \\
122.0 \\
122.0 \\
122.0 \\
122.0 \\
122,0 \\
122.0 \\
122.0\end{array}$ & $\begin{array}{l}1.9 \\
2.1 \\
4.2 \\
6.3 \\
5.4 \\
10.5 \\
12.5 \\
14.7 \\
16.4 \\
23.0 \\
25.2 \\
29.1 \\
33.6 \\
37.0 \\
42.2\end{array}$ & $\begin{array}{l}10204 \\
12255 \\
15554 \\
11191 \\
10255 \\
10824 \\
12036 \\
11474 \\
11308 \\
11905 \\
10115 \\
10959 \\
9977 \\
11272 \\
9593\end{array}$ & 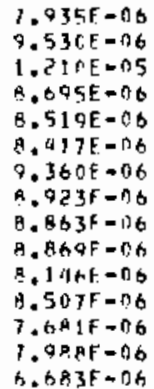 & $\begin{array}{l}2.64 B E=05 \\
3.240 E=05 \\
1.112 E=05 \\
2.956 E=05 \\
2.897 E=05 \\
2.962 E=05 \\
3.1 B 2 E=05 \\
3.034 E=05 \\
3.014 E=05 \\
3.015 E=05 \\
2.769 E=05 \\
2.892 E=05 \\
2.611 E=05 \\
2.716 E=05 \\
2.272 E=05\end{array}$ & $\begin{array}{l}900 \\
800 \\
900 \\
000 \\
800 \\
800 \\
800 \\
800 \\
100 \\
800 \\
800 \\
800 \\
800 \\
000 \\
800\end{array}$ \\
\hline
\end{tabular}

\begin{tabular}{|c|c|c|c|c|c|}
\hline $\begin{array}{l}\text { AIINUTH } \\
\text { OE TRFES }\end{array}$ & $\begin{array}{l}\text { MEIFHT } \\
\text { YETEQS }\end{array}$ & $\begin{array}{c}\text { EXPDSURF } \\
\text { GU-SEC }>C(1-N \\
\times 10 E+b\end{array}$ & $\begin{array}{c}F / C_{i} \\
s F+C=0\end{array}$ & $\begin{array}{c}E U / Q \\
1 / S y_{*}, M\end{array}$ & $\begin{array}{l}\text { DISTANCE } \\
\text { METEFS }\end{array}$ \\
\hline
\end{tabular}

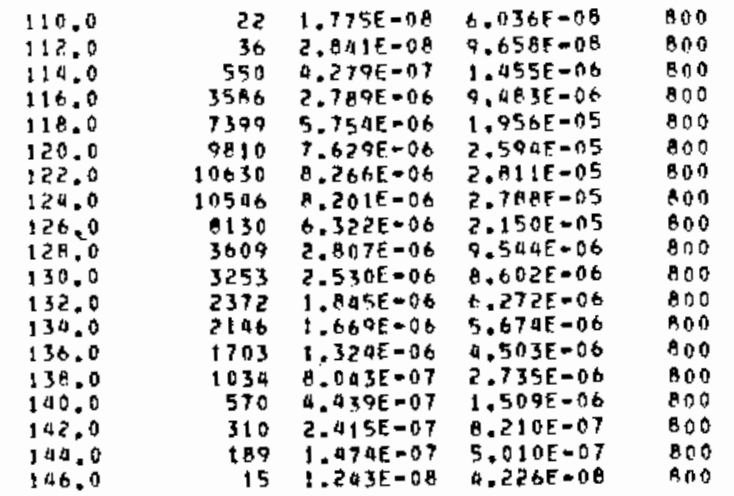

\begin{tabular}{|c|c|c|c|c|c|}
\hline $\begin{array}{l}130.0 \\
130.0 \\
130.0 \\
130.0 \\
130.0 \\
130.0 \\
130.0 \\
130.0 \\
130.0 \\
130.0 \\
130.0 \\
130.0 \\
130.0 \\
130.0 \\
130.0\end{array}$ & $\begin{array}{l}1.0 \\
2.1 \\
4.2 \\
0.3 \\
4.4 \\
10.5 \\
12.5 \\
14.7 \\
16.4 \\
21.9 \\
25.2 \\
29.4 \\
33.4 \\
37.4 \\
92.0\end{array}$ & $\begin{array}{l}2591 \\
2705 \\
3259 \\
2299 \\
3258 \\
3575 \\
3994 \\
3806 \\
3478 \\
4375 \\
3777 \\
4037 \\
4262 \\
4353 \\
4710\end{array}$ & $\begin{array}{l}2.015 E-06 \\
2.104 E=06 \\
2.528 F=06 \\
2.255 E=06 \\
2.534 E=06 \\
3.791 F=06 \\
3.090 E=06 \\
2.991 E=06 \\
2.705 E=06 \\
3.402 E=06 \\
2.938 E=06 \\
3.140 E=06 \\
3.3111 E=06 \\
3.385 E=06 \\
3.656 E=06\end{array}$ & $\begin{array}{l}6.852 E=06 \\
7.154 E=06 \\
8.595 E-06 \\
7.667 E=06 \\
8.615 E=06 \\
9.454 E-06 \\
1.054 E=09 \\
1.017 E=05 \\
9.198 E=06 \\
1.157 E-05 \\
9.988 E-06 \\
1.068 E=05 \\
1.127 E=05 \\
1.151 E=05 \\
1.247 E=05\end{array}$ & $\begin{array}{l}800 \\
800 \\
800 \\
400 \\
800 \\
800 \\
000 \\
800 \\
000 \\
800 \\
800 \\
800 \\
000 \\
800 \\
000\end{array}$ \\
\hline
\end{tabular}

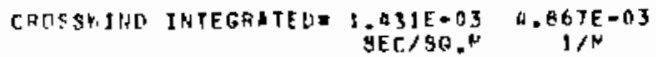




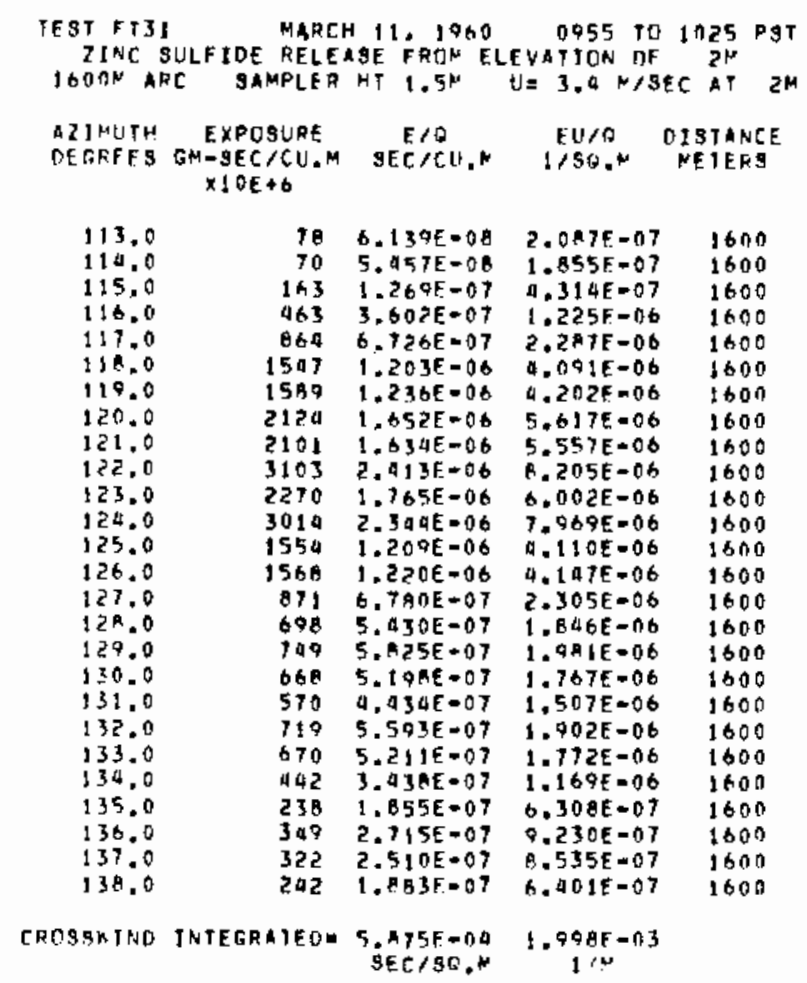

TOWEF T.ATA FOLLLIW....

\begin{tabular}{|c|c|c|c|c|c|}
\hline $\begin{array}{l}\text { AZINUTH } \\
\text { DEGKFES }\end{array}$ & $\begin{array}{l}\text { HE IGHF } \\
\text { METERS }\end{array}$ & $\begin{array}{c}\text { EXPOSURE } \\
G M=-3 E C, E U, M \\
\times 10 E+6\end{array}$ & $\begin{array}{c}E / Q \\
S E C / C U . M\end{array}$ & $\begin{array}{c}E \cup / O \\
1 / \$ 0.4\end{array}$ & $\begin{array}{c}\text { DISTANCE } \\
\text { METERS }\end{array}$ \\
\hline $\begin{array}{l}122.0 \\
122.0 \\
122.0 \\
122.0 \\
122.0 \\
122.0 \\
122.0 \\
122.0 \\
122.0 \\
122.0 \\
122.0 \\
122.0 \\
122.0 \\
122.0 \\
122.0\end{array}$ & $\begin{array}{r}1.5 \\
3.1 \\
6.2 \\
9.3 \\
12.4 \\
15.5 \\
18.4 \\
21.7 \\
24.9 \\
31.9 \\
37.9 \\
43.9 \\
49.4 \\
55.9 \\
62.7\end{array}$ & $\begin{array}{l}2655 \\
2515 \\
2424 \\
2694 \\
2416 \\
2691 \\
3472 \\
3232 \\
3151 \\
2819 \\
2642 \\
2569 \\
2470 \\
2000 \\
1960\end{array}$ & 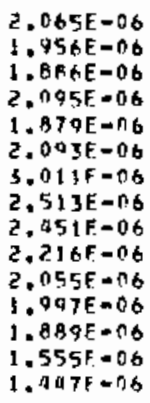 & $\begin{array}{l}7.021 E=06 \\
6.650 E-06 \\
6.011 E=06 \\
1.123 E=06 \\
6.309 E=06 \\
1.115 E=06 \\
1.024 E=05 \\
6.546 E=06 \\
8.333 E=06 \\
7.533 E=06 \\
6.987 E-06 \\
6.790 E=06 \\
6.421 E-06 \\
5.28 \mathrm{EE}=06 \\
4.919 E=06\end{array}$ & $\begin{array}{l}1600 \\
1600 \\
1600 \\
1600 \\
1600 \\
1600 \\
1600 \\
1000 \\
1600 \\
1600 \\
1600 \\
1600 \\
1600 \\
1600 \\
1600\end{array}$ \\
\hline
\end{tabular}

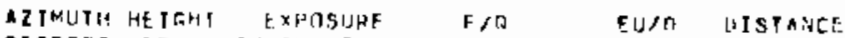
OFGREES METERS GM-gEC/CII.* SETICH, IJSO.M HETFAS $\times 1$ OE $\rightarrow h$

\begin{tabular}{|c|c|c|c|c|c|}
\hline $\begin{array}{l}130.0 \\
130.0 \\
130.0 \\
130.0 \\
130.0 \\
130.0 \\
130.0 \\
130.0 \\
130.0 \\
130.0 \\
130.0 \\
130.0 \\
130.0 \\
130.0 \\
130.0\end{array}$ & $\begin{array}{l}1.5 \\
3.5 \\
6.7 \\
9.3 \\
12.4 \\
15.5 \\
18.5 \\
21.7 \\
24.8 \\
31.0 \\
37.7 \\
43.9 \\
49.4 \\
55.8 \\
62.0\end{array}$ & $\begin{array}{r}433 \\
570 \\
632 \\
229 \\
1051 \\
1915 \\
910 \\
915 \\
910 \\
103 \\
644 \\
1095 \\
994 \\
596 \\
1365\end{array}$ & 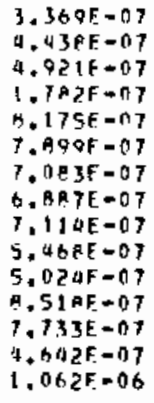 & 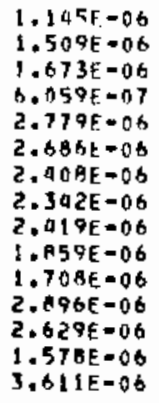 & $\begin{array}{l}1600 \\
1600 \\
1600 \\
1600 \\
1600 \\
1600 \\
1600 \\
1600 \\
1600 \\
1600 \\
1600 \\
1600 \\
1600 \\
1600 \\
1600\end{array}$ \\
\hline
\end{tabular}

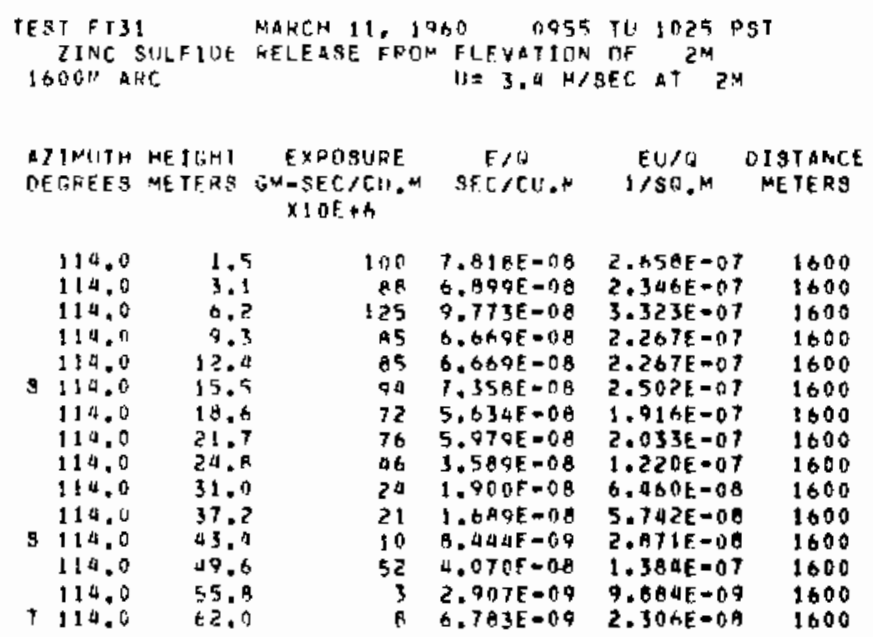




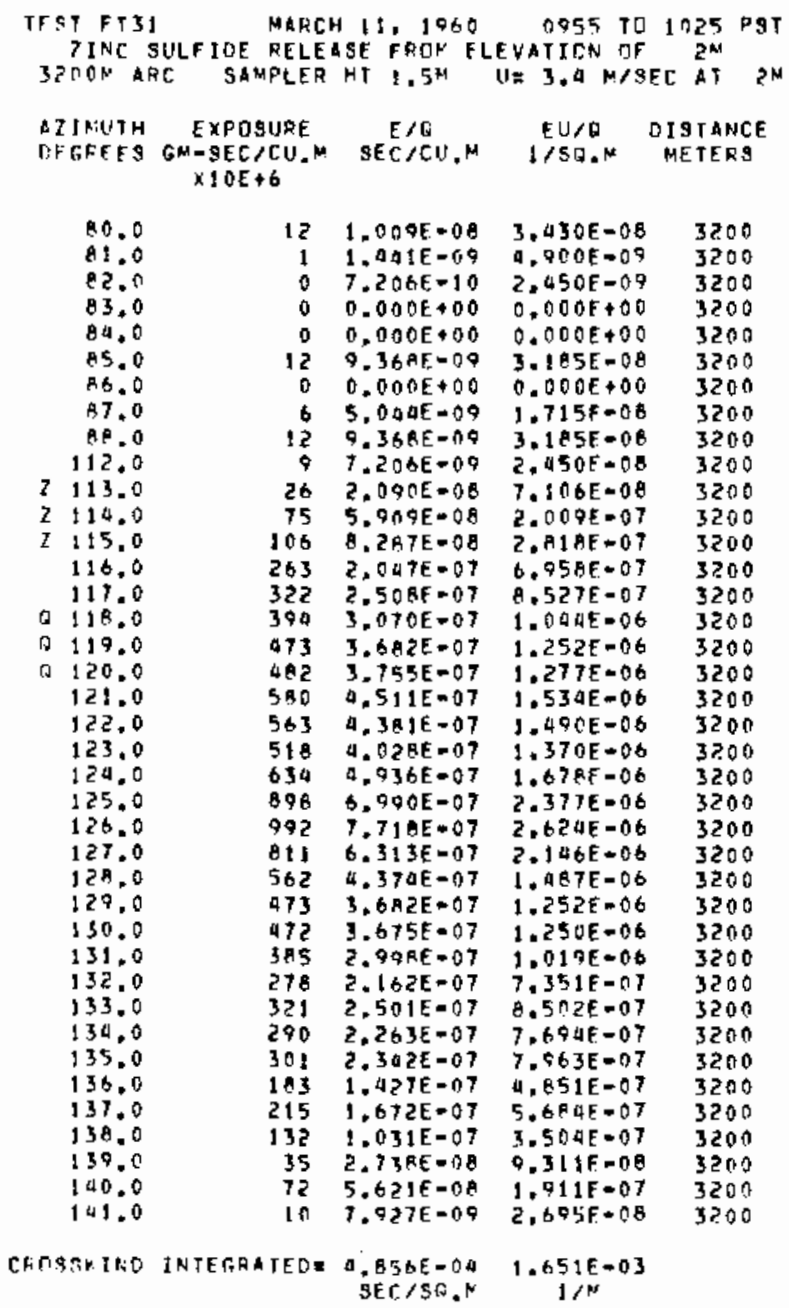

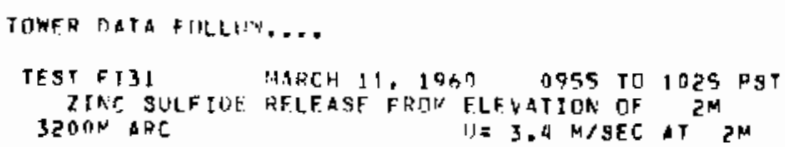

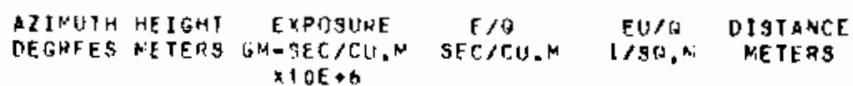

\begin{tabular}{|c|c|c|c|c|c|}
\hline 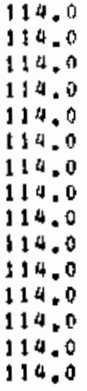 & $\begin{array}{l}1.5 \\
3.1 \\
6.7 \\
9.7 \\
12.9 \\
15.5 \\
16.4 \\
29.7 \\
24.1 \\
31.0 \\
37.2 \\
43.9 \\
49.6 \\
54.8 \\
62.8\end{array}$ & $\begin{array}{l}71 \\
31 \\
65 \\
41 \\
75 \\
32 \\
50 \\
96 \\
39 \\
13 \\
12 \\
18 \\
59 \\
29 \\
11\end{array}$ & 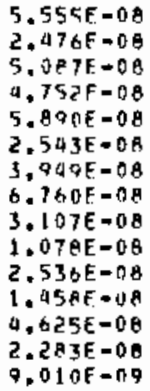 & $\begin{array}{l}1.8 B 9 E=07 \\
8.020 E=08 \\
1.729 E=07 \\
1.616 E=07 \\
2.003 E=07 \\
8.647 E-08 \\
1.343 E=07 \\
2.29 B E=07 \\
1.056 E=07 \\
3.665 E=08 \\
6.623 E=08 \\
4.958 E-08 \\
1.573 E-07 \\
7.761 E=08 \\
3.063 E=08\end{array}$ & $\begin{array}{l}3200 \\
3200 \\
3200 \\
3200 \\
3200 \\
3200 \\
3200 \\
3200 \\
3200 \\
3200 \\
3200 \\
3200 \\
3200 \\
3200 \\
3200\end{array}$ \\
\hline
\end{tabular}

AZIMITH HEISTY FXPQISURE E CEGHEES METERS GM-SEC/CII, SFC/CU.K: 1/SA.M METERS
X1OE+O

\begin{tabular}{|c|c|c|c|c|c|}
\hline $\begin{array}{l}122.0 \\
122.0 \\
122.0 \\
122.0 \\
122.0 \\
122.0 \\
122.0 \\
122.0 \\
122.0 \\
122.0 \\
122.0 \\
122.0 \\
122.0 \\
122.0 \\
122.0\end{array}$ & $\begin{array}{l}1.5 \\
3.1 \\
0.2 \\
9.3 \\
12.9 \\
15.5 \\
18.6 \\
21.7 \\
24.9 \\
31.9 \\
37.2 \\
43.9 \\
49.6 \\
55.2 \\
62.0\end{array}$ & $\begin{array}{l}307 \\
503 \\
724 \\
669 \\
685 \\
778 \\
614 \\
715 \\
511 \\
545 \\
576 \\
499 \\
417 \\
545 \\
6 \times 2\end{array}$ & 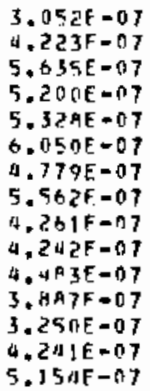 & 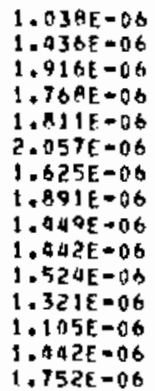 & $\begin{array}{l}3200 \\
3200 \\
3200 \\
3200 \\
3200 \\
3200 \\
3200 \\
3200 \\
3200 \\
3200 \\
3200 \\
3200 \\
3200 \\
3200 \\
3200\end{array}$ \\
\hline
\end{tabular}

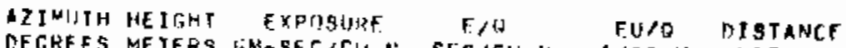
DECKEES METERS GM-SEC/CH.N SEC/CU.M IISA.M METERG

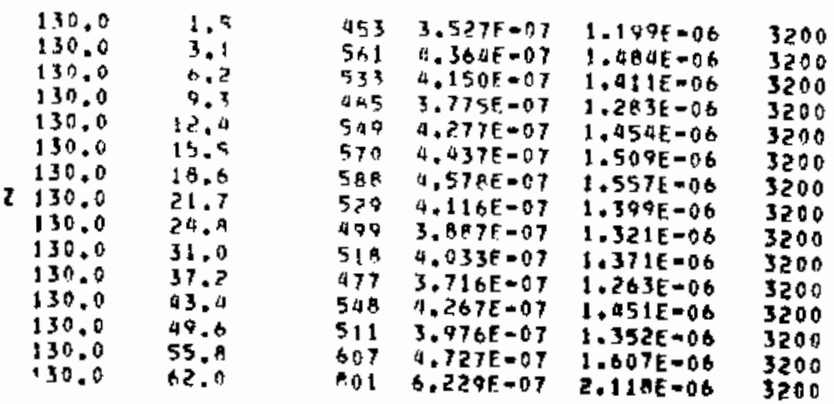


30-SERIES EXPERIMENT: 40 (FT-AD)

GROUND LEVEL AND TOWER SAMPLING 200-3200 M ARCS

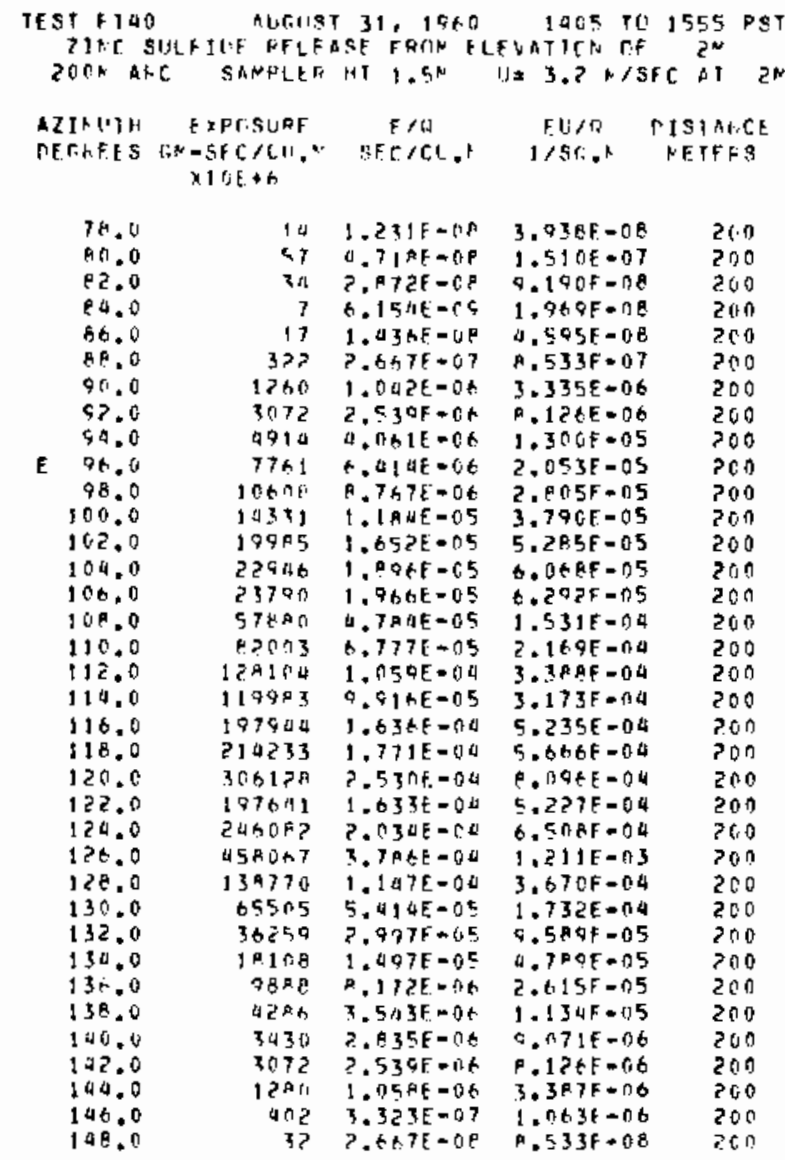

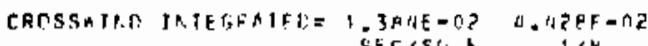

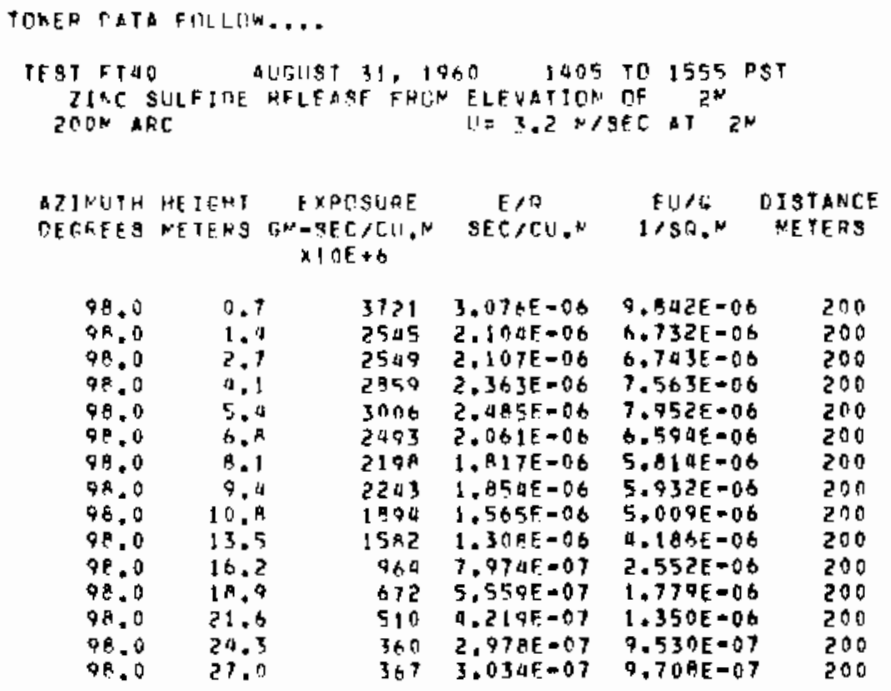

\begin{tabular}{|c|c|c|c|c|c|}
\hline $\begin{array}{l}\text { DIJMUTH } \\
\text { DEGRFES }\end{array}$ & $\begin{array}{l}\text { HEIGHT } \\
\text { METERS }\end{array}$ & $\begin{array}{c}\text { EXPOSURE } \\
G \times-S E C / C 11 . \\
\times 10 F+6\end{array}$ & $\begin{array}{c}F / B \\
S E[/ C H, N\end{array}$ & $\begin{array}{l}E \cup / C \\
1 / S 0 . N\end{array}$ & $\begin{array}{c}\text { DISTANCE } \\
\text { NETERS }\end{array}$ \\
\hline $10+. n$ & 0.7 & 10025 & A. $780 \mathrm{~F}-06$ & $2.809 E-65$ & 200 \\
\hline 106.0 & 1.4 & $63 n 6$ & $5.212 E-06$ & 1.66 RE $=05$ & 200 \\
\hline $10+.0$ & 2,7 & $\times 145$ & $0.749 \mathrm{~F}=06$ & $2.160 E=05$ & 200 \\
\hline 106.0 & $=1$ & 10345 & $0.550 E=010$ & $2.73 \mathrm{BE}=05$ & 200 \\
\hline $1 \mathrm{~PB} .0$ & 5.4 & $\begin{array}{l}7587 \\
\text { ค. } 75 \mathrm{~A}\end{array}$ & $\begin{array}{l}6.2795=166 \\
6.325 F=06\end{array}$ & $\begin{array}{l}2.009 E-05 \\
2.1 \text { ALE }=0.5\end{array}$ & $\begin{array}{l}200 \\
200\end{array}$ \\
\hline $\begin{array}{l}156.0 \\
106.0\end{array}$ & $\begin{array}{l}6.9 \\
8.1\end{array}$ & $\begin{array}{l}A \geq 59 \\
951 b\end{array}$ & $\begin{array}{l}6.325 F=06 \\
7.865 F=06\end{array}$ & $\begin{array}{l}2.164 t=05 \\
2.517 t-05\end{array}$ & $\begin{array}{l}200 \\
200\end{array}$ \\
\hline 106,0 & 9.4 & +723 & $5.550 F-06$ & $1.77 A E=05$ & 200 \\
\hline 106.0 & 10.8 & 7530 & $0.22 \Delta F=00$ & $1.792 E=05$ & 200 \\
\hline 106.0 & 13.5 & 4702 & $3.8865-06$ & $1.244 F=05$ & 200 \\
\hline int. o & $16 . ?$ & aमA & $5.043 E=06$ & $1.160 f-c 5$ & 200 \\
\hline 106.0 & 18.9 & 4781 & $3.9605=066$ & $1.267 F-05$ & 200 \\
\hline & $>1.6$ & $105 ?$ & $2.702 E=07$ & $2.785 E=0 n$ & 200 \\
\hline $10 R .0$ & 24.7 & PमQ2 & $2.275 \mathrm{~F}=\mathrm{nt}$ & $7.12^{n E}=0 h$ & 200 \\
\hline $16 \pi, 0$ & 27.0 & $17 \times 4$ & $1.47 A F=06$ & $9.724 E+06$ & 200 \\
\hline
\end{tabular}

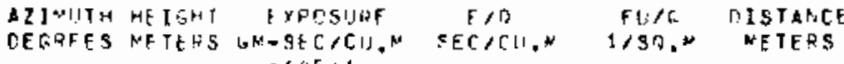
II OE +h

\begin{tabular}{|c|c|c|c|c|c|}
\hline 114.9 & 0.7 & $569 n x$ & $4.70 A E=05$ & $1.507 t=00$ & 200 \\
\hline 110,10 & 1.0 & 35970 & $2.199 E-115$ & $0.957 \mathrm{E}=05$ & 200 \\
\hline 11 14. & 2.7 & 34461 & Q Q Q $E=\cap 5$ & $0.1146=05$ & 200 \\
\hline $11 a, c$ & 4,8 & 39731 & $3.201 F=05$ & $1.024 E=04$ & 200 \\
\hline $19+0^{2}$ & 5.4 & $350>0$ & $2.894 E=0.5$ & $2625-05$ & 200 \\
\hline 114.17 & 6.8 & 3จ4จ० & $2 A Q F+15$ & $1.044 E-04$ & 200 \\
\hline 110.0 & B. 1 & $3434 !$ & Q $32 E=175$ & $9.082 E=05$ & 200 \\
\hline 110.0 & 9.4 & 22161 & $59 E-05$ & GEMOS & 200 \\
\hline 114.0 & $10 .{ }^{n}$ & 28782 & $379 E=05$ & $7.612 E=05$ & 200 \\
\hline 124.6 & 13 & 2 पene & $050 E-05$ & $501 E-05$ & 200 \\
\hline 114.11 & 16 & 20317 & $670 f=05$ & $3 E-05$ & 00 \\
\hline $11 " 0$ & IB & $1.33 \mathrm{~g}$ & $103 E-05$ & 05 & 00 \\
\hline 114.0 & 21.6 & 12020 & $.935 E=06$ & $3.179 E-05$ & 200 \\
\hline 144.0 & 24.3 & 11519 & $521 E-06$ & $3.047 E-05$ & 200 \\
\hline 114.0 & $27 . n$ & 6037 & $B 9 E=06$ & $1.597 E=05$ & 200 \\
\hline
\end{tabular}




\begin{tabular}{|c|c|c|c|c|c|}
\hline $\begin{array}{l}\text { AIINIITH } \\
\text { OFTEEES }\end{array}$ & $\begin{array}{l}\text { HE IGH T } \\
\text { NETERS }\end{array}$ & $\begin{array}{c}\text { EXPOSURE } \\
\text { SW }=5 E C / C W \\
x 10 F+6\end{array}$ & $\begin{array}{c}E / C \\
S E C / C H, N\end{array}$ & $\begin{array}{l}E \cup / 6 \\
1 / S C . N\end{array}$ & $\begin{array}{l}\text { DISTANCE } \\
\text { METERS }\end{array}$ \\
\hline 122.11 & 0.7 & 71921 & $5.936 E-05$ & $1 . B 9 Q E=04$ & 200 \\
\hline $12 ? .0$ & 1.4 & $5 ? 254$ & $4,335 F=05$ & $1.387 E-04$ & 200 \\
\hline 122.0 & 2.7 & 63090 & $5.20_{1}=05$ & $1.690 E-04$ & 200 \\
\hline 122,0 & 4. 1 & 710 at & $5.875 t-05$ & $1,880 E=04$ & 200 \\
\hline 122,0 & 5.1 & 536.4 & $4 .+35 F=0.5$ & $1.419 \mathrm{E}-0.4$ & 200 \\
\hline 122.0 & 6.3 & 54239 & $4.493 E-05$ & $1.434 E=04$ & 200 \\
\hline 122.0 & 8.1 & 492.74 & $4.072 E=05$ & $1.303 \varepsilon-04$ & 200 \\
\hline & 9.1 & 1. 3957 & $3.635 f=05$ & $1.163 E=04$ & 200 \\
\hline & 10.5 & 32520 & 2.6 eef -05 & $B .600 E=05$ & 200 \\
\hline & 13.5 & 30724 & $2.535 E=05$ & $8.125 t-0.5$ & 200 \\
\hline .0 & 16.2 & 77035 & $2.234 F-0.5$ & $7.150 E-05$ & 200 \\
\hline .11 & 18.9 & 18574 & $1.535 \xi=0.5$ & $a .912 E-05$ & 200 \\
\hline & $2-14$ & 16325 & $1.349 \mathrm{E}-05$ & $4.317 E=0.5$ & 200 \\
\hline & 34 & 3994 & प3 $4 \mathrm{E}=06$ & $2.3796=05$ & 200 \\
\hline & 27 & $7 \pi 96$ & $5.967 E-0.6$ & $1.877 E-05$ & 200 \\
\hline
\end{tabular}

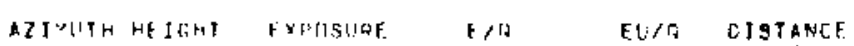

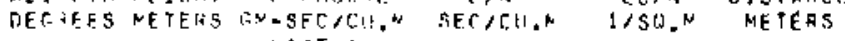
$\times 135+6$

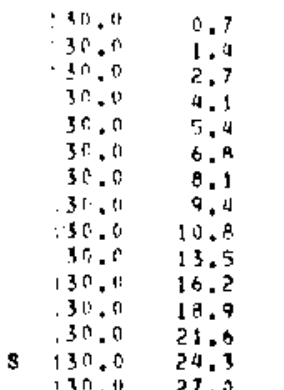
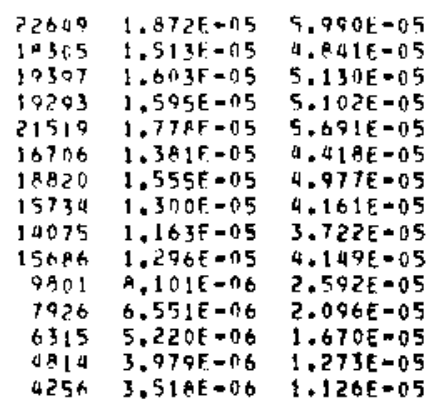

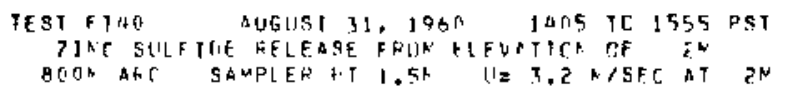

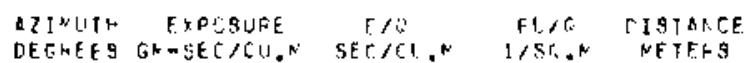

$$
\begin{aligned}
& \text { i) } 15+6
\end{aligned}
$$

\begin{tabular}{|c|c|c|c|c|}
\hline $96 . ?$ & $3 A$ & $3.175 t=0 ?$ & $1.01 t E-0.7$ & PCO \\
\hline 98.0 & 1 an & $1.3>Q \mathrm{~F}=07$ & 4.25 at -07 & 900 \\
\hline 100.0 & $2 A 5$ & $2.361 \mathrm{E}=07$ & $7.55 t F=0.7$ & Q00 \\
\hline 102.0 & 490 & औ. 12$] E-[j]$ & $1.321 E=06$ & 600 \\
\hline 104,0 & 470 & 3.PAGF $=07$ & $1.245 E-06$ & 800 \\
\hline 100.0 & Q? & $7 . A \subseteq \& E=C 7$ & $2.451 E=06$ & 300 \\
\hline 108.0 & 1035 & $1.351 E=06$ & $9.324 E=06$ & $8 \cap 0$ \\
\hline 110.0 & 2938 & z. & $7.772 F=06$ & 900 \\
\hline 112.0 & 7494 & $3.719 E-06$ & $1.1908+05$ & aOO \\
\hline 114.0 & $79 ? 0$ & $6,546 E=06$ & $2.095 \mathrm{f}=05$ & 800 \\
\hline 116.0 & $113 \times 7$ & Q. $a ! 1 E=n E$ & $3.012 E-05$ & 800 \\
\hline 118.0 & 14455 & $1,105 E-05$ & $3.023 F-05$ & 800 \\
\hline 120.0 & 11387 & "IIE 06 & $3.012 E-05$ & $\operatorname{AnO}$ \\
\hline 122.0 & $97 A 3$ & $8.0 \times 6 E-6 t$ & $2.587 E=05$ & 800 \\
\hline 12 & 13092 & $1.0 \times 2 E=05$ & $3.462 \mathrm{E}=05$ & 800 \\
\hline 126.0 & 7474 & $6.1778=08$ & $1.977 E=05$ & 000 \\
\hline 128.0 & 5474 & $9.524 E-0 t$ & $1.448 E=05$ & 800 \\
\hline 130.0 & 2384 & $1.970 E=06$ & $0.305 E-06$ & 800 \\
\hline 132.0 & 794 & H. 56 BE $=07$ & $2.102 \mathrm{~F}=06$ & 800 \\
\hline 134.0 & 127 & $1.052 E=07$ & $3.365 E-07$ & 000 \\
\hline 136,0 & 74 & $.15 I E=08$ & $1.968 E-07$ & 800 \\
\hline & 31 & $570 E-0^{\circ}$ & $P .254 E-08$ & 000 \\
\hline
\end{tabular}

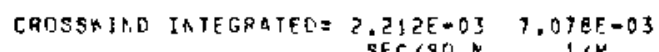

200

200

200

200
200

200

300

200

200

200

200

200

200

200

200

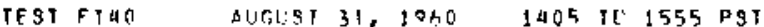 IINC SULFIDE RFLFASE FER:N FLEVATIOA OF ${ }^{N}$ BOON ARC U $=3.2 \mathrm{~N} / \mathrm{SEC}$ AT $2 \mathrm{~N}$

AIJMUTH HEIGHT EXPOSURE F/TJ ELIO OISTAREE DEGHEFS METERS GH-SEC/CH,N SEC/CLIN 1/50, METERS $\times 105+6$

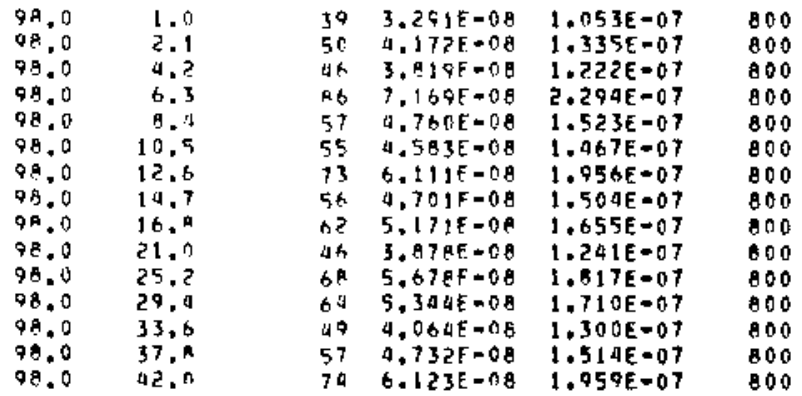

AZIMUTH HEIGHT EXPISURE E/O EUIO DISTANCE DEGREES METEAS GM-SEC/CU,N SEC/CU.N 1/SR.M METERS $x 105+6$

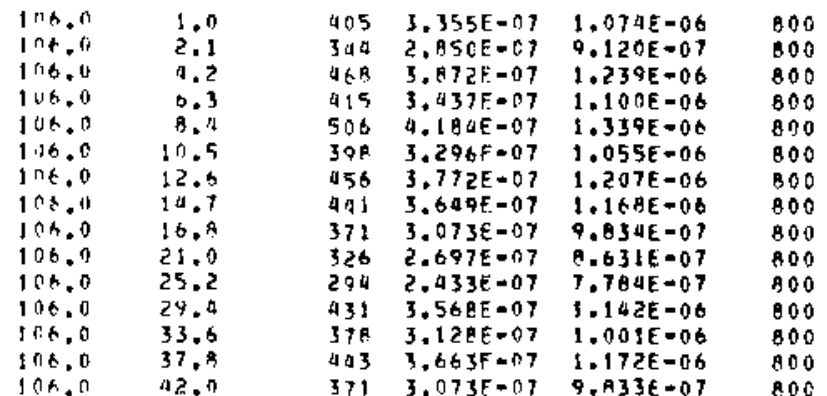

ALIMUTH HEIGHT EXPOSUIEE E/O DUIO DISTANCE DEEREFS METEAS GM-gFC/CII.N SEC/CH!N I/ST.N METERS $x \in E+6$

\begin{tabular}{|c|c|c|c|c|c|}
\hline 8139.6 & 1.0 & 1781 & $1.473 E=06$ & $9.712 E-06$ & 800 \\
\hline 114.0 & 2.1 & $18: 3$ & $1.333 E=06$ & $4,26 B E-06$ & 800 \\
\hline 114.11 & 4.2 & 2927 & $2.419 E=06$ & $7.7 a 1 E=06$ & 800 \\
\hline 11 iो, 0 & 6.3 & $2 ? 74$ & 1.8 A तE $=06$ & $6.015 E-06$ & 100 \\
\hline 113.13 & 6.0 & 2322 & $1.920 F=106$ & $6.143 E-06$ & 800 \\
\hline $114, n$ & 10.5 & 2147 & $1.775 E=06$ & $5.6791=06$ & 000 \\
\hline 114.0 & 12.3 & 1968 & $1.02+f=06$ & $5.205 t=0.6$ & 800 \\
\hline 110,0 & 14.7 & 2401 & $1.98 \mathrm{aE}=0 \mathrm{6}$ & $0.35 \cap E=06$ & 800 \\
\hline 114.0 & 16.9 & 2133 & $1.763 E-06$ & $643 E-06$ & 800 \\
\hline $1: 4.0$ & $2 ! .0$ & 2120 & 1.7565 .06 & $5.618 F-06$ & 800 \\
\hline $11 . n$ & 25,2 & 1476 & $220 \varepsilon=06$ & iE -06 & 800 \\
\hline 114.17 & 29.4 & 1707 & $1.4 ! 1 F=06$ & $0.516 E=06$ & 800 \\
\hline 110.0 & 33.6 & 1950 & $1.6125=06$ & $5.159 E-06$ & 600 \\
\hline $1 ! d$, , & 37.9 & 1600 & $1.326 E=06$ & $243 E=06$ & 600 \\
\hline$f 1$ th & & 1040 & $.190 E-06$ & $08 E-06$ & 300 \\
\hline
\end{tabular}




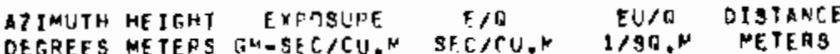
$x) D E+b$

\begin{tabular}{|c|c|c|c|c|c|}
\hline $\begin{array}{l}122.0 \\
122.0 \\
122.0 \\
122.0 \\
122.0 \\
122.0 \\
122.0 \\
122.0 \\
122.0 \\
12200 \\
122.0 \\
122.0 \\
122.1 \\
122.0 \\
122.0\end{array}$ & $\begin{array}{r}1.9 \\
2.1 \\
4.2 \\
6.3 \\
2.11 \\
10.5 \\
12.6 \\
14.7 \\
16.9 \\
21.9 \\
25.2 \\
29.0 \\
33.6 \\
37.8 \\
42.0\end{array}$ & $\begin{array}{l}560 \\
844 \\
720 \\
856 \\
853 \\
848 \\
1291 \\
855 \\
851 \\
947 \\
853 \\
569 \\
501 \\
436 \\
423\end{array}$ & $\begin{array}{l}4.636 \mathrm{E}=07 \\
6.061 \mathrm{~F}=07 \\
5.05 \mathrm{~F}=07 \\
7.075 \mathrm{E}=07 \\
7.057 \mathrm{~F}-07 \\
7.016 \mathrm{E}-07 \\
1.059 \mathrm{~F}=06 \\
7.069 \mathrm{E}-07 \\
7.034 \mathrm{E}=07 \\
7.027 \mathrm{E}-07 \\
7.053 \mathrm{E}=07 \\
4.709 \mathrm{E}=07 \\
4.192 \mathrm{E}=07 \\
3.607 \mathrm{E}=07 \\
3.501 \mathrm{E}=07\end{array}$ & $\begin{array}{l}1.4 B 4 E=06 \\
2.234 E-06 \\
1.907 E=06 \\
2.260 E-06 \\
2.258 E-06 \\
2.245 E-06 \\
3.390 E-06 \\
2.262 E=06 \\
2.251 E-06 \\
2.505 E=06 \\
2.257 E-06 \\
1.507 E=06 \\
1.325 E=06 \\
1.150 E=06 \\
1.120 E=06\end{array}$ & $\begin{array}{l}800 \\
800 \\
000 \\
000 \\
000 \\
800 \\
800 \\
800 \\
000 \\
000 \\
000 \\
000 \\
800 \\
000 \\
000\end{array}$ \\
\hline
\end{tabular}

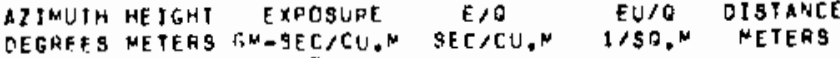 $X \perp 0 E+A$}

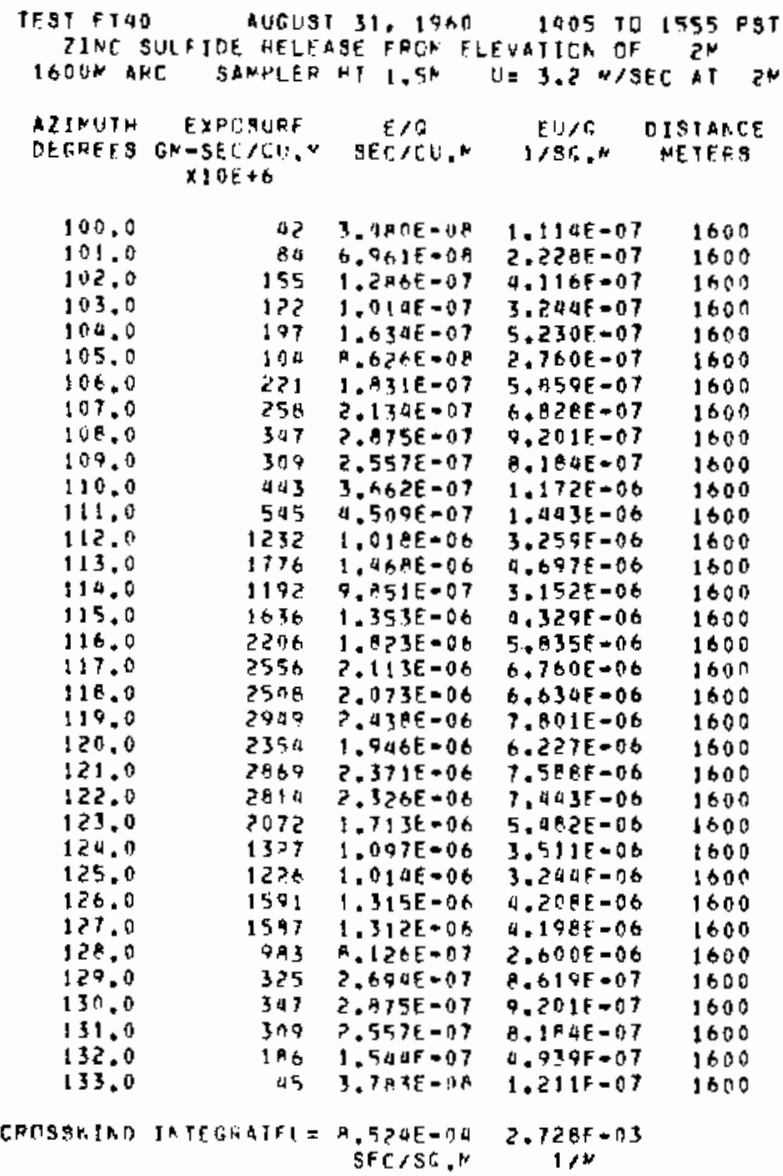

TOMFF DATA FUELCH....

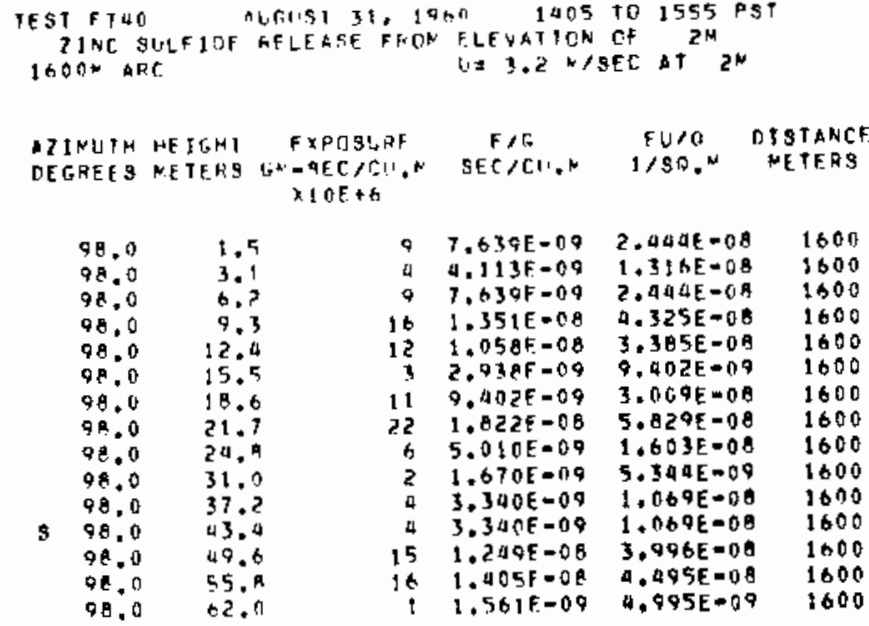




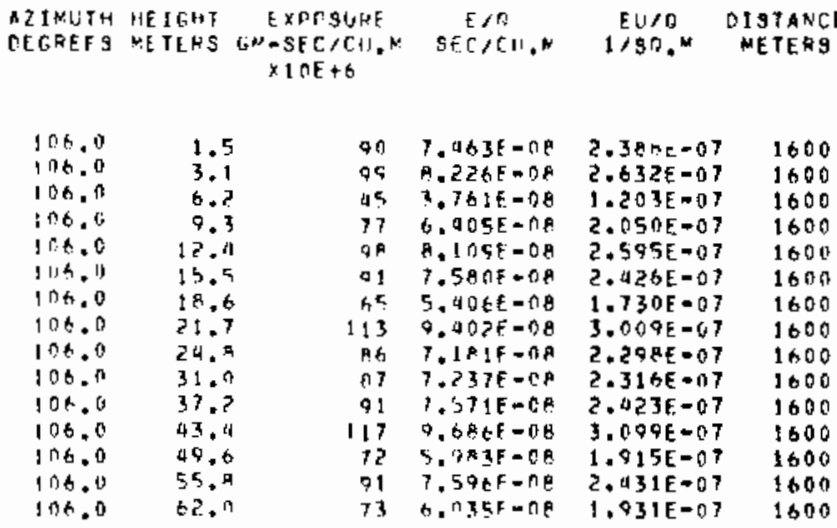

A7IVUTH HEIGHT FXPESLPF E/G EU/O OISTATCE

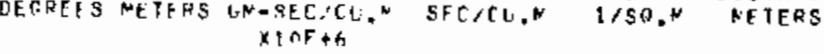

$\begin{array}{ll}15+.0 & 1.5 \\ 139.0 & 3.1 \\ 130.0 & 0.2 \\ 130.0 & 9.3 \\ 130.0 & 12.4 \\ 156.0 & 15.5 \\ 130.0 & 14.6 \\ 130.0 & 21.7 \\ 130.0 & 24.8 \\ 130.01 & 31.0 \\ 130.0 & 17.2 \\ 130.0 & 03.0 \\ 130.0 & 49.6 \\ 130.13 & 55.8 \\ 130.0 & 62.0\end{array}$

\begin{tabular}{|c|c|c|c|}
\hline $\begin{array}{r}60 \\
112\end{array}$ & $\begin{array}{l}5.758 \mathrm{~F}-\mathrm{CB} \\
9.28 \mathrm{of}=\mathrm{nB}\end{array}$ & $\begin{array}{l}1.043 E=07 \\
? .07 J E=07\end{array}$ & $\begin{array}{l}1600 \\
1600\end{array}$ \\
\hline 108 & $8.000 E-O B$ & $2.877 E-07$ & 1600 \\
\hline 50 & $4.172 E=0 E$ & $355=07$ & 1600 \\
\hline $\begin{array}{l}157 \\
117\end{array}$ & $\begin{array}{l}1.301 E=07 \\
9.695 E=08\end{array}$ & $\begin{array}{l}4.174 \mathrm{E}-07 \\
3.103 \mathrm{E}-07\end{array}$ & 1600 \\
\hline 116 & $9.637 \mathrm{E}=0 \mathrm{~F}$ & $3.084 t=07$ & 1600 \\
\hline $\begin{array}{r}70 \\
1.5\end{array}$ & $5.8175=08$ & $62 E-0.7$ & 1600 \\
\hline $\begin{array}{l}115 \\
103\end{array}$ & $75 F-08$ & $3.064 E-07$ & 1600 \\
\hline 96 & $0.016 E=08$ & $\begin{array}{l}725 E-07 \\
565 E-07\end{array}$ & 1600 \\
\hline 104 & $8.62 \mathrm{EE}=0 \mathrm{~A}$ & $761 E-07$ & 1600 \\
\hline 62 & $5.203 E=08$ & $665 E-07$ & 1600 \\
\hline 13 & $145 E-00$ & $3 E * 0 \theta$ & 1600 \\
\hline 76 & $295 E-08$ & $014 E=07$ & 1600 \\
\hline
\end{tabular}

\begin{tabular}{|c|c|c|c|c|c|}
\hline $\begin{array}{l}\text { BIT NUTH } \\
\text { DETEEES }\end{array}$ & $\begin{array}{l}\text { MEIGHT } \\
\text { PETELS }\end{array}$ & $\begin{array}{c}\text { EXPOSURF } \\
\text { GN-SEC/CL. } \\
X 1 \cap F+6\end{array}$ & $\begin{array}{c}E / O \\
\operatorname{SEC} C / C C^{2}, N\end{array}$ & 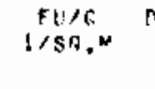 & $\begin{array}{c}\text { DISTANCE } \\
\text { NETERS }\end{array}$ \\
\hline 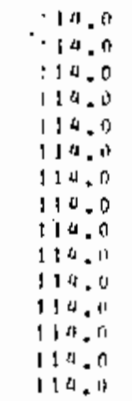 & $\begin{array}{l}1.5 \\
3.1 \\
6.2 \\
9.3 \\
12.4 \\
15.5 \\
19.5 \\
21.7 \\
24.8 \\
31.0 \\
37.7 \\
43.1 \\
49.6 \\
55.9 \\
62.0\end{array}$ & $\begin{array}{l}573 \\
505 \\
7+0 \\
470 \\
739 \\
754 \\
821 \\
870 \\
695 \\
767 \\
835 \\
343 \\
7>0 \\
531 \\
495\end{array}$ & $\begin{array}{l}4.742 F=07 \\
4.172 E=07 \\
6.2 A 1 E=07 \\
7.192 E-07 \\
6.115 F=07 \\
6.20 E E-07 \\
6.787 E=07 \\
6.810 E=07 \\
5.750 E=07 \\
6.340 E=07 \\
6.906 E=07 \\
3.006 E=07 \\
5.952 E=07 \\
4.396 E=07 \\
9.094 E=07\end{array}$ & $\begin{array}{l}1.517 E=06 \\
1.337 E=06 \\
2.0110 E=06 \\
2.302 E=06 \\
1.956 E-06 \\
1.999 E=06 \\
2.172 E=06 \\
2.179 E=06 \\
1.840 E=06 \\
2.029 E=06 \\
2.211 E=06 \\
9.610 E=07 \\
1.905 E=06 \\
1.907 E-06 \\
1.310 E=06\end{array}$ & $\begin{array}{l}1600 \\
1600 \\
1600 \\
1600 \\
1600 \\
1600 \\
1600 \\
1600 \\
1600 \\
1600 \\
1600 \\
1600 \\
1600 \\
1600 \\
1600\end{array}$ \\
\hline
\end{tabular}

AZIMUIH HEIGHT EXPOSLFE E/O EU/O CISTANCE DEGREFS METEHS GN-SECJLU.M SECICU,N IISO.N METERS $X 10 E+B$

\begin{tabular}{|c|c|c|c|c|c|}
\hline $\begin{array}{l}122.0 \\
122.0 \\
122.0 \\
122.0 \\
122.0 \\
122.0 \\
172.0 \\
122.0 \\
122.0 \\
122.0 \\
122.0 \\
122.0 \\
1270 \\
122.0 \\
122.0\end{array}$ & $\begin{array}{l}1.5 \\
3.1 \\
0.7 \\
9.3 \\
12.4 \\
15.5 \\
18.6 \\
25.7 \\
24.8 \\
31.0 \\
37.2 \\
43.0 \\
49.6 \\
55.0 \\
62.0\end{array}$ & $\begin{array}{l}914 \\
1197 \\
1375 \\
1193 \\
1030 \\
1100 \\
1224 \\
955 \\
1306 \\
876 \\
863 \\
1136 \\
825 \\
727 \\
851\end{array}$ & $\begin{array}{l}8.132 E-07 \\
9.095 E-07 \\
1.136 E-06 \\
9.866 E=07 \\
9.514 E=07 \\
1.108 E=06 \\
1.012 E=06 \\
7.097 E=07 \\
1.070 E=06 \\
7.248 E=07 \\
7.13 t E=07 \\
9.396 E=07 \\
6.857 E=07 \\
6.014 E=07 \\
7.034 E=07\end{array}$ & $\begin{array}{l}2.602 E=06 \\
3.166 E=06 \\
3.637 E=06 \\
3.157 E=06 \\
2.725 E=06 \\
3.546 E=06 \\
3.238 E=06 \\
2.527 E=06 \\
3.454 E=06 \\
2.317 F=06 \\
2.284 E=06 \\
3.007 \bar{C}=06 \\
2.193 E-06 \\
1.925 E=06 \\
2.251 E=06\end{array}$ & $\begin{array}{l}1600 \\
1600 \\
1600 \\
1600 \\
1600 \\
1600 \\
1600 \\
1600 \\
1500 \\
1600 \\
1600 \\
1600 \\
1600 \\
1600 \\
1600\end{array}$ \\
\hline
\end{tabular}

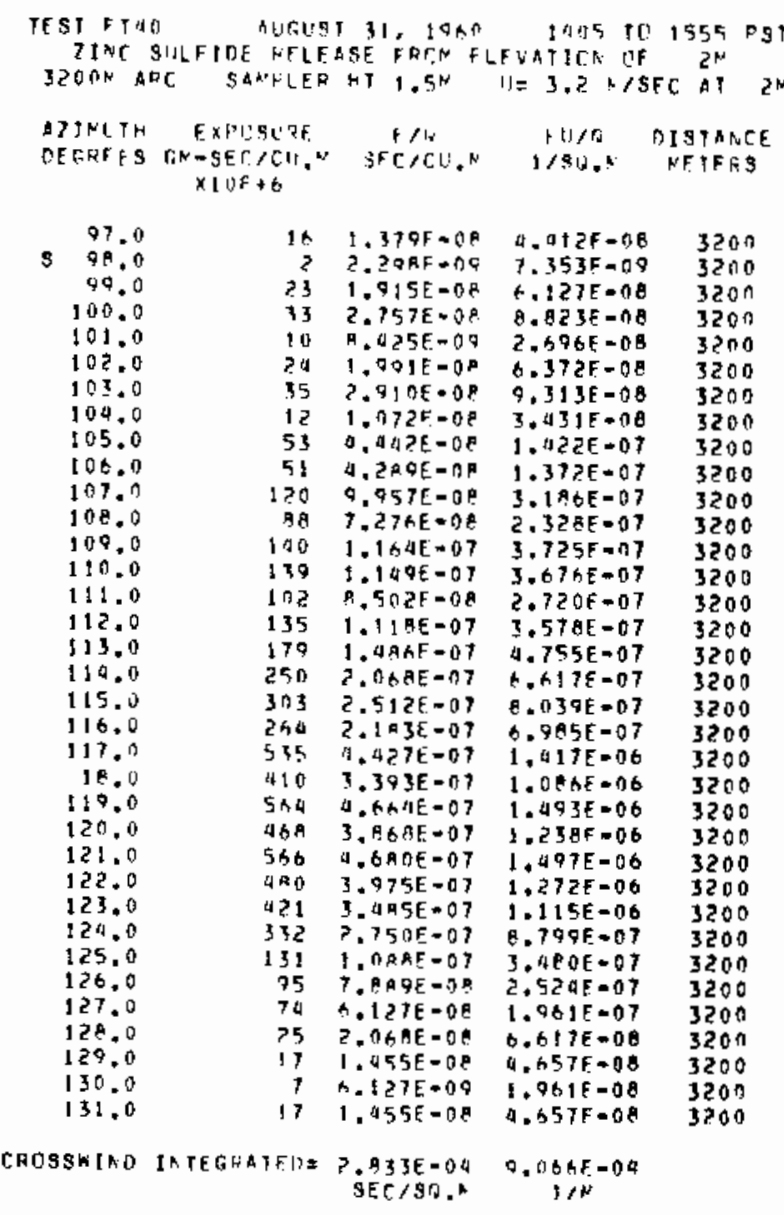


IDWEP DATA EDLLOW...

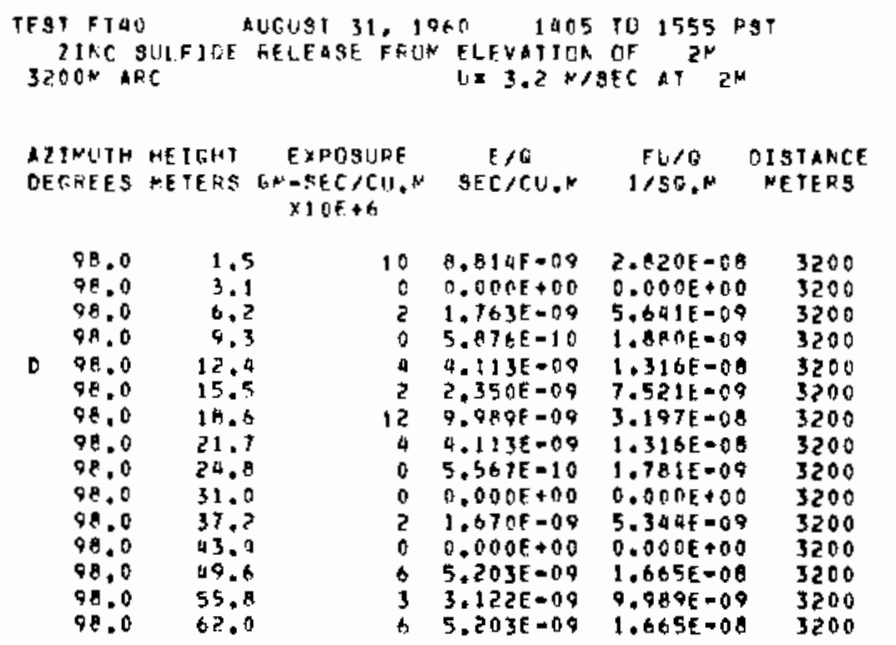

AZINUITH HETGHT EXPUSLEE F/Q EU/C DISTANCE OETREES METERS GNOGEC/CU.N SEC/CU.N 1/50.N NETERS $X] \cap E+G$

\begin{tabular}{|c|c|c|c|c|c|}
\hline $\begin{array}{l}122.0 \\
122.0 \\
122.0 \\
122.0 \\
122.0 \\
122.0 \\
122.0 \\
122.0 \\
122.0 \\
122.0 \\
122.0 \\
122.0 \\
122.0 \\
122.0 \\
122.0\end{array}$ & $\begin{array}{r}1.5 \\
3.1 \\
6.2 \\
9.3 \\
12.0 \\
15.5 \\
18.6 \\
21.7 \\
24.8 \\
31.0 \\
37.2 \\
43.9 \\
49.6 \\
55.8 \\
62.0\end{array}$ & $\begin{array}{l}273 \\
406 \\
351 \\
517 \\
439 \\
385 \\
400 \\
407 \\
284 \\
478 \\
460 \\
361 \\
904 \\
490 \\
400\end{array}$ & 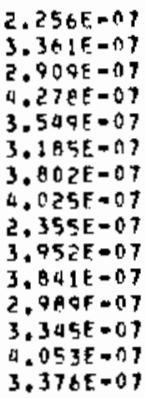 & $\begin{array}{l}7.220 E-07 \\
1.076 E-06 \\
9.308 E=07 \\
1.369 E-06 \\
1.136 E=06 \\
1.019 E-06 \\
1.217 E-06 \\
1.288 E=06 \\
7.535 E=07 \\
1.265 E=06 \\
1.229 E=06 \\
9.566 E-07 \\
1.07 n E-06 \\
1.297 E=06 \\
1.080 E-06\end{array}$ & $\begin{array}{l}3200 \\
3200 \\
3200 \\
3200 \\
3200 \\
3200 \\
3200 \\
3200 \\
3200 \\
3200 \\
3200 \\
3200 \\
3200 \\
3200 \\
3200\end{array}$ \\
\hline
\end{tabular}

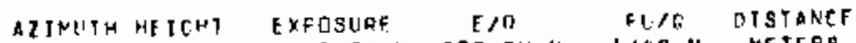
DEGLEFS METERS GM+SEC/CIIN SEC/CU,N H/SN.M NETERS $\times 1 \cap E+6$

\begin{tabular}{|c|c|c|c|c|c|}
\hline $\begin{array}{l}\text { AZINUTH } \\
\text { DETREES }\end{array}$ & $\begin{array}{l}\text { HE GGHT } \\
\text { METEHS }\end{array}$ & $\begin{array}{c}\text { EXPGSUAF } \\
\text { GW-SEC/CU.M } \\
\times 10 E+6\end{array}$ & $\begin{array}{c}E / G \\
S E C / C H, N\end{array}$ & $\begin{array}{l}\text { EU/O } \\
1 / 20, N\end{array}$ & $\begin{array}{r}\text { OISTANC } \\
\text { METERS }\end{array}$ \\
\hline $\begin{array}{l}106.0 \\
102.0 \\
106.0 \\
100.0 \\
106.0 \\
106.0 \\
106.0 \\
106.0 \\
104.0 \\
106.0 \\
104.0 \\
11060 \\
106.0 \\
106.0 \\
106.0\end{array}$ & $\begin{array}{l}1.5 \\
3.1 \\
6.7 \\
9.3 \\
12.4 \\
15.5 \\
18.6 \\
21.7 \\
29.8 \\
31.0 \\
37.2 \\
43.0 \\
49.6 \\
55.8 \\
62,0\end{array}$ & $\begin{array}{r}45 \\
27 \\
111 \\
61 \\
96 \\
81 \\
115 \\
95 \\
94 \\
112 \\
96 \\
133 \\
166 \\
101 \\
16\end{array}$ & $\begin{array}{l}3.761 E=08 \\
2.292 F=08 \\
9.225 E=08 \\
5.053 E=08 \\
7.991 E=08 \\
.757 E=08 \\
9.57 E E-08 \\
7.874 E=08 \\
7.793 F=08 \\
8.2965=08 \\
7.1 A 1 F=08 \\
1.102 F=07 \\
1.379 E=07 \\
.376 F=08 \\
1.405 F=08\end{array}$ & $\begin{array}{l}1.203 \mathrm{E}-07 \\
7.333 \mathrm{E}-08 \\
2.952 \mathrm{E}-07 \\
1.017 \mathrm{E}-07 \\
2.557 \mathrm{E}-07 \\
2.162 \mathrm{E}-07 \\
3.065 \mathrm{E}-07 \\
2.52 \mathrm{E}=07 \\
2.494 \mathrm{E}-07 \\
2.975 \mathrm{E}=07 \\
2.29 \mathrm{RE}-07 \\
3.527 \mathrm{E}-07 \\
4.012 \mathrm{E}-07 \\
2.6 \mathrm{OE}-07 \\
0.495 \mathrm{E}-\mathrm{C}\end{array}$ & $\begin{array}{l}3200 \\
3200 \\
3200 \\
3200 \\
3200 \\
3200 \\
3200 \\
3200 \\
3200 \\
3200 \\
3200 \\
3200 \\
3200 \\
3200 \\
3200\end{array}$ \\
\hline
\end{tabular}

AITHITH HEJGHI EXPTSLRF E/R FU/G OISTANCF

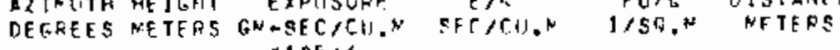
$\times 10 \mathrm{E}+6$

\begin{tabular}{|c|c|c|c|c|c|}
\hline 119.0 & 1.5 & 243 & $2.01 \mathrm{CF}=07$ & $0.031 E=07$ & 3200 \\
\hline $114 . n$ & 3.1 & 198 & $1.569 E=07$ & $5.020 E-07$ & 3200 \\
\hline 110,0 & h. & 300 & $2.48 \in E-07$ & $7.450 \mathrm{E}-0.7$ & 3200 \\
\hline 114.0 & 9.1 & 2.95 & $362 F=07$ & $7.559 \mathrm{E}-0.7$ & 3200 \\
\hline $11 * 0$ & 18.4 & 329 & $726 E-0.7$ & $725 E-07$ & 3200 \\
\hline 119.0 & 15.5 & $2 \times 3$ & $2.180 . E=C 7$ & $6.976 E-07$ & 3200 \\
\hline 110.11 & 18. & 285 & $356 \%-07$ & $7.540 t-07$ & 3200 \\
\hline 114.0 & 21.7 & $34 !$ & $6 E=07$ & $9.0446=07$ & 3200 \\
\hline 114.0 & 24.9 & $43 A$ & $24 E=07$ & $1.160 E=06$ & 3200 \\
\hline 110.0 & 31.0 & 340 & $817 E-07$ & $9.013 E=07$ & 3200 \\
\hline 114,0 & 37.2 & 278 & $305 \varepsilon-0 ?$ & $7.375 E=07$ & 3200 \\
\hline $11 \% .0$ & 43.0 & 290 & $3995-07$ & $7.677 \mathrm{E}-07$ & 3200 \\
\hline 114,0 & 49.6 & 300 & 4.2E-07 & $7.941 E-07$ & 3200 \\
\hline 114.0 & 55.8 & 400 & $30,5=07$ & $1.059 \mathrm{E}=06$ & 3200 \\
\hline 114,5 & 62. & 265 & $190 \mathrm{E}-07$ & $009 E=07$ & 3200 \\
\hline
\end{tabular}

\begin{tabular}{|c|c|c|c|c|c|}
\hline $13 n .0$ & 1.5 & $\vec{z}$ & $2.3505=518$ & $7.521 \leq-09$ & 3200 \\
\hline $13 n \cdot c^{n}$ & 3.1 & 13 & $1.116 F-06$ & $E=0 B$ & 3200 \\
\hline $13 r .0$ & 0.2 & 3 & $2.83 \mu \mathrm{F}=1.9$ & $9.002 E=09$ & 3200 \\
\hline ;30.0 & 9,3 & $1 \%$ & $1.17 \subseteq F \circ 08$ & $3,761 F=0, E$ & 3200 \\
\hline 130.0 & $\$ 2.4$ & 10 & $8,8\} 4 E=09$ & $2.820 E-00$ & 3200 \\
\hline 130.0 & 15,5 & 17 & $1.910 F=0.8$ & $513 E=08$ & 3200 \\
\hline 130.0 & 18.6 & 5 & $0,701 E-09$ & $1.504 E-08$ & 3200 \\
\hline $13 n .0$ & 21.7 & 12 & $5.9895-09$ & $97 E=08$ & 3200 \\
\hline 130.0 & z0, & 9 & $7.793 \mathrm{E}=09$ & $\Delta E=08$ & 3200 \\
\hline $13 n . n$ & 31.0 & 22 & $1,837 \mathrm{E}=0 \mathrm{~B}$ & $8 E=0 B$ & 3200 \\
\hline $136 . n$ & 37.2 & 30 & $2,501 \mathrm{E}=00$ & $8.199 E \div 08$ & 3200 \\
\hline 130.0 & 43.4 & 20 & $1.670 E-08$ & $5.344 E=08$ & 3200 \\
\hline 330,0 & 09.1 & 1 ล & $1.509 E=00$ & $0,82 \mathrm{BE}-0 \mathrm{~B}$ & 3200 \\
\hline 130.0 & 55.8 & 24 & $3.029 E=08$ & $6.493 \mathrm{E}=08$ & 3200 \\
\hline & 62.0 & $2^{4}$ & $2.3915=0 \mathrm{~B}$ & $7.092 E=00$ & 200 \\
\hline
\end{tabular}




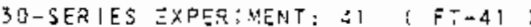

GROUNO LEVE! AHD TOWER SAMLL ING $200-3200$ ! AOCS. MANY OE THE

FILJERS OH THE TOWEPS SHCHED SIGNS OF SEATHERIIIG.

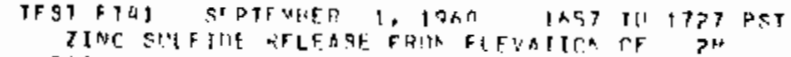

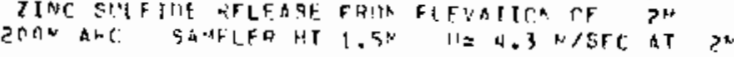

\begin{tabular}{|c|c|c|c|c|c|}
\hline \multicolumn{2}{|c|}{$\begin{array}{l}\text { ALINIJTH } \\
\text { DFFAETS }\end{array}$} & 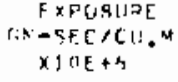 & $\begin{array}{c}4>0 \\
\text { sect! }\end{array}$ & $\begin{array}{c}F \|_{1 / \gamma} \\
1 / \$ ?+k\end{array}$ & $\begin{array}{l}\text { NISIAPCF } \\
\text { NFTFE. }\end{array}$ \\
\hline & $8.4,0$ & ?? & 1.9 ममE $=0 \mathrm{~F}$ & $P, 367 F=00$ & 200 \\
\hline & 80.0 & n & ח त ती & $n . \cap \cap n F+00$ & ?ח \\
\hline & s & 15 & $1.297 \mathrm{E}-0 \mathrm{~A}$ & $5.578 F=48$ & 200 \\
\hline & 90.0 & 9 & A. $1 \cap 7 E=09$ & 3. $\triangle A G F=O B$ & 200 \\
\hline & 92.0 & 11 & $\nabla, 7 ? 7 E=09$ & $A, 193 F-08$ & 200 \\
\hline & 94.0 & 18 & $1.0 \geqslant \mid E-6$, & $6.07 ? \mathrm{~F}=08$ & 200 \\
\hline & 46.0 & 40 & $5.199 E=08$ & $2.2315-07$ & 200 \\
\hline & $P A .0$ & 37 & $3, ?\lrcorner 3 E=0 A$ & $1.3911 E=07$ & 200 \\
\hline & 100.0 & 33 & $2.919 E=O A$ & $1.255 F-07$ & 200 \\
\hline & 102.0 & 26 & $3.27 \cap F=0 P$ & OE & $20 n$ \\
\hline & 104.0 & 17 & $3.2+3 E=O A$ & $1.3945=0.7$ & 20 \\
\hline & 106.0 & 33 & 3.7198 & 1.2 & $20 n$ \\
\hline & 10 & 175 & $1.5 \cap A E-07$ & $6.4 \times \Delta F=07$ & ?० \\
\hline & 110.0 & 90 & $9.590 \mathrm{~F}=0 \mathrm{R}$ & $3 .+95,-07$ & 200 \\
\hline & 113.0 & 300 & $3.437 E=0.7$ & $1.4785=06$ & 200 \\
\hline & 114.0 & 510 & $304 E=07$ & $1 . R+Q E-06$ & 200 \\
\hline & 116.0 & 1722 & 1. $\triangle A \Delta E=0 \Delta$ & $6.320 F=06$ & 200 \\
\hline & 114.0 & 9100 & $7.916 E=0$ & 3.140 & 200 \\
\hline & 120.0 & 27155 & $2.130 \varepsilon=05$ & $1.0065=04$ & 200 \\
\hline E & 0 & 45176 & $3.501 F=05$ & 1.67 & 200 \\
\hline & & 131019 & $1.170 E-04$ & $4.753 F=04$ & 200 \\
\hline$s$ & 12 & $129+94$ & $1.119 E=0.1$ & $4 . A 11 E-0 H$ & 200 \\
\hline & 12 & 77 & $2.336 E=04$ & $1.0+4 E=03$ & 200 \\
\hline & 130.0 & 235 & $2.061 E=04$ & F.961F-04 & 200 \\
\hline & 132.0 & 230137 & $: .982 E=0$ & B. $524 \mathrm{E}=04$ & 200 \\
\hline & 134.0 & 185738 & 1.6005 & A.A79E $=04$ & 200 \\
\hline & & 109905 & $9.4 A G E=05$ & $4.072 E=04$ & 200 \\
\hline & & 39603 & $3.411 F=05$ & $1.467 E=0.4$ & 200 \\
\hline & 140.0 & 15338 & $1.321 E-05$ & $5.681 \mathrm{E}-05$ & 200 \\
\hline & & & 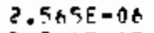 & $1.1035-05$ & 200 \\
\hline & & 604 & $a E-07$ & $2.405 F .06$ & $\geq 0 n$ \\
\hline & & 50 & $9 \mathbf{F}$ & $31 E=07$ & \\
\hline
\end{tabular}

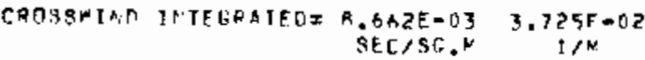

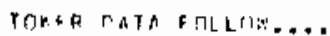

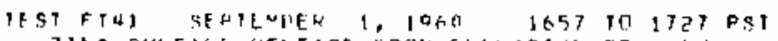

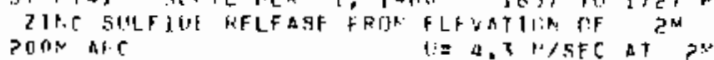

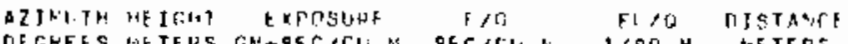

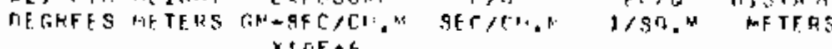
$x \geqslant 5: E$

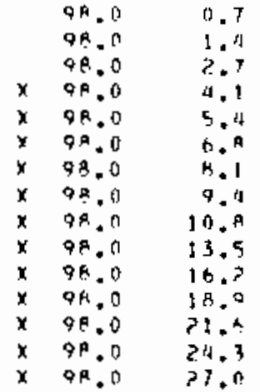

\begin{tabular}{|c|c|c|}
\hline 5 & - & \\
\hline 10 & $7.051 F=09$ & \\
\hline 9 & a. $14+F=19$ & \\
\hline n & T. $000 \mathrm{~F} \cdot 00$ & \\
\hline 5 & 4.5 ? Af $=00$ & SE \\
\hline n & $0.00 D E+00$ & Quite \\
\hline 3 & $3.105 F-09$ & 5 \\
\hline 15 & $1.131 \mathrm{~F}=0 \mathrm{~A}$ & \\
\hline 2 & $3 E=09$ & \\
\hline 0 & $=10$ & \\
\hline$n$ & 10 & \\
\hline 3 & $2.715 \mathrm{E}=09$ & $\mathbf{E}$ \\
\hline 0 & $4.526 E-10$ & 46 \\
\hline 5 & $4.95 a f=00$ & 3 ? \\
\hline & -109 & \\
\hline
\end{tabular}

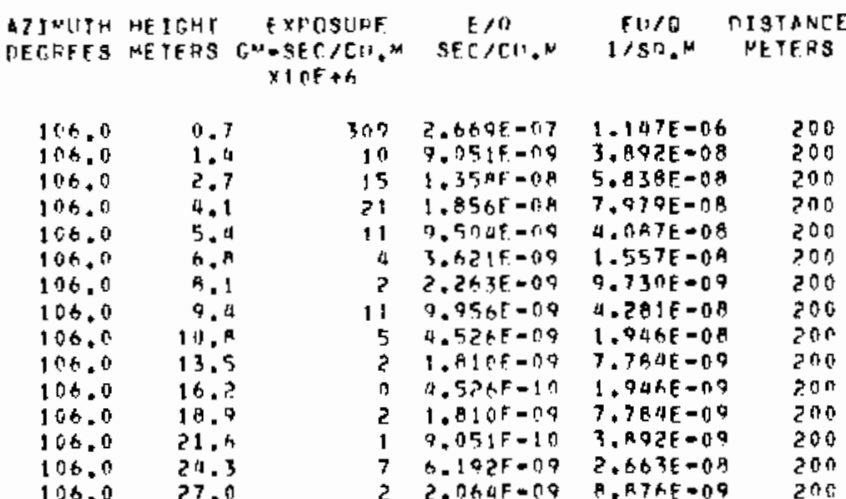

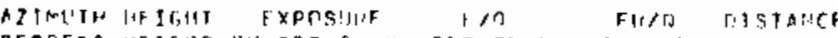

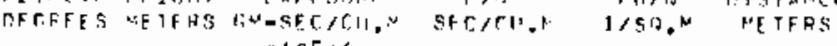
$\times \ln F+4$

\begin{tabular}{|c|c|c|c|c|c|}
\hline 114.0 & 0.7 & 154 & $\therefore, A 77 F=\{17$ & $1.237 F=n B$ & חִ \\
\hline 114.0 & 1. 1 & F & $7.377 F=\cap 9$ & $3.173 E=07$ & 213 \\
\hline 114.0 & 3.7 & $1>\mathrm{A}$ & $1.1 \cap 5 F-\cap 7$ & a. 7 TAE $=6.7$ & $\operatorname{xn}$ \\
\hline 114.0 & 4 & $1+?$ & $1.413 F-07$ & $6.0335=07$ & 200 \\
\hline $114 . n$ & 5.11 & 396 & $3.331 F=n 7$ & $1.432 t-c^{x}$ & 200 \\
\hline 114.0 & b. .9 & 213 & 1. $837 F=07$ & $7.901 f=67$ & 200 \\
\hline 114.0 & ค. 1 & 209 & $2.15 \cap F=87$ & $9.2411 E-6.7$ & วクई \\
\hline 114.0 & 9.14 & $3+.7$ & $3.1035-07$ & $1.3605=06$ & 300 \\
\hline 114.0 & 10.9 & $2>1$ & $? .335 \mathrm{E}=07$ & $1.0 C 0 E-06$ & 290 \\
\hline 114.0 & 13.5 & 310 & $2.6065=07$ & $1.1465=06$ & $2 \cap n$ \\
\hline 114.0 & 16.2 & 214 & $\left.1.806^{\circ}=0\right\}$ & $7.940 \mathrm{E}-0.7$ & 200 \\
\hline 110.0 & 18.7 & 105 & $9.051 \mathrm{f}-\mathrm{rin}$ & $3,992 E-07$ & 200 \\
\hline 114.0 & 21.6 & 50 & $4,390 \mathrm{E}-n \mathrm{~B}$ & 1.AAAE $=07$ & 200 \\
\hline $\begin{array}{l}115.0 \\
110.0\end{array}$ & $24 \cdot 3$ & $\begin{array}{l}19 \\
4 !\end{array}$ & 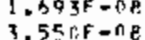 & $\begin{array}{l}7.27 R E=0 B \\
1.527 E-07\end{array}$ & 200 \\
\hline 134.0 & 3. & $4:$ & , SSTit $=11 \mathrm{C}$ & $1-327 t-07$ & 200 \\
\hline
\end{tabular}

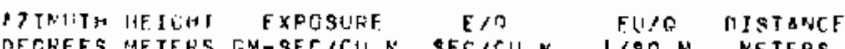
AFGRFFS METENS GM-SEC/CU!N SFC/TII. I/SD.M NETFRS $x \perp 0 F+0$

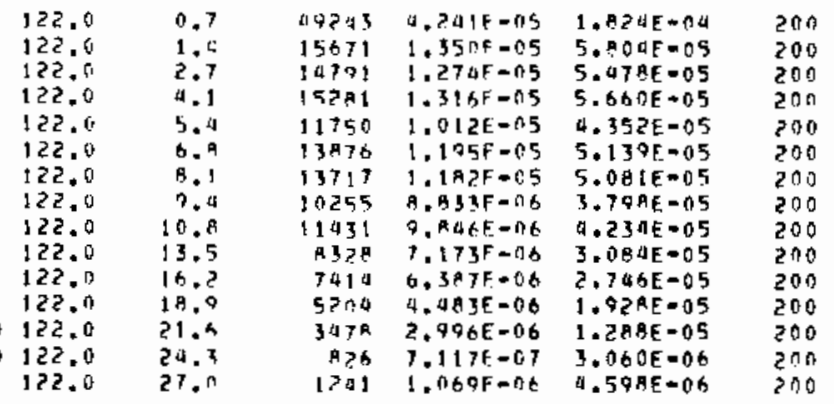




\begin{tabular}{|c|c|c|c|c|c|c|c|c|c|c|c|}
\hline $\begin{array}{l}\text { AZIA:HIPH } \\
\text { RFTHEFS }\end{array}$ & $\begin{array}{l}\text { HE I LIHT } \\
\text { NEETERS }\end{array}$ & 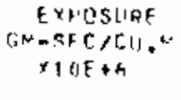 & 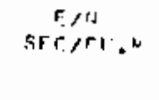 & $\begin{array}{l}\mathrm{K}_{1} / 6 \\
1 / 512 . \mathrm{N}\end{array}$ & $\begin{array}{l}\text { DISTANC:" } \\
\text { MFTFRS }\end{array}$ & $\begin{array}{l}\text { ATINDTH } \\
\text { DEREEES }\end{array}$ & $\begin{array}{l}\text { HE IGHT } \\
\text { METEAS }\end{array}$ & $\begin{array}{c}\text { EXPOSURE } \\
\text { SNESEC }=\text { CIJ.N } \\
X I D E+h\end{array}$ & 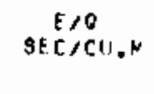 & $\begin{array}{c}E L ! / 0 \\
1 / S O . M\end{array}$ & $\begin{array}{l}\text { DISTANCE } \\
\text { METFRS }\end{array}$ \\
\hline 130.0 & 0.7 & 207907 & $3.5 \times 7 f=14$ & $1.1 a A F=01$ & $\ln n$ & 114.0 & 1.0 & 16 & $1.401 E=0 B$ & $6.0245=08$ & 800 \\
\hline $\begin{array}{l}130.8 \\
130.8\end{array}$ & $\begin{array}{l}1.14 \\
3.7\end{array}$ & 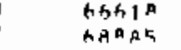 & $\begin{array}{l}5.73 A F=05 \\
5.933 F=05\end{array}$ & $\begin{array}{l}2,4 R 7 t=04 \\
7.551 E=04\end{array}$ & $\begin{array}{l}200 \\
200\end{array}$ & $\begin{array}{l}114.0 \\
114.0\end{array}$ & $\begin{array}{l}2.1 \\
4.2\end{array}$ & 13 & 1.182 & $E=68$ & 000 \\
\hline $13 n, 0$ & 4.1 & ร月ค5ท & $5.0607+05$ & 2. $1 E \cap E-0 \&$ & 200 & E 110.0 & 6,3 & 13 & $\begin{array}{l}A .318 E=09 \\
1.13 A F=O A\end{array}$ & $\begin{array}{l}-0 A \\
-04\end{array}$ & $\begin{array}{l}000 \\
000\end{array}$ \\
\hline 130.5 & 5.1 & 6.7756 & $5.5 \times+F=05$ & द. $.5 \cap 9 E=04$ & 200 & 110,0 & 8.4 & is & $1.445 E-08$ & $6.212 E-08$ & 800 \\
\hline 130.11 & h. $R$ & $\cos \theta a$ & $2.213 f=175$ & $7.517 t=05$ & $2 n n$ & 114.0 & 10.5 & h & $5.691 E-10$ & $2.6 a+c=08$ & 800 \\
\hline $1 \geq 0,0$ & 9.1 & यP. की & 4.1 a C.F $=05$ & $1.7 A \cap E-04$ & 200 & 114.0 & $12 . \mathrm{A}$ & 15 & $1.1 A 2 E=08$ & $5.083 E-00$ & 800 \\
\hline $\begin{array}{l}130.01 \\
130.0\end{array}$ & T. & 45711 & $3.940 F=05$ & 1.60 वE $-0 A$ & 201 & 114.0 & 14.7 & 2 & $2.189 F=09$ & $9.412 E=09$ & AOO \\
\hline 135.6 & $\begin{array}{l}13.8 \\
13.5\end{array}$ & $\begin{array}{l}4503 n \\
37917\end{array}$ & $\begin{array}{l}3 . P / 5 F-155 \\
3.257 F=05\end{array}$ & $\begin{array}{l}1.668 E=0 A \\
1.401 E-04\end{array}$ & $\begin{array}{l}25 n \\
200\end{array}$ & 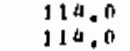 & $\begin{array}{l}16.9 \\
21.0\end{array}$ & $?$ & $6.129 F=n 0$ & $=04$ & A O O \\
\hline $\begin{array}{l}130.0 \\
130.0\end{array}$ & $\begin{array}{l}1+? \\
1 R \cdot 0\end{array}$ & $\begin{array}{l}19151 \\
250130\end{array}$ & $\begin{array}{l}? .65 A F-15 \\
? .161 F=05\end{array}$ & $\begin{array}{l}7.003 E=05 \\
9.292 E=05\end{array}$ & $\begin{array}{l}200 \\
200\end{array}$ & $\begin{array}{l}114.0 \\
110.0 \\
110.0\end{array}$ & $\begin{array}{l}21.0 \\
25.2 \\
29.4\end{array}$ & $\begin{array}{l}? \\
? \\
1\end{array}$ & $\begin{array}{l}2.199 E-09 \\
1.997 F=00\end{array}$ & $\begin{array}{l}9.412 E=09 \\
A .5 B 6 E=09\end{array}$ & $\begin{array}{l}100 \\
1000\end{array}$ \\
\hline & 21.4 & 10653 & $8.17 t f-06$ & $3.946 F=05$ & 2n & 114.0 & $\begin{array}{l}29.4 \\
33.6\end{array}$ & $\begin{array}{l}1 \\
0\end{array}$ & $\begin{array}{l}1.198 E-19 \\
0.000 E+00\end{array}$ & $\begin{array}{l}5.151 E=09 \\
0.000 E+00\end{array}$ & $\begin{array}{l}\text { POO } \\
\text { ROO }\end{array}$ \\
\hline $\begin{array}{l}135.0 \\
130.0\end{array}$ & $\begin{array}{l}23.3 \\
27.6\end{array}$ & $\begin{array}{l}\text { 93AA } \\
\text { 45A1 }\end{array}$ & $\begin{array}{l}2,053 E=06 \\
3.7 a t E=06\end{array}$ & $\begin{array}{l}3.076 E=05 \\
1.607 E=05\end{array}$ & $\begin{array}{l}200 \\
200\end{array}$ & $\begin{array}{l}110.0 \\
119.0\end{array}$ & $\begin{array}{l}37.8 \\
42 .\end{array}$ & h & $\begin{array}{l}5.1015=09 \\
5.900 F=09\end{array}$ & $\begin{array}{l}2.232 E=08 \\
2.576 F=06\end{array}$ & $\begin{array}{l}\text { AOO } \\
\text { COOO }\end{array}$ \\
\hline
\end{tabular}

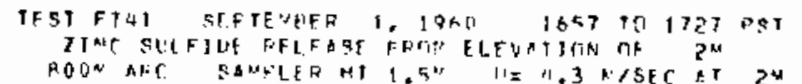

\begin{tabular}{|c|c|c|c|c|}
\hline 107.0 & 23 & $2 . \cap T Q E=\cap B$ & $A, 7 K A F=0 B$ & ano \\
\hline 104.0 & 0 & F. OCOF+OO & $\therefore . A D O F+D O$ & P.00 \\
\hline 178.6 & $? 0$ & $1.725 \mathrm{~F}=7 \mathrm{R}$ & $7.419 E=08$ & $\sin 0$ \\
\hline 1080 & 6 & n. ninf ton & 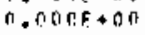 & $\operatorname{Ron}$ \\
\hline $11 n .0$ & id & 1. $2 \sigma_{5} \subset F=O A$ & $5.365 F-08$ & $\times 00$ \\
\hline 112.0 & $3 n$ & $2.66 \mathrm{hF}=0$ & $1.147 F=07$ & $\sin 0$ \\
\hline 114.0 & 43 & $3.7 \times 45-179$ & $1.6195-127$ & ano \\
\hline 116.0 & 14 & $6.4315=08$ & $? .765 F-07$ & sin: \\
\hline $11 A 0$ & 120 & $1.0 .35 E-107$ & j. $2515-0,7$ & $\operatorname{RnO}$ \\
\hline 120.0 & $2 ? 8$ & $1.9765=07$ & ค. $098 F=07$ & $\sin 0$ \\
\hline 122.0 & 970 & F.611E=07 & $3.703 E=00$ & 900 \\
\hline 124.0 & 5,011 & a. $313 \mathrm{kF}=0 \mathrm{~A}$ & $1.8+3 E-0) 5$ & $\operatorname{lno}$ \\
\hline 126.0 & 8490 & $2,175 E=n t$ & $.515 \mathrm{E}-05$ & Ann \\
\hline $12^{9} \cdot 0$ & $P 14 \times 1$ & $1.847 F=05$ & $7 . \square$ a $\cap E=05$ & $A O O$ \\
\hline $130 \cdot 0$ & 47832 & $1.5365=05$ & $1.6055=05$ & 800 \\
\hline 13 & 7445 & $h=013 F-0 t$ & $2.758 F=05$ & $A \cap D$ \\
\hline 134.0 & $\begin{array}{l}415 \mathrm{~A} \\
1700\end{array}$ & $\begin{array}{l}3.571 F=c h \\
5\end{array}$ & $1.53 \times F=05$ & $\begin{array}{l}100 \\
800\end{array}$ \\
\hline 139 & 254 & ?. 196E-D? & $0 . \triangle 42 E=07$ & \\
\hline
\end{tabular}

CROSSRTAD IHTELEAIFC= 1.659F-03 7.135E-03

$$
\text { SEC } 1 \text { SR. } 1 / \mathrm{N}
$$

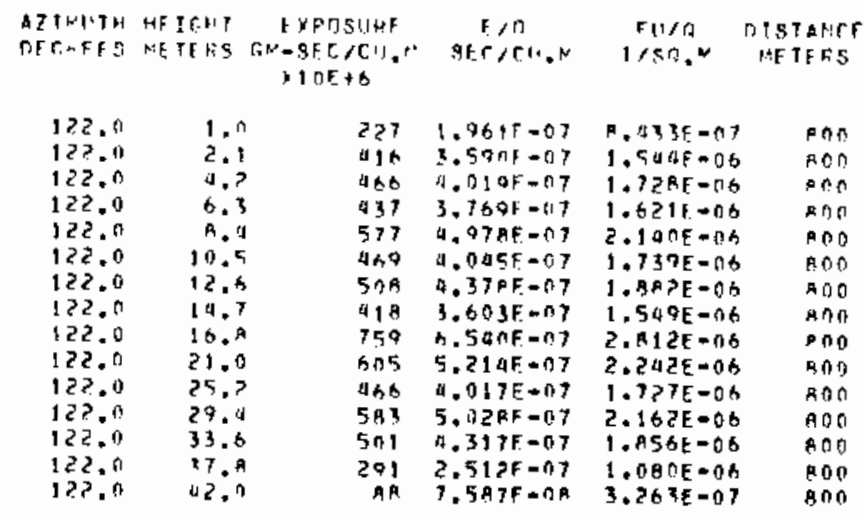

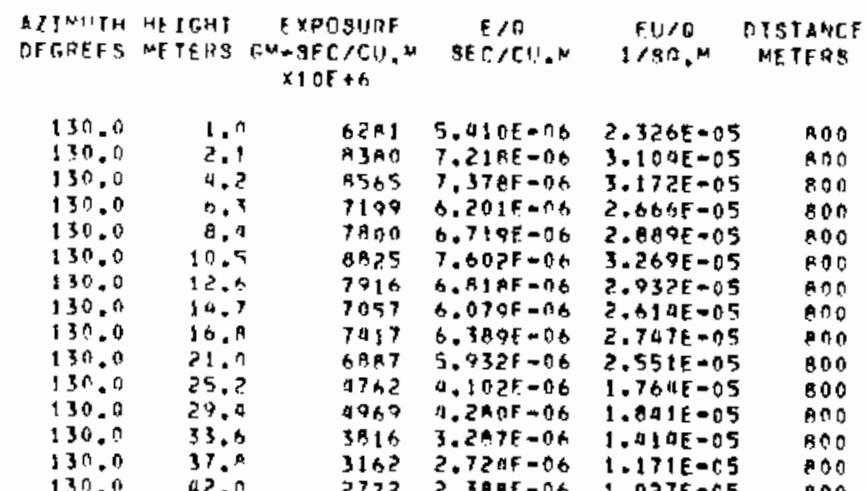

277? 2.3ARF-06 $1.027 E=05$ \$OO

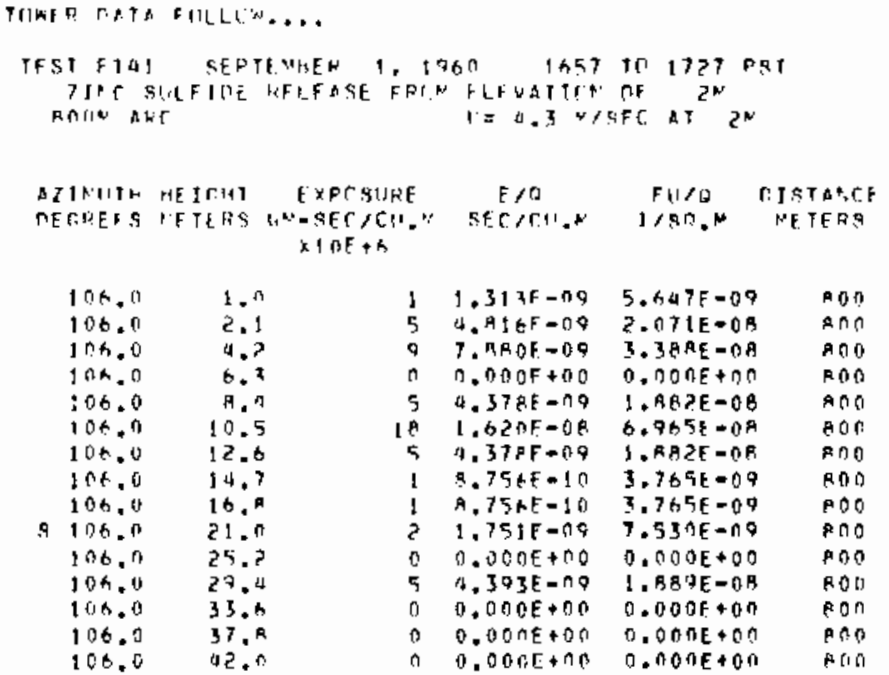




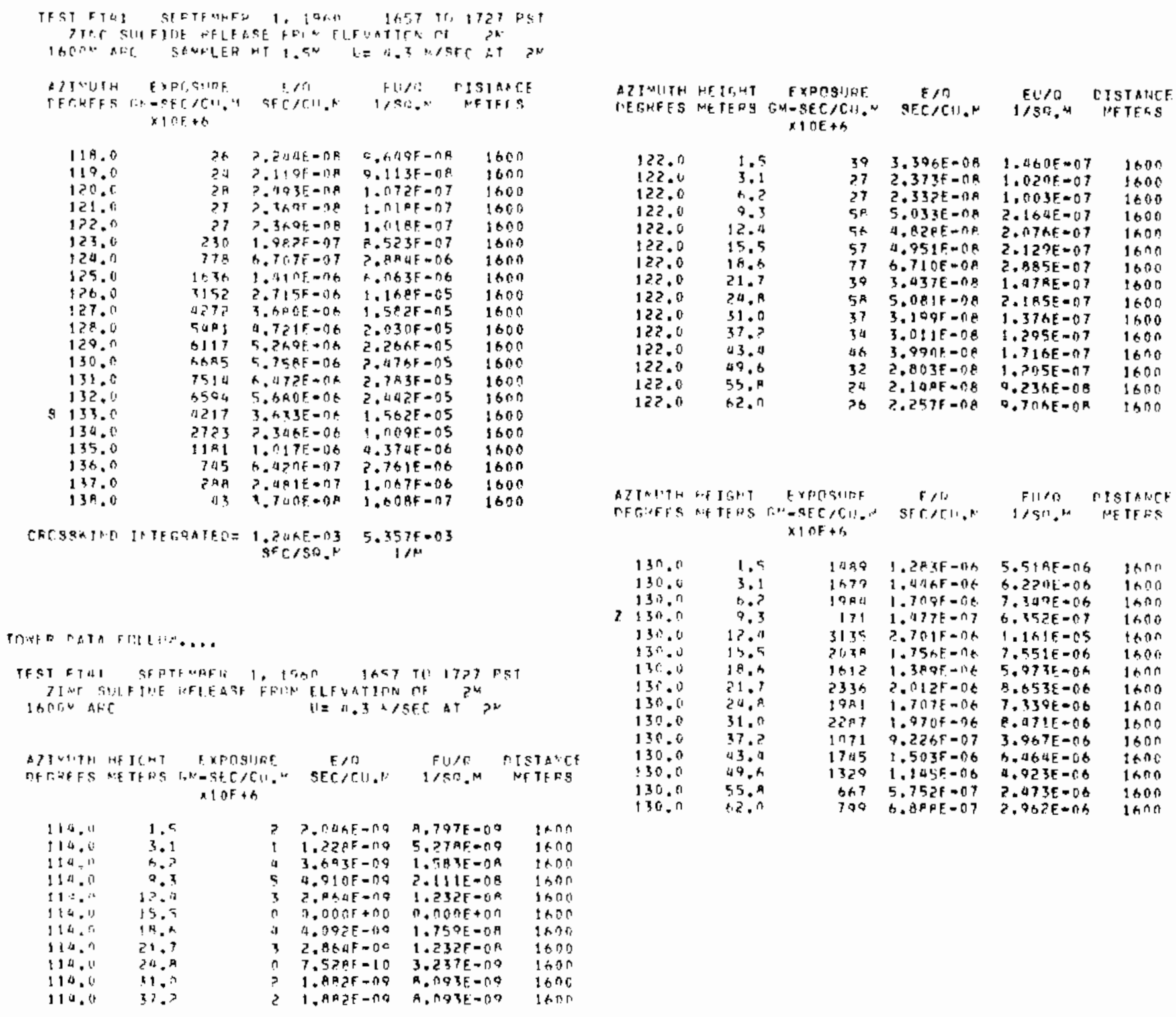




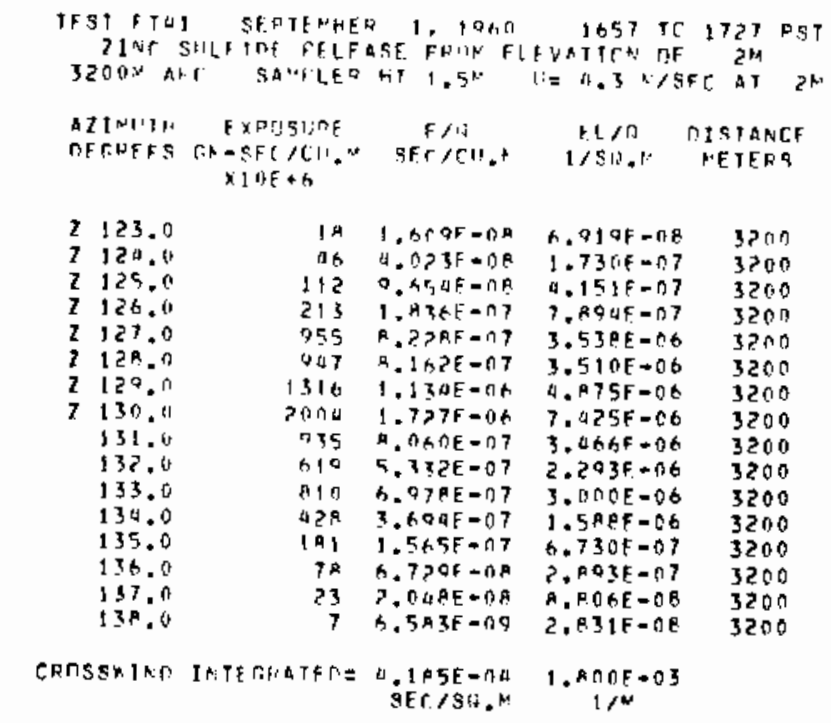

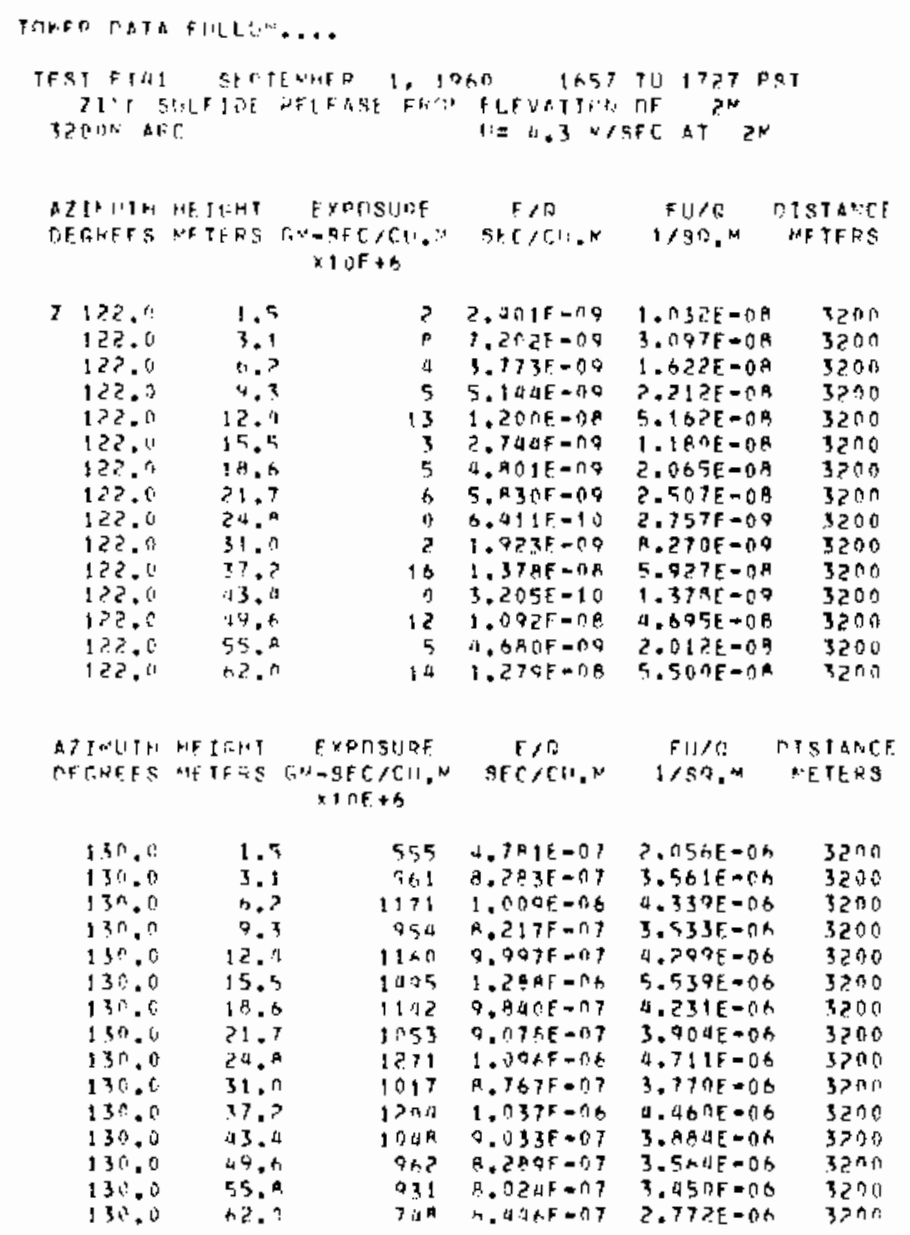


30-5ERIES EXPERIMENT: 42 ; FT-42;

GROUND LEVEL ANC TOWER SAMPLIMG $200-3250$ ARSS. IAAMV OFF THE

FILTERS ON THE TOWERS SHOXED SIGNS OF : EATHERING.

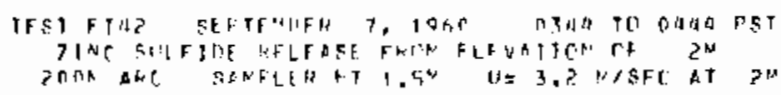

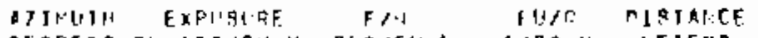

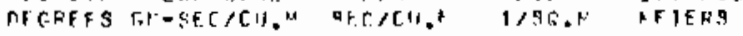
$x \in 11+$ th

\begin{tabular}{|c|c|c|c|c|}
\hline e. ח. & $? 0$ & $4.632 F=08$ & $1.12+21=07$ & 250 \\
\hline 82.11 & $? ?$ & $? .071 F=78$ & $1.112 F+n 7$ & 200 \\
\hline 84.0 & ; & C.OCOE+ $3 A$ & O. $\cap \cap O F+\cap$ ? & 2 䖶 \\
\hline $8+.1$ & 22 & $7.474 f=118$ & $1.11 \sum F=0 ?$ & 200 \\
\hline$P P . O$ & Th & $1.103 f=0 ?$ & $3.9295=07$ & 200 \\
\hline 9 त. & $2>1$ & $3.4 \leq 5 F-07$ & $1.0 .98 F-06$ & $2 \pi 0$ \\
\hline 92.0 & 3906 & 14. $a \cap C E-1, b$ & $1.53 \times E=\cap 5$ & 200 \\
\hline 04.0 & 2731 & $3.9+A F-05$ & $1,1105=04$ & 200 \\
\hline 96.0 & 74232 & $1.154 \mathrm{~F}=.3 \mathrm{a}$ & $3.8935-04$ & 200 \\
\hline 98.9 & $18 \times 354$ & ?. $\mathrm{B} 5 \mathrm{PE}=\mathrm{n} \mathrm{a}$ & $9.1255=04$ & 200 \\
\hline $1 \times 0.0$ & $2463 \times 3$ & $\square . A 31 E=04$ & $1.226 F=03$ & 200 \\
\hline 102.0 & $3 \cos 8.7$ & $4.772 \mathrm{~F}-\mathrm{nu}$ & $1.527 E=03$ & 200 \\
\hline 101.0 & $271 \mathrm{HAA}$ & $a .2>A F-04$ & $53 F=03$ & 200 \\
\hline 106.0 & $1+3 t=15$ & $2.5 \| \subseteq F=n u$ & $a, 103 F-0 a$ & 200 \\
\hline $10^{\circ} \cdot 0$ & 179713 & $2.017 \mathrm{~F}=0 \mathrm{a}$ & 0.4555 .04 & 200 \\
\hline 110.0 & Qnतs) & $1.10 \cap E-\cap 4$ & $4.781 F=04$ & 200 \\
\hline 113.0 & $42 \cap 145$ & $1.452 \mathrm{E} * 1.4$ & 4. SA 1E $=04$ & 200 \\
\hline 114.0 & 510410 & $1.717 f=00$ & $5.495 F-04$ & $20 n$ \\
\hline 116.0 & 130085 & $? .029 \mathrm{E}=0 \mathrm{~d}$ & $6.094 E-194$ & 200 \\
\hline 118.0 & 131964 & 2. กรEF-0A & $6.567 E-114$ & 207 \\
\hline 120.0 & 91271 & $1.4+0 E=04$ & $4.547 F=04$ & 200 \\
\hline 122.0 & 71830 & $1.4 ?$ ? AE $=04$ & $4.570 E=04$ & 200 \\
\hline 120.0 & 03918 & $1.4 \times 1 E=04$ & $4.6745=04$ & 200 \\
\hline 120.0 & $9.3 ? 25$ & $1.4971=04$ & $\triangle, 7 R 9 \mathrm{E}-04$ & 200 \\
\hline 128.0 & 570.4 & $A . A 7 B E=05$ & $2,041 E=04$ & 200 \\
\hline 150.0 & 27873 & 2. 6UAE $=05$ & $1.4 A T E-04$ & 200 \\
\hline 152.0 & 14656 & $2.2705=05$ & $7.290 E-155$ & 200 \\
\hline 130.0 & $11+23$ & $6.257 F=0 \mathrm{~B}$ & $2.002 F=05$ & 200 \\
\hline 130.0 & $5 \times 3$ & $9.071 F-17$ & $2,903 F=00$ & 200 \\
\hline $13 R . n$ & 114 & $1.7 T 6 F=07$ & $5.6 R_{2} F=0 y$ & 200 \\
\hline & & $\begin{array}{l}652 E-02 \\
\text { SEC } 150 .\end{array}$ & $\begin{array}{c}485 E-02 \\
1 / N\end{array}$ & \\
\hline
\end{tabular}

THETE THT \&MLLI!W...

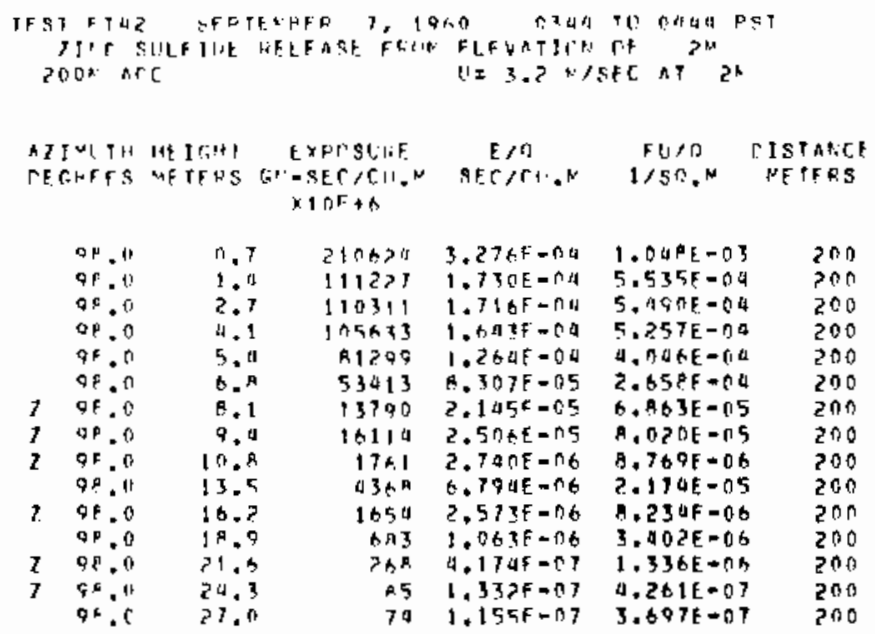

\begin{tabular}{|c|c|c|c|c|c|c|}
\hline \multicolumn{2}{|c|}{$\begin{array}{l}\text { AZINUTM } \\
\text { DE GOFFS }\end{array}$} & $\begin{array}{l}\text { HE IGHT } \\
\text { NFTERS }\end{array}$ & 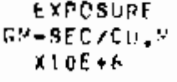 & $\begin{array}{c}F / 7 \\
S E C / t i !, N\end{array}$ & $\stackrel{F U / C}{1 / S O N}$ & $\begin{array}{l}\text { DYSTENCF } \\
\text { NETERS }\end{array}$ \\
\hline & 306.0 & 0.7 & 265391 & $4.12 T F=04$ & $1.3205=03$ & 200 \\
\hline & 106.0 & 1.0 & 128276 & $1.995 F=04$ & $n .384 E-04$ & 200 \\
\hline & $10 t .0$ & 2,7 & 1 己2725 & $1.974 E=04$ & $6.333 \varepsilon=04$ & 200 \\
\hline & 106.0 & 0.1 & 124193 & $1.931 \mathrm{~F}=0.4$ & $6.181 F=00$ & 200 \\
\hline & 106.0 & 5.4 & 135123 & $2,10, F=04$ & $6.725 E=04$ & $20 n$ \\
\hline & .7 & b. 8 & 91917 & $1.430 \mathrm{E}=0 \mathrm{a}$ & 4.57 वE $=04$ & 700 \\
\hline$z$ & 108.0 & 8.1 & 7 ती & 1. $\ln \vec{z} F=\{1\}$ & $3.52 \times E-94$ & 200 \\
\hline & 156,0 & 9.4 & $\begin{array}{ll}48 \\
A 08\end{array}$ & $7.698 F=05$ & 43 \&E $\rightarrow 4$ & 200 \\
\hline & 106.11 & 10.8 & SOANA & $9.46+1=05$ & $0 ? 9 F=04$ & हती \\
\hline 2 & 1060 & 13.3 & 3791 & $4.8677=05$ & $1.557 E=04$ & $20 n$ \\
\hline r & $10 t .0$ & 18.9 & $\begin{array}{l}9420 \\
4453\end{array}$ & $\begin{array}{l}1.4811=15 \\
6.927 F=0 t\end{array}$ & $\begin{array}{l}4.13 R E=05 \\
2.3 \mathrm{H}=05\end{array}$ & 200 \\
\hline & $\operatorname{lng} .0$ & 21.5 & $I C B$ & $3.09 a F=07$ & $900 E=07$ & 200 \\
\hline 7 & 106.0 & $24 . x$ & 172 & $2.6 A 3 F=07$ & $0.545 F=0.7$ & 200 \\
\hline & $10 \times .0$ & $27 . n$ & $0 *$ & 1. $\Delta 4 Q F=07$ & $4.637 E=07$ & $2 \cap 0$ \\
\hline
\end{tabular}

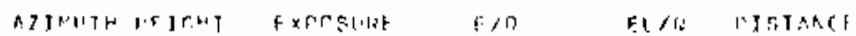

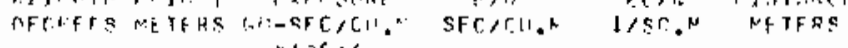
$x y+i f+6$

\begin{tabular}{|c|c|c|c|c|c|c|}
\hline & $114 . n$ & 0.7 & $1011 \times 9$ & $1.5737=5.4$ & $5 . n 35 F-1,4$ & $? \cap n$ \\
\hline & 114,14 & 1.4 & $5 / 371$ & म. $748 t=15$ & 2. .970 Ot -04 & $2 n \theta$ \\
\hline & 114.4 & 2.7 & 65473 & $1 . n_{1}>\mathrm{F}=04$ & $3 .>565=010$ & 276 \\
\hline 8 & 114.0 & 4.1 & $51 \cap 05$ & $7.939 \mathrm{~F}-195$ & $2.5 \operatorname{sen} t=04$ & 200 \\
\hline & $119=6$ & 5.4 & UR $3+h$ & $7,522 F=15$ & $2.407 t=04$ & $20 \%$ \\
\hline 2. & 114,11 & b. . & 232115 & $3, \operatorname{RnQF}=0.5$ & $1.155 t-614$ & 200 \\
\hline & $114=0$ & $M .1$ & $375 n \%$ & $5 .+33 F=0 S$ & $1 . A_{6}+F-(14$ & $2 n 0$ \\
\hline & 110.0 & 9.4 & 33211 & $5.1 \times 5 F=05$ & $1.653 \mathrm{E}-0 \mathrm{~d}$ & $? 00$ \\
\hline & 114.0 & 10.8 & 77447 & $.09 F-05$ & $1.3+0 E-04$ & 200 \\
\hline & 134.0 & 13.5 & $\operatorname{ID\cap AA}$ & $9 F=04$ & $5.021 E-05$ & 200 \\
\hline & 1140 & $16 . ?$ & 4676 & $7,3055=0$. & $2.337 \mathrm{E}-05$ & 200 \\
\hline & 110 & 12.9 & 2270 & $3.531 \mathrm{~F}+0 \mathrm{~A}$ & $1.130 E=\$ 5$ & 200 \\
\hline 7 & 114.0 & $21 . h$ & $\mathrm{~A}_{3} 1$ & $1,3 \cap Q F=O A$ & $\triangle .1 B A F=0 B$ & 200 \\
\hline 7 & 114.0 & 24.3 & 312 & $a .057 F=07$ & $1.554 E-016$ & ats \\
\hline 7 & 1 & 27.1 & 154 & $09 \mathrm{Q}=07$ & $70 R E \backsim 07$ & 300 \\
\hline
\end{tabular}

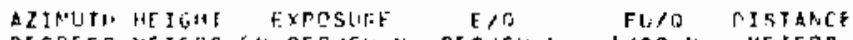

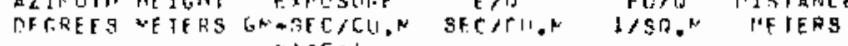
$X 1 \mathrm{CE}+\mathrm{h}$

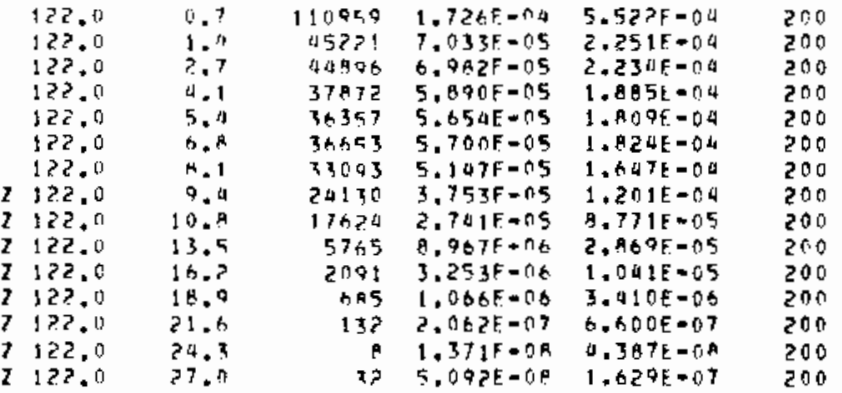




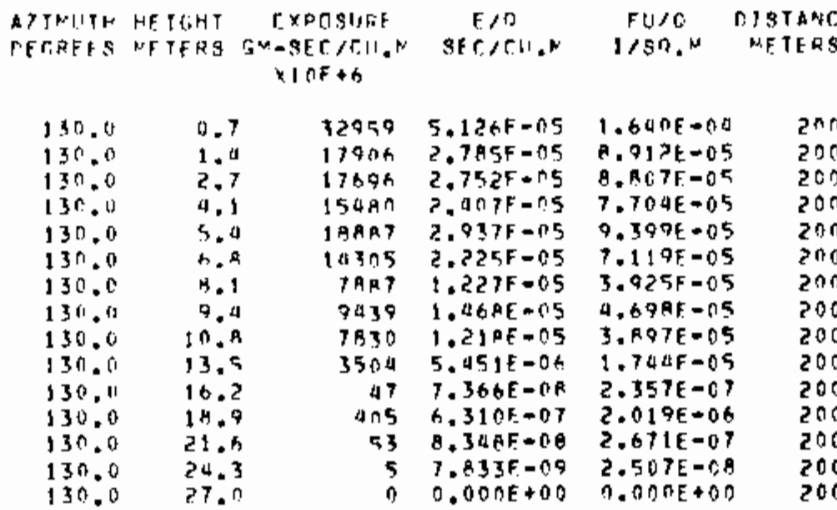

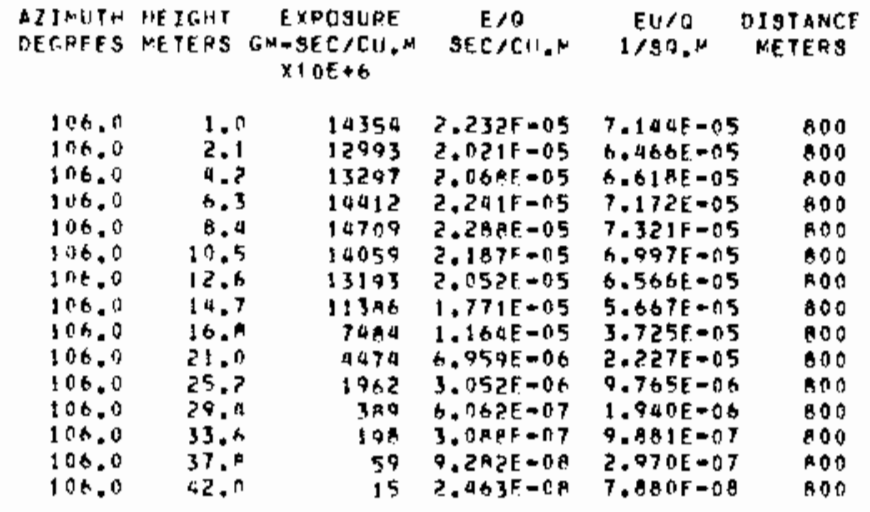

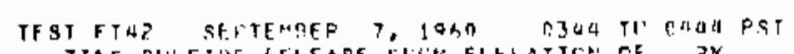

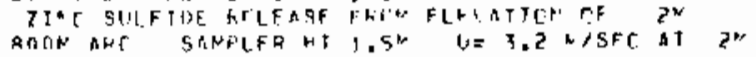

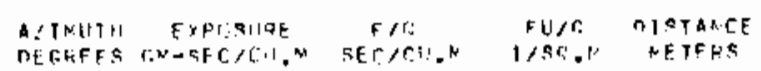
$\times 1 O F+6$

\begin{tabular}{|c|c|c|c|c|c|}
\hline$s$ & $\begin{array}{l}90.0 \\
0.2 .0\end{array}$ & 7 & $\begin{array}{l}1,1>n F=n A \\
11-55 a F=n 7\end{array}$ & $\begin{array}{l}3.5 R 5 F=08 \\
1.5 R F=06\end{array}$ & $A \cap \pi$ \\
\hline & 94.6 & $\begin{array}{l}\text { sq5 } \\
30>1\end{array}$ & 1.5 SIRF=0A & $\begin{array}{l}1.45 R F=06 \\
1.455 F=05\end{array}$ & ano \\
\hline & 74.0 & 16508 & $>.5 x>7=115$ & $\begin{array}{l}1.437 t=13 \\
0,216 F=05\end{array}$ & $\begin{array}{l}800 \\
\text { RnO }\end{array}$ \\
\hline & $9 P, 0$ & $2 \times 268$ & $3.152 \mathrm{E}-12 \mathrm{~S}$ & $1.009 F-n 4$ & Ban \\
\hline & 100.0 & 25001 & $3.8 Q 4 F+n 5$ & $1.24 G E-A 4$ & $A 00$ \\
\hline & 102.0 & 25639 & $3 . \triangle A 7 E-05$ & $1.276 E+04$ & 800 \\
\hline & 104.0 & $2 \times 6 \times 3$ & $3.150 \mathrm{~F}=05$ & 1.3287 .04 & 800 \\
\hline & 106.0 & 16544 & 2.57 aF $=05$ & $9.236 \%-15$ & 800 \\
\hline & 108.0 & 69.95 & $1.070 F-05$ & $3.43+F=05$ & 800 \\
\hline & 110.0 & 9747 & 1.516 .05 & $A . A 51 E-05$ & ROA \\
\hline & 112.0 & $110 \mathrm{A1}$ & $1.7>0 A F-05$ & $5.505 \mathrm{~F}-15$ & 000 \\
\hline & 110,0 & 14896 & $2.311+=05$ & $7.3^{\circ} \triangle E=05$ & 800 \\
\hline & $11+.9$ & $106>1$ & $1.652 f-05$ & 5.2 A -05 & A00 \\
\hline $\mathbf{s}$ & 118.0 & 2422 & $3.7 \mathrm{APE}-\mathrm{DA}$ & $1.20 \Delta E=05$ & Bon \\
\hline & 120.0 & 3ค?? & $5.956 \mathrm{E}-06$ & $1.906 \mathrm{~F}=05$ & Bon \\
\hline & 122.0 & 7300 & $1.151 \varepsilon=05$ & $3.685 E-05$ & 800 \\
\hline & 124.0 & 11135 & $1.732 \mathrm{~F}=05$ & $5.542 F=05$ & 800 \\
\hline & 126.0 & 9041 & 1. $4 A B F=05$ & $4.505 E-05$ & 100 \\
\hline & 125.0 & 6705 & $1.052 F-05$ & $3.367 E-05$ & 900 \\
\hline & 130.0 & $? 715$ & $\triangle .2 \cap R_{E}=06$ & $1,307 E-05$ & 800 \\
\hline & 132.0 & ond & $1.031 E=06$ & $3.28 \mathrm{eE}-06$ & $\mathrm{BCO}$ \\
\hline & 134.0 & $1>4$ & $1.5 \triangle 2 E-07$ & $6.213 F-07$ & AOO \\
\hline & & & SET.190.N & $2 \prod_{1 / N}$ & \\
\hline
\end{tabular}

TIREF TATA FOLLIA....

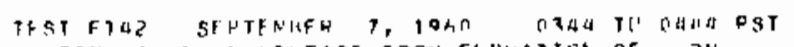

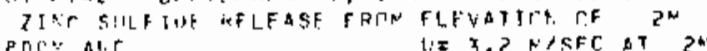

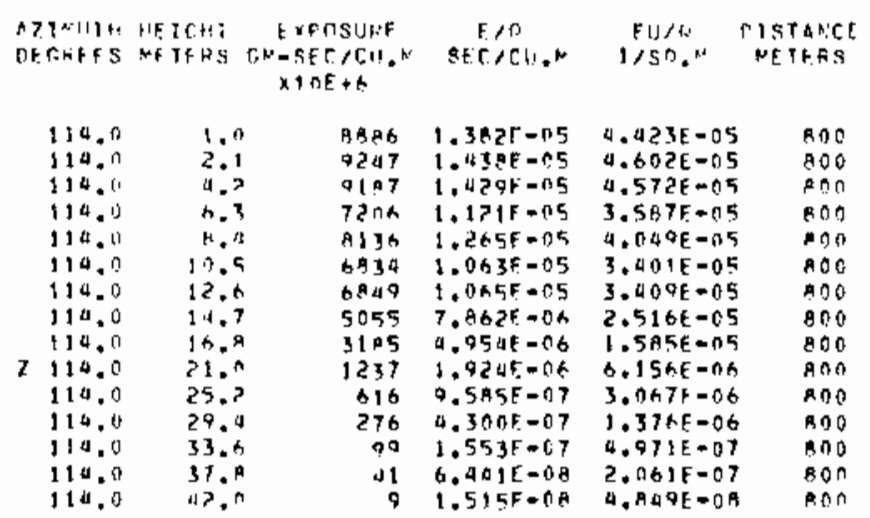

\begin{tabular}{|c|c|c|c|c|c|}
\hline $\begin{array}{l}\text { AZIRITH } \\
\text { DE GRFFS }\end{array}$ & $\begin{array}{l}\text { ME TSHA } \\
\text { METERSS }\end{array}$ & $\begin{array}{c}\text { EXPOSURE } \\
G 4=-3 E C / C U . N \\
\times 10 E+6\end{array}$ & $\begin{array}{c}E / A \\
\operatorname{SEC} / \mathrm{CL} \text {. }\end{array}$ & $\begin{array}{c}E 4 / 0 \\
1 / \$ 0 . \mu\end{array}$ & $\begin{array}{l}\text { DISTANCE } \\
\text { NETERS }\end{array}$ \\
\hline 122.0 & 1.0 & 4 a 19 & $6.874 F=06$ & $2.200 E-05$ & 800 \\
\hline & 2.1 & 31 & $7.204 E-0 \mathrm{~b}$ & $2.305 E-05$ & $\begin{array}{l}800 \\
800\end{array}$ \\
\hline $\begin{array}{l}122.0 \\
122.0\end{array}$ & $\begin{array}{l}4.3 \\
6.3\end{array}$ & $\begin{array}{l}5 \ln 0 \\
3744\end{array}$ & $\begin{array}{l}7.933 E=06 \\
5.820 E=06\end{array}$ & $\begin{array}{l}3.539 \mathrm{E}=05 \\
1.064 \mathrm{E}=05\end{array}$ & $\begin{array}{l}\ln 0 \\
A D D\end{array}$ \\
\hline 122.0 & A. a & $57 n 4$ & $A . B 71 F=06$ & $2.839 E-05$ & 800 \\
\hline 122.0 & 10.5 & $43 / 43$ & $6.755 E-06$ & $2,162 E=05$ & $9 \cap 0$ \\
\hline 122.0 & $12, \mathrm{~A}$ & 4551 & 7.07 PF-G6 & $2.265 E-05$ & eno \\
\hline & 14.7 & 3365 & $5.235 \mathrm{~F}-$ & $1.675 F-05$ & an \\
\hline 122.0 & $16 . \mathrm{R}$ & 2976 & $4.629 F=06$ & $1 . A B 1 E=05$ & 8Do \\
\hline 2122.0 & 21.0 & 1473 & $2.292 \mathrm{~F}=0 \mathrm{~b}$ & $7.330 \mathrm{E}=00$ & 800 \\
\hline 122.0 & $25 . ?$ & 487 & $7.577 E-57$ & $2.425 E-00$ & 400 \\
\hline 122 & 29.9 & 140 & $2.254 F-07$ & $7.213 E-07$ & $B O 0$ \\
\hline 122.11 & 33.6 & 54 & $8.524 \mathrm{~F}=0 \mathrm{~A}$ & $2.72 B \mathrm{~F}=07$ & 600 \\
\hline 122,0 & 37.8 & 41 & 6.4415 .08 & $2,061 E-017$ & ROO \\
\hline 122.0 & 4?. 1 & $n$ & $.00 \cap F+00$ & $0.00 \cap E+00$ & 900 \\
\hline
\end{tabular}

\begin{tabular}{|c|c|c|c|c|c|c|c|c|c|c|c|c|}
\hline \multicolumn{2}{|c|}{ 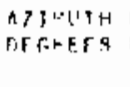 } & $\begin{array}{l}\text { HE I I FHI } \\
\text { PETE TSS }\end{array}$ & 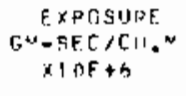 & $\begin{array}{c}\text { E/R } \\
\text { ser.rug }\end{array}$ & $\begin{array}{l}F u / 1+ \\
1 / 50 . \mathrm{N}\end{array}$ & $\begin{array}{c}\text { NISTANCE } \\
\text { PFTERS }\end{array}$ & 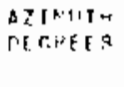 & 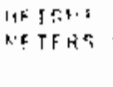 & 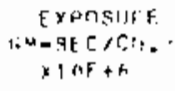 & $\begin{array}{c}k>0 \\
\operatorname{sic} / \mathrm{cll} . *\end{array}$ & $\begin{array}{c}F 11 / 6 \\
1 / s C^{\prime \prime m}\end{array}$ & $\begin{array}{l}\text { OBStant } \\
\text { PETras }\end{array}$ \\
\hline & 00.0 & $1 . n$ & 11793 & $1.834 E=05$ & $5.869 F=05$ & Ann & 155.11 & $1 . n$ & 2278 & .4619 & $1.107 E-05$ & ROO \\
\hline & $4 P .0$ & $? .1$ & $15 y 08$ & $2.3955-05$ & $7.063 \mathrm{E}=05$ & $P O$ & $13 n .4$ & 2. 1 & 19 & & $t=n t$ & \\
\hline & & $4 . ?$ & 16707 & $2.570 \mathrm{~F}=05$ & A.315t-05 & An: & 130.0 & a.? & $13<3$ & $2.10 \pi k=n$ & $0.734 \mathrm{E}=06$ & AOU \\
\hline & 9 P. & 0.3 & $133 \mathrm{~Kb}$ & $2.0635=05$ & $6, A \cap>E=05$ & Ro & & 0.3 & IRE, & T.PAIE-AC & $0,2195-\pi n$ & $\operatorname{mon}$ \\
\hline & 85.0 & 3.1 & 12500 & 1.94 & $6.224 E-05$ & an & & ?.i & je & 2.95 & $9.455 \mathrm{E}-\mathrm{nA}$ & An 0 \\
\hline & 98.0 & 10.5 & 91 & $F=0 \leq$ & $=05$ & $B$ & $\therefore$ & 10.5 & 1770 & 2.76 & R. HFAE $=$ DS & 900 \\
\hline & 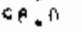 & $12, \mathrm{~h}$ & 7057 & $1.098 F=05$ & $3.512 E-05$ & 90 & I th. t? & i 2.4 & $1^{\text {n }}$ & & $9.09 n E-06$ & 900 \\
\hline & 08.0 & 10,7 & 3294 & $5.123 f=06$ & $1.639 \mathrm{k}=05$ & AO & 130.0 & 14.7 & 130.0 & 2.1 & $6.71 \geq E-0 b$ & \\
\hline & QA. 0 & & $17 \geq 1$ & 2.677 & A. $56 \mathrm{hE}=\mathrm{fh}$ & 80 & & 16.8 & 1317 & $-c t$ & $9 F-06$ & A0 \\
\hline & $9 p .0$ & 21.0 & $5 A 1$ & $9.0495-n 7$ & $2 . A 96 F=06$ & 0 & 130.0 & 21.0 & 954 & $1.4 A$ & $n F=06$ & ADO \\
\hline & 88.0 & 25.2 & Aम & 1,3 R E E $=07$ & 4.42 & 6 & & 25 & 174 & 2.70 & $8.66 A_{E}=0.7$ & \\
\hline & & .1 & $?$ & $0.725 \mathrm{~F}=0 \mathrm{~F}$ & $1.576 E=07$ & A 0 & & 28 & $16 \mathrm{~F}$ & 2.59 & $8.3056-07$ & \\
\hline 7 & 98.0 & 33.6 & 1 & $1.834 F=00$ & $6.0625=09$ & 6n & 136.0 & & a 4 & B. DOCUF $=07$ & $3.213 E-07$ & Aon \\
\hline & 88.0 & $37 . R$ & n & $0.000 .5+00$ & $0.000 E+00$ & 80 & 136.0 & $37 .^{\text {R }}$ & 26 & 4.3 & $1.334 E=07$ & 800 \\
\hline & QR. 0 & $42, \pi$ & $n$ & $0.0005+00$ & $0.000 E+00$ & 800 & 130.0 & $42 . n$ & c & $0.0 n n F+n \pi$ & $0.000 E+00$ & 200 \\
\hline
\end{tabular}




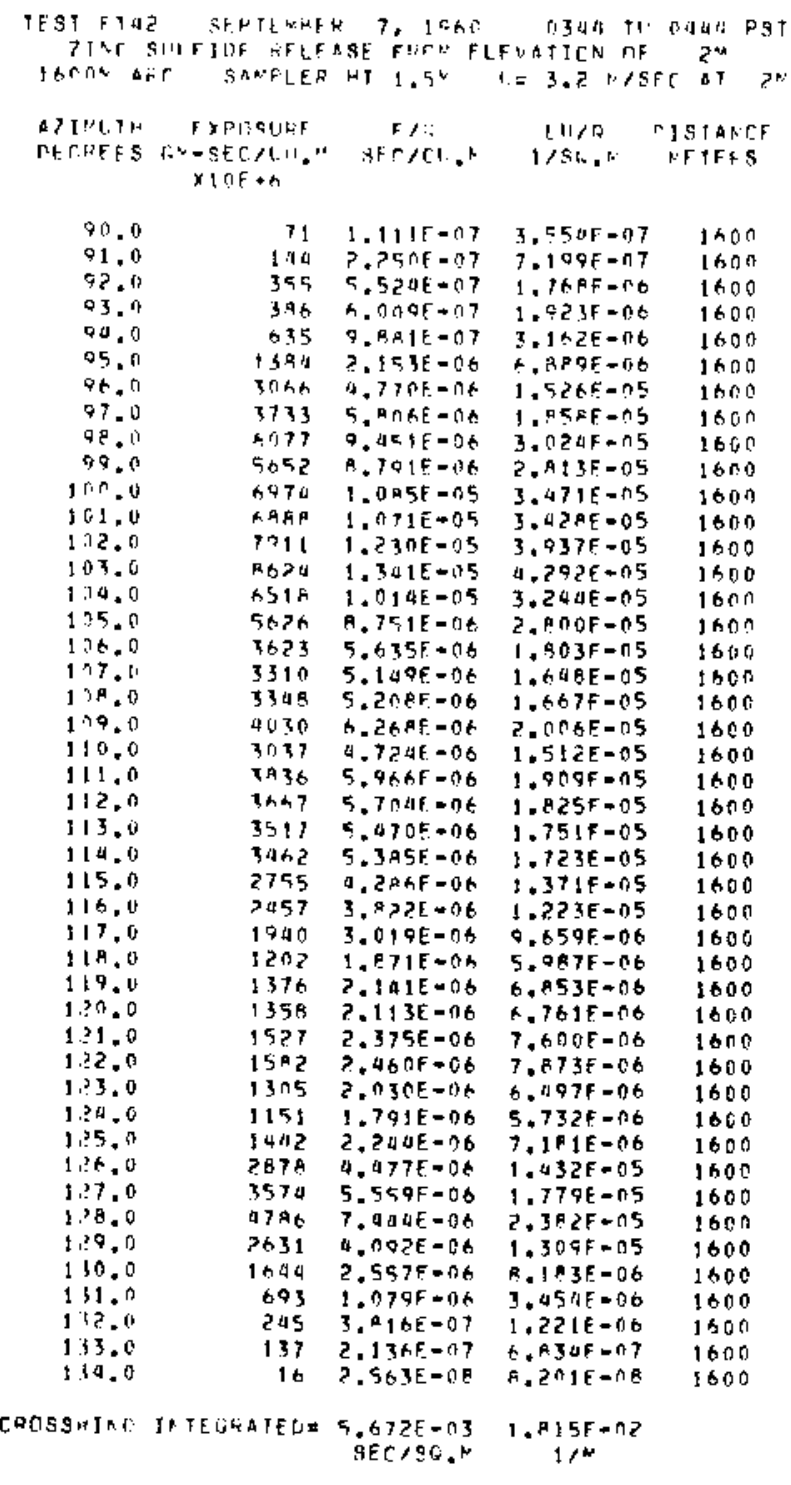

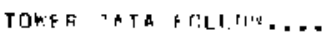

IFST IIUZ SEPTEAHER 7,1960 O34A TC OALA PST

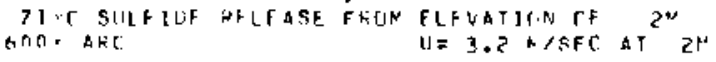

\begin{tabular}{|c|c|c|c|c|c|c|}
\hline \multicolumn{2}{|c|}{$\begin{array}{l}\text { AZN ITH } \\
\text { TRENGS }\end{array}$} & $\begin{array}{l}\text { ME J J,HT } \\
\text { NETENG }\end{array}$ & 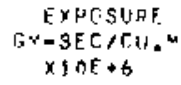 & $\begin{array}{c}E / \mathrm{ij} \\
\mathrm{SF} / \mathrm{CH}, \mathrm{N}\end{array}$ & $\begin{array}{l}F \cup / 12 \\
1 / \mathrm{sn} .\end{array}$ & $\begin{array}{l}\text { DISTANC } \\
\text { METEAS }\end{array}$ \\
\hline & 09.0 & 1.5 & 4500 & $7.01 \mathrm{AE}=n \mathrm{~A}$ & $2.2 \Delta 4 E-05$ & 1600 \\
\hline & 99.0 & 3.1 & 4364 & $0,7 \mathrm{AF}-\mathrm{C}_{6}$ & $2.172 \mathrm{E}=05$ & 1600 \\
\hline & & $0 . ?$ & $337 \mathrm{~K}$ & $5.254 \mathrm{~F}-\mathrm{n}_{6}$ & $3.6+1 E-05$ & 1600 \\
\hline & 92.0 & 9.3 & $3 \times 73$ & $6.023 F=06$ & $1.927 E-05$ & 1600 \\
\hline & 92.0 & 12. & 29114 & $0.5 \mathrm{BOF}=06$ & $1.465 \mathrm{E}-05$ & 1600 \\
\hline & & 15.9 & 17,4 & $2,667 E-06$ & A. $53 a F-06$ & $160 n$ \\
\hline & Q9. & $10 . \mathrm{t}$ & คn 5 & $1.252 E=06$ & $A .000 E=06$ & 1600 \\
\hline 7 & 42.0 & 21.7 & 390 & S.066E $=07$ & $1.941 E=06$ & 1600 \\
\hline & & 24,8 & 100 & $3.1025=07$ & $9.025 E-07$ & 1600 \\
\hline & 99.0 & 31.0 & 20 & $A .637 F-A B$ & 1.48 यद-C? & 1600 \\
\hline & & $37, ?$ & 0 & $0.000 E+00$ & $0.000 E+00$ & \\
\hline & & 43,4 & 4 & $6.395 \mathrm{~F}-100$ & $2.046 E-00$ & 1600 \\
\hline & 99.0 & 49.6 & 0 & $1.406 E-69$ & $4.500 E=09$ & 1600 \\
\hline & & 55 & 0 & $1.406 f-00$ & $4.500 E-09$ & \\
\hline & & & 0 & $.00 C F+00$ & $0.00 D E+00$ & 1600 \\
\hline
\end{tabular}

\begin{tabular}{|c|c|c|c|c|c|}
\hline $\begin{array}{l}\text { AZTEUTH } \\
\text { DEFEETS }\end{array}$ & $\begin{array}{l}\text { HE IGHT } \\
\text { ME TERS }\end{array}$ & $\begin{array}{c}\text { EXPOSURE } \\
\text { GM-SEC/CH.N } \\
x ! O E+b\end{array}$ & $\begin{array}{c}E / 0 \\
\mathrm{SEC} / \mathrm{CH}, \mathrm{N}\end{array}$ & $\begin{array}{l}\text { EU/D } \\
1 / \mathrm{SO}, \mathrm{M}\end{array}$ & $\begin{array}{l}\text { DISTANCE } \\
\text { METFRS }\end{array}$ \\
\hline $\begin{array}{l}100.0 \\
100.0\end{array}$ & $\begin{array}{l}1.5 \\
3.1\end{array}$ & $\begin{array}{l}3279 \\
3921\end{array}$ & $\begin{array}{l}5.101 \mathrm{~F}-06 \\
6.099 \mathrm{E}-06\end{array}$ & $\begin{array}{l}1.632 E=05 \\
1.952 E=05\end{array}$ & $\begin{array}{l}1600 \\
1600\end{array}$ \\
\hline 106.0 & 6.2 & 4369 & $6.787 \mathrm{E}-06$ & $2.172 \mathrm{E}-05$ & 1600 \\
\hline 106,0 & 4.3 & 3956 & $6,153 E-06$ & $1.969 \mathrm{E}-05$ & 1000 \\
\hline 106.0 & 12.4 & 4352 & S.77OE -06 & $2.166 \mathrm{E}-05$ & 1600 \\
\hline 105.0 & 15.5 & $42: 3$ & $6,552 E=n b$ & $2.097 \mathrm{E}=05$ & 1600 \\
\hline 106.0 & & 4195 & D. $525 \mathrm{E}=1 \mathrm{G}$ & $2.088 E-0 S$ & 1600 \\
\hline 106.0 & 21.7 & 2064 & A. $456 E-06$ & $1.026 E-05$ & 1600 \\
\hline 106.0 & 20.9 & 1 คด & $2,862 E-06$ & 9.15 AE-OH & 1600 \\
\hline 106.0 & 31.0 & 471 & $7.339 E=07$ & $2.348 E=06$ & 1600 \\
\hline $10 \mathrm{n} .0$ & 37.2 & 105 & 2.A7AF -07 & $9.209 E=07$ & 1600 \\
\hline 106.0 & 43. & 34 & $5.436 \mathrm{E}=0 \mathrm{~A}$ & $1,7 \triangle A E=07$ & 1600 \\
\hline$s 106.0$ & 49.6 & 0 & $0.000 F+00$ & $0.000 E+00$ & 1600 \\
\hline 106.0 & $55 . A$ & $n$ & $0.0 O A E+00$ & $0.000 E+00$ & 1600 \\
\hline 106. & he. & 0 & O. NODOE+TO & $0.000 E+00$ & 1600 \\
\hline
\end{tabular}

\begin{tabular}{|c|c|c|c|c|c|}
\hline $\begin{array}{l}\text { A Z TH:UTh } \\
\text { DF GHE ES }\end{array}$ & $\begin{array}{l}\text { WE IFHF } \\
\text { "ETERS }\end{array}$ & $\begin{array}{l}\text { FPOSUEF } \\
\text { GN-SEC/CI!=N } \\
\times 10 E+6\end{array}$ & $\begin{array}{c}F / O \\
\text { SEr } / C l \cdot \text {. }\end{array}$ & $\begin{array}{c}F \cup / r \\
1 / S n . M\end{array}$ & $\begin{array}{l}\text { ISTANCF } \\
\text { NETERS }\end{array}$ \\
\hline 110,0 & 1.5 & 3226 & $5.01 \mathrm{AF}=0 \mathrm{~K}$ & $1.606 E=05$ & 1000 \\
\hline 114.0 & 3.1 & & $3.91+E=0 t$ & 1.2 & 1600 \\
\hline 114.0 & b.? & 397 & $6.107 \mathrm{~F}=\mathrm{nG}$ & $1.954 E=15$ & 1601 \\
\hline 114.0 & 9.3 & 2990 & $0.65 Y E=06$ & $1.090 E-05$ & inon \\
\hline 110.0 & 12.4 & 3096 & $F=06$ & $1.536 E-0.5$ & 1600 \\
\hline 114.9 & & 24 & 3.78 & 1.2 & 1600 \\
\hline 114.0 & 18.6 & $17 n 1$ & $2.045 \mathrm{E}-\mathrm{n6}$ & $8.065 \mathrm{E}=06$ & $160 \mathrm{n}$ \\
\hline 114.0 & 21.7 & 1332 & $2.072 F=06$ & $E=0 \mathrm{~B}$ & 1600 \\
\hline 110 & 24 & 653 & 1.01 & 3.2 & 1600 \\
\hline 114.17 & $31, n$ & $21 \%$ & $3.405 \mathrm{f}-07$ & $1.000 E-O B$ & 1600 \\
\hline 114.0 & 37 & B & $1.375 \mathrm{E}$ & $4.400 E=07$ & 1600 \\
\hline 190.1 & 43.9 & 50 & 7.83 of $=08$ & $2.507 \mathrm{~F}=07$ & 1000 \\
\hline 2114.0 & & A & $1.26+5=08$ & a. $05 \cap E-D B$ & 10 no \\
\hline 114.0 & 53 & 2 & $19 E-05$ & $1.350 E=O A$ & 1600 \\
\hline $1: 4.0$ & $62 . n$ & 0 & $0.0 \cap \cap E+O \cap$ & $0.000 \mathrm{~F}+00$ & 1500 \\
\hline
\end{tabular}

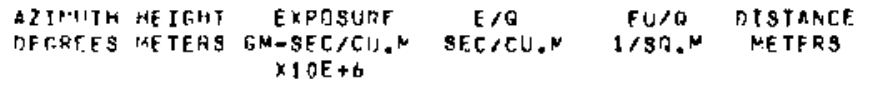

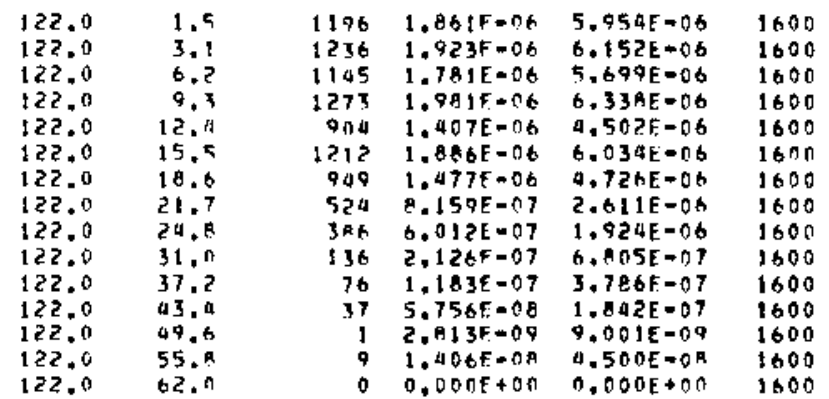

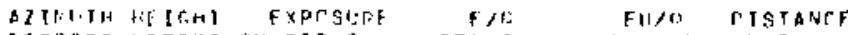

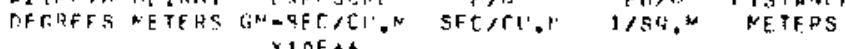

\begin{tabular}{|c|c|c|c|c|c|c|}
\hline & $1 ; 0] .0$ & 1.5 & 742 & $F=7.6$ & a. GAPE $=0 \mathrm{H}$ & \\
\hline & $\$ 30,0$ & 3.1 & 1256 & $1.950 \mathrm{oF}=0 \mathrm{t}$ & $0.251 E-06$ & 1600 \\
\hline & 130.0 & 6.2 & 1203 & $2.012 F-0.6$ & $6.037 E-06$ & 10.00 \\
\hline & 130.0 & 9.1 & 325 & $5.05 \mathrm{AF}-077$ & $1.6198-06$ & 1000 \\
\hline & 130.0 & 12.1 & $15 \%$ & $2,4615=0 A$ & T. GTAEE-CA & 1600 \\
\hline & 130.0 & 15.5 & 920 & $1,43 E F=06$ & $1.602 E-06$ & $100 ?$ \\
\hline & $13 n .0$ & 18. & $11 ? h$ & $1.767 \mathrm{E}-00$ & $6 E=06$ & 1600 \\
\hline$z$ & 130.0 & 21. & $74 \mathrm{~A}$ & $1.2385=0 \mathrm{~h}$ & $3.963 \mathrm{E}=06$ & 1600 \\
\hline$i$ & 130.0 & 24 & $6: 3$ & $9.505 \mathrm{f}-0.7$ & $3.050 E=06$ & 1600 \\
\hline & 130.0 & 31 & $20 a$ & $3.8054-07$ & $1.21 \mathrm{AF}=0 \mathrm{~B}$ & $16 \cap 0$ \\
\hline & 130.0 & 37.7 & $\zeta R$ & $9.1136=08$ & $2.91 \mathrm{AE}=07$ & 1600 \\
\hline & 130.0 & 43. & in & $1.50 \mathrm{NE}-0 \mathrm{~A}$ & 5.1IAE $-0 B$ & 1600 \\
\hline & 230.0 & 49. & 3 & $4,219 F=C Q$ & $1.35 \cap E=0 A$ & 1000 \\
\hline & 130.0 & & 0 & $1.4065=09$ & $a .5 n O E-0 \Omega$ & 1600 \\
\hline & & & & $\mathrm{F}$ & IE & \\
\hline
\end{tabular}




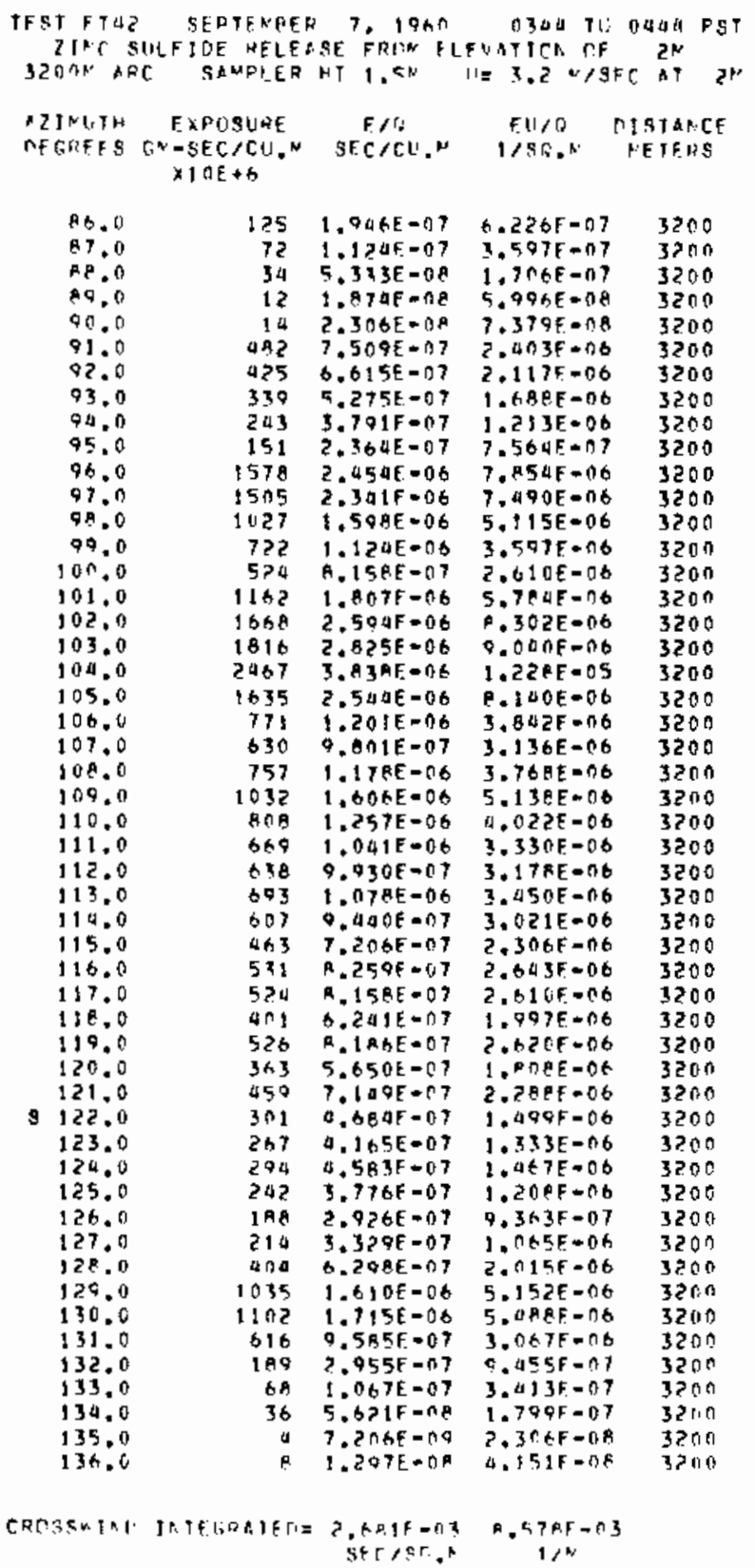

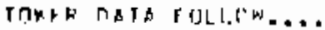

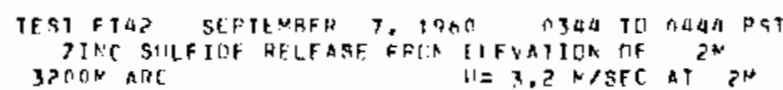

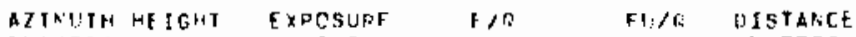

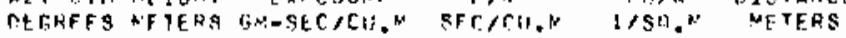
$\times 10 F+6$

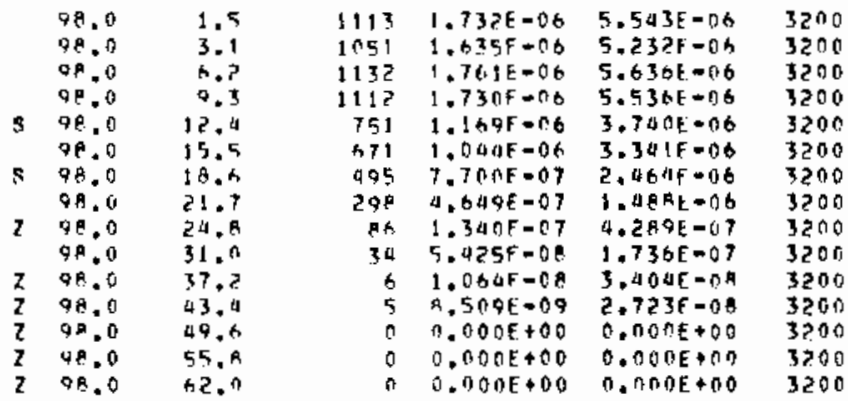

AZIMUTH HEIGHT EXPOSUPE EFQ FU/D OTSTANEE

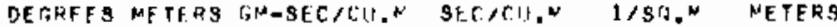
$x 10 F+6$

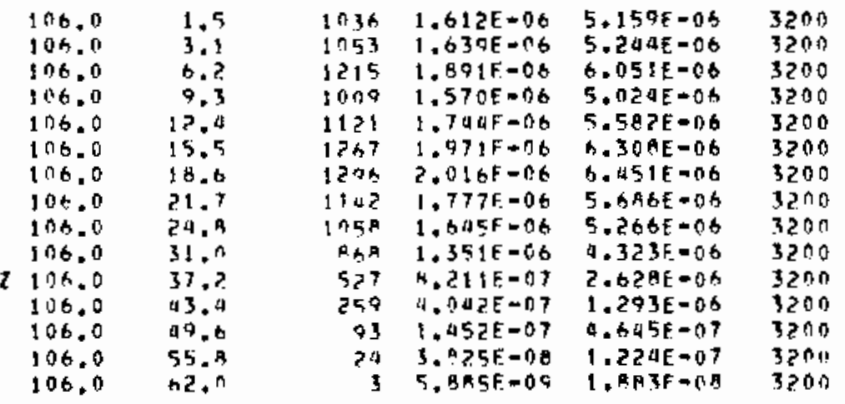

\begin{tabular}{|c|c|c|c|c|c|c|}
\hline \multicolumn{2}{|c|}{ 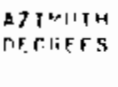 } & $\begin{array}{l}\text { HE ILHT } \\
\text { NETFKS }\end{array}$ & 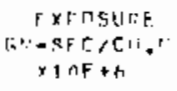 & $\begin{array}{c}F, n \\
S F[, r+1\end{array}$ & $\begin{array}{l}F U / 1 \\
1 / 5 \% .\end{array}$ & $\begin{array}{l}\text { NTSTANCE } \\
\text { NFTERS }\end{array}$ \\
\hline & 114,0 & 1.5 & $5 \times 7$ & $7, I A, F=07$ & $2.9355-06$ & $32^{n} n$ \\
\hline & 114.0 & 3.1 & 57 & $8.19 n$ & $F=06$ & 3200 \\
\hline & 1.0 & 0.7 & 472 & $7.35 ?$ & $t=06$ & $32 n 0$ \\
\hline & 110.0 & $4 . x$ & $7 \times 3$ & $1.171 \mathrm{E}=0.6$ & $E=0 A$ & $32 n n$ \\
\hline & 110.0 & 12.11 & 650 & 9.9 .1 & 3.1 म, 30.06 & $32 \cap 0$ \\
\hline$z$ & 11 & 15.5 & 412 & 6.415 & $F=0 h$ & $2 n n$ \\
\hline & & h & UA & 7.50 & $0301-06$ & 3200 \\
\hline 7 & 0.0 & 21.7 & 3 a & $6.003 F=07$ & $.93115=06$ & 3? ก \\
\hline & & 24.8 & 27 & 1.212 & . 3 UAC $=$ त 6 & 3) ती \\
\hline & 1 & $31 . n$ & 250 & $A .04>E-0.7$ & $.295 \mathrm{~F}=0 \mathrm{~s}$ & $320 n$ \\
\hline & & 37.7 & no & $1 . n 7$ UF -07 & $. \triangle 3 \mathrm{BE}-07$ & 3200 \\
\hline 7 & & 43.4 & 53 & $0.246 F-08$ & $.655 E-07$ & 3200 \\
\hline & j & & 15 & $2.45 z E-0 A$ & 7. तथ $\triangle E E=O B$ & 3200 \\
\hline $\mathbf{F}$ & 1 & & 11 & $1.863 E-08$ & $5.963 F=0 A$ & 5200 \\
\hline & & & R & $1 . x 73 F-C: A$ & $3,394 \mathrm{UE}=\mathrm{RA}$ & $32 \pi$ \\
\hline
\end{tabular}




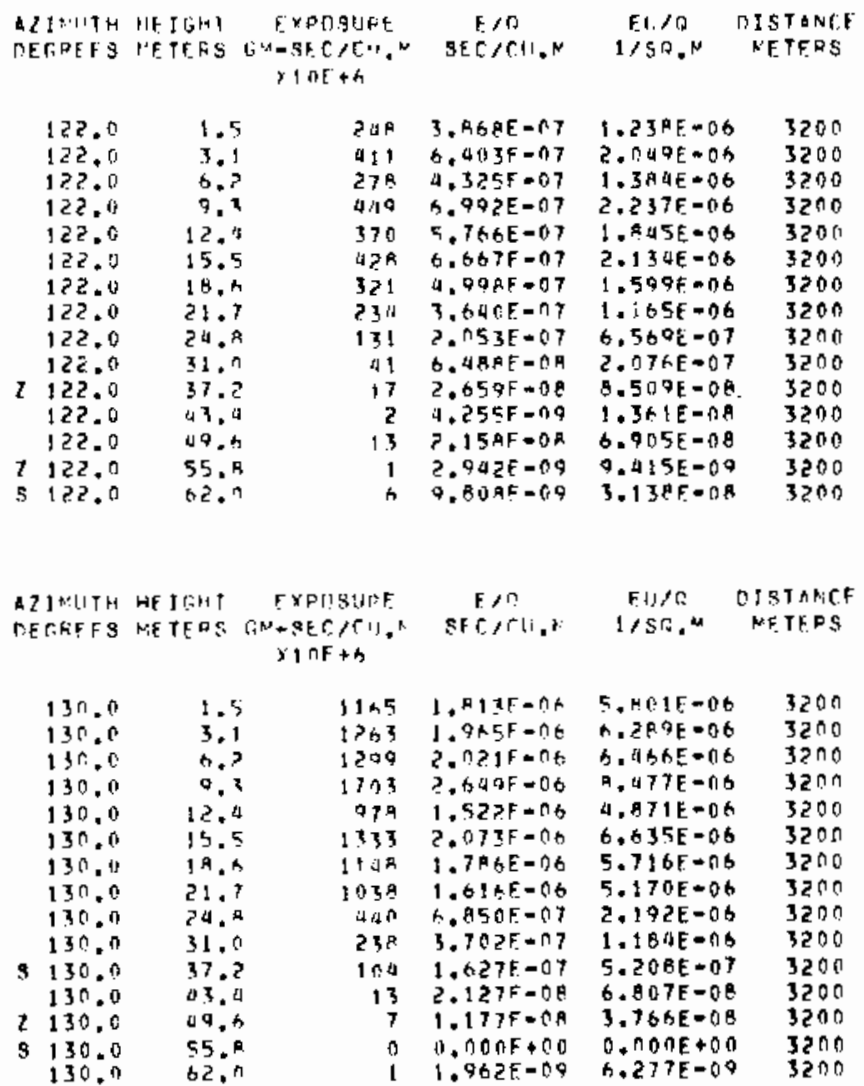


30-SERIES EXPERINENT: 43 (FT-43)

GROUND LEYEL ANO TOWER SAHPL ING 200-3200 M ARC5

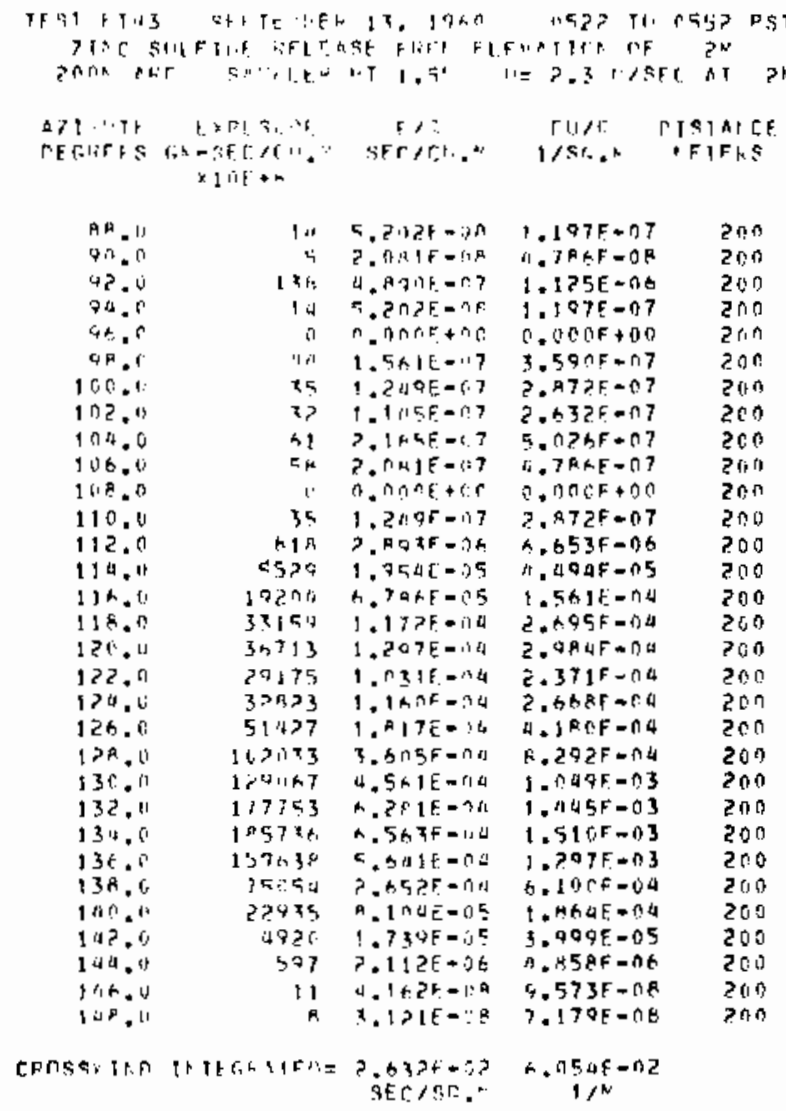

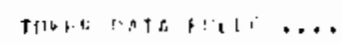

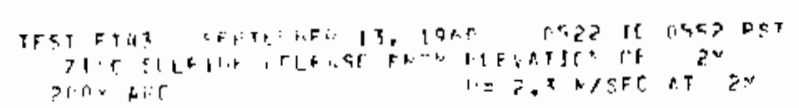

\begin{tabular}{|c|c|c|c|c|c|}
\hline $4 \times$ & $1, .7$ & 1,3 & $\therefore=+0 \cap F=n 7$ & 1. $" 1 ? \mathrm{f}=\mathrm{C} \cdot \mathrm{b}$ & $2 n n$ \\
\hline 94.0 & $1 . i$ & 15 & 1. H1AFAC. & $3.720 t-67$ & $2^{n} n$ \\
\hline 95.6 & 2.7 & 2 & $7,555 f=19$ & $1 .+9 P E-0 B$ & $\vec{E}(10)$ \\
\hline 94.6 & 4.1 & 83 & $3 . x 16 F=07$ & $7.613 E=07$ & $2^{n+i}$ \\
\hline $9 \pm-6$ & 4.1 & 31 & $+.527 k=6 . A$ & $2.03 n t=1,7$ & $20 \mathrm{C}$ \\
\hline $4 ?, 0$ & n. & 17 & $H_{1} .+13 \mathrm{~F}=\mathrm{r} \mathrm{E}$ & $1.015 F=07$ & $20 \%$ \\
\hline $2 a-2$ & $r .1$ & 4 & $1.471 E-C E$ & $3.30 t=0=0$. & $2 \uparrow 0$ \\
\hline 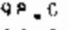 & 7. 2 & 31 & $1.103 F=17$ & $2.533^{A}=0.7$ & 2. 00 \\
\hline SA 0 & 19.6 & 24 & $7.355 \mathrm{~F}=\mathrm{NO}$ & $1.692 \mathrm{~F}=07$ & 5 \\
\hline 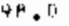 & 17.5 & 47 & $\because .913 E-68$ & $\therefore, C 15 E-c 7$ & 200 \\
\hline$\in R^{\circ}$ & $1 n . ?$ & 1is & $5.197 F=C 8$ & $1.184 E=17$ & $2 \cap 0$ \\
\hline 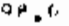 & 19.7 & $1^{\circ}$ & $A . A D C F=\pi A$ & $1.523 t-0.7$ & $a^{n} n$ \\
\hline
\end{tabular}

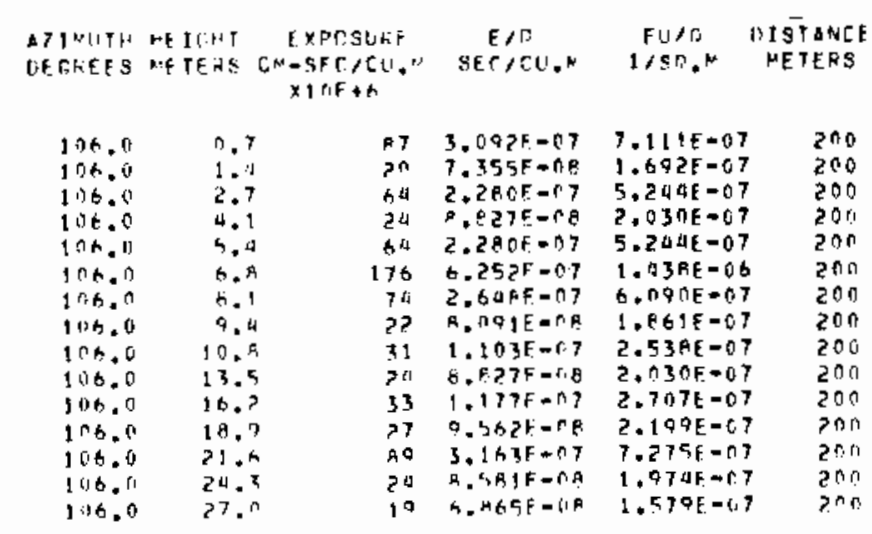

\begin{tabular}{|c|c|c|c|c|c|}
\hline$A Z 1 \cdots 1$ Y & $r \cdot E[1 ;-\pi T$ & 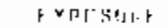 & $+1 n$ & F $1 /$ & istanct \\
\hline PFFEFS & 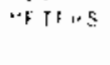 & 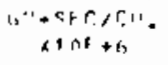 & sf $t / C \mathrm{l}$. & $1 / \leq n$, & "FTES \\
\hline 130.0 & 97 & $\cos 16$ & ?. $2 x \in 5=05$ & $5 . ! 50 F=04$ & $3 \Gamma \mathrm{n}$ \\
\hline 114.0 & 1.1 & 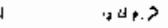 & $1,5175-55$ & $3 . \hbar_{\zeta}>F=015$ & 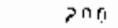 \\
\hline $11 \% .0$ & 3.7 & $? 955$ & $1.0+B+F=1 S$ & $7.407 t=05$ & 20,3 \\
\hline 114.0 & 4.1 & 3 Dh? & $1.3 \mathrm{AOE}=0 \mathbf{5}$ & $2.509 F=0.4$ & 210 \\
\hline $11+.6$ & 'i. & 2916 & $9.03, F-0 t$ & $2 . \supset A A F=\{15$ & 200 \\
\hline 110.0 & 6.5 & $17: 46$ & $h .171 E=n t$ & $1.41 \% E=05$ & 200: \\
\hline $1 ! 4.11$ & \pm .1 & 1719 & $A .076 F=5 A$ & $1.307 E-05$ & 200 \\
\hline 114.0 & 4.1 & 1255 & $\therefore 45$ C.F $=00$ & 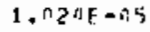 & 201 \\
\hline $11 * 0$ & (1) & 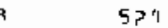 & $1.55 a F=06$ & $4.263 E=0 h$ & 2nt: \\
\hline 114.0 & 13.5 & 191 & $5.1 \times 9 F-07$ & $1.735 F=n h$ & 200 \\
\hline $11 \%, 0$ & $1 \mathrm{~h}, \mathrm{~T}$ & 12 & $b,+2$ lif $=\cap A$ & $1.5338-177$ & 706 \\
\hline 114.0 & 1月. & 4 & $\sigma_{1} .100 \cap E+00$ & ח. OOOEE+OO & 200 \\
\hline 114.0 & 21.4 & $r$ & n. nOOF + nn & $0.0 O A E+00$ & $20 n$ \\
\hline$\$ 1+4$ & $2,1.2$ & 1 & $5.721 F=09$ & $1.316 E=0.9$ & 200 \\
\hline $11^{i 1,0}$ & $=7.1$ & $\mathrm{n}$ & $0.000 F+00$ & D. กUतEद & 200 \\
\hline
\end{tabular}

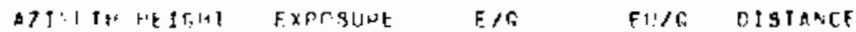

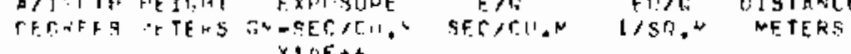

\begin{tabular}{|c|c|c|c|c|c|}
\hline 122.0 & 11.7 & $41: 153$ & $1.065 E=144$ & $3.369 \varepsilon=04$ & 200 \\
\hline 122.0 & 1.4 & $23 A 73$ & $9.430 F-0.5$ & $n E-n d$ & 2 t0 \\
\hline 122.0 & 2.7 & 19123 & $6.757 F=05$ & $1.55 \mathrm{AE}=0 \mathrm{~A}$ & 200 \\
\hline 23.0 & +.1 & 17375 & $6.140 E=05$ & $1.0125=04$ & 200 \\
\hline$n$ & 5.1 & $1557 \mathrm{~h}$ & $5.485 F=0.5$ & $1.262 F=04$ & 200 \\
\hline & $A .3$ & 11580 & $4.0155 F=09$ & $0.41 A F=05$ & $5 n 0$ \\
\hline 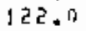 & $\therefore .1$ & 120,1 & 4. टे घ $F=t, 5$ & $9.77 ? E=05$ & 200 \\
\hline & 9.15 & $\sin 3$ & $3.136 .5=05$ & $1.712 E=05$ & 200 \\
\hline & 10.8 & 7600 & $2.717=05$ & $6.349 E-05$ & 200 \\
\hline & 13.5 & 2506 & $9.0110=10$ & $2 . n 725=05$ & 200 \\
\hline & 16.7 & P $\underline{\underline{t}} \overrightarrow{7}$ & $2.9425=0 x$ & $0.707 E=05$ & 200 \\
\hline & 14.9 & 14 & ?. $6485-\hat{0}, 7$ & $0.087 E=07$ & 200 \\
\hline & 21.5 & t? & $4.413 E-0 \mathrm{~A}$ & $1,015 E=07$ & 200 \\
\hline & 24.3 & $\hat{\imath}$ & $0.000 F+0 r_{1}$ & D. On?E + OD & 200 \\
\hline & 77.7 & ^ & J $n 0 r_{i} F_{-}+10$ & $n . n O B E+00$ & $2 n 6$ \\
\hline
\end{tabular}




\begin{tabular}{|c|c|c|c|c|c|c|c|c|c|c|c|}
\hline 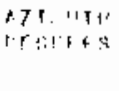 & 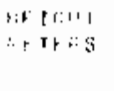 & 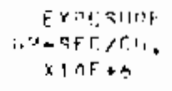 & 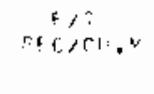 & $\begin{array}{c}511 / \mathrm{T} . \\
1 / 50.2\end{array}$ & 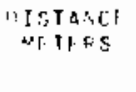 & 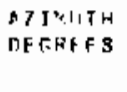 & $\begin{array}{l}\text { HE ITHT } \\
\text { PETERS }\end{array}$ & 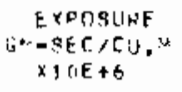 & 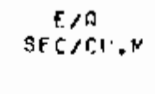 & $\begin{array}{c}E \cup / B \\
j / S C, N\end{array}$ & $\begin{array}{l}\text { DISTANCE } \\
\text { METEAS }\end{array}$ \\
\hline 126.11 & 4.7 & 1 गा?口内 & t. 07 F F o r d & $1.1 A$ A -03 & $2^{n} n$ & $10 k .0$ & 1.0 & h & $2,135 \mathrm{~F}=08$ & $\triangle .909 E=0 A$ & AOO \\
\hline $1: x^{i 1} \cdot 11$ & 1. & 6.617 & $3.210 \mathrm{~F}-104$ & $7.343 \overline{\mathrm{c}}=\mathbf{1} a$ & 200 & 106.11 & 2.1 & 0 & $0.0 O A F+10$ & $0.0 \| B E \leftarrow 00$ & AOO \\
\hline $190.0^{\circ}$ & 2.7 & 27437 & $3, I 0 B F=1.4$ & $7.151+=6$ is & 300 & 1 th. ? & 4.? & 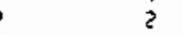 & $7.1155 \cdot n 9$ & $1+\cos A=0$. & $A 00$ \\
\hline $131,-11$ & 4.1 & คSA 51 & $2.32 \mathrm{c}+1=0 \mathrm{a}$ & $5.33 \mathrm{ht}=0.4$ & $2 n n$ & $1 \cap 5.0$ & 0.3 & jr & $3.55 \&=-08$ & $3.182 F=0 B$ & AOO \\
\hline $13 n \cdot 6$ & $5 . "$ & 75517 & $7.3625=14$ & $6.1371=0.7$ & ח ח 2 & 106.0 & B. 11 & ia & $n, \operatorname{tec} \Delta[-t, 8$ & $1.473 E \cdot 07$ & an \\
\hline 135.0 & $\leftrightarrow, 5$ & A. 12 C & $>.>71 F-1):$ & $5.223 f=0.4$ & 206 & $1 \mathrm{nn} .0$ & 10.5 & 14 & $1.9 \times[F-09$ & 1.1 IAE $=07$ & คุ० \\
\hline $13 \cdot 0$ & $\mathrm{H} .1$ & $5 \cap A ว 7$ & $1.79 A F=14$ & $4.1375=54$ & $20 n$ & 106.0 & $1 ? .4$ & $i^{k}$ & $A, 4 n \Delta F=A B$ & $1.473 \mathrm{~F}=07$ & B०0 \\
\hline $13^{n} \cdot 0^{1}$ & 17.8 & i4 12.75 & $1.6505-0.4$ & $3.35 \mathrm{hE}=0.2$ & 200 & 106.0 & 14.7 & it & 1. $2 R I F \rightarrow C T$ & $2.94 B E=07$ & 000 \\
\hline 150.1 & $1 n \cdot 8$ & $x+4,2 x$ & $1.21 \leq 15=14$ & $2.7 \div 3 \mathrm{k}-04$ & $2 n 0$ & 106.6 & 10.9 & in & 3.55 A $\varepsilon=\cap A$ & $A, t g 2 F=\cap B$ & ROA \\
\hline 130.1 & 13.5 & 16.137 & $4.215 F=n 5$ & $1.335 F=\hat{A}$ & 200 & 186.0 & $21 . n$ & 30 & $1.067 \mathrm{E}-5.7$ & $2.455 E=07$ & 800 \\
\hline $130^{\circ}=6$ & 10.2 & 1433 & $1.3: 75=0.5$ & $4.0005=05$ & 200 & 106.0 & ?5.? & s & $2 . ? 14 \mathrm{~F}=0 \mathrm{e}$ & $5.091 E=019$ & POO \\
\hline 130.5 & $1 H^{2}=0$ & 1335 & $1.7 r: C F=06$ & $1.081 E \times 05$ & 200 & 106.0 & 29. 1 & ${ }^{9}$ & $3.32 \times E=08$ & $7.637 \mathrm{E}-0 \mathrm{~A}$ & ROE. \\
\hline 135.0 & $21 . *$ & 104 & S. $+4\{E-n$ & $1.5735=06$ & 200 & 306.0 & 33.6 & 1 & $5.53 \Delta E=09$ & $5.2735-09$ & A ON1 \\
\hline $131^{\circ} .6$ & ?1. & s. & $3.24=F=04$ & $5.263 E-C . R$ & 200 & 130.0 & 37.9 & 3 & $1.1075=0 \%$ & $2.5456-58$ & P 00 \\
\hline 130.0 & 27.4 & a & $\therefore .86 \Gamma 5=\pi 7$ & $5.579 E-1 . A$ & 200 & 106.0 & $42 . n$ & 10 & $3.87 \| F=1.5$ & $9.909 E=\cap 8$ & Ron \\
\hline
\end{tabular}

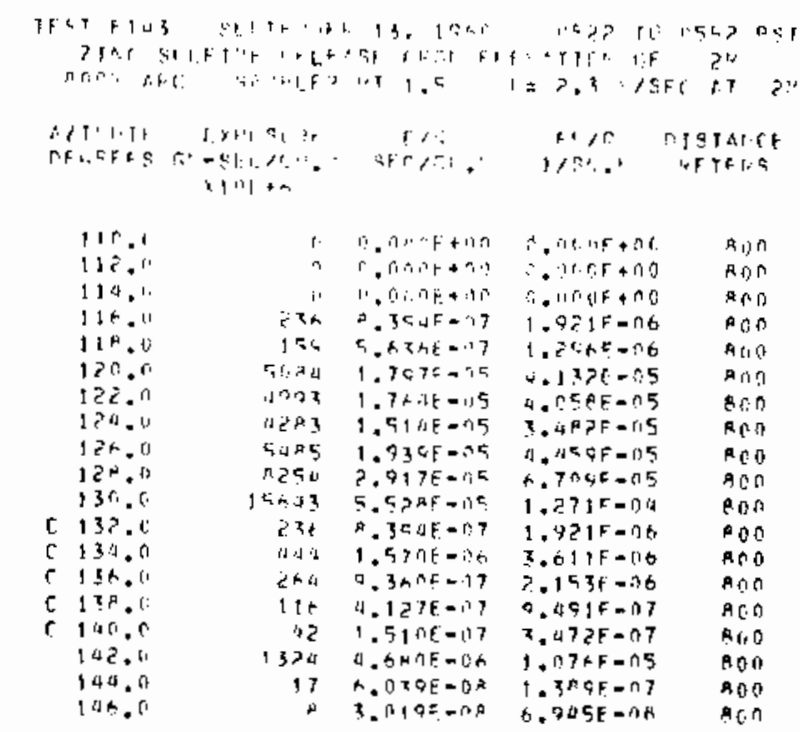

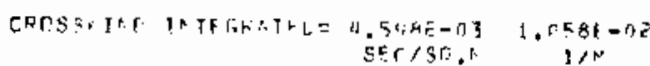

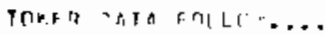

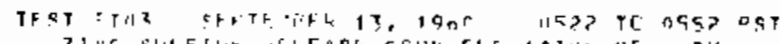

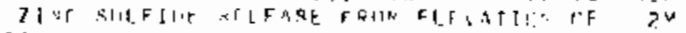

\begin{tabular}{|c|c|c|c|c|c|}
\hline $\begin{array}{l}\text { AIJ II } \\
\text { RFGH SFS }\end{array}$ & $\begin{array}{l}\text { ME IEl: } \\
\text { o Tk.t:s }\end{array}$ & 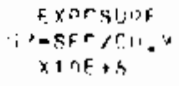 & 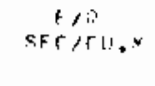 & 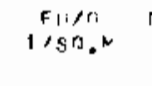 & $\begin{array}{l}\text { PISTANCF } \\
\text { MFTFRS }\end{array}$ \\
\hline 92.6 & 1.3 & 4 & 1. गट3F $=68$ & $3.2735+0.9$ & $A 00$ \\
\hline 97.0 & $2 . t$ & 4 & $1.423 \mathrm{r}=0 \mathrm{P}$ & $3.273 E-2 A$ & an \\
\hline 03.1 & 4.2 & 37 & $1.13 \times 5-17$ & $2.618 \varepsilon=07$ & 800 \\
\hline $9 ; .4$ & 6.3 & t. & $? .13 \angle E=n A$ & $4.909 E-09$ & 800 \\
\hline 930 & 4. 4 & $u$ & $1.425 E+08$ & $3.273 \mathrm{E}-0.5$ & AOO \\
\hline 9,0 & 10.5 & $\mathbf{x p}$ & $1.3527=0.7$ & $3.109 \mathrm{E}-07$ & Aก \\
\hline 72.6 & 12.4 & $2^{n}$ & $1.115 \mathrm{~F}=\mathrm{nH}$ & $1.63 \mathrm{KF}-07$ & मी० \\
\hline $4.8 \times 6$ & 14.7 & 22 & 7 ด ควบร $=98$ & $1.8 \cap 0 F=n 7$ & $80 \cap$ \\
\hline$O^{H} \cdot 10$ & $1 \mathrm{~h} . \mathrm{f}$ & h & $2.135 \mathrm{~F}=\mathrm{ng}$ & $4.9\{19 E=0)$ & Anc \\
\hline 9,11 & 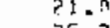 & R & $2.83 \times 5=0.9$ & 6.54 the $=0.9$ & 100 \\
\hline $9: .9$ & $25 . ?$ & 3 & $1.1075=00$ & $2.546 E-09$ & AOC \\
\hline 94.5 & 24.5 & 4 & 1. nof:E $=0 ?$ & $3.81]^{A} E=0 \%$ & 800 \\
\hline 919.0 & 33.6 & 1 & $5.53115=n 9$ & $5.273 \mathrm{E}=0 \mathrm{~B}$ & BCo \\
\hline$Q^{4}=n$ & $37 . \AA$ & 14 & $4.990, F=0.0$ & $1.145 f-07$ & 900 \\
\hline 94.0 & 42.7 & 3 & $1, J \times 7 F=0.0$ & $2.546 E-0.9$ & $\operatorname{A\theta C}$ \\
\hline
\end{tabular}

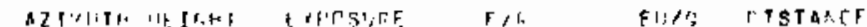

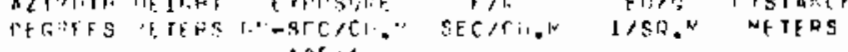
infte

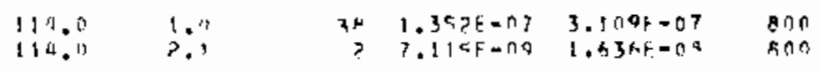

\begin{tabular}{|c|c|c|c|c|}
\hline$A Z I=1 ! T+$ H & FУFRS!JRF & $8>0$ & $f(1) / c_{0}$ & DISTARCE \\
\hline 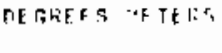 & 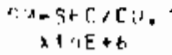 & at $t, 0+$. & $1 / 50$. & NFTFHS \\
\hline
\end{tabular}

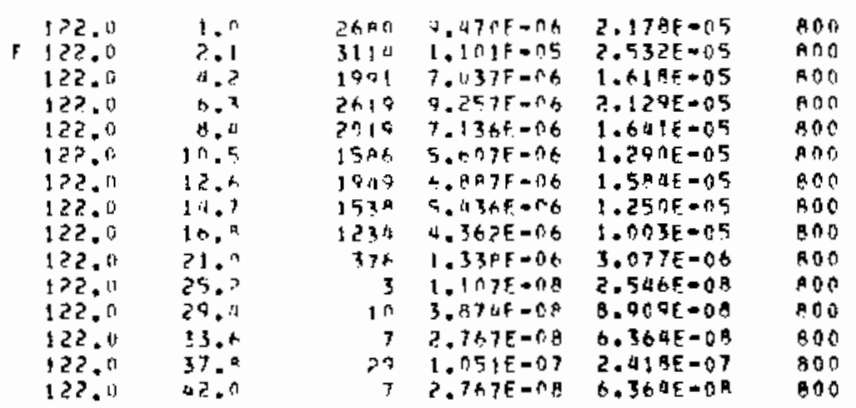

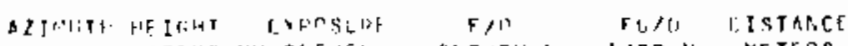

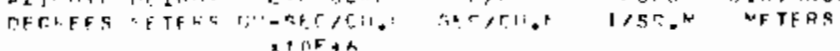

\begin{tabular}{|c|c|c|c|c|c|}
\hline $13 n .0$ & $1 . ?$ & 2711 & $3.932 f=05$ & $7 . A 93 t+05$ & $2, n$ \\
\hline 310.0 & 2.1 & $97+77$ & $\therefore .5265=015$ & ค. $109 E=05$ & pon \\
\hline 130.0 & $4 . ?$ & ming & 2. คร F.F $=145$ & $6.556 E=05$ & BAn \\
\hline 130.01 & 6.2 & $72 \times n$ & $3.1001 F=05$ & $7.315 \mathrm{E}=05$ & BO? \\
\hline 130.0 & $M .: 1$ & $x \times 5$ & C. 40दF $=05$ & $5.531 \mathrm{E}=05$ & son \\
\hline 130.0 & 14.5 & 5.91 & $2.29 n F \rightarrow \Gamma 5$ & $5.2 b \mathrm{RF}=05$ & 800 \\
\hline$c$ & $1 ? .2$ & $A, 5 \times 5$ & $2.310 .6-15$ & 5,31 \& $f=05$ & $A 00$ \\
\hline .0 & 14.7 & is 1 a th & $1,471 k=05$ & $3.384 E-05$ & Bof \\
\hline 130.6 & 10.9 & 3173 & $1.120 \mathrm{E}=015$ & $2.5955=05$ & $B \cap 0$ \\
\hline 130.0 & ? 1 ? & 1417 & $5,004 E-56$ & $1.15 \geq E=05$ & 800 \\
\hline & & $? 03$ & $7.1945=07$ & $5 E-06$ & 800 \\
\hline 13 & 29.1 & $2 n$ & $7.1945=0 \mathrm{R}$ & $1.455 E=01$ & 800 \\
\hline & 33.5 & 1 & $5,534 E=\cap 0$ & $1.273 \epsilon=0.8$ & Anr \\
\hline
\end{tabular}




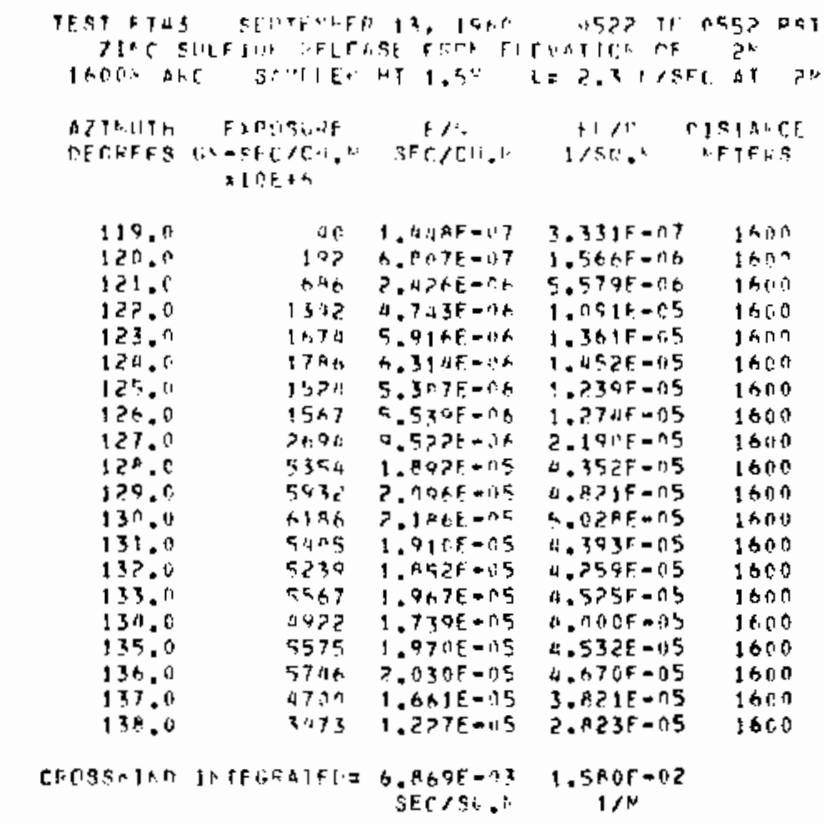

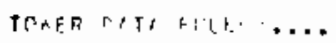

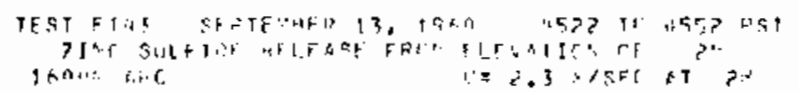

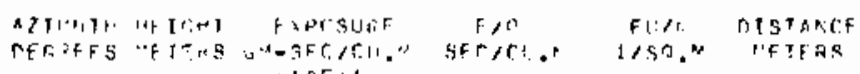
$\operatorname{lin}+\mathrm{A}$

\begin{tabular}{|c|c|c|c|c|c|}
\hline I1", & 1.5 & द? & 1.4 उन्स $=117$ & $a .22^{A} F=07$ & $10 n$ \\
\hline 114.0 & 3.1 & $n$ & $n$. InnF + nn & $n .+n n E E+n)$ & 100 \\
\hline 114.0 & 6.7 & 1 & $5.5711=09$ & $3.2 B 1 E-A B$ & the \\
\hline 110.0 & 7.7 & 9 & n. nof 5 , no & n. nOOH $\rightarrow 00$ & 160 \\
\hline 114.0 & 12.4 & 75 & $8.91 \times 5-n$ & $2.0506=07$ & 160 \\
\hline 114.0 & 15.5 & $2^{\mathrm{r}}$ & $T, 242 F-0 ?$ & $1.66 \mathrm{hF}=0.7$ & 160 \\
\hline 114.0 & $1 \% .5$ & $n$ & 3.510 C.F + 00 & $0.500+400$ & $16 n$ \\
\hline 11 ts. & 21.7 & $1 \%$ & U1. $457 F=0 P$ & $1.025 F-07$ & \\
\hline 114.6 & 34.7 & $\stackrel{n}{n}$ & {$[1 ., 30 \cap E+00]$} & $0.00 \cap E+00$ & 60 \\
\hline 114.0 & 31.7 & 3 & $11905=08$ & $0.035 \mathrm{~F}-108$ & bo \\
\hline & & & $4.44 Q E=n Q$ & & \\
\hline
\end{tabular}

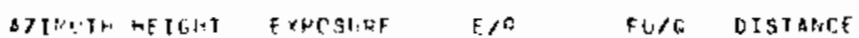

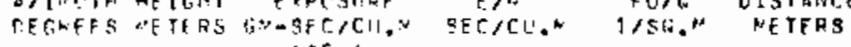

\begin{tabular}{|c|c|c|c|c|c|}
\hline 122.0 & 1,5 & $112 \pi$ & $3.961 F=06$ & $9.1105-06$ & 1600 \\
\hline 122,9 & 3,1 & 1148 & $\begin{array}{l}1-2345=n \theta \\
\therefore=179 E-10\end{array}$ & $\begin{array}{l}9.73 \mathrm{BE}=0 \mathrm{BS} \\
1.03 \mathrm{OE}=05\end{array}$ & $\begin{array}{l}1600 \\
1600\end{array}$ \\
\hline , i? & 3.3 & 1148 & $\therefore$ ijol $F=n s$ & $9.5416=06$ & 1600 \\
\hline $12 ? .11$ & 12.4 & 1054 & $3.727 \mathrm{~F}=0 \mathrm{~b}$ & $9.5 \times 2 F=1,6$ & 1000 \\
\hline 12 & 15 & 1307 & 1.91 of $=n h$ & $1.131 E=0 ?$ & $1 \in \cap n$ \\
\hline 12 & 18.5 & A द 0 & $2.9 A \triangle E=06$ & 6. A 1 GF $=\mathrm{CH}$ & $100 \mathrm{n}$ \\
\hline & 21,7 & 317 & $? .535 \mathrm{~F}=0 \mathrm{t}$ & $5.83 \cap F=06$ & $16 n 0$ \\
\hline 12 & $24 . R$ & 374 & $1.300 \mathrm{E}=0.6$ & - nI tE $=n h$ & 1600 \\
\hline & 31.6 & 71 & $=51<F+r 7$ & $.794 E-07$ & 1600 \\
\hline 16 & 37.2 & 1 & $1.4905-1.3$ & $1.035 E-0 A$ & $\sin 0$ \\
\hline & & $n$ & i. & $n, n \in C, a+00$ & 1600 \\
\hline
\end{tabular}

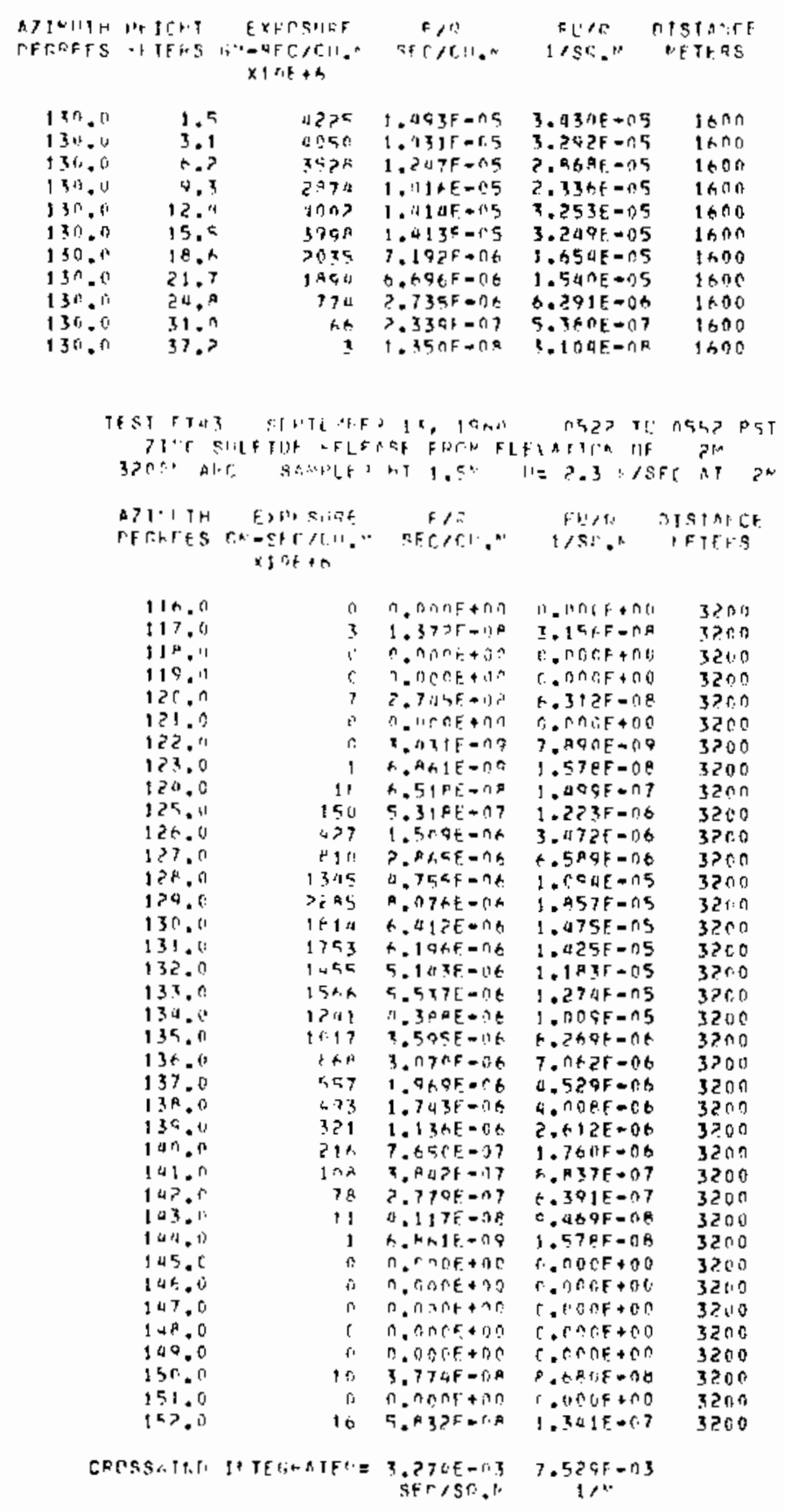




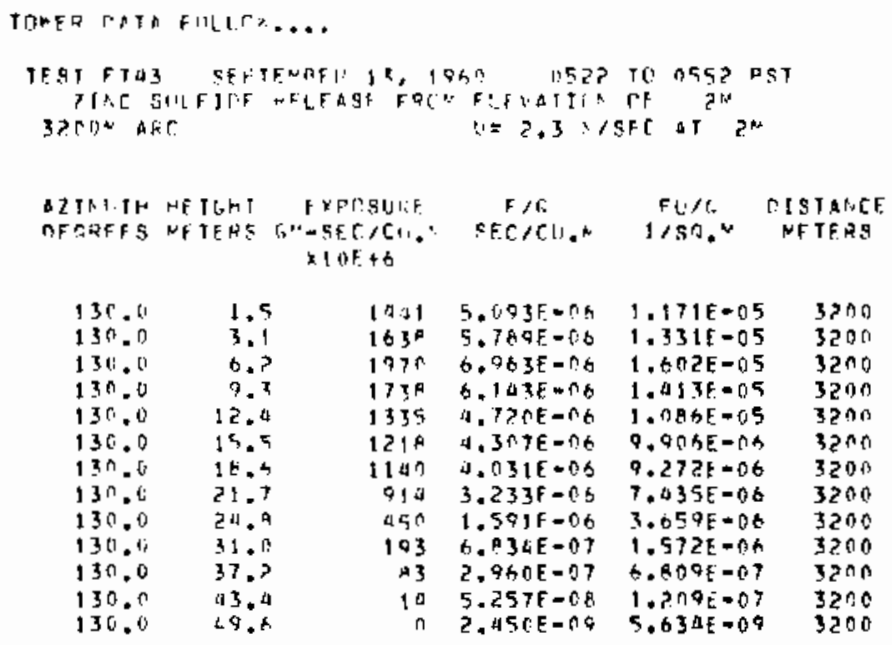


30-SERIES EXPERIMENT: 44 (FT-44)

GROUNO LEVEL AND TOWER SAMPLING 200-3200 M ARCS. EVERY OTHER

GROUNO LEVEL SAMPLER WAS ROTATEO 1 gO DEGREES, 5O AS TO FACE AWAY

FROM THE TRACER SOURCE.

TEST FT44 SEWTEAFR, 27,1960 OU57 RO 0557 PST ZINC STLLFIDE STFLESE FRON FLEVATICN OF ZN

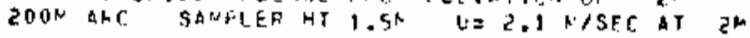

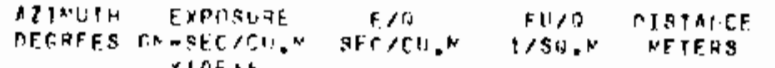
$x \in \cap+h$

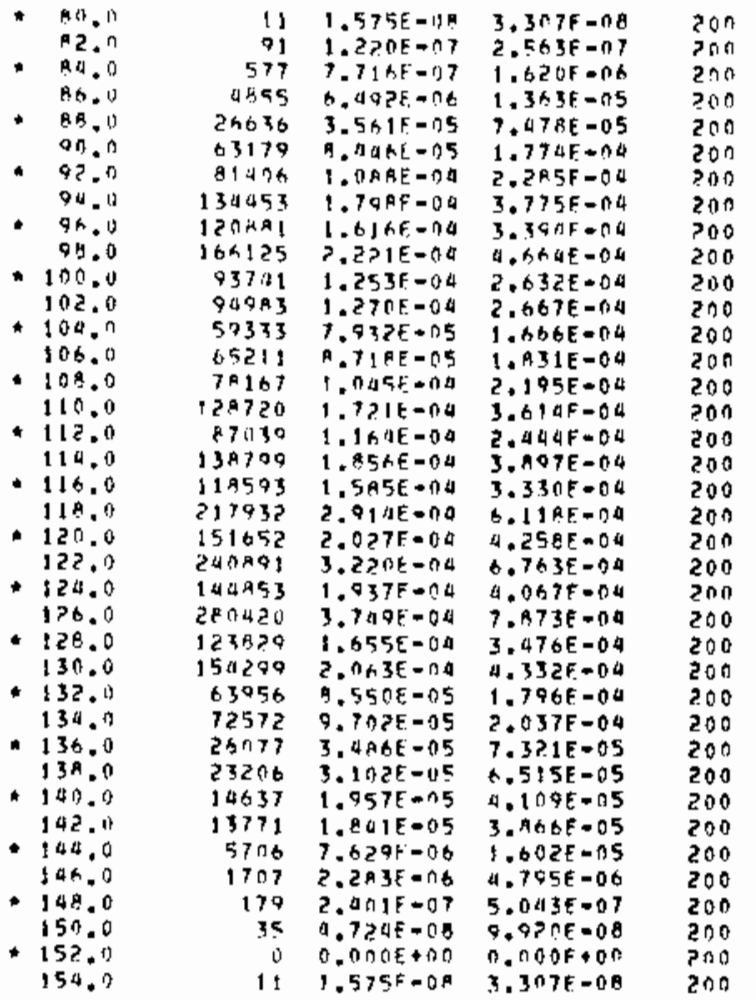

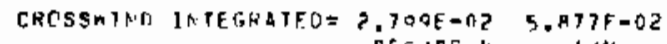
Sf $t>2 \sqrt{2}$

$1 / 2$

TOWSR TATA RIJLLTX....

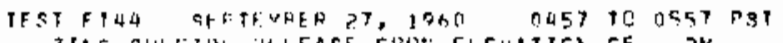

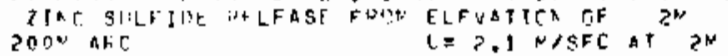

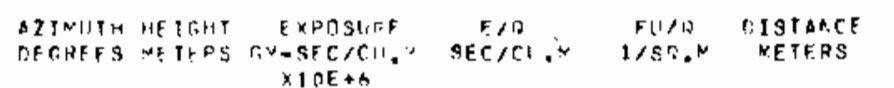

\begin{tabular}{|c|c|c|c|c|}
\hline QR. & 9.7 & $\begin{array}{l}17+905 \\
615 p ?\end{array}$ & $\begin{array}{l}1.83(1, E-\cap 4 \\
A-233 F=05\end{array}$ & $\begin{array}{l}\text { 3. Aa } 45-04 \\
729 E=04\end{array}$ \\
\hline 98.0 & $\begin{array}{l}1.11 \\
2.7\end{array}$ & $\begin{array}{l}615 \mathrm{~A} 2 \\
5,5169\end{array}$ & $\begin{array}{l}1.253 t=05 \\
6.034 F=1: 5\end{array}$ & 1.2 B AE $=04$ \\
\hline 8.0 & 4.1 & $467 \div 1$ & $6.754 F=.15$ & $1.313 \mathrm{E}=04$ \\
\hline$F .0$ & $5 . \pi$ & 40778 & $5.452 E=115$ & $1.1 \triangle 5 E=04$ \\
\hline 0 & 促 & 49772 & $A E=015$ & $1.3974=04$ \\
\hline 1 & 8,1 & 31173 & $4.16 A 5=05$ & $52 \xi-c 5$ \\
\hline 99.4 & 9.7 & 15142 & $2.0245=05$ & $4.251 E-05$ \\
\hline 98,0 & 10.8 & $135>t$ & 1. AOAE -05 & QAE- CS \\
\hline .0 & 13.5 & 3 A 30 & $5.121 F-06$ & $1.075 E-05$ \\
\hline .0 & 16.7 & 1 ate & 97 a -06 & $145 E=06$ \\
\hline .0 & & $2+2$ & $50 t E-07$ & $3 E-07$ \\
\hline & 2 & $4 \mathrm{n}$ & $493 E=0 \mathrm{~A}$ & $45-07$ \\
\hline$=0$ & 20 & 14 & $9795=188$ & $156 \mathrm{E}-0 \mathrm{~B}$ \\
\hline & $27 . n$ & 3 & $4.567 E=10$ & $9.591 E=09$ \\
\hline
\end{tabular}

200
200
200
200
200
200
200
200
200
200
200
200
200
200
200

$13 n .0$

130.0

130.0

130.0

130.0

130.0

130.0

130.0

130.0

130.0
130.0

130.0

0.7
1.9
2.7
4.1
5.11
6.9
8.1
9.9
10.8
13.5
16.2
18.9
21.5
24.3
27.5

EXPDSULF $x \neq 0 \mathrm{E}+6$

$\begin{array}{ll}106.0 & 0.7 \\ 106.0 & 1.4 \\ 10.00 & 2.7 \\ 106.0 & 4.1 \\ 106.0 & 5.4 \\ 106.0 & 6.9 \\ 106.0 & 9.1 \\ 106.0 & 9.1 \\ 106.0 & 10.9 \\ 104.0 & 13.5 \\ 106.0 & 16.2 \\ 106.0 & 10.0 \\ 106.0 & 21.4 \\ 106.0 & 24.3 \\ 100.0 & 27.0\end{array}$

$$
\begin{array}{rl}
71303 & 9 \\
38421 & 5 \\
31242 & 4 \\
39206 & 5 \\
34231 & 9 \\
34397 & 4 \\
35957 & 4 \\
22566 & 3 \\
23970 & 3 \\
13048 & 1 \\
8690 & 1 \\
2470 & 3 \\
667 & 8 \\
231 & 3 \\
46 & 50
\end{array}
$$

$E>0$ EU/O OISTANCE Cr.t.

$\sin 20$

TERS

$9.532 F-05$
$5.137 F-05$ $4.177 \mathrm{E}-15$ $5.242 E-15$

$2.002 \varepsilon-04$ 1. $079 E-04$ $0.771 E-05$ $1,101 E-04$ Q. B OEE-05 $9.610 E=05$
$1.022 E=04$ $1.010 E-04$ $0.336 E-05$ $0.702 E-05$ $3.663 E=05$ $3.663 E=05$
$2.437 E=05$ $6.936 \mathrm{E}=06$ $1.073 E-06$

6. $.90 E=01$ $1.311 E=0$ :

200
200
200
200
200
200
200
200
200
200
200
200
200
200
200

DINIITH HFIGHI EXPTSURE F/O EUAO TISTANTF

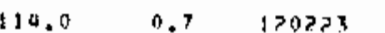

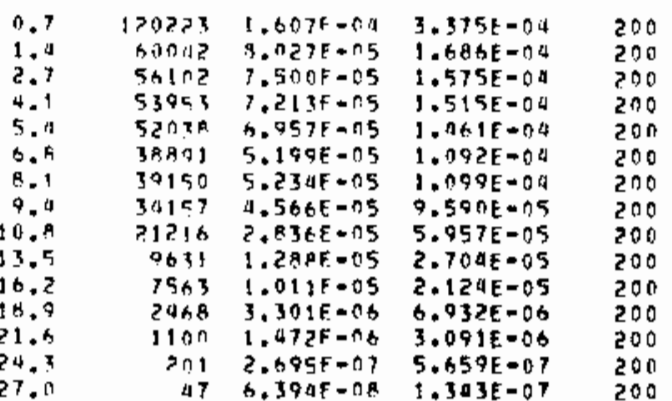
27.

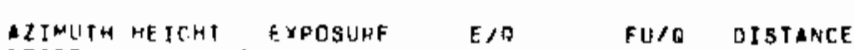

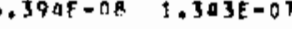

200 $\times 1$ (IE, +6

$$
\begin{aligned}
& \text { 1月61กร } \\
& 111137 \\
& \begin{array}{r}
116015 \\
90548
\end{array} \\
& 79776 \\
& \text { A7०र } 4 \\
& 56163 \quad 7,509 F=05 \\
& 77234 \quad 3.601 \mathrm{~F}=05 \\
& 6331 \quad 0.465 F=06
\end{aligned}
$$

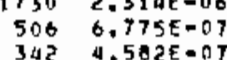$$
5.927 E-05
$$$$
36956 \quad 9.941 \mathrm{E}-05
$$$$
11912 \quad 1.593 E=05
$$$$
1730 \quad 2.31 \mathrm{aE}=0
$$
FU/O DISTANCE
I/SON METERS

$525 E-04$

$3.120 E-04$ $3+257 E-04$ ट. $542 \mathrm{E}=04$ 2.240E-04 $2.443 E=09$ $1.2495=04$ $1.577 E=04$ $1.038 E=04$ $7.646 E=05$ $3.344 \mathrm{E}=05$ 1.770E-05 4. $959 E=06$ $-07 \quad 1.023 E-06 \quad 200$ 37

EXPП 91,0

\begin{abstract}
E 1 í
\end{abstract}
ELIO DISTANCE 1/SR.N NETERS $\times 10 F+6$
0

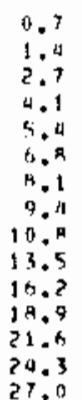

$$
\begin{aligned}
& 11092 \text { ? } \\
& 723 \times 9 \quad 0.67 \mathrm{EE}-0.5 \quad 2.032 \mathrm{E}=04 \\
& 57144 \text { 1.640E-05 1.6OAE }-04 \\
& 0.175 \text { } 5.37 A E=35 \quad 1.12 B E=04 \\
& 1725 R \text { 2.30TE-05 A.845E-05 } \\
& 26114 \text { 3. यह } 3 E-06 \quad 7.333 E-0 B \\
& \begin{array}{rll}
12 \cap 4 & 1.610 E-06 & 3.382 E=06 \\
2 A ? & 3.775 F=07 & 7.920 E=07
\end{array}
\end{aligned}
$$$$
\text { 1. } 4 \text { CAF }-00 \quad 3.116 E=01
$$$$
44533 \quad 5.954 F-n 5 \quad 1.250 E-04
$$$$
44372 \quad 5.925 E-05 \quad 1.204 E=00
$$$$
\begin{array}{lll}
\triangle 0175 & 5.37 A E-75 & 1.12 B E-04 \\
31279 & 9.176 F-05 & 8.771 E-05
\end{array}
$$$$
0071 \quad 8.117 E-66 \quad 1.704 E=05
$$$$
1204 \text { 1.6105-06 3.3A2E-06 }
$$$$
111 \text { 1.492E-07 } 3,133 F=07
$$

200
200
200
200
200
200
200
200
200
200
200
200
200
200
200




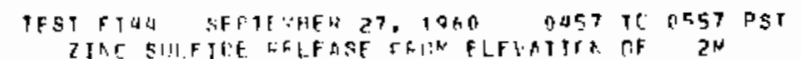

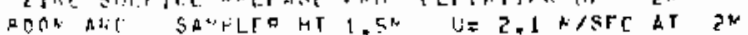

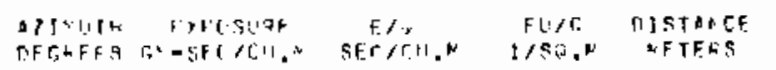
XI $\cap F+n$

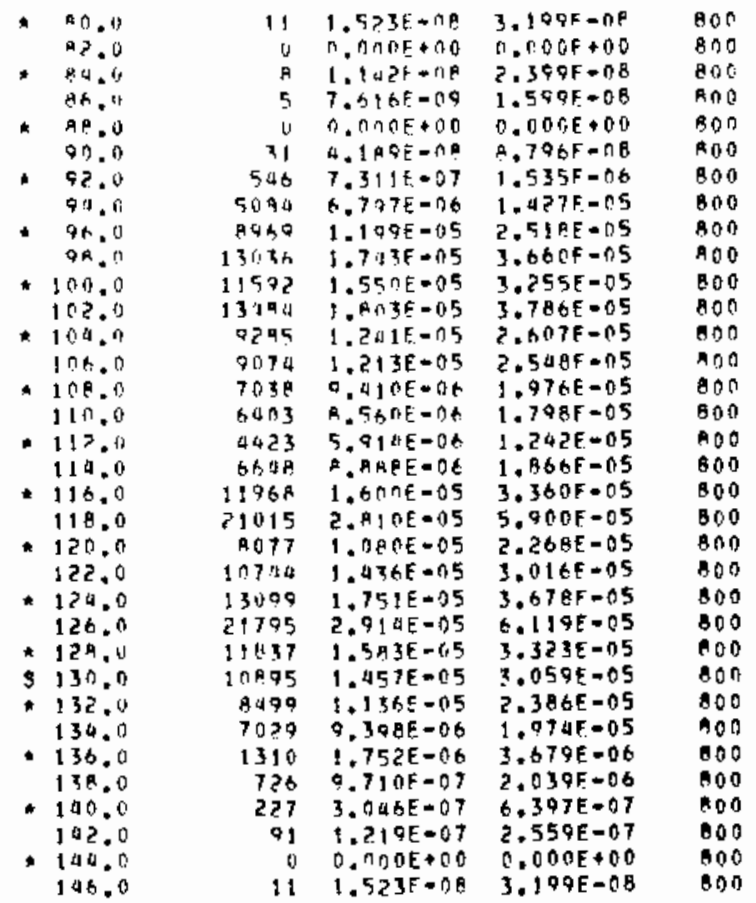

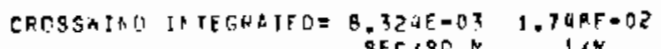

TBHFR DATA FOLLL.....

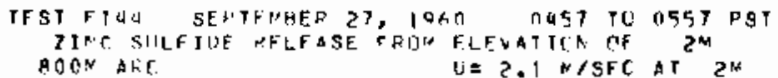

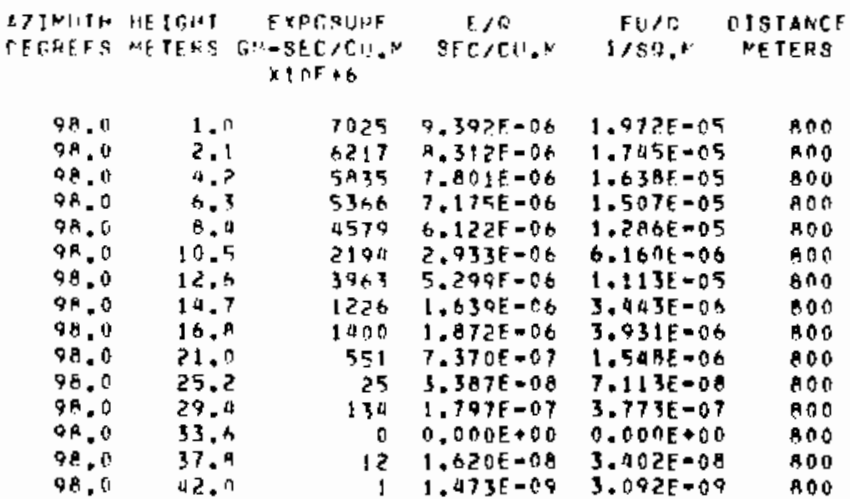

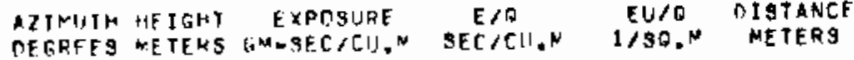
$x \in$, If $+b$

\begin{tabular}{|c|c|c|c|c|}
\hline $\begin{array}{l}106.0 \\
106.0 \\
106.0 \\
100.0 \\
106.0 \\
106.0 \\
106.0 \\
106.0 \\
106.0 \\
106.0 \\
106.0 \\
106.0 \\
106.0 \\
106.0 \\
106.0\end{array}$ & $\begin{array}{l}1.0 \\
2.1 \\
4.2 \\
6.3 \\
0.4 \\
10.5 \\
12.4 \\
14.7 \\
16.9 \\
21.0 \\
25.2 \\
29.11 \\
33.6 \\
37.8 \\
42.0\end{array}$ & $\begin{array}{l}5146 \\
5379 \\
5155 \\
2734 \\
4726 \\
6513 \\
4435 \\
5781 \\
5548 \\
2679 \\
1449 \\
666 \\
338 \\
52 \\
52\end{array}$ & $\begin{array}{l}3.800 F=06 \\
7.192 \mathrm{E}=06 \\
6.892 \mathrm{E}-06 \\
3.656 \mathrm{E}=06 \\
6.319 \mathrm{E}=06 \\
8.708 \mathrm{E}-06 \\
5.929 \mathrm{E}=06 \\
7.702 \mathrm{E}=06 \\
7.41 \mathrm{EE}=06 \\
3.552 \mathrm{E}=06 \\
1.93 \mathrm{AE}=06 \\
9.909 \mathrm{E}=07 \\
1.521 \mathrm{E}=07 \\
7.069 \mathrm{E}-0 \mathrm{O} \\
1.069 \mathrm{~F}=08\end{array}$ & $\begin{array}{l}1.045 E-05 \\
1.510 E-05 \\
1.047 E=05 \\
7.677 E-06 \\
1.327 E-05 \\
1.029 E-05 \\
1.245 E-05 \\
1.626 E=05 \\
1.556 E-05 \\
7.523 E-06 \\
4.079 E-06 \\
1.071 E=06 \\
9.094 E=07 \\
1.484 E=07 \\
1.404 E=07\end{array}$ \\
\hline
\end{tabular}

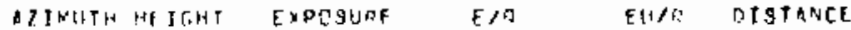
CFFEFS IETERS ISA-SEC,CL, SEC/CU,N 1/SD,N METFRS $x 1$ ก +5

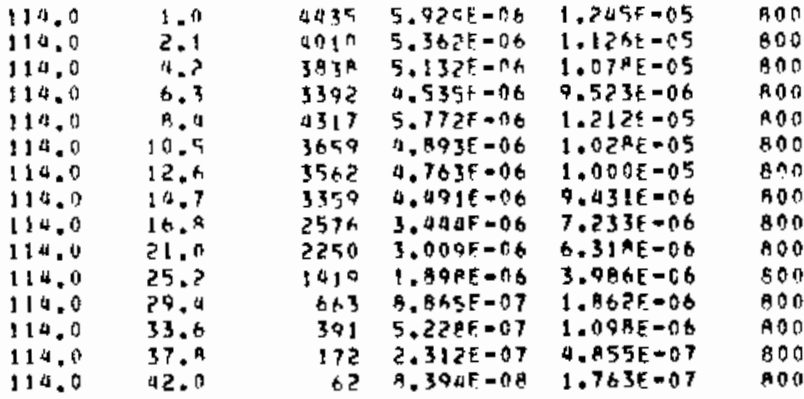

MTENITH HEIGHT EXPOGURE FAO EUTO DISTANCE DERAEFS METERS THASECICU, SECICU.N 1/9O.M METERS $\times 10 E+6$

\begin{tabular}{|c|c|c|c|c|}
\hline $\begin{array}{l}122.0 \\
122.0 \\
122.0 \\
122.0 \\
122.0 \\
122.0 \\
122.0 \\
122.0 \\
122.0 \\
122.0 \\
122.0 \\
122.0 \\
122.0 \\
122.0 \\
122.0\end{array}$ & $\begin{array}{l}1.0 \\
2.1 \\
4.2 \\
6.3 \\
0.4 \\
10.5 \\
12.6 \\
14.7 \\
16.0 \\
21.0 \\
25.2 \\
29.4 \\
33.8 \\
37.9 \\
42.0\end{array}$ & $\begin{array}{l}6987 \\
5492 \\
6967 \\
7244 \\
9103 \\
3724 \\
9312 \\
6113 \\
4114 \\
4101 \\
2046 \\
1027 \\
391 \\
371 \\
215\end{array}$ & $\begin{array}{l}9.200 E=06 \\
7.330 E=06 \\
9.151 F=06 \\
9.685 E=06 \\
1.217 E=05 \\
4.979 F=06 \\
1.111 E=05 \\
8.170 E=06 \\
5.500 F=06 \\
5.483 E=06 \\
2.736 E=06 \\
1.370 E=06 \\
5.095 F=07 \\
9.963 F=07 \\
2.086 E=07\end{array}$ & $\begin{array}{l}1.934 E=05 \\
1.539 E=05 \\
1.929 E=05 \\
2.039 E=05 \\
2.556 E=05 \\
1.046 E=05 \\
2.334 E=05 \\
1.716 E=05 \\
1.155 E=05 \\
1.151 E=05 \\
5.746 E=06 \\
2.045 E=06 \\
1.070 E=06 \\
1.042 E=06 \\
6.061 E=07\end{array}$ \\
\hline
\end{tabular}

\begin{tabular}{|c|c|c|c|c|c|}
\hline $\begin{array}{l}\text { AZJMLIT } \\
\text { OE GRE }\end{array}$ & $\begin{array}{l}\text { Iff Jinl } \\
\text { P TEPS }\end{array}$ & 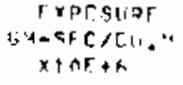 & $\begin{array}{c}\text { e/ } \\
\text { ger.ri... }\end{array}$ & $\begin{array}{c}E U / 6 \\
1 / 5 r_{i} .\end{array}$ & $\begin{array}{l}\text { DISTANCF } \\
\text { MFIERS }\end{array}$ \\
\hline $\begin{array}{l}1310.0 \\
130.0 \\
130.0 \\
130.0 \\
130.0 \\
130.0 \\
130.0 \\
130.0 \\
130.0 \\
130.0 \\
130.0 \\
130.0 \\
130.0 \\
130.0 \\
130.0\end{array}$ & $\begin{array}{l}1.0 \\
2.1 \\
4.2 \\
0.7 \\
4.9 \\
10.5 \\
12.4 \\
14.7 \\
10.4 \\
21.0 \\
25.2 \\
29.4 \\
33.4 \\
37.8 \\
42.0\end{array}$ & $\begin{array}{l}557 \\
7 \ln 6 \\
4370 \\
1973 \\
5090 \\
5310 \\
5401 \\
4049 \\
2156 \\
1960 \\
889 \\
639 \\
312 \\
245 \\
72\end{array}$ & $\begin{array}{l}7.453 F=07 \\
9.5034=06 \\
9.25 C E=06 \\
6.650 F=06 \\
7.313 E=06 \\
7.10 C F=06 \\
7.2215=06 \\
5.414 E=06 \\
2.803 F=06 \\
2.487 E=06 \\
1.188 E=06 \\
9.556 E=07 \\
4.182 E=07 \\
3.280-07 \\
9.710 F=08\end{array}$ & $\begin{array}{l}1.565 E=06 \\
1.995 E=05 \\
1.775 E=05 \\
1.396 E-05 \\
1.534 E=05 \\
1.091 F=05 \\
1.516 E=05 \\
1.137 E=05 \\
6.054 E-06 \\
5.223 F=06 \\
2.096 E-06 \\
1.797 E=06 \\
8.783 E-07 \\
6.896 E=07 \\
2.041 E=07\end{array}$ & $\begin{array}{l}A 00 \\
A \cap 0 \\
A 00 \\
800 \\
800 \\
800 \\
B 00 \\
900 \\
600 \\
800 \\
800 \\
800 \\
800 \\
800 \\
800\end{array}$ \\
\hline
\end{tabular}




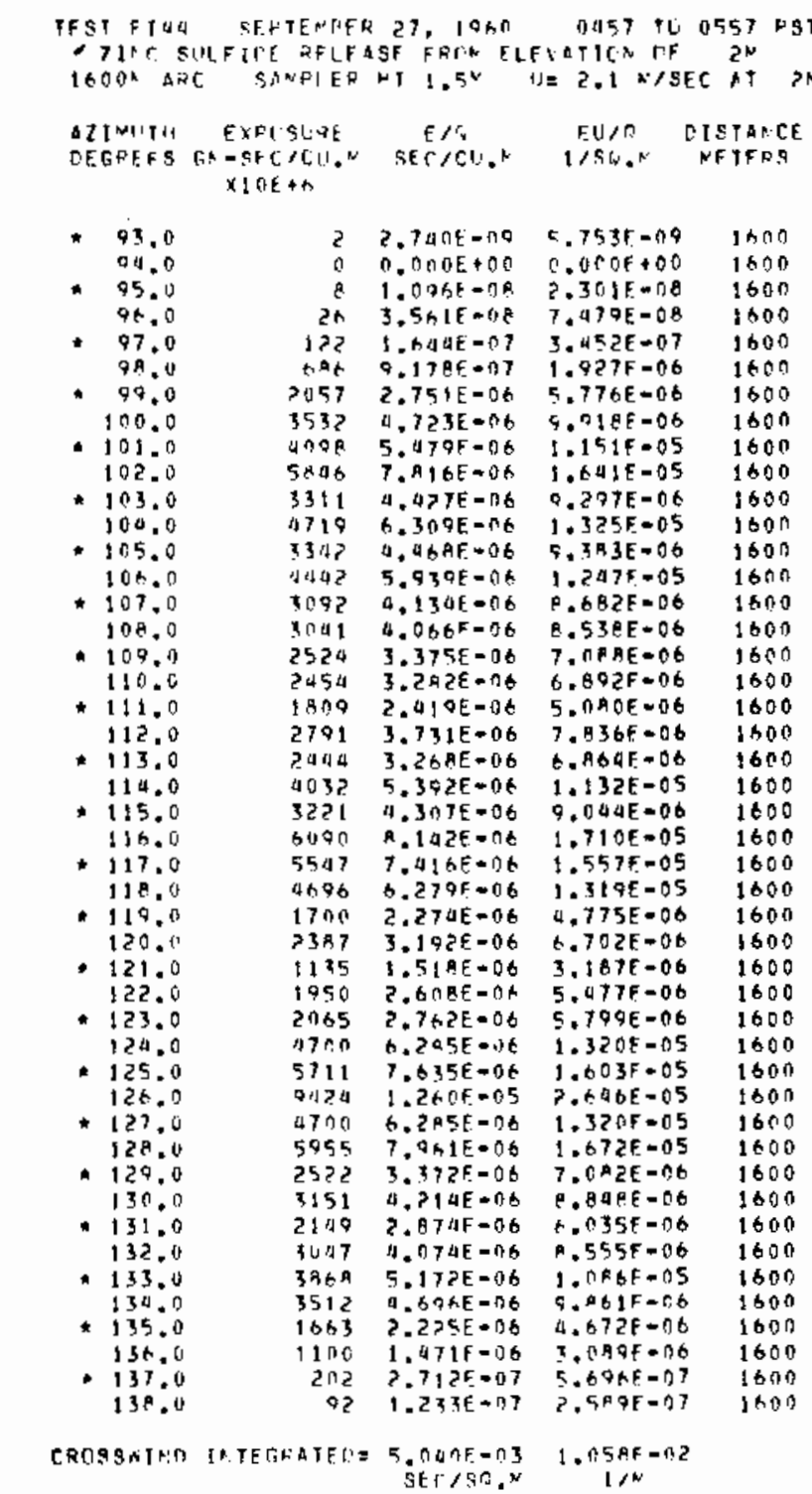

TORER TATA FILLLC:....

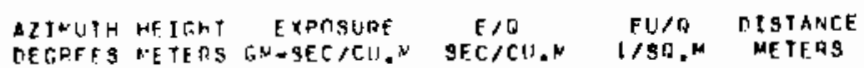
$\times 10 \mathrm{E}+\mathrm{A}$

\begin{tabular}{|c|c|c|c|c|c|}
\hline 106.0 & 1.5 & 7540 & $3.407 E=06$ & $7.155 E-06$ & 1600 \\
\hline$i^{n}$ & 3. & 2455 & $3,283 \mathrm{E}=06$ & $0.895 E=06$ & 1600 \\
\hline 100.0 & 0.2 & 2351 & $3.194 F=06$ & $6.601, E-06$ & 1600 \\
\hline 106.0 & 9.3 & 3120 & $4.1775-06$ & $0.771 \mathrm{E}-06$ & 1600 \\
\hline 106.0 & 12.8 & 2218 & $2.967 \mathrm{E}=06$ & $6.230 E-06$ & 1600 \\
\hline 106.0 & 15.5 & $255 \%$ & $3.421 E=06$ & 7.18 \&F -06 & 1000 \\
\hline 106.0 & 18.6 & 2745 & $3.004 E-16$ & $1.98 A E-06$ & 1600 \\
\hline 106.0 & 21 & $206 \mathrm{P}$ & $2.7667-00$ & $5 . A Q B E=D 6$ & 1600 \\
\hline 106.0 & $20, R$ & 1231 & $1,647 E-16$ & $3.45 B E-06$ & 1600 \\
\hline 100. & 31.0 & 662 & $8.657 F=07$ & $1.800 E=06$ & 1600 \\
\hline 106.0 & $37 . ?$ & 209 & $2.801 F-07$ & $5.082 E=07$ & 1000 \\
\hline 106.0 & 43 & 36 & $4.921 E=08$ & $1.033 \mathrm{~F}=07$ & 1600 \\
\hline 100 & 49 & 11 & $1.561 \mathrm{~F}-08$ & $3.2790-08$ & 1600 \\
\hline 10 & 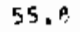 & 0 & $0,000 E+0 \mathrm{~A}$ & 0, DOOE $\$ 00$ & 1600 \\
\hline
\end{tabular}

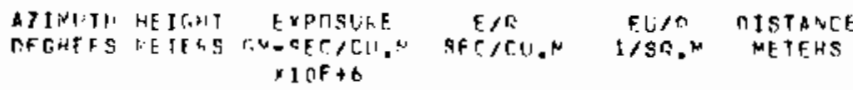

\begin{tabular}{|c|c|c|c|c|c|}
\hline 114.0 & 1.5 & 409 & $5.4005-n$ ? & $1.151 E=06$ & $160 \%$ \\
\hline 114.0 & 3.1 & 1339 & $1.790 \mathrm{cF}-06$ & $3.760 t-06$ & 1500 \\
\hline 114.0 & 0.2 & $19+9$ & $2.553 F=n 6$ & $5.361 \mathrm{E}-06$ & 1enn \\
\hline 114.0 & 9.7 & If 15 & $2.427 \mathrm{~F}-0 \mathrm{G}$ & $5.097 \mathrm{E}=06$ & 1600 \\
\hline 119.0 & [2. $t]$ & A 71 & $1.169 \mathrm{E}=00$ & $2.455 E=0 \mathrm{~A}$ & 1600 \\
\hline $1 \pm 4.0$ & 15.5 & 1538 & $2.0545=06$ & $a, 314 E=06$ & $\operatorname{lon} 6$ \\
\hline 114.01 & $1 B . t$ & 720 & $0.033 F-07$ & $2.023 \mathrm{E}-116$ & inno \\
\hline $11 a, n$ & 21.7 & 1203 & $1.1296=0 \mathrm{~b}$ & $3.631 \mathrm{E}=06$ & 1600 \\
\hline 114.0 & 24.9 & 996 & $1.332 f-60$ & $2.79 B E=06$ & 16010 \\
\hline 114.0 & 31.1 & 622 & $0.327 E-07$ & $1.749 \mathrm{E}=0 \mathrm{6}$ & 1600 \\
\hline 114.0 & 37.2 & $4=4$ & $6.082 E-117$ & $1.277 E=06$ & 1600 \\
\hline 114.0 & 43,4 & 215 & Z.ATIE-0? & $6.041 \mathrm{E}=07$ & 1600 \\
\hline 114.0 & 49.6 & 45 & $6.141 F=08$ & $1.290 \mathrm{E}=07$ & 1000 \\
\hline 114.0 & $55 .{ }^{\circ}$ & 7 & $9.36 \mathrm{FF}=09$ & $1.867 \mathrm{E}=0 \mathrm{~A}$ & $1+00$ \\
\hline 116.0 & 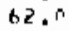 & a & $00 \cap F+00$ & 0, OOOE +00 & 1600 \\
\hline
\end{tabular}

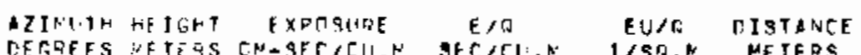

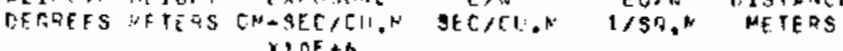

\begin{tabular}{|c|c|c|c|c|c|}
\hline 122.0 & 1.5 & A24 & $1.1035-06$ & $2.316 E-06$ & 1600 \\
\hline 127.0 & 3.1 & 11 ib & $1.493 E-06$ & $3.135 E=06$ & 1600 \\
\hline 122.0 & 0.2 & $10 R 9$ & $1.457 E=0 E$ & $3.059 \mathrm{E}=06$ & 1600 \\
\hline 122.0 & 9,3 & $11 \times 4$ & $1.516 E=06$ & $3.185 E-06$ & 1600 \\
\hline 122.0 & 12. & 1345 & $1.065 E-06$ & $3.917 \mathrm{E}=0 \mathrm{~b}$ & 1600 \\
\hline 122,0 & 15.5 & 1419 & $1,898 E-06$ & $3.985 E=0 \mathrm{n}$ & $1 \in 00$ \\
\hline 127.0 & $1 \mathrm{~F}$ & 1409 & $1.8 \triangle D E-0.6$ & $3.957 E-06$ & 1000 \\
\hline 122.0 & 21.7 & 1330 & $1.790 E=06$ & $3.76 \cap E-06$ & 1600 \\
\hline $1<2.0$ & 24.9 & $102 B$ & $1.3755=06$ & $Z . B \triangle A E=06$ & 1600 \\
\hline 122.0 & $31 . n$ & 941 & $9.9171=07$ & $Z . \cap B 3 F=06$ & 1800 \\
\hline 122.0 & 37.2 & 3 व & $4.580 E-07$ & $9.01 \times 1 \times-07$ & 1600 \\
\hline 122.0 & 43 & 95 & $1.274 f-07$ & $2.67 \delta E=07$ & 1600 \\
\hline 122 & 49 & 18 & 2. $.99 E-O B$ & $5.246 \mathrm{E}=O \mathrm{~A}$ & 1600 \\
\hline 122 & & 0 & $.041 E=09$ & $1 E G E-09$ & inOrj \\
\hline & & & not & $00 \mathrm{E}$ & \\
\hline
\end{tabular}

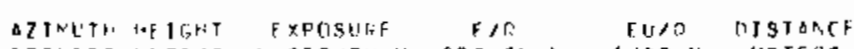

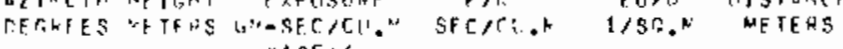

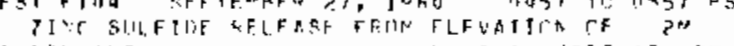

\begin{tabular}{|c|c|}
\hline 130.4. & 1,5 \\
\hline 130,0 & 3.1 \\
\hline 130.0 & $0 . ?$ \\
\hline 136.0 & \\
\hline 130.0 & 12.4 \\
\hline $13 n .0$ & 15.9 \\
\hline $43 \pi .0$ & $1 \mathrm{A.A}$ \\
\hline 130.0 & 21.7 \\
\hline 130.0 & $34 . \mathrm{P}$ \\
\hline 130.0 & $31 . r$ \\
\hline 130.0 & $37 . ?$ \\
\hline 136.0 & 43.4 \\
\hline 130.0 & 48.0 \\
\hline 130.0 & \\
\hline i3 & \\
\hline
\end{tabular}
$\times 1 \cap F+6$ 


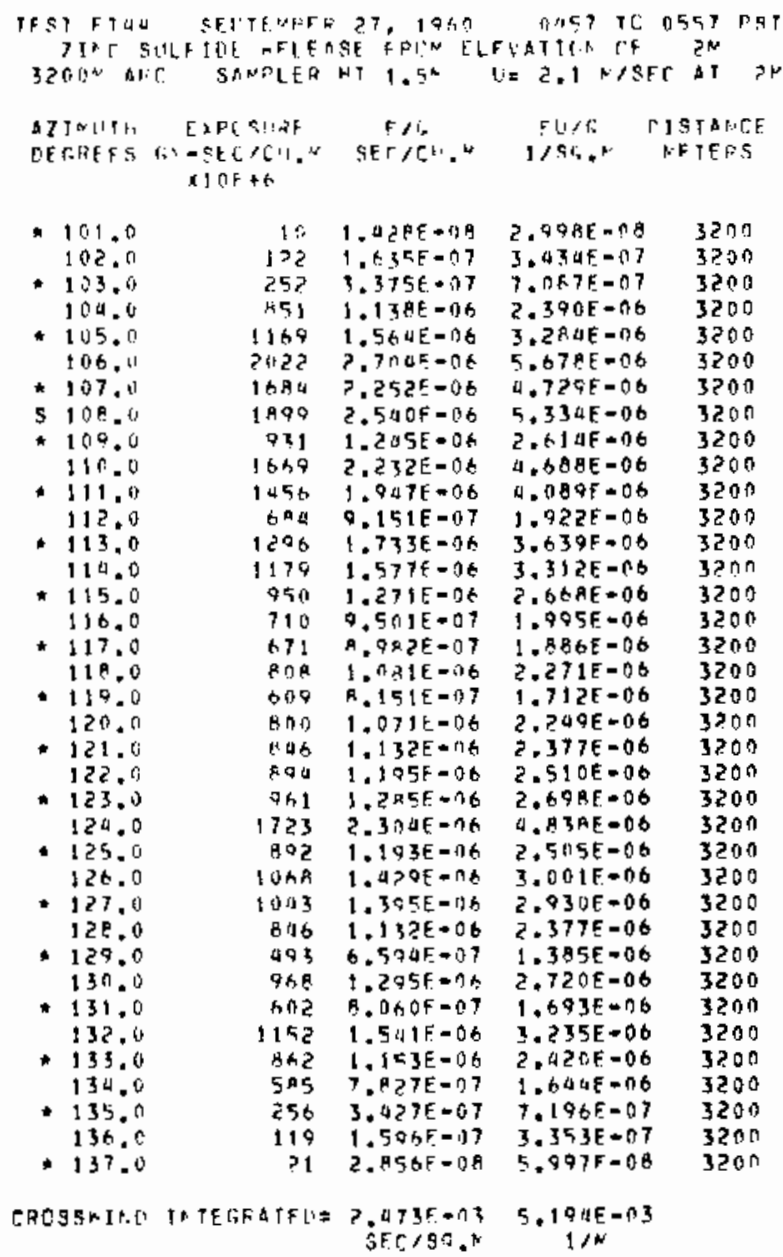

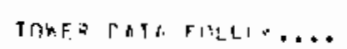

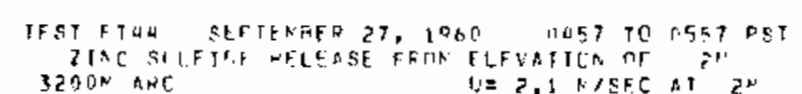

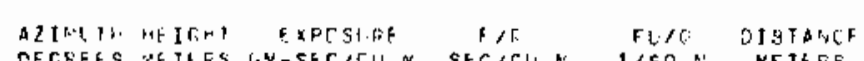

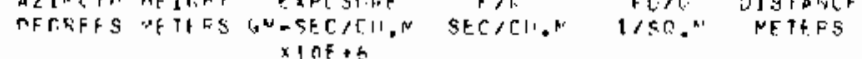

\begin{tabular}{|c|c|c|c|c|c|}
\hline 100.0 & 1.5 & $15 ? 2$ & $2.03+E=C E$ & $4.7 .15 E=06$ & 3200 \\
\hline 106.0 & 3,1 & 1677 & $2.242 E+0 t$ & $4.70 A_{1}=\{6$ & 3270 \\
\hline 100.8 & 6.2 & 1736 & $2.322 F=0 b$ & $a .876 F-0 b$ & 3200 \\
\hline 106.0 & 9.1 & 1353 & $1, D^{2} 1$ D $E=c t$ & $3.8 \cap 1 E-06$ & उशी \\
\hline 166.0 & 12,0 & $11 \% 0$ & $1.565 E-00$ & 3. $. P A B E=\cap B$ & 3200 \\
\hline $10 \mathrm{~h} . \mathrm{C}$ & 15.5 & $1,5=3$ & 1. $\triangle A P E=56$ & $2.757 E-016$ & 3200 \\
\hline 100.0 & $1 \%$ & 1276 & $1.7 D A E=C G$ & VE $=0 \mathrm{~A}$ & 3200 \\
\hline $10 t .0$ & 21.7 & 1068 & $1,430 E=O A$ & $3.003 E-06$ & $320 \mathrm{n}$ \\
\hline 1 i है, i & & 618 & $8.27,2 E-0$ ? & $1.737 E-06$ & 3200 \\
\hline 176.0 & 31.0 & 45 A & t. 1 ह \& $F=0\}$ & $E E=0 H$ & 3200 \\
\hline 104.6 & 37.2 & $7 \div 7$ & $2.772 \mathrm{~F}=07$ & $5.821 E=07$ & 3200 \\
\hline 100.0 & 43.4 & 90 & $1.2045=07$ & $2.52 \mathrm{AE}=07$ & $32 \cap 0$ \\
\hline $10+.3$ & & 30 & $5,2: 5=0$ ) & $1.095 E-07$ & 3200 \\
\hline 106.0 & 55.2 & 20 & $2.7615=00$ & $5.78^{A E}=08$ & 3200 \\
\hline$A .0$ & $n$ & n & $O \cap O E+\cap C$ & $0.00 O E+00$ & $3>00$ \\
\hline
\end{tabular}

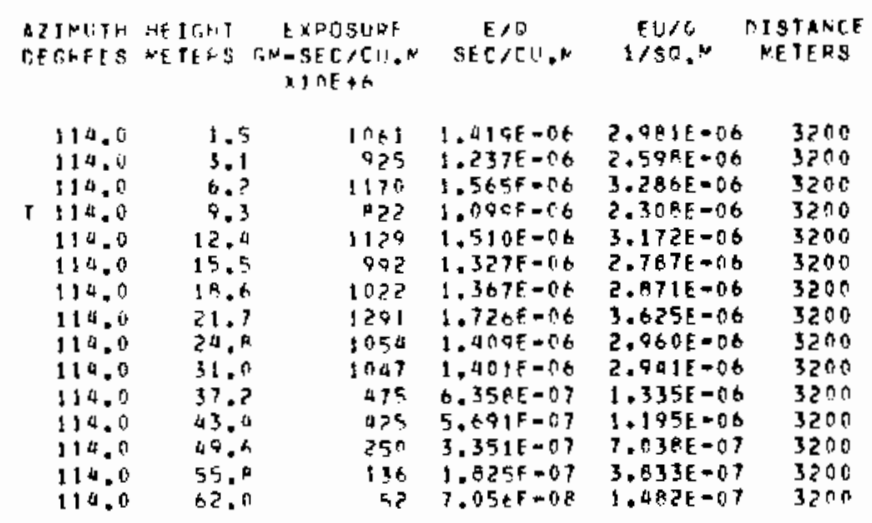

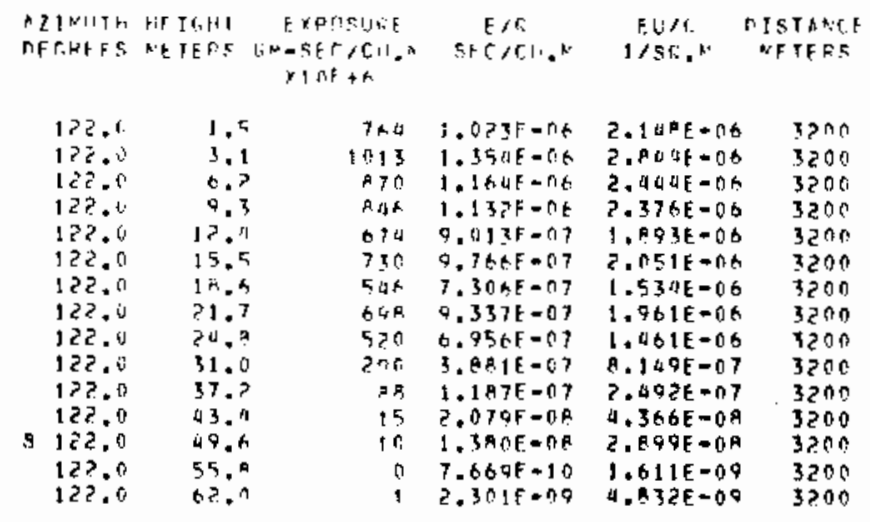

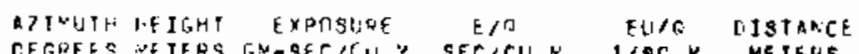

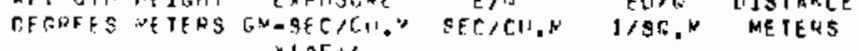 $x \cap 0 \mathrm{E}+6$

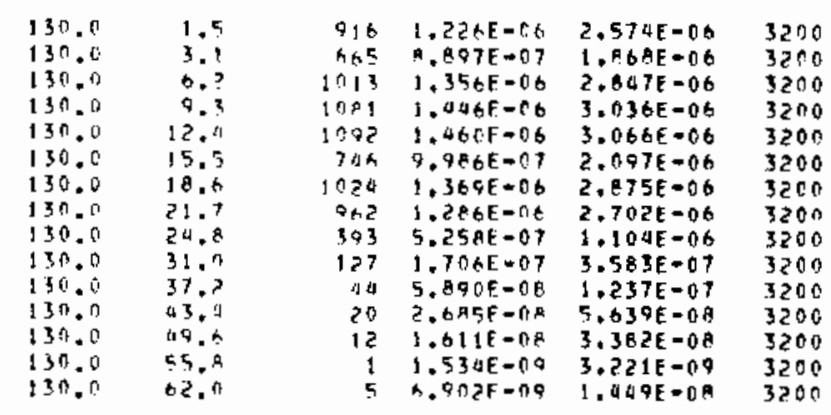


30-SERIES EXPERIMENT: 45, FT=45

GROUNO LEVEL ANO TONER SAMPLING 200-12800 Y ARCS. EVERY OTHER GROLNO LEYEL SAMPLER WAS ROTATED IBC DEGREES, SO AS TO FACE AWAY FROM THE TRACER SOURCE.

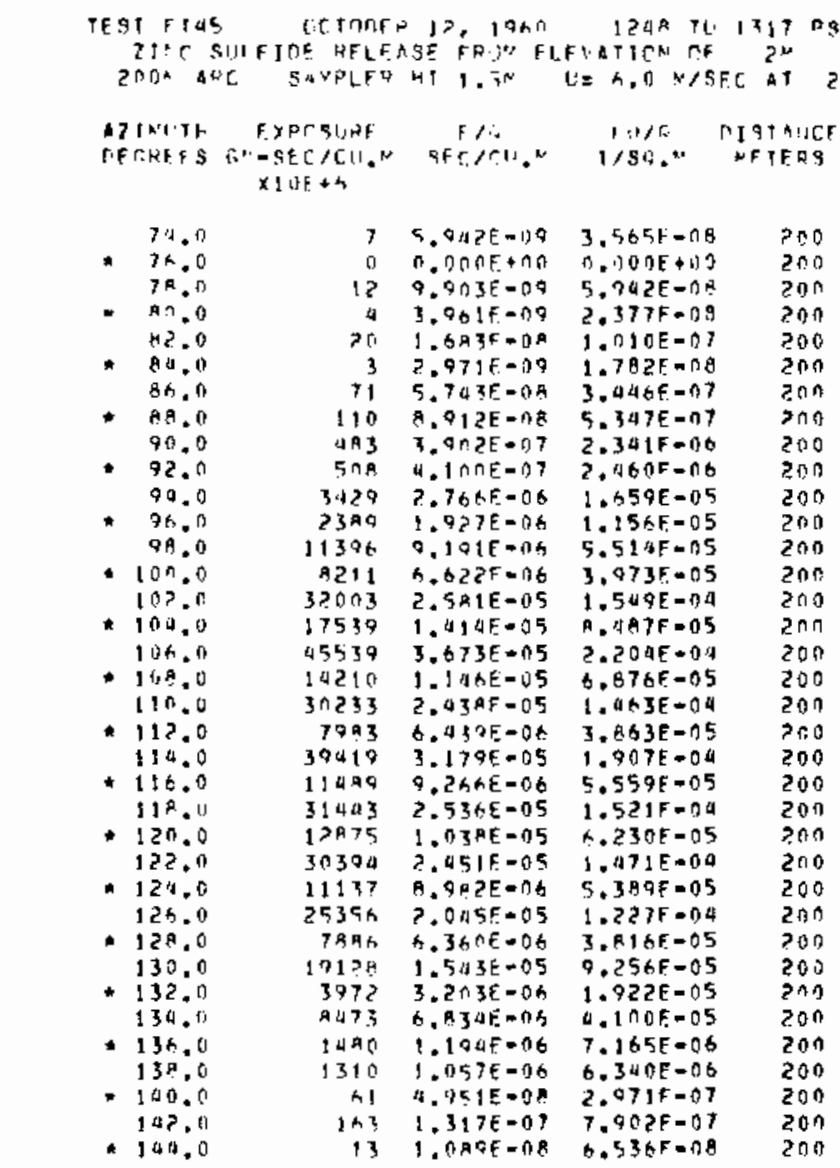

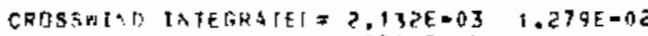

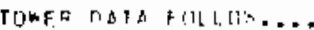

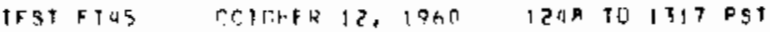

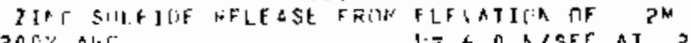

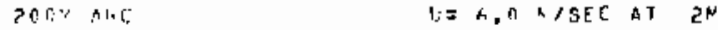

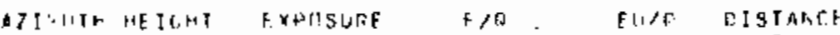

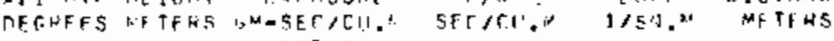
$>1 \cap E+6$

\begin{tabular}{|c|c|c|c|c|c|}
\hline 98,0 & 11.7 & $x 72 \times 0$ & $3.16+E=015$ & $1.90 \cap E=04$ & $=00$ \\
\hline $9 \% .5$ & 1.4 & 8913 & B. A $A 6 B=D E$ & $\Delta, 119 t=05$ & $2 \cap 0$ \\
\hline 2.0 & 2.7 & Ran? & 5 . $\angle Z A F-166$ & 4. $097 E=05$ & 200 \\
\hline 98.0 & 4.1 & $6 x_{0}=h_{1}$ & $5.392 F=016$ & $3.235 E=05$ & 200 \\
\hline 90.0 & 5.4 & 2503 & $6 . B O \cap E=C O$ & 4.13 HE -05 & 200 \\
\hline - & 6.9 & 8745 & $7.085 \mathrm{E}=06$ & $4.251 f=05$ & 200 \\
\hline .0 & 1 & 6557 & $230 E-08$ & $3.17 \div f-05$ & 200 \\
\hline 98.0 & 9.4 & $66>0$ & $5,3 a b r=06$ & 3.2 त $8 \varepsilon=05$ & 200 \\
\hline 99,0 & $10, A$ & 0267 & 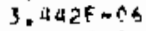 & $2.065 E-05$ & 200 \\
\hline 9.11 & 13.5 & 3057 & $2.46+6=96$ & $1.479 \varepsilon-05$ & 205 \\
\hline 98.0 & 10.2 & 2004 & $1.9357=06$ & $1.1+3 E-D 5$ & 200 \\
\hline 98.0 & 19 & 1903 & $1.602 E-08$ & $64 A C-06$ & 200 \\
\hline & & 1979 & $516 \mathrm{~F}-06$ & $095 E-06$ & $2 \pi n$ \\
\hline 98. & 24.3 & 900 & $990 E=07$ & $4.790 E-06$ & 706 \\
\hline
\end{tabular}

\begin{tabular}{|c|c|c|c|c|c|}
\hline $\begin{array}{l}\text { AZT:DUTH } \\
\text { DE GRE E } 3\end{array}$ & $\begin{array}{l}\text { WE IGW? } \\
\text { WE TERS }\end{array}$ & $\begin{array}{c}\text { EXPUSURE } \\
\text { B. }- \text { SEC/CU.M } \\
\times 10 E+6\end{array}$ & $\begin{array}{c}E / A \\
S E C / C U . N\end{array}$ & $\begin{array}{c}E \cup / 0 \\
1 / 50 .\end{array}$ & $\begin{array}{l}\text { DI GTANCE } \\
\text { METERS }\end{array}$ \\
\hline $\begin{array}{l}106.0 \\
106.0 \\
106.0 \\
106.0 \\
106.0 \\
106.0 \\
106.0 \\
106.0 \\
106.0 \\
106.0 \\
106.0 \\
7106.0 \\
7106.0 \\
106.0 \\
106.0\end{array}$ & $\begin{array}{l}0.7 \\
1.5 \\
2.1 \\
4.1 \\
5.4 \\
6.1 \\
8.1 \\
9.9 \\
10.8 \\
13.5 \\
16.7 \\
18.0 \\
21.6 \\
24.7 \\
27.0\end{array}$ & $\begin{array}{l}117900 \\
26171 \\
19343 \\
16333 \\
22198 \\
20076 \\
18395 \\
17254 \\
16010 \\
10842 \\
8619 \\
4958 \\
3438 \\
4996 \\
2876\end{array}$ & 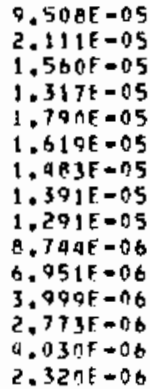 & $\begin{array}{l}5.705 E=04 \\
1.26 B E=04 \\
9.360 E=05 \\
7.903 E=05 \\
1.074 E=04 \\
9.714 E=05 \\
6.896 E=05 \\
8.349 E=05 \\
7.747 E=05 \\
5.20 \mathrm{BE}=05 \\
4.171 E=05 \\
2.399 E=05 \\
1.662 E=05 \\
2.41 \mathrm{BE}=05 \\
1.3921=05\end{array}$ & $\begin{array}{l}200 \\
200 \\
200 \\
200 \\
200 \\
200 \\
200 \\
200 \\
200 \\
200 \\
200 \\
200 \\
200 \\
200 \\
200\end{array}$ \\
\hline
\end{tabular}

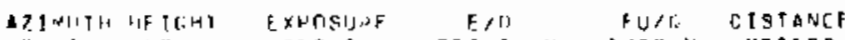

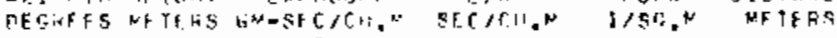
$x \mid t, F+6$

\begin{tabular}{|c|c|c|c|c|c|}
\hline 114.0 & 0.7 & 93962 & $5.771 \mathrm{~F}=05$ & $4.0635=04$ & D? \\
\hline 114.0 & 1.0 & $1+70 ?$ & $1.351 F=0.5$ & F. $10+F=05$ & 200 \\
\hline $11^{4} \cdot 4$ & 2.7 & $1540 \%$ & $1.2+3 t=0.5$ & $7.056 E-05$ & 200 \\
\hline 114.0 & a. 1 & 25037 & 2. DI\&E $=05$ & $1.211 E-04$ & 200 \\
\hline 114.0 & 5,1 & 24179 & 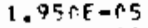 & $1.170 \mathrm{E}-04$ & 200 \\
\hline 19.0 & 6.8 & 187 ดค & $1.515 k-05$ & $9.001 E=05$ & 200 \\
\hline .0 & A. 1 & $1397 ?$ & $1.127 F=05$ & $6.7+1 E=05$ & 200 \\
\hline 4.0 & 9.4 & 12332 & $9 a+E=0 b$ & $5.967 f=05$ & $20 \pi$ \\
\hline 19.0 & $10 . R^{2}$ & 12091 & $1 F-6 b$ & $950-05$ & $e^{n}$ त \\
\hline 14.0 & 15,5 & 11692 & $9,428 E-60$ & $5.65 B E=05$ & 200 \\
\hline 14.0 & 16.2 & 0919 & $7.994 \mathrm{E}-06$ & $4.7705-05$ & $2 \times 0$ \\
\hline 14.0 & 18.9 & 6976 & $5.545 \mathrm{~F}=0 \mathrm{t}$ & $3.327 E-05$ & 200 \\
\hline 0 & 21,6 & $59 \times 4$ & - $810 F-016$ & $.896 \mathrm{k}-05$ & 200 \\
\hline & 24.3 & $4 ? ! 4$ & $3.309 f=06$ & $2.039 E=0.5$ & 200 \\
\hline & & 4692 & le af -06 & $270 E-05$ & 20 \\
\hline
\end{tabular}

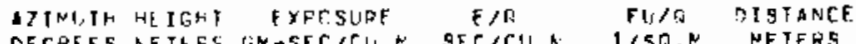
MERAFFS RETESS GNOSEC/CI, SET/CU,N 1/SD.N NETFES

\begin{tabular}{|c|c|c|c|c|c|}
\hline 22.0 & $\begin{array}{r}0.7 \\
1.4 \\
2.7 \\
4.1 \\
5.4 \\
6.8 \\
8.1 \\
9.4 \\
10.8 \\
13.5 \\
16.2 \\
18.9 \\
21.5 \\
29.3 \\
27.9\end{array}$ & $\begin{array}{l}97493 \\
15000 \\
19102 \\
15436 \\
19570 \\
20711 \\
16561 \\
15251 \\
1749 \\
10639 \\
7345 \\
6571 \\
0480 \\
5806 \\
4900\end{array}$ & $\begin{array}{l}1.862 F=05 \\
1.210 F=05 \\
1.540 F=05 \\
1.205 E=05 \\
1.480 E-05 \\
1.673 F=05 \\
1.33 E E=05 \\
1.210 E=05 \\
6.750 F=06 \\
1.580 E=06 \\
5.924 E=06 \\
5.249 E=06 \\
5.234 E=06 \\
3.070 E=06 \\
3.952 F=06\end{array}$ & 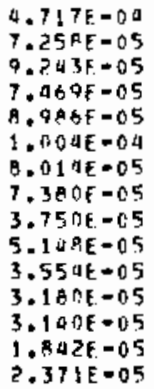 & $\begin{array}{l}200 \\
200 \\
200 \\
200 \\
200 \\
200 \\
200 \\
200 \\
200 \\
200 \\
200 \\
200 \\
200 \\
200 \\
200\end{array}$ \\
\hline
\end{tabular}




\begin{tabular}{|c|c|c|c|c|c|}
\hline 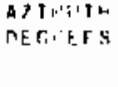 & $\begin{array}{l}\text { NE J IiH } \\
\text { NEIfHS }\end{array}$ & 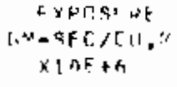 & $\begin{array}{c}f / 6 \\
s+c 11, N\end{array}$ & $\begin{array}{c}F 1110 \\
1 / 5 n .\end{array}$ & $\begin{array}{r}\text { P. ISIAPC } \\
\text { NE.T+RS }\end{array}$ \\
\hline 150.17 & $n \cdot ?$ & 55177 & $\therefore .450 F=05$ & $2.67 \cap F=04$ & 20 \\
\hline 135,0 & 1. & 10กम0 & $R, 136 \mathrm{CF}=\mathrm{Ag}$ & $1,0705=05$ & \\
\hline $13 n .4$ & 2.7 & $90 ? 3$ & $7.2775=06$ & $4.36+5=115$ & 5 \\
\hline $13 n=13$ & id. 1 & $1251^{\circ}$ & $1.010 \mathrm{of}=05$ & $6.05 \mu_{t}-05$ & $2 \cap n$ \\
\hline 150.1 & 5. & 7501 & $F .0 A 9 F=1: t$ & $3.63)=05$ & 200 \\
\hline $13 \mathrm{~m}, 1$ & h. & 12358 & $9.95 \pm f=1.6$ & $5.7700-015$ & 200 \\
\hline 130.0 & $r .1$ & 10717 & $E, k 43 F-10 b$ & $5.1 \times 6 F=0.5$ & 24 \\
\hline $13 n .0$ & 7. & $16 h, 89$ & $A .61 \mathrm{cF}=1 \mathrm{~h}$ & $5.17>\mathrm{F}=05$ & \\
\hline 13 in. 0 & 11.7 & 73 ค9 & $5,959 f-06$ & $3.57+E=0.5$ & 2 \\
\hline 156.0 & 13. & $\cos 3$ & $1.0035-06$ & $4.202,-65$ & \\
\hline 130,0 & 16,7 & 5745 & $a .633 \mathrm{E}=0 \mathrm{~F}$ & $2.7 A 0 E-05$ & \\
\hline $13 n .0$ & 18,7 & 7160 & $5,774 E-106$ & $3.46 \mathrm{AF}=015$ & 20 \\
\hline 130.0 & 21.6 & 5408 & $4,3945=06$ & $2.6365=0.5$ & 2n 0 \\
\hline 130.0 & 24.7 & $37+1$ & $3.033 E=06$ & $1.920 \mathrm{k}=05$ & 20 \\
\hline & 27. & $25 \times 3$ & $2,068 F=06$ & $1.241 E=05$ & 5 \\
\hline
\end{tabular}

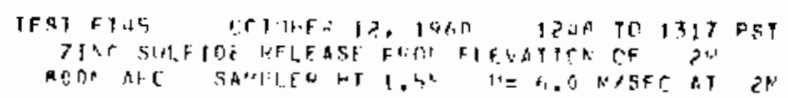

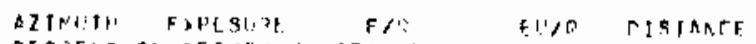

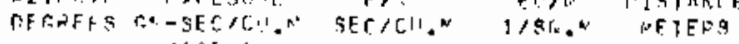
$\times 10 E+h$

\begin{tabular}{|c|c|c|c|c|c|}
\hline - & $A A={ }^{n}$ & 1 & $P . A 7 \| F=D O$ & $1.73 A=\cap A$ & and \\
\hline & Q:. 0 & $\underline{x}$ & $2.94 f=09$ & $1.72 A \mathrm{~F}-\mathrm{NA}$ & AOn \\
\hline & $9 \vec{x} \cdot 0$ & IA & I. त1, 5 F $=0.0$ & $1.93 \times c-n 7$ & 800 \\
\hline & 94.0 & 291 & $7.270 \mathrm{E}+17$ & $1.3+2 t-n t$ & 500 \\
\hline & 90.0 & 125 & $1.40 t \mathrm{E}-07$ & $8.966 F-07$ & 900 \\
\hline & 98.11 & $15.1 \mathrm{P}$ & $1.2+1 \mathrm{E}=06$ & $7.495 F=016$ & AOO \\
\hline$\star$ & 100.0 & $n+4$ & $4.952 E-0.7$ & $2.0715-06$ & 900 \\
\hline & $10 \vec{\angle}, 0$ & 1424 & $1.1005-0 E$ & $6.591 \neq-06$ & $\operatorname{son}$ \\
\hline * & 104.0 & 4.75 & $1.8 \times 5 E-07$ & $2.731 E=06$ & 800 \\
\hline & 106.01 & 2136 & $1.723 E=06$ & & 800 \\
\hline & 9080 & 730 & $5.991 f=07$ & $\begin{array}{l}3.5355-106 \\
0.7075=06\end{array}$ & 800 \\
\hline & 110.0 & $\begin{array}{r}2021 \\
532\end{array}$ & $1.630 \mathrm{E}=06$ & $\begin{array}{l}9.702 E=06 \\
2.575 F=06\end{array}$ & $\begin{array}{l}8 \cap 0 \\
\operatorname{ang} 0\end{array}$ \\
\hline & 114,0 & 1635 & $1.343 E=\cap b$ & $P .058 F=06$ & ดก \\
\hline ^ & 110.0 & 401 & 3.9 ABE -0.7 & $2.370 F-06$ & 000 \\
\hline & 112,0 & 1433 & $1.15 \mathrm{kF}=06$ & $6.2375=06$ & 900 \\
\hline & $12 n .0$ & 874 & $7.0504-07$ & $4.230 E-06$ & 800 \\
\hline & 122,0 & 3760 & 2.2? $6 E=06$ & $1.3366-105$ & 000 \\
\hline & 120.0 & 767 & $N .20, E=0.7$ & $3.724 E-06$ & 000 \\
\hline & 126.0 & 1648 & $1.331 f=06$ & $0.006=-106$ & 800 \\
\hline m & 128.0 & 450 & $3 O E-07$ & $E .17 A E-06$ & 800 \\
\hline & $13 n .0$ & 1126 & $9.0 \times 1 E=07$ & $5.447,5=06$ & 900 \\
\hline a & 132.0 & 213 & $1.7 ? 4 E=07$ & $1.035 F-136$ & 800 \\
\hline & 134.0 & 559 & $4.5 \pm \geq E-07$ & $2.707 E-06$ & AOO \\
\hline & 136.0 & HA & $3.927 \mathrm{E}=\mathrm{DP}$ & $2.356 E-07$ & 100 \\
\hline & $\begin{array}{l}139.16 \\
140.0\end{array}$ & $\begin{array}{r}23 \\
2\end{array}$ & $\begin{array}{l}1.816 E=08 \\
1.9165=08\end{array}$ & $\begin{array}{l}1.148 F-07 \\
1.148 E=0.8\end{array}$ & $\begin{array}{l}800 \\
800\end{array}$ \\
\hline
\end{tabular}

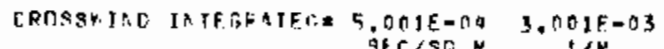

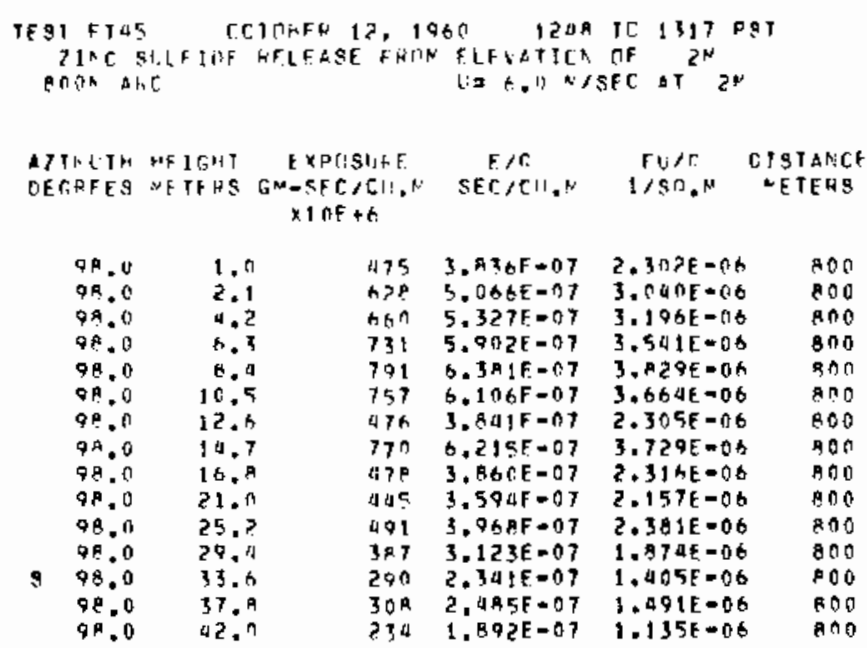

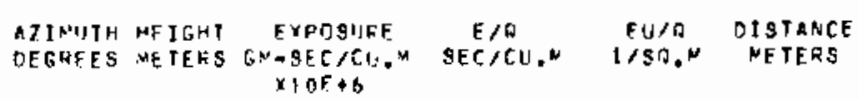

\begin{tabular}{|c|c|c|c|c|c|}
\hline $\begin{array}{l}106.0 \\
104.0 \\
106.0 \\
106.0 \\
106.0 \\
106.0 \\
106.0 \\
106.0 \\
106.0 \\
106.0 \\
106.0 \\
106.0 \\
106.0 \\
106.0 \\
106.0\end{array}$ & $\begin{array}{l}1.0 \\
2.1 \\
4.2 \\
6.3 \\
8.4 \\
10.5 \\
12.6 \\
14.7 \\
16.2 \\
21.4 \\
25.9 \\
29.1 \\
33.6 \\
37.8 \\
42.7\end{array}$ & $\begin{array}{r}956 \\
400 \\
1405 \\
1275 \\
1502 \\
1388 \\
1517 \\
1541 \\
1342 \\
1439 \\
983 \\
698 \\
1061 \\
1047 \\
603\end{array}$ & $\begin{array}{l}7.716 E=07 \\
3.955 E=07 \\
1.134 F=06 \\
1.02 A E=06 \\
1.276 F=06 \\
1.120 E=06 \\
1.224 E=06 \\
1.243 E=06 \\
1.003 E=06 \\
1.1615=06 \\
7.931 E=07 \\
5.630 E=07 \\
9.564 E=07 \\
9.009 E=07 \\
5.509 F=07\end{array}$ & $\begin{array}{l}9.629 E-06 \\
2.373 E=06 \\
6.803 E=06 \\
6.171 E=06 \\
7.659 E=06 \\
6.716 E=06 \\
7.349 E=06 \\
7.058 E=06 \\
6.095 E=06 \\
6.966 E=06 \\
4.750 E=06 \\
3.378 E=06 \\
5.139 E=06 \\
5.166 E=06 \\
3.305 E=06\end{array}$ & $\begin{array}{l}800 \\
800 \\
800 \\
800 \\
800 \\
800 \\
800 \\
800 \\
800 \\
000 \\
800 \\
000 \\
800 \\
000 \\
000\end{array}$ \\
\hline
\end{tabular}

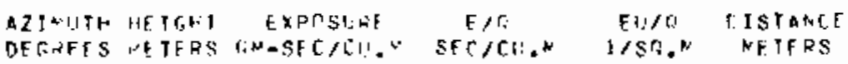
$x \in+b$

\begin{tabular}{|c|c|c|c|c|c|}
\hline 114,8 & 1.1 & A 76 & $7.065=07$ & $4.339 E-06$ & $B O C$ \\
\hline 114.0 & 2.1 & 1445 & $1.167 \mathrm{t}=0 \mathrm{~b}$ & $7.0 \cap 0 \mathrm{DF}-0 \mathrm{~S}$ & ann \\
\hline 114.0 & $4, ?$ & 1251 & $1.0005=06$ & $6.05 t 16-016$ & ROS \\
\hline 114,0 & 6.3 & 991 & $7.99+5=0.7$ & 4.79 पE $=06$ & POO \\
\hline 114.0 & 8.4 & 933 & $7.531 f=07$ & $4.5 \int^{A F}=\Gamma G$ & $A \cap 0$ \\
\hline 114.0 & 10.5 & $11 \ln _{2}$ & Q $E=07$ & $5.721 \mathrm{~F}-0 \mathrm{k}$ & Roc \\
\hline $11^{4} .0$ & $1 \overrightarrow{2} \cdot 6$ & 1450 & $1.169 E-0 t$ & $7.017 t=0.6$ & 200 \\
\hline 110.0 & 14.7 & $11 \leqslant 2$ & 9.37 IE-07 & 5.62 af -06 & 600 \\
\hline 114,0 & 16.9 & 912 & $7.84 a E-\pi 7$ & $4.706 E=06$ & 800 \\
\hline 114.0 & 21.0 & 1126 & $.083 E-07$ & $5.450 E-06$ & 800 \\
\hline 114.0 & 25.2 & 710 & $5.725 F-07$ & $3.437 E=06$ & 800 \\
\hline 114,0 & 29.1 & 971 & $A+E=07$ & $4.702 f=06$ & คด ก \\
\hline 114.0 & 33.6 & 1114 & $9915-07$ & $5.395 E-06$ & Aon \\
\hline $1 \mid 4.0$ & 37.4 & 1277 & $1.030 E=06$ & $6.17^{\circ} \mathrm{E}-06$ & 800 \\
\hline & & 1190 & $0 ?, \mathrm{E}-\mathrm{Cr}$ & $5.761 E=06$ & 800 \\
\hline
\end{tabular}




\begin{tabular}{|c|c|c|c|c|c|}
\hline $\begin{array}{l}\text { ZINITH } \\
\text { EREFS }\end{array}$ & $\begin{array}{l}\text { HE IGHT } \\
\text { X'ETEAS }\end{array}$ & $\begin{array}{c}\text { EXPOSUDF } \\
G M=S F C>C U * N \\
x \notin N F+6\end{array}$ & $\begin{array}{c}E / \theta \\
\sec (\mathrm{C}), \mathrm{N}\end{array}$ & $\begin{array}{l}E U / 0 \\
1 / S D, M\end{array}$ & $\begin{array}{l}\text { DISTANCE } \\
\text { METERS }\end{array}$ \\
\hline $\begin{array}{l}122.0 \\
122.0 \\
122.0 \\
122.0 \\
122.0 \\
122.0 \\
122.0 \\
122.0 \\
122.0 \\
122.0 \\
122.0 \\
122.0 \\
122.0 \\
122.0 \\
122.0\end{array}$ & $\begin{array}{l}1.0 \\
2.1 \\
4.3 \\
6.3 \\
8.5 \\
10.5 \\
12.6 \\
14.7 \\
10.8 \\
21.0 \\
25.7 \\
29.4 \\
33.6 \\
37.8 \\
42.0\end{array}$ & $\begin{array}{l}1268 \\
1309 \\
1627 \\
1587 \\
1352 \\
1109 \\
1392 \\
1640 \\
1509 \\
1182 \\
937 \\
1258 \\
750 \\
776 \\
700\end{array}$ & $\begin{array}{l}1.023 E=06 \\
1.056 E-06 \\
1.312 E=06 \\
1.200 E=06 \\
1.091 E=06 \\
1.950 E-07 \\
1.123 E=06 \\
1.323 E-06 \\
1.217 E=06 \\
9.534 E=07 \\
7.55 E E=07 \\
1.015 E-06 \\
6.053 E=07 \\
6.264 E=07 \\
5.072 E=07\end{array}$ & $\begin{array}{l}6.139 E=06 \\
6.336 E=06 \\
7.879 E=06 \\
7.681 E=06 \\
6.544 E=06 \\
5.370 E=06 \\
6.736 E=06 \\
7.940 E=06 \\
7.305 E=06 \\
5.721 E=06 \\
4.535 E=06 \\
6.08 R E=06 \\
3.632 E=06 \\
3.758 E=06 \\
3.583 E=06\end{array}$ & $\begin{array}{l}800 \\
800 \\
800 \\
800 \\
800 \\
800 \\
800 \\
000 \\
800 \\
000 \\
800 \\
800 \\
000 \\
100 \\
800\end{array}$ \\
\hline $\begin{array}{l}\text { ITUUTी: } \\
\text { ERTIFFS }\end{array}$ & $\begin{array}{l}\text { HEICHT } \\
\text { NETEKS }\end{array}$ & $\begin{array}{c}\text { EXPOSUIZF } \\
C \times+S F C, C: 4,2 \\
\times 10 E+B\end{array}$ & $\begin{array}{c}E / F \\
\text { SEC }\end{array}$ & $\begin{array}{c}F 1_{6} / 0 \\
1 / 5 r^{2} \times\end{array}$ & $\begin{array}{l}\text { PISTANCF } \\
\text { WETfES }\end{array}$ \\
\hline $\begin{array}{l}130.0 \\
130.01 \\
130.0 \\
13500 \\
136.0 \\
130.0 \\
130.0 \\
130.0 \\
130.0 \\
130.0 \\
130.0 \\
130.0 \\
131.0 \\
130.0\end{array}$ & $\begin{array}{l}1.11 \\
2.1 \\
0.2 \\
0.3 \\
0.4 \\
10.5 \\
12.5 \\
14.7 \\
16.8 \\
21.0 \\
25.2 \\
13.5 \\
37.2 \\
42.0\end{array}$ & $\begin{array}{l}300 \\
709 \\
705 \\
601 \\
690 \\
700 \\
771 \\
750 \\
710 \\
596 \\
307 \\
391 \\
306 \\
379\end{array}$ & 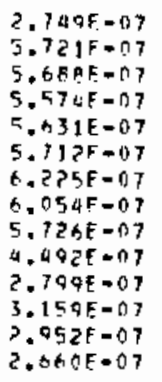 & $\begin{array}{l}1.450 E=1.6 \\
3.133 E=06 \\
3.413 E=06 \\
3.315 E=06 \\
3.374 E=06 \\
3.427 E=06 \\
3.735 E=06 \\
3.632 E=06 \\
3.036 E=06 \\
2.695 E=06 \\
1.580 E=06 \\
1.895 E=06 \\
1.771 E-06 \\
1.596 E-06\end{array}$ & 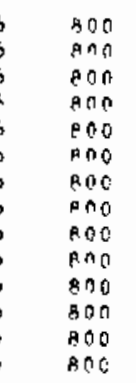 \\
\hline
\end{tabular}

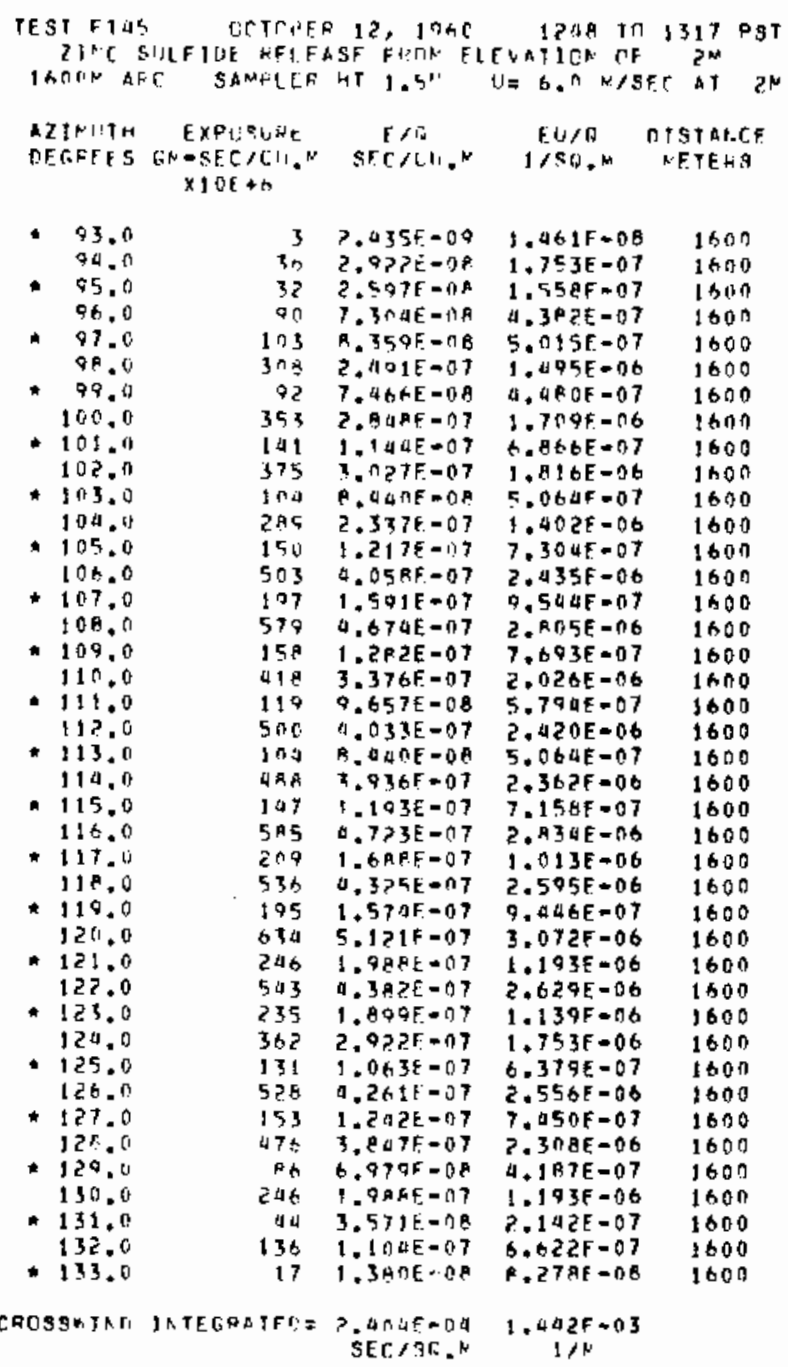

TONFR TATA FIDLLE, E.

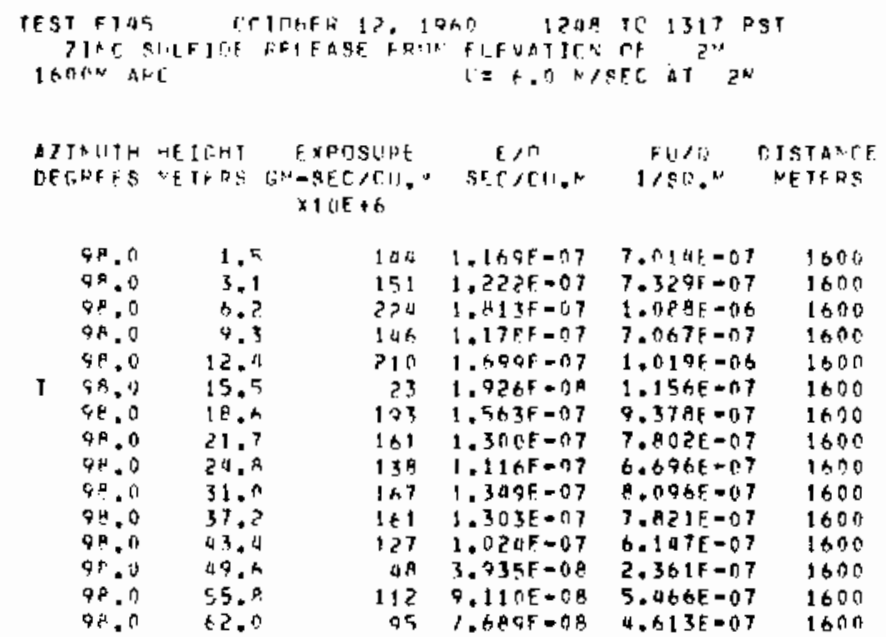




\begin{tabular}{|c|c|c|c|c|c|}
\hline $\begin{array}{l}2 \text { INUTH } \\
\text { EGREFS }\end{array}$ & $\begin{array}{l}\text { MEIFHI } \\
\text { METERS }\end{array}$ & $\begin{array}{c}\text { EXPOSLRE } \\
\text { GN=SEC/CU. } \\
Y 1 O F+G\end{array}$ & $\begin{array}{c}E / R \\
S E C / C L, N\end{array}$ & $\begin{array}{c}F \cup / \pi \\
1 / S \cap .\end{array}$ & $\begin{array}{l}\text { OISTANCE } \\
\text { NETERS }\end{array}$ \\
\hline $\begin{array}{l}100.0 \\
100.0 \\
106.0 \\
106.0 \\
10 \% .0 \\
100.0 \\
106.0 \\
100.0 \\
106.0 \\
106.0 \\
106.0 \\
100.0 \\
106.0 \\
100.0 \\
100.0\end{array}$ & $\begin{array}{l}1.5 \\
3.1 \\
6 . ? \\
9.3 \\
12.4 \\
15.5 \\
18.4 \\
21.7 \\
24.8 \\
31.0 \\
31.2 \\
43.11 \\
49.5 \\
55.7 \\
62.7\end{array}$ & $\begin{array}{l}147 \\
204 \\
133 \\
263 \\
270 \\
310 \\
3140 \\
245 \\
212 \\
377 \\
189 \\
284 \\
149 \\
195 \\
167\end{array}$ & $\begin{array}{l}1.197 F-07 \\
1.064 F-07 \\
1.122 F-07 \\
2.1235-07 \\
2.195 F-07 \\
2.5040-07 \\
2.793 E=07 \\
1.070 E=07 \\
1.717 F=07 \\
3.048 F-07 \\
1.607 F=07 \\
2.205 E=07 \\
1.200 E-197 \\
1.570 E-07 \\
1.340 E=07\end{array}$ & $\begin{array}{l}7.119 E=07 \\
9.982 E=07 \\
7.933 E=07 \\
1.2711 F=06 \\
1.311 E-06 \\
1.503 E=06 \\
1.674 E=06 \\
1.187 E=06 \\
1.027 E=06 \\
1.029 E=06 \\
9.845 E=07 \\
1.377 F=06 \\
7.237 E=07 \\
9.045 E-07 \\
0.09 F=07\end{array}$ & $\begin{array}{l}1600 \\
1600 \\
1600 \\
1600 \\
1600 \\
1600 \\
1600 \\
1600 \\
1600 \\
1600 \\
1600 \\
1600 \\
1600 \\
1600 \\
1600\end{array}$ \\
\hline
\end{tabular}

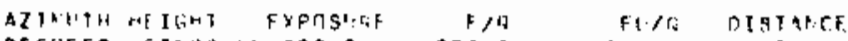

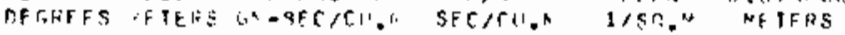
$\times 10 \mathrm{~F}+6$

\begin{tabular}{|c|c|c|c|c|c|}
\hline 110.0 & 1.5 & $1 \times n$ & $1 .(4515=97$ & $6 . x-5 F-07$ & $1+\cap 0$ \\
\hline 114.0 & 3.1 & 105 & $1.173 \mathrm{~F}=07$ & $7.0 \Delta n t-B .7$ & $1+10$ \\
\hline $11 \% .0$ & h.? & 103 & $1.5635=07$ & 9.37 ac. -07 & $160 n$ \\
\hline 114.0 & 7.3 & 2nt. & $? .145 E=5 . ?$ & $1.287 F=06$ & 1600 \\
\hline 114.0 & 12.1 & 253 & $2.0458=0.7$ & $1.227 F=06$ & 1600 \\
\hline 14.0 & 15.5 & $2 ! 4$ & $1.7295 \cdot 07$ & $1.03 \mathrm{~B} .06$ & 1600 \\
\hline 10,0 & 15 & 2Aa & $2405=0 ?$ & $377 E=06$ & 1600 \\
\hline 114.6 & 24.7 & 232 & $1.781 \mathrm{E}=0.7$ & 1.07 ilf $=06$ & 1600 \\
\hline 114.0 & 25 & 170 & $1.3745=07$ & $8.246 E=07$ & 1500 \\
\hline 114.0 & $31 . n$ & 162 & $1.3: 2 E-07$ & $1 E-07$ & $161) 0$ \\
\hline $1+4.0$ & 37.2 & 118 & $0 E=O B$ & $.0226-07$ & 1600 \\
\hline 114.0 & 43.4 & 155 & $1.2535-07$ & $.521 f=07$ & 1600 \\
\hline 110,0 & 49.5 & 170 & $1.00 \geq E=07$ & $6.253 E-07$ & 1600 \\
\hline $114=0$ & 55.4 & $1+14$ & $1.16 z F=07$ & 6.9745 .07 & 1600 \\
\hline$\cdot y$ & & 126 & $12 \cap E=n 7$ & $6.1225=07$ & 1600 \\
\hline
\end{tabular}

DTINITTH HE IGHT EXPOSURE ETO EL/O DISTANCE

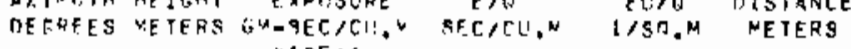

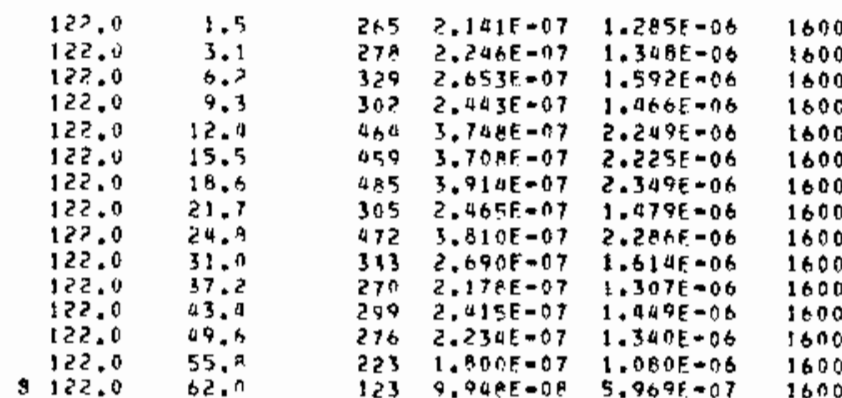

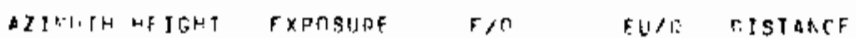

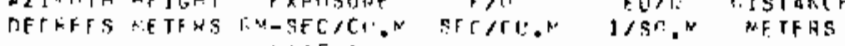
$x+M E+B$

\begin{tabular}{|c|c|c|c|c|c|}
\hline 130.0 & 1.5 & 7 म & h. $3055=$ ate & $3.7+3 F=0.7$ & $1+00$ \\
\hline $13 \% .0$ & 3.1 & 117 & $9.4578=08$ & $5.670 F=07$ & 1600 \\
\hline $13 \%$. & $6 . ?$ & $11^{8}$ & $7.545 \mathrm{E}=\mathrm{C}=$ & $27 E-07$ & 1600 \\
\hline 130.0 & 9.3 & 48 & $5.94 \mathrm{CE}-\mathrm{CE}$ & $36 \pi E-07$ & 1600 \\
\hline 1311.0 & 12.11 & 135 & $1.000 E-07$ & $6.541 E=07$ & 1600 \\
\hline 130.0 & & 201 & $1.62 g E=07$ & $7 ? E-07$ & 1600 \\
\hline 0 & & 111 & $\mathrm{E} .975 \mathrm{~F}=0 \mathrm{~B}$ & $385[-07$ & 1 t 00 \\
\hline 130.0 & 21.7 & 211 & $1.70 F E=07$ & $1.025 \mathrm{~F}-0.6$ & 1600 \\
\hline 136.0 & & 132 & $1.070 F-07$ & $25-07$ & $1+00$ \\
\hline $13(1.0$ & $31 . n$ & 128 & $1, n a 15=07$ & $17 E-07$ & 1600 \\
\hline 130.0 & 37.7 & $15 ?$ & $1.066 E+07$ & $6.397 \mathrm{E}=07$ & 1800 \\
\hline & $\begin{array}{l}33.14 \\
49.4\end{array}$ & $\int_{1}^{R}$ & $0.578 E-0 B$ & $7 E=07$ & 1600 \\
\hline - 11 & & $\ln 7$ & $672=08$ & $036-07$ & 1000 \\
\hline & 6. 2.0 & $\begin{array}{l}95 \\
95\end{array}$ & $0.1024=06$ & 4.497007 & 1600 \\
\hline
\end{tabular}

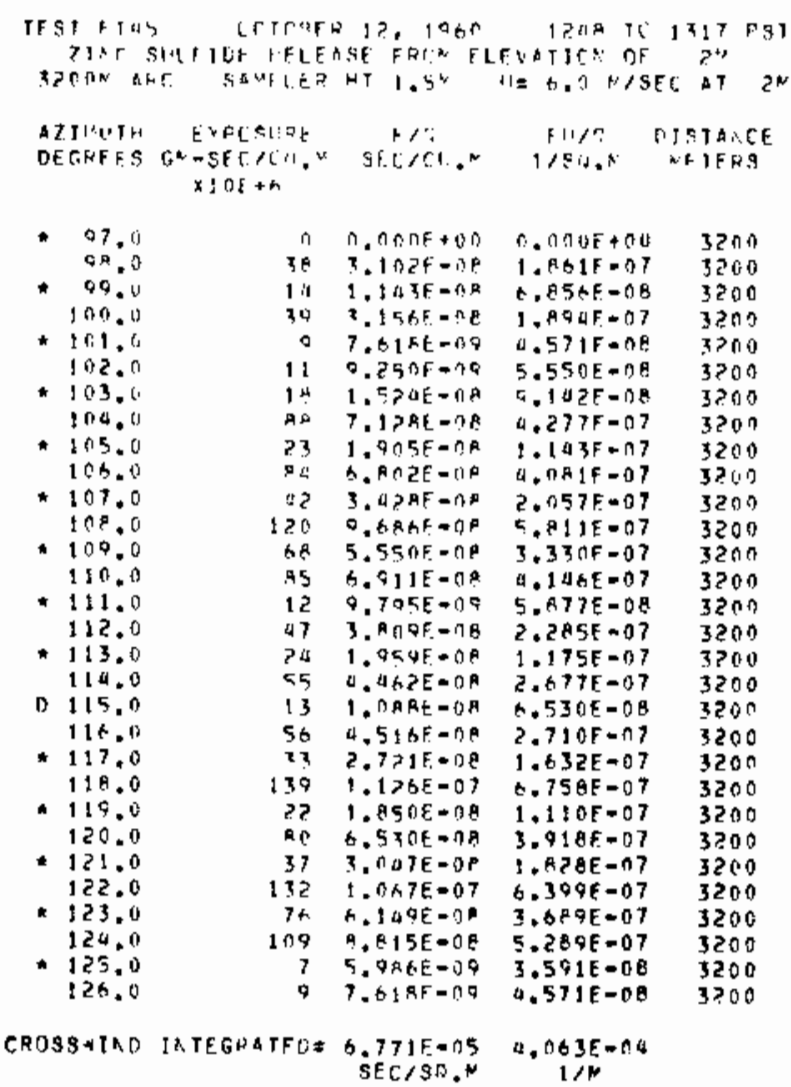

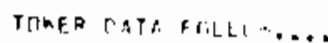

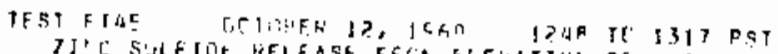

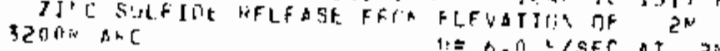

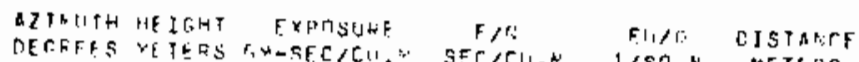

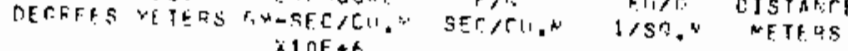

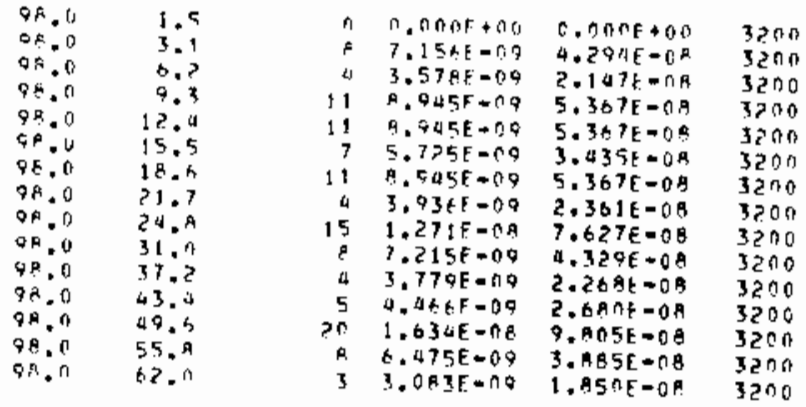




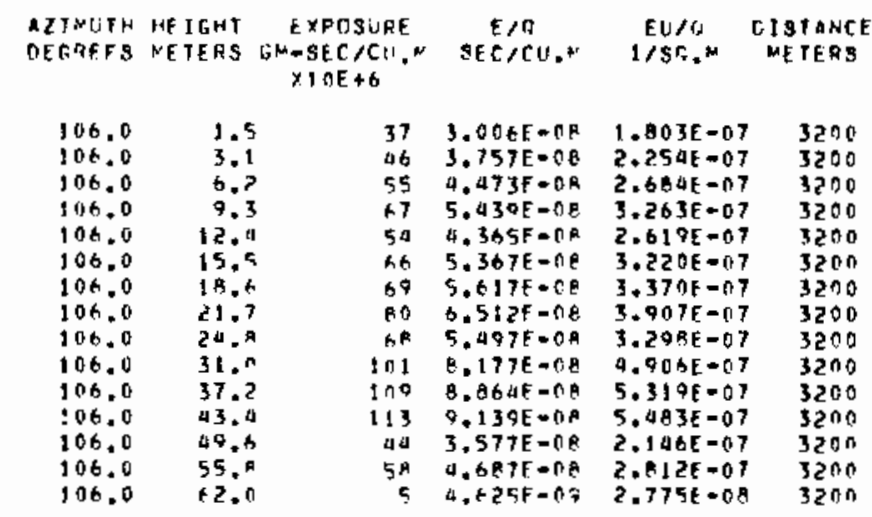

\begin{tabular}{|c|c|c|c|c|c|}
\hline $\begin{array}{l}\text { AZIS:HTH } \\
\text { DECFEFS }\end{array}$ & $\begin{array}{l}\text { HE IFHI } \\
\text { METERS }\end{array}$ & $\begin{array}{c}\text { EXPOSUFF } \\
G \times=8 F / C+1 . \\
\times 1 \cap F+h\end{array}$ & $\begin{array}{c}5 / n \\
\text { sec } / C 1: k\end{array}$ & $\begin{array}{l}511<0 \\
1<50 . N\end{array}$ & $\begin{array}{c}\text { TISTANCE } \\
\text { PETFPS }\end{array}$ \\
\hline 114.0 & 1.5 & 55 & $1.437 \mathrm{~F}-0 \mathrm{e}$ & $2.662 E-07$ & 3200 \\
\hline 110.0 & 3.1 & $? 4$ & 2. OA $A F=n A$ & $1.202 E-07$ & 3200 \\
\hline 114.0 & $0 . ?$ & 49 & $3.972 \mathrm{~F}-\mathrm{CC}$ & $2.383 E-07$ & 3200 \\
\hline 114.0 & 9.3 & 23 & 1.AGAE $=R A$ & $1.138 t=07$ & 3200 \\
\hline 114.0 & 12.4 & 36 & $2.97 n E=0 B$ & $1.78 \mathrm{PF}-07$ & $320 \%$ \\
\hline 114.0 & 15.5 & 31 & $2.576 f=0 \mathrm{~A}$ & $1.546 E-07$ & 3200 \\
\hline 114.0 & 13.6 & $3 k$ & $3.113 f-08$ & 1.86 मF $=07$ & 3200 \\
\hline 914.0 & 21.7 & 27 & $2.218 E-O A$ & $1.331 t=07$ & 3200 \\
\hline 114.0 & 24.9 & 3 ค & $3.127 F=08$ & $1.876 E-07$ & $32 \cap 0$ \\
\hline 114.3 & 31.0 & 39 & $3.161 \mathrm{~F}-08$ & $1.897 E=07$ & $320 n$ \\
\hline $1: 4.0$ & $37 . ?$ & 26 & $? .165 F=08$ & $1.299 E-07$ & 3200 \\
\hline 114.0 & 43.4 & 35 & $2.08 \cap E=08$ & $1.608 E=07$ & 3200 \\
\hline 114.0 & 44.5 & 32 & $2.65 \mathrm{cF}=0 \mathrm{~F}$ & $1.591 \mathrm{E}=0.7$ & 3200 \\
\hline 114,0 & $55 . R$ & 29 & $2.405 E=08$ & $1.443 E-07$ & 3200 \\
\hline 2114.0 & 62.0 & $3 h$ & $2.928 f=0.9$ & $1.758 F=07$ & 3 हो \\
\hline
\end{tabular}

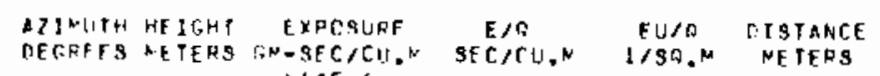
$\times 10 \mathrm{~F}+6$

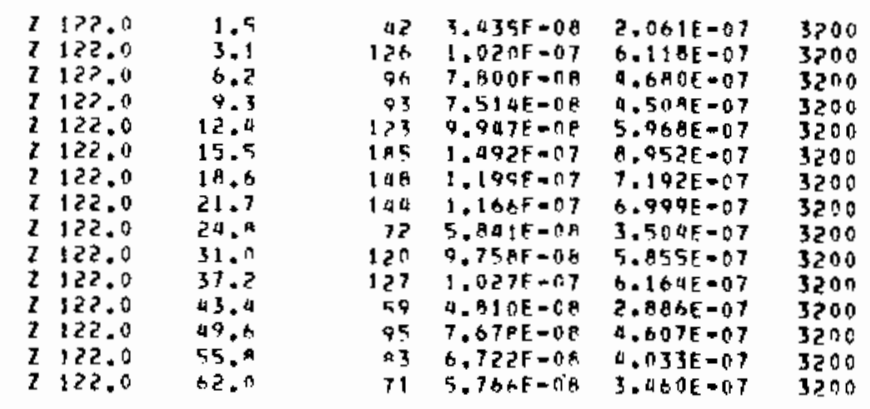


30-5ERIES EXPER HENT: 46 (FT-46)

GROUNO LEVEL AND TOWER SAMPLING 200-12800 M ARCS. EYERY OTHEA GROUNO LEVEL SAHPLER WAS ROTATED 180 OEGREES, SO AS TO FACE AWAY FROM THE TRACER SOURCE.

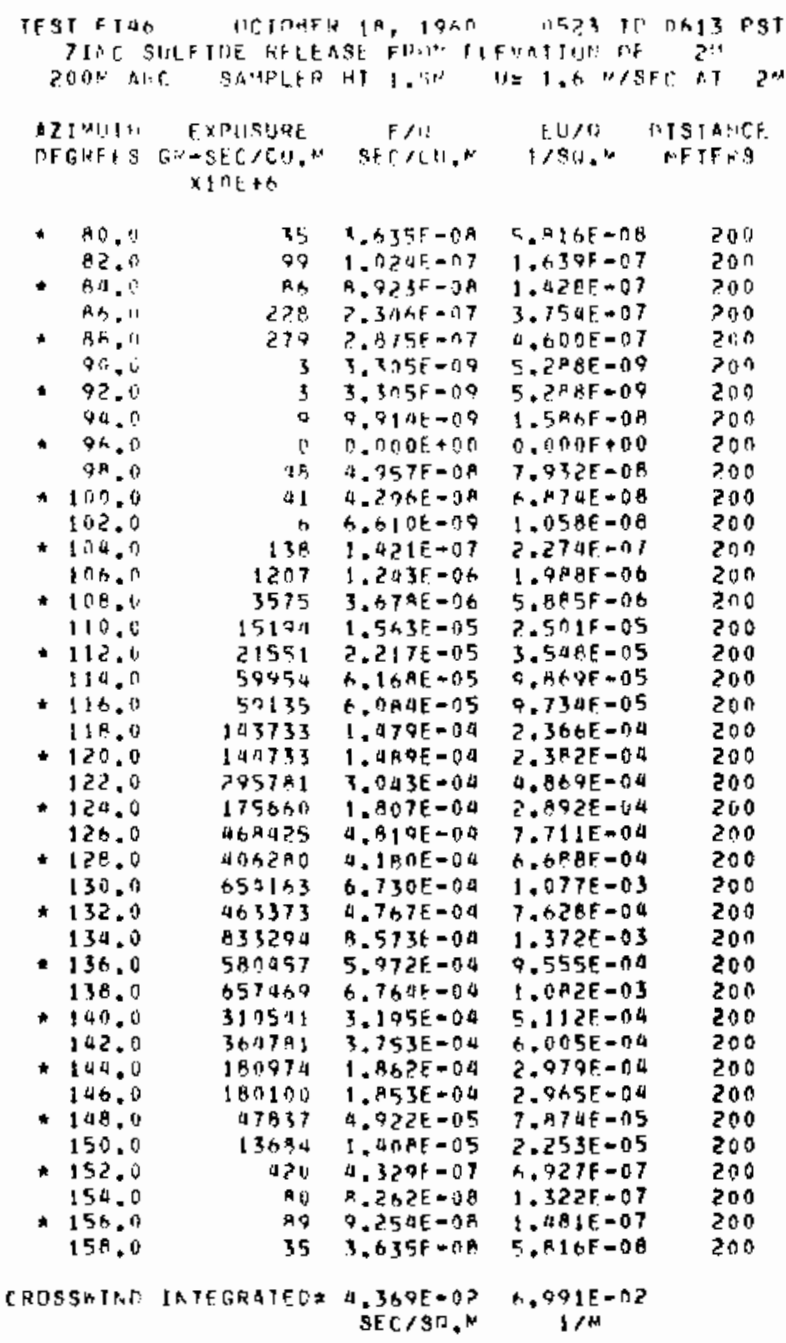

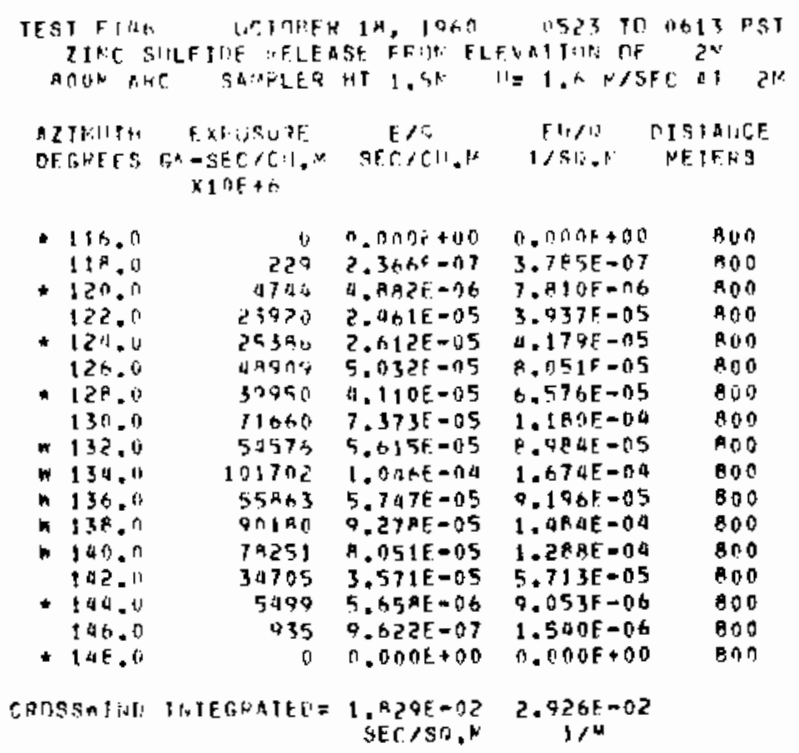

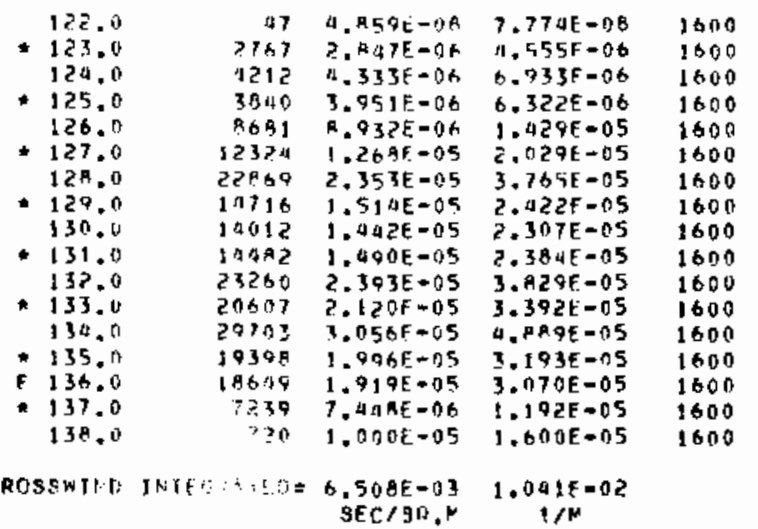




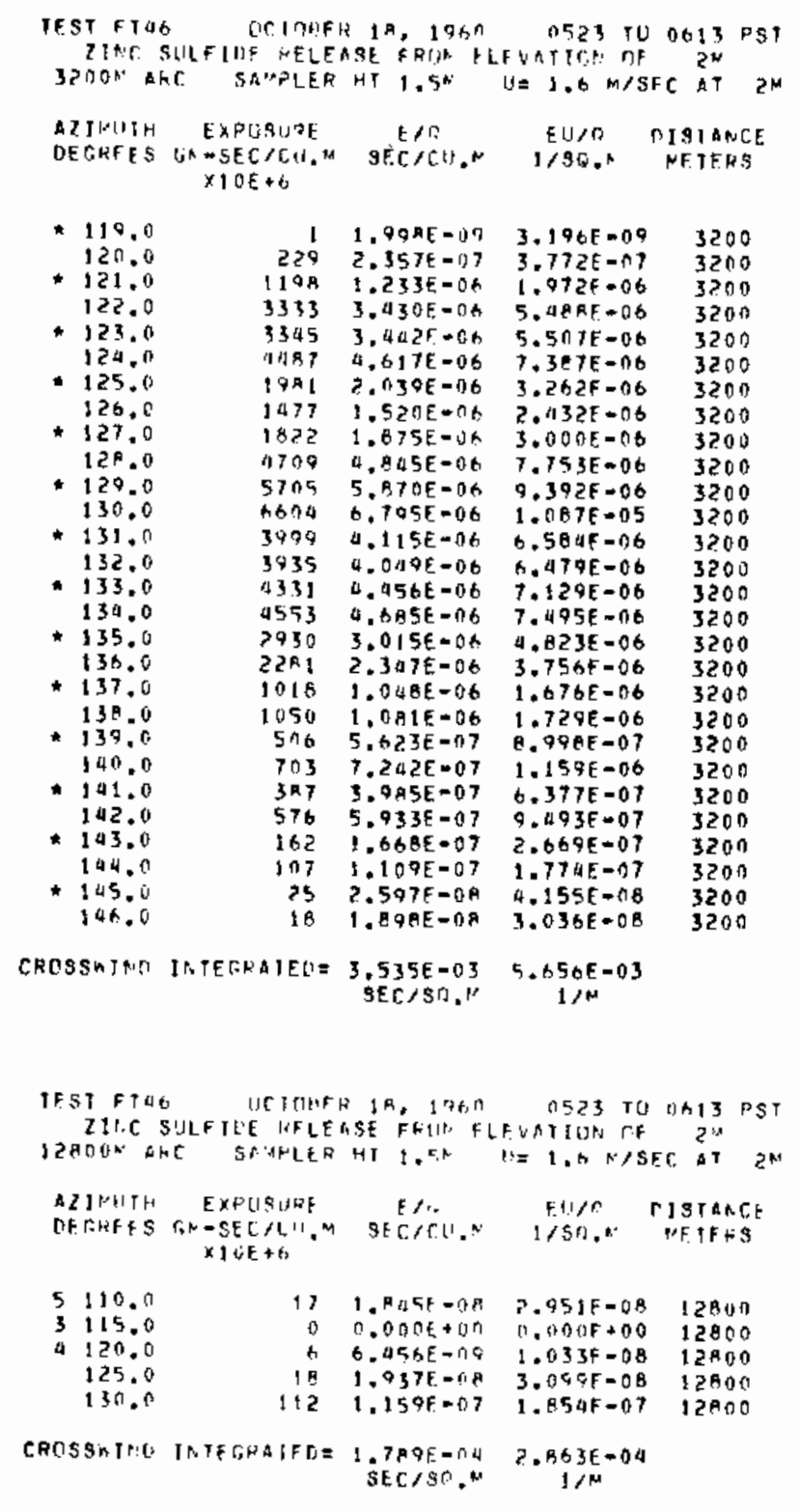


30-5ERISS EXPERIMENT: 47 I FT-47

GROUNC LEVEL AND TOWEZ SAMPLING $200-3200$ A ARCS

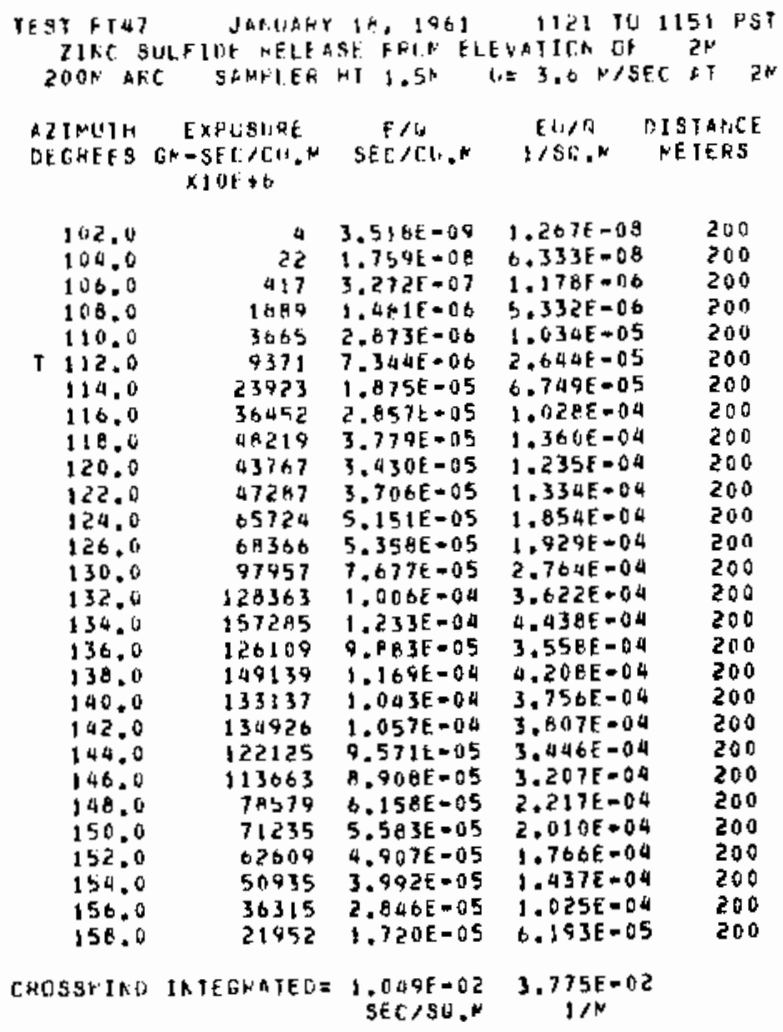

THEFE TATA FULLCH....

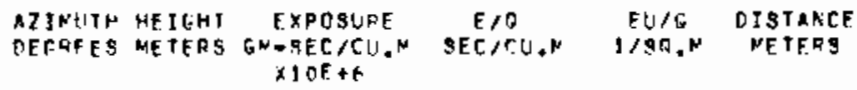

\begin{tabular}{|c|c|c|c|c|c|}
\hline $\begin{array}{l}\text { AZINUTH } \\
\text { DFCRFFS }\end{array}$ & $\begin{array}{l}\text { HE IGFiT } \\
\text { NE TFES }\end{array}$ & 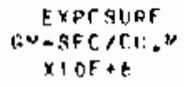 & $\begin{array}{c}\text { Sertri, } \\
E / A\end{array}$ & $\begin{array}{c}F(: / 6) \\
1 / S 0, N\end{array}$ & $\begin{array}{l}\text { DISTARCE } \\
\text { METERS }\end{array}$ \\
\hline 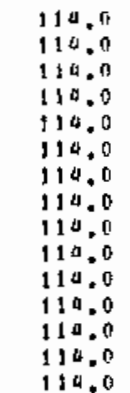 & $\begin{array}{l}0.7 \\
1.0 \\
2.7 \\
4.5 \\
5.5 \\
5.1 \\
9.2 \\
10.5 \\
13 . \\
18 . \\
21 . \\
24 . \\
27 .\end{array}$ & $\begin{array}{l}25210 \\
19420 \\
20909 \\
70225 \\
22501 \\
72957 \\
26139 \\
16596 \\
13597 \\
13347 \\
9349 \\
9054 \\
6012 \\
11609 \\
0924\end{array}$ & 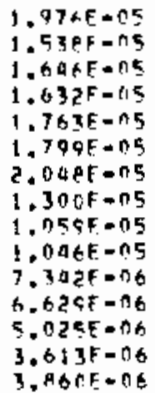 & $\begin{array}{l}7.113 E=05 \\
5.537 E-05 \\
5.025 E=05 \\
5.876 E=05 \\
6.34 A E-05 \\
6.477 E=05 \\
7.374 E=05 \\
1.680 E=05 \\
3.814 E=05 \\
3.766 E=05 \\
2.643 E=05 \\
2.386 E=05 \\
1.809 E=05 \\
1.301 E=05 \\
1.389 E=05\end{array}$ & $\begin{array}{l}200 \\
200 \\
200 \\
200 \\
200 \\
200 \\
200 \\
200 \\
200 \\
200 \\
200 \\
200 \\
200 \\
200 \\
200\end{array}$ \\
\hline
\end{tabular}

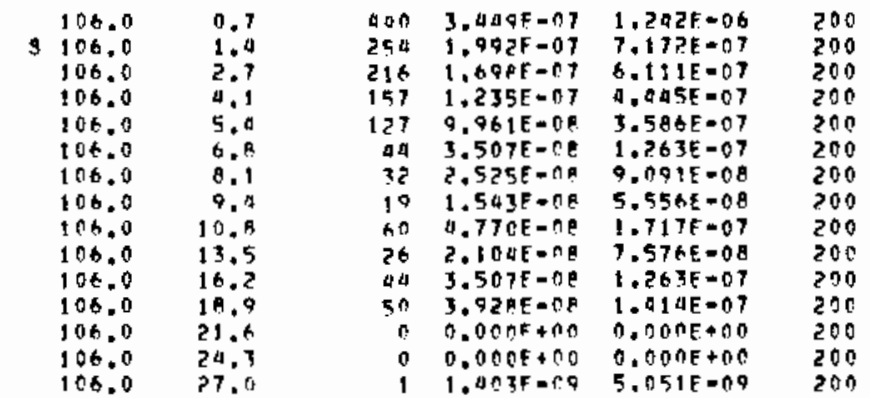

AZJNUTH HEIGHT EXPCSLRE FIR FU/O CISTANCE

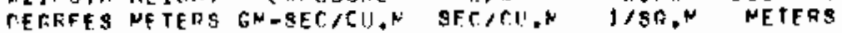
$\times 10 F+6$

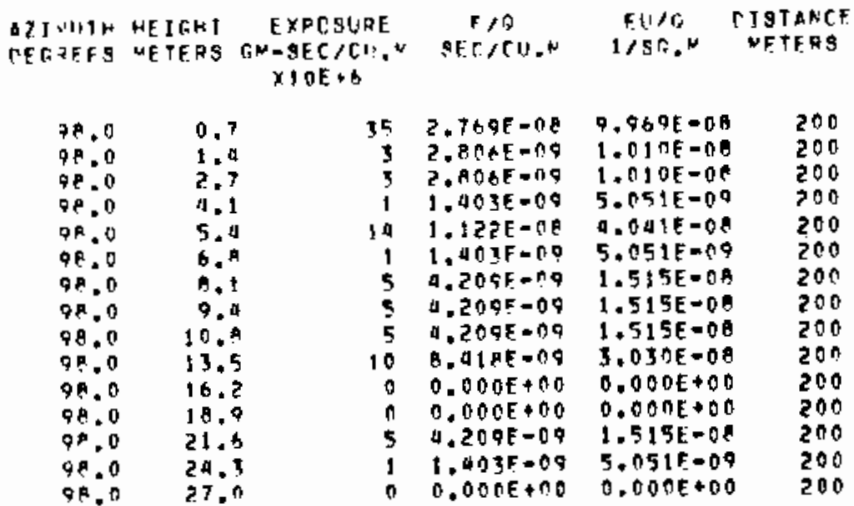




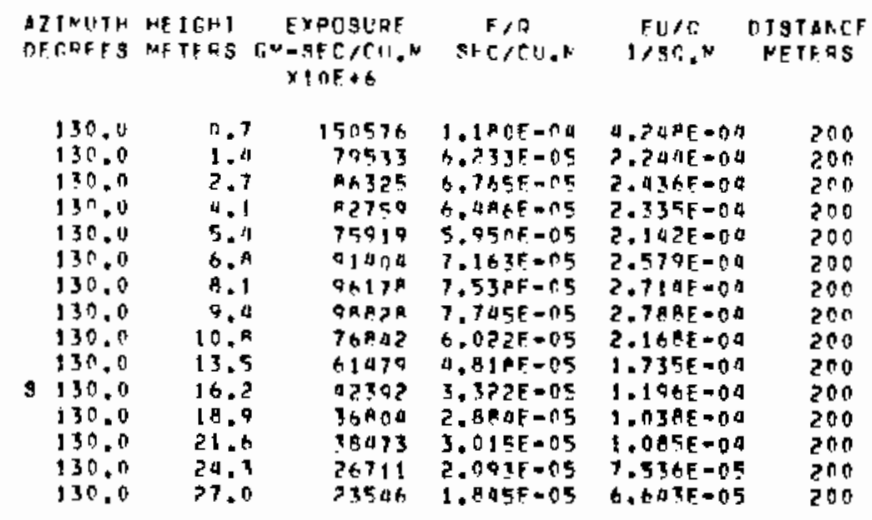

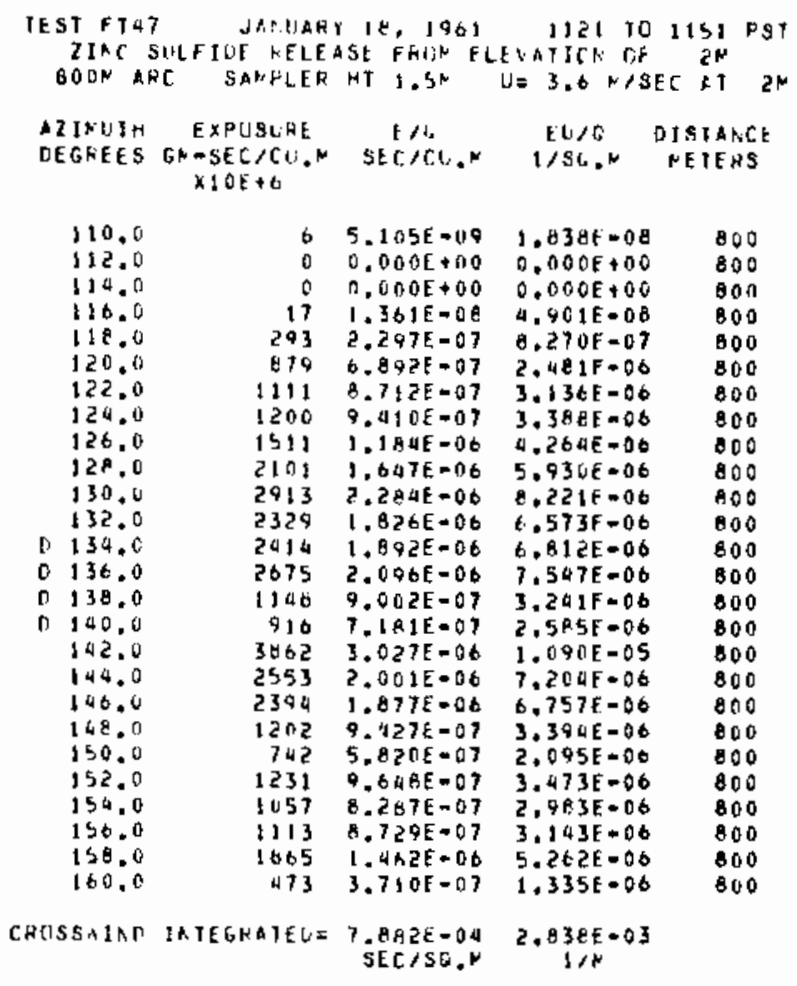

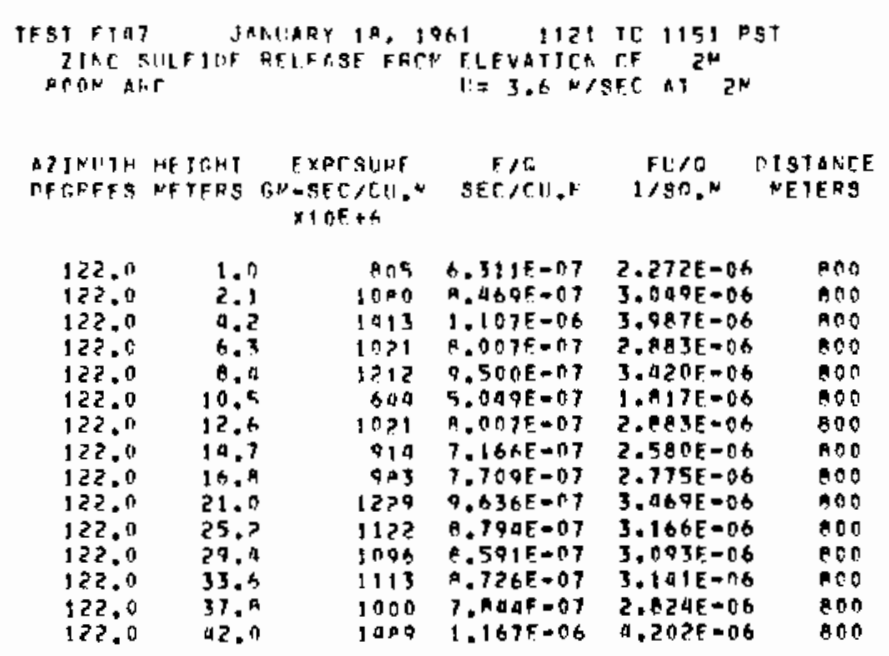
AZINLTH HEIGMT EXPOSURE E/O FU/D DISTANCE REFRFES NETERS GN-SEC/CU.N SECICU.N $1 / 80 . N$ METERS $\times 10 F+A$

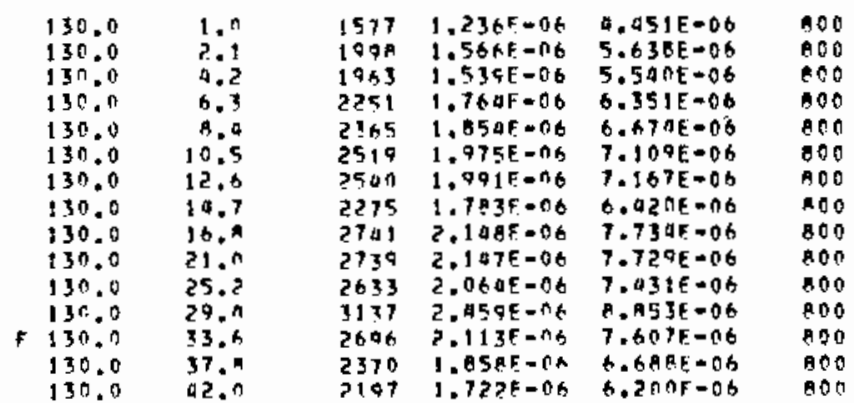

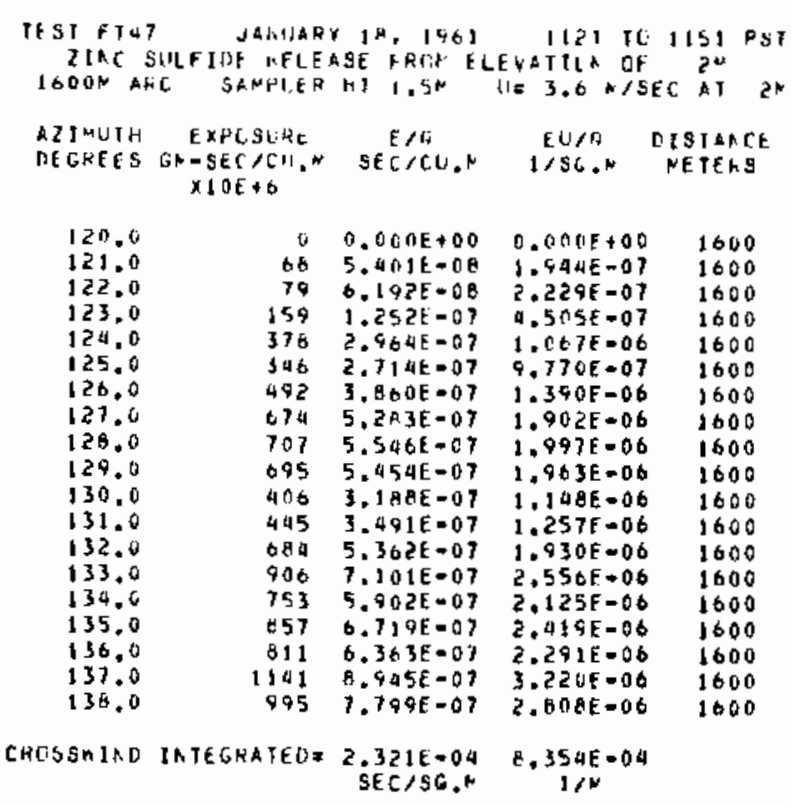




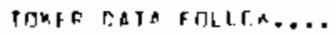

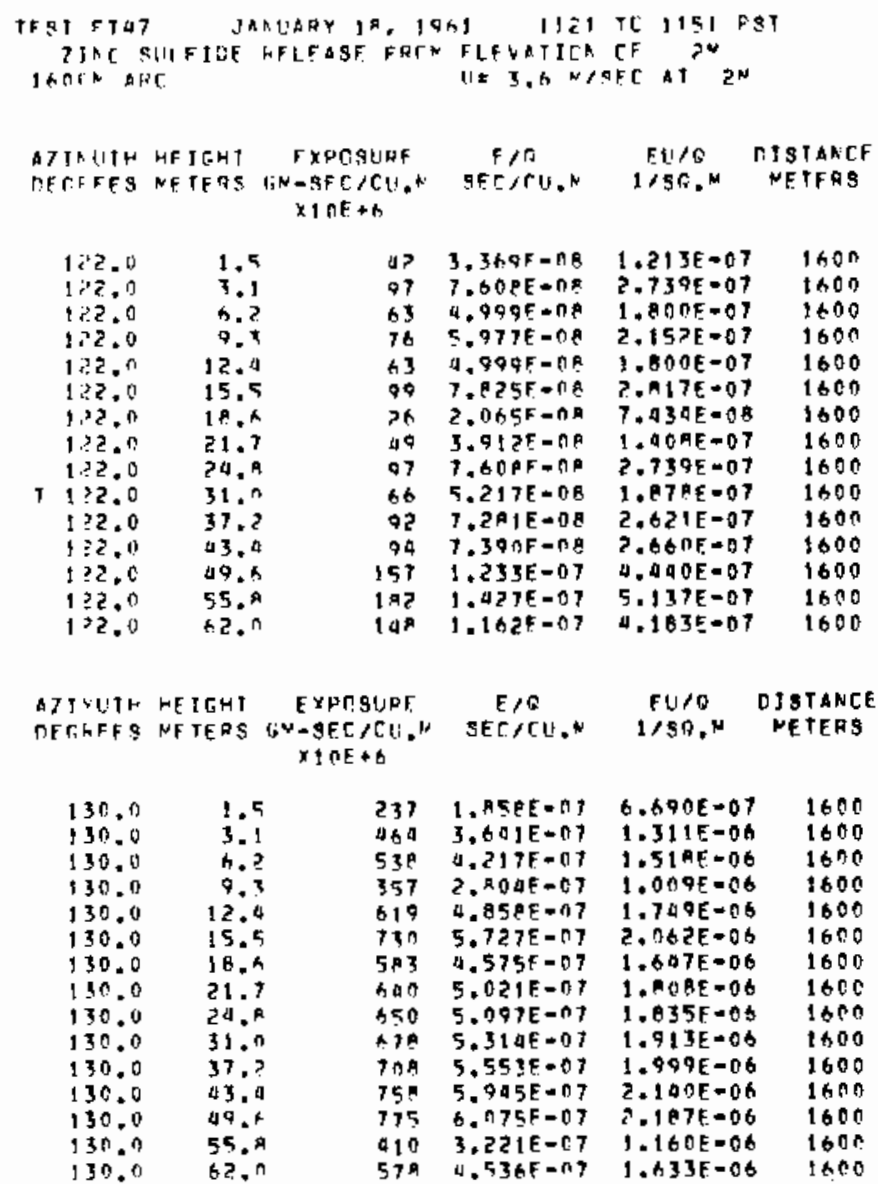

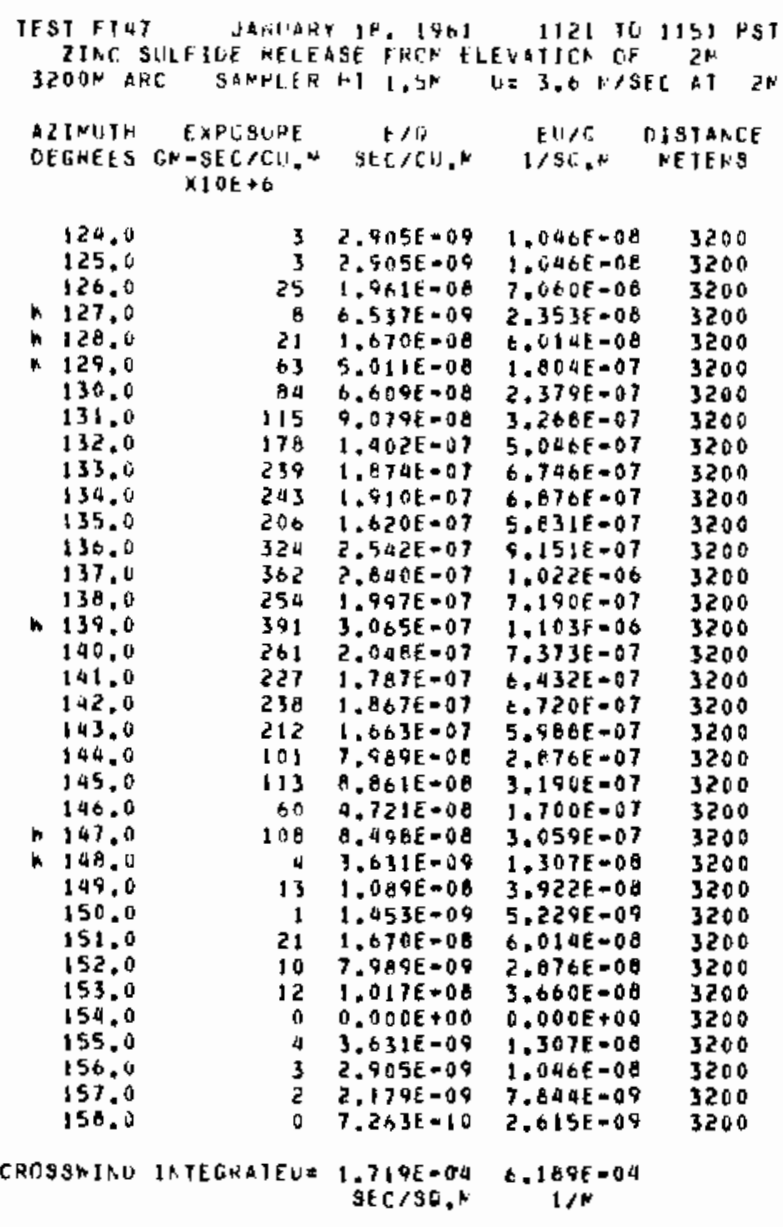

TOKER T:ATA FOLLO',...

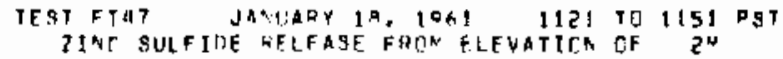
35RON ART

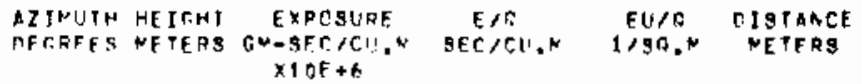

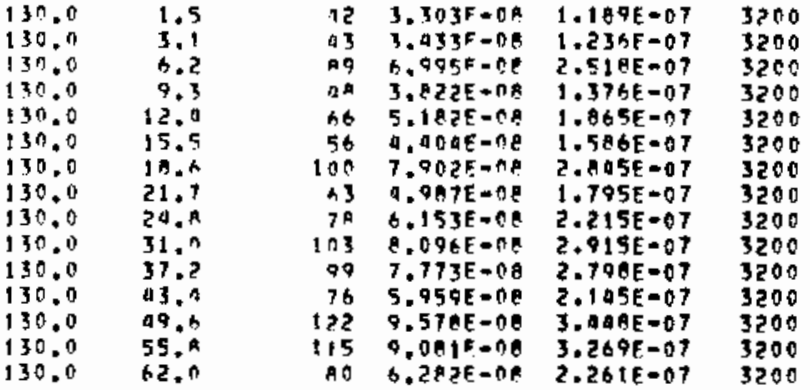


30-SERIES EXFERINENT: 48 (FT-48)

GROUHD LEVE! AND TOWER SAMPL ING 200-3200 ' A ARCS, HANY OF THE

FILTERS ON THE TOWERS SHOWED SIGNS OF WEATHERING.

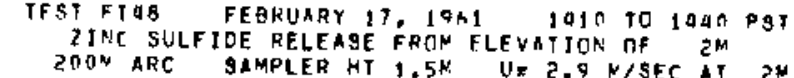

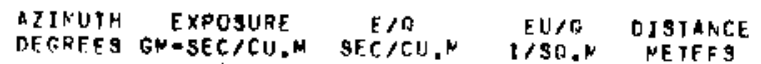
$X 1 O E+6$

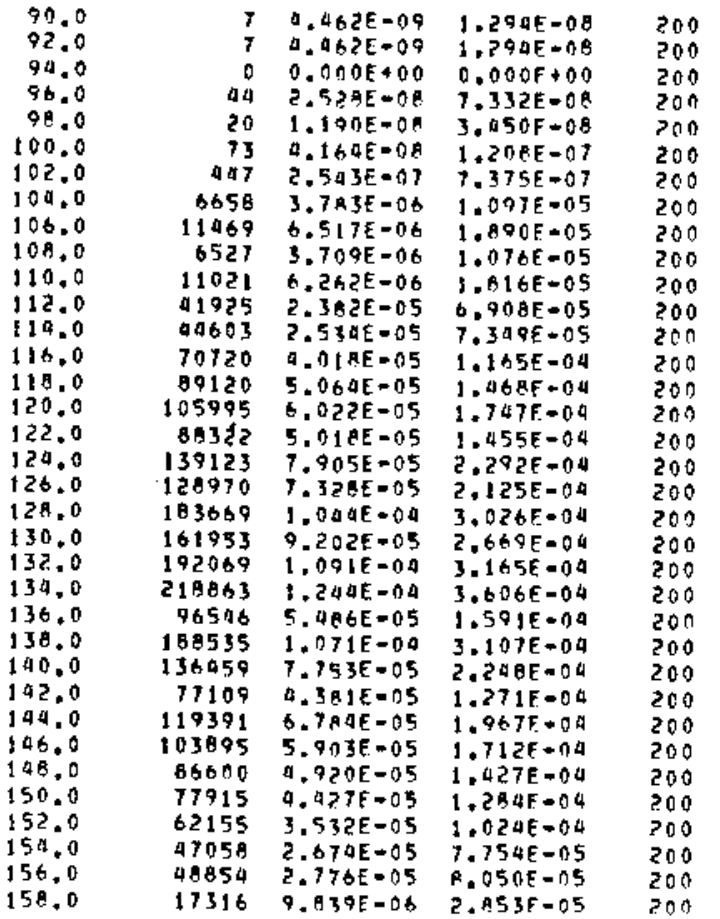

CROSBKIND INTEGRAIEDE 1.097E-DE 2.90QF-02

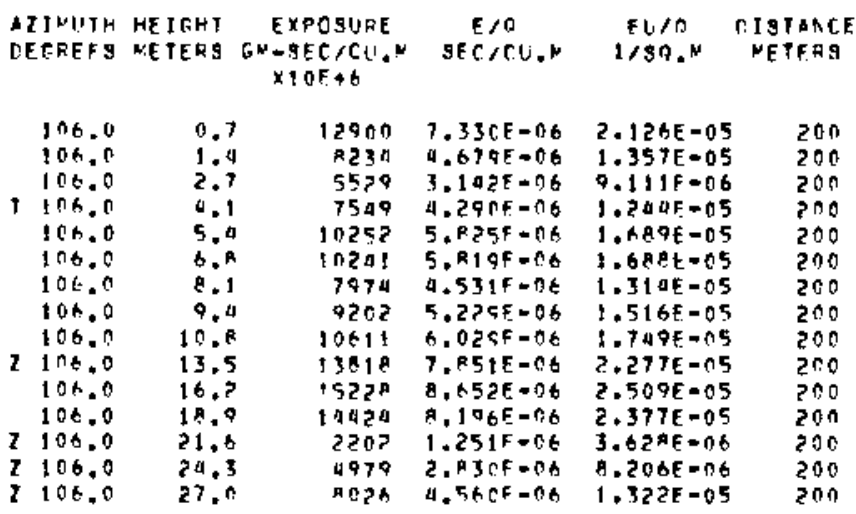

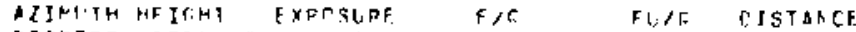

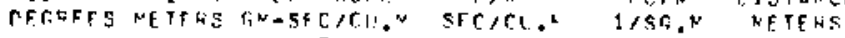

\begin{tabular}{|c|c|c|c|c|c|c|}
\hline & $\begin{array}{l}114.0 \\
110.6\end{array}$ & $\because .7$ & $\begin{array}{l}a n ?+6 \\
10 p 10\end{array}$ & $\begin{array}{l}3 .+25 F=n 5 \\
3.2^{2}+25=c 5\end{array}$ & $\begin{array}{l}7.623 E=05 \\
6.5 t 0 f=05\end{array}$ & $\begin{array}{l}200 \\
200\end{array}$ \\
\hline$t$ & 114,0 & ?. 7 & $70 \times 05$ & $1.517 F=5.5$ & $4.390 \mathrm{f}-75$ & $\geqslant \pi s$ \\
\hline 7 & $\$ 1 \%, 0$ & $a .1$ & 15011 & $P . D C F=1.6$ & $2.5726=0.5$ & $25 n$ \\
\hline 7 & 113,0 & 5.1 & $771 n 9$ & $1.5005-15$ & $4.4+74-05$ & 200 \\
\hline & $11 \% 0$ & & $2 \times 591$ & $1.02^{C F}=05$ & $4.711 t-n 5$ & 200 \\
\hline & 114.0 & & 10251 & $1.1 \div 45=0.5$ & 3.2RAC $\circ 5$ & $20 n$ \\
\hline 7 & 114.0 & 9.0 & $>01 A 3$ & $1.1 \triangle 7 F-0.5$ & $3.326 f=05$ & 200 \\
\hline 7 & $11 \% n$ & 10.9 & 17277 & $9.5175-16$ & $7 £-05$ & 200 \\
\hline 3 & 114.0 & 13.5 & 11 กลก & $\therefore .294 F=06$ & 1. В $225=05$ & 200 \\
\hline & 114.0 & $16 . ?$ & ग1मद? & $1.230 E=05$ & $3.568 E=09$ & 200 \\
\hline 2 & 1140 & 10,0 & 9425 & $355 F=06$ & $1.553 \mathrm{~F} .05$ & 200 \\
\hline & 114.0 & 21.8 & $77 n z$ & $377 F=06$ & $269 F=05$ & 200 \\
\hline$Z$ & 1100 & $5^{20} \cdot 7$ & 6898 & $3.0195=n 6$ & $1.136 E=05$ & 500 \\
\hline & (1) & 21 & 12017 & $B$. करेPF $=$ (16 & QnE $=05$ & ?nn \\
\hline
\end{tabular}

A2INUTH HEICHT FYPCSUEF FA FUAG DISTANCF

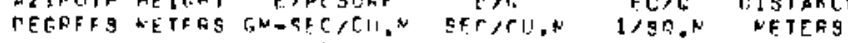

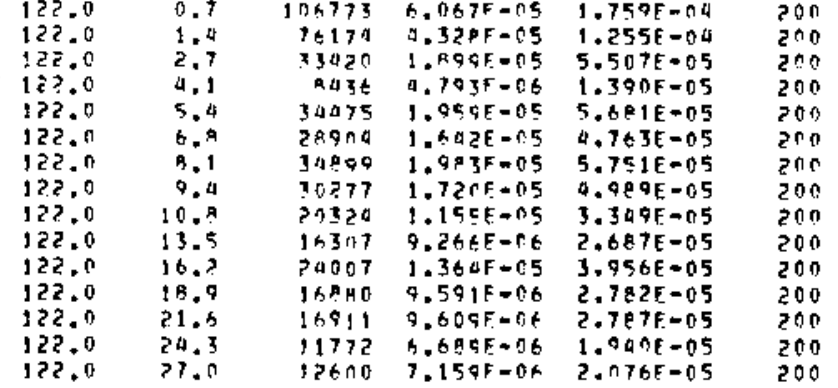

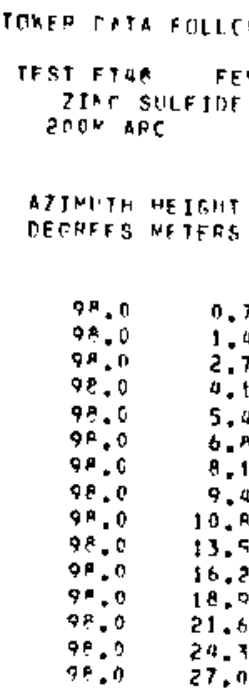

1470 te 1 and

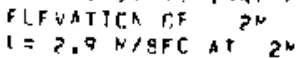

FYP 3 UUE

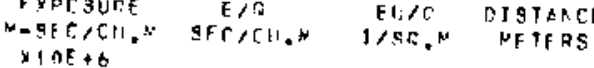

$$
\begin{aligned}
& 3
\end{aligned}
$$

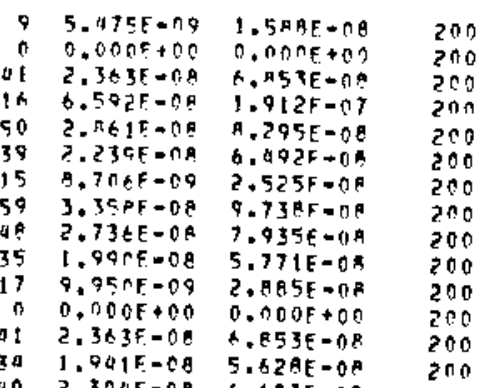




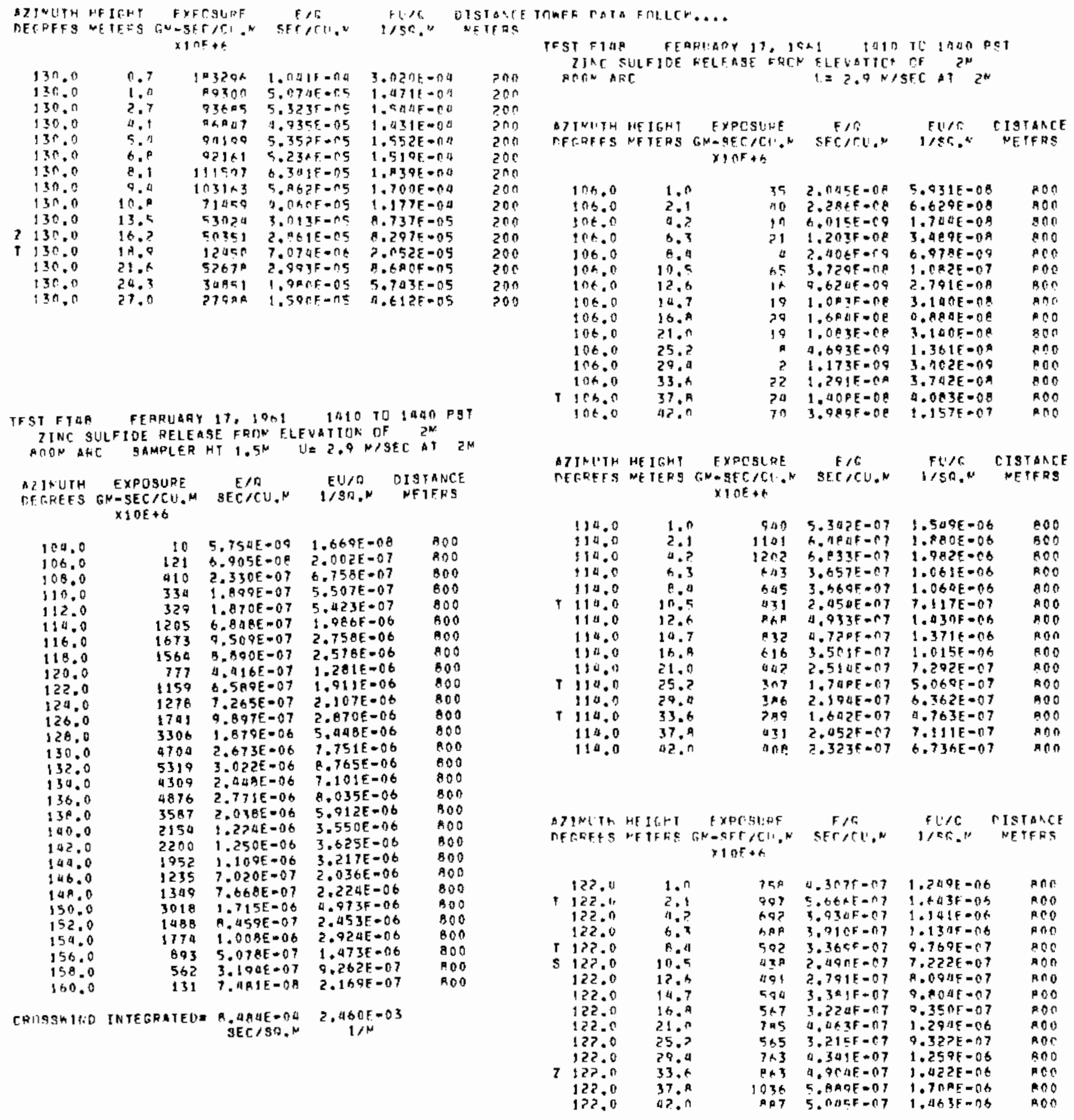




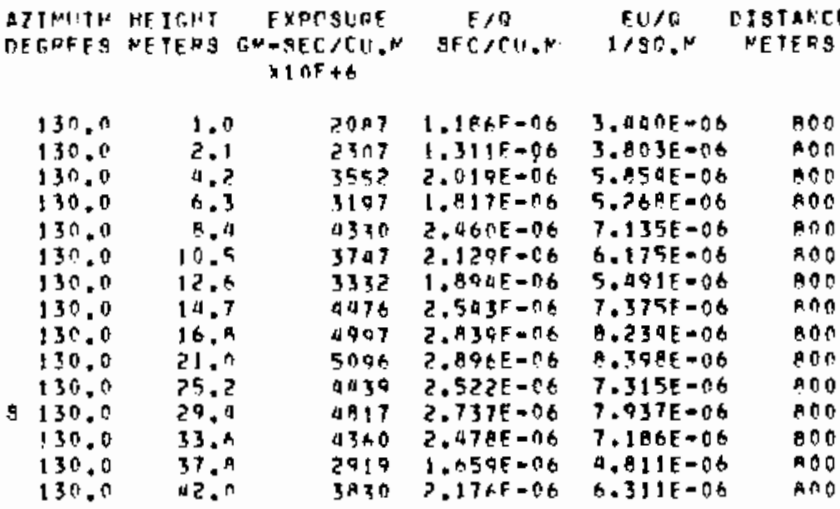

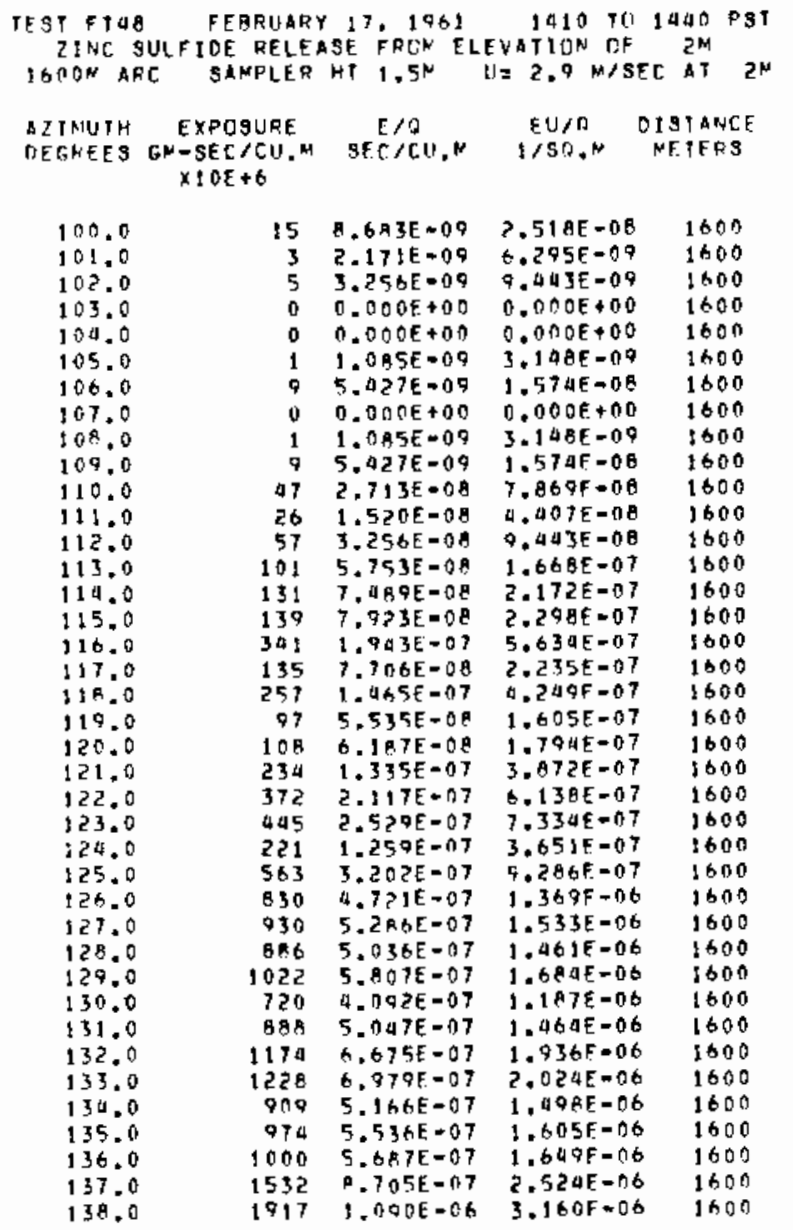

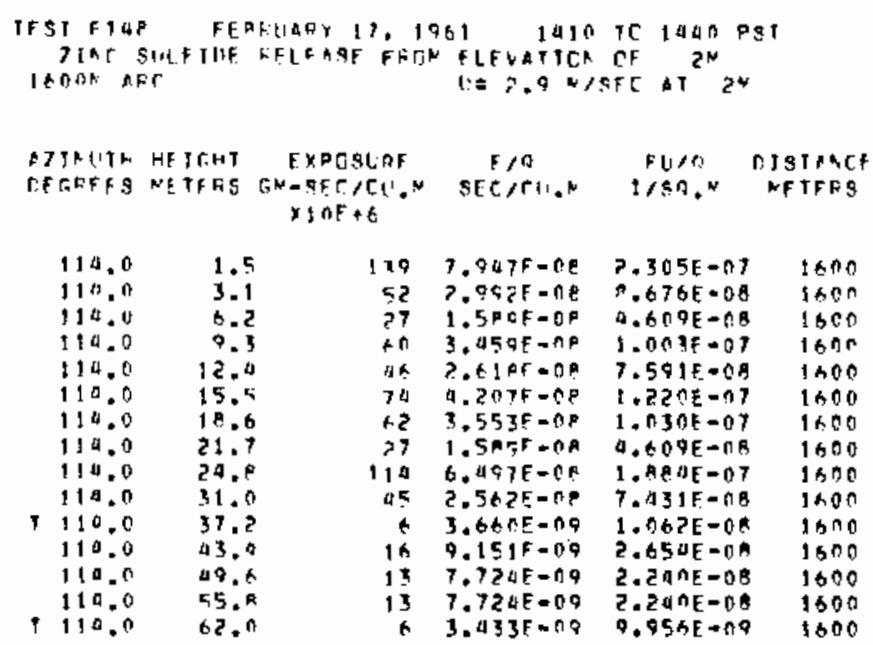

AITNITH HEIGHT EXVCSURE ETE EUR DISTARCE DEGFEES NETFHS GNABEC/CU.N SECIC!!N 1/SH.N NETERS

\begin{tabular}{|c|c|c|c|c|c|}
\hline $\begin{array}{l}122.0 \\
122.0 \\
122.0 \\
122.0 \\
122.0 \\
122.0 \\
122.0 \\
12200 \\
122.0 \\
122.0 \\
122.0 \\
122.0 \\
122.0 \\
122.0 \\
122.0\end{array}$ & $\begin{array}{l}1.5 \\
3.1 \\
6.7 \\
9.3 \\
12.4 \\
15.5 \\
18.6 \\
71.7 \\
24.8 \\
31.0 \\
37.2 \\
113.4 \\
49.6 \\
55.8 \\
62.0\end{array}$ & $\begin{array}{r}118 \\
106 \\
70 \\
09 \\
95 \\
48 \\
28 \\
110 \\
125 \\
82 \\
102 \\
117 \\
78 \\
75 \\
30\end{array}$ & 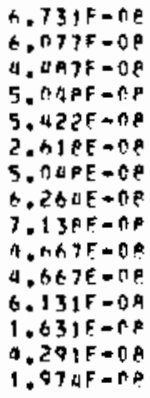 & 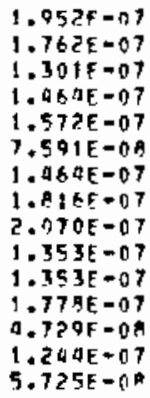 & $\begin{array}{l}1600 \\
1600 \\
1600 \\
1600 \\
1600 \\
1600 \\
1600 \\
1600 \\
1600 \\
1600 \\
1600 \\
1690 \\
1600 \\
1600 \\
1600\end{array}$ \\
\hline
\end{tabular}

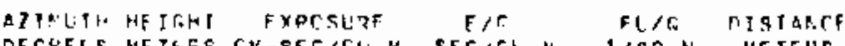

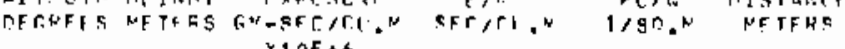

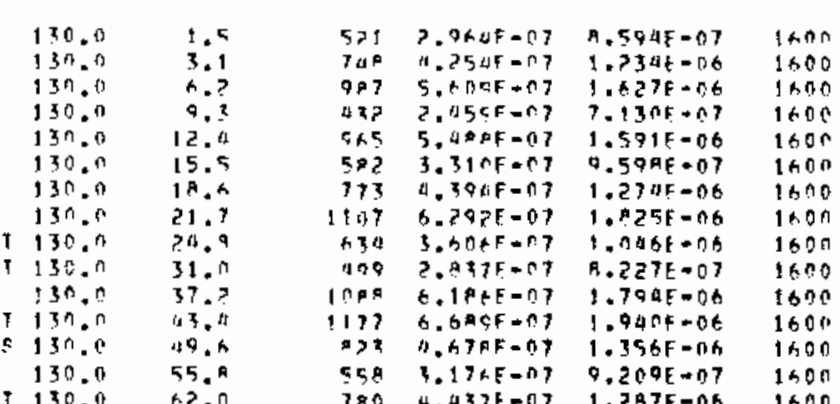

CROSSHIHT IATEGRAIED $=2.752 E-5907.98 .2 F-04$ 


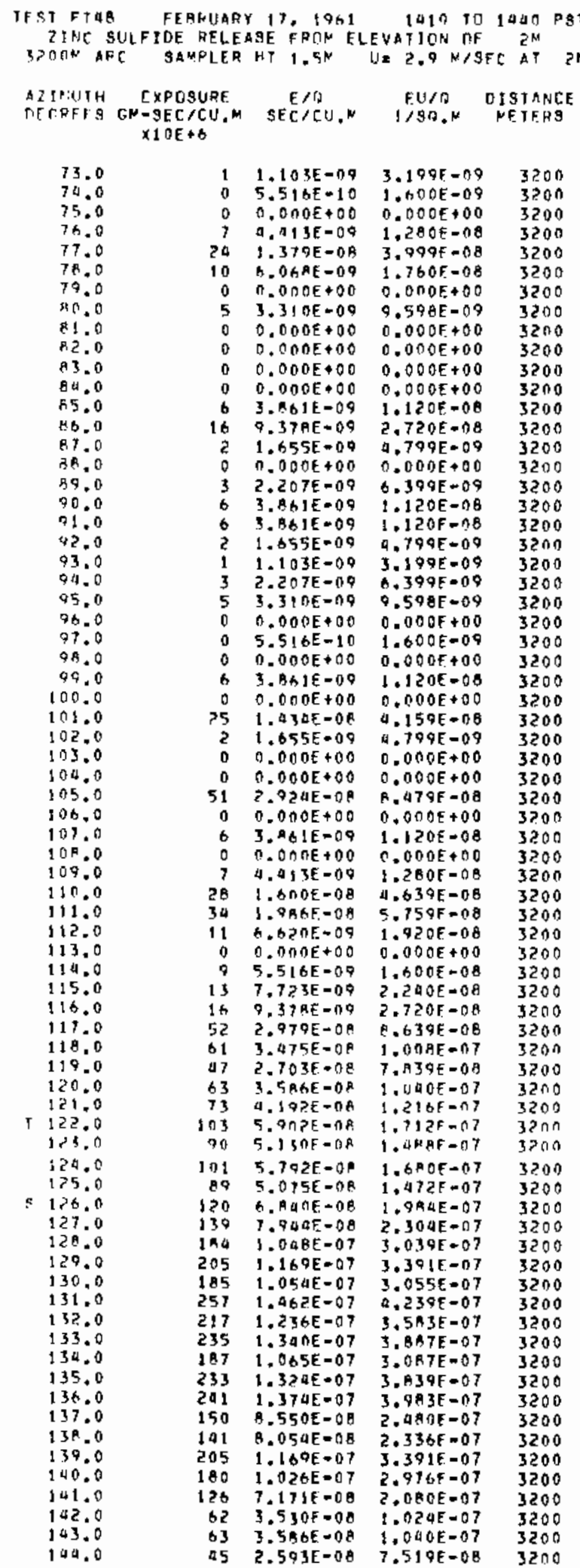

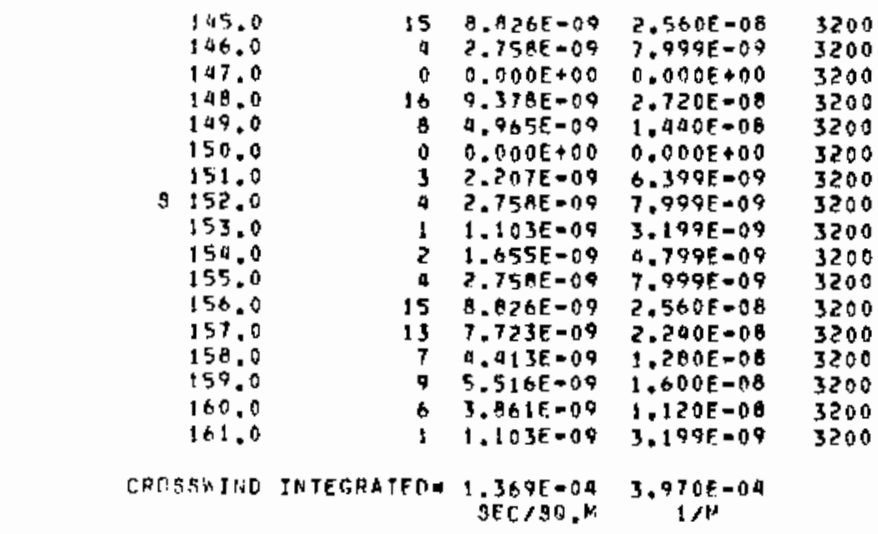

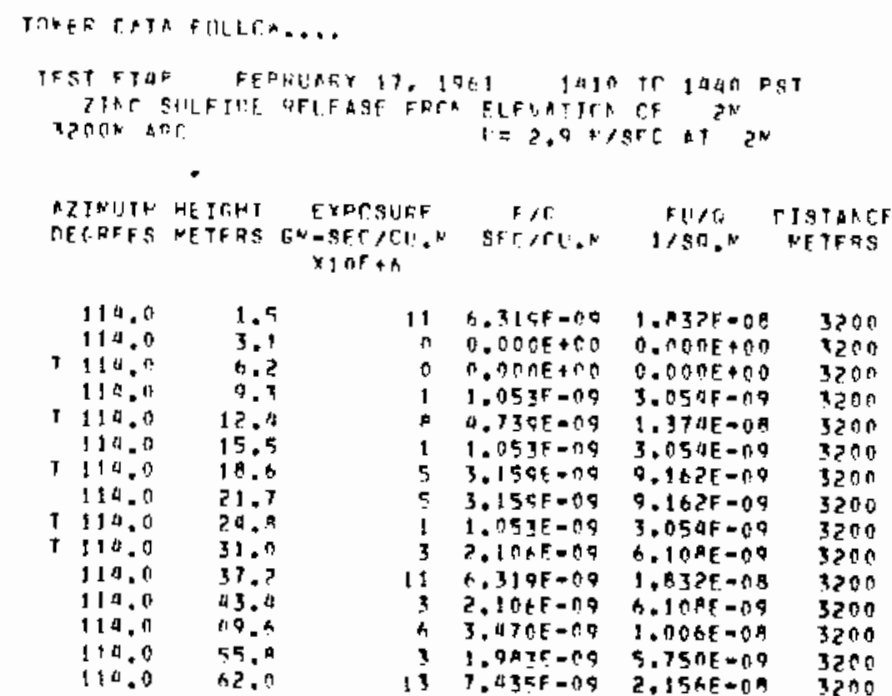

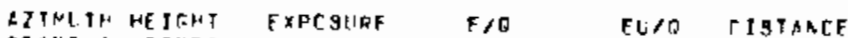

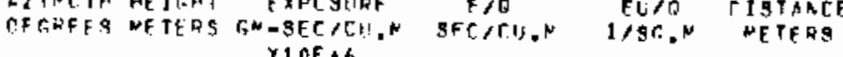
$\times 1 \mathrm{nF}+\mathrm{t}$

\begin{tabular}{|c|c|c|c|c|c|}
\hline $\begin{array}{l}122.0 \\
122.0 \\
122.0 \\
127.0\end{array}$ & $\begin{array}{l}1.9 \\
3.1 \\
6.7 \\
9.7\end{array}$ & $\begin{array}{r}68 \\
70 \\
117 \\
149\end{array}$ & $\begin{array}{l}3.897 E=0 R \\
1.902 F=08 \\
6.8 Q 7 E=0 E \\
9.47 F F=08\end{array}$ & $\begin{array}{l}1.130 E-07 \\
1.161 E=07 \\
1.939 E=07 \\
2.459 F-07\end{array}$ & $\begin{array}{l}3200 \\
3200 \\
3200 \\
3200\end{array}$ \\
\hline $\begin{array}{l}122.0 \\
132.0\end{array}$ & $\begin{array}{l}12.9 \\
15.5\end{array}$ & $\begin{array}{l}100 \\
107\end{array}$ & $\begin{array}{l}5.4 A 7 F=08 \\
9.37 ? E=08\end{array}$ & $\begin{array}{l}1 . \Delta 40 E-07 \\
2 . \Delta 2 \text { DE }=01\end{array}$ & $\begin{array}{l}3200 \\
3200\end{array}$ \\
\hline 122.0 & 18.6 & 117 & $6.687 E=08$ & $1.939 F-07$ & 3200 \\
\hline $\begin{array}{l}122.0 \\
122.0\end{array}$ & $\begin{array}{l}21.7 \\
24.9\end{array}$ & $\begin{array}{l}112 \\
1 \geq 9\end{array}$ & $\begin{array}{l}0.371 E-O B \\
7.372 E-O E\end{array}$ & $\begin{array}{l}1.04 A E=07 \\
2.13 \cap E=07\end{array}$ & $\begin{array}{l}3200 \\
3200\end{array}$ \\
\hline 122.0 & 31.0 & 104 & $5.95 C E-0 A$ & $1.726 E-07$ & 3200 \\
\hline & $\begin{array}{l}37.7 \\
43.4 \\
09.4\end{array}$ & $\begin{array}{r}134 \\
97\end{array}$ & $\begin{array}{l}7.635 E=08 \\
5.529 E=0 A\end{array}$ & $\begin{array}{l}2.714 E-07 \\
1.603 E-07 \\
7115=07\end{array}$ & $\begin{array}{l}3200 \\
3200\end{array}$ \\
\hline 12 & 55.7 & 123 & 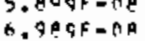 & $\begin{array}{l}1.711 E-07 \\
2.5775=0 ?\end{array}$ & $\begin{array}{l}3200 \\
3200\end{array}$ \\
\hline & A. . $n$ & $A 2$ & 11. 30 CE- ती & $1.36 \mathrm{BE}=07$ & $3 ? 00$ \\
\hline
\end{tabular}




\begin{tabular}{|c|c|c|c|c|c|c|}
\hline \multicolumn{2}{|c|}{$\begin{array}{l}\triangle 7 I N 11 T h \\
\text { FFFEF }\end{array}$} & $\begin{array}{l}\text { HE I I, H T } \\
\text { NE TFitS }\end{array}$ & $\begin{array}{l}\text { EPCSLPF } \\
G^{N}=-5 E C / C I: N \\
\times 1 \cap F+6\end{array}$ & $\begin{array}{c}F / s \\
\text { sferden }\end{array}$ & $\begin{array}{c}F U / C_{i} \\
1 / S^{N} .\end{array}$ & $\begin{array}{l}\text { P. TSTARCF } \\
\text { NETFRS }\end{array}$ \\
\hline & $13 n .0$ & 1.5 & $2 \mathrm{da}$ & 1. $3 \cap \cap F=\cap 7$ & $1.001 E=07$ & 3201 \\
\hline & $13 n .0$ & 3.1 & 1,9 & $6.7 \Delta C F=n R$ & $1.055 \mathrm{E}=07$ & 3200 \\
\hline $\mathbf{5}$ & 150.0 & h. & 213 & $1.21\{\neq-0.7$ & $3.51 ? 5=07$ & 1700 \\
\hline$r$ & $1 \leq 0.0$ & 9.7 & 100 & $h, 2 I J F=\hat{A}$ & 1. 10 OPE $=07$ & 37ח \\
\hline & $13 c \cdot n$ & 12.0 & $23:$ & $1.31+5=07$ & 3. P $\mid$ PE $=07$ & 3200 \\
\hline & $130 . n$ & 15.5 & $2+2$ & $1.108 \mathrm{E}=07$ & $3,3295=07$ & 3200 \\
\hline & $13 n .0$ & Ia. & $1 \cap p$ & $6.1615=0.8$ & $1.707 F=07$ & $3 \geqslant 00$ \\
\hline & $13 n . n$ & 21.7 & $13 ?$ & $7.5 A 2 F+0 A$ & $2.189 \mathrm{E}=07$ & 3200 \\
\hline & .0 & $a^{4}$ & $25 \pi$ & $1.400 \mathrm{C}=\mathrm{Cl}$ & $0.251 E=0 ?$ & 3700 \\
\hline & 130.0 & 3 & 213 & $1.211 \mathrm{~F}=07$ & $3.512 E-07$ & 3200 \\
\hline & 130.0 & 37.7 & $16 t$ & $9.47 P E-08$ & $2.749 \mathrm{E}-1.7$ & 3200 \\
\hline & $13 n .0$ & 43.6 & 213 & $1,211 E=.7$ & $3.512 F=07$ & $320 n$ \\
\hline & 130.0 & 49 & A) & $4.75 \gamma=0.08$ & 1.43 A $E=07$ & 3200 \\
\hline & 130.0 & & $0 ?$ & $5.254 \mathrm{~F}=0 \mathrm{E}$ & $1.527 E-07$ & 3200 \\
\hline r & $13 n$ & & 102 & $5, \operatorname{lnc}(-0)$ & hP $2 \mathrm{~F}-\cap 7$ & $3>00$ \\
\hline
\end{tabular}


JD-SERIES EXPERIHE:TT: 49 \& FT-d9:

GROUND LEYEL AND TUIER 5AMPLING $2 J 0-3200$ A ARCS. YANY JF THE

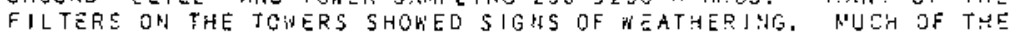

PLUME PASSED TO THE SUUTH OF THE SRID.

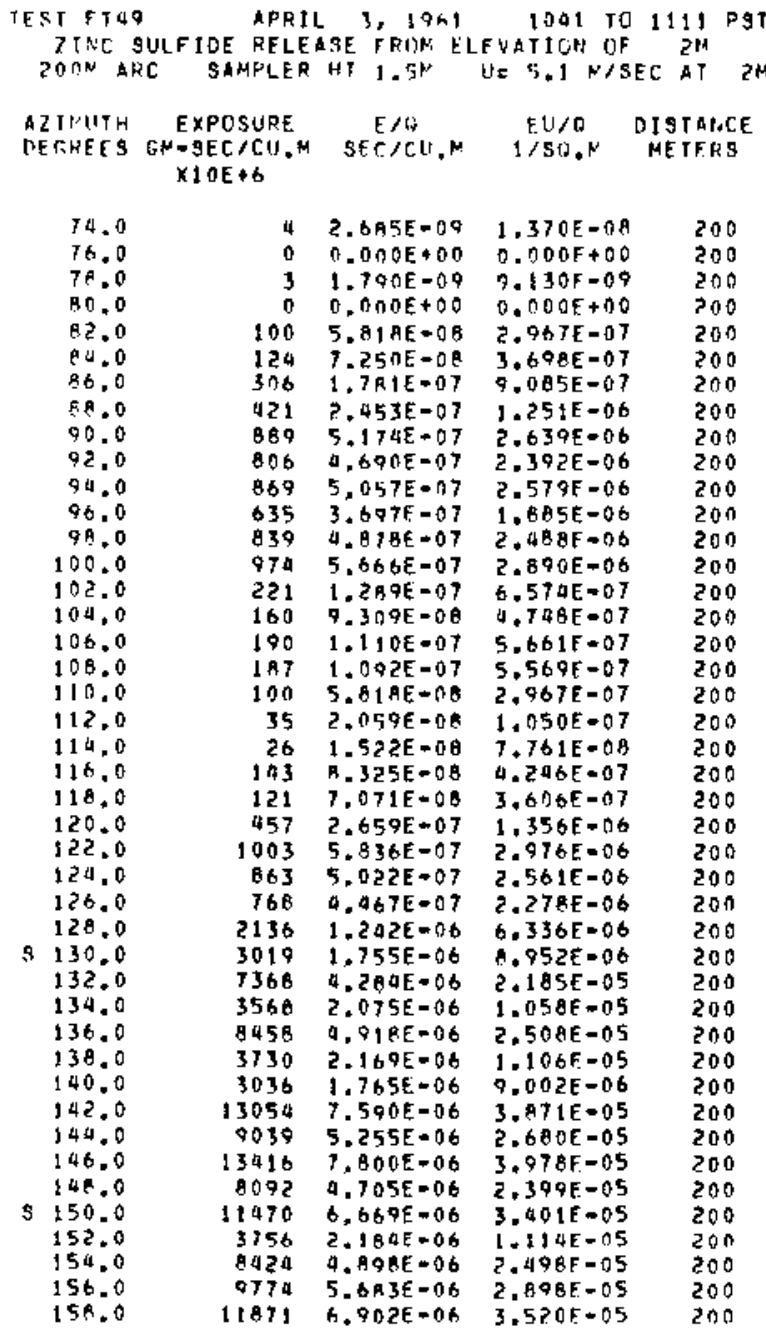

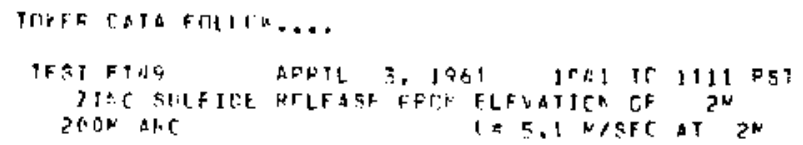

\begin{tabular}{|c|c|c|c|c|c|}
\hline 100.0 & $\begin{array}{l}0.7 \\
1.9 \\
2.7 \\
4.1 \\
5.9 \\
6.4 \\
9.1 \\
9.7 \\
10.9 \\
13.5 \\
16.2 \\
18.9 \\
21.6 \\
24.3 \\
27.0\end{array}$ & $\begin{array}{r}172 \\
119 \\
69 \\
191 \\
19 \\
39 \\
47 \\
27 \\
13 \\
0 \\
20 \\
30 \\
0 \\
37 \\
57\end{array}$ & $\begin{array}{l}1.003 E-07 \\
6.94 E E-08 \\
4.043 E=08 \\
1.116 E=07 \\
4.612 E=08 \\
2.278 E=08 \\
3.733 E=08 \\
1.594 F=08 \\
3.701 E=08 \\
0.000 E+00 \\
1.19 t E=08 \\
1.765 E-08 \\
0.000 E+00 \\
2.197 E=08 \\
3.343 E=08\end{array}$ & $\begin{array}{l}5.113 E-07 \\
3.543 E=07 \\
2.062 E=07 \\
5.691 E-07 \\
2.352 E-07 \\
1.162 E=07 \\
1.390 E=07 \\
8.131 E=07 \\
1.807 E=07 \\
0.000 E+00 \\
6.09 B E=08 \\
9.002 E=08 \\
0.000 E+00 \\
1.120 E=07 \\
1.705 E-07\end{array}$ & $\begin{array}{l}200 \\
200 \\
200 \\
200 \\
200 \\
200 \\
200 \\
200 \\
200 \\
200 \\
200 \\
200 \\
200 \\
200 \\
200\end{array}$ \\
\hline
\end{tabular}

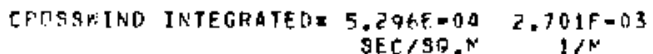




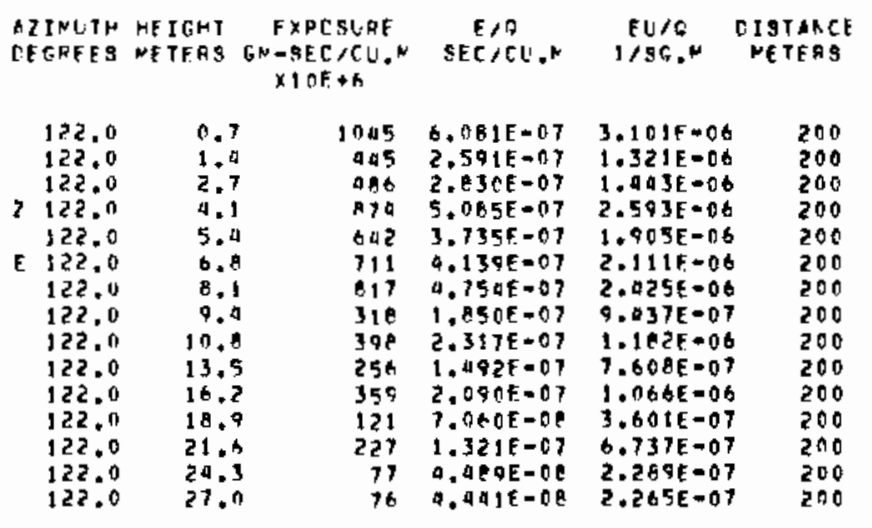

\begin{tabular}{|c|c|c|c|c|c|}
\hline $\begin{array}{l}\text { ATINLTH } \\
\text { DEGREES }\end{array}$ & $\begin{array}{l}\text { HE JGH } \\
\text { NF TERS }\end{array}$ & 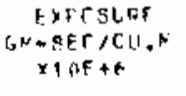 & $\begin{array}{c}E / G \\
\text { stc }<>t+1, N\end{array}$ & $\begin{array}{c}F L / R \\
1 / S C . N\end{array}$ & $\begin{array}{l}\text { I I TALICE } \\
\text { NETEFG }\end{array}$ \\
\hline $\begin{array}{l}130.0 \\
130.0 \\
130.0 \\
130.0 \\
130.0 \\
130.0 \\
130.0 \\
130.0 \\
130.0 \\
130.0 \\
130.0 \\
130.0 \\
130.0 \\
130.0 \\
130.0\end{array}$ & $\begin{array}{l}1.7 \\
1.9 \\
2.7 \\
4.1 \\
5.4 \\
6.8 \\
8.1 \\
9.4 \\
10.8 \\
13.5 \\
10.2 \\
18.0 \\
21.4 \\
24.3 \\
27.0\end{array}$ & $\begin{array}{r}76 P 3 \\
771 \\
978 \\
1624 \\
2595 \\
1100 \\
1196 \\
1704 \\
503 \\
628 \\
1903 \\
1700 \\
1922 \\
469 \\
040\end{array}$ & 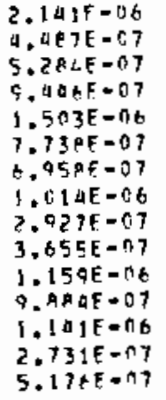 & $\begin{array}{l}1.092 E=05 \\
2.288 E=06 \\
2.645 \mathrm{~F}=06 \\
0.817 \mathrm{E}=06 \\
7.666 \mathrm{E}=06 \\
3.946 \mathrm{E}=06 \\
3.508 \mathrm{E}=06 \\
5.172 \mathrm{E}=06 \\
1.093 \mathrm{E}=06 \\
1.864 \mathrm{E}=06 \\
5.012 \mathrm{E}=06 \\
5.041 \mathrm{E}=06 \\
5.019 \mathrm{E}=06 \\
1.393 \mathrm{E}=06 \\
2.640 \mathrm{E}=06\end{array}$ & $\begin{array}{l}200 \\
200 \\
200 \\
200 \\
200 \\
200 \\
200 \\
200 \\
200 \\
200 \\
200 \\
200 \\
200 \\
200 \\
200\end{array}$ \\
\hline
\end{tabular}

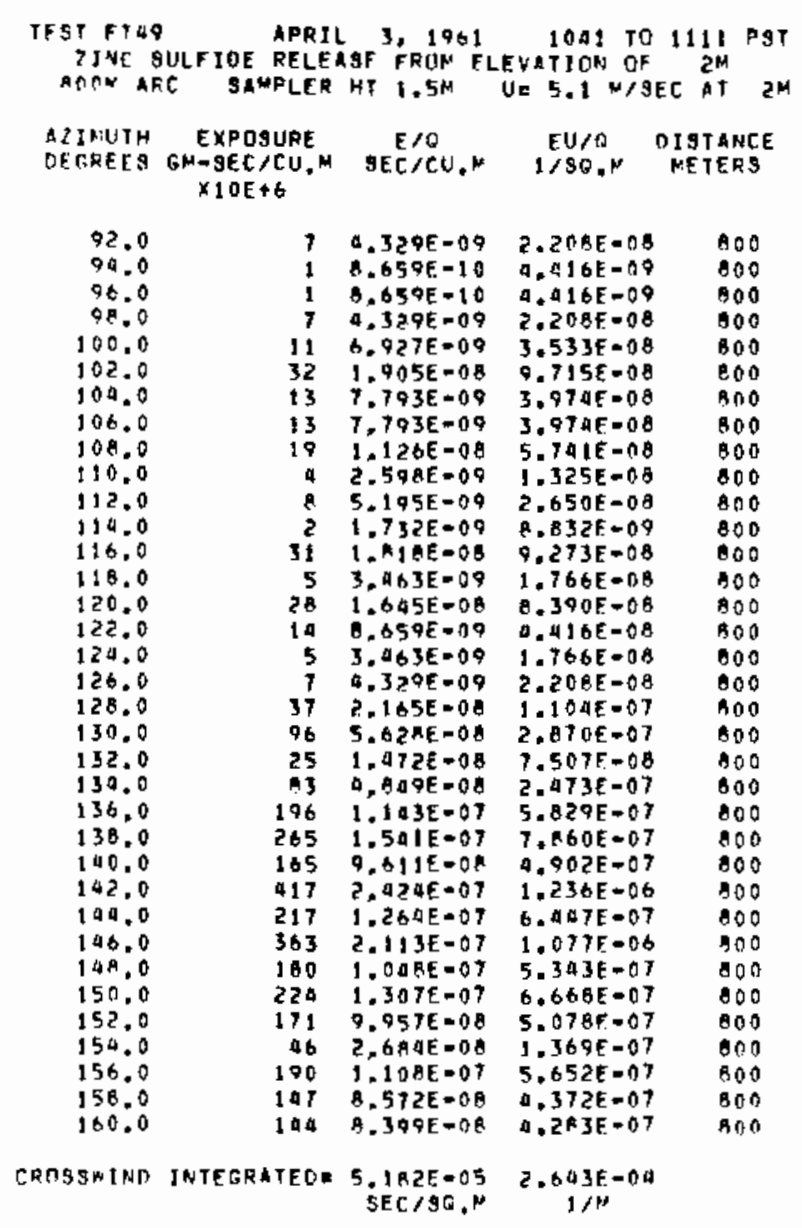

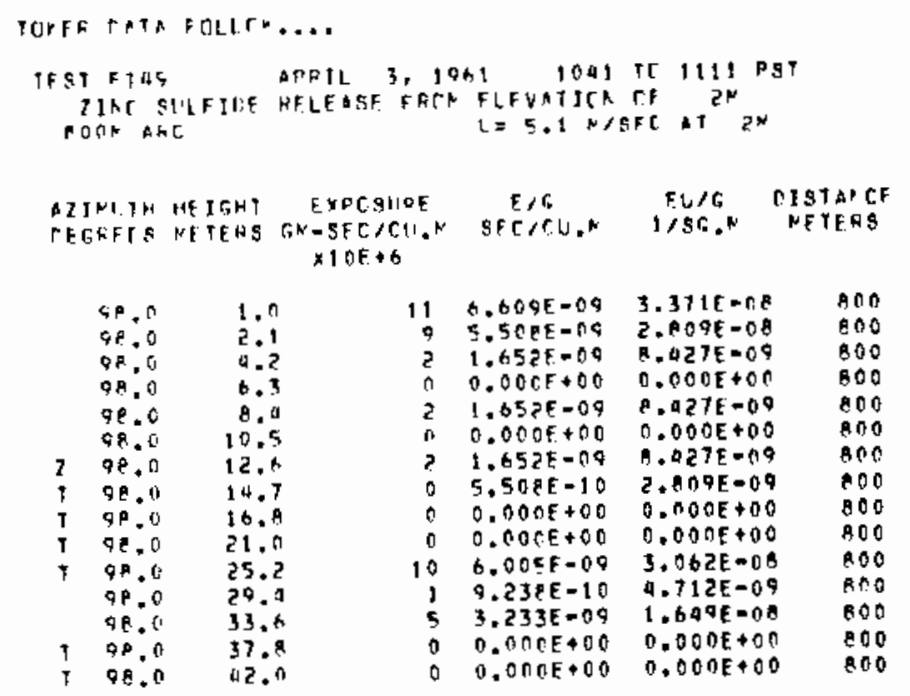




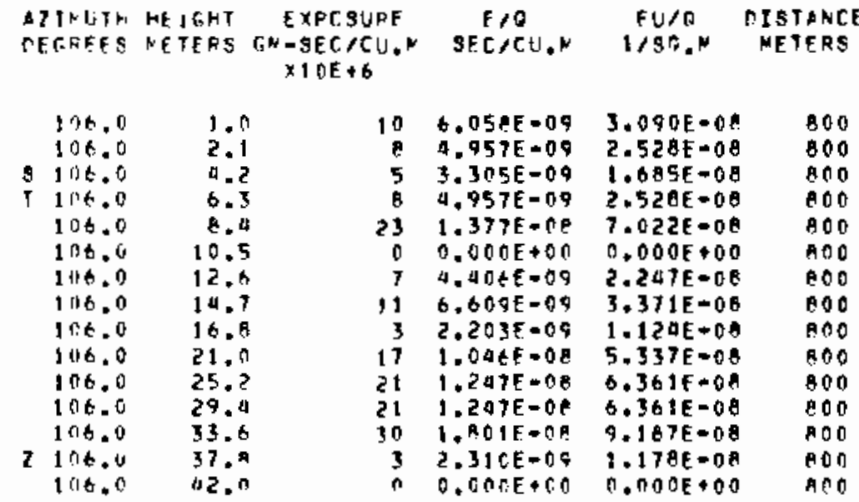

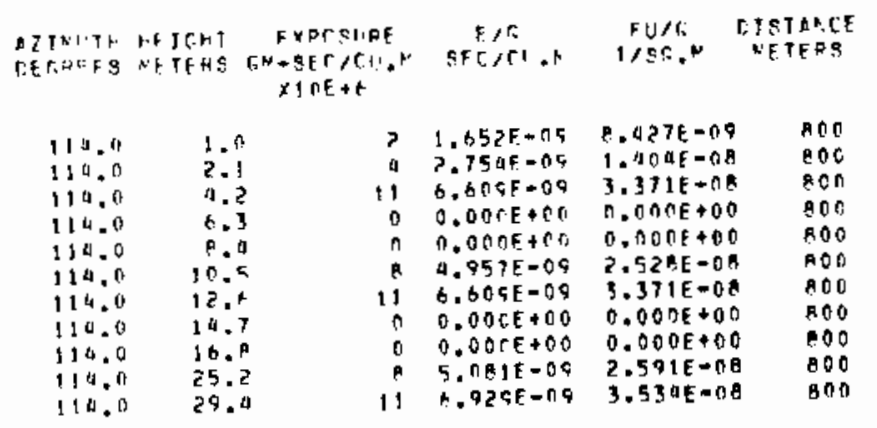

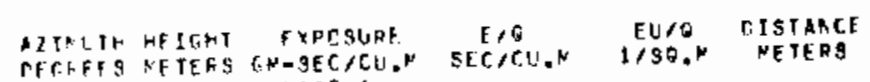

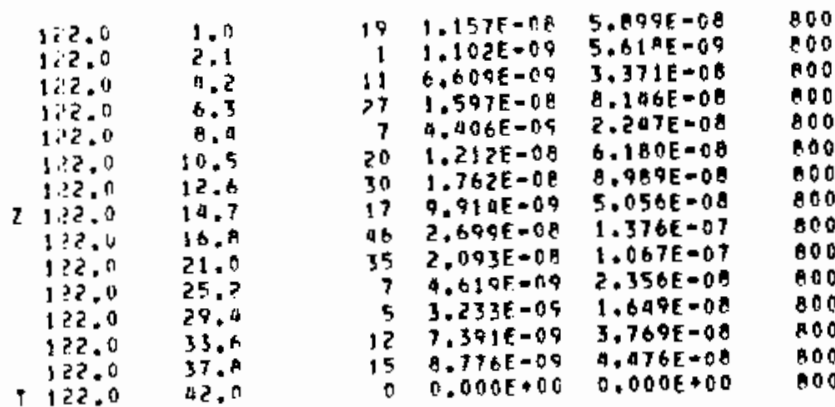

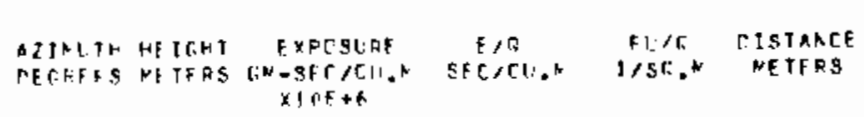

\begin{tabular}{|c|c|c|c|c|c|}
\hline 110.0 & 1.6 & 15 & A.A $A$ IF $=09$ & $4.494 E-O A$ & 800 \\
\hline $11 r .0$ & 2.1 & ค? & $9.797 \mathrm{E}=0 \mathrm{E}$ & $2.4 \Delta a E=C ?$ & Ano \\
\hline $1 \geq 0.9$ & 4.7 & 112 & $6.550 \mathrm{E}=0 \mathrm{P}$ & $3.343 E-07$ & A00 \\
\hline 76.0 & 0.3 & 35 & $1.928 \mathrm{~F}=0 \mathrm{C}$ & $9.5318-06$ & AnO \\
\hline 10.0 & 8.0 & 9 & $8 E=69$ & $2+209 E-08$ & 800 \\
\hline $\begin{array}{r}10.0 \\
1.0 .0\end{array}$ & 10.5 & 14 & $1.102 E-n A$ & $5.618 E-08$ & 800 \\
\hline $\begin{array}{l}C .0 \\
0.6\end{array}$ & 12.6 & 45 & $2, \operatorname{sant}=68$ & $1.34 \mathrm{AE}=07$ & 200 \\
\hline 0 & 14.7 & 73 & $1.3775=08$ & $7.022 E=05$ & 200 \\
\hline$\therefore$ & $16 .{ }^{9}$ & 59 & $3, a 7 r t=60$ & $\begin{array}{l}1.7700=07 \\
5.3375=08\end{array}$ & 10 \\
\hline .0 & 21.13 & 35 & $1.295 E-N E$ & $0.596 t=08$ & 800 \\
\hline 30.0 & 29.4 & 18 & $1.06 \bar{E} E=n E$ & $5.01 E E-08$ & 800 \\
\hline 0 & 33.6 & 7 & $4.157 E=09$ & $2.120 E=08$ & AC \\
\hline & 37.4 & 35 & $? .019 \mathrm{E}-0 \mathrm{e}$ & $1.060 \mathrm{E}-0 \mathrm{~T}$ & 80 \\
\hline .6 & A & 70 & $1.4325=0$ & $303 E-00$ & A 0 \\
\hline
\end{tabular}

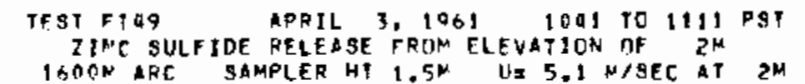

DIIFIUTH EXFOSURE
DEGREES GN $=$ SEC $/ C$ E
XIOE $\$ 6$

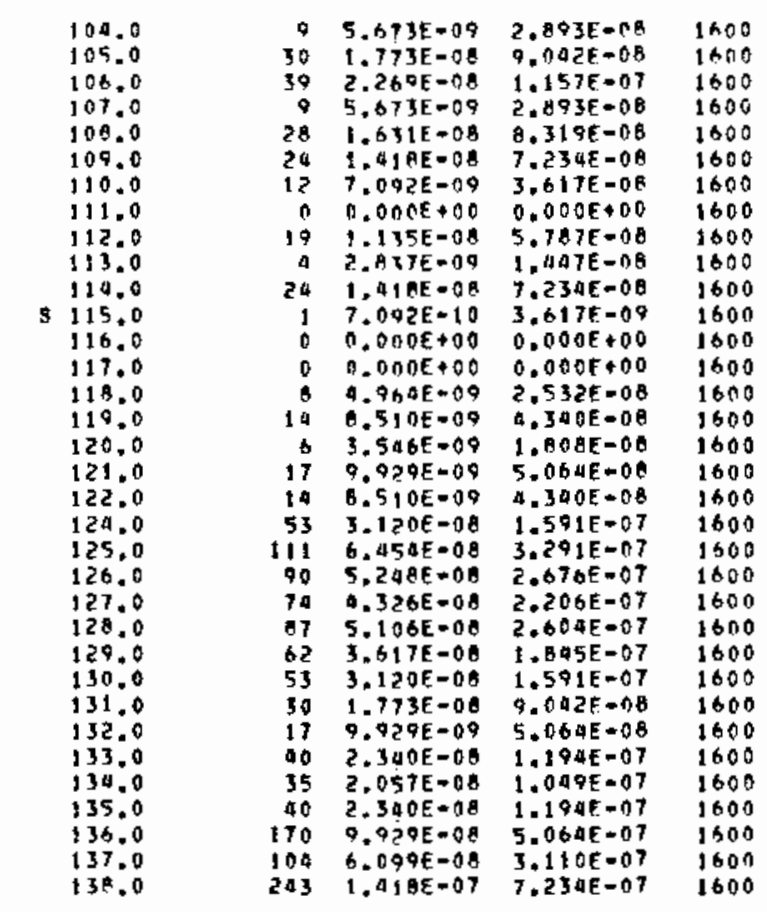

CROSSHIND INTEGRATEDE ? $960 E-05 \quad 1.254 E=04$

TOKFE rAto FULLCK...

TFSTFIMO AFHIL 3,1061 JOOT IC 1 t11 PST

ZIAR SILLJUE HFLESSE FRCN FLFUATICA CF ZN

$L=5.1$ N

AZINUTH HEIGHI EXPESLAF F/G FU/G CISTANCE

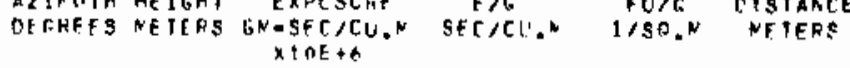

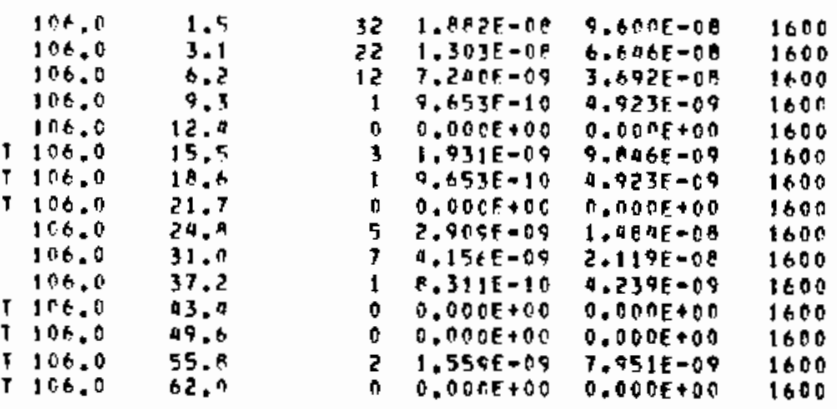




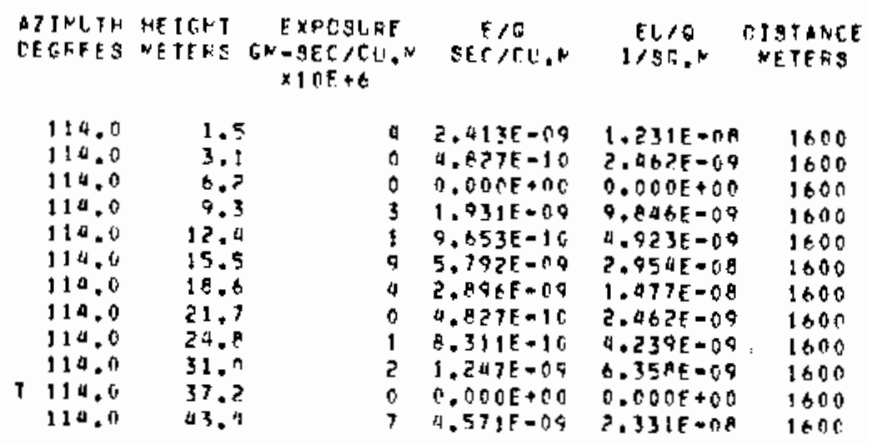

AZJHITH FEIGH EXPTSLRF F/5 FLF CISTANCF

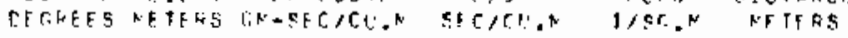
$+n F+t$

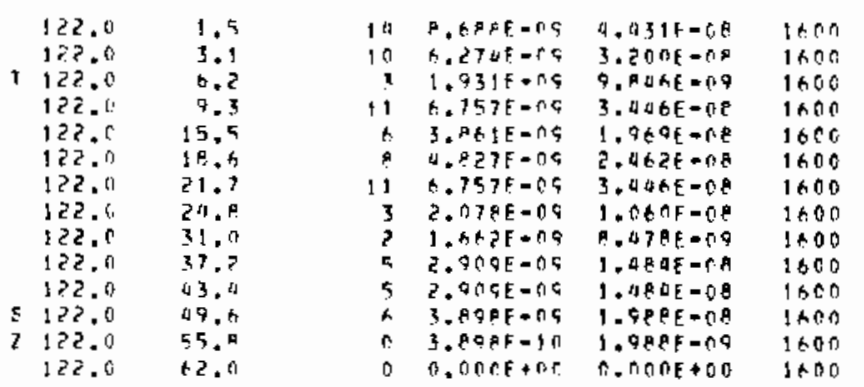

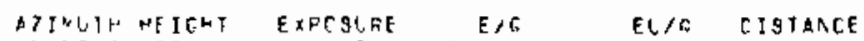

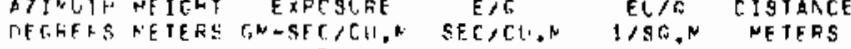
in 0

\begin{tabular}{|c|c|c|c|c|c|}
\hline 130.0 & 1.5 & 13 & $7.722 F=08$ & $3.93 \mathrm{AF}=0 \mathrm{~B}$ & $1+00$ \\
\hline 130.0 & 3.1 & 3 & $1.931 E=n c$ & - CABf-CA & 1600 \\
\hline 130.0 & 6.2 & 13 & $7.722 \mathrm{E}=\mathrm{n} 9$ & $3.93 \mathrm{~B}=0 \mathrm{~A}$ & 1600 \\
\hline 130.0 & 9.3 & 1 & $9.651 \mathrm{~F}-10$ & $4.9235=09$ & $1 \in 0 \mathrm{C}$ \\
\hline 130.0 & 12.4 & 19 & $\mathrm{E}=0 \mathrm{C}$ & $5.908 \mathrm{E}-08$ & 1600 \\
\hline 130.0 & 15.5 & 12 & $7.2 \Delta 05=09$ & $3.642 \mathrm{E}=0.8$ & 1600 \\
\hline 130.0 & 13.5 & 21 & $1.255 \%=01$ & $6.4 B O E=0 B$ & 1 की ก \\
\hline 130,0 & 21.7 & 30 & 1. $7 P E E=C 8$ & $9.10 A E-O B$ & 1600 \\
\hline 130.0 & 24.8 & 37 & $2.2075=00$ & $1.123 \leqslant-07$ & 1800 \\
\hline 130.0 & 31.0 & 12 & $0 \div 5 E=09$ & $3.6035=08$ & 1600 \\
\hline 130.0 & 43.4 & 13 & $7.896 E-09$ & $4.027 E=0.8$ & 1 binc \\
\hline 130.0 & & 23 & $1.247 E-6:$ & $t .361 E=0 e$ & $100 ?$ \\
\hline 130.0 & 55.8 & 2 & $1.55 \zeta E-C G$ & $7.951 E=08$ & 1600 \\
\hline $13 r .0$ & $\in \bar{c}, n$ & 5 & $x, 11 E E+15$ & $1,5 \subseteq$ \& $F=O A$ & 1600 \\
\hline
\end{tabular}

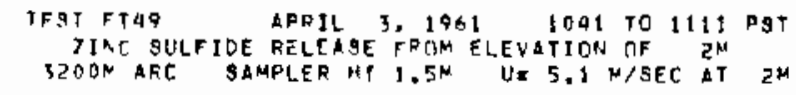

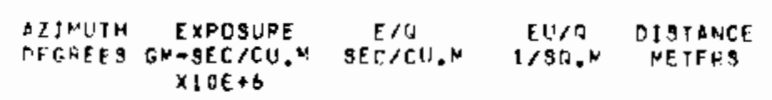

122.0

135.0

124.0

125.0

$12 \mathrm{~A} .0$

127.0

128,0

129.0

130.0

$131: 0$

$13 ? .0$

133.0

134.0

135.0

136.0

137.0

139.0

139.0

140,0

141.0

142.0

9144.0

145.0

146.0

147.0

148,0

149.0

150.0

151.0

152.0

153.0

150.0

155.0

156.0

157.0

150.0

159,0

160.0

6 3.9REE-IO

$3,03 a E=08 \quad 3 ? 00$

$2.938 E=08 \quad 3200$

$30 \quad 1.772 E=08 \quad 1.130 E=08 \quad 3200$

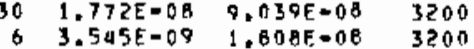

$3 \quad 2.215 E-00 \quad 1.130 E-08$ 3?00

B $974 E-00 \quad 2.4 A 6 E-08$ 3200

3 2.215E-0Q 1.130E-08 3200

$5 \quad 3.101 E=09 \quad 1.582 E=08 \quad 3200$

2 $1.379 F-09 \quad 6.779 F-09 \quad 3200$

2 $1.379 E-09 \quad 6.779 E-09 \quad 3200$

$3 \quad 1.712 E=09 \quad 0.039 E=09 \quad 3200$

$2 \quad 1.329 E-00 \quad 6.779 \%-09 \quad 3 \geq 00$

$0 \quad 4.43 I E-10 \quad 2.260 E-09 \quad 3200$

$3 \quad 2.215 E=09 \quad 1.130 E-08 \quad 3200$

$2 \quad 1.3295-09 \quad 0.779 E-1993200$

5 3.101E-0Q $1.582 E=0 B \quad 3200$

$\begin{array}{llll}5 & 3.101 E=08 & 1.582 E-18 & 3200 \\ 4 & 2.65 P E-09 & 1.356 F-1.8 & 3200\end{array}$

$\begin{array}{llll}4 & 2.65 \\ 6 & 1.9 B E-09 & 1.3560-09 & 3200\end{array}$

$35 \quad 2.030 E-00 \quad 1.039 E=07 \quad 3200$

$12 \quad 7.532 E-0 Q \quad 3.0 A 1 E-\cap 8 \quad 3200$

$7 \quad 0.911 E-09 \quad 2.260 E-08 \quad 3200$

$19 \quad 1.100 E=08 \quad 5.629 E+08 \quad 3200$

$\checkmark \quad 5.760 E-09 \quad 2.938 E-08$ $3>00$

$12 \quad 7.080 \mathrm{~F}=09 \quad 3.615 \mathrm{E}-08 \quad 3200$

$9 \quad 5.760 E=09 \quad 2.038 E-08 \quad 3200$

$9.760 E-09$
$1.772 F=09$

$28 \quad 1.4 A A E-0 B \quad 0.587 E-08 \quad 3200$

$10 \quad 6.203 E=09 \quad 3.163 F=08 \quad 3200$

0 a.43TE-10 2.2605-09 3?00

1 A.A.TE-10 $4.519 E-09 \quad 3200$

28 1.6AUE-OA B.SPTF-OQ 3700

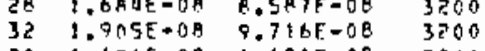

$\begin{array}{llll}32 & 1.905 E-0 B & 9.716 F=08 & 3700 \\ 20 & 1.19 A E-0 B & 6.101 \mathrm{~F}=A B & 3>00\end{array}$

$37 \quad 2.171 \mathrm{E}=0 \mathrm{~A} \quad 1.107 \mathrm{~F}-07 \quad 3700$

$16 \quad 0.747 E=09 \quad 0.971 \mathrm{E}=08 \quad 3 ? 00$

$25 \quad 1.506 E=0 B \quad 7.6 A 3 F=08 \quad 3200$

$127.532 \mathrm{E}-08 \quad 3.891 E=08 \quad 3200$

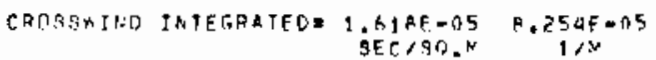

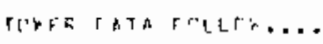

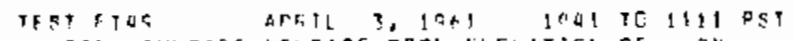

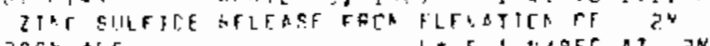

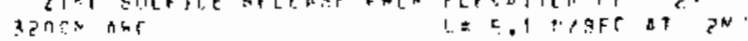

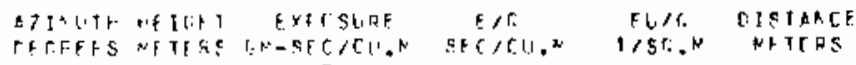
$\times 1 \cap r+\theta$

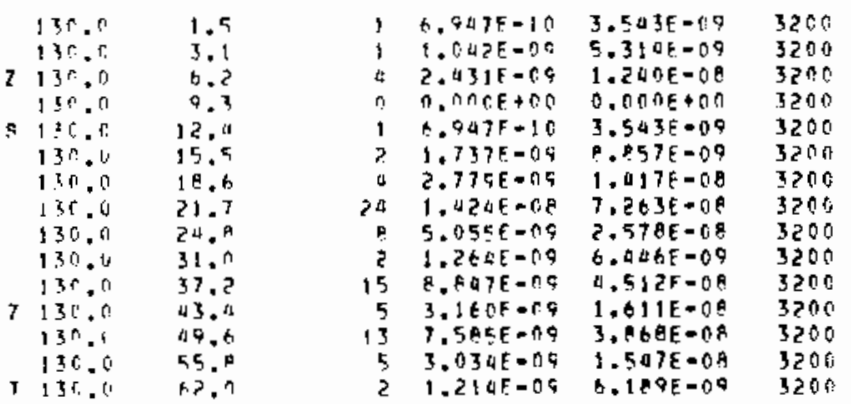


30-SERIES EXPER:HEN" : 50 \& FT-5J)

GPOLND LEYEL RND TOWER SAMPLING 200-12800 Y ARCE.

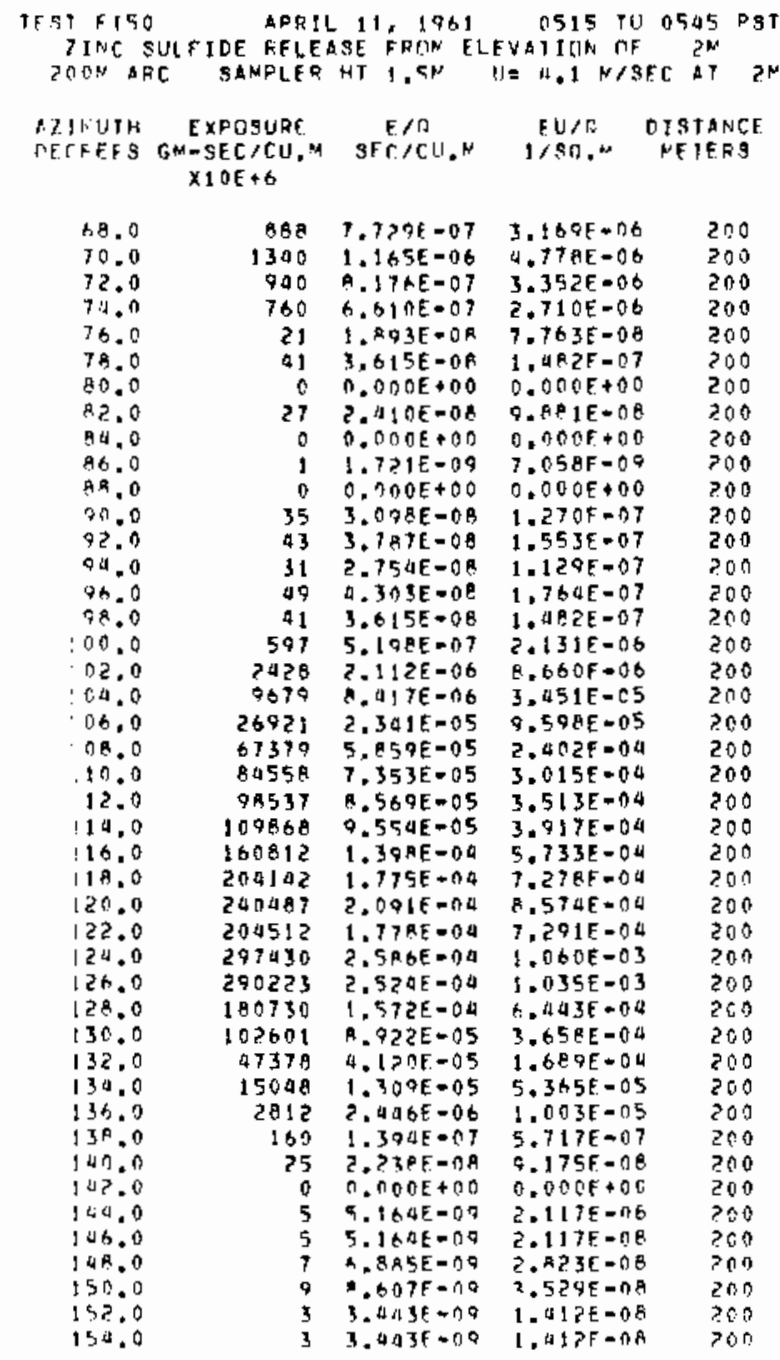

CROSSRTED INTEGRATEOA= $1.34 K E-02 \quad 5.3535-02$

TOWFR FATA FTLLOW....

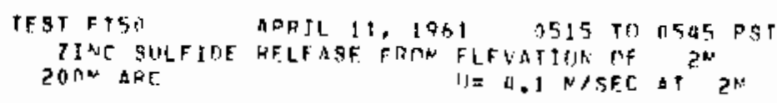

AZINIFTH HEITHT EYOTQUHF T/O TISTANCE

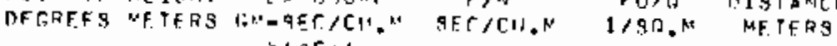
I 1 af $F$

\begin{tabular}{|c|c|c|c|c|}
\hline Q日, & 7.7 & 21 & 1. A 7 OF - त . & $7.66 A F=n A$ \\
\hline $\begin{array}{l}39=0 \\
99=0 \\
98.0\end{array}$ & $\begin{array}{l}1.1 \\
2.7 \\
4.1\end{array}$ & $\begin{array}{r}11 \\
30 \\
507\end{array}$ & $\begin{array}{l}1.0365=08 \\
2.6371=08 \\
2.5425=07\end{array}$ & $\begin{array}{l}4.247 E=0 B \\
1.081 E=07\end{array}$ \\
\hline 980 & 5.9 & की & $\begin{array}{l}5.342 E=17 \\
5.273 E=n A \\
3.4 B \Delta F=O E\end{array}$ & $\begin{array}{l}1.042 E=06 \\
2.152 E=07 \\
1.429 E=07\end{array}$ \\
\hline$P A, 0$ & $A=1$ & 63 & $5.556 F=08$ & $2.27 A E-07$ \\
\hline $\begin{array}{l}P A .0 \\
Q R .0 \\
P R .0\end{array}$ & $\begin{array}{r}9.4 \\
10.4 \\
13.5\end{array}$ & $\begin{array}{r}108 \\
115 \\
78\end{array}$ & $\begin{array}{l}9.417 E-\cap B \\
1.0 \cap P E-07 \\
2.4 A B F=\cap A\end{array}$ & $\begin{array}{l}3.861 E-07 \\
4.131 E=07 \\
1.004 E=07\end{array}$ \\
\hline $\begin{array}{l}9 A .0 \\
9 A .0 \\
9 A .0 \\
9 A .0\end{array}$ & $\begin{array}{l}16.2 \\
18.9 \\
21.4 \\
21.3\end{array}$ & $\begin{array}{r}14 \\
5 \\
5 \\
0\end{array}$ & $\begin{array}{l}1.224 E=00 \\
2.125 E=10 \\
5.650 E=09 \\
7.701 F=10\end{array}$ & $\begin{array}{l}5.019 E=O B \\
1.15 A E=O A \\
2.316 E=0 B \\
3.159 F=09\end{array}$ \\
\hline
\end{tabular}

AIIMITH HEIGHT EXPTSUPE F/B FI/Q IISTANCF DERREES METENS GH-SEC/CH.N SEC/CIJ.N 1/SO,N METFRS $\times 10 E+b$

\begin{tabular}{|c|c|c|c|c|}
\hline $\begin{array}{l}106.0 \\
106.0 \\
106.0 \\
106.0 \\
100.0 \\
106.0 \\
106.0 \\
106.0 \\
106.0 \\
196.0 \\
106.0 \\
106.0 \\
106.0 \\
106.0 \\
106.0\end{array}$ & $\begin{array}{l}0.7 \\
1.9 \\
2.7 \\
4.1 \\
5.9 \\
6.9 \\
0.1 \\
9.9 \\
10.9 \\
13.5 \\
16.8 \\
18.9 \\
21.5 \\
24.3 \\
27.0\end{array}$ & $\begin{array}{l}36519 \\
13848 \\
15449 \\
15593 \\
10849 \\
12406 \\
12100 \\
11510 \\
12719 \\
1345 \\
6960 \\
5012 \\
1496 \\
1140 \\
645\end{array}$ & $\begin{array}{l}3.175 E=05 \\
1.204 F=05 \\
1.343 E=05 \\
1.356 F=05 \\
1.2018=05 \\
1.100 E=05 \\
1.054 F=05 \\
1.001=-05 \\
1.106 E=05 \\
7.257 F=06 \\
6.053 E=06 \\
1.364 E=06 \\
1.301 F=06 \\
9.850 E=07 \\
5.5948=07\end{array}$ & 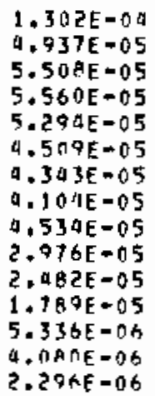 \\
\hline
\end{tabular}

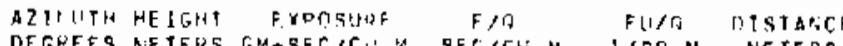

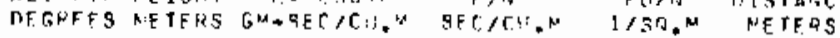
$1 \mathrm{OF}+\mathrm{K}$

\begin{tabular}{|c|c|c|c|c|c|}
\hline $1 ! 4.0$ & 0.7 & $14001 \mathrm{~h}$ & $1.2185=04$ & $4.992 f-04$ & 200 \\
\hline 119,6 & 1. 3 & $61 P+5$ & $5,3 \&$ ก $=05$ & $2.206 E=0.4$ & $2 \cap 0$ \\
\hline 114.0 & 2.7 & 6038 & $5.250 F=05$ & $2.153 E=0.4$ & 200 \\
\hline 114.0 & 1.1 & 64330 & $5.504 F=05$ & $? .297 E=04$ & 200 \\
\hline 114.0 & 5.4 & 45135 & $5.66414=015$ & $322 E-n 4$ & 200 \\
\hline .0 & 7.9 & a 2216 & $4.1035-05$ & $719 \mathrm{E}-0 \mathrm{O}$ & 200 \\
\hline 110.0 & 5.1 & 57703 & $5.0215=05$ & $059 E-04$ & 200 \\
\hline 114.0 & 9.4 & 49814 & $332 E=05$ & $76 E \div 04$ & 200 \\
\hline 114.0 & 10.8 & d 14337 & $5 F-05$ & $1 E-00$ & 200 \\
\hline 14.0 & 13.5 & 33522 & $5 F-05$ & -04 & 200 \\
\hline 14.0 & 10,2 & 19419 & $1.689 F-0.5$ & यद $=05$ & 200 \\
\hline 14.0 & $1 \% .9$ & 12949 & $1.126 E=05$ & $9.617 E-05$ & 200 \\
\hline a. & $? 1.6$ & 5675 & 37 AE- 06 & -05 & 200 \\
\hline 1.0 & $2 \pi, 3$ & 2778 & $2.4165=06$ & $9.905 E-06$ & 200 \\
\hline & $27 . n$ & $14 ? 5$ & - ? $30 E=0 h$ & $5.081 \varepsilon=06$ & 200 \\
\hline
\end{tabular}




\begin{tabular}{|c|c|c|c|c|c|}
\hline $\begin{array}{l}\text { ZIMUTH } \\
\text { EGREFS }\end{array}$ & $\begin{array}{l}\text { ME IGHT } \\
\text { MFTERS }\end{array}$ & $\begin{array}{c}\text { EXPOSUAE } \\
64=9 E C / C 11 . M \\
\times 10 E+6\end{array}$ & $\begin{array}{c}E / R \\
\text { SE.C/RU.M }\end{array}$ & $\begin{array}{c}E U / G \\
1 / S A, M\end{array}$ & $\begin{array}{l}\text { DISYANE } \\
\text { METEFS }\end{array}$ \\
\hline $\begin{array}{r}122.0 \\
122.0 \\
122.0 \\
122.0 \\
122.0 \\
122.0 \\
122.0 \\
122.0 \\
122.0 \\
122.0 \\
122.0 \\
122.0 \\
122.0 \\
122.0 \\
122.0\end{array}$ & $\begin{array}{r}0.7 \\
1.9 \\
2.7 \\
4.1 \\
5.4 \\
6.8 \\
8.1 \\
7.9 \\
10.8 \\
13.5 \\
16.2 \\
18.9 \\
25.6\end{array}$ & $\begin{array}{r}344183 \\
191158 \\
141351 \\
156459 \\
92516 \\
126413 \\
115402 \\
107325 \\
72272 \\
50409 \\
31716 \\
17354 \\
9873 \\
3306 \\
1077\end{array}$ & $\begin{array}{l}2.993 E=04 \\
1.227 E=04 \\
1.229 E=04 \\
1.364 F=04 \\
7.175 E=05 \\
1.100 E-04 \\
1.004 F=04 \\
9.333 F=05 \\
6.285 F=05 \\
4.383 E=05 \\
2.758 E=05 \\
1.509 E=05 \\
8.506 E=06 \\
2.875 E=06 \\
1.650 E=06\end{array}$ & $\begin{array}{l}1.227 E=03 \\
5.033 E=04 \\
5.039 E=04 \\
5.592 E=00 \\
2.942 E=00 \\
4.500 E=04 \\
4.119 E=04 \\
3.826 E=04 \\
2.577 E=04 \\
1.797 E=04 \\
1.131 E=04 \\
6.187 E=05 \\
3.520 E=05 \\
1.179 E=05 \\
6.767 E=05\end{array}$ & $\begin{array}{l}200 \\
200 \\
200 \\
200 \\
200 \\
200 \\
200 \\
200 \\
200 \\
200 \\
200 \\
200 \\
200 \\
200 \\
200\end{array}$ \\
\hline
\end{tabular}

AZTHITH HEIGHT EXDIISIMRF FID FE FU/O DISTENCF

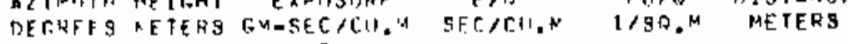
$\times 1 \cap E+5$

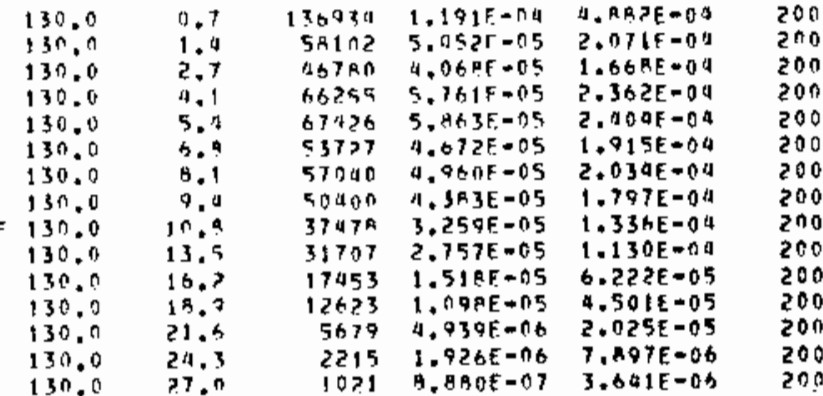

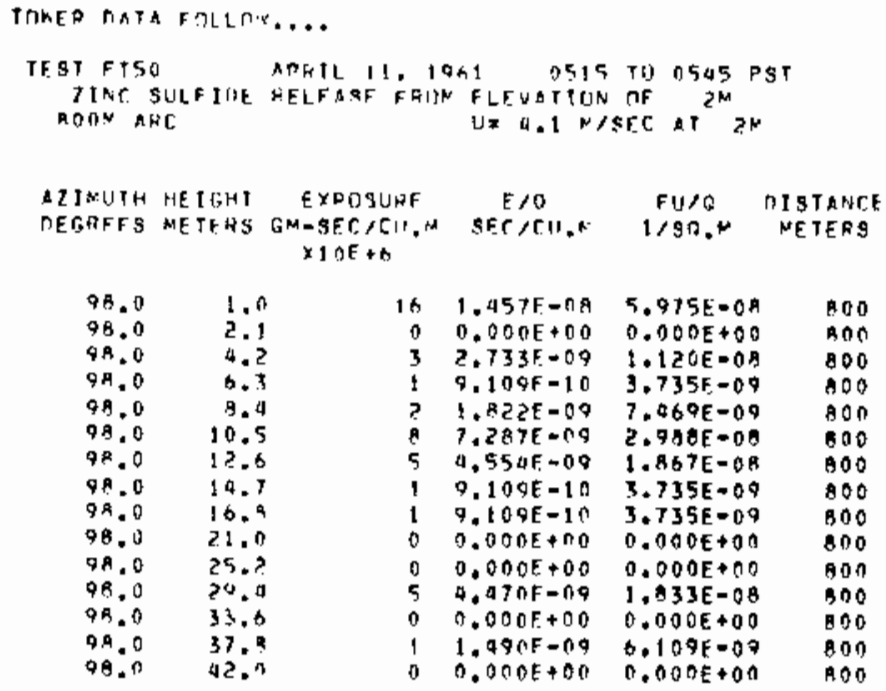




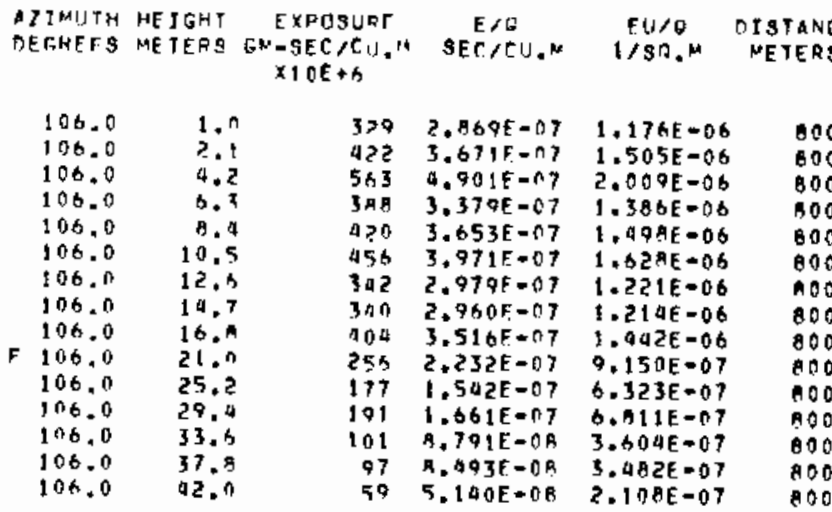

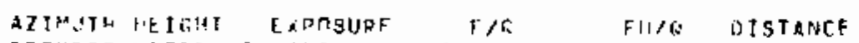

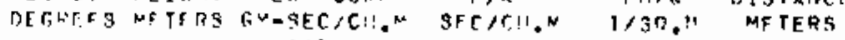
at tof +5

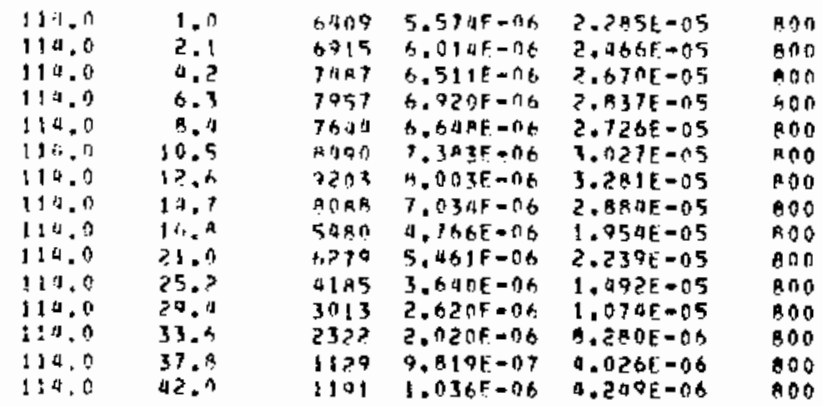

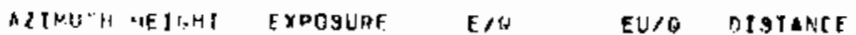

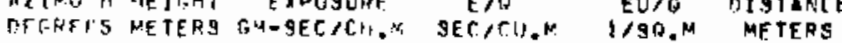
$x+O E+B$

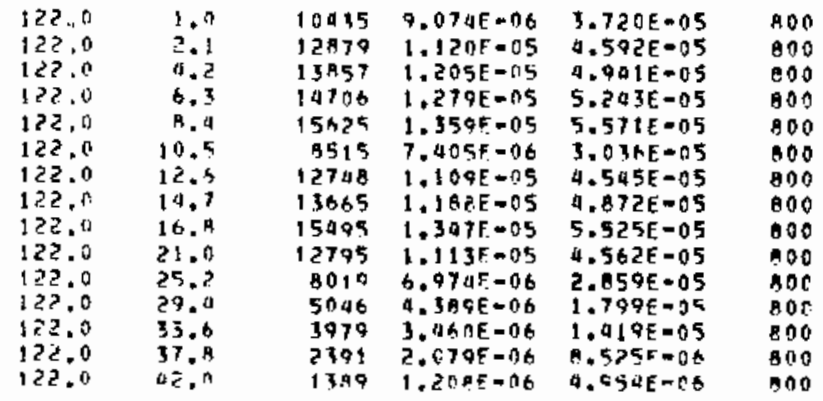

A7TKLTH HEJGHT EXPOSURF F/O FU/D DISTANCE

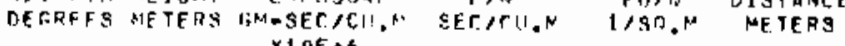

\begin{tabular}{|c|c|c|c|c|c|}
\hline $\begin{array}{l}130.0 \\
130.0 \\
136.0 \\
130.0 \\
130.0 \\
130.0 \\
136.0 \\
130.0 \\
130.0 \\
130.0 \\
13600 \\
130.0 \\
130.0 \\
130.0 \\
130.0\end{array}$ & $\begin{array}{l}1.0 \\
2.1 \\
4.2 \\
6.3 \\
9.0 \\
10.5 \\
12.6 \\
14.7 \\
16.1 \\
21.0 \\
25.2 \\
29.4 \\
33.6 \\
37.9 \\
42.1\end{array}$ & 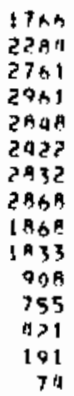 & $\begin{array}{l}1.536 F=06 \\
1.987 E=06 \\
2.401 E=06 \\
2.575 F=06 \\
2.477 E=06 \\
2.107 F=06 \\
? .463 E=06 \\
2.494 E=06 \\
1.625 E-06 \\
1.594 E-06 \\
7.397 F=07 \\
6.571 E=07 \\
3.665 F-07 \\
1.6695=07 \\
6.485 E=08\end{array}$ & $\begin{array}{l}A .297 E-06 \\
9.145 E=06 \\
9.344 E=06 \\
1.056 E-05 \\
1.015 E=05 \\
0.63 B E=06 \\
1.010 E=05 \\
1.023 E=05 \\
6.663 E=06 \\
6.536 E=06 \\
3.238 E=06 \\
2.694 E=06 \\
1.503 E=06 \\
6.842 E=07 \\
2.657 E=07\end{array}$ & $\begin{array}{l}900 \\
100 \\
800 \\
800 \\
800 \\
800 \\
800 \\
000 \\
000 \\
000 \\
800 \\
800 \\
800 \\
800 \\
800\end{array}$ \\
\hline
\end{tabular}

IFST FISO AREIL 11. 19A1 0515 TO 0545 F\$T ZINC SULFJTF AFIEASE FRON ELFWATHC TF $2 \mathrm{~N}$

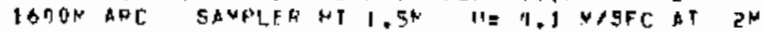

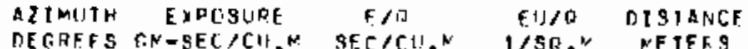
$x \in 0 E+b$

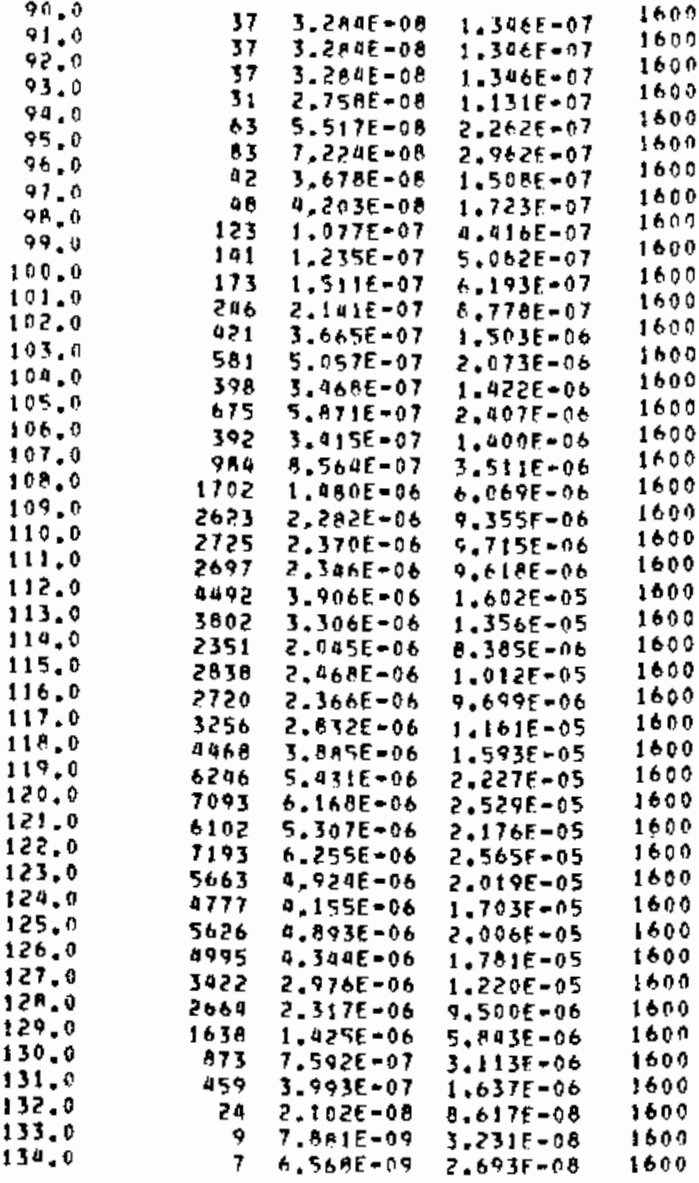

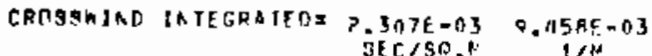

THWER TATA FIILLIT,...

TEST FISO APATL 11, 100 0.5:5 TO 0545 PST

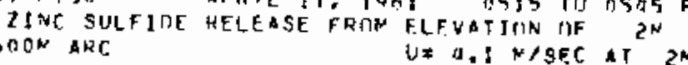

AITMITHH HEIGHT EXPOSURF E/N EUIO DISTANCE DECRFES NETERS GM-SEC/CU.H SEC/CH.N J/SH, MU METEOS

\begin{tabular}{|c|c|c|c|c|c|}
\hline $\begin{array}{l}98.0 \\
98.0 \\
9 R .0 \\
98.0 \\
98.0 \\
99.0 \\
98.0 \\
98.0 \\
98.0 \\
99.0 \\
98.0 \\
98.0 \\
98.0\end{array}$ & $\begin{array}{l}1.5 \\
3.1 \\
6.2 \\
9.3 \\
12.0 \\
15.9 \\
18.6 \\
21.7 \\
24.9 \\
31.0 \\
37.2 \\
93.4 \\
49.8\end{array}$ & $\begin{array}{l}35 \\
38 \\
50 \\
82 \\
37 \\
35 \\
36 \\
66 \\
25 \\
6 \\
9 \\
10 \\
12\end{array}$ & $\begin{array}{l}3.067 E=0 R \\
3.381 E-08 \\
4.403 E=08 \\
7.156 E=08 \\
3.303 E-0 A \\
3.067 F=08 \\
3.105 F=08 \\
5.919 E=08 \\
2.253 E=08 \\
5.302 E=09 \\
7.953 E=09 \\
9.278 E=09 \\
1.09 Z E=0 A\end{array}$ & 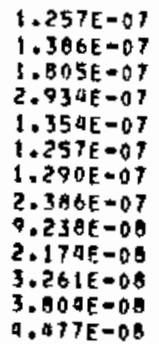 & $\begin{array}{l}1600 \\
1600 \\
1600 \\
1600 \\
1600 \\
1600 \\
1600 \\
1600 \\
1600 \\
1600 \\
1600 \\
1600 \\
1600\end{array}$ \\
\hline
\end{tabular}




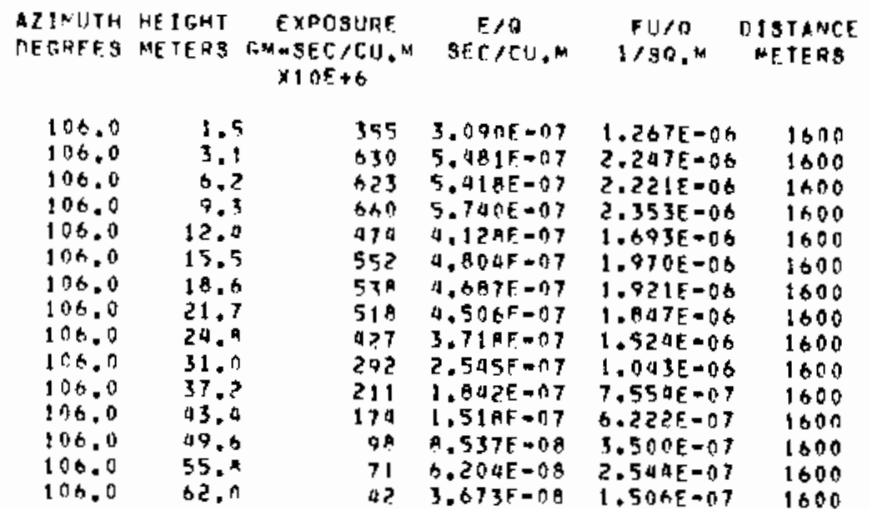

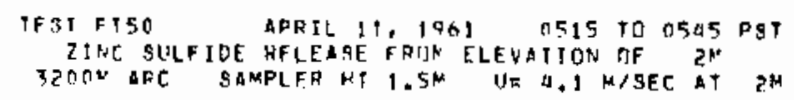

AZINITH HEITHT EXPIISUHE F/D FUIO DISTANCE

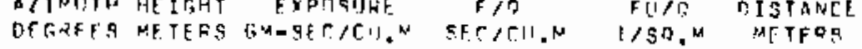
IOF. of

\begin{tabular}{|c|c|c|c|c|c|}
\hline $1[4=13$ & 1.5 & 1511 & $1.314 E=n A_{1}$ & $5.387 t=0 \mathrm{~S}$ & 1600 \\
\hline 114.0 & 3,1 & I उसम & $1.204 F=06$ & $9.936 \mathrm{~F}-06$ & 1600 \\
\hline 114.0 & 6.? & ?ก8? & $1.8 \div 1 F=06$ & $7.425+=06$ & 1000 \\
\hline 114.0 & 9.5 & 2159 & $A 77 F=\cap A$ & $1.696 E=0 \mathrm{~A}$ & 0 \\
\hline 114.0 & 12. 2.1 & 2? 19 & $1.9295-06$ & $7.90 A E=06$ & 1) \\
\hline 14.0 & 15.5 & $19 n 7$ & 1.55 RE $=06$ & $6.799 E-06$ & n \\
\hline 154.0 & 18 & 2007 & 1. & $7.4 A \cap E-04$ & 400 \\
\hline 110.0 & 21.7 & $10 \times 5$ & 1. $275 E=10$ & $5.226 F=06$ & $\$ 0$ \\
\hline 114.0 & A & 1249 & $1.1215-06$ & $0.597 F-06$ & 160 \\
\hline 114.0 & 31.0 & $1 \geq 5 \AA$ & $1.0947-06$ & $4.486 E=06$ & 1000 \\
\hline 114,0 & $37 . ?$ & $112 B$ & $9,795 E=\cap y$ & $4.016 E-06$ & 1600 \\
\hline .0 & 43 & 757 & $5 A 7 F=07$ & $2.701 \mathrm{E}=0 \mathrm{~S}$ & 1600 \\
\hline 14.0 & 49 & 415 & $119 k-07$ & $1 . \Delta B 1 E=06$ & $16 \cap 0$ \\
\hline 0 & 55 & $30 \pi$ & 3. यूी $0=07$ & $1.394 E-0,1$ & 160 \\
\hline 14.0 & 62 & 30 & $.55 .=07$ & $1.105 E=06$ & 160 \\
\hline
\end{tabular}

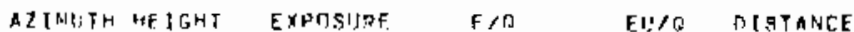

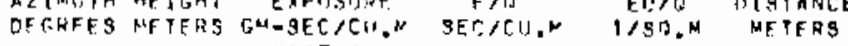

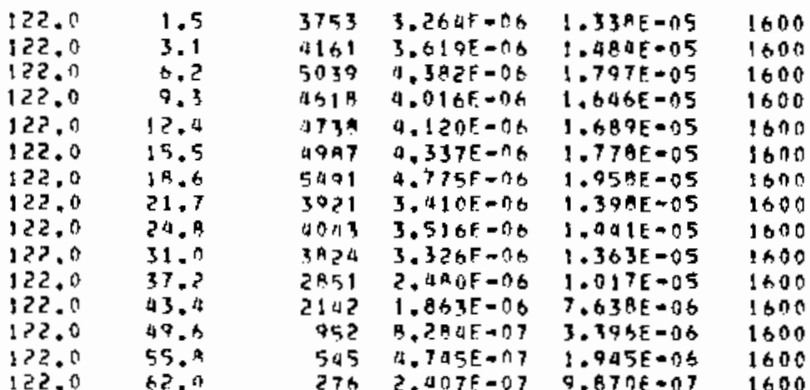

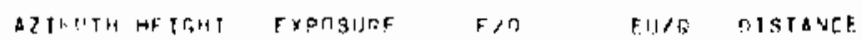

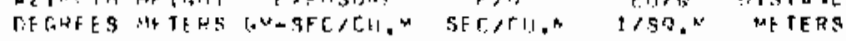
$x \geqslant 0+6$

\begin{tabular}{|c|c|c|c|c|c|}
\hline $130 . n$ & 1.5 & $4>7$ & $3.718 \mathrm{~F}=07$ & $1.5254=006$ & 1600 \\
\hline 130.0 & 3.1 & $4+0$ & H. $002 E-177$ & $1.601 \mathrm{E}=0 \mathrm{~h}$ & 1600 \\
\hline 30.0 & 6.3 & 450 & $3.905 \mathrm{~F}=17$ & $1.639 E=06$ & 1600 \\
\hline 130.0 & 9.7 & 277 & $2.414 \mathrm{~F}=1 ! 7$ & $9.89 \mathrm{AF}=07$ & 1600 \\
\hline $13 n \cdot 0$ & 12.4 & 713 & H. $200 \mathrm{H}=8.7$ & $2.54+E=06$ & 1690 \\
\hline $3 n \cdot n$ & 15.5 & 651 & $5.7 \triangle A F-07$ & $2.357 t-0 h$ & 1600 \\
\hline 130.0 & 18.6 & 709 & $5.175=07$ & $531 E-06$ & 1600 \\
\hline $13 n .13$ & 21.7 & $\ln 2$ & $6,9 A 3 E-n 7$ & $2.963 E-06$ & 1600 \\
\hline $13 n .0$ & $2^{a} \cdot 2$ & she & $.7795=07$ & $369 E-06$ & 1600 \\
\hline 139.0 & 31.0 & $4 a n$ & $3,4 b_{5}=07$ & $1.4295=06$ & 1600 \\
\hline$+3 \pi .0$ & 37.0 & $3 \cap 7$ & $2.671 f=0.7$ & $1.095 E-06$ & 1600 \\
\hline 150.0 & $53 . t$ & 351 & $1.955 F-67$ & A. $01 B E=0 ?$ & 1600 \\
\hline & 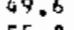 & 101 & $8.795 E=08$ & $2 E-09$ & 1600 \\
\hline $\begin{array}{l}130.0 \\
130.0\end{array}$ & $\begin{array}{l}55 .{ }^{a} \\
62.0\end{array}$ & $\begin{array}{r}76 \\
5\end{array}$ & $\begin{array}{l}2,353 f=08 \\
4,363 F=09\end{array}$ & $\begin{array}{l}2.564 E=0 B \\
2.035 E=0 B\end{array}$ & $\begin{array}{l}1600 \\
1600\end{array}$ \\
\hline
\end{tabular}

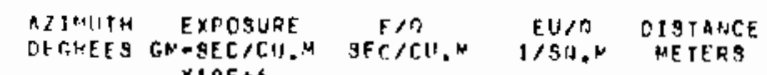
$\times 10 E+6$

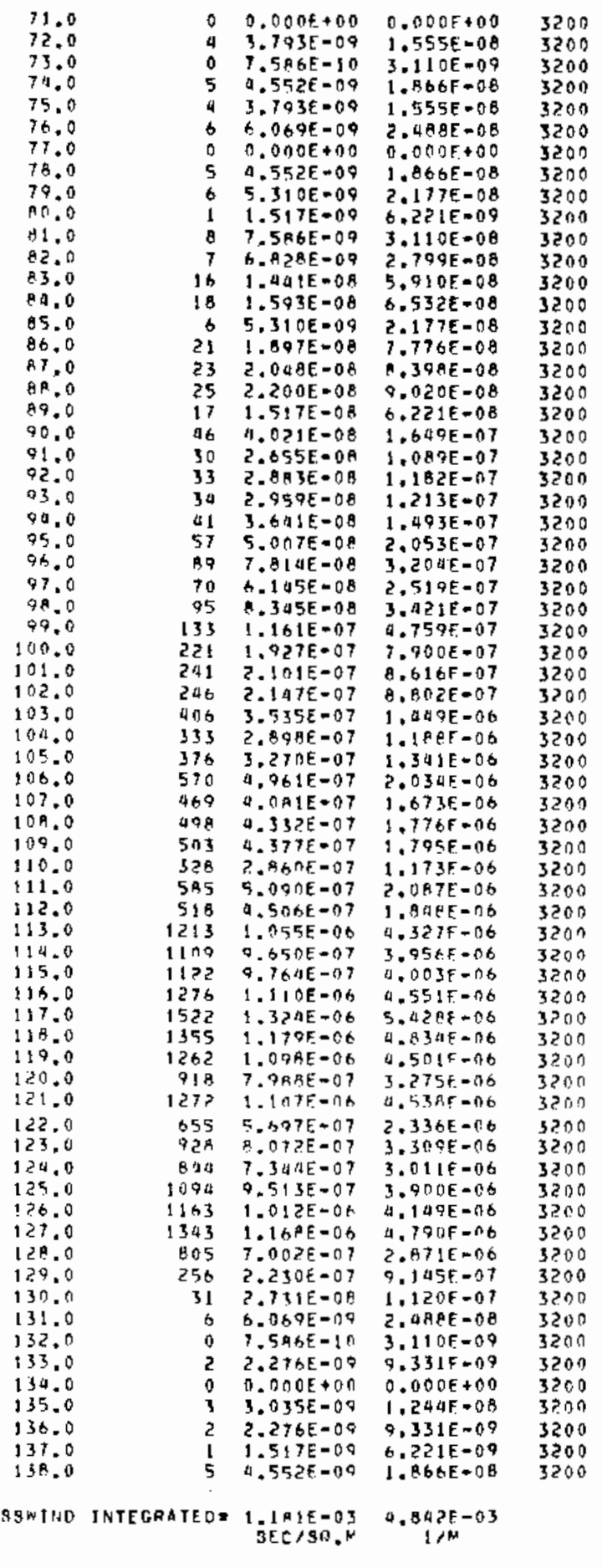


TOKFE CATA FIILLWW...

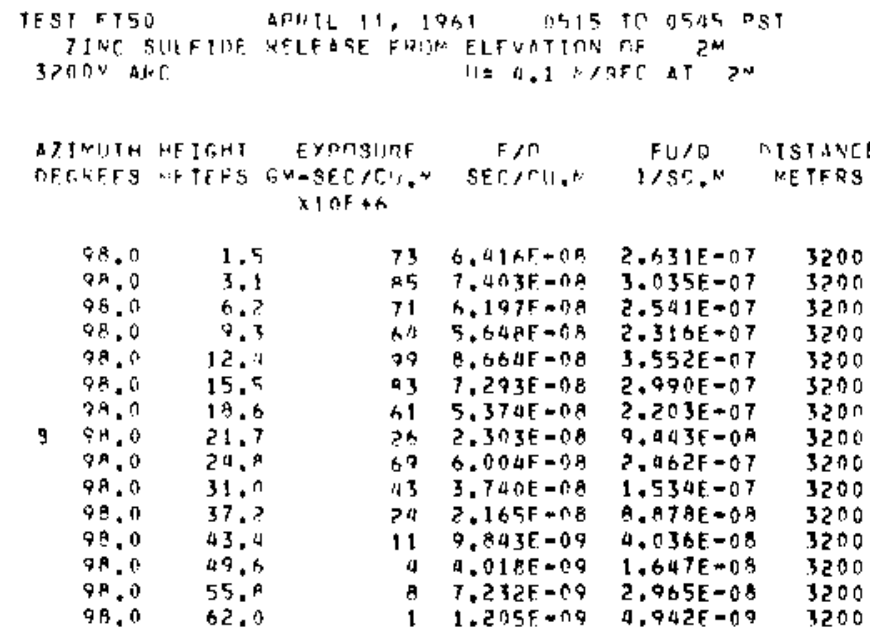

ATIMITH HEIGHT EXPOSURE F/D FU/R DISTANCE

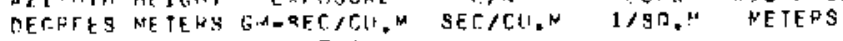
$x 10 F+6$

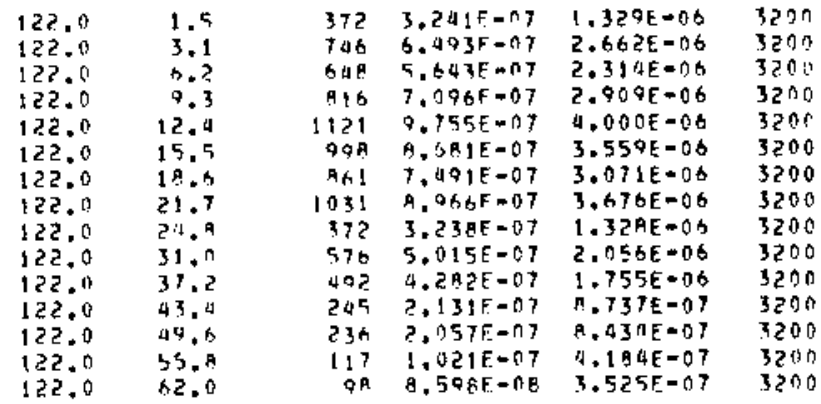

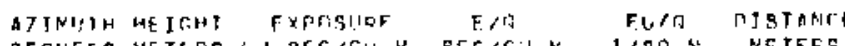

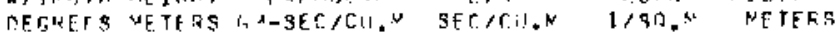
I I F, 6

\begin{tabular}{|c|c|c|c|c|c|}
\hline 130.0 & 1.5 & $2 n$ & 1. $\triangle \triangle \cap E=\cap A$ & $7.719 \mathrm{~F}=0 \mathrm{~A}$ & 3200 \\
\hline 130.0 & 3.1 & 41 & 3.51 QF $=A B$ & $1.4 \mathrm{H} A \mathrm{~A} \rightarrow 07$ & 3200 \\
\hline 130.0 & $0 . ?$ & $3 n$ & $2.037 E+08$ & $1.070 E=07$ & 3200 \\
\hline 130.0 & 9.3 & 11 & $1.042^{\xi}=0.8$ & a. $27 ? \mathrm{E}-0 \mathrm{R}$ & 3200 \\
\hline 130.0 & 12.4 & 17 & $1.4 A 1 F=0$ A & $B .070 E-08$ & 3201 \\
\hline 130.0 & 15.5 & is 4 & $3.2305=0$ A & $1.574 E-67$ & 3200 \\
\hline 1319,0 & 18.6 & 35 & $3.1265-08$ & $1.252 E-07$ & 3200 \\
\hline 130.0 & 21.7 & 47 & $1.16 \mathrm{FF}=0 \mathrm{~B}$ & $1.709 \mathrm{E}=07$ & 3200 \\
\hline 130.0 & 24.1 & 15 & $1.47 B E=O R$ & $6.0536=01$ & 3200 \\
\hline 130.0 & $31 . n$ & 50 & $5.167 E-O B$ & $2.119 E=07$ & 3200 \\
\hline 130.0 & 37.2 & 41 & $3.593 \mathrm{~F}=0 \mathrm{~B}$ & $1.073 E-07$ & 3200 \\
\hline 130.0 & 43.7 & 30 & $2 . B 5 A F=0 A$ & $1.090 \mathrm{E}=07$ & 3200 \\
\hline 130.0 & $40 . t$ & 12 & $1.0 \times 5 F=0 A$ & 4. DAAE $=0.9$ & 3200 \\
\hline 130.0 & 55.8 & 13 & $1,165 \mathrm{f} .0 \mathrm{~A}$ & $4.7775=09$ & 3200 \\
\hline 130.0 & & 9 & $436 E-709$ & $3.205 \mathrm{E}-0 . \mathrm{A}$ & 3200 \\
\hline
\end{tabular}

TFST FTSO APRIL 11,1941 0515 TO 0545 PST 7 INE SIJLIDE RELEASE FROMN ELEVATIUN TF $2^{\mathrm{N}}$

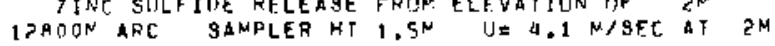

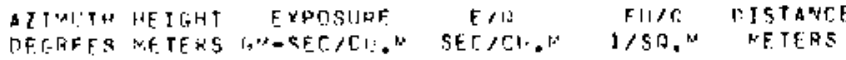
$\times 1 \cap c+b$

\begin{tabular}{|c|c|c|c|c|c|}
\hline 114.6 & 1. 5 & ARQ & $5.994 F=07$ & ?. $457 F-06$ & $32^{n n}$ \\
\hline 114.0 & $3 . t$ & 798 & $4.691 \mathrm{E}=07$ & $3.5595-06$ & $320 \pi$ \\
\hline 1140 & 6.7 & 235 & $7.266=-17$ & $079 E-0 B$ & 3230 \\
\hline 154.0 & 9.2 & $x+2$ & $7.3265=07$ & $3 . \cap \cap \Delta E=06$ & 3?0: \\
\hline 114.0 & 12.4 & 761 & 6.62 if $=07$ & $2.716 t=06$ & 3200 \\
\hline 114.0 & 15.5 & A.? & $7.1785=07$ & $843 E=016$ & 3200 \\
\hline $114 . ?$ & $1^{A} \cdot \mathrm{K}$ & BAR & $643 F-07$ & 31 UE 0 OE & 3200 \\
\hline$\therefore 14.6$ & 21.7 & $5 \mathrm{kh}$ & $5.100 \mathrm{O}=07$ & $091 E-06$ & 3200 \\
\hline 114.0 & $24 \cdot a$ & 592 & $5.153 f-07$ & $2.1 ! 3 E=06$ & 3200 \\
\hline 114.0 & 31.0 & 384 & $3,3 a>E-07$ & $372 E=06$ & 3200 \\
\hline 114.0 & 37.2 & 680 & $5,9 ?\{1 F-n ?$ & $.427 E=06$ & 3200 \\
\hline 116,0 & 43.4 & $6 ? 1$ & $5.404 E=07$ & 21 RE -06 & 3200 \\
\hline 114,0 & $4 \% .5$ & 512 & $4.05 h F=07$ & 1.A27E-06 & 3200 \\
\hline $1 ! 4.0$ & $5 \% .8$ & $2>8$ & $1.9895=07$ & 15 af $=01$ & 3200 \\
\hline 114.19 & $\hat{H} \vec{E}+\hat{r}$ & $30 \mathrm{~h}$ & $B A B F=07$ & $n 9 ? 6=18$ & 3200 \\
\hline
\end{tabular}

\begin{tabular}{|c|c|c|c|c|}
\hline $\begin{array}{l}\text { A.TMUTH } \\
\text { TESAEES }\end{array}$ & $\begin{array}{c}\text { EXPOSURE } \\
\text { GH-SEC/CU.N } \\
\times 1 O E+6\end{array}$ & $\begin{array}{c}E / D \\
\text { SFC }\end{array}$ & $\begin{array}{l}E U / 0 \\
1 / S^{\circ} .\end{array}$ & $\begin{array}{l}\text { DISTANCE } \\
\text { METFES }\end{array}$ \\
\hline
\end{tabular}

$$
\begin{array}{ll} 
& 100.0 \\
? & 102.0 \\
? & 105.0 \\
4 & 107.0 \\
1 & 110.0 \\
2 & 112.0 \\
2 & 115.0 \\
T & 120.0 \\
2 & 125.0 \\
& 135.0
\end{array}
$$

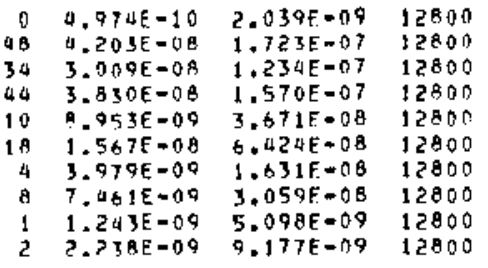

CRCS3WIAD IATETRATEDE $9.336 E-05 \quad 3.82 A E-O 4$ 
30-SERIES EXPERINENT: 5 I FT-51;

GROUND LEVEL AND TOWER SAMPLING $200-3200$ M ARCS. VANY OF THE
FILTERS ON THE TOWERS SHOWED SIGNS OF WEATHERING.

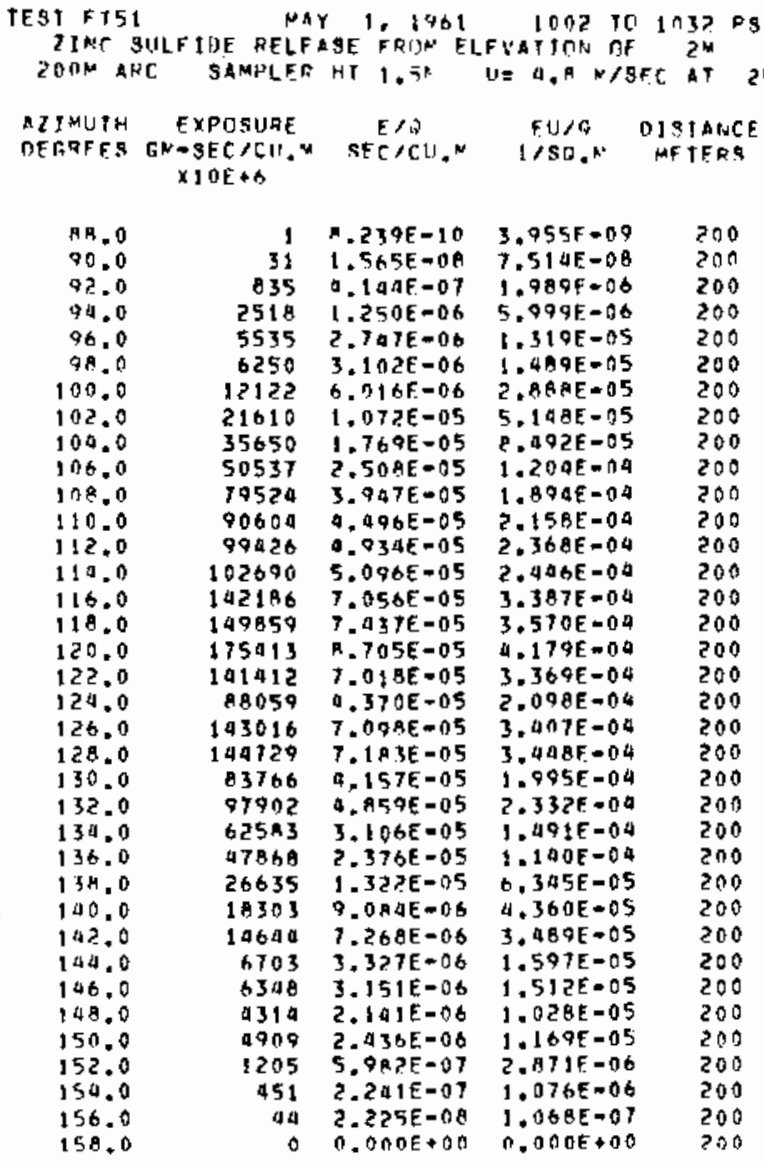

CANSSIYND TNTEGRATFDE $6.071 E-03 \quad 3,106 F-02$

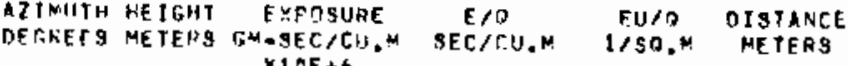
$\times 10 F+6$

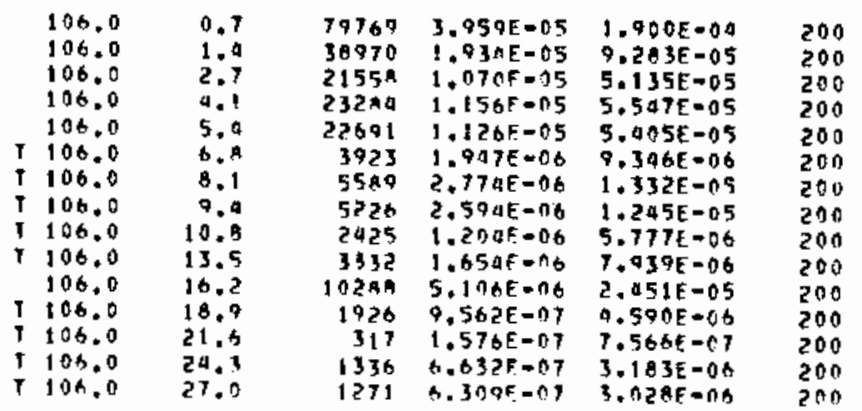

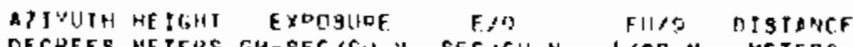

DEFHFES METEMS GM-SEC/CI, Y SFC/CU,N $1 /$ ST.M WETERG $X 1 \cap F+S$

\begin{tabular}{|c|c|c|c|c|c|c|}
\hline & 114,0 & 0.7 & 172150 & A. $543 F-05$ & $a .10 ! E-0, a$ & 200 \\
\hline & 114.0 & 1.4 & 91104 & $4.025 r-05$ & $1.932 E-00$ & $20 \pi$ \\
\hline 7 & 114.0 & 2.7 & $5>n 92$ & $5.96 E-1.5$ & $1.2 A 1 \mathrm{E}=04$ & 200 \\
\hline 9 & 113,0 & a. 1 & $12 n 95$ & $6.1003 \mathrm{~F}=\mathrm{Nh}$ & $1 E-\pi 5$ & 200 \\
\hline$T$ & 114,0 & 5.1 & 10317 & $7.121 F-06$ & $2.45 \mathrm{AE}=05$ & 290 \\
\hline T & 114.0 & 6.7 & IП7क & 5.59 व $=0 \mathrm{~b}$ & $565 E-05$ & 200 \\
\hline & 114.0 & 1 & 23315 & $1+157 F=n 5$ & $4 E=05$ & 2 ก \\
\hline & 114.11 & 0 & 15035 & $0626=06$ & $3+502 f=05$ & 200 \\
\hline $\mathbf{3}$ & 114.0 & 10.9 & 19469 & $6625=06$ & $4.638 E-05$ & 200 \\
\hline $\mathbf{T}$ & $\$ 10.0$ & 1 & 4291 & $2.131 E=06$ & $1.02 \mathrm{TE}-05$ & 200 \\
\hline & 114.0 & $16 . ?$ & 21605 & $1.072 F=05$ & $5.147 F=05$ & 200 \\
\hline$\$$ & $11 \% .0$ & 18 & 11353 & $5.635 E-06$ & $2.705 E-05$ & 200 \\
\hline$\$$ & 114.0 & 21.6 & 12910 & $5.4075=06$ & $3.075 E-n 5$ & 200 \\
\hline 1 & $\begin{array}{l}114.0 \\
114.0\end{array}$ & $\begin{array}{l}24.3 \\
27.0\end{array}$ & $\begin{array}{l}3 \text { I } 4 \text { स } \\
\text { A } 472\end{array}$ & $\begin{array}{l}1.581 E=06 \\
4.205 F=06\end{array}$ & $\begin{array}{l}7.597 E=06 \\
2.710 E=05\end{array}$ & $\begin{array}{l}200 \\
200\end{array}$ \\
\hline
\end{tabular}

AITMUTH HETGHT EXPOSURE E/O FUIO DISTANCE DEIFEF METERS GM-SEC/CI, SE SEICU.N IISA.M METERS

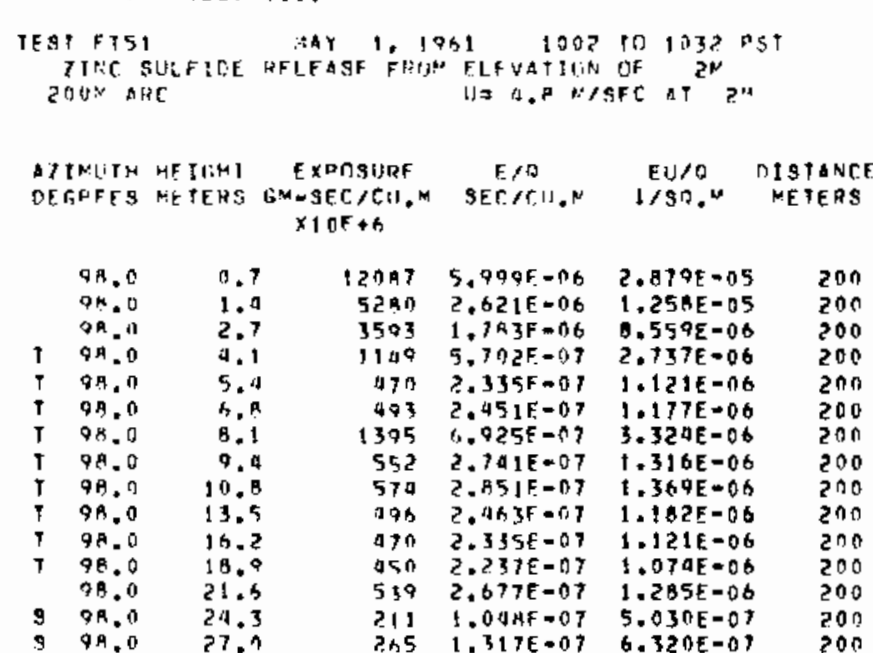

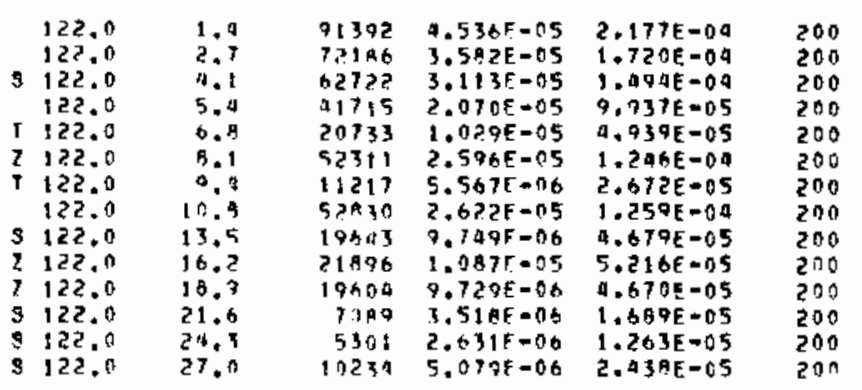




\begin{tabular}{|c|c|c|c|c|c|c|}
\hline \multicolumn{2}{|c|}{$\begin{array}{l}\text { DINAITH } \\
\text { WFREFS }\end{array}$} & $\begin{array}{l}\text { HE FGHT } \\
\text { IAE TF, RS }\end{array}$ & $\begin{array}{c}\text { EXPCHOLF } \\
\text { GM-SEC } / C 11 . " \\
\times 1 \text { OF }+4\end{array}$ & $\begin{array}{c}\text { SEC/CH.N } \\
\text { F/N }\end{array}$ & $\begin{array}{l}F \cup / 0 \\
3 / 30, \mathrm{~m}\end{array}$ & $\begin{array}{l}\text { MISTANCE } \\
\text { HETERS }\end{array}$ \\
\hline & $\begin{array}{l}130.0 \\
130.0 \\
130.0 \\
130.0 \\
130.0 \\
130.0 \\
130.0 \\
130.0 \\
130.0 \\
130.0 \\
130.0 \\
130.0 \\
130.0 \\
130.0 \\
130.0\end{array}$ & $\begin{array}{l}0.7 \\
1.0 \\
2.7 \\
4.1 \\
5.4 \\
6.8 \\
8.1 \\
9.4 \\
10.8 \\
13.5 \\
10.7 \\
15.9 \\
21.6 \\
24.3 \\
27.0\end{array}$ & $\begin{array}{l}167736 \\
69558 \\
59678 \\
58531 \\
52872 \\
43106 \\
17849 \\
29149 \\
37171 \\
53959 \\
34551 \\
22369 \\
14707 \\
4912 \\
13955\end{array}$ & 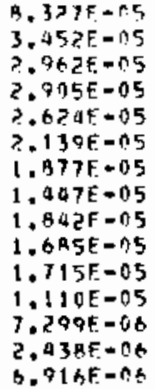 & $\begin{array}{l}3.997 E=07 \\
1.657 E=04 \\
1.022 E=04 \\
1.394 E=04 \\
1.200 E=04 \\
1.027 E=04 \\
9.009 E=05 \\
6.944 E=05 \\
8.043 E=05 \\
8.090 E=05 \\
8.231 E=05 \\
5.329 E=05 \\
3.503 E=05 \\
1.170 E=05 \\
3.320 E=05\end{array}$ & $\begin{array}{l}200 \\
200 \\
200 \\
200 \\
200 \\
200 \\
200 \\
200 \\
200 \\
200 \\
200 \\
200 \\
200 \\
200 \\
200\end{array}$ \\
\hline
\end{tabular}

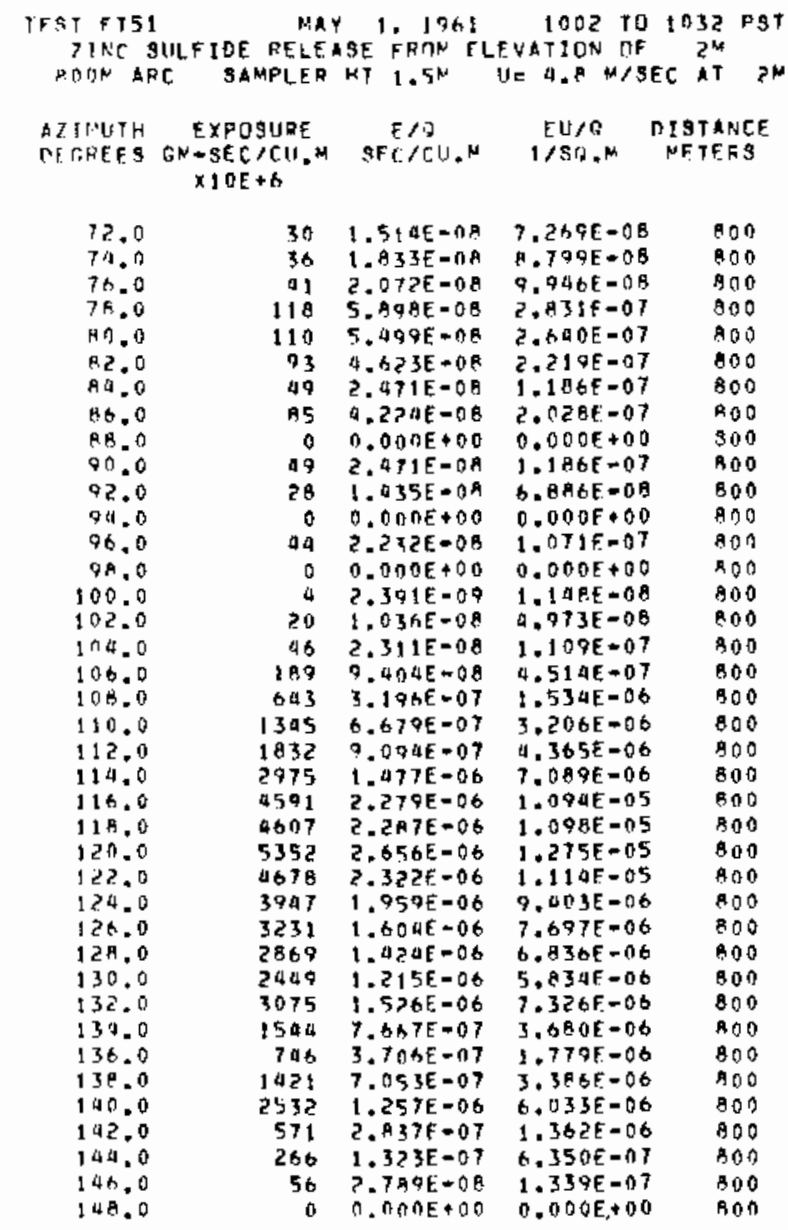

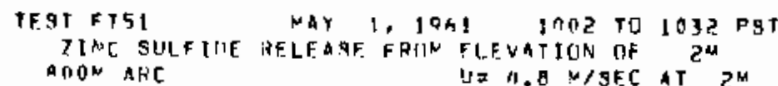

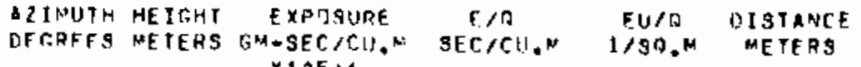
$X 10 F+b$

\begin{tabular}{|c|c|c|c|c|c|c|}
\hline 3 & $\begin{array}{l}106.0 \\
106.0 \\
106.0 \\
108.0\end{array}$ & $\begin{array}{l}1.1 \\
2.1 \\
4.2 \\
6.3\end{array}$ & $\begin{array}{l}211 \\
192 \\
116 \\
140\end{array}$ & $\begin{array}{l}1.0 \angle A E=07 \\
9.86 B E=0 B \\
5.77 \angle E=08 \\
7.400 E=08\end{array}$ & $\begin{array}{l}5.032 E=07 \\
4.736 E=07 \\
2.771 E=07 \\
3.552 E=07\end{array}$ & $\begin{array}{l}000 \\
800 \\
800 \\
000\end{array}$ \\
\hline & 106.0 & g. a & 116 & $5.77 a f=06$ & $2.771 \mathrm{E}=07$ & 000 \\
\hline$t$ & 106.0 & 10.5 & 63 & $3.1595-01$ & $1.507 E-07$ & 800 \\
\hline 9 & $\begin{array}{l}106.0 \\
106.0\end{array}$ & & $\begin{array}{l}115 \\
492\end{array}$ & $\begin{array}{l}5.71 \text { AF }=018 \\
1.9965=07\end{array}$ & $\begin{array}{l}2.745 E=07 \\
9.570 E=07\end{array}$ & $\begin{array}{l}900 \\
800\end{array}$ \\
\hline T & 106.0 & 16.7 & 84 & $4.200 F-08$ & $2.018 E-07$ & 100 \\
\hline $\mathbf{T}$ & 106.0 & .9 & 159 & $7.904 F_{-}=08$ & $790 \mathrm{~A}=07$ & 000 \\
\hline 9 & 106.17 & $25 . ?$ & 173 & $3.630 F-08$ & $\{42 E-07$ & 500 \\
\hline & 19 & & $2 \times 2$ & 40 JE -07 & $737 E-07$ & 800 \\
\hline 2 & 106 & & 270 & . $440 \mathrm{E}-\pi 7$ & $12 E-07$ & 100 \\
\hline 7 & 106.0 & & 349 & $929 E-07$ & $1 E=07$ & 000 \\
\hline 1 & 100.0 & & $\$ 1$ & $79 E-08$ & $1 E-08$ & 800 \\
\hline
\end{tabular}

AZJMITH HETGHT EXPISURE E/O EU/O OISTANCE BFRAEES METERS GM-SEC/CH.M SEC/CU.M I/SO,N METERS $\times 1 O E+B$

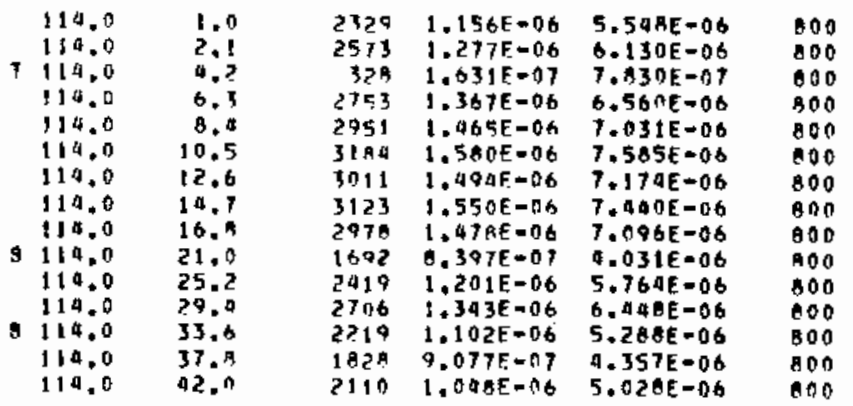

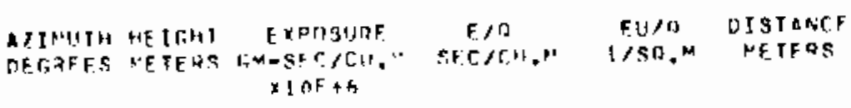

\begin{tabular}{|c|c|c|c|c|c|}
\hline $\begin{array}{l}122.0 \\
122.0\end{array}$ & $1 . n$ & 2.14\% & $\begin{array}{l}1.240 F=n A \\
\text { A } 10 F=n 6\end{array}$ & $\begin{array}{l}5.95 \geq E=0 A \\
\text { A. } 6 \text { A } 6 E=06\end{array}$ & $\begin{array}{l}\text { BกO } \\
\text { AOก }\end{array}$ \\
\hline $\begin{array}{l}122.0 \\
122.0\end{array}$ & $\begin{array}{l}2.1 \\
4.2\end{array}$ & $\begin{array}{l}3616 \\
1192\end{array}$ & $5.527 F=07$ & $2.45 \cap E=06$ & $A 00$ \\
\hline 122.0 & $6, x$ & $a d b 1$ & $2.214 E-116$ & $1.0635=05$ & AOO \\
\hline \pm 22.0 & 8.1 & 4506 & $2.281 F=106$ & $1.095 \xi-05$ & 800 \\
\hline .0 & 10.5 & 1772 & $95 F=07$ & $\begin{array}{l}4.222 \varepsilon-06 \\
9.332 \varepsilon=06\end{array}$ & 800 \\
\hline $\begin{array}{l}122.0 \\
122.0\end{array}$ & 12.4 & 3917 & $\begin{array}{l}94 \Delta E=06 \\
915 E=07\end{array}$ & $\begin{array}{l}9.332 E=06 \\
1.399 E-06\end{array}$ & R01 \\
\hline 122,0 & 16.9 & 447 & B $915 E-06$ & $\begin{array}{l}1.399 E-66 \\
A .979 E-06\end{array}$ & $\begin{array}{ll}80 \text { ถ } \\
0\end{array}$ \\
\hline $122=0$ & $21 . !+1$ & 4177 & $7.0735=06$ & $9.950 E-06$ & 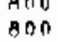 \\
\hline 122.0 & 25.7 & 3777 & $1.8755=00$ & $3.99 A E-06$ & 800 \\
\hline 0 & 29.4 & 3713 & $1.843 \mathrm{~F}=06$ & $8 . B \cup 6 E=06$ & 800 \\
\hline & 33.6 & 2879 & - AO $\triangle F=O \mathrm{~h}$ & $6.740 E-06$ & 800 \\
\hline & 37.4 & 2761 & $\begin{array}{l}1.37 \text { तF }-06 \\
4.325 F-07\end{array}$ & $6.57 \mathrm{BF}=06$ & ano \\
\hline 1 & & 871 & & $076 \mathrm{E}=68$ & 300 \\
\hline
\end{tabular}

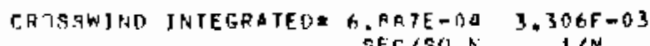




\begin{tabular}{|c|c|c|c|c|c|}
\hline $\begin{array}{l}\text { AZINUTH } \\
\text { DETREES }\end{array}$ & $\begin{array}{l}\text { HE IGHT } \\
\text { METER }\end{array}$ & 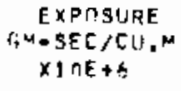 & $\begin{array}{c}E / O \\
S E C / C H, N\end{array}$ & $\begin{array}{c}E U / 1 \\
1 / 80 . M\end{array}$ & $\begin{array}{l}\text { DISTANCE } \\
\text { METENSS }\end{array}$ \\
\hline 130.0 & 1.0 & $9 ? 5$ & h.501E-07 & $2.204 E=06$ & Roo \\
\hline $13 n$ & 3,1 & 5111 & $=-n 7$ & $4.076 \mathrm{E}=06$ & 800 \\
\hline 130.0 & 4,2 & Ias: & $7.2545-07$ & $3.432 \mathrm{E}-06$ & 800 \\
\hline 130.0 & 6.3 & $\$ 125$ & $5.583 f-07$ & $2.680 \mathrm{E}=06$ & AOO \\
\hline 130.0 & 8.4 & 2430 & $1.206 E-06$ & $5.790 E=0 B$ & BOO \\
\hline 130.0 & 10.5 & 2125 & $1.055 E=06$ & $5.064 E=06$ & 800 \\
\hline 130.0 & 12,0 & 2791 & $1.3155-06$ & $6.648 \mathrm{E}-0 \mathrm{~B}$ & 800 \\
\hline 130.0 & 14.7 & 1396 & $0.929 F=07$ & $3.326 E=0 S$ & 800 \\
\hline 130.0 & $16 . \mathrm{A}$ & अ०? & $1.8335=n 6$ & $A .798 E=106$ & 800 \\
\hline 130.0 & 21.0 & 2178 & $1.0818-06$ & $5.190 E-06$ & $B 00$ \\
\hline & & 2032 & $1.000 E-06$ & $4 . A 1 B E-O B$ & 800 \\
\hline $13 n .0$ & 29.4 & Zhosa & $1.313 \mathrm{EE}-06$ & $6,301 E=06$ & 200 \\
\hline 130.0 & 33.6 & 2101 & $1.093 \mathrm{E}-06$ & $5.19 B E-06$ & 800 \\
\hline 13 & 37.8 & $146 ?$ & $7.257 f=07$ & $3.493 E=06$ & 800 \\
\hline 130.0 & $42, n$ & 2692 & $1.336 f-06$ & $6.413 E=06$ & $A 06$ \\
\hline
\end{tabular}

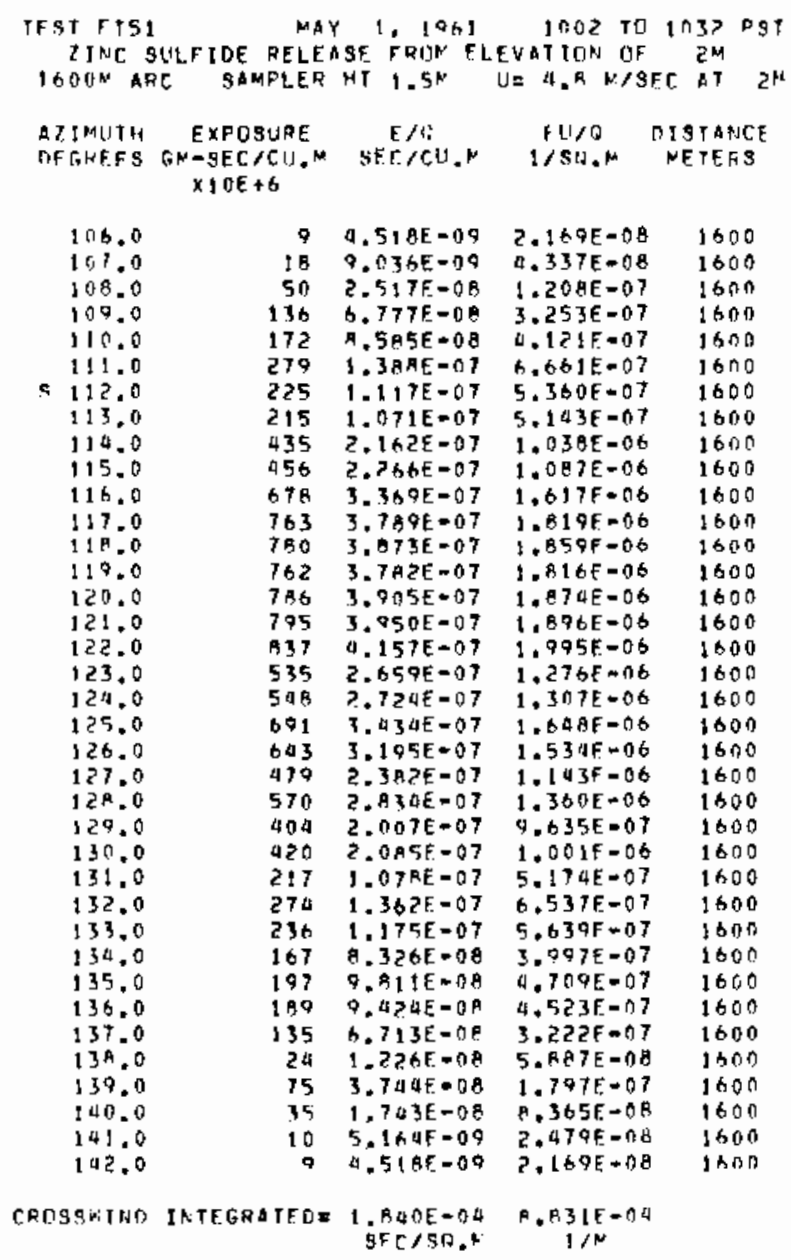

TOMER TATA FULLOHW....

TFST FTSI MAY 1. 19 I 1002 TO IA37 PST

ZINC SULFIUE HELEASE FACIM FLFVATTON OF ZM

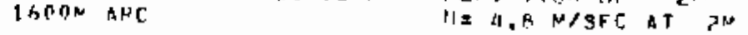

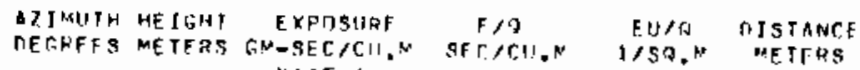
$x \quad 10 E+6$

\begin{tabular}{|c|c|c|c|c|c|}
\hline $\begin{array}{l}114.0 \\
114.0 \\
114.0 \\
11 \%, 0\end{array}$ & $\begin{array}{l}1.5 \\
3.5 \\
6.2 \\
9.3\end{array}$ & $\begin{array}{l}279 \\
150 \\
260 \\
273\end{array}$ & $\begin{array}{l}1.3 A F E=07 \\
9.374 F=00 \\
1.311 E=07 \\
1.110 E=07\end{array}$ & $\begin{array}{l}6.601 E=07 \\
4.019 E=197 \\
6.293 E=07 \\
5.329 E=07\end{array}$ & $\begin{array}{l}1600 \\
1600 \\
1600 \\
1600\end{array}$ \\
\hline $\begin{array}{l}110.0 \\
119.0\end{array}$ & $\begin{array}{l}12.9 \\
15.5\end{array}$ & $\begin{array}{l}45 \\
23\end{array}$ & $\begin{array}{l}2.209 F=08 \\
3.15 P E=08\end{array}$ & $\begin{array}{l}1.079 E-07 \\
1.516 E-0.7\end{array}$ & $\begin{array}{l}1600 \\
1600\end{array}$ \\
\hline 114.0 & 18.6 & lna & $5+16 F E=08$ & $2.481 E=07$ & 1600 \\
\hline $\begin{array}{l}11+0.0 \\
114.0\end{array}$ & $\begin{array}{l}21.7 \\
24.7\end{array}$ & 57 & $\begin{array}{l}2.871 E-08 \\
105 E-00\end{array}$ & $1.378 E=07$ & 1500 \\
\hline 114.0 & 31.0 & $A B$ & $3.276 \mathrm{~F}=0 \mathrm{n}$ & $1.5735=07$ & 1600 \\
\hline 114,0 & $37 . ?$ & $5 \AA$ & $2,917 E-08$ & $1.400 E-07$ & 1600 \\
\hline 114.0 & 43.11 & 70 & $500 E=0 B$ & $1.6 R \cap E=07$ & 16 ดी \\
\hline 114.0 & 49.5 & 17 & $A 39 E=09$ & $4.243 \mathrm{E}-0$ म & $1 \times 00$ \\
\hline 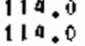 & $\begin{array}{l}55.9 \\
\times 2.9\end{array}$ & $\begin{array}{l}16 \\
47\end{array}$ & $\begin{array}{l}8.41 \mathrm{AE}=09 \\
2.357 \mathrm{~F}=08\end{array}$ & $\begin{array}{c}4.041 E=08 \\
111 E=0 ?\end{array}$ & 1600 \\
\hline
\end{tabular}

ALIMUIH HE IGHT EXPOSUPE E/O EU/O DISTANCE DFFHEES METEAS FM-SFC/CII," $S E C / C H, N$ 1/SO.M METERS $\times 10 \mathrm{~F}+6$

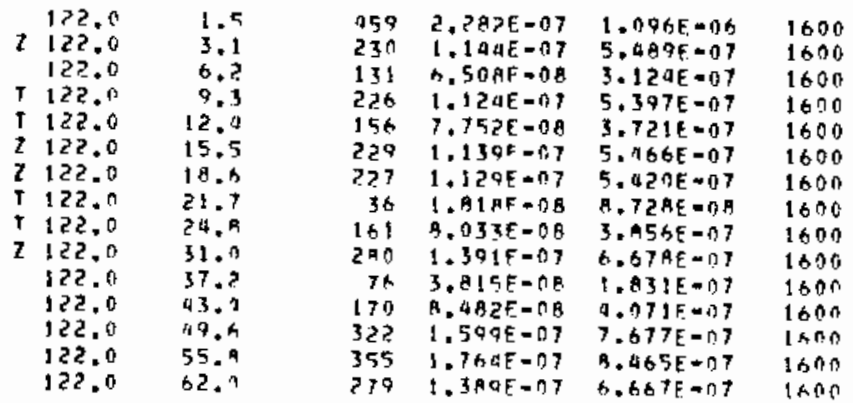

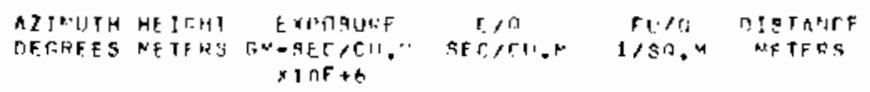

\begin{tabular}{|c|c|c|c|c|c|c|}
\hline & 130.4 & 1.5 & $2+5$ & $1.3 \ln E-0.7$ & $=.716 E-07$ & $\ln \cap 0$ \\
\hline & $13^{n} \cdot 0^{\circ}$ & 3.1 & 1 se & $\forall, 379 F=n A$ & $1.50 \mathrm{CF}=07$ & $1+000$ \\
\hline 7 & 130.4 & $b .>$ & קנ & $? .105 F=\cap R$ & J.A $A 1 E=07$ & IAB \\
\hline$T$ & 130.0 & 8,4 & 1 & $3.570 F-10$ & $4.59+E=09$ & 1500 \\
\hline$T$ & 130.4 & 12.4 & 91 & $2,05 B E-\cap 8$ & $9 . A 76 \varepsilon=0$ म & $1+\pi 0$ \\
\hline 1 & 130.0 & 15.5 & 12 & $3.584 \mathrm{E}=0 \mathrm{e}$ & $1.7 \geq I E=07$ & 1600 \\
\hline & 130.0 & $1 B .5$ & 100 & 9.97 a & 4.5 A $A E=07$ & 1600 \\
\hline $\mathbf{Z}$ & 130.0 & 21.7 & ?.6n & $1.292 E=017$ & $6.201 E=07$ & 1000 \\
\hline T & 130.0 & $2^{4} \cdot a^{a}$ & $a ?$ & ?. In $95=08$ & $1.01 ? E-07$ & 1600 \\
\hline $\mathbf{T}$ & 130.0 & 31.2 & 56 & ?. 7 ด्र $F=0 \mathrm{H}$ & $1,33+E=0.7$ & $16 \% 0$ \\
\hline $\boldsymbol{\top}$ & 1314.1 & 37.7 & $\operatorname{lng}$ & $5.27+5=08$ & $2.502 \mathrm{E}-01$ & 1600 \\
\hline & 530.0 & 43.1 & 262 & $1.301 F=01$ & $6.2 \Delta 7 F=07$ & 1600 \\
\hline 7 & $13 n, n$ & 4.7 .4 & Ith? & $O R 1 E=A a$ & ค) $A E=07$ & 1600 \\
\hline $\begin{array}{l}\top \\
\end{array}$ & 150.0 & 55.2 & 19 & $9.64,1=09$ & $4.647 E-08$ & 1600 \\
\hline & & +2 & $3 t 5$ & $1.56+F=07$ & $7.515 E-07$ & 1500 \\
\hline
\end{tabular}




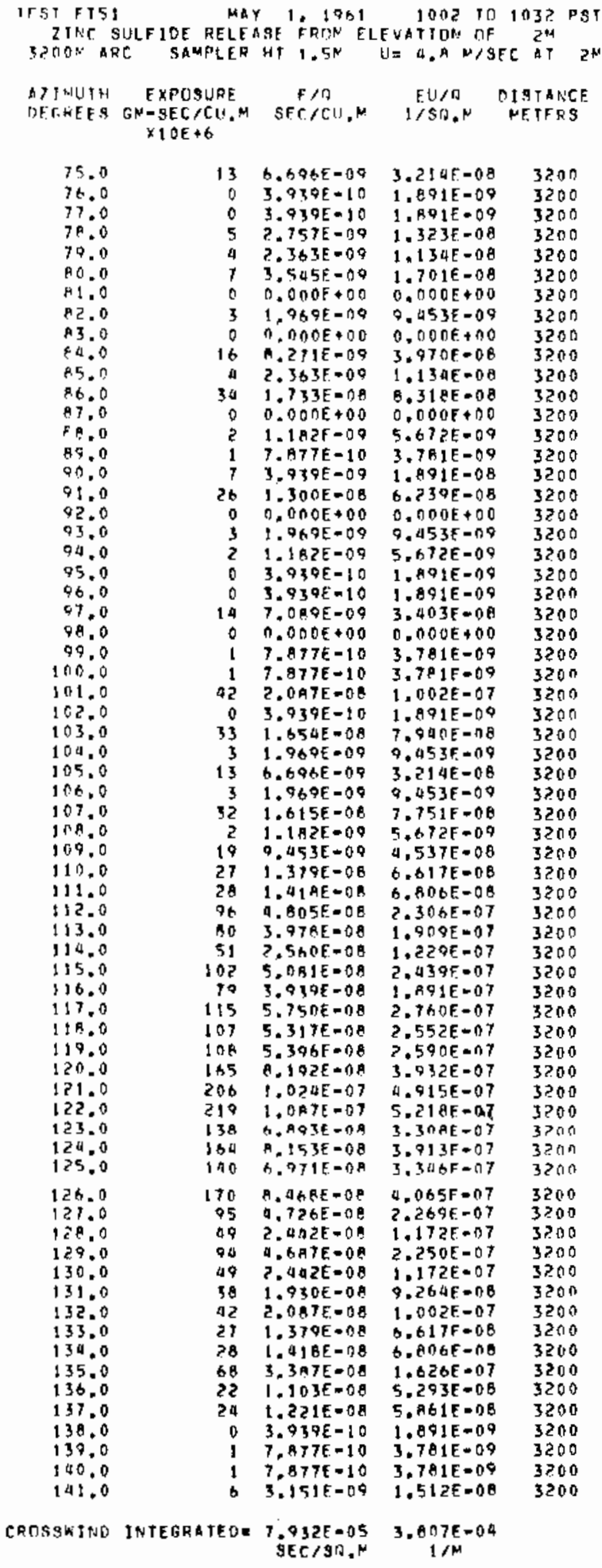

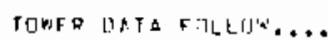

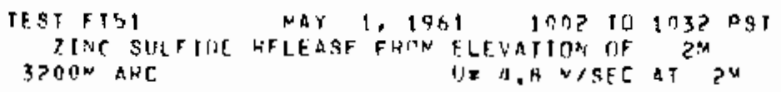

\begin{tabular}{|c|c|c|c|c|c|c|}
\hline & 114.0 & $\begin{array}{l}1.5 \\
3.1\end{array}$ & 30 & $\begin{array}{l}1.4995=08 \\
3.2595=09\end{array}$ & $\begin{array}{l}1.196 E-O B \\
1.564 E-O B\end{array}$ & $\begin{array}{l}3200 \\
3500\end{array}$ \\
\hline 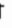 & 114.0 & a. 2 & A & $3.25 \% 5=109$ & $1.564 E=08$ & 3200 \\
\hline I & 124.0 & 9,3 & 17 & $O O E=189$ & $E E=\cap B$ & 320 \\
\hline I & 114.0 & 12.4 & 13 & $6.5 t e F=07$ & $3.129 E=O B$ & $3 \geq 1>0$ \\
\hline & 114.0 & 1 A. 6 & 3 & $1.530 F=09$ & $2 E=09$ & 320 \\
\hline I & 114.4 & 21.7 & 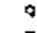 & $1.8 A 95=09$ & $\overline{I \varepsilon-0 h}$ & 3200 \\
\hline $\mathbf{I}$ & $1: 4.0$ & 24.9 & 5 & $2.504 F=09$ & $1.202 E-O B$ & 3 \\
\hline I & 114.0 & 31.0 & 7 & $3.756 E=09$ & $03 E-08$ & 200 \\
\hline & 114.0 & 3 & की 9 & $411 E=08$ & $\{1 E-0\}$ & 320 \\
\hline i & $1 \leq 4,0$ & 43.7 & 18 & $076 E=09$ & $35 B E=0 B$ & 3 \\
\hline $\mathbf{T}$ & 114.0 & 4 & it & $0215-09$ & $10 E=03$ & \\
\hline$\$$ & & & $d t$ & $B \Delta F=0 A$ & AE -07 & \\
\hline
\end{tabular}

AZIMITH HEIGHT EXPRSUHE E/T DEFHEFS NETERS GM-3EC/CH, SH SFE/CU,N I/SO.M METERS $X 1 O E+6$

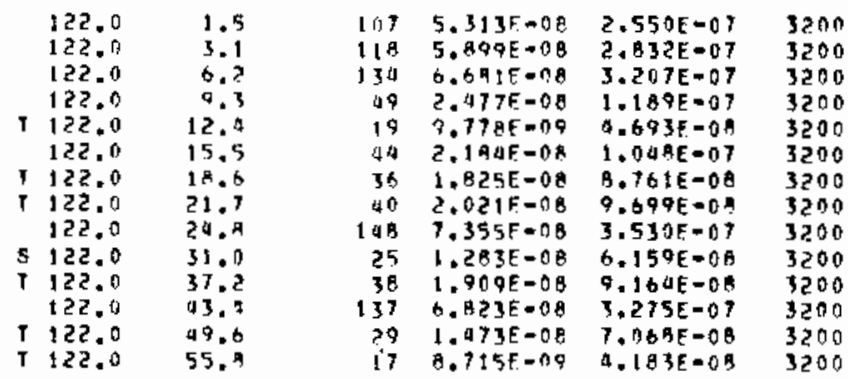

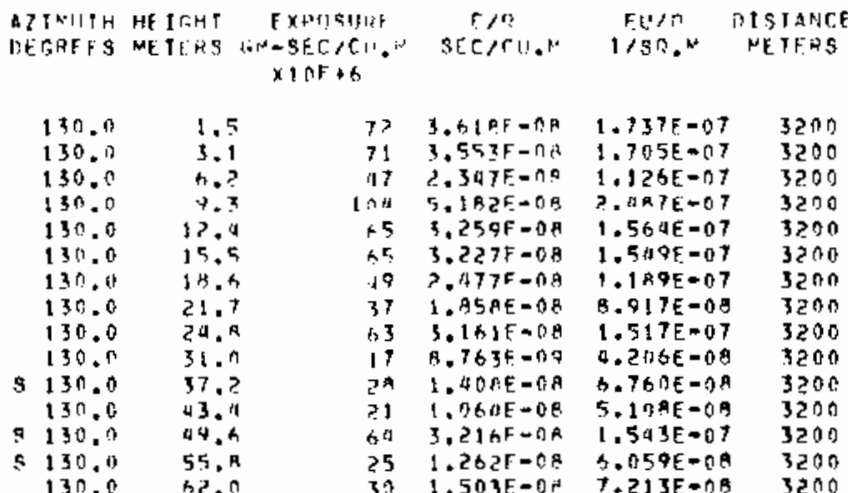


30-SERIES EXPERIMENT: 52 \& FT-52,

GROUND LEYEL AND TOWER SAMPLING 200-3200 M ARCS. WANY OF THE

FILTERS ON THE TOWERS SHOHED 5 IGNS OF WEATHERING.

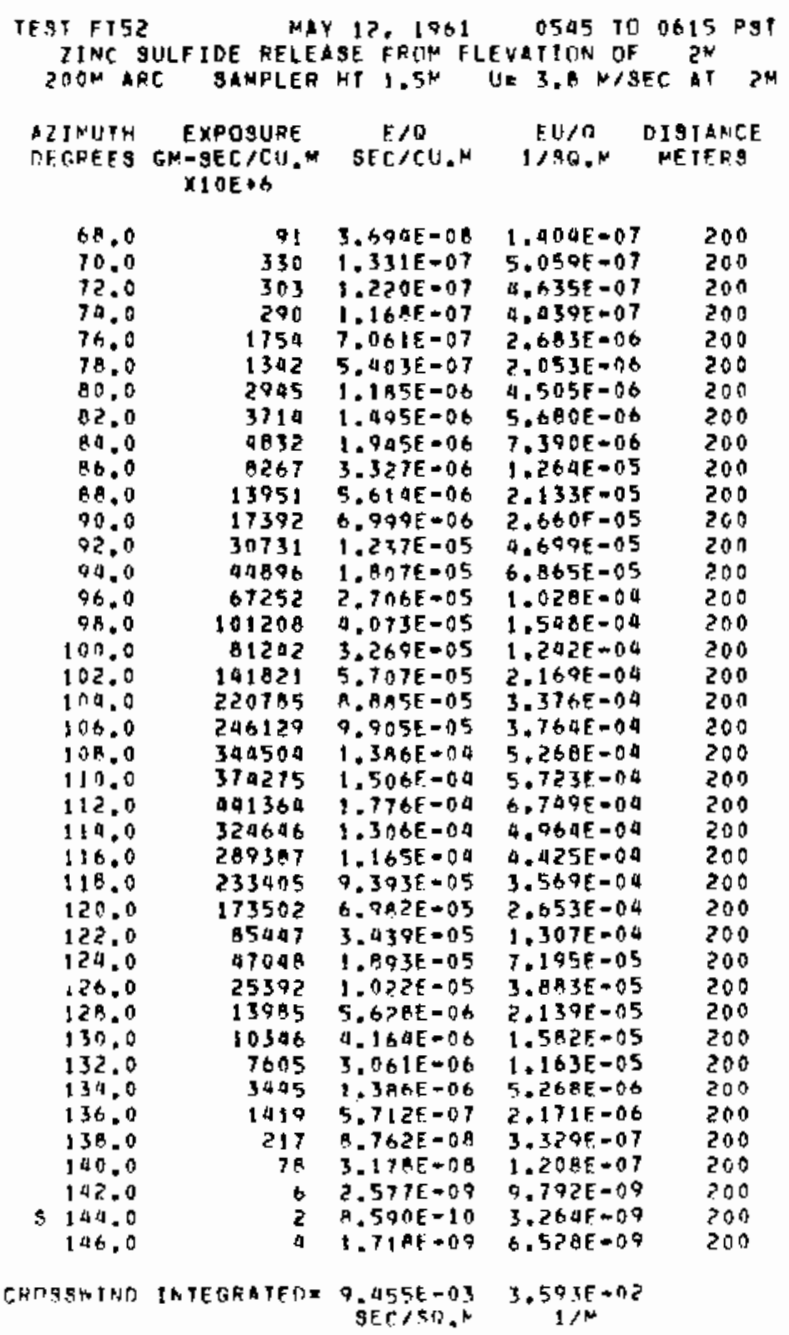

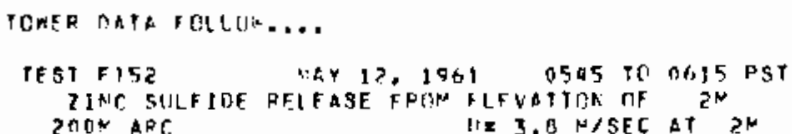




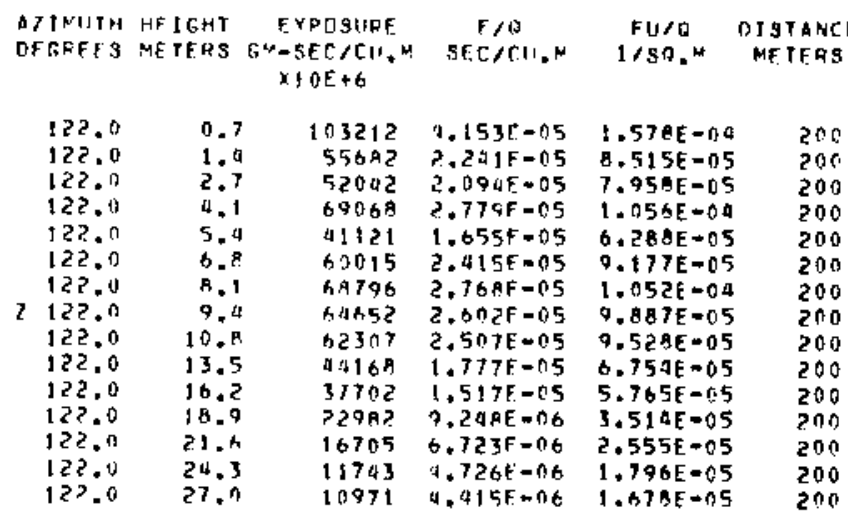

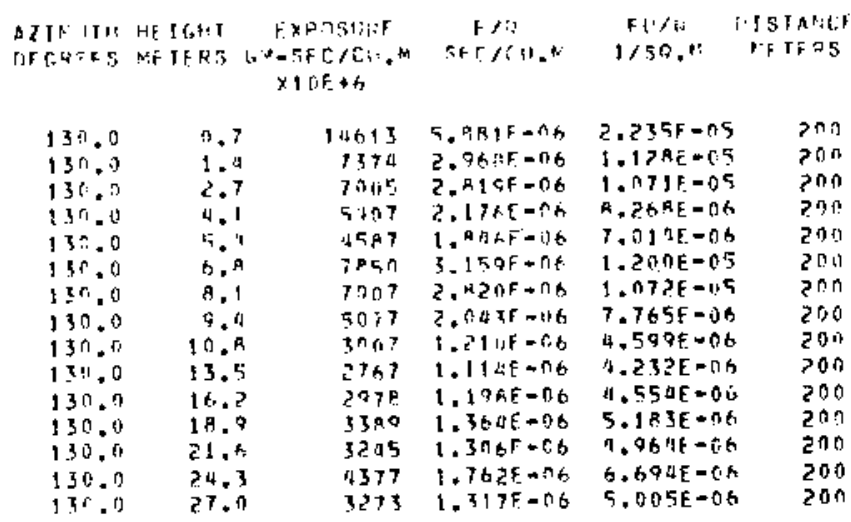

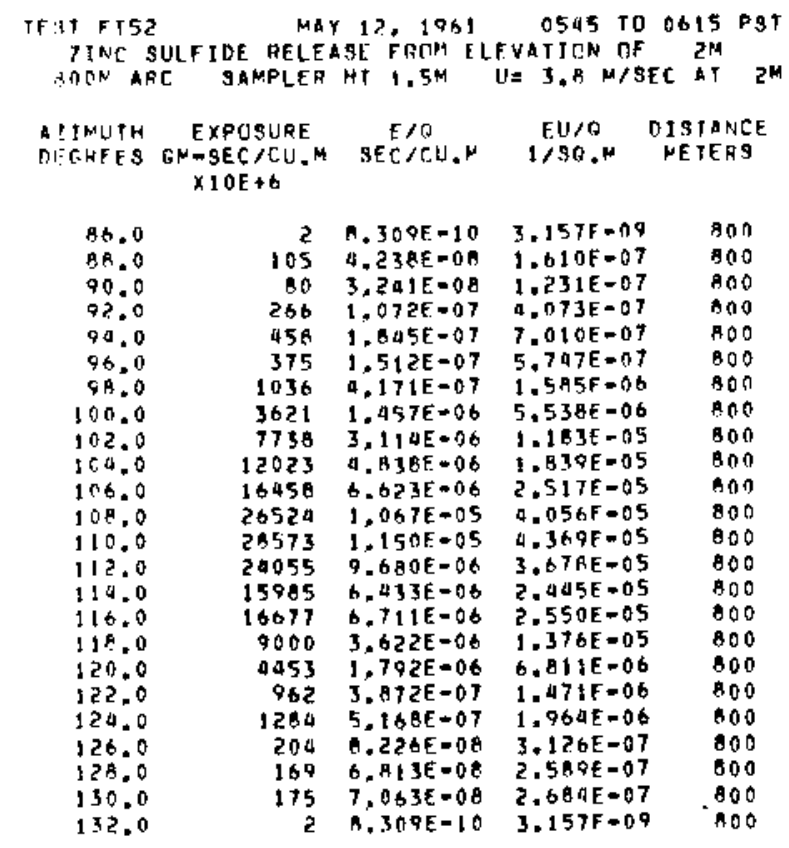

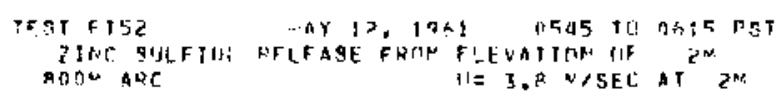

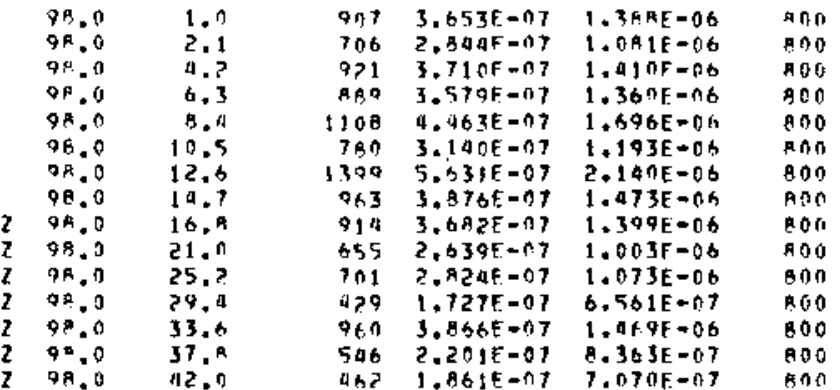

AZIMUTH HEIGHT EXPISUVE E/O FUIO OISTANCF DEGREFS METERS GNOSEC/CIN.N SEC/CU.N IISG.M METERS

\begin{tabular}{|c|c|c|c|c|c|}
\hline $\begin{array}{l}106.0 \\
106.0 \\
106.0 \\
106.0 \\
106.0 \\
106.0 \\
106.0 \\
106.0 \\
106.0 \\
106.0 \\
106.0 \\
106.0 \\
106.0 \\
106.0 \\
106.0\end{array}$ & $\begin{array}{l}1.0 \\
2.1 \\
4.2 \\
6.3 \\
8.9 \\
10.5 \\
12.8 \\
14.7 \\
16.8 \\
21.0 \\
25.2 \\
29.9 \\
33.6 \\
37.8 \\
42.0\end{array}$ & $\begin{array}{r}1>061 \\
11507 \\
12665 \\
9143 \\
10522 \\
15334 \\
2017 \\
13346 \\
12705 \\
11369 \\
9923 \\
7276 \\
5545 \\
5373 \\
9996\end{array}$ & $\begin{array}{l}9.954 E-06 \\
9.067 E=06 \\
5.097 F=06 \\
3.679 F=06 \\
5.804 F=06 \\
6.815 F=06 \\
9.201 E=07 \\
5.372 E=06 \\
5.113 F=06 \\
4.575 E=06 \\
3.993 F=06 \\
2.92 A E=06 \\
2.233 E=06 \\
2.162 E=06 \\
1.970 F=06\end{array}$ & $\begin{array}{l}1.844 E-05 \\
1.774 E=05 \\
1.937 E=05 \\
1.398 E=05 \\
2.221 E=05 \\
2.590 E=05 \\
3.132 E=06 \\
2.041 E=05 \\
1.943 E=05 \\
1.759 E=05 \\
1.518 E=05 \\
1.113 E=05 \\
8.405 E=06 \\
8.216 E=06 \\
7.08 B E=06\end{array}$ & $\begin{array}{l}800 \\
800 \\
100 \\
800 \\
800 \\
600 \\
800 \\
800 \\
000 \\
100 \\
800 \\
800 \\
800 \\
100 \\
B 00\end{array}$ \\
\hline
\end{tabular}

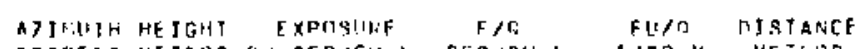

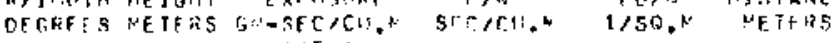
$x+0 F+3$

\begin{tabular}{|c|c|c|c|c|c|c|}
\hline & 114.0 & 1.9 & 12757 & $5.1345=\pi, 6$ & $1.951 E=05$ & $8 \cap \mathrm{fi}$ \\
\hline & 114.0 & 2.1 & $1561 \mathrm{~K}$ & $4.675 r-06$ & $766-05$ & son \\
\hline & 114.0 & $4 . ?$ & $134 ? ?$ & $5.401 f=06$ & $2.052 E-05$ & son \\
\hline & 104.0 & 6.3 & $1200 n$ & $1,829 E-06$ & $35 F=05$ & $A 00$ \\
\hline & $144 . ?$ & 8.9 & IS7RR. & $7.561 f=06$ & A $73 f-05$ & 100 \\
\hline & 114.0 & 10.5 & $13 n<5$ & $25 \mathrm{AF}-\mathrm{nt}$ & $1.99 A E-05$ & 800 \\
\hline 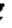 & 194.0 & 12.4 & 11903 & $D .79 A E=0$. & $1.92 \cap E \rightarrow 05$ & $A 00$ \\
\hline ? & 5190 & 14,7 & $110 \mathrm{AP}$ & $\pi .430 \mathrm{~F}-\pi \mathrm{s}$ & $1.683 E-05$ & $B 00$ \\
\hline & 114.0 & 16.5 & 16001 & $6.430 \mathrm{E}=0.6$ & $2.04 J E-05$ & AOO \\
\hline & 113.0 & $21 \cdot n$ & 10675 & $4.290 \mathrm{~F}=06$ & $1.633 E * 65$ & 800 \\
\hline l & 114.0 & $25 . ?$ & 1830 & $3.155 F+106$ & $1.199 \mathrm{E}=05$ & 80 \\
\hline 7 & 114.0 & $2^{4} \cdot 1$ & es4a & 3. dact $=176$ & $1.307 E=05$ & aon \\
\hline & 114,0 & 33.6 & 9101 & $3.260 f=n .6$ & $39 E-05$ & 900 \\
\hline & 114.0 & 57.9 & 5077 & $3.613 F-116$ & $1.373 E=05$ & 800 \\
\hline & 3 & $42, \pi$ & 7707 & $015 E=1.0$ & $146 \mathrm{E}-015$ & AOE \\
\hline
\end{tabular}




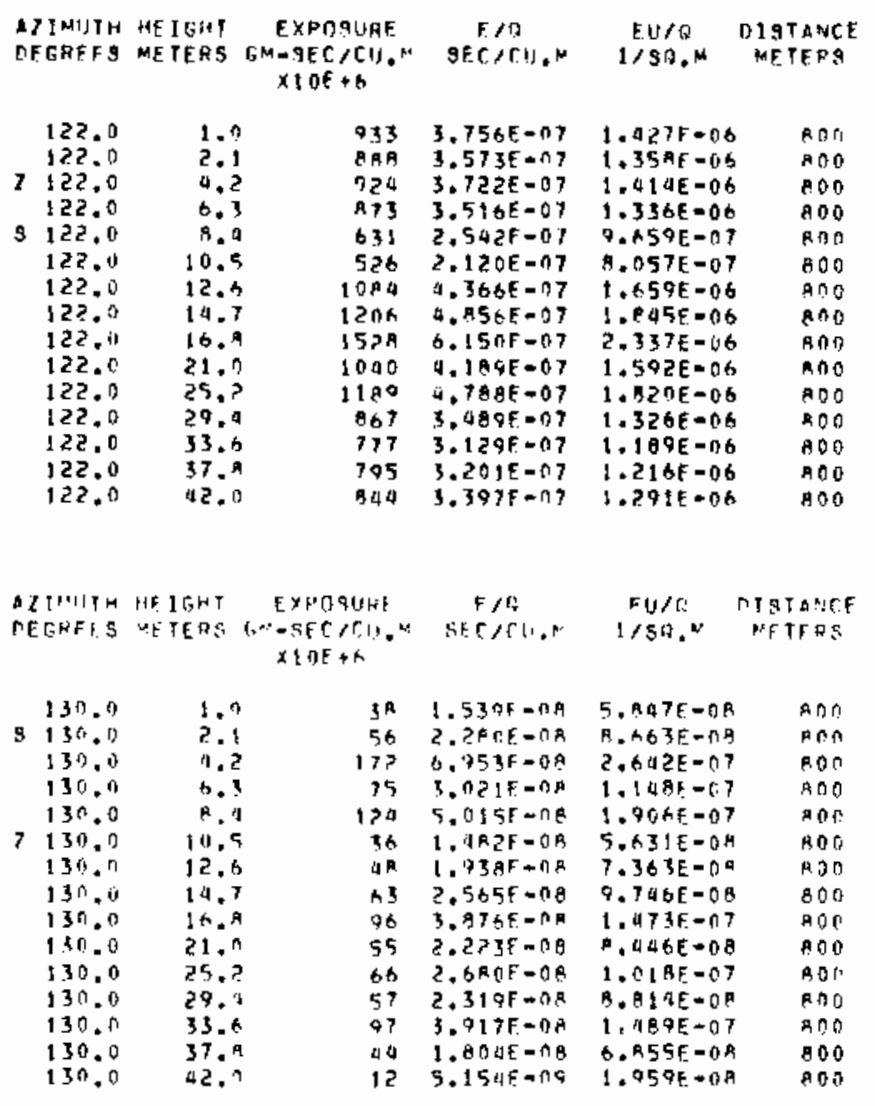

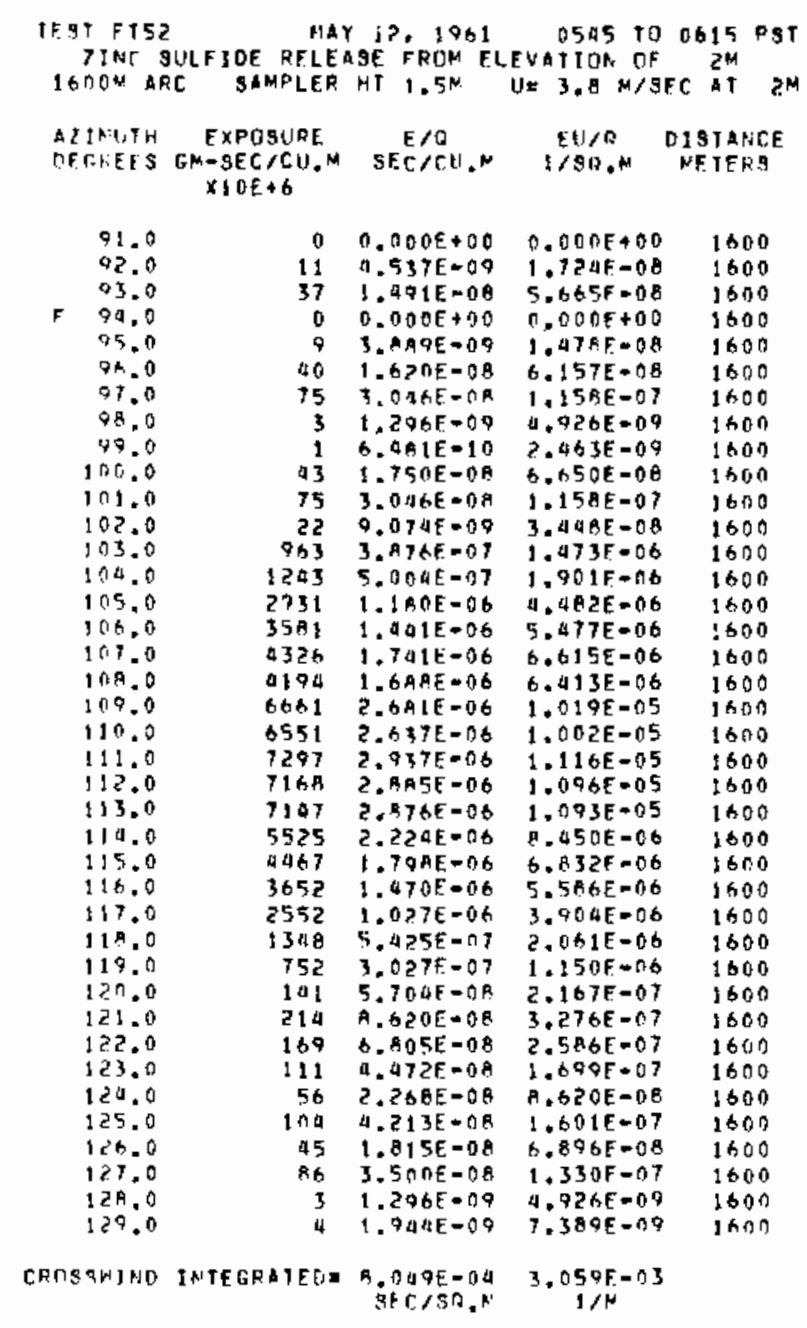

Iחמש

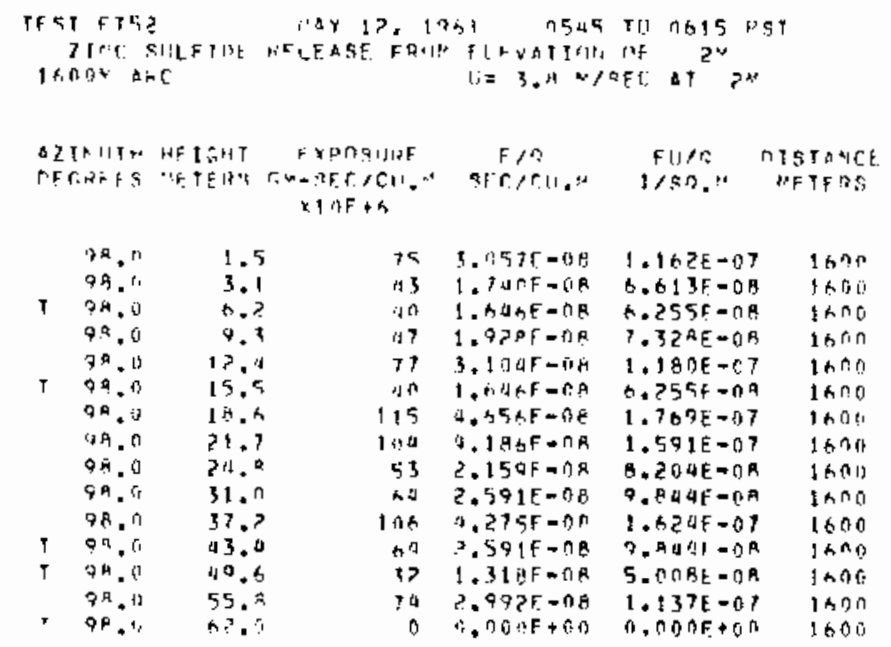




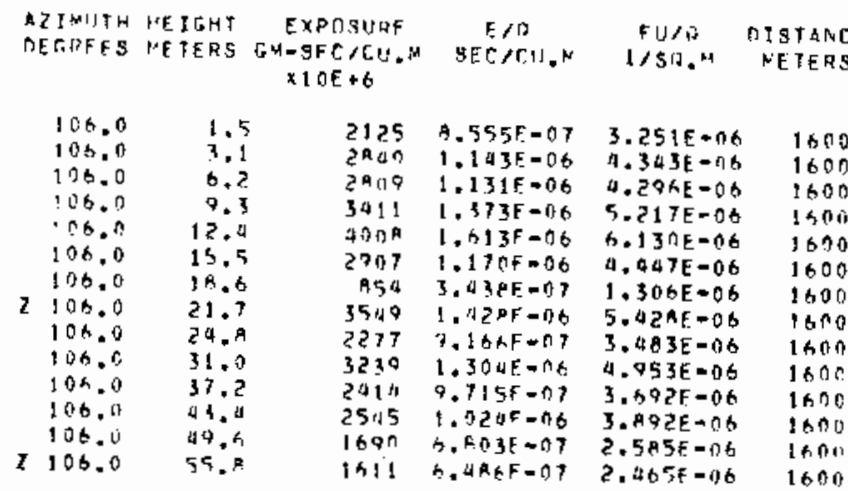

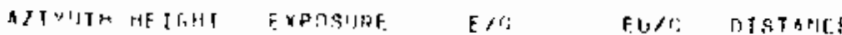

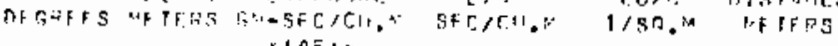
$\times 10 \bar{C}+\rightarrow$

\begin{tabular}{|c|c|c|c|c|c|c|}
\hline & 114.0 & 1.5 & 4297 & $1.1305-01$ & $6.575 \mathrm{~F}=0 \mathrm{~h}$ & $1+n$ \\
\hline & 114.0 & 3.1 & 1361 & $1.755 F-10$ & $x_{1} .670 \mathrm{E}-06$ & \\
\hline & $\$ 14.0$ & A.? & $14>5$ & $1.7705=06$ & $A .759 E=0 \mathrm{~B}$ & \\
\hline & 110.0 & 9.3 & 5546 & 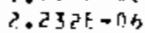 & A. 4 AZE-06 & In \\
\hline & 114.17 & 12.4 & 3914 & $1.585 F=06$ & $6.023 E-n G$ & 16 \\
\hline & 11.3 .13 & 13.5 & 4534 & $1.425 F=06$ & $6.83 \Delta \varepsilon=0 \mathrm{~s}$ & 160 \\
\hline & 111.1 & $1 \mathrm{H} . \mathrm{h}$ & 56014 & $2.355 E=04$ & $A .570 \mathrm{E}-116$ & int \\
\hline$?$ & 114.0 & 21.7 & d $11 \mathrm{~h}$ & $1 . n S A F=n t$ & $6 . P Q 5 E=06$ & h) \\
\hline & 114.0 & 24.8 & 58 ? ? & $1.5 \cap 3 F=n B$ & $S .00 C F=0 S$ & . \\
\hline & 114.5 & 31.0 & 111,3 & $1.675 F-56$ & $6.360 E-06$ & 1600 \\
\hline & 114.0 & $37 . ?$ & $44 \leq 7$ & $1.7 A+E=06$ & $6.7865-08$ & 60 \\
\hline 7 & 110 & 45.9 & 2971 & $1+196 \mathrm{~F}=106$ & $4.543 \mathrm{E}-06$ & 60 \\
\hline & 114.0 & 55.9 & 2231 & R. $38 \cap 5-07$ & $3,012 E=06$ & 160 \\
\hline 7 & $1 ! 4,0$ & 62.0 & 3284 & $1,32 \geqslant 0=06$ & $5.023 E+06$ & 100 \\
\hline
\end{tabular}

\begin{tabular}{|c|c|c|c|c|c|c|}
\hline & $\begin{array}{l}\text { TREAER } \\
\text { INIITH }\end{array}$ & $\begin{array}{l}\text { MEIGHI } \\
\text { METEHS }\end{array}$ & $\begin{array}{c}\text { EXPDSURE } \\
r, N=S E C / C, N=N^{\circ} \\
\times 1 O E+\theta\end{array}$ & $\begin{array}{c}E / ? \\
S F C / C 1, N\end{array}$ & $\begin{array}{c}F U / O \\
1 / S 0 . M\end{array}$ & $\begin{array}{l}\text { ISTANCE } \\
\text { METFRS }\end{array}$ \\
\hline & $\begin{array}{l}122.0 \\
122.0 \\
122.0 \\
122.0 \\
122.0 \\
122.0 \\
122.0 \\
122.0 \\
122.0 \\
122.0 \\
122.0 \\
122.0 \\
122.0 \\
122.0 \\
122.0\end{array}$ & $\begin{array}{r}1.5 \\
3.1 \\
6.2 \\
9.3 \\
12.0 \\
15.5 \\
18.6 \\
21.7 \\
24.7\end{array}$ & $\begin{array}{l}70 \\
54 \\
128 \\
139 \\
141 \\
137 \\
140 \\
126 \\
143 \\
172 \\
135 \\
120 \\
58 \\
119 \\
119\end{array}$ & $\begin{array}{l}2 . A 22 F=0 A \\
2.210 E=0 A \\
5.174 F=0 A \\
5.2218=0 B \\
5.691 F=08 \\
5.550 E=0 A \\
5.6411 E=08 \\
5.079 F=08 \\
5.7 A 6 E=08 \\
6.952 E=08 \\
5.440 E=08 \\
0.836 E=0 B \\
2.173 E=08 \\
2.895 E=0 B \\
1.452 E=0 B\end{array}$ & $\begin{array}{l}1.072 E-07 \\
A .400 E=09 \\
1.966 E=07 \\
1.984 E=07 \\
2.163 E=07 \\
2.109 E=07 \\
2.145 E=07 \\
1.930 E=07 \\
2.199 \mathrm{E}=07 \\
2.642 \mathrm{E}=07 \\
2.067 \mathrm{~F}=07 \\
1.839 \mathrm{E}-07 \\
1.257 \mathrm{E}=08 \\
1.096 \mathrm{~F}-07 \\
1.692 \mathrm{E}=07\end{array}$ & $\begin{array}{l}1600 \\
1600 \\
1600 \\
1600 \\
1600 \\
1600 \\
1600 \\
1600 \\
1600 \\
1600 \\
1600 \\
1600 \\
1600 \\
1600 \\
1600\end{array}$ \\
\hline
\end{tabular}

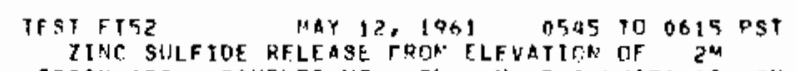
3RTON AFE SAMPLER HT $1.5 \mathrm{~K}$ DE $3.8 \mathrm{M} / \mathrm{SEC}$ AT IM $^{\mathrm{M}}$

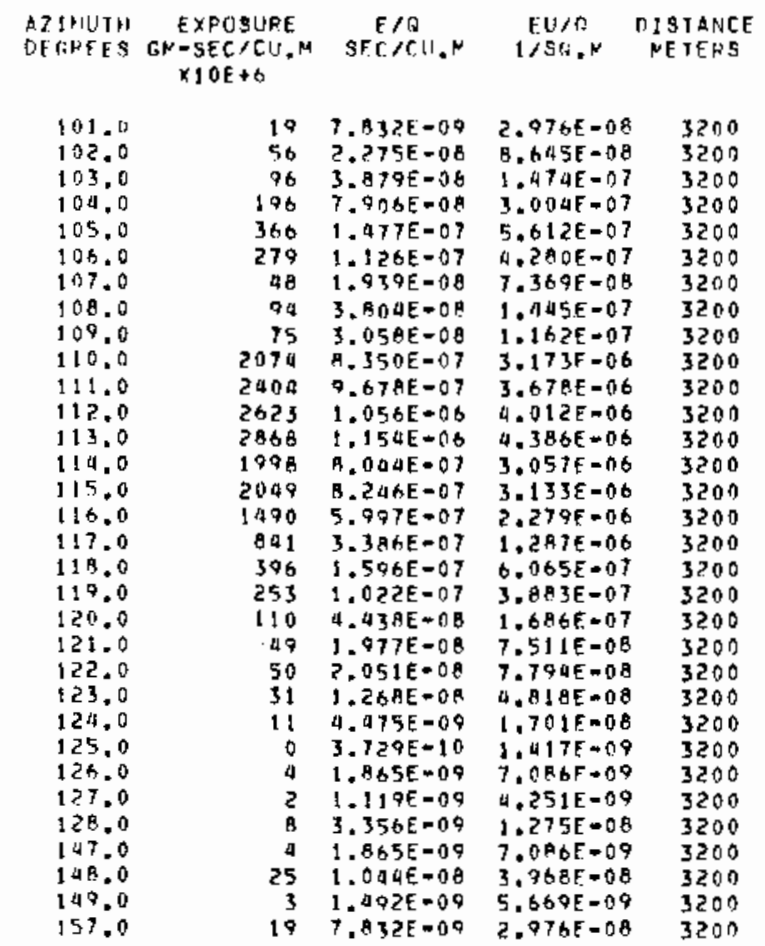

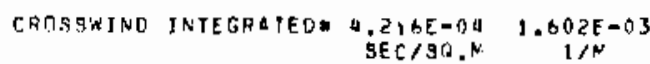

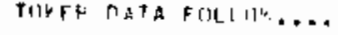

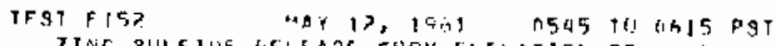

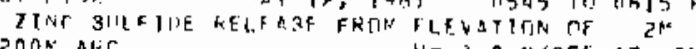

wOON AlOC $H=3 . A$ NISFC AT $2 N$

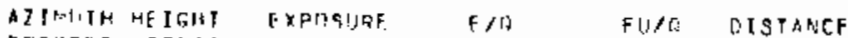

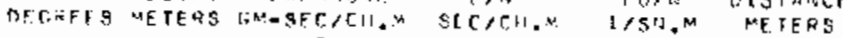
$x+$ of $+b$

\begin{tabular}{|c|c|c|c|c|c|}
\hline $1(16.9$ & 1.5 & 1436 & $1.7545-077$ & $6.369 \mathrm{E}=07$ & 3200 \\
\hline 100.0 & 3.1 & 384 & $1.303 \mathrm{~F}-0$ ) & $6.091 E=07$ & 3200 \\
\hline 106.8 & b.? & 517 & $2.4 P E E-07$ & 0.4 \& AE $=07$ & 3200 \\
\hline 104.05 & 9.5 & 150 & $3,099 \mathrm{~F}=0 \mathrm{~A}$ & $2.303 E=07$ & $3 \geqslant 00$ \\
\hline $\begin{array}{l}105.0 \\
104.0\end{array}$ & $\begin{array}{l}12.4 \\
15.5\end{array}$ & $\begin{array}{l}308 \\
138\end{array}$ & $\begin{array}{l}2.587 E-08 \\
5\end{array}$ & $\begin{array}{l}3.1876=07 \\
3,135=07\end{array}$ & 3200 \\
\hline $11+2.0$ & $I \theta, 6$ & 23 ค & $9.5 \times 1 F=08$ & $3.641 f=07$ & 3500 \\
\hline 106.0 & 21.7 & $69 ?$ & 2,7 PE $=07$ & 1.05 A $=06$ & 3200 \\
\hline 106.6 & 24.9 & 554 & $1.436 E-07$ & $5.459 F-0.7$ & 3200 \\
\hline 106.0 & 51.3 & IAQ & $1.948 F=07$ & $7.403 f=0.7$ & 3200 \\
\hline $10,6 \cdot 0$ & 37.7 & ब中 & $1.965 E-07$ & $7.460 E-07$ & 3200 \\
\hline & 43.4 & 457 & $1.809 E-07$ & $7.027 E-07$ & 3200 \\
\hline 10 & & $2 A 13$ & $1.1205=0.7$ & $4.291 f=07$ & 3200 \\
\hline 106.0 & 55.0 & 203 & $1.5 A 5 F=0.7$ & $6.0225=07$ & 3 3า \\
\hline & & $\sqrt{2}$ & $5,007 E=09$ & $1.403 E=08$ & 3200 \\
\hline
\end{tabular}




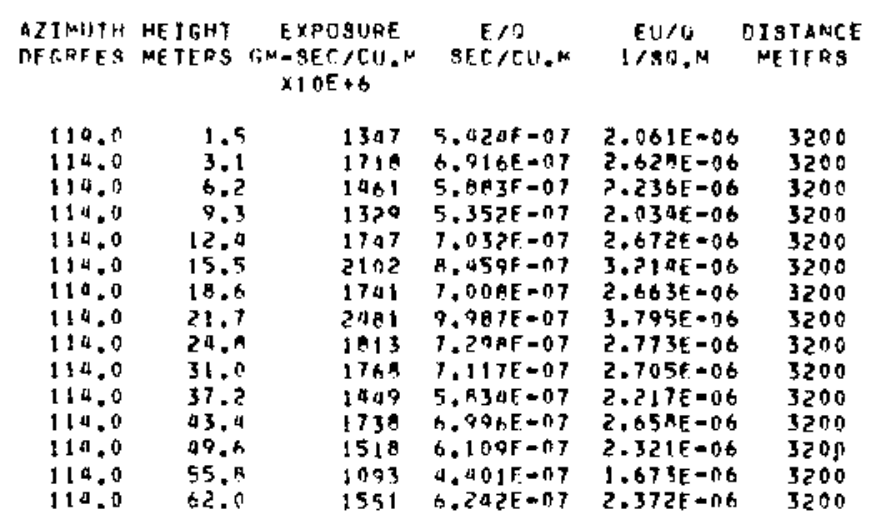

\begin{tabular}{|c|c|c|c|c|c|}
\hline 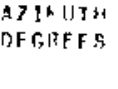 & 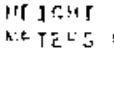 & 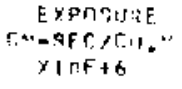 & 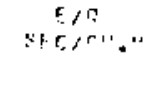 & $\begin{array}{c}e+1 /: \\
1 / 5 \% *\end{array}$ & 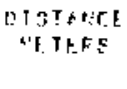 \\
\hline $\begin{array}{l}122.01 \\
122.0\end{array}$ & 1.5 & i & 4. ная下 -09 & $1.4755=09$ & 3200 \\
\hline 122.0 & $\begin{array}{l}3.9 \\
6 . ?\end{array}$ & $\begin{array}{l}17 \\
31\end{array}$ & $\begin{array}{l}\text { A. } .45 E=0 Q \\
1.254 F=0 A\end{array}$ & $\begin{array}{l}2.600 E-0 A \\
4.76,2-08\end{array}$ & $\begin{array}{l}3200 \\
3200\end{array}$ \\
\hline 122.0 & 7.3 & 74 & З. 9स5F. -0म & 1.13 : $6=07$ & 32 in \\
\hline $\begin{array}{l}122,0 \\
12 ? .0\end{array}$ & $\begin{array}{l}12.4 \\
15.5\end{array}$ & $\begin{array}{l}30 \\
14\end{array}$ & $\begin{array}{l}1.224 t=0, \\
5.0711=00\end{array}$ & $4 .+500=0,9$ & $35^{n} 1$ \\
\hline 127.0 & 13.4 & $\begin{array}{l}14 \\
2^{n}\end{array}$ & $2.150 F-69$ & $\begin{array}{l}353 F=04 \\
3.962 E=09\end{array}$ & $\begin{array}{l}3200 \\
3 c^{3} \text { ?0 }\end{array}$ \\
\hline $\begin{array}{l}122.9 \\
122.0 \\
122.0\end{array}$ & $\begin{array}{l}21.3 \\
24.9 \\
31.9\end{array}$ & $\begin{array}{l}03 \\
51 \\
53\end{array}$ & $\begin{array}{l}1.761 F-c . p \\
2.0975=08\end{array}$ & $\begin{array}{l}4.692 \mathrm{E}-10 \\
7.951 \mathrm{E}=08\end{array}$ & $\begin{array}{l}3200 \\
320 n\end{array}$ \\
\hline $\begin{array}{l}128.0 \\
172.0 \\
125.6 \\
122.0\end{array}$ & $\begin{array}{l}37.7 \\
37.9 \\
43.4 \\
49.9\end{array}$ & $\begin{array}{l}53 \\
90 \\
13 \\
52\end{array}$ & $\begin{array}{l}2,147 E=06 \\
1.6125=05 \\
5.3735=09 \\
2.174=08\end{array}$ & $\begin{array}{l}B .164 E=0 A \\
A .125 E=0 A \\
2.042 E=08 \\
A .012 E=08\end{array}$ & $\begin{array}{l}3200 \\
3200 \\
3200 \\
3200\end{array}$ \\
\hline $\begin{array}{l}122.0 \\
122.0\end{array}$ & $\begin{array}{l}55.7 \\
62.13\end{array}$ & $\begin{array}{l}27 \\
77\end{array}$ & $\begin{array}{l}1.127 \%=0 B \\
1.5 n 5 E-9 A\end{array}$ & $\begin{array}{l}4.2 \times 1 E-C B \\
5.7 \cap A F-O B\end{array}$ & $\begin{array}{l}3200 \\
3000\end{array}$ \\
\hline
\end{tabular}


30-SER1ES EXPERIMENT: 53 (FT-53)

GROUND LEVEL AMD TONER SAMPLIMG 200-12800 M ARCS

\begin{abstract}
TFET F T 53 JUNE 27, 1961 0550 TU 061 \& PST

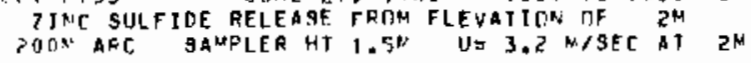

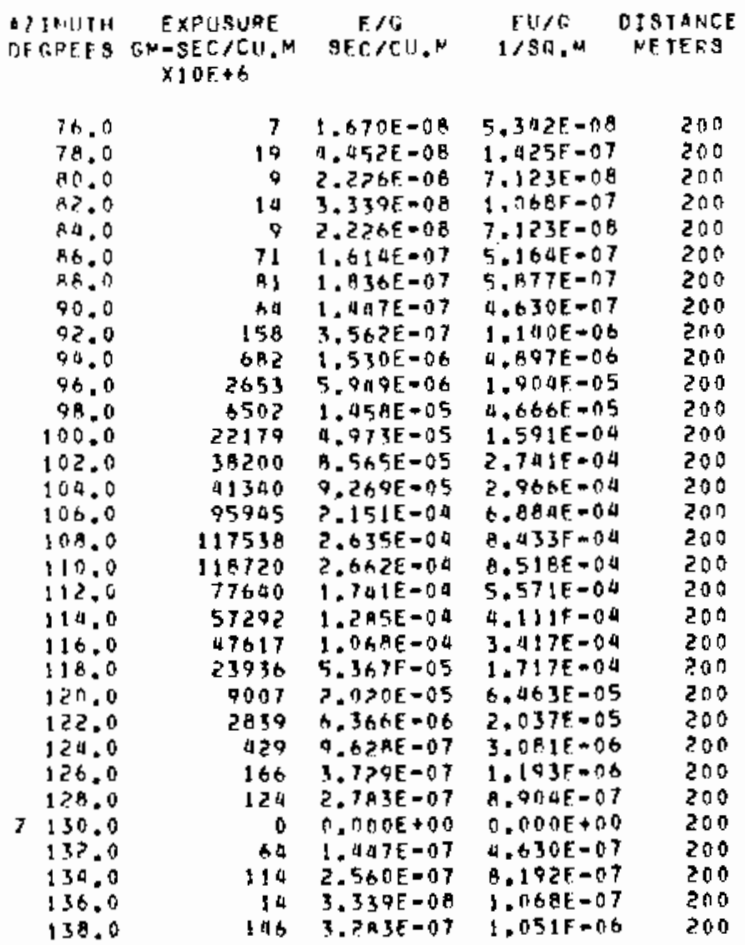

CDOBSWIAD INTEGRATERA 1.036E-02 3.316E-02

TOWER VATA FILLIIW....

TEST E१53 JUME Z7, 1961 C550 TO 0611 PQT

2 I'C gULFIDE RELEASE FRIJN ELEVATITN OF $2 M$

$200 \mathrm{~N}$ ARC

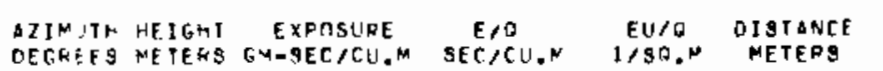
$\times 10 E+6$

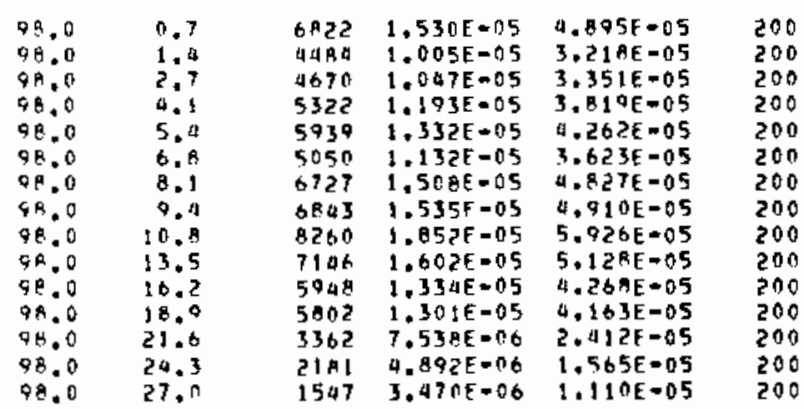

A7ERUTH HEIGHT EXPOSURE E/O EU/O DISTANCE DEGPEES METERS GM-SECIEU.M SEC/CII, 1/5O."A METERS $\times 10 E+6$

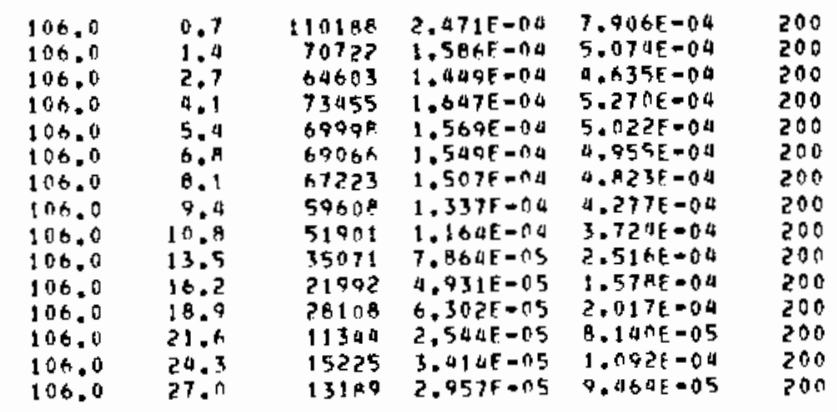

\begin{tabular}{|c|c|c|c|c|c|}
\hline $\begin{array}{l}\text { DIJUTH } \\
\text { DEGKFES }\end{array}$ & $\begin{array}{l}\text { HE IGHT } \\
\text { METERS }\end{array}$ & $\begin{array}{c}\text { EXPOSURE } \\
\text { GH }=S E C / C 1 . M \\
\times 1 O E+6\end{array}$ & $\begin{array}{c}E / R \\
S E C / C L, N\end{array}$ & $\begin{array}{c}F_{1 !} / r_{1} \\
1 / 5 \cap .\end{array}$ & $\begin{array}{l}\text { CISTANCE } \\
\text { METERS }\end{array}$ \\
\hline $\begin{array}{l}114.0 \\
114.0 \\
114.0 \\
114.0 \\
114.0 \\
114.0 \\
114.0 \\
114.0 \\
114.0 \\
114.0 \\
114.0 \\
114.0 \\
114.0 \\
114.0 \\
114.0\end{array}$ & $\begin{array}{r}0.7 \\
1.4 \\
2.7 \\
4.1 \\
5.4 \\
6.9 \\
8.1 \\
9.9 \\
10.0 \\
13.5 \\
16.2 \\
18.0 \\
21.6 \\
24.3 \\
27.0\end{array}$ & $\begin{array}{l}92314 \\
46994 \\
53967 \\
1607 \\
1917 \\
1575 \\
1913 \\
2409 \\
2409 \\
2506 \\
1716 \\
2044 \\
1847 \\
1105 \\
140\end{array}$ & $\begin{array}{l}2.070 E-04 \\
1.05 a E-04 \\
1.210 F=04 \\
3.005 E-06 \\
9.299 E=06 \\
3.532 E-06 \\
9.291 E-06 \\
5.403 E-06 \\
5.491 E=06 \\
5.619 E=06 \\
3.849 E=06 \\
4.584 F=06 \\
9.187 F-06 \\
2.478 E-06 \\
1.902 E-06\end{array}$ & $\begin{array}{l}6.623 E-04 \\
3.372 E-04 \\
3.072 E-09 \\
1.218 E=05 \\
1.376 E=05 \\
1.130 E-05 \\
1.373 E-05 \\
1.729 E=05 \\
1.757 E=05 \\
1.798 E=05 \\
1.232 E=05 \\
1.467 E-05 \\
1.340 E-05 \\
7.929 E-06 \\
6.0 R 5 E-06\end{array}$ & $\begin{array}{l}200 \\
200 \\
200 \\
200 \\
200 \\
200 \\
200 \\
200 \\
200 \\
200 \\
200 \\
200 \\
200 \\
200 \\
200\end{array}$ \\
\hline
\end{tabular}




\begin{tabular}{|c|c|c|c|c|c|c|c|c|c|c|c|}
\hline $\begin{array}{l}\text { AZIM(ITH } \\
\text { DEGREFS }\end{array}$ & $\begin{array}{l}\text { HEIGHT } \\
\text { NEYERS }\end{array}$ & 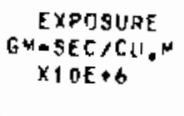 & $\begin{array}{c}E / 0 \\
\$ E C / C U, N\end{array}$ & $\begin{array}{c}E U / Q \\
1 / S D . M\end{array}$ & $\begin{array}{c}\text { DISTANCE } \\
\text { NETERS }\end{array}$ & $\begin{array}{l}\text { DZIKIITH } \\
\text { DETREES }\end{array}$ & $\begin{array}{l}\text { HE IGHT } \\
\text { METERS }\end{array}$ & $\begin{array}{l}\text { EXPOSURE } \\
G M-3 E C / C U, N \\
\times 1 O E+6\end{array}$ & $\begin{array}{c}E / Q \\
S E C / C, N . N\end{array}$ & $\begin{array}{c}E U / 0 \\
1 / S O . M\end{array}$ & $\begin{array}{l}\text { DISTANCE } \\
\text { METERS }\end{array}$ \\
\hline $\begin{array}{l}130.0 \\
130.0 \\
130.0 \\
130.0 \\
130.0 \\
130.0 \\
130.0 \\
130.0 \\
130.0 \\
130.0 \\
130.0 \\
130.0 \\
130.0 \\
130.0\end{array}$ & $\begin{array}{l}0.7 \\
2.7 \\
4.1 \\
5.4 \\
6.8 \\
8.1 \\
9.4 \\
10.8 \\
13.5 \\
16.2 \\
18.9 \\
21.6 \\
24.3 \\
27.0\end{array}$ & $\begin{array}{l}144 \\
109 \\
62 \\
41 \\
32 \\
26 \\
46 \\
5 \\
21 \\
25 \\
23 \\
1 \\
4 \\
8\end{array}$ & $\begin{array}{l}3.24) E=07 \\
2.007 E=07 \\
1.005 E=07 \\
9.232 E=08 \\
7.225 E=08 \\
6.021 E=08 \\
1.004 E=07 \\
1.204 E=08 \\
4.617 E=08 \\
5.619 E=08 \\
5.218 E=08 \\
9.014 E=09 \\
9.604 F=09 \\
1.921 E=08\end{array}$ & $\begin{array}{l}1.037 E-06 \\
6.422 E=07 \\
4.496 E=07 \\
2.954 E=07 \\
2.312 E=07 \\
1.927 E=07 \\
3.340 E=07 \\
3.853 E=08 \\
1.541 E=07 \\
1.79 A E=07 \\
1.670 E=07 \\
1.2 A 4 E=08 \\
3.073 E=08 \\
6.107 E=0 B\end{array}$ & $\begin{array}{l}200 \\
200 \\
200 \\
200 \\
200 \\
200 \\
200 \\
200 \\
200 \\
200 \\
200 \\
200 \\
200 \\
200\end{array}$ & $\begin{array}{l}106.0 \\
106.0 \\
106.0 \\
106.0 \\
106.0 \\
106.0 \\
106.0 \\
106.0 \\
106.0 \\
106.0 \\
106.0 \\
106.0 \\
105.0 \\
105.0 \\
106.0\end{array}$ & $\begin{array}{l}1.0 \\
2.1 \\
4.2 \\
6.3 \\
8.4 \\
10.5 \\
12.6 \\
14.7 \\
16.9 \\
21.0 \\
25.2 \\
29.0 \\
33.6 \\
37.8 \\
42.0\end{array}$ & $\begin{array}{l}7683 \\
1205 \\
9939 \\
7725 \\
9797 \\
9579 \\
9093 \\
9653 \\
9179 \\
8764 \\
6931 \\
4289 \\
5355 \\
4175 \\
3490\end{array}$ & $\begin{array}{l}1.723 F-0.5 \\
2.702 E-06 \\
2.229 E=05 \\
1.732 E=05 \\
2.195 F-05 \\
2.198 E-05 \\
2.039 E=05 \\
2.209 F=05 \\
2.058 E-05 \\
1.965 E=05 \\
1.532 F=05 \\
9.619 E=06 \\
1.201 E=05 \\
9.361 E-06 \\
1.710 E=06\end{array}$ & $\begin{array}{l}5.513 E=05 \\
8.64 B F=06 \\
7.13 Z E=05 \\
5.543 E=05 \\
7.022 E-05 \\
6.873 E=05 \\
6.524 E=05 \\
7.070 E=05 \\
6.585 E=05 \\
6.28 A E=05 \\
9.901 E=05 \\
3.078 E=05 \\
3.043 E=05 \\
2.996 E=05 \\
2.468 E=05\end{array}$ & $\begin{array}{l}800 \\
100 \\
800 \\
300 \\
800 \\
900 \\
800 \\
000 \\
800 \\
800 \\
000 \\
800 \\
800 \\
800 \\
900\end{array}$ \\
\hline
\end{tabular}

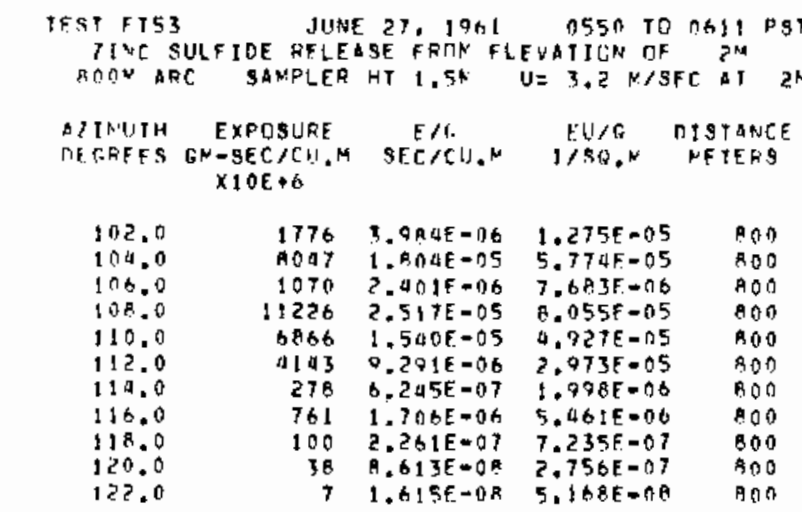

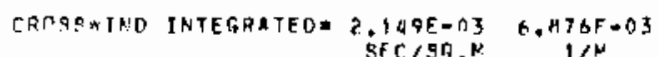

TOREA CAIA FITLLOW.,...

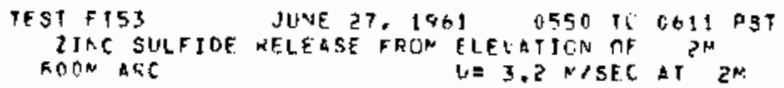

\begin{tabular}{|c|c|c|c|c|c|}
\hline $\begin{array}{l}98.0 \\
98.0 \\
9 A .0 \\
9 A .0 \\
98.0 \\
48.0 \\
98.0 \\
98.0 \\
98.0 \\
98.0 \\
98.0 \\
98.0 \\
98.0 \\
98.0 \\
98.0\end{array}$ & $\begin{array}{l}1.0 \\
2.1 \\
4.2 \\
6.3 \\
8.4 \\
10.5 \\
12.6 \\
14.7 \\
16.8 \\
21.0 \\
25.2 \\
29.4 \\
33.6 \\
37.8 \\
42.0\end{array}$ & $\begin{array}{r}17 \\
5 \\
38 \\
31 \\
17 \\
25 \\
12 \\
8 \\
20 \\
86 \\
23 \\
9 \\
0 \\
15 \\
0\end{array}$ & $\begin{array}{l}3.8 A 3 E=08 \\
1.165 E=08 \\
7.542 E=08 \\
6.989 E=0 R \\
3.8 B 3 E=0 B \\
5 . A 24 E=08 \\
2.718 F=08 \\
1.9015=08 \\
4.650 E=08 \\
1.941 E=07 \\
5.260 E=08 \\
2.168 E=08 \\
0.000 E+00 \\
3.406 E=08 \\
0.000 E+00\end{array}$ & $\begin{array}{l}1.242 E=07 \\
3.727 E=08 \\
2.733 E=07 \\
2.236 E=07 \\
1.242 E=07 \\
1.964 E=07 \\
2.697 E=08 \\
6.212 E=00 \\
1.491 E=07 \\
6.212 E=07 \\
1.685 E=07 \\
6.937 E=08 \\
0.000 E+00 \\
1.090 E=07 \\
0.000 E+00\end{array}$ & $\begin{array}{l}800 \\
800 \\
800 \\
800 \\
800 \\
800 \\
800 \\
800 \\
800 \\
800 \\
800 \\
800 \\
800 \\
800 \\
800\end{array}$ \\
\hline
\end{tabular}

\begin{tabular}{|c|c|c|c|c|c|}
\hline $\begin{array}{l}\text { DZIAUTH } \\
\text { DEGHEES }\end{array}$ & $\begin{array}{l}\text { HEITHT } \\
\text { METERS }\end{array}$ & $\begin{array}{c}\text { EXPCSURE } \\
G \times-9 E C / C: 1 . N \\
\times 10 E+6\end{array}$ & $\begin{array}{c}E / R \\
\sec C / \mathrm{C} \|, \mathrm{S}\end{array}$ & $\begin{array}{c}E L / R \\
1 / S R . N\end{array}$ & $\begin{array}{l}\text { DISTANCE } \\
\text { METERS }\end{array}$ \\
\hline 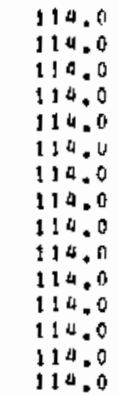 & $\begin{array}{l}1.5 \\
2.1 \\
4.2 \\
6.3 \\
8.0 \\
10.5 \\
12.6 \\
14.7 \\
16.8 \\
21.0 \\
25.2 \\
29.4 \\
33.6 \\
37.8 \\
42.0\end{array}$ & $\begin{array}{l}1229 \\
178 \\
1075 \\
1219 \\
1720 \\
1712 \\
1762 \\
1800 \\
1802 \\
2349 \\
1691 \\
1502 \\
1407 \\
2024 \\
2012\end{array}$ & $\begin{array}{l}3.757 E-06 \\
3.339 E=07 \\
2.411 E=06 \\
2.733 E=06 \\
3.879 E=06 \\
3.800 E=06 \\
3.053 F=06 \\
4.03 P E=06 \\
4.244 E=06 \\
5.269 F=06 \\
3.793 E=06 \\
3.549 E=06 \\
3.156 E-06 \\
4.540 E=06 \\
4.512 E=06\end{array}$ & $\begin{array}{l}9.922 E=06 \\
1.069 E-06 \\
7.716 E=06 \\
8.747 E-06 \\
1.241 E-05 \\
1.229 E=05 \\
1.265 E=05 \\
1.292 E=05 \\
1.359 E=05 \\
1.68 A E=05 \\
1.214 E=05 \\
1.13 \mathrm{HE}=05 \\
1.010 E-05 \\
1.453 E=05 \\
1.004 E=05\end{array}$ & $\begin{array}{l}800 \\
800 \\
900 \\
800 \\
600 \\
800 \\
800 \\
600 \\
100 \\
800 \\
000 \\
800 \\
800 \\
800 \\
000\end{array}$ \\
\hline
\end{tabular}

DZTNUTH HEIGHT EXPOSURE ETQ FU/O DISTANCE DEGREES METERS GMOSEC/CU.M SEC/CU.N 1/SH,M METERS $\times 10 E+6$

\begin{tabular}{|c|c|c|c|c|c|}
\hline $\begin{array}{l}122.0 \\
122.0 \\
122.0 \\
122.0 \\
122.0 \\
122.0 \\
122.0 \\
122.0 \\
122.0 \\
122.0 \\
122.0 \\
122.0 \\
122.0 \\
122.0 \\
122.0\end{array}$ & $\begin{array}{r}1.0 \\
2.1 \\
4.2 \\
6.3 \\
8.0 \\
10.5 \\
12.6 \\
14.7 \\
16.0 \\
21.0 \\
25.2 \\
29.4 \\
33.6 \\
37.8 \\
42.0\end{array}$ & $\begin{array}{r}0 \\
5 \\
8 \\
0 \\
9 \\
5 \\
15 \\
10 \\
5 \\
6 \\
41 \\
22 \\
30 \\
26 \\
35\end{array}$ & $\begin{array}{l}0.900 E+00 \\
1.165 E=0 B \\
1.941 E=08 \\
0.000 F+00 \\
1.941 E=08 \\
1.165 E=08 \\
3.494 E=08 \\
2.330 E=08 \\
1.165 E=08 \\
1.553 F=08 \\
9.290 E=08 \\
4.955 F=08 \\
7.742 E=0 A \\
5.684 E=08 \\
9.051 E=0 B\end{array}$ & $\begin{array}{l}0.00 \cap E+00 \\
3.727 E=00 \\
6.212 E=08 \\
0.00 \cap E+00 \\
6.212 E=08 \\
3.727 E=00 \\
1.118 E=07 \\
7.955 E=08 \\
3.727 E=08 \\
9.970 E=08 \\
2.973 E=07 \\
1.586 E=07 \\
2.477 E=07 \\
1.583 E=07 \\
2.576 E=0.7\end{array}$ & $\begin{array}{l}800 \\
800 \\
800 \\
800 \\
800 \\
800 \\
000 \\
800 \\
800 \\
800 \\
800 \\
800 \\
800 \\
800 \\
800\end{array}$ \\
\hline
\end{tabular}




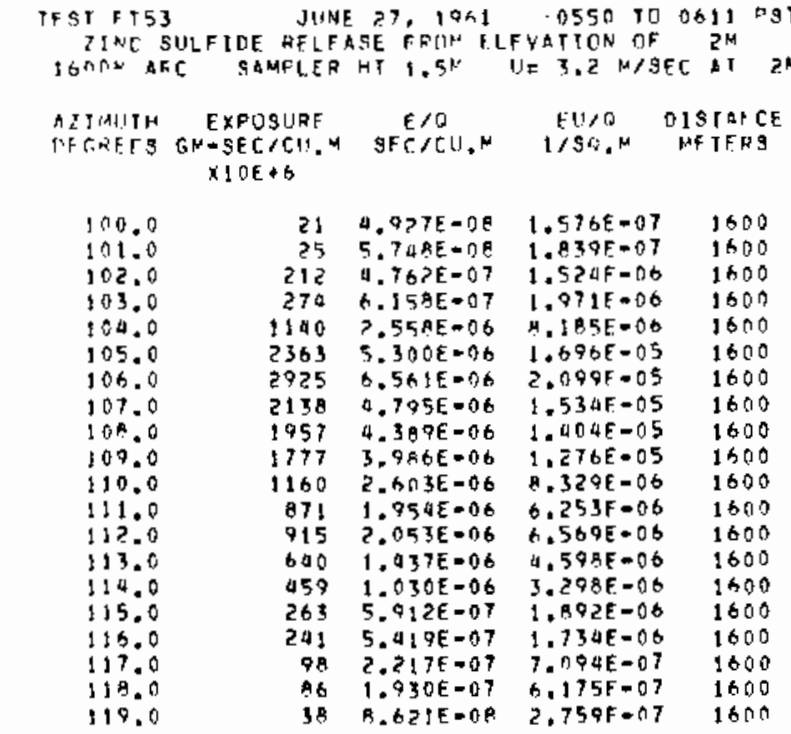

EROSSWIT:D IATEGRATED= $1.103 E-03 \quad 3,530 F=03$

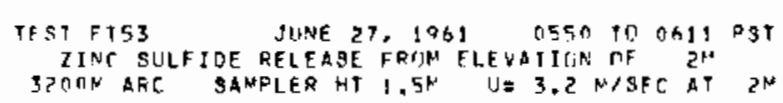

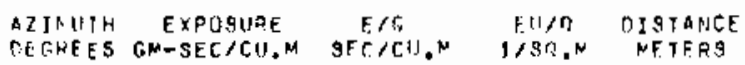
$\times 10 \mathrm{E}+6$

101.0
102.0
103.0
104.0
105.0
106.0
107.0
108.0
109.0
110.0
111.0
112.0
113.0
111.0
115.0
119.0
117.0
118.0
119.0

\begin{tabular}{|c|c|}
\hline $\begin{array}{l}? \\
9\end{array}$ & $\begin{array}{l}\text { R. } 2 \times \triangle E=08 \\
2.0 T P E=08\end{array}$ \\
\hline 78 & $1.7+6 F=07$ \\
\hline 196 & $11.405 \mathrm{E}=07$ \\
\hline 410 & P. 2ЯRE $=07$ \\
\hline $\begin{array}{l}5 P_{6} \\
1002\end{array}$ & $\begin{array}{l}1.3156 .06 \\
? .24 \text { RE }-06\end{array}$ \\
\hline $6 \geqslant 0$ & $1.392 E=06$ \\
\hline 7a? & 1.6 \\
\hline $54 !$ & $13 E=06$ \\
\hline 432 & $0.7 \cap \Delta E=0.7$ \\
\hline 377 & $A .657 E=07$ \\
\hline 397 & $P E-0.7$ \\
\hline 164 & $3.678 E=07$ \\
\hline $1 \geq B$ & $2 . A H 7 E=07$ \\
\hline $2 B$ & 6. A ISE $=0$ A \\
\hline 14 & $3,3>5 E=190$ \\
\hline & $1.455 E=0 B$ \\
\hline & $15 \mu F=09$ \\
\hline
\end{tabular}

$1.995 E-08$

$6.600 E=08$

$5.452 F=17$

$1.410 E-06$

$2.972 E=06$

7. $209 \mathrm{E}=0 \mathrm{O}$

$7.195 \mathrm{E}=06$

a. $455 E-0 B$

$5.3060-06$

3. $18.3 E=06$

$3.1055=06$

2.706E=06

2.20 सE $=06$
$1.177 \mathrm{~F} \rightarrow 06$

1. $177 F \rightarrow 06$
9. 11 AE $=07$

$2.061 E-07$

1.0645 .07

$4.654 F-0 B$

3200

3200

3200

3200
3200

3200

$3>00$

3200

3200

$3 \geq 00$
3200

3200

3200

3200

3500

3200

300

$3>00$

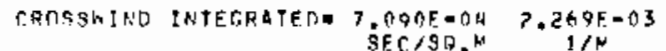

TOWES TATA EOLLLCN....

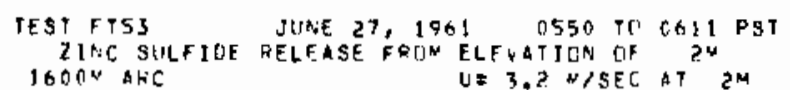

TONER DAIA FIJLLOLW...

TEST FIS3 JUNE 27,1931 O550 TO OBII PST TINC SULFIOE RELEAGE FRCM ELEVATION OF ZM 320 ON AHC UE $3.2 \mathrm{~N}$ SEC AT $2 \mathrm{~N}$

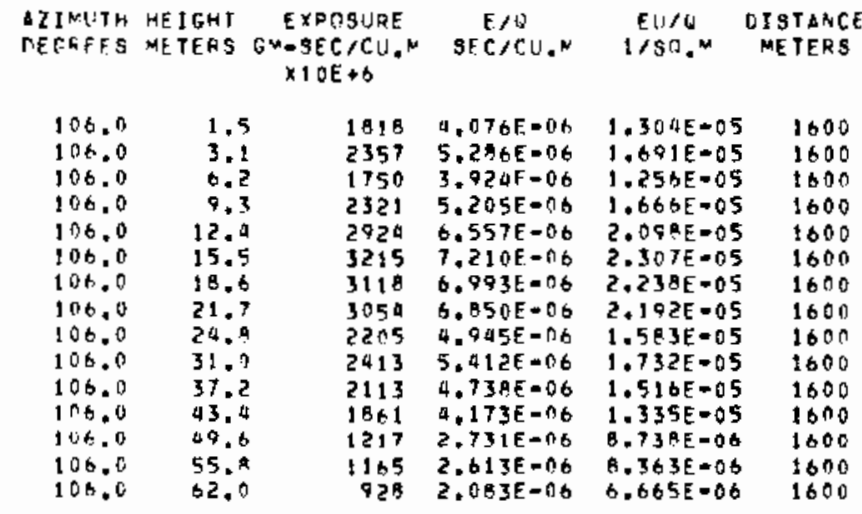

AZIMIITH HEIGHT EXPOSURE $\begin{aligned} & M=S E C, C 11 \\ & \times 1 O E+6\end{aligned}$

$\begin{array}{ll}106.0 & 1.5 \\ 100.0 & 3.1 \\ 100.0 & 0.2 \\ 106.0 & 9.3 \\ 100.0 & 12.4 \\ 106.0 & 15.5 \\ 106.0 & 18.4 \\ 100.0 & 21.7 \\ 106.0 & 29.8 \\ 106.0 & 31.0 \\ 106.0 & 37.2 \\ 104.0 & 43.4 \\ 106.0 & 49.6 \\ 106.0 & 55.8 \\ 106.0 & 62.0\end{array}$

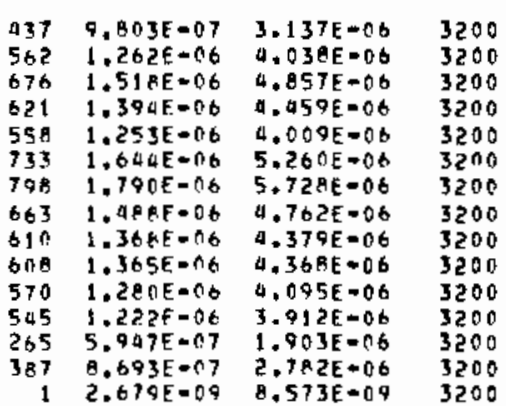

AZINUTH HEIGHT EXPDSURE E/A EU/Q DISTANEE DE GRFES METERS GMOSEC/CL.N SEC/CU.N 1/30.M METERS $\times 10 \mathrm{E}+6$
AZTNUTHF HEIGHT EXPOSURE DETREES METERS GN-SEC $/ C 11$.
XIOE $\$ 6$

$\begin{array}{ll}114.0 & 1.5 \\ 114.0 & 3.1 \\ 114.0 & 6.2 \\ 114.0 & 9.3 \\ 114.0 & 12.0 \\ 114.0 & 15.5 \\ 114.0 & 18.6 \\ 114.0 & 21.7 \\ 114.0 & 24.8 \\ 114.0 & 31.0 \\ 114.0 & 37.2 \\ 114.0 & 43.0 \\ 119.0 & 49.5 \\ 119.0 & 55.7 \\ 114.0 & 62.0\end{array}$

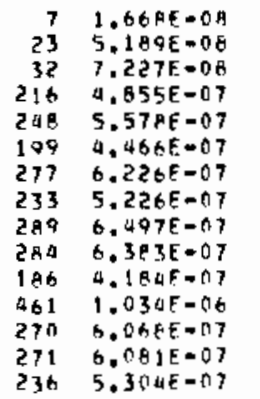

$5.337 \mathrm{E}-0 \mathrm{~B}$

$1.660 \mathrm{HE}=07$

$2.313 E=07$

$1.554 E=06$

$1.785 E-06$

$1.429 E=08$

$1.992 \mathrm{E}=06$

$1.77 E-06$
$.079 E-06$

$2.079 E-06$

$2.002 E-06$

$1.339 \mathrm{t}=0 \mathrm{~A}$

3. $30 B E-110$

$1.902 \mathrm{E}-06$

$1.94 \mathrm{AE}-00$

$1.697 \mathrm{E}=06$
DISTANCE 


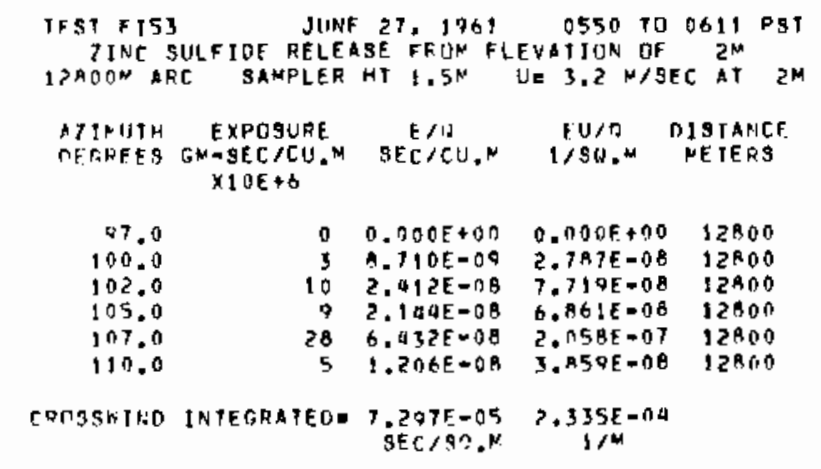


30-SERIES EXPERIMENT: 54 (FT-54)

GROUND LEVEL AND TOWER SAMPL ING 200-3200 N ARCS

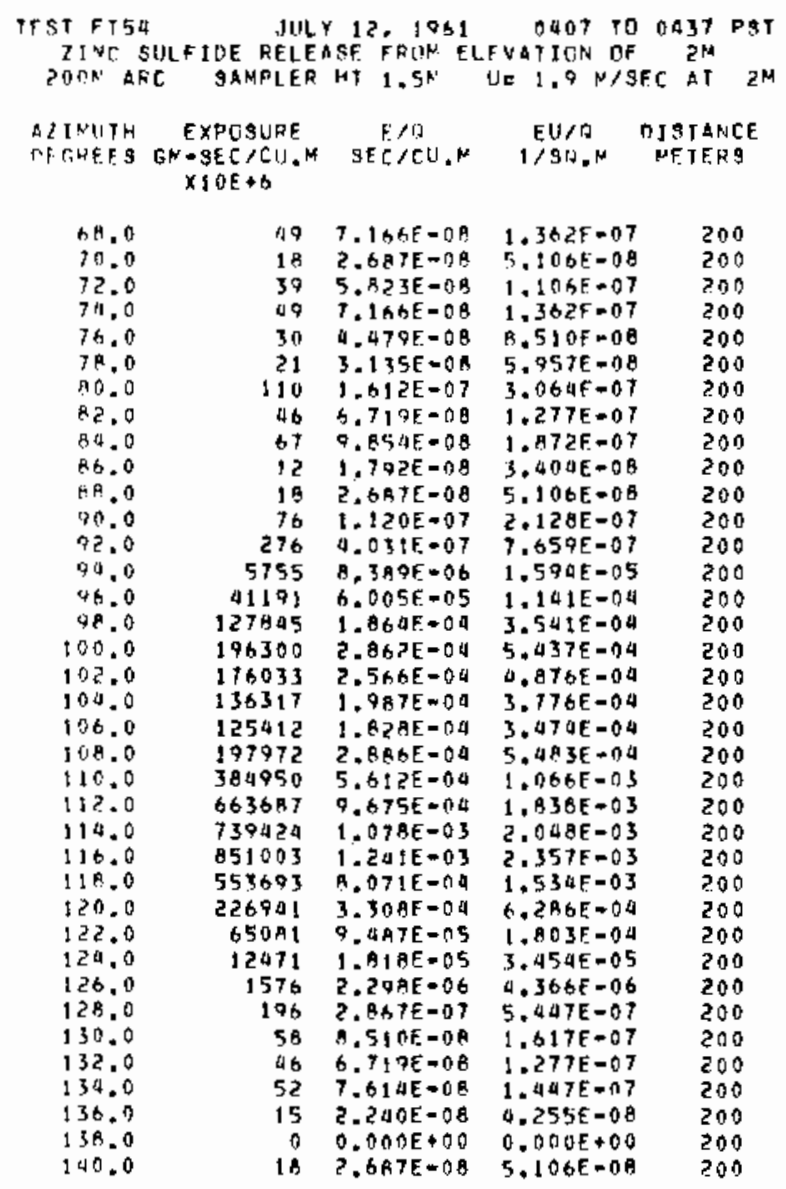

\begin{tabular}{|c|c|c|c|c|c|}
\hline $\begin{array}{l}\text { DZJNIYH } \\
\text { DEGRESS }\end{array}$ & $\begin{array}{l}\text { ME IGHI } \\
\text { WE TERS }\end{array}$ & $\begin{array}{c}\text { EXPOSUOF } \\
\text { GW- } 9 E C>C 1 . \\
\times 10 E+6\end{array}$ & $\begin{array}{c}E / F \\
3 E C / C U . N\end{array}$ & $\begin{array}{c}F \cup / Q \\
1 / S C_{0}{ }^{N}\end{array}$ & $\begin{array}{l}\text { DISTANCE } \\
\text { NETEAS }\end{array}$ \\
\hline $\begin{array}{l}106.0 \\
106.0 \\
106.0 \\
106.0 \\
108.0 \\
106.0 \\
106.0 \\
103.0 \\
104.0 \\
106.0 \\
106.0 \\
106.0\end{array}$ & $\begin{array}{l}0.7 \\
1.9 \\
2.7 \\
9.1 \\
5.9 \\
6.8 \\
8.1 \\
9.4 \\
10.9 \\
13.5 \\
16.8 \\
18.9\end{array}$ & $\begin{array}{r}136409 \\
7909 A \\
91571 \\
77999 \\
67909 \\
64004 \\
55039 \\
29693 \\
70353 \\
4971 \\
911 \\
96\end{array}$ & 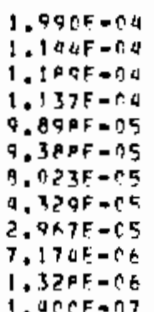 & 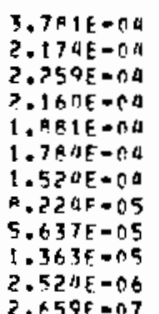 & $\begin{array}{l}200 \\
200 \\
200 \\
200 \\
200 \\
200 \\
200 \\
200 \\
200 \\
200 \\
200 \\
200\end{array}$ \\
\hline
\end{tabular}

\begin{tabular}{|c|c|c|c|c|c|}
\hline $\begin{array}{l}\text { ZTMUITH } \\
\text { OFGFF S }\end{array}$ & $\begin{array}{l}\text { MEIGHT } \\
\text { METERS }\end{array}$ & 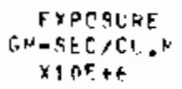 & $\begin{array}{c}F / r \\
\text { SFC } / \mathrm{Cl}^{\circ}\end{array}$ & $\begin{array}{c}E 1,16 \\
1 / 55, N\end{array}$ & $\begin{array}{c}\text { CISTANCE } \\
\text { NETFNS }\end{array}$ \\
\hline 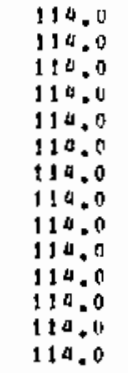 & $\begin{array}{l}0.7 \\
1.4 \\
2.7 \\
4.1 \\
5.4 \\
6.8 \\
8.1 \\
9.4 \\
10.8 \\
13.5 \\
16.2 \\
18.9 \\
21.4 \\
24.3\end{array}$ & $\begin{array}{r}940266 \\
477746 \\
494903 \\
1369>9 \\
339286 \\
240334 \\
194789 \\
118277 \\
59118 \\
15707 \\
3645 \\
918 \\
113 \\
70\end{array}$ & $\begin{array}{l}1.3715=03 \\
6.965 F=04 \\
7.985 F=04 \\
6.3695=04 \\
4.931 F=04 \\
3.503 F=04 \\
2.716 E=04 \\
1.724 E=04 \\
9.610 F=05 \\
2.733 F=05 \\
5.387 F=06 \\
1.19 a F=06 \\
1.654 E=07 \\
2.999 E=08\end{array}$ & $\begin{array}{l}? .604 F=03 \\
1.32 Z E=03 \\
1.346 F=03 \\
1.210 E=03 \\
9.369 E=04 \\
6.657 E=04 \\
5.16 A E=04 \\
3.276 E=00 \\
1.637 E=00 \\
5.193 E=05 \\
1.024 E=05 \\
2.267 E=06 \\
3.143 E=07 \\
5.699 E=01\end{array}$ & $\begin{array}{l}200 \\
200 \\
200 \\
200 \\
200 \\
200 \\
200 \\
200 \\
200 \\
200 \\
200 \\
200 \\
200 \\
200\end{array}$ \\
\hline
\end{tabular}

A7INUTH HEIGH EXPCSURE EIO FI'G DISTANCE DFFREFS NETERS GN-SEC/CIIN SEC/CU.N 1/SO.N METFRS

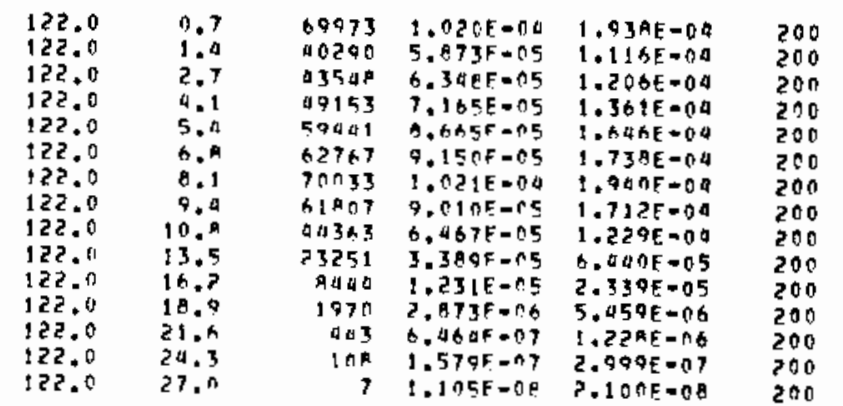

TOEF TIOTA FILLIN, ...

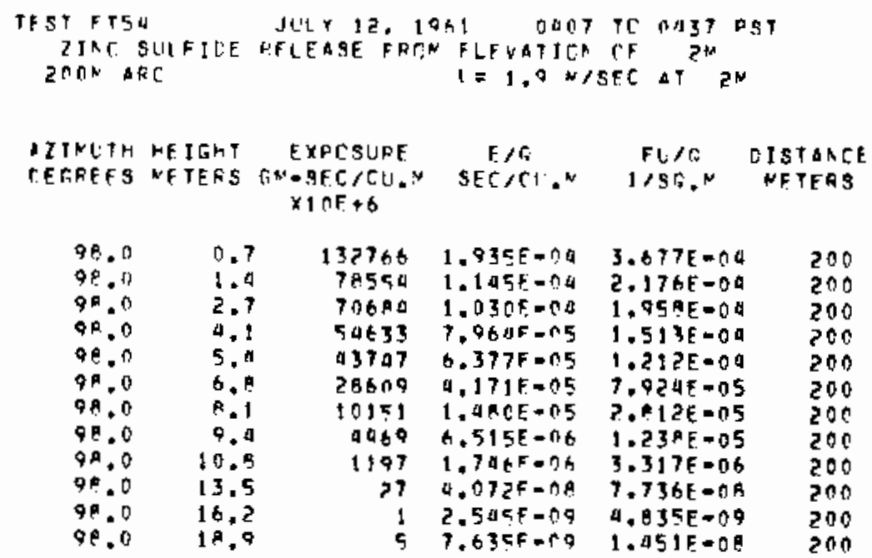




\begin{tabular}{|c|c|c|c|c|c|}
\hline $\begin{array}{l}\text { DINLITH } \\
\text { DECDE S }\end{array}$ & $\begin{array}{l}\text { HE IGHT } \\
\text { METERS }\end{array}$ & $\begin{array}{c}\text { EXPCSURE } \\
\text { GN-SEC } / \mathrm{CH} . \\
\times 10 E+4\end{array}$ & $\begin{array}{c}F / R \\
S F C / C H . *\end{array}$ & $\begin{array}{l}F \|, \pi \\
1 / \$ 0,\end{array}$ & $\begin{array}{l}\text { PISTARCE } \\
\text { NETERS }\end{array}$ \\
\hline 130.0 & 0,7 & $4 n$ & $5.8 \times 7 F=0 A$ & $\left.1.11 K^{K E} E-0\right\}$ & \\
\hline 130. & 1. & 27 & $\Delta .072 F=c e$ & $7.736 \mathrm{E}-08$ & 200 \\
\hline 130.0 & ?. 1 & $10 a$ & $2.11 \geqslant \mathrm{~F}-\mathrm{rq}$ & $0.013 E-07$ & On \\
\hline 130.0 & 4.1 & Q ? & $1.34 G \mathrm{~F}=17$ & $2.563 E=n 7$ & $2 n n$ \\
\hline 130.0 & 5.9 & 130 & $1.9 n O E-n 7$ & $3.62 \mathrm{RE}=07$ & 200 \\
\hline 130.0 & 6.8 & 169 & $2.069 E=07$ & $4.69 \cap E=07$ & \\
\hline 130.0 & 8.1 & 71 & $1.043 F=n ?$ & $1.982 E-07$ & 200 \\
\hline $\begin{array}{l}13 \% .0 \\
130.0\end{array}$ & $\begin{array}{r}9.0 \\
10.9\end{array}$ & $\begin{array}{l}185 \\
209\end{array}$ & $\begin{array}{l}2.699 \mathrm{E}=7 \\
3.05 \mathrm{BF}=\mathrm{n}\end{array}$ & $\begin{array}{l}5.125 E=07 \\
5.0 \cap 2 E=07\end{array}$ & $\begin{array}{l}200 \\
200\end{array}$ \\
\hline 130,0 & 13.5 & 78 & $\begin{array}{l}0.145 E-n 7 \\
1.07\end{array}$ & $2.176 E-07$ & 200 \\
\hline 130.0 & 16.2 & 26 & $3.817 E-08$ & $7.253 E-08$ & 200 \\
\hline 130.0 & 18 & 6 & $1.019 \mathrm{~F}-0 \mathrm{~g}$ & $1.930 \mathrm{~g}=08$ & 200 \\
\hline 130.0 & 21 & 3 & $5,090 E=09$ & $9.670 E-09$ & 200 \\
\hline $\begin{array}{l}130.0 \\
130.0\end{array}$ & $\begin{array}{l}24.3 \\
? 7.0\end{array}$ & 1 & $\begin{array}{l}1.579 E=09 \\
6.314 F=\wedge 9\end{array}$ & $\begin{array}{l}2.999 \mathrm{E}=09 \\
1.200 \mathrm{E}=0 \mathrm{P}\end{array}$ & 200 \\
\hline & & & & $1,2 \times n E=0 \mathrm{R}$ & 200 \\
\hline
\end{tabular}

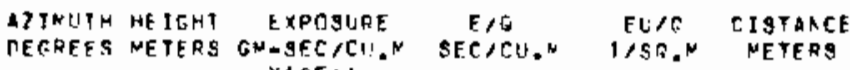
$\times 10 F+6$

\begin{tabular}{|c|c|c|c|c|}
\hline $\begin{array}{l}106.0 \\
106.0 \\
106.0 \\
106.0 \\
106.0 \\
106.0 \\
106.0 \\
106.0 \\
104.01 \\
106.01 \\
106.0\end{array}$ & $\begin{array}{l}1.0 \\
2.1 \\
4.2 \\
6.3 \\
0.4 \\
10.5 \\
12.6 \\
14.7 \\
16.4 \\
21.0 \\
25.7\end{array}$ & $\begin{array}{r}1082 \\
709 \\
4139 \\
5405 \\
6219 \\
5481 \\
4554 \\
2659 \\
1097 \\
271 \\
11\end{array}$ & 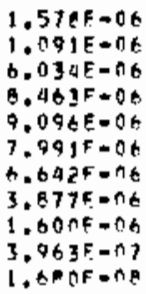 & $\begin{array}{l}2.94 A E=06 \\
2.072 E=06 \\
1.146 E=05 \\
1.00 A E=05 \\
1.72 A E=05 \\
1.519 E=05 \\
1.262 E=05 \\
7.367 E=06 \\
3.040 E=06 \\
7.530 E=07 \\
3.191 E=0 A\end{array}$ \\
\hline
\end{tabular}

AZIMUTH HEIGHT EXPCSURE F/S FI. F, TISTAREF

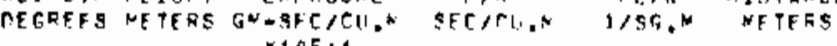

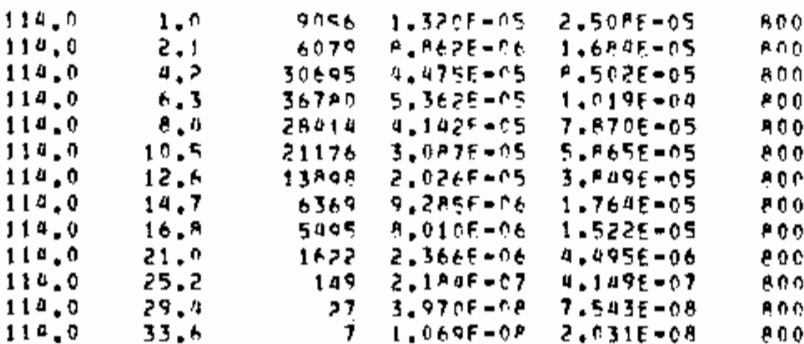

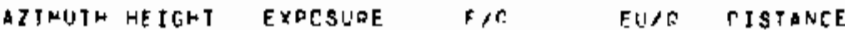
EEGQFE MFTEPS GM-GEC/CI:A SEC/CH, 1/SR.N NFTERS $X ! O E+t$

\begin{tabular}{|c|c|c|c|c|}
\hline $\begin{array}{l}122.0 \\
12200 \\
122.0 \\
122.0 \\
12200 \\
12200 \\
12200 \\
12200 \\
122.0 \\
12200 \\
12200 \\
12200 \\
122.0 \\
122.0 \\
122.0\end{array}$ & $\begin{array}{l}1.0 \\
2.1 \\
4 . ? \\
6.3 \\
1.0 \\
10.5 \\
12.6 \\
14.7 \\
16.9 \\
21.0 \\
25.2 \\
29.4 \\
33.5 \\
37.9 \\
42.5\end{array}$ & $\begin{array}{r}148 \\
109 \\
1330 \\
2433 \\
3458 \\
1145 \\
4873 \\
5582 \\
5307 \\
4904 \\
1123 \\
301 \\
75 \\
5 \\
5\end{array}$ & 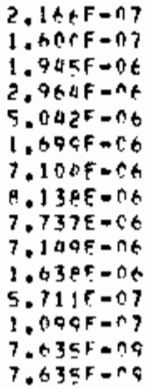 & $\begin{array}{l}4.116 E=07 \\
3.040 E=07 \\
3.695 E=06 \\
5.631 E=06 \\
9.579 E=06 \\
3.227 E=06 \\
1.350 E=05 \\
1.546 E=05 \\
1.470 E=05 \\
1.351 E=05 \\
3.113 E=06 \\
1.085 E=06 \\
2.029 E=07 \\
1.451 E=08 \\
1.451 E=0 B\end{array}$ \\
\hline
\end{tabular}

TOMER DATA FOLLCW....

TEST FT54 JULY IZ, 1961 0467 TC 0437 PST ZIRC SIJLFIUE HFLEASF. FRCN FLFVATICA TF 2 " AOQN AFC UIE

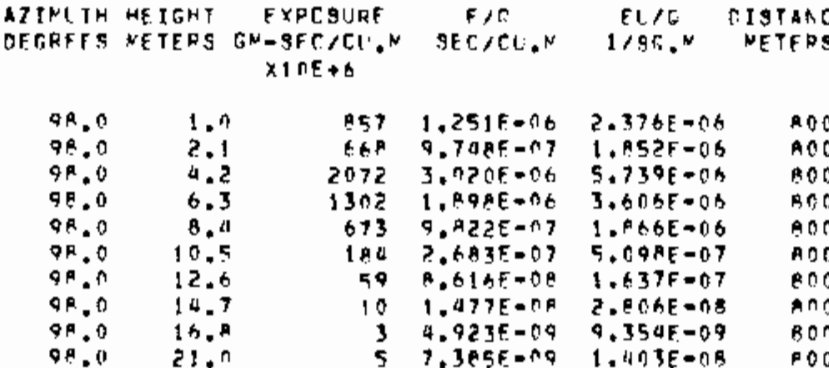

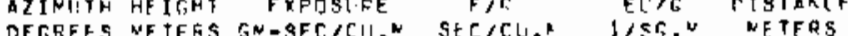
$\times \rightarrow \operatorname{cit}+6$

\begin{tabular}{|c|c|c|c|c|c|}
\hline $13 n .0$ & $1 \cdot n$ & 15 & $\begin{array}{c}5.000 F+00 \\
3.51+F=06\end{array}$ & $\begin{array}{l}1.209 F=0 P \\
0 . A C A F+00\end{array}$ & $\begin{array}{l}R 00 \\
800\end{array}$ \\
\hline $\begin{array}{l}130.0 \\
130.0\end{array}$ & $\begin{array}{l}2.1 \\
4.2\end{array}$ & $3^{n}$ & $4,125 \mathrm{~F}=0$ A & $7.951 F=08$ & 800 \\
\hline 130.0 & 6,3 & 5 & $7.3958=04$ & $1.403 E=09$ & $\mathrm{BOC}$ \\
\hline 130.0 & 8.4 & 16 & $2.442 F=0 F$ & $4.677 E=08$ & ROD \\
\hline $\begin{array}{l}130.0 \\
130.0\end{array}$ & $\begin{array}{l}10.5 \\
12,4\end{array}$ & $\begin{array}{l}77 \\
35\end{array}$ & $\begin{array}{l}3.930 F=08 \\
5.170 E=0.8\end{array}$ & 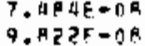 & $\begin{array}{l}A 00 \\
0.00\end{array}$ \\
\hline 130.0 & 14.7 & 21 & $3,20 C E=G B$ & $6.0806-08$ & e00 \\
\hline 13 & 16.8 & $? \pi$ & $2.954 F=02$ & $5 . t 13 \pm-0 A$ & AOO \\
\hline .9 & 21.0 & 6 & $9.847 \mathrm{E}-1.9$ & $1 . A 7\{5=08$ & A00 \\
\hline 0 & ? & 10 & $1.5275=0$ & $\begin{array}{l}3.909 E=09 \\
1951 \mathrm{E}=09\end{array}$ & 200 \\
\hline & & 3 & $254=04$ & $1.451 t=04$ & 200 \\
\hline
\end{tabular}




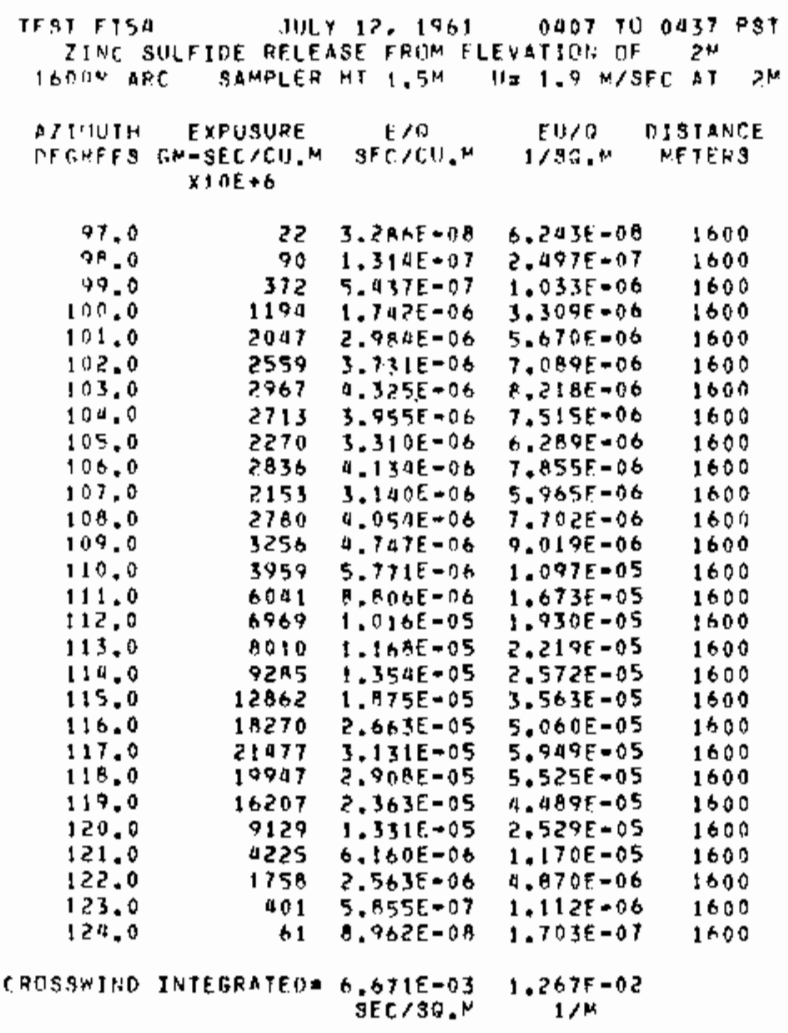

TOMEF r.TA FOLLCH....
AZINLIH HEIRHT EYPCSLRE FIT E DE OISTARCE

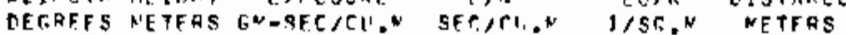
$x 1 \cap F+6$

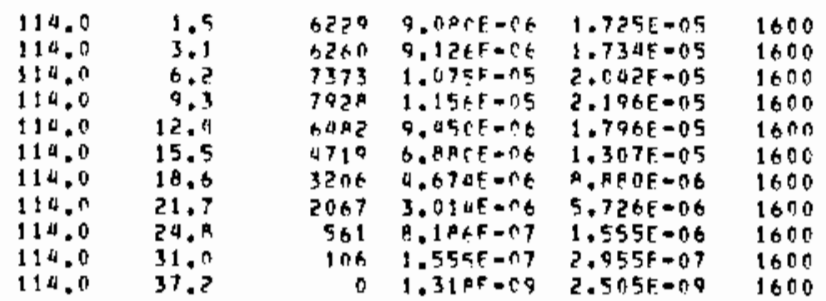

AZINIITH HEIGHT EXPOSLRE E/R FUIO CISTANCE DEGREES NETERS GM-GEC/CU." SFC/rU,N 1/SE.N METERS $\times 10 F+6$

\begin{tabular}{|c|c|c|c|c|c|}
\hline $\begin{array}{l}122.0 \\
122.0 \\
122.0 \\
122.0 \\
122.0 \\
122.0 \\
122.0 \\
122.0 \\
122.0 \\
122.0 \\
122.0 \\
122.0\end{array}$ & $\begin{array}{r}1.5 \\
3.1 \\
6.2 \\
9.3 \\
12.4 \\
15.5 \\
19.6 \\
21.7 \\
24.4 \\
31.0 \\
37.2 \\
43.4 \\
49.4\end{array}$ & $\begin{array}{r}847 \\
1073 \\
1179 \\
1349 \\
2689 \\
2910 \\
3794 \\
2371 \\
1518 \\
404 \\
71 \\
15 \\
0\end{array}$ & $\begin{array}{l}1.235 E-116 \\
1.565 E=06 \\
1.720 E-06 \\
1.967 E=06 \\
3.920 F-08 \\
4.240 F-06 \\
5.517 E-06 \\
3.457 E-06 \\
2.213 E-06 \\
5.892 E=07 \\
1.001 E-07 \\
2.241 E=08 \\
1.042 F=09\end{array}$ & $\begin{array}{l}2.546 E=06 \\
2.974 E=06 \\
3.267 E=06 \\
3.737 E=06 \\
7.048 E=06 \\
9.072 E=06 \\
1.048 E=05 \\
6.568 E=06 \\
4.205 E=06 \\
1.120 E=06 \\
1.979 E=07 \\
4.258 E=08 \\
1.980 E=09\end{array}$ & $\begin{array}{l}1600 \\
1600 \\
1600 \\
1600 \\
1600 \\
1600 \\
1600 \\
1600 \\
1600 \\
1600 \\
1600 \\
1600 \\
1600\end{array}$ \\
\hline
\end{tabular}

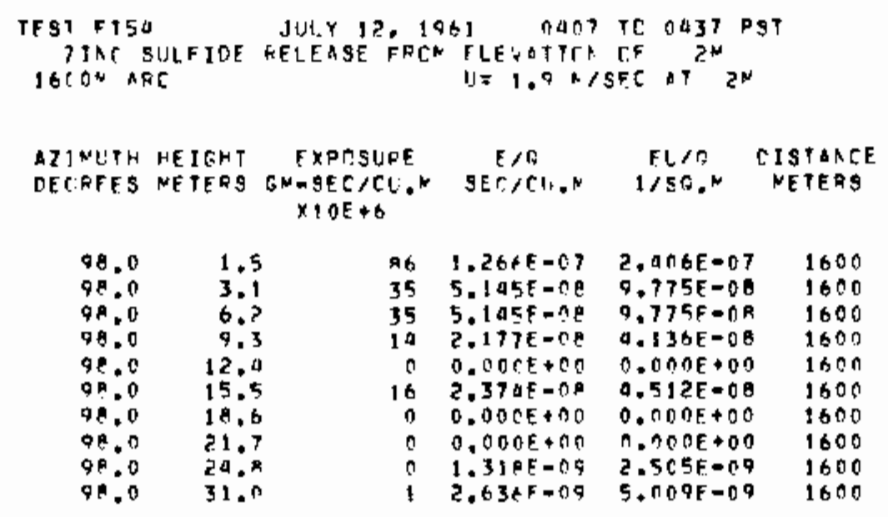




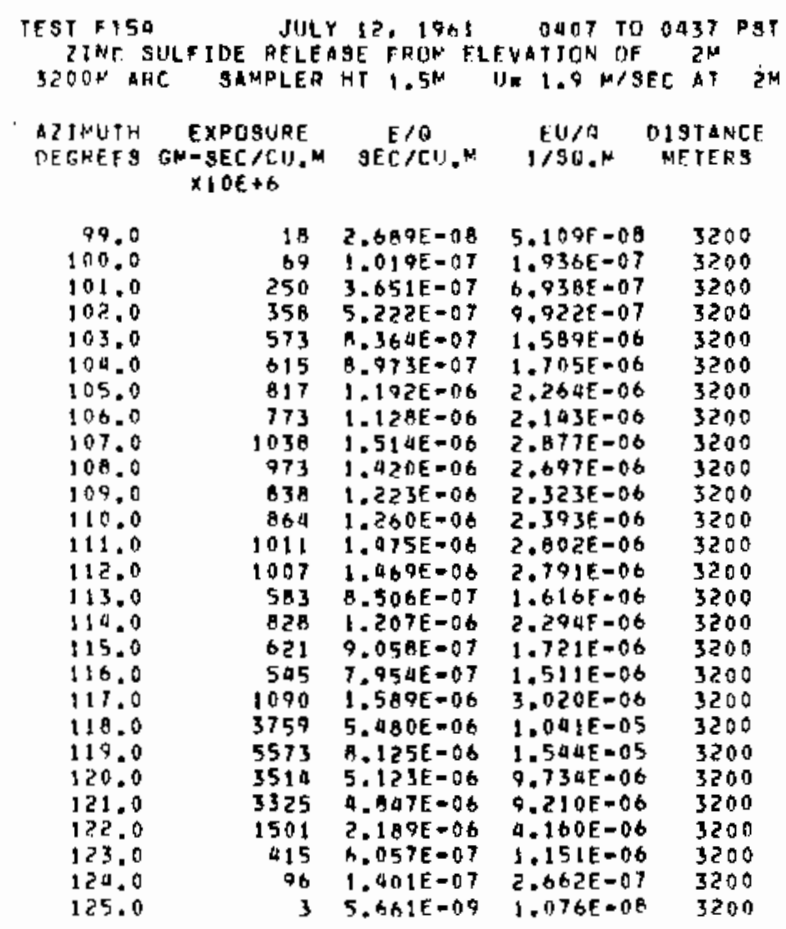

CROSAN1NO INTEGRATEDE ?.530E-03 A. AOSE-03

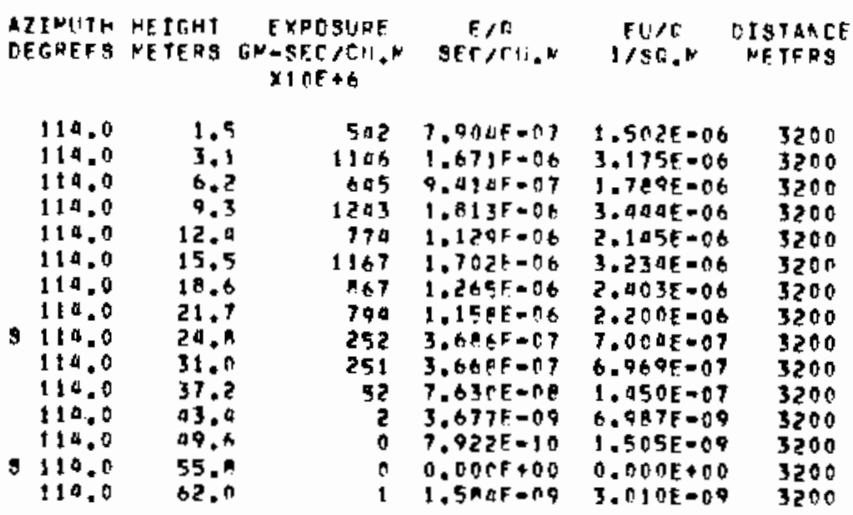

\begin{tabular}{|c|c|c|c|c|c|}
\hline $\begin{array}{l}\text { IMYITH } \\
\text { ERRFES }\end{array}$ & $\begin{array}{l}\text { HE IGH T } \\
\text { METERS }\end{array}$ & $\begin{array}{c}\text { EXPOSURE } \\
\text { GN- }-9 E C>C L+N \\
\times 1 O E+G\end{array}$ & $\begin{array}{c}E>r^{\prime} \\
\operatorname{set} / \mathrm{CL}^{\circ}\end{array}$ & $\begin{array}{c}E U>0 \\
1<E 0, N\end{array}$ & $\begin{array}{l}\text { TISTANCE } \\
\text { NETERS }\end{array}$ \\
\hline $\begin{array}{l}122.0 \\
122.0 \\
122.0 \\
122.0 \\
122.0 \\
122.0 \\
122.0 \\
122.0 \\
122.0 \\
122.0 \\
122.0 \\
122.0 \\
122.0 \\
122.0 \\
122.0\end{array}$ & $\begin{array}{r}1.5 \\
3.5 \\
6.2 \\
9.3 \\
12.0 \\
15.5 \\
18.6 \\
21.7 \\
24.8 \\
31.0 \\
37.2 \\
43.4 \\
49.6\end{array}$ & $\begin{array}{r}923 \\
1469 \\
1542 \\
2223 \\
2015 \\
2192 \\
2504 \\
2915 \\
1263 \\
959 \\
101 \\
25 \\
17 \\
3 \\
4\end{array}$ & 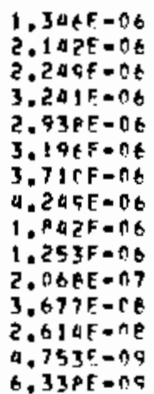 & $\begin{array}{l}2.556 E=06 \\
4.070 E=06 \\
0.273 E=06 \\
6.150 E=06 \\
5.593 E=06 \\
6.073 E=06 \\
7.002 E=06 \\
.079 E=06 \\
3.500 E=06 \\
2.381 E=06 \\
3.930 E=07 \\
6.987 E=08 \\
4.967 E=08 \\
9.031 E=06 \\
1.200 E=0 R\end{array}$ & $\begin{array}{l}3200 \\
3200 \\
3200 \\
3200 \\
3200 \\
3200 \\
3200 \\
3200 \\
3200 \\
3200 \\
3200 \\
3200 \\
3200 \\
3200 \\
3200\end{array}$ \\
\hline
\end{tabular}

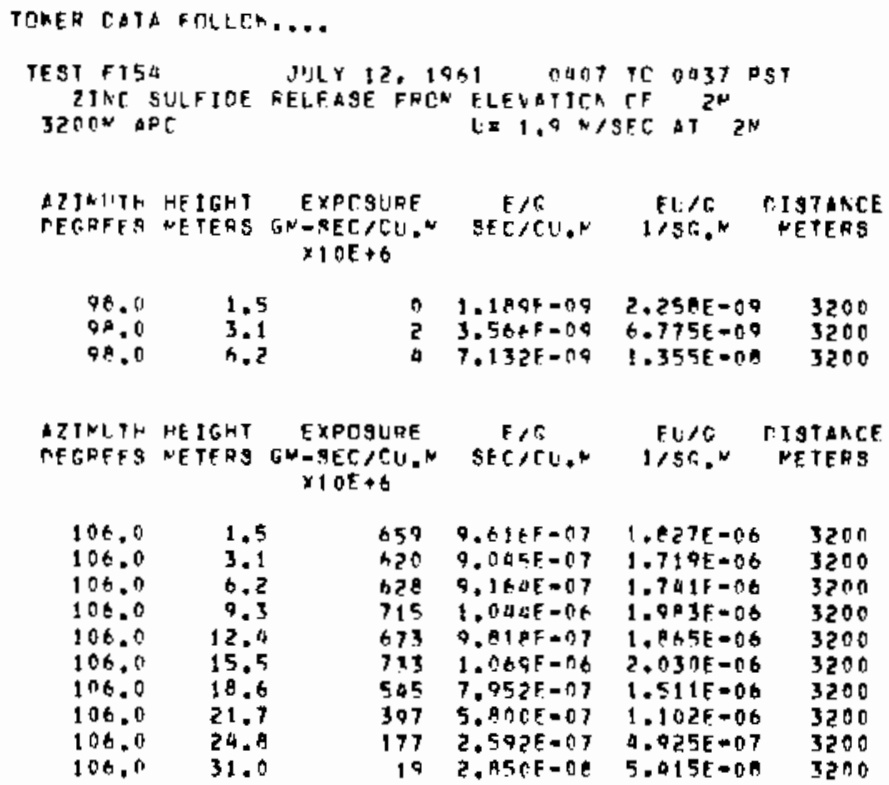


30-SER IES EXPERIMENT: 55 ( FT-55)

GROUNO LEYEL, AND TOWER SAMPLING 200-3200 M ARCS

\begin{tabular}{|c|c|c|c|c|c|}
\hline \multicolumn{6}{|c|}{ 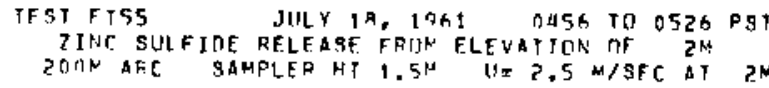 } \\
\hline 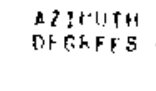 & $\begin{array}{l}\text { EXPUSURE } \\
\text { EM }+S E C / C 11.4 \\
\times 10 E+6\end{array}$ & SEe/C(1, & $\begin{array}{c}\text { EU/R } \\
\text { DST.N }\end{array}$ & $\begin{array}{l}\text { DTSTANC } \\
\text { METFRS }\end{array}$ & $\begin{array}{l}\text { ICE } \\
\text { is }\end{array}$ \\
\hline $7 n .0$ & ? & $2.110 E=09$ & $5.2745 * 09$ & & \\
\hline 72.0 & i) & ח.OONE + OO & $0.000 E+00$ & 200 & 0 \\
\hline & 25 & $1.899 E=08$ & $4.747 F=08$ & 200 & \\
\hline 76.0 & 22 & $1.6 R Q E=D Q$ & $4.218 E-08$ & 200 & 0 \\
\hline 78.0 & 19 & $3.4 Y 7 E=D Q$ & $3,6926=08$ & 200 & \\
\hline RO. 0 & 39 & $2.953 E-08$ & $7.3 E 3 E=08$ & 200 & \\
\hline 82.0 & A2 & $4.641 E=0 \mathrm{~A}$ & $1.160 E=07$ & 200 & \\
\hline$\varepsilon 4.0$ & 67 & $5.063 E-D A B$ & $1.266 E=07$ & 200 & \\
\hline 86.0 & 350 & $2.616 E-07$ & $6.5400-07$ & 200 & \\
\hline AP. 0 & 87 & $A, 540 E=0 A$ & $3.635 E-07$ & 300 & \\
\hline 90.0 & 322 & $2.405 E=07$ & $6.012 F-07$ & 200 & \\
\hline 72.0 & 1255 & $0.36+E=07$ & $2.342 E=06$ & 200 & \\
\hline 94.0 & jo7a1 & $0.046 E=06$ & $2.051 E=05$ & 200 & \\
\hline 96.0 & 97207 & $3.523 E-05$ & Q.AOTE-OS & $? 00$ & \\
\hline 90.0 & 123365 & $9.206 \mathrm{~F}-05$ & $2.302 E=14$ & 200 & \\
\hline 100.0 & 278348 & $2.077 E-04$ & $5.193 \mathrm{E}=04$ & 200 & 60 \\
\hline 102.0 & 406301 & $3.032 \mathrm{E}=0 \mathrm{~A}$ & $7.5 A 0 F=04$ & 200 & 60 \\
\hline 104.0 & 459605 & $3.422 E=04$ & Q.556E.04 & 200 & 0 \\
\hline $10 \in .0$ & 400767 & $3.5 B E E-00$ & $0.970 \mathrm{E}=0.4$ & 200 & 0 \\
\hline $10 B .0$ & 610818 & $4.550 E-04$ & $1.100 \mathrm{E}-03$ & 200 & 0 \\
\hline 110.0 & 652728 & D.ABIE-OA & $1.218 \mathrm{~F}-03$ & 200 & \\
\hline 112.0 & 572392 & $4.2+2 E=04$ & $1.068 E-03$ & 200 & \\
\hline 114.0 & 326709 & $2.43 A E-04$ & $0.095 E-00$ & $? 00$ & \\
\hline 116.0 & 144323 & $1.077 E-04$ & $2.693 E=04$ & 200 & \\
\hline 118.0 & 69524 & A.B15E.05 & $1.2 n a E-04$ & 200 & 0 \\
\hline 120.0 & 13619 & $1,016 E=05$ & $2.541 F-05$ & 200 & \\
\hline 122.0 & 163 & $5.696 E+07$ & $1.424 E=O B$ & 200 & \\
\hline 124.0 & 39 & $2.953 E=08$ & $7.3 \div 35.08$ & 200 & \\
\hline 126.0 & & $2.110 E=0 Q$ & $5.274 E=09$ & 200 & \\
\hline 128.0 & 19 & $1.05 S E-0 B$ & $2.637 E=08$ & 200 & \\
\hline 130.0 & 26 & $2.110 E=08$ & $5.274 E=08$ & 200 & \\
\hline 132.0 & $B$ & $6.3296-09$ & $1.582 E-08$ & 200 & 0 \\
\hline 134.0 & 19 & $1.477 \mathrm{E}-0 \mathrm{~A}$ & $3.092 E=08$ & 200 & \\
\hline 136.0 & 0 & $0.000 E+00$ & $0.000 E+00$ & 200 & \\
\hline 138.0 & 5 & $4.219 E=09$ & $1,055 E-08$ & & \\
\hline 140.0 & $B$ & $0.3 ? 9 E=09$ & $1.5 A 2 E-0 B$ & 200 & 0 \\
\hline 0 & $D=$ & $\begin{array}{c}P .1 \text { म5E-OZ } \\
\text { SEC S SO.N }\end{array}$ & $5.41,2 E=02$ & & \\
\hline
\end{tabular}

\footnotetext{
TFST FT55 JULY 18, 1961 NAS6 TC 0526 PST

TIAC SULFIDE RELEASE. FRON ELEVATICN DF $2 \mathrm{~N}$

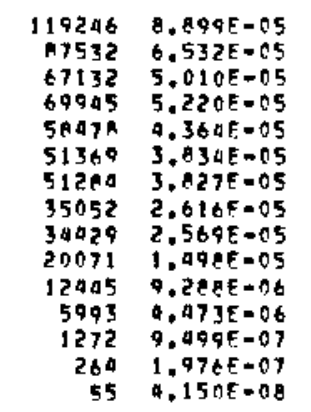

$2+225 E-04$ $1.633 E=0.0$ $1.252 \mathrm{E}-04$ $1.305 E * 04$ $1.091 E=04$ $9.5 A 4 E-05$ $9.56 \mathrm{AE}=05$

$6.500 E-05$

$6.423 E * 05$ $3.745 E-05$ $2.322 E-05$ $1.11 \mathrm{AE}=05$ $2.375 \mathrm{E}-06$ 4. $941 \mathrm{E}-07$ $1.030 E+07$
DIINITH HEIEHT EXPOSURE DFGREES METEES GNOSEC/CU.N SER F FU/Q DISTANCE $x \leqslant 0 E+6$

106.0
106.0
106.0
106.0
106.0
106.0
106.0
106.0
106.0
196.0
106.0
106.0
106.0
106.0
106.0

0.7
1.4
2.7
9.1
5.4
6.9
0.1
9.4
10.9
13.5
16.7
18.9
21.4
24.3
27.0

514103
340612
157990
251900
313745
201930
205063
292913
210742
130495
83770
50958
19541
14047
819

$3.837 E-C A$ $2.572 E=00$ $1.03 \cap E=00$ $1.879 E=04$ 2.301E-04 $1.506 E=04$ $1.832 F=04$ $2+1965-04$ $1.575 E-04$

$0.7627-15$

$0.25 \geq E-05$ $3.203 F-05$
$1.06 O E-05$ $1.04 \times F=05$ $6.107 \mathrm{E}-0.6$

\begin{tabular}{|c|c|}
\hline $\begin{array}{l}9.592 E=04 \\
6.429 E=0 A \\
2.574 E=04 \\
4.699 E=04 \\
5.053 E=04 \\
3.766 E=04 \\
4.580 E=04 \\
5.466 E=04 \\
3.932 E=04 \\
2.402 E=04 \\
1.563 E=04 \\
9.507 E=05 \\
3.650 E=05 \\
2.621 E=05 \\
1.527 E-05\end{array}$ & $\begin{array}{l}200 \\
200 \\
200 \\
200 \\
200 \\
200 \\
200 \\
200 \\
200 \\
200 \\
200 \\
200 \\
200 \\
200 \\
200\end{array}$ \\
\hline
\end{tabular}

\begin{tabular}{|c|c|c|c|c|c|}
\hline $\begin{array}{l}\text { AZIFUTH } \\
\text { MEGREFS }\end{array}$ & $\begin{array}{l}\text { HE IGHT } \\
\text { METERS }\end{array}$ & $\begin{array}{c}\text { EXPCSUAE } \\
\text { GN-SEC }>C U . N \\
\times 1 O E+6\end{array}$ & $\begin{array}{c}E / 6 \\
\mathrm{sF} / \mathrm{CU}, \mathrm{N}\end{array}$ & $\begin{array}{c}E \cup / G \\
1 / S D .\end{array}$ & $\begin{array}{l}\text { CISTANCE } \\
\text { NFTEAS }\end{array}$ \\
\hline $\begin{array}{l}114.0 \\
114.0 \\
110.0 \\
114.0 \\
119.0 \\
110.0 \\
114.0 \\
110.0 \\
110.0 \\
110.0 \\
110.0 \\
110.0 \\
11400 \\
110.0 \\
110.0\end{array}$ & $\begin{array}{r}0.7 \\
1.0 \\
2.7 \\
4.1 \\
5.0 \\
6.1 \\
8.1 \\
9.0 \\
10.1 \\
13.5 \\
16.2 \\
18.9 \\
21.0 \\
29.3 \\
27.0\end{array}$ & $\begin{array}{r}287805 \\
161650 \\
166203 \\
177605 \\
180592 \\
79005 \\
193026 \\
182093 \\
152990 \\
128000 \\
53992 \\
15281 \\
39500 \\
12131 \\
5916\end{array}$ & $\begin{array}{l}2.14 E E-0 A \\
1.206 E=00 \\
1.201 E=0 a \\
1.326 E=00 \\
1.377 E=00 \\
5.963 E=05 \\
1.366 E=04 \\
1.362 E=00 \\
1.192 E-00 \\
9.556 E=05 \\
0.029 E=05 \\
6.360 E=05 \\
2.511 E=05 \\
9.053 E=06 \\
4.015 E=06\end{array}$ & 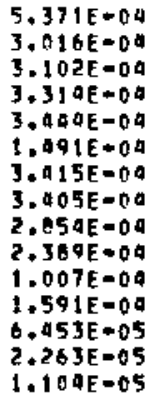 & $\begin{array}{l}200 \\
200 \\
200 \\
200 \\
200 \\
200 \\
200 \\
200 \\
200 \\
200 \\
200 \\
200 \\
200 \\
200 \\
200\end{array}$ \\
\hline
\end{tabular}

AZINUTH REIGHT EXPCSURE E/R DISTARCE DERREES METERS GN-SEC/CU.N SEC/CU.N 1/\$O.N NETERG $\times 10 E+6$
122.0

122,0

125.0

122.0

125.0

122.0

122.0

122.0

122.0

122.0

122.0

122.0

$152=0$

$\$ 22.0$

122.0

0.7
1.9
2.7
4.1
5.4
6.8
9.1
9.9
10.8
13.5
16.2
18.9
21.9
24.3
27.0

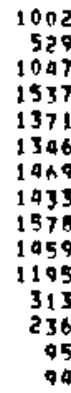

$7.479 E-0.7$

\section{$1.070 E-06$}

$9.186 E-07$

$1.954 \mathrm{E}=06$

2. $869 E=06$

$2.558 E=06$

$2.512 E-06$

$2.742 E=06$

$2.675 E \cdot 06$

$2.946 \mathrm{E}=06$

$2.722 E-06$

$2.231 E+0 B$

$5.045 E-67$

4. $409 E=07$

$1.770 \mathrm{E}=07$

$1,754 E=07$
200

200

200
200

200

200

200

200
200

200

200

200

200
200

200 


\begin{tabular}{|c|c|c|c|c|c|}
\hline $\begin{array}{l}\text { AZIMUTH } \\
\text { DEGREFS }\end{array}$ & $\begin{array}{l}\text { HE IGHT } \\
\text { HETERS }\end{array}$ & $\begin{array}{c}\text { EXPOSURF } \\
\text { GN-SEC/CU. } \\
\times 1 O F+6\end{array}$ & $\begin{array}{c}F / G \\
S E C / C U, N\end{array}$ & $\begin{array}{c}E \cup / 0 \\
1 / \$ 0 . N\end{array}$ & $\begin{array}{l}\text { DISTAKCE } \\
\text { METERS }\end{array}$ \\
\hline $\begin{array}{l}130.0 \\
130.0 \\
130.0 \\
130.0 \\
130.0 \\
130.0 \\
130.0 \\
130.0 \\
130.0 \\
130.0 \\
130.0 \\
130.0 \\
130.0 \\
130.0 \\
130.0\end{array}$ & $\begin{array}{r}0.7 \\
1.4 \\
2.7 \\
4.1 \\
5.4 \\
6.1 \\
9.1 \\
9.0 \\
10.8 \\
13.5 \\
16.2 \\
18.0 \\
21.6 \\
24.3 \\
27.0\end{array}$ & $\begin{array}{r}35 \\
5 \\
66 \\
25 \\
103 \\
134 \\
50 \\
54 \\
34 \\
44 \\
32 \\
10 \\
23 \\
7 \\
5\end{array}$ & $\begin{array}{l}2.637 E=0 E \\
4.00 P F=09 \\
4.903 E=08 \\
1.870 E=08 \\
1.043 E=07 \\
1.015 F=07 \\
3.741 E=08 \\
4.400 E=0 P \\
2.53 E E=08 \\
3.340 E=08 \\
2.405 E=08 \\
0.016 E=09 \\
1.737 E=08 \\
5.929 E=09 \\
3.952 E=09\end{array}$ & $\begin{array}{l}6.592 E=0 A \\
1.002 F=0 B \\
1.236 E-07 \\
4.676 E=08 \\
3.607 E=07 \\
2.535 E=07 \\
9.352 E=08 \\
1.102 E=07 \\
6.346 E=08 \\
9.350 E=08 \\
6.012 E=08 \\
2.004 E=08 \\
4.302 E=08 \\
1.082 E-08 \\
9.0 B 1 E=09\end{array}$ & $\begin{array}{l}200 \\
200 \\
200 \\
200 \\
200 \\
200 \\
200 \\
200 \\
200 \\
200 \\
200 \\
200 \\
200 \\
200 \\
200\end{array}$ \\
\hline
\end{tabular}

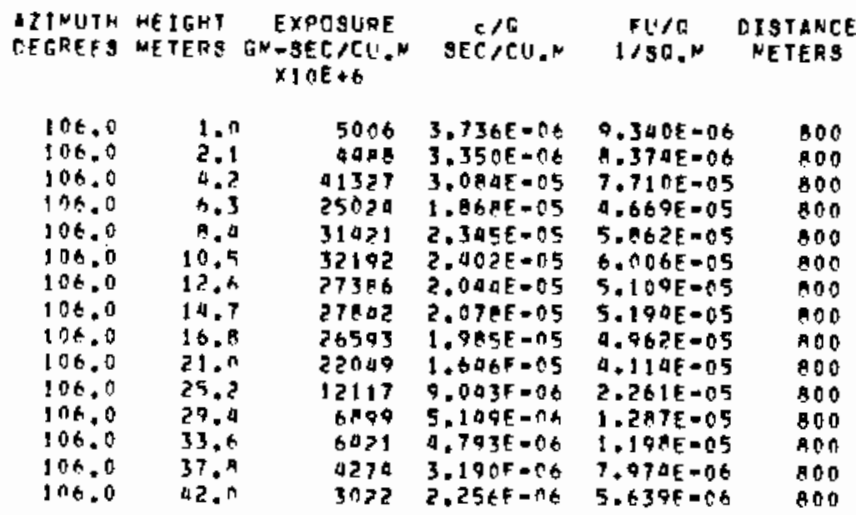

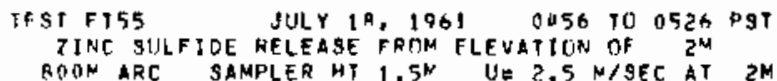
$\times 10 \mathrm{E}+6$

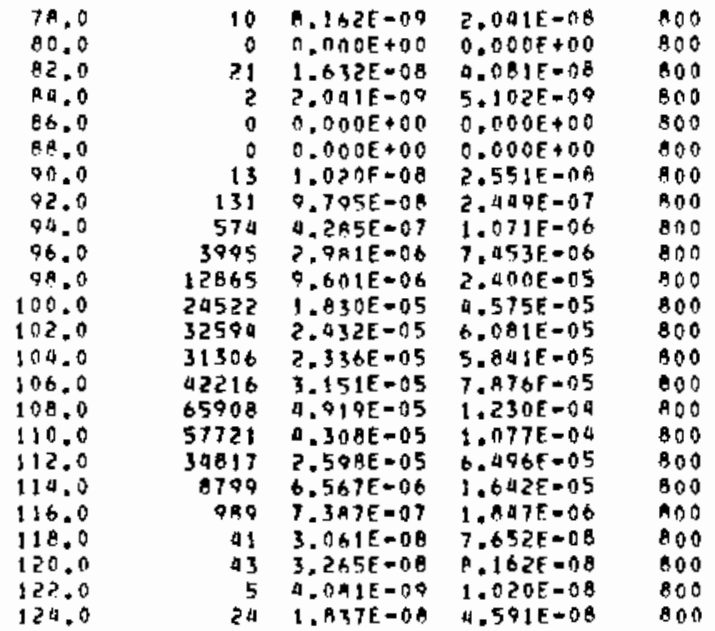

CRTISTIND INTEGRATEOE 6.59RE-03 1.650E-02

TORER TATA FOLLEM....

FFST FT55 SULY 18. 1961 OA56 T0 0526 PST

ZINE SULFIDE RELFASE FRON ELEVATICA DF $2 \mathrm{H}$

ROON ARE

$L=2,5 N /$ SC AT $2 N$

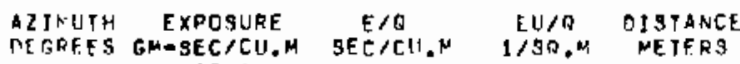

AZIMLTH HEIGHT EXPQSLIEE F/O FL/O CISTANCE

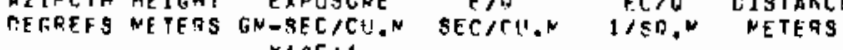
$\times 10 E+6$

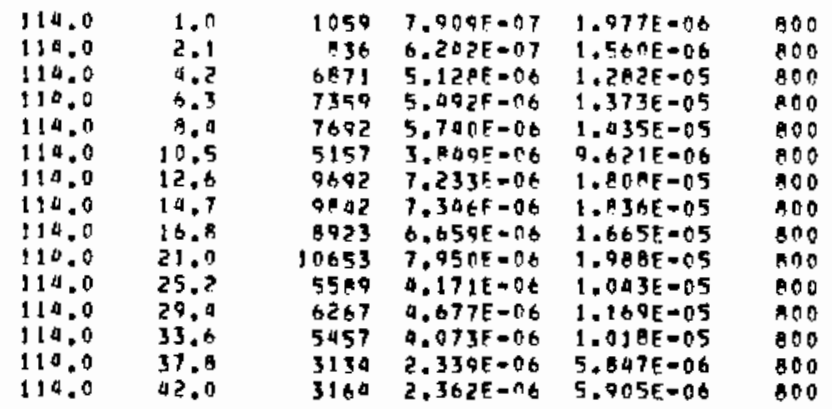

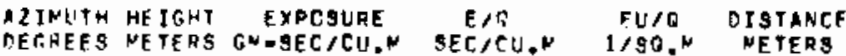 $x \in O E$ \&}

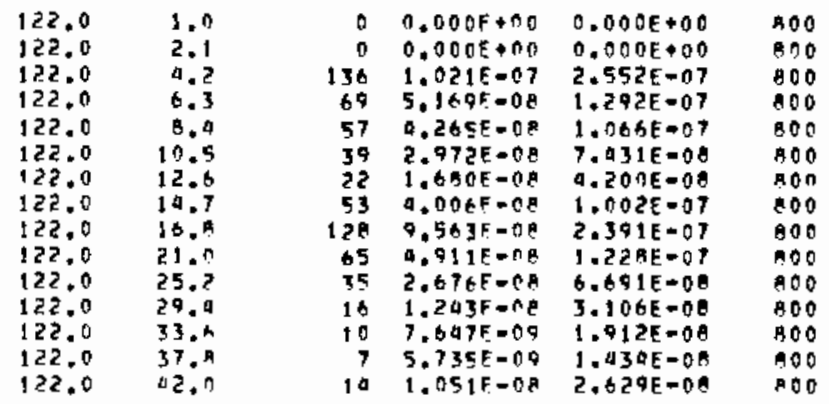

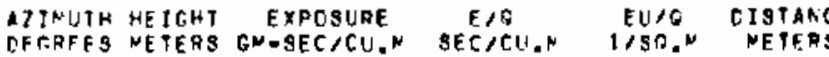
$x+0 E \nrightarrow 6$

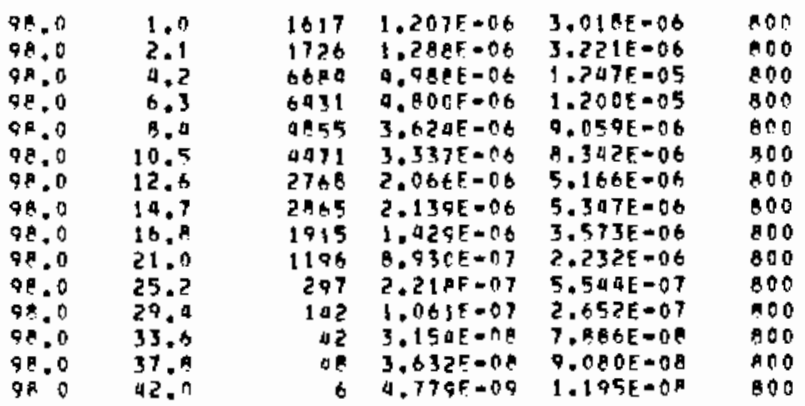

AZIMUTH HEIGHT EXPCSURE E/R FU/O CISTANCE $X \perp O E+B$

$\begin{array}{ll}130.0 & 1.0 \\ 130.0 & 2.1 \\ 130.0 & 0.2 \\ 130.0 & 6.3 \\ 130.0 & 8.0 \\ 130.0 & 10.5 \\ 130.0 & 12.6 \\ 130.0 & 10.7 \\ 130.0 & 16.9 \\ 130.0 & 21.0 \\ 130.0 & 25.2 \\ 130.0 & 29.4 \\ 130.0 & 33.6 \\ 130.0 & 37.0 \\ 130.0 & 42.0\end{array}$




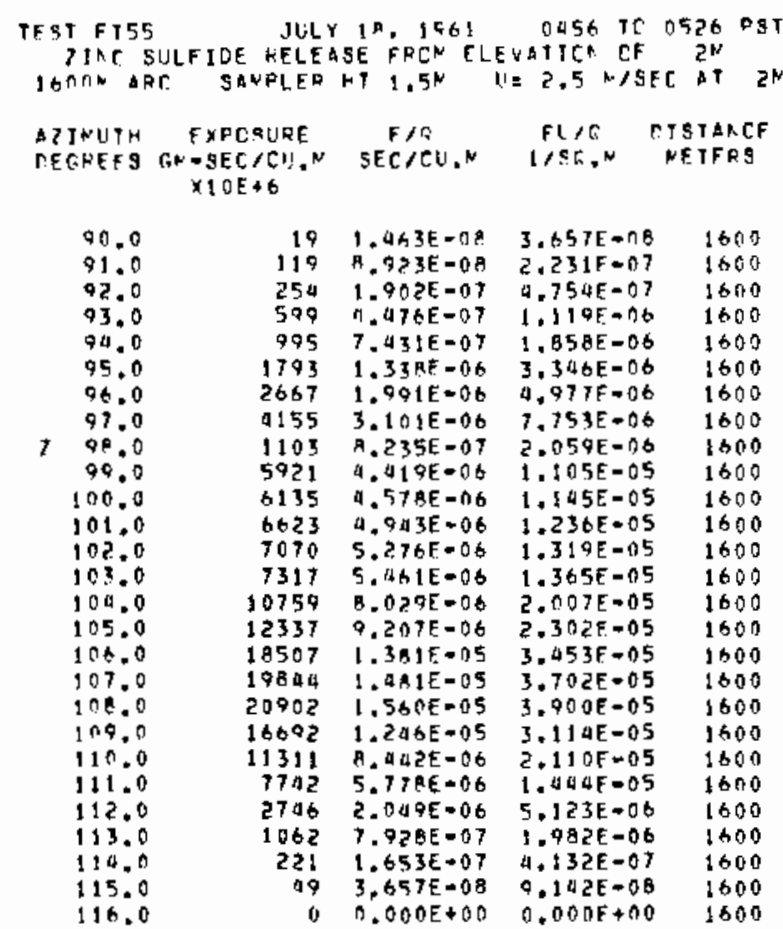

CRESSKTAR INTEGRATEC= 3.479E-03 8.698E-03

TOWEF DATA FOLLCK.....

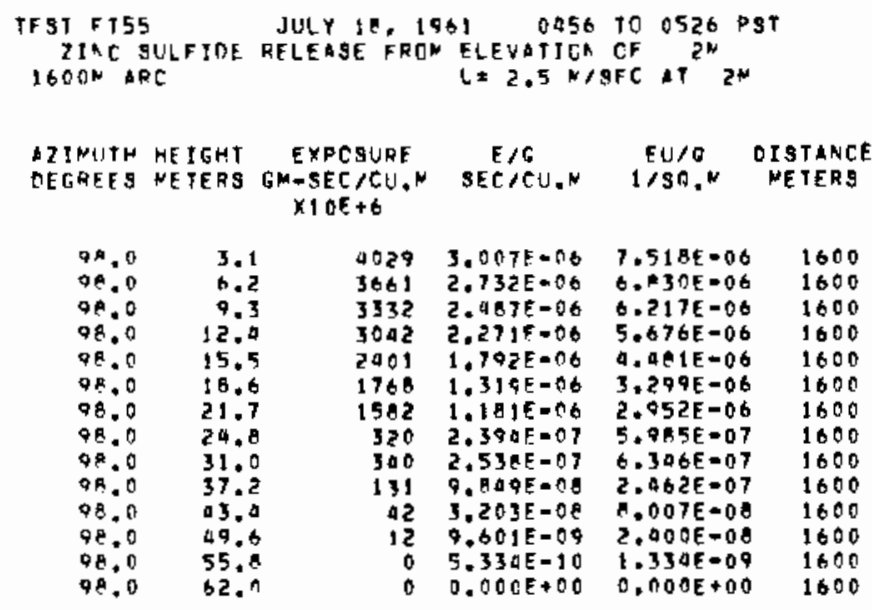

\begin{tabular}{|c|c|c|c|c|c|}
\hline $\begin{array}{l}\text { AZINIITH } \\
\text { PERAEES }\end{array}$ & $\begin{array}{l}\text { HE IGH } \\
\text { METERS }\end{array}$ & 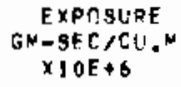 & $\begin{array}{c}E / C \\
S E C / C U, N\end{array}$ & $\begin{array}{c}E U / Q \\
1 / B Q_{0} N\end{array}$ & $\begin{array}{l}\text { DISTANCE } \\
\text { METERS }\end{array}$ \\
\hline $\begin{array}{l}10+.0 \\
106.0 \\
106.0 \\
106.0 \\
106.0 \\
106.0 \\
106.0 \\
106.0 \\
106.0 \\
105.0 \\
106.0 \\
106.0 \\
106.0 \\
106.0 \\
106.0\end{array}$ & $\begin{array}{l}1.5 \\
3.1 \\
6.2 \\
9.3 \\
12.9 \\
15.5 \\
10.6 \\
21.7 \\
24.9 \\
31.0 \\
37.2 \\
43.9 \\
49.4 \\
55.8 \\
62.0\end{array}$ & $\begin{array}{r}10190 \\
13011 \\
3023 \\
14287 \\
12770 \\
13515 \\
13365 \\
9094 \\
7375 \\
5050 \\
1430 \\
2703 \\
901 \\
609 \\
102\end{array}$ & $\begin{array}{l}7.600 F=06 \\
1.031 E=05 \\
2.256 E-06 \\
1.066 E=05 \\
9.530 E-06 \\
1.009 E=05 \\
9.825 E=06 \\
6.707 E=06 \\
5.504 E=06 \\
3.760 E-06 \\
1.070 E=06 \\
2.01 P E=06 \\
6.726 E=07 \\
5.102 F=07 \\
3.003 F=07\end{array}$ & 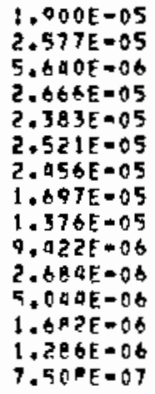 & $\begin{array}{l}1600 \\
1600 \\
1600 \\
1600 \\
1600 \\
1600 \\
1600 \\
1600 \\
1600 \\
1600 \\
1600 \\
1600 \\
1600 \\
1600 \\
1600\end{array}$ \\
\hline $\begin{array}{l}\text { A2TNLITH } \\
\text { DEREEES }\end{array}$ & $\begin{array}{l}\text { HEIGHT } \\
\text { NETERS }\end{array}$ & $\begin{array}{c}\text { EXPQSURE } \\
\text { GN-SEC } \\
\times 10 \mathrm{C}, \mathrm{N}\end{array}$ & $\begin{array}{c}E / G \\
S E C / C U, N\end{array}$ & $\begin{array}{l}E U / B \\
1 / S O . M\end{array}$ & $\begin{array}{l}\text { CISTANCE } \\
\text { NETEAS }\end{array}$ \\
\hline $\begin{array}{l}114.0 \\
1110.0 \\
110.0 \\
114.0 \\
110.0 \\
114.0 \\
114.0 \\
114.0 \\
119.0 \\
119.0 \\
114.0 \\
114.0 \\
114.0 \\
114.0 \\
114.0\end{array}$ & $\begin{array}{r}1.5 \\
3.1 \\
6.2 \\
9.3 \\
12.0 \\
15.5 \\
18.6 \\
21.7 \\
20.9 \\
31.9 \\
37.2 \\
43.9 \\
49.6 \\
55.9 \\
62.0\end{array}$ & $\begin{array}{l}163 \\
270 \\
270 \\
210 \\
201 \\
710 \\
367 \\
371 \\
906 \\
957 \\
1705 \\
1862 \\
828 \\
548 \\
781\end{array}$ & $\begin{array}{l}1.221 \mathrm{~F}-07 \\
2.018 \mathrm{E}-07 \\
2.009 \mathrm{~F}=07 \\
1.600 \mathrm{E}-07 \\
1.501 \mathrm{E}=07 \\
5.290 \mathrm{E}=07 \\
2.702 \mathrm{E}-07 \\
2.773 \mathrm{E}=07 \\
7.350 \mathrm{E}=07 \\
7.102 \mathrm{E}-07 \\
1.303 \mathrm{E}-06 \\
1.390 \mathrm{E}=106 \\
6.182 \mathrm{E}-07 \\
4.201 \mathrm{E}-07 \\
5.030 \mathrm{0}-07\end{array}$ & 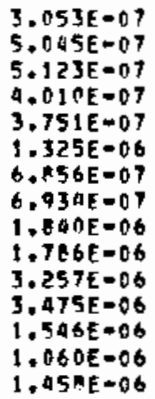 & $\begin{array}{l}1600 \\
1600 \\
1600 \\
1600 \\
1600 \\
1600 \\
1600 \\
1600 \\
1600 \\
1600 \\
1600 \\
1600 \\
1600 \\
1600 \\
1600\end{array}$ \\
\hline
\end{tabular}

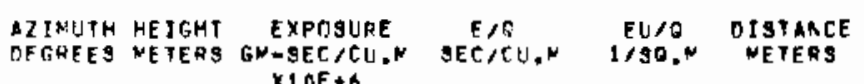
$\times 10 \mathrm{OE}+6$

\begin{tabular}{|c|c|c|c|c|c|}
\hline $\begin{array}{l}122.0 \\
122.0 \\
122.0 \\
122.0 \\
122.0 \\
122.0 \\
122.0 \\
122.0 \\
122.0 \\
122.0 \\
122.0 \\
122.0 \\
122.0 \\
122.0\end{array}$ & $\begin{array}{r}3.1 \\
6.2 \\
9.3 \\
12.4 \\
15.5 \\
18.6 \\
21.7 \\
24.8 \\
31.0 \\
37.2 \\
43.4 \\
49.6 \\
55.8 \\
62.0\end{array}$ & $\begin{array}{l}52 \\
13 \\
13 \\
26 \\
29 \\
16 \\
22 \\
15 \\
20 \\
59 \\
25 \\
12 \\
8 \\
7\end{array}$ & $\begin{array}{l}3.933 E=08 \\
1.035 E=08 \\
1.035 F=08 \\
1.966 E=08 \\
2.173 E=08 \\
1.242 E=08 \\
1.656 E=08 \\
1.121 E=08 \\
1.842 E=08 \\
4.401 E=08 \\
1.922 E=08 \\
9.601 E=09 \\
6.401 F=09 \\
5.868 E=00\end{array}$ & 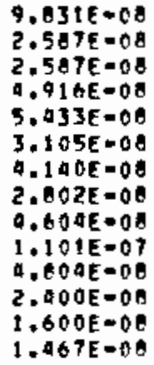 & $\begin{array}{l}1600 \\
1600 \\
1600 \\
1600 \\
1600 \\
1600 \\
1600 \\
1600 \\
1600 \\
1600 \\
1600 \\
1600 \\
1600 \\
1600\end{array}$ \\
\hline
\end{tabular}

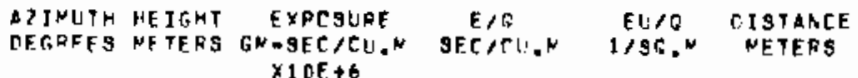

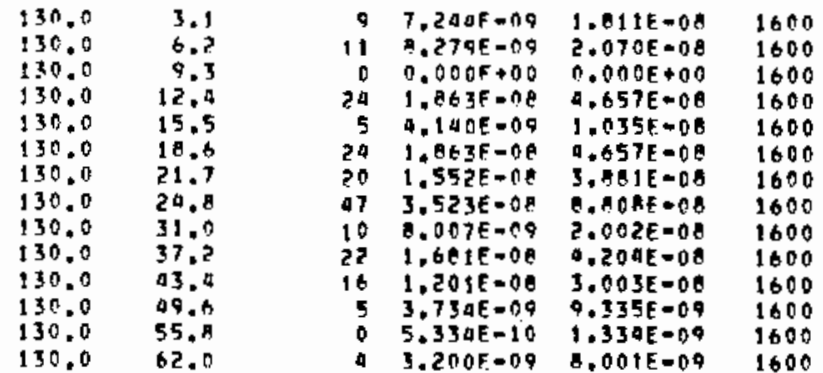




\begin{tabular}{|c|c|c|c|c|}
\hline $\begin{array}{l}\text { BZIMUTH } \\
\text { DECREFS }\end{array}$ & $\begin{array}{l}\text { EXPOSURE } \\
\text { GM }-S E C / C U . M \\
\times I D E+6\end{array}$ & $\begin{array}{c}E / \theta \\
\operatorname{SEc} / C(1)\end{array}$ & $\begin{array}{l}E U / 0 \\
1 / 30 . M\end{array}$ & $\begin{array}{l}\text { DISTANCE } \\
\text { METERS }\end{array}$ \\
\hline 81.0 & 5 & $9.307 E=09$ & $1.087 E=00$ & 3200 \\
\hline 82.0 & 18 & $1.377 E=08$ & $3.402 E-0 \theta$ & 3200 \\
\hline 83.0 & 10 & $7.970 E-09$ & $1.9825-08$ & 3200 \\
\hline 84.0 & 37 & $2.826 E=0$ A & $7.064 E=08$ & 3200 \\
\hline 85.0 & 50 & $3.76 B E=08$ & $9.419 E=08$ & 3200 \\
\hline 86.0 & 55 & $0.130 \mathrm{E}-0 \mathrm{~B}$ & $1.032 \mathrm{E}-07$ & 3200 \\
\hline 87.0 & 133 & $9.976 E-08$ & Z. $4 B 2 E-07$ & 3200 \\
\hline B. 0 & 97 & $0.525 E=08$ & $1.630 \mathrm{E}-07$ & 3200 \\
\hline 87.0 & $1 \geq 6$ & $9.419 \mathrm{E}=0 \mathrm{~B}$ & $2,355 E-07$ & 3200 \\
\hline 90.0 & $19 \%$ & $1.478 E-07$ & $3.695 E=07$ & 3200 \\
\hline 91.0 & 437 & $3.26 A E-07$ & $8,169 E=07$ & 3200 \\
\hline 92.0 & 1263 & 9.57 AE -07 & $2.395 E-06$ & 3200 \\
\hline 93.0 & 1713 & $1.279 E-06$ & $3.107 E=06$ & 3200 \\
\hline 94.0 & 2257 & 1.6 A5E -06 & $4.211 \varepsilon=00$ & 3200 \\
\hline 95.0 & 1818 & $1.3577-06$ & $3.393 E=06$ & 3200 \\
\hline 98.0 & $215 \mathrm{~h}$ & $1.30 Q E-06$ & $4.023 E=06$ & 3200 \\
\hline 97.0 & 2648 & $1.977 E-08$ & a. $901 E=06$ & 3200 \\
\hline 88.0 & 2091 & $1.551 \mathrm{~F}-06$ & 3.9025 .06 & 3200 \\
\hline 99.0 & 27 & $2.029 \mathrm{E}-0 \mathrm{~B}$ & $5.012 E=08$ & 3200 \\
\hline 100.0 & 3199 & $2.3 \times O E-06$ & $5.950 E-06$ & 3200 \\
\hline 101.0 & 2969 & $2.216 E-08$ & $5.541 E-06$ & 3200 \\
\hline 102.0 & 2827 & $2.155 E-06$ & $5.387 E-06$ & 3200 \\
\hline 103.0 & 3110 & $2.3 \geq 1 E-0 b$ & S.A01E-06 & 3200 \\
\hline 104.0 & 3346 & $2.497 E-06$ & $6.244 E=06$ & 3200 \\
\hline 105.0 & 0002 & 2.9 A7E 006 & $7.468 \mathrm{E}-0.0$ & 3200 \\
\hline 100.0 & 4057 & $3.699 E-06$ & $9.249 \mathrm{E}=06$ & 3200 \\
\hline 107.0 & 9658 & $3.476 E-06$ & $8.691 E=06$ & 3200 \\
\hline 100.0 & 393 & $2.934 \mathrm{E}=07$ & $7.330 E-07$ & 3200 \\
\hline 109.0 & 2231 & $1.6 \mathrm{GAE}=06$ & $4,161 E=06$ & 3200 \\
\hline 110.0 & 931 & A. 94 AAE -07 & $1.737 \mathrm{~F}-0.6$ & 3200 \\
\hline 111.0 & ono & $0.4 A 5 E=07$ & $1.121 \mathrm{E}-06$ & 3200 \\
\hline 112.0 & 1 a 8 & $1.109 E-07$ & $2.771 E=07$ & 3200 \\
\hline 113.0 & $B$ & $6.5218-09$ & $1.630 E-08$ & 3200 \\
\hline 114.0 & 0 & $7.245 E-10$ & $1.8117=09$ & 3200 \\
\hline 115.0 & 14 & $1.0 B 7 E-0 B$ & $2.7178-08$ & 3200 \\
\hline 116.0 & 0 & $0.000 E+00$ & $0.000 E+00$ & $3 \geq 00$ \\
\hline 117.0 & 3 & $\supset A \triangle A E-09$ & $1.245 E=09$ & 3700 \\
\hline Canssitud & INTEGRATEDE & 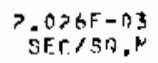 & $5.065 E_{1 / N}-03$ & \\
\hline
\end{tabular}

TOHE PATA FOLLCH...

\begin{tabular}{|c|c|c|c|c|c|}
\hline $\begin{array}{l}\text { AZINLTH } \\
\text { MEGREF }\end{array}$ & $\begin{array}{l}\text { WE IGHT } \\
\text { NETERS }\end{array}$ & $\begin{array}{c}\text { EXPOSURE } \\
\text { GM-SEC } \\
\times 1 O E+6\end{array}$ & $\begin{array}{c}E / 0 \\
S E[/ C U, k\end{array}$ & $\begin{array}{l}E U / C \\
1>30.4\end{array}$ & $\begin{array}{l}\text { DISTANCE } \\
\text { NETERS }\end{array}$ \\
\hline $\begin{array}{l}106.0 \\
106.0 \\
106.0 \\
106.0 \\
106.0 \\
104.0 \\
106.0 \\
106.0 \\
106.0 \\
106.0 \\
106.0 \\
106.0 \\
106.0 \\
106.0 \\
106.0\end{array}$ & $\begin{array}{r}1.5 \\
3.1 \\
6.2 \\
9.3 \\
12.0 \\
15.5 \\
10.6 \\
21.7 \\
24.8 \\
31.0 \\
37.2 \\
43.4 \\
49.6 \\
55.9 \\
62.0\end{array}$ & $\begin{array}{l}3950 \\
4571 \\
4007 \\
0229 \\
5120 \\
5125 \\
5705 \\
5026 \\
3361 \\
5795 \\
3163 \\
3002 \\
854 \\
1114 \\
26\end{array}$ & $\begin{array}{l}2.954 F=06 \\
3.411 E=06 \\
3.289 E=06 \\
3.157 F=06 \\
3.827 F=06 \\
3.925 E=06 \\
0.325 E=06 \\
3.751 F=06 \\
2.509 E=06 \\
2.832 F=06 \\
2.361 E=06 \\
2.241 E=06 \\
6.375 F=07 \\
0.837 F=07 \\
1.907 E-02\end{array}$ & $\begin{array}{l}7.386 E=06 \\
7.529 E=06 \\
9.223 E=06 \\
7.992 E=06 \\
9.568 E=06 \\
9.563 E=06 \\
1.091 E=05 \\
9.379 E=06 \\
6.272 E=06 \\
7.081 E=06 \\
5.902 E=06 \\
5.602 E=06 \\
1.590 E=06 \\
2.209 E=06 \\
0.0+7 E=01\end{array}$ & $\begin{array}{l}3200 \\
3200 \\
3200 \\
3200 \\
3200 \\
3200 \\
3200 \\
3200 \\
3200 \\
3200 \\
3200 \\
3200 \\
3200 \\
3200 \\
3200\end{array}$ \\
\hline
\end{tabular}
AZILUTH HEIGH EXPOSUAE E/D ELIO DISTANCE AETEEFS NETERS GN-SEC/CU.N SEC/CU.N IISO.N METERS $x 10 \mathrm{E} * 6$

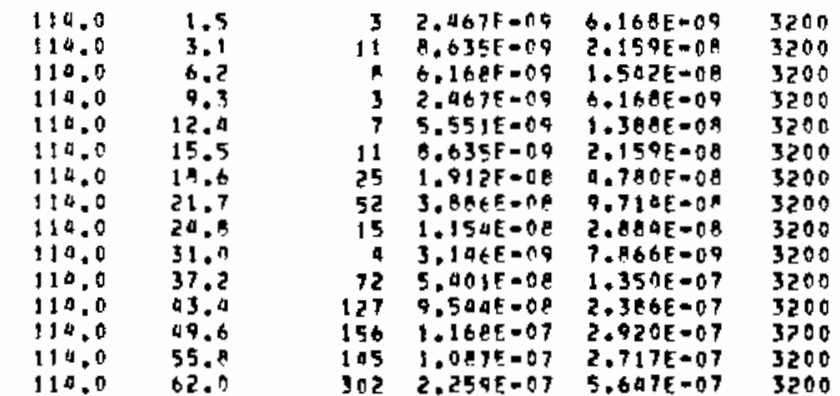

AZTNUTH HETGHT EXPOSURE ETC EU/O CISTANCE DERREES METERS GNESEC/CU.N SECICU.N IISR.M NETERS $\times 10 \mathrm{OE} \rightarrow 6$

$\begin{array}{llllll}122.0 & 3.1 & 6.168 E-09 & 1.542 E-0 . & 3200 \\ 122.0 & 6.2 & 9 & 6.795 F-09 & 1.696 E-00 & 3200\end{array}$

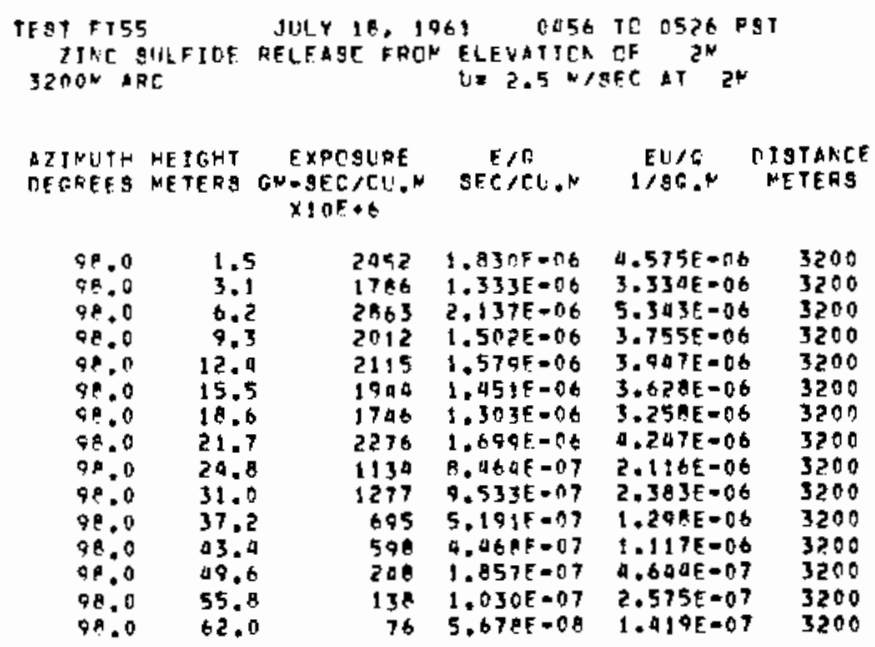


TO-5ERIES EXPE: IMENT: 56 \& FT-56,

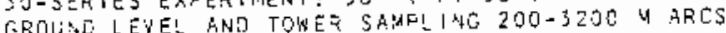

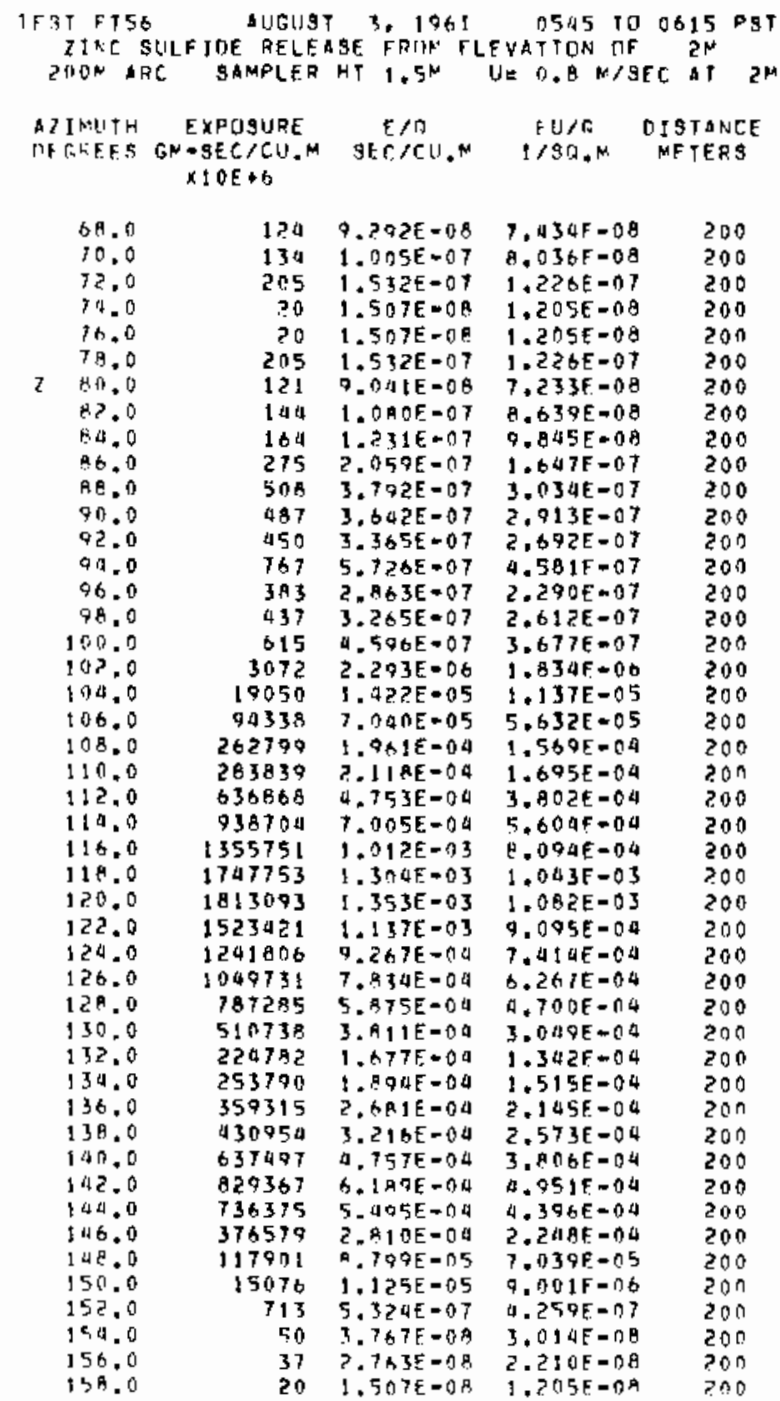

TOKER CATA FULLCW....

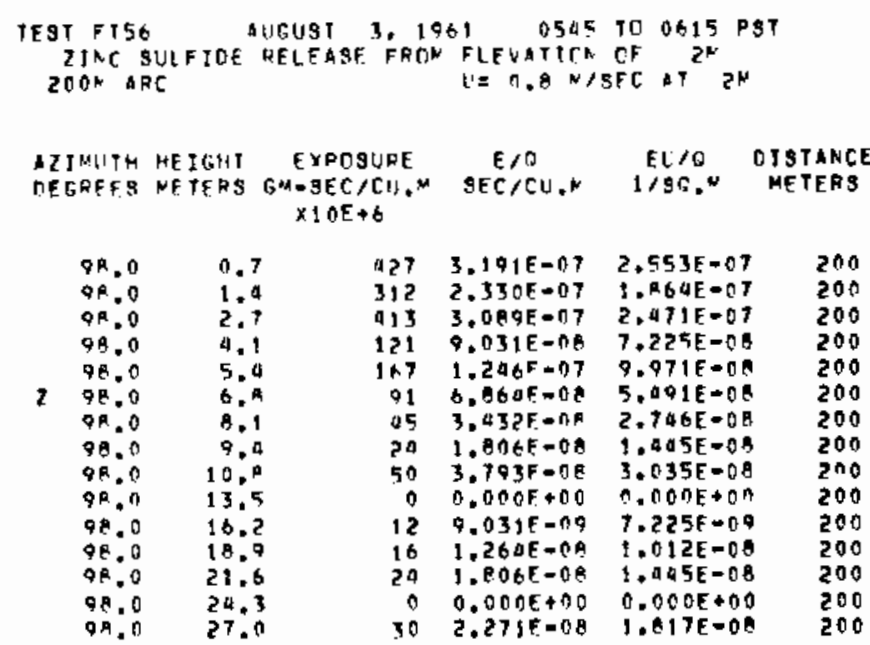

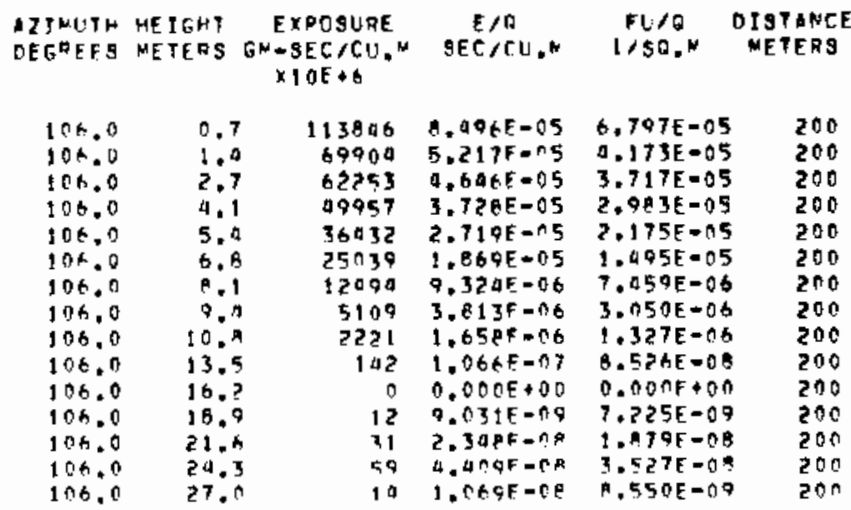

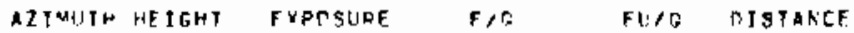
DEGEFFS NETERS GM-SEC/CH." SFCJEI!.N 1/SG,N NETFES $\times 10 F+6$

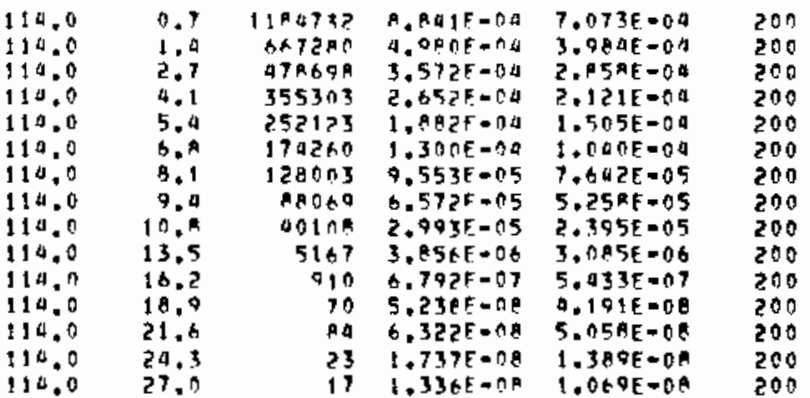




\begin{tabular}{|c|c|c|c|c|c|}
\hline $\begin{array}{l}\text { ZJNITH } \\
\text { EGREES }\end{array}$ & $\begin{array}{l}\text { HE IGHT } \\
\text { NETERS }\end{array}$ & $\begin{array}{c}\text { EXPQSURF } \\
\text { GN=SEC } \\
\times 1 O E+G\end{array}$ & $\begin{array}{c}\varepsilon / \theta \\
g E \tau /(1) . N\end{array}$ & $\begin{array}{c}F U / G \\
1 / S D, M\end{array}$ & $\begin{array}{l}\text { OISTANCE } \\
\text { NETERS }\end{array}$ \\
\hline $\begin{array}{l}122.0 \\
12200 \\
122.0 \\
122.0 \\
122.0 \\
12200 \\
12200 \\
122.0 \\
122.0 \\
122.0 \\
122.0 \\
122.0 \\
122.0 \\
122.0 \\
122.0\end{array}$ & $\begin{array}{l}0.7 \\
1.9 \\
2.7 \\
4.1 \\
5.9 \\
6.8 \\
8.1 \\
9.9 \\
10.9 \\
13.5 \\
16.2 \\
18.9 \\
21.8 \\
24.1\end{array}$ & $\begin{array}{r}1077903 \\
1055803 \\
956097 \\
969307 \\
026154 \\
397901 \\
407813 \\
702530 \\
126926 \\
52259 \\
13975 \\
2231 \\
510 \\
60 \\
2 A\end{array}$ & 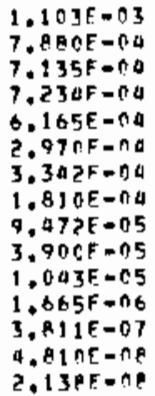 & 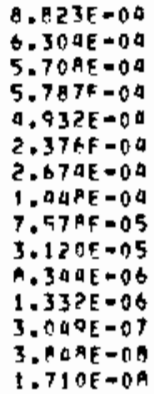 & $\begin{array}{l}200 \\
200 \\
200 \\
200 \\
200 \\
200 \\
200 \\
200 \\
200 \\
200 \\
200 \\
200 \\
200 \\
200 \\
200\end{array}$ \\
\hline
\end{tabular}

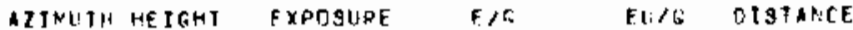
DETRFFS METERS GN-SECICU.N SECICU.N IISO.N NETERS $x \quad \cap \mathrm{OF}+6$

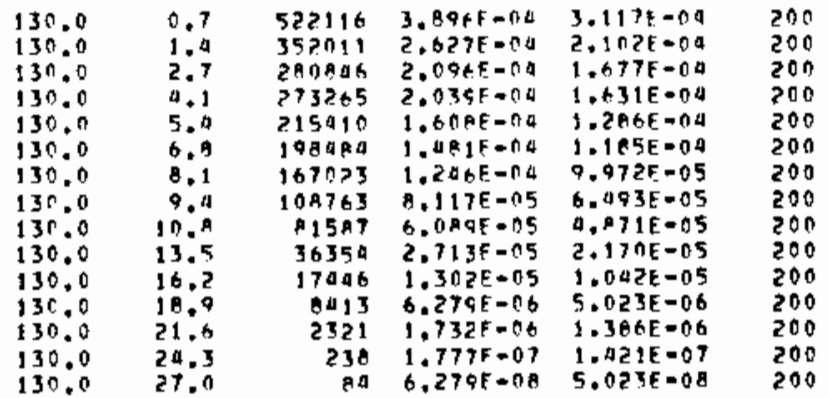
if $\$$ T FTS6
AUGUST 3, 1961 OSA5 TO OH15 PST ZINC SULFIDE RFLEASE FRUM FLEVATICN OF 2 MON

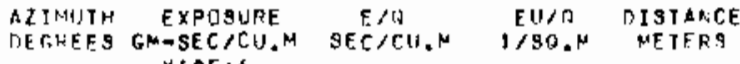
$\times 10 E+6$

\begin{tabular}{|c|c|c|c|c|}
\hline $\begin{array}{l}70.0 \\
72.0\end{array}$ & $\begin{array}{l}9 \\
9\end{array}$ & 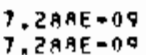 & $\begin{array}{l}5.830 E=09 \\
5.830 E=09\end{array}$ & $\begin{array}{l}\text { AOO } \\
\text { BnO }\end{array}$ \\
\hline 74.0 & 26 & $1.03 E-0 B$ & $1.555 \mathrm{E}-0 \mathrm{~B}$ & 800 \\
\hline 76.0 & 26 & $1.943 E-08$ & $1.555 \mathrm{~F}=00$ & 800 \\
\hline 78.0 & 45 & $3.901 E=00$ & $2.721 E=08$ & 800 \\
\hline $2 n .0$ & 39 & $2.915 E-08$ & $332 E=0.8$ & 800 \\
\hline ค?. 0 & 143 & $1.0 .9 E-07$ & 515.08 & $A O O$ \\
\hline 84.0 & 45 & $3.0015-09$ & $2.721 \mathrm{~F}=0 \mathrm{~B}$ & 800 \\
\hline 86.0 & 81 & $0.073 E-0.6$ & $\triangle, B 59 E=0 B$ & $8 D 0$ \\
\hline 99.0 & 65 & Q. $859 E-0 B$ & $3.887 \mathrm{f}-08$ & 800 \\
\hline $9 n .0$ & 81 & $6.073 E-08$ & $4.859 E-08$ & on \\
\hline 92.0 & ay & $6.559 E-00$ & $5.247 \mathrm{E}=08$ & 800 \\
\hline 9 a. 0 & 240 & $1.798 \mathrm{gE}-07$ & $1,438 E-0 ?$ & 800 \\
\hline 96.0 & 402 & $3,304 E-07$ & $2.603 E-07$ & 800 \\
\hline 98.9 & 908 & $E=07$ & $5.422 E=0 ?$ & 800 \\
\hline 100.0 & 1968 & $1.394 E=06$ & $1.216 E=06$ & 900 \\
\hline 102.0 & 3909 & 2.91BE-06 & $2.334 \mathrm{E}-06$ & 800 \\
\hline 104.0 & 5781 & $4.3145=06$ & $3.452 F=06$ & 800 \\
\hline 106.0 & 9876 & $7.3 \mathrm{ASE}=06$ & $908 E=06$ & 800 \\
\hline 108.0 & 11500 & $B .583 \varepsilon-06$ & $960 E-06$ & 800 \\
\hline 110.0 & 11269 & $A-410 E=0 B$ & $6.728 E+06$ & 800 \\
\hline 112.0 & $1077 \theta$ & $03 t=08$ & $5 E=016$ & 800 \\
\hline 114.0 & 10540 & $1.089 E=05$ & $8,711 \mathrm{E}=0 \mathrm{~b}$ & 800 \\
\hline 116.0 & 41706 & $3.132 E=05$ & $2.490 F=05$ & 800 \\
\hline 118.0 & 146191 & $1.001 E=04$ & A. $728=05$ & 800 \\
\hline 120.0 & 295159 & $2.203 E-04$ & $2.762 E=04$ & 300 \\
\hline 122.0 & 301273 & $248 E-04$ & $1.7995=0.4$ & 800 \\
\hline 124.0 & 89412 & $6.673 E=05$ & $5.33 A E-05$ & 800 \\
\hline 126.0 & 26192 & $5 E-05$ & SUE-OS & 300 \\
\hline 123.0 & 15693 & $1.17 ! E=05$ & $369 \mathrm{E}=06$ & 800 \\
\hline 130.0 & 17519 & $07 E=05$ & 1. $0465-05$ & 800 \\
\hline 132.0 & 15308 & $1.125 E-05$ & $9.1635=06$ & 800 \\
\hline 134.0 & $18 B$ & $1.409 E-07$ & $1.127 \mathrm{~F}=07$ & ROO \\
\hline 136.0 & 22523 & $1.6 A 1 E-05$ & $1.345 \mathrm{~F}-05$ & 800 \\
\hline $13 * 0$ & 20200 & $1.807 E-05$ & $1.425 \mathrm{E}=05$ & 800 \\
\hline 140.0 & 29740 & $2.219 E=05$ & $1.776 E=05$ & 800 \\
\hline 142.0 & 37400 & $192 F_{-}=05$ & $233 F-05$ & 800 \\
\hline .0 & 40202 & $3.000 E-05$ & $2.400 E=05$ & 300 \\
\hline 146.0 & $2 ? 640$ & $1.600 \mathrm{E}-05$ & $1.352 F-05$ & gon \\
\hline 108.0 & $22 A 5$ & $1.705 E+06$ & $1.36 \mathrm{AE}=06$ & 800 \\
\hline 150.0 & 52 & - 8R>E $=0 E$ & $3.110 F=08$ & 300 \\
\hline 152.0 & 3 & $.429 E-09$ & $1.743 \mathrm{E}-09$ & 800 \\
\hline 15 a. & 3 & $a>95-09$ & $943 \mathrm{E}-09$ & 800 \\
\hline & 0 & $000 E+00$ & $000 \mathrm{E}+00$ & 800 \\
\hline $15 A \cdot ?$ & 3 & $2.4 ? 9 F=09$ & $1.9435-09$ & A00 \\
\hline 160.17 & 19 & $\triangle 5 \mathrm{AE}=0 \mathrm{~A}$ & $1.166 f-128$ & 900 \\
\hline
\end{tabular}

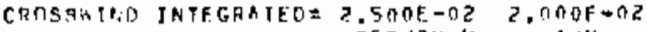


TOMFP DATA FOLLCW....

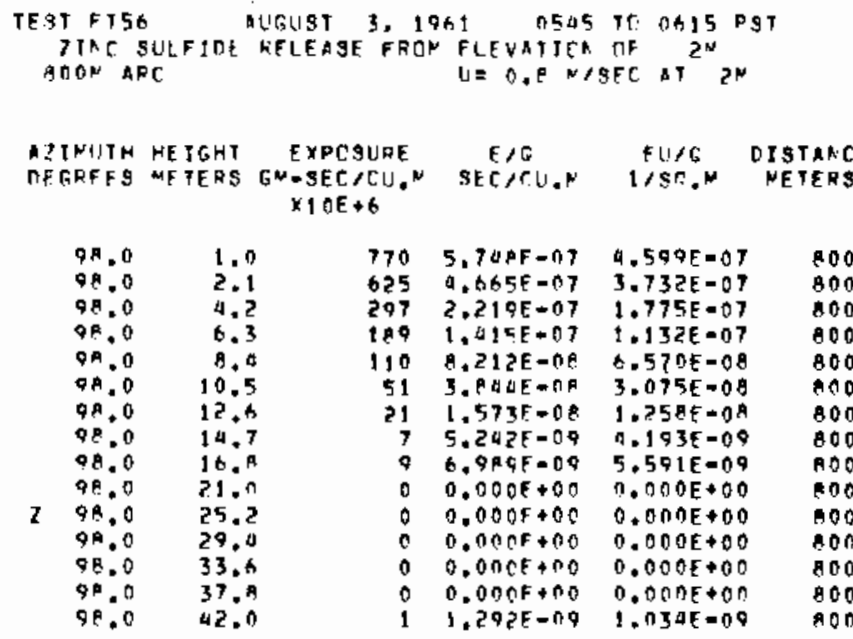

\begin{tabular}{|c|c|c|c|c|c|}
\hline $\begin{array}{l}\text { AZINUTH } \\
\text { DETREFS }\end{array}$ & $\begin{array}{l}\text { HE IGHT } \\
\text { METEFS }\end{array}$ & $\begin{array}{c}\text { EXPCSURE } \\
\text { GN-SEC/CU,N } \\
\times 1 O E+6\end{array}$ & $\begin{array}{c}E>C \\
S E C>C H\end{array}$ & $\begin{array}{l}E U / O \\
2 / 50 . N\end{array}$ & $\begin{array}{l}\text { DISTANCE } \\
\text { METEAS }\end{array}$ \\
\hline $\begin{array}{l}122.0 \\
122.0 \\
122.0 \\
122.0 \\
122.0 \\
122.0 \\
122.0 \\
122.0 \\
122.0 \\
122.0 \\
122.0 \\
122.0 \\
122.0 \\
122.0 \\
122.0\end{array}$ & $\begin{array}{l}1.0 \\
2.1 \\
4 . \\
6 . \\
8.5 \\
10.5 \\
12 . \\
19 . \\
16 . \\
21 . \\
25 . \\
29 . \\
33 . \\
37 . \\
42 .\end{array}$ & $\begin{array}{r}172440 \\
221062 \\
211019 \\
163552 \\
110259 \\
16641 \\
69605 \\
56521 \\
96996 \\
15040 \\
8097 \\
272 ? \\
926 \\
359 \\
51\end{array}$ & 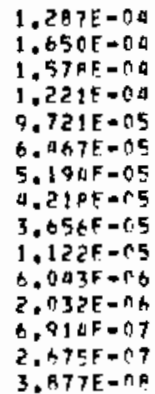 & 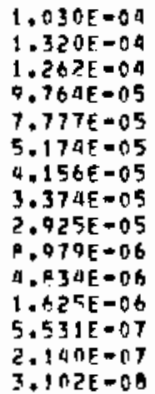 & $\begin{array}{l}B 00 \\
800 \\
100 \\
100 \\
900 \\
800 \\
800 \\
800 \\
800 \\
800 \\
800 \\
000 \\
000 \\
800 \\
000\end{array}$ \\
\hline
\end{tabular}

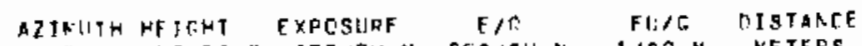
PETREFS NETERS GH-SET/CL!. SECITIIN 1/SA.N NETERS $x+\cap F+6$

\begin{tabular}{|c|c|c|c|c|c|}
\hline $\begin{array}{l}7 \\
0 \\
0 \\
0 \\
0 \\
0 \\
0 \\
0 \\
0 \\
0 \\
0 \\
0 \\
0\end{array}$ & $\begin{array}{l}1.0 \\
2.1 \\
4.2 \\
6.3 \\
8.0 \\
10.5 \\
12.6 \\
14.7 \\
16.8 \\
21.0 \\
25.2 \\
29.4 \\
53.6 \\
37.4 \\
42.0\end{array}$ & $\begin{array}{l}9433 \\
8946 \\
0917 \\
1100 \\
7855 \\
4755 \\
5111 \\
6611 \\
1970 \\
2308 \\
976 \\
540 \\
53 \\
12 \\
13\end{array}$ & 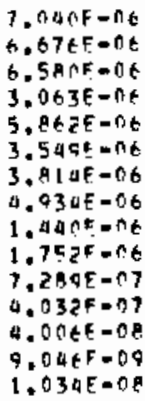 & $\begin{array}{l}5.632 E=06 \\
5.361 E-06 \\
5.264 E-06 \\
2.050 E-06 \\
4.690 E-06 \\
2.839 E-06 \\
3.051 E=06 \\
3.907 E=06 \\
1.152 E=06 \\
1.402 E=06 \\
5.231 E=07 \\
3.226 E=07 \\
3.205 E=08 \\
7.237 E-09 \\
0.271 E=09\end{array}$ & $\begin{array}{l}800 \\
800 \\
800 \\
800 \\
100 \\
800 \\
900 \\
000 \\
100 \\
800 \\
000 \\
000 \\
800 \\
000 \\
100\end{array}$ \\
\hline
\end{tabular}

AYIMITH HEIGHT EXPCSURE E/F FI:IT OJSTANCE

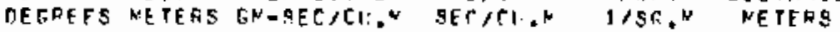
$\times 1 O E+6$

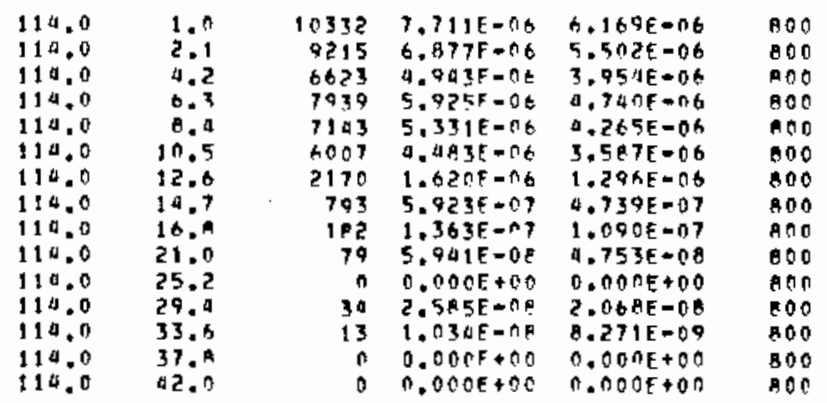




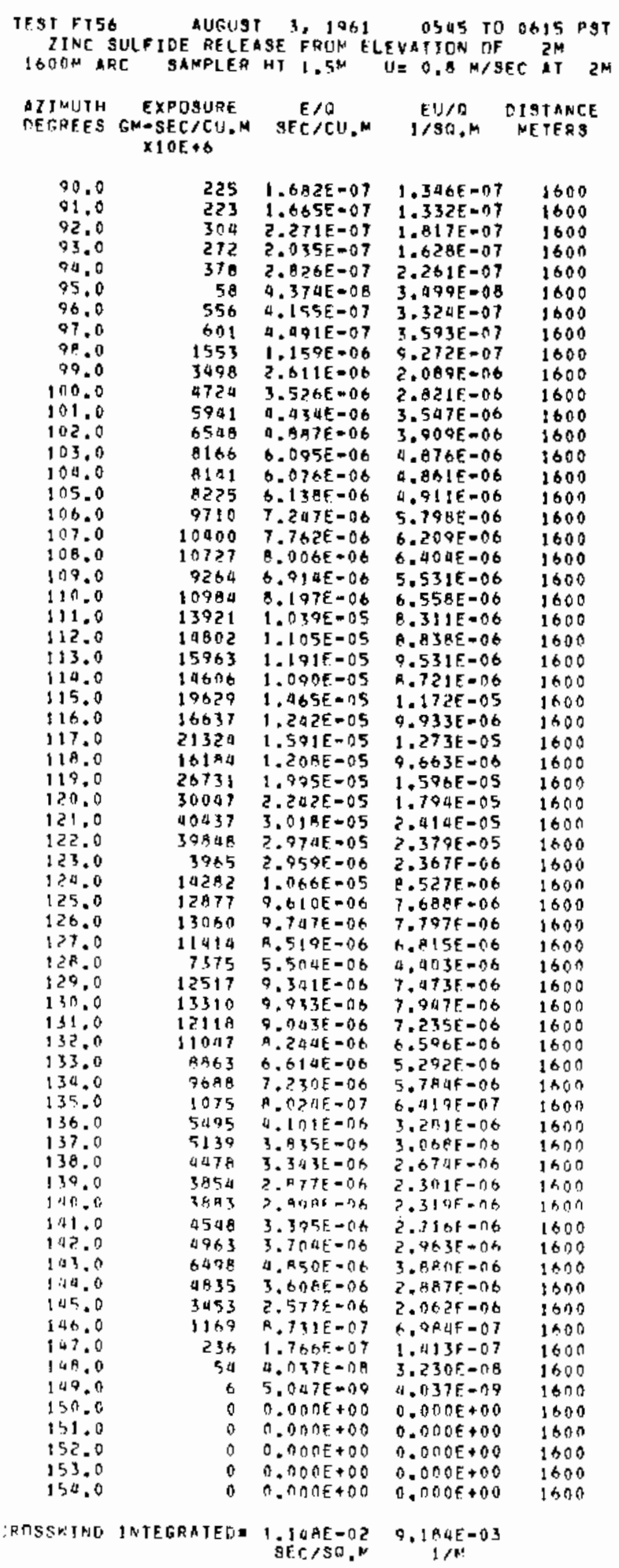

TOMER TAT \& FOLLW....

TESTFT56 DUCUSI 3,1001 ก545 TC $0615 \mathrm{OgT}$

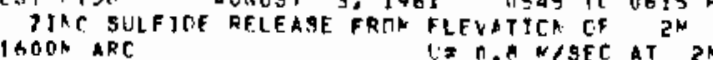

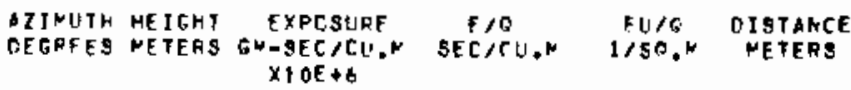
$X+O E+6$

\begin{tabular}{|c|c|c|c|c|c|}
\hline $\begin{array}{l}98.0 \\
98.0 \\
98.0 \\
9.00 \\
99.0 \\
99.01 \\
98.0 \\
99.0 \\
9.00 \\
91.0 \\
98.0 \\
98.0 \\
98.0 \\
98.0 \\
99.0\end{array}$ & $\begin{array}{l}1.5 \\
3.1 \\
6.2 \\
9.3 \\
12.0 \\
15.5 \\
18.6 \\
21.7 \\
24.9 \\
31.0 \\
37.2 \\
43.0 \\
49.6 \\
55.8 \\
62.0\end{array}$ & $\begin{array}{r}964 \\
906 \\
490 \\
268 \\
195 \\
73 \\
60 \\
55 \\
6 \\
15 \\
5 \\
0 \\
0 \\
0 \\
1\end{array}$ & 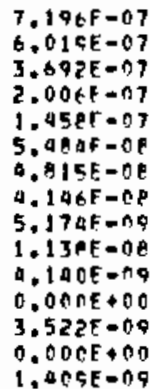 & $\begin{array}{l}5.757 E=07 \\
9.815 E-07 \\
2.953 E=07 \\
1.005 E=07 \\
1.166 E-07 \\
4.387 E=08 \\
3.052 E=08 \\
3.317 E=09 \\
4.100 E=09 \\
9.107 E=09 \\
3.312 E=09 \\
0.000 E+00 \\
2.017 E=09 \\
0.00 N E+00 \\
1.127 E=09\end{array}$ & $\begin{array}{l}1600 \\
1600 \\
1600 \\
1600 \\
1600 \\
1600 \\
1600 \\
1600 \\
1600 \\
1600 \\
1600 \\
1600 \\
1600 \\
1600 \\
1600\end{array}$ \\
\hline
\end{tabular}
$\times 1 O E+b$

\begin{tabular}{|c|c|c|c|c|c|}
\hline $\begin{array}{l}106.0 \\
106.0 \\
106.0 \\
106.0 \\
106.0 \\
106.0 \\
106.0 \\
106.0 \\
106.0 \\
106.0 \\
106.0 \\
106.0 \\
106.0 \\
106.0 \\
106.0\end{array}$ & $\begin{array}{r}1.5 \\
3.1 \\
6.2 \\
9.3 \\
12.0 \\
15.5 \\
18.5 \\
21.7 \\
24.8 \\
31.0 \\
37.2 \\
43.0 \\
09.9 \\
55.2 \\
52.0\end{array}$ & $\begin{array}{r}5301 \\
6065 \\
519 \\
2600 \\
1327 \\
376 \\
09 \\
32 \\
0 \\
0 \\
0 \\
0 \\
0 \\
0 \\
0\end{array}$ & 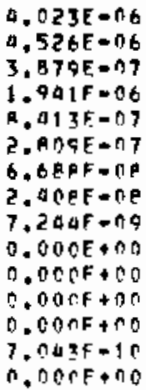 & 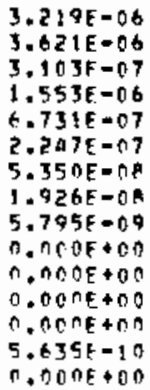 & $\begin{array}{l}1600 \\
1600 \\
1600 \\
1600 \\
1600 \\
1600 \\
1600 \\
1600 \\
1600 \\
1600 \\
1600 \\
1600 \\
1600 \\
1600 \\
1600\end{array}$ \\
\hline
\end{tabular}

AZINUTH HEIGHT EXPCSURE ETO FLF DISTANCE

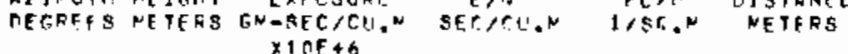

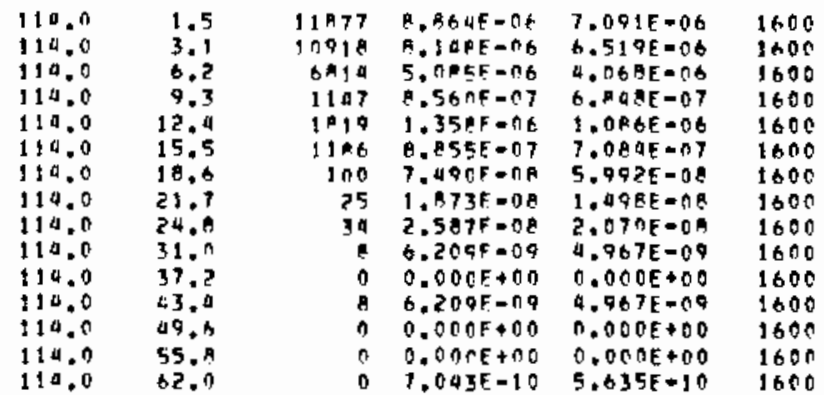




\begin{tabular}{|c|c|c|c|c|c|}
\hline $\begin{array}{l}\text { DINALTH } \\
\text { DE TREES }\end{array}$ & $\begin{array}{l}\text { HE TGHT } \\
\text { NETERS }\end{array}$ & $\begin{array}{c}\text { EXPOSLAE } \\
\text { GN-SEC/CU. } \\
\times 1 O F+6\end{array}$ & $\begin{array}{c}\varepsilon / 0 \\
\sec >\cos .\end{array}$ & $\begin{array}{l}E L / 6 \\
2 / 8 \pi, N\end{array}$ & $\begin{array}{l}\text { STANCE } \\
\text { E TERS }\end{array}$ \\
\hline $\begin{array}{r}122.0 \\
122.0 \\
122.0 \\
122.0 \\
1122.0 \\
122.0 \\
122.0 \\
122.0 \\
122.0 \\
122.0 \\
122.0 \\
122.0 \\
122.0 \\
122.0 \\
122.0\end{array}$ & $\begin{array}{r}1.5 \\
3.1 \\
6.2 \\
9.3 \\
12,4 \\
15.5 \\
18.6 \\
21.7 \\
21.10 \\
31.0 \\
37.2 \\
43.0 \\
49.6 \\
55.9 \\
42.7\end{array}$ & $\begin{array}{r}21835 \\
33557 \\
36560 \\
39906 \\
36731 \\
31998 \\
23967 \\
10 \times 97 \\
10401 \\
3399 \\
535 \\
135 \\
6 \\
4 \\
9\end{array}$ & $\begin{array}{l}1.630 F=05 \\
2.501 F=05 \\
2.724 F=05 \\
2.981 E=05 \\
2.74 J F=05 \\
2.310 E=05 \\
2.162 F=05 \\
1.47 C F=05 \\
7.763 E=06 \\
2.520 E=06 \\
3.095 F=07 \\
1.014 F=07 \\
4.930 F=09 \\
3.522 F=09 \\
7.043 E=09\end{array}$ & $\begin{array}{l}1.3 C A E=05 \\
2.003 E=05 \\
2.183 E=05 \\
2.3 E 5 E=05 \\
2.193 E=05 \\
1.910 E=05 \\
1.729 E=05 \\
1.174 E=05 \\
6.210 E=06 \\
2.023 E=06 \\
3.194 E=07 \\
9.113 E=00 \\
1.940 E=09 \\
2.017 E=09 \\
5.635 E=09\end{array}$ & $\begin{array}{l}1600 \\
1600 \\
1600 \\
1600 \\
1600 \\
1600 \\
1600 \\
1600 \\
1600 \\
1600 \\
1600 \\
1600 \\
1600 \\
1600 \\
1600\end{array}$ \\
\hline
\end{tabular}

BIINUTH HEIGHT EXPCSURE F/F FLIR DISTANCE

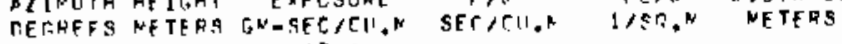
inE +6

\begin{tabular}{|c|c|c|c|c|c|}
\hline $\begin{array}{l}130.0 \\
30.0 \\
30.0\end{array}$ & $\begin{array}{l}1.5 \\
3.1 \\
0.2\end{array}$ & $\begin{array}{l}59 \times 4 \\
5059 \\
7321\end{array}$ & $\begin{array}{l}4.453 E=06 \\
0.372 F-06 \\
5.464 E-16\end{array}$ & $\begin{array}{l}3.542 E=06 \\
3.49 A E=06 \\
0.371 E-66 \\
.975 F=06\end{array}$ & $\begin{array}{l}1600 \\
1600 \\
1600 \\
1600\end{array}$ \\
\hline 130.0 & 9.3 & $\begin{array}{l}3274 \\
4250\end{array}$ & $\begin{array}{l}2.40 E E-06 \\
3.170 E=0 E\end{array}$ & $\begin{array}{l}1.9255=06 \\
2.5035-00\end{array}$ & $\begin{array}{l}1600 \\
1600\end{array}$ \\
\hline $\begin{array}{l}130.0 \\
130.0\end{array}$ & $\begin{array}{l}12.4 \\
15.5\end{array}$ & 2016 & $2.17 \in E=06$ & $\begin{array}{l}1.741 E-06 \\
10 F-06\end{array}$ & $\begin{array}{l}1600 \\
1600\end{array}$ \\
\hline 130.0 & $\begin{array}{l}18.6 \\
21.7\end{array}$ & $\begin{array}{l}1449 \\
1202\end{array}$ & $\begin{array}{l}1,26 \mathrm{e}=116 \\
8,975 \mathrm{E}=07\end{array}$ & 7.1 AीE $=07$ & 1600 \\
\hline$\therefore 30,0$ & 24.1 & 507 & $4,4295-07$ & $3.505 E=07$ & 1670 \\
\hline$: 30.0$ & 31 & $15 t$ & $1.12 P E=07$ & $.02 A E=08$ & 1600 \\
\hline 30.0 & 3 & 40 & $3.0 \cap 1 E-08$ & $2.001 E=0 \%$ & 1600 \\
\hline$\therefore .0$ & 43 & 20 & $1.9665=08$ & $\begin{array}{r}1.573 E=00 \\
1.690 E=09\end{array}$ & $\begin{array}{l}1600 \\
1600\end{array}$ \\
\hline 30 & 55 & 0 & $0.00 A E 400$ & $0.000 E+00$ & 1600 \\
\hline & & 10 & $7.147 \mathrm{E}=09$ & $-19 B E=09$ & 1600 \\
\hline
\end{tabular}

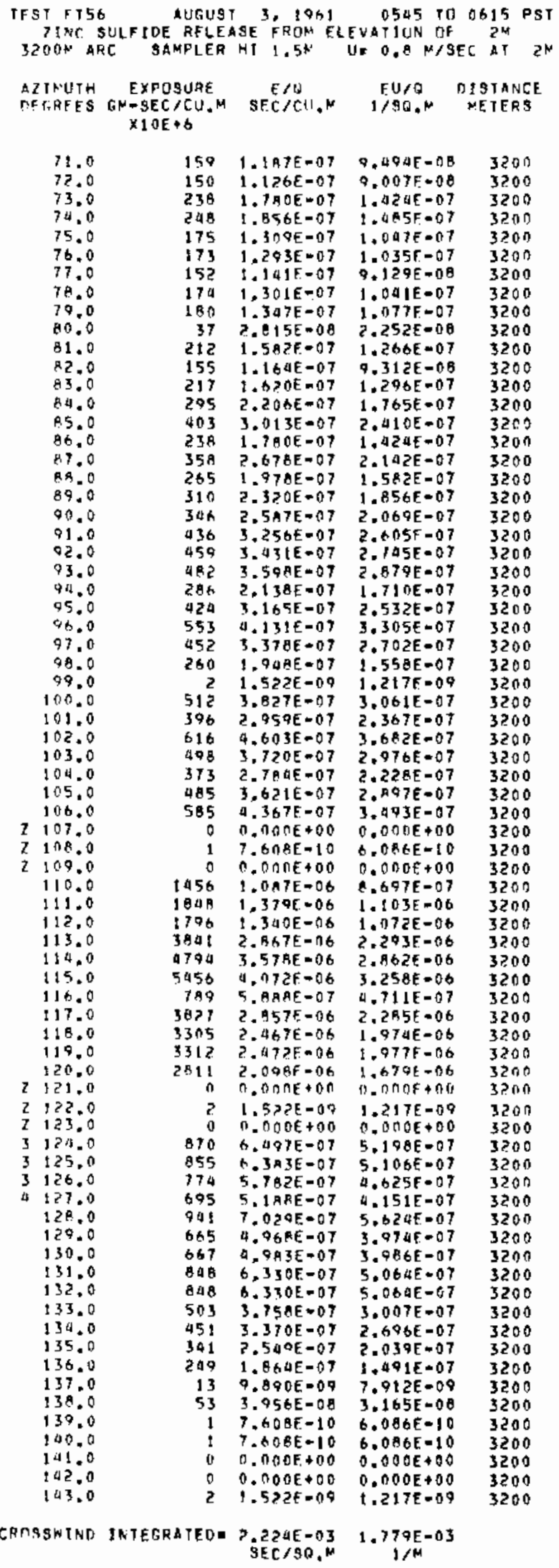


TONEA DATA FOLLCH....

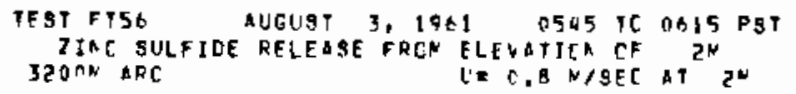

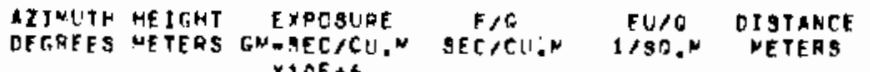
$\times 10 E+6$

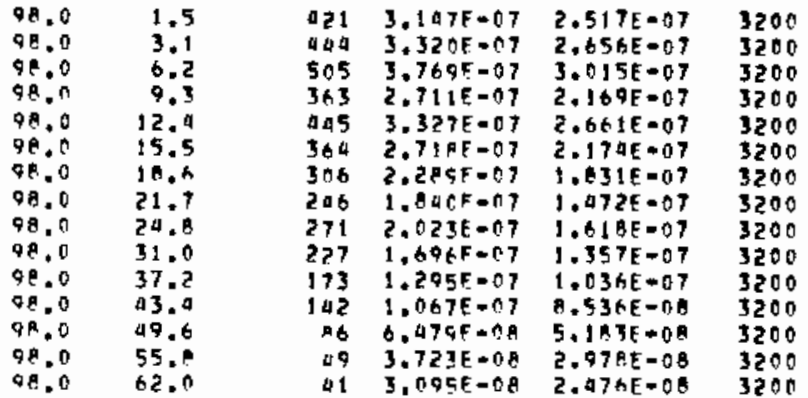

\begin{tabular}{|c|c|c|c|c|c|}
\hline $\begin{array}{l}\text { DIFLITH } \\
\text { DECOPEFS }\end{array}$ & $\begin{array}{l}\text { HE IGHT } \\
\text { NETERS }\end{array}$ & $\begin{array}{c}\text { EXPCSURE } \\
\text { GK-9EC }>C 11 . N \\
\times 1 \cap E+6\end{array}$ & $\begin{array}{c}E / 0 \\
\sec / C 1 \%, N\end{array}$ & $\begin{array}{c}F \mathrm{~b} / \mathrm{Q} \\
1 / \mathrm{S} N \mathrm{~N}^{N}\end{array}$ & $\begin{array}{l}\text { CISTHNCE } \\
\text { NETERS }\end{array}$ \\
\hline $\begin{array}{l}100.0 \\
100.0 \\
106.0 \\
106.0 \\
108.0 \\
100.0 \\
106.0 \\
106.0 \\
106.0 \\
104.0 \\
100.0 \\
106.0 \\
106.0 \\
106.0 \\
100.0\end{array}$ & $\begin{array}{l}1.5 \\
3.1 \\
0.2 \\
9.3 \\
12.4 \\
15.5 \\
18.4 \\
21.7 \\
24.9 \\
31.7 \\
37.2 \\
43.4 \\
09.4 \\
55.9 \\
62.7\end{array}$ & $\begin{array}{r}624 \\
701 \\
410 \\
029 \\
55 \\
432 \\
570 \\
519 \\
436 \\
374 \\
275 \\
191 \\
5 \\
46 \\
?\end{array}$ & $\begin{array}{l}4.661 F=07 \\
5.2358=07 \\
3.5825=07 \\
3.202 E=07 \\
4.150 F=08 \\
3.230 F=07 \\
3.009 F=07 \\
3.073 F=07 \\
3.251 F=07 \\
2.006 F=07 \\
2.054 F=07 \\
1.431 F=07 \\
4.3527=09 \\
7.204 F=02 \\
1.934 F=00\end{array}$ & $\begin{array}{l}3.729 E-07 \\
4.189 E=07 \\
2.066 E-07 \\
2.562 E-07 \\
3.320 E=08 \\
2.520 E-07 \\
3.159 E=07 \\
3.099 F=07 \\
2.605 E-07 \\
2.245 E=07 \\
1.643 F=07 \\
1.105 E=07 \\
3.421 E=09 \\
5.7+4 E=08 \\
1.547 F=09\end{array}$ & $\begin{array}{l}3200 \\
3200 \\
3200 \\
3200 \\
3200 \\
3200 \\
3200 \\
3200 \\
3200 \\
3200 \\
3200 \\
3200 \\
3200 \\
3200 \\
3200\end{array}$ \\
\hline
\end{tabular}

1ZINUTH HEIGHT EXPOSUFF ET6 EITO DISTANCE

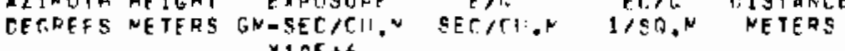

\begin{tabular}{|c|c|c|c|c|c|}
\hline 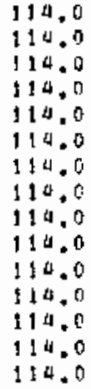 & $\begin{array}{l}1.5 \\
3.1 \\
6.2 \\
9.3 \\
12.4 \\
15.5 \\
19.8 \\
21.9 \\
24.8 \\
11.0 \\
37.2 \\
43.9 \\
49.4 \\
55.8 \\
62.0\end{array}$ & $\begin{array}{l}4209 \\
3056 \\
3045 \\
2471 \\
33+5 \\
2501 \\
1846 \\
1409 \\
979 \\
31 \\
212 \\
159 \\
72 \\
27 \\
15\end{array}$ & $\begin{array}{l}3.20 P F=16 \\
2.729 F=06 \\
2.943 F=06 \\
2.219 F=06 \\
2.511 E=60 \\
1.912 E=0 E \\
1.37 P E=06 \\
1.110 E=06 \\
6.934 E=07 \\
2.344 F=09 \\
1.585 F=07 \\
1.190 E=07 \\
5.415 F=08 \\
2.031 E=08 \\
1.160 F=08\end{array}$ & $\begin{array}{l}2.567 E=06 \\
2.183 E=06 \\
2.354 E=06 \\
1.774 E=06 \\
2.009 E=06 \\
1.529 E=06 \\
1.103 E=06 \\
8.952 E=07 \\
5.451 E=07 \\
1.075 E=08 \\
1.269 E=07 \\
9.523 E=08 \\
4.332 E=0 A \\
1.625 E=08 \\
9.2 P 4 E=09\end{array}$ & $\begin{array}{l}3200 \\
3200 \\
3200 \\
3200 \\
3200 \\
3200 \\
3200 \\
3200 \\
3200 \\
3200 \\
3200 \\
3200 \\
3200 \\
3200 \\
3200\end{array}$ \\
\hline
\end{tabular}

ATIMUTH HEIGHT EXPOSUAE

$E / Q$

EUIO DISTANCE $\times 10 E+6$

122.0
122.0
122.0
122.0
122.0
122.0
122.0
122.0
122.0
122.0
122.0
122.0
122.0
122.0
122.0

1.5
3.1
6.2
9.3
12.8
15.5
18.6
21.7
24.7
31.0
37.2
43.4
49.6
55.1
62.7

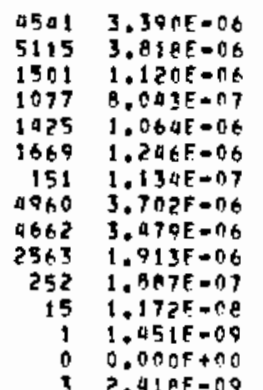

$2.712 E-06$ $0.063=07 \quad 3200$ $-07 \quad 3200$ $0.435 E=07 \quad 3200$ $0.509 E-07 \quad 3200$ $9.965 \mathrm{~F}-07 \quad 3200$ $9,074 E-00 \quad 3200$ $2.962 E-06 \quad 3200$ $2,703 E=06 \quad 3200$ $\begin{array}{ll}3.531 E-06 & 3200\end{array}$ $\begin{array}{ll}1.531 E=06 & 3200 \\ 1.510 E-07 & 3200\end{array}$ $\begin{array}{ll}1.510 E=07 & 3200 \\ 9,375 E=09 \quad 3200\end{array}$ $1.160 E=09 \quad 3 \geqslant 00$ $0.000 E+003200$ $1.9306-09 \quad 3200$

AZINISTH HEIGH EXPCSURE F/F EU/E DISTANCE DEGRFES NETEFS GN-SEC/CL.N SECICU.N 1/SF.N NETEFS $\times 10 F+2$

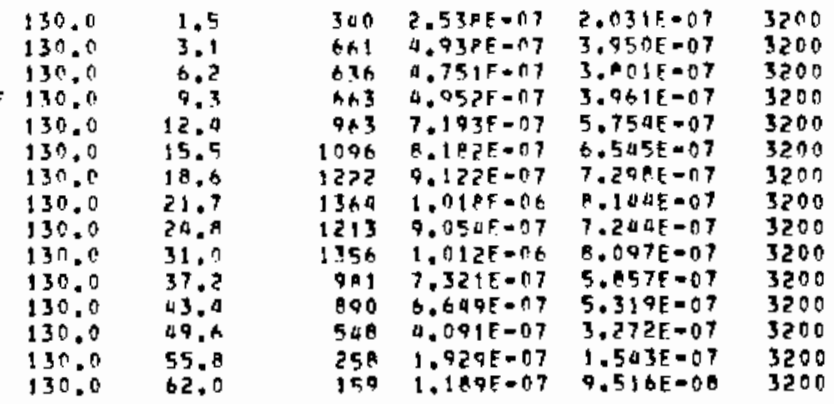


30-SERIES EXIERIAEMT: 57 (FT-57!

GROUND LEYEL AND TOWER SAMPL INS $290-3200$ H ARCS

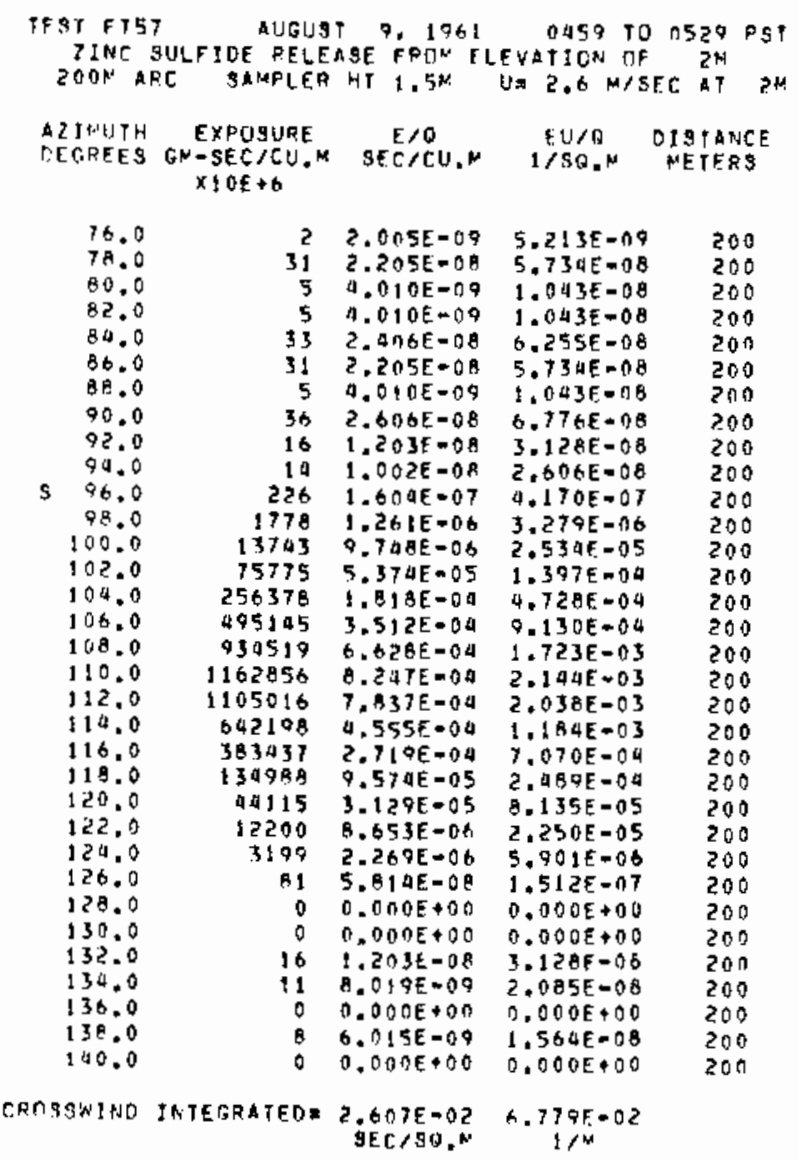

TOWER CATA FOLLOW....

TEST FT57 QUGLIST P, 1961 DU59 TO 0529 PST

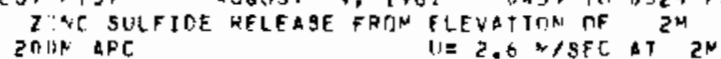

ATJMITH HEIGHT EXPOSURE E/G EU/O OISTANCE DEGHEES METEAS GM-SEC/CU.N SEC/CU.N I/SO. M METEAS $\times 10 E+6$

\begin{tabular}{|c|c|c|c|c|c|}
\hline 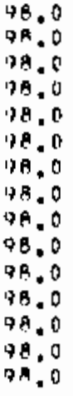 & $\begin{array}{l}0.7 \\
1.4 \\
2.7 \\
4.1 \\
5.4 \\
6.9 \\
6.1 \\
9.9 \\
10.1 \\
13.5 \\
16.2 \\
18.9 \\
21.6 \\
24.3 \\
27.0\end{array}$ & $\begin{array}{r}1879 \\
1296 \\
1516 \\
1954 \\
1537 \\
322 \\
673 \\
1172 \\
1180 \\
1267 \\
379 \\
118 \\
192 \\
92 \\
24\end{array}$ & 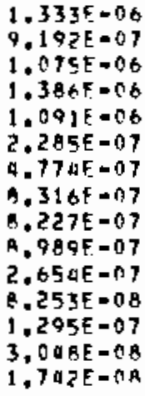 & $\begin{array}{l}3.965 E-06 \\
2.390 E-06 \\
2.796 E-06 \\
3.605 E-06 \\
2.836 E=06 \\
5.942 E=07 \\
1.241 E=06 \\
2.162 E=06 \\
2.139 E=06 \\
2.337 E=06 \\
6.899 E=07 \\
2.146 E=07 \\
3.367 E=07 \\
7.925 E=08 \\
0.528 E=08\end{array}$ & $\begin{array}{l}200 \\
200 \\
200 \\
200 \\
200 \\
200 \\
200 \\
200 \\
200 \\
200 \\
200 \\
200 \\
200 \\
200 \\
200\end{array}$ \\
\hline
\end{tabular}

AZIMUTH HEJGHT EXPOSURE EAT FU/G DISTANCE DEGREES METERS GMASEC/CU,M SER/CII, IISR.M METERS $\times 1 O E+6$

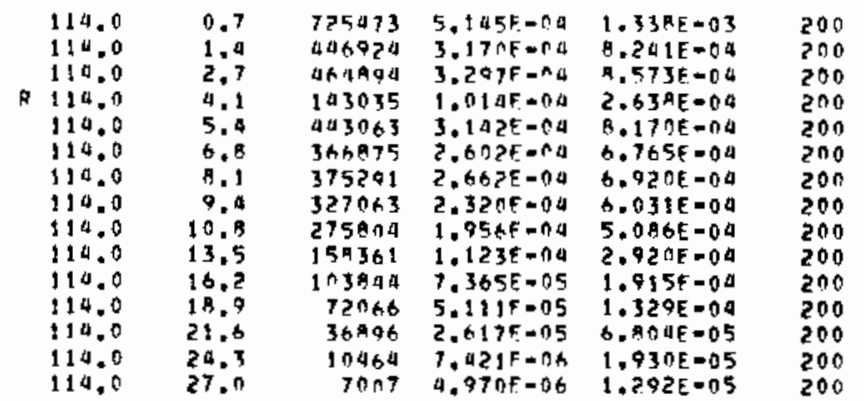

AZIMIITH HEZGHT EXPNGURE E/A FU/O DISTANEE DEGRFFS METEHS GM-SEC/CU.M SEC/CH.N IISH.N METERS

\begin{tabular}{|c|c|c|c|c|c|}
\hline $\begin{array}{l}122.0 \\
122.0 \\
122.0 \\
122.0 \\
122.0 \\
122.0 \\
122.0 \\
122.0 \\
122.0 \\
122.0 \\
122.0 \\
122.0 \\
122.0 \\
122.0 \\
122.0\end{array}$ & $\begin{array}{r}0.7 \\
1.4 \\
2.7 \\
4.1 \\
5.4 \\
6.8 \\
8.1 \\
4.7 \\
10.7 \\
13.5 \\
16.2 \\
18.0 \\
21.6 \\
24.3 \\
27.0\end{array}$ & $\begin{array}{r}1257 ? \\
0507 \\
7984 \\
8897 \\
6209 \\
5168 \\
3413 \\
3653 \\
2695 \\
1747 \\
657 \\
721 \\
409 \\
103 \\
99\end{array}$ & $\begin{array}{l}9.917 E=06 \\
6.033 E=06 \\
5.663 E=06 \\
6.310 E=06 \\
4.468 E=06 \\
3.665 E=06 \\
2.421 E=06 \\
2.591 E=06 \\
1.904 E=96 \\
1.239 E=06 \\
4.660 E=07 \\
5.117 E=07 \\
2.9 n 7 E=07 \\
7.315 F=08 \\
3.135 E=08\end{array}$ & $\begin{array}{l}2.31 R E=05 \\
1.569 F=05 \\
1.472 E=05 \\
1.641 E=05 \\
1.162 E=05 \\
9.531 E=06 \\
6.205 E=06 \\
6.738 E=06 \\
0.952 E=06 \\
3.222 E=06 \\
1.211 E=06 \\
1.330 E=06 \\
7.559 E=07 \\
1.902 E=07 \\
1.151 E=08\end{array}$ & $\begin{array}{l}200 \\
200 \\
200 \\
200 \\
200 \\
200 \\
200 \\
200 \\
200 \\
2100 \\
200 \\
200 \\
200 \\
200 \\
200\end{array}$ \\
\hline
\end{tabular}

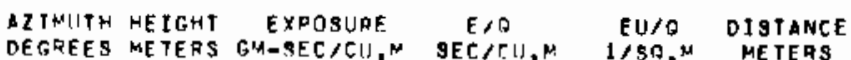
$\times 10 E+6$

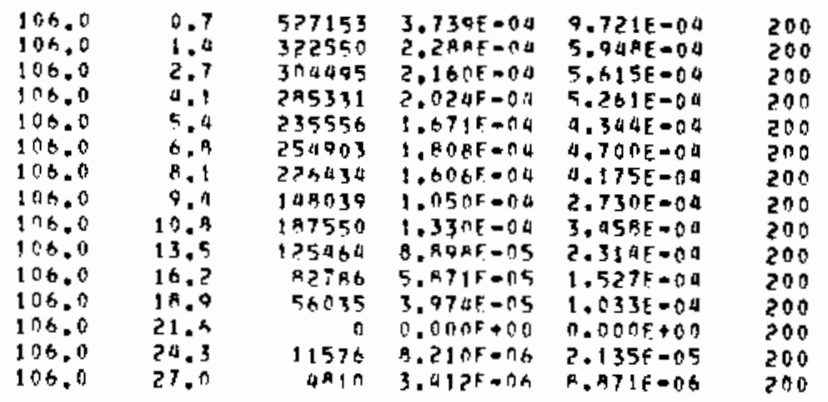




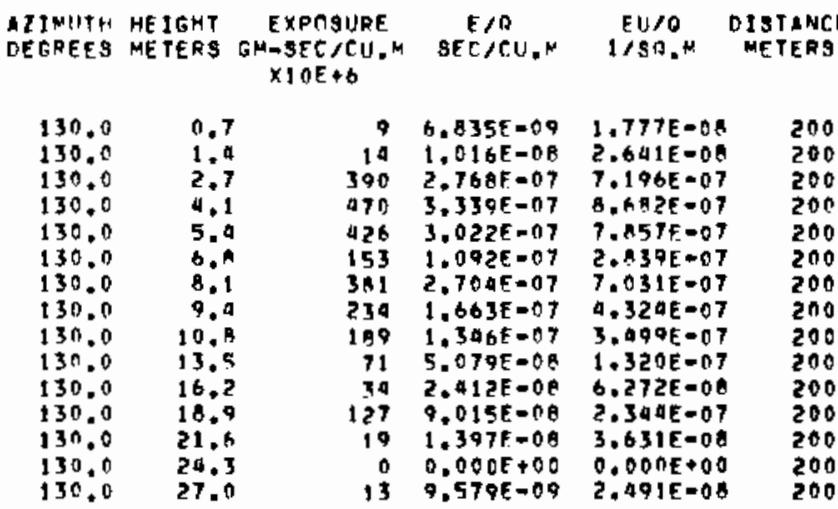

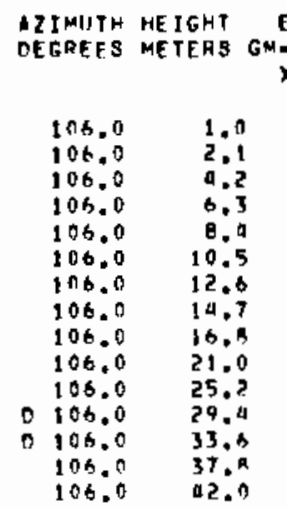

EXPOSUAF

$E / 0$

EUPO OISTANCE $\times 10 \mathrm{E}+6$

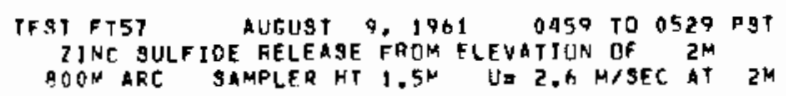

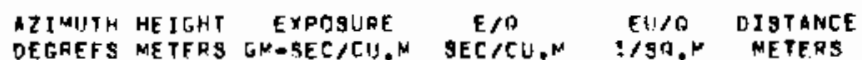

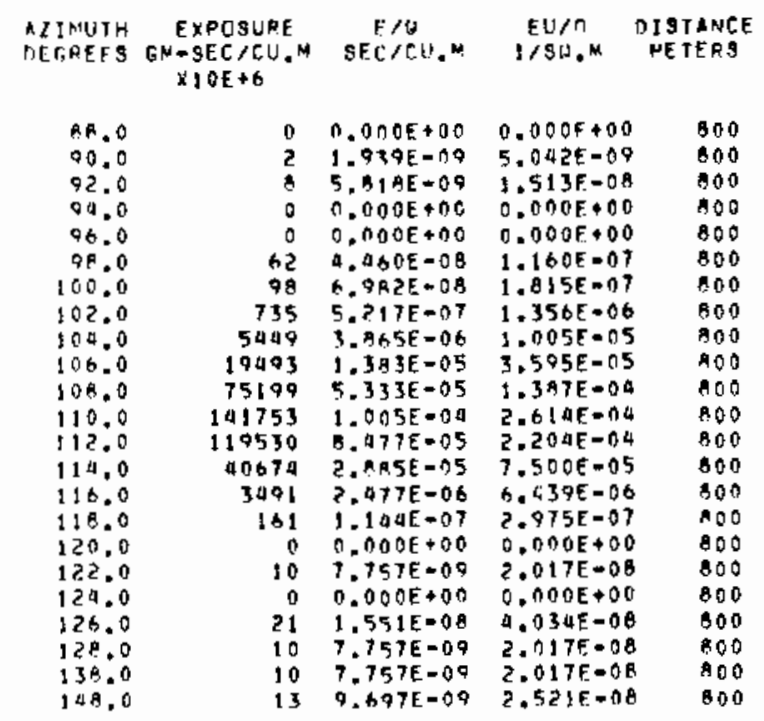

CAOSSAIHD INTEGRATEOE B.058E-03 $2.095 E-02$

TOWER DATA FOLLOW....

TESTFTS7 AUGUST 9,1961 0459 TO O529 OST

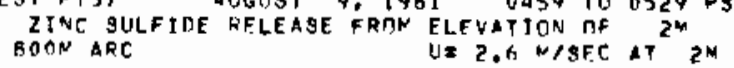

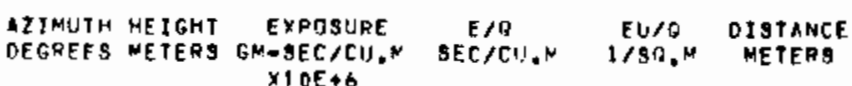

A2JNUTH HE IGHT EXPOSURE

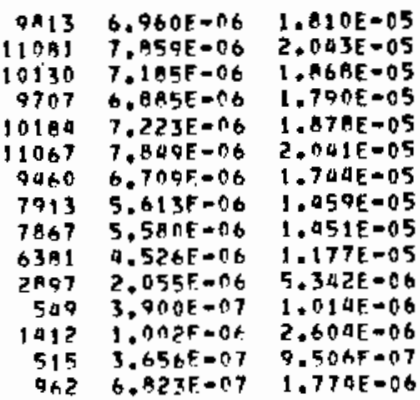

$\$ 00$

000

000

600

100

800

800

800

ค 00

800

A

OEGFEFS METFRS GR-SEC/CIS.M SEC/CU.M I/SO.F METERS

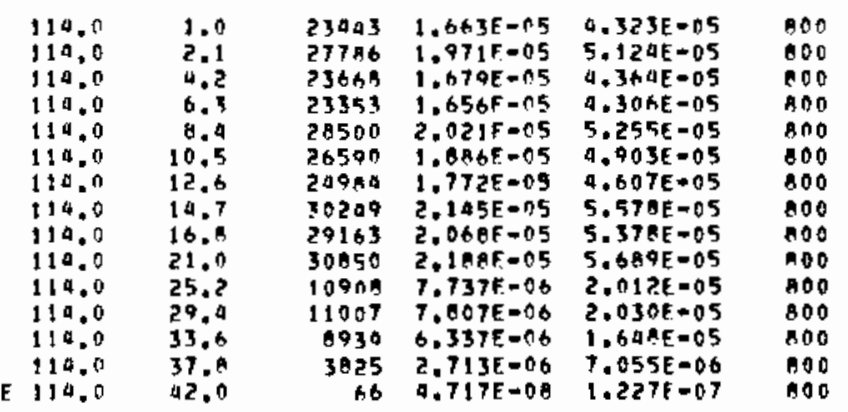

AZIMITH HEIGHT EXPISURE E.O EU/O OISTANCE DEGREES METERS GM-SEC/CU.M SFC/EH.M 1/SO.M METERS

\begin{tabular}{|c|c|c|c|c|c|}
\hline $\begin{array}{l}122.0 \\
122.0 \\
122.0 \\
122.0 \\
122.0 \\
122.0 \\
122.0 \\
122.0 \\
122.0 \\
122.0 \\
122.0 \\
122.0 \\
122.0 \\
122.0 \\
122.0\end{array}$ & $\begin{array}{l}1.9 \\
2.1 \\
4.2 \\
6.3 \\
8.9 \\
10.5 \\
12.8 \\
14.7 \\
16.8 \\
21.0 \\
25.2 \\
29.0 \\
33.8 \\
37.2 \\
42.8\end{array}$ & $\begin{array}{r}5 \\
187 \\
204 \\
161 \\
93 \\
34 \\
131 \\
05 \\
79 \\
57 \\
62 \\
09 \\
4 \\
16 \\
11\end{array}$ & $\begin{array}{l}3.604 E=09 \\
1.191 E=07 \\
1.732 E=07 \\
1.142 E=07 \\
6.632 E=08 \\
2.456 E=08 \\
9.334 F=07 \\
3.193 E=08 \\
5.650 E=08 \\
9.053 F=08 \\
4.465 E=08 \\
3.538 E=0 A \\
3.370 E=08 \\
1.179 F=08 \\
7.582 E=09\end{array}$ & $\begin{array}{l}9.5 A 0 E-09 \\
3.097 E=07 \\
4.502 E=07 \\
2.970 E=07 \\
1.720 E=07 \\
6.386 E=01 \\
2.027 E=07 \\
8.302 E=08 \\
1.469 E-07 \\
1.050 E=07 \\
1.161 E=07 \\
9.199 E=08 \\
8.761 E=09 \\
3.064 E=08 \\
1.071 E=0 A\end{array}$ & $\begin{array}{l}800 \\
800 \\
800 \\
800 \\
100 \\
800 \\
800 \\
800 \\
500 \\
800 \\
100 \\
000 \\
000 \\
800 \\
100\end{array}$ \\
\hline
\end{tabular}

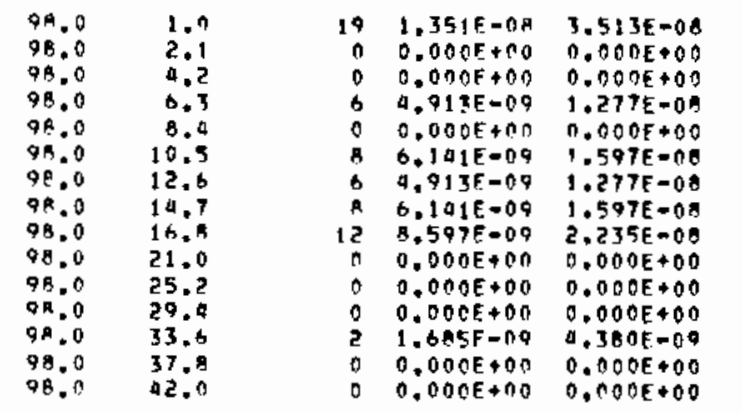

$\begin{array}{lll}800 & 130.0 & 1.0 \\ 800 & 130.0 & 2.1 \\ 800 & 130.0 & 9.2 \\ 000 & 130.0 & 6.3 \\ 800 & 130.0 & 0.0 \\ 000 & 130.0 & 10.5 \\ 100 & 130.0 & 12.6 \\ 000 & 130.0 & 19.7 \\ 100 & 130.0 & 21.0 \\ 000 & 130.0 & 25.2 \\ 800 & 130.0 & 29.4 \\ 100 & 130.0 & 33.6 \\ 100 & 130.0 & 37.9 \\ 800 & 130.0 & 42.0 \\ 000 & & \end{array}$
$\times 10 E+6$ 


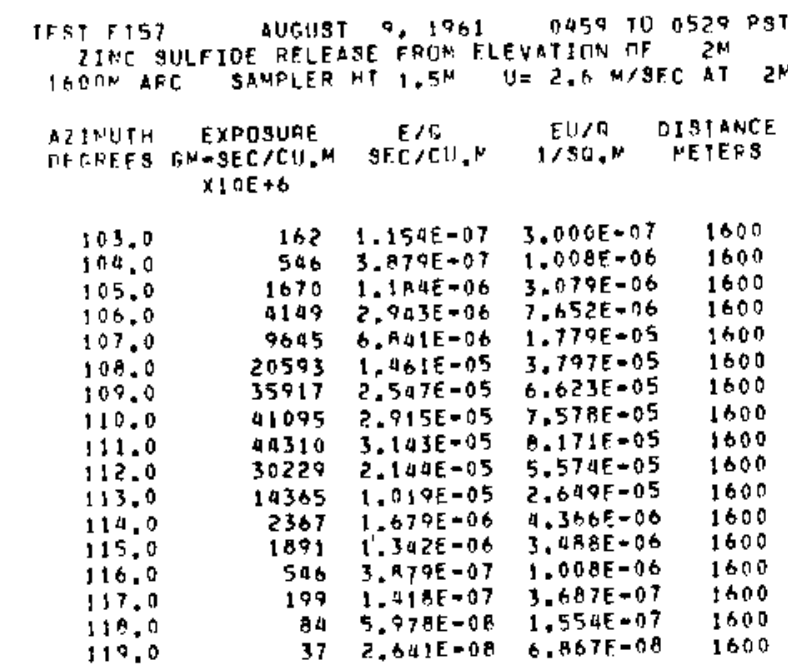

CRM95WIND INTEGRATEDA A.116E-03 1.07 OE-02

TONER DATA FOLLCW....

TEST FTST SUSUST 9,1961 OA59 TO O529 PST

IINC SLLFIDE RELEASE FRON FLEVATION OF ZM

$1600 \mathrm{~N} A \mathrm{KC} \quad U=2.6 \mathrm{~N} / \mathrm{SEC} A T^{2} 2^{M}$

\begin{tabular}{|c|c|c|c|c|c|}
\hline $\begin{array}{l}\text { AZTNHTH } \\
\text { DEGQEES }\end{array}$ & $\begin{array}{l}\text { HEIGH T } \\
\text { METERS }\end{array}$ & $\begin{array}{c}\text { EXPOSURE } \\
\text { GM-SEC } / C 1]+M \\
\times 10 E+6\end{array}$ & $\begin{array}{c}E / D \\
\text { SEC } / C \cup, N\end{array}$ & $\begin{array}{c}E U / C \\
1 / 3 A_{0} M\end{array}$ & $\begin{array}{l}\text { ISTANCE } \\
\text { METERS }\end{array}$ \\
\hline $\begin{array}{l}106.0 \\
106.0 \\
106.0 \\
106.0 \\
106.0 \\
106.0 \\
106.0 \\
106.0 \\
106.0 \\
106.0 \\
106.0 \\
106.0 \\
106.0 \\
106.0 \\
106.0\end{array}$ & $\begin{array}{l}1.5 \\
3.1 \\
6.2 \\
9.3 \\
12.0 \\
15.5 \\
18.6 \\
21.2 \\
24.8 \\
31.0 \\
37.2 \\
43.0 \\
49.6 \\
55.8 \\
62.0\end{array}$ & $\begin{array}{r}2220 \\
2401 \\
502 \\
2411 \\
2102 \\
1618 \\
503 \\
1339 \\
955 \\
550 \\
164 \\
73 \\
28 \\
12 \\
5\end{array}$ & $\begin{array}{l}1.575 E=06 \\
1.703 E=06 \\
3.846 E=07 \\
1.710 E=06 \\
1.491 E=06 \\
1.198 E-06 \\
9.149 E=07 \\
9.501 E=07 \\
6.760 E=07 \\
3.904 E=07 \\
1.1635=07 \\
5.210 E=08 \\
2.047 E=00 \\
9.100 E=09 \\
9.095 E=09\end{array}$ & $\begin{array}{l}0.094 E=06 \\
4.429 E=06 \\
9.990 E=07 \\
0.449 E=06 \\
3.477 E=06 \\
2.984 E=06 \\
1.077 E=06 \\
2.475 E=06 \\
1.763 E=06 \\
1.015 E=06 \\
3.025 E=07 \\
1.355 E=07 \\
5.323 E=08 \\
2.366 E-08 \\
1.065 E-08\end{array}$ & $\begin{array}{l}1600 \\
1600 \\
1600 \\
1600 \\
1600 \\
1600 \\
1600 \\
1600 \\
1600 \\
1600 \\
1600 \\
1600 \\
1600 \\
1600 \\
1600\end{array}$ \\
\hline
\end{tabular}

\begin{tabular}{|c|c|c|c|c|c|}
\hline $\begin{array}{l}\text { DISMUTH } \\
\text { DEGREES }\end{array}$ & $\begin{array}{l}\text { HE IGHT } \\
\text { NETERS }\end{array}$ & $\begin{array}{c}\text { EXPOSUAE } \\
G \mathrm{M}-\mathrm{SEC} / \mathrm{CU}, \mathrm{M} \\
\times 10 E+6\end{array}$ & $\begin{array}{c}E / 0 \\
\mathrm{SEC} / \mathrm{CU}, \mathrm{M}\end{array}$ & $\begin{array}{c}E U / B \\
1 / 30 . N\end{array}$ & $\begin{array}{l}\text { OISTANCE } \\
\text { METERS }\end{array}$ \\
\hline 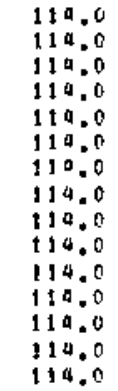 & $\begin{array}{r}1.5 \\
3.1 \\
6.2 \\
9.3 \\
12.4 \\
15.5 \\
18.6 \\
21.9 \\
24.8 \\
31.0 \\
37.2 \\
43.9 \\
49.6 \\
55.8 \\
62.0\end{array}$ & $\begin{array}{l}4165 \\
9215 \\
5820 \\
1596 \\
6397 \\
7725 \\
3120 \\
3215 \\
5609 \\
3751 \\
9499 \\
4200 \\
2015 \\
941 \\
517\end{array}$ & 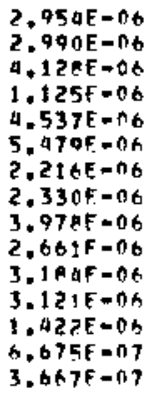 & $\begin{array}{l}7.662 E=06 \\
7.774 E=06 \\
1.073 E=05 \\
2.925 E=06 \\
1.180 F=05 \\
1.425 E=05 \\
5.761 E=06 \\
6.05 A E=06 \\
1.034 E=05 \\
6.91 A E=06 \\
1.27 A E=06 \\
8.114 E=06 \\
3.69 A E=06 \\
1.735 E=06 \\
9.535 E=07\end{array}$ & $\begin{array}{l}1600 \\
1600 \\
1600 \\
1600 \\
1600 \\
1600 \\
1600 \\
1600 \\
1600 \\
1600 \\
1600 \\
1600 \\
1600 \\
1600 \\
1600\end{array}$ \\
\hline
\end{tabular}

\begin{tabular}{|c|c|c|c|c|c|}
\hline $\begin{array}{l}\text { AIIHUTH } \\
\text { DE GREFS }\end{array}$ & $\begin{array}{l}\text { HE I GHT } \\
\text { ME TERS }\end{array}$ & $\begin{array}{c}\text { EXPOSURE } \\
\text { GM-SEC/C(1.N } \\
\times 1 O E+G\end{array}$ & $\begin{array}{c}E / C_{i} \\
S E E / C U, H\end{array}$ & $\begin{array}{c}E U / O \\
1 / S O . M\end{array}$ & $\begin{array}{l}\text { DI ST ANCE } \\
\text { METFRS }\end{array}$ \\
\hline $\begin{array}{l}122.0 \\
122.0 \\
122.0 \\
122.0 \\
122.0 \\
122.0 \\
122.0 \\
122.0 \\
12200 \\
122.0 \\
122.0 \\
122.0 \\
122.0 \\
122.0 \\
122.0\end{array}$ & $\begin{array}{r}1.5 \\
3.1 \\
6.2 \\
9.3 \\
12.0 \\
15.5 \\
18.6 \\
21.7 \\
24.8 \\
31.0 \\
37.2 \\
43.0 \\
49.6 \\
55.0 \\
62.0\end{array}$ & $\begin{array}{r}0 \\
18 \\
42 \\
26 \\
47 \\
20 \\
92 \\
66 \\
53 \\
28 \\
36 \\
5 \\
5 \\
10 \\
3\end{array}$ & $\begin{array}{l}0.000 F+00 \\
1.279 F=08 \\
3.049 F=0 A \\
1.069 E=08 \\
3.34 a F=0 A \\
1.475 E=08 \\
6.589 E=0 A \\
4.721 E=08 \\
3.783 E=0 B \\
1.90 P E=08 \\
2.569 E=08 \\
3.56 R E=09 \\
3.640 F=09 \\
7.260 E=09 \\
2.275 F=09\end{array}$ & $\begin{array}{l}0.000 E+00 \\
3.329 E=08 \\
7.927 E=00 \\
4.050 E=08 \\
8.690 E=00 \\
3.036 E=00 \\
1.713 E=07 \\
1.227 E=07 \\
9.835 E=00 \\
5.196 E=08 \\
6.680 E-08 \\
9.27 A E-09 \\
9.064 E-09 \\
1.093 E-08 \\
5.915 E=00\end{array}$ & $\begin{array}{l}1600 \\
1600 \\
1600 \\
1600 \\
1600 \\
1600 \\
1600 \\
1600 \\
1600 \\
1600 \\
1600 \\
1600 \\
1600 \\
1600 \\
1600\end{array}$ \\
\hline
\end{tabular}
TFST FIST AUGHST 0,1961 O459 PO 0529 PST 7 INC SULFIDE RELEASE FRIMM ELEVATIUN OF ?M $3200 \mathrm{H}$ ARC SAMPLER HT $1,5 \mathrm{~N} U=2,6 \mathrm{M} / \mathrm{SEC}$ \& $2^{M}$

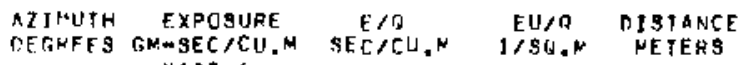
$\times 10 \mathrm{E}+6$

\begin{tabular}{|c|c|c|c|c|}
\hline $\begin{array}{l}101.0 \\
102.0 \\
103.0 \\
104.0 \\
105.0 \\
106.0 \\
107.0 \\
108.0 \\
109.0 \\
119.0 \\
111.0 \\
112.0 \\
113.0 \\
11140 \\
115.0 \\
116.0 \\
117.0 \\
118.0 \\
119.0 \\
120.0\end{array}$ & $\begin{array}{r}0 \\
37 \\
88 \\
300 \\
482 \\
1519 \\
3623 \\
5293 \\
6018 \\
8411 \\
7824 \\
5165 \\
1815 \\
267 \\
90 \\
0 \\
0 \\
0 \\
0 \\
1\end{array}$ & $\begin{array}{l}0.000 E+00 \\
2.685 E-00 \\
6.266 E-08 \\
2.115 E-07 \\
3.022 E=07 \\
1.074 E=06 \\
2.570 E=06 \\
3.754 E-06 \\
5.6 A 7 E=06 \\
5.966 E-06 \\
5.549 E=06 \\
3.663 E=06 \\
1.2 A A E=06 \\
1.900 E=07 \\
6.404 E=0 A \\
0.000 E+00 \\
0.000 E+00 \\
0.000 E+00 \\
0.000 E+00 \\
1.377 E=09\end{array}$ & $\begin{array}{l}0.05,0 E+00 \\
6.982 E=00 \\
1.629 E=07 \\
5.550 E=07 \\
3.898 F=07 \\
2.793 E=06 \\
6.681 E=06 \\
9.761 E=06 \\
1.479 E=05 \\
1.551 E=05 \\
1.443 E=05 \\
9.524 E=06 \\
3.308 E=06 \\
4.941 E=07 \\
1.665 E=07 \\
0.000 E+00 \\
0.000 E+00 \\
0.000 E+00 \\
0.000 E+00 \\
3.501 E=00\end{array}$ & $\begin{array}{l}3200 \\
3200 \\
3200 \\
3200 \\
3200 \\
3200 \\
3200 \\
3700 \\
3200 \\
3200 \\
3200 \\
3200 \\
3200 \\
3200 \\
3200 \\
3200 \\
3200 \\
3200 \\
3200 \\
3200\end{array}$ \\
\hline
\end{tabular}

CROSSUIND INTEGRATED= $1.701 E=03$ 4.422E-03

$$
\text { SEC/SG. }
$$

TOHER OATA FOLLOW...

TEST FT5T AUGUST 9,1961 OA59 TO 0529 PST ZINC SULFIDE RELERSE FRTM ELEVATION TF $2 \mathrm{M}$ $3200 M$ ARC WO 20 W/SEC AT $2 M$

AZIMUTH HEIGHT EXPOSURE E/R EU/O DISTANCE DEGREES METERS GM-SEC/CII, SEC/CU.M 1/30.N METERS

\begin{tabular}{|c|c|c|c|c|c|}
\hline $\begin{array}{l}106.0 \\
106.0 \\
106.0 \\
106.0 \\
106.0 \\
106.0 \\
106.0 \\
106.0 \\
106.0 \\
106.0 \\
106.0 \\
106.0 \\
106.0 \\
106.0 \\
106.0\end{array}$ & $\begin{array}{l}1.5 \\
3.1 \\
6.2 \\
9.3 \\
12.4 \\
15.5 \\
18.6 \\
21.7 \\
24.6 \\
31.0 \\
37.2 \\
43.4 \\
99.6 \\
55.4 \\
62.0\end{array}$ & $\begin{array}{r}1158 \\
1661 \\
1715 \\
1769 \\
491 \\
1568 \\
1340 \\
1352 \\
767 \\
181 \\
272 \\
22 \\
17 \\
51 \\
3\end{array}$ & $\begin{array}{l}8.218 E=07 \\
1.178 E=06 \\
1.217 E=06 \\
1.255 E=06 \\
3.400 E=07 \\
1.113 E=06 \\
9.508 E=07 \\
9.595 E=07 \\
5.406 E=07 \\
1.287 E=07 \\
1.933 E=07 \\
1.579 E=08 \\
1.225 E=08 \\
3.671 F=08 \\
2,161 E=09\end{array}$ & $\begin{array}{l}2.137 E=06 \\
3.063 E=06 \\
3.169 E=06 \\
3.263 E=06 \\
9.068 E=07 \\
2.093 E=06 \\
2.472 E=06 \\
2.045 E=06 \\
1.016 E=06 \\
3.347 E=07 \\
5.027 E=07 \\
4.106 E=08 \\
3.104 E=08 \\
9.553 E=08 \\
5.620 E=09\end{array}$ & $\begin{array}{l}3200 \\
3200 \\
3200 \\
3200 \\
3200 \\
3200 \\
3200 \\
3200 \\
3200 \\
3200 \\
3200 \\
3200 \\
3200 \\
3200 \\
3200\end{array}$ \\
\hline
\end{tabular}




\begin{tabular}{|c|c|c|c|c|c|}
\hline $\begin{array}{l}\text { AZIMUJTH } \\
\text { DEGREES }\end{array}$ & $\begin{array}{l}\text { HE IGHT } \\
\text { WE TERS }\end{array}$ & $\begin{array}{c}\text { EXPOSURE } \\
\text { GN=SEC } / C U, M \\
\times 1 O E+6\end{array}$ & $\begin{array}{c}E / \theta \\
S E C / C U . M\end{array}$ & $\begin{array}{c}E U / 0 \\
1 / \$ Q . M\end{array}$ & $\begin{array}{l}\text { DISTANCE } \\
\text { METERS }\end{array}$ \\
\hline 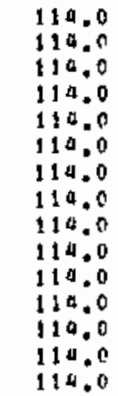 & $\begin{array}{l}1.5 \\
3.1 \\
6.2 \\
9.3 \\
12.5 \\
15.6 \\
21.7 \\
24.5 \\
31.0 \\
37.2 \\
43.2 \\
49.6 \\
55.1 \\
62.0\end{array}$ & $\begin{array}{l}912 \\
557 \\
480 \\
920 \\
1167 \\
1332 \\
1317 \\
400 \\
033 \\
223 \\
2236 \\
2702 \\
1397 \\
1036 \\
1040\end{array}$ & $\begin{array}{l}2.925 E-07 \\
3.9575-07 \\
3.406 E=07 \\
6.553 E-07 \\
8.282 E-07 \\
9.406 F-07 \\
9.303 E-07 \\
2.037 E-07 \\
5.915 E-07 \\
1.584 F-07 \\
1.586 F-06 \\
1.945 E-06 \\
1.306 F-06 \\
7.340 F=07 \\
7.522 E-07\end{array}$ & $\begin{array}{l}7.605 E=07 \\
1.029 E=06 \\
8.855 E=07 \\
1.704 E=06 \\
2.153 E=06 \\
2.457 E=06 \\
2.429 E-06 \\
7.376 E=07 \\
1.53 R E=06 \\
4.11 R E=07 \\
9.123 E=06 \\
5.050 E=06 \\
3.099 E=06 \\
1.911 E=06 \\
1.95 A E=06\end{array}$ & $\begin{array}{l}3200 \\
3200 \\
3200 \\
3200 \\
3200 \\
3200 \\
3200 \\
3200 \\
3200 \\
3200 \\
3200 \\
3200 \\
3200 \\
3200 \\
3200\end{array}$ \\
\hline
\end{tabular}

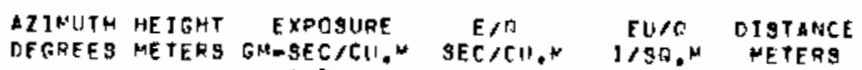
$\times 10 E+6$
}

\begin{tabular}{|c|c|c|c|c|c|}
\hline $\begin{array}{l}122.0 \\
122.0 \\
122.0 \\
122.0 \\
122.0 \\
122.0 \\
122.0 \\
122.0 \\
122.0 \\
122.0 \\
122.0 \\
122.0 \\
122.0 \\
122.0 \\
122.0\end{array}$ & $\begin{array}{r}1.5 \\
3.1 \\
6.2 \\
9.3 \\
12.0 \\
15.5 \\
18.6 \\
21.7 \\
24.8 \\
31.0 \\
37.2 \\
43.4 \\
49.6 \\
55.8 \\
62.0\end{array}$ & $\begin{array}{r}2 \\
2 \\
10 \\
1 \\
14 \\
12 \\
4 \\
13 \\
14 \\
2 \\
1 \\
11 \\
4 \\
13\end{array}$ & $\begin{array}{l}1.75 E F=09 \\
1.758 F=09 \\
7.620 E=09 \\
1.172 E=09 \\
1.055 E=0 A \\
8.782 E=08 \\
2.931 E=09 \\
9.379 E=09 \\
1.053 E=08 \\
1.456 E=09 \\
9.571 E=10 \\
9.135 F=09 \\
2.802 E=09 \\
6.124 E=09 \\
9.726 E=09\end{array}$ & $\begin{array}{l}4.572 E=09 \\
9.572 E=09 \\
1.981 E=08 \\
3.04 A E=09 \\
2.743 E=08 \\
2.286 E=08 \\
7.620 E-09 \\
2.438 E=08 \\
2.737 E=00 \\
3.733 E=09 \\
2.488 E=09 \\
2.115 E=08 \\
7.993 E=08 \\
1.592 E=08 \\
7.529 E-00\end{array}$ & $\begin{array}{l}3200 \\
3200 \\
3200 \\
3200 \\
3200 \\
3200 \\
3200 \\
3200 \\
3200 \\
3200 \\
3200 \\
3200 \\
3200 \\
3200 \\
3200\end{array}$ \\
\hline
\end{tabular}


3O-SERIES EXPERIMENT: 5 A $(E T-5 a j$

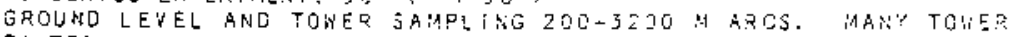

FILTERS HERE RUPTUREL.

TF.T FY58 DECEMAER 5. 1961 0053 TO 100A PST IINC SULFIDE HELEASE FRON ELEVATION OF $2^{N}$
2 OON ARC SANPLER HT $1.5^{\mathrm{N}}$ UE 1 , O N/SEC AT 2M

A.THUTH EXPUSURE E/O EU/D DISTARCE TETHFES GN-SEC/CII.M SECICU.N T/SO.N NETFRS $x \equiv D E+6$

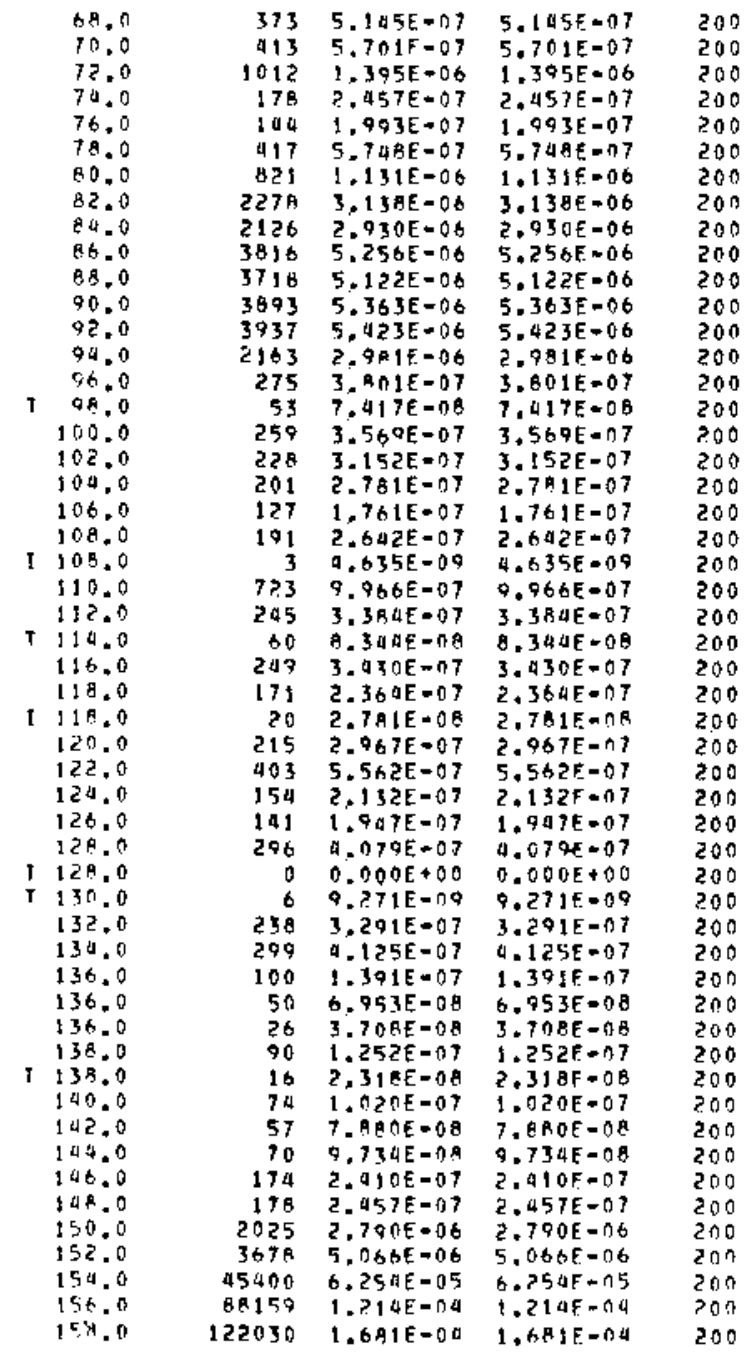

CROSSWIMD IATEGRATEDR $2.803 E-03$ 2.8O3E+ 3
TORER nATA fOLlÓ,...

TFST FT5B DECENBER 5, 1901 0953 TO $100 \mathrm{~B}$ PST ZINC SIILFIDE RELEASE FRON ELFHATICK DF ZN 200 ARC

$U=1.0 \mathrm{~N} / \mathrm{SEC}$ AT $2 \mathrm{~W}$

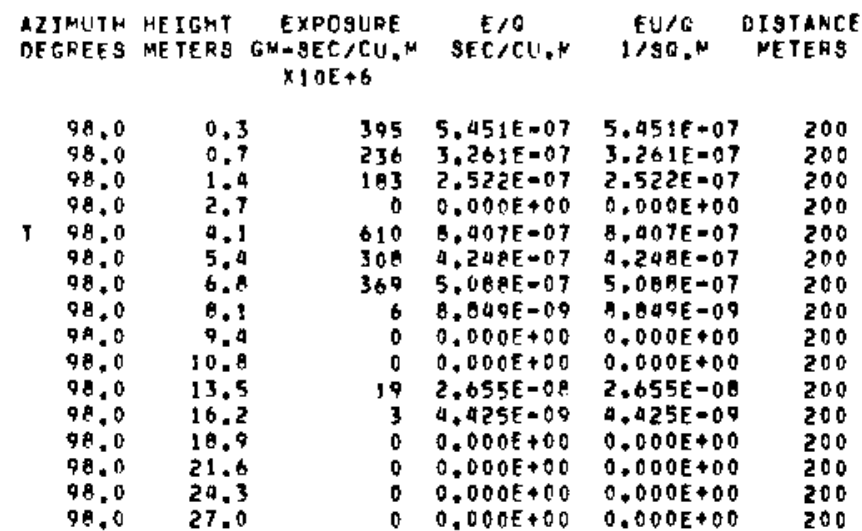

AZIMUTH HEIGHT EXPOSURE E/O EU/O DISTANCE DEGREES METERS GM-SEC/CU.N SEC/CL.N 1 $x \perp 0 E+C$

\begin{tabular}{|c|c|c|c|c|}
\hline $\begin{array}{l}106.0 \\
106.0 \\
106.0 \\
106.0 \\
106.0 \\
106.0 \\
106.0 \\
106.0 \\
106.0 \\
106.0 \\
106.0 \\
100.0 \\
106.0 \\
106.0 \\
106.0 \\
100.0\end{array}$ & $\begin{array}{r}0.3 \\
0.7 \\
1.4 \\
2.7 \\
4.1 \\
5.9 \\
6.1 \\
8.1 \\
9.4 \\
10.4 \\
13.5 \\
16.2 \\
18.9 \\
21.6 \\
24.5 \\
27.0\end{array}$ & $\begin{array}{r}254 \\
293 \\
3 \times 1 \\
0 \\
0 \\
221 \\
221 \\
0 \\
0 \\
0 \\
0 \\
0 \\
0 \\
0 \\
0 \\
0\end{array}$ & $\begin{array}{l}3.500 E=07 \\
9.000 E=07 \\
5.354 E=07 \\
0.000 E+00 \\
0.000 E+00 \\
3.053 E=07 \\
3.053 E=07 \\
1.327 F=00 \\
1.327 E=00 \\
0.000 E+00 \\
0.000 E+00 \\
3.000 F=09 \\
0.000 E+D 0 \\
0.000 E+00 \\
0.000 F+00 \\
0.000 E+00\end{array}$ & $\begin{array}{l}3.50 a E=07 \\
4.040 E=07 \\
5.354 E=07 \\
0.000 E+00 \\
0.000 E+00 \\
3.053 E=07 \\
3.053 E=07 \\
1.327 E=00 \\
1.327 E=00 \\
0.000 E+00 \\
0.000 E+00 \\
0.0 \Delta Q E=09 \\
0.000 E+00 \\
0.0 D O E+00 \\
0.000 E+00 \\
0.000 E+00\end{array}$ \\
\hline
\end{tabular}

\begin{tabular}{|c|c|c|c|c|c|}
\hline $\begin{array}{l}\text { ZINUTH } \\
\text { EGREE } 9\end{array}$ & $\begin{array}{l}\text { ME IGHT } \\
\text { HETERS }\end{array}$ & $\begin{array}{c}\text { EXPOSLRE } \\
\text { GN=SEC }=\text { CLIN } \\
\times 10 F+6\end{array}$ & $\begin{array}{c}F / Q \\
\text { SEC } / C, U, H\end{array}$ & $\begin{array}{l}F U / 0 \\
1 / S R,\end{array}$ & $\begin{array}{l}\text { DI STANCE } \\
\text { NETERS }\end{array}$ \\
\hline $\begin{array}{l}114.0 \\
114.0 \\
114.0 \\
114.0 \\
114.0 \\
119.0 \\
114.0 \\
114.0 \\
114.0 \\
114.0 \\
119.0 \\
119.0 \\
114.0 \\
114.0 \\
114.0 \\
119.0\end{array}$ & $\begin{array}{l}0.3 \\
0.7 \\
1.4 \\
2.7 \\
4.1 \\
5.4 \\
6.8 \\
0.1 \\
9.4 \\
10.8 \\
13.5 \\
16.2 \\
18.9 \\
21.6 \\
24.3 \\
27.0\end{array}$ & $\begin{array}{r}515 \\
002 \\
094 \\
67 \\
0 \\
67 \\
12 \\
0 \\
0 \\
0 \\
0 \\
0 \\
0 \\
0 \\
0 \\
10\end{array}$ & $\begin{array}{l}7.106 E=07 \\
5.544 E-07 \\
1.164 E-00 \\
9.292 E=08 \\
0.000 E+00 \\
9.292 E=08 \\
1.770 E=08 \\
0.000 E+00 \\
0.000 E+00 \\
0.000 E+00 \\
0.000 E+00 \\
0.000 E+00 \\
0.000 E+00 \\
0.000 F+00 \\
0.000 E+00 \\
2.726 E=00\end{array}$ & $\begin{array}{l}7.106 E=07 \\
5.509 E=07 \\
1.109 E=06 \\
9.292 E=08 \\
0.000 E+00 \\
9.292 E=00 \\
1.770 E-08 \\
0.000 E+00 \\
0.000 E+00 \\
0.000 E+00 \\
0.000 E+00 \\
0.000 E+00 \\
0.000 E+00 \\
0.000 E+00 \\
0.000 E+00 \\
2.726 E=00\end{array}$ & $\begin{array}{l}200 \\
200 \\
200 \\
200 \\
200 \\
200 \\
200 \\
200 \\
200 \\
200 \\
200 \\
200 \\
200 \\
200 \\
200 \\
200\end{array}$ \\
\hline
\end{tabular}




\begin{tabular}{|c|c|c|c|c|c|}
\hline $\begin{array}{l}\text { IZIMUTH } \\
\text { DEGREES }\end{array}$ & $\begin{array}{l}\text { HE IGHT } \\
\text { ME TERS }\end{array}$ & $\begin{array}{c}\text { EXPOSURE } \\
G M-\$ E C / C U . M \\
\times 1 O E+b\end{array}$ & $\begin{array}{c}E / 0 \\
\sec / \mathrm{CU}, \mathrm{k}\end{array}$ & $\begin{array}{c}E U / 0 \\
1>30 . N\end{array}$ & $\begin{array}{l}\text { DISTANCE } \\
\text { NETERS }\end{array}$ \\
\hline $\begin{array}{l}122.0 \\
122.0 \\
122.0 \\
122.0 \\
122.0 \\
122.0 \\
122.0 \\
122.0 \\
122.0 \\
122.0 \\
122.0 \\
122.0 \\
122.0 \\
122.0 \\
\$ 22.0 \\
122.0\end{array}$ & $\begin{array}{l}0.3 \\
0.7 \\
1.4 \\
2.7 \\
0.1 \\
5.0 \\
6.8 \\
8.1 \\
9.4 \\
10.8 \\
13.5 \\
16.2 \\
19.9 \\
21.6 \\
24.3 \\
27.0\end{array}$ & $\begin{array}{r}159 \\
201 \\
340 \\
372 \\
0 \\
32 \\
16 \\
35 \\
9 \\
25 \\
6 \\
0 \\
22 \\
3 \\
5 \\
0\end{array}$ & $\begin{array}{l}2.190 E=07 \\
2.770 E=07 \\
4.640 E=07 \\
5.133 E=07 \\
0.000 E+00 \\
0.025 E-08 \\
2.212 E=06 \\
4.867 E=08 \\
1.327 E=08 \\
3.500 F=08 \\
8.800 E=09 \\
0.000 E+00 \\
3.097 E=08 \\
0.425 E=09 \\
7.787 E=09 \\
0.000 F+00\end{array}$ & $\begin{array}{l}2.190 E=07 \\
2.770 E=07 \\
9.690 E=07 \\
5.133 E=07 \\
0.000 E+00 \\
4.025 E=00 \\
2.212 E=08 \\
4.067 E=08 \\
1.327 E=08 \\
3.500 E=0 A \\
0.809 E=00 \\
0.000 E+00 \\
3.097 E=00 \\
1.025 E=09 \\
7.787 E=09 \\
0.000 E+00\end{array}$ & $\begin{array}{l}200 \\
200 \\
200 \\
200 \\
200 \\
200 \\
200 \\
200 \\
200 \\
200 \\
200 \\
200 \\
200 \\
200 \\
200 \\
200\end{array}$ \\
\hline $\begin{array}{l}\text { AZIMUTH } \\
\text { OEGREES }\end{array}$ & $\begin{array}{l}\text { HEIGHT } \\
\text { METERS }\end{array}$ & $\begin{array}{c}\text { EXPQSURE } \\
\text { GHE } \\
\times 10 E+6, W\end{array}$ & $\begin{array}{c}E / 0 \\
\operatorname{seC} / C{ }_{1} N\end{array}$ & $\begin{array}{l}E L / O \\
1 / 30 . N\end{array}$ & $\begin{array}{l}\text { CISTANCE } \\
\text { NETERS }\end{array}$ \\
\hline $\begin{array}{l}130.0 \\
130.0 \\
130.0 \\
130.0 \\
130.0 \\
130.0 \\
130.0 \\
130.0 \\
130.0 \\
130.0 \\
130.0 \\
130.0 \\
130.0 \\
130.0 \\
130.0 \\
130.0\end{array}$ & $\begin{array}{l}0.3 \\
0.7 \\
1.9 \\
2.7 \\
4.1 \\
5.9 \\
6.8 \\
6.1 \\
9.4 \\
10.8 \\
13.5 \\
16.2 \\
18.9 \\
21.6 \\
24.3 \\
27.0\end{array}$ & $\begin{array}{r}190 \\
191 \\
199 \\
120 \\
94 \\
64 \\
9 \\
12 \\
12 \\
0 \\
0 \\
0 \\
6 \\
12 \\
0 \\
0\end{array}$ & $\begin{array}{l}2.620 E=07 \\
1.947 E=07 \\
2.743 E=07 \\
1.770 E=07 \\
6.195 E=08 \\
8.009 E=08 \\
1.327 E=08 \\
1.770 E=08 \\
1.770 E=08 \\
0.000 E+00 \\
0.000 E+00 \\
0.000 E+00 \\
1.009 E=09 \\
1.770 E=08 \\
0.000 E+00 \\
0.000 E+00\end{array}$ & $\begin{array}{l}2.620 E=07 \\
1.947 E=07 \\
2.743 E=07 \\
1.770 E=07 \\
6.195 E=08 \\
6.849 E+08 \\
1.327 E=08 \\
1.77 \text { EE=08 } \\
1.770 E=08 \\
0.000 E+00 \\
0.000 E+00 \\
0.000 E+00 \\
1.049 E=09 \\
1.770 E=00 \\
0.000 E+00 \\
0.000 E+00\end{array}$ & $\begin{array}{l}200 \\
200 \\
200 \\
200 \\
200 \\
200 \\
200 \\
200 \\
200 \\
200 \\
200 \\
200 \\
200 \\
200 \\
200 \\
200\end{array}$ \\
\hline
\end{tabular}

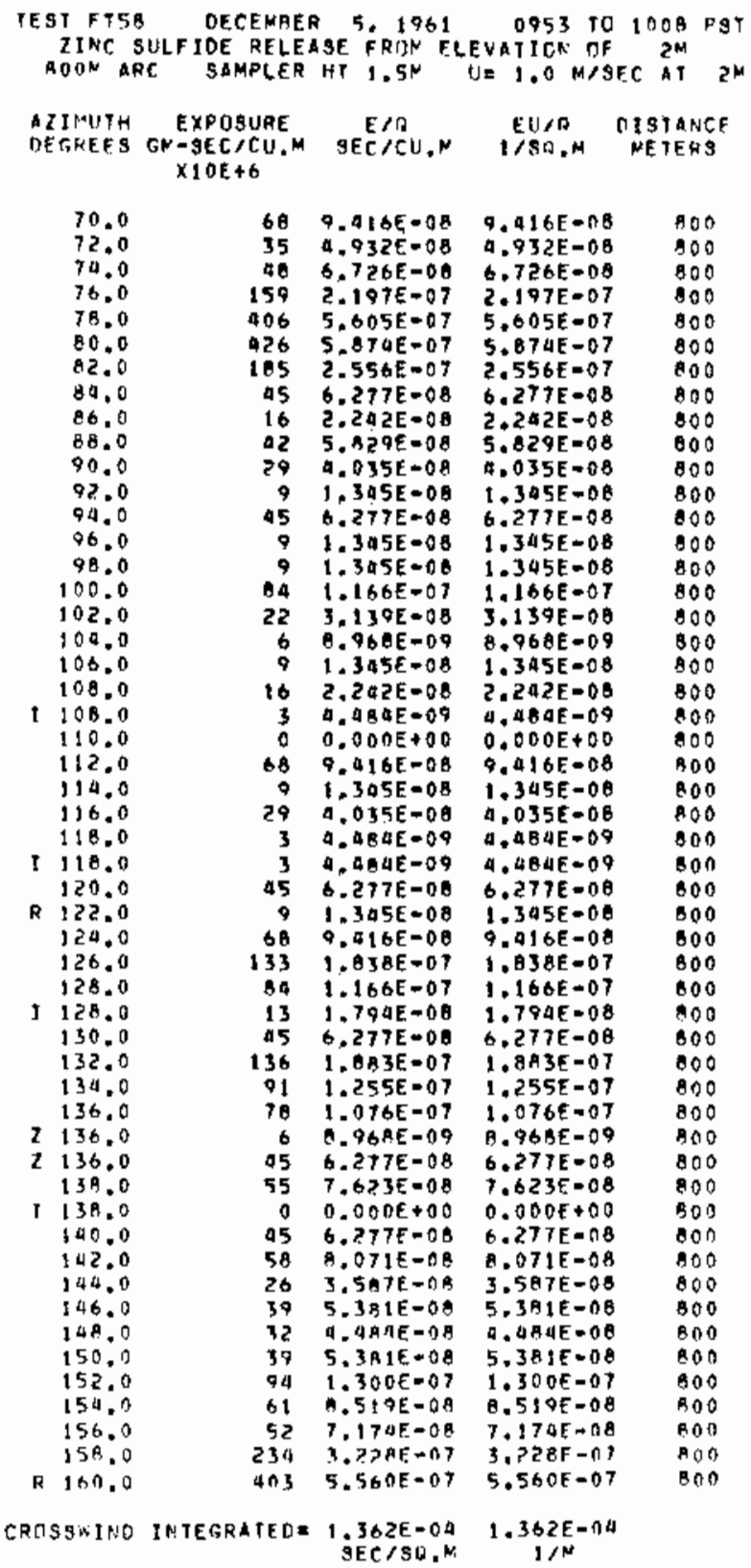


TONER TATA FOLLCK....

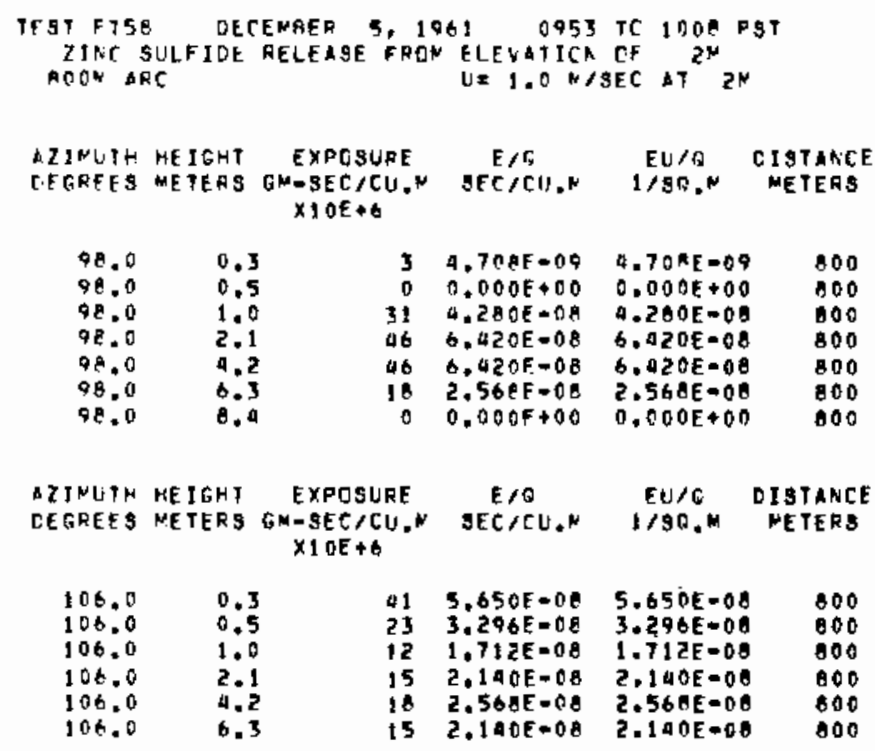

\begin{tabular}{|c|c|c|c|c|c|}
\hline $\begin{array}{l}\text { AIMUTTS } \\
\text { OEGREES }\end{array}$ & $\begin{array}{l}\text { HE IGHT } \\
\text { ME TERS }\end{array}$ & $\begin{array}{l}\text { EXPCSURE } \\
\text { GN-SEC }-C U . N \\
\times 1 O E+6\end{array}$ & $\begin{array}{c}E / R \\
S E C / C U, N\end{array}$ & $\begin{array}{c}E U / 6 \\
1 / \$ 0, N\end{array}$ & $\begin{array}{c}\text { DISIANCE } \\
\text { NETERS }\end{array}$ \\
\hline $\begin{array}{l}114.0 \\
114.0 \\
114.0 \\
114.0 \\
119.0 \\
114.0\end{array}$ & $\begin{array}{l}0 . \\
0 . \\
1 . \\
2 . \\
4 .\end{array}$ & $\begin{array}{r}51 \\
23 \\
49 \\
59 \\
46 \\
0\end{array}$ & $\begin{array}{l}7.0 E P E=0 B \\
3.296 E=0 A \\
6.04 A E=08 \\
6.132 E=08 \\
6.020 E=08 \\
0.000 E+00\end{array}$ & $\begin{array}{l}7.062 E=08 \\
3.296 E=08 \\
6.848 E=08 \\
8.132 E=08 \\
6.420 E=08 \\
0.000 E+00\end{array}$ & $\begin{array}{l}800 \\
800 \\
800 \\
000 \\
900 \\
800\end{array}$ \\
\hline
\end{tabular}

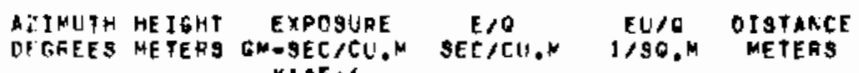
$x>0+6$

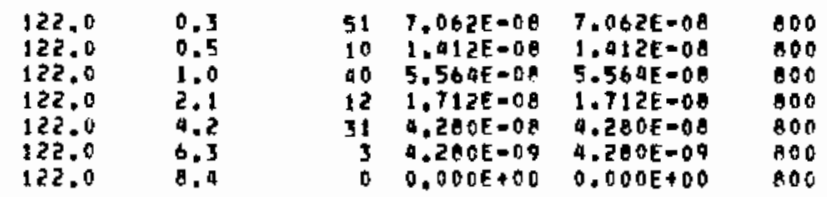

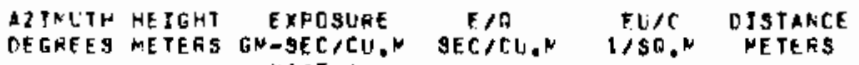
$\times 10 E+6$

\begin{tabular}{|c|c|c|c|c|c|}
\hline $\begin{array}{l}30.0 \\
30.0 \\
30.0 \\
30.0 \\
30.0 \\
30.0\end{array}$ & $\begin{array}{l}0.3 \\
0.5 \\
1.0 \\
2.1 \\
9.2 \\
6.3\end{array}$ & $\begin{array}{l}37 \\
34 \\
21 \\
55 \\
34 \\
49\end{array}$ & $\begin{array}{l}5.179 E=0 B \\
9.700 E=0 E \\
2.996 E=0 E \\
7.704 F=08 \\
4.70 \angle E=08 \\
6.840 E=0 B\end{array}$ & $\begin{array}{l}5.179 E=08 \\
0.70 A E=08 \\
2.496 E=08 \\
7.70 A E=08 \\
0.700 E=00 \\
0.048 E=08\end{array}$ & $\begin{array}{l}000 \\
800 \\
000 \\
000 \\
000 \\
800\end{array}$ \\
\hline
\end{tabular}




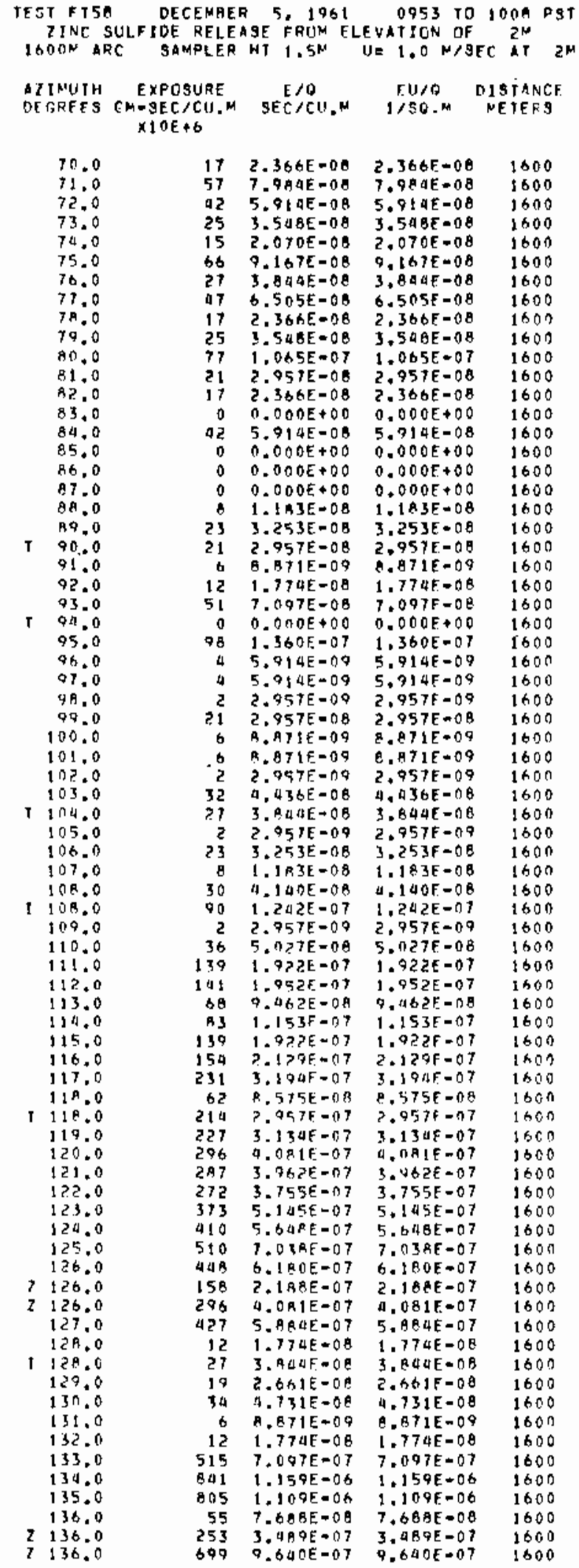

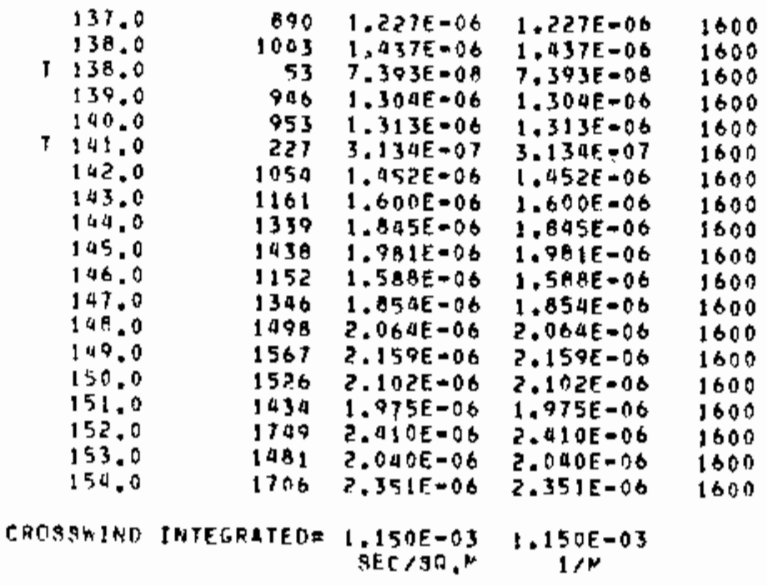

TOMFE CATA FOLLOH....

TEST FTSB DECEMBER 5. 1961 0953 TE 1 10OB PST ZINC SULFIOE RELEISE FROH ELEVATICA OF $2^{\text {mo }}$ I6OON APC $U * 1.0 \mathrm{~N} / \mathrm{BEC} A T^{2 N}$

\begin{tabular}{|c|c|c|c|c|c|}
\hline $\begin{array}{l}\text { AZIMUTH } \\
\text { CEGFEFS }\end{array}$ & $\begin{array}{l}\text { HEIGHT } \\
\text { METERS }\end{array}$ & $\begin{array}{c}\text { EXPOSUAF } \\
\text { GN=SEC }- \text { EU.W } \\
\times 1 O E+6\end{array}$ & $\begin{array}{c}E \neq \theta \\
s \in[/ C U . N\end{array}$ & $\begin{array}{l}E .10 \\
1 / 50 .\end{array}$ & $\begin{array}{l}\text { DISTANCE } \\
\text { NETERS }\end{array}$ \\
\hline $\begin{array}{l}91.0 \\
98.0 \\
98.0 \\
9 \% .0\end{array}$ & $\begin{array}{l}0.2 \\
0.4 \\
0.8 \\
1.5\end{array}$ & $\begin{array}{l}0 \\
20 \\
20 \\
1\end{array}$ & $\begin{array}{l}0.000 E+00 \\
2.790 E=08 \\
2.794 E=0 E \\
1.103 E=08\end{array}$ & $\begin{array}{l}0.000 E+00 \\
2.79 \Delta E=00 \\
2.794 E=08 \\
1.183 E=08\end{array}$ & $\begin{array}{l}1600 \\
1600 \\
1600 \\
1600\end{array}$ \\
\hline
\end{tabular}

\begin{tabular}{|c|c|c|c|c|c|}
\hline $\begin{array}{l}\text { AZINLETH } \\
\text { DERREES }\end{array}$ & $\begin{array}{l}\text { HE IGH } \\
\text { METERS }\end{array}$ & $\begin{array}{c}\text { EXPDSUAE } \\
\text { GN }=9 E C / C U . N \\
X 10 E+6\end{array}$ & $\begin{array}{c}E>0 \\
\operatorname{EC} / \mathrm{CU}, \mathrm{r}\end{array}$ & $\begin{array}{l}E U / 0 \\
1 / 30 . N\end{array}$ & $\begin{array}{c}\text { OISTAMCE } \\
\text { METERS }\end{array}$ \\
\hline $\begin{array}{l}106.0 \\
106.0 \\
106.0 \\
106.0\end{array}$ & $\begin{array}{l}0.2 \\
0.0 \\
0.8 \\
1.5\end{array}$ & $\begin{array}{l}24 \\
22 \\
31 \\
23\end{array}$ & $\begin{array}{l}3.415 F=0 E \\
3.105 E=08 \\
4.3 a Y E=08 \\
3.253 E=08\end{array}$ & $\begin{array}{l}3.415 E=00 \\
3.105 E=00 \\
0.347 E=08 \\
3.253 E=08\end{array}$ & $\begin{array}{l}1600 \\
1600 \\
1600 \\
1600\end{array}$ \\
\hline
\end{tabular}

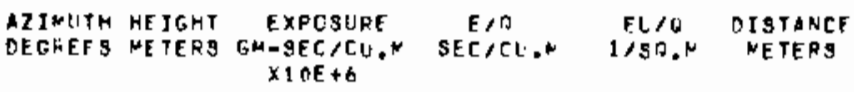

\begin{tabular}{|c|c|c|c|c|c|}
\hline 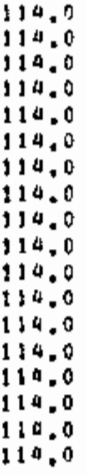 & $\begin{array}{l}0.2 \\
0.0 \\
0.8 \\
1.5 \\
3.1 \\
6.2 \\
9.3 \\
12.0 \\
15.5 \\
16.6 \\
21.7 \\
24.0 \\
31.0 \\
37.2 \\
43.0 \\
49.6 \\
55.8 \\
62.0\end{array}$ & $\begin{array}{l}119 \\
109 \\
106 \\
66 \\
70 \\
57 \\
27 \\
130 \\
105 \\
55 \\
40 \\
17 \\
5 \\
0 \\
11 \\
16 \\
9 \\
12\end{array}$ & $\begin{array}{l}1.64 t E=07 \\
2.329 E-07 \\
2.29 E E=07 \\
9.167 E=08 \\
9.758 E-08 \\
7.984 E=08 \\
3.844 E=08 \\
1.804 E=07 \\
1.444 E=07 \\
7.682 E=0 E \\
5.618 E=08 \\
2.430 E=08 \\
5.40 C E=09 \\
0.000 E+00 \\
1.620 E-08 \\
2.270 E=08 \\
1.261 E-08 \\
1.765 E=08\end{array}$ & $\begin{array}{l}1.60 B E=07 \\
2.320 E=07 \\
2.29 B E=07 \\
9.167 E=0 B \\
9.75 A E=00 \\
7.984 E=0 B \\
3.040 E=08 \\
1.800 E=07 \\
1.409 E=07 \\
7.680 E=08 \\
5.618 E=08 \\
2.030 E=08 \\
5.000 E=09 \\
0.000 E+00 \\
1.620 E=08 \\
2.270 E=0 E \\
1.261 E=00 \\
1.765 E=00\end{array}$ & $\begin{array}{l}1600 \\
1600 \\
1600 \\
1600 \\
1600 \\
1600 \\
1600 \\
1600 \\
1600 \\
1600 \\
1600 \\
1600 \\
1600 \\
1600 \\
1600 \\
1600 \\
1600 \\
1600\end{array}$ \\
\hline
\end{tabular}




\begin{tabular}{|c|c|c|c|c|c|}
\hline $\begin{array}{l}\text { AZINUTH } \\
\text { DEGHEES }\end{array}$ & $\begin{array}{l}\text { HEIGHT } \\
\text { METERS }\end{array}$ & $\begin{array}{c}\text { EXPOSURE } \\
\text { GM }=\text { SEC } / C U \text {.M } \\
\times 1 O E+6\end{array}$ & $\begin{array}{c}E / 0 \\
9 E C / C L, N\end{array}$ & $\begin{array}{l}E U / O \\
1 / S H_{0}\end{array}$ & $\begin{array}{l}\text { OISTANEE } \\
\text { NETERS }\end{array}$ \\
\hline $\begin{array}{l}122.0 \\
122.0 \\
122.0 \\
122.0\end{array}$ & $\begin{array}{l}0.2 \\
0.4 \\
0.8 \\
1.5\end{array}$ & $\begin{array}{l}180 \\
268 \\
281 \\
296\end{array}$ & $\begin{array}{l}2.489 E=07 \\
3.645 E-07 \\
3.881 E=07 \\
4.081 E=07\end{array}$ & $\begin{array}{l}2.084 E-07 \\
3.695 E=07 \\
3.081 E=07 \\
4.001 E-07\end{array}$ & $\begin{array}{l}1600 \\
1600 \\
1600 \\
1600\end{array}$ \\
\hline $\begin{array}{l}\text { AZINUTH } \\
\text { DEGREFS }\end{array}$ & $\begin{array}{l}\text { HE IGHT } \\
\text { ME TEAS }\end{array}$ & $\begin{array}{c}\text { EXPOSURE } \\
\text { GM- }=5 E C / C U . * \\
\times 1 O E+6\end{array}$ & $\begin{array}{c}E, / \delta \\
\operatorname{se} E / C L,\end{array}$ & $\begin{array}{c}F U / 6 \\
1 / S O . N\end{array}$ & $\begin{array}{l}\text { CISTANCE } \\
\text { METERS }\end{array}$ \\
\hline $\begin{array}{l}130.0 \\
130.0 \\
130.0 \\
130.0\end{array}$ & $\begin{array}{l}0.2 \\
0.4 \\
0.1 \\
1.5\end{array}$ & $\begin{array}{r}49 \\
6 \\
0 \\
796\end{array}$ & $\begin{array}{l}6.831 E=08 \\
9.315 E=09 \\
6.210 E=09 \\
1.097 E=06\end{array}$ & $\begin{array}{l}6.831 E=08 \\
9.315 E-09 \\
6.210 E=09 \\
1.097 E-06\end{array}$ & $\begin{array}{l}1600 \\
1600 \\
1600 \\
1600\end{array}$ \\
\hline
\end{tabular}

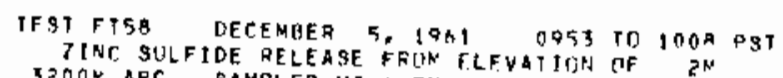

S200 ARC SAMPLER HT $1.5 \mathrm{M}$ UE $1.0 \mathrm{M} / S F C$ AT $2 \mathrm{M}$

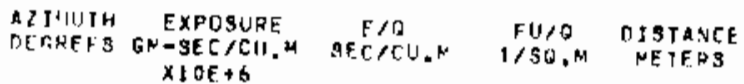

\begin{tabular}{|c|c|c|c|c|}
\hline 73.0 & 10 & $1.40 Q E=08$ & $\begin{array}{l}7.021 E-09 \\
1.00 H E=00\end{array}$ & 3200 \\
\hline 74.0 & 20 & $2.208 E=0$. & $2.808 F=08$ & $\begin{array}{l}3200 \\
3200\end{array}$ \\
\hline 75.0 & 20 & $3.808 \mathrm{E}=O B$ & $2.808 E=08$ & 3200 \\
\hline 76.0 & 35 & $4.915 E=0 B$ & $4.915 E=08$ & 3200 \\
\hline 77.0 & 24 & $3.570 E-08$ & $3.370 \mathrm{~F}=08$ & 3200 \\
\hline 78.0 & 32 & $4.993 \mathrm{E}=\mathrm{OB}$ & $4.493 E=188$ & 3200 \\
\hline 79.0 & 19 & $2.66 K E=00$ & $2.668 \mathrm{E}-08$ & 3200 \\
\hline 80.0 & 23 & $3.230 E=08$ & $3.250 F=08$ & 3200 \\
\hline 81.0 & 14 & $1,966 E=08$ & $1.966 E-08$ & 3200 \\
\hline $\begin{array}{l}82.0 \\
83.0\end{array}$ & 21 & $2.949 E-08$ & $2.949 E=08$ & 3200 \\
\hline 84.0 & $\begin{array}{r}5 \\
14\end{array}$ & $\begin{array}{l}7.071 E=09 \\
1.96 B E=0 A\end{array}$ & $7.021 E=09$ & 3200 \\
\hline 85.0 & 1 & $\begin{array}{l}1.96 B E=O A \\
1.90 \Delta E=0 Q\end{array}$ & $1.9666=08$ & 3200 \\
\hline 86.0 & 3 & $\begin{array}{l}1.40 \Delta E=09 \\
4.212 E=09\end{array}$ & $1.404 E-09$ & 3200 \\
\hline 97.0 & 15 & $\begin{array}{l}a .212 E=09 \\
2.10 b E=08\end{array}$ & $4.212 E-09$ & 3200 \\
\hline$A B .0$ & 12 & $\begin{array}{l}2.10 B E=08 \\
1.6 A 5 E=08\end{array}$ & $2,106 E=08$ & 3200 \\
\hline 89.0 & 10 & $\begin{array}{l}1.6 A 5 E=08 \\
1.4 \cap 4 E-D A\end{array}$ & $\therefore .685 E=08$ & 3200 \\
\hline 30.0 & 0 & $\begin{array}{l}\text { 1. } 4 O A E=0 A \\
0.0 O O E+0 D\end{array}$ & $1.404 E=08$ & 3200 \\
\hline 91.0 & $!$ & $\begin{array}{l}0.000 E+00 \\
1.404 E=09\end{array}$ & $0.000 E+00$ & 3200 \\
\hline 92.0 & 17 & & $1.404 E-09$ & 3200 \\
\hline 93.0 & 5 & $\begin{array}{l}2.367 E=08 \\
7.021 E=09\end{array}$ & & 3200 \\
\hline 94.0 & 6 & $0.425 E=09$ & & 3200 \\
\hline 95.0 & 5 & 1.0215 .09 & $7.021 E-09$ & $\begin{array}{l}3200 \\
3200\end{array}$ \\
\hline 94.0 & $\mathbf{5}$ & $7.071 E=09$ & $7.021 E=09$ & $\begin{array}{l}3200 \\
3200\end{array}$ \\
\hline 97,0 & 18 & $2.527 E-08$ & $2.527 \epsilon=08$ & 3200 \\
\hline 98.0 & 11 & $1.545 E=00$ & $2.545 E-08$ & 3200 \\
\hline 99.0 & 37 & $5.195 E-08$ & $5.195 E-08$ & 3200 \\
\hline 100.0 & 30 & $4.212 E-0 B$ & $4.212 E-0 B$ & 3200 \\
\hline 101.0 & 55 & $7.5 A 2 E=0.8$ & $7.5+2 E=08$ & 3200 \\
\hline 102.0 & 50 & $7.0 \geq 1 E=0 A$ & $7.021 E=00$ & 3200 \\
\hline 103.0 & 67 & $9.2675=08$ & 9.2675 .08 & 3200 \\
\hline 104,0 & 100 & $1.300 E-07$ & $1.390 E-07$ & 3200 \\
\hline 105.0 & 122 & $1.485 E-07$ & $1.685 E-07$ & 3200 \\
\hline 106,0 & 98 & $1.376 E=07$ & $1.376 E=07$ & 3200 \\
\hline 107.0 & 11 & $1.545 E=0 B$ & $1.545 E-08$ & 3200 \\
\hline 100.0 & 6 & ค. $425 E=09$ & $8.425 E-09$ & $3 \geq 00$ \\
\hline 109.0 & $\theta$ & $1.123 \mathrm{E}=0.8$ & $1.123 E-08$ & 3200 \\
\hline & 2 & $O E=09$ & $2.808 E-09$ & 3200 \\
\hline 110.0 & 120 & $1.557 E-07$ & $1.657 E-07$ & 3200 \\
\hline 111.0 & 214 & $? .949 E=07$ & $4 Q E=07$ & 3200 \\
\hline 112.0 & 192 & $2.654 E-07$ & $2.654 \mathrm{E}=07$ & 3200 \\
\hline 113.0 & 304 & $4.269 E-07$ & $4.2695-07$ & 3200 \\
\hline 114.0 & 374 & $5.153 \mathrm{~F}-07$ & $5.153 E-07$ & 3200 \\
\hline $1: 5.0$ & $45 ?$ & 6.7 उ यE $=07$ & $6.2341 E=0.7$ & 3200 \\
\hline 116.0 & 59 & A. $1 \triangle A E=O B$ & $B=144 \mathrm{E}-O B$ & 3200 \\
\hline 117.0 & $3 B 1$ & $5.2525-07$ & $5.252 F-17$ & 3200 \\
\hline 118.0 & 436 & IOE $=07$ & $6.010 E-07$ & 3200 \\
\hline 119.0 & 4 & $5.417 F=09$ & $5.617 F=0.3$ & $3 ? 00$ \\
\hline 119.0 & 638 & A. 7 DOE. $-1,7$ & $F .740,=07$ & 3?.n \\
\hline
\end{tabular}

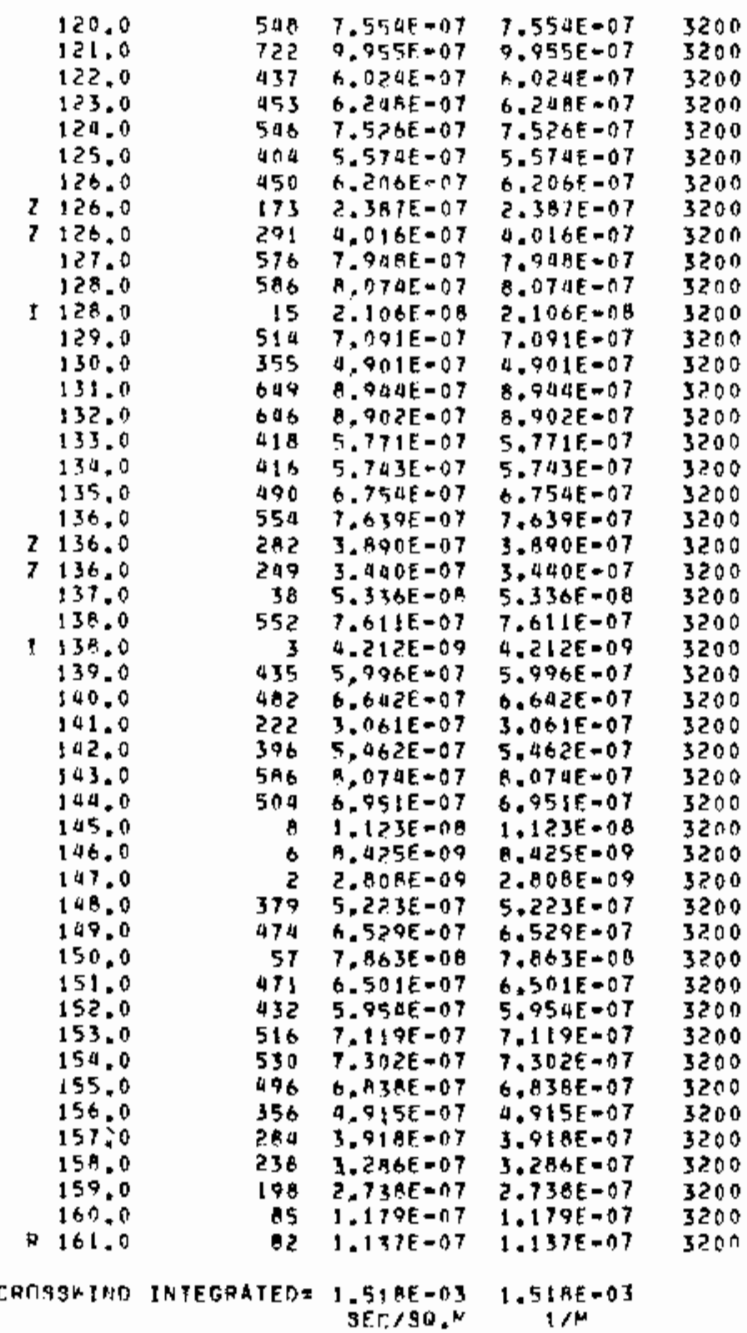

TOUEF CATA FOLLOW....

TEST FT5R DECEMPER 5, $196 \mathrm{t}$ 0953 TO 1008 PGT ZIRC SULFIDE RELFASE FRON ELEVATICN OF $2 M$ 32 OON AFC UE $1.0 \mathrm{~N} / \mathrm{SEC}$ AT $2 \mathrm{M}$

\begin{tabular}{|c|c|c|c|c|c|}
\hline $\begin{array}{l}\text { ZINUTH } \\
\text { EGREES }\end{array}$ & $\begin{array}{l}\text { ME TGHT } \\
\text { ME TEAS }\end{array}$ & $\begin{array}{l}\text { EXPOSURE } \\
\text { GN-SEC } / C U, M \\
\times 1 O E+6\end{array}$ & $\begin{array}{c}E / 0 \\
S E C / C U, N\end{array}$ & $\begin{array}{l}E I / 0 \\
1 / S B_{0}\end{array}$ & $\begin{array}{l}\text { OISTANCE } \\
\text { METERS }\end{array}$ \\
\hline $\begin{array}{l}98.0 \\
98.0 \\
98.0 \\
98.0 \\
98.0 \\
98.0 \\
98.0 \\
98.0 \\
98.0 \\
98.0 \\
98.0 \\
98.0 \\
98.0 \\
98.0 \\
98.0 \\
98.0 \\
98.0 \\
98.0\end{array}$ & $\begin{array}{l}0.2 \\
0.4 \\
0.8 \\
1.5 \\
3.1 \\
6.2 \\
9.3 \\
12.4 \\
15.5 \\
16.6 \\
21.7 \\
24.8 \\
31.0 \\
37.2 \\
43.4 \\
49.6 \\
55.8 \\
62.0\end{array}$ & $\begin{array}{r}7 \\
11 \\
0 \\
13 \\
15 \\
4 \\
4 \\
14 \\
9 \\
15 \\
8 \\
0 \\
0 \\
1 \\
1 \\
0 \\
0 \\
1\end{array}$ & $\begin{array}{l}9.629 E=09 \\
1.545 E=08 \\
0.000 E+00 \\
1.025 E=08 \\
2.100 E=08 \\
5.617 E=09 \\
5.617 E=09 \\
2.527 E=08 \\
1.264 E=08 \\
2.106 E=08 \\
1.123 E=08 \\
0.000 E+00 \\
1.337 E=09 \\
2.675 E=09 \\
2.675 E=09 \\
0.000 E+00 \\
0.000 E+00 \\
2.553 E=09\end{array}$ & $\begin{array}{l}9.829 E=09 \\
1.545 E=00 \\
0.000 E+00 \\
1.825 E=08 \\
2.106 E=08 \\
5.617 E=09 \\
5.617 E=00 \\
2.527 E=08 \\
1.264 E=08 \\
2.106 E=08 \\
1.123 E=08 \\
0.000 E+00 \\
1.337 E=09 \\
2.675 E=09 \\
2.675 E=09 \\
0.000 E+00 \\
0.000 E+00 \\
2.553 E=09\end{array}$ & $\begin{array}{l}3200 \\
3200 \\
3200 \\
3200 \\
3200 \\
3200 \\
3200 \\
3200 \\
3200 \\
3200 \\
3200 \\
3200 \\
3200 \\
3200 \\
3200 \\
3200 \\
3200 \\
3200\end{array}$ \\
\hline
\end{tabular}




\begin{tabular}{|c|c|c|c|c|c|}
\hline $\begin{array}{l}\text { AZINLTH } \\
\text { DEGPEES }\end{array}$ & $\begin{array}{l}\text { ME IGH T } \\
\text { ME TENS }\end{array}$ & $\begin{array}{c}\text { EXPCSURE } \\
\text { GW- } 9 E C / C U \text {. } \\
\times 10 E+6\end{array}$ & $\begin{array}{c}E / \theta \\
B E C / C U, H\end{array}$ & $\begin{array}{l}E \cup / 0 \\
1 / 90 .\end{array}$ & $\begin{array}{l}\text { STANCE } \\
\text { ETEAS }\end{array}$ \\
\hline $\begin{array}{l}106.0 \\
106.0 \\
106.0 \\
106.0 \\
106.0 \\
106.0 \\
106.0 \\
106.0 \\
106.0 \\
106.0 \\
106.0 \\
106.0\end{array}$ & $\begin{array}{l}0.2 \\
0.4 \\
0.8 \\
1.5 \\
3.1 \\
6.2 \\
9.3 \\
12.9 \\
15.5 \\
18.6 \\
21.7 \\
24.8\end{array}$ & $\begin{array}{r}138 \\
88 \\
95 \\
113 \\
138 \\
145 \\
110 \\
120 \\
98 \\
103 \\
113 \\
15\end{array}$ & $\begin{array}{l}1.910 E=07 \\
1.222 E=07 \\
1.320 E=07 \\
1.559 E-07 \\
1.950 E=07 \\
2.008 E=07 \\
1.573 E=07 \\
1.657 E=07 \\
1.362 E=07 \\
1.432 E=07 \\
1.559 f=07 \\
2.140 E=08\end{array}$ & $\begin{array}{l}1.91 \mathrm{AE}=07 \\
1.222 \mathrm{E}=07 \\
1.320 \mathrm{E}=07 \\
1.559 \mathrm{E}=07 \\
1.910 \mathrm{E}=07 \\
2.008 \mathrm{E}=07 \\
1.573 \mathrm{E}=07 \\
1.65 \mathrm{E}=07 \\
1.362 \mathrm{E}=07 \\
1.432 \mathrm{E}=07 \\
1.559 \mathrm{E}=07 \\
2.140 \mathrm{E}=08\end{array}$ & $\begin{array}{l}3200 \\
3200 \\
3200 \\
3200 \\
3200 \\
3200 \\
3200 \\
3200 \\
3200 \\
3200 \\
3200 \\
3200\end{array}$ \\
\hline
\end{tabular}

\begin{tabular}{|c|c|c|c|c|c|}
\hline $\begin{array}{l}\text { AIINUTH } \\
\text { DEGREES }\end{array}$ & $\begin{array}{l}\text { HE IGHT } \\
\text { ME TERS }\end{array}$ & $\begin{array}{c}\text { EXPQSURE } \\
\text { GM-SEC } 1 C U, M \\
\times 10 E+6\end{array}$ & $\begin{array}{c}E / 0 \\
\sec / \mathrm{CU} .\end{array}$ & $\begin{array}{l}F U / C \\
1 / S Q^{\circ}\end{array}$ & $\begin{array}{l}\text { TANCE } \\
\text { TERB }\end{array}$ \\
\hline 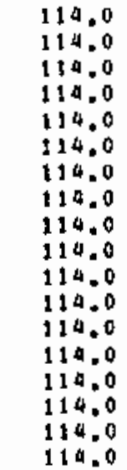 & $\begin{array}{r}0.2 \\
0.0 \\
0.8 \\
1.5 \\
3.1 \\
6.2 \\
9.3 \\
12.0 \\
15.5 \\
18.6 \\
21.7 \\
29.8 \\
31.0 \\
37.2 \\
43.4 \\
49.6 \\
55.0 \\
62.0\end{array}$ & $\begin{array}{r}357 \\
362 \\
366 \\
380 \\
303 \\
945 \\
406 \\
408 \\
342 \\
376 \\
341 \\
293 \\
106 \\
81 \\
0 \\
1 \\
13 \\
0\end{array}$ & $\begin{array}{l}4.929 E=07 \\
4.994 E=07 \\
5.055 E=07 \\
5.23 F E=07 \\
5.200 E=07 \\
6.136 E=07 \\
5.603 E=07 \\
5.631 E=07 \\
4.71 E E=07 \\
5.101 E=07 \\
4.700 E=07 \\
4.034 E=07 \\
2.56 E E=07 \\
1.123 E=07 \\
0.000 E+00 \\
2.553 E=09 \\
1.915 E=08 \\
1.277 E=09\end{array}$ & $\begin{array}{l}4.929 E=07 \\
9.099 E=07 \\
5.055 E=07 \\
5.238 E=07 \\
5.280 E=07 \\
6.136 E=07 \\
5.603 E=07 \\
5.631 E=07 \\
0.718 E=07 \\
5.105 E=07 \\
4.700 E=07 \\
0.039 E=07 \\
2.568 E=07 \\
1.123 E=07 \\
0.000 E+00 \\
2.553 E=09 \\
1.915 E=08 \\
1.277 E=09\end{array}$ & $\begin{array}{l}3200 \\
3200 \\
3200 \\
3200 \\
3200 \\
3200 \\
3200 \\
3200 \\
3200 \\
3200 \\
3200 \\
3200 \\
3200 \\
3200 \\
3200 \\
3200 \\
3200 \\
3200\end{array}$ \\
\hline
\end{tabular}

\begin{tabular}{|c|c|c|c|c|c|}
\hline $\begin{array}{l}\text { AZINUTH } \\
\text { OEGREES }\end{array}$ & $\begin{array}{l}\text { HE IGHT } \\
\text { WE TERS }\end{array}$ & $\begin{array}{c}\text { EXPQSURE } \\
\text { GW }=3 E C / C U . \\
\times 10 E+6\end{array}$ & $\begin{array}{c}E / A \\
S E C / C D, N\end{array}$ & $\begin{array}{l}E U / O \\
1 / S B . M\end{array}$ & $\begin{array}{c}\text { DISTANCE } \\
\text { NETERS }\end{array}$ \\
\hline $\begin{array}{l}122.0 \\
122.0 \\
122.0 \\
122.0\end{array}$ & $\begin{array}{l}0.2 \\
0.2 \\
0.8 \\
1.2\end{array}$ & $\begin{array}{l}524 \\
417 \\
603 \\
461\end{array}$ & $\begin{array}{l}7.231 E-07 \\
5.757 E=07 \\
0.315 E=07 \\
6.361 E=07\end{array}$ & $\begin{array}{l}7.23 t E=07 \\
5.757 E=07 \\
8.313 E=07 \\
6.361 E=07\end{array}$ & $\begin{array}{l}3200 \\
3200 \\
3200 \\
3200\end{array}$ \\
\hline
\end{tabular}

\begin{tabular}{|c|c|c|c|c|c|}
\hline $\begin{array}{l}\text { AZINUTH } \\
\text { DE GRFES }\end{array}$ & $\begin{array}{l}\text { HE IGHT } \\
\text { NETERS }\end{array}$ & $\begin{array}{c}\text { EXPQSURF } \\
\text { GN-SEC/CI!.M } \\
\times 1 O E+6\end{array}$ & $\begin{array}{c}E / D \\
S E C / C H, N\end{array}$ & $\begin{array}{l}E U / O \\
1 / 30 . M\end{array}$ & $\begin{array}{l}\text { DISTANCE } \\
\text { METERS }\end{array}$ \\
\hline $\begin{array}{l}130.0 \\
130.0 \\
130.0 \\
130.0\end{array}$ & $\begin{array}{l}0.2 \\
0.4 \\
0.8 \\
1.5\end{array}$ & $\begin{array}{l}506 \\
028 \\
605 \\
476\end{array}$ & $\begin{array}{l}6.974 F=07 \\
5.697 F=07 \\
6.5415=07 \\
6.557 E=07\end{array}$ & $\begin{array}{l}6.979 E=07 \\
5.897 E=07 \\
8.391 E=07 \\
6.557 E=07\end{array}$ & $\begin{array}{l}3200 \\
3200 \\
3200 \\
3200\end{array}$ \\
\hline
\end{tabular}


30-5ERIES EXPERIMENT: 59 (FT-59)

GROUND LEVEL AND TOWER SAMPLING 200-3200 M ARCS

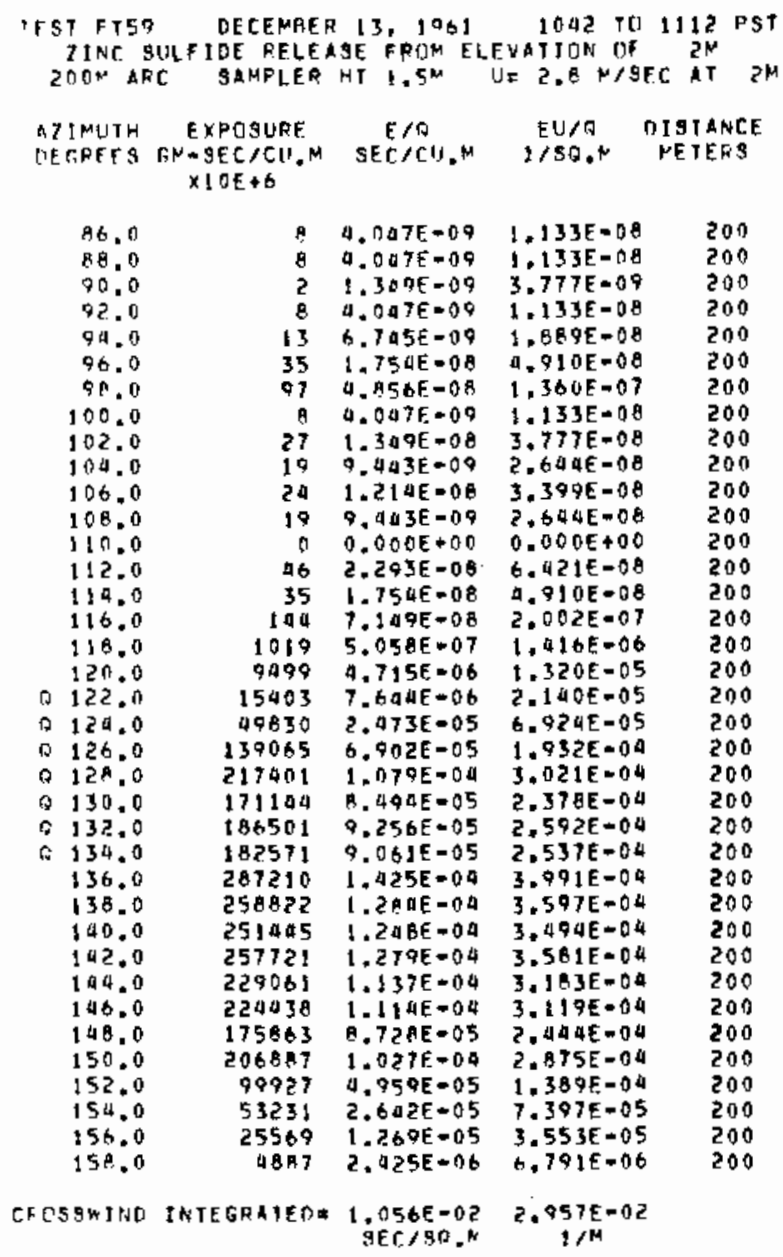

TOWER DATA FOLLOW....
TEST FT59 DECEMBER 13. 1961 10A? TO 1112 PST ZINC SULFIDE RELEASE FAOM ELEVATION DF $2 \mathrm{M}$ 2OOM ARC

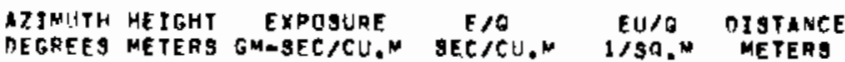
$x]$ OE $\$ 6$

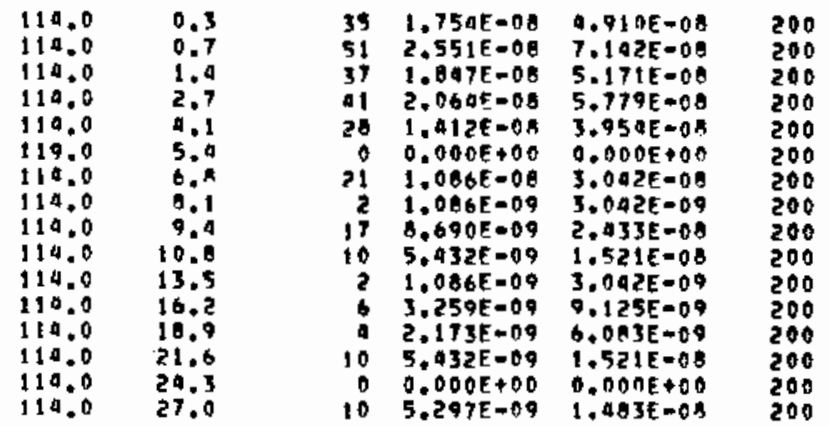

\begin{tabular}{|c|c|c|c|c|c|}
\hline $\begin{array}{l}\text { AZIMUTH } \\
\text { DETPEES }\end{array}$ & $\begin{array}{l}\text { HEIGH } \\
\text { NETERS }\end{array}$ & $\begin{array}{c}\text { EXPOSURE } \\
\text { GM-SEC } C U . M \\
\times 10 E+6\end{array}$ & $\begin{array}{c}E / A \\
\Delta E C / C U, \mu\end{array}$ & $\begin{array}{c}F \cup / O \\
1 / 90 . N\end{array}$ & $\begin{array}{l}\text { ANCE } \\
\text { EAS }\end{array}$ \\
\hline
\end{tabular}

\begin{tabular}{|c|c|c|c|c|c|}
\hline $\begin{array}{l}122.0 \\
122.0 \\
122.0 \\
122.0 \\
122.0 \\
122.0 \\
122.0 \\
122.0 \\
122.0 \\
122.0 \\
122.0 \\
122.0 \\
122.0 \\
122.0 \\
122.0 \\
122.0\end{array}$ & $\begin{array}{l}0.3 \\
0.7 \\
1.4 \\
2.7 \\
0.1 \\
5.4 \\
6.9 \\
8.1 \\
9.4 \\
10.4 \\
13.5 \\
16.2 \\
18.9 \\
21.6 \\
28.3 \\
27.0\end{array}$ & $\begin{array}{r}7943 \\
9569 \\
1632 \\
3571 \\
356 \\
7308 \\
20799 \\
7596 \\
17397 \\
9066 \\
7856 \\
5005 \\
11327 \\
5910 \\
19233 \\
19777\end{array}$ & $\begin{array}{l}3.942 E-06 \\
9.749 E=06 \\
5.104 E=07 \\
1.520 E=06 \\
1.771 E=07 \\
3.647 E=06 \\
1.032 E-05 \\
3.765 E=06 \\
3.634 E=06 \\
4.499 E=06 \\
3.899 E=06 \\
2.504 E-06 \\
5.622 E=06 \\
2.937 E-06 \\
7.064 E-06 \\
7.3345=06\end{array}$ & $\begin{array}{l}1.104 E=05 \\
1.330 E=05 \\
2.269 E=06 \\
4.267 E=06 \\
1.959 E=07 \\
1.021 E=05 \\
2.090 E=05 \\
1.050 E=05 \\
2.418 E=05 \\
1.260 E=05 \\
1.092 E=05 \\
7.011 E=06 \\
1.579 E=05 \\
8.225 E=06 \\
1.975 E=05 \\
2.053 E=05\end{array}$ & $\begin{array}{l}200 \\
200 \\
200 \\
200 \\
200 \\
200 \\
200 \\
200 \\
200 \\
200 \\
200 \\
200 \\
200 \\
200 \\
200 \\
200\end{array}$ \\
\hline
\end{tabular}

DITUTH HEIGHT EXPOSURE E/O EU/A OISTANCE DEGREES METERS GM-SEC/CU.M IEC/CL.M 1/30.M METERS

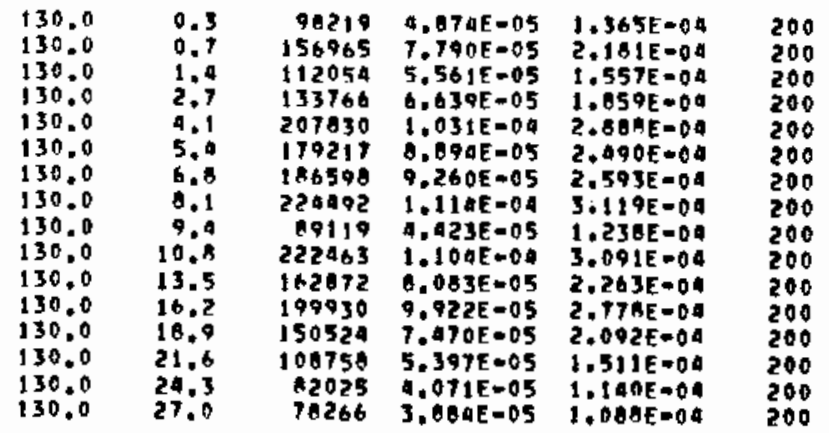




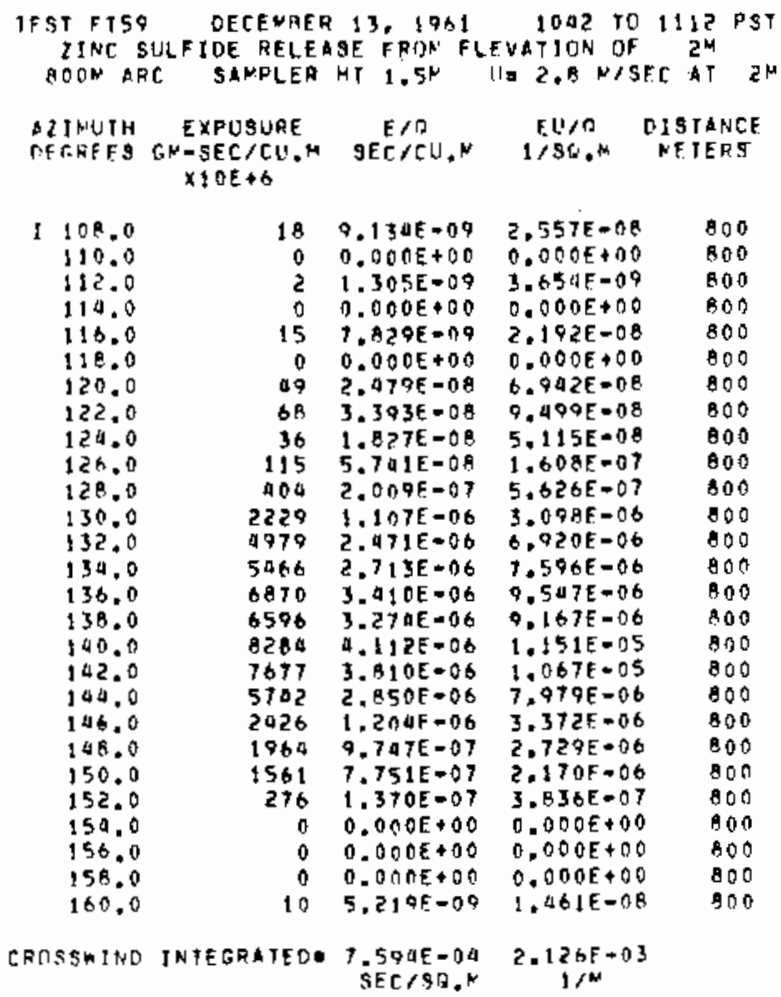

TOWER MATA FRLLDH....

TEST FTS9 DECEMBER 13, 1901 1042 TO 1112 PST 7 INC SULFIDE RELEASE FROM FLEVATIDN OF ZM BOON ARC UE $2 . B N / S E C$ AT $2 M$

\begin{tabular}{|c|c|c|c|c|c|}
\hline $\begin{array}{l}\text { AZINIITH } \\
\text { DEGEEES }\end{array}$ & $\begin{array}{l}\text { HE IGHT } \\
\text { METERS }\end{array}$ & $\begin{array}{c}\text { EXPQSURE } \\
\text { GM-SEC } / C U . M \\
\times 10 E \$ 6\end{array}$ & $\begin{array}{c}E / Q \\
S E C / C U, M\end{array}$ & $\begin{array}{l}F U / 8 \\
1 / 98 . M\end{array}$ & $\begin{array}{l}\text { DISTANEE } \\
\text { METERS }\end{array}$ \\
\hline $\begin{array}{l}122.0 \\
127.0 \\
122.0 \\
122.0 \\
122.0 \\
122.0 \\
122.0 \\
122.0 \\
122.0 \\
122.0 \\
122.0 \\
122.0 \\
122.0 \\
122.0 \\
122.0 \\
122.0 \\
122.0\end{array}$ & $\begin{array}{r}0.3 \\
0.5 \\
1.0 \\
2.1 \\
9.2 \\
0.7 \\
8.0 \\
10.5 \\
12.8 \\
14.7 \\
16.8 \\
21.0 \\
25.2 \\
29.0 \\
33.6 \\
37.8 \\
92.0\end{array}$ & $\begin{array}{r}34 \\
03 \\
4 \\
27 \\
76 \\
14 \\
12 \\
0 \\
21 \\
2 \\
0 \\
6 \\
0 \\
0 \\
14 \\
0\end{array}$ & $\begin{array}{l}1.696 E=00 \\
9.160 E=00 \\
2.102 E=09 \\
1.366 E=08 \\
3.703 E=08 \\
7.356 E=09 \\
6.305 E=09 \\
0.000 E+00 \\
1.051 E=00 \\
1.051 E=09 \\
0.000 E+00 \\
3.152 E=09 \\
4.099 E=09 \\
2.049 F=09 \\
0.099 E=09 \\
7.173 F=09 \\
0.000 F+00\end{array}$ & $\begin{array}{l}9.750 E=00 \\
1.168 E=07 \\
5.985 E=09 \\
3.925 E=08 \\
1.059 E=07 \\
2.067 E=08 \\
1.765 E=08 \\
0.000 E+00 \\
2.942 E=08 \\
2.942 E=09 \\
0.000 E+00 \\
8.027 E=09 \\
1.198 E=08 \\
5.738 E=09 \\
1.14 B E=08 \\
2.000 E=08 \\
0.000 E+00\end{array}$ & $\begin{array}{l}800 \\
000 \\
800 \\
800 \\
800 \\
000 \\
800 \\
500 \\
100 \\
800 \\
800 \\
800 \\
800 \\
800 \\
800 \\
800 \\
000\end{array}$ \\
\hline
\end{tabular}

AZIMUTH HEIGHT EXPOSURE E/O EU/O DISTANCE TEGRES METERS GM-SEC/CU,N SEC/CL,N IISO.N METERS $\times 10 E+6$

\begin{tabular}{|c|c|c|c|c|c|}
\hline $\begin{array}{l}130.0 \\
130.0 \\
130.0 \\
130.0 \\
130.0 \\
130.0 \\
130.0 \\
130.0 \\
130.0 \\
130.0 \\
130.0 \\
130.0 \\
130.0 \\
130.0 \\
130.0 \\
130.0 \\
130.0\end{array}$ & $\begin{array}{r}0.3 \\
0.5 \\
1.0 \\
2.1 \\
4.2 \\
6.3 \\
8.4 \\
10.5 \\
12.6 \\
14.7 \\
16.8 \\
21.0 \\
25.2 \\
29.4 \\
33.4 \\
37.9 \\
42.0\end{array}$ & $\begin{array}{l}1810 \\
1326 \\
671 \\
736 \\
838 \\
912 \\
196 \\
110 \\
910 \\
311 \\
283 \\
692 \\
212 \\
398 \\
489 \\
935 \\
97\end{array}$ & $\begin{array}{l}0.376 F-07 \\
6.585 E-07 \\
3.331 E=07 \\
3.657 E=07 \\
0.161 E=07 \\
2.099 E=07 \\
9.247 E=08 \\
5.469 E=08 \\
2.039 E=07 \\
1.545 E=07 \\
1.408 E-07 \\
3.036 E=07 \\
1.004 E=07 \\
1.978 E-07 \\
2.029 E-07 \\
2.162 E=07 \\
0.016 E=08\end{array}$ & $\begin{array}{l}2.625 E=06 \\
1.044 E=06 \\
9.327 E-07 \\
1.024 E=06 \\
1.165 E=06 \\
5.737 E=07 \\
2.589 E=07 \\
1.530 E=07 \\
5.700 E=07 \\
4.325 E=07 \\
3.903 E=07 \\
9.621 E=07 \\
3.931 E-07 \\
5.338 E=07 \\
6.000 E=07 \\
6.050 E=07 \\
1.349 E=07\end{array}$ & $\begin{array}{l}000 \\
000 \\
000 \\
000 \\
000 \\
000 \\
800 \\
000 \\
000 \\
000 \\
000 \\
100 \\
100 \\
000 \\
800 \\
800 \\
800\end{array}$ \\
\hline
\end{tabular}

\begin{tabular}{|c|c|c|c|c|c|}
\hline $\begin{array}{l}\text { AZTMUTH } \\
\text { OEGREES }\end{array}$ & $\begin{array}{l}\text { HE TGHT } \\
\text { METERS }\end{array}$ & $\begin{array}{l}\text { EXPกSUAE } \\
\text { GM } \triangle A E C>C U, M \\
\times 1 O E+B\end{array}$ & $\begin{array}{c}E / Q \\
S E C / C U_{.}\end{array}$ & $\begin{array}{l}E U / \theta \\
1 / 80 . \mu\end{array}$ & $\begin{array}{l}\text { DISTANCE } \\
\text { WETERS }\end{array}$ \\
\hline 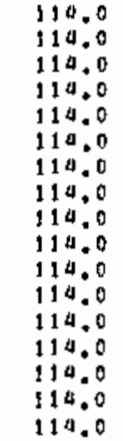 & $\begin{array}{l}0.3 \\
0.5 \\
1.0 \\
2.1 \\
4.2 \\
6.3 \\
8.0 \\
10.5 \\
12.6 \\
14.7 \\
16.9 \\
21.0 \\
25.2 \\
29.4 \\
33.6 \\
37.8 \\
42.0\end{array}$ & $\begin{array}{r}12 \\
21 \\
16 \\
6 \\
0 \\
16 \\
0 \\
0 \\
10 \\
0 \\
0 \\
2 \\
6 \\
0 \\
0 \\
0 \\
0\end{array}$ & $\begin{array}{l}6.160 E=109 \\
1.079 E=00 \\
0.406 E=09 \\
3.152 E=09 \\
0.203 E=09 \\
0.400 E=09 \\
0.000 E+00 \\
0.000 E+00 \\
5.254 E=09 \\
0.000 E+00 \\
0.000 E+00 \\
1.051 E=09 \\
3.070 E=09 \\
0.000 E+00 \\
0.000 E+00 \\
0.000 E+00 \\
0.000 E+00\end{array}$ & $\begin{array}{l}1.727 E=08 \\
3.022 E=08 \\
2.350 E=08 \\
0.827 E=09 \\
1.177 E=08 \\
2.350 E=01 \\
0.000 E+00 \\
0.000 E+00 \\
1.4711 E=09 \\
0.000 E+00 \\
0.000 E+00 \\
2.942 E=09 \\
1.000 E=00 \\
0.000 E+00 \\
0.000 E+00 \\
0.000 E+00 \\
0.000 E+00\end{array}$ & $\begin{array}{l}800 \\
800 \\
100 \\
100 \\
000 \\
000 \\
000 \\
000 \\
000 \\
000 \\
000 \\
000 \\
000 \\
800 \\
500 \\
000 \\
000\end{array}$ \\
\hline
\end{tabular}




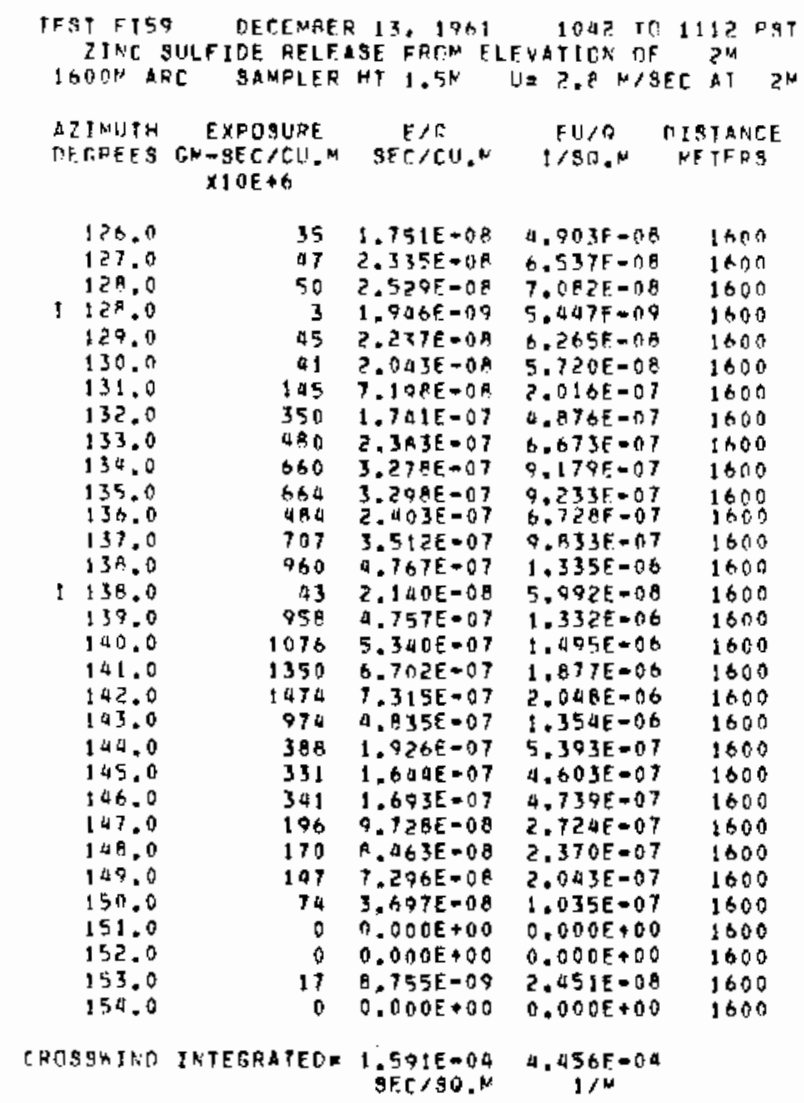

TOWER DATA FILLOW....

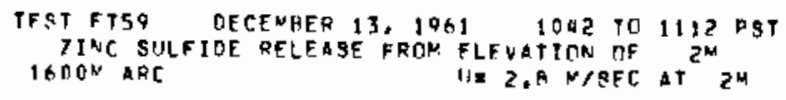

\begin{tabular}{|c|c|c|c|c|c|}
\hline $\begin{array}{l}130.0 \\
130.0 \\
130.0 \\
130.0 \\
130.0 \\
130.0 \\
130.0 \\
30.0 \\
30.0 \\
30.0 \\
130.0 \\
130.0 \\
130.0 \\
130.0 \\
130.0 \\
130.0 \\
130.0 \\
130.0\end{array}$ & $\begin{array}{r}0.2 \\
0.9 \\
0.9 \\
1.5 \\
3.1 \\
6.2 \\
9.3 \\
12.0 \\
15.5 \\
10.6 \\
21.7 \\
24.8 \\
31.0 \\
37.2 \\
43.4 \\
49.4 \\
55.8 \\
62.0\end{array}$ & $\begin{array}{r}66 \\
34 \\
79 \\
17 \\
54 \\
8 ? \\
8 \\
29 \\
52 \\
49 \\
57 \\
17 \\
167 \\
178 \\
70 \\
190 \\
543 \\
138\end{array}$ & $\begin{array}{l}3.303 E=08 \\
1.705 E=08 \\
3.942 E=08 \\
1.97 P E=08 \\
2.695 E=08 \\
3.103 F=08 \\
4.003 E=09 \\
1.225 E=08 \\
2.613 E=08 \\
2.450 E=08 \\
2.850 E=08 \\
9.792 E=09 \\
6.313 E=08 \\
8.872 E=08 \\
3.517 E=08 \\
9.879 E=08 \\
1.750 E=07 \\
6.893 E=0 B\end{array}$ & 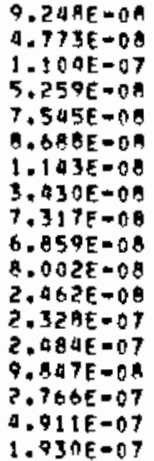 & $\begin{array}{l}1600 \\
1600 \\
1600 \\
1600 \\
1600 \\
1600 \\
1600 \\
1600 \\
1600 \\
1600 \\
1600 \\
1600 \\
1600 \\
1600 \\
1600 \\
1600 \\
1600 \\
1600\end{array}$ \\
\hline
\end{tabular}

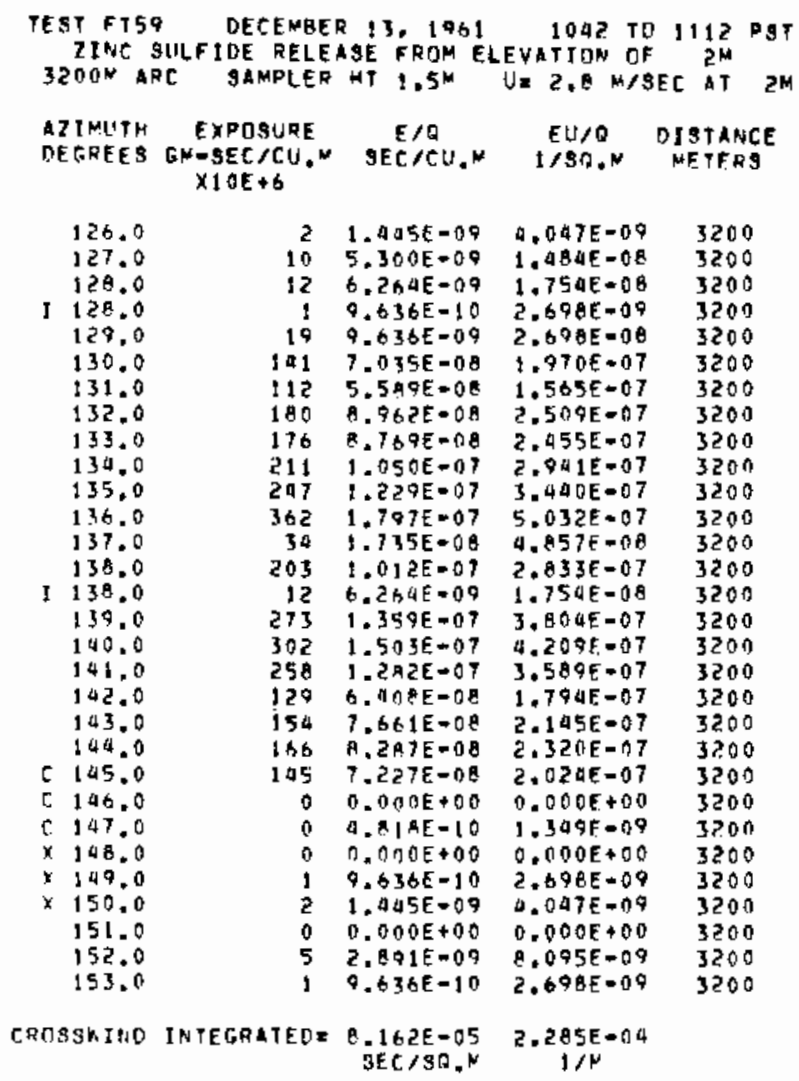

TOWER DATA FOLLOW....

TEST F\$59 DECEMBER 13.1961 1OAZ TO 1112 PST ZINC SULFIOE RELEASE FRON ELFVATION OF $2 \mathrm{M}$ $3200^{N}$ ARC UE ?, B N/SEC AT $2^{\mathrm{M}}$

\begin{tabular}{|c|c|c|c|c|c|}
\hline $\begin{array}{l}\text { AZIMUTH } \\
\text { CETREES }\end{array}$ & $\begin{array}{l}\text { MEIGHT } \\
\text { METERS }\end{array}$ & $\begin{array}{l}\text { EXPOSURE } \\
\text { GM-SEC /CU, } \\
\times 1 O E+6\end{array}$ & $\begin{array}{c}E / 0 \\
\sec / C U_{0}\end{array}$ & $\begin{array}{l}E U / C \\
1 / 39, M\end{array}$ & $\begin{array}{c}\text { DISTANCE } \\
\text { WETERS }\end{array}$ \\
\hline $\begin{array}{l}130.0 \\
130.0 \\
130.0 \\
130.0 \\
130.0 \\
130.0 \\
130.0 \\
130.0 \\
130.0 \\
130.0 \\
130.0 \\
130.0 \\
130.0 \\
130.0 \\
130.0 \\
130.0 \\
130.0 \\
130.0\end{array}$ & $\begin{array}{l}0.2 \\
0.4 \\
0.9 \\
1.5 \\
3.1 \\
6.2 \\
9.3 \\
12.4 \\
15.5 \\
19.6 \\
21.7 \\
24.0 \\
31.0 \\
37.2 \\
43.4 \\
49.6 \\
55.8 \\
62.0\end{array}$ & $\begin{array}{r}51 \\
10 \\
45 \\
20 \\
5 \\
41 \\
5 \\
4 \\
15 \\
14 \\
10 \\
2 \\
23 \\
20 \\
4 \\
3 \\
36 \\
4\end{array}$ & $\begin{array}{l}2.500 E=08 \\
5.050 E=09 \\
2.277 E=08 \\
1.012 E=08 \\
2.760 E=09 \\
2.070 E=08 \\
2.760 E=09 \\
2.300 E=09 \\
7.010 E=09 \\
7.350 E=09 \\
5.050 E=09 \\
1.380 E=09 \\
1.150 E=08 \\
1.012 E=08 \\
2.300 E=09 \\
1.755 E=09 \\
1.799 E=08 \\
9.309 E=09\end{array}$ & $\begin{array}{l}7.224 E=08 \\
1.017 E=08 \\
6.375 E=08 \\
3.433 E=09 \\
7.727 E=09 \\
5.795 E=00 \\
7.727 E=09 \\
6.039 E=09 \\
2.189 E=08 \\
2.060 E=08 \\
1.017 E=08 \\
3.063 E=09 \\
3.219 E=08 \\
2.033 E=08 \\
6.439 E=09 \\
4.915 E=09 \\
5.038 E=08 \\
1.229 E=0 B\end{array}$ & $\begin{array}{l}3200 \\
3200 \\
3200 \\
3200 \\
3200 \\
3200 \\
3200 \\
3200 \\
3200 \\
3200 \\
3200 \\
3200 \\
3200 \\
3200 \\
3200 \\
3200 \\
3200 \\
3200\end{array}$ \\
\hline
\end{tabular}


30-SER IES EXPERIMENT: 60 (FT-60)

GROUND LEVEL ANO TOXER SAMPLING 200-?200 HA ARCS

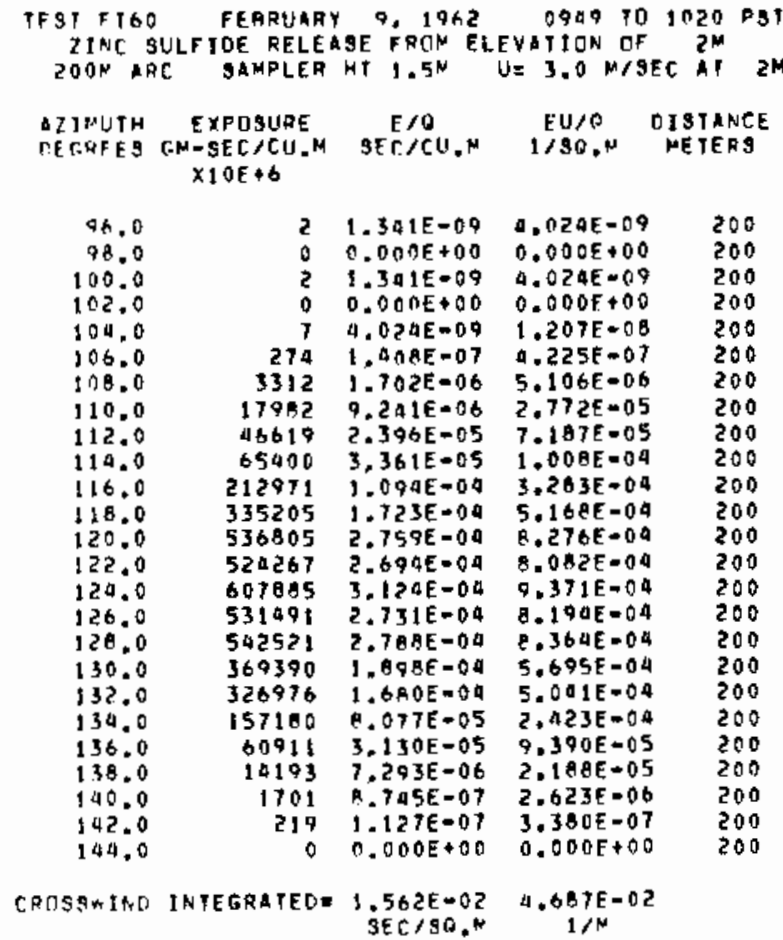

TONER DATA FOLLOH....

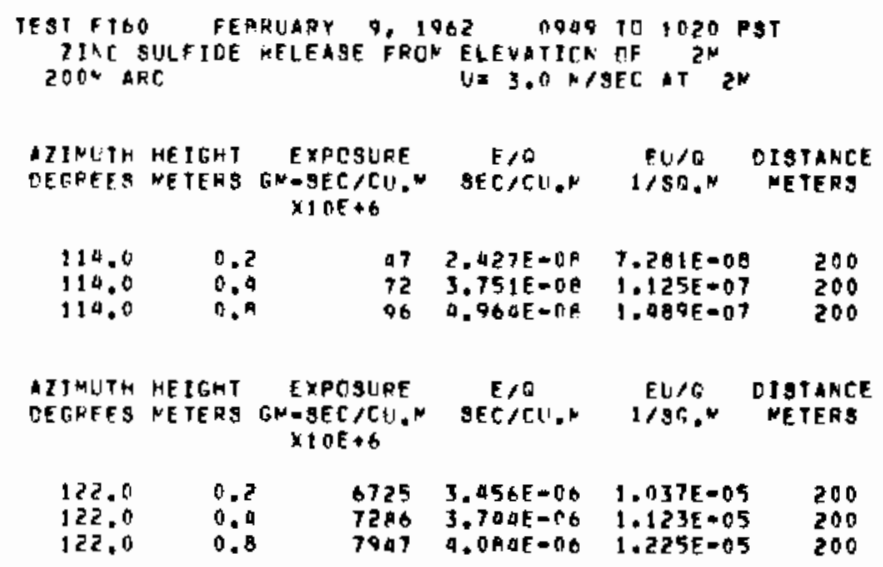

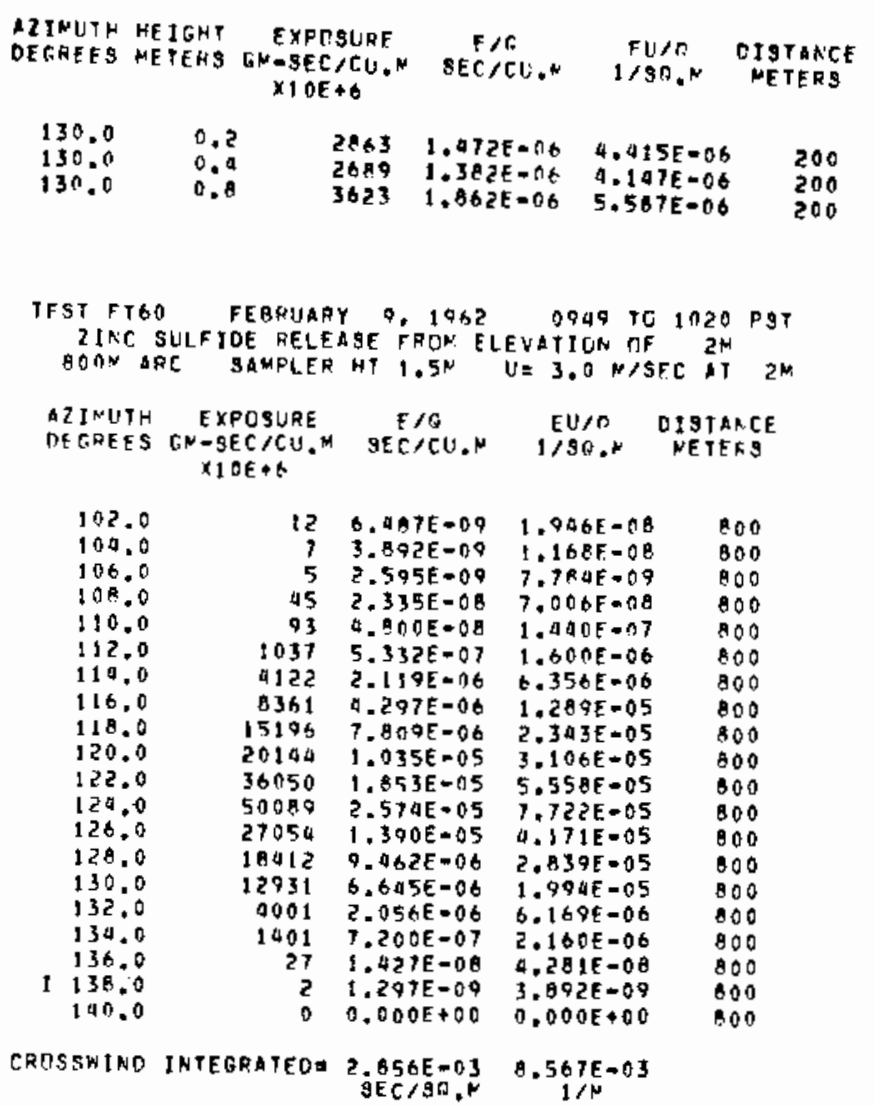

TIKER DATA FQLLCW....

TEST FTOO FEPRUARY 9. J962 094S TC 1 DEN PST ZINE SULFIOE RELEASE FRON ELEVATICA OF
MOON AFC

RZINUTH HEIGHT EXPOSUPE E/O EU/F CISTANCE DEGREFS METERS GN-SECACU.N SECICU.N HISO,N METERS $\times 1$ OE +6

$\begin{array}{llllll}130.0 & 0.2 & 278 & 1.430 E-07 & 9.290 E-07 & 000 \\ 130.0 & 0.4 & 325 & 1.980 E-07 & 5.900 E-07 & 000 \\ 130.0 & 0.8 & 445 & 2.289 E-07 & 6.808 E-07 & 000\end{array}$




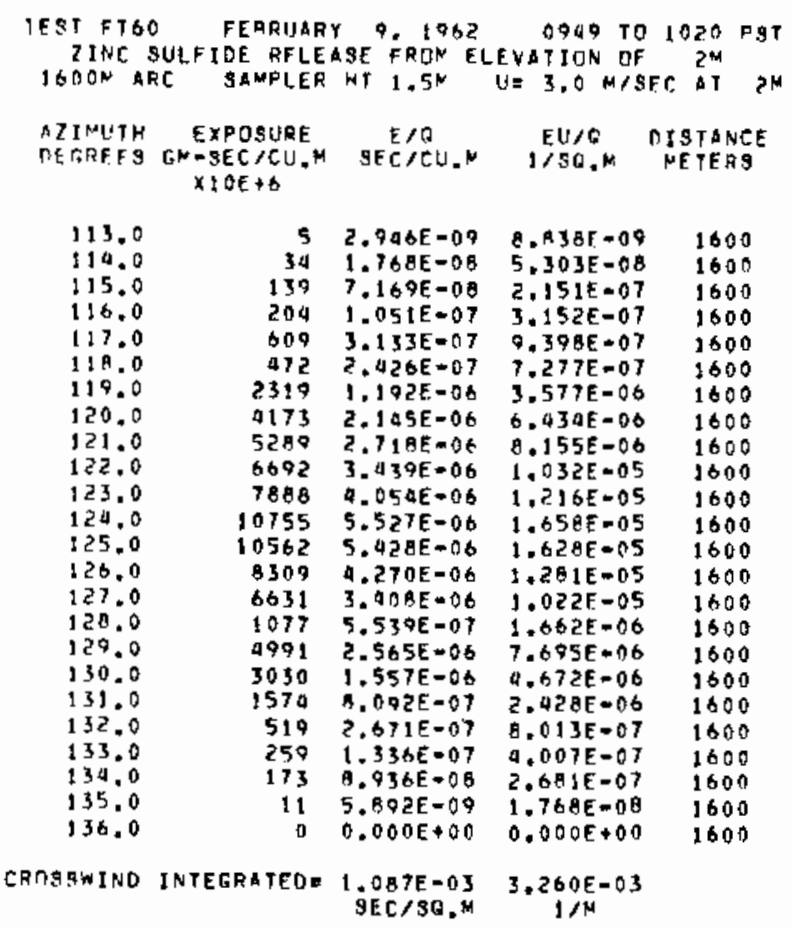

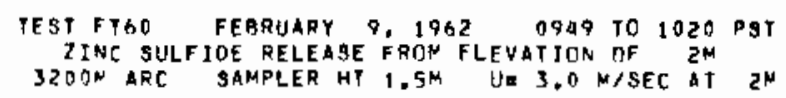

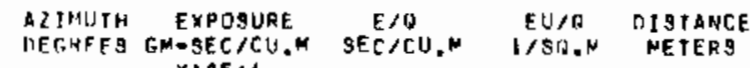
$\times 10 E+6$

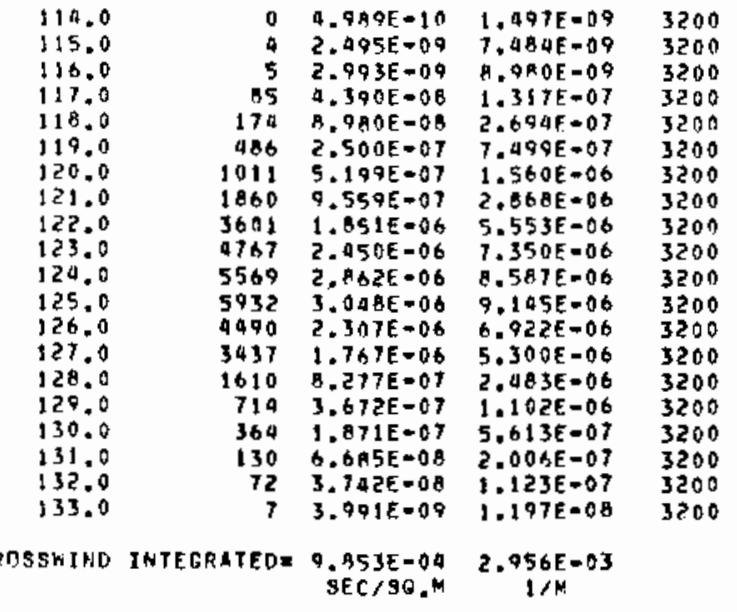


30-5ERIES EXPER INENT: E: (FT-51

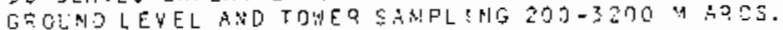

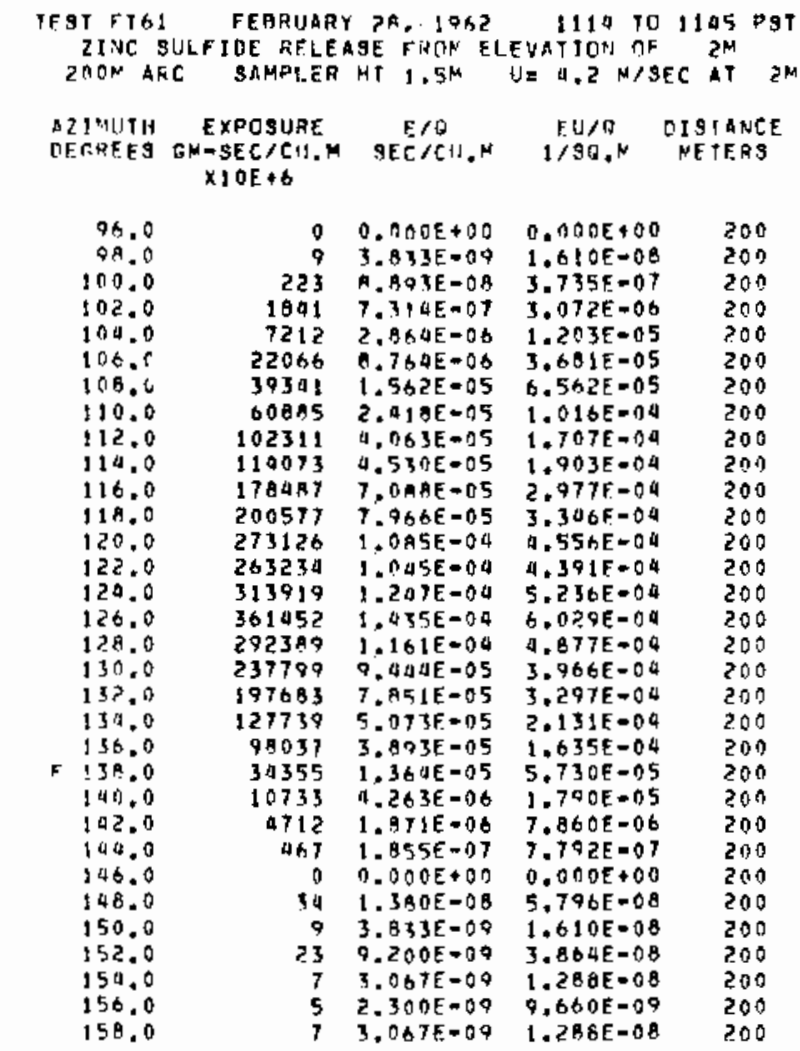

CRTSTHIHD ZHTEGRATEO= $8 . \$ 59 E-03 \quad 3.427 E-02$
TONER PATA FOLLCW...

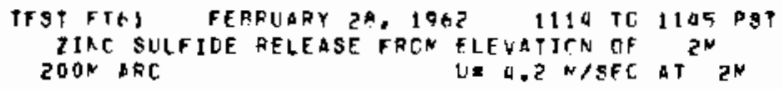

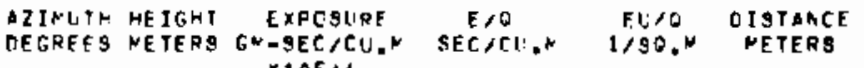
$x+0 E+t$

\begin{tabular}{|c|c|c|c|c|c|}
\hline $\begin{array}{l}98.0 \\
98.0 \\
98.0 \\
98.0 \\
98.0 \\
98.0 \\
98.0 \\
98.0 \\
98.0 \\
98.0 \\
98.0 \\
98.0 \\
98.0 \\
98.0 \\
98.0 \\
98.0 \\
98.0\end{array}$ & $\begin{array}{l}4.1 \\
0.3 \\
0.7 \\
1.9 \\
2.7 \\
4.1 \\
5.9 \\
6.8 \\
8.1 \\
9.0 \\
10.8 \\
13.5 \\
16.2 \\
16.9 \\
21.6 \\
24.3 \\
27.0\end{array}$ & $\begin{array}{r}3 \\
18347 \\
20870 \\
9375 \\
11544 \\
7620 \\
1670 \\
0903 \\
8895 \\
6996 \\
5963 \\
1579 \\
3725 \\
2660 \\
1075 \\
549 \\
390\end{array}$ & $\begin{array}{l}1.578 E=09 \\
7.2 A 7 E=06 \\
8.290 E=06 \\
3.726 E=06 \\
4.585 E=06 \\
3.026 E=06 \\
6.536 E=07 \\
3.536 E=06 \\
3.533 E=06 \\
2.779 E=06 \\
2.36 P E-06 \\
6.273 E=07 \\
1.480 E=06 \\
1.056 F=06 \\
4.27 C E=07 \\
2.18 C E=07 \\
1.553 E=07\end{array}$ & $\begin{array}{l}6.626 E=09 \\
3.060 E=05 \\
3.482 E=05 \\
1.565 E=05 \\
1.926 E=05 \\
1.271 E=05 \\
2.787 E=06 \\
1.485 E=05 \\
1.484 E=05 \\
1.167 E=05 \\
9.947 E=06 \\
2.635 E=06 \\
6.215 E=06 \\
4.437 E=06 \\
1.793 E=06 \\
9.158 E=07 \\
6.521 E=07\end{array}$ & $\begin{array}{l}200 \\
200 \\
200 \\
200 \\
200 \\
200 \\
200 \\
200 \\
200 \\
200 \\
200 \\
200 \\
200 \\
200 \\
200 \\
200 \\
200\end{array}$ \\
\hline
\end{tabular}

\begin{tabular}{|c|c|c|c|c|c|}
\hline $\begin{array}{l}2 I N 11 \mathrm{H} \\
\text { EGREES }\end{array}$ & $\begin{array}{l}\text { HEIGHT } \\
\text { METERS }\end{array}$ & 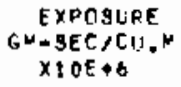 & $\begin{array}{c}E / Q \\
S E C / C H, W\end{array}$ & $\begin{array}{c}E U / 0 \\
1 / 30 . N\end{array}$ & $\begin{array}{l}\text { DISTANCE } \\
\text { WETERS }\end{array}$ \\
\hline 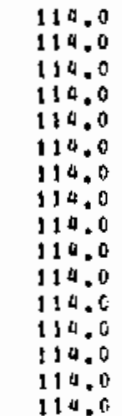 & $\begin{array}{l}0.3 \\
0.7 \\
1.4 \\
2.7 \\
4.1 \\
5.7 \\
6.9 \\
8.1 \\
9.9 \\
10.1 \\
13.5 \\
16.2 \\
18.9 \\
21.6 \\
24.3 \\
27.0\end{array}$ & $\begin{array}{l}99153 \\
97325 \\
26677 \\
02507 \\
33400 \\
22304 \\
15642 \\
15320 \\
14174 \\
13492 \\
8043 \\
12196 \\
5756 \\
1159 \\
3094 \\
2569\end{array}$ & 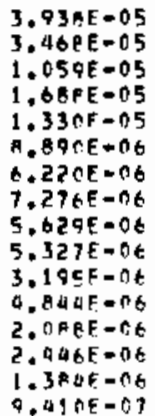 & $\begin{array}{l}1.654 E=04 \\
1.457 E=04 \\
0.450 E=05 \\
7.090 E=05 \\
5.555 E=05 \\
3.734 E=05 \\
2.613 E=05 \\
3.056 E=05 \\
2.364 E=05 \\
2.237 E=05 \\
1.342 E=05 \\
2.034 E=05 \\
0.76 R E=06 \\
1.027 E=05 \\
5.013 E=06 \\
3.952 E=06\end{array}$ & $\begin{array}{l}200 \\
200 \\
200 \\
200 \\
200 \\
200 \\
200 \\
200 \\
200 \\
200 \\
200 \\
200 \\
200 \\
200 \\
200 \\
200\end{array}$ \\
\hline
\end{tabular}

\begin{tabular}{|c|c|c|c|c|c|}
\hline $\begin{array}{l}\text { A7INLTH } \\
\text { DEGREE }\end{array}$ & $\begin{array}{l}\text { KETGHT } \\
\text { METERS }\end{array}$ & $\begin{array}{c}\text { EXPOSURE } \\
\text { GU }=5 E C / C U . N \\
\times 10 E+6\end{array}$ & $\begin{array}{c}E / 0 \\
S E C / C U, k\end{array}$ & $\begin{array}{c}F 1 / 6 \\
1 / 90.2\end{array}$ & $\begin{array}{l}\text { DISTANCE } \\
\text { NETERS }\end{array}$ \\
\hline $\begin{array}{l}122.0 \\
122.0 \\
122.0 \\
122.0 \\
122.0 \\
122.0 \\
122.0 \\
122.0 \\
122.0 \\
122.0 \\
122.0 \\
122.0 \\
122.0 \\
122.0 \\
122.0 \\
122.0\end{array}$ & $\begin{array}{l}0.3 \\
0.7 \\
1.4 \\
2.7 \\
4.1 \\
5.9 \\
6.9 \\
6.1 \\
9.9 \\
10.8 \\
13.5 \\
16.2 \\
10.9 \\
21.6 \\
24.3 \\
27.0\end{array}$ & $\begin{array}{r}176534 \\
233159 \\
12471 \\
00685 \\
137103 \\
106842 \\
119425 \\
97057 \\
56270 \\
18340 \\
75904 \\
18401 \\
53214 \\
26087 \\
4218 \\
5537\end{array}$ & $\begin{array}{l}7.011 E=05 \\
9.260 E=05 \\
3.275 E=05 \\
3.204 F=05 \\
5.405 E-05 \\
4.244 E=05 \\
4.759 E=05 \\
3.055 E-05 \\
2.235 E=05 \\
2.716 E=05 \\
3.016 E=05 \\
7.324 E=06 \\
2.113 E=05 \\
1.036 E-05 \\
1.676 E=06 \\
2.194 E=06\end{array}$ & $\begin{array}{l}2.925 E=04 \\
3.289 E=04 \\
1.376 E=04 \\
1.346 E=04 \\
2.287 E=04 \\
1.782 E=04 \\
1.999 E=04 \\
1.619 E=0 A \\
9.386 E=05 \\
1.191 E=04 \\
1.267 E=0 A \\
3.076 E=05 \\
8.876 E=05 \\
4.351 E-05 \\
7.037 E=06 \\
9.237 E=06\end{array}$ & $\begin{array}{l}200 \\
200 \\
200 \\
200 \\
200 \\
200 \\
200 \\
200 \\
200 \\
200 \\
200 \\
200 \\
200 \\
200 \\
200 \\
200\end{array}$ \\
\hline
\end{tabular}

AZINITTM HEIGHT EXPQSLRE E/O EU/Q DISTANCE CEGEFS METERS GM-SEC/CI.N SEC/CU.N I/SO.N NETERS

\begin{tabular}{|c|c|c|c|c|c|}
\hline $\begin{array}{l}130.0 \\
130.0 \\
130.0 \\
130.0 \\
130.0 \\
130.0 \\
130.0 \\
130.0 \\
130.0 \\
130.0 \\
130.0 \\
130.0 \\
130.0 \\
130.0 \\
130.0 \\
130.0\end{array}$ & $\begin{array}{r}0.3 \\
0.7 \\
1.4 \\
2.7 \\
4.1 \\
5.4 \\
6.8 \\
8.1 \\
9.4 \\
10.8 \\
13.5 \\
16.2 \\
18.9 \\
21.6 \\
24.3 \\
27.7\end{array}$ & $\begin{array}{r}161005 \\
150748 \\
06190 \\
66955 \\
53200 \\
57373 \\
49492 \\
46680 \\
17017 \\
39341 \\
30224 \\
70676 \\
12927 \\
8464 \\
5856 \\
4840\end{array}$ & $\begin{array}{l}6.410 E=05 \\
5.987 E=05 \\
1.835 E=05 \\
2.740 F=05 \\
2.113 E=05 \\
2.275 E=05 \\
1.974 E=05 \\
1.854 E=05 \\
6.75 P E=06 \\
1.562 E=05 \\
1.200 E=05 \\
8.212 E=06 \\
5.134 E=06 \\
3.363 E=06 \\
2.326 E=06 \\
1.922 E=06\end{array}$ & 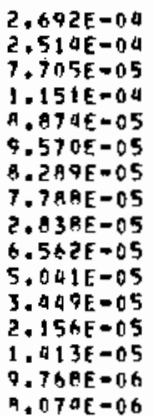 & $\begin{array}{l}200 \\
200 \\
200 \\
200 \\
200 \\
200 \\
200 \\
200 \\
200 \\
200 \\
200 \\
200 \\
200 \\
200 \\
200 \\
200\end{array}$ \\
\hline
\end{tabular}




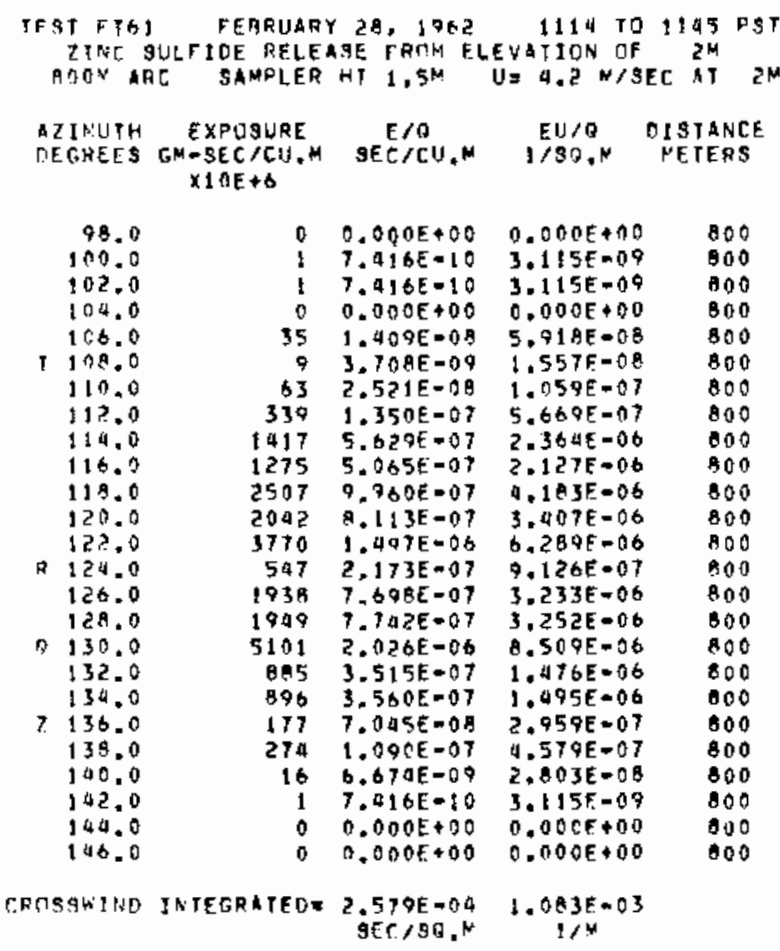

TEMER CATA FOLLCW...

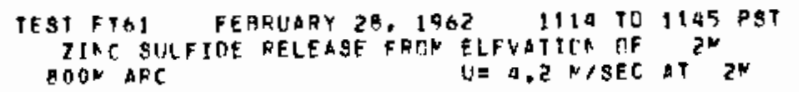

AZINUTN HEIGHI EXPTSURE EIG FU/D DISTINCE

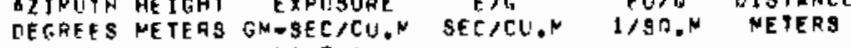
$\times 10 E+6$

\begin{tabular}{|c|c|c|c|c|c|}
\hline $\begin{array}{l}106.0 \\
106.0 \\
106.0 \\
106.0 \\
106.0 \\
0106.0 \\
106.0 \\
106.0 \\
106.0 \\
100.0 \\
106.0 \\
106.0 \\
106.0 \\
106.0 \\
106.0 \\
106.0 \\
106.0\end{array}$ & $\begin{array}{l}0.3 \\
0.5 \\
1.0 \\
2.1 \\
4.2 \\
6.3 \\
8.4 \\
10.5 \\
12.6 \\
14.7 \\
16.8 \\
21.0 \\
25.7 \\
29.9 \\
33.6 \\
37.9 \\
12.0\end{array}$ & $\begin{array}{r}108 \\
79 \\
60 \\
0 \\
2 \\
7 \\
11 \\
7 \\
12 \\
0 \\
0 \\
0 \\
0 \\
0 \\
0 \\
0 \\
0\end{array}$ & $\begin{array}{l}A .299 E=08 \\
3.167 E=08 \\
2.391 E=08 \\
0.000 E+00 \\
1.017 E=09 \\
3.052 E-09 \\
4.572 E=09 \\
3.052 E=09 \\
5.087 E=09 \\
0.000 E+00 \\
0.000 E+00 \\
0.000 E+00 \\
0.000 E+00 \\
0.000 E+00 \\
0.000 E+00 \\
0.000 E+00 \\
0.000 E+00\end{array}$ & $\begin{array}{l}1.005 E=07 \\
1.330 E=07 \\
1.000 E=07 \\
0.000 E+00 \\
1.273 E=08 \\
1.202 E-08 \\
1.923 E=08 \\
1.282 E+00 \\
2.136 E=08 \\
0.000 E+00 \\
0.000 E+00 \\
0.000 E+00 \\
0.000 E+00 \\
0.000 E+00 \\
0.000 E+00 \\
0.000 E+00 \\
0.000 E+00\end{array}$ & $\begin{array}{l}000 \\
000 \\
000 \\
100 \\
000 \\
000 \\
000 \\
000 \\
800 \\
800 \\
800 \\
800 \\
800 \\
000 \\
800 \\
100 \\
800\end{array}$ \\
\hline
\end{tabular}

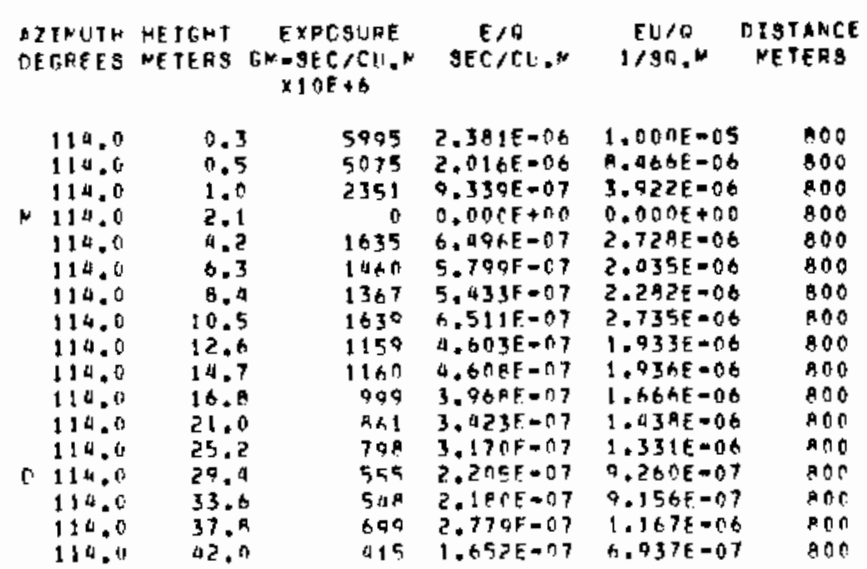

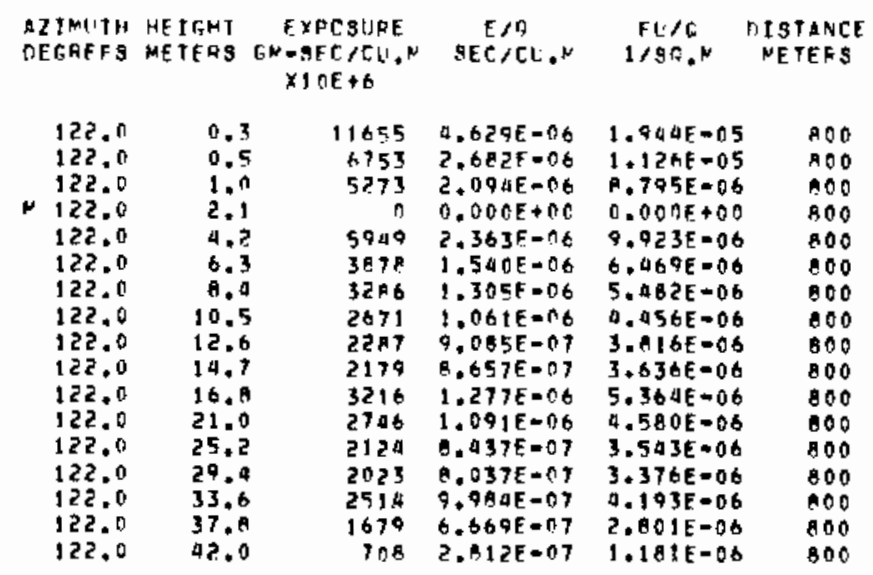

AZINITH HEIGHT EXPOSLRF EAG EU/G DISTANLE DEGREES NETERG GN-SEC/CI.N SEC/CII.N 1/SR.N NETERS $X I O E+6$

\begin{tabular}{|c|c|c|c|c|c|}
\hline $\begin{array}{l}130.0 \\
130.0\end{array}$ & $\begin{array}{l}0.3 \\
0.5\end{array}$ & $\begin{array}{l}6750 \\
705 \%\end{array}$ & $\begin{array}{l}2.681 E=06 \\
2.803 F=06\end{array}$ & $\begin{array}{l}1.126 E=05 \\
1.175=05\end{array}$ & $\begin{array}{l}100 \\
800\end{array}$ \\
\hline 130.0 & 1.0 & 2903 & $1.14 O E=0 B$ & $4.026 E=06$ & 800 \\
\hline 130.0 & 2.1 & A & $0,000 F+00$ & $0.000 E+00$ & 000 \\
\hline 130.0 & $4 . ?$ & SBA & 1. 46 LE $=06$ & $0.149 \mathrm{E}=06$ & 000 \\
\hline 130.0 & 6.3 & 67 & $1.8 \times 2 F=08$ & $7.905 E=08$ & 800 \\
\hline 130.0 & 0.4 & 23 & $9.156 E-09$ & 3. $845 E=08$ & 000 \\
\hline 130.0 & 10.5 & 0 & $0.0 \cap B E+\cap 0$ & $0.000 E+00$ & 000 \\
\hline 130.0 & 12.6 & 6 & $2.543 E=19$ & $1.068 E=00$ & 800 \\
\hline 130.0 & 10.7 & 21 & $8.647 E=09$ & $3.632 E=08$ & AOO \\
\hline 130.0 & 16.9 & $\ln 8$ & $324 E-n g$ & $1.16 E-07$ & 600 \\
\hline 130.0 & 21.0 & 10 & $6.613 F=04$ & $2.777 \mathrm{E}=00$ & 100 \\
\hline $13 n .0$ & 25.2 & 30 & $1.206 F=08$ & $5.067 \mathrm{~F}=0 \mathrm{~B}$ & 800 \\
\hline & & 47 & $1.872 E=08$ & $.9635=08$ & $\operatorname{lno} 0$ \\
\hline 130.0 & 33.8 & FO & $2035-08$ & $1.345 E-07$ & AOn \\
\hline 130.0 & & 30 & $.373 E-0 A$ & $5.760 E=08$ & 800 \\
\hline 130.0 & 42.0 & 28 & $1,123 E=0 A$ & $4.71 \mathrm{AE}=0 \mathrm{O}$ & 100 \\
\hline
\end{tabular}




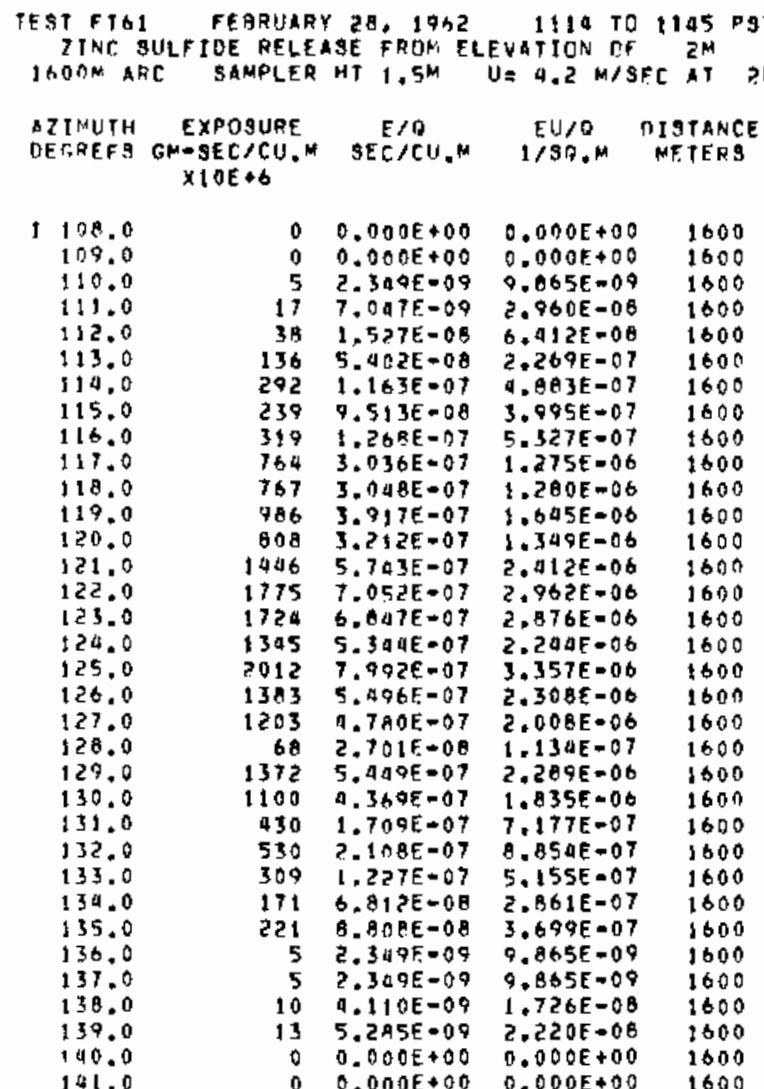

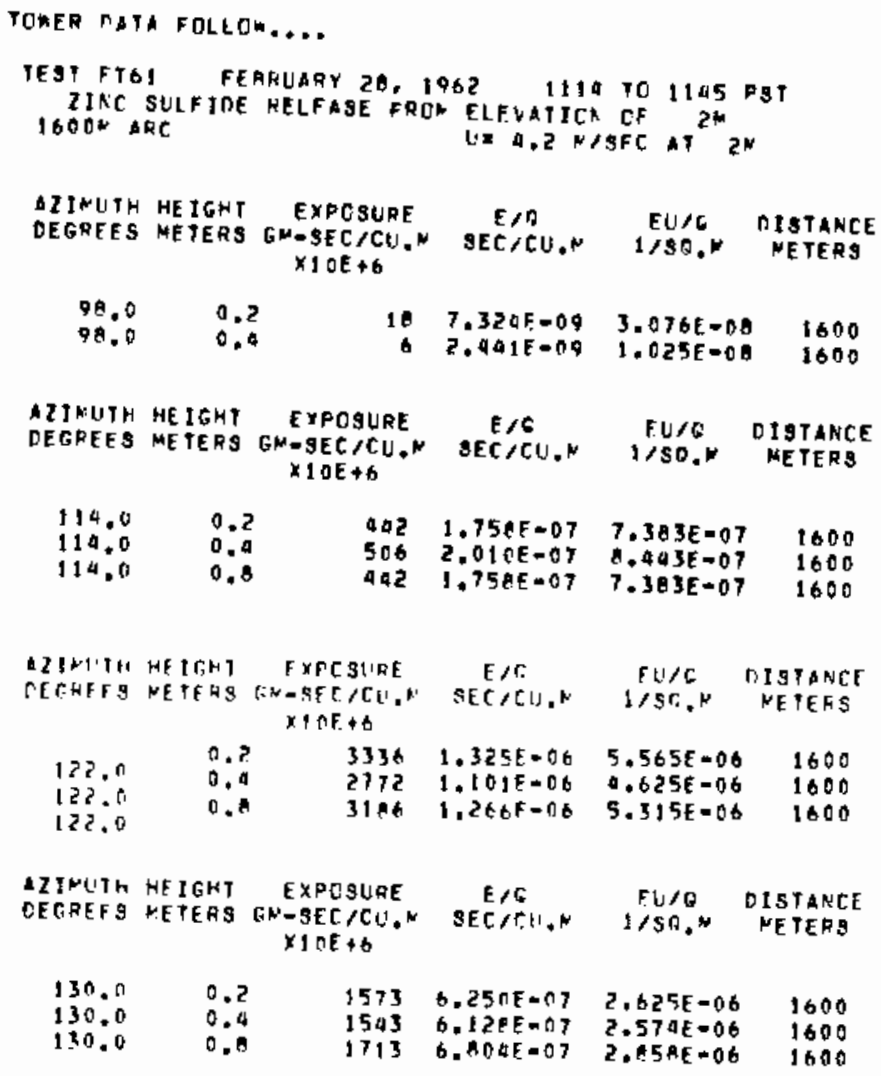

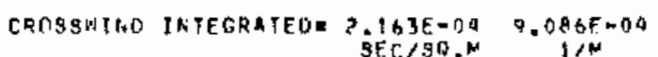




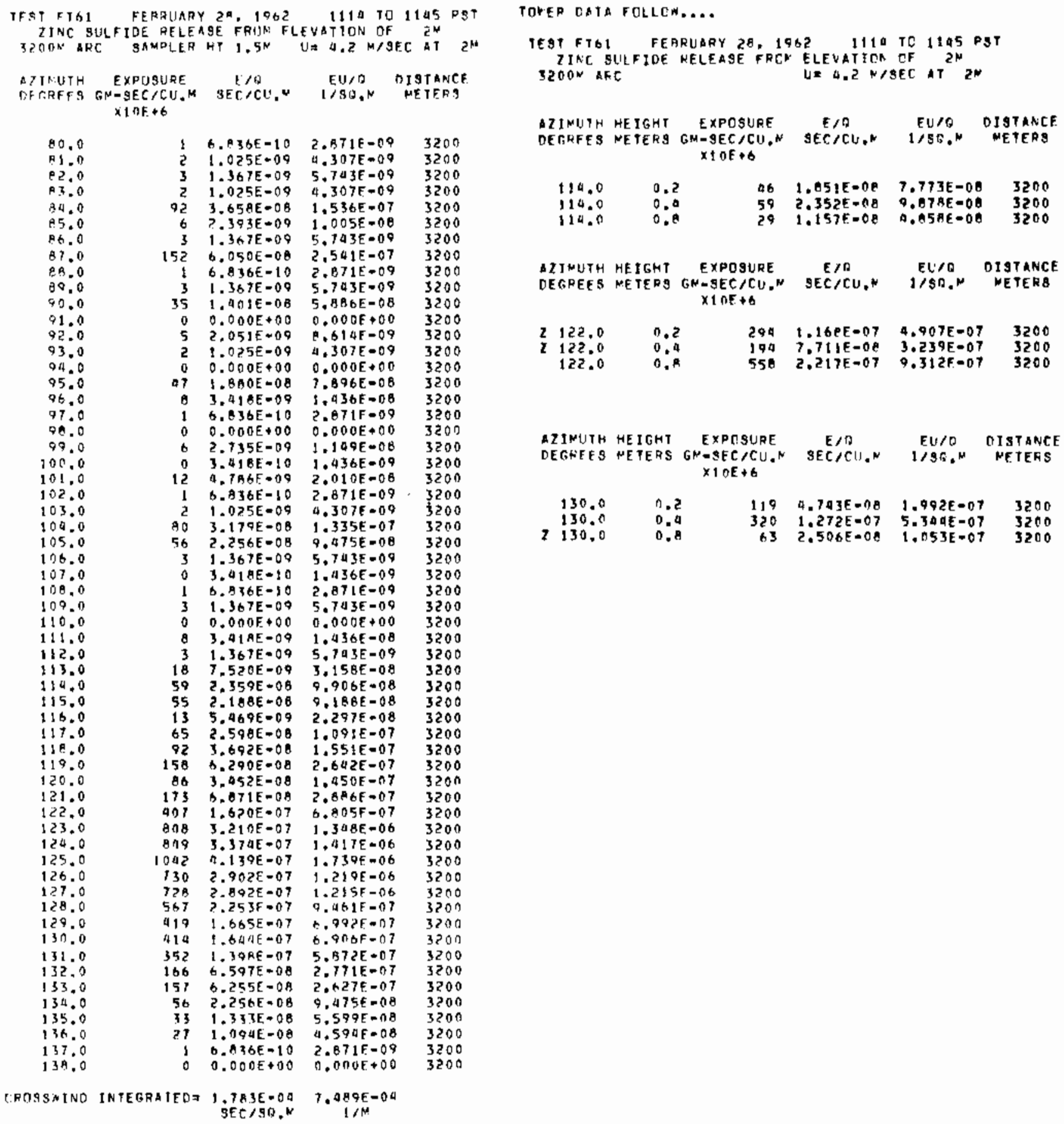


30-5EPIES EXPERIMENT: 62 \{FT-62,

GROUND LEYEL AND TOWER SAMP! IYG 200-3200 M ARCS. MLCH OF THE PLUME PASSED TO THE NORTH OF THE GRID.

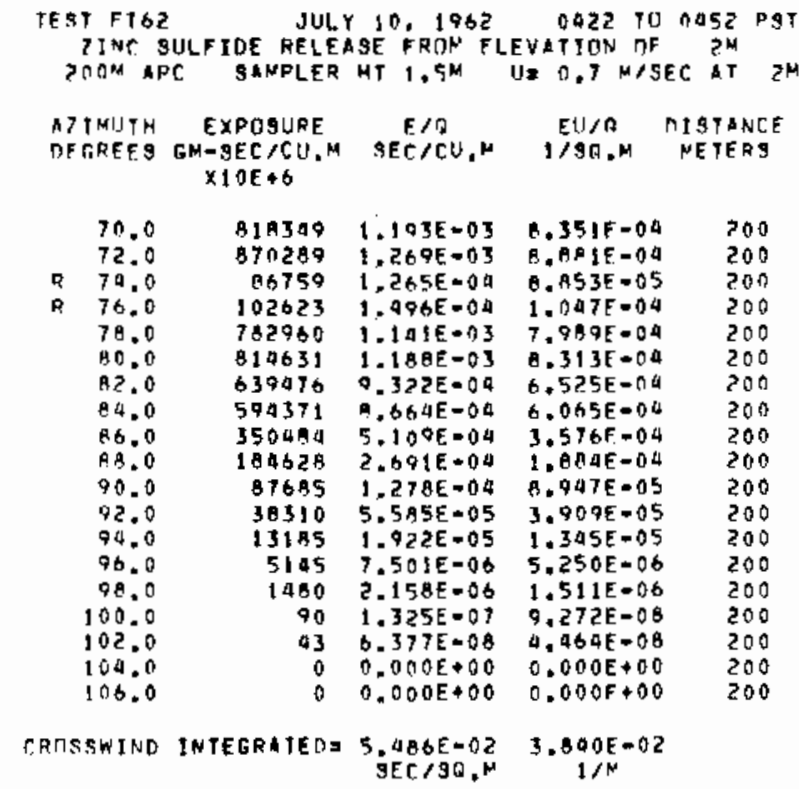

TUMFR PATA FRLLCW...

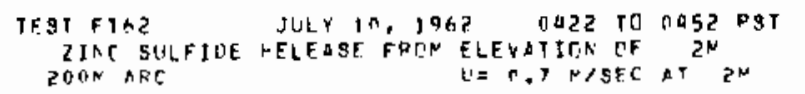

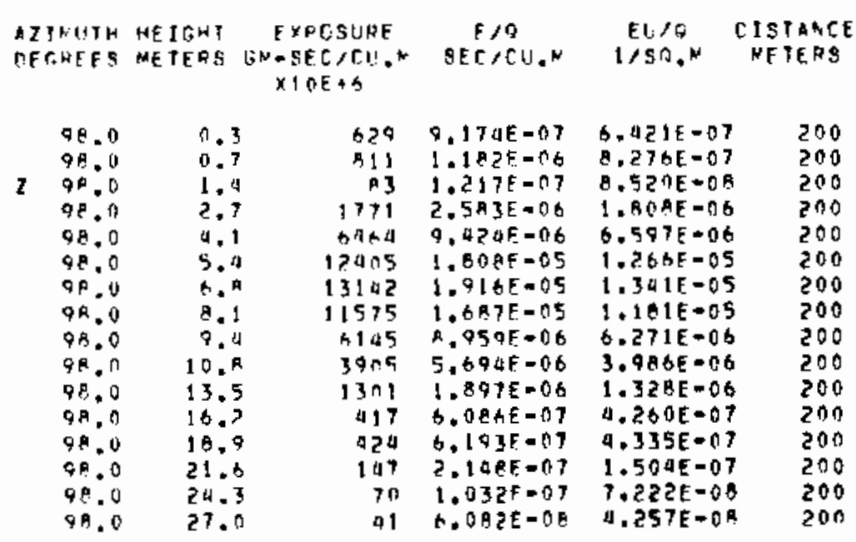

\begin{tabular}{|c|c|c|c|c|c|}
\hline $\begin{array}{l}\text { AZINI:TH } \\
\text { DETRFES }\end{array}$ & $\begin{array}{l}\text { HE IGH? } \\
\text { ME TERS }\end{array}$ & $\begin{array}{c}\text { EXPOSURE } \\
\text { EN-SEC CU.N } \\
\times 10 E+6\end{array}$ & $\begin{array}{c}E / 0 \\
\operatorname{SEC} / \mathrm{CU}, N\end{array}$ & $\begin{array}{c}E U / O \\
1 / S 0 . M\end{array}$ & $\begin{array}{c}\text { OISTAMCE } \\
\text { NETERS }\end{array}$ \\
\hline $\begin{array}{l}106.0 \\
106.0 \\
108.0 \\
106.0 \\
106.0 \\
106.0 \\
106.0 \\
106.0 \\
106.0 \\
106.0 \\
106.0 \\
106.0 \\
106.0 \\
106.0 \\
106.0 \\
106.0\end{array}$ & $\begin{array}{l}0.3 \\
0.7 \\
1.9 \\
2.7 \\
4.1 \\
5.15 \\
6.9 \\
6.1 \\
9.4 \\
10.4 \\
13.5 \\
16.2 \\
16.9 \\
21.4 \\
24.3 \\
27.0\end{array}$ & $\begin{array}{r}20 \\
94 \\
0 \\
18 \\
9 \\
306 \\
279 \\
766 \\
1191 \\
397 \\
273 \\
14 \\
132 \\
58 \\
77 \\
15\end{array}$ & $\begin{array}{l}2.943 E=08 \\
1.374 E=0 ? \\
0.000 E+00 \\
2.085 E=06 \\
1.432 E=08 \\
5.78 Z E=07 \\
9.081 E=07 \\
1.117 E=06 \\
1.73+E=06 \\
5.794 E=07 \\
3.258 E=07 \\
1.235 E=07 \\
1.933 E=07 \\
8.592 E=08 \\
4.018 E=0 B \\
2.2 P 1 E=0 A\end{array}$ & $\begin{array}{l}2.060 E=08 \\
9.615 E=08 \\
0.000 E+00 \\
1.679 E-08 \\
1.002 E-08 \\
1.047 E=07 \\
2.157 E=07 \\
7.819 E-07 \\
1.215 E=06 \\
4.060 E=07 \\
2.280 E=07 \\
1.646 E-0 A \\
1.353 E-07 \\
6.014 E=08 \\
2.813 E-08 \\
1.597 E-0 B\end{array}$ & $\begin{array}{l}200 \\
200 \\
200 \\
200 \\
200 \\
200 \\
200 \\
200 \\
200 \\
200 \\
200 \\
200 \\
200 \\
200 \\
200 \\
200\end{array}$ \\
\hline
\end{tabular}

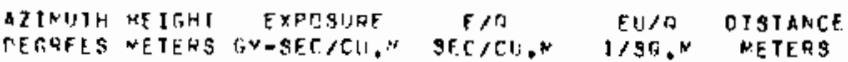
$x \cap \cap E+6$

\begin{tabular}{|c|c|c|c|c|c|}
\hline $12 ? .0$ & 12,3 & 16 & $2.453 E=08$ & $1.717 E=O H$ & 200 \\
\hline & 0.7 & $n$ & $0.000 F+00$ & $0.00 \cap E+00$ & 200 \\
\hline 122.0 & 1.1 & 17 & $2.1425=08$ & $1.5045=0.3$ & 200 \\
\hline 122.0 & 2.7 & 0 & $0.000 F+00$ & $0.000 E+00$ & 200 \\
\hline 122.0 & 4.1 & 3 & $5.370 F=n 9$ & $3.759 E-09$ & 200 \\
\hline $12 ? .0$ & 5.4 & $n$ & $9.000 F+00$ & $0.000 F+00$ & 200 \\
\hline & $h .5$ & 8 & $1.253 F-018$ & $8.771 \varepsilon=07$ & 200 \\
\hline 122.0 & 8. 1 & 0 & $0,000 E+00$ & $0.000 E+00$ & 200 \\
\hline & 9.11 & 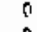 & $0.000 E+00$ & $0.0 \cap 0 E+00$ & 200 \\
\hline & 10,8 & 0 & $0.0 \cap C E+00$ & $0.000 E+00$ & 200 \\
\hline $\begin{array}{l}122.0 \\
122.0 \\
122.0\end{array}$ & $\begin{array}{l}13.5 \\
16 . ? \\
14.9\end{array}$ & $\begin{array}{l}9 \\
0 \\
2\end{array}$ & $\begin{array}{l}1.432 E-10 \\
0.000 E+00 \\
3.5 A O F=09\end{array}$ & $\begin{array}{l}1.002 E=0 B \\
0.000 E+00 \\
2.506 E=0 Q\end{array}$ & $\begin{array}{l}200 \\
200 \\
200\end{array}$ \\
\hline 122,0 & 21.4 & $n$ & $0.000 E+00$ & $0.000 E+00$ & 200 \\
\hline 122.0 & 24,3 & $n$ & $0.000 E+00$ & $0.000 \mathrm{E}+00$ & 200 \\
\hline & 27.1 & 2 & $4.3 \Delta \Delta \mathrm{F}=100$ & $3.041 \mathrm{E}=09$ & 200 \\
\hline
\end{tabular}

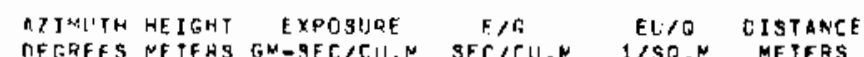
DECRFES NETEHS GM-AECICU.N SECICU,N 1/SO.N MEYEAS

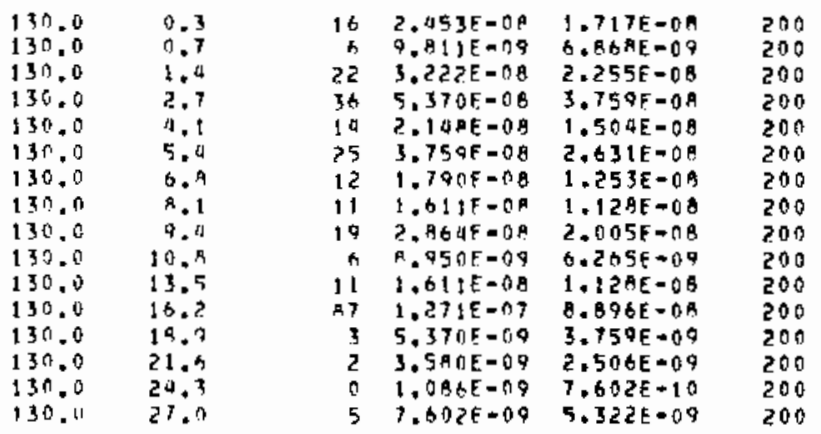




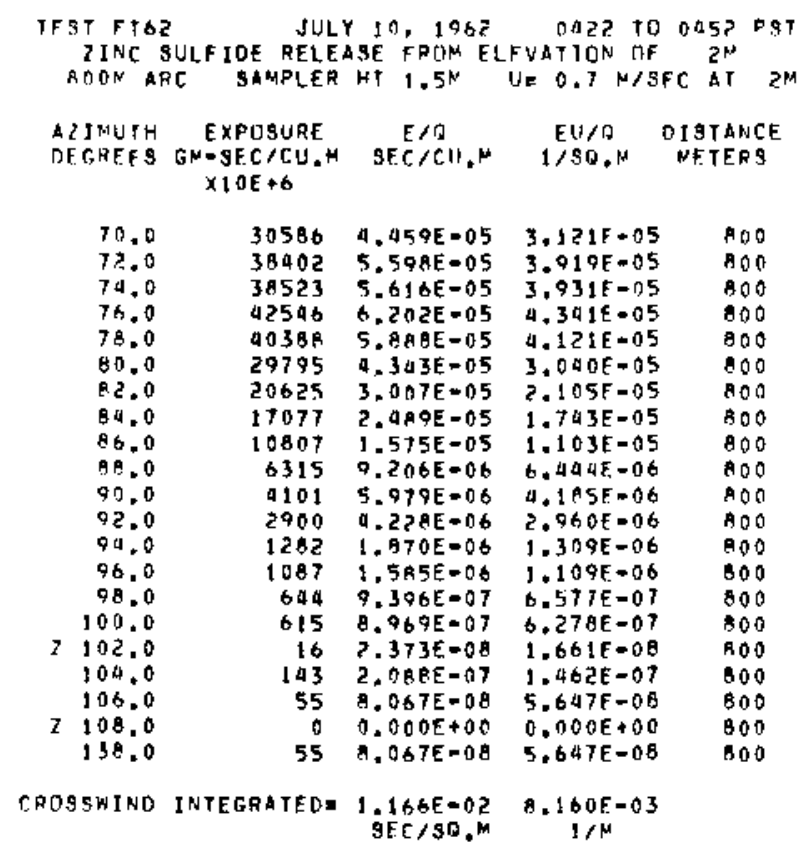

TOLFR TATA FOLLOW....

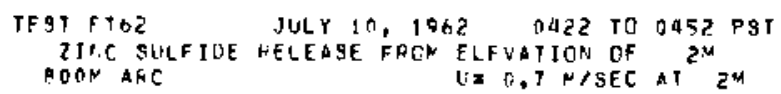

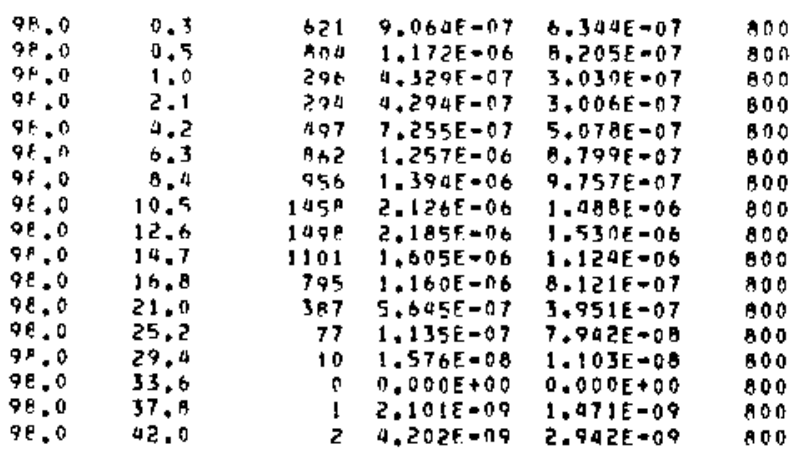

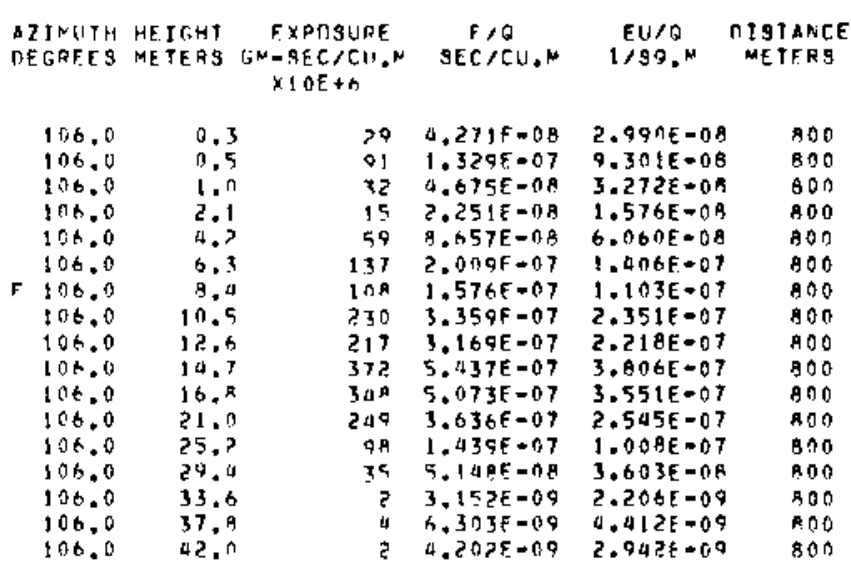

\begin{tabular}{|c|c|c|c|c|c|}
\hline $\begin{array}{l}\text { AZINUTH } \\
\text { DEGREES }\end{array}$ & $\begin{array}{l}\text { HETGHT } \\
\text { METFRS }\end{array}$ & $\begin{array}{c}\text { EXPRSUPE } \\
\text { GN }=\text { SEC }>C I: * \\
\text { MIOE }\end{array}$ & $\begin{array}{c}E / D \\
S E C / C U, N\end{array}$ & $\begin{array}{c}E(1 / 0) \\
1 / 50 . N\end{array}$ & $\begin{array}{l}\text { OISTANCE } \\
\text { NETEAS }\end{array}$ \\
\hline 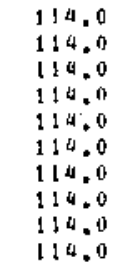 & $\begin{array}{l}10.5 \\
12.6 \\
14.7 \\
16.5 \\
21.0 \\
25.2 \\
29.4 \\
33.8 \\
37.9 \\
42.0\end{array}$ & $\begin{array}{r}0 \\
4 \\
34 \\
5 ? \\
49 \\
11 \\
18 \\
10 \\
7 \\
7\end{array}$ & $\begin{array}{l}0.000 E+D O \\
6.026 F=09 \\
5.021 E=0 A \\
7.01 A E=0 B \\
1.272 E=0 B \\
1.061 E=08 \\
2.731 E=08 \\
1.576 E=0 B \\
0.000 F+00 \\
1.051 F=08\end{array}$ & $\begin{array}{l}0.00 O E+O O \\
2.8 U E E=0 Q \\
3.515 E=08 \\
5.333 E-00 \\
5.091 E=06 \\
1.177 E=0 B \\
1.912 E=0 B \\
1.103 E=08 \\
0.000 E+00 \\
7.354 E=00\end{array}$ & $\begin{array}{l}100 \\
000 \\
100 \\
000 \\
600 \\
000 \\
800 \\
800 \\
800 \\
600\end{array}$ \\
\hline
\end{tabular}

\begin{tabular}{|c|c|c|c|c|c|}
\hline $\begin{array}{l}\text { AIIMEYTH } \\
\text { DEGHEES }\end{array}$ & $\begin{array}{l}\text { HEIGHT } \\
\text { METERS }\end{array}$ & $\begin{array}{c}\text { EXPOSUAE } \\
\text { GN-SEC } / C U . \\
\times 1 O E+6\end{array}$ & $\begin{array}{c}E / O \\
S E C / C U . M\end{array}$ & $\begin{array}{l}E \cup / O \\
1 / S Q . N\end{array}$ & $\begin{array}{l}\text { DISTANCE } \\
\text { NETERS }\end{array}$ \\
\hline $\begin{array}{l}122.0 \\
122.0 \\
122.0 \\
122.0 \\
122.0 \\
122.0 \\
122.0 \\
122.0 \\
122.0 \\
122.0 \\
122.0 \\
122.0 \\
122.0 \\
122.0 \\
122.0 \\
122.0 \\
122.0\end{array}$ & $\begin{array}{l}0.3 \\
0.5 \\
1.0 \\
2.1 \\
4.2 \\
6.3 \\
6.1 \\
10.5 \\
12.6 \\
14.7 \\
10.8 \\
21.0 \\
25.2 \\
29.1 \\
33.6 \\
37.0 \\
42.0\end{array}$ & $\begin{array}{l}6 \\
0 \\
0 \\
0 \\
0 \\
0 \\
0 \\
0 \\
0 \\
0 \\
0 \\
0 \\
0 \\
4 \\
0 \\
0 \\
0\end{array}$ & $\begin{array}{l}9.491 F=09 \\
0.000 E+00 \\
0.000 E+00 \\
0.000 E+00 \\
6.026 E=09 \\
0.000 E+100 \\
6.926 E=09 \\
0.000 E+00 \\
0.000 E+100 \\
0.000 E+00 \\
3.463 F=09 \\
0.000 E+00 \\
6.303 E=09 \\
0.000 E+00 \\
0.000 E+00 \\
0.000 E+00 \\
2.101 E=09\end{array}$ & $\begin{array}{l}6.643 E-09 \\
0.000 E+00 \\
0.000 E+00 \\
0.000 E+00 \\
4.048 E-09 \\
0.000 E+00 \\
4.046 E=00 \\
0.000 E+00 \\
0.000 E+00 \\
0.000 E+00 \\
2.424 E=09 \\
0.000 E+00 \\
9.412 E=09 \\
0.000 E+00 \\
0.000 E+00 \\
0.000 E+00 \\
1.071 E=09\end{array}$ & $\begin{array}{l}800 \\
800 \\
800 \\
800 \\
800 \\
000 \\
800 \\
000 \\
800 \\
800 \\
000 \\
800 \\
800 \\
800 \\
800 \\
800 \\
800\end{array}$ \\
\hline
\end{tabular}

DITMLIYH HEIGHT EXPTSURE EAD EU/O DISTANCE

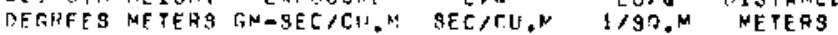
$x \rightarrow O E+h$

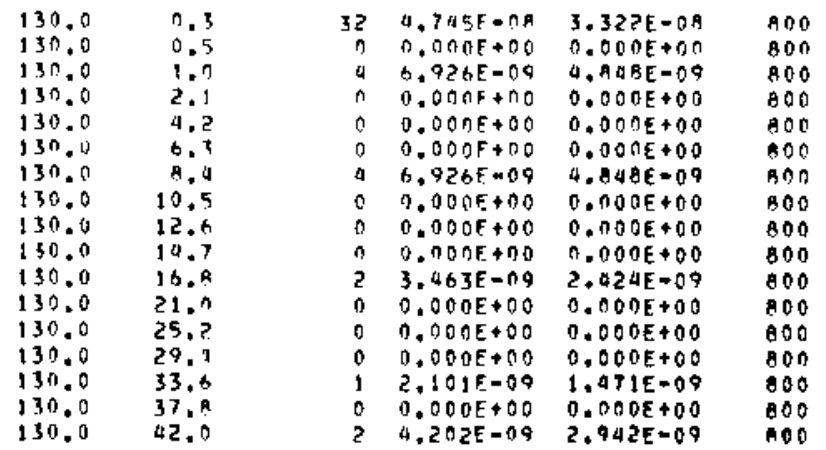




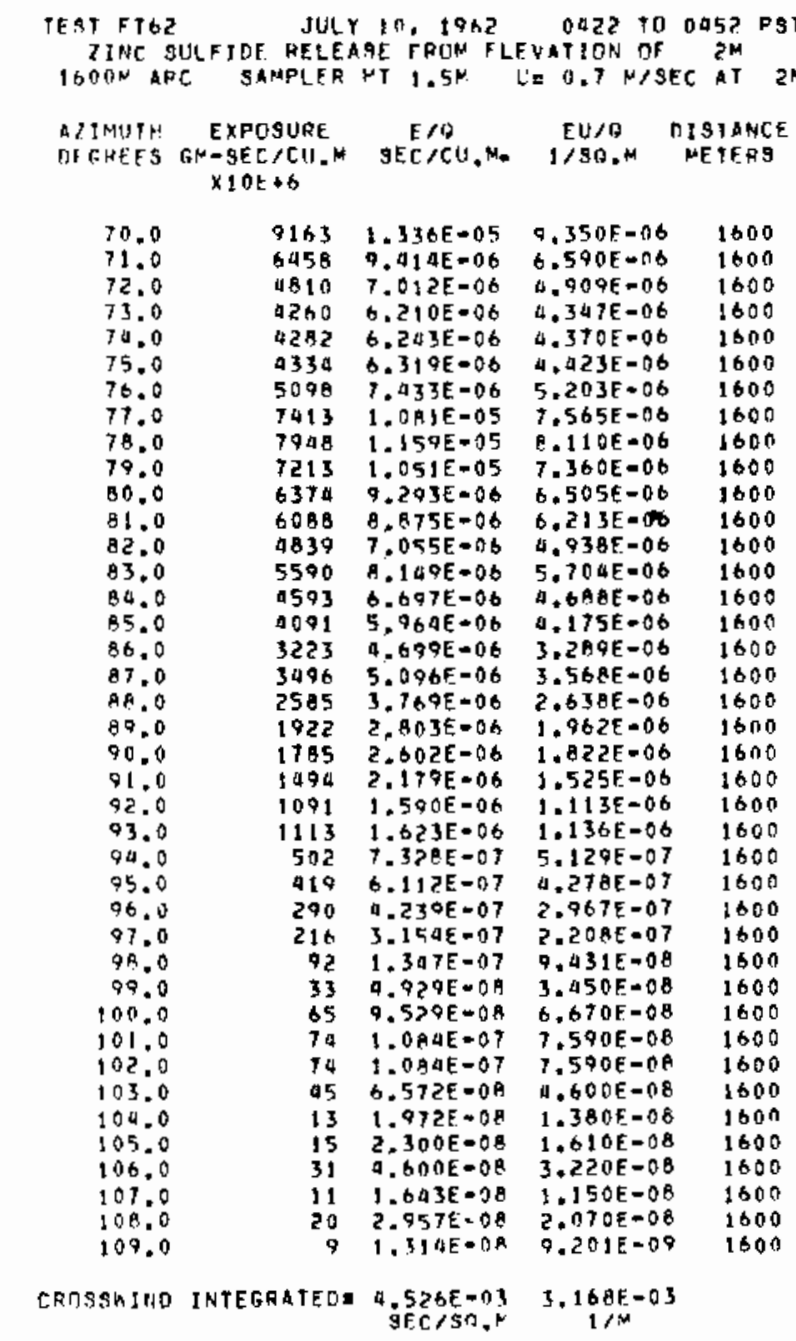

TOWFE DATA FULLCV....

TFSTFYOZ JULY 1D, 1962 OAZ2 TO 0452 PST TINC SULFJDE HELEASE FRTN ELEVATION CF

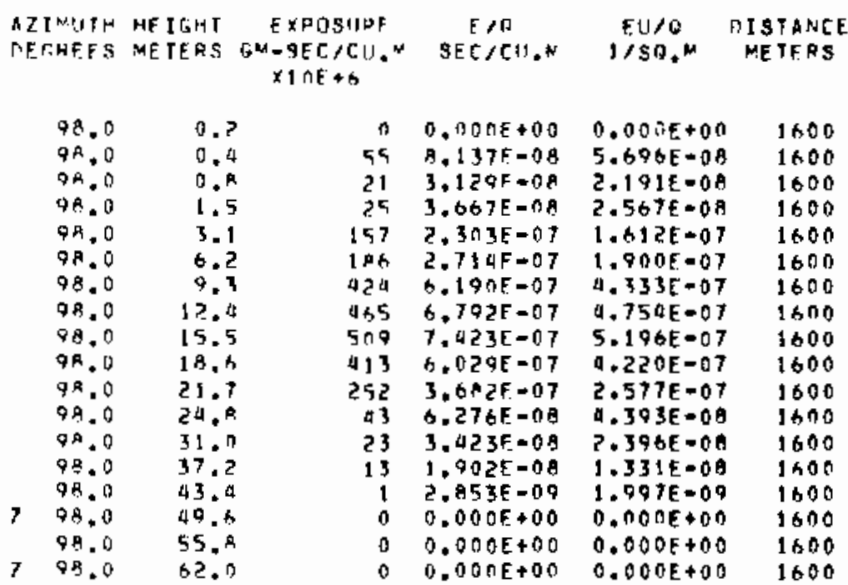

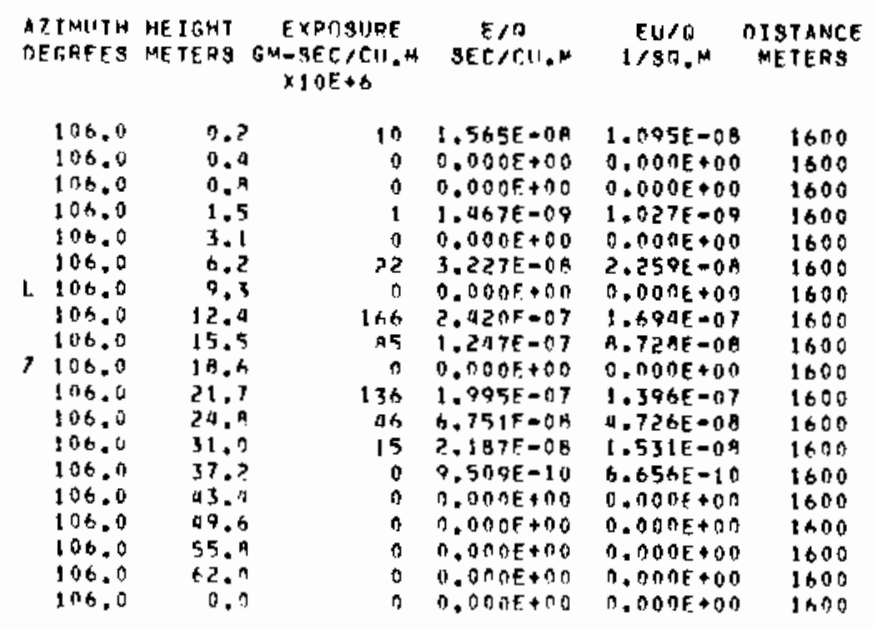

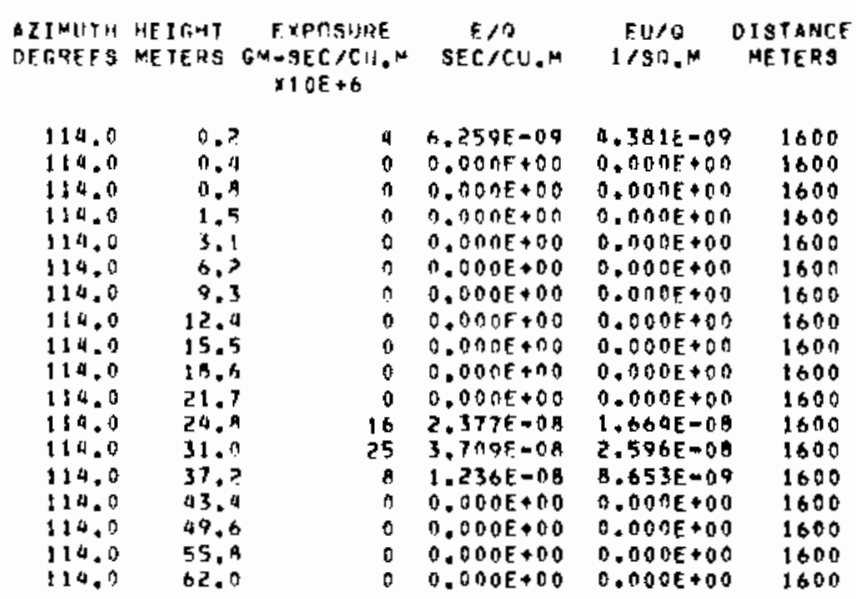

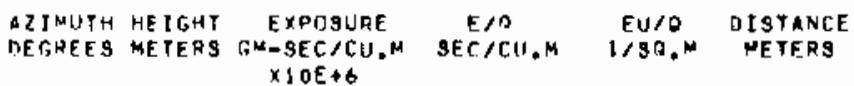

$\begin{array}{llllll}122.0 & 0.2 & 0 & 0.000 E+00 & 0.000 E+00 & 1600 \\ 122.0 & 0.4 & 0 & 0.000 E+00 & 0.000 E+00 & 1600 \\ 122.0 & 0.8 & 27 & 4.068 E-08 & 2.040 E-08 & 1600 \\ 122.0 & 1.5 & 0 & 0.000 E+00 & 0.000 E+00 & 1600 \\ 122.0 & 3.1 & 0 & 0.000 E+00 & 0.000 E+00 & 1600 \\ 122.0 & 6.2 & 0 & 0.000 E+00 & 0.000 E+00 & 1600 \\ 122.0 & 9.3 & 0 & 0.000 F+00 & 0.000 E+00 & 1600 \\ 122.0 & 12.4 & 0 & 0.000 E+00 & 0.000 E+00 & 1600 \\ 122.0 & 15.5 & 1 & 1.067 E-00 & 1.027 E+09 & 1600 \\ 122.0 & 18.6 & 0 & 0.000 E+00 & 0.000 E+00 & 1600 \\ 122.0 & 21.7 & 0 & 0.000 E+00 & 0.000 E+00 & 1600 \\ 122.0 & 24.9 & 0 & 0.000 E+00 & 0.000 E+00 & 1600 \\ 122.0 & 31.0 & 0 & 0.000 E+00 & 0.000 E+00 & 1600 \\ 122.0 & 37.7 & 0 & 0.000 E+00 & 0.000 E+00 & 1600 \\ 122.0 & 43.4 & 0 & 0.000 E+00 & 0.000 E+00 & 1600 \\ 122.0 & 49.8 & 0 & 0.000 E+00 & 0.000 E+00 & 1600 \\ 122.0 & 55 . A & 0 & 0.000 E+00 & 0.000 E+00 & 1600 \\ 122.0 & 62.0 & 0 & 0.000 E+00 & 0.000 E+00 & 1600\end{array}$




\begin{tabular}{|c|c|c|c|c|c|}
\hline $\begin{array}{l}\text { A7IUITH } \\
\text { PEGK:F. }\end{array}$ & $\begin{array}{l}\text { HE IGHT } \\
\text { ME TE:RS }\end{array}$ & 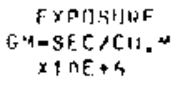 & 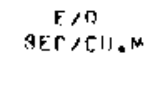 & $\begin{array}{c}\mathrm{Eu} / \mathrm{O} \\
1 / \mathrm{sin} \times\end{array}$ & $\begin{array}{l}\text { ISTANTE. } \\
\text { METFRS }\end{array}$ \\
\hline $13 \% .0$ & 0.7 & t) & $0.9 \cap \cap F+n D$ & $0.000 \mathrm{E}+00$ & 1600 \\
\hline $13 ?, 4$ & 0.4 & 0 & $0.0 n 0$ of +00 & $0.000 E+00$ & 1690 \\
\hline 159.0 & $n \cdot A$ & 70 & $1.033 F=07$ & $7.22^{\circ} t=0.8$ & 1400 \\
\hline 133.0 & 1.5 & $n$ & n. OOCOE + & $0.000 E+00$ & 1600 \\
\hline$\$ 37.0$ & 3.1 & 0 & $0.00 \mathrm{E}+00$ & $0.000 \mathrm{E}+00$ & 1600 \\
\hline 137.0 & 6.3 & n & $0.000 F+00$ & $0.00 \mathrm{OE}+00$ & 1600 \\
\hline 132.0 & 9.3 & 0 & $0.00 n F+00$ & $0.00 D E+00$ & 1600 \\
\hline 137.0 & 12.4 & (1) & $0.7 \cap \cap F+00$ & $0.000 E+00$ & 1600 \\
\hline & 15.5 & $n$ & $0.000 F+00$ & $0.000 E+00$ & 1600 \\
\hline & 18.5 & 0 & $0.000 F+100$ & $0.00 \cap E+00$ & 1600 \\
\hline & 21.7 & 0 & $0.00 \cap E+00$ & $0.000 \mathrm{E}+00$ & 1600 \\
\hline & 24.1 & 0 & $0.000 E+00$ & $0,00 \cap \mathrm{E}+00$ & 1600 \\
\hline & 31.0 & 0 & $0.000 F+00$ & $0.000 \mathrm{E}+00$ & 1600 \\
\hline 13 & 37,2 & n & $0.000 \mathrm{~F}+00$ & $0.000 \mathrm{E} \bullet 00$ & 1600 \\
\hline & 43.0 & 24 & $3,613 F-08$ & $2.529 E-08$ & 1600 \\
\hline & & 0 & $0.000 \mathrm{~F}+00$ & $0.000 \mathrm{E}+00$ & 1600 \\
\hline $\begin{array}{l}13 \\
13\end{array}$ & & 0 & $0.000 E+00$ & $0.000 E+00$ & 1600 \\
\hline & & $n$ & $0.000 \mathrm{~F}+00$ & $0.000 E+00$ & 1600 \\
\hline
\end{tabular}

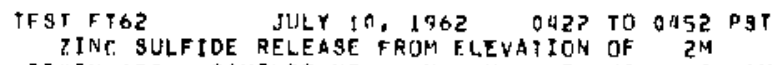
32 HON ARC $\triangle A M P L E R$ HT $1.5 \mathrm{M}$ UN 0.7 M/SEC AT $2 M$
TOWFR MATA FULLUH....

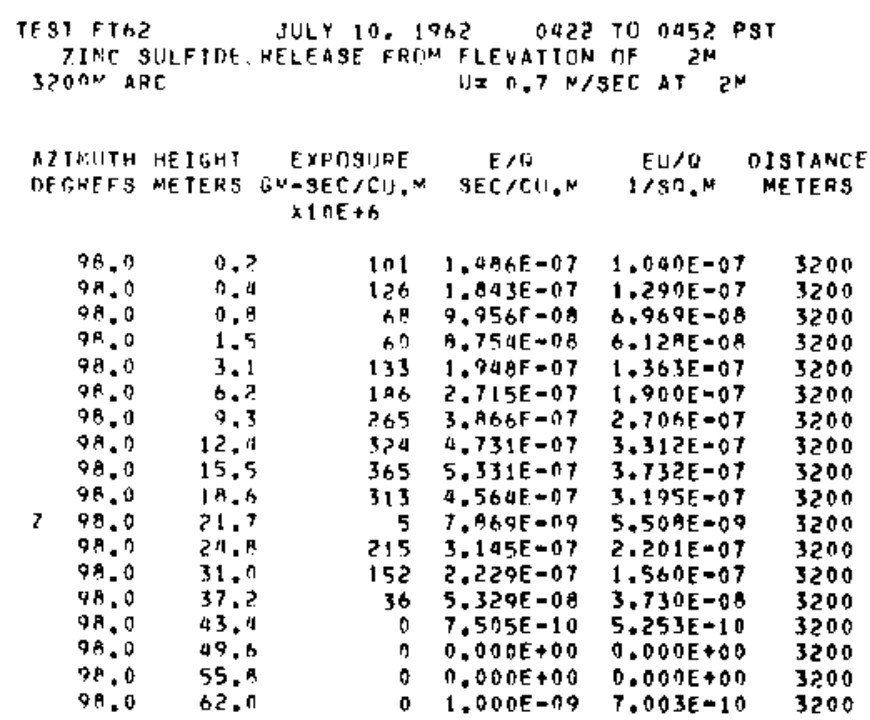

AZIMUTH HEIGHT EXPOSUIDE E/A DU DUTO DISTACE OFCREFS METFHS GN-SEC/CI,M SEC/CII, 1/9N.M MEFERG $x \rightarrow 0 E+6$

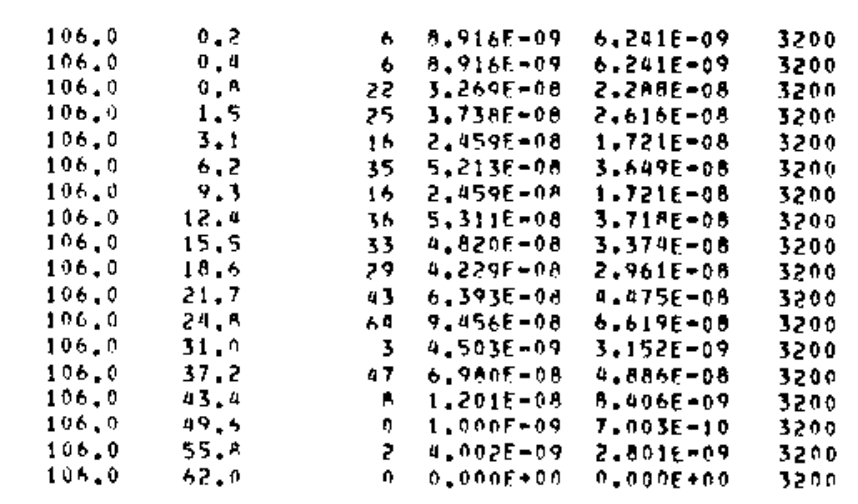

AZTMUTH HEIGHT EXPOSURE F/O FU/B DISTANCE DEGHEFS N'ETERS GNoSEC/CU.M SEC/CH.N 1/SO,M METERS AI $\mathrm{CE}$ +

\begin{tabular}{|c|c|c|c|c|c|}
\hline $15 n .6$ & 0.3 & 0 & $0.0 \cap \mathrm{BE}+00$ & $0.000 E+00$ & 3200 \\
\hline 130.0 & A. 4 & n & 0.0 DOF +00 & $0.00 \cap E+00$ & 3200 \\
\hline 130.0 & 0. & y & 14.45 AE $=09$ & $3.121 E-09$ & 3200 \\
\hline 130.0 & 1.5 & 0 & $1.000 \mathrm{~F}+00$ & 0. OOAE + ח & 3200 \\
\hline 130.0 & 3.1 & $1 \mathrm{~A}$ & $2.754 F=n B$ & $1.928 E=00$ & 3200 \\
\hline $15 n .0$ & $3 \cdot$ & 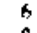 & 9. A SKE- O9 & $6.885 E=09$ & 3200 \\
\hline . 0 & & 6) & $0.000 E+00$ & $0.000 \mathrm{E}+0.0$ & 3200 \\
\hline 130.0 & 12.4 & 0 & $9.000 E+00$ & $0.000 E+00$ & 3200 \\
\hline 130.0 & 15.5 & 3 & $4,9 ! R F=09$ & $3.403 E=09$ & 3200 \\
\hline $136 \cdot 0$ & 14.5 & 0 & $0.000 E+00$ & $0.00 n E+0 n$ & 3200 \\
\hline 130.0 & 23.7 & 8 & $1.180 F=00$ & $8,262 E-09$ & 3200 \\
\hline 0 & 24 & 1 & 1. $501 E=09$ & $1.051 E=09$ & 3200 \\
\hline & $31 \cdot n$ & d & $6.004 E=09$ & $203 E=09$ & 3200 \\
\hline $\begin{array}{l}130.0 \\
130.0\end{array}$ & $\begin{array}{l}37.2 \\
43.4\end{array}$ & $\begin{array}{l}0 \\
a\end{array}$ & $\begin{array}{l}0.00 D E+00 \\
6.00 \mathrm{aE}=0 .\end{array}$ & $\begin{array}{l}0.000 E+00 \\
4.203 E=09\end{array}$ & $\begin{array}{l}3200 \\
3200\end{array}$ \\
\hline 130.0 & 49.6 & 6 & $1.005 E-O B$ & $7.003 E=09$ & 3200 \\
\hline 0 & A & 5 & $9,003 E=09$ & $5.602 E=09$ & 3200 \\
\hline & 62 & 0 & $0.000 E+00$ & $0.000 E+00$ & 3200 \\
\hline
\end{tabular}

3200

3200

3200

3200

3200

3200

3200

3200

3200

3200

3200

3200

3200

3200

3200

3200

3200

3200

3200

3200

3200

5200

3200

3200

3200

3200

3200

3200

3200

3200

$320 n$

3200

3200

\begin{tabular}{|c|c|c|c|}
\hline & $\begin{array}{l}5.350 E=00 \\
2.954 E=06\end{array}$ & $\begin{array}{l}3.745 E=08 \\
2.06 B E=06\end{array}$ & $\begin{array}{l}3200 \\
3200\end{array}$ \\
\hline & $\begin{array}{l}2.7 A Q E=06 \\
2.03 E=06\end{array}$ & $\begin{array}{l}1.952 f-06 \\
1990 E=06\end{array}$ & $\begin{array}{l}3200 \\
3200\end{array}$ \\
\hline 2228 & $3.2 \mathrm{ABE}=06$ & $2.274 E=06$ & 3200 \\
\hline $\begin{array}{l}2223 \\
2160\end{array}$ & $\begin{array}{l}3.241 E-06 \\
3.109 \varepsilon=06\end{array}$ & $\begin{array}{l}2.269 E=06 \\
2.200 E=06\end{array}$ & $\begin{array}{l}3200 \\
3200\end{array}$ \\
\hline 2086 & $3.042 E=06$ & $2.129 E=06$ & 3200 \\
\hline 1932 & $2.8: B E=06$ & $\therefore .972 \mathrm{E}-06$ & 3200 \\
\hline 1791 & $2.6 ! 1 \varepsilon-06$ & $1, A 2 B E=06$ & 3200 \\
\hline 1022 & $1.49 \mathrm{gE}=06$ & $1.043 E-06$ & 3200 \\
\hline 1630 & 2.3765 .06 & $1.663 E-06$ & 3200 \\
\hline $19 A 1$ & $? .8$ म $9 E=06$ & $2.022 E-06$ & 3200 \\
\hline 2038 & $2.972 E-06$ & $2.080 E=00$ & 3200 \\
\hline 2162 & 3. $152 E=06$ & $2,206 E=06$ & 3200 \\
\hline 1685 & $2.456 E=06$ & $1.719 \mathrm{E}-06$ & 3200 \\
\hline SA: & $1.43: E=06$ & $1.002 E-06$ & 3200 \\
\hline 1523 & $2.220 E-06$ & $1.55 \times E=06$ & 3200 \\
\hline 1201 & $1 . A 1 O E=0 B$ & $1.267 E-06$ & 3200 \\
\hline 1196 & $1.795 E-06$ & $1.2215=06$ & 3200 \\
\hline 1022 & $1.490 E=06$ & 1.0436 .06 & 3200 \\
\hline 1135 & $1.655 E=06$ & $1.1599=06$ & 3200 \\
\hline 839 & $1.224 \mathrm{E}-06$ & A. $57: 15-07$ & 3200 \\
\hline 739 & $1.077 E-06$ & $7.542 E=07$ & 3200 \\
\hline 528 & $7.698 E-07$ & $5.38 B E=07$ & 3200 \\
\hline 193 & P. $2 \geq 3 E=07$ & $1.976 E-07$ & 3200 \\
\hline 221 & $3.225 E-07$ & $2.257 E-07$ & 3200 \\
\hline 30 & a. $45 \mathrm{AE}=08$ & $3.121 E=00$ & 3200 \\
\hline 11 & $1.635 E=08$ & $1.144 \mathrm{EE}-0 \mathrm{~B}$ & 3200 \\
\hline 6 & A.936E-09 & $6.241 \mathrm{~F}=09$ & 3200 \\
\hline 115 & $1.679 E=07$ & $1.175 E-07$ & 5200 \\
\hline 191 & $2,104 \mathrm{E}=07$ & $1.956 \mathrm{E}-07$ & 3200 \\
\hline 120 & $1.754 \mathrm{E}-07$ & $1.227 E-07$ & 3200 \\
\hline Bo & $1.17 \triangle E=07$ & $A, 218 E-0 B$ & 3200 \\
\hline 64 & $9.362 \mathrm{E}=00$ & $6.553 \mathrm{E}-08$ & 3200 \\
\hline 3 & 4. $45 B E=09$ & $3,121 E=09$ & 3200 \\
\hline$? 2$ & $3.249 E=08$ & 2.2 BGF-08 & 320 ค \\
\hline ti & $1.635 F=08$ & $1.144 E-\cap 8$ & 3200 \\
\hline 13 & $1.93 Z E=O B$ & $1.352 \mathrm{E}=188$ & 3200 \\
\hline 14 & 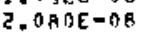 & $1.456 E-08$ & 3200 \\
\hline 37 & $5.4 \div 8 \mathrm{BE}-0 \mathrm{~B}$ & $3,849 E=08$ & 3200 \\
\hline 3 & $4.45 A E=09$ & 3.1PIE-0Q & 3200 \\
\hline 0 & $0.000 E+00$ & $0.000 E+00$ & 3200 \\
\hline 1 & $1.9 \times 6 E_{0}=09$ & $1.040 F-09$ & $320 n$ \\
\hline
\end{tabular}

1 
SO-SERIES EXPERINENT: 63 \& FT-53 ।

GROUND LEYEL AND TOWER SAMPLING 200 ANO 200 M ARCS. GROIND LEVEL.

SAMPLING ONLY 1600-12800 M RRCS. DUST AND CARBON CEPCSITS ON THE

FILTERS COLLECTED FROM THE 12800 M ARC.

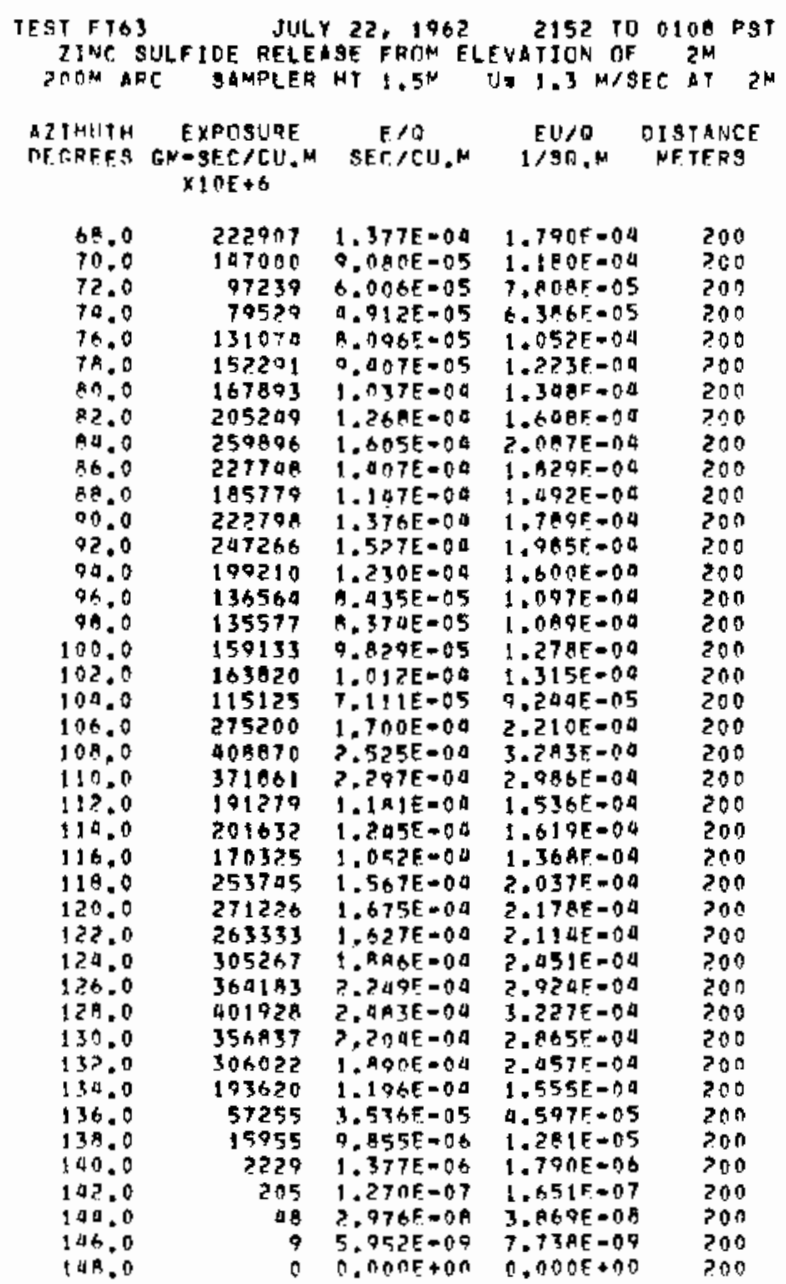

TOWER TATA FOLLOW....

TFSTFIT3 IULY 23, 1942 215? TO OLOA PST $2 J+3 C$ SIILFIDE RFLEASE FRTIN ELEVATITN OF $5^{\mathrm{N}}$

2OON AEC III $1.3 \mathrm{~N} / \mathrm{SFT}$ AT $2 M$

\begin{tabular}{|c|c|c|c|c|c|}
\hline $\begin{array}{l}2 \text { INLITH } \\
\text { EGNEES }\end{array}$ & $\begin{array}{l}\text { HEIGHT } \\
\text { NEYERS }\end{array}$ & 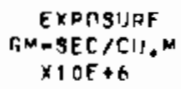 & $\begin{array}{c}E / Q \\
S E C / C 11, M\end{array}$ & $\begin{array}{c}E 10 / 0 \\
1 / 30,0\end{array}$ & $\begin{array}{l}\text { DI STANCE } \\
\text { METERS }\end{array}$ \\
\hline 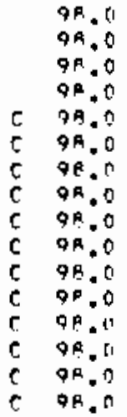 & $\begin{array}{l}0.7 \\
0.7 \\
1.0 \\
2.7 \\
4.1 \\
5.4 \\
6.8 \\
9.1 \\
9.7 \\
10.0 \\
13.5 \\
16.0 \\
18.0 \\
21.5\end{array}$ & $\begin{array}{r}142131 \\
104278 \\
84477 \\
103819 \\
113334 \\
177250 \\
115464 \\
66969 \\
19291 \\
76509 \\
6592 \\
3900 \\
1498 \\
497 \\
112 \\
13\end{array}$ & $\begin{array}{l}8.779 E=05 \\
8.912 E=05 \\
5.212 E=05 \\
6.413 E=05 \\
7.000 E=05 \\
7.860 E=05 \\
7.132 E=05 \\
0.136 F=05 \\
1.191 E=05 \\
1.637 F=05 \\
4.072 E=06 \\
2.465 E=06 \\
9.193 E=07 \\
3.073 E=07 \\
6.955 F=01 \\
0.346 E=08\end{array}$ & $\begin{array}{l}1.141 E=04 \\
1.159 E=04 \\
6.783 E=05 \\
B .336 E=05 \\
9.100 E=05 \\
1.022 E=04 \\
9.271 E=05 \\
5.377 E=05 \\
1.543 E=05 \\
2.129 E=05 \\
5.293 E=06 \\
3.204 E=06 \\
1.195 E=06 \\
3.995 E=07 \\
9.042 E=08 \\
1.085 E=0 .\end{array}$ & $\begin{array}{l}200 \\
200 \\
200 \\
200 \\
200 \\
200 \\
200 \\
200 \\
200 \\
200 \\
200 \\
200 \\
200 \\
200 \\
200 \\
200\end{array}$ \\
\hline
\end{tabular}

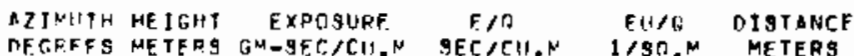
$x \quad 0 E+6$

\begin{tabular}{|c|c|c|c|c|c|}
\hline $\begin{array}{l}10 \mathrm{~h} .0 \\
104.0\end{array}$ & 0.3 & $\begin{array}{l}264.381 \\
295536\end{array}$ & $\begin{array}{l}1.7575=04 \\
1.925 F=04\end{array}$ & $\begin{array}{l}2.2+3 F=0 a \\
2.373 F=04\end{array}$ & $\begin{array}{l}200 \\
200\end{array}$ \\
\hline $10 \mathrm{~m} . \mathrm{C}$ & 1.4 & 205172 & $1.257 f=n a$ & $1.607 \mathrm{~F}=04$ & 200 \\
\hline $\begin{array}{l}104.0 \\
106.0\end{array}$ & $\begin{array}{l}2.7 \\
4.7\end{array}$ & $\begin{array}{l}1578 \times 2 \\
170033\end{array}$ & $\begin{array}{l}9.151 F=05 \\
1.108 F=04\end{array}$ & $\begin{array}{l}1.26 A E=04 \\
1.0 N 1 E=04\end{array}$ & $\begin{array}{l}200 \\
200\end{array}$ \\
\hline $10 \mathrm{a} .0$ & 5.4 & $1550 \geq 0$ & $58=0.5$ & $205 E-0.4$ & 200 \\
\hline 1 nh. 0 & 6.9 & $137 \geqslant 73$ & $R .470 \mathrm{~F}=\mathrm{NS}$ & $1.102 \mathrm{E}=04$ & 200 \\
\hline $10 n .0$ & 8.1 & $93{ }^{\circ} 63$ & $5.79 \mathrm{PE}-115$ & $7 E=05$ & 200 \\
\hline 104.11 & 9.4 & 76042 & $4,7 \geqslant 2 F=05$ & 3 AE -05 & $20 n$ \\
\hline 106.0 & $10, A$ & $5 \cap 3 \times 1$ & $.111 E=05$ & a $A F=05$ & 200 \\
\hline $106, \pi$ & 13.5 & $16+11$ & $1.014 F=05$ & $1.3]$ A $=05$ & 200 \\
\hline $1 \times n, n$ & $1 \mathrm{~h} \cdot 2$ & 7031 & $4.3 a_{3} F=A_{B}$ & $5.64 \mathrm{AF}=0 \mathrm{6}$ & 200 \\
\hline $0 \mathrm{n} .0$ & $1^{4}, 0$ & 2631 & $1.625 E=06$ & $2.113 f=06$ & $2 \cap 0$ \\
\hline 106.0 & 21.6 & 670 & $4.140 F-07$ & $5.3 A>F=07$ & 200 \\
\hline 106.0 & & 200 & $261 E-07$ & $1.640 E-07$ & 200 \\
\hline $10 n .0$ & 77.9 & $\ln R$ & $n .677 F=n A$ & D.ASAF $O$ OA & $2 n n$ \\
\hline
\end{tabular}

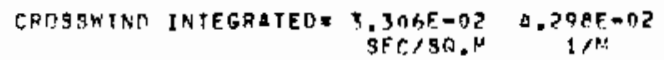

AZIMIITH HEZGHT FXPTSURE FID FU/O DISTANCE

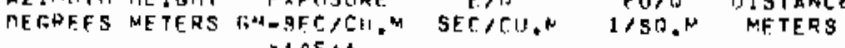

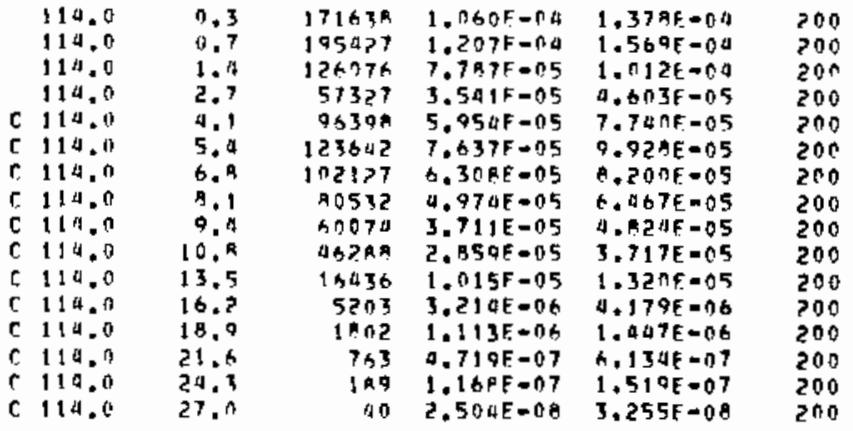




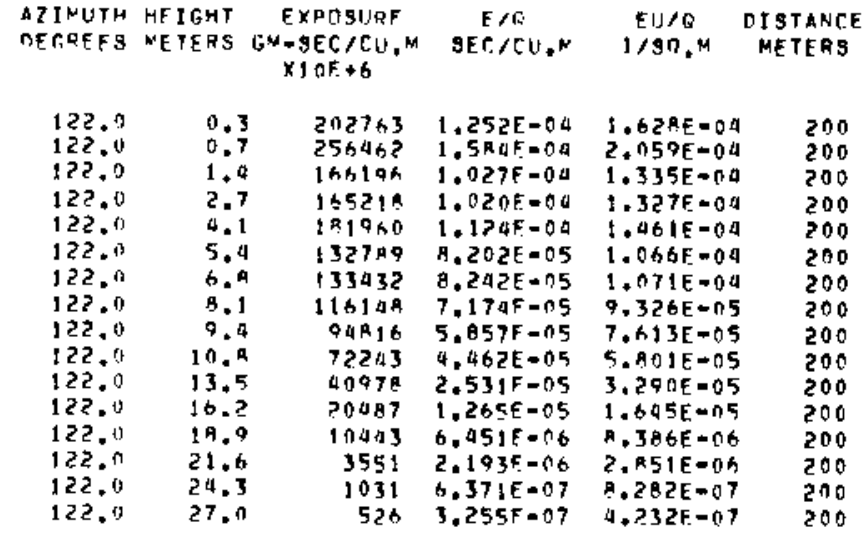

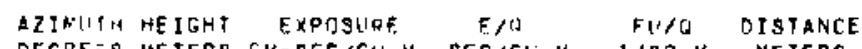

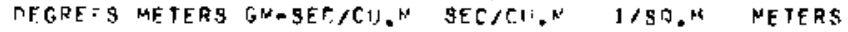

$$
\times 1 \cap E+6
$$

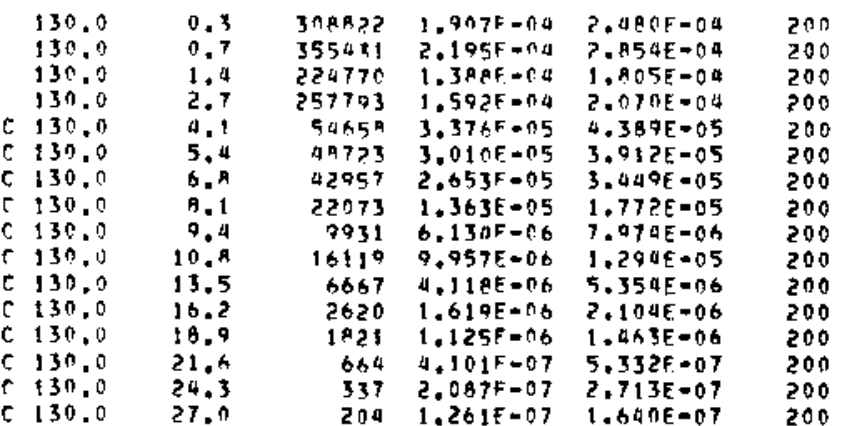

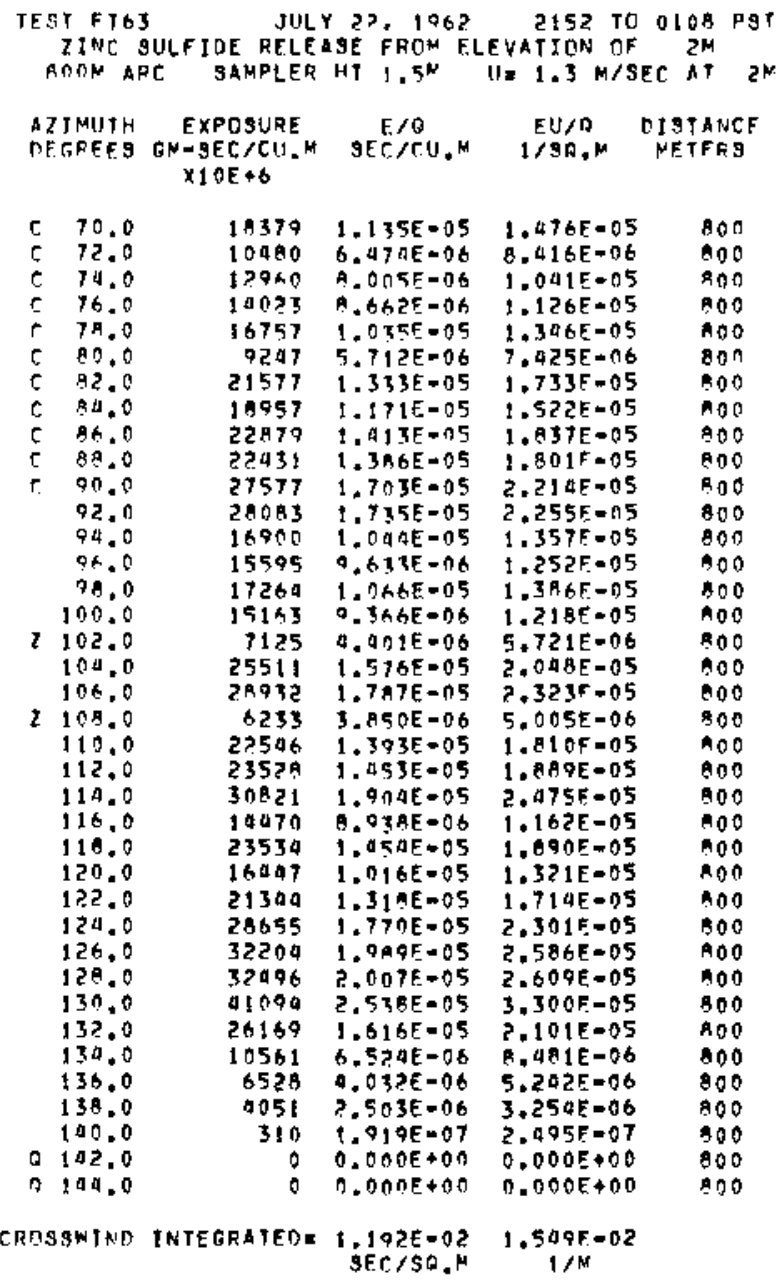

TOWFR DATA FCLLOW....

TEST FT6 JULY Z2, 1962 5152 TO OIAB PST ZINC SULFIJE RELEAGE FAOM ELEVATJTH TF ZM $U=1.3 \% / \operatorname{SEC}$ \& ${ }^{2 M}$

\begin{tabular}{|c|c|c|c|c|c|}
\hline $\begin{array}{l}\text { INUTH } \\
\text { EREESS }\end{array}$ & $\begin{array}{l}\text { HE IGHT } \\
\text { METERS }\end{array}$ & $\begin{array}{c}\text { EXPOSUPE } \\
\text { GM-SEC } / \mathrm{CU}, \mathrm{M} \\
\times I O F+6\end{array}$ & $\begin{array}{c}E / O \\
S E C / C U, N\end{array}$ & $\begin{array}{l}F U / 0 \\
1 / S O . M\end{array}$ & $\begin{array}{l}\text { DI I TANCE } \\
\text { METERS }\end{array}$ \\
\hline $\begin{array}{l}98.0 \\
98.0 \\
98.0 \\
98.0 \\
98.0 \\
9 A .0 \\
9 R .0 \\
98.0 \\
99.0 \\
99.0 \\
98.0 \\
98.0 \\
98.0 \\
99.0 \\
98.0 \\
99.0 \\
99.0\end{array}$ & $\begin{array}{r}0.3 \\
0.5 \\
1.0 \\
2.1 \\
4.2 \\
6.3 \\
8.5 \\
10.5 \\
12.4 \\
14.7 \\
16.5 \\
21.0 \\
25.2 \\
29.4 \\
33.6 \\
37.7 \\
42.0\end{array}$ & $\begin{array}{r}13515 \\
14206 \\
9922 \\
10555 \\
8660 \\
9912 \\
9077 \\
7909 \\
5943 \\
3911 \\
2762 \\
1089 \\
518 \\
109 \\
50 \\
26 \\
10\end{array}$ & $\begin{array}{l}8.348 F=06 \\
8.775 E=06 \\
6.127 F=06 \\
6.520 F=06 \\
5.349 E=06 \\
6.123 E=06 \\
5.607 E=06 \\
4.885 F=06 \\
3.634 E=06 \\
2.350 E=06 \\
1.706 F=06 \\
6.728 E=07 \\
1.965 E=07 \\
9.240 E=08 \\
3.100 E=08 \\
1.615 F=08 \\
6.279 E=09\end{array}$ & 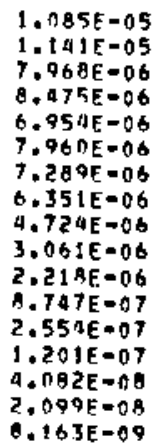 & $\begin{array}{l}800 \\
000 \\
000 \\
000 \\
800 \\
000 \\
800 \\
000 \\
800 \\
100 \\
000 \\
000 \\
000 \\
000 \\
000 \\
000 \\
800\end{array}$ \\
\hline
\end{tabular}




\begin{tabular}{|c|c|c|c|c|c|}
\hline $\begin{array}{l}\text { AZTMEITH } \\
\text { DETREES }\end{array}$ & $\begin{array}{l}\text { HE IGHT } \\
\text { ME TERS }\end{array}$ & $\begin{array}{c}\text { EXPNSURE } \\
G \mathrm{MEC} / \mathrm{C} ! \mathrm{M} \\
x+0 E+b\end{array}$ & $\begin{array}{c}E / H \\
S E C / C U, N\end{array}$ & $\begin{array}{c}E \cup / 0 \\
1 / S 0, N\end{array}$ & DISTAN \\
\hline $\begin{array}{l}106.0 \\
106.0 \\
106.0 \\
106.0 \\
106.0 \\
106.0 \\
105.0 \\
106.0 \\
106.0 \\
106.0 \\
106.0 \\
106.0 \\
104.0 \\
106.0 \\
106.0 \\
104.0 \\
104.0\end{array}$ & $\begin{array}{r}0.3 \\
0.5 \\
1.13 \\
2.1 \\
4.2 \\
6.3 \\
8.4 \\
10.5 \\
12.4 \\
14.7 \\
16.8 \\
21.1 \\
25.2 \\
29.9 \\
33.8 \\
37.8 \\
42.1\end{array}$ & $\begin{array}{l}26546 \\
24711 \\
19026 \\
19155 \\
19676 \\
19538 \\
15198 \\
13496 \\
10182 \\
6630 \\
5335 \\
2216 \\
750 \\
176 \\
141 \\
65 \\
\times n\end{array}$ & 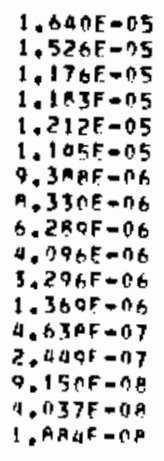 & 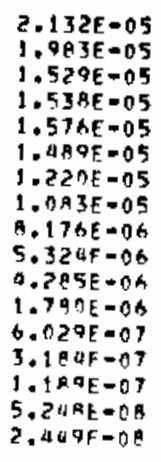 & $\begin{array}{l}800 \\
800 \\
000 \\
000 \\
900 \\
800 \\
800 \\
800 \\
800 \\
100 \\
000 \\
800 \\
800 \\
800 \\
900 \\
000 \\
000\end{array}$ \\
\hline $\begin{array}{l}\text { IMUTH } \\
\text { GREFS }\end{array}$ & $\begin{array}{l}\text { HETGHT } \\
\text { METERS }\end{array}$ & $\begin{array}{c}\text { EXPGSURF } \\
\text { G:A-SFC } \\
\times 1 \text { OE }+ \text { A }\end{array}$ & $\begin{array}{c}E / 1 \\
5 E R / C U, N\end{array}$ & $\begin{array}{c}E U / C \\
1 / 39.19\end{array}$ & $\begin{array}{l}\text { DISTANCE } \\
\text { NETERS }\end{array}$ \\
\hline 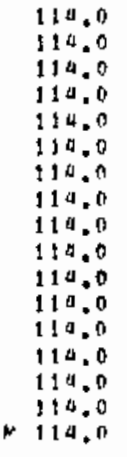 & $\begin{array}{l}0.3 \\
0.5 \\
1.7 \\
2.1 \\
4.7 \\
0.5 \\
8.9 \\
10.5 \\
12.6 \\
14.7 \\
16.8 \\
21.7 \\
25.2 \\
29.9 \\
33.6 \\
37.2 \\
42.0\end{array}$ & $\begin{array}{r}27793 \\
29346 \\
16076 \\
96916 \\
20524 \\
19713 \\
11660 \\
10595 \\
7901 \\
5970 \\
3263 \\
1657 \\
949 \\
393 \\
251 \\
170 \\
0\end{array}$ & 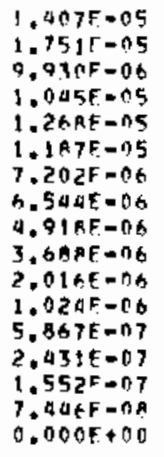 & $\begin{array}{l}1.829 E=05 \\
2.276 E=05 \\
1.291 E=05 \\
1.35 A F=05 \\
1.65 B E=05 \\
1.543 E=05 \\
9.363 F=06 \\
8.50 A E=06 \\
6.393 E=06 \\
1.794 E=06 \\
2.621 E=06 \\
1.331 E=06 \\
7.627 E=07 \\
3.160 E=07 \\
2.017 E=07 \\
9.679 F=08 \\
0.000 E=00\end{array}$ & $\begin{array}{l}600 \\
800 \\
000 \\
000 \\
000 \\
R 00 \\
000 \\
100 \\
000 \\
800 \\
000 \\
000 \\
800 \\
000 \\
100 \\
800 \\
000\end{array}$ \\
\hline $\begin{array}{l}\text { AZINUTH } \\
\text { DEGREES }\end{array}$ & $\begin{array}{l}\text { HEITHT } \\
\text { METEQS }\end{array}$ & $\begin{array}{c}\text { EXPDSURF } \\
C M-S E C / C O=N \\
\times 10 E+6\end{array}$ & $\begin{array}{c}E / 0 \\
9 E C / C+1 . R\end{array}$ & $\begin{array}{l}E(1 / A \\
1 / \$ \cap, M\end{array}$ & $\begin{array}{l}\text { DISTANEE } \\
\text { METERS }\end{array}$ \\
\hline $\begin{array}{l}122.0 \\
122.0 \\
122.0 \\
122.0 \\
122.0 \\
122.0 \\
122.0 \\
122.0 \\
122.0 \\
122.0 \\
122.0 \\
122.0 \\
122.0 \\
122.0 \\
122.0 \\
122.0 \\
122.0\end{array}$ & 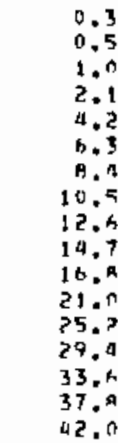 & $\begin{array}{l}189 A 6 \\
17923 \\
1347 A \\
14590 \\
13796 \\
12687 \\
12226 \\
10424 \\
9024 \\
6003 \\
6125 \\
4236 \\
1646 \\
1009 \\
514 \\
201 \\
96\end{array}$ & $\begin{array}{l}1.102 E=05 \\
1.107 F=05 \\
8.325 F=06 \\
9.012 E=06 \\
9.401 F=06 \\
7.037 F=06 \\
7.552 F=06 \\
6.439 E=06 \\
5.574 F=06 \\
4.203 E=06 \\
3.821 F=06 \\
2.617 F=06 \\
1.017 F=06 \\
8.234 F=07 \\
3.202 F=07 \\
1.207 F=07 \\
3.090 F=08\end{array}$ & $\begin{array}{l}1.0 B 4 E=105 \\
1.039 E=05 \\
1.082 F=05 \\
1.172 E=05 \\
1.104 E=155 \\
1.019 E=05 \\
9.217 E=06 \\
1.37 \cap E=06 \\
7.247 E=06 \\
5.463 E=06 \\
4.967 E=06 \\
3.402 E=06 \\
1.322 E=06 \\
9.105 E=07 \\
0.163 F=07 \\
1.621 E-07 \\
4.54 A F=0 B\end{array}$ & $\begin{array}{l}800 \\
A 00 \\
800 \\
800 \\
800 \\
B 00 \\
0100 \\
B 00 \\
800 \\
100 \\
800 \\
800 \\
800 \\
B 00 \\
800 \\
800 \\
000\end{array}$ \\
\hline
\end{tabular}




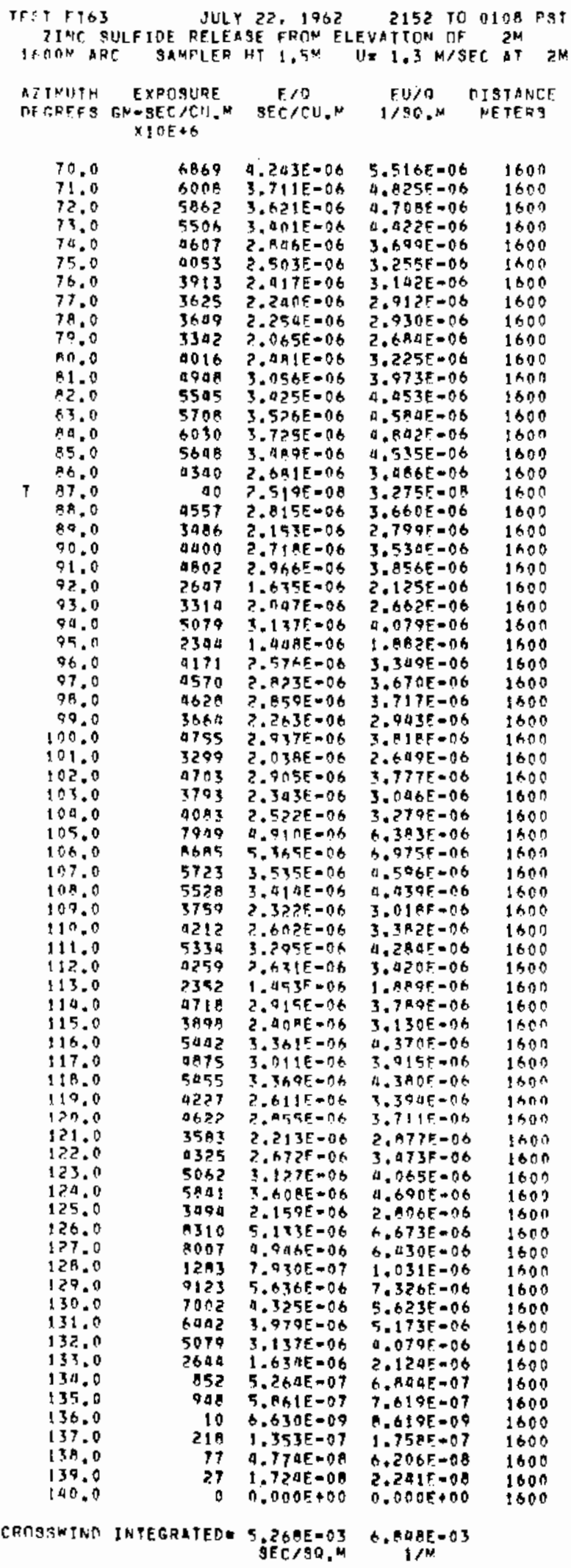




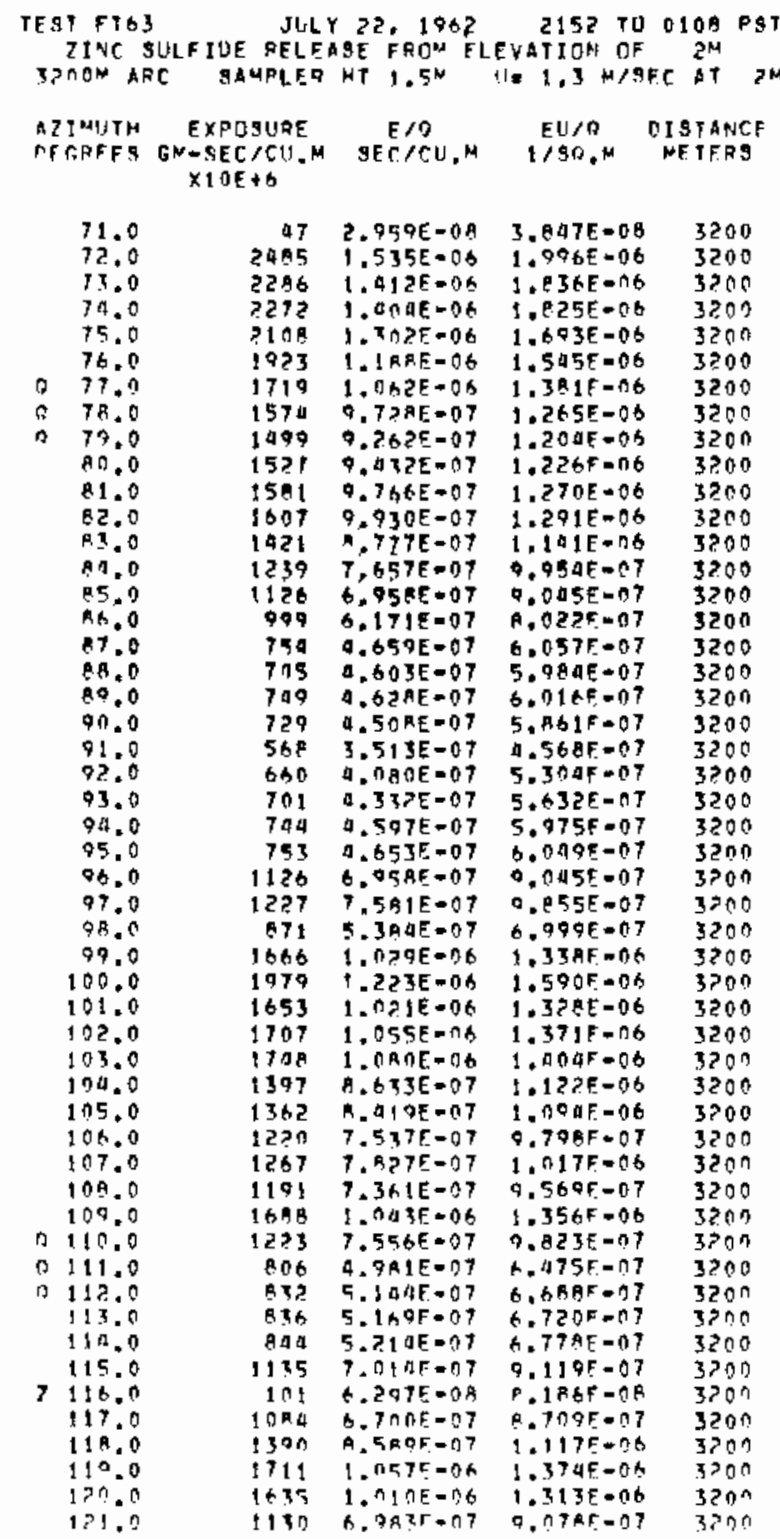

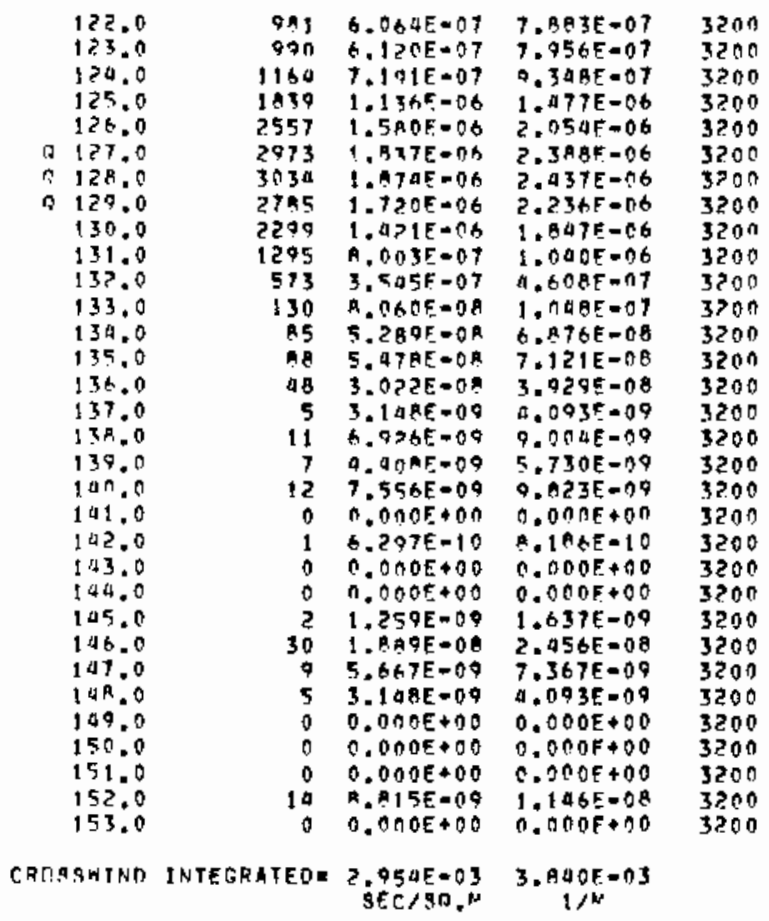




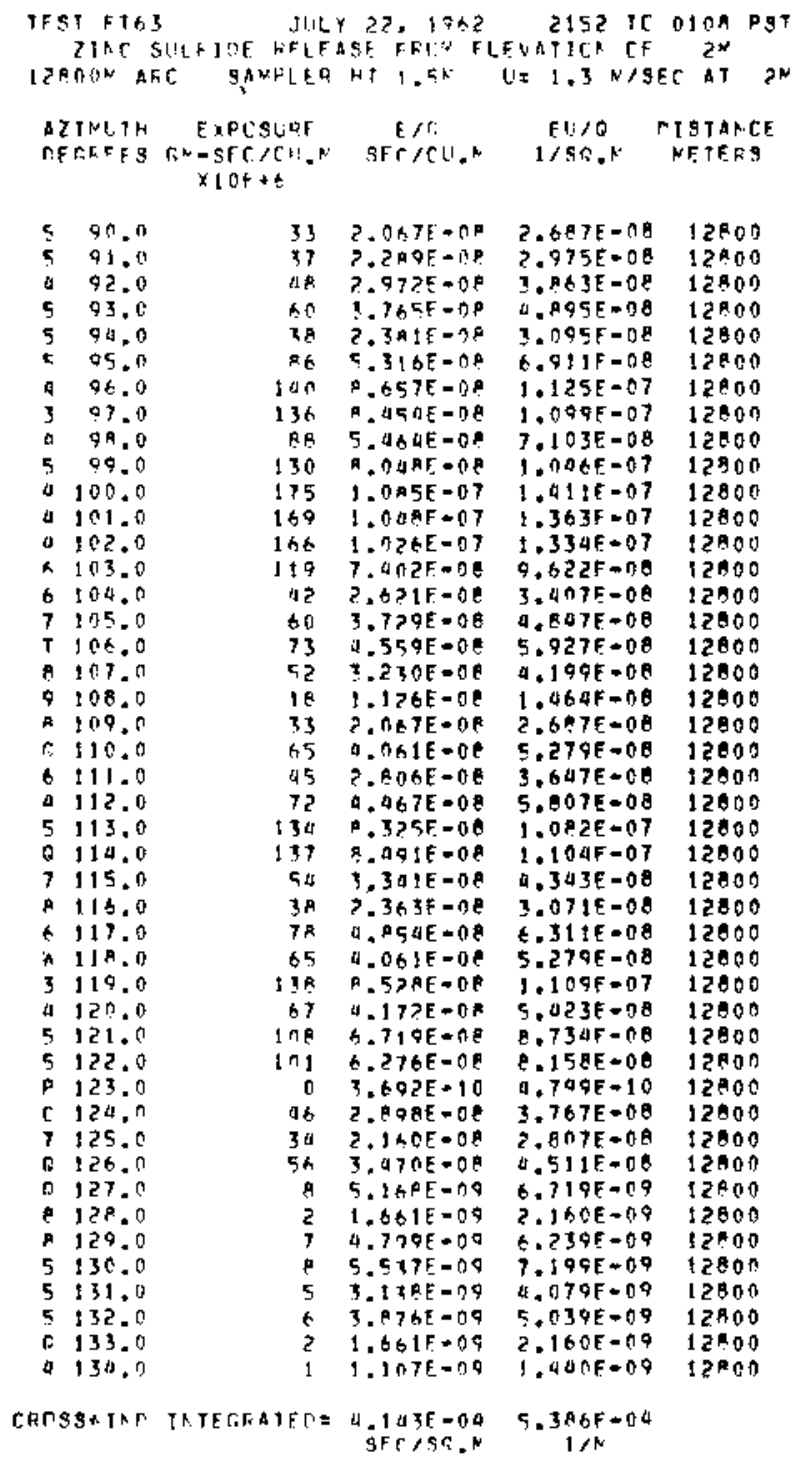


3D-SERIES EXPERIMENT: 64 : FT-64 J

GROUND LEYEL AND TOWER SAMPL ING 200 ANO 800 M ARCS. GROUNC LE:EL

SAMPLING ONLY ON THE 1600 AND 3200 M ARCS.

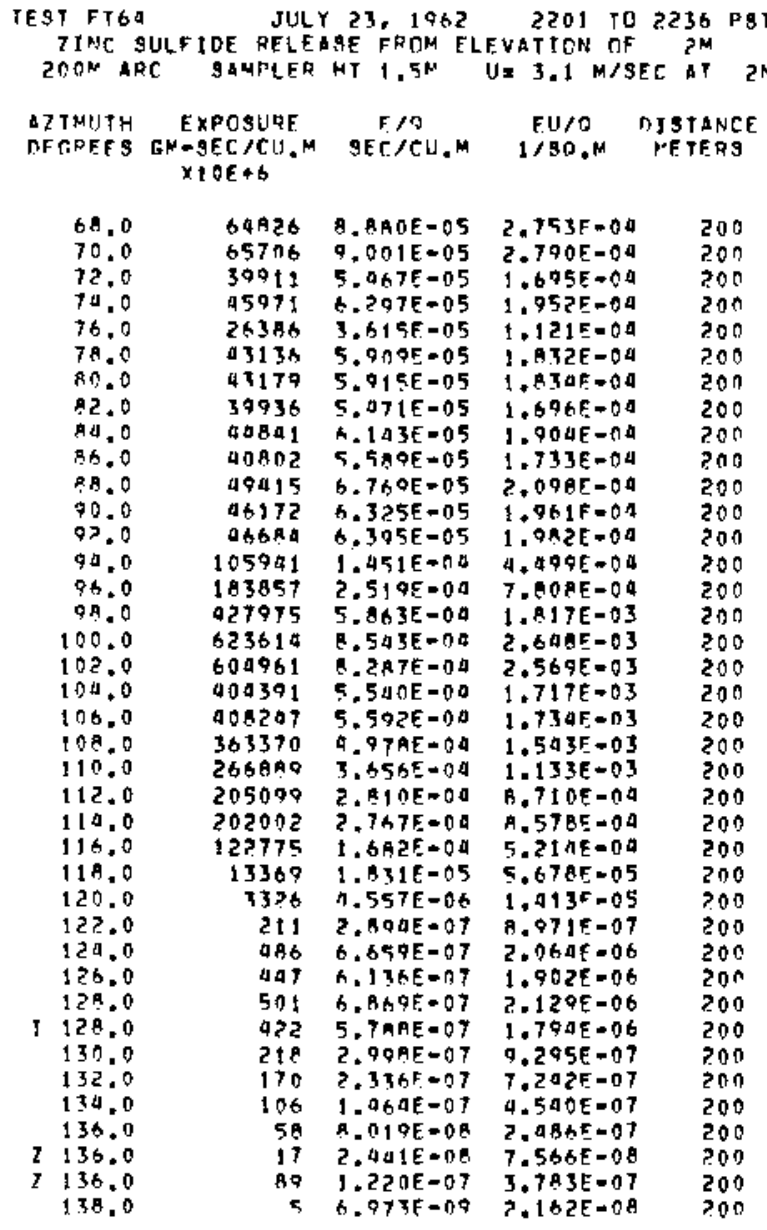

TOMER CATA FOLEOH,...

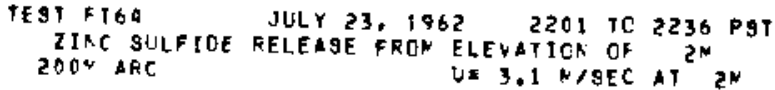

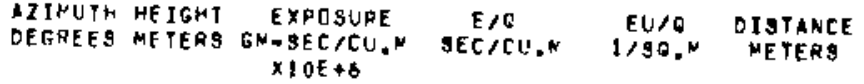

\begin{tabular}{|c|c|c|c|c|c|}
\hline $\begin{array}{l}98.0 \\
98.0 \\
98.0 \\
98.0 \\
98.0 \\
98.0 \\
98.0 \\
98.0 \\
98.0 \\
98.0 \\
98.0 \\
98.0 \\
98.0 \\
98.0 \\
9.00 \\
98.0\end{array}$ & $\begin{array}{l}0.3 \\
0.7 \\
1.4 \\
2.7 \\
4.1 \\
5.9 \\
6.9 \\
8.1 \\
9.0 \\
10.0 \\
13.5 \\
16.2 \\
18.9 \\
21.6 \\
24.3 \\
27.0\end{array}$ & $\begin{array}{r}545874 \\
975377 \\
373027 \\
355017 \\
354016 \\
207930 \\
182589 \\
133229 \\
81113 \\
45721 \\
16572 \\
5165 \\
965 \\
53 \\
0 \\
0\end{array}$ & $\begin{array}{l}7.472 E=04 \\
1.512 E=04 \\
5.115 E=04 \\
4.864 E=04 \\
4.904 E=04 \\
3.396 E=04 \\
2.501 E=04 \\
1.025 E=04 \\
1.111 E=04 \\
.263 E=05 \\
2.270 E=05 \\
1.076 F=06 \\
1.323 E=06 \\
7.204 E=08 \\
0.000 E+00 \\
0.000 E+00\end{array}$ & $\begin{array}{l}2.318 E=03 \\
2.019 E=03 \\
1.586 E=03 \\
1.508 E=03 \\
1.520 E=03 \\
1.053 E=03 \\
7.754 E=04 \\
5.658 E=04 \\
3.445 E=00 \\
1.942 E=00 \\
7.038 E=05 \\
2.193 E=05 \\
1.101 E=06 \\
2.261 E=07 \\
0.000 E+00 \\
0.000 E+00\end{array}$ & $\begin{array}{l}200 \\
200 \\
200 \\
200 \\
200 \\
200 \\
200 \\
200 \\
200 \\
200 \\
200 \\
200 \\
200 \\
200 \\
200 \\
200\end{array}$ \\
\hline
\end{tabular}

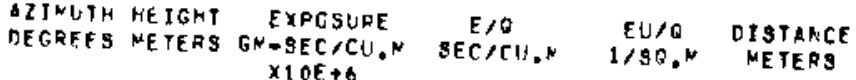

106.0

106.0

106.0

106.0

106.0

$10 t .0$

$10 t .0$

106,0

100.0

106.0

106.0

106.0

106.0

106.0

0.3
0.7
1.4
2.7
4.1
5.9
6.9
8.1
10.4
13.5
16.2
18.9
21.4
24.3
27.0

433566

$5.939 E=04 \quad 1.041 E-03$

200

$230103 \quad 3.153 E-04 \quad 9.775 F=04 \quad 200$

422648 5.79CE-04 $1.795 E-03200$

$325469 \quad 0.45 \mathrm{FE}=04 \quad 1.362 \mathrm{E}-03 \quad 200$

$329310 \quad 4.511 E-04 \quad 1.398 E-03 \quad 200$

2.701E-0 $1.147 \mathrm{~F}-03200$

208596 2.857E-tid .85EF-04 2nO

$1994312.595 F-04$ A. OAUE=0A

$157235 \quad 2.154 E-14 \quad 6.677 E-04 \quad 200$

$143697 \quad 1.96 P F=04 \quad 6.102 E=04 \quad 200$

$\begin{array}{llll}74541 \quad 1.021 E-04 & 3.165 E=04 & 200\end{array}$

$26372 \quad 3.887 F=05 \quad 1.205 E-04 \quad 200$

$11000 \quad 1.507 F-05 \quad 4.672 E-05 \quad 300$

$3109 \quad 4.3 .3 E=C B \quad 1.359 E=05 \quad 200$

6B) $9.142 F=07$ 2.034F=0

$\begin{array}{lll}175 \quad 2.402 E-07 \quad 7.946 E-07 & 200\end{array}$ 


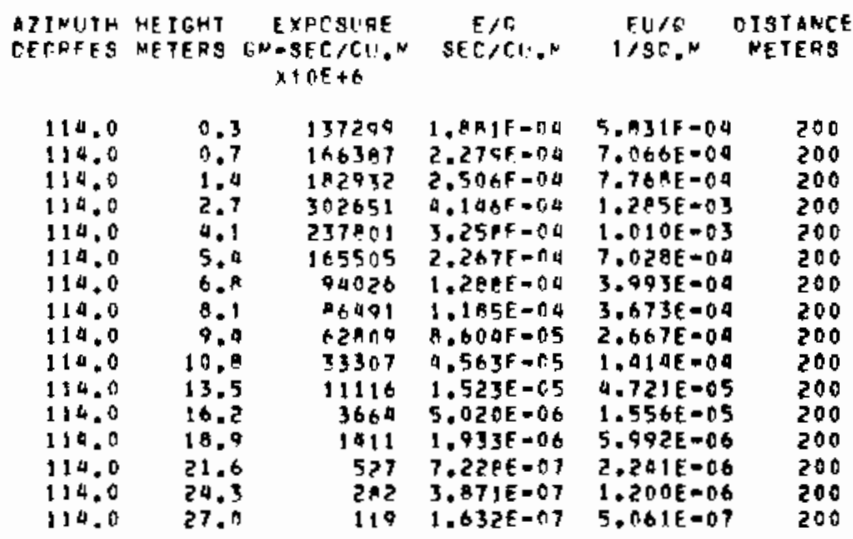

DIRUTH HEIGHT EXPCSURE E/G EU/O DISTANCE DEGPEY 3 METERS GM-SEC/CU,H SEC/CI, N 1/3Q,M NETEAS $\times 10 \mathrm{O}+6$

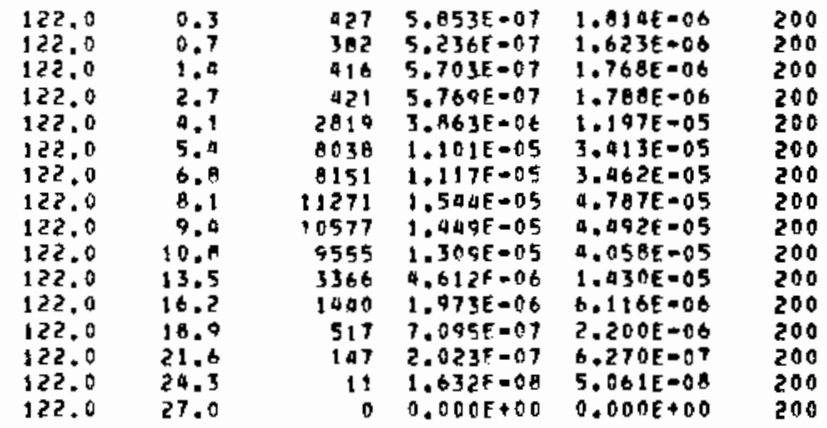

ATINUTH HEIGHT EXPCQURE E/G TINO DISTANCE CEGRFP HETEFS GN-SEC/CU.N SEC/CI!.N IJSON NETERS $\times 10 E+6$

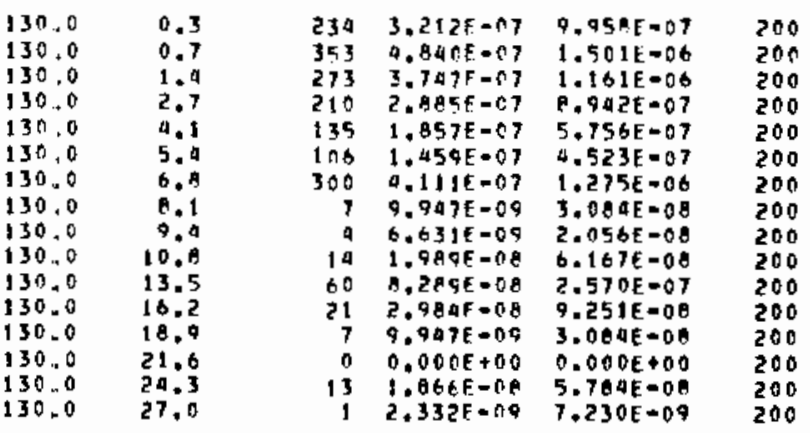

\begin{abstract}
IFST FT64
JULY 25, 1962 2201 TO 2236 PST TINC SULFIDE RELEASE FROM ELEVATION TF $2 \mathrm{M}$ ROON ARC GAMPLER HT $1.5 \mathrm{M} U=$ T.T M/SEC AT $P$
\end{abstract}

AZTMIIM EXPOSURE
DFTREFS GM-GEC/CIU.M SEC/CU,N TU/O DISTANCE $\times 10 E+6$

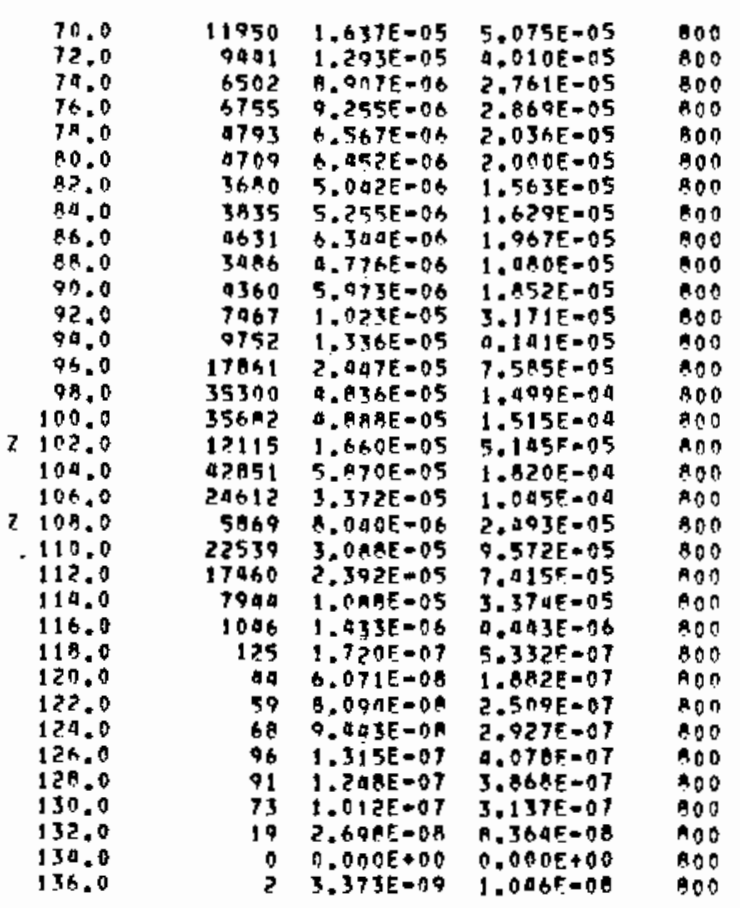

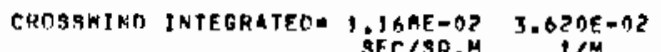

TOHER DATA FOLLOW....

TEST FTOA JULY 23,1962 2201 TO 2236 PST

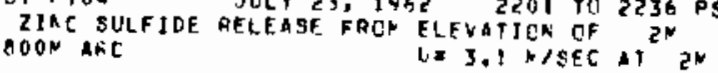

AZIMUTH HEIGHT EXPOSURE E/O EU/O OISTANCE

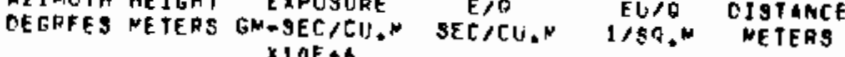

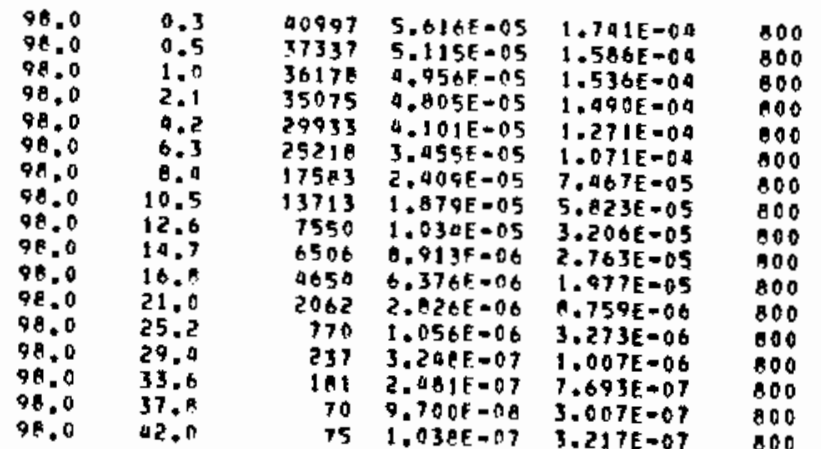




\begin{tabular}{|c|c|c|c|c|c|c|c|c|c|c|c|}
\hline $\begin{array}{l}\text { SIINLTH } \\
\text { CEGREES }\end{array}$ & $\begin{array}{l}\text { HEIGHT } \\
\text { WEYERS }\end{array}$ & $\begin{array}{c}\text { EXPOSUAE } \\
G M=9 E C / C U, N \\
\times 10 E+6\end{array}$ & $\begin{array}{c}E, f \\
S E C / C U, N\end{array}$ & $\begin{array}{l}f \cup / 0 \\
1 / 50 . M\end{array}$ & $\begin{array}{l}\text { DISTANCE } \\
\text { WETEWS }\end{array}$ & $\begin{array}{l}\text { DINUTH } \\
\text { DEGFEES }\end{array}$ & $\begin{array}{l}\text { HE IGHT } \\
\text { NETERS }\end{array}$ & $\begin{array}{c}\text { EXPCSURE } \\
\text { GN= } 9 E C>C U . N \\
\times 10 E+6\end{array}$ & $\begin{array}{c}\mathrm{sFC} / \mathrm{Cu} \\
\mathrm{sen}\end{array}$ & $\begin{array}{c}F L / C \\
1,50 . N\end{array}$ & $\begin{array}{l}\text { DISTANCE } \\
\text { NETERS }\end{array}$ \\
\hline $\begin{array}{l}106.0 \\
106.0 \\
106.0 \\
100.0 \\
106.0 \\
106.0 \\
106.0 \\
106.0 \\
104.0 \\
106.0 \\
106.0 \\
106.0 \\
106.0 \\
106.0 \\
106.0 \\
106.0 \\
106.0\end{array}$ & $\begin{array}{l}0.3 \\
0.5 \\
1.0 \\
2.1 \\
4.2 \\
6.7 \\
8.0 \\
10.5 \\
12.6 \\
14.7 \\
16.7 \\
21.0 \\
25.2 \\
29.0 \\
33.6 \\
37.2 \\
42.0\end{array}$ & 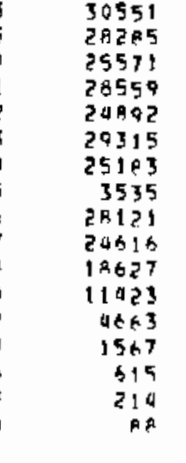 & $\begin{array}{l}4.185 E=05 \\
3.875 E=05 \\
3.50 Z E=05 \\
3.912 F=05 \\
3.410 F=05 \\
4.01 E E=05 \\
3.450 E=05 \\
3.843 E=06 \\
3.852 F=05 \\
3.372 F=05 \\
2.552 F=05 \\
1.565 E=05 \\
6.309 F=01 \\
2.34 F F=06 \\
9.437 F=07 \\
2.933 E-07 \\
1.24 F F=07\end{array}$ & $\begin{array}{l}1.297 E=04 \\
1.201 E=04 \\
1.086 E=04 \\
1.213 E=04 \\
1.057 E=04 \\
1.245 E=04 \\
1.069 E=04 \\
1.501 E=05 \\
1.150 E=04 \\
1.045 E=04 \\
7.810 E=05 \\
4.851 E=05 \\
1.980 E=05 \\
6.65 R E=06 \\
2.415 E=06 \\
9.091 E=07 \\
3.776 E=07\end{array}$ & 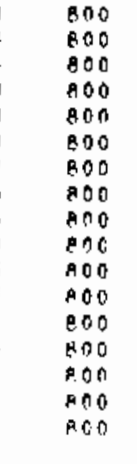 & $\begin{array}{l}130.0 \\
130.0 \\
130.0 \\
130.0 \\
130.0 \\
130.0 \\
130.0 \\
130.0 \\
130.0 \\
130.0 \\
130.0 \\
130.0 \\
130.0 \\
130.0 \\
130.0 \\
130.0 \\
130.0\end{array}$ & $\begin{array}{l}0.3 \\
0.5 \\
1.0 \\
2.1 \\
4.2 \\
6.3 \\
8.4 \\
10.5 \\
12.6 \\
10.7 \\
16.8 \\
21.0 \\
25.2 \\
29.4 \\
33.5 \\
37.8 \\
42.0\end{array}$ & $\begin{array}{r}55 \\
93 \\
590 \\
60 \\
70 \\
35 \\
10 \\
30 \\
0 \\
18 \\
0 \\
44 \\
6 \\
1 \\
1 \\
1 \\
13\end{array}$ & $\begin{array}{l}7.662 E=08 \\
1.277 F=07 \\
7.409 F=07 \\
8.355 E=08 \\
1.090 E=07 \\
4.011 E=08 \\
2.560 E=08 \\
5.052 F=08 \\
0.000 E+00 \\
2.566 E=08 \\
0.000 E+00 \\
6.090 E=08 \\
9.020 E=09 \\
2.256 E=09 \\
2.256 E=09 \\
2.256 E=09 \\
1.005 E=08\end{array}$ & $\begin{array}{l}2.175 E=07 \\
3.959 E=07 \\
2.297 E-06 \\
2.565 E=07 \\
3.300 E=07 \\
1.491 E=07 \\
7.954 E=0 B \\
1.490 E=07 \\
0.000 E+00 \\
7.950 E=08 \\
0.000 E+00 \\
1.989 E=07 \\
2.797 E=06 \\
6.993 E=09 \\
6.993 E=09 \\
6.993 E=09 \\
5.595 E=08\end{array}$ & $\begin{array}{l}800 \\
800 \\
800 \\
800 \\
800 \\
800 \\
900 \\
800 \\
800 \\
800 \\
800 \\
000 \\
800 \\
800 \\
800 \\
800 \\
800\end{array}$ \\
\hline
\end{tabular}

\begin{tabular}{|c|c|c|c|c|c|}
\hline $\begin{array}{l}\text { Z1NU1? } \\
\text { EERFES }\end{array}$ & $\begin{array}{l}\text { ME JGH' } \\
\text { METEAS }\end{array}$ & $\begin{array}{c}\text { EXPOSURE } \\
\text { GN-SEC }>C H \text { * } \\
\times 10 E+6\end{array}$ & $\begin{array}{c}F / C \\
S E C>C 11 . N\end{array}$ & $\begin{array}{l}F L / C \\
1 / 50.2\end{array}$ & $\begin{array}{r}\text { DISTARC } \\
\text { NETEAS }\end{array}$ \\
\hline $\begin{array}{l}119.0 \\
119.0 \\
114.0 \\
110.0 \\
114.0 \\
114.0 \\
114.0 \\
114.0 \\
114.0 \\
114.0 \\
114.0 \\
114.0 \\
114.0 \\
114.0 \\
114.0 \\
114.0 \\
114.0\end{array}$ & $\begin{array}{l}0.3 \\
0.5 \\
1.0 \\
2.1 \\
4.2 \\
6.3 \\
8.9 \\
10.5 \\
12.6 \\
14.7 \\
16.8 \\
21.0 \\
25.2 \\
29.4 \\
33.6 \\
37.0 \\
42.0\end{array}$ & $\begin{array}{r}5701 \\
8932 \\
7269 \\
7805 \\
17639 \\
17590 \\
11413 \\
10114 \\
6572 \\
5525 \\
3680 \\
1890 \\
469 \\
283 \\
158 \\
39 \\
23\end{array}$ & $\begin{array}{l}7.011 F=06 \\
9.496 F=06 \\
9.959 F=06 \\
1.069 E=05 \\
2.41 t E=05 \\
2.410 F=05 \\
1.564 F=05 \\
1.396 E=05 \\
9.003 E=06 \\
7.569 E=06 \\
5.055 E=06 \\
2.601 E=06 \\
6.429 E=07 \\
3.880 E=07 \\
2.164 F=07 \\
5.414 E=08 \\
3.158 F=08\end{array}$ & 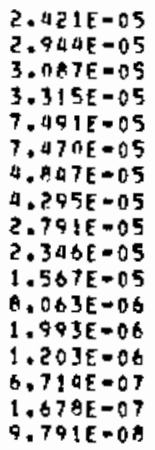 & $\begin{array}{l}A 00 \\
800 \\
800 \\
000 \\
000 \\
800 \\
000 \\
800 \\
800 \\
800 \\
800 \\
000 \\
800 \\
100 \\
800 \\
800 \\
800\end{array}$ \\
\hline
\end{tabular}

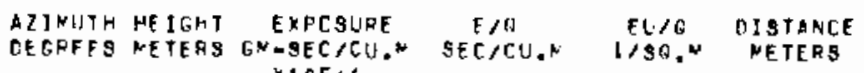
$\times 10 E+6$

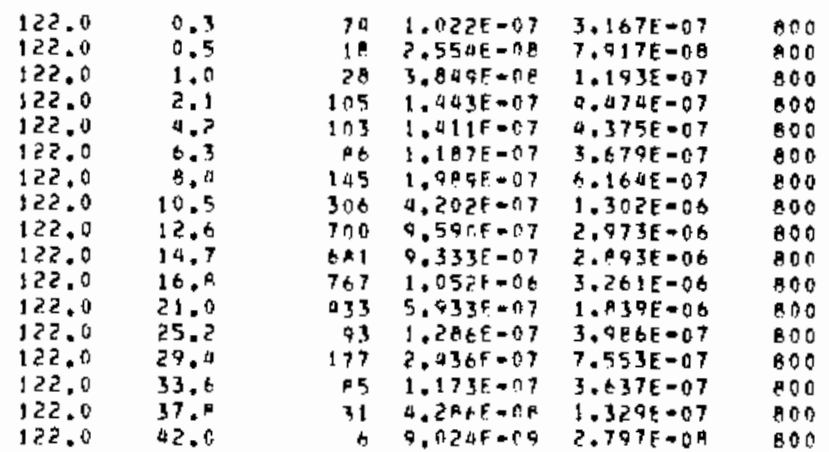




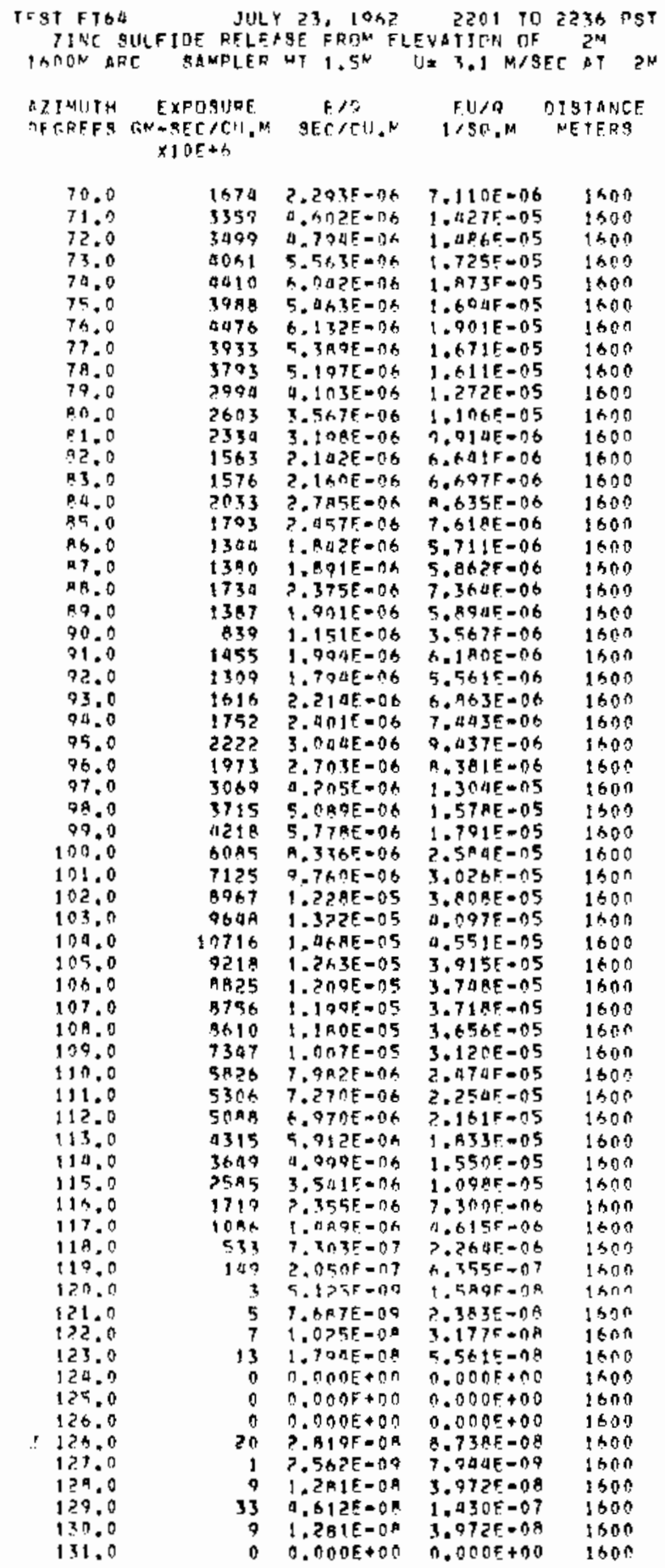

TEST FT64 JULY 23. 1962 2201 TO 2236 PST ZINE SULFTOE RELEASE FRIJN FLFVATION TOF SN $^{\text {N }}$ SONAM ARC SANFLER HT 1 .5N UN 3.1 N/SFC AT ?M

\begin{tabular}{|c|c|c|c|c|}
\hline $\begin{array}{l}\text { AITHUTH } \\
\text { AFTREFS }\end{array}$ & $\begin{array}{l}\text { FXFCSIJPE } \\
G M-S E C / C U . M \\
\times 1 O E+6^{\circ}\end{array}$ & $\begin{array}{c}E / D \\
\text { SEC } / C U, N\end{array}$ & $\begin{array}{c}5,100 \\
1 / 50.2\end{array}$ & $\begin{array}{l}\text { OISTANCE } \\
\text { NFTFHS }\end{array}$ \\
\hline
\end{tabular}

\begin{tabular}{|c|c|c|c|c|}
\hline $\begin{array}{l}71.0 \\
72.0 \\
73.0\end{array}$ & $\begin{array}{r}0 \\
21 \\
50 \\
70\end{array}$ & $\begin{array}{l}0.0 n C E+00 \\
2.02 \cap E=08 \\
6.9 A 2 E=0 B \\
102 F=07\end{array}$ & $\begin{array}{l}0.000 E+00 \\
9.052 E-08 \\
2.165 E=07 \\
3.395 E=07\end{array}$ & $\begin{array}{l}3 ? 00 \\
3200 \\
3200 \\
3200\end{array}$ \\
\hline 70.0 & $\begin{array}{r}79 \\
3 n x\end{array}$ & $1.0025=07$ & $\begin{array}{l}3.3 \text {.5E }=07 \\
1.291 F=06\end{array}$ & $\begin{array}{l}3200 \\
3200\end{array}$ \\
\hline $\begin{array}{l}75.0 \\
75.0\end{array}$ & $\begin{array}{l}3 \pi x \\
572\end{array}$ & $\begin{array}{l}\text { Q. } 1 \text { A AE }=07 \\
7 . \text { BAC - } 07\end{array}$ & $2.4 \leq 2 E=06$ & $\begin{array}{l}3200 \\
3200\end{array}$ \\
\hline 77.0 & 1087 & 1.40 ก E $=06$ & $4.6200=06$ & 3200 \\
\hline 78.0 & $159 a$ & & $\begin{array}{l}6.7735=06 \\
7.1235=0.6\end{array}$ & $\begin{array}{l}3200 \\
3200\end{array}$ \\
\hline $\begin{array}{l}79.0 \\
80.0\end{array}$ & $\begin{array}{l}1677 \\
2239\end{array}$ & $\begin{array}{l}2.798 E=06 \\
3 . \text { TRSE-DA }\end{array}$ & $\begin{array}{l}7.1635=010 \\
0.5125=06\end{array}$ & $\begin{array}{l}3200 \\
37.00\end{array}$ \\
\hline R. 1.0 & 1799 & $2.465 F=06$ & $7.6435=06$ & $3 ? 00$ \\
\hline $\begin{array}{l}8.2 .0 \\
03.0\end{array}$ & $\begin{array}{l}2020 \\
215 ?\end{array}$ & $\begin{array}{l}2.773 E=06 \\
? .949 E=06\end{array}$ & $\begin{array}{l}9.595 E-06 \\
9.1 \text { A2E }=06\end{array}$ & $\begin{array}{l}3200 \\
3 ? 00\end{array}$ \\
\hline 94.0 & $213 n$ & $7.719 E-08$ & $9.042 E=06$ & $3 ? 00$ \\
\hline A5.0 & 1727 & $2.3665=06$ & $7,3365=05$ & 3200 \\
\hline An, 0 & 1359 & $1.862=0.76$ & $5.773 \mathrm{E} .0 \mathrm{~B}$ & 3200 \\
\hline 4. $7 . ?$ & 1355 & $1.957 F=06$ & $5.75 \mathrm{RE}=06$ & 3200 \\
\hline ค ค. 0 & $13 n 7$ & $1.900 E-06$ & $5.891 \mathrm{~F}=0.6$ & 3200 \\
\hline 99. 0 & 1357 & 1.PBOE-OG & $5.7655=06$ & 3200 \\
\hline 90.0 & 1235 & $1 . t ? \geqslant E-O B$ & $5.246 E=06$ & $32 \cap 0$ \\
\hline 91.0 & 10.90 & $1.0935=06$ & $4.62 B E-06$ & 3200 \\
\hline 82,0 & $9 \times 2$ & $1.346 E-06$ & $11.172 E-06$ & 3200 \\
\hline 73.0 & 1160 & $1.5 \% 9 E-06$ & $4.927 E-08$ & 3200 \\
\hline $0+1.0$ & 835 & $1.1 \Delta 5 E=0$, & $3.550 F-0 \mathrm{~b}$ & 3200 \\
\hline $95 . n$ & 10 ค5 & 1. $4 R 7 E-06$ & 0.60 ดุe $=06$ & $320 ?$ \\
\hline 96.0 & 1101 & $1.5135=06$ & $a, 69 ! 5=06$ & 3700 \\
\hline 87.0 & 1070 & $1 . \operatorname{Ang} 6=06$ & $0.505 E-96$ & 3200 \\
\hline 09,0 & 1319 & I. $P \cap A E-O A$ & $5.604 E-96$ & 3200 \\
\hline 99.0 & $19 ! 3$ & $1.936 E-134$ & $6.00 ? E-06$ & 3300 \\
\hline 100.0 & 112.3 & $1,5395-06$ & $4.770 E \rightarrow \cap B$ & $3 ? 00$ \\
\hline 101.0 & 1320 & $1 . B 1 \mathrm{aF}=\mathrm{OH}$ & $5.620 F=06$ & $320 \pi$ \\
\hline 102.0 & 1412 & $1.935 \mathrm{E} \times 0 \mathrm{H}$ & $5.9985-06$ & $32 \div 2$ \\
\hline 103.0 & 1232 & 1.6 A $9=06$ & $5.2345=06$ & 3700 \\
\hline 104.9 & 1129 & . $5 \triangle A E-D A$ & $4,797 F=06$ & $3 ? 00$ \\
\hline 105,0 & 1264 & $1.7 \times 25-06$ & $5 . x+25=0 b$ & 3200 \\
\hline 106.0 & 1.370 & 1. PTAE-06 & $5, A \geq 1 E=06$ & 3200 \\
\hline 107.0 & 1405 & $976 E_{2}=06$ & $5.9705-06$ & 3201 \\
\hline IOR.0 & $97 ?$ & $1.3 \times 2 E=0 A$ & $A .128 E=06$ & 3200 \\
\hline 109.0 & 1100 & $S 1 A E=O B$ & $0.6095-08$ & $3>0$ \\
\hline 110.0 & 1255 & $1.7705=06$ & $5.333 F-06$ & $320 \mathrm{n}$ \\
\hline 111.0 & $9 \pi 7$ & $1.243 E-06$ & $3.953 E=06$ & $\operatorname{ling}$ \\
\hline 112.0 & 970 & $1.3>7 E-D 6$ & $0.120 F=06$ & $3>n n$ \\
\hline 113.0 & 997 & $3+6 E=06$ & $4.235 \mathrm{E}=\mathrm{nb}$ & $320 n$ \\
\hline 110,0 & 6ค9 & $405 f-07$ & $92 \times 5=n t$ & ח? \\
\hline 115.0 & $\operatorname{san}$ & $9.0525-07$ & $2+906=-96$ & I300 \\
\hline 116.0 & 1 Я & $2.5395=0 A$ & $7.871 E-08$ & xpon \\
\hline 117.0 & s! & $0.370 \mathrm{OF}=? .9$ & $7.5975-17$ & 3 ?อก \\
\hline 118.0 & 3 & 5.07 A $=0.9$ & $1.57 a F_{-} \rightarrow 08$ & $3 ? 0 \mathrm{~N}$ \\
\hline 119.0 & $?$ & $3, x+9 F=00^{\circ}$ & $1.18\} F=09$ & $320 \mathrm{n}$ \\
\hline 120.0 & $?$ & 3. ACOE $=0 \%$ & $1 A 1 F-\cap A$ & $3>n$ \\
\hline 121.0 & $?$ & $3,8 n \geqslant F=00$ & $1, I^{A} I^{-1 F-0 A}$ & $32 \mathrm{n}$ \\
\hline 122.0 & 4 & $6.3 \triangle \mathrm{BE}=09$ & $1.96 \mathrm{AF}=0 \mathrm{C}$ & 3200 \\
\hline 123.0 & 17 & $2.012 E \cdot 00$ & $477 E=08$ & 3200 \\
\hline 129.0 & 5 & $7.6575=09$ & $2.3615-0 A$ & 3200 \\
\hline .0 & 0 & $.000 E+00$ & $0.000 E+00$ & 3200 \\
\hline & 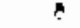 & $1 \triangle 3 E-0 A$ & $.542 E=08$ & 3200 \\
\hline
\end{tabular}

CROSSUIND INTEGRATEN T.891E-03 $1.237 E-02$ 
5D-SER IES EXPERIMENT: 65 $($ FT-65)

GROUND LEVEL AND TOWER SAMPLING 200 AND 800 M ARCS. GROUND LEVEL

SAMPL ING ONLY $1600+12800$ M ARCS.

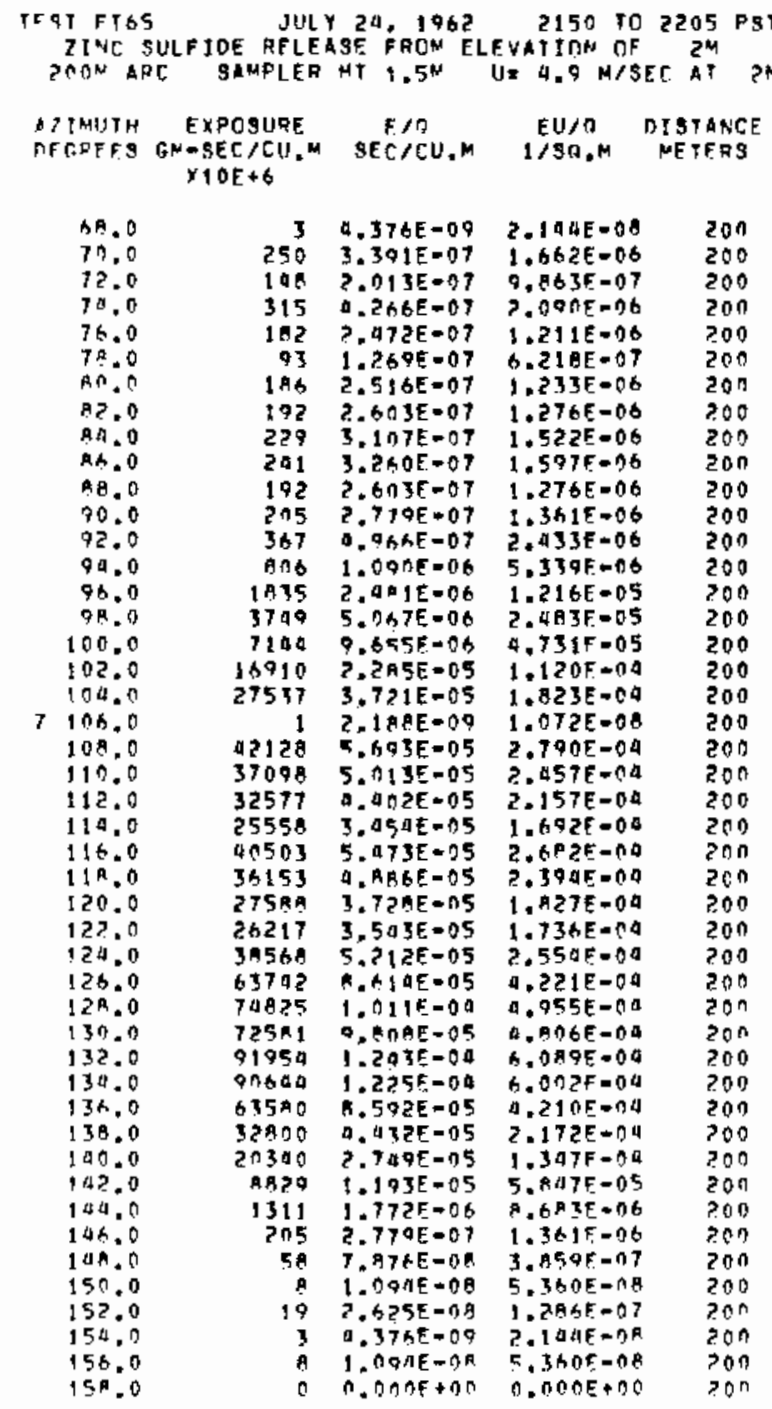

TOKEP C.ATA FOLLCW....

TF\$T F165 JULY 24,1962 2ISO TC 2205 PgT IINC SULFIDE HELEASE FRON ELEVATICA CF $2 \mathrm{~N}$

\begin{tabular}{|c|c|c|c|c|c|}
\hline $\begin{array}{l}\text { DIFUIM } \\
\text { DEGAEES }\end{array}$ & $\begin{array}{l}\text { HE IGH } \\
\text { ME TEFS }\end{array}$ & $\begin{array}{c}\text { EXPOSURE } \\
\text { GN-SEE }-\mathrm{CU}, \mathrm{N} \\
\times 1 O E+6\end{array}$ & $\begin{array}{c}E / B \\
S E C / C U . N\end{array}$ & $\begin{array}{c}\varepsilon U / G \\
1 / 5 \sigma^{N}\end{array}$ & $\begin{array}{l}\text { DI TIANCE } \\
\text { WETERS }\end{array}$ \\
\hline $\begin{array}{l}90.0 \\
98.0 \\
90.0 \\
96.0\end{array}$ & $\begin{array}{l}0.3 \\
0.7 \\
1.4 \\
2.7\end{array}$ & $\begin{array}{l}7541 \\
7080 \\
2023 \\
3368\end{array}$ & $\begin{array}{l}1.019 E=05 \\
9.573 E=06 \\
3.81 \in E=06 \\
0.552 E=06\end{array}$ & $\begin{array}{l}9.990 E-05 \\
9.691 E=05 \\
1.070 E-05 \\
2.231 E=05\end{array}$ & $\begin{array}{l}200 \\
200 \\
200 \\
200\end{array}$ \\
\hline $\begin{array}{l}98.0 \\
96.0 \\
98.0\end{array}$ & $\begin{array}{l}4.1 \\
5.9 \\
6.0\end{array}$ & $\begin{array}{l}3015 \\
3082 \\
3019\end{array}$ & $\begin{array}{l}4.103 E=06 \\
0.170 E=06 \\
0.073 E=06\end{array}$ & $\begin{array}{l}2.010 E-05 \\
2.045 E=05 \\
1.996 E-05\end{array}$ & $\begin{array}{l}200 \\
200 \\
200\end{array}$ \\
\hline $\begin{array}{l}98.0 \\
98.0\end{array}$ & $\begin{array}{l}8.1 \\
9.0\end{array}$ & $\begin{array}{l}3031 \\
2108\end{array}$ & $\begin{array}{l}4.007 E-06 \\
2.809 E=06\end{array}$ & $\begin{array}{l}2.000 E-05 \\
1.396 E-05\end{array}$ & $\begin{array}{l}200 \\
200\end{array}$ \\
\hline 98.0 & 10.0 & 2274 & $3,070 E-06$ & $1.506 \mathrm{E}=05$ & 200 \\
\hline 98.0 & 13.5 & 1903 & $2,572 E-06$ & $1+260 E=05$ & \\
\hline 98.0 & 16.2 & 1340 & $1.817 E-06$ & $0.906 E-06$ & \\
\hline 98.0 & & 1413 & $1.911 E=06$ & $9.363 E=06$ & 200 \\
\hline 98.0 & 21,6 & $\begin{array}{l}934 \\
402\end{array}$ & $\begin{array}{l}1.262 E=06 \\
5.44 \angle E=07\end{array}$ & $\begin{array}{l}6.155 E=06 \\
2.6605=06\end{array}$ & $\begin{array}{l}200 \\
200\end{array}$ \\
\hline 7 & & & $160=01$ & $752 E-06$ & \\
\hline
\end{tabular}

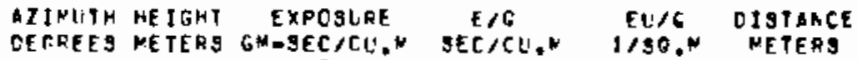

\begin{tabular}{|c|c|c|c|c|c|}
\hline $\begin{array}{l}106.0 \\
100.0 \\
106.0 \\
106.0 \\
106.0 \\
106.0 \\
106.0 \\
106.0 \\
106.0 \\
106.0 \\
106.0 \\
106.0 \\
106.0 \\
106.0 \\
106.0 \\
106.0\end{array}$ & $\begin{array}{l}0.3 \\
0.7 \\
1.4 \\
2.7 \\
9.1 \\
5.4 \\
6.8 \\
8.1 \\
9.4 \\
10.8 \\
13.5 \\
16.2 \\
19.9 \\
21.6 \\
24.3 \\
27.0\end{array}$ & 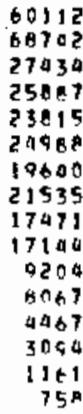 & $\begin{array}{l}6.123 E=05 \\
9.29 C E=05 \\
3.707 E=05 \\
3.49 E E=05 \\
3.21 E E=05 \\
3.377 E=05 \\
2.65 A E=05 \\
2.91 C E=05 \\
2.361 F=05 \\
2.317 F=05 \\
1.241 E=05 \\
1.040 F=05 \\
6.037 F=05 \\
4.122 E=06 \\
1.57 F E=06 \\
1.025 E=06\end{array}$ & $\begin{array}{l}3.980 E=04 \\
4.552 E=04 \\
1.017 E=04 \\
1.719 E=04 \\
1.577 E=04 \\
1.655 E=04 \\
1.301 E=04 \\
1.726 E=04 \\
1.157 E=04 \\
1.135 E=04 \\
6.095 E=05 \\
5.342 E=05 \\
2.559 E=05 \\
2.049 E=05 \\
7.691 E=06 \\
5.023 E=06\end{array}$ & $\begin{array}{l}200 \\
200 \\
200 \\
200 \\
200 \\
200 \\
200 \\
200 \\
200 \\
200 \\
200 \\
200 \\
200 \\
200 \\
200 \\
200\end{array}$ \\
\hline
\end{tabular}

AZINTITH HEICHT EXPESLRE F/ DEGPEF NETERS GV-SFCICU." SETICU.N IISG.N METFRS $X ! 0 E+6$

\begin{tabular}{|c|c|c|c|c|}
\hline 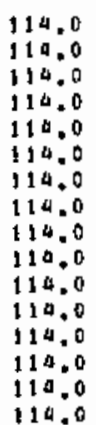 & $\begin{array}{l}0.3 \\
0.7 \\
1.4 \\
2.7 \\
0.1 \\
5.4 \\
6.1 \\
8.1 \\
9.4 \\
11.8 \\
13.5 \\
16.2 \\
18.9 \\
21.6 \\
24.3 \\
27.0\end{array}$ & $\begin{array}{l}17622 \\
47750 \\
16092 \\
10897 \\
22970 \\
19670 \\
12317 \\
16555 \\
15073 \\
14219 \\
10630 \\
7189 \\
5139 \\
3501 \\
1084 \\
1200\end{array}$ & $\begin{array}{l}2.3 E 1 F=05 \\
6.453 E=05 \\
2.175 F=05 \\
2.277 F=05 \\
3.105 E=05 \\
2.65 P E=05 \\
1.665 E=05 \\
2.237 E=05 \\
2.195 E=05 \\
1.927 E=05 \\
1.439 F=05 \\
9.714 E=06 \\
6.946 E=06 \\
0.732 E=06 \\
1.46 t E=06 \\
1.730 F=06\end{array}$ & $\begin{array}{l}1.167 F=04 \\
3.162 E=04 \\
1.066 E=04 \\
1.116 E=04 \\
1.521 E=00 \\
1.303 E=04 \\
6.156 E=05 \\
1.096 E=0 A \\
1.051 E=04 \\
9.416 E=05 \\
7.045 E-05 \\
9.760 E=05 \\
3.403 E=05 \\
2.318 E=05 \\
7.183 E=06 \\
8.476 E=06\end{array}$ \\
\hline
\end{tabular}




\begin{tabular}{|c|c|c|c|c|c|}
\hline $\begin{array}{l}\text { A2INUTH } \\
\text { DEGREES }\end{array}$ & $\begin{array}{l}\text { HE IGHT } \\
\text { ME TERS }\end{array}$ & $\begin{array}{c}\text { EXFCSURE } \\
G M=\$ E C / C U . N \\
\times 10 E+6\end{array}$ & $\begin{array}{c}E / 6 \\
S E C / C U, k\end{array}$ & $\begin{array}{l}E U / O \\
I / S B . N\end{array}$ & $\begin{array}{l}\text { CISTANC } \\
\text { NETERS }\end{array}$ \\
\hline $\begin{array}{l}6+122.0 \\
6122.0 \\
6.122 .0 \\
6 \quad 122.0 \\
122.0 \\
122.0 \\
122.0 \\
122.0 \\
122.0 \\
122.0 \\
122.0 \\
122.0 \\
122.0 \\
122.0 \\
122.0 \\
122.0\end{array}$ & $\begin{array}{l}0.3 \\
0.7 \\
1.9 \\
2.7 \\
4.1 \\
5.0 \\
6.8 \\
8.1 \\
9.4 \\
10.8 \\
13.5 \\
16.2 \\
10.9 \\
21.6 \\
20.3 \\
27.0\end{array}$ & $\begin{array}{l}37661 \\
95193 \\
18643 \\
20406 \\
18026 \\
19721 \\
19092 \\
19201 \\
15350 \\
14213 \\
11240 \\
8257 \\
5596 \\
3231 \\
1223 \\
1173\end{array}$ & 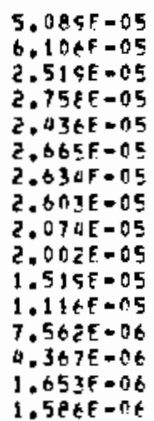 & $\begin{array}{l}2.494 E=04 \\
2.992 E=04 \\
1.235 E=04 \\
1.351 E=04 \\
1.190 E=04 \\
1.306 E=04 \\
1.291 E=04 \\
1.275 E=04 \\
1.016 E=04 \\
9.009 E=05 \\
7.043 E=05 \\
5.469 E=05 \\
3.705 E=05 \\
2.140 E=05 \\
9.099 E=06 \\
7.769 E=06\end{array}$ & $\begin{array}{l}200 \\
200 \\
200 \\
200 \\
200 \\
200 \\
200 \\
200 \\
200 \\
200 \\
200 \\
200 \\
200 \\
200 \\
200 \\
200\end{array}$ \\
\hline
\end{tabular}

ARIMLTH HEIGMT EXPOSURE E, T EUTG OISTARCE DEGREES NETERS GN-SEC/CU.N SECACU.A 1/SG.N NETERS $\times 1$ of $\uparrow b$

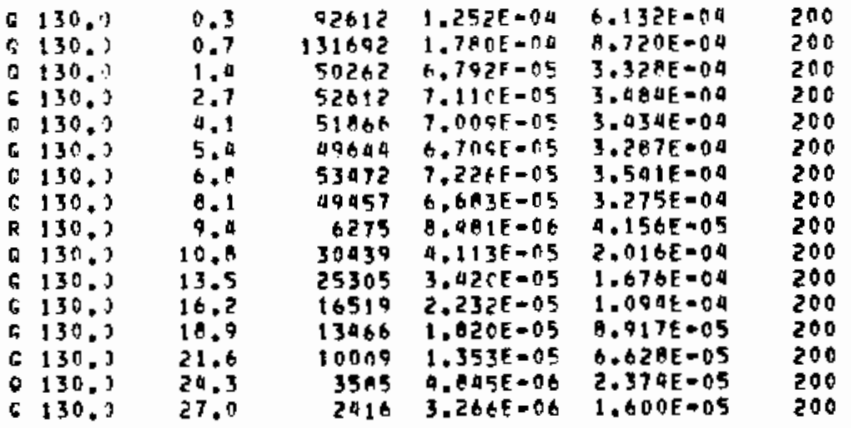

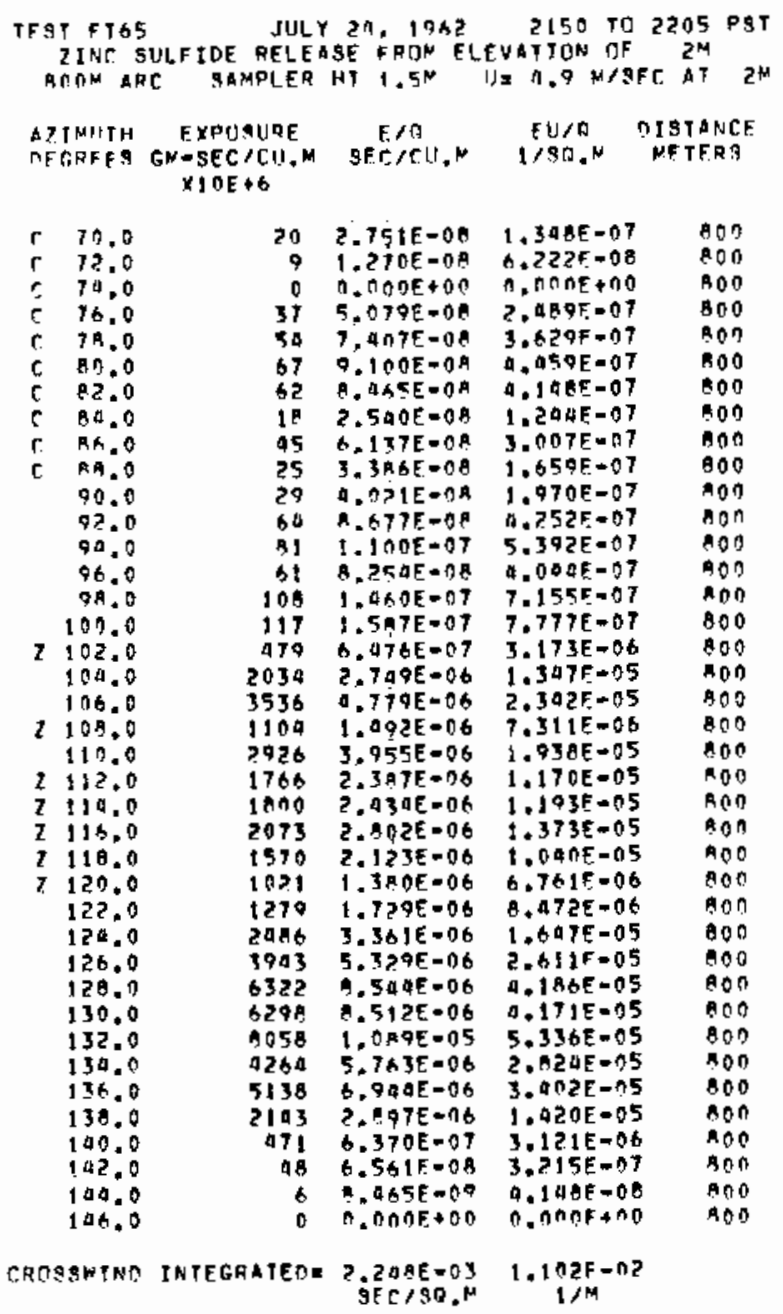

TOKER CATA FOLLEW....

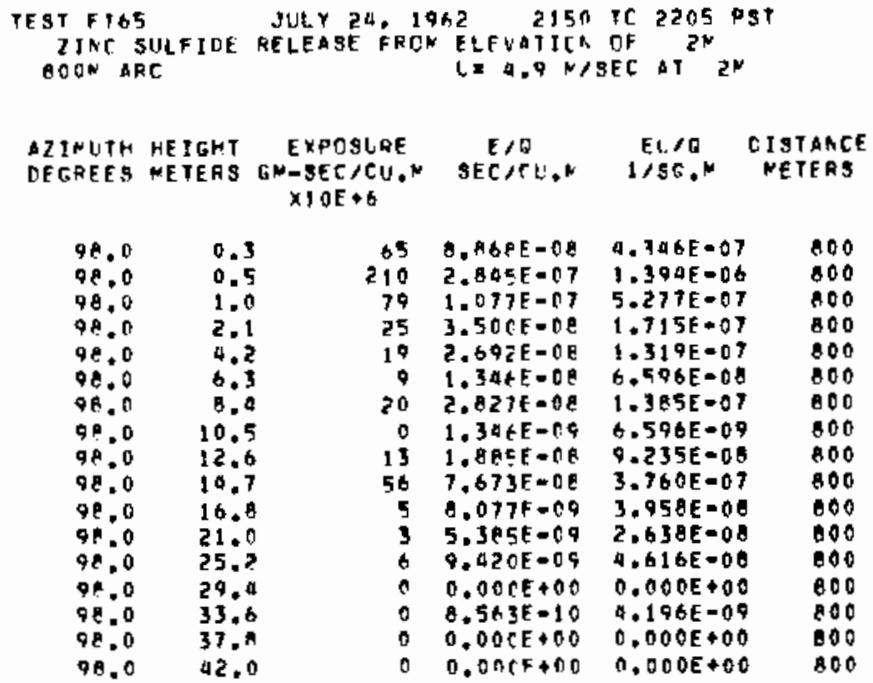




\begin{tabular}{|c|c|c|c|c|c|c|c|c|c|c|c|}
\hline $\begin{array}{l}\text { DIINLTH } \\
\text { CETREES }\end{array}$ & $\begin{array}{l}\text { HEIGHT } \\
\text { METEFS }\end{array}$ & $\begin{array}{c}\text { EXPOSURE } \\
\text { GN-SEC/CU.N } \\
\times 10 E+6\end{array}$ & $\begin{array}{c}E>0 \\
\operatorname{SEC} / \mathrm{CU}, *\end{array}$ & $\begin{array}{l}E L / 0 \\
1 / 30 . N\end{array}$ & $\begin{array}{l}\text { DISTAATE } \\
\text { NETEAS }\end{array}$ & $\begin{array}{l}\text { AZINUTH } \\
\text { DEGRFES }\end{array}$ & $\begin{array}{l}\text { HEIGHT } \\
\text { METEAS }\end{array}$ & $\begin{array}{c}\text { EXPCSURE } \\
\text { GN-9EC } \\
\times 1 O E+B\end{array}$ & $\begin{array}{c}E / G \\
\text { SEC } / C U\end{array}$ & $\begin{array}{l}F L / 0 \\
1 / 50 . N\end{array}$ & $\begin{array}{l}\text { CISTANCE } \\
\text { NETERS }\end{array}$ \\
\hline $\begin{array}{l}106.0 \\
106.0 \\
106.0 \\
106.0 \\
106.0 \\
106.0 \\
106.0 \\
106.0 \\
106.0 \\
106.0 \\
106.0 \\
106.0 \\
106.0 \\
106.0 \\
106.0 \\
106.0 \\
106.0\end{array}$ & $\begin{array}{r}0.3 \\
0.5 \\
1.0 \\
2.1 \\
4.2 \\
6.1 \\
8.9 \\
10.5 \\
12.6 \\
14.7 \\
16.8 \\
21.0 \\
25.2 \\
29.9 \\
33.6 \\
37.8 \\
42.0\end{array}$ & $\begin{array}{l}4952 \\
5530 \\
2390 \\
2769 \\
2789 \\
2753 \\
2728 \\
2608 \\
2604 \\
2222 \\
2650 \\
1926 \\
1150 \\
734 \\
529 \\
571 \\
448\end{array}$ & 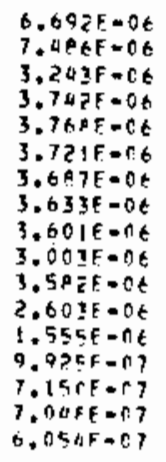 & $\begin{array}{l}3.279 F=05 \\
3.688 E=05 \\
1.588 E=05 \\
1.034 E=05 \\
1.846 E=05 \\
1.823 E=05 \\
1.007 E=05 \\
1.700 E=05 \\
1.764 E=05 \\
1.072 E=05 \\
1.755 E=05 \\
1.276 E=05 \\
7.620 E=06 \\
0.862 E=06 \\
3.509=06 \\
3.153 E=06 \\
2.867 E=06\end{array}$ & $\begin{array}{l}800 \\
800 \\
800 \\
A 00 \\
800 \\
800 \\
A 00 \\
800 \\
800 \\
800 \\
A 00 \\
A 00 \\
800 \\
A 00 \\
B 00 \\
A O C \\
A C O\end{array}$ & $\begin{array}{l}130.0 \\
130.0 \\
130.0 \\
130.0 \\
130.0 \\
130.0 \\
130.0 \\
130.0 \\
130.0 \\
130.0 \\
130.0 \\
130.0 \\
130.0 \\
130.0 \\
130.0 \\
130.0 \\
130.0\end{array}$ & $\begin{array}{r}0.3 \\
0.5 \\
1.0 \\
2.1 \\
4.2 \\
6.3 \\
0.4 \\
10.5 \\
12.6 \\
14.7 \\
16.8 \\
21.0 \\
25.2 \\
29.4 \\
33.6 \\
37.8 \\
42.0\end{array}$ & $\begin{array}{l}7572 \\
6819 \\
3708 \\
4108 \\
5290 \\
5097 \\
5172 \\
4749 \\
4617 \\
5219 \\
4568 \\
4800 \\
2789 \\
2577 \\
1681 \\
1631 \\
1200\end{array}$ & $\begin{array}{l}9.062 E=06 \\
5.216 E=06 \\
5.012 E=C 6 \\
5.552 E=C 6 \\
7.149 E-0 t \\
7.029 E=06 \\
6.999 E=06 \\
6.418 E=0 t \\
6.235 E=06 \\
7.054 E=66 \\
6.173 E=06 \\
6.541 E=06 \\
3.770 E=06 \\
3.484 E=06 \\
2.272 E=06 \\
2.204 E=06 \\
1.622 E=0 t\end{array}$ & $\begin{array}{l}4 . A Q 1 E=05 \\
4.516 E=05 \\
2.456 E=05 \\
2.720 E=05 \\
3.503 E=05 \\
3.640 E=05 \\
3.425 E=05 \\
3.145 E=05 \\
3.057 E=05 \\
3.456 E=05 \\
3.025 E=05 \\
3.205 E=05 \\
1.047 E=05 \\
1.707 E=05 \\
1.113 E=05 \\
1.000 E=05 \\
7.907 E=06\end{array}$ & $\begin{array}{l}800 \\
000 \\
000 \\
800 \\
000 \\
000 \\
000 \\
000 \\
000 \\
100 \\
000 \\
800 \\
800 \\
800 \\
800 \\
000 \\
000\end{array}$ \\
\hline
\end{tabular}

AZINUTH HEIGHT EXPCSLIE F, E TISTAACE DETREES METEAS GN-SEC/TU,N SET,CI.N $1 / S R$.N METERS
XIOE +6

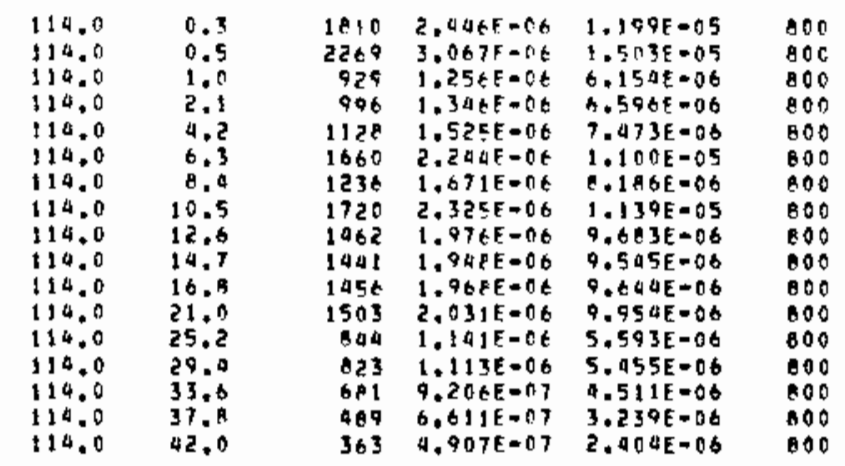

ATINUTH HEIGHT EXPCSURE E/O ELIO OISTANCE

DEGREES METEAS GN-SEC/CU,N SEC/CL,N 1/SO.N NETERS

\begin{tabular}{|c|c|c|c|c|c|}
\hline $\begin{array}{l}122.0 \\
122.0 \\
122.0 \\
122.0 \\
122.0 \\
122.0 \\
122.0 \\
122.0 \\
122.0 \\
122.0 \\
122.0 \\
122.0 \\
122.0 \\
122.0 \\
122.0 \\
122.0 \\
122\end{array}$ & $\begin{array}{r}0.3 \\
0.5 \\
1.0 \\
2.1 \\
4.2 \\
6.3 \\
8.4 \\
10.5 \\
12.6 \\
14.7 \\
16.0 \\
21.0 \\
25.2 \\
29.4 \\
33.6 \\
37.8 \\
42.0\end{array}$ & $\begin{array}{l}1875 \\
1901 \\
405 \\
1053 \\
1063 \\
1016 \\
1155 \\
1369 \\
1365 \\
1441 \\
1136 \\
1040 \\
676 \\
561 \\
471 \\
201 \\
250\end{array}$ & 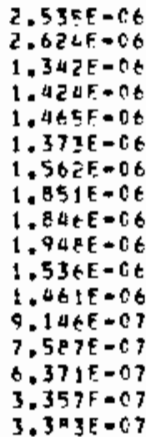 & $\begin{array}{l}1.242 E=05 \\
1.286 E=05 \\
6.576 E=06 \\
6.979 E=06 \\
7.177 E=06 \\
6.727 E=06 \\
7.652 E=06 \\
9.070 E=08 \\
9.043 E=06 \\
9.545 E=06 \\
7.526 E=06 \\
7.157 E=06 \\
9.911 E=06 \\
3.718 E=06 \\
3.122 E=06 \\
1.645 E=06 \\
1.657 E=06\end{array}$ & $\begin{array}{l}800 \\
000 \\
800 \\
800 \\
800 \\
800 \\
800 \\
000 \\
800 \\
800 \\
000 \\
000 \\
000 \\
000 \\
000 \\
800 \\
800\end{array}$ \\
\hline
\end{tabular}




\begin{abstract}
If it 5765
JULY $2 \%$ 175Z 2450 ID 2205 PST IINC SULFIDE RELESSE FRC' FLEVATIOP! OF RS

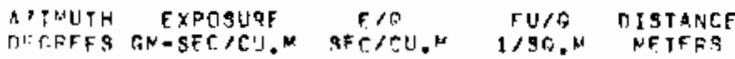
$\times 10 E+6$
\end{abstract}

\begin{tabular}{|c|c|c|c|c|c|}
\hline & 70.0 & 3 & $5.1 A: E-09$ & $2.529 F=08$ & $1+00$ \\
\hline & $7 a . ?$ & 7 & 1. $\cap \backslash Z E=0 A$ & $5.05 A E=0 \mathrm{~B}$ & 1500 \\
\hline & 76.0 & 7 & $1.0325-0 \mathrm{~g}$ & $5.05 A F=0 R$ & $1 \sin 0$ \\
\hline & 77.0 & in & $1.3765=0 \mathrm{~A}$ & $6.7445=08$ & 1600 \\
\hline & 79.0 & P & 1. $2 \cap A E=09$ & $5.971 E-08$ & 1600 \\
\hline & 79.0 & 10 & 1.37 LE - OA & $6.7445 \rightarrow 0 R$ & 1500 \\
\hline & A. . . & 12 & $1.7 \geqslant ? 5=09$ & $A, A Y C E-$ ก B & 1600 \\
\hline & A 1.0 & 5 & 6. 8 म $? E=09$ & $3.372 F=08$ & 1600 \\
\hline & ค. ह & 12 & $1.7>0 E-08$ & $A, 130 E=D B$ & 1500 \\
\hline & 83,0 & 17 & $? . A \cap 9 E-0 P$ & 1.1 ACE-NT & 1600 \\
\hline & 84,0 & 6 & $.40 \geqslant 5=09$ & $4.215 E-0$ ) & 1600 \\
\hline & 85.7 & $P Z$ & $3.097 \mathrm{E}-08$ & $1.517 \mathrm{E}=07$ & 1600 \\
\hline & $A 6.0$ & 3 & $5.161 \mathrm{E}-09$ & $2.529 F-08$ & 1600 \\
\hline & e. 7.0 & 8 & $1.204 F=0.9$ & $5.9017=08$ & 1600 \\
\hline & $A R=0$ & 2 & $3, a a 1 E-09$ & $1.6 R G E=0 B$ & $1 \leq 03$ \\
\hline & 80.0 & $4 Z$ & $5.677 \mathrm{E}=0 \mathrm{~B}$ & $2.7 A 2 E-07$ & 1600 \\
\hline & 70.0 & 7 & $1.072 E=0$. & $5.058 F-0 B$ & 1600 \\
\hline & 81.0 & 5 & A. А म $2 E=09$ & $3.372 \mathrm{~F}-0 \mathrm{~A}$ & 1000 \\
\hline & 92.0 & 22 & $3.097 E=0 P$ & $1.517 \varepsilon-0.7$ & 160 ? \\
\hline & 93.0 & $? 0$ & $2.753 \mathrm{E}=0$ & $1.3995=07$ & 2000 \\
\hline & 94.0 & 28 & $3.7 A 5 E-09$ & $1,8555-07$ & 1600 \\
\hline & 85.0 & 19 & $2.5815=0$ & $1.260 E=07$ & 1600 \\
\hline & 96.0 & 14 & I. $A 02 E=0.9$ & $0.273 F-0$. & 1600 \\
\hline & 97.0 & 19 & ?.5म」E-0म & $1.26 a F=07$ & 1600 \\
\hline & 78.0 & ? P & 3.7 A5E - OP & 1. $A 55 F=07$ & $16 n 0$ \\
\hline & 99.0 & 31 & $\because .301 \mathrm{~F}=O \mathrm{~A}$ & $2.107 F=07$ & $160 n$ \\
\hline & 170.0 & 10 & $1.376 E=08$ & 6. 7 व A $\varepsilon=08$ & $160 \%$ \\
\hline & 101.0 & 26 & $3.6135-08$ & $1.770 F=07$ & 1600 \\
\hline & $10 ? .0$ & 178 & $1.73 \cdot E=07$ & 0.51 UE $=07$ & 1600 \\
\hline & 103.0 & $\begin{array}{l}417 \\
\text { वी }\end{array}$ & $5.6 A 3 E-07$ & $2.765 F-06$ & 1500 \\
\hline & 100.0 & 982 & 6.5 म $9=07$ & $3.229 \mathrm{E}=0 \mathrm{6}$ & 1300 \\
\hline & 105.0 & & 1.378E-16 & $\begin{array}{c}6-509 E-08 \\
0\end{array}$ & 1600 \\
\hline & $\begin{array}{l}1+5.0 \\
107.0\end{array}$ & 1504 & $1.973 E=06$ & $\begin{array}{l}9,425=-108 \\
9-97+5=0\end{array}$ & 10000 \\
\hline & 108.0 & 1354 & $\because 35=019$ & R. $769 F=06$ & 1500 \\
\hline f & $1: 3.0$ & 34 & $\begin{array}{l}. .530 E=0 \\
4.875 E-0.8\end{array}$ & $3.276 E=07$ & 1600 \\
\hline & 100.0 & +247 & $1.6 A \times E-06$ & $A .261 F-06$ & 1600 \\
\hline & 110.0 & 831 & $1.123 E-06$ & $5.5 \cap 5 E=06$ & 1600 \\
\hline & 111.0 & 622 & A. A $13 E-0\}$ & $0.1275-06$ & 1600 \\
\hline & 112.0 & 556 & 7.51 A $=07$ & $3.6 P \triangle E=0 B$ & 1600 \\
\hline & 113.0 & 577 & $7.811 E=07$ & 3.APTE $=06$ & 1600 \\
\hline & 114.0 & $49 !$ & $5.970 E-07$ & 2.9257 .06 & 1600 \\
\hline & 115,0 & 227 & .7AnE-07 & $8325=06$ & 1500 \\
\hline & $1 ! 6.0$ & 343 & $8.645 F=07$ & $2.276 F=06$ & 1500 \\
\hline & 117.0 & 391 1 & $5.1+1 E=07$ & $2.529 E-06$ & $160 \%$ \\
\hline & 128.0 & 1136 & $5.911 E-07$ & $7.891 F-06$ & 1500 \\
\hline I & $11^{\mu} \cdot 0$ & 14 & $1.5 \Rightarrow B F-08$ & $7.5^{\circ} 7 E-99$ & $160 n$ \\
\hline & 119.0 & 353 & $n .7 A 3 E-0.7$ & $2.34 A 5=06$ & $1 \leqslant 00$ \\
\hline & 120.0 & 276 & $3.723 E=07$ & $1 . A 29 E-D B$ & 1 sen \\
\hline & 121.0 & 254 & $3.4 a 15-01$ & 1. SARE = ?A & 160n \\
\hline & 122.0 & 342 & $A>A F=07$ & $O A F E=O B$ & $130 \pi$ \\
\hline & $\begin{array}{l}123.0 \\
129.0\end{array}$ & $\begin{array}{l}305 \\
585\end{array}$ & $\begin{array}{l}4.139 E=07 \\
7.639 E=07\end{array}$ & $\begin{array}{l}2.0235=06 \\
3.7935=06\end{array}$ & $\begin{array}{l}1500 \\
1500\end{array}$ \\
\hline & 125.0 & 903 & $1.2>1 E-06$ & $5.965 \mathrm{E}-116$ & 1600 \\
\hline & 126.0 & 1153 & $1.57 \geqslant E=06$ & $7.705 E-06$ & 1500 \\
\hline 2 & $1 \geq 6.0$ & 168 & $2.2715=07$ & $1 . t 13 F=06$ & 1609 \\
\hline 7 & 126.0 & 497 & $7>7 E=07$ & $3.7965=06$ & 1600 \\
\hline & $\$ 27.0$ & 1290 & $1.74 a E=06$ & $A .50 B E=06$ & 1600 \\
\hline & $1 \geq 9.0$ & 753 & $1.01 \mathrm{AE}=06$ & $99 \cap 5=06$ & 3600 \\
\hline & 120.0 & 1721 & $376 E=06$ & $1.100 E=05$ & 1600 \\
\hline & 130.0 & 1376 & 1. $A A O E=06$ & $9.1135-06$ & 1500 \\
\hline & 131.8 & 1630 & $21 \Delta E-06$ & $1.085 F=05$ & 1600 \\
\hline & 132.0 & 2016 & $725 E=196$ & $1.335 E=05$ & 1800 \\
\hline & 133.0 & 2425 & $277 E-06$ & $1.60 \in E=05$ & 1600 \\
\hline & 130.0 & 2003 & TOBE-0S & $1.327 \mathrm{E}=05$ & 1500 \\
\hline & 135.0 & $16 ? B$ & $200 E-06$ & $1.076 F-015$ & 1600 \\
\hline & 136.0 & 309 & 4.1 AE- 07 & 2.0 CBE $=6$ & 1600 \\
\hline & 137.0 & $5 n 8$ & $7.9 \div 55-07$ & $3.633 E=06$ & 1600 \\
\hline & 138.0 & 322 & $4.353 E-07$ & $2.1335=06$ & 1600 \\
\hline $\mathbf{P}$ & 139.0 & 24 & $269 E-08$ & $1.002 E-07$ & 1600 \\
\hline$p$ & in & 15 & $O S A E=0 B$ & $012 E=07$ & 1600 \\
\hline P & 101 & 0 & $200 E+00$ & $000 E+00$ & 1600 \\
\hline
\end{tabular}

IFST FT65 JULY 20, 1962 Z\$50 TO 2205 PST

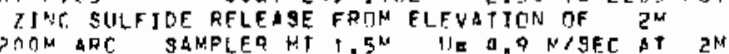
HTNITH EXPCISURE EID FIID OISTANCF $\times 10 \mathrm{O}+6$

81.0

ค2. 0

L 83.0

r. 94.0

r. 85.0

a. 0

Q.7.0

ค. 0

90.0

\$1. 0

क?. 0

73.0

จ4. 0

\$5. 0

\$6. 0

97.0

Q. 0

79.0

$1 \cap 0.0$

101.0

102.0

103.0

104.0

105.0

106.0

107.0

$1 C 8.9$

109.0

110.0

$11 ! .0$

$+12.0$

155.0

114.0

115.0

136.0

117.0

11 月. 0

119.0

$12 \mathrm{n} .0$

121.0

127.0

123.0

129.0
$r \quad 125.0$

r. 125.0

127.0

128.0

129.0

130.0

131.0

132.0

$13.3, n$

$13 n .0$

135.0

136.0

137.0

$13 \mathrm{C} .0$

139.0

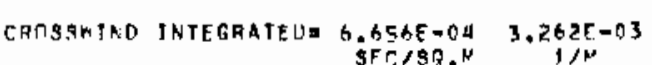

t>on

3200

3200

$3 ? 00$

3200

3200

3201

3200

32.00

3200

3200

3700

3200

3200

3700

32 ता 0

3200

3200

3200

3200

3200

3200

3200

3 ? 00

3200

$320 ?$

3200

3300

$3>0 n$

3200

3700

3200

370 ?

5200

$3 \geq 00$

3200

3200

3200

3700

3 ?००

32 กn

3200

3,00

$320 \%$

37.00

$3 ? 0 n$
$3>00$

3200

$3>00$

3200

3200

3 ?0?

320 ?

3200

3200

3700 


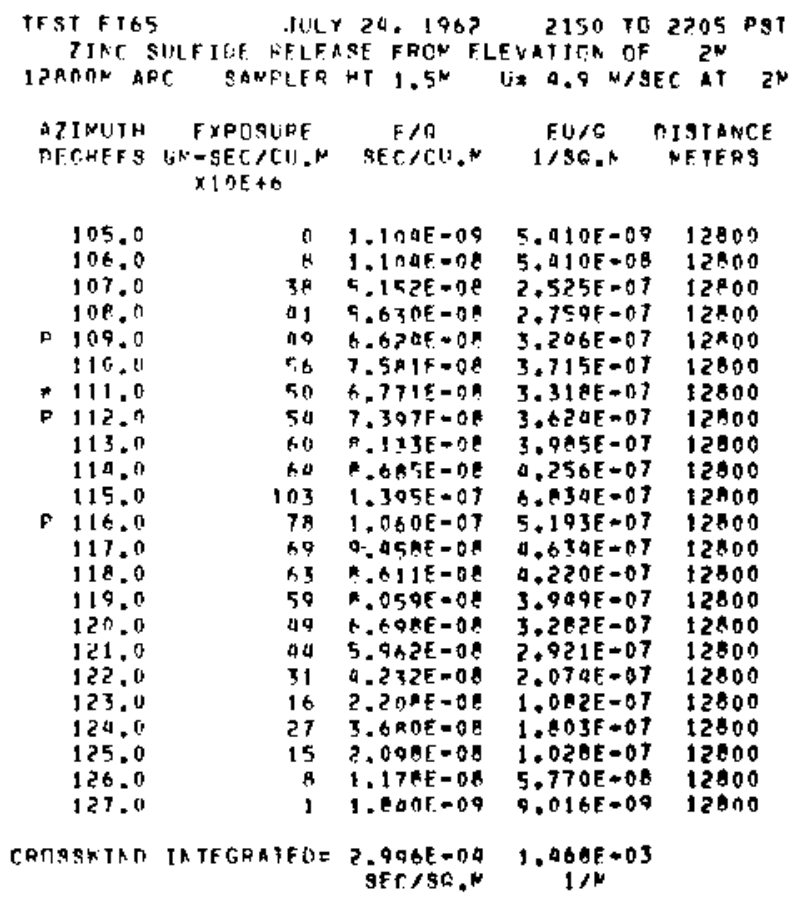


30-SERIES EXPER H MENT: 66 ; FT-66 3

GROUUTD LEVEL AHD TOWER SAMPLING 200 ANO 800 M ARCS. GROUND LEVEL

SAMPL ING ONLY 1600-12800:A ARCS. DUST ANO CAREON DEPOSATS ON THE

FILTERS COLLECTED FROM THE 12800 M ARC.

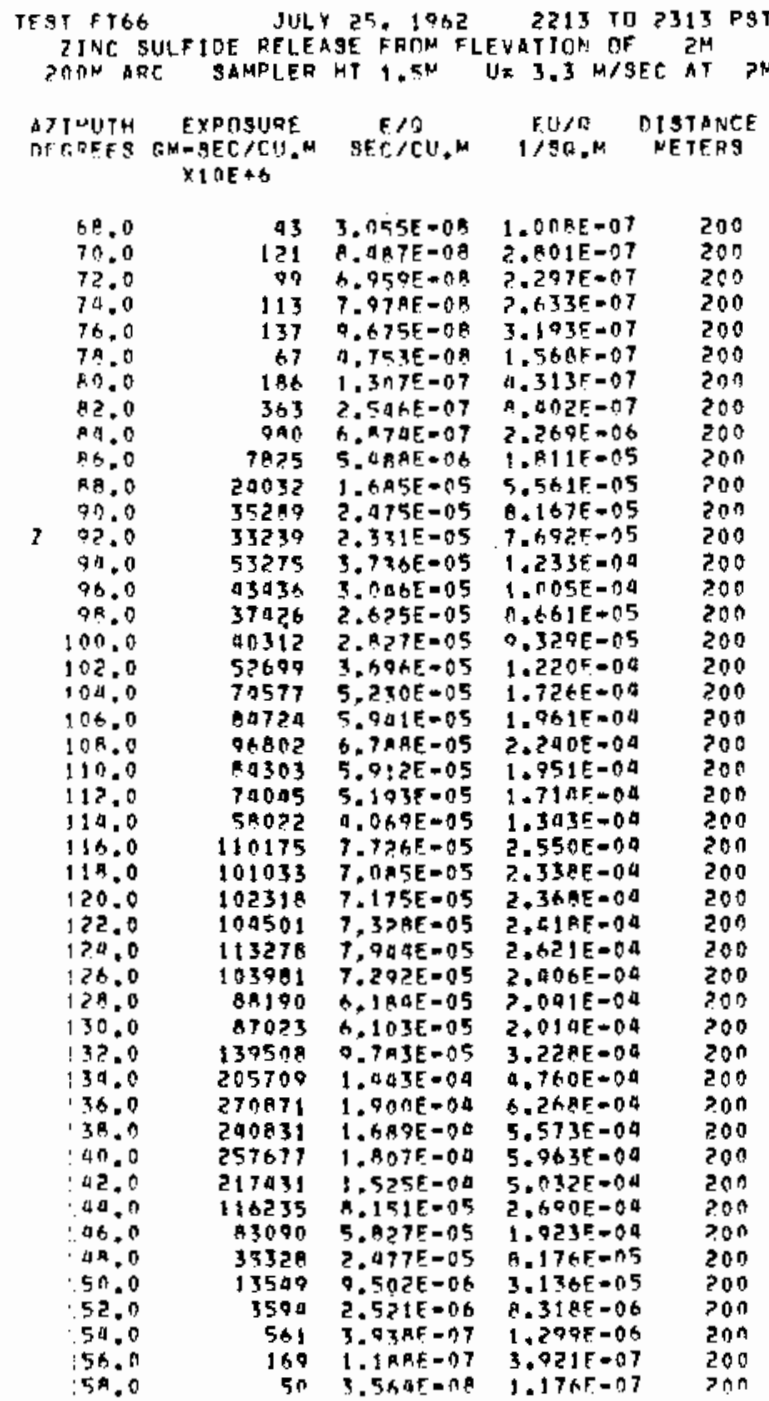

TOWER DATA FDLLOW....

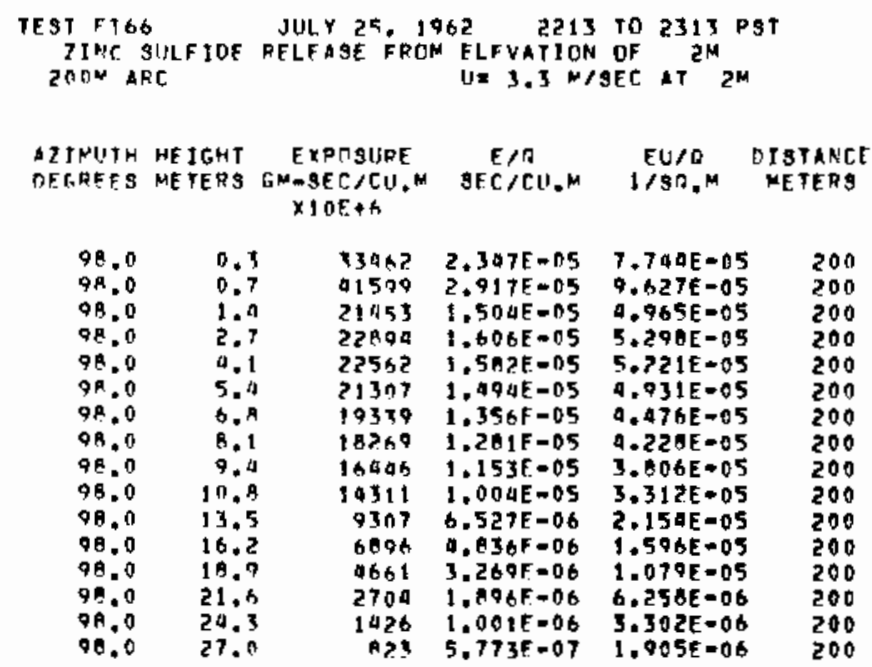

\begin{tabular}{|c|c|c|c|c|c|}
\hline $\begin{array}{l}\text { DIRIITH } \\
\text { DE RREES }\end{array}$ & $\begin{array}{l}\text { HE IGHT } \\
\text { ME PERS }\end{array}$ & $\begin{array}{c}\text { ExPOSURE } \\
G M-3 E C / C I, M \\
\times ! n E+6\end{array}$ & $\begin{array}{c}\text { E } / \theta \\
\text { SEC/rU.M }\end{array}$ & $\begin{array}{l}E U / D \\
I / S O . M\end{array}$ & $\begin{array}{c}\text { OI ST ANCE } \\
\text { METERS }\end{array}$ \\
\hline
\end{tabular}

\begin{tabular}{|c|c|c|c|c|c|}
\hline $\begin{array}{l}106.0 \\
106.0 \\
106.0 \\
106.0 \\
106.0 \\
106.0 \\
106.0 \\
106.0 \\
106.0 \\
106.0 \\
106.0 \\
106.0 \\
106.0 \\
106.0 \\
106.0 \\
106.0\end{array}$ & $\begin{array}{r}0.3 \\
0.7 \\
1.9 \\
2.7 \\
4.1 \\
5.9 \\
6.1 \\
8.1 \\
9.4 \\
10.1 \\
13.5 \\
16.2 \\
18.9 \\
21.6 \\
24.3 \\
27.0\end{array}$ & $\begin{array}{r}99131 \\
197156 \\
51331 \\
50286 \\
95936 \\
46111 \\
38629 \\
34787 \\
20006 \\
30093 \\
20915 \\
10036 \\
0013 \\
5572 \\
2363 \\
1227\end{array}$ & $\begin{array}{l}6.250 E-05 \\
7.515 E=05 \\
3.600 E=05 \\
3.520 E=05 \\
3.221 E=05 \\
3.234 E=05 \\
2.709 E=05 \\
2.490 E=05 \\
2.037 E=05 \\
2.110 F=05 \\
1.432 F=05 \\
7.038 E=06 \\
5.619 E=06 \\
3.90 E F=06 \\
1.658 E=06 \\
6.616 E=07\end{array}$ & $\begin{array}{l}2.063 E=04 \\
2.400 E=04 \\
1.188 E=04 \\
1.164 E=04 \\
1.063 E=04 \\
1.067 E=04 \\
6.939 E=05 \\
0.050 E=05 \\
6.723 E=05 \\
6.962 E=05 \\
9.725 E=05 \\
2.323 E=05 \\
1.050 E=05 \\
1.290 E=05 \\
5.470 E=06 \\
2.940 E=06\end{array}$ & $\begin{array}{l}200 \\
200 \\
200 \\
200 \\
200 \\
200 \\
200 \\
200 \\
200 \\
200 \\
200 \\
200 \\
200 \\
200 \\
200 \\
200\end{array}$ \\
\hline
\end{tabular}

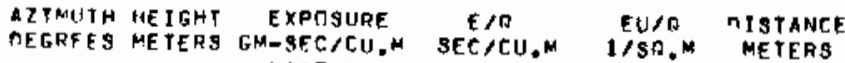
$x \geq 0 E+6$

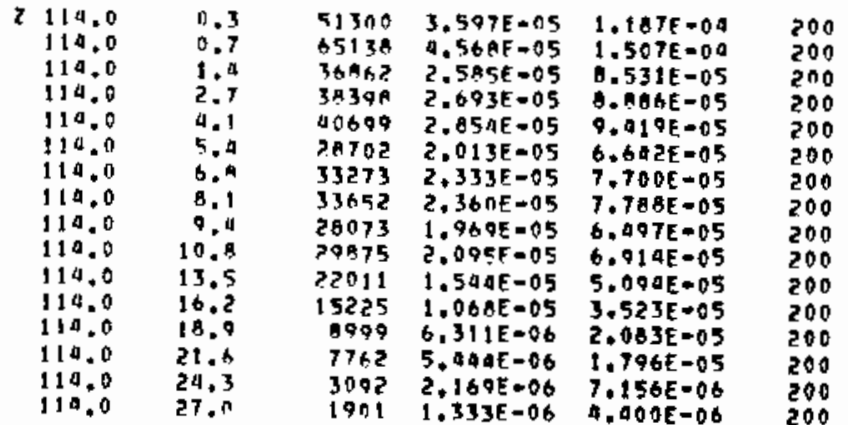


-2IMUTH HE IGH: DEGREES METERS GM-SECICU $\times 10 E+6$

122.0
122.0
122.0
122.0
122.0
122.0
122.0
122.0
122.0
122.0
122.0
122.0
122.0
122.0
122.0
122.0

$E>0$

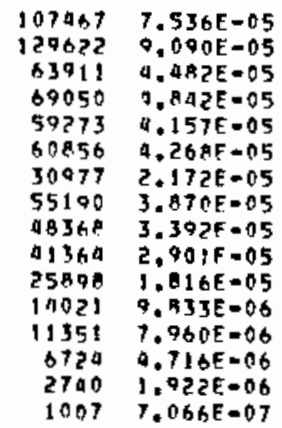

ev/o distance

$2.487 E=04$

$3.000 E-00$ $1.479 E=04$ $1.590 E-04$ 1.372E-04 $1.0 O A E=0 a$ $7+169 E=05$ $1.277 \mathrm{E}-0.4$ 1.119E-OA $9,5725=05$ $5.993 \mathrm{E}=05$ $3.245 F-05$ $2,627 E-05$ 1. $556 \varepsilon-05$ $6.3 A 3 E=06$ $2.3325-06$

ATIMITH HEIGHT EXPISURE E/O EU/O DISTANCE DEGRFES QFTERS TOM-SECJCU.N SEE/CU.N 1/SO.M METFRS $x+0 E+6$

$130 . n$

130.0

130.0

130.0

130.0

130.0

130.0

130.0

130.0

130.0

130.0

130.0

130.0

130.0

130.0

130.0

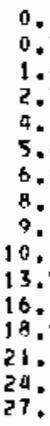

0.3
0.7
1.0
2.7
4.1
5.9
6.9
8.1
9.9
10.8
13.5
16.2
19.9
21.6
24.3
27.7
Q676T 6,7RBE-OS

118 A96 $\quad .33 \%=05$

D. $330 F-05$ 2. $0.751 E-04$

$55523 \quad 3.834 E=05$ 1.2E $5 E=0$ a

50890 3.569E-05 1.17 AE 04

A989R 3.4QQE-05 1.:55E-0U

$56367 \quad 3.953 E-05 \quad 1.304 E=00$

$50454 \quad 3.552 E=05 \quad 1.172 F=0$

$84395.918 E-06 \quad 1.953 E-05$

$33430 \quad 2.345 E-05 \quad 7.737 E-05$

$312 ? 9$ 2.190E-195 T.227E-05

20073 1.408E-05 $4.645 E-05$

$10907 \quad 7.677 E-06 \quad 2.533 E-05$

$8005 \quad 5.677 E=06 \quad 1.873 E-05$

$350 n \quad 2.45 E E-06 \quad 0.111 E-06$
200

500
500

sin

200

200

200

300

$5 \cap 0$

200

200

200

200

200

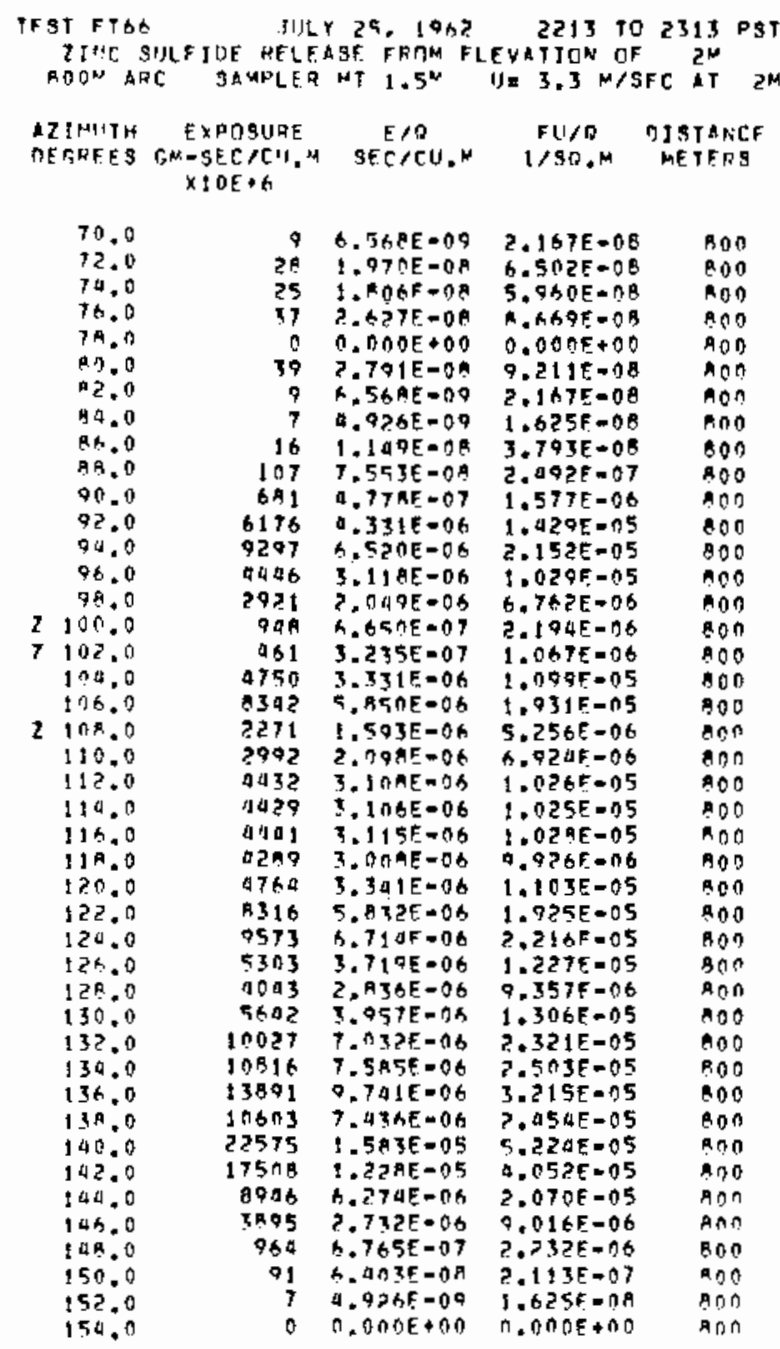

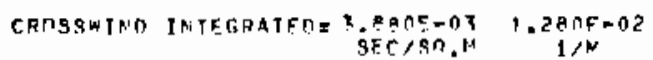

TOKEP OATA FOLLOW....

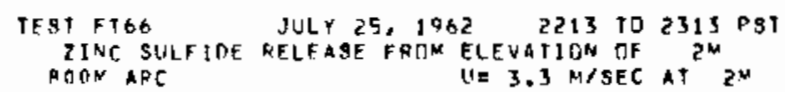

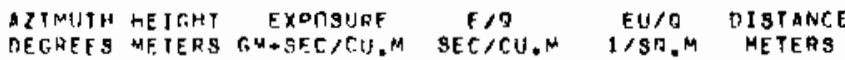
$\times 1 O E+S$

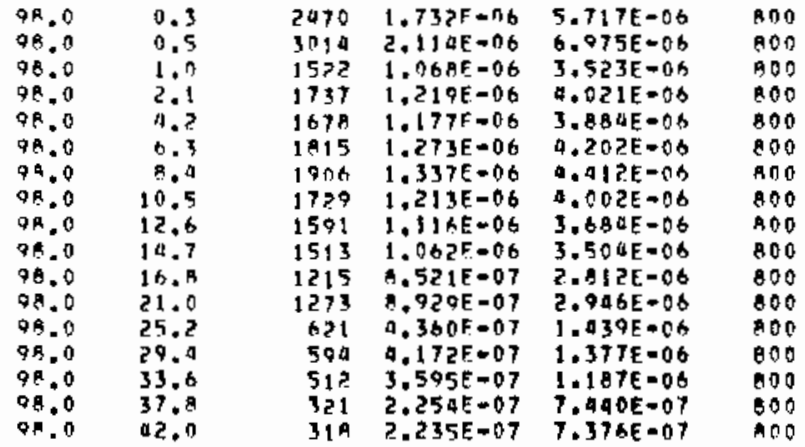




\begin{tabular}{|c|c|c|c|c|c|}
\hline $\begin{array}{l}\text { ZI"'TH } \\
\text { EGRES }\end{array}$ & $\begin{array}{l}\text { HEIGHT } \\
\text { METERS }\end{array}$ & $\begin{array}{c}\text { EXPOSURE } \\
\text { GM=SEC }=\text { CI! } \\
\times 10 E \$ 6\end{array}$ & $\begin{array}{c}E / / 0 \\
\mathrm{SEC} / \mathrm{CU} . M\end{array}$ & $\begin{array}{l}E U / Q \\
L / 9 R, M\end{array}$ & $\begin{array}{l}\text { DISTANCE } \\
\text { METERS }\end{array}$ \\
\hline $\begin{array}{l}105.0 \\
105.0 \\
106.0 \\
106.0 \\
106.0 \\
106.0 \\
105.0 \\
106.0 \\
106.0 \\
106.0 \\
106.0 \\
108.0 \\
106.0 \\
106.0 \\
106.0 \\
106.0 \\
106.0\end{array}$ & $\begin{array}{l}0.3 \\
0.5 \\
1.0 \\
2.8 \\
4.2 \\
6.3 \\
3.0 \\
10.5 \\
12.8 \\
14.7 \\
16.8 \\
21.0 \\
25.2 \\
29.4 \\
33.0 \\
37.9 \\
42.0\end{array}$ & $\begin{array}{l}8470 \\
8246 \\
3171 \\
4908 \\
4948 \\
6102 \\
5396 \\
6058 \\
5996 \\
4693 \\
1910 \\
3716 \\
2097 \\
1585 \\
1516 \\
820 \\
566\end{array}$ & $\begin{array}{l}5.940 E-06 \\
6.200 E-176 \\
2.224 E-06 \\
3.942 E-06 \\
3.470 E=06 \\
4.260 E=06 \\
3.777 E-06 \\
9.256 E=06 \\
4.19 A F=06 \\
3.2 A 4 E-06 \\
2.742 F=06 \\
2.606 E=06 \\
1.695 E-06 \\
1.112 E=06 \\
1.060 E-06 \\
6.174 E=07 \\
3.971 F=07\end{array}$ & 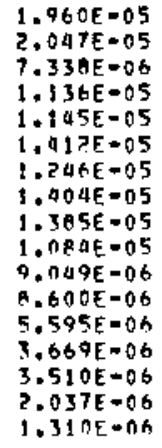 & $\begin{array}{l}000 \\
000 \\
800 \\
A 00 \\
000 \\
000 \\
000 \\
000 \\
000 \\
000 \\
800 \\
800 \\
800 \\
900 \\
890 \\
000 \\
900\end{array}$ \\
\hline $\begin{array}{l}\text { ATINIITH } \\
\text { DERQEES }\end{array}$ & $\begin{array}{l}\text { HE ITHT } \\
\text { NETERS }\end{array}$ & $\begin{array}{c}\text { EXPDSURE } \\
G N-5 E C>C H, M \\
\times 1 N E+6\end{array}$ & $\begin{array}{c}f / B \\
A F[C(1) \mu\end{array}$ & $\begin{array}{c}\text { EU/n } \\
1 / \text { SO. }\end{array}$ & $\begin{array}{l}\text { DISTANCE } \\
\text { METFPS }\end{array}$ \\
\hline 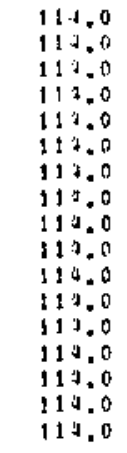 & $\begin{array}{l}0.3 \\
0.5 \\
1.0 \\
2.1 \\
9.2 \\
6.7 \\
8.0 \\
10.5 \\
12.6 \\
21.7 \\
16.7 \\
21.0 \\
25.2 \\
29.9 \\
33.6 \\
37.9 \\
47.0\end{array}$ & $\begin{array}{l}4909 \\
4240 \\
245 ? \\
2114 \\
2954 \\
3003 \\
2513 \\
3474 \\
3212 \\
3013 \\
3519 \\
2617 \\
1631 \\
1802 \\
699 \\
762 \\
650\end{array}$ & $\begin{array}{l}3.493 E=06 \\
2.987 F=06 \\
1.720 F=06 \\
1.533 F=06 \\
2.972 E=06 \\
2.387 F=06 \\
1.763 E=06 \\
2.436 E=06 \\
2.253 E=06 \\
2.113 F=06 \\
2.46 A E=06 \\
1.835 E=06 \\
1.3495=06 \\
1.269 E=06 \\
4.904 E=07 \\
5.345 E=07 \\
4.561 E=07\end{array}$ & $\begin{array}{l}1.136 E=05 \\
9.859 E=06 \\
5.677 E=06 \\
5.060 E=06 \\
6.837 E=06 \\
7.076 E=06 \\
5.818 E=06 \\
8.040 E=06 \\
7.035 E=06 \\
6.975 E=06 \\
8.145 E=06 \\
6.057 E=06 \\
3.793 E=06 \\
4.171 E=06 \\
1.61 A E=06 \\
1.764 E=06 \\
1.505 E=06\end{array}$ & $\begin{array}{l}000 \\
800 \\
800 \\
800 \\
900 \\
800 \\
000 \\
800 \\
000 \\
900 \\
800 \\
000 \\
800 \\
000 \\
000 \\
800 \\
000\end{array}$ \\
\hline $\begin{array}{l}\text { AZIN JTH } \\
\text { DEGRTFS }\end{array}$ & $\begin{array}{l}\text { HEIGHT } \\
\text { ME TERS }\end{array}$ & $\begin{array}{c}\text { EXPOSIJRE } \\
\text { GM-SEC } \\
\times 1 O E+G\end{array}$ & $\begin{array}{c}E / Q \\
S E C / C U, H\end{array}$ & $\begin{array}{l}E \cup / 0 \\
1 / 90 . M\end{array}$ & $\begin{array}{l}\text { DISTANTE } \\
\text { METERS }\end{array}$ \\
\hline $\begin{array}{l}122.0 \\
122.0 \\
122.00 \\
122.0 \\
122.0 \\
12300 \\
122.0 \\
122.0 \\
122.0 \\
122.0 \\
122.0 \\
123.0 \\
122.0 \\
122.0 \\
122.0 \\
122.0 \\
122.0\end{array}$ & $\begin{array}{r}0.3 \\
0.5 \\
1.0 \\
2.1 \\
0.2 \\
6.3 \\
3.0 \\
10.5 \\
12.0 \\
14.7 \\
16.8 \\
21.0 \\
25.2 \\
29.0 \\
33.6 \\
37.0 \\
02.0\end{array}$ & $\begin{array}{l}7911 \\
0094 \\
3312 \\
0588 \\
5784 \\
5063 \\
5026 \\
4719 \\
4195 \\
4659 \\
3490 \\
3159 \\
1535 \\
1377 \\
836 \\
859 \\
1573\end{array}$ & $\begin{array}{l}5.548 E=06 \\
5.676 E=06 \\
2,323 E=06 \\
3.218 E=06 \\
4.056 E=06 \\
3.551 E=06 \\
3.525 E=06 \\
3.309 E=06 \\
2.935 E=06 \\
3.268 E=06 \\
2.459 E=06 \\
2.215 E=06 \\
1.077 E=06 \\
9.659 E=07 \\
5,363 E=07 \\
6.025 E=07 \\
1.103 E=06\end{array}$ & $\begin{array}{l}1.031 E=05 \\
1.073 E=05 \\
? .666 E=06 \\
1.002 E=05 \\
1.339 E=05 \\
1.172 E=05 \\
1.163 E=05 \\
1.192 E=05 \\
9.685 E=06 \\
1.070 E=05 \\
1.099 E=06 \\
7.309 E=06 \\
3.553 E=06 \\
3.18 A E=06 \\
1.935 E=06 \\
1.980 E=06 \\
3.641 E=06\end{array}$ & $\begin{array}{l}180 \\
800 \\
800 \\
800 \\
800 \\
000 \\
100 \\
000 \\
500 \\
800 \\
800 \\
800 \\
800 \\
800 \\
800 \\
800 \\
800\end{array}$ \\
\hline
\end{tabular}




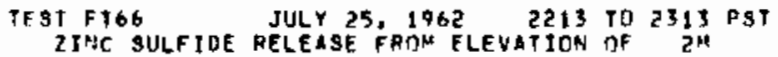

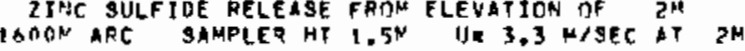

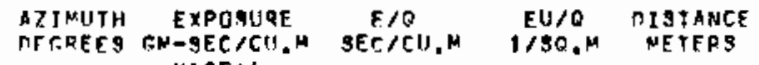
$\times 10 E+6$

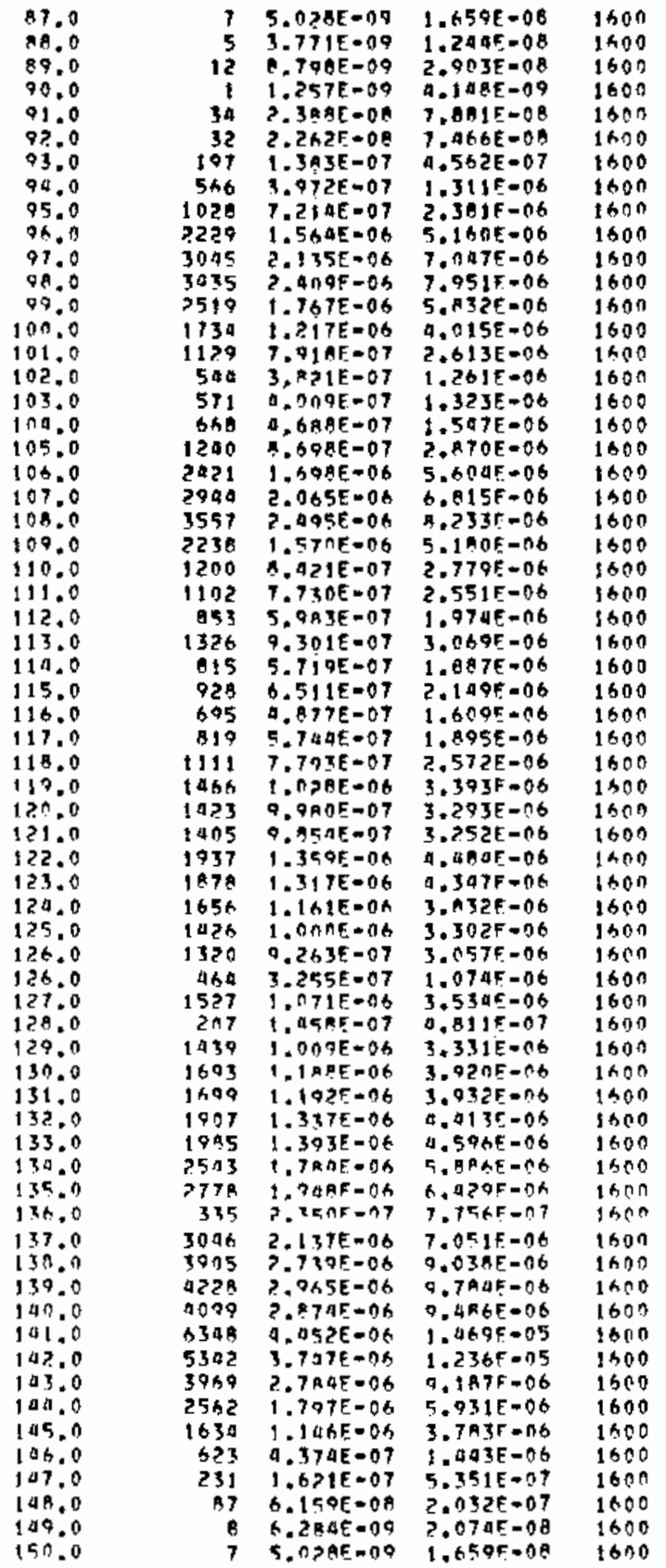

CRIISANTINO INTEGRATEDE 2.023E-03 6.677E-ก!3

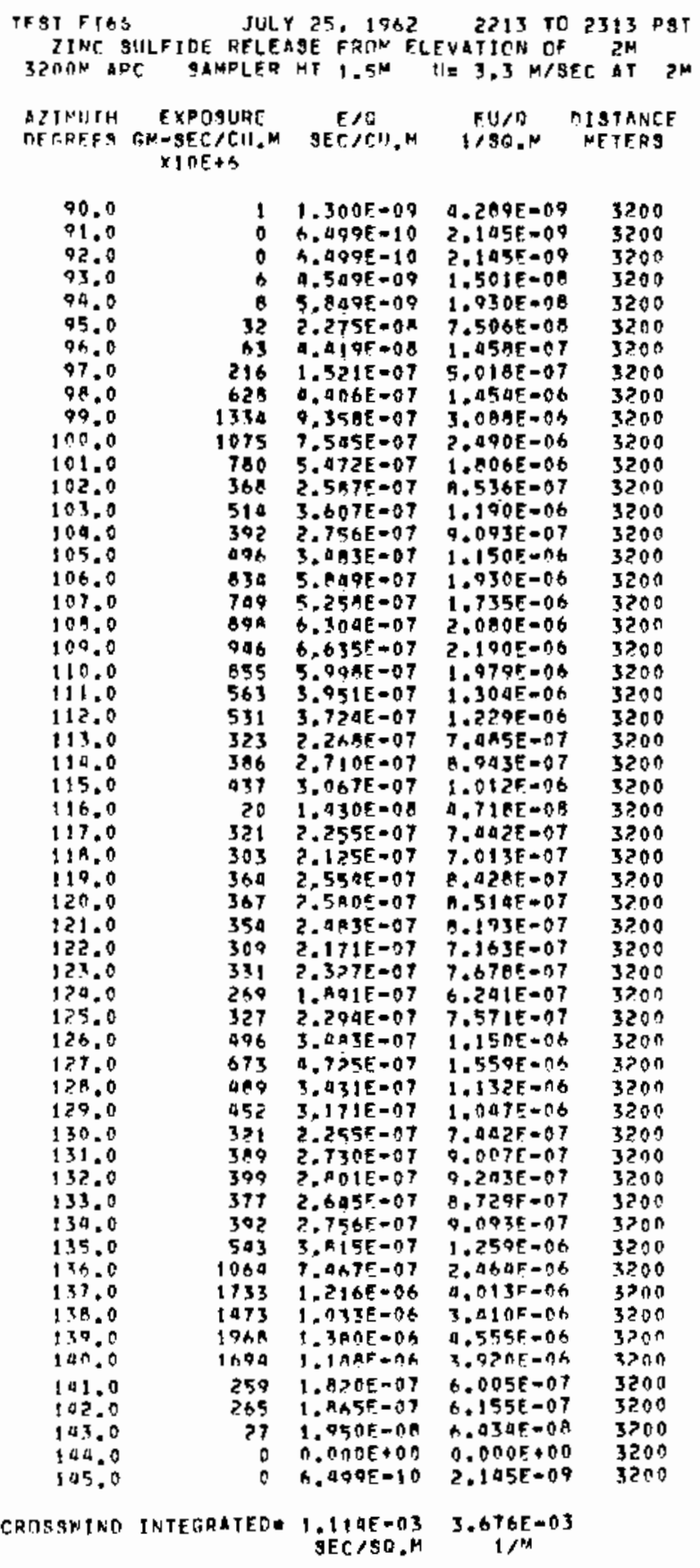




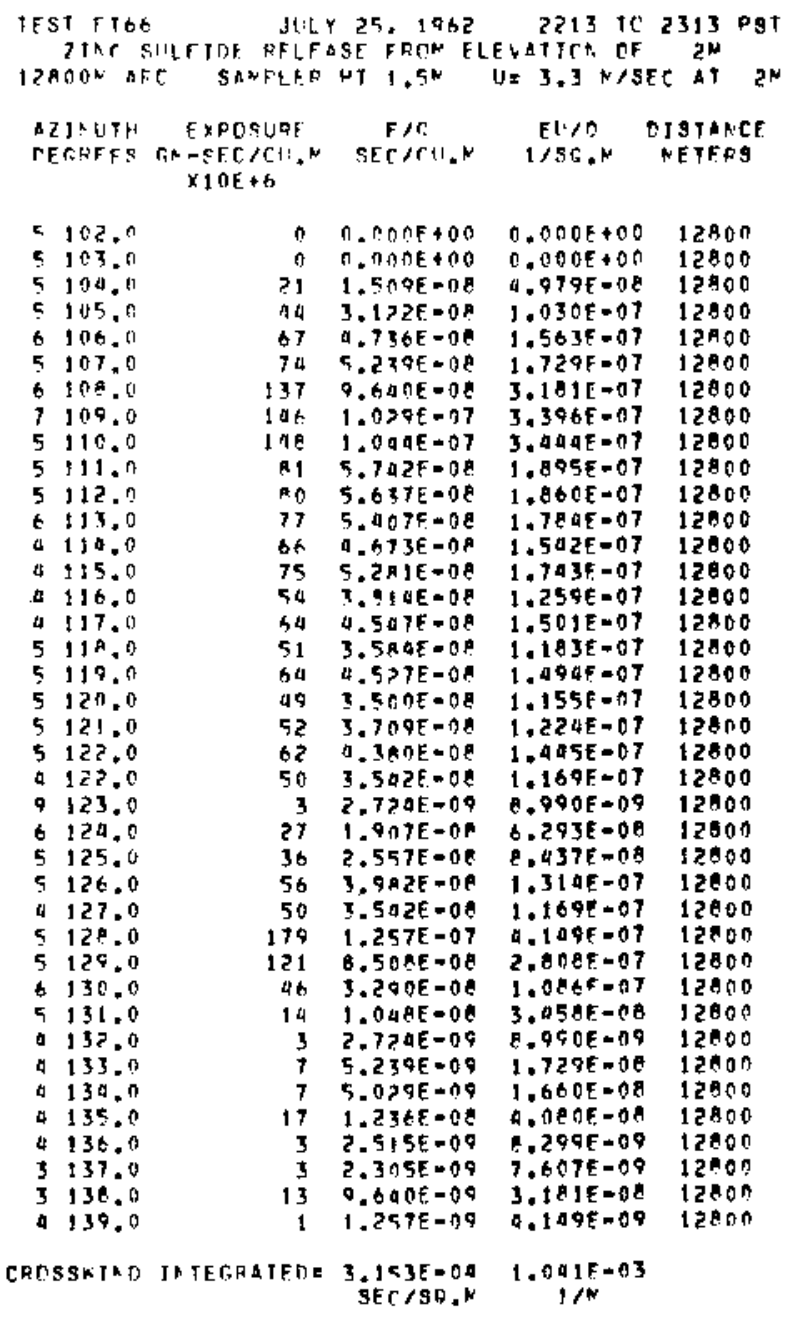


30-SERIES EXPERIMENT: $67:$ FT-67;

GROUND LEVEL ANL TGWER SAMPL FNG 200 AND BOI M ARCS. GROUND LEVEL

SAMPLING ONLY 1600-3200 M ARCS. A PORTIOH OF THE PLUME PASSED TO

THE NORTH OF THREE OF THE FOUR ARCS.

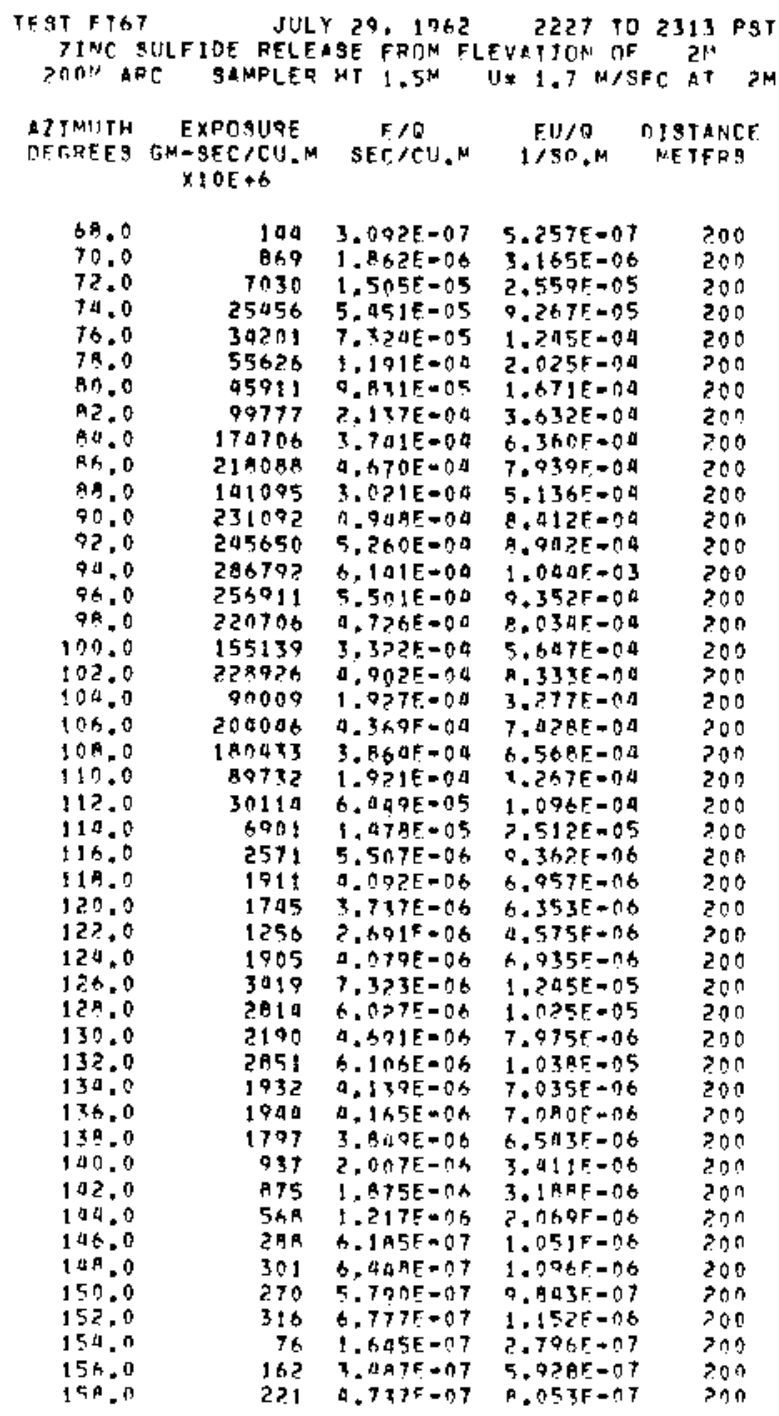

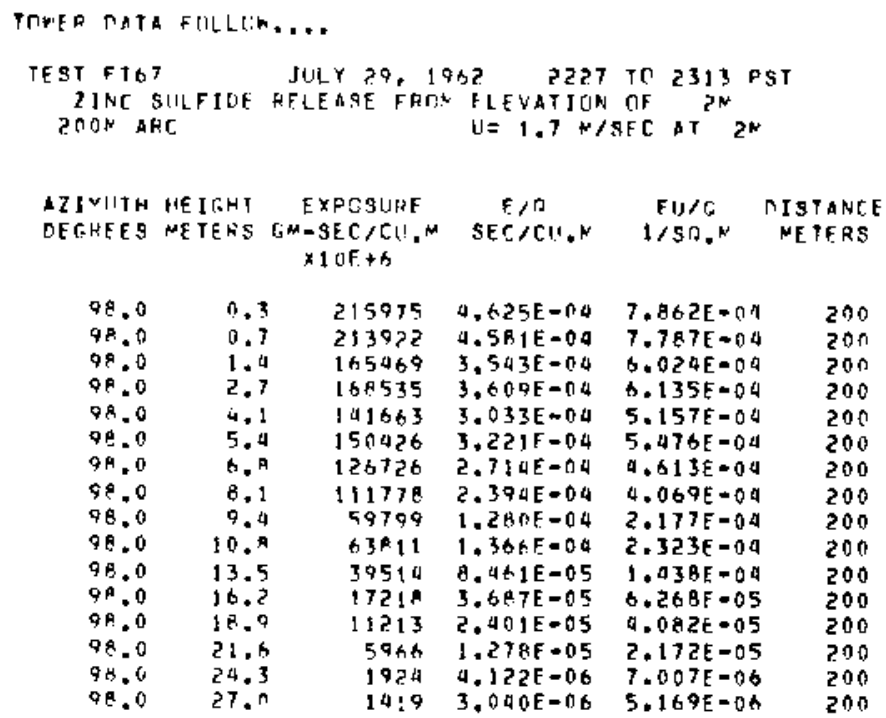

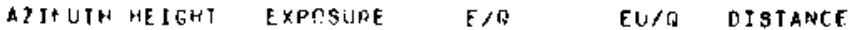
DEREFS METEFS GM-SEC/CU." SEC/CU.N 1/SO.M NETERS $\times 10 \mathrm{E}+6$

\begin{tabular}{|c|c|c|c|c|c|}
\hline $\begin{array}{l}106.0 \\
106.0\end{array}$ & 0.3 & $\begin{array}{l}211311 \\
239232\end{array}$ & $\begin{array}{l}1.525 E=04 \\
5.123 E=04\end{array}$ & $\begin{array}{l}7.692 \mathrm{E}=04 \\
9.709 \mathrm{E}=0 \mathrm{O}\end{array}$ & $\begin{array}{l}200 \\
200\end{array}$ \\
\hline 100.0 & 1.4 & 95123 & $1,823 \mathrm{E}=04$ & $3.090 E-04$ & 200 \\
\hline $\begin{array}{l}106.0 \\
106.0\end{array}$ & $\begin{array}{l}2.7 \\
0.1\end{array}$ & $\begin{array}{l}163479 \\
1602+7\end{array}$ & $\begin{array}{l}3.501 \mathrm{~F}=04 \\
3.432 \mathrm{~F}=00\end{array}$ & $\begin{array}{l}5.951 E=04 \\
5.834 E=04\end{array}$ & $\begin{array}{l}200 \\
200\end{array}$ \\
\hline 106.0 & 5.4 & $13063 ?$ & $2.969 E=04$ & $5.047 E=04$ & 200 \\
\hline 106.0 & $D, R$ & 134579 & $2.8829=00$ & $4.8 \% 9 E=04$ & 200 \\
\hline 106.0 & A.t & $957+1$ & $2.051 \mathrm{E}=04$ & 3. $186 E=04$ & 300 \\
\hline 106.0 & 0.4 & A5567 & $1.852 \mathrm{~F}=02$ & $3.115 E=00$ & 200 \\
\hline 106.0 & $10 .{ }^{2}$ & 76902 & $1.6478=04$ & $2.799 \mathrm{E}=04$ & 200 \\
\hline 100.0 & 13.5 & a $100 \mathrm{~A}$ & $A$, eOOF $=05$ & $1.49 B E=04$ & 200 \\
\hline 306.0 & 16.7 & 18478 & $3.956 F-05$ & $6.720 E=05$ & $20 n$ \\
\hline 100.0 & $18 . ?$ & 7904 & $1.693 \mathrm{E}=05$ & $2.878 E-05$ & 200 \\
\hline 10 & 21.6 & 3474 & $7.440 E=06$ & $1.265 E-05$ & 200 \\
\hline 100,0 & 24,3 & 1100 & $.527 F=04$ & $4.29 \mathrm{BF}=06$ & $2 \cap 0$ \\
\hline $\mathbf{1}$ & 37 & $5 \div 1$ & $137 F=06$ & $.933 E-06$ & 200 \\
\hline
\end{tabular}

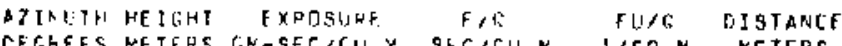

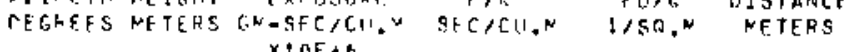

CRTGQATHA INTEGRATFDE $0.574 F-02 \quad 7.775 F-02$

\begin{tabular}{|c|c|c|c|c|c|}
\hline 19.0 & 0.3 & $\Delta a+5$ & $7.565 E=06$ & $1.626 E-05$ & 200 \\
\hline 110.0 & 0.7 & 5354 & $1.106 F=05$ & $1.8497=105$ & 200 \\
\hline 119.0 & 1.4 & 5005 & 1. DASE- OS & $1,0.44 E=05$ & 200 \\
\hline 14.0 & 2.7 & 5902 & 1.2 ค $5 E-05$ & $2.182 E=05$ & 200 \\
\hline 14.0 & 4.1 & $953 ?$ & $1.998 E=05$ & $197 \mathrm{E}-05$ & 200 \\
\hline 114.0 & 5.4 & 10452 & $2.270 \mathrm{f}=105$ & 3.87 aE -05 & 200 \\
\hline 14.0 & 6.7 & 11598 & $2.4 A \triangle F=05$ & $a .222 \mathrm{E}-05$ & 200 \\
\hline 110.6 & A. 1 & $10 \sin$ & $2.276 E-05$ & $3.070=05$ & 200 \\
\hline 14.0 & 9.4 & 12302 & $2.032 \mathrm{~F}=05$ & $4.075 \mathrm{E}=05$ & 200 \\
\hline 14,0 & 10.8 & 11504 & $2.4637=05$ & $4.1 B A E-05$ & 200 \\
\hline 110.0 & 13.5 & 10130 & $2.160 E=0.5$ & $3.6 e$ AE -05 & 200 \\
\hline 114,0 & 16.2 & 5660 & $1.212 E=05$ & $2.060 \mathrm{E}=05$ & 20 ก \\
\hline 14.0 & 18.9 & 3125 & $69 z t=06$ & $1.13 B E=05$ & 200 \\
\hline 14.0 & 21.6 & 1701 & 73 CE- O6 & $6.311 E-06$ & 200 \\
\hline 0 & 1,3 & $50 n$ & $071 E-06$ & $1.821 E=06$ & 200 \\
\hline 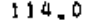 & & 145 & $20 E-07$ & $304 E-07$ & 200 \\
\hline
\end{tabular}




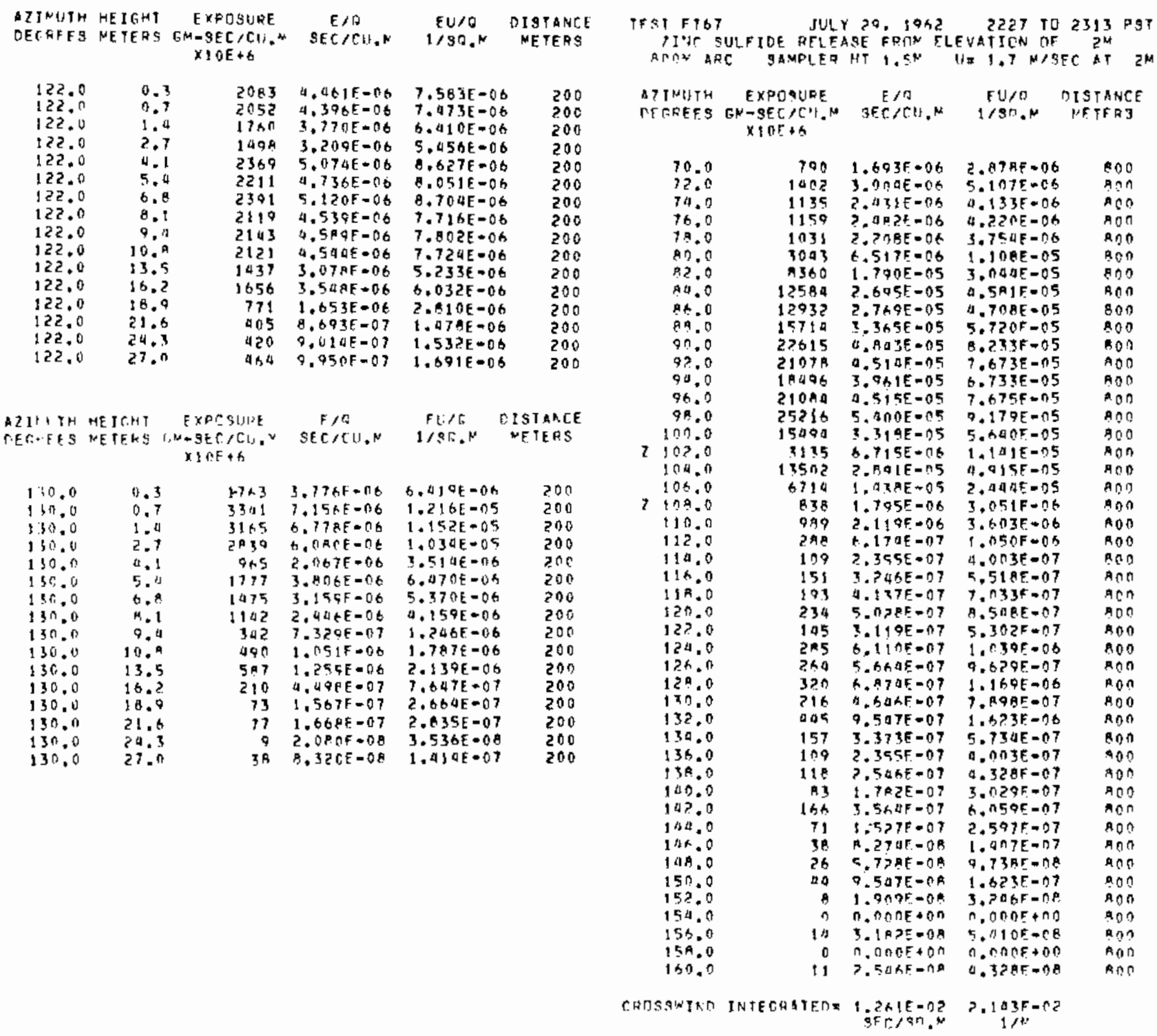


TONER CATA FOLLCW....

TEST FIOT JITE SULFIUE RELEASE FRTN 1962 22Z7 TC 2313 PST ZIRC SULFJUE RELEASE FROH ELEVATION OF ZN

\begin{tabular}{|c|c|c|c|c|c|}
\hline $\begin{array}{l}\text { DZINUTH } \\
\text { DEGREES }\end{array}$ & $\begin{array}{l}\text { HE IGHT } \\
\text { ME TERS }\end{array}$ & $\begin{array}{c}\text { F XPGSURE } \\
\text { GN }=9 E C / C U . N \\
\times 10 E+b\end{array}$ & $\begin{array}{c}\epsilon / D \\
\sec / C !, *\end{array}$ & $\begin{array}{l}E U / O \\
\because / S O . N\end{array}$ & $\begin{array}{l}\text { DISTANCE } \\
\text { METERS }\end{array}$ \\
\hline $\begin{array}{r}98.0 \\
99.0 \\
98.0 \\
98.0 \\
9.00 \\
98.0 \\
98.0 \\
98.0 \\
98.0 \\
98.0 \\
98.0 \\
9 \% .0 \\
98.0 \\
98.0 \\
9.00 \\
98.0 \\
98.0\end{array}$ & $\begin{array}{r}0.3 \\
0.5 \\
1.0 \\
2.1 \\
4.5 \\
6.3 \\
8.5 \\
10.5 \\
12.6 \\
14.7 \\
16.8 \\
21.0 \\
25.2 \\
29.4 \\
33.6 \\
37.0 \\
42.0\end{array}$ & $\begin{array}{r}22562 \\
24301 \\
20530 \\
19105 \\
20135 \\
17621 \\
16503 \\
16831 \\
11384 \\
10619 \\
11009 \\
6870 \\
4172 \\
0 \\
0 \\
0 \\
440\end{array}$ & $\begin{array}{l}4.831 E=05 \\
5.223 E=05 \\
4.396 E=05 \\
4.091 E-05 \\
4.312 E=05 \\
3.773 E=05 \\
3.534 E=05 \\
3.006 F=05 \\
2.43 E E=05 \\
2.270 F=05 \\
2.35 E E=05 \\
1.071 E=05 \\
8.930 E-06 \\
0.00 C E+00 \\
0.000 F+00 \\
0.000 E+00 \\
9.423 E=07\end{array}$ & $\begin{array}{l}8.213 E=05 \\
8.070 E=05 \\
7.474 E=05 \\
6.055 E=05 \\
7.330 E=05 \\
6.015 E=05 \\
6.000 E=05 \\
6.127 E=05 \\
4.104 E=05 \\
3.060 E=05 \\
4.000 E=05 \\
2.501 E=05 \\
1.519 E=05 \\
0.000 E+00 \\
0.000 E+00 \\
0.000 E+00 \\
1.602 E=06\end{array}$ & $\begin{array}{l}800 \\
600 \\
800 \\
800 \\
000 \\
000 \\
800 \\
000 \\
000 \\
000 \\
000 \\
000 \\
000 \\
000 \\
000 \\
000 \\
800\end{array}$ \\
\hline
\end{tabular}

AZIMHTH HEIGHT EXPQSURE ERY EUTG DISTANCE DEGFFES METEPS GN-SEC/CU.N SEC/CU.N 1/SG.N NETERS

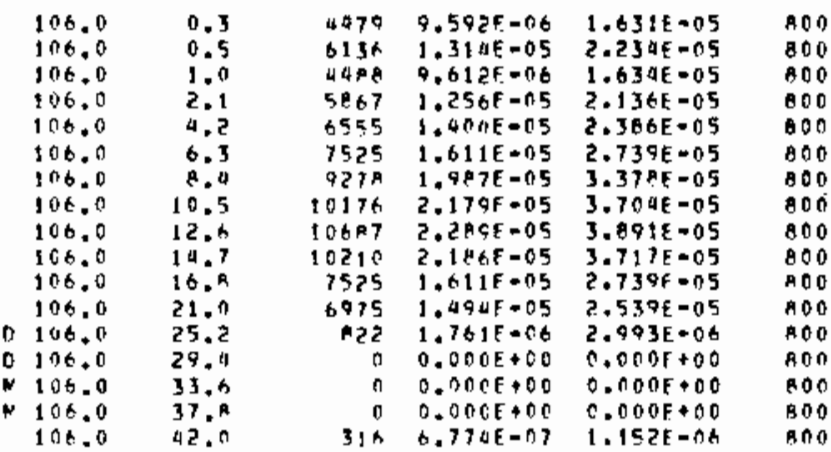

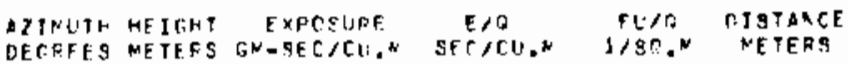
$x: 9 \mathbf{F}+6$

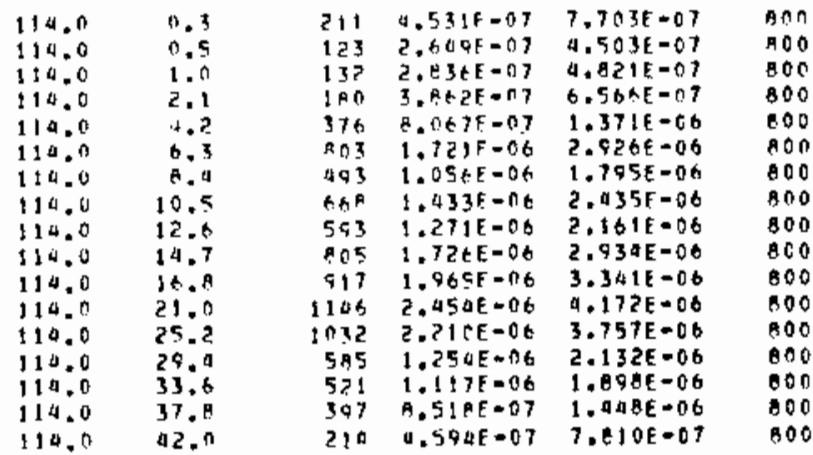

12IHUTH HEIGHI EXPCSLRE E/A EU/F OISTANCE DEGREES METERS GNOSECICU.N SECICEIN IISO,N METEAS $x 1$ OF +6

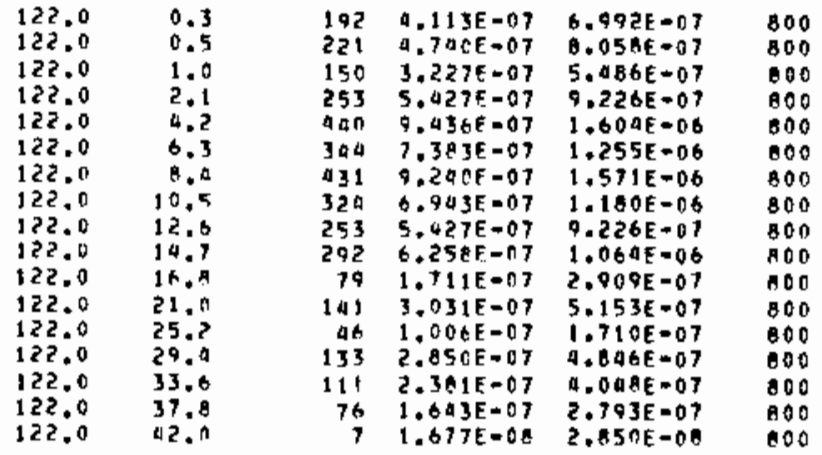

AZIMUTH HEIGHT EXPQSURE E/O EU/C DISTANCE CEGHES PETEAS GN-9EC/EU.N SEC/CI.N I/SG.M RETERS $x+0 F+6$

\begin{tabular}{|c|c|c|c|c|c|}
\hline $\begin{array}{l}130.0 \\
130.0 \\
130.0 \\
130.0\end{array}$ & $\begin{array}{l}0.3 \\
0.5 \\
1.0 \\
2.1\end{array}$ & $\begin{array}{l}273 \\
289 \\
269 \\
299\end{array}$ & $\begin{array}{l}5.855 E=07 \\
0.200 E=07 \\
5.765 E=07 \\
6.005 E-07\end{array}$ & $\begin{array}{l}9.959 E=07 \\
1.055 E=06 \\
9.008 E=07 \\
1.0 B 9 E=06\end{array}$ & $\begin{array}{l}800 \\
800 \\
800 \\
800\end{array}$ \\
\hline 130.0 & 4.2 & 479 & $1.027 \mathrm{~F}-06$ & $5.745 E=06$ & An 0 \\
\hline $\begin{array}{l}130.0 \\
130.0\end{array}$ & $\begin{array}{l}6.3 \\
8.9\end{array}$ & $\begin{array}{l}397 \\
397\end{array}$ & $\begin{array}{l}8.5075-07 \\
8.503 E-07\end{array}$ & $\begin{array}{l}1.946 E-06 \\
1.446 E-06\end{array}$ & $\begin{array}{l}100 \\
800\end{array}$ \\
\hline $\begin{array}{l}130.0 \\
130.0 \\
130.0\end{array}$ & $\begin{array}{l}10,5 \\
12,6 \\
14,7\end{array}$ & $\begin{array}{l}354 \\
529 \\
940\end{array}$ & $\begin{array}{l}7.676 E=07 \\
1.134 E=06 \\
9.936 E=07\end{array}$ & $\begin{array}{l}1.305 E=06 \\
1.928 E=06 \\
1.604 E-06\end{array}$ & $\begin{array}{l}100 \\
800 \\
800\end{array}$ \\
\hline $\begin{array}{l}130.0 \\
130.0 \\
130.0\end{array}$ & $\begin{array}{l}16.8 \\
21.0 \\
25.2\end{array}$ & $\begin{array}{l}2,7 \\
305 \\
217\end{array}$ & $\begin{array}{l}6.160 E=07 \\
6.551 E=07 \\
0.661 E=07\end{array}$ & $\begin{array}{l}1.047 \mathrm{E}=06 \\
1.114 \mathrm{EE}=06 \\
1.92 \mathrm{AE}=07\end{array}$ & $\begin{array}{l}800 \\
800 \\
800\end{array}$ \\
\hline $\begin{array}{l}130.0 \\
130.0 \\
130.0 \\
130.0\end{array}$ & $\begin{array}{l}29.6 \\
33.6 \\
37.8 \\
42.0\end{array}$ & $\begin{array}{l}1 \text { 月6 } \\
155 \\
142 \\
122\end{array}$ & $\begin{array}{l}3.991 E=07 \\
3.320 E=07 \\
3.052 E=07 \\
2.616 E=07\end{array}$ & $\begin{array}{l}6.704 E-07 \\
5.644 E-07 \\
5.188 E-07 \\
9.947 E-07\end{array}$ & $\begin{array}{l}800 \\
800 \\
800 \\
800\end{array}$ \\
\hline
\end{tabular}




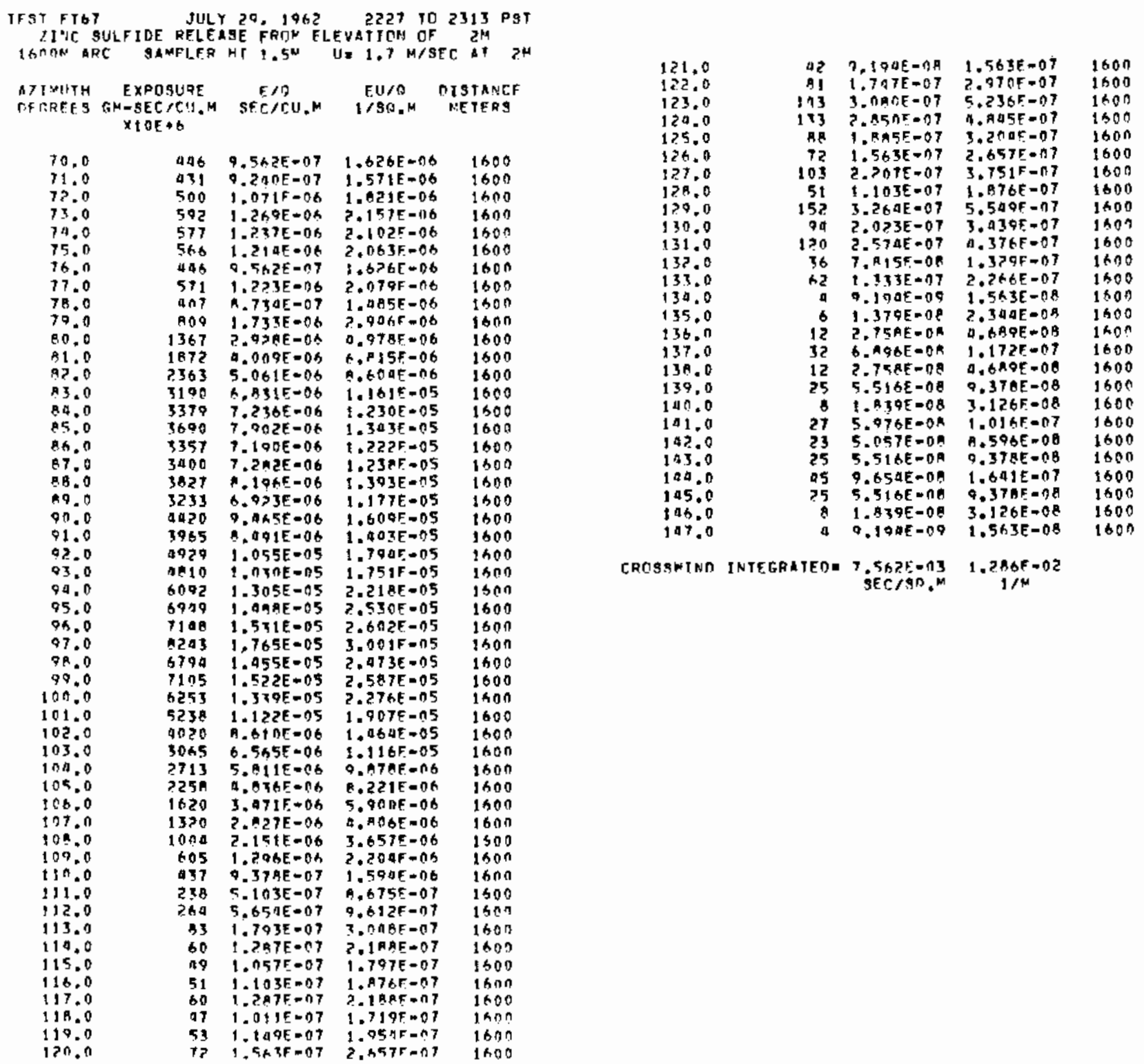




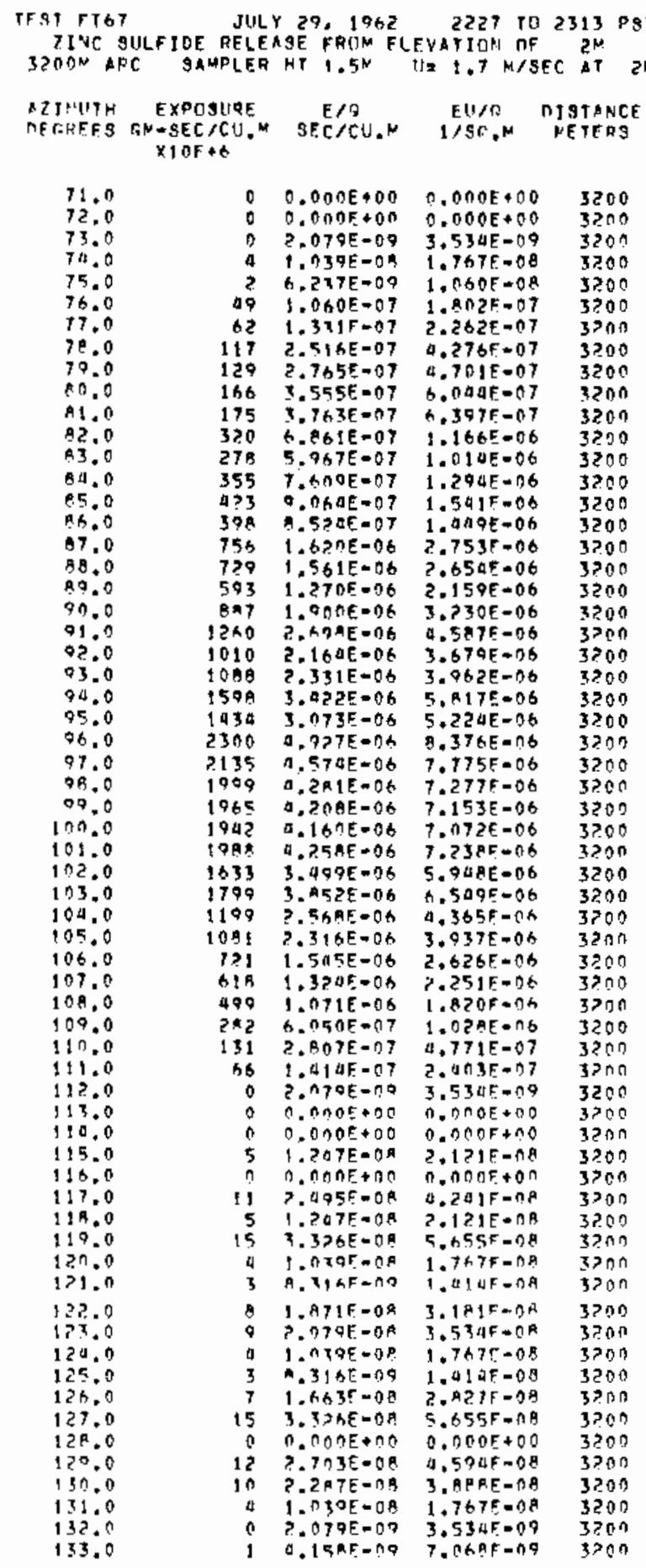

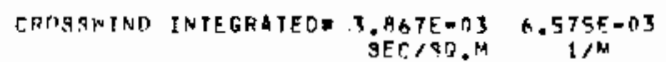




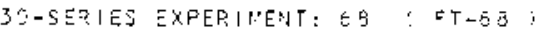

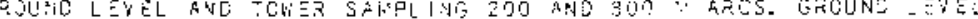

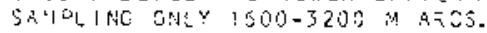

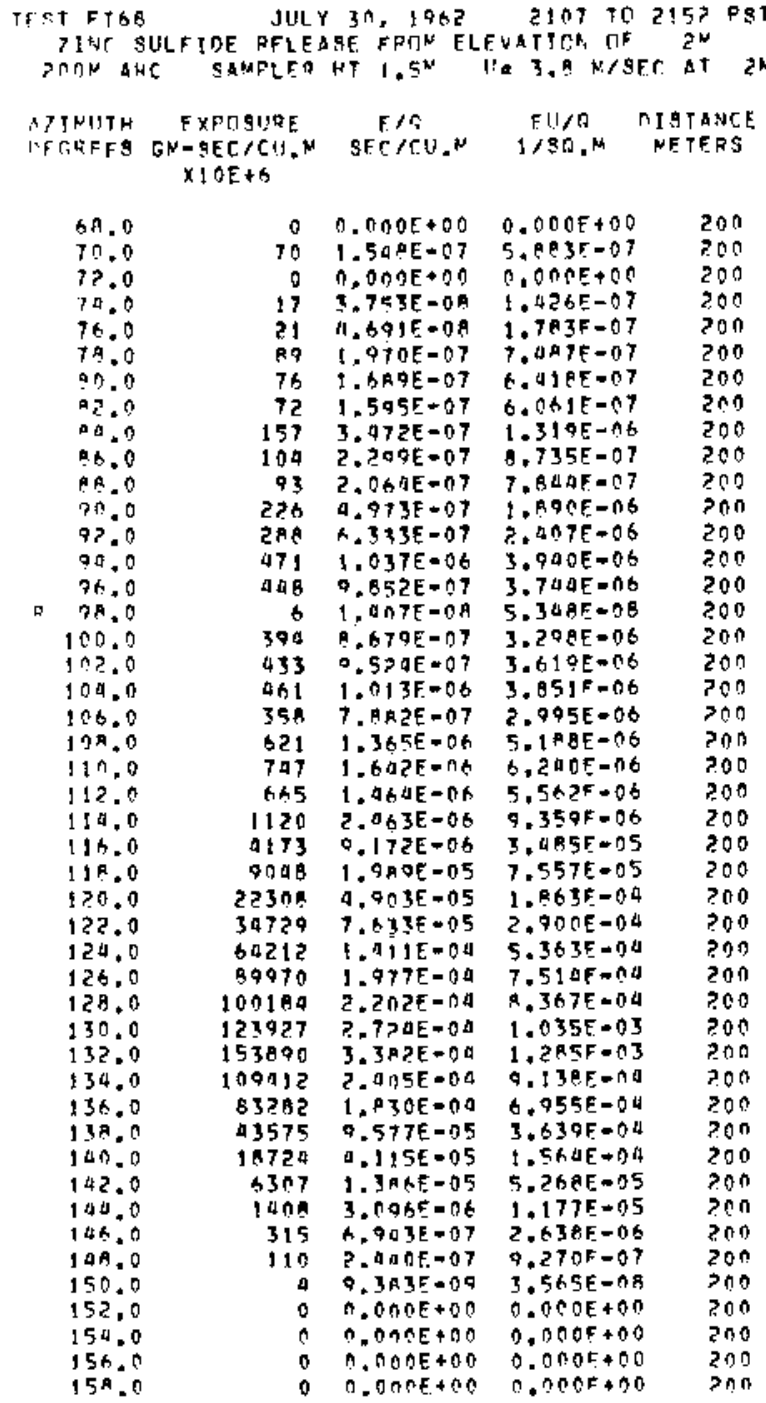

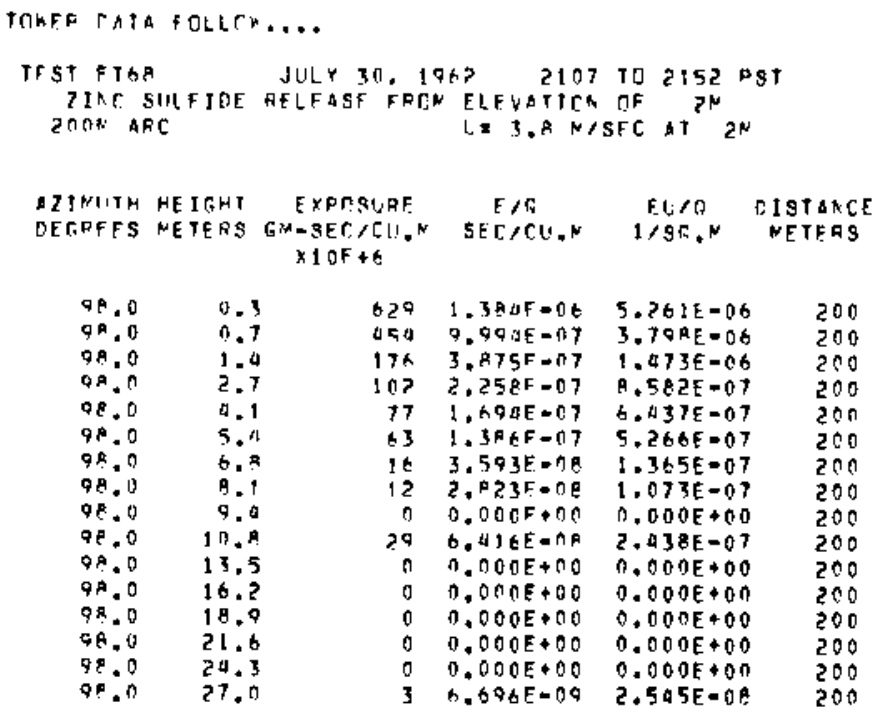

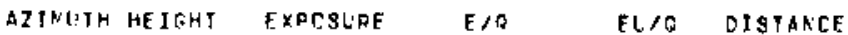

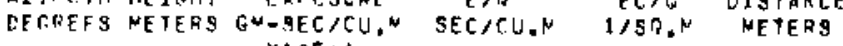
×) $\cap$ E +6
\end{abstract}

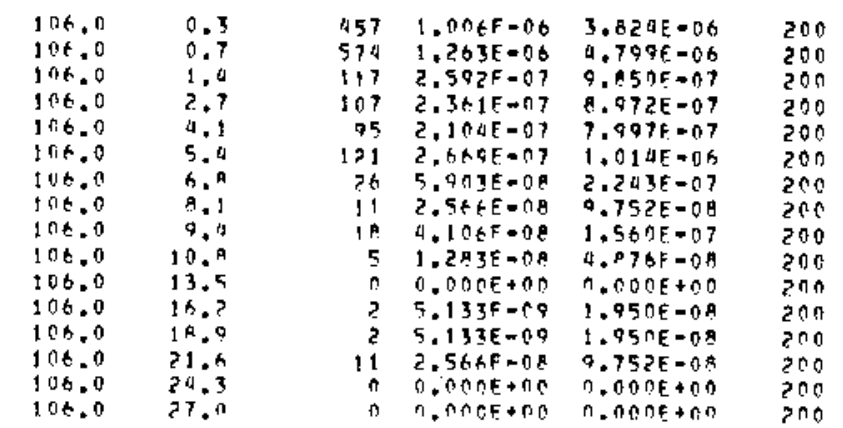

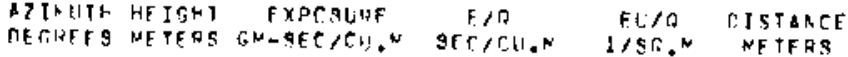
$\times 10 \mathrm{~F}+6$

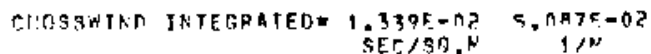




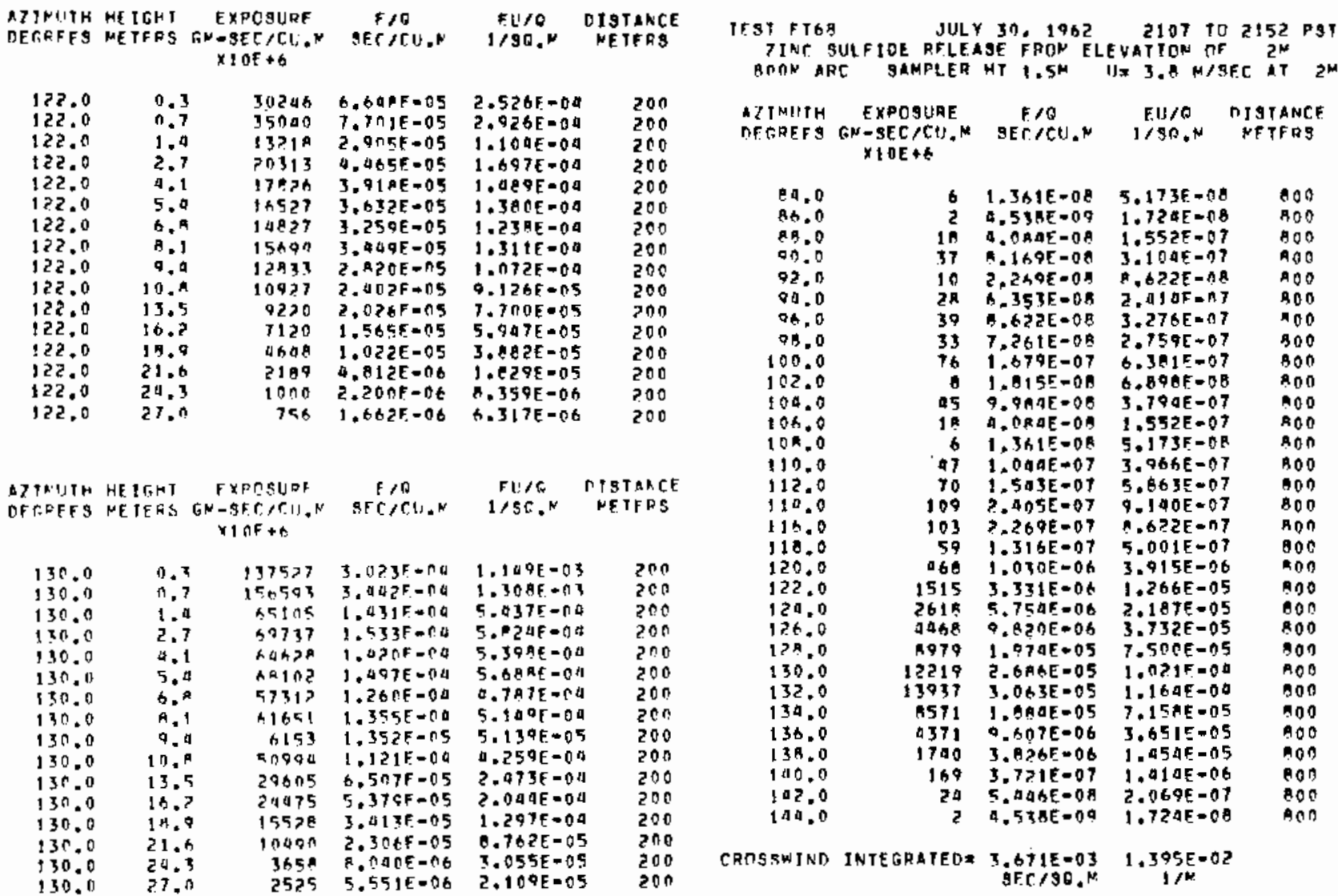

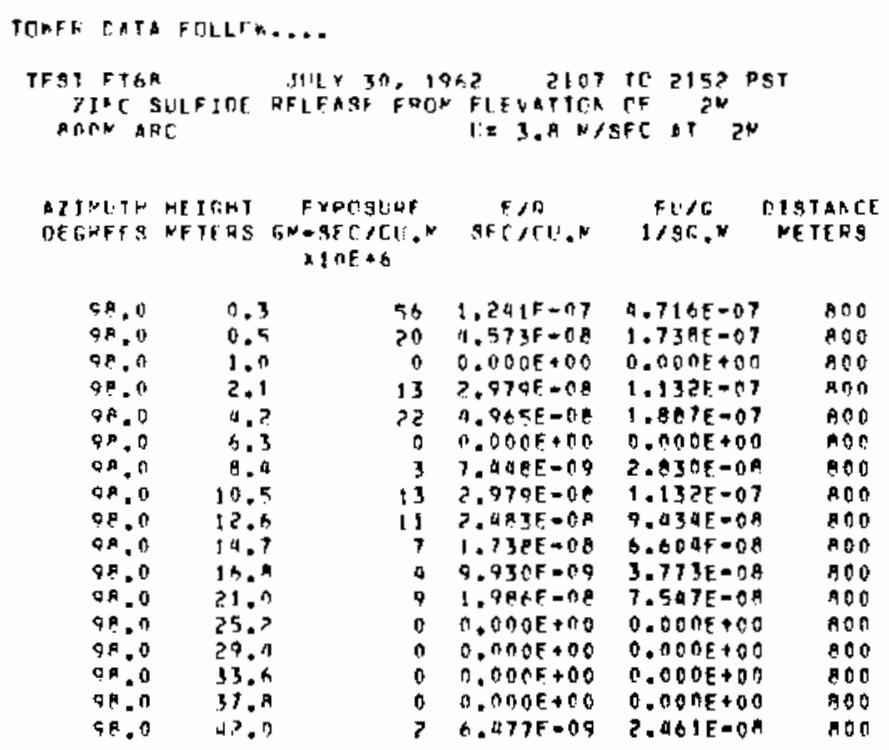




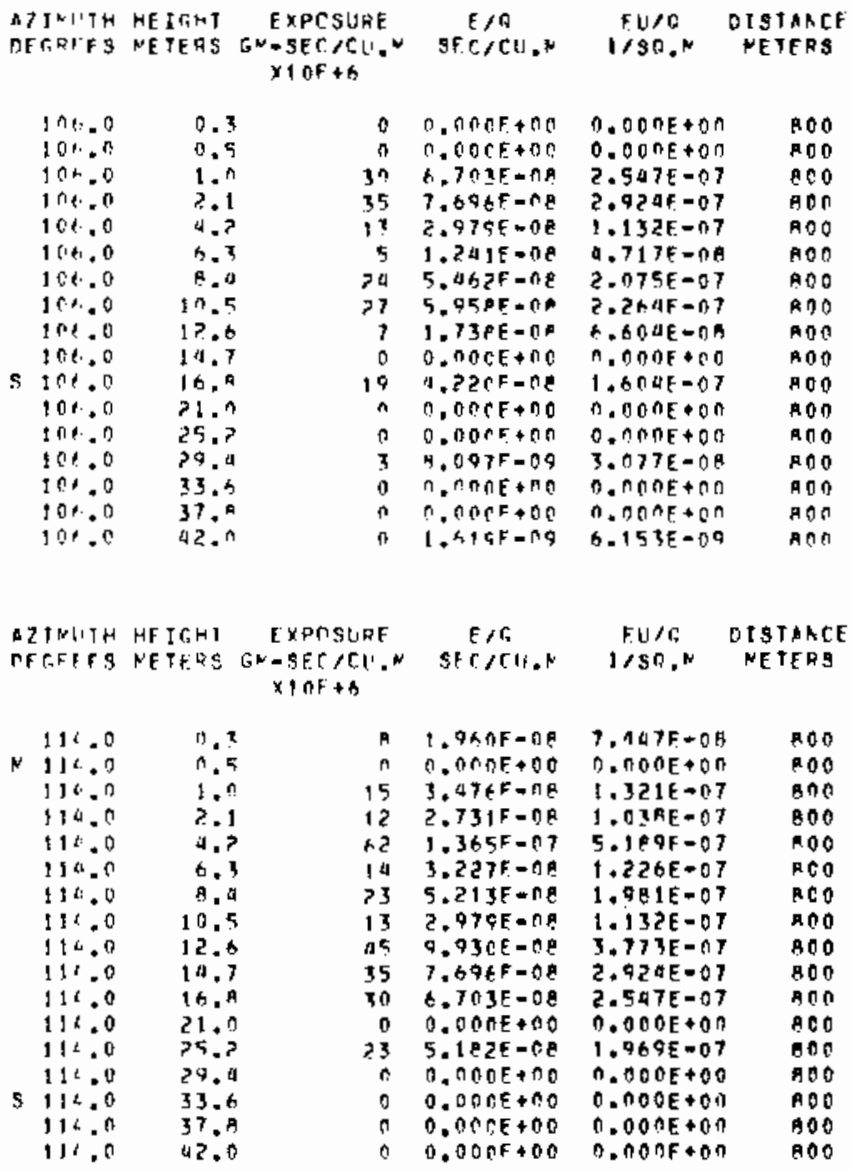

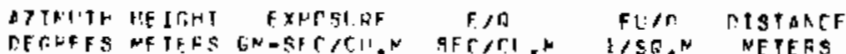

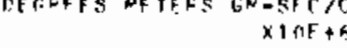

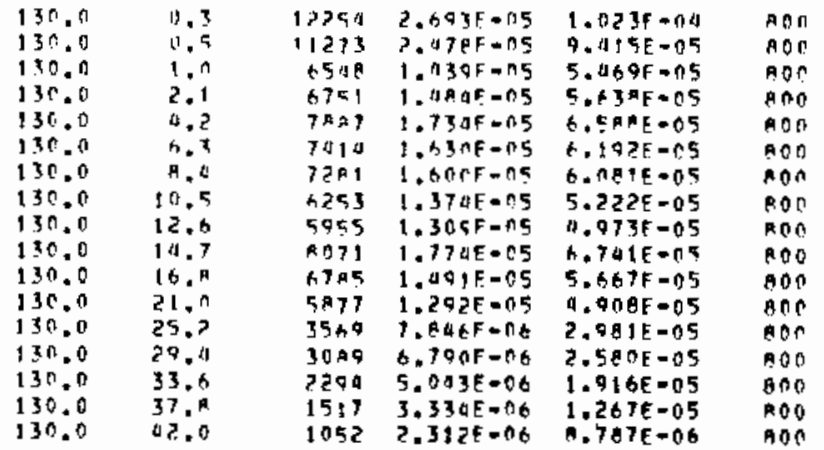

AZINLTH HEIGHT FXPCSURE E/O FU/O TISTARCE TEEFFFS HETENS GN-SEC/CII,N SEC/CIJ.N 1/SA.N NETERS

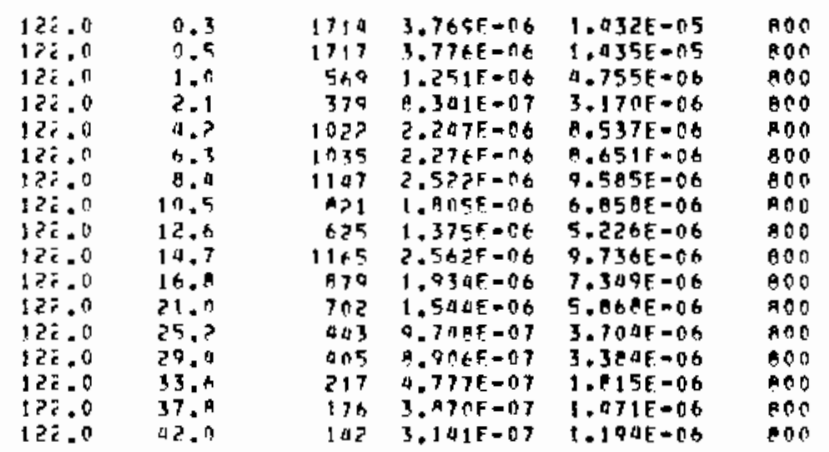




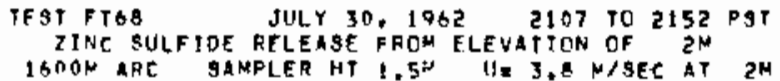

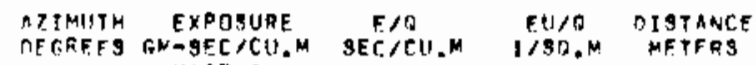
$\times 10 E+6$

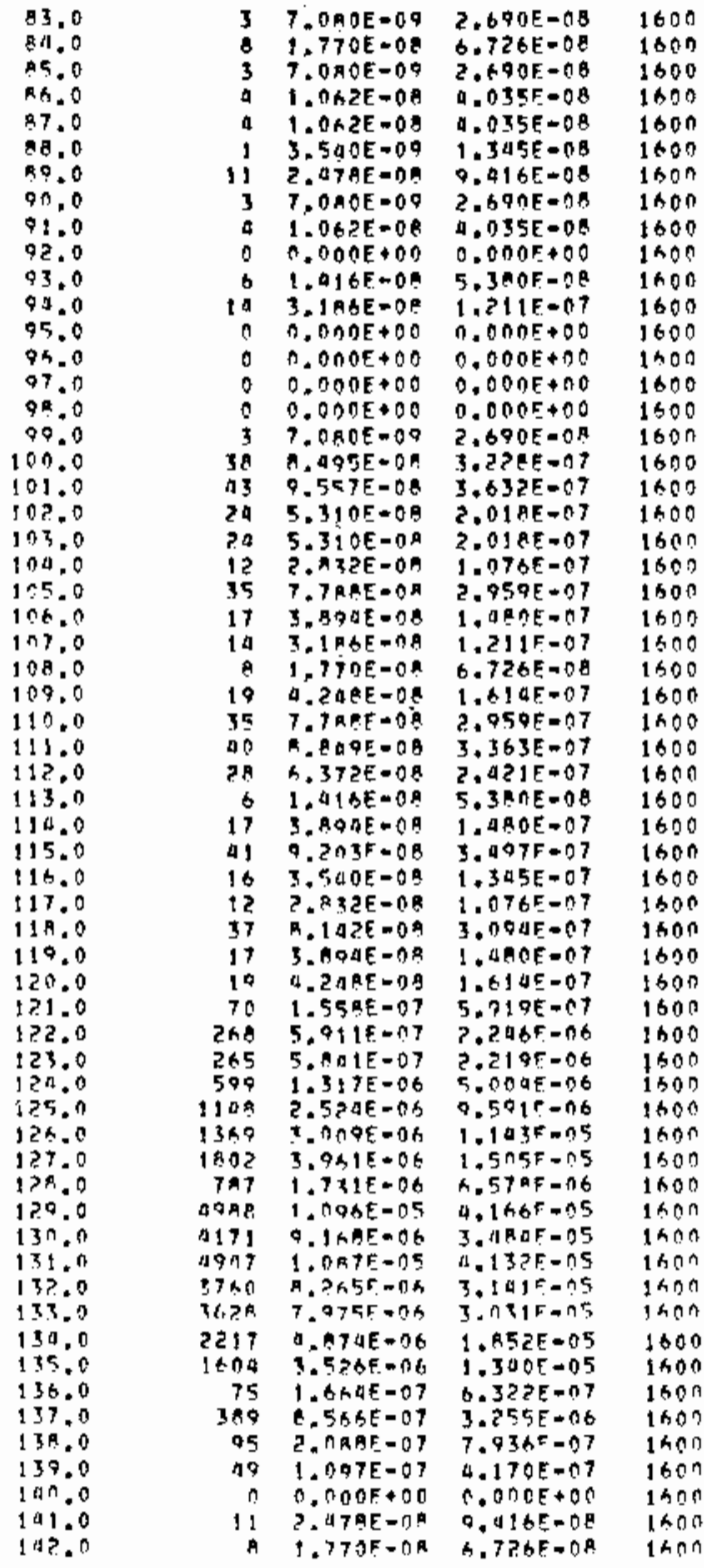

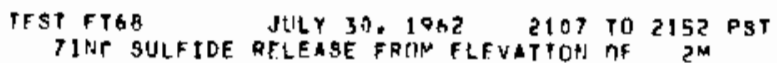

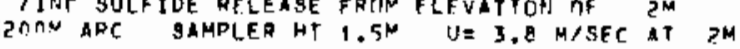

MLIMUTH EXPOSURE EIT EIIT BISTANCE

AFTREFS GM-SEC/CU.M SEC/CU.M 1/SA.N METFRS $\times 10 E+6$

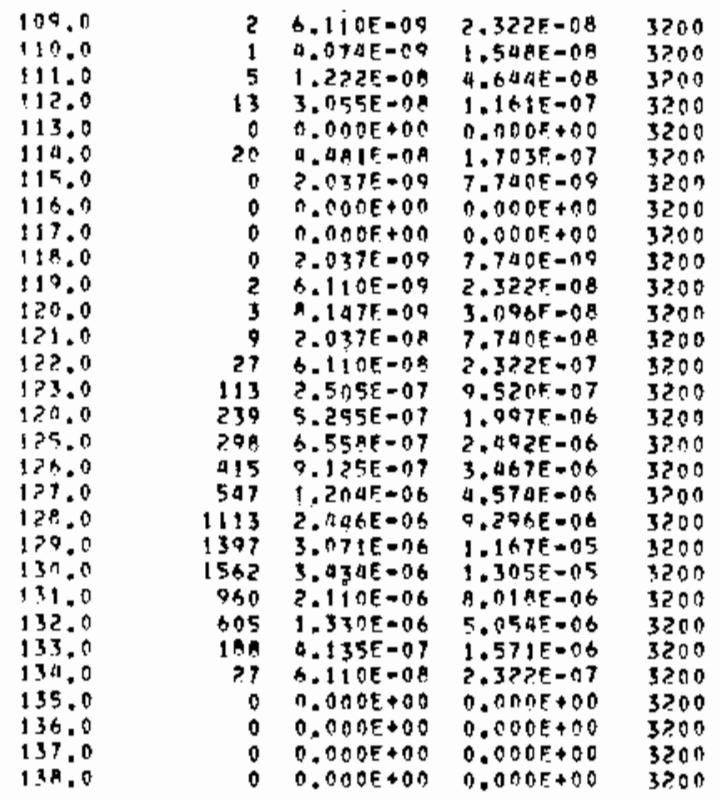

CADSSWIND INTEGRATEOA O.Z7AE-04 3.520E-03 
30-5ERIES EXPERINEYT: 69 : FT-69:

GPOUNO LEVEL ANO TONER SAMPLING 200 ASTD BOO HARCS. GFOLNO LEYE!

SARPLING CNLY :SOO-12800 H ARCS. DUST AN2 CARBCN DEPOSITS JN THE

FILTERS COLLECTED FPOCI THE 12900 " ARC.

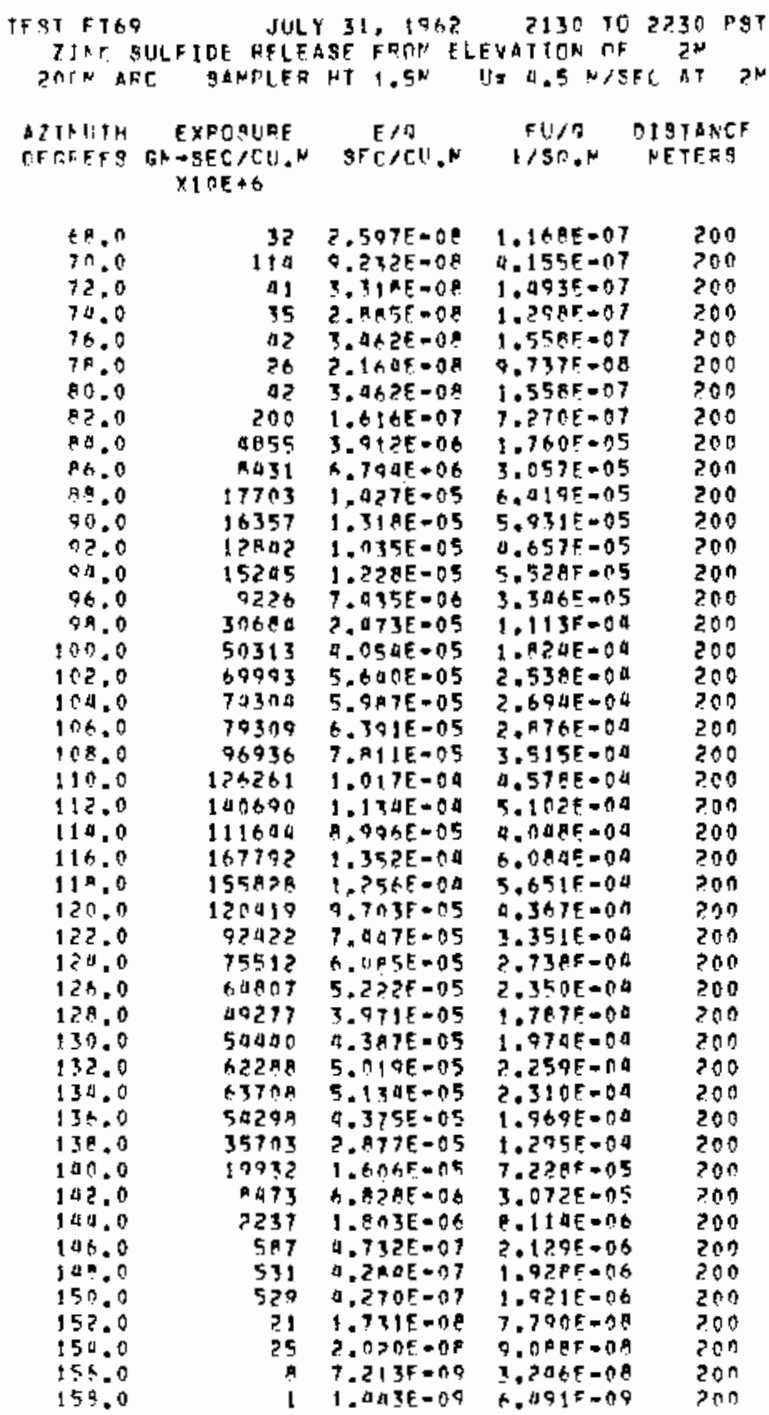

CRCSSHTAD IRTEGRATER= 1.06AE-0? A.795F-0?
TOMER DATA FOLLOHA....

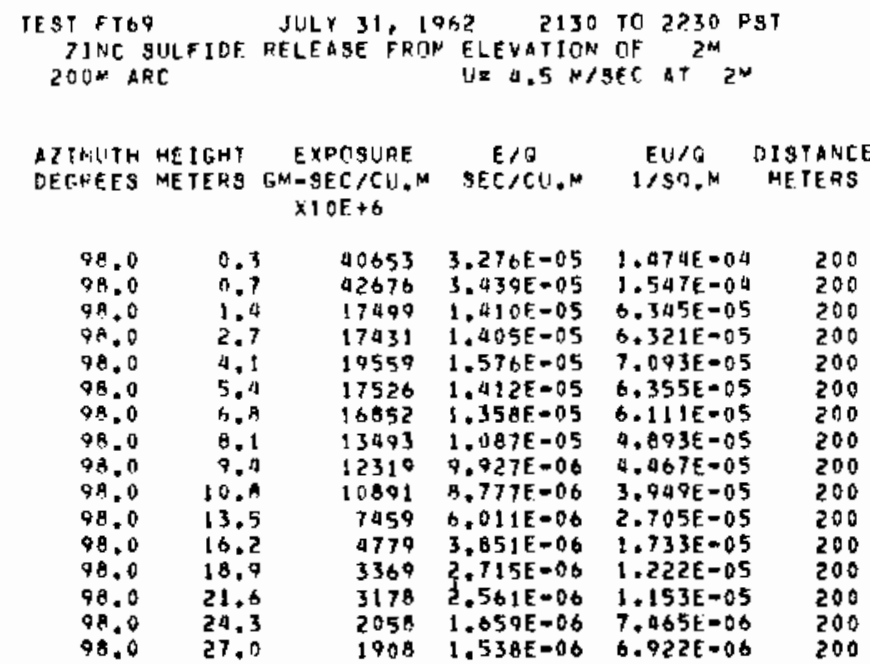

\begin{tabular}{|c|c|c|c|c|c|}
\hline $\begin{array}{l}\text { IZIMUTH } \\
\text { DEGREES }\end{array}$ & $\begin{array}{l}\text { HE IGHT } \\
\text { ME TERS }\end{array}$ & $\begin{array}{c}\text { EXPQSURE } \\
\text { GM-SEC/CU.M } \\
\times 1 O E+6\end{array}$ & $\begin{array}{c}E / Q \\
S E[/ C U, M\end{array}$ & $\begin{array}{c}E U / Q \\
1 / S Q, M\end{array}$ & $\begin{array}{l}\text { DISTANCE } \\
\text { METERS }\end{array}$ \\
\hline $\begin{array}{l}106.0 \\
106.0 \\
106.0 \\
106.0 \\
106.0 \\
106.0 \\
106.0 \\
100.0 \\
106.0 \\
106.0 \\
106.0 \\
106.0 \\
106.0 \\
106.0 \\
106.0 \\
106.0\end{array}$ & $\begin{array}{l}0.3 \\
0.7 \\
1.4 \\
2.7 \\
9.1 \\
5.4 \\
6.8 \\
0.1 \\
9.9 \\
10.1 \\
13.5 \\
16.2 \\
18.9 \\
21.6 \\
24.5 \\
27.0\end{array}$ & $\begin{array}{r}127789 \\
73255 \\
41695 \\
52554 \\
47888 \\
41645 \\
44039 \\
39380 \\
35824 \\
34539 \\
22504 \\
16099 \\
11232 \\
7962 \\
3793 \\
2985\end{array}$ & $\begin{array}{l}1.030 E=04 \\
5.903 E=05 \\
3.360 E=05 \\
4.235 E=05 \\
3.850 E=05 \\
3.35 A E=05 \\
3.540 E=05 \\
3.170 E=05 \\
2.007 E=05 \\
2.783 E=05 \\
1.013 E=05 \\
1.207 E=05 \\
9.052 E-06 \\
6.418 E=00 \\
3.056 E=06 \\
2.000 E=06\end{array}$ & $\begin{array}{l}1.659 E=04 \\
2.656 E=04 \\
1.512 E=04 \\
1.906 E=04 \\
1.736 E=04 \\
1.510 E=04 \\
1.597 E=04 \\
1.42 B E=04 \\
1.299 E=04 \\
1.252 E=04 \\
6.161 E=05 \\
5.33 E E=05 \\
2.073 E=05 \\
2.887 E=05 \\
1.375 E=05 \\
1.083 E=05\end{array}$ & $\begin{array}{l}200 \\
200 \\
200 \\
200 \\
200 \\
200 \\
200 \\
200 \\
200 \\
200 \\
200 \\
200 \\
200 \\
200 \\
200 \\
200\end{array}$ \\
\hline
\end{tabular}

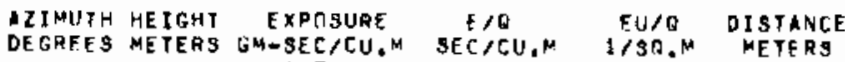
$\times 10 E+6$

\begin{tabular}{|c|c|c|c|c|}
\hline 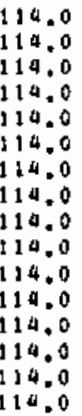 & $\begin{array}{r}0.3 \\
0.7 \\
1.9 \\
2.7 \\
4.1 \\
5.4 \\
0.8 \\
8.1 \\
9.4 \\
10.8 \\
13.5 \\
16.2 \\
18.9 \\
21.6 \\
24.3 \\
27.0\end{array}$ & $\begin{array}{r}121232 \\
148242 \\
70030 \\
11927 \\
73348 \\
71893 \\
71955 \\
66410 \\
44696 \\
47915 \\
91605 \\
23634 \\
19198 \\
10798 \\
5196 \\
3752\end{array}$ & $\begin{array}{l}9.769 E=05 \\
1.195 E=04 \\
5.603 E=05 \\
6.602 E=05 \\
5.910 E=05 \\
5.793 E=05 \\
6.040 E=05 \\
5.351 E=05 \\
3.924 E=05 \\
3.861 E=05 \\
3.353 F=05 \\
1.905 E=05 \\
1.507 E=05 \\
8.702 E=06 \\
4.187 E=06 \\
3.024 E=00\end{array}$ & $\begin{array}{l}2.396 E=04 \\
5.375 E=04 \\
2.539 E=04 \\
2.971 E=04 \\
2.660 E=04 \\
2.607 E=04 \\
2.718 E=04 \\
2.406 E=04 \\
1.766 E=04 \\
1.737 E=04 \\
1.509 E=04 \\
0.570 E=05 \\
6.962 E=05 \\
3.916 E=05 \\
1.884 E=05 \\
1.361 E=05\end{array}$ \\
\hline
\end{tabular}




\begin{tabular}{|c|c|c|c|c|c|}
\hline $\begin{array}{l}\text { A. IMUTH } \\
\text { DECREES }\end{array}$ & $\begin{array}{l}\text { HE IGHT } \\
\text { HE TERS }\end{array}$ & $\begin{array}{c}\text { EXPOQURE } \\
\text { GM-SEE } \\
\times 1 O U \text { - } \\
\times 1 O E\end{array}$ & $\begin{array}{c}E / 0 \\
S E C / C U, M\end{array}$ & $\begin{array}{l}E U / 0 \\
1 / 90 . \mathrm{m}\end{array}$ & $\begin{array}{c}\text { D.ISTANCE } \\
\text { METERS }\end{array}$ \\
\hline $\begin{array}{l}122.0 \\
122.0 \\
122.0 \\
122.0 \\
122.0 \\
122.0 \\
122.0 \\
122.0 \\
122.0 \\
122.0 \\
122.0 \\
122.0 \\
122.0 \\
122.0 \\
122.0 \\
122.0\end{array}$ & $\begin{array}{l}0.3 \\
0.7 \\
1.9 \\
2.7 \\
9.1 \\
5.4 \\
6.9 \\
8.1 \\
9.9 \\
10.9 \\
13.5 \\
16.2 \\
18.9 \\
21.6 \\
24.3 \\
27.0\end{array}$ & $\begin{array}{l}130213 \\
155251 \\
63584 \\
57638 \\
47603 \\
62034 \\
60049 \\
51569 \\
00203 \\
40055 \\
27798 \\
10986 \\
13729 \\
10971 \\
5376 \\
4943\end{array}$ & $\begin{array}{l}1.040 E-04 \\
1.251 E-04 \\
5.120 E-05 \\
4.661 E-05 \\
3.836 E-05 \\
4.994 E-05 \\
4.830 E-05 \\
4.155 E=05 \\
3.200 E=05 \\
3.292 E=05 \\
2.240 E=05 \\
1.530 E-05 \\
1.106 E=05 \\
8.800 E-06 \\
4.333 E=06 \\
3.501 E=06\end{array}$ & $\begin{array}{l}9.722 E=04 \\
5.630 E=04 \\
2.300 E=04 \\
2.097 E=04 \\
1.726 E=04 \\
2.250 E=04 \\
2.177 E=04 \\
1.070 E=04 \\
1.450 E=04 \\
1.011 E=04 \\
1.000 E=04 \\
6.805 E=05 \\
4.970 E=05 \\
3.978 E=05 \\
1.050 E=05 \\
1.011 E-05\end{array}$ & $\begin{array}{l}200 \\
200 \\
200 \\
200 \\
200 \\
200 \\
200 \\
200 \\
200 \\
200 \\
200 \\
200 \\
200 \\
200 \\
200 \\
200\end{array}$ \\
\hline
\end{tabular}

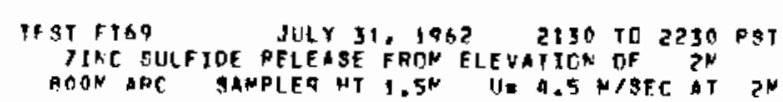

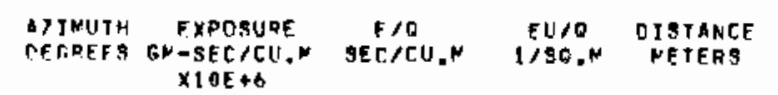

\begin{tabular}{|c|c|c|c|c|}
\hline $\begin{array}{l}82.0 \\
80.0 \\
18.0 \\
18.0 \\
90.0 \\
09.0 \\
94.0 \\
96.0 \\
98.0 \\
100.0 \\
102.0 \\
109.0 \\
108.0 \\
10100 \\
110.0 \\
112.0 \\
119.0 \\
116.0 \\
118.0 \\
120.0 \\
122.0 \\
129.0 \\
126.0 \\
128.0 \\
130.0 \\
132.0 \\
130.0 \\
138.0 \\
138.0 \\
100.0 \\
102.0 \\
144.0\end{array}$ & 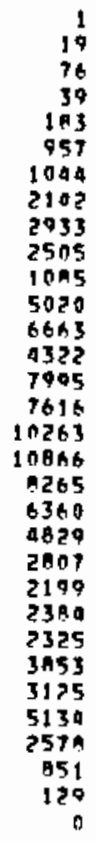 & 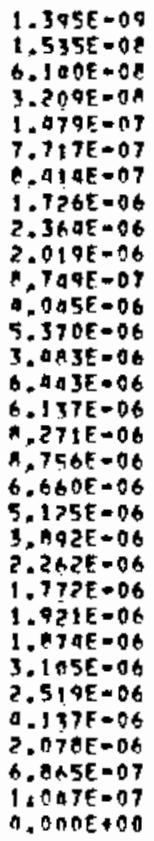 & 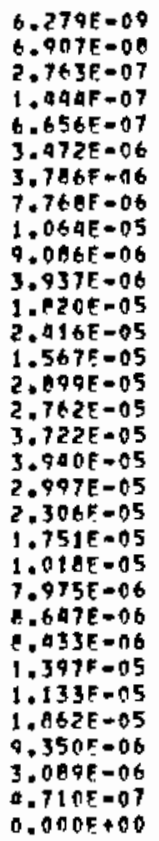 & 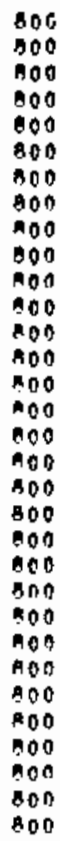 \\
\hline
\end{tabular}

CRCASKIAD IATEGRATEDE 3.0A3E-03 $1+100 F=02$

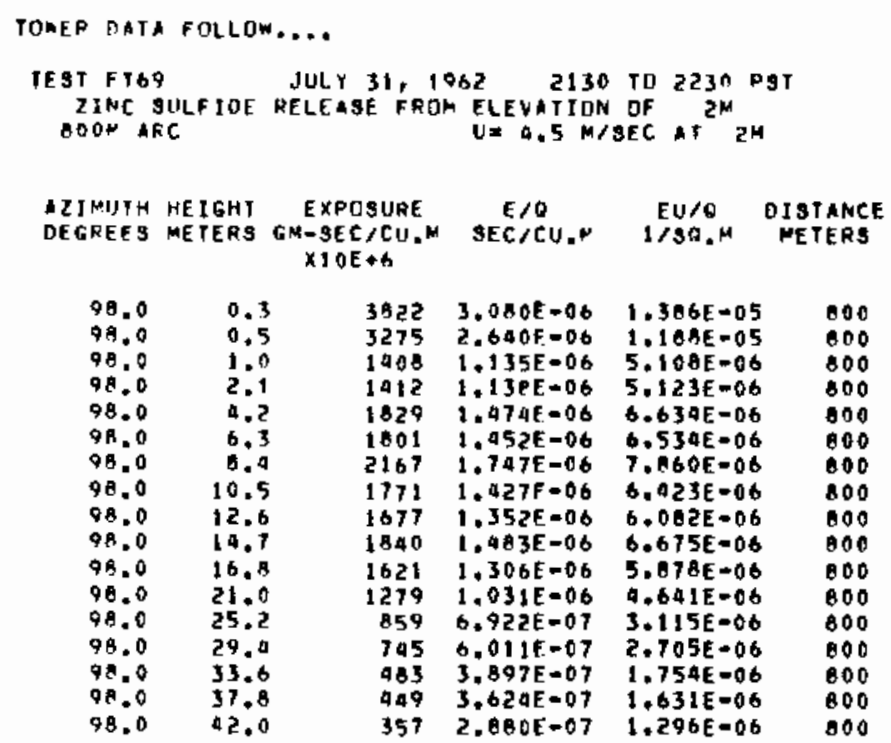




\begin{tabular}{|c|c|c|c|c|c|c|c|c|c|c|c|}
\hline $\begin{array}{l}\text { AIIMUTH } \\
\text { DEGKEES }\end{array}$ & $\begin{array}{l}\text { HE IGHT } \\
\text { METER }\end{array}$ & $\begin{array}{c}\text { EXPOSURE } \\
\text { GM }=3 E C / C U, M \\
\times 10 E+6\end{array}$ & $\begin{array}{c}E / O \\
S E C / C U . M\end{array}$ & $\begin{array}{c}E U / A \\
1 / S 0 . M\end{array}$ & $\begin{array}{l}\text { DISTANCE } \\
\text { METERS }\end{array}$ & $\begin{array}{l}\text { AZIMIIYH } \\
\text { DEGREES }\end{array}$ & $\begin{array}{l}\text { HE IGHT } \\
\text { ME TERS }\end{array}$ & $\begin{array}{l}\text { EXPOSUAE } \\
\text { GM-SEC/C } \\
\times 10 E+6\end{array}$ & $\begin{array}{c}E / B \\
S E C / C U_{*} N\end{array}$ & $\begin{array}{l}E U / O \\
1 / S^{\circ}, M\end{array}$ & $\begin{array}{l}\text { DIST ANCE } \\
\text { METERS }\end{array}$ \\
\hline 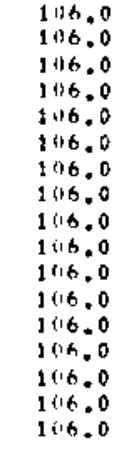 & $\begin{array}{l}0.3 \\
0.5 \\
1.0 \\
2.1 \\
4.2 \\
6.3 \\
8.4 \\
10.5 \\
12.6 \\
14.7 \\
16.8 \\
21.0 \\
25.2 \\
29.4 \\
33.6 \\
37.8 \\
42.0\end{array}$ & $\begin{array}{l}7511 \\
7787 \\
3510 \\
4112 \\
4514 \\
4926 \\
4711 \\
4811 \\
4261 \\
4736 \\
4720 \\
3161 \\
2420 \\
2160 \\
1651 \\
1270 \\
1916\end{array}$ & $\begin{array}{l}6.053 E=106 \\
6.275 E-06 \\
3.829 E=06 \\
3.319 E=06 \\
3.637 F=06 \\
3.979 E=06 \\
3.796 E=06 \\
3.077 F=06 \\
3.434 E=06 \\
3.317 E=06 \\
3.852 F=06 \\
2.549 E=06 \\
1.951 E-06 \\
1.741 E=06 \\
1.330 E=06 \\
1.031 E=06 \\
8.18 A E=07\end{array}$ & $\begin{array}{l}2.720 \mathrm{E}=05 \\
2.824 \mathrm{E}-05 \\
1.273 \mathrm{E}-05 \\
1.491 \mathrm{E}-05 \\
1.637 \mathrm{E}=05 \\
1.786 \mathrm{E}=05 \\
1.708 \mathrm{E}-05 \\
1.745 \mathrm{E}=05 \\
1.545 \mathrm{E}=05 \\
1.716 \mathrm{E}-05 \\
1.730 \mathrm{E}=05 \\
1.146 \mathrm{E}-05 \\
1.778 \mathrm{E}-06 \\
7.835 \mathrm{E}=06 \\
5.987 \mathrm{E}-06 \\
4.639 \mathrm{~F}-06 \\
3.685 \mathrm{E}-06\end{array}$ & $\begin{array}{l}800 \\
800 \\
000 \\
800 \\
000 \\
000 \\
000 \\
000 \\
800 \\
800 \\
800 \\
800 \\
800 \\
100 \\
100 \\
000 \\
200\end{array}$ & $\begin{array}{l}130.0 \\
130.0 \\
130.0 \\
130.0 \\
130.0 \\
130.0 \\
130.0 \\
130.0 \\
130.0 \\
130.0 \\
130.0 \\
130.0 \\
130.0 \\
130.0 \\
130.0 \\
130.0 \\
130.0\end{array}$ & $\begin{array}{l}0.3 \\
0.5 \\
1.0 \\
2.1 \\
4.2 \\
6.3 \\
0.9 \\
10.5 \\
12.5 \\
19.7 \\
16.8 \\
21.0 \\
25.2 \\
29.4 \\
33.6 \\
37.0 \\
42.0\end{array}$ & $\begin{array}{l}3039 \\
2842 \\
571 \\
1517 \\
1796 \\
1000 \\
2060 \\
1638 \\
2076 \\
2002 \\
1633 \\
1793 \\
1166 \\
910 \\
827 \\
822 \\
904\end{array}$ & $\begin{array}{l}2.449 E=06 \\
2.291 F=06 \\
4.601 E=07 \\
1.223 F=06 \\
1.498 E=06 \\
1.451 E=06 \\
1.667 E=06 \\
1.482 F=06 \\
1.673 E=06 \\
1.613 E=06 \\
1.316 E=06 \\
1.445 E=06 \\
9.396 E=07 \\
7.335 E=07 \\
6.667 E=07 \\
6.626 E=07 \\
7.28 E E=07\end{array}$ & $\begin{array}{l}1.102 E=05 \\
1.031 E=05 \\
2.071 E=06 \\
5.50 \mathrm{AE}=06 \\
6.51 \mathrm{BE}=06 \\
6.530 E-06 \\
7.501 E=06 \\
6.667 E=06 \\
7.530 E=06 \\
7.260 E=06 \\
5.923 E=06 \\
6.500 E=06 \\
4.220 E=06 \\
3.301 E=06 \\
3.000 E=06 \\
2.902 E=06 \\
3.200 E=06\end{array}$ & $\begin{array}{l}800 \\
800 \\
800 \\
800 \\
000 \\
800 \\
000 \\
000 \\
800 \\
800 \\
800 \\
800 \\
800 \\
000 \\
800 \\
000 \\
000\end{array}$ \\
\hline
\end{tabular}

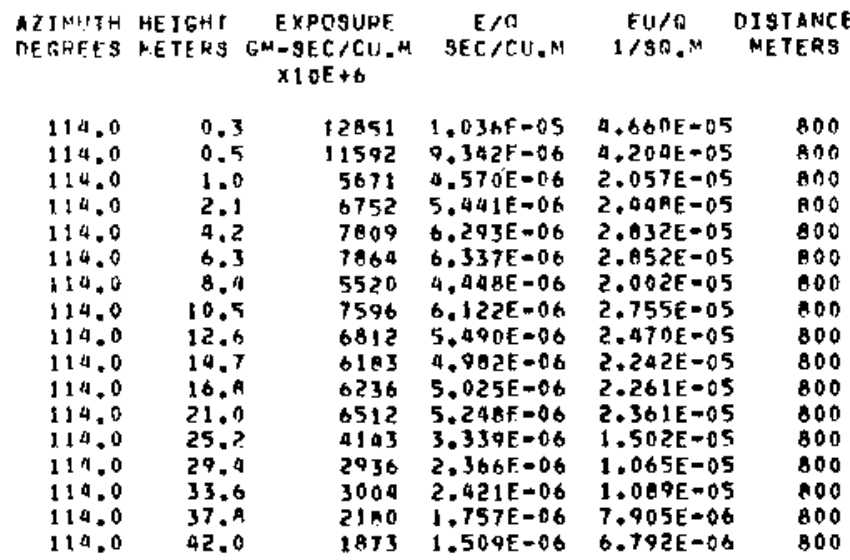
DZINUTH HEIGHT EXPOSURE
DEGFEES METERS GM-SEC/CU,M SEC/CU,M

\begin{tabular}{|c|c|c|c|c|c|}
\hline $\begin{array}{l}1<2.0 \\
122.0 \\
122.0 \\
122.0 \\
122.0 \\
122.0 \\
122.0 \\
1<2.0 \\
122.0 \\
122.0 \\
122.0 \\
1<2.0 \\
1<2.0 \\
1<2.0 \\
1<2.0 \\
1<2.0 \\
1<2.0\end{array}$ & $\begin{array}{l}0.3 \\
0.5 \\
1.0 \\
2.1 \\
4.2 \\
6.3 \\
0.4 \\
10.5 \\
12.6 \\
14.7 \\
16.8 \\
21.0 \\
25.2 \\
29.0 \\
33.6 \\
37.8 \\
42.0\end{array}$ & $\begin{array}{l}5636 \\
5078 \\
2502 \\
3024 \\
2955 \\
2797 \\
3330 \\
3260 \\
3021 \\
3100 \\
2825 \\
3058 \\
2199 \\
1639 \\
1609 \\
1110 \\
1439\end{array}$ & $\begin{array}{l}4.542 E=06 \\
4.092 E=06 \\
2.017 E=06 \\
2.437 E=06 \\
2.391 E=06 \\
2.255 E=06 \\
2.003 E=06 \\
2.627 E=06 \\
2.435 E-06 \\
2.096 E=06 \\
2.277 E=06 \\
2.464 E=06 \\
1.732 E=06 \\
1.403 E-06 \\
1.297 E=06 \\
8.949 E-07 \\
1.160 E=06\end{array}$ & $\begin{array}{l}2.044 \mathrm{E}-05 \\
1.042 \mathrm{E}-05 \\
9.075 \mathrm{E}-06 \\
1.097 \mathrm{E}-05 \\
1.072 \mathrm{E}-05 \\
1.015 \mathrm{E}-05 \\
1.200 \mathrm{E}=05 \\
1.102 \mathrm{E}=05 \\
1.096 \mathrm{E}-05 \\
1.120 \mathrm{E}=05 \\
1.025 \mathrm{E}-05 \\
1.109 \mathrm{E}-05 \\
7.796 \mathrm{E}-06 \\
6.672 \mathrm{E}-06 \\
5.836 \mathrm{E}-06 \\
4.027 \mathrm{E}-06 \\
5.221 \mathrm{E}-06\end{array}$ & $\begin{array}{l}800 \\
800 \\
800 \\
800 \\
800 \\
100 \\
800 \\
000 \\
800 \\
800 \\
800 \\
800 \\
800 \\
800 \\
800 \\
800 \\
800\end{array}$ \\
\hline
\end{tabular}




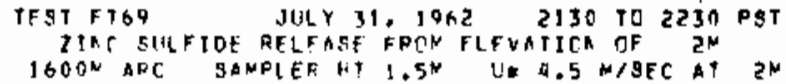

AIINLTH EXPOSLRE DFIO EU/R DISTARCE

IEGREF GNAGECCH.M SECICU,N I/SG,M NETERS $X \perp D E+6$

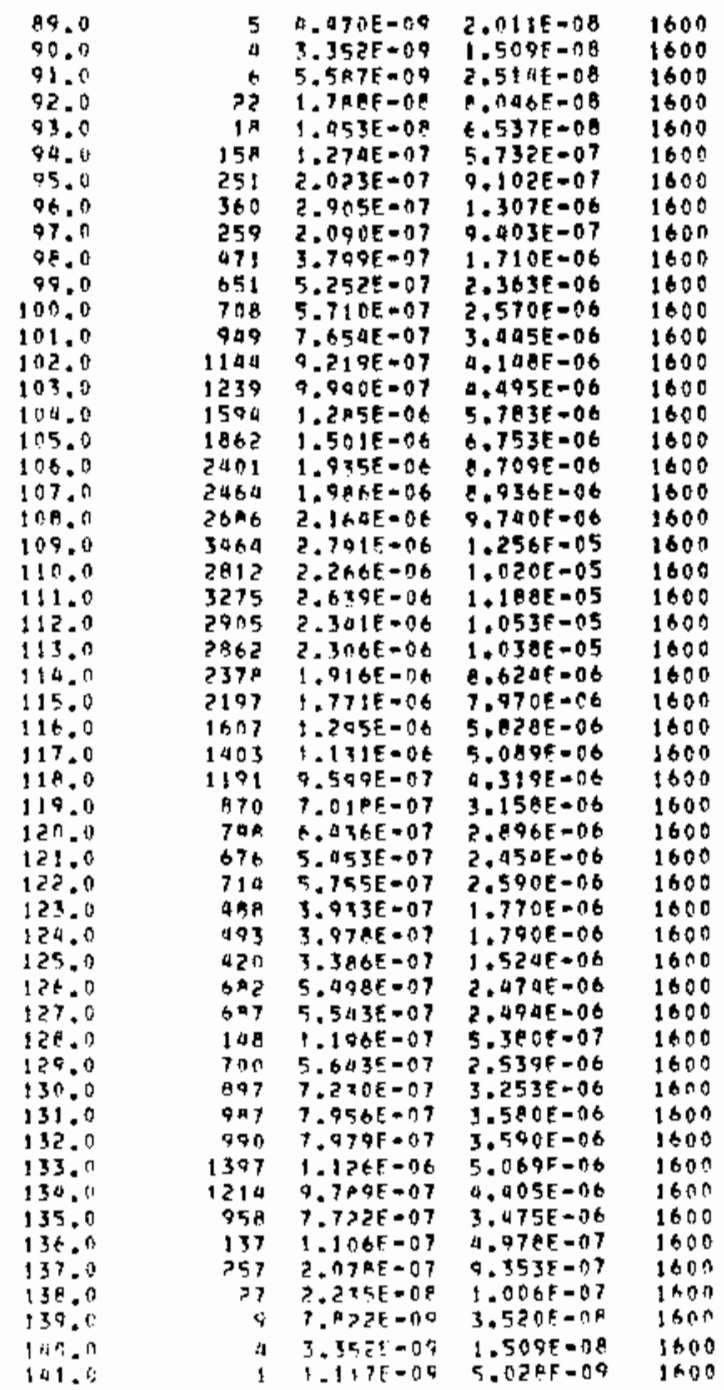

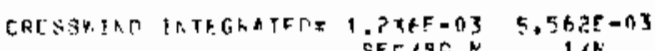

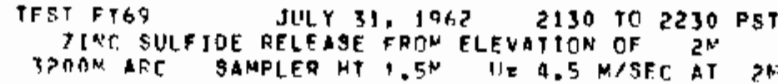

ATMUITH EXPDSURE F/g EU/O DISTARICE CFRPEF GH-SEC/CU.M SEC/CU.N I/SG,N NETERS $\times 10 F+6$

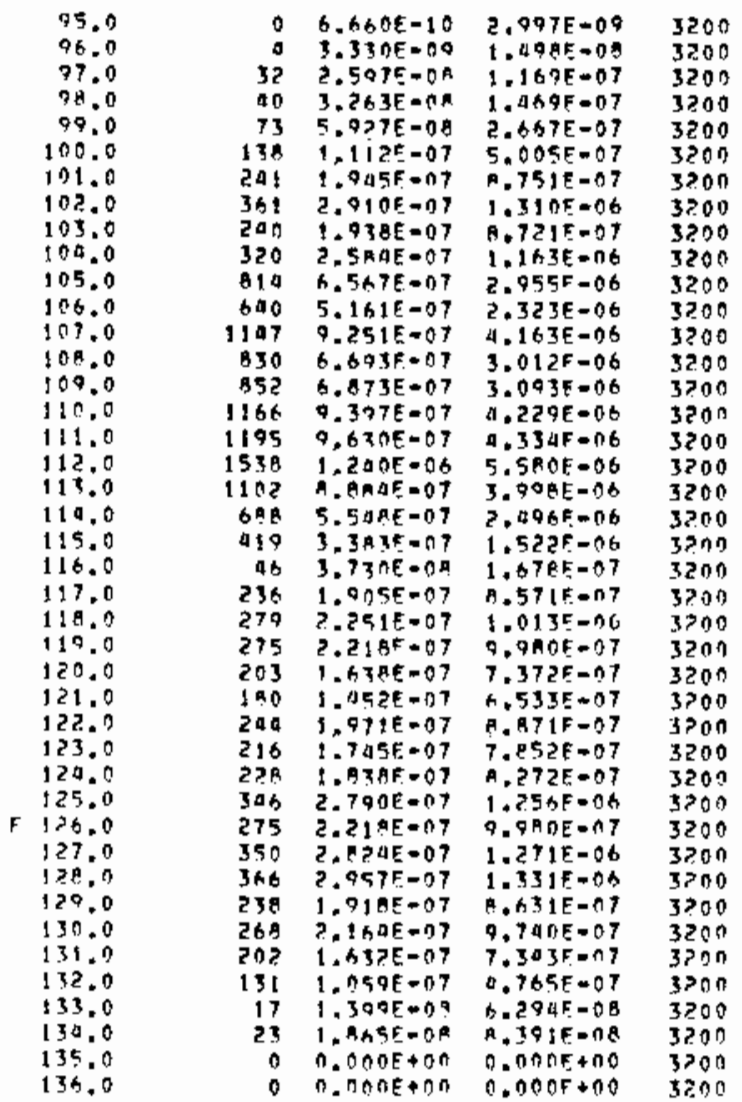

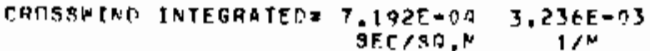




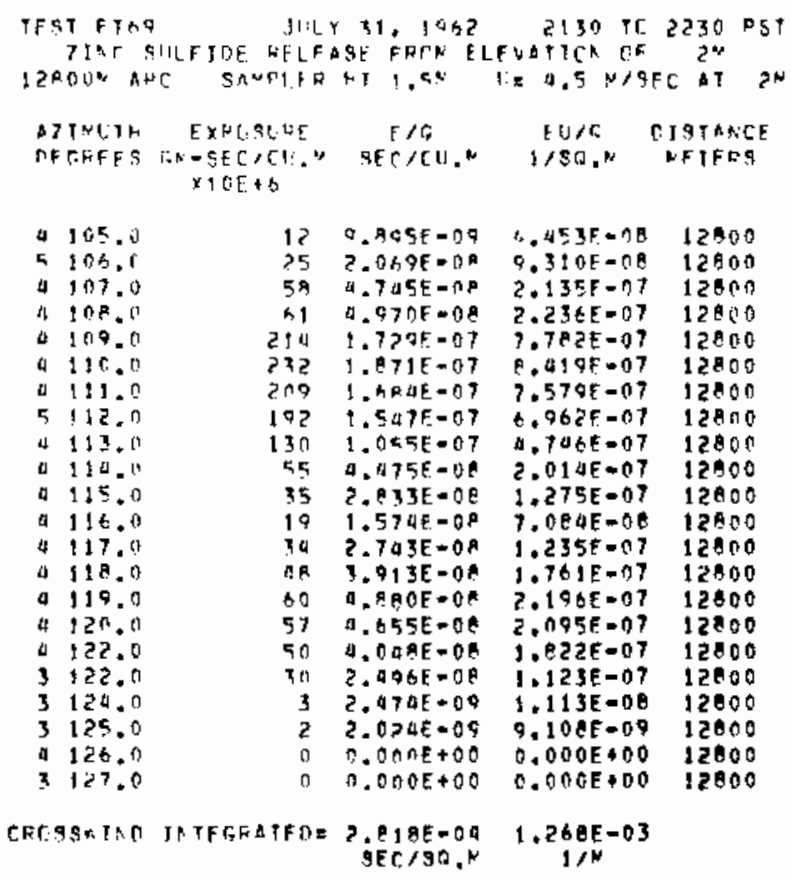


30- SERIES EXPERIMENT: 70 ( $\mathrm{FT}-70$

GROUND LEVEL AND TOWER SAMPL ING 200 AND 900 A ARCS, GROUND LEYE!

SRMPLING ONIY 1600-12800 M ARCS. DUST ANO CAREON DEPOSITS ON THE

F1LTERS COLLECTED FROM THE 12800 M AKC.

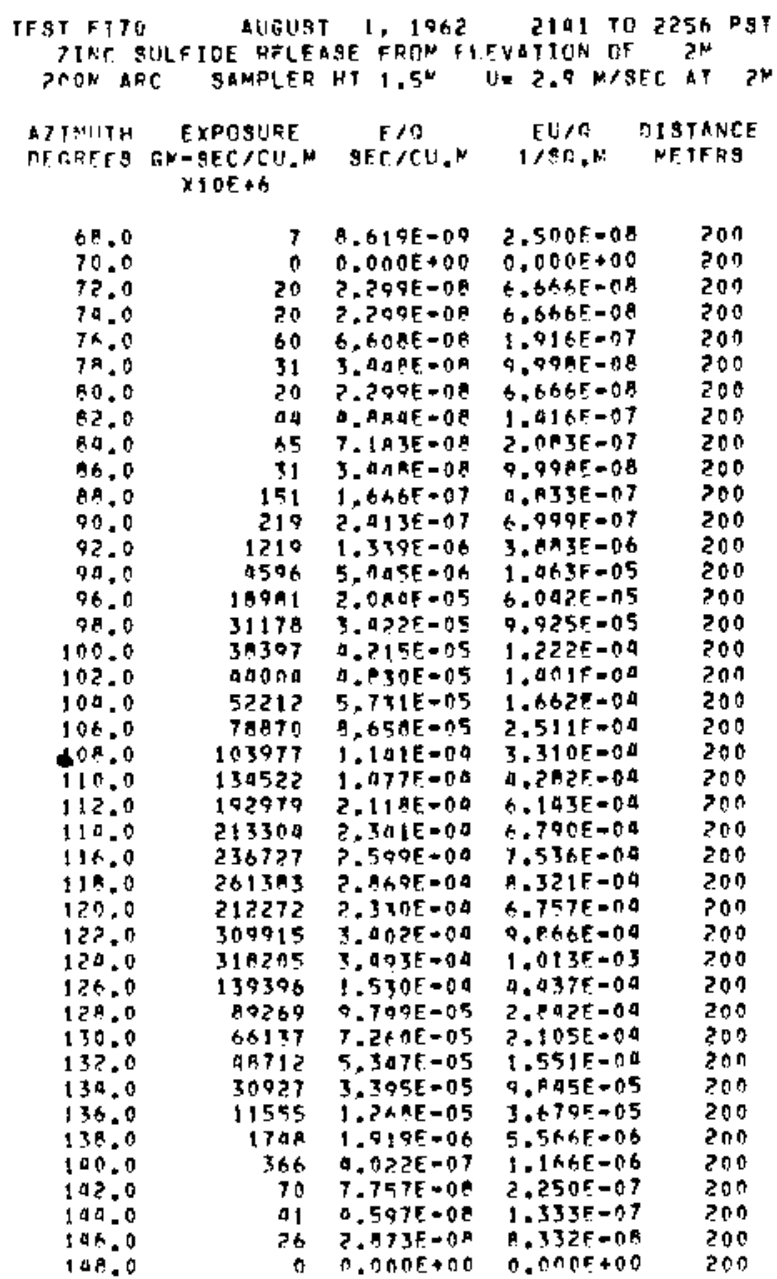

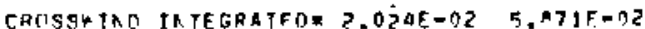

TORER DATH FOLLCW....

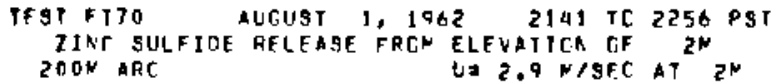

AZINITTH HEIGHT EXPCSURF E/G EU/O OISTANCE DEGREES METEHS GM-SEC/CU.N SEC/CU.N IISO.N NETERS $\times 1 \cap E+6$

\begin{tabular}{|c|c|c|c|c|c|}
\hline $\begin{array}{l}98.0 \\
98.0 \\
98.0 \\
98.0 \\
98.0 \\
98.0 \\
98.0 \\
98.0 \\
98.0 \\
98.0 \\
98.0 \\
98.0 \\
98.0 \\
98.0 \\
9.00 \\
9.0\end{array}$ & $\begin{array}{l}0.3 \\
0.7 \\
1.4 \\
2.7 \\
9.1 \\
5.9 \\
6.1 \\
8.1 \\
9.9 \\
10.8 \\
13.5 \\
16.2 \\
18.9 \\
21.6 \\
29.3 \\
27.4\end{array}$ & $\begin{array}{l}37256 \\
37522 \\
21537 \\
20745 \\
17405 \\
16540 \\
14203 \\
7737 \\
9577 \\
10417 \\
7910 \\
5525 \\
2756 \\
1926 \\
576 \\
252\end{array}$ & 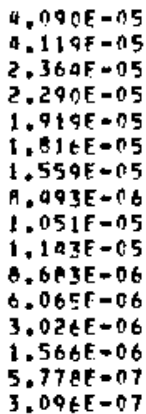 & $\begin{array}{l}1.166 E=04 \\
1.194 E=04 \\
6.856 E=05 \\
0.642 E=05 \\
5.566 E=05 \\
5.267 E=05 \\
4.521 E=05 \\
2.063 E=05 \\
3.049 E=05 \\
3.316 E=05 \\
2.518 E=05 \\
1.759 E=05 \\
0.775 E=06 \\
0.540 E=06 \\
1.675 E=06 \\
0.979 E=07\end{array}$ & $\begin{array}{l}200 \\
200 \\
200 \\
200 \\
200 \\
200 \\
200 \\
200 \\
200 \\
200 \\
200 \\
200 \\
200 \\
200 \\
200 \\
200\end{array}$ \\
\hline
\end{tabular}

AZINUTH HEIGHT EXPCSURF E/O EU/O DISTANCE

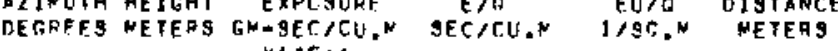
$x \geq 0 \%+6$

\begin{tabular}{|c|c|c|c|c|c|}
\hline $\begin{array}{l}106.0 \\
106.0 \\
106.0 \\
106.0 \\
106.0 \\
105.0 \\
106.0 \\
106.0 \\
106.0 \\
106.0 \\
106.0 \\
106.0 \\
106.0 \\
106.0 \\
106.0 \\
108.0\end{array}$ & $\begin{array}{l}0.3 \\
0.7 \\
1.9 \\
2.7 \\
9.1 \\
5.4 \\
6.9 \\
8.1 \\
9.9 \\
10.9 \\
13.5 \\
16.2 \\
18.9 \\
21.4 \\
29.3 \\
27.0\end{array}$ & $\begin{array}{l}95106 \\
97779 \\
42582 \\
43917 \\
5981 ! \\
56023 \\
50609 \\
49140 \\
15094 \\
39949 \\
26998 \\
16307 \\
10712 \\
5399 \\
2594 \\
1453\end{array}$ & $\begin{array}{l}9.302 F=05 \\
9.635 E=05 \\
5.772 E=05 \\
9.81 C E=05 \\
6.56+E=05 \\
6.150 E=05 \\
5.562 E=05 \\
5.3 E 1 E=05 \\
3.939 F=05 \\
0.3 A 5 E=05 \\
2.962 E=05 \\
1.79 C F=05 \\
1.176 E=05 \\
5.916 E=06 \\
2.633 F=06 \\
1.595 E=06\end{array}$ & $\begin{array}{l}2.709 E=04 \\
2.790 E=04 \\
1.674 E=00 \\
1.395 E=04 \\
1.904 E=04 \\
1.703 E=0 A \\
1.613 E=04 \\
1.561 E=04 \\
1.192 E=04 \\
1.272 E=04 \\
8.591 E=05 \\
5.191 E=05 \\
3.010 E=05 \\
1.716 E=05 \\
7.636 E=06 \\
4.626 E=06\end{array}$ & $\begin{array}{l}200 \\
200 \\
200 \\
200 \\
200 \\
200 \\
200 \\
200 \\
200 \\
200 \\
200 \\
200 \\
200 \\
200 \\
200 \\
200\end{array}$ \\
\hline
\end{tabular}

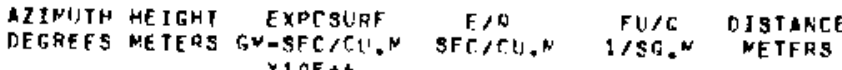
$\times 1$ I F +

\begin{tabular}{|c|c|c|c|c|c|}
\hline 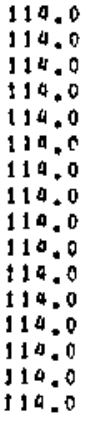 & $\begin{array}{l}7.3 \\
0.7 \\
1.4 \\
2.7 \\
4.7 \\
5.4 \\
6.4 \\
3.1 \\
9.4 \\
17.4 \\
13.5 \\
10.2 \\
18.9 \\
21.6 \\
24.3 \\
27.0\end{array}$ & $\begin{array}{l}166775 \\
730053 \\
141615 \\
104476 \\
150913 \\
140967 \\
117755 \\
171350 \\
107854 \\
96774 \\
75643 \\
49901 \\
33570 \\
19877 \\
10610 \\
5513\end{array}$ & $\begin{array}{l}1.831 E=04 \\
2.613 F=04 \\
1.555 F=04 \\
1.805 F=04 \\
1.657 E=04 \\
1.547 F=04 \\
1.293 E=04 \\
1.332 F=04 \\
1.114 E=04 \\
1.062 E=09 \\
1.306 E=05 \\
5.478 E=05 \\
3.60 C E=05 \\
2.182 E=05 \\
1.165 E=05 \\
6.053 E=06\end{array}$ & $\begin{array}{l}5.309 E=04 \\
7.57 A E=04 \\
0.50 A E=04 \\
5.235 E=00 \\
4.004 E=04 \\
4.487 E=04 \\
3.749 E=00 \\
3.863 E=04 \\
3.433 E=04 \\
3.079 E=04 \\
2.009 E=04 \\
1.589 E=04 \\
1.067 E=04 \\
6.328 E=05 \\
3.379 E=05 \\
1.755 E=05\end{array}$ & $\begin{array}{l}200 \\
200 \\
200 \\
200 \\
200 \\
200 \\
200 \\
200 \\
200 \\
200 \\
200 \\
200 \\
200 \\
200 \\
200 \\
200\end{array}$ \\
\hline
\end{tabular}




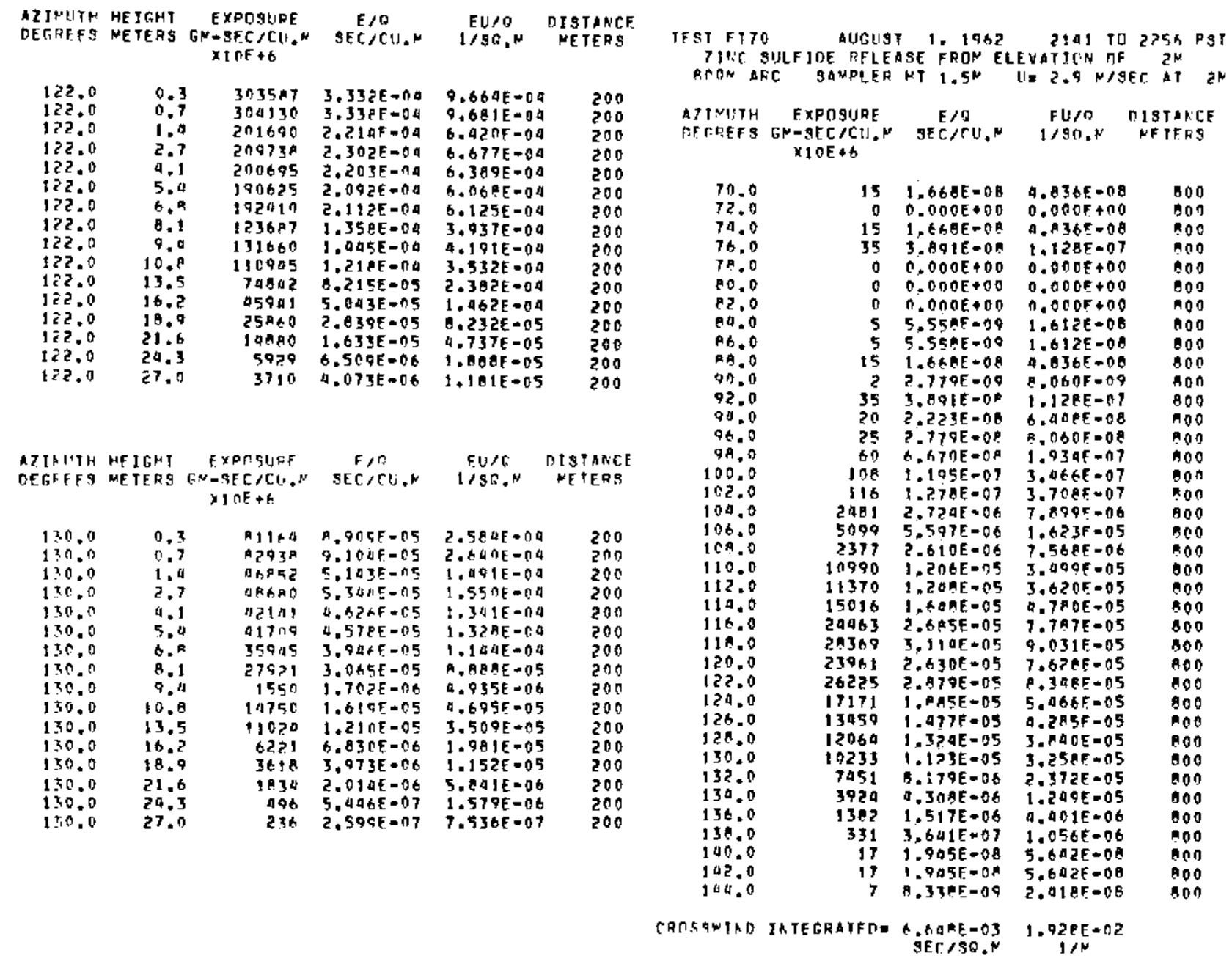

TOKER COA A FOLEC....

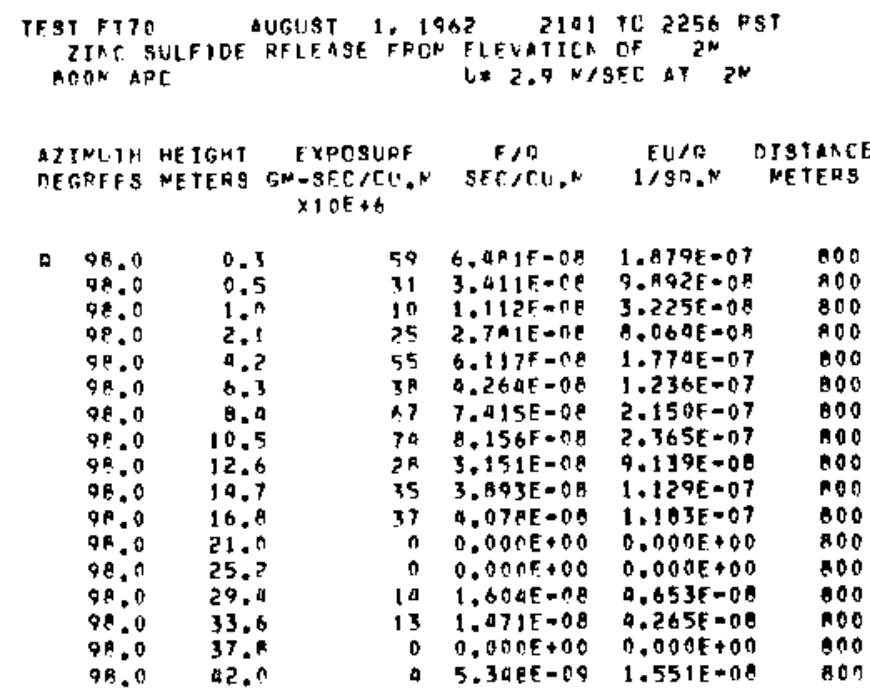




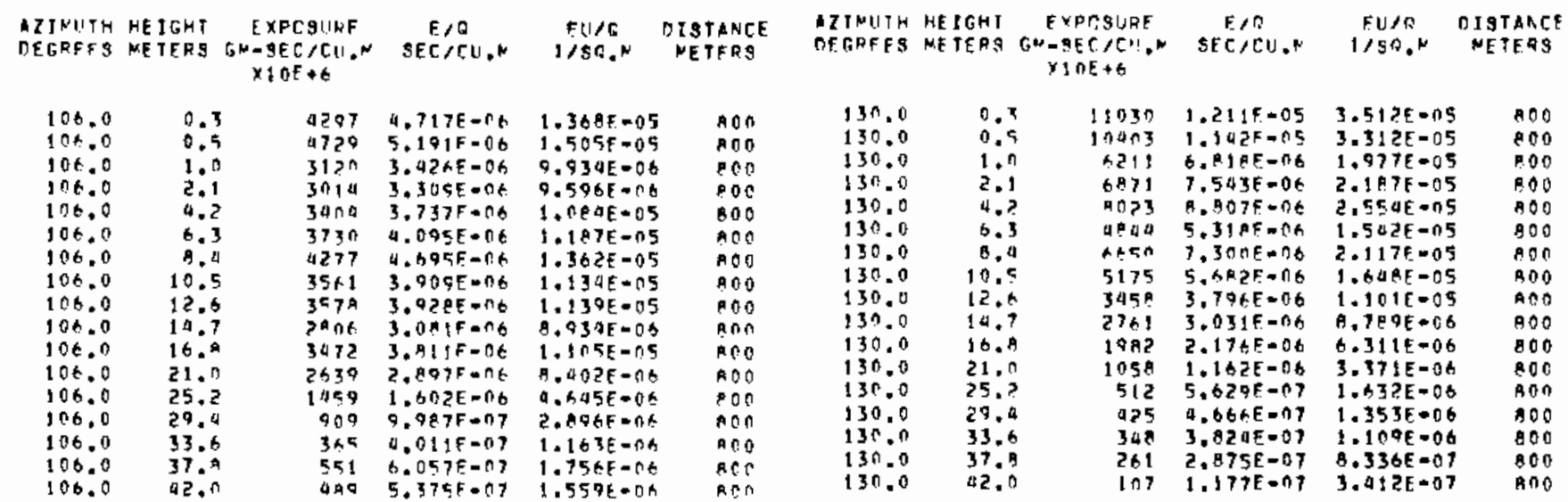

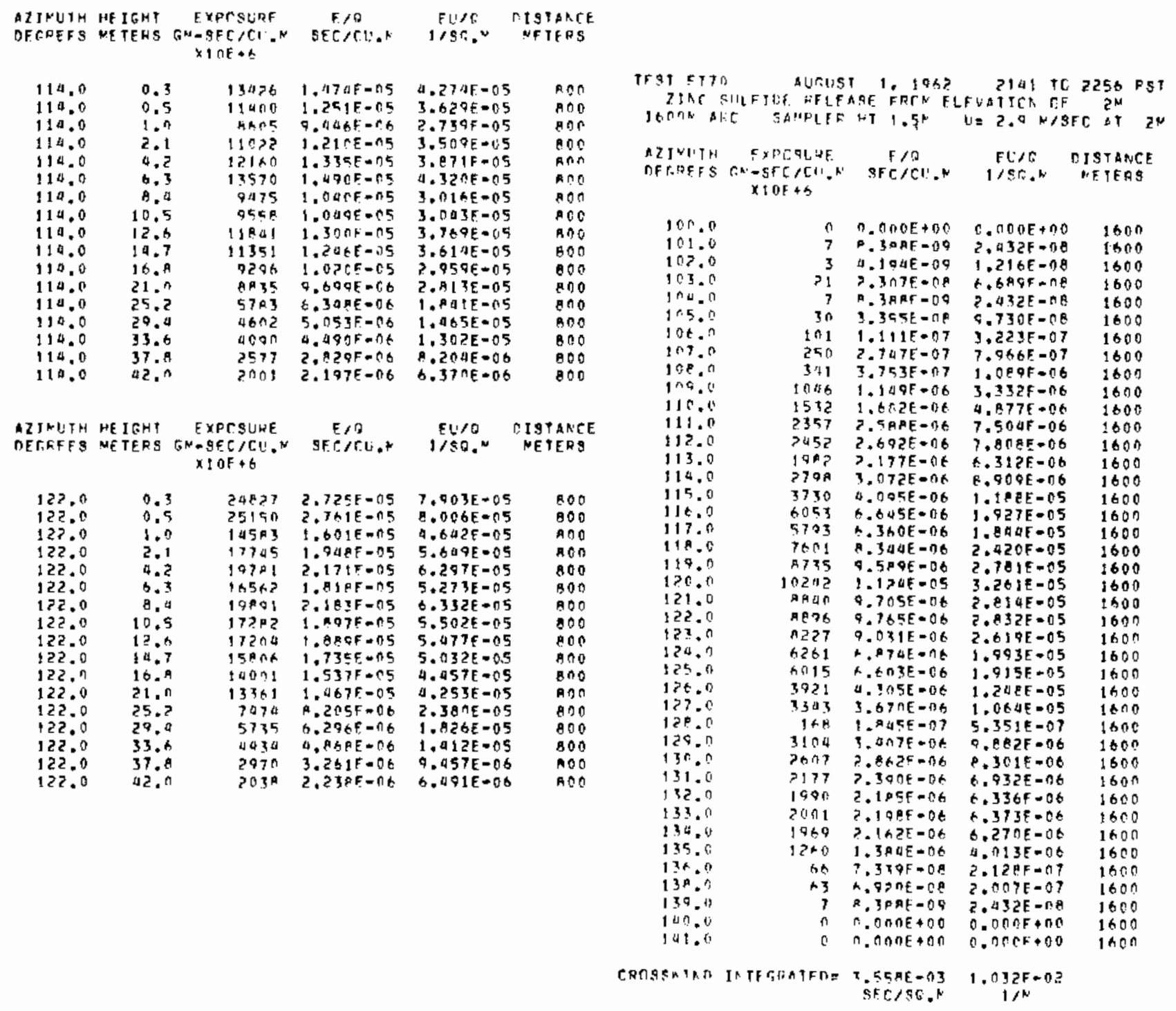




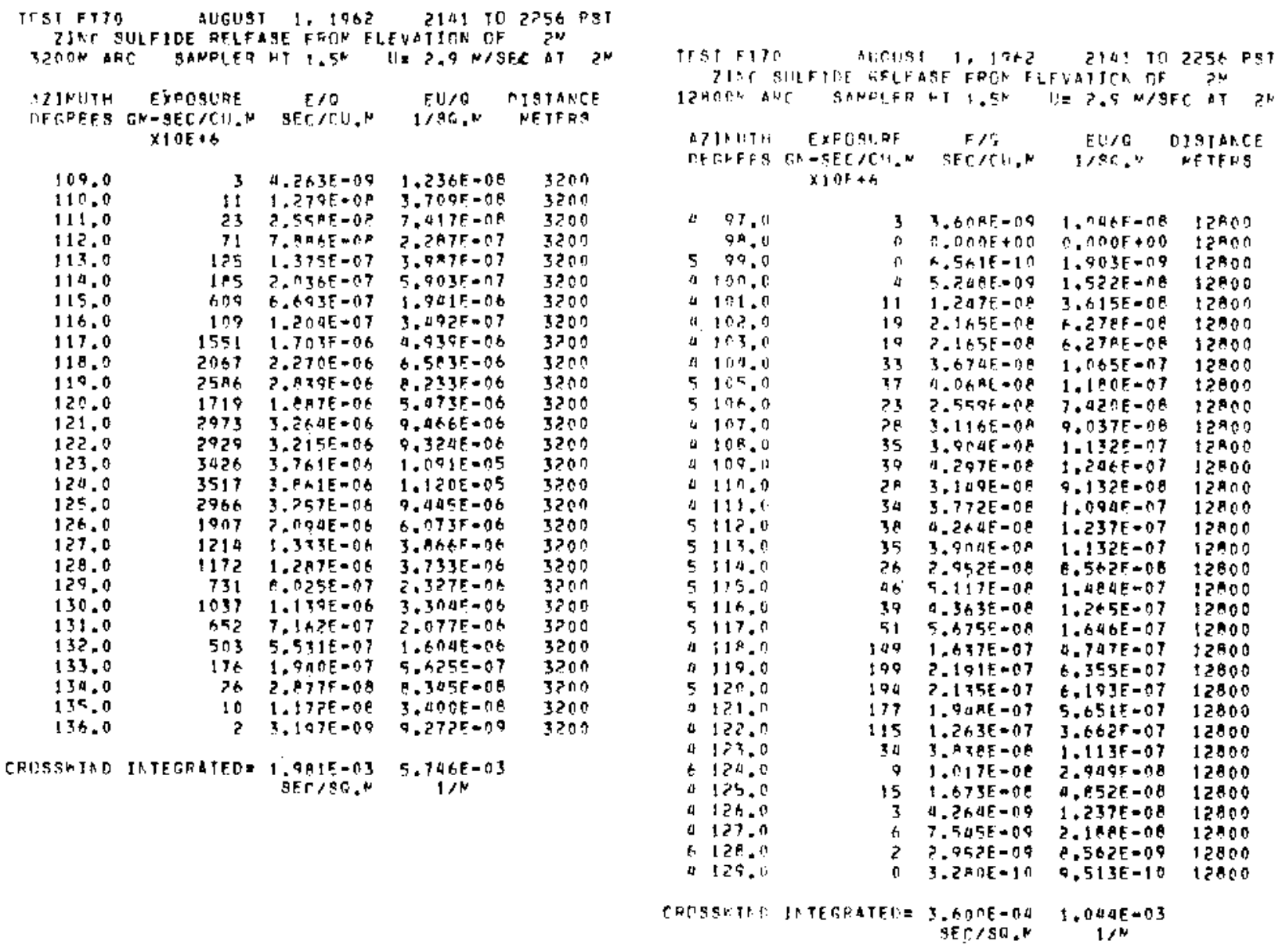


30-SERIES EXPERLMENT: 71 \& FT-71 ?

GROUND LEYEL AND TOWER SAMPLING 200 AND DOO $A$ ARCS. GROUND LEVEL SAMPLING ONLY $1600-3200$ M APCS.

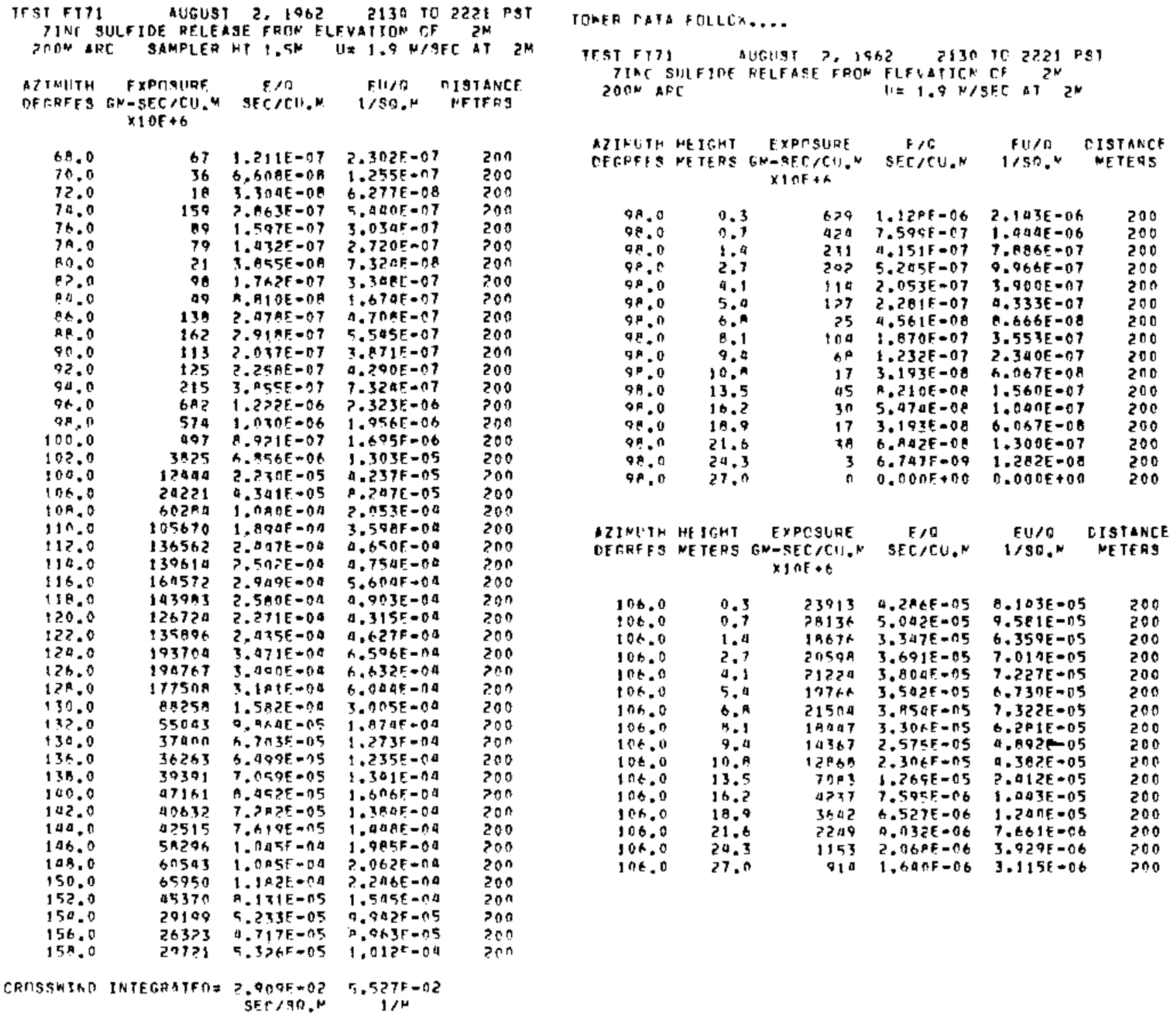




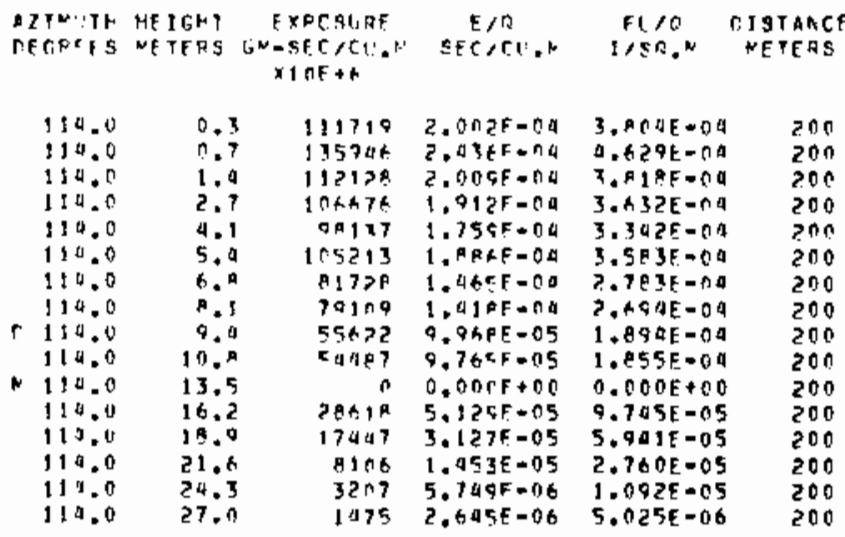

A7IMITH HEIGHT EXPOSLPE F/O FI/O DIGTARCE DEGFEG METERS GM-AEC/CU.N SEC/CU.N HOS.N METERS $\times 10 F+6$

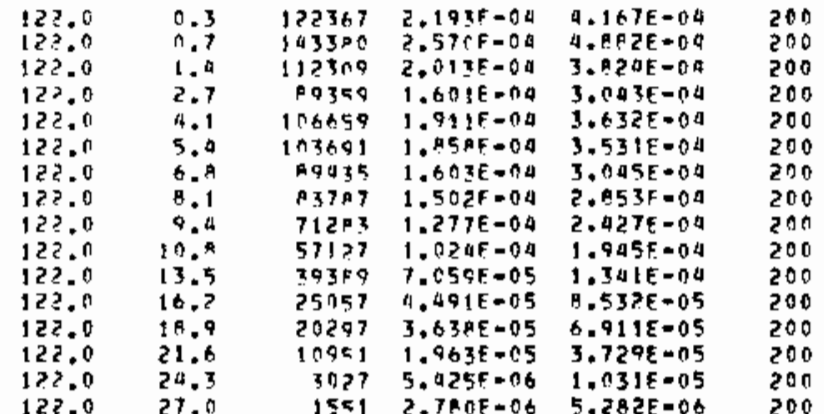

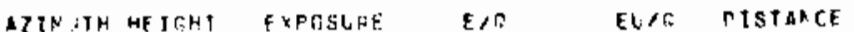

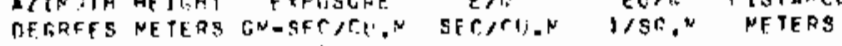
$x+c t h$

\begin{tabular}{|c|c|c|c|c|c|}
\hline $13 n .0$ & $n, 3$ & $y 57+5$ & $1.35 \times F=04$ & $z+5 \times \cap \mathrm{F}=0 \mathrm{a}$ & 200 \\
\hline $13 n .0$ & $\Uparrow .7$ & 20115 & $1.5985-n d$ & $3.035 F+04$ & 200 \\
\hline 150.0 & 1.1 & $67 n: 2$ & $? 0: E=04$ & $2 A 2 E=04$ & $20 n$ \\
\hline 130.0 & 2.7 & $542+1$ & 1. $\cap O P F=\cap A$ & $1.916 E=04$ & 200 \\
\hline 130.0 & 1,1 & $577+1$ & I. $.0 z+F=04$ & $1.950 E=04$ & 200 \\
\hline$x n=n$ & 5.1 & $6337 n$ & $1.1364=04$ & $2.15 A E-n d$ & ? 10 \\
\hline 130.0 & 6.19 & 59704 & $1.050 F-04$ & $3.00>E-04$ & 200 \\
\hline 130.0 & A. 1 & 52517 & $8.41 ? F-05$ & $1.78 R E-04$ & $2 \pi 0$ \\
\hline 30.0 & 9.9 & 32906 & $5.897 \mathrm{E}=0.5$ & $1.12 A E-04$ & 200 \\
\hline 0.0 & $10 .{ }^{A}$ & 35500 & $B .37 A F=05$ & $212 E-04$ & 200 \\
\hline $3 n .0$ & 13.5 & 22376 & 4. $001 F-05$ & $7.602 E-05$ & 200 \\
\hline & 16.2 & 13401 & $2.41, F=0.5$ & $4.591 E=05$ & 200 \\
\hline & & $9>0$ & $1.4 A \in E=05$ & - $823 E=05$ & 200 \\
\hline .0 & $? 1.5$ & $440 ?$ & $6.051 E-06$ & $1.530 E-05$ & 200 \\
\hline & 24.3 & 1317 & $362 F-06$ & $467 \mathrm{E}-16$ & 200 \\
\hline & & 1157 & $075 E-06$ & $.942 E-106$ & 201 \\
\hline
\end{tabular}

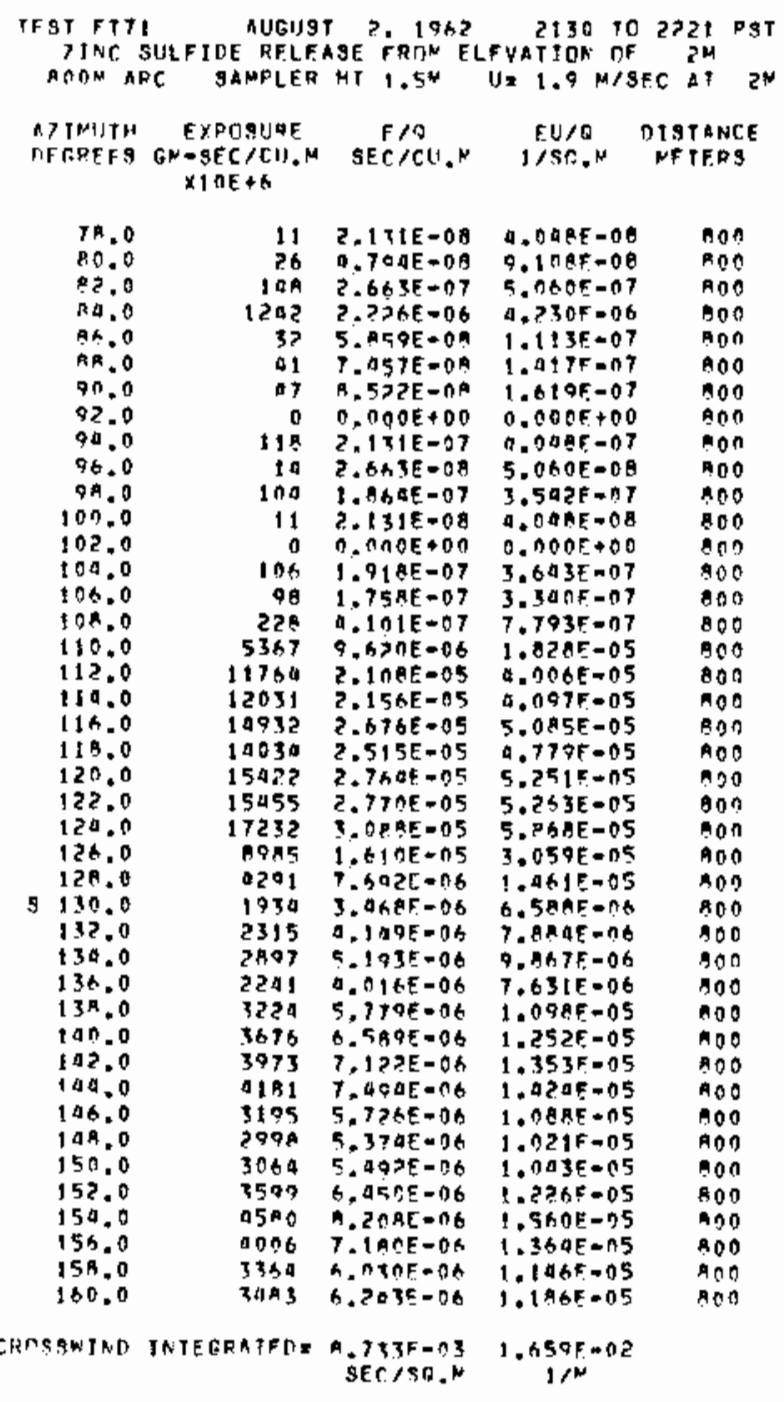


TOMER DATA FOLLEM....

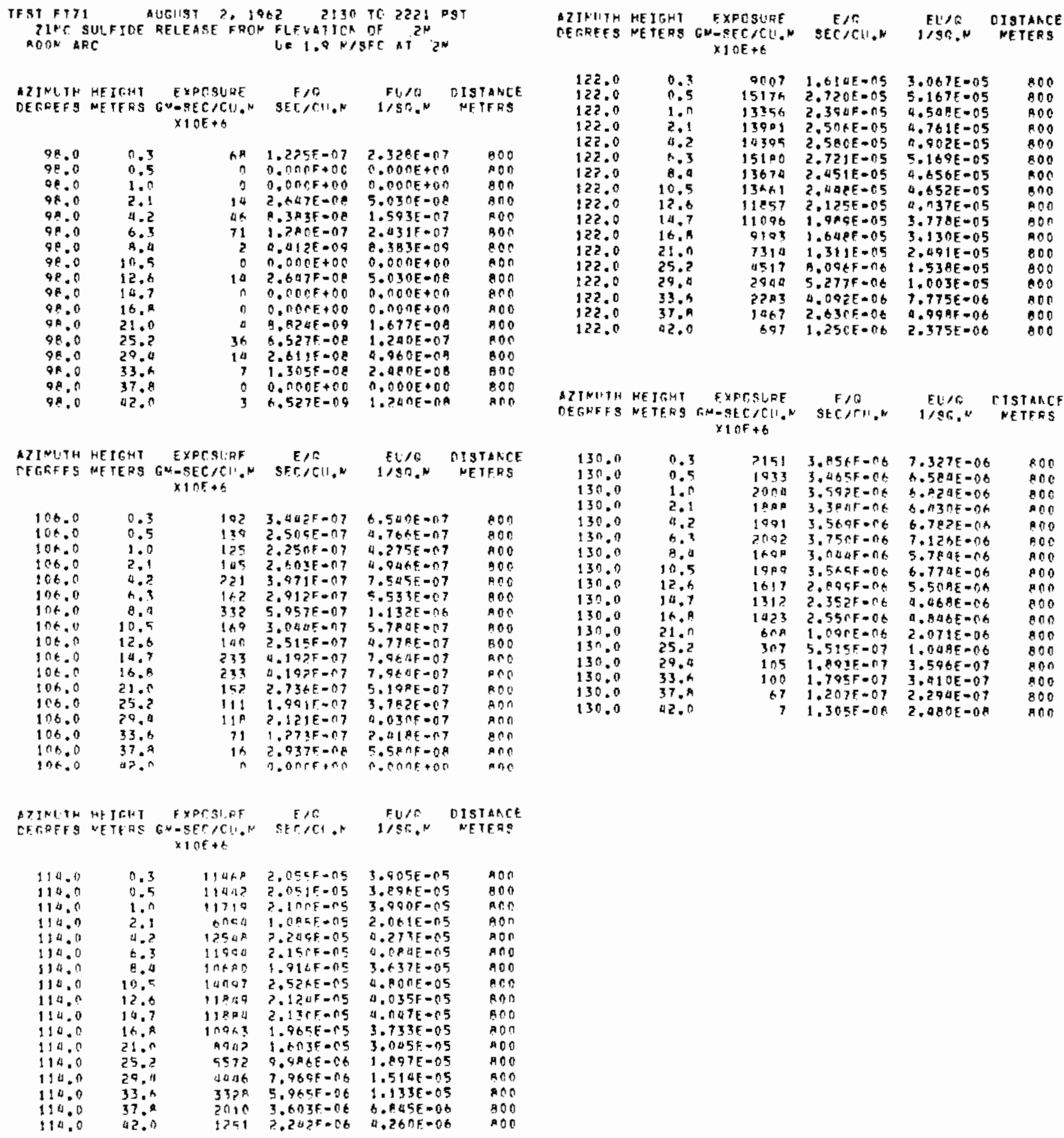




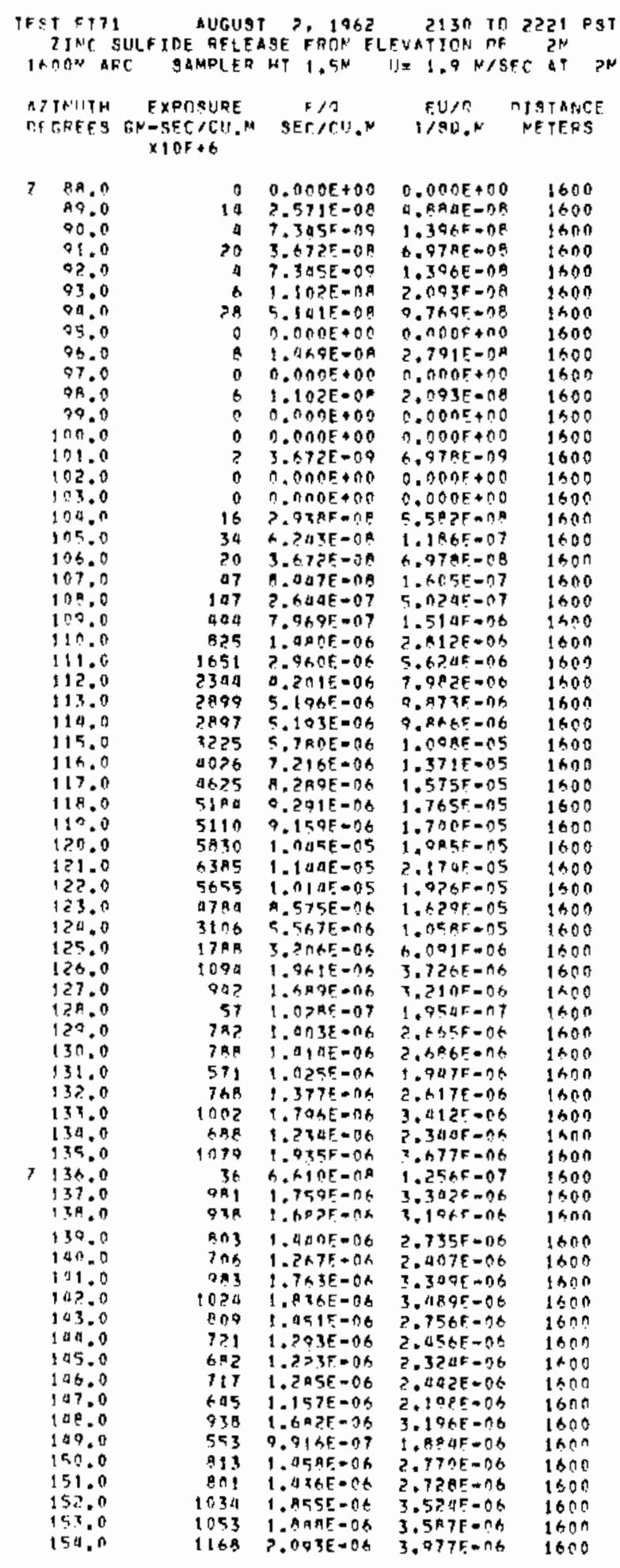

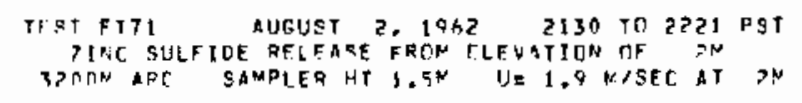

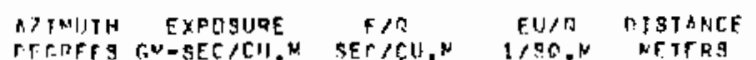
$\times 1 \cap E+6$

95.0
98.0
49.0
190.0
101.0
102.0
103.0

10 iा.

105.0

100.0

107.0

108.0

100.0

110.0

111.0

112.0

113.0

114.0

115.0

116.0

117.0

$11 \mathrm{~A} .0$

119.0

127.0

$1 ? 1.0$

127.0

123.0

120.0

125.0

126.0

127.0

1 ह 8.0

129.0

131.0

$13: .0$

132.0

133.0

130.0

135.0

136.0

137.0

13 ค. 0

139.0

140.0

141.0

147.0

103.0

1 a 4.0

105.0

$10+0$

147.0

1 นค. ก

149.0

150.0

151.0

$15 \% .0$

153.0

154.0

155.0

156.0

157.0

$15 A .0$

$+59.0$

$1+0.0$

$12 \quad 3.2 B 3 E-0 C$

A. $29 A E-08$ $0.0 A O F+00 \quad 0.000 F+00$

O O.ONOE + OO O. OOOE $\$ 00$

11 2.DRCE-0B $3.067 \mathrm{~F}=0 \mathrm{~B}$

0 C. CnOf 400 O. nONF +00

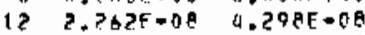

$6 \quad 1.21 A E-O Q \quad 2.314 F-O B \quad 3 \geqslant 00$

4 $.700 F=09 \quad 1.653 E=08 \quad 3200$

$13.400=09 \quad$ h.612E-09 3200

$3 \quad 3.960 E-09 \quad 1.322 E=08 \quad 3201$

$15 \quad 2.794 E-\cap A \quad 5.2 A Q E-08 \quad 3200$

53 9.57OF-OP $1.81 P E=07$ 320ी

5.710E=07 3200

TA7 1.411F-06 2.6BIF=06 3?0त

$\begin{array}{llll}7 B 7 & 1.411 F-06 & 2.681 F=06 & 3200 \\ 860 & 1.542 E-05 & 2.929 E-06 & 3200\end{array}$

7 I $1.334 E-06$ Z.559E-OG 3200

$992 \quad 1.77 R E-06 \quad 3.3795-06 \quad 3700$

71 1.2XAE-07 $7.446 E-07 \quad 3200$

96t $1.731 E-06$ 3.2A9E-06 3200

1643 2.946E-OA 5.597E-06 3200

$2933 \quad 5.25 \mathrm{AE}-0 \mathrm{~A} \quad 9.9 \mathrm{TE}=116 \quad 3200$

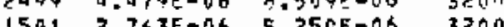

3200

$972 \quad 1.743 E-06 \quad 3.312 E-166 \quad 3200$

UA7 7.3 AAE $=07 \quad 1.388 F=06 \quad 3200$

$\begin{array}{llll}350 & 6.2 R 1 E-07 & 1.193 E-06 & 3700 \\ 330 & 6.090 F-07 & 1.157 F-06 & 3>00\end{array}$

$332 \quad 5.9515-07 \quad 1.1336=06 \quad 3700$

$431 \quad 7.5515-07 \quad 1.935 F-06 \quad 3700$

$\begin{array}{llll}421 & 7.551 E-07 & 1.035 E-06 & 3700 \\ 300 & 5.304 E-07 & 1.025 E-06 & 3200\end{array}$

211 2.795E-07 7.207E-07 3?0ी

27A A. Q9aF-07 9.AB 8F-B) 3200

252 Q.53AE-07 R.505E-07 3203

225 A. $037 E=07 \quad 7.670 F-07 \quad 3700$

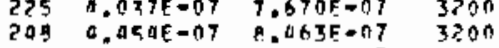

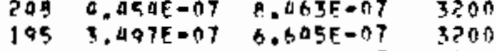

त. $3.671 E-17 \quad 6.975 F-07 \quad 3700$

$210 \quad 345 E-079.3065-07 \quad 3200$

$198 \quad 3.5095-07 \quad 6.744 F-07 \quad 3200$

$\begin{array}{llll}198 & 3.509 F-07 & 6.794 F-07 & 3200 \\ 139 & 2.505 F=07 & 9.760 F=07 & 3700\end{array}$

$\begin{array}{llll}139 & 2.5 n E F=07 & 9.760 F-07 & 3700 \\ 109 & 1.965 E-07 & 3.736 F-n 7 & 3200\end{array}$

Ih2 2.006F-07 5.52IE-97. 3200

उ9 $7.134 F=08 \quad 355 F=07 \quad 3200$

टी $4.332 E+17 \quad 8.232 E-07 \quad 3200$

$\begin{array}{llll}241 & 4.332 E-1) 7 & 8.232 E-07 & 3200 \\ 211 & 1.7035-07 & 7.207 F=07 & 3200\end{array}$

203 3.65AE-07 6.8U25-n7 3?00

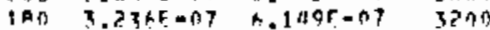

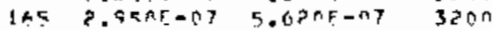

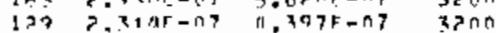

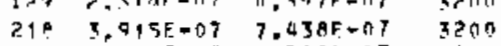

ग5i $\quad 0.5 n b E-0 \%$ Q.562F-07 3207

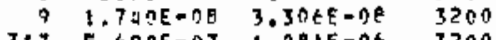

233 a. $1037-07 \quad 7.967 \mathrm{~F}-07 \quad 3200$

$\begin{array}{llll}233 & 0.1035-07 & 7.967 F-07 & 3200 \\ 313 & 5.030-07 & 1.068 E-06 & 3200\end{array}$

$\begin{array}{llll}313 & 5.620 E-07 & 1.068 E-96 & 3200 \\ 307 & 7.315 E-07 & 1.352 E-06 & 3200\end{array}$

5A $\quad .9 A \cap E-07 \quad 1.322 F=A B \quad 3200$

UQA $A .0 \cap 4 E=0 ? \quad 1.571 F=06 \quad 3700$

$497 \quad$ QRAE $=07 \quad 1.693 E-06 \quad 3200$

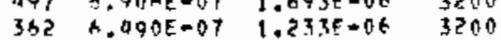

$\begin{array}{llll}362 & 1.090 E-07 & 1.233 E=06 & 3200 \\ 5 A 7 & 1.053 E-06 & 2.0018=06 & 3200\end{array}$

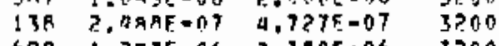

161,0

$600 \quad 1.2535=06$

$2.300 E-\pi h$

3200 
UNSTABLE SERIES EXPERIMENT: 2 (

DATA FROM EXPERIMENT UI IS NOT AVAILABLE, GROUND LEVEL SAMPLING

100- 1600 M ARCS. TRACER DISPER5AL DIFFICULTIES MAY HAVE RESULTEO

IN A VARIABLE RELEASE RATE DURING EXPERIMENTS U-I THROUGH U-TO.

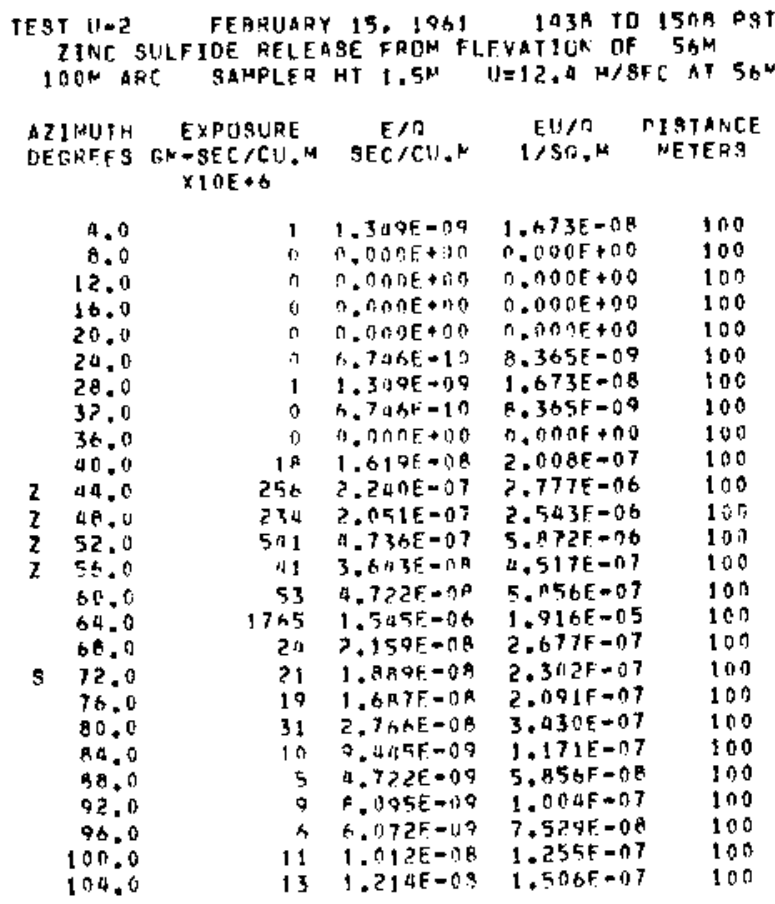

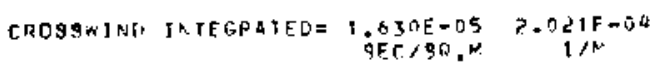

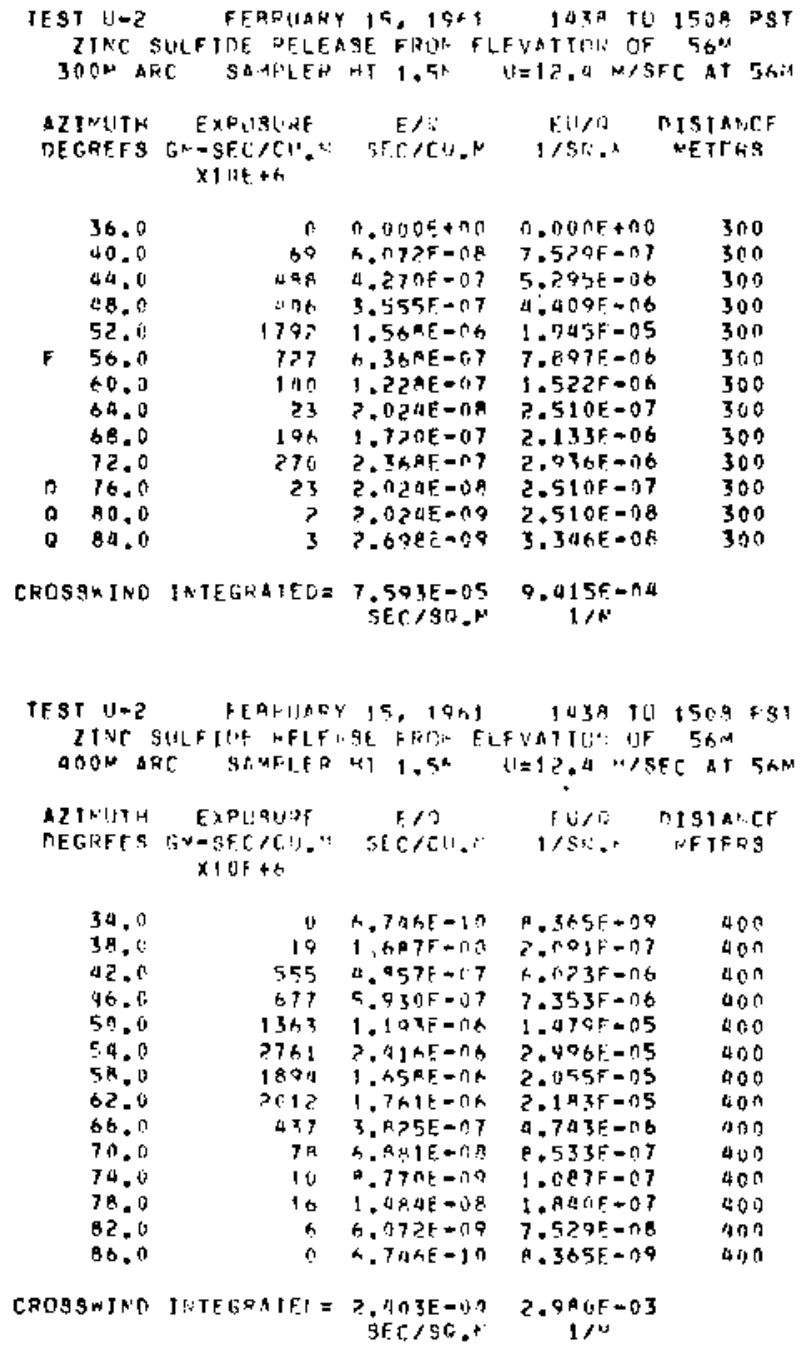




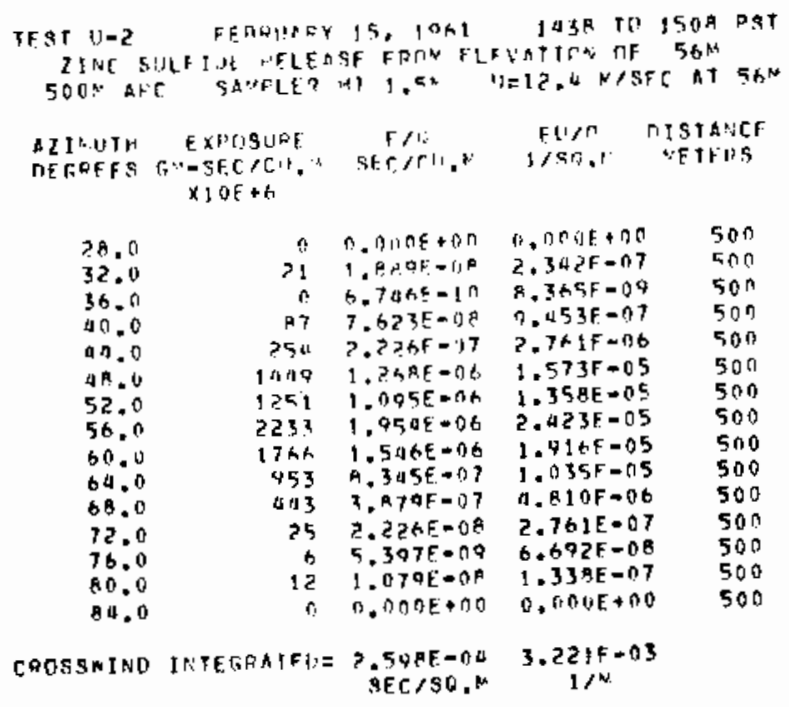

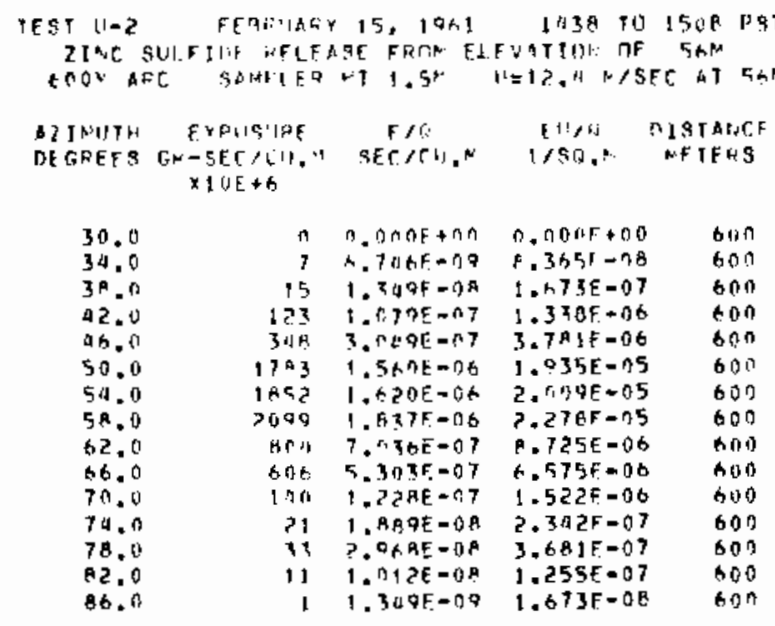

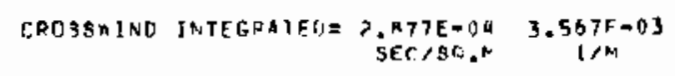

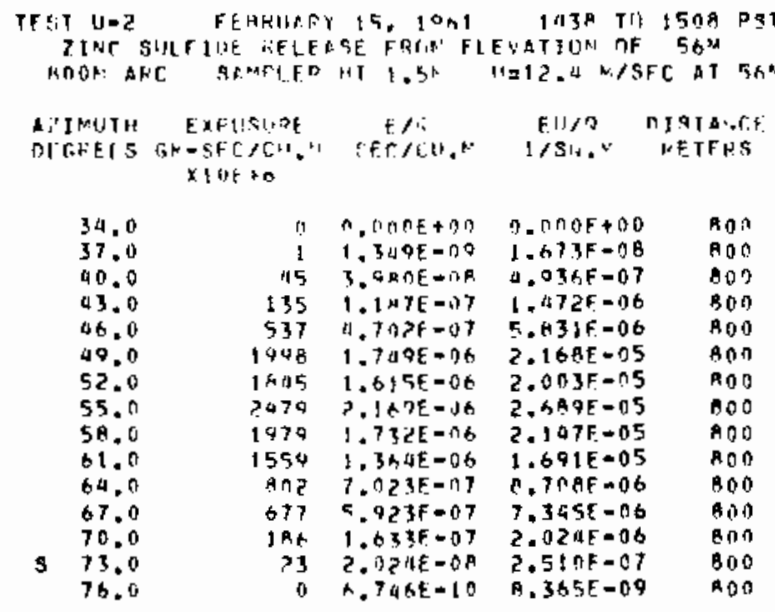

CROSSWIHD JNTEGRATFI $=4$, DQRE-OA 5.577E-03

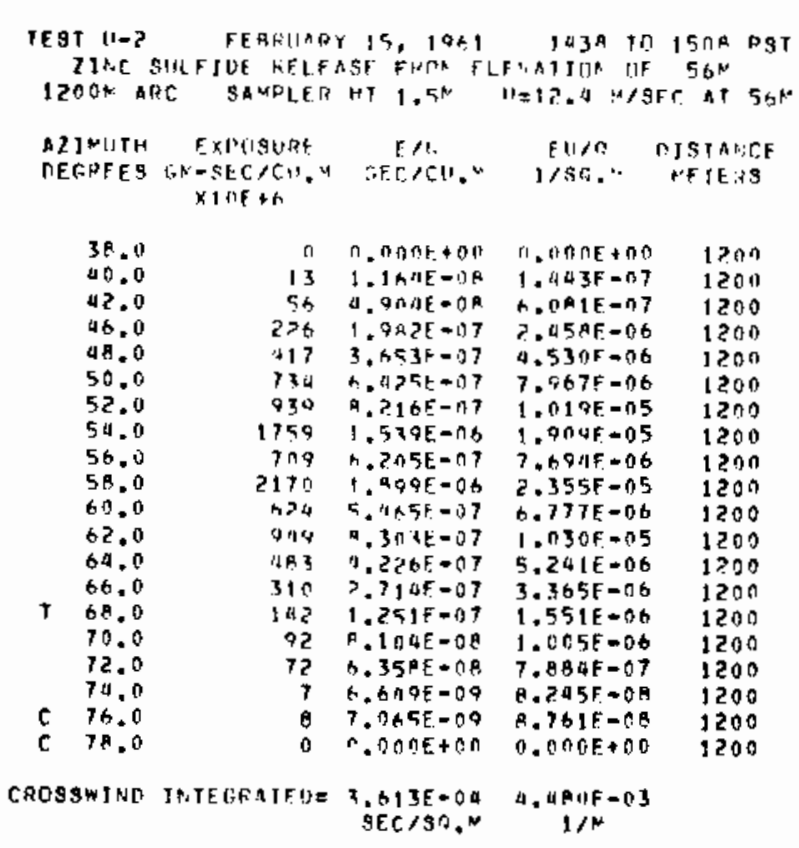

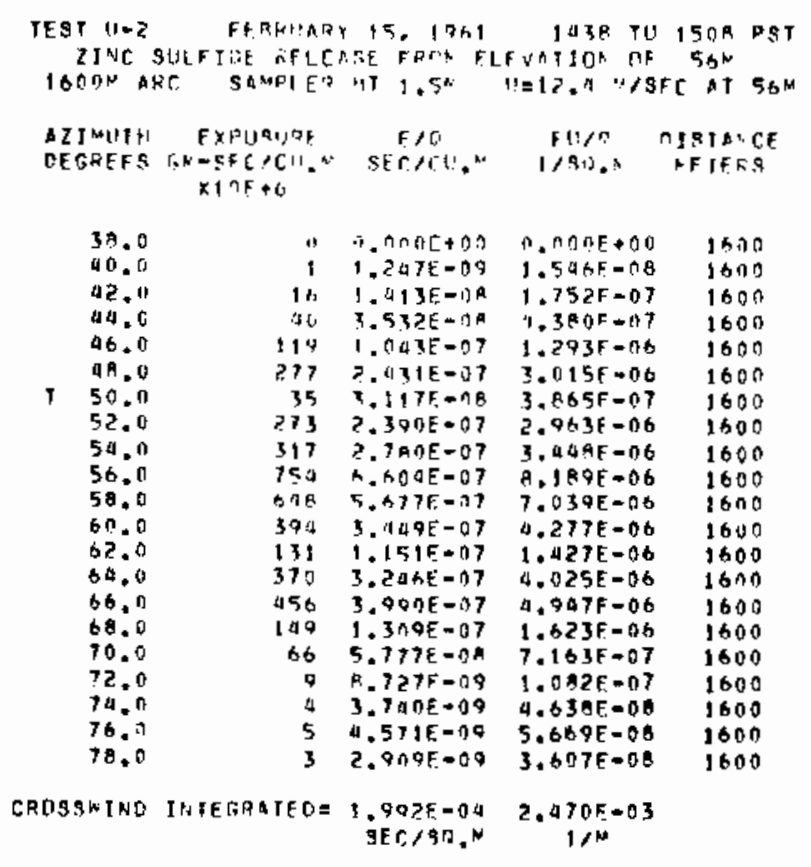


UNSTABLE SERIES EXPERINENT: 5 (U-5)

GROUND LEVEL SAMPLING 100-1600 $\because$ ARCS. THE TRACER HAS HOT

DETECTEO OH THE 100 M ARC. TRACER DISPERSAL OIFFICULTIES MAY

HAYE RESULTED IN A YARIAELE RELEASE RATE DURIPSG EXPERI:ZENTS U-:

THROUGH $U-10$.

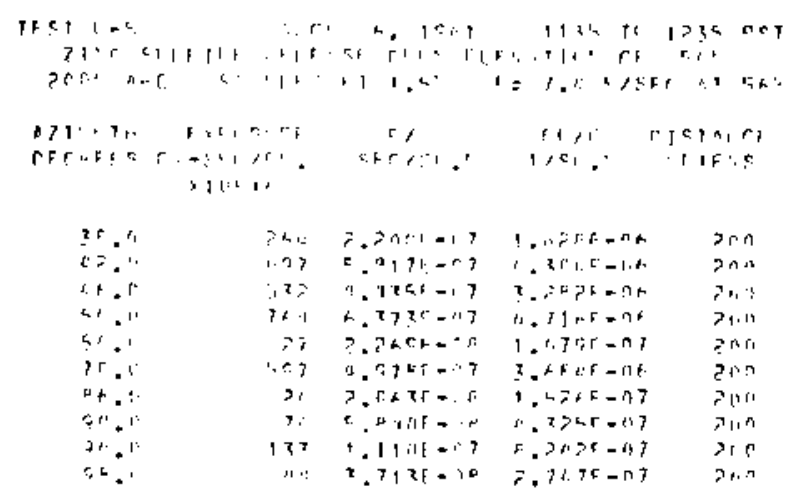

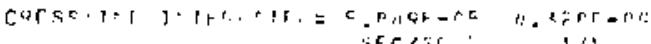

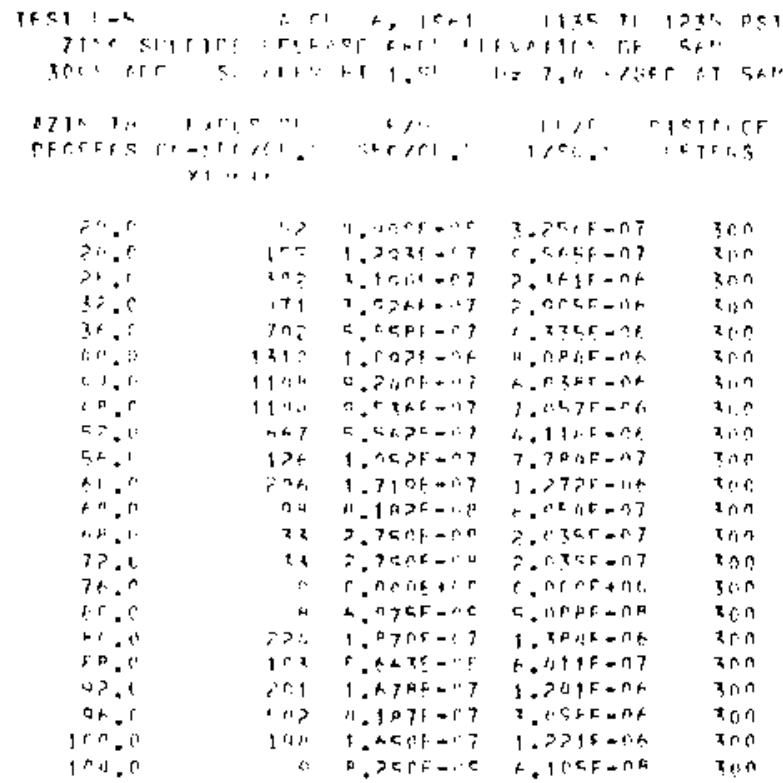

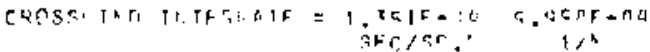

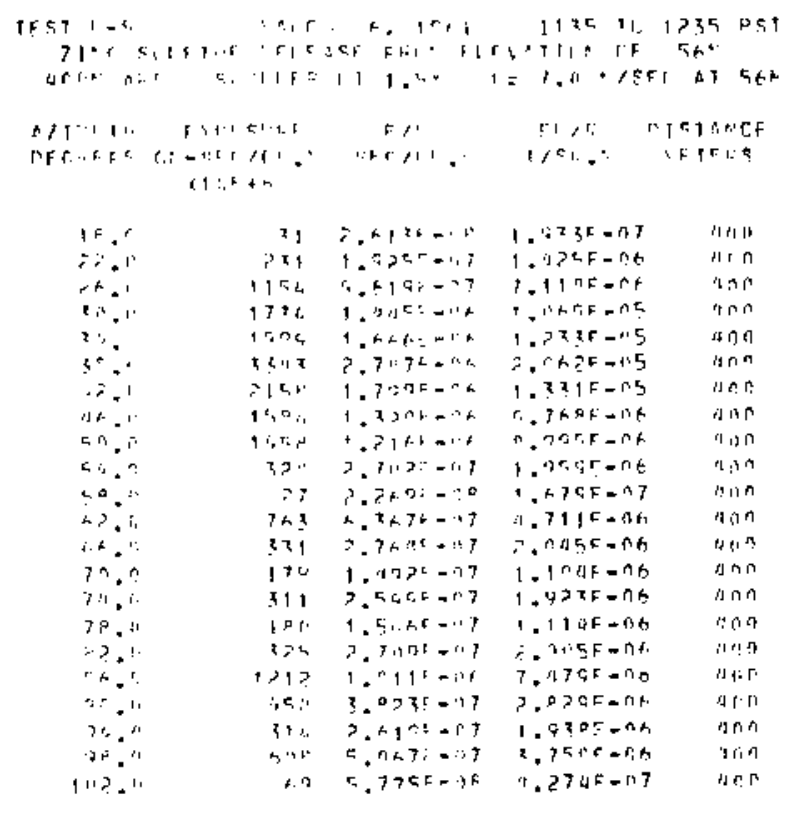

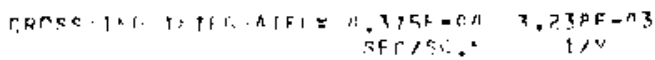

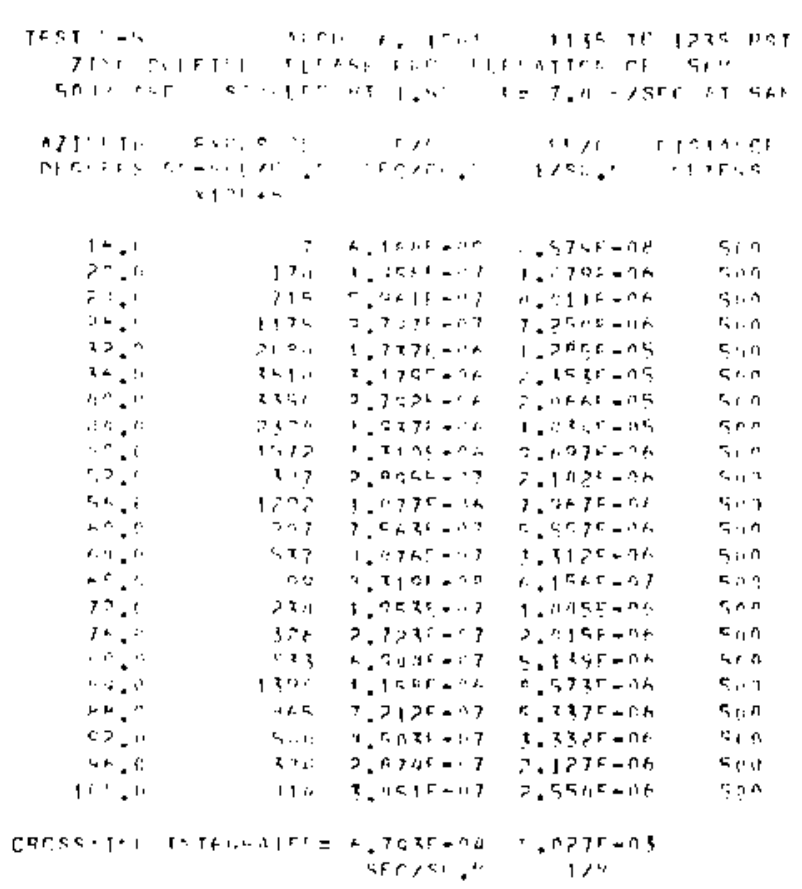




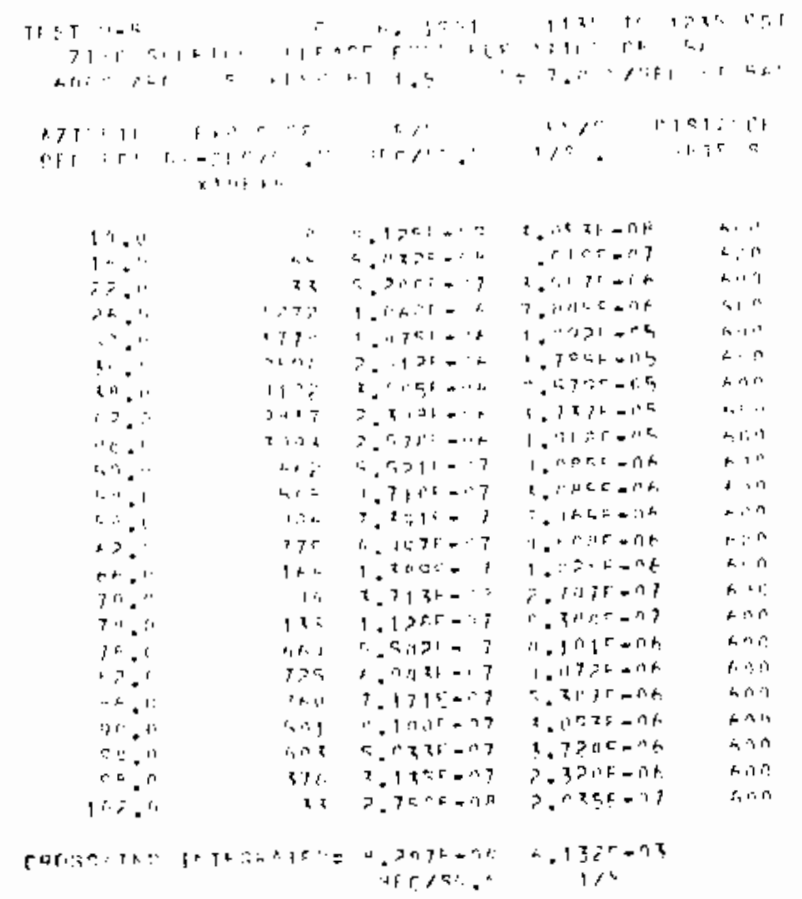

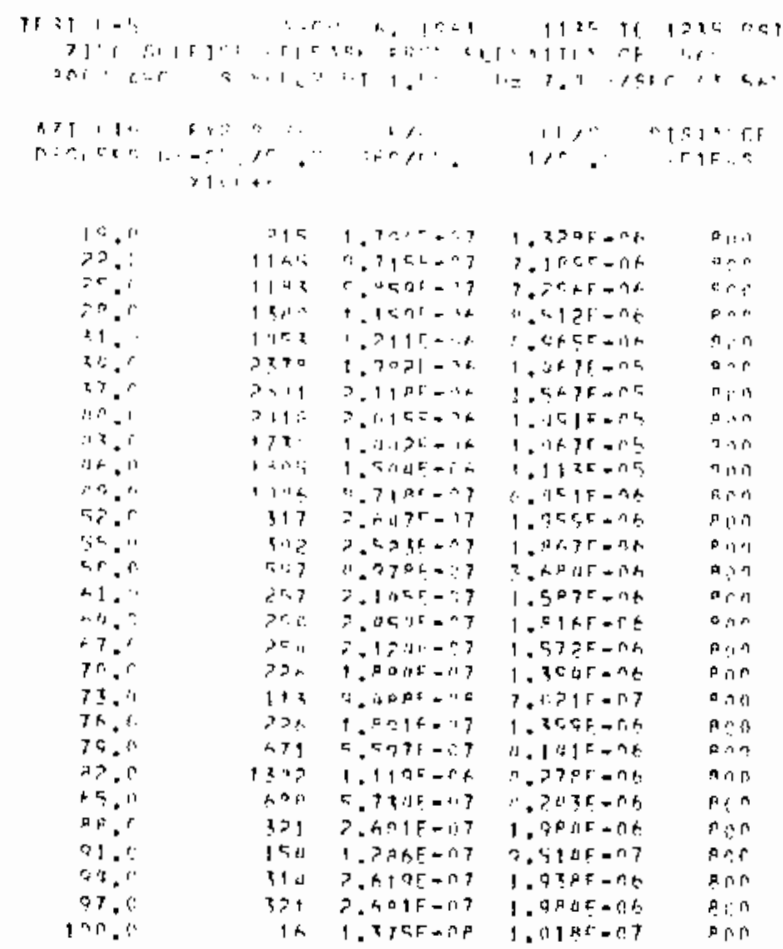

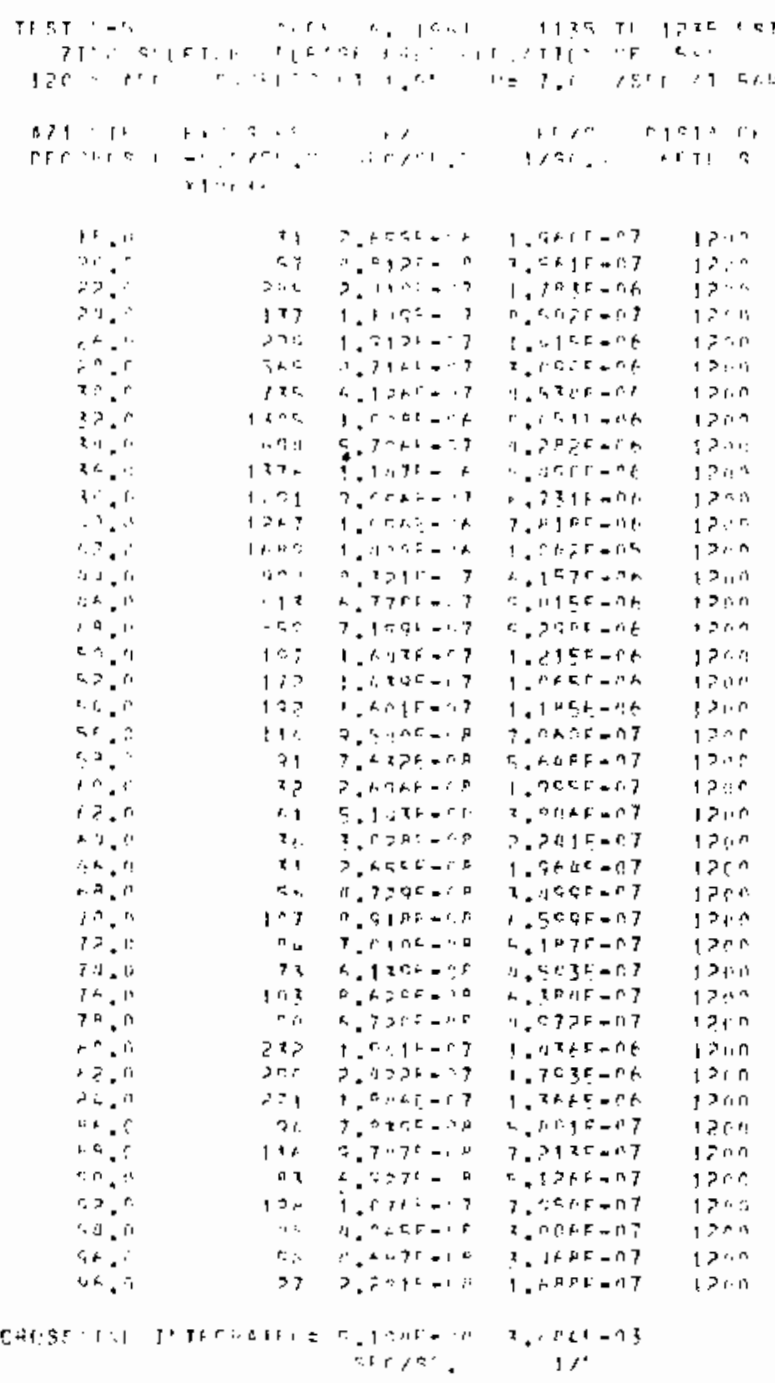

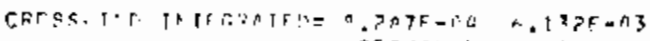




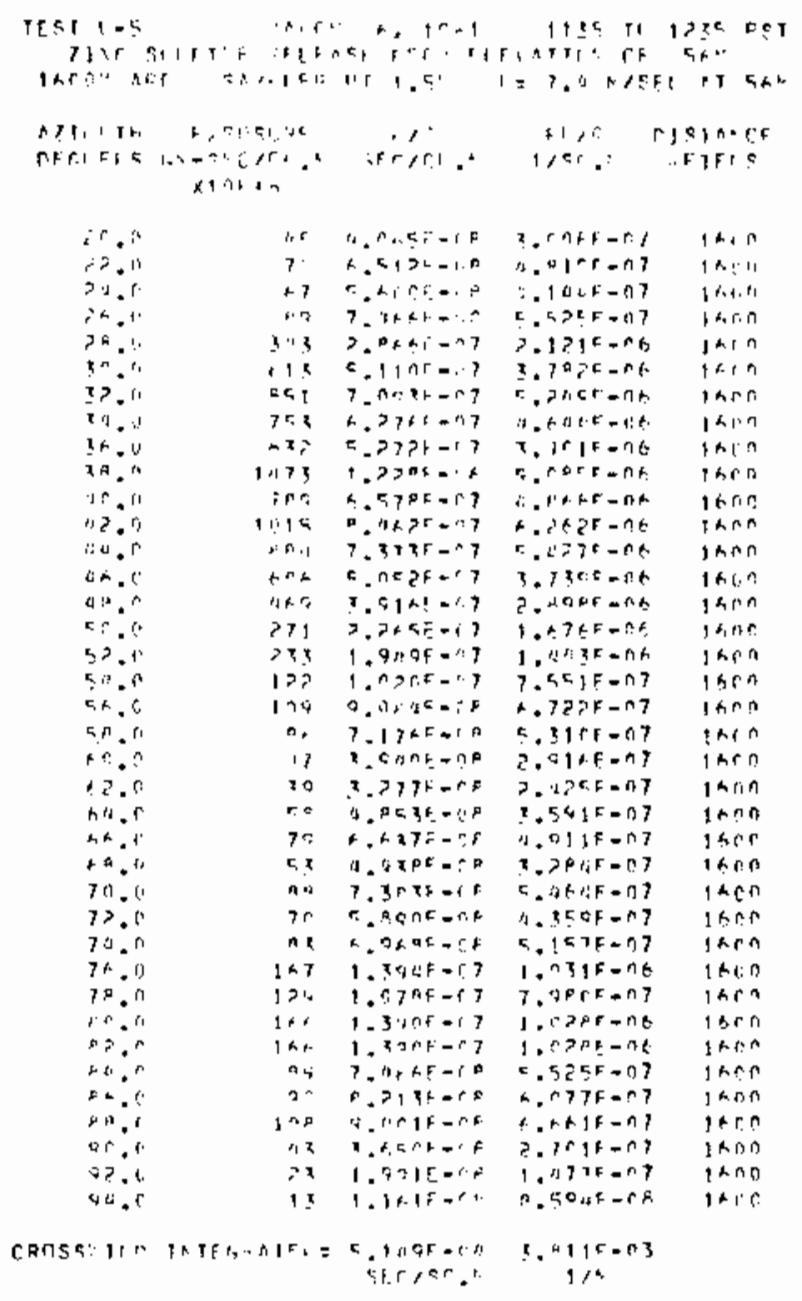


UNSTABLE SERIES EXPERIMENT: 6 (U-6)

GROLNO LEVEL SAMPLING 100-1500 M ARCS, TRACER CONTAMINATION ON

I00-400 M ARCS. CONTAMINATION ON 100 AND 200 M ARCS IS 50 SEYERE THAT DATA FROM THESE ARCS ARE NOT PRESENTEO. TRACER JISPERSAL DIFFICULTIES MAY HAVE RESULTED IN A VARIAGLE RELEASE RATE OURING EXPERIMENTS U-1 THROUGH U-10.

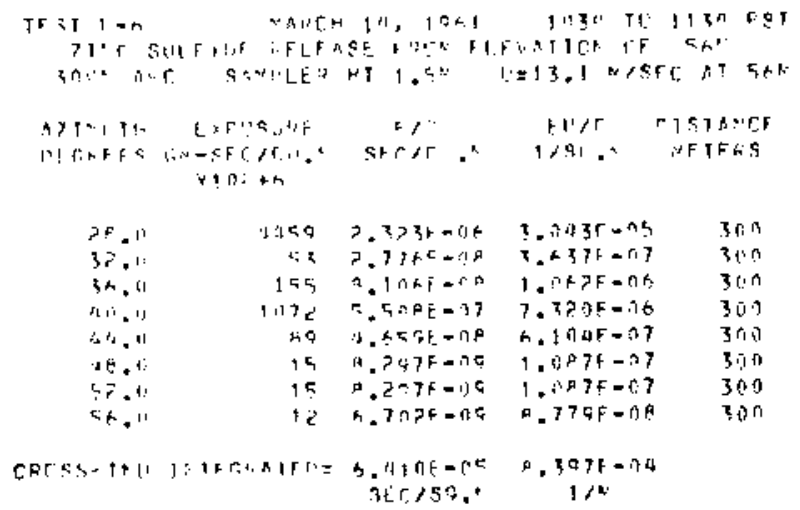

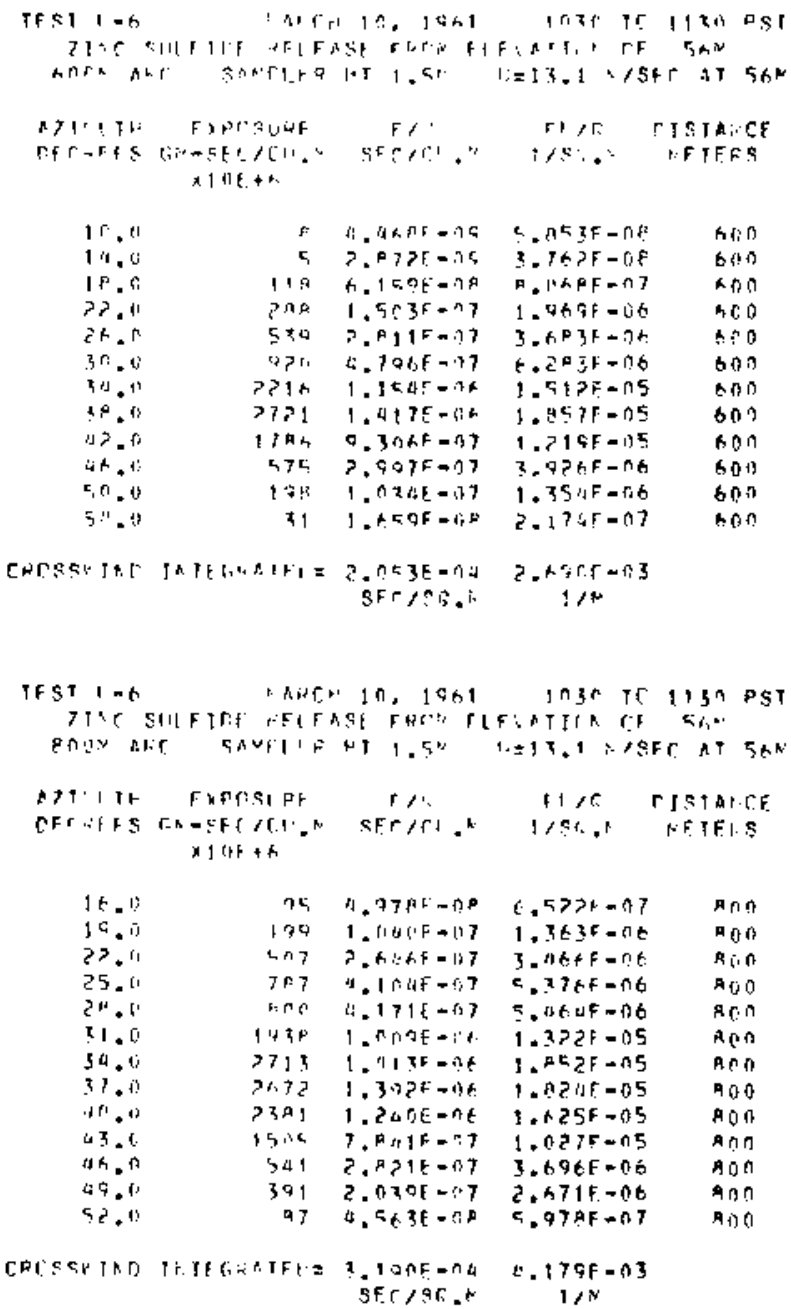




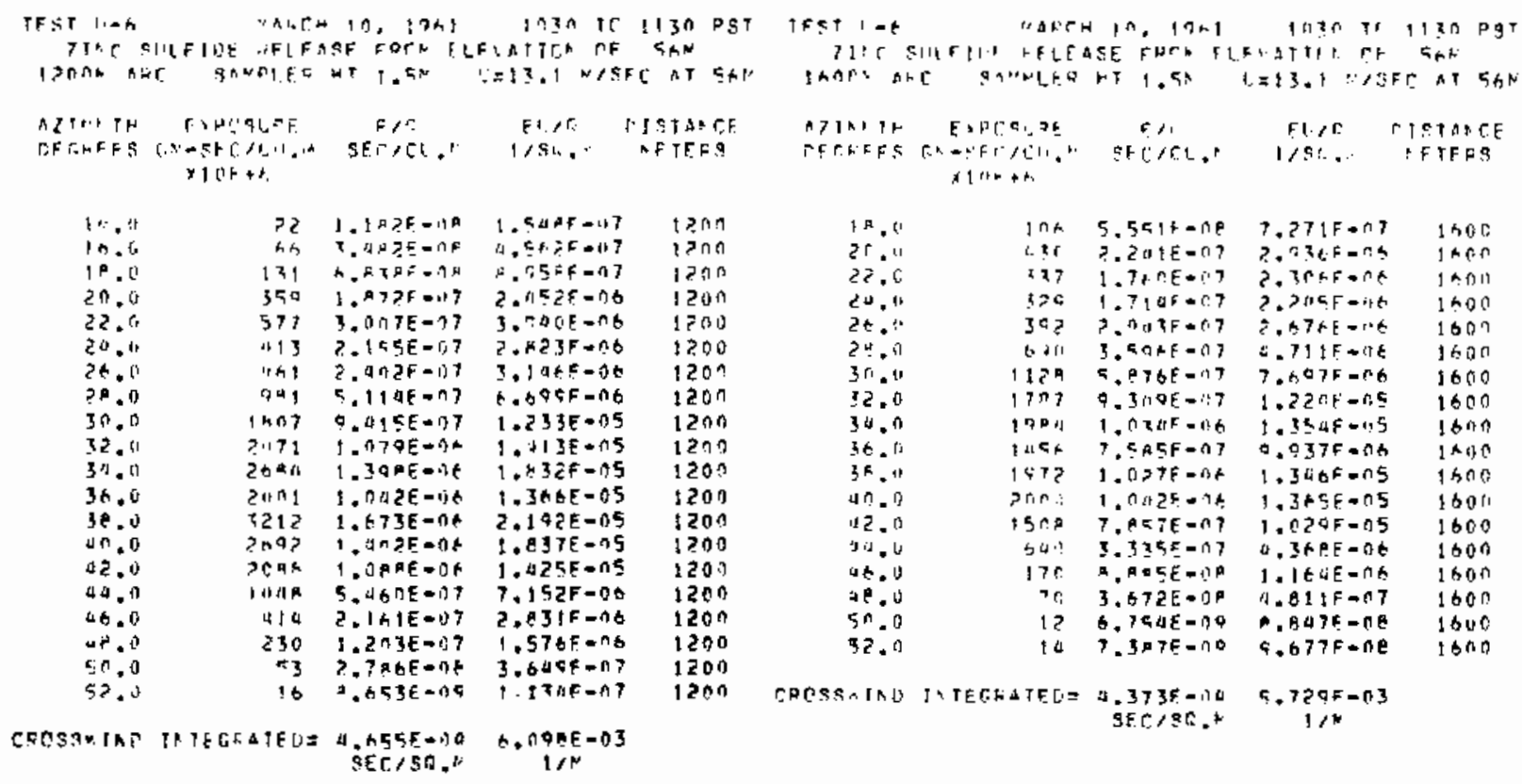


UNSTABLE SERIES EXPERIMENT: 7 ( U-7)

GROUND LEVEL SAHPLING $100-1600$ M ARCS. TRACER DISPERSAL

DIFFICULTIES MAY HAVE RESULTED IN A YARIABLE RELEASE RATE OURING

EXPERIMENTS $U-1$ THROUGH U-1O.

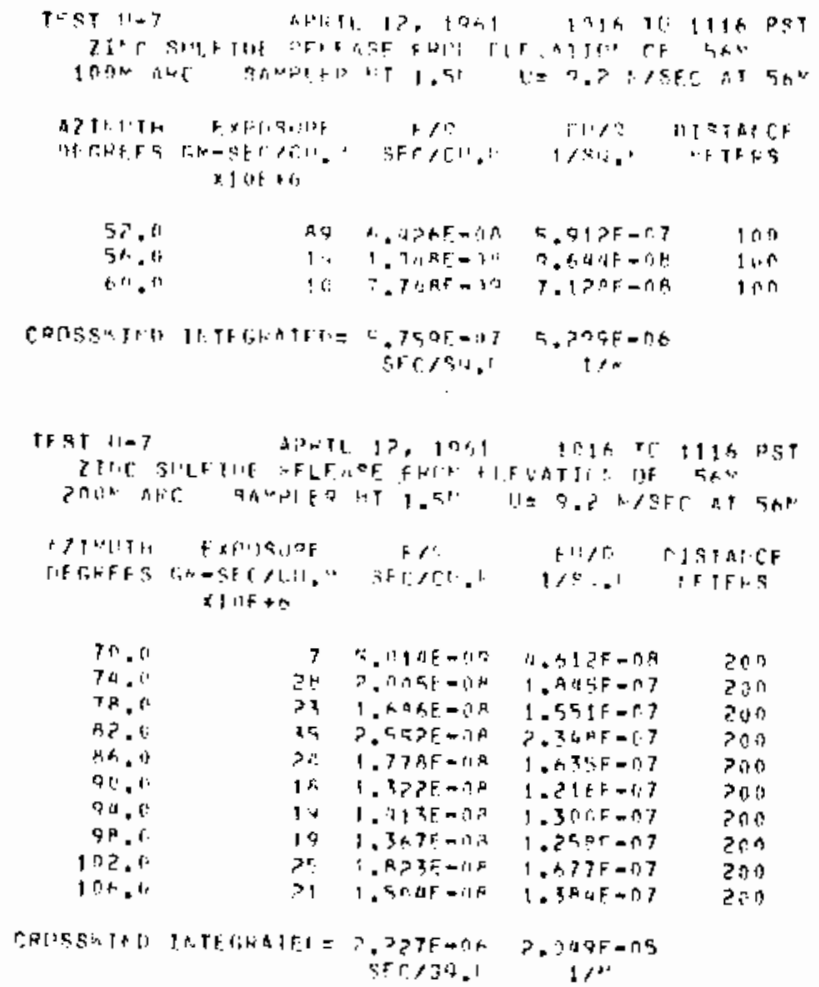




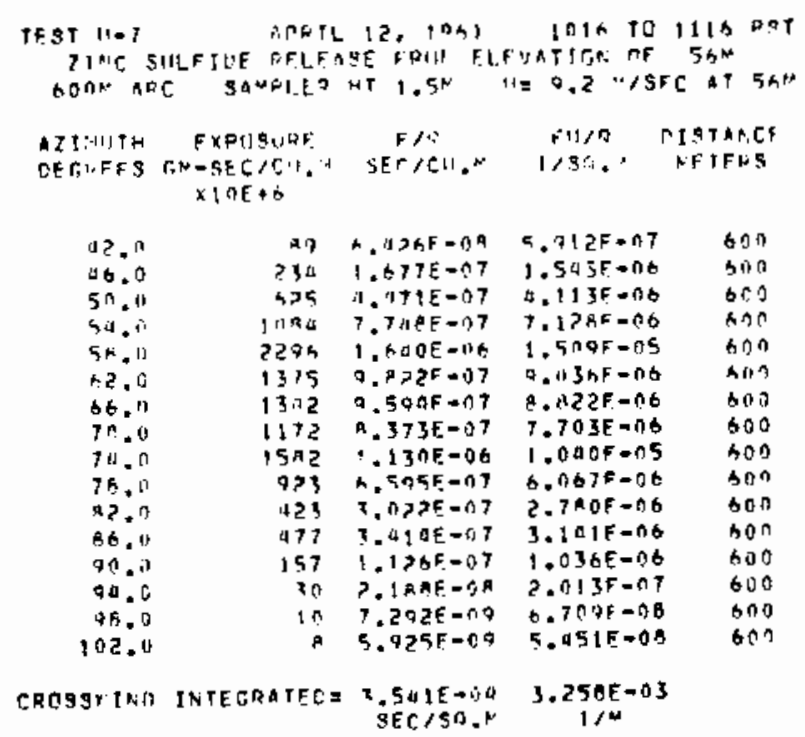

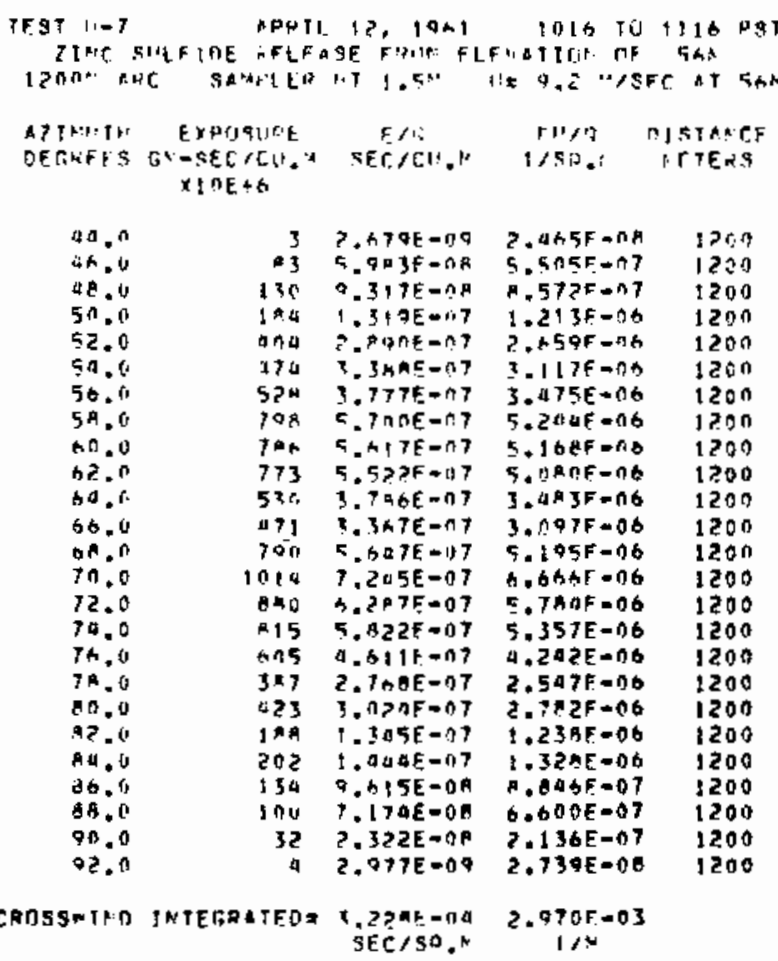

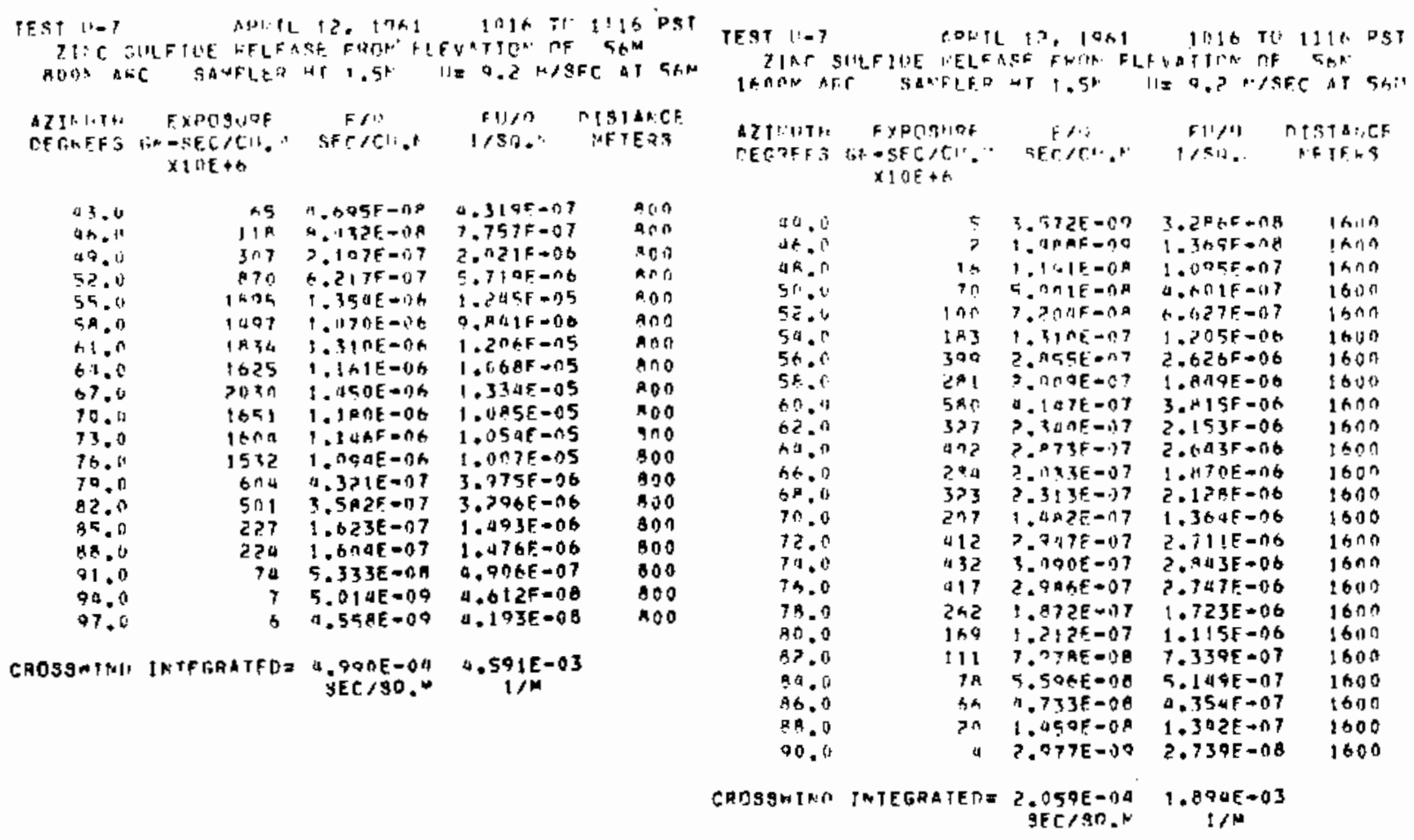


UNSTABLE SERIES EXPERIMENT: 8 ( $U$-8 $)$

GROUND LEYEL SAMPIING $100-1600$ M ARCS. TRACER DISPERSAL

DIFFICULTIES MAY HAVE RESULTEO IN A YARIABLE RELEASE RATE DURING

EXPERIMENTS U-1 THROUGH U-10.

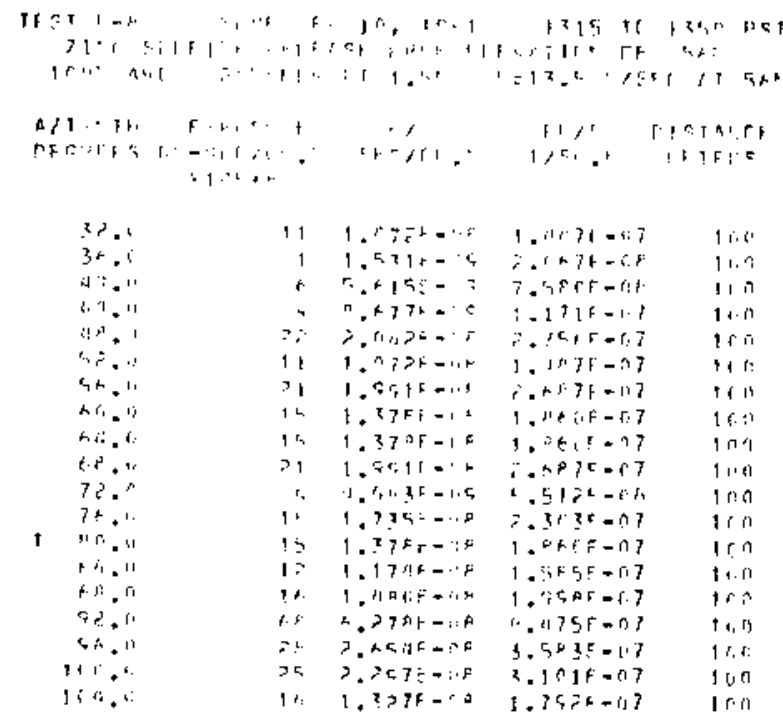

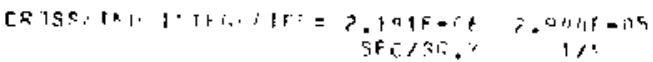

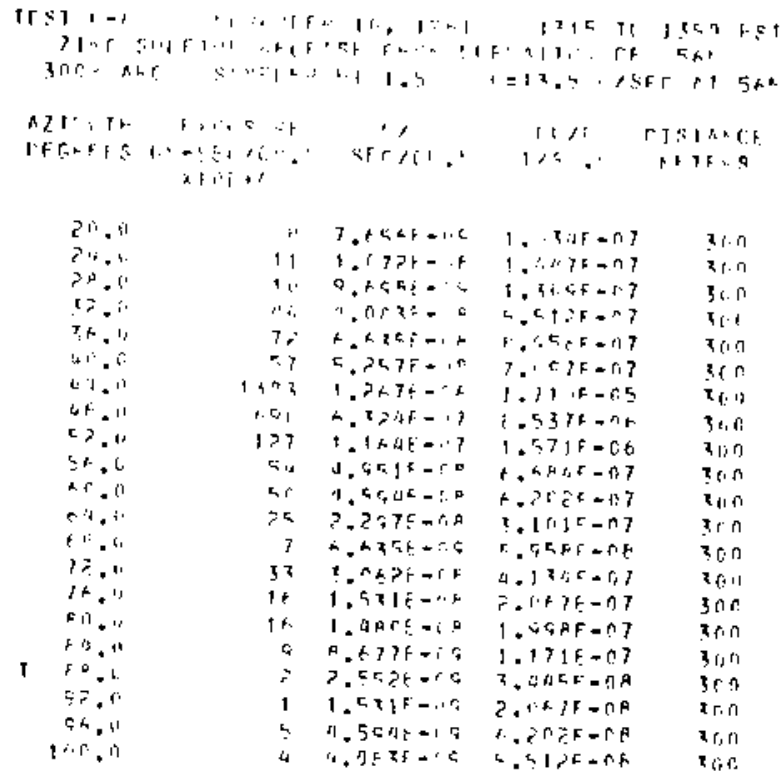

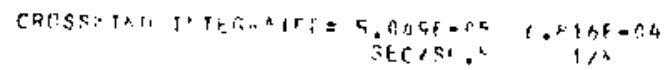

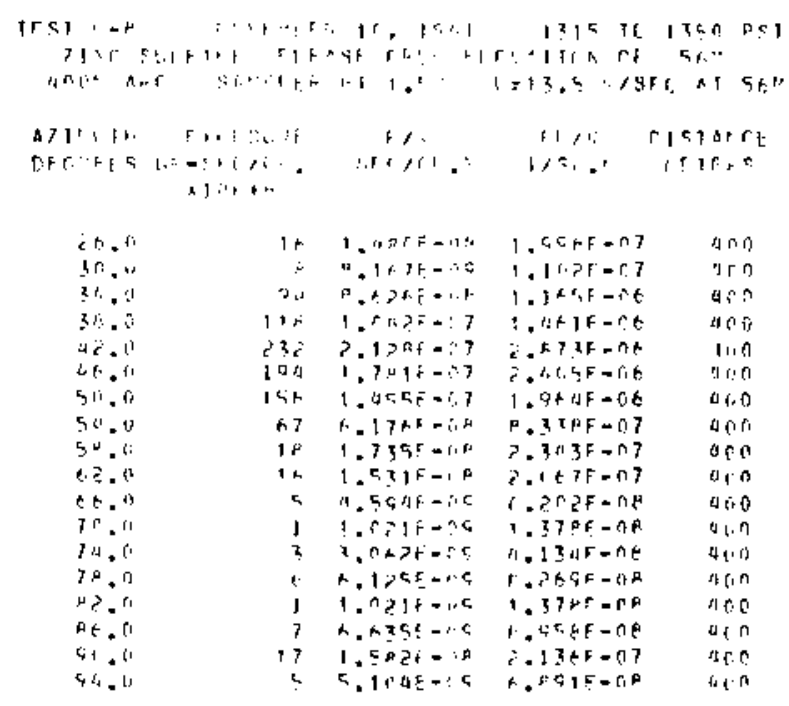

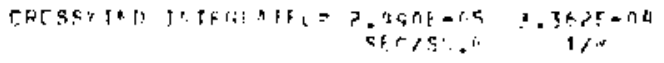

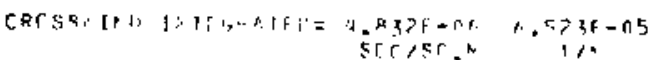




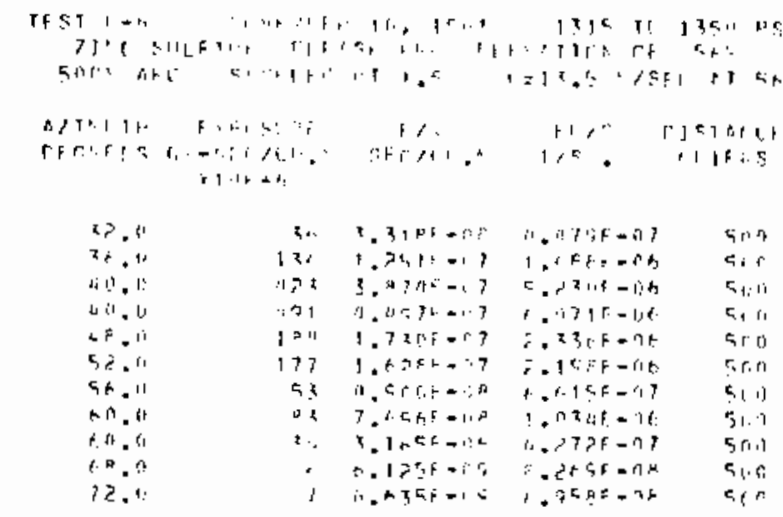

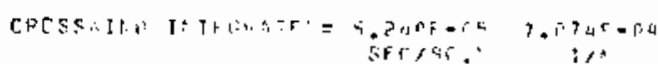

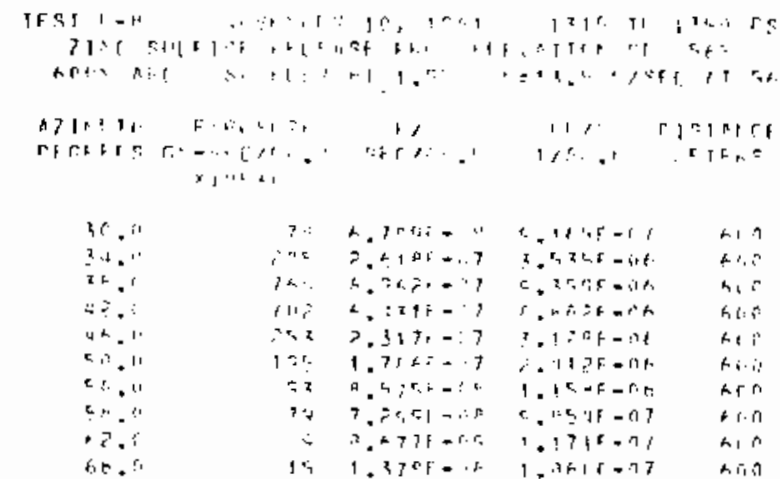

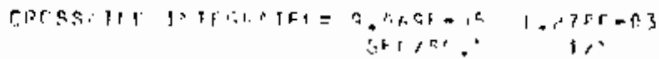

HS

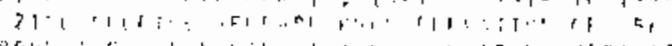

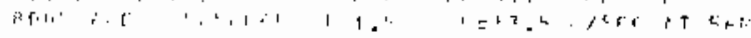

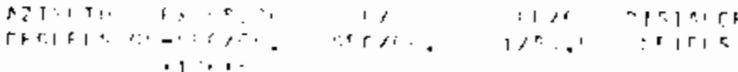

\begin{tabular}{|c|c|c|c|c|}
\hline 31.1 & , & $A, 1,-4=$ & $\therefore \ldots+r \in$. & $n+1$. \\
\hline 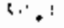 & $\therefore$ & $n, z=r,-c !$ & $\therefore .126=07$ & H \\
\hline . & $\vdots !$ & $\therefore \therefore i r \cdots r$ & $x: T+[+\infty$ & $c_{1}, 1$ \\
\hline$\therefore .11$ & $\cdots$ & 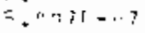 & $\therefore . P 1=-r . x$ & $n_{1} \cdot$, \\
\hline$x^{2}:$ & $1: ?$ & $1, x=1+\cdots$ & $\therefore \quad \therefore F-1.4$ & י: \\
\hline $1 .+\ldots !$ & $\therefore$ & $2,2 \cdot 1:-7$ & $\therefore+r \&=n$ & Air: \\
\hline$\therefore$, & $\cdots \cdot$ & $4.2+1-7$ & $\Rightarrow x \cdot 1-1 t$ & Fi \\
\hline 5.7. & $\because \mathrm{r}$ & $1<t+-7$ & $1.1=+t=r t$ & $\therefore i$ \\
\hline$\because 9.4$ & .7 & $\left.\because x_{-}\right]=7$ & $\therefore, \cdots=1=0$ & $m i:$ \\
\hline 3 & 11. & $t: ?+7^{-}=?$ & $\therefore A: 1=r$. & al $<$ : \\
\hline$+1 .:$ & $?$ & $7.1 \cdot 75-1.8$ & $\because T 1,1=1=1$ & Dif $r$ \\
\hline-14 & 2. & 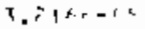 & I x $1 f=$ fit & म \\
\hline+7. & $1: i$ & $1,7,20.6$ & 1. $2 \bar{r} 3 t-n, 7$ & Sit? \\
\hline 35.1 & $\because$ & $10910+16$ & $.4,1=0,12$ & D $A$ \\
\hline & $\because$ & i $\mathrm{t}$ & 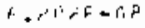 & $a_{1} 11$ \\
\hline
\end{tabular}

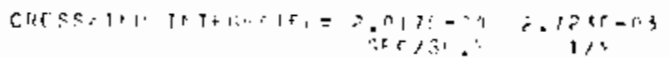

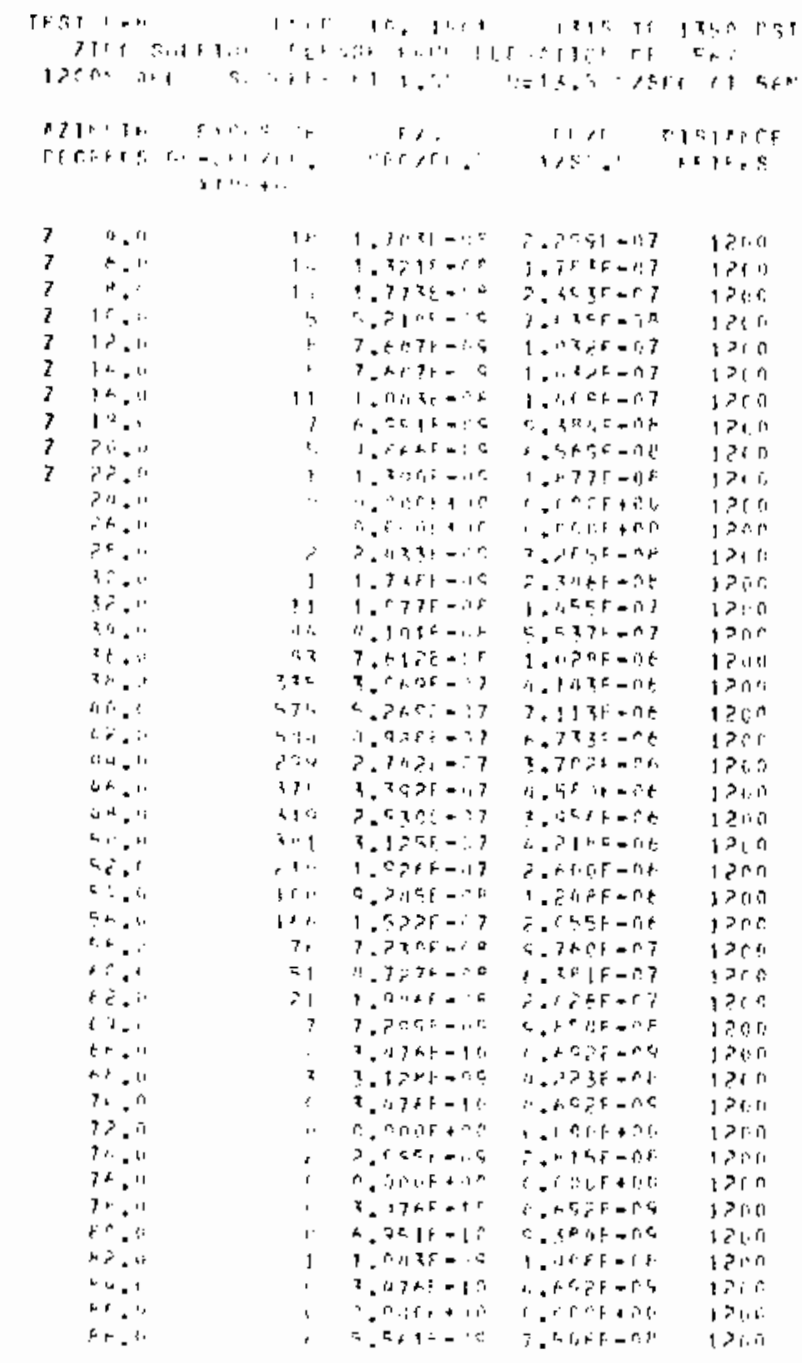

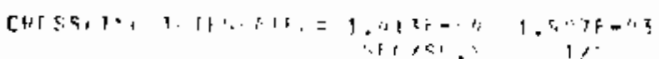




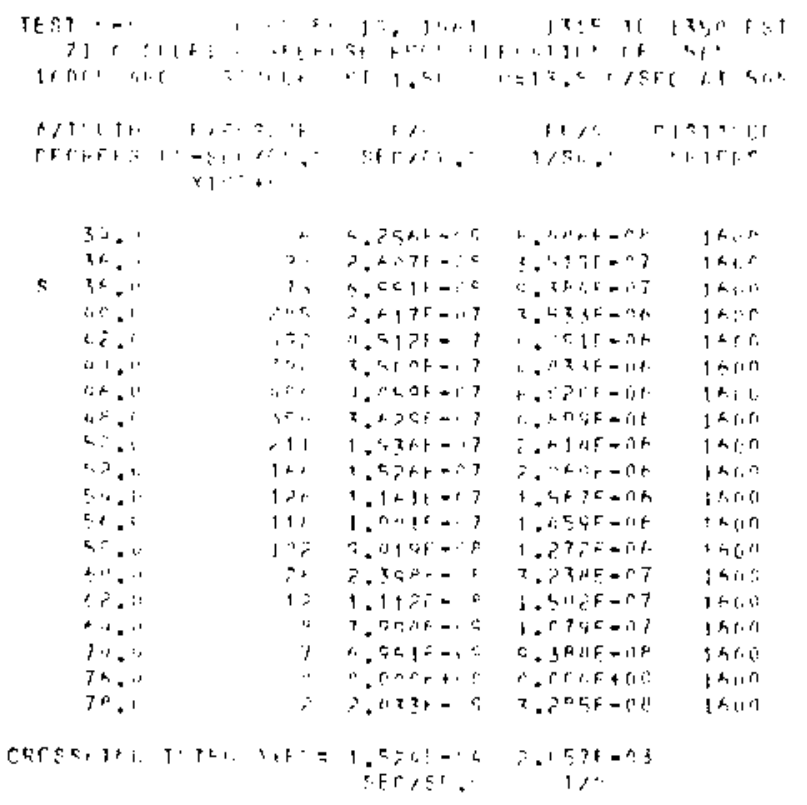


UNSTABLE SERIES EXPERIIIENT: 9 \& U-9 )

GROUND LEVEL SA:PLING 100-1600 :A ARCS. THF TPACER WAS NOT

OETECTED ON THE 100 HA ARC. TRACEP DISPERSAL DIFFICULTIES HAY

HAVE RESULTED IN A VARTABLE RELEASE RATE DUPING EXPERILENTS U-1

THROUGH U-1O. A LIGHT PAIN FELL DURING THE EXPERIHENT

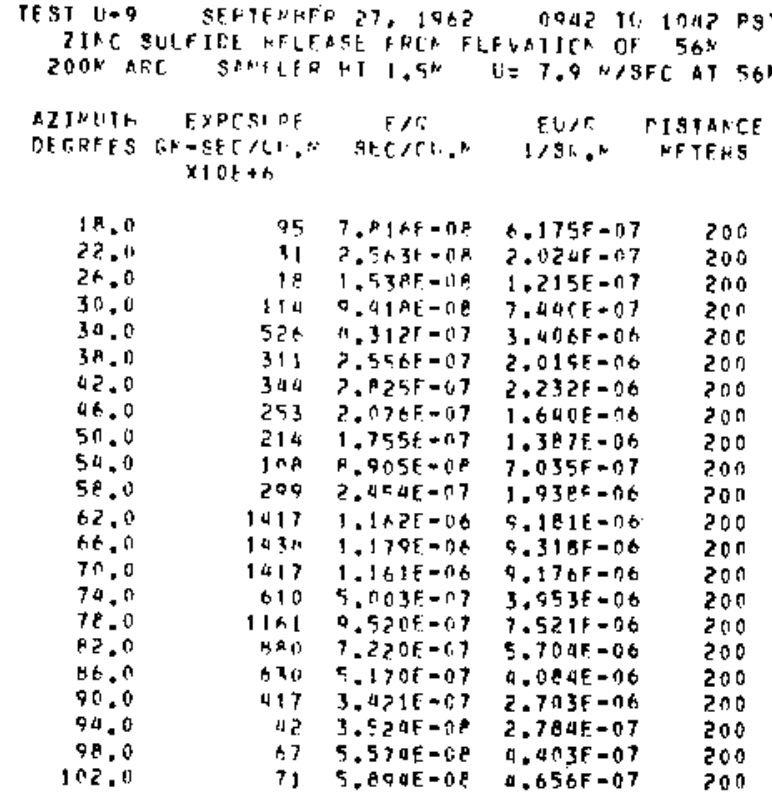

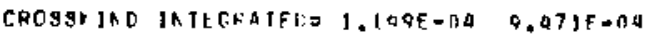

SEC/90.M $1 / \mathrm{N}$

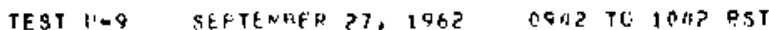
ZIAC SULFIOE NFLEASE FRC ELEVATTCA R.F $56^{\circ}$

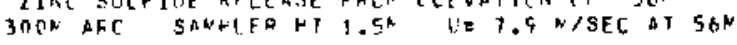

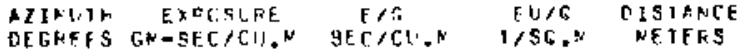
$\times 10 E+6$

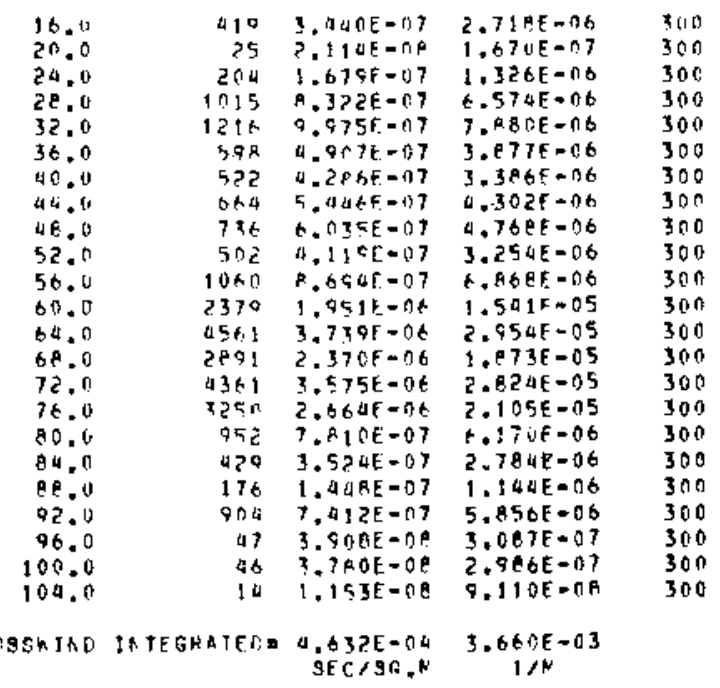

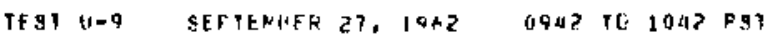
ZIAC SULFIDE HFLEASE FREF ELEVAT]Ch CF 5 SW

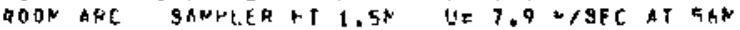

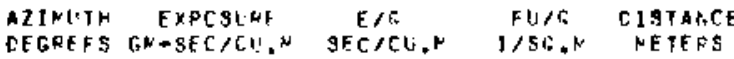
$x \backslash \cap E+b$

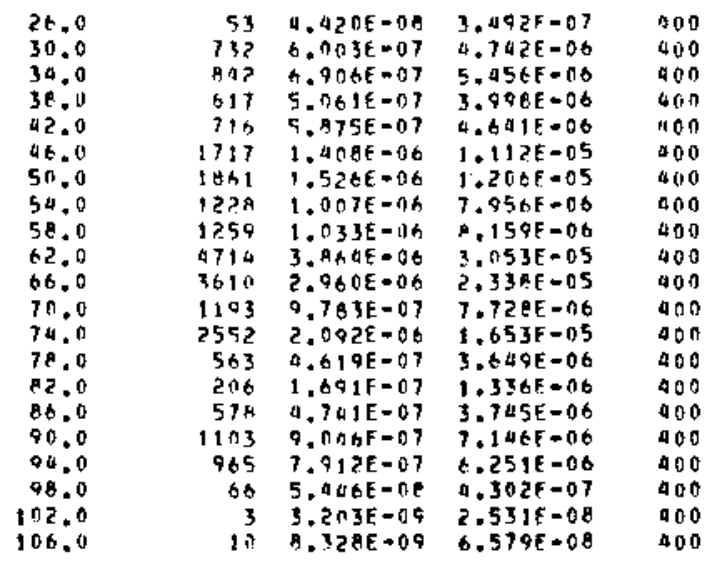

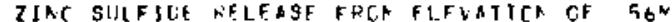
SOON AFC SANFIEA HT I.SH $U=7.0$ Y.SFC AT $5 \mathrm{SH}$

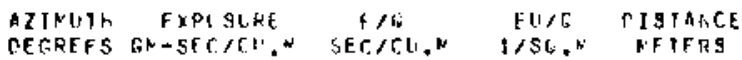
$x ! D E+6$

\begin{tabular}{|c|c|c|c|c|}
\hline $2 a .0$ & 3 & $2.563 E-09$ & $\bar{c}-02$ aE $=$ - & $50 n$ \\
\hline 29.0 & 106 & 1. $2 n \Delta E=07$ & $9.51 \leq F=07$ & 5010 \\
\hline 32.0 & 13 & $1.009+68$ & $A, 6 \cap A F=0 A$ & $5+0$ \\
\hline 36.1 & $4 \cap ?$ & $3.358 \mathrm{f}-07$ & $2.0375=06$ & 500 \\
\hline 40.0 & 411 & $3.376 E-07$ & $2.667 F-126$ & 500 \\
\hline 44.0 & 15.49 & $1.270 F=06$ & $1,003 \mathrm{~F} * 0 \mathrm{~S}$ & 500 \\
\hline 48.0 & 2132 & $1.74 A F-06$ & 1.3 A $1 E=05$ & 500 \\
\hline 52.0 & 944 & $7.739 k-07$ & $t .11 a F=n 6$ & 500 \\
\hline $5 t .0$ & 2203 & 1. An $B E=0 t$ & $1.427 F=05$ & 500 \\
\hline 60.0 & 2520 & $2.066 f=06$ & $1.632 E-05$ & $5 n 0$ \\
\hline 64.0 & 2773 & $? .273 E-0 t$ & $1.796 E=0.5$ & 500 \\
\hline$B e . n$ & 2192 & 1.7 A $9 E-66$ & $1.914 \mathrm{~F}-05$ & 500 \\
\hline 72.0 & $113 n$ & $9.270 \mathrm{E}=0.7$ & $7.3235=08$ & $50 n$ \\
\hline 76.0 & 845 & $7.0096-07$ & $5.537 E .06$ & 500 \\
\hline 00.7 & 364 & 2. SA9E-07 & $2.358 \mathrm{~F}=0 \mathrm{t}$ & 500 \\
\hline .0 & 474 & ค. ค $95-07$ & $.072 F-00$ & $5 n 0$ \\
\hline 88.0 & 1118 & $7.10 \mathrm{dF}=07$ & $.182 E-06$ & 500 \\
\hline 92,0 & $7 R 1$ & 6. 4 त $6 E=0$ ? & $5.061 E=06$ & 500 \\
\hline 96.0 & 297 & $434 E=07$ & $1.923 \mathrm{E}=06$ & 500 \\
\hline 100.0 & 10 & उ2 मE -09 & $; 79 F-08$ & 500 \\
\hline 104.0 & $\underline{3}$ & $3.2 \pi 3 E-09$ & $2.531 \mathrm{E}=0.0$ & 500 \\
\hline
\end{tabular}

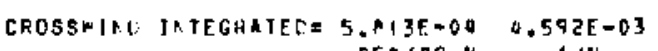




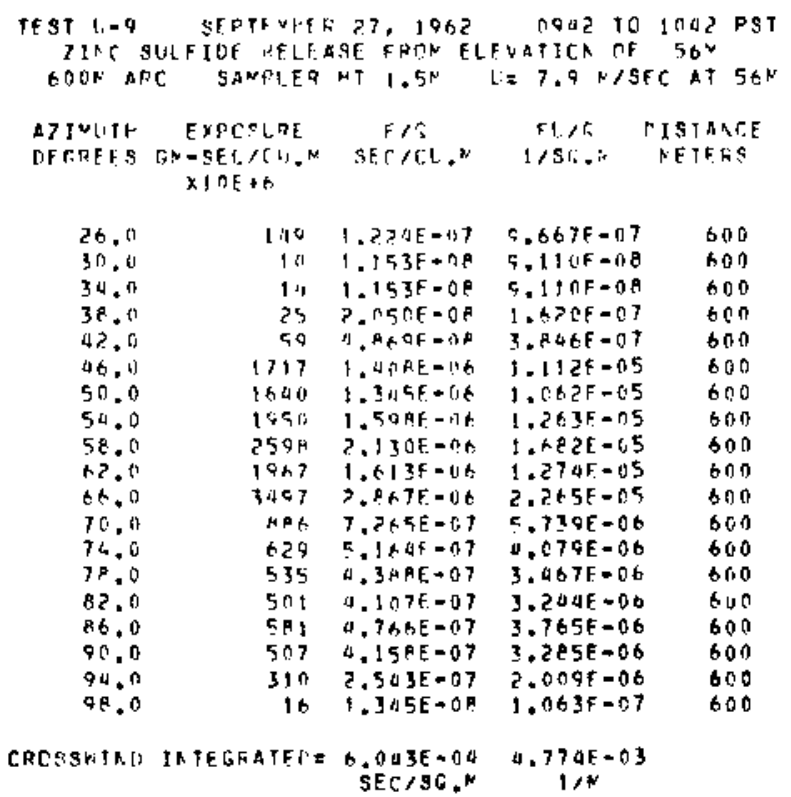

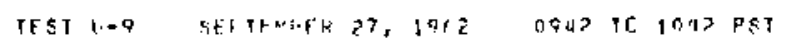
JIHC SULFIUF RELFOSE FDCN FIFUATILP CF 56.

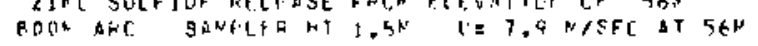

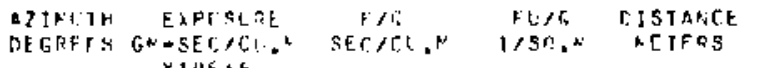
$\times 10 E+8$

\begin{tabular}{|c|c|c|c|c|}
\hline 18.0 & $2 h$ & $? .17 \% 5=0 P$ & $1.721 F=07$ & ADO \\
\hline 22.0 & n & n. nrot +120 & $0.0000+00$ & $A 00$ \\
\hline 25.0 & 5 & $1.4 F 5 E=09$ & $7.5+3 E-00$ & $\operatorname{snn}$ \\
\hline 250 & $1>5$ & $1.025 E-07$ & $A .0 \angle A E=07$ & स०० 0 \\
\hline 31,0 & 457 & $3.7 \mathrm{tAF}=07$ & $\approx .961 F=06$ & Pon \\
\hline 30.0 & $47 !$ & $3,863 E-117$ & $3.0152 E=06$ & 800 \\
\hline 37.0 & 355 & $3.915 f+177$ & $2.3035=08$ & $A \cap n$ \\
\hline 40.0 & 149 & $1.22 H E-07$ & $9 . x \in 7 t=07$ & AnO \\
\hline $4 \geq .0$ & $1 P ?$ & $1.995 E=07$ & 1.1 a af $=0 b$ & AnO \\
\hline 40.0 & 739 & $6.0<1 \varepsilon=07$ & $0.7 A B E=06$ & R०० 0 \\
\hline 45.0 & 15 A 3 & $1, I, \Delta E=D A$ & e. $950 F=06$ & 800 \\
\hline 52.0 & $172 \mathrm{~B}$ & $1.500 E-0.6$ & $1.2 .95-65$ & 900 \\
\hline 55.0 & 2173 & $1.7+2 F=00$ & $1.1108 \mathrm{~F}-05$ & Bon \\
\hline 58.0 & 2551 & $2.071 E=06$ & $1.652 E-05$ & 800 \\
\hline 61.0 & 2791 & $2,2 A F E-D G$ & 1.80 A $=05$ & 000 \\
\hline $\begin{array}{l}4.0 \\
67.0\end{array}$ & $\begin{array}{l}1941 \\
1070\end{array}$ & $\begin{array}{l}1.591 E=0 t \\
A .00 F=07\end{array}$ & $\begin{array}{l}1.257 \varepsilon=05 \\
6.059 E=06\end{array}$ & $\begin{array}{l}\text { BOO } \\
\text { BOA }\end{array}$ \\
\hline 70.0 & 553 & $4.536 E=017$ & $3.5 \times 3 E=06$ & 800 \\
\hline 73,0 & 470 & $3.9276=07$ & $3.102 E=06$ & $8 \cap 0$ \\
\hline 76.0 & 300 & 2. $A B \cap E=\{7$ & $1.9435=06$ & 900 \\
\hline 79.0 & 261 & $? .100 E-07$ & $1.690 E-06$ & $80 n$ \\
\hline A. 0 & 3 स4 & 2. $851 E-07$ & $2.252 \mathrm{~F}-06$ & 800 \\
\hline (25.,0 & 371 & $3.043 f=-6.7$ & $2.40,4 E=06$ & $\sin 9$ \\
\hline 82.0 & 343 & Z.PISE-QT & $2.2276=06$ & $8 \cap n$ \\
\hline 91.0 & 629 & $9.157 E-07$ & 4.07 HE $=06$ & 800 \\
\hline 94 & 206 & $1.691 \varepsilon-07$ & $1.336 F=06$ & 800 \\
\hline & 42 & $3.524 E=08$ & $7 P$ \&F $=07$ & $A D C$ \\
\hline
\end{tabular}

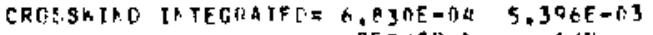

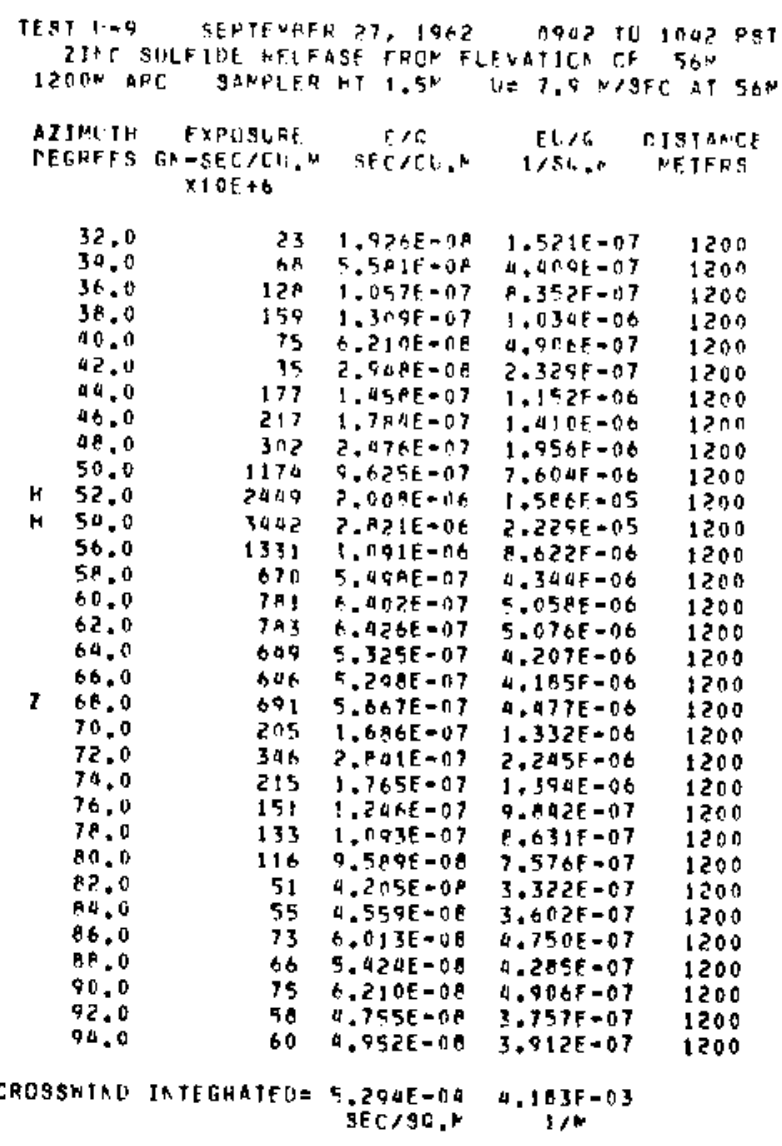




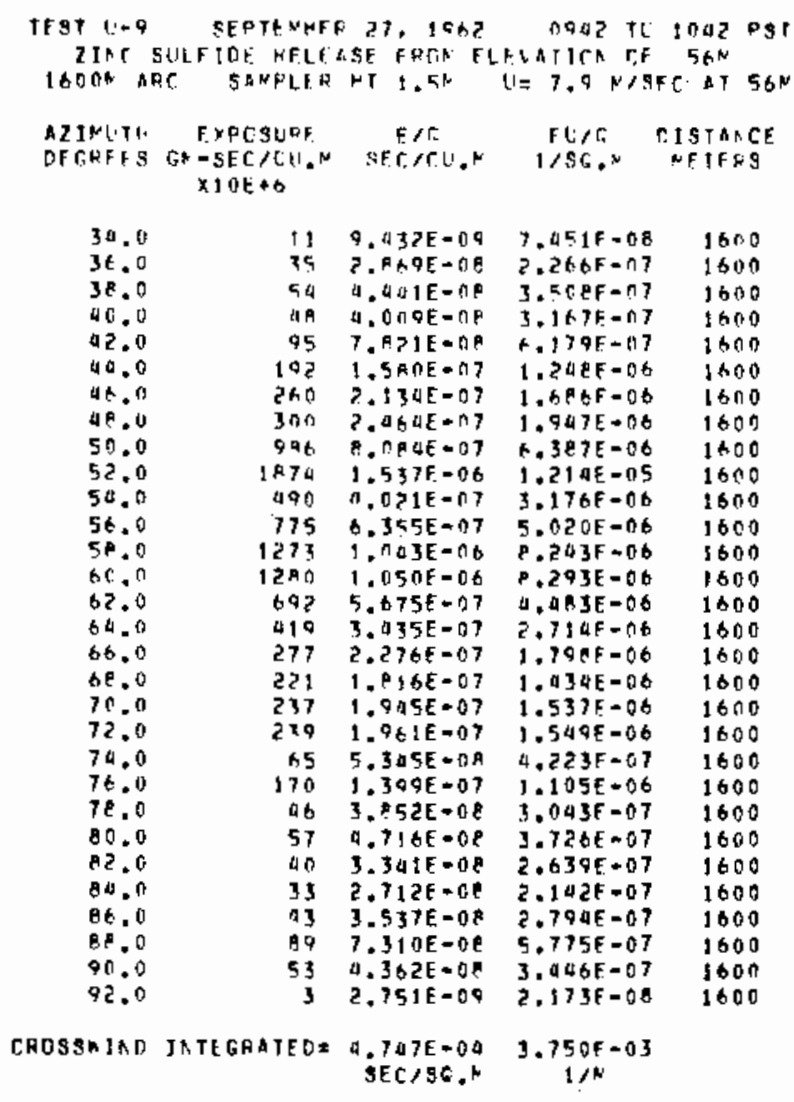




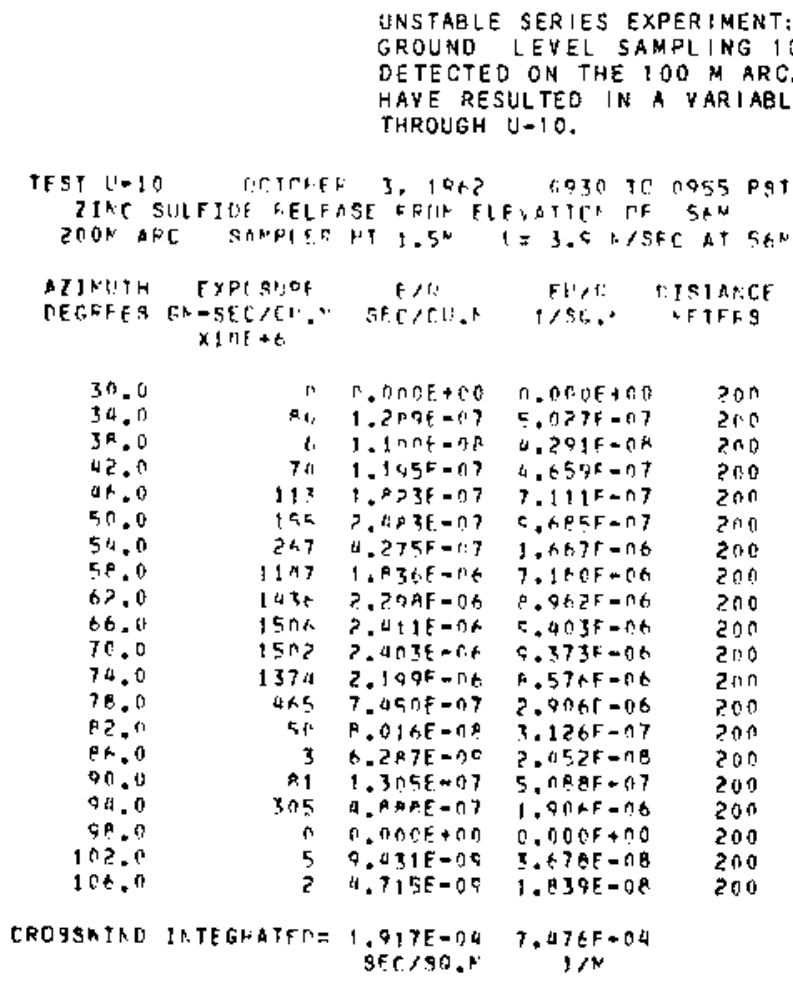

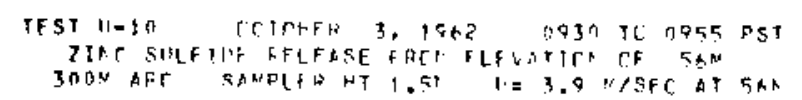

\begin{tabular}{|c|c|c|c|c|}
\hline $\begin{array}{l}\text { AZINUTH } \\
\text { CEGEEES }\end{array}$ & 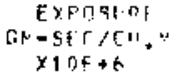 & 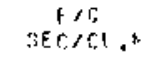 & $\begin{array}{c}F U / r \\
1 / \mathrm{SI} .\end{array}$ & $\begin{array}{l}\text { MISIAREF } \\
\text { NFTFW }\end{array}$ \\
\hline 24.0 & $a$ & 7. $A 59 E-\cap C$ & $3.0,655-05$ & 300 \\
\hline 28.0 & 17 & $\therefore . P>05=0 \mathrm{H}$ & $1.1035=n 7$ & $3 \cap n$ \\
\hline $32 \cdot 0$ & 07 & $1.40 .7 F-n T$ & 9. $.23 F=.77$ & $30 n$ \\
\hline 36.0 & IPAB & $? \cdot 901 F=11 t$ & $1.127 \mathrm{~F}=0.5$ & $3 n n$ \\
\hline $4 n . n$ & 3240 & 5 . I A5F $=01$ & $3.072 E-15$ & $3 n ?$ \\
\hline $\begin{array}{l}44.0 \\
48.0\end{array}$ & $\begin{array}{l}3 \times 31 \\
35 \times 9\end{array}$ & $\begin{array}{l}5.7056=17 t \\
=.7125=0 t\end{array}$ & $\begin{array}{l}2.26 \text { CF }=05 \\
? .2 P F F-05\end{array}$ & $\begin{array}{l}300 \\
3 n \pi\end{array}$ \\
\hline 52.0 & 3875 & $6.2 C 11=06$ & $3.418 F-15$ & $3 \cap n$ \\
\hline $\begin{array}{l}5.00 \\
60.0 \\
64.0\end{array}$ & $\begin{array}{l}1287 \\
5575 \\
5427\end{array}$ & $\begin{array}{l}6.950 E=01 \\
0.971 E=04 \\
8.0 n 5 E=06\end{array}$ & $\begin{array}{l}2.6755=05 \\
3.470 F=05 \\
3.512 E=05\end{array}$ & $\begin{array}{l}300 \\
300 \\
300\end{array}$ \\
\hline 68.0 & $\begin{array}{l}4757 \\
3275\end{array}$ & 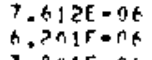 & $\begin{array}{l}2.8605=05 \\
\hat{2} .498 E=05\end{array}$ & $30 n$ \\
\hline $7+0$ & ?द्य & 1. A. $\mid 1 E=0$ & $1.00 \mathrm{PF}=\mathrm{n5}$ & $3 \cap n$ \\
\hline 80.0 & 960 & $1.377 E-0 t$ & $5.37 \cap E-06$ & 300 \\
\hline 84.0 & $3+10$ & $5.054 E-n 7$ & $2.127 f+06$ & 300 \\
\hline A . 0 & $17 n 1$ & $2.72 ? E=06$ & $1.0625=05$ & 300 \\
\hline 92.0 & $?$ & $3.3575=06$ & $1+3 c 9 f=115$ & 300 \\
\hline 100.0 & $\begin{array}{r}2048 \\
773\end{array}$ & $\begin{array}{l}3.277 E=06 \\
1.257 E=06\end{array}$ & $\begin{array}{l}1.27 F E=05 \\
4.224 E=06\end{array}$ & $\begin{array}{l}300 \\
300\end{array}$ \\
\hline 104.0 & 253 & $4.055 E-07$ & $1.5 e 25-06$ & 300 \\
\hline
\end{tabular}

CROBSEISH INTEGFATEDE $1.703 E-03 \quad 6.093 E-03$

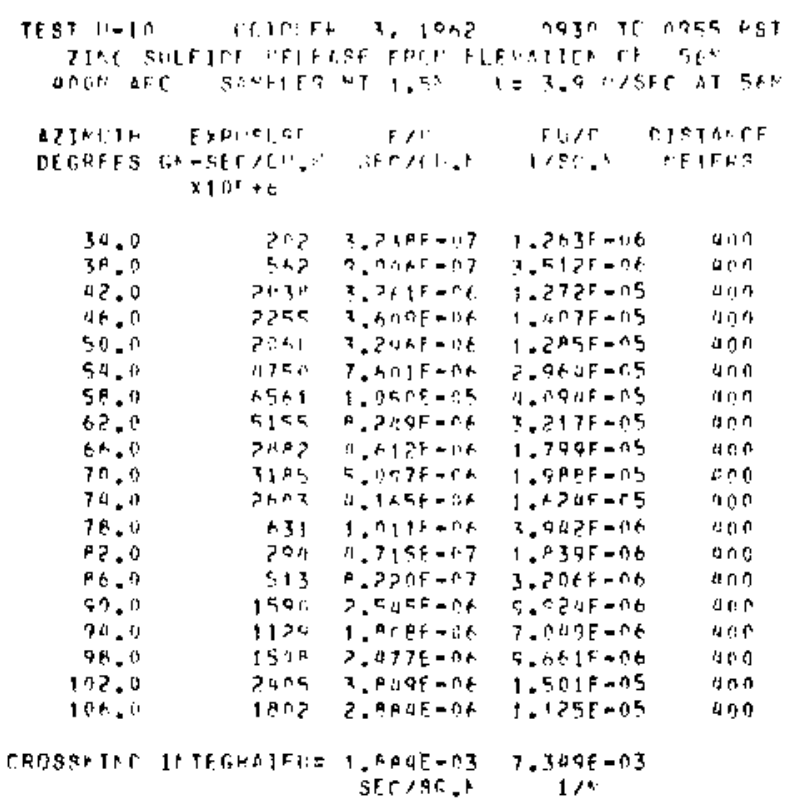

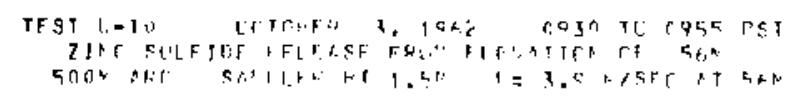

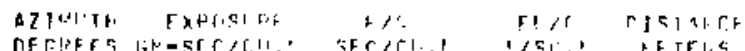

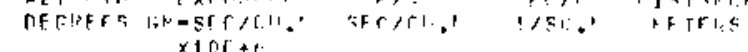

\begin{tabular}{|c|c|c|c|c|}
\hline $3+.6$ & $\because$ & $1.572 E-1: 5$ & $6.13 \cap 5-69$ & $5 n n$ \\
\hline औ & 16,6 & $7 . x<x F=07$ & $1.13 \in 5=\pi t$ & $5 n n$ \\
\hline 44.0 & $12^{x_{i}} 3$ & P.ryOF= ist & P. $.73+=0.6$ & $5 n n$ \\
\hline $4^{p} \cdot n$ & 1 a 5 & $=3 H_{1}, 2 F=\pi$ & $A, P x \mu F=n t$ & $5 r n$ \\
\hline $5 ? .0$ & 2791 & A. $47^{c t}=r . t$ & $1.1156=0.5$ & $5(1)$ \\
\hline 56.0 & $47 \times 2$ & $P=h>P^{\prime}=\ldots+$ & ․ $072 f-05$ & $5 n n$ \\
\hline $\mathrm{na} \cdot \mathrm{C}$ & 74,47 & $5 .+7 t+5=3 t$ & ?.214A - A.5 & $51 \cdot n$ \\
\hline 5.0 & $>4>5$ & य. & $1 .+255=05$ & Fon \\
\hline EP. ${ }^{n}$ & $\overrightarrow{t 101}$ & $2.210 \mathrm{~F}-.3 \mathrm{t}$ & $1.755 \mathrm{x}=05$ & $50 n$ \\
\hline $7 ? .0$ & $71 H_{1}$ & x. 401E $=7 t$ & $1.3615=05$ & 50 \\
\hline $7 \mathrm{~h} .1$ & $17 n \pi$ & $7.7 \times 95=0 t$ & 1. Ittaf - is & ritre \\
\hline $5 ? .0$ & $4>3$ & $0.77+5=17$ & J.A $A 2 \beta=0 \theta$ & $50 t$ \\
\hline P. . . 0 & $5+0$ & $a .915 F-37$ & $3.5 \cap G F=C B$ & $5(1+1$ \\
\hline 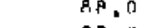 & $5+7$ & $9 . n \times 5 F-n 7$ & $3.5113+-06$ & 5010 \\
\hline 02.0 & 11 d & 1.A3 $3: t=06$ & $7.1355-16$ & $50 n$ \\
\hline $9+.9$ & $15>\mathrm{r}$ & $\vec{A} \| t / \mathbf{Z}=0 \mathrm{~h}$ & $0.53+5=n t_{1}$ & $50 n$ \\
\hline $10 \% .0$ & $10 \times 2$ & $5.1+7 F_{0}=18$ & $1.2+2 t-0.5$ & 500 \\
\hline $10 \mathrm{~s} \cdot \mathrm{n}$ & 1287 & $1.90 G E=0 t$ & $7, J A 5 F=06$ & $5 \cap 0$ \\
\hline wtr. & ] IFGF $\triangle T F I=$ & 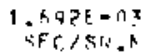 & $\begin{array}{c}6.597 f-03 \\
1 / 4\end{array}$ & \\
\hline
\end{tabular}




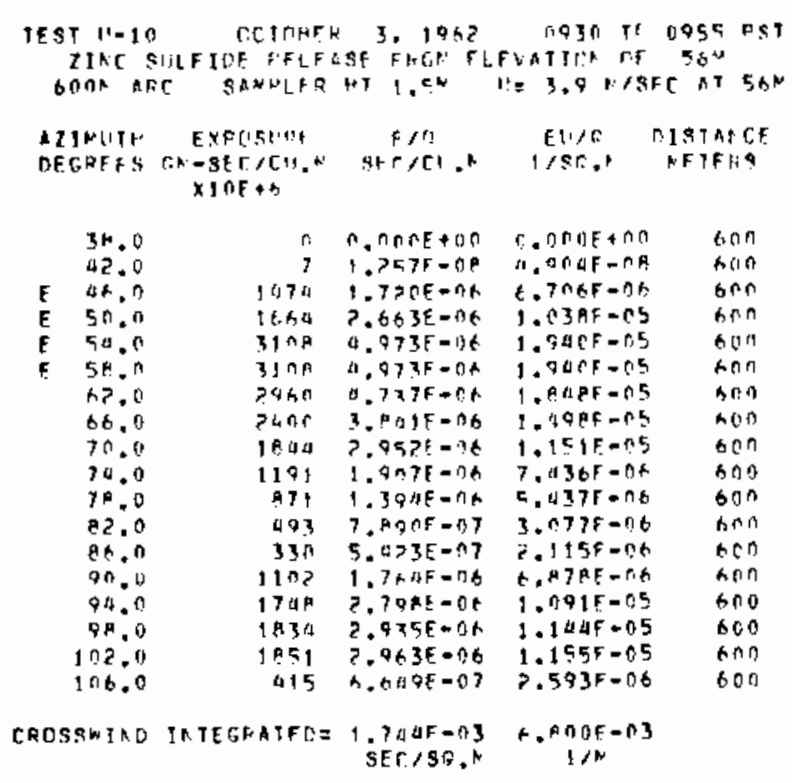

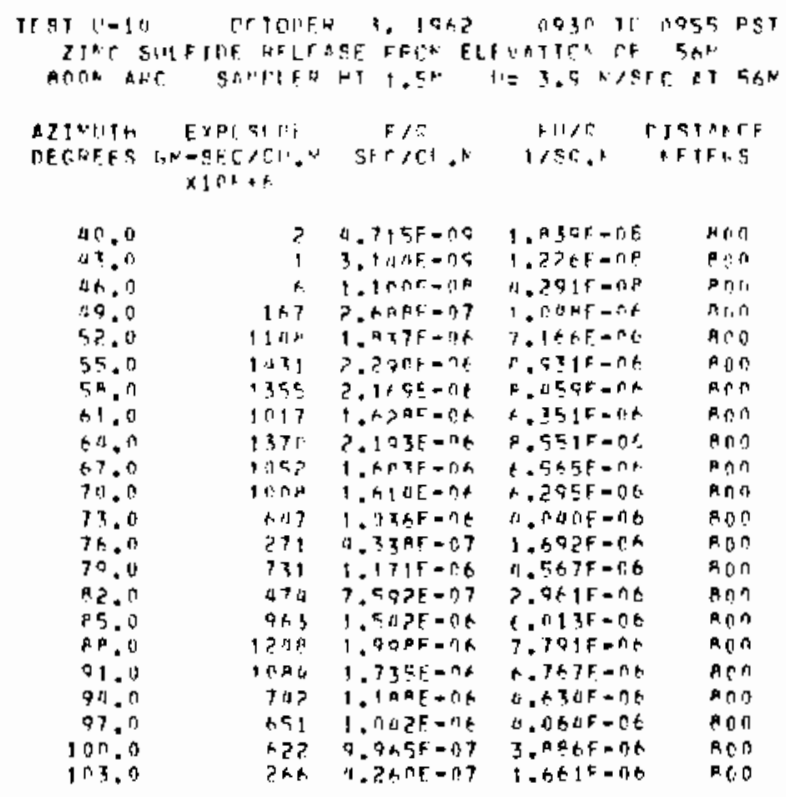

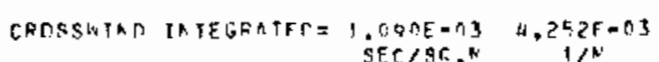

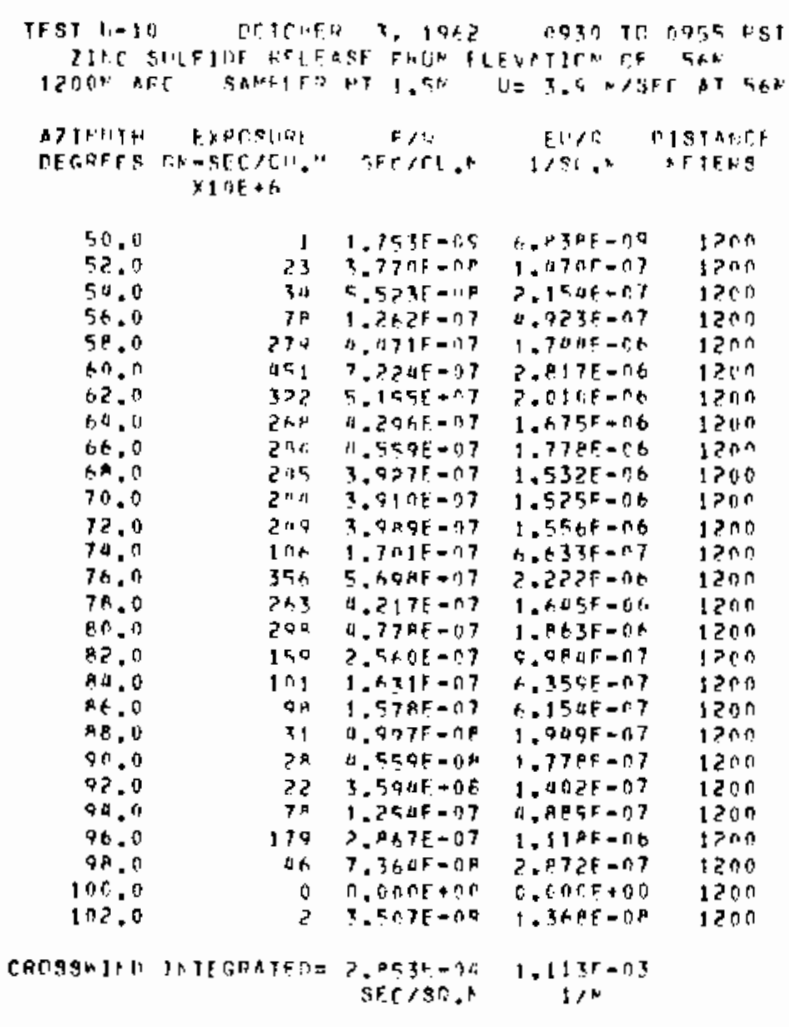

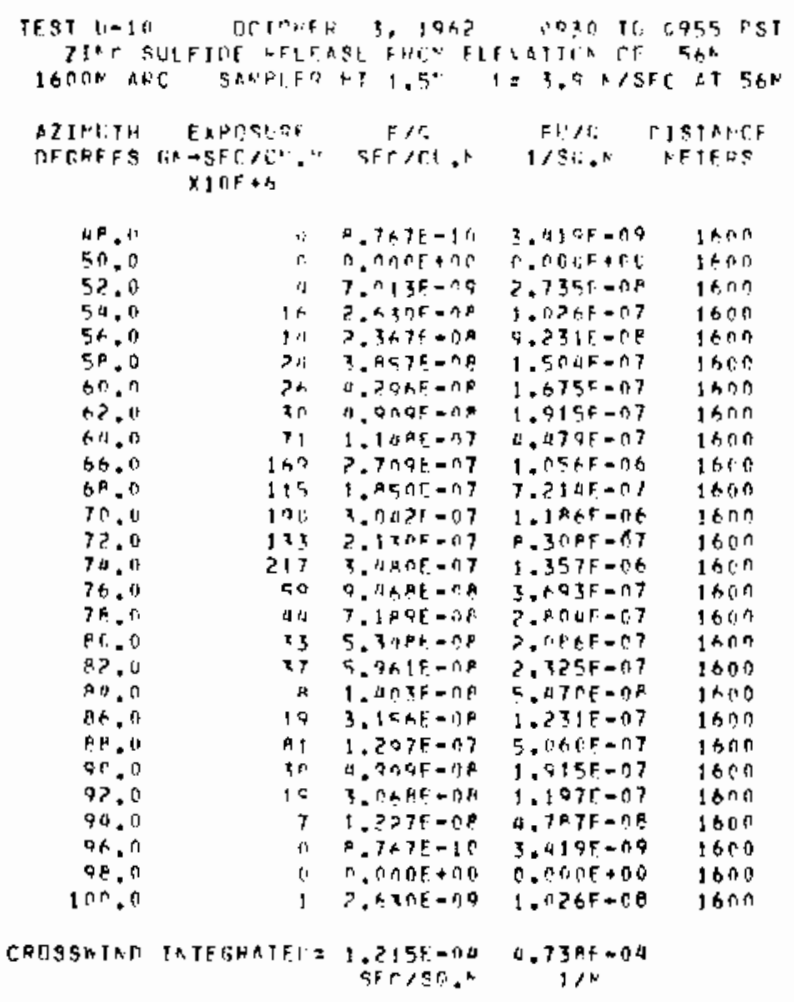


UNSTAELE SERIES EXPERIMENT: $11: U-11 ;$

GROUND LEVEL SAMPLING 100-i600 M ARCS.

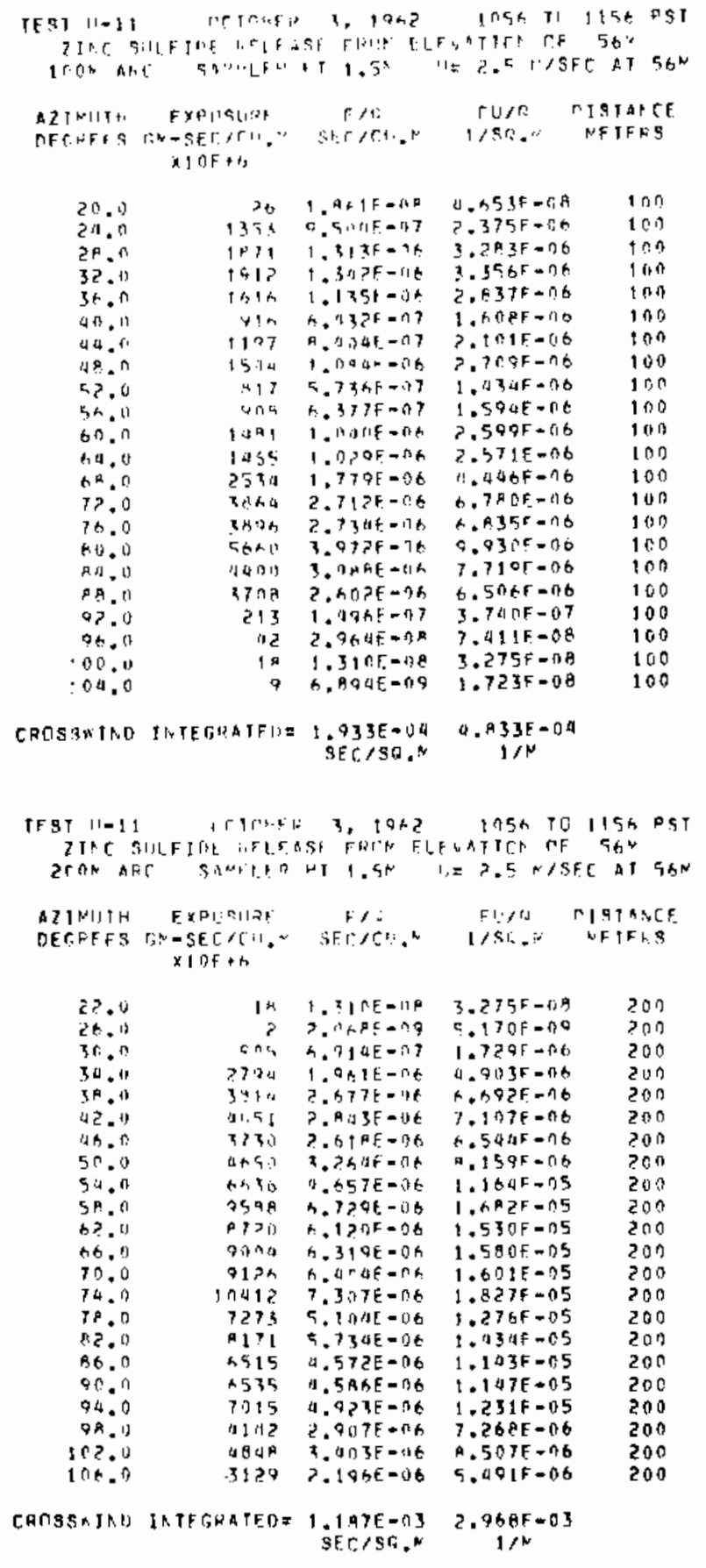

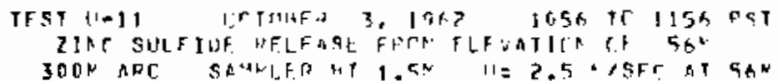

A21R.1JTH EXPITIFF F/: FIIR CIQ1ACF

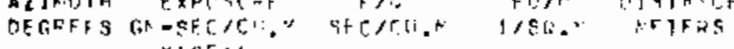
$x i+1:+8$

\begin{tabular}{|c|c|c|c|c|}
\hline 12.0 & 9 & S. $2 \cap \operatorname{ll}=00$ & $1.551 F=0 A$ & $30 \%$ \\
\hline 16.0 & $5 \%$ & $x .51 \leqslant F=0 A$ & $H .790 F=r_{1} \beta$ & $30 \%$ \\
\hline 20.10 & 1 & $5.7>P E-n P$ & $1.430 F=177$ & 300 \\
\hline 24,0 & ५ร & T. Fक $1 E=($ A & $9.551 F=08$ & 300 \\
\hline $2 A .0$ & $\ln ^{n}$ & $7.1015=68$ & $1.1755=07$ & $30 n$ \\
\hline 32.0 & 279 & $1.85,0 E=\{7$ & $4.895+-07$ & $3 \cap 0$ \\
\hline 36.0 & $7>t$ & $5.02 \times 5=07$ & $1.2725=00$ & $36 n$ \\
\hline 40.0 & 1577 & $1,1 n A E=n$ & $2.754 \mathrm{f}=0 \mathrm{t}$ & $3 n n$ \\
\hline a 4.0 & $\geq 3>9$ & $1.774 E=n R$ & $4.436 F=06$ & 300 \\
\hline$\Delta A, 0$ & $\times 2.73$ & $2.277 F+n b$ & $5.743 F=106$ & 340 \\
\hline 52.0 & 3615 & $2.559 E-1 ! 6$ & $n .3965=n 6$ & 300 \\
\hline 56.0 & क3 $35 n$ & $0.457 k-06$ & 1.11 UF $=0.05$ & 300 \\
\hline 60.0 & 45118 & $6.701 E=0 A$ & $1.675 F-185$ & 300 \\
\hline 00.0 & $35,2 ?$ & $6.725 E=46$ & 1.6 . $1 F-05$ & 500 \\
\hline 69.0 & $\ln 718$ & $7.52 Z 2 E-6 \mathrm{~A}$ & 1. 4 A OF $=05$ & 300 \\
\hline 72.0 & $\ln 172$ & $7.1395-36$ & $1.785 \mathrm{~F}=05$ & $3 \div n$ \\
\hline 76.0 & 9701 & $A .1 \cap 7 E=0 t$ & $1.527 c=05$ & 300 \\
\hline 80.0 & 1931 & $3.0 n 1 E-116$ & $A .552 f-0 b$ & 300 \\
\hline 84.0 & 110 ? & $7.7635=0$ & $1.901 F=05$ & 301 \\
\hline AA. 0 & $108 \mathrm{no}$ & $7.45 ? F-0 t$ & $1.913 F=.05$ & 300 \\
\hline 42.0 & 13356 & $9.373 \mathrm{E}=0 \mathrm{~A}$ & $2.34 y=15$ & 300 \\
\hline $9 n, 0$ & I4 $2 \div A$ & $9.9795-106$ & $2.500 F-05$ & 300 \\
\hline 100.0 & คดทด & 5.51 dE $=9 A$ & 1.40 at $=05$ & .500 \\
\hline 104.0 & क141 & $4.310 f=06$ & $1.077 \mathrm{E}=15$ & 300 \\
\hline$S S$ J A I & INTEGLA IELI= & $\begin{array}{c}1.999 E-0.3 \\
S E E, 90.2\end{array}$ & $\begin{array}{c}11.999 E=03 \\
1 / N\end{array}$ & \\
\hline
\end{tabular}

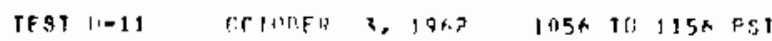

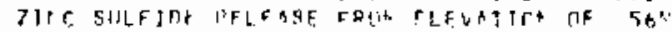

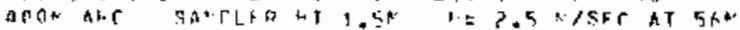

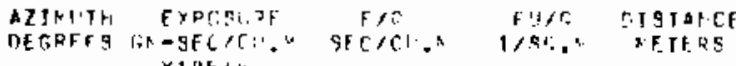
$\times 1$ IIf + .

\begin{tabular}{|c|c|c|c|c|}
\hline $\begin{array}{l}1 P .0 \\
27.0\end{array}$ & 2 & $2 \cdot n+0=00$ & $5.17 \pi F=09$ & Nun \\
\hline 26.0 & 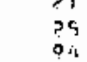 & 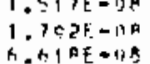 & $\begin{array}{l}3.792 F=48 \\
1.0915+r A \\
1.655 F=07\end{array}$ & $\begin{array}{l}400 \\
4110 \\
400\end{array}$ \\
\hline 34.0 & 276 & 1. पै पKE- U? & $\begin{array}{l}1.655 \mathrm{E}=17 \\
3.619 \mathrm{~F}=07\end{array}$ & 406 \\
\hline $3^{A}=0$ & IVA & $7.597 x=117$ & $1 . A \square Q F=n b$ & 400 \\
\hline 47.0 & $1 R 71$ & $1.213 \mathrm{~F}=16$ & $3.2 A 3 E-00$ & 400 \\
\hline 46.0 & 2189 & $.5 n 15-n B$ & $3.754 F=06$ & $40 \%$ \\
\hline 50.0 & $9 \times 3$ & $5.7635+07$ & $1.691 F=06$ & 4 ही \\
\hline $5 \Delta .0$ & र 41 ? & $395 f-76$ & $87 E=00$ & 400 \\
\hline $5 e .0$ & 4105 & $5 E-n s$ & $7.3635+06$ & 400 \\
\hline 62.0 & 6033 & $1.555 \bar{f}=06$ & $1.160 F-05$ & 400 \\
\hline 66.0 & 50517 & $t E=06$ & 1. $8 \Delta 5 F=05$ & 400 \\
\hline 70.0 & Q 104 & $A \cdot 3$ S $9 E=6 x$ & $1.5975=05$ & 400 \\
\hline 74.0 & $10<19$ & $7.172 E=06$ & $1.793 F=05$ & 400 \\
\hline 78.0 & 12704 & $n \Rightarrow 2 E=n E$ & $2.270 \mathrm{E}-05$ & 000 \\
\hline 0 & 2100 & $1.577 E-0 E$ & - $A 42 F=06$ & 100 \\
\hline 8h. 0 & A 49.9 & $5.7 B 4 E-06$ & $1.491 \mathrm{E}=5$ & 400 \\
\hline 0 & $457 n$ & $4.615 E=08$ & $1.1545-05$ & 400 \\
\hline 94.0 & $57 ? 7$ & $19 E=06$ & $005 E-05$ & 900 \\
\hline$Q R .0$ & 6059 & $25>E=06$ & $1.063 \mathrm{~F}=05$ & 000 \\
\hline 02.0 & 5983 & $190 E=00$ & $1.050 E=05$ & 400 \\
\hline & 3160 & $73 F=06$ & $05 F=06$ & 400 \\
\hline
\end{tabular}

CROSSKIH.D IATFGPATFI $=1.972 E-03 \quad \triangle .930 F=03$ 


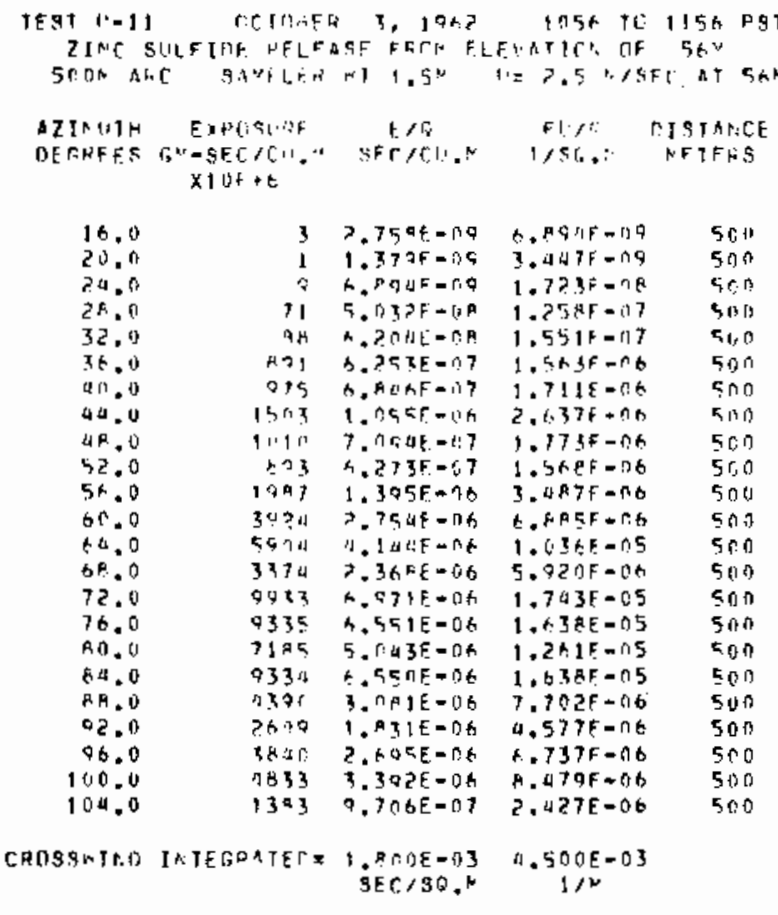

TEST 11-11 LiC.17GFW 3, 19h2 1956 TE 1156 PST

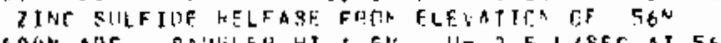

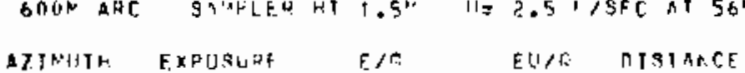

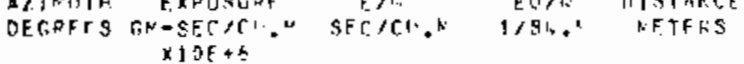

\begin{tabular}{|c|c|c|c|c|}
\hline $\begin{array}{l}18.0 \\
28.0\end{array}$ & $\begin{array}{r}2 \\
20\end{array}$ & $\begin{array}{l}? .06 R F=019 \\
1.4996=01\end{array}$ & $\begin{array}{l}5.17 \text { DF }=09 \\
4.94 \mathrm{HF}=76\end{array}$ & $\begin{array}{l}b \\
b \leqslant 0\end{array}$ \\
\hline $26, n$ & 12 & $\because . a+2 F=19$ & $2.2+10=08$ & AD \\
\hline 30.0 & $\underline{x}$ & $P .413 t=0 A$ & $A .032 E=\square A$ & 600 \\
\hline $34.1+$ & $1>4$ & $A .755 E-O B$ & $2.1 A Q F=07$ & 600 \\
\hline $3 \mathrm{H} .0$ & 5.25 & 1. onat $=07$ & $2.7 C F-07$ & 600 \\
\hline 42.0 & $5: 13$ & $3,9,2 E=117$ & $0.531 F-07$ & 600 \\
\hline 46.0 & 405 & $n .35 \mathrm{hE}=07$ & $1.589 F-n B$ & thos \\
\hline 50.0 & 16.94 & $7.1>1 E=07$ & $1.78 \cap \mathrm{F}-06$ & 600 \\
\hline $\begin{array}{l}54.0 \\
58.0\end{array}$ & $\begin{array}{r}612 \\
1427\end{array}$ & $\begin{array}{l}11.35 .7 E=07 \\
1.359 E=06\end{array}$ & $\begin{array}{l}1.075 E-06 \\
3.394 F-0.6\end{array}$ & $\begin{array}{l}600 \\
600\end{array}$ \\
\hline 62.0 & $42 n 1$ & $2,9 \triangle R E=O A$ & $7.3715=06$ & $\operatorname{son}$ \\
\hline 00.0 & 9613 & $3.7 \times 7 E=1.6$ & P. $1193 \mathrm{~F}=\mathrm{ng}$ & 600 \\
\hline 710.0 & $\cap \cup Q P$ & ?. . $7 \mathrm{MF}-176$ & $7.190,5=06$ & 600 \\
\hline 74,0 & 5375 & $x .7 A d E=0 . t$ & $9.360 F=0 t$ & 1000 \\
\hline 72.0 & 5957 & $a, 1=\dot{U} E=\square t$ & $1.0 .45 F=05$ & 600 \\
\hline 82.0 & $7+27$ & $9.35 P E-0 A$ & $1.3 I B F=05$ & 600 \\
\hline$F+, 11$ & 4,472 & $4.5 n>E-n x^{2}$ & $1.127 E=0.5$ & 600 \\
\hline 90.0 & 1401 & $1.309 E-196$ & $3.4995=06$ & ton \\
\hline 94.17 & 7985 & 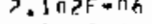 & $2555-06$ & 600 \\
\hline 98.0 & 2477 & $1.739 E-11 t$ & $4.347 E-n E$ & 600 \\
\hline 102.0 & $31: 4$ & $1.505 \varepsilon-0.6$ & $3.762 F=06$ & 600 \\
\hline 106.0 & $4>5$ & $2.985 f-07$ & $7.4635=07$ & 600 \\
\hline & & $\begin{array}{r}1.5 R R E-\{13 \\
\text { SEC } / 9 R, K\end{array}$ & $\begin{array}{c}3.871 f=-93 \\
1 / H\end{array}$ & \\
\hline
\end{tabular}

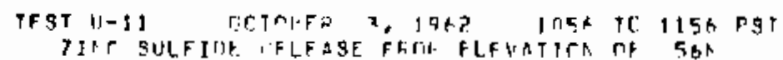

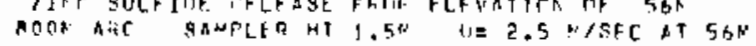

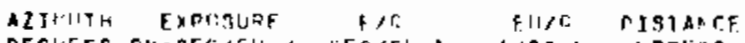

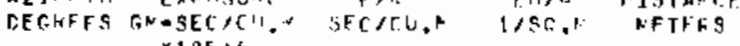
$\times 10 \mathrm{Q}+6$

\begin{tabular}{|c|c|c|c|c|c|}
\hline & 22.0 & $1 \mathrm{~A}$ & $1.3, \cap F-\cap A$ & $3.275 F-0 \mathrm{H}$ & $A \cap 0$ \\
\hline & 25.0 & 7 & $A, P S \triangle E=\cap T$ & $1.723 F=18 A$ & $\operatorname{R\cap 0}$ \\
\hline & $2^{2} \cdot 0$ & 33 & $7.344 E=4 P$ & $5.460 E-13 B$ & $80 n$ \\
\hline & 31.11 & 60 & $\| .905 \mathrm{~F}=1 \mathrm{~F}$ & $1.2205-n y$ & and \\
\hline & 34.0 & 45 & X. จ२०E - 0? & $9.9 ?+5,=8$ & 800 \\
\hline & 37.0 & 117 & ค. $273 F+f R E$ & $2.560 F=07$ & $P \cap 0$ \\
\hline & 40.0 & $2 \cap 7$ & $1.755 E-37$ & $3.6365=07$ & Ron \\
\hline & 43.0 & 297 & $1.9 \cap G E=07$ & $4.519 F=07$ & A\& 4 \\
\hline & 46.0 & $4 \pi x$ & $3 . \ln 98-n 7$ & $7.7735=n 7$ & 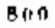 \\
\hline & 60.7 & 251 & $1.7+55-1.7$ & $4.012 \mathrm{~F}-0.7$ & Anก \\
\hline & 52.0 & $3 \cap 8$ & $2.165 E-6.7$ & $5.412 F=177$ & AnO \\
\hline & 55.0 & 252 & $1.772 \mathrm{E}-197$ & $9.424 \mathrm{~F}=07$ & 300 \\
\hline & $5 \rho, 0$ & At? & $5.701 f-n 7$ & $1.425 F=06$ & 800 \\
\hline & +1.0 & 10,7 & $7.2>55=07$ & $1.806 E=06$ & $R_{00}$ \\
\hline & 64.0 & $193 A$ & $1.35 \cap F-76$ & $3 . a \cap C F=06$ & $A 00$ \\
\hline & 67.0 & 3005 & $2.17 ? \mathrm{~F}-11 \mathrm{~B}$ & $5.331 \mathrm{~F}=00$ & Rno \\
\hline & 7 th. 0 & $22 \times 3$ & $1.603 E-106$ & $5.01175-06$ & 500 \\
\hline & 73.0 & 1559 & $1.0 Q \square E=I A$ & $2.737 \mathrm{~F}=06$ & 800 \\
\hline & $7+.0$ & 3055 & $1 . a$ is $2 F-O B$ & $3.605 F=00$ & $0 \cap 0$ \\
\hline & 79.41 & 3552 & 2. $453 E-6 G$ & $4.232 E=06$ & 800 \\
\hline & 22.0 & 2890 & $2.035 E=0.6$ & $5, D P A F-76$ & $A 00$ \\
\hline & 85.0 & 2364 & $1.6 B 2 E=06$ & $6.155 E-26$ & 800 \\
\hline & 88,0 & 1314 & $9.151 E-\pi 7$ & c. $3635=06$ & 800 \\
\hline & 91.0 & 2454 & $2.07 \Delta \varepsilon=176$ & 5. 1 A Q $\angle F=06$ & Pon \\
\hline & 94.0 & $2 Z A B$ & $1.605[-c t$ & $4.012 F=\pi 0$ & BOO \\
\hline & 97.0 & $2 \ln 0$ & $1.479 \mathrm{E}=\mathrm{nb}_{6}$ & $3,699 F=56$ & 800 \\
\hline & 100.0 & 150.9 & $1.059 E-116$ & $2.6475=06$ & 800 \\
\hline & 103.0 & 1243 & A. $659 \mathrm{~F}=177$ & $2.135 F-106$ & 800 \\
\hline & 100.0 & 1001 & $7.307 E-12 ?$ & $1.027 E=06$ & $\ln n$ \\
\hline & 109.0 & 628 & $5 . A) 1 E=107$ & $1.453 E=06$ & $\$ 00$ \\
\hline$F$ & 912.0 & x & $2.551 E=08$ & $6.3775-08$ & $A 00$ \\
\hline & & TESOHATES:= & 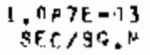 & $\begin{array}{c}2+x 17 F=173 \\
1 / \mathrm{N}\end{array}$ & \\
\hline
\end{tabular}




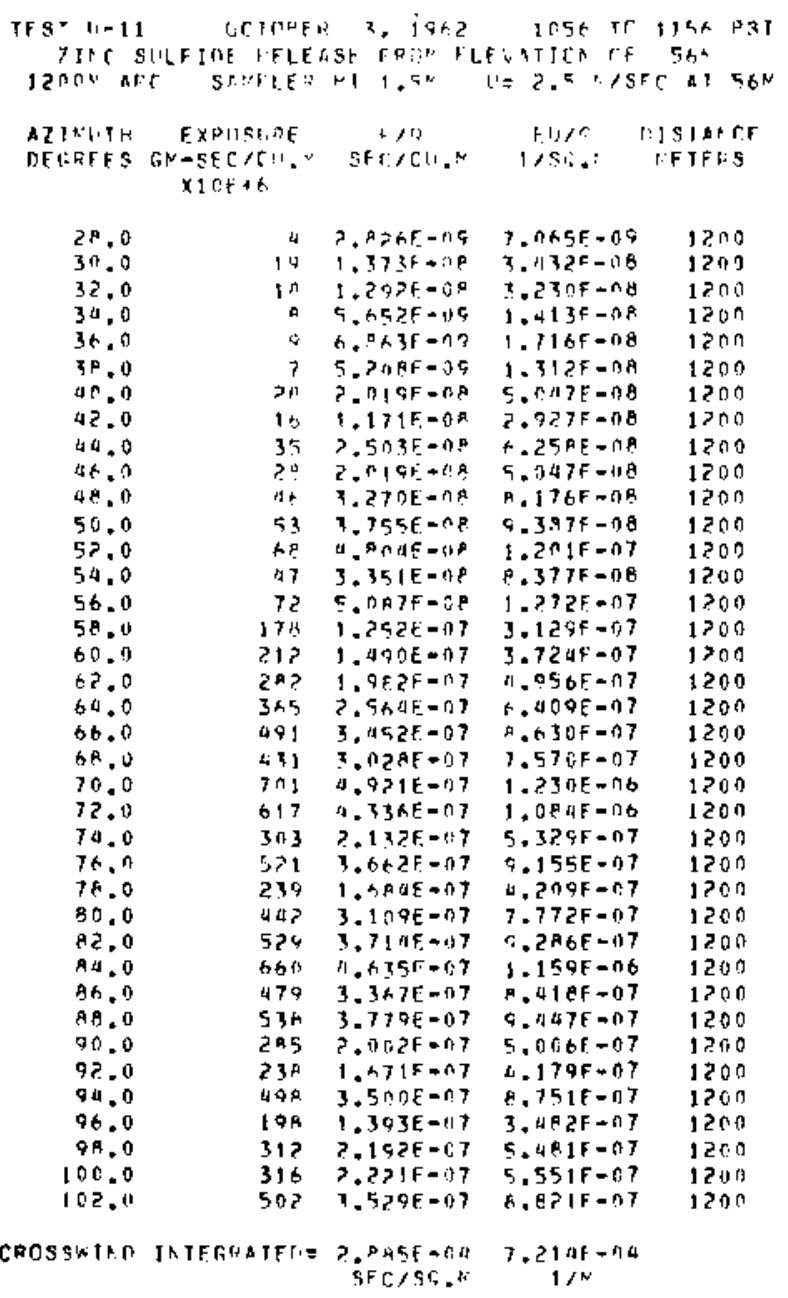

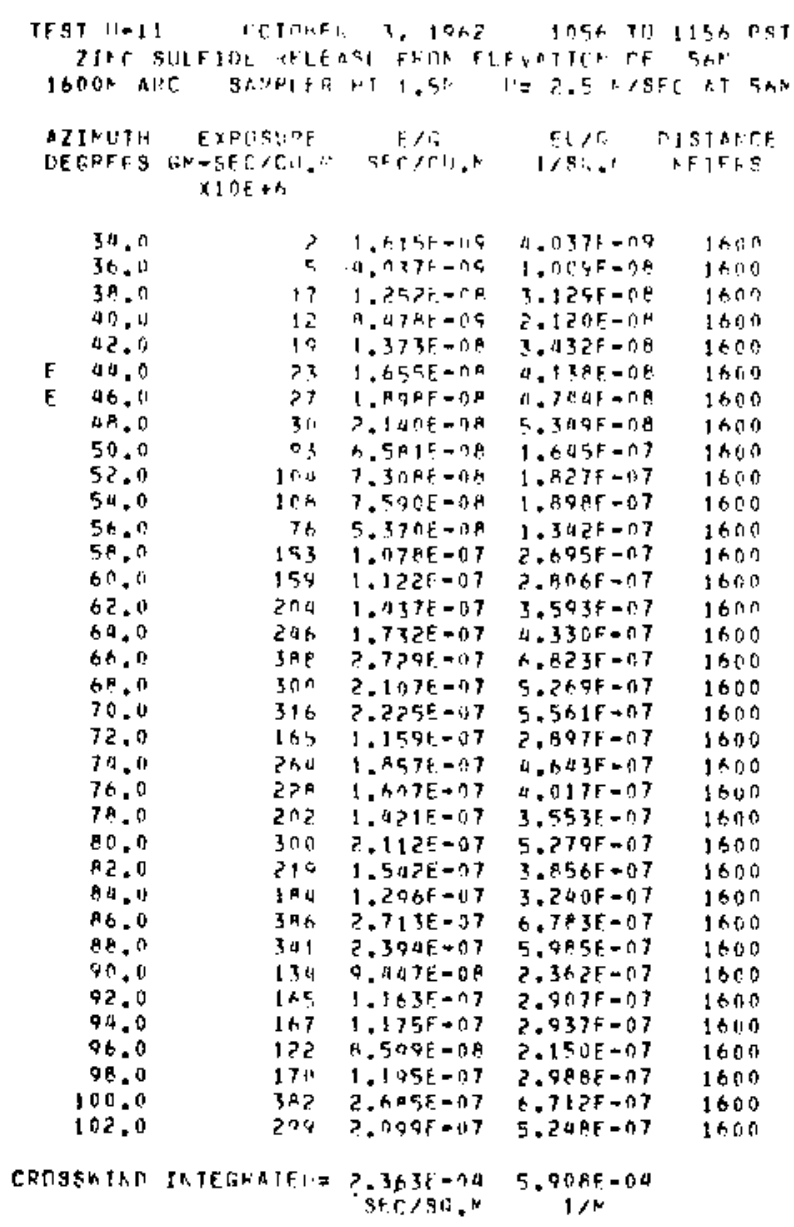


UNSTABLE SERIES EXPERIMENT; $12(\mathrm{~J}=12)$

GROUND LEVEL SAMPLING 100-1600 M ARCS. FOR EASE IN STATISTICAL

ANALYSIS, ALL SAMPLER AZJMUTHS BAVE BEEN INCREASED BY 30 DEGREES

FROM THEIR ACTUAL POSITIONS.

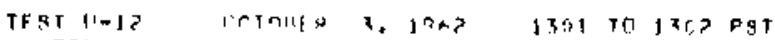

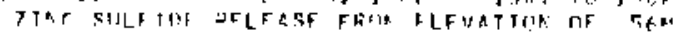

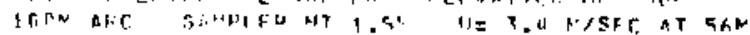

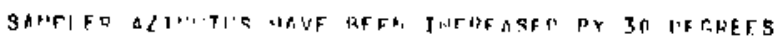

\begin{tabular}{|c|c|c|c|c|}
\hline $\begin{array}{l}\text { ATIFHTH } \\
\text { AETHFFS }\end{array}$ & 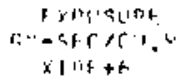 & 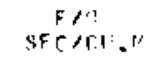 & $\begin{array}{c}\Gamma / 1 / r \\
1 / s_{i}, r\end{array}$ & $\begin{array}{l}\text { MIETAR.EE } \\
\text { MFTFFIS }\end{array}$ \\
\hline $1 \cap .0$ & $4 t$ & $5.16 \Delta 1=.3 n$ & $1.75+F=07$ & $1 n n$ \\
\hline 14.1 & $I^{2} f_{1}$ & 7 notar-ef & 7. $n \geq\{15-07$ & $\ln n$ \\
\hline $1 A \cdot r$ & HIII & $0 . ?+>\mathrm{f}=5.7$ & $3.1425=16$ & $10 n$ \\
\hline 27.0 & 1230 & $1.30 n t=86$ & a). $75 \mathrm{~A}-\mathrm{r}=\mathrm{6}$ & $\operatorname{lon}$ \\
\hline$\dot{c}^{4} .0$ & $20>2$ & $? .301-x^{2}$ & 7. HAOF $=$ nG & 100 \\
\hline 30.2 & $\pi 0_{p}$ & 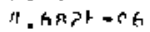 & $1.59>5-05$ & $10 n$ \\
\hline 31.0 & $\operatorname{sen} \pi$ & $t .120 \mathrm{~F}=1 \mathrm{~A}$ & $1 . \operatorname{1n} 25-15$ & 100 \\
\hline $3 A .0$ & $\sin x$ & $n .512 k=2 k$ & $3.2++F=05$ & ln \\
\hline $42, n$ & 7049 & $A, n$ 가 $a f=b$ & $2.73 k F-05$ & 100 \\
\hline यद, n & SF:7 & $1.1>x F=n F$ & $3.813 F=05$ & 100 \\
\hline 50.0 & 10351 & $1.1 P \times c=.15$ & $1 . n>25=05$ & $10 \pi$ \\
\hline 540 & $1 \cap 5 \times 4$ & $1.1+7+=5$ & $3.0675=05$ & $\ln n$ \\
\hline$\therefore 4.0$ & 9310 & $0.503 F=n A$ & I.? $215-05$ & 10 ก \\
\hline$+? .0$ & 7111 & $A, 1>M E=m A$ & $2.77 k 5=05$ & $1 \cap 0$ \\
\hline+2.0 & $55 \times 1$ & $4.3>4+-14$ & $>.15 \cap F=05$ & 100 \\
\hline $76 \cdot n$ & $7+3 t=$ & $R .7>R E=n k$ & $2.067 F-05$ & 100 \\
\hline $7 \unlhd$ & $750+$ & $\therefore .4 n \pi F=n \theta$ & $? .8715=05$ & $\ln n$ \\
\hline 70.0 & 13วว & $5 . n>75=n h$ & $1.7 \cap 35=05$ & $\operatorname{lon}$ \\
\hline A?. I I & $37: n$ & $a .275 F=$ is & $1.054 E-05$ & $\ln n$ \\
\hline H. & $4 ? P_{1}$ & $7.179 \mathrm{~F}-2 \mathrm{~A}$ & 2. 0 U $\} F=05$ & $1 \cap 0$ \\
\hline$\because n .0$ & +117 & $6.971 F=ก$ & $? .3775-155$ & $10 n$ \\
\hline $1 ; 4=0$ & $17 k 3$ & a) $A>B F=0 B$ & $1.1 \times 7 F-05$ & 100 \\
\hline ח & 11997 & $5.213 F-n h$ & $1.723 F+15$ & in \\
\hline 192.0 & lat? & $5.1075-16$ & $1.735 E-05$ & $\ln n$ \\
\hline $10 . t .0$ & $\ln x \pi$ & A. hasF $=11 h$ & $1.5 \times 7 f=05$ & 100 \\
\hline 110.0 & TP1t & $x . \leftrightarrow T+E-1 n$ & $1.2505=05$ & 100 \\
\hline 114.0 & 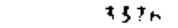 & X.AС QF $=A A$ & $1 . x 1 \geq F=05$ & 100 \\
\hline $11^{2} .0$ & $2 \cap 27$ & $? .317 F=116$ & $7.0795=106$ & 1 ก \\
\hline $1 \geq 2 . n$ & $n \pi 20$ & 5. A $17 F=U E$ & 1.97 AF $=05$ & פח 1 \\
\hline 126.0 & 132,9 & $3.05 A 5=$ th & $1.6 A$ AF $=05$ & $1 \cap 0$ \\
\hline $13 n .0$ & $7+3.3$ & 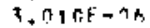 & $1.0235=05$ & 100 \\
\hline $134 . n$ & $15 \mathrm{nn}$ & $1.7 A A F-i A$ & ᄃ.A $>0 F-16$ & $\operatorname{lnn}$ \\
\hline
\end{tabular}

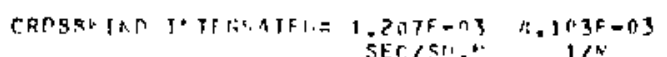

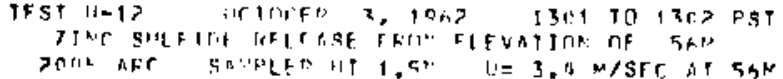

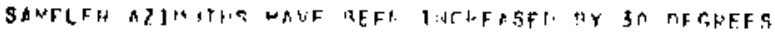

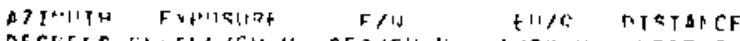

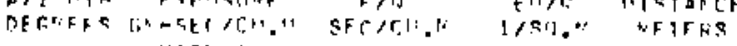
$\times 1 \times 4+6$

\begin{tabular}{|c|c|c|c|c|}
\hline $1>0$ & $H \geqslant 5$ & $9.431 t=17$ & $3.2 n+. F=00$ & 300 \\
\hline 16,0 & $14 z^{2}$ & $1 . h>A F-1) h$ & $5.535 \mathrm{~F}=1 \mathrm{~A}$ & 200 \\
\hline 211.0 & D & $A, A>7 F=2 A$ & $1.5735=05$ & $\operatorname{sen}$ \\
\hline 21.0 & $42^{n} x$ & $7 . n 95 t=06$ & $2.11325=05$ & $30 n$ \\
\hline$P H, O$ & 1725 & $5 . a 13 F-3 A$ & J. $A \Delta \cap F \rightarrow n S$ & 200 \\
\hline $3 ? .0$ & मयन & $7.9975-1 \mathrm{~h}$ & $3.713 F=05$ & 200 \\
\hline $3+.0$ & म55 को & $0.7795-1 h$ & $3.325 F-n 5$ & 300 \\
\hline U. 0 & मenk & D. T795 $=0 \mathrm{n}$ & $3.1 \times 9 E-05$ & ח \\
\hline $4 " .0$ & $15 n$ & 1.7 叶 $=7$, & $5.8 \Delta \cap F=07$ & 200 \\
\hline औค. ก & $11+13$ & 1. . КБคГ-NT & $5.611 F=07$ & $2 \cap n$ \\
\hline 52.5 & 95 & $9 . \operatorname{th} 70=0 \mathrm{~A}$ & $3,321 \mathrm{~F}=07$ & ?חn \\
\hline 54.0 & 47 & $5.3895=98$ & $1 . P 3>F=07$ & $\ln n$ \\
\hline$+\cap .0$ & 7735 & $9, P ? 0 F=16$ & $3.01025-05$ & ton \\
\hline ta. 0 & $1 \geqslant \int^{2} 2$ & $1.392 F-45$ & $0.750 \mathrm{~F}=05$ & $20 n$ \\
\hline$A B=0$ & $7 ? \cap n$ & $9.2365=16$ & z. Bent $=05$ & 300 \\
\hline 72.0 & Quat & 1. DA?F.-75 & 3.67 AF -05 & 200 \\
\hline $7 A .0$ & 7hath & $1 . \ln 20-45$ & 3.74 AF $=05$ & $\geq 0 n$ \\
\hline $8 \cap .1 i$ & 11657 & $1.3 \times 75=135$ & $4.5 \times 0 F=05$ & 200 \\
\hline 50.0 & $11 \geq 31$ & $1 . P \triangle A E=.15$ & $t, 3+4 F=05$ & $2 n 0$ \\
\hline$\therefore A$ & Qวค? & $1.13>F-35$ & $3, A O A F=n 5$ & 200 \\
\hline $7 ?, 0$ & $\times 3 \times 1$ & $7.293 \mathrm{E}=\mathrm{nt}$ & $2.080 F-05$ & 200 \\
\hline on 0 & 5976 & $3.71 \mathrm{GF}=2 \mathrm{~h}$ & $7.2^{2} 35=\pi 5$ & $30 n$ \\
\hline 100.0 & 3 in $\times$ A & $3.979 F=36$ & $1.336 .0-05$ & 300 \\
\hline $1 \times a .0$ & $7+59$ & $3, n 305=76$ & $1.035 \mathrm{~F}=05$ & $7 \cap 0$ \\
\hline $1+a, 0$ & 4271 & $A .8 \times 2 F=A h$ & $1 . t+0 F-n 5$ & 200 \\
\hline $11>0$ & गคс 1 & 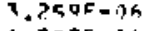 & 1.1 ก AF $\bullet 05$ & $\ln n$ \\
\hline $\begin{array}{l}116.0 \\
120.0\end{array}$ & $\begin{array}{r}1519 \\
479\end{array}$ & $\begin{array}{l}1.737 E=06 \\
5.473 F=17\end{array}$ & $\begin{array}{l}5.905 F=166 \\
\text { PUAF }=106\end{array}$ & $\begin{array}{l}2 \times n \\
200\end{array}$ \\
\hline 120.0 & 1151 & 1.31 AE $=136$ & $4.474 F=06$ & $2 \cap 0$ \\
\hline $12^{A}, 0$ & $? 33$ & P.KT>E-J7 & $0.0 \times 55=07$ & $? 00$ \\
\hline 132.0 & 153 & $1.751 \mathrm{E}-27$ & $5.955 F-07$ & 300 \\
\hline $1 \times x, 0$ & $\mid+1$ & 1. $A_{a 1}=-07$ & $A, 2+0=07$ & ?ח \\
\hline
\end{tabular}

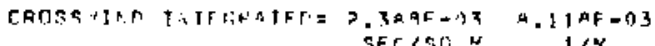




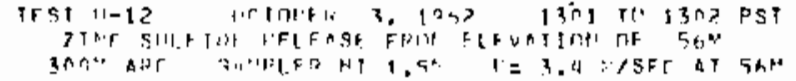

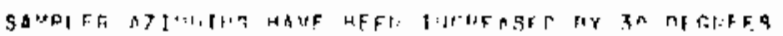

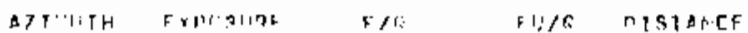

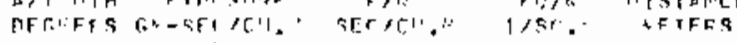
xing $+h$

\begin{tabular}{|c|c|c|c|c|}
\hline $1 \cap .1$. & 5 & $1.2 П>E-71$ & $8.71+46=06$ & $3 \cap n$ \\
\hline 14.11 & 795 & 7. $715 \mathrm{~F}-\mathrm{H}, 7$ & $5.0+5 F-06$ & $3 \cap n$ \\
\hline $1 x^{2} \cdot 1$ & $\sin x$ & 3 poutals & $7.799 \mathrm{~F}=1.6$ & $x \cap 0$ \\
\hline $2 ? . \mathrm{n}$ & כด & $3 . x>55-34$ & $\because 1, x, F=05$ & (n) \\
\hline$=6.11$ & 1 . & ?. nว7k = :h & 7. $1 x(1 F-\cap 6$ & $\sin$ \\
\hline in $\cdot c$ & 1341 & $1.5 \times 4 E=.1$. & $\because .710 F=06$ & 300 \\
\hline 54.0 & 2327 & $3, h \times$ AIE $=14$ & $? . n A 3 F=n B$ & 3 in \\
\hline $3 p=0$ & 1237 & 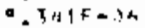 & P.PThF-O5 & 300 \\
\hline ता $>0$ & 716 & A. $A\}$ a $=96$ & JOQAF- 15 & $\sin 0$ \\
\hline Ha. $n$ & bilia & $A, R A \geqslant f-\cdots A$ & $P .333 F-n 5$ & 300 \\
\hline $50, n$ & 7317 & $P, A, A, F=O A$ & $1.3 n 1 t=05$ & $3 \cap 0$ \\
\hline$=0$ & Тни兵 & IJ.35 of -15 & $1.1182 F-15$ & $30 n$ \\
\hline ון . מ & lish? & $A . A B P F=\Omega A$ & $1.570,5=15$ & $x+4$ 形 \\
\hline m? & $3 \&<2$ & $11.313 f=3 x$ & $1.5 n$ J $F-05$ & $30 n$ \\
\hline$t: t, 0)$ & $7 \times a 9$ & 3.07 af $=16$ & $1.045 F=05$ & 300 \\
\hline 70.0 & 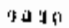 & $5.0754=n x$ & $1.725 \mathrm{~F}=05$ & 200 \\
\hline 74.11 & 5125 & $n$, ?, SกF $=n g$ & $3.135 F+05$ & $3 \pi n$ \\
\hline 18.6 & $\operatorname{l7\cap A}$ & $R . A 1, T=3 a$ & $2.905 F=175$ & $3 n 0$ \\
\hline P2. 0 & $r_{H}$ ild & $A .7 \times>C=3 A$ & $3 . \cap U Q F-\cap 5$ & $3 \times 0$ \\
\hline$R A$. & 4919 & $7.1515 .3 n$ & $7.523 \mathrm{~F}=05$ & $30 n$ \\
\hline $2 n .0$ & 1511 & $5.1575=1.6$ & $1.753=0.05$ & 300 \\
\hline 51.0 & 131 \& & $7.9 \times 5 F=n A$ & $1 .$, 1. AE $=05$ & tho \\
\hline $8 \times .0$ & $10+?$ & $1.9+2 F=36$ & A. $5 n 1 F=0 h$ & $x \cap 0$ \\
\hline $10 ? .0$ & 1587 & 1. $5 A 5 F=n A$ & $5.390 \mathrm{E}-00$ & $3 \cap 0$ \\
\hline 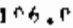 & $790 A$ & 1. $3>45=06$ & 1.13 Of $=05$ & 307 \\
\hline 110.0 & 3254 & $2.5795=10 \mathrm{~h}$ & $A .7 B A F=06$ & 3 in $n$ \\
\hline 194.0 & 555 & A. $10 \times 7 F=07$ & $2.1995=06$ & 300 \\
\hline 11 ค. 0 & 77 & $A, A+O E-1) A$ & $x, 01$ B $=-07$ & $30 \mathrm{~A}$ \\
\hline+22.0 & 910 & 1. $.145 F=96$ & $3.550 F=06$ & 300 \\
\hline 126.0 & $5>0$ & $A, D, A F=7$ & $D, N A B F=N B$ & $30 \mathrm{n}$ \\
\hline $33 n$ & $7>3$ & $9.7+35=117$ & J. $A \cap Q F-O B$ & $3 n n$ \\
\hline 132 & 520 & $A_{-}\left\{A_{1}, F=n T\right.$ & $3, n 9+5=0 B$ & $\operatorname{lon}$ \\
\hline
\end{tabular}

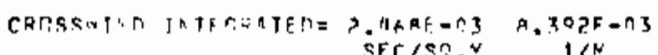

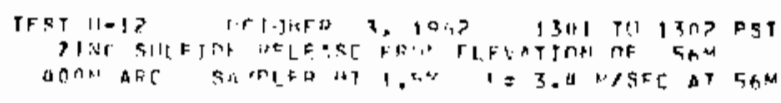

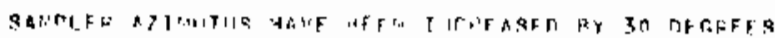

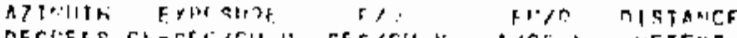
$x+1,+0$

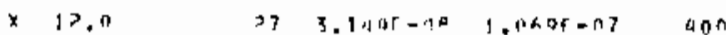

$x \quad 20.0$

$5 \%, 0$

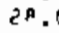

3.2 .0

36.8

16.0

4.

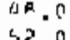

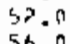

60.0

1. 4.0

$1,8.0$

$7 ? .0$

76.0

หก. 0

A. 10

Ha. 0

72.1

$96, .9$

110.0

$1 \cap 4.0$

1 คค. 0

117.0

$11+.0$

120.0

124.0

12 ค. 1

132.0

$35 \quad 3.1405-10 \quad 1.0605=07$ 40

3) T.917F-10 1.20AF-07 HOB

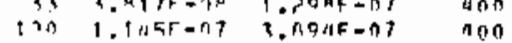

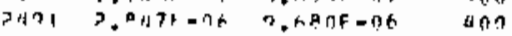

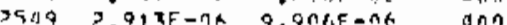

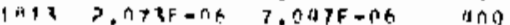

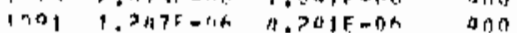

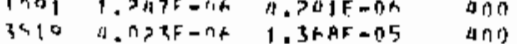

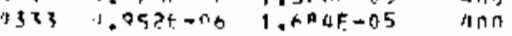

3737 :. $128 \mathrm{~F}=A 6$ 1. fIRQF-05

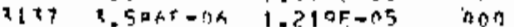

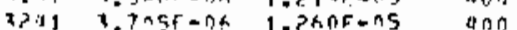

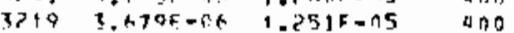

$19>4>1974-145$ 7.J78F-06 300

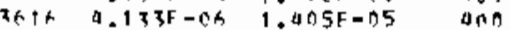

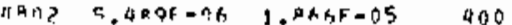

G>5A T.153F-nA P.A3PF-05 $A 00$

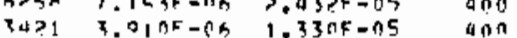

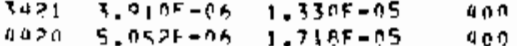

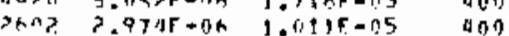

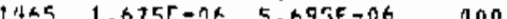

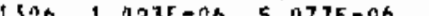

879 I.005F-76 3.016F-06

$1942,730 F-06 \quad 7.547 F=06$ ano

Q13 $0.991 F=07 \quad 3.393 F-06 \quad 400$

$317 \quad 3.626 F=07 \quad 1.333 F+16$

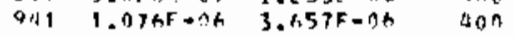

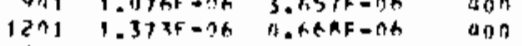

8QA $5.023 F-n h \quad T .377 E-n B$ 000

$1116 \quad 1.277 E=16 \quad 4.340 F=06 \quad 400$

$1191 \quad 1.351 \mathrm{~F}-36 \quad 4.5975=06 \quad$ non

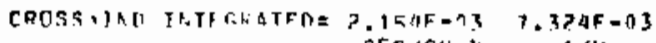

$1 / \mathrm{m}$ 


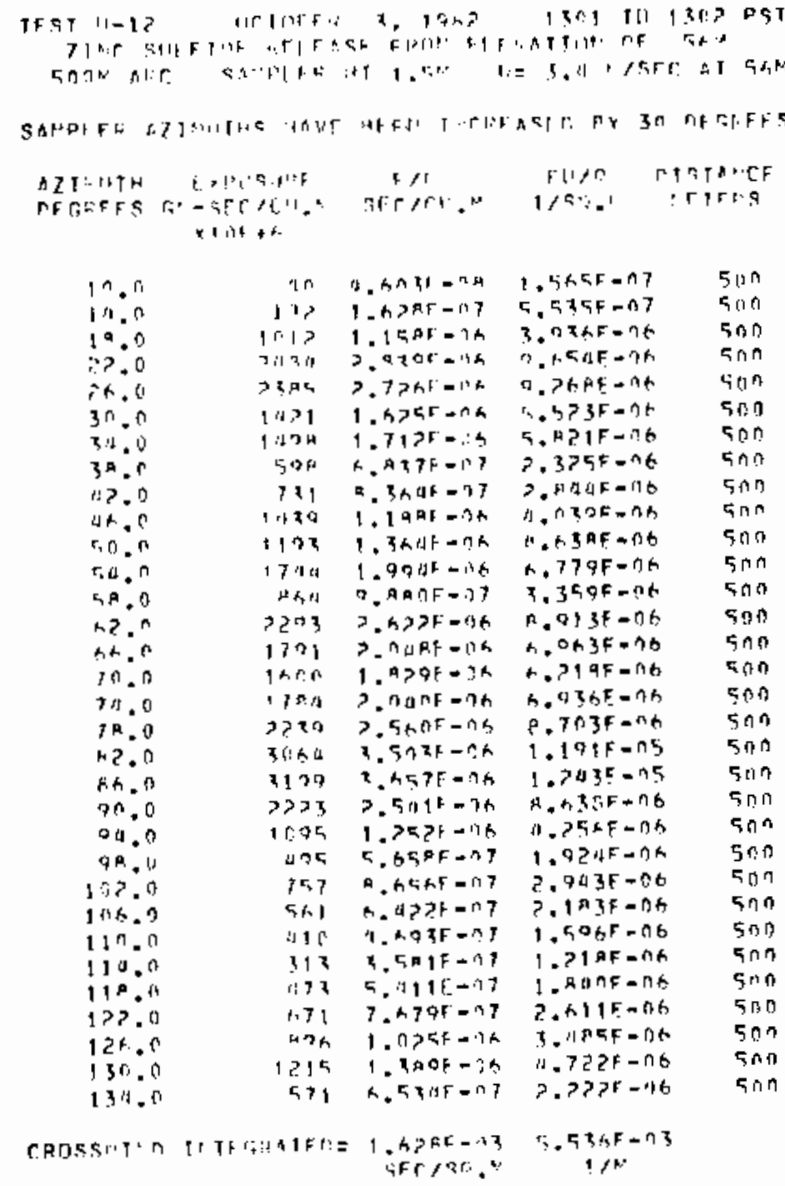

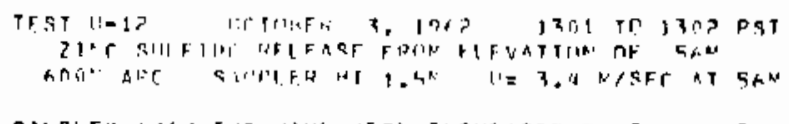

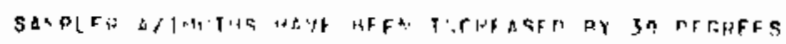

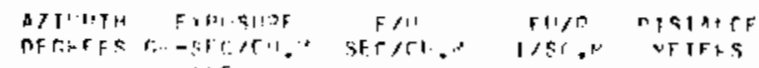
rin $1+5+1$

\begin{tabular}{|c|c|c|c|c|c|}
\hline & $+2 . n$ & 3 & $3.7 \cap 51=n R$ & 1. PAns $=\cap 7$ & $40 n$ \\
\hline & $1+.0$ & 54 & H. $2 A 7 F-11 A$ & 7.1 Kaf -07 & Mat \\
\hline & $? n \cdot n$ & $3 \cap ?$ & 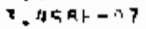 & $1.17+5=176$ & 601 \\
\hline & ¿1 0 & ya & - - द厶كF-ก7 & $\therefore$ OnOF-nA & $\operatorname{tin}$ \\
\hline & $>9.11$ & $321 x$ & $7.5>0 F-9 B$ & A. $6 \cap \cap 7=06$ & $\operatorname{tin}$ \\
\hline & 37.61 & $1 x>P$. & 1. $A h 1 F=n a$ & $5.3296=110$ & $A D O$ \\
\hline & Sh. 0 & 1212 & 1 . IJAF $=7 \mathrm{H}$ & $1 .+4555=06$ & $3 \cap 0$ \\
\hline & 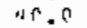 & 354 & R.APF-17? & ?. Qx? $5-6 x$ & סמא \\
\hline & stis, & 477 & $5, \|=65-177$ & 1. & $\sin$ \\
\hline & is n. (1 & 590 & $A .175 E-H T$ & ?. NOCF=nG & $\operatorname{snO}$ \\
\hline & 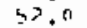 & $1+20$ & $1.635 F-n h$ & $5.55 H F=n h$ & and \\
\hline & 54.13 & A 55 & $0.503 t=137$ & $3.305 F=96$ & hon \\
\hline & nn.? & 041 & 1. AnOF $-1 A$ & $3.7 \times 7 E-n E$ & $60 n$ \\
\hline & his. & tentid & 1. $A 731=3 \mathrm{~A}$ & $0.396 f=n t$ & A. 10 \\
\hline & ar. 0 & $1 \ln p$ & $1.153 t-n b$ & $3.327 F=06$ & $A=D$ \\
\hline & 13.0 & 1078 & $1.109 F=0 A$ & $s=A>7 F=n x$ & $\operatorname{mon}$ \\
\hline$x$ & in. $n$ & 24 & 1. $n+A F=n T$ & $3 . h+\Delta F=n$ ? & $n \in n$ \\
\hline$x$ & ar. & $? 05$ & 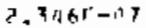 & $7.978[-07$ & ton \\
\hline$x$ & 8 il . 0 & 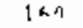 & $1 . A \zeta A F=0\}$ & h.P>? $=0$ ? & $6 \cap 5$ \\
\hline$x$ & 48,7 & 24 & $\Rightarrow .35 A F-5 R$ & $\therefore, \cap 1 A F=\cap A$ & $\sin \pi$ \\
\hline & $7 ? . n$ & $11 \times 7$ & $1.3140=5 \mathrm{~h}$ & $\| .535 F=n 6$ & hกn \\
\hline & $9+.11$ & $k+7$ & $7.6\rangle\langle F=07$ & $? .502 F=? h$ & 609 \\
\hline & 1 nn." & 507 & $5, \operatorname{RndF}-\Omega 7$ & $1.27 \times F=06$ & $\operatorname{son}$ \\
\hline & 1 fiti. 1 ? & $23 n$ & $7.7 \cap A F=17$ & $0.109 \mathrm{~F}=07$ & $\sin$ \\
\hline & $\ln \cdot n$ & र५ว & $4.0 \mathrm{NE}-\mathrm{f:7}$ & $1.370 F=n t$ & 400 \\
\hline & $11 ? .0$ & $1 \times 7$ & 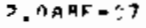 & $7.100=-0.7$ & 600 \\
\hline & $11 \% .0$ & 2 ant & $3 . n A 3 E-27$ & $1.034 F-n t$ & 600 \\
\hline & $1>n, n$ & 657 & $7.5115=0.7$ & 2.55tr $=00 h$ & $\operatorname{sen}$ \\
\hline & 170.0 & 556 & $A .366 F=07$ & $P . \$+\$ F+n 6$ & 6019 \\
\hline & 127.0 & 1070 & $1 .>3 \pi F=16$ & $4.105 F-3 x$ & An \\
\hline & 153.5 & 517 & $7.3005-n 7$ & $? .51+E-n t$ & $\operatorname{ann}$ \\
\hline & $1 ?+.0$ & $1+1 / 4$ & $1.65 \cap F=17$ & $5.611 F=07$ & his? \\
\hline & & $:=$ & 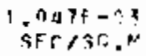 & $\begin{array}{c}x .5+1 F-n 3 \\
1 / N\end{array}$ & \\
\hline
\end{tabular}




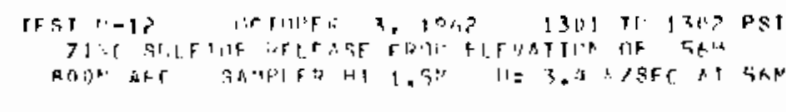

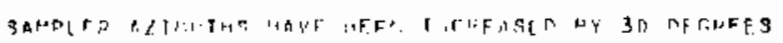

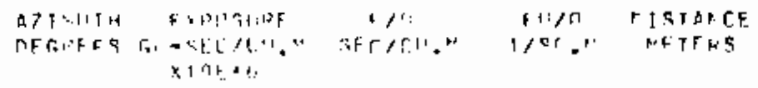

\begin{tabular}{|c|c|c|c|c|}
\hline 10.0 & 18 & 1. Innt $=n$ s & $1.382 F-n \theta$ & A $\mathrm{a}(\mathrm{O}$ \\
\hline 13.0 & 11 & $1,3 a 75=0$ & $(1.5,215=3 A$ & ann \\
\hline $1 \mathrm{H}$. & Gh & $4.51 ? E=18$ & $7.2 !$ is $5=n 7$ & $A 00$ \\
\hline $10 . n$ & id & $5.75,75-48$ & 1.71 PF-n & Aก \\
\hline 27.9 & 120 & Y. AGAF $=17$ & $7.12+5=07$ & ann \\
\hline 25,0 & けフ & $3 . ? 215-77$ & $7.5905-07$ & $\operatorname{Ann}$ \\
\hline $2^{2} .8$ & $15 t$ & $1.7 D h 5-1.7$ & $\mathrm{n}, 107 \mathrm{f}=07$ & Rin \\
\hline $31 . n$ & 277 & $5.1+4+5=7$ & $1,07 t, F=n 6$ & Pro \\
\hline $3.4,11$ & 47 & K. $+19 E-n)$ & P. $? \mathrm{UUPF}=\mathrm{AN}$ & 200 \\
\hline 37.8 & 417 & $0.36 \times 5-17$ & $3.1^{A}:=5=0$ & at 0 \\
\hline $\operatorname{lin} .0$ & 517 & $5.9 A \cap E-A 7$ & $1.903 F=60$ & $\operatorname{Ann}$ \\
\hline$\because 3.7$ & 277 & * & $1.15>F=n 0$ & ann \\
\hline ita & 201 & $3.6715=117$ & $1.748 F-06$ & ann \\
\hline$=17.0$ & $2 \pi k$ & $x .731+=61\}$ & $1.031 \mathrm{~F}-n \mathrm{t}$ & D n \\
\hline 53.0 & $\sqrt{2} \pi$ & tt $.917 F=17$ & $1.672 F=0 B$ & 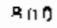 \\
\hline 55,4 & $2 \uparrow \%$ & $2.7176[-97$ & $7.5 n 55-\pi 7$ & $817 n$ \\
\hline 59.6 & $? 2,3$ & $3.215 F=17$ & $1,103 F=0.6$ & $\left.x_{1}\right)$ \\
\hline का. & 㑊 5 & $5.007 F=01$ & $1.723 F-0.6$ & 800 \\
\hline $6,1,0$ & $1 n$ & $5.7>\mathrm{mF}-197$ & $1.947 F=06$ & $\operatorname{ann}$ \\
\hline 6.0 & 314 & $3.7 \Perp 1 F-n 7$ & $1.300 F=n \theta$ & $=0$ \\
\hline $7 n . ?$ & 534 & $5.1 \cap ? P_{-}-27$ & $2.0775=10$ & $\operatorname{Rnn}$ \\
\hline $7 x .0$ & $1 \times 9$ & $A^{2} .15 \mathrm{a} F-A 7$ & $? \cdot D^{D} T A F=n h$ & $\operatorname{Arn}$ \\
\hline $7 n .0$ & 937 & $1 . B>15=06$ & $3.0125=06$ & $R_{1}: 0$ \\
\hline 79.0 & $7 \% 4$ & $0.0875=07$ & T.DARF-DG & $A \div 0$ \\
\hline 27.0 & ! na $x$ & $1 . ? \geq P F-\pi x$ & $11.210, F-n 6$ & an \\
\hline 45. 0 & 300 & $1.13 A E=A B$ & $3,055 F=176$ & मn० \\
\hline 20 & 430 & $5.4 .7 \times F=07$ & 1. AA IJF $=06$ & $a+0$ \\
\hline$? 1.7$ & Eanto & a. acht -17 & $\therefore .195 F-16$ & 800 \\
\hline Q4. 0 & than & $7, A \cap 15=n$ & $P .50 a r=n h$ & $\min 0$ \\
\hline 37.11 & 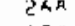 & $3 . n_{4} 5 F=17$ & $1.154>F=06$ & ron \\
\hline$r, n, 0$ & 171 & $? .27 x F-17$ & $7.5585=07$ & aco \\
\hline $\ln x \cdot 0$ & 250 & ?. $9 \times n=07$ & $Q .9 \times 7 F=n 7$ & $R \cap 0$ \\
\hline 116.0 & $3 \mathrm{~A}$ & $1.1 \cos =07$ & $2.7+1 \mathrm{~F}=07$ & Ano \\
\hline 100.0 & $1 ? 1$ & $1.30>5-77$ & $a .7335=07$ & an \\
\hline 112.0 & 117 & $1.347 F=07$ & ".5ค1F-n7 & ano \\
\hline 115.0 & 372 & a. $2555-117$ & $1.11475-176$ & $\cos$ \\
\hline $11^{R} \cdot n$ & $2 \cap \mathrm{d}$ & $2.325 F=17$ & $7.94115-07$ & मดกn \\
\hline $1 \geq 1.0$ & 247 & $\operatorname{I.nE} 15=07$ & $1.73 A F=06$ & $\operatorname{ann}$ \\
\hline $124 \cdot n$ & 22 & $? .6 x 75=17$ & $\pi \cdot \theta>>C=n 7$ & Ann \\
\hline 177.7 & $3 \times 3$ & $4.37 \% f-07$ & $1, H A \cap F=n b$ & 2na \\
\hline $13 n=$ & 100 & $3.7 .70 \mathrm{~F}-177$ & $7.747 \mathrm{~F}-177$ & $A \cap n$ \\
\hline 132.9 & $\ln 5$ & $1,2>5, F=1.7$ & $11.1895=17$ & 200 \\
\hline 36.9 & $? \geq 1$ & $? .5>61=5 ?$ & $P .5 P Q F-n 7$ & ${ }^{2} \mathrm{c}$ \\
\hline & $? 57$ & $7.7415-107$ & $1, n \cap a F=n g$ & \\
\hline
\end{tabular}

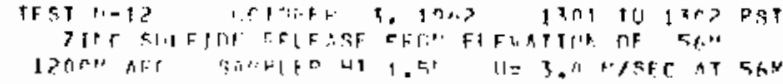

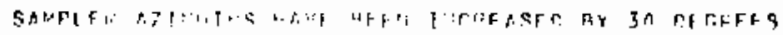

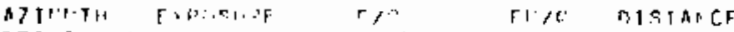

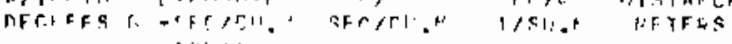
alitith

\begin{tabular}{|c|c|c|c|c|}
\hline $3 n .0$ & 3 & $1 . n 5,2 x-n 7$ & $3.5375=07$ & $1>4 n$ \\
\hline $3>0$ & 55 & 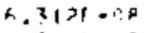 & $?, 10+F=n 7$ & IPAC \\
\hline & 50 & $A .3+>R-! A$ & $2.14+5-n 7$ & $1>010$ \\
\hline $3 h .0$ & 77 & $\Rightarrow$. $x: \cap n=n \theta$ & $1.475 F-n 7$ & $12 \cap \cap$ \\
\hline 3.0 & 38 & $7.7971=08$ & $2.5311-07$ & $12 \ln$ \\
\hline$\pi r .0$ & 113 & $1.3 n>8=.7$ & a. $M S A F-n]$ & $17 \cap n$ \\
\hline it> 70 & 23 & $1.07>6-127$ & $x \cdot+\| d \&=07$ & $1 ? 0.0$ \\
\hline 4 is. 0 & $1 \cdot 7$ & $1.2 \times 15-i ! 7$ & $H .18 n 5=n 7$ & $1>09$ \\
\hline 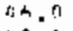 & 67 & $7.75 Q E-N A$ & 7.63 누 $=77$ & $1>00$ \\
\hline$\angle A .0$ & $2 t$ & $9, \alpha \times 5 F-? 2$ & $x-2^{2}+5=07$ & $1>00$ \\
\hline$=n .0$ & 71 & ${ }^{n} .>125-A_{i}$ & $P, 70 a f=n 7$ & 1200 \\
\hline ५? & 175 & $>.9 \Omega 55=n 7$ & A.P $\{$ PF $-n 7$ & $1 ? n \mathrm{n}$ \\
\hline 50.4 & a.s. & $0.7 \times 1 F=08$ & $3 . ? \cap Q F=0 ?$ & $1 ? 0 \pi$ \\
\hline $5+.0$ & $! 7$ & I. $3 \|^{\circ} \mathrm{F}=\cap \mathrm{A}$ & $1.1155=0.7$ & $1 ? 0 \pi$ \\
\hline c. & 74 & $0.0 n 9 f-$ in & $3.9+35=n 7$ & i? 00 \\
\hline$b^{n}=n$ & 45 & $7.29 \times 5=66^{2}$ & $>, 500=07$ & 1300 \\
\hline 4.7. 0 & $1>x$ & $1.21 S F=n 7$ & $\triangle . A n t, F=n 7$ & $120 n$ \\
\hline tid +0 & $\sqrt{3}$ & $x .979 F=119$ & $1.3100-17$ & (n) \\
\hline nt. 0 & 100 & $1 . ?+25-117$ & $1 . ? 207 \mathrm{~F}-07$ & $120 n$ \\
\hline 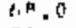 & 22 & $0.4 \cap ? E-\cap A$ & $3.1975=07$ & $120 \%$ \\
\hline 70.0 & 120 & $2.375 F-0 ?$ & $7.7355-07$ & $12 n 0$ \\
\hline 72.0 & 235 & $? .609 F=97$ & $0.103 F=n t$ & 1200 \\
\hline $7+4=0$ & 352 & 7. Anst $=167$ & $1.297 \mathrm{~F}=06$ & 1700 \\
\hline 74.0 & 233 & $3.353 F=07$ & $1.113015=106$ & 1300 \\
\hline $7^{\circ} \cdot 0$ & $1 \times 9$ & $1.94 n F-57$ & $+.505 F=n 7$ & 1300 \\
\hline$A 1.0$ & 171. & $1.9 a+E=r 1$ & $+.017 F=n 7$ & $120 n$ \\
\hline 27.0 & 214 & $7.95,25=97$ & A. 33 AF $=07$ & $1>00$ \\
\hline 44.0 & $P \pi n$ & $P .+x N F=n y$ & $8.9425-07$ & 1 ? ก ก \\
\hline$A+\ldots$ & $2 \times 5$ & $3.6+94-n 7$ & $?, 1 d \underline{T} F-n 7$ & $1>n n$ \\
\hline P占 & 120 & $1.5785=07$ & $5,3 \mathrm{~A} 5 \mathrm{~F}=\mathrm{n} 7$ & $\operatorname{lon}$ \\
\hline $\sin 6$ & 41 & ${ }^{t \mid} \cdot 7 x f \mid f=r a$ & $1, A I \cap F=7$ & 1200 \\
\hline 47,0 & $i 1$ & $1.3154=08$ & if. $47 \mathrm{ff}=08$ & 130 ก \\
\hline $\mathrm{CH}, 0$ & 11 & $1.3155=0.0$ & $4.471 F-08$ & 12010 \\
\hline $0 t .0$ & 3.7 & $3.15 x^{2}=00$ & $1.0735=07$ & $130 n$ \\
\hline $4, P, 1+$ & 75 & $7, A 23=0$ & $r, A Y H F=\cap P$ & $3>n$ \\
\hline $1 n^{2} \cdot 0$ & 27 & $x, \cap \nabla^{x}[-11, A$ & $1, r i=1 F-n 7$ & 170 ก \\
\hline " & 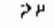 & $3 .>2, P F-6.9$ & $1.11255-0.7$ & $1 ? \mathrm{nn}$ \\
\hline $16,4.0$ & $1 !$ & $1.3158=n p$ & $\therefore .471 \mathrm{~F}-\cap 8$ & $130 \%$ \\
\hline Ifim. & 23 & $P .+3 n 5=11 \mu$ & $51.2 a c^{2}-0.8$ & $! 2 \cap ?$ \\
\hline $1 ?$ a & $p 0$ & र. $5095-1: 0$ & $\dagger .11 r F-n\rangle$ & $13 \times n$ \\
\hline $11 \div .0$ & 12 & $3.7 \cup x=f \cdot 2$ & 1.27 \& $5-n 7$ & 1200 \\
\hline $112 . n$ & 55 & h. T7AF-i A & $?+161=0=7$ & $1 ? 63$ \\
\hline $11 \% .0$ & $1+3$ & $1.2751=47$ & 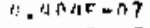 & $y>0 n$ \\
\hline 118,6 & $\ln 3$ & $1.1015-6.7$ & a. $0 ? t, 5=n 7$ & $1 ? 0$ \\
\hline & $\ln 4$ & 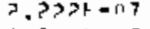 & $7.5=55=\hat{7}$ & $1>n$ \\
\hline 122.11 & 73 & $\Rightarrow 36 n+-11 a$ & $\bar{r} \cdot r^{n} x^{13}=n z$ & $1>0 n$ \\
\hline 1840 & a1 & $2,7+5 \times=1.0$ & T. $13 ! 5-07$ & $3>n n$ \\
\hline 124.7 & 107 & $1.1771=: 87$ & $\mathrm{ii} \cdot \sim \Gamma_{i} \rho-n \gamma$ & 17011 \\
\hline 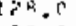 & 100 & 1. Iदान $=A 7$ & $\because, \alpha \uparrow \sigma_{j} F-n T$ & $1 ? n \pi$ \\
\hline $\begin{array}{l}3 \\
3\end{array}$ & a? & $1.03 \times+-[7$ & $3 .\{9>1=n 7$ & $1 ? 00$ \\
\hline 13 & $1+7$ & $1.030+-17$ & $x, 5>K E=n T$ & $1>00$ \\
\hline
\end{tabular}

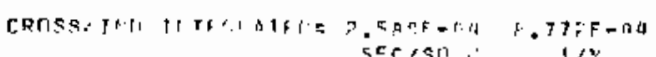




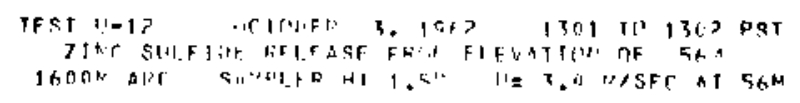

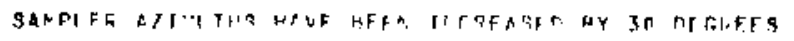

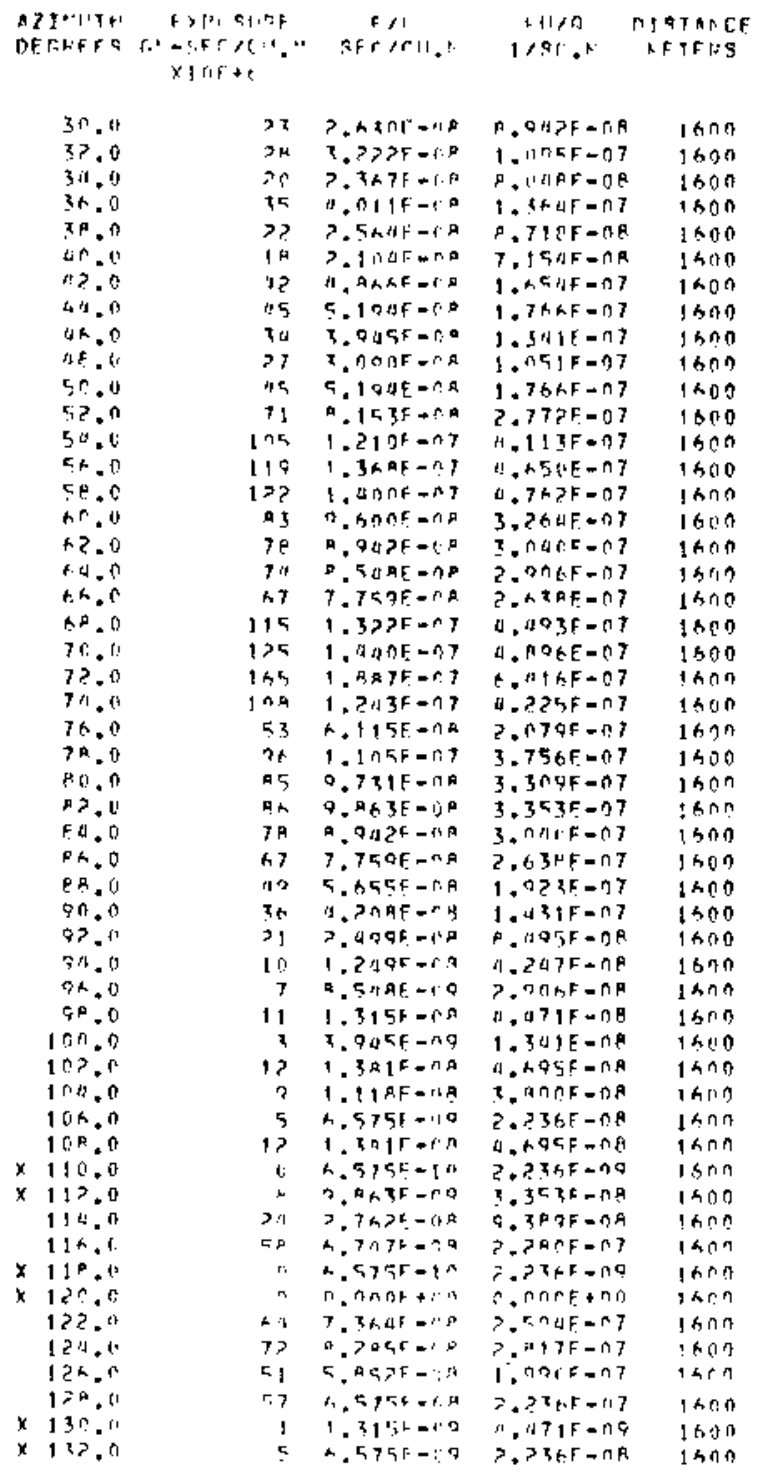

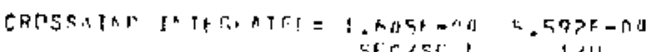

Sertaril 
UNSTABLE SERIES EXPERIMENT: 13 ( U-13)

GROUNO LEVEL SAMPL ING 100-1600 M ARCS. FOR EASE IN STATISTICAL

ANALYSIS, ALL SAMPLER RZIMUTHS HAVE BEEN INCREASED BY 30 DEGREES

FROM THEIR ACTUAL POSITIONS.

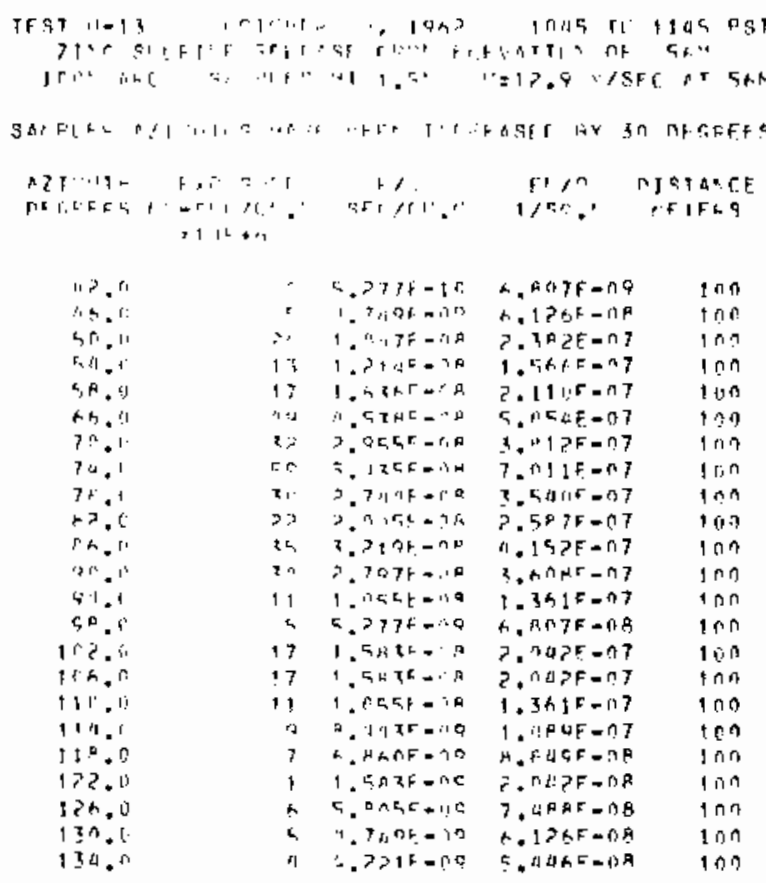

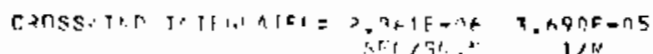

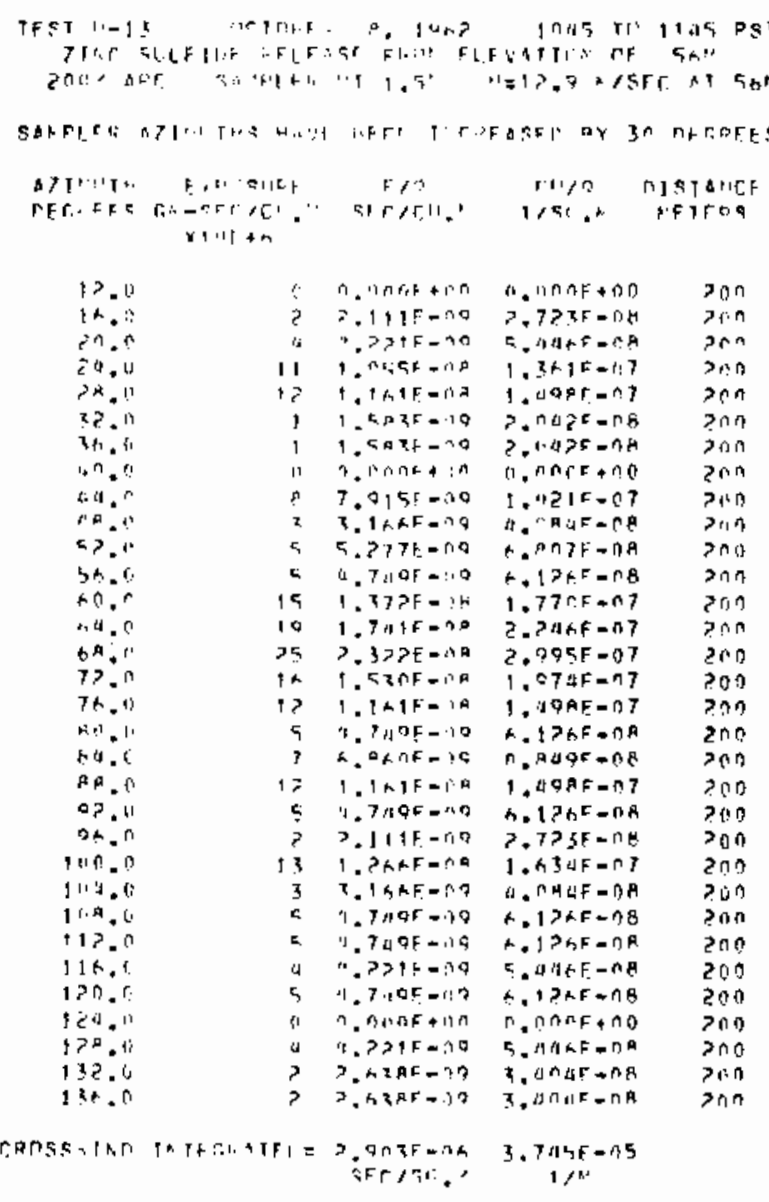




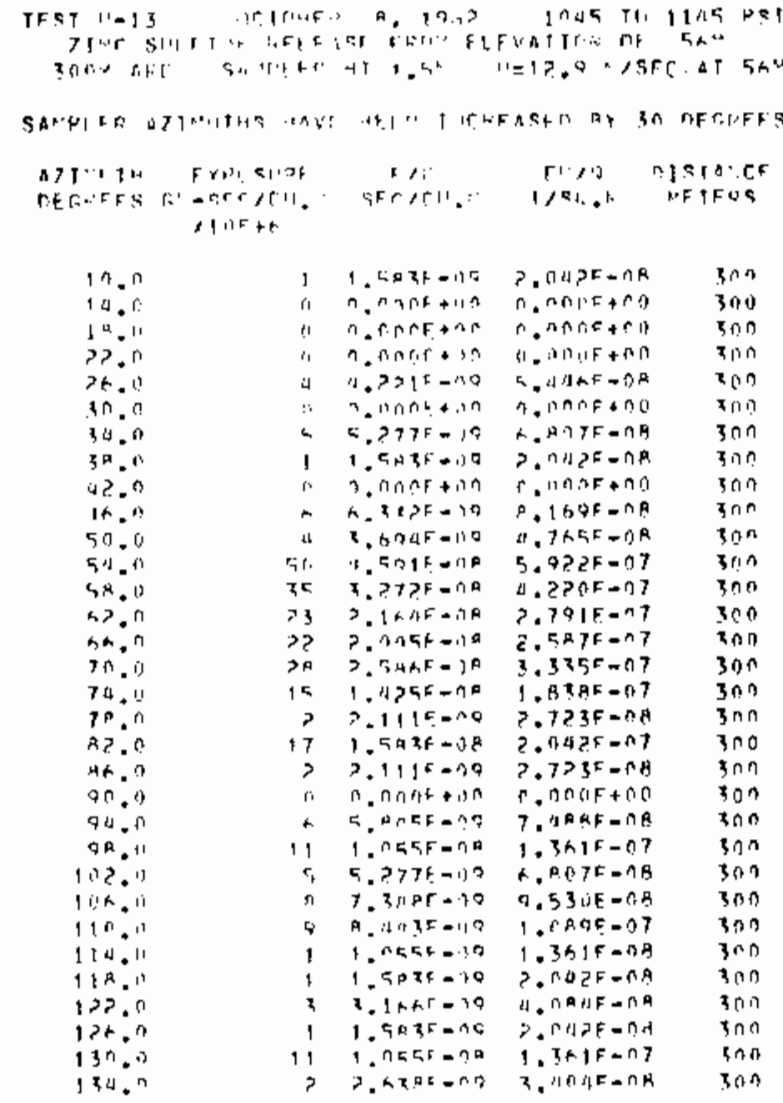

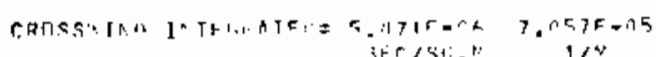

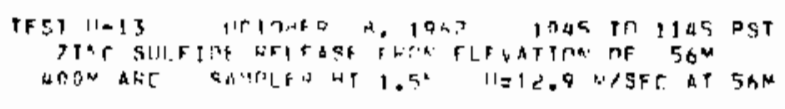

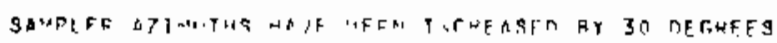

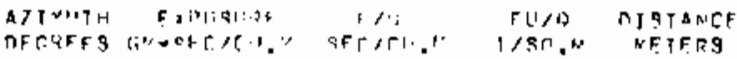
$x \perp 1++\infty$

\begin{tabular}{|c|c|c|c|}
\hline$? \omega .0$ & 5 & 3. IKAS +108 & $\triangle, \cap$ 口 \\
\hline $24 \cdot 7$ & $n$ & $? .3 \times 9 F=$ ก & $a .53 \cap F=0 A$ \\
\hline $3 ? .12$ & 11 & $n \cdot n n n<+11 n$ & $n \cdot 1, n n k+n 0$ \\
\hline$(t, 0)$ & $n$ & $1 . \ln n \mathrm{r}+n \pi$ & $n \cdot \ln n F+n 0$ \\
\hline$a^{n} .0$ & 1 & $1 . n 55 F=n Q$ & $1.36,15=n g$ \\
\hline ia & i & $4 . \supset \supset J F=n$ & $5.4 d \leqslant F=\cap A$ \\
\hline$\Delta n, 16$ & $>t$ & $2.1 \mathrm{n} H \mathrm{H}=\mathrm{i}, \mathrm{s}$ & $7.7015=07$ \\
\hline 42.0 & $2+5$ & $7.4175=07$ & 3,11 AF $=n 6$ \\
\hline $56 . n$ & $41: 1$ & $3.73 \mathrm{AL}=07$ & 4.P1DF $-A B$ \\
\hline $6 ? .0$ & $37 k$ & $3 ., 2,5=07$ & $2.670 F=06$ \\
\hline sa.n & $37 r$ & 7. 11 ว $=$ - 7 & a. $911 F=06$ \\
\hline$\left.x^{2 x}=1\right]$ & 121 & $1.65 ?+21) 7$ & $7.1515=06$ \\
\hline 72.7 & 375 & $1.5895-1.7$ & $7 . n+3 E=n 6$ \\
\hline 74.0 & $I^{n}$ & $3.9575=n p$ & $1.746 \mathrm{~F}=06$ \\
\hline $8 n .0$ & 8 & a. $2715=09$ & $1.1575-07$ \\
\hline 84.7 & 1 & $1.9525=08$ & $7.519 F=1.7$ \\
\hline 9.0 & 33 & $?+\left.11\right|^{x}=n$ & $2.7735=07$ \\
\hline $92 \cdot n$ & ฯ & $5.2775=07$ & $B .807 F=n$ \\
\hline $96 . ?$ & to & $5.52>5-79$ & $A, 16 Q E=\cap 8$ \\
\hline $\ln n \cdot 0^{2}$ & 3 & T. 1 hat $=$ ? 9 & $A . \cap A \& F=\cap B$ \\
\hline 104.9 & 4 & ".? • & $5.4 d B F-n g$ \\
\hline $10 \mathrm{~A} . \mathrm{n}^{n}$ & i. & $n$. nnF +nn? & $\cap .11 \cap \cap E+00$ \\
\hline 112,0 & 1 & $1.255 \mathrm{E}=110$ & 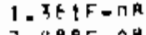 \\
\hline 115.0 & to & $5.005 F=00$ & $7 . \backsim A A F=\cap \theta$ \\
\hline $12 n .4$ & 3 & $x .1+A F=10$ & 4. กคด \\
\hline 120.11 & " & $n .0 \cap \cap 5+9 n$ & $n=A \cap n F+n O$ \\
\hline $12^{2} \cdot 0$ & ij & $7 x+x=0$ & $5 . \Delta 4 M F=08$ \\
\hline 137.0 & $?$ & $3.4321=00$ & $3.4 \cap 45=n 8$ \\
\hline & $1=$ & 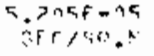 & $\begin{array}{c}0.710 F=n d \\
1 / \mathrm{m}\end{array}$ \\
\hline
\end{tabular}

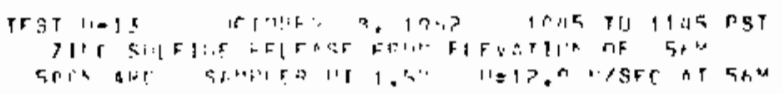




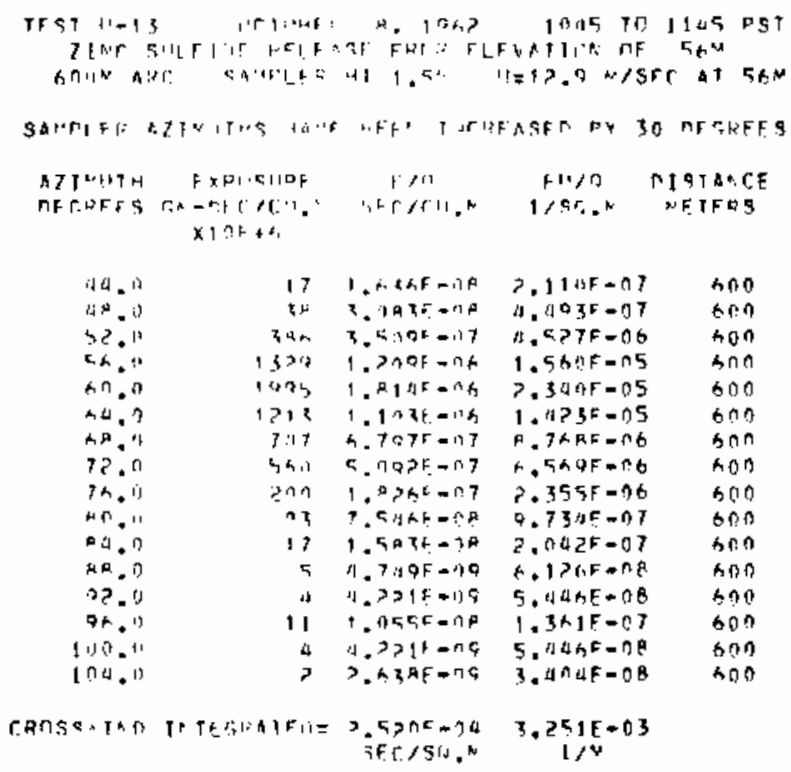

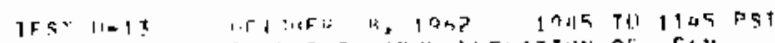

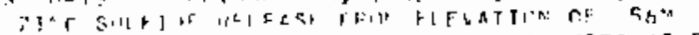

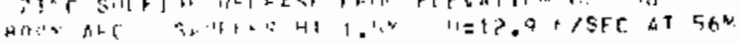

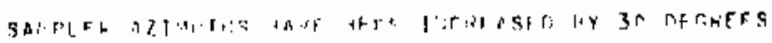

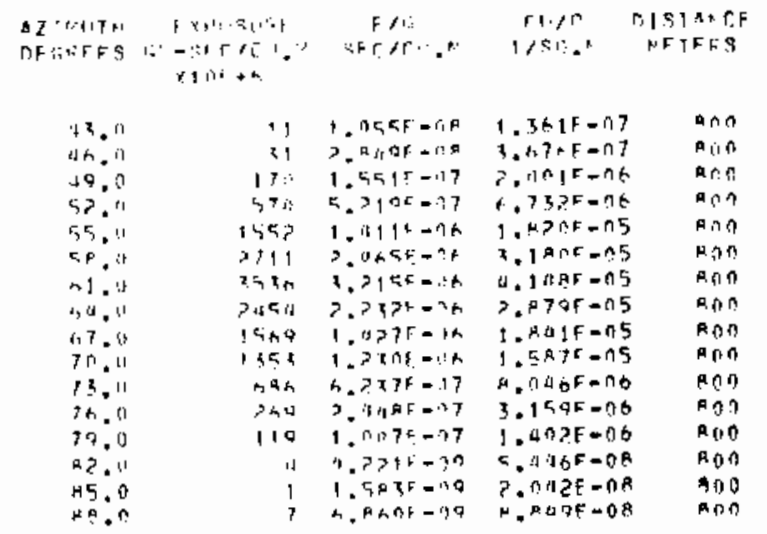

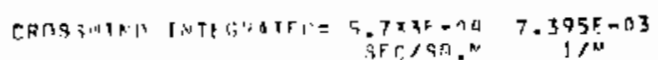

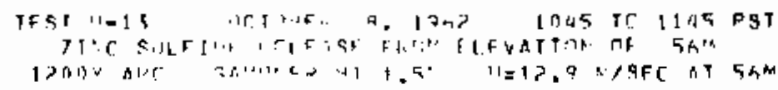

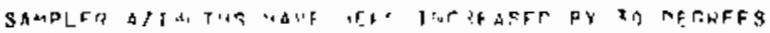

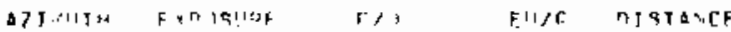
nferafs r. $x \ln$

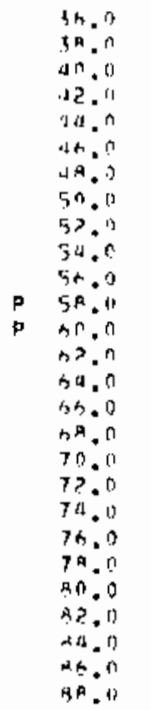

\begin{tabular}{|c|c|c|c|}
\hline$n$ & $7 . n \in 7^{2}-1 n$ & $A .159 F=n Q$ & $1>n \pi$ \\
\hline 1 & $1.4185-\therefore q$ & 1. P\1F-กम & $120 \%$ \\
\hline 8 & $n$ n & $\therefore . \cap \cap O F+n O$ & 1200 \\
\hline i & $n$. nn nF+. nn & D) $\cap A F+\cap 0$ & 1200 \\
\hline 14 & 1. Jсск- in & $1 .+177+-1.7$ & 1200 \\
\hline 1 & $\Rightarrow F 15=n$ B & KaรF-n 7 & 1200 \\
\hline 2.3 & $A, \partial=\{[=19$ & $1025=0: 7$ & $1>0 n$ \\
\hline 102 & $t . x+145=17$ & - $137 f=n t$ & $1 ? \cap 0$ \\
\hline 278 & $1.1544^{6}=17$ & $6175=06$ & $1 \geqslant 0 n$ \\
\hline รก & 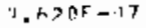 & ZABF $=\because h$ & $1 ? 00$ \\
\hline 1757 & $1.502 \mathrm{~F}=.16$ & $055 \mathrm{~F}=0.5$ & $1 ? 00$ \\
\hline 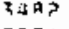 & $A F=. J h$ & $-\cap 5$ & 1300 \\
\hline $2>91$ & -96 & 05 & $1>0 n$ \\
\hline $1 \times 3 A$ & UPAF $=n A$ & $19 F-n 5$ & $1>0 n$ \\
\hline Isa! & $1.7 \times R E=7 A$ & $597 F=15$ & 1200 \\
\hline $155 \mathrm{~h}$ & $1.3155=34$ & . $25 F=05$ & $1 ? 00$ \\
\hline 751 & 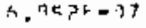 & R. & $1>0$ \\
\hline $10+1$ & 0.7 is $a F=37$ & $1.257 E=05$ & $1 ? 00$ \\
\hline 517 & ב. $74.9=07$ & $1175 F=06$ & $1 ? 00$ \\
\hline 310 & $3.7>06=147$ & $5235-06$ & 1300 \\
\hline 115 & $1.0575=37$ & $1.36+F=176$ & 1200 \\
\hline 11 & $\therefore$ - 1 A & $3735-07$ & $1 \geq 00$ \\
\hline 3 & $7 F=-10$ & 7 AF $=98$ & $1>n 0$ \\
\hline 7 & & $n$ & $1>00$ \\
\hline 7 & $7.9775=19$ & $\div .155 \%=08$ & 1200 \\
\hline :! & T. $5.19 \%=10$ & 97 मP $=19$ & 1200 \\
\hline 5 & $5.3>3 F=10$ & $A, H B 7 E-O H$ & $120 \pi$ \\
\hline
\end{tabular}

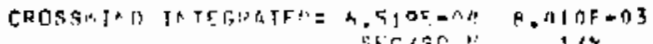

iदि

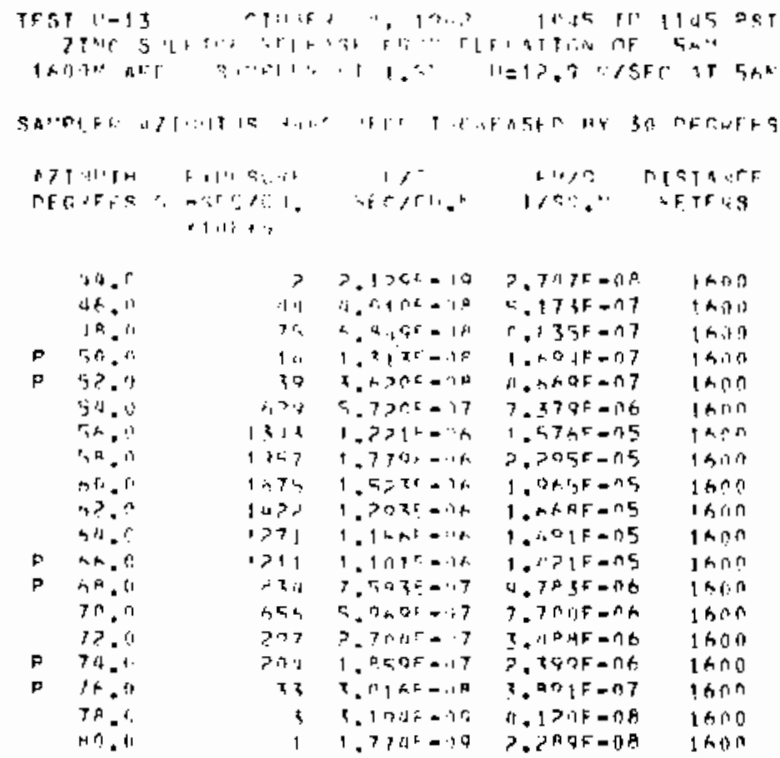

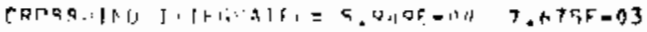


UNSTRELE SERIES EXPERIMENT: I4 (U=14)

GROUND LEVEL SAMPLING 100-1600 M ARCS.

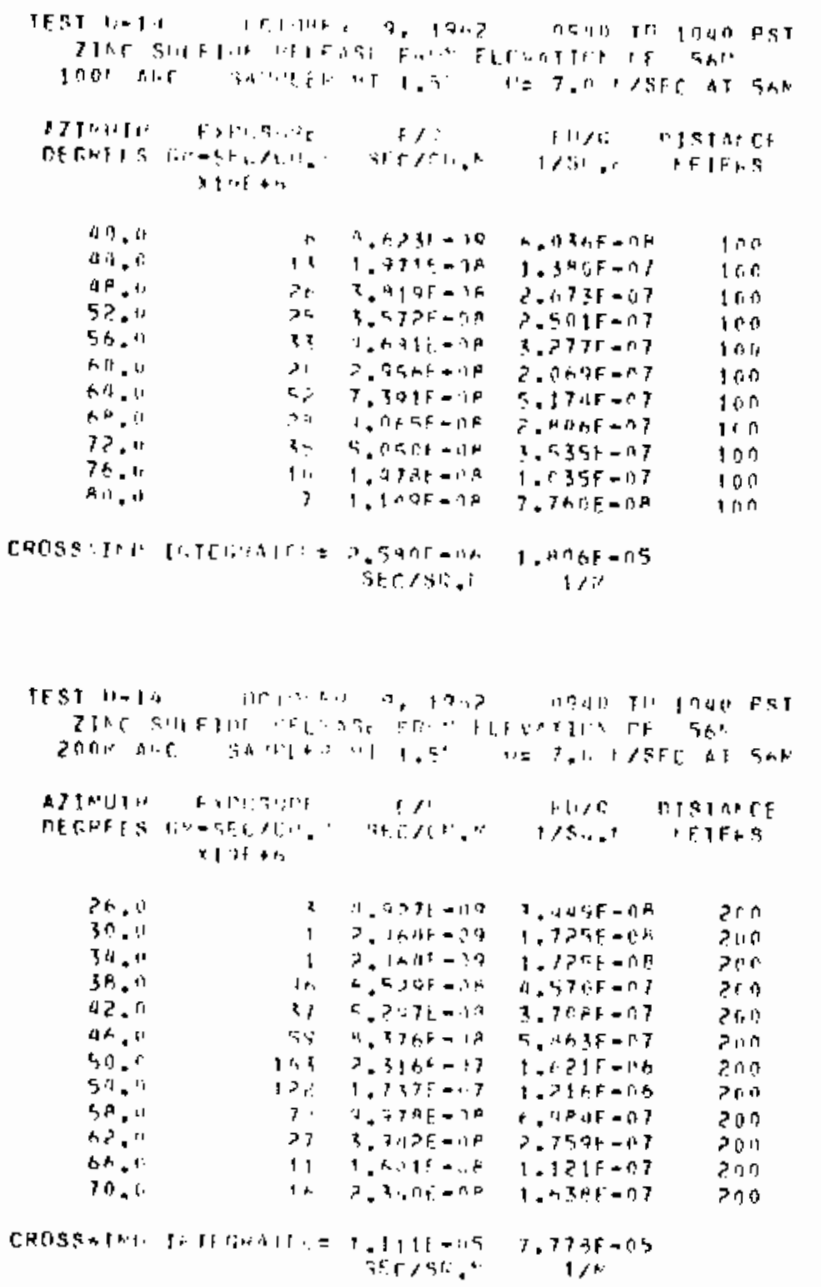

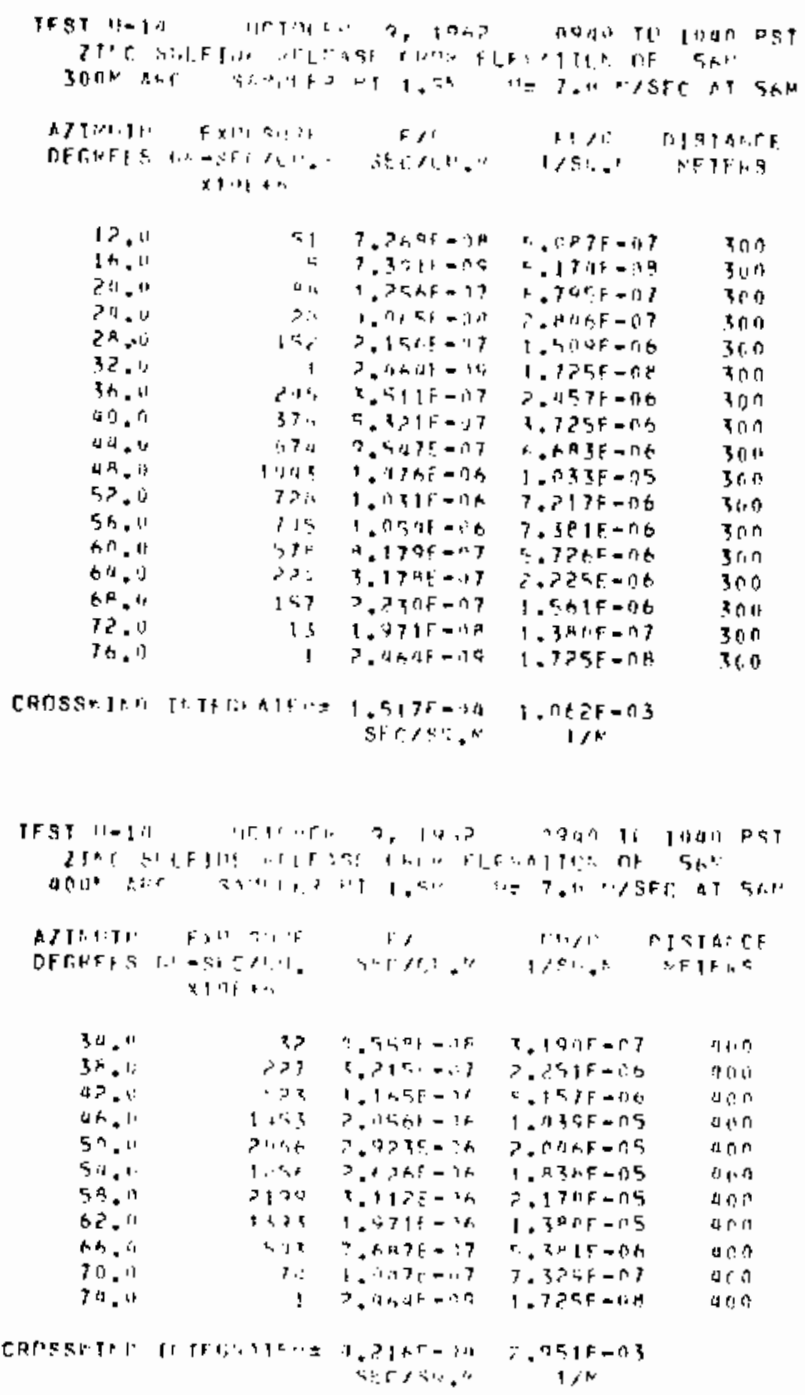




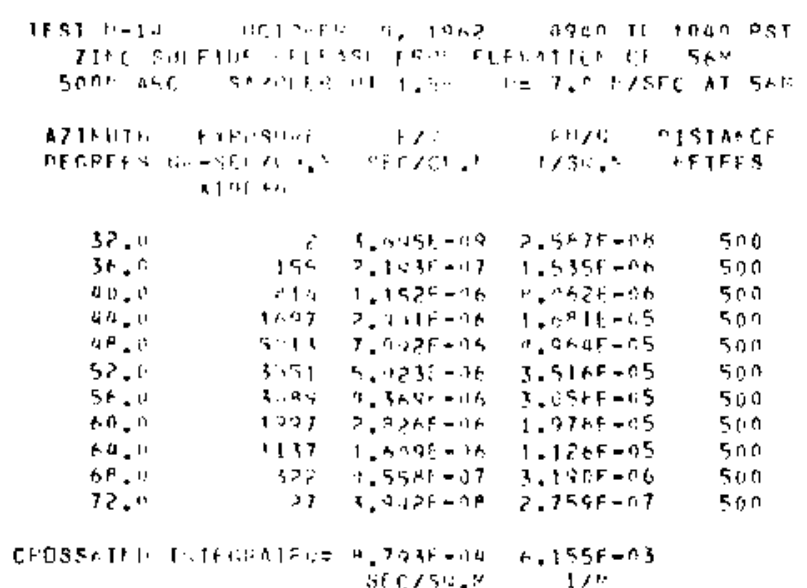

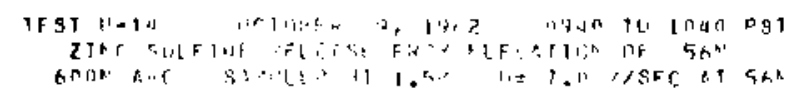

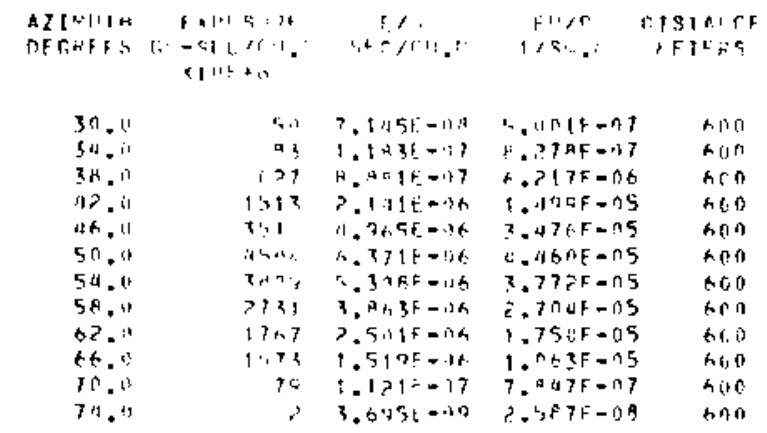

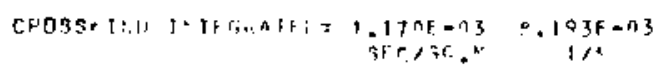

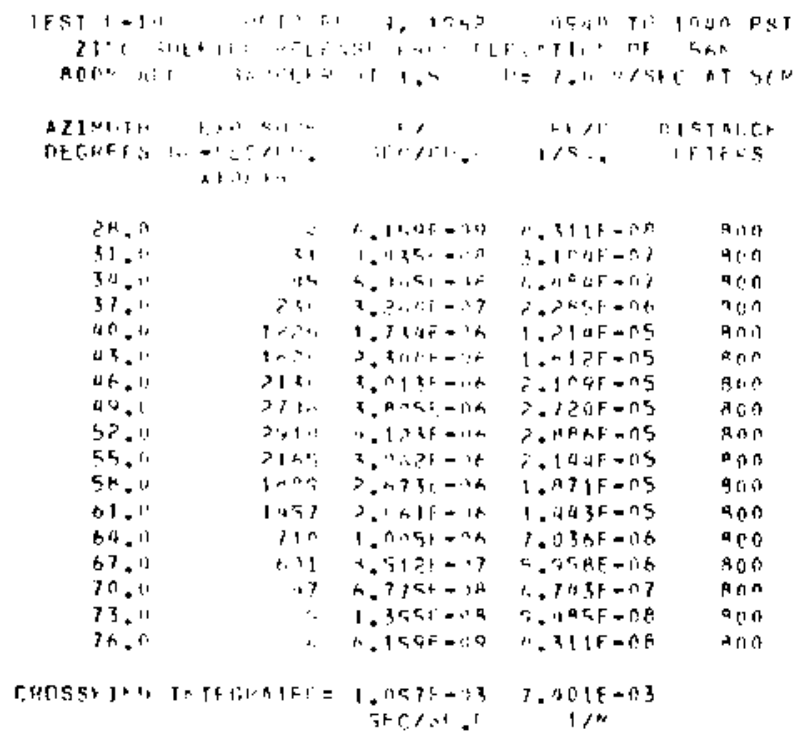

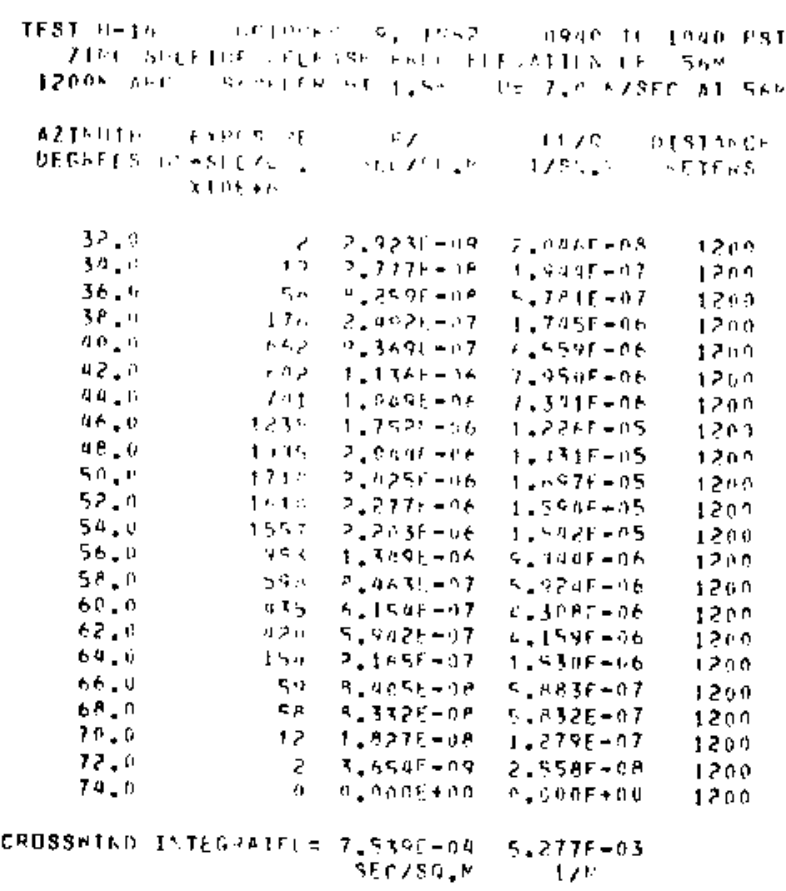

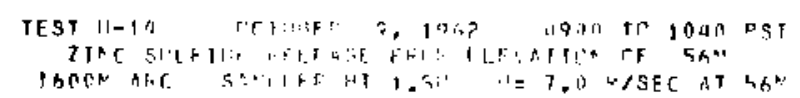


UNSTAQLE SERIES EXPERIMENT: $15,(\mathrm{U}-15)$

GROUND LEVEL SAMPLING 100-1500 M ARCS.

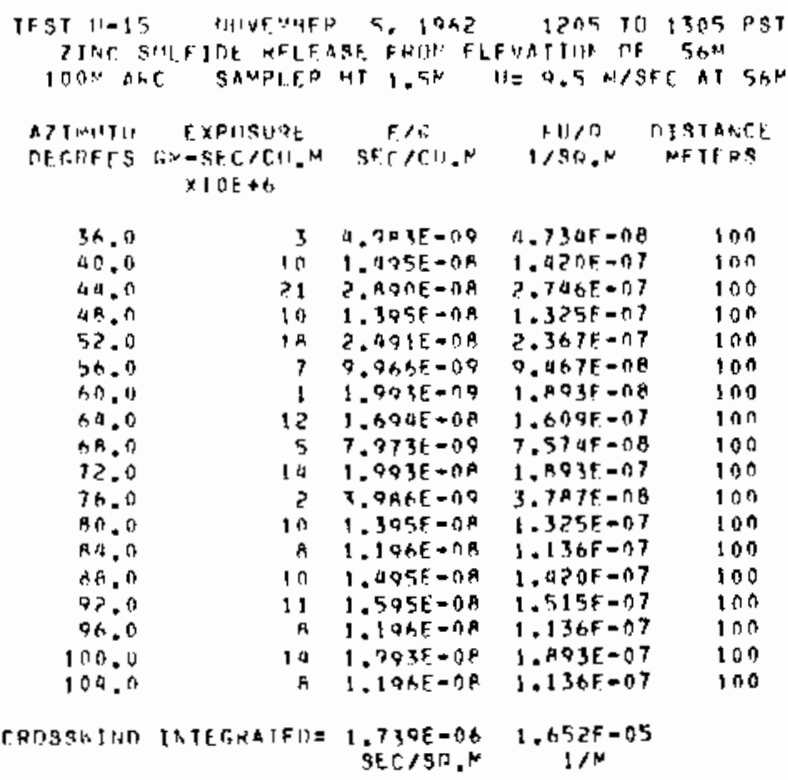

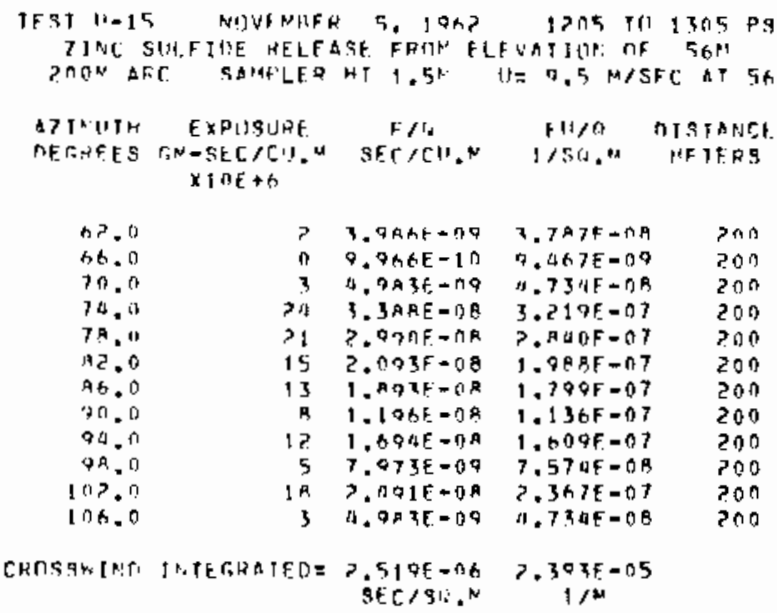

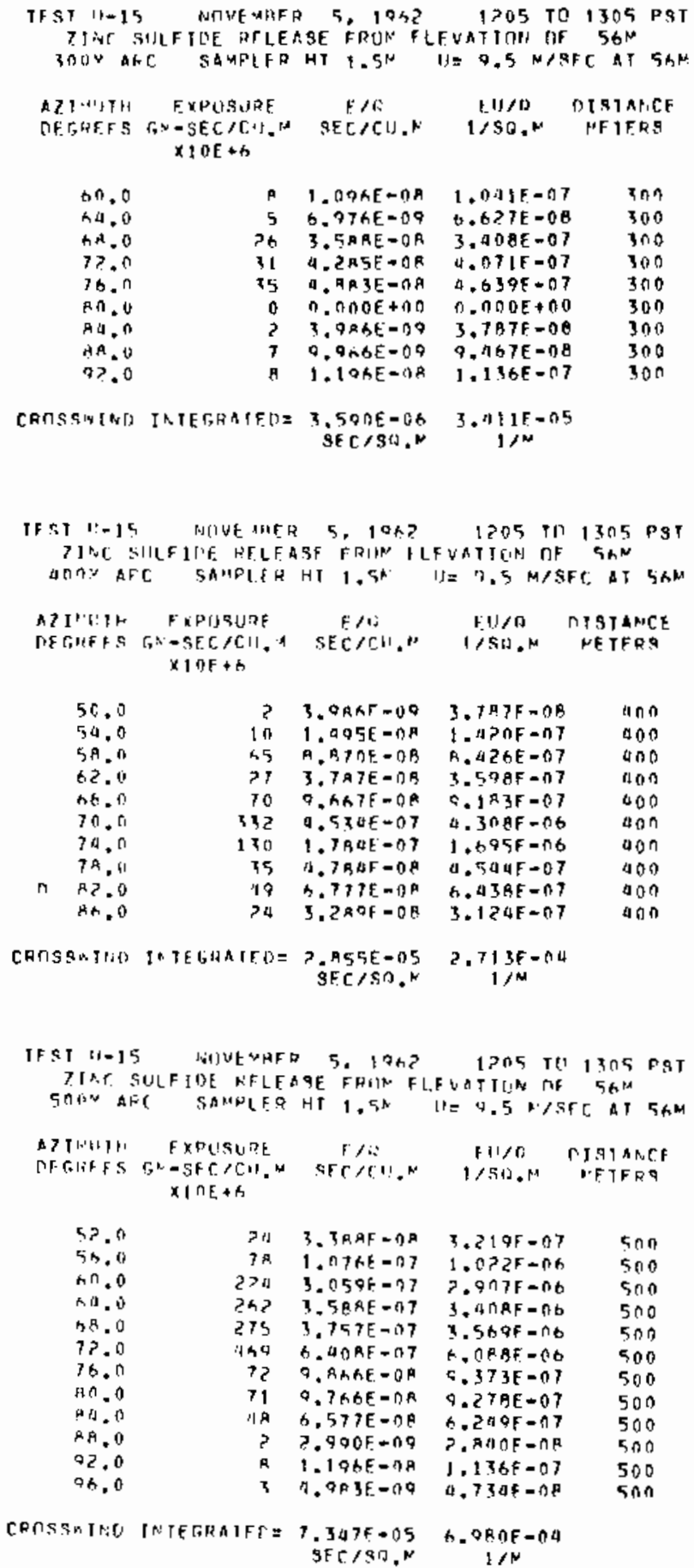




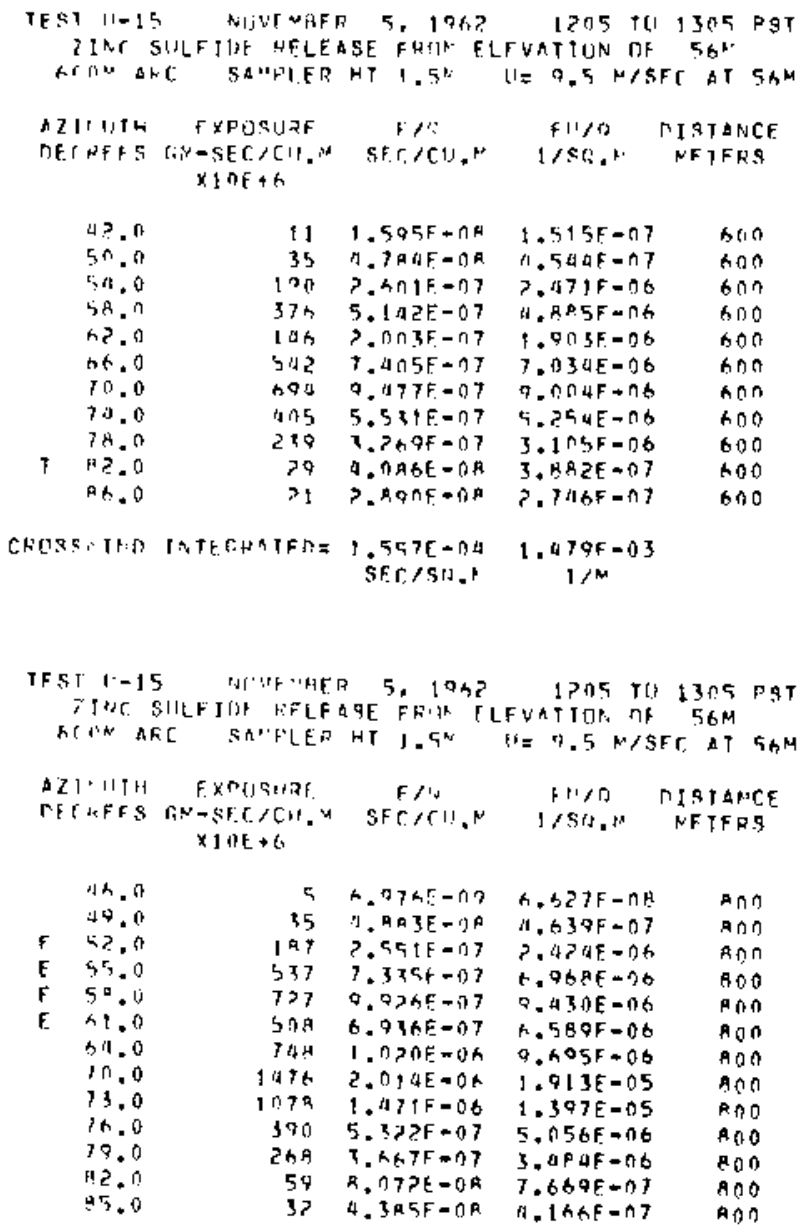

\begin{tabular}{|c|c|c|c|c|}
\hline $4 ? .0$ & $\{1$ & $1.505 \mathrm{~F}+\mathrm{nA}$ & $1.515 \mathrm{~F}-07$ & $s+10$ \\
\hline $5 n .0$ & 35 & 7.7 a & $1.5 a d F=07$ & hon \\
\hline $5 n, 0$ & 120 & $? .4 \cap 1 F=07$ & $7.45715=06$ & $60 n$ \\
\hline 58.7 & $37 \mathrm{~h}$ & $5.142 E-07$ & H. RP $5 F=n G$ & 600 \\
\hline n?. 0 & I at & ?. $\cap \cap 3 F-\cap 7$ & $1.905 F=06$ & 600 \\
\hline $6 t .0$ & 34 & $7.405 F-n 7$ & 7. D3 यE=06 & SnO \\
\hline 10.0 & 595 & $9.177 F=07$ & $7.0 \cap 4 F+n b$ & non \\
\hline 73.0 & 205 & $5.5315=07$ & 4. $254 E-06$ & 600 \\
\hline 0 & 258 & $1.7+95=07$ & $3.1 \cap 5 F=06$ & 600 \\
\hline 0 & 79 & OABE $=O A$ & 3, BRZE $\rightarrow 07$ & 100 \\
\hline 6.0 & 31 & $P . R Q \cap F=A B$ & $7.7165=07$ & $6 \cap 0$ \\
\hline
\end{tabular}

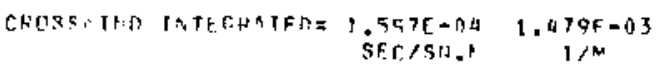

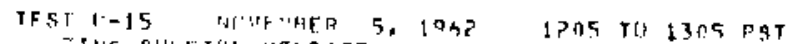

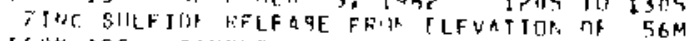
HC ANE SAPFLER HT J.GN

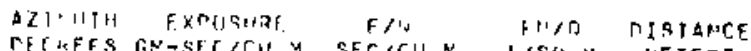

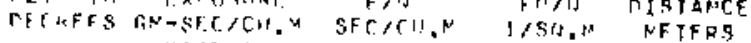
$\times 1 \mathrm{AE} \rightarrow \mathrm{C}$

\begin{tabular}{|c|c|c|c|c|}
\hline 小h. त & 5 & $+.745-107$ & A. $327 \mathrm{~F}-\mathrm{nH}$ & $A \cap n$ \\
\hline 40.0 & 35 & $\because A A 3 E-D A$ & $1.639 F-07$ & anก \\
\hline 52,6 & Iay & $? .55 t E=07$ & $2.472 \mathrm{E}=06$ & ADก \\
\hline 55.0 & 537 & $7.3 \times 5+-07$ & $1.9685-96$ & 800 \\
\hline$\because 4$ & $7>7$ & $0.9765-97$ & $9.430 \mathrm{E}=06$ & Ann \\
\hline aid 0 & 50 की & $6.916 E-07$ & $\mathrm{~h} .589 \mathrm{~F}=06$ & Aon \\
\hline $\begin{array}{l}3 \pi .0 \\
10.0\end{array}$ & 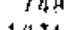 & $1.0>0 E=O A$ & $9.605 \mathrm{~F}+0.6$ & Aon \\
\hline 73.0 & 1976 & $014 E=0 \mathrm{~A}$ & $1.913 E-05$ & An \\
\hline $\ln .0$ & to & $1.471+=00$ & $1.397 \mathrm{E}=0 \mathrm{~S}$ & $R \cap 0$ \\
\hline 79.0 & $3 \mathrm{ha}$ & $7=30 \mathrm{~T}-11$ & $=.1156 \mathrm{G}-006$ & A 10 \\
\hline 492.0 & 50 & - & $5.0+4=-60$ & 800 \\
\hline 95.0 & 37 & $43 \times 5 F=0 A$ & $1.004 \mathrm{k}=07$ & 800 \\
\hline
\end{tabular}

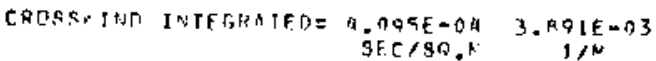

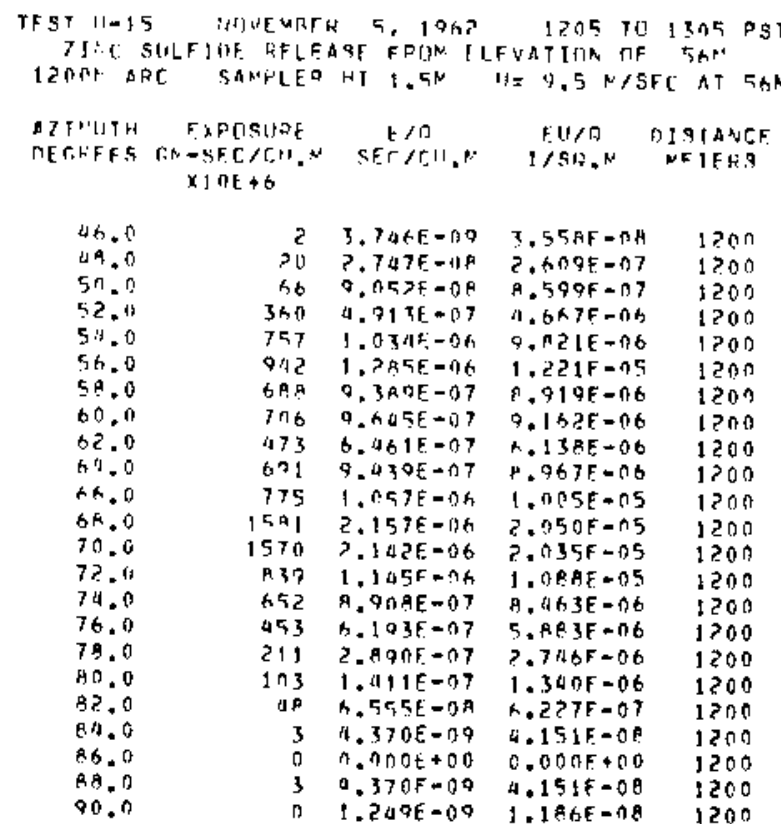

CROSSWIND INTEGRATEOE $4.259 E-04 \quad 5.047 E-03$ SEC $130 . M \quad, M$

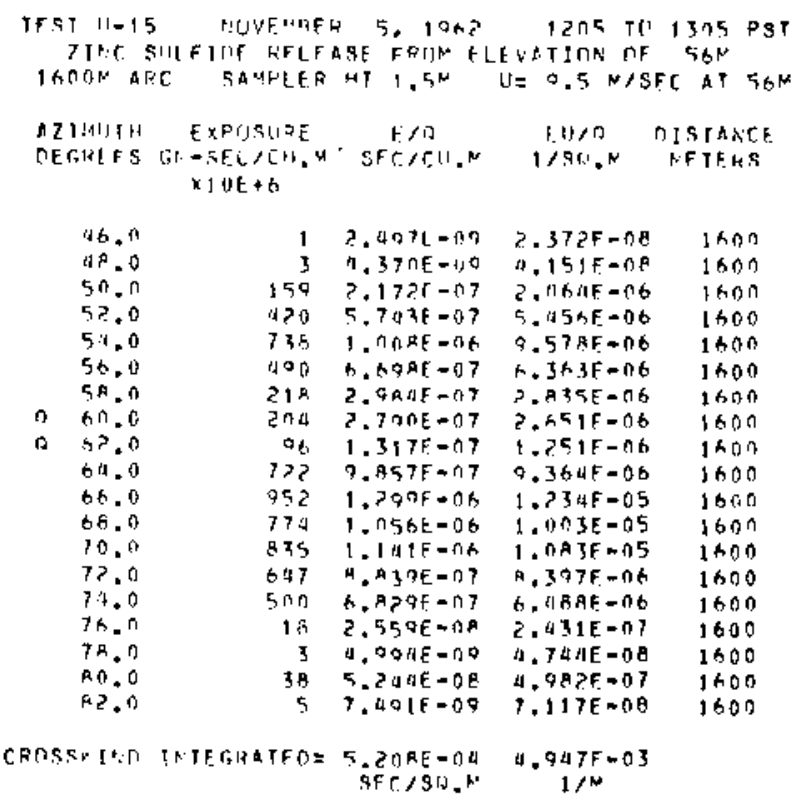


UNSTABLE SERJES EXPERIMENT: 16 ( J-16)

GROUND LEVEL SAMPLING 100-1600 M ARCS. THE TRACER WAS NOT

DETECTED ON THE 100 OR 200 M ARCS.

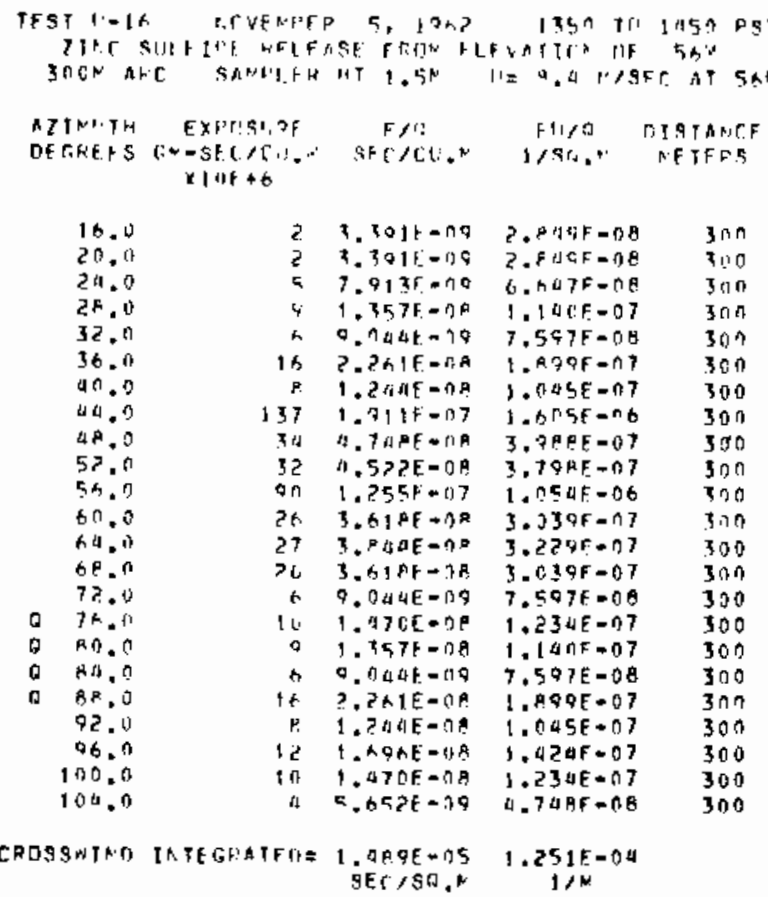

TEST 1-1A ARWENHFR C, 14A? I35 TC 1AGA PST ZIAC SIHFTHE KELFASE FHIIN FLFVATICR OF $5 \mathrm{AN}$

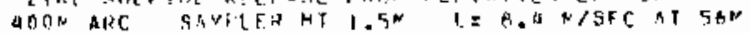

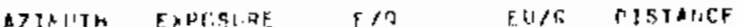

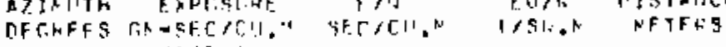
(i) 1 i + h

\begin{tabular}{|c|c|c|c|c|}
\hline 50.0 & ! & $3.261+=00$ & 1. $A Q \cap F=D A$ & 400 \\
\hline 42.6 & Jid & $3.37 \div 6-18$ & ?. $R=9 F=07$ & 400 \\
\hline$\Delta h .1$ & 571 & $5.155 \mathrm{~F}-1) 7$ & $\therefore, 33 \cup 5=176$ & $40 \pi$ \\
\hline 58.0 & 195 & 1. यद्व $95-17$ & $1.725 F=00$ & ח \\
\hline 54.6 & 37. & $5.4725=17$ & $a=5 Q g F=n t$ & anon \\
\hline$S^{A}=0$ & 157 & $>, 1 P>E-1) 7$ & $1.533 F-50$ & 400 \\
\hline 62.0 & IhH & $7.2 \times\{1 F-\cap 7$ & $1.918 F=06$ & 400 \\
\hline $6 h .0$ & $7 n$ & $Y . A \mid F E=O A$ & $3.0335=07$ & 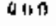 \\
\hline 70.7 & A & $5 .+5 Z E-D S$ & 4.7 गAF - OB & 400 \\
\hline $74 . n$ & 11 & $1.5 \mathrm{AF}=0 \mathrm{O}$ & $1.3295=0 ?$ & 400 \\
\hline 38.4 & 5 & $913 E-09$ & $+.647 E-18$ & $\operatorname{ano}$ \\
\hline 0 & 7 & $1.017+=0$ A & $0.547 F=08$ & 400 \\
\hline An, in & 0 & $n, 000 E+00$ & $c .000 E+100$ & 400 \\
\hline 80,11 & a & $1.13 \cap E-08$ & $9,445 F=08$ & $40 \cap$ \\
\hline 911.13 & $?$ & $3.391 F=00$ & $2.849 F-09$ & 400 \\
\hline 48.0 & (1) & $13 C E=09$ & $0.496 F=09$ & 400 \\
\hline
\end{tabular}

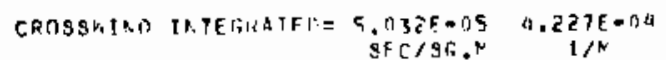

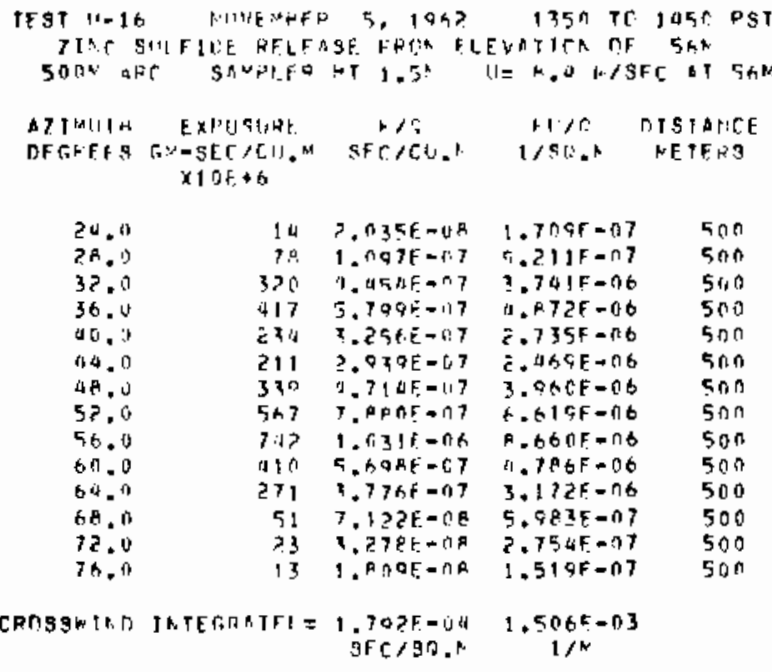

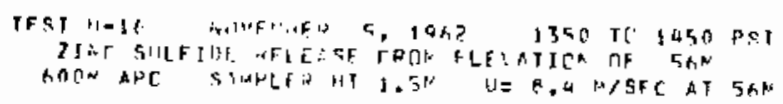

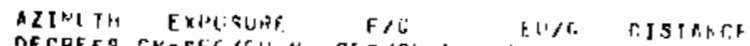

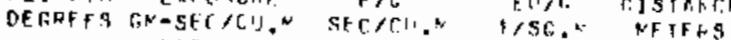
$x(1)+\infty$

\begin{tabular}{|c|c|c|c|c|}
\hline 22.17 & $? 0$ & $3.7 \mathrm{f} F=11 \mathrm{~A}$ & 3.5745 .07 & niso \\
\hline & 30 & $5,4 \geqslant t \in[-1) P$ & a. $55.8 F-07$ & $b: n$ \\
\hline $3 \sqrt[3]{3}$ & 271 & $3 . T A 5 E-1) 7$ & $3.360 \%=0 t$ & $\sin n$ \\
\hline 34.0 & 57 & A. OरAE -07 & $+.75,2 F=06$ & bon \\
\hline 3 A. $V$ & $5 x_{1}$ & $9.2915-177$ & $4.4 U य F=06$ & 600 \\
\hline 4?.0 & $3 i k$ & $5.257 f-07$ & $4 . \Delta 1 A F=n b$ & 600 \\
\hline 44.0 & 2.73 & $4.070 f-37$ & $3.4: 50=06$ & AOO \\
\hline $5 n .11$ & 117 & $0.971 \mathrm{f}=0.7$ & $A .37 b E-0 B$ & son \\
\hline 50.1 & $14>1$ & $1.975 F=180$ & $1.649 f=115$ & too \\
\hline $5 p .0$ & $7 \% 7$ & $t .052 \mathrm{t}=0 \mathrm{~A}$ & $\therefore, 901 F=06$ & $a \cap 0$ \\
\hline 52.0 & $13+2$ & $0.037 F=07$ & $5.071 \mathrm{~F}=06$ & 6on \\
\hline 60,11 & 190 & $2.56+. E=67$ & $2 \cdot 15$ t $F-06$ & 600 \\
\hline 70.0 & 72 & $1 . n \pi \in[=07$ & $P .1525=n 7$ & 600 \\
\hline 74,4 & ? & $3.3015-00$ & $Z . A \Delta O E-A B$ & 600 \\
\hline 78 & 7 & $1.017 E-A A$ & A. $547 \mathrm{f}=08$ & $x \cap 0$ \\
\hline
\end{tabular}

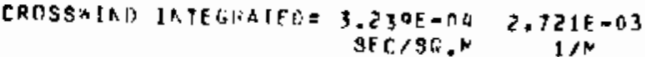




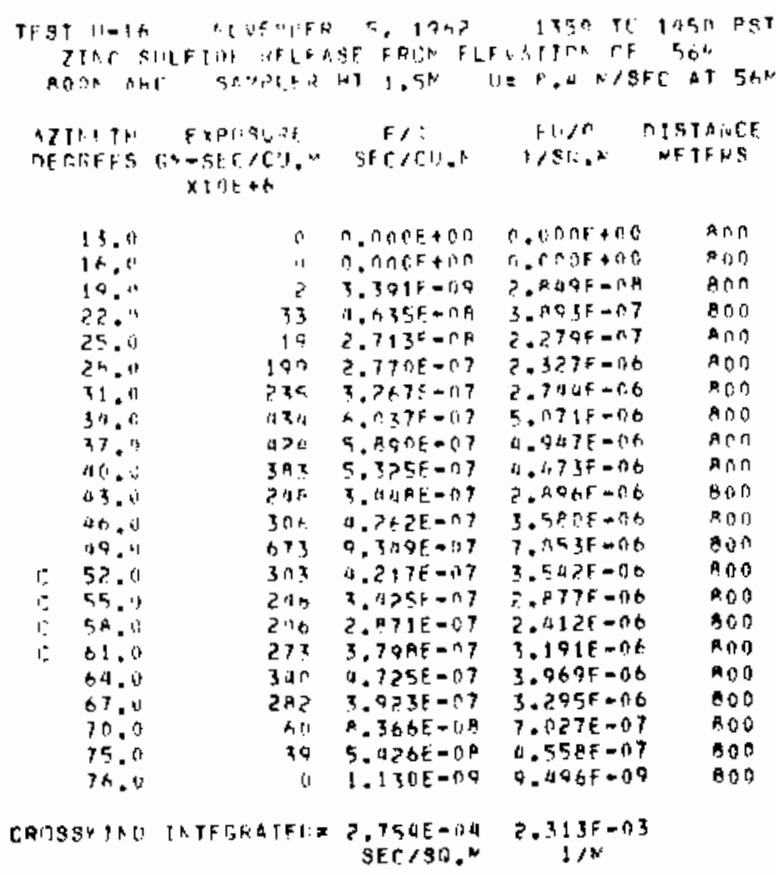

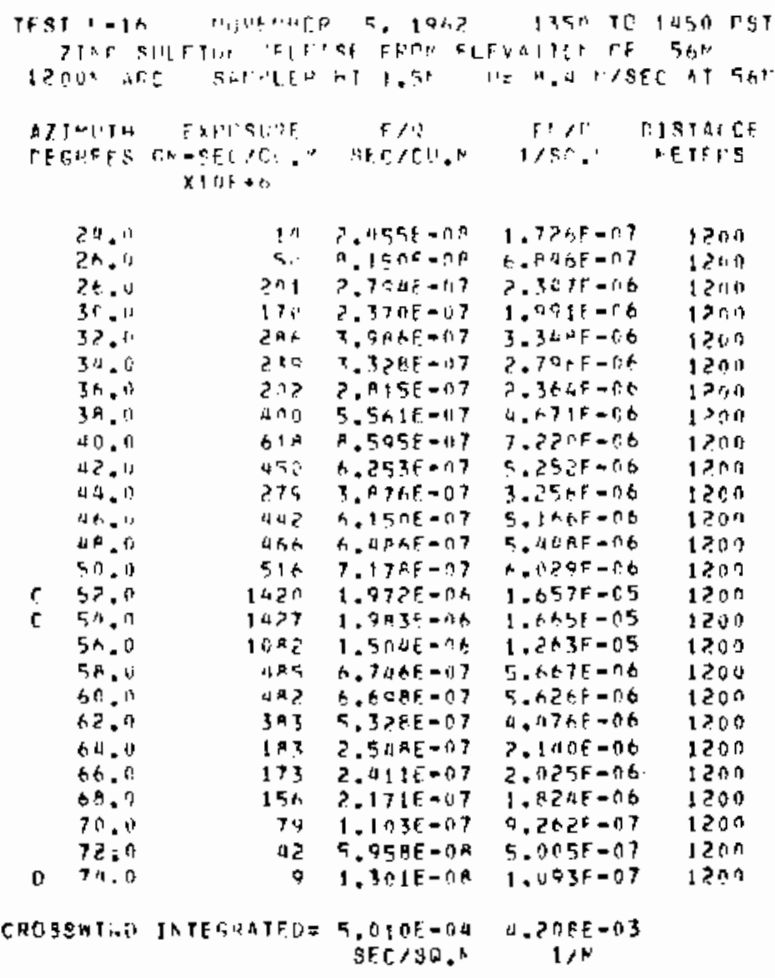

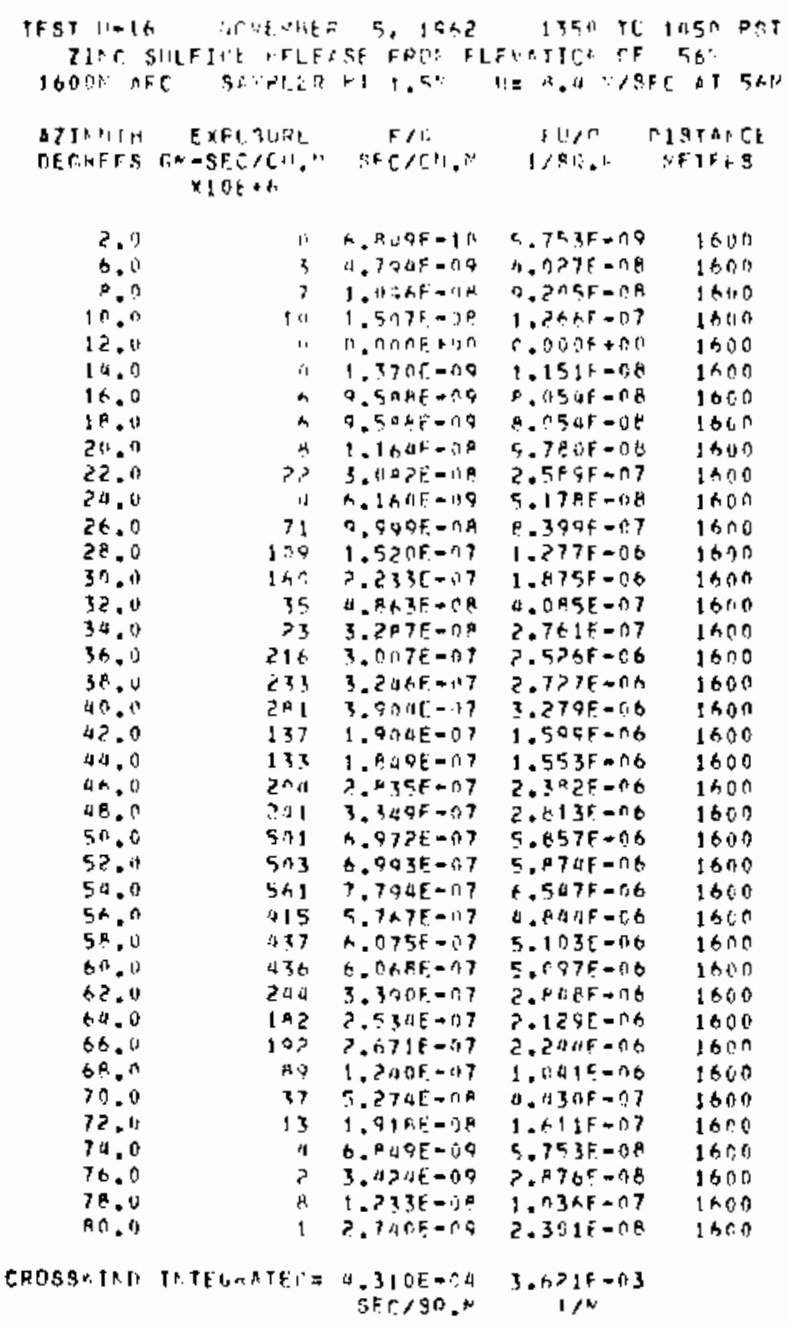


UNSTABLE SERIES EXPERIMENT: 17 (U-17)

GROUND LEVEL SAMPLING 300-1600 M ARCS. TRACER CONTAMINATION ON 300 AND 400 M ARCS.

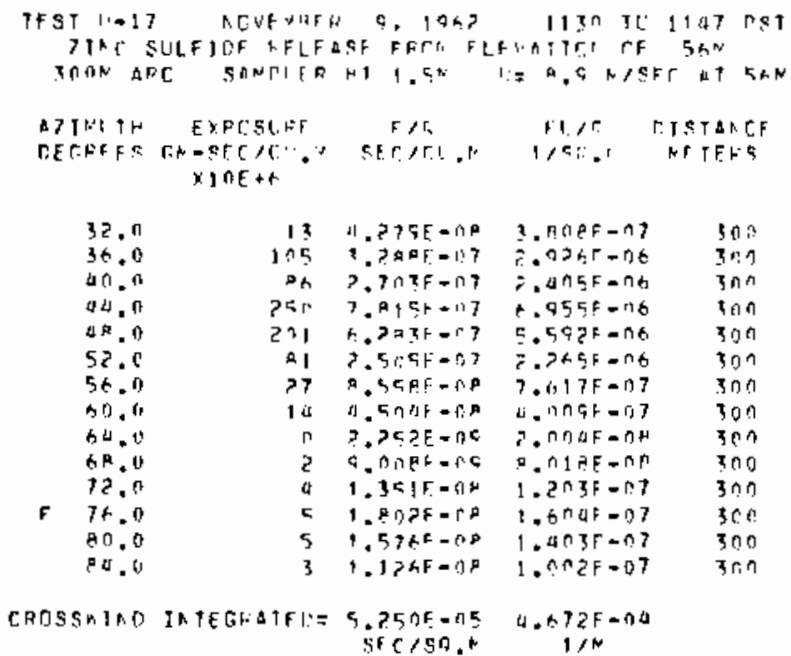

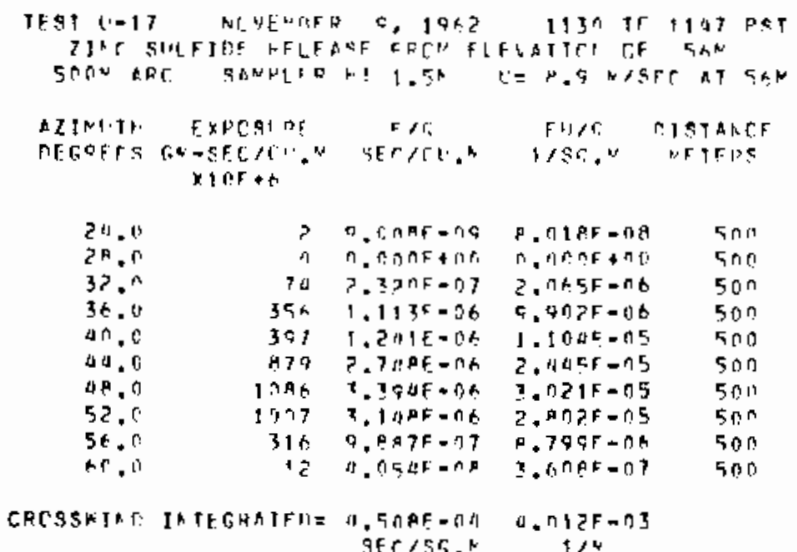




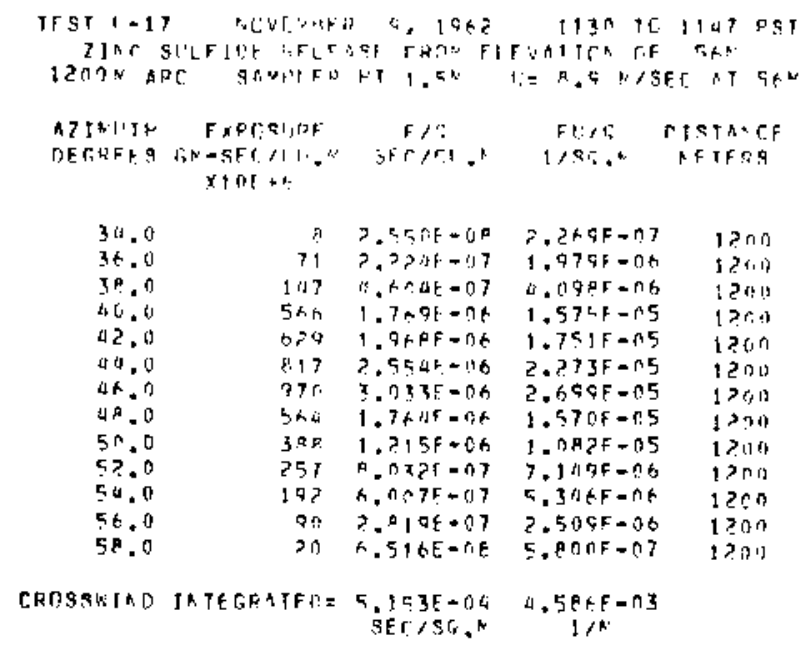

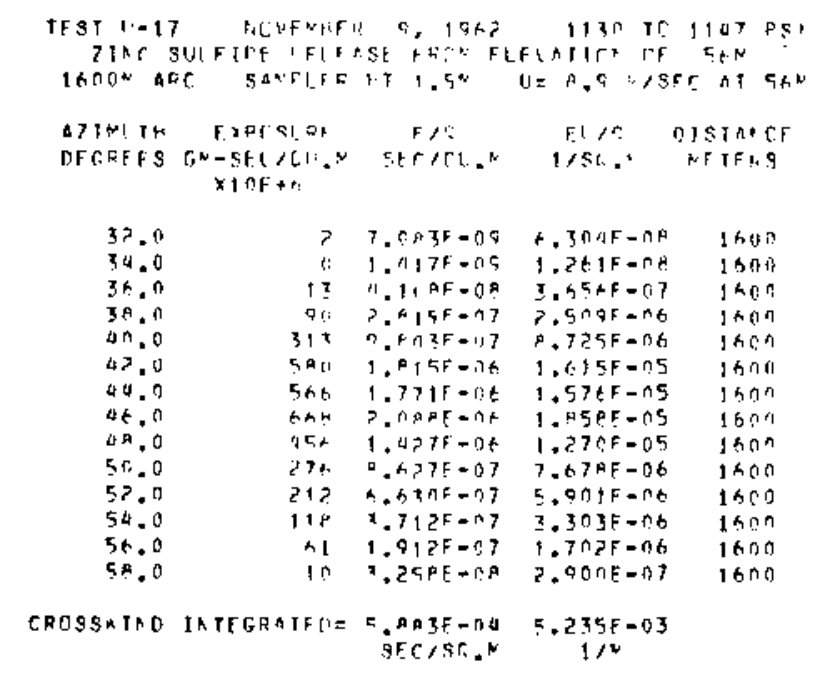


UNSTABLE SERIES EXPERIFEHT: 18 ( U-18)

GROUHD LEVEL SAMPLING 100-1000 HARCS. THE TRACER WAS HOT

DETECTED ON THE 100 OP, 200 l.1 ARCS.

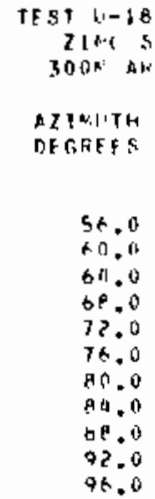

\begin{tabular}{|c|c|c|c|c|}
\hline 42.4 & ? & $P . n \times n+=n O$ & 1. $7 n 5 F=0 A$ & $\Delta 0$ त \\
\hline 50.0 & $\gamma$ & $5.523 \mathrm{H}-100$ & $4.689 F=\pi 8$ & 400 \\
\hline 54.0 & ? & $1.523 E=09$ & $t .2795-0.8$ & 100 \\
\hline $5 P .0$ & $>0$ & $1 . H \geqslant I E-D R$ & $1.104 \mathrm{~F}-077$ & 400 \\
\hline 62.11 & $1 \mathrm{~h} 7$ & $1.17\}\{-07$ & $9.891 F=07$ & 400 \\
\hline 60.0 & 105 & $7.11 B 11=11 A$ & $0.267 f=07$ & 400 \\
\hline 10.0 & 451 & $3,177 \mathrm{~F}-1\} 7$ & $2.069 f-06$ & 400 \\
\hline 74.0 & $3>1$ & $2+48=07$ & $1.9 n 1 E=00$ & don \\
\hline $7 \rho, 0$ & I thit & $1.157 \mathrm{E}=07$ & $4.7205-07$ & $4 n n$ \\
\hline R.2.0 0 & कर & $4.415 t=68$ & $3.7095-107$ & 400 \\
\hline 86.0 & ॥ & 7. $1105 E-09$ & $7.55 \mathrm{AF}=0 \mathrm{\theta}$ & 400 \\
\hline 9 & $z$ & $1.5>3 F-D G$ & $1.2795=18$ & 400 \\
\hline $911=0$ & ? & $7.930 E-04$ & $1.705 F=08$ & 400 \\
\hline
\end{tabular}

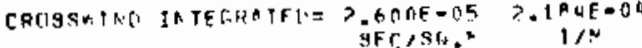

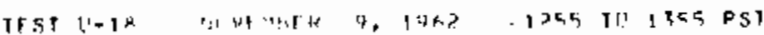

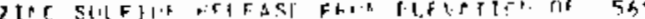

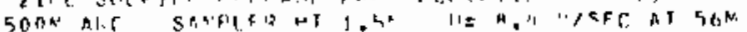

\begin{tabular}{|c|c|c|c|c|}
\hline 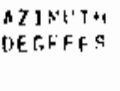 & 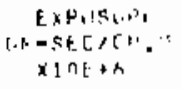 & 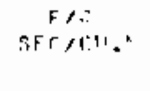 & $\begin{array}{l}1618 \\
1 / 5: 2:\end{array}$ & $\begin{array}{l}\text { PISIARCE } \\
\text { OFIEISS }\end{array}$ \\
\hline 52.0 & 15 & $B . \operatorname{In} B \mid=D A$ & $4,75 ?[-0 R$ & $50 n$ \\
\hline $5 t, 1$ & 43 & $x . n d 5 t-n p$ & 子. T $\{E, F=n\}$ & $5 \operatorname{trn}$ \\
\hline 61:. is & $\bar{c}^{2}$ & $1.5,4 \uparrow=1,7$ & $1,: 111+-0, b$ & $5 n n$ \\
\hline 50.0 & $351:$ & $1.7+1 E-07$ & $1.37 \mathrm{CF}=1) b$ & 5011 \\
\hline$\neq 0.0$ & $147 x$ & $1 . n+E E-A B$ & D. $411+5=0 b$ & $5+2$ \\
\hline 72,0 & 557 & $3.97 \times E-\hat{H}$ & $3.2755=116$ & $50 \%$ \\
\hline $76-11$ & 1119 & $A . A>D E=07$ & $+.7 a t F=0 b$ & 500 \\
\hline$A[1,1$ & $5>\mathrm{H}$ & $3.7>018-07$ & $I .125 E=106$ & 500 \\
\hline a 0 & 1.5 & T. J1OE $=38$ & $=.224 F=07$ & $5 n 0$ \\
\hline .0 & u & 3.0 , $45 E-118$ & $3.5584-08$ & 506 \\
\hline
\end{tabular}

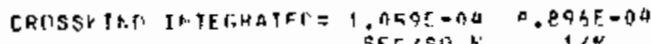

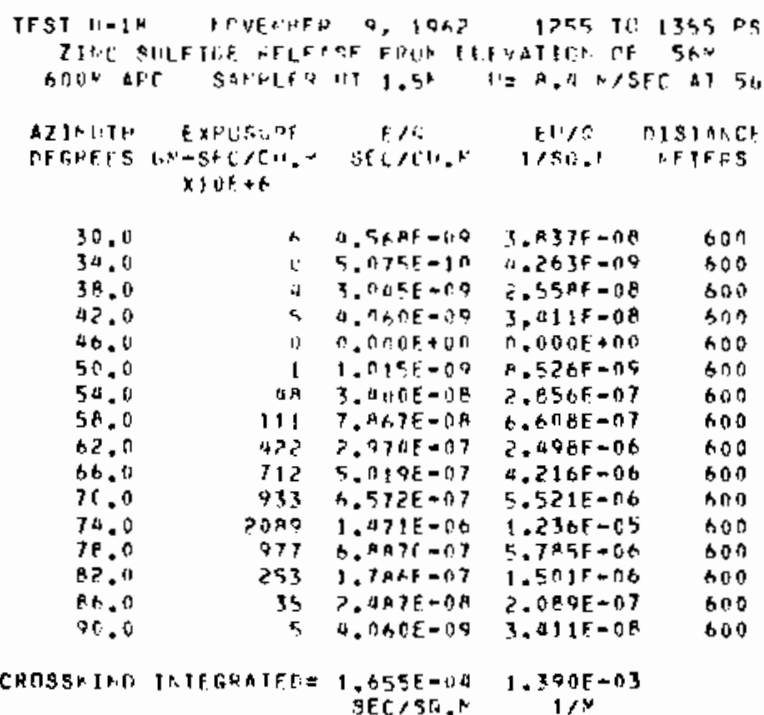

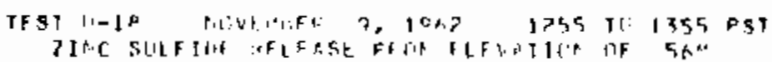

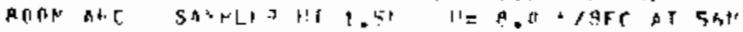

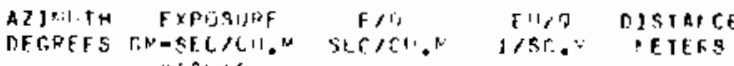
$\times j 0 t+6$

\begin{tabular}{|c|c|c|c|c|}
\hline 46.0 & 5 & $3.55 ? 5=n ?$ & $2.9 P$ b $F=08$ & Fis \\
\hline 45.0 & t. & $5.9756-18$ & $4.2+35-1) 4$ & Alin \\
\hline 52.0 & 71 & $5 . n \geq 4 f=n f$ & $4.22 ! E=97$ & $A 00$ \\
\hline 55.0 & $1+1$ & 1. $2 \cap 5 . E=07$ & $1.015 F=06$ & $A_{1} \cap$ \\
\hline $58, n$ & 34.3 & $1, P 5>E-\cap 7$ & $1.556 F-0.6$ & Reo \\
\hline 61.0 & $65 c^{2}$ & $595 E-117$ & $3.862 E-176$ & $A 00$ \\
\hline 60.0 & 415 & $\triangle C E=11$ ? & $4.8225-006$ & 000 \\
\hline 67.0 & ?Ins & $14 F-1,6$ & $X E-0.5$ & sin \\
\hline 70.0 & 2944 & $D, \cap P Q E=\cap C$ & $1.754 \mathrm{~F}=\mathrm{i}_{\mathrm{s}} \mathrm{S}$ & $\theta \cap 0$ \\
\hline 73.0 & $33>3$ & $? .3+\cap \cap 5=116$ & $1.96+F=05$ & ant \\
\hline $7 \epsilon .0$ & 1350 & $.5 a 1 \leq-n 7$ & $A .015 F=0.6$ & Ron \\
\hline .0 & $9>8$ & $5378-177$ & $5.491 f=06$ & $8 \cap 0$ \\
\hline .0 & 470 & $0,=117$ & $2.830 E=06$ & 800 \\
\hline & 10.8 & $?-A 13 E=0 \Omega$ & $6.395 E-07$ & son \\
\hline$B P .0$ & $\%$ & A. $598 E-C E$ & $5.542 F=0 B$ & BOO \\
\hline
\end{tabular}

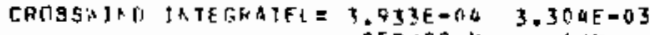




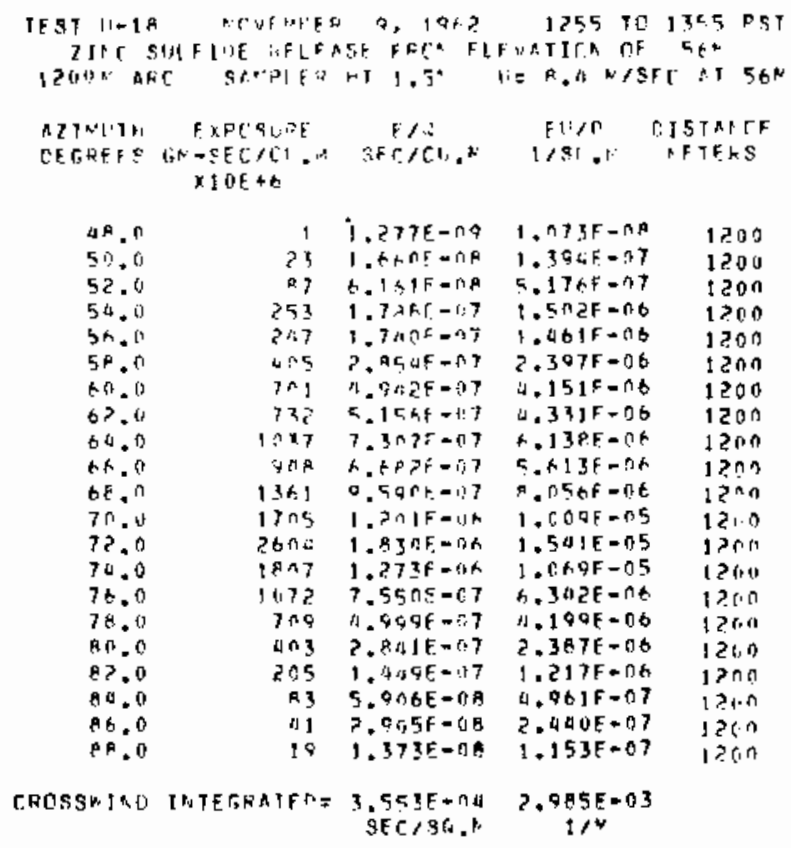

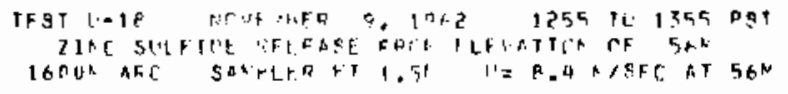

\begin{tabular}{|c|c|c|c|c|}
\hline$a 0.0$ & \& & $x .53+E-100$ & $3.21 P F-0 P$ & $1 \tan 0$ \\
\hline 42.6 & 1 & $r$. inforton & $4.0005+\pi 0$ & $1+n n$ \\
\hline 40.0 & $1^{4}$ & $1.341 E-63$ & $1.12+5-07$ & $1 \ln 10 n$ \\
\hline $4+0$ & $\therefore$ & 1. $T Q O E=I S C$ & $11.0225=0 . \theta$ & 16,0 \\
\hline$M A .0$ & 2 & $1.591 . \mathrm{E} \rightarrow \mathrm{nO}$ & 1. $341 E-08$ & 1600 \\
\hline 50.0 & 77 & $1.547 F=0 A$ & $1.036 E=f: 7$ & 1600 \\
\hline 52.0 & ha & $1.5 \because T E=\cap P$ & $3 ., 7,2 E=0,7$ & 1600 \\
\hline 50.0 & 167 & $1.170 E-37$ & $9.895 F=07$ & 1600 \\
\hline $5 n, 0$ & $19 \%$ & $1.370 \mathrm{r}_{-}-0.7$ & $1.150 F=n 6$ & 1000 \\
\hline $5 e .0$ & $14 \mathrm{~A}$ & $1 . n+7 E=37$ & R. $.796 E-077$ & 1600 \\
\hline (9.0 & ZAQ & $? .637 \mathrm{E}=07$ & $1,711 \varepsilon=06$ & 1600 \\
\hline 62. 0 & $2 \geqslant ?$ & $0.457 E-107$ & $3.7445-108$ & 1600 \\
\hline 60.0 & 701 & $5.574 E-37$ & 4. $A$ A $2 E-16$ & 1600 \\
\hline 60.0 & 914 & $\therefore$ मAC & $5.7 \mathrm{~A} 36=0.6$ & 1600 \\
\hline 68.0 & $1 \backsim \cap n$ & 7. naGE-i) & $5.921 E=90$ & 1600 \\
\hline 70.0 & 163,1 & $7.255+07$ & $E .119 E=6 t 6$ & 1660 \\
\hline 72.0 & 1112 & $P .0 \leqq / F-57$ & $5.763 E-16$ & 1600 \\
\hline 70.0 & $16 \mathrm{bll}$ & $7.0775-\hat{n}$ & $t .280 \mathrm{E}=0 \mathrm{t}$ & 1600 \\
\hline 76.9 & RAI & $5.2 \cap 9 E-07$ & $5.216 E=06$ & 1600 \\
\hline $7 e .0$ & $5+5$ & $7.57 \mathrm{hE}=07$ & $2.104 E=08$ & 1600 \\
\hline 80.0 & $2 \pi 4$ & $1.475 F=07$ & $1,235 E-0.6$ & 1600 \\
\hline 82.0 & 147 & $1,0 \cap E E=37$ & P.447t-07 & 1600 \\
\hline Q.1 0 & 11 & $2.905 \mathrm{E}=08$ & $2.000 E-n 7$ & 1600 \\
\hline 9.0 & 2 & $A .3 * 5 E-79$ & $5.3+3 E=08$ & 1600 \\
\hline-1 & $1 \tilde{c}$ & $\mathrm{k} .939 \mathrm{E}=03$ & $7.509 F=\hat{A}$ & 1600 \\
\hline & & $\begin{array}{l}3.6>9 E-125 \\
8 F, / 30.12\end{array}$ & $\begin{array}{c}3.0, a 9 F=0.3 \\
1 / x\end{array}$ & \\
\hline
\end{tabular}


UHSTAELE SERIES EXPERIHEHT: 19 (U-19)

GROUNO LEVEL SAMPLING 1 G0-3200 M ARCS. A SIGE:IFICANT PORTION OF

THE PLUME PASSED TO THE NORTH OF THE GRID.

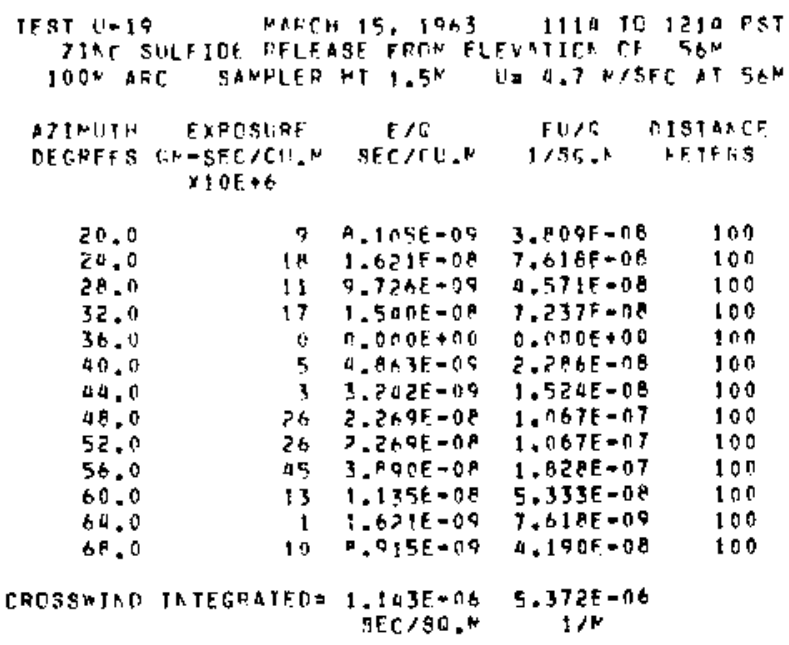

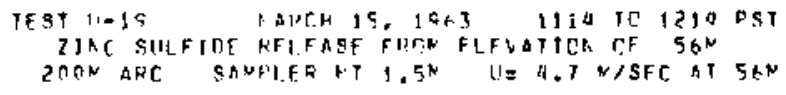

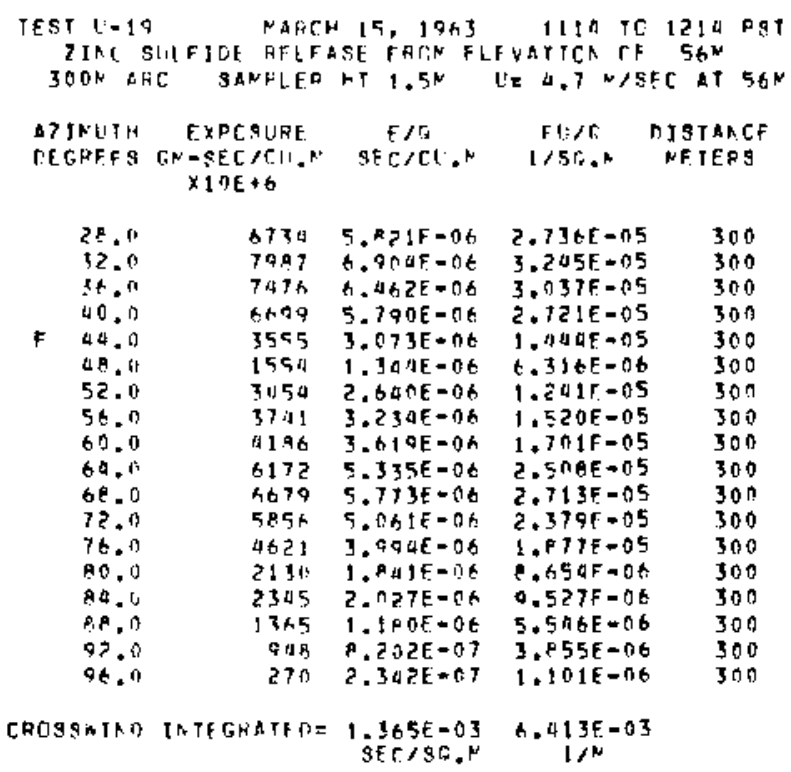

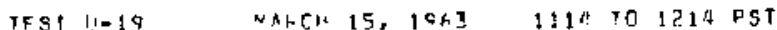
IJAC SUIFJDF HFIFASE FWTN ELFUATICA OF $5 \mathrm{GN}$ AOBN MAR SANELFR HT $1.5 \mathrm{~N} \quad \mathrm{~V}=4.7 \mathrm{~N} / 5 \mathrm{FC}$ AI $56 \mathrm{~N}$

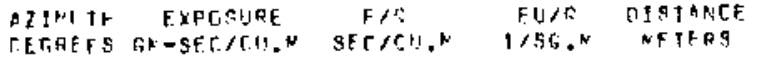

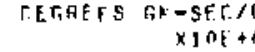

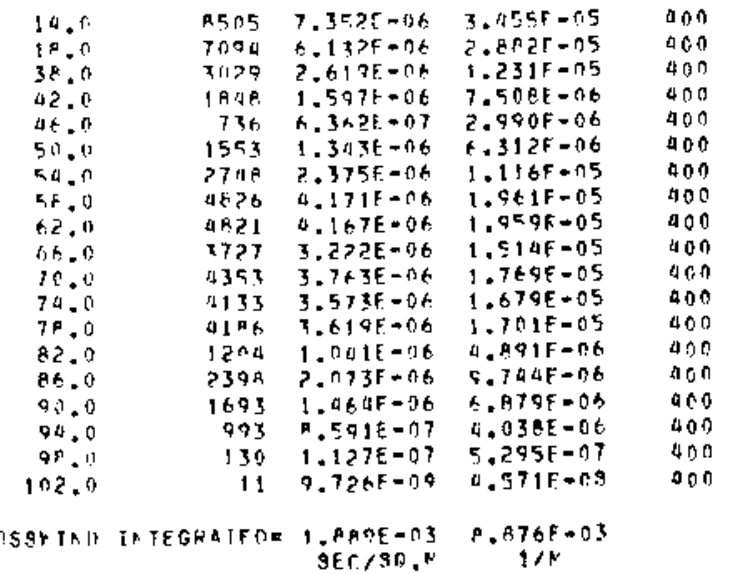




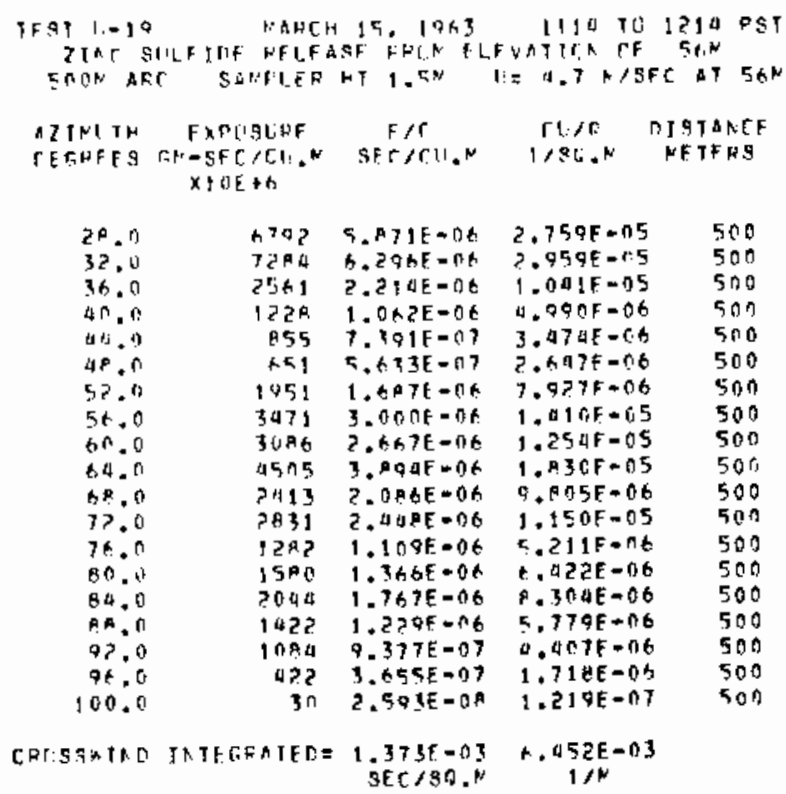

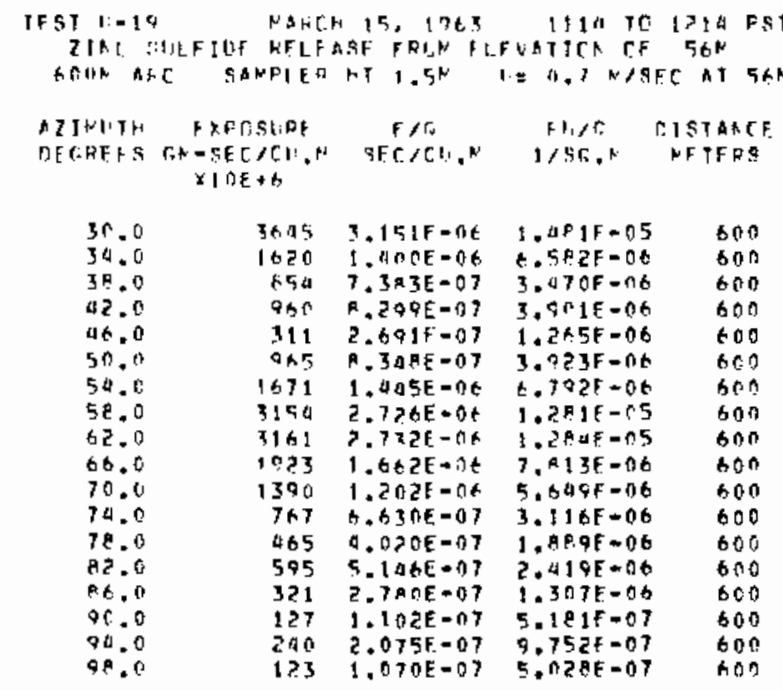

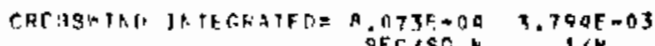

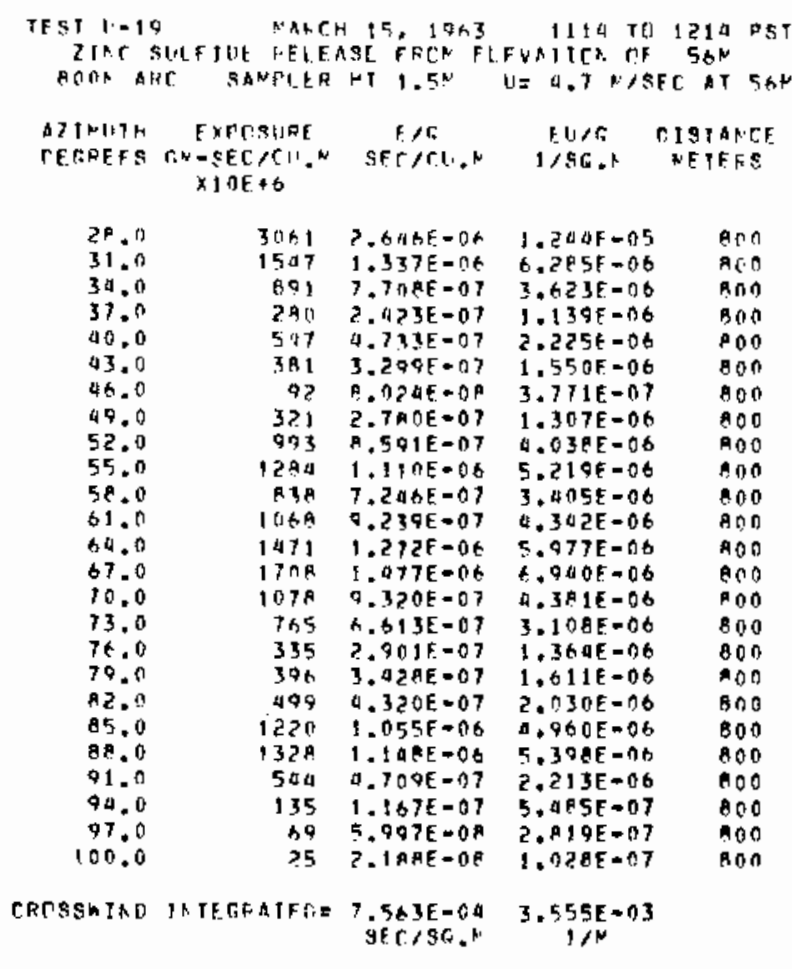




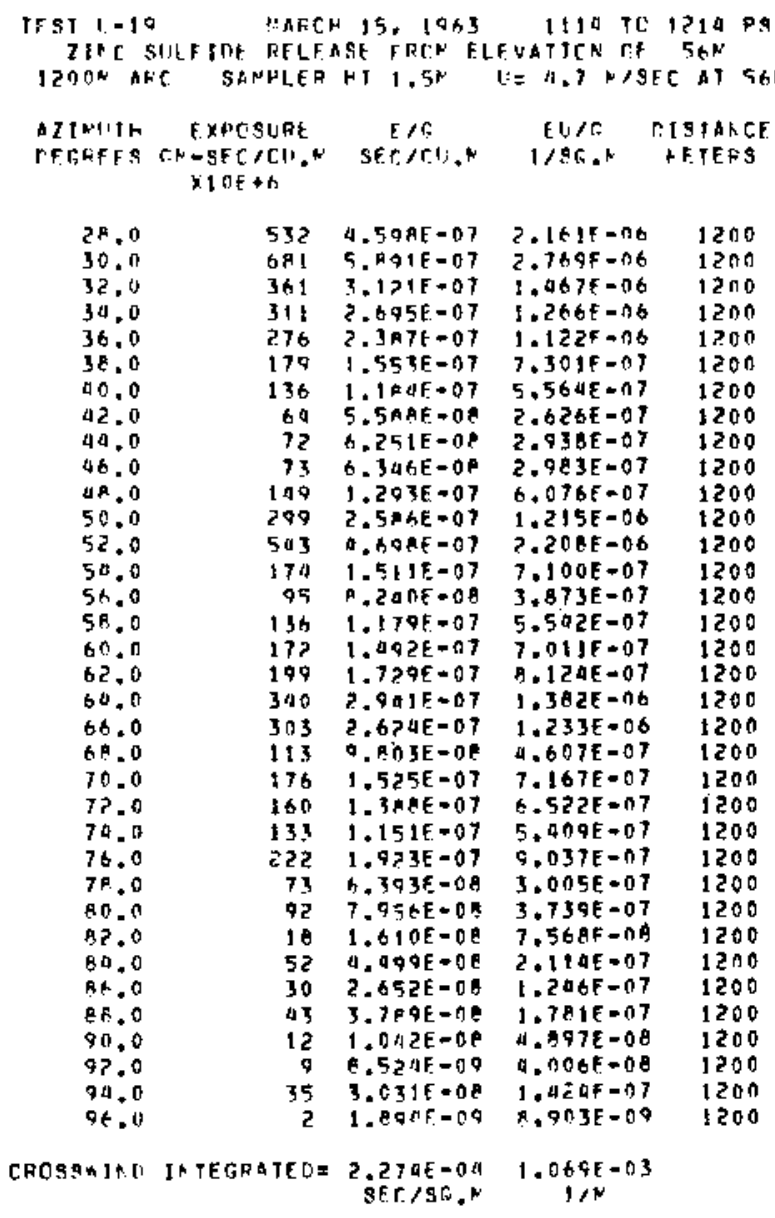

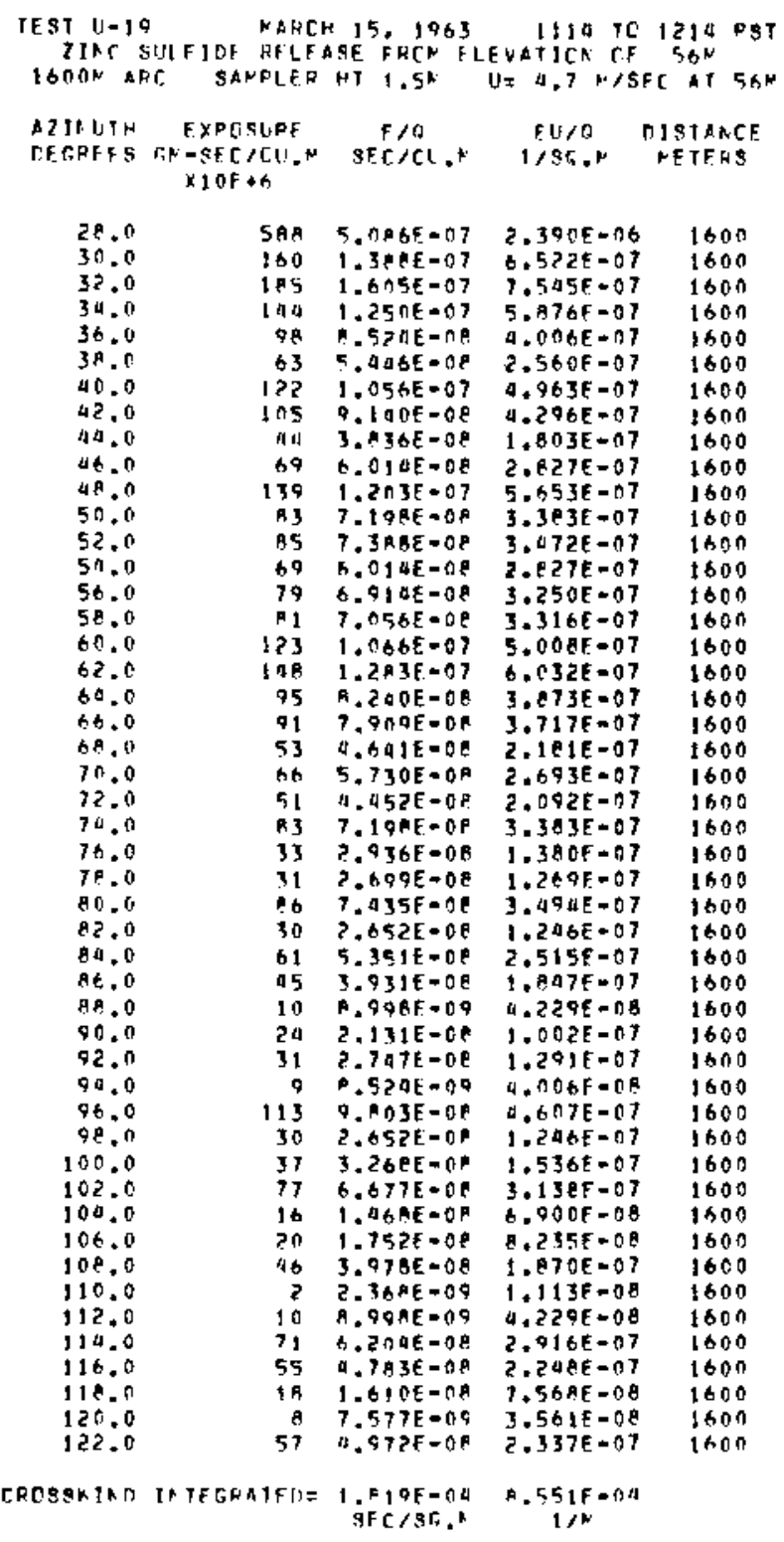




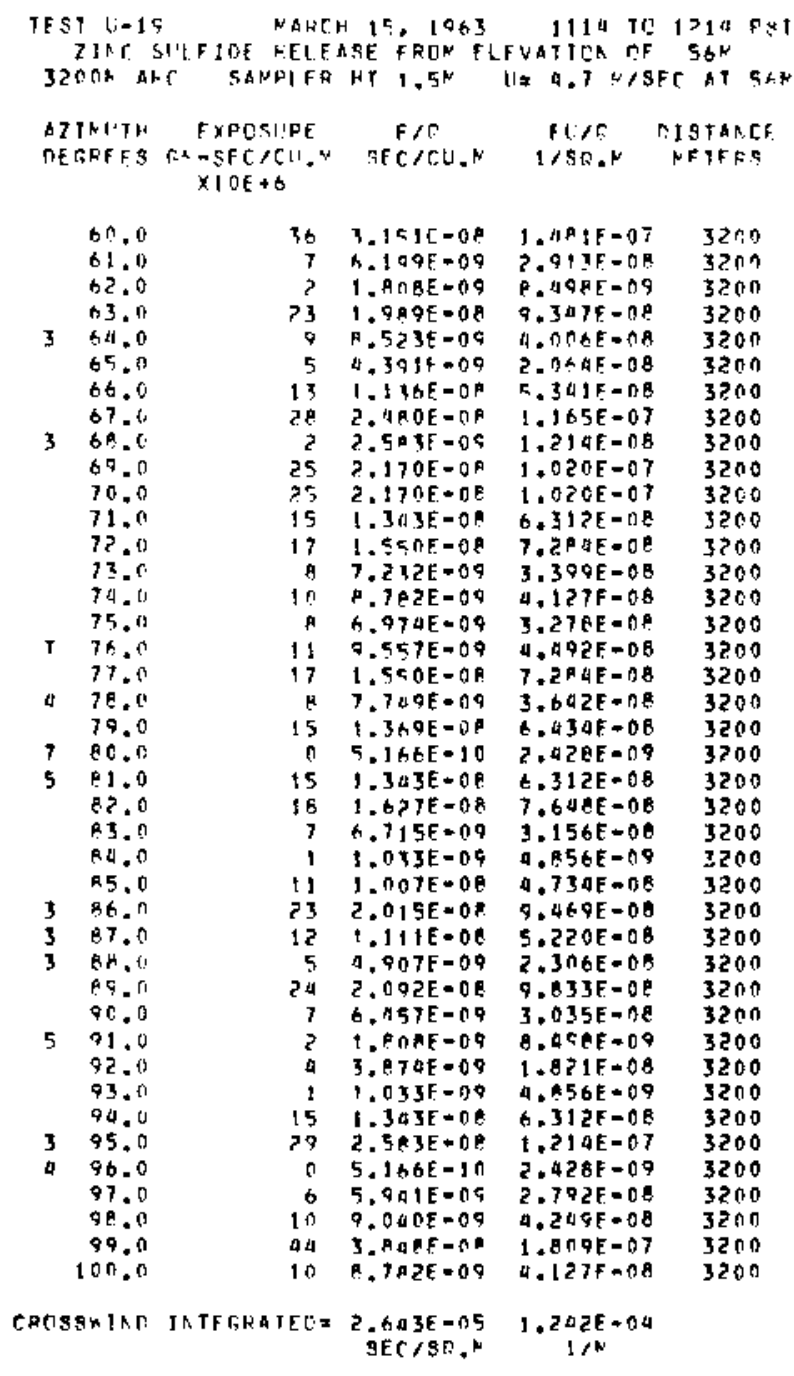


UNSTABLE 5ERIES EXPERI HENT: 20 ( U-20)

GROUND LEVEL SAMPLING TOO-1600 M ARCS.

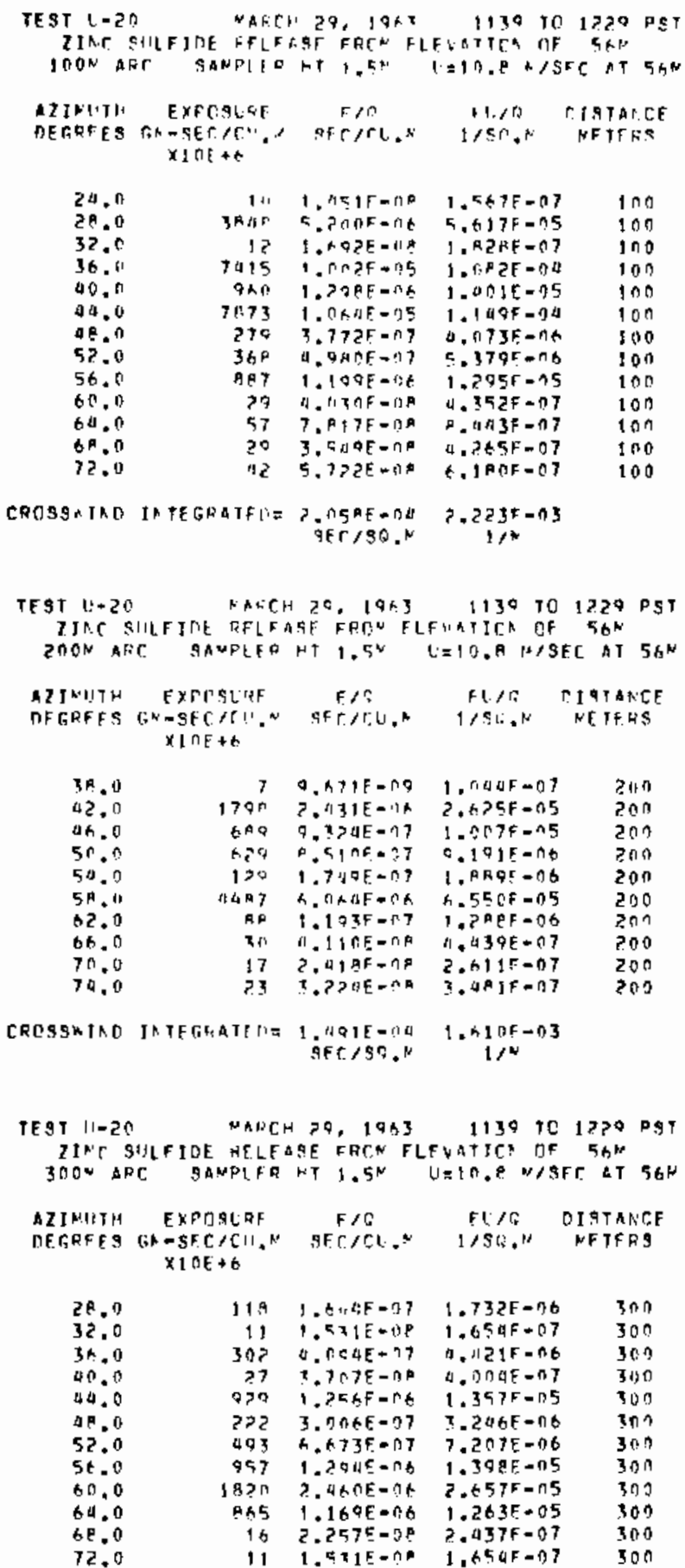

\begin{tabular}{|c|c|c|c|c|}
\hline $\begin{array}{l}28.0 \\
32.0\end{array}$ & $\begin{array}{r}119 \\
11\end{array}$ & $\left\{\begin{array}{l}6+t, E=? 7 \\
5 \times 1 E-0 p\end{array}\right.$ & $\begin{array}{l}1.732 F-76 \\
1.654 F-07\end{array}$ & $\begin{array}{l}300 \\
300\end{array}$ \\
\hline $3 \ldots, 0$ & 307 & $0.7<4 E+77$ & a. $12 द 1 F=06$ & 300 \\
\hline 40.0 & 27 & TAOTE-AP & $4.1) \cap Q E=n 7$ & 3ivo 0 \\
\hline 44.0 & $Q>Q$ & $1.2565=106$ & $1.3575=05$ & 300 \\
\hline$\triangle P .0$ & 202 & $3.5 n \in E=37$ & $3.246 F=106$ & $\ln n$ \\
\hline 52.0 & 493 & $.073 F-17$ & $7.207 E-06$ & $30 ?$ \\
\hline $5 t .0$ & 957 & $1.20 H E=n b$ & $1.398 F-05$ & $30 n$ \\
\hline 60,0 & חק? & ?. $4 A \cap E=0 A$ & $z, t 57 F=15$ & 300 \\
\hline 64.0 & 605 & $1.169 E=06$ & $1.263 E-05$ & 309 \\
\hline 0 & 16 & $57 E-5 P$ & $037 F=0.7$ & 0.0 \\
\hline & 11 & $1.5 \times 1 E=0$ & $1,65 a F=A 7$ & 300 \\
\hline
\end{tabular}

CROGGKINO ITTEFAATFE $=1.635 E-94 \quad 1.7665-03$

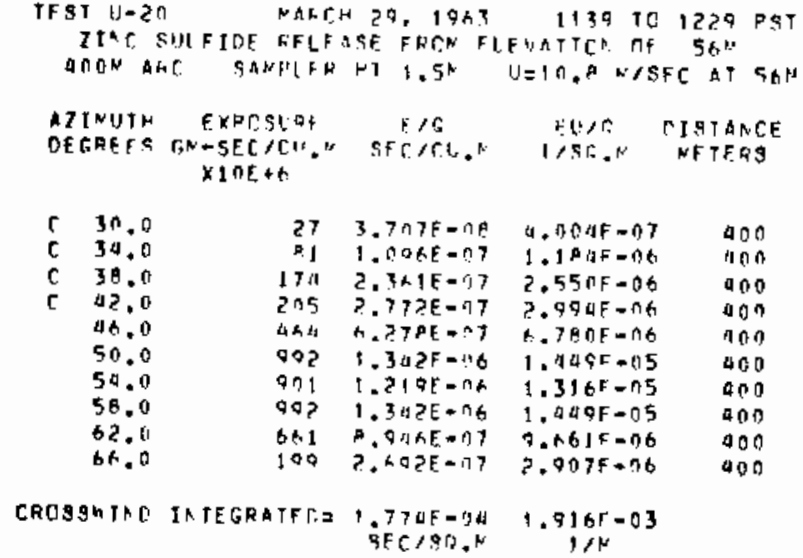




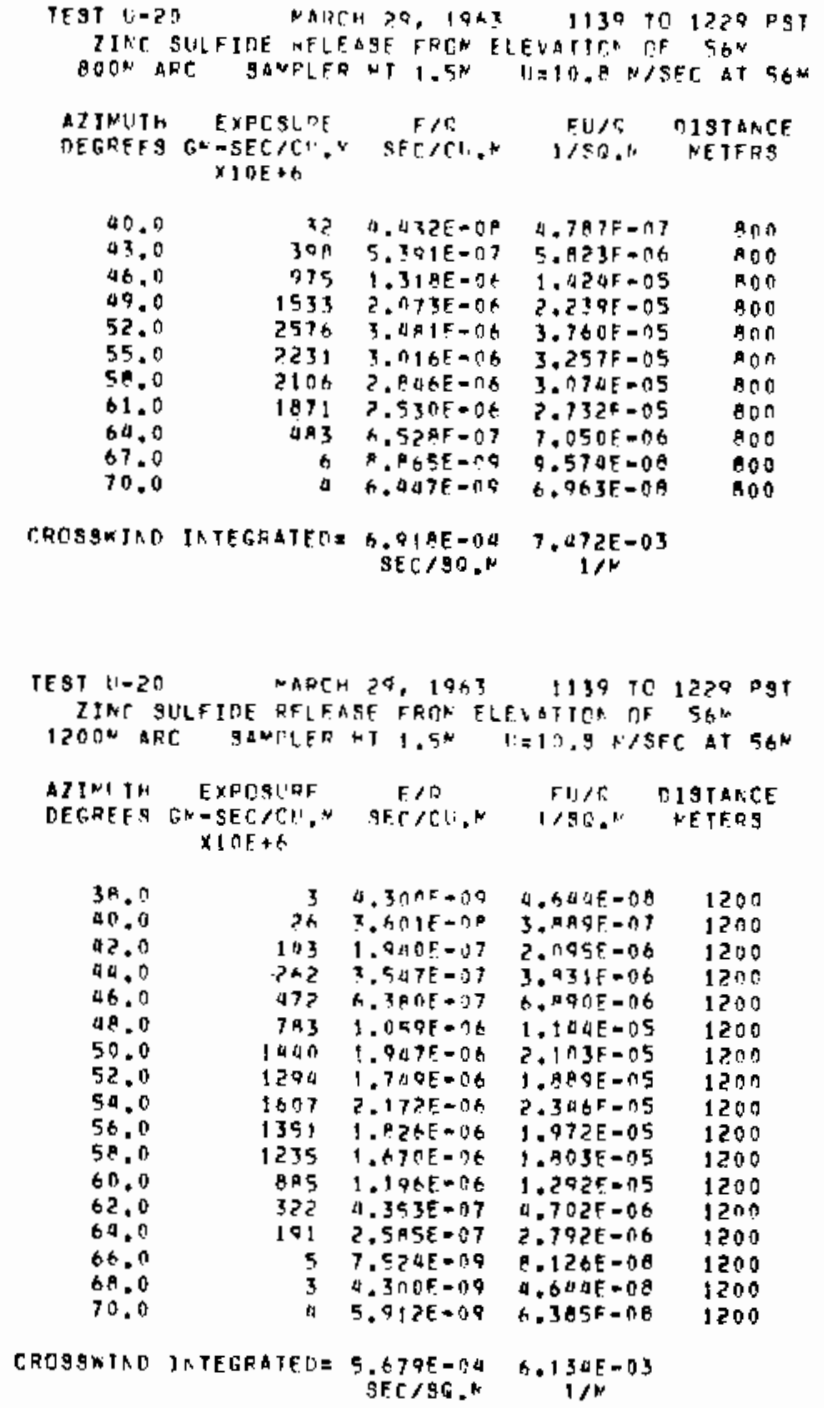

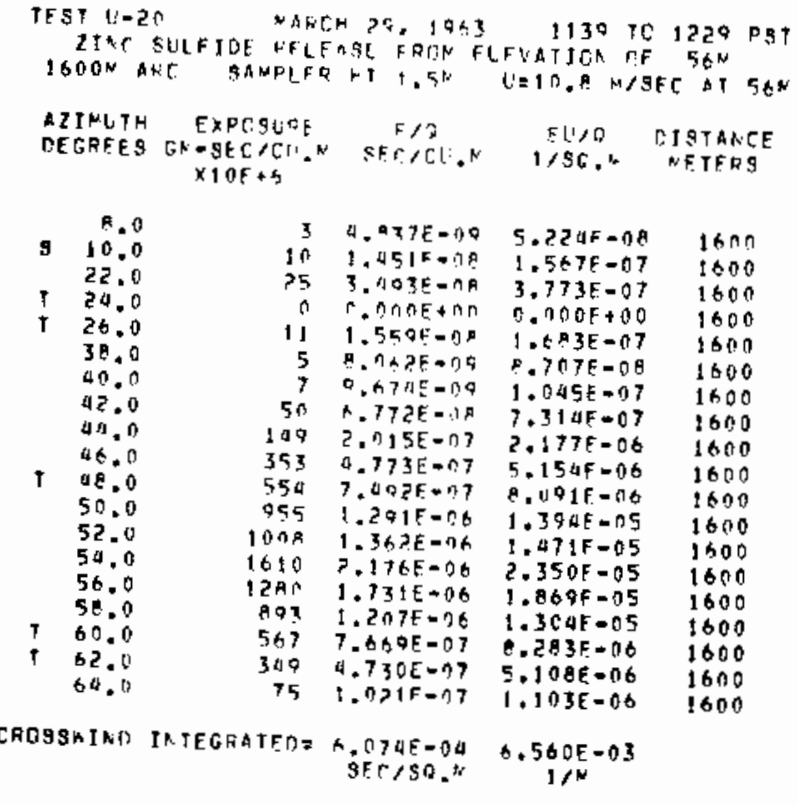


UNSTABLE SER IES EXPERIMENT: 2; ( U-21)

GROUND LEYEL SAMPLING $100-1600$ M ARCS. THE TRACER WAS NOT DETECTED ON THE TOO OR $200 \mathrm{M}$ ARCS.

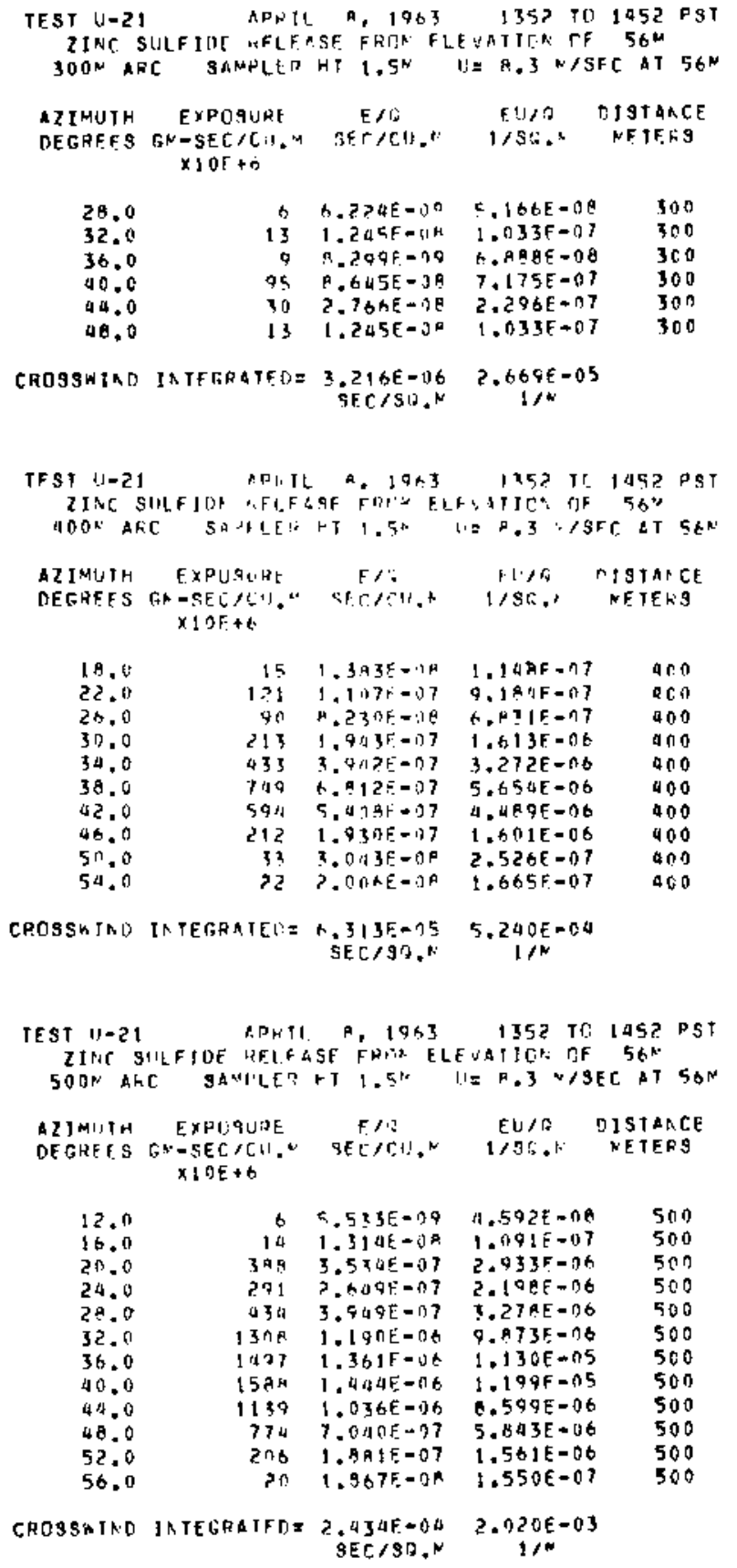

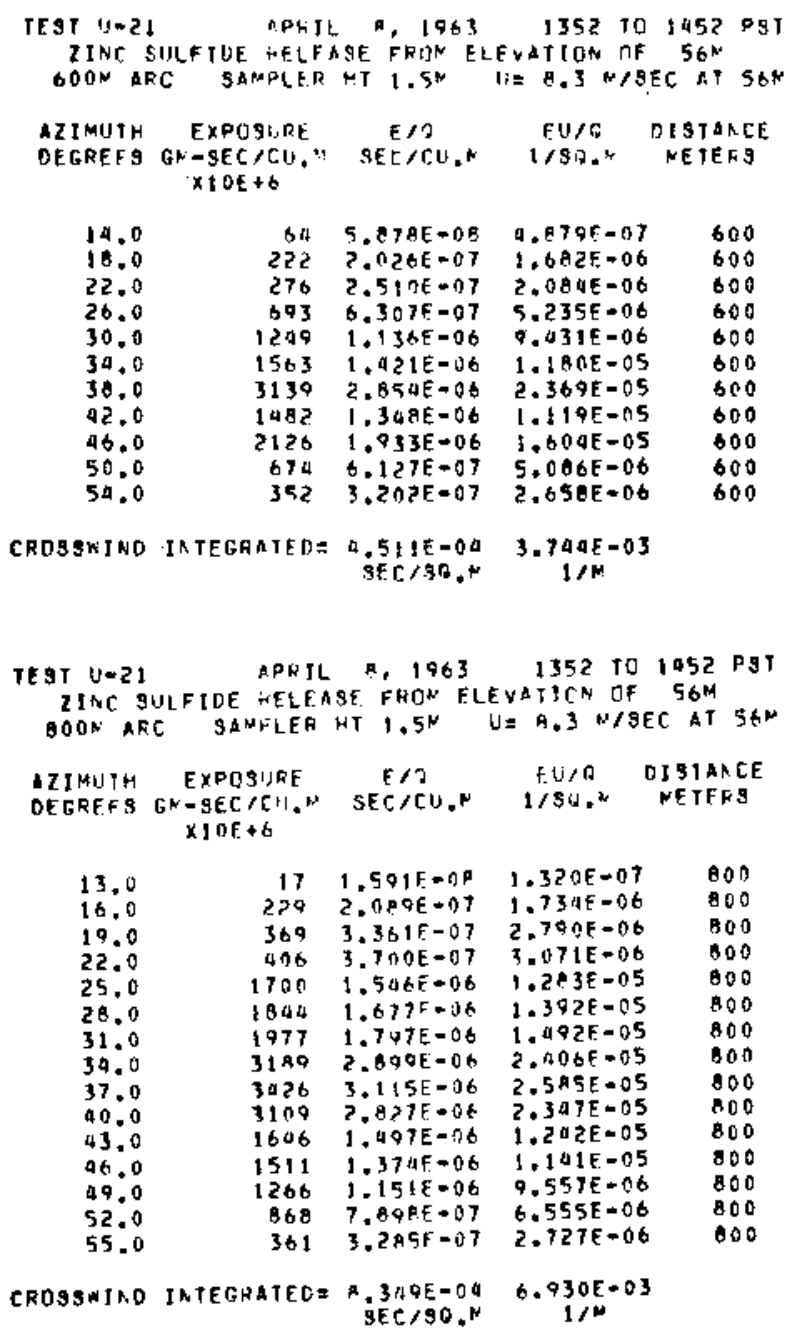




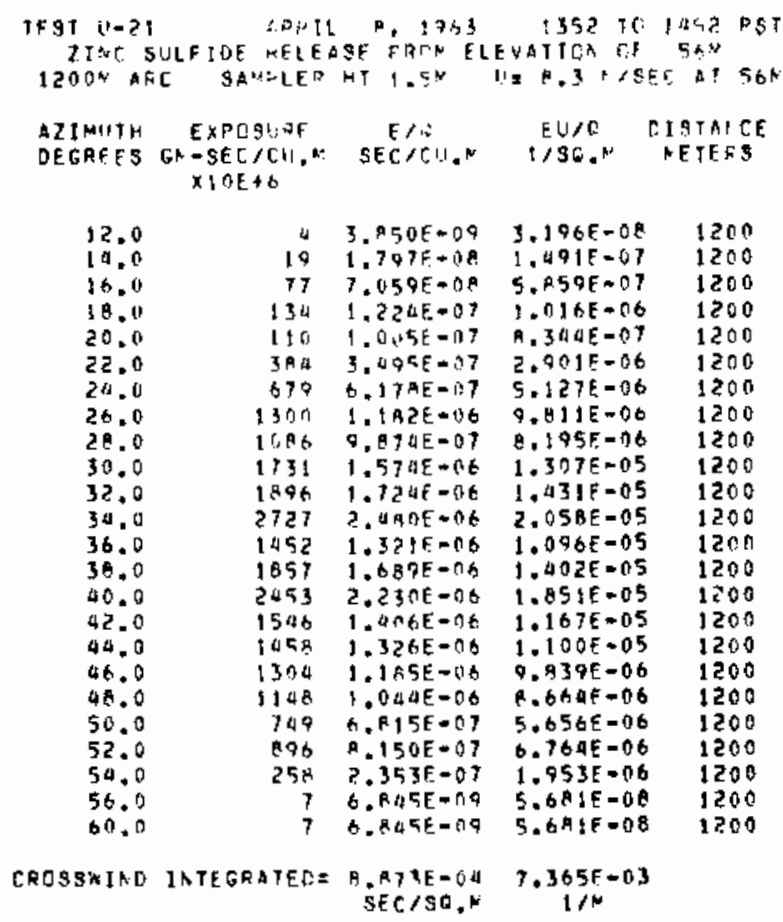

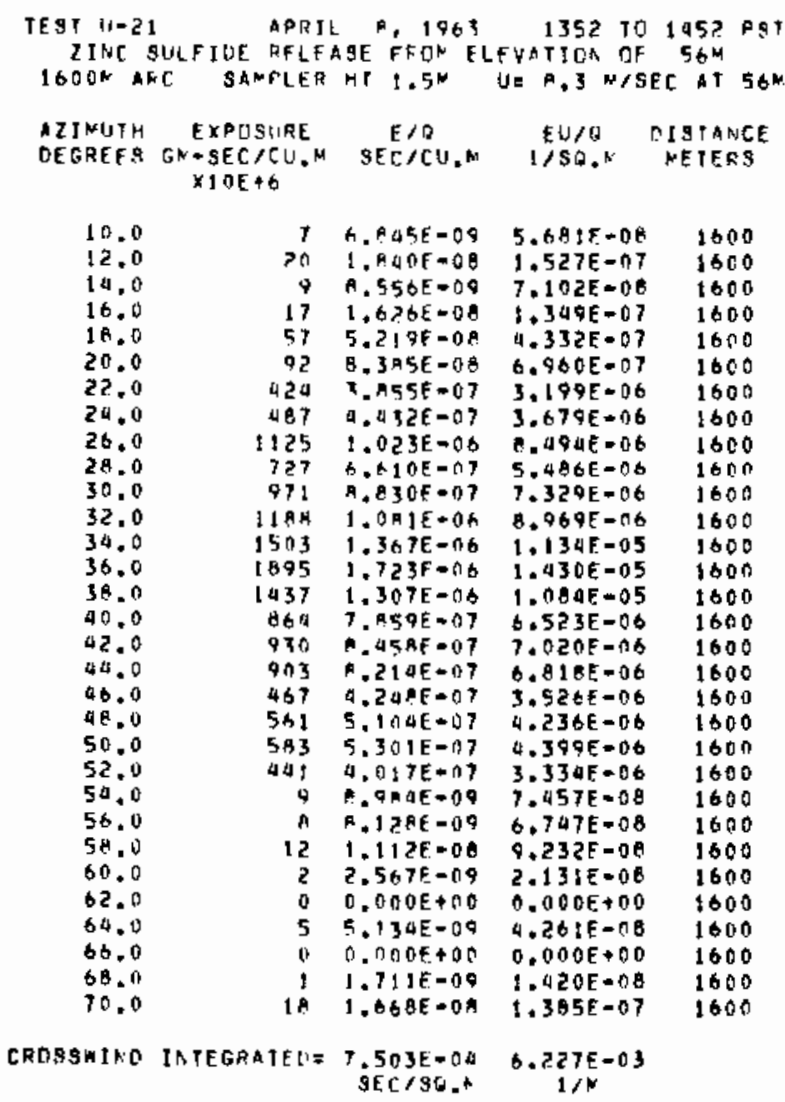


UNSTABLE SERIES EXPER|MENT: 22 \{ U-22 \}

GROUND LEVEL SAMPLING 100-1600 M ARCE.

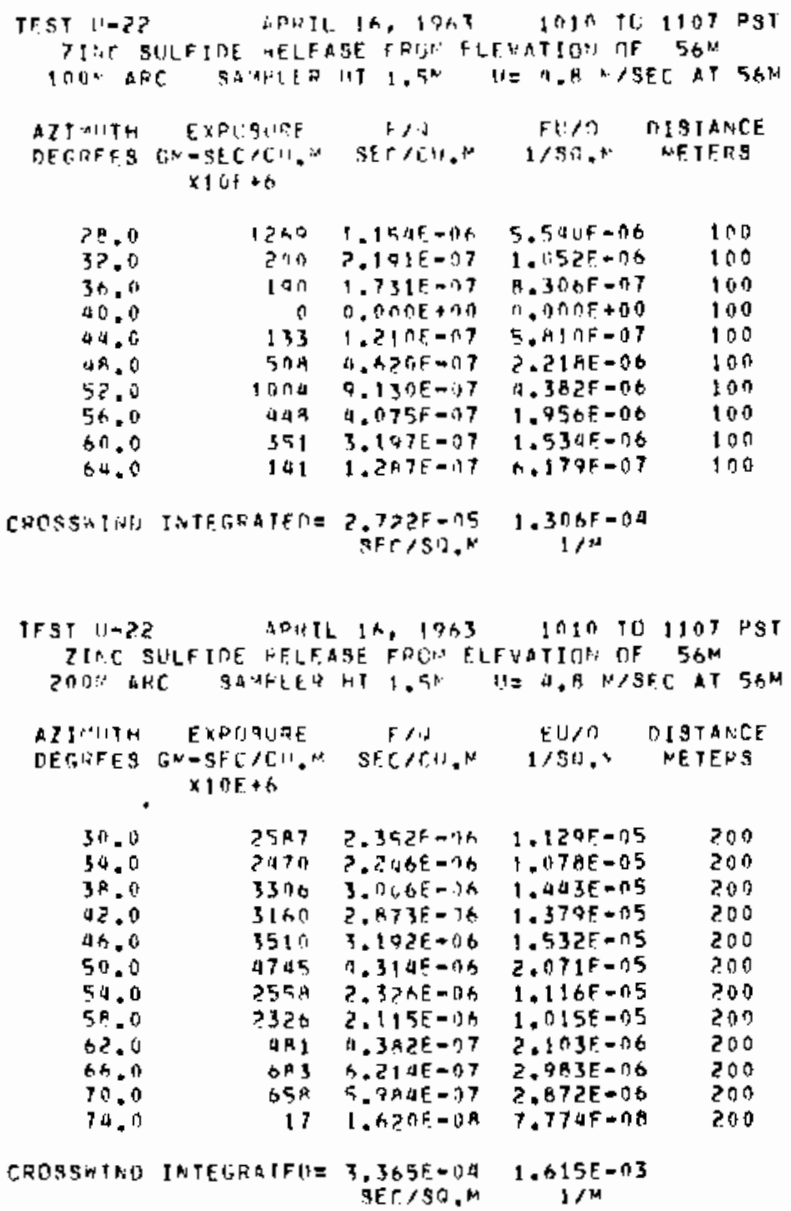

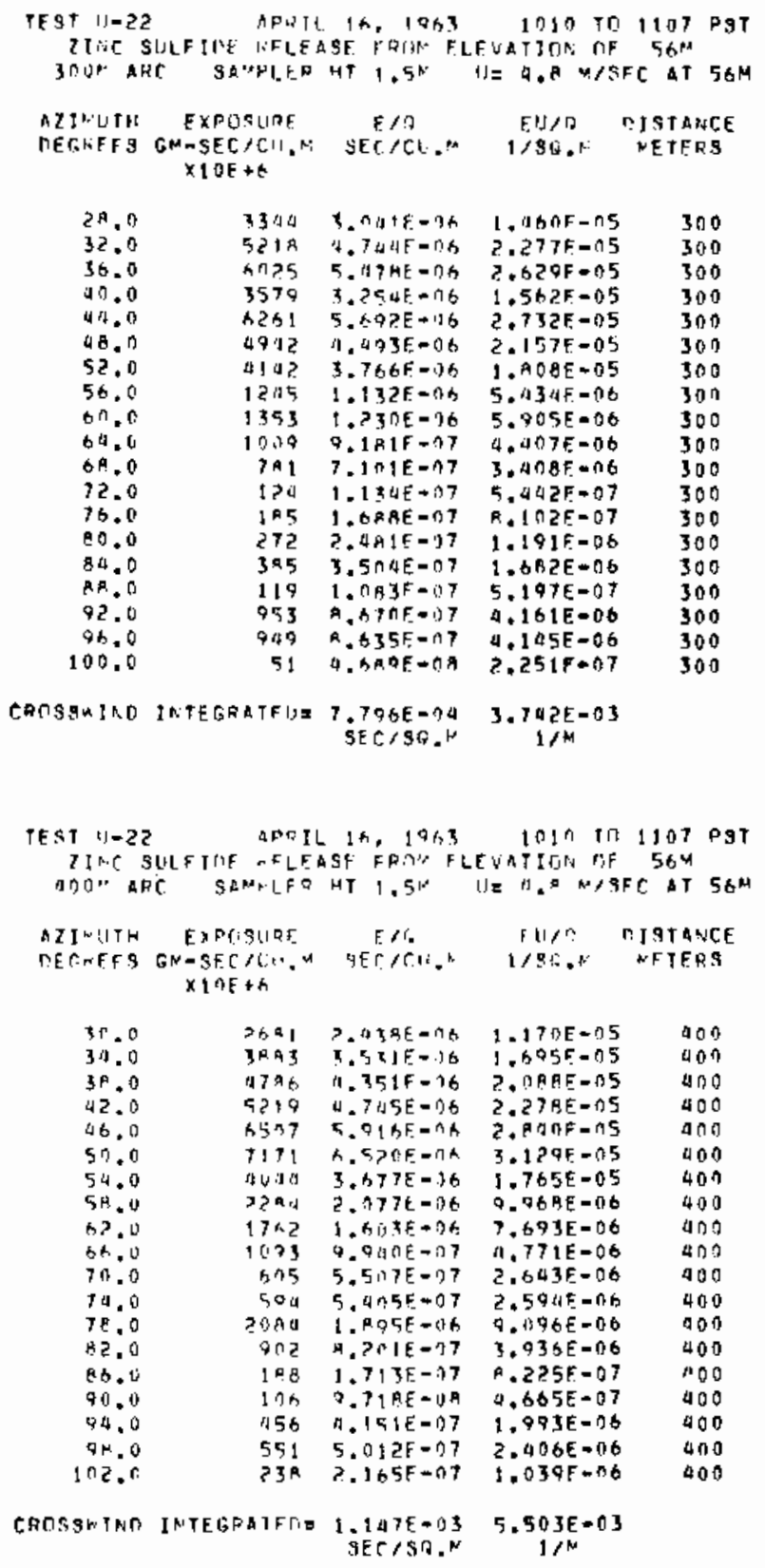




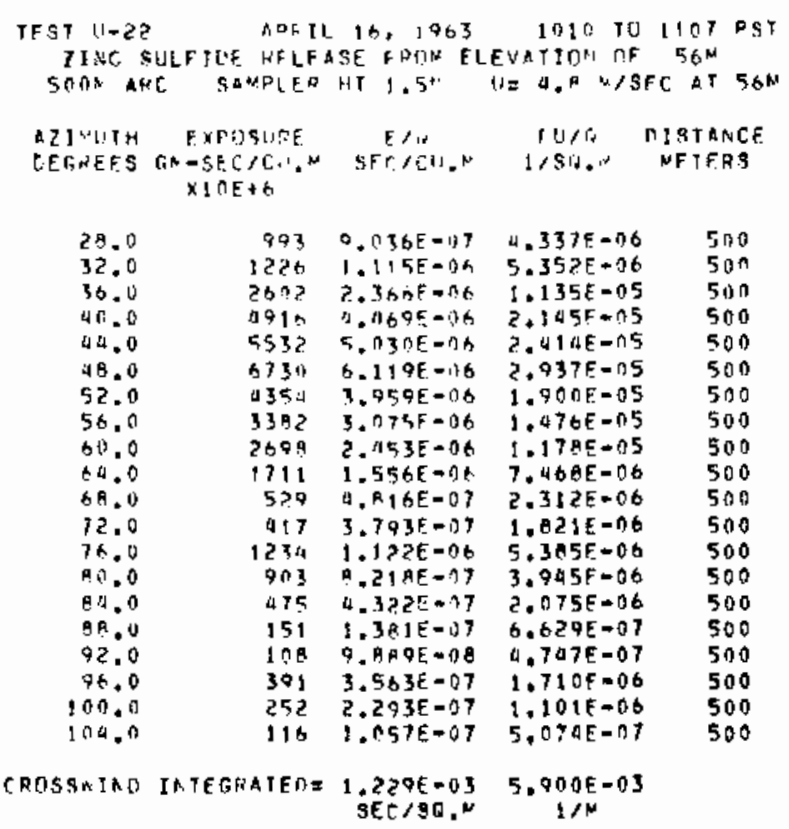

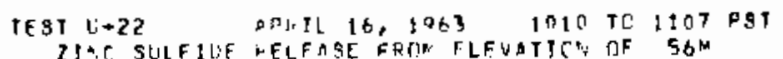

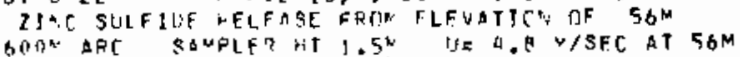

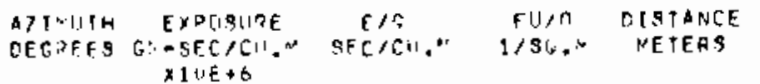

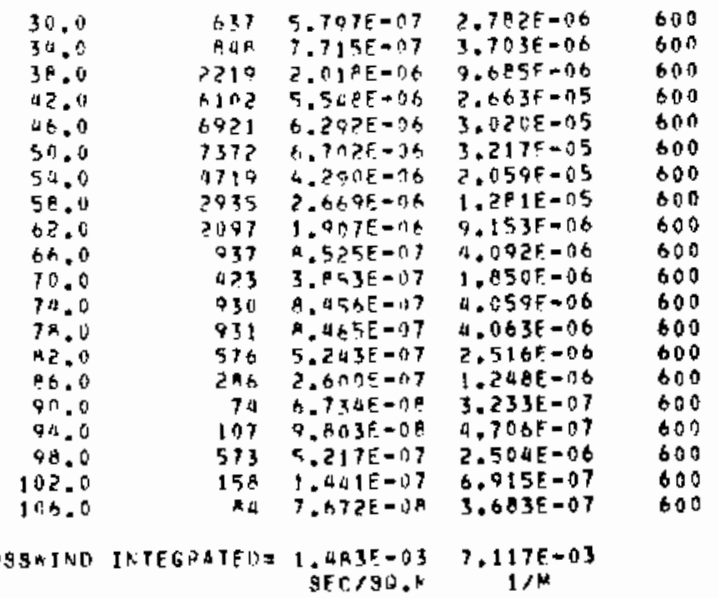

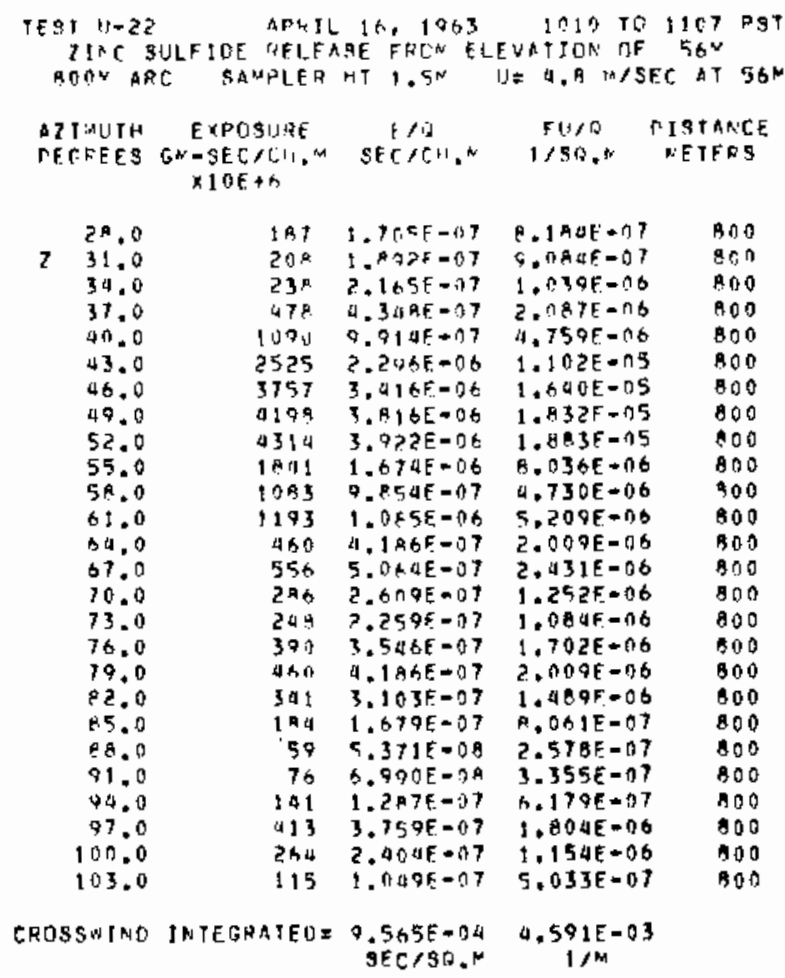




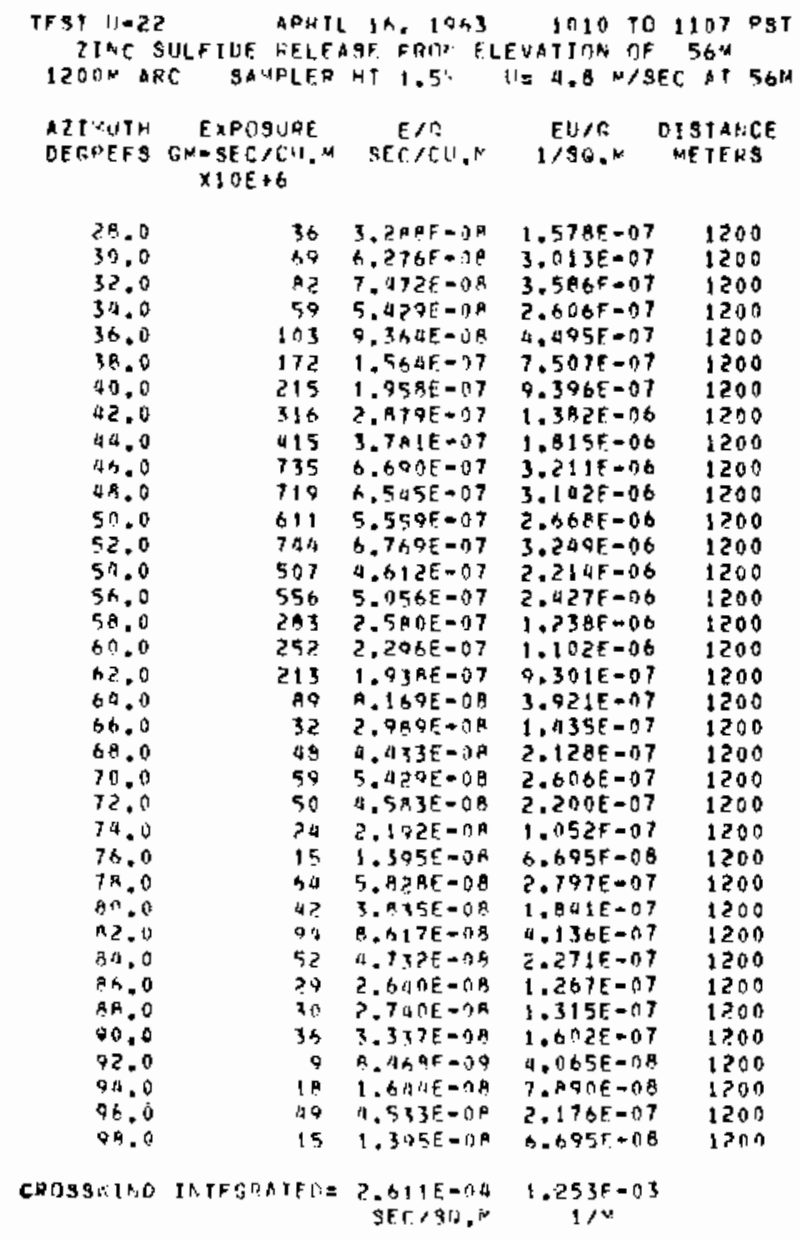

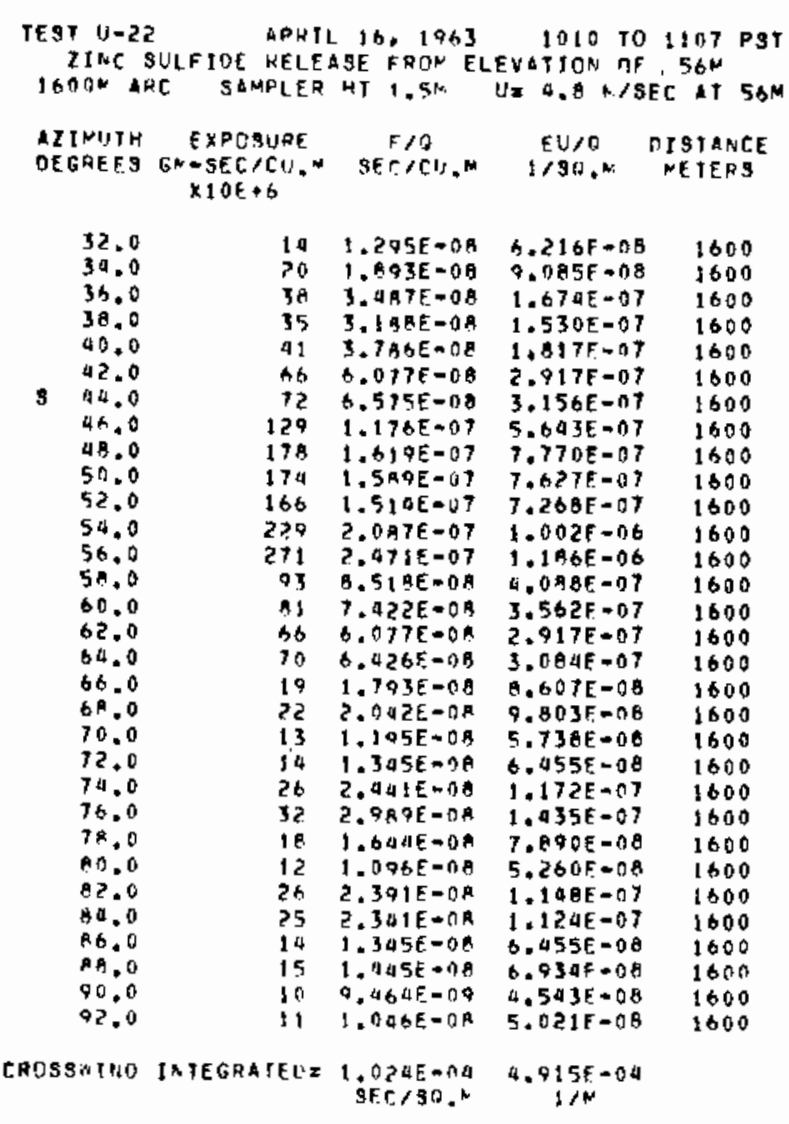


UNSTAELE SERIES EXPERIMENT: 23 ( U-23;

GROUNO LEVEL SAMPLING 100-1500 M ARCS.

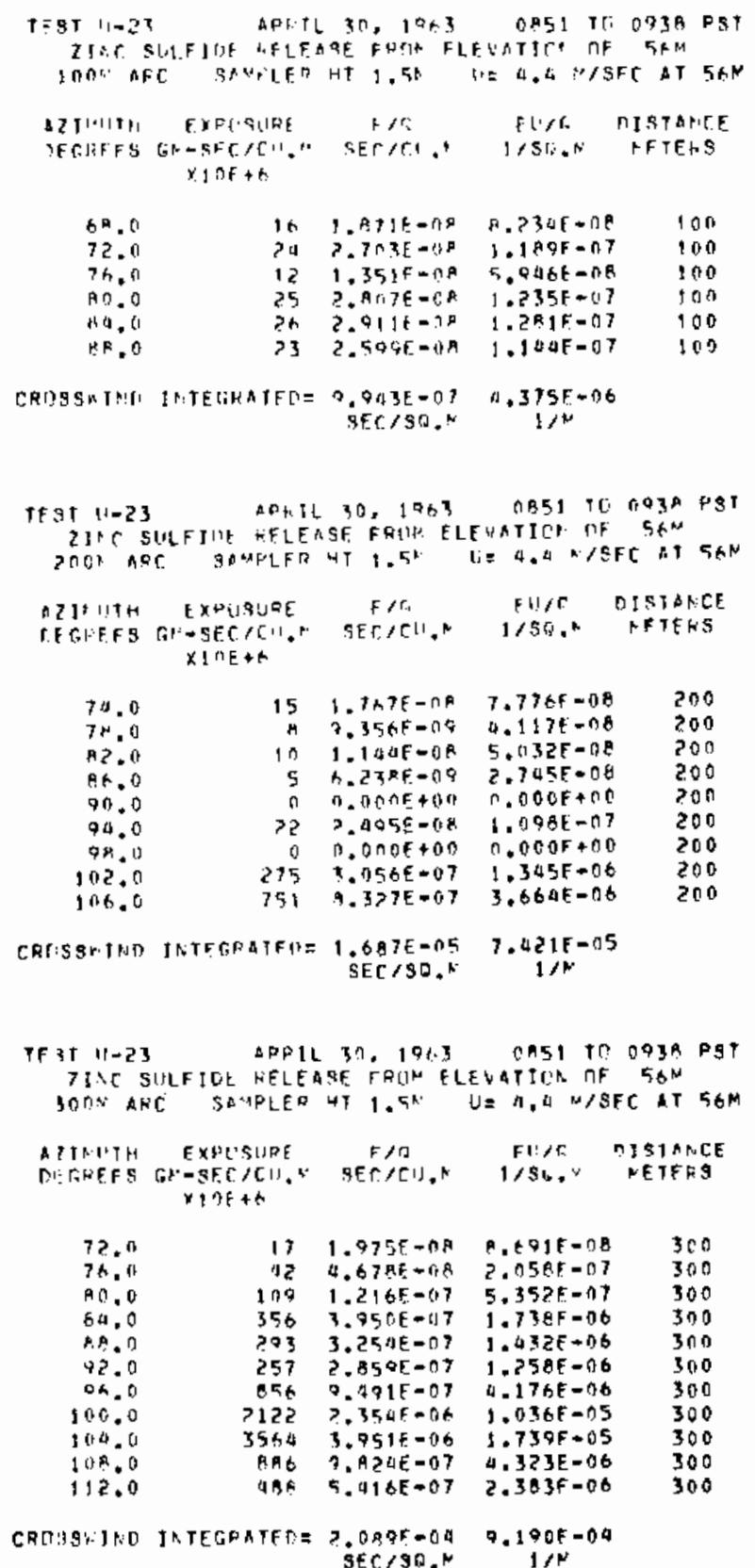

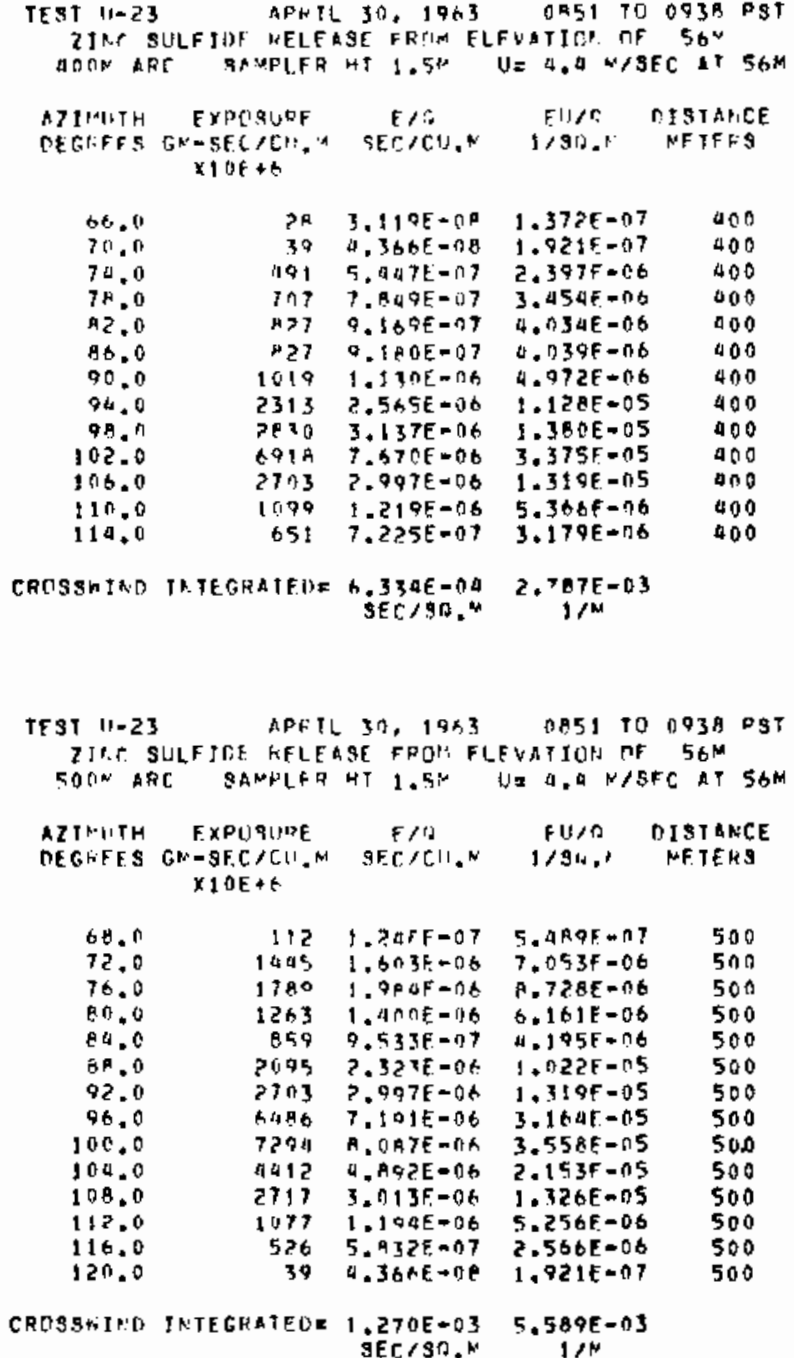




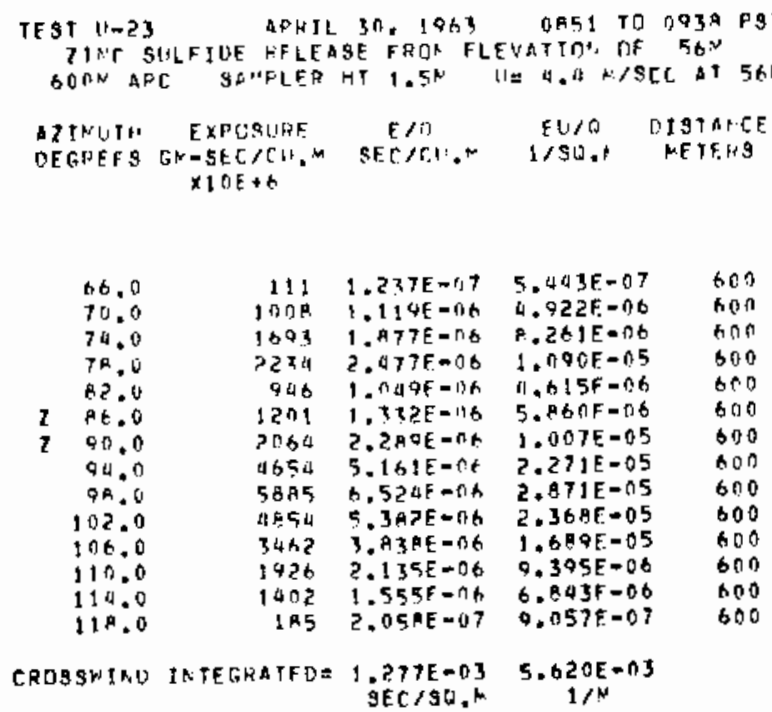

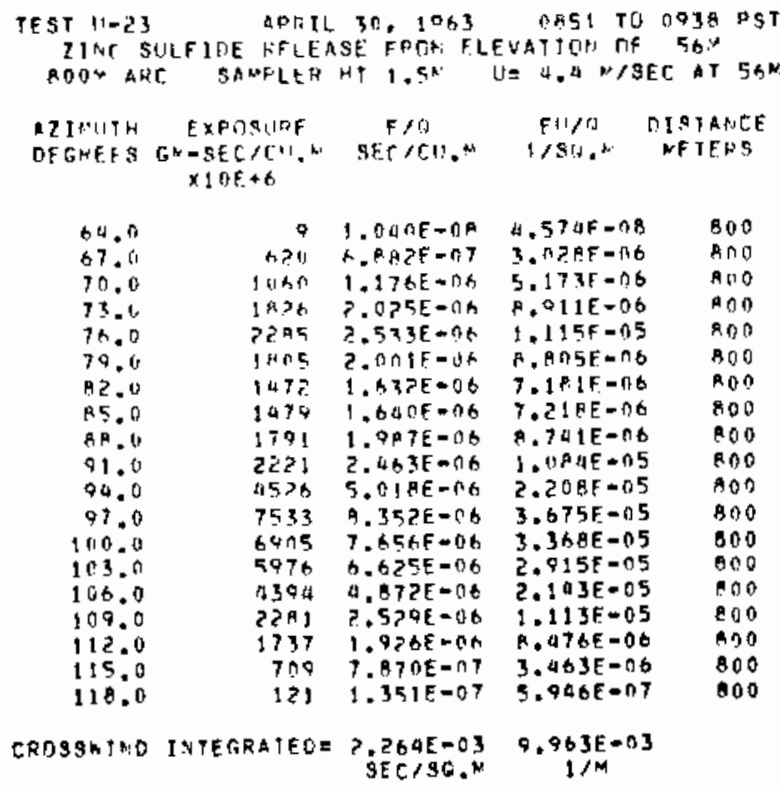

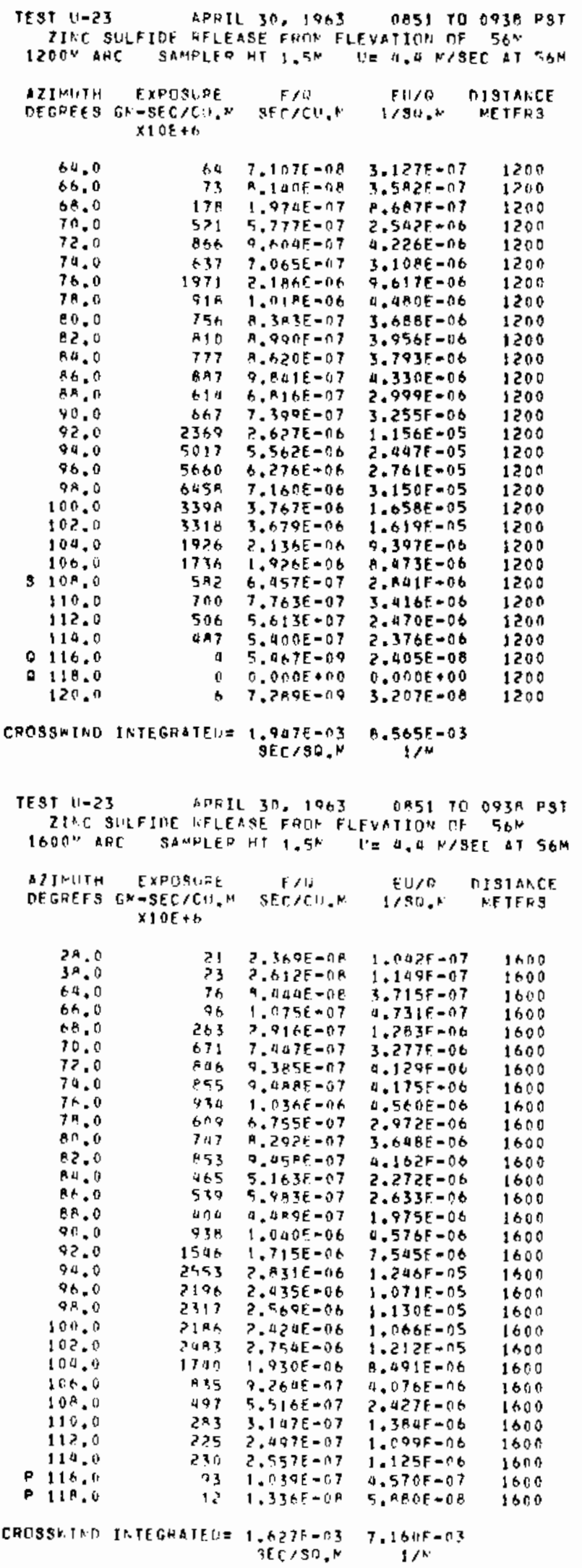


UNSTABLE SERIES EXPERIMENT: $24 \div \mathrm{U}-24$;

GROUND LEVEL SAMPL ING 100-1600 M ARCS.

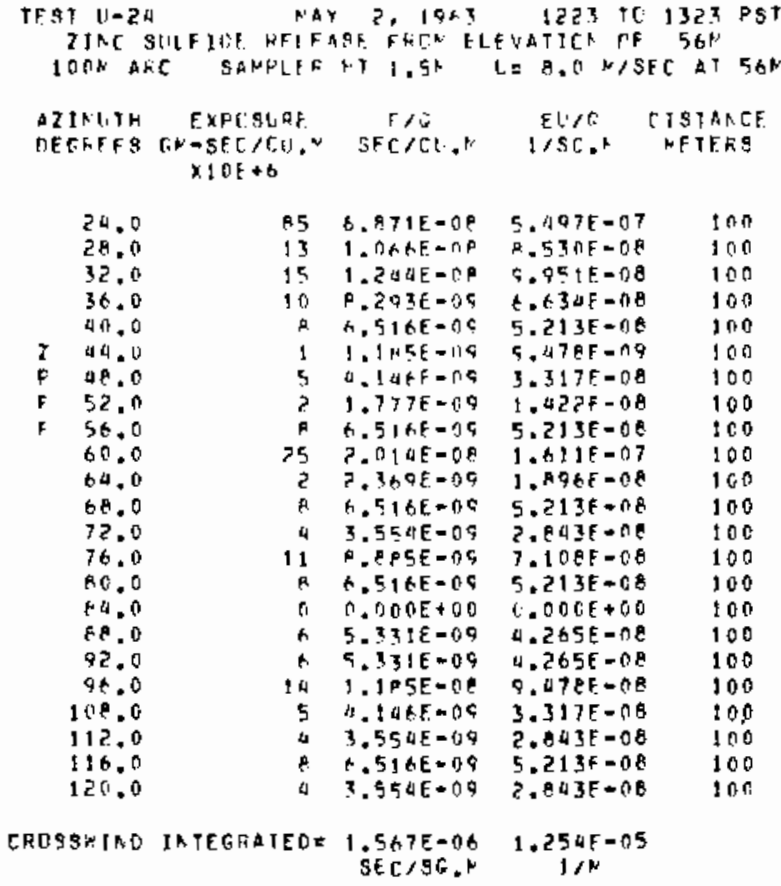

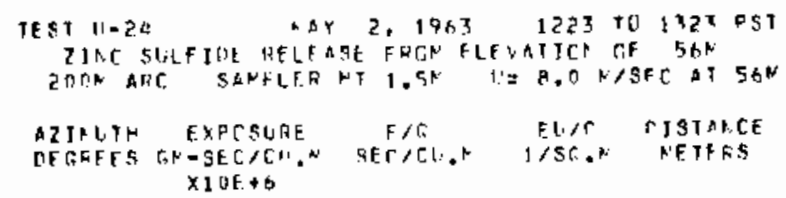

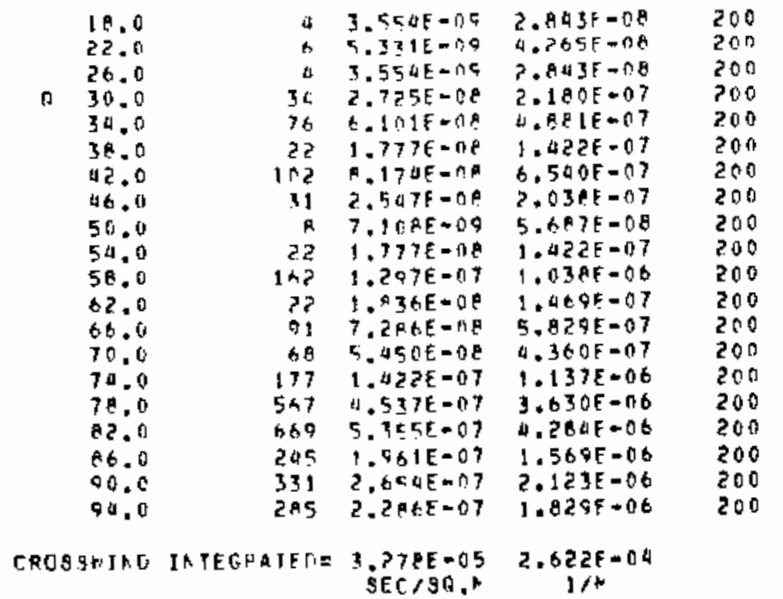

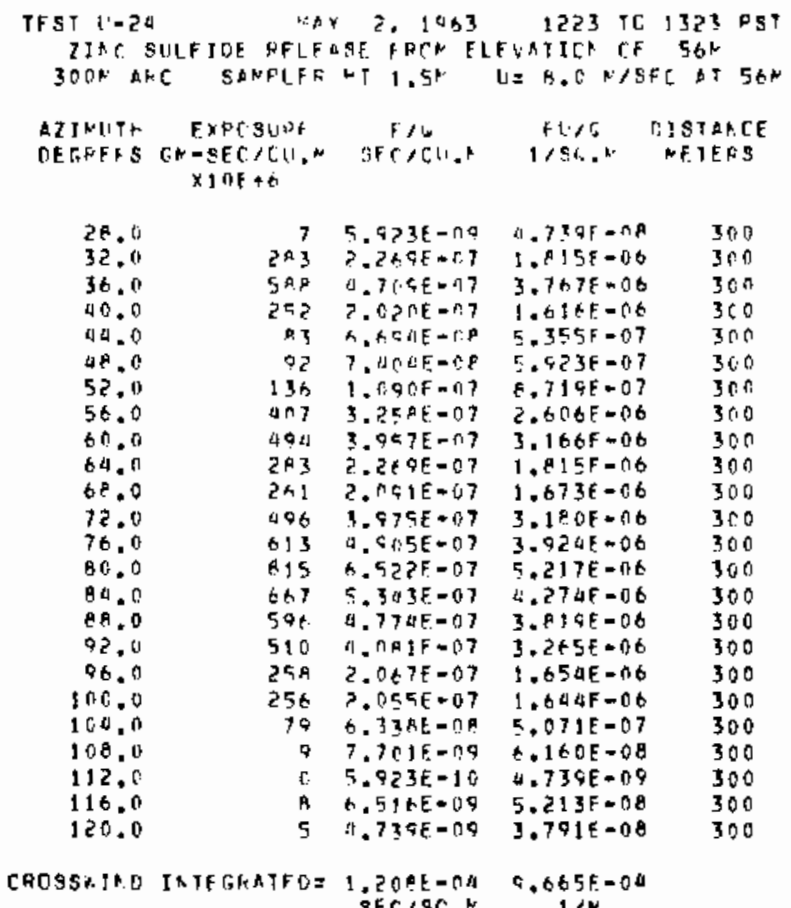

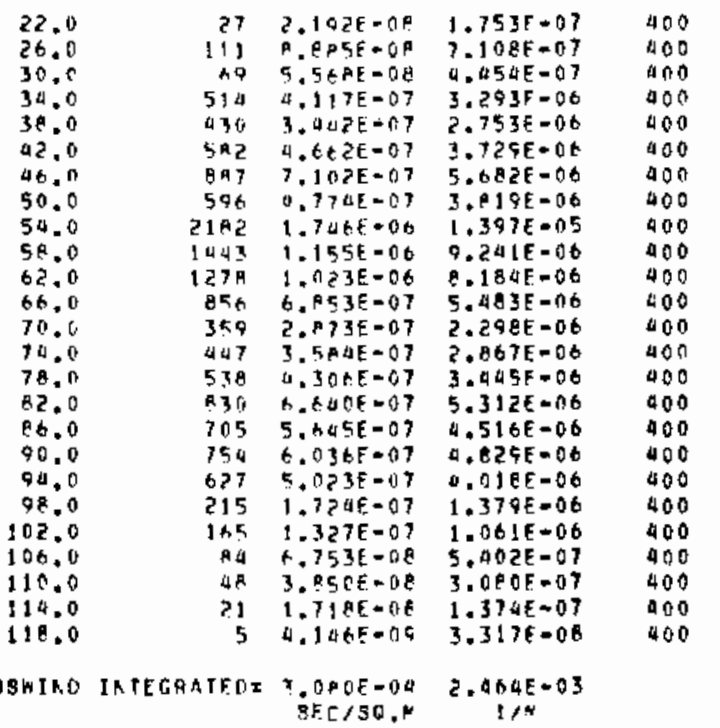




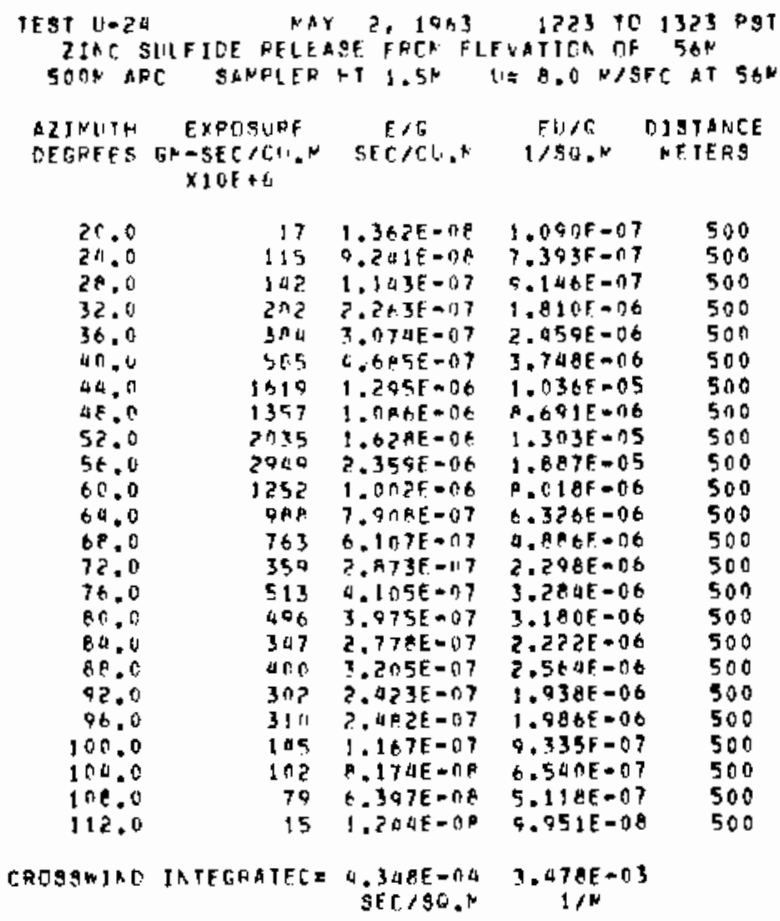

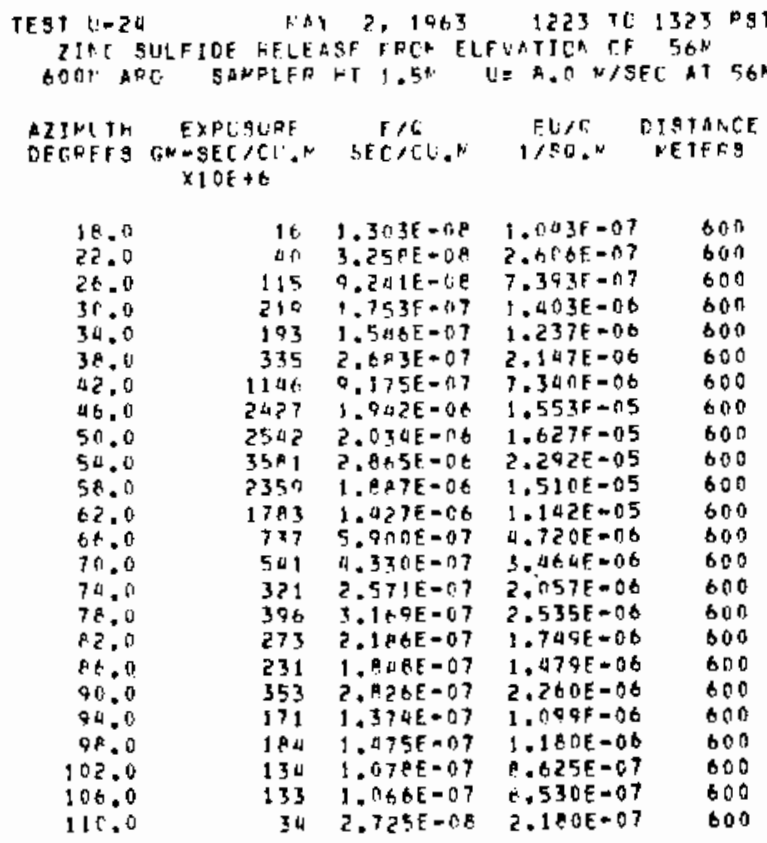

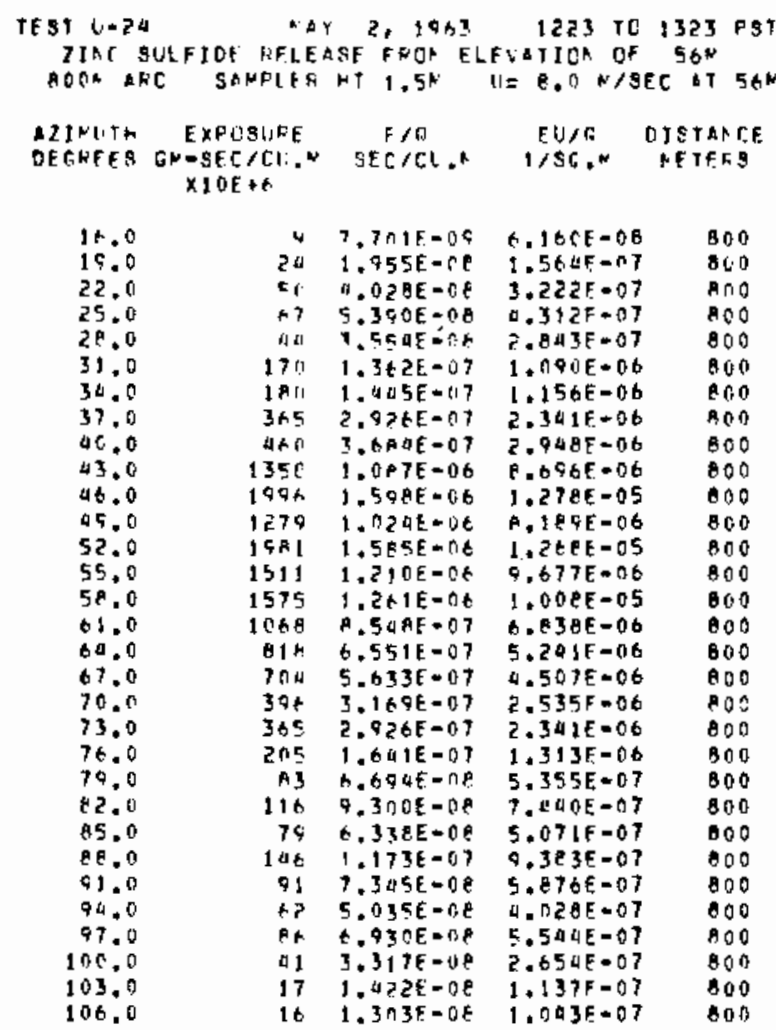

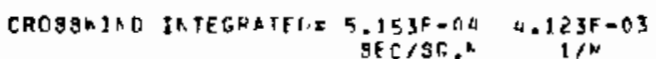

CROSERIAD IRTEGHATELE 6.123E-04 4.89QE-03

SEt $190^{\circ} \quad 1 / N$ 


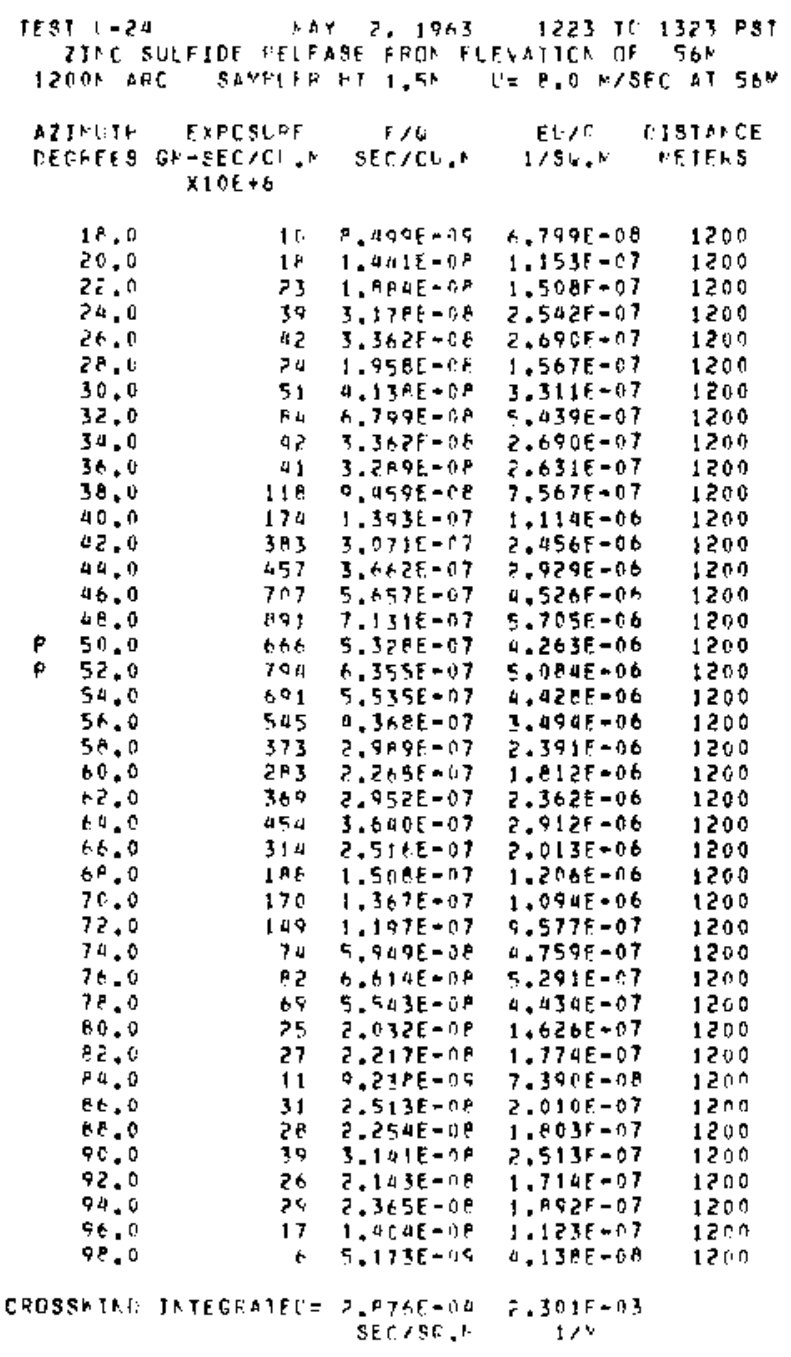

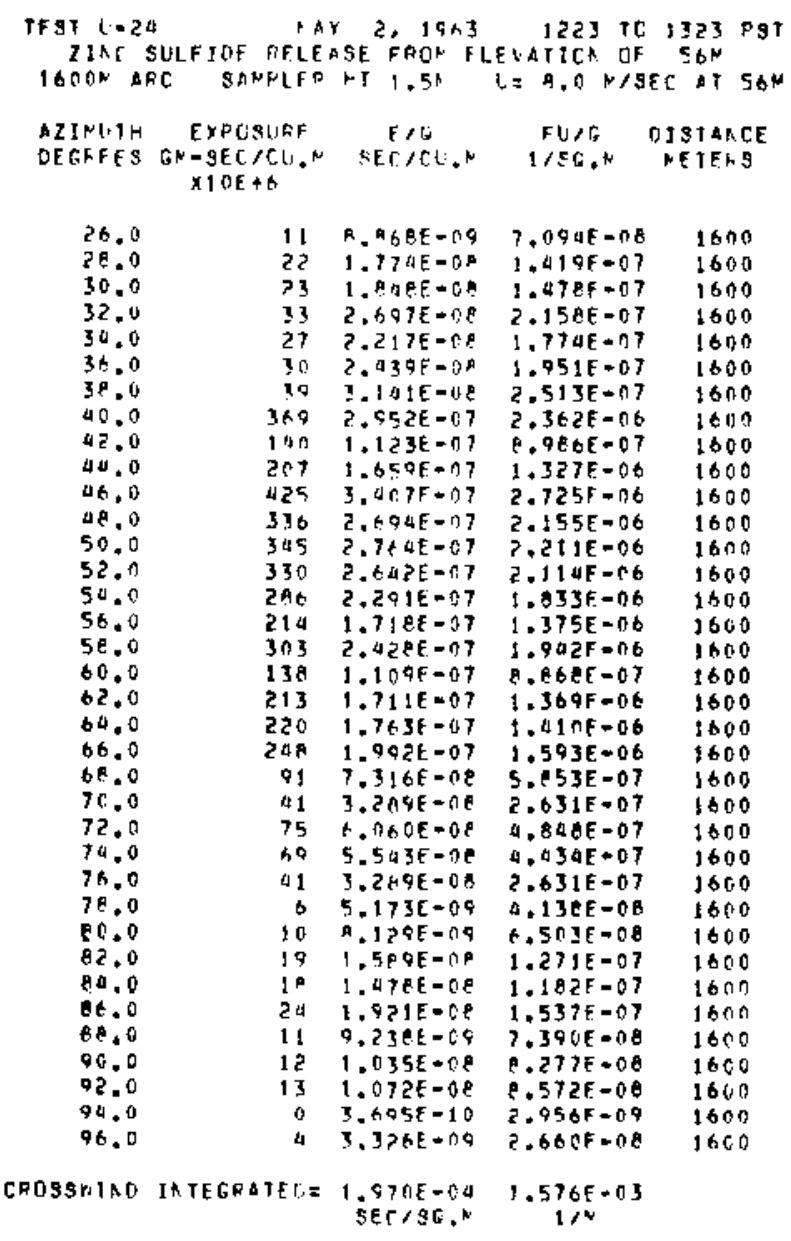


UNSTABLE SERIES EXPERIMENT: 25 ( U-25)

GROUND LEVEL SAMPLING 100-1600 M ARCS. FOR EASE IN STATISTICAL

ANALYS $\$ 5$, ALL SAMPLER AZIMUTHS HAVE BEEN INCREASED BY 30 DEGREES

FROM THEIR ACTUAL POSITIONS.THE TRACER WAS NOT DETECTED ON THE

100 M ARC. A PORTION OF THE PLUME PASSED TO THE NORTH OF THE

GRID.

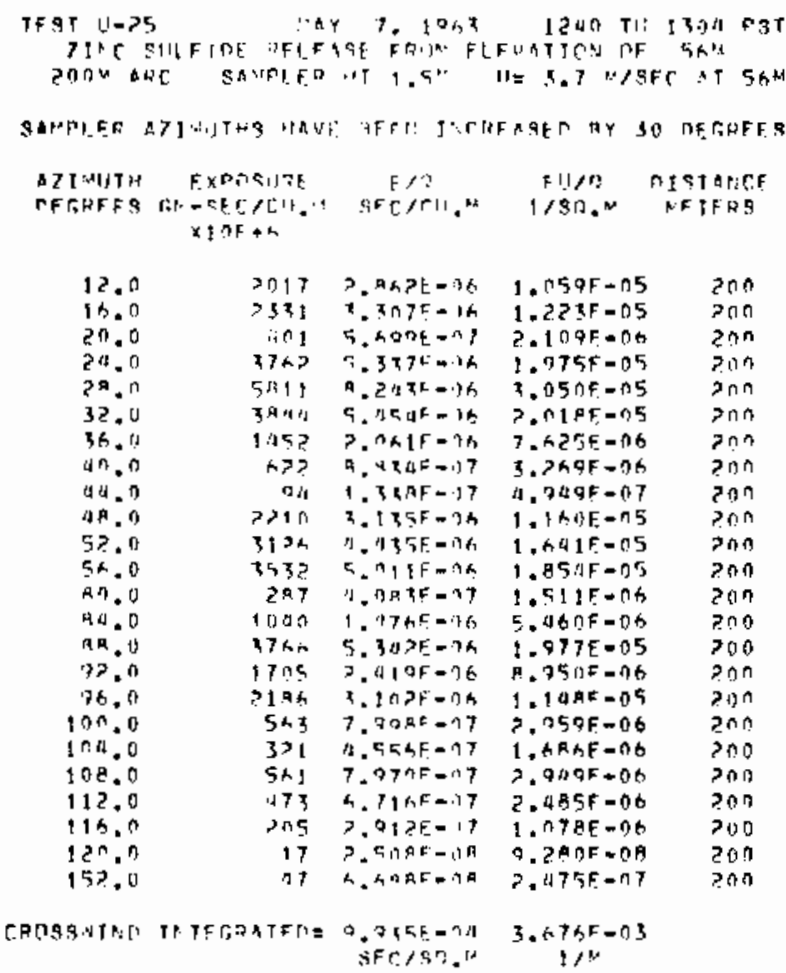

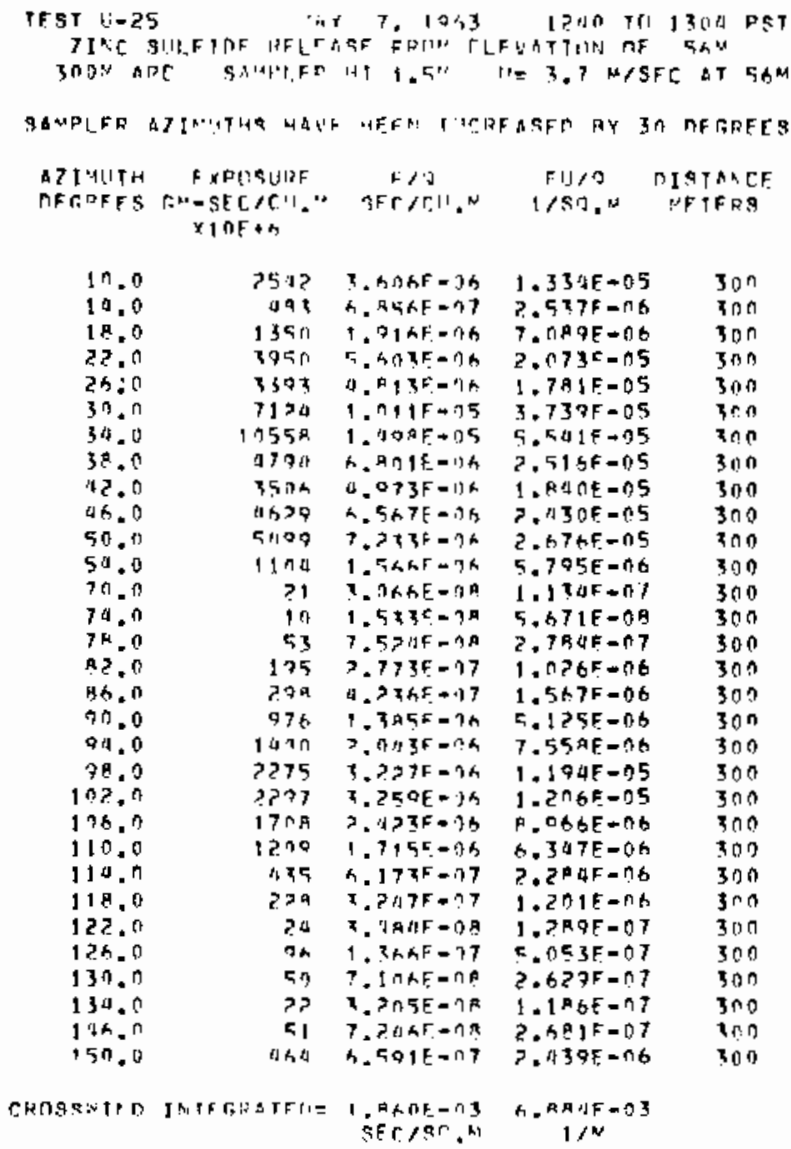




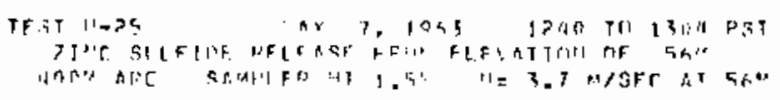

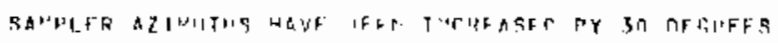

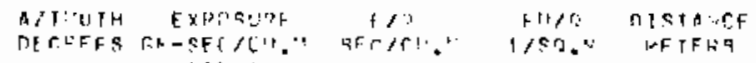
xint 4

\begin{tabular}{|c|c|c|c|c|}
\hline 12.0 & 195 & $0.71 \mathrm{dF}=1.7$ & $3.495 \mathrm{~F}=\pi \mathrm{b}$ & $\ln n$ \\
\hline $16 \cdot n$ & 0 & 7. XAGC $=07$ & $2.7335-176$ & an \\
\hline $2^{n} .0$ & inn & $7.1 \times 5 F=n y$ & 2. $6+105=16$ & In? \\
\hline$>11.0$ & 3517 & $4.7 P A F-9 \alpha$ & $1.50 \Delta F-05$ & 400 \\
\hline$? A, 0$ & 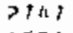 & 3. คै כ $7 E=$ th & 1. $142 F=n 5$ & $\operatorname{acn}$ \\
\hline 32.0 & +553 & $\because$ ?ARE $=00$ & $3.439 F=05$ & I) \\
\hline $36 . n$ & 5124 & $7.37 \cap E=3 \%$ & $7.727 \mathrm{~F}-05$ & Iח \\
\hline यn. & $5, a A\rangle$ & $R=31 R=0 A$ & $3.1977 f=n 5$ & $A \cap n$ \\
\hline 1 4. U & 4217 & $5.7 A \geq A=1 B$ & $2.213 F-05$ & 1007 \\
\hline "क. 0 & 2105 & 1. Anafon & $1 . x 3 \cap F-25$ & 100 \\
\hline 52.0 & 2018 & 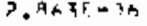 & $1.059 \mathrm{~F}=05$ & $40 n$ \\
\hline $5+, 0$ & 70 & 3. WA B $-9 n$ & $1.2945-n 7$ & $4 \cap A$ \\
\hline AR. 0 & $1 r_{i}$ & $5.713 F=n A$ & $2,11 t+\mathrm{F}=n 7$ & 400 \\
\hline 37.0 & 35 & $h, d 1 D E=? a$ & $7.37 ? F=n 7$ & 100 \\
\hline 94.0 & a? & $1.17 n F-n 7$ & $4.33 \pm t=07$ & an \\
\hline 900.0 & 1174 & $1.4 h, 7 f=n h$ & $A, 1+A=0=0$ & 400 \\
\hline 104.0 & 613 & R. $7 \mathrm{nQE}=\Omega 7$ & $3 . ? 225=06$ & $4<0$ \\
\hline $1 \cap \mathrm{p}=0$ & 773 & $1.9776=9 \mathrm{~s}$ & $A, \cap 5 R F=16$ & $40 n$ \\
\hline 112.0 & 377 & $1.7035+197$ & $1.7715=06$ & $4(1 \pi$ \\
\hline 116.0 & ?3h & ?. $75 A F-n ?$ & $1.2035=06$ & 450 \\
\hline 120.0 & $1+3$ & $1.063 E=37$ & $5.413 f-37$ & AD \\
\hline $13+10$ & 108 & $1.5 \times 35-117$ & $x+15=07$ & $\ln 0$ \\
\hline $1 ? ?^{4}, 0$ & 1 nd & 1. $4>>F=n t$ & $5.365 E-177$ & 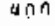 \\
\hline $15>0$ & Is st & h. ${ }^{A}>A F=n \cap$ & $7.4265=07$ & $\operatorname{lin} \pi$ \\
\hline 134.9 & 12 & 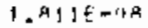 & $5.705 ?-90$ & 10ก \\
\hline 1 in 0 & $a$ & $4.3 A 7 E-10$ & $2.57 R F=\cap A$ & 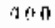 \\
\hline 14.0 & $5 ?$ & $7.3 A 5 F=n A$ & $3.733 E-07$ & 4 पก \\
\hline ! WR. 0 & 1 मद & $1.1915=07$ & $.517 F=07$ & 450 \\
\hline$=42,13$ & QRa & $1.32 A 5=146$ & $1 B S E-116$ & 400 \\
\hline
\end{tabular}

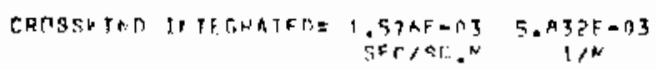

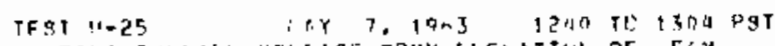

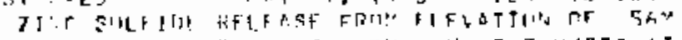

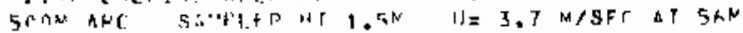

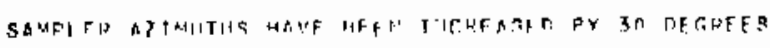

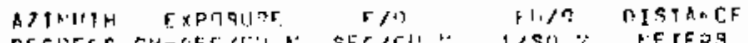

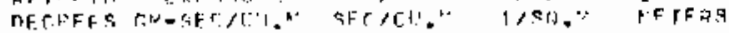
$x+101+h$

\begin{tabular}{|c|c|c|c|c|}
\hline 10.0 & 4 A7 & $5.211+-37$ & $2.597 F=076$ & $50, n$ \\
\hline 14.0 & $5+1$ & $3.4015=117$ & $1.3+6 F=06$ & द ती सी \\
\hline $1 A .7$ & 143 & $5.7 ? 7 F=n]$ & $? .119 F-1 \mathrm{CB}$ & $50 n$ \\
\hline टౌ.0 & $13 \times 7$ & $1.9 h R F=n h$ & $7.3 A \cap F=00$ & 500 \\
\hline $2+. n$ & $1 ?^{A} \mathrm{I}$ & 1. $P A_{1} T E=\cap A$ & $A .7>55=06$ & $50 n$ \\
\hline $3 n, 0$ & 3357 & $11.7 \times 3[-5)$ & $1.26 .78-0.5$ & $5 \cap n$ \\
\hline 34.0 & 5710 & $5.557^{5}=06$ & $055 F=05$ & $\sin 0$ \\
\hline 39.0 & $31 \% 7$ & I) $=14,5 r=014$ & $1.6525=05$ & $50 \sqrt{3}$ \\
\hline 42.0 & タखा & $1.17 \% 5=14$ & $4,3 \in 25=06$ & 500 \\
\hline $4 h .0$ & †口只 & 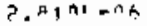 & $1.042 F-25$ & 5 กी \\
\hline 57.0 & 1100 & $1, h A M E=11 \mathrm{~h}$ & $A+13 \cap F=06$ & $\sin$ \\
\hline 50.0 & $22 k$ & $3, ? 1^{0} \mathrm{k}_{-}=01$ & $1.191 F-06$ & 50 ती \\
\hline 59,0 & 1? & 1. ${ }^{A}: 1 F=U n$ & $A .7$ ก ZE-08 & 500 \\
\hline $1 \cap h .0$ & 1 & $2.7 n>5=37$ & $1.0318=0$ A & 500 \\
\hline 10.0 & $1 \mathrm{AR}$ & $1.5 \cap 5 E-n 7$ & $5.568 F-07$ & 500 \\
\hline $1 \pm 4 . r$ & $1 \mathrm{A3}$ & $7 . A B A F=D$ & $8.001 F=07$ & 500 \\
\hline $1: 1,0$ & $h>R$ & Я . 918Е $=\cap 7$ & $3.300 F=06$ & $5 n 0$ \\
\hline 122.0 & $10 \%$ & ?. $t 1 \times E-n T$ & $7.8378-67$ & $50 n$ \\
\hline 124.0 & प? & $1.170 \mathrm{~F}=0.7$ & $4.331 F=07$ & $50 n$ \\
\hline 135.0 & 20 & A. IRAF A AR & $1.547 E+07$ & 500 \\
\hline 134.0 & In & $1.535=0 \mathrm{~A}$ & $5.671 F+08$ & 500 \\
\hline 138.0 & 53 & $7.5>45-1 A$ & $2.784 \mathrm{~F}=07$ & 500 \\
\hline $1+2.0$ & 20 & $A .1$ म $\cap F=11 B$ & $1.507 E=07$ & 500 \\
\hline $1 \div 6.0$ & 20 & $5.5719=79$ & $2.0107 E=07$ & 500 \\
\hline $15 n .7$ & ath & $1.3 \times<F=17\}$ & $053 F-07$ & 500 \\
\hline
\end{tabular}

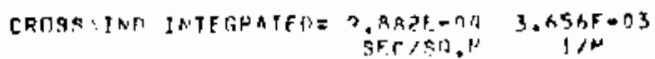

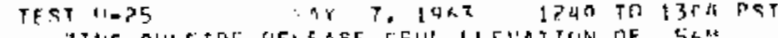

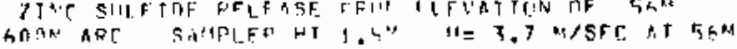

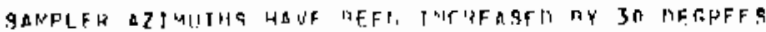

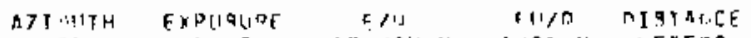

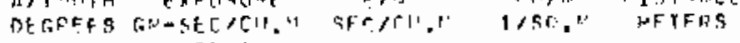
$\times 1$ If +4

\begin{tabular}{|c|c|c|c|c|}
\hline 12.0 & 253 & $x .505 f=117$ & $1.33 A F=0 b$ & hol \\
\hline $1 \mathrm{~h} .0$ & ii 70 & F. $=0.0 F=17$ & $2.516 \mathrm{~F}-16$ & tinn \\
\hline 20.0 & 9 & $1.104 F=06$ & $4,114=06$ & 600 \\
\hline $24, n$ & 1110 & $1.575 \%=11 \mathrm{~A}$ & $S . A 26 E=06$ & AOD \\
\hline $2+0$ & $10 \times 4$ & $\Rightarrow .707 \mathrm{~F}-1 \mathrm{~h}$ & $1.033 F-05$ & $\sin n$ \\
\hline $3 z, 0$ & 3225 & $\because A X \odot F-A x$ & $1.730 F=05$ & $\operatorname{ann}$ \\
\hline 36.0 & $35>11$ & $5 . D \cap \cap F=7 A$ & $1.050 F-05$ & 600 \\
\hline at? 4 & $\ln \cap Q$ & $? . ? \times 7 F=16$ & A, $445 F=06$ & $A \cap \pi$ \\
\hline 40.0 & 1009 & $1.517 E-A B$ & $5.6: 5 E-06$ & ton? \\
\hline 48.0 & $A \times 9$ & 1.? $33 E-1) h$ & $96.35-00$ & 600 \\
\hline $5 ? .0$ & וח & $>A 5_{3} 7 F+37$ & $1.057 \mathrm{~F}=00$ & hen \\
\hline 56.0 & 57 & n. $2>1 F \mathrm{~m}, 9$ & 3. $.0 \mathrm{ALE}=07$ & hon \\
\hline hn. $n$ & $t a$ & $1.5 \times 3 E-A R$ & $5, A 71 F=\cap B$ & 600 \\
\hline $1 \geq n, 0$ & $2+1$ & $1.33 \mathrm{AE}-17$ & $1,949 \mathrm{E}=07$ & $A \cap 0$ \\
\hline $12 \pi, 0$ & נג נ] & $A .270 F=A R$ & $2.320 E=07$ & 600 \\
\hline $1>9.0$ & 21 & A. $A 5 P F-11 A$ & $1.65015-n 7$ & $\operatorname{sen}$ \\
\hline 132.0 & 11 & $1.47>F-48$ & $A . J A>F=\cap A$ & 600 \\
\hline 134.0 & a? & A. 9 (AF & $3.3005-017$ & कती \\
\hline 140.0 & 37 & 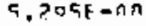 & $1.459 E=07$ & x \\
\hline $1 \mathrm{du} \cdot 0$ & 10 & $1.5 \times * F=0 R$ & $5.6715=08$ & $6 \cap 7$ \\
\hline 149.0 & 32 & $4.5 O A F=0 A$ & $1.702 E=0 ?$ & 600 \\
\hline 157.0 & $17 h$ & ?. $S \cap P E=\cap 7$ & PADE $=07$ & 600 \\
\hline
\end{tabular}

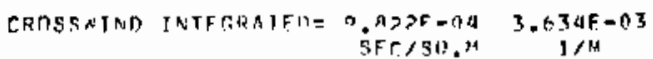

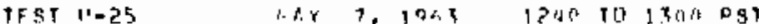

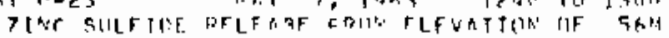

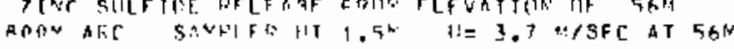

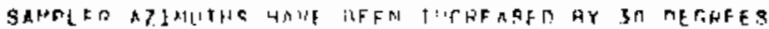

DIJMTH EYPLEIJZF FID FUIO MISTAUEF

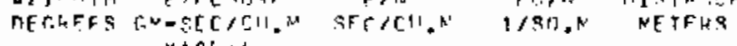
$x+0$ th

\begin{tabular}{|c|c|c|c|c|}
\hline 13.0 & 11 & 1. $\mathrm{A}$ ? & 6.1 1 $7 F=08$ & $90 n$ \\
\hline 15.0 & ba 45 & $0.155 \mathrm{~F}-17$ & $3.3975-06$ & $A_{t} \cap 0$ \\
\hline 10.0 & 370 & $5 .>535=07$ & $1.704 \mathrm{~F}-106$ & ann \\
\hline $2 ? .0$ & $2 \cap 5$ & $>.71>5-17$ & $1 . \cap 7 P F=D G$ & Aon \\
\hline 25.0 & 413 & A. JRUT $=n 7$ & $2.375 \mathrm{~F}=0 \mathrm{~m}$ & $R \cap \cap$ \\
\hline $2 A .0$ & 530 & $7.5 ? A F=1: 7$ & $2.7^{24}+5=36$ & $\operatorname{s} 00$ \\
\hline 31.0 & 21163 & $3,0, a n r_{-}-1 ; t$ & $1.125 \mathrm{~F}=175$ & $\operatorname{AnO}$ \\
\hline 30.0 & खटा & $3.1 \times 7 F=A A$ & $1.1615=05$ & no? \\
\hline 37.0 & 19 AR & >. KTRE $=0 \mathrm{E}$ & $9.9095=06$ & AOO \\
\hline 40.0 & 1504 & $>.1 \times 5 \mathrm{E}=3 \mathrm{~h}$ & $7 . P Q R F=D \theta$ & 800 \\
\hline 03,0 & $105 ?$ & $1.4925-76$ & $5.522 F=00$ & मำ? \\
\hline 44.0 & $\sin 1$ & 9.37 AF $-n ?$ & 3.07 तf -00 & AOO \\
\hline $49, \pi$ & $3^{n} n_{h}$ & $4.347 F-0.7$ & $1.609 F=06$ & Ans \\
\hline 53 & I5 A & $3 .>113 F=167$ & A. $3 \cap 1 F=n 7$ & ano \\
\hline & 15 & $A . \| \cap E=A B$ & $2,372 \mathrm{E}-07$ & 900 \\
\hline.$n$ & 30 & $A .3>0 E-0 A$ & $1.59 A F=07$ & $\operatorname{snn}$ \\
\hline$A 1.0$ & is & $1 . S>\geqslant F=61$ & $6.187 E-08$ & $\operatorname{Ron}$ \\
\hline$x a, 0$ & b & $n .7545=09$ & $3.6 \cap 47=08$ & Ron \\
\hline+7.0 & חִ & $n .00 n+\pi n$ & ODOF+0O & $\operatorname{Rn} 0$ \\
\hline & 5 & $9 x^{2}+15=110$ & $.0935=78$ & 900 \\
\hline 47.0 & 9 & $1,3 \geqslant x=-1, \pi$ & $5.156 F=08$ & 900 \\
\hline 45.0 & 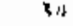 & 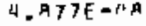 & $1 . A \cap \Delta F=07$ & 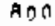 \\
\hline 0 & $n$ & $n, n+n \in+0 n$ & n. nons t no & AQO \\
\hline 5 & 83 & $1,3, \pi E-ก 7$ & Aุव $A F=\cap 7$ & An \\
\hline
\end{tabular}

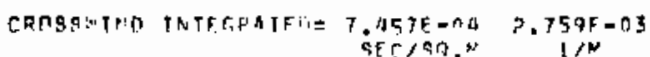




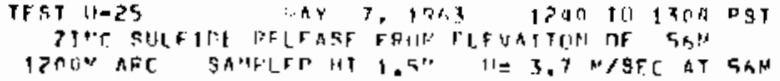

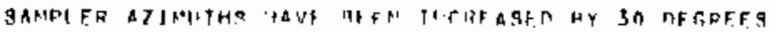

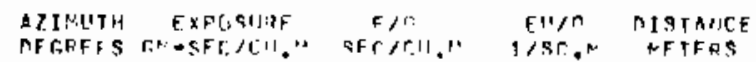
$\times 101+h$

\begin{tabular}{|c|c|c|c|c|c|}
\hline & 30.0 & 437 & $x, P$ IAf $=$ il & 2. $>7 A F=06$ & $1>00$ \\
\hline & $3 ? .9$ & 470 & h. $+47 x-01$ & $? .4 \times 75=06$ & $1 ? 00$ \\
\hline & 34.0 & 507 & $7,76,98-017$ & $2 . A 7 \Delta F=06$ & $1>00$ \\
\hline & 36.0 & $4<7$ & 6. $47 \mathrm{AE}=09$ & $2.11035-76$ & $12 n n$ \\
\hline 2 & 38.0 & In? & $5.7 \cap A F-1) 7$ & $2.111 F=16$ & $1>n$ \\
\hline$Z$ & $40, n$ & $5 \div 5$ & $A \cdot 1 \times 9 F-\cap 7$ & $3.022 F=06$ & 1700 \\
\hline & $4 \bar{z} . n$ & 670 & $0.5075=117$ & $3.51 A F+n 6$ & 1200 \\
\hline & 44.0 & 377 & $5.3 A 1 F=07$ & $1.974 F-106$ & 1200 \\
\hline$s$ & 40.0 & $4 \pi \mathrm{A}$ & $6.3 \mathrm{~h} 5 \mathrm{~F}-97$ & $2.3555-06$ & 1200 \\
\hline & aै. त & $5 x x$ & $\gamma .8 \times 0 F=11 ?$ & $2.95 B E-06$ & 1300 \\
\hline & 50,0 & 352 & $5.141 E=17$ & $1.9 n 2 E-06$ & 1200 \\
\hline & $2 ? 00$ & 125 & $3.76 \mathrm{AF}=-177$ & $1.02 a 5-06$ & 1200 \\
\hline & 54.0 & $1>1$ & $1.73 \cap 5=197$ & $5.4 n[F=07$ & 1200 \\
\hline 9 & $5 h .17$ & 72 & $1 . n>P F=07$ & 3. $R \cap 45=07$ & 1200 \\
\hline & $5 \mathrm{~A}, 0$ & +2. & $A, A \triangle 5 F=\cap A$ & $3.791 F=07$ & 1200 \\
\hline & 60.0 & 27 & $1.2 a n F=n 7$ & 4.5 A $Q F-n 7$ & 1200 \\
\hline s & 52.0 & 23 & $4, T \times 3 E=\cap R$ & $1.751 \mathrm{E}-0.7$ & 1200 \\
\hline & 60.0 & $P \mathrm{~s}$ & $x .7545=04$ & $1.3995=07$ & 1200 \\
\hline & $h=.0$ & 57 & $5.4 x 1+=02$ & $2.0935-07$ & 1200 \\
\hline & $6^{6} \cdot 0$ & 71 & $3 . n+9 F=11$ A & $1.117 F-n 7$ & 1200 \\
\hline & $7 \pi \cdot 0$ & 100 & $7.3,98=07$ & $P .877 F=0.7$ & 1200 \\
\hline & $7 ? .0$ & रम & G. $U A \cap F=t h A$ & $3.0>3 f-07$ & 1200 \\
\hline 3 & 74,0 & 10 & $>.775 x-10.7$ & $1.0276=07$ & $\$ 200$ \\
\hline 7 & 76.10 & 8 & n. $0 \cap n+$ an & $n \cdot 0 \cap n F+00$ & 1200 \\
\hline $\mathbf{T}$ & 7月, 0 & 7 & $1 . n+1 E \rightarrow n A$ & $3.975 F=18$ & 1300 \\
\hline & AO 0 & 5 & $5 . n+0) F=n$ & $1.872 F-07$ & $1 \geqslant 00$ \\
\hline & 22.0 & 13 & $1 . R 7 Z K=A R$ & $\therefore .945 F=08$ & 1 ? \\
\hline $\mathrm{s}$ & $R n+?$ & h & $9.7035-18$ & $3.623 k=O R$ & 1800 \\
\hline & 26.0 & $t$ & A. $5 ? \mathrm{PAF}=\mathrm{nO}$ & $2.116 \mathrm{~F}-08$ & 1200 \\
\hline & $A^{A}=0$ & ? & d. $n p n c=1 ; 0$ & $1.51 \cap \mathrm{F}=\mathrm{n} \mathrm{A}$ & $1 \geq 00$ \\
\hline & 146.0 & 10 & $1.55 n E-58$ & $5.737 f-n: 8$ & 1200 \\
\hline & $14 \mathrm{~A}, 0$ & $n$ & n. AnnE+An & $=00 \cap F+00$ & 1200 \\
\hline & 150.0 & 4 & ค. $57 \propto E=0.7$ & $2.416 E-0$ O & 1205 \\
\hline & 152.0 & an & $Q .505 F=0 A$ & $3.4725=n 7$ & $120 n$ \\
\hline & & & 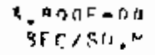 & $\begin{array}{c}1.450 F=03 \\
1,2\end{array}$ & \\
\hline
\end{tabular}

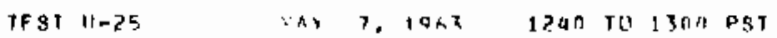

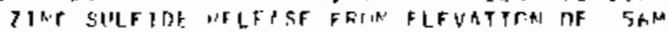

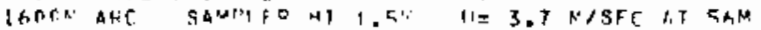

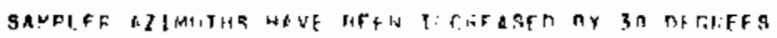

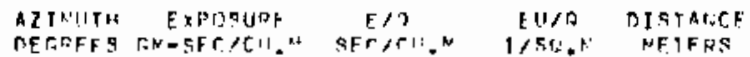
$\times 1 \circ r+\varepsilon$

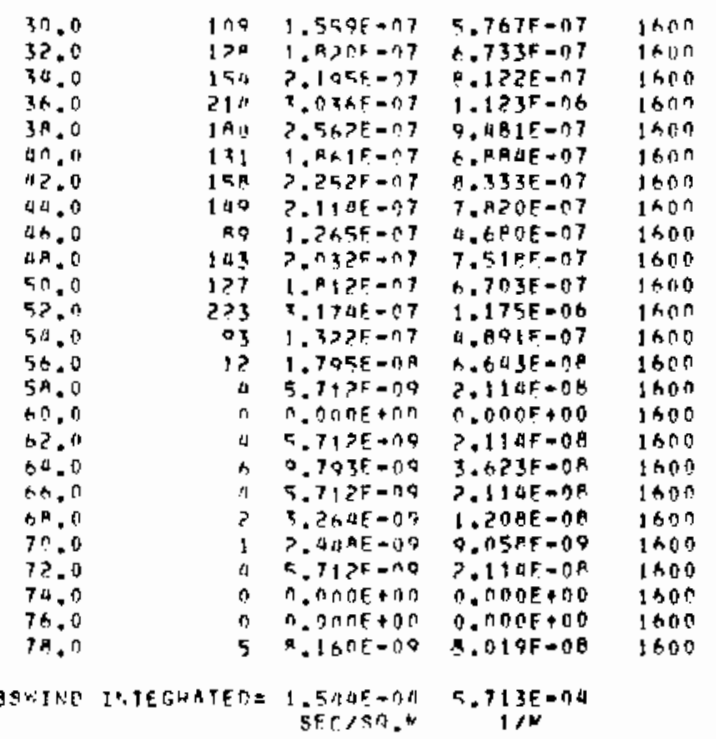




\begin{abstract}
UNSTABLE SERIES EXPERIMENT: $26, \mathrm{U}-26$,
GROUND LEYEI SAHPLING $100-1600$ M ARCS. FOR EASE IN STATISTICAL

ANALYSIS. ALL SAMPLER AZTMUTHS HAYE BEEN INCREASED BY 100 DEGREFS

FROM THEIR ACTUAL POSITIONS. THE TRACER WAS NOT DETECTED ON THE
\end{abstract}

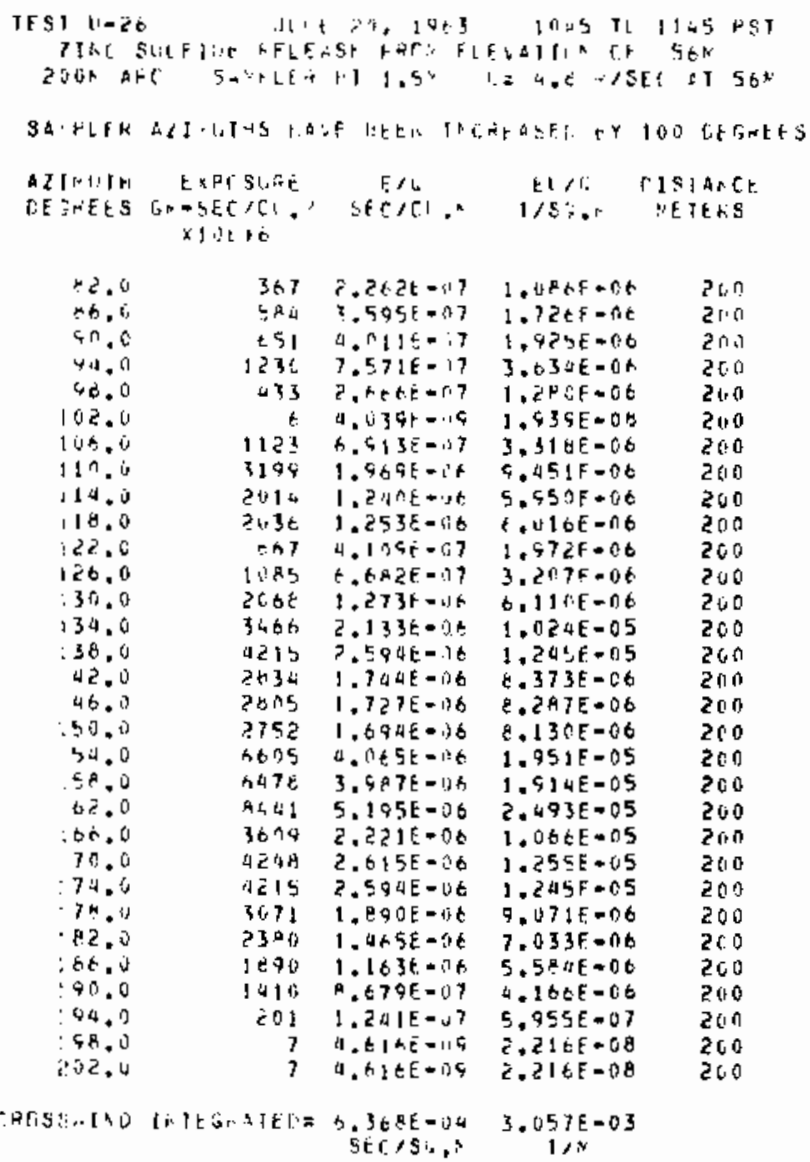

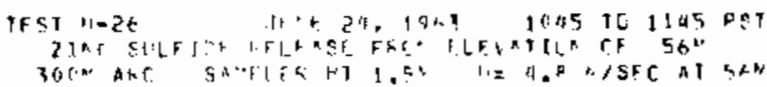




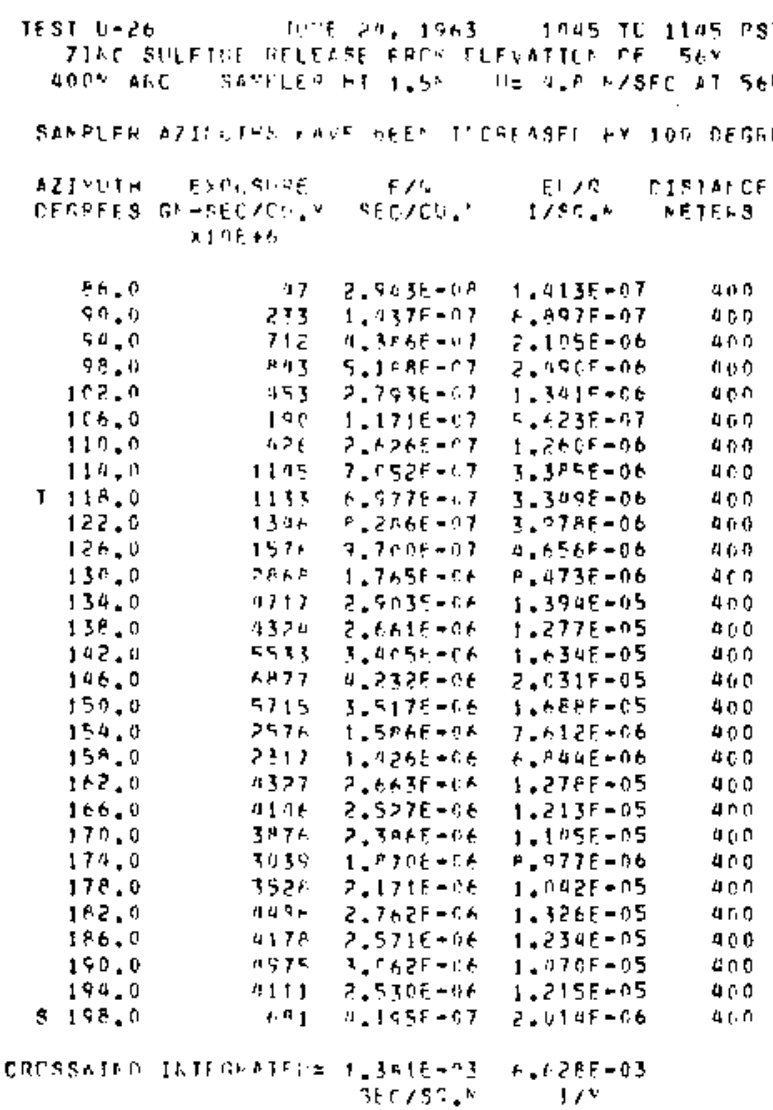

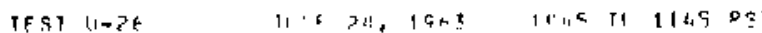

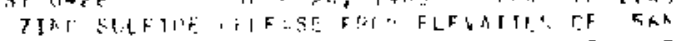

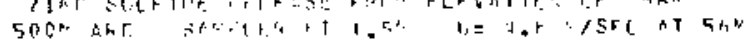

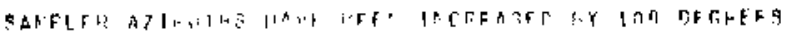

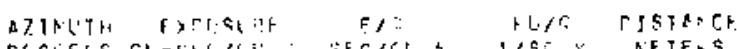

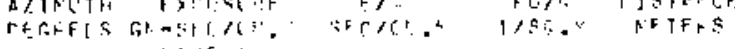

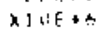

\begin{tabular}{|c|c|c|c|c|c|}
\hline & SP. $\hat{v}$ & $7: \mathrm{i}$ & 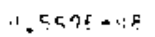 & $2 \cdot 1+5[-617$ & $5, n$ \\
\hline & $4 ? .1$ & $1 \cdots 2$ & $\Rightarrow$ Donted & 4.jues-ny & sin \\
\hline & 96.0 & an & $5.1820=07$ & $\therefore, H+2 F=c h$ & 500 \\
\hline & $\operatorname{lin} 0.0$ & $5>$ & $x, B A \cap=B, ?$ & 1. chate-lit & $5(1, n$ \\
\hline & $1: 4.1$ & 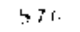 & $3.50 .06=0.7$ & $1 .+B D=D O=D O$ & $5 i n$ \\
\hline & JE. & 24 & 1.15 IE $=97$ & $0.58\{5=07$ & $50 \pi$ \\
\hline & $11 ? .0$ & $34 \mathrm{c}$ & $? .7515=07$ & $1.0 P A F=06$ & 503 \\
\hline & $11 \mathrm{~h} .11$ & は1 & $4.7205-77$ & $2.5945=016$ & 502 \\
\hline$s$ & 1213. & $13 \% 5$ & $=.529+-.97$ & $4 . \therefore Q L E=n E$ & 50 \\
\hline & 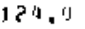 & nat & $5.2494=87$ & $2.424 F-O R$ & 5110 \\
\hline & 引方。 & 1311 & A. $>5 A E-W$ & $x .964=00$ & 50 \\
\hline & 132.0 & $351 \%$ & $\partial . \mid A J E=i j h$ & 1. nUEF-n & 400 \\
\hline & 136.1 & 1 1) & $? .5+3 t-0 \mathrm{r}$ & $1.245 F-155$ & $50 n$ \\
\hline & 140.0 & Thant: & $2,3,8 F=16$ & $1.141 E-05$ & 500 \\
\hline & $14 \pi, 0$ & 5119 & $3 . t \leq \mid E-1) t$ & $1.512 E=05$ & $\sin 0$ \\
\hline & 146.11 & 354 & $2.17^{i 1} t=n t$ & $1.044 E-05$ & 503 \\
\hline & $15 ? .0$ & $2 \pi$ & $1.397+04$ & $0.7 C \in E-06$ & 500 \\
\hline & $15 n .6$ & $1.17,$. & $A, A 15 F=0 ; 7$ & $4.1355=06$ & $5 \cap 0$ \\
\hline 7 & $1+i$ & 1127 & $7.0 \mathrm{nnE}-97$ & $3.360,0=00$ & 51,0 \\
\hline 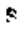 & 164.0 & 534. & $3.209 E-61 \mathrm{~A}$ & $1.5 A_{4}=\cap 5$ & $50 \pi$ \\
\hline & $1+8.0$ & i3 3 ?? & D.AOOE $=n$ A & $1.277 F=n s$ & $50 \%$ \\
\hline & 172.0 & $?: 1$ & $1, x>7 F-10$ & n.t>cen-nt & sits \\
\hline & 173.1 & 2275 & $1.3+8 F+01 h$ & $t .5735-06$ & 500 \\
\hline & $18 \%, 0$ & $32 \%$ & $1.9016=04$ & G.55\$F- 016 & 500 \\
\hline & $18: .8$ & $314:$ & 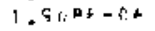 & $C .3 * B F=\cap \delta$ & $5 ; 0$ \\
\hline & $1 \mathrm{FF}$.! & 3150 & $1.9375-12 t$ & $4.773 F-06$ & $50: 0$ \\
\hline & 192,5 & 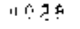 & $=.6795006$ & $1.190 \mathrm{E}=0 \mathrm{~S}$ & $5 \mathrm{ln}$ \\
\hline$s$ & $19 t .0$ & $17: 16$ & $1.2 \cos c=n t$ & $5.755 E-06$ & 500 \\
\hline & $? 6 n .0$ & 251 & $1.5475-197$ & $7.4235-17$ & $50 n$ \\
\hline & 294.0 & 111 & $1.1915+00$ & $5.505 F-\cap 8$ & $56^{A}$ \\
\hline & $1 \mathrm{p}$ & & 182 & 4 & \\
\hline
\end{tabular}

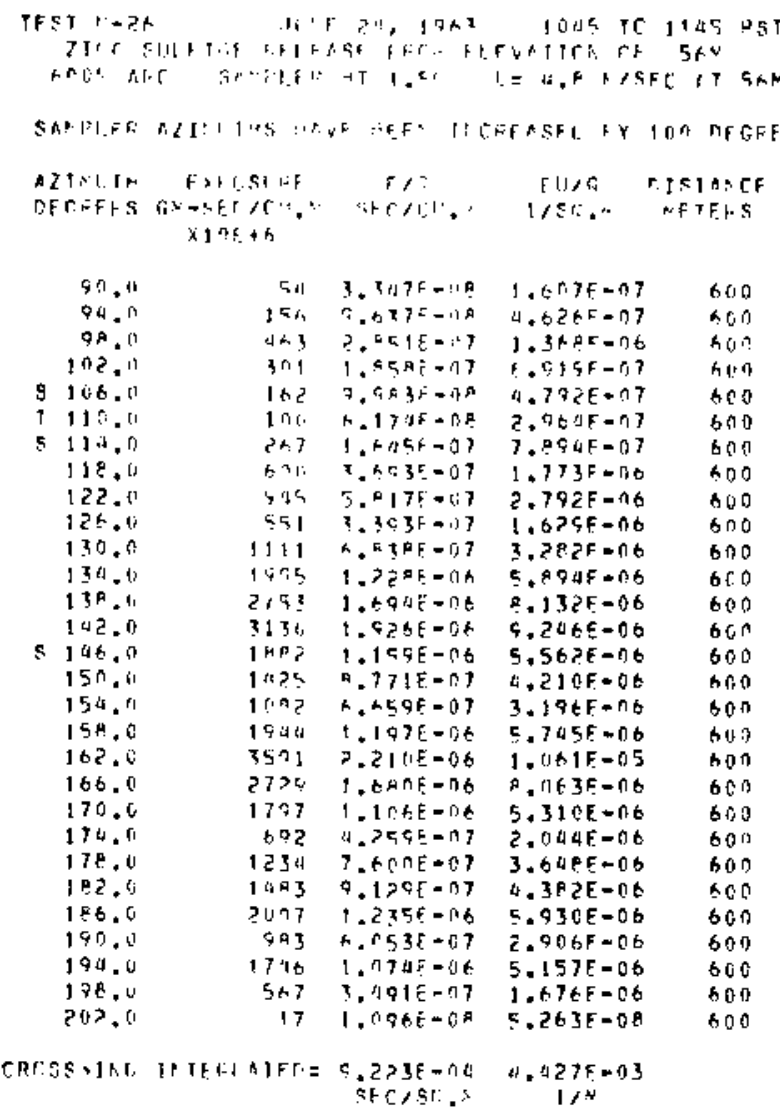

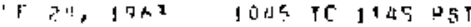

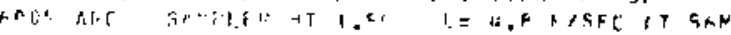

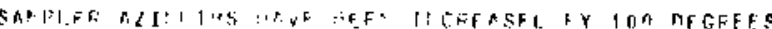




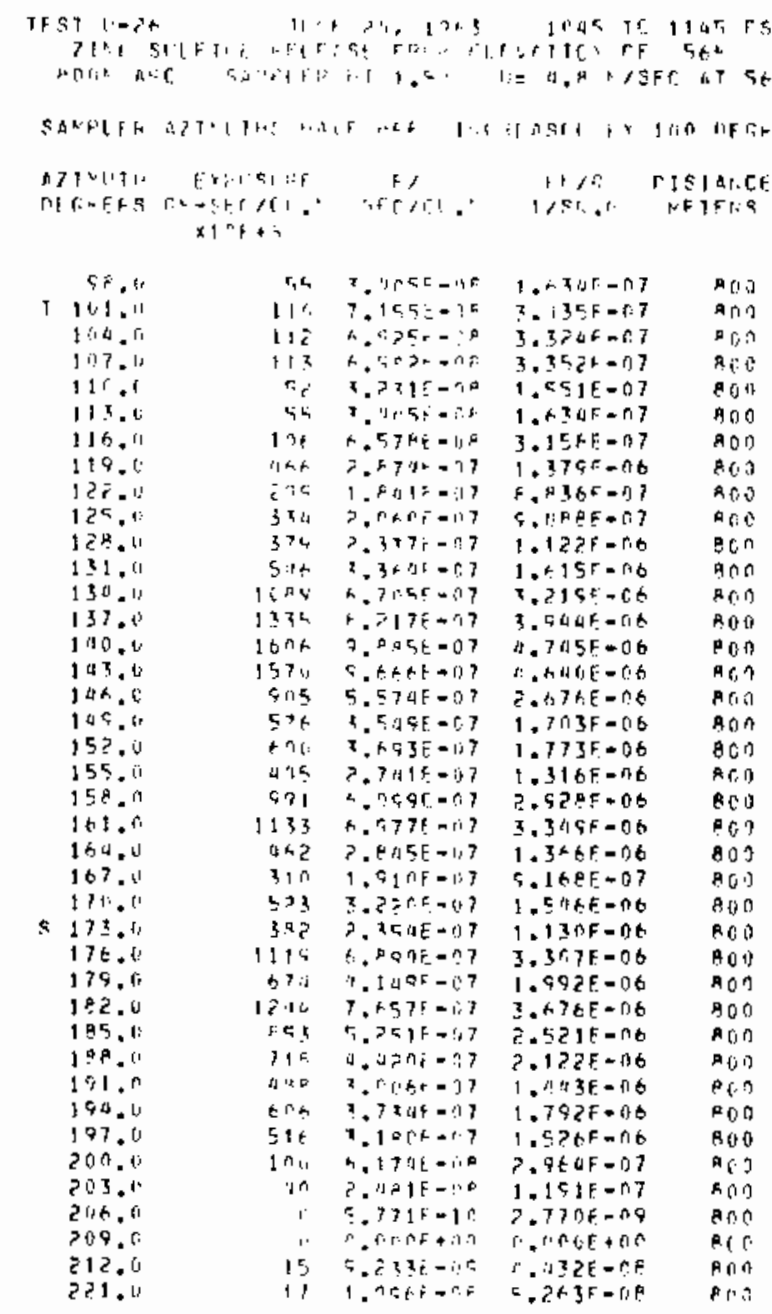

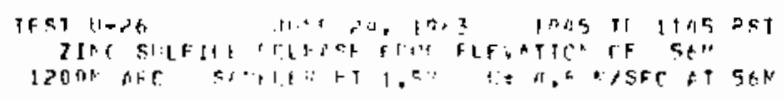

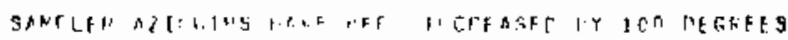

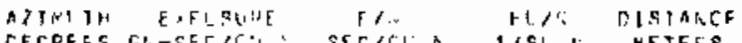

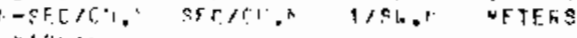
$x \in t u+r$

\begin{tabular}{|c|c|c|c|c|c|}
\hline & 104.0 & : & 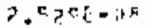 & 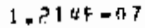 & 1200 \\
\hline & $\cot 0$ & 32 & $4 \cos r-c{ }^{\circ}$ & $9.545 F=0 B$ & $1 ? 00$ \\
\hline & 10.00 & 31 & $4.656=0.28$ & $9.5 \% 7 F=n A$ & 1200 \\
\hline & 110.0 & xo & $\therefore=2 a 2^{2}-78$ & $20.055-\{.7$ & $1>00$ \\
\hline & 112,0 & $a t$ & 5.7CAEAD & $2,541 F=07$ & 1200 \\
\hline \multirow[t]{5}{*}{$\mathbf{T}$} & 120.19 & ? 11 & $1, ? 4 F \dot{E}=0 t P$ & $5.9 F P E=\cap A$ & 1200 \\
\hline & $11+.6$ & tet & U. & 1.05 a -5.7 & 1200 \\
\hline & 118.0 & 45 & 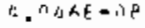 & $1.042 \mathrm{r}-0.7$ & 1200 \\
\hline & 120.0 & 3 & $=.125 F-1, P$ & $2.4+n E-0 ?$ & 1207 \\
\hline & !2?." & $1 \geq 3$ & $5.1<3\ulcorner=0$ & $3.933 \mathrm{E}=07$ & 1200 \\
\hline 7 & $12 \%, "$ & 115 & $7.1155-1: ?$ & $3 .: 15 F=07$ & 1200 \\
\hline \multirow[t]{5}{*}{7} & 126.0 & 193 & a $5005=0$ ? & $4.22+E-07$ & 1200 \\
\hline & $1 ?$ P. 0 & 215 & $1.3>85 .=07$ & $6.377 f=n 7$ & 1200 \\
\hline & 1310.4 & $17 \%$ & न. $3 \zeta\left\{[-1)^{\circ}\right.$ & $t, 0,30 E=07$ & J?6? \\
\hline & 132.0 & 1 I I & $1.12 r=-117$ & $5.4225-07$ & 1203 \\
\hline & 134.0 & 245 & $1.521 F-177$ & $7.54 E F=07$ & 1200 \\
\hline$s$ & $13 t .11$ & 170 & $1.173 E=07$ & $5.2425=07$ & 1200 \\
\hline \multirow[t]{2}{*}{$s$} & 138.11 & 375 & $1.95+F-07$ & $E-n 7$ & 1200 \\
\hline & 100.11 & 976 & $5.75+F-17$ & CAA?QF — OB & $1 ? 00$ \\
\hline \multirow[t]{4}{*}{$s$} & $14 ? .0$ & $5 \mu_{4}$ & $1,-118=07$ & $1.733 E-7 h$ & 1200 \\
\hline & 104.01 & 353 & $2.1755=07$ & $1.0146=06$ & 1200 \\
\hline & $\operatorname{tat}, 0$ & 207 & $1 .+11 \mathrm{c}=07$ & $P .7 A \& F=07$ & 1200 \\
\hline & 1 4e.: & 341 & $? .1 \times 4 \mathrm{E}=07$ & $1 . ; 1 C E-n b$ & 1200 \\
\hline \multirow[t]{12}{*}{$s$} & 150.0 & $2 n$ & $1.755,5=07$ & $t, 1+t+F+0) 7$ & 1200 \\
\hline & 152.0 & $1: 5$ & $n .935 E=0 P$ & 4. टेA GE $=07$ & 1200 \\
\hline & 150.0 & $2: 4$ & $1.53 P F-117$ & $7.3 \times C F=07$ & 1200 \\
\hline & $156 . n$ & 6911 & $A .272 E-6.7$ & $2.051 F=06$ & 1200 \\
\hline & $150.0^{\circ}$ & 33.0 & $? .58$ a $5=17$ & $1.0 \cap 0 F=0 t$ & 1200 \\
\hline & 160.0 & $3 y^{n}$ & $P .3 \Delta 7 F=U 7$ & $1.1265=06$ & 1200 \\
\hline & 167.0 & $2 \mathbf{v}_{t}$ & 1.0575 .07 & $6.3025=07$ & 1200 \\
\hline & 164.0 & 111 & A. $F>A F-i) A$ & $3.3025=07$ & 1200 \\
\hline & 166.0 & $9{ }^{\prime \prime}$ & $5.57,7 F-3 F$ & $7.0 F 7 F-n ?$ & 1200 \\
\hline & 168 & 178 & 1. $[1,75=197$ & $5 . C \subset A F=n 7$ & 1209 \\
\hline & $170 . \mathrm{r}$ & 171 & $2 E-? A$ & $3 . R A 4 F=n 7$ & 1700 \\
\hline & 172.0 & 13.6 & $2.1: 1 D E * 7$ & $5.006,+n ?$ & 170 \\
\hline s & 170.0 & $5 n 4$. & $3 ., P A F=17$ & $1.741 \mathrm{~F}=\mathrm{nh}$ & 1200 \\
\hline$s$ & $17 \mathrm{~h} . \mathrm{c}$ & $\| A>$ & $7.5 A 7 E-07$ & $1.4 ? \Delta \mathrm{F}-\mathrm{n} \theta$ & $220 n$ \\
\hline \multirow[t]{10}{*}{$s$} & 178,11 & 467 & $=. n>+5=n ?$ & $1 . x^{*} 1 E=n t$ & $1 ? 0 n$ \\
\hline & 180.5 & $\Leftrightarrow>1$ & ?. $2>75-1 ! ?$ & $1 .+37 F=06$ & 120.3 \\
\hline & $3 \times 2.5$ & 525 & $3-2748=017$ & 1. $r 5 ? 5=06$ & 1250 \\
\hline & $1+4 a$. & 35,1 & 3. ${ }^{2}=4, F=17$ & $1 .+\hat{z} 7 F-n b$ & 32110 \\
\hline & 106,0 & $5 ! c$ & ni & $1.5365=f .6$ & $1>010$ \\
\hline & $I^{P P} .:$ & $\forall x \underline{z}$ & $7.977 F=07$ & $1 . j \gg G F=n t$ & $1 ? 00$ \\
\hline & 190.4 & 352 & $2.16 .2 f-i 1 ?$ & $1.1111 F=n A$ & 1200 \\
\hline & 192.6 & 351 & $>, 1+5 t-n ?$ & $1 . " 139 F=0.4$ & $124: 9$ \\
\hline & 194,0 & $12 ?$ & $1.1 \nabla a f=37$ & $5.725 F=07$ & $1 ? t, \hat{t}$ \\
\hline & 184.1 & $10^{\circ}$ & $1.146=0$ & $=.495-57$ & $1 \geq 0$ \\
\hline \multirow[t]{3}{*}{8} & $19 R \cdot r:$ & $1, t$ & 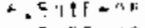 & $3.1+0,-12 ?$ & $1 ? 0$ \\
\hline & & at & $4 F-18$ & $\therefore \cdot r>3+-5 ?$ & 1? 2117 \\
\hline & 242.0 & 3 & 1. & $\therefore .1+1, F=0 k$ & J, $310 \pi$ \\
\hline
\end{tabular}

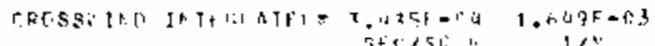




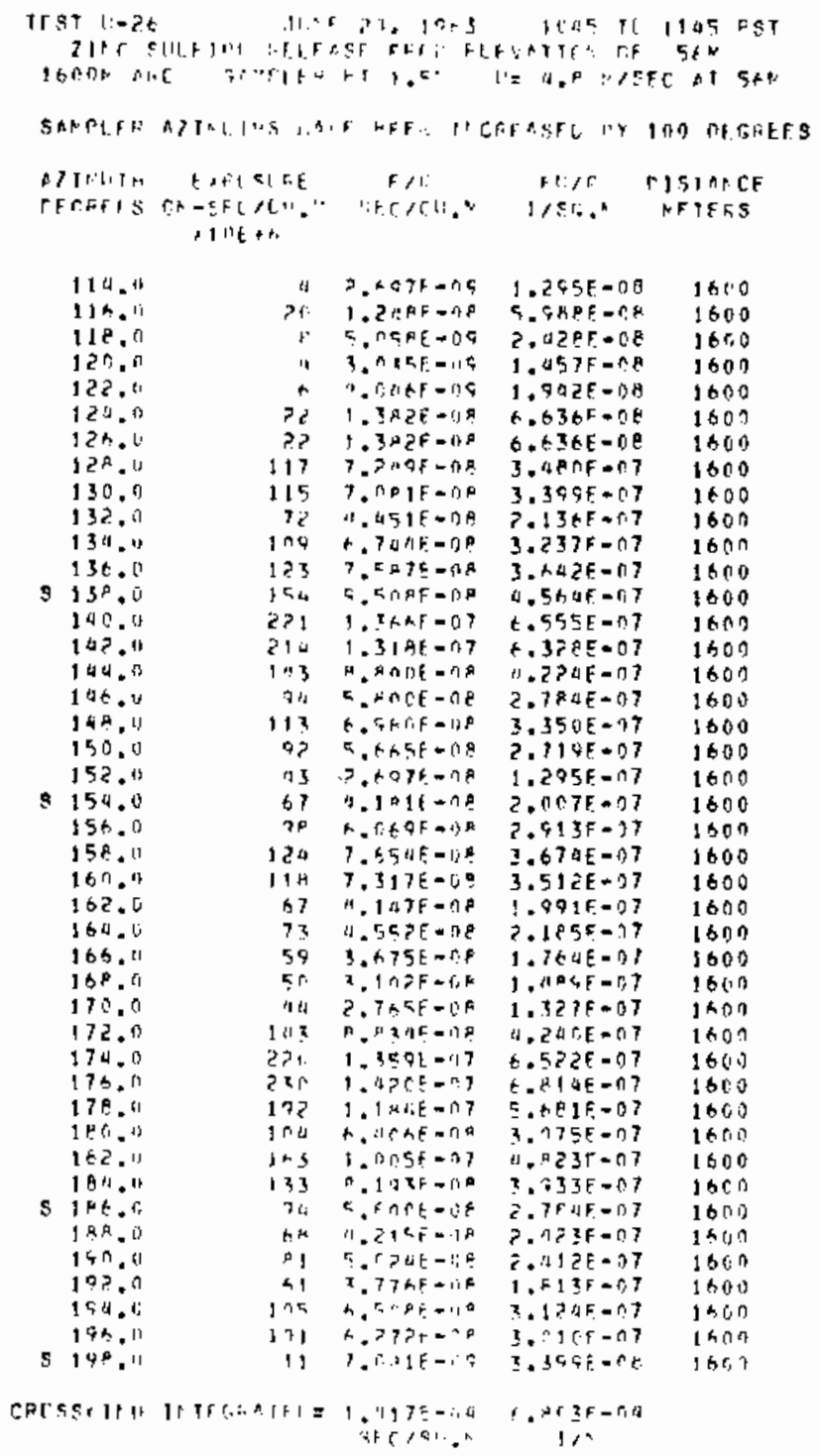


UNSTABLE SERIES EXPERIMENT: 27 ( $\mathrm{J}-27$ )

GROUND LEYEL SAMPLING 100-1600 M ARCS. FOR EASE IN STATISTICAL ANALYSIS. ALL SAMPLER AZIMUTHS HAYE BEEN INCREASED BY 30 DEGREES

FRON THEIR ACTUAL POSITIONS.

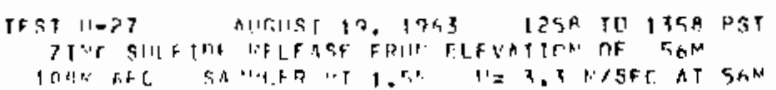

\begin{tabular}{|c|c|c|c|c|}
\hline $1 n .0$ & य० $3 x+1$ & $>A \geqslant 1 F=05$ & $9.5 n 7 f=05$ & 100 \\
\hline 14,0 & $\triangle A W R_{0}$, & $\Rightarrow$ A 7 S 5 - 05 & $9 .+1+1<-05$ & $1 \cap n$ \\
\hline 10.0 & 30093 & P.PPFF -14 & 7.35 if $=015$ & 100 \\
\hline 2.0 & $31>4$ & 1.7 APE $=7 \mathrm{H}$ & $5.0 \cap 25=106$ & 100 \\
\hline$p+.0$ & $=2$ thints & 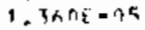 & U. IRQF $=05$ & $\ln 9$ \\
\hline $\sin .0$ & $1+2^{n} 7$ & 1. AnOC $=185$ & $3.0 \times 3 F=05$ & 100 \\
\hline $3 a$ & $17 a n ?$ & Q. 2งn厂 - $0 \mathrm{n}$ & $3.2 P, 2 F=05$ & $\ln n$ \\
\hline $3 A .0$ & $1 \cap B 7 h$ & $A .215 F=9 A$ & $2.051 E=05$ & $10 n$ \\
\hline 42.0 & $\ln 541$ & $2.035 \mathrm{~F}=1 \mathrm{n}$ & $1.992 F-05$ & 100 \\
\hline at. . & 1 XSA & $? .1 P A F=n A$ & $2.3745-155$ & $10 n$ \\
\hline 50.0 & 1 I पA 1 & $7.76 .2 F=3 A$ & $2.542 F-75$ & $10 n$ \\
\hline $5 \pi .0$ & 21719 & $1.3+\Delta F=35$ & $a .5+7 F-05$ & ton \\
\hline $5^{2} \cdot 0$ & 24,120 & $1.3775=35$ & 1). $9 A 0 F=05$ & 100 \\
\hline $42, \pi$ & $\ln 16 \mathrm{~h}$ & 5. ROQE $=06$ & $1.917 F=05$ & $1 \cap 0$ \\
\hline $\begin{array}{ll}66 \\
700\end{array}$ & $\begin{array}{l}12574 \\
2>509\end{array}$ & $\begin{array}{l}1.1>5 E-175 \\
1.2 A 125=05\end{array}$ & $\begin{array}{l}3.71 \text { 4F }-05 \\
0.252 f-05\end{array}$ & $\begin{array}{l}100 \\
100\end{array}$ \\
\hline 74.0 & $2397 \mathrm{~A}$ & $1.3 n \Delta F=05$ & $a, a n t 5=05$ & 109 \\
\hline $7 H .0$ & 25915 & 1. 0 वा:-0.05 & U.RA7E- I5 & 100 \\
\hline$\mu>0$ & PAtat & $1.5 \times A,-05$ & $5.034 \mathrm{~F}=\mathrm{nS}$ & $10 n$ \\
\hline$m+\ldots$ & $315 \times 2$ & 1. $3 n \geqslant F=05$ & 5. OHEF $=05$ & $\operatorname{lon}$ \\
\hline Q & 12004 & $7.75+15-10$ & 2.39 af $=05$ & 100 \\
\hline 34.0 & 1237 & $1.1 \times 5=n A$ & $1.3+5 x=05$ & 109 \\
\hline QR. 0 & $a>n 2$ & $3 . h \times 7 F-\pi t$ & A. AA AE $=16$ & 100 \\
\hline $0 ? .9$ & 5015 & $\Rightarrow$. QQर $=0 G$ & $9.51 d E=06$ & 100 \\
\hline 0.0 & $3[47$ & $A . M \times A E-1)$ & 1.3 & 100 \\
\hline 10.0 & 1 nuls & 5.75 if $-0.0 \mathrm{~A}$ & $1.96 \Delta F=05$ & $1 \cap n$ \\
\hline 114.0 & $11>53$ & $6.1131 f=06$ & $2.1225-05$ & 100 \\
\hline 119.0 & 7238 & $5.2796=06$ & $1.742 F+05$ & $\ln 0$ \\
\hline 122,0 & nQna & $5.3 \times 95-06$ & $1.6795-115$ & 100 \\
\hline $12 k .0$ & Онद八 & $5.0111-016$ & 1. म5AF $=05$ & 100 \\
\hline $13 n .0$ & 7214 & $\Rightarrow .1>3 F-n A$ & $1.3+10+15$ & $10 \%$ \\
\hline 137.0 & $703 \mathrm{~A}$ & " $=15 F=n R$ & $1.3257=n 5$ & $10 n$ \\
\hline 15 म .0 & $53 \pi 5$ & 3.05 tf $=17 x$ & $1, \cap \cap B F=05$ & 100 \\
\hline & $>4 \times 3$ & $1.4 c a r=n k$ & $11.040 F=06$ & $1 \cap 0$ \\
\hline 140,0 & $A \cap 3$ & $3 . t \Delta 75-i 7$ & $1.137 f=0.6$ & $\ln n$ \\
\hline $15 n .0$ & +7 & $3 . \sin 3(-1) A$ & 1.1 AhF $=n 7$ & $\ln n$ \\
\hline
\end{tabular}

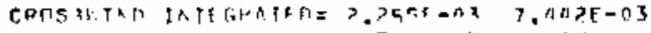

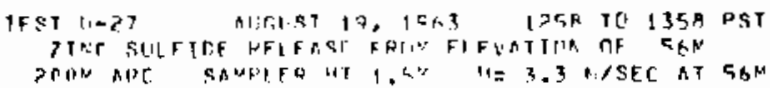




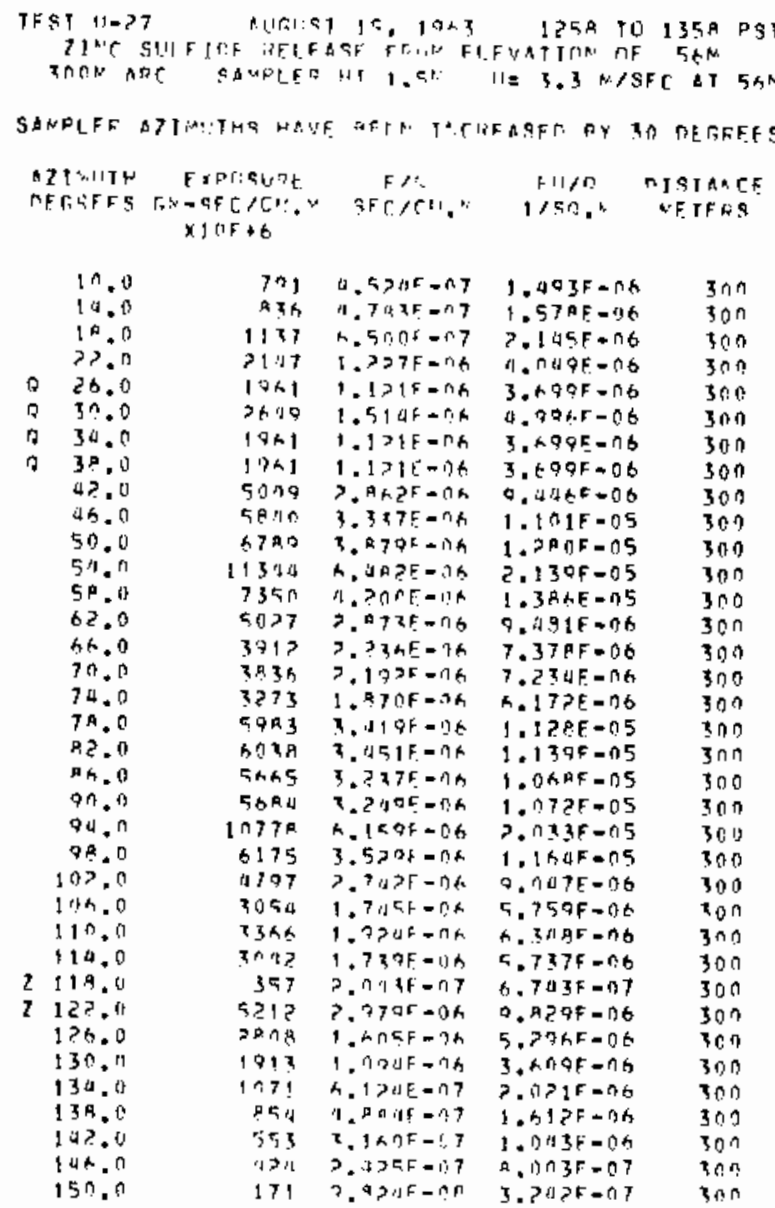

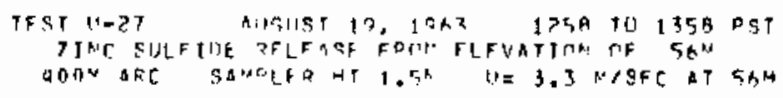

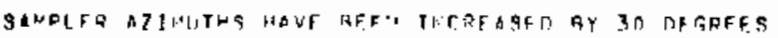

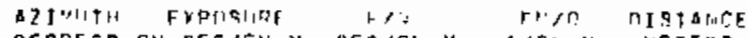

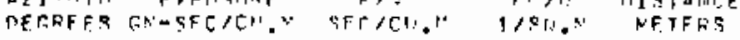
$\times 1 \cap F+5$

\begin{tabular}{|c|c|c|c|c|}
\hline 12.0 & (מנד & 1. $7: 16 \mathrm{a}=n)$ & c. .7 IJ $5=07$ & an \\
\hline $15 . n$ & 130 & 7 . thate =na & 2. $4 x \cdot 5=\cap 7$ & 400 \\
\hline 20.0 & $3 ! n$ & 1. Dnat $=$ की & $5.9+55=07$ & 400 \\
\hline 20.0 & 1017 & 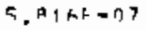 & $1.91 \mathrm{GF}=\mathrm{AB}$ & 4 औก \\
\hline 20.0 & $\ln 16$ & $5.2105=07$ & $1.017 \mathrm{~F}-106$ & $40 n$ \\
\hline 32.0 & $1 ? n a$ & 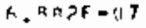 & $2.2715=06$ & $\Delta \cap 0$ \\
\hline 36.0 & 710 & $1.958 E-07$ & $1.3305-106$ & 400 \\
\hline 49.0 & $37 \mathrm{R} G$ & $1.7 \times 1 F-116$ & $5.25 D E-\pi 6$ & य०? \\
\hline 14.0 & 5979 & x.417F-06 & 1.12 PE $=$ ก 5 & $\Delta 0 \cap$ \\
\hline 42,0 & 4213 & 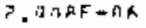 & $7.915 E-00$ & 400 \\
\hline 52.0 & $32>4$ & $1, A_{4}, F=\beta B$ & $A, 0 R 0 F=D G$ & $4 \cap 0$ \\
\hline 56.0 & $48 \times 3$ & ?. 9 a $\cap F-D A$ & $9.39 A F=06$ & 400 \\
\hline 60.0 & a6ว & J. Sis $5 t=10$ & $1.729 \mathrm{~F}=106$ & 400 \\
\hline 34,0 & उค? & $=.170 F_{-}=16 t$ & $7.22 A . F=0 B$ & 400 \\
\hline 6,0 & $37 \times 7$ & $1.593 \mathrm{~F}=\mathrm{Rt}$ & $5.257 \mathrm{E}=06$ & $40 n$ \\
\hline $7 ? .0$ & a732 & $\supset .7 \cap \Delta F_{-}=\cap n$ & $A .9 ? 3 F=06$ & $\operatorname{ann} 0$ \\
\hline 15.0 & $\operatorname{~मकव~}$ & $3.3 \times g F=C h$ & $7.717 E=26$ & यह \\
\hline$A n, 0$ & a9:9 & ?. POKE-DE & $9.25 .95-06$ & $4 \cap 0$ \\
\hline$p=0$ & $24 \times 3$ & 1. RouE $-i n t$. & $5.599 F \bullet 176$ & 400 \\
\hline Pค. 0 & nops & $3.95 A F=1.4$ & $1.3 \cap 65-75$ & an \\
\hline $9 ? .0$ & $777 n$ & А. मगम $F=n A$ & 1. atset - क 5 & $\operatorname{ag} 0$ \\
\hline 96.0 & $x y>\beta$ & 4. $21 \mathrm{~A} F=\mathrm{r}_{\mathrm{A}}$ & $1.5 \times 0 F=05$ & ann \\
\hline 100.0 & $>243$ & 1. $3 \cap 55=0 \%$ & $4.305 E=16$ & 100 \\
\hline $15,0.0$ & $30>5$ & $1.15 B F=D A$ & 3, 月 $>0 F=06$ & 400 \\
\hline 108.0 & \{ith2 & $A .350 F \rightarrow n 7$ & 3.75 P. -126 & don \\
\hline 117.0 & $(3,7)$ & $7.5715=07$ & $? .198 F=06$ & 40? \\
\hline 11 A .0 & $30>1$ & $1.3 a 11 F-0 b$ & $11.5+5 F-106$ & $a \cap n$ \\
\hline 120.0 & $29>3$ & $1, A, 7 ! F=A B$ & $5.513 F=06$ & $d \cap \eta$ \\
\hline $1 \overrightarrow{2}+.6$ & 4153 & $2.3735=11 \mathrm{t}$ & $7.832 F=16$ & 400 \\
\hline $12 \times .0$ & $>0 \geq n$ & $1.3 \times 7 t=13 A$ & $4,57 \mathrm{mE}=16$ & $4 \pi n$ \\
\hline 132.7 & 1704 & $1 . n \geqslant 7 F=n h$ & $3.38 P F=06$ & d \\
\hline 136.0 & 1159 & $4.6>15=07$ & $2.1 P A F=0$. & 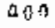 \\
\hline 1 in 6 & $7: 13$ & $11, ?(9)+-1) 7$ & $1.11025=06$ & 400 \\
\hline $\operatorname{lag}$. & 779 & $1.5 \mu \geq F=07$ & $5+P ?+F=07$ & an? \\
\hline 142.0 & $7.7 \%$ & $1.5+145=07$ & $5.0945-n 7$ & $40 \%$ \\
\hline 153.0 & 177 & $1.71+5=07$ & $3.89 x F-07$ & แก \\
\hline & & 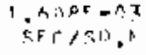 & $\begin{array}{c}5,306=03 \\
1 / 2\end{array}$ & \\
\hline
\end{tabular}




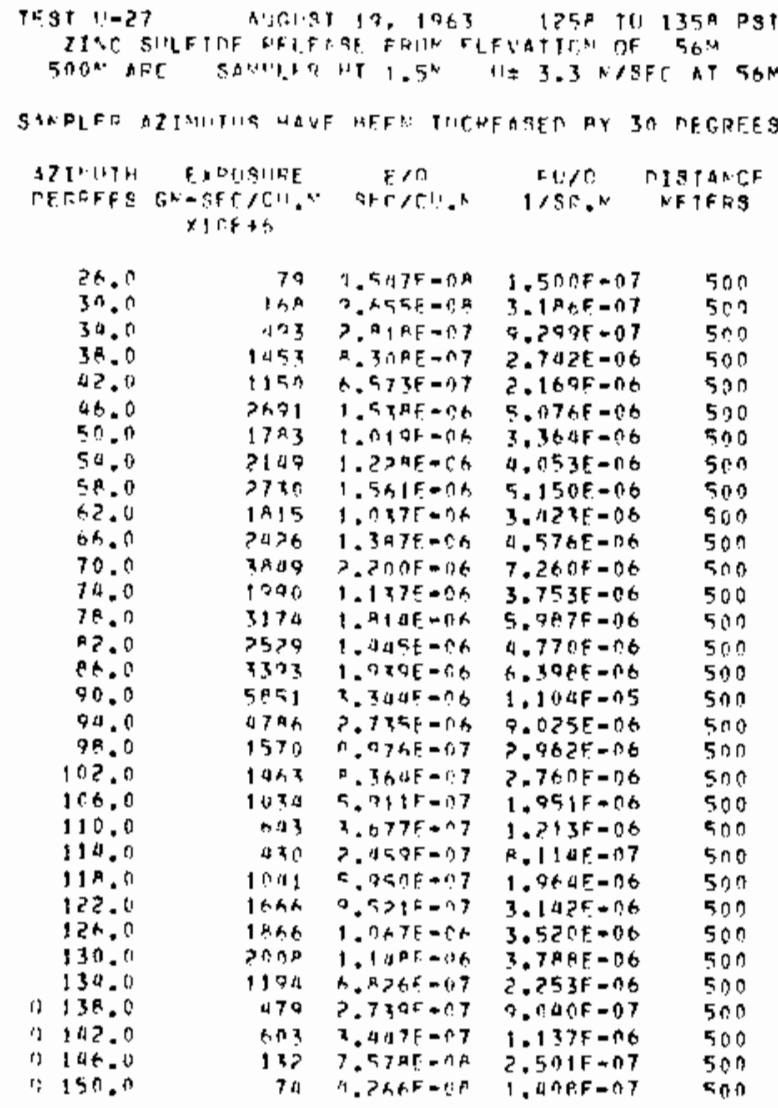

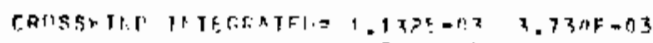

\begin{tabular}{|c|c|c|c|c|}
\hline 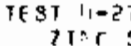 & 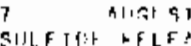 & 17, 170.2 & 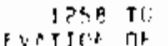 & $\begin{array}{r}1358 \\
5,11\end{array}$ \\
\hline $600, N$ AF & HC SADFEIS & $141.4^{\circ}$ & $11=3.3 \% / 5 F$ & $\mathrm{FC} A T$ \\
\hline$S \triangle M P L F B$ & A7 INA Ha Whif & F MFI. T'Г & BSEn ay 30 & \\
\hline $\begin{array}{l}\text { DXINITH } \\
\text { DETFFES }\end{array}$ & 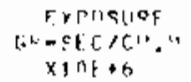 & 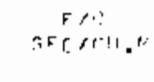 & 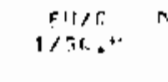 & $\begin{array}{l}\text { ISTALCE } \\
\text { MTFHQ }\end{array}$ \\
\hline 24.0 & it & จ. דis $>0$ & 3.1 Jणf $=$ กम & 800 \\
\hline PA. 0 & 14 & $7.5,2 F-n g$ & $3.1495=08$ & hin \\
\hline 32.0 & $2 p$ & $1 . h>A E=(1 A$ & $5.37 ? F=08$ & hon \\
\hline 36.1$)$ & 275 & 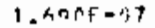 & $5.576,5-17$ & hon \\
\hline 40.0 & $7+7$ & $1.59 x=07$ & $5 .>24 \mathrm{~F}=.177$ & $A+r$ \\
\hline 44.0 & 557 & x. $10,05=7$ & $1 \cdot C 5 \bar{F}=\cap 6$ & 500 \\
\hline $4{ }^{2} .0$ & 1074 & $+.1315=1: 7$ & $2 \cdot+27 F=n h$ & 600 \\
\hline 52.0 & AR2 & $5, D \geqslant\{F=\{1\}$ & 1.nBt]F=n6 & $6 \cap 7$ \\
\hline $5 \times .0$ & 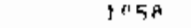 & $\alpha, 0 i \Delta,-07$ & $1.0055-16$ & $x \cap \pi$ \\
\hline 60.0 & $18+9$ & 1. n\&AF $=n h$ & $3.5235=06$ & hon \\
\hline 64.0 & 1779 & $1.917 F-196$ & $3.395 F=06$ & $H 00$ \\
\hline$G R \cdot O$ & $1 \mathrm{R} a_{n}$ & 1. $n p$ य $=n h$ & $3.577 F=06$ & sחn \\
\hline 72.0 & Plns & $1.7 P U E=176$ & $3.9725=06$ & $6 \pi n$ \\
\hline 75.0 & $P 3$ : 15 & $1.5 \sin =5 x$ & a) $429 F+n t$ & $b 013$ \\
\hline 80.0 & $19 \times 5$ & $1.133 F=06$ & 3.7075 .06 & 600 \\
\hline Aa. $n$ & 1630 & 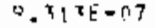 & $3.075 \mathrm{~F}=0 \mathrm{~h}$ & $6 \cap n$ \\
\hline A. . 0 & $3+79$ & 1. वRRF-OA & $6.561 \mathrm{E}=106$ & $5 \cap 0$ \\
\hline $9 ? .0$ & 2650 & $1.515 E=n A$ & $4.4 D P . F=1 B$ & $\sin$ \\
\hline 96.0 & 1047 & $\therefore .11 \times 5=n x$ & $3.672 F=n h$ & 600 \\
\hline $10 n .0$ & 972 & $4.327 \mathrm{~F}=\mathrm{n}]$ & $1.758 F=06$ & 600 \\
\hline 160,0 & 736 & $t .15, A F-[1\}$ & $1.371 F=n t$ & $x=0$ \\
\hline $\sec 0$ & $4 \times 0$ & $?, k \times 2+=3 ?$ & $R .6$ RF $=07$ & han \\
\hline 112.0 & 573 & $\because .27 A F=17$ & $1.0 A 2 E=06$ & $b \cap 5$ \\
\hline $11 k .0$ & 6,45 & $3 . \mathrm{SP} P \mathrm{~F}-1: 7$ & $1.217 F=$ nt & 6010 \\
\hline $12 n .0$ & $35 \mathrm{~s}$ & 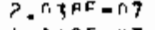 & $6.724 F=07$ & $6 \cap n$ \\
\hline 120.0 & 1653 & A. NIFF=MT & $1.9 A \& F=06$ & 800 \\
\hline $12 a .0$ & 9.22 & $5.1031=07$ & $1 . \operatorname{xnd} 6-50$ & 600 \\
\hline $13 ?, n$ & 332 & $1-8375-77$ & $4.261 E=07$ & 600 \\
\hline 136.0 & 71 & ". ^ $Q A \Gamma=0 A$ & $1.352 F=17$ & 600 \\
\hline & $T *$ & 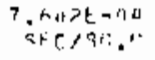 & $\begin{array}{c}2.527 \mathrm{~F}=\mathrm{n3} \\
1 \mathrm{k}\end{array}$ & \\
\hline
\end{tabular}




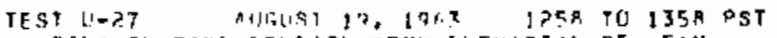

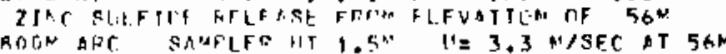

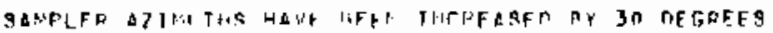

\begin{tabular}{|c|c|c|c|c|}
\hline $\begin{array}{l}\text { AZTA IITH } \\
\text { RECEFFS }\end{array}$ & 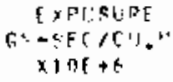 & $\begin{array}{c}F / 4 \\
\text { cerlu.l }\end{array}$ & $\begin{array}{c}F \mid j / 0 \\
1 / 81 . .6\end{array}$ & $\begin{array}{l}\text { I T ARECE } \\
\text { NFTFSS }\end{array}$ \\
\hline $1 \times .0$ & $2 \pi$ & $1.179 \mathrm{E}=\mathrm{nP}$ & उ. A०तF $=Я$ В & R⿻n n \\
\hline 16.0 & $3 n$ & $1.177 E=\cap P$ & 3. $A Q C F=O A$ & $\operatorname{ann}$ \\
\hline $18 . \pi$ & 45 & $3.5 P 75=19$ & $P .52\{5=0 \theta$ & ron \\
\hline 22.0 & 17 & $1 . n \cap \cap F=n d$ & $3.3 \times 45=0 A$ & RoO \\
\hline 25.0 & 67 & $x, A>X F=\pi A$ & $1.2785-07$ & คon \\
\hline 28.0 & 72 & $5.27 \gamma k=\cap A$ & $1.7 \| 1 F-07$ & ADO \\
\hline 31.0 & $1 \times 7$ & $7.850 F=n R$ & $z, 59: F=07$ & 800 \\
\hline 34.0 & 161 & $\therefore .>n+F=n p$ & $3.038 F=07$ & $A_{00}$ \\
\hline 37.0 & 147 & $A .4>05=0^{\circ}$ & $2.7795-07$ & 100 \\
\hline 40.0 & $\ln 2$ & $1,7>0 \Gamma-07$ & $5.7065-097$ & non \\
\hline 43.51 & 325 & 1. $A<R F=07$ & $0.1 \leq 25=07$ & $\operatorname{Ann}$ \\
\hline na. & $2>0$ & $1.257 F=07$ & A. $1505=07$ & A 00 \\
\hline 59.0 & $3 ? 2$ & $1.975 F=07$ & 5.1 \& $7=07$ & AOO \\
\hline 52.0 & 77 & $5.557^{\mathrm{F}}=17 \mathrm{~A}$ & 1. $8305=0$ ? & Rnn \\
\hline 55.0 & $5 \cap n$ & $2.257 F=07$ & $9.429 E-07$ & A0O \\
\hline $5 A, 0$ & GAA & $7.9355=07$ & $1.299 F=06$ & AOO \\
\hline 61.0 & 500 & 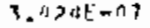 & $1.1305=06$ & 800 \\
\hline 60.0 & 918 & $5,37>\mathrm{F}=n 7$ & $1.773 \mathrm{E}=0.6$ & Ano \\
\hline 67.0 & 723 & A. औमกร $=07$ & $1.47 A F=06$ & $\operatorname{Ron}$ \\
\hline 70.0 & 714 & $H=f_{1} 91 \mathrm{~F}-07$ & $1.3475=06$ & $6 n n$ \\
\hline 73.0 & 1237 & ᄀ. $T$ T3E $=7$ & $2.334 E=196$ & $A 00$ \\
\hline 76.0 & SAh & 5. $1 \cap \cap A F=07$ & $1.760 F=0 B$ & $5 \times 9$ \\
\hline 70.0 & 989 & $=\{x \in>5-1] 7$ & $1.771 F=\hat{0} b$ & AnO \\
\hline 52.0 & $4>5$ & 2. $13: F=07$ & A. $02 \leq E-07$ & $A \cap O$ \\
\hline 95.8 & $\sin \theta$ & $? .9: 3 F=07$ & 9.61 यE $=n 7$ & A त n \\
\hline 88,0 & 7 7に & H.? $2 x=n$ & $1.307 \mathrm{~F}=06$ & 900 \\
\hline 91.0 & 1002 & $5.95 \times 5=07$ & $1.9+5=06$ & AoO \\
\hline 94.0 & 809 & $5.7 \cap 8 f-67$ & $1 . B A \triangle F=06$ & ano \\
\hline 87.0 & G.A & $3.975 F=147$ & $1.7085=76$ & RoO \\
\hline 100,0 & $36 \mathrm{~A}$ & $? .1 \cap 5 E-\cap 7$ & $0.947 E-n 7$ & मn \\
\hline $10,3,6$ & $1+6$ & a. $1+a r=0$ & 2.7 HOE $=4.7$ & An \\
\hline $10 t .0$ & 159 & $0.043+15=6 ; 8$ & $3.0 A 1 F=07$ & $R_{0}{ }^{n}$ \\
\hline 500.0 & 147 & $0.5 A_{1} \times f-0 R$ & $3.109 \mathrm{~F}=07$ & $A 00$ \\
\hline 112.5 & $15\}^{\prime}$ & $D . A_{3} 5 F=0 \mathrm{n}$ & $P . n 53 F=07$ & ann \\
\hline 115.0 & $7+4$ & 1. AnQR $=n 7$ & $4.650 .5=07$ & 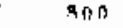 \\
\hline 158.0 & 101 & $7.751^{5-i . j 7}$ & $5.0775-197$ & $9 n n$ \\
\hline 121.0 & $4>A$ & $2.497 t-n 7$ & $8.077 \mathrm{E}=07$ & $90 \%$ \\
\hline 124.6 & $5+7$ & $x .3455=n 7$ & $1 . n 71 F=n \theta$ & $\mathrm{A}_{0} 0$ \\
\hline $127 . n$ & $4 \pi 9$ & $2.2 \|[+-10]$ & $7.725 \%=07$ & $\operatorname{RAB}$ \\
\hline 136.0 & $\ln \theta$ & $n . n A x=-n p$ & $>, \cap \cap / F=n 7$ & Bun \\
\hline 133.0 & $7 ?$ & $\because .>775=\Pi \pi$ & $1.741 F-07$ & $x_{n} \cap$ \\
\hline 136.0 & 72 & $9.15314-05$ & $1.3715=07$ & pon \\
\hline 139.1 & ว月 & $1, A P P F=\Gamma \cdot F$ & $5.37 P^{5}=08$ & ann \\
\hline
\end{tabular}

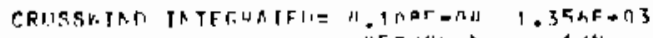

TFST U-27 BITHST 10,1943 125A TO $135 \mathrm{R}$ PST

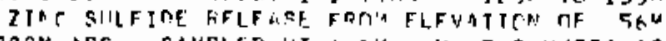

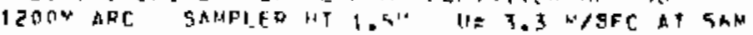

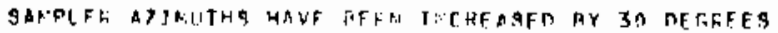

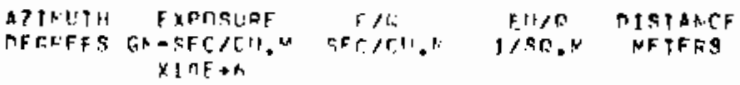

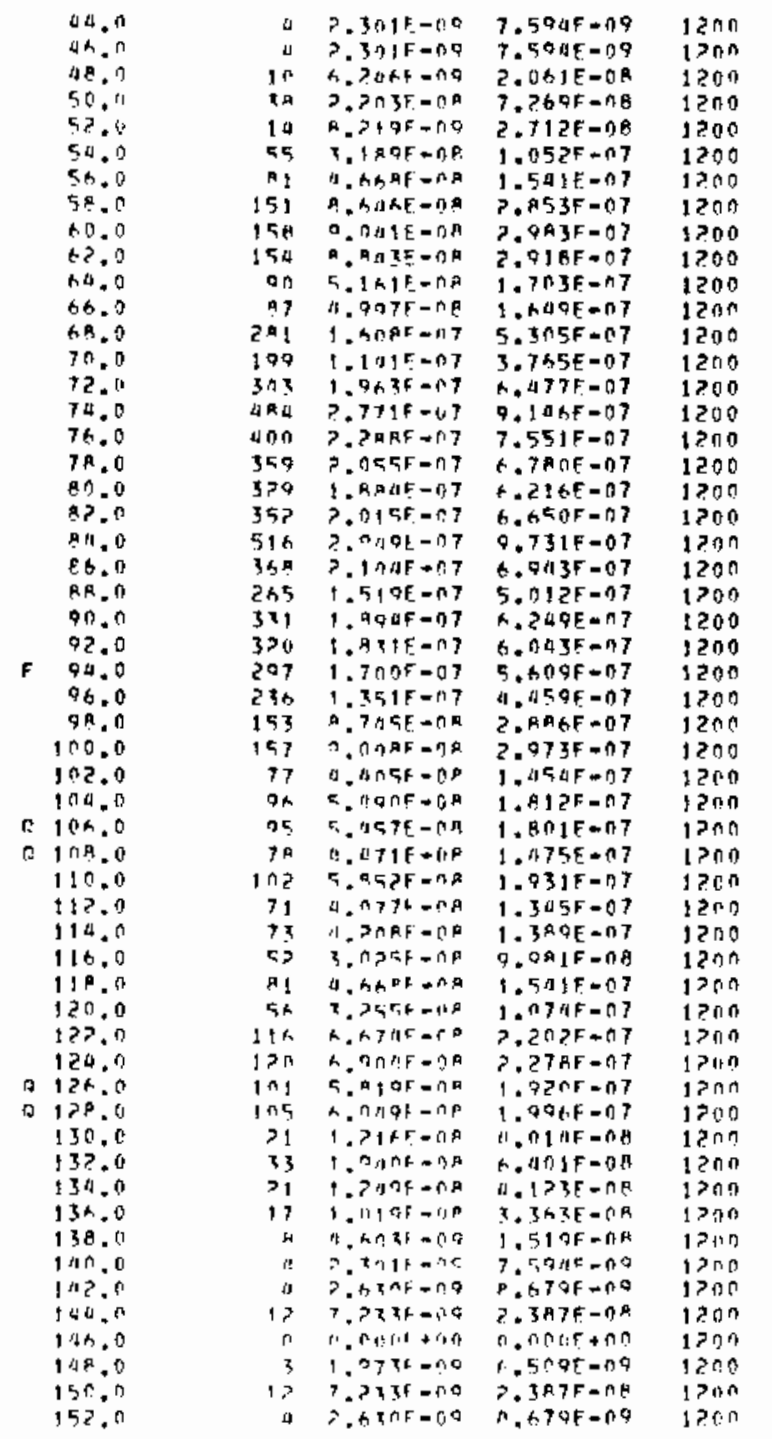

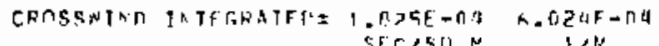




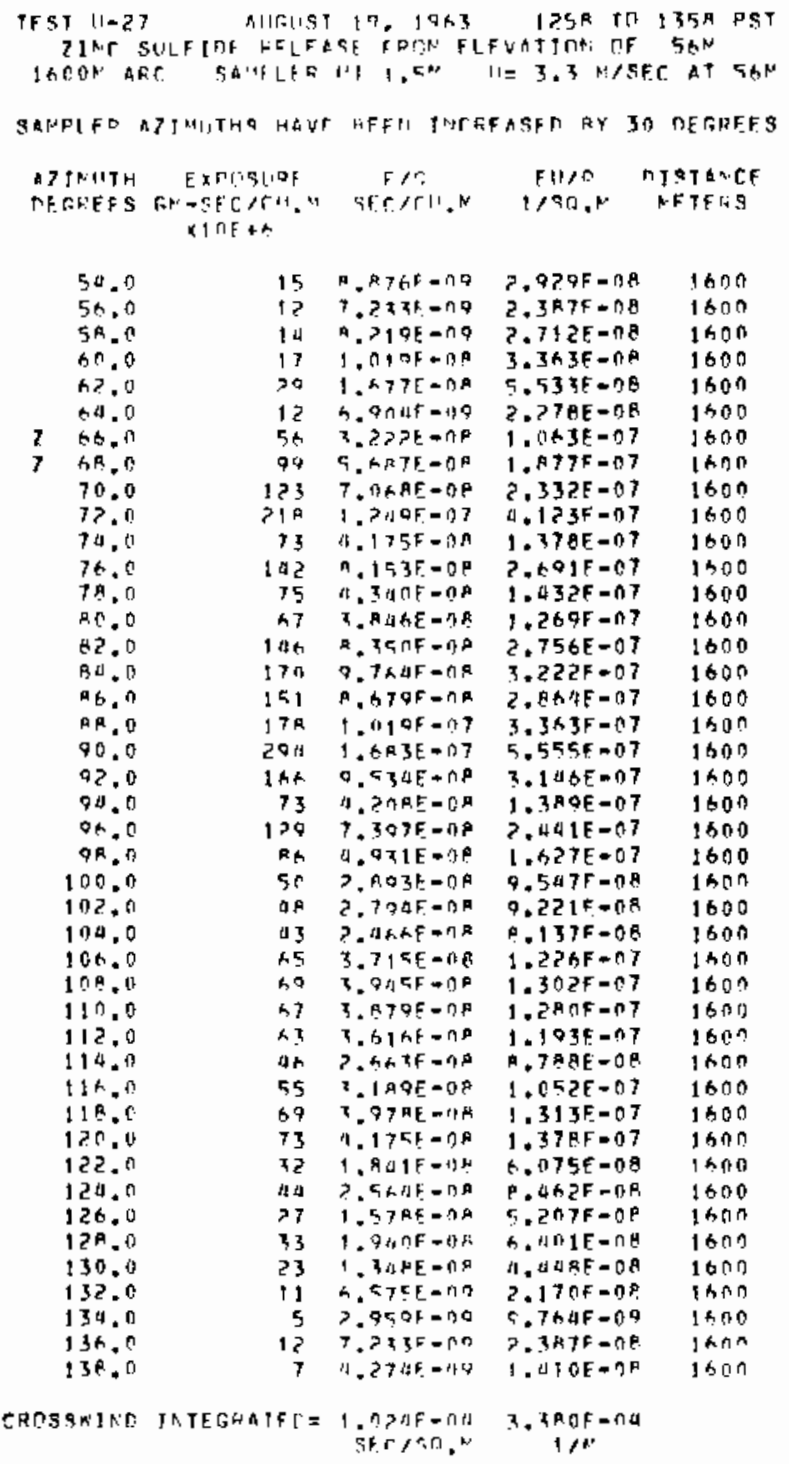


INSTABLE SERIES EXPERIMENT: 28 ( $\mathrm{U}-28)$

GROLJND LEVEL SAMPLING 100-1600 M ARCS. THE TRACER WAS NOT

DETECTED ON THE 100 OR 200 A ARCS.

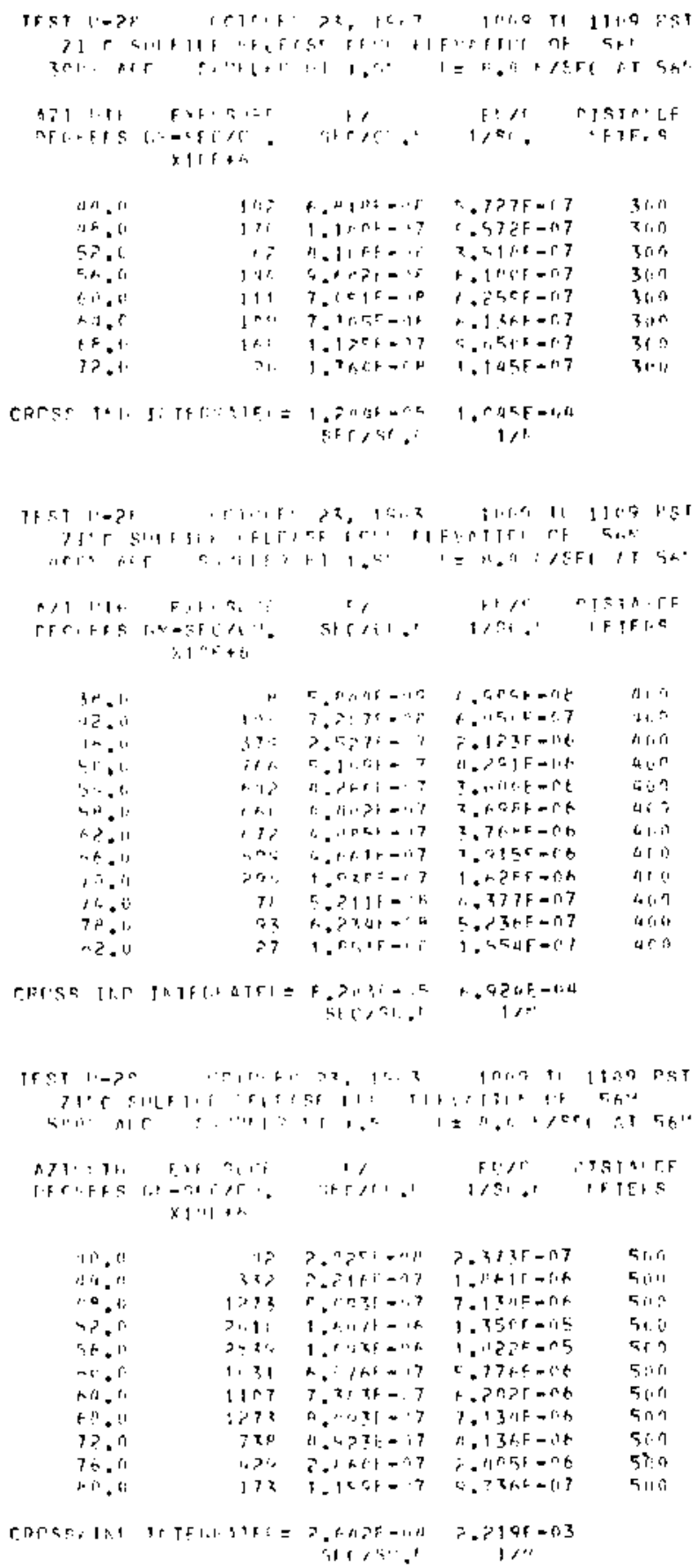

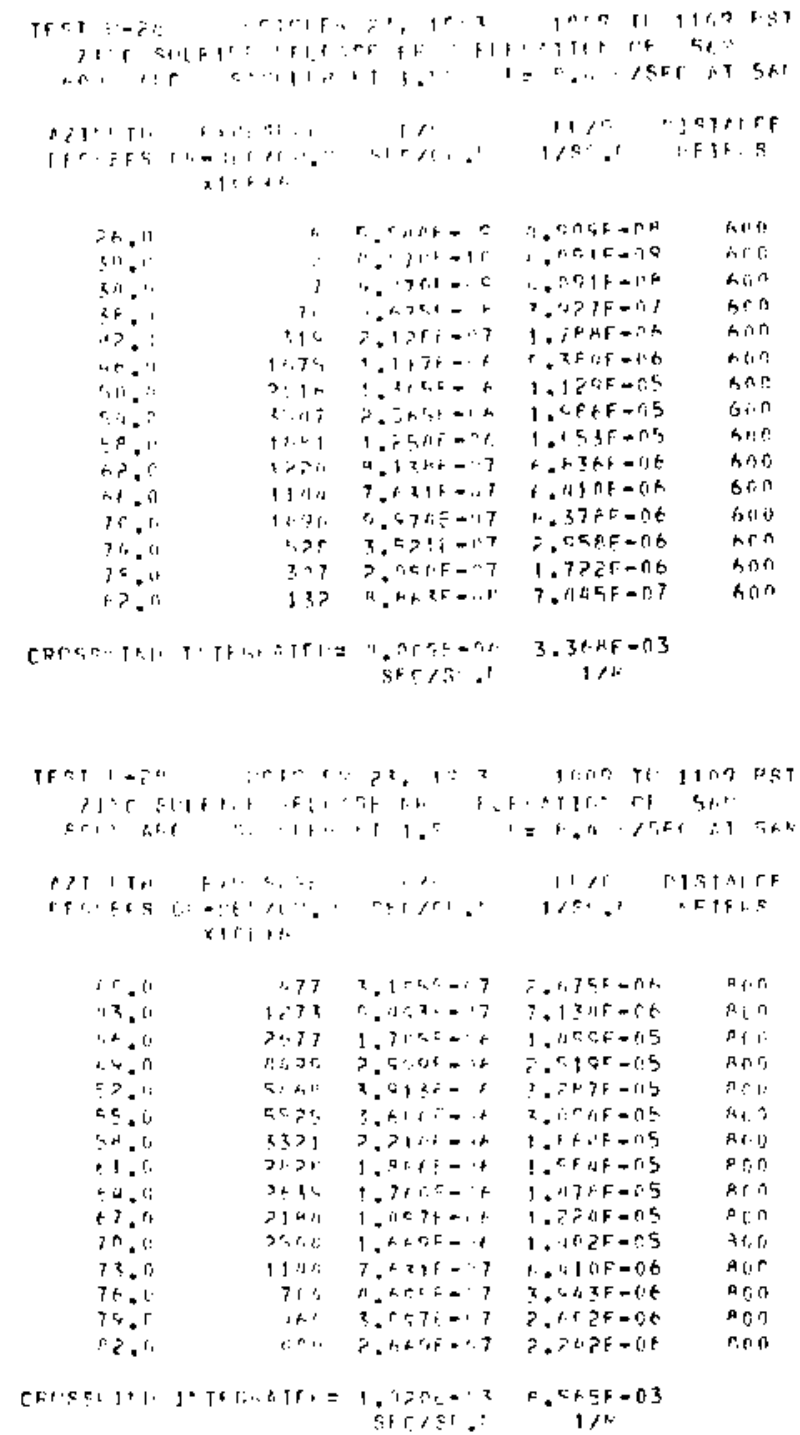




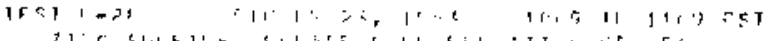

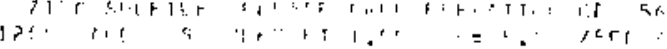

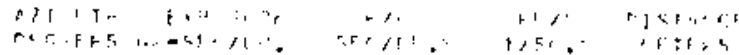

$$
\begin{aligned}
& \times 1 !+
\end{aligned}
$$

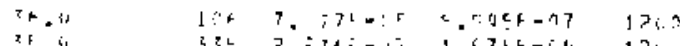

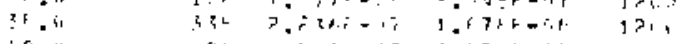

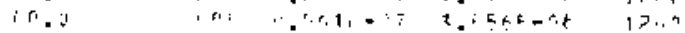

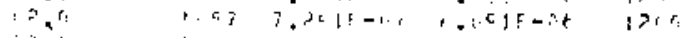

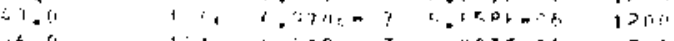

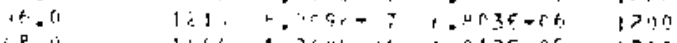

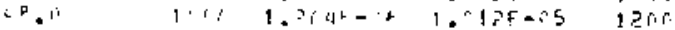

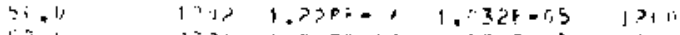

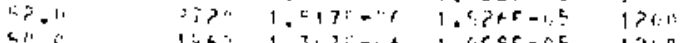

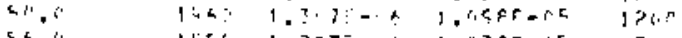

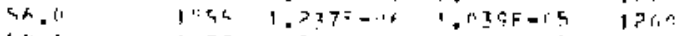

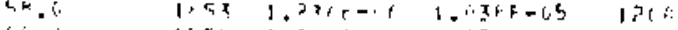

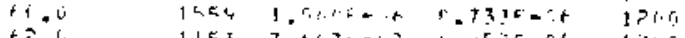

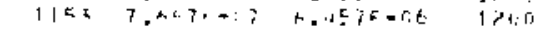

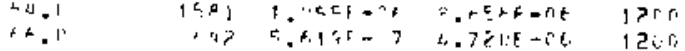

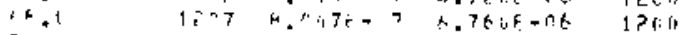

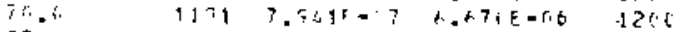

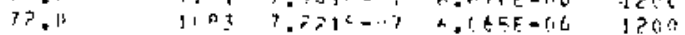

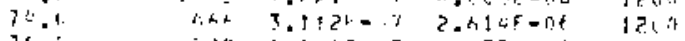

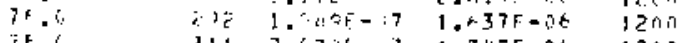

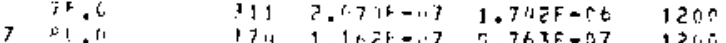

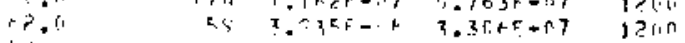

$$
\begin{aligned}
& \text { मझा }
\end{aligned}
$$

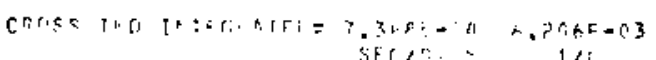

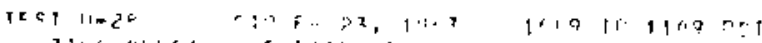

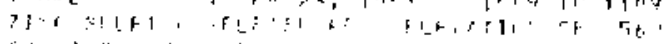

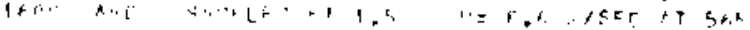

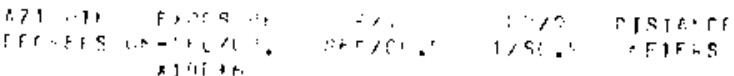

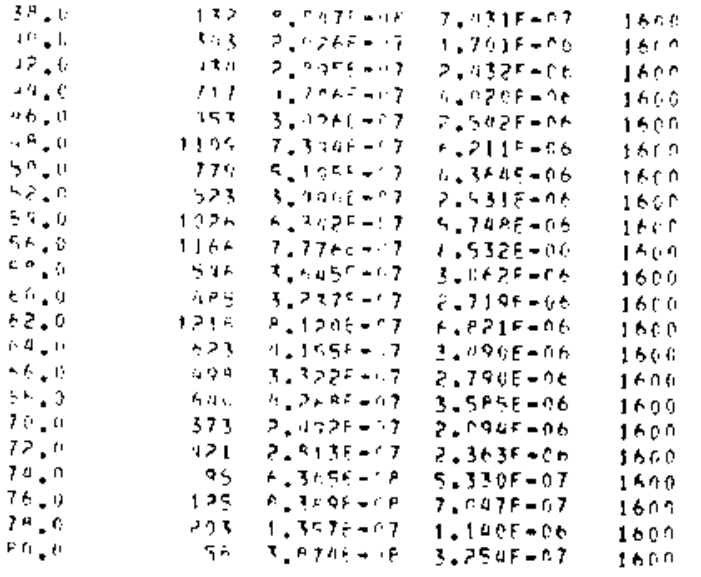


UNSTAELE SERIES EXPERIMENT: 29 ( U-29)

GROUND LEYEL SAMPLING $100-1600$ M ARCS. FOR EASE IN STATISTICAL

ANALYSIS, ALL SAMPLER AZI HUTHS HAVE BEEN INCREASED BY 30 DEGREEJ

FROM THEIR ACTUAL POSITIONS.

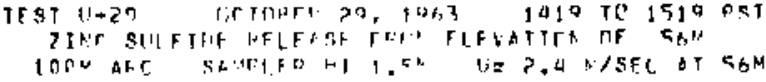

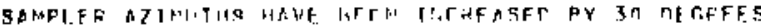

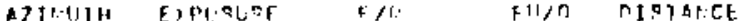

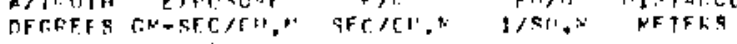
$x \mid n+t$

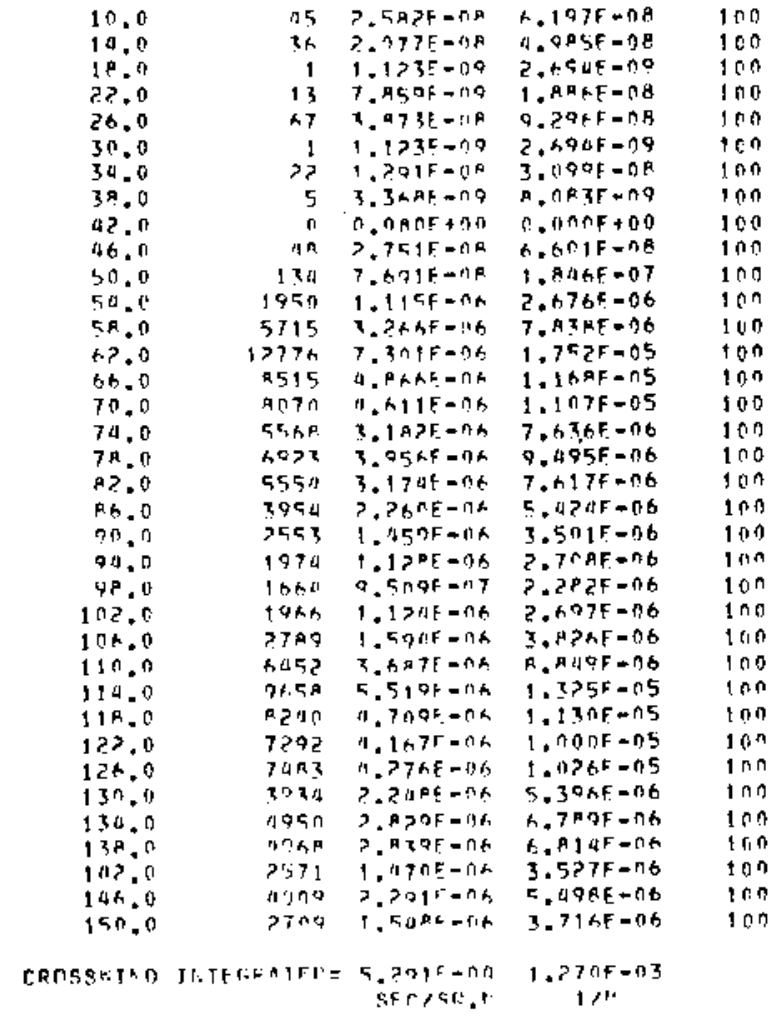

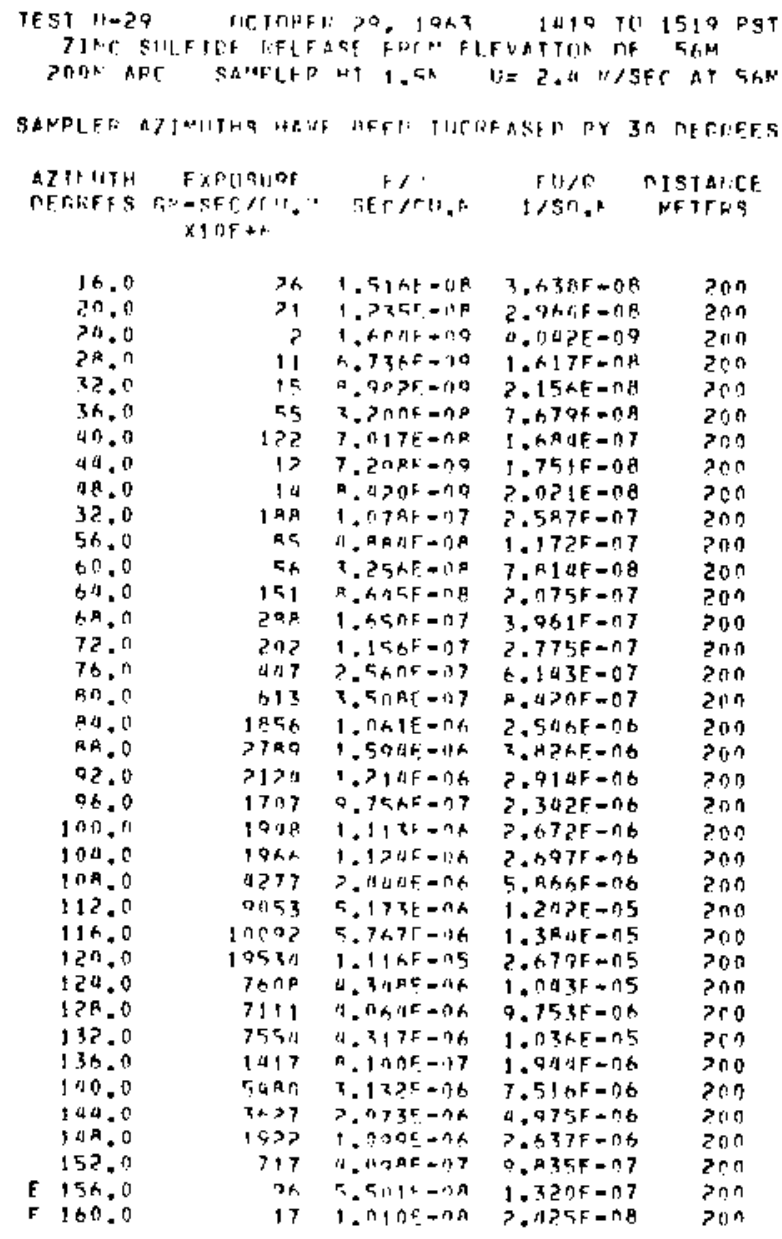

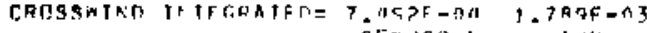




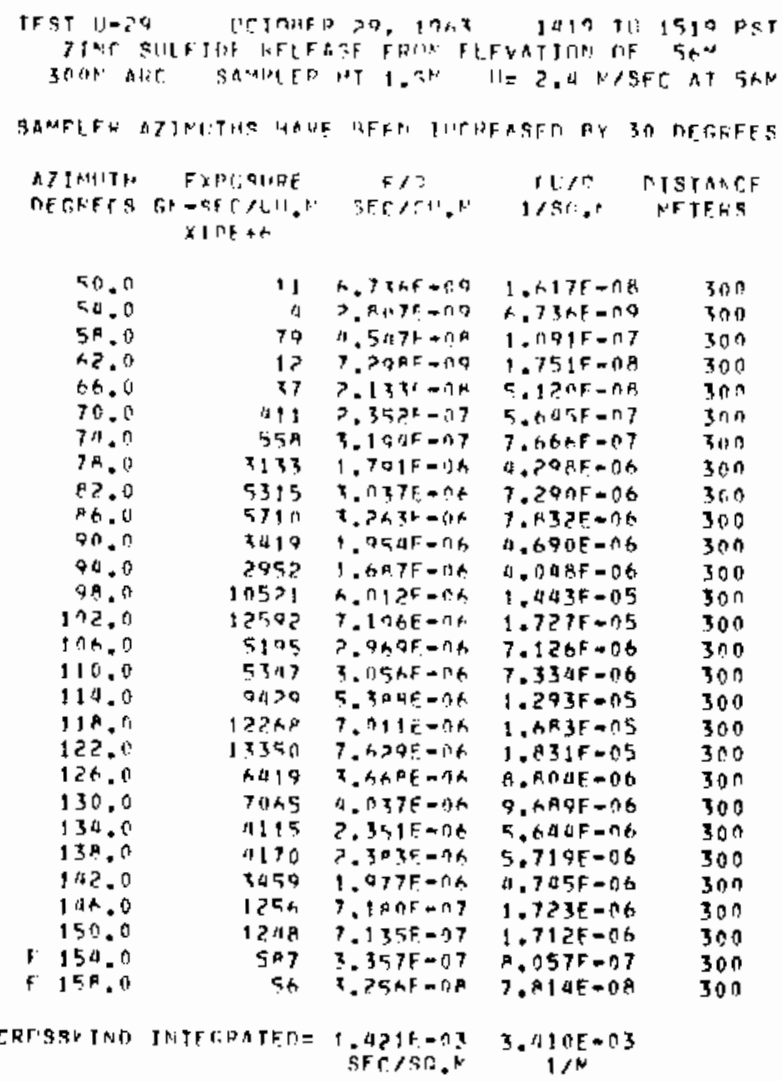

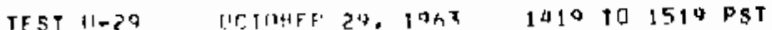

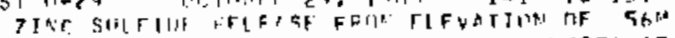

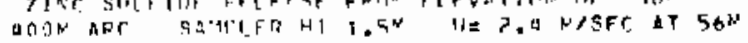

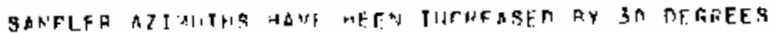

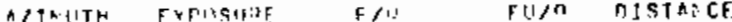

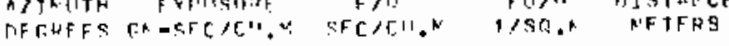
al if + +h

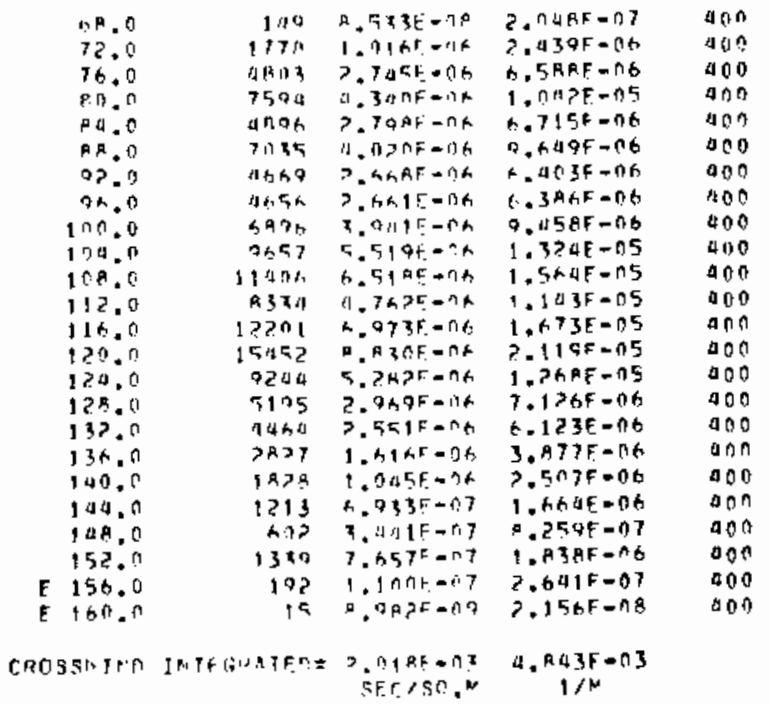

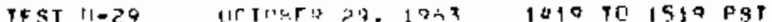

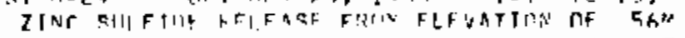

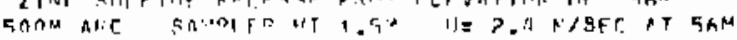

SAMPLFD ATJMITHS MAUE HFEH I TREASFT AY 30 DEREFES

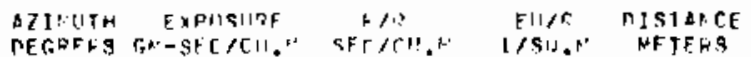
$x+1 \cap \mathrm{E}+4$

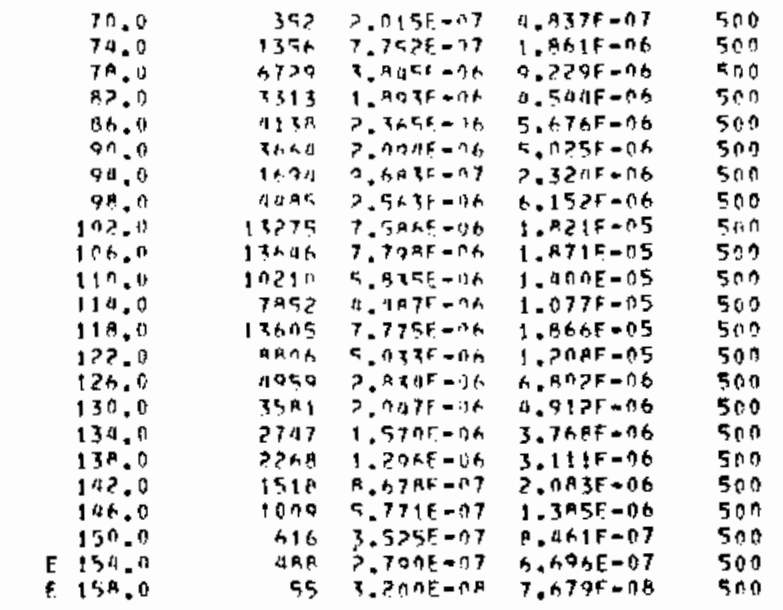

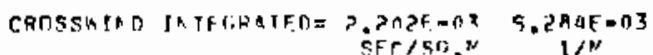

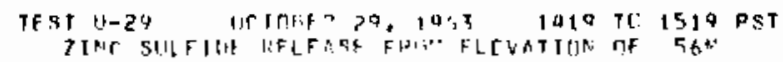

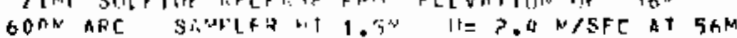

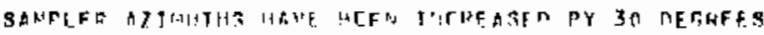

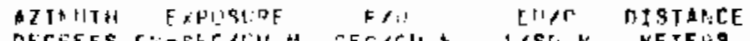

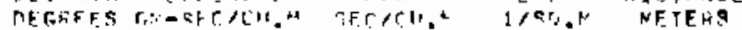
$x ! 11+t$

\begin{tabular}{|c|c|c|c|}
\hline $\begin{array}{l}72.0 \\
72,0\end{array}$ & $\begin{array}{r}153 \\
12 ? 54\end{array}$ & $\begin{array}{l}9.757 F=1 p \\
7.0 \times 7 b^{2}-97\end{array}$ & $\begin{array}{l}7.1025=07 \\
1.67+F=06\end{array}$ \\
\hline 2n. & 2307 & $1,370 \mathrm{~F}-\mathrm{nh}$ & $3.790 F=06$ \\
\hline 20.0 & $A N+t$. & $7.7 A \mid F=? t$ & $6.6 .7 \mathrm{LF}-0.6$ \\
\hline BA." & 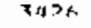 & †. गदAF $\triangle A A$ & $1,609 E-06$ \\
\hline 97.0 & PA 17 & $3 . A \mid \cap F=1 R$ & $3.564 F-06$ \\
\hline 96.0 & 1937 & 1.1 a $1 \mathrm{~F}=n \mathrm{~s}$ & $2.739 \mathrm{E}=1,6$ \\
\hline $1+n, 0$ & 7324 & $\Rightarrow .2 P n E_{-}-n G$ & $1.013 F=05$ \\
\hline 100.0 & $91>0$ & $5.21)+-16$ & $1.251 F=05$ \\
\hline Ine.0 & 7755 & ". घวOCE=nh & $1, D+1 F=\hat{A}$ \\
\hline 112.0 & 11255 & $A .77$ तE- $=0 A$ & $1.6265=05$ \\
\hline $11 \% .0$ & Oons & $=14 x+=n x$ & $35 F-n 5$ \\
\hline 120.0 & $A, 05$ & $4.90 \cap E=6 t$ & 1.1RAF-DS \\
\hline 124.0 & 6.3nR & $3 .+30 f=n h$ & $0.734 F=06$ \\
\hline $12^{\mu} \cdot 0$ & 3113 & $1.951 k=0 \mathrm{~B}$ & $4 . A 8 Z E=06$ \\
\hline 132.0 & 1053 & $1.117 t=n t$ & 2. hA CAF $=06$ \\
\hline$\$ 36.0$ & 1767 & $1.01 \mathrm{OF}=\mathrm{Bh}$ & $2.1125 E-06$ \\
\hline 140.0 & SAI & $3.20 A E-17$ & $9.350 \mathrm{n}=07$ \\
\hline 144.0 & $4 \times 5$ & $? .6631 F=07$ & $6.3805-07$ \\
\hline $10 A .0$ & Shat & $>.18035=07$ & $1.990 F=07$ \\
\hline 152.0 & $2 ? 9$ & $1.31 \mathrm{dE}=07$ & $3.153 F=07$ \\
\hline 150.0 & Ah & W. $D A \cap E=U A$ & $1.1^{R_{6}}=07$ \\
\hline & & 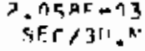 & $\begin{array}{c}4.930 F=03 \\
1 / M\end{array}$ \\
\hline
\end{tabular}




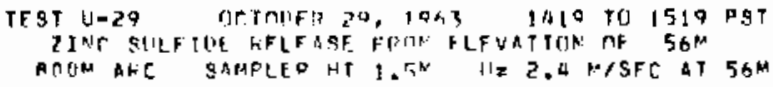

SAMPLEF AIIHUTHS HAUF AEFF: TIJTIFASED AY 30 AFGFEES

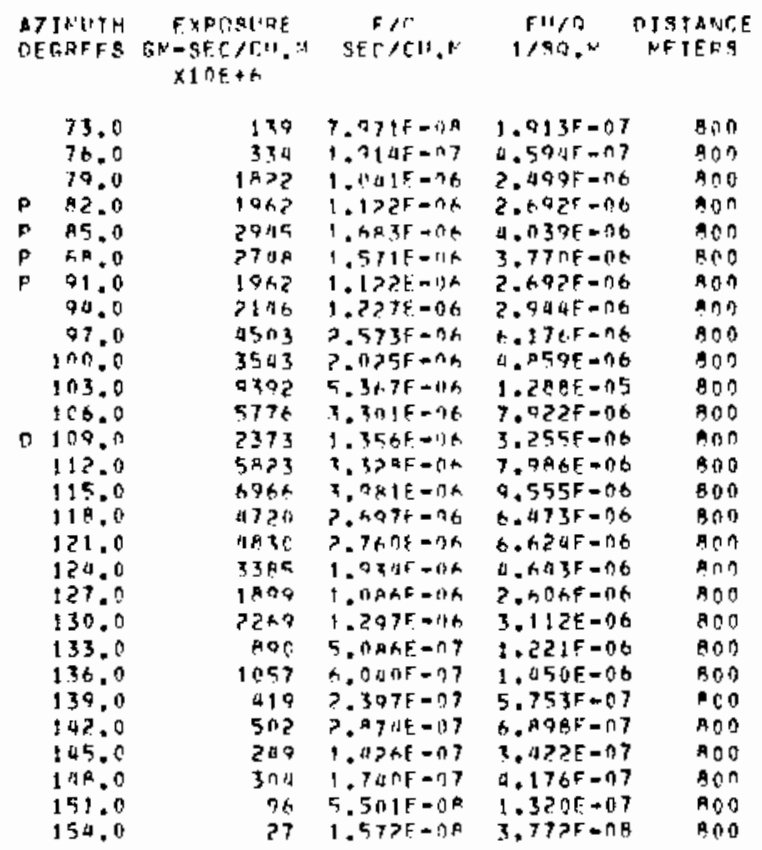

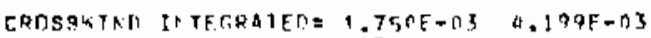

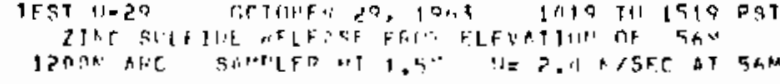

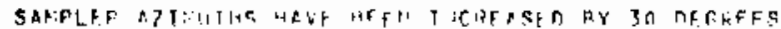

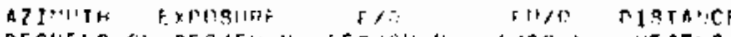

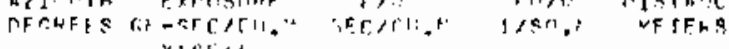
$\times 10+6$

\begin{tabular}{|c|c|c|c|c|c|}
\hline \multicolumn{2}{|c|}{74.0} & 11 & $4.5751-10$ & 1. $\triangle 7 A F=A R$ & $12 \pi n$ \\
\hline & $7 h .0$ & 93 & $5.3 ? \mathrm{PE}=\mathrm{OV} \mathrm{A}$ & $1.27 A F=07$ & $1 \geq n \pi$ \\
\hline & $7 \times 0$ & $1+7$ & $4.5 \cap n F=n s$ & $7.3045-07$ & 1200 \\
\hline & 4n. 1 & 390 & $11.517 t-97$ & $1 . A B D E=r B$ & $12 \cap n$ \\
\hline & 62,0 & 532 & Bnalons? & 7. PQAF $=07$ & $1>0$ \\
\hline & $R_{4} . n$ & 675 & $\because A+3 E=07$ & $0.2 .71 F=07$ & 1300 \\
\hline & $8 x, n$ & $61 t$ & :.5>1t-5,7 & P. $.4505=07$ & $i>n n$ \\
\hline & $A R=0$ & $5 \times 3$ & 3 तथ & $7.3>>F=97$ & $120 n$ \\
\hline & 90.0 & $52 \pi$ & $P .924 F-\cap 7$ & 7.1 वaf-n? & 1200 \\
\hline & $9 ? .0$ & $7 ? ?$ & 4.1 OFF -177 & $1, n \cap 5 F=0$ & ? ? 4 ก \\
\hline & $Q: 0$ & 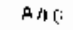 & $4.9031-07$ & $1.153 \mathrm{E}=00 \mathrm{0}$ & $12 n n$ \\
\hline & $Q A .0$ & 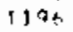 & $A, \cap \geq B !=\cap T$ & $1 . A A Y F=D 0$ & 1200 \\
\hline & $9 F=0$ & $85 ?$ & $5.44 \Delta F=117$ & $1,307 f=n h$ & $120 n$ \\
\hline & $1 \cap n, 0$ & $10>7$ & $9.3 \cap \cap E-07$ & $7.2 \geq 2 F-06$ & 1700 \\
\hline & 132.0 & วด 3 & 1.1918 .16 & ?. H5AF $=n \theta$ & 1200 \\
\hline & 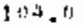 & $17 \times 2$ & จ. $R \cap \Omega R=07$ & $2.37+F=06$ & 1700 \\
\hline & 106,0 & 3337 & $1.3341=06$ & $3.2065-106$ & 1200 \\
\hline & 108,0 & 3 उח & 1. $1 A K F=M H$ & 2.75117 .06 & 1200 \\
\hline & 110,0 & 1370 & $7 . Q A T F=07$ & $1.8925=06$ & 1200 \\
\hline & 112.0 & $10 \times 3$ & 1.1055 .06 & $2 .+51 \mathrm{~F}=0 \mathrm{th}$ & 1200 \\
\hline & 114.0 & 1675 & $0.541 F=01$ & $7.23 f \cdot F=106$ & $12 n 0$ \\
\hline & 116.0 & $\times 110$ & 1. Jnat - Jh & $2.8945-06$ & $1>00$ \\
\hline & $11^{2} \cdot 0$ & 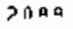 & $1.1335=0 \mathrm{~h}$ & ?. $A+4 F-n h$ & $1>0 ?$ \\
\hline & $12^{16}=0$ & 1511 & 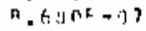 & $2.573 f=06$ & 1700 \\
\hline & 132.0 & 1204 & $7 . x \cap 7 F-17$ & $1.775 F=16$ & $1>n$ \\
\hline & 124.0 & 1137 & $k .5131=117$ & 1.5635000 & 1200 \\
\hline & $126 . ?$ & 1123 & $0.417 f=97$ & $1,5 \theta) F-0 b$ & 1200 \\
\hline & 126.0 & $45 n$ & $7, \alpha>\beta K=A 7$ & $\mathrm{H} . \mathrm{Z}^{\mathrm{A}} \mathrm{HF}=\mathrm{n} 7$ & $1 ? 00$ \\
\hline & 130.0 & 375 & $>147 \mathrm{k}=n 7$ & 5.19 PF $=07$ & $1>n n$ \\
\hline & 132.0 & $3 n h$ & $7.2+05=n 7$ & $\{, n ! 1+5=0\}$ & $120 n$ \\
\hline & 134.0 & $3 ? 9$ & 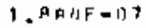 & $4.5>1 F-07$ & $1 \geqslant n \pi$ \\
\hline & 136.0 & $1+7$ & $D . A \cap \cap F=\cap A$ & $7.30115-17$ & 1200 \\
\hline & 139.0 & $3 \div 5$ & כ. AT? & $\because, A 7, E=07$ & 1700 \\
\hline & 140.0 & $30: 1$ & $\supset . ? 5>F-07$ & $5.4055-107$ & 1200 \\
\hline & $142, n$ & $2\left\{\begin{array}{l}3 \\
2\end{array}\right.$ & $1.3415-37$ & $3.7+25=n 7$ & 1700 \\
\hline & 144.9 & $h x$ & T. MIAt $=00^{\circ}$ & $A .67: 35=n e$ & $1>00$ \\
\hline 6 & 145.0 & $5 b$ & 3. $?>P P=14$ & $7.7 Y, 7 F=n H$ & $1 \geqslant 00$ \\
\hline & foten 0 & tit. & $2 . n>1 F-\eta p$ & $A .971 F=n A$ & 1200 \\
\hline & & 37 & $\Rightarrow+1>n f=0 F$ & $5,2 \cap ? 5=\cap 8$ & $1 ? 0 ?$ \\
\hline & & 79 & $1 . A 77 F=00$ & $\because, 6) \rightarrow B F-C A$ & $1>n n$ \\
\hline
\end{tabular}

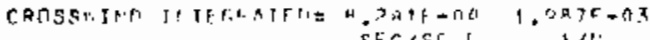




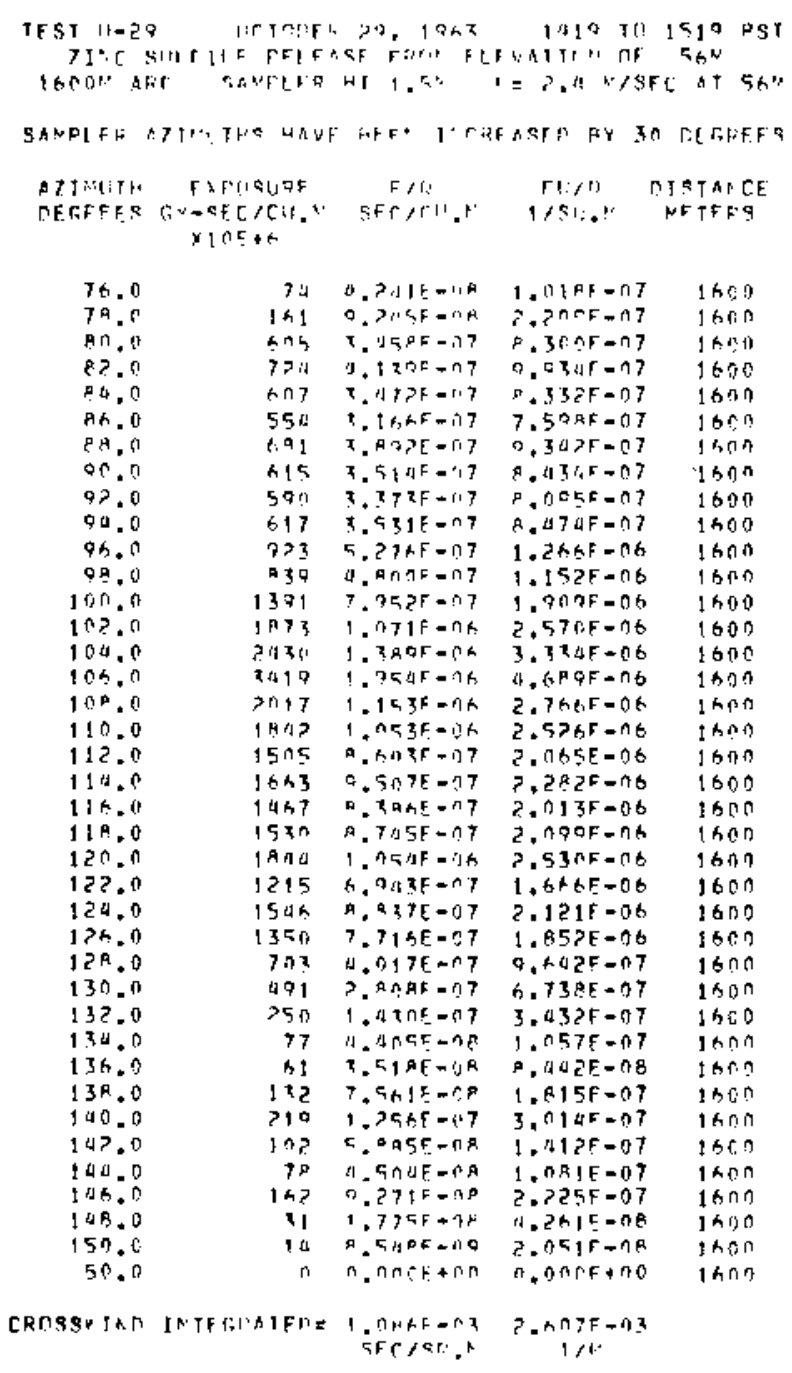


UNSTABLE SERIES EXPERIMENT: 30 (U-30,

GROUNO LEYEL SAMPLING $100-3200$ M ARCS. THE TRACER WAS NOT

DETECTED ON THE $100 \mathrm{M}$ ARC.

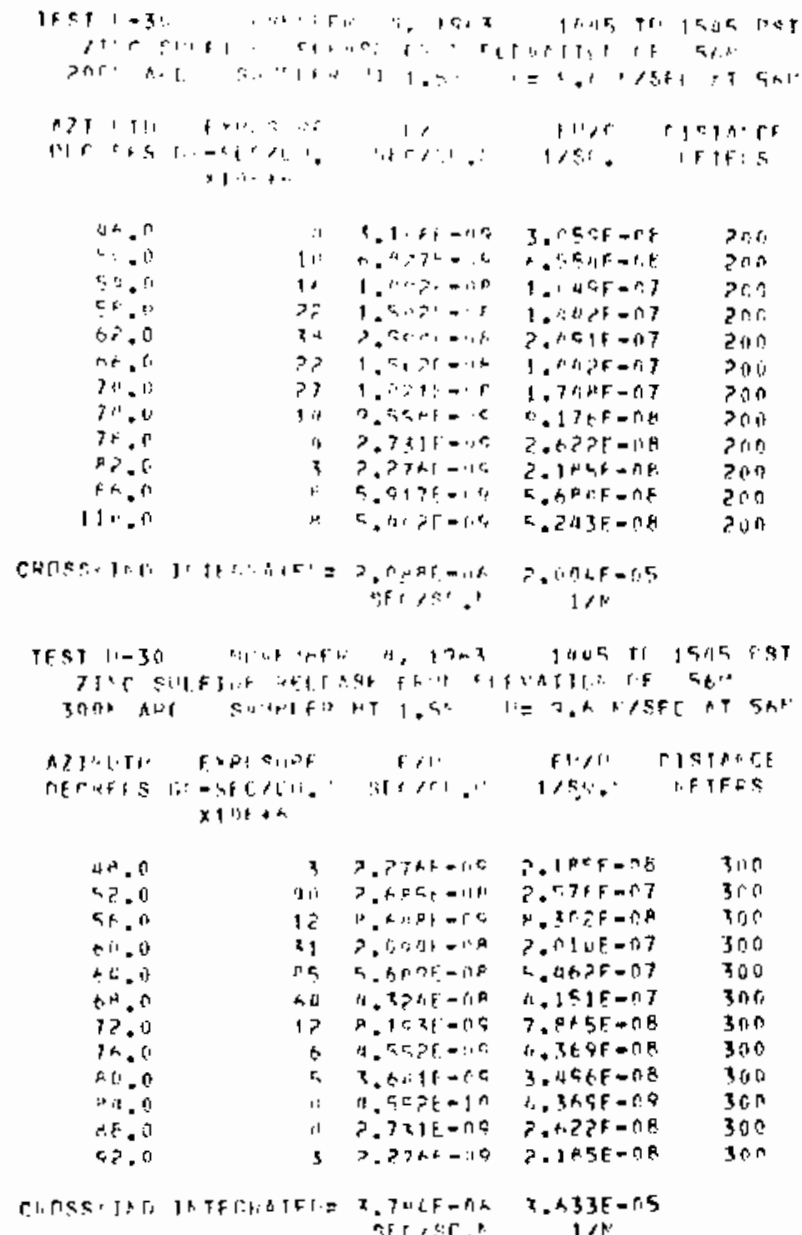

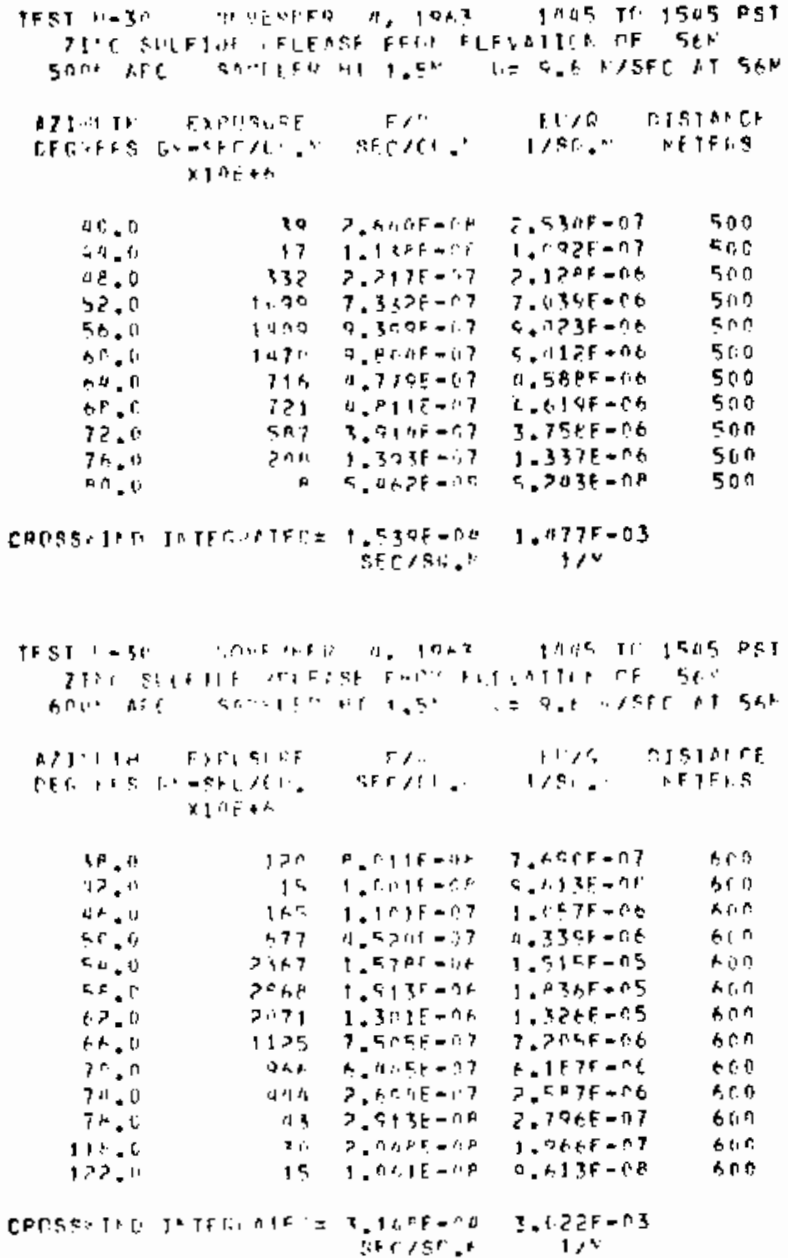




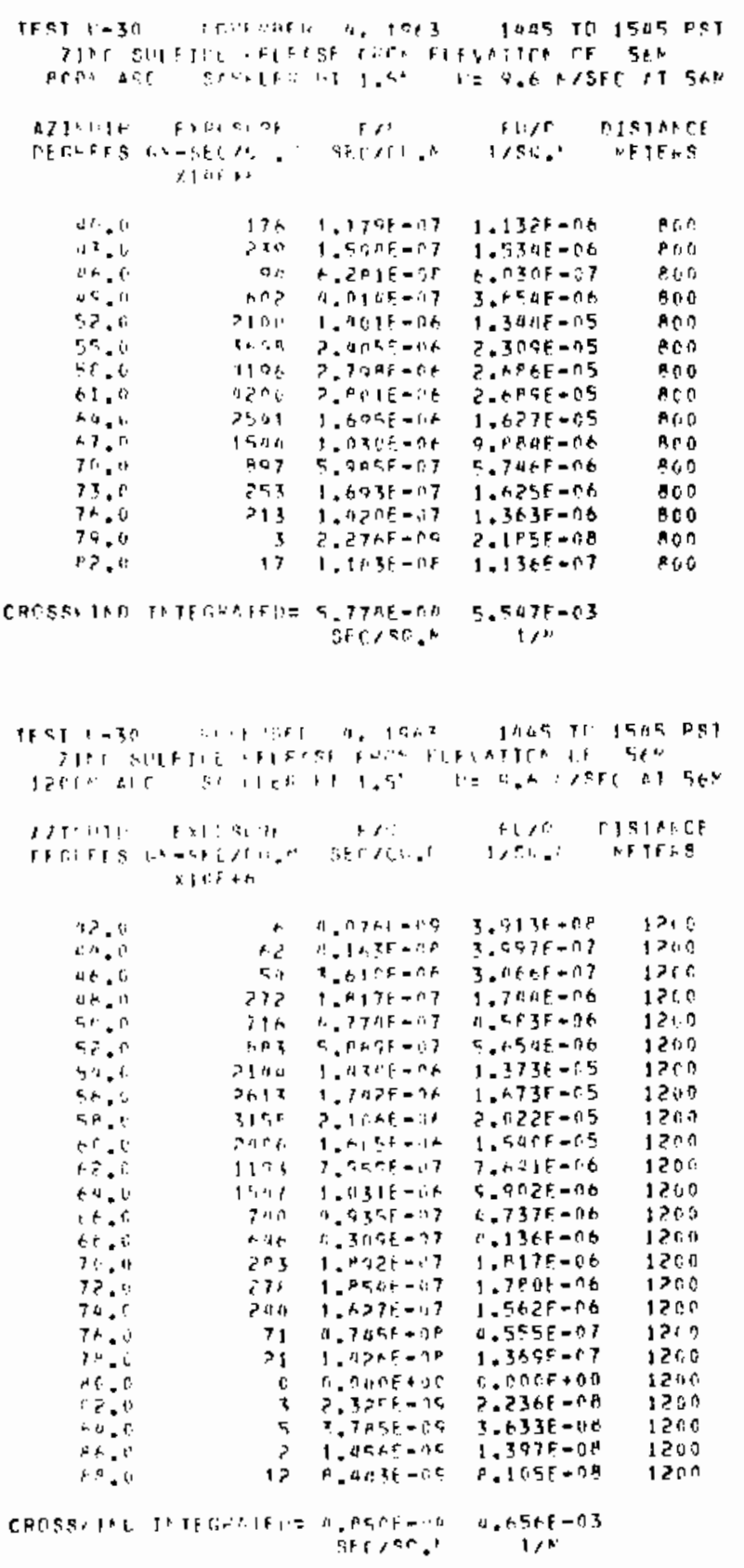

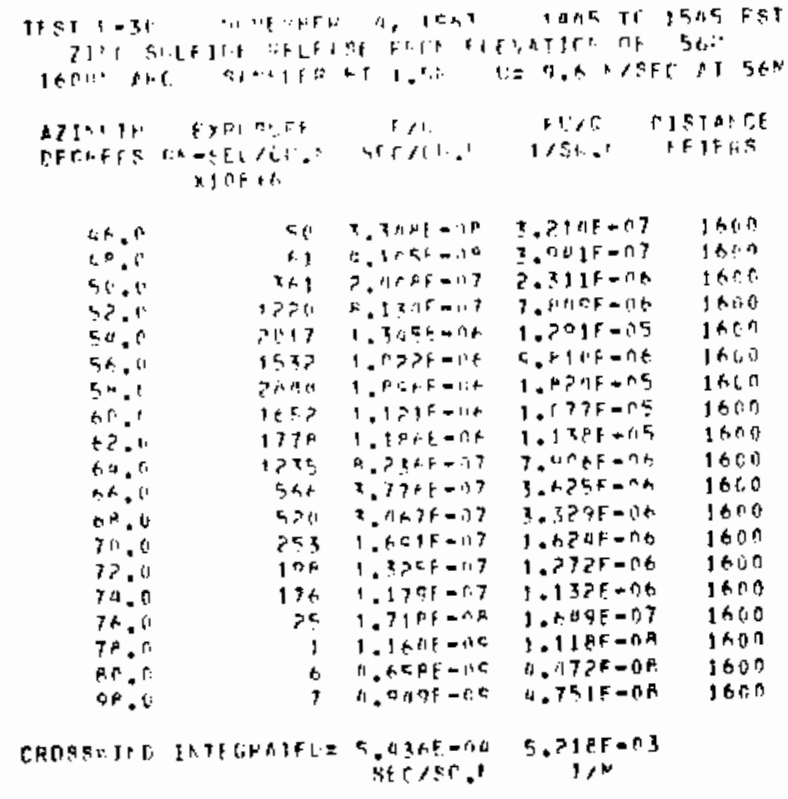

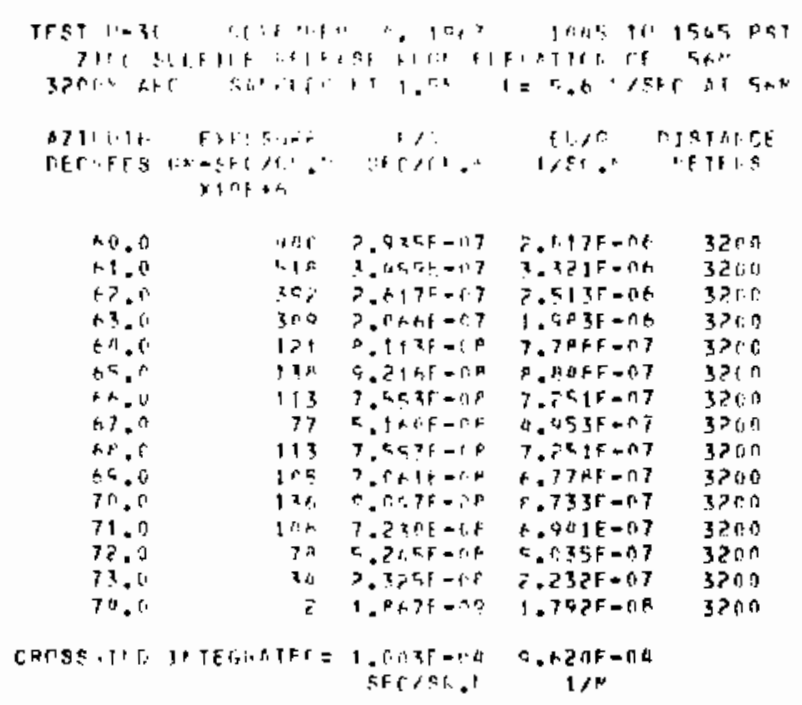




\begin{abstract}
LNSTABLE SERIES EXPER1MENT: 31 ( U-31) GROUND LEYEL SAMPLING 100-1600 M ARCS. THE TRACER WAS NCT DETECTED ON THE 100 Y ARC.
\end{abstract}

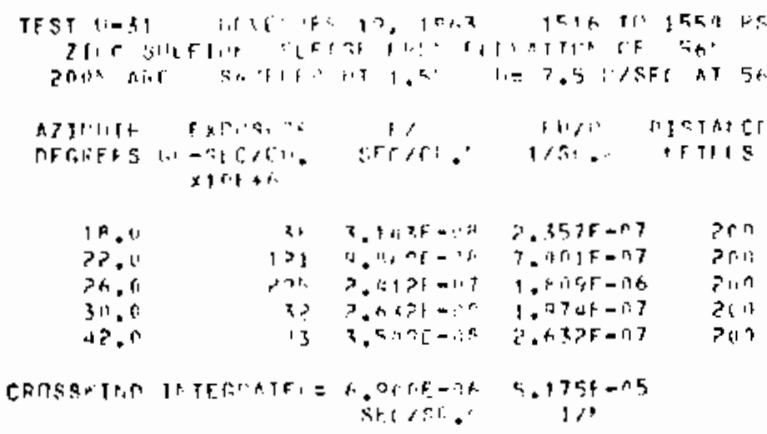

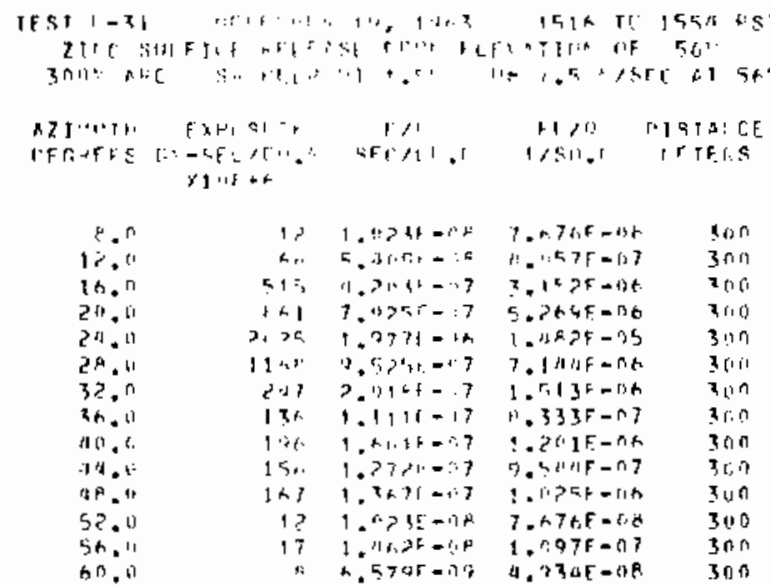

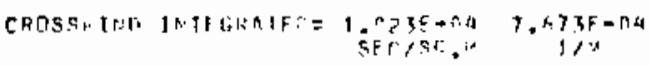

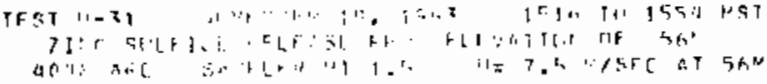

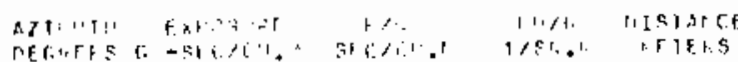
$\times 111+1$

\begin{tabular}{|c|c|c|c|c|}
\hline 10.11 & $\therefore$ & $7.1: 3, F-1: 4$ & 1. mist $=n ?$ & Mr.? \\
\hline 14,1 & 17 & $=27+F=8$ & $\Rightarrow, 1+c, f=n 7$ & बis \\
\hline 140 & $3 \%$ & $? .7+5=17$ & $7 . r 8.35=96$ & $4 \|$ \\
\hline 22.0 & $12 \div 1$ & $1 . r+? 1=: 14$ & $\gamma .5 i p t-n t$ & $\therefore n$ \\
\hline $2 h, 1$ & $1 \therefore 1+$ & 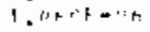 & $2.111 F=05$ & $\Delta \mathrm{t} \cdot \mathrm{n}$ \\
\hline $3 n, 0$ & 1575 & $1.6345=+4$ & $7.7+5 f-66$ & $\theta 0$ \\
\hline 3id. iो & $1: 5$ & $\therefore 4 F-.7$ & ",$Y 7 L F=14$ & $2 !$ \\
\hline $3{ }^{A} \cdot 11$ & 011 & r., 27710.7 & $3 . r_{1} p+1, f=0$ & aर \\
\hline $4 ? .6$ & 126 & 4. $\because 778=77$ & ii. $.1725=11 \mathrm{~h}$ & $4\{n$ \\
\hline Wh, 0 & i. $4:$ & 3.7 is $x+=3$ & $t^{2} .407 \mathrm{~F}=12 \mathrm{t}$ & 49,11 \\
\hline 50.6 & $1>7$ & $1 . \sin 05-117$ & $7.7+5, F=0,7$ & $\operatorname{ten} n$ \\
\hline $5: 1.0$ & $i_{i}$ & $7.3 r 46=17$ & $1.7 \hat{\mathbf{c}} 7 \mathrm{f}-0 \mathrm{6}$ & $t_{1}^{2}+15$ \\
\hline 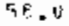 & 54 & iA. $r^{\prime}-5 i-\therefore, 1$ & $x^{2}+.9 A F=07$ & $s_{\Delta} 0^{\circ}{ }_{1}$ \\
\hline
\end{tabular}

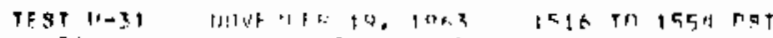

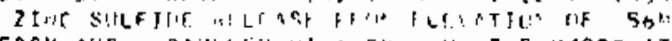

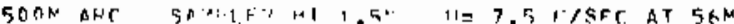

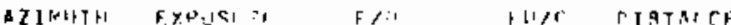

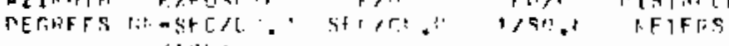
xi11t: + .

\begin{tabular}{|c|c|c|c|c|}
\hline 12.4 & S & $\supset . n+I t-14 n$ & $1.535 F-07$ & $50 n$ \\
\hline $1+0$ & $14 c$ & $1,10>1-n 7$ & $4.317 t=07$ & $50 ?$ \\
\hline 20.0 & ?3\% & $3.77 \cap[-! i]$ & $\because$ ONAF $=n b$ & 500 \\
\hline 24.0 & $\therefore 47$ & 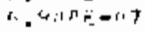 & 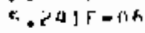 & 500 \\
\hline $2 ?, 0$ & $1,15 c$; & $5,+4 \geq\{-117$ & $4.179 \mathrm{~F}=\mathrm{B}$ & 500 \\
\hline 32.0 & 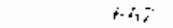 & 7. & $4.1152 F=06$ & 5 if 0 \\
\hline $3+.0$ & $112 \mathrm{c}$ & $0.7 ! 56,-17$ & $1.296 F=08$ & 500 \\
\hline 40.0 & $\log [\mathrm{in}$ & $1 . x, n+=. x$ & $\because \cdot 25 F=106$ & 500 \\
\hline 44.1 & $n ?$ & $751,5=1 ; 7$ & 5. $\cap R A F=06$ & 500 \\
\hline $4 \mathrm{H}, 0$ & 677 & $3,02,8=i, 7$ & $3.9) 75=06$ & 500 \\
\hline 58.4 & 521 & $\{x a i-t\}$ & $3.351 F-06$ & 500 \\
\hline 56.0 & ETO & $7.3+1 F=177$ & $1.711 \mathrm{E}=06$ & 500 \\
\hline$A=n \cdot 11$ & 110 & $9.7>2 r-11$ & $7.2^{\circ} 2 F=07$ & 501 \\
\hline & TEl:= & 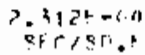 & $\begin{array}{c}1.73 \text { uF }=\cap 3 \\
1 / N\end{array}$ & \\
\hline
\end{tabular}

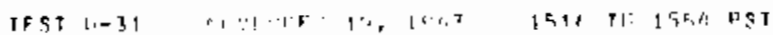

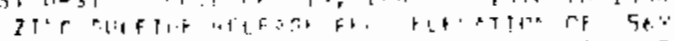

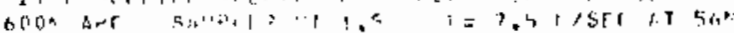

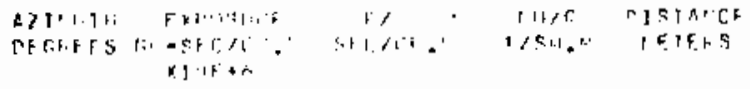

\begin{tabular}{|c|c|c|c|c|}
\hline 140 & $1 \%$ & i. & $7.6 .7 .+5-1.8$ & thin \\
\hline 18,1 & $15 \%$ & 1. $20 ; 6,5-11 y$ & $\because 7 f_{1} \ldots F-117$ & $6,5 n$ \\
\hline $3 ? .4$ & 30. & $7.77 r_{1}:-=7$ & $7.1 .75 F=0.6$. & $n \in$ \\
\hline 25. & .7 & $5.5: 1:-1.7$ & $i_{1} .151: c=00$ & hi.il \\
\hline $30^{\prime \prime}+11$ & 323 & $5.12,21-7$ & $i .5 .78=6$ & tot. \\
\hline $3+11$ & $3 \% .1$ & $7.1+76=7$ & $1.18 C+=1.0$ & $\left.f, t_{i} 1\right)$ \\
\hline $3 \% .1$ & $112 x$ & $1,2,11,-6$ & $\because .15: 5-6 h$ & 5. .7 \\
\hline 12.6 & $107 x$ & $1.41: 7+=0$ & 1. $2 \times x-15$ & ha? \\
\hline $1+.0$ & D. 13 & $5.2 n 5 F=17$ & $3.5+1 ; F=n t$ & $t, r, n$ \\
\hline 50.2 & ot & $5.28,+5 .-1: 7$ & $\therefore \therefore c, 5 F=r$. & की 0 \\
\hline $5 \% .0$ & lets & $1.11,3 F_{-}=\pi / k$ & $7 . * 71 . F=n .6$ & tolo \\
\hline 58.0 & 2.74 & ?. & $1 .+31 E=n 6$ & $\tan \theta$ \\
\hline $4>$, iो & 12 & $1.0>x-\Gamma A$ & $7, A .7 B F=\cap A$ & fo: \\
\hline
\end{tabular}

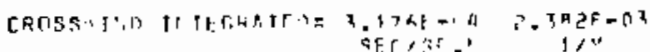

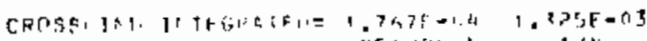




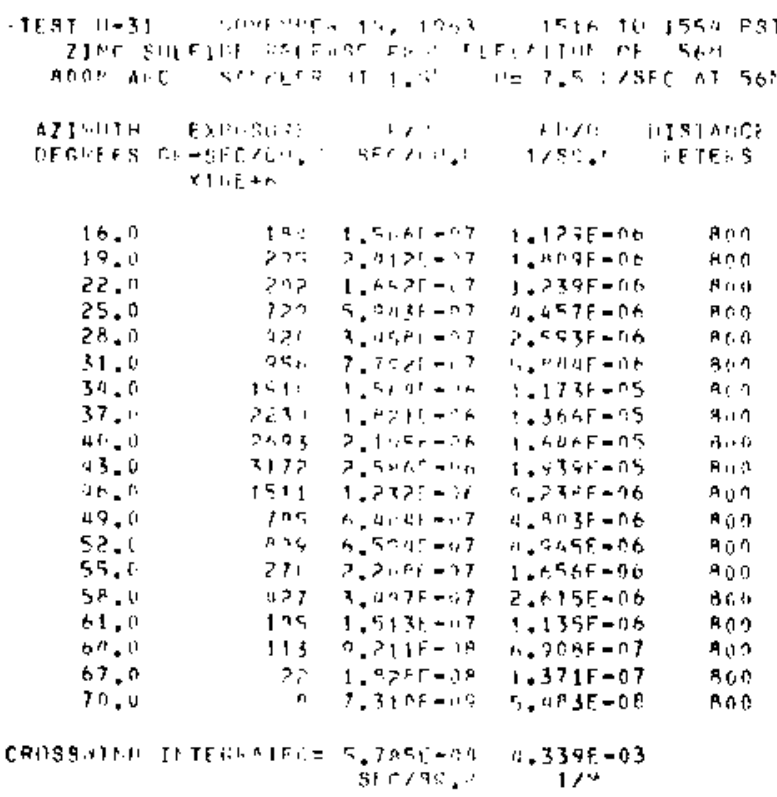

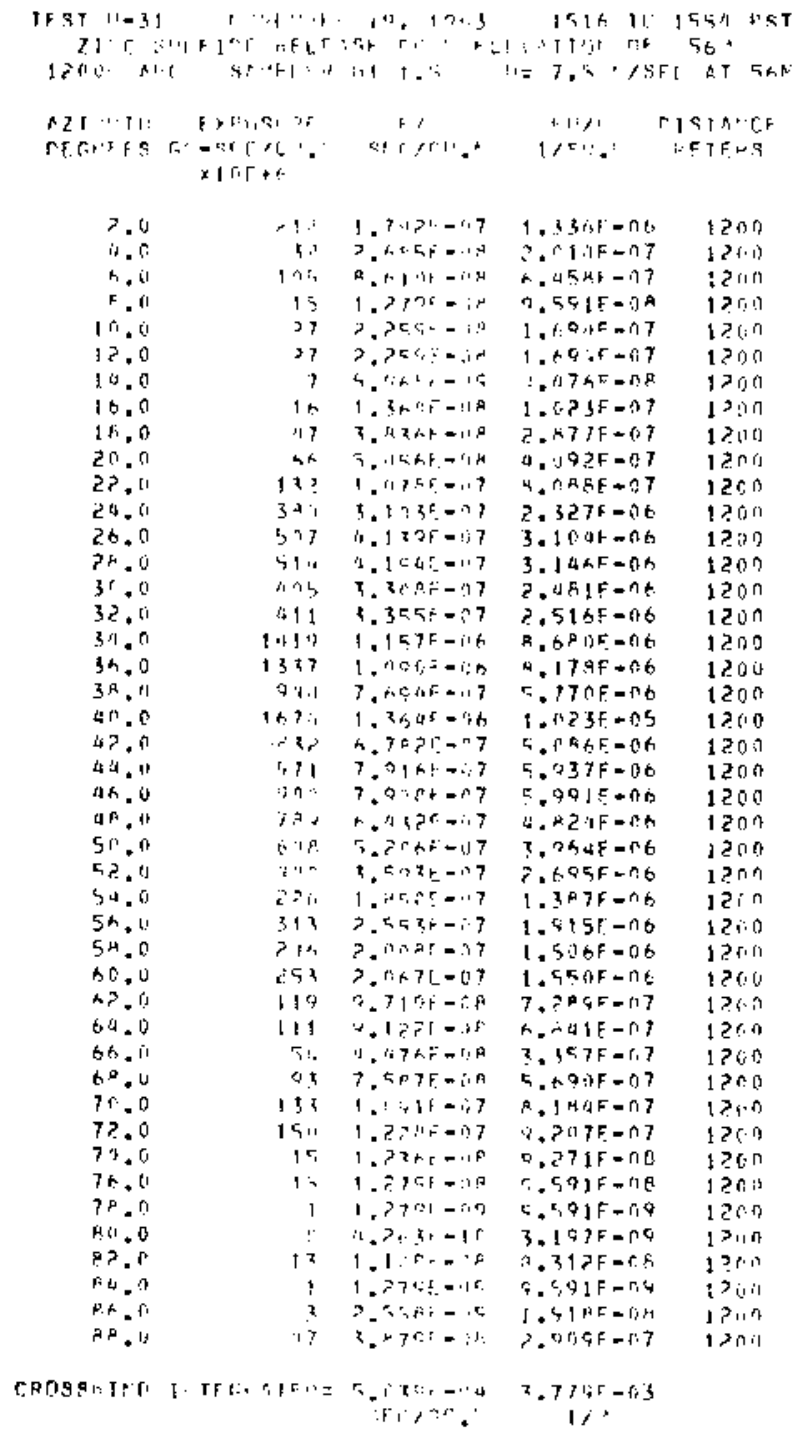




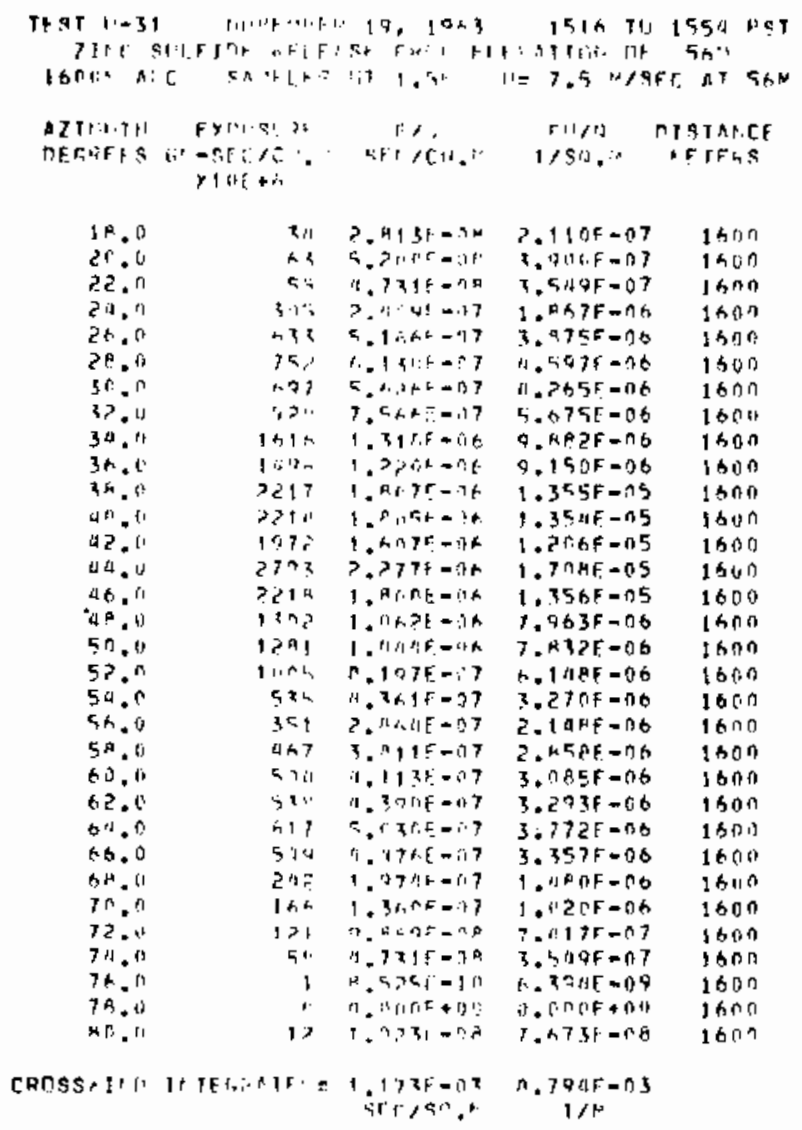


UNSTABLE SERIES EXPERIMENT: $32(\mathrm{U}-32$ )

GROUND LEVEL SAMPLING 100-1600 M ARCS. FOR EASE IN STATISTICAL ANALYSIS, ALL SAMPLER AZIMUTHS HAVE BEEN INCREASED BY 30 DEGREES FROM THEIR ACTUAL POSITIONS.

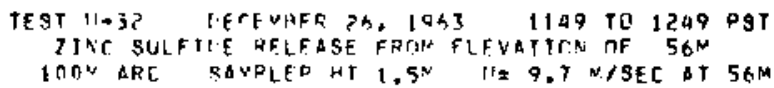

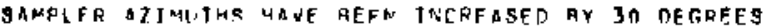

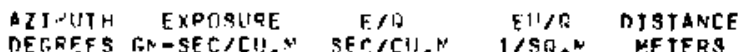
$x \geq 0 E+6$

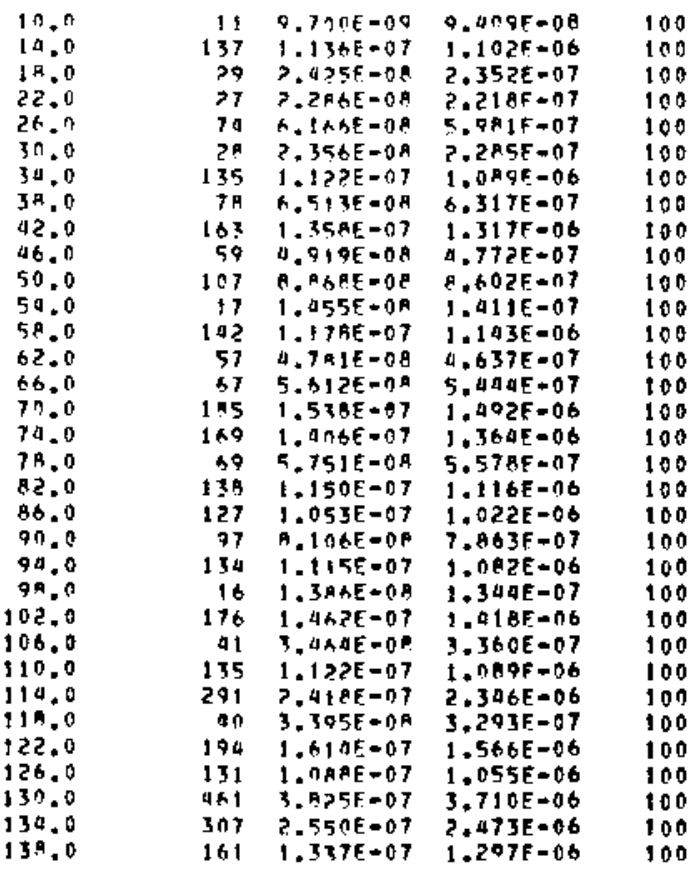

CAOSS HTND IATEGPATEN= 2.326E-05 2.356F-04

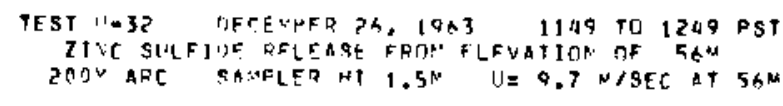

3ANPLEP AIIMUTHS TAVE TEFM TWCAEASFO AY 3 O MEREEES

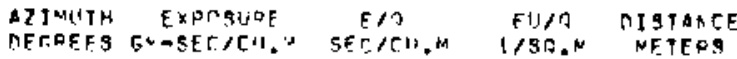
$x: \cap E+0$

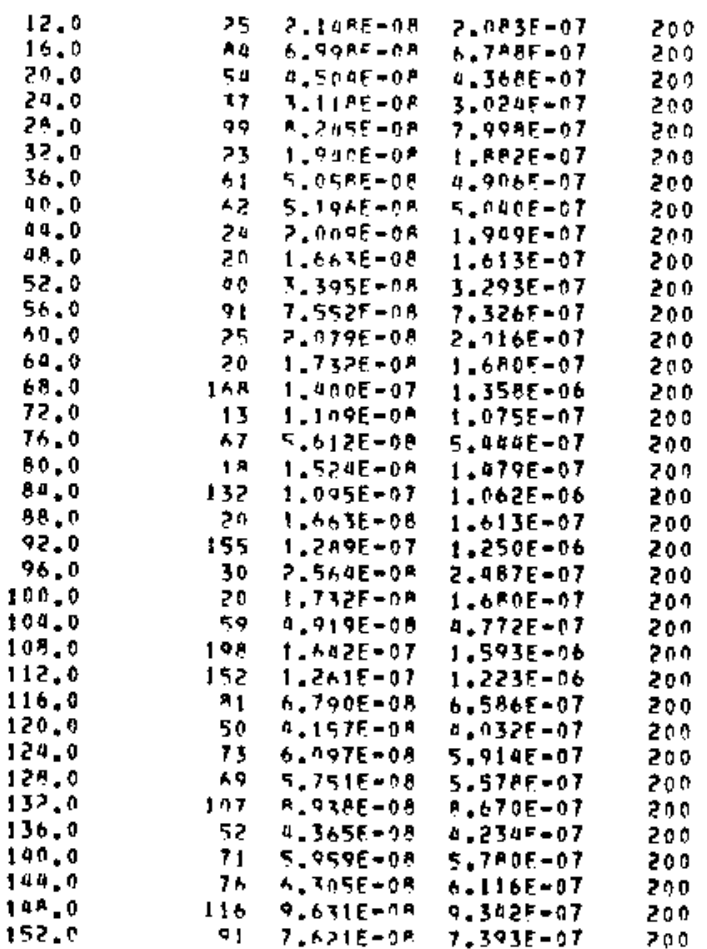

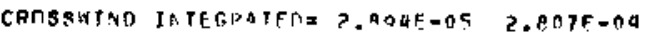


TE97 11.32 UETEHPER 26,1963 1149 TO 1299 PST ZINC SIJLFIDE RFLEASE FRON ELFVATION OF $56 \mathrm{~N}$

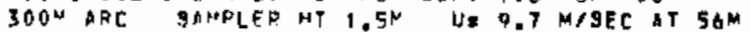

SAMPLER AZIMUTHS HAVE AEEN! INCAEAGET RY JO DEGREES

\begin{tabular}{|c|c|c|c|c|}
\hline $\begin{array}{l}\text { MINUTH } \\
\text { DEGRFF }\end{array}$ & $\begin{array}{c}\text { EXPQSUAE } \\
\text { GN-SEC }>C ! 1 . N \\
\times 10 E+6\end{array}$ & $\begin{array}{c}E / Q \\
S E C / C L\end{array}$ & $\begin{array}{c}\varepsilon \cup / 0 \\
1 / 90 . N\end{array}$ & $\begin{array}{l}\text { DTSTANCE } \\
\text { FETERS }\end{array}$ \\
\hline 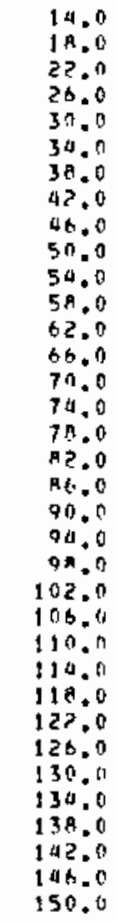 & $\begin{array}{r}30 \\
5 \\
5 \\
0 \\
0 \\
0 \\
49 \\
70 \\
193 \\
192 \\
172 \\
35 \\
106 \\
17 \\
97 \\
0 \\
40 \\
5 \\
0 \\
3 \\
3 \\
0 \\
0 \\
13 \\
31 \\
52 \\
21 \\
13 \\
4 \\
78 \\
90 \\
35 \\
47 \\
05 \\
155\end{array}$ & 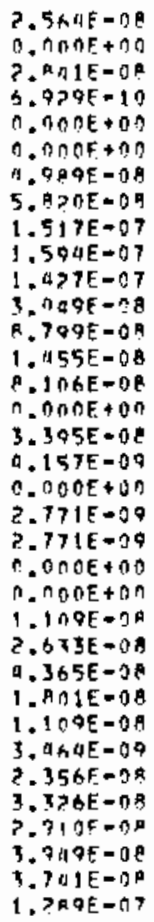 & 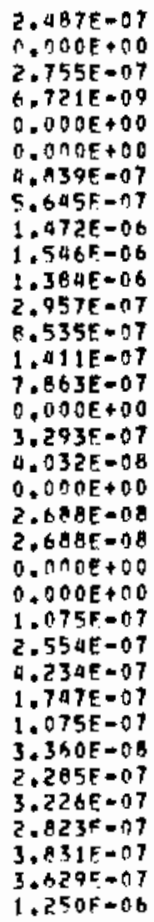 & $\begin{array}{l}300 \\
300 \\
300 \\
300 \\
300 \\
300 \\
300 \\
300 \\
300 \\
300 \\
300 \\
300 \\
300 \\
300 \\
300 \\
300 \\
300 \\
300 \\
300 \\
300 \\
300 \\
300 \\
300 \\
300 \\
300 \\
300 \\
300 \\
300 \\
300 \\
300 \\
300 \\
300 \\
300 \\
300 \\
300\end{array}$ \\
\hline
\end{tabular}

CROS9HIND INIEGHATEIIE-0.912E-OK - 4,765E-OS

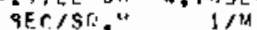

TFST 11-32 OECEAAER Zh, 19M 1149 TO 1240 PST

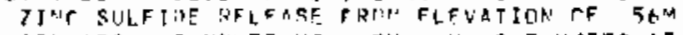

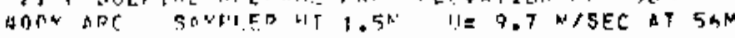

SAMFLEF AZIMIITSS HAUF REF: INCRFAGEM EY 3O DEFREES

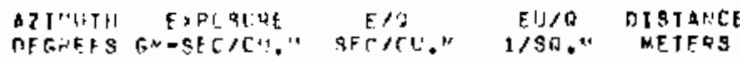
$\times 1$ if +6 .

\begin{tabular}{|c|c|c|c|c|}
\hline 32.0 & Al & $5.1>7 F=n$. & $4.7736-07$ & an \\
\hline $3+.0$ & 7 & $3.27 A F=00$ & $A . \cap \triangle 9 E=0 A$ & aon \\
\hline 49.0 & $1 n n$ & A. $799 E-19 P$ & $x .535 F=07$ & 400 \\
\hline 44.0 & 511 & $1.023 E-1) 7$ & $4.3105-06$ & 400 \\
\hline 47.0 & $A Q R$ & 7.0 thaF $=37$ & $7.225 E=106$ & 000 \\
\hline $5 ? . n$ & $24 \leqslant$ & $3.679 E=07$ & $569 F=106$ & 900 \\
\hline $5 n .0$ & 607 & $5.1151 E-77$ & $\triangle, A 99 E=06$ & 100 \\
\hline 60.0 & 231 & $1.81 \cap F=n 7$ & $1+2625=06$ & 000 \\
\hline 60.11 & 197 & $1.235 E-37$ & $5 A B E-06$ & 400 \\
\hline 69.0 & SO & $20 Q E=2 A$ & $3 Q E=n 7$ & $a \cap 0$ \\
\hline $7 ?$ & 31 & $A \geq 3 F=O R$ & $54 E-07$ & 400 \\
\hline
\end{tabular}

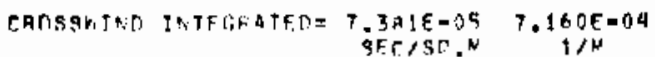

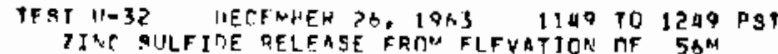
IINC SULFIDE RELEASE FRON FLFVATION HF $5 \mathrm{SM}^{\mathrm{M}}$

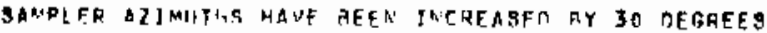

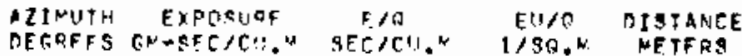
$x, \cap E+6$

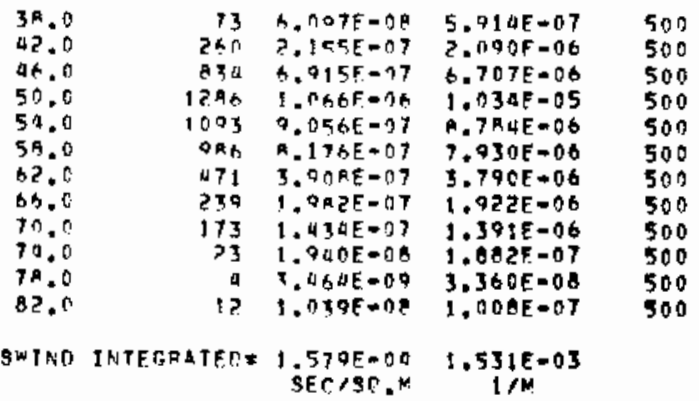

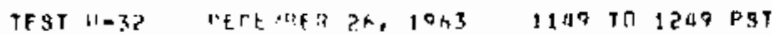

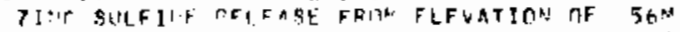

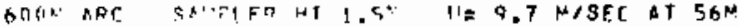

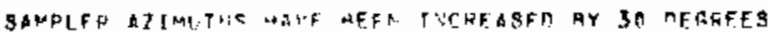

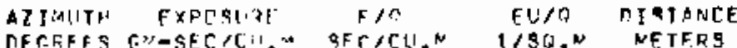
$\times 10 \mathrm{OH}$

\begin{tabular}{|c|c|c|c|c|}
\hline$a^{n}, 0$ & 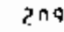 & $1.730 E-117$ & $1.687 \mathrm{~F}=06$ & $b \cap 0$ \\
\hline & 1031 & A. $5035-8: 7$ & $7 E-0 b$ & 500 \\
\hline $4 x$ & 7287 & $1, A 05 E-06$ & $1 . E 38 F=05$ & 600 \\
\hline & 1972 & 1. $h \times \Delta E=I h$ & $585 E-175$ & 600 \\
\hline 5 & 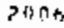 & $1 . A B \geq E=n A$ & $1.6: 2 F=05$ & ton \\
\hline no. o & $1 \times 5$ & 1. $5+2 \varepsilon=n k$ & $1.516 \mathrm{~F}-05$ & 600 \\
\hline n & M6t & $17 R E=07$ & $E-n \in$ & 00 \\
\hline 69,0 & $4 \div 5$ & $6105=07$ & $501 E-06$ & 609 \\
\hline 72.0 & $\operatorname{lng}$ & $0.765=0 A$ & $B O \Delta E=07$ & 600 \\
\hline 76.0 & at & 3. ChUE $=10$ & $3605-\cap 8$ & 600 \\
\hline$R$ & ? & $P . \cap \cap O F=D A$ & $.9495=07$ & 600 \\
\hline
\end{tabular}

CAOSSHTNO INTFGPATFOE X TGOE-OA 3.647E-03

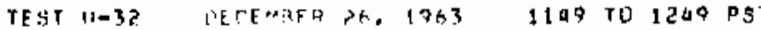

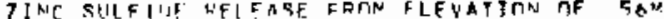

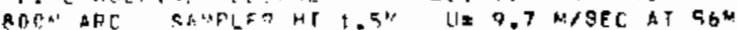

SANDLFR AINITHO UAVF IAEFH TNEREASFP RY 3 OE DREES AZIFITH EXPNELIE FIT EU/A DISTAACE $X I D E+6$

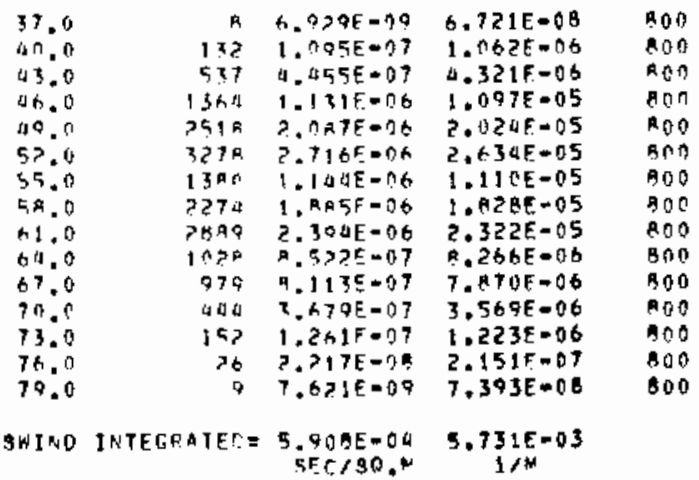


UNSTAELE SERIES EXPERIMENT: 33 (U-33)

GROUND LEVEL SAMPLING 100-1500 M ARCS. FOR EASE IN STATISTICAL

ANALYSIS, ALL SAMPLER AZINUTHS HAVE BEEN INCREASED BY 30 DEGREES

FROM THEIR ACTUAL POSITIONS.

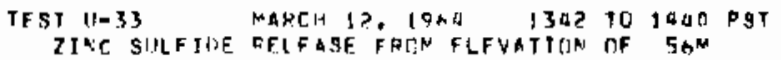

IOON ARC SAMPLER HT $1.5 \%$ UE A, 1 M/SFC AT $56 \mathrm{~F}$

SAMPIFP AZIMUTMS HAVF HEFT. TMCAEAGFB DY 30 DFGREEG

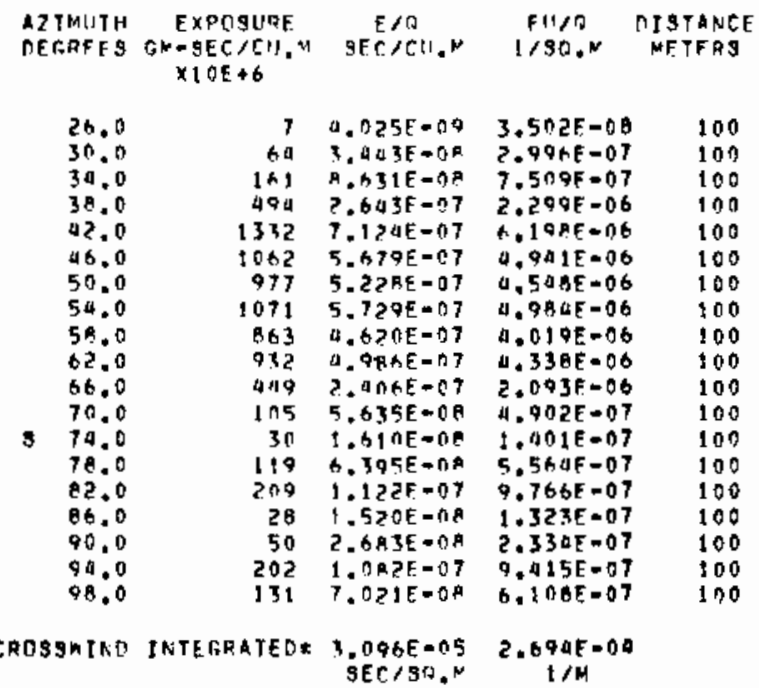

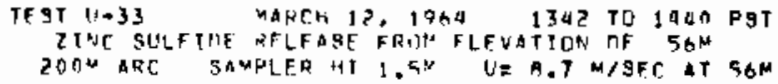

SANPLFR AZJNITHS HAUE REFM INCRFASED RY 30 DEGREES

AZIMUTH EXPOIBUDE EIE EU/D DISTANCE DEREFFS FN-SFC/CH.M BEC/CU.N 1/GA.M HETFRS
XIOETG

\begin{tabular}{|c|c|c|c|}
\hline $\begin{array}{l}36.0 \\
40.0 \\
04.0 \\
48.0 \\
52.0 \\
56.0 \\
60.0 \\
64.0 \\
69.0 \\
72.0 \\
76.0 \\
10.0 \\
04.0 \\
8.00 \\
92.0 \\
96.0 \\
00.0\end{array}$ & $\begin{array}{r}P 6 \\
91 \\
291 \\
918 \\
1018 \\
1670 \\
3159 \\
1033 \\
8 \geqslant 0 \\
176 \\
293 \\
219 \\
196 \\
206 \\
918 \\
17 \\
5\end{array}$ & 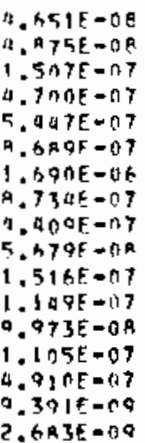 & $\begin{array}{l}4.046 F-07 \\
0.219 \varepsilon=07 \\
1.311 E=06 \\
9.089 F=06 \\
4.739 F=06 \\
7.560 F=06 \\
1.970 E=05 \\
7.59 A E=06 \\
3.836 E=06 \\
9.941 E=07 \\
1.319 E=06 \\
9.999 F=07 \\
9.676 E=07 \\
9.610 E=07 \\
4.272 E=06 \\
0.170 E=06 \\
2.334 E=08\end{array}$ \\
\hline
\end{tabular}

CROSSWINO INTFGRATFOE A. B15E-0.5 7.495E-04
TEST U-33 MARCH 1? 1704 1342 TO 1440 PST ZTNC SULFIDE RELEASE FQON FLEVATYON OF $56 \mathrm{M}$ $300 M$ ARC SAMPLER MT $1.5 \mathrm{M}$ U. $9.7 \mathrm{M} / \mathrm{SEC}$ AT $56 \mathrm{M}$

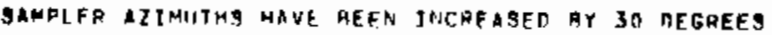

\begin{tabular}{|c|c|c|c|c|}
\hline $\begin{array}{l}\text { IZIMUTH } \\
\text { DEGAEES }\end{array}$ & 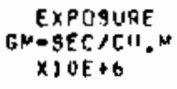 & $\begin{array}{c}E / \theta \\
S F[/ C 1] . \mu\end{array}$ & $\begin{array}{l}50 / 0 \\
1 / 80.4\end{array}$ & $\begin{array}{l}\text { OISTANCE } \\
\text { NETERS }\end{array}$ \\
\hline $\begin{array}{l}38.0 \\
42.0 \\
46.0 \\
50.0 \\
54.0 \\
58.0 \\
62.0 \\
66.0 \\
70.0 \\
74.0 \\
70.0 \\
82.0 \\
86.0 \\
91.0 \\
94.0 \\
98.0\end{array}$ & $\begin{array}{r}61 \\
158 \\
200 \\
402 \\
1270 \\
21140 \\
1951 \\
1506 \\
1496 \\
1578 \\
1393 \\
1564 \\
423 \\
49 \\
205 \\
750\end{array}$ & 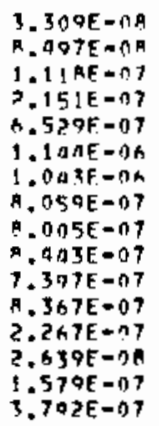 & $\begin{array}{l}2.879 E=07 \\
7.392 F=07 \\
9.777 E=07 \\
1.871 E=06 \\
5.080 F=06 \\
9.956 E-06 \\
9.077 F=06 \\
7.011 E=06 \\
6.964 E=06 \\
7.346 E=06 \\
6.935 E=06 \\
7.279 F=06 \\
1.973 E=06 \\
2.295 F=07 \\
1.373 E=06 \\
3.299 E=06\end{array}$ & $\begin{array}{l}300 \\
300 \\
300 \\
300 \\
300 \\
300 \\
300 \\
300 \\
300 \\
300 \\
300 \\
300 \\
300 \\
300 \\
300 \\
300\end{array}$ \\
\hline & 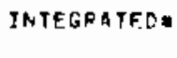 & $\begin{array}{l}\text {. } 697 \\
\text { SEC }\end{array}$ & $\begin{array}{c}1.476 E-0 \\
t / m\end{array}$ & \\
\hline
\end{tabular}

TEST 11-33 HARCH 12, 1964 1342 PO 1440 EST

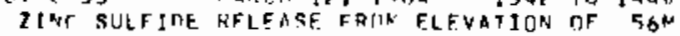
$400 \mathrm{~N}$ AHC SAMPLEO HT $1.5 \mathrm{M}$ JE R.7 M/SEC AT $5 \mathrm{SM}$

SAKPLEQ AIIMUTHS HAVE HEFM TSICRFARED AY 30 DEGREES

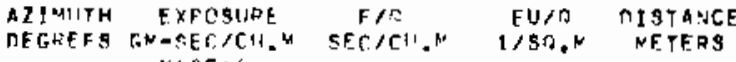
$X 10 F+6$

\begin{tabular}{|c|c|c|c|c|}
\hline $\begin{array}{l}4 \% .0 \\
44.0\end{array}$ & $\begin{array}{r}14 \\
2 n 1\end{array}$ & $\begin{array}{l}7 . A n Z F=00 \\
1.07 A F=37\end{array}$ & $\begin{array}{l}0.614 F=19 \\
9.377 E=07\end{array}$ & $\begin{array}{l}\Delta \cap 0 \\
a 00\end{array}$ \\
\hline$\triangle 8.0$ & 108 & $1.000 E-07$ & $9.2216-07$ & a 00 \\
\hline 52.0 & $\$ 217$ & $2.511 E=07$ & $5.665 F=06$ & 000 \\
\hline 56.0 & 1561 & 7. $2 A \cap \cap F=07$ & $6.334 E-06$ & 000 \\
\hline 00.0 & $12 \cap 4$ & A. $940 E-J 7$ & $5.603 F=06$ & 400 \\
\hline 64.0 & 2222 & $1.199 F-76$ & 1.03 af $=05$ & 000 \\
\hline 68.0 & $14 \cap 7$ & 7.5 KAF $=67$ & $6.548 \mathrm{~F}-06$ & 400 \\
\hline $\begin{array}{l}72.0 \\
76.0\end{array}$ & 2536 & $1.35 A E=06$ & 1.1 AOE $=05$ & 400 \\
\hline 80.0 & $\begin{array}{r}9187 \\
979\end{array}$ & 4.96 AF $=07$ & $\begin{array}{l}1.318 t=113 \\
4.323 F-06\end{array}$ & a 00 \\
\hline$B 4.0$ & 3248 & $1.7 .77 f=0 x_{1}$ & $1.511 E=05$ & 400 \\
\hline 8.8 .0 & $? 354$ & $1.259 \mathrm{~F}=\mathrm{nA}$ & $1.096 \varepsilon-05$ & 400 \\
\hline 92.0 & 172 & $0.21 P F=0 R$ & $A .015 E-1) 7$ & 400 \\
\hline 96.0 & 451 & ?. $415 F=07$ & $2.1 A 1 E=06$ & 400 \\
\hline 100.0 & 90 & $a . R 30 E=0.8$ & $4.202 E-07$ & 900 \\
\hline 1040 & 52 & $3.897 E-08$ & $2.0517=07$ & 000 \\
\hline & & $\begin{array}{r}3.154 F=04 \\
\text { SEE } / 3 D . M\end{array}$ & $\begin{array}{c}3.7 A A E-03 \\
1 / N\end{array}$ & \\
\hline
\end{tabular}




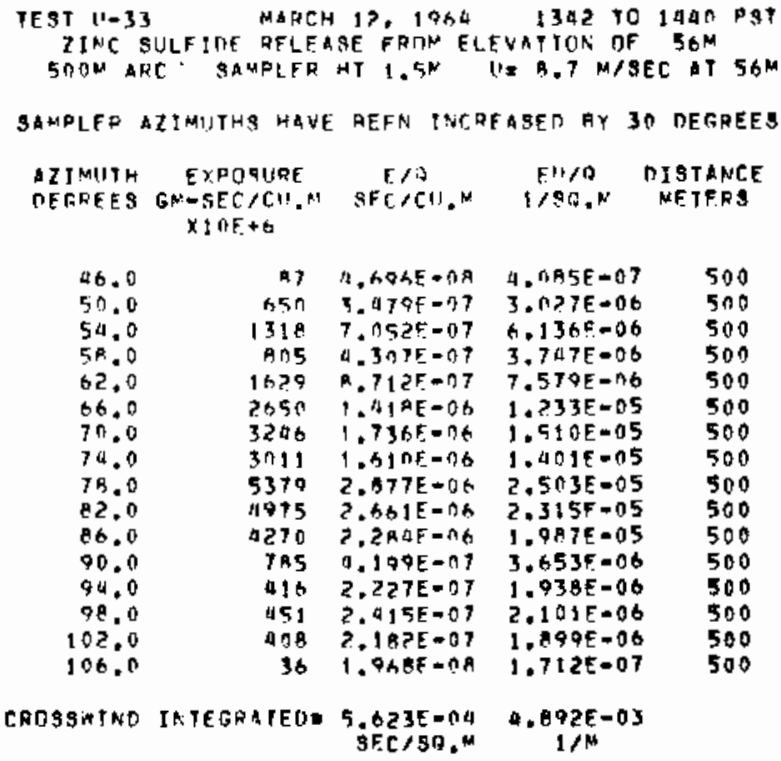

TF 11033 MAPCH 13, 1964 1342 TO 1440 PGT II NC SULFIDE RFLEASF FRIIF ELFVATION DF SON DOGM ARC SANOLER HF $1.5 \%$ Ua $0.7 \mathrm{M} / \mathrm{SEC}$ AT $56 \mathrm{M}$

SAMPLER AZIMIITHS HAVE REFN IIUCREABED AY JO OEGREES

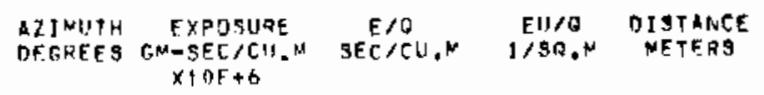

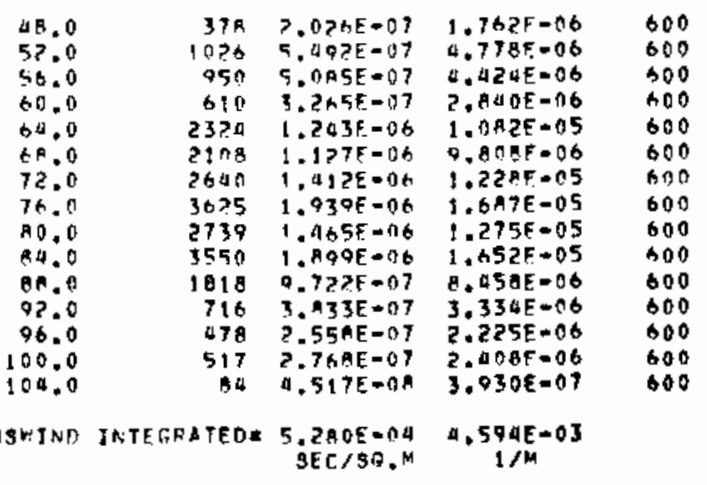

\footnotetext{
CROASUIND INTEGRATEDE 5.2AOE-OA A, $594 E-03$
}

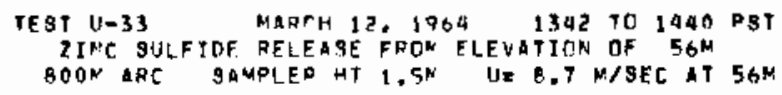

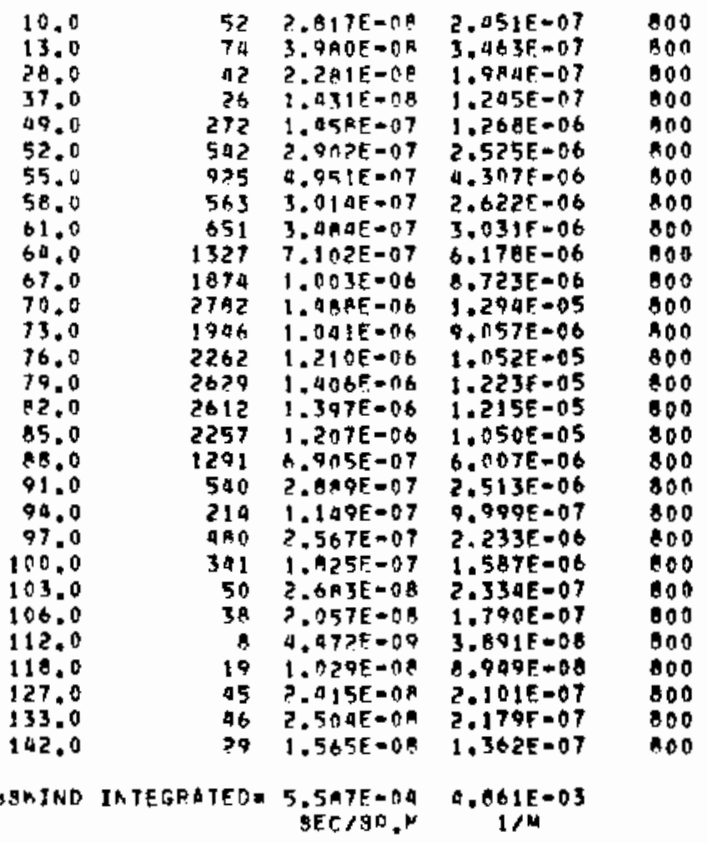




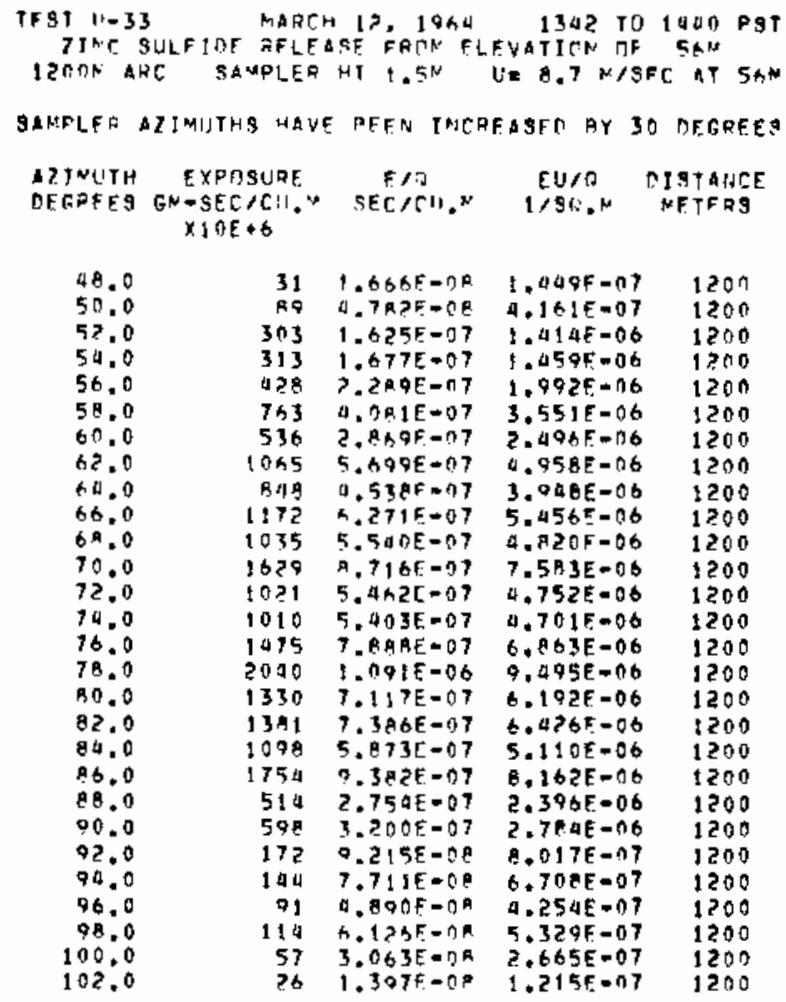

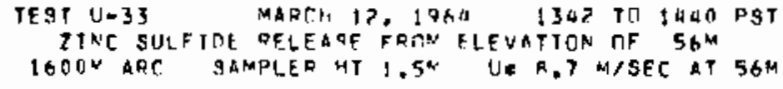

SAMPLER AZJMUTHS HAVF BEFA: IACRFASED FY IO TEGREES

\begin{tabular}{|c|c|c|c|c|}
\hline $\begin{array}{l}\text { DZINETTM } \\
\text { DEGHFES }\end{array}$ & $\begin{array}{c}\text { EXPOSUDE } \\
\text { GN-SEC } \\
X I O E+6\end{array}$ & $\begin{array}{c}E / 0 \\
\sec / \mathrm{CU}, N\end{array}$ & $\begin{array}{l}E \cup / 8 \\
1 / 96.2\end{array}$ & $\begin{array}{l}\text { DIMTANCE } \\
\text { METERS }\end{array}$ \\
\hline $\begin{array}{l}38.0 \\
44.0 \\
46.0 \\
48.0 \\
50.0 \\
52.0 \\
54.0 \\
56.0 \\
58.0 \\
60.0 \\
62.0 \\
64.0 \\
66.0 \\
68.0 \\
70.0 \\
72.0 \\
74.0 \\
78.0 \\
78.0 \\
80.0 \\
82.0 \\
90.0 \\
96.0 \\
88.0 \\
90.0 \\
92.0 \\
94.0 \\
96.0 \\
98.0 \\
100.0 \\
102.0 \\
104.0 \\
106.0 \\
108.0 \\
110.0\end{array}$ & $\begin{array}{r}175 \\
5 \\
4 \\
5 \\
29 \\
50 \\
114 \\
140 \\
356 \\
144 \\
243 \\
403 \\
357 \\
411 \\
244 \\
311 \\
595 \\
373 \\
551 \\
440 \\
413 \\
320 \\
299 \\
202 \\
123 \\
145 \\
49 \\
37 \\
44 \\
25 \\
6 \\
5 \\
21 \\
8 \\
23\end{array}$ & 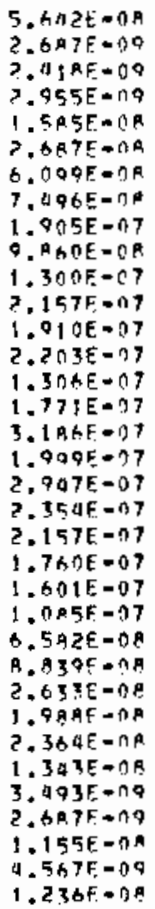 & 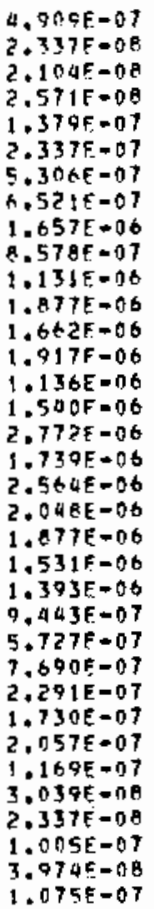 & $\begin{array}{l}1600 \\
1600 \\
1600 \\
1600 \\
1600 \\
1600 \\
1600 \\
1609 \\
1600 \\
1600 \\
1600 \\
1600 \\
1600 \\
1600 \\
1600 \\
1600 \\
1600 \\
1600 \\
1600 \\
1600 \\
1600 \\
1600 \\
1600 \\
1600 \\
1600 \\
1600 \\
1600 \\
1600 \\
1600 \\
1600 \\
1600 \\
1600 \\
1600 \\
1600 \\
1600\end{array}$ \\
\hline $\sin \sin$ & INTEGFATFD = & $\begin{array}{l}2 . n \in 3 F-\cap 1 \\
\text { SEC }\end{array}$ & $\begin{array}{c}1.705 E-03 \\
1 / 4\end{array}$ & \\
\hline
\end{tabular}


UNSTABLE SERIES EXPER MENT; $34(U-34)$

GROUND LEVEL SAMPLING 100-3200 M ARCS.

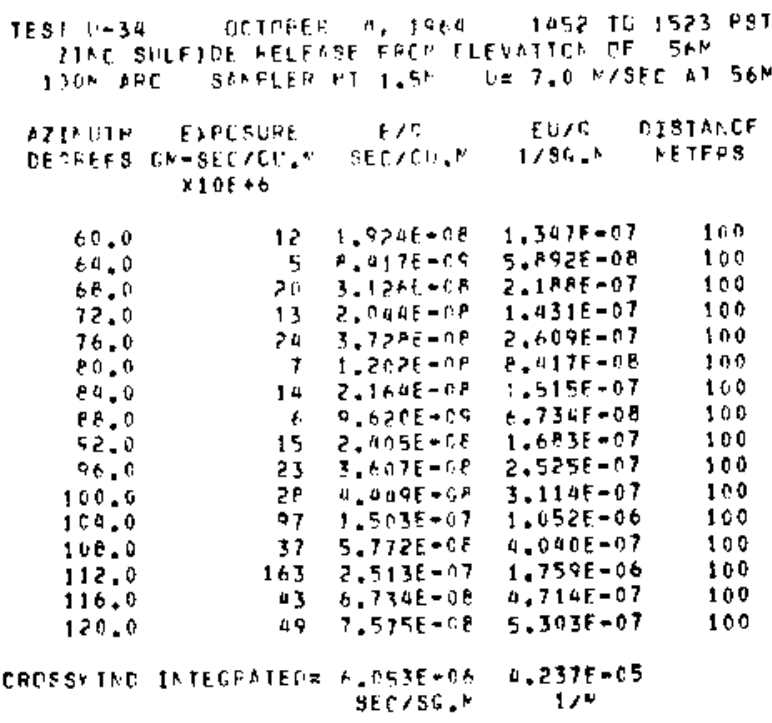

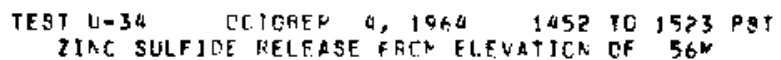
ZIAC SULFJDE TELEASE FECN ELEVATICR CF $56 \mathrm{~N}$
$20 O \mathrm{~N}$ ARC SANPLER HT $1.5^{\mathrm{N}}$ U. $7.0 \mathrm{~N} / \mathrm{SEC}$ AT $56 \mathrm{~N}$ A2INITH EXPCSURE E/A EUIG DISTANCE OEREEES GR-SEC/CH." SEC/CL.R 1/SG.N NETERS $\times 10 \mathrm{E}+6$

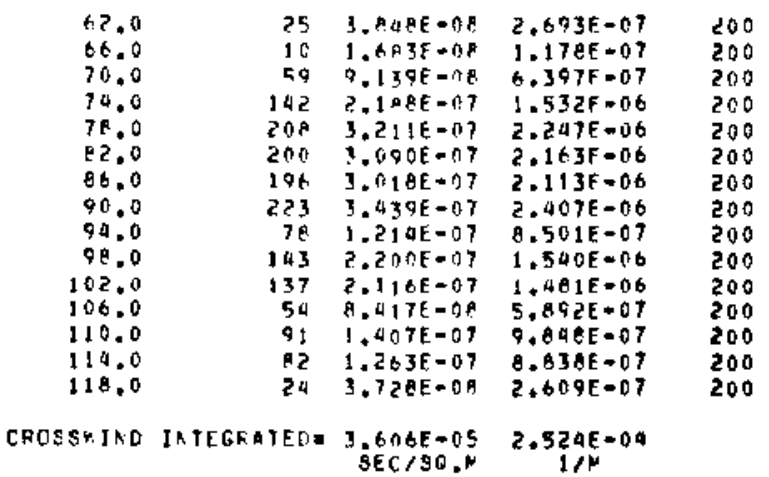

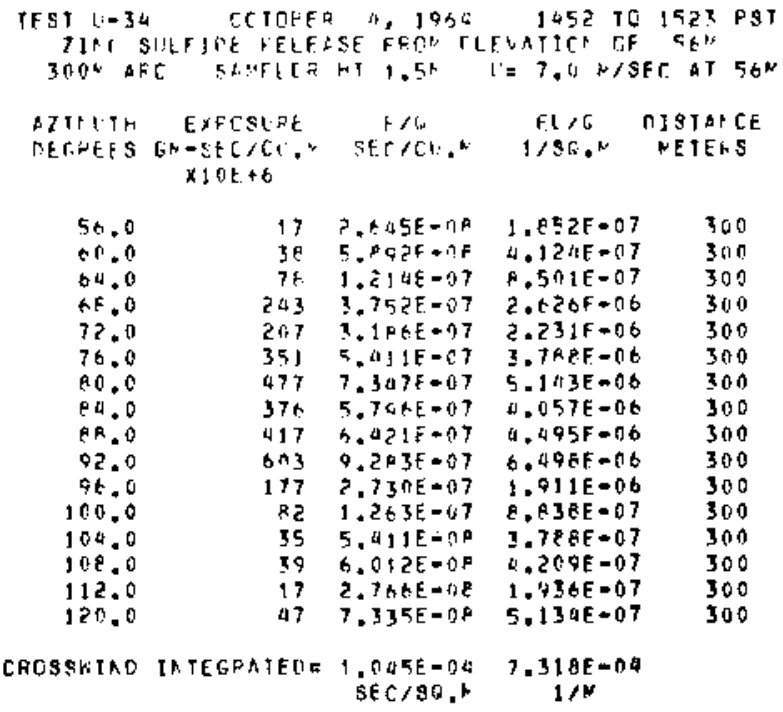

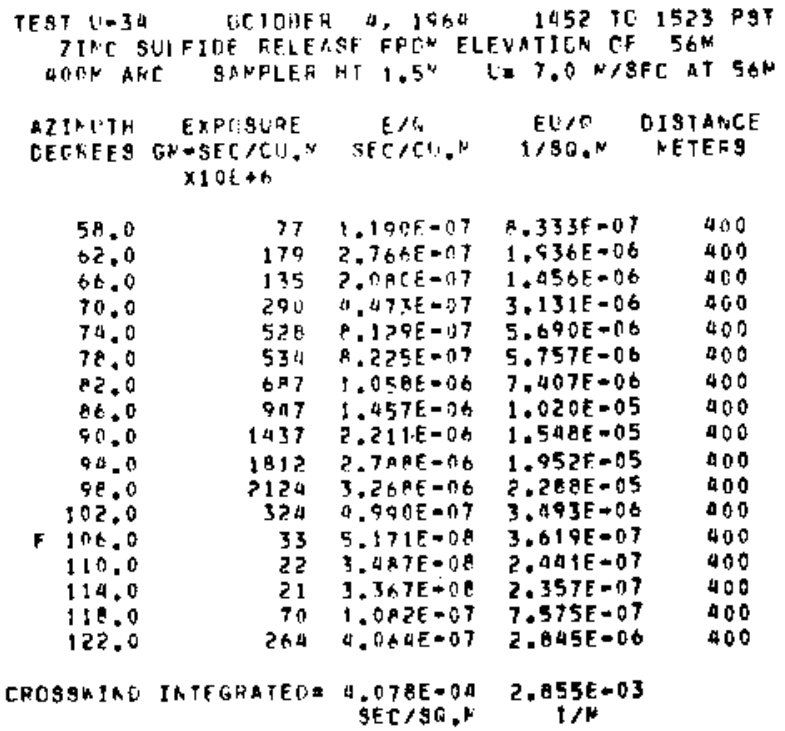




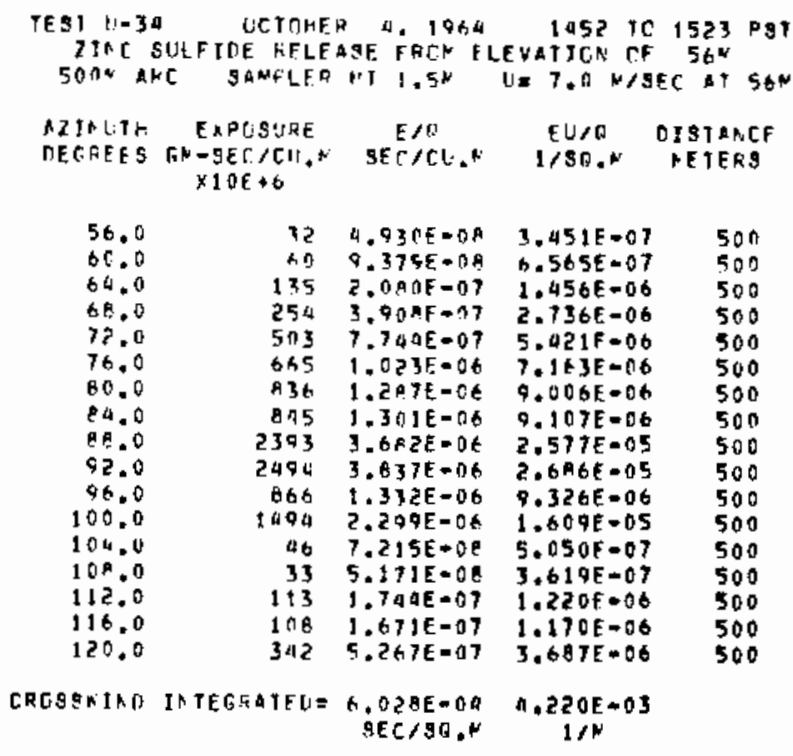

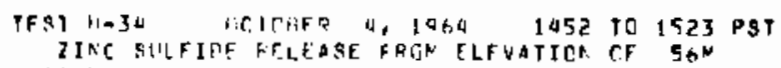

\begin{tabular}{|c|c|c|c|c|}
\hline $5 n$. & 4 & $1.403 F=O A$ & $1.020 F=07$ & 600 \\
\hline 0.0 & I 0 , & $\therefore, \cos E-6,8$ & $1.178 F=07$ & 600 \\
\hline $5 \% .0$ & 62 & $Q, A 2 D F=08$ & $6.734 E=07$ & 600 \\
\hline 62.0 & 57 & A.7TRE - OA & $0.145 E+07$ & 600 \\
\hline 60.0 & 112 & $1.732 E-197$ & $1.212 E-06$ & 600 \\
\hline 70.0 & 300 & $0.012 F-07$ & $0,209 E=06$ & 600 \\
\hline 74.0 & $5 \times 3$ & $\triangle, T \triangle 2 E=07$ & 6. 2 ACAF $=06$ & 600 \\
\hline 78,0 & 1572 & $\therefore \triangle 19 E=O B$ & $: .694 E=05$ & 600 \\
\hline 82.0 & 935 & $1.430 E=08$ & $1.008 E=05$ & 600 \\
\hline 86.0 & $1 n \geqslant 1$ & $1.57>E-106$ & $1.100 E-05$ & 600 \\
\hline $90 \div 0$ & 2011 & 3. CऽUE-DA & $2.166 E=05$ & 60.0 \\
\hline 84.0 & 243 & $1.2516-06$ & 0.75 QE $=06$ & 600 \\
\hline $98 . n$ & 1150 & 1.7 AE $=06$ & $1.249 E-05$ & 600 \\
\hline 102.0 & 1 月9 & $=910 E-07$ & $2.037 t-06$ & 600 \\
\hline 100.0 & 1011 & $1.5395-07$ & $1.0775=06$ & 600 \\
\hline 10,0 & $\leq 5$ & $5.53 t E=0 \mathrm{~A}$ & $3.872 F=07$ & 600 \\
\hline 114,0 & 14 & $1.299 E=07$ & 9.09 IE -07 & 600 \\
\hline 112.0 & 134 & 7,06 AE $=07$ & $1.44 B E=06$ & 601 \\
\hline 122.0 & 193 & $2.9 \times 2 E=07$ & $2,087 E-06$ & 600 \\
\hline
\end{tabular}

CRDSSHIRD JATEGHATEDE $6,10 A E-n Q$ M.

\begin{tabular}{|c|c|c|c|c|}
\hline 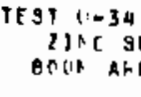 & $\begin{array}{l}\text { WCTRAFD } \\
\text { ULFIIE HELFA } \\
\text { S SAMFI.ED }\end{array}$ & 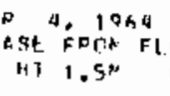 & 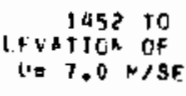 & $\begin{array}{r}1523 \mathrm{P} \\
56 \mathrm{~N} \\
\text { EEC AT } 5\end{array}$ \\
\hline $\begin{array}{l}\text { AZJMIII } \\
\text { DEEHEFS }\end{array}$ & $\begin{array}{c}\text { EXHCSUPE } \\
\text { GR }=5 E L C C O N \\
\times 1 O E+6\end{array}$ & $\begin{array}{c}E /: \\
\text { SEC/CU.r }\end{array}$ & $\begin{array}{c}E(1 / 6 \\
1 / 50 . k\end{array}$ & $\begin{array}{l}\text { DISIANCE } \\
\text { NEYERS }\end{array}$ \\
\hline 46.0 & 0 & $1.2025-09$ & $0.4178-09$ & 800 \\
\hline 52.0 & $\begin{array}{l}16 \\
12\end{array}$ & $2.5 ? 5 E-0 \mathrm{~A}$ & $1.768 \mathrm{BE}=07$ & 800 \\
\hline 55.0 & $\begin{array}{l}12 \\
18\end{array}$ & $1.974 E=0 \%$ & $1,347 \varepsilon-07$ & 100 \\
\hline $5+.0$ & $\begin{array}{l}18 \\
39\end{array}$ & 2. ARAE- UA & $2.020 E-0 ?$ & 000 \\
\hline 61.0 & 50 & & $0.124 F=07$ & 600 \\
\hline 84.0 & 30 & & $5.387 E=07$ & 800 \\
\hline 67.0 & 126 & $\begin{array}{l}5.652 E-08 \\
1.94 A E=07\end{array}$ & $3.956 E=07$ & 000 \\
\hline 70.0 & 224 & & $1.36 a E \rightarrow 06$ & 000 \\
\hline 73.0 & by & $\begin{array}{l}3.451 E=07 \\
9.84 A E=67\end{array}$ & $2.916 E=06$ & 800 \\
\hline 76.0 & 530 & $\begin{array}{l}.2 Q R E=t) 7 \\
R .2 P 5 E=07\end{array}$ & $\begin{array}{l}6.894 E-06 \\
5.7095-06\end{array}$ & 800 \\
\hline 70.0 & 494 & $8,9 \geqslant B E=07$ & $\begin{array}{l}5.799 E=06 \\
4.890 E=116\end{array}$ & 800 \\
\hline 82.0 & 1214 & $1.8 B Q E=0 B$ & $1.3085=05$ & 800 \\
\hline 85.0 & 852 & 1.31 PE=06 & $9.1 B 3 E-06$ & 800 \\
\hline QP. 0 & 894 & $1.377 E=0 \%$ & $0.638 E=06$ & $\begin{array}{l}800 \\
000\end{array}$ \\
\hline 91.0 & $6 \times 9$ & $1.3 \pi 7 E=06$ & $5.360 \mathrm{E}=06$ & 800 \\
\hline 94.0 & and & $6.042 E=07$ & $4.709 E=06$ & 800 \\
\hline 47.0 & 537 & $0.2735=07$ & $5.79 \mathrm{tE}=\mathrm{C6}$ & 000 \\
\hline 100.0 & 312 & $5.729 \mathrm{9E}-07$ & $4.007 E-06$ & 800 \\
\hline 103.0 & $15 i$ & $2.333 \mathrm{E}-07$ & $1.633 \mathrm{E}=06$ & 000 \\
\hline 106.0 & $7 i$ & $1.004, E=07$ & $7.660 E=07$ & 100 \\
\hline 109.0 & 39 & 9. $\triangle Q 2 E-C \theta$ & $a .124 E=07$ & 100 \\
\hline 112.0 & 44 & $0.8505=08$ & $9.790 E-07$ & 000 \\
\hline 115.0 & 35 & $5.531 E+0 E$ & $3.872 E=07$ & 000 \\
\hline 118.0 & 87 & $1.347 E-077$ & $9.4276-07$ & 800 \\
\hline & $5 n$ & A.ASRE $O O A$ & n.060E $=07$ & 800 \\
\hline & & $\begin{array}{l}\text { a } 045 E=04 \\
\text { SECASTr.N }\end{array}$ & $3.531 f=03$ & \\
\hline
\end{tabular}

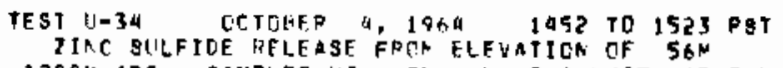
$1200 \mathrm{~N}$ ARC SANPLER HP $1.5 \mathrm{~N}$ U. $7.0 \mathrm{M} / \mathrm{MEE}$ AT $56 \mathrm{M}$

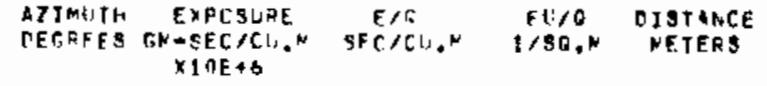

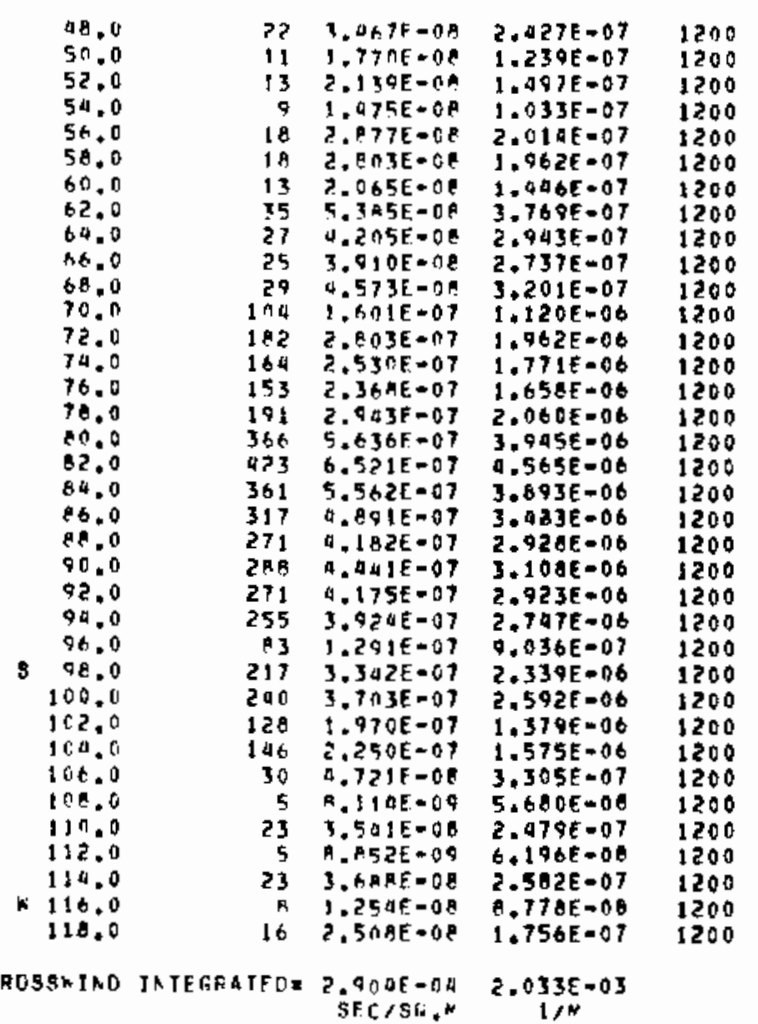




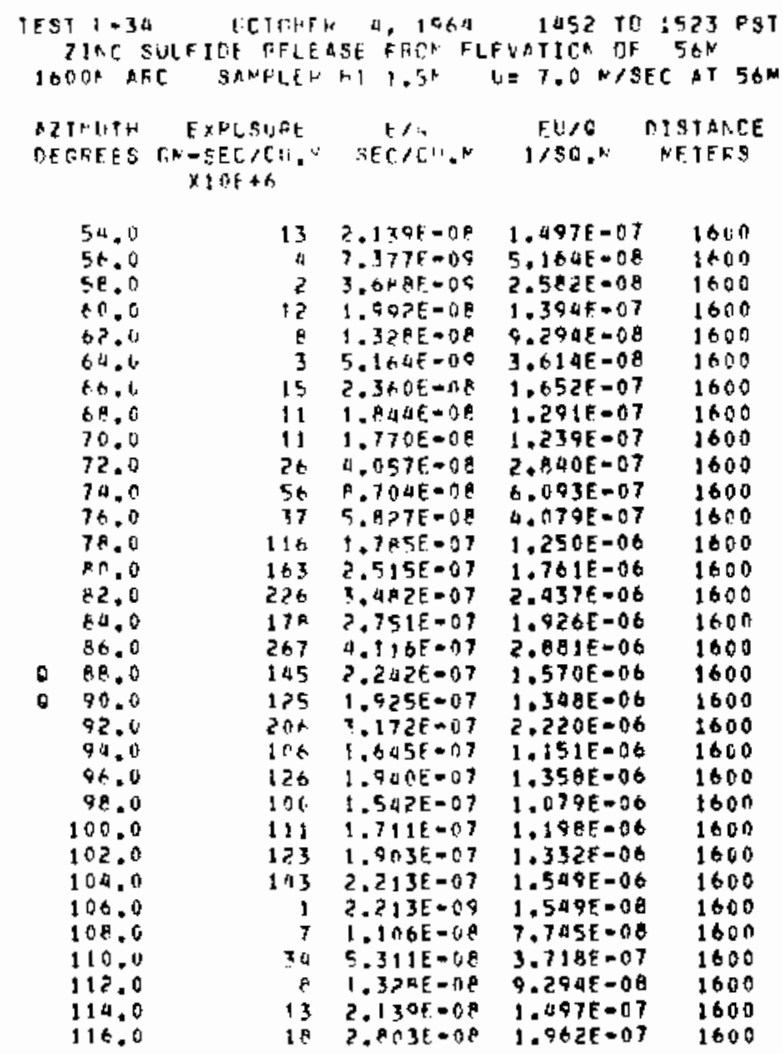

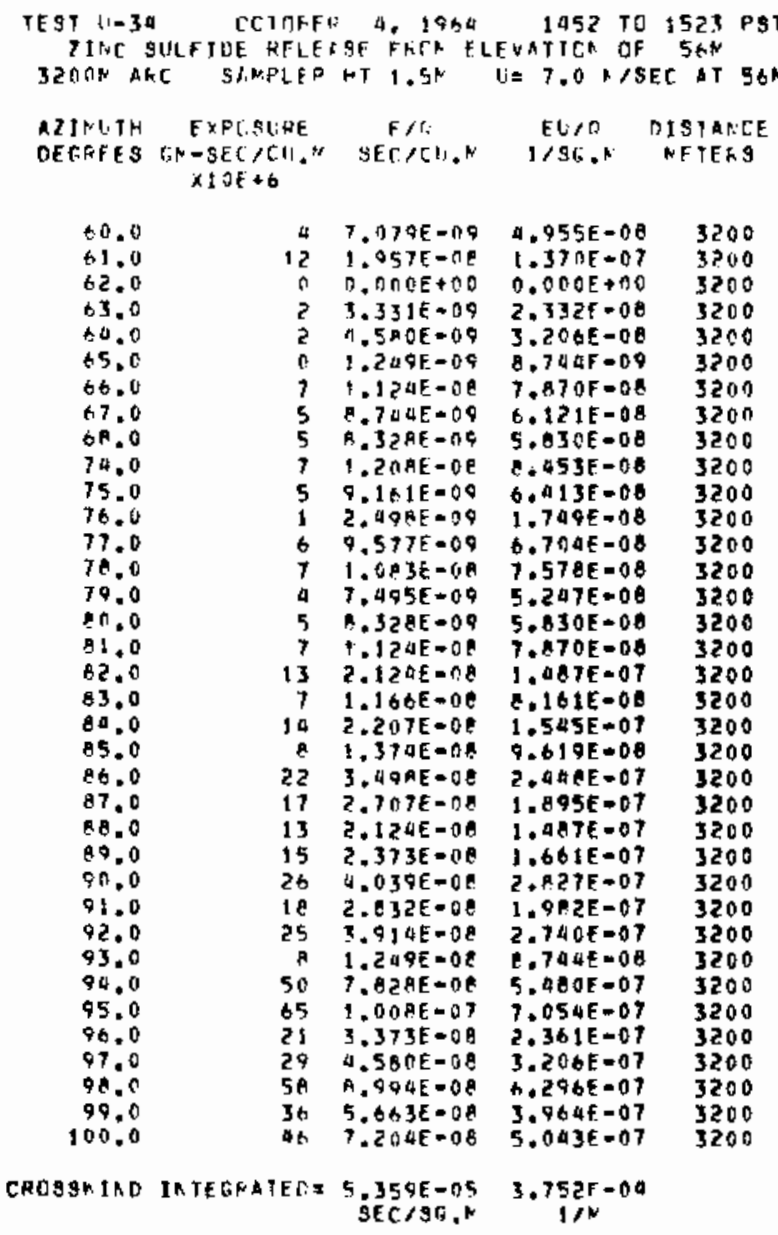


UNSTABLE SERIE5 EXPERIMENT: 35 (U-35)

GROUND LEVEL SAMPLING 100-1600 M ARCS.

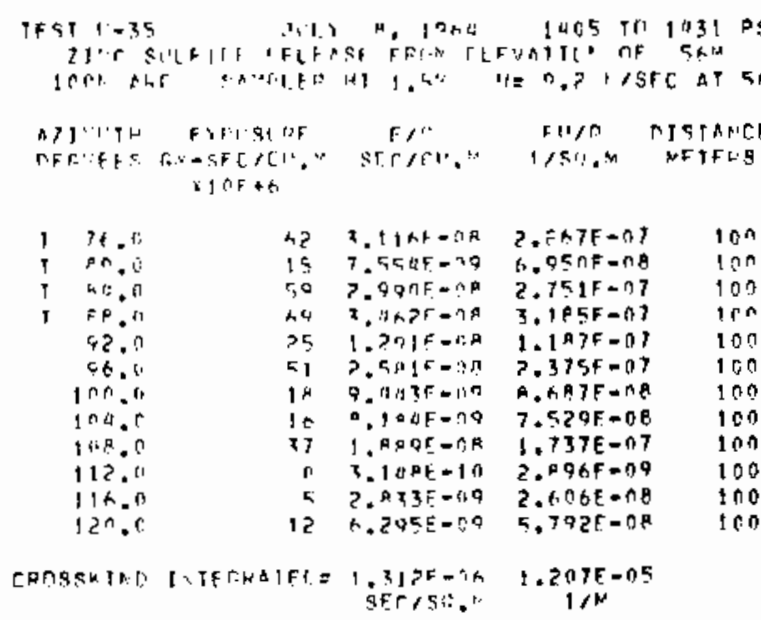

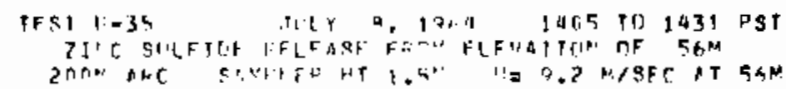

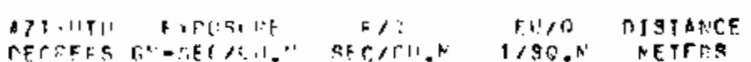
$\times 1$ in +

\begin{tabular}{|c|c|c|c|c|}
\hline A? ${ }^{\circ}$ & 17 & D. $\left.{ }^{1} 1\right\} F=19$ & $P, 108 F=08$ & ? \\
\hline th. $1:$ & $1 ! 3$ & $5.79 \geqslant F-f R$ & $5.32 A F=n 7$ & 200 \\
\hline $7 r, 6$ & $h \in$ & 3. $n$, > & $? .7 A \cup F=\wedge .7$ & 260 \\
\hline $7 d .5$ & S17 & ?. 5 (Pf $=18$ A & $P .317 F=07$ & ?ith \\
\hline $7 P .4$ & $>5$ & 1. $759 k-1.0$ & $1,15 \mu \mathrm{F}=01$ & $? \times 0$ \\
\hline n?. & $\times 7$ & 1 , A, Q & $1.717 t=07$ & $20 \hat{0}$ \\
\hline wh. & 107 & $0.2578=12$ & $9 . n A B F=07$ & $2 n n$ \\
\hline $0 n .0$ & 7 & $x .7775=00$ & $3.475 F=08$ & 200 \\
\hline 40.0 & 3 & $1.53: 1 f-10$ & $1.44 R E-A B$ & 20 ह \\
\hline a日. & $1 \sqrt{b}$ & $5.17\} 1 . F=0$ & $4.4335=08$ & 700 \\
\hline 107.11 & 17 & a. ${ }^{2}, 35=09$ & R.jOBE- 08 & 2010 \\
\hline $\operatorname{lig} 0$ & 70 & $1.757 F=n A$ & $1.7955=07$ & 200 \\
\hline $111^{n}$ & 11 & $5.240 f=110$ & $5.50 Z F=n E$ & 200 \\
\hline $11 d .6$ & $\hat{\imath} \vec{e}$ & $6.325 E-10$ & $5.702 F=08$ & 200 \\
\hline $11^{R} \cdot 1$ & 5 & $7.91 A E=09$ & $? .3+7 F=98$ & 300 \\
\hline & & 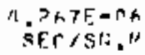 & $\begin{array}{c}92 \mathrm{CHE}=05 \\
\mathrm{~J} / \mathrm{N}\end{array}$ & \\
\hline
\end{tabular}

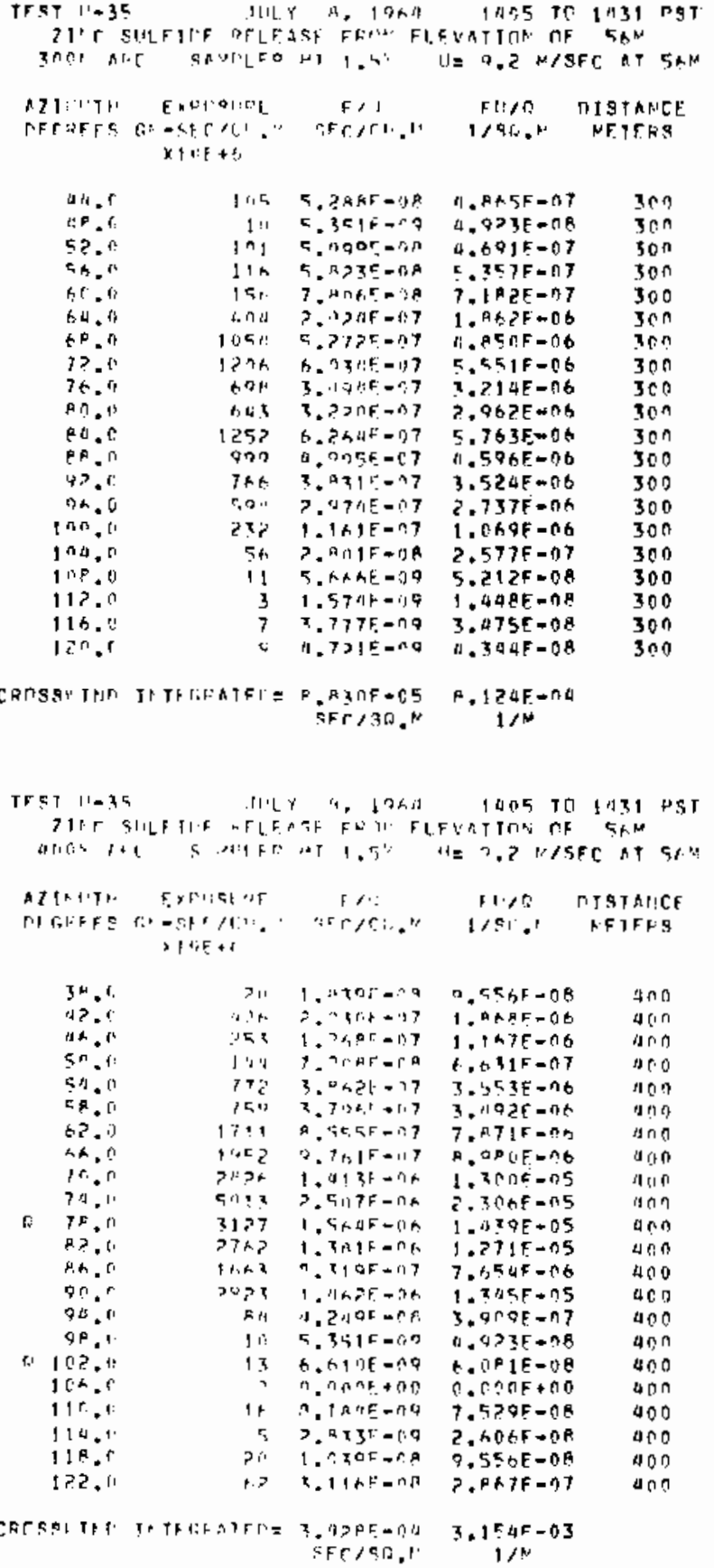




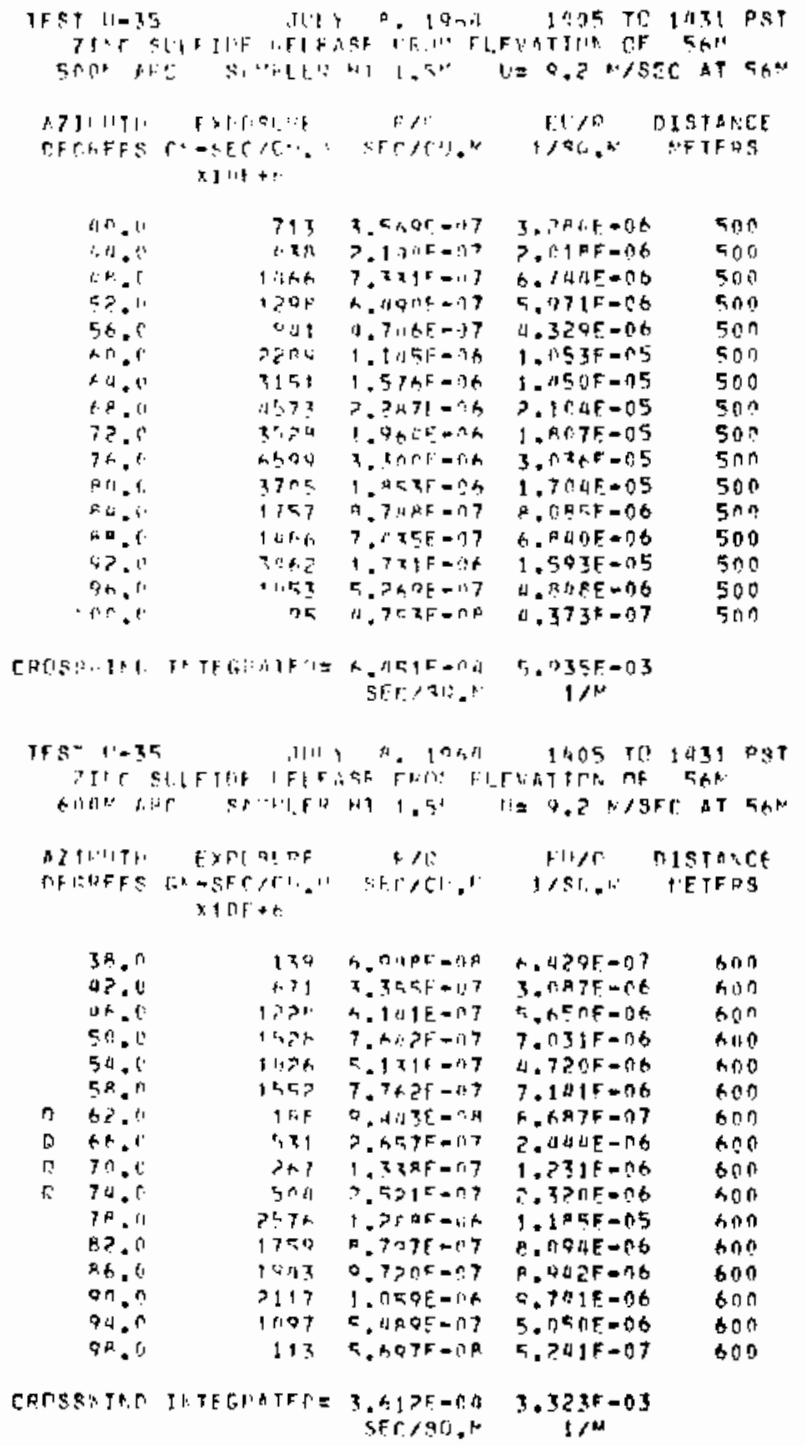
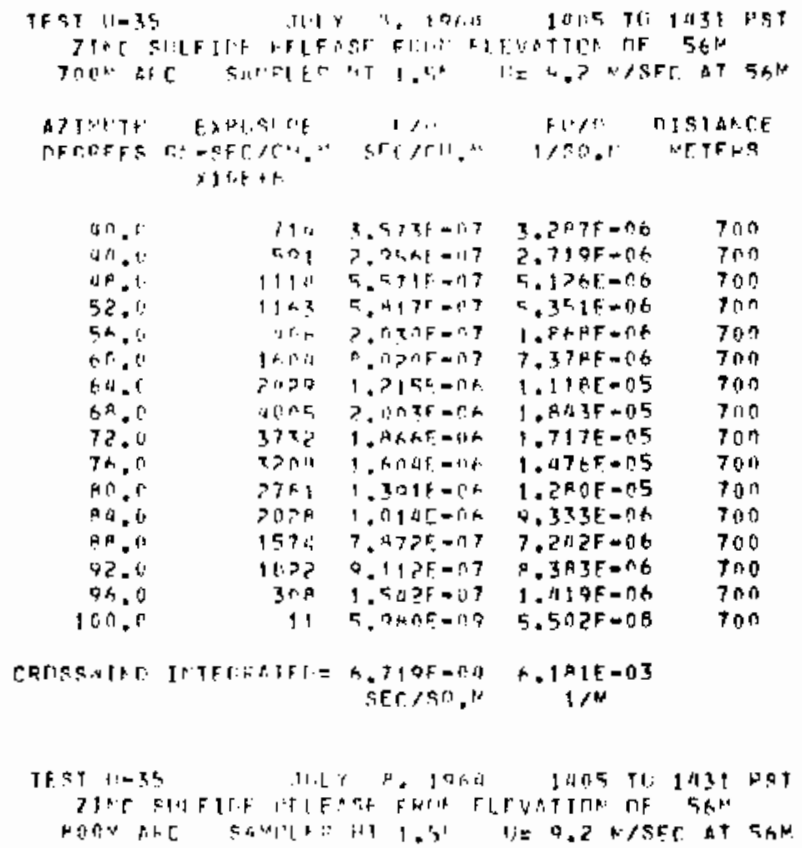


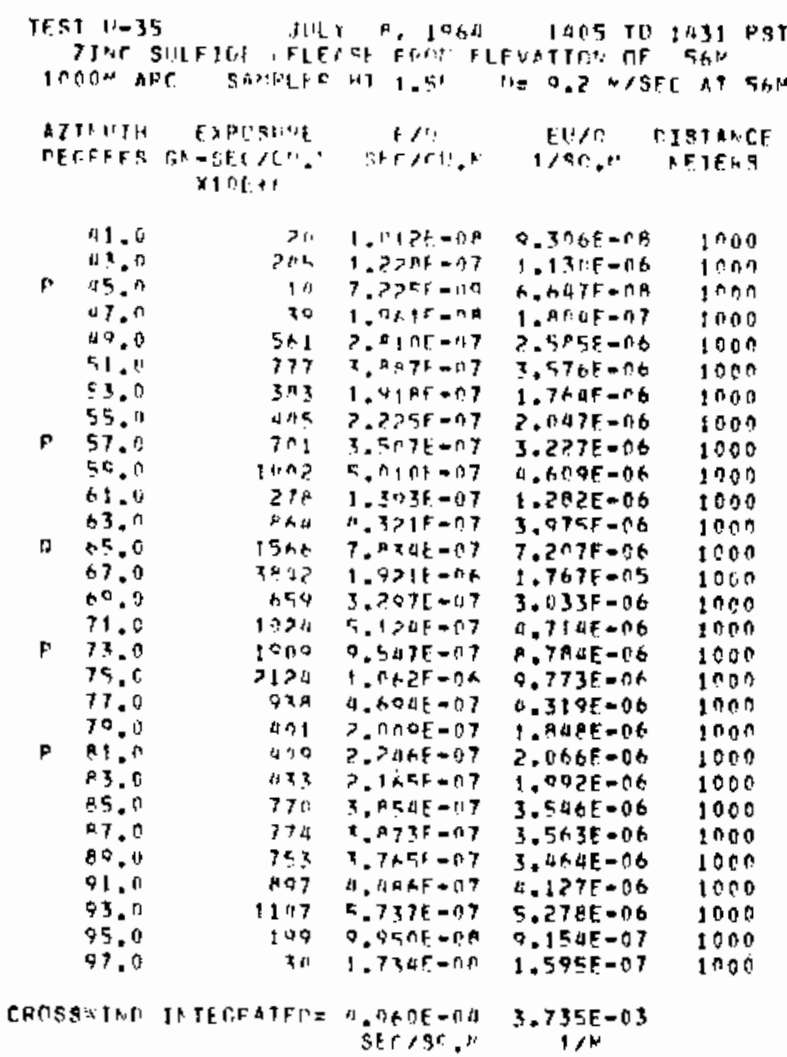

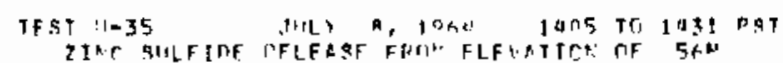

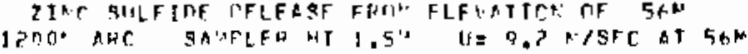

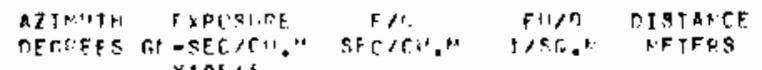
$x i$ or 15

\begin{tabular}{|c|c|c|c|c|}
\hline $4 \pi .0$ & 50 & $2,0 r_{3} 3 t=0 R$ & $3.7548=017$ & 1700 \\
\hline$\Delta h, n$ & of & $\forall, G Q R F=\mathrm{Bl}$ & $11.59 \times 5=07$ & 1700 \\
\hline पa. & $A=8$ & $x .7095-07$ & $0 \leq 1 F=n t$ & 1200 \\
\hline $50 . n$ & $6 .>5$ & $3.1>55=07$ & $2.875 f=n 6$ & 1200 \\
\hline 52.0 & SA. & $7.5 \Delta x E-07$ & $3.340 E-06$ & $120 n$ \\
\hline 50.0 & 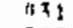 & $7.1595=09$ & $A 7 E=16$ & $120 n$ \\
\hline $5+.0$ & $35 ?$ & $1.7 A A F-n 7$ & $645 \mathrm{E}=06$ & 1200 \\
\hline 5म, 1] & 628 & 3. यव $5 \mathrm{~F}=07$ & $3.215 E-06$ & $120 n$ \\
\hline 60.6 & $P n_{B}$ & $1.3 \times 3 E=07$ & $1.318 F=06$ & $t \geqslant 00$ \\
\hline 62.0 & 284 & $1.47>F=n$ & $1.354 E=106$ & 1200 \\
\hline 60.9 & $\operatorname{anz}$ & i. .51 SE $=07$ & $a, 1545.06$ & 1200 \\
\hline $6 h .0$ & $7>09$ & $1.1>9 E=A B$ & QF. 05 & 1200 \\
\hline 20 & 1907 & จ. $7 a 5 F=n ?$ & $186 F=06$ & 1200 \\
\hline 70.0 & $12 \geqslant 4$ & $A .1>1 F=0.7$ & $5.601 F=06$ & $120 n$ \\
\hline 73.0 & 1258 & $4.25 .25-07$ & $5.778 F=06$ & $120 n$ \\
\hline 14.9 & $t ? 17$ & h. I) gat -07 & $590 F-76$ & 1200 \\
\hline $7 h .0$ & $15 \%$ & 7. $0.31 E+07$ & $306 E=06$ & $1 ? 00$ \\
\hline $7^{9} .0$ & $\operatorname{Tas}$ & $F 41 E-07$ & $4545=106$ & 1200 \\
\hline An, & 1037 & 5. IARE $=C 7$ & $4.7735=06$ & $12 n n$ \\
\hline$A_{2} .0$ & $77 ?$ & $3.9645=07$ & $5 E-06$ & $1 \geq 00$ \\
\hline$A=0$ & 619 & 3. กQRE $=0$ ? & $9 E-06$ & 1200 \\
\hline en. 0 & 697 & $3.0 \times 7 E-07$ & $3.162 f=06$ & 1200 \\
\hline AR. 0 & 1105 & $5.57 \mathrm{PF}=07$ & $5.0 B 6 E-06$ & 1200 \\
\hline 90,0 & 911 & $8,056, f=07$ & $.7325-08$ & 1200 \\
\hline & $7+4$ & $3.73^{0}=07$ & $426 E-06$ & 1200 \\
\hline 94.0 & 508 & $1.4635-07$ & $.373 E-08$ & 1200 \\
\hline $9 * .0$ & $15 !$ & $7.576 \mathrm{~F}=\mathrm{nA}$ & $0705-07$ & $170 n$ \\
\hline $8-\mathrm{c}$ & $75^{\circ}$ & 3. $5 x \cap E=0$ X & 20 a $=07$ & 1200 \\
\hline & k. & $3.0765=08$ & Cथ $\triangle 9 E-0 E$ & 1200 \\
\hline & h & $10 K F=19$ & $249 E-08$ & 1200 \\
\hline
\end{tabular}

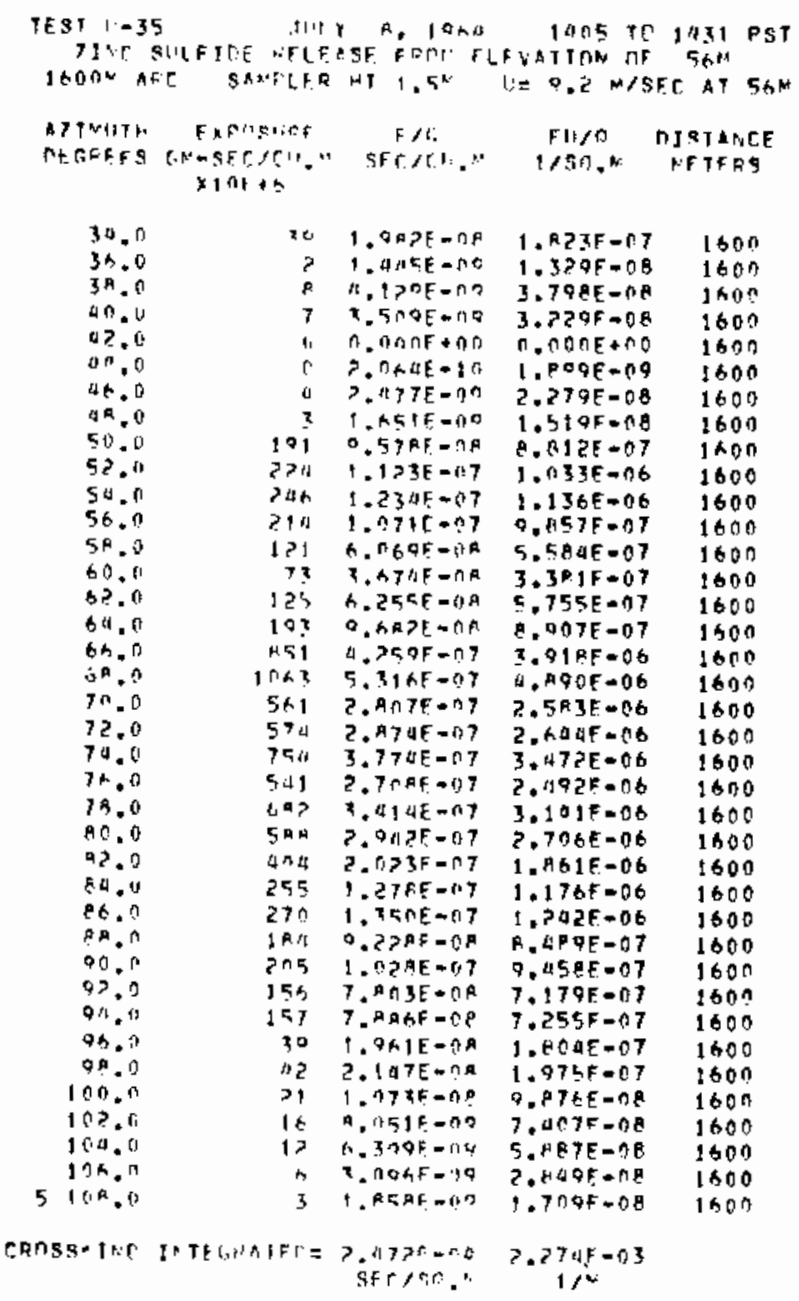

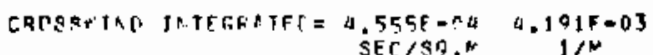


UNSTABLE SERIES EXPER MENT: $36(\mathrm{U}-36)$

GROUND LEVEL SAMPL ING $100-1600 \mathrm{M}$ ARCS.

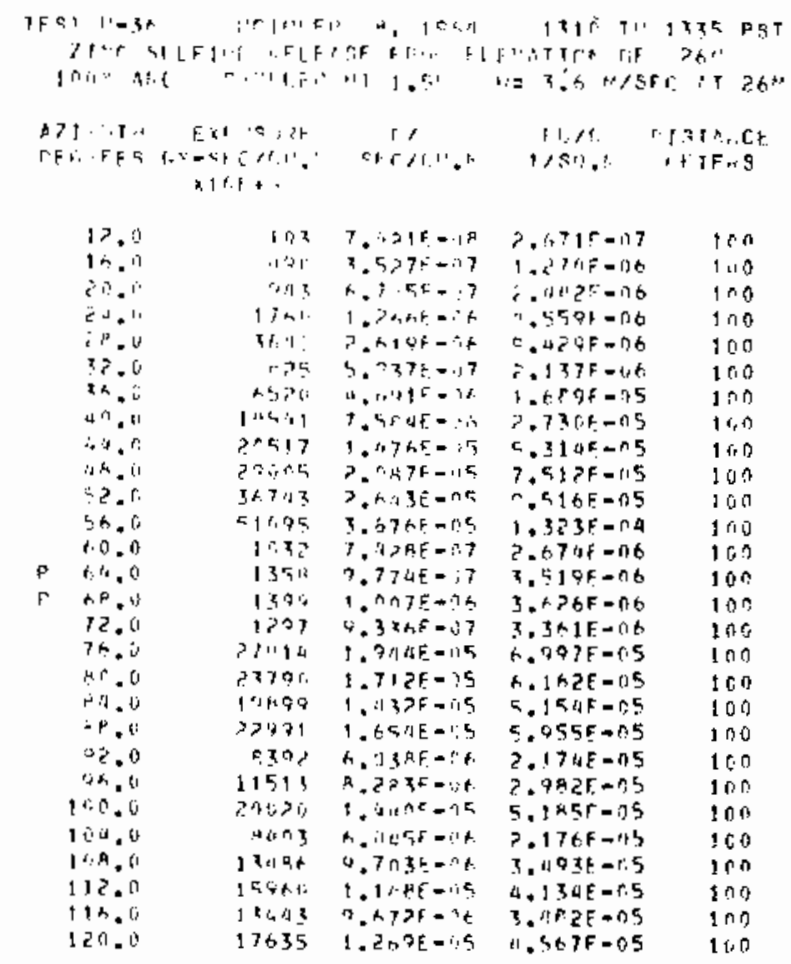

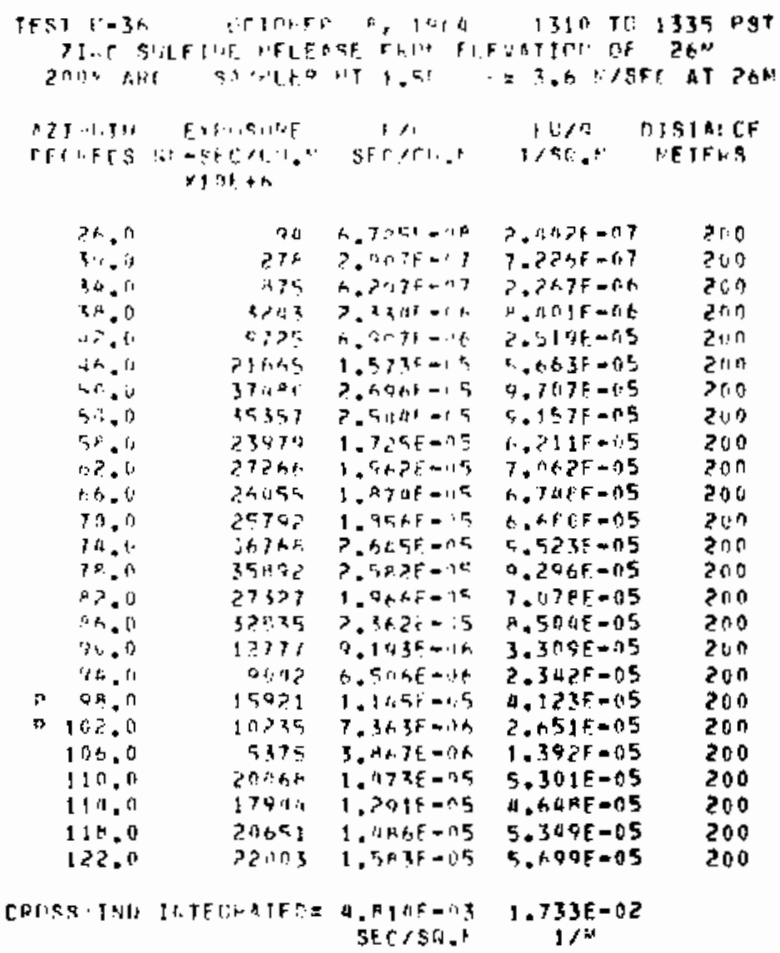

CRTSSHING IATEGIIAIFI =

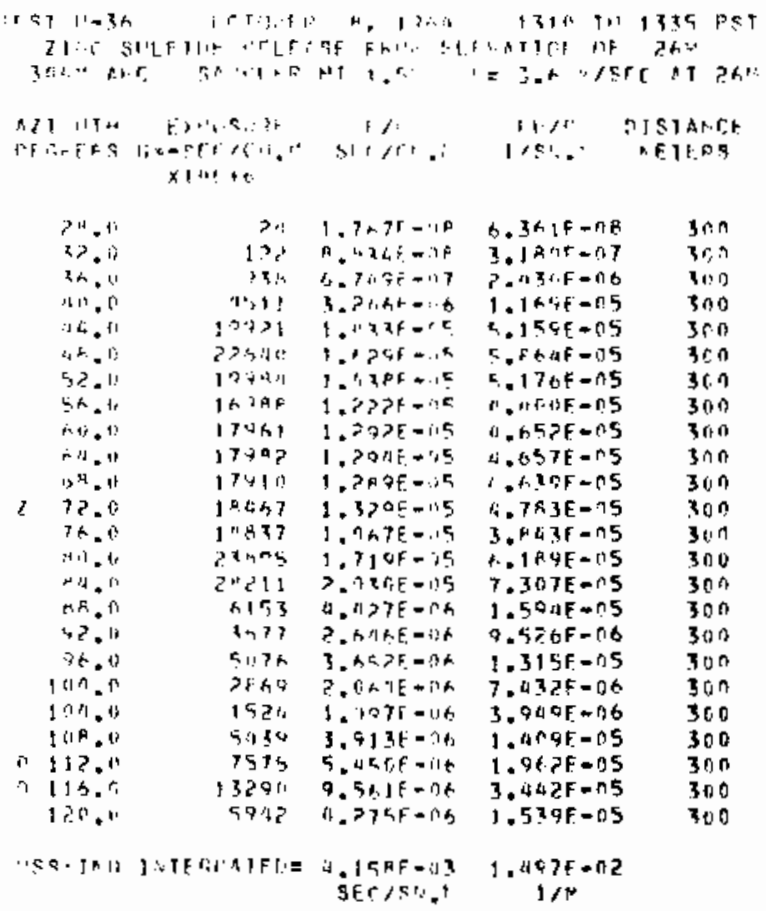




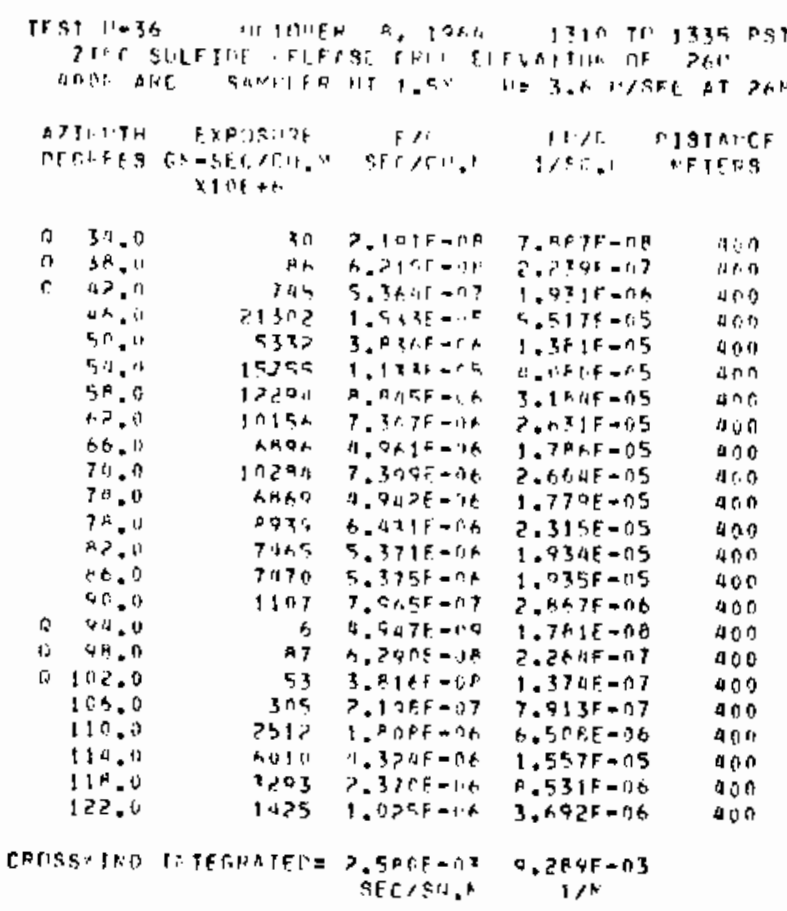

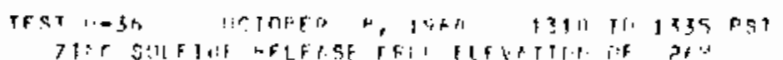

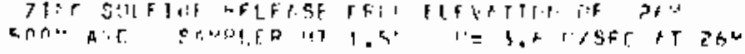

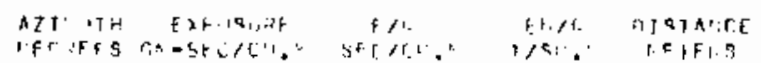
$x$ id $\mathrm{f}+\mathrm{t}$

\begin{tabular}{|c|c|c|c|c|}
\hline 36.0 & $4 \mathrm{CO}$ & $3.520 \mathrm{n}-0\}$. & 1. introns & $5 \pi n$ \\
\hline ac. n & 25 & 1. $h F 1+F=n h$ & $+. n A M F=n \theta$ & 503 \\
\hline $4 i .0$ & 1)小al & R. $9475=12$ & $\pi .72$ : $=-05$ & $5 x+\pi$ \\
\hline . 3 th & $\ln 22^{2}$ & $7.307 i=i 1 \mathrm{~h}$ & $2 . x+35=x^{2}$ & $50 n$ \\
\hline $5 p . v$ & 4778 & $B .4+R E=A A_{1}$ & $? .325 F-6.5$ & $50, n$ \\
\hline$=A .0$ & $53 n t$ & 3. Р $\mid \Delta \ddot{\Delta}=1: t$ & $1.3737=0,5$ & $50+1$ \\
\hline $6 n, 13$ & 5018 & $4.2505=16$ & 1.5335 .05 & 500 \\
\hline & 3435 & $7.756 f=0 t$ & $4.9335=0$. & 540 \\
\hline tis & $7+18$ & $5, \cap E N F=\Lambda A$ & $1 . n$ I HF $=05$ & 500 \\
\hline 72.0 & A110.4 & $3.5 \times 7+=11 t$ & $1.574 \mathrm{~F}-05$ & $50 ?$ \\
\hline $7 h . n$ & 1145 & $x, 1$ SAF $=0 A$ & $1.151 E=05$ & $5 n n$ \\
\hline$A 0,0$ & 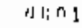 & ?. A $) G F=$ it & $1,03 A F=05$ & 500 \\
\hline 84 & $7+39$ & $2.7 k 2[=.74$ & $0,9135=0 \mathrm{~B}$ & 500 \\
\hline ré & 1389 & $9.559 \mathrm{t}-.97$ & $x, \cap A_{1}=06$ & $S 00$ \\
\hline 0.4 & $2+2$ & 1. AA 7 E $=17$ ? & $0.7035=0 ?$ & 500 \\
\hline $9+, 0$ & $3 \times 3$ & $2.7565=17$ & $0.9235=07$ & 500 \\
\hline 150.0 & 1035 & 7. य小 DE $=0$ ? & $2.682 f+6 h$ & 500 \\
\hline 104.0 & $1: 57$ & $7.45 .40=137$ & $2.73 \mathrm{FF}=06$ & 500 \\
\hline $1 \cup^{P} \cdot 0$ & 1157 & A. $?+\cap \mathrm{FF}=? 7$ & . $\triangle Q$ पE $-\pi B$ & 500 \\
\hline 112.0 & 1594 & $1.1475-i 16$ & $4.1295=n 6$ & 500 \\
\hline 116.0 & $1 \div 79$ & $1.4 \cap P F=3 \alpha$ & $5 . \cap A R E-06$ & 500 \\
\hline .0 & $10 \times 4$ & k.tif 1 - T & 75 AE $=00$ & 500 \\
\hline
\end{tabular}

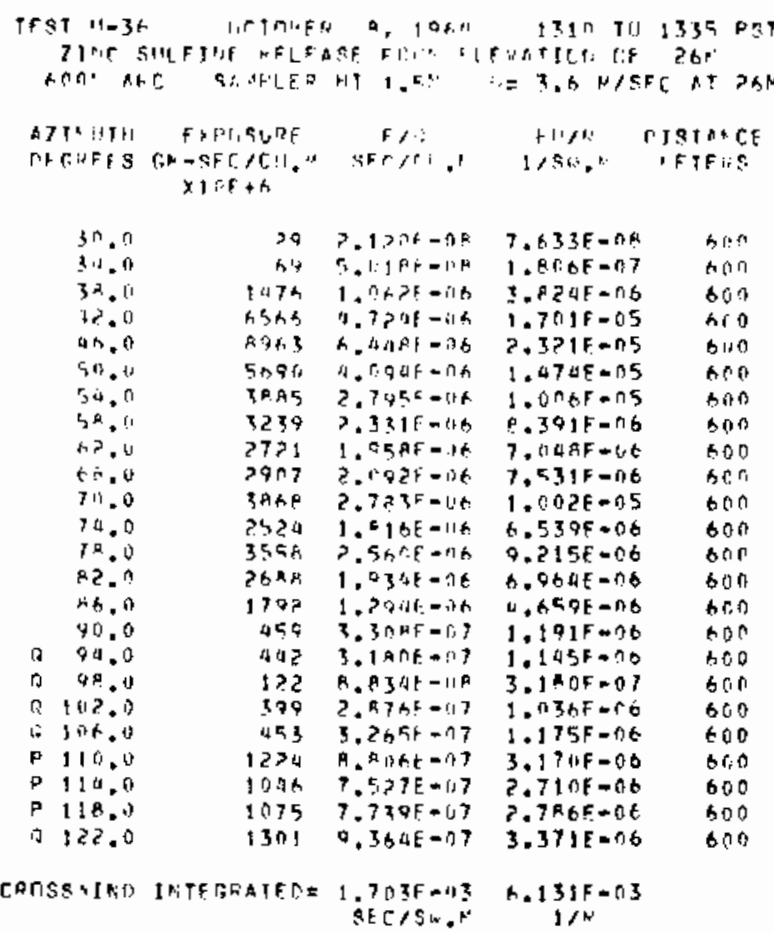

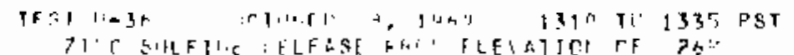

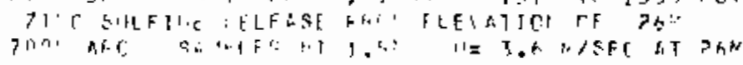

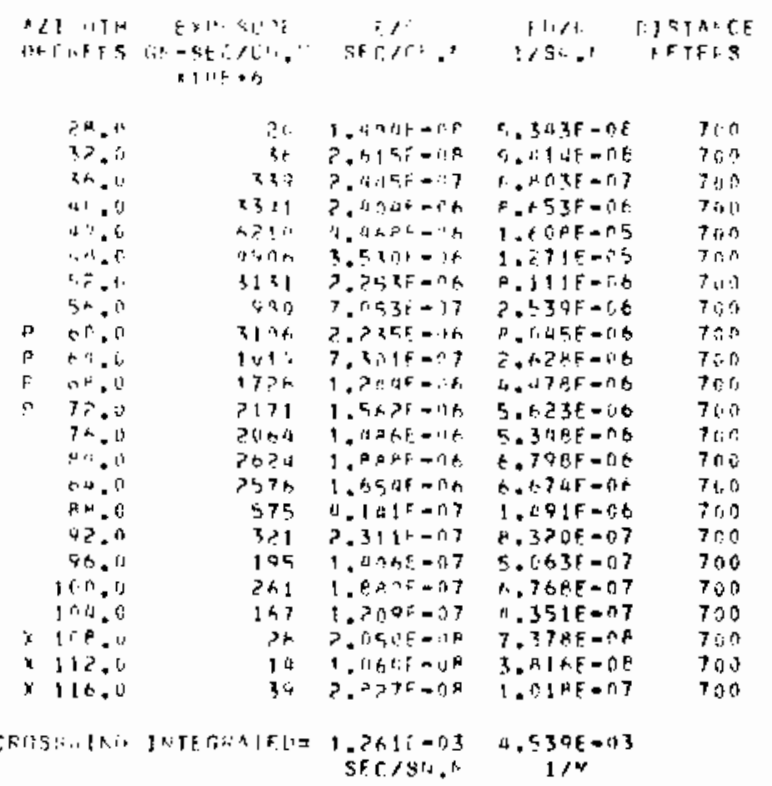




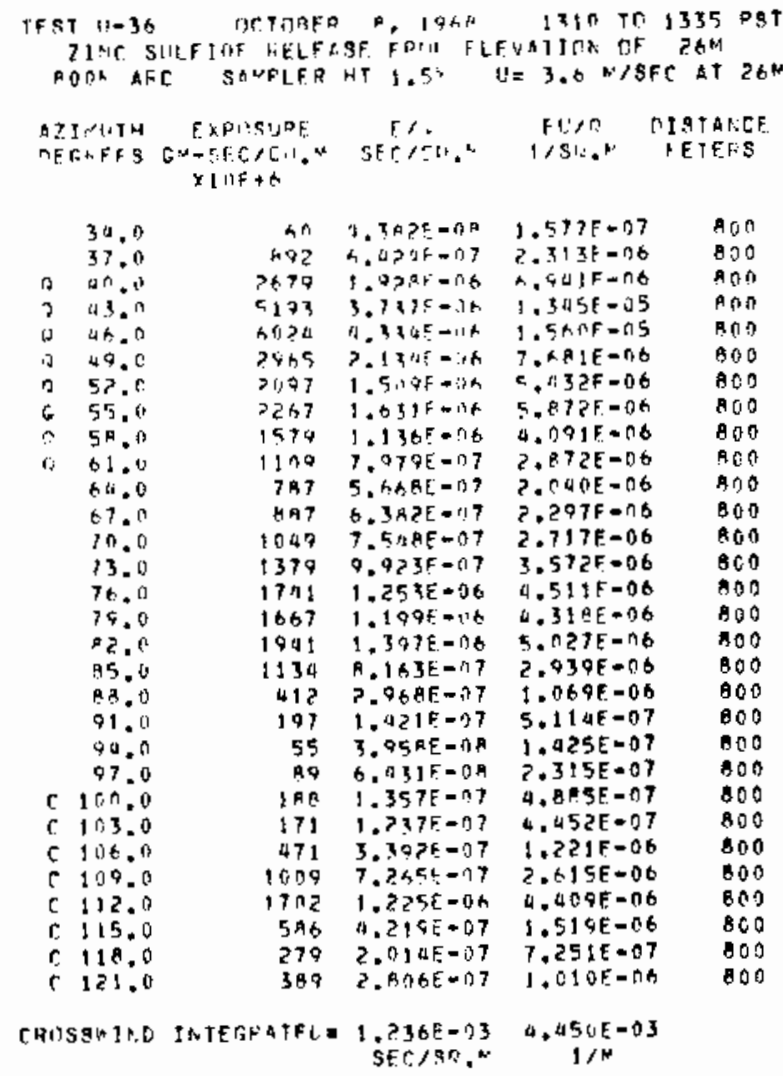

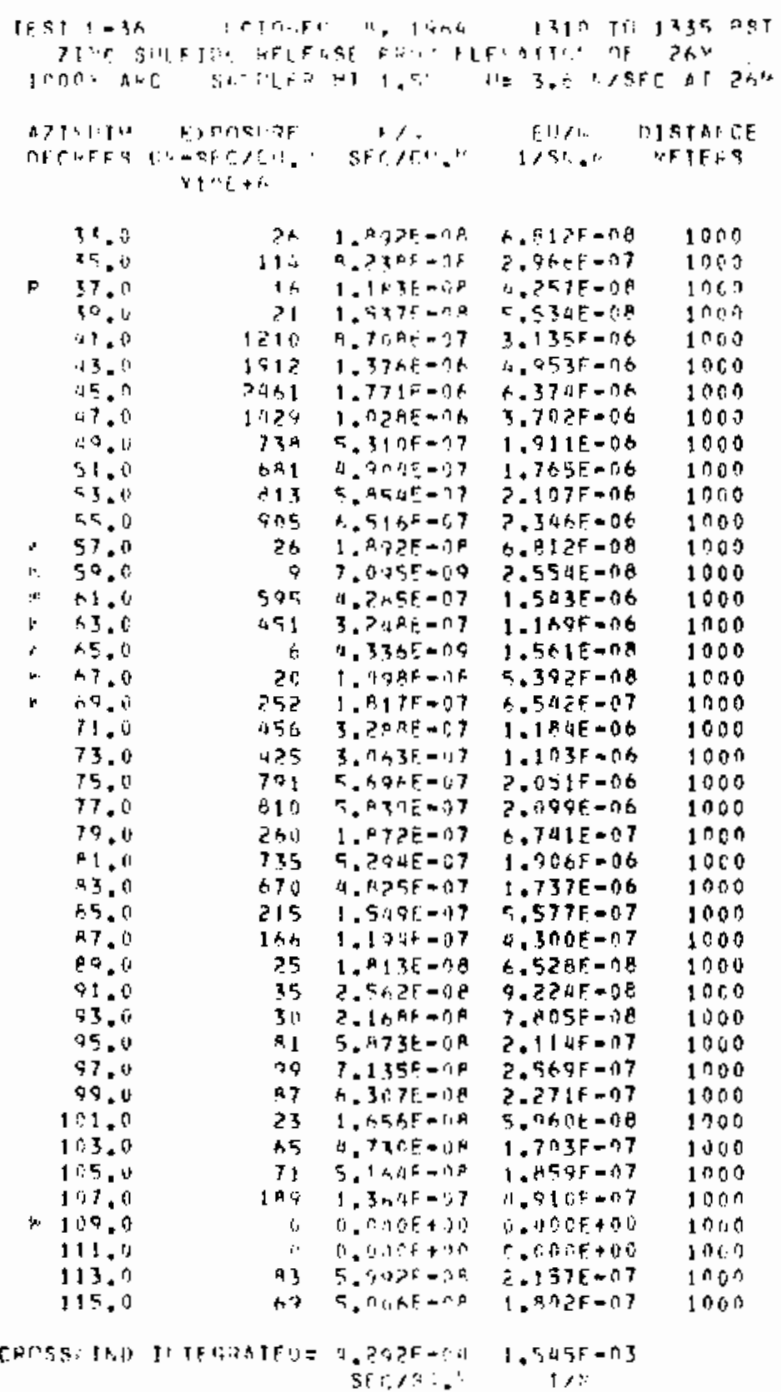




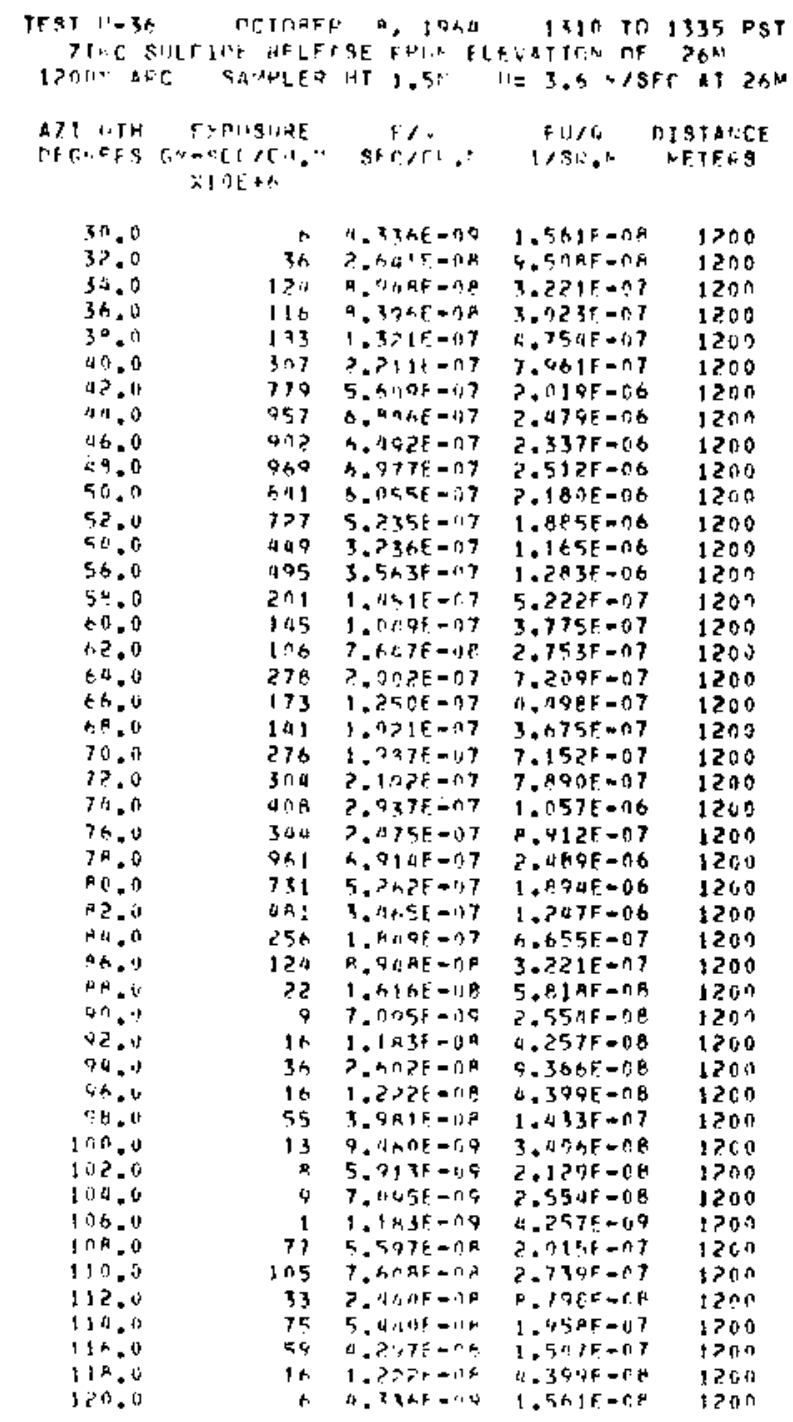

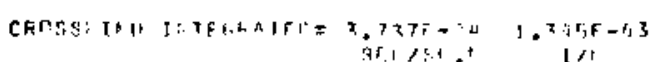

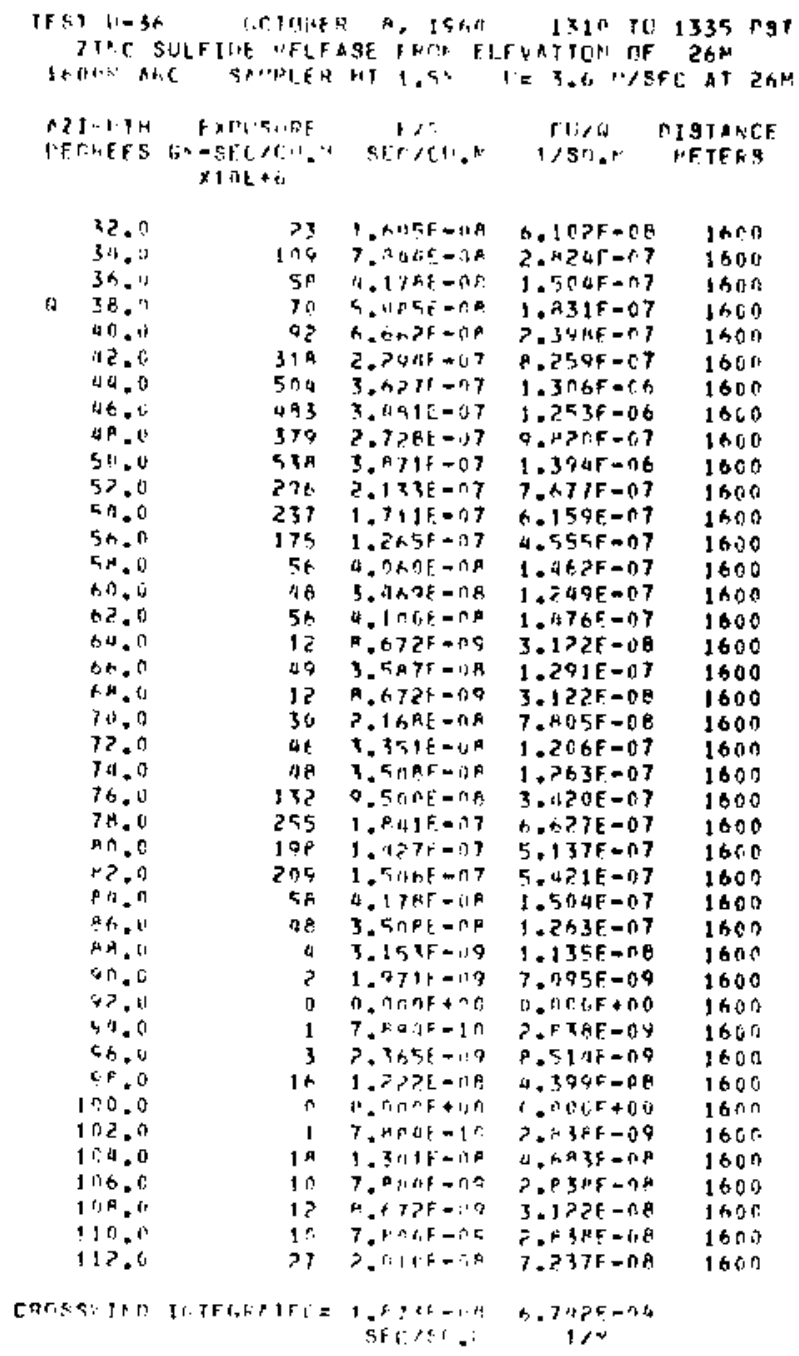


UNSTABLE SERIES EXPERIMENT: 37 ( U-37)

GROUND LEVEL SAMPL ING 100-3200 M ARCS. FOR EASE IN STATISTICAL ANALYSIS, AEL SAMPLER AZIMUTHS HAYE BEEN INCREASED BY 30 DEGREES FPOM THEIR ACTUA POSITIONS THE TRACER WAS NOT DETECTED ON THE 100 OR 200 M ARCS. SOME FILTERS KERE ONLY EXPOSED FOR A SHORT PERIOD OF TIME.

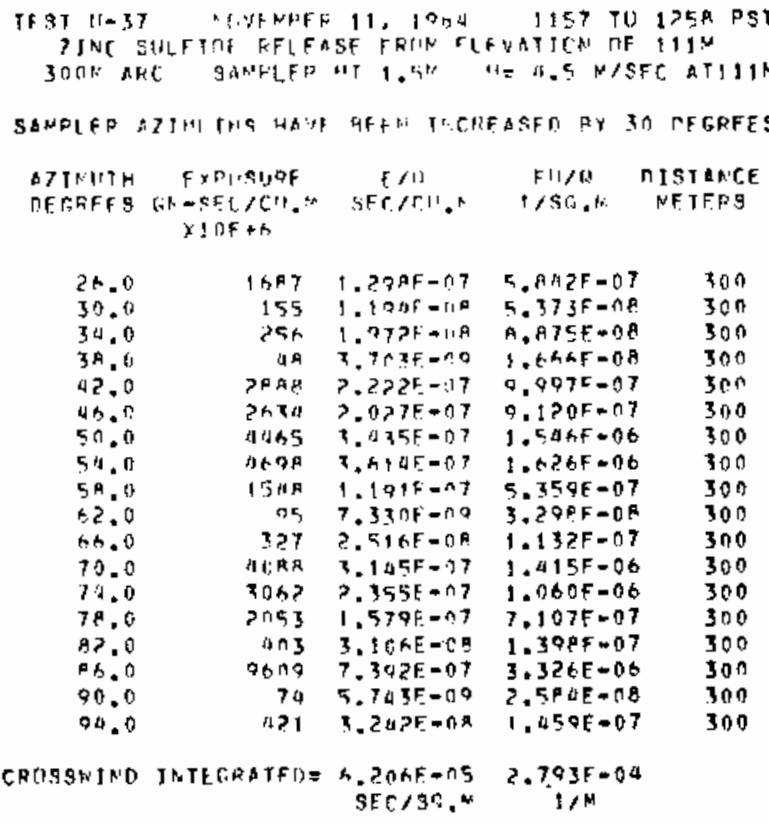

TEST 11037 NUVFMER 11, 10KU 1157 TO 1?5A PST

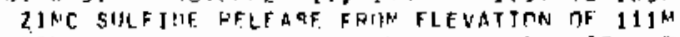

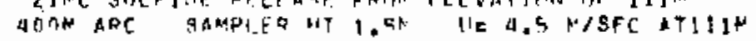

SANPLFR AZIMIIHQ HAVE HEFA INCRFASFD PY 3 T DEGFEFS

\begin{tabular}{|c|c|c|c|c|c|}
\hline \multicolumn{2}{|c|}{$\begin{array}{l}\text { AZINHITH } \\
\text { DEFFEES }\end{array}$} & $\begin{array}{c}\text { EXO1JSUDE } \\
\text { GHOSEC/CII.N } \\
\times 1 O F+6\end{array}$ & $\begin{array}{c}E / B \\
\text { SECCR!I } N\end{array}$ & $\begin{array}{l}f(1 / 1) \\
1 / 86.2\end{array}$ & $\begin{array}{l}\text { CISTANCE } \\
\text { NFFPS }\end{array}$ \\
\hline & $\begin{array}{l}20.0 \\
28.0 \\
32.0 \\
36.0 \\
40.0\end{array}$ & $\begin{array}{r}1294 \\
16109 \\
9205 \\
2233 \\
1784\end{array}$ & $\begin{array}{l}9.937 F-O A A \\
1.242 F-0 A \\
7.150 E-07 \\
1.712 F=07 \\
1.373 F=637\end{array}$ & $\begin{array}{l}4.472 F=07 \\
5.500 F=06 \\
3.2105=06 \\
7.733 E=07 \\
5.170 E=07\end{array}$ & $\begin{array}{l}400 \\
400 \\
000 \\
400 \\
000\end{array}$ \\
\hline D & $\begin{array}{l}49.0 \\
46.0 \\
52.0 \\
56.0\end{array}$ & $\begin{array}{r}0 \\
4008 \\
1835 \\
2536\end{array}$ & $\begin{array}{l}0.0 \cap \cap 5+00 \\
3.11 \Delta F=77 \\
1.41 \partial F=11 ? \\
4.9 n \Delta F=07\end{array}$ & $\begin{array}{l}0.000 F+00 \\
1.401 F=06 \\
6.352 F=07 \\
0.746 F=07\end{array}$ & $\begin{array}{l}400 \\
400 \\
000 \\
000\end{array}$ \\
\hline & 60.0 & $\begin{array}{l}2390 \\
4997\end{array}$ & 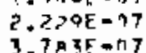 & $1.003 F=06$ & 400 \\
\hline & $\begin{array}{l}68.0 \\
72.0\end{array}$ & $\begin{array}{l}2745 \\
\operatorname{nin} 79\end{array}$ & $\begin{array}{l}2.112 F=07 \\
5.221 E=17\end{array}$ & $\begin{array}{l}9.5045=07 \\
2.3495=06\end{array}$ & $\begin{array}{l}\text { aOn } \\
\text { a0n }\end{array}$ \\
\hline & $\begin{array}{l}76.0 \\
80.0 \\
14.0 \\
89.0\end{array}$ & $\begin{array}{l}5517 \\
5391 \\
1241 \\
2 \times 3\end{array}$ & $\begin{array}{l}1.2 A 7 E=07 \\
4.1 \text { AOE }=07 \\
0.552 E=18 \\
1.564 E=08\end{array}$ & $\begin{array}{l}1.930 E=06 \\
1.863 E=06 \\
4.298 E=07 \\
7.039 E=08\end{array}$ & $\begin{array}{l}000 \\
400 \\
400 \\
900\end{array}$ \\
\hline D & $\begin{array}{r}92.0 \\
96.0 \\
100.0 \\
104.0\end{array}$ & $\begin{array}{l}35 \\
35 \\
48\end{array}$ & $\begin{array}{l}0.000 E+00 \\
? .720 E=09 \\
2.191 E=09 \\
3.703 E=09\end{array}$ & $\begin{array}{l}0.000 E+00 \\
1.22 A E=00 \\
9.061 E=09 \\
1.666 E=00\end{array}$ & $\begin{array}{l}400 \\
000 \\
400 \\
000\end{array}$ \\
\hline & 6 & EGRATFD & 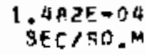 & $\begin{array}{c}B .670 E-0.4 \\
1 / \mathrm{M}\end{array}$ & \\
\hline
\end{tabular}

TEST H-37 ILIERAEN 11, 1964 1157 TO $125 \mathrm{~A}$ PST 7 INC SUEFIDF BFLFASE FACIN FLEVATIMN DF $111 \mathrm{M}$ SOON ARE $3 \mathrm{AMPLFO} H \mathrm{HT} 1.5^{\mathrm{M}}$ Ii= $0.5 \mathrm{~N} / \mathrm{SEC}$ ATI11M

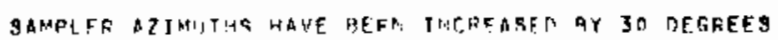

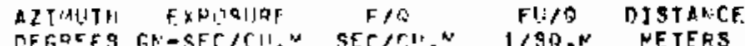

$$
\begin{aligned}
& \rightarrow i n+6
\end{aligned}
$$

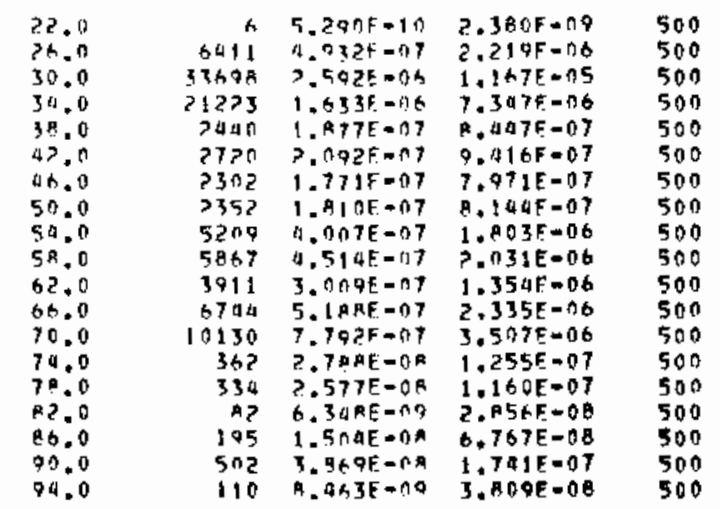

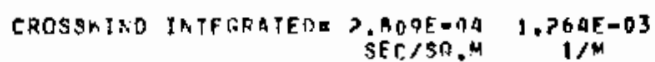

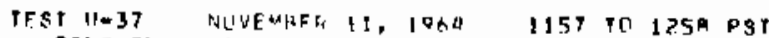
7 INT. SULTIJE RELFASE FROF FLFVATITA OF IIIM toON AFC SAMQLER HT $1.5 \mathrm{~V} \quad \mathrm{H}=4.5 \mathrm{M} / \mathrm{SEC}$ ATIIIM SAMPIFP AZIBIITHQ HAJE FEFN TNCAFASFT aY 30 DEgREEG

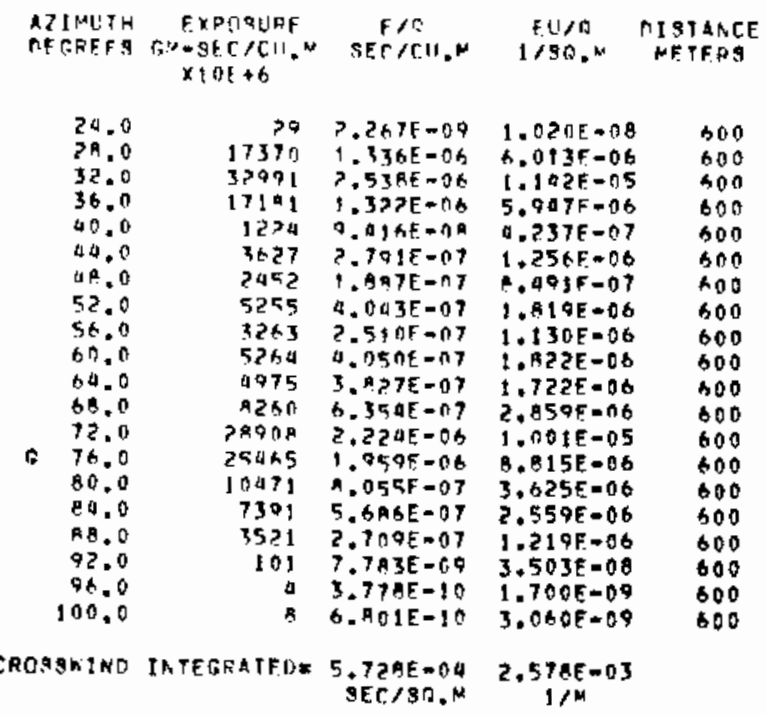




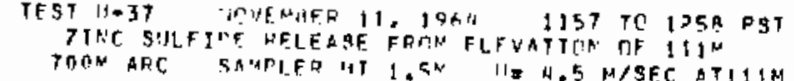

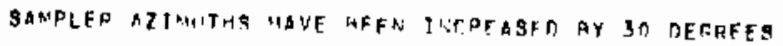

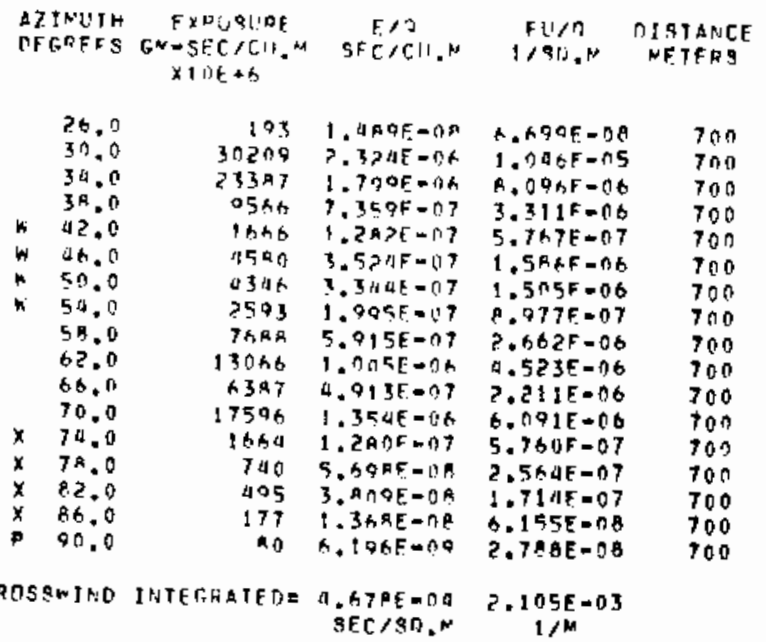

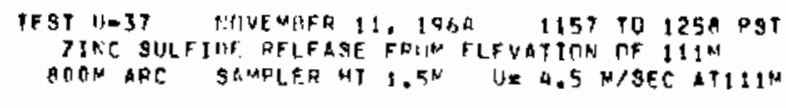

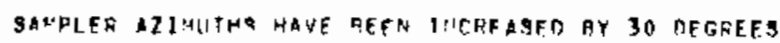

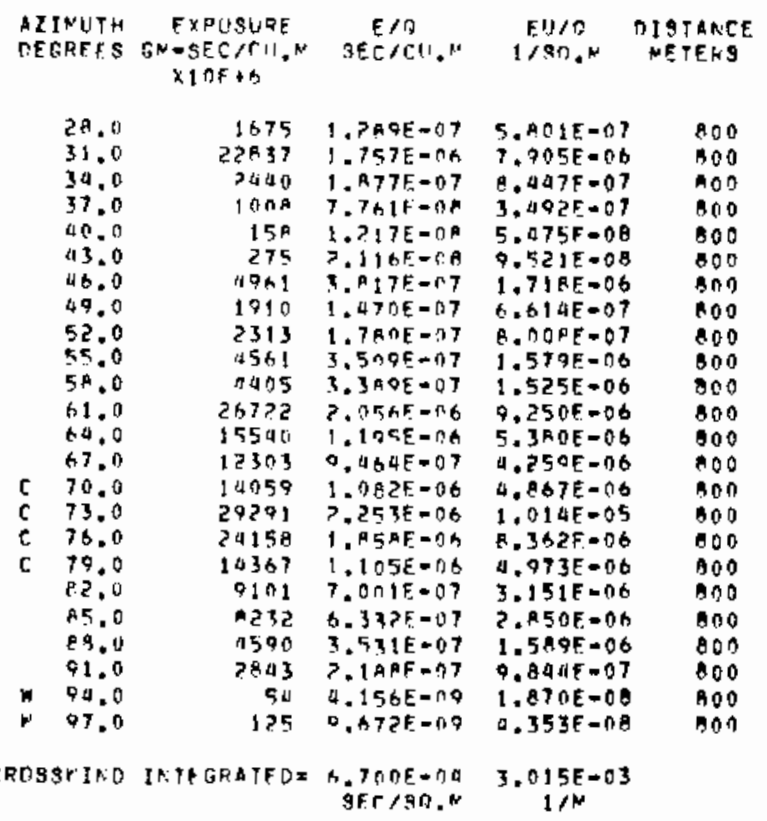

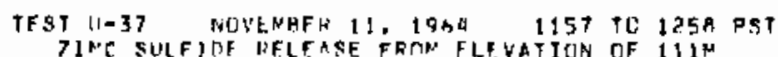

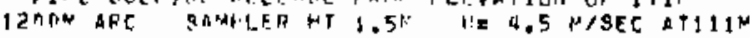

SAMPLFT MZINUITHA HAVE GEEN THERFAGET PY 30 DEGKEES

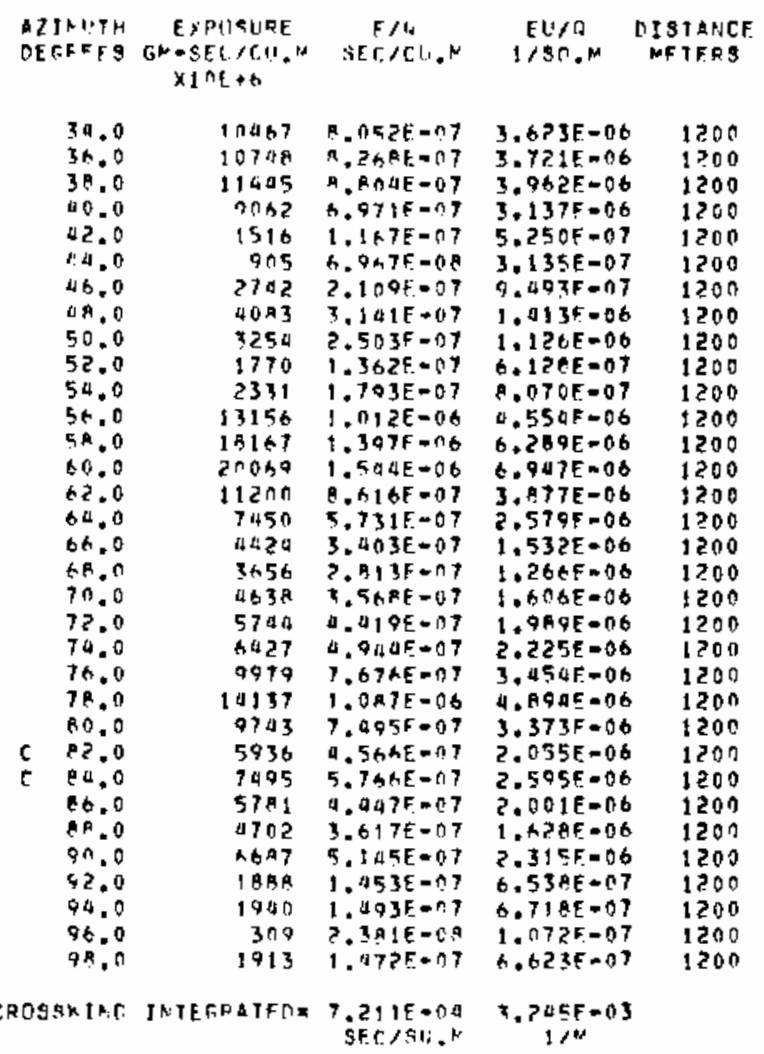




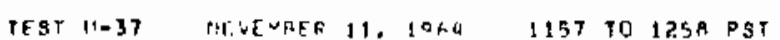

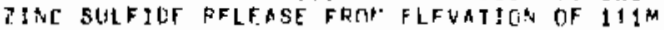

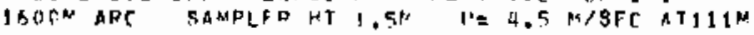

SANPLER AZIMLTHS MAVE PEFM JNTREASFM FY 30 DERREES

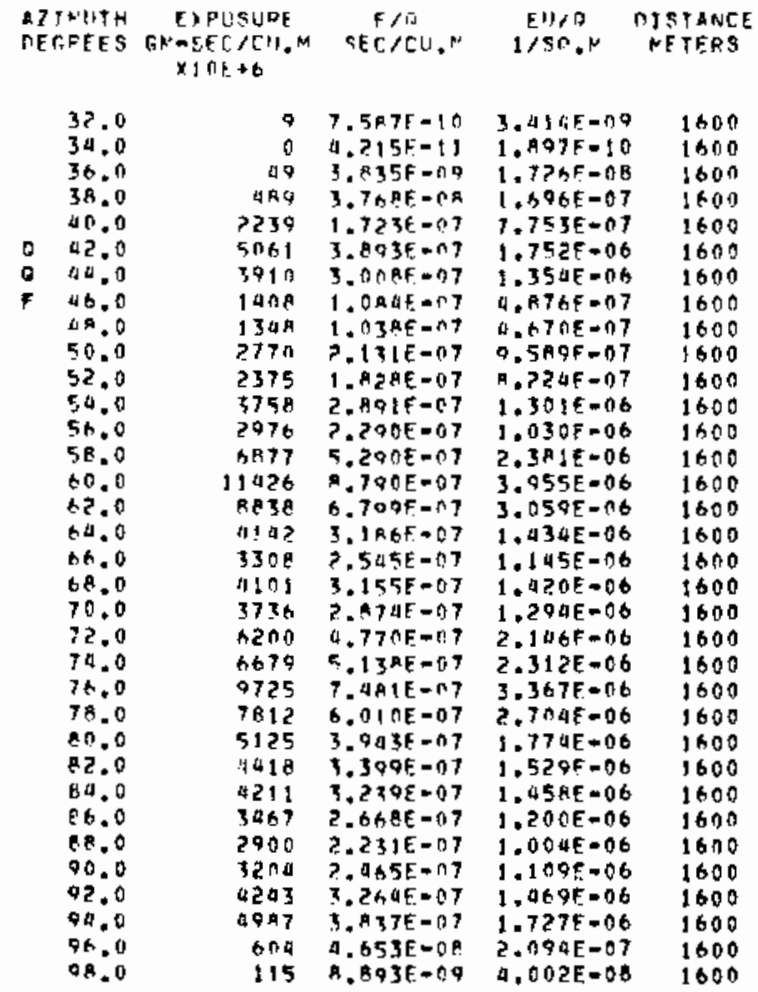

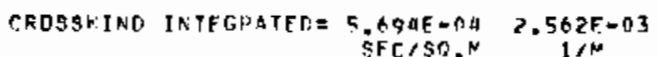

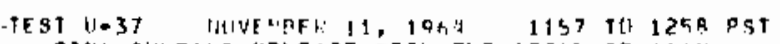

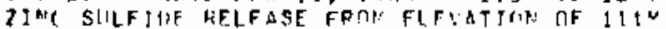
3 POON SFC SAMPLEP HF $1.5 \mathrm{~N} \quad 11=4.5 \mathrm{~N} / \mathrm{SFC} \mathrm{ATIIIN}$

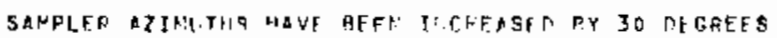

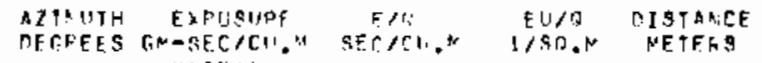
$x$ Int th

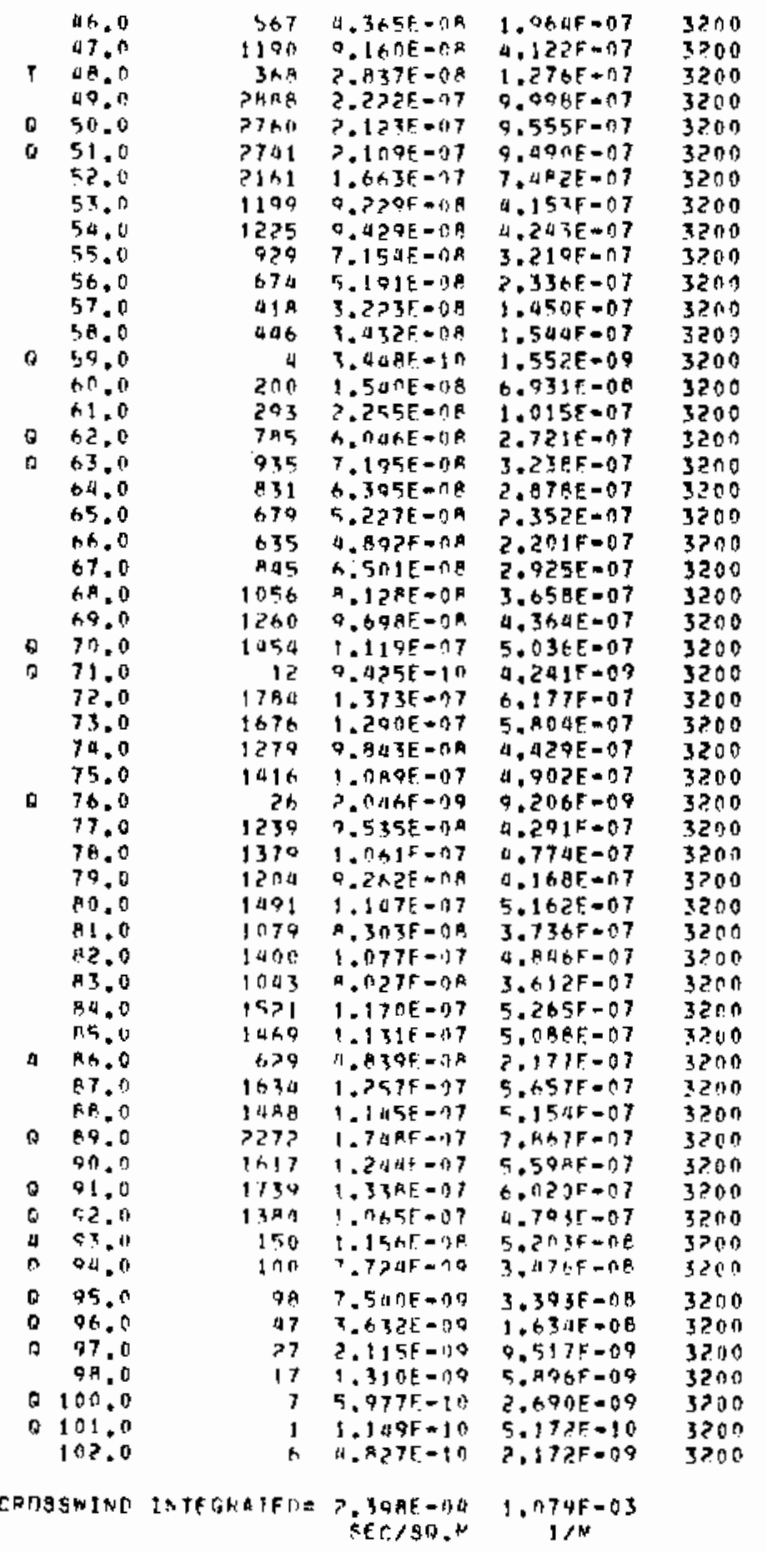


UNSTABLE SERIES EXPERHMENT: 38 (U-3B)

GROUND LEVEL SAIPLING 100-1600 ARCS.

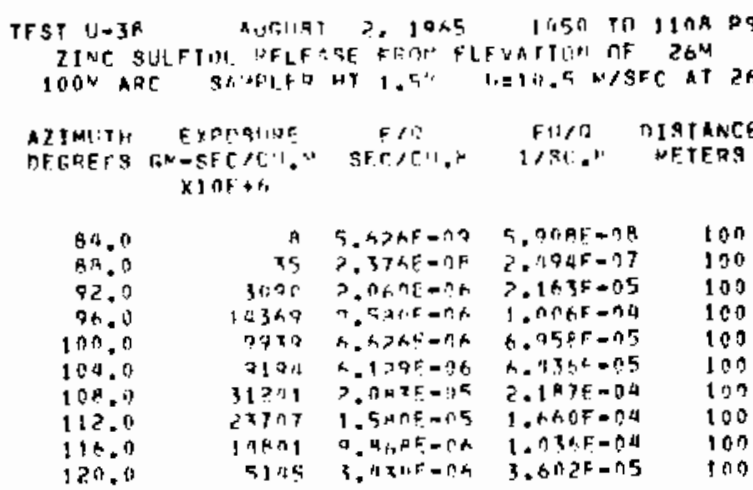

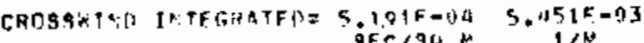

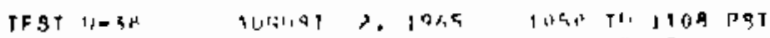

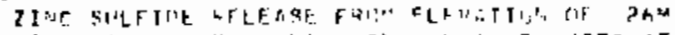

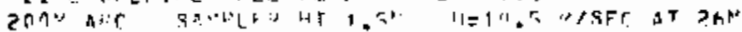

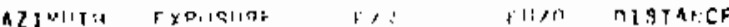

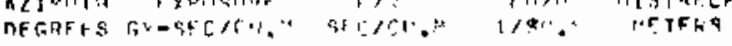
$x \operatorname{lig} r+h$

\begin{tabular}{|c|c|c|c|c|}
\hline 94. 11 & $+5: 4$ & 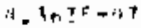 & $\|, 5 R>P=06$ & $2+1 n$ \\
\hline 90,0 & 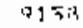 & $A, 1$ is $5=3 A$ & $n, \$ 1, F=n 5$ & מח? \\
\hline 94.6 & $4>737$ & $3.7 \div 05=04$ & $3.978 \mathrm{~F}=30$ & 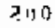 \\
\hline จP. & $3<0 \leqslant 3$ & $? .4 x+5=145$ & P. $\left.5 a_{1+t}-1\right) 4$ & 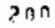 \\
\hline $10 ? .0$ & $=1 \times 390$ & 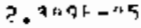 & $3+1 E=04$ & $2 \pi n$ \\
\hline $10 t .4$ & $n>005$ & $9 s, 4 \leq-115$ & $=0$ & कृत \\
\hline 110,5 & ג & $A_{i}>\mathrm{F}-2 \times 5$ & $n z F=04$ & $20 n$ \\
\hline & $3+107+1$ & $J>2>15$ & $535=114$ & $30 n$ \\
\hline 112.6 & itang & $1.1705-.95$ & $1 . A B A F=n U$ & 300 \\
\hline 122.11 & 15396 & $1.11>0.5-1) 5$ & $1.47 \mathrm{AF}=0 \mathrm{~A}$ & מת ? ? \\
\hline
\end{tabular}

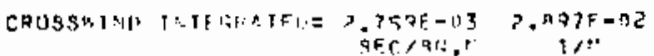

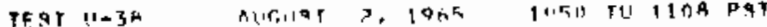

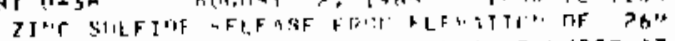

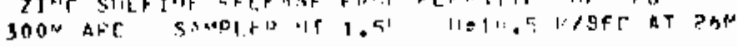

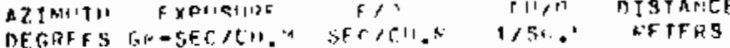
$x \perp \cap F+6$

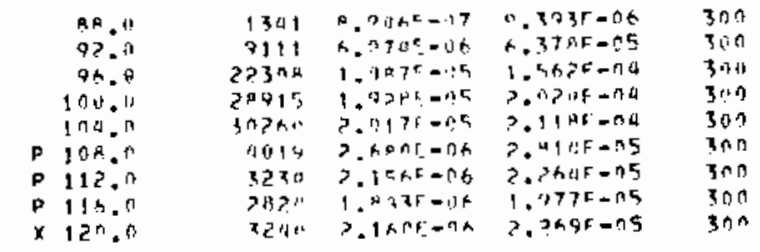

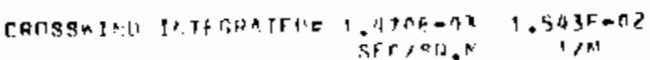

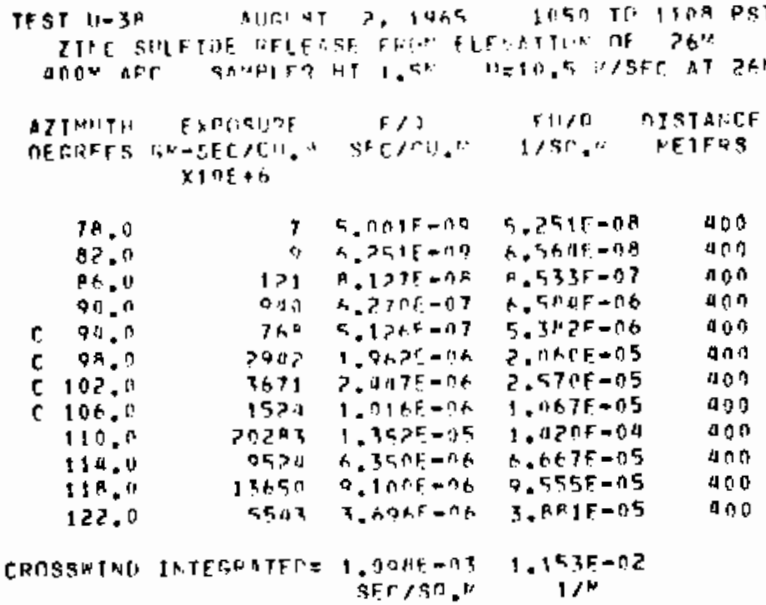

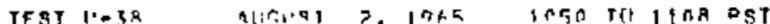

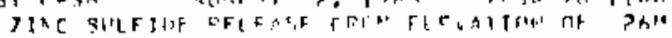

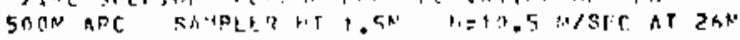

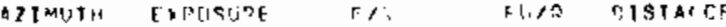

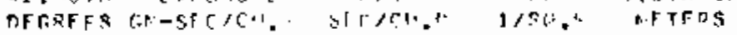
iा $10+h$

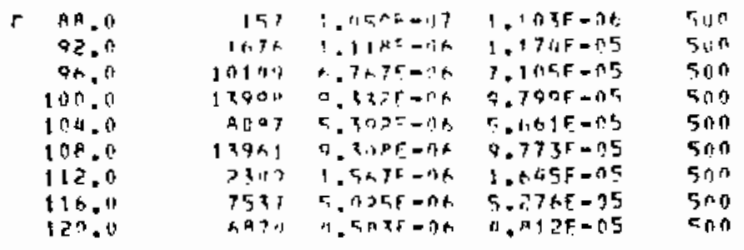

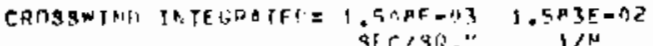

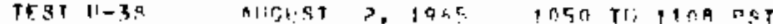

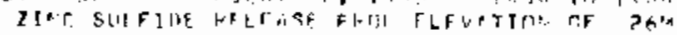

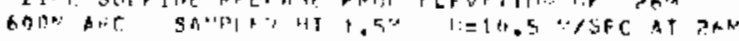

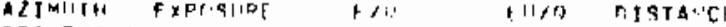

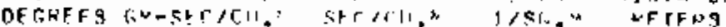
$\times 10 \mathrm{OH}$,

\begin{tabular}{|c|c|c|c|c|}
\hline 90,0 & 58 & $x, 0 \geq 5 r=18$ & $0.135 \mathrm{~F}+17$ & hND \\
\hline 94.0 & 6112 & 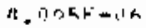 & $=.3 n \cup F=n S$ & 600 \\
\hline Q日.: & $111 \mathrm{A1}$ & 7.4 यन $=0 \mathrm{~A}$ & $? .+13 F=0 \mathrm{~S}$ & sin \\
\hline $1 \cap 2$. & RQ, 5 & $5.177 k F-17 h$ & t.?t if $=105$ & $\operatorname{sno}$ \\
\hline 106.0 & huks & $\eta n+3 F=3 h$ & $1.74+5 \cdot 05$ & $h 00$ \\
\hline 119.0 & 77712 & $4.1+4+193$ & $5.4055-05$ & 600 \\
\hline 114.0 & $4 \mathrm{bt}: \mathrm{M}$ & A. $5 \times 5 \%=0 x$ & $0.700 \mathrm{~F}=05$ & sol \\
\hline 11 年 & the & $? .9065=n A$ & $3.135 F=05$ & b) 0 \\
\hline 127.1 & 236 & $1.5 \mathrm{~A} 0 \mathrm{E}=n 7$ & $1.6 \triangle A F=06$ & 600 \\
\hline
\end{tabular}

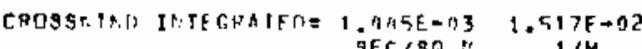




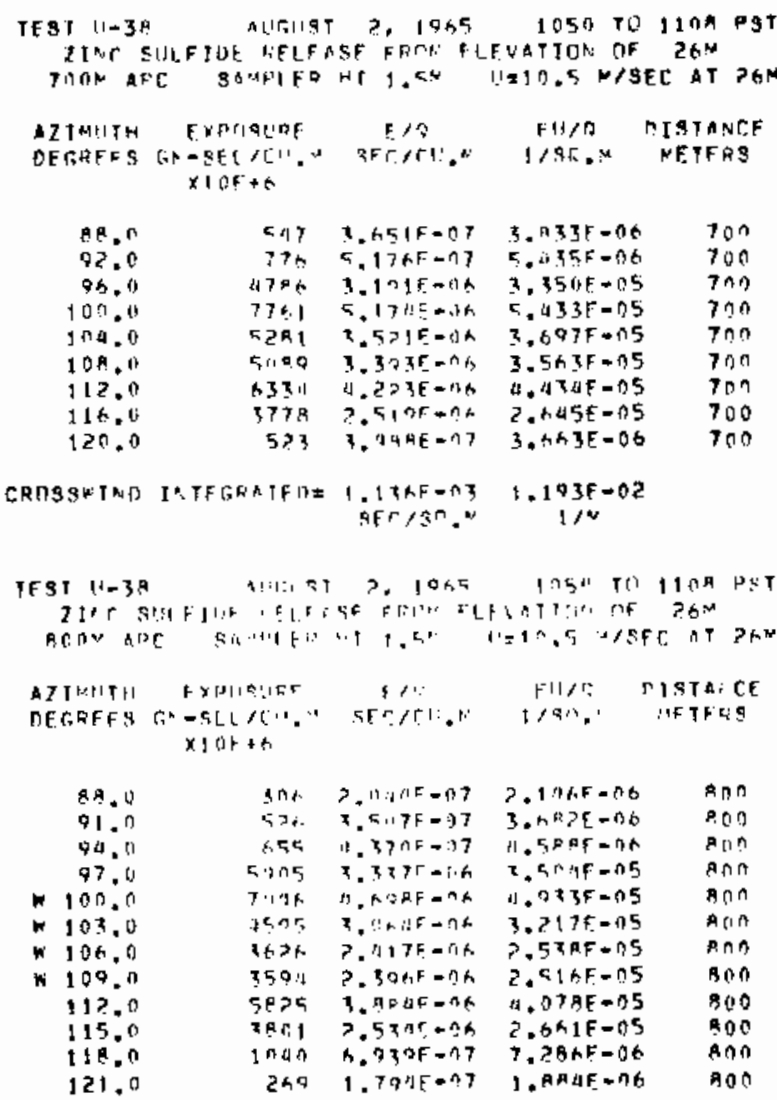

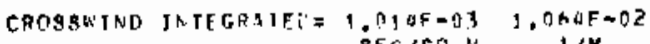

TEgT 11-38 ALIIIST $?$, 1965 1050 TO 1109 PST ZINE SULFIIIE LFLFLAT. FRIIN FLEUATIOI! TF $26 \mathrm{H}$

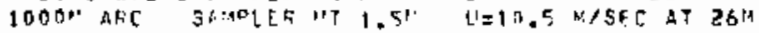

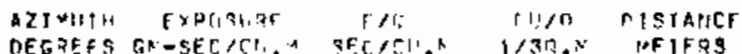

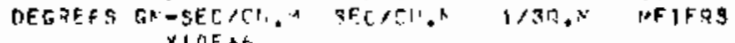

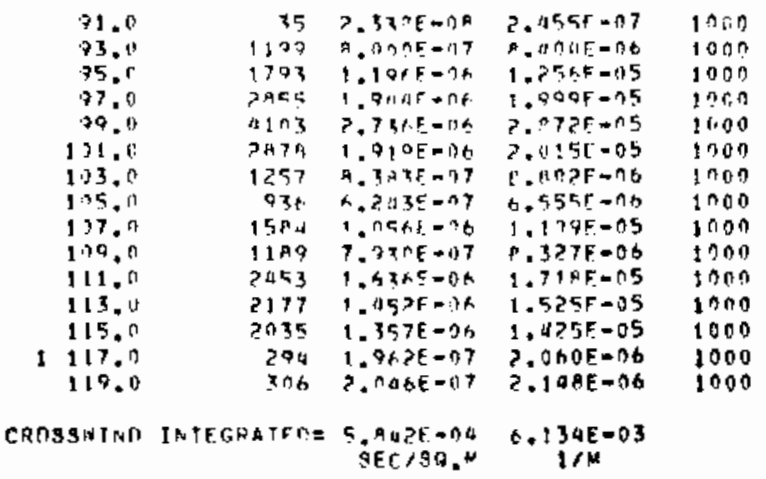

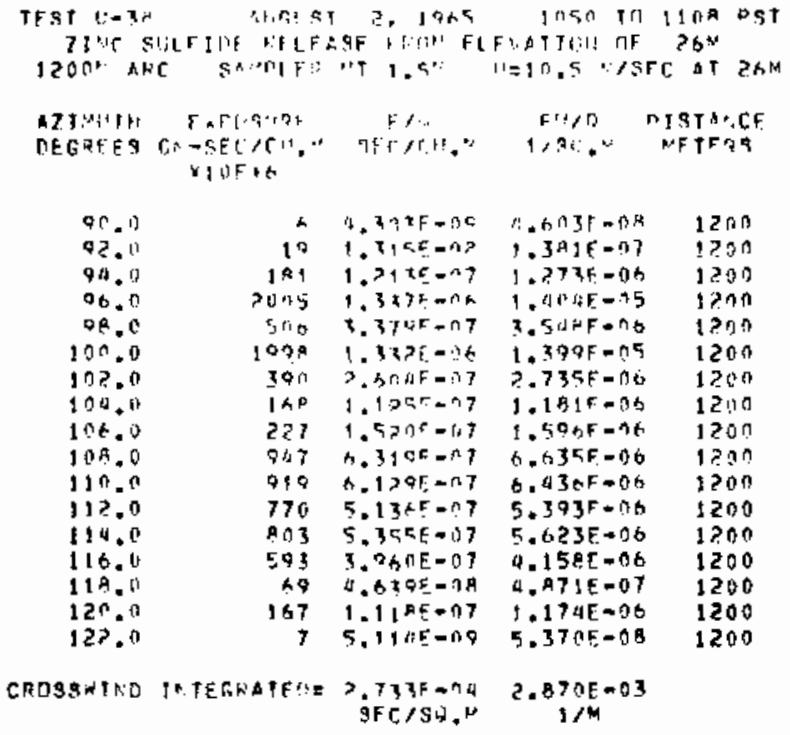

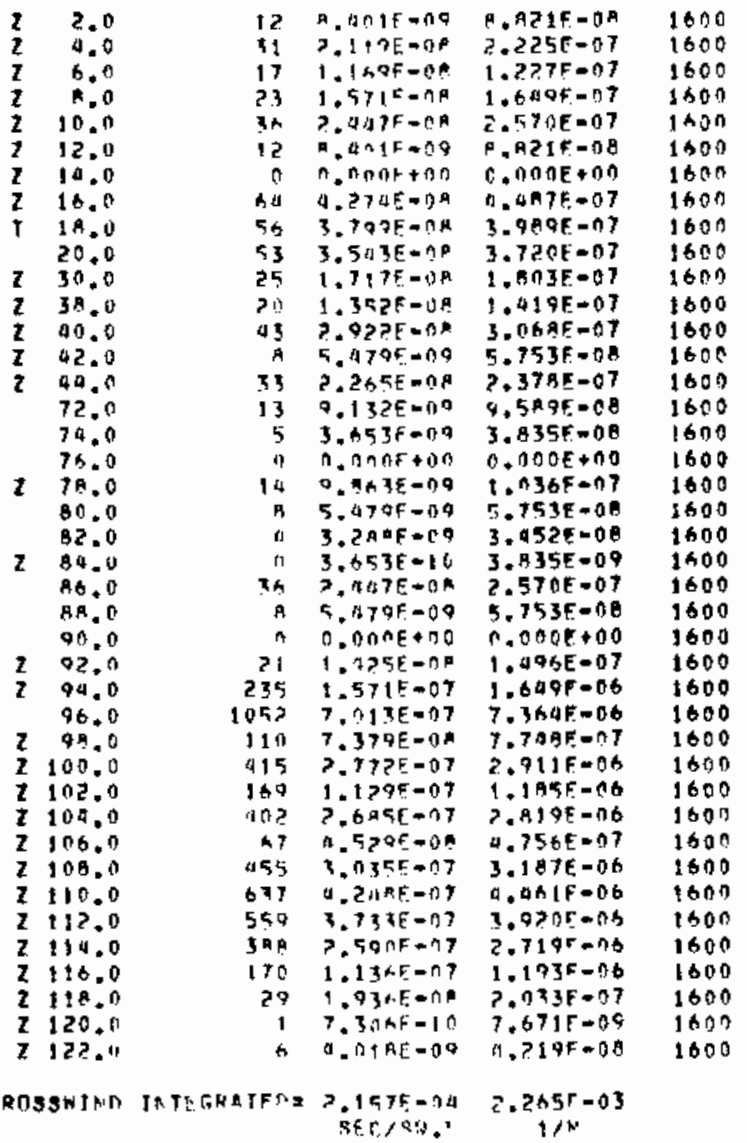


UNSTABLE SERIES EXPERIMENT: 39 ( J-39)

GROUND LEYEL SAMPLING 100-1500 M ARCS.

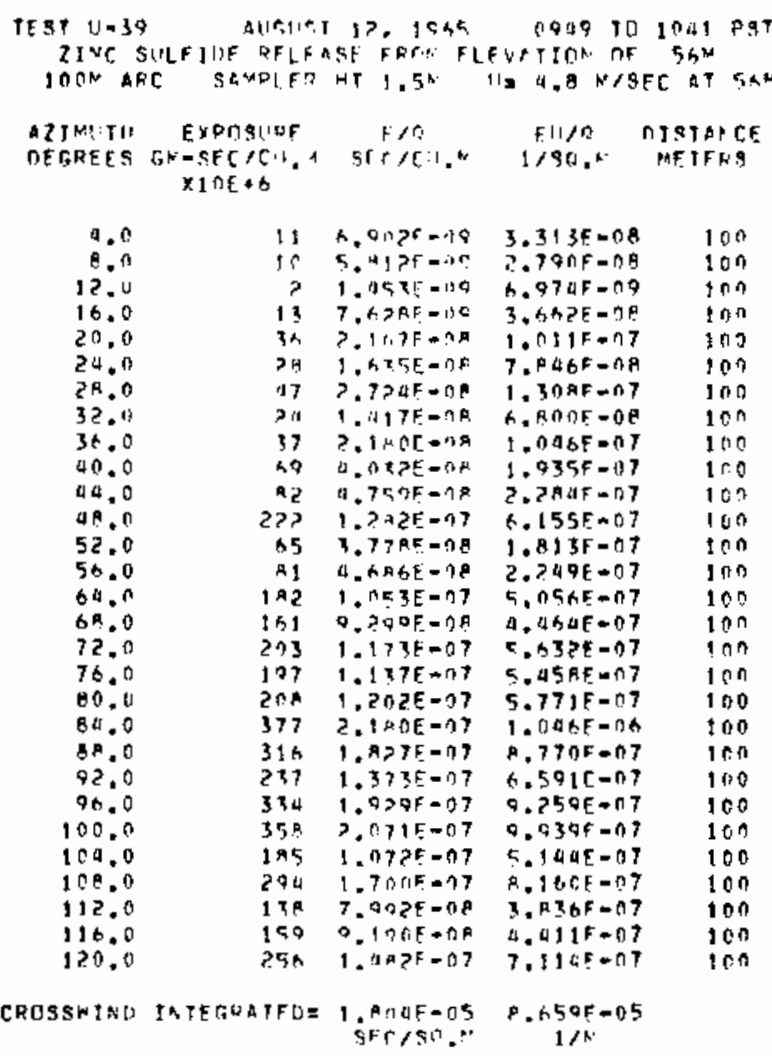

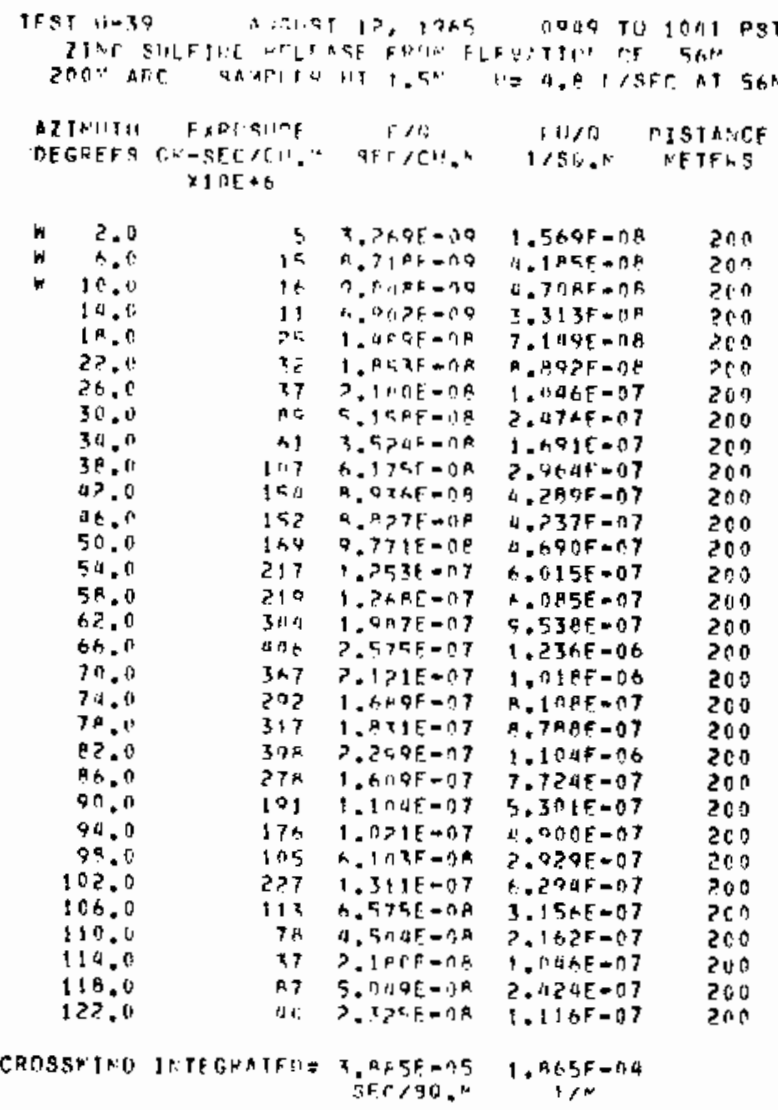




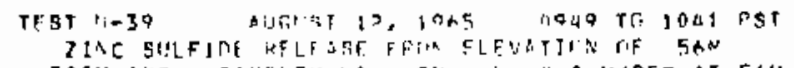

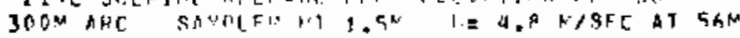

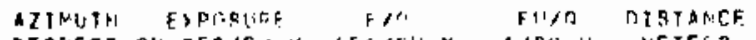

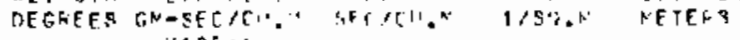
$x 10+4$

\begin{tabular}{|c|c|c|c|}
\hline 12.8 & 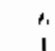 & $\begin{array}{l}1.4 \times 5 t-i, 0 \\
1000=40\end{array}$ & $\begin{array}{l}1.14 \Delta F=\cap 4 \\
5 .>21[=-1]\end{array}$ \\
\hline 20.0 & $\gamma$ & A. 3रSE= & פ \\
\hline 24,0 & 24 & $1.17 f=ก A$ & 6. $H C O F-O A$ \\
\hline 28.0 & 110 & $6.201 F=140$ & $3 . \therefore+95=07$ \\
\hline 32.1 & $11 \%$ & 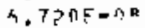 & $3 . ? 2+5=07$ \\
\hline 36.6 & 11 i & 6.7 的 & $3 . P 3+F=: 7 ?$ \\
\hline 40.0 & $\$ 11$ & ค. $ว * \cap 5=0 P$ & $3+n B+F=07$ \\
\hline 44.0 & 151 & $D, T: \Delta f=\cap A$ & 4. $\vec{P}+\hat{c} t=07$ \\
\hline 48.0 & $2 \cap 3$ & $1.1>35=017$ & $5.63 z F=07$ \\
\hline 52.0 & 159 & $8.704 E=1.8$ & $4.202 E-07$ \\
\hline 56.0 & 258 & 1. $4 k>E-n, 7$ & $7.114 F=07$ \\
\hline 60.0 & Zn ب & $551 F=47$ & 7. A4 $45 F=17$ \\
\hline OR. & Pי & $1.2759=07$ & $6.126 E=07$ \\
\hline $7 ? .9$ & $2 \times 2$ & $1,3,8 E+0 ?$ & c. $469 F=01$ \\
\hline $7 h, 0$ & 508 & $731 E-197$ & $1 . \Delta 07 E=06$ \\
\hline e. 0 & 335 & $1.9 x+F-07$ & $9.793 F-17$ \\
\hline$(0.1), 0$ & 256 & $1.4 T A E=M T$ & $7.077 E=07$ \\
\hline R. .0 & 10.3 & $5.957 \mathrm{~F}=19.8$ & ?. $=$ पh \\
\hline 92.0 & 125 & $7.2>9 E-08$ & $3.07 \cup f=07$ \\
\hline 06.0 & 711 & 4. $2 \mathrm{HBF}=0 \mathrm{~A}$ & $2.057 t-0.7$ \\
\hline 100,0 & A 6 & $5, n \vee \in E=0.0$ & $2.451 t=07$ \\
\hline 104.0 & op & $5 .+a 7 f=0 ?$ & $2.7 \geqslant 0.5=07$ \\
\hline 100.0 & 117 & h. $75, A E=J R$ & $3,243 E-07$ \\
\hline 112.0 & 100 & $5 . A 12 E=0 A$ & $2.790 F=07$ \\
\hline 16.0 & ${ }^{2} 1$ & $4.6 \times \mathrm{BE}-\mathrm{OAP}$ & $2.209 \mathrm{~F}-07$ \\
\hline & 52 & {$[1 F-3 f$} & $\triangle A 5 E=07$ \\
\hline
\end{tabular}

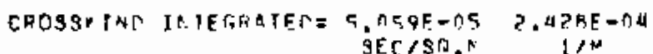

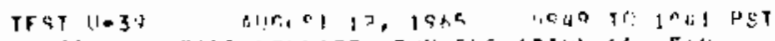

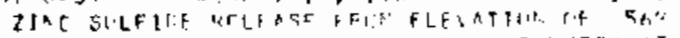

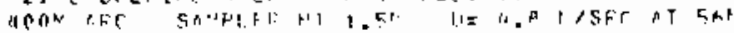

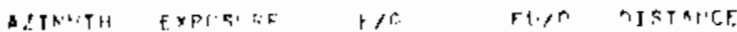

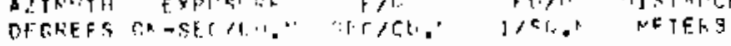
X $j \xi 1 F+\div$.

\begin{tabular}{|c|c|c|c|c|}
\hline 1 ค. 9 & n & $5 . r i r+f=05$ & 2. iक $1 F=08$ & 469 \\
\hline 27.4 & t & $3,4+A-=10$ & $\begin{array}{l}1 . \angle 1 P E=0 B \\
19 F E=07\end{array}$ & $\begin{array}{l}400 \\
400\end{array}$ \\
\hline 26.0 & $\begin{array}{l}14 \\
7,2\end{array}$ & $\begin{array}{l}3 A H F-10 \\
M E D F-N P\end{array}$ & $\begin{array}{l}1.19+5=07 \\
1.5+F=07\end{array}$ & $\begin{array}{l}400 \\
400\end{array}$ \\
\hline $3 n \cdot 0$ & 135 & 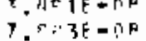 & 3.7 A $1=07$ & $40 ?$ \\
\hline 38.0 & 117 & $h, r, r E-O P$ & $3.121 F=1.7$ & 400 \\
\hline 42.0 & $1+5$ & व. $40 \mathrm{~B}=0 \mathrm{~A}$ & $7.5335=07$ & 000 \\
\hline 46.0 & 251 & $1.2475=07$ & $A=416 F=07$ & 400 \\
\hline 50.0 & $3 n_{4}$ & $1.736 F-.97$ & 0.33 d $=07$ & J6n \\
\hline 54.0 & 193 & 的, 537 F $-\mathrm{FA}$ & $3.1345=07$ & 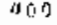 \\
\hline 58.0 & $2: 7$ & $1.0>P 5-\pi 7$ & t. . $+5 E=0.7$ & $40 . n$ \\
\hline +2. 0 & 1511 & $5.6 P ? F=0$ P & $4.167 F_{-}-n 7$ & $a 0 n$ \\
\hline 60.0 & 1 st & $x .277 t-0 x$ & il $0: 055-07$ & $4 \cap 0$ \\
\hline & $15 t$ & $0.150 F=0$ ? & $4.394 \mathrm{E}=07$ & 400 \\
\hline 74.0 & $1 \times 1$ & $5, \therefore H R E-C^{\circ}$ & $? .207 F=07$ & $4 c^{n}$ \\
\hline $7 x \cdot 0$ & 123 & $7.9106=0 R$ & $3.0015=07$ & 400 \\
\hline A2. & 115 & $A, A^{A} E=0 A$ & $3.191 \mathrm{E}=0 ?$ & $a 0 n$ \\
\hline 86.0 & $1+H$ & $9.509 E-48$ & $4.0555=.77$ & 450 \\
\hline 90.0 & का? & $=x \times 35=0,8$ & $1.7 \Delta \Delta \mathrm{F} \cdot 07$ & 400 \\
\hline 94.0 & $\gamma$ & $4,35,75=1: 9$ & $2.1+92 E-0 B$ & 400 \\
\hline 98.0 & 53 & $1.12 K A E=08$ & $1.4 \mathrm{e}, \mathrm{F}=\mathrm{n} ?$ & 400 \\
\hline 02.0 & 91 & $3+35-7 n$ & $2.546 E-0,7$ & 400 \\
\hline 100.0 & ס กู & $7 \times 3 \underline{5}=0$ & $2.7378-0.7$ & ac) \\
\hline & $7 \mathrm{~s}$ & $A=305 E-08$ & $2.11 \mathrm{CE}=37$ & $40 n$ \\
\hline 114.0 & 233 & 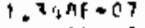 & $2.40 \mathrm{G}=0 \mathrm{~T}$ & 400 \\
\hline 118.01 & 77 & $4.4 A \times E=0 ?$ & $2.195 E-07$ & 000 \\
\hline & $\rightarrow t$ & $.1095-1) F$ & $5.754 \mathrm{~F}=08 \mathrm{~B}$ & and \\
\hline
\end{tabular}

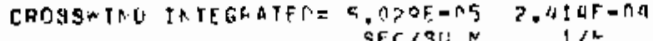

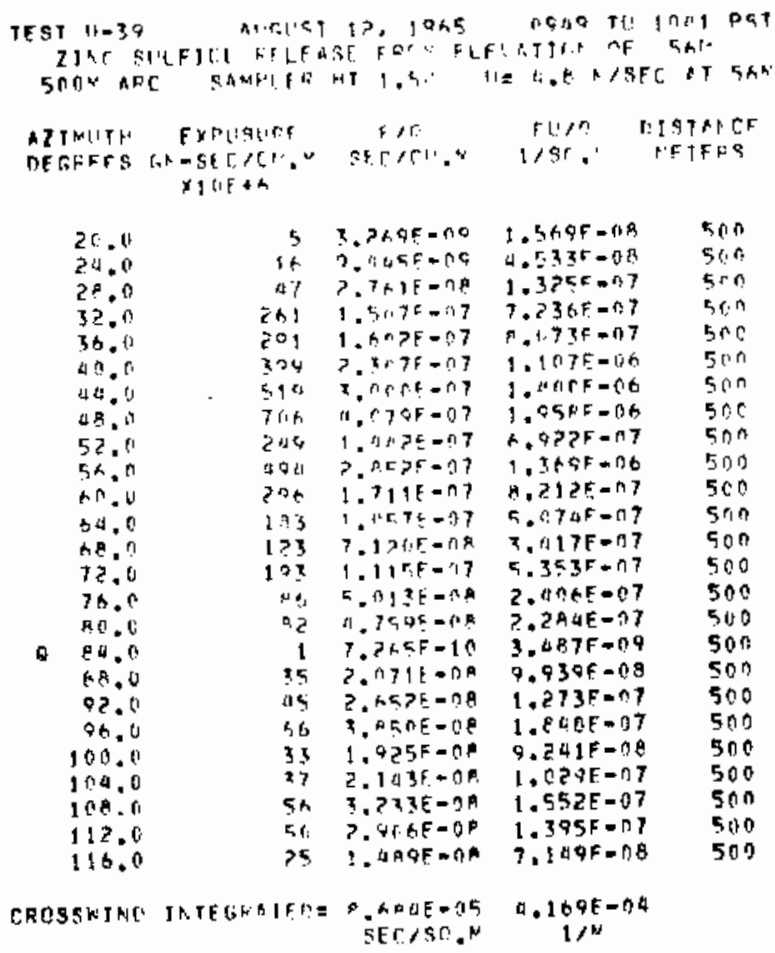

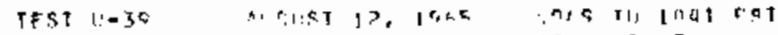

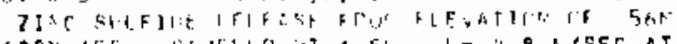

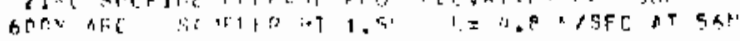

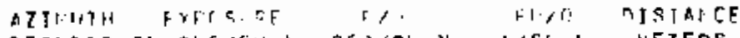

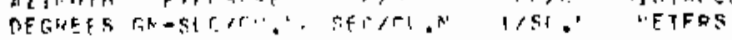
$x i l i t$

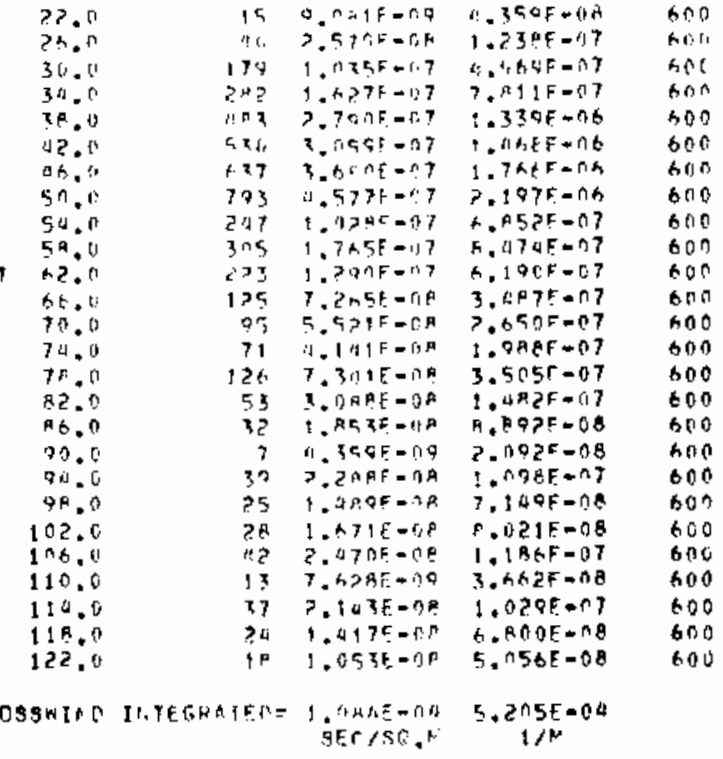




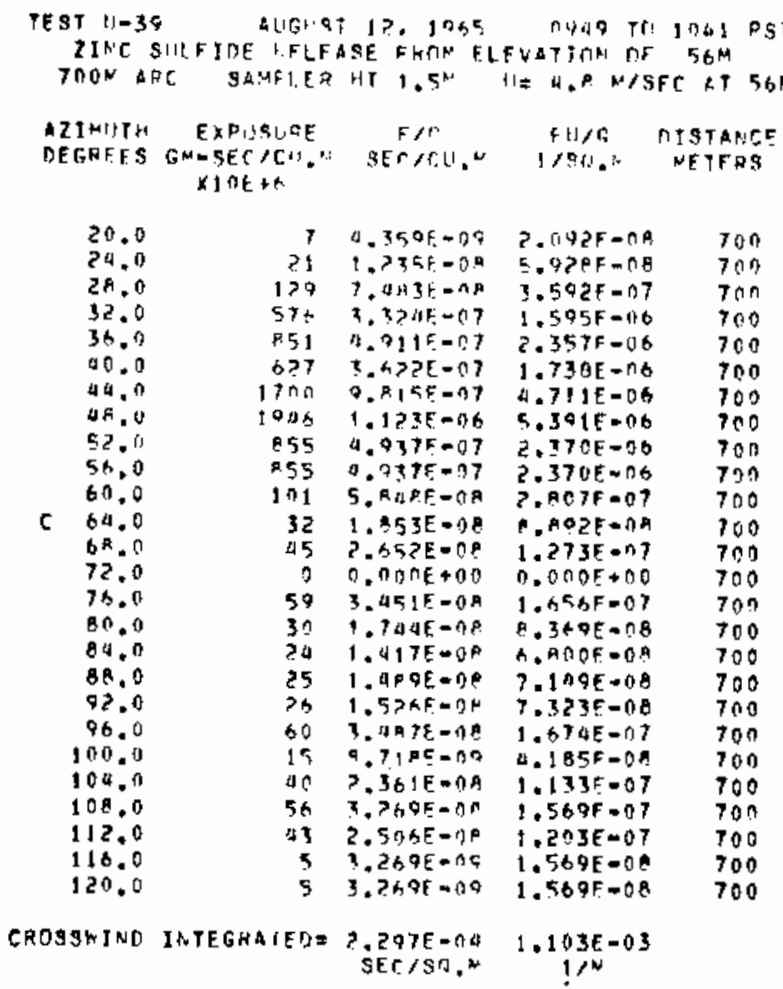

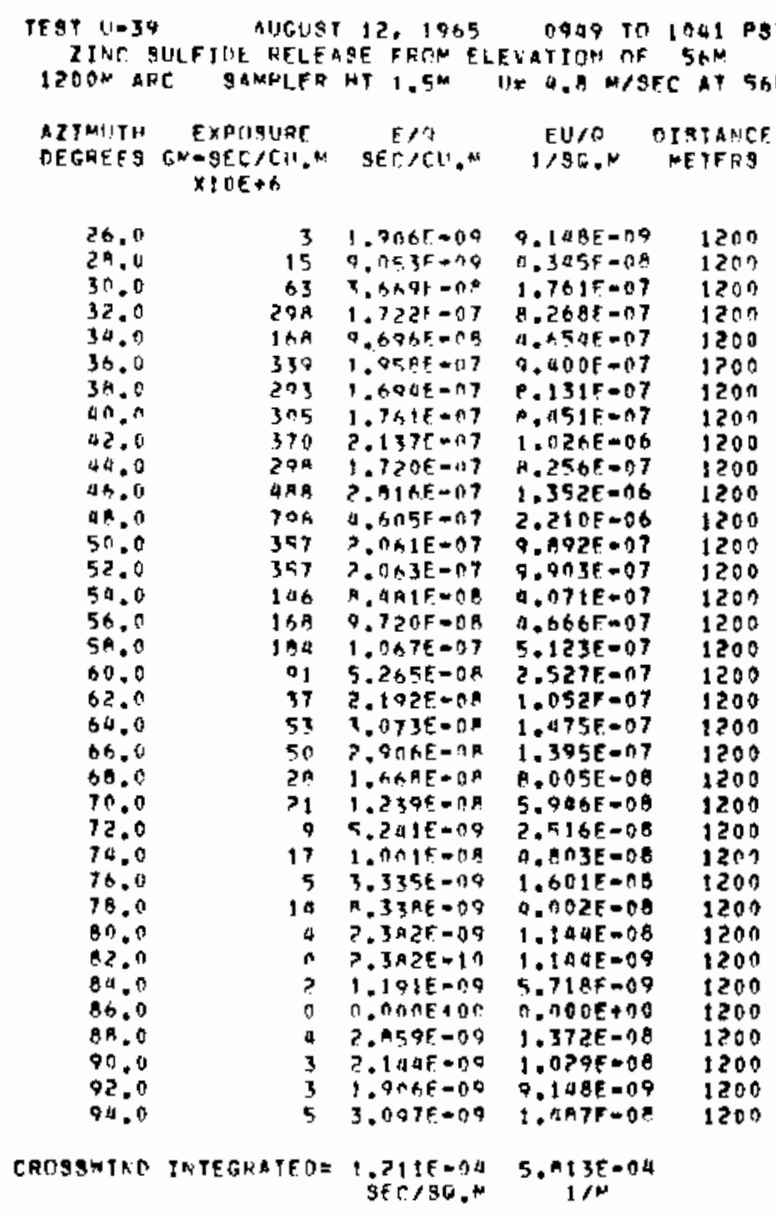

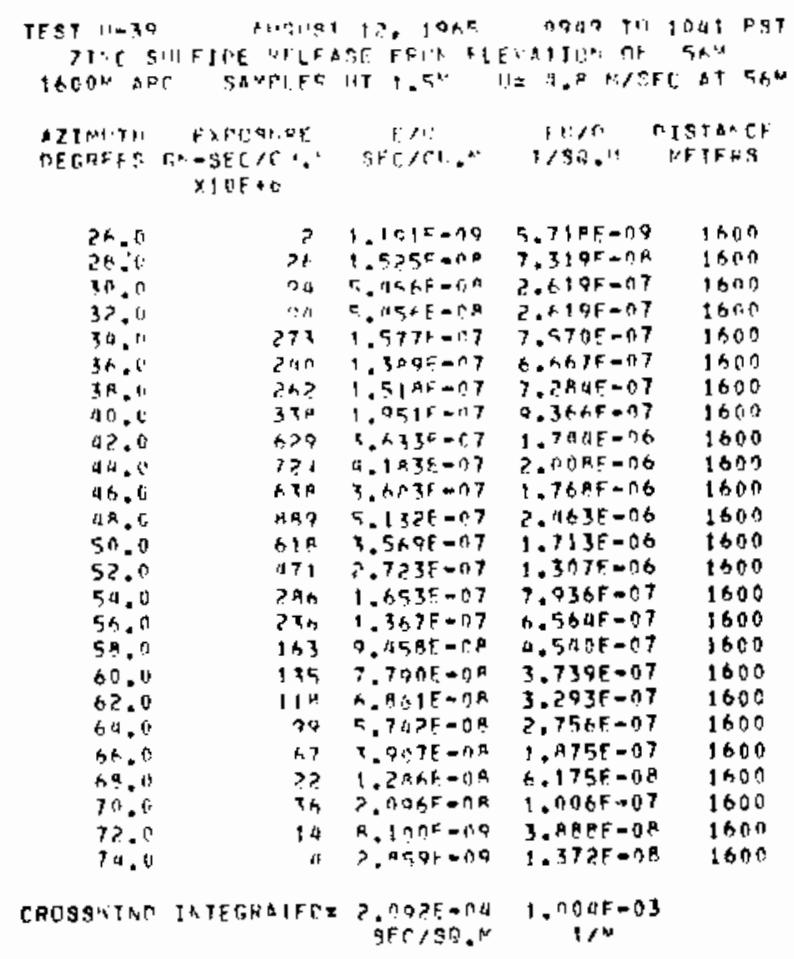


UNSTABLE SERIES EXPER IMENT: $40(U \rightarrow 40)$

GROUNO LEVEL SAMPLING 100-3200 A ARCS.

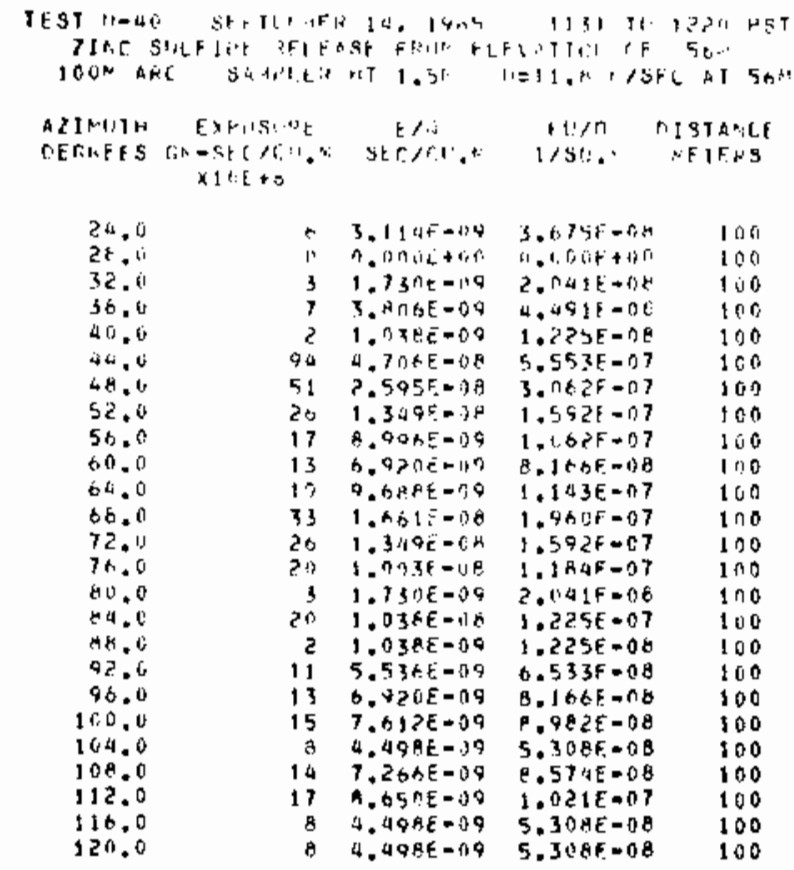

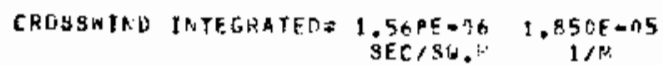

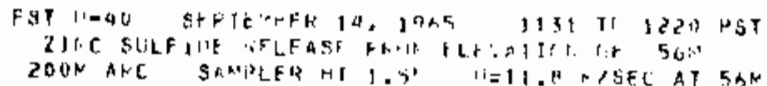

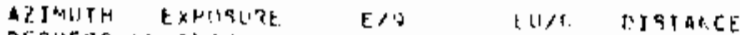

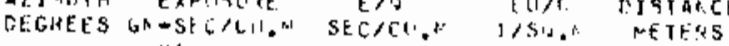
$x \geq$ ut $+n$

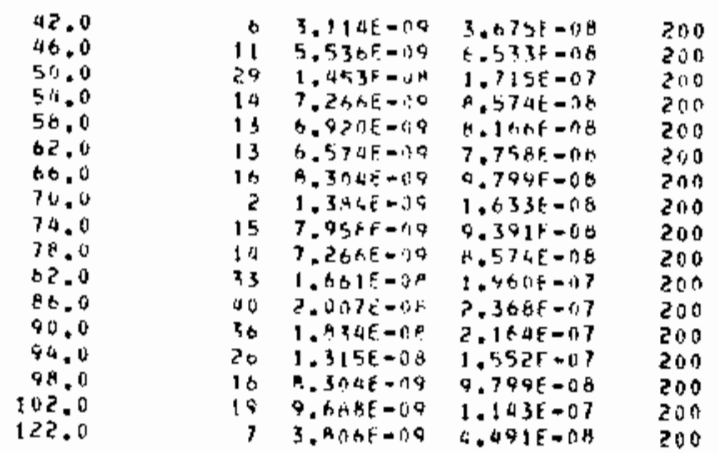

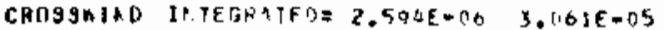
0 0 0 0 100

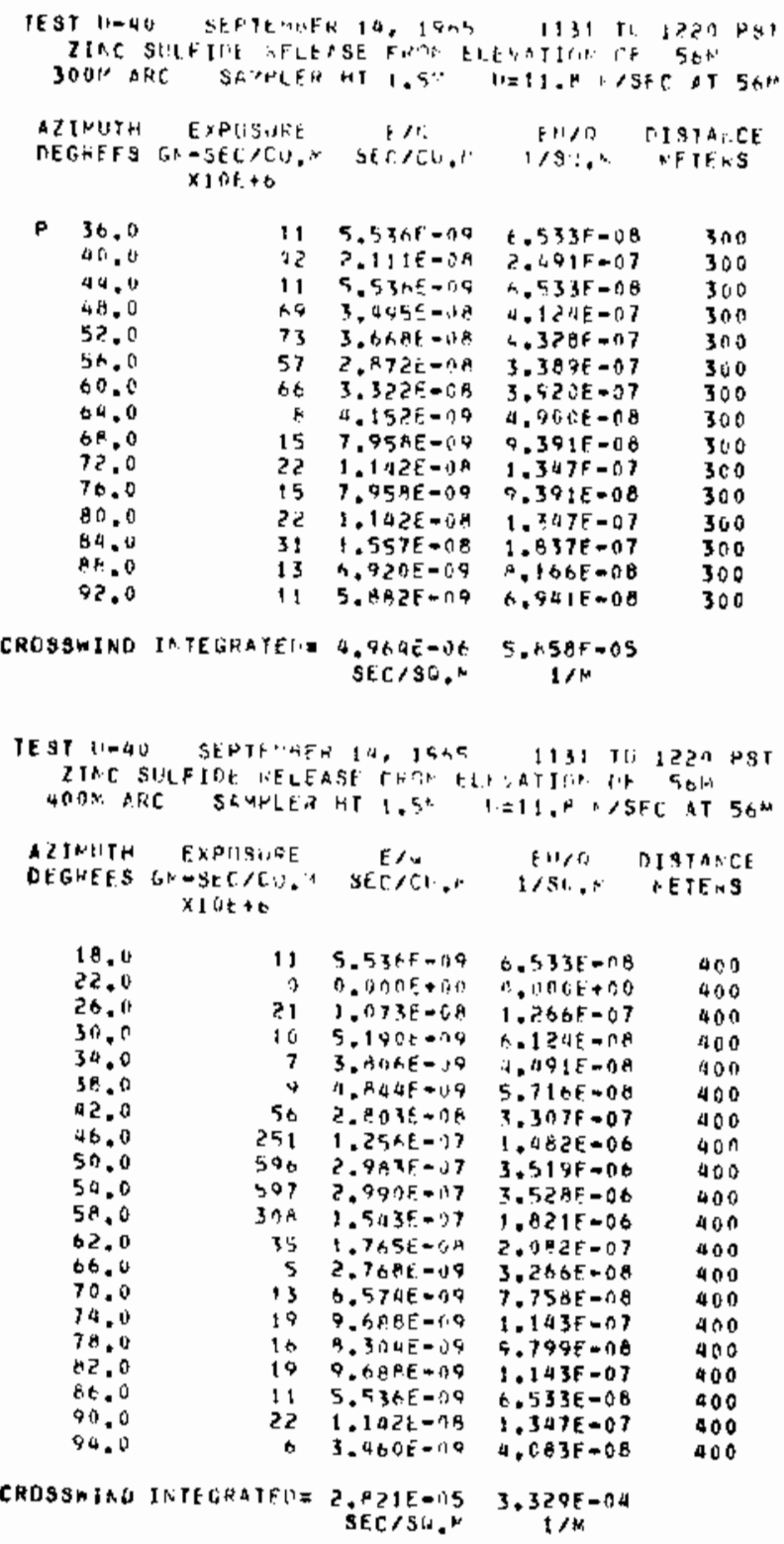
$5 E C / C U, *$ SES.CU. 


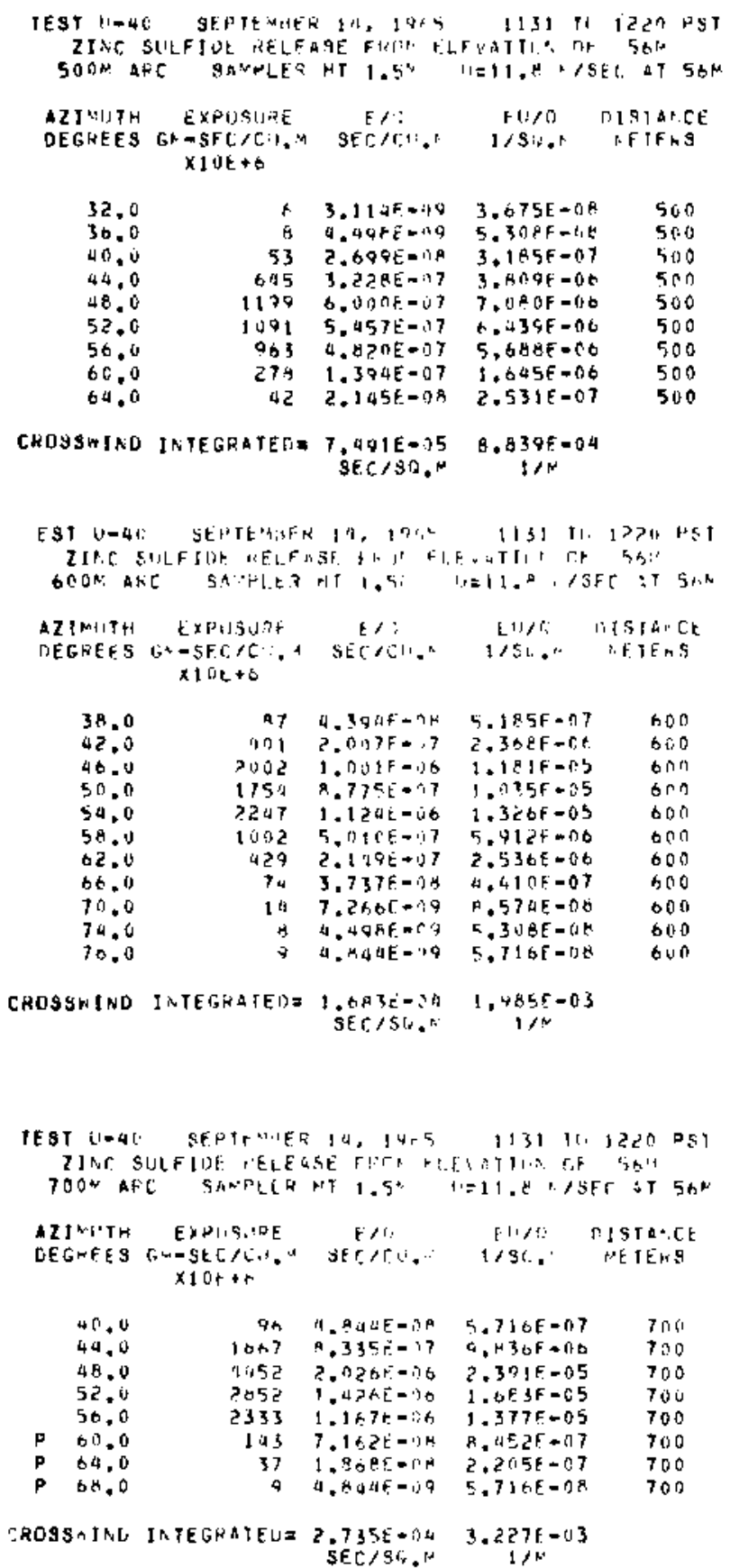

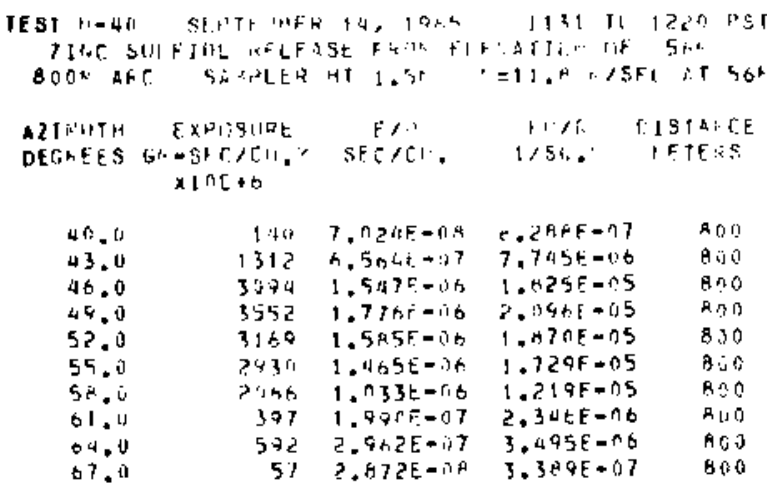




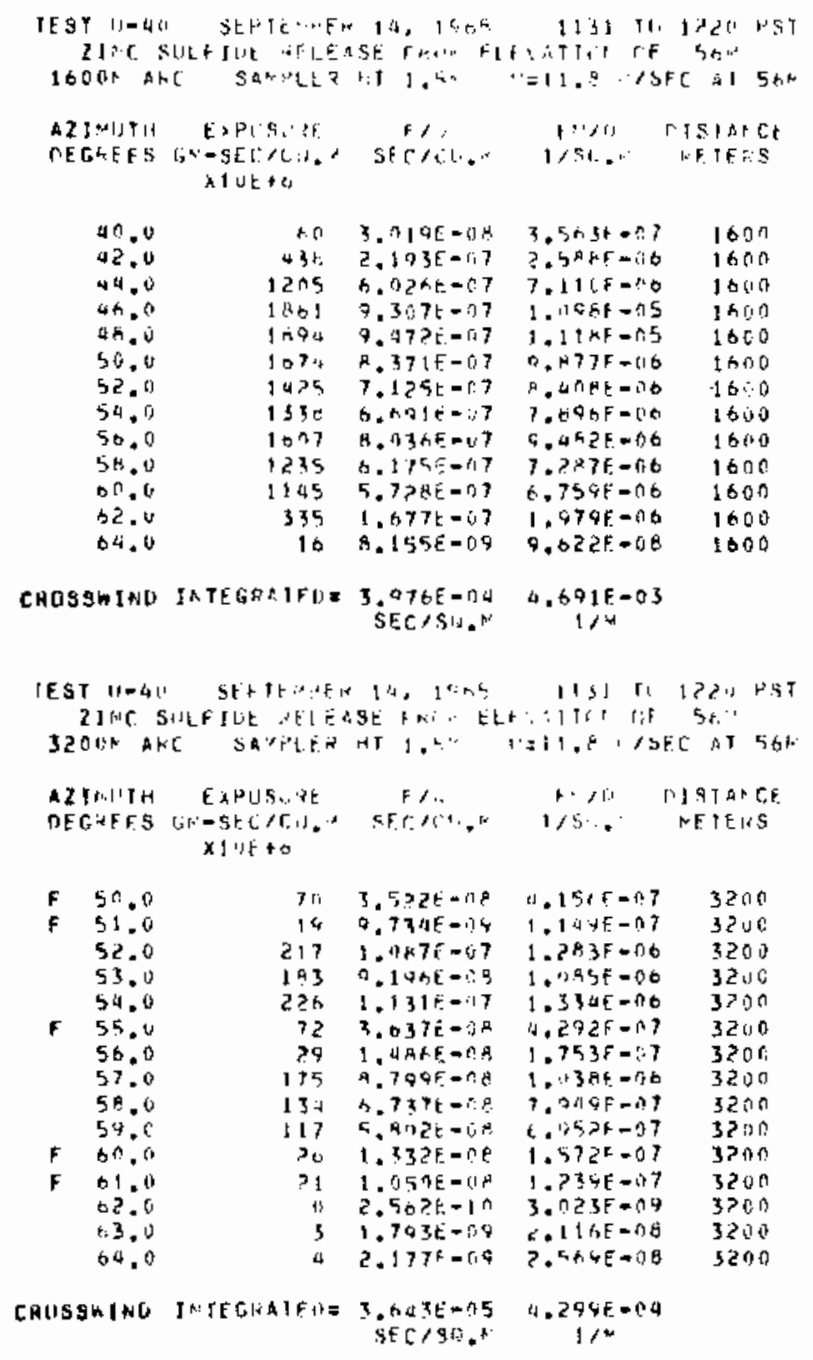




\begin{abstract}
UNSTABLE SERIES EXPERIMENT: 41 ( U-4t)
GROUND LEYEL SAMPLING 100-1600 M ARCS. FOR EASE IN STATISTICAL ANALYSIS, ALL SAMPLER AZIMUTHS HAVE BEEN INCREASED BY JO DEGREES
\end{abstract} FROM THEIR ACTUAL POSITIONS.

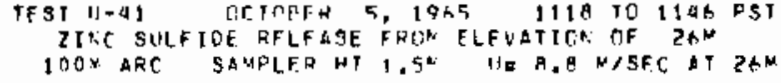

BAMPLER AIIMITHAG HAVE AFFN I HCREASFP AY 30 DETREES

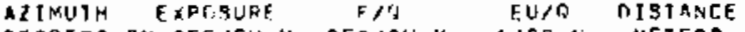
DEGPEES GNESEC/CU.M SEC/C11.N 1/SR.N METFRS $\times I O E+6$

\begin{tabular}{|c|c|c|c|c|}
\hline $\begin{array}{l}58.0 \\
62.0 \\
66.0 \\
70.0\end{array}$ & $\begin{array}{r}47 \\
957 \\
354 \\
78\end{array}$ & $\begin{array}{l}9.512 F=0 A \\
1.931 E=06 \\
7.3 P 1 E=07 \\
1.591 E=07\end{array}$ & $\begin{array}{l}8.311 F=07 \\
1.699 F=05 \\
1.319 E=06 \\
1.391 F=06\end{array}$ & $\begin{array}{l}100 \\
100 \\
100 \\
100\end{array}$ \\
\hline 74.0 & 231 & $4 . K T 6 F-07$ & a. $115 F-0 b$ & 100 \\
\hline $7 R .0$ & 17 . & 3.6 COE $=06$ & $3.1715=05$ & 100 \\
\hline 82.0 & 172 & $3.470 E=07$ & $3.054 F=06$ & 100 \\
\hline 86.0 & $2 n 1$ & $5.265 \mathrm{E}-0.7$ & $4.6335=06$ & 100 \\
\hline 90.0 & 270 & $5.453 \mathrm{t} \cdot 17$ & $4.70 \mathrm{BF}=06$ & 100 \\
\hline 0 & 554 & $1.119 E=06$ & $9.8045-06$ & 100 \\
\hline 0 & 7 & $1 . A \cap A E=O B$ & $1.415 E=07$ & 100 \\
\hline 2,0 & 10 & $2.10 \angle F=0 A$ & $6 F-07$ & 100 \\
\hline 106.0 & h & $1.3 \angle O E=O R$ & $1.179 F-07$ & 100 \\
\hline $11 n .0$ & 9 & $1 . A 7 A F=0 \%$ & $1.651 E=07$ & 100 \\
\hline 314.0 & 4 & 9.37 AF $=09$ & $9.253 E-08$ & 100 \\
\hline 118.0 & 1 & $4.010 E=09$ & $3.537 \varepsilon-08$ & 100 \\
\hline 122.0 & 15 & $3.215 F=08$ & $A 30 E-07$ & 100 \\
\hline 126.0 & 15 & $3.0 R 1 E=08$ & $\begin{array}{l}2.712 E=07 \\
1\end{array}$ & $\ln 0$ \\
\hline & 10 & - JaLE+11R & $\begin{array}{l}1.0 \mathrm{HOE}=07 \\
4.00 A F=C 7\end{array}$ & $\begin{array}{l}100 \\
100\end{array}$ \\
\hline 0 & $? ?$ & $\begin{array}{l}4.5556=0 A \\
474 E=02\end{array}$ & $1.297 E-07$ & 100 \\
\hline 0 & a & $1.747 F=08$ & $1.533 E=07$ & 100 \\
\hline 0 & 35 & $7.1 \cap ! E=\cap n$ & $5.249 E=07$ & 100 \\
\hline 5 & 29 & $5.7 B 1 E=0 P$ & $5.0705-07$ & 100 \\
\hline
\end{tabular}

CROSSNIND INTEREATERE 6.901E-05 $6.073 F=00$

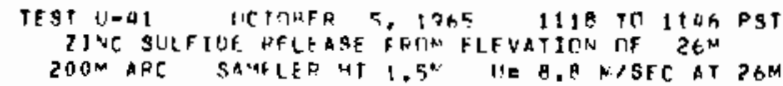

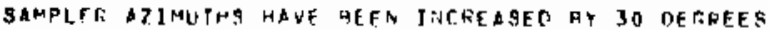

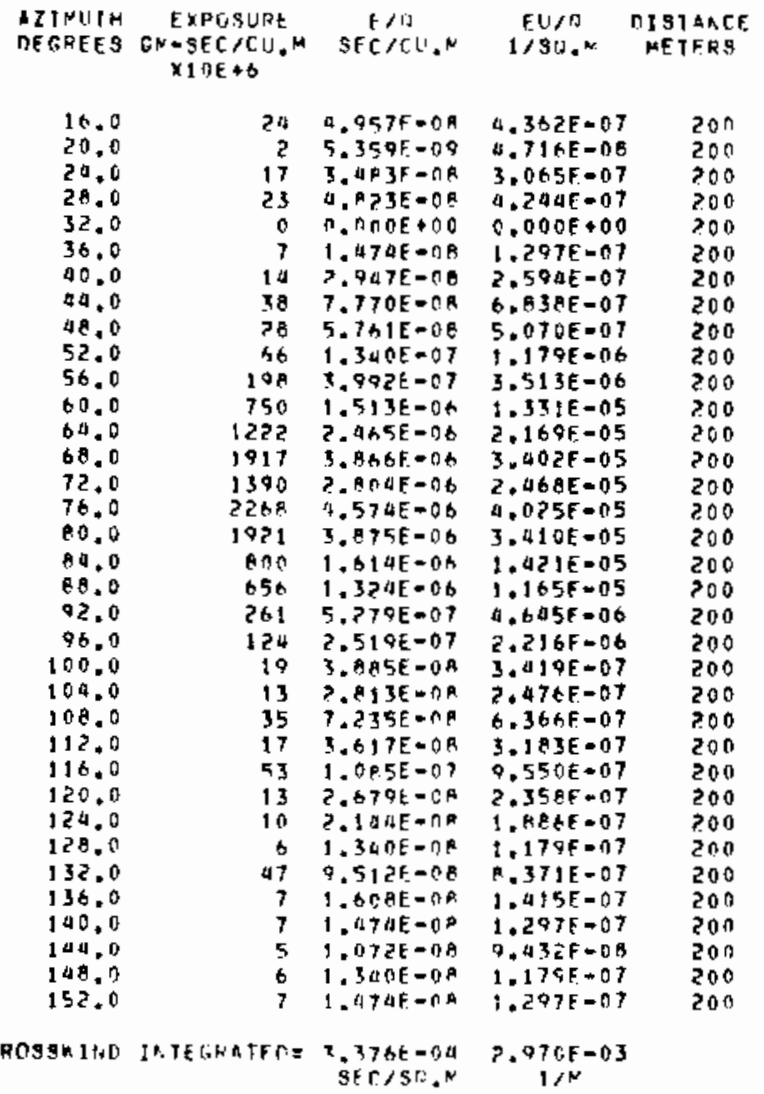




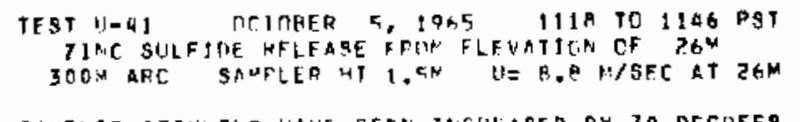

SAMPLER AZIMUTHS HAVE REFP JTCHEASEN AY 30 DEGREES

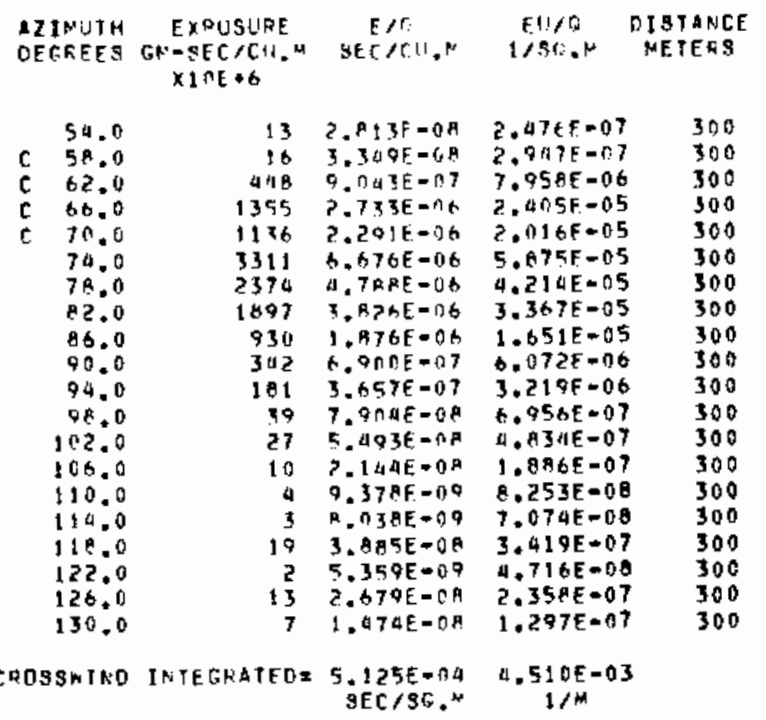

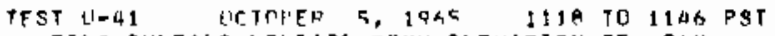

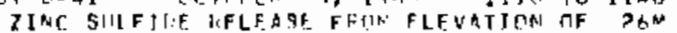
WOON AFE SAMFLER HT 1.5\% US R.A M/SFC AT $26 \mathrm{M}$

SANPLER AZIMLITHS WAVE AEEN INTKFASED GY 30 DERGEES

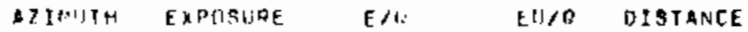

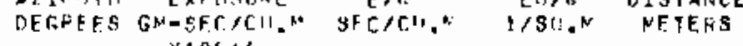
$x 1 \cap E+6$

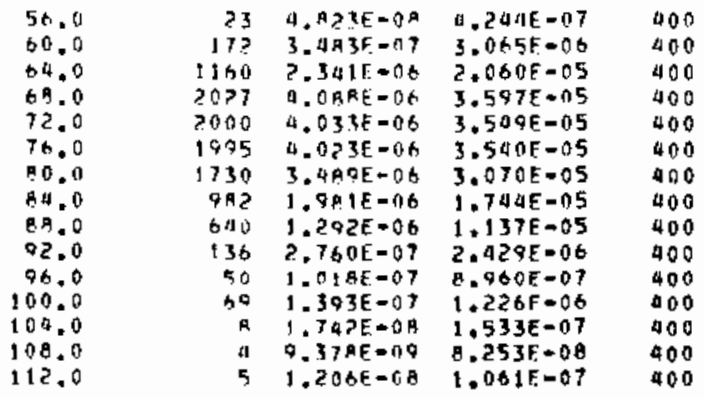

CRJSSHIND JNTEGEATELX 6.199E-OA 5.455E-03

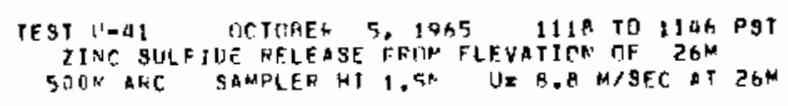

GARPLFR AIIMUITHS WAVE BEFN THCREASED GY 30 DEGREES

\begin{tabular}{|c|c|c|c|c|}
\hline $\begin{array}{l}\text { AIIMUTH } \\
\text { DFEREES }\end{array}$ & $\begin{array}{l}\text { EXPOSURE } \\
\text { GN=SEC CII. } \\
\times 10 E+6\end{array}$ & $\begin{array}{c}E / O \\
S E C / C U . M\end{array}$ & $\begin{array}{c}F U / 0 \\
1 / 30 . \mathrm{K}\end{array}$ & $\begin{array}{l}\text { DI STANCE } \\
\text { METER S }\end{array}$ \\
\hline $\begin{array}{l}54.0 \\
58.0 \\
62.0 \\
66.0 \\
70.0 \\
14.0 \\
79.0 \\
12.0 \\
86.0 \\
90.0 \\
94.0 \\
96.0 \\
102.0\end{array}$ & $\begin{array}{l}15 \\
176 \\
263 \\
1693 \\
1310 \\
1940 \\
1281 \\
1224 \\
684 \\
219 \\
122 \\
158 \\
11\end{array}$ & 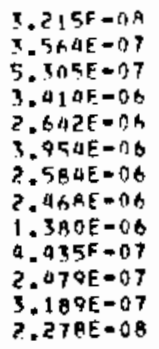 & $\begin{array}{l}2.830 E-07 \\
3.13 \mathrm{HE}=06 \\
9.660 E-06 \\
3.00 \mathrm{EE}=05 \\
2.325 E-05 \\
3.079 E-05 \\
2.27 \mathrm{AE}=05 \\
2.172 E=05 \\
1.214 E-05 \\
3.002 E=06 \\
2.185 E-06 \\
2.006 E-06 \\
2.004 E-07\end{array}$ & $\begin{array}{l}500 \\
500 \\
500 \\
500 \\
500 \\
500 \\
500 \\
500 \\
500 \\
500 \\
500 \\
500 \\
500\end{array}$ \\
\hline 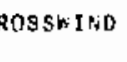 & INTEGR ATED & $\begin{array}{l}6.420 E=04 \\
3 E C / 90 . M\end{array}$ & $\begin{array}{c}5.650 \mathrm{~g}-03 \\
1 / \mathrm{M}\end{array}$ & \\
\hline
\end{tabular}

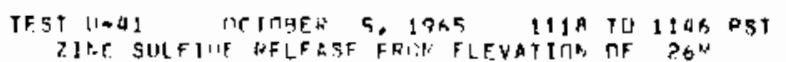

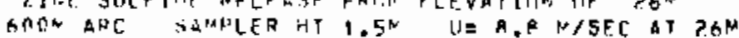

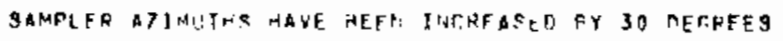

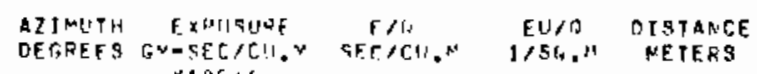
x $10 F+6$

\begin{tabular}{|c|c|c|c|c|}
\hline $\begin{array}{l}50.0 \\
60.0 \\
64.0 \\
68.0 \\
12.0 \\
16.0 \\
90.0 \\
84.0 \\
8.0 \\
92.0 \\
96.0 \\
100.0 \\
104.0 \\
108.0\end{array}$ & $\begin{array}{r}31 \\
114 \\
049 \\
1017 \\
994 \\
1034 \\
090 \\
610 \\
518 \\
134 \\
149 \\
23 \\
7 \\
7\end{array}$ & 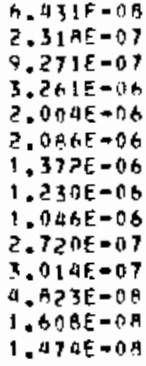 & $\begin{array}{l}5.659 F=07 \\
2.040 E=06 \\
8.15 A E-06 \\
2.870 E=05 \\
1.764 E=05 \\
1.636 E-05 \\
1.207 F=05 \\
1.082 E=05 \\
9.20 B E=06 \\
2.393 E=06 \\
2.653 E=06 \\
0.244 F=07 \\
1.415 E=07 \\
1.297 F=07\end{array}$ & $\begin{array}{l}600 \\
600 \\
600 \\
600 \\
600 \\
600 \\
600 \\
600 \\
600 \\
600 \\
600 \\
600 \\
600 \\
600\end{array}$ \\
\hline
\end{tabular}

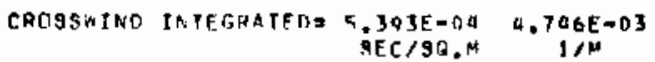




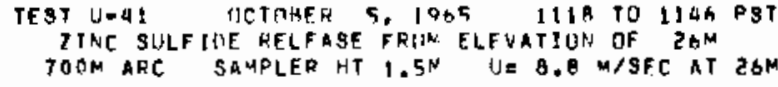

SAMPLER AZIAUTHS HAVE BEEN IITCREAGED AY 3 OH DEGREES

\begin{tabular}{|c|c|c|c|c|}
\hline $\begin{array}{l}\text { ZISUTH } \\
\text { ESREES }\end{array}$ & $\begin{array}{c}\text { EXPOSURE } \\
\text { GN-SEC/CH, } \\
\times 10 E+6\end{array}$ & $\begin{array}{c}E / C \\
S E C / C H, F\end{array}$ & $\begin{array}{c}E \backslash / A \\
1 / S A, N\end{array}$ & $\begin{array}{l}\text { DIST ANCE } \\
\text { NETFRS }\end{array}$ \\
\hline $\begin{array}{l}50.0 \\
54.0 \\
58.0 \\
62.0 \\
66.0 \\
70.0 \\
70.0 \\
79.0 \\
82.0 \\
96.0 \\
91.0 \\
90.0 \\
98.0 \\
102.0 \\
106.0 \\
110.0\end{array}$ & $\begin{array}{r}21 \\
18 \\
11 \\
243 \\
661 \\
550 \\
1053 \\
653 \\
255 \\
543 \\
273 \\
201 \\
33 \\
5 \\
79 \\
10\end{array}$ & $\begin{array}{l}0.421 E=0 R \\
3.751 E=0 B \\
8.30 A E=08 \\
4.903 E=017 \\
1.330 E=0 A \\
1.109 E=0 . A \\
2.123 E=06 \\
1.31 A E=06 \\
5.105 E=07 \\
1.176 E=06 \\
4.502 E=07 \\
4.059 E=07 \\
1.033 E=08 \\
1.206 E=08 \\
1.608 E=07 \\
2.144 E=0 A\end{array}$ & $\begin{array}{l}3.891 E=07 \\
3.301 E=07 \\
7.310 E=07 \\
4.315 E=06 \\
1.174 E=05 \\
9.7 \$ 2 F=06 \\
1.869 F=05 \\
1.160 E=05 \\
4.527 E=06 \\
1.035 E=05 \\
3.061 F=06 \\
3.572 E=06 \\
6.013 E=07 \\
1.061 E=07 \\
1.415 E=06 \\
1.086 E=07\end{array}$ & $\begin{array}{l}700 \\
700 \\
700 \\
700 \\
700 \\
700 \\
700 \\
700 \\
700 \\
700 \\
700 \\
700 \\
700 \\
700 \\
700 \\
700\end{array}$ \\
\hline W IND & INTELINAT & 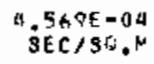 & $\begin{array}{c}4.021 E=03 \\
1 / N\end{array}$ & \\
\hline
\end{tabular}

TEST (1-41 DCTOPFH S. 12X5 1)IA TO 1146 P9T

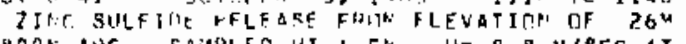
HOON AYC SAMPLF HT $1.5 \mathrm{~N}$ UI O.P N/SEC AT $26 \mathrm{M}$

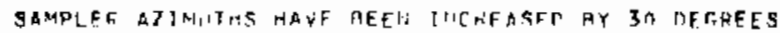

\begin{tabular}{|c|c|c|c|c|}
\hline $\begin{array}{l}\text { A I N } N T \text { TH } \\
\text { DE } r, R E F S\end{array}$ & $\begin{array}{c}E, P_{1}: Q \Delta R^{2} F \\
G^{N}-S E C / C \%, \\
\times 10 E+b\end{array}$ & $\begin{array}{c}E / 2 \\
S E C / C^{\prime \prime} .\end{array}$ & $\begin{array}{c}\mathrm{F} 1 / \mathrm{C} \\
1 / \mathrm{S} 1 .\end{array}$ & $\begin{array}{l}\text { DISTAACE } \\
\text { NTEK3 }\end{array}$ \\
\hline म万. 0 & $1 A$ & $3.751 E_{r}-08$ & $3.30: E-07$ & 800 \\
\hline$\$ 5.0$ & i & $1.470 F_{0} \rightarrow 12$ & $1.297 \mathrm{~F}=07$ & .00 \\
\hline 58.0 & 21 & $4.421 E=0.9$ & $3.2015-07$ & 900 \\
\hline$\times 1.0$ & 1 ho & $3.242 \mathrm{~F}=07$ & $\therefore .853 \mathrm{~F}=\mathrm{ns}$ & Bon \\
\hline+4.0 & 178 & $3.002 \mathrm{~F} .07$ & $3.513 \mathrm{~F}+06$ & 300 \\
\hline 67.0 & 436 & $a . A+2 F=07$ & $7.781 F=06$ & $A_{00}$ \\
\hline $7 n, 0$ & 790 & $1.514 t=06$ & $1.332 f=05$ & 200 \\
\hline 73.0 & स12 & $1.637 E=n_{t}$ & $1.4 \Delta 1 F=05$ & 200 \\
\hline 76.0 & HAI & 1.73 AE $=96$ & $1.528 E-05$ & 900 \\
\hline 70.0 & 55? & 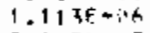 & $9.7975=06$ & 100 \\
\hline$H \geq 0$ & $40 a$ & A. $159 E=177$ & 7. 1PCE-06 & ADO \\
\hline .5 .0 & $5 \cap 3$ & $1.0[4 E=n A$ & R.925F-106 & POD 0 \\
\hline 88.0 & 279 & $5.0275-07$ & $4.952 F-116$ & $a 00$ \\
\hline 91.0 & $1 \cap ?$ & $P .077 E=0.7$ & $1.9>75=06$ & BOO \\
\hline 94.0 & 112 & 2. $2.7 \mathrm{AE}=07$ & $2.000 \mathrm{~F}-06$ & $R_{0} 0$ \\
\hline 97.0 & 07 & $1.95 \mathrm{BF}-1.7$ & $1.3215-06$ & $\ln 0$ \\
\hline 190.0 & $? 7$ & $5.4 ? 7 E=n B$ & $4.952 \varepsilon-0.7$ & 800 \\
\hline 103.0 & 11 & $>. ? 78 F+18$ & 2. $0 \cap \Delta F=07$ & AOO \\
\hline 106.0 & 12 & $3.545 r-79$ & $2.200 E=07$ & $8 \cap 0$ \\
\hline 109.0 & 13 & ?.A79F-AR & $2.35 B F=07$ & 360 \\
\hline 115.0 & 71 & $1.0215-\sqrt{1} A$ & 3. $091 \mathrm{f}=07$ & non \\
\hline 118.0 & 11 & $P .41 Z E=1) A$ & $2,122 f-07$ & 800 \\
\hline 121,0 & 7 & $1.474 E-\pi R$ & $1.297 E-07$ & 900 \\
\hline 124.0 & 79 & $5 . A 05 E=1.9$ & 5.1 A. $=37$ & 900 \\
\hline
\end{tabular}

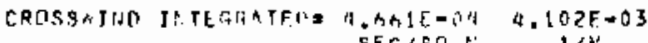

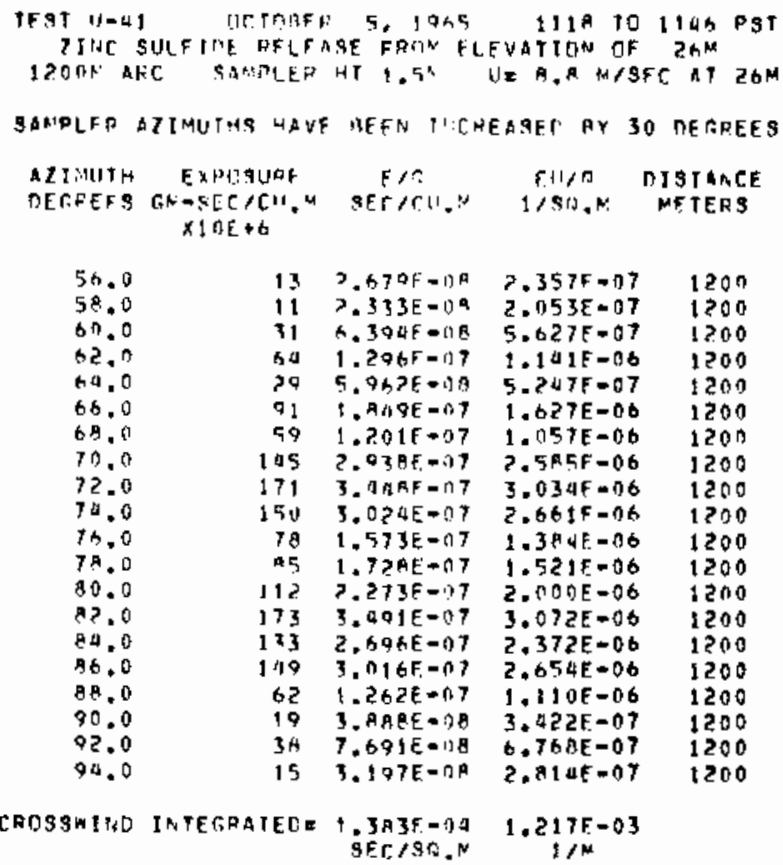




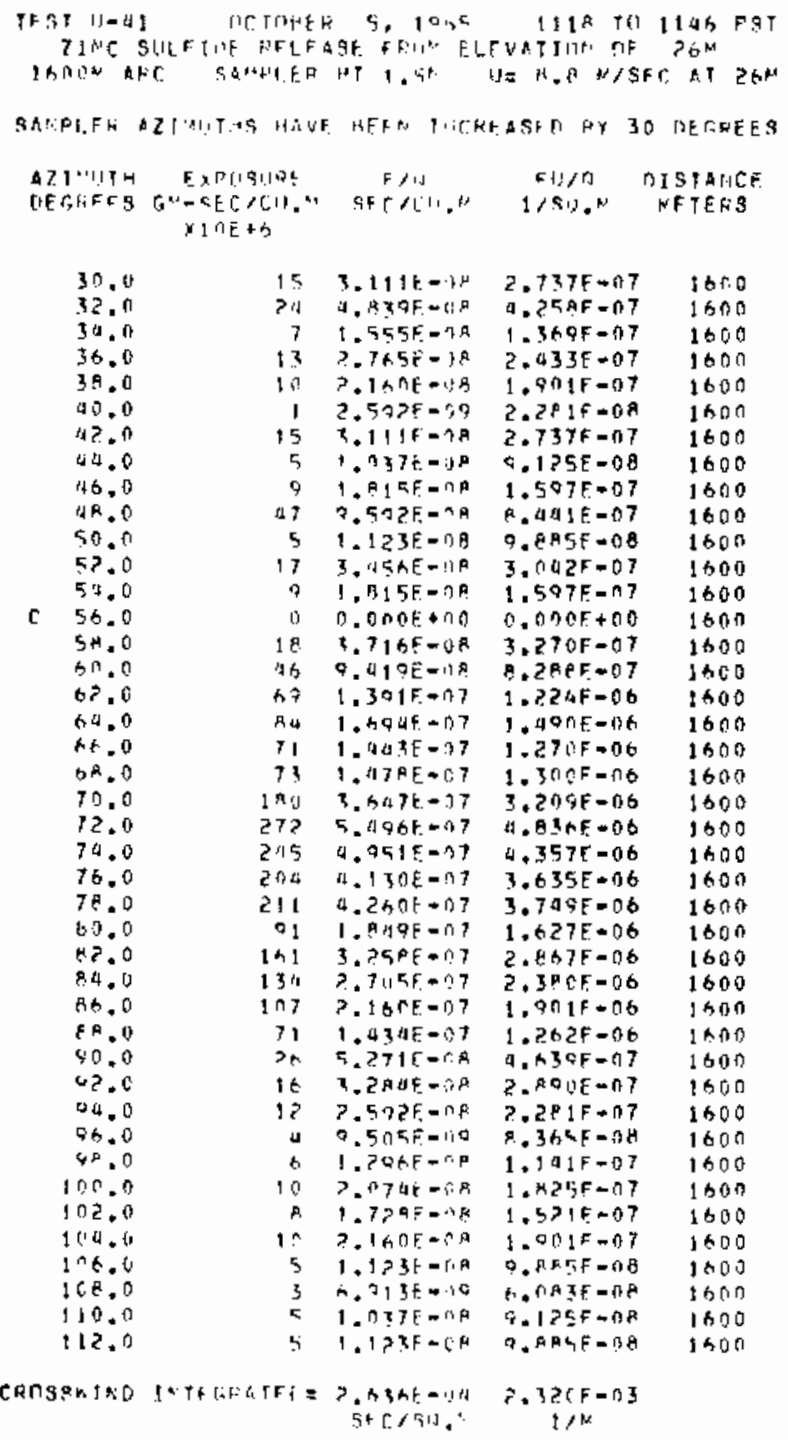


UNSTABLE SERIES EXPERIMENT: $42(\mathrm{U}-42$,

GROUND LEVEL 5AMPLING 400-3200 M ARCS.

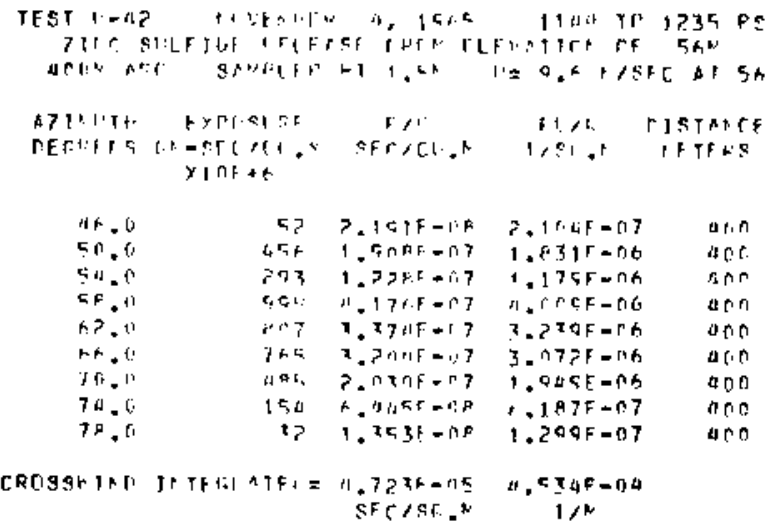

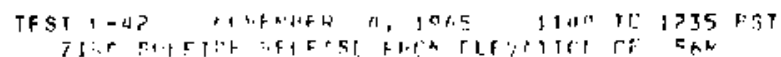

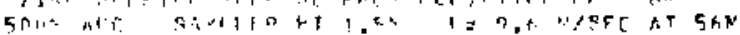

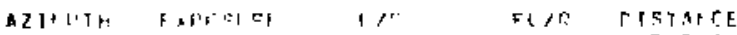

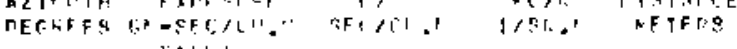
i) 1 it $+r$

\begin{tabular}{|c|c|c|c|}
\hline कS 0 f & 13 & 5. $\mathrm{A} \cap \cap \mathrm{F}-\cap 9$ & 5.5 न \\
\hline If $P .4$ & 1 & $7.57 .7 F=1.6$ & $7.2+501-n$. \\
\hline & $5 \cap \%$ & $7.19 r i=07$ & $2.034 \mathrm{f}=\mathrm{nh}$ \\
\hline 50.4 & 13,2 & $=.4$ ar -17 & $=. P A A F=0 B$ \\
\hline+5.11 & 15,40 & *. $+01+07$ & $1.37 \times 5-106$ \\
\hline 6.4 .11 & $15 x$ & $+.5571-0^{2} 7$ & H. $>05.5=0 t$ \\
\hline sp & pian & $0.1 \times 7,5=07$ & $4.70 .7 F=0 B$ \\
\hline ". & $7 \cdot 3$ & $3 . \partial>\left[\begin{array}{l}3 \\
3\end{array}\right.$ & $3.1+2=0=10$ \\
\hline $7 *$ & จ7 & ก૭วГ $=n$ ก & X. จPCF $=07$ \\
\hline
\end{tabular}

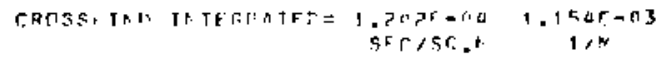

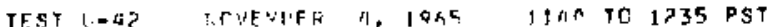

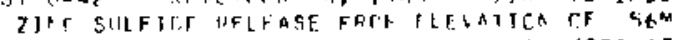

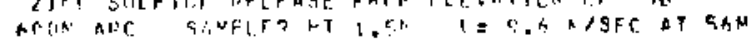

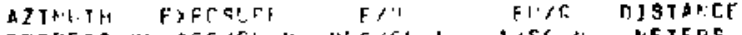

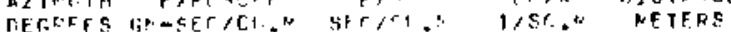
$\times 3$ iE +6

\begin{tabular}{|c|c|c|c|c|}
\hline$a+.0$ & 311 & 1. $3 \cap \overrightarrow{r F}=n$ ? & 1. दcef-Ot & ton \\
\hline ค. & 219 & $9.1 A f F=n p$ & $P . A T A F=07$ & 600 \\
\hline 5,1 & 1577 & $+.53^{7} F=1: ?$ & $t .3295-06$ & 600 \\
\hline 5. & .950 & $1.2 \geq 36-016$ & $1.181,5=05$ & $\operatorname{ten} 0$ \\
\hline 52.0 & व4:34 & $\ln x>5-0 x$ & $1.870 f-05$ & har \\
\hline $6 t, 1$ & 1290 & cantert & $2.9 ? 0 F+05$ & 600 \\
\hline 70.8 & 5737 & $3536=96$ & $1.2045=05$ & 600 \\
\hline $7 \mathrm{~h} . \mathrm{O}$ & 797 & $20+5=11 ?$ & $3.15 P F=16$ & 600 \\
\hline & 71 & $497 F+n e$ & $2.9775=07$ & $\sin \theta$ \\
\hline
\end{tabular}

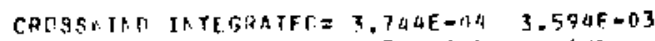

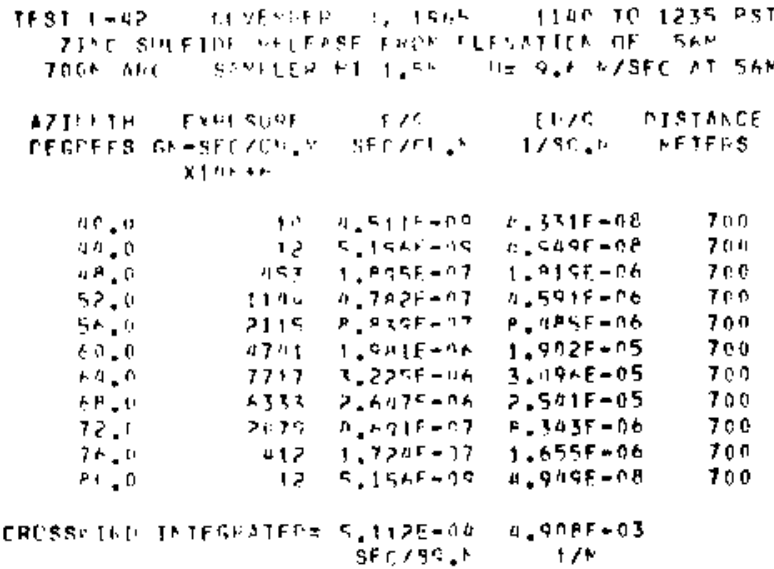

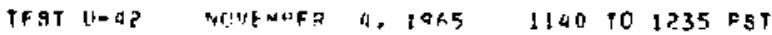

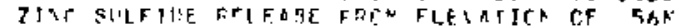

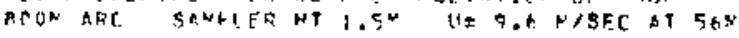

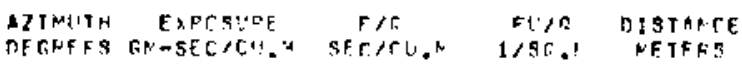
$x \notin \cap F+6$

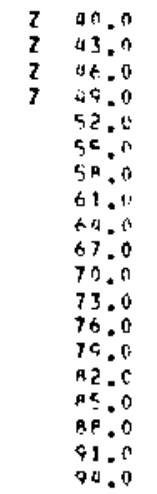

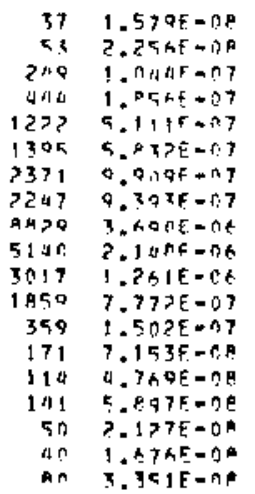

$1.516 F-0.7$ $305-0,100$ 1 , $012 \mathrm{~F}-\mathrm{CO}$ की ती $1.7 P 2 F-106$ $0.9 n \in 5-10$ an $5.599 F=16$ an 9.512E-06 800 $5.0175=06 \quad$ on T.5u2f-nS POO $3.062 F-05 \quad 800$ $1.3105=05 \quad 000$ $\begin{array}{ll}1.2105=95 & 00 \\ 1.1918-06 & 000\end{array}$ $\begin{array}{ll}7.1618-06 & 00 \\ 1.0107-06 & 100\end{array}$ $6.8675-07 \quad 100$ $0.578 F=07 \quad 000$ $5,6015-07$ 5.600 800 $2.0425-C 7$ $1.6095-07 \quad 800$

CRCSSATHD IATERRAIETE A.RTIF-C.4 $4.676 E-03$ SFr/SO. ${ }^{\mathrm{N}}$ 
UNSTAELE SERIES EXPERIMENT: $43(U-43)$ GROUNO LEYEL SAMPLING 100-1000 M ARCS.

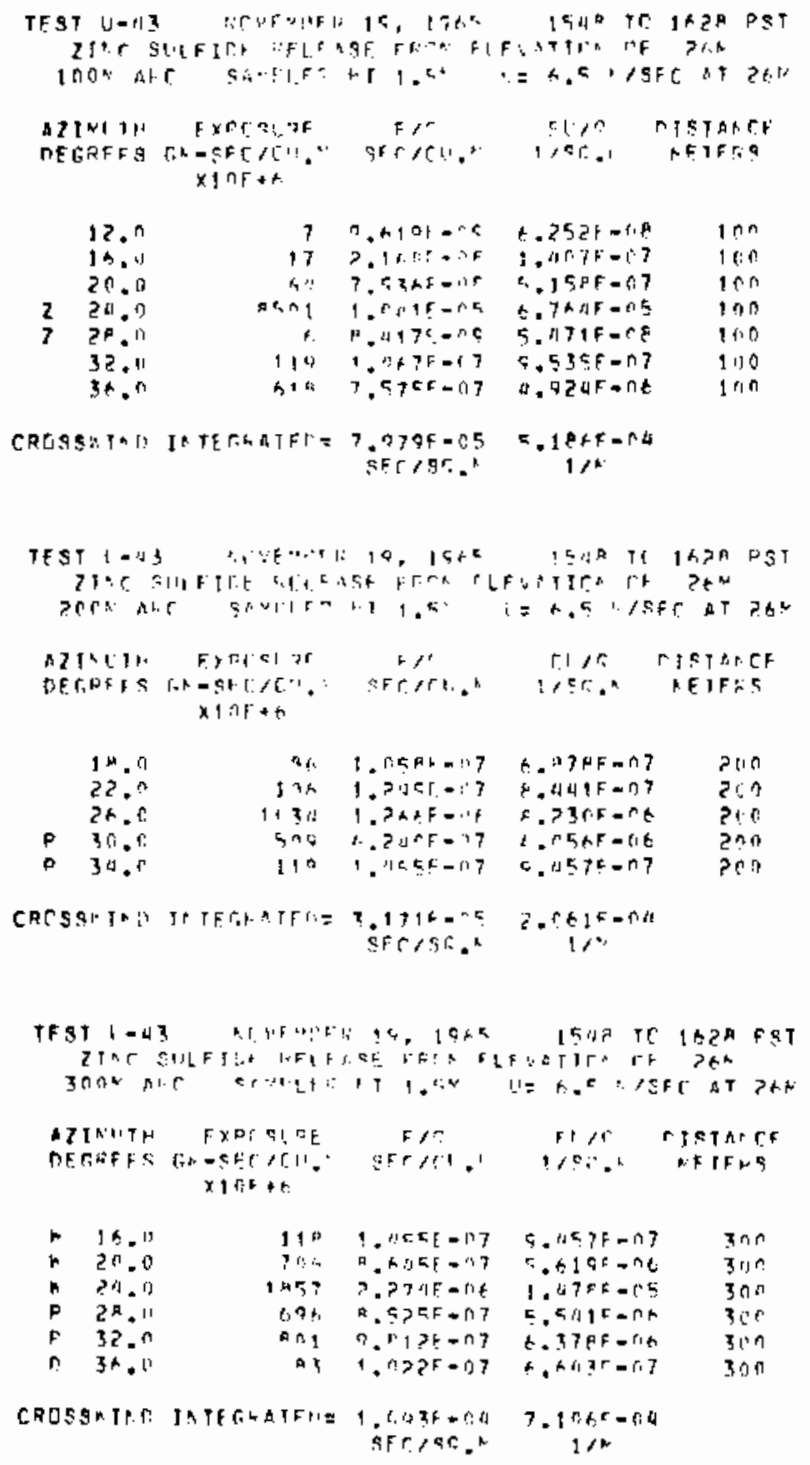

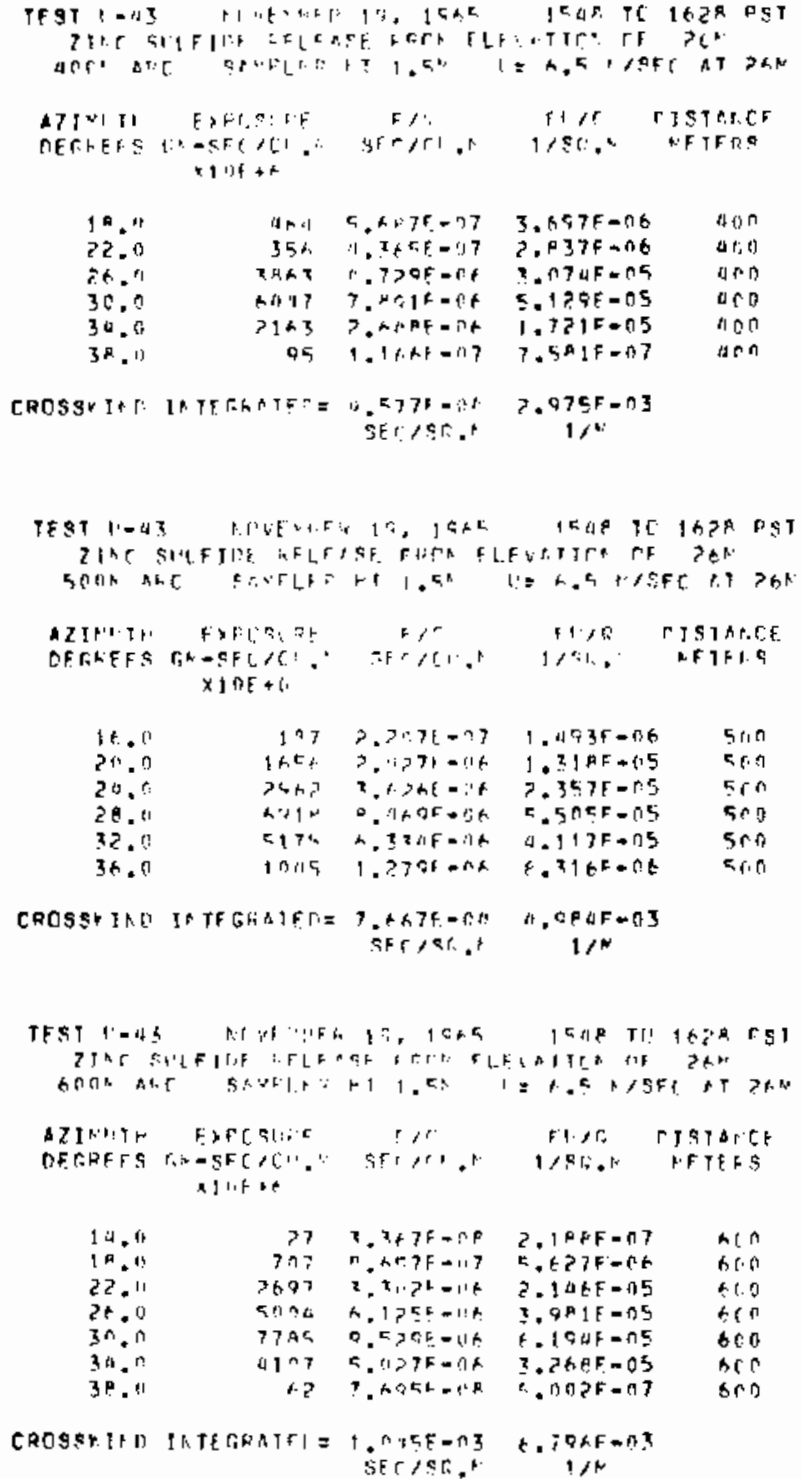




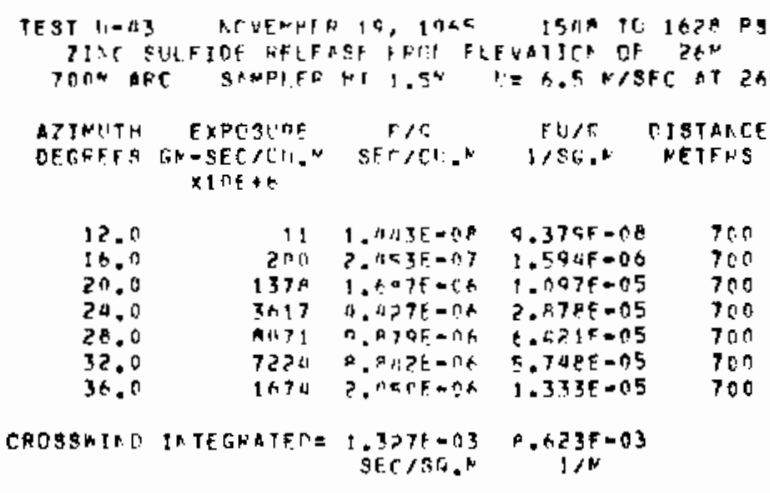

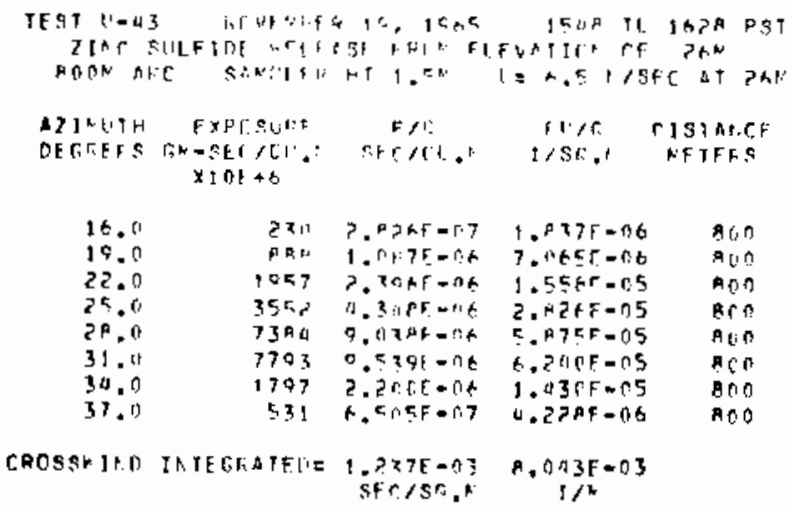

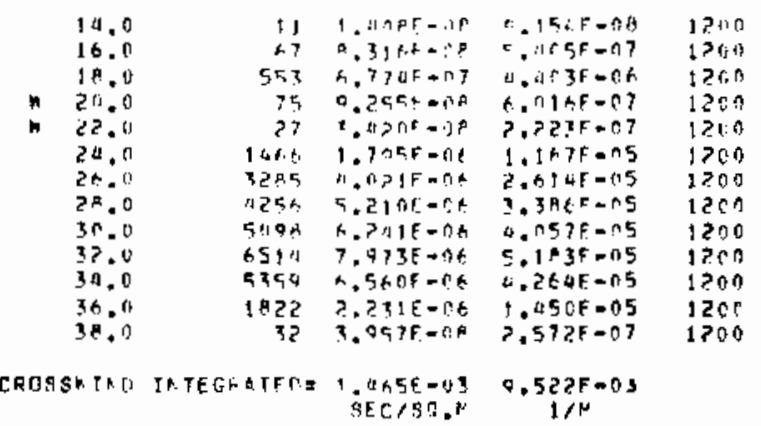

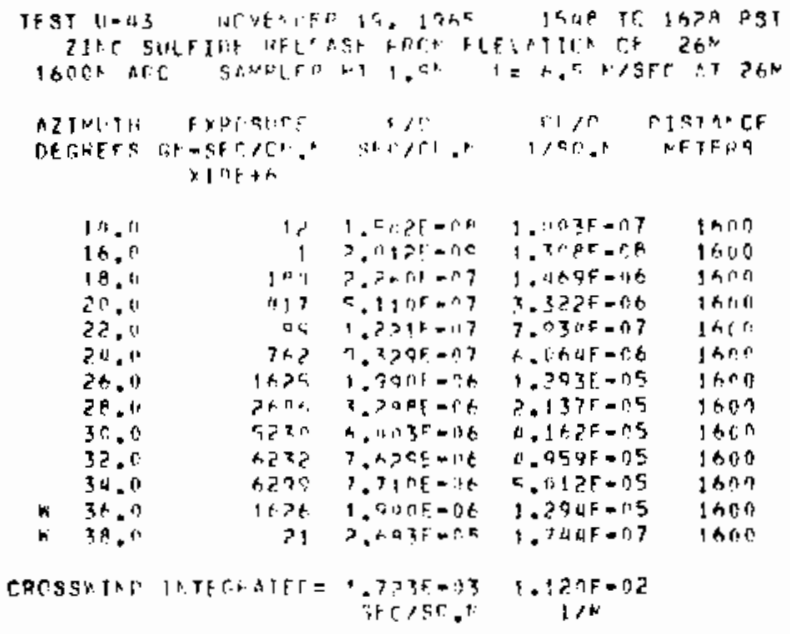

\begin{tabular}{|c|c|c|c|c|c|}
\hline & $1 * .11$ & اه & 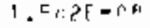 & $1 .: 10 \geq 0=07$ & $\ln n \pi$ \\
\hline & 16.8 & 1 & $>, n i z l-n c$ & 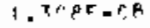 & 1600 \\
\hline & 18.6 & 101 & $z . z+n i l=07$ & $1.4094=116$ & Sant \\
\hline & 20.01 & 417 & $=.1,0507$ & $3.322 F=06$ & $|x| 1 \mid$ \\
\hline & 22,0 & $0 \&$ & $1.2 .31 k=117$ & 7.03 in $=0.07$ & lat 6 \\
\hline & 20.10 & $7+?$ & 7.2095 .97 & A. $l: h d F=r, 6$ & J han \\
\hline & $2 t .0$ & $1+25$ & $1.39 n f=n t$ & $1.3035-105$ & If०a \\
\hline & $2 B .16$ & $7+\pi 6$. & ?. Jaff $=f t$ & $2.1375=0.5$ & 1600 \\
\hline & 36.0 & $722 n$ & $4.1635=136$ & $0,1+2, F=0.5$ & $\sec n$ \\
\hline & 32.0 & $x-2 \geq 2$ & $7,4>C E-14$ & $0.959 F=05$ & 1600 \\
\hline & 34.0 & कर 20 & 3.7 , IE $=: t$ & C. 位 $12 F-05$ & 1607 \\
\hline w & $3 t .9$ & $1 \mathrm{t} .2 \mathrm{t}$ & $1.51205=0 t$ & $1.294 F=115$ & $\ln 0$ ? \\
\hline n & 39.9 & 71 & $2.493 F$ F. & 1.74 a $F=07$ & 16n:0 \\
\hline & & 年 & 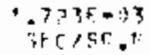 & $\begin{array}{c}127 F=02 \\
1 / N\end{array}$ & \\
\hline
\end{tabular}


UNSTABLE SERIES EXPERIMENT: 44 ( U-44)

GROUND LEVEL \$ANPLING \$00-1600 M ARCS. FOR EASE IN STATISTICAL

AHALYSIS, ALL SAMPLER NZIMUTHS HAYE BEEN INCAEASED BY 30 DEGREES

FROM THEIR ACTUAL POSITIOHS.

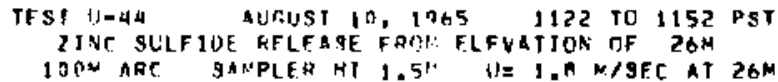

\begin{tabular}{|c|c|c|c|c|}
\hline 10.0 & $\begin{array}{r}114 \\
652 \\
67 \\
8 \\
044 \\
306 \\
200 \\
2027 \\
3624 \\
3204 \\
2663 \\
3728 \\
3704 \\
2032 \\
3571 \\
3633 \\
1540 \\
3811 \\
5793 \\
3755 \\
2040 \\
2139 \\
1458 \\
1032 \\
279 \\
151 \\
29 \\
8 \\
53 \\
2 \\
86 \\
20\end{array}$ & 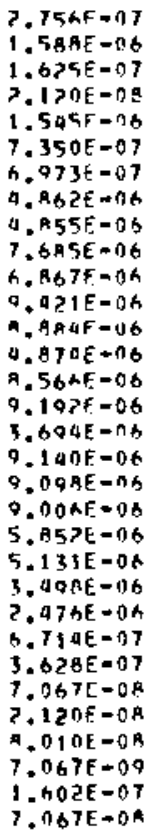 & 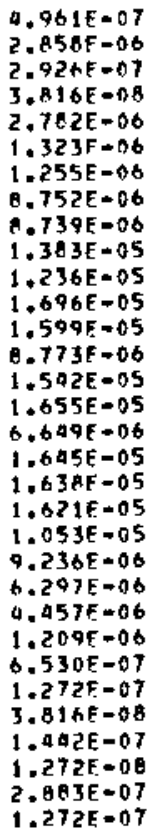 & $\begin{array}{l}100 \\
100 \\
100 \\
100 \\
100 \\
100 \\
100 \\
100 \\
100 \\
100 \\
100 \\
100 \\
100 \\
100 \\
100 \\
100 \\
100 \\
100 \\
100 \\
100 \\
100 \\
100 \\
100 \\
100 \\
100 \\
100 \\
100 \\
100 \\
100 \\
100 \\
100 \\
100\end{array}$ \\
\hline
\end{tabular}

CROSSWIND INTEGRATED = R.36?F-n:4 $1.505 E-03$

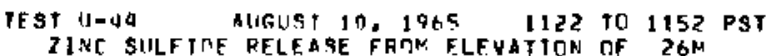
200 HAC SAMFLER HT $1.5 \mathrm{H}^{\circ} \mathrm{U}=1.6 \mathrm{M} / \mathrm{SEC}$ TT $26 \mathrm{M}$

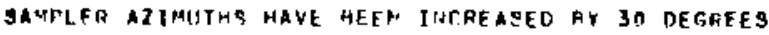

\begin{tabular}{|c|c|c|c|c|c|}
\hline \multicolumn{2}{|c|}{21 MUTH } & $\begin{array}{c}\text { EXPOSURE } \\
\text { GR } \\
\times 1 O C>C U .1 \\
X 1 O E+b\end{array}$ & $\begin{array}{c}5 / 0 \\
\operatorname{ser} / \mathrm{CH}^{+H}\end{array}$ & $\begin{array}{l}E U / 0 \\
1 / 30, k\end{array}$ & $\begin{array}{l}\text { DISTANCE } \\
\text { RETERS }\end{array}$ \\
\hline & 12.0 & ?644 & $6.3 a 2 E=06$ & $1.142 E-05$ & 200 \\
\hline & 16.0 & 5371 & 1. RAAE $=05$ & $2.319 E-05$ & 200 \\
\hline & 20.0 & 1801 & $4.417 E=06$ & $7.951 E=06$ & 200 \\
\hline & 24.0 & 1554 & $3.727 E=0 A$ & $6.708 E-06$ & 200 \\
\hline & 28.0 & 772 & $1.952 E-0 B$ & $3.3335=06$ & 200 \\
\hline & 32.0 & 221 & $5.301 \mathrm{E}=07$ & $9.541 E=07$ & 200 \\
\hline & 36.0 & 1094 & $2.601 E=06$ & $4.681 F=0.6$ & 200 \\
\hline & $a n, 0$ & 1165 & $2.790 \mathrm{E}-116$ & $5.029 E=06$ & 200 \\
\hline & 44,0 & 1712 & $106 \mathrm{E}-06$ & $7.391 \epsilon-06$ & 200 \\
\hline & 49.0 & 24月1 & $5.951 E=06$ & $1.0716=05$ & 200 \\
\hline & 52.0 & 496 & $1.190 E=06$ & $2.191 E=06$ & 200 \\
\hline & 56.0 & 1428 & $3.425 E-06$ & $6.166 E-06$ & 200 \\
\hline & 60.0 & 2792 & $A .698 E-06$ & $1.206 E=05$ & 200 \\
\hline & 69.0 & 6207 & $1.49 \mathrm{AE}-05$ & $2.697 E-05$ & 200 \\
\hline & 68.0 & 5113 & $1.226 E=05$ & $2.207 E-05$ & 200 \\
\hline & 72.0 & 0724 & $1.133 E=05$ & $2.034 E-05$ & 200 \\
\hline & 76.0 & 3936 & 9. $4 \triangle A \mathrm{AE}=06$ & $1.699 E-05$ & 200 \\
\hline & 80.0 & $40 B 4$ & $9.795 E-0 B$ & $1.763 E=05$ & 200 \\
\hline 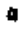 & 84.0 & 916 & $2.365 E-06$ & $4.257 E-06$ & 200 \\
\hline & 88.0 & $22 \div 6$ & $5.2018-06$ & 9.52 AE - 06 & 200 \\
\hline & 92.0 & 1509 & $3.621 E=06$ & $6.518 E=06$ & 200 \\
\hline & 96.0 & $5 t a$ & $1.23 \mathrm{AE}=06$ & $2.222 E=06$ & 200 \\
\hline 3 & 100.0 & 27 & $4.59 A E=08$ & $1.187 E-07$ & 200 \\
\hline & 104.0 & 56 & $1.36 \times E=07$ & $2.959 E-07$ & 200 \\
\hline & 100.0 & 12 & $3.063 E=01$ & $5.513 E=08$ & 200 \\
\hline & $112+0$ & 0 & $0.000 E+00$ & $0.000 E+00$ & 200 \\
\hline & 116.0 & 11 & $2.127 E-08$ & $5.060 E-00$ & 200 \\
\hline & 120.0 & 21 & $5.1 \mathrm{AB}=0 \mathrm{~A}$ & $9.3295=08$ & 200 \\
\hline & 129.0 & 14 & $3.534 E=00$ & $.361 E-08$ & 200 \\
\hline & 129.0 & 0 & $0.100 E+00$ & $0.000 E+00$ & 200 \\
\hline & 132.0 & 31 & 7.53 OE - OA & $1.357 \mathrm{E}-07$ & 200 \\
\hline & 152.0 & 15 & $3.769 E-06$ & $6.785 E-08$ & 200 \\
\hline
\end{tabular}

CAMSGWINO IHTEGRATEDE 1.7N1E-03 3.205E-03 


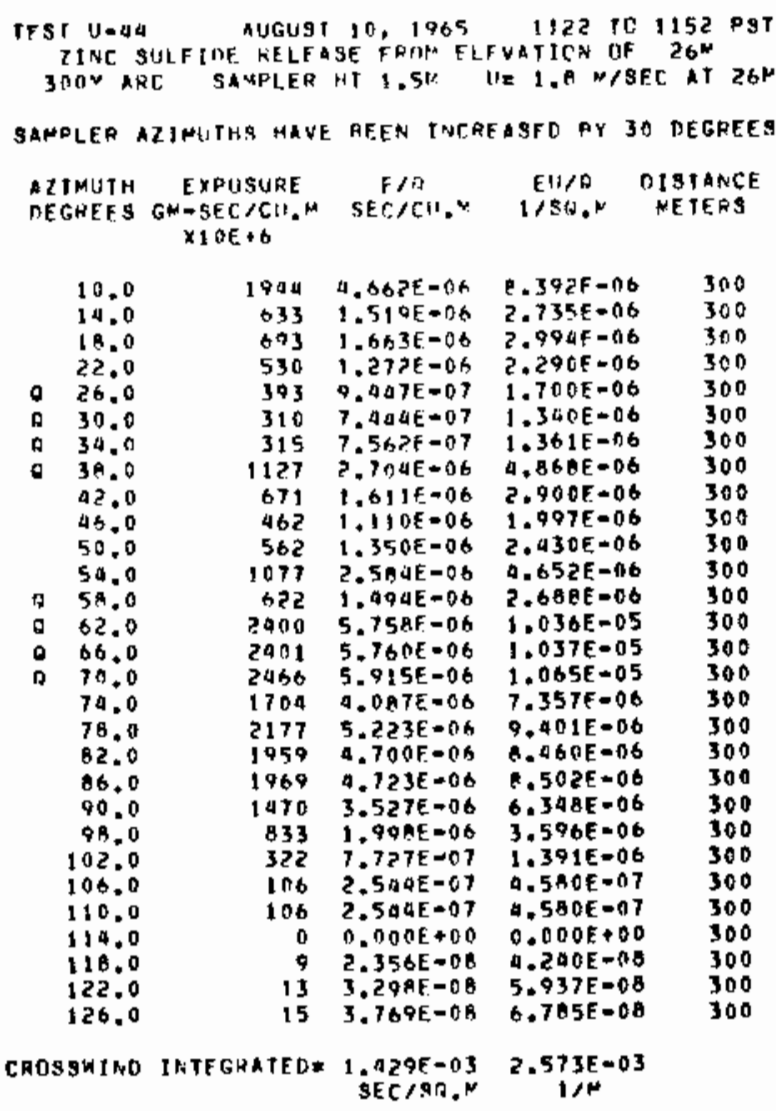
TEST U-44 AERUST 10 , 10R5 1 I2Z TO 1152 PST ZINC SULFIUE RELEASE FROP” FLFUATITM OF TMM WOOW AFC SAMPLER HF $1.5 \mathrm{~K} \quad H=1$. $\mathrm{M} / \mathrm{SEC}$ AT $26 \mathrm{M}$

SANPLER AZIMUTHA HAVE BEET, INCHEASE AY 30 DEGFEES

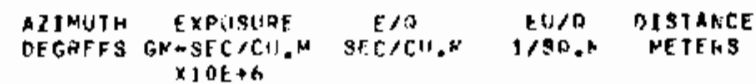

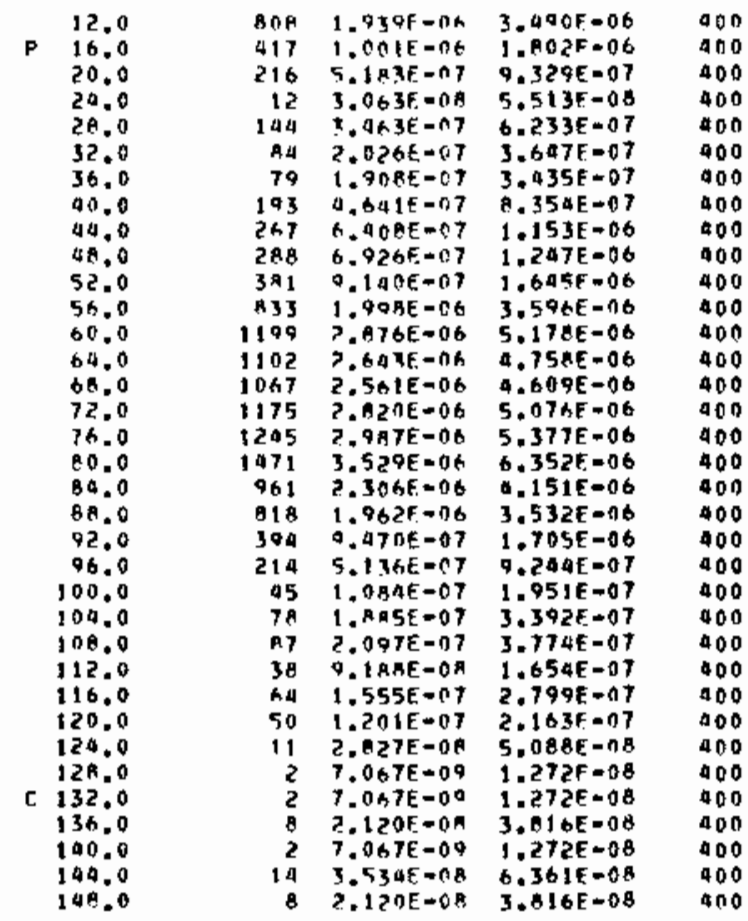

CRNSSWINO INTEGRATED $=9.239 E-0.40635-03$ 


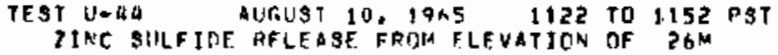
IINC SIILFIDE RFLEASE. FRDIA FLEVATICN OF $26 \mathrm{M}$
$500 \mathrm{M}$ AFC SAMPLER. HT $1,5 \mathrm{~N}$ UE 1 , M/SEC AF $26 \mathrm{M}$

SANPLER AZIMHTHS HAVE GEEN TIERFASED FY 30 DEREEES AZIMUTH EXPUSUAE
DEGREES GN-SEC/CU.M GECICU.N TISG.M MISTANCE $x \backslash 0 \mathrm{E}+6$

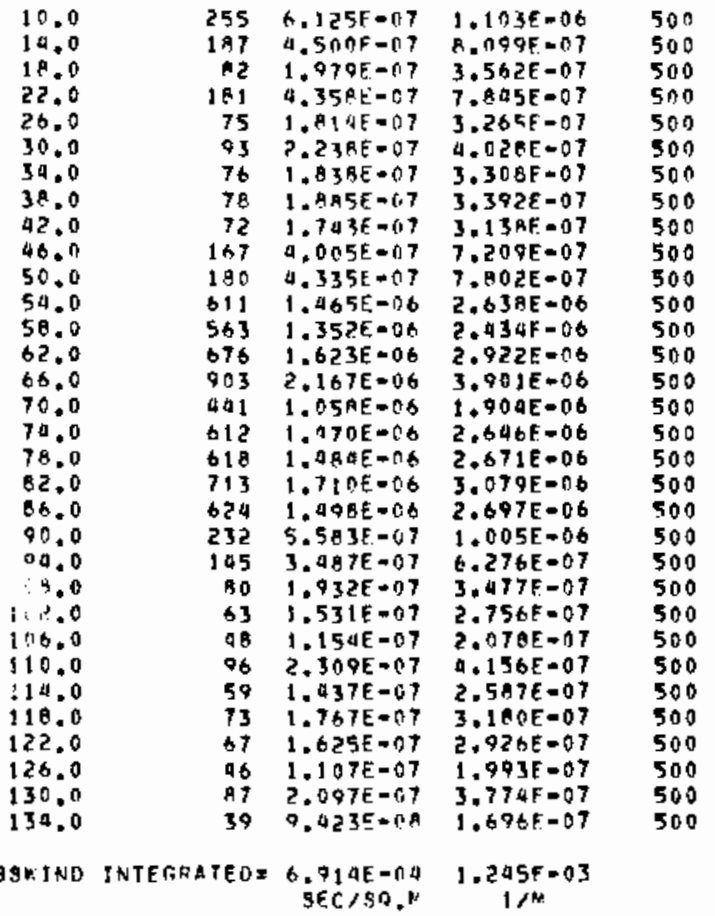

IEST II-4D AUSUAT IA, 19R5 112? TO 115? PST ZINC SULFIJE RELFAGE FROM FLEVATICV OF $26 \mathrm{~N}$ 6004 ARC SAPIFLER MP $1.5 \mathrm{M}$ LF 1 . 9 M/SFC AT $26 \mathrm{M}$

SAMPLFR ATIMLITHS HAVE REFN ITECREASED FY 30 DERREE

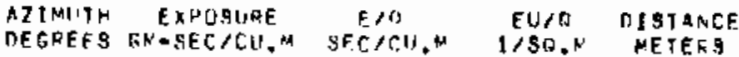
$x] \cap E+b$

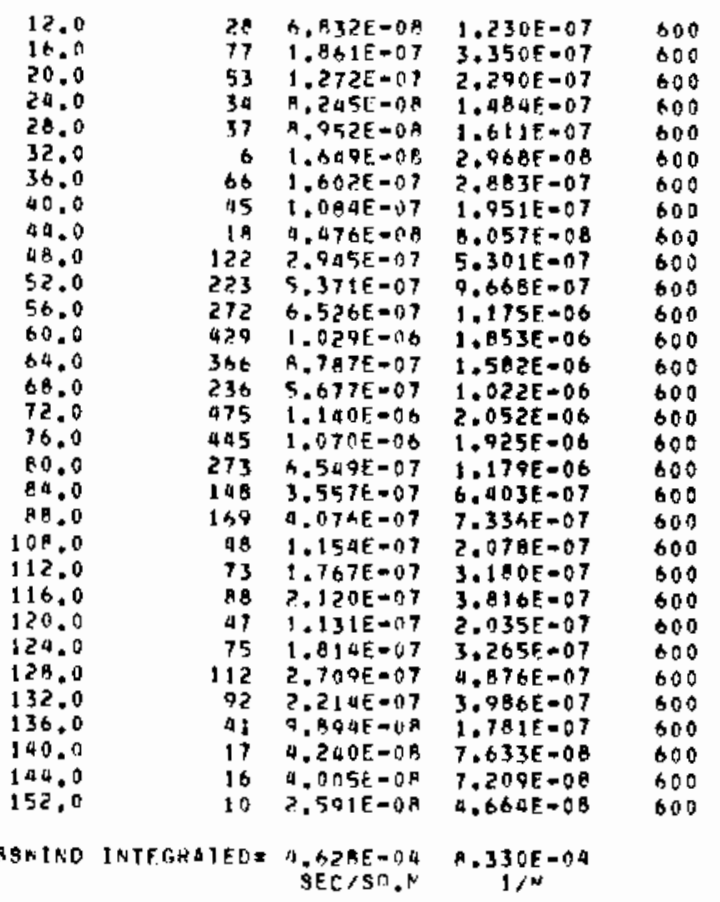




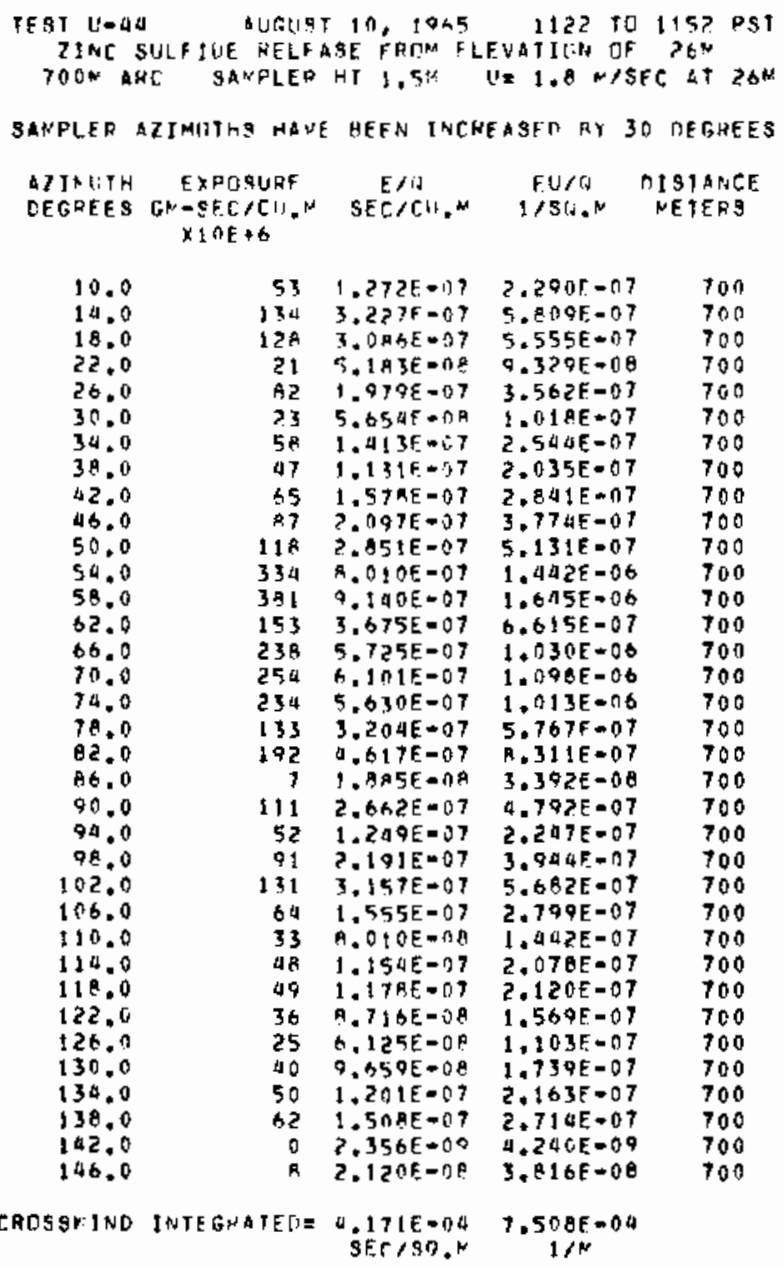

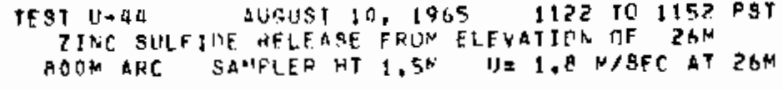

SANPLER AZIMUTHO HAVE AEFH THCREASET PY 30 CETERES

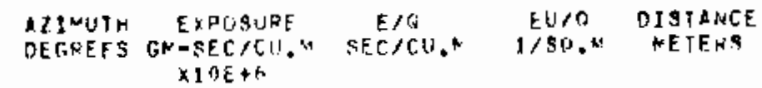

\begin{tabular}{|c|c|c|c|c|}
\hline $\begin{array}{l}10.0 \\
13.0 \\
16.0 \\
19.0 \\
22.0 \\
25.0 \\
28.0 \\
31.0 \\
34.0 \\
37.0 \\
40.0 \\
43.0 \\
46.0 \\
49.0 \\
52.0 \\
55.0 \\
58.0 \\
61.0 \\
64.0 \\
67.0 \\
70.0 \\
82.0 \\
85.0 \\
88.0 \\
91.0 \\
94.0 \\
97.0 \\
100.0 \\
103.0 \\
106.0 \\
109.0 \\
112.0 \\
115.0 \\
118.0 \\
121.0 \\
120.0 \\
127.0 \\
130.0 \\
133.0 \\
136.0 \\
139.0\end{array}$ & $\begin{array}{r}104 \\
86 \\
131 \\
76 \\
68 \\
92 \\
54 \\
95 \\
91 \\
63 \\
65 \\
156 \\
83 \\
204 \\
238 \\
340 \\
127 \\
375 \\
222 \\
192 \\
10 \\
222 \\
111 \\
98 \\
216 \\
94 \\
92 \\
64 \\
67 \\
75 \\
42 \\
44 \\
46 \\
33 \\
27 \\
41 \\
22 \\
16 \\
47 \\
23 \\
28\end{array}$ & 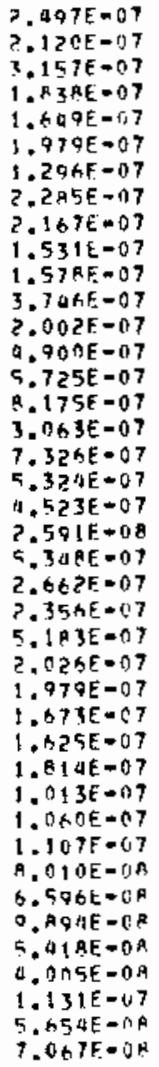 & 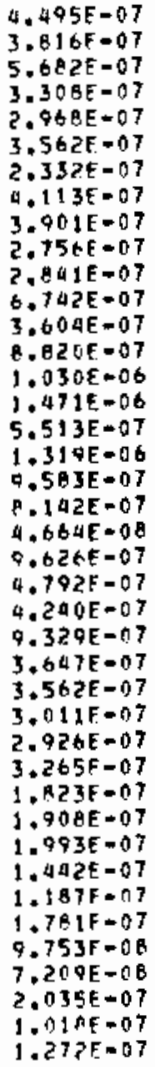 & $\begin{array}{l}100 \\
800 \\
800 \\
800 \\
800 \\
800 \\
800 \\
800 \\
800 \\
800 \\
800 \\
800 \\
800 \\
800 \\
800 \\
800 \\
800 \\
000 \\
800 \\
800 \\
800 \\
800 \\
800 \\
800 \\
000 \\
800 \\
100 \\
100 \\
800 \\
800 \\
800 \\
800 \\
800 \\
800 \\
800 \\
800 \\
800 \\
000 \\
800 \\
800 \\
800\end{array}$ \\
\hline asskIND & TEGIFATEC & 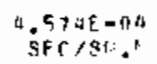 & $\begin{array}{c}A .233 E=04 \\
1 / 1\end{array}$ & \\
\hline
\end{tabular}




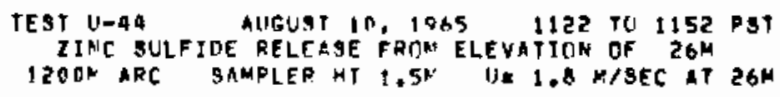

SAMPLER AZIMUTHS MQVE REEN INCREASED BY 30 DEGREES

\begin{tabular}{|c|c|c|c|c|}
\hline $\begin{array}{l}\text { AZIMUTH } \\
\text { DEGAEES }\end{array}$ & $\begin{array}{c}\text { EXPOSURE } \\
\text { GNESEC/C11.M } \\
\times 10 E+6\end{array}$ & $\begin{array}{c}E / A \\
S E C / C U, M\end{array}$ & $\begin{array}{l}E l 1 / 0 \\
1 / 30, M\end{array}$ & $\begin{array}{l}\text { DIOTANCE } \\
\text { METERS }\end{array}$ \\
\hline $\begin{array}{l}30.0 \\
32.0 \\
34.0 \\
36.0 \\
38.0 \\
40.0 \\
42.0 \\
44.0 \\
96.0 \\
0.00 \\
54.0 \\
56.0 \\
58.0 \\
60.0 \\
62.0 \\
60.0 \\
66.0 \\
68.0 \\
70.0 \\
72.0 \\
70.0 \\
75.0 \\
78.0 \\
10.0 \\
62.0 \\
84.0 \\
86.0 \\
88.0 \\
40.0 \\
42.0 \\
90.0 \\
96.0 \\
98.0 \\
100.0 \\
102.0 \\
104.0 \\
106.0 \\
108.0 \\
110.0 \\
112.0 \\
119.0 \\
118.0 \\
120.0 \\
122.0 \\
124.0 \\
126.0 \\
128.0 \\
130.0\end{array}$ & $\begin{array}{r}79 \\
50 \\
98 \\
97 \\
158 \\
73 \\
124 \\
147 \\
192 \\
128 \\
255 \\
223 \\
245 \\
196 \\
178 \\
111 \\
154 \\
157 \\
169 \\
13 \\
113 \\
65 \\
145 \\
56 \\
22 \\
60 \\
40 \\
55 \\
73 \\
41 \\
43 \\
29 \\
28 \\
18 \\
27 \\
18 \\
90 \\
38 \\
61 \\
52 \\
16 \\
38 \\
19 \\
39 \\
70 \\
16 \\
15 \\
55\end{array}$ & 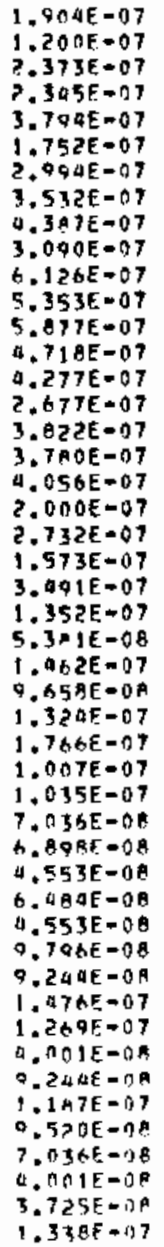 & 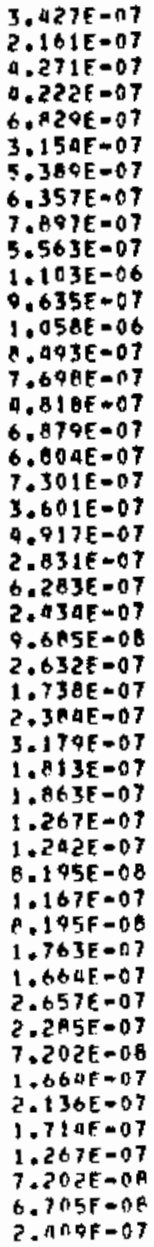 & $\begin{array}{l}1200 \\
1200 \\
1200 \\
1200 \\
1200 \\
1200 \\
1200 \\
1200 \\
1200 \\
1200 \\
1200 \\
1200 \\
1200 \\
1200 \\
1200 \\
1200 \\
1200 \\
1200 \\
1200 \\
1200 \\
1200 \\
1200 \\
1200 \\
1200 \\
1200 \\
1200 \\
1200 \\
1200 \\
1200 \\
1200 \\
1200 \\
1200 \\
1200 \\
1200 \\
1200 \\
1200 \\
1200 \\
1200 \\
1200 \\
1200 \\
1200 \\
1200 \\
1200 \\
1200 \\
1200 \\
1200 \\
1200 \\
1200\end{array}$ \\
\hline
\end{tabular}

TEST UEA UUGUST 10,1965 1 122 TO 3152 PST IINC SILLIDE RELEASE FPON ELEVATION OF $20 \mathrm{M}$ $1600 \mathrm{~K}$ ARC SAPPLER HT $1.5 \%$ U. $1.8 \mathrm{M} / \mathrm{SEC}$ AT $26 \mathrm{M}$

SAMPLER AZIMUTHS HAVE REEN IHCREASF BY 30 DEGREES

\begin{tabular}{|c|c|c|c|c|}
\hline $\begin{array}{l}\text { DINUTH } \\
\text { DEGREES }\end{array}$ & $\begin{array}{c}\text { EXPOSURE } \\
\text { GW } \rightarrow \text { SEC } / C U \cdot M \\
\text { XIOE }+6\end{array}$ & $\begin{array}{c}E / 0 \\
\sec /(1) *\end{array}$ & $\begin{array}{l}E U / O \\
1 / 3 a_{*} M\end{array}$ & $\begin{array}{l}\text { OISTANCE } \\
\text { METERS }\end{array}$ \\
\hline $\begin{array}{l}30.0 \\
32.0 \\
34.0 \\
36.0 \\
38.0 \\
40.0 \\
42.0 \\
40.0 \\
46.0 \\
48.0 \\
50.0 \\
52.0 \\
54.0 \\
56.0 \\
58.0 \\
60.0 \\
62.0 \\
64.0 \\
66.0 \\
64.0 \\
70.0 \\
72.0 \\
74.0 \\
76.0 \\
70.0 \\
80.0 \\
82.0 \\
84.0 \\
86.0 \\
88.0 \\
40.0 \\
92.0 \\
04.0 \\
96.0 \\
98.0 \\
100.0 \\
102.0 \\
104.0 \\
110.0 \\
114.0 \\
116.0 \\
118.0 \\
122.0 \\
124.0 \\
126.0 \\
120.0 \\
130.0 \\
134.0 \\
136.0\end{array}$ & $\begin{array}{r}37 \\
42 \\
58 \\
66 \\
70 \\
71 \\
28 \\
6 \\
61 \\
53 \\
63 \\
98 \\
105 \\
40 \\
65 \\
6 \\
66 \\
50 \\
41 \\
39 \\
49 \\
41 \\
40 \\
40 \\
24 \\
36 \\
24 \\
16 \\
27 \\
20 \\
9 \\
14 \\
11 \\
23 \\
17 \\
25 \\
20 \\
16 \\
11 \\
10 \\
10 \\
17 \\
18 \\
21 \\
12 \\
14 \\
20 \\
9 \\
9\end{array}$ & 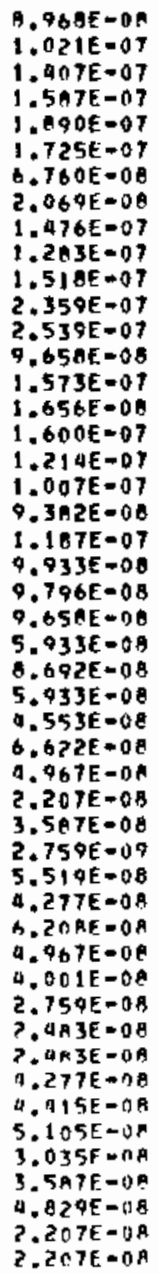 & 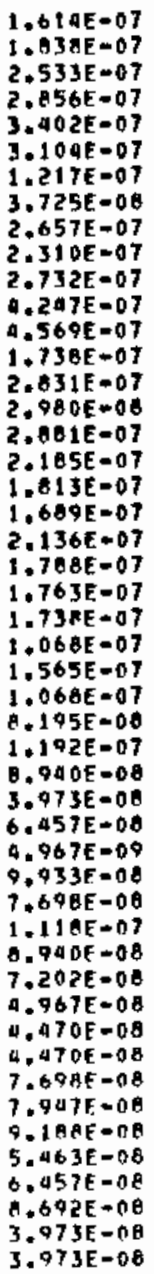 & $\begin{array}{l}1600 \\
1600 \\
1600 \\
1600 \\
1600 \\
1600 \\
1600 \\
1600 \\
1600 \\
1600 \\
1600 \\
1600 \\
1600 \\
1600 \\
1600 \\
1600 \\
1600 \\
1600 \\
1600 \\
1600 \\
1600 \\
1600 \\
1600 \\
1600 \\
1600 \\
1600 \\
1600 \\
1600 \\
1600 \\
1600 \\
1600 \\
1600 \\
1600 \\
1600 \\
1600 \\
1600 \\
1600 \\
1600 \\
1600 \\
1600 \\
1600 \\
1600 \\
1600 \\
1600 \\
1600 \\
1600 \\
1600 \\
1600 \\
1600\end{array}$ \\
\hline & RTEFRATEDT & $\begin{array}{l}P .375 E=04 \\
S F C / S U . N\end{array}$ & $4.275 \mathrm{~F}=00$ & \\
\hline
\end{tabular}


UASTABLE SERIES RXPERTMEAT: 45 (J-45)

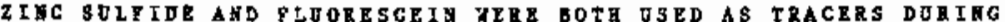

EIPBRTHEGT 45. ZIHC SOLFIDB RESTLTS ARE PRESBRTED GEBE. GROUND

LEVRL SAYPLI\&G 200-i600 B ABC.

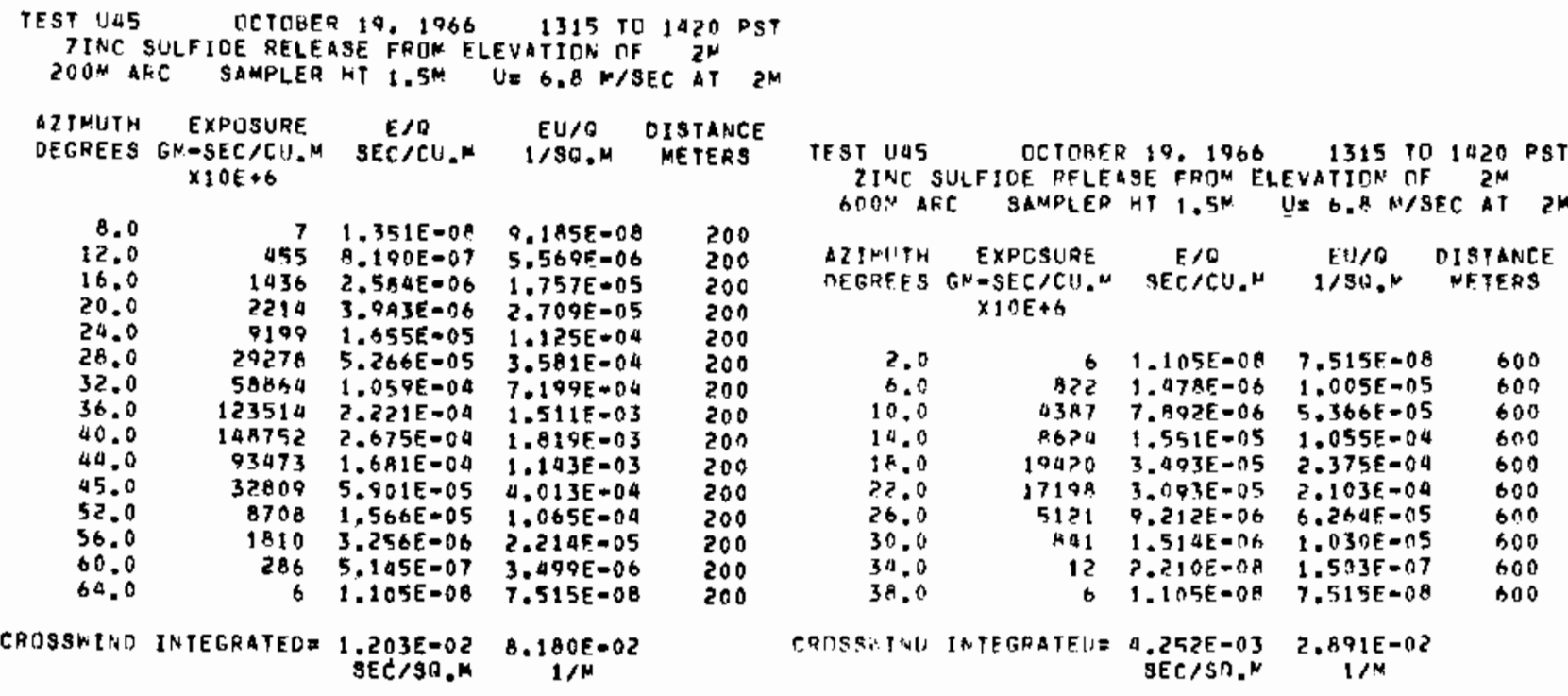

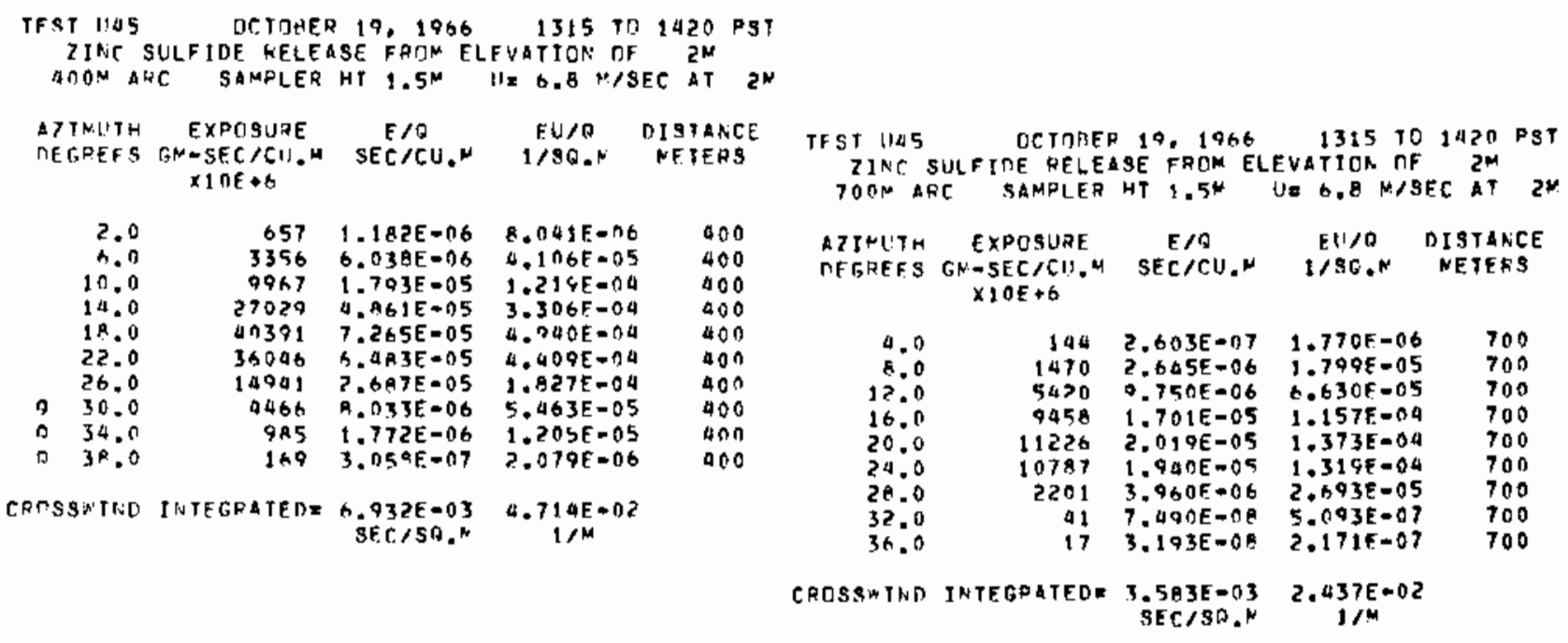




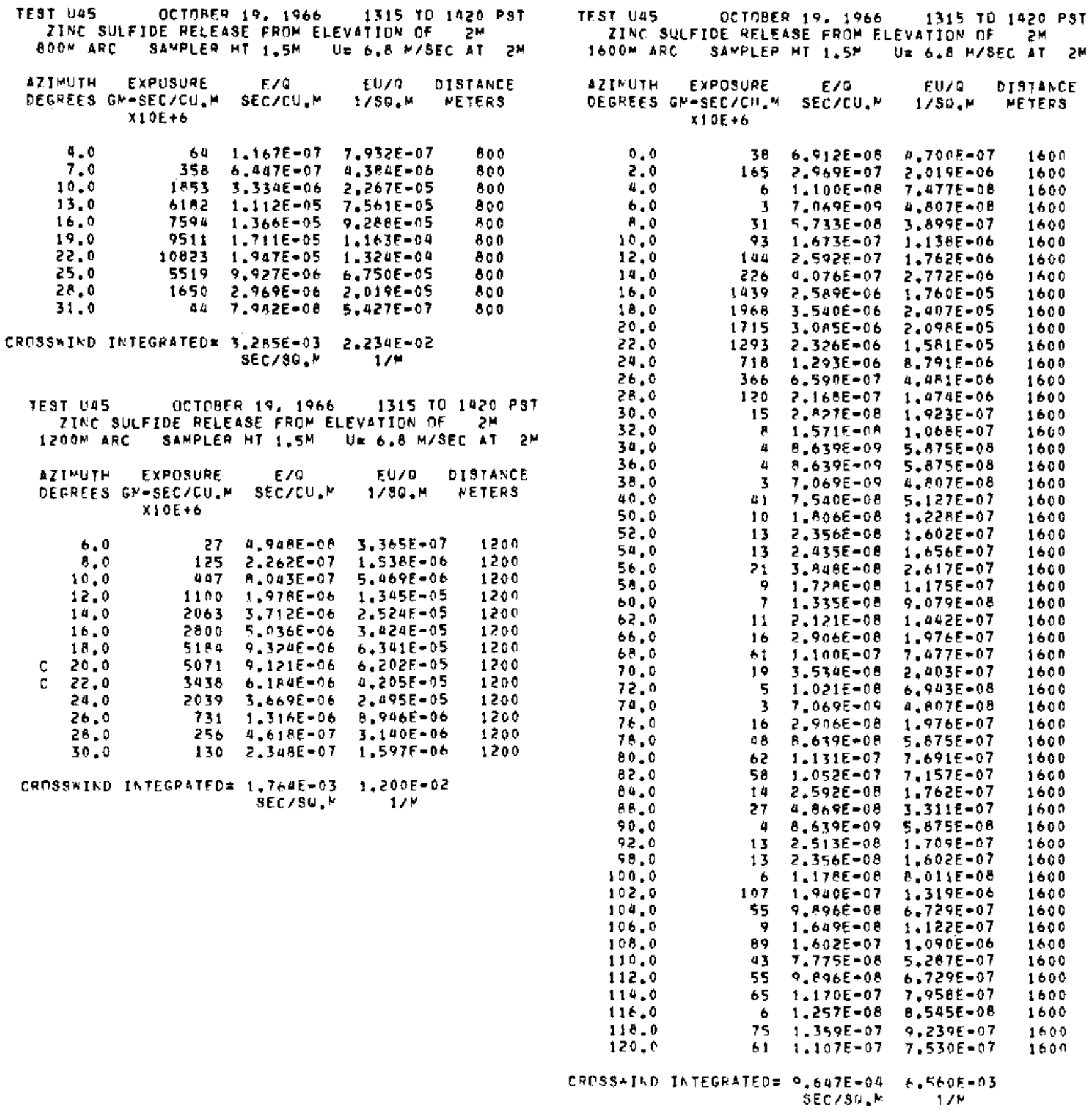


TNSTABLE SERIES EXPEREMENT: 45F ( T-45F)

ZINC SOLFIDE AD PIJORESCEIN URBE 30TH TSED AS TZACERE DORING

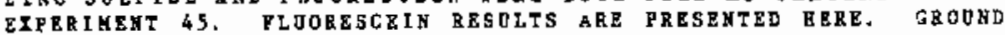

LEVEL SAHPLIEG $200-1600$ M ARC.

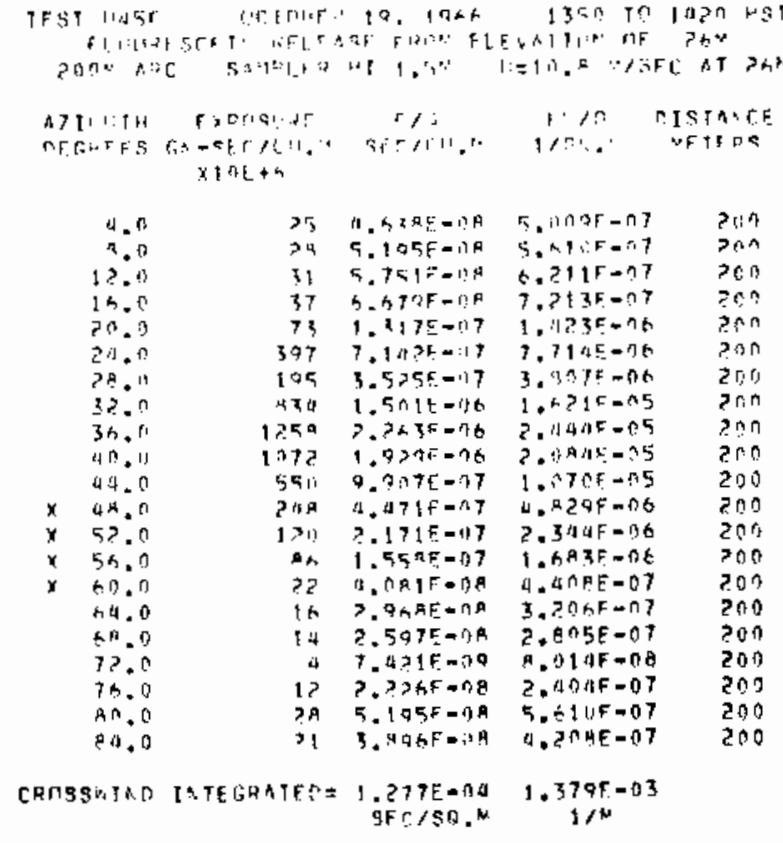

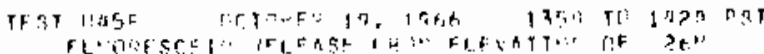

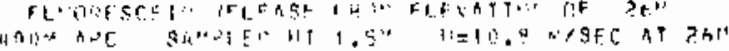

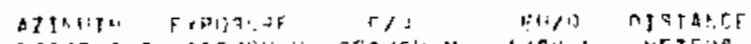

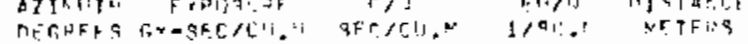
$x$ in +0

\begin{tabular}{|c|c|c|c|c|c|}
\hline & a. $n$ & 174 & 1. .87$\lrcorner 5=07$ & 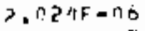 & $f(?) \pi$ \\
\hline & H.O & וד & $1.1775=96$ & $1,595 F=15$ & $f \circ 00$ \\
\hline & 12.0 & $1+12$ & $2.95 \cap F-1) 6$ & 5.1 RूE $=15$ & 46." \\
\hline & $1+.6$ & $25 \times 7$ & $1.5 n$ या $=0 \rightarrow$ & $a .0 ? 95=05$ & $3 n_{i 1}$ \\
\hline & $2 n .0$ & $\tan x 1$ & $7,73 \cap f=36$ & $A .994 F=55$ & ann \\
\hline & 24.0 & $41+2$ & "A $A>F=9 A$ & $9.5175-105$ & ד \\
\hline & $?^{P} \cdot 0$ & 75,54 & $4,4+15=136$ & $4.969 F=195$ & חִ \\
\hline 7 & 32.0 & 4,5 & $f .197 \mathrm{E}=? \mathrm{H}$ & $1.2025=05$ & aof \\
\hline 7 & 34.0 & 110 & $1.9 \times 5 E=07$ & $2.14+5=08$ & 407 \\
\hline 7 & $4 n, 0$ & ? & $5.1 \cap 5 F, n A$ & $5.6105=07$ & $a 00$ \\
\hline$z$ & 44.2 & $P 6$ & a. $2>3 F=0 A$ & $5.209 \mathrm{~F}=07$ & 100 \\
\hline & 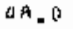 & .5 & A. ANAE $=0$, & $5,1) \cap Q E-07$ & 100 \\
\hline & 57.0 & 23 & $1.247 E=0 R$ & d. $\{\cap 9 E-\cap 7$ & $\operatorname{ann}$ \\
\hline & & 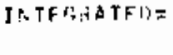 & 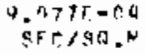 & 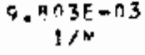 & \\
\hline
\end{tabular}

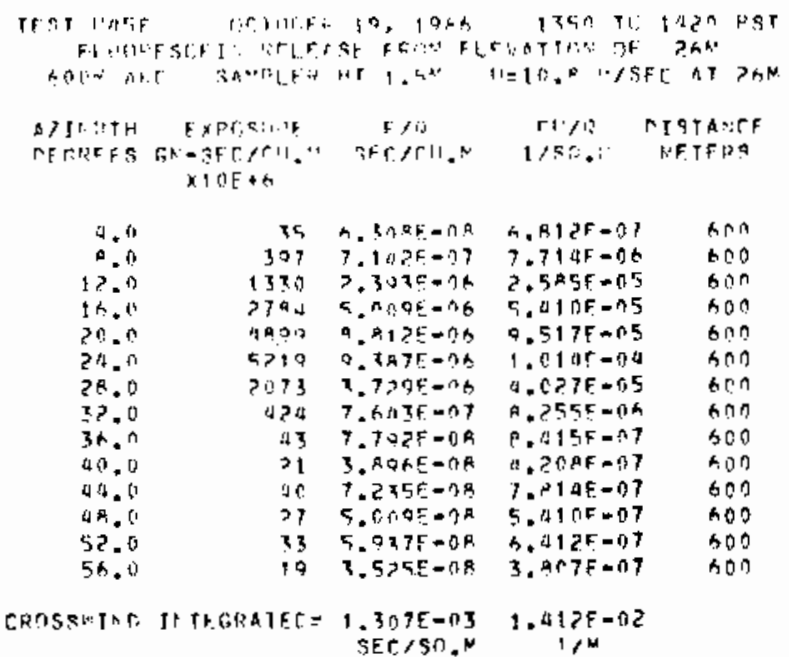

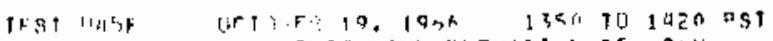

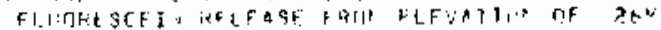

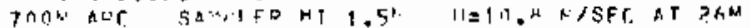

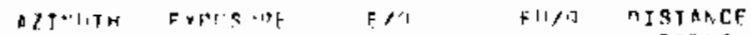

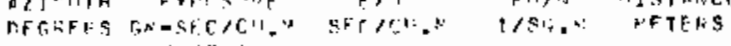
$\times 1 M E+6$

\begin{tabular}{|c|c|c|c|}
\hline 4.0 & 259 & $4.4758=6.7$ & $5 . P 119=06$ \\
\hline P. 0 & an? & A.7J日F $=07$ & $8.93 \times 5=16$ \\
\hline 12,0 & 1621 & T. $n+>E-n: A$ & $3.2 A+E=05$ \\
\hline 16.0 & रव वर्प & $A .1, P E=0 A$ & $a .+12 F=05$ \\
\hline 20.0 & Q74: & 9.53 aE $=\| \mathrm{H}$ & ?TE-0S \\
\hline 24.6 & ד & $A .3 n$ AF $=D A$ & $A . A 12 F-05$ \\
\hline $2 A .11$ & (⿻4 ? ? & $1.1566=176$ & $248 E=05$ \\
\hline 32.0 & 125 & $D A E-0.7$ & $A \cap \Delta F=0 b$ \\
\hline 35.0 & 75 & $4, x \neq A \varepsilon=\sigma_{1} R$ & $0 \cap 9 F-67$ \\
\hline
\end{tabular}

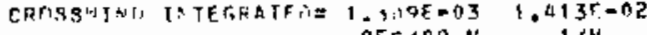




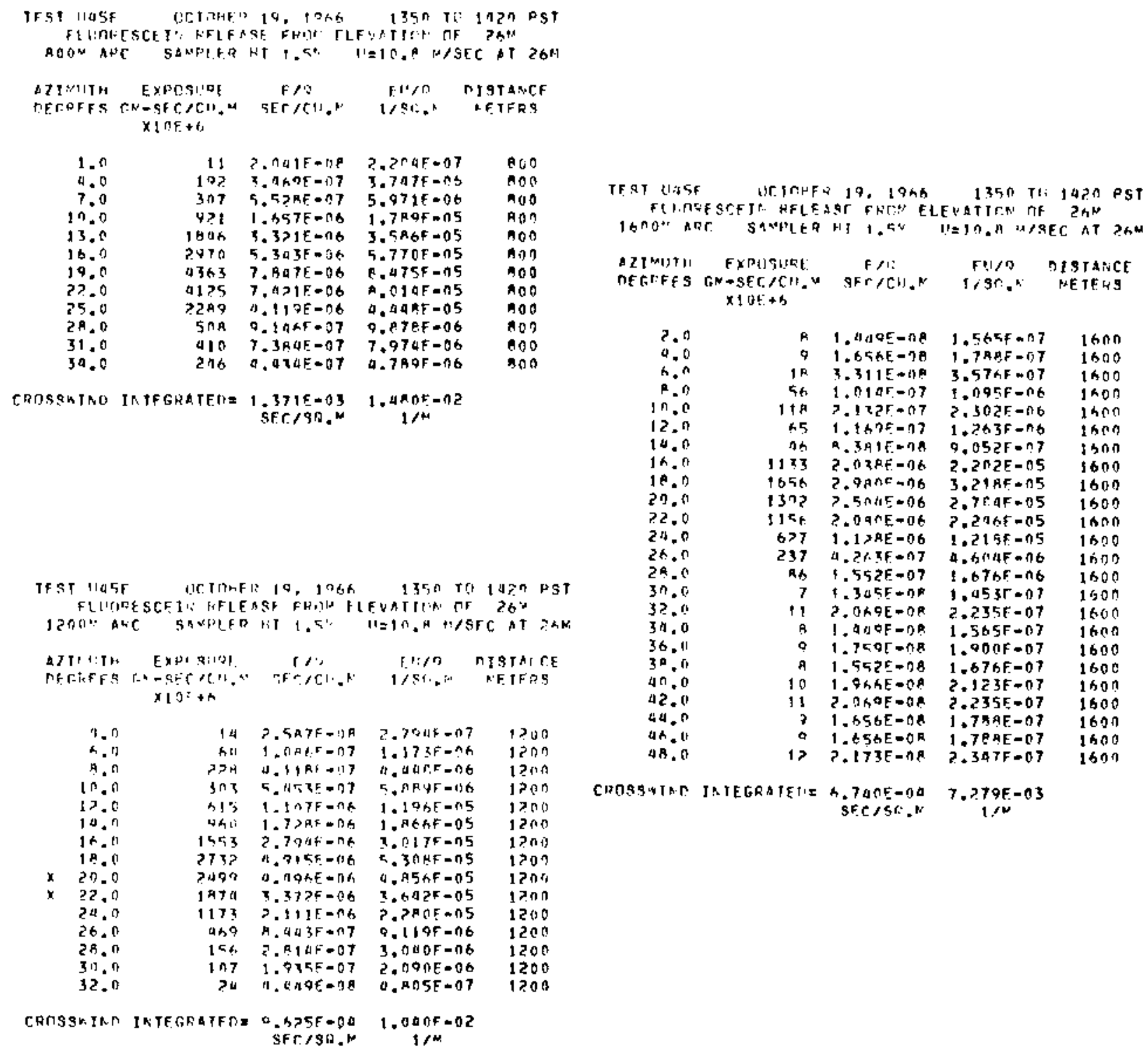


UESTABLE SERIES EXPEEIYBNT: 46 ( $0-46)$

ZIHC STLFIDB ARD FLOORESCEIN WRRE BOTR OSED AS TRACERS DORIRG

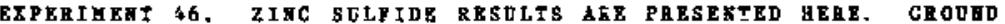
LEVIL SAMPLIBG 200-1600 KARE.

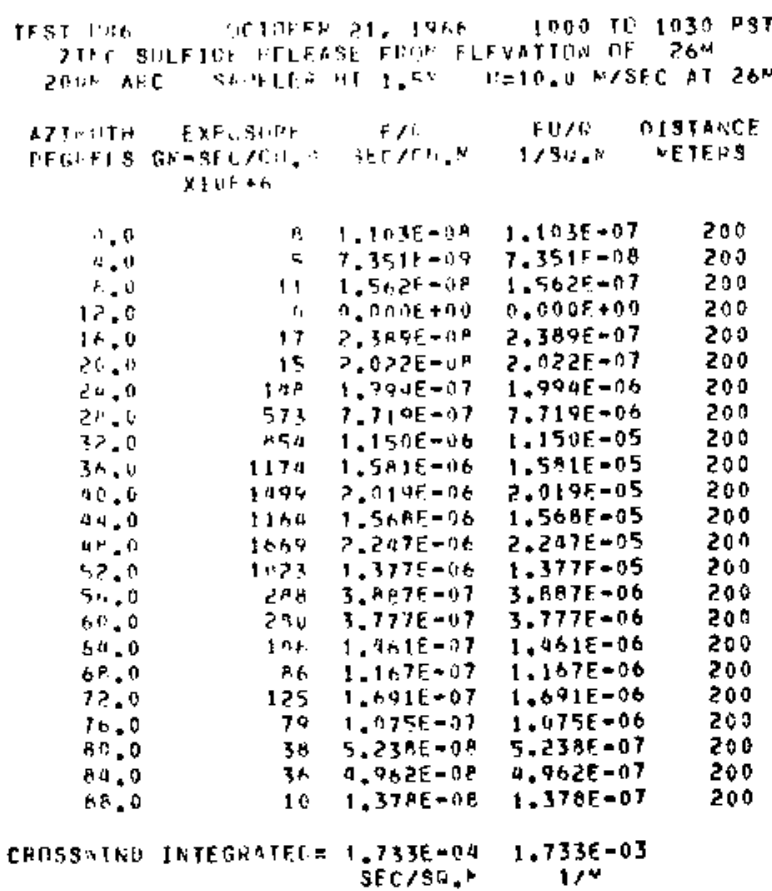

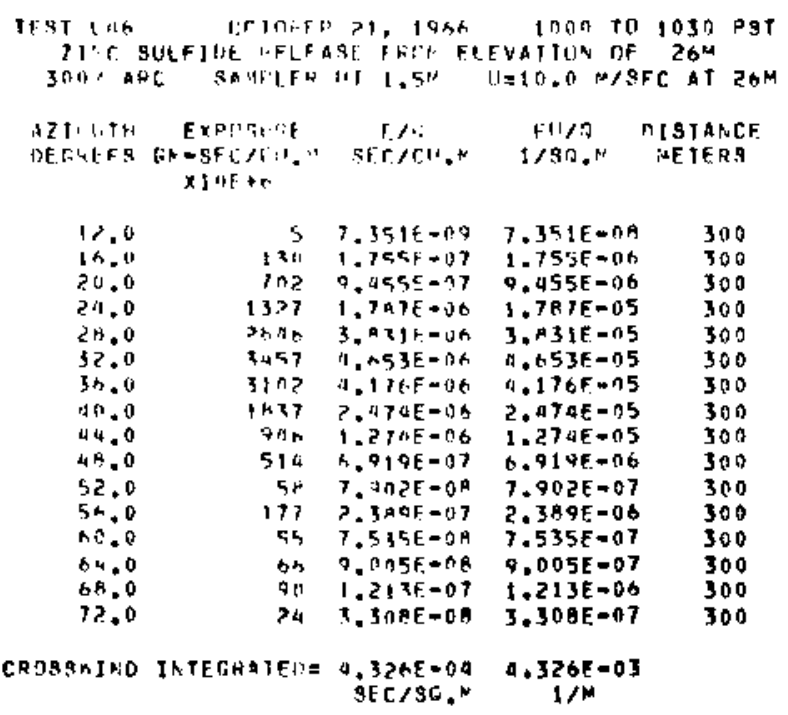

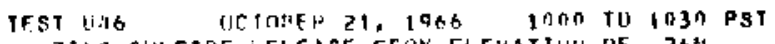
7IT.C SULFIDE VELEAGE FKON FLEVATIUH DF $2 b^{* 4}$

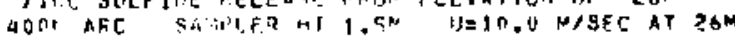

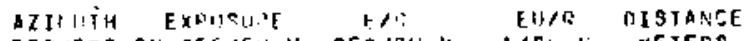

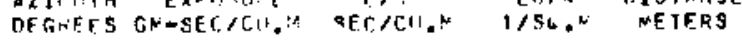
$\times \operatorname{In}+6$

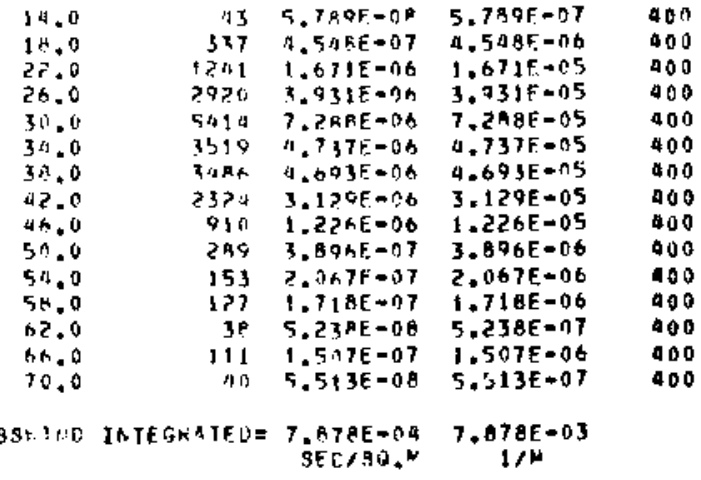

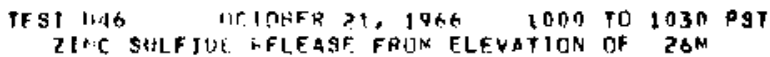

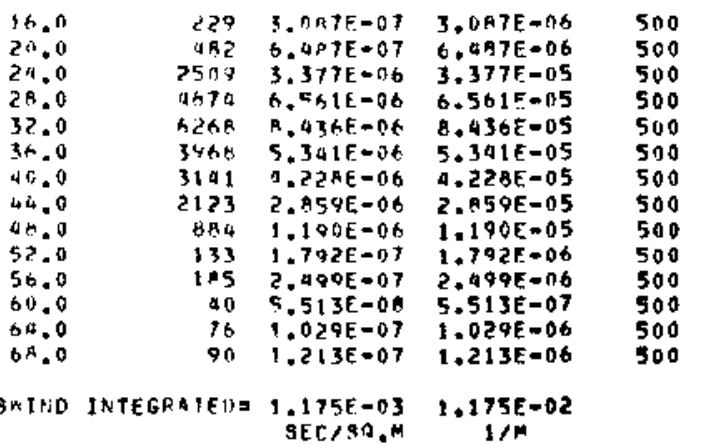




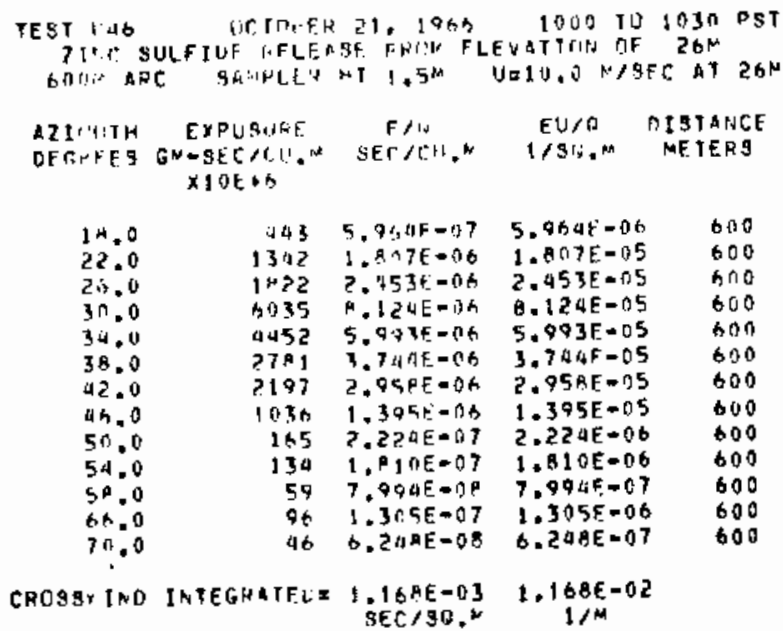

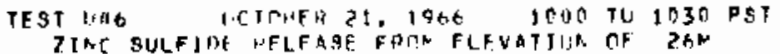

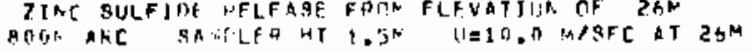

\begin{tabular}{|c|c|c|c|c|}
\hline $\begin{array}{l}\text { AZINALTH } \\
\text { TEFOEESS }\end{array}$ & 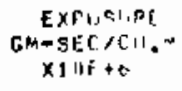 & $\begin{array}{c}f / l ! \\
s F c / C(1 .\end{array}$ & $\begin{array}{c}E 1 />0 \\
1 / 214,\end{array}$ & $\begin{array}{l}\text { PISTANCE } \\
\text { METEAS }\end{array}$ \\
\hline 13.0 & 5 & $7.351 t-09$ & $7.351 \mathrm{~F}=08$ & $\operatorname{son}$ \\
\hline $1+.0$ & 34 & \#. 6 AGE $=0 A$ & A. GASE - O? & 800 \\
\hline 10.0 & 450 & $3.757 \mathrm{E}=07$ & $0.757 \mathrm{E}=00$ & 100 \\
\hline$? .0$ & 614 & $4.27 M E=07$ & $2.270 E-06$ & $a 00$ \\
\hline 24.0 & $12 \leqslant 2$ & $1.609 E=3 h$ & $1.6997-05$ & 800 \\
\hline & 2201 & $2.0+2 E=-6$ & $2.9 A 7,=05$ & 000 \\
\hline 31.0 & $25>0$ & $3.397 \mathrm{E}-0 \mathrm{~b}$ & $3.307 \varepsilon-05$ & 000 \\
\hline 34.0 & $25 n 3$ & $3.37 \mathrm{AF}-06$ & $3.370 f-05$ & 100 \\
\hline 37.0 & $2+40$ & $3.554 F=06$ & J.5S UE -05 & 000 \\
\hline & 772 & 1. $0 A B E=0 \theta$ & $1.040 E=05$ & $\sin 0$ \\
\hline 43.0 & 627 & A. $3 \operatorname{Ln} E=07$ & $A .3 B .0 E-n b$ & 000 \\
\hline 46.0 & 320 & $4.392 \mathrm{~F}=07$ & $4.39 ? \mathrm{E}-06$ & 800 \\
\hline 10.0 & 124 & $1.072 E=07$ & $1.6725=06$ & 800 \\
\hline 52,0 & $A$ & $1.185 E=07$ & $1.195 F \cdot 06$ & 800 \\
\hline .0 & 54 & $7.351 \mathrm{E}=18$ & $7.351 E-07$ & 800 \\
\hline 58.0 & 47 & $6.4325=08$ & $6.032 E=07$ & 800 \\
\hline 01.0 & hos & $9.913 \mathrm{r}=08$ & $0.913 F-177$ & मOO \\
\hline ho. 0 & 8 & $1.135 E=0 A$ & $1.195 t-07$ & 100 \\
\hline 67.0 & 65 & $n .821 \mathrm{E}-0$ & -A? If $f-07$ & 000 \\
\hline 70.0 & 32 & $4.3] \mathrm{OE}=0$. & $4.3 \pm 9 E=07$ & 000 \\
\hline 73.0 & 3 & $4.5941 E=09$ & $4.594 E-08$ & 000 \\
\hline 0 & e & $1.195 \mathrm{E}-0 \mathrm{~B}$ & $1.1058-n 7$ & 000 \\
\hline
\end{tabular}

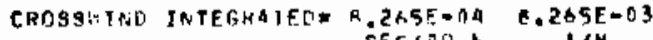

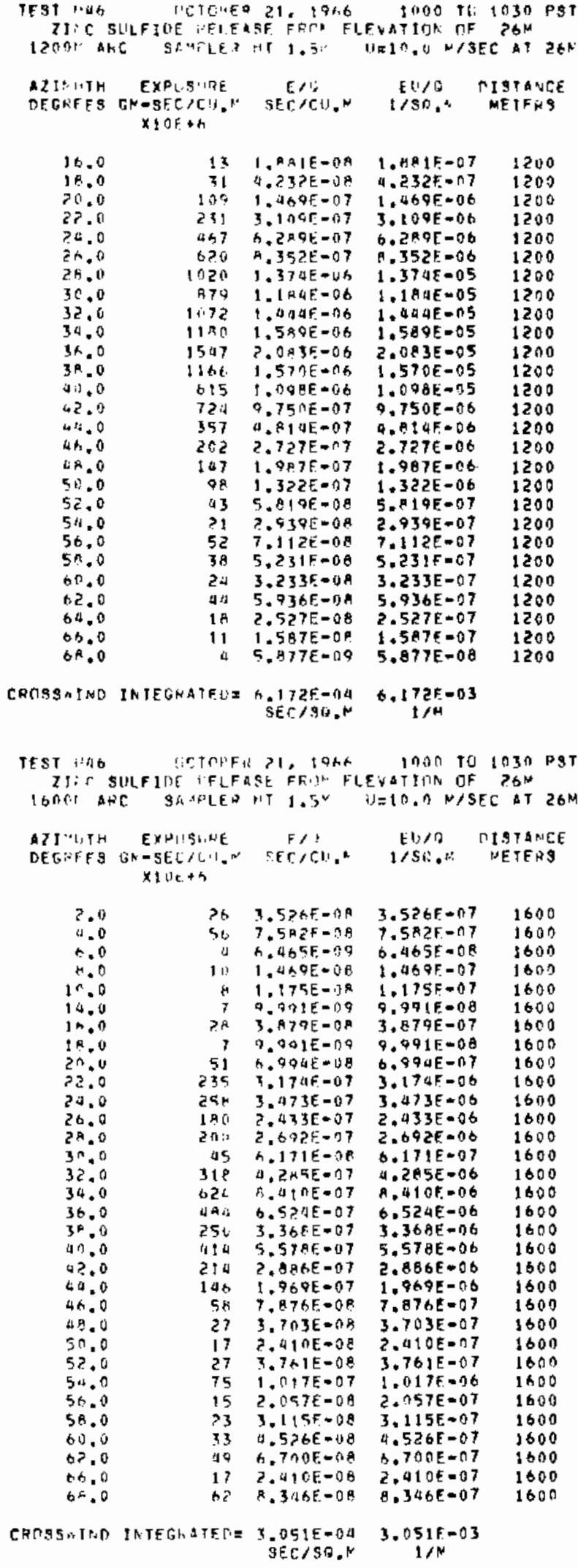


ONSTABLE SERIES EXPERIMENT: $46 \%$ ( $\mathrm{U}-46 \mathrm{~F}$ )

ZINC SUEFIDE AFD FLUORESCEIS WRRE BOTH USED AS IEACEES DURIGG RXFERIMENT 46, FLUORESCEIN KRESLTS ARR PRESENTED BERE. GROUND

LEVEL SAMPLING 200-1EU0 Y ARC.

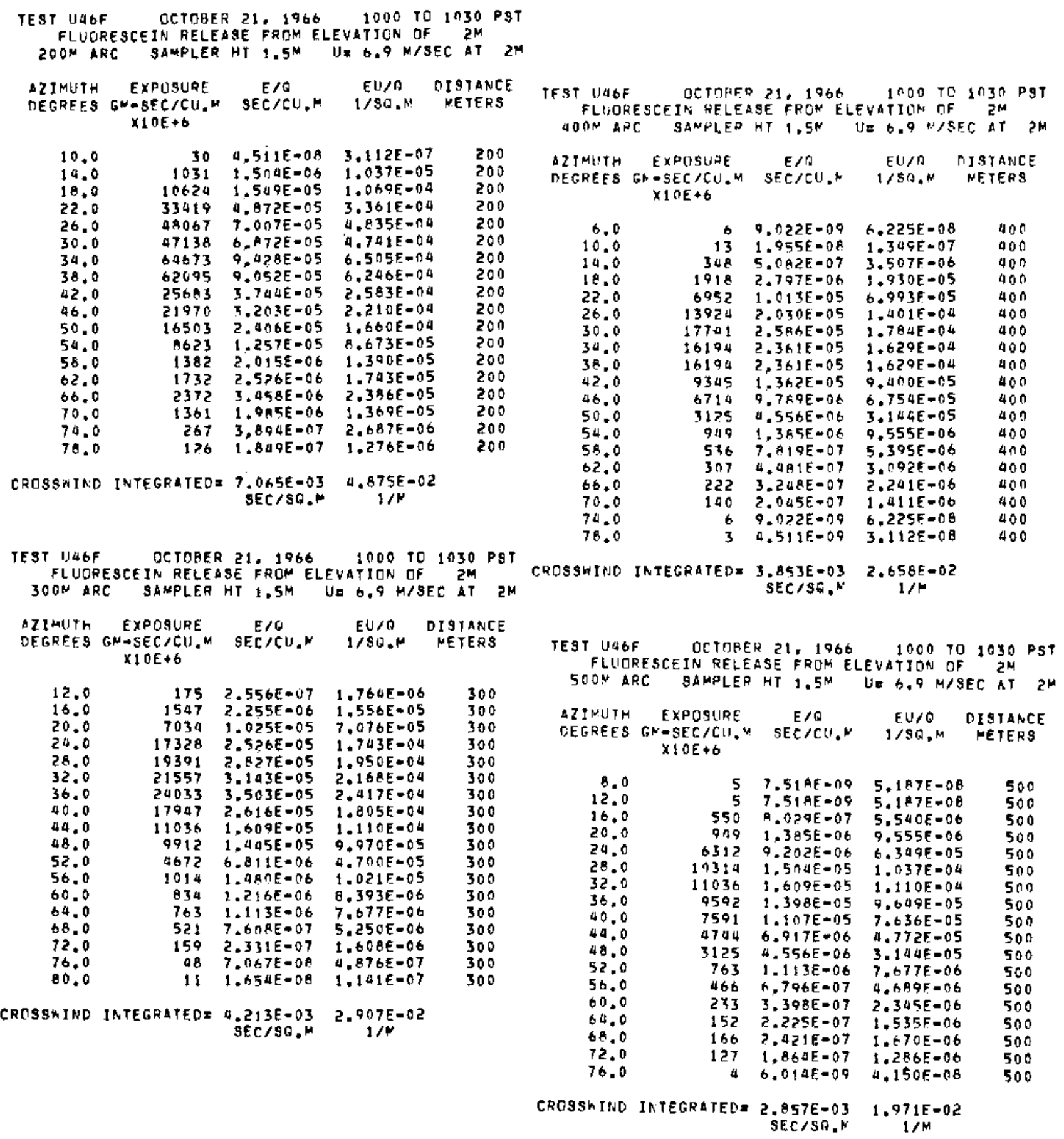




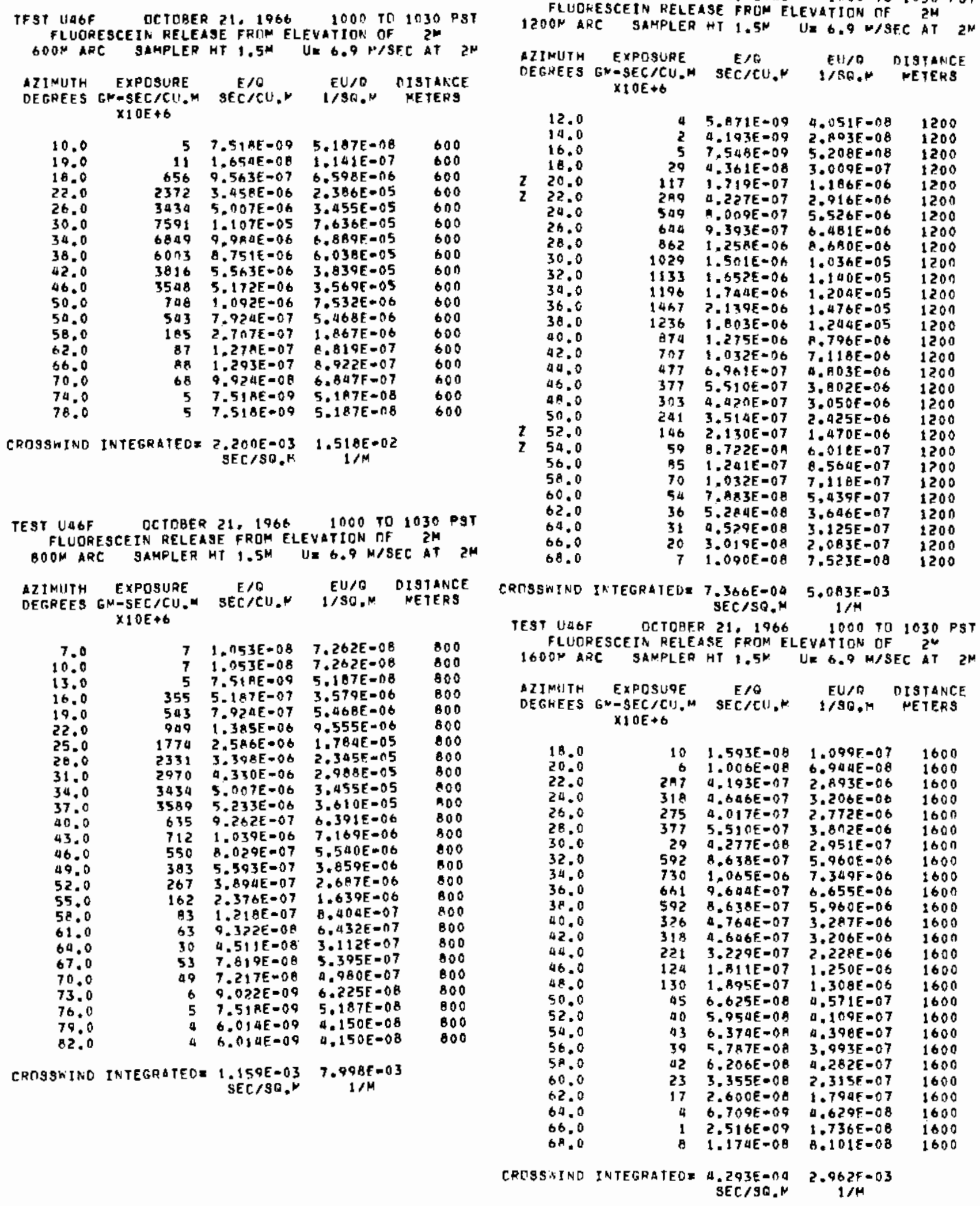


UHSTABLE SBEIBS EIPQRIMENT: 47 (

ZISC SULPIDE ARD FIDORESCETA URRE BOIH JSQD AS TRACERS DERIHC

EXPERINEHT 47. ZIIC SOLFIDB RESULTS ARE PRESEHTED GERB. GROUYO

LEVEL SAFPLIAG 100-1600 A ARC.

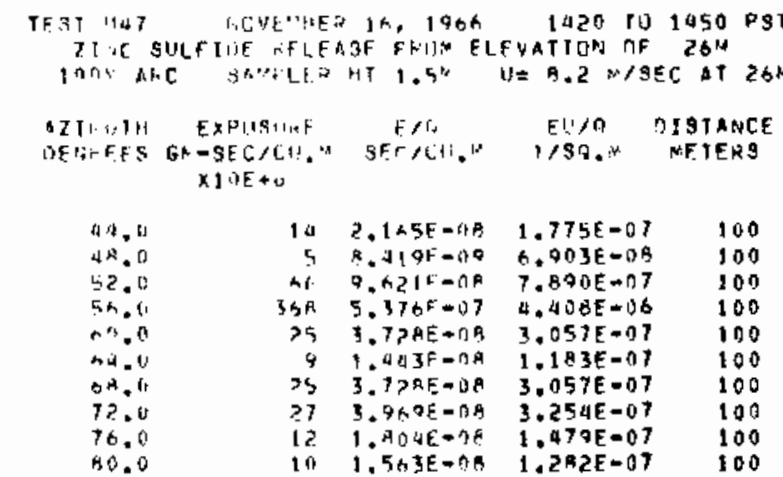

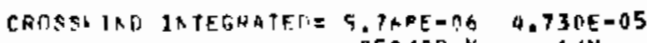

SFC $190 . \mathrm{N} \quad 1 / \mathrm{M}$

TEST 1147 NHIEFE 14, 1800 1420 TO 1450 P3T

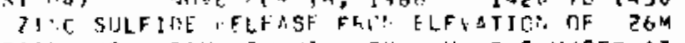

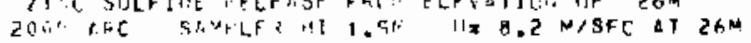

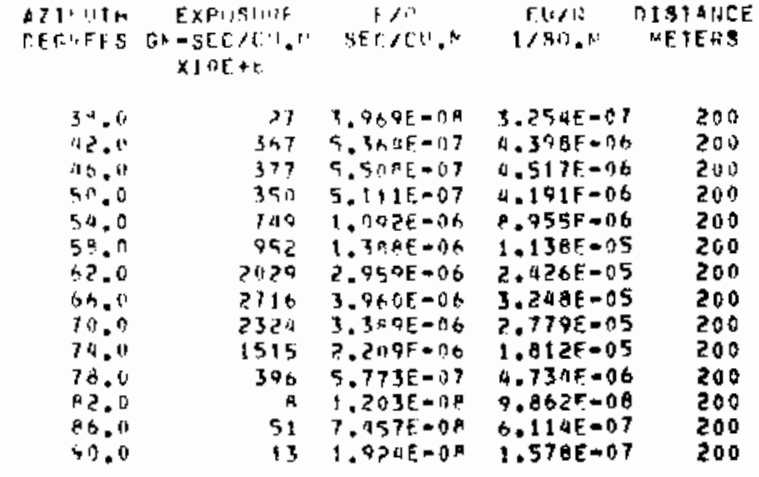

CACSB. IS.O IATEGRATED $=2, \triangle A E=04 \quad 1.983 E-03$

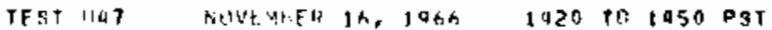
2INE: SULFIUE WFLEASE FRIN FLFUATION OF $26 \mathrm{~K}$

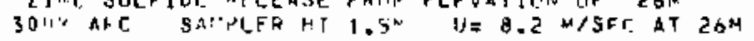

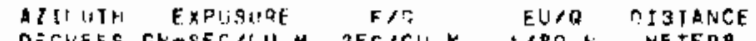
DEGHEES GN-SEC/C"M BECJCU.K J\$OQR METERS $x \mid 0 E+3$

\begin{tabular}{|c|c|c|c|c|}
\hline 40.0 & 36 & A. $+A B E=07$ & $3.6 \mathrm{APF}=100$ & 300 \\
\hline 44.0 & 610 & $A$ DCOE-07 & $7.298 \%=06$ & 300 \\
\hline 40.0 & 731 & $1.007 t-36$ & $8.7 A B E=0 B$ & \\
\hline 52.4 & 855 & $0.5 x, 15=07$ & $7.840 F=06$ & 30 \\
\hline 56.6 & 115 & $1.64 f, E-1.7$ & $1.351 \mathrm{~F}=06$ & 30 \\
\hline av. & 1397 & $7.023 E-10$ & $1.659 \mathrm{E}-05$ & \\
\hline+5.0 & 3709 & $9.4 \pi 7 F=5,6$ & $4 F=05$ & 30 \\
\hline $\begin{array}{l}68.0 \\
72.0\end{array}$ & $\begin{array}{l}5230 \\
1360\end{array}$ & $\begin{array}{l}? .63+E=06 \\
6,3+6 E=116\end{array}$ & $\begin{array}{l}6.261 E=05 \\
5.220 E=05\end{array}$ & 30 \\
\hline 76.0 & 1072 & $2.43 A E-06$ & $1.999 E=05$ & 30 \\
\hline H9. 0 & 702 & $1.02 \mathrm{JE}=06$ & $0.393 E-06$ & 30 \\
\hline P 4.0 & 344 & $5,077 E=07$ & $4.122 E=06$ & \\
\hline 89.0 & 174 & 2.53 AE-137 & $2.081 \mathrm{E}=06$ & 30 \\
\hline 92.0 & 10 & $1.5 B 3 E=09$ & $1.202 E-07$ & 300 \\
\hline
\end{tabular}

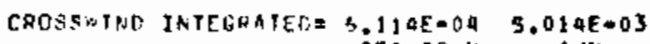

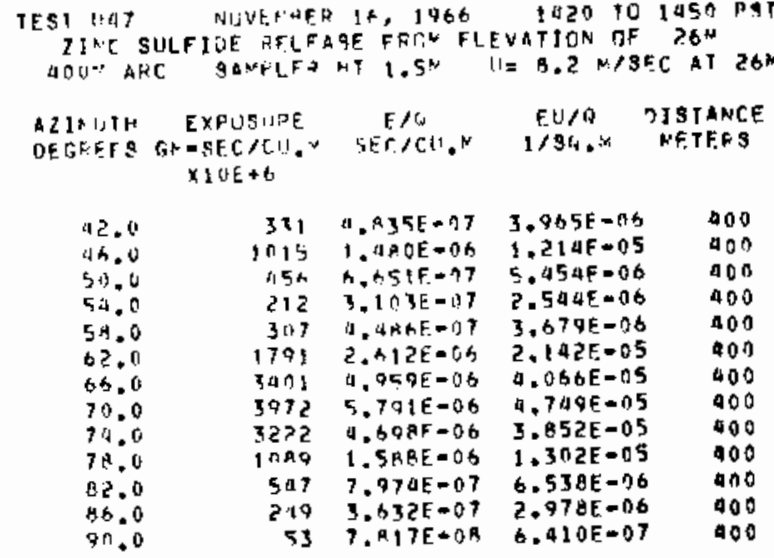

CRDSBWIR:D INTEGKATELX S.7TEE-OA S.558E-OS

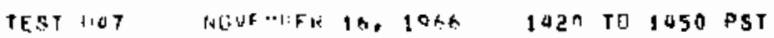

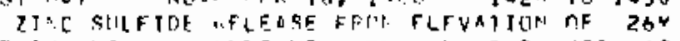

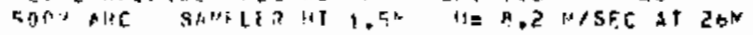

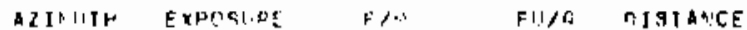

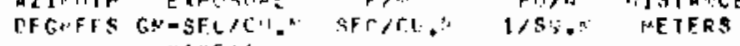
X10E+ +6

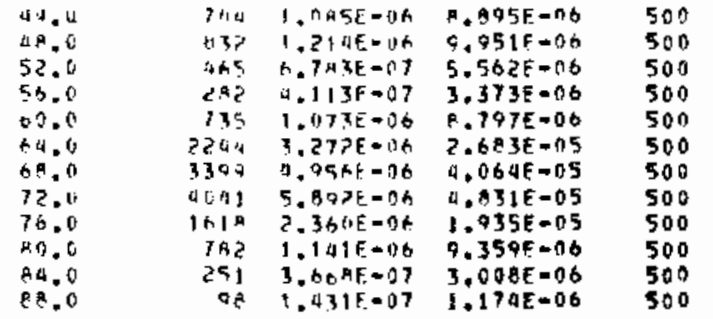

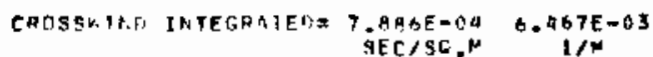

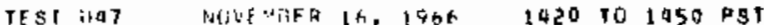
IIST SULFINE HFLFASE FTETH FLEVATIDN CF 364 DODE AFC SANFLED HT $1,5 \mathrm{~K} I=$ I.Z NISEC AT $26 \mathrm{M}$

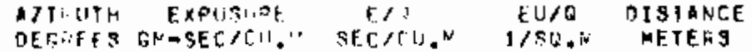
$x\}$ II +6

\begin{tabular}{|c|c|c|c|c|}
\hline $\begin{array}{l}42.0 \\
46.0 \\
50.0 \\
54.0 \\
58.0 \\
62.0 \\
66.0 \\
10.0 \\
24.0 \\
79.0 \\
82.0 \\
85.0\end{array}$ & $\begin{array}{r}134 \\
1057 \\
064 \\
314 \\
217 \\
1251 \\
2226 \\
3273 \\
2456 \\
1042 \\
177 \\
146\end{array}$ & 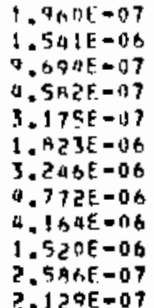 & $\begin{array}{l}1.608 E=16 \\
1.263 E=05 \\
7.949 E-06 \\
3.757 E=06 \\
2.604 E=06 \\
1.495 E-05 \\
2.662 E=05 \\
3.913 E=05 \\
3.414 E=05 \\
1.247 E-05 \\
2.120 E-06 \\
1.746 E=06\end{array}$ & $\begin{array}{l}600 \\
600 \\
600 \\
600 \\
600 \\
600 \\
600 \\
600 \\
600 \\
600 \\
600 \\
600\end{array}$ \\
\hline
\end{tabular}

CRCSS 


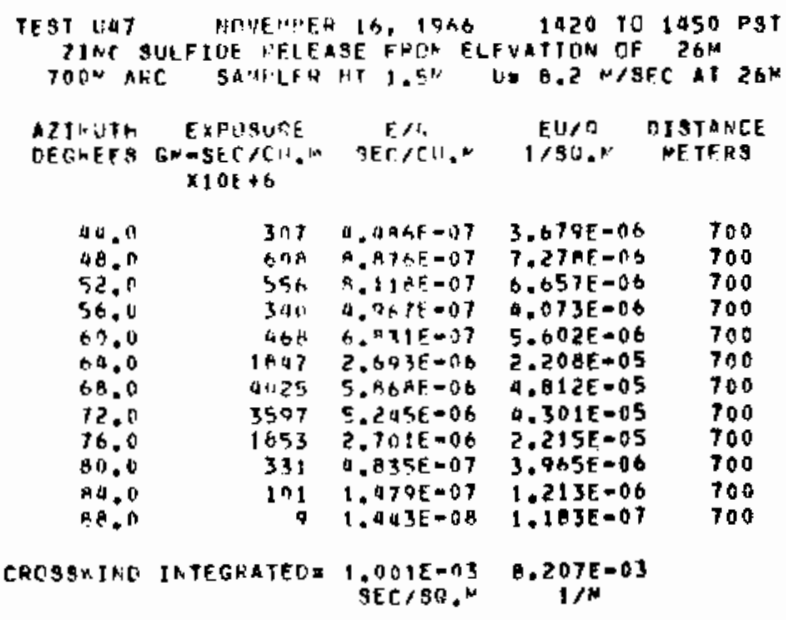

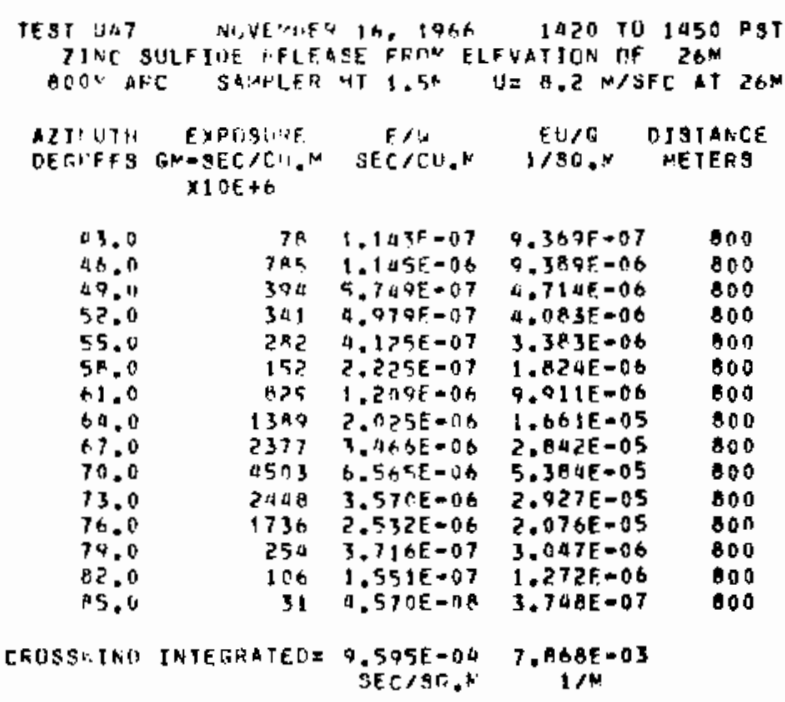

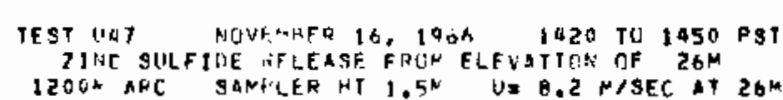

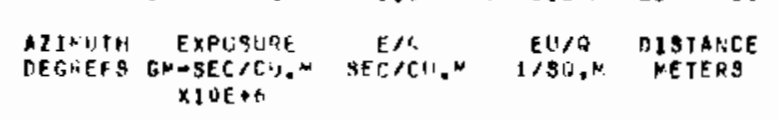

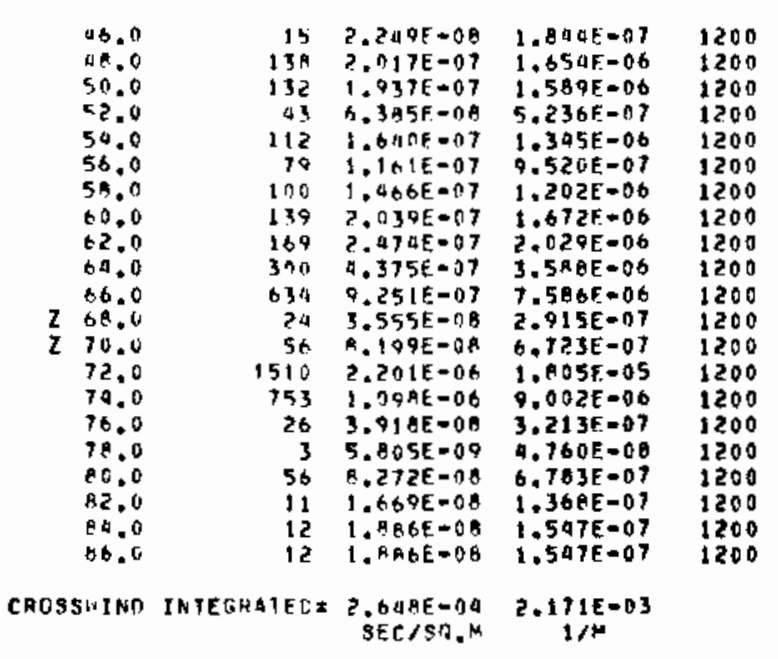

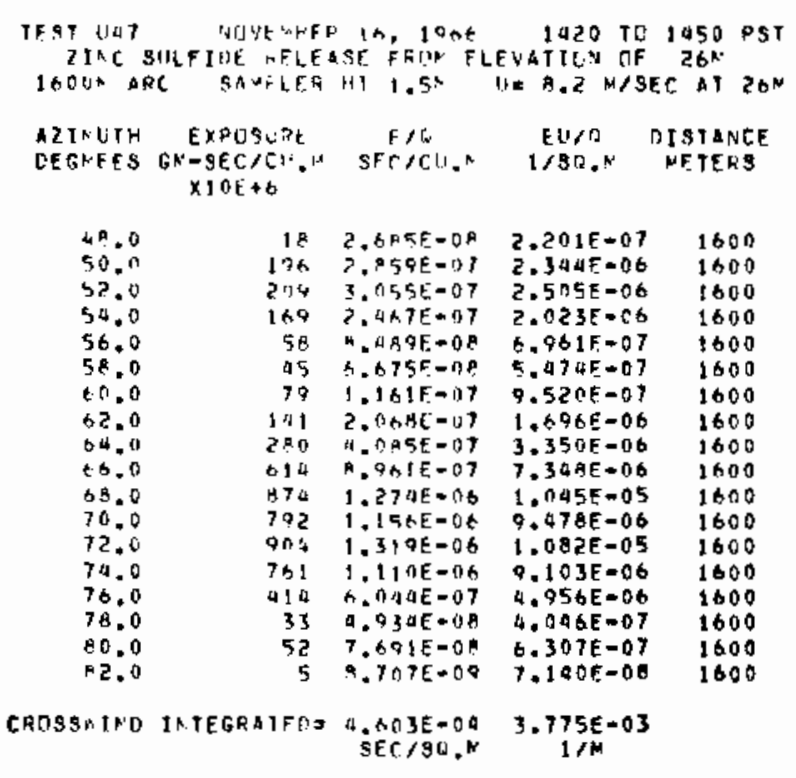




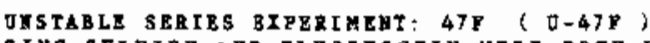

2IHC GULRIDE ARD FLUORESCEIN WERE BOTE OSEDAS TRACERS DORING EIREIMEHT 47, FLUORESCEIM BBSDLTS ARR PRESEMTED HERE. GRODED LEVEL SAXPLIHG 100-1600 KARC.

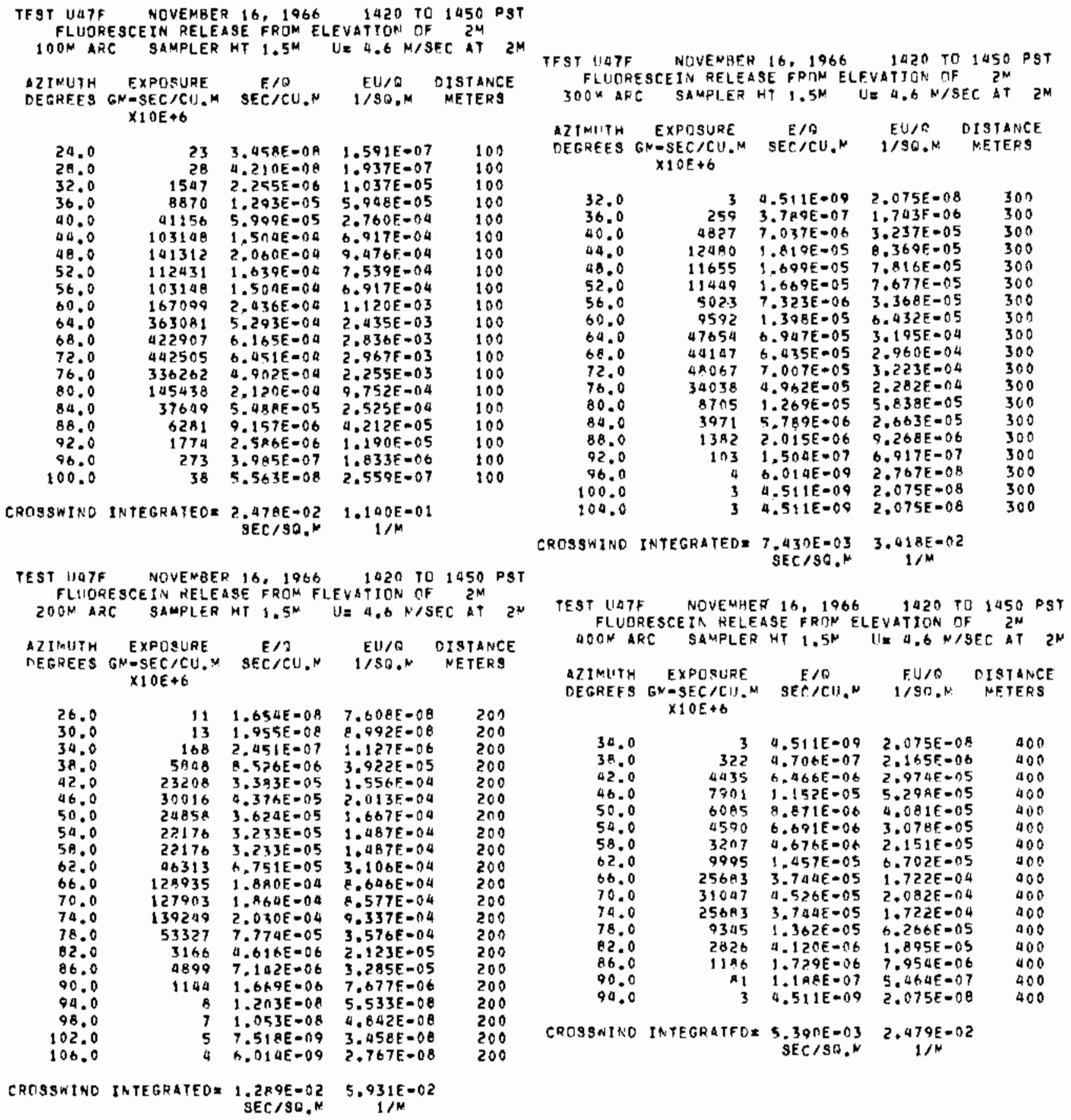




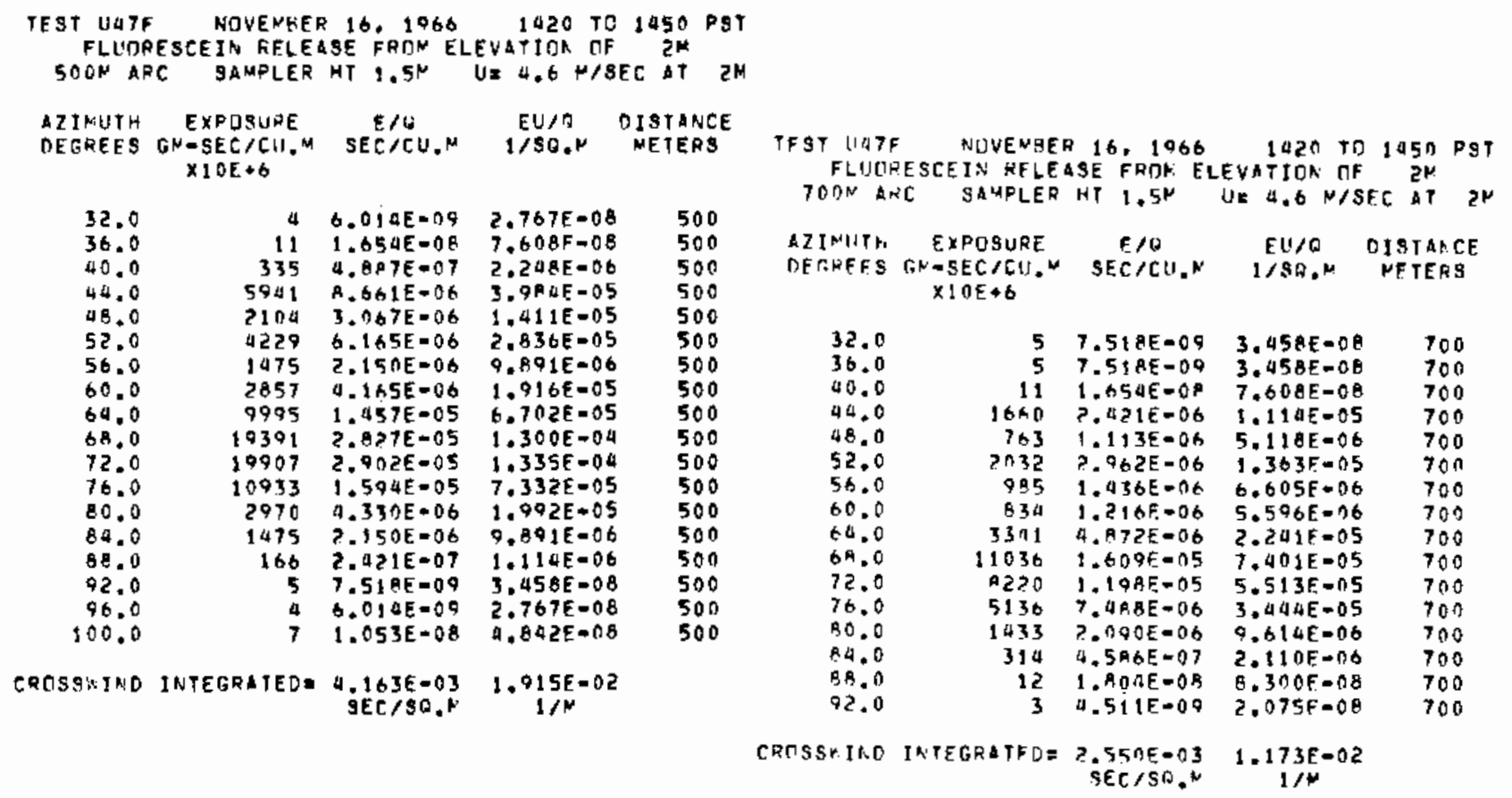

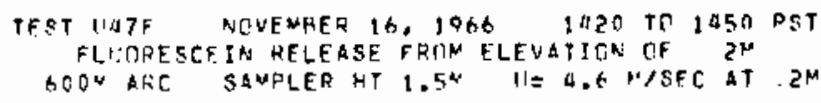

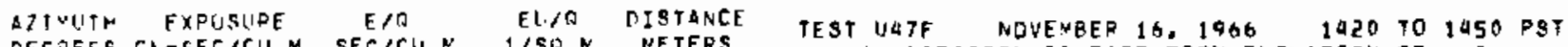

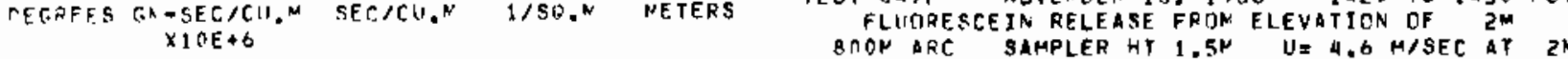

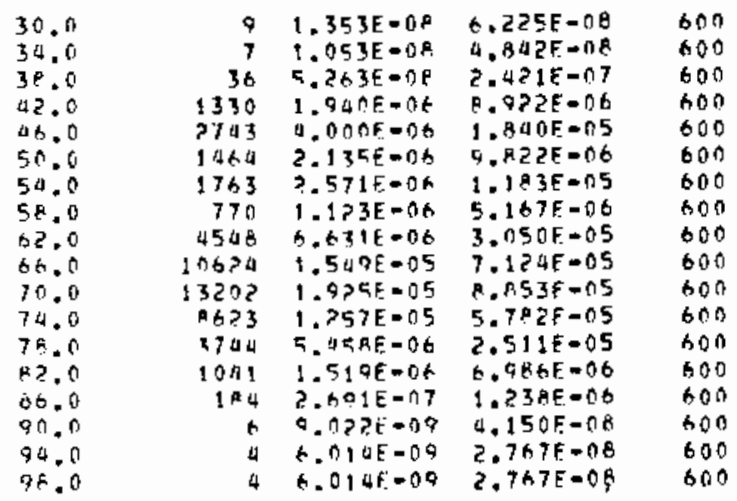

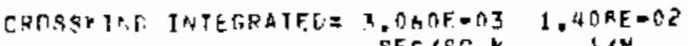
SEC SQ, $\quad 1, N$

\begin{tabular}{|c|c|c|c|c|}
\hline $\begin{array}{l}\text { DTIMUTH } \\
\text { DEGREES }\end{array}$ & $\begin{array}{c}\text { EXPDSURE } \\
\text { GNESEC } \angle C U, M \\
\times 1 O E+6\end{array}$ & $\begin{array}{c}E / G \\
S E C / C U . N\end{array}$ & $\begin{array}{l}E U / O \\
1 / 80 . M\end{array}$ & $\begin{array}{l}\text { DISTAR:CE } \\
\text { HETEAS }\end{array}$ \\
\hline $\begin{array}{l}37.0 \\
40.0 \\
43.0 \\
46.0 \\
49.0 \\
52.0 \\
55.0 \\
58.0 \\
61.0 \\
64.0 \\
67.0 \\
79.0 \\
73.0 \\
76.0 \\
79.0 \\
82.0 \\
85.0 \\
88.0\end{array}$ & $\begin{array}{r}3 \\
38 \\
199 \\
1807 \\
508 \\
433 \\
921 \\
166 \\
1134 \\
3393 \\
5611 \\
9345 \\
5064 \\
4125 \\
1217 \\
321 \\
68 \\
3\end{array}$ & $\begin{array}{l}4.511 E=09 \\
5.56 J E=08 \\
2.002 E=07 \\
2.767 E=06 \\
7.413 E=07 \\
2.090 E=06 \\
1.303 E=06 \\
2.421 E=07 \\
1.654 E=06 \\
4.947 E=06 \\
2.190 E=06 \\
1.362 E=05 \\
7.3 A 3 E=06 \\
6.014 E=06 \\
1.774 E=06 \\
0.601 E=07 \\
0.974 E=08 \\
4.511 E=09\end{array}$ & $\begin{array}{l}2.075 E=08 \\
2.559 E=07 \\
1.335 E=06 \\
1.273 E=05 \\
3.410 E=06 \\
9.614 E=06 \\
6.177 E=06 \\
1.114 E=06 \\
7.608 F=06 \\
2.276 E=05 \\
3.763 E=05 \\
6.266 E=05 \\
3.396 E=05 \\
2.767 E=05 \\
8.162 E=06 \\
2.158 E=06 \\
9.565 E=07 \\
2.075 E=08\end{array}$ & $\begin{array}{l}800 \\
800 \\
800 \\
100 \\
800 \\
800 \\
800 \\
800 \\
800 \\
800 \\
800 \\
000 \\
800 \\
800 \\
800 \\
000 \\
000 \\
800\end{array}$ \\
\hline 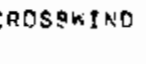 & INTEGRATED & $\begin{array}{l}? .165 E-0 J \\
S E C / S 0 . K\end{array}$ & $\begin{array}{c}9.958 E=03 \\
1 / M\end{array}$ & \\
\hline
\end{tabular}




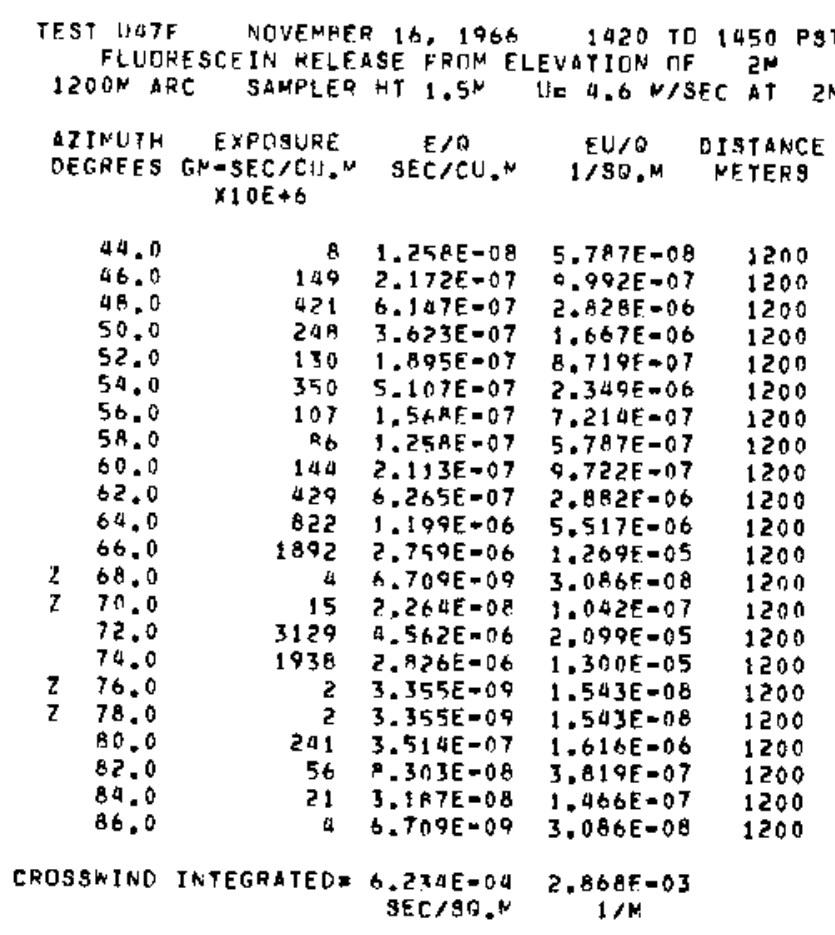

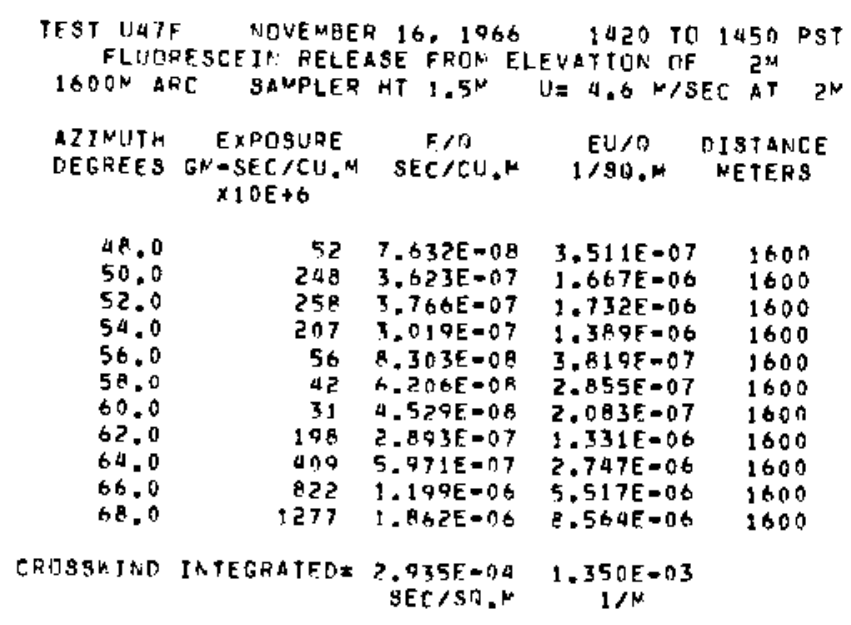


DHSTABLE SERIES EXPERIMBAT: 49 ( $0-48$ )

ZINC SULFIDE AND PIUCRESGEIH WBRB DOTB OSED AS TRACERS DERING

ZXPERIMEST 48. ZIMC SOLZIDE RBSULTS ARE PRESERTED BERE. GROUND

LEVEL SAKPLIXG 100-1600 HAEC.

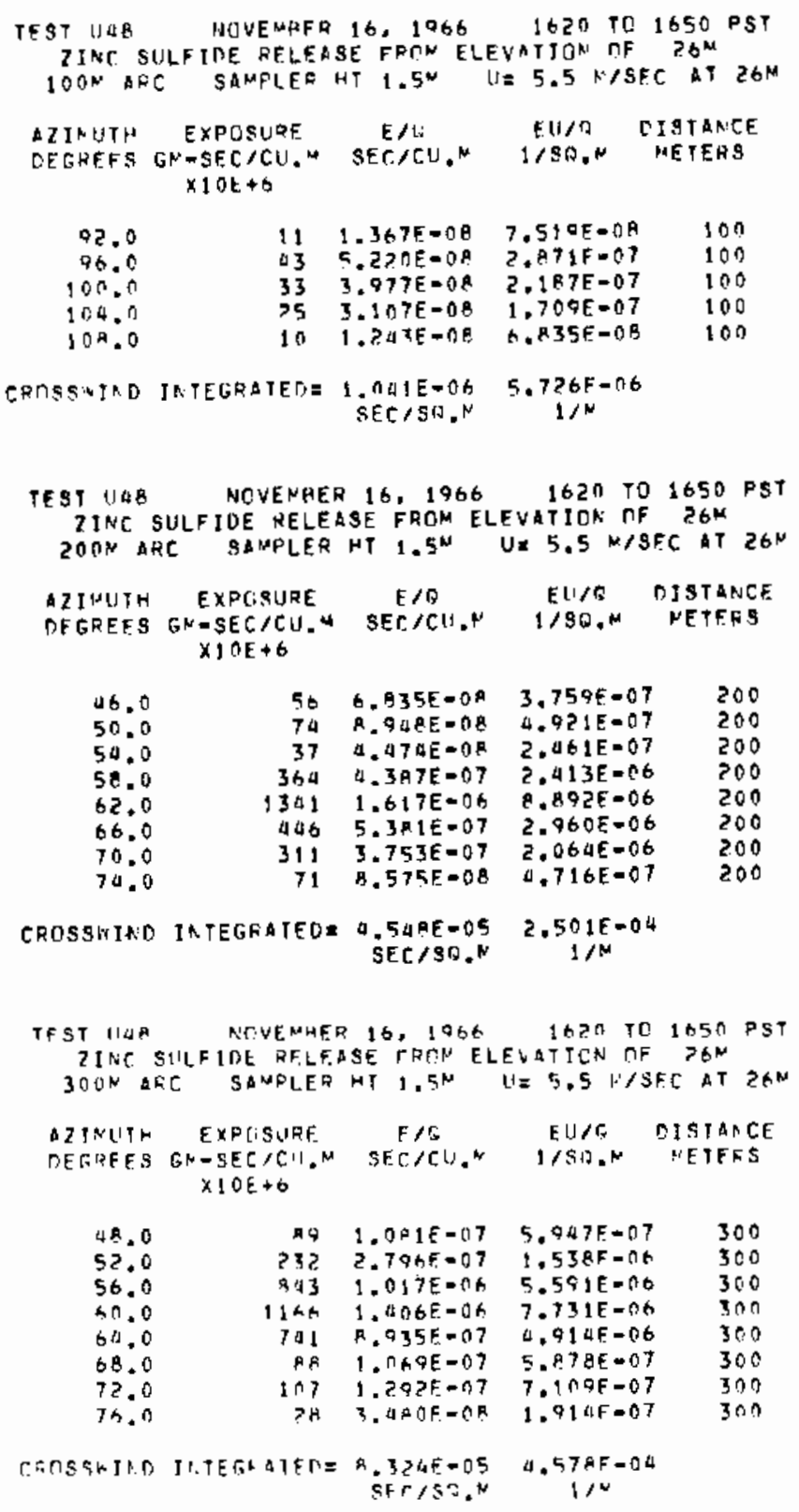

TEST UAG NOVERRER 16,1966 1620 TO 1650 PST
ZINC SULFIDE RFLEASE FDAN ELEVATION DF 264
4OON AKC SAMPLER HT $1.5 \mathrm{~N}$ UE $5.5 \mathrm{M} / \mathrm{SEC}$ AT $26 \%$

AZIMUTH EXPOSURE E/G DU/O DISIANCE DEGREES GN-SEC/CU.M SEC/CU.N 1/SO,N NETERS $X 10 E+6$

\begin{tabular}{|c|c|c|c|c|}
\hline $\begin{array}{l}96.0 \\
50.0 \\
50.0 \\
58.0 \\
62.0 \\
66.0 \\
70.0 \\
70.0 \\
70.0\end{array}$ & $\begin{array}{r}95 \\
634 \\
1180 \\
1600 \\
1042 \\
1005 \\
774 \\
233 \\
17\end{array}$ & $\begin{array}{l}1.15 E E=07 \\
7.623 E=07 \\
1.42 Z E=06 \\
2.170 E=06 \\
1.256 E=06 \\
1.212 E=06 \\
9.333 E=07 \\
2.809 E=07 \\
2.113 E=08\end{array}$ & $\begin{array}{l}6.357 F=07 \\
4.20 A E=06 \\
7.819 E=06 \\
1.193 E=05 \\
6.910 E=06 \\
6.664 E=06 \\
5.133 E=06 \\
1.545 E=06 \\
1.162 F=07\end{array}$ & $\begin{array}{l}400 \\
400 \\
400 \\
400 \\
400 \\
000 \\
400 \\
400 \\
400\end{array}$ \\
\hline
\end{tabular}

CROSSUIND INTEGRATEDI 2.2A3E-04 1.256E-03 SEC $/ S O . N \quad 1 / M$

TFST UL8 NOVENEER 16,1966 1620 TO 1650 PST ZINC SULFIDE RFLEASE FPD ELEVATJON TF $26 \mathrm{M}$ 5NOW ARC SAMPLER HT $1.5 \mathrm{SM} U=5.5 \mathrm{~N} / \mathrm{SEC} A T 26 \mathrm{M}$

IZINLTH EXPTSURE E/G EUTR DISTANCE DEGREES GW-SEC/CU.M SEC/CU.N 1/SO,M NETERS $x: 0 E+6$

\begin{tabular}{|c|c|c|c|}
\hline $\begin{array}{l}46.0 \\
52.0 \\
5 \% .0 \\
60.0 \\
60.0 \\
60.0 \\
72.0\end{array}$ & $\begin{array}{r}239 \\
937 \\
1667 \\
1551 \\
90 \\
1199 \\
731 \\
75\end{array}$ & $\begin{array}{l}? .893 E=07 \\
1.130 E=06 \\
2.010 E=06 \\
1.06 Q E=06 \\
1.019 E=07 \\
1.033 E=06 \\
8.811 E=07 \\
0.07 ? E=08\end{array}$ & $\begin{array}{l}1.586 E=06 \\
6.213 E=06 \\
1.105 E=05 \\
1.028 E=05 \\
5.605 E=07 \\
7.881 E=06 \\
1.846 E=06 \\
4.990 E=07\end{array}$ \\
\hline
\end{tabular}

CAOSSTIT.D INTEGRATFRIE 2.72UE-04 1.4QAE=03 $S E C / 9 r_{4}, N \quad 1 / N$

TEST LISO NOVENAER 16, 1966 1620 TO 3650 PST ZIN.C SULFIDE RELEASE FRTMi ELEVATION OF $26 \mathrm{M}$ $600 \mathrm{M} A K C$ SAMPLER MT $1.5 \mathrm{M} \quad \mathrm{M}=5.5 \mathrm{~N} / \mathrm{SEC}$ AT $26 \mathrm{M}$ AZIMUTH EXPCSURE E/G EL/Q OISTAHCE NEGREES GN-SEC/CIIM SEC/CU.N 1/SQ.M METERS $\times 10 \mathrm{E}+6$

$\begin{array}{lllll}50.0 & 1003 & 1.209 E-06 & 6.051 E-06 & 600 \\ 50.0 & 1425 & 1.717 F-06 & 9.406 E-06 & 600 \\ 50.0 & 1795 & 2.164 E-06 & 1.190 E-05 & 600 \\ 02.0 & 1056 & 1.273 E-06 & 6.999 E-06 & 600 \\ 66.0 & 1648 & 2.011 E=06 & 1.106 E-05 & 600 \\ 70.0 & 1924 & 2.319 E-06 & 1.275 E=05 & 600 \\ 74.0 & 403 & 5.340 E-07 & 2.939 E-06 & 600\end{array}$

CROSSWIND INTEGRATED=4.7N3E-TH 2.587E-03 


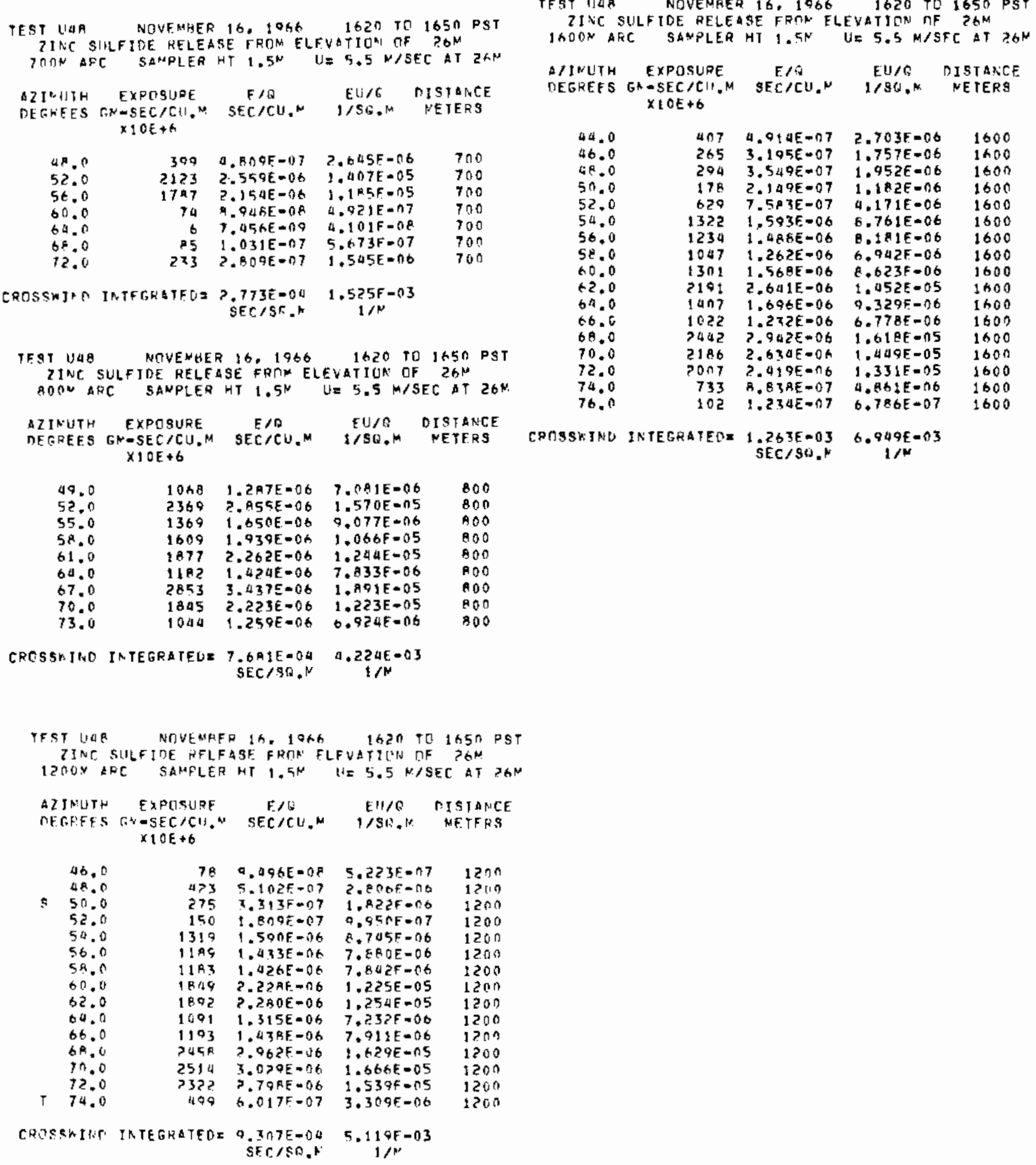




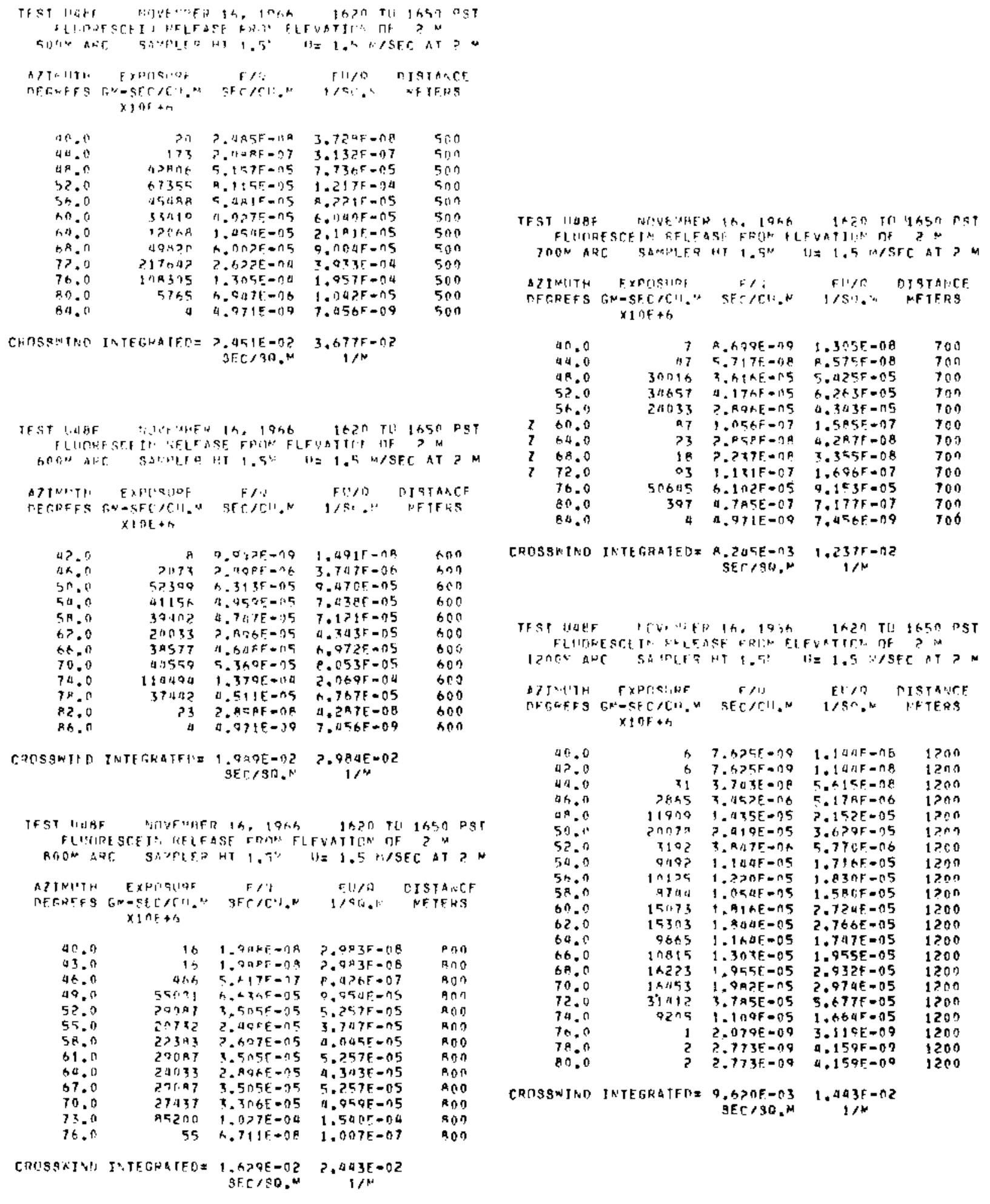




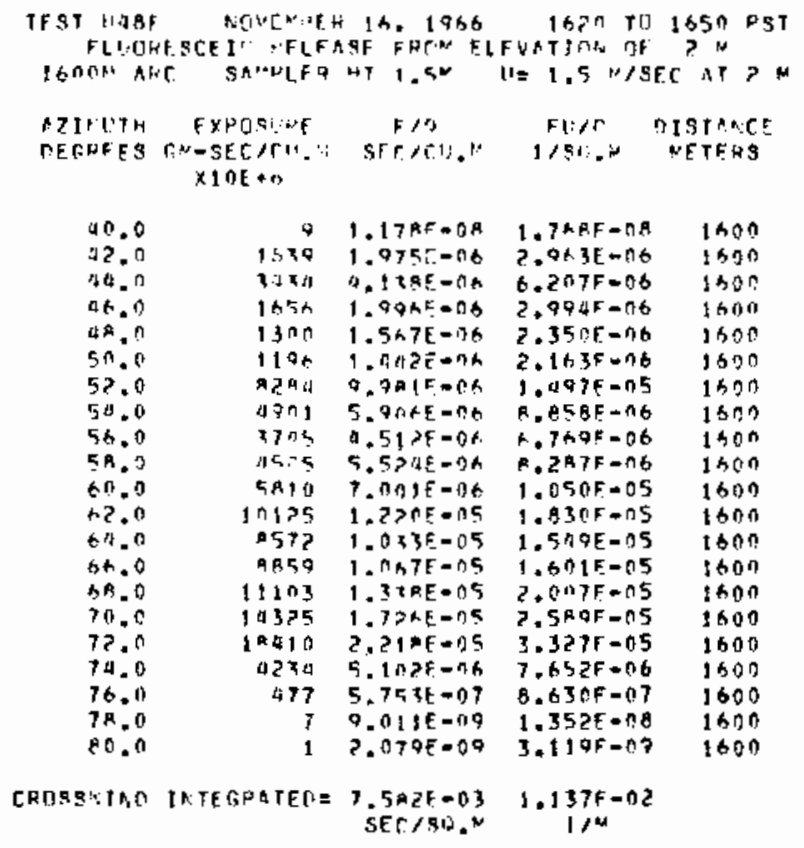


OMSTABL SEIIS EXPERIMEH: 49 (

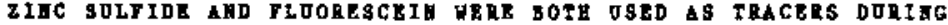

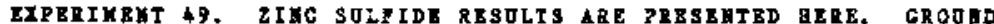

LEVEL SAMPLIAG 100-1600 A ACC. A SICHIFICATT FORTIOS OR TRE

PLJKE PASSBD TO THE SOUTE OF THE GRTD.

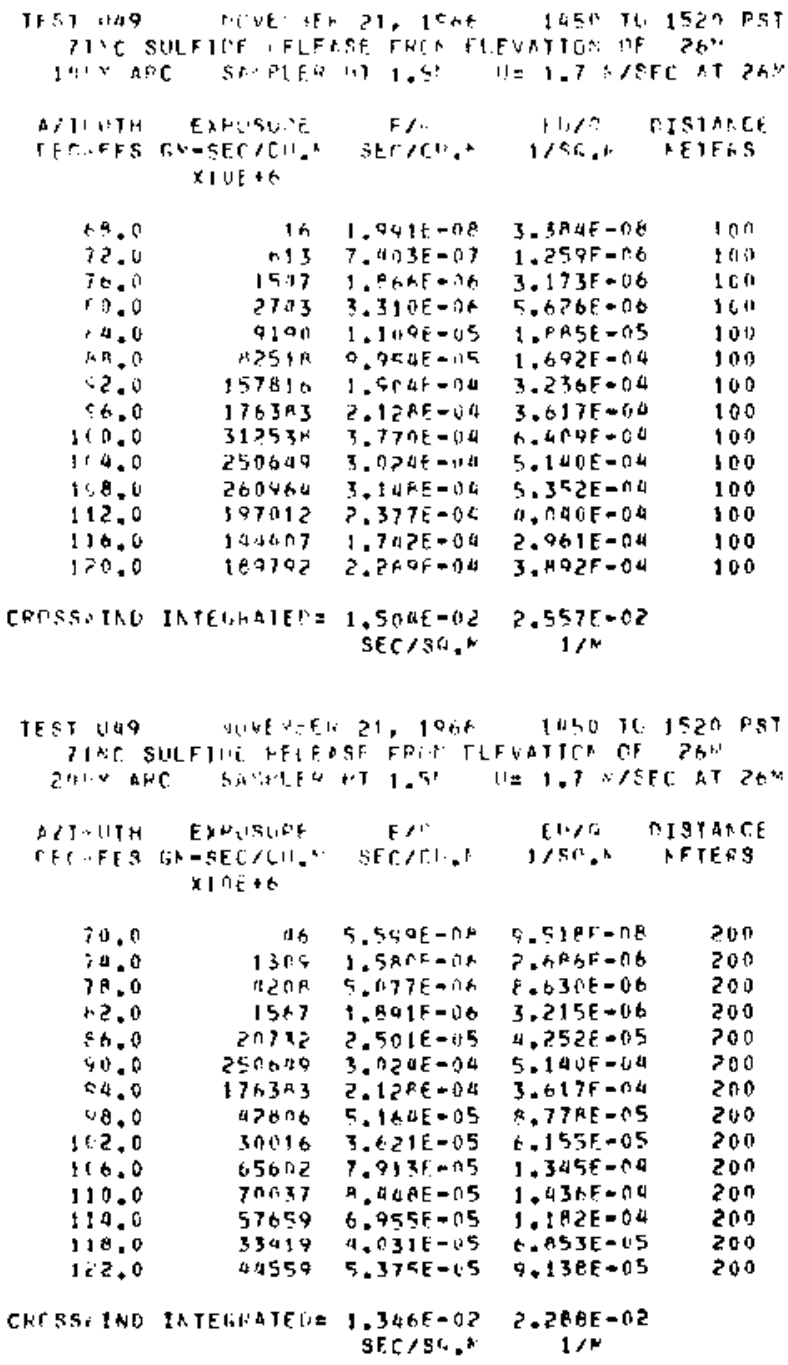

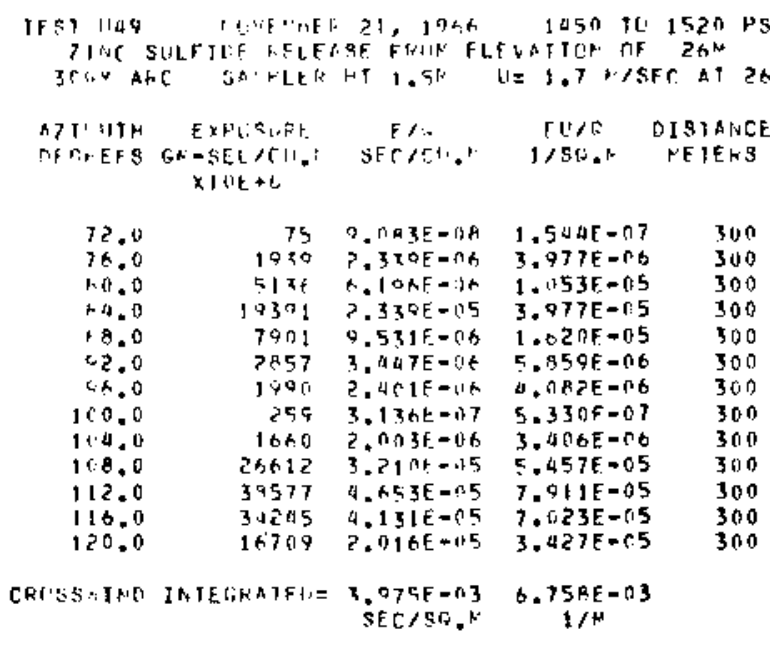

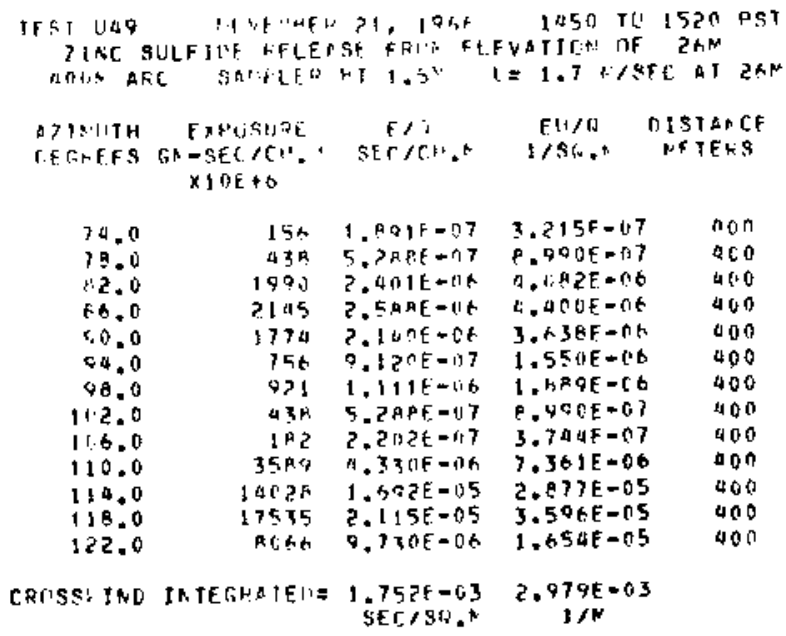

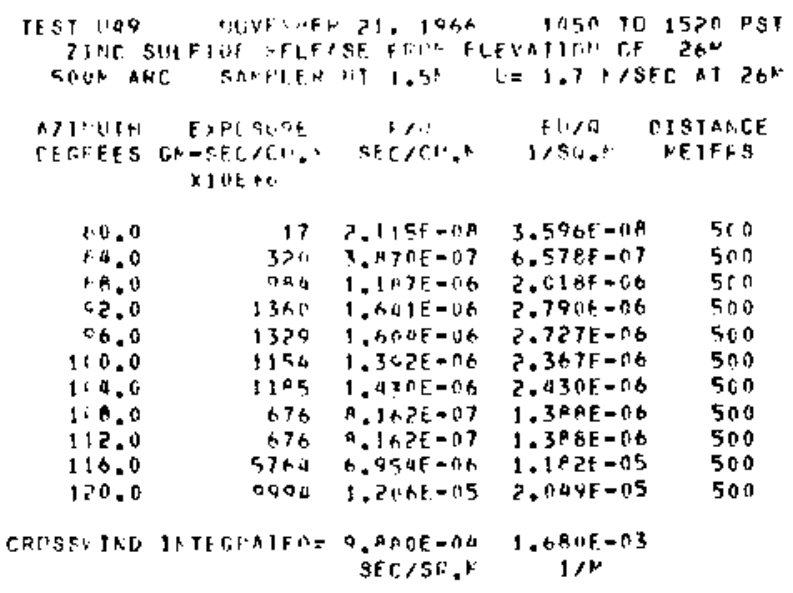




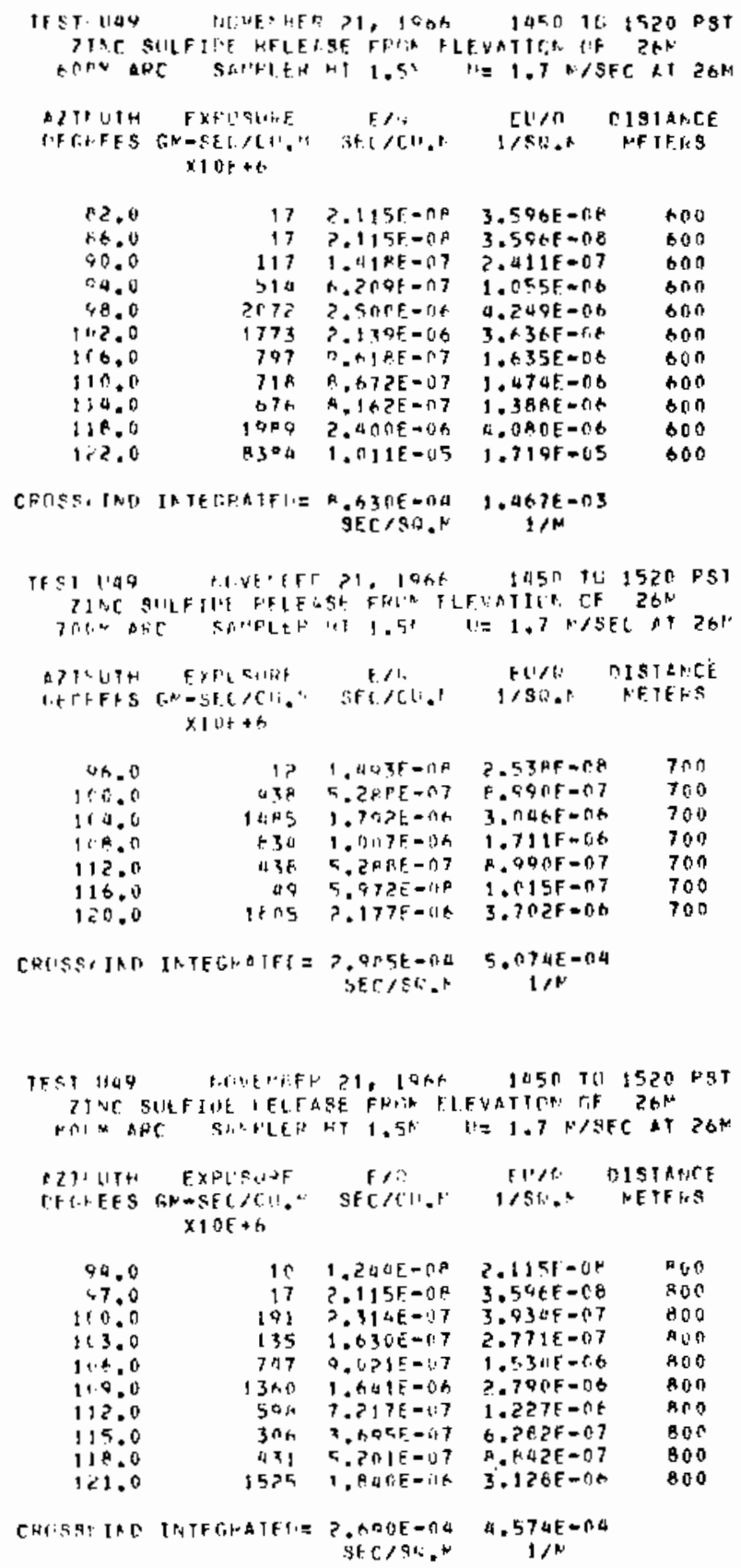

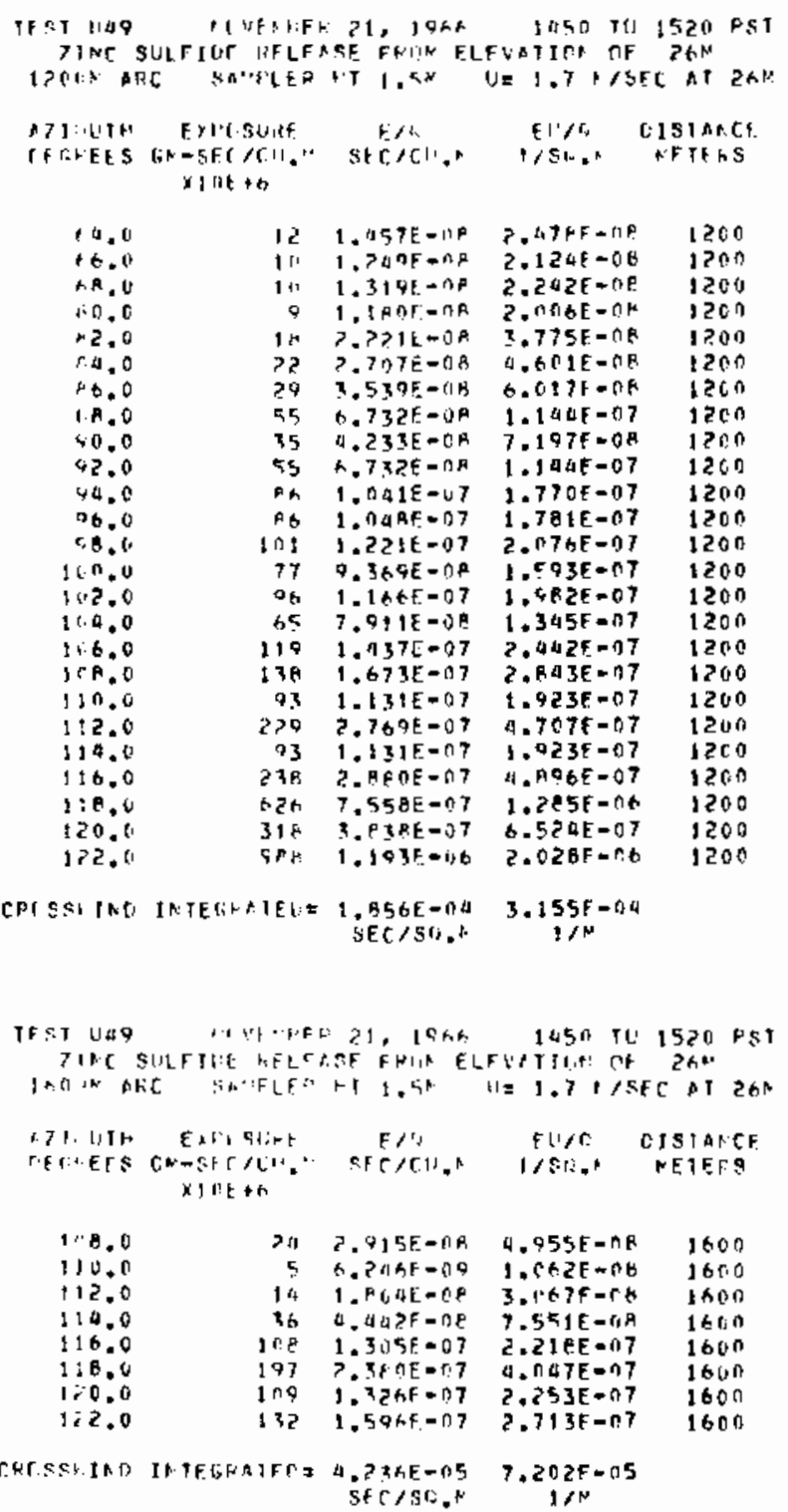


OSBTABLB BELIES EIPEBIHEYT: 49P (

ZITC SOLPTDE AXD FLJORESCEIX UZRB DOTH OSED AS TRACERS DURIBG

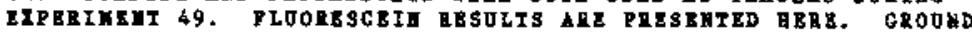

LEFEL SAMPLING 100-1600 CARG. A SIGHIPICAKT PORTION OP TBR

PLUKE EASSBD TO TER SOOTH OF TEB GRID.

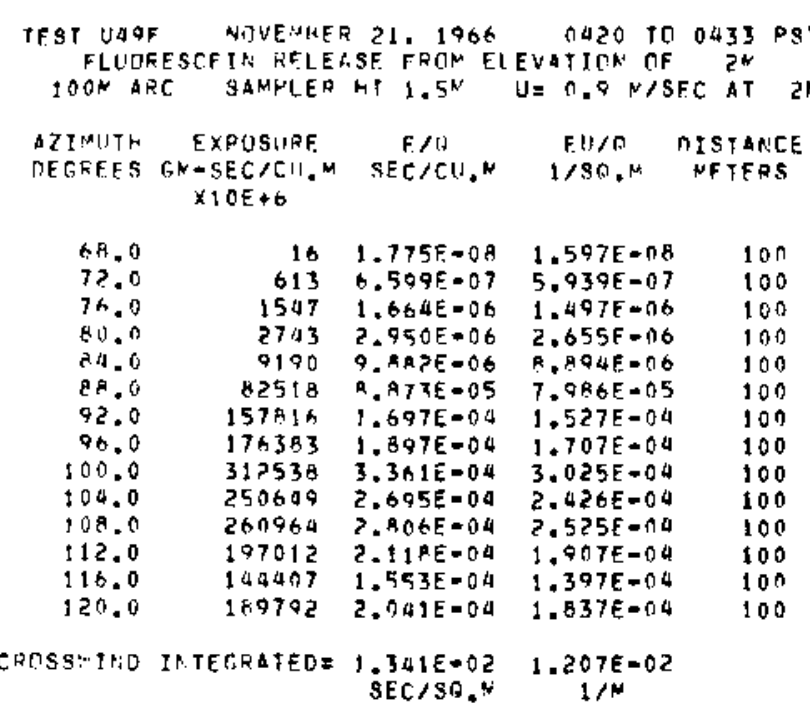

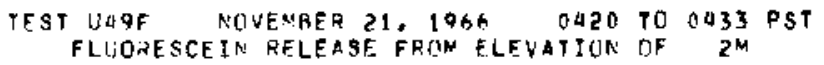
2NON ATC SANPLER HF $1.5 \mathrm{M} U=0.9 \mathrm{M} / \mathrm{SEC} A T$ IM

\begin{tabular}{|c|c|c|c|c|}
\hline $\begin{array}{l}\text { ATRDLTH } \\
\text { DETREES }\end{array}$ & $\begin{array}{c}\text { EXPUAURE } \\
\text { GN }=5 E C / C I, M \\
\times 10 E+6\end{array}$ & $\begin{array}{c}E / O \\
S E[/ C U . *\end{array}$ & $\begin{array}{c}E U / D \\
1 / 80 . \mu\end{array}$ & $\begin{array}{l}\text { DISTANCE } \\
\text { NFTERS }\end{array}$ \\
\hline
\end{tabular}

\begin{tabular}{|c|c|c|c|}
\hline $\begin{array}{l}50.0 \\
54.0 \\
58.0 \\
62.0 \\
66.0 \\
70.0 \\
74.0 \\
78.0 \\
42.0 \\
86.0 \\
90.0 \\
94.0 \\
99.0 \\
102.0 \\
106.0 \\
110.0 \\
114.0 \\
118.0 \\
122.0\end{array}$ & $\begin{array}{r}10 \\
13 \\
7 \\
7 \\
9 \\
96 \\
1309 \\
4298 \\
1567 \\
20732 \\
250699 \\
174393 \\
42806 \\
30016 \\
65602 \\
70037 \\
57659 \\
33419 \\
40559\end{array}$ & $\begin{array}{l}1.109 E=08 \\
1.442 E=08 \\
7.764 E=09 \\
7.764 E=09 \\
9.873 E=09 \\
9.901 E=08 \\
1.409 E=04 \\
4.525 E=06 \\
1.6 A 6 E=06 \\
2.279 E=05 \\
7.695 E=04 \\
1.807 E=04 \\
1.673 E=05 \\
3.729 E=05 \\
7.054 E=05 \\
7.531 E=05 \\
6.200 E=05 \\
3.594 E=05 \\
0.791 E=05\end{array}$ & 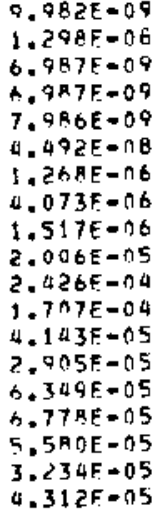 \\
\hline $\operatorname{CnSBR} I \wedge D$ & INTEGNATEDI & $\begin{array}{c}1=200 E-02 \\
S E C / 50^{*}\end{array}$ & $\begin{array}{c}1.080 F=02 \\
1 / M\end{array}$ \\
\hline
\end{tabular}

TFST ULQF NOVENBEA $71,106 \mathrm{~h}$ 0420 TO 0433 PST FLUORE SCEIN RFLEASF FPIVM ELEVATION DF $2 \mathrm{~N}$ JTON ARC SAMPLER HT $1.5 \mathrm{~N} U=0.0 \mathrm{~N} / \mathrm{SEC}$ AT $2^{\mathrm{N}}$

\begin{tabular}{|c|c|c|c|c|}
\hline $\begin{array}{l}\text { AZINIIYH } \\
\text { DEGPEES }\end{array}$ & $\begin{array}{c}\text { EXPOSURF } \\
\text { GN= } 5 E C / C \div . " \\
\times 10 E+6\end{array}$ & $\begin{array}{c}E / O \\
S E C / C U, N\end{array}$ & $\begin{array}{c}E U / 0 \\
1 / S N .\end{array}$ & $\begin{array}{l}\text { CISYANCE } \\
\text { METEFS }\end{array}$ \\
\hline $\begin{array}{l}56.0 \\
60.0 \\
64.0 \\
69.0 \\
72.0 \\
76.0 \\
80.0 \\
84.0 \\
8.00 \\
92.0 \\
96.0 \\
100.0 \\
104.0 \\
109.0 \\
112.0 \\
116.0 \\
120.0\end{array}$ & $\begin{array}{r}10 \\
5 \\
8 \\
5 \\
75 \\
1938 \\
5176 \\
19391 \\
7901 \\
2857 \\
1990 \\
259 \\
1060 \\
26612 \\
38577 \\
34205 \\
16709\end{array}$ & $\begin{array}{l}1.109 E=08 \\
5.546 E=09 \\
R .873 E=09 \\
5.50 A E=08 \\
8.097 E=00 \\
2.095 E=06 \\
5.523 E=06 \\
2.085 E=05 \\
9.496 E=06 \\
3.072 E=06 \\
2.141 E=06 \\
2.795 E=07 \\
1.7 R 6 E=06 \\
2.942 E=05 \\
0.20 A E=05 \\
3.692 E=05 \\
1.797 E=05\end{array}$ & $\begin{array}{l}9.982 E=09 \\
4.991 E=09 \\
7.9 R 6 E=09 \\
4.991 E=09 \\
7.287 E=08 \\
1.977 E=06 \\
1.971 E=06 \\
1.877 E=05 \\
7.646 E=06 \\
2.765 E=06 \\
1.927 E=06 \\
2.515 E=07 \\
1.607 E=06 \\
2.575 E=05 \\
3.133 E=05 \\
3.314 E=05 \\
1.617 E=05\end{array}$ & $\begin{array}{l}300 \\
300 \\
300 \\
300 \\
300 \\
300 \\
300 \\
300 \\
300 \\
300 \\
300 \\
300 \\
300 \\
300 \\
300 \\
300 \\
300\end{array}$ \\
\hline
\end{tabular}

CROSSUIND INTEGRATEO= 3.544E-0J 3.190E-03

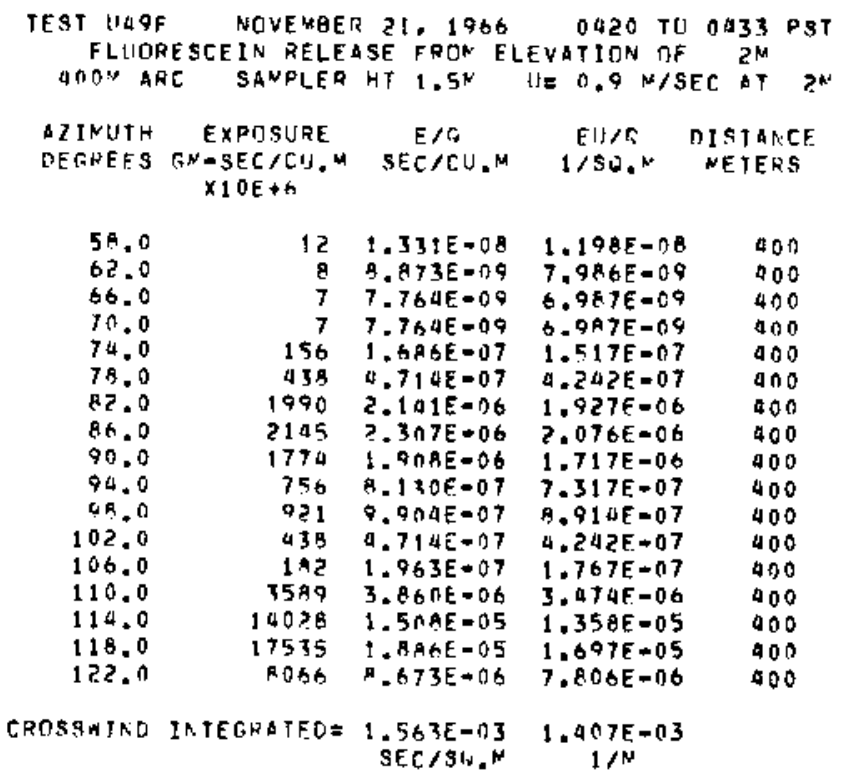




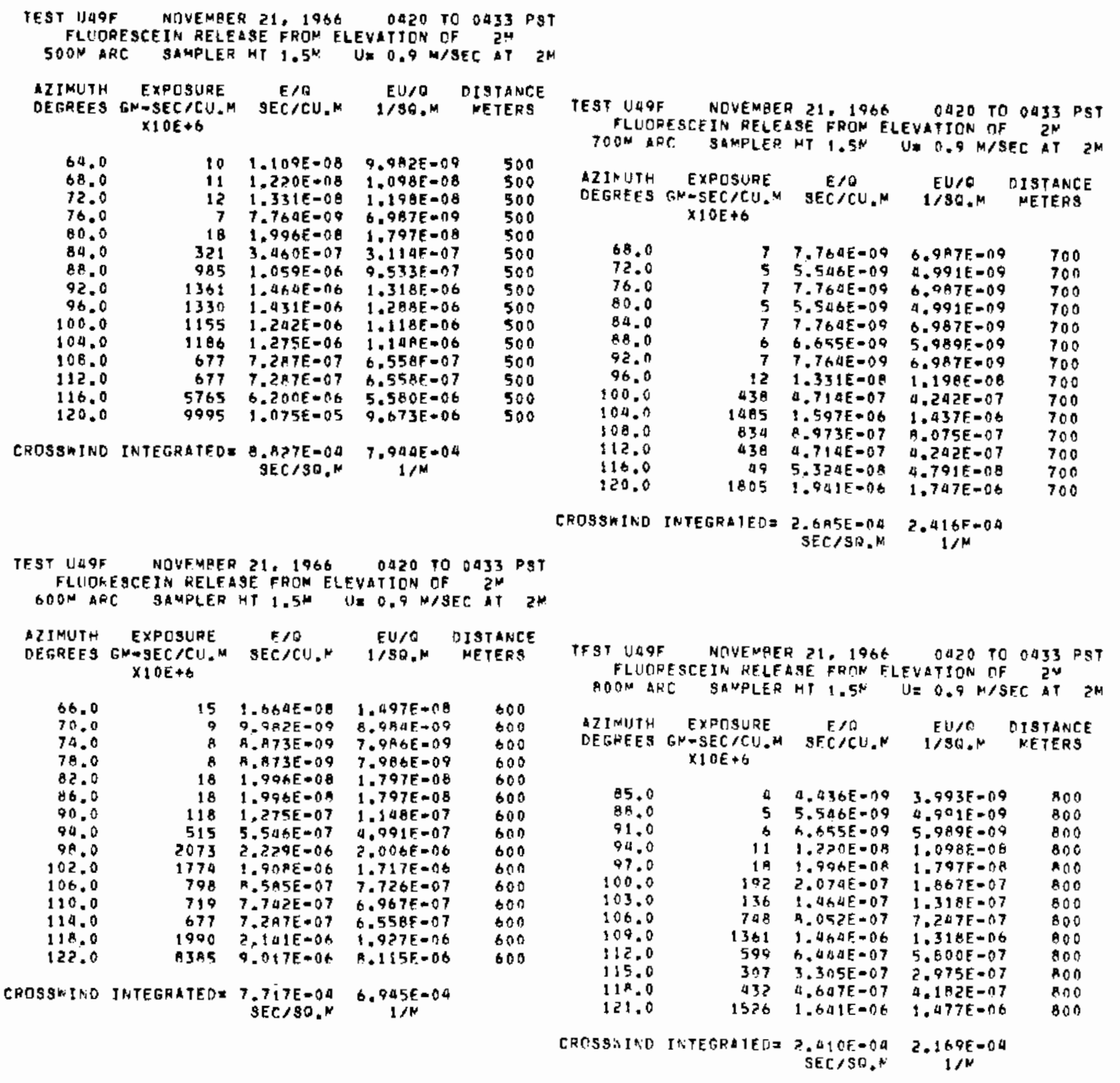




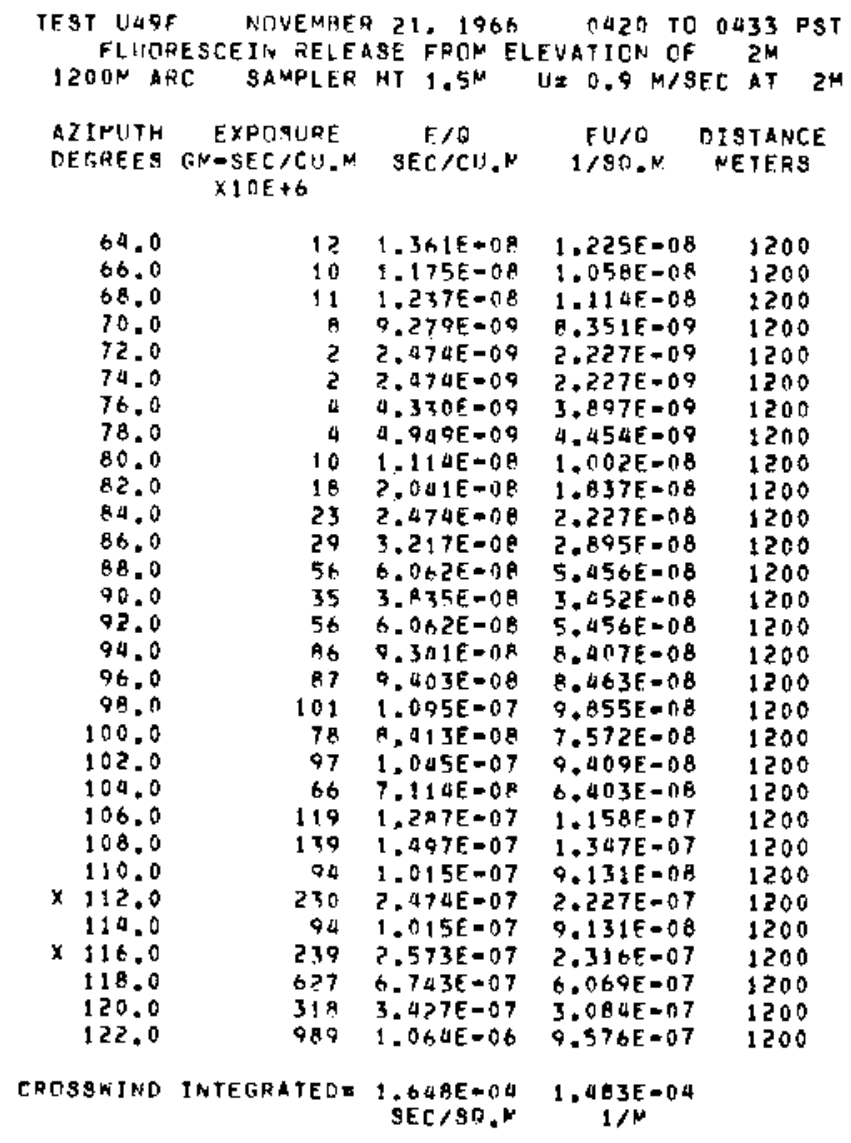


UNSTAQLE SERIES EXPERIMENT:50 ( U-50)

GROUND LEVEL SAMPLING $100-1600$ H ARCS.

TEST U50 DECENBEA 5,1966 1110 TO 1140 PST
ZIAC SULFIOE RELEASE FPTH ELEVATICN OF 26\% IOON AAC SAMPLER HT $1.5 \mathrm{~N}$ U. $5.7 \mathrm{~N} / \mathrm{SEC}$ T $26 \mathrm{~m}$

\begin{tabular}{|c|c|c|c|c|}
\hline $\begin{array}{l}\text { AZIRUFH } \\
\text { CEGREFS }\end{array}$ & $\begin{array}{c}\text { EXPCSURE } \\
\text { GN=SEC/CIJ.N } \\
\times 10 E \bullet 6\end{array}$ & $\begin{array}{c}E / G \\
S E C / C U, N\end{array}$ & $\begin{array}{c}E U / C \\
1 / 30 .\end{array}$ & $\begin{array}{l}\text { DISTANCE } \\
\text { NETERS }\end{array}$ \\
\hline $\begin{array}{l}16.0 \\
20.0 \\
29.0 \\
28.0 \\
32.0 \\
36.0 \\
40.0 \\
44.0 \\
46.0\end{array}$ & $\begin{array}{r}0 \\
0 \\
331 \\
68 \\
130 \\
59 \\
132 \\
39 \\
0\end{array}$ & $\begin{array}{l}0.000 E+00 \\
0.000 E+00 \\
4.840 E=07 \\
9.500 E=08 \\
1.832 E=07 \\
0.365 E=08 \\
1.062 E=07 \\
5.576 E=08 \\
0.000 E+00\end{array}$ & $\begin{array}{l}0.000 E+00 \\
0.000 E+00 \\
2.645 E=06 \\
5.449 E=07 \\
1.004 E=06 \\
4.76 B E=07 \\
1.061 E=06 \\
3.179 E=07 \\
0.000 E+00\end{array}$ & $\begin{array}{l}100 \\
100 \\
100 \\
100 \\
100 \\
100 \\
100 \\
100 \\
100\end{array}$ \\
\hline Dove & TEGRATEO & $\begin{array}{l}7.450 E=06 \\
\text { SEC } / 30, N\end{array}$ & $a+252 E=05$ & \\
\hline
\end{tabular}

\begin{abstract}
TEgT U5O DECFNAF 5,1366 11:A TE 1140 PST IIAC SULF IOE RELFASE FRO FLEVATTCA TF $26 \mathrm{~K}$ $200 \mathrm{~N}$ ARC SANPLEA HT $1.5 \mathrm{SN} U=5.7 \mathrm{~N} / \mathrm{SEC}$ AT $20 \mathrm{~N}$
\end{abstract}

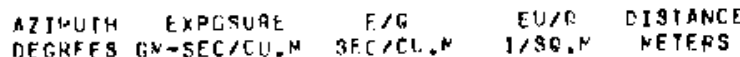
$\times 10 \mathrm{~F}+6$
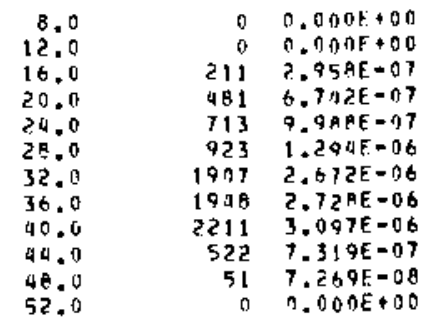

C. $000+00$

$0.00 \mathrm{OC} \rightarrow \mathrm{AOO}$

$1.606 \mathrm{E}=06$

$3.843 E=0.6$

$5.6937=06$

$7.3736=06$

$1.523 E=05$

$1.555 E-05$

$1.765 E-105$

$0.172 E-06$

4. $143 E-07$

$0.0007+00$

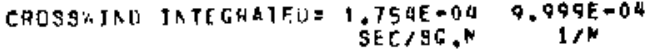

TEST U50 DECENLER 5, $1966 \quad 1110$ TO 1140 PST ZIAC SULFIOF RELEAGE FRCN ELFVATICA DF $26 \mathrm{~N}$ $30 O \mathrm{~N}$ AFC SAMPLER HT $1.5 \mathrm{~N} U=5.7 \mathrm{~K} / 9 \mathrm{SC}$ AT $26 \mathrm{M}$

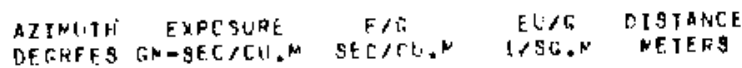
$\times 108+6$

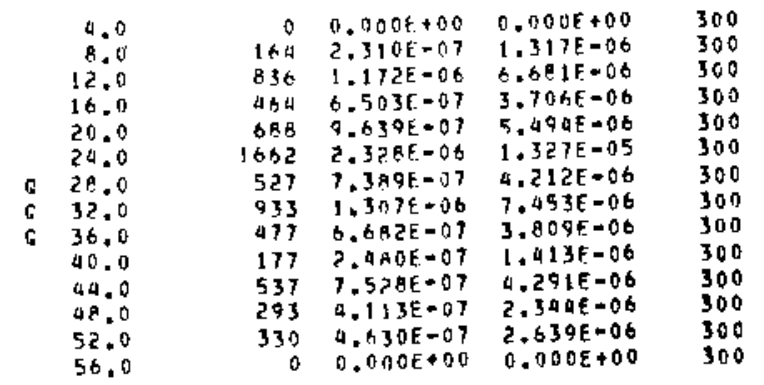

CROSSNIND INTEGAATED $=2.081 E-04 \quad 1.186 E-03$

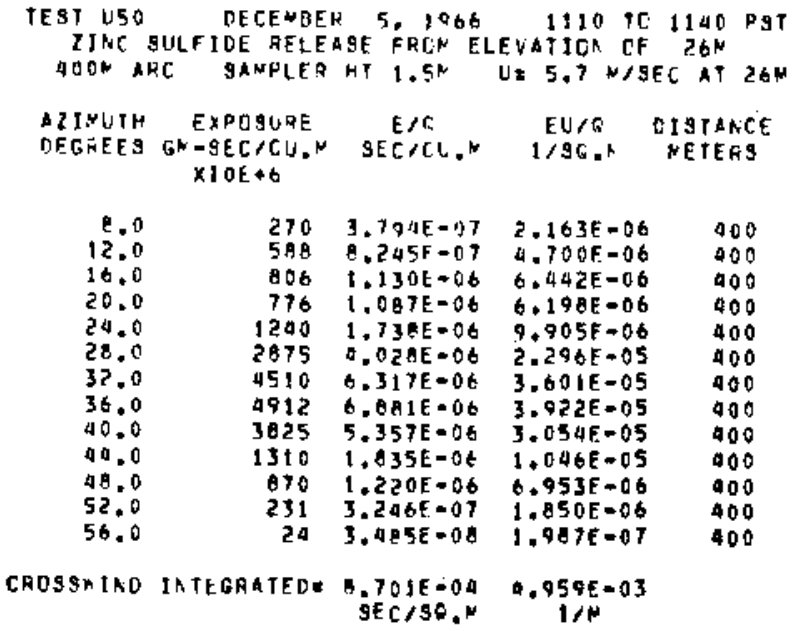

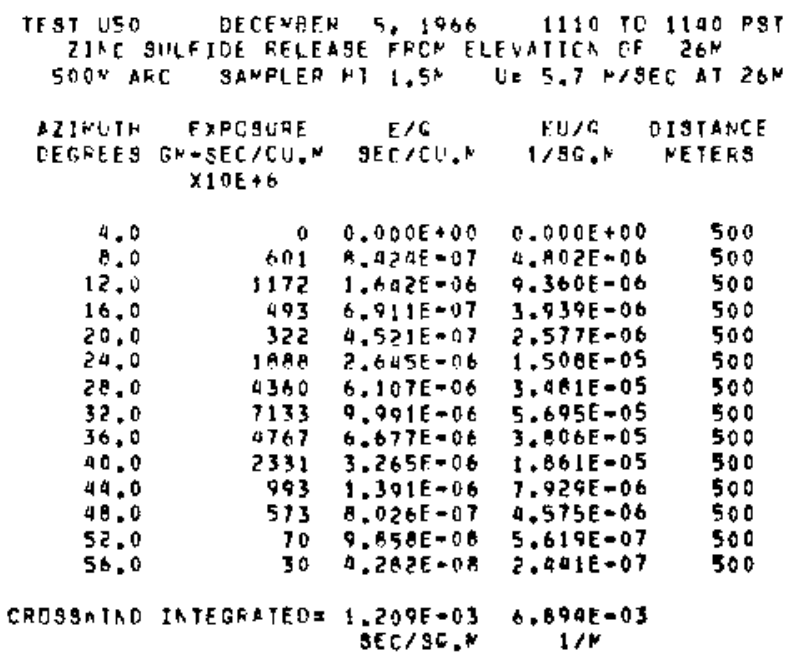

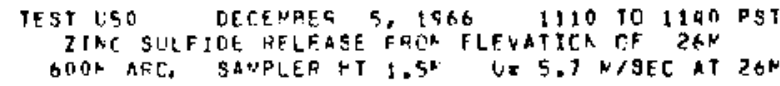

AZJNUTH EXFLSURE FI: EU/G DISTANCE

DEGHFES GNEEC/CG,N SFC/CU,F $1 / 90 . N$ NETEGS $x \leq 0 E+6$

\begin{tabular}{|c|c|c|c|c|}
\hline $\begin{array}{l}6.0 \\
10.0 \\
14.0 \\
19.0 \\
22.0 \\
26.0 \\
30.0 \\
34.0 \\
39.0 \\
42.0 \\
46.0 \\
50.0\end{array}$ & $\begin{array}{l}160 \\
897 \\
542 \\
322 \\
610 \\
2492 \\
4078 \\
4374 \\
2657 \\
912 \\
558 \\
17\end{array}$ & $\begin{array}{l}? .250 E-07 \\
1.257 E=06 \\
8.156 E-07 \\
4.511 E=07 \\
A .54 \Delta E=07 \\
3.400 E=06 \\
0.832 E=06 \\
6.127 E=06 \\
3.722 E=06 \\
1.278 E=06 \\
7.827 E=07 \\
2.190 E=08\end{array}$ & $\begin{array}{l}1.293 E=06 \\
1.163 E=06 \\
4.649 E-06 \\
2.571 E=06 \\
4.670 E=06 \\
1.989 E=05 \\
3.894 E=05 \\
3.492 E=05 \\
2.122 E=05 \\
1.282 E=06 \\
1.461 E=06 \\
1.362 E=07\end{array}$ & $\begin{array}{l}400 \\
600 \\
600 \\
600 \\
600 \\
600 \\
600 \\
600 \\
600 \\
600 \\
600 \\
600\end{array}$ \\
\hline
\end{tabular}

CROS9KIAD IATEGPATED= $1.083 E=03 \quad 6.1$ TUE-03 


$$
\begin{aligned}
& \text { TFST } 150 \text { DECENBER } 5,1066 \text { 1110 TC } 1140 \text { PST } \\
& \text { ZIAC SLILFIUE HF.LFASE FRCN ELFVATICA EF } 2 \mathrm{O}^{\mathrm{K}} \\
& 7 \text { OON ANE SANLLER HT } 1.5^{\mathrm{N}} \text { WE } 5.7 \text { MISEC AT ZEN }
\end{aligned}
$$

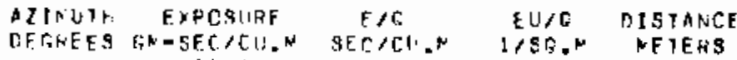

$$
\begin{aligned}
& X 10 E+6
\end{aligned}
$$

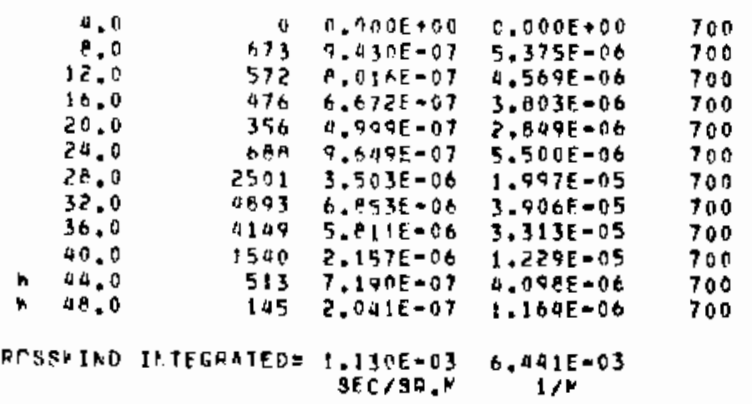

PEST USO DECENHFA 5,1906 1110 TO 1140 PST

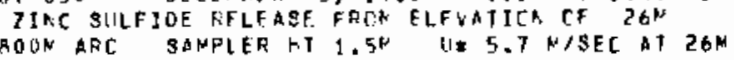
ATINUTH EXPCSURF FAT EUIC DISTAACE

\begin{tabular}{|c|c|c|c|c|}
\hline $\begin{array}{l}7.0 \\
10.0 \\
13.0 \\
16.0 \\
15.0 \\
22.0 \\
25.0 \\
28.0 \\
31.0 \\
34.0 \\
37.0 \\
40.0 \\
43.0 \\
46.0\end{array}$ & $\begin{array}{l}64 \\
272 \\
588 \\
445 \\
386 \\
298 \\
760 \\
2386 \\
4410 \\
4554 \\
3096 \\
1308 \\
000 \\
345\end{array}$ & $\begin{array}{l}9.0+2 E=08 \\
3 . A 14 E=07 \\
9.245 E=07 \\
6.7 .74 E=07 \\
5.407 E=07 \\
0.1 A 2 F=07 \\
1.005 E=06 \\
3.342 E=06 \\
6.177 E=06 \\
6.379 E=06 \\
9.337 E=0 E \\
1.032 E=06 \\
6.414 E=07 \\
5.537 E=07\end{array}$ & $\begin{array}{l}5.165 E=07 \\
2.174 \mathrm{E}=06 \\
4.700 \mathrm{E}=06 \\
\pm .553 \mathrm{E}=06 \\
3.062 \mathrm{E}=06 \\
2.364 \mathrm{E}=06 \\
0.068 \mathrm{E}=06 \\
1.905 \mathrm{E}=05 \\
3.521 \mathrm{E}=05 \\
3.636 \mathrm{E}=05 \\
2.472 \mathrm{E}=05 \\
1.044 \mathrm{E}=05 \\
4.796 \mathrm{E}=06 \\
3.156 \mathrm{E}=06\end{array}$ & $\begin{array}{l}800 \\
800 \\
800 \\
800 \\
800 \\
800 \\
100 \\
800 \\
800 \\
800 \\
800 \\
800 \\
800 \\
800\end{array}$ \\
\hline
\end{tabular}
OESREES GN-SEC/CU.N SERICL.N 1/81\%,N RETERS $X 1 D E$ th

CAOSGHIAD IATEGRATEUE $1.148 E-03 \quad 6.543 E-03$

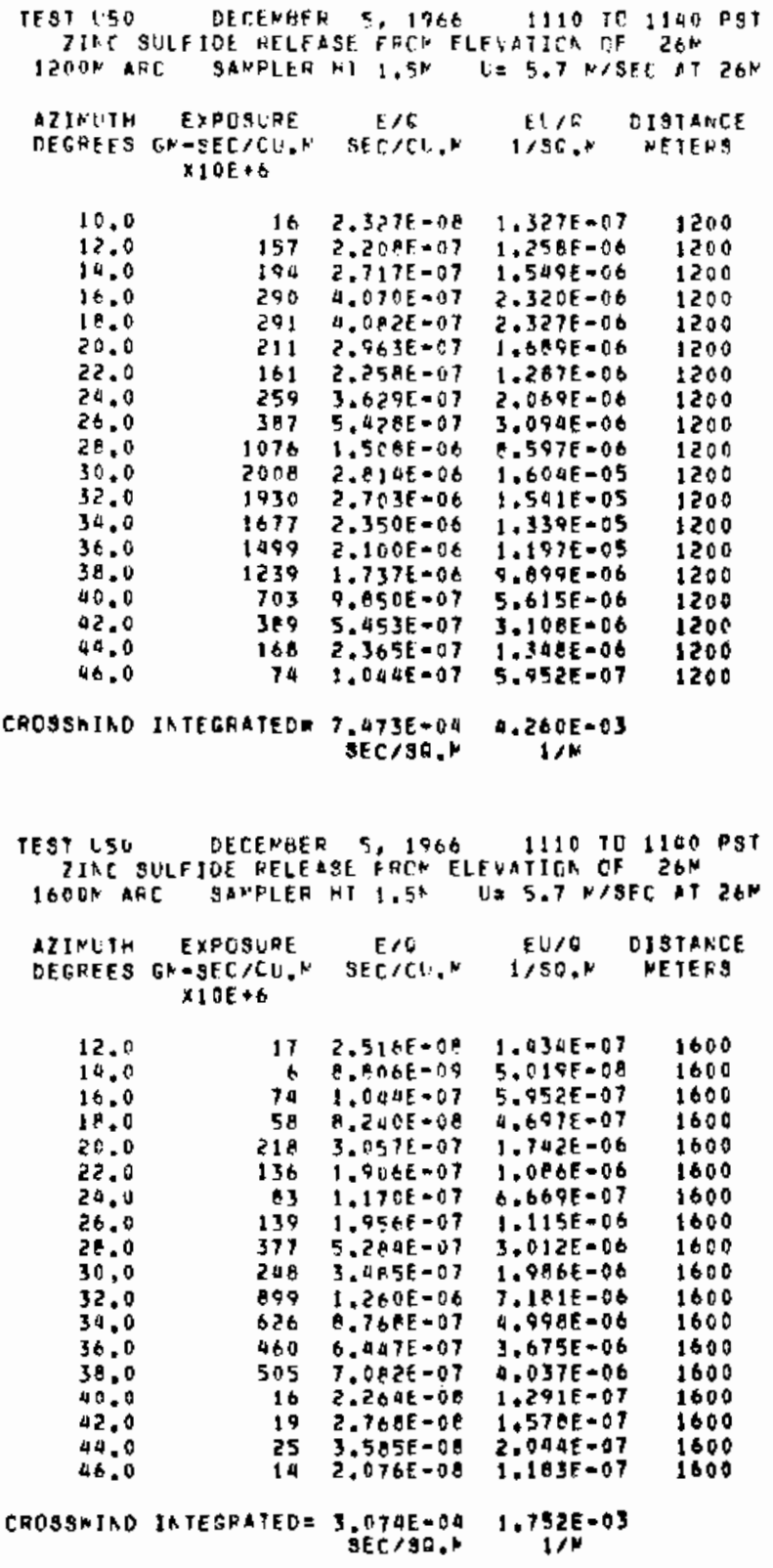


UNSTABLE SERIES EXPERIMENT: SOF (

$Z$ IHC SULFIDE ANO FLUORESCEIN WERE BOTH USEO AS TRACERS OURING

EXPERIMENT 51. FLUORESCEIN RESULTS ARE PRESENTED HERE. GROUND

LEVEL SAMPLING 100-1600 M ARC.

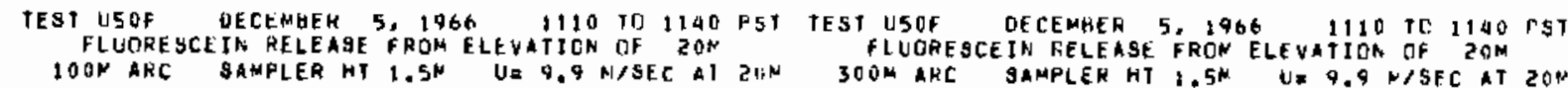

SAMPLEH AZIMUTHS HAYE GEEN INCREASED BF 30 DEGREES SAMPLER AZIMUTHS HAVE BEEA INCREASED HY 30 DEGREES

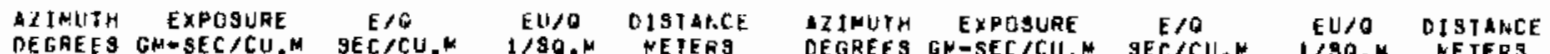
$X 10 E+6$
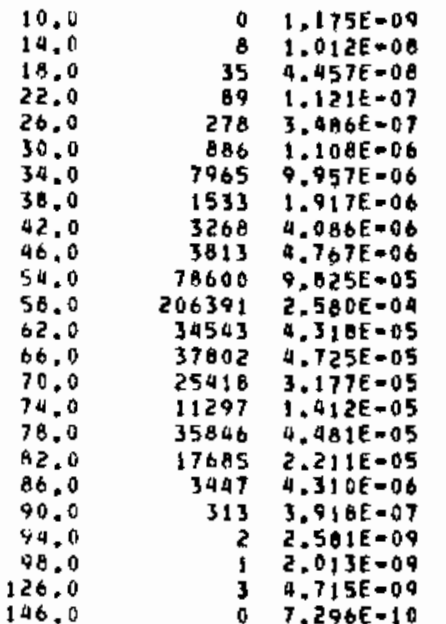

$1.164 E-00$

$1.0025=07$

$4.413 \mathrm{E}=07$

$1.110 \mathrm{E}-06$

$3.451 E=00$

$1.097 f=05$

$9.858 E-05$

$1.896 \mathrm{E}=05$

$4,045 E-05$

4. $719 \mathrm{E}-05$

$9.727 E-04$

$2.554 \mathrm{E}-03$

$4.275 E=04$

$4.678 E=04$

3. $1460=04$

$1.3965=04$

$4,436 E-04$

2. $189 E=04$

$4.267 E=05$

$3.879 \mathrm{E}=06$

$2,555 E=00$

$1.993 E-08$

146.0

$0 \quad 7.296 E-10 \quad 7.223 E=09$

$$
\begin{aligned}
& 100 \\
& 100 \\
& 100 \\
& 100 \\
& 100 \\
& 100 \\
& 100 \\
& 100 \\
& 100 \\
& 100 \\
& 100 \\
& 100 \\
& 100 \\
& 100 \\
& 100 \\
& 100 \\
& 100 \\
& 100 \\
& 100 \\
& 100 \\
& 100 \\
& 100 \\
& 100 \\
& 100
\end{aligned}
$$

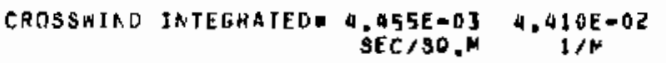

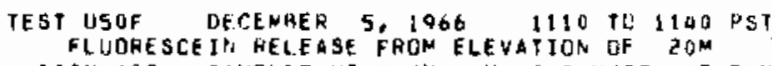

ZOON ARC SAMPLER MT $1.5 \%$ UE $9.9 \mathrm{~N} / \mathrm{SEC}$ AT $20 \mathrm{~N}$

SARPLEF ALIWUTS HAVE GEEA INCAEASEO OY 30 DEGREES

DECENLER $5,1960 \quad 1110$ 10 $1140 \mathrm{PST}$ FLUURFSCEIN RELEASE FROM ELEVATIOR CIF $20+1$

$400 \mathrm{~N} A R C$ SAMHLER HI, $5 \mathrm{~N} U=9.9 \mathrm{M} / \mathrm{SEC}$ AT $20 \mathrm{M}$

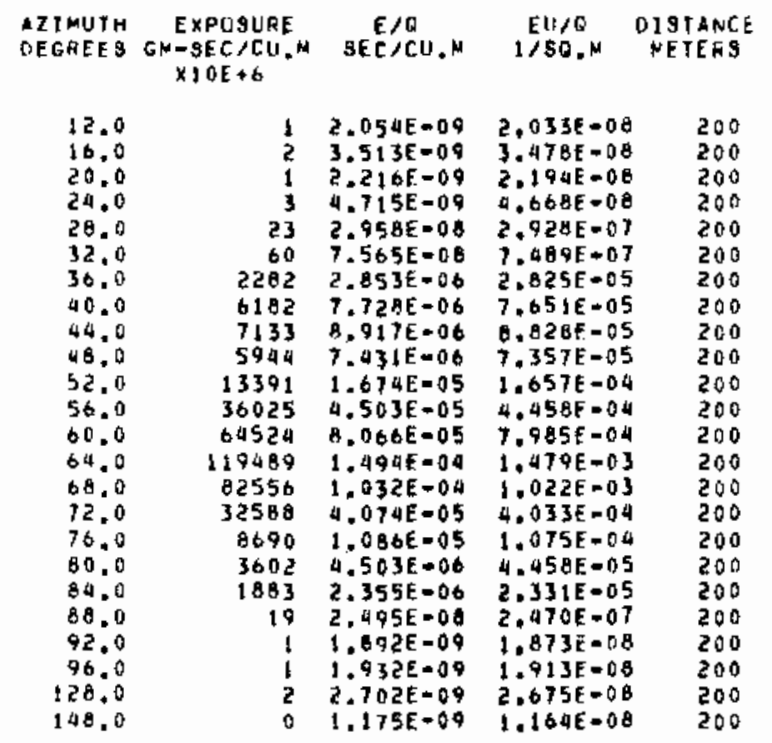

SAMPLER AZIMUTHS HAVE GEEM INCREABED BY 30 DEGREES

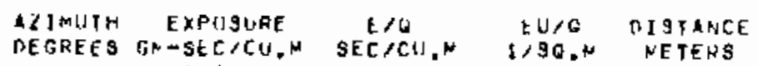

023.0

$0 \quad 60.0$

70.0

18.0

B2.0

66. 0

90.0

98.19

102.0 $\times 10 \mathrm{E}+6$

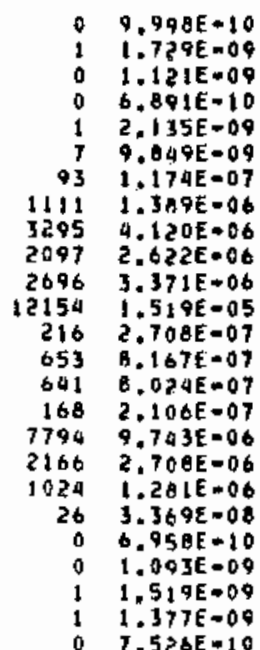

$9.89 \mathrm{BE}-09$

300

6.0

34.0

42.0

0.0

.0

$X 10 \mathrm{~F}+6$

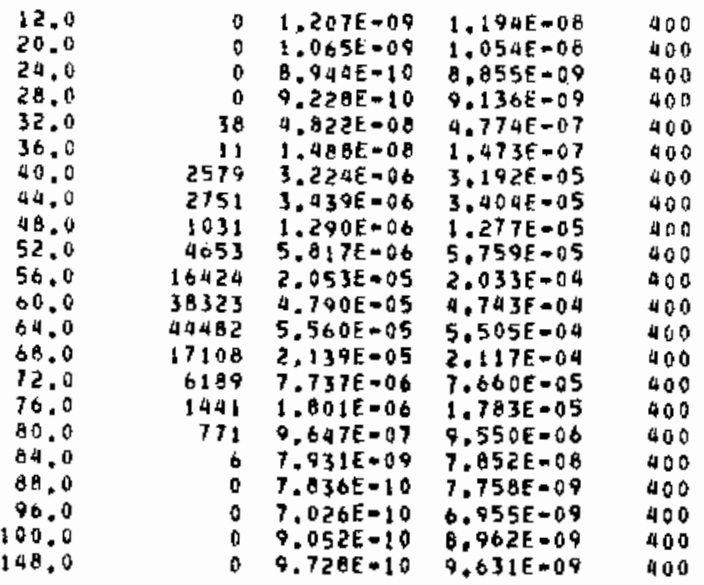

CNOSSMIAD INTEGRATEOE 6.71 DE-03 b.643E-02

CROSSIIND IKTEGRATETE $4.741 E-03$ 4.694E-02

SEC $190, N$ M. 


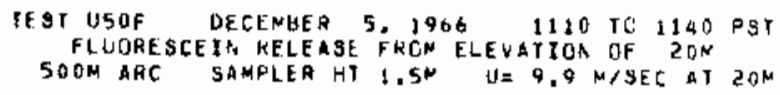

SAMPLER AZIMUTHS HAVE GEEA INCAEASEO GY 30 OELREES

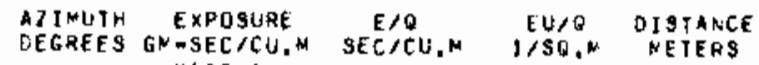
$\times 10 E+6$

10.0
14.0
18.0
22.0
26.0
30.0
34.0
38.0
42.0
46.0
50.0
54.0
58.0
62.0
66.0
70.0
74.0
78.0
82.0
86.0
90.0
94.0
98.0
902.0
146.0

500
500
500
500
500
500
500
500
500
500
500
500
500
500
500
500
500
500
500
500
500
500
500
500
500

IEST USOF OECEMBER 5.9906 1110 101140 PST FLLOFESCEIN RELEASE FRON ELEVATIDN OF ZON TOON AÑC SAMPLER MT 1.SH U 9.9 H/SEC AT ZDN

SAMPLER AZIMUTHS HAVE GEEN IKCREASED EY 30 DEGREES

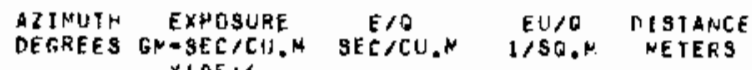
$x \perp 0 \varepsilon+6$

\begin{tabular}{|c|c|c|c|}
\hline $\begin{array}{r}0 \\
0 \\
1 \\
1 \\
0 \\
1 \\
47 \\
37 \\
22 \\
52 \\
286 \\
526 \\
499 \\
66 \\
54 \\
45 \\
0 \\
0 \\
0 \\
0 \\
0\end{array}$ & $\begin{array}{l}9.052 E-10 \\
0.485 E=10 \\
1.459 E=09 \\
2.108 E=09 \\
1.216 E=09 \\
6.215 E-09 \\
5.945 E=08 \\
1.729 E=08 \\
2.937 E=08 \\
6.620 E=08 \\
3.607 E=07 \\
6.587 E=07 \\
6.249 E=07 \\
2.077 E=07 \\
6.755 E=08 \\
1.024 E=07 \\
1.013 E=09 \\
9.964 E=10 \\
9.284 E=10 \\
0.000 E+00 \\
6.755 E=10\end{array}$ & $\begin{array}{l}8.962 E=09 \\
6.420 E=09 \\
1.445 E=08 \\
2.087 E=08 \\
1.204 E=08 \\
6.153 E=08 \\
5.865 E=07 \\
4.682 E=07 \\
2.809 E=07 \\
6.554 E=07 \\
3.571 E=06 \\
6.521 E=06 \\
6.1 B 6 E=06 \\
2.057 E=06 \\
6.08 B E=07 \\
1.806 E=06 \\
1.003 E=08 \\
9.845 E=09 \\
9.196 E=09 \\
0.000 E+00 \\
6.08 B E=09\end{array}$ & $\begin{array}{l}700 \\
700 \\
700 \\
700 \\
700 \\
700 \\
700 \\
700 \\
700 \\
700 \\
700 \\
700 \\
700 \\
700 \\
700 \\
700 \\
700 \\
700 \\
700 \\
700 \\
700\end{array}$ \\
\hline
\end{tabular}

CROSSNIND INTEGRATED= $1.137 E=04 \quad 1.126 E=03$
CHOSSWIND INTEGKATED $=2.753 E-03 \quad 2,726 E=02$

IEST USOF DECEMAER S. 1966 1110 T0 1140 PST OOON ARE SAMPLER MT $1.5 \mathrm{~N}$ UI $9.9 \mathrm{M} / \mathrm{SEC}$ AT $20 \mathrm{M}$

SAHPLER AZIMUTHS HAVE GEEN INCREASED EY 30 DEGREES

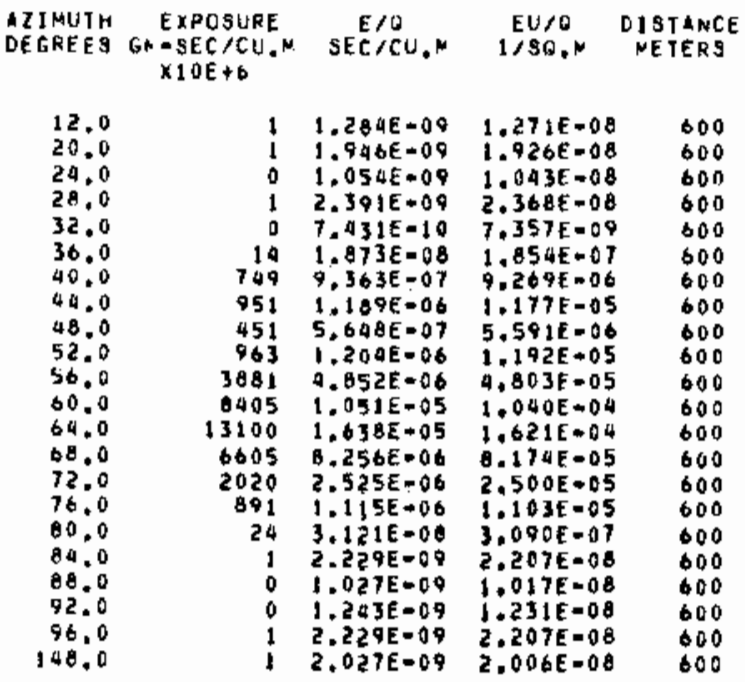

CROSSNIAD INTEGRATED 1.995E-03 1.975E-02
TEST LI5OF DECEMBER 5, 1960 1110 TO 1340 PST FLUOFESCEIN RELEASE FRON ELEVATION OF ZAM 8OON AFC SAMPLER MT $1.5 \mathrm{~N}$ UI $9.9 \mathrm{~N} / \mathrm{SEC}$ AT $20 \mathrm{M}$

SALPLEH AZIMUFMS MAVE BEEN IMCREASED GY 30 DEgAEES

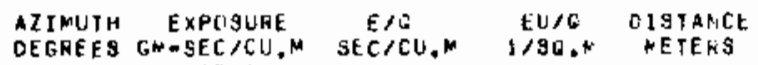
$\begin{array}{r}\times 1 O E \rightarrow 6 \\ \hline\end{array}$

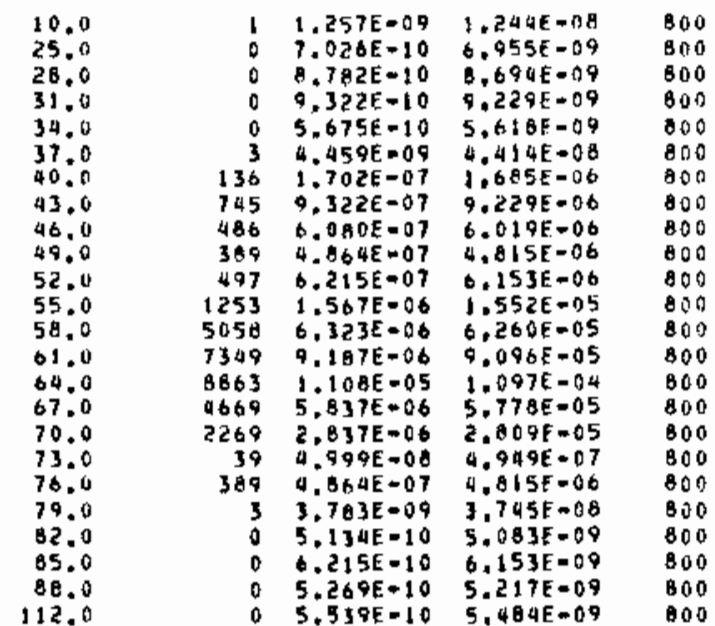

CRDSSWIAG INTEGHATED= $1.664 E-03 \quad 1,667 E=02$ 


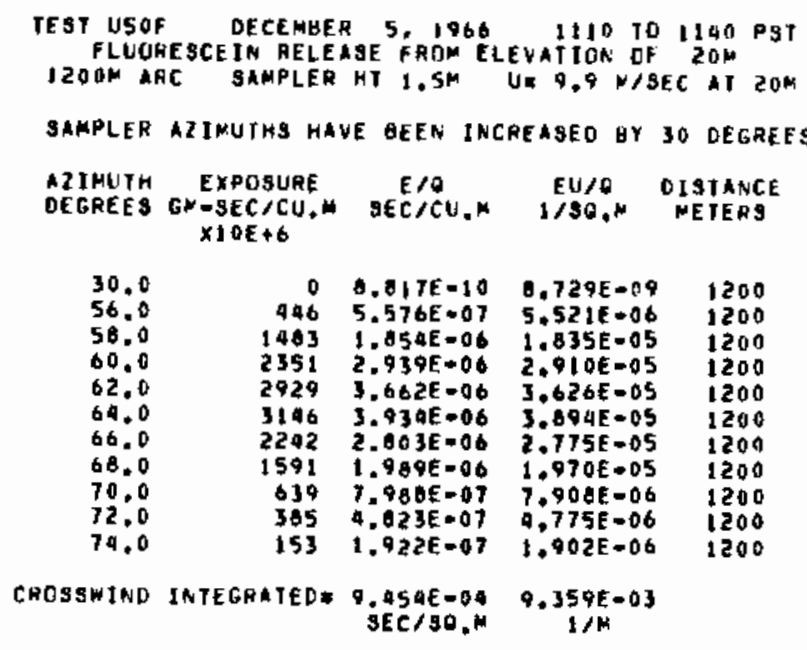

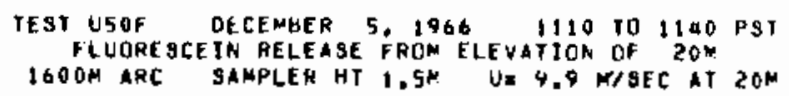


UNSTABLE 5ERIES EXPERIMENT: $\$ 1$ (U-51)

UNSTABLE SERIES EXPERIMENT: SN WERE BOTH USED AS TRACERS DURING ZINC SULFIDE AND FLUORESCE IN WERE BOTH PRE LEVEL SAMPL ING $100-1600$ M ARC.

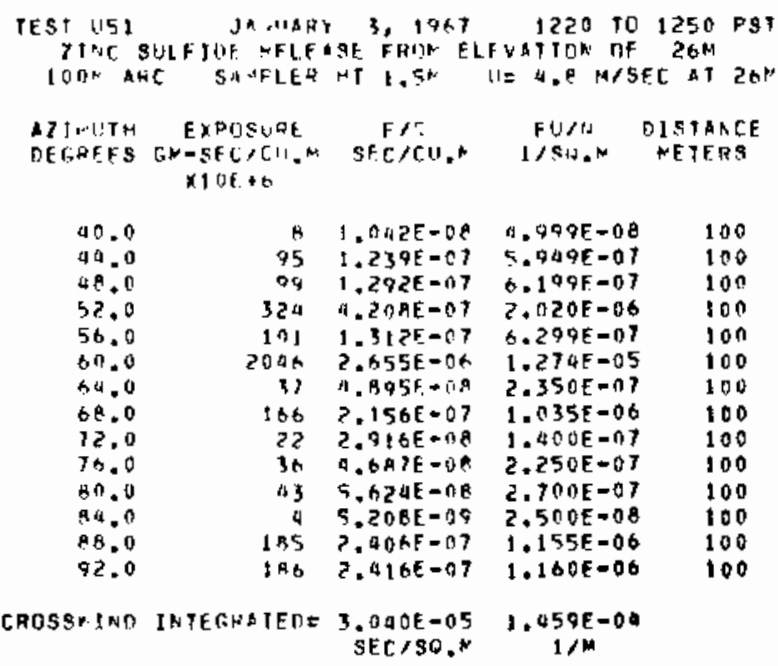

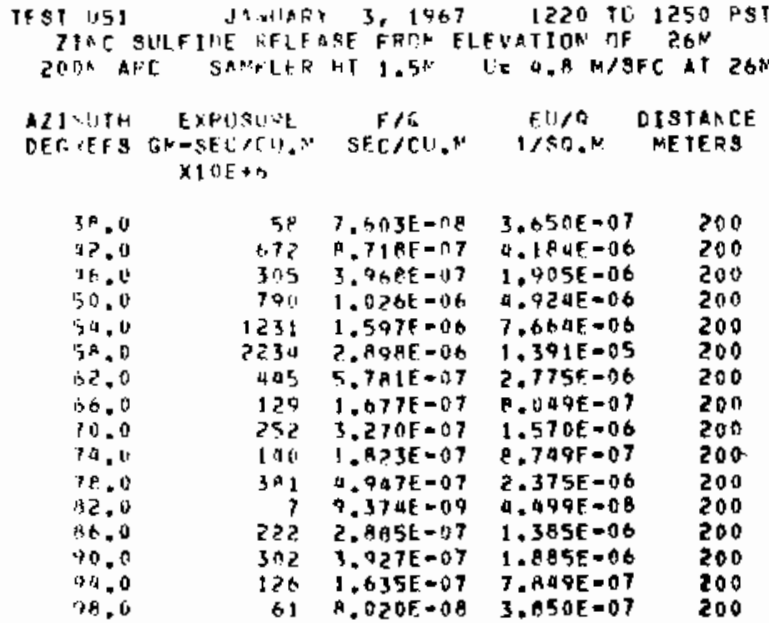

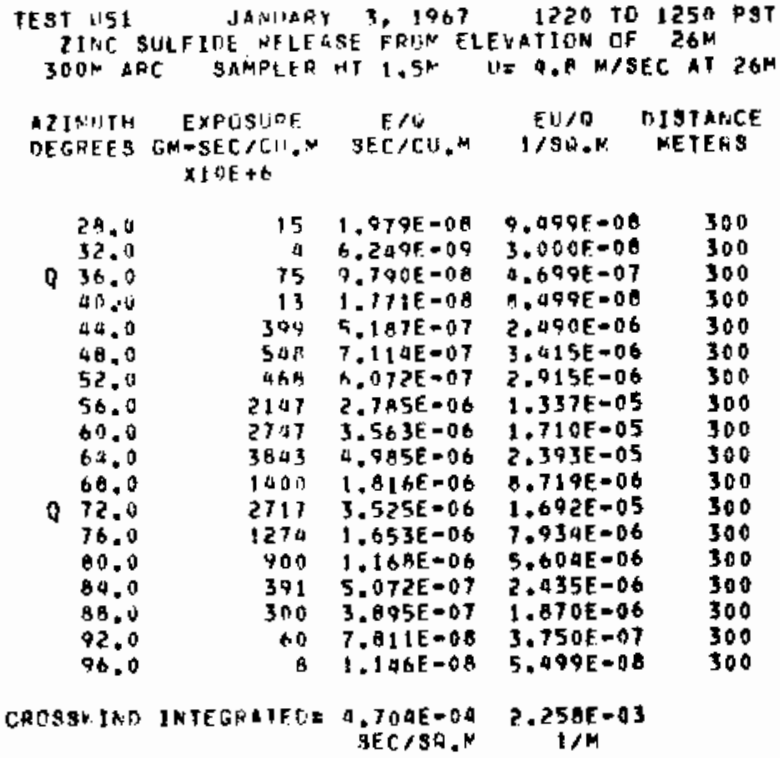

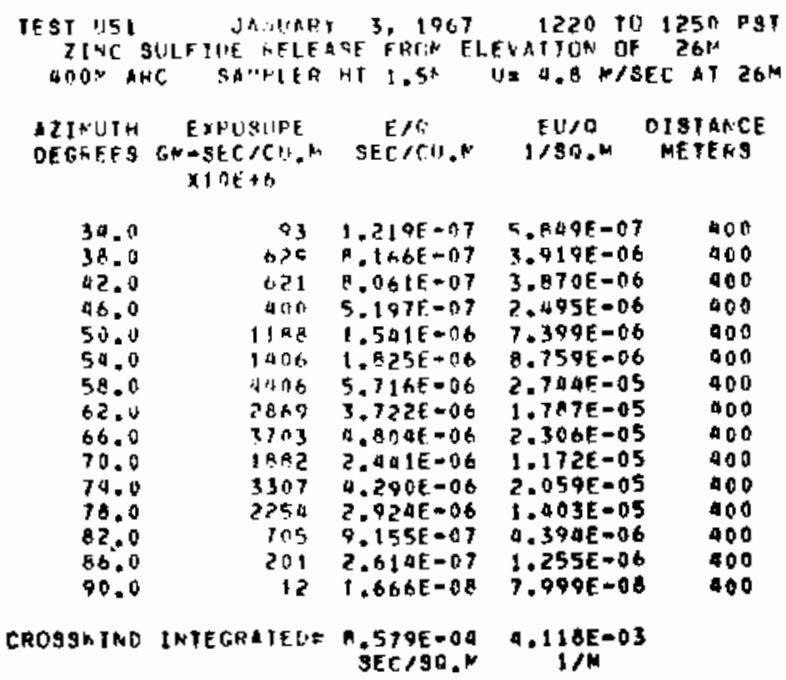




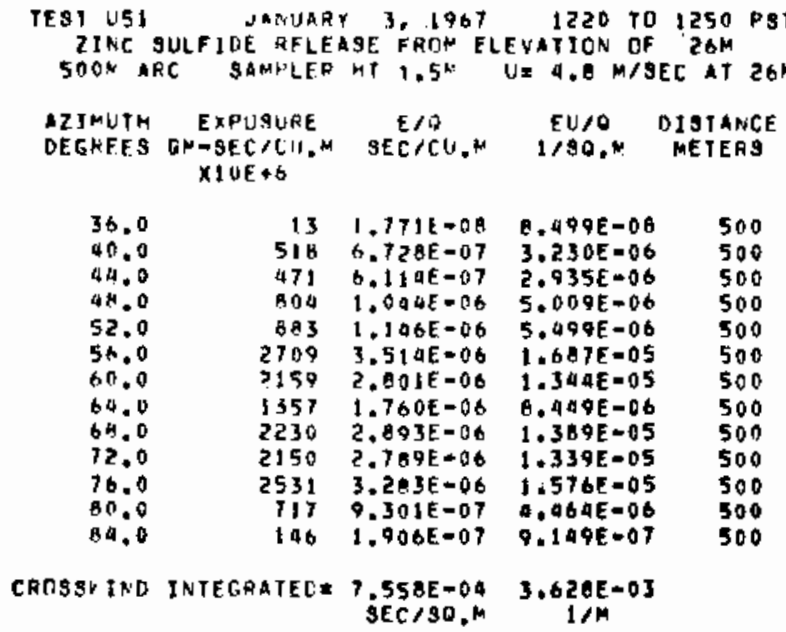

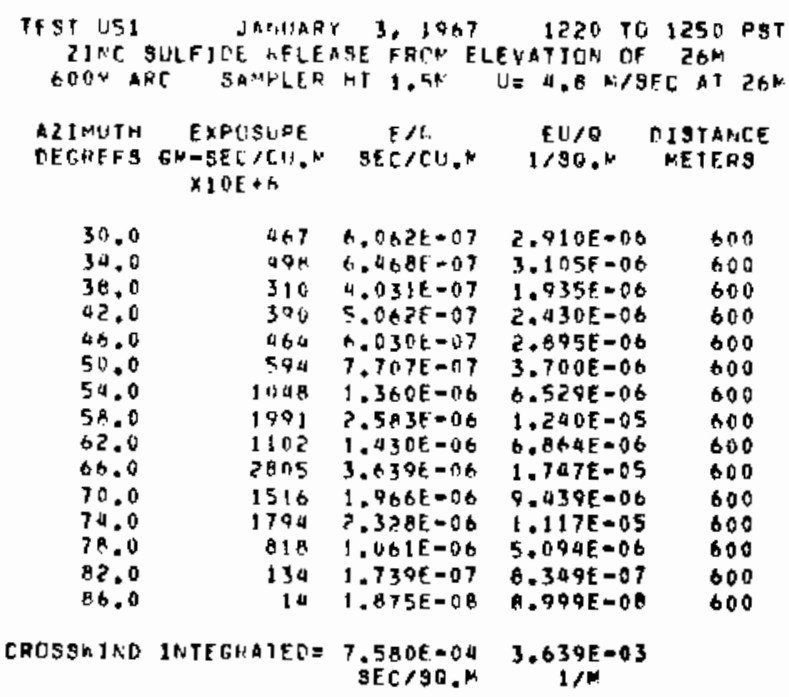

TEST U5I JAIVUALY 3, 1967 1220 TO 1250 FST ZJNC SULFILE TFLFAGE FRIIH FLEVATION TIF 26 M

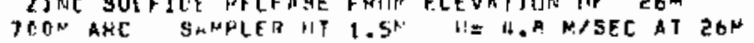

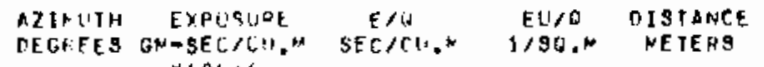
$\times j 0 t \cdot 6$

\begin{tabular}{|c|c|c|c|c|}
\hline 40.0 & 7 & $3.370 \mathrm{E}-00$ & $4.499 \mathrm{E}-\mathrm{AQ}$ & 760 \\
\hline 44,0 & $\ln 9$ & 1. $11+E-137$ & $0.799 E=07$ & 700 \\
\hline 18.0 & $\begin{array}{l}344 \\
343\end{array}$ & $\begin{array}{l}4.4 \text { AE }=07 \\
5.79 E=07\end{array}$ & $\begin{array}{l}2.145 E=06 \\
2.760 E=06\end{array}$ & $\begin{array}{l}700 \\
700\end{array}$ \\
\hline 56.0 & 1142 & 1. यहतE- Ot & $7.114 \mathrm{E}=00$ & 700 \\
\hline+6.0 & 8.96 & $1.143 F-66$ & $5.484 E-06$ & 700 \\
\hline 64.0 & 1262. & $1.6375=0 t$ & $7.859 E=06$ & 700 \\
\hline$O H .0$ & 1252 & $1.625 E=0 A$ & $7.799 E-06$ & 700 \\
\hline 72.0 & $12 a 2$ & $1.611 \mathrm{E}=46$ & 7.73 aE $=06$ & 700 \\
\hline & 741 & $.613 E-07$ & $0.619 E-06$ & 700 \\
\hline 60.0 & $\begin{array}{r}182 \\
71\end{array}$ & $\begin{array}{l}2.360 E-07 \\
9,2+0 E-O B\end{array}$ & $\begin{array}{l}1.135 E=06 \\
4.469 E=07\end{array}$ & $\begin{array}{l}700 \\
700\end{array}$ \\
\hline
\end{tabular}

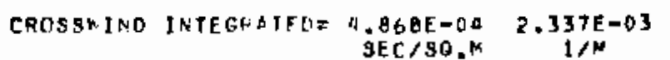

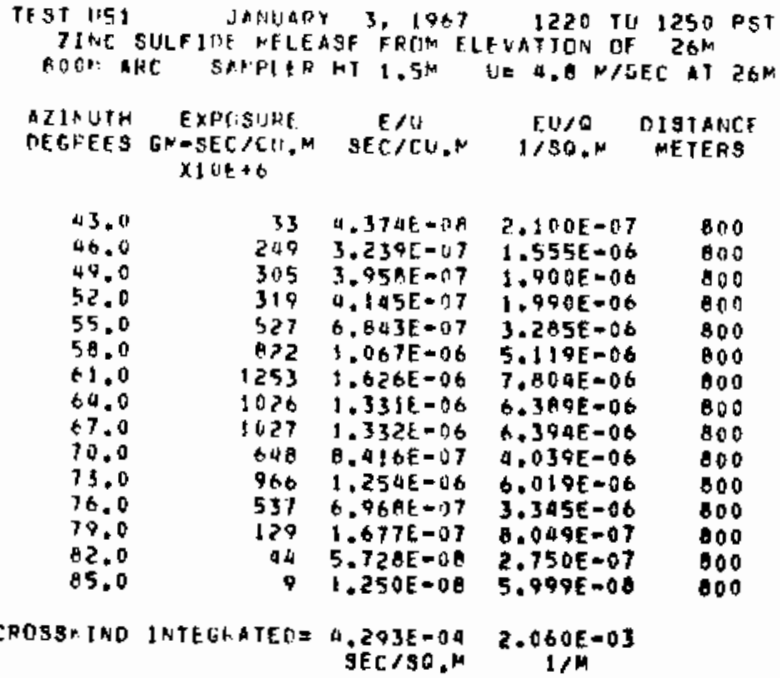

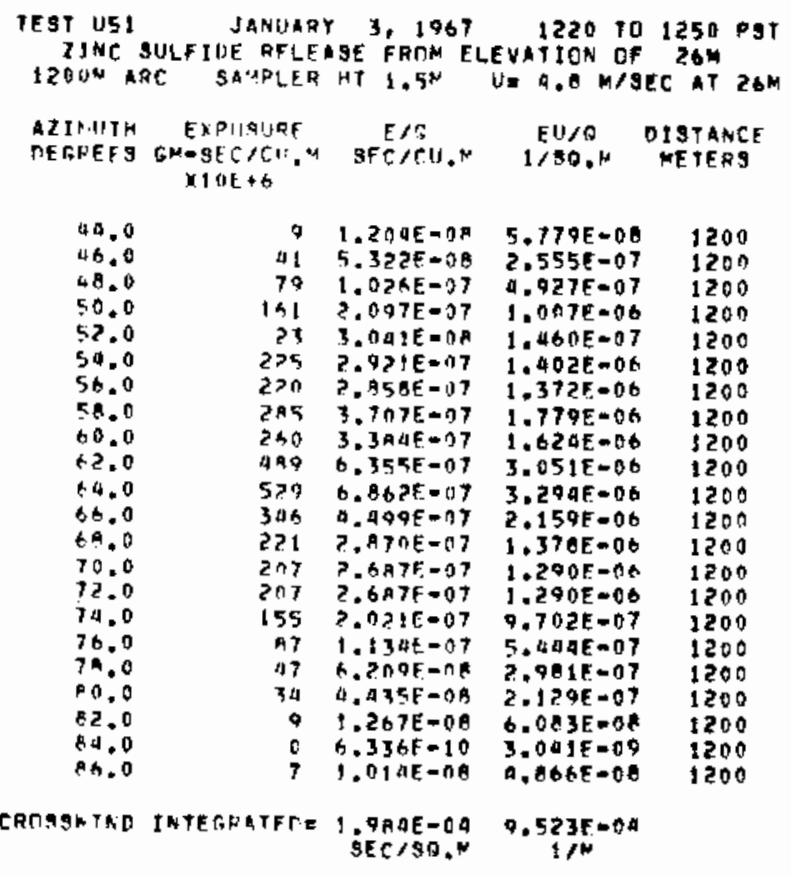




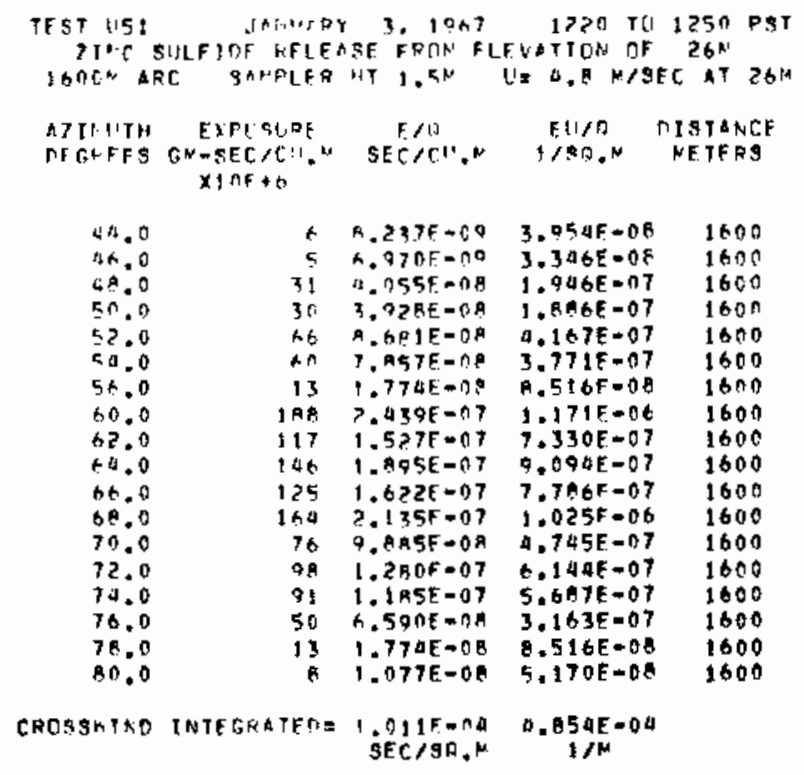


UNSTABLE SERIES EXPERIMENT: $51 \mathrm{~F}$ ( U-51F)

ZINC SLILFIOE AND FLLORESCEIN WERE BOTH USED AS TRACERS DURING EXPERIMENT 51. FLUORESCEIN RESULTS ARE PRESENTED HERE. GROUND LEVEL SAMPL ING ON ONLY THE $1600 \mathrm{M}$ ARC.

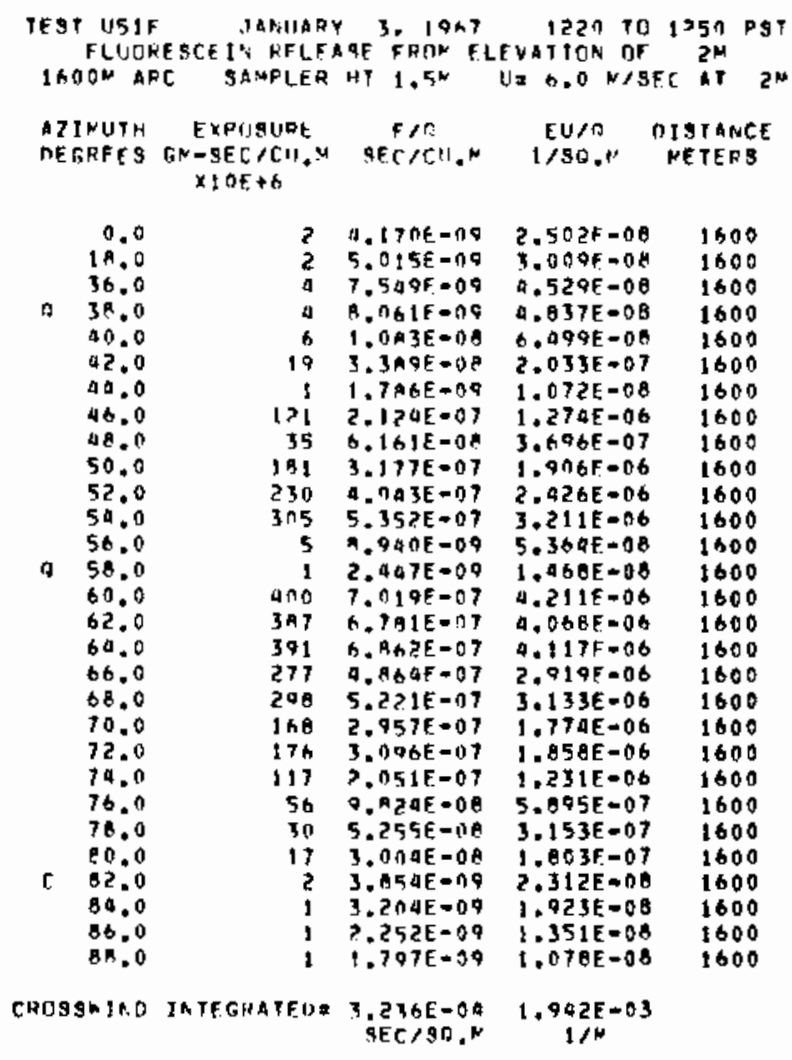


UNSTABLE SERIES EXPERITENT: 52 (U-52)

ZINC SULFIDE AND FLUORESCEIN WERE BOTH USED AS TRACERS OURIWC

EXPERI IENT $5 Z$. ZINC SULFIDE RESULTS RRE PRESENTED HERE. GROUND

LEYEL SAMPLING 1 CO-1600 i. ARCS.

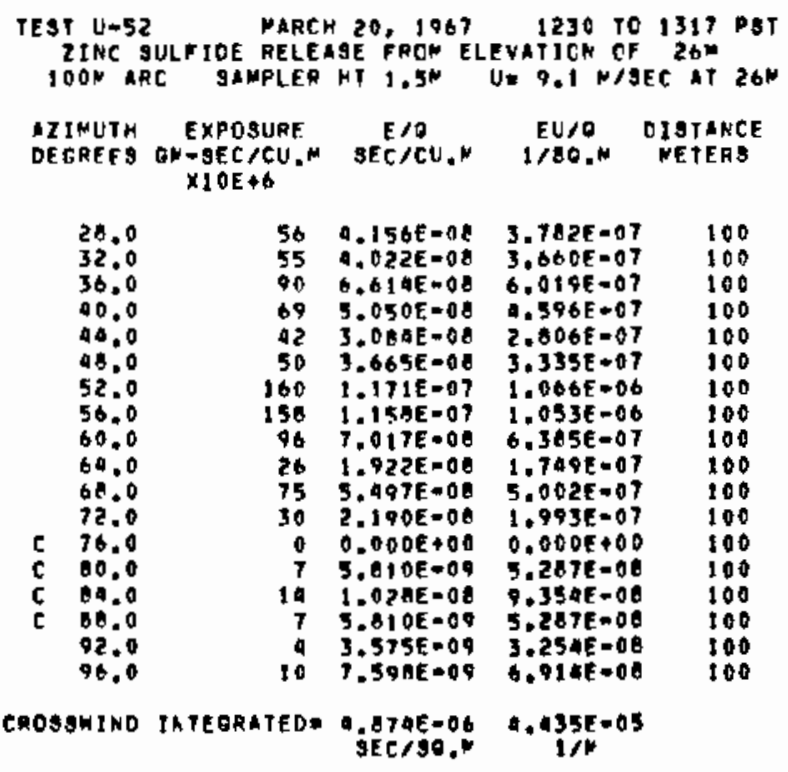

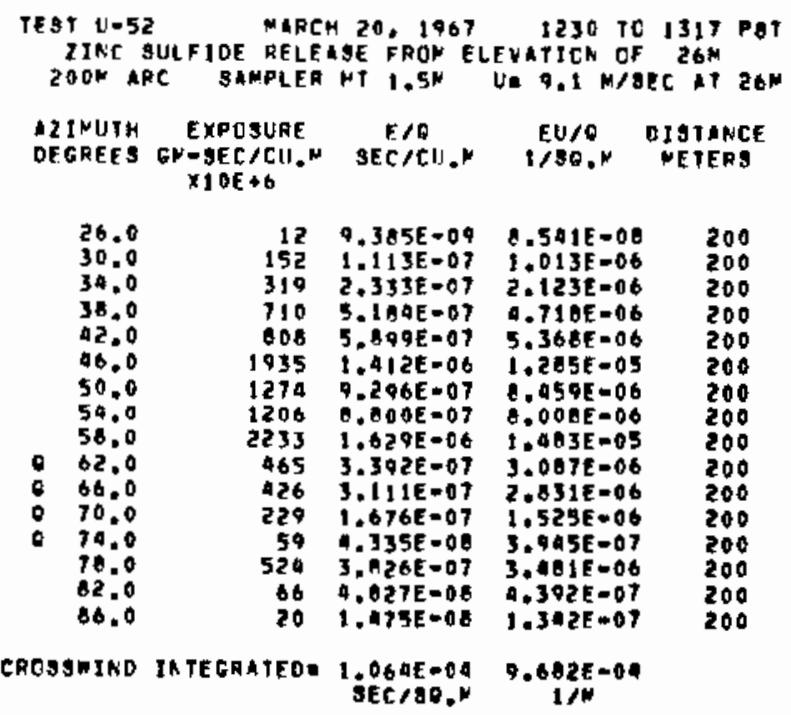

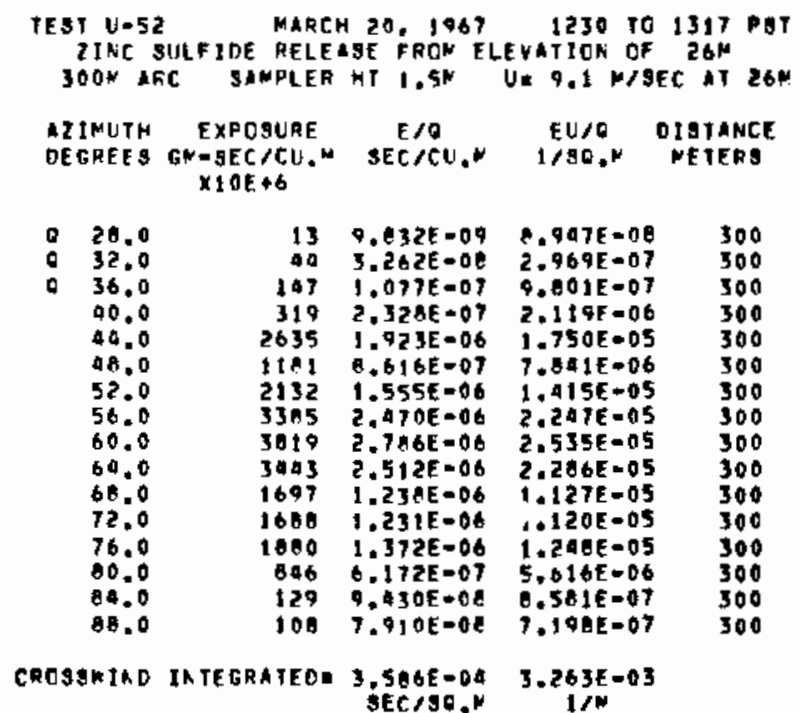




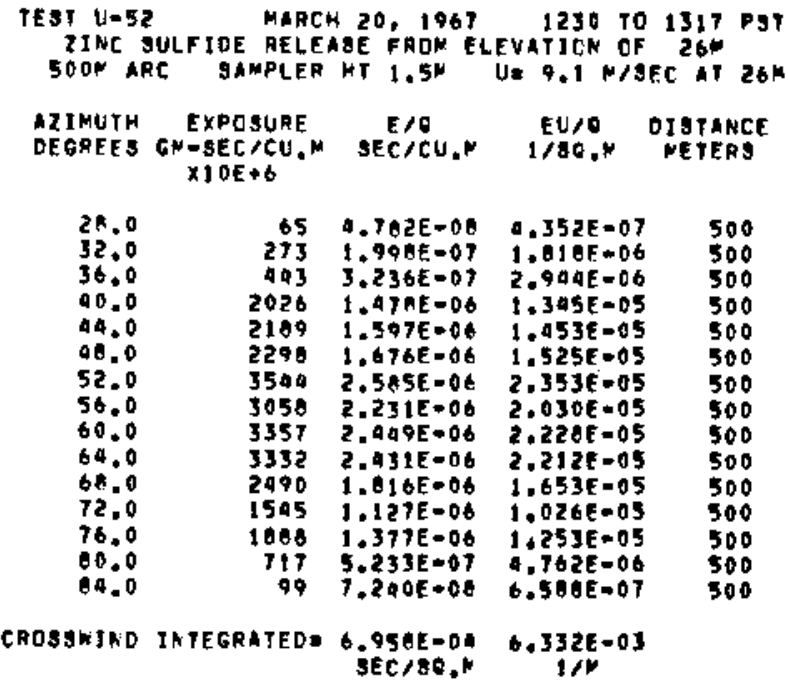

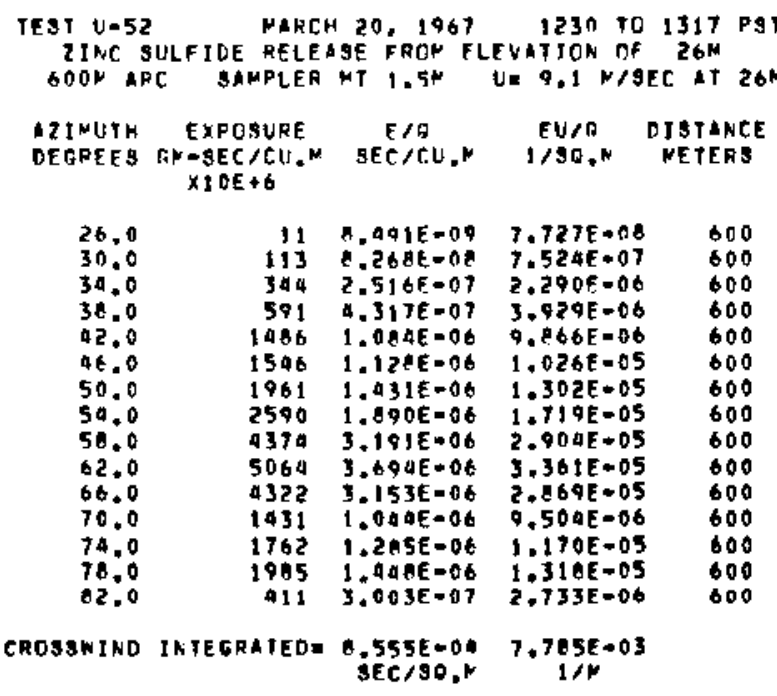

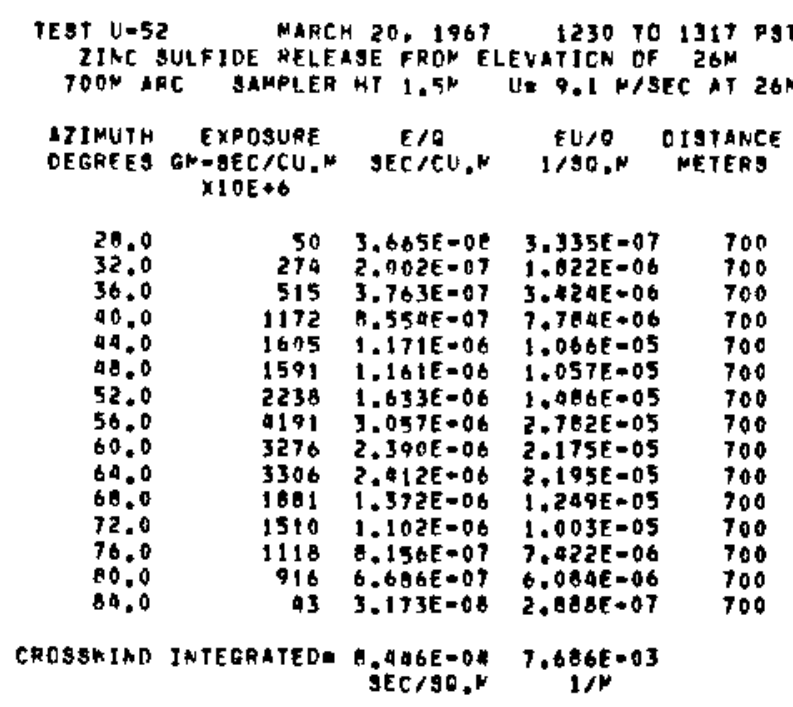

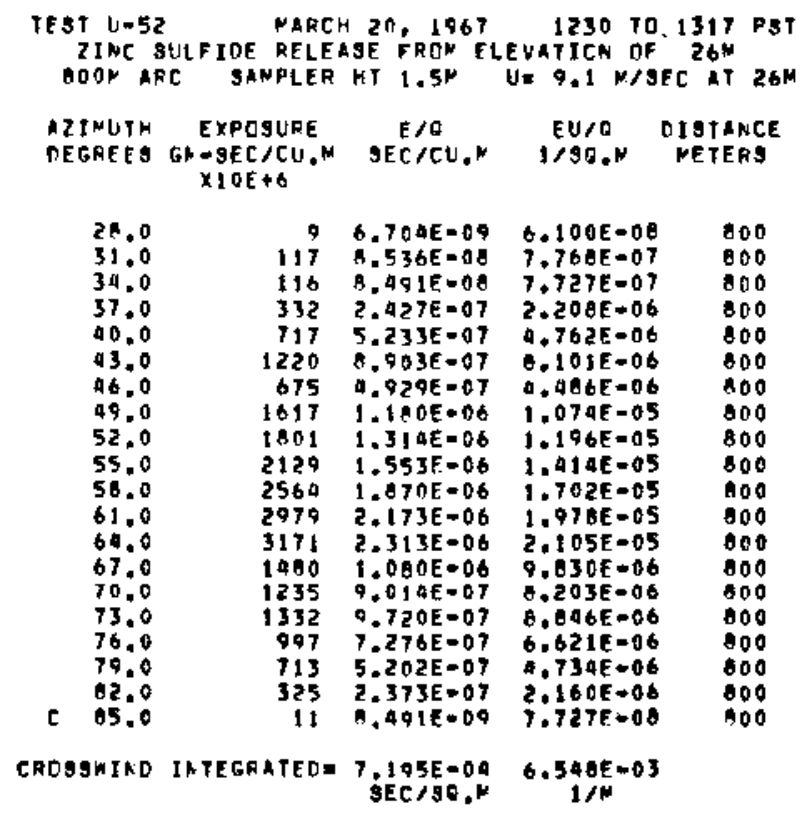




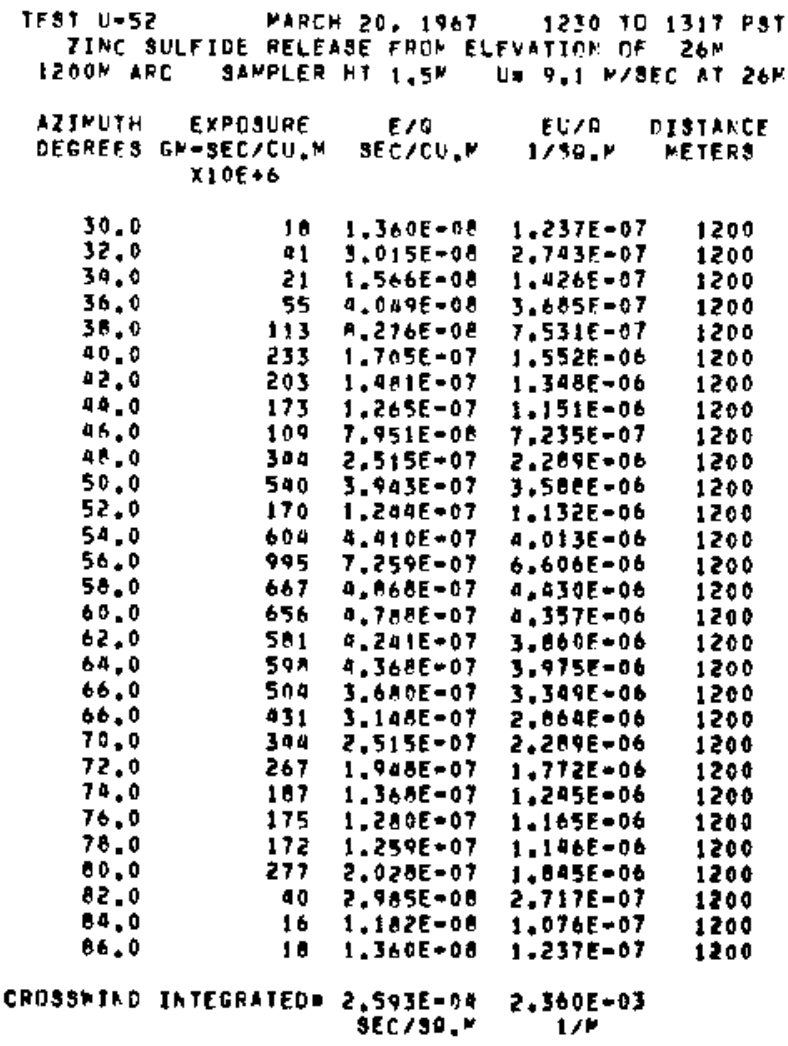

\begin{tabular}{|c|c|c|c|c|}
\hline $\begin{array}{r}\text { TESI U-52 } \\
\text { IINC } 9 \\
1600 \mathrm{NR}\end{array}$ & $\begin{array}{l}\text { Z MARCH } \\
\text { gULFIDE RELE } \\
\text { RC SAWPLER }\end{array}$ & $\begin{array}{l}\text { H Z0, } 1967 \\
\text { AE FRCH EL } \\
\text { HT } 1.5 \%\end{array}$ & $\begin{array}{l}1230 \text { TO } \\
\text { ULVATIEN OF } \\
9.1 \mathrm{M}\end{array}$ & $\begin{array}{l}1317 \mathrm{PST} \\
2 \mathrm{MM} \\
\text { EC HT } 26 \mathrm{M}\end{array}$ \\
\hline $\begin{array}{l}\text { DINUTH } \\
\text { DEGREES }\end{array}$ & $\begin{array}{c}\text { EXPOSURE } \\
\text { GN-SEC } \triangle C U * N \\
\times 1 O E+6\end{array}$ & $\begin{array}{c}E / 0 \\
\text { sec }<U_{0} k\end{array}$ & $\begin{array}{c}E U / 0 \\
1 / 90, N\end{array}$ & $\begin{array}{l}\text { DISTARCE } \\
\text { NETERS }\end{array}$ \\
\hline $\begin{array}{l}32.0 \\
34.0 \\
36.0 \\
30.0 \\
40.0 \\
42.0 \\
44.0 \\
46.0 \\
40.0 \\
50.0 \\
52.0 \\
54.0 \\
56.0 \\
58.0 \\
60.0 \\
62.0 \\
64.0 \\
66.0 \\
68.0 \\
70.0 \\
72.0 \\
74.0 \\
76.0 \\
70.0 \\
60.0 \\
62.0\end{array}$ & $\begin{array}{l}11 \\
15 \\
23 \\
43 \\
117 \\
141 \\
158 \\
149 \\
230 \\
273 \\
259 \\
290 \\
523 \\
561 \\
456 \\
501 \\
438 \\
414 \\
538 \\
335 \\
194 \\
104 \\
134 \\
87 \\
199 \\
4\end{array}$ & 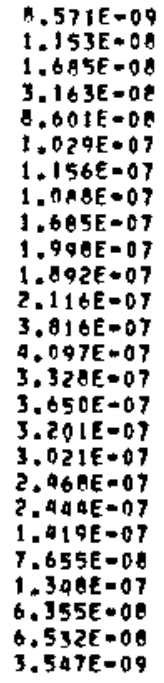 & 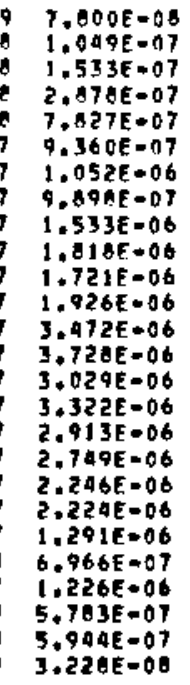 & $\begin{array}{l}1600 \\
1600 \\
1600 \\
1600 \\
1600 \\
1600 \\
1600 \\
1600 \\
1600 \\
1600 \\
1600 \\
1600 \\
1600 \\
1600 \\
1600 \\
1600 \\
1600 \\
1600 \\
1600 \\
1600 \\
1600 \\
1600 \\
1600 \\
1600 \\
1600 \\
1600\end{array}$ \\
\hline DSSW IND & INTEGALTEDE & $\begin{array}{l}2.423 E-04 \\
\text { SEC/SO. }\end{array}$ & $2.203 E-03$ & \\
\hline
\end{tabular}

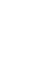


UNSTABLE SERIES EXPERIMENT: $52 \mathrm{~F}$ (U-52F)

ZINC SULFIOE AND FLUORESCEIN WERE BOTH USED AS TRACERS DURING

EXPERIMENT 52. FLUORESCEIN RESULTS ARE PRESENTED HERE. GROUND

LEVEL SAMPLING 100-1200 H ARC.

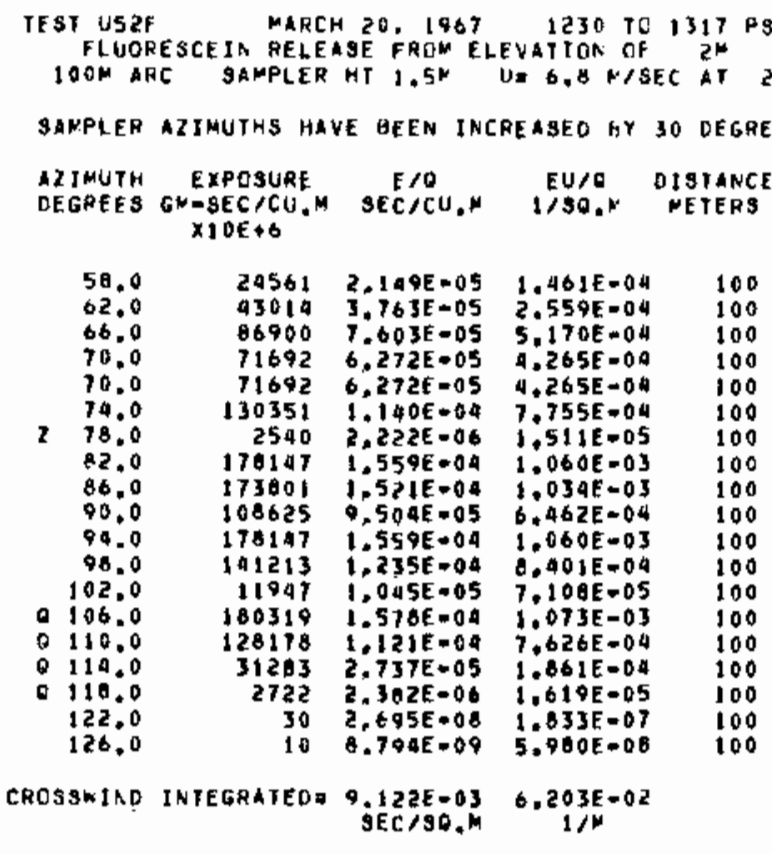

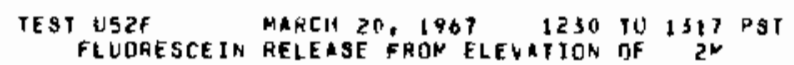
20ON ARC GAMPLER HT $1.5 \mathrm{~N} U=0.0 \mathrm{~K} / \mathrm{SEC}$ AT $2 \mathrm{H}$

GAMPLER AZIMUTHS HUV OEEM TNCREABED HY 30 DEGREES

RZINUIH EXPOSIJRE ETO EUTQ OISTINCE DEEREES CNASEC/CU.M SECICU.N I/SO,N HETERS $\times 10 \mathrm{O}+6$

\begin{tabular}{|c|c|c|c|c|}
\hline $\begin{array}{l}46.0 \\
52.0 \\
56.0 \\
60.0 \\
64.0 \\
68.0 \\
72.0 \\
76.0 \\
80.0 \\
84.0 \\
68.0 \\
92.0 \\
96.0 \\
100.0 \\
104.0 \\
108.0 \\
112.0 \\
116.0 \\
120.0\end{array}$ & $\begin{array}{r}11 \\
820 \\
1460 \\
7167 \\
12164 \\
28676 \\
31934 \\
41059 \\
5017 \\
44970 \\
55398 \\
1282 \\
1032 \\
925 \\
841 \\
45621 \\
2031 \\
647 \\
8\end{array}$ & 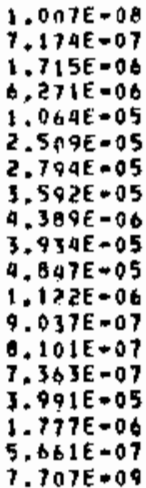 & $\begin{array}{l}0.848 E=08 \\
4.878 E=06 \\
1.166 E=05 \\
4.264 E=05 \\
7.237 E=05 \\
1.706 E=04 \\
1.900 E=04 \\
2.443 E=04 \\
2.905 E=05 \\
2.675 E=04 \\
3.296 E=04 \\
7.631 E=06 \\
6.145 E=06 \\
5.509 E=06 \\
5.007 E=06 \\
2.714 E=04 \\
1.209 E=05 \\
3.850 E=06 \\
5.241 E=06\end{array}$ & $\begin{array}{l}200 \\
200 \\
200 \\
200 \\
200 \\
200 \\
200 \\
200 \\
200 \\
200 \\
200 \\
200 \\
200 \\
200 \\
200 \\
200 \\
200 \\
200 \\
200\end{array}$ \\
\hline
\end{tabular}

CROSSWIND IATEGRATEDE $\begin{array}{r}3,440 E-03 \\ \text { SEC } 2,30,49 E-02\end{array}$

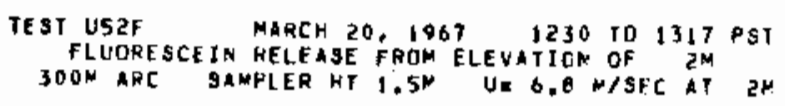

SAMPLER AZIMUTHG HAVE BEEN INCREASED EY JO BEGREES

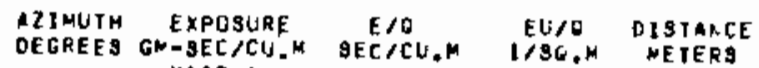
$\times 10 E+6$

\begin{tabular}{|c|c|c|c|c|}
\hline $\begin{array}{l}46.0 \\
50.0 \\
54.0 \\
50.0 \\
62.0 \\
66.0 \\
70.0 \\
74.0 \\
78.0 \\
62.0 \\
66.0 \\
90.0 \\
94.0 \\
98.0 \\
102.0 \\
106.0 \\
110.0 \\
114.0 \\
118.0 \\
122.0 \\
126.0\end{array}$ & $\begin{array}{r}0 \\
38 \\
615 \\
2 \\
94 \\
32 \\
177 \\
14736 \\
12771 \\
31657 \\
29474 \\
32749 \\
26342 \\
14409 \\
13666 \\
25544 \\
11134 \\
1069 \\
12 \\
4 \\
3\end{array}$ & $\begin{array}{l}0.000 E+00 \\
3.33 A E=00 \\
5.3 A 4 E=07 \\
2.360 E=09 \\
3,905 E=00 \\
2.065 E=00 \\
1.554 E=07 \\
1.269 E=05 \\
1.117 E=05 \\
2.770 E=05 \\
2.570 E=05 \\
2.865 E=05 \\
2.463 E=05 \\
1.261 E=05 \\
1.633 E=05 \\
2.235 E=05 \\
9.701 E=06 \\
9.355 E=07 \\
1.125 E=00 \\
0.066 E=09 \\
3.026 E=09\end{array}$ & $\begin{array}{l}0.000 E+00 \\
2.270 E=07 \\
3.661 E=06 \\
1.609 E=08 \\
2.656 E=07 \\
1.996 E=07 \\
1.057 E=06 \\
8.767 E=05 \\
7.598 E=05 \\
1.803 E=04 \\
1.754 E=04 \\
1.946 E=04 \\
1.689 E=04 \\
8.573 E=05 \\
1.111 E=04 \\
1.520 E=04 \\
6.624 E=05 \\
6.362 E=06 \\
7.652 E=08 \\
2.765 E=08 \\
2.058 E=08\end{array}$ & $\begin{array}{l}300 \\
300 \\
300 \\
300 \\
300 \\
300 \\
300 \\
300 \\
300 \\
300 \\
300 \\
300 \\
300 \\
300 \\
300 \\
300 \\
300 \\
300 \\
300 \\
300 \\
300\end{array}$ \\
\hline In & R ATEO & $\begin{array}{l}\text { A. } 059 E-03 \\
\text { SEC } / 90 . \mathrm{m}\end{array}$ & $2.760 \mathrm{E}=02$ & \\
\hline
\end{tabular}

TEST USEF MARCH 20,1967 1230 IO 1317 PST FLUORESCEIN RELEASE FRDN ELEVATIOR OF $5^{H}$ 40ON ARC SAKPLER HT 1.SN U* 6.8 N/SEC AT $2 \mathrm{~N}$

SAMPLER AZIMUTHS HAVE BEEN INCREASEO HY 30 DEGREES

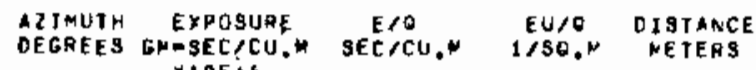
$x 10 \mathrm{E}+6$

\begin{tabular}{|c|c|c|c|c|}
\hline $\begin{array}{l}40.0 \\
52.0 \\
56.0 \\
60.0 \\
60.0 \\
68.0 \\
72.0 \\
76.0 \\
80.0 \\
64.0 \\
08.0 \\
92.0 \\
96.0 \\
100.0 \\
104.0 \\
102.0 \\
112.0 \\
116.0 \\
120.0\end{array}$ & $\begin{array}{r}2 \\
55 \\
615 \\
1274 \\
1474 \\
5894 \\
8514 \\
5676 \\
7859 \\
12449 \\
17029 \\
16702 \\
13754 \\
10007 \\
11462 \\
12117 \\
2450 \\
32 \\
9\end{array}$ & $\begin{array}{l}1,901 E=08 \\
4,866 E=06 \\
5.305 E=07 \\
1.115 E=06 \\
1.290 E=06 \\
5.157 E=06 \\
7.449 E=06 \\
4,966 E=06 \\
6.876 E=06 \\
1.089 E=05 \\
1.490 E=05 \\
1.461 E=05 \\
1.203 E=05 \\
9.455 E=06 \\
1.003 E=05 \\
1.060 E=05 \\
2.144 E-06 \\
2.880 E=08 \\
4.123 E=09\end{array}$ & $\begin{array}{l}1.293 E-00 \\
3.300 E-07 \\
3.662 E=06 \\
7.584 E-06 \\
8.770 E=06 \\
3.507 E=05 \\
5.065 E-05 \\
3.377 E=05 \\
4.676 E=05 \\
7.404 E=05 \\
1.013 E=09 \\
9.936 E=05 \\
6.183 E=05 \\
6.429 E=05 \\
6.819 E=05 \\
7.209 E=05 \\
1.458 E=05 \\
1.959 E=07 \\
2.804 E=08\end{array}$ & $\begin{array}{l}400 \\
400 \\
400 \\
900 \\
400 \\
900 \\
900 \\
400 \\
400 \\
400 \\
400 \\
400 \\
400 \\
400 \\
400 \\
400 \\
400 \\
400 \\
400\end{array}$ \\
\hline
\end{tabular}

CHOSSHIND IATEGHATEDE $3,1316=03 \quad 2,129 E+02$ 


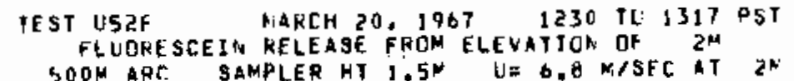
SAMPLER AZIHUIHS MAVE DEEN INCREAGEO HY 30 DEGHEE

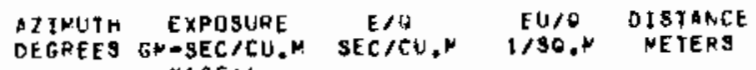
$x \in$ O $\$$

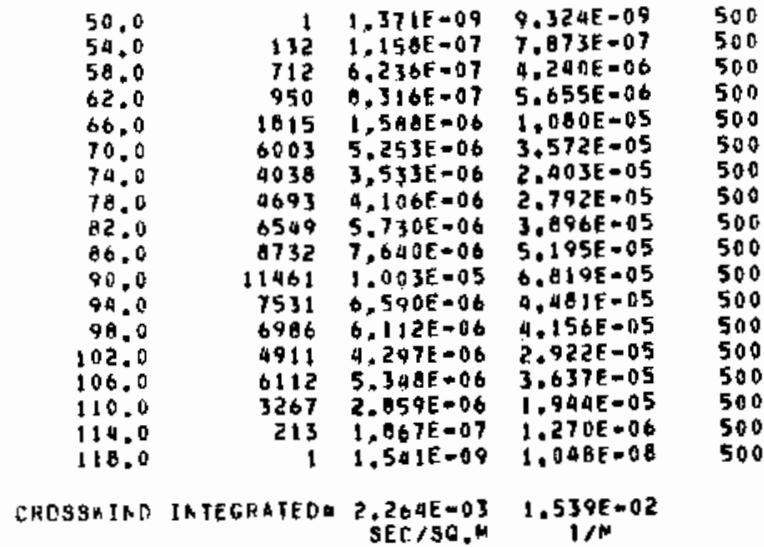

TEST USZF

FLSORESTEIN 1230 TO 1317 PS

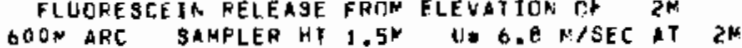

SAMPLER AZIMUTHS HAVE GEEN INCREAGED FY YO DEGREES

\begin{tabular}{|c|c|c|c|c|}
\hline $\begin{array}{l}l \text { IHUIम } \\
\text { EGREES }\end{array}$ & $\begin{array}{c}\text { EXPOSUAE } \\
\text { GN-\$EC CCU.M } \\
\times I O E+6\end{array}$ & $\begin{array}{c}E / \theta \\
s E C / C U, m\end{array}$ & $\begin{array}{l}E U / O \\
1 / 90, M\end{array}$ & $\begin{array}{l}\text { DISIANCE } \\
\text { NETERS }\end{array}$ \\
\hline $\begin{array}{l}48.0 \\
52.0 \\
50.0 \\
60.0 \\
64.0 \\
60.0 \\
72.0 \\
76.0 \\
80.0 \\
94.0 \\
88.0 \\
92.0 \\
96.0 \\
100.0 \\
104.0 \\
108.0 \\
112.0 \\
116.0 \\
120.0\end{array}$ & $\begin{array}{r}0 \\
0 \\
167 \\
550 \\
476 \\
2723 \\
2815 \\
3165 \\
2837 \\
4802 \\
7204 \\
0077 \\
5566 \\
3020 \\
3601 \\
4357 \\
975 \\
39 \\
0\end{array}$ & 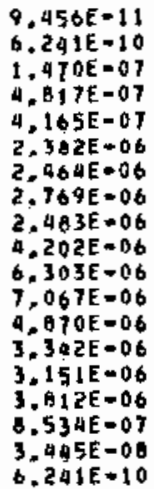 & $\begin{array}{l}0.430 E=10 \\
4.244 E=09 \\
9.995 E=07 \\
3.276 E-06 \\
2.832 E=06 \\
1.620 E=05 \\
1.675 E=05 \\
1.003 E=05 \\
1.608 E=05 \\
2.057 E=05 \\
9.286 E=05 \\
4.006 E=05 \\
3.312 E=05 \\
2.273 E=05 \\
2.143 E=05 \\
2.592 E=05 \\
5.003 E=06 \\
2.343 E=07 \\
4.204 E=09\end{array}$ & $\begin{array}{l}600 \\
600 \\
600 \\
600 \\
600 \\
600 \\
600 \\
600 \\
600 \\
600 \\
600 \\
600 \\
600 \\
600 \\
600 \\
600 \\
600 \\
600 \\
600\end{array}$ \\
\hline
\end{tabular}

CROSGNIAD IATEGHATED 1. ETOE-03 $1.276 E-02$
TEST USZF MAREH 20, 1967 1230 TO 1317 PST FLUORESCEIN HELEASE FRON ELEVATIGN TIF $3 \mathrm{M}$ TOON ARC SAMPLER HT $1.5 \mathrm{~N}$ U. 6.8 N/SEC AT $2 \mathrm{~N}$

SAMPLER AZIMUTHS HAVE BEEN INCREASF D EY 30 DEGREES

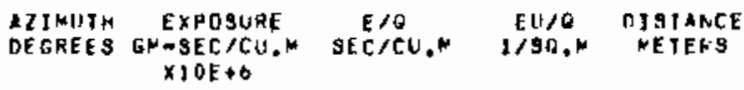

\begin{tabular}{|c|c|c|c|c|}
\hline $\begin{array}{l}59.0 \\
58.0 \\
62.0 \\
66.0 \\
70.0 \\
74.0 \\
78.0 \\
82.0 \\
86.0 \\
90.0 \\
94.0 \\
90.0 \\
102.0 \\
106.0 \\
110.0 \\
114.0\end{array}$ & $\begin{array}{r}1 \\
129 \\
465 \\
464 \\
2314 \\
2314 \\
2319 \\
2723 \\
4357 \\
4357 \\
4357 \\
3680 \\
2382 \\
2246 \\
2587 \\
126\end{array}$ & $\begin{array}{l}1.966 E=09 \\
1.131 E=07 \\
4.252 E=07 \\
4.062 E=07 \\
2.025 E=06 \\
2.025 E=06 \\
2.025 E=06 \\
2.383 E=06 \\
3.812 E=06 \\
3.012 E=06 \\
3.812 E=06 \\
3.395 E=06 \\
2.015 E=06 \\
1.966 E=06 \\
2.263 E=06 \\
1.103 E=07\end{array}$ & $\begin{array}{l}9.967 E=09 \\
7.691 E=07 \\
2.891 E=06 \\
2.762 E=06 \\
1.377 E=05 \\
1.377 E=05 \\
1.377 E=05 \\
1.620 E=05 \\
2.592 E=05 \\
2.592 E=05 \\
2.592 E=05 \\
2.309 E=05 \\
1.918 E=05 \\
1.337 E=05 \\
1.539 E=05 \\
7.499 E=07\end{array}$ & $\begin{array}{l}700 \\
700 \\
700 \\
700 \\
700 \\
700 \\
700 \\
700 \\
700 \\
700 \\
700 \\
700 \\
700 \\
700 \\
700 \\
700\end{array}$ \\
\hline
\end{tabular}

CROSBNIND INTEGRATEDE 1,

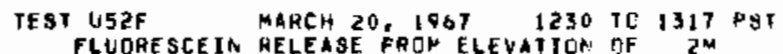
FLUDRESCEIN AELEASE FROK ELEVATIOA DF
800M ARE SAMPLER HT $1.5 \mathrm{~K}$ LN O.8 M/SEC AT $2 \mathrm{M}$ SAMPLEF AZIMUTHS HAVE GEEN IRCREASED GY 30 DEGREES

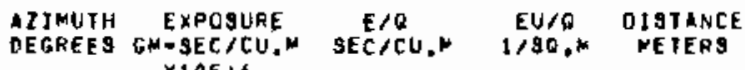
$X 10 E+6$

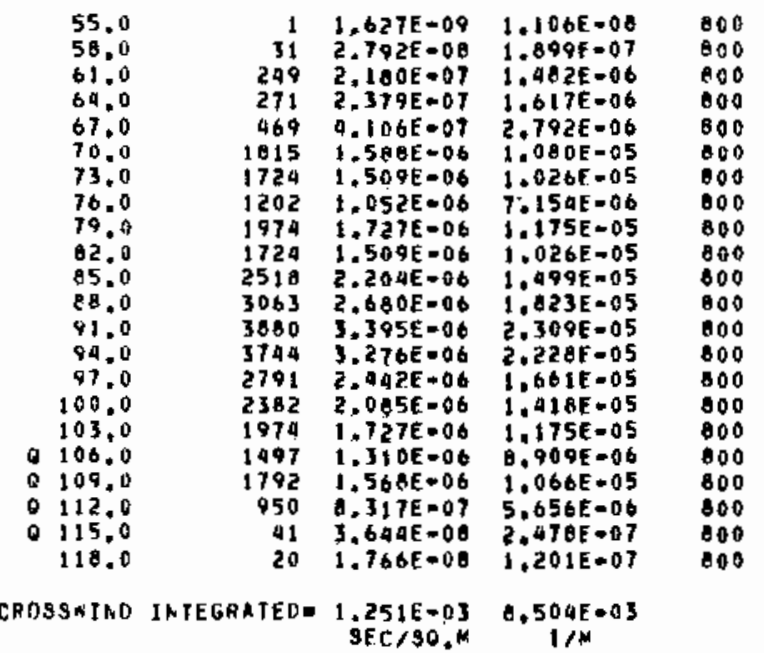




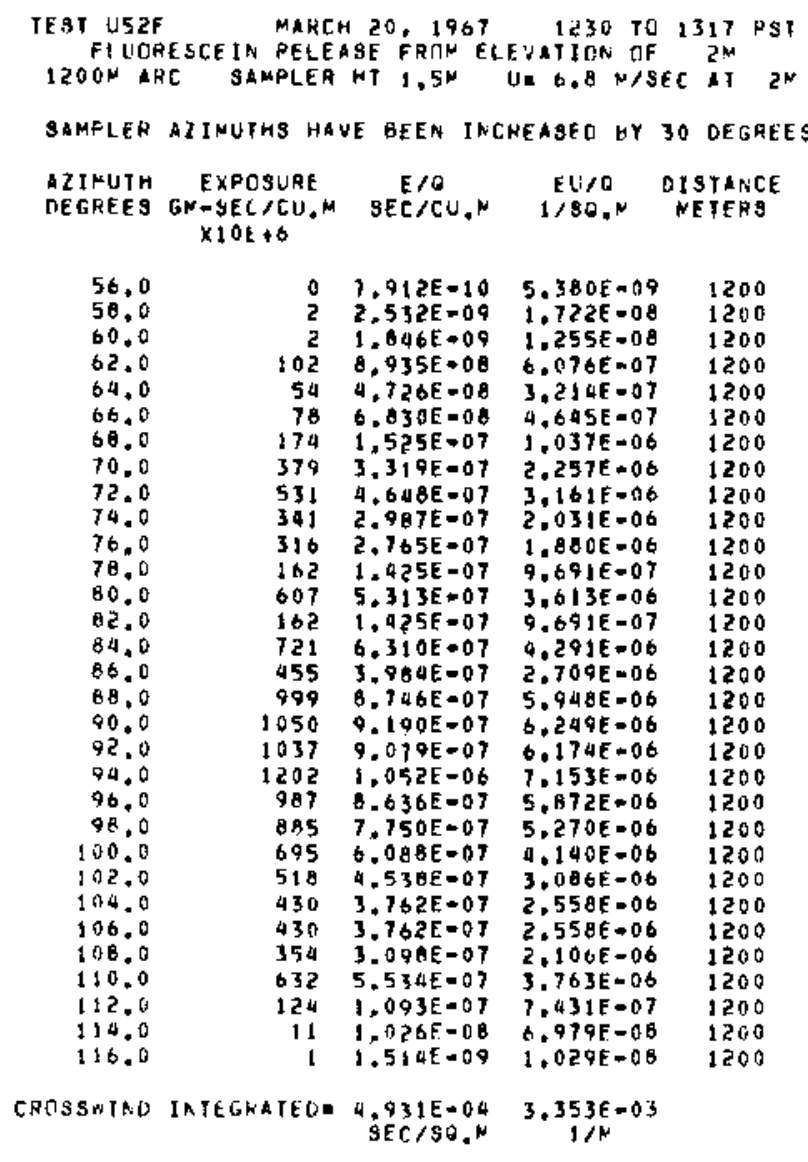


UNSTABLE SERIES EXPERIHENT: 53 (U-53)

ZINC SULFIDE AND FLUORESCEIN WERE BOTH JSED AS TRACERS DURING EXPERIMENT 53. ZINC SULFIDE RESULTS ARE PRESENTED HERE, GROUHD

LEVEL SAMPL ING 100-1600 M ARC.

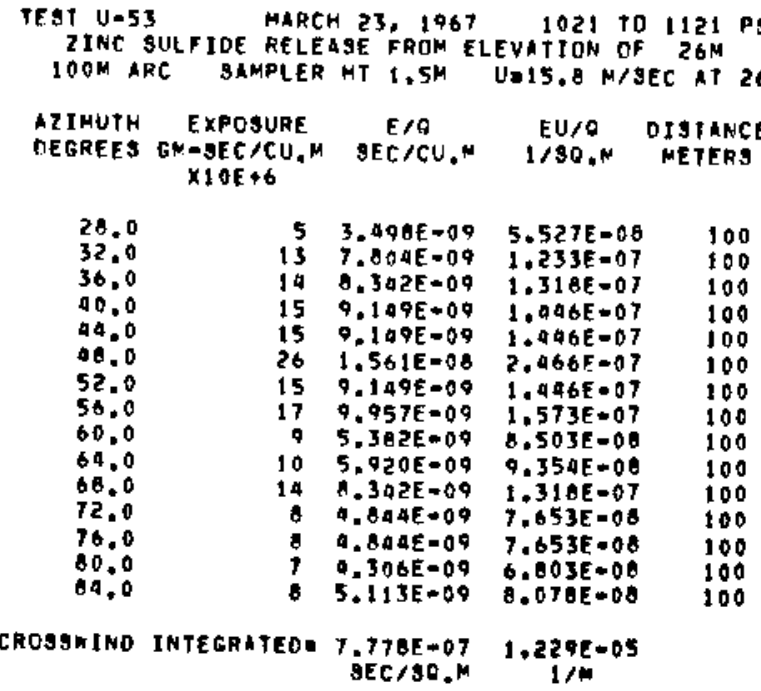

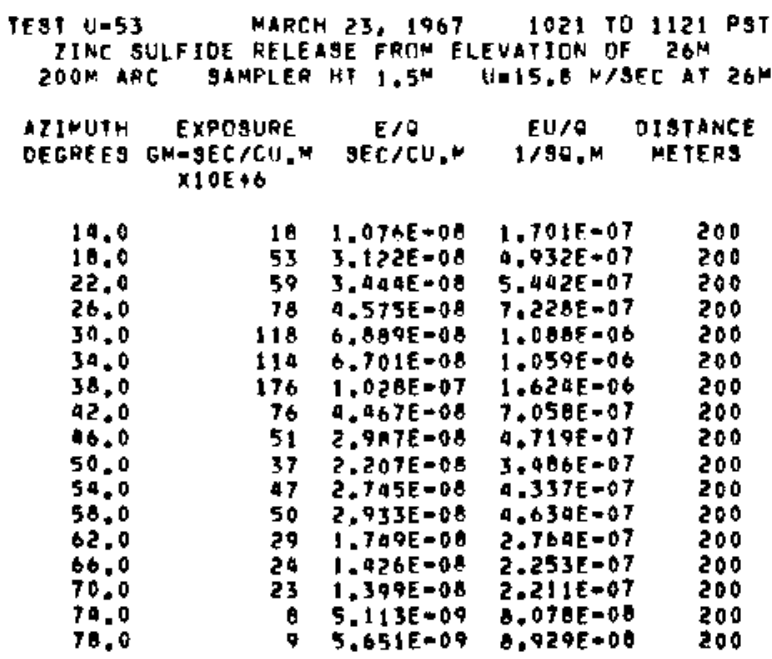

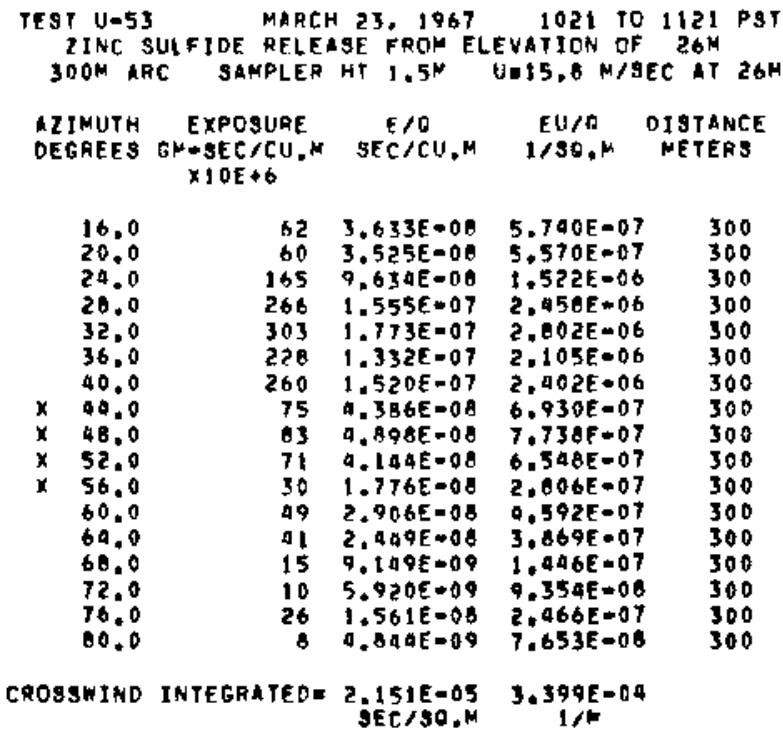

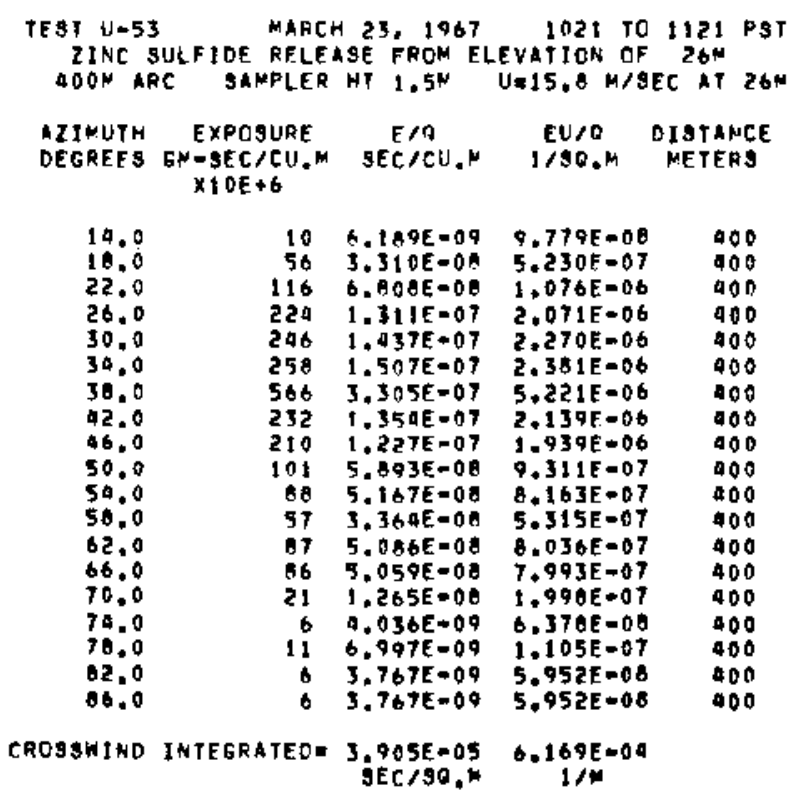




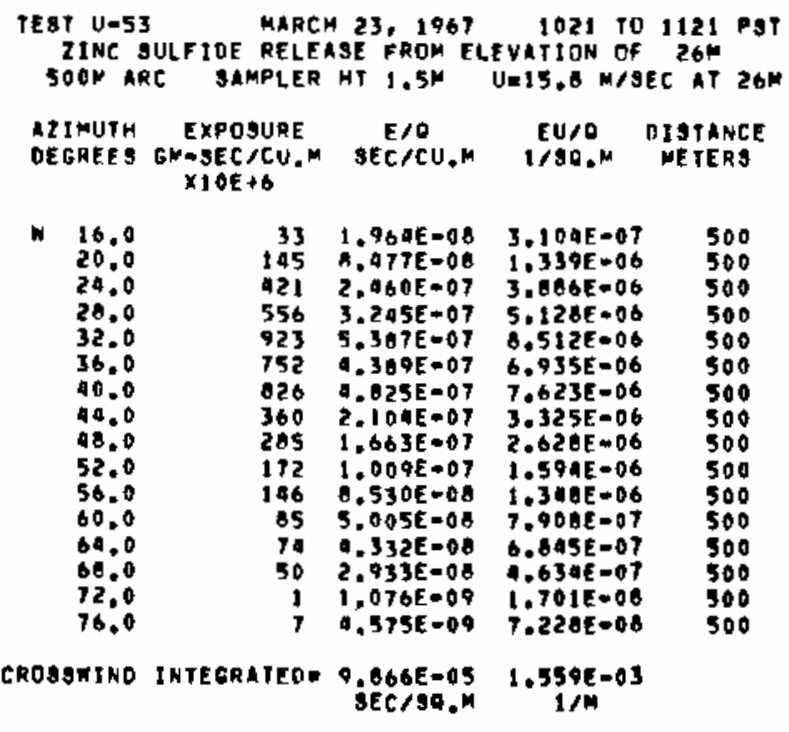

TEST U-53 MARCH 23, 1967 1021 TO 1121 PST IINC SULFIDE RELEASE FRO ELEVATION OF $26 \mathrm{M}$ GOOM ARC SAMPLEF HI $1.5 \mathrm{M}$ UE $15.6 \mathrm{M} / \mathrm{SEC}$ AT $26 \mathrm{M}$

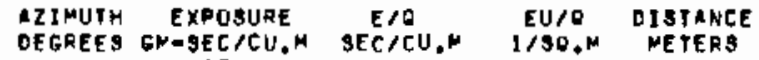
$\times 10 E 46$

\begin{tabular}{|c|c|c|c|c|}
\hline $\begin{array}{l}18.0 \\
22.0 \\
26.0 \\
30.0 \\
34.0 \\
38.0 \\
92.0 \\
46.0 \\
50.0 \\
54.0 \\
38.0 \\
62.0 \\
66.0\end{array}$ & $\begin{array}{r}97 \\
240 \\
586 \\
902 \\
1039 \\
720 \\
436 \\
137 \\
295 \\
159 \\
130 \\
37 \\
100\end{array}$ & 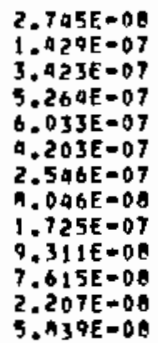 & $\begin{array}{l}4.337 E=07 \\
2.256 E=06 \\
5.400 E=06 \\
0.316 E=06 \\
9.332 E=06 \\
6.691 E=06 \\
4.022 E=06 \\
1.271 E=06 \\
2.725 E=06 \\
1.471 E=06 \\
1.203 E=06 \\
3.466 E=07 \\
9.226 E=07\end{array}$ & $\begin{array}{l}600 \\
600 \\
600 \\
600 \\
600 \\
600 \\
600 \\
600 \\
600 \\
600 \\
600 \\
600 \\
600\end{array}$ \\
\hline & $E$ & 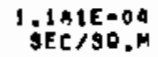 & $1.866 E=03$ & \\
\hline
\end{tabular}

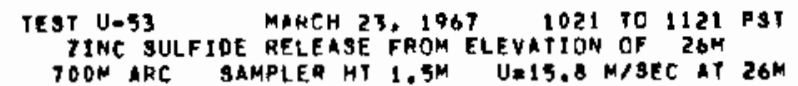
IIIMUTH EXPOSUAE
OEGRES GM-SEC/CU." SEC $\times 10 E+6$

\begin{tabular}{|c|c|c|c|c|}
\hline $\begin{array}{l}16.0 \\
20.0 \\
24.0 \\
20.0 \\
32.0 \\
36.0 \\
40.0 \\
14.0 \\
48.0 \\
52.0 \\
56.0 \\
60.0 \\
64.0 \\
68.0\end{array}$ & $\begin{array}{r}19 \\
190 \\
502 \\
795 \\
1918 \\
010 \\
609 \\
300 \\
209 \\
250 \\
102 \\
39 \\
77 \\
12\end{array}$ & $\begin{array}{l}1.157 E=00 \\
1.157 E=07 \\
3.399 E=07 \\
4.6 a 2 E=07 \\
9.277 E=07 \\
4.720 E=07 \\
9.020 E=07 \\
1.752 E=07 \\
1.453 E=07 \\
1.469 E=07 \\
0.315 E=08 \\
2.207 E=00 \\
4.548 E=08 \\
7.535 E=09\end{array}$ & $\begin{array}{l}1.028 E-07 \\
1.028 E=06 \\
5.370 E=06 \\
7.334 E=06 \\
1.308 E=05 \\
7.470 E=06 \\
6.352 E=06 \\
2.768 E=06 \\
2.296 E=06 \\
2.313 E-06 \\
1.314 E-06 \\
3.614 E=07 \\
7.185 E-07 \\
1.190 E=07\end{array}$ & $\begin{array}{l}700 \\
700 \\
700 \\
700 \\
700 \\
700 \\
700 \\
700 \\
700 \\
700 \\
700 \\
700 \\
700 \\
700\end{array}$ \\
\hline
\end{tabular}

CROSSMINO INTEGRATEDE $1.5936-04 \quad 2.517 E-03$

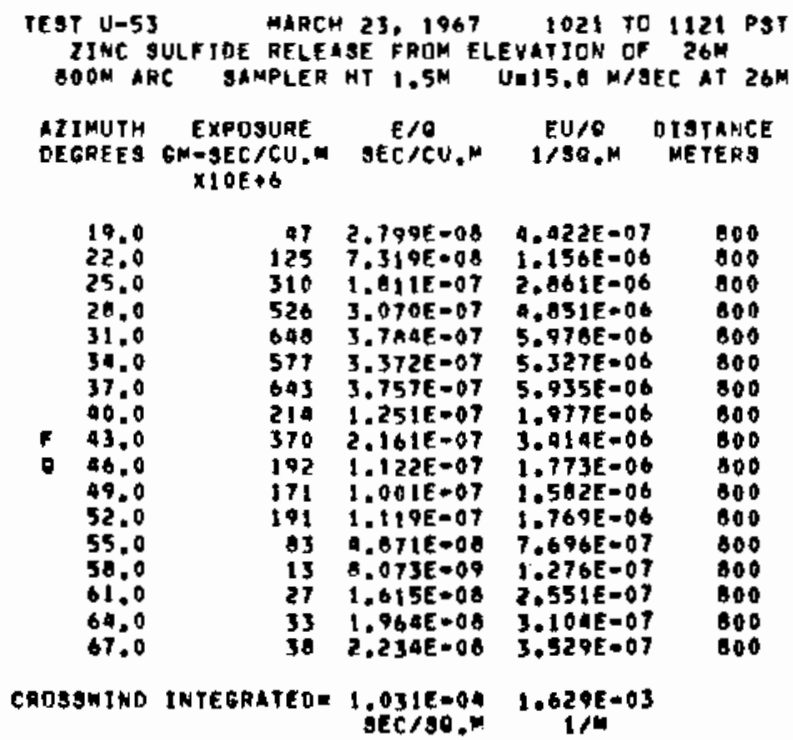

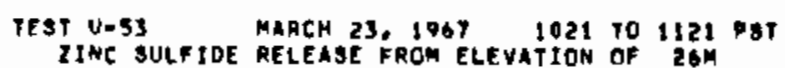
IINC SULFIDE RELEASE FROM ELEVATION OF $26 \mathrm{M}$.
AZJMUTH EXPOSURE E/O EU/O DISTANCE DEGREES GM-SEC/CU,M SECICU.M 1/SO,M METERS $\times 10 E \div 6$

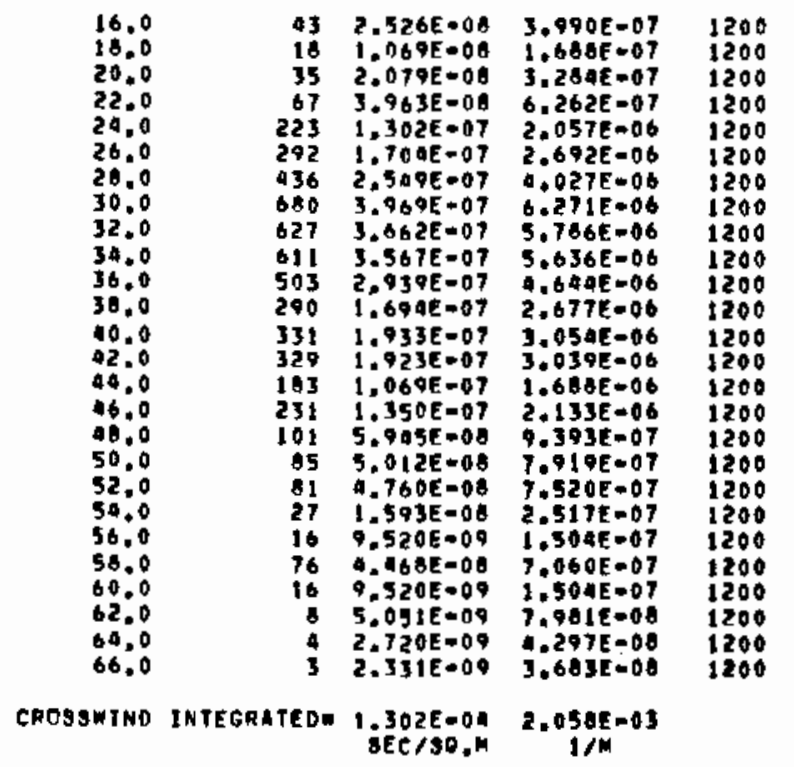




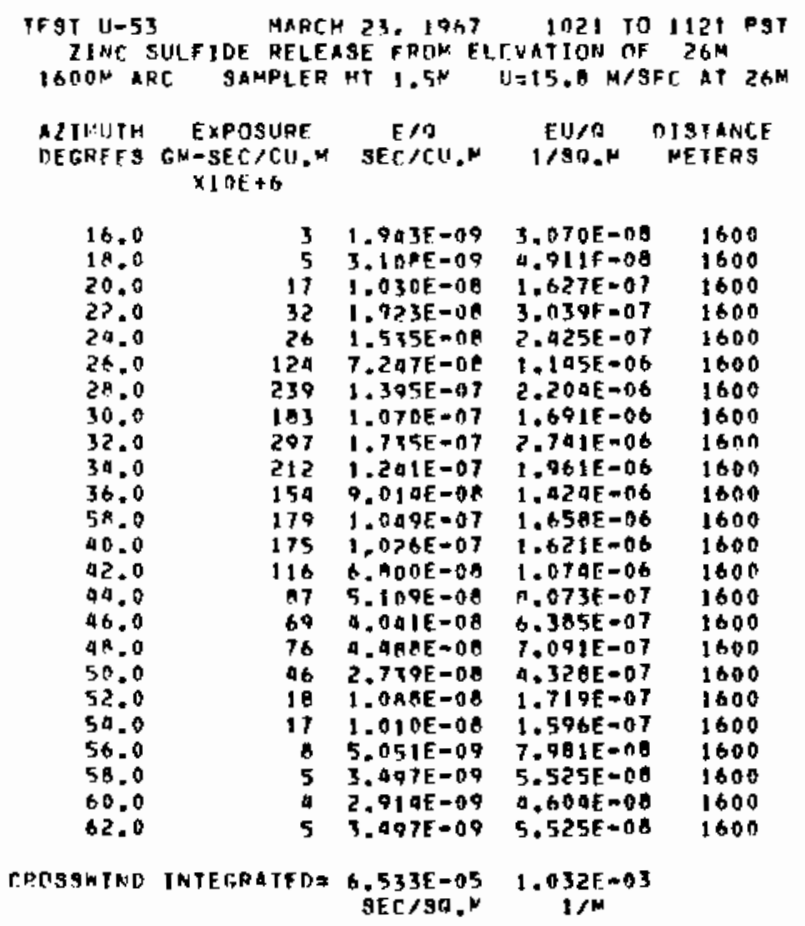


UNSTABLE SERIES EXPERIMENT: $53 \mathrm{~F}$ (U-53F)

ZINC SULFIDE AND FLUORESCEIN WERE BOTH USED AS TRACERS OURING

EXPERIMENT 51. FLUORESCEIN RESULTS ARE PRESENTED HERE. GROUND

LEYEL SAMPLING 100-1200 M ARC.

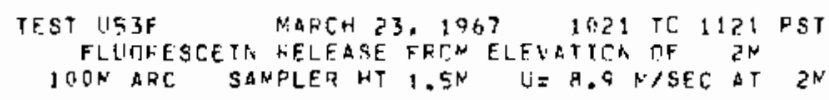
SANPLER AZINUTHS HAVE GEEN INGFEASFD BY 30 TIEGREES

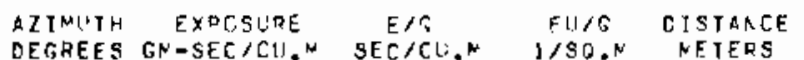
$\times 10 \mathrm{E}+6$

\begin{tabular}{|c|c|c|c|c|}
\hline 42.0 & 1722 & $1.13 R F=16$ & $1.013 F=05$ & 100 \\
\hline 46.0 & 7312 & 4. $A 3 \cap E-N E$ & $4.2905=05$ & 100 \\
\hline 50.0 & $33 \times 1$ & $2.2 \times 4 E-100$ & $1.4 \times 6 E=05$ & 100 \\
\hline 50.0 & 3176 & 1. 4 प $5 F=n b$ & 1. $2 n \cap F-\cap 5$ & 100 \\
\hline $5 P \cdot 0$ & 1132 & $7.4 A>E-157$ & $6.6595=06$ & 100 \\
\hline 62.0 & 7679 & $5.04 \theta E=96$ & $4.4916=175$ & 10 ? \\
\hline he. 0 & 7202 & $4.75 A E=7 A$ & $11.234 F=05$ & 100 \\
\hline 70.0 & $4: 4 \div 2$ & $2.9675=06$ & $2 \cdot A 41 F=05$ & 100 \\
\hline 74.0 & 3198 & $2.11 \geq F=06$ & 1. $P$ POE $П 5$ & 100 \\
\hline 78.0 & 2653 & $1.753 E=06$ & 1. 5 GOF $=05$ & 100 \\
\hline 82.0 & 1399 & ค. 0 Q $1 E-07$ & $7.993=-06$ & 100 \\
\hline 86.0 & $1 \mathrm{H}$ & $5.3 \times 3 F=07$ & $4.791 \mathrm{E}=06$ & 100 \\
\hline & 13425 & R. $P A B E-O E$ & $7.292 F-105$ & 100 \\
\hline 94.0 & $\operatorname{tac} 1$ & $3.171 \mathrm{E} \rightarrow \mathrm{DD}$ & $2.022 E-05$ & 100 \\
\hline 99.0 & 0015 & $2.652 E=06$ & $2.360 F=05$ & $\ln 0$ \\
\hline 102.0 & 1790 & $1.193 \mathrm{~F}=36$ & $1.153 f=115$ & $10 n$ \\
\hline 106.0 & 951 & $6,2 A 2 \xi-A 7$ & $5.591 E=106$ & 100 \\
\hline 10.0 & 133 & म. $B 5 \cap E=\cap A$ & 7. $A 7 B F=07$ & $\ln 0$ \\
\hline 14.0 & 38 & ?.567E-08 & $2.2855-17$ & 100 \\
\hline 0 & 18 & $5.2116 E-08$ & $1.10 Q F=07$ & 100 \\
\hline & 7 & $5.183 E=09$ & $0.013 F=08$ & 100 \\
\hline $\operatorname{CIND}$ & TEG & $\begin{array}{r}3.14 P E-O D \\
S E C / S O . N\end{array}$ & $\begin{array}{c}2.802 \mathrm{E}=03 \\
1 / N\end{array}$ & \\
\hline
\end{tabular}

TEST U53F MARCH 23. 1967 1D21 TC 1121 PST

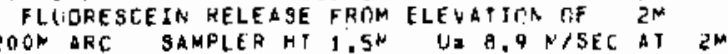

SAMPLER AZIMUTHS HAVE UEEN INCREASED HY 30 DFGAES

\begin{tabular}{|c|c|c|c|c|}
\hline $\begin{array}{l}l \mathrm{INUTH} \\
\text { ESTEES }\end{array}$ & $\begin{array}{c}\text { EXPOSURE } \\
\text { GN }- \text { SEC }>C U 1, \\
\times 1 O E+6\end{array}$ & $\begin{array}{c}E / O \\
\text { SEC } / C O . H\end{array}$ & $\begin{array}{c}E, 1 / 0 \\
1 / 9 G . M\end{array}$ & $\begin{array}{l}\text { OISYANCC } \\
\text { METERG }\end{array}$ \\
\hline $\begin{array}{l}36.0 \\
40.0 \\
44.0 \\
48.0 \\
52.0 \\
50.0 \\
60.0 \\
64.0 \\
68.0 \\
72.0 \\
76.0 \\
60.0 \\
84.0 \\
88.0 \\
92.0 \\
96.0 \\
100.0 \\
104.0 \\
108.0 \\
82.0 \\
86.0\end{array}$ & $\begin{array}{r}3 \\
141 \\
625 \\
0 \\
12816 \\
35193 \\
48228 \\
50144 \\
49532 \\
35845 \\
16292 \\
4365 \\
6875 \\
3164 \\
1406 \\
784 \\
369 \\
358 \\
84 \\
7 \\
6\end{array}$ & $\begin{array}{l}2.540 E=09 \\
9.31 \mathrm{AE}=08 \\
4.130 E=07 \\
0.000 E+00 \\
9.465 E=06 \\
2.325 E=05 \\
3.186 E=05 \\
3.315 E=05 \\
3.272 E=05 \\
2.36 B E=05 \\
1.076 E=05 \\
2.0 B 3 E=06 \\
4,542 E=06 \\
2.090 E=06 \\
9.293 E=07 \\
5.181 E=07 \\
2.441 E=07 \\
2.369 E=07 \\
5.602 E=08 \\
5.190 E=09 \\
4.048 E=09\end{array}$ & $\begin{array}{l}2.268 E-08 \\
2.290 E-07 \\
3.676 E=06 \\
0.000 E+00 \\
7.534 E=05 \\
2.069 E=09 \\
2.035 E=04 \\
2.950 E=04 \\
2.912 E=04 \\
2.107 E=04 \\
9.577 E=05 \\
2.566 E=05 \\
4.042 E=05 \\
1.860 E=05 \\
8.269 E=06 \\
4.611 E=06 \\
2.172 E=06 \\
2.108 E=06 \\
4.966 E=07 \\
4.619 E=08 \\
3.003 E=08\end{array}$ & $\begin{array}{l}200 \\
200 \\
200 \\
200 \\
200 \\
200 \\
200 \\
200 \\
200 \\
200 \\
200 \\
200 \\
200 \\
200 \\
200 \\
200 \\
200 \\
200 \\
200 \\
200 \\
200\end{array}$ \\
\hline
\end{tabular}

CANSSNIAD INFEGRATED= 2.452E-03 2.1836 .02
TEST U53F MARCK 23,1967 1921 TO 1123 PST FL LQRESCEIN RELEASE FFON FLEVATICR OF ZN

SAMPLER IZINUTHS HAVE DEEN INCREASED BY 30 DEGREES

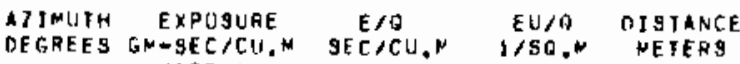
$x: 0 E+6$

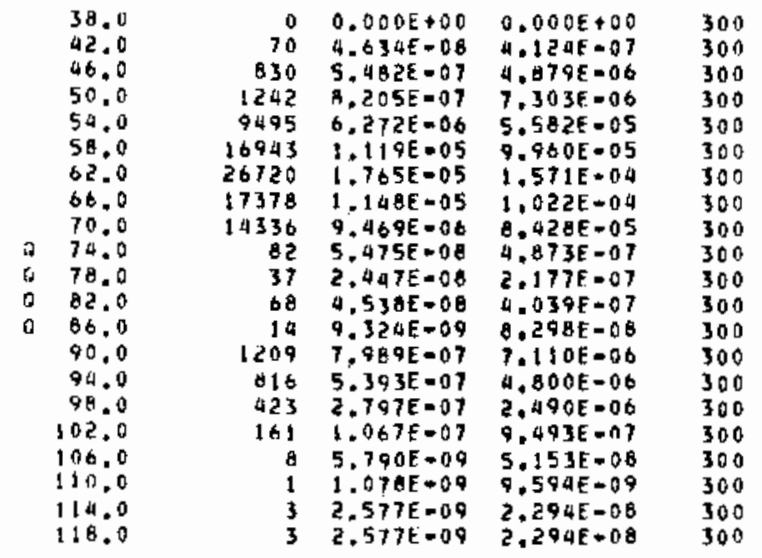

CROSSKTMA IATEGRATED $1.243 E-03 \quad 1.106 E=02$
SER SSQ.N TEST US3F MARCH 23,1907 1021 TC IIZS PST
FLLORESCEIN RELERSE FRON ELEVOTION OF 2 HA $40 O N$ ARC SAMPLEA MT $1.5 \mathrm{~N} \quad \mathrm{O}=8.9 \mathrm{M} / \mathrm{SFC}$ AT $2 \mathrm{M}$

SAMPLER AZINUTHS HAVF UEEN IHCREASEU HY 30 OEGREES

AZIMUTH EXPOSURE E/O EU/O DISTARCE DEGREES GNOSECJCO.M SECICU,N 1/SG,M NETFGS $X: 0 E+6$

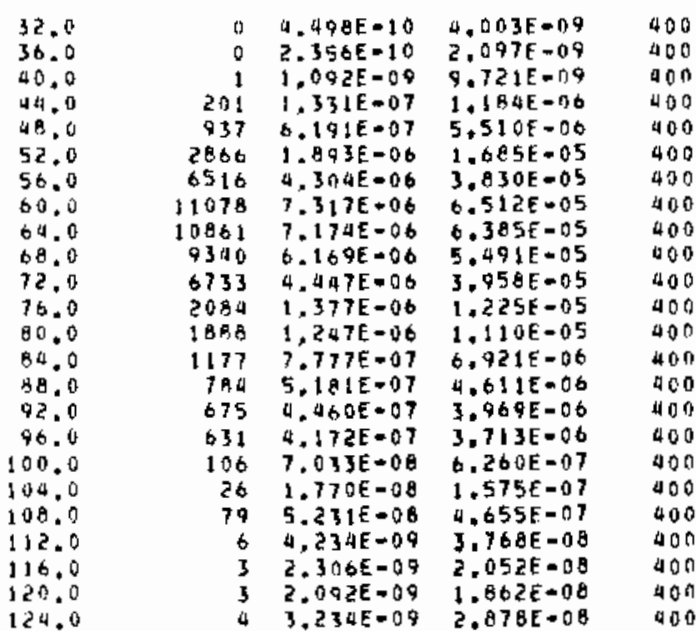

CRCASEIND IATEGHATEO= $\begin{array}{r}1,033 E-03 \\ \text { SECISQ, }\end{array}$ 


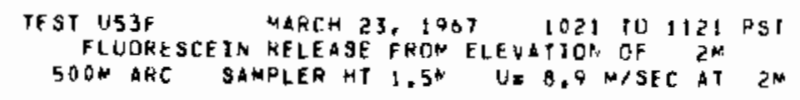
SAMPLEF AZTMUTHS HAVE BEEN INCREASFD BY 30 DEGRES

\begin{tabular}{|c|c|c|c|c|}
\hline $\begin{array}{l}\text { AZJMUTH } \\
\text { DEGREFS }\end{array}$ & $\begin{array}{c}\text { EXPOSUAE } \\
\text { GN-SEC/CU.M } \\
\times J O E+6\end{array}$ & $\begin{array}{c}E / O \\
S E C / C U,\end{array}$ & $\begin{array}{l}E U / G \\
1 / S Q, F\end{array}$ & $\begin{array}{c}\text { CISTANCE } \\
\text { NETERS }\end{array}$ \\
\hline $\begin{array}{l}38.0 \\
42,0 \\
46.0 \\
50.0 \\
54.0 \\
58.0 \\
62.0 \\
68.0 \\
72.0 \\
74.0 \\
78.0 \\
82.0 \\
86,0 \\
90.0 \\
94.0 \\
98.0 \\
102.0 \\
106.0 \\
110.0 \\
114.0 \\
118.0 \\
122.0\end{array}$ & $\begin{array}{r}9 \\
6 \\
92 \\
387 \\
8688 \\
9774 \\
8905 \\
8254 \\
6036 \\
3273 \\
1887 \\
1046 \\
784 \\
456 \\
347 \\
240 \\
22 \\
10 \\
1 \\
1 \\
1 \\
0\end{array}$ & 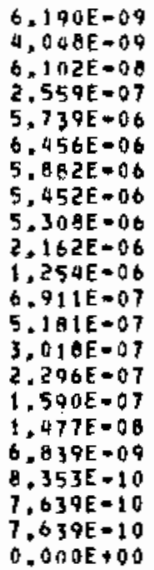 & $\begin{array}{l}5.509 E-08 \\
3.603 E=08 \\
5.431 E=07 \\
2.278 E=06 \\
5.108 E=05 \\
5.746 E=0 S \\
5.235 E=05 \\
4.052 E=05 \\
4.724 E=05 \\
1.924 E=05 \\
1.116 E=05 \\
6.151 E=08 \\
4.611 E=06 \\
2.686 E=06 \\
2.044 E=06 \\
1.415 E=06 \\
1.315 E=07 \\
0.087 E=08 \\
7.434 E=09 \\
6.799 E=09 \\
6.799 E=08 \\
0.000 E+00\end{array}$ & $\begin{array}{l}500 \\
500 \\
500 \\
500 \\
500 \\
500 \\
500 \\
500 \\
500 \\
500 \\
500 \\
500 \\
500 \\
500 \\
500 \\
500 \\
500 \\
500 \\
500 \\
500 \\
500 \\
500\end{array}$ \\
\hline
\end{tabular}

CROSSHIAD IATEGRATEDE 1, Z33E-03 $1.097 E-02$

IEST U53F MAACH 23, 1967 1021 IU 1121 HST FLLIURESCEIN RELEASE FRDN ELFUATICA OF $2 \mathrm{~K}$ bOON ARC SAMPLER HT I.SN IJW $9,9 \mathrm{~K} / \mathrm{SEC}$ AT

SAMFLER AZINUTHS HAVE GEE INCHEASEC EY 30 DEGREES

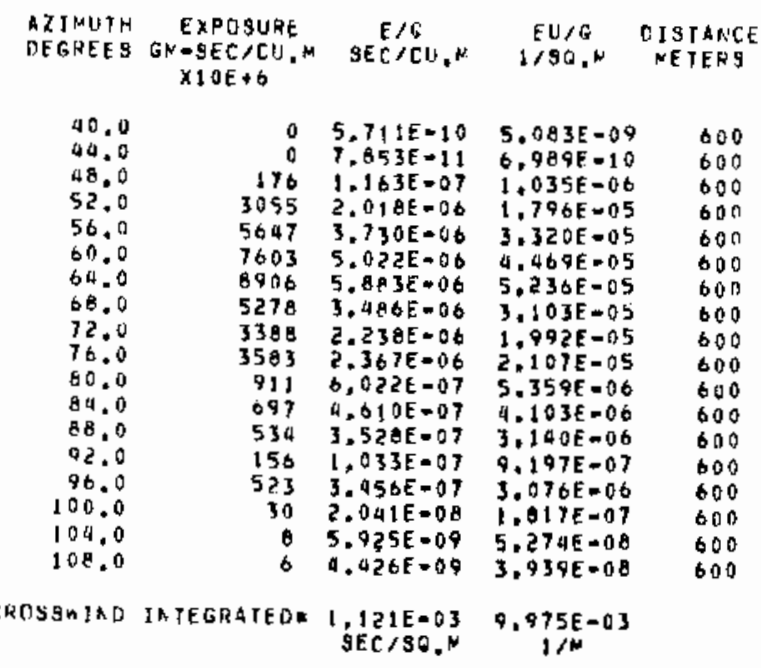

TEST U53F MKHCH 23, 1967 102! TO 1121 WST FLUORESCEIN RELEASE FRUN ELEVATION OF ZM $70 O M$ ANC SAMPLER HT $1.5 \mathrm{~N} U=8,9 \mathrm{~N} / \mathrm{NEC}$ AT $2^{\mathrm{N}}$

SAMPLER AZINUTHS HAVE HEEN INCAEABEE BY 30 DEGREES

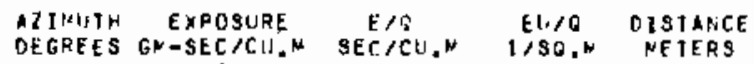
$\times 10 \mathrm{O} \phi 6$

\begin{tabular}{|c|c|c|c|c|}
\hline $\begin{array}{l}38.0 \\
42.0 \\
46.0 \\
50.0 \\
54.0 \\
58.0 \\
62.0 \\
66.0 \\
70.0 \\
74.0 \\
76.0 \\
82.0 \\
86.0 \\
90.0 \\
94.0 \\
98.0 \\
102.0 \\
06.0 \\
110.0\end{array}$ & $\begin{array}{r}0 \\
1 \\
51 \\
517 \\
2259 \\
6877 \\
4038 \\
5457 \\
4366 \\
1800 \\
840 \\
348 \\
512 \\
129 \\
145 \\
58 \\
2 \\
2 \\
1\end{array}$ & $\begin{array}{l}7.853 E-11 \\
1,171 E=09 \\
3,390 E=06 \\
3,352 E-07 \\
1.402 E=06 \\
0.542 E=06 \\
2,668 E=06 \\
3.605 E=06 \\
2,894 E=06 \\
1,189 E=06 \\
5,548 E=07 \\
2,304 E=07 \\
3,385 E=07 \\
8,530 E=08 \\
9,601 E=08 \\
3,889 E=08 \\
1.913 E=09 \\
1.414 E=09 \\
1.299 E=09\end{array}$ & $\begin{array}{l}6.989 E-10 \\
1.042 E=08 \\
3.017 E=07 \\
2.983 E=06 \\
1.328 E=05 \\
4.043 E=05 \\
2.374 E=05 \\
3.208 E=05 \\
2.567 E=05 \\
1.059 E=05 \\
4.938 E=00 \\
2.050 E=08 \\
3.013 E=06 \\
7.592 E=07 \\
3.545 E=07 \\
3.062 E=07 \\
1.703 E=08 \\
1.250 E=08 \\
1.156 E=08\end{array}$ & $\begin{array}{l}700 \\
700 \\
700 \\
700 \\
700 \\
700 \\
700 \\
700 \\
700 \\
700 \\
700 \\
700 \\
700 \\
700 \\
700 \\
700 \\
700 \\
700 \\
700\end{array}$ \\
\hline
\end{tabular}

CROSGMIND INTEGHATEO= "TZOEEOA T,76BE-O3

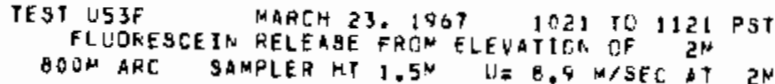
SAMPLER AZINLTHS HAVE BEEN IACREABEL AY 30 TEGREES AZIHIYM EXPCSURE ESB EUIG DISTANCE DELHEES GN-SEC/CU.M SEC/CU.M I/SG.K NETERS
XIOE+6

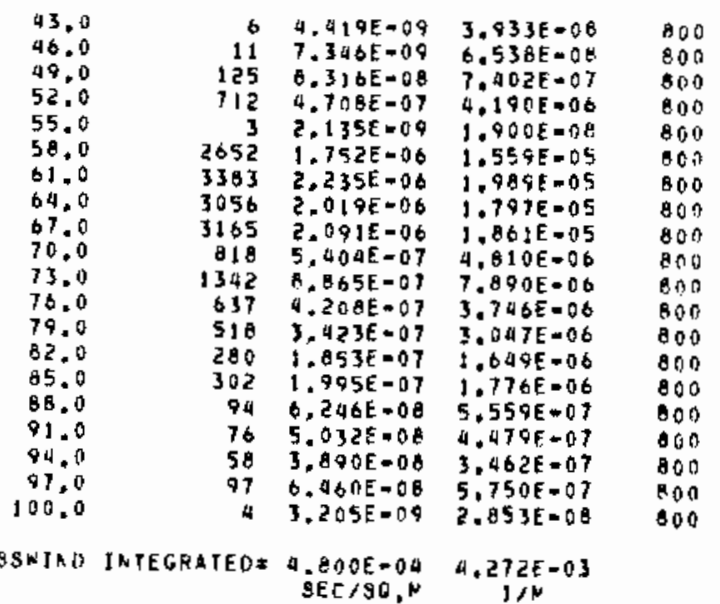




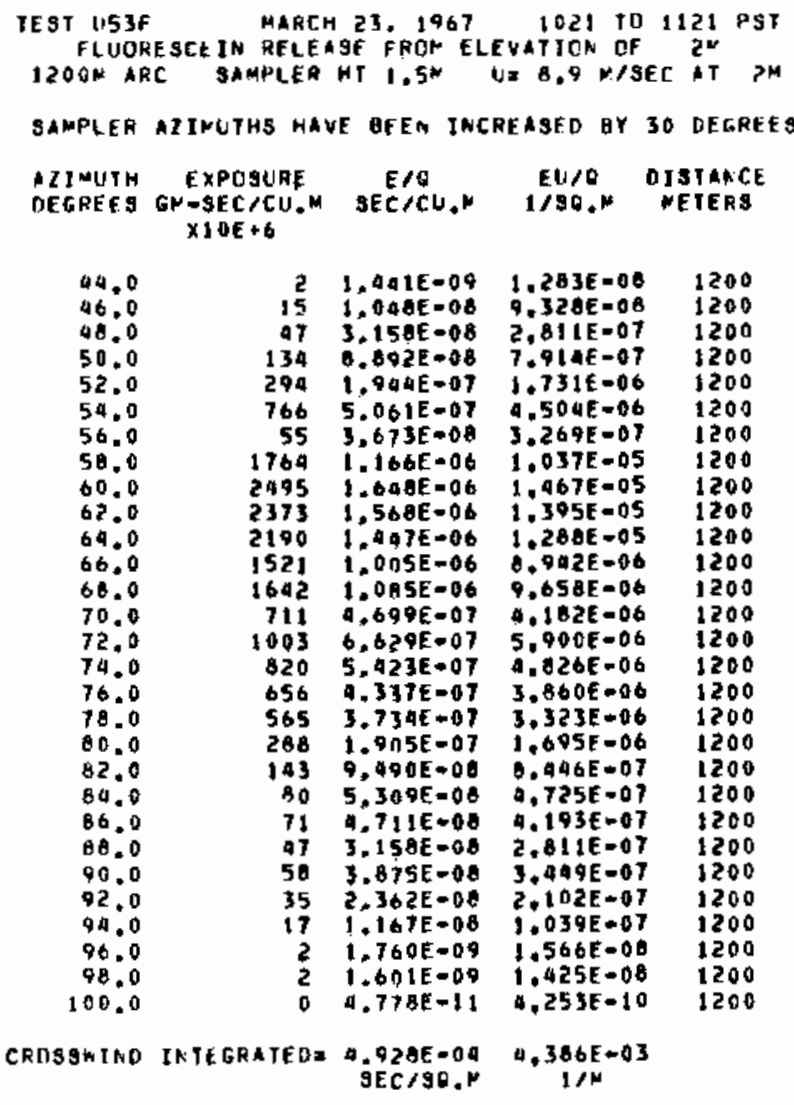

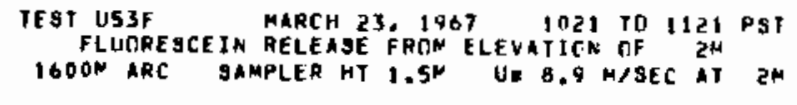
SAMPLER AZIMUTHS HAVE GEEM INCREASED GY 30 DEGREES

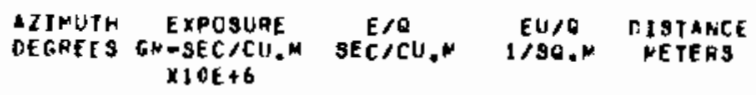

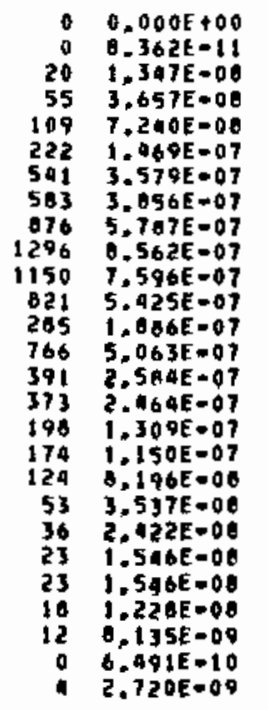

$2.720 E=09$

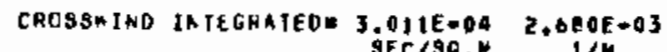


UNST ABLE SERIES EXPERI HENT: $54, \mathrm{U}-54$;

ZINC SULFIDE AND FLUORESCEIN WERE BOTH USED AS TRACERS DURING EXPERIMENT 54, ZINC SULFIDE RESULTS ARE PRESENTED HERE. GROUNO

LEVEL SAMPL ING 100-1600 M ARC.

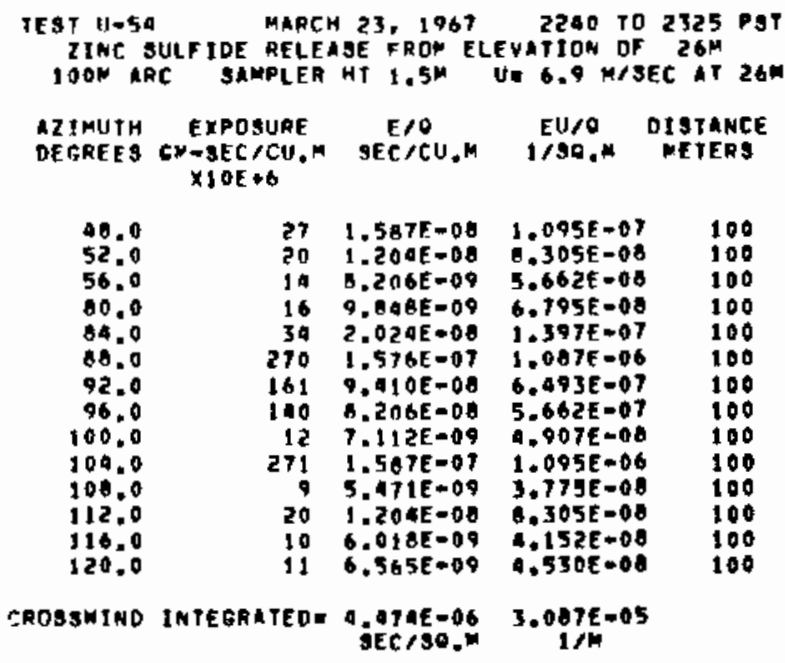

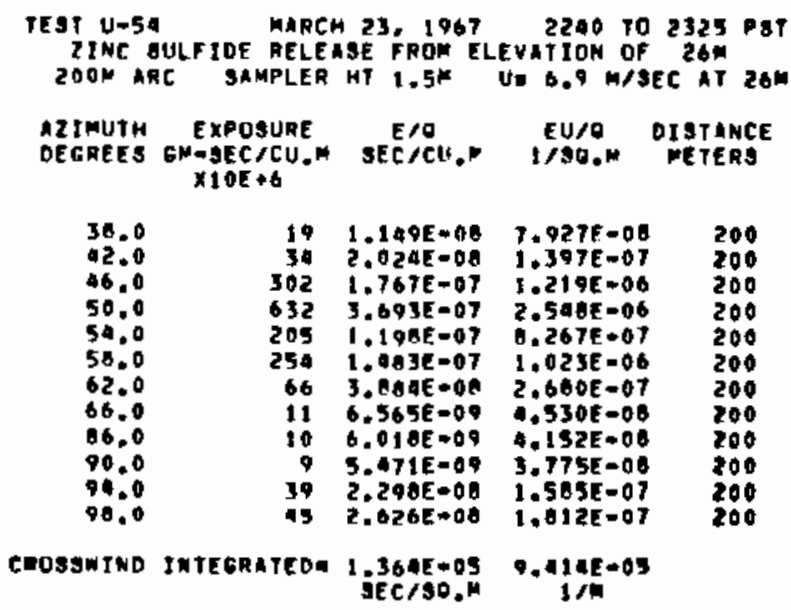

TEST U-SA MARCH 23, 1967 2240 TO 2325 PST IINC. SULFIDE RELEAGE FROM ELEVATION OF $26 \mathrm{M}$ $300 \mathrm{~N}$ ARC SAMPLER MT $1.5 \mathrm{H}$ UE $6.9 \mathrm{M} / \mathrm{SEC}$ AT $26 \mathrm{M}$

MZJMUTH EXPQSURE EF EU/O DISTANCE DEGREES SK-SEC/CU.M SEC/CU.M 1/30," METERG $\times 10 E+6$

$\begin{array}{lrlll}36.0 & 20 & 1.641 E-08 & 1.132 E-07 & 300 \\ 40.0 & 377 & 3.205 E-07 & 1.521 E-06 & 300 \\ 44.0 & 302 & 2.232 E-07 & 1.540 E-06 & 300 \\ 40.0 & 111 & 0.732 E-07 & 3.265 E-06 & 300 \\ 52.0 & 1659 & 9.613 E-07 & 6.682 E-06 & 300 \\ 56.0 & 10 \$ B & 5.943 E-07 & 9.100 E-06 & 300 \\ 60.0 & 000 & 1.672 E-07 & 3.224 E-06 & 300 \\ 64.0 & 395 & 2.309 E-07 & 1.593 E-06 & 300 \\ 60.0 & 264 & 1.543 E-07 & 1.065 E-06 & 300 \\ 12.0 & 02 & 4.014 E-00 & 3.322 E-07 & 300\end{array}$

CROSAHIND INTEGRATED= 7.113E-05 A.900C-04

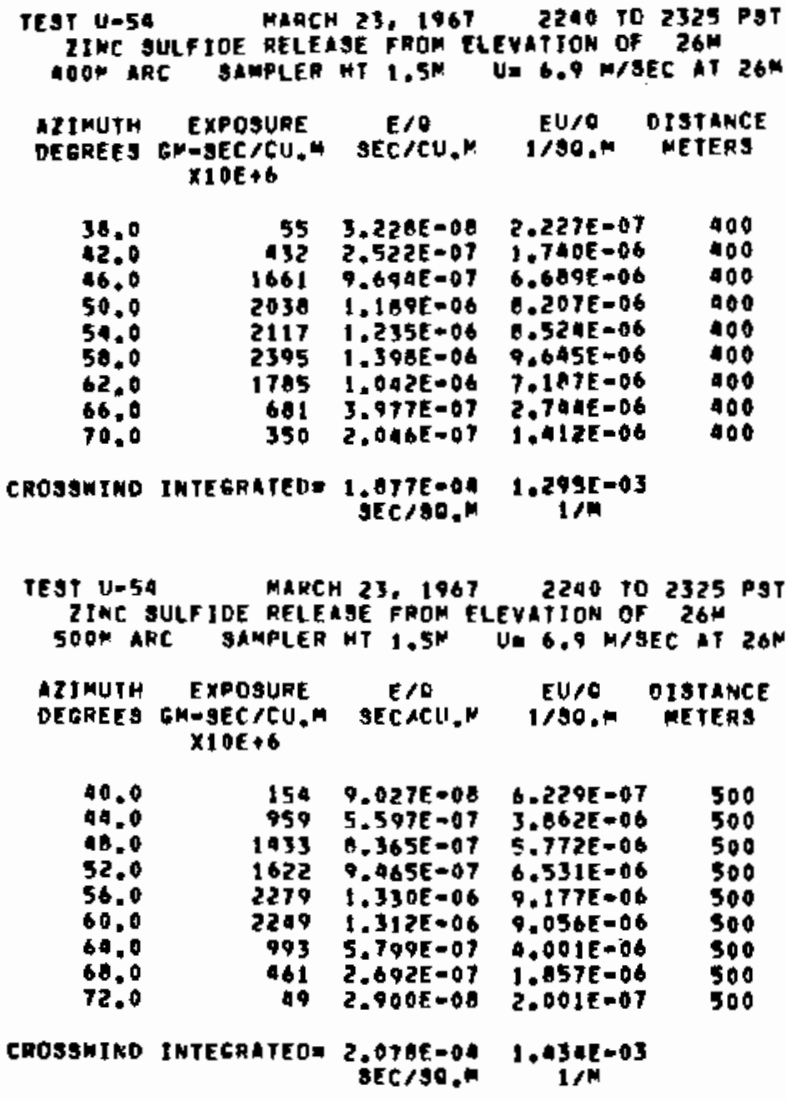

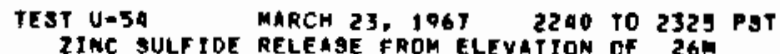
ZINC SULF IDE RELEABE FROM ELEVATION OF 26I AZIMUTH EXPOSURE E/O EU/O DISTANCE DEGREES GWABEC/CU.M SEC/CU.M I/SO.M DETERS $\times 10 E \$ 6$

\begin{tabular}{|c|c|c|c|c|c|}
\hline $\mathbf{F}$ & $\begin{array}{l}92.0 \\
16.0 \\
50.0 \\
34.0 \\
50.0 \\
62.0 \\
66.0 \\
70.0\end{array}$ & $\begin{array}{r}556 \\
1309 \\
1679 \\
2708 \\
3007 \\
1440 \\
695 \\
235\end{array}$ & $\begin{array}{l}3.250 E=07 \\
7.613 E=07 \\
9.771 E=07 \\
1.627 E=06 \\
2.221 E=06 \\
0.403 E=07 \\
4.059 E=07 \\
1.373 E=07\end{array}$ & $\begin{array}{l}2.242 E-06 \\
5.27 A E-06 \\
6.742 E-06 \\
1.123 E-05 \\
1.933 E=05 \\
5.798 E-06 \\
2.001 E=06 \\
9.475 E=07\end{array}$ & $\begin{array}{l}600 \\
600 \\
600 \\
600 \\
600 \\
600 \\
600 \\
600\end{array}$ \\
\hline & 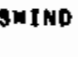 & FEGRATEO & $\begin{array}{r}3.057 E-04 \\
\text { sec } / 90.0\end{array}$ & $2.109 E=03$ & \\
\hline
\end{tabular}




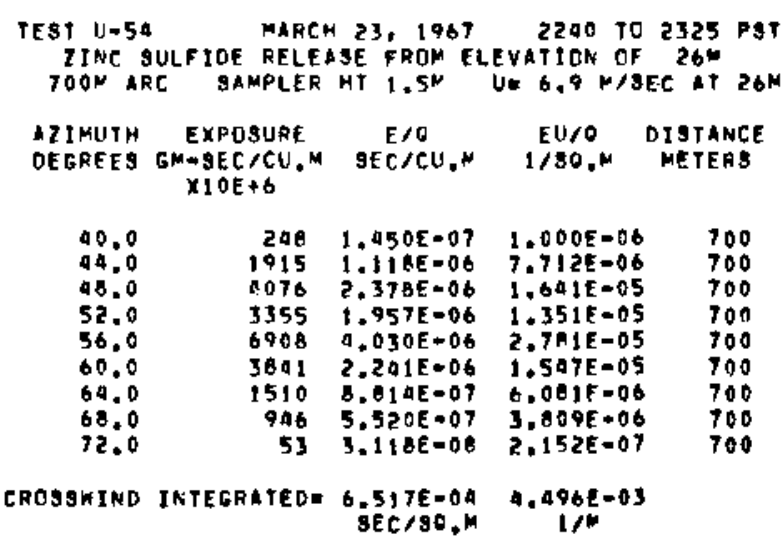

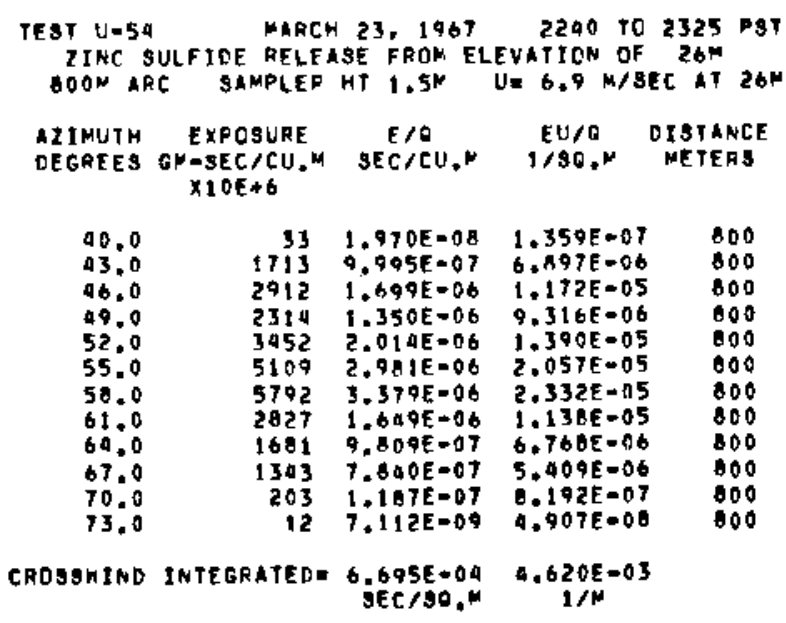

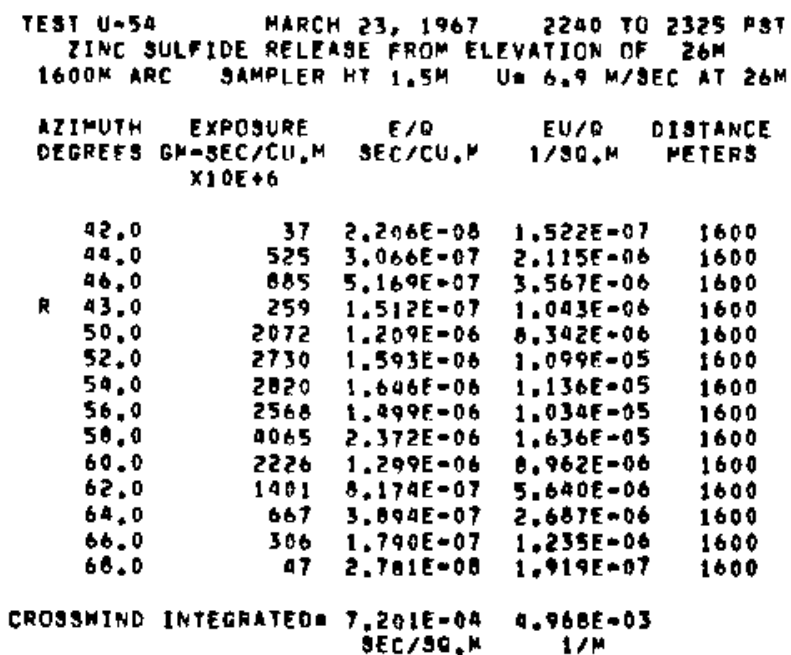


UNSTAQLE SERIES EXPERIMENT: $54 F$ ( U-5 4F)

ZINC SULFIDE AND FULORESCE IN WERE BOTH USED AS TRACERS CLRING

EXOERI WENT 54. FLJORESCEIN RESULTS ARE TRESEYTED TIERE. GROUIO

LEYEL SAMPLING $100-1600$ :H ARC.

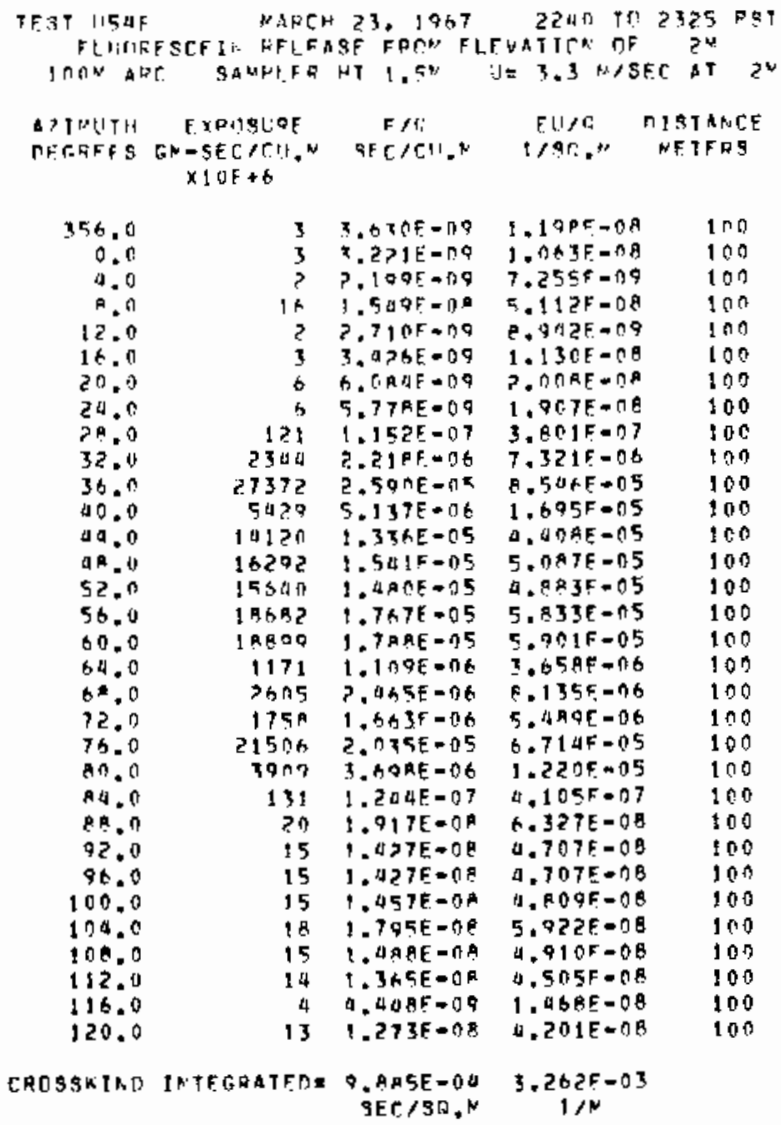

\footnotetext{
TFST I:SUF FALCH 23,1967 22A TO 2325 PST

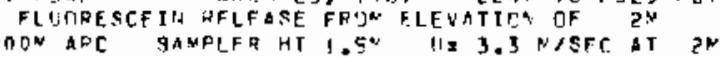

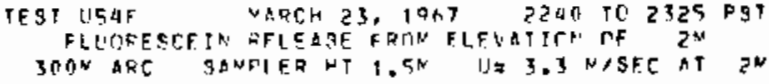




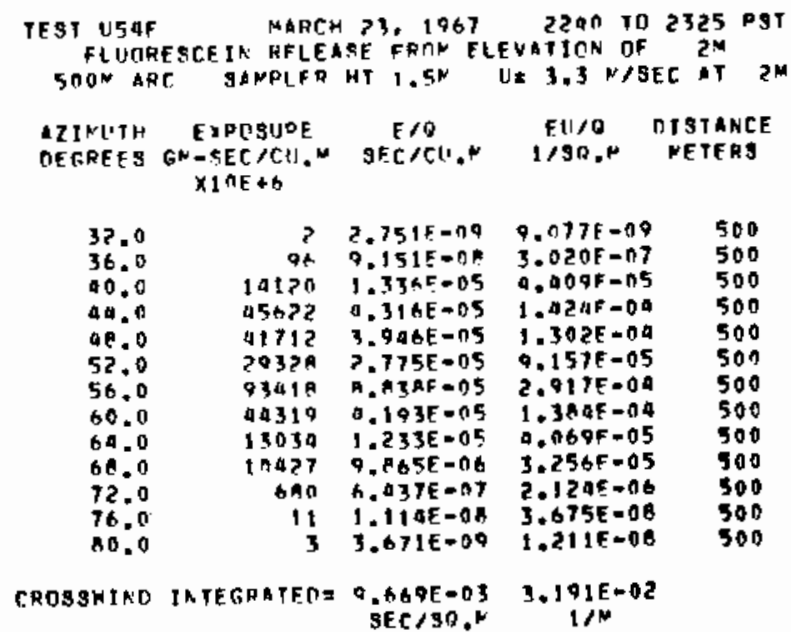

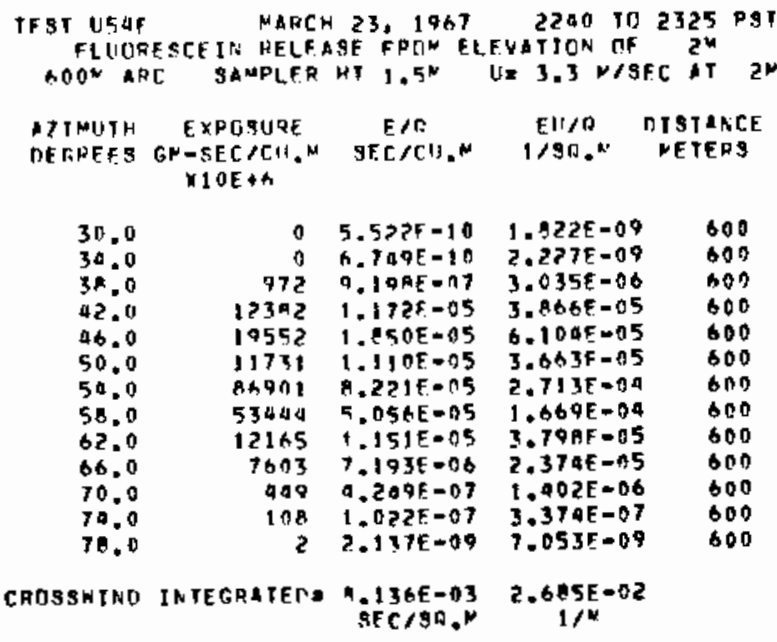

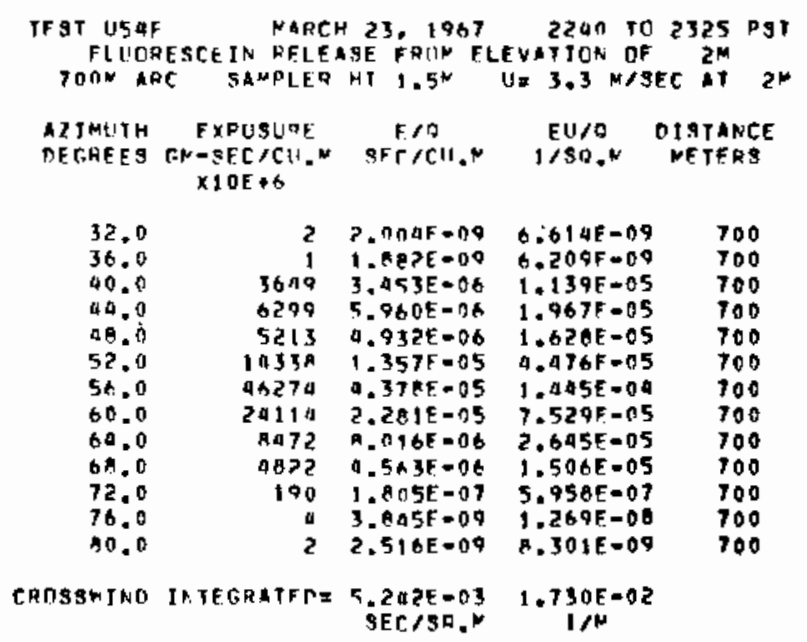

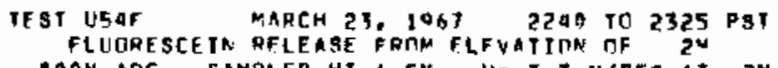
MOON ARC SAMPLER HT $1.5^{\mathrm{N}} \mathrm{U}=3.3 \mathrm{~K} / \mathrm{SEC}$ AT $2 \mathrm{M}$

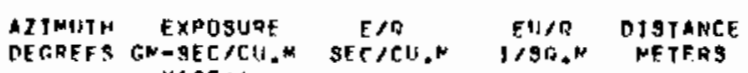
X10E+G

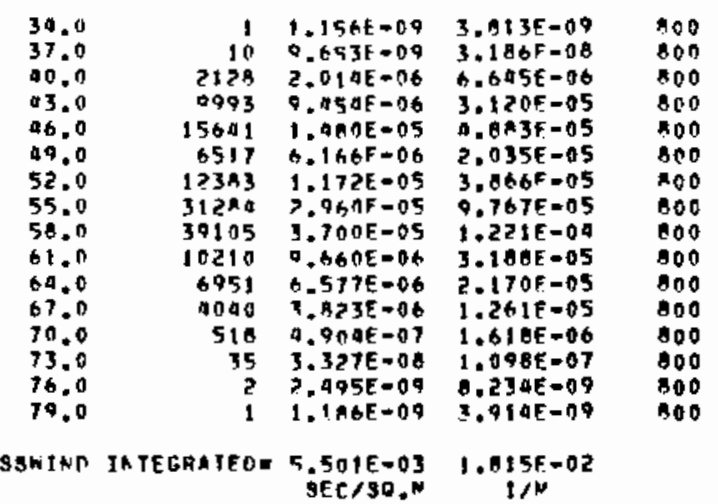

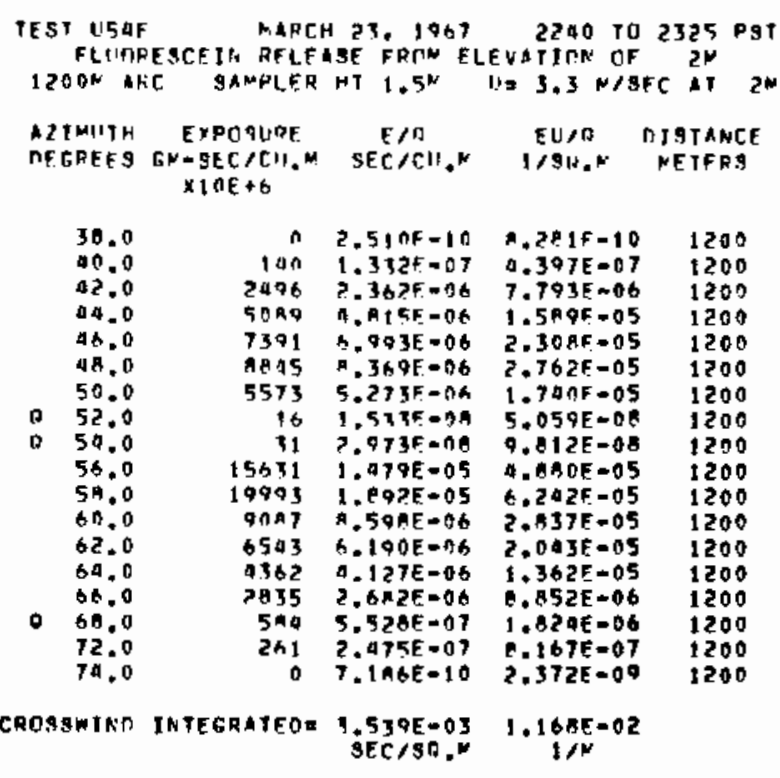




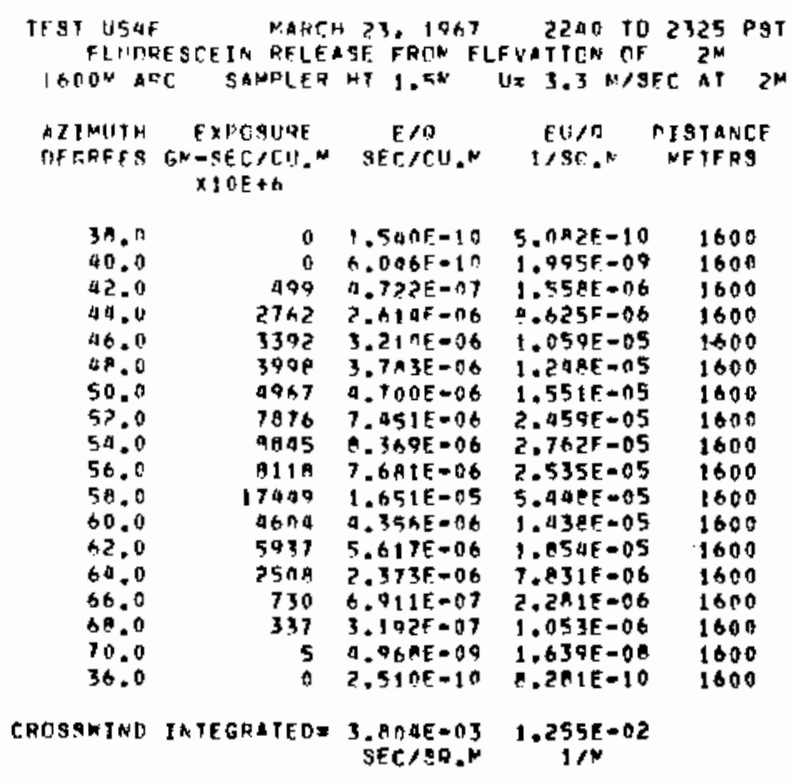


UNSTABLE SERIES EXPER HHENT: 55 (U 55 )

GROUNO LEYEL SAHPLING $100-1600$ M ARCS. FOR EASE IN STATISTICAL ANALYSIS, ALL SAMPLER AZIMUTHS HAVE BEEN INCREASEO BY 30 DEGREES

FROM THEIR ACTUAL POSITIONS.

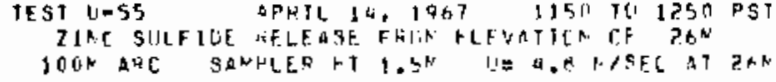

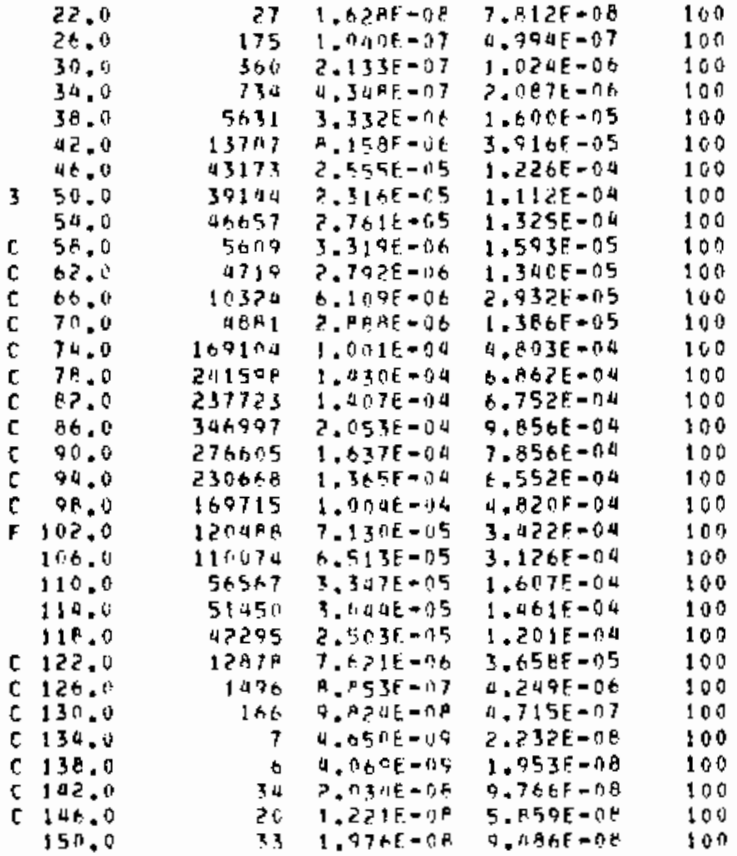

CHOSSMINE. IRTEGHATET = 9.2AGE-U3 A. DLEE-02

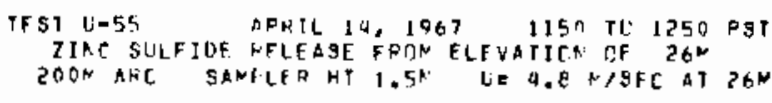

SANFLEF DZINLTHG HAVE BEEN IHCREASET GY 3O IEGREES

AlTVUTR EXDUSURE EAT FUIO DISTARCE

DECHEFS GK-SEC/CII." SEC/CI." IISCN N NETFES $\times 10 E+6$

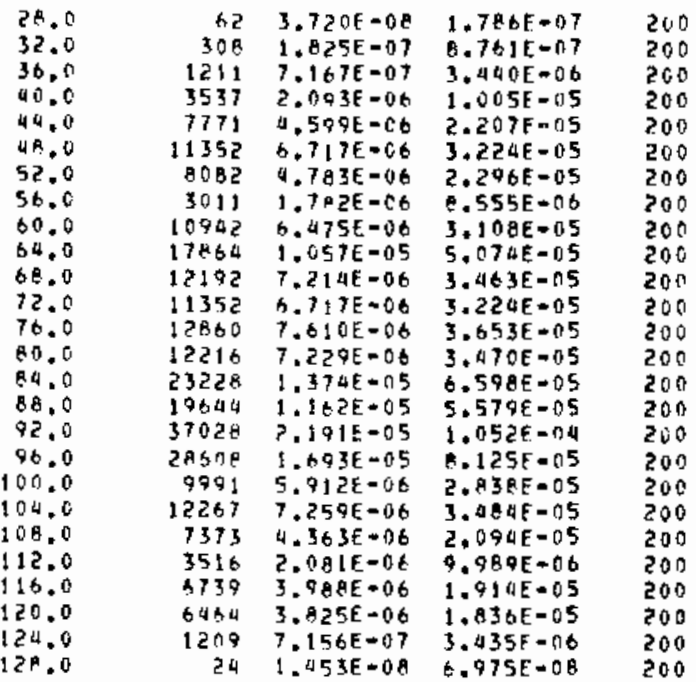

CROSSAIND INTEGRATET:= 2.22IE $\rightarrow 03$ S.06EE-02

$B \in C / 20 . \mathrm{N} \quad 1, \mathrm{~m}$ 


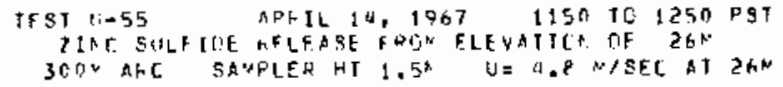

SARPLEF AZIALITHS NAUE GEES IHCREABFD PY 30 DEGFEES

AZJXUTH EKPUSURE EZM TLO OISTARE

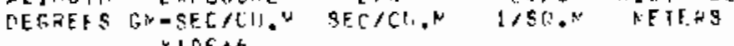
$\times 1 D E+6$

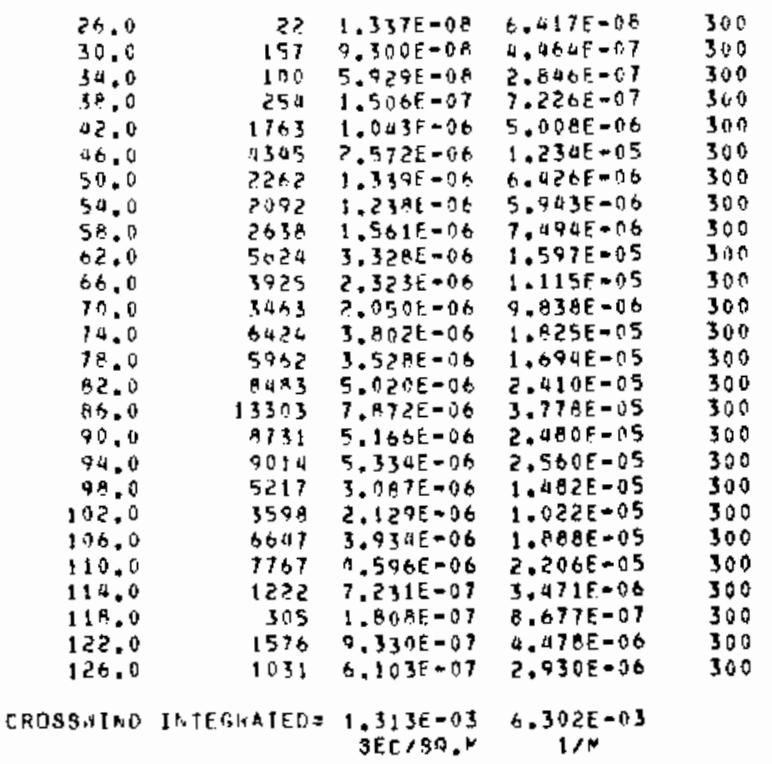

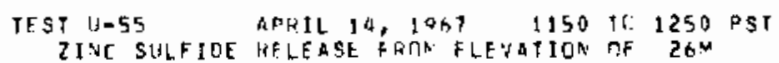

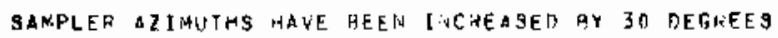

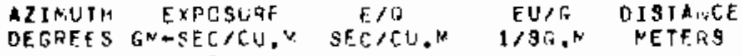
$X \perp$ OE +6

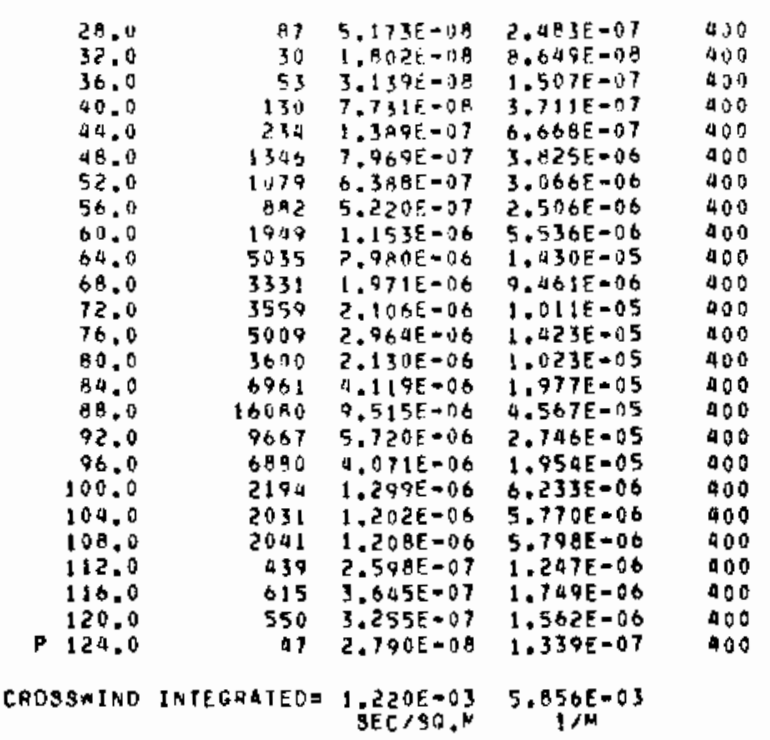

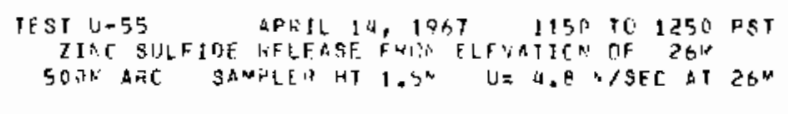

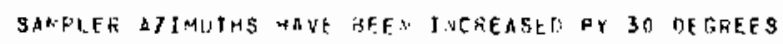

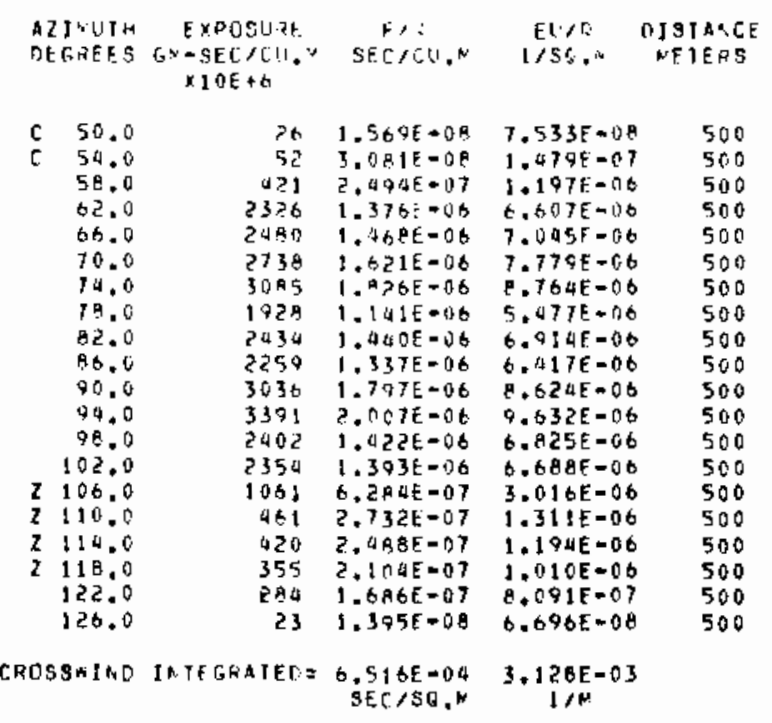

TEST 1.55 APKIL $14,1867 \quad 1150$ T0 1250 PGT ZINC SULFIDE RELEASE FRFIN FLEYATIUIN DF $26 \mathrm{~N}$ GOON AFC SANFLER HT 1 , SN UN

9AMPLER AZIMUTHS HAUE HEER INCAEASED FY 30 DEGREES AZIMUTH EXPESURE E/O EU/G DISIAACE

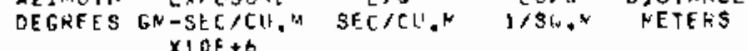

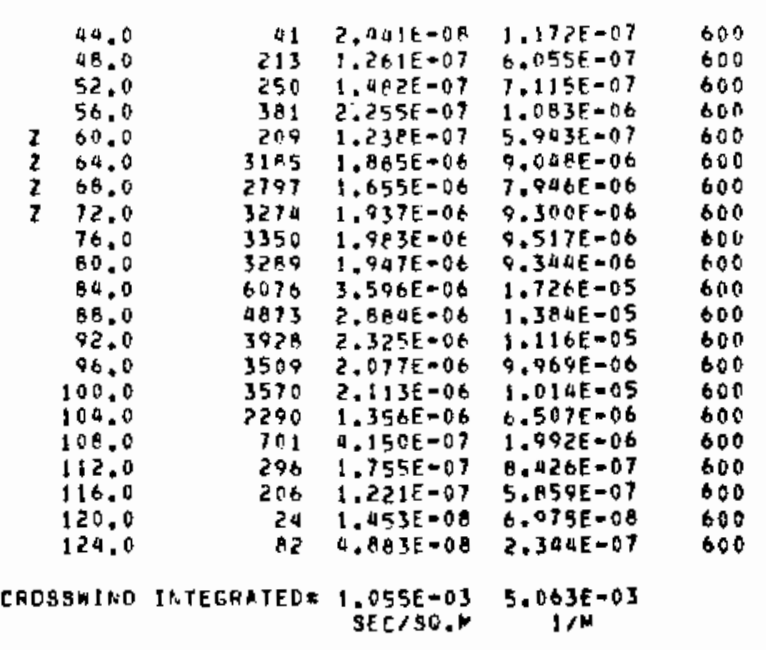


TEST U-S5 APRIL 19, 1967 1150 TO $1250 \mathrm{PSY}$ ZINC SLLFIOE RELFASE FRIN ELEVATION THF ZGN:

7 CON ARL SAMPLER HT J.SM UIE 4.8 K/SEC AT $26 M$

SHMFLER AZIHUTHS HAVE HEEN INCREASED BY 30 DERREES

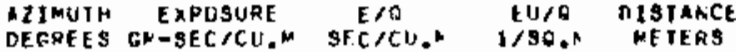
$\times 10 E+6$

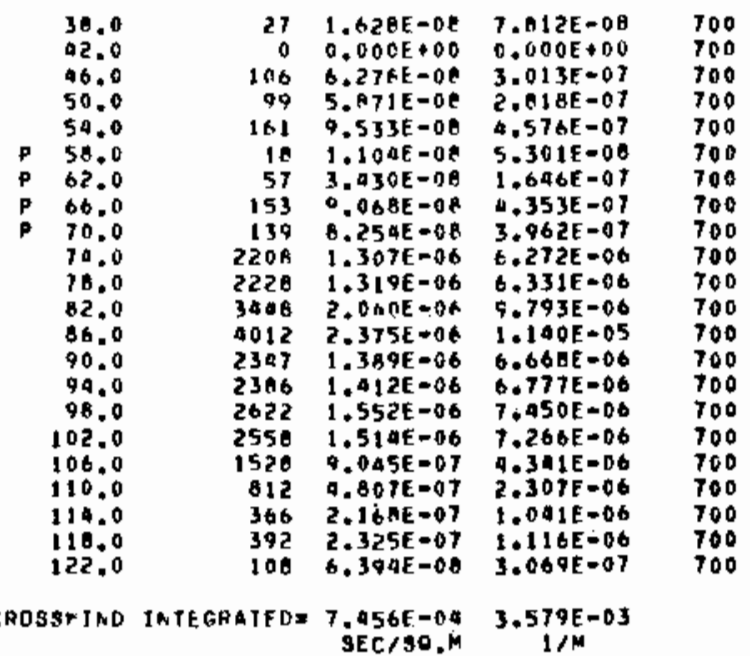

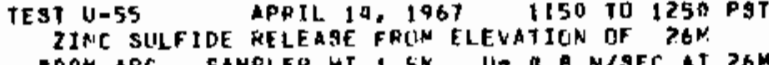
DOOH ARC SAMPLEP HT $1 . \mathrm{SN}$ DO $4.8 \mathrm{M} / \mathrm{SEC}$ AT $26 \mathrm{M}$

SAMPLER AZIMUTHS HAVE GEFM INCPEASED PY 30 DEGREE AZINUTH EXPOSURE
DEGREES GKEEC/CIJ, SECA $\times 10 E+6$

\begin{tabular}{|c|c|c|c|}
\hline $\begin{array}{l}96.0 \\
49.0 \\
52.0 \\
55.0 \\
50.0 \\
61.0 \\
64.0 \\
67.0 \\
70.0 \\
73.0 \\
76.0 \\
79.0 \\
02.0 \\
05.0 \\
88.0 \\
91.0 \\
94.0 \\
97.0 \\
100.0 \\
103.0 \\
106.0 \\
109.0 \\
112.0 \\
115.0 \\
118.0 \\
121.0\end{array}$ & $\begin{array}{l}26 \\
23 \\
100 \\
151 \\
266 \\
403 \\
633 \\
1492 \\
1497 \\
1377 \\
2160 \\
2354 \\
2168 \\
2515 \\
2312 \\
1634 \\
2163 \\
2507 \\
1662 \\
1673 \\
1236 \\
745 \\
255 \\
353 \\
167 \\
154\end{array}$ & 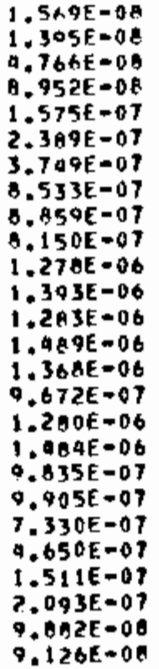 & $\begin{array}{l}7.533 E=08 \\
6.696 E=08 \\
2.268 E-07 \\
4.297 E-07 \\
7.561 E=07 \\
1.147 E=06 \\
1.000 E-06 \\
4.046 E=06 \\
4.252 E=06 \\
3.912 E=06 \\
6.136 E=06 \\
6.600 E=06 \\
6.154 E=06 \\
7.146 E=06 \\
6.568 E-06 \\
4.643 E=06 \\
6.144 E-06 \\
7.123 E-06 \\
4.725 E=06 \\
4.754 E=06 \\
3.518 E-06 \\
2.232 E=06 \\
7.254 E-07 \\
1.004 E-06 \\
4.743 E=07 \\
4.301 E=07\end{array}$ \\
\hline
\end{tabular}

CROSSWIND INTEGRATEOC 7.43AE-0A 3.570E-03
TEST $U-55$ APRIL $14,1967 \quad 1150$ TO 1250 PST

2IHC SULFIDE RELEASE FRIJM ELEVATION OF $26 \mathrm{M}$ I200\% ARC SAMPLER HT I.5W UE $7.8 \mathrm{M} / \mathrm{SFC}$ AT $26 \mathrm{M}$

SAMPLER AZIMUTHS HAYE HEFN INCREASED AY SO DEGREES

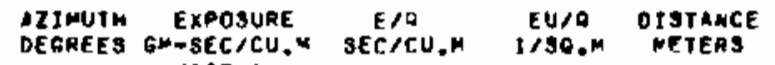
$\times 10 E+6$

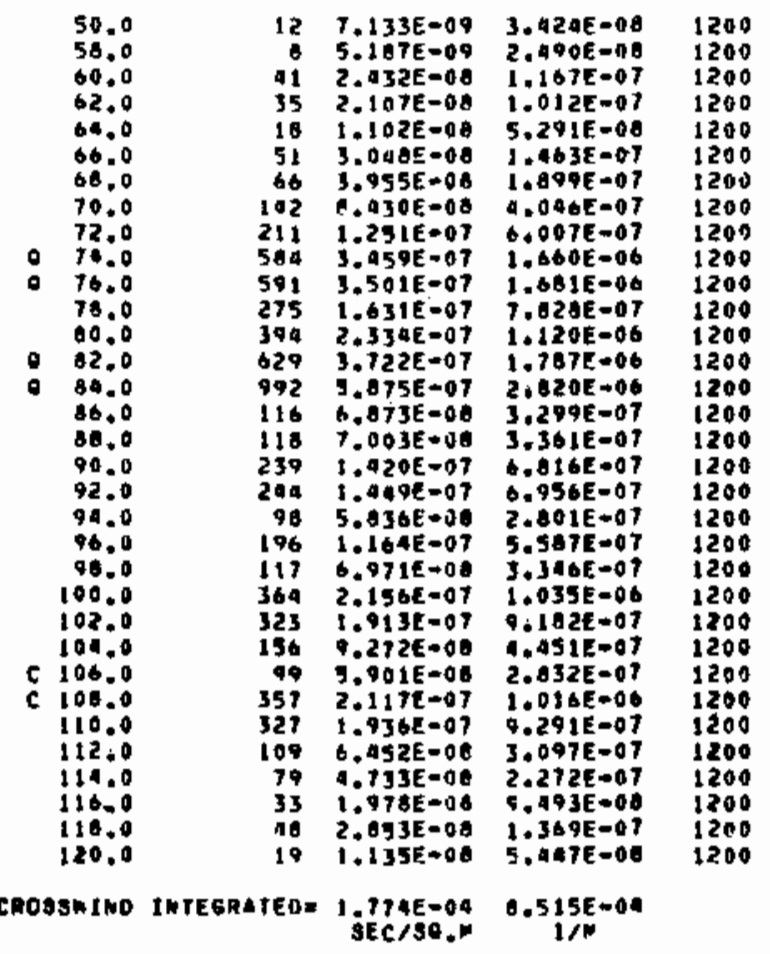




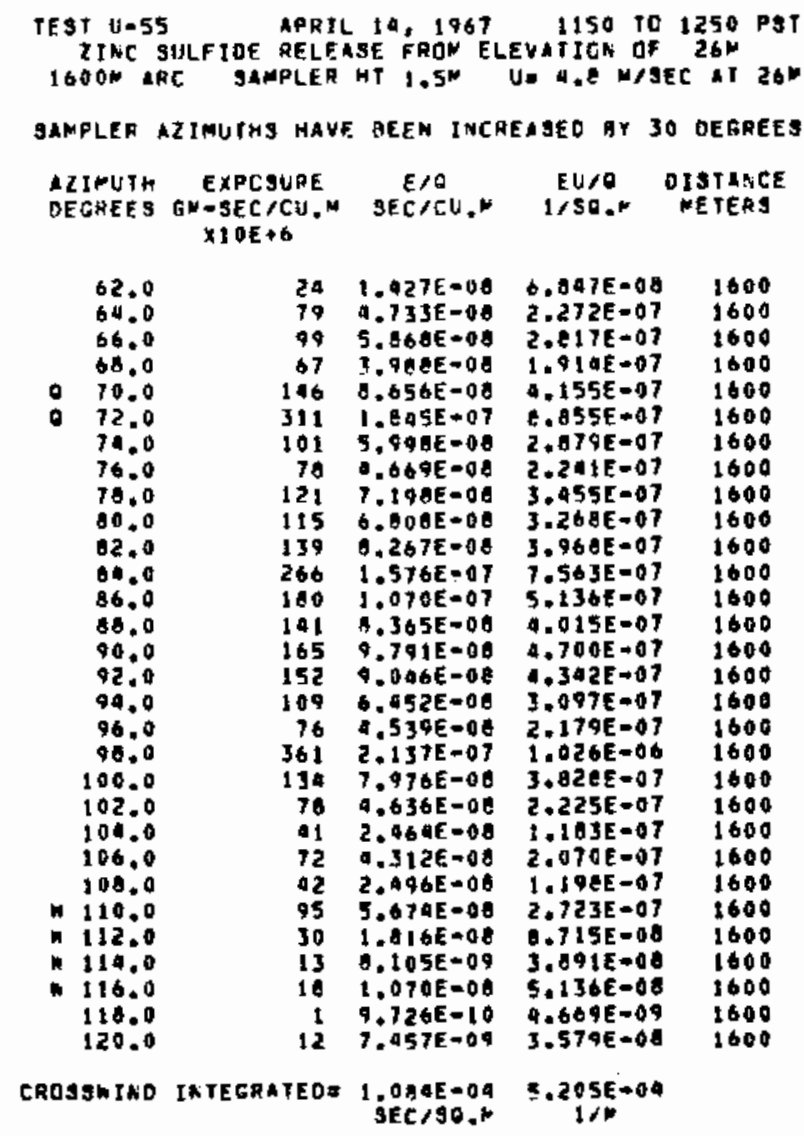


UNSTABLE SERIES EXPERIMENT: $55 \mathrm{~F}$ ( U-55F)

ZINC SULFIDE AND FLUORESCEIN WERE BOTH USED AS TRACERS DURING

EXPERIMENT 51. FLUORESCE IN RESULTS ARE PLESENTED HERE. GROUNO

LEVEL SAMPL ING $100-1600$ \& ARC.

TEST L55F
FLUTAESCEIN RERIL 14.1967 RE 1150 TO 1250 PST IOON AHC SAMPLER HT $1.5 \mathrm{~N} U=3.0 \mathrm{M} / \mathrm{SEC}$ MT $2 \mathrm{~N}$

SANPIER AZINUTHS hAVE GEEN INCREASED BY 30 DEgREFS

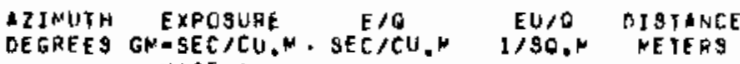
$\times 106+6$

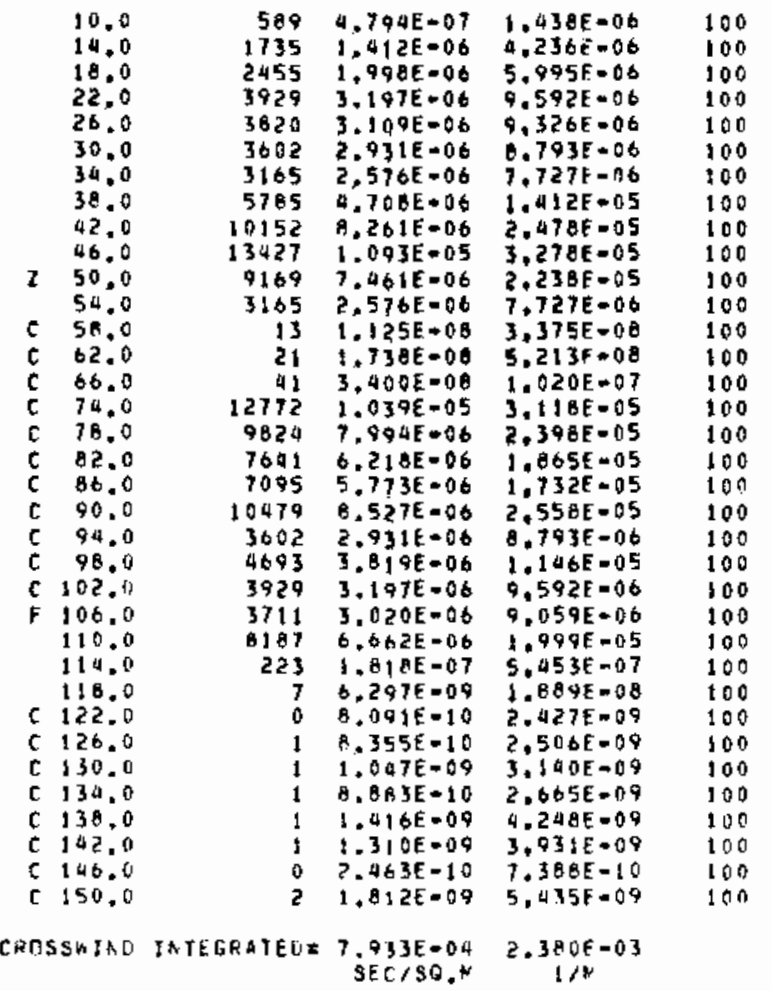

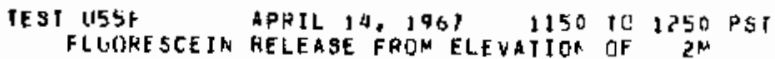
ZOON ARC SAMPLER HT $1.5 \mathrm{~N}$ L: $3.0 \mathrm{M} / \mathrm{SEC}$ AT $2 \mathrm{M}$

SANPLER RIMUTHS HAYE BEEN INCREASF OY 30 DEGHEES

\begin{tabular}{|c|c|c|c|c|}
\hline $\begin{array}{l}\text { ZINUTH } \\
\text { EGREFS }\end{array}$ & $\begin{array}{c}\text { EXPQSURE } \\
\text { GN }- \text { SEC } / C U . M \\
X 10 E+6\end{array}$ & $\begin{array}{c}E / Q \\
S E C / C U, M\end{array}$ & $\begin{array}{l}E U / O \\
1 / S O . M\end{array}$ & $\begin{array}{l}\text { DISTANCE } \\
\text { NETERS }\end{array}$ \\
\hline $\begin{array}{l}24.0 \\
20.0 \\
32.0 \\
36.0 \\
40.0 \\
44.0 \\
40.0 \\
52.0 \\
56.0 \\
60.0 \\
60.0 \\
68.0 \\
72.0 \\
76.0 \\
80.0 \\
84.0 \\
68.0 \\
92.0 \\
96.0 \\
100.0 \\
104.0 \\
108.0 \\
112.0 \\
116.0 \\
120.0 \\
124.0 \\
128.0 \\
13200 \\
136.0 \\
140.0\end{array}$ & $\begin{array}{r}600 \\
604 \\
421 \\
1866 \\
2259 \\
3929 \\
2324 \\
3383 \\
3602 \\
6656 \\
1296 \\
3383 \\
1899 \\
3711 \\
5021 \\
5457 \\
7532 \\
12117 \\
11134 \\
8842 \\
10807 \\
7095 \\
875 \\
907 \\
56 \\
572 \\
12 \\
3 \\
3 \\
2\end{array}$ & 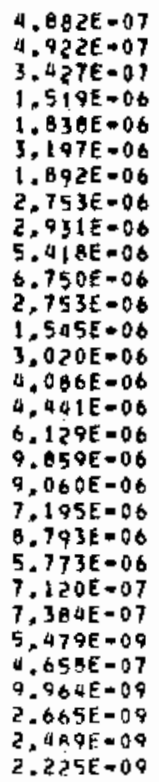 & $\begin{array}{l}1.465 E=06 \\
1.477 E=06 \\
1.028 E=06 \\
4.556 E=06 \\
5.515 E=06 \\
9.592 E=06 \\
5.675 E=06 \\
1.260 E=06 \\
9.793 E=06 \\
1.625 E=05 \\
2.025 E=05 \\
8.260 E=06 \\
4.636 E=06 \\
9.059 E=06 \\
1.226 E=05 \\
1.332 E=05 \\
1.839 E=05 \\
2.958 E=05 \\
2.718 E=05 \\
2.158 E=05 \\
2.658 E=05 \\
1.732 E=05 \\
2.136 E=06 \\
2.215 E=06 \\
1.644 E=08 \\
1.397 E=06 \\
2.969 E=08 \\
7.994 E=08 \\
1.467 E=09 \\
0.675 E=09\end{array}$ & $\begin{array}{l}200 \\
200 \\
200 \\
200 \\
200 \\
200 \\
200 \\
200 \\
200 \\
200 \\
200 \\
200 \\
200 \\
200 \\
200 \\
200 \\
200 \\
200 \\
200 \\
200 \\
200 \\
200 \\
200 \\
200 \\
200 \\
200 \\
200 \\
200 \\
200 \\
200\end{array}$ \\
\hline
\end{tabular}

CHOSSLINL INTEGRATED= I.ZAAE-03 3, BOJE-03 


\begin{abstract}
TEST 1155R APRIL $14,1967 \quad 1150$ T11 1290 PST

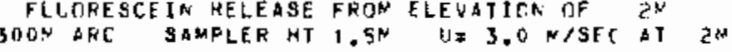

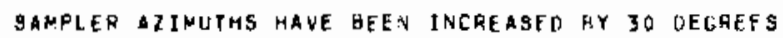

AZINUTH EXPOSURE EAR EUAG DISTANCE

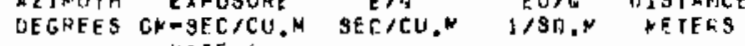
$\times 10 E+6$

18.0
22.0
26.0
30.0
34.0
38.0
42.0
46.0
50.0
58.0
62.0
66.0
70.0
74.0
78.0
82.0
86.0
90.0
44.0
99.0
102.0
106.0
110.0
114.0
118.0
122.0
126.0
130.0

\begin{tabular}{|c|c|c|}
\hline & & $E * 09$ \\
\hline & $3.25 \mathrm{AE}-10$ & \\
\hline 323 & $2.635 E-07$ & $7.906 \varepsilon=07$ \\
\hline 50 & $E=07$ & $=0$ \\
\hline 39 & 3. & $3 E=07$ \\
\hline 42 & & \\
\hline 30 & $b$ & 06 \\
\hline 190 & & -06 \\
\hline $\begin{array}{l}1838 \\
3540\end{array}$ & 1.8 & \\
\hline 57115 & & \\
\hline 458 & & \\
\hline 232 & 06 & 00 \\
\hline 37 & & \\
\hline & & \\
\hline $73: 3$ & 6 & 05 \\
\hline 1012 & 5 & \\
\hline 982 & & \\
\hline 1015 & .2 & \\
\hline & & \\
\hline 6446 & & \\
\hline 764 & i & \\
\hline & & \\
\hline 04 & & \\
\hline & 00 & 6 \\
\hline & & \\
\hline & & \\
\hline & 09 & 700 \\
\hline
\end{tabular}

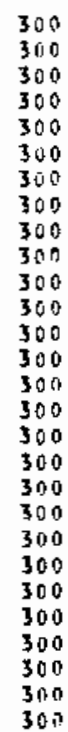

$30 \%$

CHESSRIAD IATEGRATEDE 1 B.BZE-03 $5.466 E-03$

\begin{abstract}
TEST U55F APHIL $10.1967 \quad 1150$ T1 1250 P9T FL UORESCEIN KELEASE FRON ELEVATIOR OF $2{ }^{\circ}$ AOUN AFE SAMPLER HF $1,5 \mathrm{~N} U=3,0 \mathrm{~m} / \mathrm{SEC}$ at $\mathrm{Z}^{\mathrm{N}}$
\end{abstract}

MARPLEK AZIMUTHG HAVE REEN JHCREASEL EY 30 DEGREES

DIJULIH EXPQSURE E/O EIIA DISTAR,CE MEGEEFS GN-SECJCH.M SECICISN I/SO,H NETERS X) $0 \mathrm{E}+\mathrm{b}$

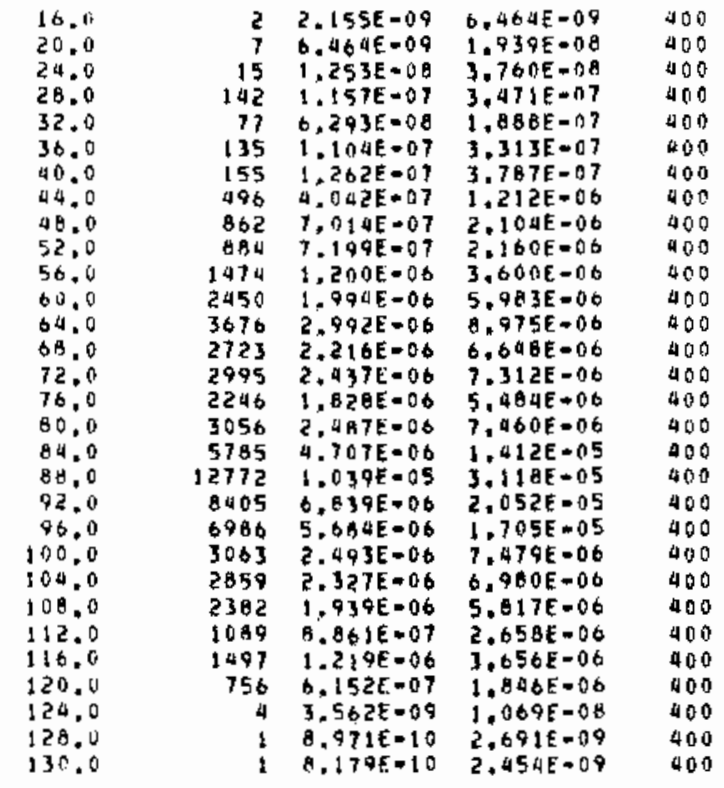

CPTSSNINO INTEGAATED= $1.523 E-03 \quad 4.560 E-03$

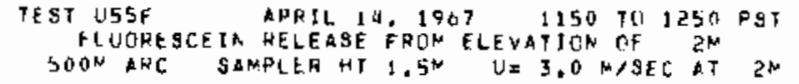

SAMMLER AZINUTHS HAVE GEEN IACFEASED BY 30 DEGAEES

ATINUTH EXPOSURE E/A EL:OQ FISTALCE CEEREES GN-SEC/C11.M SEC/CU.N 1/SG,N NETERS $\times] O E+6$

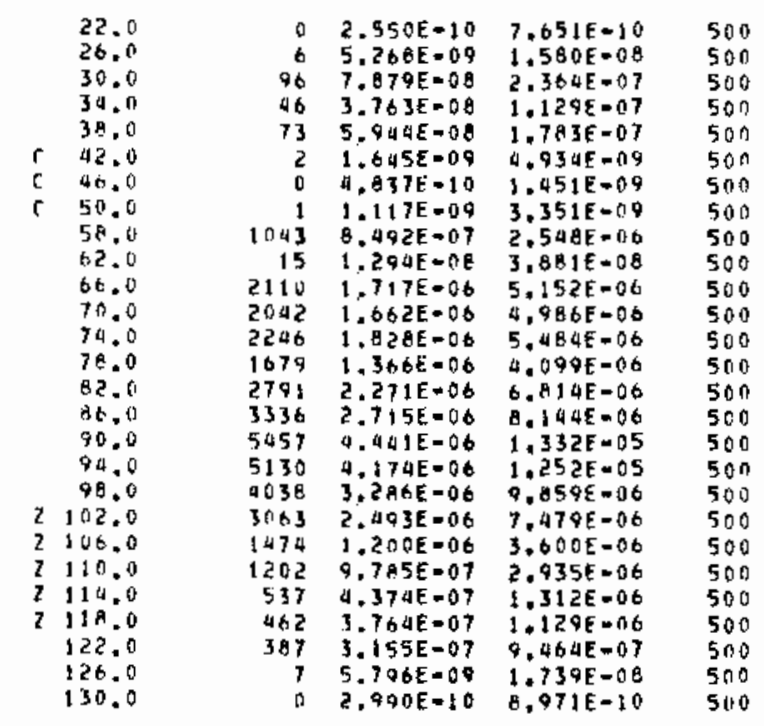

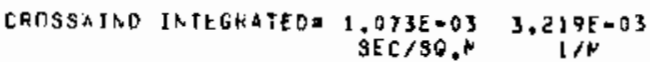

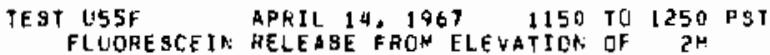
GOON ARC SAMPLER HT. $1.5^{\mathrm{N}} \mathrm{LE} 3.0 \mathrm{M} / \mathrm{SEC}$ AT $2 \mathrm{M}$

SAMPLER AZIMUTHS HAVE BEEN INCAELGF OY 30 DEGREES

AZINUTH EXPOSURE EIO EUIO DISIANCE DEGREES GNASEC/CU.M SEC/CU.N 1/SG.M NETERS $\times 10 E+6$

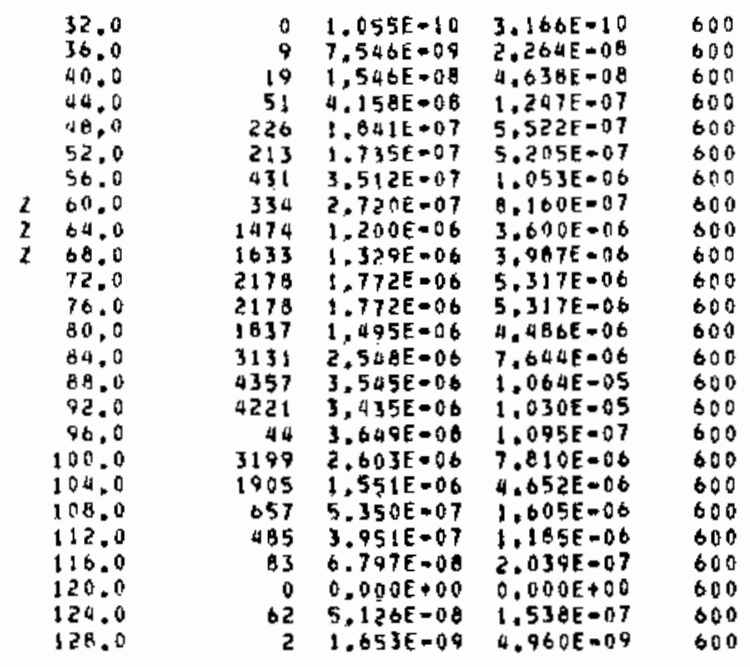

CHOSSIVIND INTEGAATEDF $9.795 E-04 \quad 2.939 E-03$ 


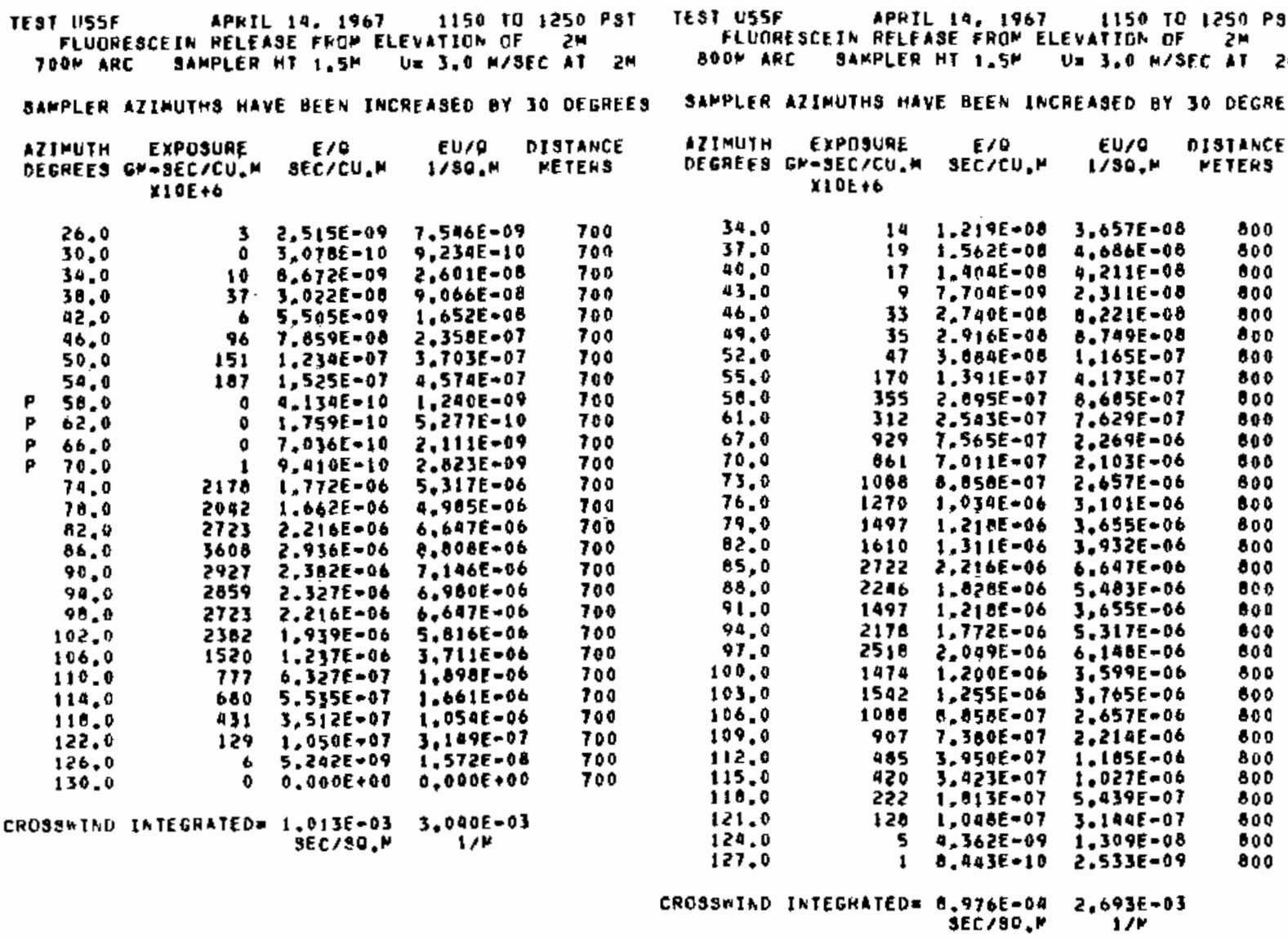




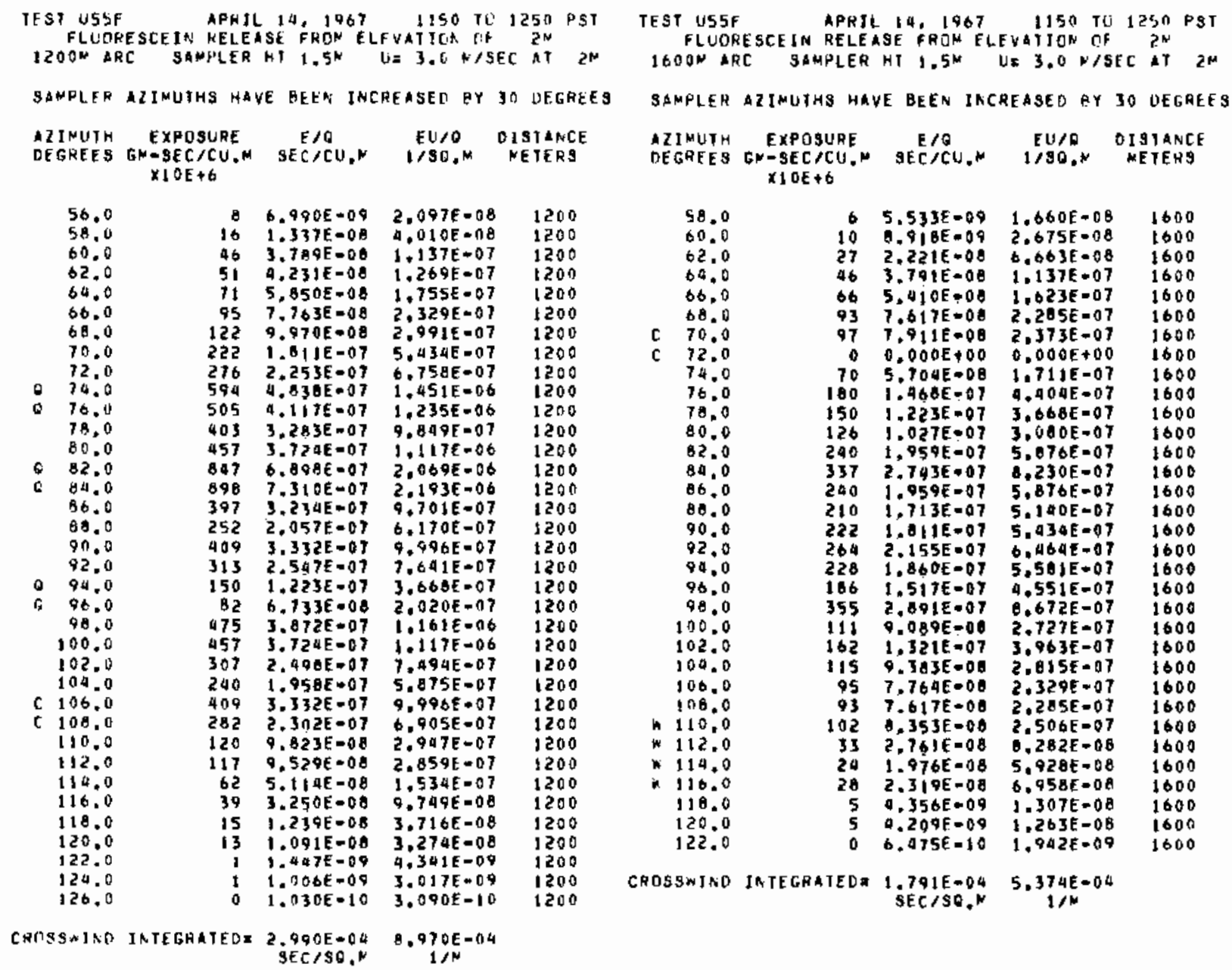


SERIES 163 EXPERIMENT: 1 ( FT-1)

GROUND LEVEL AND TOWER SAHFLING 200-3200 M ARCS. A SIGNIFICANT

PORTION OF THE PLUME NEVER PASSEO OVER THE GRID.

\begin{abstract}
TF ST FT1
APRIL 23, 1963

0516 TO 0601 PST TINC SULFIDE FELEASE FROK ELEYATTON COF TM
\end{abstract}

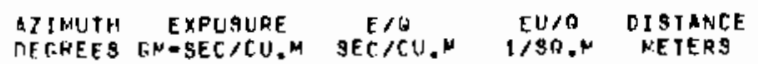
$\times 10 E+6$

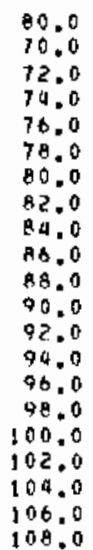

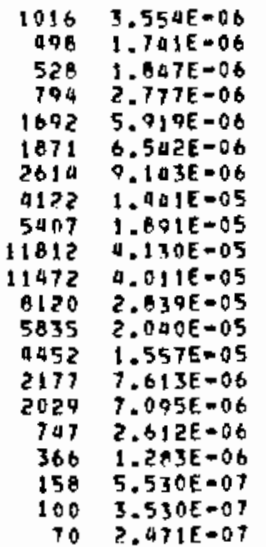

$5.330 E=06$
$2.612 E=06$
$2.771 E=06$
$4.165 E-06$
$6.878 E=06$
$9.813 E=06$
$1.371 E-05$
$2.162 E=05$
$2.036 E=05$
$6.195 E-05$
$6.017 E=05$
$4.259 E=05$
$3.061 E=05$
$2.335 E=05$
$1.142 E-05$
$1.064 E-05$
$3.918 E=06$
$1.924 E-06$
$8.295 E=07$
$5.295 E=07$
$3.700 E=07$

200 200 200 (2000 200 200 200 200 200 200 200 200 200 200 200 200 206 200 200

CHOSSWIND INTEGRATED= $1.423 E-03 \quad 2.135 E-03$

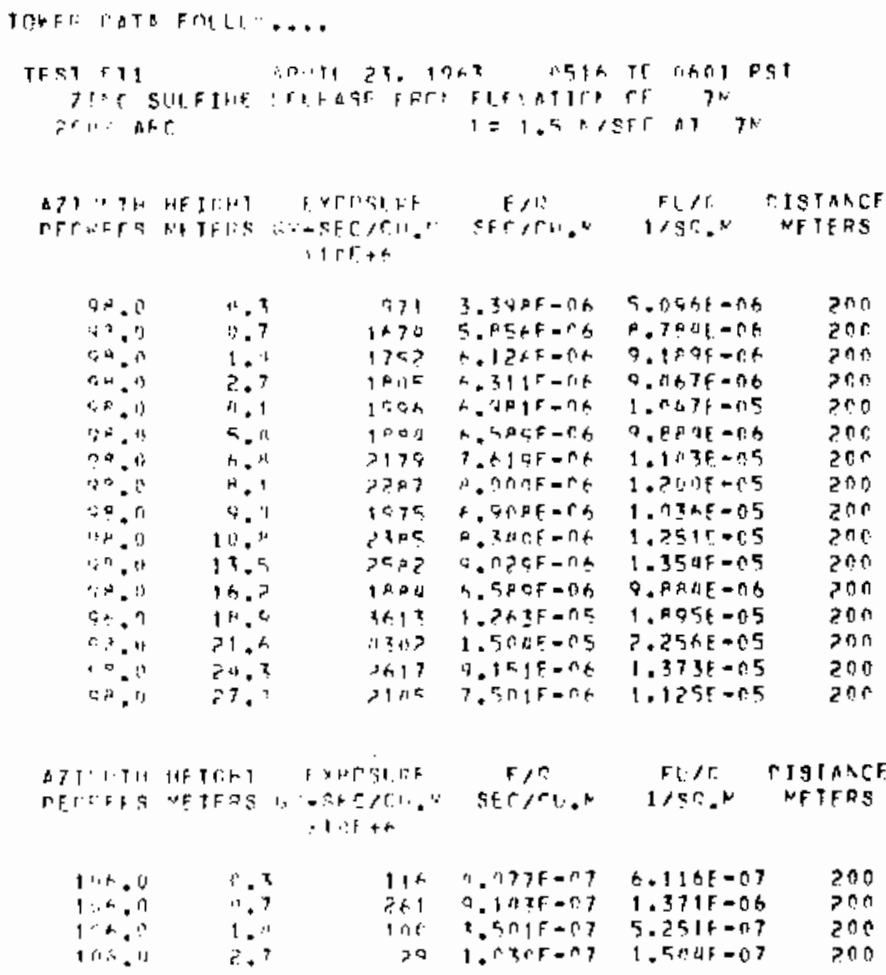

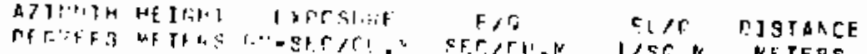

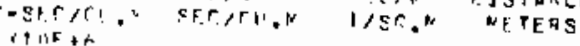

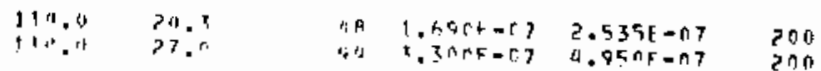

Rกn 


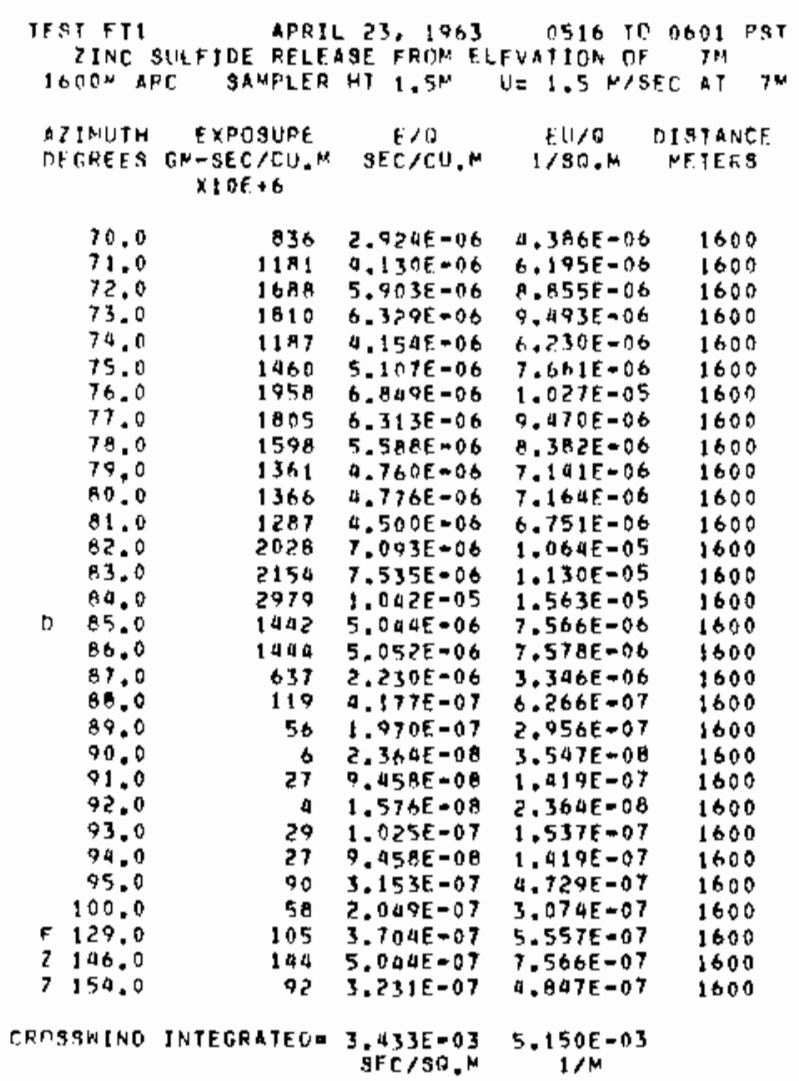

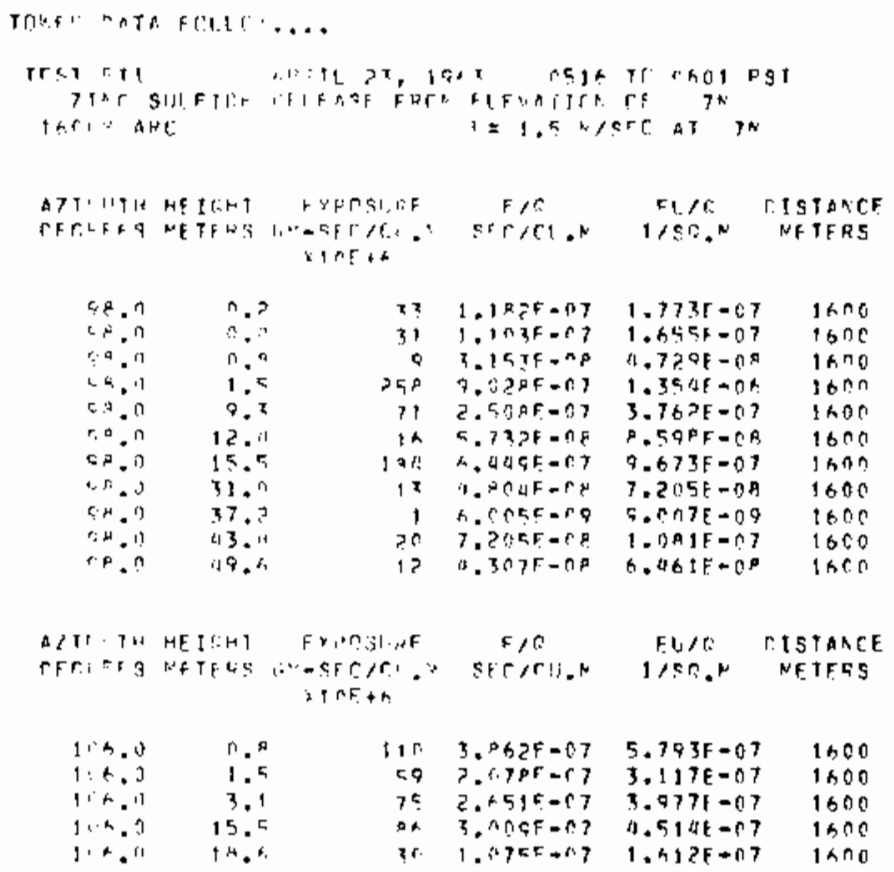

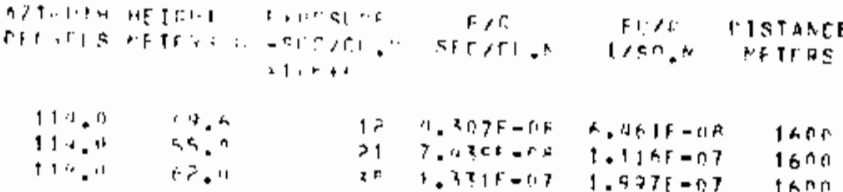




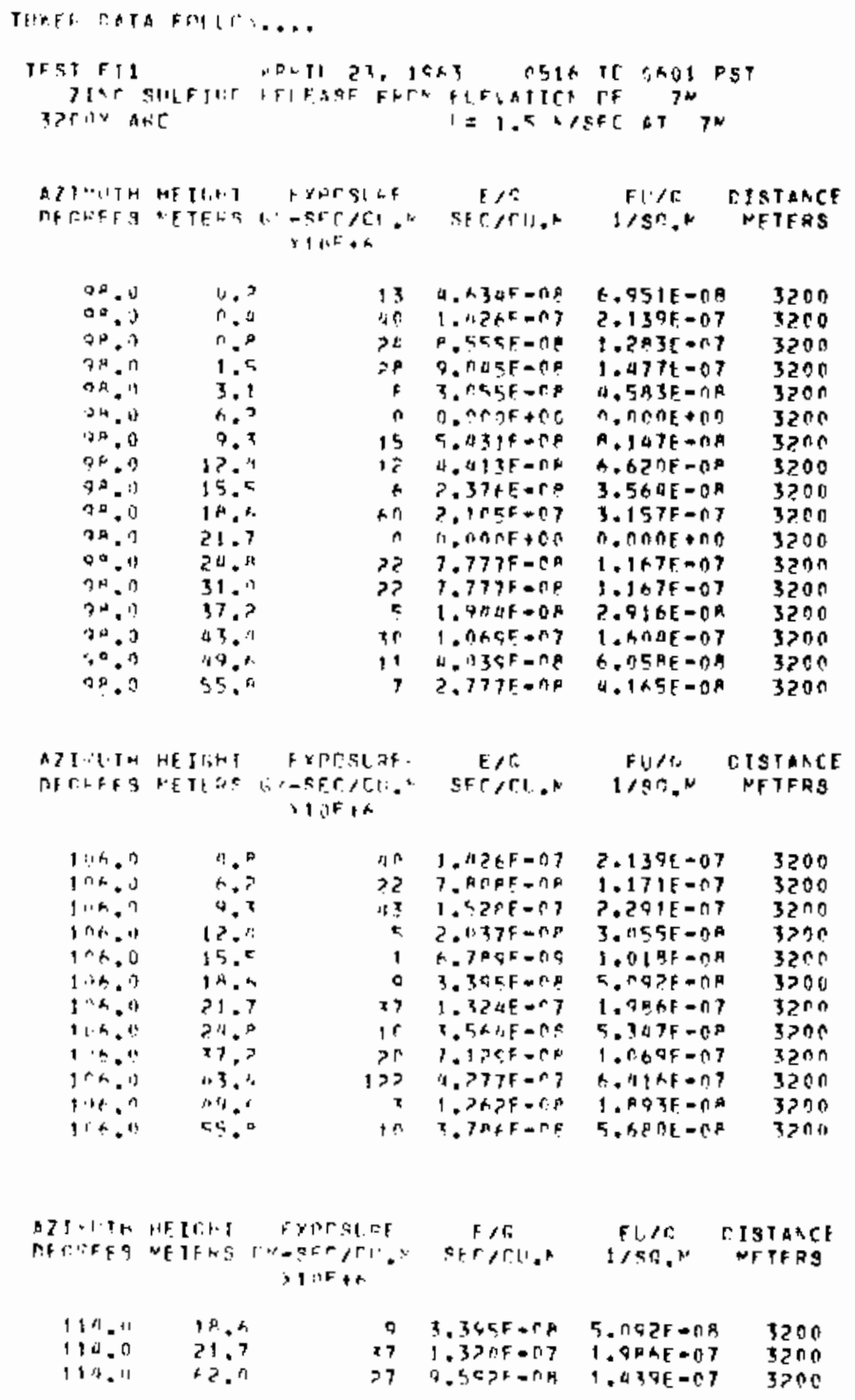


SERIES 163 EXPERIMENT: 3 (FT-3)

GROUND LEYEL AND TOWER SAMPLING 200-3200 H ARCS. A SIGNIFICANT

PORTION OF THE PLUME NEVER PASSED OYER THE GRID.

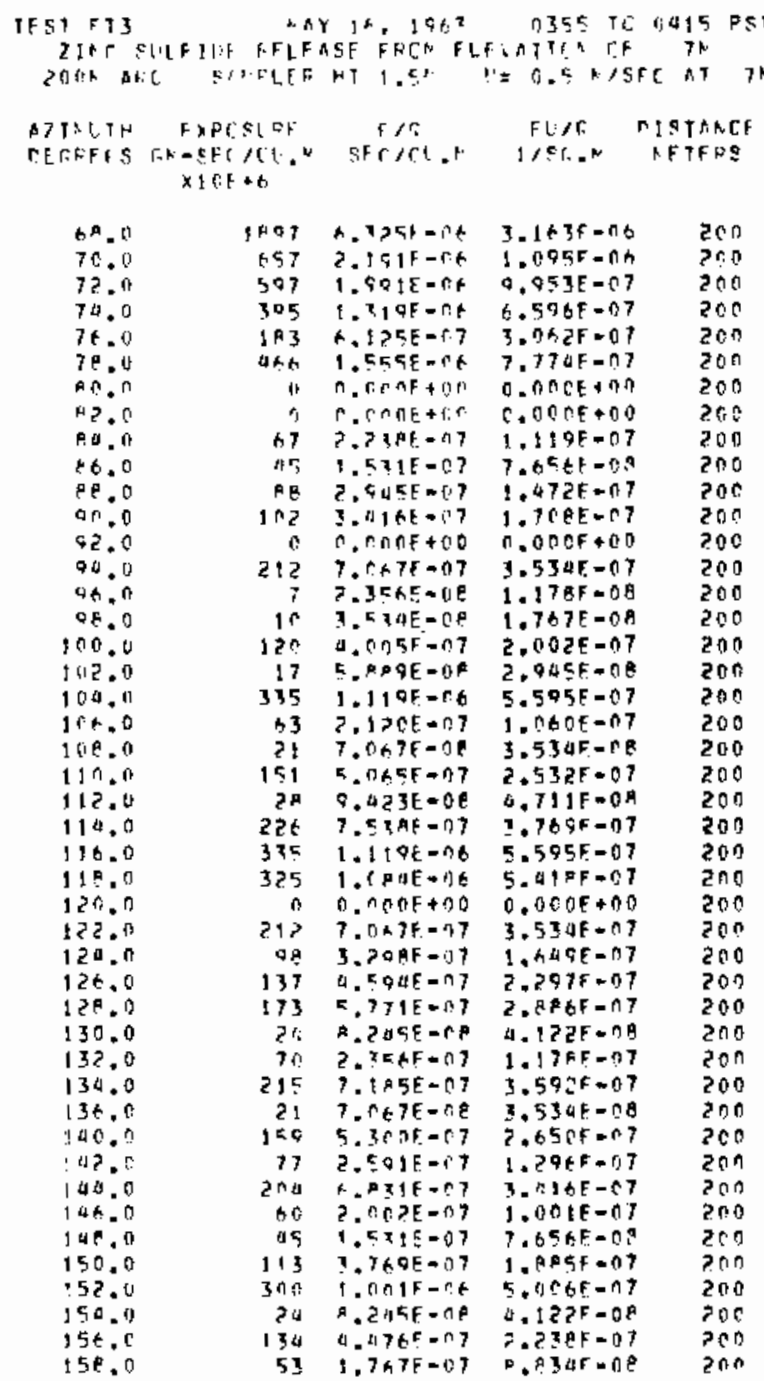

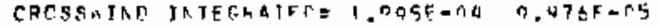

TOYFR IASTA FILLCH....

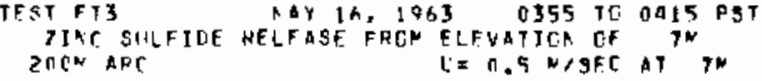

\begin{tabular}{|c|c|c|c|c|c|}
\hline $\begin{array}{l}106.0 \\
106.0 \\
106.0 \\
106.0 \\
106.0 \\
106.0 \\
106.0 \\
106.0 \\
106.0 \\
106.0 \\
106.0 \\
106.0 \\
106.0 \\
106.0\end{array}$ & $\begin{array}{r}0.7 \\
1.9 \\
2.7 \\
4.1 \\
5.4 \\
6.9 \\
8.1 \\
10.8 \\
43.5 \\
16.2 \\
18.9 \\
21.6 \\
24.3 \\
27.0\end{array}$ & $\begin{array}{r}0 \\
38 \\
201 \\
118 \\
49 \\
35 \\
05 \\
28 \\
10 \\
177 \\
3401 \\
49190 \\
3308 \\
3607\end{array}$ & $\begin{array}{l}0.000 E+00 \\
1.296 E=07 \\
6.710 E=07 \\
3.8 A 7 E=07 \\
1.609 E=07 \\
1.178 E=07 \\
1.531 F=07 \\
9.923 E=08 \\
3.530 E-08 \\
3.591 E=06 \\
1.164 E=05 \\
1.615=-05 \\
1.1335=05 \\
1.211 E=05\end{array}$ & $\begin{array}{l}0.000 E+00 \\
6.470 E=08 \\
3.357 E=07 \\
1.903 E=07 \\
2.245 E=08 \\
5.859 E-08 \\
7.656 E=08 \\
9.711 E-08 \\
1.767 E-08 \\
1.296 E=06 \\
5.019 E-06 \\
0.174 F-06 \\
5.664 E-06 \\
3.055 E-06\end{array}$ & $\begin{array}{l}200 \\
200 \\
200 \\
200 \\
200 \\
200 \\
200 \\
200 \\
200 \\
200 \\
200 \\
200 \\
200 \\
200\end{array}$ \\
\hline
\end{tabular}

AZINLTH HEIGHT EXPESUMF ENO EL/O OISTARCE DEGPFES METEAS GM-SFCICU.N IET/CIIN 1/SO, N NETEFS $x \perp O E+t$

\begin{tabular}{|c|c|c|c|c|c|}
\hline 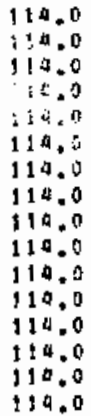 & $\begin{array}{l}0.3 \\
0.7 \\
1.0 \\
2.7 \\
4.1 \\
5.4 \\
6.9 \\
8.1 \\
9.0 \\
10.8 \\
13.5 \\
16.2 \\
18.9 \\
21.6 \\
24.3 \\
21.0\end{array}$ & $\begin{array}{r}120 \\
250 \\
212 \\
137 \\
084 \\
162 \\
170 \\
7 \\
74 \\
268 \\
1927 \\
3166 \\
5045 \\
6109 \\
10404 \\
8798\end{array}$ & 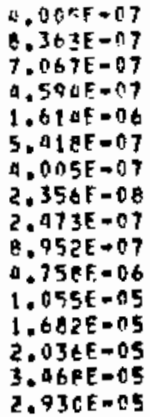 & $\begin{array}{l}2.002 E=07 \\
4.181 E=07 \\
3.534 E=07 \\
2.297 E=07 \\
0.068 E=07 \\
2.709 E=07 \\
2.002 E=07 \\
1.178 E=08 \\
1.237 E=07 \\
4.476 E=07 \\
2.379 E=06 \\
5.277 E=06 \\
8.410 E=06 \\
1.015 E=05 \\
1.734 E=05 \\
1.065 E=05\end{array}$ & $\begin{array}{l}200 \\
200 \\
200 \\
200 \\
200 \\
200 \\
200 \\
200 \\
200 \\
200 \\
200 \\
200 \\
200 \\
200 \\
200 \\
200\end{array}$ \\
\hline
\end{tabular}




\begin{tabular}{|c|c|c|c|c|c|}
\hline $\begin{array}{l}\text { DZIKUTH } \\
\text { DEGKEES }\end{array}$ & $\begin{array}{l}\text { HE IGHT } \\
\text { NE TERS }\end{array}$ & $\begin{array}{c}\text { EXPCSURE } \\
\text { GNASEC } / C U . N \\
\times S O E+t\end{array}$ & $\begin{array}{c}E / 0 \\
\sec / C \mathrm{Cu}\end{array}$ & $\begin{array}{l}E L / 0 \\
1 / 50 . N\end{array}$ & $\begin{array}{c}\text { DISTANCE } \\
\text { METERS }\end{array}$ \\
\hline $\begin{array}{l}122.0 \\
122.0 \\
122.0 \\
122.0 \\
122.0 \\
122.0 \\
122.0 \\
122.0 \\
122.0 \\
122.0 \\
122.0 \\
122.0 \\
12200 \\
122.0 \\
122.0\end{array}$ & $\begin{array}{r}0.7 \\
1.0 \\
2.7 \\
4.1 \\
5.4 \\
6.8 \\
8.1 \\
9.9 \\
10.8 \\
13.5 \\
16.2 \\
19.8 \\
21.8 \\
24.3 \\
27.0\end{array}$ & $\begin{array}{l}250 \\
201 \\
392 \\
791 \\
346 \\
541 \\
190 \\
487 \\
1268 \\
3678 \\
9738 \\
18794 \\
16292 \\
4709 \\
2775\end{array}$ & $\begin{array}{l}0.400 E=07 \\
6.71 \text { AE }=07 \\
1.307 E=06 \\
2.632 E=06 \\
1.154 F=06 \\
1.837 E=06 \\
6.360 E=07 \\
1.625 E=06 \\
4.221 E=06 \\
1.210 E=05 \\
3.246 F=05 \\
6.265 E=05 \\
5.427 E-05 \\
1.570 E=05 \\
4.251 F=06\end{array}$ & $\begin{array}{l}4.290 E=07 \\
3.357 E=07 \\
6.537 E=07 \\
1.319 E=08 \\
5.771 E=07 \\
9.197 E=07 \\
3.100 E=07 \\
1.127 E=07 \\
2.119 E=06 \\
6.048 E=06 \\
1.623 E=05 \\
3.132 E=05 \\
2.719 E=05 \\
7.809 E=06 \\
4.626 E=06\end{array}$ & $\begin{array}{l}200 \\
200 \\
200 \\
200 \\
200 \\
200 \\
200 \\
200 \\
200 \\
200 \\
200 \\
200 \\
200 \\
200 \\
200\end{array}$ \\
\hline
\end{tabular}

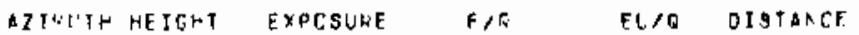
OEGREES NETERS GN-SEC/CL." SEC/CU.N I/SG," METERS $\times 1 \cap F+b$

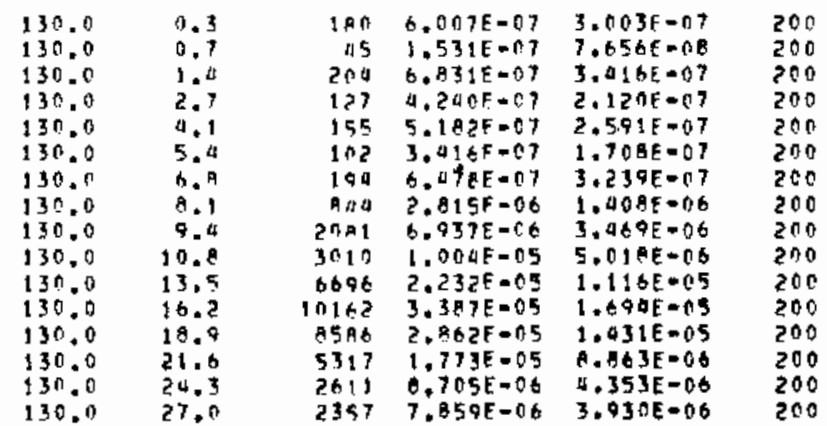

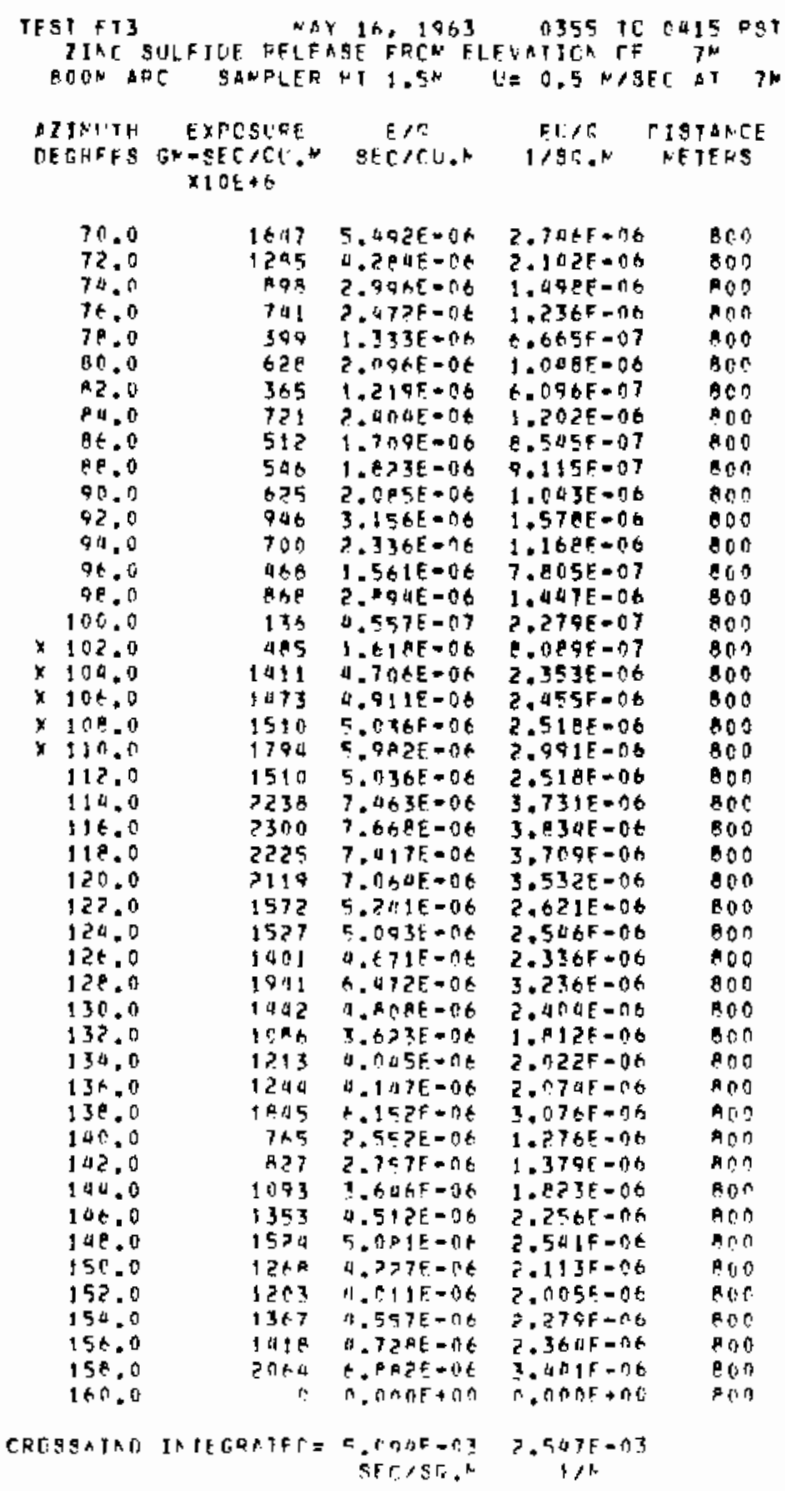


TOLFF I:ATH FOLLCW...

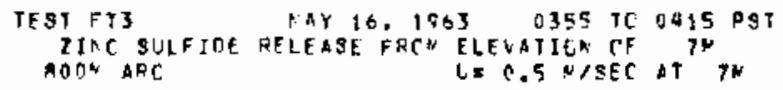

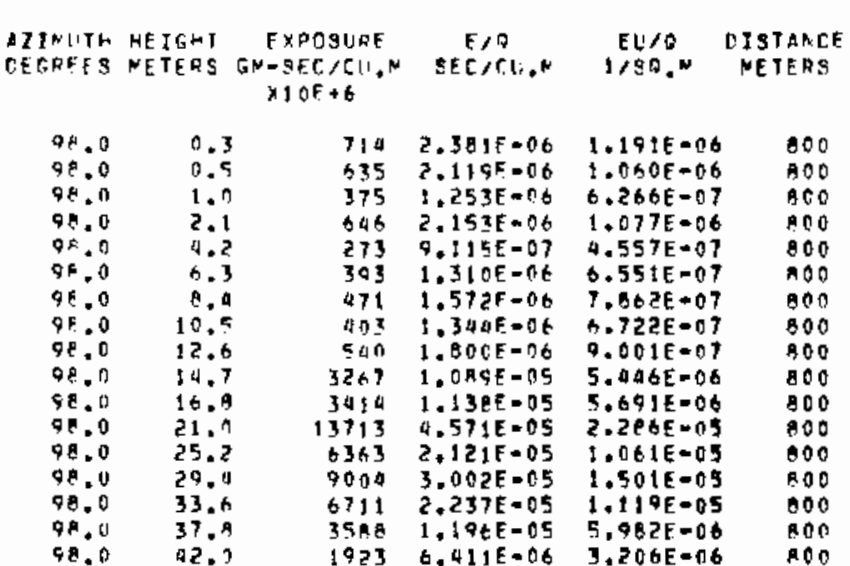

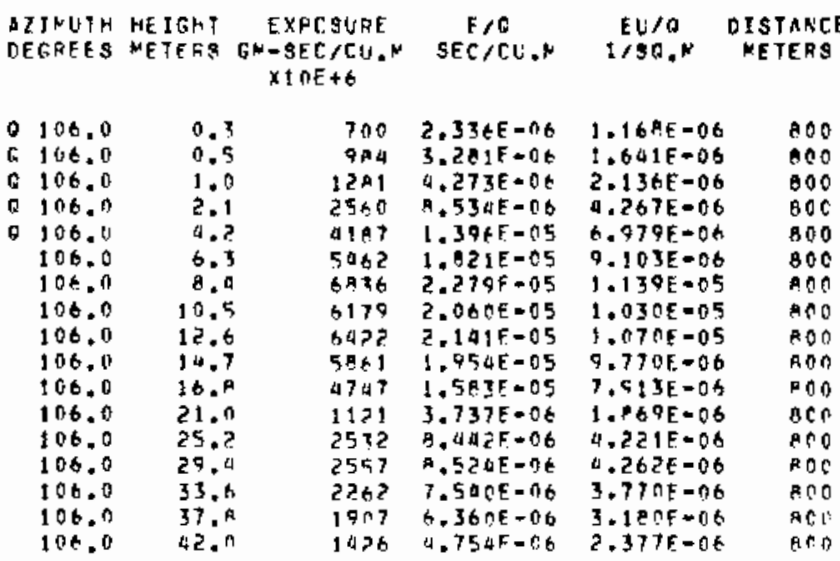

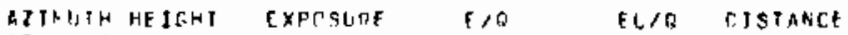

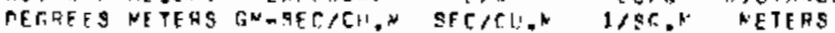
$\times 106+6$

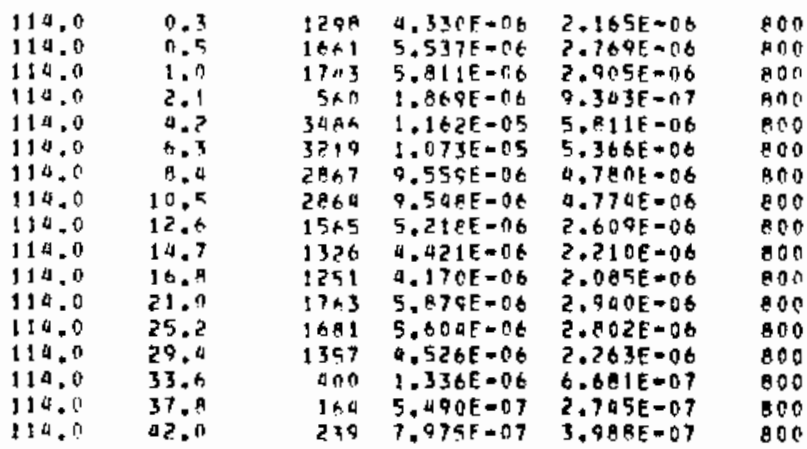

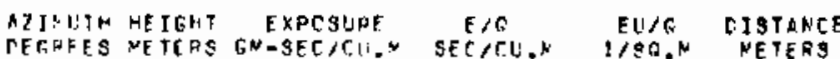
$\times 10 F+6$

\begin{tabular}{|c|c|c|c|c|c|}
\hline $\begin{array}{l}122.0 \\
122.0 \\
122.0 \\
122.0 \\
122.0 \\
122.0 \\
122.0 \\
122.0 \\
122.0 \\
122.0 \\
122.0 \\
122.0 \\
122.0 \\
122.0 \\
122.0 \\
122.0 \\
122.0\end{array}$ & $\begin{array}{l}0.3 \\
0.5 \\
1.0 \\
2.1 \\
0.2 \\
6.3 \\
8.4 \\
10.5 \\
12.6 \\
14.7 \\
16.8 \\
21.0 \\
25.2 \\
29.4 \\
33.4 \\
37.9 \\
42.7\end{array}$ & $\begin{array}{l}1407 \\
1596 \\
2135 \\
1739 \\
1019 \\
1917 \\
1504 \\
1926 \\
1104 \\
7 \geqslant 1 \\
104 \\
51 \\
12 \\
71 \\
79 \\
120\end{array}$ & 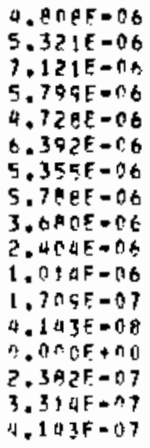 & $\begin{array}{l}2.004 E=06 \\
2.660 E=06 \\
3.560 E=06 \\
2.900 E=06 \\
2.364 E=06 \\
3.196 E=06 \\
2.677 E=08 \\
2.894 E=06 \\
1.840 E=06 \\
1.202 E=06 \\
5.070 E=07 \\
0.505 E=08 \\
2.072 E=08 \\
0.000 E+00 \\
1.191 E=07 \\
1.657 E=07 \\
2.07 ? E=07\end{array}$ & 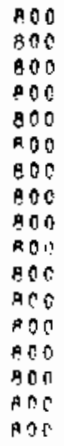 \\
\hline
\end{tabular}

DZINHTH HEIGHT EXPQSLAE FIO FIJG DISTANCE DETOFES METERE GNOSECICII.N SECICU.N IISO,N NETERS $\times 1 \cap E+A$

\begin{tabular}{|c|c|c|c|c|c|}
\hline $\begin{array}{l}130.0 \\
130.0 \\
130.0 \\
130.0 \\
130.0 \\
130.0 \\
130.0 \\
130.0 \\
130.0 \\
130.0\end{array}$ & $\begin{array}{r}0.3 \\
0.5 \\
1.0 \\
2.1 \\
4.7 \\
6.3 \\
8.0 \\
10.5 \\
12.6 \\
14.7\end{array}$ & $\begin{array}{r}1689 \\
2047 \\
1674 \\
2037 \\
1145 \\
1360 \\
5 \times 0 \\
542 \\
2 \leq 2 \\
41\end{array}$ & $\begin{array}{l}5.622 E=06 \\
3.625 E=06 \\
5.5 B 1 E=06 \\
6.791 E-06 \\
3.917 E-106 \\
0.535 E-06 \\
1.965 E=06 \\
1.709 E=06 \\
7.708 E=07 \\
1.367 E=07\end{array}$ & $\begin{array}{l}2.914 E=06 \\
3.412 E=06 \\
2.791 E=06 \\
3.395 E=06 \\
1.908 E=06 \\
2.267 E=06 \\
9.343 E=07 \\
8.505 E=07 \\
3.074 E=07 \\
6.936 E-08\end{array}$ & $\begin{array}{l}800 \\
800 \\
800 \\
800 \\
900 \\
800 \\
500 \\
800 \\
100 \\
900\end{array}$ \\
\hline
\end{tabular}




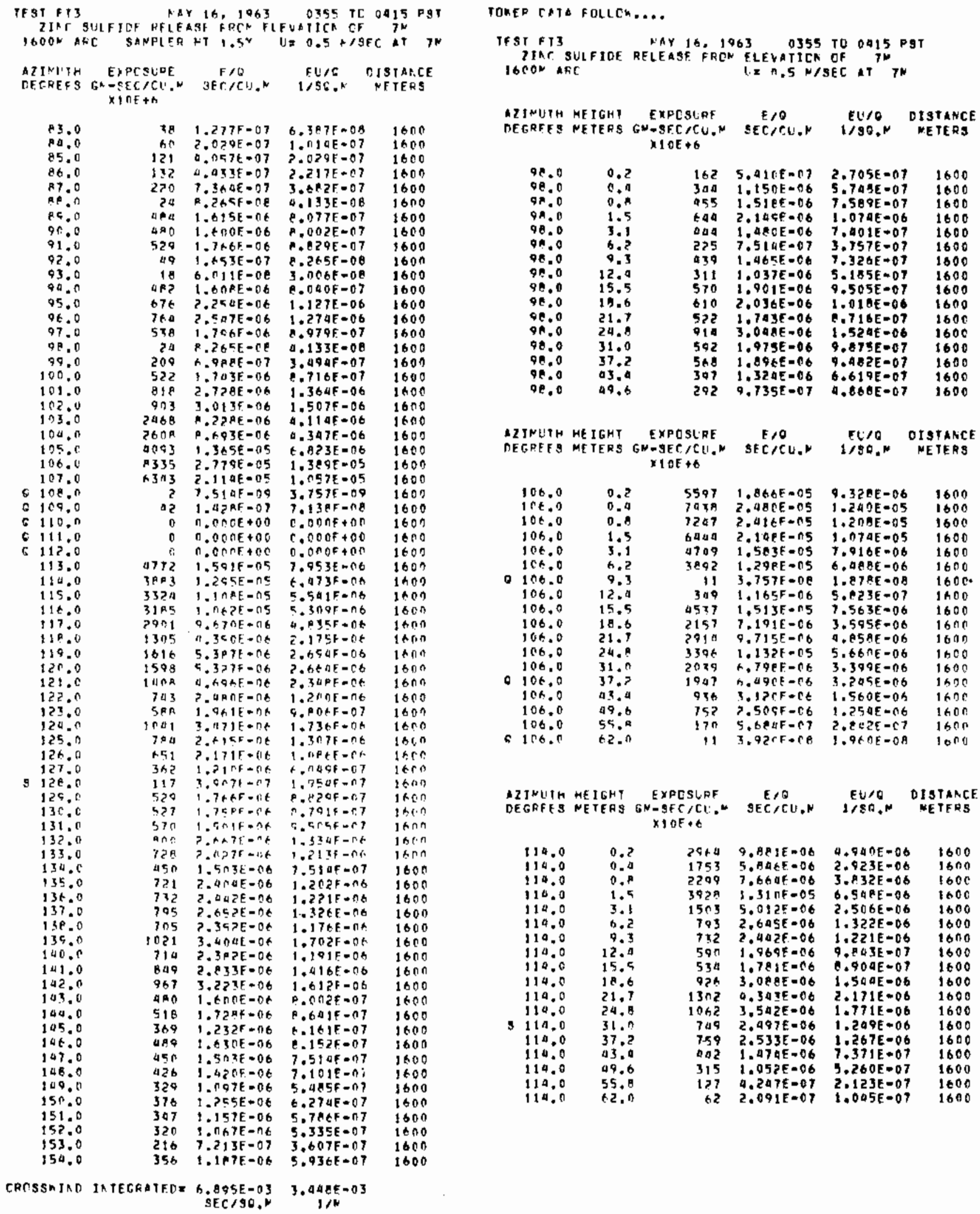




$\begin{array}{rr}\text { AIINUTH HEIGHI } \\ \text { DEGREFS HEYEAS G } \\ 122.0 & 0.2 \\ 122.0 & 0.9 \\ 122.0 & 0.9 \\ 122.0 & 1.5 \\ 122.0 & 3.1 \\ 122.0 & 6.2 \\ 122.0 & 9.3 \\ 122.0 & 12.4 \\ 122.0 & 15.5 \\ 122.0 & 18.6 \\ 122.0 & 21.7 \\ 122.0 & 24.8 \\ 122.0 & 31.0 \\ 122.0 & 37.2 \\ 122.0 & 62.0\end{array}$

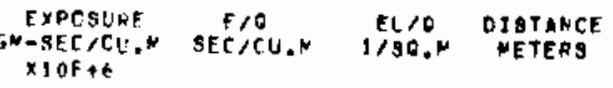

\begin{tabular}{|c|c|}
\hline 737 & $2.457 F=00$ \\
\hline 434 & $=-430$ \\
\hline 797 & $660 E=06$ \\
\hline 563 & $37 E F=06$ \\
\hline 696 & $327 E=06$ \\
\hline 471 & $570 F-06$ \\
\hline 38 & $1.277 E=07$ \\
\hline 396 & $1.322 E-C 6$ \\
\hline 13 & \\
\hline 169 & $635 E-07$ \\
\hline 250 & $693 E=07$ \\
\hline 105 & $3.506 E-07$ \\
\hline 70 & $.361 E-07$ \\
\hline 23 & $172 E-C B$ \\
\hline 1 & $53 a F=00$ \\
\hline
\end{tabular}

$1.229 E * 06$

1600

1.3 $30 E=06$

$9.392 E=07$

$1.161 E-06$

$7.052 E-07$

$0.387 k=08$
$0.612 E-07$

$2.254 \mathrm{E}-08$

2. $A 1 A E-07$

a. $046 \mathrm{E}-07$

$1.753 E=07$

$1.181 E-0$ ?

$3.9362=00$
$3,26=08$

1600

1600

1600

1600

1600

1600

1000

1600

1600

1600

1600

\begin{tabular}{|c|c|c|c|c|c|}
\hline $\begin{array}{l}\text { XNL.1H } \\
\text { FGEFSS }\end{array}$ & $\begin{array}{l}\text { HEIGHT } \\
\text { METEHS }\end{array}$ & $\begin{array}{c}\text { EXPCSURE } \\
\qquad M-S E C / C L+N \\
\times 1 \text { DE }\end{array}$ & $\begin{array}{c}E / F_{i} \\
S F E, r u, k\end{array}$ & 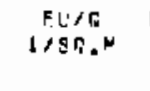 & $\begin{array}{l}\text { DISIAACE } \\
\text { NETFRS }\end{array}$ \\
\hline $\begin{array}{l}130.0 \\
130.0 \\
130.0 \\
130.0 \\
130.0 \\
130.0 \\
130.0 \\
130.0 \\
130.0\end{array}$ & $\begin{array}{l}0.2 \\
0.0 \\
0.7 \\
1.5 \\
3.1 \\
6.2 \\
9.3 \\
12.0 \\
15.5\end{array}$ & $\begin{array}{l}725 \\
5 \times 1 \\
426 \\
595 \\
324 \\
45 \\
17 \\
42 \\
67\end{array}$ & $\begin{array}{l}2.415 E-16 \\
1.935 E=06 \\
1.49 E E=06 \\
1.990 E=06 \\
1.082 E=06 \\
2.179 E=07 \\
2.930 E=07 \\
1.428 E=07 \\
2.250 F=07\end{array}$ & $\begin{array}{l}1.210 E=06 \\
9.893 E=07 \\
7.430 E=07 \\
9.910 E=07 \\
5.410 E=07 \\
1.090 E-07 \\
1.465 E=07 \\
7.138 E=00 \\
1.127 E=07\end{array}$ & $\begin{array}{l}1600 \\
1600 \\
1600 \\
1600 \\
1600 \\
1600 \\
1600 \\
1600 \\
1600\end{array}$ \\
\hline
\end{tabular}

1600

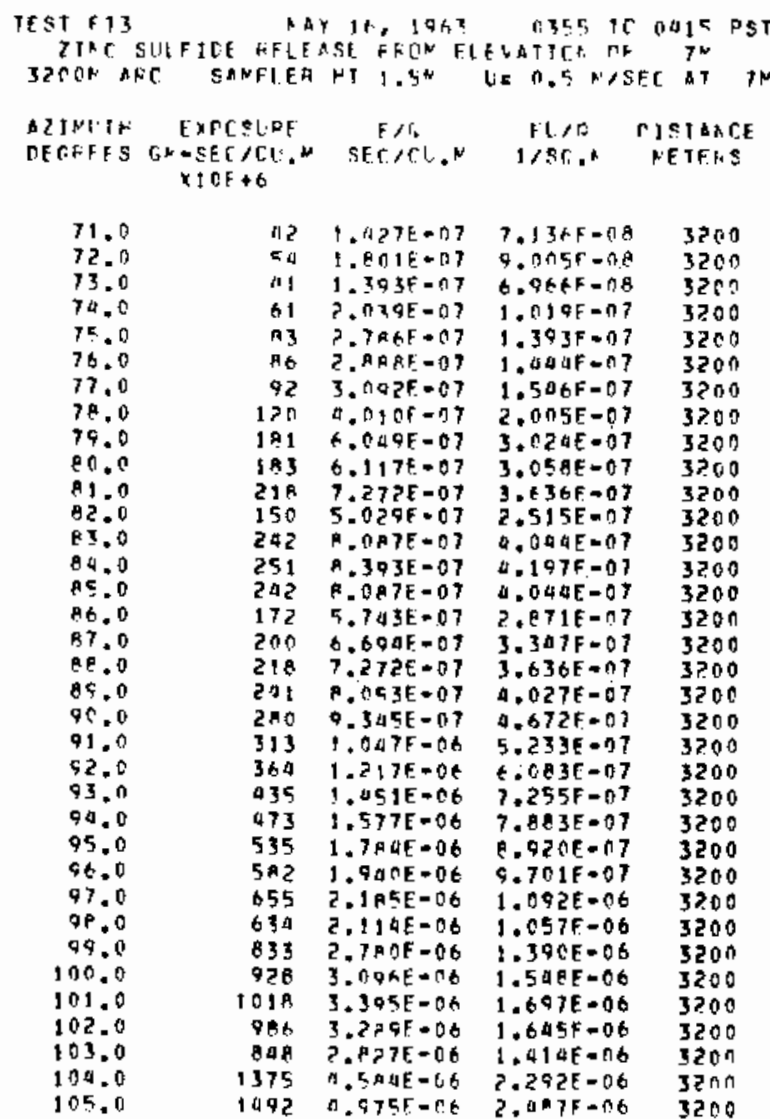

(1)

7106.0

107.0

$10 \% 0$

1189.0

$11 \% .0$

111.0

112.0

113.0

114.0

115.0

116.0

117.0

118.0

119.

120.0

121.0

122.0

$123 . ?$

120.0

$\$ 25.0$

126.0

127.0

t 2 e. 0

$12 \% .0$

130.0

131.0

137.0

133.0

134.0

135.0

136.0

137.0

138.0

$13 \% .0$

140.0

141.0

142.0

103.0

144.0

145.0

146.0

147.0

1 पै ह तो

149.0

150.0

$151.0^{\circ}$

152.0

153.0

154.0

155.0

$15 \mathrm{~h}, 0$

157.0

$15 \mathrm{~A} .0$

159.0

160.0

161.0

CRCssint $T$

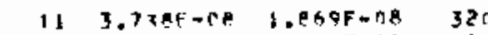

$16075.354500 \quad 2.579 F=06 \quad 3200$

1430 4.767E-RE 2.3P4E-06 32तA

.277 9 $752 E-06$ Z.528F-06 3200

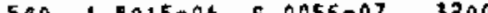

6 TO ?.20OE-06 1.100F-00 3200

$970 \quad 3.255 F-116 \quad 1.62 \mathrm{eF}=0.6 \quad 3 ? 00$

Q0) $3.004 E=n G \quad 1.502 F=1,6 \quad 3 ? 0 A$

812 ?.7NPF-116 $1.354 F-16 \quad 32 C 0$

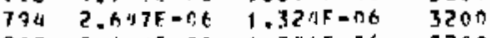

AC7 $2.601 E-96 \quad 1.346 F-r 6 \quad 3700$

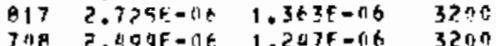

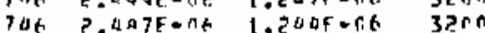

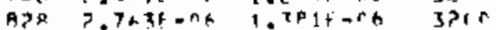

$530 \quad 1.747 E=106$ P. $35 E-C 7$ उ20A

$670 \quad 2.2365=06 \quad 1.118 F-16 \quad 3200$

$5531.845 E+00 \quad 9.2205-07 \quad 3200$

यत) $1.3345-06 \quad$ H. SQ4F-C7 3200

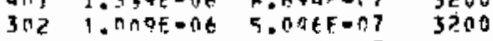

$\begin{array}{rrrr}216 & 7.2045-07 & 3.602 F-07 & 3200 \\ 2 & 6.796 E-09 & 3.396 E-09 & 3200\end{array}$

101 3. 30QE-07 $1.699 \mathrm{~F}-07$ 3200

74 2.4स1E-07 $1.240 E-07$ उ200

$973.262 k-07 \quad 1.6318-07 \quad 3200$

$110 \quad 3.670 \mathrm{E}-0) \quad 1.835 \mathrm{E}-[1 \quad 32 \mathrm{C} 0$

Qb 3.2 ?AE-07 $1.614 F-07 \quad 3700$

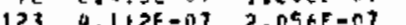

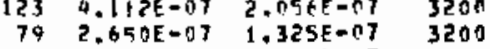

$79 \quad 3.370 F-07 \quad 1.6456-07 \quad 3200$

$97 \quad 3.262 E-0 ? \quad 1.631 F-07 \quad 3200$

90 3.02AE-07 $1.512 E-67 \quad 3200$

a) $3.262 E-07 \quad 1.631 E-07 \quad 3200$

$\begin{array}{llll}72 & 2.453 E-07 & 1.204 E-07 & 3200 \\ 84 & 7.050 E-07 & 1.410 E=07 & 3200\end{array}$

$125 \quad 9.100 E=07 \quad 2.090 E-07 \quad 3700$

$99 \quad 3.330 \mathrm{E}-07 \quad 1.665 \mathrm{E}-07 \quad 3200$

1 I $3.56 \mathrm{CE}-07 \quad 1.7 \mathrm{AAE}-07 \quad 3200$

90 3.024E-07 1.512E-07 3200

$03 \quad 2.107 E-0 ? \quad 1.053 E-07 \quad 3200$

$0.7 \times 6 E=07 \quad 1.393 E-07$
122

$169 \quad 5.601 E=07 \quad 2.820 E=07 \quad 3200$

$193 \quad 6.117 \mathrm{E}-07 \quad 3.058 \mathrm{C}=07 \quad 3200$

$\begin{array}{llll}123 & 6.117 E-07 & 3.05 E E-07 & 3200 \\ 227 & 7.578 E-07 & 3.789 E-07 & 3200\end{array}$

$278 \quad 9.209 E=07 \quad 4.000 E=07 \quad 3200$

$27 \quad 9.243 E-07 \quad 4.621 F-07 \quad 3200$

$246 \quad 0.223 E-07 \quad 4.112 E-07 \quad 3200$

$300 \quad 1.002 E-06 \quad 5.012 E-07 \quad 3200$

$\begin{array}{llll}300 & 1.002 E-06 & 5.012 E-07 & 3200 \\ 231 & 7.710 E-07 & 3.057 F-n 7 & 3200\end{array}$

$187 \quad 6.252 E-07 \quad 3.126 E-07 \quad 3200$

ITA $5.947 E-07 \quad 2.973 E-07 \quad 3200$

$134 \quad 4.0 A 5 E-07 \quad 2.243 F=07$

$\begin{array}{cc}\text { A TED } 6.71 E-03 & 3.135 E-03 \\ \text { SEC } 150 . N & 1 / N\end{array}$ 
TOMER TATA FDLLEM....

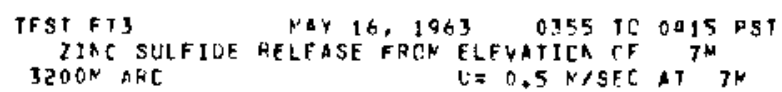

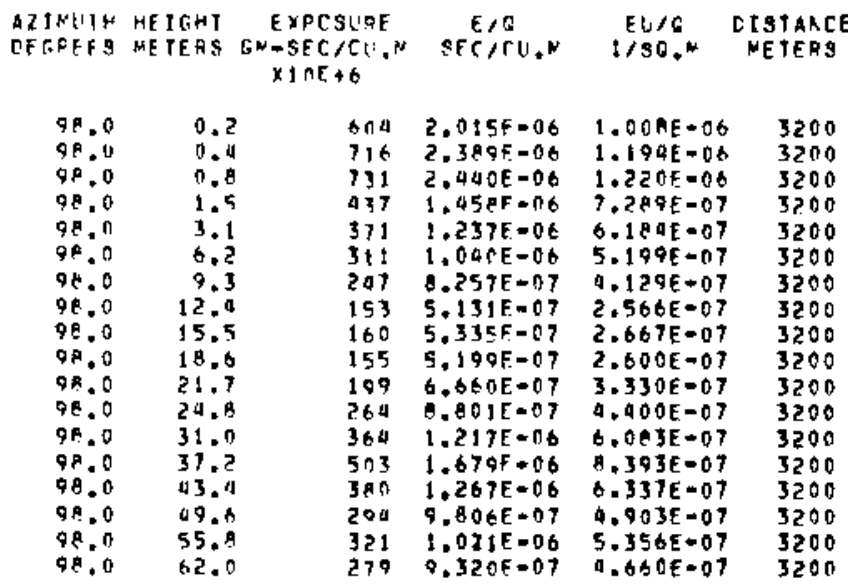

AZTNUTH HEIGHT EXPOSURE E/G EUTO CISTANCE DERRES METERS GNOSECIEU.M SECICU.N IIST.M NETERS $\times 10 E+6$

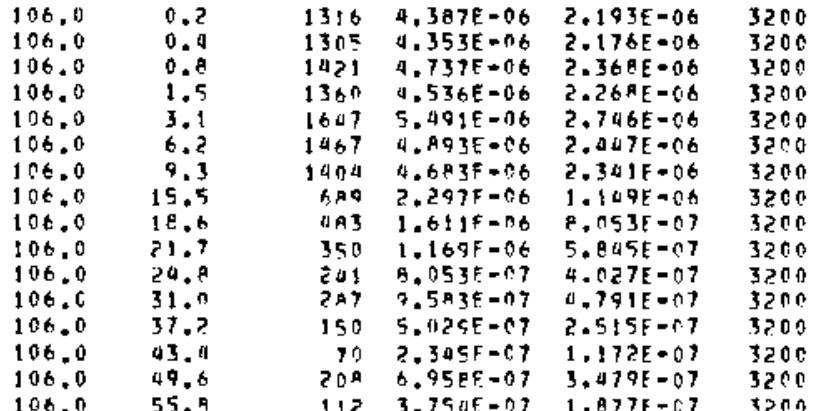

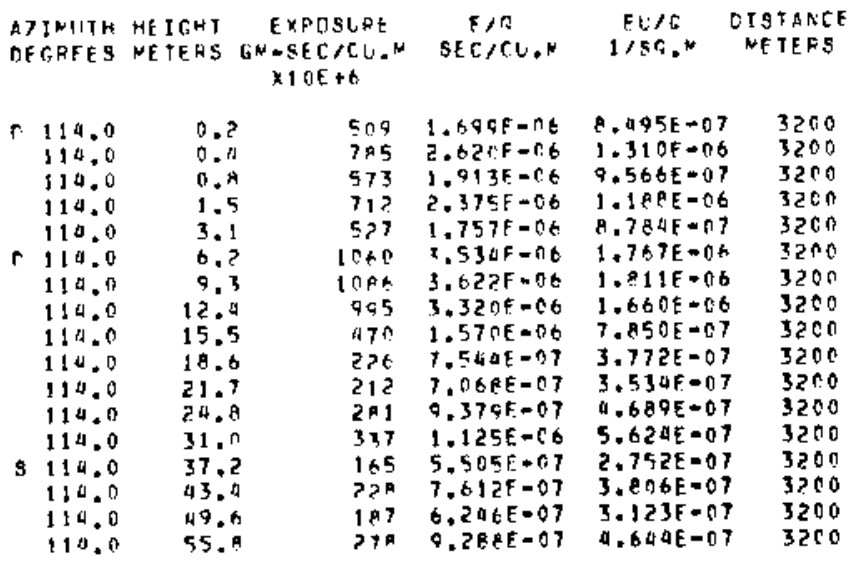

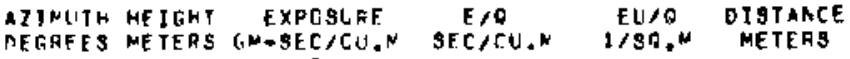
$\times 10 \mathrm{O}+\mathrm{B}$

\begin{tabular}{|c|c|c|c|c|c|}
\hline $\begin{array}{l}122.0 \\
122.0 \\
122.0 \\
122.0 \\
122.0 \\
122.0 \\
122.0 \\
122.0 \\
122.0 \\
122.0 \\
122.0 \\
122.0 \\
12200 \\
122.0 \\
122.0 \\
122.0 \\
122.0\end{array}$ & $\begin{array}{l}0.2 \\
0.0 \\
0.8 \\
1.5 \\
3.1 \\
6.2 \\
9.3 \\
12.0 \\
15.5 \\
18.5 \\
21.7 \\
24.8 \\
31.0 \\
37.2 \\
49.6 \\
55.8 \\
67.0\end{array}$ & $\begin{array}{l}713 \\
358 \\
567 \\
052 \\
633 \\
645 \\
399 \\
205 \\
66 \\
27 \\
15 \\
12 \\
6 \\
9 \\
9 \\
32 \\
15\end{array}$ & $\begin{array}{l}2.379 E=06 \\
1.196 E=06 \\
1.093 E-06 \\
1.505 E=06 \\
2.110 E=06 \\
2.219 E=06 \\
1.29 E E=06 \\
6.844 F=07 \\
2.204 E=07 \\
9.175 E=08 \\
5.097 E=08 \\
1.07 E E=08 \\
2.039 F=08 \\
3.058 E=08 \\
3.23 E E=08 \\
1.068 E=07 \\
1.197 E=07\end{array}$ & 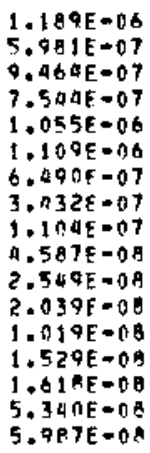 & $\begin{array}{l}3200 \\
3200 \\
3200 \\
3200 \\
3200 \\
3200 \\
3200 \\
3200 \\
3200 \\
3200 \\
3200 \\
3200 \\
3200 \\
3200 \\
3200 \\
3200 \\
3200\end{array}$ \\
\hline
\end{tabular}

QZISTITH MEIGHT EXPCSLRF F/R ELIG TANCE

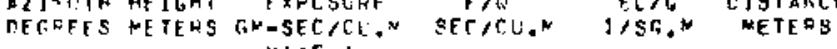
$x \in \mathbb{E}+6$

\begin{tabular}{|c|c|c|c|c|}
\hline $\begin{array}{l}130.0 \\
130.0 \\
130.0 \\
130.0 \\
130.0 \\
130.0 \\
130.0 \\
130.0 \\
130.0\end{array}$ & $\begin{array}{r}0.2 \\
0.4 \\
0.4 \\
1.5 \\
3.1 \\
6.2 \\
9.3 \\
12.4 \\
15.5\end{array}$ & $\begin{array}{l}73 \\
68 \\
53 \\
47 \\
34 \\
45 \\
53 \\
47 \\
43\end{array}$ & $\begin{array}{l}2.447 E=07 \\
2.277 F=07 \\
2.107 E-07 \\
2.073 F=07 \\
1.155 E-07 \\
1.035 E=07 \\
1.767 E=07 \\
1.427 E=07 \\
1.417 E=08\end{array}$ & $\begin{array}{l}1.223 E=07 \\
1.13 B E=07 \\
1.053 E=07 \\
1.036 E=07 \\
5.777 E=08 \\
9.175 E=00 \\
.835 E=06 \\
7.136 E=08 \\
2.20 Q E=08\end{array}$ \\
\hline
\end{tabular}


SERIES 163 EXPERIMENT: 4 (FT-4)

GROUND LEYEL AND TOWER SAMPLING 200-3200 M ARCS.

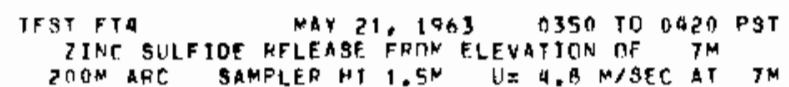

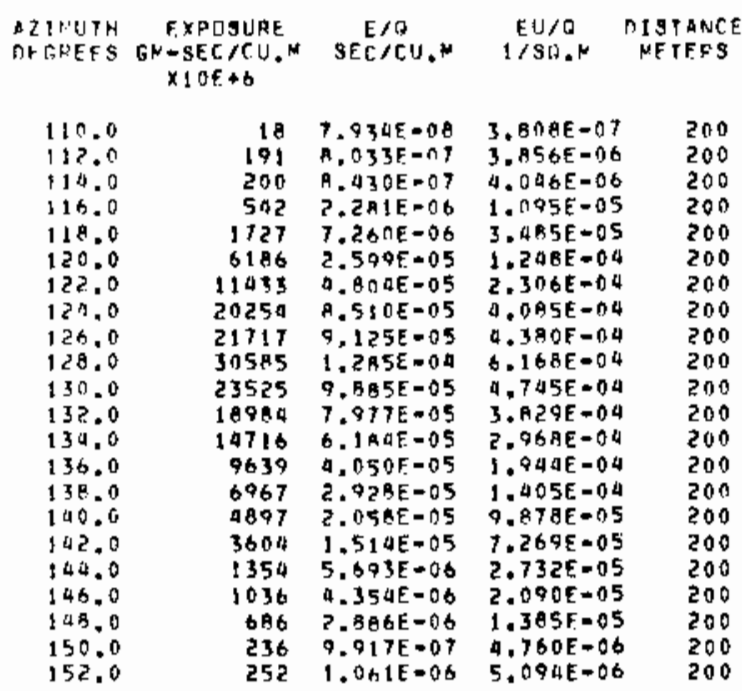

CHISSAIND INTEGRA FED= 5.2AME-03 $2.517 E-02$

TOKFE TATA FOLLLEA....

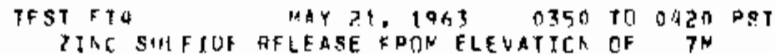

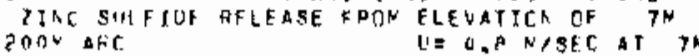

ATINLTH HEITHT EXPNDIJAE E/G ELAE DISTALCE NEGKFE METERS GN-SFC/CI: N SEC/CLA 1/SG,M NETERS $\times 10 \mathrm{E}+6$

$59 \% 0$ H. 0.3 2.545-07 $1.231 E-08$ 200

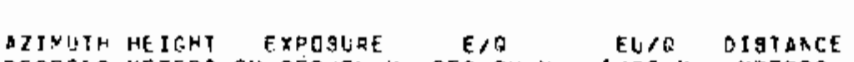
DEGEEYS NETFRS GM-SEC/CU.S SFC/CU,N 1/SO.N METERS $\times 10 F+6$

$106.0 \quad 0.3 \quad$ In $2.969 \varepsilon-07 \quad 1.425 E-06 \quad 200$

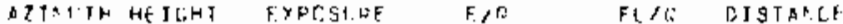

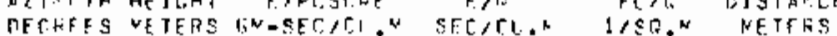
$\times 1 \cap \mathrm{AF}+\mathrm{A}$

\begin{tabular}{|c|c|c|c|c|c|}
\hline 110.0 & 12.3 & 227 & $0.9 Q 8 F=1] 7$ & $0.7951+06$ & $2^{n} \theta^{0}$ \\
\hline 114.0 & 0.7 & 376 & $1.161 f=06$ & $5.572 f=06$ & 200 \\
\hline 114.0 & 1. & 1.15 & A. $13 ; E=07$ & $2,5435=06$ & $20 n$ \\
\hline 114.0 & 2.7 & 179 & $7.5415-0.7$ & $3.42 \cap E=n A$ & 200 \\
\hline 110.0 & 4.1 & 1 A $\mathrm{P}$ & $7.9215-07$ & $3.80 ? E-06$ & 2 ก1 \\
\hline$\$ 14.0$ & 5.14 & 109 & $4.5115=07$ & 2.21 \&F $=16$ & $2 \cap 0$ \\
\hline 110.0 & A. A & ? : & $9.00 t E=.57$ & $4.3 Z 3 E=06$ & $2 \cap 0$ \\
\hline \pm 14.0 & $8 . t$ & 123 & 5.2 . & $7.500 E=00$ & 200 \\
\hline 114.0 & 9.4 & 105 & $0.131 F-07$ & $2.903 E=00$ & 200 \\
\hline 110.0 & 10. . & 250 & $1.996 E=06$ & $5.2608-06$ & 200 \\
\hline 110.0 & 13.5 & 236 & $928 E=07$ & $4.766 \mathrm{E}=0 \mathrm{~K}$ & 200 \\
\hline 114.0 & $10 . ?$ & 109 & $6.293 F-n 7$ & $3.02 \leq E-06$ & 2ก0 \\
\hline 110.0 & 18.9 & 74 & $3.1475-07$ & $1.510 \varepsilon-C 6$ & 200 \\
\hline 114.0 & 21.4 & $1 ?$ & $5.425 E-0 E$ & $2.6045=0 ?$ & 200 \\
\hline 10.0 & $24 . \pi$ & 12 & $.130 F=n \theta$ & $2.4675=07$ & 200 \\
\hline 1.4 .0 & 27.0 & 5 & $2.112 E=\pi B$ & 1. CIAE-OT & 201 \\
\hline
\end{tabular}

A7INITH HEIGHT EXPOSUNE E/D EUPO DISTANCE DFEFF $\times 1$ of +6

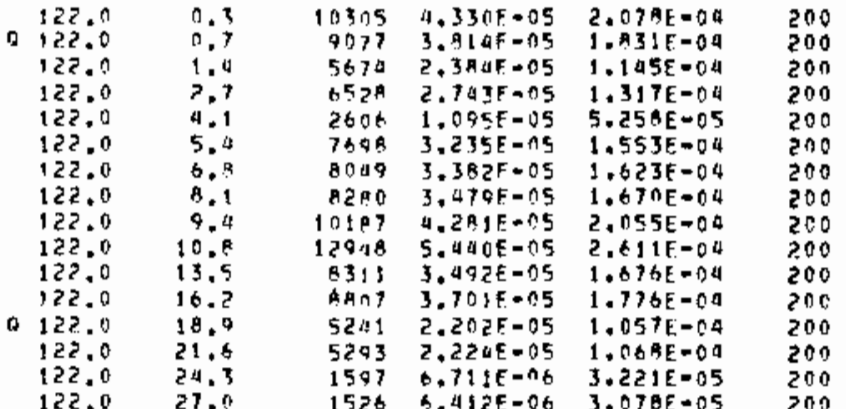

AZINETH HEIGHT EXPCSUPF F/G EL/G TISTAMCE

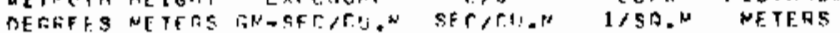

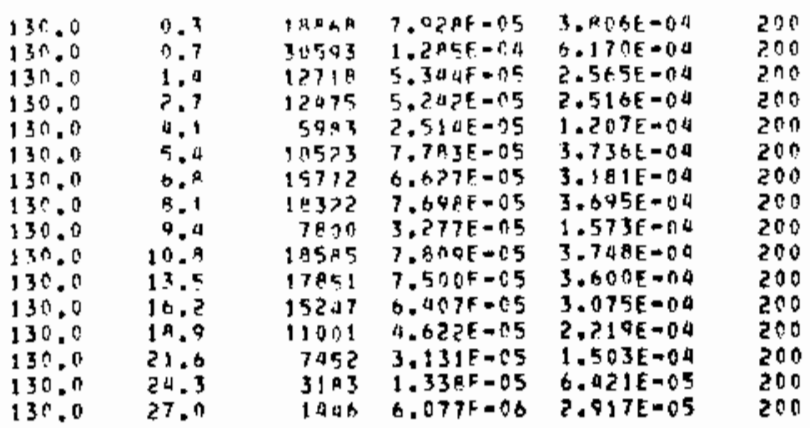




\begin{tabular}{|c|c|c|c|c|}
\hline $\begin{array}{l}\text { EST FTA } \\
\text { ZINE S } \\
\text { SCON AR }\end{array}$ & $\begin{array}{l}\text { HAY } \\
\text { ULFIOE RELEA } \\
\text { C MAMPLER }\end{array}$ & $\begin{array}{l}21,1963 \\
\mathrm{SE} F \mathrm{FPOM} \\
\mathrm{KT} 1.5 \mathrm{~F}\end{array}$ & $\begin{array}{rl}0350 & T 0 \\
\text { EVHTON DF } \\
\text { WE } 4,8 \mathrm{M} / \mathrm{SE}\end{array}$ & $\begin{array}{r}0420 \\
7 \mathrm{~m} \\
\text { EC AT }\end{array}$ \\
\hline $\begin{array}{l}\text { AI INIUTH } \\
\text { OEGREES }\end{array}$ & $\begin{array}{c}\text { EXPCSURE } \\
G M-S E C / C U, M \\
\times 1 O E+b\end{array}$ & $\begin{array}{c}E / Q \\
s E C / C U, M\end{array}$ & $\begin{array}{l}\text { EU/B } \\
1 / 30 . \mu\end{array}$ & $\begin{array}{l}\text { DIPTANCE } \\
\text { METERG }\end{array}$ \\
\hline $\begin{array}{l}120.0 \\
122.0 \\
124.0 \\
126.0 \\
128.0 \\
130.0 \\
132.0 \\
134.0 \\
136.0 \\
130.0 \\
140.0 \\
142.0 \\
104.0 \\
196.0 \\
148.0 \\
150.0 \\
160.0\end{array}$ & $\begin{array}{l}162 \\
557 \\
1502 \\
4000 \\
6726 \\
5892 \\
5892 \\
3035 \\
2042 \\
1525 \\
436 \\
335 \\
335 \\
296 \\
187 \\
65 \\
807\end{array}$ & 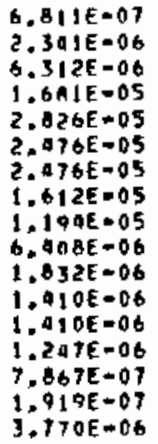 & 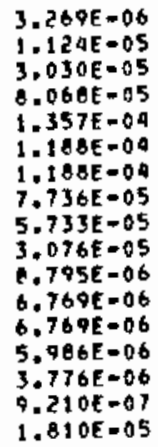 & $\begin{array}{l}800 \\
800 \\
800 \\
800 \\
100 \\
800 \\
800 \\
800 \\
800 \\
800 \\
800 \\
000 \\
800 \\
800 \\
800 \\
600 \\
000\end{array}$ \\
\hline $\sin (1)$ & TEGRATEDE & $\begin{array}{c}383 E-0 \\
3 E C / \$ 4 .\end{array}$ & $2.104 \mathrm{AE}-02$ & \\
\hline
\end{tabular}

TOWER TIATA FIJLLC:Y...

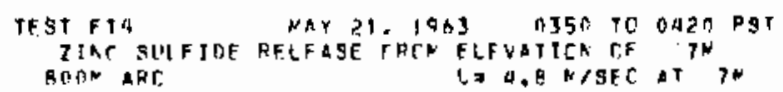

\begin{tabular}{|c|c|c|c|c|}
\hline 172.0 & 0.3 & $2+1$ & $1.097 E-06$ & $5.264 E-00$ \\
\hline 122.0 & 0.5 & 279 & $1.175 F=0 b$ & $5.0 A \cap E=06$ \\
\hline 122.0 & $1 . n$ & 2 कर & $1.102 F=0 E$ & $5,290 E-06$ \\
\hline 122.0 & 2,1 & 159 & $0.717 \mathrm{E} \cdot 07$ & $3.220 E-06$ \\
\hline 122.10 & 4,2 & 257 & $1.0 A J E=A G$ & $5.189 E-06$ \\
\hline 122.0 & 0,3 & 260 & $1.134 \mathrm{E}=0 \mathrm{~b}$ & $5.941 E-06$ \\
\hline 122.0 & 9.4 & 239 & $9 . R B \in E-07$ & $0.7365=06$ \\
\hline 172.0 & 10.5 & $31 k$ & $1.422 \mathrm{~F}-0 \mathrm{t}$ & $6.027 E=06$ \\
\hline 122.0 & 12.6 & 167 & $7,032 \leqslant-07$ & $3.376 E-n 6$ \\
\hline 122.0 & 14.7 & 252 & $1.060 E-06$ & $5.088 \varepsilon=06$ \\
\hline 122.0 & $10 . \mathrm{A}$ & 290 & $1.2348=06$ & $5.945 E-06$ \\
\hline 123.0 & 21.0 & 296 & $1.202 F=06$ & $5.769 E-06$ \\
\hline 122.0 & 25.7 & 123 & $5,190 E-07$ & $2.494 \mathrm{E}=06$ \\
\hline 122.0 & 29.0 & 99 & $0.17 \Delta E=07$ & $2.003 E=06$ \\
\hline 122.0 & 33.6 & 61 & $2.59 M E-07$ & $1.207 E-06$ \\
\hline 122.0 & 37.9 & 50 & 2.:02E-07 & $1 . \operatorname{cose}-06$ \\
\hline 120.0 & 02.0 & 50 & $2,306 E=07$ & $1.107 E-06$ \\
\hline
\end{tabular}

AZINUTH HETTMT EXPESHRE E/Q EUTG DISTARCE

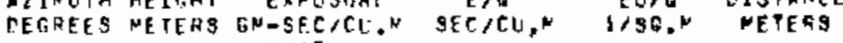

\begin{tabular}{|c|c|c|c|c|}
\hline 130.0 & 0.3 & 5204 & $2.225 E-05$ & $1.06 \mathrm{AE}-0$ \\
\hline 130.0 & 0.5 & 5926 & $2.4 \triangle P F-C 5$ & $1.375 E-14$ \\
\hline 130.0 & 1.0 & 2079 & $A, 73 \& E-\pi E$ & $0.19 A E-05$ \\
\hline 130.0 & 2.1 & 309 & $1.3 C 2 E-85$ & $6.250 \mathrm{E}-0 \mathrm{5}$ \\
\hline $1 \times 0.0$ & $4 . ?$ & 0325 & $2.65 P E=05$ & $1.27 \mathrm{bE}-0.4$ \\
\hline $130 \%$ & 6.3 & $39 \mathbb{u}^{2}$ & $1.666 E-05$ & $7.095 E=05$ \\
\hline 130,0 & 0.4 & $340 ?$ & $1.470+0.05$ & $7.050 E=05$ \\
\hline 130.0 & 10.5 & 2117 & $\therefore .995 \mathrm{E}-\mathrm{Cb}$ & $4.270 \mathrm{E}-05$ \\
\hline $35: 0$ & 13.6 & $2 \times 83$ & $1.212 \mathrm{~F}-\mathrm{r} 5$ & $5.81 \mathrm{GE}=015$ \\
\hline 130.0 & 14,7 & 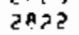 & 1.) JeEF=ns & $5.6910-05$ \\
\hline 130.0 & ito. & 5231 & $1.770 F=n s$ & $A .53$ U1. 05 \\
\hline 1300 & $21 . n$ & Ptan & $1.196 f=n 5$ & $5.74 t \varepsilon-05$ \\
\hline $13 x=\pi$ & 75,7 & $7 \times 3$ & $3, m p \in f=n t$ & $j .4 P A F-015$ \\
\hline $13 n .0$ & 29,4 & ian & 3.2 JF-CE & $1.575 t+05$ \\
\hline 130.0 & 33.6 & $7 \times 19$ & $3,103=06$ & $1 . \wedge Q[-r 5$ \\
\hline 130.0 & 37. & 419 & $1,76 C F+C E$ & C. Ad \\
\hline 130.0 & 42.7 & $1 \neq 1$ & $6,7725=0.7$ & $3.25^{n} \mathrm{E}-06$ \\
\hline
\end{tabular}

THST FIA I $600 \mathrm{~N}$ ARC AAMPLER HT $1.5 \mathrm{~N}$ UE $4.8 \mathrm{M} / \mathrm{SEC}$ AT $\mathrm{MM}$ RIMUTH EXPOSURE
DEGRES GM-SEC/CU.M SE/D
XIOE+6

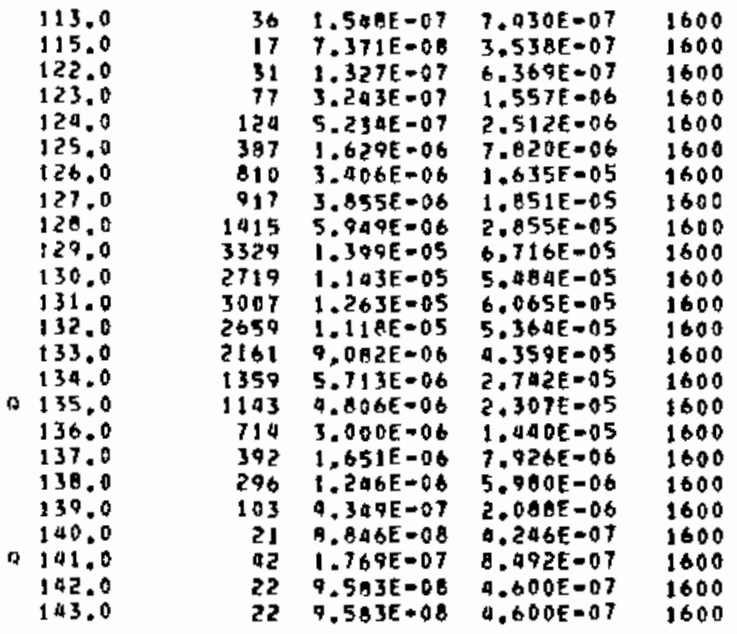

CRNSSNINO JNTEGRATEOE $2.582 E-03 \quad 1.240 E-02$ SEC/98.N. IN

TOYFF TATA GCLLCA...

IFOT FT4 FAY $21,1963 \quad 0350$ TC $0420 \mathrm{PgT}$

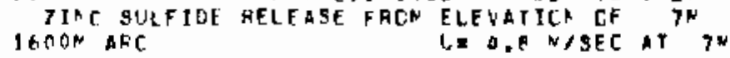

\begin{tabular}{|c|c|c|c|c|c|}
\hline $\begin{array}{l}\text { AITIIIH } \\
\text { DEGTFES }\end{array}$ & $\begin{array}{l}\text { HE IGNT } \\
\text { NETERS }\end{array}$ & $\begin{array}{c}\text { EXPCSLPE } \\
G N=S E C / C(1-* \\
\times 10 E+t\end{array}$ & $\begin{array}{c}E / B \\
\operatorname{sEC} / \mathrm{TU} .\end{array}$ & $\begin{array}{c}E v / 6 \\
1 / 80 . *\end{array}$ & $\begin{array}{l}\text { DISTANCE } \\
\text { METERS }\end{array}$ \\
\hline $\begin{array}{l}122.0 \\
122.0 \\
122.0 \\
122.0 \\
122.0 \\
122.0 \\
122.0 \\
122.0 \\
12200 \\
122.0\end{array}$ & $\begin{array}{r}1.5 \\
3.1 \\
6.2 \\
8.3 \\
12.0 \\
15.5 \\
18.6 \\
21.7 \\
24.8 \\
31.0\end{array}$ & $\begin{array}{r}20 \\
24 \\
? \\
22 \\
1 ? \\
9 \\
? \\
?\end{array}$ & 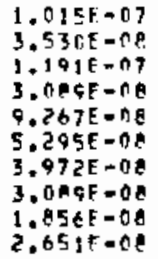 & 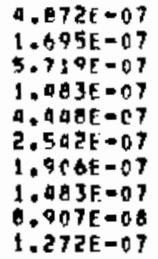 & $\begin{array}{l}1600 \\
1600 \\
1000 \\
1600 \\
1600 \\
1600 \\
1600 \\
1600 \\
1600 \\
1600\end{array}$ \\
\hline
\end{tabular}

AZJMLTM HETGHT EXPCSUPE E/G EU/O DISTANCE

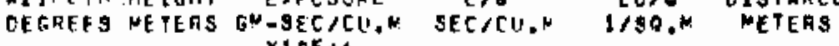

\begin{tabular}{|c|c|c|c|c|c|}
\hline $\begin{array}{l}130.0 \\
130.0\end{array}$ & 0.2 & $\begin{array}{l}2548 \\
2449\end{array}$ & $\begin{array}{l}1.071 \mathrm{E}=05 \\
1.236 \mathrm{E}=05\end{array}$ & $\begin{array}{l}5.1396-05 \\
5.540 \mathrm{E}-05\end{array}$ & $\begin{array}{l}1600 \\
1600\end{array}$ \\
\hline $\begin{array}{l}130.0 \\
130.0\end{array}$ & 0.4 & $\begin{array}{l}2449 \\
3256\end{array}$ & $\begin{array}{l}1.236 E=05 \\
1.36 E F=05\end{array}$ & $\begin{array}{l}3,50 E=05 \\
6.563 E=05\end{array}$ & $\begin{array}{l}1600 \\
1600\end{array}$ \\
\hline 130.0 & 1,5 & 40 & $1.7 ? 1 F=07$ & $0.261 \varepsilon-07$ & 1600 \\
\hline 130.0 & $3 .:$ & 1639 & $6 . \sec E=06$ & $3.306 E-05$ & 1600 \\
\hline 130.0 & 0.2 & 1515 & $6.360 E-16$ & $3.057 E .05$ & 1600 \\
\hline 130.0 & 9.1 & 3 & $t, 32 \& F=0 k$ & 0.35 aE $=08$ & 1600 \\
\hline $13 n+0$ & 12. & 14 & $5.9575-07$ & ROOE $=06$ & 1600 \\
\hline $13 n .0$ & 15. & 805 & $3.760 E-016$ & $1.207 \mathrm{E}=05$ & 1600 \\
\hline 30. & 10 & $\ln 19$ & $7.643 E=0 \mathrm{~A}$ & $3.669 E-0.5$ & 1600 \\
\hline 130.0 & 21.7 & 28 & $1.191 E=07$ & $5,719 E=07$ & 1600 \\
\hline 130.0 & $2 n$ & 874 & $3.674 \mathrm{~F}-16$ & $1.764 E-05$ & lono \\
\hline 130 & $3 i$ & 511 & $2.147 F-10$ & $1.031 E-05$ & 1600 \\
\hline & 37. & & $5.4 A E E-\cap E$ & $1.654 \mathrm{E}=07$ & 1600 \\
\hline 130.0 & 43. & 201 & $1.015 \mathrm{~F}-06$ & $4.86 t \xi=06$ & 1600 \\
\hline $13 n .0$ & & 107 & $6.2036-07$ & $2.971 E-06$ & 1600 \\
\hline 130 & & AQ & $7645-07$ & COTE-06 & 1600 \\
\hline $13 n$. & & 14 & $.431 E=07$ & $.071 E-07$ & 1600 \\
\hline
\end{tabular}




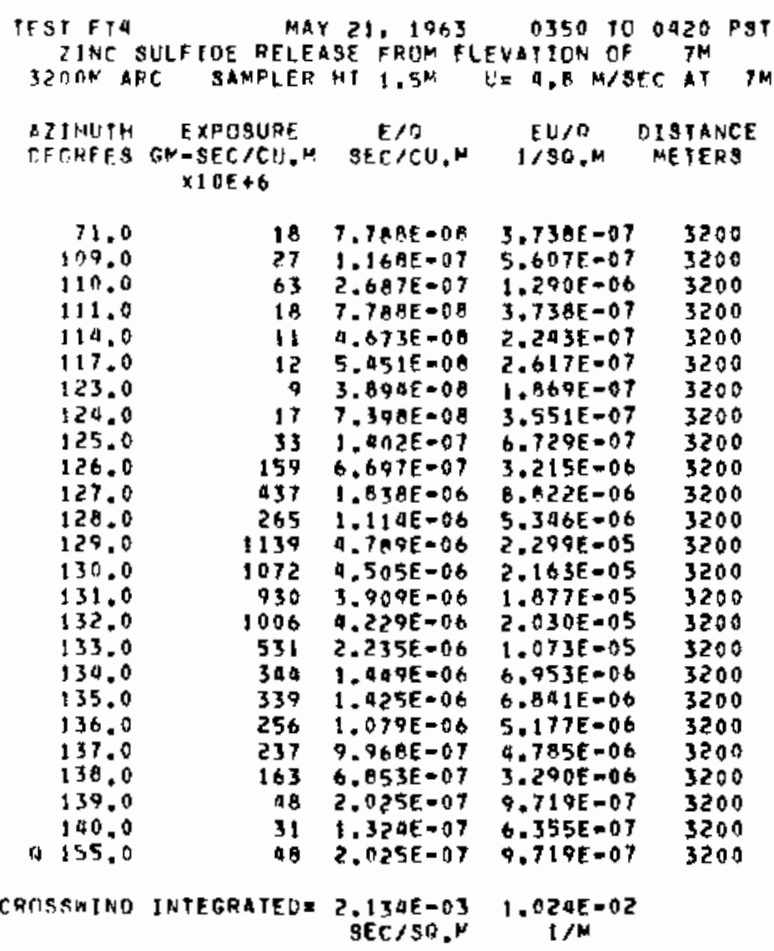

BHAER TAT HOLLCb....

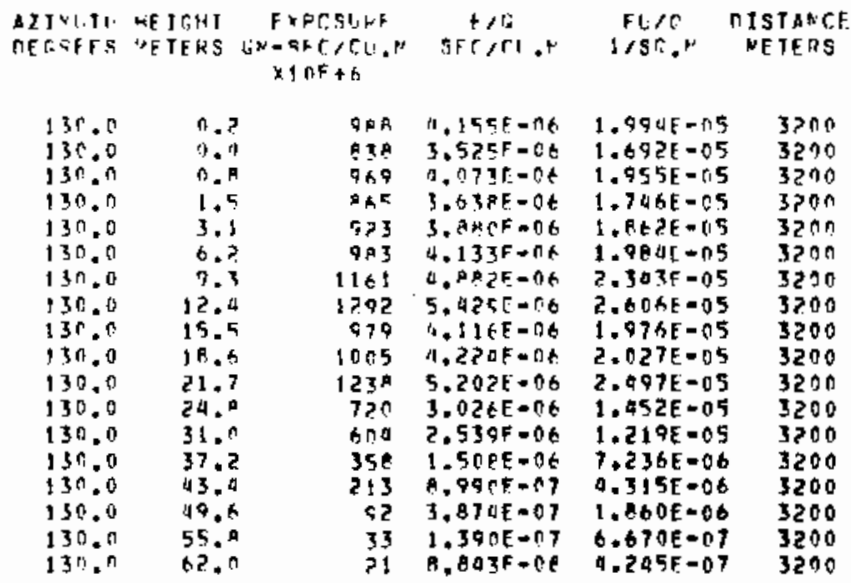

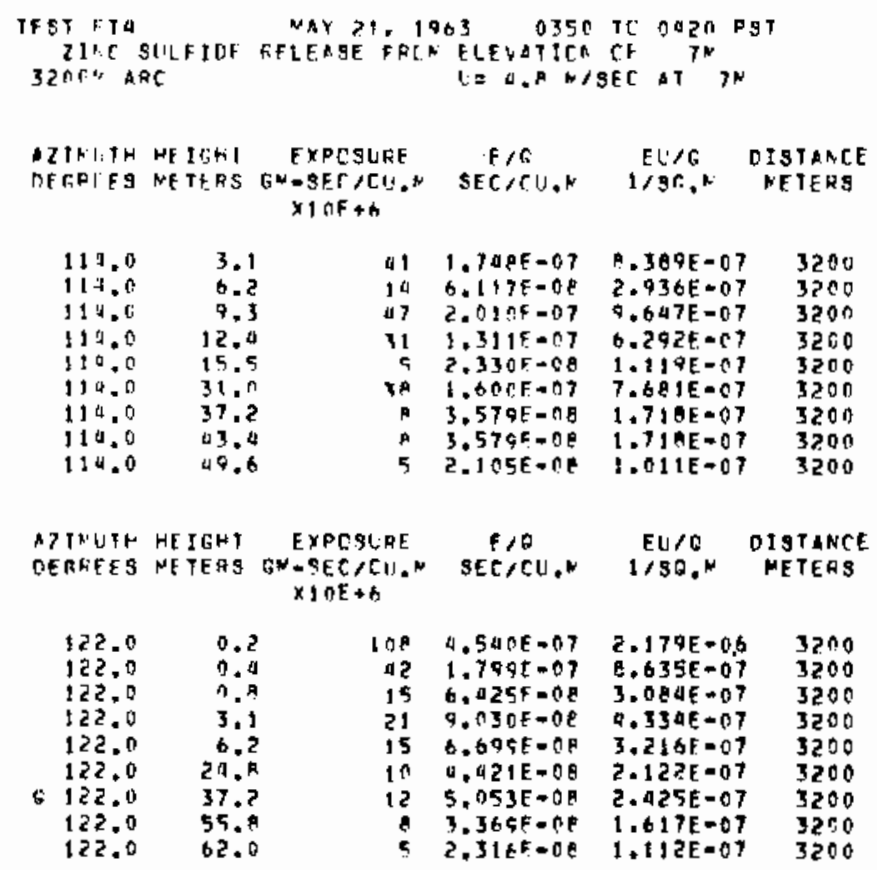


SERIES 163 EXPERIMENT: 5 ( FT-5)

GROJND LEYEL AMD TONER SAMPLING 200-3200 M ARCS.

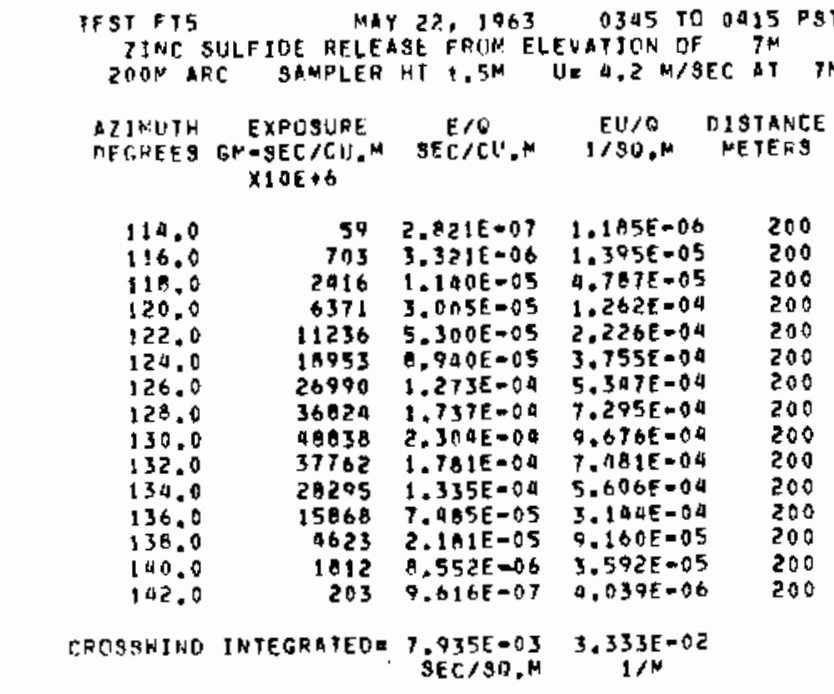

TOWER DAT FDLLOW....

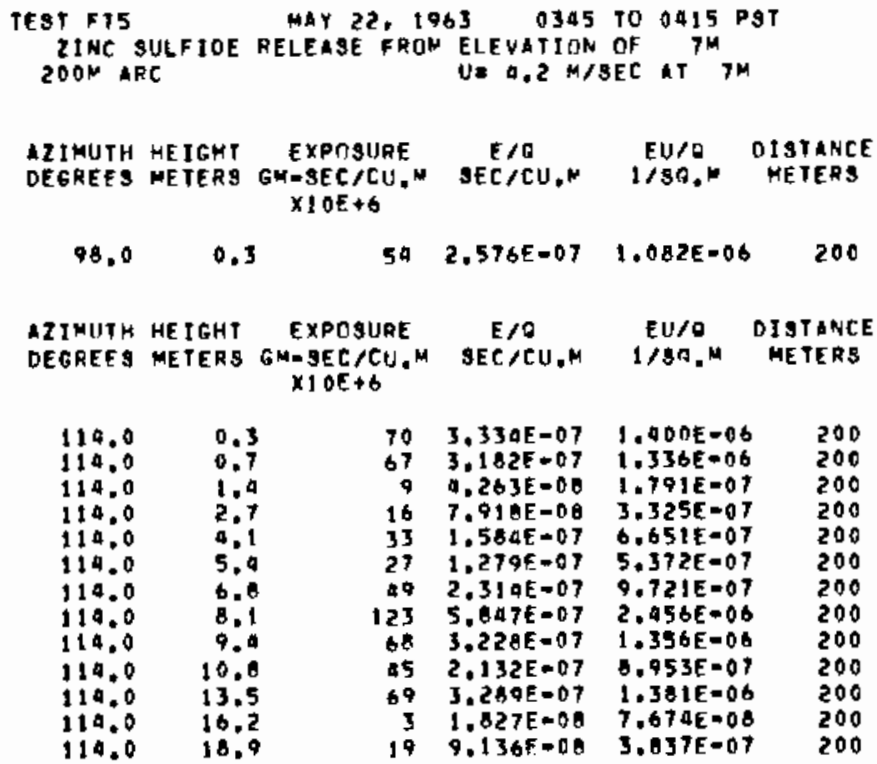

\begin{tabular}{|c|c|c|c|c|c|}
\hline $\begin{array}{l}\text { AIIMUYH } \\
\text { OEGREES }\end{array}$ & $\begin{array}{l}\text { HE IEHT } \\
\text { METERS }\end{array}$ & $\begin{array}{c}\text { EXPOSURE } \\
\text { GW-SEC CW.M } \\
\times 10 E+6\end{array}$ & $\begin{array}{c}E / 0 \\
s E C / C U . M\end{array}$ & $\begin{array}{l}E \cup / 0 \\
1 / 50 . M\end{array}$ & $\begin{array}{l}\text { DISTANCE } \\
\text { METERS }\end{array}$ \\
\hline $\begin{array}{l}122.0 \\
122.0 \\
122.0 \\
122.0 \\
122.0 \\
122.0 \\
122.0 \\
122.0 \\
122.0 \\
122.0 \\
122.0 \\
122.0 \\
122.0 \\
122.0 \\
122.0 \\
122.0\end{array}$ & $\begin{array}{l}0.3 \\
0.7 \\
1.0 \\
2.7 \\
4.1 \\
5.4 \\
6.8 \\
0.1 \\
9.4 \\
10.8 \\
13.5 \\
16.2 \\
18.9 \\
21.6 \\
24.3 \\
27.0\end{array}$ & $\begin{array}{r}5460 \\
12351 \\
3961 \\
4570 \\
5549 \\
6370 \\
6972 \\
7934 \\
7034 \\
2861 \\
6865 \\
7113 \\
6010 \\
3011 \\
963 \\
355\end{array}$ & $\begin{array}{l}2.577 E=05 \\
5.828 E=05 \\
1.860 E=05 \\
2.156 E=05 \\
2.618 E=05 \\
3.005 E=05 \\
3.209 E=05 \\
3.743 E=05 \\
3.318 E=05 \\
1.350 E=05 \\
3.238 E=05 \\
3.355 E=05 \\
2.835 E=05 \\
1.420 E=05 \\
9.543 E=06 \\
1.677 E=06\end{array}$ & $\begin{array}{l}1.003 E=04 \\
2.407 E=04 \\
7.04 B E=05 \\
9.056 E=05 \\
1.099 E=04 \\
1.262 E=04 \\
1.381 E=04 \\
1.572 E=04 \\
1.394 E=04 \\
5.669 E=05 \\
1.360 E=04 \\
1.409 E=04 \\
1.191 E=04 \\
5.965 E=05 \\
1.908 E=05 \\
7.043 E=06\end{array}$ & $\begin{array}{l}200 \\
200 \\
200 \\
200 \\
200 \\
200 \\
200 \\
200 \\
200 \\
200 \\
200 \\
200 \\
200 \\
200 \\
200 \\
200\end{array}$ \\
\hline $\begin{array}{l}\text { AZIMUTM } \\
\text { DEGREES }\end{array}$ & $\begin{array}{l}\text { HE IGHT } \\
\text { ME TERS }\end{array}$ & $\begin{array}{c}\text { EXPOSURE } \\
\text { GM+SEC } / C U . M \\
\times 10 E+6\end{array}$ & $\begin{array}{c}E / \theta \\
\text { SEC/CU,M }\end{array}$ & $\begin{array}{l}E U / 0 \\
1 / 30 . m\end{array}$ & $\begin{array}{l}\text { DISTRNCE } \\
\text { METERS }\end{array}$ \\
\hline $\begin{array}{l}130.0 \\
130.0 \\
130.0 \\
130.0 \\
130.0 \\
130.0 \\
130.0 \\
130.0 \\
130.0 \\
130.0 \\
130.0 \\
130.0 \\
130.0 \\
130.0 \\
130.0 \\
130.0\end{array}$ & $\begin{array}{l}0.3 \\
0.7 \\
1.4 \\
2.7 \\
0.1 \\
5.4 \\
6.8 \\
8.1 \\
9.9 \\
10.9 \\
13.5 \\
16.2 \\
18.9 \\
21.6 \\
29.3 \\
27.0\end{array}$ & $\begin{array}{l}39830 \\
49985 \\
11086 \\
23204 \\
16460 \\
30807 \\
27728 \\
34139 \\
34206 \\
20395 \\
29487 \\
18478 \\
13989 \\
8427 \\
2300 \\
1090\end{array}$ & $\begin{array}{l}1.879 E=04 \\
2.334 E=04 \\
5.229 E=05 \\
1.099 E-04 \\
7.764 E=05 \\
1.453 E=04 \\
1.308 E=04 \\
1.610 E=04 \\
1.614 E=04 \\
1.387 E=04 \\
1.391 E=04 \\
8.952 E=05 \\
6.599 E=05 \\
3.975 E=05 \\
1.095 E=05 \\
5.146 E=06\end{array}$ & $\begin{array}{l}7.893 E=04 \\
9.00 A E=04 \\
2.196 E=04 \\
4.615 E=04 \\
3.261 E=04 \\
6.103 E=04 \\
5.493 E=04 \\
6.764 E=04 \\
6.777 E=04 \\
5.024 E=04 \\
5.842 E=04 \\
3.760 E=04 \\
2.771 E=04 \\
1.670 E=04 \\
4.557 E=05 \\
2.161 E=05\end{array}$ & $\begin{array}{l}200 \\
200 \\
200 \\
200 \\
200 \\
200 \\
200 \\
200 \\
200 \\
200 \\
200 \\
200 \\
200 \\
200 \\
200 \\
200\end{array}$ \\
\hline
\end{tabular}




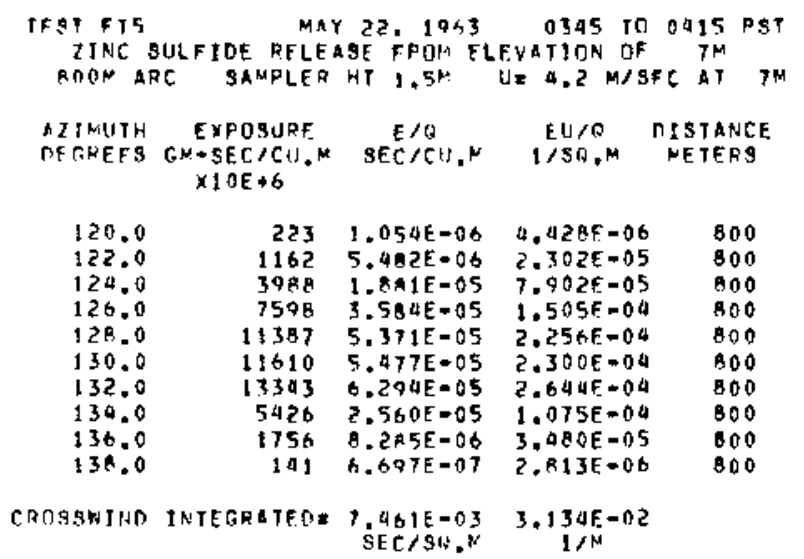

TOWER DATA FOLLOW....

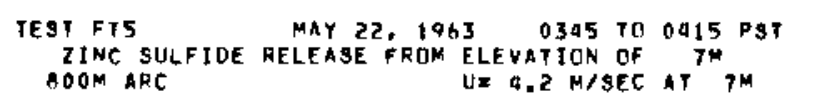

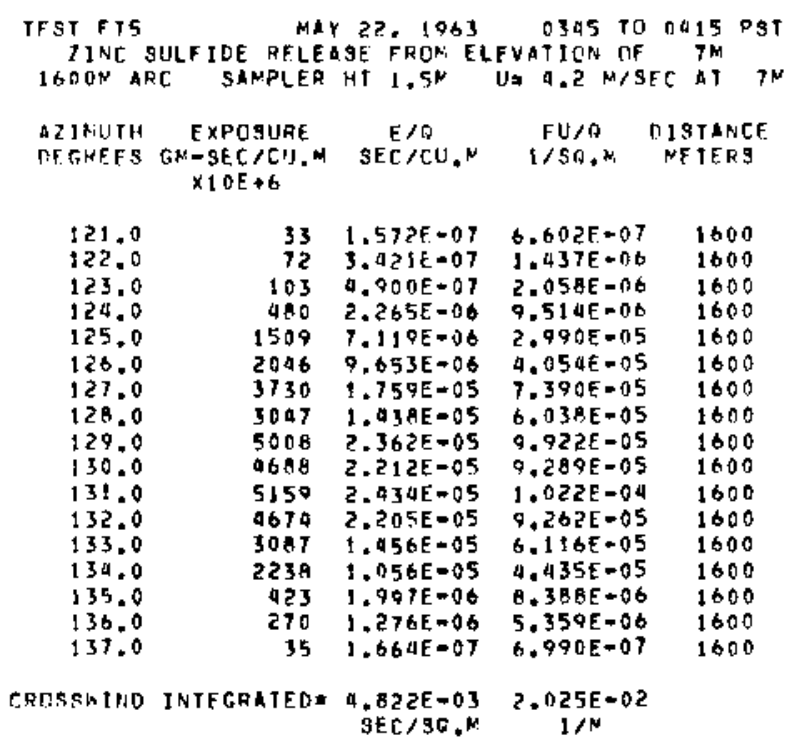

\begin{tabular}{|c|c|c|c|c|c|}
\hline $\begin{array}{l}\text { AZIMUTH } \\
\text { OEGREES }\end{array}$ & $\begin{array}{l}\text { HE IGHT } \\
\text { ME TEAS }\end{array}$ & $\begin{array}{c}\text { EXPOSURE } \\
\text { GM-SEC } C U . M \\
\times 10 E \$ 6\end{array}$ & $\begin{array}{c}E / Q \\
S E[/ C U, M\end{array}$ & $\begin{array}{l}E U / O \\
1 / 90 . M\end{array}$ & $\begin{array}{l}\text { DI ST ANC } \\
\text { METERS }\end{array}$ \\
\hline $\begin{array}{l}122.0 \\
122.0 \\
122.0 \\
122.0 \\
122.0 \\
0122.0 \\
122.0 \\
122.0 \\
122.0 \\
122.0 \\
122.0 \\
122.0 \\
122.0 \\
122.0 \\
122.0 \\
122.0 \\
122.0\end{array}$ & 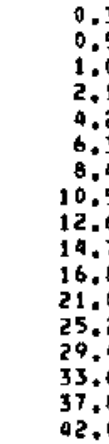 & $\begin{array}{r}1634 \\
1263 \\
690 \\
196 \\
635 \\
226 \\
623 \\
1151 \\
813 \\
573 \\
806 \\
536 \\
213 \\
93 \\
6 \\
2 \\
21\end{array}$ & $\begin{array}{l}7.710 E=06 \\
6.053 E=06 \\
3.258 E=06 \\
3.759 E=06 \\
3.941 E=06 \\
1.066 E=06 \\
2.940 E=06 \\
5.032 E=06 \\
3.035 E=06 \\
2.704 E=06 \\
3.812 E=06 \\
2.539 E=06 \\
1.009 E=06 \\
4.424 E=07 \\
2.949 E=08 \\
1.311 E=08 \\
1.016 E=07\end{array}$ & $\begin{array}{l}3.23 B E=05 \\
2.542 E=05 \\
1.368 E=05 \\
1.579 E=05 \\
1.655 E=05 \\
4.979 E=06 \\
1.235 E=05 \\
2.281 E=05 \\
1.611 E=05 \\
1.136 E=05 \\
1.601 E=05 \\
1.066 E=05 \\
4.239 E=06 \\
1.056 E=06 \\
1.239 E=07 \\
5.505 E-08 \\
4.266 E-07\end{array}$ & $\begin{array}{l}00 \\
10 \\
00 \\
00 \\
00 \\
00 \\
00 \\
00 \\
00\end{array}$ \\
\hline
\end{tabular}

AZIMUTH HEIGHJ EXPOSURE E/Q EU/O DIOTANCE OEGREES METERS GM-SEC/CU,M SEC/CU,M I/SQ,M METERS $\times 10 E+6$

\begin{tabular}{|c|c|c|c|c|c|}
\hline $\begin{array}{l}130.0 \\
130.0 \\
130.0 \\
130.0 \\
130.0 \\
130.0 \\
130.0 \\
130.0 \\
130.0 \\
130.0 \\
130.0 \\
130.0 \\
130.0 \\
130.0 \\
130.0 \\
130.0 \\
130.0\end{array}$ & $\begin{array}{l}0.3 \\
0.5 \\
1.0 \\
2.1 \\
4.2 \\
6.3 \\
8.4 \\
10.5 \\
12.6 \\
19.7 \\
16.8 \\
21.0 \\
25.2 \\
29.4 \\
33.6 \\
37.8 \\
92.0\end{array}$ & $\begin{array}{r}11500 \\
10431 \\
5039 \\
6401 \\
7409 \\
6491 \\
5380 \\
6747 \\
7876 \\
5944 \\
4700 \\
0404 \\
1880 \\
623 \\
192 \\
29 \\
6\end{array}$ & $\begin{array}{l}5.09 A F=05 \\
4.920 E=05 \\
2.377 E=05 \\
3.019 E=05 \\
3.533 E=05 \\
3.057 E=05 \\
2.500 E=05 \\
3.103 E=05 \\
3.715 E=05 \\
2.823 E=05 \\
2.217 E=05 \\
2.077 E=05 \\
0.870 E=06 \\
2.939 F=06 \\
9.077 E=07 \\
1.409 E=07 \\
3.277 E=08\end{array}$ & $\begin{array}{l}2.206 E=04 \\
2.067 E=04 \\
9.985 E=05 \\
1.268 E=04 \\
1.404 E=04 \\
1.284 E=04 \\
1.067 E=04 \\
1.337 E=04 \\
1.560 E=04 \\
1.186 E=04 \\
9.311 E=05 \\
6.725 E=05 \\
3.726 E=05 \\
1.235 E=05 \\
3.012 E=06 \\
5.917 E=07 \\
1.376 E=07\end{array}$ & $\begin{array}{l}000 \\
000 \\
100 \\
000 \\
000 \\
000 \\
800 \\
000 \\
800 \\
800 \\
800 \\
800 \\
000 \\
800 \\
800 \\
800 \\
000\end{array}$ \\
\hline
\end{tabular}

TOWER DATA FalLOW....

TEST FTS MAY 22, 1963 OS45 TO 0415 PGT ZINE SULFIDE RELEASE FROM ELEVATION OF $7 \mathrm{M}$ $1600 \mathrm{M}$ ARC UE $0.2 \mathrm{M} / \mathrm{SEC}$ AT TM ALIPUTH HEIGHT EXPOSURE E E/O
DEGREES METERS GM-SEC/CU.M SEC/CU,M I/SA,M DISTANCE $\times 10 E+6$

\begin{tabular}{|c|c|c|c|c|c|}
\hline $\begin{array}{l}122.0 \\
122.0 \\
122.0 \\
122.0 \\
122.0 \\
122.0 \\
122.0 \\
122.0 \\
122.0 \\
122.0 \\
122.0 \\
122.0 \\
122.0\end{array}$ & $\begin{array}{l}0.2 \\
0.4 \\
0.8 \\
1.5 \\
3.1 \\
6.2 \\
9.3 \\
12.4 \\
15.5 \\
10.6 \\
21.7 \\
24.8 \\
31.0\end{array}$ & $\begin{array}{l}47 \\
25 \\
23 \\
45 \\
40 \\
34 \\
25 \\
19 \\
29 \\
34 \\
15 \\
10 \\
6\end{array}$ & $\begin{array}{l}2.220 E-07 \\
1.215 E-07 \\
1.114 E=07 \\
2.130 E=07 \\
1.932 E-07 \\
1.635 E-07 \\
1.149 E-07 \\
9.413 E=08 \\
1.367 E-07 \\
1.635 E-07 \\
7.431 E=08 \\
4.762 E=08 \\
2.976 E=08\end{array}$ & $\begin{array}{l}9.357 E=07 \\
5.104 E-07 \\
4.676 E=07 \\
0.947 E=07 \\
0.115 E=07 \\
6.866 E=07 \\
0.994 E=07 \\
3.953 E=07 \\
5.026 E=07 \\
6.866 E=07 \\
3.121 E=07 \\
2.000 E=07 \\
1.250 E=07\end{array}$ & $\begin{array}{l}1600 \\
1600 \\
1600 \\
1600 \\
1600 \\
1600 \\
1600 \\
1600 \\
1600 \\
1600 \\
1600 \\
1600 \\
1600\end{array}$ \\
\hline
\end{tabular}

AZIMUTH HEIGHT EXPOSURE E/O EU/O DISTANCE DEGREES METERS GM-SET/CU.M SEC/CU,M I/SO,M MEIERS $X 10 E+6$

$\begin{array}{llllll}130.0 & 0.2 & 3705 & 1.740 E-05 & 7.341 E-05 & 1600 \\ 130.0 & 0.4 & 0403 & 2.077 E-05 & 0.723 E-05 & 1600 \\ 130.0 & 0.8 & 3424 & 1.615 E-05 & 6.784 E-05 & 1600 \\ 130.0 & 1.5 & 1836 & 5.665 E-06 & 3.639 E-05 & 1600 \\ 130.0 & 3.1 & 2547 & 1.202 E-05 & 5.040 E-05 & 1600 \\ 130.0 & 6.2 & 2671 & 1.260 E-05 & 5.293 E-05 & 1600 \\ 130.0 & 9.3 & 2422 & 1.143 E-05 & 4.000 E-05 & 1600 \\ 130.0 & 12.4 & 1219 & 5.752 E-76 & 2.416 E-05 & 1600 \\ 130.0 & 15.5 & 2857 & 1.340 E-05 & 5.662 E-05 & 1600 \\ 130.0 & 18.6 & 1649 & 7.783 E-06 & 3.260 E-05 & 1600 \\ 130.0 & 21.7 & 1204 & 5.871 E-06 & 2.466 E-05 & 1600 \\ 130.0 & 24.8 & 859 & 0.056 E-06 & 1.709 E-05 & 1600 \\ 130.0 & 31.0 & 172 & 8.124 E-07 & 3.412 E-06 & 1600 \\ 130.0 & 37.2 & 30 & 1.428 E-07 & 1.000 E-07 & 1600\end{array}$




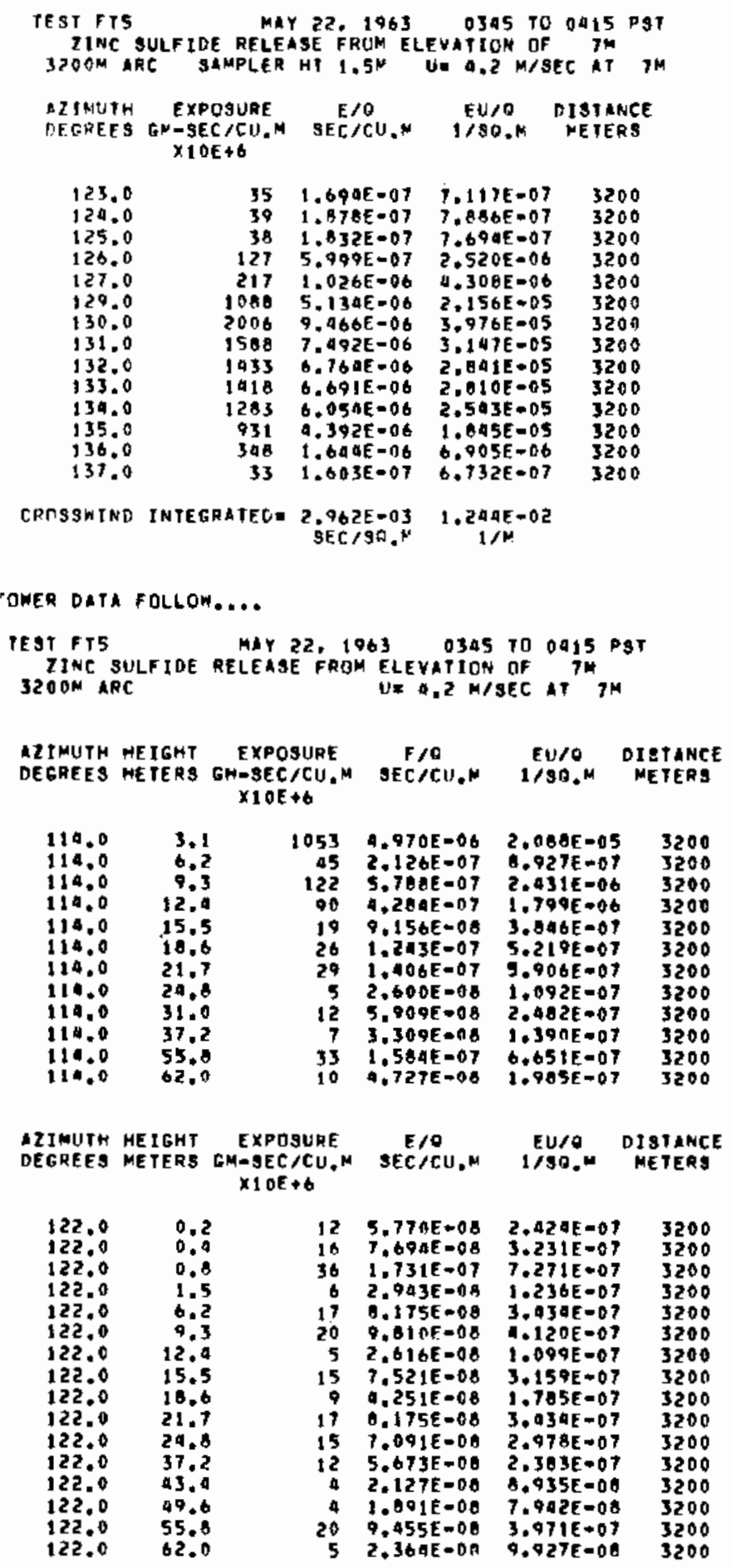


SERIES 163 EXPERIMENT: 6 ( FT-6)

GROUND LEVEL ANO TOWER SAMPLING 200-3200 M ARCS. A LARGE PORTION

OF THE PLUME PASSED TO THE NORTH OF THE GRI'.

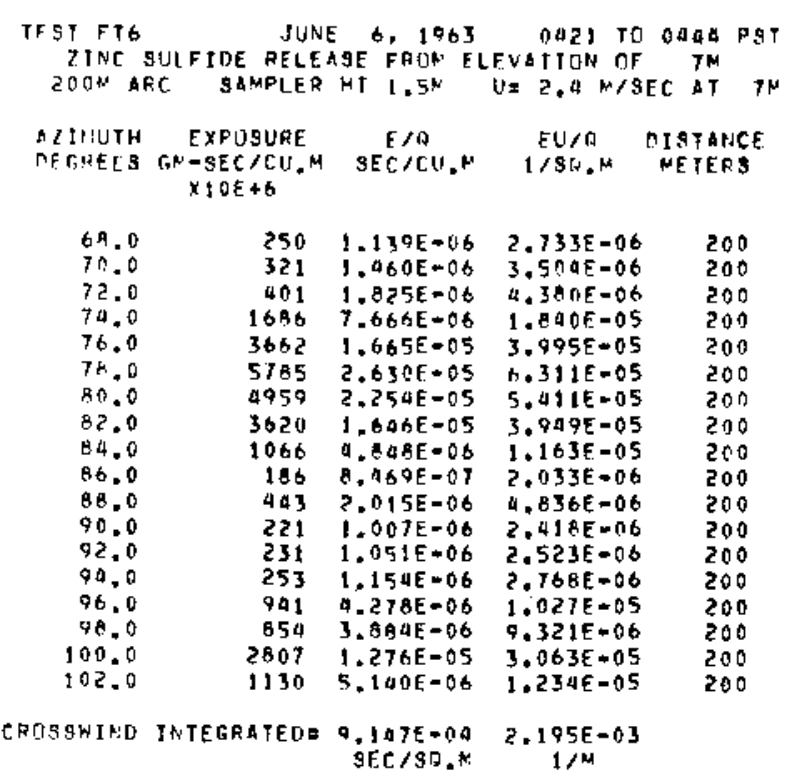

TOMFR TATA FIILLTIY...

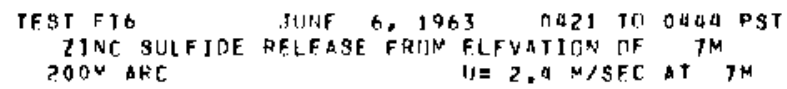

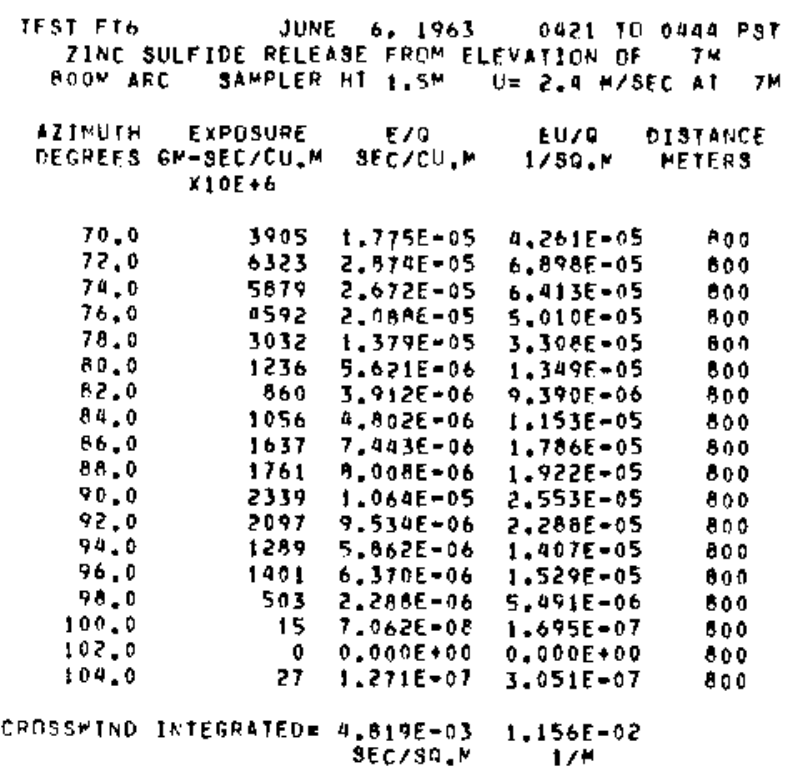

TOWFP DATA F HILLIL!...

TFSTFTO JUNE $A, 1963$ OUZ1 TO 0 व4य PST ZINC SULFJOE RFLFASF FRTN ELEVATIOA OF IN RODN APC

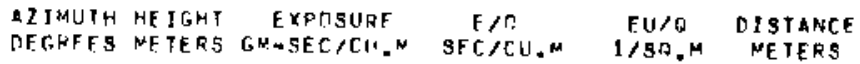
$X 1 \cap F+6$

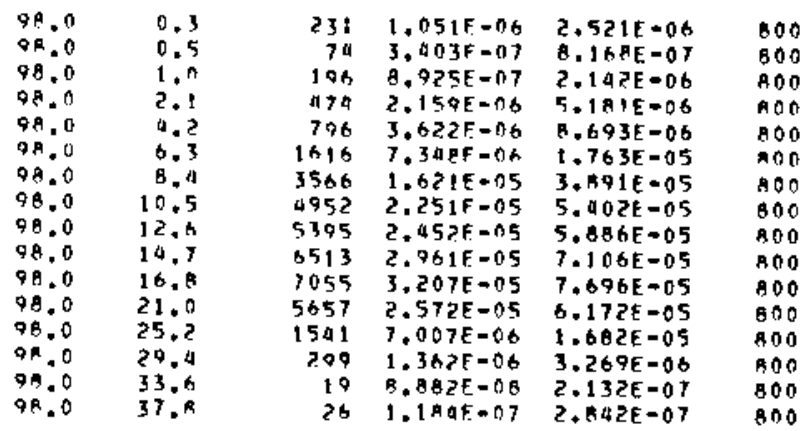




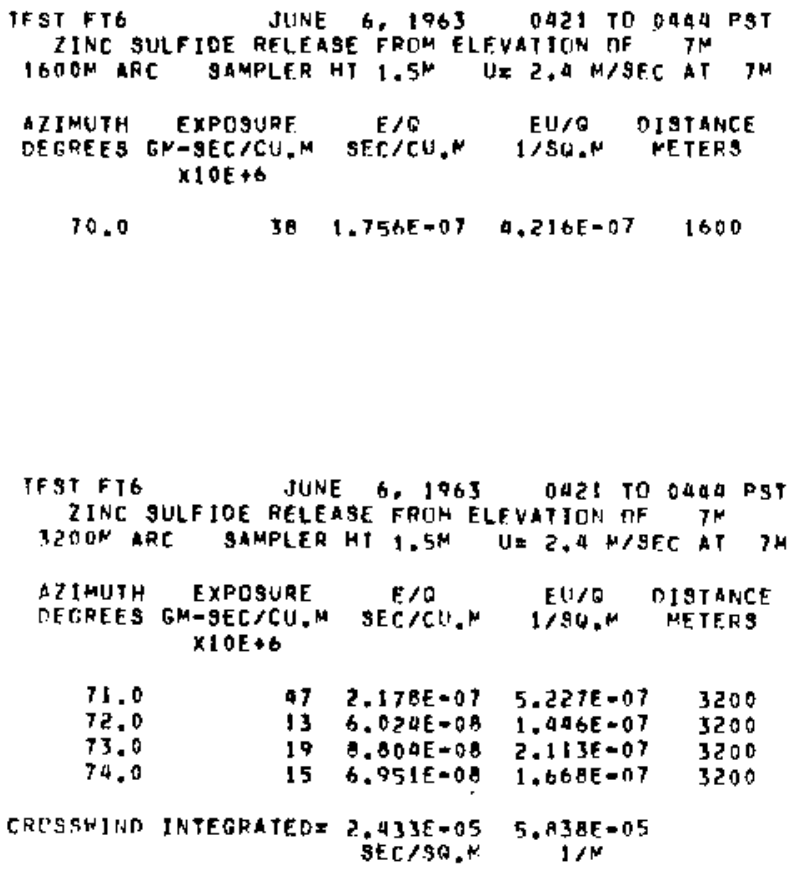


SERIES 163 EXPERIMENT: 7 ( FT-7)

GROUNO LEYEL AND TOWER SAMPLING 200-3200 M ARCS.

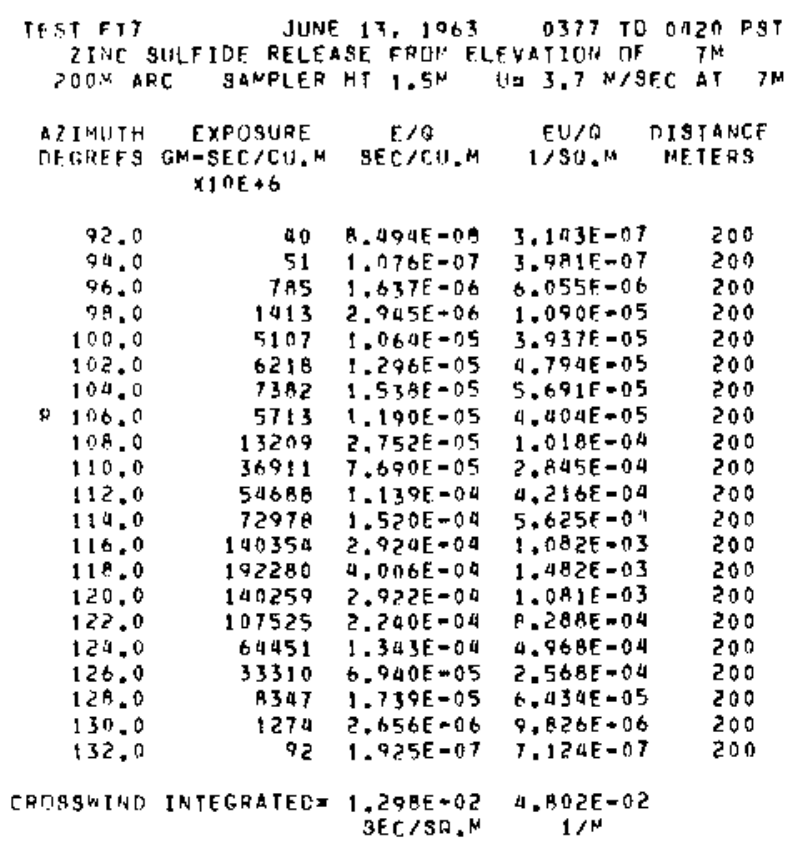

TOHER DATA FULLOW....
TFSTFTT JUNE 13, 1963 O37T TO OA2 PGT ZINC SULFIDE HELEASE FRTM FLFVATION OF TM
20 IIM ARC

ATIMHTH HEITHT FXPOSURE E/J FU/O DISTANCF DEGFEFS METFRS [OM-SEC/CII.N SFC/CII.M 1/SD.M NETERS xi $\cap \mathrm{E}+6$

\begin{tabular}{|c|c|c|c|c|}
\hline 98,0 & 0.3 & 1607 & $3.339 \mathrm{~F}-\mathrm{n} 6$ & $1.23 b F-05$ \\
\hline . 1.0 & 0.7 & 1157 & $2.416 \mathrm{~F}-106$ & QE -06 \\
\hline अA . 0 & 1.0 & 766 & $5976-06$ & $5.90 A E-06$ \\
\hline H. 0 & & 703 & 1. 4 ABE -06 & $423 E=0.6$ \\
\hline$\Delta A, 0$ & 4.1 & $\ln 36$ & $2.16 \mathrm{aE}=06$ & $0.007 E=08$ \\
\hline 98.0 & 5.4 & 1625 & $6 E=106$ & $253 E-05$ \\
\hline 48.0 & A & 1675 & $E=06$ & $2 E=05$ \\
\hline 98.0 & a. 1 & 1777 & $3.702 E-06$ & $1.370 E-05$ \\
\hline 92.0 & 9.4 & 2964 & $6.1765=06$ & $2.285 E-05$ \\
\hline $2 A .0$ & 10.8 & 363 A & 7. $\operatorname{SBnE}=06$ & $104 E=05$ \\
\hline 19.0 & 13.5 & 3386 & $7.056 E-06$ & $.6 ! 1 E-05$ \\
\hline 28.0 & 16.2 & $22 A 6$ & $64 E-06$ & $1.763 E-05$ \\
\hline 178.0 & 18.9 & $19 \times n$ & 4. OA IIF $=06$ & $1.511 \mathrm{E}-05$ \\
\hline 178.0 & 21.6 & 1534 & $3.197 E-08$ & $1.103 E-05$ \\
\hline 198.0 & 24,3 & $x \in 6$ & 1.38 \& -06 & $5.135 E-06$ \\
\hline 138.0 & 27.0 & 672 & $1.0016-06$ & C 193E-06 \\
\hline
\end{tabular}

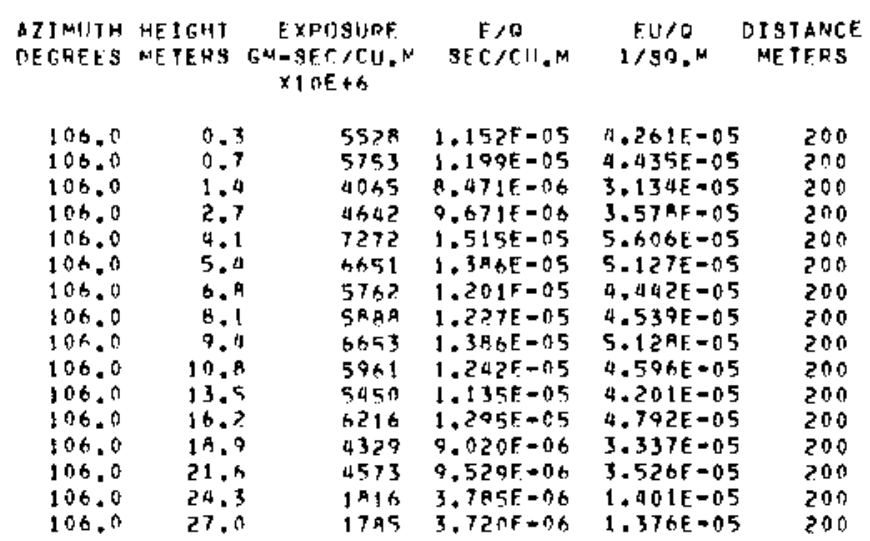

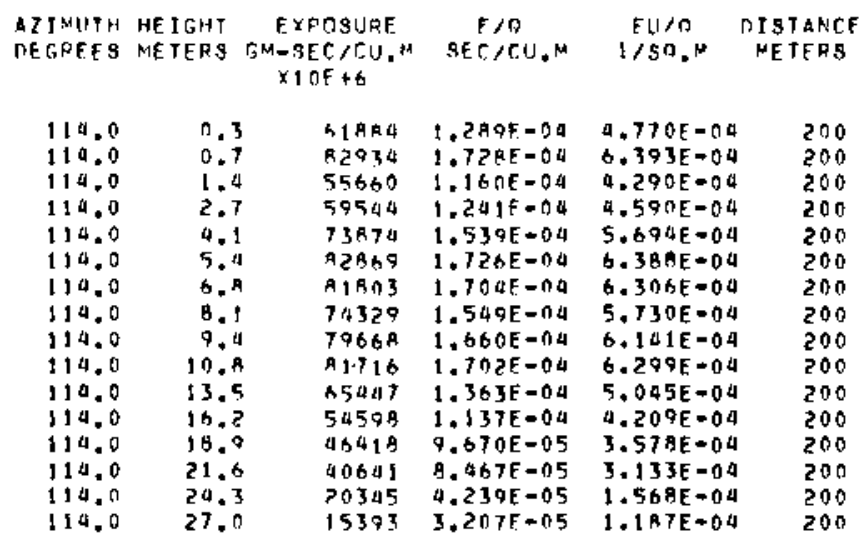

\begin{tabular}{|c|c|c|c|c|c|}
\hline $\begin{array}{l}\text { AZTMUITH } \\
\text { DE GREFS }\end{array}$ & $\begin{array}{l}\text { HE IGHT } \\
\text { ME TERS }\end{array}$ & $\begin{array}{c}\text { EXPCSUAE } \\
G M-3 E C / C 11 . M \\
\times 10 E+6\end{array}$ & $\begin{array}{c}E / D \\
\operatorname{set}(\mathrm{CH}, \mathrm{N}\end{array}$ & $\begin{array}{c}F U / O \\
1 / S O, M\end{array}$ & $\begin{array}{l}\text { DISTANCE } \\
\text { METERS }\end{array}$ \\
\hline $\begin{array}{r}122.0 \\
122.0 \\
122.0 \\
122.0 \\
122.0 \\
122.0 \\
122.0 \\
122.0 \\
122.0 \\
122.0 \\
122.0 \\
122.0 \\
122.0 \\
122.0 \\
122.0 \\
122.0\end{array}$ & $\begin{array}{l}0.3 \\
0.7 \\
1.9 \\
2.7 \\
4.1 \\
5.9 \\
6.9 \\
8.1 \\
9.9 \\
10.9 \\
13.5 \\
16.2 \\
18.0 \\
21.6 \\
24.7 \\
27.0\end{array}$ & $\begin{array}{l}R 2302 \\
114496 \\
78297 \\
64070 \\
76112 \\
84676 \\
34272 \\
99264 \\
99492 \\
97990 \\
66952 \\
57212 \\
39892 \\
32684 \\
19093 \\
13877\end{array}$ & $\begin{array}{l}1.715 F=04 \\
2.3 A 5 E=04 \\
1.631 F=04 \\
1.33 \text { UE=04 } \\
1.58 \text { GE }=04 \\
1.764 E=04 \\
7.130 F=05 \\
1.860 E=04 \\
2.06 D E=04 \\
2.031 E=04 \\
1.395 E=04 \\
1.192 F=04 \\
A .309 E=05 \\
3.809 E=05 \\
3.976 F=05 \\
2.891 E=05\end{array}$ & $\begin{array}{l}6.344 E=04 \\
8.825 E=04 \\
6.035 E=04 \\
1.935 E=04 \\
5.867 E=04 \\
6.527 E=04 \\
2.638 E=04 \\
6.081 E=04 \\
7.623 E=04 \\
7.515 E=04 \\
5.161 E=04 \\
1.010 E=04 \\
3.074 E=04 \\
2.519 E=04 \\
1.071 E=04 \\
1.070 E=04\end{array}$ & $\begin{array}{l}200 \\
200 \\
200 \\
200 \\
200 \\
200 \\
200 \\
200 \\
200 \\
200 \\
200 \\
200 \\
200 \\
200 \\
200 \\
200\end{array}$ \\
\hline
\end{tabular}




\begin{tabular}{|c|c|c|c|c|c|c|c|c|c|c|c|}
\hline $\begin{array}{l}\text { AZTMUTH } \\
\text { DET.AEFS }\end{array}$ & $\begin{array}{l}\text { HE ISHT } \\
\text { METERS }\end{array}$ & $\begin{array}{c}\text { EXPDSIJAE } \\
G \times-\$ E C / C 11, N \\
\times 1 \cap F+6\end{array}$ & $\begin{array}{c}E / B \\
\operatorname{set} / C A, N\end{array}$ & $\begin{array}{c}E \cup / 0 \\
1 / S O M\end{array}$ & $\begin{array}{l}\text { DISTANCE } \\
\text { METEAS }\end{array}$ & $\begin{array}{l}\text { AZIMUTH } \\
\text { OEGREFS }\end{array}$ & $\begin{array}{l}\text { ME ISHT } \\
\text { METERS }\end{array}$ & $\begin{array}{c}\text { EXPOSURE } \\
\text { GNE }=S E C / C U, M \\
\times 1 O E+G\end{array}$ & $\begin{array}{c}E / 0 \\
S E C / C U, M\end{array}$ & $\begin{array}{l}E \cup / Q \\
1 / S 0, M\end{array}$ & $\begin{array}{c}\text { DISTHNE } \\
\text { METERS }\end{array}$ \\
\hline $\begin{array}{l}130.0 \\
130.0 \\
130.0 \\
130.0 \\
130.0 \\
130.0 \\
130.0 \\
130.0 \\
130.0 \\
130.0 \\
130.0 \\
130.0 \\
130.0 \\
130.0 \\
130.0 \\
130.0\end{array}$ & $\begin{array}{l}0.7 \\
0.7 \\
1.4 \\
2.7 \\
4.1 \\
5.4 \\
6.9 \\
9.1 \\
9.4 \\
10.8 \\
13.5 \\
16.2 \\
18.0 \\
21.6 \\
24.3 \\
27.0\end{array}$ & $\begin{array}{l}1117 \\
1150 \\
411 \\
534 \\
342 \\
371 \\
445 \\
754 \\
1498 \\
577 \\
943 \\
347 \\
350 \\
343 \\
144 \\
244\end{array}$ & $\begin{array}{l}2.329 F=06 \\
2.396 E-06 \\
1.273 E=06 \\
1.113 E=06 \\
7.965 E=07 \\
1.295 E=06 \\
9.274 E=07 \\
1.571 F=06 \\
1.01 E E=06 \\
1.09 A F=06 \\
9.23 E E-07 \\
7.237 F=07 \\
7.310 E=07 \\
7.165 E=07 \\
3.017 F=07 \\
5.099 E-07\end{array}$ & $\begin{array}{l}8.617 E=06 \\
3.165 E=06 \\
1.71 \text { AE=06 } \\
4.11 B E=06 \\
2.947 E=06 \\
4.791 E=06 \\
3.432 E=06 \\
5.813 E=06 \\
3.761 E=06 \\
1.064 E=06 \\
3.018 E=06 \\
2.678 E=06 \\
2.705 E=06 \\
2.651 E=06 \\
1.116 E=06 \\
1.083 E=06\end{array}$ & $\begin{array}{l}200 \\
200 \\
200 \\
200 \\
200 \\
200 \\
200 \\
200 \\
200 \\
200 \\
200 \\
200 \\
200 \\
200 \\
200 \\
200\end{array}$ & $\begin{array}{l}106.0 \\
106.0 \\
106.0 \\
106.0 \\
106.0 \\
106.0 \\
106.0 \\
106.0 \\
106.0 \\
106.0 \\
106.0 \\
106.0 \\
106.0 \\
106.0 \\
106.0 \\
106.0 \\
106.0\end{array}$ & $\begin{array}{l}0.3 \\
0.5 \\
1.0 \\
2.1 \\
4.2 \\
6.3 \\
6.0 \\
10.5 \\
12.6 \\
11.7 \\
16.0 \\
21.0 \\
25.2 \\
29.8 \\
33.6 \\
37.8 \\
42.7\end{array}$ & $\begin{array}{l}201 \\
791 \\
427 \\
665 \\
156 \\
542 \\
7 A 6 \\
7 \times 1 \\
746 \\
8 \times 3 \\
979 \\
842 \\
532 \\
498 \\
939 \\
327 \\
? 13\end{array}$ & $\begin{array}{l}4.208 E=07 \\
1.567 E=06 \\
8.901 E=07 \\
1.3 B 6 E=06 \\
1.576 E=06 \\
1.210 E=06 \\
1.639 E=06 \\
1.625 E=06 \\
1.555 E-06 \\
1.840 E=06 \\
2.041 F=06 \\
1.756 E=06 \\
1.109 E=06 \\
1.038 E=196 \\
9.161 E=07 \\
0.926 E=07 \\
1.491 E=07\end{array}$ & $\begin{array}{l}1.557 E=06 \\
5.796 E=06 \\
3.293 E=06 \\
5.129 E=06 \\
5.032 E=06 \\
4.091 E=06 \\
6.066 E=06 \\
6.014 E=06 \\
5.754 E=06 \\
9.008 E=06 \\
7.550 E=06 \\
6.096 E=06 \\
9.103 E=06 \\
3.340 E=06 \\
3.389 E=06 \\
2.526 E=06 \\
1.643 E=06\end{array}$ & $\begin{array}{l}800 \\
800 \\
0100 \\
000 \\
800 \\
000 \\
000 \\
000 \\
000 \\
800 \\
800 \\
000 \\
000 \\
000 \\
000 \\
000 \\
000\end{array}$ \\
\hline
\end{tabular}

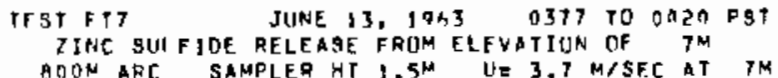
AZTHUTH EXPOSURE E/O EU/R OISTANCE DEGREES GR-SEC/CIIM SEE/CI,N I/SG.M MFTERS $x ! O E+b$

\begin{tabular}{|c|c|c|c|c|}
\hline $\begin{array}{l}100.0 \\
102.0 \\
100.0 \\
106.0 \\
108.0 \\
110.0 \\
112.0 \\
114.0 \\
116.0 \\
118.0 \\
120.0 \\
122.0 \\
124.0 \\
126.0 \\
128.0 \\
130.0 \\
132.0\end{array}$ & $\begin{array}{r}55 \\
712 \\
775 \\
893 \\
1014 \\
1856 \\
7427 \\
19125 \\
38176 \\
28800 \\
13806 \\
9818 \\
5737 \\
3954 \\
2303 \\
720 \\
76\end{array}$ & 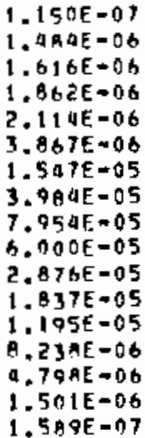 & $\begin{array}{l}4.256 E-07 \\
5.992 E-06 \\
5.979 E-06 \\
6.891 E=08 \\
7.823 E-06 \\
1.431 E=05 \\
5.726 E=05 \\
1.474 E-04 \\
2.943 E=04 \\
2.220 E=04 \\
1.064 E=04 \\
6.798 E=05 \\
4.422 E=05 \\
3.098 E=05 \\
1.775 E-05 \\
5.553 E=06 \\
5.878 E=07\end{array}$ & $\begin{array}{l}800 \\
800 \\
800 \\
800 \\
800 \\
800 \\
800 \\
800 \\
800 \\
800 \\
800 \\
800 \\
800 \\
800 \\
800 \\
800 \\
800\end{array}$ \\
\hline
\end{tabular}

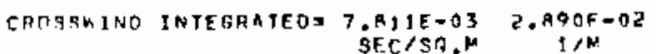

TOKER DETA FOLLOH....

TEST FTT JUPE 13, 1963 OS 0377 TO 0420 PST ZJNC SULFJDE HELEATF FRDM FLFVATION OF
ADON ARC.

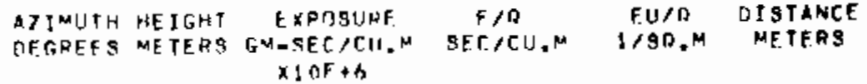
$\begin{array}{llllll}98.0 & 14.7 & 27 & 5.629 F-0 B & 2.083 E-07 & 800 \\ 99.0 & 21.0 & 30 & 6.333 E=08 & 2.343 E-07 & 800\end{array}$

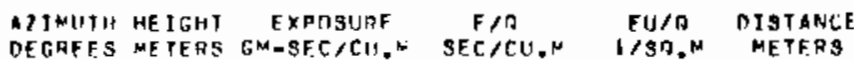
$\times 1 \cap E+6$

$$
\begin{aligned}
& 110.0 \\
& 114.0 \\
& 119.0 \\
& 110.0 \\
& 114.0 \\
& 114.0 \\
& 114.0 \\
& 114.0 \\
& 114.0 \\
& 114.0 \\
& 114.0 \\
& 114.0 \\
& 114.0 \\
& 119.0 \\
& 114.0 \\
& 114.0 \\
& 110.0
\end{aligned}
$$
0,3
10.5
5,7

0.5

2.1

4.?

$0, a$

12.4

14.7

16..

21.0

29.4

37.5

a. 0

$$
1713 n
$$

$14523 \quad 3.026 E=15$ 1.320E-04

2.737F-15 $1.013 E-0 A$

717 A $1.496 F=05 \quad 5,534 E=05$

$13656 \quad 2.805 E=05 \quad 1.053 E=04$

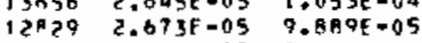

$12 A n 9$ 2.627E-05 $9.720 E-05$

$1372: 2.900 F=05 \quad 1.073 E-00$

$141072.958 E-05 \quad 1.094 E=09$

$114692.390 \mathrm{~F}-05 \quad 0.841 \mathrm{E}=05$

$\begin{array}{rll}111169 & 2.390 \mathrm{~F}=05 & 0.841 \mathrm{E}-05 \\ 9426 & 1.964 \mathrm{~F}=05 & 7.260 \mathrm{E}-05\end{array}$

5812 1.211F-05 A.481E-0

a163 8.673E-06 $3.209 E-05$

27 ID $5.63 P E-06 \quad 2.086 E-05$

$2250 \quad 4.689 \mathrm{E}=06 \quad 1.735 E=05$

$\begin{array}{lll}2250 & 4.689 E-06 & 1.735 E=05 \\ 1939 & 4.040 F-06 & 1.495 E-05\end{array}$

R.8 $1.852 \mathrm{~F}-06 \quad 6.054 \mathrm{E}=06$

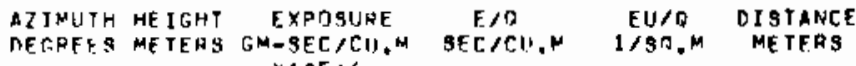
$\times 10 F+G$

$\begin{array}{rr}122.0 & 0.3 \\ 122.0 & 0.5 \\ 122.0 & 1.0 \\ 122.0 & 2.1 \\ 122.0 & 4.2 \\ 122.0 & 6.3 \\ 122.0 & 0.0 \\ 122.0 & 10.5 \\ 122.0 & 12.3 \\ 122.0 & 14.7 \\ 122.0 & 16.8 \\ 122.0 & 21.0 \\ 122.0 & 25.2 \\ 122.0 & 29.1 \\ 122.0 & 33.6 \\ 122.0 & 37.1 \\ 122.0 & 42.0\end{array}$

POO

AOO

800

600

800

600

000

800

800

800

900

800

$B 00$

000

000

800

800 


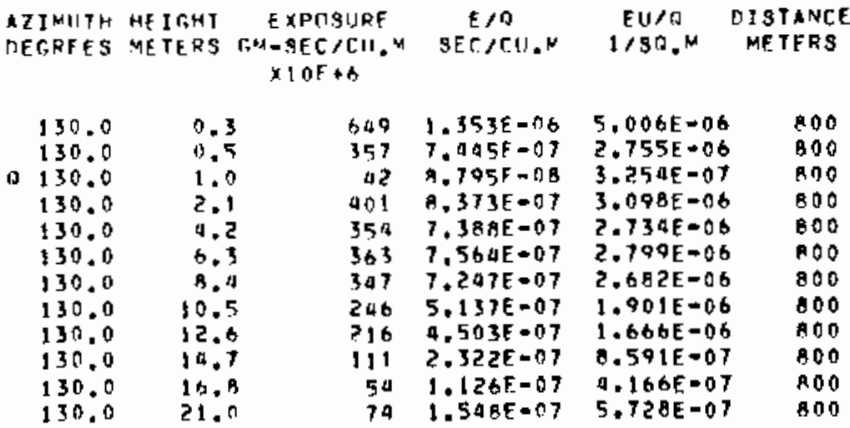

TF ST FI7 JUNE 13,1963 O377 TO 0420 PST ZINC SULFIDE RFLEASE FRDM ELEVATION DF TM
SOOM ARC SAMPLER HT I.5N UM

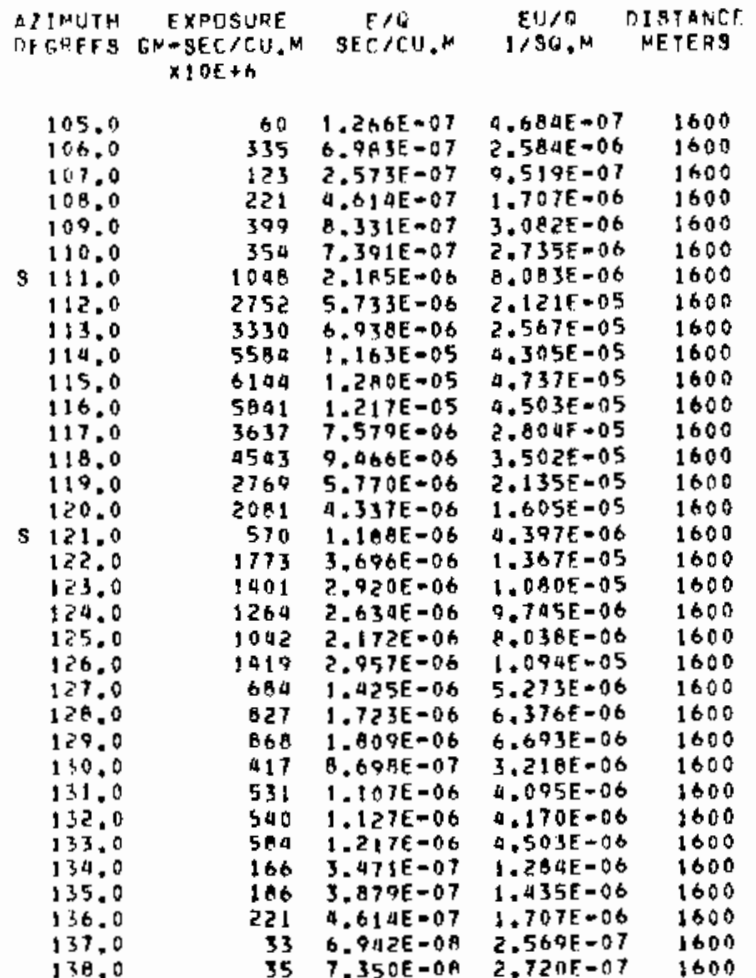

CRPSBHITE INTEGRATEDE 3,013E-03 $1,115 \mathrm{~F}-02$

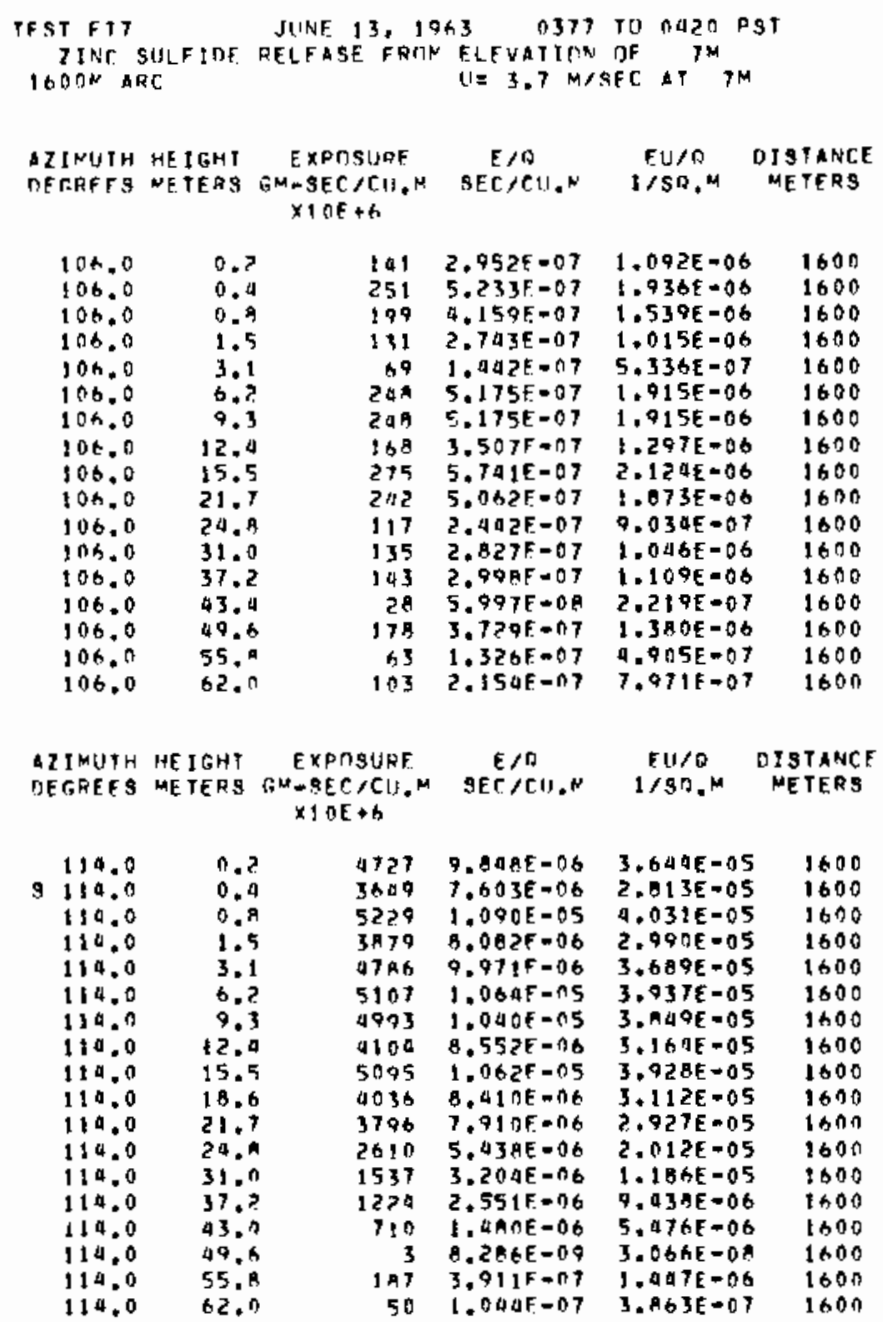

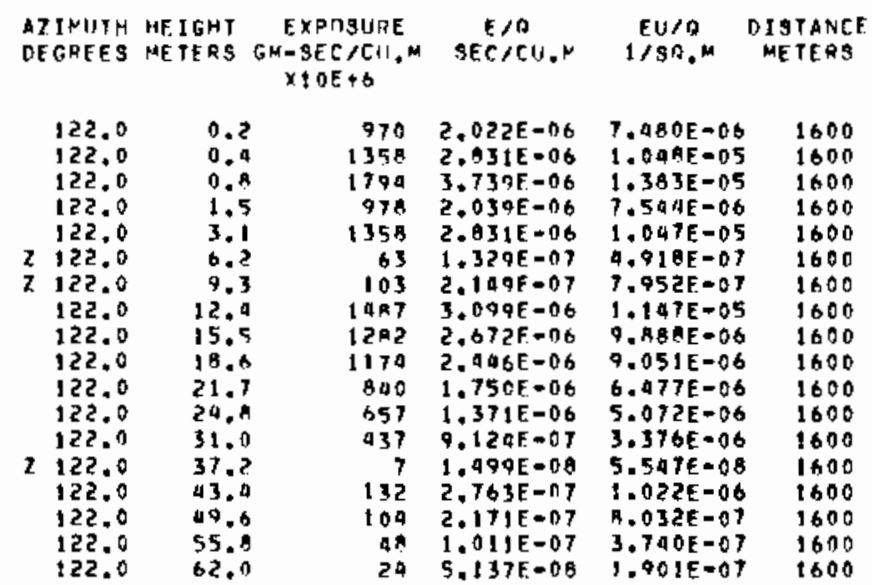




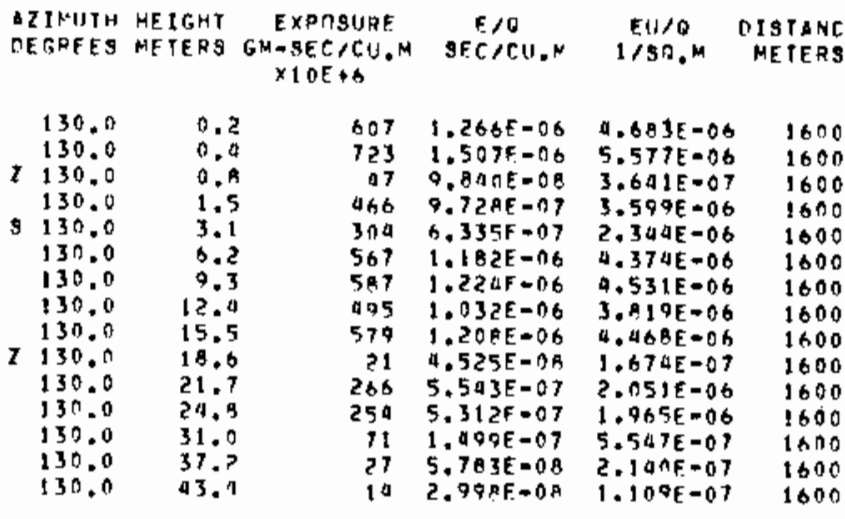

TEST FIT JUNE 13,1963 O377 TO OQZO PST ZINC SULFIDE RELEASE FPIJM ELEVATTUN OF TM $320 \mathrm{~N}$ ARC SAMFLER HT $1.5 \mathrm{~N} U=3.7 \mathrm{M} / \mathrm{SEC}$ AT $7 \mathrm{M}$

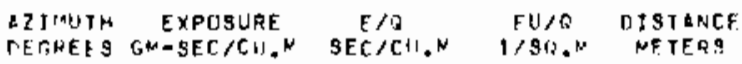
$\times 10 \varepsilon+6$

105.0
108.0
107.0
108.0
109.0
110.0
111.0
112.0
113.0
114.0
115.0
116.0
117.0
118.0
119.0
120.0
121.0
122.0
123.0
124.0
125.0
126.0
127.0
128.0
129.0
130.0
131.0
132.0
133.0
134.0
135.0
136.0
137.0
138.0
139.0
140.0
141.0
142.0
143.0
144.0

\begin{tabular}{|c|c|}
\hline 24 & $\begin{array}{l}1.011 E=0 A \\
5.057 E=08\end{array}$ \\
\hline 23 & 4. A $54 E=0 B$ \\
\hline 118 & 2. $A B A E=07$ \\
\hline 118 & $3.005 \mathrm{E}=07$ \\
\hline 97 & $2.023 E=07$ \\
\hline 110 & $2.306 E-07$ \\
\hline 210 & $3 A 9 E=07$ \\
\hline 49 & $1.032 \mathrm{E}=07$ \\
\hline 1088 & $267 E-06$ \\
\hline $14 \nabla 8$ & S. $12+E=06$ \\
\hline 192 & $1.650 E=06$ \\
\hline 1457 & $3.036 E=06$ \\
\hline 1434 & $2.98 \nabla E-06$ \\
\hline 1360 & $2.034 E=06$ \\
\hline 1053 & $175 E=06$ \\
\hline 601 & 1.25 AE $=06$ \\
\hline 473 & $971 E-07$ \\
\hline 333 & 6.93 HE - D7 \\
\hline 363 & $7.585 E-07$ \\
\hline $\begin{array}{l}352 \\
270\end{array}$ & $\begin{array}{l}7.3 \Delta 2 \varepsilon=07 \\
5.73 \Delta E=07\end{array}$ \\
\hline 213 & $4.450 \mathrm{E}-07$ \\
\hline 180 & $3 . S E 3 E=07$ \\
\hline 228 & $0.753 E-0.7$ \\
\hline 152 & $3.176 \mathrm{E}=07$ \\
\hline 189 & $9 \triangle 4 E-07$ \\
\hline 110 & $306 E-07$ \\
\hline 156 & $3.297 \mathrm{E}-07$ \\
\hline 122 & $549 E-07$ \\
\hline & $002 E-07$ \\
\hline 150 & $.216 E-07$ \\
\hline 112 & $3 \triangle \Delta E=07$ \\
\hline 120 & 50 RE-07 \\
\hline 133 & $2.791 \epsilon=07$ \\
\hline 100 & $2.104 \mathrm{E}=07$ \\
\hline 60 & $1.335 E-07$ \\
\hline 11 & $. A Z T E=O A$ \\
\hline 11 & $2.9 \angle 7 E-D B$ \\
\hline g & $23 E=0 P$ \\
\hline
\end{tabular}

3.742E-DA 3200 $1.871 E \rightarrow 07 \quad 3200$ 1.7 DEE-07 37.00 $9.130 E-n Y \quad 3200$ $1.145 E-06 \quad 3200$ $7.1484 \mathrm{E}=07 \quad 3200$ $0.532 E-07 \quad 3200$ $1.6245=06 \quad 3200$ $3,817 \varepsilon=07 \quad 3200$ Q.389E-06 3200 $1.155 E-05 \quad 3200$ $6.107 E-06 \quad 3200$ $1.123 E-05 \quad 3200$ $1.106 \mathrm{E}-0 \mathrm{~S} \quad 3200$ 1. $0486-05 \quad 3200$ B. $1205-06 \quad 3200$ $0.640 E-06 \quad 3200$ $3.652 E-06 \quad 3200$ $2.567 E-06 \quad 3200$ $2.7995+06 \quad 3200$ $2.7175 .06 \quad 3200$ 2.11BE-06 3200 $1.640 E-06 \quad 3200$ $1.437 E-06 \quad 3200$ $1.759 \mathrm{~F}+106 \quad 3200$ $1.175 \mathrm{E}-06 \quad 3200$ $1.459 E-06 \quad 3200$ $8.532 \mathrm{E}-07 \quad 3200$ $1.220 E-06 \quad 3200$ $9.430 E-07 \quad 3200$ $7.4095-07 \quad 3200$ $1.1900=06 \quad 3200$ Q.6A1E-07 3200 Q. टसतE-07 उ200 $1.033 \mathrm{E}-06 \quad 3200$ $7.783 E-07 \quad 3200$ $4.939 E-07 \quad 3200$ Q.SAJF-กB 3200 ค. 9 BIE-กB 3200

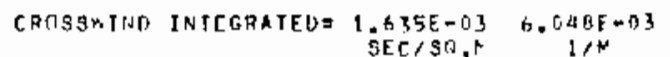

320,0

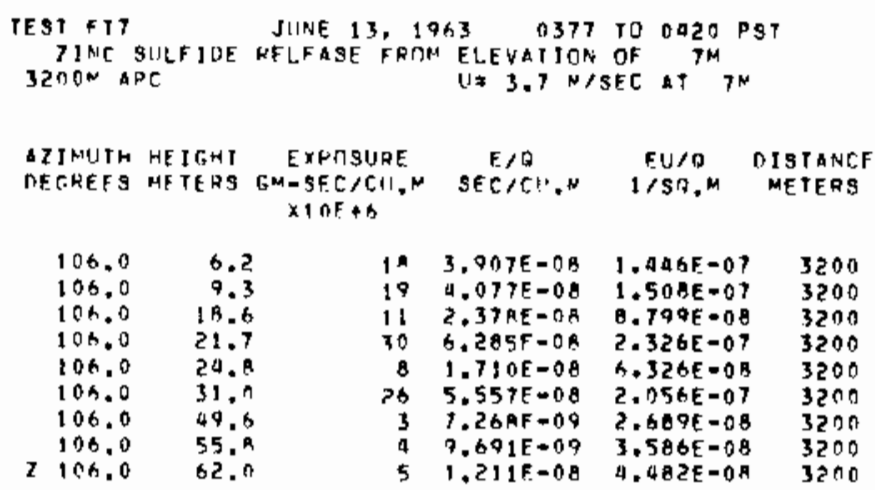

DITMUITH HETGHT EXPOSUIRE E/Q DUTO DISTANCE DERAEES NETERS F, M-SEC/CU,N SEC/CU.N $1 / S O$.M PFTERS $x \cap \cap+6$

\begin{tabular}{|c|c|c|c|c|c|}
\hline 114.0 & $0, ?$ & 1333 & $2.77 \times E=06$ & $1.028 E-05$ & 3200 \\
\hline 2114.0 & n. 5 & is & $2.761 E=08$ & $22 E=07$ & 3200 \\
\hline 114,0 & ).. & 1677 & $2.453 F=06$ & $9.076 E=06$ & 3200 \\
\hline 1140 & 1.5 & 1142 & $2.380 E=06$ & $A .806 \varepsilon=06$ & 3200 \\
\hline 114,0 & 3.1 & 1302 & $2.713 F=06$ & $1.00 \mathrm{aE}=0 \mathrm{~S}$ & $32 \cap 0$ \\
\hline 1111,0 & 0.2 & 1437 & $5 E=06$ & 1.10 AE $=05$ & 3200 \\
\hline 110,0 & 9.3 & 1603 & $3.341 E=06$ & $236 F=05$ & 3200 \\
\hline$Z 114.0$ & 12.4 & 13 & AREE - OB & $68 E-07$ & 3200 \\
\hline 114.0 & 15.5 & 13 an & $793 E-06$ & $1.0336-05$ & 3200 \\
\hline 110.0 & 18,3 & 1012 & $.942 E-06$ & 1. ПB9E $=05$ & 3200 \\
\hline 114.0 & 21.7 & $15 n 7$ & $141 E-106$ & $1+162 E=05$ & 3200 \\
\hline 114.0 & ค & 9 9 0 & $042 E-D B$ & $555 E=06$ & 3200 \\
\hline 114.0 & 31.0 & 1157 & $11 E=16$ & OE $=06$ & 200 \\
\hline 114.0 & 37.7 & ค7 7 & $1.92 B E-176$ & 6.76 DE $=06$ & 3200 \\
\hline 114.0 & 43.4 & 525 & $1.096 E=06$ & $n, 05 \triangle E=06$ & 3200 \\
\hline $11 \% .0$ & $55 . A$ & 131 & $3.98 p 5=07$ & $1.079 E=06$ & 3200 \\
\hline
\end{tabular}
ATIMUTH HEIGHT EXPOSIJPF
DECREFS METERS GM-SEC/CH,iA SEC/CU,H

\begin{tabular}{|c|c|c|c|c|c|}
\hline $12 ? . n$ & 0.2 & औUA & $9.3+5 F=07$ & $3.45 A t=06$ & 3200 \\
\hline 122.0 & 0.9 & AA 9 & $1.019 F=06$ & $3.772 E=06$ & $320 n$ \\
\hline 122.11 & 0,1 & $26^{\circ}$ & $5.607 E=07$ & $2.075 E-06$ & 3200 \\
\hline .0 & 1.5 & 440 & $9.173 E-177$ & $3.394 E-06$ & $3 ? 00$ \\
\hline . & 3.1 & 452 & $9.428 E-07$ & 3.48 AE $=0 \mathrm{~B}$ & 3200 \\
\hline - & b.? & 523 & $1.091 E=06$ & a. $135 F-06$ & 3200 \\
\hline & 9.3 & 434 & $0.0545-07$ & $3.350 \mathrm{E}=0 \mathrm{G}$ & 3200 \\
\hline & 12.4 & $5+9$ & $1.385 E=0.6$ & $5.16 n E-06$ & 3200 \\
\hline & 15.5 & 353 & $355 E=07$ & $2.722 E-06$ & 3200 \\
\hline & $1 B=6$ & 464 & $9.7685=07$ & $3.6 ! 0 \mathrm{DE}=06$ & 3200 \\
\hline & 21.7 & 4 a 3 & $1.007 E=0 D$ & $3.727 E=06$ & 3200 \\
\hline & & 453 & $61 f=07$ & $537 t=06$ & 3200 \\
\hline & 31.1 & 4ad & $1.010 \mathrm{E}=0 \mathrm{~B}$ & $3.73 B E-06$ & 3200 \\
\hline & 37.2 & 332 & $6.9255-07$ & $2.562 E=06$ & 3200 \\
\hline & 43.9 & 282 & $5.885 E-07$ & $2.177 E-n 6$ & 3200 \\
\hline & & $2 \cap 6$ & $.300 \mathrm{~F}=07$ & $591 \mathrm{E}=0 \mathrm{6}$ & 3200 \\
\hline 0 & 55.9 & 180 & $\begin{array}{l}3.767 E=07 \\
5 \text { A }=07\end{array}$ & $1.394 E=06$ & 3200 \\
\hline & & 6 & 107 & arecon & $3<00$ \\
\hline
\end{tabular}$$
X \cap \cap E+6
$$ 


\begin{tabular}{|c|c|c|c|c|c|}
\hline $\begin{array}{l}\text { ATMUTH } \\
\text { DEGREFS }\end{array}$ & $\begin{array}{l}\text { HE I CHT } \\
\text { NF TFRS }\end{array}$ & $\begin{array}{c}\text { EXPOSURF } \\
\text { GM-SEC } \\
\times 1 O=\$ B\end{array}$ & $\begin{array}{c}E / n \\
\operatorname{ser} / C H . N\end{array}$ & $\begin{array}{l}E U / D \\
1 / S A . M\end{array}$ & $\begin{array}{l}\text { DISTANCE } \\
\text { METERS }\end{array}$ \\
\hline $\begin{array}{l}130.0 \\
130.0 \\
130.0 \\
130.0 \\
130.0 \\
130.0 \\
130.0 \\
130.0 \\
130.0 \\
130.0 \\
130.0 \\
130.0 \\
130.0 \\
130.0 \\
130.0 \\
130.0 \\
130.0 \\
130.0\end{array}$ & $\begin{array}{l}0.7 \\
0.7 \\
0.8 \\
1.5 \\
3.1 \\
6.2 \\
9.1 \\
12.4 \\
15.5 \\
18.5 \\
21.7 \\
24.9 \\
31.0 \\
37.2 \\
43.9 \\
49.6 \\
55.0 \\
02.0\end{array}$ & $\begin{array}{r}136 \\
153 \\
224 \\
107 \\
203 \\
225 \\
228 \\
219 \\
159 \\
90 \\
757 \\
129 \\
94 \\
91 \\
97 \\
27 \\
11 \\
6\end{array}$ & 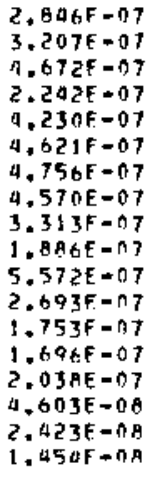 & $\begin{array}{l}1.053 \mathrm{E}=06 \\
1.167 \mathrm{E}-06 \\
1.729 \mathrm{E}-06 \\
8.297 \mathrm{E}=07 \\
1.565 \mathrm{E}=06 \\
1.710 \mathrm{E}-06 \\
1.760 \mathrm{E}=06 \\
1.691 \mathrm{E}=06 \\
1.226 \mathrm{E}=06 \\
6.977 \mathrm{E}=07 \\
2.067 \mathrm{E}=06 \\
9.96 \mathrm{AE}=07 \\
6.485 \mathrm{E}=07 \\
6.271 \mathrm{~F}=07 \\
7.539 \mathrm{E}=07 \\
1.703 \mathrm{E}=07 \\
8.964 \mathrm{E}=08 \\
5.379 \mathrm{~F}=08\end{array}$ & $\begin{array}{l}3200 \\
3200 \\
3200 \\
3200 \\
3200 \\
3200 \\
3200 \\
3200 \\
3200 \\
3200 \\
3200 \\
3200 \\
3200 \\
3200 \\
3200 \\
3200 \\
3200 \\
3200\end{array}$ \\
\hline
\end{tabular}


SERIES 163 EXPERIMENT: 8 ( FT-8)

GROUNO LEYEL AND TOWER SAMPL ING 200-3200 H ARCS. MANY OF THE FILTERS ON THE TOHERS MERE DAMAGED.

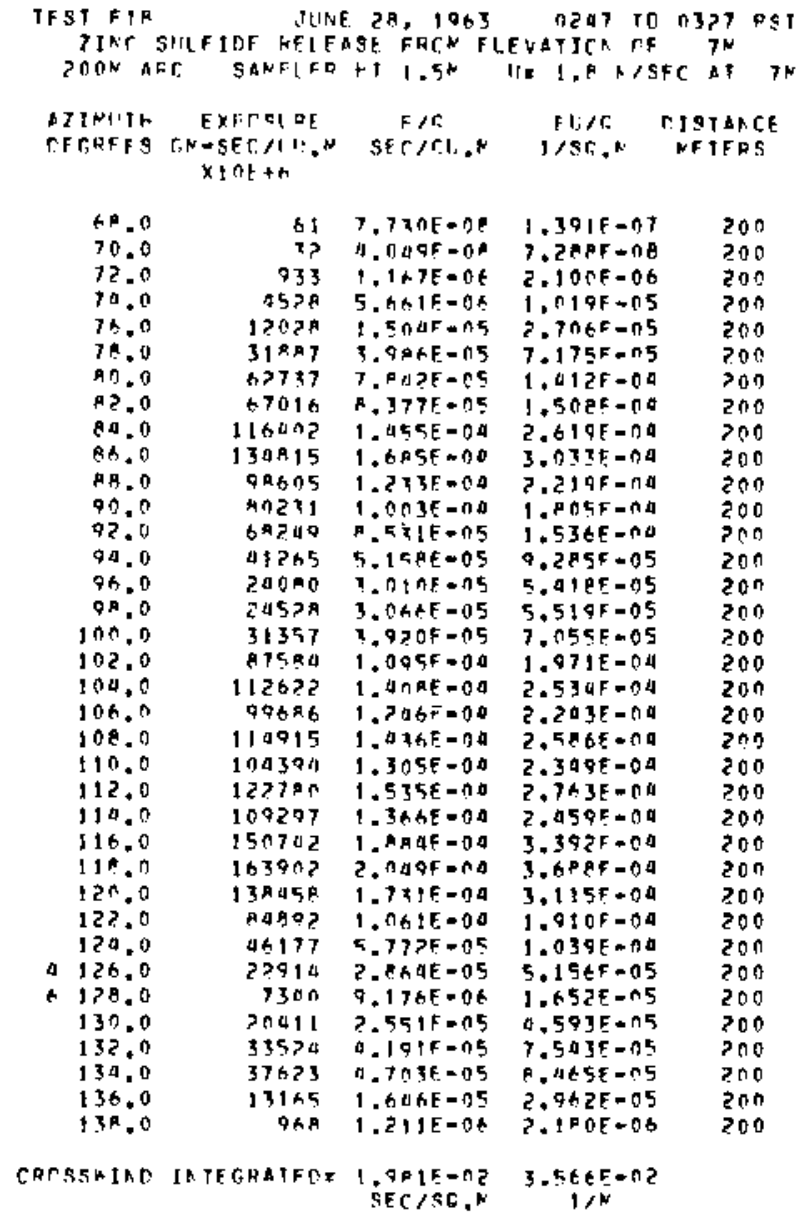

TONER DSTH FOLCH...

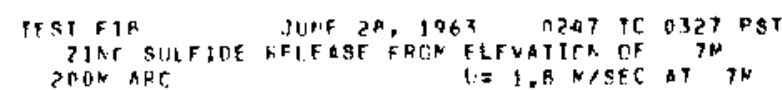

\begin{tabular}{|c|c|c|c|c|c|c|}
\hline & $\begin{array}{l}\text { IIH } \\
\text { IIES }\end{array}$ & $\begin{array}{l}\text { HE ILHT } \\
\text { ME TEHS }\end{array}$ & $\begin{array}{c}\text { FPCSURE } \\
\text { GN-SFC }>C U .: \\
x: n F+6\end{array}$ & $\begin{array}{c}F / D \\
S E C / C U . N\end{array}$ & $\begin{array}{l}F 0 / 0 \\
1 / 50 . N\end{array}$ & $\begin{array}{l}\text { PISTAMCE } \\
\text { WFTFRS }\end{array}$ \\
\hline $\mathbf{S}$ & $\begin{array}{l}98.0 \\
98.0 \\
98.0 \\
98.0 \\
98.0 \\
98.0 \\
98.0 \\
98.0\end{array}$ & $\begin{array}{l}0.3 \\
0.7 \\
1.0 \\
4.1 \\
5.2 \\
9 .\end{array}$ & $\begin{array}{l}73759 \\
22579 \\
25252 \\
19072 \\
19107 \\
9364 \\
17158 \\
12764\end{array}$ & $\begin{array}{l}2.860 F=05 \\
2.222 f=05 \\
3.157 f=05 \\
2.378 F=05 \\
2.275 F=05 \\
1.171 E=05 \\
2.145 F=05 \\
1.59+F=05\end{array}$ & $\begin{array}{l}5.305 F=05 \\
5.019 E=05 \\
5.6 A 7 E=05 \\
0.200 E=05 \\
0.092 E=05 \\
2.108 E=05 \\
3.061 F=05 \\
2.077 F=05\end{array}$ & $\begin{array}{l}200 \\
200 \\
200 \\
200 \\
200 \\
200 \\
200 \\
200\end{array}$ \\
\hline
\end{tabular}

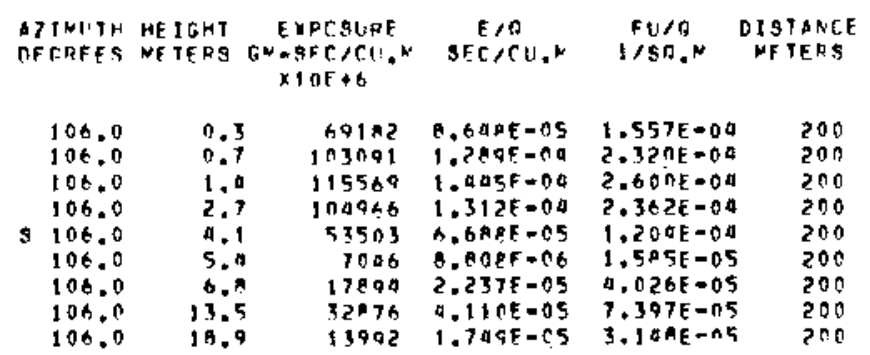

\begin{tabular}{|c|c|c|c|c|c|}
\hline $\begin{array}{l}\text { AZTUUTH } \\
\text { NETOFF }\end{array}$ & $\begin{array}{l}\text { HE IGHT } \\
\text { NETERE }\end{array}$ & $\begin{array}{c}\text { YPCCSURF } \\
\{N=S F C / C 11=N \\
x \in O E+6\end{array}$ & $\begin{array}{c}* / 0 \\
\operatorname{SEC} / \mathrm{CU}_{0} *\end{array}$ & $\begin{array}{c}F \mathrm{~L} / 0 \\
1 / 30 .\end{array}$ & $\begin{array}{l}\text { CISTAACE } \\
\text { NE TERS }\end{array}$ \\
\hline $\begin{array}{l}110.0 \\
110.00 \\
110.0 \\
110.0 \\
110.0 \\
110.0 \\
1100\end{array}$ & $\begin{array}{l}0.1 \\
0.7 \\
1.2 \\
4.1 \\
6.5 \\
9.0\end{array}$ & $\begin{array}{l}1234>0 \\
129734 \\
120908 \\
90717 \\
90952 \\
99708 \\
0+93 k\end{array}$ & $\begin{array}{l}1.545 F=0 a \\
1.609 F=04 \\
1.510 E=01 \\
1.130 F=04 \\
1.006 E=0 a \\
1.122 F=00 \\
5.86+F=05\end{array}$ & $\begin{array}{l}2.7 P 2 F=04 \\
2.897 E=04 \\
2.71 A E=04 \\
2.041 F=04 \\
1.810 E=04 \\
2.019 E=04 \\
1.056 E=00\end{array}$ & $\begin{array}{l}300 \\
500 \\
500 \\
500 \\
500 \\
500 \\
300\end{array}$ \\
\hline
\end{tabular}

MIINUTH HEIGMT EXPCSURE EIG FLIO DISTANCF.

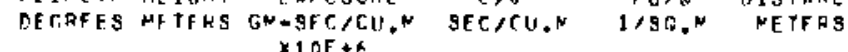

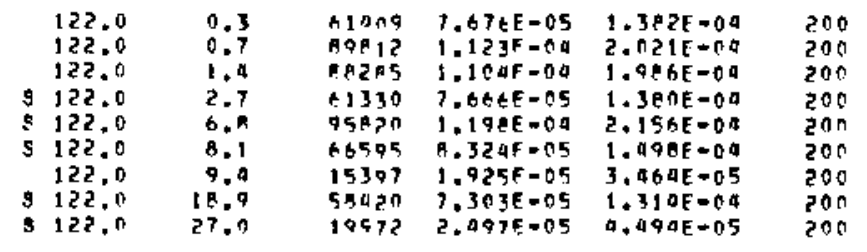

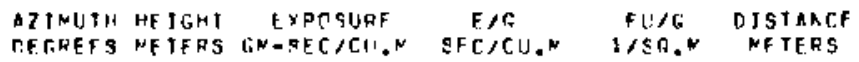
$x \perp$ OF +4

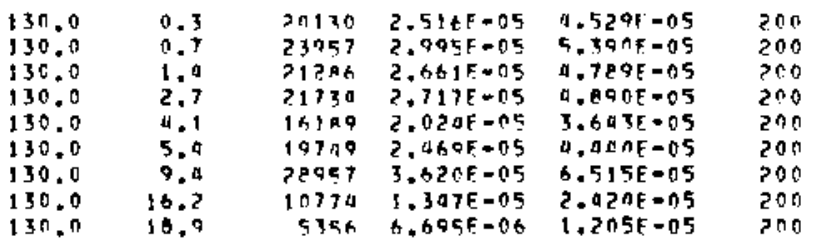




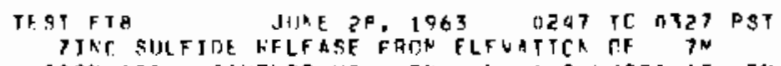

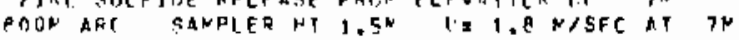

\begin{tabular}{|c|c|c|c|c|}
\hline $\begin{array}{l}\text { AINUTTH } \\
\text { IFTCEFS }\end{array}$ & $\begin{array}{c}\text { EXPCOADE } \\
G N=S E C / C H=N \\
\times 1 D E+6\end{array}$ & $\begin{array}{c}F / G \\
\text { SFC } / R L^{\prime}, N\end{array}$ & $\begin{array}{c}F \cup / O \\
1 / \$ 6, W\end{array}$ & $\begin{array}{c}\text { MISIANCF } \\
\text { WFIERS }\end{array}$ \\
\hline
\end{tabular}

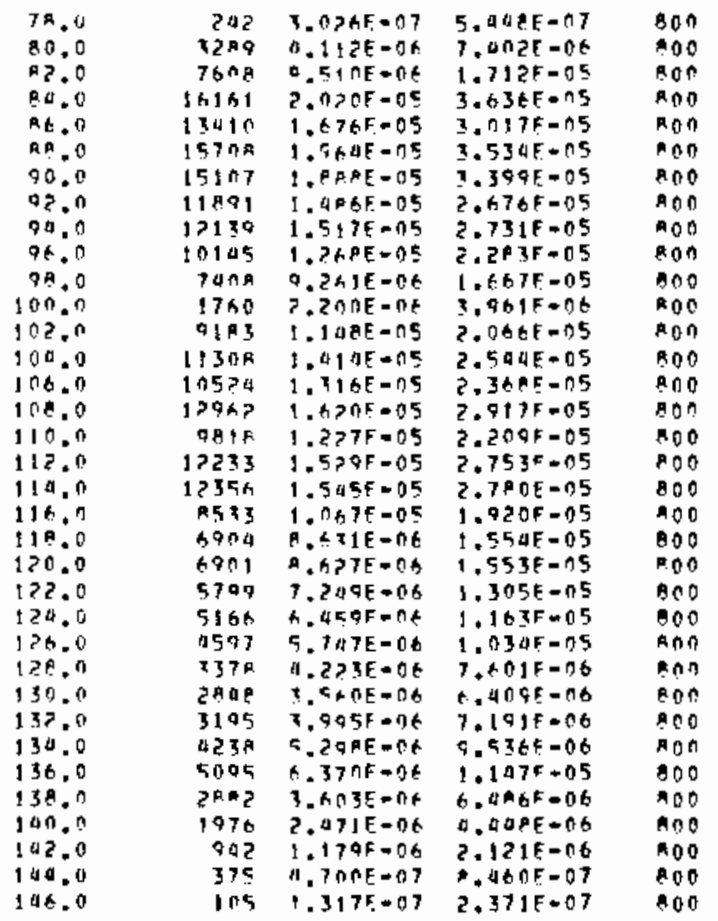

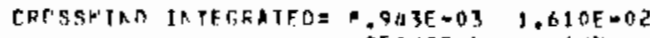

A7INUTH HEIGHT EXPCSUPE E/R FU/ TISTARCF

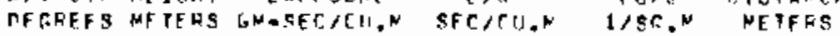
$x 1$ of +6

\begin{tabular}{|c|c|c|c|c|c|}
\hline 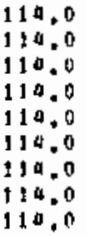 & $\begin{array}{l}0.3 \\
0.5 \\
1.7 \\
2.1 \\
6.7 \\
12.8 \\
14.7 \\
16.8 \\
37.9\end{array}$ & $\begin{array}{r}7981 \\
7919 \\
9123 \\
9160 \\
12094 \\
6331 \\
4708 \\
3415 \\
7406\end{array}$ & $\begin{array}{l}9.851 F-06 \\
9.770 F-06 \\
1.100 F=05 \\
1.008 F=05 \\
1.5075=05 \\
7.915 F=06 \\
5.935 F=06 \\
4.269 F-06 \\
3.05 P F=06\end{array}$ & $\begin{array}{l}1.773 E=05 \\
1.759 E=05 \\
2.053 E=05 \\
1.019 E=05 \\
2.703 E=05 \\
1.025 E=05 \\
1.067 E=05 \\
7.604 E=06 \\
5.505 E=06\end{array}$ & $\begin{array}{l}A 00 \\
900 \\
000 \\
800 \\
B 00 \\
900 \\
800 \\
900 \\
010\end{array}$ \\
\hline
\end{tabular}

AZINITH HEJGHT EXPCSURE FIR ELID OISTANCE PECHFES METERS GN-SECICIJ,N SECJCL,N 1/BR, N NETERS $\times] 0 F+6$

\begin{tabular}{|c|c|c|c|c|c|}
\hline $\begin{array}{l}122.0 \\
122.0 \\
122.0 \\
122.0 \\
122.0 \\
122.0 \\
122.0 \\
122.0\end{array}$ & $\begin{array}{r}0.3 \\
0.5 \\
1.0 \\
2.1 \\
4.2 \\
6.3 \\
12.4 \\
37.8\end{array}$ & $\begin{array}{l}4977 \\
5586 \\
4910 \\
6595 \\
7720 \\
4097 \\
1711 \\
713\end{array}$ & $\begin{array}{l}6.222 F=06 \\
6.983 E=06 \\
6.138 E=06 \\
A .232 E=06 \\
9.656 F=06 \\
5.059 E=06 \\
2.140 E=06 \\
9.913 E=07\end{array}$ & $\begin{array}{l}1.120 E-05 \\
1.257 E=05 \\
1.105 E=05 \\
1.082 E=05 \\
1.738 E=05 \\
9.107 E=06 \\
3.052 E=06 \\
1.604 E=06\end{array}$ & $\begin{array}{l}800 \\
n 00 \\
800 \\
100 \\
800 \\
000 \\
n 00 \\
n=0\end{array}$ \\
\hline
\end{tabular}

MINUTH HFIEHT FXPESLIFF F/R FU/G PISTARCF

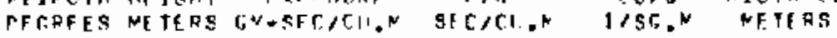
$\times 10 F+6$

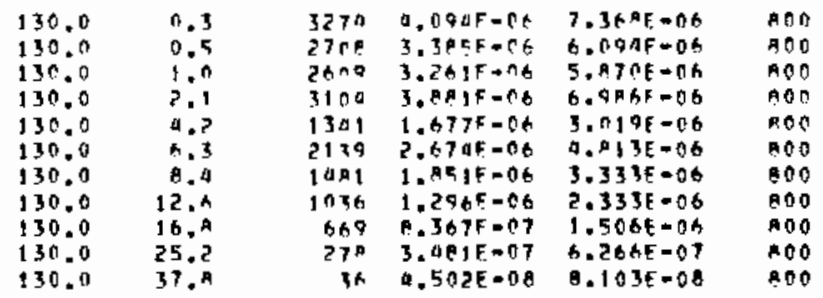

TOMER CATA F CLLCW...

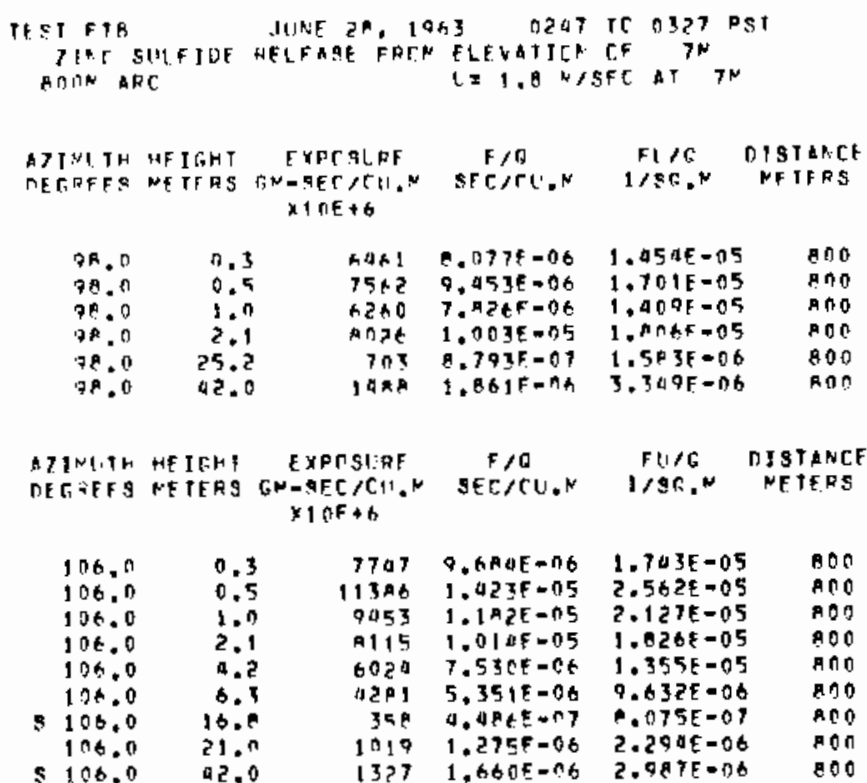




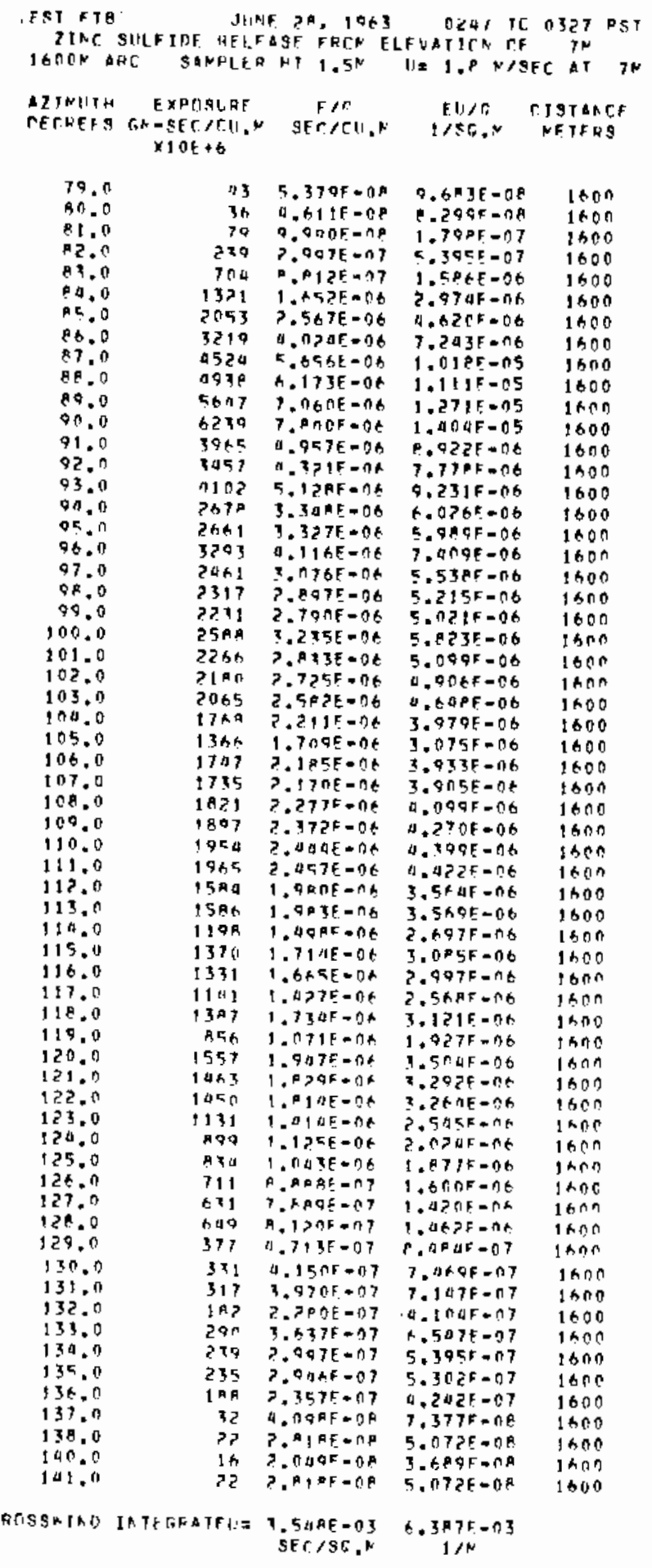

TORER TOTA FGLLCH....

IFST FIE JUME ?P, 19K3 0247 TE 0327 PST

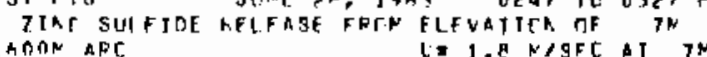

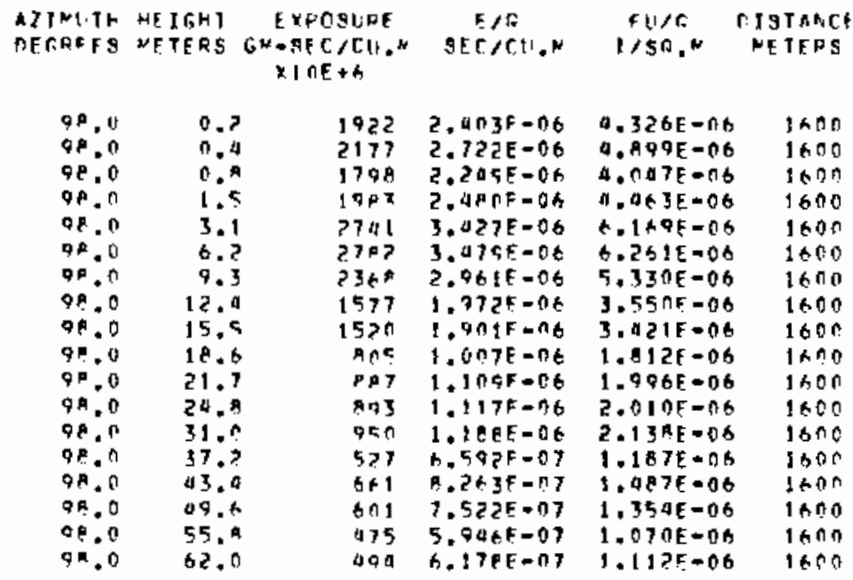

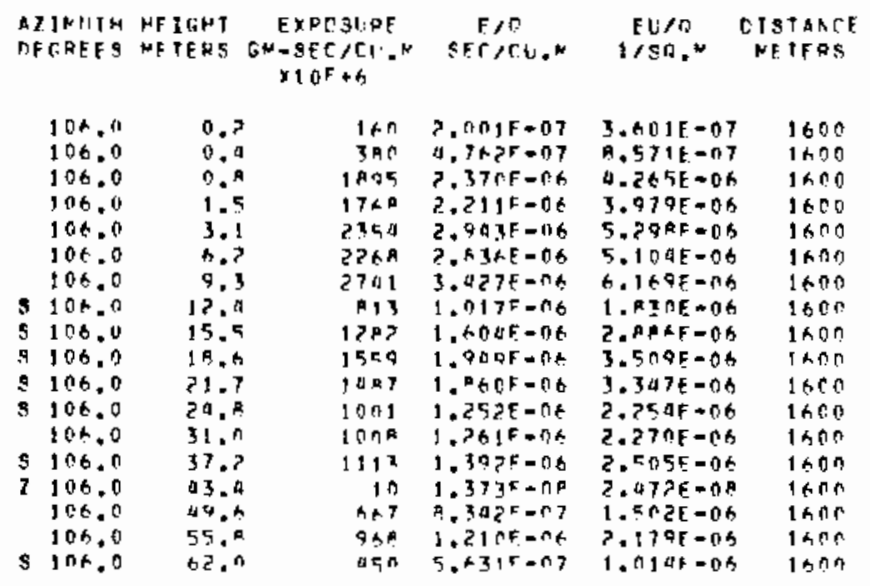

\begin{tabular}{|c|c|c|c|c|c|c|}
\hline & 134.0 & 0.2 & 1260 & $1 . \Delta\ulcorner 1 F=0 t$ & $?-+1 ? F=n h$ & 1000 \\
\hline & 114.0 & 0.2 & $1+00$ & $1 . \triangle A F=06$ & $2 . A 79 E-06$ & 1600 \\
\hline & 114.0 & $0 . P$ & $10<2$ & 1.31 \& $=0 \mathrm{t}$ & $2.369 \mathrm{~F}=06$ & 1000 \\
\hline & $1 \pm 4.0$ & 1,5 & $11 P n$ & $1.075 F-166$ & $2.65 \mathrm{AE}=0.6$ & 1600 \\
\hline & 114.0 & 3.1 & 1706 & $1.575 F=06$ & $2, A 36 F=n 6$ & 1600 \\
\hline & 154.0 & 6. & 1784 & $23 a F=06$ & $a .0 Z 1 E-C B$ & 1600 \\
\hline & 110.0 & & 1729 & $1625-06$ & $1 E=06$ & 1000 \\
\hline & 114.0 & 12.0 & 1477 & Ea> $=0 b$ & $3.324 E-\pi n$ & 1600 \\
\hline & $1 \pm 0.0$ & & $6+5$ & $2.3745-07$ & SRE $=06$ & $\sin 00$ \\
\hline & 124.0 & & 1230 & $1.550 F=06$ & $2.79 n E=00$ & $1 \times 00$ \\
\hline & 110.0 & 21.7 & 702 & E. $78+5-07$ & $1.581 \mathrm{E}=06$ & 1600 \\
\hline & $11 \mathrm{~d} .0$ & 74.9 & e 16 & $1.021 F=06$ & $1.037 f=06$ & 2600 \\
\hline 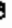 & 114.0 & 51.0 & +92 & $\neq 585-07$ & $1.557 E-06$ & $\ln n \pi$ \\
\hline 9 & 114,0 & 37.2 & $+0 n$ & $p .62<F=07$ & $1.553 F-06$ & 1600 \\
\hline & 114.0 & 43,9 & 015 & $5.19+E-1.7$ & $352 E=07$ & 1600 \\
\hline & $11 \div .0$ & & $3: 4$ & $3.929 \mathrm{E}-177$ & $173 E=07$ & $1+00$ \\
\hline & 130.0 & $\alpha$ & 132 & $1680,=07$ & $98 \mathrm{~A}-07$ & $160 ?$ \\
\hline & & & 49 & $A C E=n T$ & $231 E-07$ & 1600 \\
\hline
\end{tabular}




\begin{tabular}{|c|c|c|c|c|c|c|}
\hline \multicolumn{2}{|c|}{$\begin{array}{l}\text { AINNTTH } \\
\text { TECEFE }\end{array}$} & $\begin{array}{l}\text { HE IGHT } \\
\text { ME TERS }\end{array}$ & $\begin{array}{c}\text { ExaCSLDF } \\
\text { GN=SFC } \\
\times 10 \mathrm{C}+6\end{array}$ & $\begin{array}{c}E>C \\
\text { SEC }\end{array}$ & $\begin{array}{c}\mathrm{FL} / 0 \\
1 / \mathrm{ST} .\end{array}$ & $\begin{array}{l}\text { PISTARC } \\
\text { NETFRS }\end{array}$ \\
\hline & 122,0 & 0.2 & $7 \times 4$ & $\begin{array}{l}0.55 \bar{c}=07 \\
0.00 F=07\end{array}$ & $\begin{array}{l}1.719 E-06 \\
A .825 E=07\end{array}$ & \\
\hline I & $\begin{array}{l}122.0 \\
122.0\end{array}$ & $0 . \mathrm{A}$ & $\begin{array}{l}392 \\
707\end{array}$ & $\begin{array}{l}1.903 F=07 \\
\text { e. RAPF }=07\end{array}$ & $\begin{array}{l}A .825 E=07 \\
1.593 E=06\end{array}$ & \\
\hline & 122.0 & 1.5 & $10 \times 1$ & $1.352 F=106$ & $2.43 A E=06$ & \\
\hline & 122.0 & 3.1 & 1305 & $1.6375=06$ & $2.937 \mathrm{E}=06$ & \\
\hline & 122.0 & 6.2 & 1375 & $1.7105=06$ & $3.090 E=06$ & \\
\hline 7 & 12.2 .0 & 9.3 & 130 & 1.7 प $2 F=C 7$ & $3.135 E=07$ & \\
\hline & 122.0 & 2.4 & उद? & औ. $\Delta \cap B F=07$ & $F=07$ & \\
\hline & .0 & 15,5 & a 23 & $1.0 \geq n f-06$ & $1.054 E=06$ & \\
\hline & 0 & $18, A$ & $6>0$ & 7.94 यद $=07$ & $1, \triangle 15 E=O A$ & \\
\hline & .0 & 21.7 & 605 & $9.064 F=07$ & $1.452 \mathrm{~F}=0 \mathrm{~B}$ & \\
\hline & 12 & 21.5 & a $5 n$ & $5.030,5=07$ & $1.013 E=06$ & \\
\hline$T$ & 127.0 & 31.0 & 51 & $6.405 F=00$ & $1.1511 E-07$ & \\
\hline & 122.0 & 37.2 & 311 & $3 . R 915=07$ & $7.004 E-07$ & \\
\hline $\mathrm{s}$ & 122.0 & a 3.0 & 111 & $1.39+5-17$ & $2.5135=07$ & \\
\hline & & 40.6 & 80 & $1.110 \%-n 7$ & $2,005 E=07$ & \\
\hline & & 55. & 67 & $8.4 n<F=n ?$ & $1.513 \mathrm{E}-0.7$ & \\
\hline
\end{tabular}

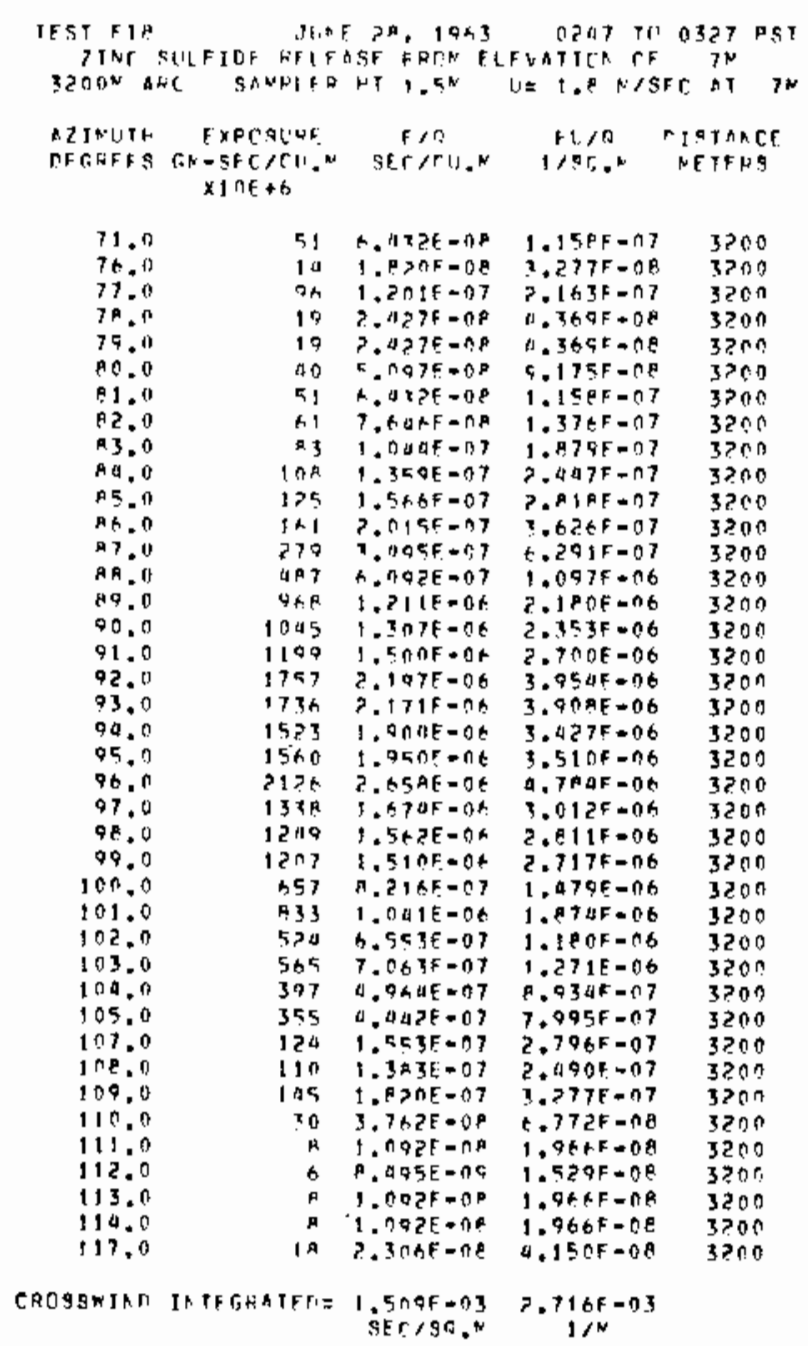

POKFF CATO FOLLTW....

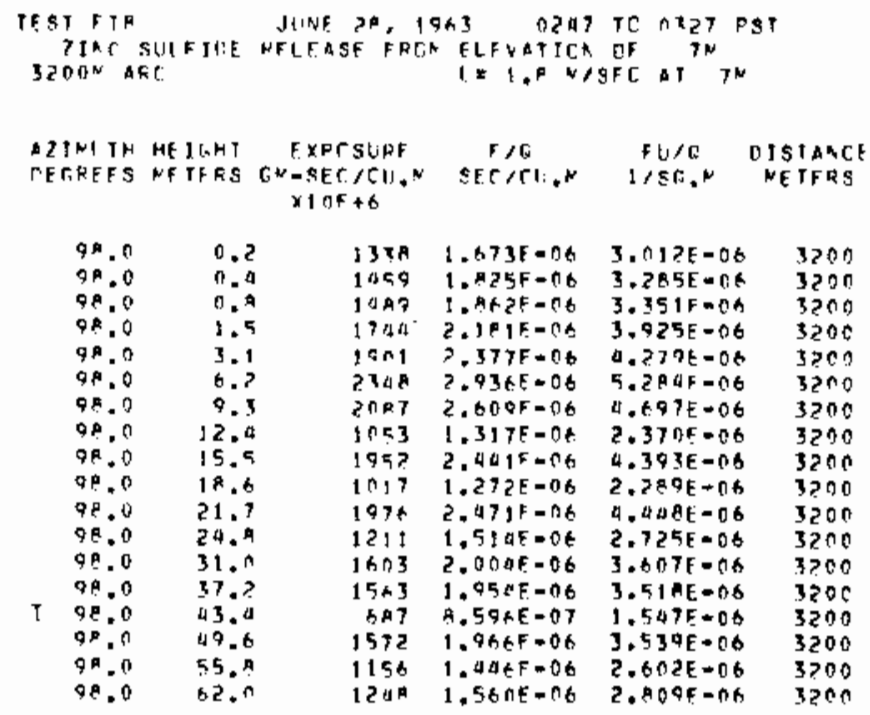




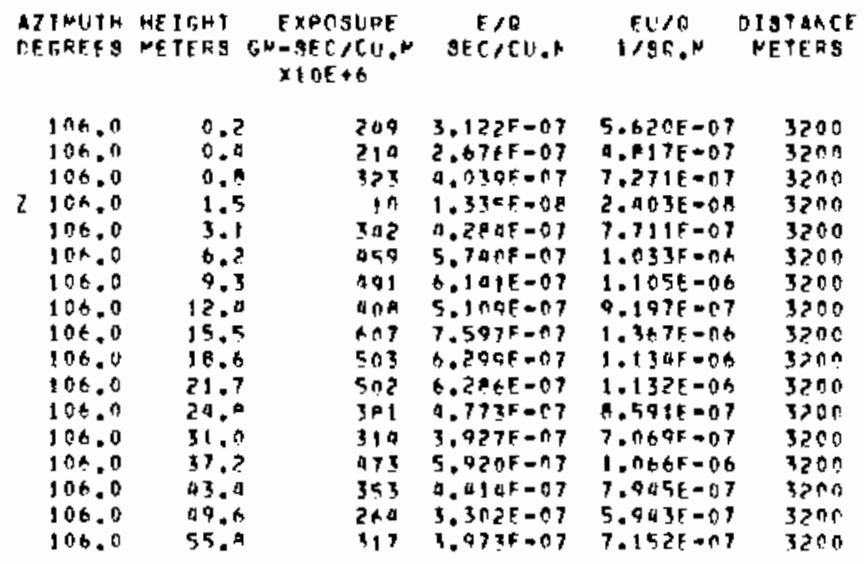

\begin{tabular}{|c|c|c|c|c|c|}
\hline $\begin{array}{l}\text { DITNUTH } \\
\text { MFCEFES }\end{array}$ & $\begin{array}{l}\text { HE ICRT } \\
\text { ME TSRS }\end{array}$ & $\begin{array}{c}\text { EXFSSLRE } \\
G^{N}=S F C / C ! 1 . N \\
\times 10 F+6\end{array}$ & $\begin{array}{c}f / 5 \\
s F[/ C U . N\end{array}$ & $\begin{array}{c}F(1 / 0) \\
1 / 26 \%\end{array}$ & $\begin{array}{l}\text { C. ISTAREE } \\
\text { VEFARS }\end{array}$ \\
\hline 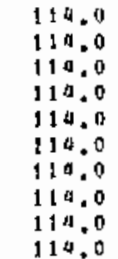 & $\begin{array}{l}0.9 \\
0.9 \\
3.1 \\
18.7 \\
21.7 \\
29.7 \\
31.7 \\
37 . ? \\
63.9 \\
55.9\end{array}$ & $\begin{array}{l}27 \\
55 \\
17 \\
20 \\
30 \\
10 \\
14 \\
9 \\
0 \\
9\end{array}$ & 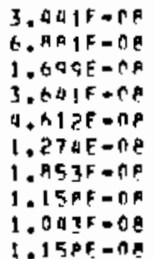 & $\begin{array}{l}6.193 F=08 \\
1.239 E=07 \\
3.75 \times F=O A \\
6.553 F=0 A \\
9.3 \cap 1 E=0 A \\
2.299 E=0 R \\
3.336 E=0 A \\
2.085 E=08 \\
1.277 E=O A \\
2.085 E=O A\end{array}$ & $\begin{array}{l}3500 \\
3200 \\
3200 \\
3200 \\
1200 \\
3200 \\
3200 \\
3200 \\
3200 \\
3>000\end{array}$ \\
\hline
\end{tabular}

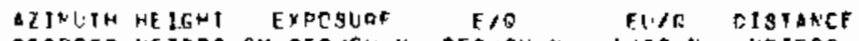
TEGREFS NETERS GN-SECICU.N SECICU,N IISA,N NETFFS $X ! \cap E+G$

\begin{tabular}{|c|c|c|c|c|}
\hline $\begin{array}{l}122.0 \\
122.0 \\
122.0 \\
127.0\end{array}$ & $\begin{array}{l}0.2 \\
0.4 \\
0.2 \\
9.3\end{array}$ & in & $\begin{array}{l}1.27 \Delta F=08 \\
2.739 F=08 \\
8.537 F=08 \\
230 \times F=00\end{array}$ & $\begin{array}{l}2.294 E=0 A \\
3.670 E=09 \\
1.537 \mathrm{E}=07\end{array}$ \\
\hline
\end{tabular}


SERIES 163 EXPERIMENT: 9 (FT-9)

GROUNO LEYEL ANO TOWER SAMPLING $200-5200$ M ARCS.

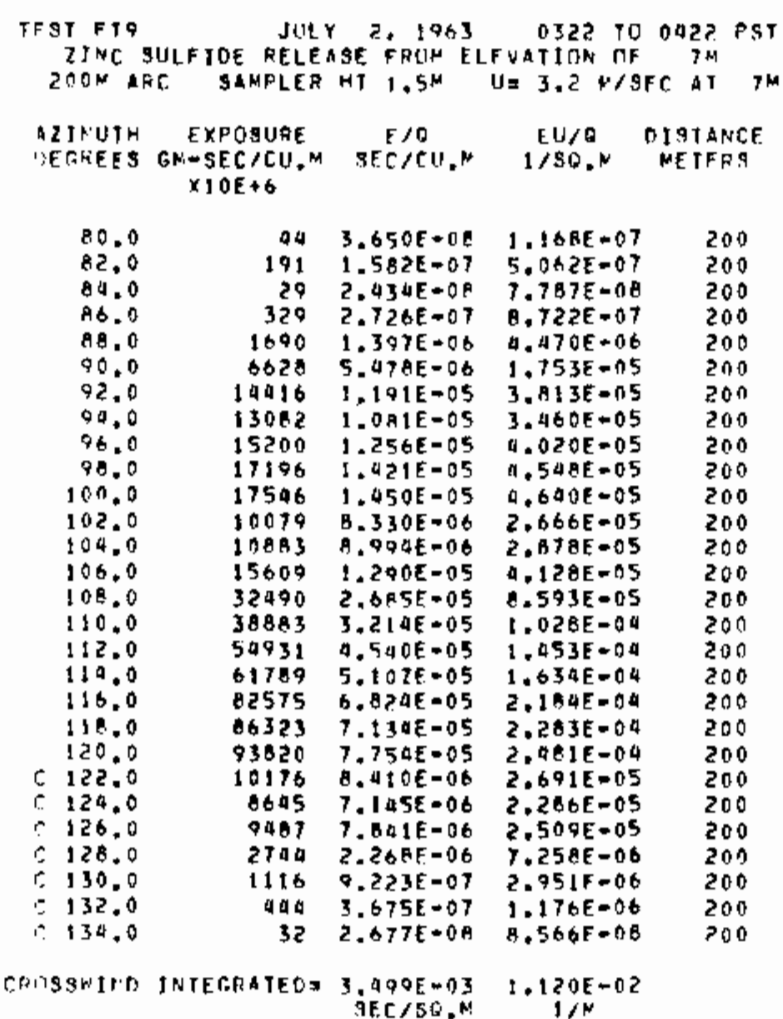

THWf DATA FOLLCR....

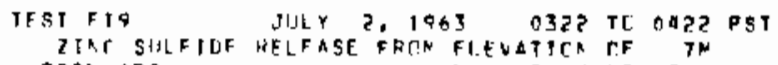

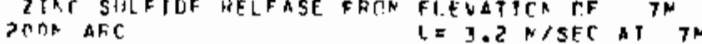

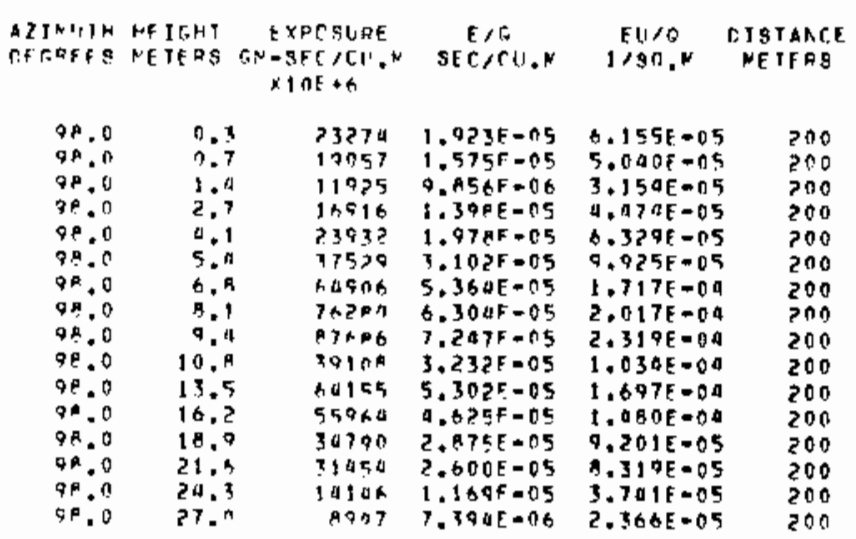

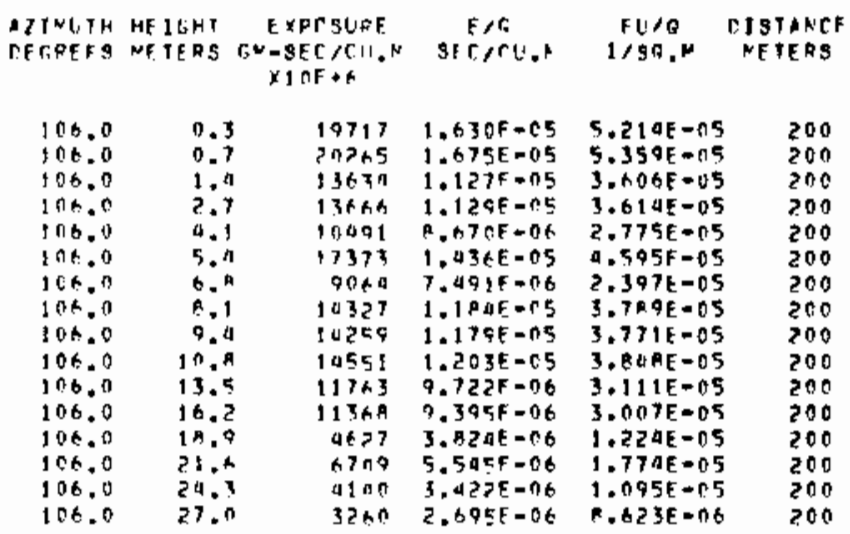

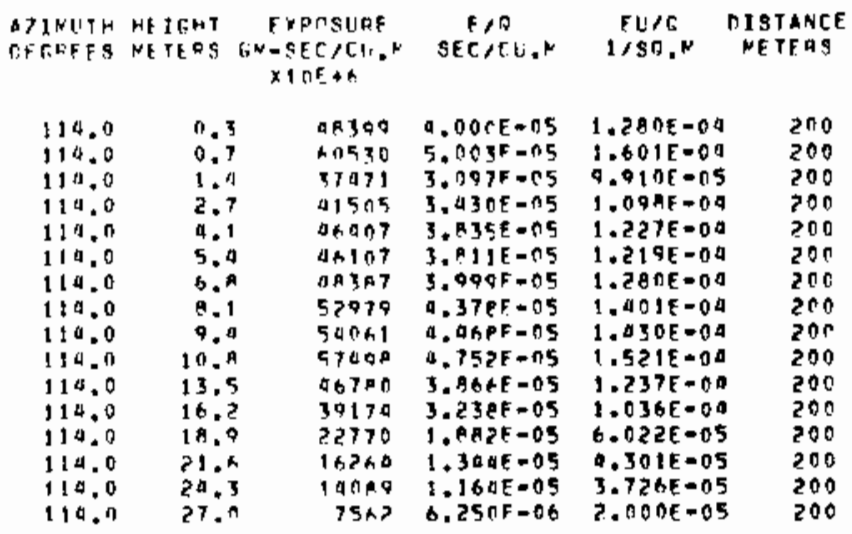

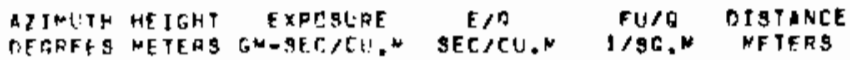

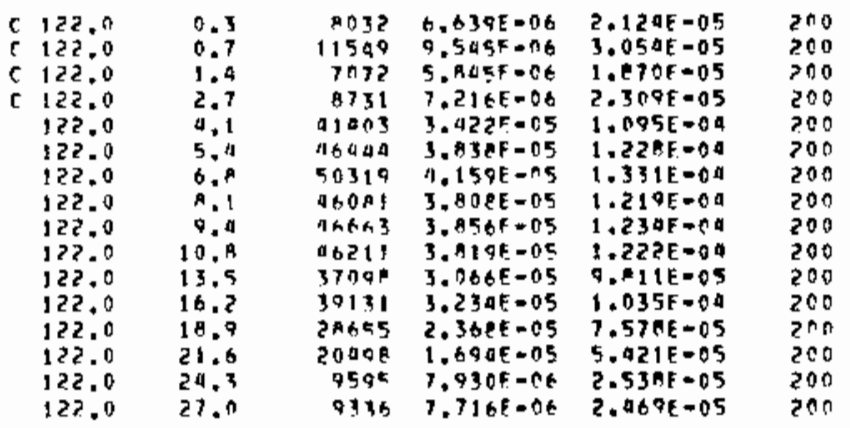




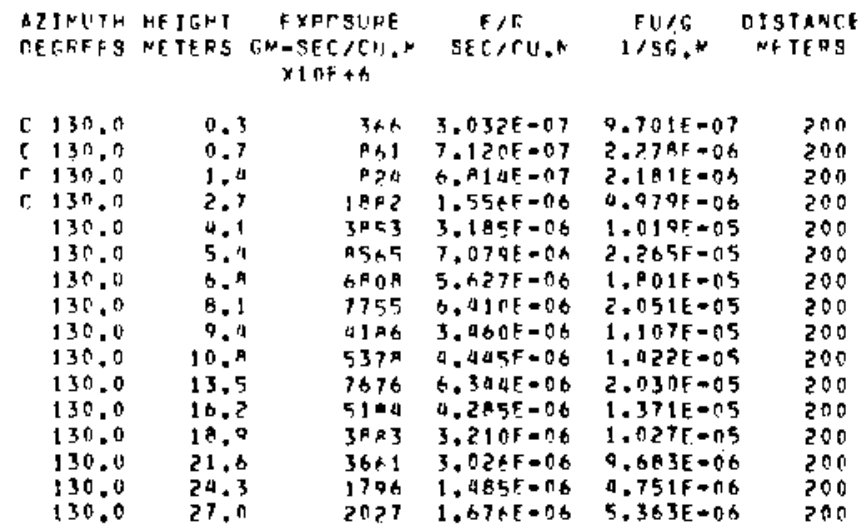

\begin{abstract}
TEST F19 JULY ?, 19630322 TO RAZ2 PST

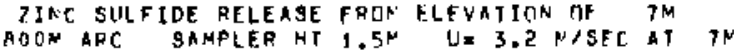

\begin{tabular}{|c|c|c|c|c|}
\hline $\begin{array}{l}\text { A7IMUTH } \\
\text { PFGRES }\end{array}$ & $\begin{array}{c}\text { EXPOQURE } \\
\text { GN-SEC/CU.M } \\
\times 10 E+6\end{array}$ & $\begin{array}{c}E / C \\
\text { SEC/CU.M }\end{array}$ & $\underset{1 / S U, N}{F U / O}$ & $\begin{array}{l}\text { DISTANCF } \\
\text { NETER }\end{array}$ \\
\hline 86.0 & 11 & $5.895 E-08$ & 1. AESE-0? & 800 \\
\hline AB. 0 & 2360 & $1.956 \mathrm{E}-06$ & $6.200 E=06$ & 900 \\
\hline 90.0 & 8103 & $6.697 E=06$ & $2.143 E-05$ & 800 \\
\hline 92.0 & 7847 & $6.4 A 5 E=0 B$ & $2.075 E-05$ & $B 00$ \\
\hline 94.0 & 9693 & $8.011 E=06$ & $2.563 E-05$ & BOO \\
\hline $\begin{array}{l}96.0 \\
90.0\end{array}$ & $\begin{array}{r}0097 \\
11897\end{array}$ & $\begin{array}{l}0.692 \varepsilon=06 \\
9.833 E=06\end{array}$ & $\begin{array}{l}2.142 E=05 \\
3.106 E=05\end{array}$ & $\begin{array}{l}000 \\
800\end{array}$ \\
\hline 100.0 & 1731 & $1.431 \mathrm{E}-06$ & a. $580 \mathrm{E}=06$ & 800 \\
\hline 102,0 & 4887 & $4.000 E-06$ & $1.293 \mathrm{E}-05$ & 800 \\
\hline 104.0 & 5152 & $0,25 A E=06$ & $1.363 \mathrm{JE}=05$ & 800 \\
\hline 106.0 & 1022 & $A, 451 E=07$ & $2.704 E=06$ & 800 \\
\hline 108.0 & 1683 & $1.391 E=06$ & $0.452 E-06$ & 800 \\
\hline 110.0 & 9657 & $3.800 E-06$ & $1.232 \mathrm{E}-05$ & 800 \\
\hline 112.0 & 9319 & 7.7 RE-06 & $2.465 E=05$ & 800 \\
\hline 114.0 & 0650 & $7.149 E-06$ & $2.280 \mathrm{c}-05$ & 800 \\
\hline 116.0 & 14586 & $1.205 E-05$ & $3.858 E=05$ & 300 \\
\hline 118.0 & 18070 & $1.493 \varepsilon=05$ & $4.7 T 9 E-05$ & 800 \\
\hline 120.0 & 9912 & $9.1026-06$ & $2.621 \mathrm{E}-05$ & 800 \\
\hline 122.0 & 10313 & $B .524 E=06$ & $2.7 P B E=05$ & 800 \\
\hline 120.0 & 9627 & $7.957 \mathrm{E}=06$ & $2.540 E-05$ & 800 \\
\hline 126.0 & 5201 & $4.298 E=06$ & $1.376 E-05$ & 800 \\
\hline 128.0 & 979 & A.098E-07 & $2.501 \mathrm{E}-06$ & 800 \\
\hline
\end{tabular}

CROSAWIND INTEGRAIELI $3.551 E-03 \quad 1.136 E-02$

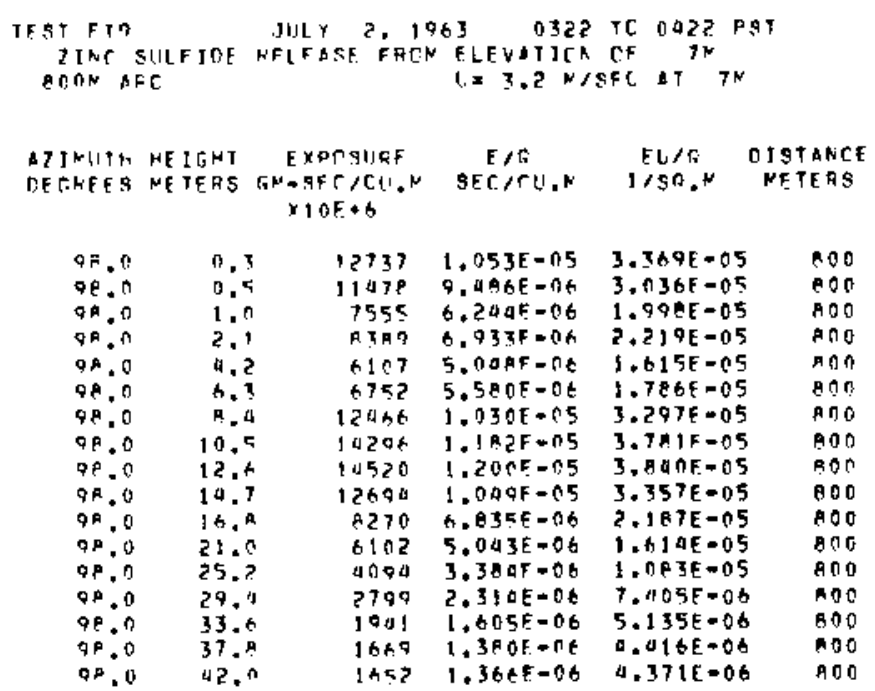

AZIMITH HEITHI EXPCSURE E/F FL/O OISTANCF DEFREFS METERS G,N-SEC/CU,N SEC/CLON 1/SO.N NETEFS $\times 10 \%+6$

\begin{tabular}{|c|c|c|c|c|c|}
\hline 100.0 & 0.3 & 963 & $7.363 \mathrm{~F}=17$ & $2.50 R E-06$ & $B 00$ \\
\hline $10 t .0$ & 1.5 & & T.RA3E -07 & $2.522 F-06$ & 200 \\
\hline 106.0 & $1 . n$ & 702 & $5.8000-n 7$ & $1,059 E=06$ & ADO \\
\hline $10 \div .0$ & 2.1 & $2 \geqslant 4$ & $6.817 E-07$ & $2,182 E=0 B$ & 200 \\
\hline int. & a. & $10 \times 2$ & R. $533 F=07$ & $2.731 E=06$ & 800 \\
\hline 106.0 & 6.7 & 1138 & $9.421 F=07$ & $3.015 E=06$ & मत \\
\hline 106.0 & 9.7 & 675 & $5.5 B 3 F=07$ & $1.787 E-06$ & 900 \\
\hline 106.0 & 10.9 & R6t & $7.164 \mathrm{~F}=07$ & $2.292 E=06$ & $9 \cap 0$ \\
\hline 104.0 & $12 . \mathrm{K}$ & 1 nat & $9.653 E-17$ & $2.76^{\circ} \mathrm{E}=0 \mathrm{OS}$ & 900 \\
\hline $10+.0$ & 14,7 & 1230 & $1.017 F-16$ & $3.2 \subseteq 5 E-06$ & $A 00$ \\
\hline 1 1 it. 0 & 16.9 & 1047 & $A, A 53 F=07$ & $2,769 E=0 \mathrm{~B}$ & $A 00$ \\
\hline $10 \% .0$ & $21 . n$ & 575 & $7.7390=07$ & $2.316 F=0 b$ & ano \\
\hline 1 ne. of & 25,7 & $5+9$ & $4.705 \mathrm{f}=\mathrm{n} 7$ & $1.50 \mathrm{BE}=0 \mathrm{~B}$ & RחO \\
\hline 106.0 & 29.0 & 558 & $4.612 \mathrm{E}-\mathrm{C}^{3}$ & $1.076 F=0 A$ & ROO \\
\hline $10 \mathrm{An}$ & 33.5 & 516 & $0.2725-07$ & $1.367 E=0 A$ & noO \\
\hline 106.0 & $37 .^{A}$ & 257 & $2.135 E=07$ & A.R $\backslash 7 E=07$ & 500 \\
\hline $1(15,0$ & 42.0 & 201 & $2.491 F=07$ & 7.7168 .07 & Ron \\
\hline
\end{tabular}

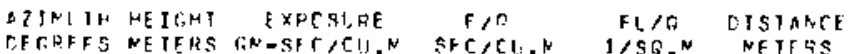
$\times 1$ intt

\begin{tabular}{|c|c|c|c|c|c|}
\hline 0.0 & 0.3 & AG $x_{1}$ & $7.406 \mathrm{~F}=00$ & $2.370 E-05$ & Ron \\
\hline 14.0 & 0,9 & 4717 & $7.205 f=06$ & $2.305 F=05$ & ROC \\
\hline 114.0 & $1, n$ & $n n 1 t$ & $4.977 f=06$ & $1.591 E=05$ & $P \cap 0$ \\
\hline 114.0 & 2.1 & $154 n$ & $0.232 E-06$ & $1.904 E-05$ & oOn \\
\hline 114.0 & $4 . ?$ & 6713 & $5.949 F \cdot 76$ & $1.77 \% E-05$ & son \\
\hline 110.0 & b. $\mathrm{T}$ & $20>7$ & $\therefore, A 7 A F=O A$ & $2.13+E=05$ & Ano \\
\hline 110.0 & a.t & 6799 & $5,+155=06$ & $1.70 A E=05$ & AOO \\
\hline 110.0 & 10.5 & $\mathrm{H} 2 \pi \mathrm{Y}$ & $6 . A 1 E F=010$ & $2.181 F=05$ & $R D 0$ \\
\hline 114.0 & 12.6 & 7136 & $5 . P Q P E=O B$ & $1.887 E-05$ & ROO \\
\hline 114.0 & 14.7 & 5925 & $4 . A Q 7 E-00$ & $1.567 \mathrm{E}-05$ & Boo \\
\hline 110.0 & 16.9 & $60+6$ & 5.510 .06 & $1.763 \mathrm{E}=05$ & S00 \\
\hline 110.0 & $21, n$ & 4774 & $3, Q \Delta \& F=0 t$ & $1.263 E=05$ & 100 \\
\hline $1 ! 4,0$ & $25 . ?$ & CA $3 A$ & $2.3 d+F=116$ & $7.506 E=06$ & $\operatorname{Ann}$ \\
\hline 114.0 & 29.4 & $250^{\circ}$ & $2.0735=06$ & $A .633 E=06$ & R.0 0 \\
\hline 14.0 & h & 1705 & $\triangle A B E=0 b$ & $10.749 E-06$ & ROC \\
\hline 114.0 & 7.9 & 1175 & $.715 \mathrm{~F} \cdot \mathrm{n} 7$ & $3,109 E-06$ & son \\
\hline & 12.0 & & $737 F$ & $2.476 E$ & in \\
\hline
\end{tabular}




\begin{tabular}{|c|c|c|c|c|c|}
\hline $\begin{array}{l}\text { AZTNIJTH } \\
\text { DETHEES }\end{array}$ & $\begin{array}{l}\text { HE IEMT } \\
\text { NETERS }\end{array}$ & $\begin{array}{c}E \times P C S U R E \\
\text { EN }=3 E C / C(1 . N \\
\times 1 \cap E+6\end{array}$ & $\begin{array}{c}E / \pi \\
S E C / C 11 . x\end{array}$ & $\begin{array}{c}E U / G \\
1 / 9 G_{.}\end{array}$ & $\begin{array}{l}\text { DISTANCE } \\
\text { METERS }\end{array}$ \\
\hline $\begin{array}{l}122.0 \\
122.0 \\
122.0 \\
12200 \\
122.0 \\
122.0 \\
12200 \\
12200 \\
122.0 \\
122.0 \\
127.0 \\
122.0 \\
122.0 \\
122.0 \\
122.0 \\
122.0 \\
122.0\end{array}$ & $\begin{array}{l}0.3 \\
0.5 \\
1.0 \\
2.1 \\
4.7 \\
5.7 \\
8.4 \\
10.5 \\
12.5 \\
10.7 \\
16.9 \\
21.0 \\
25.7 \\
29.9 \\
33.5 \\
37.4 \\
42.0\end{array}$ & 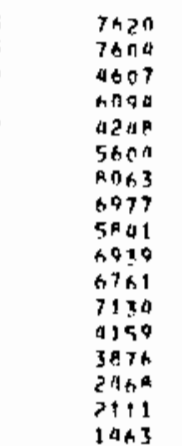 & 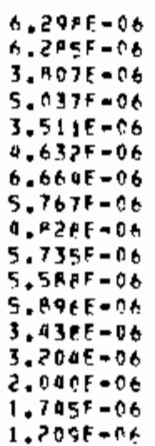 & 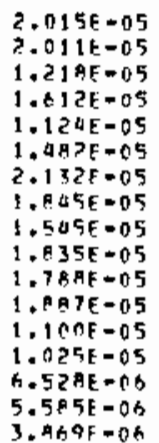 & 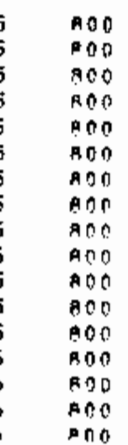 \\
\hline
\end{tabular}

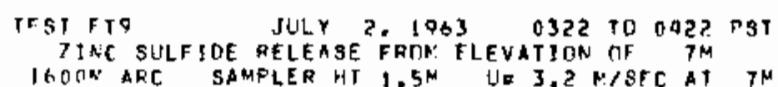
AZIMUITH EXPOSURE

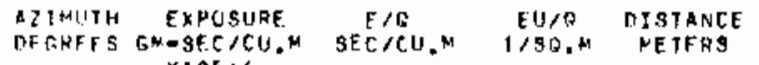
$\times 10 E+6$

AZINUTH HETEH EXPCSLIRF F/G GLAE DISTAMCF

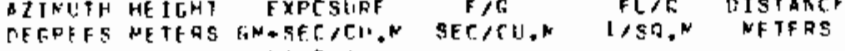
$\times 1$ CE +6

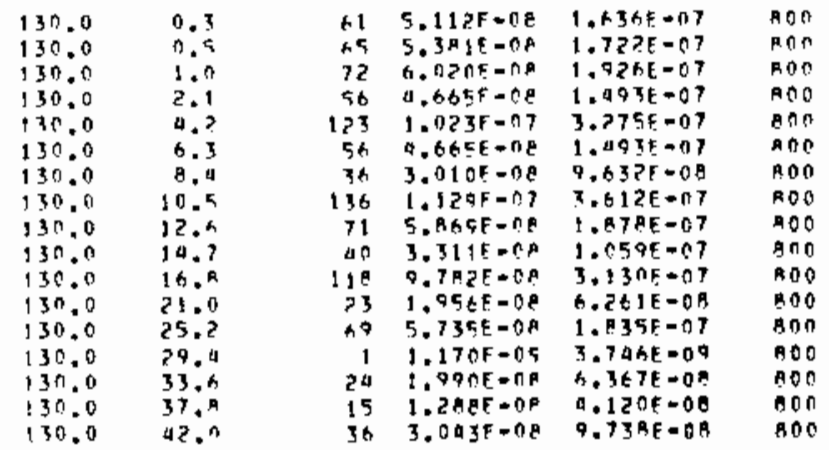

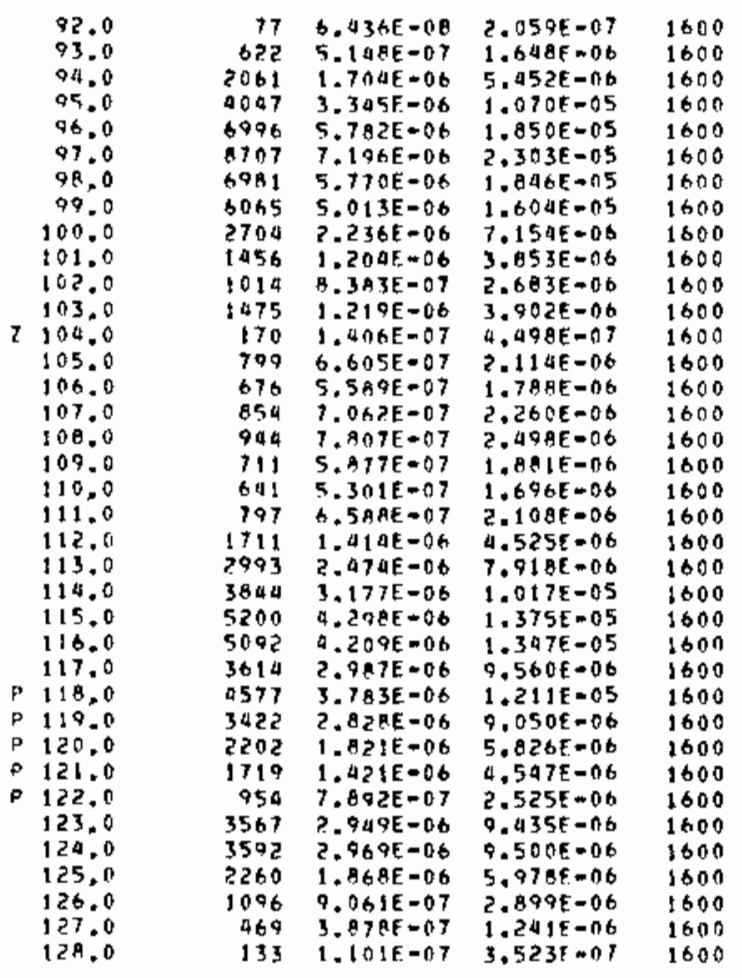

COASBWILD INTEGPRTED= $2.175 E-03 \quad h .961 E-03$

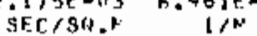

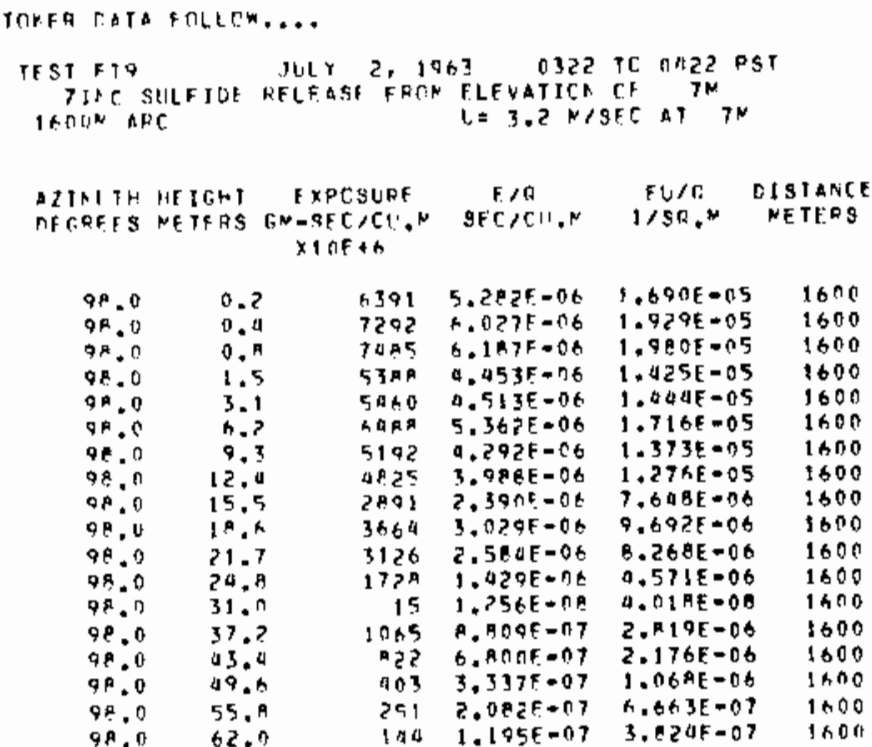




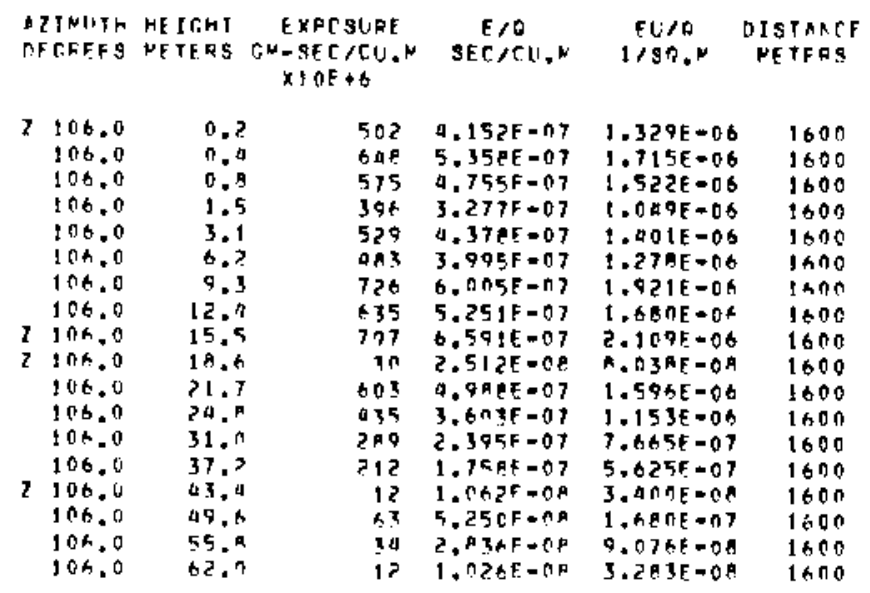

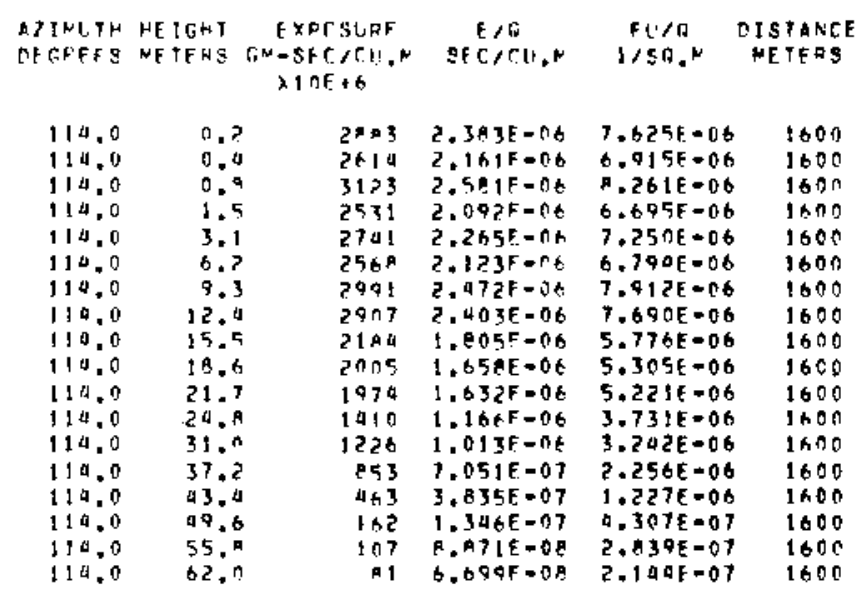

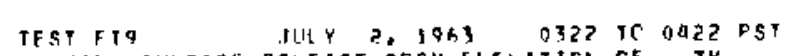

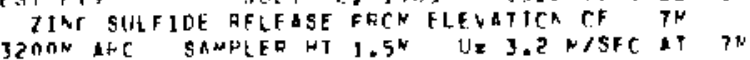

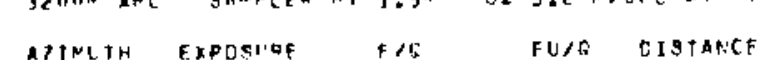

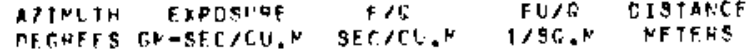
$\times 10 \mathrm{E}+\mathrm{h}$

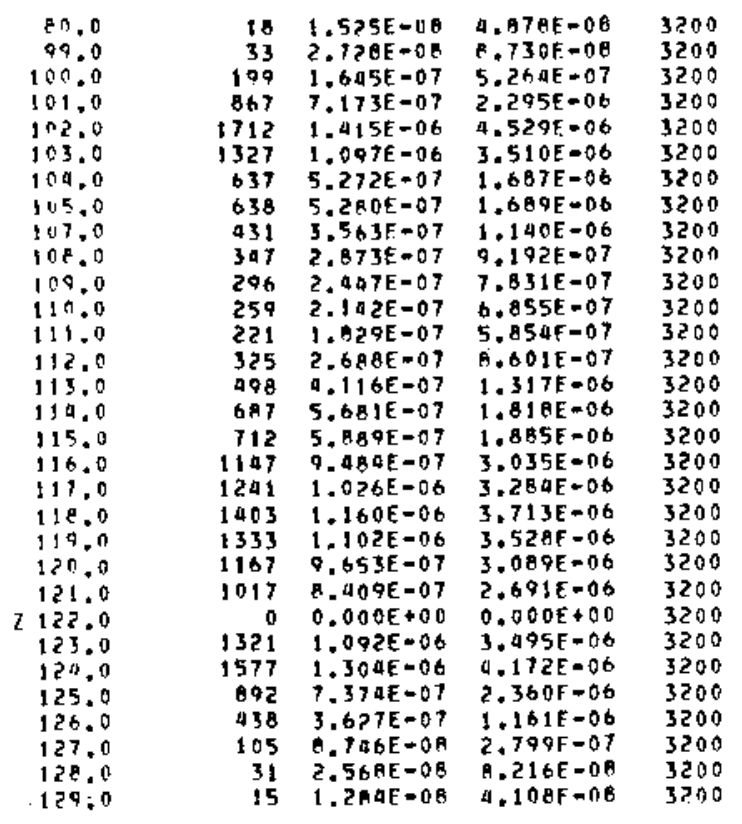

$$
\text { CRCSSHIAR IATEGRATED= } 1.019 E-03 \quad 3.260 E-03
$$

ITRER TOTA FCLLLE....

TFST TOQ JULY 3,1943 O3Z2 TC 0AZ? PST

7JKC SULFIDE HFLFASE FRCF ELFWATTCA CF $7 \mathrm{M}$

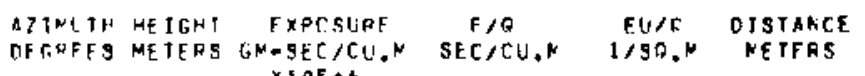
$X Y O E \bullet B$

\begin{tabular}{|c|c|c|c|c|c|}
\hline $\begin{array}{l}122.0 \\
0122.0 \\
122.0 \\
122.0 \\
122.0 \\
122.0 \\
122.0 \\
127.0 \\
122.0 \\
12200 \\
122.0 \\
122.0 \\
122.0 \\
122.0 \\
122.0 \\
122.0 \\
122.0\end{array}$ & $\begin{array}{l}0.2 \\
9.8 \\
1.5 \\
3.1 \\
6.2 \\
9.3 \\
12.5 \\
15.5 \\
18.6 \\
21.7 \\
20.9 \\
31.0 \\
37.2 \\
43.9 \\
49.6 \\
55.8 \\
62.0\end{array}$ & $\begin{array}{l}757 \\
676 \\
1998 \\
1851 \\
1978 \\
2713 \\
2020 \\
2127 \\
1470 \\
2059 \\
1141 \\
1072 \\
1097 \\
1109 \\
476 \\
314 \\
129\end{array}$ & $\begin{array}{l}6.2 A 3 E=07 \\
5.5 F 0 E=07 \\
1.503 F=06 \\
1.53 C E-06 \\
1.635 E=06 \\
2.243 E=06 \\
1.673 E=06 \\
1.75 E E=06 \\
1.719 F=06 \\
1.702 E=06 \\
9.037 E=07 \\
1.217 E=06 \\
1.233 F=06 \\
9.0945=07 \\
3.935 E=07 \\
2.801 E=07 \\
1.0685=07\end{array}$ & 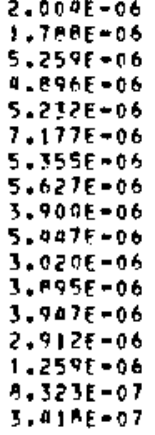 & $\begin{array}{l}1600 \\
1600 \\
1600 \\
1600 \\
1600 \\
1600 \\
1600 \\
1600 \\
1600 \\
1600 \\
1600 \\
1600 \\
1600 \\
1600 \\
1600 \\
1600 \\
1600\end{array}$ \\
\hline
\end{tabular}
JZOON AFC U. 3,2 NSEC AT TN

\begin{tabular}{|c|c|c|c|c|c|}
\hline $\begin{array}{l}\text { ARINLTH } \\
\text { DEREEFS }\end{array}$ & $\begin{array}{l}\text { HE IGHT } \\
\text { METERS }\end{array}$ & $\begin{array}{c}\text { EXPCSLRF } \\
G N+S E C / C U \text { - } \\
\text { : } 1 \cap E+6\end{array}$ & 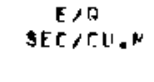 & $\begin{array}{l}F \cup 10 \\
1 / 50 . N\end{array}$ & $\begin{array}{l}\text { CISTAACE } \\
\text { NETERS }\end{array}$ \\
\hline $\begin{array}{l}98.0 \\
98.0 \\
98.0 \\
98.0 \\
98.0 \\
9 A .0 \\
08.0 \\
98.0 \\
9.00 \\
98.0 \\
98.0\end{array}$ & $\begin{array}{r}6.7 \\
9.3 \\
12.7 \\
15.5 \\
18.6 \\
21.7 \\
24.8 \\
31.7 \\
37.7 \\
43.9 \\
49.6\end{array}$ & $\begin{array}{r}59 \\
11 \\
7 \\
24 \\
19 \\
21 \\
7 \\
7 \\
12 \\
14 \\
14\end{array}$ & $\begin{array}{l}9.802 E=0 . \\
9.825 E=00 \\
6.316 E=09 \\
2.035 E=08 \\
1.610 E=08 \\
1.754 E=08 \\
1.130 E=09 \\
1.130 E=09 \\
1.002 E=08 \\
1.226 E=08 \\
1.224 E=08\end{array}$ & 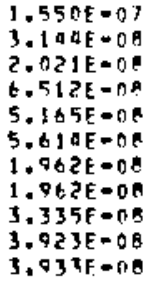 & $\begin{array}{l}3200 \\
3500 \\
3200 \\
3500 \\
32500 \\
3200 \\
3500 \\
3200 \\
3200 \\
3200 \\
3200\end{array}$ \\
\hline
\end{tabular}

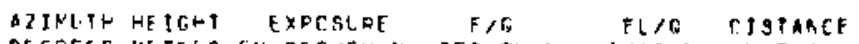 DEROFFS NETEHS GW $\times 1$ तE +6
$\begin{array}{llllll}130.0 & 21.7 & 24 & 2.033 E-08 & 6.507 E-09 & 1600 \\ 130.0 & 31.0 & 17 & 1.449 F=08 & 4.6365-00 & 1600\end{array}$




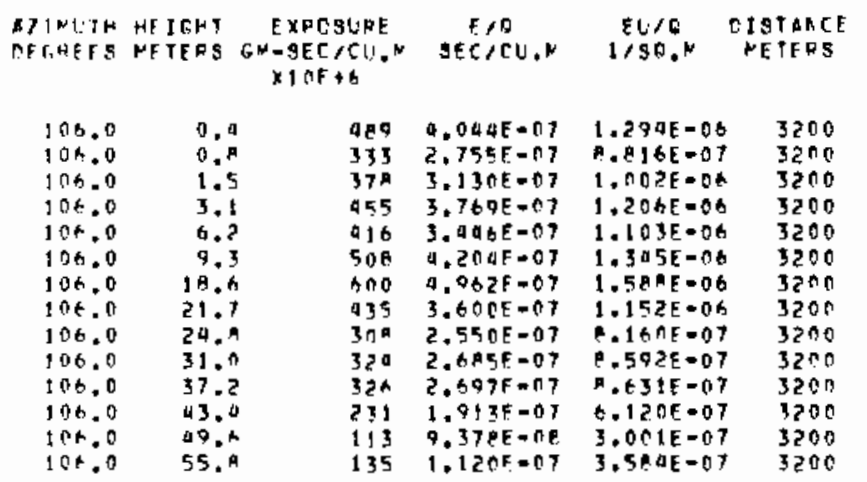

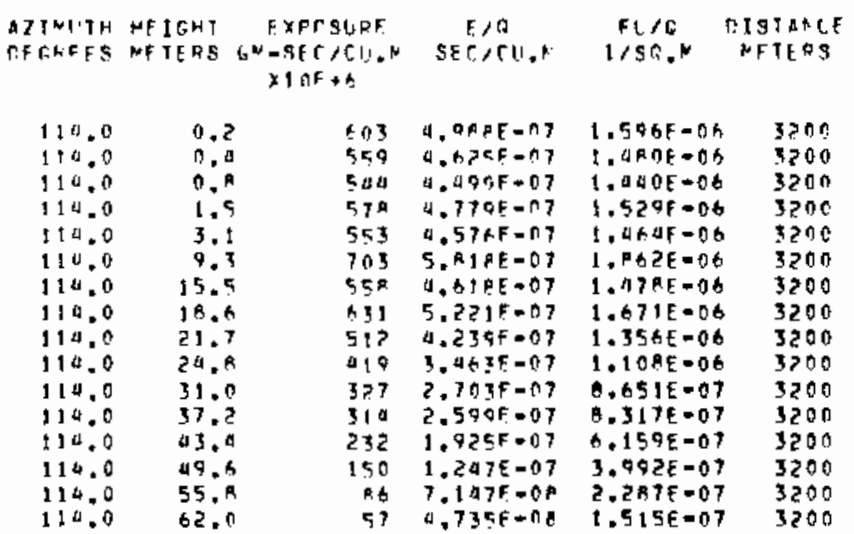

MIUITH HEIGHT EXPCSLRE E/Q FU/G DISTANCE DFTRFF NETFAS GN-SEC/CU.N SEC/CLAN I/SO.N NETERS

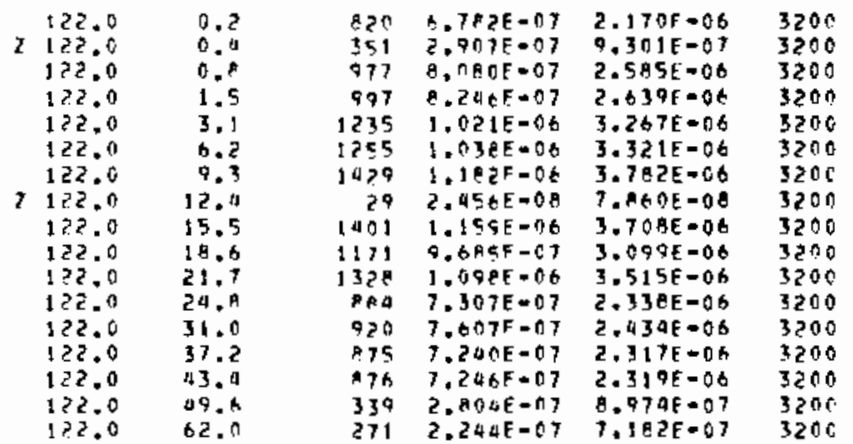


SERIES 163 EXPERIWENT: 10 （FT-10)

GROUND LEVEL AND TOHER SAMPLING 200-3200 M ARCS.

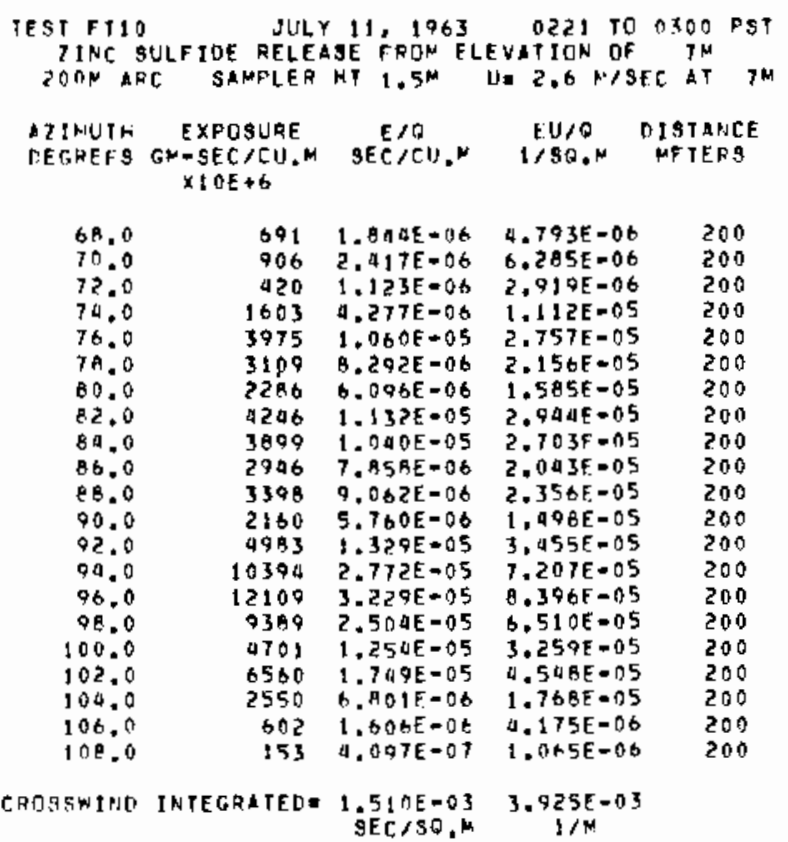

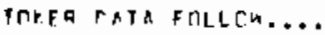

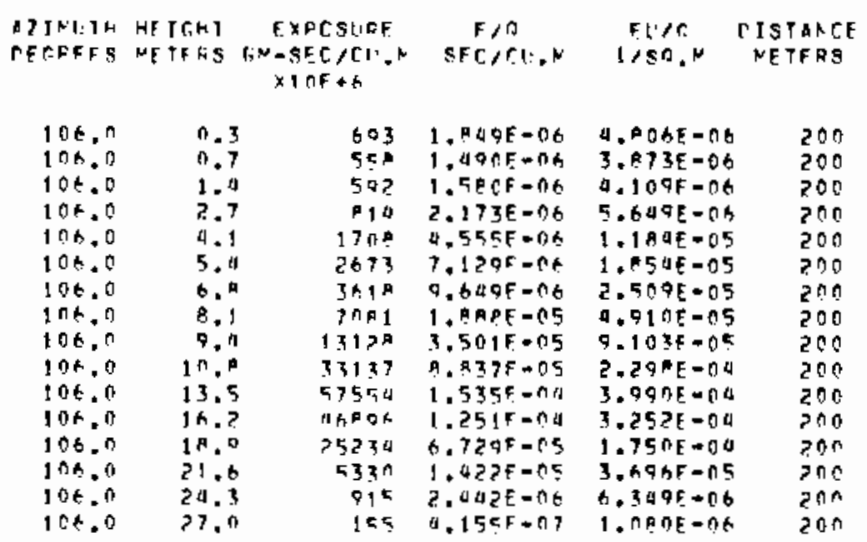

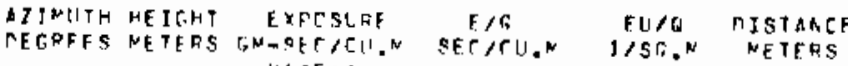
$\times \rightarrow \cap F+6$

\begin{tabular}{|c|c|c|c|c|c|}
\hline 122.0 & 4. 1 & $\times 5$ & $9.577 E=00$ & $2.49 \cap E-\cap 7$ & $\operatorname{lnn}$ \\
\hline $\begin{array}{l}\text { MINUITH } \\
\text { RECPEFS }\end{array}$ & $\begin{array}{l}\text { HEJCHT } \\
\text { NETERS }\end{array}$ & $\begin{array}{c}\text { EXPNSUFF } \\
G N=S F C, C 11, N \\
\times 1 O E+E\end{array}$ & $\begin{array}{c}E>n \\
g=r / r i, n\end{array}$ & $\begin{array}{c}F U / 0 \\
1 / S \cap . N\end{array}$ & $\begin{array}{l}\text { DISTABCEE } \\
\text { NETFRS }\end{array}$ \\
\hline $13 n, 0$ & $10 . A^{2}$ & 71 & $1.815 F=07$ & $4.980 E-07$ & 200 \\
\hline
\end{tabular}

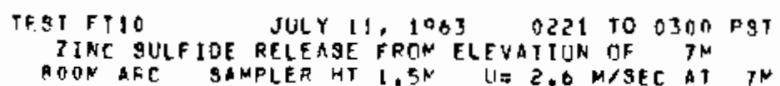

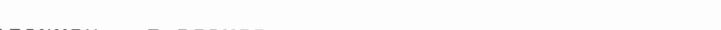

BZTNUTH EXPOSUAE E/O EU/O OISTANCE DFGREES GNOSEC/CU.M SECICU.N I/ST.N NEIERS
XIOE+6

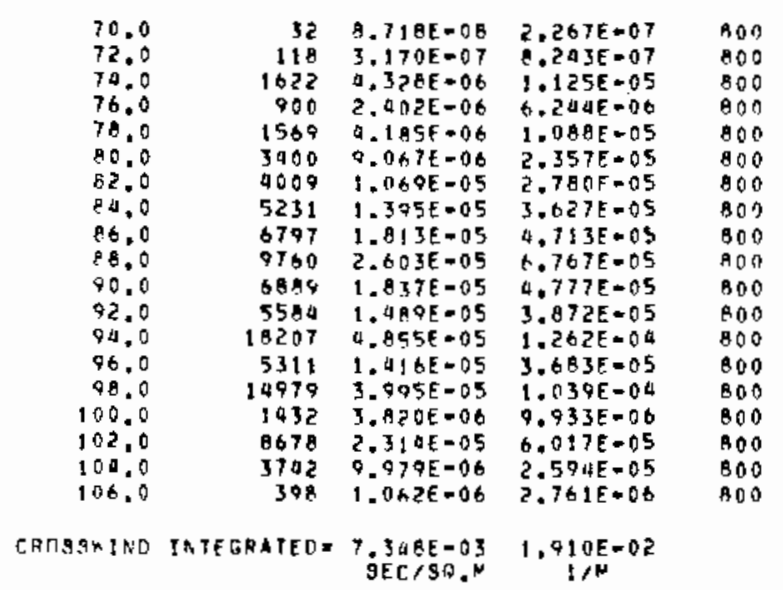


TRAFR TATA IRLLCH...

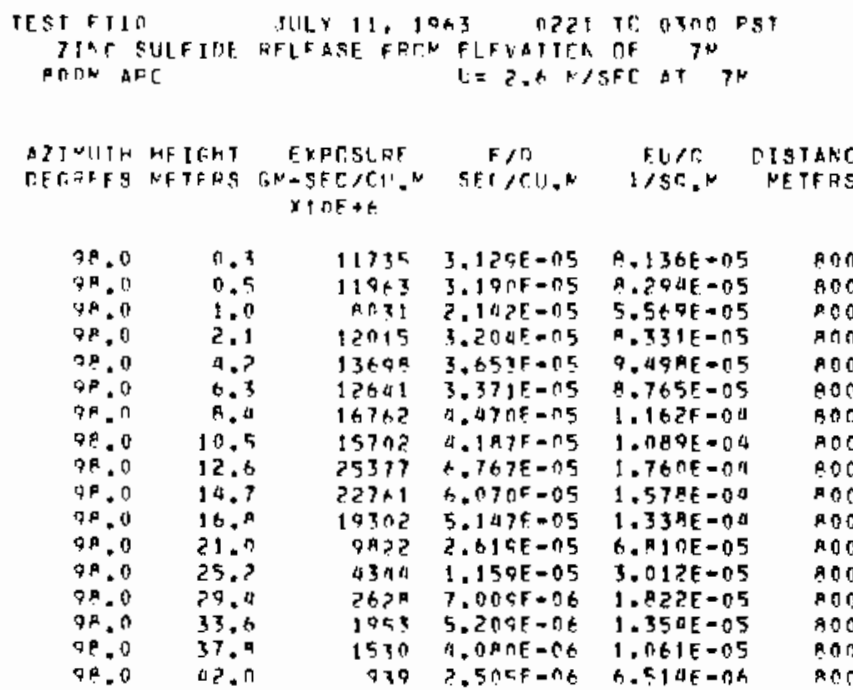

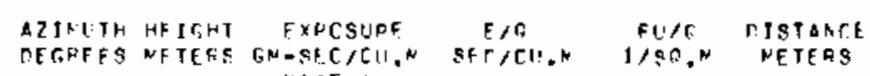
$\operatorname{lin}+6$

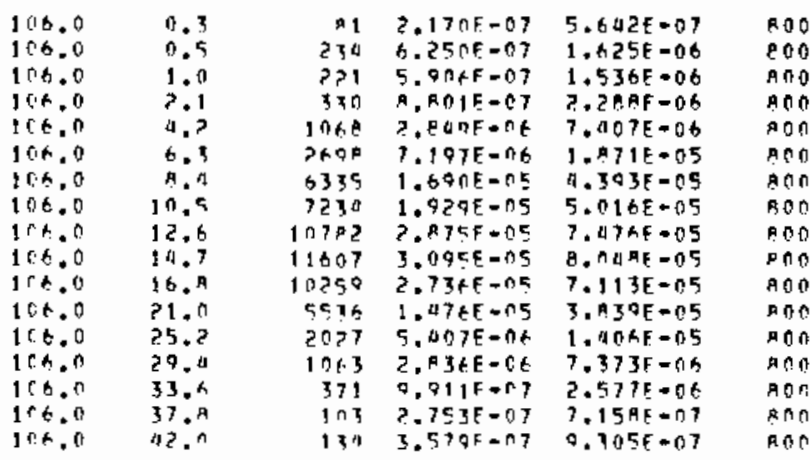

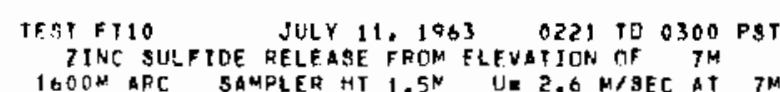

AZIMUTH EXPOSURE
DEFREFS GMESECICU.M SEC/CU.N TH/O DISTANCE $\times 10 E$ \& 6

\begin{tabular}{|c|c|c|c|c|c|}
\hline 2 & $\begin{array}{l}70.0 \\
71.0 \\
72.0 \\
73.0 \\
74.0 \\
75.0 \\
76.0 \\
77.0 \\
78.0 \\
79.0 \\
80.0 \\
81.0 \\
87.0 \\
83.0 \\
89.0 \\
85.0 \\
86.0 \\
87.0 \\
88.0 \\
89.0 \\
90.0 \\
91.0 \\
92.0 \\
93.0 \\
94.0 \\
95.0 \\
96.0 \\
97.0 \\
98.0 \\
99.0 \\
100.0 \\
126.0\end{array}$ & $\begin{array}{r}62 \\
68 \\
60 \\
90 \\
53 \\
113 \\
221 \\
575 \\
794 \\
1331 \\
1122 \\
598 \\
1468 \\
1674 \\
1831 \\
3175 \\
3920 \\
9452 \\
5519 \\
5255 \\
6077 \\
8502 \\
8555 \\
7269 \\
6135 \\
5146 \\
4473 \\
2810 \\
1663 \\
521 \\
25 \\
34\end{array}$ & 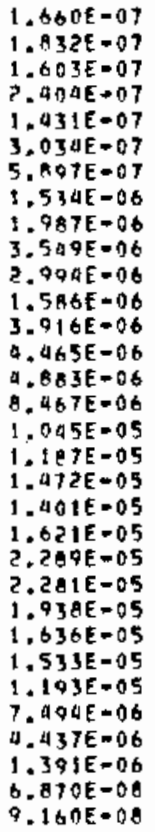 & $\begin{array}{l}4.317 E=07 \\
4.763 E=07 \\
4.16 B E=07 \\
6.251 E-07 \\
3.721 E=07 \\
7.889 E=07 \\
1.533 E=06 \\
3.989 E=06 \\
5.165 E=06 \\
9.22 B E=06 \\
7.785 E-06 \\
4.123 E=06 \\
1.018 E=05 \\
1.161 E=05 \\
1.270 E=05 \\
2.201 E=05 \\
2.718 E=05 \\
3.0 B 7 E=05 \\
3.827 E=05 \\
3.644 E=05 \\
4.219 E-05 \\
5.951 E=05 \\
5.931 E=05 \\
5.040 E=05 \\
4.254 E=05 \\
3.965 E=05 \\
3.102 E=05 \\
1.948 E=05 \\
1.154 E=05 \\
3.617 E-06 \\
1.786 E=07 \\
2.382 E=07\end{array}$ & $\begin{array}{l}1600 \\
1600 \\
1600 \\
1600 \\
1600 \\
1600 \\
1600 \\
1600 \\
1600 \\
1600 \\
1600 \\
1600 \\
1600 \\
1600 \\
1600 \\
1600 \\
1600 \\
1600 \\
1600 \\
1600 \\
1600 \\
1600 \\
1600 \\
1600 \\
1600 \\
1600 \\
1600 \\
1600 \\
1600 \\
1600 \\
1600 \\
1600\end{array}$ \\
\hline & 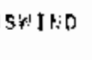 & TEGR & $\begin{array}{c}6.32 R E-03 \\
\text { STC C B B. }\end{array}$ & $1.645 E=02$ & \\
\hline
\end{tabular}

TOKFE TATA FOLLEW....

TFST FI1D JULY 11. 1963 0221 TL OSOO PST

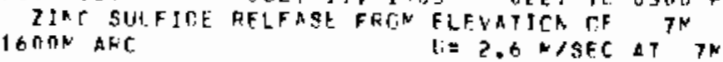

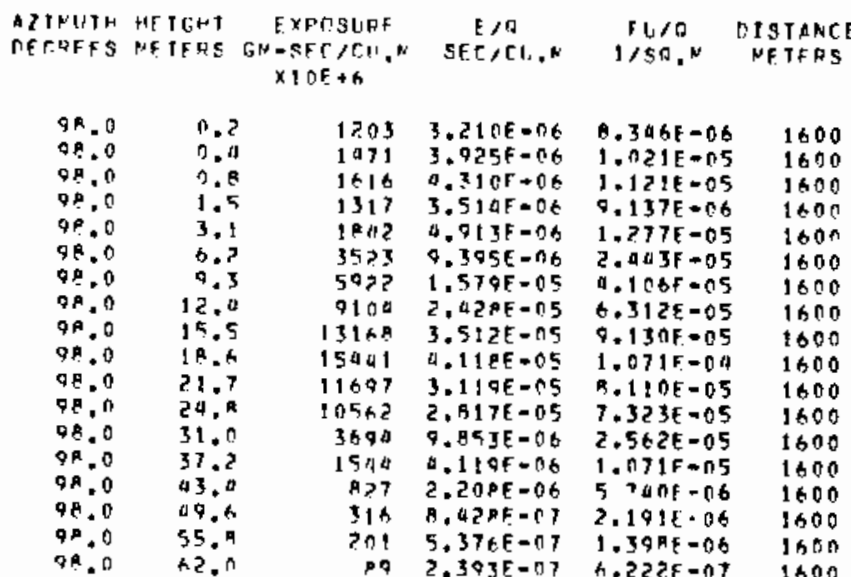




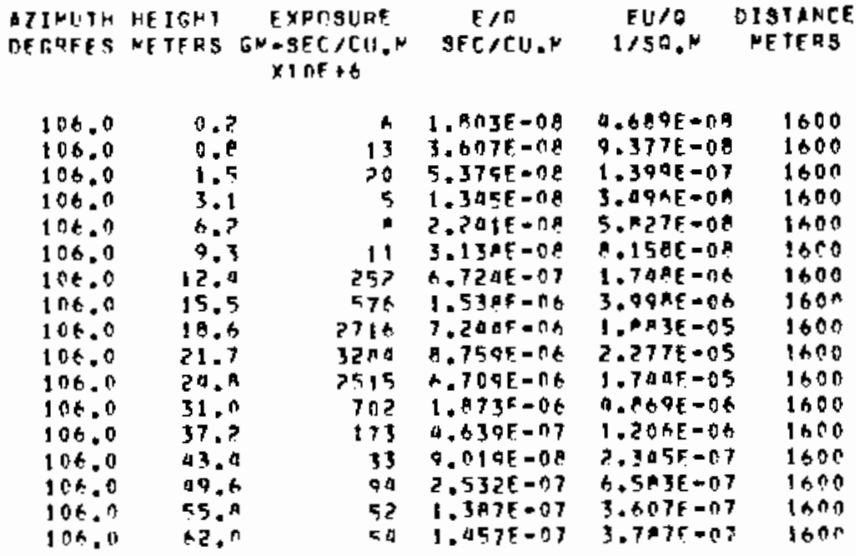

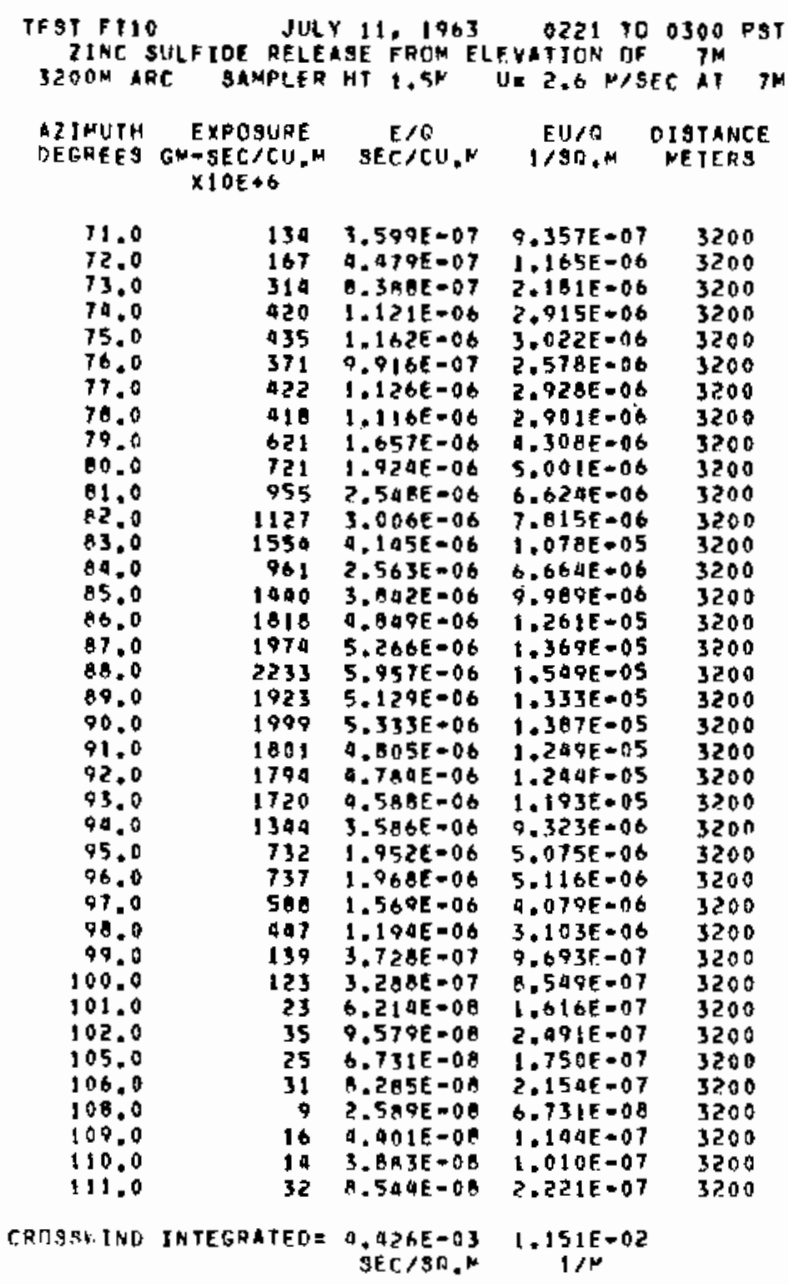


SERIES 163 EXPERIMENT: † \& FT-1 \&

GROUND LEVEL AND TOWER SAMPLING 200-3200 M ARCS.

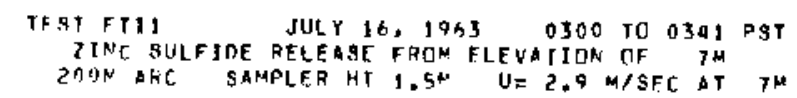

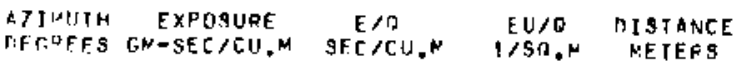
$\times \perp O E+6$

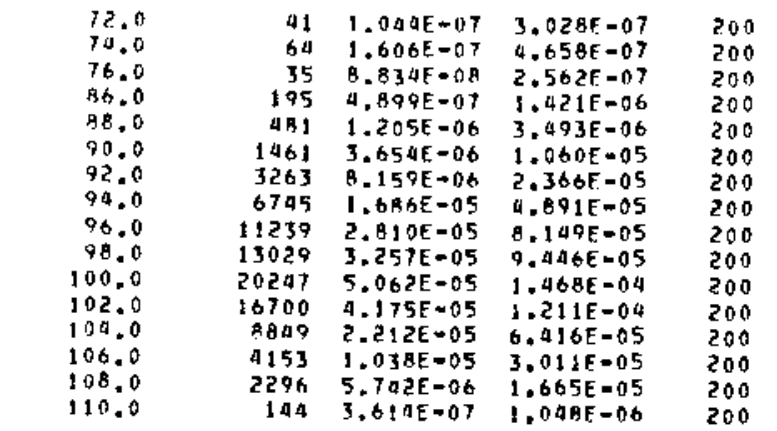

CROSSWITD INTESRATED $=1.56 I E-03 \quad 0.526 E-03$

\begin{tabular}{|c|c|c|c|c|c|}
\hline $\begin{array}{l}\text { AZJNLYH } \\
\text { DEGREFS }\end{array}$ & $\begin{array}{l}\text { ME IEHT } \\
\text { ME TERS }\end{array}$ & $\begin{array}{c}\text { FXPOSURF } \\
\text { GN.SEC/CU.N } \\
\times 10 E+6\end{array}$ & $\begin{array}{c}E<6 \\
\& E C / C U . N\end{array}$ & $\begin{array}{l}E \mathrm{~L} / 6 \\
\mathrm{t} / \mathrm{si} . \mathrm{F}\end{array}$ & $\begin{array}{l}\text { DI STANCE } \\
\text { NETERS }\end{array}$ \\
\hline $\begin{array}{l}106.0 \\
106.0 \\
106.0 \\
106.0 \\
106.0 \\
106.0 \\
106.0 \\
106.0 \\
106.0\end{array}$ & $\begin{array}{l}0.3 \\
0.7 \\
1.7 \\
2.7 \\
4.1 \\
5.1 \\
6.8 \\
8.1 \\
9.4\end{array}$ & $\begin{array}{l}2675 \\
3946 \\
3078 \\
4592 \\
9179 \\
14896 \\
16192 \\
32517 \\
52636\end{array}$ & $\begin{array}{l}6.6 A A E-06 \\
9.616 F=06 \\
7.572 E=06 \\
1.142 F=05 \\
2.245 E-05 \\
3.724 F=05 \\
4.04 E E=05 \\
8.124 F=05 \\
1.31+E=00\end{array}$ & $\begin{array}{l}1.900 E=05 \\
2.760 E=05 \\
2.196 E=05 \\
3.330 E=05 \\
6.655 E=05 \\
1.080 E=04 \\
1.179 E=04 \\
2.358 E=04 \\
3.816 E=04\end{array}$ & $\begin{array}{l}200 \\
200 \\
200 \\
200 \\
200 \\
200 \\
200 \\
200 \\
200\end{array}$ \\
\hline $\begin{array}{l}106.0 \\
106.0 \\
106.0 \\
106.0 \\
106.0 \\
106.0 \\
106.0\end{array}$ & $\begin{array}{l}10.9 \\
13.5 \\
16.2 \\
18.9 \\
21.4 \\
24.3 \\
27.0\end{array}$ & $\begin{array}{r}93402 \\
137451 \\
133978 \\
\times 7942 \\
11859 \\
2057 \\
572\end{array}$ & $\begin{array}{l}2.335 E=04 \\
3.436 E=04 \\
3.34 P F=04 \\
1.695 F=04 \\
2.965 E=05 \\
5.1445=06 \\
1.307 F=06\end{array}$ & $\begin{array}{l}6.772 E=04 \\
9.965 E=04 \\
9.710 E=04 \\
4.927 E=04 \\
6.597 E=05 \\
1.492 E-05 \\
3.791 E=06\end{array}$ & $\begin{array}{l}200 \\
200 \\
200 \\
200 \\
200 \\
200 \\
200\end{array}$ \\
\hline $\begin{array}{l}Z I N U T H \\
\text { FGEES }\end{array}$ & $\begin{array}{l}\text { HE IFH } \\
\text { NE TERS }\end{array}$ & 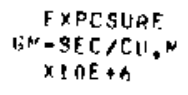 & $\begin{array}{c}\text { SEC } \\
\text { E }\end{array}$ & $\begin{array}{c}F \| / C \\
1 / 59 . N\end{array}$ & $\begin{array}{l}\text { OISTARCE } \\
\text { METERS }\end{array}$ \\
\hline $\begin{array}{l}113.0 \\
114.0\end{array}$ & $\begin{array}{l}2.7 \\
0.1\end{array}$ & $\begin{array}{l}27 \\
40\end{array}$ & $\begin{array}{l}6.75+E=0.9 \\
1.013 F=07\end{array}$ & $\begin{array}{l}1.959 E=07 \\
2.939 E-07\end{array}$ & $\begin{array}{l}200 \\
200\end{array}$ \\
\hline
\end{tabular}

TEREF CAIA FALLCH...

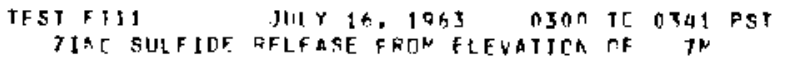

7 IAC SULFIDE. FFLFASE FRON FLEVATJCA CE TH
2OCN ARC

ATIULIH HEIGHT EXPGSURE ER TE DURO DISACE

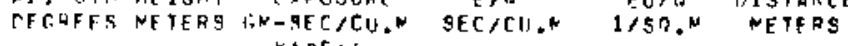
$x+n E+6$

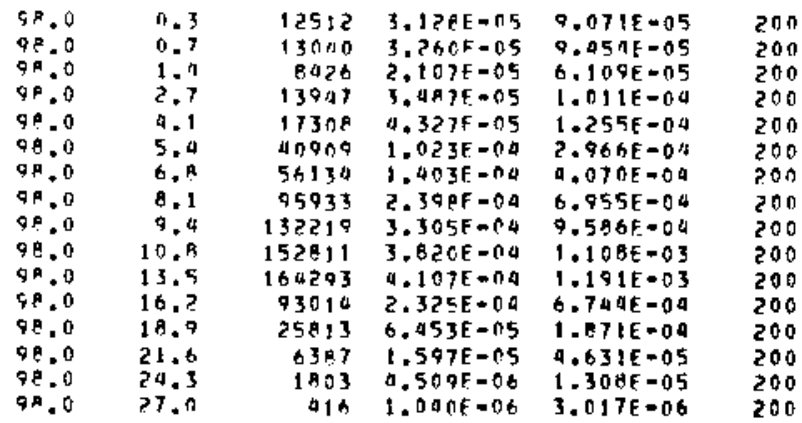

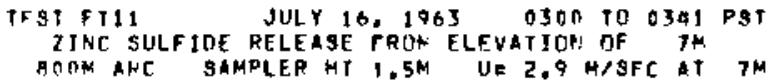

DFFHEES GM-SEC/CU.M SEC/CU,N I/SQ.M METEES $\times 10 E+6$

$\begin{array}{rrrrr}A 2.0 & 159 & 3.962 E-07 & 1.199 E-06 & 800 \\ 90.0 & 1031 & 2.530 E-06 & 7.979 E-06 & 800 \\ 92.0 & 3992 & 9.982 E-06 & 2.895 E=05 & 000 \\ 90.0 & 16910 & 9.279 E-05 & 1.226 E-04 & 800 \\ 96.0 & 30187 & 7.547 E-05 & 2.189 E-04 & 000 \\ 98.0 & 39030 & 9.756 E-05 & 2.030 E-04 & 000 \\ 100.0 & 9291 & 1.073 E-05 & 3.111 E-05 & 800 \\ 102.0 & 38223 & 9.556 E-05 & 2.771 E-04 & 800 \\ 100.0 & 20309 & 5.077 E-05 & 1.472 E-04 & 800 \\ 106.0 & 5391 & 1.348 E-05 & 3.909 E-05 & 000 \\ 108.0 & 155 & 3.884 E-07 & 1.126 \mathrm{~F}-06 & 000\end{array}$

CANSSW]ND INTEGRATEDE $1.115 E-02 \quad 3.233 E-02$
ATJMUITH EXPOSURE E/Q EU/O DISTANCE 
TUKFF OATA FOLLE',...

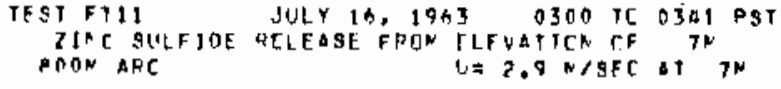

AZIKITIH HETGHT EXPCSURF F/D TE TLG TISTANCE DECREFS NETEAS GM-SEC/CU.N SEC/CU.N 1/SK." NETERS $\times 10 \mathrm{~F}+6$

\begin{tabular}{|c|c|c|c|c|c|}
\hline $\begin{array}{l}9 R .0 \\
9 A .0 \\
98.0 \\
9 A .0 \\
9 E .0 \\
9 A .0 \\
99.0 \\
98.0 \\
98.0 \\
98.0 \\
98.0 \\
98.0 \\
99.0 \\
98.0 \\
98.0 \\
9 \% .0 \\
9 R .0\end{array}$ & $\begin{array}{r}0.5 \\
0.5 \\
1.0 \\
2.1 \\
4.2 \\
6.3 \\
8.0 \\
10.5 \\
12.4 \\
14.7 \\
16.4 \\
21.0 \\
25.7 \\
29.4 \\
33.4 \\
37.9 \\
42.0\end{array}$ & $\begin{array}{l}31902 \\
29421 \\
190 \times 3 \\
24361 \\
31923 \\
35428 \\
09901 \\
46378 \\
26073 \\
13976 \\
5714 \\
1249 \\
409 \\
100 \\
145 \\
104 \\
95\end{array}$ & 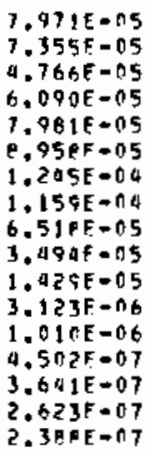 & $\begin{array}{l}2.311 E=00 \\
2.133 E=00 \\
1.3 A 2 E=04 \\
1.766 E=04 \\
2.314 E=04 \\
2.598 E=04 \\
3.611 E=04 \\
3.362 E=04 \\
1.095 E=04 \\
1.013 E=04 \\
1.103 E=05 \\
9.057 E=06 \\
2.929 E=06 \\
1.306 E=06 \\
1.056 E=06 \\
7.607 E=07 \\
0.926 E-07\end{array}$ & $\begin{array}{l}000 \\
000 \\
000 \\
800 \\
000 \\
000 \\
800 \\
800 \\
100 \\
000 \\
000 \\
900 \\
100 \\
000 \\
800 \\
000 \\
100\end{array}$ \\
\hline
\end{tabular}

ATINLTH HEIEHF FXPCSURE E/Q FU/O TISTANCE

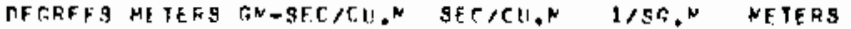
YIOE+G

\begin{tabular}{|c|c|c|c|c|}
\hline $\begin{array}{l}106.0 \\
106.0 \\
106.0 \\
106.0 \\
106.0 \\
106.0 \\
106.0 \\
106.0 \\
106.0 \\
106.0 \\
106.0 \\
106.0 \\
106.0 \\
106.0 \\
106.0 \\
106.0 \\
106.0\end{array}$ & $\begin{array}{r}0.3 \\
0.5 \\
1.0 \\
2.1 \\
0.2 \\
6.3 \\
8.0 \\
10.5 \\
12.6 \\
14.7 \\
16.5 \\
21.0 \\
25.2 \\
29.4 \\
33.6 \\
37.9 \\
42.0\end{array}$ & $\begin{array}{r}9087 \\
3160 \\
2307 \\
2786 \\
6300 \\
91407 \\
13792 \\
17955 \\
19975 \\
25774 \\
28713 \\
15433 \\
4906 \\
1970 \\
815 \\
374 \\
114\end{array}$ & 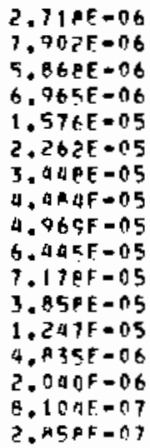 & 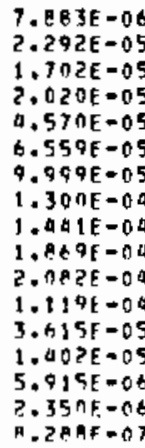 \\
\hline
\end{tabular}

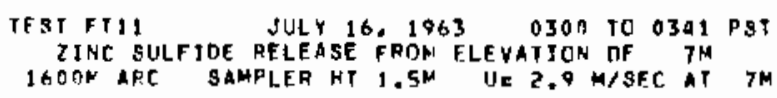

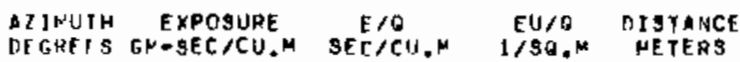
$X 1 O E+6$

\begin{tabular}{|c|c|c|c|c|}
\hline $\begin{array}{l}87.0 \\
88.0 \\
89.0 \\
90.0\end{array}$ & $\begin{array}{l}212 \\
377 \\
558 \\
790\end{array}$ & $\begin{array}{l}5.313 E-07 \\
9.446 E=07 \\
1.395 E=06 \\
975 E=06\end{array}$ & $\begin{array}{l}1.541 E=06 \\
2.739 E=06 \\
4.047 E=06 \\
5.730 F=06\end{array}$ & $\begin{array}{l}1600 \\
1600 \\
1600 \\
1600\end{array}$ \\
\hline 91.0 & 1646 & $0.116 E-06$ & $1.194 E-05$ & 1600 \\
\hline 92.0 & 17 & $4.290 E-06$ & $i .245 E-07$ & 1600 \\
\hline 93.0 & 9248 & $1.062 E=05$ & $3.090 E-05$ & 1600 \\
\hline 94.0 & 1020 & $2.005 \mathrm{E}-05$ & $5.815 E-05$ & 1600 \\
\hline 95.0 & $1000 a$ & 2. $101 E-05$ & $7.834 E-05$ & 1600 \\
\hline 90.0 & 12905 & $6 E=05$ & $15 E=05$ & 1600 \\
\hline 97.0 & 12560 & $1 \triangle O E-05$ & $9.107 E=05$ & 1600 \\
\hline 3.0 & 11053 & $763 E-05$ & 0.01 LE- 05 & 1600 \\
\hline 99.0 & 12088 & $022 E-05$ & $0.764 E-05$ & 1600 \\
\hline 100.0 & 11725 & $931 E=05$ & $B+501 E=05$ & 1600 \\
\hline $10 ! .0$ & 12084 & $021 E-05$ & $761 E=05$ & 1600 \\
\hline 102.0 & 13544 & $366 E-05$ & Q2OE-05 & 1600 \\
\hline 103.0 & 13101 & $.295 E-05$ & $56 E=05$ & 1600 \\
\hline & 6150 & $1.536 E=05$ & $59 E-05$ & 1600 \\
\hline 105.0 & 1665 & $165 E-06$ & $00 E-05$ & 1600 \\
\hline 106.0 & 513 & $36 E-07$ & $72 E=06$ & 1600 \\
\hline 107.0 & 36 & $124 E-01$ & $696 E-07$ & 1600 \\
\hline
\end{tabular}

CROSSWIND TMTEGRATEDE 9.357E-0.3 2, $713 E-02$

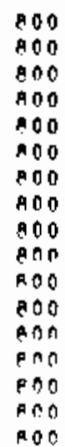

TOUER ratA FOLLCH....

TFST \&TII

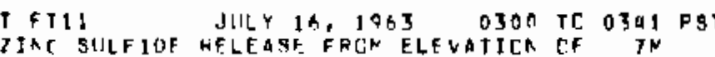
$1600 \mathrm{~N}$ AGE
LZ ?.9 NGSFC AT TH

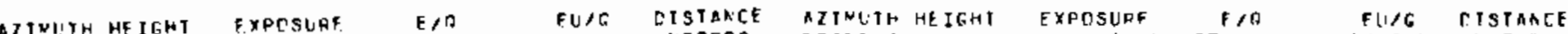

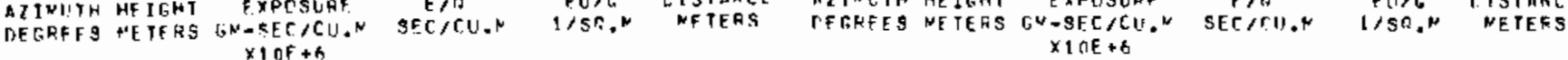

$110.0 \quad 4.7 \quad 46 \quad 1.167 \mathrm{~F}=07 \quad 3.395 \mathrm{~F}=07 \quad 800$

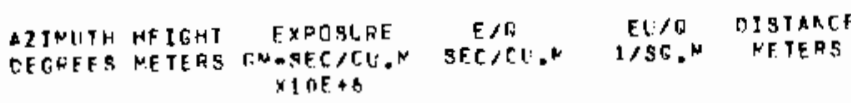
$522.0 \quad 4.7 \quad 78 \quad 7.002 \mathrm{~F}=0 \mathrm{E} \quad 2.031 \mathrm{E}=07$ *0त

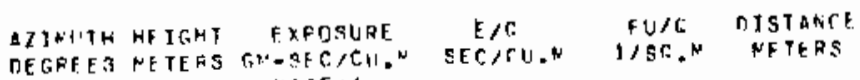
$\times 1$ nf +6

135.0 4.? at $1.027 F=072.9798-07$ aOD

\begin{tabular}{|c|c|c|c|c|c|}
\hline $\begin{array}{l}94.0 \\
98.0 \\
98.0 \\
98.0 \\
98.0 \\
98.0 \\
98.0 \\
98.0 \\
99.0 \\
98.0 \\
98.0 \\
91.0 \\
98.0 \\
98.0 \\
98.0 \\
98.0 \\
94.0\end{array}$ & $\begin{array}{l}0.2 \\
0.4 \\
0.8 \\
1.5 \\
3.1 \\
6.2 \\
9.7 \\
12.9 \\
15.5 \\
18.4 \\
21.7 \\
24.8 \\
31.0 \\
37.7 \\
43.4 \\
49.6 \\
55.8\end{array}$ & $\begin{array}{r}11730 \\
12441 \\
9945 \\
4309 \\
9911 \\
10224 \\
9721 \\
9355 \\
5135 \\
2845 \\
1626 \\
724 \\
246 \\
100 \\
61 \\
10 \\
7\end{array}$ & 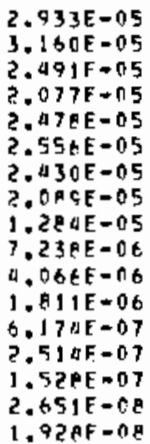 & $\begin{array}{l}8.505 E-05 \\
9.165 E-05 \\
7.225 E=05 \\
6.025 E=05 \\
7.186 E=05 \\
7.413 E=05 \\
7.048 E=05 \\
6.058 E=05 \\
3.723 E=05 \\
2.089 E-05 \\
1.179 E=05 \\
5.252 E=06 \\
1.791 E=06 \\
7.292 E=07 \\
4.430 E=07 \\
7.689 E=0 A \\
5.592 E=0 A\end{array}$ & $\begin{array}{l}1600 \\
1600 \\
1600 \\
1600 \\
1600 \\
1600 \\
1600 \\
1600 \\
1600 \\
1600 \\
1600 \\
1600 \\
1600 \\
1600 \\
1600 \\
1600 \\
1600\end{array}$ \\
\hline
\end{tabular}




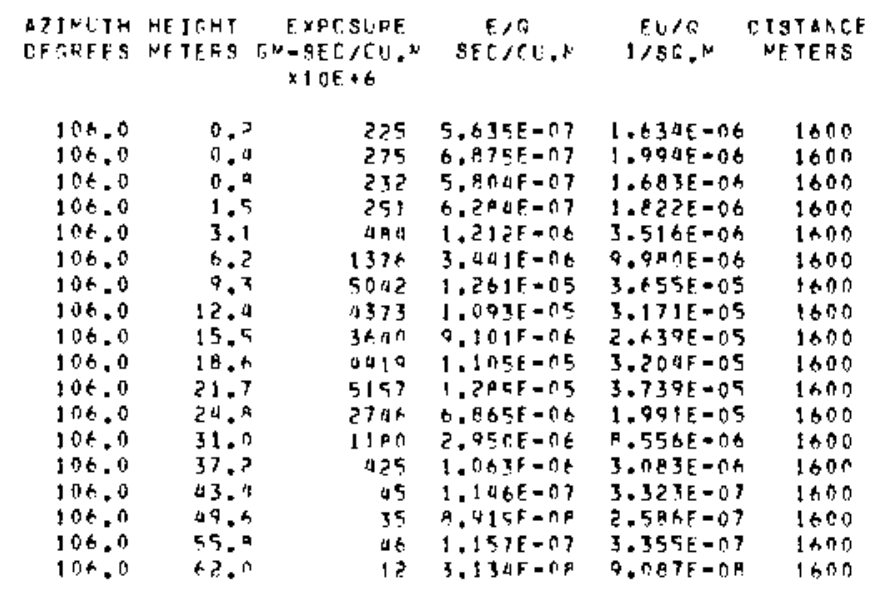

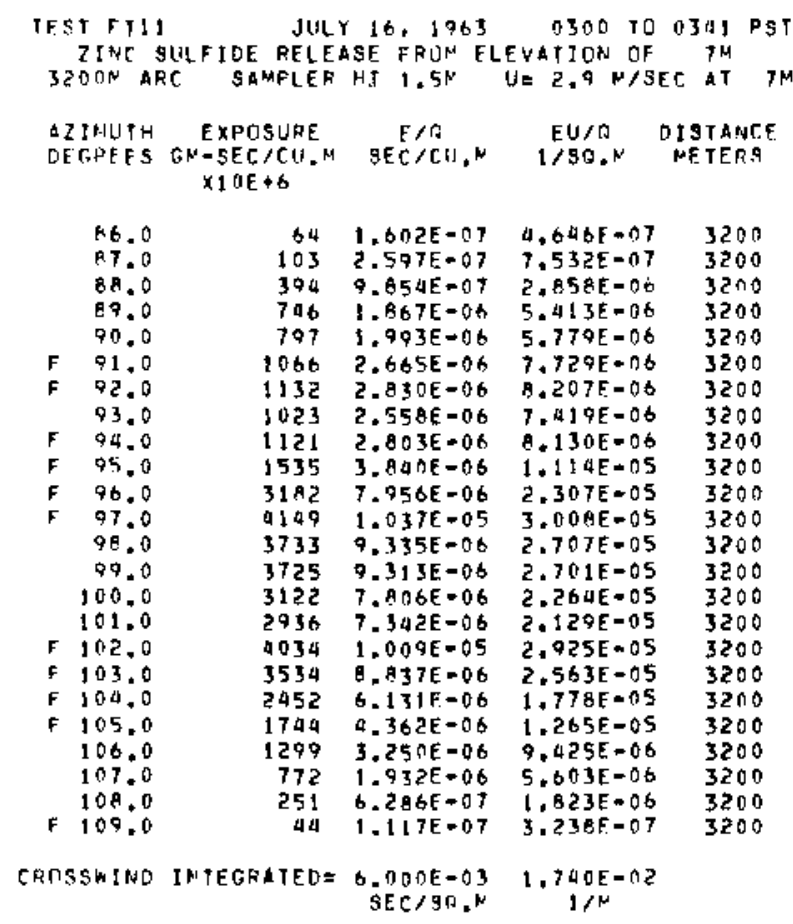

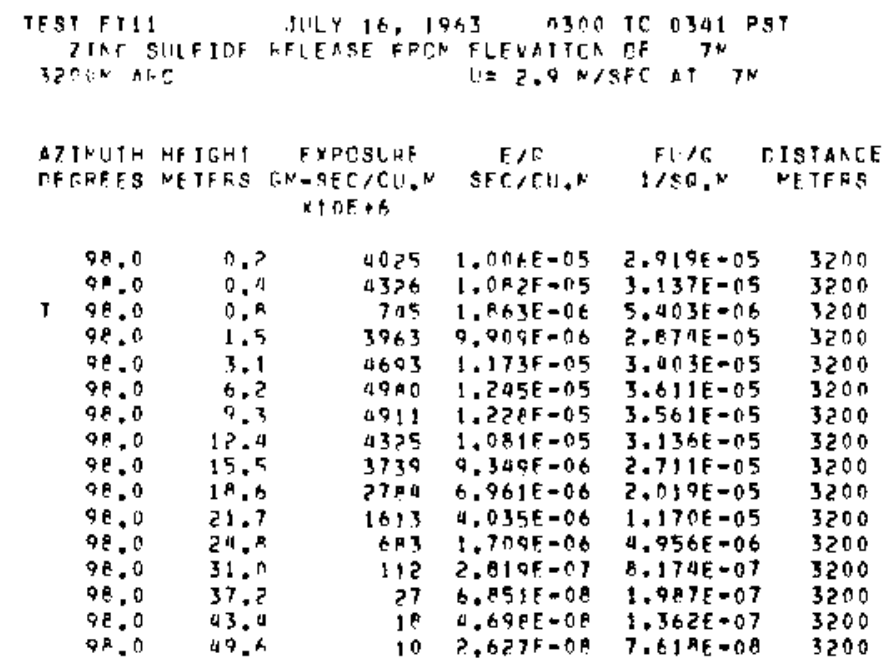

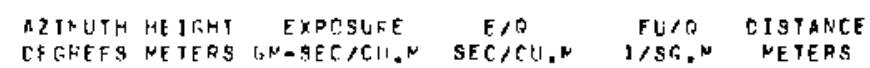

\begin{tabular}{|c|c|c|c|c|c|}
\hline 106.0 & 0.7 & $14 n \pi$ & $3.517 \mathrm{E}-0.0$ & $1.020 E-05$ & 3200 \\
\hline 106.0 & 0.4 & 1281 & $2 \cap \Delta F=0 t$ & $9.290 E-06$ & 3200 \\
\hline 106,0 & $0,{ }^{\circ}$ & 1400 & $3.502 E=n E$ & $1.015 \varepsilon-05$ & 3200 \\
\hline 106.0 & 1.7 & 1373 & $3.434 F=06$ & $9.959 E=06$ & 3200 \\
\hline 106.0 & 3.1 & 1727 & $4.319 f-06$ & $1.252 \mathrm{E}=05$ & 3200 \\
\hline $10 t .0$ & 6.7 & 2022 & $5.057 E=06$ & $1.466 E=05$ & $32 \cap 0$ \\
\hline 106.0 & 9.3 & 2331 & $824 E-06$ & $1.690 \mathrm{n}=05$ & 3200 \\
\hline $10 t, 0$ & 12.1 & 11 & $707 E-0 E$ & $11 \geqslant f-0 B$ & 3200 \\
\hline $1 n t .0$ & 15 & 3750 & GPE- 06 & nf. -05 & 200 \\
\hline 106.0 & 18.6 & 3025 & $7,5645=06$ & $2.193 E-05$ & 3200 \\
\hline 106.0 & 21.7 & $22 n d$ & $5 \pm 1 E-c t$ & $2 E-05$ & 3200 \\
\hline 106.0 & 24 & 1164 & ot & HU1E-06 & 000 \\
\hline 106,0 & $31, n$ & 260 & $6.590 .5-07$ & $1.890 E-06$ & 3200 \\
\hline $10 \times, 0$ & 37.2 & 150 & $.75 \mathrm{PE}=07$ & $1.090 \mathrm{E}=\mathrm{DB}$ & 3200 \\
\hline 106.0 & 43.1 & $\ln 5$ & $2.643 E=\cap 7$ & $7.663 \mathrm{E}=07$ & 3200 \\
\hline 106.0 & 49 & 44 & 1. $10 \cap E=\cap 7$ & $3.19 D E-1) 7$ & 3200 \\
\hline $1 \cap t, 0$ & & 70 & $? 3 द F=\cap A$ & $1.009 F-07$ & $x>00$ \\
\hline
\end{tabular}


SERIES 163 EXPERIMENT: 12 (FT=12,

GROUND LEVEL AND TOWER SAHPLING 200-3200 M ARCS. A SIGNIFICANT

PORTION OF THE PLUHE PASSED TO THE NORTH OF THE GRID.

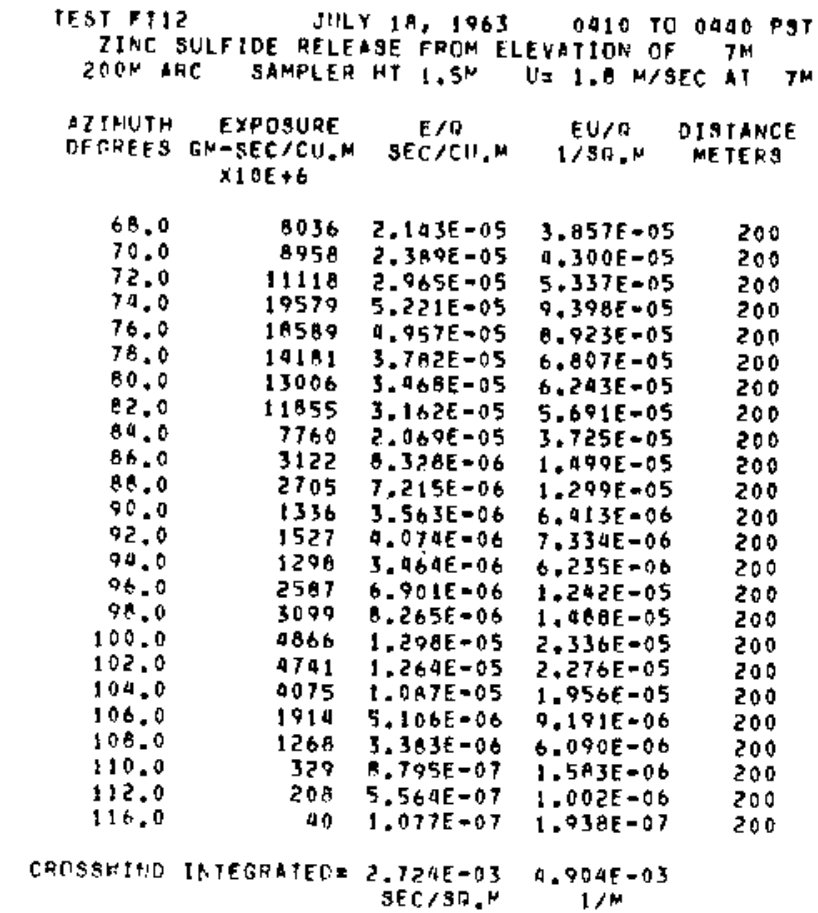

TOUFR CATA FOLLCK...

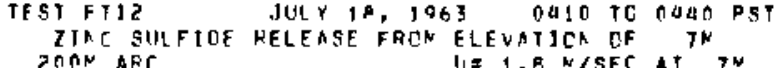

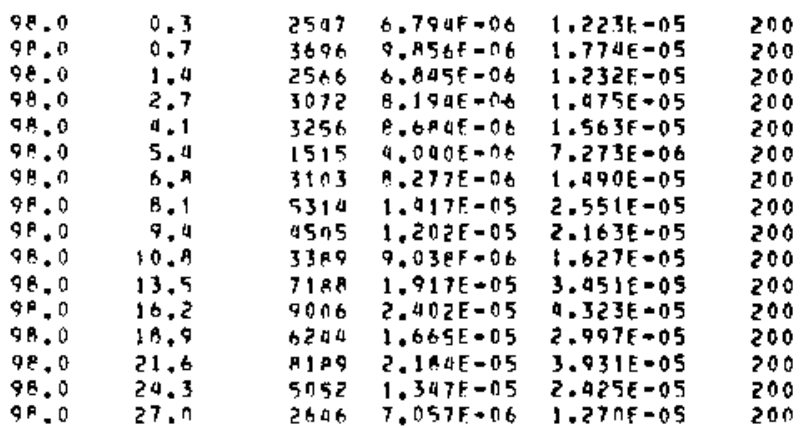

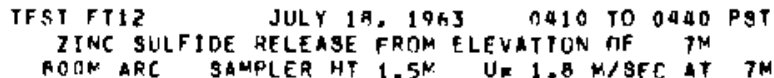
AZIMITH EXPOSURE E/Q EU/O DISTANCE CEGREES GM-SEC/CU.M SEC/EU.M I/SA,M METERS $\times 10 E+6$

\begin{tabular}{|c|c|c|c|c|}
\hline $\begin{array}{l}70.0 \\
72.0 \\
79.0 \\
76.0 \\
78.0 \\
80.0 \\
82.0 \\
84.0 \\
86.0 \\
88.0 \\
90.0\end{array}$ & $\begin{array}{l}7933 \\
9173 \\
6974 \\
6164 \\
7968 \\
5667 \\
5004 \\
4089 \\
2168 \\
817 \\
117\end{array}$ & $\begin{array}{l}2.115 E-05 \\
2.446 E-05 \\
1.727 E-05 \\
2.177 E-05 \\
2.125 E-05 \\
1.511 E-05 \\
1.548 E-05 \\
1.304 E-05 \\
5.781 E-06 \\
2.179 E-06 \\
5.125 E-07\end{array}$ & $\begin{array}{l}3.806 E=05 \\
4.403 E=05 \\
3.106 E=05 \\
3.919 E=05 \\
3.825 E-05 \\
2.720 E=05 \\
2.786 E=05 \\
2.347 E=05 \\
1.041 E-05 \\
3.922 E=06 \\
5.625 E=07\end{array}$ & $\begin{array}{l}800 \\
800 \\
800 \\
800 \\
800 \\
800 \\
800 \\
800 \\
800 \\
800 \\
800\end{array}$ \\
\hline ISAW]ND & INTEGRATE[S= & $\begin{array}{l}A \text { A JOTE-03 } \\
\text { SEC } / 3 Q . K\end{array}$ & $\begin{array}{c}7.932 E-03 \\
1 / M\end{array}$ & \\
\hline
\end{tabular}

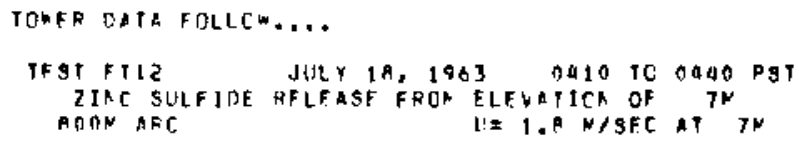

\begin{tabular}{|c|c|c|c|c|c|}
\hline 98.0 & $\begin{array}{r}0.3 \\
0.5 \\
1.0 \\
2.1 \\
4.2 \\
6.3 \\
0.4 \\
10.5 \\
12.6 \\
14.7 \\
16.9 \\
21.0 \\
25.2 \\
29.4 \\
33.4 \\
37.4 \\
42.0\end{array}$ & $\begin{array}{r}51 \\
10 \\
13 \\
2 \\
43 \\
27 \\
507 \\
1066 \\
1590 \\
1111 \\
1151 \\
159 \\
303 \\
306 \\
229 \\
335\end{array}$ & $\begin{array}{l}1.367 E=07 \\
2.734 E-08 \\
3.64 E F-08 \\
7.292 E-09 \\
1.167 F-07 \\
2.333 E-07 \\
7.219 F-07 \\
1.610 E-06 \\
2.044 E-06 \\
4.25 \mathrm{tE}-06 \\
3.150 E-06 \\
3.07 \cap E-06 \\
2.026 \mathrm{~F}-06 \\
1.023 E-06 \\
1.057 E-06 \\
6.127 E-07 \\
9.954 F=07\end{array}$ & $\begin{array}{l}2.961 E=07 \\
4.922 E=08 \\
6.563 E-08 \\
1.313 E=08 \\
2.100 E=07 \\
4.200 E=07 \\
1.290 E=06 \\
2.914 E-06 \\
5.119 E-06 \\
7.652 E-06 \\
5.670 E=06 \\
5.526 E-06 \\
3.64 B E-06 \\
1.842 E=06 \\
1.903 E-06 \\
1.103 E=06 \\
1.612 E=06\end{array}$ & $\begin{array}{l}900 \\
000 \\
600 \\
100 \\
000 \\
800 \\
100 \\
000 \\
000 \\
000 \\
800 \\
000 \\
000 \\
000 \\
800 \\
800 \\
800\end{array}$ \\
\hline
\end{tabular}




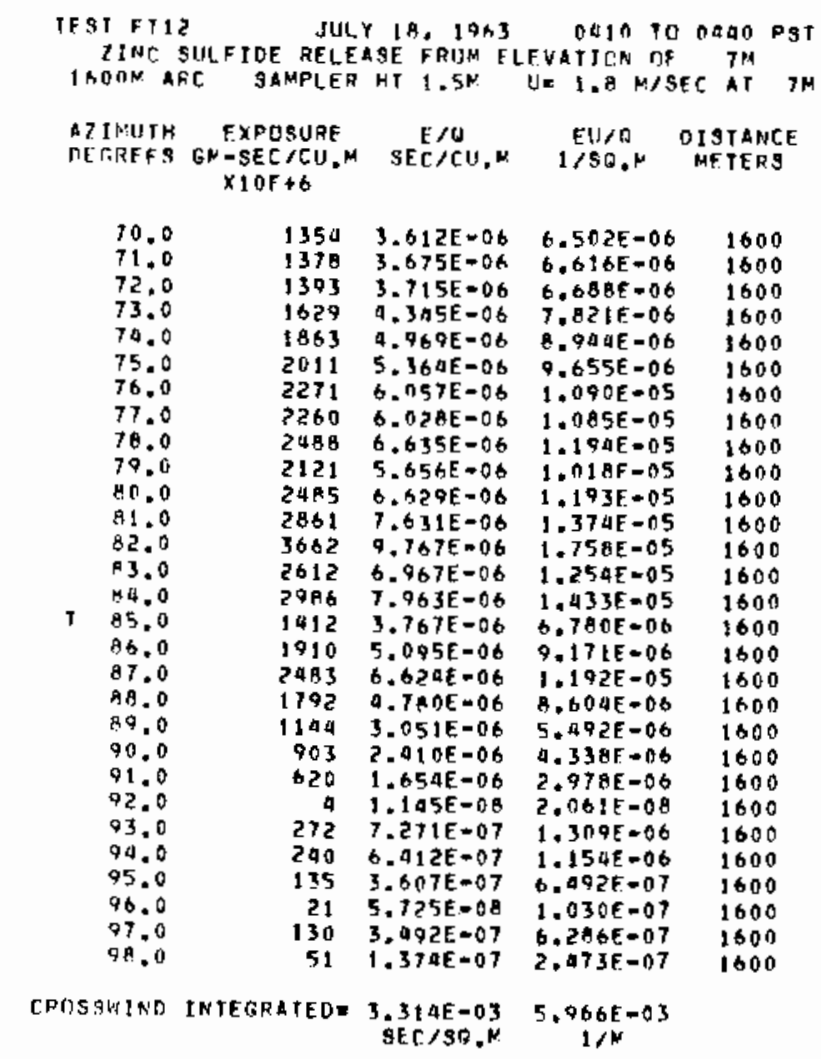

PONFR CATA FOLLCK....

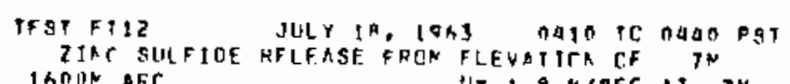

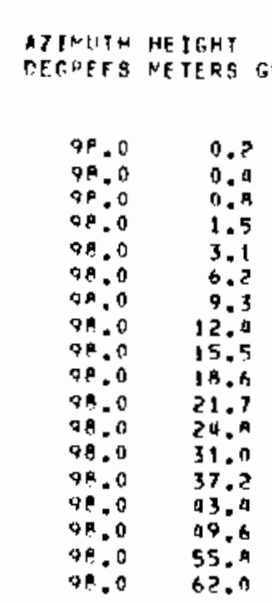

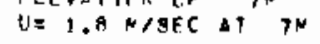




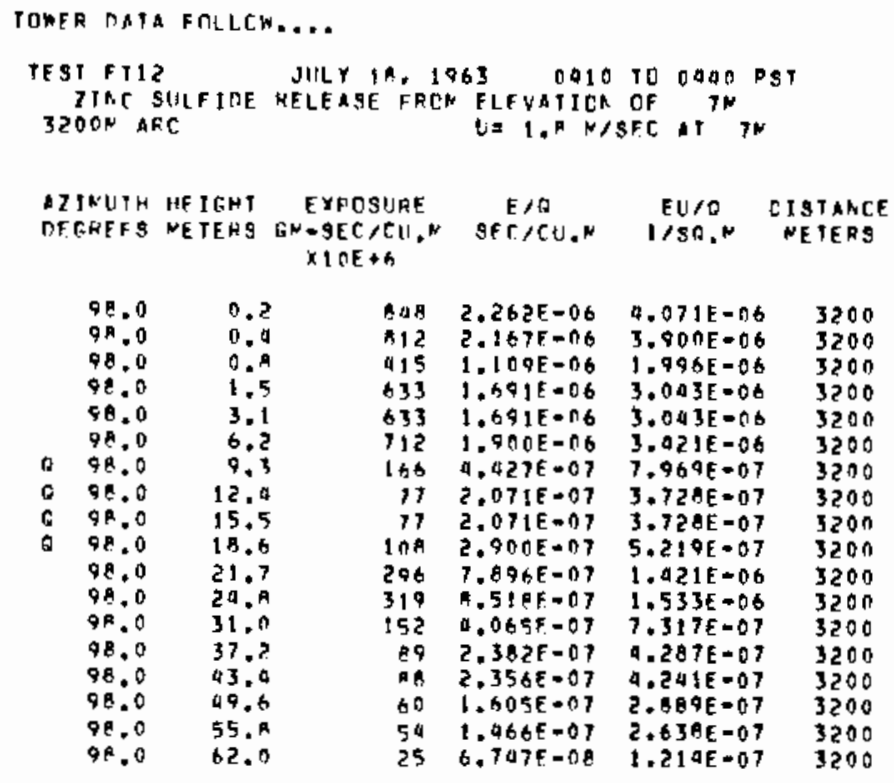


SERIES 163 EXPER!MENT: 13 (FT-13)

GROLND LEVEL ANO TOWER SAMPLING 200-3200 M ARCS. MANY OF THE

FHLTERS ON THE TOWERS WERE DAMAGEO.

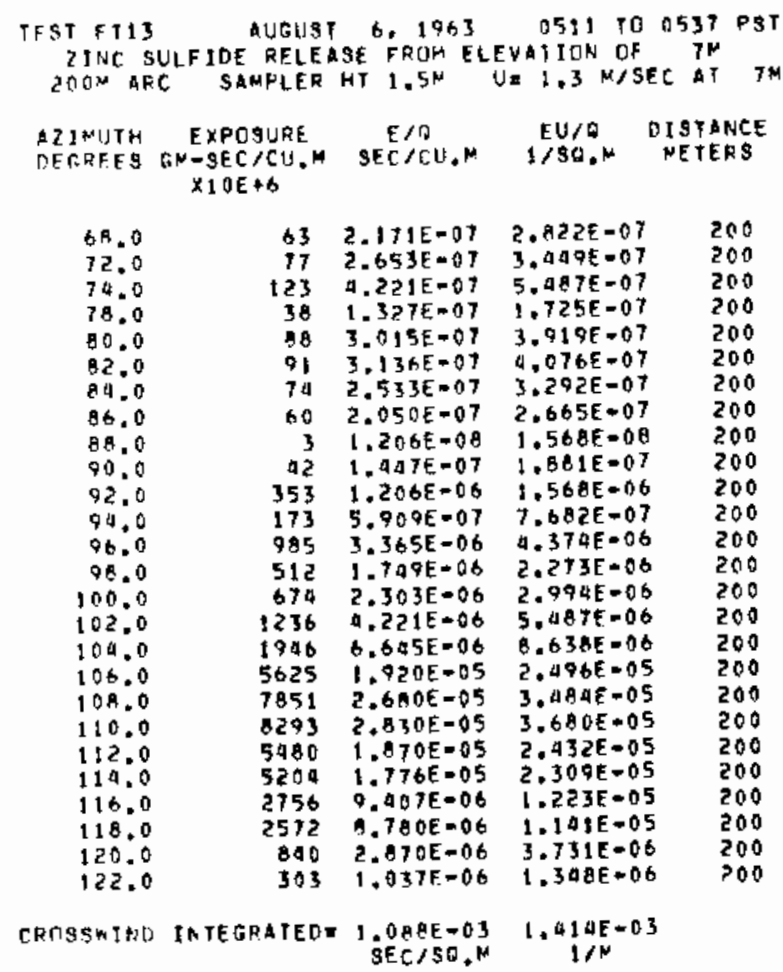

TOMEP DATA FOLLOW....

TEST FI13 AUGUST b, 1963 0511 TO 0537 FST ZIAC SULFIDE RELEASE FRQN ELEVATIOK OF $7 "$ ZOON AAC LE 1.3 N/SFC AT TN
LIC

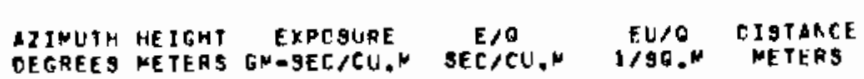
$\times 10 E \rightarrow 6$

\begin{tabular}{|c|c|c|c|c|c|}
\hline $\begin{array}{l}90.0 \\
96.0 \\
98.0 \\
98.0 \\
98.0 \\
98.0 \\
90.0 \\
90.0 \\
98.0 \\
98.0 \\
98.0 \\
98.0 \\
98.0 \\
98.0 \\
98.0 \\
98.0\end{array}$ & $\begin{array}{l}0.3 \\
0.7 \\
1.9 \\
2.7 \\
4.1 \\
5.0 \\
6.8 \\
8.1 \\
9.9 \\
10.8 \\
13.5 \\
16.2 \\
10.9 \\
21.6 \\
24.3 \\
27.0\end{array}$ & $\begin{array}{l}148 \\
260 \\
912 \\
412 \\
76 \\
1330 \\
1660 \\
4290 \\
5030 \\
3660 \\
0846 \\
0400 \\
7502 \\
5656 \\
4490 \\
3020\end{array}$ & $\begin{array}{l}5.065 E=07 \\
9.165 E=07 \\
3.115 E=06 \\
1.007 E=06 \\
2.013 E=07 \\
9.503 E=06 \\
5.608 E=06 \\
1.047 E=05 \\
1.720 E=05 \\
1.240 E=05 \\
1.654 E=05 \\
2.867 E=05 \\
2.561 E=05 \\
1.931 E=05 \\
1.519 E=05 \\
1.307 E=05\end{array}$ & $\begin{array}{l}6.585 E=07 \\
1.192 E=06 \\
9.050 E=06 \\
1.029 E=06 \\
3.397 E=07 \\
5.905 E=06 \\
7.395 E=06 \\
1.881 E=05 \\
2.235 E=05 \\
1.624 E=05 \\
2.150 E=05 \\
3.727 E=05 \\
3.329 E=05 \\
2.510 E=05 \\
1.974 E=05 \\
1.699 E=05\end{array}$ & $\begin{array}{l}200 \\
200 \\
200 \\
200 \\
200 \\
200 \\
200 \\
200 \\
200 \\
200 \\
200 \\
200 \\
200 \\
200 \\
200 \\
200\end{array}$ \\
\hline
\end{tabular}

\begin{tabular}{|c|c|c|c|c|c|}
\hline $\begin{array}{l}\text { IZINUTH } \\
\text { CEGREES }\end{array}$ & $\begin{array}{l}\text { HE IGHT } \\
\text { METERS }\end{array}$ & $\begin{array}{l}\text { EXPCSUAE } \\
\text { GM-SEC } C \text { CW.W } \\
\times 10 E+b\end{array}$ & $\begin{array}{c}E / \theta \\
S E C / C U, N\end{array}$ & $\begin{array}{c}E U / \theta \\
1 / 30, N\end{array}$ & $\begin{array}{l}\text { DISTANCE } \\
\text { NETEAS }\end{array}$ \\
\hline $\begin{array}{l}106.0 \\
106.0 \\
106.0 \\
106.0 \\
106.0 \\
106.0 \\
106.0 \\
106.0 \\
106.0 \\
106.0 \\
106.0 \\
106.0 \\
106.0 \\
106.0 \\
106.0 \\
106.0\end{array}$ & $\begin{array}{r}0.3 \\
0.7 \\
1.4 \\
2.7 \\
4.1 \\
5.4 \\
6.8 \\
5.1 \\
9.4 \\
10.8 \\
13.5 \\
16.2 \\
10.9 \\
21.6 \\
24.3 \\
27.0\end{array}$ & $\begin{array}{r}3008 \\
5328 \\
4209 \\
7517 \\
17111 \\
23539 \\
36301 \\
56570 \\
87057 \\
131499 \\
165399 \\
188363 \\
119606 \\
54554 \\
16599 \\
7464\end{array}$ & $\begin{array}{l}1.054 E=05 \\
1.019 E=05 \\
1.467 E=05 \\
2.568 E=05 \\
5.040 E=05 \\
8.034 E=05 \\
1.234 E=04 \\
1.935 E=04 \\
2.971 E=04 \\
.488 E=04 \\
5.605 E=04 \\
6.425 E=04 \\
1.082 E=04 \\
1.962 E=04 \\
5.665 E=05 \\
2.541 E=05\end{array}$ & $\begin{array}{l}1.370 E=05 \\
2.364 E=05 \\
1.907 E=05 \\
3.335 E=05 \\
7.592 E=05 \\
1.04 A E=04 \\
1.611 E=04 \\
2.510 E=04 \\
3.063 E=04 \\
5.834 E=04 \\
7.330 E=04 \\
. .357 E=04 \\
5.307 E=04 \\
2.021 E=04 \\
7.365 E=05 \\
3.312 E=05\end{array}$ & $\begin{array}{l}200 \\
200 \\
200 \\
200 \\
200 \\
200 \\
200 \\
200 \\
200 \\
200 \\
200 \\
200 \\
200 \\
200 \\
200 \\
200\end{array}$ \\
\hline
\end{tabular}

\begin{tabular}{|c|c|c|c|c|c|}
\hline $\begin{array}{l}\text { AZIMUTH } \\
\text { DEGREES }\end{array}$ & $\begin{array}{l}\text { HE IEHT } \\
\text { METERS }\end{array}$ & $\begin{array}{c}\text { EXPOSURE } \\
\text { GM-SEC }-3 C U, N \\
\times 1 O E+6\end{array}$ & $\begin{array}{c}E / \theta \\
S E C / C U, M\end{array}$ & $\operatorname{lv}_{1 / 90 .}$ & $\begin{array}{l}\text { DISTANCE } \\
\text { METERS }\end{array}$ \\
\hline 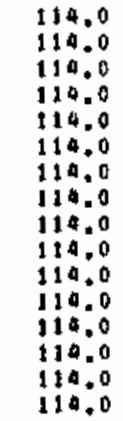 & $\begin{array}{l}0.3 \\
0.7 \\
1.9 \\
2.7 \\
4.1 \\
5.9 \\
6.8 \\
0.1 \\
9.9 \\
10.0 \\
13.5 \\
16.2 \\
18.9 \\
21.6 \\
24.3 \\
27.0\end{array}$ & $\begin{array}{l}2660 \\
3637 \\
6236 \\
9617 \\
11830 \\
21074 \\
20059 \\
30055 \\
39622 \\
41162 \\
66901 \\
69210 \\
62637 \\
29033 \\
8676 \\
3520\end{array}$ & $\begin{array}{l}9.081 E=0 . \\
1.310 E=05 \\
2.129 E-05 \\
3.280 E=05 \\
4.039 E=05 \\
7.324 E=05 \\
7.119 E=05 \\
1.026 E=04 \\
1.162 E=04 \\
1.405 E=04 \\
2.283 E=09 \\
2.362 E=04 \\
2.13 E E=00 \\
9.909 E=05 \\
2.041 E=05 \\
1.202 E=05\end{array}$ & $\begin{array}{l}1.181 E=05 \\
1.703 E=05 \\
2.767 E=05 \\
4.276 E=05 \\
5.251 E=05 \\
9.520 E=05 \\
9.255 E=05 \\
1.534 E=04 \\
1.536 E=04 \\
1.826 E=04 \\
2.968 E=04 \\
3.071 E=04 \\
2.779 E=04 \\
1.268 E=04 \\
3.850 E=05 \\
1.562 E=05\end{array}$ & $\begin{array}{l}200 \\
200 \\
200 \\
200 \\
200 \\
200 \\
200 \\
200 \\
200 \\
200 \\
200 \\
200 \\
200 \\
200 \\
200 \\
200\end{array}$ \\
\hline
\end{tabular}

ARIMUTH HEIGHT EXPOSURE EIO EU/O OISTARCE DEGREES METERS GM-SEC/CU," JECICU.N I/SO,N METERS $\times 1 O E+6$

\begin{tabular}{|c|c|c|c|c|c|}
\hline $\begin{array}{l}122.0 \\
122.0 \\
122.0 \\
122.0 \\
122.0 \\
2122.0 \\
122.0 \\
122.0 \\
122.0 \\
122.0 \\
122.0 \\
122.0 \\
122.0 \\
122.0 \\
122.0 \\
122.0\end{array}$ & $\begin{array}{r}0.5 \\
0.7 \\
1.4 \\
2.7 \\
4.1 \\
5.4 \\
6.7 \\
8.1 \\
9.4 \\
10.8 \\
13.5 \\
16.2 \\
16.9 \\
21.6 \\
24.5 \\
27.0\end{array}$ & $\begin{array}{r}149 \\
533 \\
350 \\
1375 \\
3024 \\
3692 \\
16766 \\
19525 \\
64616 \\
108113 \\
155022 \\
141013 \\
67996 \\
26601 \\
3339 \\
1407\end{array}$ & $\begin{array}{l}6.633 E=07 \\
1.021 E=06 \\
1.196 E=06 \\
9.893 E=06 \\
1.032 E=05 \\
1.260 E=05 \\
5.722 E=05 \\
1.349 E=04 \\
2.205 E=04 \\
3.690 E=04 \\
5.318 E=04 \\
1.813 E=04 \\
2.321 E=04 \\
9.079 E=05 \\
1.140 E=05 \\
4.940 E=06\end{array}$ & $\begin{array}{l}0.623 E-07 \\
2.367 E=06 \\
1.555 E=06 \\
6.101 E=06 \\
1.342 E=05 \\
1.638 E=05 \\
7.439 E-05 \\
1.754 E=04 \\
2.067 E=04 \\
9.797 E=00 \\
0.914 E=04 \\
6.257 E=04 \\
3.017 E=04 \\
1.100 E=04 \\
1.962 E=05 \\
6.022 E=06\end{array}$ & $\begin{array}{l}200 \\
200 \\
200 \\
200 \\
200 \\
200 \\
200 \\
200 \\
200 \\
200 \\
200 \\
200 \\
200 \\
200 \\
200 \\
200\end{array}$ \\
\hline
\end{tabular}




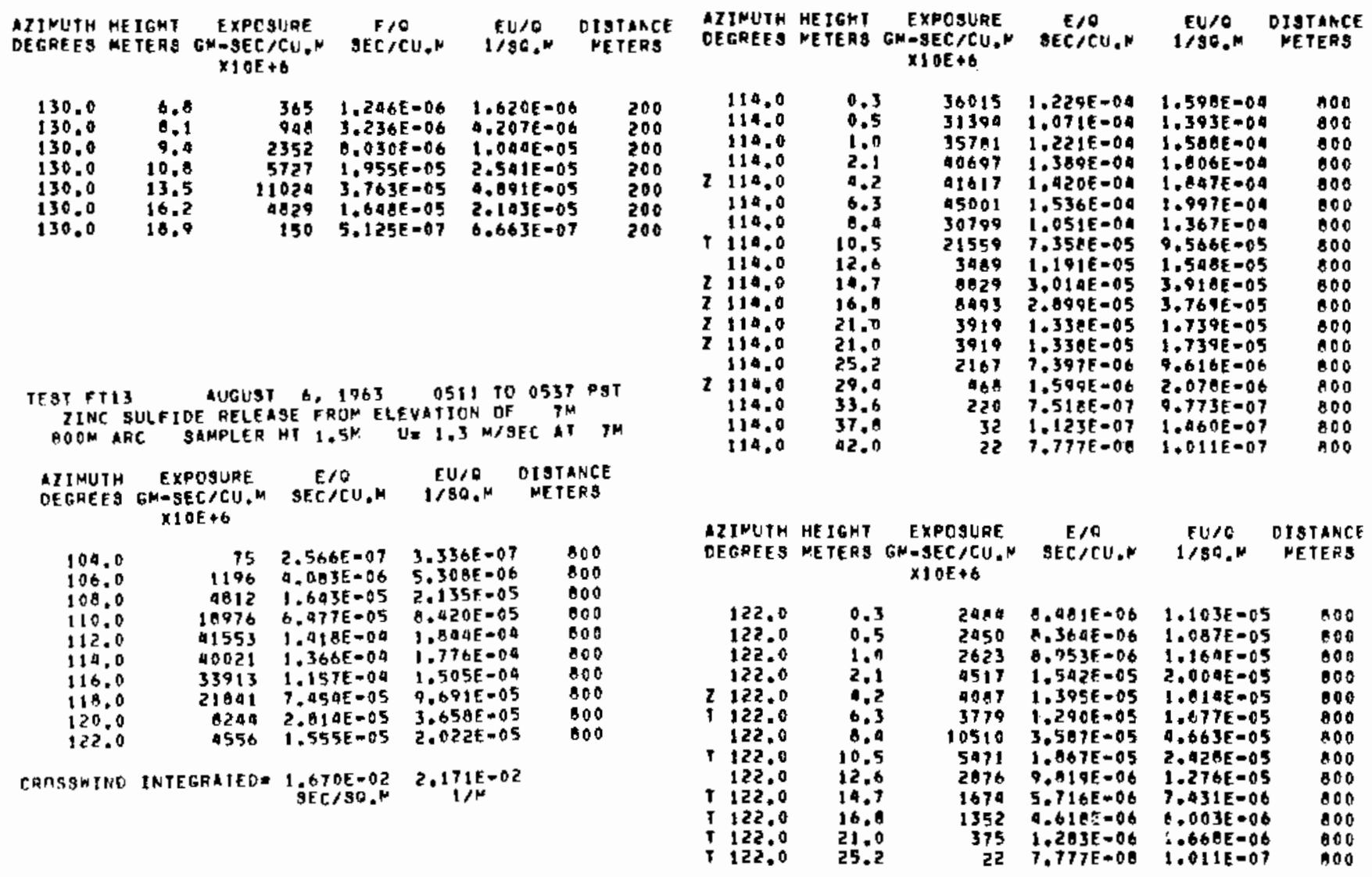

TOKER CATA FOLLOM....

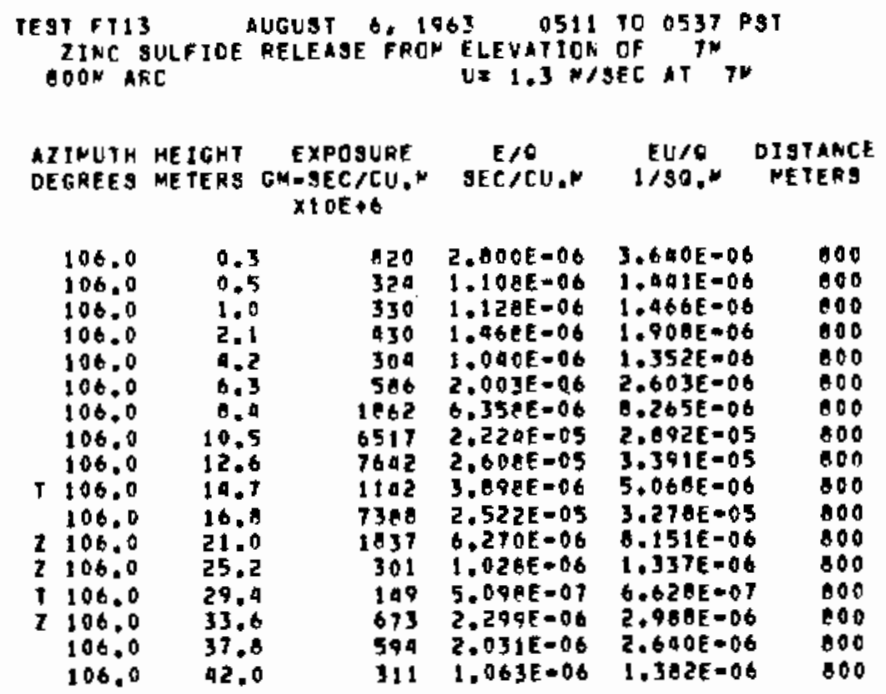




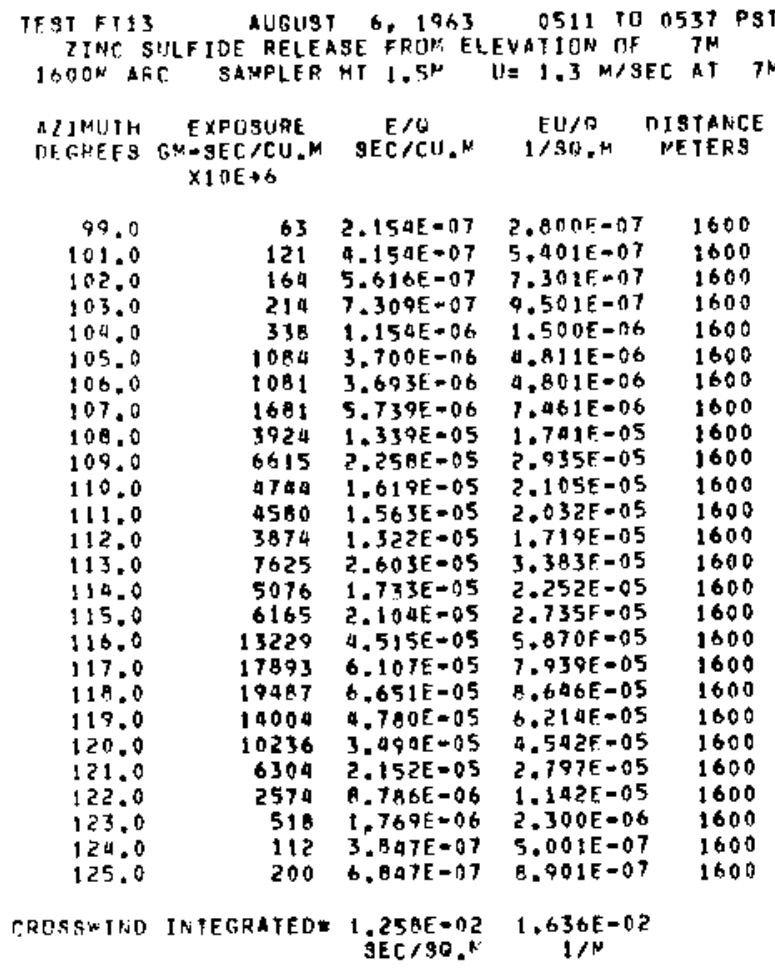

TOWER DATA FOLLOH....

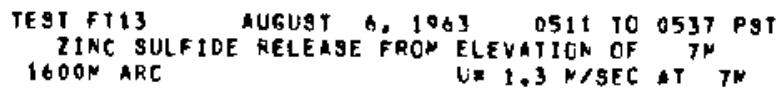

\begin{tabular}{|c|c|c|c|c|c|}
\hline $\begin{array}{l}106.0 \\
106.0 \\
106.0 \\
106.0 \\
106.0 \\
106.0 \\
106.0 \\
106.0 \\
106.0 \\
106.0 \\
106.0 \\
106.0 \\
106.0 \\
106.0 \\
106.0 \\
106.0 \\
106.0 \\
106.0\end{array}$ & $\begin{array}{l}0.2 \\
0.4 \\
0.4 \\
1.5 \\
3.1 \\
6.2 \\
9.3 \\
12.4 \\
15.5 \\
18.6 \\
21.7 \\
24.8 \\
31.0 \\
37.2 \\
43.4 \\
99.6 \\
55.0 \\
62.0\end{array}$ & $\begin{array}{l}606 \\
966 \\
741 \\
456 \\
504 \\
104 \\
206 \\
243 \\
264 \\
569 \\
229 \\
191 \\
212 \\
280 \\
9 \\
19 \\
10\end{array}$ & $\begin{array}{l}2.070 F=06 \\
1.593 E=06 \\
2.531 E=06 \\
1.560 E=06 \\
1.721 E=06 \\
3.567 E=07 \\
7.064 E=07 \\
.323 E=07 \\
9.022 E=07 \\
1.940 E=06 \\
7.033 E=07 \\
6.520 E=07 \\
7.237 E=07 \\
9.045 E=07 \\
3.260 E=00 \\
6.730 E=08 \\
2.060 E=08 \\
6.177 E=00\end{array}$ & $\begin{array}{l}2.690 E=06 \\
2.070 E=06 \\
3.290 E=06 \\
2.020 E=06 \\
2.237 E=06 \\
9.637 E=07 \\
9.183 E=07 \\
1.002 E=06 \\
1.173 E=06 \\
2.528 E=06 \\
1.010 E=06 \\
0.476 E=07 \\
9.408 E=07 \\
1.290 E=06 \\
4.238 E=08 \\
0.761 E=08 \\
3.650 E=08 \\
0.030 E=08\end{array}$ & $\begin{array}{l}1600 \\
1600 \\
1600 \\
1600 \\
1600 \\
1600 \\
1600 \\
1600 \\
1600 \\
1600 \\
1600 \\
1600 \\
1600 \\
1600 \\
1600 \\
1600 \\
1600 \\
1600\end{array}$ \\
\hline
\end{tabular}

\begin{tabular}{|c|c|c|c|c|c|}
\hline $\begin{array}{l}\text { DZINUTH } \\
\text { DEGREE }\end{array}$ & $\begin{array}{l}\text { HE IGHT } \\
\text { MEYERS }\end{array}$ & $\begin{array}{c}\text { EXPOSURE } \\
\text { GW-SEC } / C U . N \\
\times 1 O E+6\end{array}$ & $\begin{array}{c}E / Q \\
\text { GEC } / \mathrm{tu}, \mathrm{N}\end{array}$ & $\begin{array}{l}E 4 / 0 \\
1 / 90 . M\end{array}$ & $\begin{array}{l}\text { DISTANCE } \\
\text { NETERS }\end{array}$ \\
\hline $\begin{array}{r}114.0 \\
114.0 \\
114.0 \\
114.0 \\
119.0 \\
114.0 \\
114.0 \\
114.0 \\
114.0 \\
114.0 \\
114.0 \\
114.0 \\
114.0 \\
114.0 \\
119.0 \\
119.0 \\
114.0 \\
119.0\end{array}$ & $\begin{array}{l}0.2 \\
0.0 \\
0.0 \\
1.5 \\
3.1 \\
6.2 \\
9.3 \\
12.4 \\
15.5 \\
10.6 \\
21.7 \\
24.0 \\
31.0 \\
37.2 \\
42.4 \\
49.4 \\
55.8 \\
62.0\end{array}$ & $\begin{array}{l}2815 \\
4079 \\
4172 \\
4093 \\
1254 \\
5075 \\
6916 \\
8621 \\
5616 \\
5477 \\
4045 \\
2200 \\
953 \\
32 A \\
54 \\
50 \\
31 \\
57\end{array}$ & $\begin{array}{l}9.609 E=06 \\
1.392 E=05 \\
1.424 E=05 \\
1.300 E=05 \\
1.280 E=06 \\
1.731 E=05 \\
2.360 E=05 \\
2.942 E=05 \\
1.917 E=05 \\
1.064 E=05 \\
1.381 E=05 \\
7.511 E=06 \\
3.253 E=06 \\
1.121 E=06 \\
2.060 E=07 \\
2.022 E=07 \\
1.067 E=07 \\
1.06 E E=07\end{array}$ & $\begin{array}{l}1.249 E=05 \\
1.10 E=05 \\
1.051 E=05 \\
1.794 E=05 \\
5.564 E=06 \\
2.250 E=05 \\
3.069 E=05 \\
3.025 E=05 \\
2.492 E=05 \\
2.430 E=05 \\
1.795 E=05 \\
9.76 A E=06 \\
4.229 E=06 \\
1.450 E=06 \\
3.729 E=07 \\
2.620 E=07 \\
1.3 B 7 E=07 \\
2.555 F=07\end{array}$ & $\begin{array}{l}1600 \\
1600 \\
1600 \\
1600 \\
1600 \\
1600 \\
1600 \\
1600 \\
1600 \\
1600 \\
1600 \\
1600 \\
1600 \\
1600 \\
1600 \\
1600 \\
1600 \\
1600\end{array}$ \\
\hline
\end{tabular}

\begin{tabular}{|c|c|c|c|c|c|}
\hline $\begin{array}{l}\text { DIINUTH } \\
\text { DEGREE }\end{array}$ & $\begin{array}{l}\text { HE I GHT } \\
\text { ME TEFS }\end{array}$ & $\begin{array}{c}\text { EXPOSURE } \\
\text { GNESEC } 1 \text { CU. } \\
\times 10 E+6\end{array}$ & $\begin{array}{c}E / 0 \\
S E C / C U, N\end{array}$ & $\begin{array}{l}E U / O \\
1 / \$ 0 .\end{array}$ & $\begin{array}{l}\text { STANCE } \\
\text { ETERS }\end{array}$ \\
\hline $\begin{array}{l}122.0 \\
122.0 \\
122.0 \\
122.0 \\
122.0 \\
122.0 \\
122.0 \\
122.0 \\
122.0 \\
122.0 \\
122.0 \\
122.0 \\
122.0 \\
122.0\end{array}$ & $\begin{array}{r}0.2 \\
0.4 \\
0.0 \\
1.5 \\
3.1 \\
6.2 \\
9.3 \\
12.4 \\
15.5 \\
18.5 \\
21.7 \\
24.5\end{array}$ & $\begin{array}{l}2240 \\
1957 \\
1433 \\
2407 \\
3008 \\
2592 \\
4164 \\
2893 \\
2660 \\
2668 \\
1346 \\
1159 \\
26 \\
95 \\
72\end{array}$ & $\begin{array}{l}7.816 E=06 \\
6.339 E=06 \\
0.893 E=06 \\
0.211 E=06 \\
1.027 E=05 \\
0.847 E=06 \\
1.421 E=05 \\
9.875 E=06 \\
9.760 E=06 \\
9.106 E-06 \\
4.595 E=06 \\
3.957 E=06 \\
9.128 E=08 \\
3.260 E=07 \\
2.471 E=07\end{array}$ & $\begin{array}{l}1.016 E=05 \\
0.201 E=06 \\
6.361 E=06 \\
1.060 E=05 \\
1.335 E=05 \\
1.150 E=05 \\
1.048 E=05 \\
1.284 E=05 \\
1.269 E=05 \\
1.184 E=05 \\
5.474 E=06 \\
5.145 E=06 \\
1.107 E=07 \\
4.23 A E=07 \\
1.217 E=0 .\end{array}$ & $\begin{array}{l}1600 \\
1600 \\
1600 \\
1600 \\
1600 \\
1600 \\
1600 \\
1600 \\
1600 \\
1600 \\
1600 \\
1600 \\
1600 \\
1600 \\
1600\end{array}$ \\
\hline
\end{tabular}

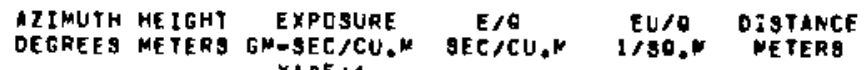
$\times 10 \mathrm{E}+6$

$\begin{array}{rrrrrr}130.0 & 0.2 & 65 & 2.231 E-07 & 2.900 E=07 & 1600 \\ 130.0 & 0.0 & 42 & 1.462 F-07 & 1.900 E-07 & 1600 \\ 130.0 & 1.5 & 03 & 1.469 E-07 & 1.909 E-07 & 1600 \\ 130.0 & 3.1 & 30 & 1.049 E-07 & 1.364 E-07 & 1600 \\ 130.0 & 6.2 & 8 & 2.798 E-00 & 3.637 E=08 & 1600 \\ 130.0 & 12.4 & 28 & 9.791 E-00 & 1.273 E=07 & 1600\end{array}$




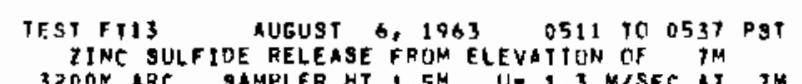
$320 O N$ ARE SAMPLER HT $1.5 \mathrm{M}$ U: $1.3 \mathrm{M} / \mathrm{SEC}$ AT TH

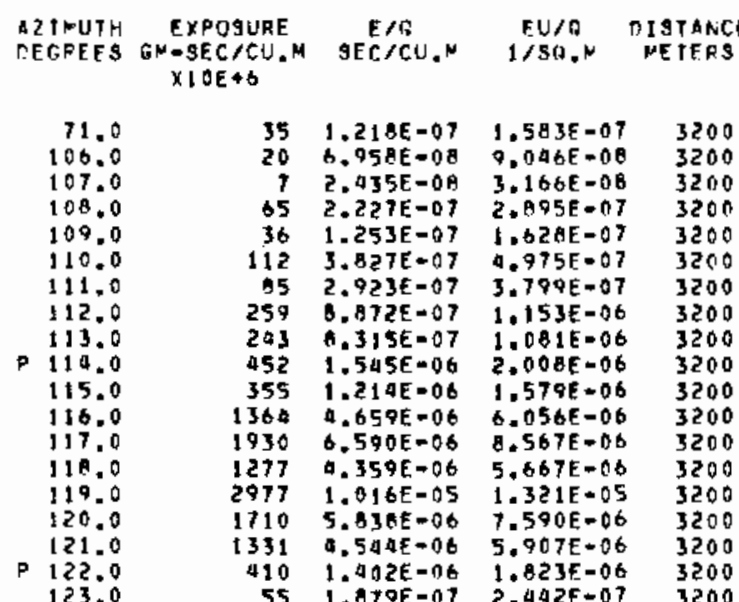

CARSSAIND INTEGRATEN= 2.72SE-03 $3.5425-03$
SEC/SG.M

TOWER CATA FQLLCW....

TEST FT13 AUFUST 6,1963 O511 TC O537 PST

ZINC SULFIDE RELEASE FRON ELEVITICA OF TW
$200 N$ ARC

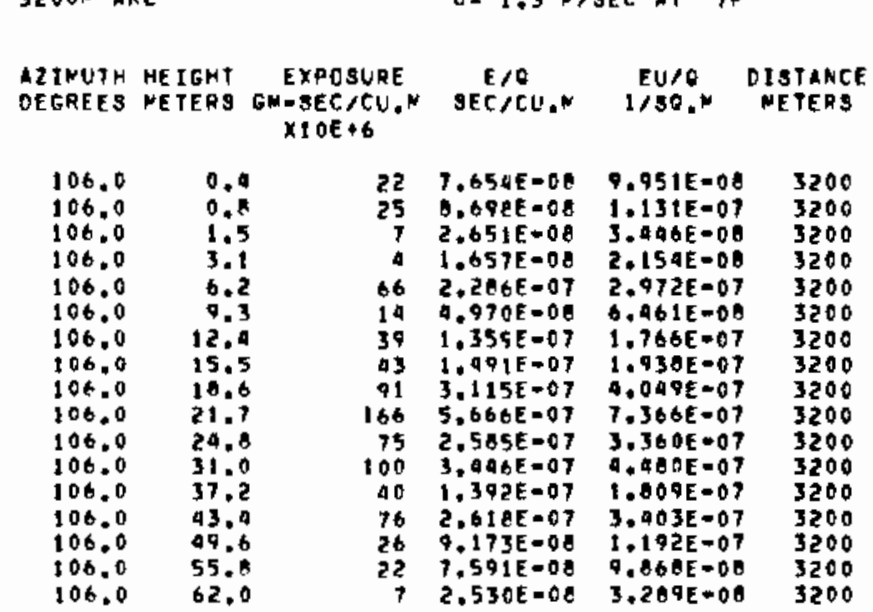

ATIMITH HEIGHT EXPOSURE E/O EU/G DISTANCE

DEGREFS NETERS GM-SEC/CU.N SEC/CU.N 1/SO,M NETERS

\begin{tabular}{|c|c|c|c|c|c|}
\hline $\begin{array}{l}119.0 \\
114.0 \\
119.0 \\
119.0 \\
119.0 \\
114.0 \\
119.0 \\
114.0 \\
114.0 \\
119.0 \\
119.0 \\
119.0 \\
119.0 \\
119.0 \\
119.0\end{array}$ & $\begin{array}{r}0.0 \\
1.5 \\
3.1 \\
6.2 \\
9.3 \\
12.4 \\
15.5 \\
18.6 \\
21.7 \\
24.9 \\
31.0 \\
37.2 \\
43.4 \\
48.6 \\
55.4\end{array}$ & $\begin{array}{r}110 \\
507 \\
447 \\
47 \\
1062 \\
1077 \\
1065 \\
1769 \\
6610 \\
1090 \\
1003 \\
570 \\
255 \\
130 \\
40\end{array}$ & 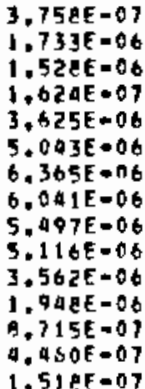 & $\begin{array}{l}4.085 E=07 \\
2.253 E=06 \\
1.906 E=06 \\
2.111 E=07 \\
4.713 E=06 \\
6.556 E=06 \\
0.275 E=06 \\
7.053 E=06 \\
7.146 E=06 \\
6.651 E=06 \\
4.631 E=06 \\
2.533 E=06 \\
1.133 E=06 \\
5.790 E=07 \\
1.974 E=07\end{array}$ & $\begin{array}{l}3200 \\
3200 \\
3200 \\
3200 \\
3200 \\
3200 \\
3200 \\
3200 \\
3200 \\
3200 \\
3200 \\
3200 \\
3200 \\
3200 \\
3200\end{array}$ \\
\hline
\end{tabular}

ATIMUTH HEIGHT EXPCSURE E/O ELTO DISTANCE DEGREES METERS GM-SEC/TU,N SEC,CU,N 1/SO,M METERS $\times 10 E+6$

$\begin{array}{rrrrrr}122.0 & 0.2 & 330 & 1.127 E-06 & 1.065 E-06 & 3200 \\ 122.0 & 0.4 & 222 & 7.505 E-07 & 9.060 E-07 & 3200 \\ 122.0 & 0.8 & 2 A 3 & 9.672 E-07 & 1.257 E-06 & 3200 \\ 122.0 & 1.5 & 261 & 9.913 E-07 & 1.159 E-06 & 3200 \\ 122.0 & 3.1 & 391 & 1.335 E-06 & 1.736 E-06 & 3200 \\ 122.0 & 6.2 & 306 & 1.047 E-06 & 1.361 E-06 & 3200 \\ 122.0 & 9.3 & 569 & 1.945 E-06 & 2.529 E-06 & 3200 \\ 122.0 & 12.4 & 11 & 3.97 E E-06 & 5.169 E-08 & 3200 \\ 122.0 & 15.5 & 699 & 2.306 E-06 & 3.101 E-06 & 3200 \\ 122.0 & 18.6 & 639 & 2.104 E-06 & 2.039 E-06 & 3200 \\ 122.0 & 21.7 & 556 & 1.905 E-06 & 2.077 E-06 & 3200 \\ 122.0 & 24.8 & 0 & 0.000 E+00 & 0.000 E+00 & 3200 \\ 122.0 & 31.0 & 0 & 0.000 E+00 & 0.000 E+00 & 3200 \\ 122.0 & 37.2 & 109 & 0.461 E-07 & 0.900 E-07 & 3200\end{array}$


SERIES 163 EXPERINENT: 1 d \& FT-14:

GROUNO

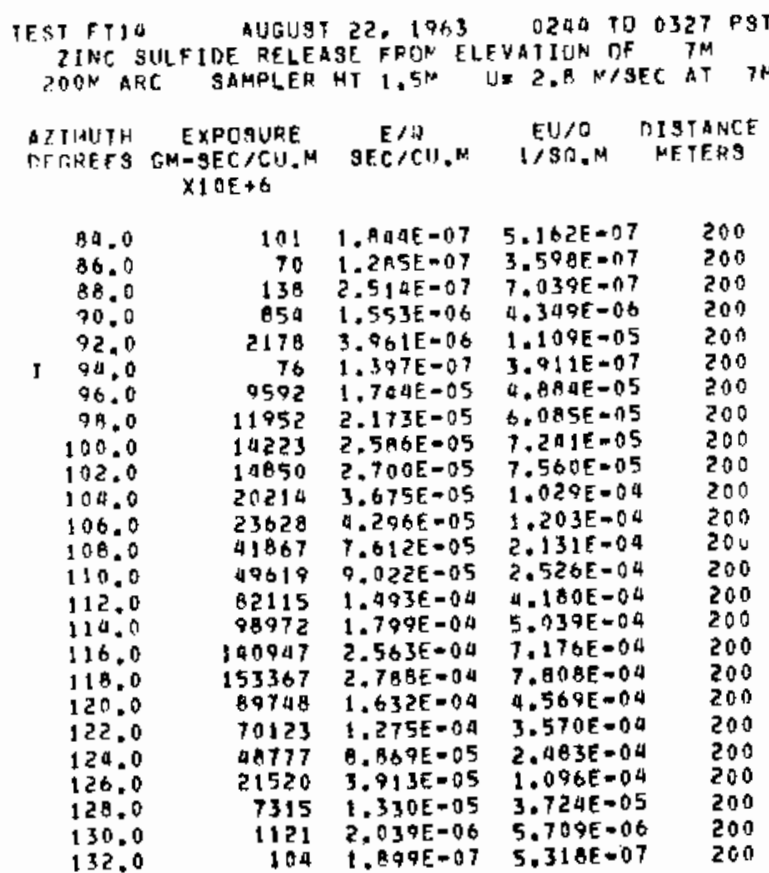

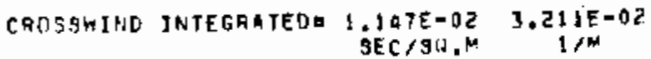

TOWER COTA FOLLOH....

TEST FIIU AUGUST 22,1963 O240 TO O327 PST IJRC SULFIBE RELEASE FRON ELEVATION EF TM

\begin{tabular}{|c|c|c|c|c|c|}
\hline $\begin{array}{l}\text { INUTH } \\
\text { EGREFS }\end{array}$ & $\begin{array}{l}\text { HE IGHT } \\
\text { METERS }\end{array}$ & $\begin{array}{c}\text { EXPOSURE } \\
\text { GM=SEC/CU.H } \\
\times 1 O E+6\end{array}$ & $\begin{array}{c}E / 0 \\
S E C / C U, N\end{array}$ & $\begin{array}{c}E U / O \\
1 / \$ Q . M\end{array}$ & $\begin{array}{l}\text { DISTANCE } \\
\text { METERS }\end{array}$ \\
\hline $\begin{array}{l}106.0 \\
106.0 \\
106.0 \\
100.0 \\
106.0 \\
106.0 \\
106.0 \\
106.0 \\
106.0 \\
106.0 \\
106.0 \\
106.0 \\
106.0 \\
106.0 \\
106.0 \\
106.0\end{array}$ & $\begin{array}{r}0.3 \\
0.7 \\
1.4 \\
2.7 \\
4.1 \\
5.9 \\
6.7 \\
8.1 \\
9.9 \\
10.8 \\
13.5 \\
16.2 \\
18.9 \\
21.6 \\
24.5\end{array}$ & $\begin{array}{l}22846 \\
25292 \\
20011 \\
17969 \\
23214 \\
23259 \\
29329 \\
35304 \\
39123 \\
03052 \\
39311 \\
26954 \\
42791 \\
12010 \\
24700 \\
22541\end{array}$ & $\begin{array}{l}0.154 E=05 \\
4.770 E=05 \\
3.638 E=05 \\
3.267 F=05 \\
9.210 F=05 \\
0.220 E=05 \\
5.333 E=05 \\
6.419 E=05 \\
6.932 E=05 \\
7.828 E=05 \\
7.108 E=05 \\
5.174 E=05 \\
1.780 E=05 \\
5.820 E=05 \\
4.491 E=05 \\
0.103 F=05\end{array}$ & 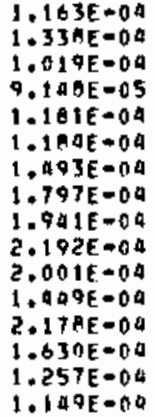 & $\begin{array}{l}200 \\
200 \\
200 \\
200 \\
200 \\
200 \\
200 \\
200 \\
200 \\
200 \\
200 \\
200 \\
200 \\
200 \\
200 \\
200\end{array}$ \\
\hline
\end{tabular}

AZIMLITH HEIGHT EXPOSURE E/O EUTO OISTANCE OEGREES METERS GN-SET/CH,M SEC/TU.N 1/SO,M METERS $x I D E+6$

\begin{tabular}{|c|c|c|c|c|}
\hline 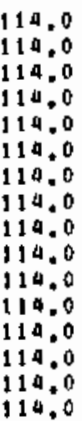 & $\begin{array}{r}0.3 \\
0.7 \\
1.4 \\
2.7 \\
0.1 \\
5.4 \\
6.8 \\
0.1 \\
9.4 \\
10.8 \\
13.5 \\
16.2 \\
18.9 \\
21.9 \\
24.3 \\
27.0\end{array}$ & $\begin{array}{l}A 1952 \\
96461 \\
7379 A \\
728 A 7 \\
99365 \\
98475 \\
98712 \\
115891 \\
100909 \\
115083 \\
126511 \\
101136 \\
93627 \\
63231 \\
91416 \\
31937\end{array}$ & $\begin{array}{l}1.481 E=04 \\
1.75 A E=04 \\
1.342 E=04 \\
1.325 E=04 \\
1.716 E=0 A \\
1.790 E=0 A \\
1.79 A E=04 \\
2.107 E=00 \\
1.826 E=00 \\
2.092 E=04 \\
2.300 E=04 \\
1.839 E=04 \\
1.520 E=04 \\
1.150 E=0 A \\
7.530 E=05 \\
5.789 E=05\end{array}$ & $\begin{array}{l}4.147 E-04 \\
4.911 E=04 \\
3.757 E=04 \\
3.711 E=04 \\
4.004 E=04 \\
5.011 E=04 \\
5.029 E=04 \\
5.199 E=04 \\
5.112 E=09 \\
5.859 E=04 \\
6.441 E=0 a \\
5.149 E=04 \\
4.257 E=0 a \\
3.219 E=0 a \\
2.108 E=04 \\
1.621 E=04\end{array}$ \\
\hline
\end{tabular}

DIJNUTH HEIGHT EXPOSUAE E/R EUIO DISTENCE DEGREES METERS GN-SEC/CW.M SEC/CU.N 1/SB.M NETSRS
XIOE+6

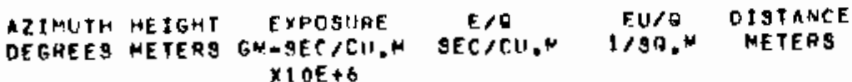

\begin{tabular}{|c|c|c|c|c|c|}
\hline $\begin{array}{l}98.0 \\
98.0 \\
90.0 \\
98.0 \\
98.0 \\
98.0 \\
98.0 \\
98.0 \\
98.0 \\
98.0 \\
98.0 \\
98.0 \\
98.0 \\
98.0 \\
98.0 \\
91.0\end{array}$ & $\begin{array}{l}0.3 \\
0.7 \\
1.4 \\
2.7 \\
0.1 \\
5.9 \\
6.6 \\
6.1 \\
9.9 \\
10.8 \\
13.5 \\
16.2 \\
10.9 \\
21.6 \\
24.3 \\
27.9\end{array}$ & $\begin{array}{l}13030 \\
15227 \\
10560 \\
10896 \\
11577 \\
13564 \\
16736 \\
10335 \\
25349 \\
22671 \\
20202 \\
11209 \\
9071 \\
9552 \\
2371 \\
1632\end{array}$ & $\begin{array}{l}2.360 E-05 \\
2.769 E=05 \\
1.920 E=05 \\
1.982 E=05 \\
2.105 E=05 \\
2.466 F=05 \\
3.043 E=05 \\
3.334 E=05 \\
1.245 E=05 \\
0.113 E=05 \\
3.673 E=05 \\
2.03 E E=05 \\
1.649 E=05 \\
8.270 E=06 \\
9.312 E=06 \\
2.967 E=06\end{array}$ & $\begin{array}{l}6.634 E=05 \\
7.752 E=05 \\
5.376 E=05 \\
5.508 E=05 \\
5.894 E=05 \\
6.905 E=05 \\
0.520 E=05 \\
9.355 E=05 \\
1.109 E=04 \\
1.152 E=04 \\
1.028 E=04 \\
5.707 E=05 \\
4.618 E=05 \\
2.310 E=05 \\
1.207 E=05 \\
0.30 A E=06\end{array}$ & $\begin{array}{l}200 \\
200 \\
200 \\
200 \\
200 \\
200 \\
200 \\
200 \\
200 \\
200 \\
200 \\
200 \\
200 \\
200 \\
200 \\
200\end{array}$ \\
\hline
\end{tabular}

122,0

122.0

122,0

122.0

122.0

122,0

R 122,0

125,0

122.0

122.0

122,0

122.0

122.0

125.0

122.0

122.0
0.
0.7
1.
2.
5.
6.
9.
10.
13.
10.
21.
24.
21.

$0.3 \quad 51330 \quad 0.333 F-05$

$.7 \quad 73665 \quad 1.339 E-04$

$1.6556791 .012 F=04$

$2.7 \quad 57101 \quad 1.03 A E=04$

$5,4 \quad 70015 \quad 1.302 E=04$

$6.8 \quad 01629 \quad 7.569 E-05$

$9.4 \quad 6009 \quad 1.602 \mathrm{E}-04$

$13.5 \quad 76832 \quad 1.397 \mathrm{E}=04$

103738 1.806 -04

$\begin{array}{rr}103728 & 1.806 E=04 \\ 95 A 12 & 1.742 F=04\end{array}$

$\begin{array}{ll}95 A 12 & 1.742 F=04 \\ 91.45 & 1.48 C E-04\end{array}$

$52251 \quad 9,5006-05$

$36071 \quad 0.558 E-05$
$0.1 \quad 71952 \quad 1,300 E=0 a$
$2.613 E=04 \quad 200$ $3.750 \mathrm{E}=04 \quad 2 \mathrm{O}$ 2.034E=04 200 $3.907 E-04 \quad 200$ $3.276 E-04 \quad 200$ $3.870 E-00 \quad 200$ $2.119 E=04 \quad 200$ $3.663 E=04 \quad 200$ $9.455 E=04 \quad 200$ $9.215 E-04 \quad 200$ $3.911 E-04 \quad 200$ $5.2816 * 04 \quad 200$ A. HTOE=0A 200 a.167E-04 200 $1.036 E=04 \quad 200$ $2.660 E-04 \quad 200$ 


\begin{tabular}{|c|c|c|c|c|c|}
\hline $\begin{array}{l}\text { DZIMUTH } \\
\text { DEGREES }\end{array}$ & $\begin{array}{l}\text { HE ITHT } \\
\text { ME TERS }\end{array}$ & $\begin{array}{c}\text { EXPDSUFE } \\
\text { GP }-S E C / C U . N \\
\times 1 O E+6\end{array}$ & $\begin{array}{c}E / \infty \\
\sec / \mathrm{CU} .\end{array}$ & $\begin{array}{c}F U / 0 \\
1 / 50, M\end{array}$ & $\begin{array}{c}\text { DISTANCE } \\
\text { METERS }\end{array}$ \\
\hline $\begin{array}{l}130.0 \\
130.0 \\
130.0 \\
130.0 \\
130.0 \\
130.0 \\
130.0 \\
130.0 \\
130.0 \\
130.0 \\
130.0 \\
130.0 \\
130.0 \\
130.0 \\
130.0 \\
130.0\end{array}$ & $\begin{array}{r}0.3 \\
0.7 \\
1.4 \\
2.7 \\
4.1 \\
5.4 \\
6.8 \\
6.1 \\
9.4 \\
10.8 \\
13.5 \\
16.2 \\
18.9 \\
21.6 \\
24.3 \\
27.0\end{array}$ & $\begin{array}{r}578 \\
1322 \\
637 \\
702 \\
1459 \\
2051 \\
2762 \\
2810 \\
4069 \\
3993 \\
6290 \\
6580 \\
6695 \\
6767 \\
5309 \\
4554\end{array}$ & $\begin{array}{l}1.052 E=06 \\
2.405 E=06 \\
1.523 E=06 \\
1.276 E=06 \\
2.653 E=06 \\
4.457 E=06 \\
5.022 E=06 \\
5.110 E=06 \\
7.399 E=06 \\
7.261 E=06 \\
1.135 E=05 \\
1.196 E=05 \\
1.217 E=05 \\
1.230 E=05 \\
9.000 E=06 \\
6.201 E=02\end{array}$ & $\begin{array}{l}2.947 E=06 \\
6.733 E=06 \\
1.265 E=06 \\
3.574 E=06 \\
7.429 E=06 \\
1.24 A E=05 \\
1.406 E=05 \\
1.431 E=05 \\
2.072 E=05 \\
2.033 E=05 \\
3.179 E=05 \\
3.350 E=05 \\
3.409 E=05 \\
3.445 E=05 \\
2.744 E=05 \\
2.319 E=05\end{array}$ & $\begin{array}{l}200 \\
200 \\
200 \\
200 \\
200 \\
200 \\
200 \\
200 \\
200 \\
200 \\
200 \\
200 \\
200 \\
200 \\
200 \\
200\end{array}$ \\
\hline
\end{tabular}
TEST FTIA AUGUST 22, 1963 024A TO 0327 PST ZINE SULFIDE RELEASE FRUM ELEVATION OF $7 M$ AZIMUTH EXPOSURE E/R EU/O OISTANCE DEGREES GN-SEC/CU.H SEC/CU.M I/SU,M MEFEAS $\times 10 E+6$

\begin{tabular}{|c|c|c|c|c|}
\hline $\begin{array}{l}66.0 \\
80.0 \\
90.0 \\
92.0 \\
40.0 \\
96.0 \\
98.0 \\
100.0 \\
102.0 \\
100.0 \\
106.0 \\
108.0 \\
110.0 \\
112.0 \\
114.0 \\
116.0 \\
118.0 \\
120.0 \\
122.0 \\
124.0 \\
126.0\end{array}$ & $\begin{array}{r}77 \\
924 \\
2229 \\
4235 \\
4684 \\
4877 \\
5308 \\
6283 \\
6458 \\
6265 \\
5882 \\
7668 \\
8182 \\
15193 \\
15122 \\
18597 \\
23311 \\
17128 \\
4620 \\
826 \\
53\end{array}$ & 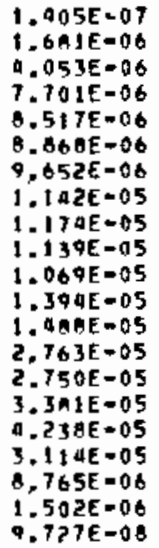 & $\begin{array}{l}3.93 \text { AE }=07 \\
0.706 E=06 \\
1.135 E=05 \\
2.150 E=05 \\
2.305 E=05 \\
2.463 E=05 \\
2.702 E=05 \\
3.199 E=05 \\
3.28 B E=05 \\
3.190 E=05 \\
2.990 E=05 \\
3.904 E=05 \\
4.166 E=05 \\
7.735 E=05 \\
7.699 E-05 \\
9.468 E=05 \\
1.107 E-04 \\
0.720 E=05 \\
2.454 E=05 \\
4.206 E=06 \\
2.724 E=07\end{array}$ & $\begin{array}{l}800 \\
000 \\
800 \\
000 \\
000 \\
000 \\
000 \\
000 \\
800 \\
000 \\
800 \\
800 \\
000 \\
800 \\
000 \\
800 \\
000 \\
000 \\
800 \\
000 \\
800\end{array}$ \\
\hline Rกsร1 & INTEGRATED & $\begin{array}{r}0.029 E-03 \\
\text { SEC/OD.M }\end{array}$ & $2+2 a B E=02$ & \\
\hline
\end{tabular}

TOWER DATA FOLLOW....

\begin{abstract}
TEST FT14 QUCUST 22,1963 O240 100327 PST 2INC SULFIDE RELEASE FRON ELEVATICN OF TK, BOOM APC U. 2 . A H SEC IT $7 M$
\end{abstract}

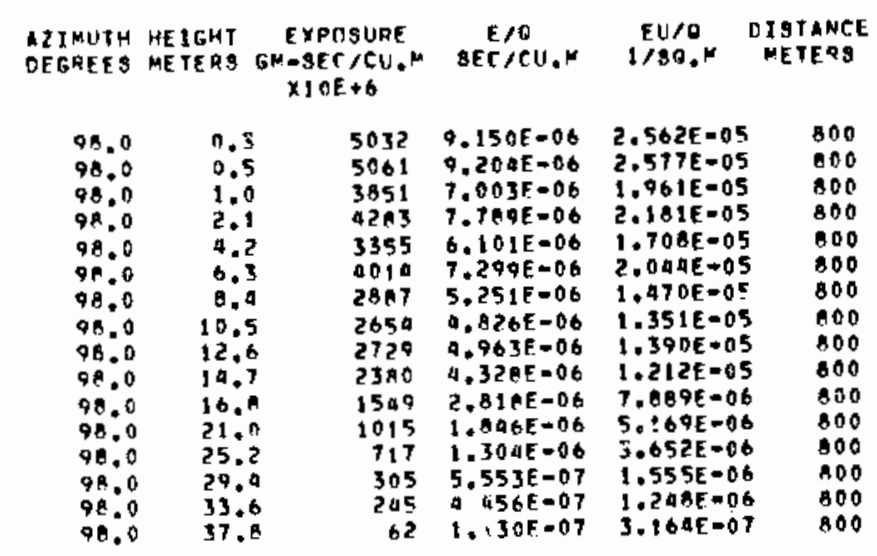

AZIMUTH HEIGHT EXPOSURE
DEGREES METERS GM-SEC/CU.K JEC/CU,N I/SO.M METERS $\times 10 E+6$

$\begin{array}{ll}106.0 & 0.3 \\ 108.0 & 0.5 \\ 106.0 & 1.0 \\ 106.0 & 2.1 \\ 106.0 & 4.2 \\ 106.0 & 6.3 \\ 106.0 & 9.4 \\ 106.0 & 10.5 \\ 106.0 & 12.5 \\ 106.0 & 14.7 \\ 106.0 & 16.8 \\ 106.0 & 21.0 \\ 106.0 & 25.2 \\ 106.0 & 29.7 \\ 106.0 & 33.6 \\ 106.0 & 37.8 \\ 106.0 & 42.0\end{array}$

\begin{tabular}{|c|c|c|c|}
\hline $\begin{array}{l}3319 \\
5039 \\
3524 \\
0217 \\
4907 \\
5350 \\
5853 \\
6296 \\
7240 \\
7055 \\
7262 \\
4379 \\
4397 \\
3818 \\
2511 \\
1909 \\
1152\end{array}$ & $\begin{array}{l}6.161 E=06 \\
9.890 E=06 \\
6.400 E=06 \\
7.667 E=06 \\
0.922 E=06 \\
9.72 B F=06 \\
1.060 E=05 \\
1.145 E=05 \\
1.316 E=05 \\
1.203 E=05 \\
1.317 E=05 \\
7.963 E=06 \\
7.904 E=06 \\
6.93 A E=06 \\
1.695 F=06 \\
3.545 F=06 \\
2.095 F=176\end{array}$ & $\begin{array}{l}1.725 E=05 \\
2.769 E=05 \\
1.790 E=05 \\
2.147 E=05 \\
2.49 \mathrm{AE}=05 \\
2.724 E=05 \\
2.9 A 0 E=05 \\
3.207 E=05 \\
3.68 \mathrm{HE}=05 \\
3.592 E=05 \\
3.687 E=05 \\
2.230 E=05 \\
2.213 E=05 \\
1.943 E=05 \\
1.514 E=05 \\
9.926 E=06 \\
5.067 E-06\end{array}$ & $\begin{array}{l}800 \\
100 \\
100 \\
800 \\
800 \\
100 \\
800 \\
000 \\
000 \\
000 \\
000 \\
000 \\
100 \\
000 \\
800 \\
100 \\
000\end{array}$ \\
\hline
\end{tabular}

AZIMUTH HEIGHT EXPDSURE FID OU/O DIGTHNCE DEGREES RETERS GM-SEC/CU.M SEC/CU,N I/SR.M METERS $\times 1 \cap E+h$

\begin{tabular}{|c|c|c|c|c|c|}
\hline 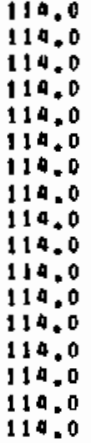 & $\begin{array}{l}0.3 \\
0.5 \\
1.0 \\
2.1 \\
0.2 \\
6.3 \\
0.4 \\
10.5 \\
12.6 \\
19.7 \\
16.8 \\
21.0 \\
25.2 \\
29.4 \\
33.6 \\
37.8 \\
42.0\end{array}$ & $\begin{array}{l}12353 \\
11559 \\
10582 \\
11419 \\
14915 \\
14325 \\
12399 \\
17291 \\
15469 \\
15592 \\
14447 \\
13421 \\
11016 \\
8230 \\
0564 \\
7919 \\
5904\end{array}$ & $\begin{array}{l}2.246 E=05 \\
2.102 E-05 \\
1.024 F=05 \\
2.076 F=05 \\
2.712 E-05 \\
2.605 E=05 \\
2.254 E=05 \\
3.144 E=05 \\
2.813 E=05 \\
2.835 E=05 \\
2.627 E=05 \\
2.440 E=05 \\
2.003 E=05 \\
1.096 E=05 \\
1.557 E=05 \\
1.453 E=05 \\
1.074 E-05\end{array}$ & $\begin{array}{l}6.209 E=05 \\
5.105 E=05 \\
5.386 E=05 \\
5.114 E=05 \\
7.593 E=05 \\
7.293 E=05 \\
6.313 E=05 \\
6.603 E=05 \\
7.076 E=05 \\
7.93 A E=05 \\
7.355 E=05 \\
6.133 E=05 \\
5.609 E=05 \\
4.190 E=05 \\
4.360 E=05 \\
4.067 E-05 \\
3.006 E=05\end{array}$ & $\begin{array}{l}100 \\
000 \\
800 \\
000 \\
000 \\
800 \\
000 \\
000 \\
000 \\
000 \\
000 \\
000 \\
000 \\
000 \\
000 \\
000 \\
000\end{array}$ \\
\hline
\end{tabular}

IZIMUTH HEIGHT EXPQSURE
DEGRE E

\begin{tabular}{|c|c|c|c|c|c|}
\hline $\begin{array}{l}122.0 \\
122.0 \\
122.0 \\
122.0 \\
122.0 \\
122.0 \\
122.0 \\
122.0 \\
122.0 \\
122.0 \\
122.0 \\
122.0 \\
122.0 \\
122.0 \\
122.0 \\
122.0 \\
122.0\end{array}$ & $\begin{array}{l}0.3 \\
0.5 \\
1.0 \\
2.1 \\
4.2 \\
6.3 \\
0.4 \\
10.5 \\
12.6 \\
14.7 \\
16.8 \\
21.0 \\
25.2 \\
29.0 \\
33.6 \\
37.8 \\
42.0\end{array}$ & $\begin{array}{l}3046 \\
3300 \\
3631 \\
4217 \\
4508 \\
5746 \\
6612 \\
8142 \\
7405 \\
8501 \\
9749 \\
9434 \\
8976 \\
8204 \\
7638 \\
6744 \\
6499\end{array}$ & $\begin{array}{l}6.339 E=06 \\
6.002 E=06 \\
6.603 E=06 \\
1.667 E=06 \\
1.190 E-06 \\
1.045 E=05 \\
1.202 E=05 \\
1.460 E=05 \\
1.346 E=05 \\
1.600 E=05 \\
1.773 E=05 \\
1.701 E=05 \\
1.632 E=05 \\
1.492 E=05 \\
1.387 E=05 \\
1.226 E=05 \\
1.192 F=05\end{array}$ & 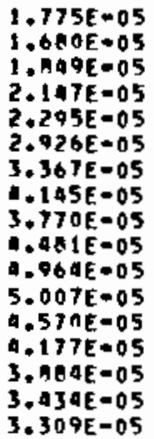 & $\begin{array}{l}800 \\
800 \\
800 \\
800 \\
500 \\
800 \\
100 \\
800 \\
800 \\
600 \\
800 \\
800 \\
800 \\
800 \\
800 \\
800 \\
800\end{array}$ \\
\hline
\end{tabular}




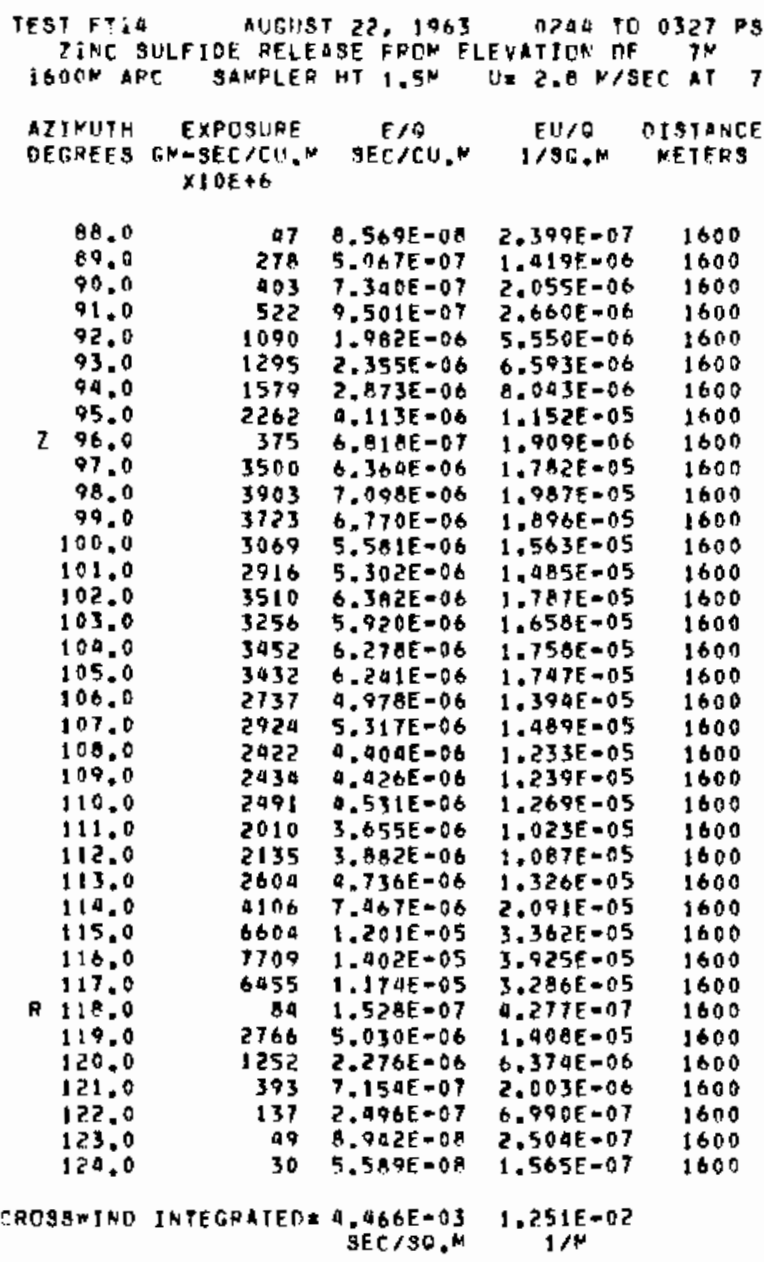

TOKER DATA FOLLOW....

\begin{abstract}
TESTFIIA AUGLIST $22,1963 \quad 0340$ TO 0327 PST

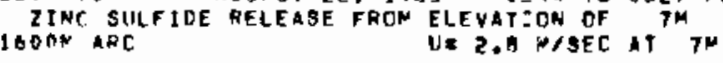

\begin{tabular}{|c|c|c|c|c|c|}
\hline $\begin{array}{l}\text { AZIMtITH } \\
\text { DEGREES }\end{array}$ & $\begin{array}{l}\text { HE IGHT } \\
\text { ME TERS }\end{array}$ & $\begin{array}{c}\text { EXPOSURE } \\
\text { GM-SEC } / C U . M \\
\times 1 O E+B\end{array}$ & $\begin{array}{c}E / O \\
\sec / C U . \mu\end{array}$ & $\begin{array}{l}E U / 0, M \\
1 / 90, M\end{array}$ & $\begin{array}{l}\text { OISTANCE } \\
\text { ME TERS }\end{array}$ \\
\hline $\begin{array}{l}98.0 \\
98.0 \\
98.0 \\
98.0 \\
98.0 \\
98.00 \\
98.0 \\
98.0 \\
98.0 \\
98.0 \\
98.0 \\
98.0 \\
98.0 \\
98.0 \\
98.0 \\
98.0\end{array}$ & $\begin{array}{l}0.2 \\
9.4 \\
0.8 \\
1.5 \\
3.1 \\
6.2 \\
9.3 \\
12.4 \\
15.5 \\
18.6 \\
21.7 \\
29.8 \\
31.0 \\
37.2 \\
43.4 \\
49.6\end{array}$ & $\begin{array}{l}3366 \\
3100 \\
3329 \\
3116 \\
2993 \\
2998 \\
1706 \\
1489 \\
631 \\
528 \\
348 \\
246 \\
45 \\
41 \\
17 \\
12\end{array}$ & 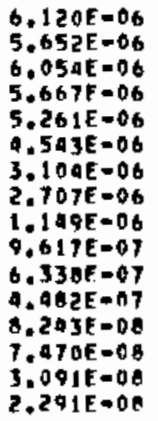 & $\begin{array}{l}1.714 E=05 \\
1.503 E=05 \\
1.695 E=05 \\
1.507 E=05 \\
1.473 E=05 \\
1.272 E=05 \\
9.690 E=06 \\
7.580 E=06 \\
3.217 E=06 \\
2.693 E=06 \\
1.775 E=06 \\
1.255 E=06 \\
2.300 E=07 \\
2.092 E=07 \\
0.655 E=08 \\
6.116 E=08\end{array}$ & $\begin{array}{l}1600 \\
1600 \\
1600 \\
1600 \\
1600 \\
1600 \\
1600 \\
1600 \\
1600 \\
1600 \\
1600 \\
1600 \\
1600 \\
1600 \\
1600 \\
1600\end{array}$ \\
\hline
\end{tabular}

\begin{tabular}{|c|c|c|c|c|c|}
\hline $\begin{array}{l}\text { DIMUTH } \\
\text { DEGREES }\end{array}$ & $\begin{array}{l}\text { HE IGHT } \\
\text { ME TERS }\end{array}$ & $\begin{array}{c}\text { EXPCSURE } \\
\text { GM }-S E E / C H, M \\
X \pm 0 E+6\end{array}$ & $\begin{array}{c}E / O \\
\text { SEC/CU.R }\end{array}$ & $\begin{array}{l}\text { EU/O } \\
\text { I/SG.M }\end{array}$ & $\begin{array}{l}\text { DISTANCE } \\
\text { METERS }\end{array}$ \\
\hline $\begin{array}{l}106.0 \\
106.0 \\
106.0 \\
106.0 \\
106.0 \\
106.0 \\
106.0 \\
106.0 \\
1106.0 \\
106.0 \\
106.0 \\
106.0 \\
106.0 \\
106.0 \\
106.0\end{array}$ & $\begin{array}{r}0.2 \\
0.4 \\
0.8 \\
1.5 \\
3.1 \\
6.2 \\
9.3 \\
12.4 \\
15.5 \\
18.6 \\
24.8 \\
31.0 \\
37.7 \\
43.0 \\
99.6\end{array}$ & $\begin{array}{l}1929 \\
2370 \\
1968 \\
1913 \\
2206 \\
2957 \\
3405 \\
3209 \\
24 \\
2557 \\
1795 \\
1594 \\
916 \\
82 \\
169\end{array}$ & $\begin{array}{l}3.509 E-06 \\
4.325 E=06 \\
3.579 E=06 \\
3.297 E-06 \\
4.012 E=06 \\
5.377 E=06 \\
6.192 F=06 \\
5.836 E=06 \\
4.371 E=08 \\
4.609 E-06 \\
3.22 \mathrm{EE}=06 \\
2.808 E=06 \\
1.667 E=06 \\
1.133 E=07 \\
3.074 E=07\end{array}$ & $\begin{array}{l}9.025 E-06 \\
1.211 E-05 \\
1.002 E-05 \\
9.232 E-06 \\
1.123 E-05 \\
1.505 E=05 \\
1.734 E=05 \\
1.634 E=05 \\
1.220 E=07 \\
1.302 E=05 \\
9.037 E=06 \\
7.8611 E=06 \\
4.665 E=06 \\
3.173 E-07 \\
9.604 E=07\end{array}$ & $\begin{array}{l}1600 \\
1600 \\
1600 \\
1600 \\
1600 \\
1600 \\
1600 \\
1600 \\
1600 \\
1600 \\
1600 \\
1600 \\
1600 \\
1600 \\
1600\end{array}$ \\
\hline
\end{tabular}

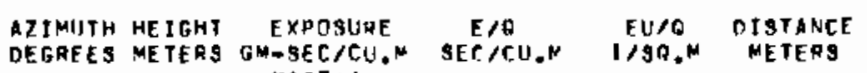
$\times 10 \mathrm{E}+6$

\begin{tabular}{|c|c|c|c|c|c|}
\hline $\begin{array}{l}114.0 \\
119.0 \\
114.0 \\
114.0 \\
114.0 \\
114.0 \\
114.0 \\
119.0 \\
114.0 \\
119.0 \\
114.0 \\
114.0 \\
114.0 \\
114.0\end{array}$ & $\begin{array}{r}0.2 \\
0.4 \\
0.4 \\
3.1 \\
6.2 \\
9.3 \\
12.4 \\
15.5 \\
21.7 \\
24.0 \\
31.0 \\
37.2 \\
99.6 \\
62.0\end{array}$ & $\begin{array}{l}3050 \\
3630 \\
3490 \\
142 \\
3091 \\
3534 \\
4009 \\
3702 \\
4420 \\
3312 \\
3110 \\
3340 \\
1704 \\
1070\end{array}$ & $\begin{array}{l}5.547 E=06 \\
6.616 E=06 \\
6.347 E=06 \\
2.592 E=07 \\
7.075 E=06 \\
6.426 E=06 \\
7.291 E=06 \\
6.732 E=06 \\
6.037 E=06 \\
6.022 E=06 \\
6.929 E=06 \\
6.082 E=06 \\
3.099 E=06 \\
1.961 E=06\end{array}$ & $\begin{array}{l}1.553 E-05 \\
1.052 E=05 \\
1.777 E=05 \\
7.256 E=07 \\
1.981 E=05 \\
1.799 E=05 \\
2.041 E-05 \\
1.065 E=05 \\
2.250 E=05 \\
1.686 E-05 \\
1.940 E=05 \\
1.703 E=05 \\
0.67 M E=06 \\
5.491 E=06\end{array}$ & $\begin{array}{l}1600 \\
1600 \\
1600 \\
1600 \\
1600 \\
1600 \\
1600 \\
1600 \\
1600 \\
1600 \\
1600 \\
1600 \\
1600 \\
1600\end{array}$ \\
\hline
\end{tabular}

AZIMUTH HEIGHT EXPOSURE E/O EUIO DISTANCE DEGREES METERS GM-SEC/CU.M SEC/CU.M 1/SA.M METERS

\begin{tabular}{|c|c|c|c|c|c|}
\hline $\begin{array}{r}122.0 \\
122.0 \\
2122.0 \\
122.0 \\
122.0 \\
122.0 \\
2122.0 \\
122.0 \\
2122.0 \\
122.0 \\
122.0 \\
122.0 \\
122.0 \\
122.0 \\
122.0 \\
122.0 \\
122.0\end{array}$ & $\begin{array}{r}0.2 \\
0.4 \\
0.8 \\
1.5 \\
3.1 \\
6.2 \\
9.3 \\
12.4 \\
19.6 \\
21.7 \\
24.4 \\
31.0 \\
37.2 \\
43.4 \\
49.6 \\
55.8 \\
62.0\end{array}$ & $\begin{array}{r}328 \\
182 \\
66 \\
317 \\
123 \\
219 \\
39 \\
518 \\
41 \\
990 \\
946 \\
1588 \\
1603 \\
1760 \\
1950 \\
1145 \\
52\end{array}$ & 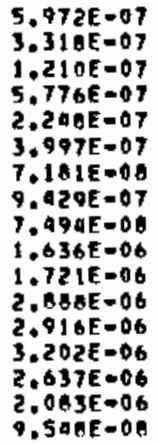 & 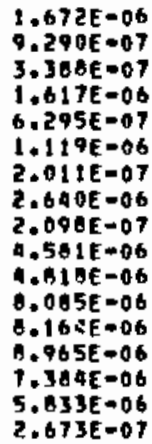 & $\begin{array}{l}1600 \\
1600 \\
1600 \\
1600 \\
1600 \\
1600 \\
1600 \\
1600 \\
1600 \\
1600 \\
1600 \\
1600 \\
1600 \\
1600 \\
1600 \\
1600 \\
1600\end{array}$ \\
\hline
\end{tabular}

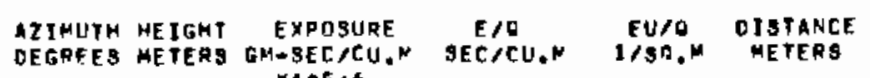
$X \operatorname{InE} \$ 6$

$\begin{array}{ll}130.0 & 0.2 \\ 130.0 & 0.4 \\ 130.0 & 0.8 \\ 130.0 & 1.5 \\ 130.0 & 3.1 \\ 130.0 & 21.7 \\ 130.0 & 24.8 \\ 130.0 & 31.0 \\ 130.0 & 37.2 \\ 130.0 & 93.4 \\ 130.0 & 99.6\end{array}$




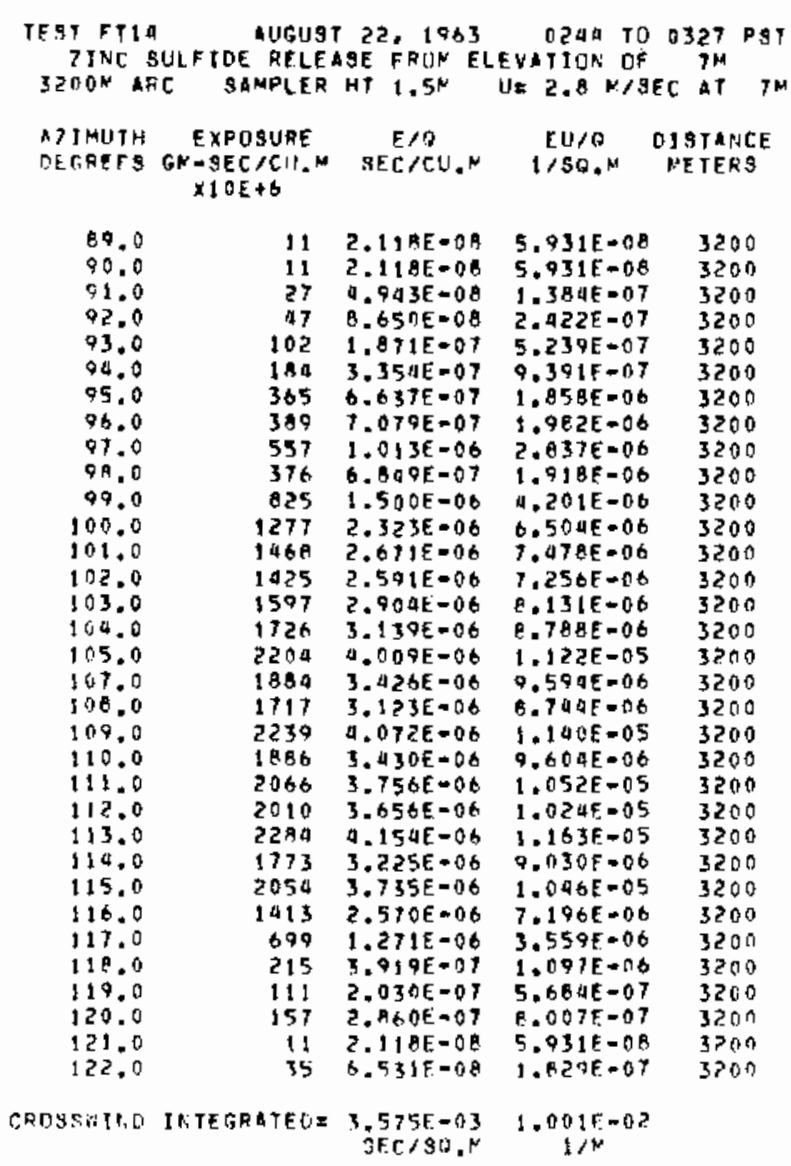

TOMER DETA FOLLIIW....

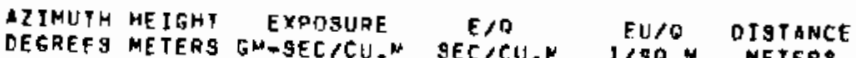
AEC/CU.N SEC/CU,N I/SB.N NETERS

2114.0 114.0

r 114.0

114,0

114.0

114.0

119.0

114.0

114.0

124.1

114.0

114.0

114.0

114.0

110.0

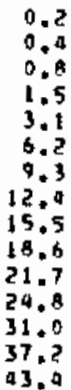

\begin{tabular}{|c|c|c|}
\hline $\begin{array}{l}2066 \\
2351 \\
1299 \\
2292 \\
2383 \\
2582 \\
2542 \\
2943 \\
2603 \\
3096 \\
2787 \\
2705 \\
2506 \\
2278 \\
1585\end{array}$ & $\begin{array}{l}3.757 E=06 \\
9.276 E=06 \\
2.363 E=06 \\
4.169 E=06 \\
4.334 E=06 \\
4.696 E=06 \\
4.622 E=06 \\
5.352 E=06 \\
4.733 E=06 \\
5.630 E=06 \\
5.068 E=06 \\
4.920 E=06 \\
4.557 E=06 \\
4.143 E=06 \\
2.03 E=06\end{array}$ & $\begin{array}{l}1.052 E=05 \\
1.197 E=05 \\
6.617 E-06 \\
1.167 E=05 \\
1.213 E=05 \\
1.315 E=05 \\
1.294 E=05 \\
1.098 E=05 \\
1.325 E=05 \\
1.576 E=05 \\
1.419 E=05 \\
1.378 E=05 \\
1.276 E=05 \\
1.160 E=05 \\
8.073 E=06\end{array}$ \\
\hline
\end{tabular}

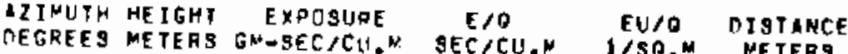
$\times 10 E+6$

125.100 .9

122.0
122.0

$193.5325=08$

$15 \quad 20064500$ Q.61E-0B 3200

$\begin{array}{llll}15 & 2.8645-08 & 0.021 E-08 & 3200\end{array}$

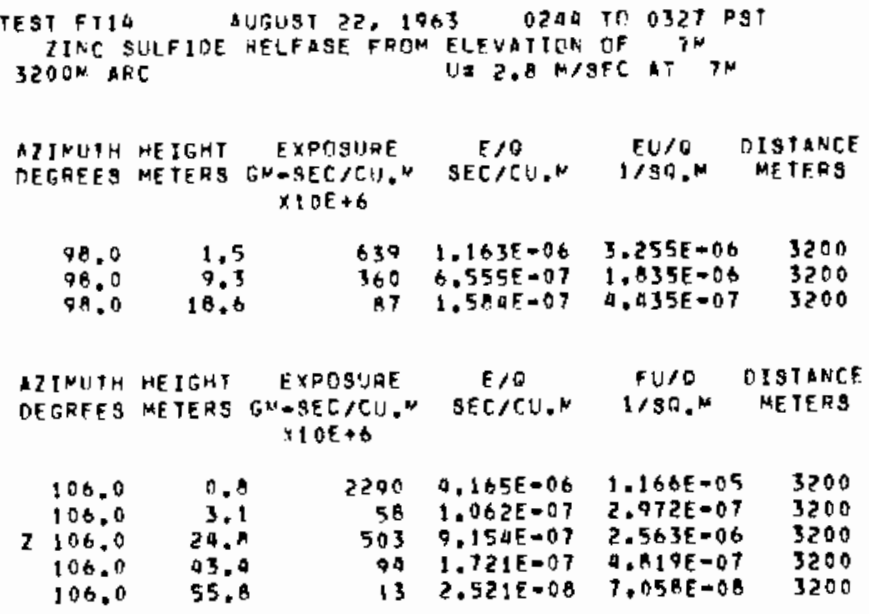


SERIES TE3 EXPERIMENT: 15 \& FT- 15

GROUND LEVEL AND TONER SAMPL ING 200-3200 M ARCS.

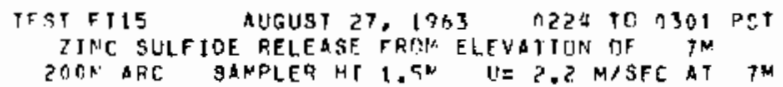

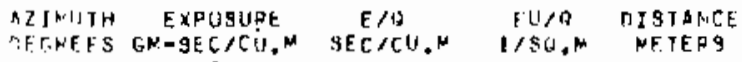
$\times 10 E+6$

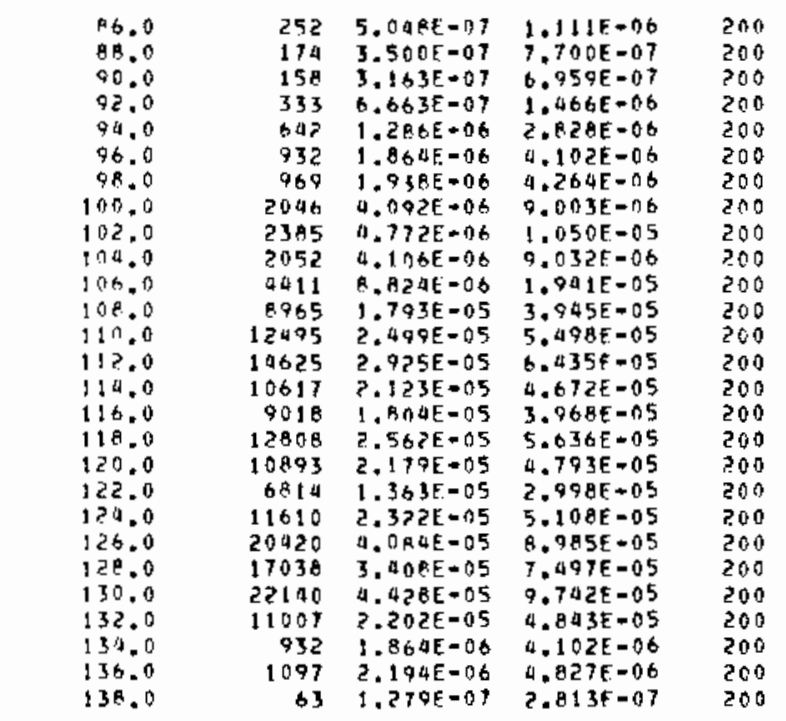

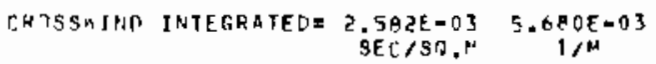

TORER DATA FDLLOW, , .

TEST FT15 QUGUST ?T, 1963 0220 TO 0301 PST ZINC SULFIOE AELEASE FRT FLEVATION DF TN 20 IN ARC UE $2.2 N / 3 F C$ AT $7 M$ A2TMUTH HEIGHT EXPOSURE
OEGHES METERS GMOSEC/CU.N SECICU.N $\times 10 E+6$

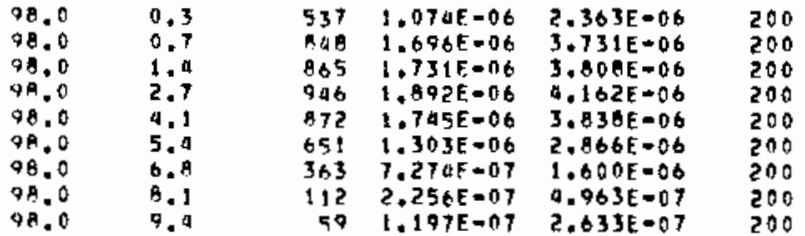

\begin{tabular}{|c|c|c|c|c|c|}
\hline $\begin{array}{l}2 \text { IMUTH } \\
\text { EGREES }\end{array}$ & $\begin{array}{l}\text { HE IGHT } \\
\text { METERS }\end{array}$ & $\begin{array}{c}\text { EXPOSURE } \\
\text { GM-SEC } / C \cup, M \\
\times 10 E+6\end{array}$ & $\begin{array}{c}E / Q \\
\mathrm{SEC} / \mathrm{Cl}^{+}, \mathrm{N}\end{array}$ & $\begin{array}{c}E U / 0 \\
1 / \$ 0^{\circ} . M\end{array}$ & $\begin{array}{l}\text { DISTANCE } \\
\text { METERS }\end{array}$ \\
\hline $\begin{array}{l}106.0 \\
106.0 \\
106.0 \\
106.0 \\
106.0 \\
106.0 \\
106.0 \\
106.0 \\
106.0 \\
106.0 \\
106.0 \\
106.0\end{array}$ & $\begin{array}{r}0.3 \\
0.7 \\
1.9 \\
2.7 \\
4.1 \\
5.4 \\
6.1 \\
8.1 \\
9.4 \\
10.4 \\
13.5 \\
16.2\end{array}$ & $\begin{array}{l}3992 \\
4380 \\
4375 \\
3709 \\
5040 \\
5066 \\
9709 \\
8258 \\
5390 \\
4911 \\
853 \\
195\end{array}$ & $\begin{array}{l}7.986 E=06 \\
8.777 E=06 \\
8.751 E=06 \\
7.499 F=06 \\
1.00 P E=05 \\
1.093 E=05 \\
1.742 E=05 \\
1.652 E=05 \\
1.000 E=05 \\
9.824 E=06 \\
1.70 A E=06 \\
2.900 E=07\end{array}$ & $\begin{array}{l}1.757 E-05 \\
1.931 E-05 \\
1.925 E=05 \\
1.650 E-05 \\
2.216 E-05 \\
2.005 E-05 \\
3.832 E=05 \\
3.634 E=05 \\
2.376 E=05 \\
2.161 E=05 \\
3.757 E-06 \\
6.300 E=07\end{array}$ & $\begin{array}{l}200 \\
200 \\
200 \\
200 \\
200 \\
200 \\
200 \\
200 \\
200 \\
200 \\
200 \\
200\end{array}$ \\
\hline
\end{tabular}

A2IMUTH HEIGHT EXPOSUEF F/O FLIA OISTANCE DEGRFES NETERS GM-SEC $\times 1 \cap E+A$

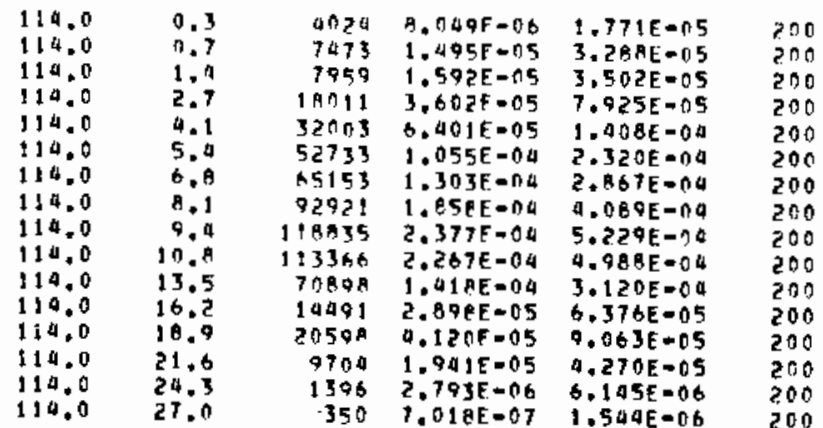
AZIMUTH HEIGHT EXPQSURE
DEGREES METERS GM-SEC/CH.N SEC/CU.N IISO,N METERS

\begin{tabular}{|c|c|c|c|c|c|}
\hline $\begin{array}{l}122.0 \\
122.0 \\
122.0 \\
122.0 \\
122.0 \\
122.0 \\
122.0 \\
122.0 \\
122.0 \\
122.0 \\
123\end{array}$ & $\begin{array}{l}0.3 \\
0.7 \\
1.4 \\
2.7 \\
4.1 \\
5.0 \\
6.8 \\
6.1 \\
9.4 \\
10.8 \\
13.5 \\
16.2 \\
10.9 \\
21.6 \\
24.3 \\
27.0\end{array}$ & $\begin{array}{r}6497 \\
5674 \\
4566 \\
7296 \\
10009 \\
14915 \\
46161 \\
60951 \\
99930 \\
146727 \\
220229 \\
155345 \\
75919 \\
23892 \\
4711 \\
2695\end{array}$ & $\begin{array}{l}1.29 E E=05 \\
1.135 E=05 \\
9.133 E=06 \\
1.459 E=05 \\
2.090 E=05 \\
2.983 E=05 \\
9.232 E=05 \\
9.217 E=04 \\
.994 E=04 \\
3.735 E=04 \\
9.405 E=04 \\
3.107 E=04 \\
1.516 E=04 \\
4.77 F E=05 \\
9.424 E=06 \\
5.391 E=06\end{array}$ & $\begin{array}{l}2.055 E=05 \\
2.097 E=05 \\
2.009 E=05 \\
3.210 E=05 \\
1.598 E=05 \\
6.563 E=05 \\
2.031 E=04 \\
2.677 E=04 \\
4.397 E=04 \\
0.216 E=04 \\
9.690 E=04 \\
0.135 E=04 \\
3.336 E=04 \\
1.051 E=04 \\
2.013 E=05 \\
1.185 E=05\end{array}$ & $\begin{array}{l}200 \\
200 \\
200 \\
200 \\
200 \\
200 \\
200 \\
200 \\
200 \\
200 \\
200 \\
200 \\
200 \\
200 \\
200 \\
200\end{array}$ \\
\hline
\end{tabular}




\begin{tabular}{|c|c|c|c|c|c|}
\hline $\begin{array}{l}\text { AZIMUTH } \\
\text { OEGREES }\end{array}$ & $\begin{array}{l}\text { HE IGHT } \\
\text { NETERS }\end{array}$ & $\begin{array}{l}\text { EXPกSUAF } \\
\text { GNESEC } \\
\times 1 \text { OE }+6\end{array}$ & $\begin{array}{c}E / \theta \\
S E C / C(1, N\end{array}$ & $\begin{array}{c}E U / 0 \\
1 / 50, M\end{array}$ & $\begin{array}{l}\text { DISTANCF } \\
\text { NETERS }\end{array}$ \\
\hline $\begin{array}{l}130.0 \\
130.0 \\
130.0 \\
130.0 \\
130.0 \\
130.0 \\
130.0 \\
130.0 \\
130.0 \\
130.0 \\
130.0 \\
130.0 \\
130.0 \\
130.0 \\
130.0 \\
130.0\end{array}$ & $\begin{array}{l}0.3 \\
0.7 \\
1.9 \\
2.7 \\
0.1 \\
5.4 \\
6.8 \\
0.1 \\
9.9 \\
10.0 \\
13.5 \\
16.2 \\
18.0 \\
21.6 \\
24.3 \\
27.0\end{array}$ & $\begin{array}{r}13271 \\
16336 \\
14316 \\
22014 \\
35920 \\
56204 \\
73676 \\
109468 \\
102161 \\
116733 \\
120271 \\
172975 \\
94049 \\
42484 \\
9907 \\
4500\end{array}$ & $\begin{array}{l}2.65 a E=05 \\
3.26 E E-05 \\
2.863 E=05 \\
0.577 E=05 \\
7.085 E=05 \\
1.126 E=04 \\
1.474 E=04 \\
2.189 E=04 \\
2.043 E=04 \\
2.335 E=04 \\
2.405 E=04 \\
3.450 E=04 \\
1.081 E=04 \\
0.498 E=05 \\
1.901 E=05 \\
9.008 E=06\end{array}$ & $\begin{array}{l}5.040 E=05 \\
7.189 E=05 \\
6.249 E=05 \\
1.007 E=04 \\
1.559 E=04 \\
2.477 E=04 \\
3.242 E=04 \\
0.817 E=04 \\
6.255 E=04 \\
5.136 E=04 \\
5.292 E=04 \\
7.589 E=04 \\
4.138 E=04 \\
1.069 E=04 \\
9.359 E=05 \\
1.982 E=05\end{array}$ & $\begin{array}{l}200 \\
200 \\
200 \\
200 \\
200 \\
200 \\
200 \\
200 \\
200 \\
200 \\
200 \\
200 \\
200 \\
200 \\
200 \\
200\end{array}$ \\
\hline
\end{tabular}

TOHER DHTA FOLLOW....

TESTFT15 AUGUST 27, 1963 O224 TO 0301 PST

IINC SULFTOE RELEASE FROM ELEVATION OF TH

NOON AHC UI 2.2 HSEC AT $7 \mathrm{M}$

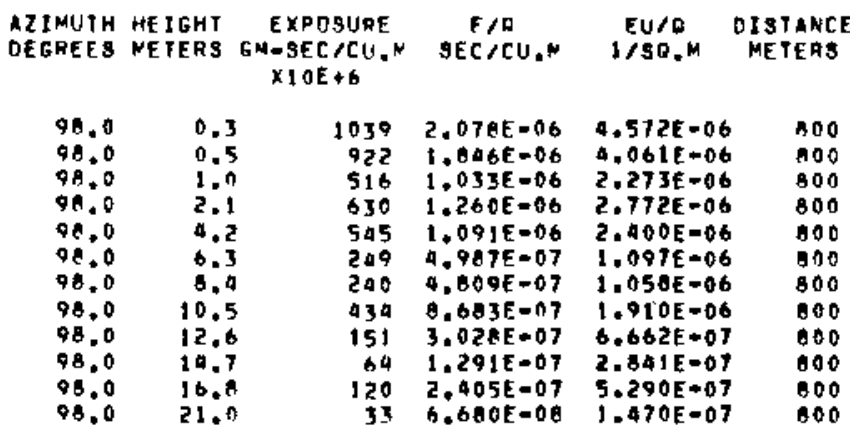

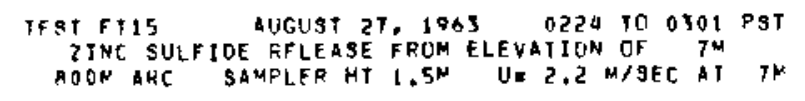

ATINUTH EXPQSURE E/O EU/R OISTANCE DFGREES GN-SEC/CU.M SEC/CU.M I/SQ.N NFTERS $\times 10 E+6$

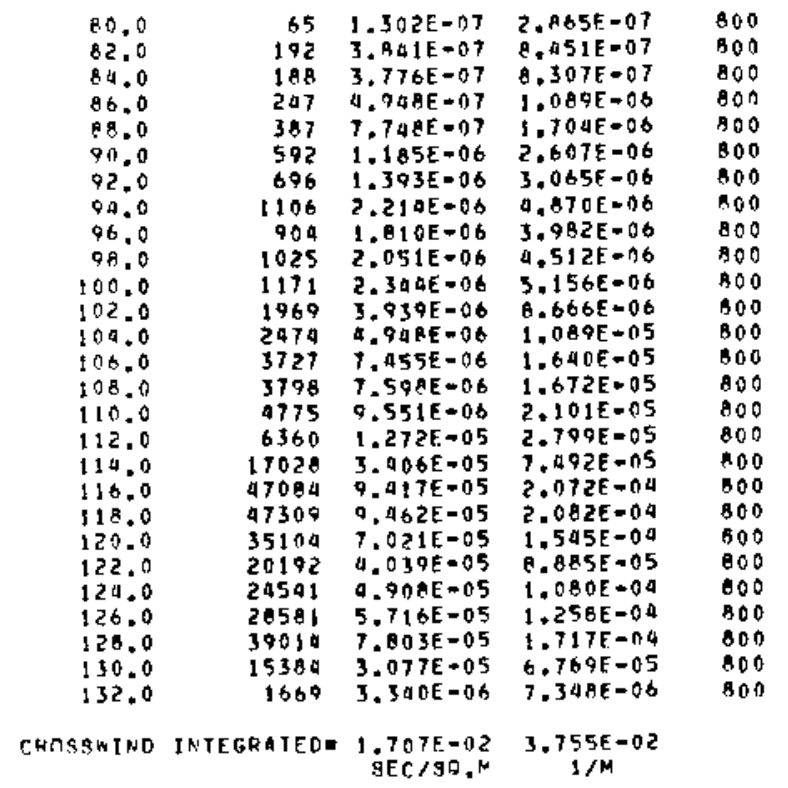

\begin{tabular}{|c|c|c|c|c|c|}
\hline $\begin{array}{l}\text { AZIMUTH } \\
\text { DEGREES }\end{array}$ & $\begin{array}{l}\text { HE IGHT } \\
\text { NETERS }\end{array}$ & $\begin{array}{c}\text { EXPOSURE } \\
\text { GM-SEC } / C U . N \\
\times 10 E+6\end{array}$ & $\begin{array}{c}E / 0 \\
\sec / \mathrm{Cu}_{4} \mathrm{~N}\end{array}$ & $\begin{array}{l}E U / O \\
1 / S A_{0}\end{array}$ & $\begin{array}{l}\text { DISTANCE } \\
\text { METERS }\end{array}$ \\
\hline $\begin{array}{l}106.0 \\
106.0 \\
106.0 \\
106.0 \\
106.0 \\
106.0 \\
106.0 \\
106.0 \\
106.0 \\
106.0 \\
106.0 \\
106.0 \\
106.0\end{array}$ & $\begin{array}{l}0.3 \\
0.5 \\
1.0 \\
2.1 \\
4.2 \\
6.3 \\
8.0 \\
10.5 \\
12.6 \\
14.7 \\
16.8 \\
21.0 \\
25.2\end{array}$ & $\begin{array}{l}1996 \\
2389 \\
1894 \\
1966 \\
1839 \\
1197 \\
907 \\
529 \\
512 \\
240 \\
130 \\
26 \\
23\end{array}$ & $\begin{array}{l}2.97 A E=06 \\
4.778 E=06 \\
3.790 E=06 \\
3.932 E=06 \\
3.67=E=06 \\
2.396 E=06 \\
1.995 E=06 \\
1.060 E=06 \\
1.024 E=06 \\
4.909 E=07 \\
2.761 E=07 \\
5.344 E=08 \\
4.728 E=08\end{array}$ & $\begin{array}{l}6.542 E=06 \\
1.051 E=05 \\
8.337 E=06 \\
8.651 E=06 \\
8.092 E=06 \\
5.271 E=06 \\
4.309 E=06 \\
2.332 E=06 \\
2.253 E=06 \\
1.058 E=06 \\
6.074 E=07 \\
1.176 E=07 \\
1.040 E=07\end{array}$ & $\begin{array}{l}800 \\
800 \\
800 \\
800 \\
800 \\
800 \\
800 \\
800 \\
800 \\
800 \\
000 \\
000 \\
000\end{array}$ \\
\hline
\end{tabular} ATIRUTH HEIGHT EXPOSURE
DEGREES METERS GM-SEC/CIJA SEC/CU,N $x \geq \cap \& 6$

\begin{tabular}{|c|c|c|c|c|c|}
\hline $\begin{array}{l}1190.0 \\
119.0 \\
1190.0 \\
110.0 \\
119.0 \\
119.0 \\
114.0 \\
119.0 \\
119.0 \\
119.0 \\
119.0 \\
114.0 \\
119.0 \\
119.0\end{array}$ & $\begin{array}{r}0.3 \\
0.5 \\
1.0 \\
2.1 \\
4.2 \\
6.3 \\
8.4 \\
10.5 \\
12.4 \\
14.7 \\
13.0 \\
21.0 \\
25.7 \\
29.4\end{array}$ & $\begin{array}{r}14766 \\
13532 \\
10547 \\
11491 \\
12257 \\
12067 \\
0542 \\
5646 \\
4288 \\
2097 \\
1013 \\
536 \\
204 \\
84\end{array}$ & $\begin{array}{l}2.957 E=05 \\
2.706 F=05 \\
2.109 E=05 \\
2.298 E=05 \\
2.451 E=05 \\
2.014 E=05 \\
1.664 E=05 \\
1.129 E=05 \\
0.577 E=06 \\
4.195 E=06 \\
2.026 E=06 \\
1.073 E=06 \\
0.0 A 7 E=07 \\
1.609 E=07\end{array}$ & $\begin{array}{l}6.506 E=05 \\
5.954 E=05 \\
4.641 E=05 \\
5.056 E=05 \\
5.393 E=05 \\
5.310 E=05 \\
3.671 E=05 \\
2.480 E=05 \\
1.087 E=05 \\
9.229 E=06 \\
4.45 E E=06 \\
2.341 E=06 \\
0.991 E=07 \\
3.715 E=07\end{array}$ & $\begin{array}{l}000 \\
000 \\
000 \\
000 \\
000 \\
000 \\
000 \\
000 \\
000 \\
000 \\
000 \\
000 \\
000 \\
000\end{array}$ \\
\hline
\end{tabular}




\begin{tabular}{|c|c|c|c|c|c|}
\hline $\begin{array}{l}\text { AZINUTH } \\
\text { DEGREES }\end{array}$ & $\begin{array}{l}\text { HEIGHT } \\
\text { METERS }\end{array}$ & $\begin{array}{c}\text { EXPCSURE } \\
\text { GM=SEC } / C U, M \\
\times 10 F+6\end{array}$ & $\begin{array}{c}E / Q \\
S E C \neq C U_{0} N\end{array}$ & $\begin{array}{l}E U / O \\
1 / S Q_{*}, M\end{array}$ & $\begin{array}{l}\text { OISTENCF. } \\
\text { METERS }\end{array}$ \\
\hline $\begin{array}{l}122.0 \\
122.0 \\
122.0 \\
122.0 \\
122.0 \\
122.0 \\
122.0 \\
122.0 \\
122.0 \\
122.0 \\
122.0 \\
122.0 \\
122.0 \\
122.0 \\
122.0 \\
122.0 \\
122.0\end{array}$ & $\begin{array}{l}0.3 \\
0.5 \\
1.0 \\
2.1 \\
4.2 \\
6.3 \\
8.4 \\
10.5 \\
12.6 \\
14.7 \\
10.8 \\
21.0 \\
25.7 \\
29.4 \\
33.6 \\
37.8 \\
92.0\end{array}$ & $\begin{array}{l}1910 ! \\
14020 \\
14559 \\
15040 \\
16966 \\
20179 \\
30339 \\
35295 \\
32943 \\
33469 \\
28593 \\
17449 \\
7975 \\
3534 \\
1442 \\
442 \\
119\end{array}$ & $\begin{array}{l}3.620 E=05 \\
2.80 A E=05 \\
2.012 E-05 \\
3.008 E=05 \\
3.343 E=05 \\
4.036 E=05 \\
6.068 E=05 \\
7.059 E=05 \\
6.549 E=05 \\
6.692 E=05 \\
5.719 E=05 \\
3.940 E=05 \\
1.595 E=05 \\
7.069 E=06 \\
2.080 E=06 \\
1.849 E=07 \\
2.3 A \Delta E=07\end{array}$ & $\begin{array}{l}7.965 E=05 \\
6.345 E=05 \\
6.006 E=05 \\
6.618 E=05 \\
7.465 E-05 \\
0.079 E=05 \\
1.335 E=04 \\
1.553 E=04 \\
1.450 E=04 \\
1.473 E=04 \\
1.250 E=04 \\
7.678 E=05 \\
3.509 E=05 \\
1.555 E=05 \\
3.346 E=06 \\
1.947 E-06 \\
5.201 E=07\end{array}$ & $\begin{array}{l}800 \\
500 \\
800 \\
800 \\
800 \\
800 \\
800 \\
000 \\
800 \\
000 \\
000 \\
800 \\
000 \\
800 \\
800 \\
100 \\
800\end{array}$ \\
\hline
\end{tabular}

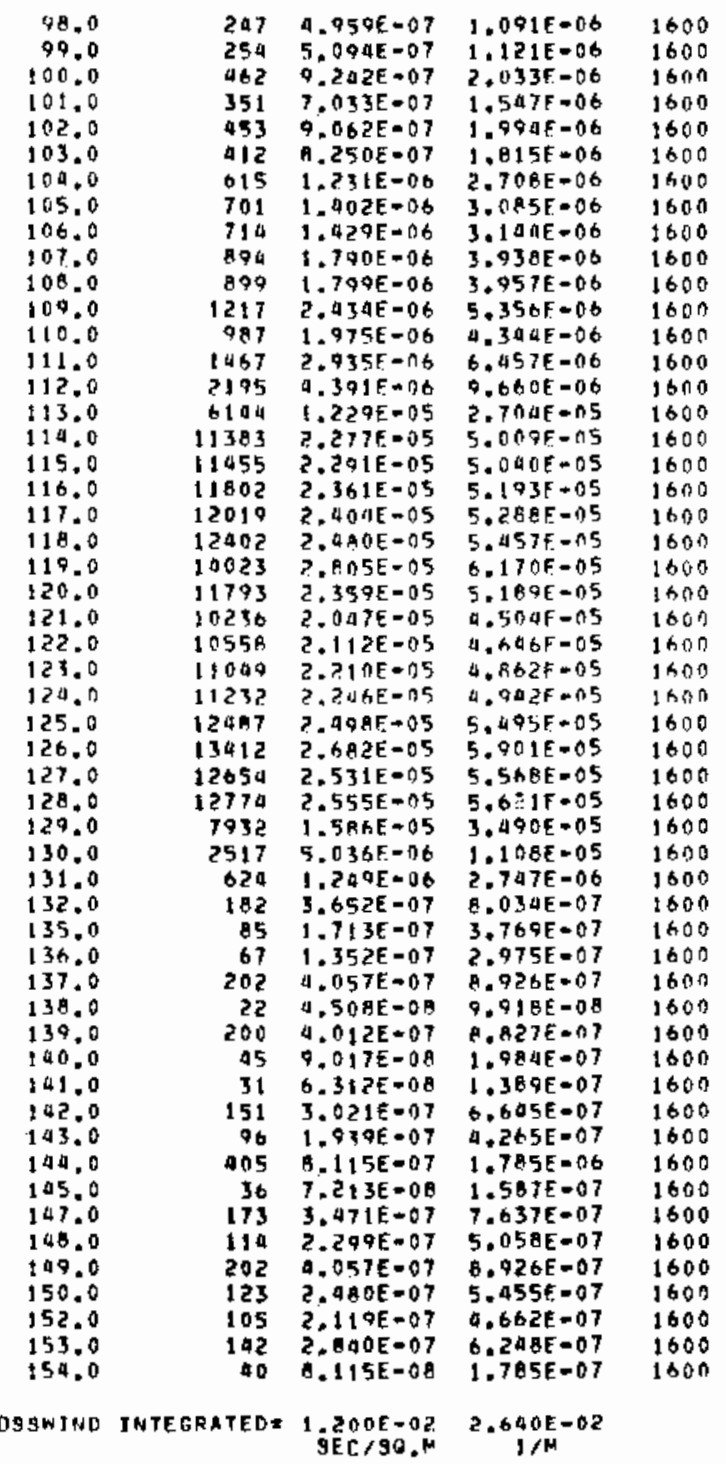

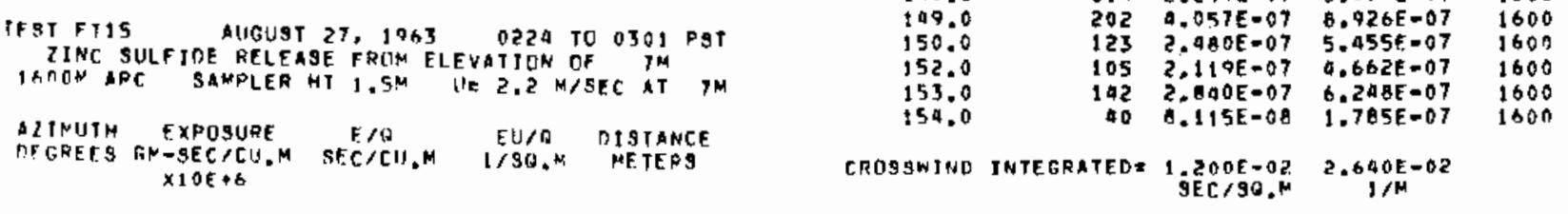

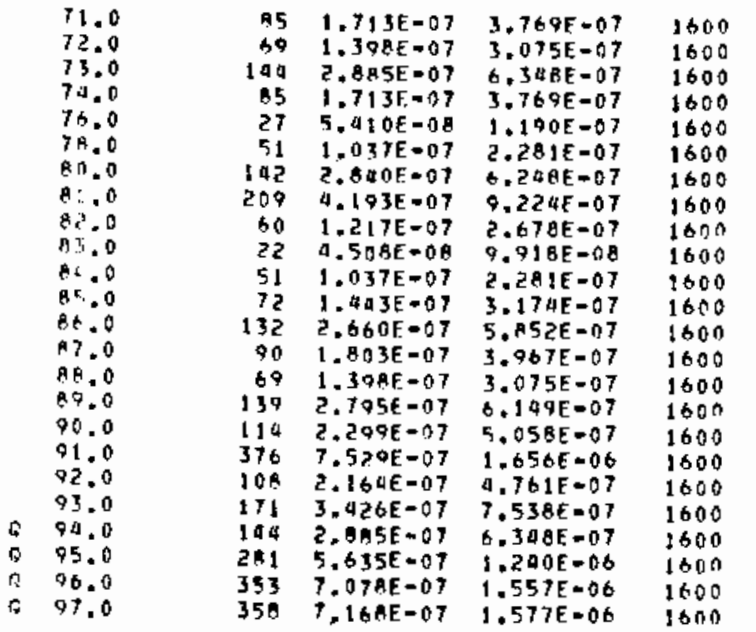

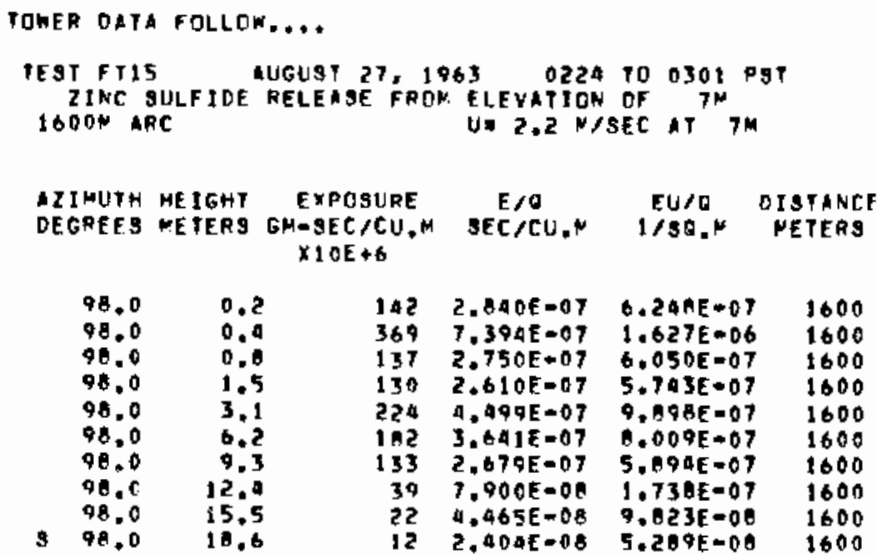




\begin{tabular}{|c|c|c|c|c|c|}
\hline $\begin{array}{l}\text { AZIMUTH } \\
\text { DEGREE } 9\end{array}$ & $\begin{array}{l}\text { HEIGHT } \\
\text { METERS }\end{array}$ & $\begin{array}{c}\text { EXPCSURE } \\
\text { GM=SEC }=\text { SU. } \\
\times 1 O E+6\end{array}$ & $\begin{array}{c}E / \theta \\
S E C / C U . N\end{array}$ & $\begin{array}{l}E U / 0 \\
1 / 90 . N\end{array}$ & $\begin{array}{l}\text { OISTANCE } \\
\text { FETERS }\end{array}$ \\
\hline $\begin{array}{l}106.0 \\
106.0 \\
106.0 \\
106.0 \\
106.0 \\
106.0 \\
106.0 \\
106.0 \\
106.0 \\
106.0 \\
106.0\end{array}$ & $\begin{array}{l}0.2 \\
0.0 \\
0.8 \\
1.5 \\
3.1 \\
6.2 \\
9.3 \\
12.9 \\
15.5 \\
18.6 \\
21.7\end{array}$ & $\begin{array}{l}550 \\
640 \\
570 \\
499 \\
530 \\
503 \\
276 \\
192 \\
109 \\
54 \\
110\end{array}$ & $\begin{array}{l}1.100 F=06 \\
1.289 E=06 \\
1.150 F=06 \\
9.995 E=07 \\
1.061 E=06 \\
1.008 E=06 \\
5.530 E=07 \\
3.847 E=07 \\
2.989 E=07 \\
1.133 E=07 \\
2.370 E=07\end{array}$ & $\begin{array}{l}2.020 E=06 \\
2.037 E=06 \\
2.529 E=06 \\
2.199 E=06 \\
2.335 E=06 \\
2.214 E=06 \\
1.217 E=06 \\
0.063 E=07 \\
6.57 a E=07 \\
2.499 E=07 \\
5.219 E=07\end{array}$ & $\begin{array}{l}1600 \\
1600 \\
1600 \\
1600 \\
1600 \\
1600 \\
1600 \\
1600 \\
1600 \\
1600 \\
1600\end{array}$ \\
\hline
\end{tabular}

\begin{tabular}{|c|c|c|c|c|c|}
\hline $\begin{array}{l}\text { LIIMUTH } \\
\text { DEGREES }\end{array}$ & $\begin{array}{l}\text { HE IGHI } \\
\text { MF TERS }\end{array}$ & $\begin{array}{c}\text { EXPRSURF } \\
\text { GH= SEC } / C 11, M \\
\times 10 E+6\end{array}$ & $\begin{array}{c}E / D \\
\operatorname{SEC} C / C U . N\end{array}$ & $\begin{array}{l}F \cup / 0 \\
1 / 50 . m\end{array}$ & $\begin{array}{l}\text { DISTINCE } \\
\text { NETERS }\end{array}$ \\
\hline 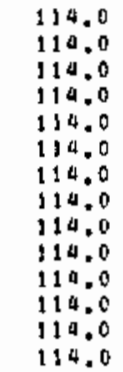 & $\begin{array}{l}0.2 \\
0.0 \\
0.8 \\
1.5 \\
3.1 \\
6.2 \\
9.3 \\
12.0 \\
15.5 \\
18.0 \\
21.7 \\
24.8 \\
31.0 \\
37.2\end{array}$ & $\begin{array}{r}8012 \\
9535 \\
7091 \\
11555 \\
660 \\
4561 \\
1294 \\
959 \\
516 \\
360 \\
319 \\
127 \\
31 \\
33\end{array}$ & $\begin{array}{l}1.600 E=05 \\
1.907 E=05 \\
1.41 A E=05 \\
2.311 F=05 \\
1.329 E=06 \\
9.122 E=06 \\
2.540 E=06 \\
1.920 E=06 \\
1.034 E=06 \\
7.213 E=07 \\
6.358 E=07 \\
2.552 E=07 \\
6.244 F=08 \\
6.787 E=0 B\end{array}$ & $\begin{array}{l}3.521 E=05 \\
4.195 E=05 \\
3.120 E=05 \\
5.084 E-05 \\
2.924 E-06 \\
2.007 E=05 \\
5.697 E=06 \\
4.224 E-06 \\
2.274 E=06 \\
1.587 E=06 \\
1.405 E-06 \\
5.614 E=07 \\
1.374 E=07 \\
1.093 E-07\end{array}$ & $\begin{array}{l}1600 \\
1600 \\
1600 \\
1600 \\
1600 \\
1600 \\
1600 \\
1600 \\
1600 \\
1600 \\
1600 \\
1600 \\
1600 \\
1600\end{array}$ \\
\hline
\end{tabular}

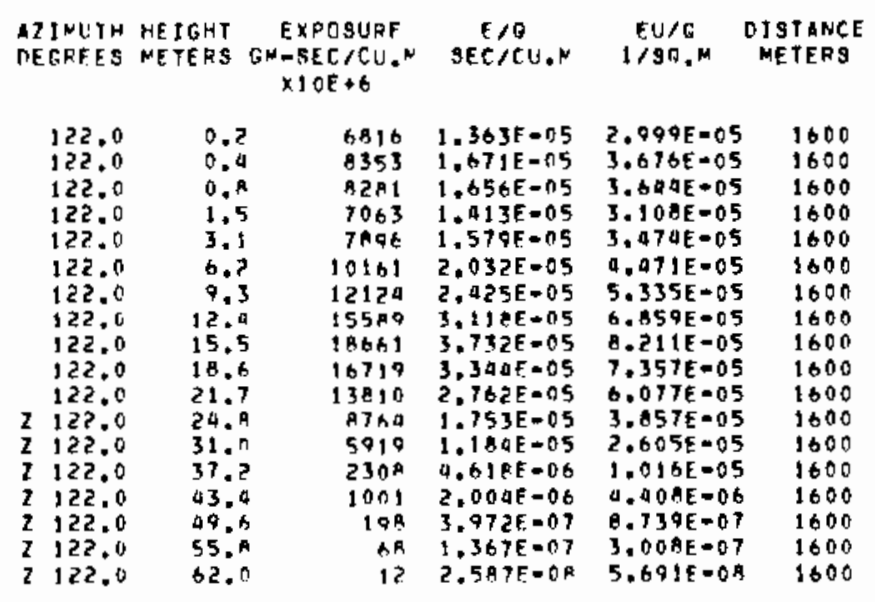

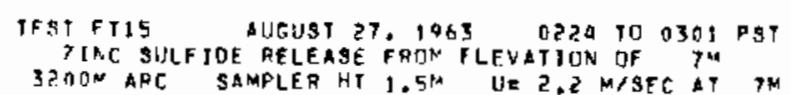

32NON APC SAMPLER HT $1.5^{\mathrm{HA}}$ UE $2.2 \mathrm{M} / \mathrm{SEC}$ AT 7

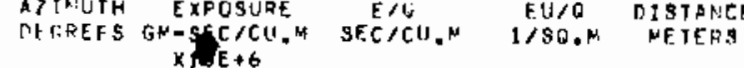

\begin{tabular}{|c|c|c|c|c|}
\hline$B \cap .0$ & 27 & $5.505 E-08$ & $\begin{array}{l}5.831 E=08 \\
1.211 E=07\end{array}$ & 3200 \\
\hline E3.0 & $9 a$ & $1 . A 96 E=07$ & 1.2171E-07 & $\begin{array}{l}3200 \\
3200\end{array}$ \\
\hline A. 5.0 & 19 & 3. AT HE $=0 B$ & B. $522 E-08$ & 3200 \\
\hline 86.0 & 17 & $3.466 \mathrm{E}-0 \mathrm{~A}$ & $7.625 E=08$ & 3200 \\
\hline 17.0 & ?. & a. $485 E-08$ & $9.868 E-08$ & 3200 \\
\hline$B R, 0$ & 12 & $2.4 a 7 E=00$ & $3 E-08$ & 3200 \\
\hline AQ. 0 & 11 & $2.243 E-08$ & $4.934 E-08$ & 3200 \\
\hline 41.0 & 15 & $3.058 E=08$ & $6.728 E=08$ & 3200 \\
\hline 93.0 & 11 & $2.243 E-06$ & $\Delta E=08$ & 3200 \\
\hline 94,0 & B & $1.631 \mathrm{E}-$ & $B E-08$ & 3200 \\
\hline 95.0 & 11 & $? .243 E=08$ & $4.934 E=08$ & 3200 \\
\hline 97.0 & 18 & $3.670 E-08$ & 6.07 AE-08 & 3200 \\
\hline 9 A. 0 & 14 & $2.854 \mathrm{E}=08$ & $6.280 E=08$ & 3200 \\
\hline 49.0 & 26 & $5,301 E-08$ & $1.166 \mathrm{E}-0 \mathrm{~T}$ & 3200 \\
\hline 100.0 & 65 & $1,305 E-07$ & $9715=07$ & 3200 \\
\hline 101.0 & to & $1.203 E=07$ & $2,6965-07$ & 3200 \\
\hline 102.0 & 34 & $6.932 \mathrm{E}-0 \mathrm{~A}$ & $1.525 E-07$ & 3200 \\
\hline 103.0 & 805 & $2.916 E-07$ & $6.414 E-07$ & 3200 \\
\hline 104.0 & 506 & $1.013 \mathrm{E}-06$ & $2.229 \mathrm{E}-06$ & 3200 \\
\hline 105.0 & 1205 & $2.9 ! 2 E=06$ & $5.306 E-06$ & 3200 \\
\hline 106.0 & 1636 & $3.2725-06$ & $7.199 E-06$ & 3200 \\
\hline 107.0 & 1254 & $2.510 E-06$ & $5.522 E-00$ & 3200 \\
\hline 108.0 & 1856 & $3.713 E=06$ & B. $16 A E-06$ & 3200 \\
\hline 109.0 & 1981 & $3 E=06$ & $0 E-06$ & 3200 \\
\hline 110.0 & 2490 & $4.9 B 1 E-0 B$ & $E-0.5$ & 3200 \\
\hline 111.0 & 2942 & 5. ABEE-OB & $1.294 E=05$ & 3200 \\
\hline 112.0 & 3641 & $7.2 A 3 E-06$ & $1.602 E-05$ & 3200 \\
\hline 113.0 & 329 & $6.59 B E=0 B$ & $1.451 F-05$ & 3200 \\
\hline 114.0 & 2619 & $5.2 a 0 E=0 B$ & $1.153 E-05$ & 3200 \\
\hline 115.0 & 1433 & $167 E=06$ & $6.307 E-06$ & 3200 \\
\hline 116.0 & 1000 & $2.0 R 2 E=0.6$ & $A .580 F=06$ & 3200 \\
\hline 117.0 & 1816 & $3.633 E=06$ & $7.943 \mathrm{E}=06$ & 3200 \\
\hline 119.0 & 2907 & $5.815 E=06$ & $1.279 E-05$ & 3200 \\
\hline 119.0 & 3044 & $6.09 B E=06$ & $1.340 F=15$ & 3200 \\
\hline 120.0 & 2805 & $5.611 E * 0.6$ & $1.234 E=05$ & 3201 \\
\hline 121.0 & 2846 & $5.602 E-06$ & $1.252 F-05$ & 3200 \\
\hline 122.0 & 23170 & $\Delta, 6 \pi 2 E=06$ & $1.0126-05$ & 3200 \\
\hline & 1614 & $3.230 \mathrm{E}-00$ & $7.1055-06$ & 3200 \\
\hline 124.0 & 1418 & $2.83+5=06$ & $6.2395=06$ & 3200 \\
\hline$\$ 25.0$ & 1322 & $2.644 E-06$ & $5.81 \mathrm{PE}=06$ & 3200 \\
\hline 126.0 & 1689 & $3.378 E-06$ & $4325=06$ & 3200 \\
\hline $1 ? 7.0$ & 1237 & $2.475 E-06$ & $5.445 E=0 \theta$ & 3200 \\
\hline .0 & 1044 & $0 Q 0 E-06$ & $0.598 \mathrm{~F}=0 \mathrm{~b}$ & 3200 \\
\hline 129.0 & 592 & $195 E-06$ & $2.6 \pi 6 f-06$ & 3200 \\
\hline .0 & 284 & SPACE- 07 & $1.251 \neq-00$ & $320 n$ \\
\hline & 25 & $\cap 97 E-\pi A$ & $1.121 \mathrm{~F}=0 \mathrm{7}$ & 3200 \\
\hline
\end{tabular}

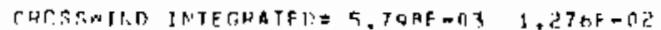

\begin{tabular}{|c|c|c|c|c|c|}
\hline $\begin{array}{l}2 \text { INUTH } \\
\text { E GREFS }\end{array}$ & $\begin{array}{l}\text { HEIEHT } \\
\text { METERS }\end{array}$ & $\begin{array}{c}\text { EXFOSURE } \\
\text { GM-SEC CU, } \\
\times 1 O E+6\end{array}$ & $\begin{array}{c}E / 0 \\
\text { SEC } / C U . R\end{array}$ & $\begin{array}{c}E U / Q \\
1 / 50 .\end{array}$ & $\begin{array}{l}\text { DISTANCE } \\
\text { METERS }\end{array}$ \\
\hline $\begin{array}{l}130.0 \\
130.0 \\
130.0 \\
130.0 \\
130.0 \\
130.0 \\
130.0 \\
130.0 \\
130.0 \\
130.0 \\
130.0 \\
130.0\end{array}$ & $\begin{array}{r}0.2 \\
0.0 \\
0.8 \\
1.5 \\
3.1 \\
6.2 \\
9.3 \\
12.4 \\
15.5 \\
18.6 \\
21.7\end{array}$ & $\begin{array}{l}2461 \\
2210 \\
2366 \\
3810 \\
2915 \\
2914 \\
1027 \\
1092 \\
571 \\
233\end{array}$ & $\begin{array}{l}0.391 E=06 \\
5.216 E=06 \\
4.723 E=06 \\
9.410 E=06 \\
4.733 E=06 \\
7.621 F=06 \\
5.832 E=06 \\
5.028 E=06 \\
3.654 E=06 \\
2.124 E=06 \\
1.104 E=06 \\
0.664 E=07\end{array}$ & $\begin{array}{l}9.660 E=06 \\
1.140 E=05 \\
1.083 E=05 \\
9.702 E=06 \\
1.041 E=05 \\
1.677 E=05 \\
1.283 E=05 \\
1.202 E=05 \\
8.040 E=06 \\
4.806 E=06 \\
2.516 E=06 \\
1.027 E=06\end{array}$ & $\begin{array}{l}1600 \\
1600 \\
1600 \\
1600 \\
1600 \\
1600 \\
1600 \\
1600 \\
1600 \\
1600 \\
1600 \\
1600\end{array}$ \\
\hline
\end{tabular}


TOWED DATA FRLLEW...

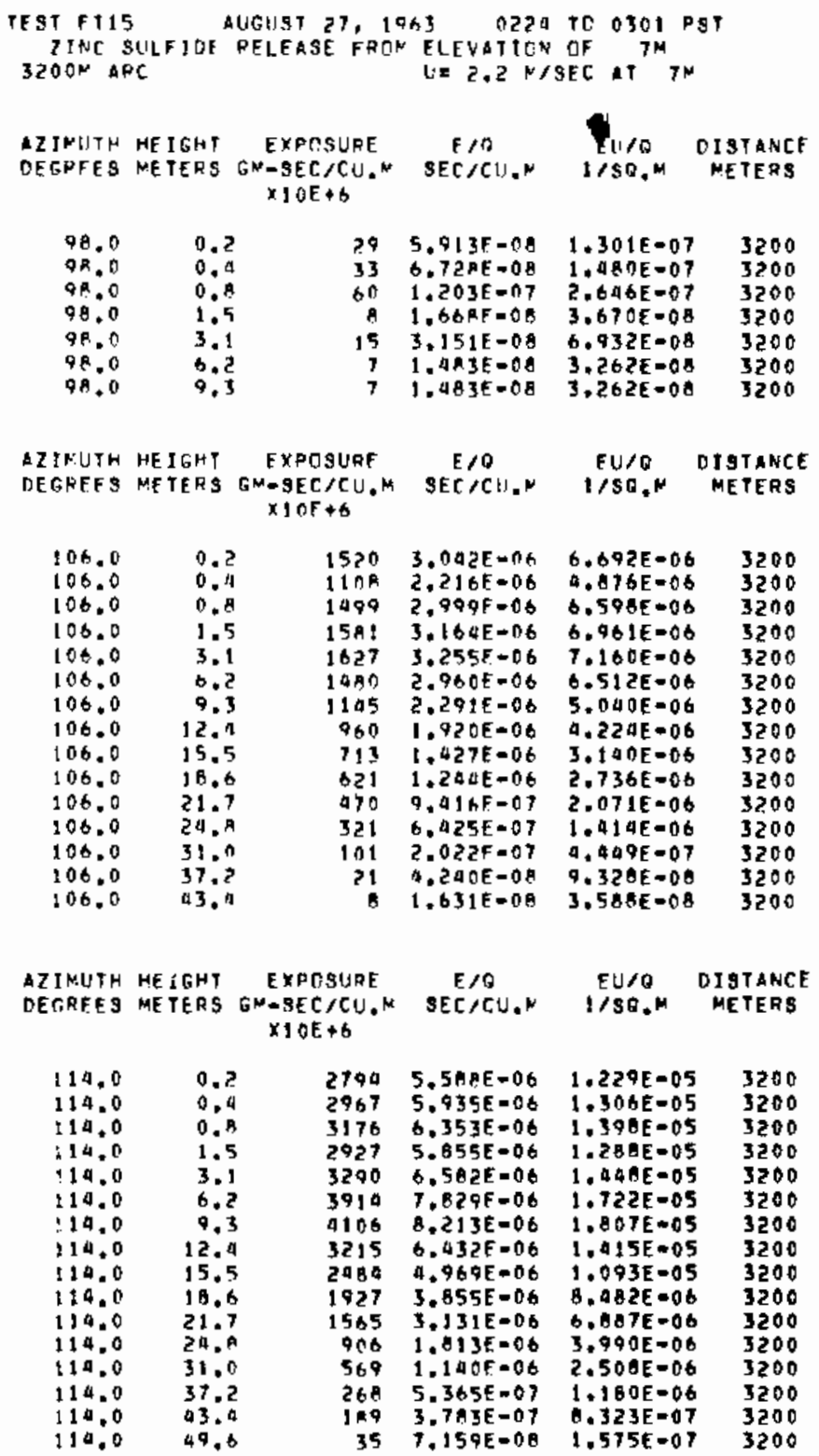

AZIMUTH HEIGHT EXPOSURE E/R EUIO DISTANCF OEGREFS METERS GH-SEC/CU,M SEC/CU.N J/SQ.N METERS $\times 10 E+6$

\begin{tabular}{|c|c|c|c|c|c|}
\hline $\begin{array}{l}122.0 \\
122.0 \\
122.0 \\
122.0 \\
122.0 \\
122.0 \\
122.0 \\
122.0 \\
122.0 \\
122.0 \\
122.0 \\
122.0 \\
122.0 \\
122.0 \\
122.0 \\
122.0 \\
122.0\end{array}$ & $\begin{array}{r}0.2 \\
0.4 \\
0.8 \\
1.5 \\
3.1 \\
6.2 \\
9.3 \\
12.4 \\
15.5 \\
18.6 \\
21.7 \\
24.9 \\
31.0 \\
37.2 \\
43.4 \\
49.6 \\
55.2 \\
62.0\end{array}$ & $\begin{array}{l}1890 \\
2151 \\
2389 \\
2258 \\
2278 \\
2562 \\
2905 \\
3023 \\
2861 \\
2139 \\
2267 \\
2040 \\
1791 \\
1070 \\
921 \\
152 \\
70 \\
105\end{array}$ & $\begin{array}{l}3.76 A E=06 \\
4.304 E=06 \\
4.770 E=06 \\
4.517 E=06 \\
4.558 E=06 \\
5.125 E=06 \\
5.811 E=06 \\
6.048 E=06 \\
5.724 F=06 \\
4.28 C E=06 \\
4.535 F=06 \\
4.012 E=06 \\
3.563 E=06 \\
2.141 E=06 \\
9.431 E=07 \\
3.043 E=07 \\
1.496 E=07 \\
9.077 E=0 A\end{array}$ & $\begin{array}{l}8.334 E=06 \\
0.469 E=06 \\
1.051 E=05 \\
9.937 E=06 \\
1.003 E=05 \\
1.127 E=05 \\
1.27 A E=05 \\
1.331 E=05 \\
1.259 E=05 \\
9.915 E=06 \\
9.978 E=06 \\
8.980 E=06 \\
7.839 E=06 \\
1.711 E=06 \\
1.855 E=06 \\
6.694 E=07 \\
3.291 E=07 \\
1.997 E=07\end{array}$ & $\begin{array}{l}3200 \\
3200 \\
3200 \\
3200 \\
3200 \\
3200 \\
3200 \\
3200 \\
3200 \\
3200 \\
3200 \\
3200 \\
3200 \\
3200 \\
3200 \\
3200 \\
3200 \\
3200\end{array}$ \\
\hline
\end{tabular}

AIINUTH HEIGHT EXPOSURF E/O FU/G DISTANCE DEGRFES METERS GM-SEC/CU,M SEC/CU.N 1/SO,M METEOS $Y 10 \mathrm{E}+6$

\begin{tabular}{|c|c|c|c|c|}
\hline $\begin{array}{r}130.0 \\
130.0 \\
2130.0 \\
130.0 \\
130.0 \\
130.0 \\
130.0 \\
130.0 \\
130.0 \\
130.0 \\
130.0\end{array}$ & $\begin{array}{l}0.7 \\
0.4 \\
0.9 \\
3.1 \\
6.2 \\
9.3 \\
12.4 \\
15.5 \\
18.6 \\
21.7 \\
37.2\end{array}$ & $\begin{array}{rl}4 & 59 \\
2 & 15 \\
130 \\
241 \\
399 \\
320 \\
281 \\
147 \\
48 \\
32 \\
90\end{array}$ & $\begin{array}{l}9.195 E=07 \\
0.302 E-07 \\
2.610 E=07 \\
4.83 A E=07 \\
1.16 E E=07 \\
6.050 E-07 \\
5.635 E=07 \\
2.047 E=07 \\
0.638 E=08 \\
6.407 E=08 \\
1.61 \text { HE }=07\end{array}$ & $\begin{array}{l}2.023 \mathrm{E}=06 \\
9.464 \mathrm{E}=07 \\
5.741 \mathrm{E}=07 \\
1.004 \mathrm{E}=06 \\
1.709 \mathrm{E}-06 \\
1.419 \mathrm{E}-06 \\
1.240 \mathrm{E}=06 \\
6.483 \mathrm{E}=07 \\
2.120 \mathrm{E}=07 \\
1.427 \mathrm{E}=07 \\
3.552 \mathrm{E}=07\end{array}$ \\
\hline
\end{tabular}


SERIES 163 EXPERIMENT: 16 , FT-16,

GROUND LEYEL AND TOWER SAMPLING 200-3200 M ARCS.

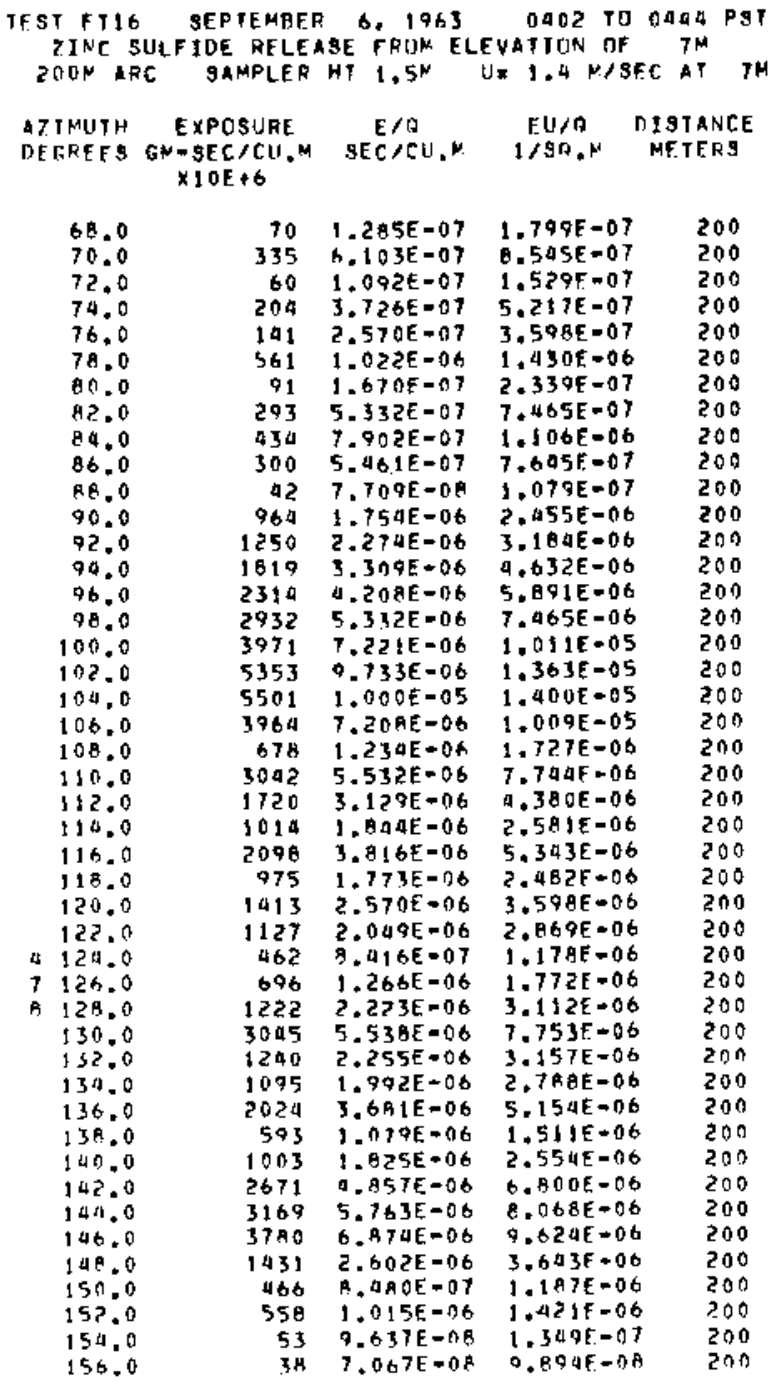

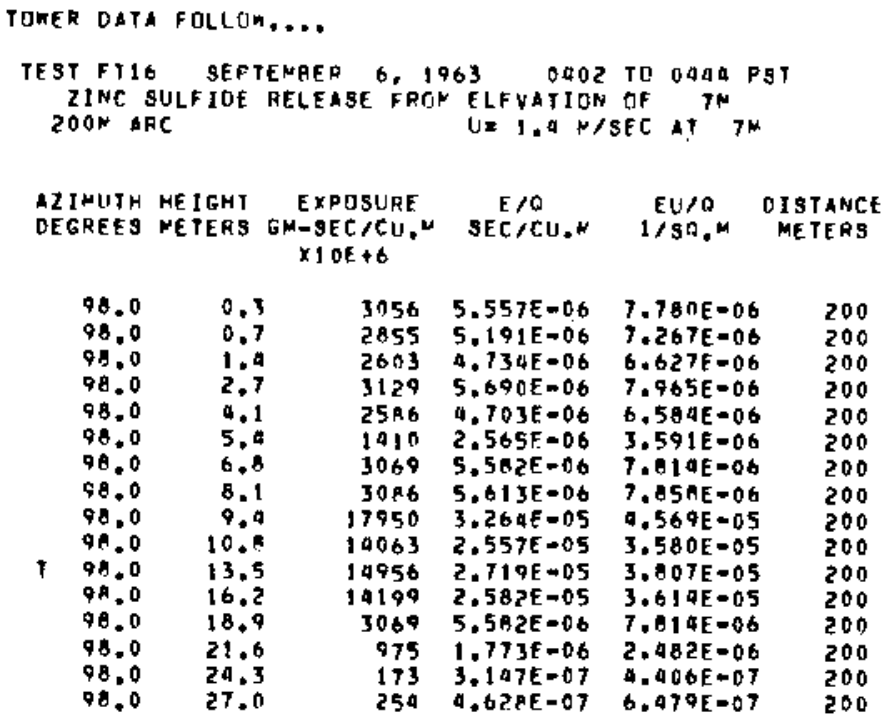

\begin{tabular}{|c|c|c|c|c|c|}
\hline $\begin{array}{l}\text { ATINITH } \\
\text { OEGREES }\end{array}$ & $\begin{array}{l}\text { HETGHT } \\
\text { METERS }\end{array}$ & $\begin{array}{c}\text { EXPOSURE } \\
\text { GM-SEC } / C U .4 \\
\times 1 \cap E+6\end{array}$ & $\begin{array}{c}E / \theta \\
\operatorname{SEC} / C U, r\end{array}$ & $\begin{array}{c}E U / Q \\
1 / S+1 . M\end{array}$ & $\begin{array}{c}\text { DISTANCE } \\
\text { METERG }\end{array}$ \\
\hline $\begin{array}{l}106.0 \\
106.0 \\
106.0 \\
106.0 \\
106.0 \\
106.0 \\
106.0 \\
106.0 \\
106.0 \\
106.0 \\
106.0 \\
106.0 \\
106.0 \\
106.0\end{array}$ & $\begin{array}{l}0.3 \\
0.7 \\
1.4 \\
2.7 \\
4.1 \\
5.9 \\
6.5 \\
8.1 \\
9.4 \\
10.6 \\
13.5 \\
16.2 \\
18.9 \\
21.6\end{array}$ & $\begin{array}{r}9392 \\
0028 \\
4196 \\
3434 \\
2383 \\
8395 \\
9401 \\
11539 \\
7748 \\
10394 \\
8121 \\
7533 \\
1503 \\
163\end{array}$ & $\begin{array}{l}7.98 G E=06 \\
7.32 A E=06 \\
7.612 E=06 \\
6.245 E=06 \\
0.333 E=06 \\
1.526 E=05 \\
1.709 E=05 \\
2.09 A E=05 \\
1.409 E=05 \\
1.890 E=05 \\
1.077 F=05 \\
1.370 E=05 \\
2.73 a E=06 \\
2.9 A 1 F=07\end{array}$ & $\begin{array}{l}1.119 E=05 \\
1.025 E=05 \\
1.066 E=05 \\
8.743 E=06 \\
6.066 E=06 \\
2.137 E=05 \\
2.393 E=05 \\
2.937 E=05 \\
1.072 E=05 \\
2.646 E=05 \\
2.067 E=05 \\
1.918 E=05 \\
3.228 E=05 \\
0.173 E=07\end{array}$ & $\begin{array}{l}200 \\
200 \\
200 \\
200 \\
200 \\
200 \\
200 \\
200 \\
200 \\
200 \\
200 \\
200 \\
200 \\
200\end{array}$ \\
\hline
\end{tabular}

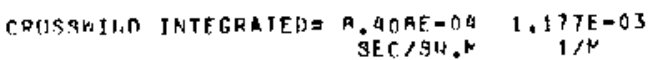

\begin{tabular}{|c|c|c|c|c|c|}
\hline $\begin{array}{l}\text { ZJMUTH } \\
\text { EGREES }\end{array}$ & $\begin{array}{l}\text { HE IGH } \\
\text { ME TEHS }\end{array}$ & $\begin{array}{c}\text { EXPQBURE } \\
\text { GNIESEC/CU.M } \\
\times 1 O E+6\end{array}$ & $\begin{array}{c}E / \theta \\
S E C / C U . N\end{array}$ & $\begin{array}{c}F U / 0 \\
1 / S B . N\end{array}$ & $\begin{array}{l}\text { OISTANCE } \\
\text { METERS }\end{array}$ \\
\hline 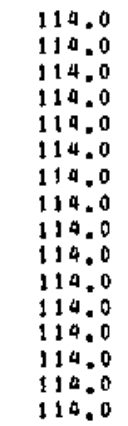 & $\begin{array}{r}0.3 \\
0.7 \\
1.4 \\
2.7 \\
0.1 \\
5.4 \\
0.8 \\
8.1 \\
9.4 \\
10.8 \\
13.5 \\
16.2 \\
16.9 \\
21.4 \\
24.3 \\
2.00\end{array}$ & $\begin{array}{r}844 \\
413 \\
658 \\
2702 \\
9055 \\
7736 \\
13526 \\
26060 \\
02998 \\
60409 \\
99433 \\
91300 \\
08646 \\
28547 \\
4184 \\
962\end{array}$ & $\begin{array}{l}1.535 E=06 \\
7.517 E-07 \\
1.198 E=06 \\
0.914 E=06 \\
7.375 E=06 \\
1.407 E=05 \\
2.059 E=05 \\
9.738 E=05 \\
7.818 E=05 \\
1.100 E=04 \\
1.808 E=04 \\
1.660 E=04 \\
8.845 E=05 \\
5.198 E=05 \\
7.608 E=06 \\
1.709 E=06\end{array}$ & $\begin{array}{l}2.150 E=06 \\
1.052 E-06 \\
1.677 E=06 \\
6.879 E=06 \\
1.033 E=05 \\
1.969 E=05 \\
3.493 E=05 \\
6.634 E=05 \\
1.095 E=09 \\
1.540 E=04 \\
2.531 E=04 \\
2.320 E=04 \\
1.237 E=04 \\
7.277 E=05 \\
1.065 E=05 \\
2.449 E=06\end{array}$ & $\begin{array}{l}200 \\
200 \\
200 \\
200 \\
200 \\
200 \\
200 \\
200 \\
200 \\
200 \\
200 \\
200 \\
200 \\
200 \\
200 \\
200\end{array}$ \\
\hline
\end{tabular}




\begin{tabular}{|c|c|c|c|c|c|}
\hline $\begin{array}{l}2 \text { INUYH } \\
\text { EGREES }\end{array}$ & $\begin{array}{l}\text { HE IGHT } \\
\text { ME TERS }\end{array}$ & $\begin{array}{c}\text { EXPDSURE } \\
\text { GM-SEC } / C H, M \\
\times 10 E+6\end{array}$ & $\begin{array}{c}E / 0 \\
S E C / C U . N\end{array}$ & $\begin{array}{c}E U / O \\
1 / 30 . N\end{array}$ & $\begin{array}{l}\text { OISTANCE } \\
\text { METERS }\end{array}$ \\
\hline $\begin{array}{l}122.0 \\
122.0 \\
122.0 \\
122.0 \\
122.0 \\
122.0 \\
122.0 \\
122.0 \\
122.0 \\
122.0 \\
122.0 \\
122.0 \\
122.0 \\
122.0 \\
122.0\end{array}$ & $\begin{array}{r}0.3 \\
0.7 \\
1.4 \\
2.7 \\
4.1 \\
5.4 \\
6.5 \\
0.1 \\
9.4 \\
10.9 \\
13.5 \\
16.2 \\
18.9 \\
21.5 \\
24.3 \\
27.5\end{array}$ & $\begin{array}{r}1293 \\
1180 \\
709 \\
1667 \\
5896 \\
13636 \\
18193 \\
21263 \\
31728 \\
62769 \\
95028 \\
99312 \\
61669 \\
20926 \\
5491 \\
7009\end{array}$ & $\begin{array}{l}2.351 E=06 \\
2.146 E=06 \\
1.290 E=06 \\
3.032 E=06 \\
1.072 E=05 \\
2.977 E=05 \\
3.318 E=05 \\
3.866 E=05 \\
5.769 E=05 \\
1.141 E=04 \\
1.72 E F=04 \\
1.806 E=04 \\
1.121 F=04 \\
3.805 E=05 \\
9.894 E-06 \\
1.274 E=05\end{array}$ & $\begin{array}{l}3.292 E=06 \\
3.004 E=06 \\
1.806 E=06 \\
4.245 E=06 \\
1.501 E=05 \\
3.071 E=05 \\
4.631 E=05 \\
5.412 E=05 \\
1.076 E=05 \\
1.59 A E=00 \\
2.019 E=04 \\
2.528 E=04 \\
1.570 E=04 \\
5.327 E=05 \\
1.385 E=05 \\
1.784 E=05\end{array}$ & $\begin{array}{l}200 \\
200 \\
200 \\
200 \\
200 \\
200 \\
200 \\
200 \\
200 \\
200 \\
200 \\
200 \\
200 \\
200 \\
200 \\
200\end{array}$ \\
\hline
\end{tabular}

\begin{tabular}{|c|c|c|c|c|c|}
\hline $\begin{array}{l}\text { IINUTH } \\
\text { EGREES }\end{array}$ & $\begin{array}{l}\text { HEIGHT } \\
\text { METFRS }\end{array}$ & $\begin{array}{l}\text { EXPOSURE } \\
\text { GM-SEC/C11.M } \\
\times 10 E+6\end{array}$ & $\begin{array}{c}E / R \\
S E[/ r J, N\end{array}$ & $\begin{array}{l}E \cup / 0 \\
1 / 50 .\end{array}$ & $\begin{array}{l}\text { ISTANC } \\
\text { NETESS }\end{array}$ \\
\hline 130 & a. & $x>3$ & 497 & $095 \mathrm{BE}-06$ & \\
\hline & & & & 06 & \\
\hline & 1 & & $=06$ & 06 & \\
\hline & 2.7 & 2 & & -06 & \\
\hline & 4.1 & a & 06 & .05 & \\
\hline 130 & 5.4 & & 21 & $1 . t$ & \\
\hline & & & 5 & $5.469 E-05$ & \\
\hline & & & & $1.941 E=04$ & \\
\hline 13 & 9.4 & 13 & 4 & $3.478 E=0$. & \\
\hline 13 & 10.8 & & 3,0 & $9.279 E=00$ & \\
\hline 130 & 13 & & 2,70 & $3.830 E-04$ & \\
\hline 130 & 16 & 94 & 1,71 & $5 E-00$ & \\
\hline 130 & & & 1.007 & $1.970 E=00$ & \\
\hline 130. & 2 & 50157 & $9.247 E-05$ & $.295 E-0 a$ & 2 \\
\hline 130.0 & 27.0 & $\begin{array}{r}17197 \\
2863\end{array}$ & $127 E=05$ & $4.376 E=0$ & \\
\hline & & & & $7.289 E=0$ & \\
\hline
\end{tabular}

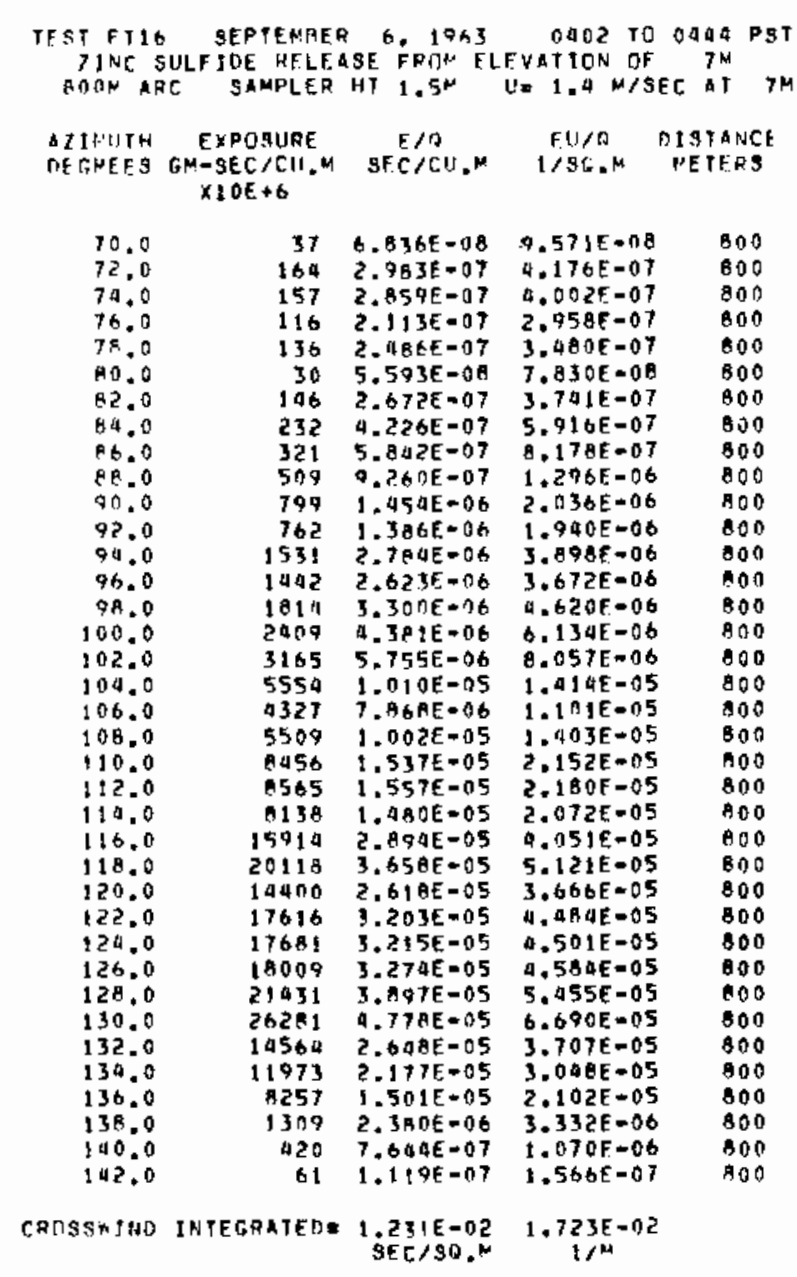

TOWER DATA FOLLOW....

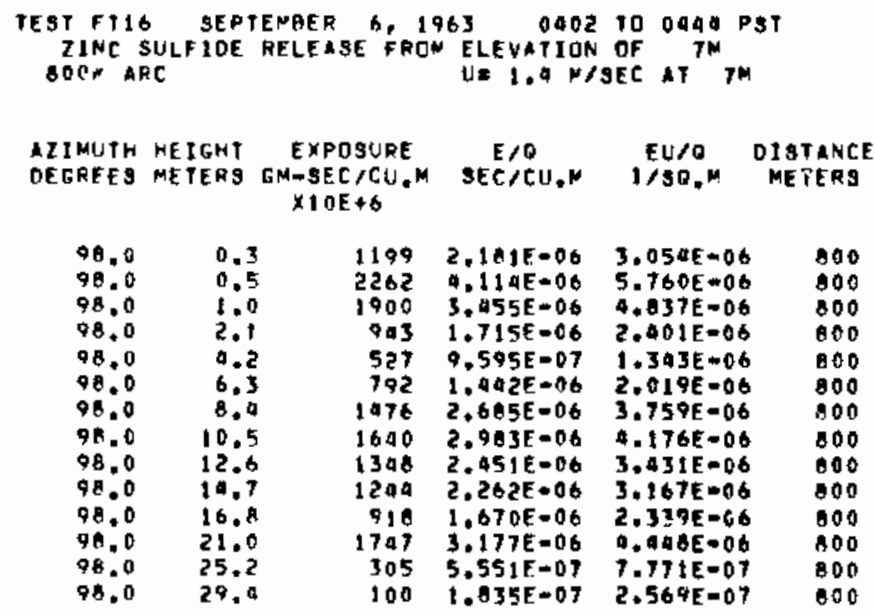




\begin{tabular}{|c|c|c|c|c|c|c|c|c|c|c|c|}
\hline $\begin{array}{l}\text { IIIMUTH } \\
\text { OEGREES }\end{array}$ & $\begin{array}{l}\text { HE IGHT } \\
\text { ME TERS }\end{array}$ & $\begin{array}{c}\text { EXPQSURE } \\
\text { GM=9EC }=\text { CU." } \\
\times 10 E+6\end{array}$ & $\begin{array}{c}E / 0 \\
S E C / C \cup, N\end{array}$ & $\begin{array}{l}E U / 0 \\
1 / S 0 .\end{array}$ & $\begin{array}{l}\text { DISTANCE } \\
\text { METERS }\end{array}$ & $\begin{array}{l}\text { AZIMUTH } \\
\text { DEGREES }\end{array}$ & $\begin{array}{l}\text { HEIGHT } \\
\text { METERS }\end{array}$ & $\begin{array}{c}\text { EXPRSHRE } \\
\text { EM- }=\text { SEC }>\text { CU. } \\
\times 10 E+6\end{array}$ & $\begin{array}{c}E / G \\
S E C / C U . M\end{array}$ & $\begin{array}{c}F U / O \\
1 / S H_{0} N\end{array}$ & $\begin{array}{l}\text { DISTANCE } \\
\text { METERS }\end{array}$ \\
\hline $\begin{array}{l}106.0 \\
106.0 \\
106.0 \\
106.0 \\
106.0 \\
106.0 \\
106.0 \\
106.0 \\
106.0 \\
106.0 \\
106.0 \\
106.0 \\
106.0 \\
106.0 \\
106.0\end{array}$ & $\begin{array}{l}0.3 \\
0.5 \\
1.0 \\
2.1 \\
4.2 \\
6.3 \\
8.0 \\
10.5 \\
12.8 \\
14.7 \\
16.8 \\
21.0 \\
25.2 \\
29.9 \\
33.8\end{array}$ & $\begin{array}{r}365 \\
5597 \\
4000 \\
3133 \\
1121 \\
768 \\
1471 \\
3073 \\
2498 \\
2989 \\
2401 \\
055 \\
295 \\
155 \\
130\end{array}$ & 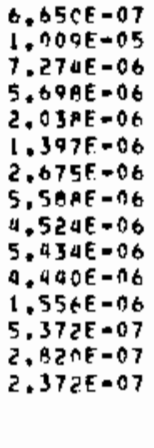 & $\begin{array}{l}9.31 \text { QE }=07 \\
1.912 E=05 \\
1.018 E=05 \\
7.977 E-06 \\
2.85 \text { AE }=06 \\
1.956 E=06 \\
3.745 E=06 \\
7.823 E=06 \\
6.339 E=06 \\
7.609 E=06 \\
0.216 E=06 \\
2.179 E-06 \\
7.520 E=07 \\
3.948 E=07 \\
3.321 E=07\end{array}$ & $\begin{array}{l}800 \\
800 \\
800 \\
100 \\
800 \\
800 \\
800 \\
800 \\
800 \\
800 \\
800 \\
800 \\
800 \\
A 00 \\
100\end{array}$ & $\begin{array}{l}130.0 \\
130.0 \\
130.0 \\
130.0 \\
130.0 \\
130.0 \\
130.0 \\
130.0 \\
130.0 \\
130.0 \\
130.0 \\
130.0 \\
130.0 \\
130.0 \\
130.0 \\
130.0 \\
130.0\end{array}$ & $\begin{array}{l}0.3 \\
0.5 \\
1.0 \\
2.1 \\
4.2 \\
6.3 \\
8.4 \\
10.5 \\
12.6 \\
19.7 \\
16.8 \\
21.0 \\
25.2 \\
29.9 \\
33.6 \\
37.8 \\
42.0\end{array}$ & $\begin{array}{l}19240 \\
16160 \\
13370 \\
23767 \\
30863 \\
20286 \\
16321 \\
19077 \\
21213 \\
22635 \\
20114 \\
14730 \\
8470 \\
4276 \\
1868 \\
573 \\
221\end{array}$ & 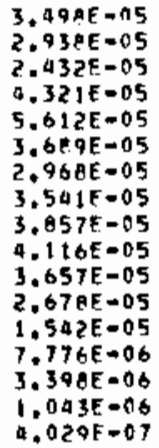 & $\begin{array}{l}4.097 E=05 \\
4.114 E=05 \\
3.004 E=05 \\
6.050 E=05 \\
7.856 E=05 \\
5.164 E=05 \\
0.155 E=05 \\
4.958 E=05 \\
5.000 E=05 \\
5.762 E=05 \\
5.120 E=05 \\
3.750 E=05 \\
2.156 E=05 \\
1.089 E=05 \\
4.757 E-06 \\
1.060 E=06 \\
5.640 E=07\end{array}$ & $\begin{array}{l}800 \\
800 \\
800 \\
800 \\
800 \\
800 \\
800 \\
800 \\
800 \\
800 \\
600 \\
800 \\
800 \\
B 00 \\
800 \\
800 \\
800\end{array}$ \\
\hline
\end{tabular}

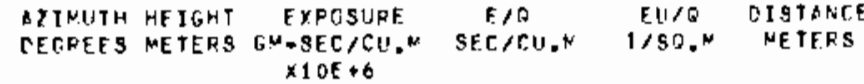

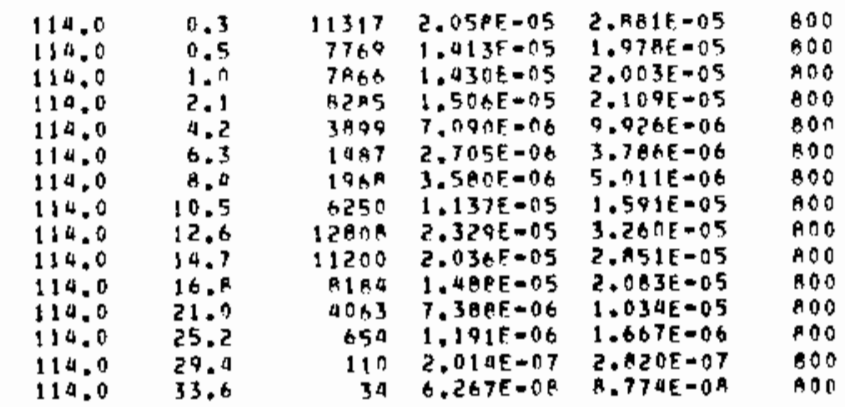

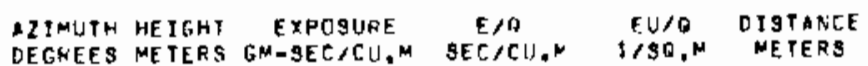

$\times 10 E+6$

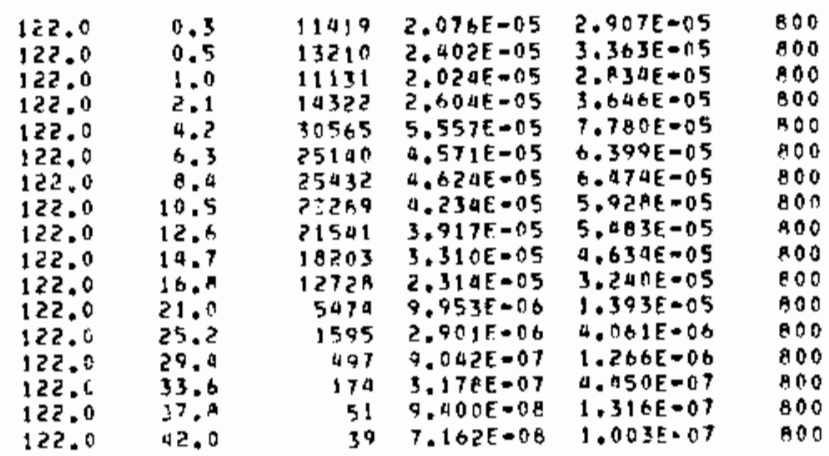




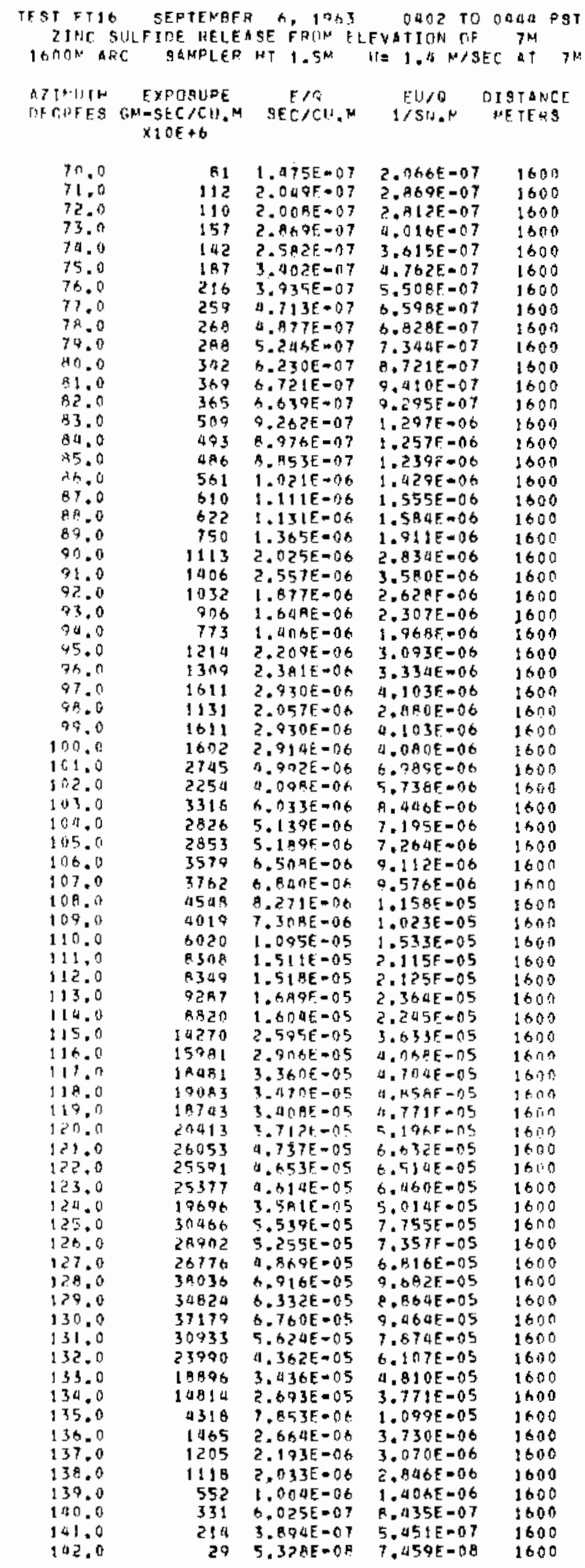

CFOSSIVTRD INTEGRATEO= $2.991 E-02 \quad 4.187 E-02$
TOWFR CATA FOLLOW....

TEST FTI6 SEPTENAER b. 1963 0402 TO 04य PST ZJNC SULFIDE RFLEASE FRIJN ELEVATICN TIF TM $1600 N$ ARE

\begin{tabular}{|c|c|c|c|c|c|}
\hline $\begin{array}{l}\text { AZIMUTH } \\
\text { DEGREES }\end{array}$ & $\begin{array}{l}\text { HE IGH } \\
\text { METERS }\end{array}$ & 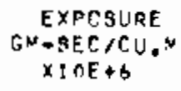 & $\begin{array}{c}E / A \\
S E C / C H, N\end{array}$ & $\begin{array}{c}E \cup / 0 \\
1 / S Q . M\end{array}$ & $\begin{array}{l}\text { OISTANCF } \\
\text { METERS }\end{array}$ \\
\hline $\begin{array}{l}98.0 \\
98.0 \\
98.0 \\
98.0 \\
98.0 \\
9 B .0\end{array}$ & $\begin{array}{l}0.2 \\
0.2 \\
0.9 \\
1.5 \\
3.1 \\
0.2\end{array}$ & $\begin{array}{r}2001 \\
1483 \\
1535 \\
807 \\
515 \\
72\end{array}$ & $\begin{array}{l}3.638 E=06 \\
2.697 E=06 \\
2.791 E=08 \\
1.468 E=06 \\
9.373 E=07 \\
1.319 E=07\end{array}$ & $\begin{array}{l}5.095 E-06 \\
3.775 E=06 \\
3.907 E=06 \\
2.056 E=06 \\
1.312 E=06 \\
1.846 E=07\end{array}$ & $\begin{array}{l}1600 \\
1600 \\
1600 \\
1600 \\
1600 \\
1600\end{array}$ \\
\hline
\end{tabular}

AZINUTH HEIGHT EXPOSURF E/D EU/O DISTANCE DEGREES METERS GN-SEC/CU,N SEC/CU.M IISO.M METERS $\times 10 \mathrm{OE}+6$

\begin{tabular}{|c|c|c|c|c|c|}
\hline $\begin{array}{l}106.0 \\
106.0 \\
106.0 \\
106.0 \\
106.0 \\
106.0 \\
106.0 \\
106.0 \\
106.0 \\
106.0 \\
106.0 \\
106.0 \\
106.0 \\
106.0\end{array}$ & $\begin{array}{l}0.7 \\
0.4 \\
0.8 \\
1.5 \\
3.1 \\
6.2 \\
9.3 \\
12.4 \\
15.5 \\
18.5 \\
21.7 \\
24.8 \\
31.0 \\
37.2\end{array}$ & $\begin{array}{l}3708 \\
4165 \\
4052 \\
2704 \\
1195 \\
486 \\
629 \\
517 \\
478 \\
411 \\
295 \\
454 \\
121 \\
24\end{array}$ & $\begin{array}{l}6.742 E=06 \\
7.574 F=06 \\
7.369 E=06 \\
4.918 E=06 \\
2.174 E=06 \\
8.63 E E=07 \\
1.144 E=06 \\
9.408 E=07 \\
8.696 E-07 \\
7.404 E=07 \\
5.381 E=07 \\
8.265 E=07 \\
2.211 E=07 \\
4.421 E=0 A\end{array}$ & $\begin{array}{l}9,439 E=06 \\
1.060 E=05 \\
1.032 E=05 \\
6.805 E=06 \\
3.049 E=06 \\
1.237 E=06 \\
1.602 E=06 \\
1.317 E=06 \\
1.217 E=06 \\
1.048 E=06 \\
7.534 E=07 \\
1.157 E=06 \\
3.095 E-07 \\
6.190 E=08\end{array}$ & $\begin{array}{l}1600 \\
1600 \\
1600 \\
1600 \\
1600 \\
1600 \\
1600 \\
1600 \\
1600 \\
1600 \\
1600 \\
1600 \\
1600 \\
1600\end{array}$ \\
\hline
\end{tabular}

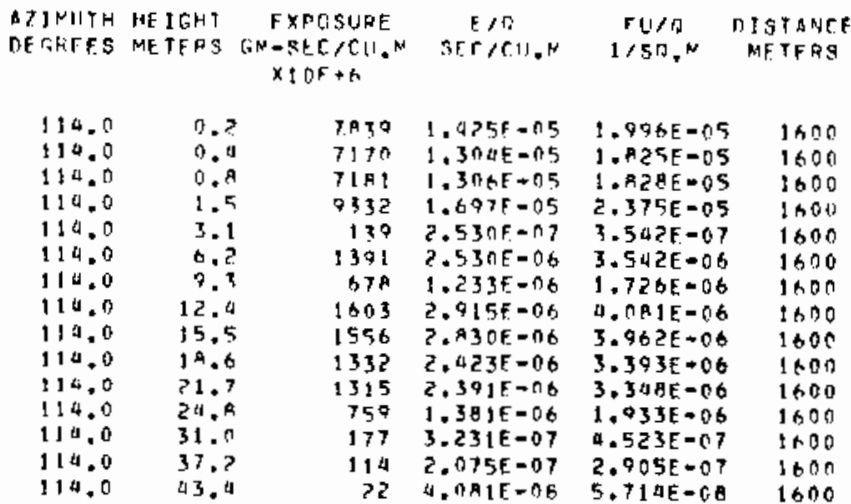

ATIMITH HEIGHT FYPOSURE E/Q EU/O DISTANCE

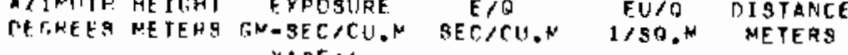
XIOE +

\begin{tabular}{|c|c|c|c|c|c|}
\hline $\begin{array}{l}122.0 \\
122.0 \\
122.0 \\
122.0 \\
122.0 \\
122.0 \\
122.0 \\
122.0 \\
122.0 \\
122.0 \\
122.0 \\
122.0 \\
122.0 \\
122.0 \\
122.0\end{array}$ & $\begin{array}{r}0.7 \\
0.9 \\
0.8 \\
1.5 \\
3.1 \\
6.2 \\
9.3 \\
12.4 \\
15.5 \\
18.6 \\
21.7 \\
24.8 \\
31.0 \\
37.2 \\
43.4\end{array}$ & $\begin{array}{l}19775 \\
19644 \\
17246 \\
19691 \\
21221 \\
8061 \\
5186 \\
6652 \\
4824 \\
2957 \\
3157 \\
4276 \\
1537 \\
920 \\
139\end{array}$ & $\begin{array}{l}3.596 E=05 \\
3.572 \mathrm{E}-05 \\
3.145 \mathrm{E}-05 \\
3.580 E=05 \\
3.859 E=05 \\
1.466 \mathrm{~F}=05 \\
9.43 \mathrm{EE}=06 \\
1.210 \mathrm{E}=05 \\
8.767 E=06 \\
5.378 \mathrm{~F}=06 \\
5.741 \mathrm{E}=06 \\
7.775 \mathrm{E}=06 \\
2.796 \mathrm{E}=06 \\
1.673 \mathrm{E}=06 \\
2.381 \mathrm{E}=07\end{array}$ & $\begin{array}{l}5.034 E-05 \\
5.001 E=05 \\
0.003 E=05 \\
5.012 E-05 \\
5.003 E-05 \\
2.052 E-05 \\
1.320 E=05 \\
1.693 E-05 \\
1.227 E=05 \\
7.529 E=06 \\
8.038 E-06 \\
1.088 E=05 \\
3.914 E=06 \\
2.343 E=06 \\
3.333 E=07\end{array}$ & $\begin{array}{l}1600 \\
1600 \\
1600 \\
1600 \\
1600 \\
1600 \\
1600 \\
1600 \\
1600 \\
1600 \\
1600 \\
1600 \\
1600 \\
1600 \\
1600\end{array}$ \\
\hline
\end{tabular}




\begin{tabular}{|c|c|c|c|c|c|}
\hline $\begin{array}{l}\text { DZJMUTH } \\
\text { DEGREES }\end{array}$ & $\begin{array}{l}\text { HE ITH T } \\
\text { ME TERS }\end{array}$ & $\begin{array}{c}\text { EXPOSUPE } \\
\text { GM= SEC } / \mathrm{CU} . M \\
\times 10 E+6\end{array}$ & $\begin{array}{c}E / D \\
\text { SEE } 2 / C U+N\end{array}$ & $\begin{array}{c}F \cup / O \\
1 / 50, M\end{array}$ & $\begin{array}{l}\text { OISTANCE } \\
\text { METEPS }\end{array}$ \\
\hline $\begin{array}{l}130.0 \\
130.0 \\
130.0 \\
130.0 \\
130.0 \\
130.0 \\
130.0 \\
130.0 \\
130.0 \\
130.0 \\
130.0 \\
130.0 \\
130.0 \\
130.0 \\
130.0\end{array}$ & $\begin{array}{r}0.2 \\
0.4 \\
0.8 \\
1.5 \\
3.1 \\
6.2 \\
9.3 \\
12.4 \\
15.5 \\
18.6 \\
21.7 \\
24.8 \\
31.0 \\
37.2 \\
43.4\end{array}$ & $\begin{array}{r}16520 \\
17187 \\
19532 \\
25910 \\
34674 \\
24070 \\
17200 \\
13693 \\
10404 \\
6676 \\
5735 \\
3630 \\
1382 \\
355 \\
50\end{array}$ & $\begin{array}{l}3.004 E=05 \\
3.125 F=05 \\
3.551 E=05 \\
a .711 E=05 \\
6.304 E=05 \\
a .376 E=05 \\
3.127 E=05 \\
2.090 E=05 \\
1.892 E=05 \\
1.210 E=05 \\
1.043 E=05 \\
6.602 E=06 \\
2.513 E=06 \\
6.462 E=07 \\
9.063 E=08\end{array}$ & $\begin{array}{l}4.205 E=05 \\
4.375 E=05 \\
4.972 E=05 \\
6.595 E-05 \\
6.826 E-05 \\
6.127 E-05 \\
9.37 B E=05 \\
3.486 E=05 \\
2.649 E=05 \\
1.699 E=05 \\
1.460 E=05 \\
9.242 E=06 \\
3.519 E=06 \\
9.047 E=07 \\
1.361 E=07\end{array}$ & $\begin{array}{l}1600 \\
1600 \\
1600 \\
1600 \\
1600 \\
1600 \\
1600 \\
1600 \\
1600 \\
1600 \\
1600 \\
1600 \\
1600 \\
1600 \\
1600\end{array}$ \\
\hline
\end{tabular}

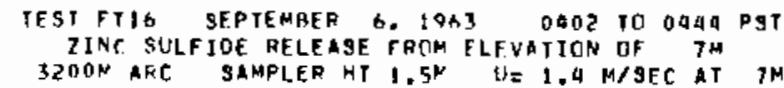
SZINUTH EXPOSURE
RFTREES EN-SEC/CU.M SEC/CH.M NU/A DISTANCE $\times 10 E+6$

71.0
72.0
73.0
74.0
75.0
76.0

$\begin{array}{rlll}75 & 1.372 E-07 & 1.920 E-07 & 3200 \\ 103 & 1.891 E-07 & 2.607 E=07 & 3200 \\ 128 & 2.335 E-07 & 3.270 E-07 & 3200 \\ 114 & 2.076 E-07 & 2.906 E-07 & 3200 \\ 110 & 2.002 E-07 & 2.802 E-07 & 3200 \\ 165 & 3.003 E-07 & 4.204 E=07 & 3200 \\ 143 & 2.613 E-07 & 3.659 E-07 & 3200\end{array}$

$78.0 \quad 215 \quad 3.911 E-07 \quad 5.475 E-07 \quad 3200$

$\begin{array}{lllll}79.0 & 239 & 4.356 E-07 & 6.098 E-07 & 3200 \\ 80.0 & 320 & 5.820 E-07 & 8.108 E-07 & 3200\end{array}$

81.0 360 6.561E-07 9.166E-07 3200

A2.0 348 6.339E-07 $0.874 E=07 \quad 3200$

83.0 $446 \quad 1.118 E-07 \quad 1.137 E=06 \quad 3200$

$64.0 \quad 454 \quad 9.267 \mathrm{E}=07 \quad 1.157 \mathrm{E}=06 \quad 3200$

$85.0 \quad 565 \quad 1.029 E-06 \quad 1.440 E-06 \quad 3200$

$86.0 \quad 720 \quad 1.310 E-06 \quad 1.835 E-06 \quad 3200$

$87.0 \quad 900 \quad 1.637 E-06 \quad 2.291 E-06 \quad 3200$

AB. $0 \quad 1068 \quad 1.942 E-06 \quad 2.719 E-06 \quad 3200$

A9.0 988 1.79RE-06 $2.517 E=06 \quad 3200$

$90.0 \quad 1158 \quad 2.106 E-06 \quad 2.94 B E-06 \quad 3200$

$\begin{array}{llll}91.0 & 1196 \quad 2.176 E-06 & 3.006 E-06 & 3200\end{array}$

$92.0 \quad 1475 \quad 2.6 A 2 E-06 \quad 3.755 E-06 \quad 3200$

$93.0 \quad 1305 \quad 2.447 E-06 \quad 3.425 E-06 \quad 3200$

$94.0 \quad 1506 \quad 2.739 E-06 \quad 3.835 E-06 \quad 3200$

$95.0 \quad 1569$ 2.85AE-06 $3.996 E-06 \quad 3200$

$96.0 \quad 1274 \quad 2.317 E-06 \quad 3.244 E-06 \quad 3200$

$97.0 \quad 2060 \quad 3.746 E-06 \quad 5.244 E-06 \quad 3200$

$98.0 \quad 1877 \quad 3.414 E=06 \quad 9.7 B 0 E-06 \quad 3200$

$99.0 \quad 1816 \quad 3.307 E-06 \quad 9.623 E-06 \quad 3200$

$100.0 \quad 2015 \quad 3.664 E-06 \quad 5.130 E-06 \quad 3200$

$101.0 \quad 2573 \quad 0.678 E-06 \quad 6.549 E-06 \quad 3200$

$102.0 \quad 2840 \quad 5.164 E-06 \quad 7.229 E-06 \quad 3200$

$103.0 \quad 3159 \quad 5.744 E-06 \quad 0.042 E=06 \quad 3200$

$104.0 \quad 209 A \quad 5.269 E-06 \quad 7.377 F=06 \quad 3200$

$105.0 \quad 2234 \quad 4.063 E-06 \quad 5.688 E-06 \quad 3200$

$106.0 \quad \$ 776 \quad 3.231 E-06 \quad 4.523 E-06 \quad 3200$

$107.0 \quad 2955 \quad 5.373 E=06 \quad 7.523 E-06 \quad 3200$

$108.0 \quad 2389 \quad 0.345 E-06 \quad 6.032 F=06 \quad 3200$

$109.0 \quad 1727 \quad 3.142 E-06 \quad a, 398 E-06 \quad 3200$

$110.0 \quad 2544 \quad 0.626 E-06 \quad 6.477 E-06 \quad 3200$

$111.0 \quad 2320 \quad 0.219 E \rightarrow 06 \quad 5.900 E-06 \quad 3200$

$112.0 \quad 1595 \quad 2.810 E-06 \quad 3.934 F+06 \quad 3200$

$113.0 \quad 2 ? 73 \quad 4.133 E=06 \quad 5.787 F-06 \quad 3200$

114.0 160R $2.925 E-06 \quad 4.095 E-06 \quad 3200$

$115.0 \quad 1787 \quad 3.614 E-06 \quad 5.06 n E=06 \quad 3200$

$116.0 \quad 916 \quad 1.666 \mathrm{E}=06 \quad 2.333 \mathrm{~F}=06 \quad 3200$

$117.0 \quad 1595 \quad 2.901 E-06 \quad 4.061 E-06 \quad 3200$

119.0 B6Q $1.572 \mathrm{E}=06 \quad 2.200 \mathrm{~F}=06 \quad 3200$

119.0 14hit $2.663 F-06 \quad 3.727 F=06 \quad 3200$

120.0 18A1 $3.3 A 4 E=06$ \$.73AF-06 3200

$121.0 \quad 974 \quad 1.909 F=06 \quad 2.533 E=06 \quad 3200$

$1.2 .0 \quad 390 \quad 7.090 \mathrm{E}-07 \quad 9.938 \mathrm{~B}-07$ 3200

$123.0 \quad 423 \quad 7.692 E-07$ 1.077E-06 3200

$124.0 \quad 551,1.003 E=06 \quad 1.404 E-06 \quad 3 \geq 00$

$125.0 \quad 335 \quad 6.098 E=07 \quad 8.537 E-07 \quad 3200$

$126.0 \quad 320 \quad 5.830 E-07 \quad 8.14$ ME $-07 \quad 3200$

$127.0 \quad 242 \quad 4.411 E-07 \quad 6.176 E-07 \quad 3200$

$128.0 \quad 127 \quad 2.317 \mathrm{E}-07 \quad 3.24 \mathrm{AE}-07 \quad 3200$

$129.0 \quad 73 \quad 1.335 E-07 \quad 1.868 E=07 \quad 3200$

$131.0 \quad 13 \quad 2.410 E=0 . \quad 3.373 E+08 \quad 3200$

$132.0 \quad 12 \quad 2,220 E=0 B \quad 3.114 E=08 \quad 3200$

CROSSKJHD INTEGRLTEDE 7.0Q2E-0.3 9.929E-03 
TOWER OATA FOLLOW....

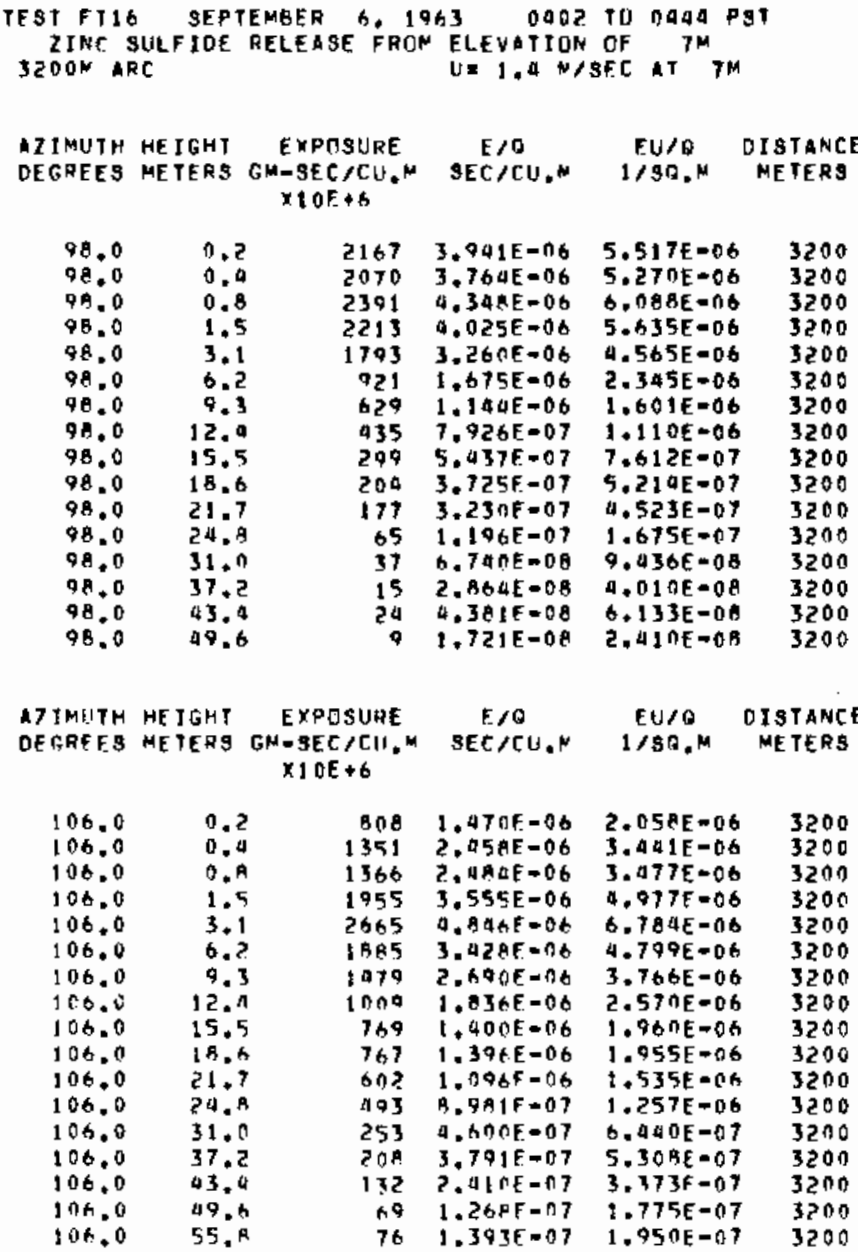

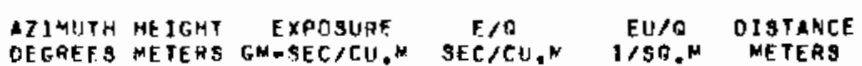
$x \perp 0 E+t$

122.0
122.0
122.0
122.0
122.0
122.0
122.0
122.0
122.0
122.0
122.0
122.0
122.0
122.0
122.0
122.0
122.0
122.0

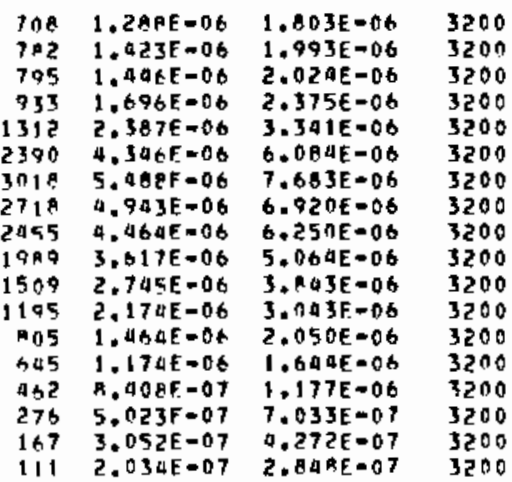

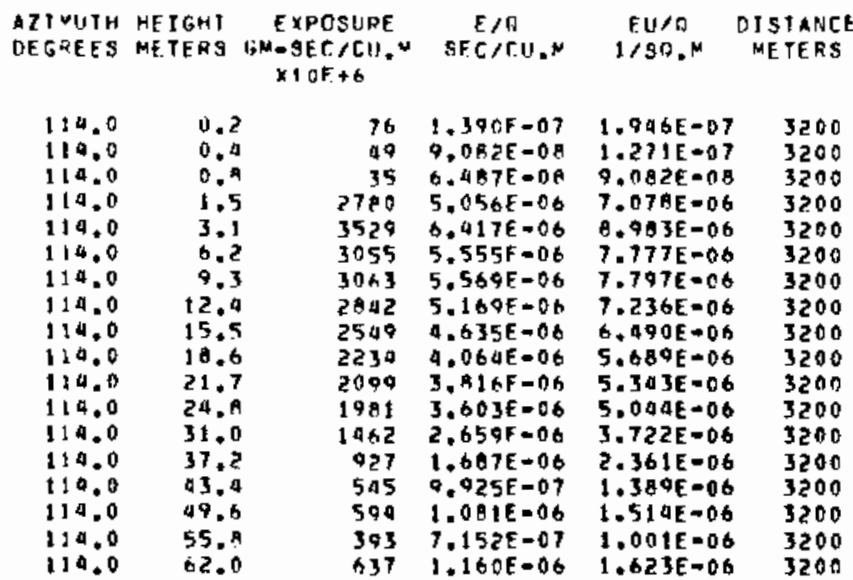


SERIES T63 EXPERIMENT: 17 ( FT-17)

GROUND LEVEL AND TOWER SAMPLING 200-3200 M ARCS. A SIGNIFICANT PORTION OF THE PLIME HEVER PASSED OVER THE GRIO.

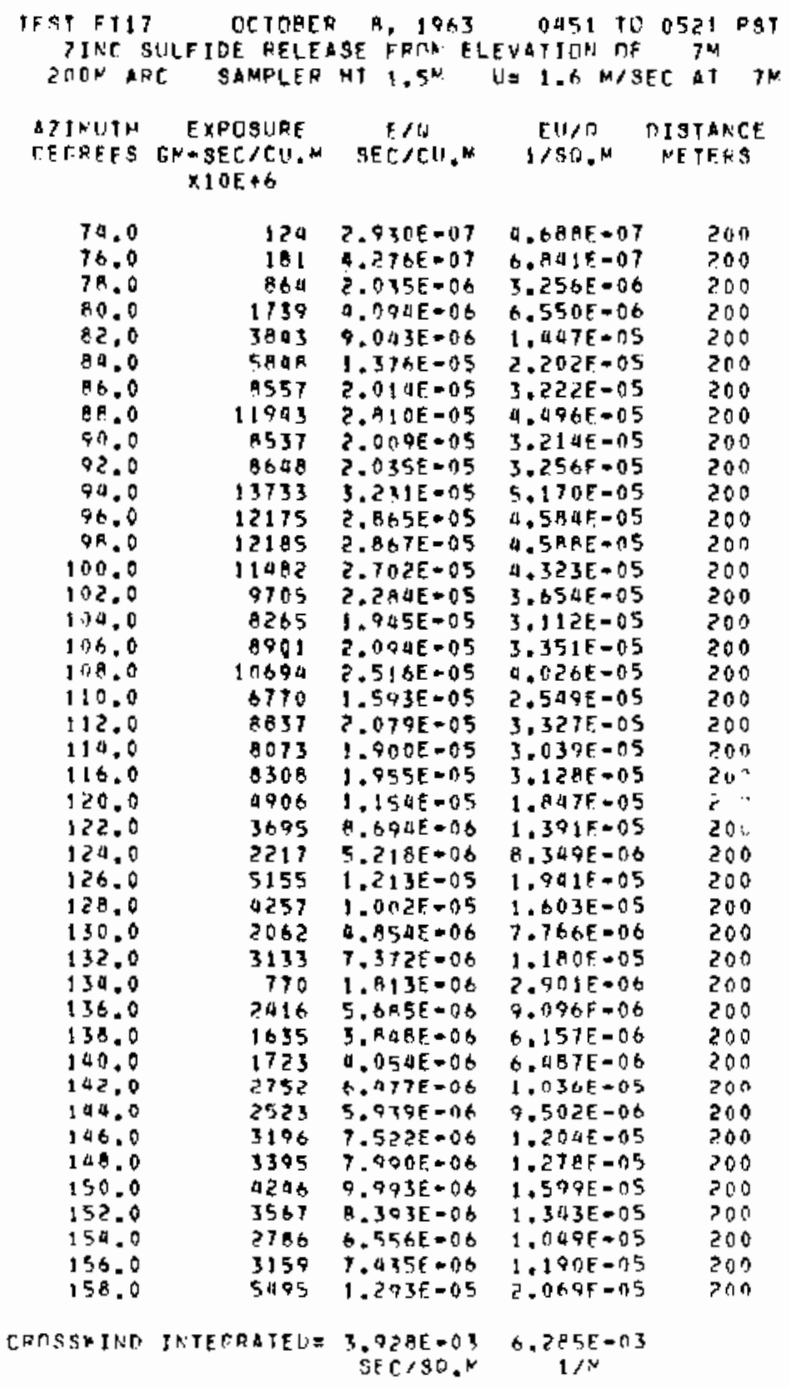

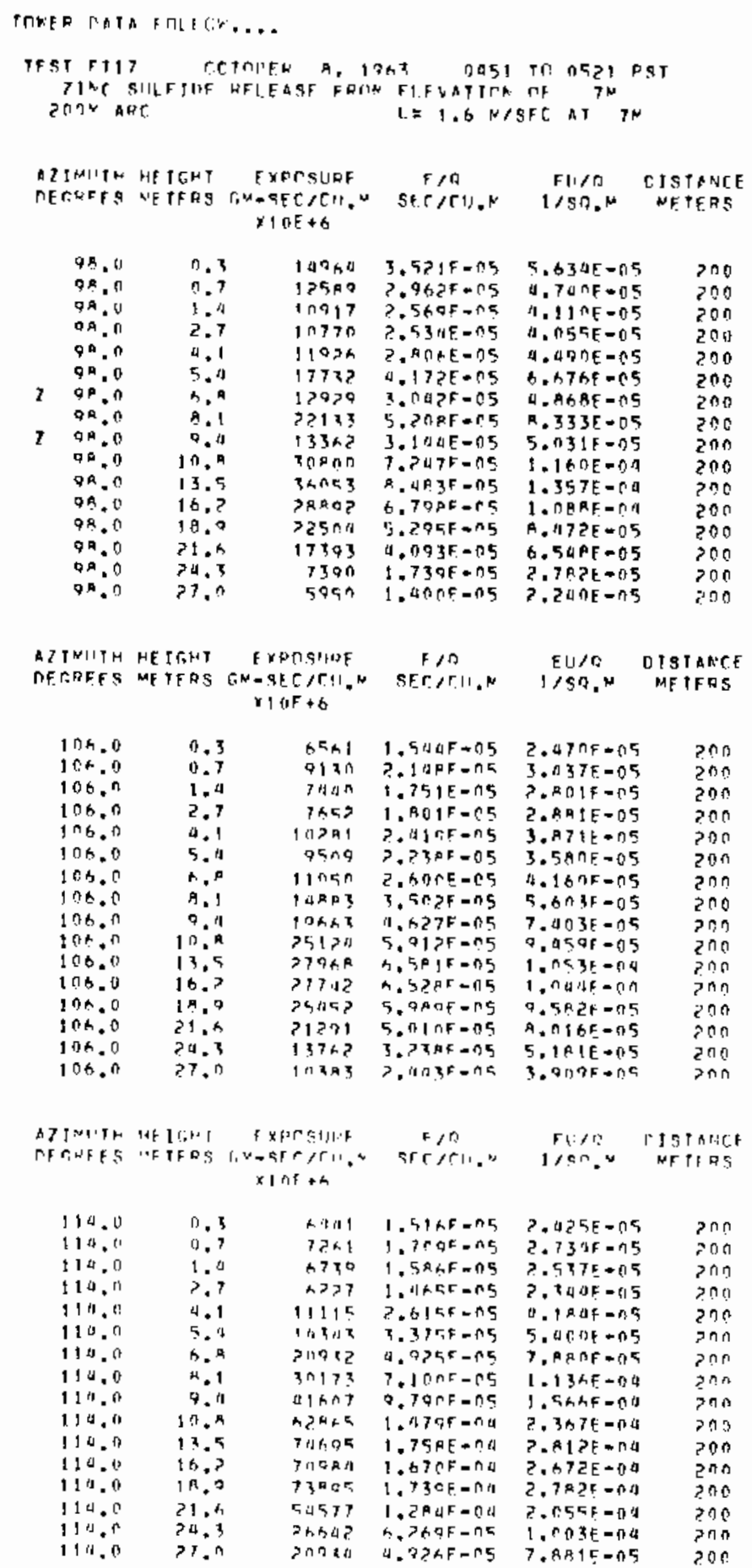




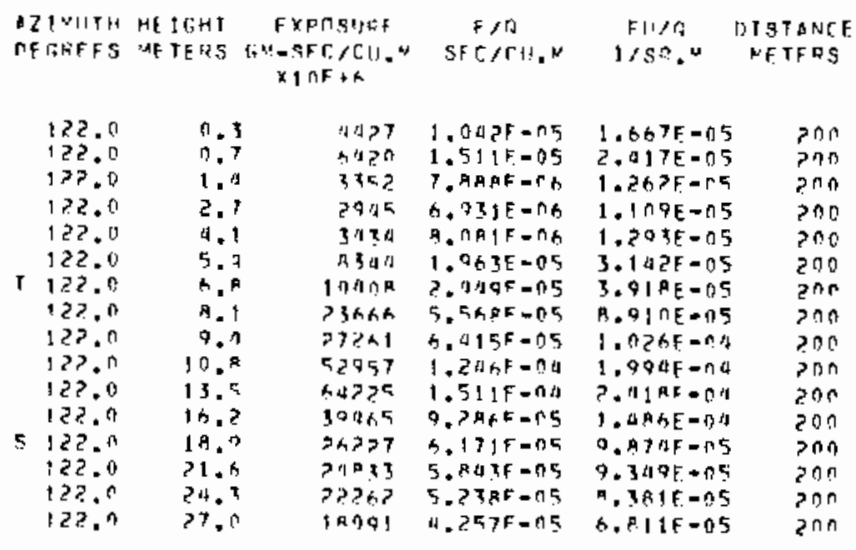

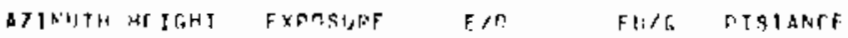

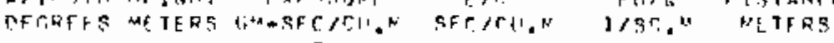
$\times 1 \cap F+h$

\begin{tabular}{|c|c|}
\hline & \\
\hline & \\
\hline $13^{n} .0$ & \\
\hline 130,0 & \\
\hline 137.0 & \\
\hline 130.0 & \\
\hline 130.0 & \\
\hline & \\
\hline & \\
\hline & \\
\hline & \\
\hline & \\
\hline & \\
\hline & \\
\hline & \\
\hline & \\
\hline & \\
\hline $1 \times n, 0$ & 0,3 \\
\hline & 0.7 \\
\hline & 1. 11 \\
\hline & ?. 1 \\
\hline & 4.1 \\
\hline & $5 . \pi$ \\
\hline 130.0 & 6.2 \\
\hline $130, n$ & 2.1 \\
\hline $13 n, n$ & 9.3 \\
\hline 130.0 & (1). \\
\hline 130.0 & 13.5 \\
\hline 1350 & $16, ?$ \\
\hline 130.0 & 18.9 \\
\hline 130.6 & 25.4 \\
\hline & \\
\hline
\end{tabular}

\begin{tabular}{|c|c|c|c|}
\hline$>030$ & A. ARE, F $=\cap_{G}$ & 1. nthat $=0.5$ & $2 n n$ \\
\hline ?22? & $5.23 \cap F=n d$ & A. $36>\varepsilon=06$ & ?חn $n$ \\
\hline$>190$ & $5.175 .50 h$ & A. 2 ANF $=$ MS & $2^{n}$ \\
\hline$I^{a}+2$ & $1.3975=7 b$ & $1.034 E=A B$ & 200 \\
\hline 5 月? & 1.2 रश $=05$ & ?. DWOE $=05$ & 200 \\
\hline 9635 & $3>F=n 5$ & $3.251 \mathrm{f}=05$ & חם מחק \\
\hline $10>1$ & QSF-AS & $25=05$ & 200 \\
\hline 545 & $258 F=05$ & A. 41 Tf $=$ - 5 & pn \\
\hline 17014 & $1.003 F=05$ & $6.405 E-05$ & $3+0$ \\
\hline 30105 & 1.09 व $=15$ & $1.133 f=04$ & $2 \cap n$ \\
\hline 37901 & A. $927 F-105$ & 1.42 AE $=0,0$ & 200 \\
\hline $201>0$ & $2 \cap 5 F=\operatorname{cs}$ & $1.473 E-04$ & 200 \\
\hline प35A? & $107 F=105$ & $1.26] 5-01$ & $2 \cap 0$ \\
\hline 13701 & $3.2245=05$ & $5.158 E-05$ & 200 \\
\hline$A 7 \div A$ & $11 F-n 5$ & r & 200 \\
\hline 2710 & $A>F=0 B$ & $1.09 R E=\cap 5$ & $? 0$ \\
\hline
\end{tabular}

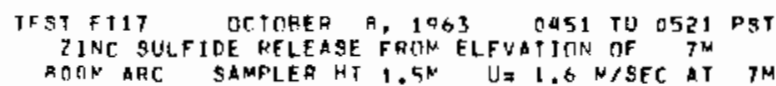

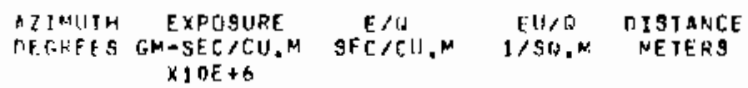

70.0
72.0
74.0
76.0
78.0

78.0

स०. 0

है. 0

84.0

8.0 .0

8.0

40.0

92.0

94.0

96.0

4 R. 0

100.0

103.0

104.0

106.0

108.0

1100

D 112,0

(1) 114,0

- 136.0

- 1180

D 120.0

122.0

120,0

126.0

128.0

130.0

132.0

130.0

134.0
136.0

138.0

140.0

142.0

144.0

146.0

$+\triangle 8.0$

150.0

152.0

154.0

156.0

158.0

100.0

$913.145 F-07 \quad 3.431 E-07 \quad 800$

94 ? 2?1F-07 $\quad .554 \mathrm{E}=07 \quad A 00$

$58 \quad 1.379 E-07 \quad 2.206 E-07 \quad 000$

$126 \quad 2.4 \mathrm{ATE}-07 \quad 4.780 E=07 \quad 800$

$71 \quad 1.685 E-07 \quad 2.696 \mathrm{E}-07 \quad 100$

$107 \quad 2.528 E-07 \quad 4.044 E-07 \quad B 00$

$97 \quad 2.298 E=07 \quad 3.677 E-07 \quad 800$

$551.302 E-07 \quad 2.083 E-07 \quad 800$

$52 \quad 1.226 E=07 \quad 1.9615-07 \quad 800$

1 QB $4.672 \mathrm{E}-0.7 \quad 7.476 \mathrm{E}-07 \quad 800$

$250 \quad 5.89 A E-07 \quad 9.436 E=07 \quad 800$

$\begin{array}{llll}279 & 6.587 E-07 & 1.054 E-06 & 800 \\ 322 & 7.583 E-07 & 1.213 E-06 & 800\end{array}$

$\begin{array}{llll}3207 & 2.842 E-06 & 4.547 E-06 & 800\end{array}$

$1943 \quad 4.573 E-06 \quad 7.316 E-06 \quad 100$

$2197 \quad 5.170 E-06 \quad$ 9.272E-06 $\quad 300$

$21124.971 E-06 \quad 7.954 E-06 \quad 800$

$2633 \quad 6.197 E-06 \quad 9.994 E=06 \quad 800$

$2900 \quad 6.925 E-06 \quad 1.092 E-05 \quad 800$

$2874 \quad 6.763 F-08 \quad 1.082 E=05 \quad 800$

33 H $7.958 E-06 \quad 1.273 E=05 \quad 800$

टी $A . A Q 4 E-0 B \quad 1.103 F=07 \quad B 00$

$19 \quad 4.596 E-08 \quad 7.353 E=0 B \quad B 00$

$\begin{array}{rlll}19 & 4.596 E-08 & 7.353 E-08 & 800 \\ 6 & 1.532 E-08 & 2.451 E-08 & 000\end{array}$

$553 \quad 1.302 E-06 \quad 2.063 E-06 \quad 000$

$123 \quad 2.911 E-07 \quad 0.657 E-07$

$0717 \quad 2.2 B 6 E-05 \quad 3.658 E-05 \quad 800$

$10993 \quad 2.5 A 7 E-05$ a.139E-05 050

16168 S.80AE-05 6.07F-05 800

17910 a.21aE-05 b.7a3E=05 800

$16299 \quad 3.835 E-05 \quad 0.136 E=05 \quad B 00$

2OBE5 $\quad .914 E-05$ 7. $663 E-05$ BOO

$19809 \quad 0.426 E-05 \quad 7.0 B J E=05 \quad 800$

$11227 \quad 2.042 E-05 \quad 4.227 E-05 \quad 800$

$19785 \quad 3.479 E-05 \quad 5.566 E-05 \quad 800$

$15381 \quad 3.619 E=05 \quad 5.791 E=05 \quad 800$

10101 3.31BE-05 5.3nQE=05 AOO

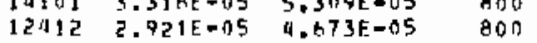

$\begin{array}{llll}2312 & 2.921 E-05 & 4.673 \mathrm{E}-05 & 800 \\ 0854 & 2.083 E-05 & 3.333 \mathrm{E}-05 & A 00\end{array}$

$6657 \cdot 1.557 \mathrm{~F}-05 \quad 2.491 \mathrm{E}-05 \quad 600$

$4365 \quad 1,0>7 E-05 \quad 1.043 E=05$

3997 9.406E-06 $1.505 \mathrm{E}-05$ 000

3356 T.B37E-06 1.264E-05 600

$3115 \quad 7.330 E=06 \quad 1.173 F=05 \quad$ AOO

25A1 $6.074 F-08 \quad 9.71 B F=16 \quad 800$

$2334 \quad 5.472 F=06 \quad$ A.7A7F $=06 \quad 800$

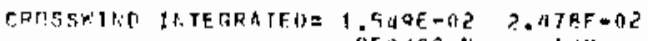




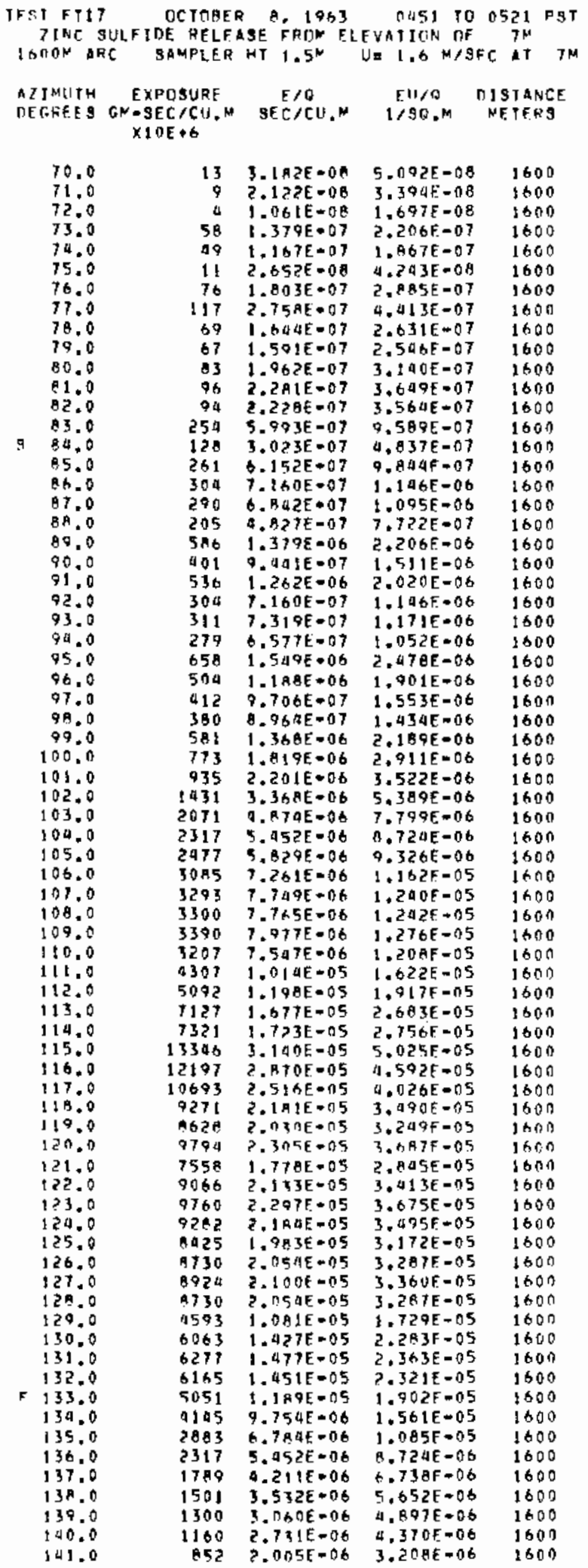

\begin{tabular}{|c|c|c|c|c|}
\hline $\begin{array}{l}142.0 \\
143.0 \\
149.0 \\
145.0 \\
148.0 \\
149.0 \\
198.0 \\
109.0 \\
150.0 \\
151.0 \\
152.0 \\
153.0 \\
159.0\end{array}$ & $\begin{array}{l}829 \\
534 \\
495 \\
302 \\
399 \\
180 \\
155 \\
189 \\
182 \\
132 \\
178 \\
155 \\
173\end{array}$ & $\begin{array}{l}1.952 E-06 \\
1.257 E-06 \\
1.167 E-06 \\
7.107 E=07 \\
.121 E-07 \\
4.243 E-07 \\
3.660 E=07 \\
4.455 E=07 \\
4.206 E=07 \\
3.129 E=07 \\
4.190 E=07 \\
3.660 E-07 \\
4.090 E=07\end{array}$ & $\begin{array}{l}3.123 E-06 \\
2.011 \mathrm{IE}=06 \\
1.067 F=06 \\
1.137 E=06 \\
1.315 E-06 \\
0.789 E=07 \\
5.055 E=07 \\
7.128 E=07 \\
6.874 E=07 \\
5.007 E=07 \\
6.704 E=07 \\
5.055 E-07 \\
6.534 E-07\end{array}$ & $\begin{array}{l}1600 \\
1600 \\
1600 \\
1600 \\
1600 \\
1600 \\
1600 \\
1600 \\
1600 \\
1600 \\
1600 \\
1600 \\
1600\end{array}$ \\
\hline $63 \mathrm{~W}$ & NTEGRATED & $\begin{array}{r}1.575 E-02 \\
3 E C / S O, N\end{array}$ & $\begin{array}{c}2.520 E-02 \\
1 / N\end{array}$ & \\
\hline
\end{tabular}

TORER MATA FIULLOW....

TFGT FTI OCTAHFR A, 1903 OUST TO O5Z PST

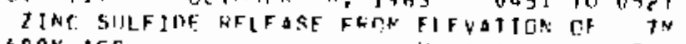
IHOON AFC IIE I, S N/SFC AT TN 
TOKFR TAIA FULLLW....

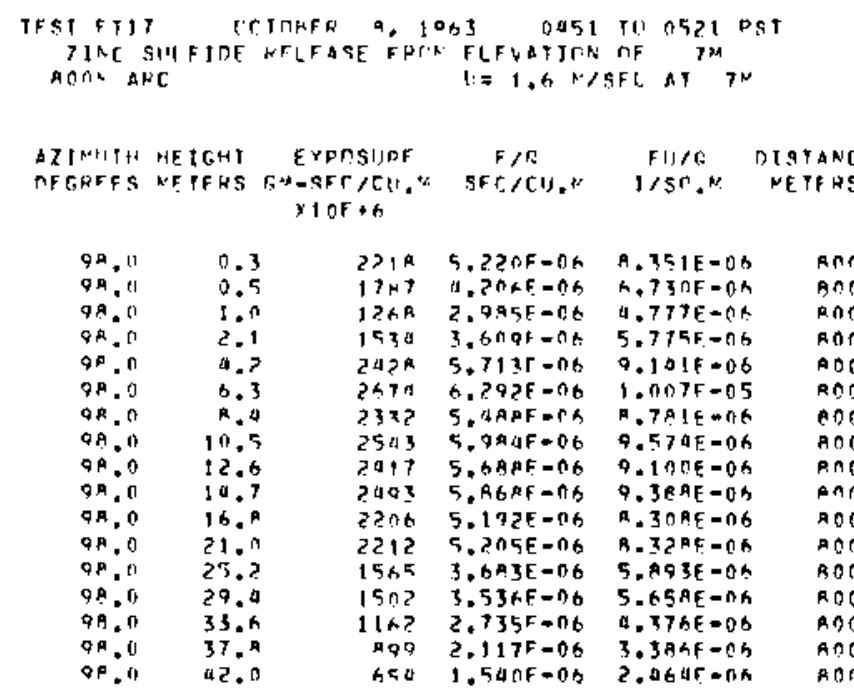

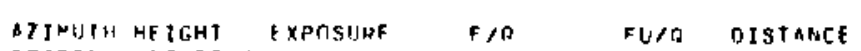

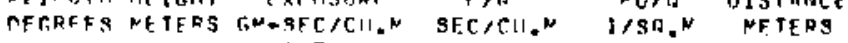
$\times 10 F+h$

\begin{tabular}{|c|c|c|c|c|c|}
\hline $\begin{array}{l}122.0 \\
122.0 \\
122.0 \\
122.0 \\
12200 \\
122.0 \\
122.0 \\
122.0 \\
127.0 \\
122.0 \\
122.0 \\
122.0 \\
122.0 \\
122.0 \\
122.0 \\
122.0 \\
122.0\end{array}$ & $\begin{array}{l}0.3 \\
0.5 \\
1.0 \\
2.1 \\
4.7 \\
6.3 \\
10.5 \\
12.6 \\
14.7 \\
14.7 \\
21.7 \\
25.7 \\
29.0 \\
33.5 \\
37.4 \\
42.0\end{array}$ & 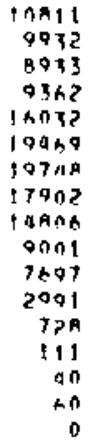 & $\begin{array}{l}2.514 F=05 \\
2.337 E=05 \\
2.102 F=05 \\
2.2035=05 \\
3.712 E=05 \\
4.581 F=05 \\
0.647 E=05 \\
4.212 E=05 \\
3.484 F=05 \\
2.118 F=05 \\
1.911 E=05 \\
7.039 F=08 \\
1.713 F=06 \\
2.019 E=07 \\
9.43 \cap F=08 \\
1.415 E-07 \\
0.000 F+00\end{array}$ & 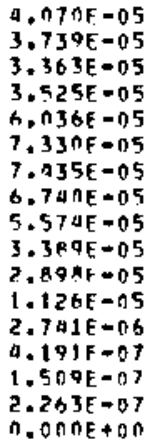 & 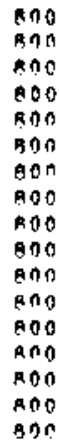 \\
\hline
\end{tabular}

ATINUTH MFITHI EXPOSIRF FAO FIIR DISTANCE

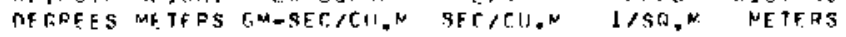

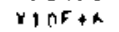

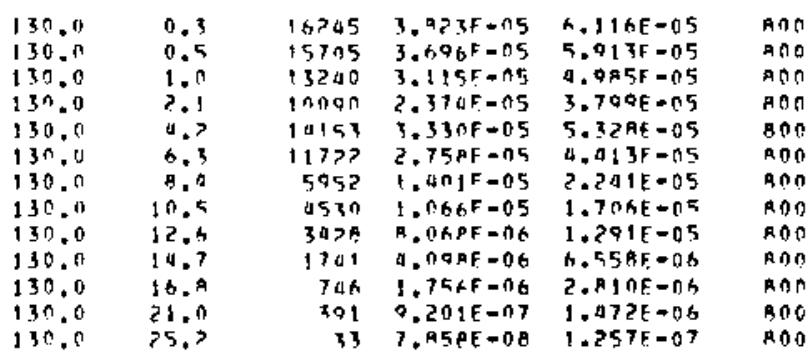

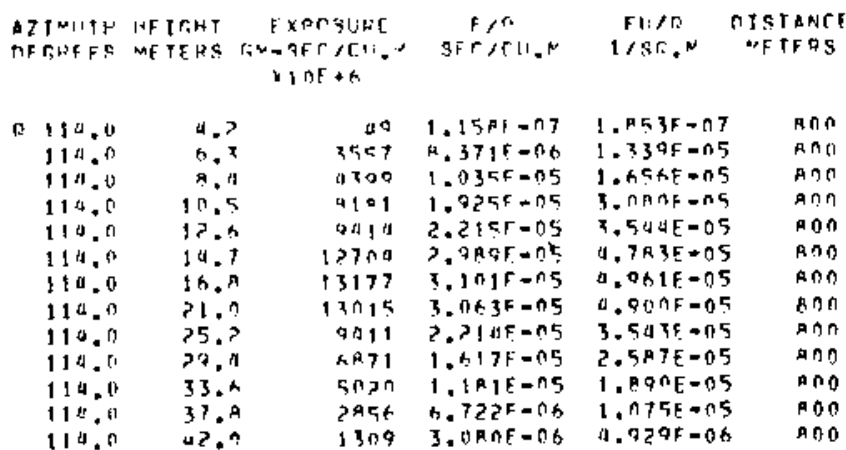




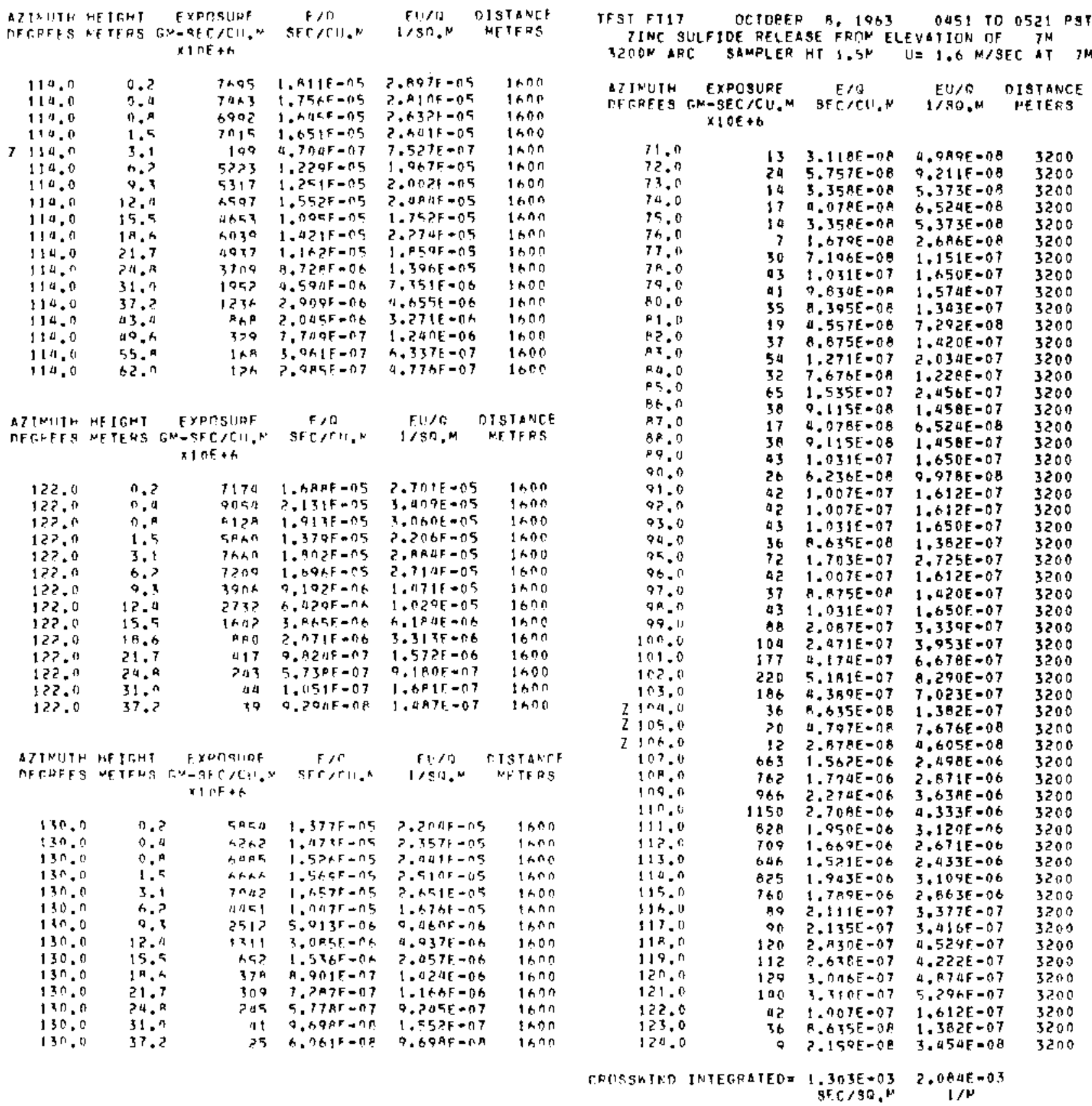


TOWFP MATA F OLIIX....

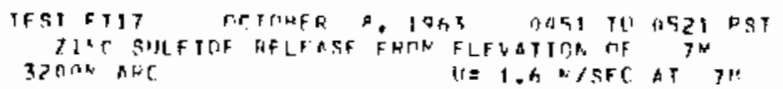

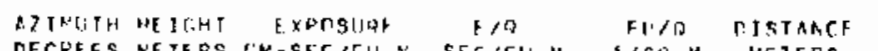

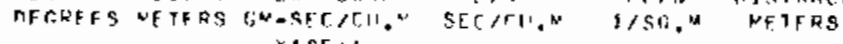
$\times 1 \cap F+6$

\begin{tabular}{|c|c|}
\hline QA. I: & 0.7 \\
\hline पA, $\mathrm{C}$ & 1). 4 \\
\hline$O R$, त & 7. 7 \\
\hline 78.7 & 1.5 \\
\hline$\nabla$ म, & 3.1 \\
\hline$Q R, 11$ & 6.7 \\
\hline ? & 9.3 \\
\hline 9 ด. & 17.1 \\
\hline $8 R .5$ & 15.5 \\
\hline$Q r .0$ & 1 स . \\
\hline पR. 0 & 31.7 \\
\hline 9.0 & 24.9 \\
\hline 92,0 & 31.0 \\
\hline$O A .11$ & 37.7 \\
\hline$Q A, O$ & 0.3 .11 \\
\hline 9. 1 & 40.6 \\
\hline q , 1" & 55.12 \\
\hline$Q A .0$ & $62 . n$ \\
\hline
\end{tabular}

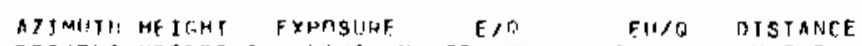

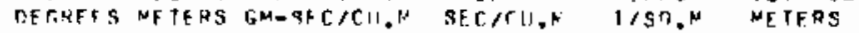
$\times 1 \cap F+h$

\begin{tabular}{|c|c|c|c|c|c|}
\hline 172.0 & $0 . ?$ & k3 & $1.4 A 7 F=07$ & $2.3795-07$ & $3>00$ \\
\hline 122.1 & 0.0 & 5 & $1.2 \Delta>5=0.7$ & $1.99 \mathrm{AF}=07$ & 3200 \\
\hline 12 & $0 . R$ & ?3 & $5.517 F=0 A$ & R. $R 27 \varepsilon=0 A$ & $\div 200$ \\
\hline 12 & 1.5 & $1>0$ & $2.924 E-177$ & $a . b 7$ AF $=07$ & $3>n 0$ \\
\hline .0 & 3.1 & 2,77 & $5.3145=0.7$ & $A .553 E=07$ & $3 ? 00$ \\
\hline 122.0 & 6.7? & $5+2$ & $1.3 \geq 3 F=06$ & $3.116 F=06$ & 3200 \\
\hline & 9.3 & ARA & 1.57 aF $=06$ & $2.5 t$ AF $=0 s$ & 3200 \\
\hline 122.0 & 12.11 & $52 ?$ & $1.2295=r .6$ & $1.9667=166$ & $3 z \cap 0$ \\
\hline & 15.5 & וד & $1.11 \mathrm{AF}=\mathrm{Ch}$ & $1.776 E=06$ & $3 \geqslant 00$ \\
\hline 12 & 18.5 & $35 \mathrm{t}$ & $270 \mathrm{~F}-177$ & $1.323 E=06$ & $320 n$ \\
\hline & 21.7 & उम & $\mathrm{A} .97 \mathrm{AF}=\mathrm{C} 7$ & $1.43 \mathrm{AF}-0 \mathrm{t}$ & $3>00$ \\
\hline & 2 & $2+5$ & $.250 F=07$ & $1,0 B 1 F-D B$ & $3>00$ \\
\hline $1,22.0$ & 31.0 & $? ? 9$ & 5.4084 .67 & $R \cdot A 53 F-n 7$ & 3200 \\
\hline 122.0 & 37.2 & 101 & 4.51 यद -07 & $7.227 F=07$ & $3 \times 00$ \\
\hline & a3. $n$ & $7 / 4$ & $1.74 a F=07$ & $2.7911=07$ & 3200 \\
\hline & 49.5 & 59 & $1.300 E-? 7$ & 7.23 AE $=\mathrm{N} ?$ & 3200 \\
\hline & $55 .{ }^{\circ}$ & 30 & $.32 a F=n P$ & $1.49>\mathrm{F}=\mathrm{n} 7$ & 3200 \\
\hline & & $>A$ & $.435 \mathrm{~F}=\mathrm{CO}$ & $1.06>E-07$ & $3>0$ \\
\hline
\end{tabular}

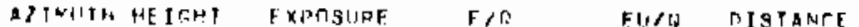
OFGRE S MF TFES GM-SFC $x+$ inth

\begin{tabular}{|c|c|c|c|c|c|}
\hline $1 \cap b .0$ & $n .2$ & $69 x$ & $1.053 t=0 t$ & $2 .+10 t=0 t$ & nח \\
\hline $\ln 6.0$ & 0.4 & $75 \%$ & $1.777 \mathrm{~F}=06$ & 2. 슨 & 3200 \\
\hline 106.0 & 0.9 & hㄱㄱ 7 & $1.47 A F=n$ & 2.3 at $=06$ & $3>0 n$ \\
\hline $10 h_{1} 0$ & 1.5 & 113 & P. . . T $3 F=07$ & a. $2765+07$ & $3>00$ \\
\hline $10 x .0$ & 3.1 & hat & $1.56 .5 \%=0 \mathrm{H}$ & $3.5 n d k=06$ & 3 2 กก \\
\hline $10 \mathrm{~m}, \mathrm{n}$ & n.? & 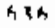 & $1.00 \mathrm{GF}=0 \mathrm{t}$ & $2.38 R F=n b$ & 3200 \\
\hline 100 & 9,3 & $5 \times 3$ & $1.35 \mathrm{AF}=\mathrm{AB}$ & D. OSOE $=0 \mathrm{~B}$ & $3>00$ \\
\hline 100.0 & 12.9 & $59 \%$ & $1.4 n 3 f-106$ & $P .7011 F=116$ & $3 ? 00$ \\
\hline 1nh, 0 & 15.5 & 479 & $t .1 \geqslant 9 F-06$ & I. RSAF $=n A$ & 32 ก \\
\hline $1 B, 0$ & 19.5 & ?०? & $A 7 A F=07$ & $1,10 \cap 5=0 \mathrm{~s}$ & $3 ? 0$ ก \\
\hline 106.0 & 21.7 & $34 \pi$ & A. $1555-07$ & $1.305 E-0 R$ & $3 p 0 n$ \\
\hline$!^{n}$ & $24 . A$ & 153 & $3.6 ? \cap 5-107$ & $5.79>0=07$ & $3>7 n$ \\
\hline$[$ nfe. 0 & $31 . n$ & 21 & $? .1<q F=07$ & $3.450 F=07$ & $x>n 0$ \\
\hline 113.0 & 37.7 & 50 & $1.39+5=07$ & $2.733 t=07$ & $3 \geqslant n n$ \\
\hline 19 & 4 & 30 & $.15 A F=n R$ & $1.0 \times 5 E=117$ & 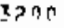 \\
\hline 100 & 40 & 10 & il. $A,>P=A A$ & $7.459 E=0 A$ & $3>0 n$ \\
\hline & & 77 & $P n_{0}: A B$ & . T⿱一⿻上丨 & 3? \\
\hline
\end{tabular}

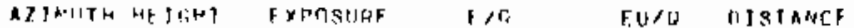

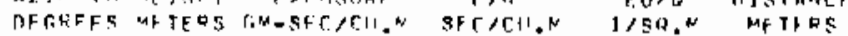
$x \rightarrow \cap E+h$

\begin{tabular}{|c|c|c|c|c|c|}
\hline $11 \%=0$ & $0, ?$ & 711 & $1.67 \triangle 5=06$ & $2.0 .79 E=n 6$ & $3,0 n$ \\
\hline$\{1 \pi, n$ & 0.7 & 605 & $1.5\} P F=06$ & 3. $424 F=06$ & $3>00$ \\
\hline 115.0 & D. 2 & 775 & $1.825=0 x$ & $7.921 E-\mathrm{ns}$ & $x \geqslant 00$ \\
\hline 14.0 & 1.5 & $8 \geqslant 0$ & $1.051 \mathrm{~F}=0 \mathrm{~s}$ & $3.12 i f=06$ & 3200 \\
\hline $114=0$ & 3.1 & 10 A & $2.55>f=0 h$ & a. $\mathrm{MPBE}=0 \mathrm{~A}$ & $x=0$ \\
\hline $11 \% .0$ & n.? & 1450 & $3.413 F=0 t$ & $5.4 x 1 E=06$ & פח 37 \\
\hline 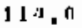 & 9.2 & $1+n ?$ & $3.77 .2 F=O A$ & $6.034 \mathrm{E}=06$ & 3200 \\
\hline $1 ! 1.0$ & 12,0 & $15 \times 5$ & $3,72 A F=n G$ & $5,961 E=0.6$ & 3200 \\
\hline 112.0 & 15.5 & 1707 & $4.113 F=06$ & $0.579 \mathrm{E}=0 \mathrm{~b}$ & 3200 \\
\hline 114.0 & $1 H$ & 1635 & $3.838 f-0 b$ & $6.14 n F=n G$ & 3700 \\
\hline $11.1 . n$ & 21.7 & 2104 & $4.9535=06$ & $7.924 E=04$ & $3>0 \mathrm{c}$ \\
\hline t] it. il & $? 4.9$ & 1900 & $4.701 \mathrm{f}=0 \mathrm{t}$ & $7.5238=06$ & $32 n$ \\
\hline 114.0 & 31.9 & 5137 & $7.3835=06$ & $1.181 E=05$ & 32 ก \\
\hline 194.0 & 37.2 & $300 ?$ & $7.065 \mathrm{E}=0 . \mathrm{r}$ & $1.13 D E=05$ & 300 \\
\hline 114.0 & 43.0 & 1612 & $3.784 E=06$ & $6.0715=06$ & $32 \times 0$ \\
\hline 114.0 & & GH? & $.405 \%=06$ & $2.56 R E_{-}=06$ & 3200 \\
\hline 14.1 & & 470 & 1. InFF $=06$ & $1.77^{n E}=06$ & 3000 \\
\hline & & $? 3 n$ & $1155=07$ & $.664 \mathrm{E}-0.7$ & 5200 \\
\hline
\end{tabular}


SERIES 163 EXPERIMENT: I\& \& FT-18,

GROUND LEVEL AND TONER SAMPLING 200-3200 M ARCS.

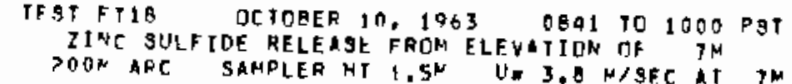

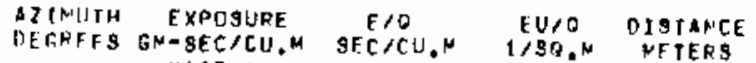

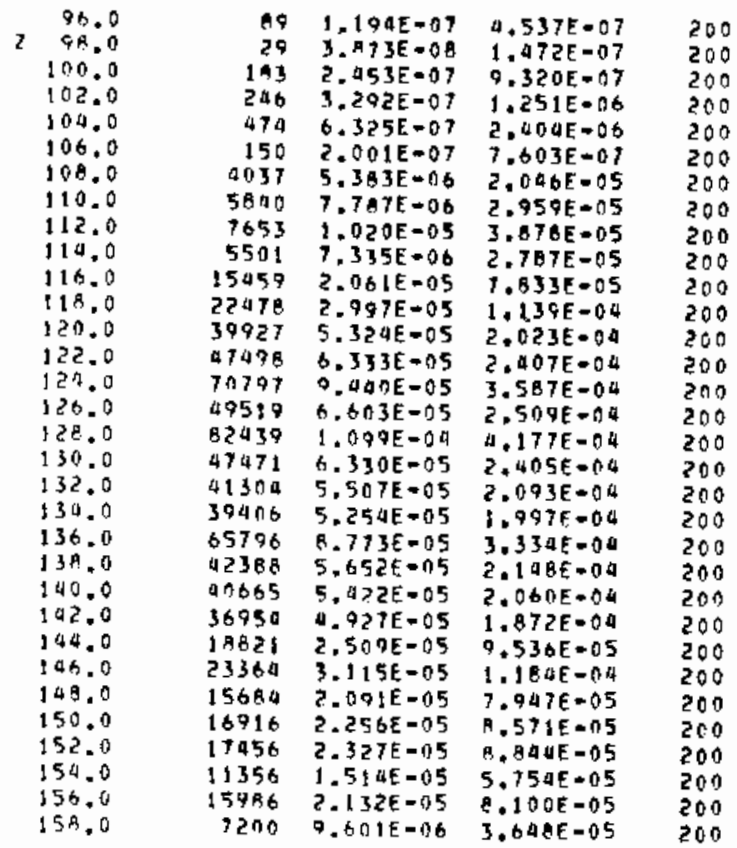

CROSSWIHD INTEGHATFD= T.3A3E-03 $2.805 E-A 2$

TOKEE HATA FULUA...

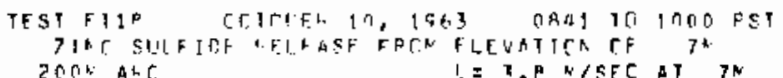

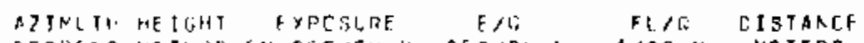

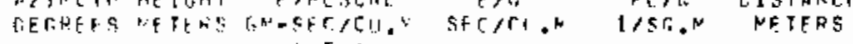
$\times 1 \cap F+5$

\begin{tabular}{|c|c|c|c|c|c|}
\hline$Q A .0$ & 0.3 & $?^{\circ}$ & $3,055 \mathrm{~F}=0 \mathrm{R}$ & $1.465 E=07$ & 200 \\
\hline 98.0 & 0.7 & 16 & $2.142 \mathrm{~F}=0 \mathrm{~A}$ & $8.133 \mathrm{E}=08$ & 200 \\
\hline 95.9 & 1.4 & 1 & $2.510 E=04$ & $9.53 \mathrm{AE}=09$ & 200 \\
\hline$\triangle A .0$ & 2. 7 & 89 & $1.3 x+5=07$ & $5.055 F=07$ & 200 \\
\hline 98.0 & 4,1 & 10.1 & $1,355.5=07$ & $5.151 t=07$ & 200 \\
\hline 9.0 & 5.4 & a & $1.255 E=00$ & $4.769 E=0 B$ & 200 \\
\hline$q A \cdot n$ & 0,4 & 9 & $1.25 L F-O E$ & $4.7605+0 \mathrm{~B}$ & $2 n n$ \\
\hline 95,11 & 8.1 & 77 & $1.024 F=07$ & $3,9,1 E-07$ & 200 \\
\hline QP. 6 & 9.1 & 32 & $4.2675=08$ & $1 . t 21 f-07$ & 200 \\
\hline 90,0 & 10.2 & 75 & $1.0 n a E=0.7$ & $3.815 F=07$ & 200 \\
\hline 98.11 & 13.5 & 47 & $0.275 F=0.8$ & $2.384 E-09$ & 200 \\
\hline 90.0 & 10.7 & 32 & $1.267 f=0 A$ & $1, \operatorname{H} 21 \mathrm{E}=07$ & 200 \\
\hline 92.0 & 1 ค. .9 & ?อ & $3.765 E-$ ती & $1.431 \mathrm{f} .07$ & 200 \\
\hline 90.0 & 21.5 & $\leqslant 4$ & $A, 53$ uf $=r 8$ & $3.2 a I E-07$ & 200 \\
\hline 98.13 & $? 4.3$ & 20.1 & $2.692 F=137$ & $1.073 E-06$ & 200 \\
\hline 98.0 & 27.7 & $14 \mathrm{t}$ & $1.95 \mathrm{AF}=07$ & $7.401 E=07$ & $>$ ก \\
\hline
\end{tabular}

\begin{tabular}{|c|c|c|c|c|c|c|}
\hline \multicolumn{2}{|c|}{$\begin{array}{l}\text { AINLTH } \\
\text { PEGRFES }\end{array}$} & $\begin{array}{l}\text { HE I GHT } \\
\text { NETEHS }\end{array}$ & $\begin{array}{c}\text { FXPQSUEF } \\
\text { CN }=S E C / C U, \\
\times 1 O F+B\end{array}$ & $\begin{array}{c}E>C \\
S E C>C(1 . N\end{array}$ & $\begin{array}{l}E L / 6 \\
1 / 50.2\end{array}$ & $\begin{array}{l}\text { OISTAACE } \\
\text { NETEAS }\end{array}$ \\
\hline$z$ & 106.0 & 9.7 & 200 & $3.4 \times 08=07$ & 1.31 AE $=0 A$ & 200 \\
\hline 2 & got, & 0.7 & $29 ?$ & 3. ค9คE $=07$ & 1. $\triangle A 1 E=06$ & 200 \\
\hline$z$ & 106.0 & 1.4 & 44 & $1.255 F=0.7$ & $4.7695-07$ & 200 \\
\hline & .6 & 2.7 & 295 & $3.741 \mathrm{E}-107$ & $1.447 \mathrm{E}-06$ & 200 \\
\hline & $10 \% .0$ & 4.1 & 2176 & $2.902 E=06$ & $1.103 E=05$ & 200 \\
\hline & .0 & 5.0 & 1012 & $417 f \cdot n b$ & $9.125 E-06$ & 200 \\
\hline & $\begin{array}{l}106.0 \\
106.0\end{array}$ & 0.8 & $\begin{array}{l}1995 \\
2375\end{array}$ & $\begin{array}{l}2.52+E=06 \\
3.16 P F=106\end{array}$ & $\begin{array}{l}9 . \sin 5=06 \\
1.204 t=05\end{array}$ & $\begin{array}{l}200 \\
200\end{array}$ \\
\hline & $10 \times .0$ & 9.4 & 239 म & 3,1 RSE $=06$ & $1.210 E=05$ & $2 n 0$ \\
\hline & & 10.8 & $23 \% 7$ & $3.1 \times 3 F=06$ & $1.2 \cap 9 \mathrm{~F}=0 \mathrm{~s}$ & 200 \\
\hline & & 3.5 & $26 ? ?$ & $50 t F=06$ & $1.332 \mathrm{E}-1,5$ & 200 \\
\hline & & & $27 \times 5$ & $R 7 F=0 t$ & $1,401 f=05$ & 200 \\
\hline & 100.0 & 18.0 & $250 \mathrm{t}$ & $3,330 \mathrm{~F}=0 \mathrm{H}$ & $1.269 \mathrm{E}=0.5$ & 200 \\
\hline & & & $P 20 A$ & $C 65 F+06$ & $\therefore=1+5 F=05$ & 200 \\
\hline & & & 2160 & g $] \mathrm{F}=0 \mathrm{t}$ & $1.095 E-05$ & 200 \\
\hline & 106.0 & 27.5 & $P_{1} A 3$ & $s 1 ; f=r o$ & $1.5^{A} E+05$ & 200 \\
\hline
\end{tabular}

\begin{tabular}{|c|c|c|c|c|c|}
\hline $\begin{array}{l}\text { MITWLTH } \\
\text { RECHEFS }\end{array}$ & $\begin{array}{l}\text { HE TCHT } \\
\text { ME TFHS }\end{array}$ & $\begin{array}{c}\text { ExPCSLAF } \\
\text { GN-SFCPCH, } \\
\times 10 F+A\end{array}$ & $\begin{array}{c}F, D \\
S E C, F 1 . \%\end{array}$ & $\begin{array}{l}F 1 / 6 \\
1 / 50 .\end{array}$ & $\begin{array}{c}\text { DISTANCE } \\
\text { HE TEFS }\end{array}$ \\
\hline 110.0 & 0.3 & 3214 & $1.2335=05$ & a. 6 A a t $=05$ & 200 \\
\hline & 0.7 & $10 \times 5 ?$ & $1.021 f=c 5$ & $5.40 \mathrm{NE}=05$ & 200 \\
\hline 114. & 1.4 & $7>31$ & $1.031 \mathrm{~F}=05$ & $3.917 E-05$ & 200 \\
\hline 114. & 2.7 & 7693 & $1.02 t F=05$ & $3.89 A E-05$ & 200 \\
\hline 11 & 4.1 & 0773 & $1.236 E-05$ & $4.698 F=05$ & 200 \\
\hline 1 & 5.4 & 13271 & $1.770 E=05$ & $6.724 k-05$ & 200 \\
\hline j & 0.8 & 13354 & $1.7 \times 1 E-n 5$ & $6 * 7665-05$ & 200 \\
\hline 1 & $\mathrm{H}, 1$ & $155 \times 6$ & $2.07 \in f=05$ & $7.027 E=05$ & 200 \\
\hline 1 & 9.1 & $1323 n$ & $1.7615=15$ & $6.7 C 3 E-05$ & 200 \\
\hline 1 & 10.9 & 15714 & $2.026 F-05$ & $1.700 E-05$ & 200 \\
\hline 11 & 13.5 & 14071 & $1.87 A F=05$ & $7.130 E+0.5$ & 200 \\
\hline 114.0 & $16 . ?$ & $1267 \mathrm{~A}$ & $1.095, E=05$ & $3.4 \hat{c}$ पF -05 & $2 \cap 0$ \\
\hline 114.0 & 19.7 & 11010 & $1.4 \times 95$ & $5.581 \mathrm{E}-05$ & 200 \\
\hline 11 & 21.6 & MSan & $1.13 c$ & $4.327 E=05$ & 200 \\
\hline & 24. 3 & 8204 & $1.106 \mathrm{E}-05$ & a.20zk-05 & 200 \\
\hline & - & 7097 & $053 \mathrm{~F}-15$ & $4.0 n \leq E=05$ & 200 \\
\hline
\end{tabular}

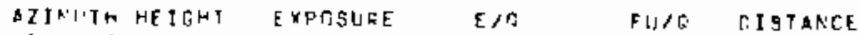

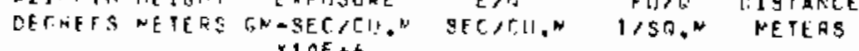

\begin{tabular}{|c|c|c|c|c|c|}
\hline $\begin{array}{l}122.0 \\
122.0 \\
122.0 \\
122\end{array}$ & $\begin{array}{l}0.3 \\
0.7 \\
1.11\end{array}$ & $\begin{array}{l}43404 \\
57869 \\
30089\end{array}$ & $\begin{array}{l}5.7 A 7 E-\cap 5 \\
7.72 C F-05 \\
9.013 F-05\end{array}$ & $\begin{array}{l}2.199 \mathrm{E}-04 \\
2.937 \mathrm{E}=04 \\
1.525 \mathrm{E}=04\end{array}$ & $\begin{array}{l}500 \\
200 \\
500\end{array}$ \\
\hline 122.0 & 2.7 & $3+570$ & $4.9295=05$ & $1.973 E=04$ & 200 \\
\hline 122,0 & $4 \cdot 1$ & 39 \& d ? & $5.25 \wedge F-05$ & $1.99 \mathrm{BF}=04$ & $\begin{array}{l}2 \cap 0 \\
2 \cap 0\end{array}$ \\
\hline $\begin{array}{l}122.0 \\
122.0\end{array}$ & 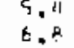 & $\begin{array}{l}45714 \\
09701\end{array}$ & $\begin{array}{l}1.075 F=05 \\
6.653 F-05\end{array}$ & $\begin{array}{l}2.31 \text { AE }-04 \\
2.520 E-04\end{array}$ & $\begin{array}{l}200 \\
200\end{array}$ \\
\hline 12 & 8.1 & $13+40$ & $5.2,26 E=0.5$ & $2.212 E-04$ & 200 \\
\hline & 9.4 & 117050 & $5.271 F=05$ & $2.383 E=04$ & 200 \\
\hline $2 \geq \hat{c} .0$ & 10.0 & $4725 \theta$ & c.3n]F= $=\{5$ & $300 f-04$ & 200 \\
\hline$c$ & 13,5 & $4 h 2 \cap ?$ & $6.16 n F=05$ & $3 \triangle 1 E=04$ & 200 \\
\hline & 16.2 & 13097 & $5.74+1=0.5$ & $2.164 E-0 A$ & 200 \\
\hline 23.0 & $1 \mathrm{~F} .0$ & 20536 & $3.805 F-05$ & $1.476 \mathrm{~F}=04$ & 200 \\
\hline D) & 21.9 & 22335 & $2.97 \mathrm{FF}=115$ & $1.132 \mathrm{E}=0 \mathrm{~J}$ & 200 \\
\hline & $2 \cdot \sqrt{3}$ & $2>0 n 7$ & $2.934 F=05$ & $1.115 t-04$ & 200 \\
\hline & $27, n$ & ??0म & $2,511<f-1 ; 5$ & $1.119 E=0 d$ & 200 \\
\hline
\end{tabular}




\begin{tabular}{|c|c|c|c|c|c|}
\hline $\begin{array}{l}\text { DIR.TIRH } \\
\text { REGREIS }\end{array}$ & $\begin{array}{l}\text { HE IEHI } \\
\text { NETENS }\end{array}$ & 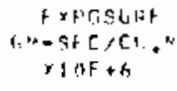 & $\begin{array}{c}F / r \\
E F C / C H A R\end{array}$ & 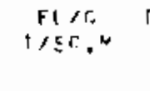 & $\begin{array}{l}\text { CISTARCE } \\
\text { NETFES }\end{array}$ \\
\hline 130.0 & 0.3 & 51000 & $\therefore .67 P F=n 5$ & $Z .5 \leq A F=\{d$ & 2 กู \\
\hline $1 \geq 6.0$ & 0.7 & 57577 & $7,6775=0.5$ & $2.817 E=04$ & 200 \\
\hline 130.0 & 1,4 & $3 \times \leq>3$ & $5.110 F=015$ & $1.942 E=04$ & 200 \\
\hline 150.6 & 2.7 & is $19 a_{1} 3$ & $5.52+F=05$ & $2.100 E-104$ & 200 \\
\hline $136 \cdot 0$ & 4.1 & Ta $34 ?$ & $5.112^{F}=05$ & $1.903 F=04$ & 200 \\
\hline 130.13 & 5.7 & 40003 & $6.50 t+1=05$ & $2.0 A 71=04$ & 200 \\
\hline 135.0 & h. $A$ & 140107 & $\therefore .4 ?+F=05$ & Z. $\square \Delta ? E=\cap A$ & 200 \\
\hline 136.0 & R. 1 & $5+4 \times 9$ & $1.87 ? 5-05$ & $2.991 \mathrm{E}=0 \mathrm{~L}$ & 200 \\
\hline $13 \% .4$ & 9.4 & 40949 & $5.4 t 0,-r .5$ & $2.075 \mathrm{r}+0.4$ & 200 \\
\hline 130.0 & $10 .{ }^{\text {म }}$ & $5536 h$ & $4.7+A F=05$ & $1.792 E=04$ & 200 \\
\hline 136.0 & 13.9 & 57314 & $6.975 F=05$ & $2.65 ! t=0 a$ & $20 n$ \\
\hline 130.6 & 16.7 & 35379 & $4.717 f=05$ & $1.792 \mathrm{E}=0.4$ & 200 \\
\hline 136.0 & 18.7 & $3035 \%$ & $4.048 F=05$ & $1.538 E=0 a$ & 200 \\
\hline 130.0 & 21.6 & $223 \cap 5$ & $2.970 f=15$ & $1.130 \mathrm{~F}=04$ & 200 \\
\hline & $24 .^{2}$ & ट 134 ה & 2. Adt $F=05$ & $1.0 \mathrm{~A} 1 \mathrm{E}=\mathrm{na}$ & 200 \\
\hline $13 \div 0$ & 27.6 & $240 \geq 2$ & $5.20 .75=05$ & $1.217 \mathrm{E}-04$ & 200 \\
\hline
\end{tabular}

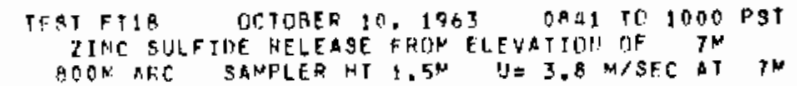
RIRMUTH EXPOSURE E/Q EU/A DISTARCE

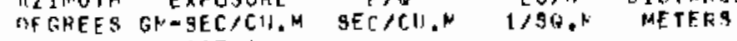
$\times 10 E+6$

\begin{tabular}{|c|c|c|c|c|}
\hline 112.0 & 53 & $1.1005-08$ & $2.728 E-07$ & 800 \\
\hline 114.0 & 154 & $2.060 E=07$ & & 800 \\
\hline 116.0 & 484 & $6.462 E-07$ & $2.456 E=06$ & 800 \\
\hline 118.0 & $10 \mathrm{BB}$ & $1.052 F=06$ & $5.516 E-06$ & 800 \\
\hline 120.0 & 1535 & $2.0 \triangle B E=O B$ & $7.782 E-06$ & BOO \\
\hline 122.0 & 1334 & $1.779 E=06$ & $6.762 \mathrm{~F}=06$ & 800 \\
\hline 120.0 & 1636 & $2,1 A>E=06$ & $8.292 E=06$ & $A 00$ \\
\hline 126.0 & 2519 & $3.359 E-06$ & $1.276 E=05$ & $B 00$ \\
\hline 128.0 & 2769 & 3.6935 .06 & $1.403 E-05$ & .00 \\
\hline 130.0 & 2320 & $3.100 E=06$ & $1,17 A E-05$ & 800 \\
\hline$P 132.0$ & 2324 & $3.100 E=06$ & $1.178 E-05$ & 800 \\
\hline$P 134.0$ & 2156 & $2.875 E-06$ & $1.093 E-05$ & 800 \\
\hline$F 136.0$ & 2055 & $2.741 E-06$ & $1.042 E=05$ & 800 \\
\hline$F 138.0$ & 1622 & $2.163 E-06$ & $9.221 E-06$ & 800 \\
\hline$F 150,0$ & 1542 & $2.057 E=06$ & $7,01 B E=06$ & AOO \\
\hline 142.0 & 810 & $1.0 \mathrm{AOE}-0 \mathrm{G}$ & $4.104 E=06$ & 301 \\
\hline 144.0 & 1587 & $2,117 E-06$ & $8.003 E-06$ & 200 \\
\hline 146.0 & 723 & $9.606 E-07$ & $3.666 \mathrm{E}-06$ & 800 \\
\hline 148.0 & 693 & $9.240 \mathrm{E}=07$ & $3.5 t 1 E-06$ & 800 \\
\hline 150.0 & 276 & 3. GA QE $=07$ & $1.400 E=06$ & 800 \\
\hline 152.0 & 170 & $2.279 E=07$ & $0.660 E-07$ & 600 \\
\hline 150.0 & 103 & $1.37 \mathrm{AE}=07$ & $5.220 \mathrm{E}-07$ & 800 \\
\hline 156.0 & 135 & $1.811 E-07$ & 6.88 OE -07 & 800 \\
\hline 158.0 & 135 & $1.911 E=07$ & $0.880 \mathrm{E}-07$ & 800 \\
\hline 160.0 & 168 & $.2 \triangle A E-07$ & $0.5418=07$ & $A O O$ \\
\hline
\end{tabular}

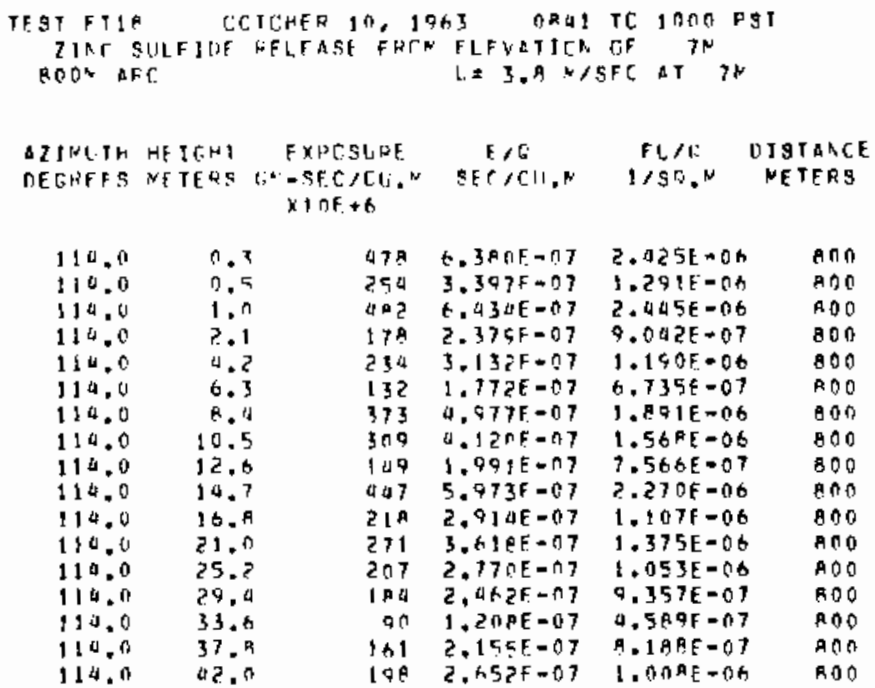

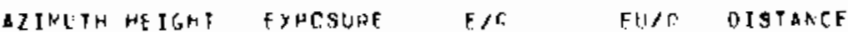

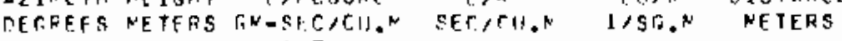
$\times 1 \cap \mathrm{F}+\mathrm{h}$

\begin{tabular}{|c|c|c|c|c|c|}
\hline 122.0 & 0.3 & 1050 & $1.4+3 F=0 A$ & $5.369 C-06$ & 800 \\
\hline 122.0 & 0.5 & 1237 & $1.703+-n_{6}$ & $6.471 t=0 \mathrm{t}$ & 600 \\
\hline 122.0 & 1,0 & 1130 & $1.513 \mathrm{~F}=00$ & $5.74 A F=06$ & 800 \\
\hline 122.0 & 2.1 & 1187 & $1.5 E 3 F=06$ & $6.016 E-n 6$ & ano \\
\hline 122.0 & 0.2 & $157 \mathrm{~A}$ & $2.105 E-10$ & $7.999 E-06$ & ano \\
\hline 122,0 & 0.3 & i32? & $1.7635=06$ & $6.69 \mathrm{AE}=00$ & ROO \\
\hline 122.0 & 8.4 & 1334 & 1. 7 ent $=16$ & $6.703 E \sim 06$ & ant \\
\hline 122.0 & 10.5 & 1509 & $2.120 E=06$ & $9.055 E-06$ & 800 \\
\hline 122.0 & 12.6 & 1210 & $1.515 F=00$ & $6.136 \mathrm{~F}-06$ & $3 \cap 0$ \\
\hline 122.0 & 14.7 & 1792 & $1.8 \Delta Z F=00$ & $7.005 E=06$ & Pnn \\
\hline 122.0 & 16.7 & 1150 & $1.542 F=0 A$ & $5.250 F-06$ & 900 \\
\hline 122.0 & $? 1,0$ & $14>0$ & $1.900 f-r .6$ & $7.196 \varepsilon=06$ & $\operatorname{mon}$ \\
\hline 122.0 & ?s & 941 & $1.255 E=0 t$ & $4.769 \mathrm{~F}=116$ & AOn \\
\hline 122.0 & 20.0 & 997 & $1.3517=176$ & $5.056 t-00$ & AOC \\
\hline 122.0 & $33 . h$ & $? 15$ & $9.5426-0.7$ & $3.6265-06$ & ROD \\
\hline $12 \%$ & 37,9 & $k>3$ & $1,1605=06$ & $4.427 f .06$ & $R D C$ \\
\hline 12 & $a 2, n$ & 640 & $\mathrm{a} .595 \mathrm{~F}=07$ & $3,3 A B E-U h$ & Anr \\
\hline
\end{tabular}

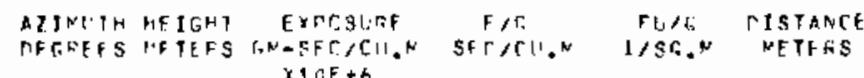

CRCISSAINO INTEGRATED= $1.05 E E=03 \quad 40.020 E-03$

\begin{tabular}{|c|c|c|c|c|c|}
\hline 130.0 & 0.7 & $19+n$ & $2.51 \Delta F=n t$ & $9.034 \mathrm{E}-06$ & Ano \\
\hline 130.0 & 0.5 & 1541 & $2.055 \mathrm{~F}=\mathrm{rb}$ & $1 . A \cap 9 t-0 n$ & aOn \\
\hline $13 n+n$ & 1.0 & 10+e & $1.4 Z \leq 5-0 h$ & $9.418 E-06$ & 200 \\
\hline$: 30,0$ & 3.9 & 1901 & $2.5 \times E-ก 6$ & $9 . A 35 E-06$ & $A 00$ \\
\hline $13 c .0$ & 0.2 & 2072 & $2,1 E 3 E+0 E$ & $1.050 \mathrm{0} 05$ & 800 \\
\hline $13 n \cdot 0$ & 6.3 & 1939 & $2.59+F=16$ & $9.826 E-06$ & 800 \\
\hline 130.0 & A. य & 2192 & $2.92 \times 5=6.6$ & $1.111 E=05$ & $A \cap O$ \\
\hline 130.0 & 10.5 & $25: 1$ & 3.34 PF $-\pi 6$ & $1.2726-05$ & soo \\
\hline 130.0 & $12, \pi$ & $3 \geq 34$ & $a .317 F-c t$ & $1.639 E-05$ & 800 \\
\hline 130.0 & 10.7 & 2090 & $3.8+1 F-06$ & $1.060 \mathrm{E}=05$ & 800 \\
\hline 130.0 & 16.9 & 2276 & $3.035 E=06$ & $1.153 \mathrm{E}-05$ & 800 \\
\hline 130.0 & 21.0 & 3550 & $0.7 \triangle 0 F=06$ & $1.803 t=05$ & P.0O \\
\hline $13 r .0$ & $75 . ?$ & 3204 & $A, 32 \mathrm{KE}=\mathrm{CE}$ & $1.64 a E-05$ & RoO \\
\hline 130.0 & 29.4 & 2713 & $3.610 \mathrm{~F}=0 \mathrm{t}$ & $: 375 \varepsilon-05$ & 800 \\
\hline 130.0 & $33 . A$ & 3217 & $4.290 F=06$ & $1.63^{\cap} F-05$ & $\operatorname{son}$ \\
\hline $13 n .0$ & 37.8 & $3: 27$ & $4,170 t=06$ & $1.58 \mathrm{AE}-0 \mathrm{~S}$ & 800 \\
\hline 130.0 & $42, n$ & 2631 & $3,5 n 5 F-06$ & $1.333 E-05$ & FOn \\
\hline
\end{tabular}




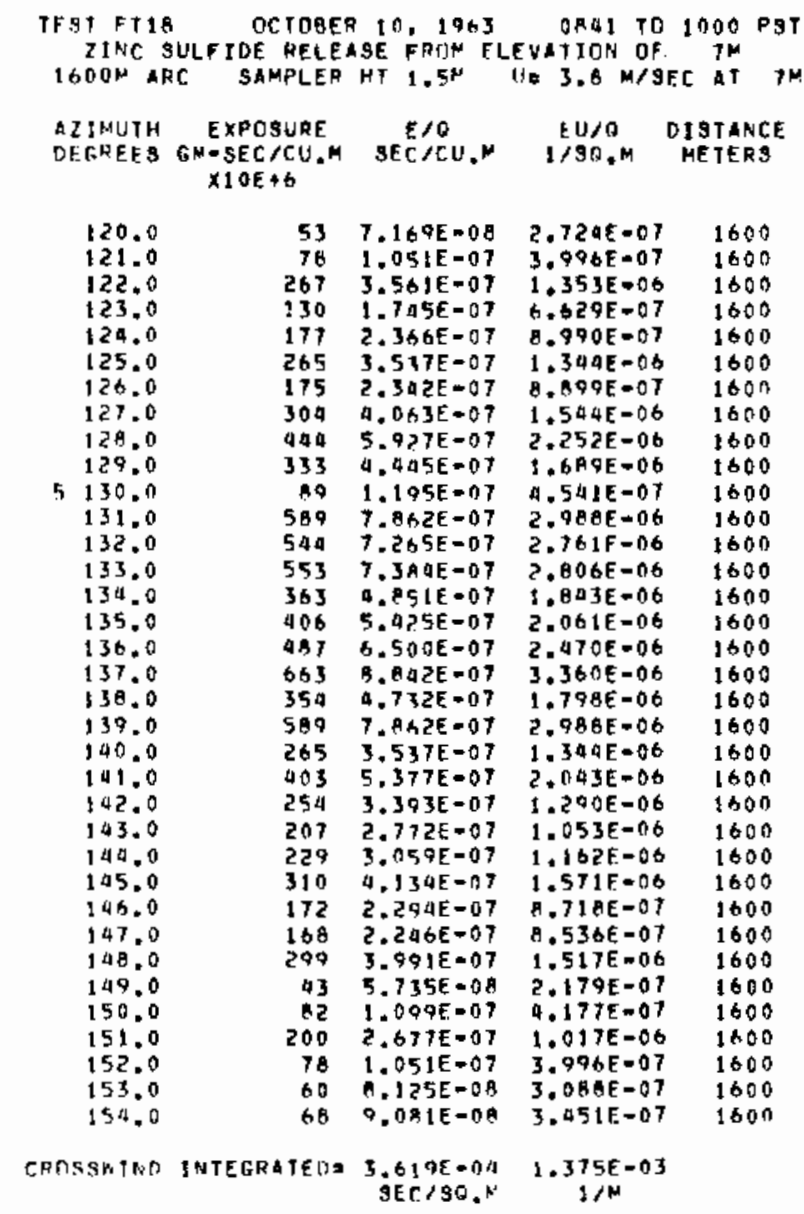

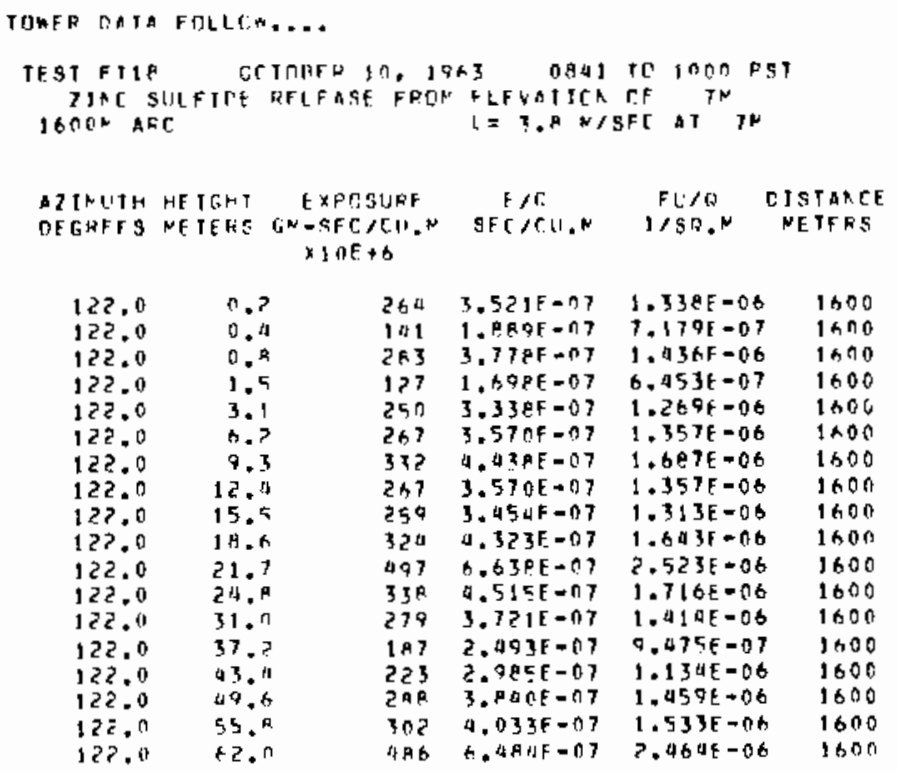

ATINUTH HEIGHT EXPOSLRE E/G DUTO DISTARCE

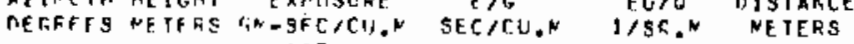
$\times \operatorname{InE}+\mathrm{B}$

\begin{tabular}{|c|c|c|c|c|c|}
\hline 130.0 & $0 . ?$ & 270 & $3.607 F=07$ & $1.3716=06$ & 1600 \\
\hline $13 n .0$ & 0.1 & 465 & $6.211 F=07$ & $2.360 \mathrm{E}=06$ & 1600 \\
\hline 130.0 & 11.8 & 465 & $6.211 \mathrm{~F}=07$ & $2.360 F=06$ & 1600 \\
\hline 130.0 & 1.5 & 344 & $9.593 F-07$ & $1.745 E-06$ & 1000 \\
\hline 130.0 & 3.1 & 343 & 4.57 a $=07$ & 1.73 AE -06 & 1600 \\
\hline $13 n \cdot 0$ & 0.2 & 366 & $4.8 P 2 F=07$ & $5 E-06$ & 1600 \\
\hline 130.0 & 9.3 & 505 & $0.735 F=07$ & $9 E-06$ & 1600 \\
\hline $\begin{array}{l}134.0 \\
130.0\end{array}$ & $\begin{array}{l}12.0 \\
15.5\end{array}$ & 399 & $5.3265=07$ & $2.024 E-06$ & $16 n 0$ \\
\hline 130.0 & I H. A & $27 n$ & 6.0.CE -177 & 8]E-06 & 1600 \\
\hline $13 n .0$ & 21.7 & She & 4 सम $38 \rightarrow 0 ?$ & $\because 4551-04$ & 1600 \\
\hline 130.0 & 24.9 & $7>1$ & $9.415, F=17$ & $3.65 \pi F=06$ & 1600 \\
\hline 130.0 & 31.0 & $14>5$ & $5.667 F=07$ & $2.153 \mathrm{E}=0 \mathrm{t}$ & 1000 \\
\hline $13 n .0$ & 37.7 & 405 & $5.7027-n 7$ & $2.053 E-06$ & $16 \pi n$ \\
\hline 130.0 & 43.7 & $\sin$ & a. $21+F=07$ & $3.38 R F=06$ & $1 \times 00$ \\
\hline $13 n .0$ & 49.5 & Ja & $5.8 A \in F=07$ & $737 \mathrm{k}-06$ & $16 \geqslant 0$ \\
\hline 130.0 & $55 . P$ & 272 & 3.52 प $F=07$ & $1.379_{E}=0 \mathrm{~h}$ & 2600 \\
\hline $13 n .0$ & $+2 . n$ & $3>3$ & $4.77 \zeta 5=67$ & 1.P92t $=3 x$ & lon \\
\hline
\end{tabular}




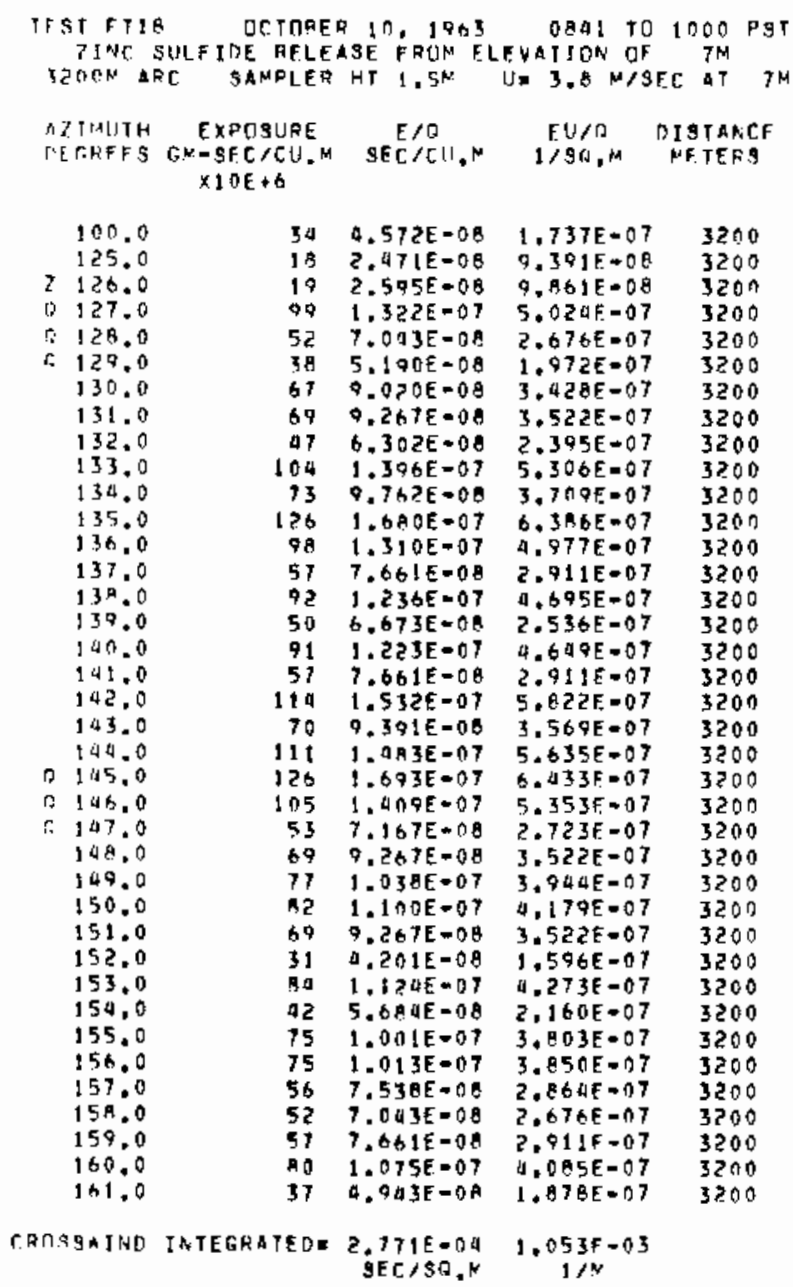

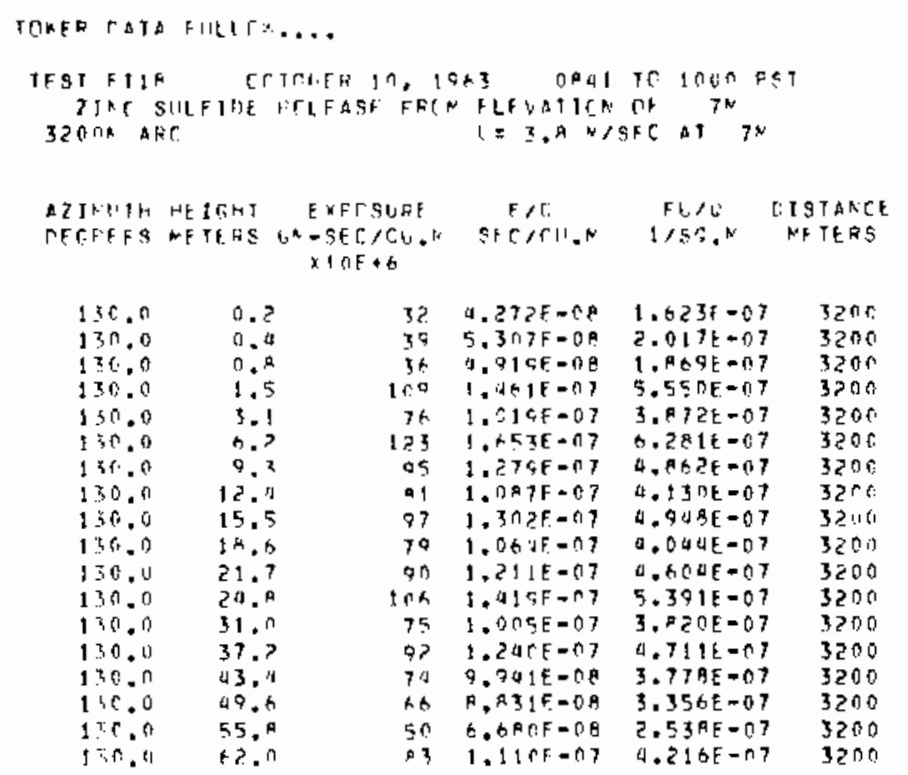


SERIES 164 EXPERIMENT: ,,$\$-1$,

GROUND LEVEL AND TOMER \$AMPLING 200-3200 H ARCS. GROUNO LEVEL

SAMPL ING ONLY ON THE $12800 \mathrm{M}$ ARC.

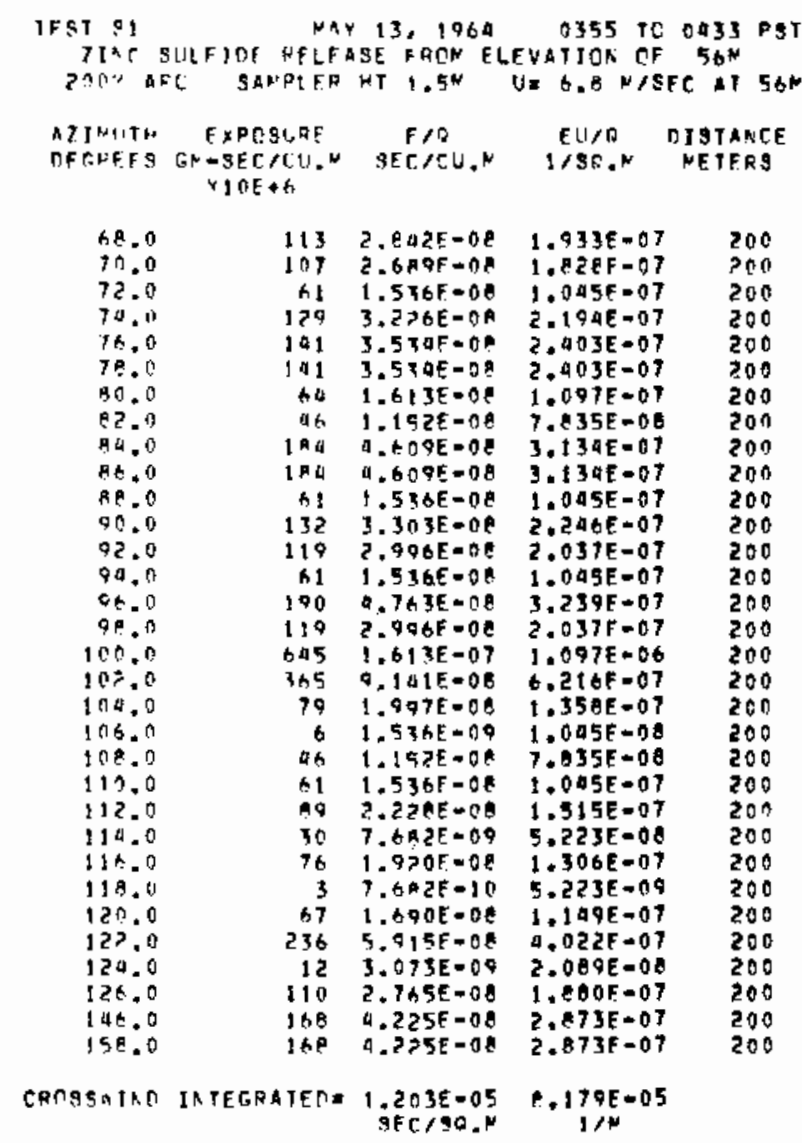

TONFR TATA FOLLLIV....

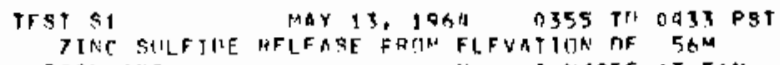

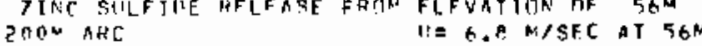

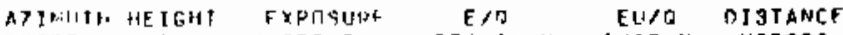

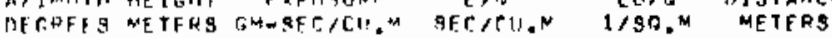
(i) int

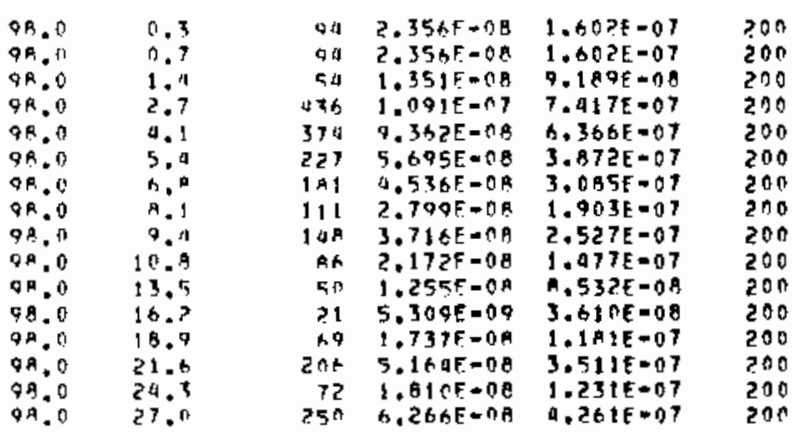

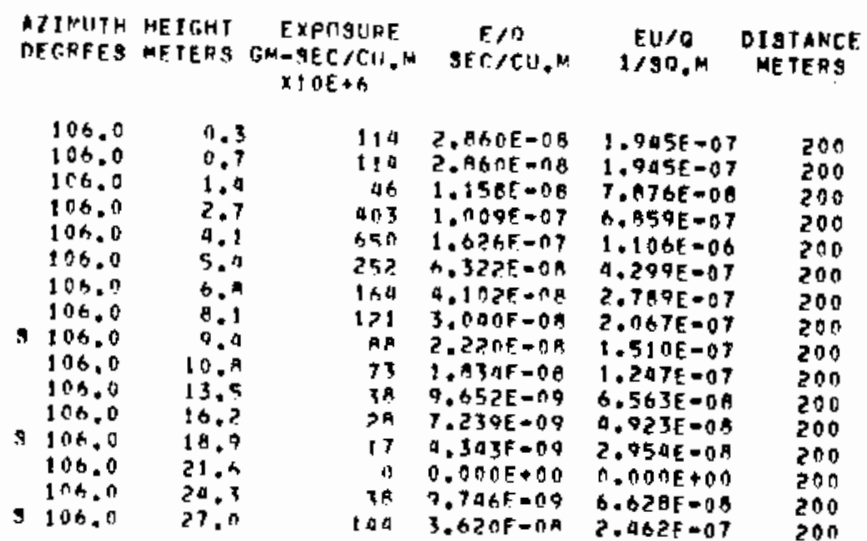

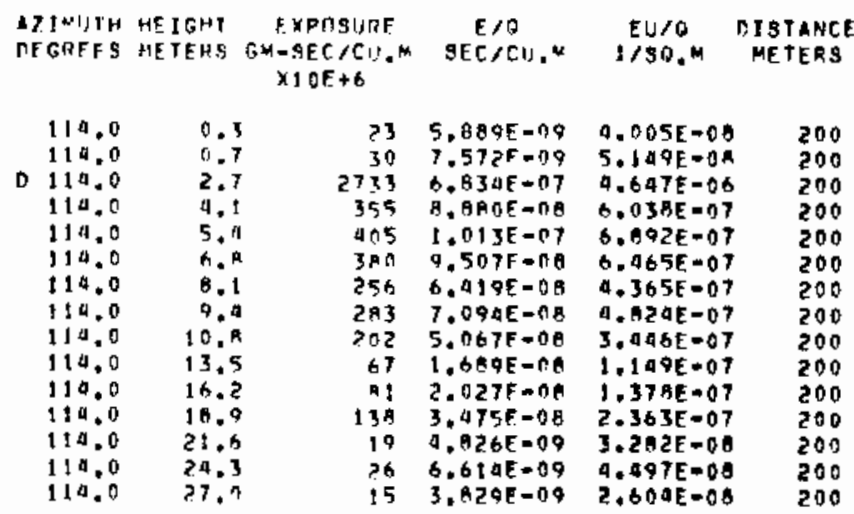

AZIMUTH HEIGHT EXPSSURE F/Q DUTO DISTANCE

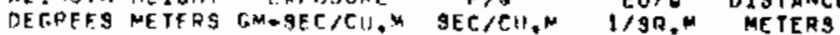
$Y \cap D F+\infty$

\begin{tabular}{|c|c|c|c|c|c|}
\hline $\begin{array}{l}122.0 \\
122.0 \\
122.0 \\
122.0 \\
122.0 \\
122.0 \\
122.0 \\
122.0 \\
122.0 \\
122.0 \\
122.0 \\
122.0 \\
122.0\end{array}$ & $\begin{array}{r}2.7 \\
9.1 \\
5.9 \\
6.9 \\
8.1 \\
9.1 \\
10.4 \\
13.5 \\
16.2 \\
18.9 \\
21.9 \\
24.3 \\
27.0\end{array}$ & $\begin{array}{l}015 \\
2114 \\
3195 \\
23 \\
212 \\
215 \\
150 \\
204 \\
94 \\
59 \\
57 \\
28 \\
6 \\
16\end{array}$ & $\begin{array}{l}1.039 F=07 \\
5.357 E=0 A \\
7.025 E=00 \\
5.936 E=08 \\
5.309 E=0 A \\
3.764 E=0 A \\
5.116 F=08 \\
2.365 E=08 \\
1.1965=08 \\
1.408 F=08 \\
9.052 F=09 \\
1.740 E-09 \\
4.177 E-09\end{array}$ & $\begin{array}{l}7.056 E=07 \\
3.643 E=07 \\
5.185 E-07 \\
9.036 E-07 \\
3.610 E=07 \\
2.560 E=07 \\
3.479 E=07 \\
1.60 A E=07 \\
1.017 E=07 \\
9.845 E=08 \\
6.563 E=08 \\
1.183 E=08 \\
2.040 E-0 B\end{array}$ & $\begin{array}{l}200 \\
200 \\
200 \\
200 \\
200 \\
200 \\
200 \\
200 \\
200 \\
200 \\
200 \\
200 \\
200\end{array}$ \\
\hline
\end{tabular}




\begin{tabular}{|c|c|c|c|c|c|}
\hline $\begin{array}{l}Z 1 \% 1 B T H \\
\text { EFHFF }\end{array}$ & $\begin{array}{l}\text { ME J JiHT } \\
\text { MF TERS }\end{array}$ & 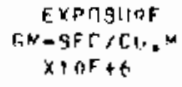 & $\begin{array}{c}E / D \\
S E C / C L, N\end{array}$ & $\begin{array}{c}F U / C \\
1 / S D . M\end{array}$ & $\begin{array}{l}\text { DISTANCE } \\
\text { METERS }\end{array}$ \\
\hline $\begin{array}{l}130.0 \\
130.0 \\
130.0 \\
130.0 \\
130.0 \\
130.0 \\
130.0 \\
130.0 \\
130.0 \\
130.0 \\
130.0 \\
130.0 \\
130.0\end{array}$ & $\begin{array}{r}1.9 \\
2.7 \\
4.1 \\
5.9 \\
6.7 \\
4.1 \\
9.9 \\
10.8 \\
13.5 \\
16.2 \\
18.0 \\
21.6 \\
27.0\end{array}$ & $\begin{aligned} 25 \\
201 \\
115 \\
201 \\
131 \\
171 \\
73 \\
110 \\
54 \\
67 \\
5 \\
72 \\
32\end{aligned}$ & $\begin{array}{l}6.27 A E=09 \\
7.094 E=08 \\
2 . A 96 F=08 \\
5.212 E=0 \% \\
3.282 E=0 A \\
3.04 A F=08 \\
1.034 E-08 \\
2.751 F=08 \\
1.351 E=08 \\
1.0 A Q F=08 \\
1.44 A F=08 \\
7.230 E=09 \\
9.006 E=09\end{array}$ & $\begin{array}{l}1.26 A E=0 A \\
4.82 A E=07 \\
1.969 E-07 \\
3.544 F=07 \\
2.232 E=07 \\
2.067 E=07 \\
1.247 E=07 \\
1.071 E=07 \\
9.159 E=08 \\
1.149 E=07 \\
9.845 E=09 \\
4.923 E-08 \\
5.444 E=00\end{array}$ & $\begin{array}{l}200 \\
200 \\
200 \\
200 \\
200 \\
200 \\
200 \\
200 \\
200 \\
200 \\
200 \\
200 \\
200\end{array}$ \\
\hline
\end{tabular}

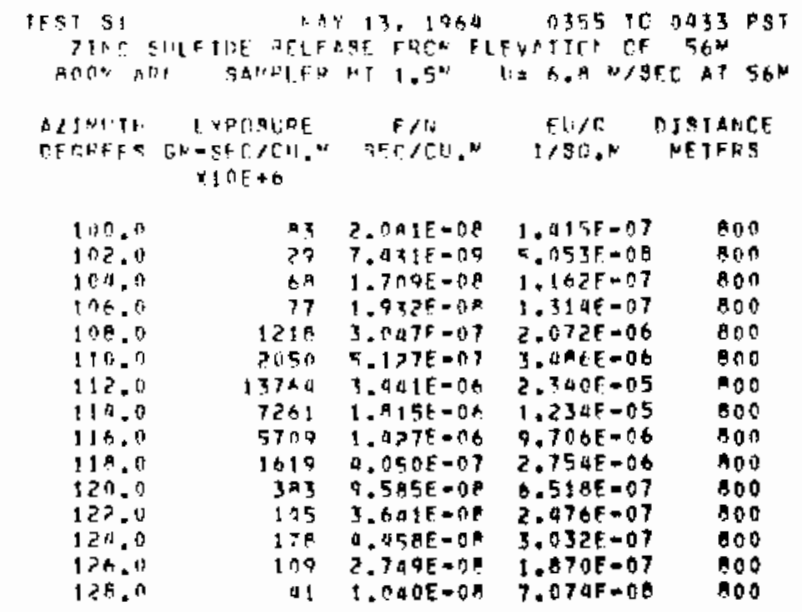

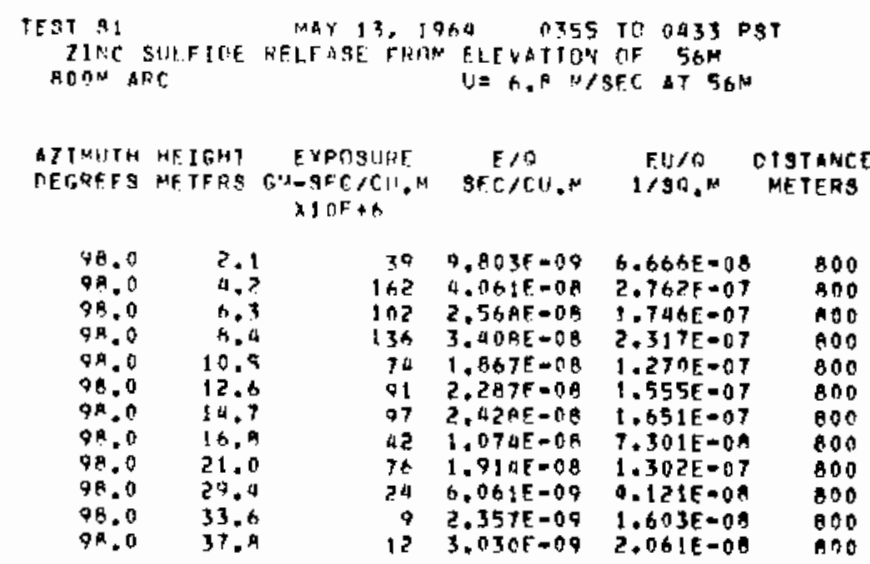

DIMIITH HEIGHT EXPNSURF F/O EU/O DISTANCE DEGREES METEHS GM-SECACH,N SFC/CI!M I/SA.M METERS $\times 10 F+6$

\begin{tabular}{|c|c|c|c|c|c|}
\hline $\begin{array}{l}106.0 \\
106.0 \\
106.0 \\
106.0 \\
106.0 \\
106.0 \\
106.0 \\
106.0 \\
106.0 \\
106.0 \\
106.0 \\
106.0 \\
106.0 \\
106.0 \\
106.0 \\
108.0 \\
106.0\end{array}$ & $\begin{array}{l}0.3 \\
0.5 \\
1.9 \\
7.1 \\
4.2 \\
4.3 \\
8.4 \\
10.5 \\
12.4 \\
14.7 \\
16.8 \\
21.9 \\
25.2 \\
29.4 \\
33.4 \\
37.4 \\
42.0\end{array}$ & $\begin{array}{l}110 \\
110 \\
178 \\
115 \\
319 \\
275 \\
255 \\
369 \\
621 \\
508 \\
302 \\
970 \\
517 \\
971 \\
1305 \\
1411 \\
1020\end{array}$ & 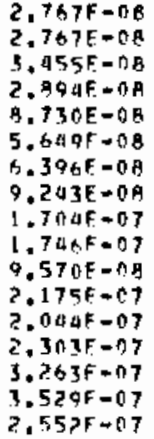 & 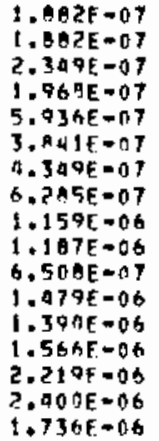 & $\begin{array}{l}100 \\
000 \\
800 \\
100 \\
000 \\
800 \\
800 \\
800 \\
000 \\
100 \\
800 \\
000 \\
000 \\
900 \\
900 \\
000 \\
000\end{array}$ \\
\hline
\end{tabular}

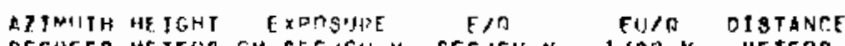

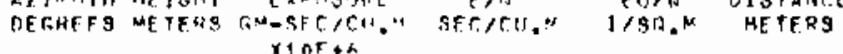

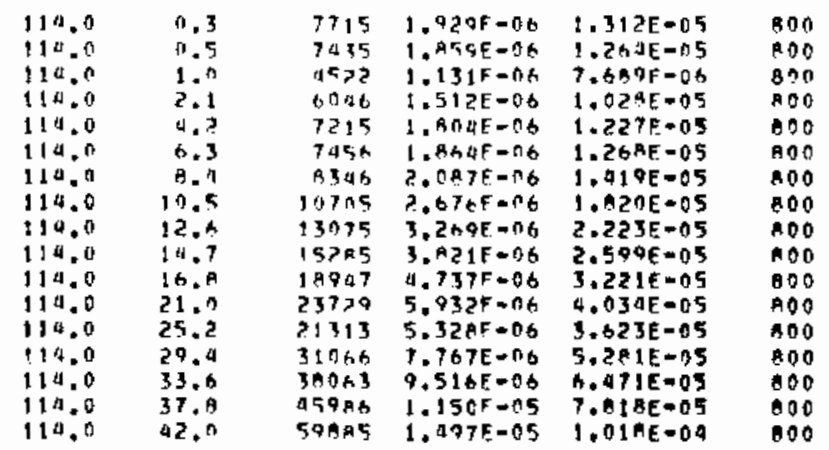




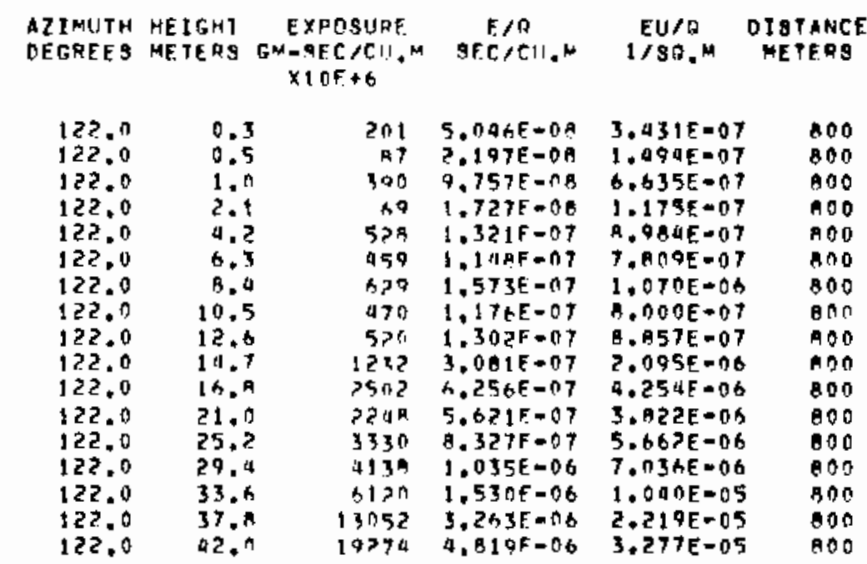

ARINITH HETGHT EXPTSUGE E/D FU/O OISTANCF DEGTEES METEHS GM-SEC/CI.H SECIRH,N 1/ST.N METERS

\begin{tabular}{|c|c|c|c|c|c|}
\hline 130.0 & $4 . ?$ & 12 & $1.8 \geqslant 1 F+0 B$ & $1.235 E-07$ & ADO \\
\hline $13 n .0$ & 6.3 & 72 & $1 . A 21 E-n B$ & $1.23 B E-07$ & 800 \\
\hline 130.0 & R. 4 & Ra & $2.102 E=0 \mathrm{~B}$ & $1.420 E-0 ?$ & 800 \\
\hline 130.0 & 10.5 & נם & $1.120 F=n A$ & $7.619 F-6$ A & 100 \\
\hline 130.4 & 12.6 & $\operatorname{lns}$ & $2 . A B, E=\cap 8$ & $1.309 \mathrm{E}-0 \mathrm{~T}$ & 800 \\
\hline 130.0 & 14.7 & 44 & $1.120 \%=0$ 月 & $7,619 \mathrm{E}-0 \mathrm{~B}$ & คดก \\
\hline $13 n . n$ & 16.9 & 12 & $1 . A \geq 1 E-08$ & $1.23 \cap E=07$ & 100 \\
\hline $13 n .0$ & 21.0 & 72 & I.BZIE-OA & $1,23 B E * 07$ & 800 \\
\hline 139.0 & $25, ?$ & 65 & $1.650 E=08$ & $1.1225-07$ & 800 \\
\hline $130 . ?$ & 29.0 & 167 & $4.175 \mathrm{~F}-18$ & $2.839 E=07$ & 800 \\
\hline 130.0 & 33.6 & 17 & $4.3775=00$ & $2.977 E-00$ & AOO \\
\hline 130.0 & $37, \mathrm{R}$ & 37 & 9.42 AF $=09$ & $6.411 E-00$ & 000 \\
\hline
\end{tabular}

TEST S1 NAY 1\$, 19RA 0355 TU 0433 PGT 21AC SULFIDE BELFASE FRON ELFYATICA OF $56 \mathrm{~K}$ 1GOUN ART. BAHPLES HT 1.5N LE G.R N/SEC AT $56 \mathrm{~N}$

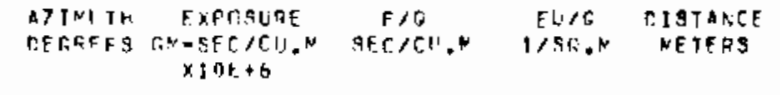

\begin{tabular}{|c|c|c|c|c|}
\hline 1100 & tia & $1.792 E-08$ & $1.504 F-0 ?$ & 1600 \\
\hline 111.0 & $2 \mathrm{R}_{6}$ & $7.1725=0 P$ & A.RTYE-07 & 1600 \\
\hline 152.0 & $95 / 1$ & $\therefore .3 A 7 E=07$ & $1.8235-06$ & 1600 \\
\hline 113.0 & 2247 & $5.6706-07$ & $3 . A 22 E=06$ & 1000 \\
\hline 114.0 & 5772 & $1.4035-528$ & $9.814 E-06$ & 1601 \\
\hline 115.0 & $6 ? 38$ & $1.5+2 F=0+$ & $1,002 F=0.5$ & 1600 \\
\hline 116.0 & 19702 & $2.6 P B E-0 t$ & $1.026 E=05$ & 1600 \\
\hline 117.0 & 13500 & $3.375 E-06$ & $2.205 E-05$ & 1600 \\
\hline 118.0 & $2426 \pi$ & $6.006 E=06$ & $4.125 E=05$ & $\$ 600$ \\
\hline 119.7 & 15733 & $3.933 E-06$ & $2.675 E=05$ & 1600 \\
\hline 120.0 & 11658 & $3.9,5 F=0$. & $1.902 E=05$ & 1600 \\
\hline 121.0 & 74146 & $1.862 E=06$ & $1.266 E=05$ & 1600 \\
\hline 122.0 & 4260 & $1.066 E-06$ & $7.250 E=06$ & 1600 \\
\hline 123.0 & 3276 & $=192 \mathrm{E}=07$ & $5.570 \mathrm{E}-06$ & 1600 \\
\hline 124.0 & 2402 & $x .1 \cap 7 E-07$ & $4,153 E-06$ & 1600 \\
\hline 125.0 & 18मक & $4.7235-07$ & $3.212 E-06$ & 1600 \\
\hline 126.0 & 1653 & $4.1345-07$ & $2.011 E-06$ & 1600 \\
\hline 127.0 & 493 & $1.209 E=07$ & $0.2218=07$ & 1600 \\
\hline 128.0 & 321 & ค. $043 E=08$ & $5.969 E-07$ & 1600 \\
\hline 120.0 & 103 & $3.5 A 6 E-08$ & $.039 E-07$ & 1600 \\
\hline 130.0 & 30 & 7,6 A5E $=09$ & $5.226 E-08$ & 1600 \\
\hline 131.0 & 60 & $1.1 \triangle 2 E=08$ & $1.1040-07$ & 1600 \\
\hline & ATEDE & 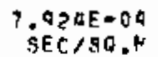 & $\begin{array}{c}5.38 B E=03 \\
1 / N\end{array}$ & \\
\hline
\end{tabular}

TOWFR rata F RLLIIW....

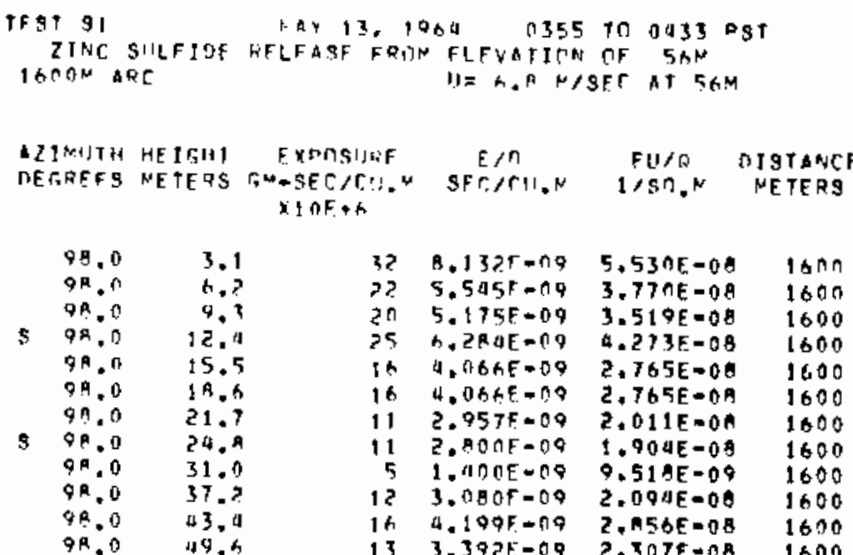

ATIMIITH HFIGHT EXPNSURE E/O EU/O DISTANCE

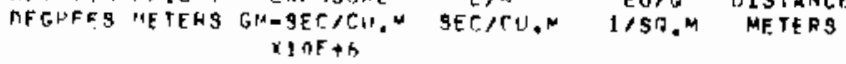

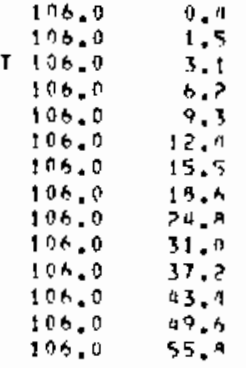

\begin{tabular}{|c|c|c|c|}
\hline 21 & $5.3675=09$ & $3.650 \mathrm{E}=0 \mathrm{R}$ & 160 \\
\hline 15 & $327 F-0.9$ & $F=O R$ & 160 \\
\hline 36 & $9.241 F=00$ & $M E=0 B$ & 1000 \\
\hline 73 & 1. MAC $+n A$ & $57 E=n 7$ & 1600 \\
\hline 30 & $7.373 f=179$ & $7 E=O A$ & 1600 \\
\hline 59 & $A F-n s$ & $D E-O A$ & 1 \\
\hline $4 !$ & $1.516 E=0 \mathrm{C}$ & 07 & 1 \\
\hline 44 & $1.109 \mathrm{~F}=0 \mathrm{~A}$ & $541 E-00$ & 1 \\
\hline 24 & $159 F=n 9$ & 4. JAAE = OA & 1600 \\
\hline 54 & $72 F-1)$ & BटAE - 08 & 1600 \\
\hline 30 & $9 A F=09$ & $663 E-O A$ & $1+00$ \\
\hline 42 & $9 B A F=08$ & $4 E=08$ & 1600 \\
\hline 1 & $41 E-10$ & $184 \mathrm{E}=09$ & 1060 \\
\hline & is $=\$ 9$ & $74 E=0$ & \\
\hline
\end{tabular}

AINWIIH HFIGHT FXIUSIIF F/O FU/O DISTANCE

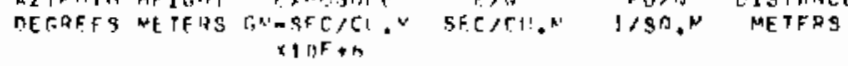

\begin{tabular}{|c|c|c|c|c|c|}
\hline $\begin{array}{l}114.7 \\
114.0\end{array}$ & $\begin{array}{l}0 \\
0.7 \\
6.7\end{array}$ & $\begin{array}{l}42 \text { iी } \\
\text { 15 का }\end{array}$ & $\begin{array}{l}1.110 F=10 \\
1.13 \text { मF }=16\end{array}$ & $\begin{array}{l}7.551 E=06 \\
7.777 f=06\end{array}$ & $\begin{array}{l}1600 \\
1600\end{array}$ \\
\hline $\begin{array}{l}110.0 \\
1[4.0\end{array}$ & 0.9 & 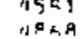 & 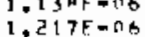 & ..777 $=06$ & $\begin{array}{l}1600 \\
1600\end{array}$ \\
\hline 1140 & 1.5 & तhकी & $1,17 \pi F-06$ & $7.95 \cap E=05$ & 1600 \\
\hline 114.0 & 3,1 & 4711 & 1.?PRF $=\mathrm{Fn}$ & $9.350 E-06$ & 1000 \\
\hline 114,6 & b.? & $5 \times 70$ & $1.06 P E-06$ & $798-06$ & 1600 \\
\hline $11 \Delta .0$ & & $54 \times 3$ & $1.00 A F=D B$ & $0.577 t=06$ & 1600 \\
\hline $11 \%, 0$ & 12.1 & 8んमA & $2,1725=06$ & $1.077 E-05$ & 1600 \\
\hline 4.0 & 15 & 7904 & a $96 F=06$ & $1.697 E-05$ & 1600 \\
\hline $11+0$ & $1 \mathrm{~A}$ & 115148 & $2.537 F=06$ & $3 E \div 05$ & 1600 \\
\hline 114,0 & 21,7 & 13551 & SRAF $=06$ & $2.3045=05$ & 1600 \\
\hline 114.0 & 29 & $12^{936}$ & $3.209 \mathrm{~F}=0 \mathrm{t}$ & $2.182 E=05$ & $\$ 600$ \\
\hline $11 \%, 0$ & $31 . ?$ & 15391 & $3.845 \mathrm{~F}=16$ & b15E=05 & 1600 \\
\hline 114.0 & $37 . ?$ & ?3ㄹำ & $5.907 E=06$ & $240 E-05$ & 1600 \\
\hline - 0 & 93 & 30702 & S77E-106 & $2245-05$ & thon \\
\hline & 49 & $\begin{array}{l}20 ? 95 \\
13020\end{array}$ & - $071 E=0 b$ & $.809 E=05$ & 1000 \\
\hline 110.0 & क., 0 & 16150 & $4.04 \cap F=06$ & $2,7 a>E$-OS & 2600 \\
\hline
\end{tabular}




\begin{tabular}{|c|c|c|c|c|c|}
\hline $\begin{array}{l}\text { AZIMUTH } \\
\text { DESREES }\end{array}$ & $\begin{array}{l}\text { HE I GHT } \\
\text { ME TEKS }\end{array}$ & $\begin{array}{c}\text { EXFกSUNF } \\
G M=9 E[/ 611 . N \\
\times 10 E+6\end{array}$ & $\begin{array}{c}\text { E/D } \\
\text { SEC } / \text { CU.N }\end{array}$ & $\begin{array}{c}E U / Q \\
1 / 50 . M\end{array}$ & $\begin{array}{l}\text { DI STANCE } \\
\text { METFES }\end{array}$ \\
\hline $\begin{array}{l}122.0 \\
122.0 \\
122.0 \\
122.0 \\
122.0 \\
122.0 \\
127.0 \\
122.0 \\
12200 \\
122.0 \\
122.0 \\
122.0 \\
122.0 \\
122.0 \\
122.0 \\
122.0 \\
122.0 \\
122.0\end{array}$ & 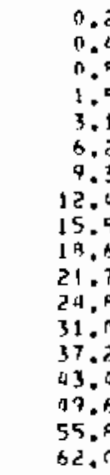 & $\begin{array}{l}3671 \\
4145 \\
4854 \\
5505 \\
3573 \\
4135 \\
5033 \\
5027 \\
9500 \\
11103 \\
10904 \\
71174 \\
9977 \\
19004 \\
15724 \\
126116 \\
11573 \\
101048\end{array}$ & 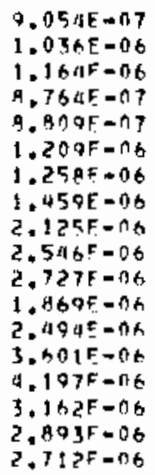 & $\begin{array}{l}6.157 E=06 \\
7.047 F=06 \\
7.912 E=06 \\
5.960 E=06 \\
5.990 E=06 \\
9.220 E=06 \\
8.556 E=06 \\
9.929 E=06 \\
1.445 E=05 \\
1.731 E=05 \\
1.054 E=05 \\
1.271 E=05 \\
1.696 E=15 \\
2.449 E=05 \\
7.654 E=05 \\
2.150 E=05 \\
1.967 E=05 \\
1.946 E=05\end{array}$ & $\begin{array}{l}1600 \\
1600 \\
1600 \\
1600 \\
1600 \\
1600 \\
1600 \\
1600 \\
1600 \\
1600 \\
1600 \\
1600 \\
1600 \\
1600 \\
1600 \\
1600 \\
1600 \\
1600\end{array}$ \\
\hline $\begin{array}{l}\text { ZJNUזH } \\
\text { FERFS }\end{array}$ & $\begin{array}{l}\text { HE ITHT } \\
\text { ME TERS }\end{array}$ & 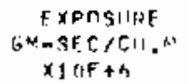 & $\begin{array}{c}E, 6 \\
S E C,(t), N\end{array}$ & $\begin{array}{l}E \cup / 1 \\
1 / 90 . N\end{array}$ & $\begin{array}{l}\text { DISTANCE } \\
\text { MF. TERS }\end{array}$ \\
\hline $\begin{array}{l}130.0 \\
130.0 \\
130.0 \\
130.0 \\
130.0 \\
130.0 \\
130.0 \\
130.0 \\
130.0 \\
130.0 \\
130.0 \\
130.0 \\
130.0 \\
130.0 \\
150.0 \\
130.0\end{array}$ & 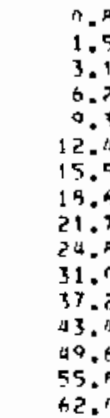 & $\begin{array}{l}35 \\
11 \\
70 \\
14 \\
17 \\
79 \\
20 \\
170 \\
43 \\
42 \\
13 \\
30 \\
50 \\
34 \\
41 \\
541\end{array}$ & 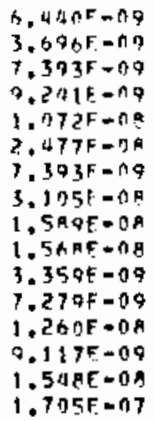 & 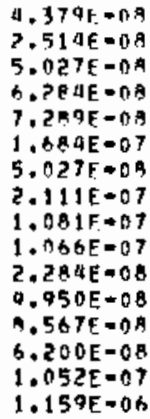 & $\begin{array}{l}1600 \\
1600 \\
1600 \\
1600 \\
1600 \\
1600 \\
1600 \\
1600 \\
1600 \\
1600 \\
1600 \\
1600 \\
1600 \\
1600 \\
1600 \\
1600\end{array}$ \\
\hline
\end{tabular}

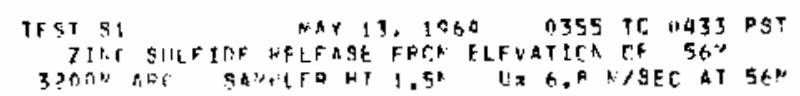

\begin{tabular}{|c|c|c|c|c|}
\hline $\begin{array}{l}\text { A IHIITH } \\
\text { AERATES }\end{array}$ & 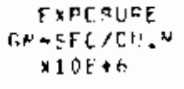 & 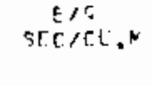 & $\begin{array}{c}F(: / Q \\
1 ; r_{5}, N\end{array}$ & $\begin{array}{l}\text { NISTAREE } \\
\text { MEIFES }\end{array}$ \\
\hline $\begin{array}{l}112.0 \\
113.0 \\
1114.00 \\
115.0 \\
116.0 \\
117.0 \\
1110.0 \\
119.0 \\
120.0 \\
121.0 \\
122.0 \\
123.0 \\
124.0 \\
125.0 \\
126.0 \\
127.0 \\
128.0 \\
129.0 \\
131.0 \\
132.0 \\
161.0\end{array}$ & $\begin{array}{r}177 \\
329 \\
982 \\
2333 \\
4694 \\
10672 \\
23796 \\
20533 \\
13017 \\
10759 \\
6151 \\
5007 \\
9490 \\
0792 \\
3039 \\
1208 \\
349 \\
72 \\
12 \\
16 \\
32\end{array}$ & 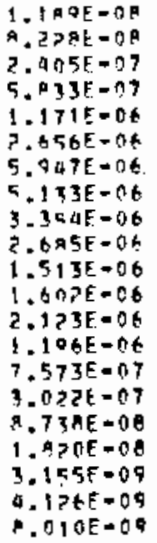 & 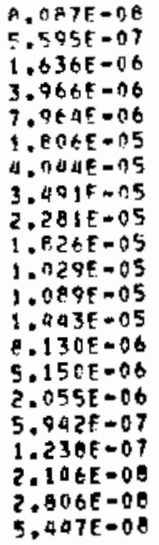 & $\begin{array}{l}3260 \\
3200 \\
3200 \\
3200 \\
3200 \\
3200 \\
3200 \\
3200 \\
3200 \\
3200 \\
3200 \\
3200 \\
3200 \\
3200 \\
3200 \\
3200 \\
3200 \\
3200 \\
3200 \\
3200 \\
3200\end{array}$ \\
\hline
\end{tabular}

TES 31 NAY I3, 1964 n355 TO DU33 PST $32 A C N$ APT.

\begin{tabular}{|c|c|c|c|c|c|}
\hline $\begin{array}{l}\text { ATTMATH } \\
\text { DEREFFS }\end{array}$ & $\begin{array}{l}\text { IIF ICHT } \\
\text { "FE TERS }\end{array}$ & 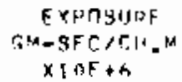 & $\begin{array}{c}E / D \\
S F r / C H, M\end{array}$ & $\begin{array}{l}F \cup / 0 \\
1 / 30.4\end{array}$ & $\begin{array}{l}\text { ISTANCE } \\
\text { METFRS }\end{array}$ \\
\hline 114.0 & 14.7 & $9 / 13$ & $? .10 A F=n 7$ & $1.433 E=O B$ & $32 n 0$ \\
\hline 114.0 & n. 4 & 690 & $1.799 F=n 7$ & $1 . \triangle A Q E=O B$ & 3200 \\
\hline 114.0 & 0. म & ARA & $2.222 F=07$ & $1.511 E=06$ & 3200 \\
\hline 114.0 & 1.5 & 713 & $1.7 A \Delta F=07$ & $213 E-06$ & 3200 \\
\hline 114.0 & 3.1 & AR 3 & $2.21 \cap E=07$ & $1.5035=06$ & 3200 \\
\hline 110.0 & h. & 015 & $B Q F-n_{7}$ & $57 \mathrm{E}=0 \mathrm{H}$ & 3200 \\
\hline $11^{\pi}, 0$ & 9.3 & 903 & $2.2595=17$ & $36 E=06$ & 3200 \\
\hline 114.0 & $12, \pi$ & $7 \cap 7$ & $1.760 E-07$ & $1.203 F=06$ & 3200 \\
\hline 114.0 & 19.5 & $71 \mathrm{~A}$ & $1.790 \mathrm{~F}-07$ & $1,217 E-06$ & 3700 \\
\hline 194.0 & 12.5 & 602 & $1.732 \mathrm{E}=07$ & $1.17 \mathrm{AE}=0 \mathrm{~B}$ & 3200 \\
\hline 1130 & 21.7 & $6 \times 7$ & 1.6万RE-A7 & $1.134 E=06$ & 3200 \\
\hline 114.0 & 24.9 & $4 a n$ & $1.15 z^{F}=07$ & $7.83 \cap\{-07$ & 3200 \\
\hline 114,0 & 31. & $3 \cap R$ & $7.797 \mathrm{E}=09$ & $241 E-07$ & 3200 \\
\hline $1: 4.0$ & 37.7 & 194 & $a, 602 F=0 A$ & $3.130 E=07$ & 3200 \\
\hline 4.0 & & 290 & $3.765 \mathrm{~F}-0.8$ & $240 E-07$ & 3200 \\
\hline 4.0 & & $9 \pi$ & $? .468 F=08$ & $678 E-07$ & 3200 \\
\hline .0 & 55.9 & $9 h$ & $2.407 F=08$ & $1.637 E-07$ & 3200 \\
\hline & 62 & 58 & $1.46 \mathrm{AE}=0 \mathrm{~A}$ & $86 F=08$ & 3200 \\
\hline
\end{tabular}

AZIMLITH HEIGHI EXPISTHA E/I EHIO OISTANCE MECREFS METERS T.M-AEC/CII, SEC/CII.M 1/SA. N METFRS $\times 10 E+6$

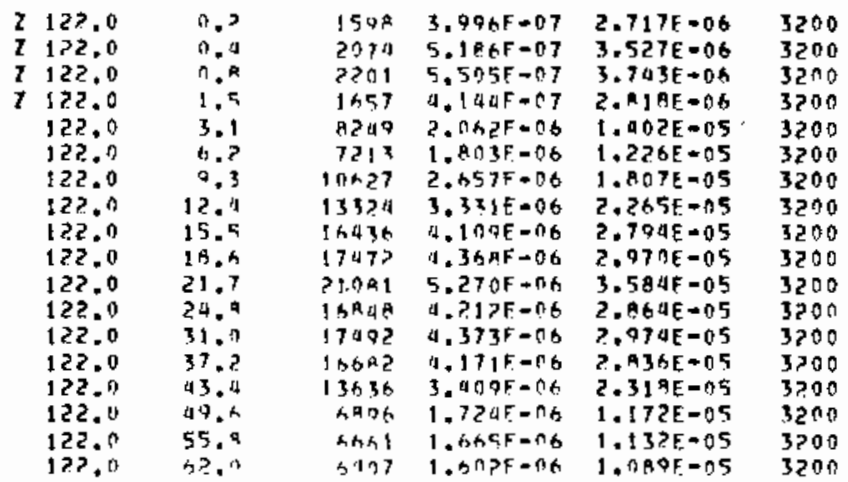

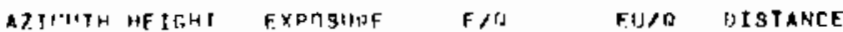
DEGRFES METEHS GM-SER/CH,D SECICU.N I/3H.M METERS $\times 1$ CE+s

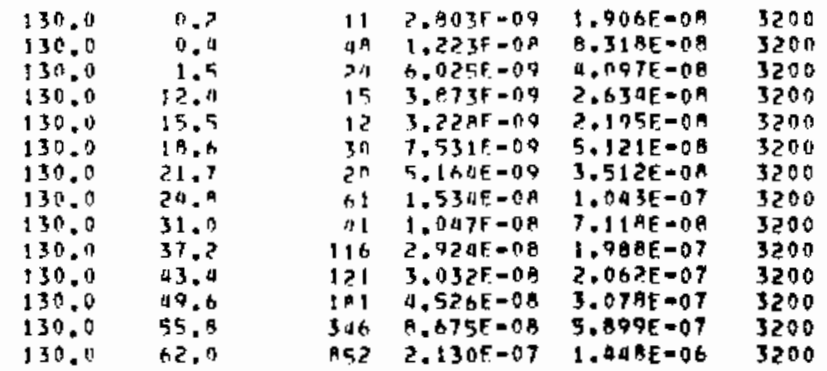

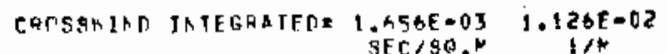




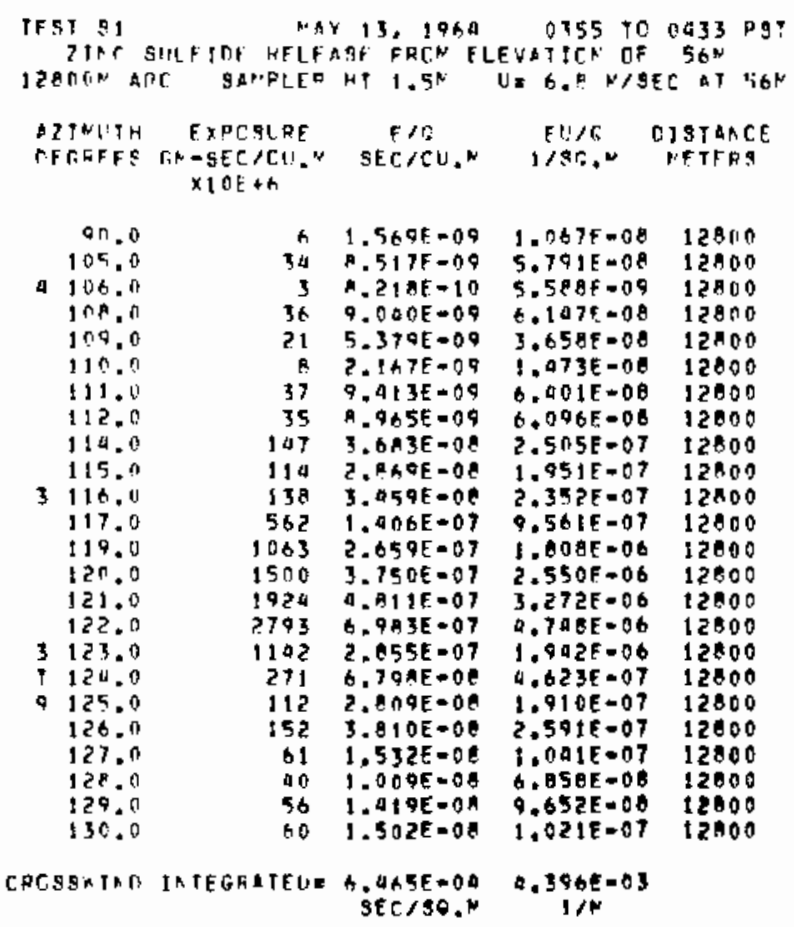


SERIES 154 EXPERIMENT: 2 (S-2)

GROUND LEYEL AND TOWER SAHPLING 200-3Z00 M ARCS. GROUND LEVEL

SAMPL I NG ONLY ON THE 12800 M ARC.

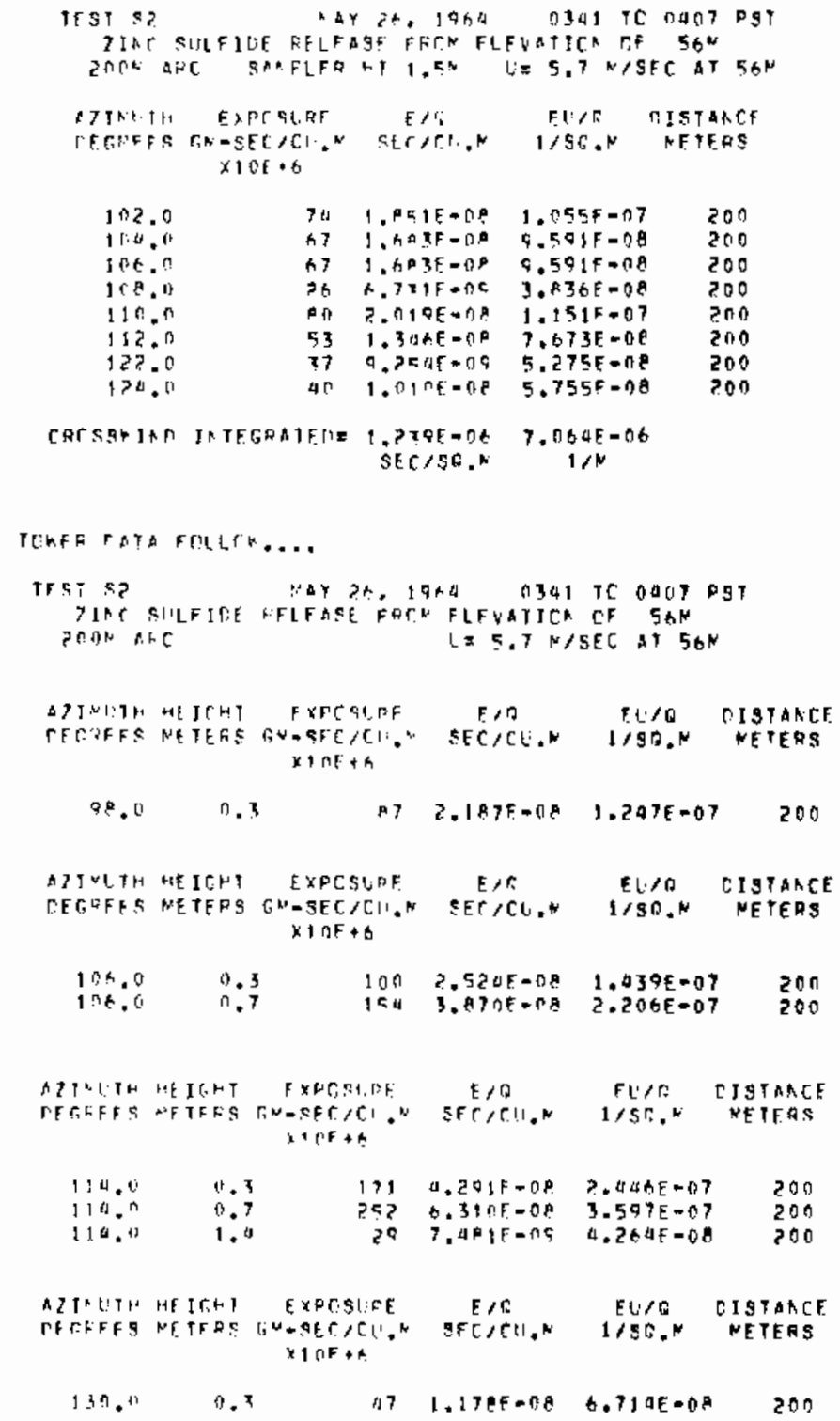

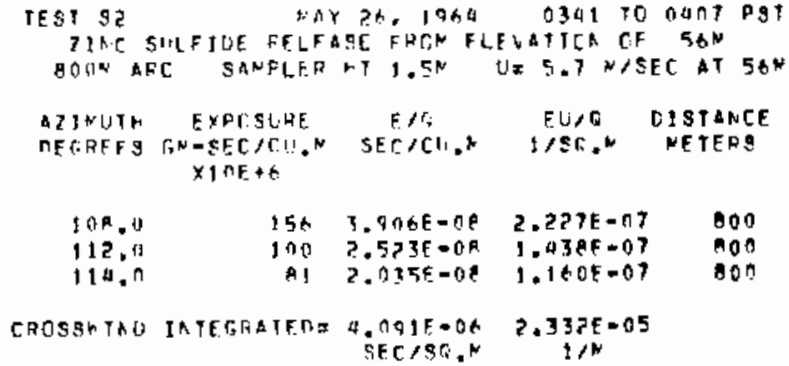




\begin{tabular}{|c|c|c|c|c|}
\hline $\begin{array}{l}\text { TEST SE } \\
\text { zIN' SU }\end{array}$ & ULFJEF FEIFY & $\begin{array}{l}26 \\
A S E \text { FRC }\end{array}$ & foust & $\begin{array}{l}0407 \text { P3T } \\
56 \%\end{array}$ \\
\hline $\begin{array}{l}\text { A7INAIT- } \\
\text { DEGAFES }\end{array}$ & $\begin{array}{c}\operatorname{EXPC9L4F} \\
r-S E C / C 11.2 \\
\times 10 E+6\end{array}$ & $\begin{array}{c}F / e \\
\text { SFr/CU, }\end{array}$ & $\begin{array}{c}F U / 6 \\
1 / 9600\end{array}$ & $\begin{array}{l}\text { DJBTANCEE } \\
\text { METFRS }\end{array}$ \\
\hline $\begin{array}{l}9 e, n \\
98.0\end{array}$ & $\begin{array}{l}117 \\
207\end{array}$ & $\begin{array}{l}2.7306-0 P \\
8.185 E-108\end{array}$ & $\begin{array}{l}1.6705-07 \\
2.955 \%-07\end{array}$ & $\begin{array}{l}1600 \\
1600\end{array}$ \\
\hline 100.0 & उ०र & $9.9 \times G E=08$ & 5.5 Q -077 & $\begin{array}{l}1600 \\
1600\end{array}$ \\
\hline 101.0 & $>7$ & R.7K2E-0G & $3.455 f-08$ & $\begin{array}{l}1600 \\
1600\end{array}$ \\
\hline 102.0 & पA? & I.2nAE $=07$ & R.A7 $\triangle E-07$ & 1600 \\
\hline 103.0 & $2 n 7$ & $7.4 \times 9 F-$ - & $4.790 F=07$ & 1600 \\
\hline 104.0 & 1 the & a. $170 E=00$ & $2.377 f-07$ & 1600 \\
\hline 105,0 & 270 & $n .7 \in 2 E=n E$ & $3.055 F-07$ & 1600 \\
\hline $10 t .4$ & 593 & $1 . a \in \cap E-B)$ & $A .319 E-07$ & $\$ 500$ \\
\hline 107.0 & 5月7 & $1.2+R 5=07$ & $7.227 E-07$ & 1600 \\
\hline 108.7 & 295 & $7.3 A 2 E-0 B$ & $4.200 E-07$ & 1600 \\
\hline $109 . n$ & 655 & $1 . A \triangle \cap E-07$ & $9.3478-07$ & 1600 \\
\hline 130.11 & $2 ? 7$ & $5.6925-09$ & $3.2005=07$ & 1600 \\
\hline 111.9 & 1731 & $1.32 \mathrm{zE}=07$ & $2.4 \times 7 F-06$ & 1600 \\
\hline 112.0 & 591 & $1.4505-07$ & $9.297 \mathrm{~F}-07$ & 1600 \\
\hline $113 . n$ & AI & $\therefore, n>Q E-O A$ & $1.15 F=07$ & 16on \\
\hline TAD & INTEGNATELI & 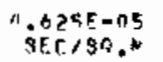 & $2.03 \operatorname{tg}=04$ & \\
\hline
\end{tabular}

THEF CATA FOLLCE....

\begin{tabular}{|c|c|c|c|c|c|}
\hline \multicolumn{2}{|c|}{$\begin{array}{l}\text { TEST S? } \\
\text { ZIR' SULEIDF } \\
\text { IARCN AFr. }\end{array}$} & $\begin{array}{l}\text { MAY } 26,1906 \\
\text { RFLFAE FRON }\end{array}$ & 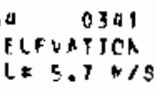 & $\begin{array}{l}\text { TC } 04079 \\
C F \quad 50 \mathrm{M} \\
\mathrm{FC} \quad 56 \mathrm{~N}\end{array}$ & PST \\
\hline $\begin{array}{l}\text { ATIVUTH } \\
\text { OEEFFES }\end{array}$ & $\begin{array}{l}\text { HE IGH } \\
\text { METERS }\end{array}$ & $\begin{array}{c}\text { EXECSURE } \\
\text { GNAFC COU.N } \\
\times 10 E+6\end{array}$ & $\begin{array}{c}E / G \\
\sec / C, N\end{array}$ & $\begin{array}{l}\text { Eu/O } \\
\text { isto, }\end{array}$ & $\begin{array}{l}\text { OISTANCE } \\
\text { NETERS }\end{array}$ \\
\hline 106.0 & $9 . ?$ & 376 & D. $171 E=0 E$ & $A .65 A E=07$ & \\
\hline 106.0 & 0.4 & 47 & & & \\
\hline 106,0 & 0.8 & aA? & $1.155 \mathrm{~F}$ & & \\
\hline $10 t$. & 1.5 & 300 & & $43 E=07$ & 1600 \\
\hline $10+.0$ & 3.1 & 602 & $1.5078-07$ & -07 & 1600 \\
\hline ict. & & 629 & $1.550 \mathrm{E}=$ & & \\
\hline 106. & 0.3 & 477 & $1,19 \cup E=$ & -07 & 1600 \\
\hline 106.0 & 12.0 & $\triangle Q A$ & $1.2 n 5 E-07$ & $7 E-07$ & 1600 \\
\hline $1^{n+.}$ & & $112 \pi$ & $2.021 E=07$ & $1.608 E=06$ & 1600 \\
\hline $10 \times$. & $1 \mathrm{~F}$ & tet. & $1.600 E=$ & $9.495 E=07$ & 1600 \\
\hline & & 2625 & $6.560 \mathrm{E}-07$ & $3.7 \mathrm{ALE}=06$ & 1600 \\
\hline $10 \%$ & & 2501 & $6.705 E-$ & $F=06$ & 1600 \\
\hline $1 n t .0$ & & OกAO & $2.27 \mathrm{CF}$ & $1.294 \mathrm{E}-05$ & \\
\hline 106.0 & 37 & 29102 & & $0.153 \mathrm{E}-05$ & \\
\hline & & 00204 & $1.007 E-05$ & $5.742 E-0 S$ & 1600 \\
\hline $\ln 6.0$ & & 13805 & $3.471 F-06$ & $1.979 E=05$ & 1600 \\
\hline 10 & & 11601 & 70 & & \\
\hline 106.8 & 62.0 & 5012 & $53 E-66$ & $93 E=0 \quad-0$ & 0 \\
\hline
\end{tabular}

AZTPUTH HETGHT FXPOSLIE E/G EG/O OTSTANCE

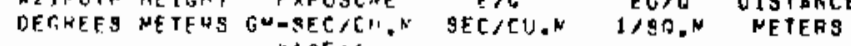

\begin{tabular}{|c|c|c|c|c|c|}
\hline 114.8 & 12.4 & 111 & $2.791 E-00$ & $1.591 E=07$ & 1600 \\
\hline 114.0 & 15.5 & $7 \in 9$ & $6.740 F=0$ & $3, B Q 2 E=07$ & 1500 \\
\hline 114.0 & $18, \mathrm{~h}$ & 703 & $1.950 E-07$ & $1.116 E-06$ & 1600 \\
\hline 114.0 & 21. & औ८०० & $1.150 F=10$ & $6.553 \mathrm{E}=0 \mathrm{~B}$ & 1600 \\
\hline 114.0 & 24.6 & $|1 n a\rangle$ & $2.767 \mathrm{~F}=06$ & $1.577 E=05$ & 1000 \\
\hline 110.0 & $31 . n$ & $4 ; *>3$ & ก०2E-05 & $1.164 E=04$ & 1600 \\
\hline 114.0 & 37.2 & 156510 & $3.853 \mathrm{~F}=05$ & $2.2300-04$ & 1600 \\
\hline 110.0 & 43.4 & $2>\sin \alpha$ & 5.64 CE.05 & $3.215 \mathrm{~F}-04$ & 1600 \\
\hline 114.0 & 49 & 96113 & $403 E=05$ & $1.3700-01$ & 1600 \\
\hline 112.0 & 55 & pount & $2.010 E=015$ & $1.146 E=04$ & 1800 \\
\hline 114. & & $7 A 070$ & $1.020 E=0 t$ & $a, n 01 E-05$ & 1600 \\
\hline
\end{tabular}

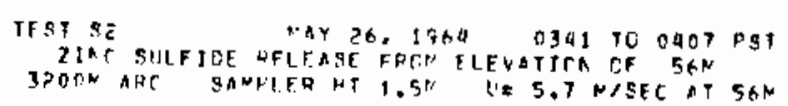

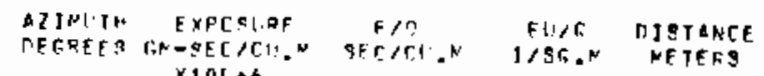
$X 10 L+h$

\begin{tabular}{|c|c|c|c|c|}
\hline 74.0 & 15 & $x, A>3 F=\pi 9$ & $2.179 E-08$ & \\
\hline 75,0 & 77 & $A P A D F=0 Q$ & $3.922 f-08$ & 3200 \\
\hline 70.0 & 11 & $?=8 \cap M F=0 Q$ & $1.59 E E-00$ & 3200 \\
\hline 77.9 & 32 & $5,+07 E-00$ & $3.196 \mathrm{~F}-08$ & 3200 \\
\hline 79.0 & 33 & $A$ AIOE-CS & $0.790 F-08$ & 3200 \\
\hline 79.11 & 31 & $7.500 E=00$ & $4.503 E-08$ & 3200 \\
\hline 0.0 & $? \AA$ & $A .4>65=00$ & $3.777=08$ & 3200 \\
\hline$P 1,0$ & 30 & $7 .+166=09$ & $4.35 t \neq=08$ & 3200 \\
\hline म2.0 & 1 & $7.9 n \cap E=08$ & $4.503 F=08$ & 3200 \\
\hline .3 .0 & 54 & $1.351 \mathrm{E}-08$ & $7,594 \mathrm{~F}=0 \mathrm{CO}$ & 3200 \\
\hline $\begin{array}{l}\text { Ha. } \\
\text { A5: }\end{array}$ & 51 & $1.3007-n e$ & 7. $009 E-00$ & 3200 \\
\hline 16:0 & $\begin{array}{l}314 \\
73\end{array}$ & $A .5650-018$ & $0.939 E-08$ & 3200 \\
\hline$\pi 7.0$ & $\begin{array}{l}73 \\
12\end{array}$ & $5 . p+2 f=n q$ & $3.3 a 1=-08$ & 3200 \\
\hline $80^{\circ}$ & $\begin{array}{l}12 \\
47\end{array}$ & P.155F-OA & $4.649 E=08$ & 3200 \\
\hline 89.0 & 1 यो & $1.19 \mathrm{PE}=0 \mathrm{O}$ & t.A2PE $=0 \mathrm{~A}$ & 3200 \\
\hline $9 n .0$ & 16 & 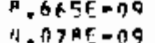 & $4,9 \geq 9 E-08$ & 3200 \\
\hline 91,0 & 11 & 7.90 ก $=09$ & $\begin{array}{l}7.324 F=08 \\
4.513=08\end{array}$ & $\begin{array}{l}3200 \\
3200\end{array}$ \\
\hline $4 \bar{\varepsilon} \cdot \pi$ & I & 4. SPTE-nG & Z.ot5E-AB & 3200 \\
\hline 93,51 & 17 & $4.3335=016$ & $3.470 E-08$ & 3200 \\
\hline 9.0 .0 & $7 n$ & $1.931 E-08$ & $1.0008=07$ & 3200 \\
\hline 95.0 & $5 \mathrm{~A}$ & $1.353 E=0 ?$ & $A, P A O F=08$ & 3200 \\
\hline $9 n .0$ & 35 & $P .970 E-04$ & 5.0PAF -08 & 3200 \\
\hline 97.0 & 20 & H. KरTE = DQ & $3.7775-08$ & 3200 \\
\hline 98.0 & 49 & $1.208 E-00$ & $7.118 E-08$ & 3200 \\
\hline 09.0 & 35 & $? .39 A E-C A$ & $1,366 \mathrm{E}-07$ & 3200 \\
\hline 100.6 & 2001 & $5 . n 2 ! F=0 P$ & $2.962 F-07$ & 3200 \\
\hline 101,0 & 4.5 & $1.014 \mathrm{E}=07$ & $5.792 E-17$ & 3200 \\
\hline $102 \cdot 0$ & $5 \times 0$ & $1 . A \cap 2 E-07$ & $7.950 E-07$ & $3 \geqslant 00$ \\
\hline 103.0 & $7 n b$ & $1,7 \mathrm{hAF}=0.7$ & $1.007 E=06$ & 3200 \\
\hline 1) 0.0 & 1220 & $3.051 \varepsilon-07$ & $1.739 \mathrm{~F}=0 \mathrm{~b}$ & $320 n$ \\
\hline $10 \times .0$ & 1512 & $x .7=25-07$ & $7.15 t E=06$ & 3200 \\
\hline 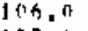 & 75 & $1 . \$ P G E \cap \cap P$ & $1.075 F-07$ & 3200 \\
\hline 107.18 & $34 / 48$ & $n \cdot 6>0 E-\{7$ & $4.9165=06$ & $3 \geq 00$ \\
\hline 108.0 & 4973 & 1 . त्वन $=0 t$ & $7.115 E-06$ & 3200 \\
\hline 109.0 & 5201 & $1.3 \geq 3 E-76$ & $7.541 E-06$ & 3200 \\
\hline 110.0 & 259 & $\angle, Q P C E=C P$ & $3.4 \mathrm{de}-07$ & 3200 \\
\hline$\$ 11.0$ & 170 & \#वसF $=n \varepsilon$ & $2.5575-07$ & 3200 \\
\hline 112.0 & 254 & $t .3715-70$ & $3.6325-177$ & 3200 \\
\hline 113.0 & 5347 & $1.94 P F=7 t$ & $7.6 A \rho F=0 B$ & $320 n$ \\
\hline 114.0 & 16301 & $4.075 E-07$ & $2.3235-0 b$ & 3200 \\
\hline 115.0 & $0 \vec{?}$ & $7.3905-18$ & $1.3225=17$ & $320 \%$ \\
\hline
\end{tabular}

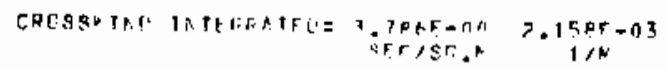

THEER CATA FDILCM....

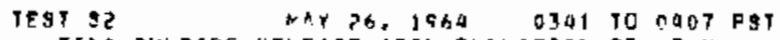

ZTAC SUT.FIDF HELFASF FREN FLEVATITR. OF $56 \mathrm{~N}$

$3200 \mathrm{~N}$ ake 1.25 .7 NISFC AT $5 \mathrm{GN}$

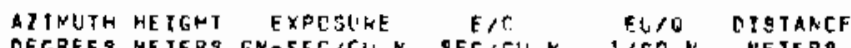
DEGREES METERS GN-SEC/CH.N SFCICH.N WISR,N NETERS $\times 1 \cap E+t$

\begin{tabular}{|c|c|c|c|c|c|}
\hline 98.0 & $0 . ?$ & ; $\beta$ & $4.5 \% 7 F=09$ & $2.615 E=00$ & 3200 \\
\hline 90.0 & 0.0 & 3) & $9.4305=09$ & $9.375 E=00$ & 5200 \\
\hline 9 9. 0 & 0.9 & ? म & $0,13+E=09$ & $9.067 E=00$ & 3200 \\
\hline SP. 0 & 1.5 & 4 & $1.594 F-08$ & $9.112 E-08$ & 3200 \\
\hline$Q R, 0$ & 3,1 & 51 & $1,2 \varsigma \geqslant E=C Q$ & $7.395 E=00$ & 3200 \\
\hline QP, $Q$ & a.? & a) & $1,1 \times 2 E-08$ & $6.735 \mathrm{E}=00$ & 3200 \\
\hline & 9,3 & 72 & $1.8 \cap 7 \varepsilon=C 8$ & $1.050 E-0 ?$ & 3200 \\
\hline 98.0 & 12.9 & 20 & $0.024 E=09$ & $3.034 E=08$ & 3200 \\
\hline 98 . & 15.5 & 47 & $1,1 \geq 2 E-0 B$ & $6.735 E-00$ & 3200 \\
\hline
\end{tabular}




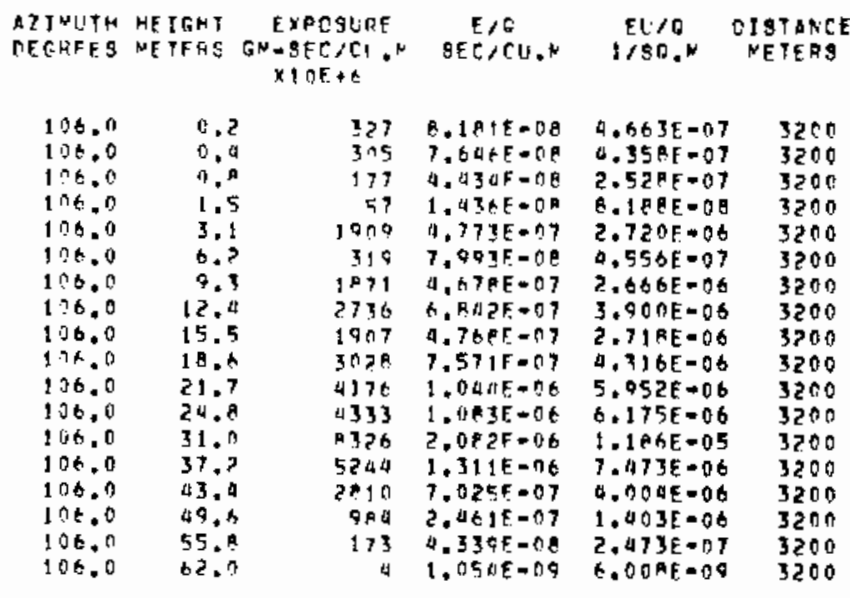

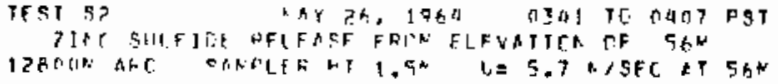

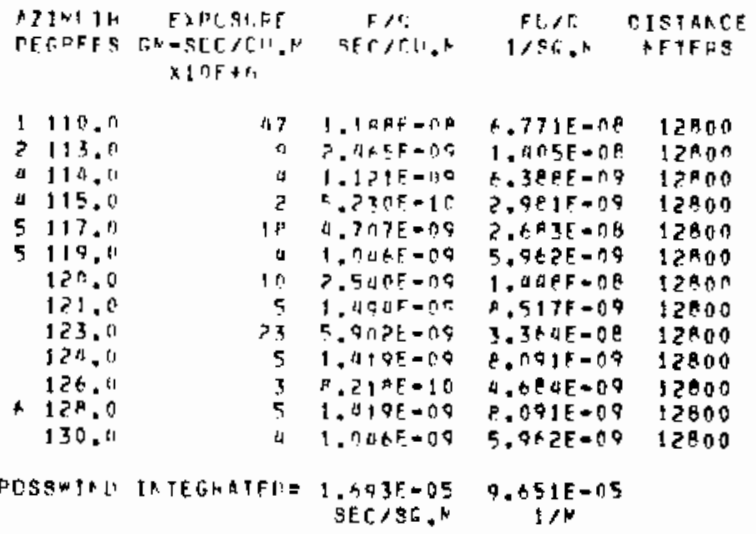

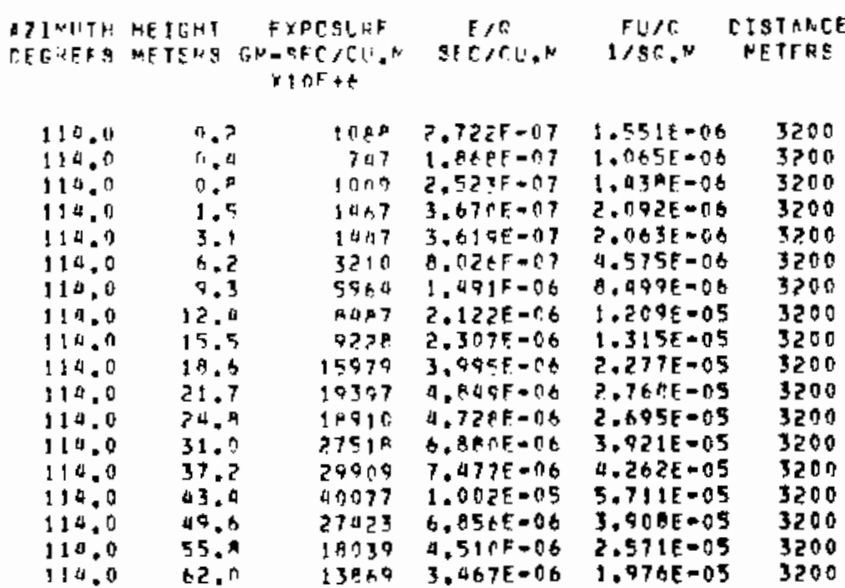

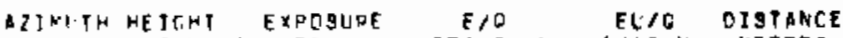
DETRFES METERS GW-SEC/CL.N BEC,CU.N 1/SO,M HETERS $\times 1$ of +6

$\begin{array}{llllll}127.0 & 0.2 & 27 & 0.891 E-09 & 3.922 E-08 & 3200 \\ 122.0 & 0.0 & 13 & 3.313 F=09 & 1.808 E=09 & 3200 \\ 122.0 & 0.2 & 30 & 7.6468 .09 & 4.358 E+08 & 3200\end{array}$




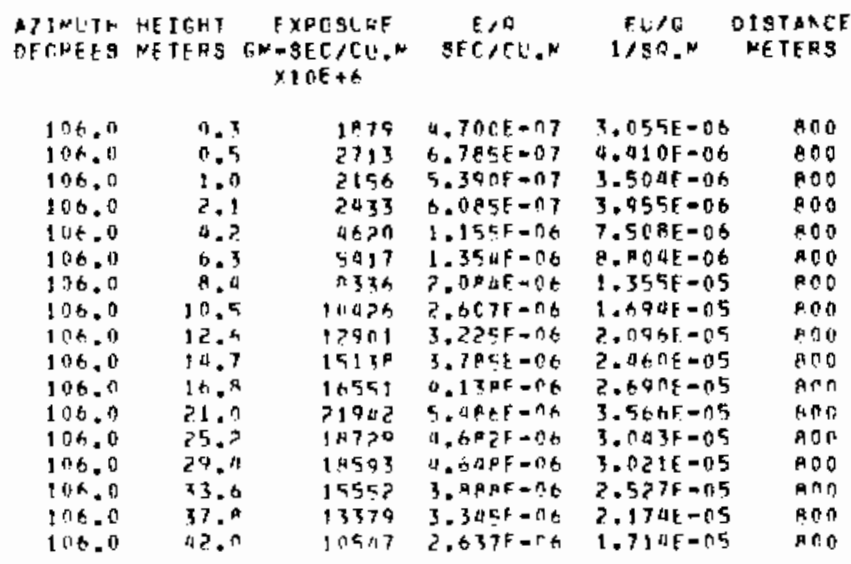

A.INITH HEIGHT FXPCGLEF E/C FU/G DISTARCF

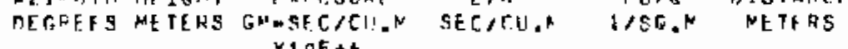

\begin{tabular}{|c|c|c|c|c|c|}
\hline $11 \%, 0$ & 0.3 & $x 7$ & G. $a \cap \cap F=\cap B$ & $6.11 D E=O B$ & $8 \cap 0$ \\
\hline 114.0 & 0.5 & <a & $1.36>7=00$ & B. AQ7E=OA & म00 \\
\hline 1$\} 4,0$ & 1.? & $A_{1} \mathrm{~B}$ & $1.7 \mathrm{tat}=0 \mathrm{~g}$ & $1.10 \mathrm{AE}-\mathrm{CC} 7$ & $\ln 0$ \\
\hline 114.0 & 7.1 & 138 & $3.471 F-\cap R$ & $2.2565=-17$ & sin \\
\hline 112.0 & 4.7 & 158 & $3.913 \mathrm{~F}-78$ & $2.544 \mathrm{~F}-107$ & $B C C$ \\
\hline $1 \pm 0.0$ & 6.3 & 179 & $1.69 e F-07$ & $1.100 E-06$ & $A D O$ \\
\hline 110.0 & A. 0 & +16 & $1.540 F=07$ & $1.001 \varepsilon=06$ & ADO \\
\hline 110.0 & 10.5 & 1560 & $3.9095=07$ & $535 E-06$ & $R$ QD \\
\hline 114.0 & 32.6 & 3007 & $E=017$ & $E=08$ & 808 \\
\hline 114.0 & 14.7 & $40+?$ & $1.216 t=0.6$ & $7.902 E-06$ & $A 00$ \\
\hline $1+4.0$ & 16.8 & 6981 & $1.75 n \mathrm{~F}-0 \mathrm{E}$ & $1.137 \mathrm{E}-05$ & $B O O$ \\
\hline 110.0 & 31.0 & 11608 & $902 F=06$ & 1. MAE $=05$ & 900 \\
\hline 134.0 & 25.2 & 15204 & $3.011 F-06$ & $2.477 E=05$ & $B 00$ \\
\hline 134.0 & 20.4 & 24902 & $2267-106$ & $4.607 \mathrm{E}-05$ & 800 \\
\hline $11+0$ & $33 . t$ & 43070 & $17 E-05$ & $999 E-05$ & 000 \\
\hline 114.0 & 37.8 & 50540 & $264 E-05$ & $8.213 E-05$ & 000 \\
\hline & & 77 & $20 F=0.5$ & $205 E=04$ & 80 \\
\hline
\end{tabular}

ATINIJH HEIFHT EXPCGURE E/O EU/O OISTANCE

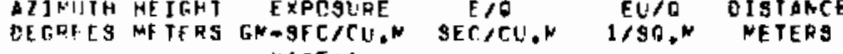
$\times 1$ of $\$ 6$

\begin{tabular}{|c|c|c|c|c|c|}
\hline $\begin{array}{l}122.0 \\
122.0 \\
122.0 \\
122.0 \\
122.0 \\
122.0 \\
122.0 \\
122.0 \\
122.0 \\
122.0\end{array}$ & $\begin{array}{l}10.5 \\
12.8 \\
14.7 \\
16.8 \\
21.0 \\
25.2 \\
29.4 \\
13.6 \\
37.8 \\
42.0\end{array}$ & $\begin{array}{r}111 \\
323 \\
300 \\
850 \\
1510 \\
3159 \\
7552 \\
12555 \\
20056 \\
48767\end{array}$ & $\begin{array}{l}2.771 E-08 \\
8.079 E=0 E \\
7.511 E=0 E \\
2.127 E=07 \\
3.800 E=07 \\
7.099 F-07 \\
1.98 F F=06 \\
3.139 E=06 \\
5.019 E=106 \\
1.219 E=05\end{array}$ & $\begin{array}{l}1.005 E=07 \\
5.751 E=07 \\
9.892 E=07 \\
1.383 E=06 \\
2.470 E=06 \\
5.134 E=06 \\
1.292 E=05 \\
2.040 E=05 \\
3.259 E=05 \\
7.925 E=05\end{array}$ & $\begin{array}{l}900 \\
800 \\
800 \\
800 \\
800 \\
800 \\
000 \\
000 \\
900 \\
000\end{array}$ \\
\hline
\end{tabular}

\footnotetext{
IIMNITM HEIGHT EXPCSLAF E/O FU/R DISTANCE DFGRES NETEFS GN-SECACI.N SEC/CU.N HISO.N NETERS $x+06+5$

$\begin{array}{llrrrr}130.0 & 37.9 & 10 & 7.6595 .09 & 9.9795-08 & 000 \\ 130.0 & 02.0 & 229 & 5.745 E-08 & 3.734 E=07 & 900\end{array}$
}

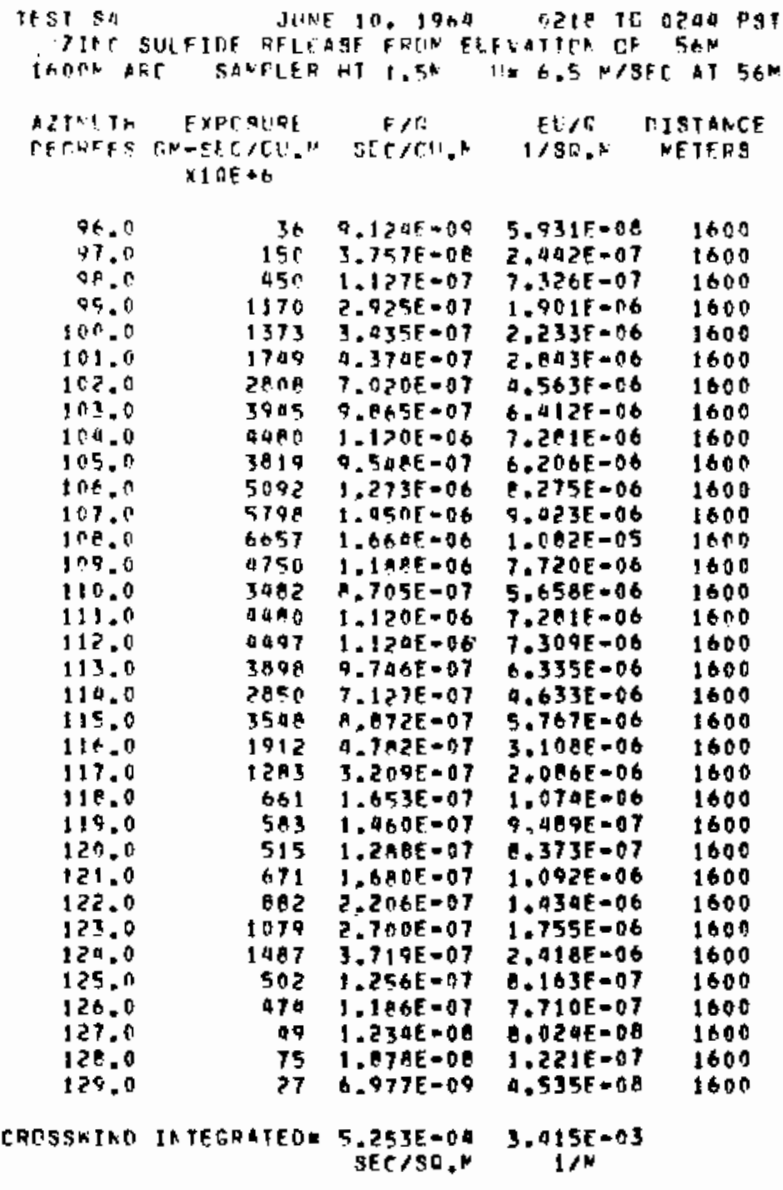

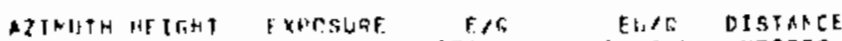

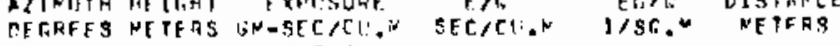
$\times 1$ OF, +4

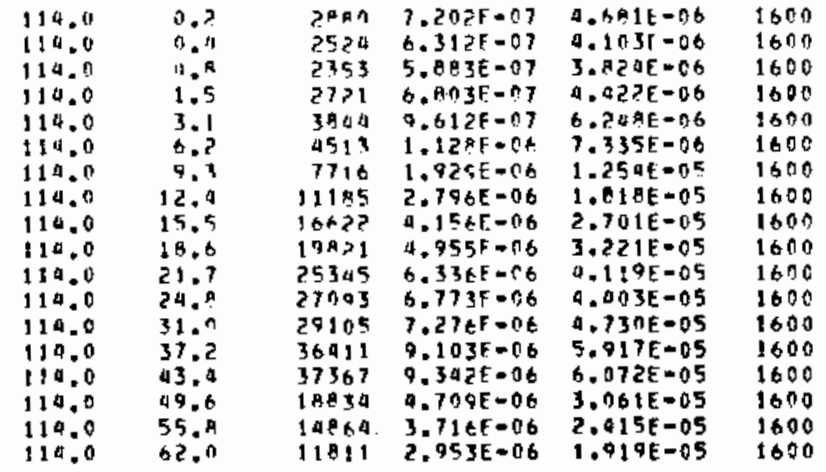


TORFP TATA FOL LCR...

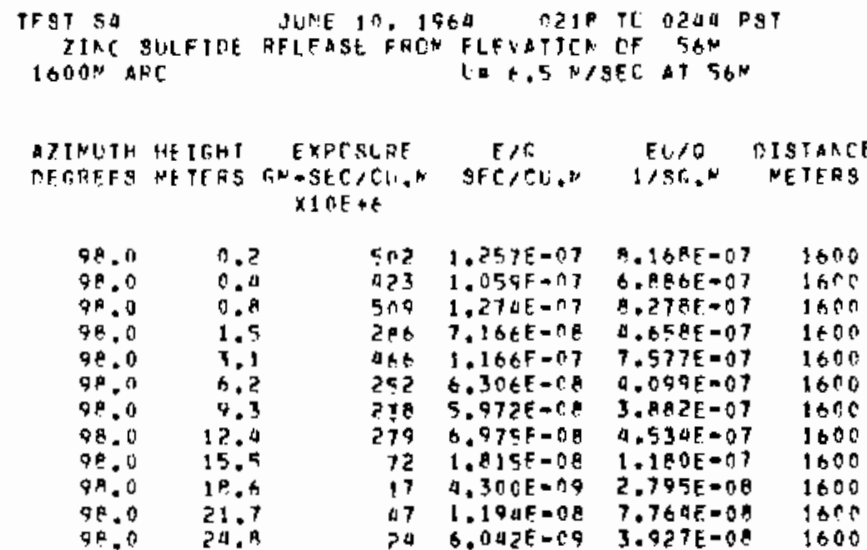

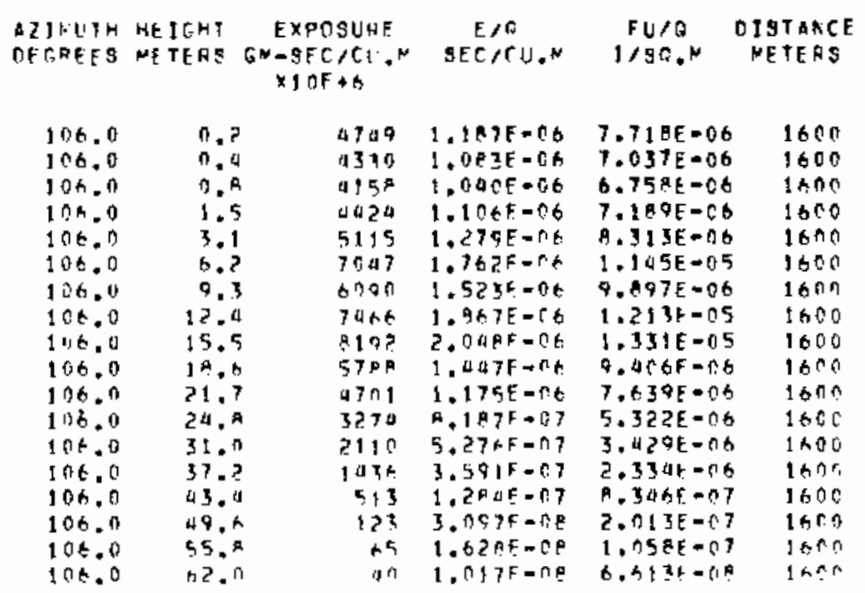

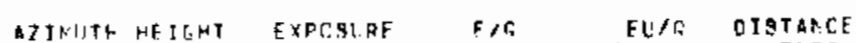

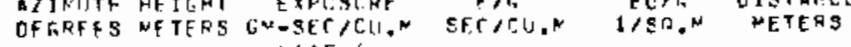
$\times 10 E+6$

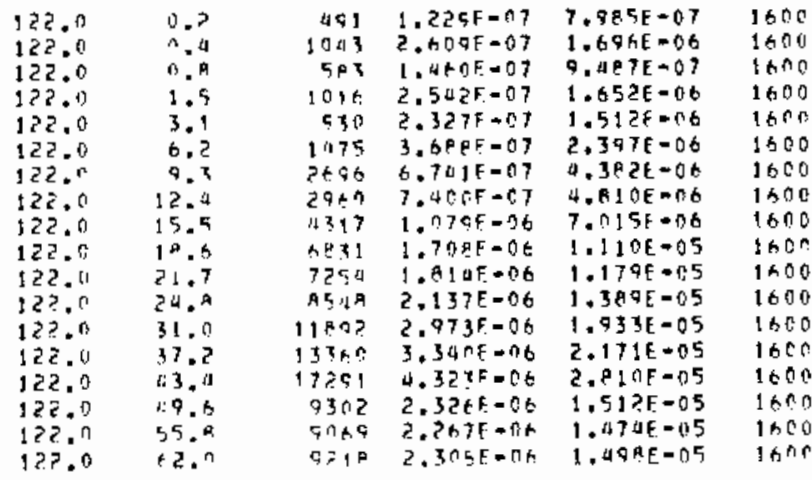

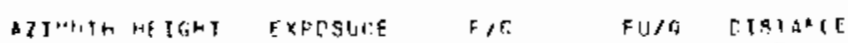

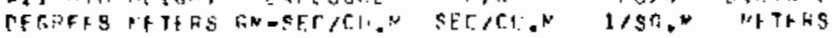
$\times 106 \rightarrow 6$

\begin{tabular}{|c|c|c|c|c|c|}
\hline $\begin{array}{l}130.0 \\
130.0 \\
130.0 \\
130.0 \\
130.0\end{array}$ & $\begin{array}{l}24.9 \\
37.2 \\
113.4 \\
49.6 \\
55.8 \\
62.0\end{array}$ & $\begin{array}{r}43 \\
5 ? \\
504 \\
755 \\
696 \\
1128\end{array}$ & 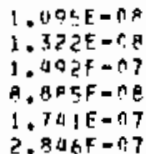 & $\begin{array}{l}7.11 A E=0 A \\
6.591 E=08 \\
9.646 E=07 \\
5.715 E=07 \\
1.131 E=06 \\
1.850 E=06\end{array}$ & $\begin{array}{l}1600 \\
1600 \\
1600 \\
1600 \\
1600 \\
1600\end{array}$ \\
\hline
\end{tabular}

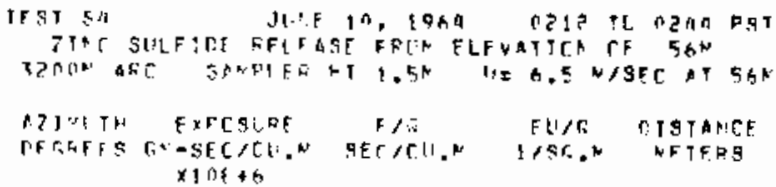

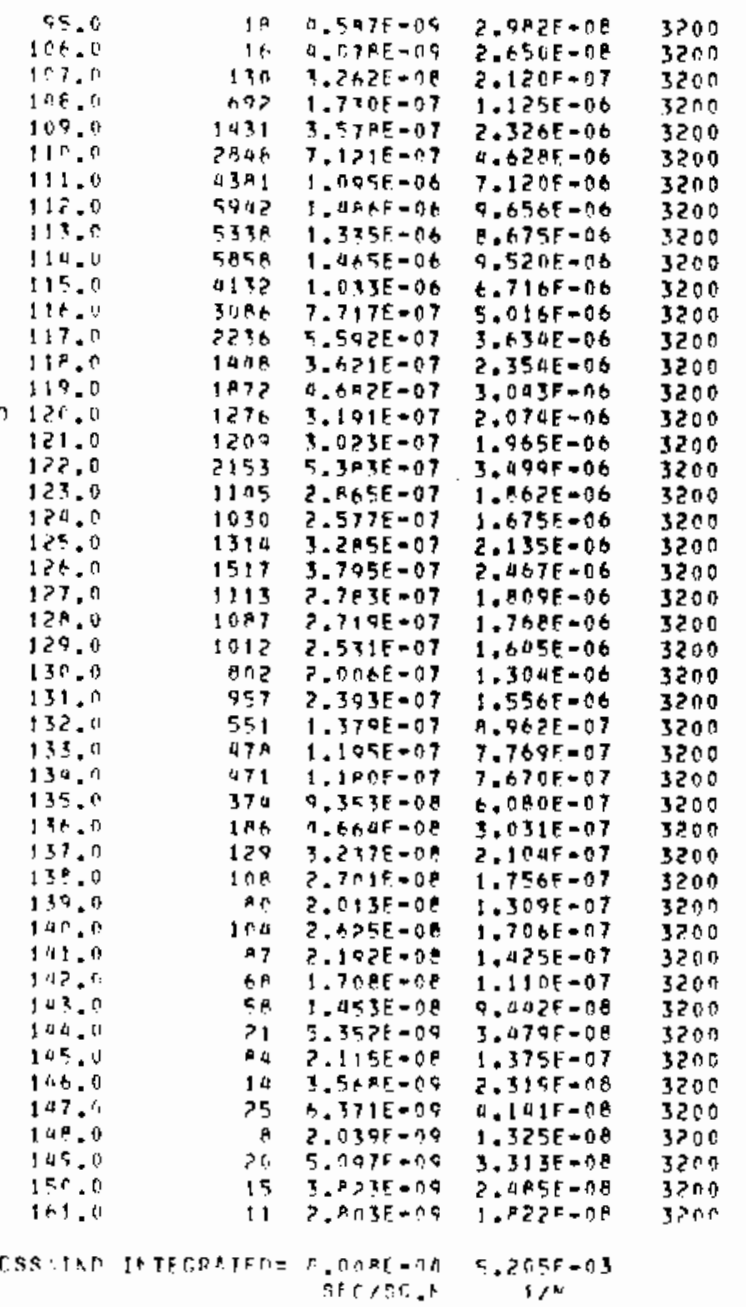




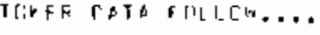

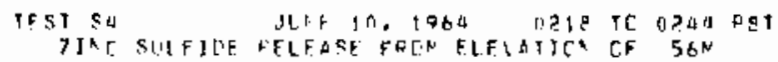

$326 \mathrm{NAFC} L=6.5 \mathrm{~N} / \mathrm{SFC} \triangle 56 \mathrm{~N}$

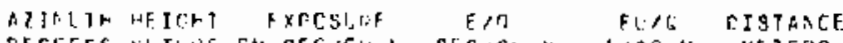

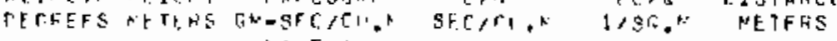
$\times 111 F+B$

\begin{tabular}{|c|c|c|c|c|c|}
\hline $1 \cap t . n$ & 8.7 & 53 & $1.336=0$ ด & $A .677 E-0 B$ & 3200 \\
\hline 300.0 & 12.0 & 74 & $1.4695=n B$ & $1.215 f-07$ & 3200 \\
\hline 106.0 & 15.5 & 67 & $1.505 F=\Gamma p$ & E2E-0B & 3200 \\
\hline $\operatorname{tros} 0$ & $1 A .6$ & a il & $1.355 \mathrm{~F}=08$ & $E \rightarrow O B$ & 3200 \\
\hline $\begin{array}{l}11:=0 \\
16 t=0 \\
10 t\end{array}$ & $\begin{array}{l}21.7 \\
24.9\end{array}$ & pa & $\begin{array}{l}5.097 \mathrm{E}-10 \\
2.399 F-09\end{array}$ & $\begin{array}{l}3.313 E=08 \\
1.553 E=08\end{array}$ & $\begin{array}{l}3200 \\
3200\end{array}$ \\
\hline & $31 . n$ & D & 2.1018 .09 & $1.41 \mathrm{AE}-08$ & 3200 \\
\hline
\end{tabular}

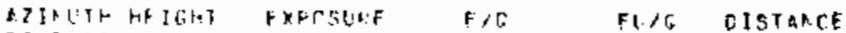

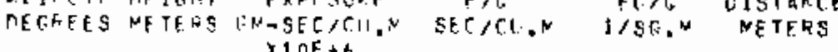
$\times 1 \cap F+t$

\begin{tabular}{|c|c|c|c|c|c|}
\hline $\begin{array}{ll}11 & 14.0 \\
1 & 14.0\end{array}$ & $\begin{array}{l}0 . ? \\
0.1\end{array}$ & $\begin{array}{l}0903 \\
0965\end{array}$ & $\begin{array}{l}1.240 E=1: 6 \\
1.2355=0.6\end{array}$ & $\begin{array}{l}8.1: 4 E=06 \\
P .05 J E=06\end{array}$ & $\begin{array}{l}3200 \\
3200\end{array}$ \\
\hline 115.0 & $0 . \mathrm{F}$ & ARก & $1.2025=06$ & 7.81 4t -06 & 3200 \\
\hline 114.0 & 1.5 & $53+5$ & $1.301 \mathrm{~F}=\mathrm{rt}$ & A. $715 E=0 B$ & (2)? \\
\hline 113.6 & 3.1 & $5 t ; 5$ & $1.25,45=016$ & $2.150 f=0 b$ & 3200 \\
\hline 114.0 & 0.7 & 7230 & $1 . P O Q F=06$ & $1.175 E=05$ & $37 n$ \\
\hline 114.0 & $9, x$ & 7433 & $1.85 \rho F=16$ & $A E=05$ & 320 \\
\hline 114.0 & 12.11 & 9 it 92 & $37 \pm f=16$ & $9.5+2 E-05$ & 3 \\
\hline 114.0 & 15.5 & 10156 & $534 F=06$ & $1.65 n E-05$ & $5 ?$ \\
\hline 114,0 & $15 \cdot t$ & $4+35$ & $2,155 F=76$ & $1.003 E=05$ & 32 \\
\hline $134=0$ & 71.7 & $07 \times 1$ & HASt: $=5.6$ & $1.614 E=05$ & \\
\hline 114.0 & $?$ & 9636 & $2.354 F=06$ & $1.533 \neq-15$ & \\
\hline 114.7 & 31.0 & $6 P+7$ & $1.711 t=0.6$ & $1.112 f=r 5$ & 3200 \\
\hline 114.0 & ? & $6 ? 115$ & $1.73 \mathrm{te}=\mathrm{nt}$ & $1+129 \mathrm{E}=05$ & 924 \\
\hline 114.0 & 43.4 & 4797 & $1.195[-0.6$ & $7.79 \mathrm{BE}=0 \mathrm{~B}$ & 35 \\
\hline 115.0 & $45^{6}$ & $247 ?$ & $t, 057 E-C)$ & $3.937 \mathrm{E}=06$ & 6 \\
\hline 110 & 55.0 & 1022 & $\begin{array}{l}? .557 F=07 \\
? 050 F=0 ?\end{array}$ & $=6678-06$ & 32 \\
\hline+2 & $E<\cdot 1$ & $0>3$ & $2,0505=0.7$ & $1.33 \mathrm{AF}=0 \mathrm{~B}$ & $32 n$ \\
\hline
\end{tabular}

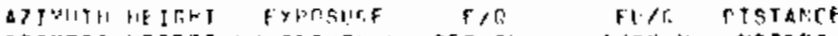

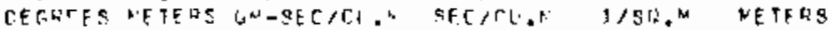
XI $\cap F+h$

\begin{tabular}{|c|c|c|c|c|c|}
\hline 123.0 & 0.2 & $? 1 \geq$ & $5+3 \cap 1 f=n P$ & 3. IUAE $=0 ?$ & 3201 \\
\hline 122,0 & 0.4 & $13 \pm$ & $3.3395=0 \mathrm{k}$ & $7.1705=07$ & 3200 \\
\hline $12 \%$ & $n .8$ & $1 \mathrm{tah}$ & $3 . \Delta 7 c F=r \varepsilon$ & $2.3955-07$ & 3200 \\
\hline 127,0 & 1.5 & +9 & $1.74 A F=08$ & $1.13+E-0 ?$ & 3200 \\
\hline 122.4 & 3.1 & 1795 & 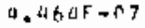 & $2.904 E-C G$ & $32 n 0$ \\
\hline 122.1 & 6. & 1901 & $4.9790-0.7$ & $236 t=06$ & 3200 \\
\hline 12 & 9.7 & 2537 & $6.31: 5 \mathrm{~F}-0.7$ & $4.12+1 E=0 A$ & 3700 \\
\hline $1 ? ?=0$ & 17.9 & 3751 & $9.379 E=07$ & $\operatorname{ton} 96^{5}=0 h$ & 3200 \\
\hline & 15 & 4407 & $1.112 F=00$ & $7 E=6 h$ & $320 \mathrm{r}$ \\
\hline 122.0 & $1 B \cdot A$ & 5916 & $1.975 F-1.6$ & $9.5\}$ a $=06$ & 3270 \\
\hline 12 & 21.7 & a $1+7$ & $2.113+E=06$ & $1.319 E-05$ & 320 t \\
\hline 12 & $2^{\prime \prime} \cdot 8$ & 7432 & $1.85 \mathrm{EF}=16$ & $1.20 R E=05$ & 3200 \\
\hline 12 & 51.13 & Het? & $z, n A+F=\hat{n}$ & $1.3 \Delta 3 E-05$ & 3200 \\
\hline 12 & 37.2 & $70>7$ & 9 ค. -0.6 & $1.28 B E=05$ & $0 n$ \\
\hline & 43.0 & 15109 & $2.547 t-106$ & $1.656 E=0.5$ & 3200 \\
\hline & $49 . \mathrm{A}$ & 4496 & $1.124 F=06$ & $7.307 E-06$ & 3200 \\
\hline & $5 \% .8$ & $69>5$ & $1.7065-06$ & $\$ .109 E-05$ & 3200 \\
\hline & 62.1 & $90 \times 3$ & $46 \in E-C h$ & $1.003 E=05$ & 3200 \\
\hline
\end{tabular}

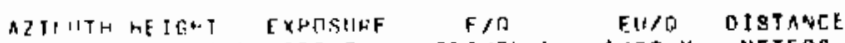

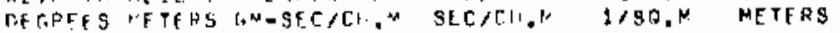
$\times 10 F+6$

\begin{tabular}{|c|c|c|c|c|c|}
\hline $13 n .0$ & ti. ? & $35 \mathrm{~A}$ & $1.896 F=07$ & $1.232 F=06$ & 3200 \\
\hline 130.0 & 0.11 & की तो & 2.25 B $=07$ & $1.463 E-76$ & 3200 \\
\hline 130.0 & $n, R$ & $52^{p}$ & $1.320 E-17$ & $A, S A 1 E=07$ & 3200 \\
\hline 130.0 & 1.5 & 1233 & $3.0855=07$ & $2.005 E-0 \%$ & 3200 \\
\hline 139.6 & 3.1 & 917 & $2,20 t r=07$ & $1.491 E-06$ & $320 n$ \\
\hline 130.0 & B.? & ??? & $1.806 f-07$ & $1.1700-06$ & 3200 \\
\hline $13^{n} \cdot 0$ &. & $A>0$ & $2.0735-07$ & $1.347 t=0 A$ & 3700 \\
\hline .0 & 12.4 & 1079 & $2,694 F-07$ & $1.754 E=06$ & 3200 \\
\hline . & 15.5 & 1100 & $3.000 \mathrm{~L}=07$ & $1.950 E-06$ & 3200 \\
\hline $13^{n} \cdot n$ & in.t. & 1709 & $4.4 A Q F=07$ & $2.917 E=06$ & 3700 \\
\hline 130.0 & 21.7 & $7+4$ & $2.410 F=07$ & $1.567 \mathrm{~F}=1.6$ & 3200 \\
\hline $13 n .0$ & 24.9 & 12 id & $3.117 F=1) 7$ & $0265=06$ & 3200 \\
\hline 130,0 & 11.7 & 1759 & $4.307 E=07$ & - ASAF $=0$. & 3200 \\
\hline $13 n .0$ & $37 . ?$ & $27: 1$ & $6.8535=07$ & $4.254 \mathrm{~F}=06$ & 3200 \\
\hline 1 & 43,4 & 2010 & $5.023 F=0.7$ & $3.265 t=0 n$ & $3, \beta^{n} 6$ \\
\hline & & 2156 & $0.13 \& F=17$ & $3.9 \times 9 E-106$ & 3200 \\
\hline 0 & $55 . A$ & 1 A $>5$ & $1, \sin t=0=07$ & $2.8 \times 7 E=0.6$ & 3700 \\
\hline & $4 ?$ & $26 \mathrm{P5}$ & $6.715 E=07$ & $36: 4 F=1: 6$ & $32 n 0$ \\
\hline
\end{tabular}

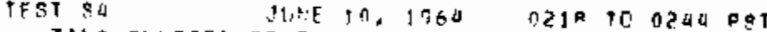

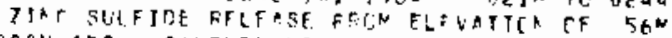
12ARA AFC SAPLER HT I. SN UN $0.52 / 5 E C$ A $56 \mathrm{M}$

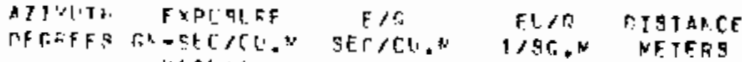
xiot $+\mathrm{H}$

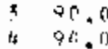

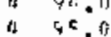

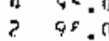

a 89.0

$+106.1$

$\rightarrow 101.0$

5105.0

$+\ln \pi 0$

a $1 \cap R, 0$

31 iी. त

5110.0

5112,0

a 113.0

a 114.0

i) $115.0^{\circ}$

4115,0

$4 \$ 17.11$

4119.0

a $11 \%, 0$

$4+2 ? 0.0$

9121.0

5122,11

4 1? .6

4124.6

7125.0

+ $1 \geq 8.0$

127.6

$712 \mathrm{a}, 0$

3129,0

130.0

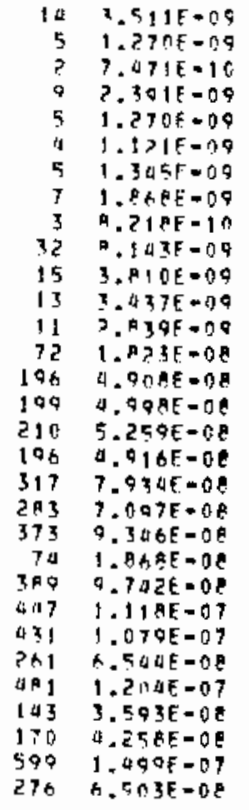

2. $2 A 7 E=08$

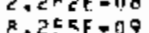

$8,255 F=09$
$a-A 56 E=10$

$1.5545=0812900$

F. $255 E+09$

$12 B 00$

7.28aE-09 12800

$P .701 F=0912$ 1200

$1.214 E-00 \quad 12800$

$5.342 \mathrm{~F}=09 \quad 12900$

$5,2936-0812800$

$2.477 E=0 B \quad 12800$

$\begin{array}{ll}2.477 E=08 & 12800 \\ 2.234 E=08 & 12800\end{array}$

$1.845 E=08 \quad 12900$

$1.1255-07$ I 2 AO

$3.1905=07 \quad 12800$

$3005-07$

$\begin{array}{ll}3.2498-07 & 12000 \\ 3.4195-07 & 12000\end{array}$

$3.1958=07 \quad 12800$

$5.1576-07 \quad 12900$

$4.6135-07 \quad 12000$

. $075 \mathrm{E}=07 \mathrm{~T}$

12800

$1.314 \mathrm{E}-07 \quad 12000$

$6.332 E-07$

$1.270 E-07$ 12600

$7.012 \mathrm{~F}=07 \quad 12800$

0.25 Uk 01 12800

$7.0=5-07 \quad 12800$

. तर

$2.336 \mathrm{~F}=07 \quad 12800$

$2.768 \mathrm{eE}=07 \quad 12000$

c. $706 E-07 \quad 12000$

CRCSBATA IATEGRATFIE $2.909 E=091.950 E-03$

SETSOA $1 / \mathrm{h}$ 
SERIES 164 EXPERIMENT: 5 ( $5-5$ )

GROUNO LEVEL ANO TOWER SAMPLING ON THE 1600 AND 3200 M ARES.

GROUNDLEYEL SAMPL ING ONLY ON THE 7000 AND 12800 M ARCS.

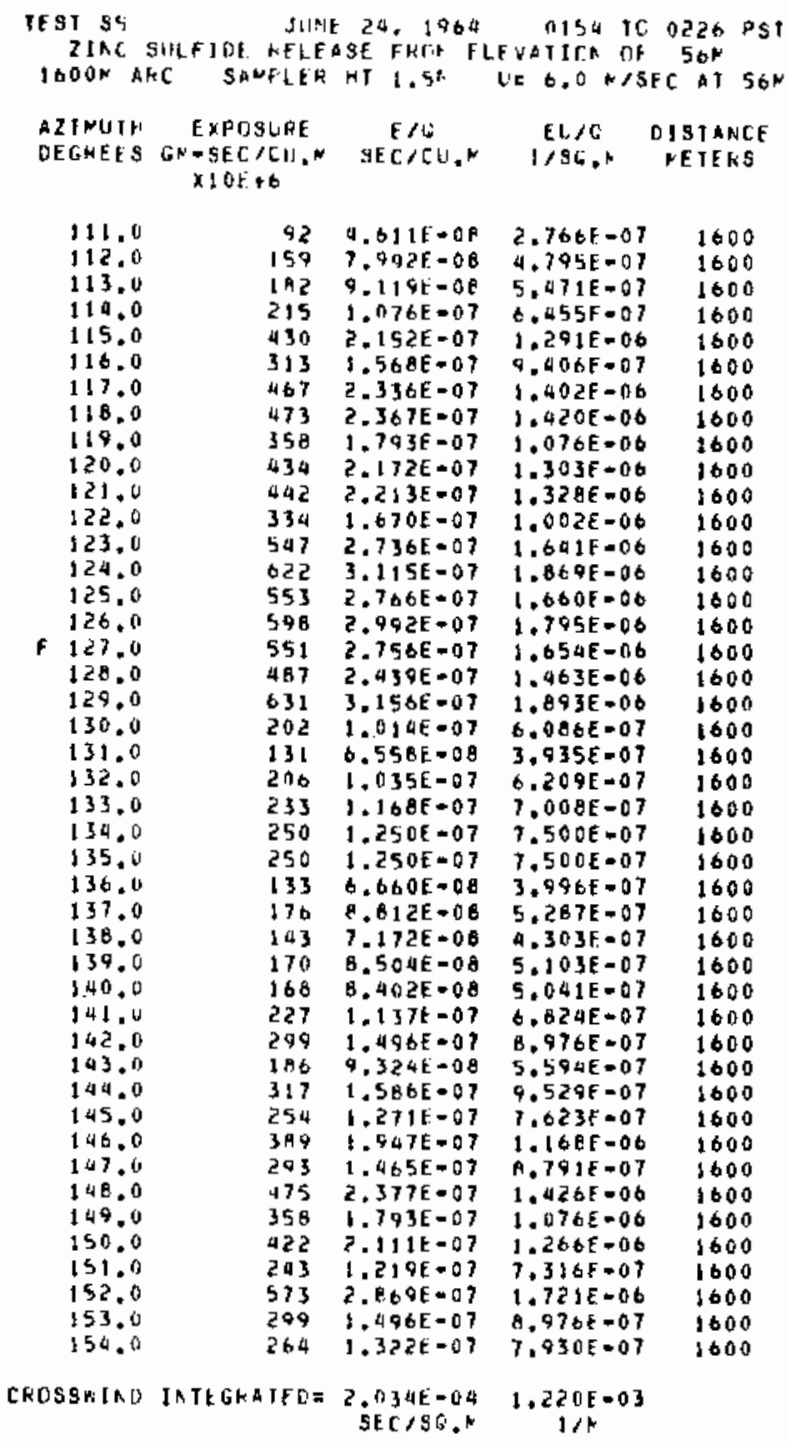




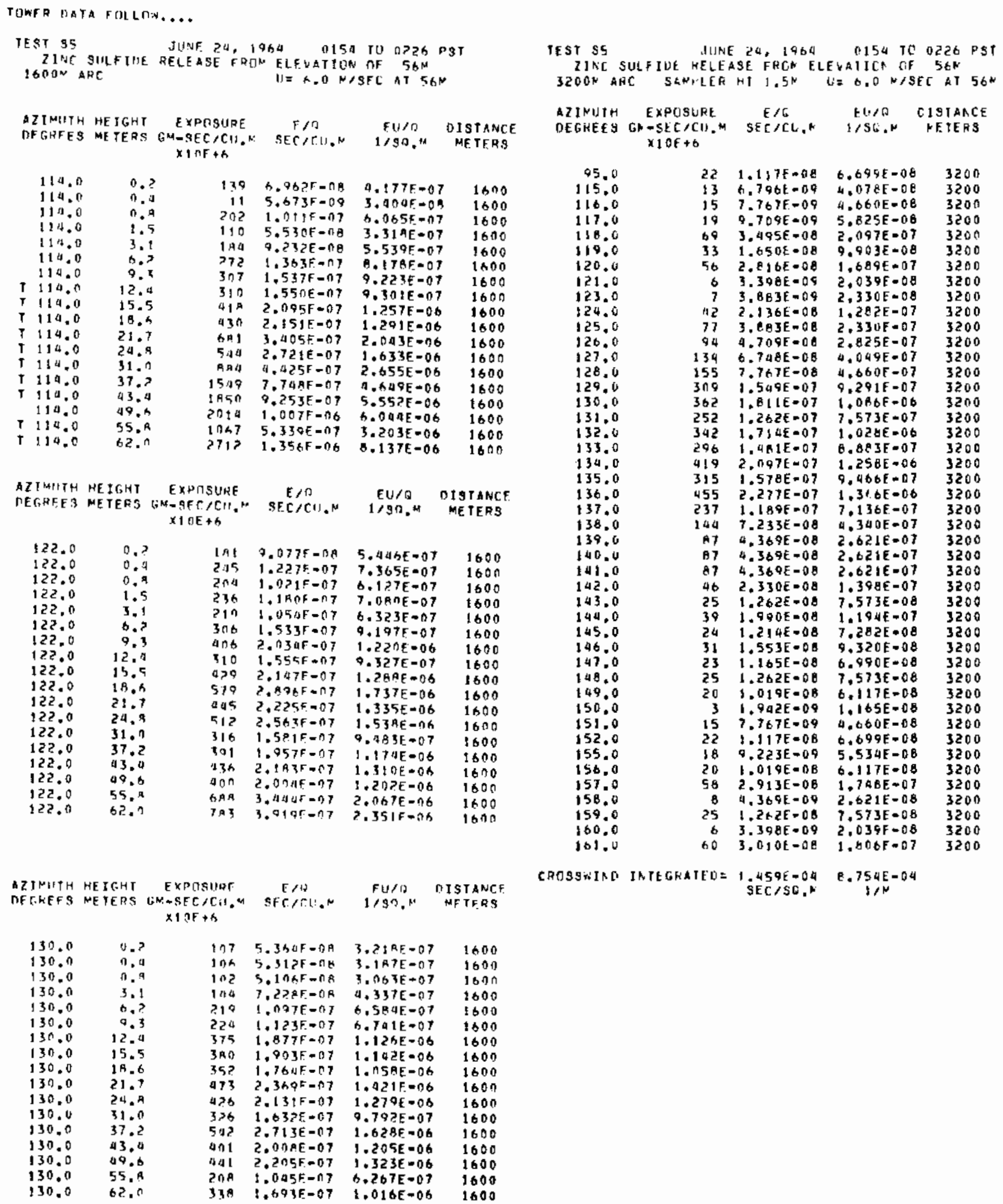


TURER DATA FILLIDH....

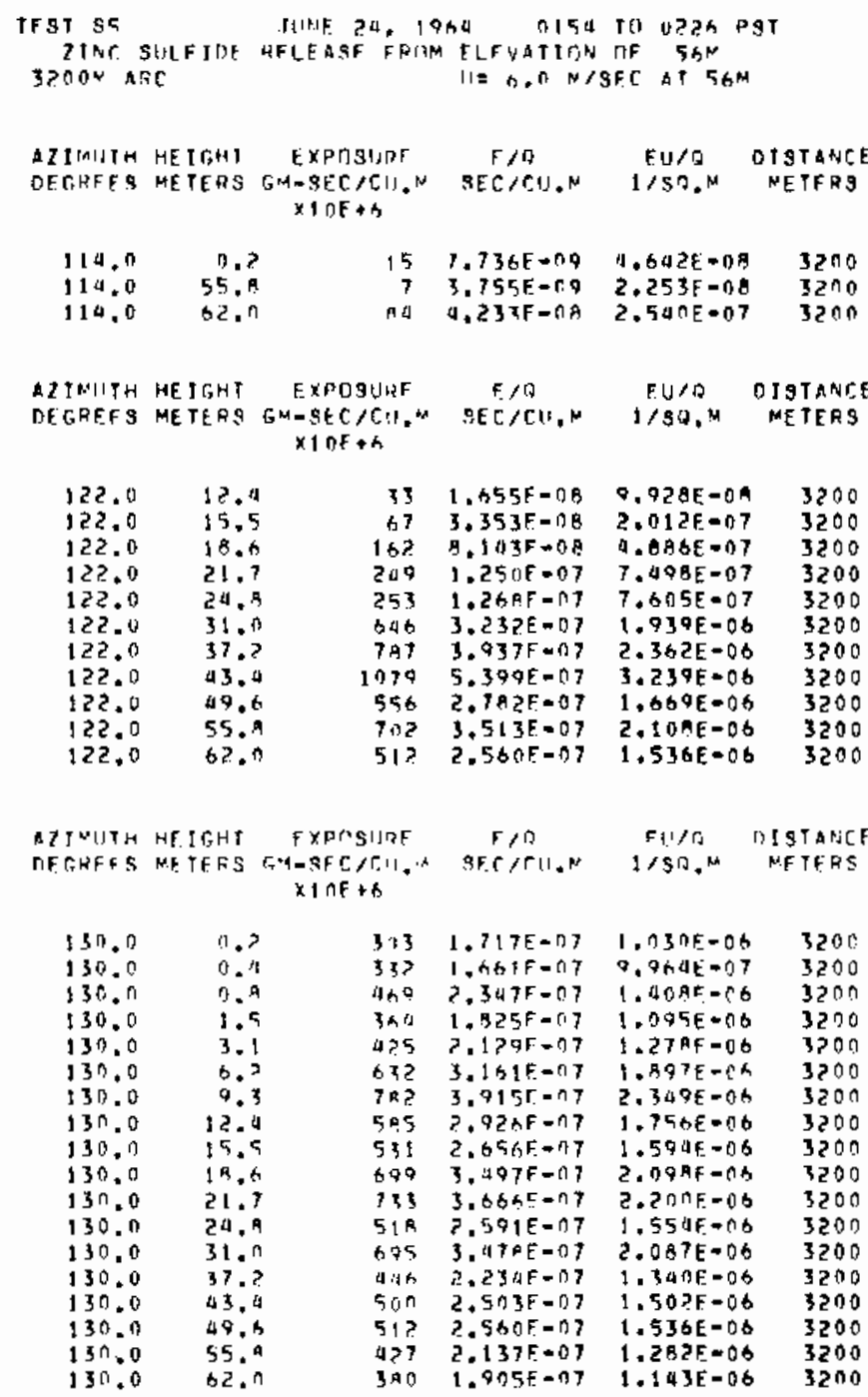

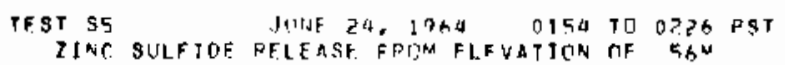

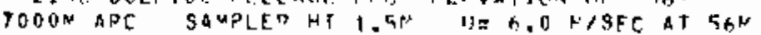

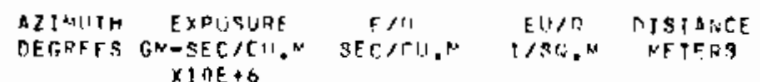




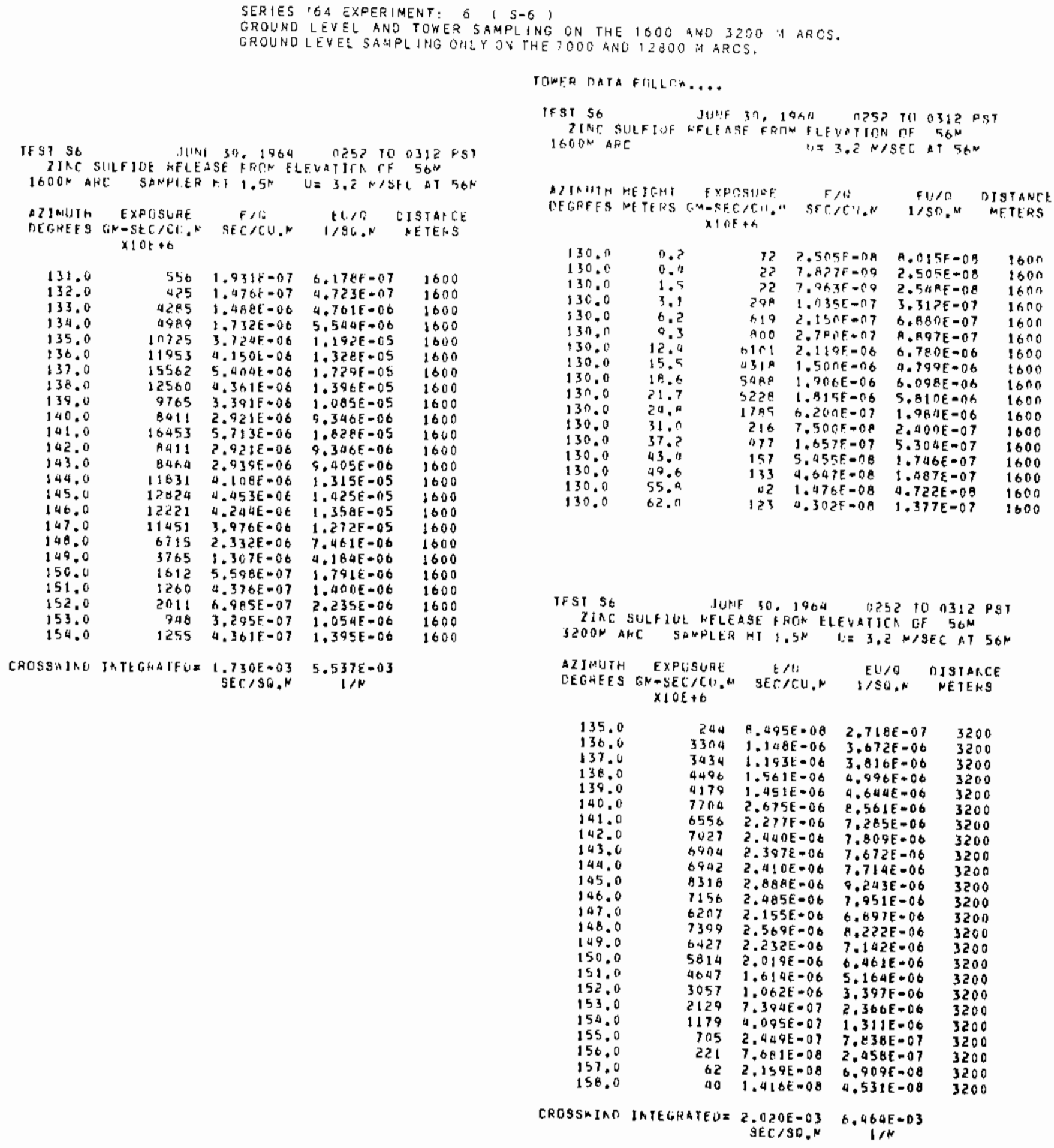




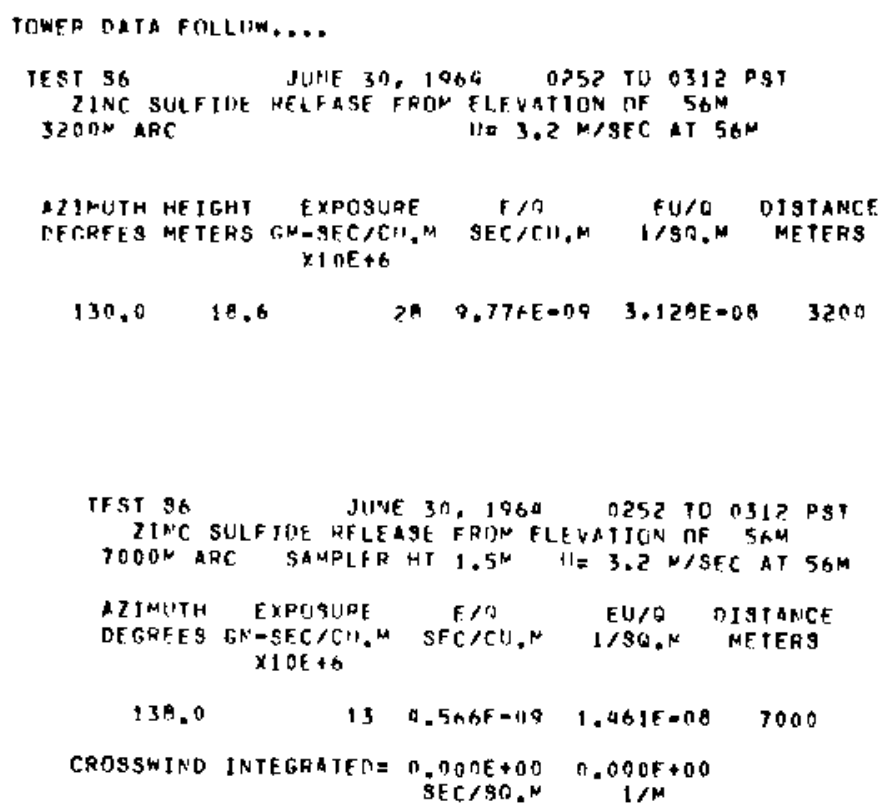

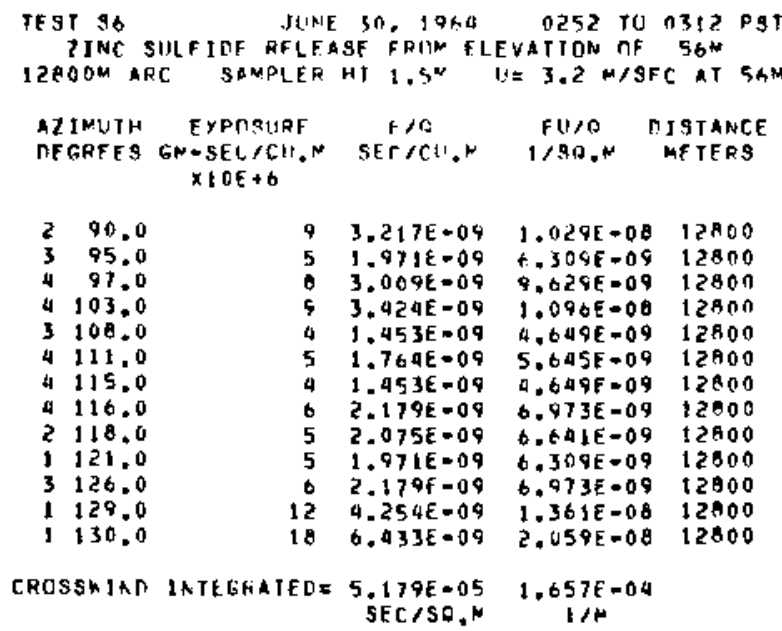


SERIES 164 EXPERIMENT: 7 \{ $\$-7$,

GROUND LEYEL AND TONER SAMPLING $200-3200$ M ARCS.

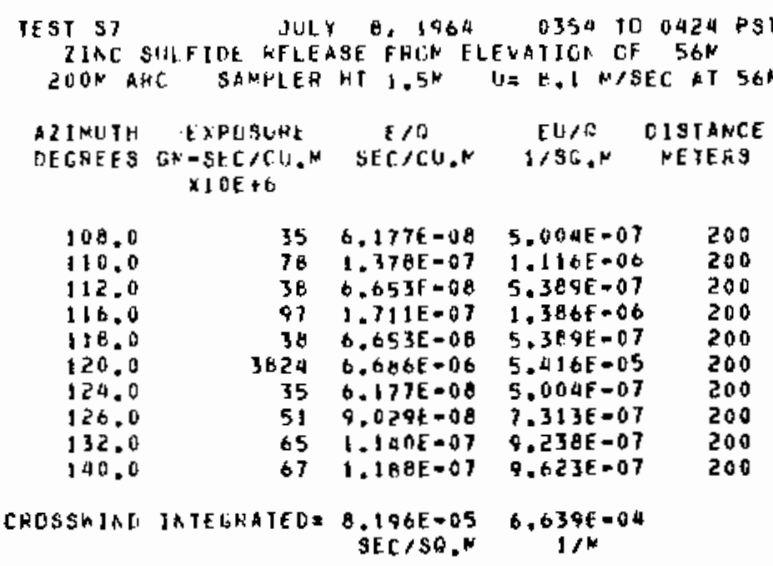

TOWF IRATA FULCUHE...

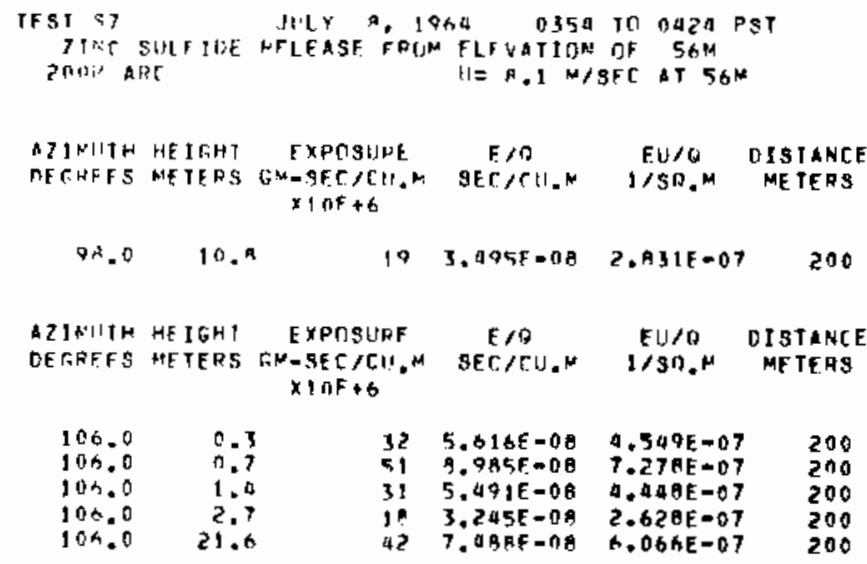

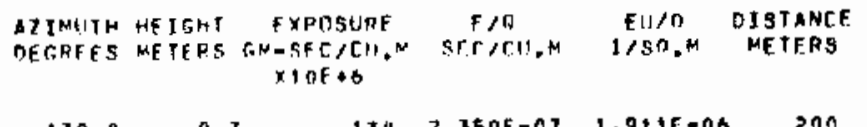
$\begin{array}{llllll}130.0 & 0.3 & 154 & 2.359 F-07 & 1.9315-0 B & 200 \\ 130.0 & 0.7 & \text { AS } & 1.5165-07 & 1.7298-06 & 200\end{array}$

TESTS7 IULY E, 1904 0354 TO 0424 PST

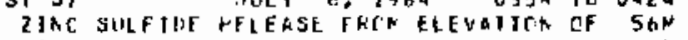
BOON AIC SAMPIER HT $1.5 \mathrm{~N} U=A .1 \mathrm{~N} / \mathrm{SEC}$ AT $50 \mathrm{~N}$

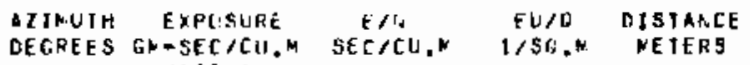
$\times \supset 0 E+6$

\begin{tabular}{|c|c|c|c|}
\hline $\begin{array}{l}100.0 \\
102.0 \\
104.0 \\
108.0 \\
110.0 \\
112.0 \\
110.0 \\
120.0 \\
130.0 \\
134.0 \\
136.0 \\
146.0\end{array}$ & $\begin{array}{r}36 \\
165 \\
63 \\
55 \\
47 \\
99 \\
126 \\
304 \\
215 \\
302 \\
89 \\
36\end{array}$ & $\begin{array}{l}6.435 E=08 \\
2.896 E-07 \\
1.103 E=07 \\
9.653 E=08 \\
0.274 E-08 \\
1.747 E=07 \\
2.206 E=07 \\
5.332 E=07 \\
3.169 F=07 \\
5.256 E=07 \\
1.563 E=07 \\
6.435 E-08\end{array}$ & $\begin{array}{l}5.213 E=07 \\
2.346 E=06 \\
8.936 E=07 \\
7.019 E=07 \\
6.702 E=07 \\
1.415 E=06 \\
1.781 E=06 \\
4.319 E=06 \\
3.053 E=06 \\
4.282 E=06 \\
1.266 E=06 \\
5.213 E=07\end{array}$ \\
\hline
\end{tabular}

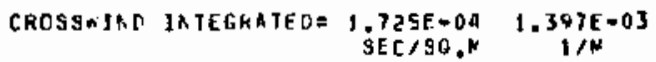

TOKFR TATA FRILEIW....

TFST 57 JILY R, 1964 OS5A TO 0 O2A PST 7JAC SULFJOE RELEAgE FRTIN ELEVATION OF $56 \mathrm{M}$ MOOM ARC IIX P.1 M/SFC AT $56 \mathrm{M}$

AZTHITH HEIGHT EXPOSUAE F/O EU/O DISTANCE DFGRFE METEPS GNASEC/CI." SEC/CI.M IISA.M METERS $X 10 E+6$

\begin{tabular}{|c|c|c|c|c|c|}
\hline $\begin{array}{l}98.0 \\
98.0 \\
98.0 \\
9 A .0 \\
98.0 \\
98.0 \\
98.0 \\
29.0\end{array}$ & $\begin{array}{l}19.7 \\
16.9 \\
21.8 \\
25.2 \\
29.9 \\
33.6 \\
37.8 \\
42.0\end{array}$ & $\begin{array}{r}18 \\
77 \\
42 \\
38 \\
27 \\
113 \\
40 \\
19\end{array}$ & $\begin{array}{l}3.3 A O E=08 \\
4.929 E=08 \\
7.4 A 5 E-0 B \\
6.454 F=08 \\
4.755 F=08 \\
1.967 E=07 \\
7.133 E=08 \\
3.397 E=08\end{array}$ & $\begin{array}{l}2.73 A E-07 \\
3.912 E-07 \\
6.063 E=07 \\
5.227 E-07 \\
3.652 E=07 \\
1.609 E=06 \\
5.778 E=07 \\
2.751 E=07\end{array}$ & $\begin{array}{l}800 \\
800 \\
800 \\
000 \\
800 \\
800 \\
800 \\
000\end{array}$ \\
\hline
\end{tabular}




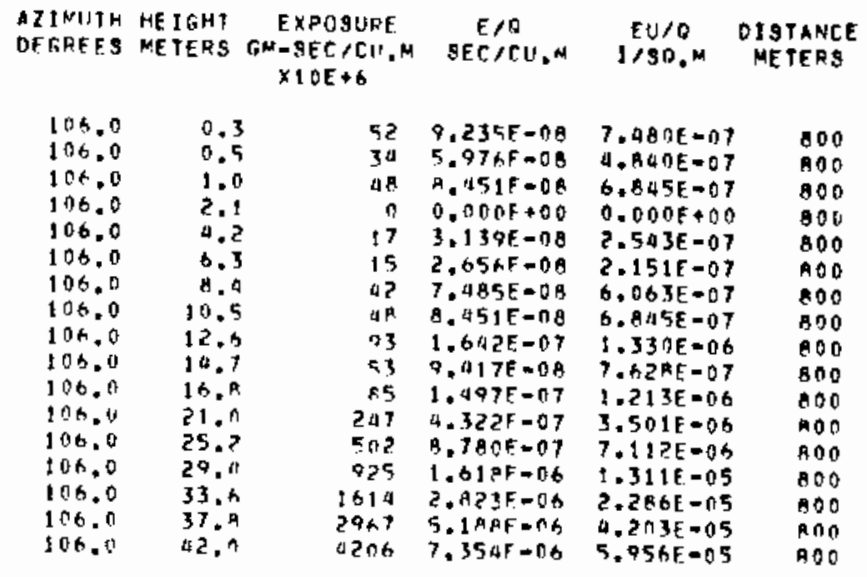

\begin{tabular}{|c|c|c|c|c|c|}
\hline $\begin{array}{l}\text { ATINITH } \\
\text { DFEAFTS }\end{array}$ & $\begin{array}{l}\text { MEIGHT } \\
\text { ME TFNS }\end{array}$ & $\begin{array}{c}\text { EXPOSUFE } \\
G M-3 E C / C 11 . N \\
\times I D E+A\end{array}$ & $\begin{array}{c}f / f] \\
\text { SEF }\end{array}$ & $\begin{array}{c}F(1 / 0 \\
1 / 5 C_{0}, 1\end{array}$ & $\begin{array}{l}\text { DISTAPCE } \\
\text { METERS }\end{array}$ \\
\hline 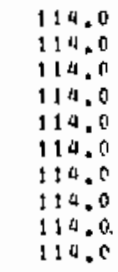 & 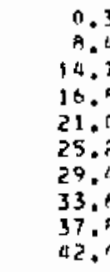 & $\begin{array}{r}124 \\
20 \\
17 \\
04 \\
109 \\
1>7 \\
745 \\
1809 \\
4 R+3 \\
7555\end{array}$ & $\begin{array}{l}2.173 F=07 \\
3.527 F=08 \\
3.139 F=08 \\
7.127 E=08 \\
1.560 F=07 \\
2.140 E=07 \\
1.3035=06 \\
3.288 E=06 \\
9.503 E=06 \\
1.321 E=05\end{array}$ & $\begin{array}{l}1.760 E=06 \\
2.934 E=07 \\
2.503 F=07 \\
6.259 E=07 \\
1.271 E=06 \\
1.733 E=06 \\
1.055 E=05 \\
2.663 E=05 \\
6.029 E=05 \\
1.070 E=04\end{array}$ & $\begin{array}{l}\text { RnO } \\
\text { POO } \\
\text { An } \\
\text { AnO } \\
\text { ROO } \\
800 \\
\text { An } \\
\text { ADO } \\
B O 0 \\
B \cap 0\end{array}$ \\
\hline $\begin{array}{l}\text { AZIMIITH } \\
\text { DETREFS }\end{array}$ & $\begin{array}{l}\text { HEIGHT } \\
\text { NETERS }\end{array}$ & 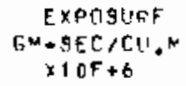 & $\begin{array}{c}E / O \\
S E C / C H . N\end{array}$ & $\begin{array}{c}F U / B \\
1 / S A, N\end{array}$ & $\begin{array}{l}\text { OISTANCE } \\
\text { METERS }\end{array}$ \\
\hline $\begin{array}{l}122.0 \\
122.0 \\
122.0 \\
122.0\end{array}$ & $\begin{array}{l}0 . \\
33, \\
37 . \\
12 .\end{array}$ & $\begin{array}{r}52 \\
22 \\
530 \\
1915\end{array}$ & $\begin{array}{l}9.235 E-00 \\
3.906 F=08 \\
9.2735=07 \\
3.17 \text { at }=06\end{array}$ & $\begin{array}{l}7.48 \cap E=07 \\
3.164 E=0.7 \\
7.511 E=06 \\
2.571 E=05\end{array}$ & $\begin{array}{l}800 \\
800 \\
800 \\
800\end{array}$ \\
\hline
\end{tabular}

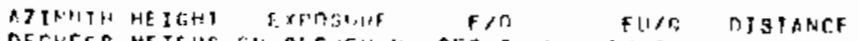

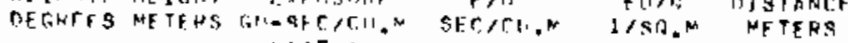 $\times \rightarrow n+A$}

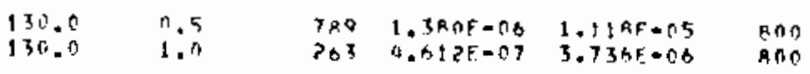

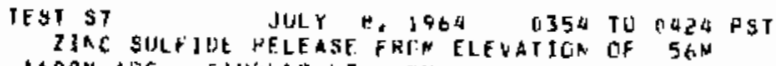
I6OON ARC SAMFLER HT $1.5 \mathrm{~N}$ LIE $8,1 \mathrm{~N} / \mathrm{SEC}$ AT $56 \mathrm{~N}$

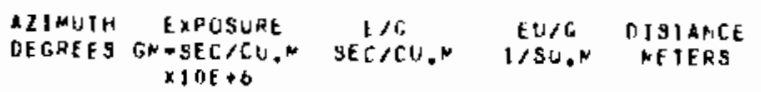

\begin{tabular}{|c|c|c|c|c|}
\hline $\begin{array}{l}100.0 \\
101.0 \\
102.0 \\
103.0 \\
104.0 \\
105.0 \\
100.0 \\
107.0 \\
108.0 \\
109.0 \\
110.0 \\
112.0 \\
112.0 \\
113.0 \\
119.0 \\
115.0 \\
116.0 \\
117.0 \\
119.0 \\
119.0\end{array}$ & $\begin{array}{r}34 \\
84 \\
339 \\
82 \\
107 \\
291 \\
194 \\
225 \\
370 \\
192 \\
119 \\
17 \\
54 \\
70 \\
19 \\
62 \\
131 \\
60 \\
31 \\
49\end{array}$ & $\begin{array}{l}6.354 E-08 \\
1.434 E=07 \\
5.928 E=07 \\
1.439 E=07 \\
1.8 E 5 E=07 \\
3.530 E=07 \\
3.221 E-07 \\
3.941 E=07 \\
6.477 E=07 \\
3.358 E=07 \\
2.090 E-07 \\
3.084 E=06 \\
9.595 E=08 \\
1.234 E=07 \\
3.427 E=08 \\
1.097 E=07 \\
2.296 E=07 \\
1.062 E=07 \\
5.483 E=08 \\
0.567 E=08\end{array}$ & $\begin{array}{l}5.551 E=07 \\
1.194 E=06 \\
4.602 E=06 \\
1.160 E=06 \\
1.527 F=06 \\
2.859 E=06 \\
2.609 E=06 \\
3.192 E=06 \\
5.246 E=06 \\
2.720 E=06 \\
1.691 E=06 \\
2.498 E=07 \\
7.772 E=07 \\
9.993 E=07 \\
2.776 E=07 \\
8.882 E-07 \\
1.660 E=06 \\
0.605 E=07 \\
1.441 E=07 \\
0.939 E=07\end{array}$ & $\begin{array}{l}1600 \\
1600 \\
1600 \\
1600 \\
1600 \\
1600 \\
1600 \\
1600 \\
1600 \\
1600 \\
1600 \\
1600 \\
1600 \\
1600 \\
1600 \\
1600 \\
1600 \\
1600 \\
1600 \\
1600\end{array}$ \\
\hline
\end{tabular}

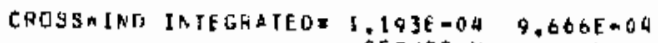
SECJSG.M

FOWER DATR FOLLLIX....

TEST S7 JILY R, 196I O35A TO 04Z4 PST

7 INL SULFIDE RELEASE FROM ELFUATION DF $56 \mathrm{M}$ ISOON ART

ATIMIJTH HEJGHT EXPTSURE FID EUTH OTSTANCE

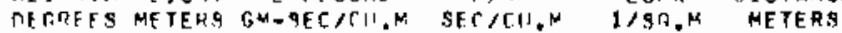
$\times 1$ त +6

\begin{tabular}{|c|c|c|c|c|c|}
\hline $\begin{array}{l}104.0 \\
106.0 \\
108.0 \\
100.0 \\
100.0 \\
106.0 \\
106.0 \\
104.0 \\
106.0 \\
106.0 \\
106.0 \\
106.0 \\
106.0 \\
100.0 \\
106.0 \\
100.0 \\
10 \times .0\end{array}$ & $\begin{array}{l}11.7 \\
0.9 \\
0.9 \\
1.5 \\
3.1 \\
6.7 \\
12.4 \\
15.5 \\
18.2 \\
21.7 \\
24.8 \\
31.0 \\
37.2 \\
43.4 \\
49.5 \\
55.8 \\
62.9\end{array}$ & $\begin{array}{l}1 \times 3 \\
1 \times 3 \\
1 \times 7 \\
91 \\
253 \\
250 \\
794 \\
695 \\
798 \\
1153 \\
932 \\
2255 \\
3573 \\
4579 \\
1505 \\
705 \\
279\end{array}$ & 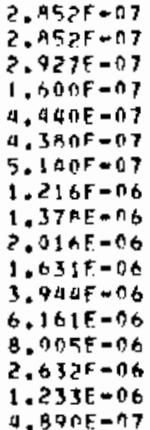 & $\begin{array}{l}2.310 E=06 \\
2.310 E=06 \\
2.371 E=06 \\
1.296 E=06 \\
3.596 E=06 \\
3.548 E=06 \\
4.163 E=06 \\
9.050 E=06 \\
1.116 E=05 \\
1.633 E=05 \\
1.321 E=05 \\
3.195 E=05 \\
4.991 E=05 \\
6.404 E=05 \\
2.132 E=05 \\
9.984 E=06 \\
3.961 E=06\end{array}$ & $\begin{array}{l}1600 \\
1600 \\
1600 \\
1600 \\
1600 \\
1600 \\
1600 \\
1600 \\
1600 \\
1600 \\
1600 \\
1600 \\
1600 \\
1600 \\
1600 \\
1600 \\
1600\end{array}$ \\
\hline
\end{tabular}




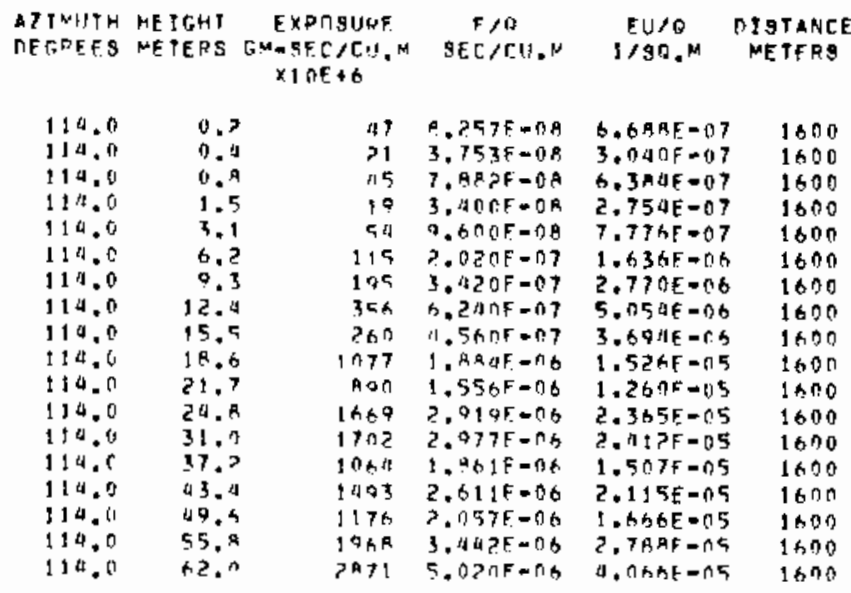

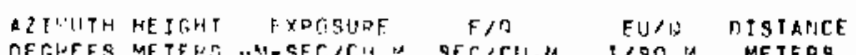

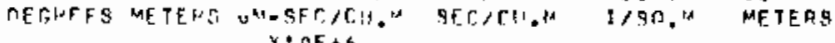
$x+\cap F+h$

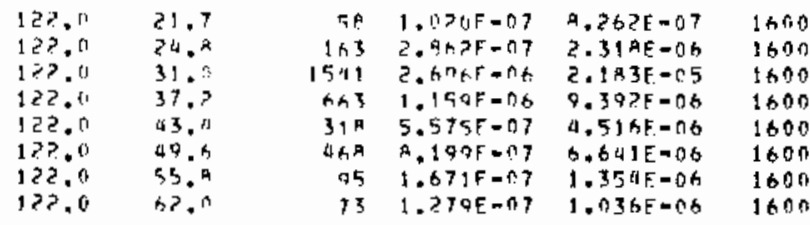

\begin{tabular}{|c|c|c|c|c|c|}
\hline $\begin{array}{l}\text { A ZIVIITH } \\
\text { DFGHES }\end{array}$ & $\begin{array}{l}\text { HE }[1,+4 T \\
\text { METETS }\end{array}$ & 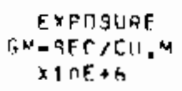 & $\begin{array}{c}E, n \\
S E C \neq[1], N\end{array}$ & $\begin{array}{c}E \| / B \\
1 / S M . M\end{array}$ & $\begin{array}{l}\text { DISTANC } \\
\text { METERS }\end{array}$ \\
\hline $\begin{array}{l}130.0 \\
130.0 \\
130.0 \\
130.0 \\
130.0 \\
130.0\end{array}$ & $\begin{array}{r}4.2 \\
37.3 \\
43.4 \\
49.4 \\
55.8 \\
62.0\end{array}$ & $\begin{array}{r}12 \\
9 \\
187 \\
105 \\
119 \\
19\end{array}$ & $\begin{array}{l}5.630 E=08 \\
1.631 E=08 \\
3.10, E=07 \\
3.021 F=07 \\
1.929 E=07 \\
3.477 F=08\end{array}$ & $\begin{array}{l}4.560 E-07 \\
1.321 E=07 \\
2.582 E=06 \\
2.771 E=06 \\
1.563 E=06 \\
2.916 E=07\end{array}$ & $\begin{array}{l}1600 \\
1600 \\
1600 \\
1600 \\
1600 \\
1600\end{array}$ \\
\hline
\end{tabular}

TESTS7 JUIYY A, 1904 OSS4 TO O424 PST ZIR.T SULFIHE RFLEASE FWTIN ELEYATICK DIF $5 \mathrm{HN}$

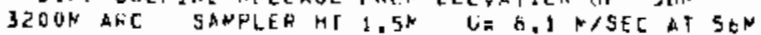

AZINUTH EXPCSURE F/A EU/G DISIAREE DEGHEES GNOSEC/CL.M SEC/CL.N J/SL.N NETERS $x+0 t+0$

\begin{tabular}{|c|c|c|c|c|}
\hline $\begin{array}{l}103.0 \\
104.0 \\
105.0 \\
106.0 \\
107.0 \\
1108.0 \\
109.0 \\
110.0 \\
111.0 \\
112.0 \\
113.0 \\
114.0 \\
115.0 \\
116.0 \\
117.0 \\
118.0 \\
119.0 \\
120.0 \\
121.0 \\
122.0 \\
123.0 \\
124.0 \\
125.0 \\
126.0\end{array}$ & $\begin{array}{r}15 \\
296 \\
405 \\
589 \\
601 \\
51 \\
521 \\
767 \\
914 \\
1165 \\
1180 \\
558 \\
314 \\
197 \\
237 \\
147 \\
70 \\
77 \\
233 \\
191 \\
299 \\
196 \\
53 \\
83\end{array}$ & $\begin{array}{l}2.710 E=00 \\
3.615 E=07 \\
7.791 E=07 \\
1.030 E=06 \\
1.052 E=06 \\
8.996 E=08 \\
9.115 E=07 \\
1.343 E=06 \\
1.599 E=06 \\
2.037 E=06 \\
2.074 E=06 \\
9.360 E=07 \\
5.448 E=07 \\
3.446 E=07 \\
4.158 E=07 \\
2.580 E=07 \\
1.239 E=07 \\
1.358 E=07 \\
4.074 E=07 \\
3.344 E=07 \\
5.220 E=07 \\
3.429 E=07 \\
9.335 E=08 \\
1.460 E=07\end{array}$ & $\begin{array}{l}2.200 \mathrm{E}=07 \\
2.928 \mathrm{E}=06 \\
6.311 \mathrm{E}=06 \\
8.345 \mathrm{E}=06 \\
8.524 \mathrm{E}=06 \\
7.207 \mathrm{E}=07 \\
7.383 \mathrm{E}=06 \\
1.087 \mathrm{E}=05 \\
1.295 \mathrm{E}=05 \\
1.650 \mathrm{E}=05 \\
1.060 \mathrm{E}=05 \\
7.905 \mathrm{E}=06 \\
4.413 \mathrm{E}=06 \\
2.791 \mathrm{E}=06 \\
3.36 \mathrm{EE}=06 \\
2.090 \mathrm{E}=06 \\
1.004 \mathrm{E}-06 \\
1.100 \mathrm{E}-06 \\
3.300 \mathrm{E}=06 \\
2.706 \mathrm{E}-06 \\
4.235 \mathrm{E}-06 \\
2.777 \mathrm{E}=06 \\
7.562 \mathrm{E}=07 \\
1.182 \mathrm{E}=06\end{array}$ & $\begin{array}{l}3200 \\
3200 \\
3200 \\
3200 \\
3200 \\
3200 \\
3200 \\
3200 \\
3200 \\
3200 \\
3200 \\
3200 \\
3200 \\
3200 \\
3200 \\
3200 \\
3200 \\
3200 \\
3200 \\
3200 \\
3200 \\
3200 \\
3200 \\
3200\end{array}$ \\
\hline & - & $\begin{array}{l}8,90 \text { AE }=0 \\
\text { SEC } / 5 C .+\end{array}$ & $\begin{array}{c}7.216 E=03 \\
1 / N\end{array}$ & \\
\hline
\end{tabular}

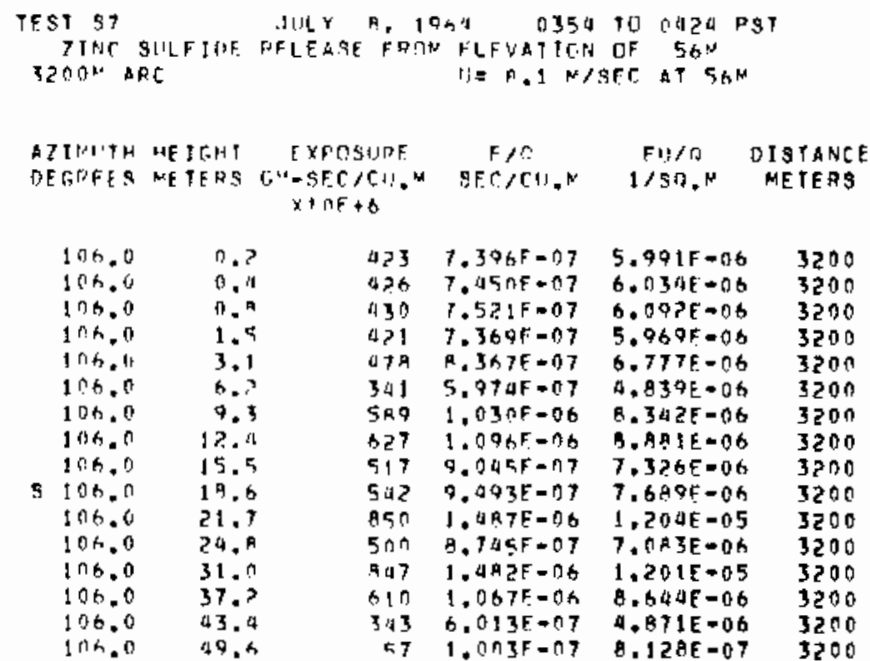

AIINITIN HEIGHT EXPDSURE F/A EUTO DISYANCE DEGRFES METERS GN-SEC/CIJ." 3ET/CU.M IIST.M METESS $\times 10 \mathrm{~F}+\mathrm{B}$

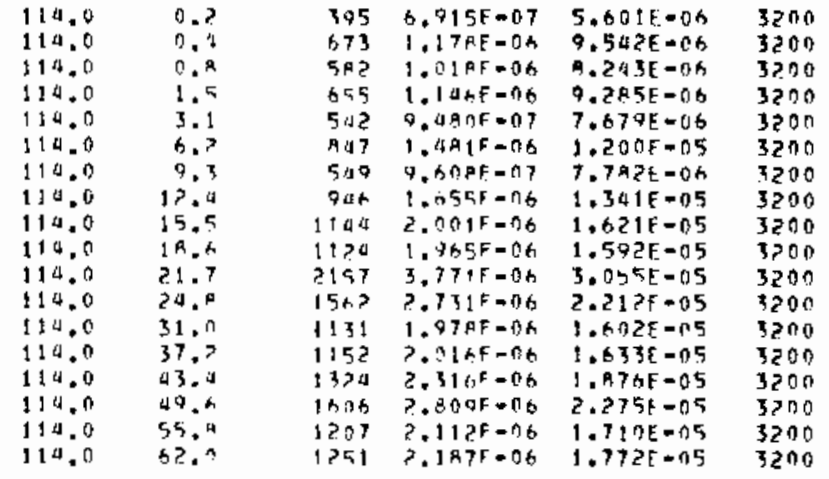

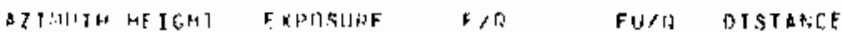

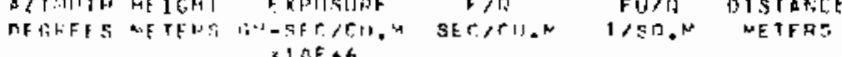

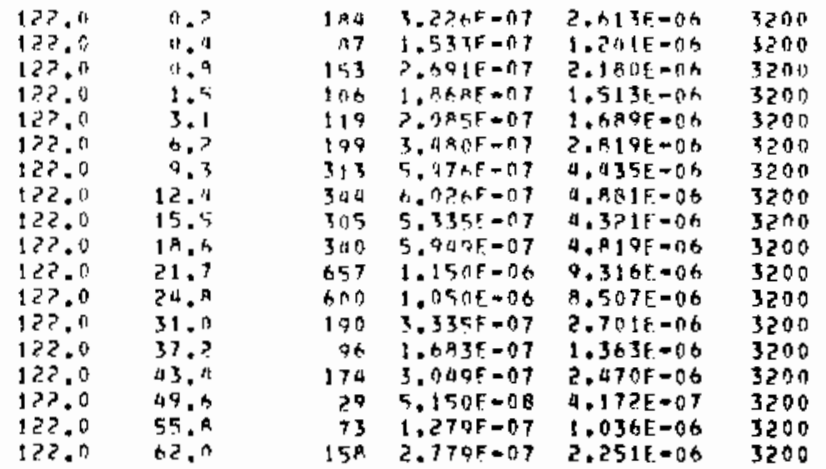


SERIES IS4 EXPERIMENT: 8 ( S-8)

GROUND LEVEL ANO TOKER SAMPLING ON THE 1600 ANO 3200 M ARCS.

GROUND LEVEL SAMPLING ONLY ON THE TOOO ANO 12800 M ARCS.

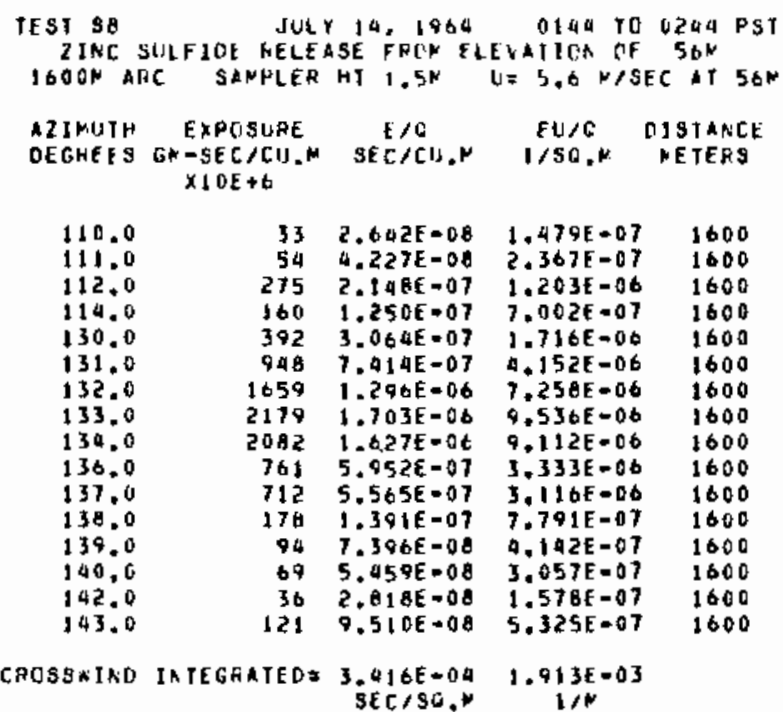

TOMER PATA FULLOH....
AZINLTH HETCHT EXPESURE EAG EU/O DISTANCE DEGPEFS METFES GM-GECJCU.N SECJCU,N 1/SA.M METERS $x \rightarrow O E+6$

$\begin{array}{llrlll}114.0 & 18.6 & 28 & 2.233 E-00 & 1.250 E-07 & 1600 \\ 114.0 & 21.7 & 233 & 1.825 E-07 & 1.022 E-06 & 1600 \\ 114.0 & 24.8 & 193 & 1.495 E-07 & 8.374 E-07 & 1600 \\ 114.0 & 31.0 & 232 & 1.813 E-07 & 1.016 E-06 & 1600 \\ 114.0 & 37.2 & 491 & 3.039 E-07 & 2.150 E-06 & 1600 \\ 114.0 & 43.4 & 1959 & 1.190 E-06 & 6.364 E-06 & 1600 \\ 114.0 & 49.6 & 1732 & 1.354 E-06 & 7.502 E-06 & 1600 \\ 119.0 & 55.9 & 2274 & 1.777 E-06 & 9.951 E-06 & 1600 \\ 114.0 & 62.0 & 3125 & 2.491 E-06 & 1.367 E-05 & 1600\end{array}$

\begin{tabular}{|c|c|c|c|c|c|}
\hline $\begin{array}{l}\text { ZINIITH } \\
\text { EGRFES }\end{array}$ & $\begin{array}{l}\text { HE JGHT } \\
\text { XETERS }\end{array}$ & 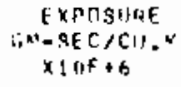 & $\begin{array}{c}\varepsilon / 2 \\
\text { sf } r / c \cup, k\end{array}$ & $\begin{array}{l}\text { FU/O } \\
1 / 80^{M}\end{array}$ & $\begin{array}{l}\text { OISTANCE } \\
\text { ME TERS }\end{array}$ \\
\hline $\begin{array}{l}122.0 \\
122.0 \\
122.0 \\
122.0 \\
122.0 \\
122.0 \\
122.0 \\
122.0 \\
122.0 \\
122.0 \\
122.0 \\
122.0\end{array}$ & $\begin{array}{l}0.7 \\
3.1 \\
15.5 \\
14.5 \\
21.7 \\
2.40 \\
31.0 \\
37.2 \\
43.0 \\
49.9 \\
55.6 \\
42.0\end{array}$ & $\begin{array}{r}51 \\
45 \\
55 \\
72 \\
57 \\
74 \\
73 \\
168 \\
357 \\
715 \\
1243 \\
2595\end{array}$ & $\begin{array}{l}4.227 F=08 \\
3.54+F=08 \\
1.33 a E=08 \\
5.647 F=08 \\
4.465 F=08 \\
5.833 F=08 \\
5.727 F=08 \\
1.315 F=07 \\
2.541 F=07 \\
5.592 F=07 \\
9.711 E=07 \\
2.254 F=06\end{array}$ & $\begin{array}{l}2.367 \mathrm{E}=07 \\
1.90 \mathrm{EE}=07 \\
2.077 \mathrm{E}=07 \\
3.162 \mathrm{E}=07 \\
3.500 \mathrm{E}=07 \\
3.266 \mathrm{E}=07 \\
3.207 \mathrm{E}=07 \\
7.360 \mathrm{E}=07 \\
1.479 \mathrm{E}=06 \\
3.132 \mathrm{E}=06 \\
5.038 \mathrm{E}=06 \\
1.262 \mathrm{E}=05\end{array}$ & $\begin{array}{l}1600 \\
1600 \\
1600 \\
1600 \\
1600 \\
1600 \\
1600 \\
1600 \\
1600 \\
1600 \\
1600 \\
1600\end{array}$ \\
\hline
\end{tabular}

ATIMUTH HEIGHT EXPOSUAFE F/O DU/O DISTANCE DEGREFS NETERS GM-SEC/CU.Y SEC/CH,M I/SO,M METERS $X 1 \cap E+6$

\begin{tabular}{|c|c|c|c|c|c|}
\hline $\begin{array}{l}130.0 \\
130.0 \\
130.0 \\
130.0 \\
130.0 \\
130.0 \\
130.0 \\
130.0 \\
130.0 \\
130.0 \\
130.0 \\
130.0 \\
130.0 \\
130.0 \\
130.0 \\
130.0 \\
130.0 \\
130.0\end{array}$ & $\begin{array}{l}9.7 \\
0.9 \\
4.8 \\
1.5 \\
3.1 \\
6.7 \\
9.3 \\
12.0 \\
15.5 \\
19.8 \\
21.7 \\
29.8 \\
11.9 \\
37.2 \\
93.4 \\
49.8 \\
55.2 \\
62.0\end{array}$ & 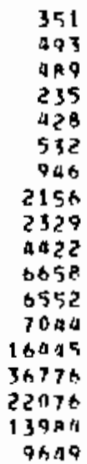 & $\begin{array}{l}2.797 E=07 \\
3.857 E=07 \\
3.821 E=07 \\
1.839 E=07 \\
3.349 E=07 \\
0.163 F=07 \\
7.394 E=07 \\
1.655 E=06 \\
1.820 F=06 \\
3.455 F=06 \\
5.207 E=06 \\
5.114 E=06 \\
5.504 E=06 \\
1.2 A 5 E-05 \\
2.873 E=05 \\
1.725 E=05 \\
1.093 F=05 \\
7.530 F=06\end{array}$ & $\begin{array}{l}1.518 E=06 \\
2.160 E=06 \\
2.140 E=06 \\
1.030 E=06 \\
1.875 E=06 \\
2.331 E=06 \\
4.140 E=06 \\
9.435 E=06 \\
1.019 E=05 \\
1.935 E=05 \\
2.913 E=05 \\
2.867 E=05 \\
3.082 E=05 \\
7.195 E=05 \\
1.609 E=09 \\
9.650 E-05 \\
6.118 E=05 \\
4.222 E=05\end{array}$ & $\begin{array}{l}1600 \\
1600 \\
1600 \\
1600 \\
1600 \\
1600 \\
1600 \\
1600 \\
1600 \\
1600 \\
1600 \\
1600 \\
1600 \\
1600 \\
1600 \\
1600 \\
1600 \\
1600\end{array}$ \\
\hline
\end{tabular}




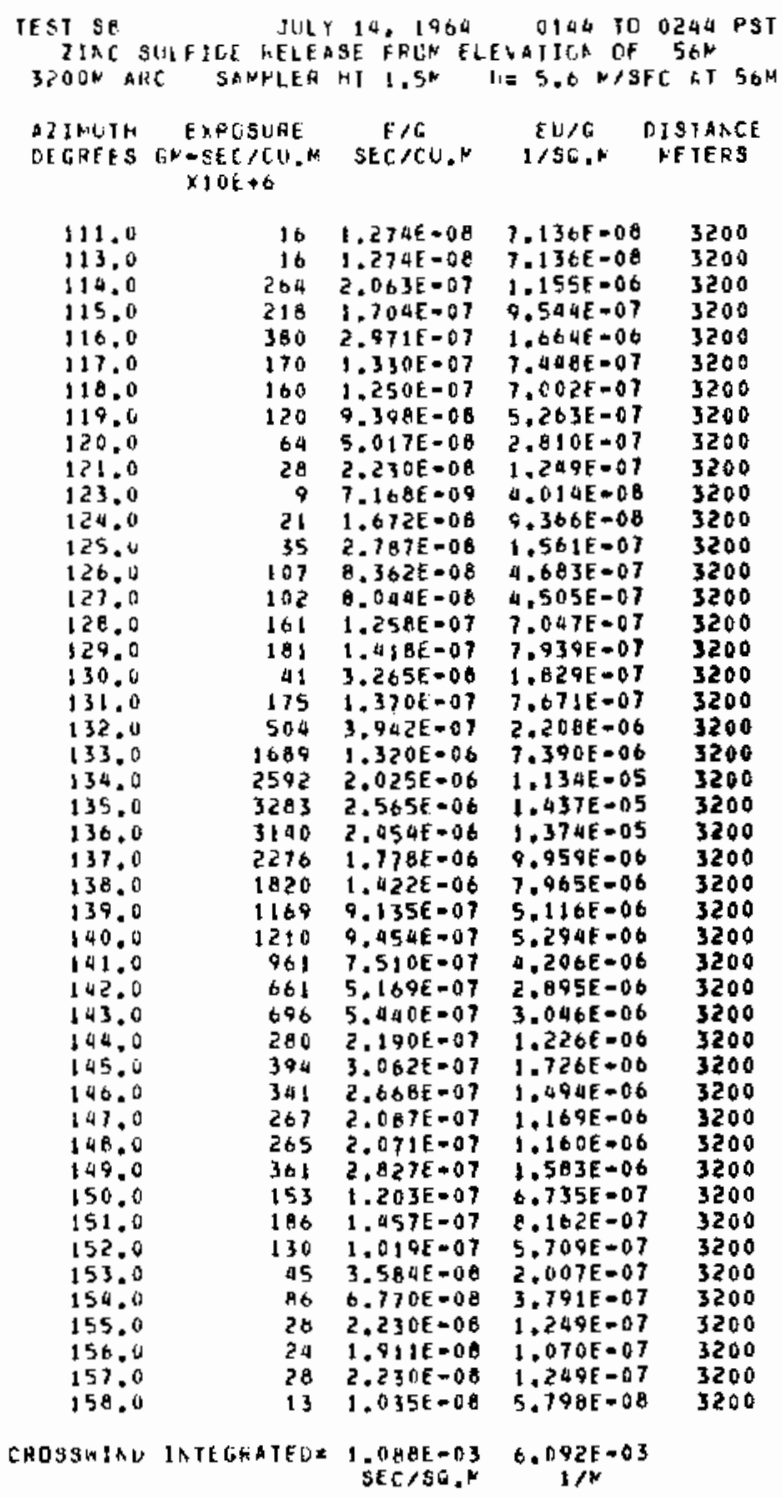

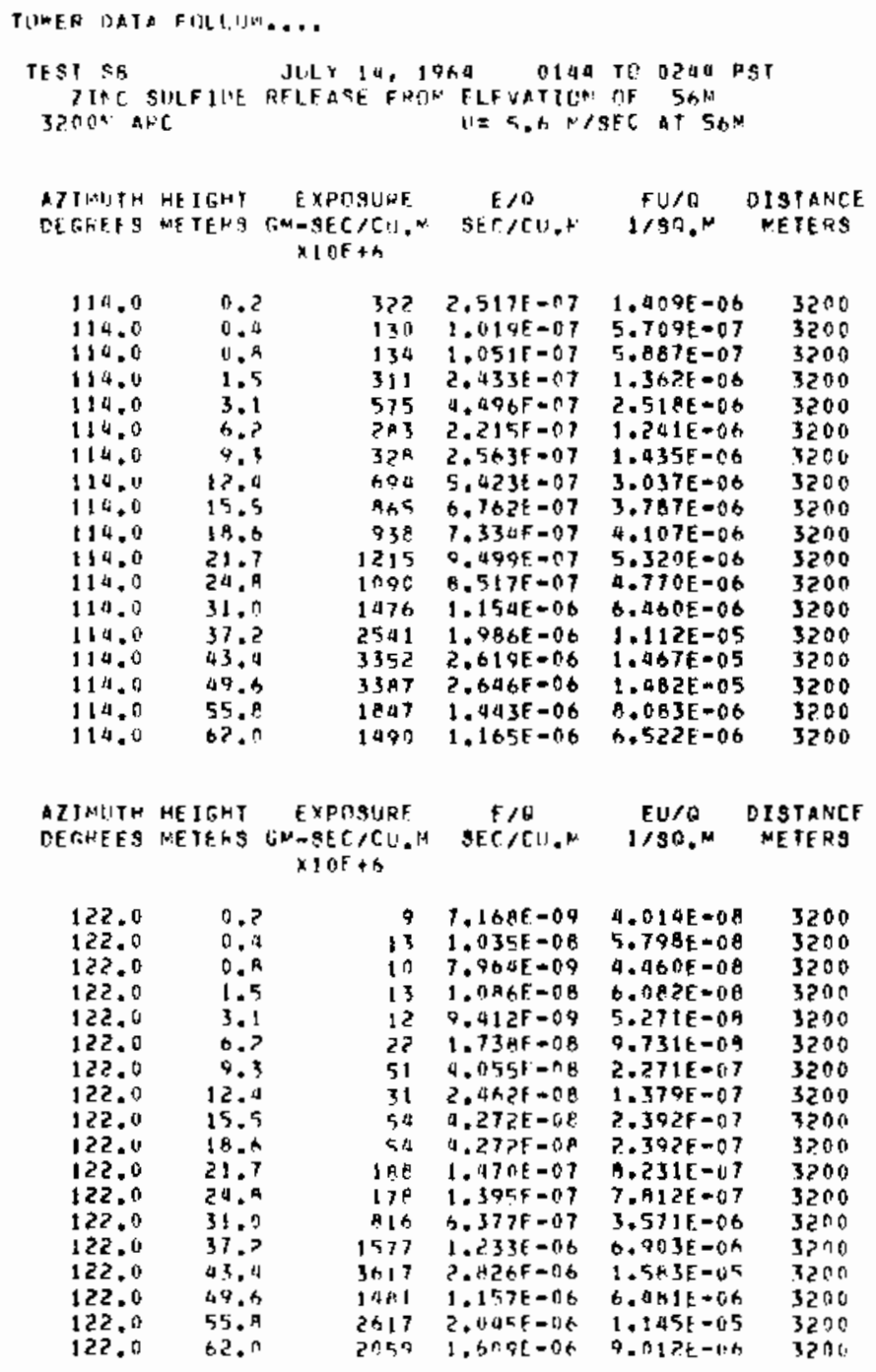

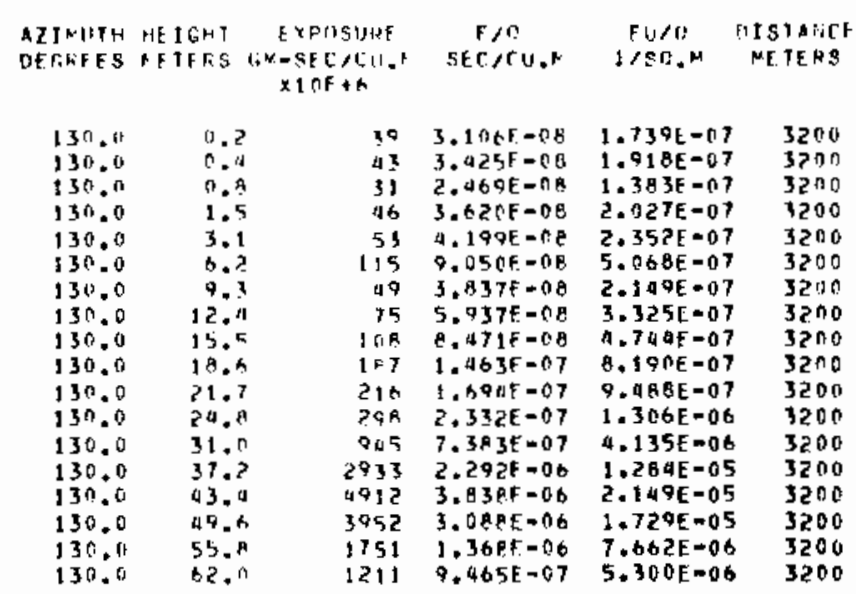




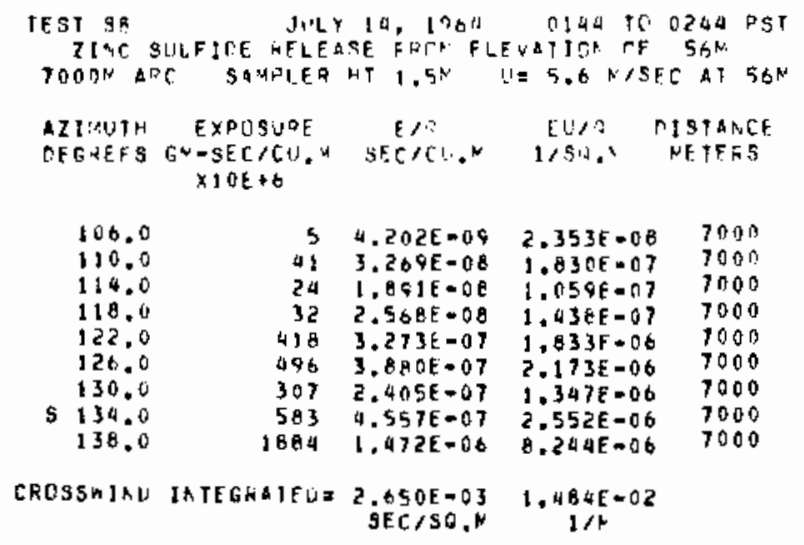

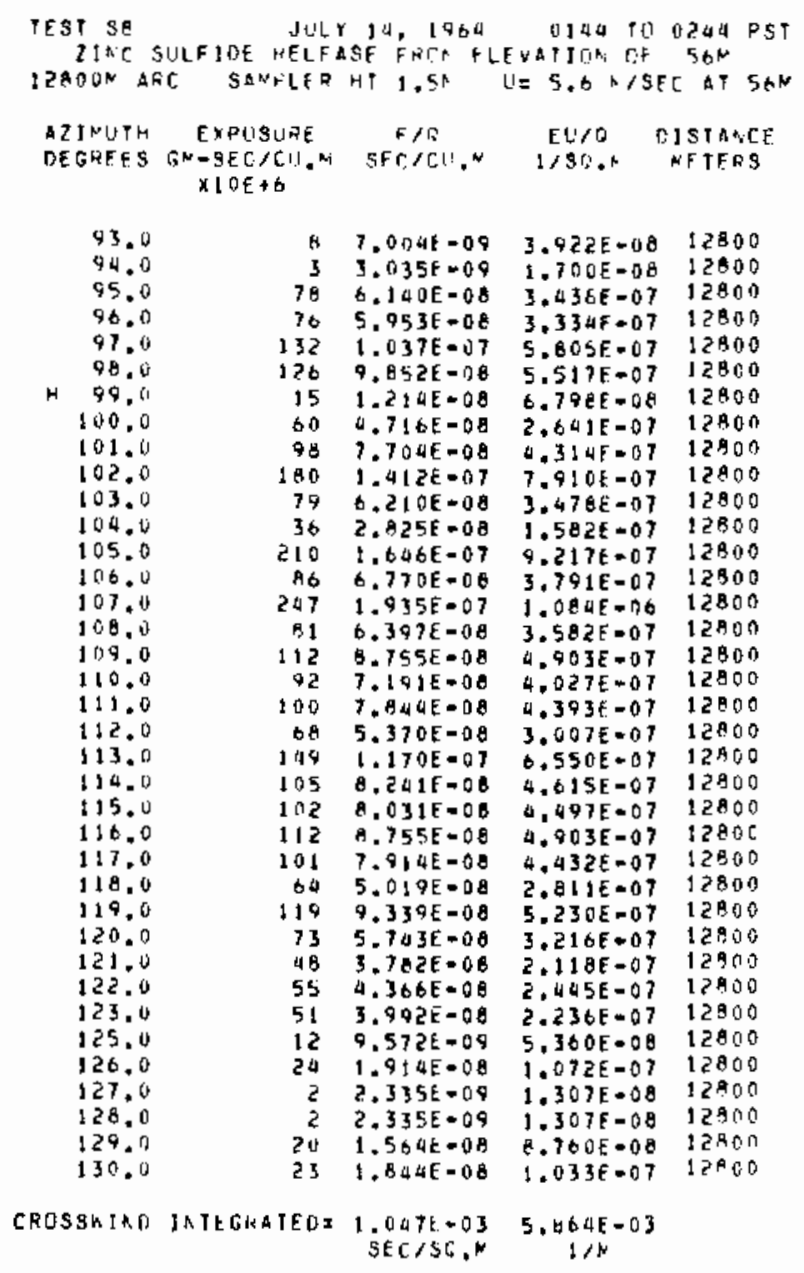


SERIES 164 EXPERIMENT: 9 ( $\$-9$ )

GROUND LEYEL ANO TOWER SAMPLENG ON THE 1600 AND 3200 M ARCS.

GROUND LEVEL SAMPLING ONLY ON THE 7 TOO ANO 12800 M ARCS.

IORED CATA FMILCH....

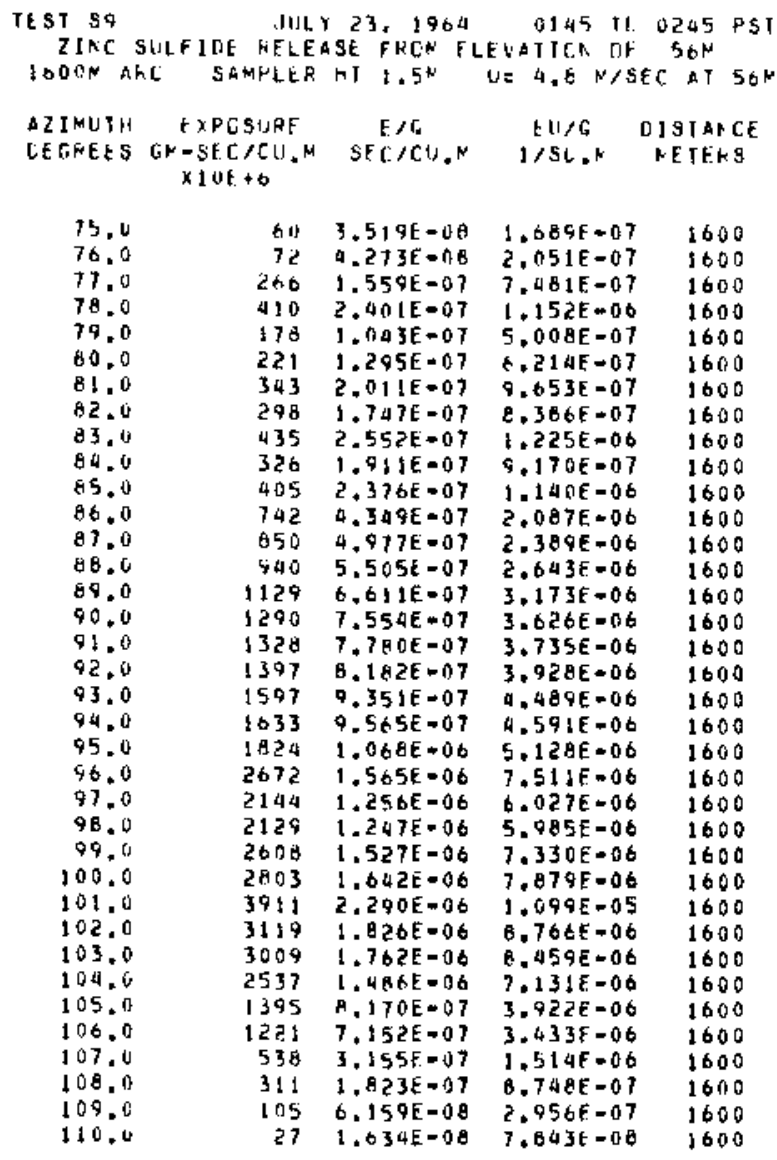
TFST 59 HWL Z3, 1340 OA45 100245 PST

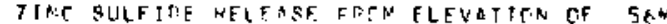 IAPIN ADC $11=0 . F \mathrm{~N} / \mathrm{SEC}$ AT $56 \mathrm{~N}$

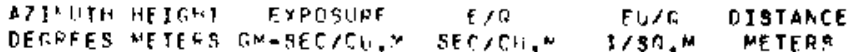
YINF

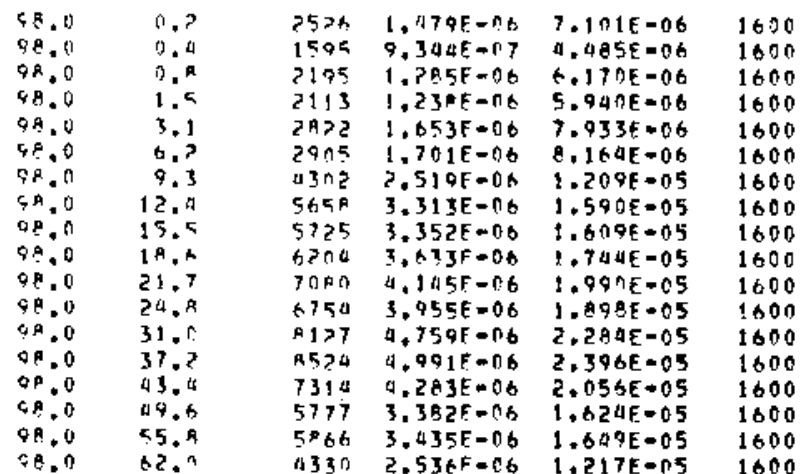

\begin{tabular}{|c|c|c|c|c|c|}
\hline $\begin{array}{l}\text { ATINI:TH } \\
\text { OFFEEES }\end{array}$ & $\begin{array}{l}\text { HE IGHT } \\
\text { METERS }\end{array}$ & $\begin{array}{c}\text { EXPCSUDE } \\
G^{N}=-9 F C C C 11 \\
\times 10 E+6\end{array}$ & $\begin{array}{c}E / \theta \\
S E C / C U, N\end{array}$ & $\begin{array}{c}E U / 0 \\
1 / \mathrm{sto}^{\circ} \mathrm{m}\end{array}$ & $\begin{array}{l}\text { DISTANCE } \\
\text { METERS }\end{array}$ \\
\hline 106.0 & 0,5 & 748 & $4,3+2 E-n 7$ & $2 \cdot \operatorname{tn} 3 t=06$ & 1600 \\
\hline $10 \mathrm{AB} .0$ & 0.4 & $n>1$ & $3.933 E-07$ & $B E=O B$ & 1600 \\
\hline 106.0 & (1. & 3054 & $6.17 \in E-07$ & $5 E=06$ & $16 \mathrm{nO}$ \\
\hline & 1.5 & 1030 & $n .035 F=0 ?$ & $7 E=0 B$ & 1600 \\
\hline 160.0 & 3.1 & 1113 & $2.516, E=07$ & $3.12 R E=06$ & 1000 \\
\hline $16 \mathrm{~h}, 0$ & 6.) & $12 \times 4$ & $7.520 \mathrm{E}=017$ & $3.612 E=06$ & $\ln 00$ \\
\hline 10 & 9.1 & 1703 & $1.050 \mathrm{C}+0 \mathrm{n}$ & $5,011 \mathrm{E}=06$ & 1600 \\
\hline 160.0 & 12.1 & 2373 & $1.19 \cap E-n E$ & $6.71 \mathrm{E}=\mathrm{Ct}$ & 1600 \\
\hline ict.o & 15.5 & $35 \times 3$ & $7.0106=06$ & $1.001 E-05$ & 1600 \\
\hline 1 n. 0 & 12.5 & 3?? 1 & $1, A R \in F=O B$ & $9 . n 5 ? t=06$ & 1500 \\
\hline 106 & $2^{5}, k$ & 4036 & $2.360 .5=0 t$ & $1.133 \%-05$ & 1600 \\
\hline 10 th 0 & 31.7 & at $1:$ & $? .70 \angle F=n A$ & 1. 29 AE $=05$ & 1600 \\
\hline $\begin{array}{l}186.0 \\
j<6.0\end{array}$ & $\begin{array}{l}37.7 \\
43.5\end{array}$ & $\begin{array}{l}1590 \\
1570 \\
150\end{array}$ & $\begin{array}{l}7.505 F-06 \\
4.271 F-06\end{array}$ & $1.203 F-05$ & 1600 \\
\hline ict & & 7167 & $4.1965=06$ & $2.014 \mathrm{~F}=0 \mathrm{C}$ & $\begin{array}{l}7600 \\
1600\end{array}$ \\
\hline & & yete? & U. $A P Q F=A B$ & $155 \%=05$ & 1600 \\
\hline $10 t, 0$ & $62, n$ & $7 n>t$ & $4.59 \Delta F=016$ & $2.157 \mathrm{E}-0.5$ & $\operatorname{lnn} 0$ \\
\hline
\end{tabular}

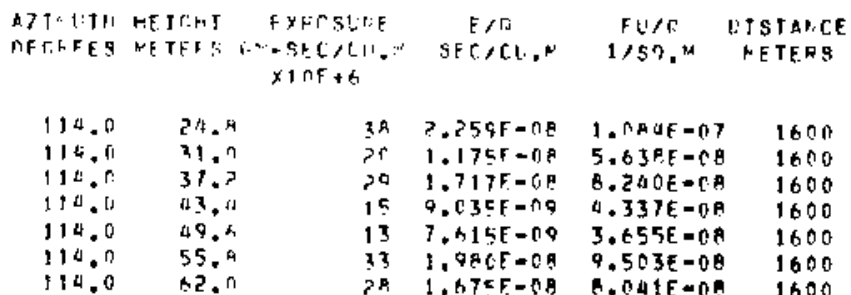




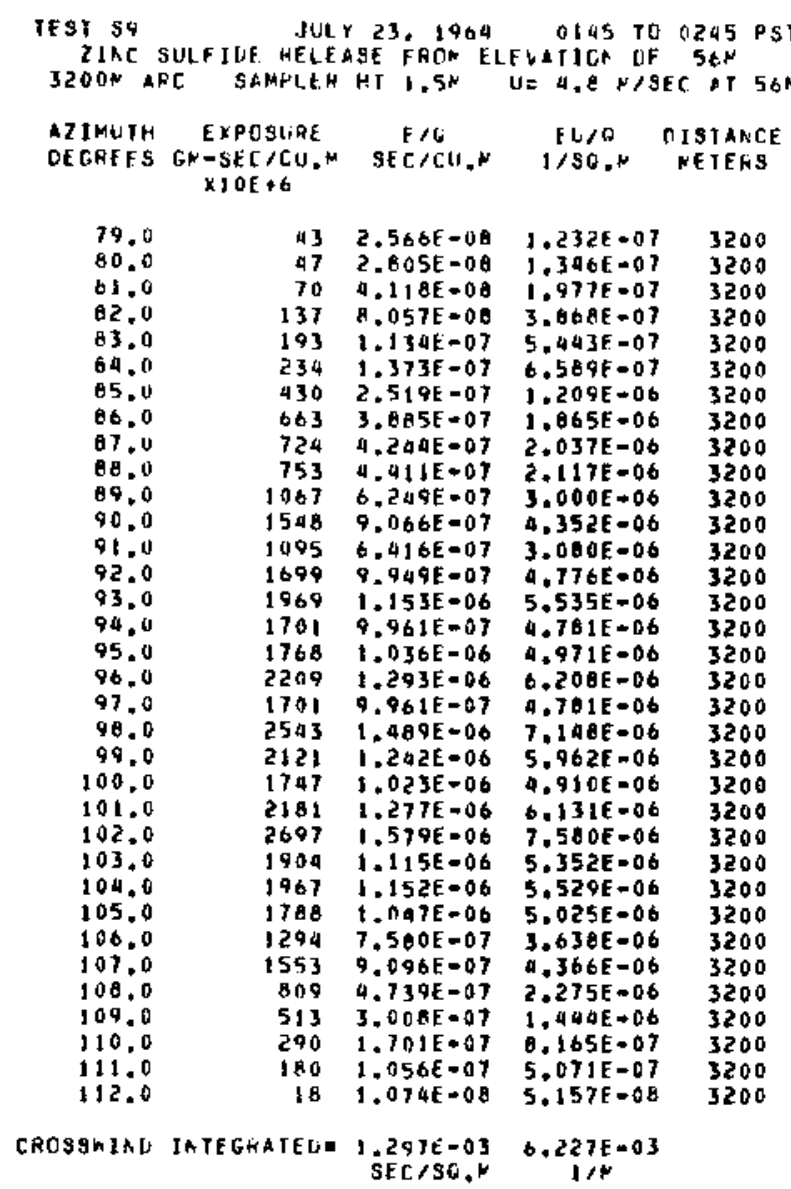

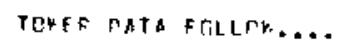

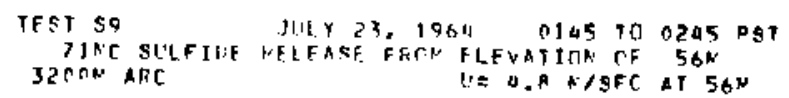

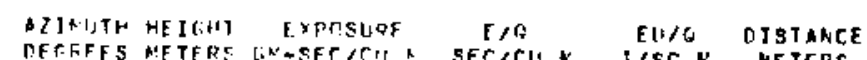

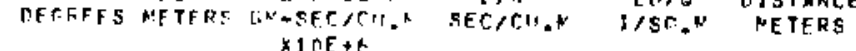

\begin{tabular}{|c|c|c|c|c|c|}
\hline 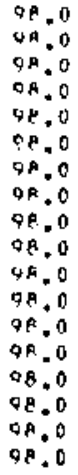 & $\begin{array}{l}0.7 \\
0.1 \\
0.8 \\
1.5 \\
3.1 \\
0.7 \\
9.3 \\
12.0 \\
15.5 \\
18.2 \\
21.7 \\
24.8 \\
31.0 \\
37.7 \\
43.4 \\
49.6 \\
55.6 \\
62.0\end{array}$ & 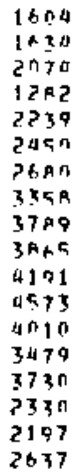 & 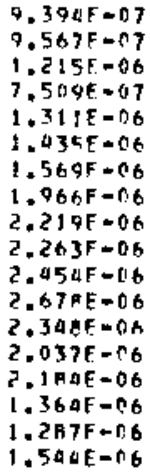 & $\begin{array}{l}9.509 E=06 \\
0.592 E=06 \\
5.930 E=06 \\
3.605 E=06 \\
6.295 E=06 \\
6.899 E=06 \\
7.532 E=06 \\
9.438 E=06 \\
1.065 E=05 \\
1.085 E=05 \\
1.178 E=05 \\
1.265 E=05 \\
1.127 E=05 \\
9.779 E=06 \\
1.04 A E=05 \\
6.508 E=06 \\
6.176 E=06 \\
7.411 E=06\end{array}$ & $\begin{array}{l}3200 \\
3200 \\
3200 \\
3200 \\
3200 \\
3200 \\
3200 \\
3200 \\
3200 \\
3200 \\
3200 \\
3200 \\
3700 \\
3200 \\
3200 \\
3200 \\
3200 \\
3200\end{array}$ \\
\hline
\end{tabular}

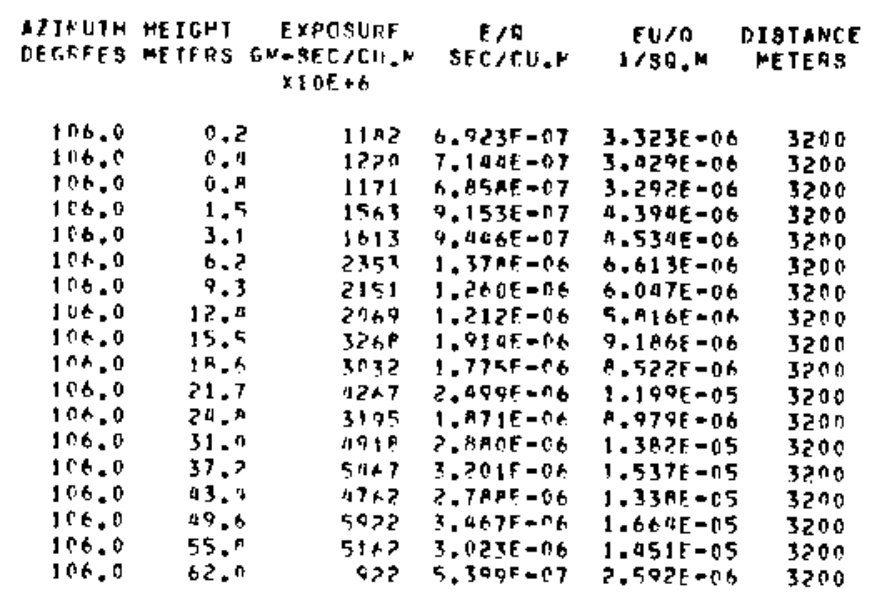

TEST 99 JULY P3, 196A 0145 TO0245 PST ZINC SULFIDE RELFASE FRAN ELEVATICN OF 5AN TOONY ARC SAMPTER HT $1.5^{*}$ U 4.0 MISEC AT $56 \mathrm{M}$

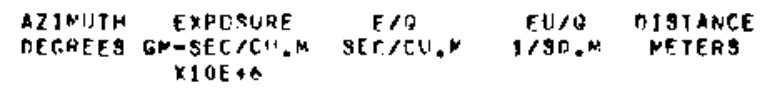

$\begin{array}{rrrrr}98.0 & 678 & 5.144 E-07 & 2.469 E-06 & 7000 \\ 102.0 & 1038 & 1.076 f-06 & 5.167 E-06 & 7000 \\ 2106.0 & 305 & 1.786 E-07 & 8.503 E-07 & 7000 \\ 110.0 & 4 & 2.949 E-09 & 1.176 E-00 & 7000\end{array}$

CROSSHIKO INTEGRAIEDE I.5A3E-03 7.601E-03

SEC $/$ SO.N

TF" 39 JLLY 23.1964 OIA5 TO O245 PST IINC SULFIDE RFLFASE FRDH ELEVATION THF 56N

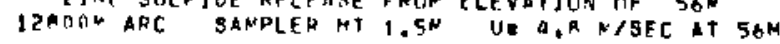

\begin{tabular}{|c|c|c|c|c|}
\hline $\begin{array}{l}\text { DINUTH } \\
\text { FETREFS }\end{array}$ & $\begin{array}{c}\text { EXPOSUPE } \\
\text { GN=SEC } / \mathrm{CI}, \mathrm{N} \\
\times \mathrm{O} O \mathrm{O}+6\end{array}$ & $\begin{array}{c}E / H \\
\text { SE }\end{array}$ & $\begin{array}{l}E \cup / 0 \\
1 / 90 .\end{array}$ & $\begin{array}{c}\text { DISTAR:CE } \\
\text { NEIERS }\end{array}$ \\
\hline
\end{tabular}

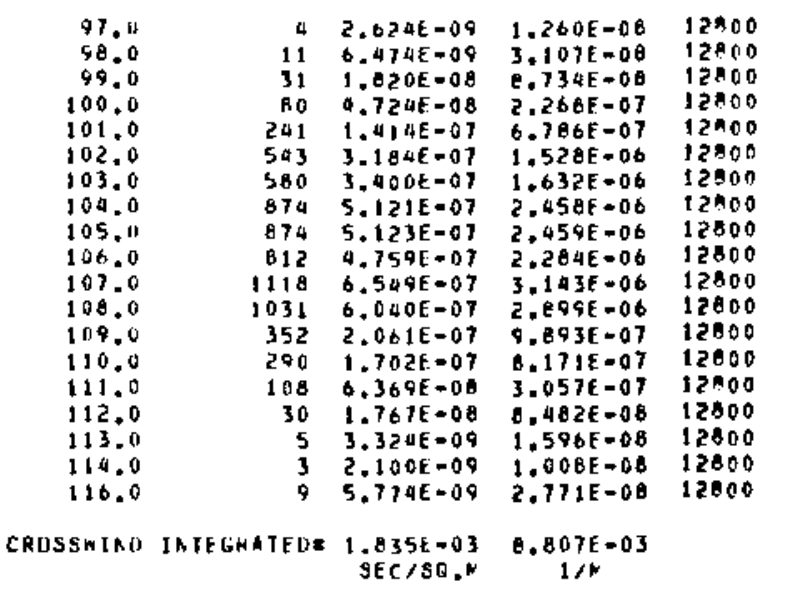


SERIES 164 EXPERIMENT: $10(S-10)$

GROUND LEVEL AND TONER SAMPLING ON THE 1600 AND 3200 M ARCS.

GROUND LEVEL SAMPL ING ONLY ON THE 7000 AND $12800 \mathrm{M}$ ARCS.

TORER DATA FOLLLW....

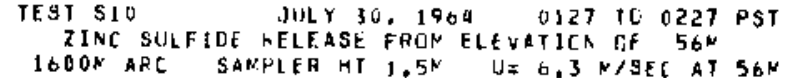

AZINUTH EXPUSURE EIC ELIG CISTARCE DFGREES GN-SEC/CU,N SECICU,N T/SG.N NETEHS $X 1$ OE +6

\begin{tabular}{|c|c|c|c|c|}
\hline $\begin{array}{l}99.0 \\
101.0 \\
102,0 \\
103.0 \\
104,0 \\
105.0 \\
100.0 \\
107.0 \\
108.0 \\
109,0 \\
110.0 \\
111.0 \\
112.0 \\
113.0 \\
114.0 \\
115.0 \\
116.0 \\
117.0 \\
118.0 \\
119.0 \\
120.0 \\
121.0 \\
122.0 \\
123.0 \\
124.0 \\
125.0 \\
126.0 \\
127.0 \\
128.0 \\
129.0 \\
130.0 \\
131.0\end{array}$ & $\begin{array}{r}33 \\
62 \\
96 \\
468 \\
533 \\
878 \\
644 \\
450 \\
498 \\
158 \\
184 \\
356 \\
758 \\
1781 \\
2095 \\
3238 \\
3473 \\
3706 \\
199 \\
319 \\
92 \\
276 \\
123 \\
1036 \\
987 \\
903 \\
1138 \\
1287 \\
797 \\
854 \\
460 \\
229\end{array}$ & $\begin{array}{l}2.083 E=08 \\
3.920 E=08 \\
6.003 E=08 \\
2.916 E=07 \\
3.332 E=07 \\
5.4 A 8 E=07 \\
9.031 E=07 \\
2.018 E=07 \\
3.050 E=07 \\
9.923 E=08 \\
1.152 E=07 \\
2.230 E=07 \\
4.741 E=07 \\
1.114 E=06 \\
1.310 E=06 \\
2.024 E=06 \\
2.171 E=06 \\
2.317 E=06 \\
1.250 E=07 \\
1.997 E=07 \\
5.750 E=08 \\
1.727 E=07 \\
7.718 E=08 \\
6.461 E=07 \\
6.174 E=07 \\
5.648 E=07 \\
7.118 E=07 \\
1.049 E=07 \\
4.986 E=07 \\
5.966 E=07 \\
2.279 E=07 \\
1.433 E=07\end{array}$ & 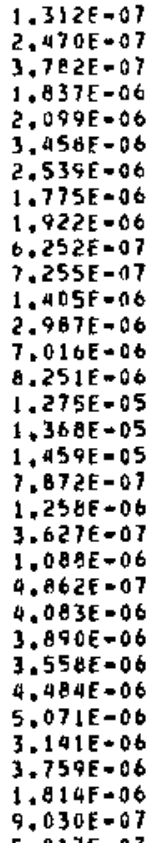 & $\begin{array}{l}1600 \\
1600 \\
1600 \\
1600 \\
1600 \\
1600 \\
1600 \\
1600 \\
1600 \\
1600 \\
1600 \\
1600 \\
1600 \\
1600 \\
1600 \\
1600 \\
1600 \\
1600 \\
1600 \\
1600 \\
1600 \\
1600 \\
1600 \\
1600 \\
1600 \\
1600 \\
1600 \\
1600 \\
1600 \\
1600 \\
1600 \\
1600\end{array}$ \\
\hline
\end{tabular}

CROSSMIAO IATEGHATEOS 4.958E-04 3.12HE-03

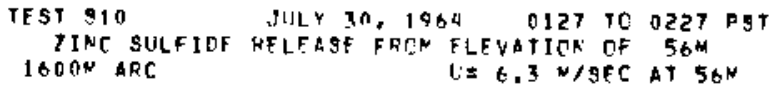

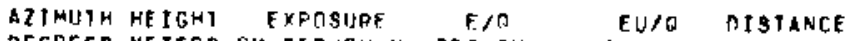

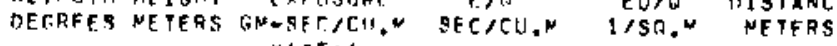
$X \cap M E+A$

\begin{tabular}{|c|c|c|c|c|c|}
\hline $\begin{array}{l}106.0 \\
106.0 \\
106.0 \\
106.0 \\
106.0 \\
106.0 \\
106.0 \\
106.0 \\
106.0 \\
106.0 \\
106.0 \\
106.0 \\
106.0 \\
106.0 \\
106.0 \\
106.0 \\
106.0 \\
106.0\end{array}$ & $\begin{array}{r}0 . ? \\
0.0 \\
0.9 \\
1.5 \\
3.1 \\
6.2 \\
9.3 \\
12.4 \\
15.5 \\
18.5 \\
21.7 \\
24.8 \\
31.0 \\
37.2 \\
43.4 \\
49.6 \\
55.8 \\
62.0\end{array}$ & $\begin{array}{l}517 \\
792 \\
674 \\
391 \\
518 \\
697 \\
762 \\
907 \\
941 \\
942 \\
1265 \\
717 \\
906 \\
694 \\
942 \\
101 \\
200 \\
110\end{array}$ & 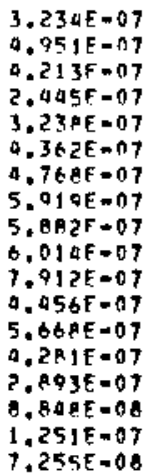 & 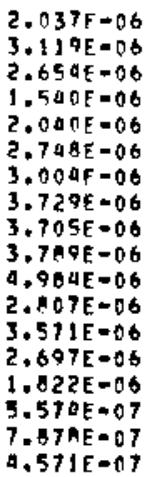 & $\begin{array}{l}1600 \\
1600 \\
1600 \\
1600 \\
1600 \\
1600 \\
1600 \\
1600 \\
1600 \\
1600 \\
1600 \\
1600 \\
1600 \\
1600 \\
1600 \\
1600 \\
1600 \\
1600\end{array}$ \\
\hline
\end{tabular}

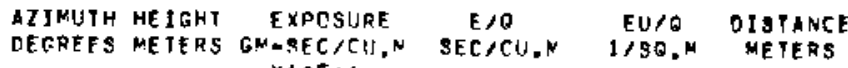
$\times 1 O E+6$

\begin{tabular}{|c|c|c|c|c|c|}
\hline 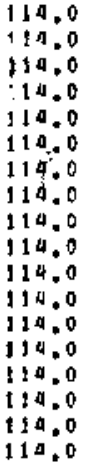 & $\begin{array}{l}0.2 \\
0.4 \\
0.8 \\
1.5 \\
3.1 \\
6.2 \\
9.3 \\
12.4 \\
15.5 \\
1.9 .5 \\
21.7 \\
20.7 \\
31.0 \\
37.7 \\
93.4 \\
49.6 \\
55.8 \\
62.7\end{array}$ & $\begin{array}{l}2002 \\
2393 \\
2200 \\
2202 \\
2583 \\
2512 \\
2909 \\
3406 \\
2645 \\
3238 \\
3098 \\
3430 \\
3440 \\
5405 \\
6107 \\
1423 \\
5301 \\
5405\end{array}$ & $\begin{array}{l}1.252 F=06 \\
1.496 E=06 \\
1.432 E=06 \\
1.37 \in E=06 \\
1.614 F=06 \\
1.57 \cap E=06 \\
1.744 E=06 \\
2.129 E=06 \\
1.679 E=06 \\
2.624 E=06 \\
1.934 E=06 \\
2.150 F=06 \\
2.275 F=06 \\
3.37 P E=06 \\
3.817 E=06 \\
2.827 F=06 \\
3.313 E=06 \\
3.37 P F=06\end{array}$ & 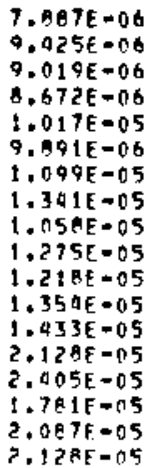 & $\begin{array}{l}1600 \\
1600 \\
1600 \\
1600 \\
1600 \\
1600 \\
1600 \\
1600 \\
1600 \\
1600 \\
1600 \\
1600 \\
1600 \\
1600 \\
1600 \\
1600 \\
1600 \\
1000\end{array}$ \\
\hline
\end{tabular}




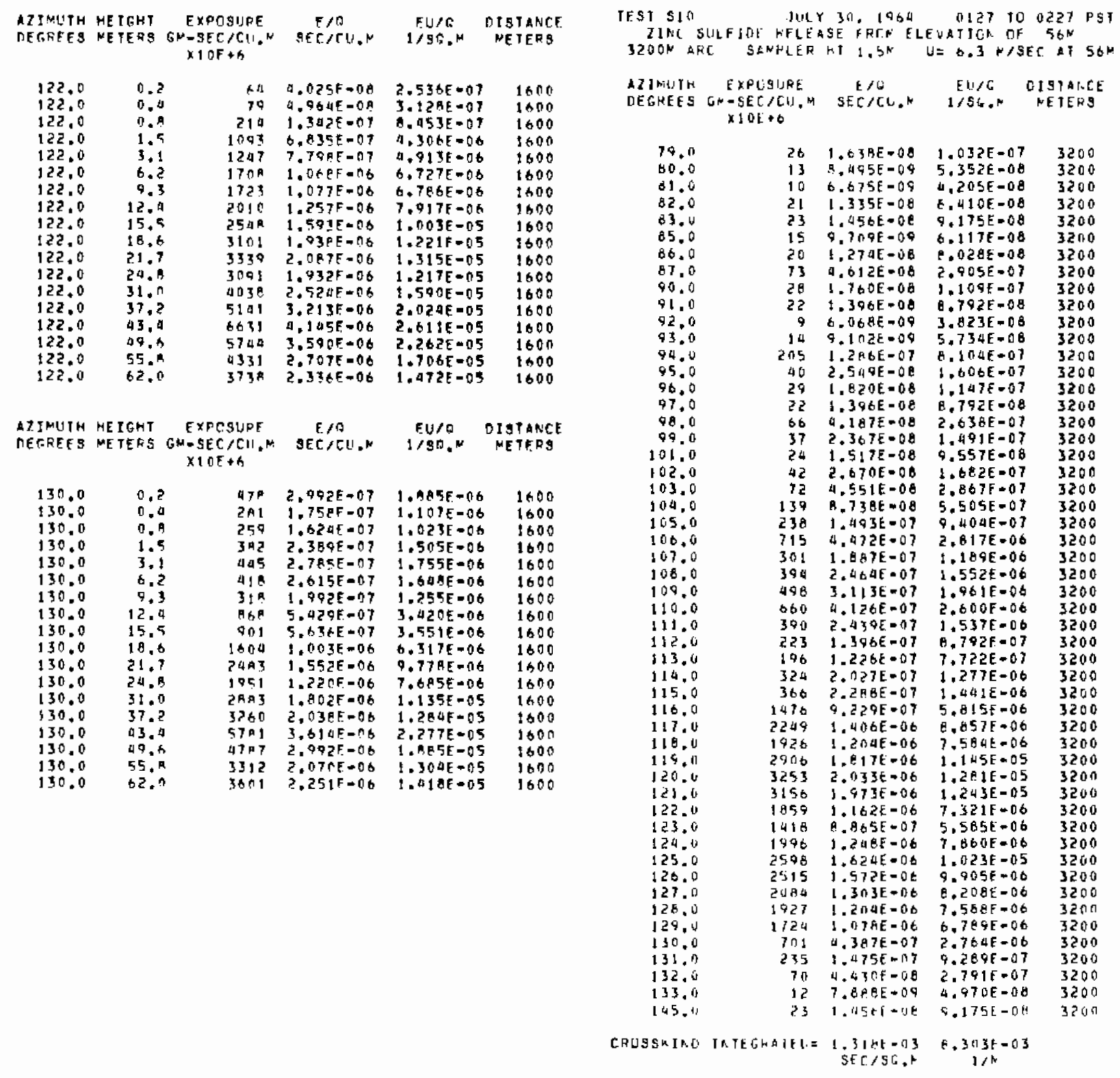


TOWER TATS FOLLCW...

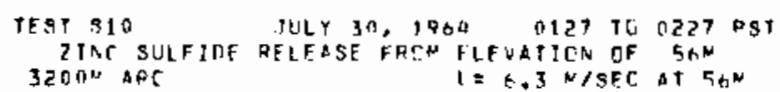

AZTMUTH HEIGHT EXFOSURF EID ELOE CISTANCE AEGRFES METERS GN-SEC/CII.N SFCITU.N IJSO.M NETERS
XIOE+G

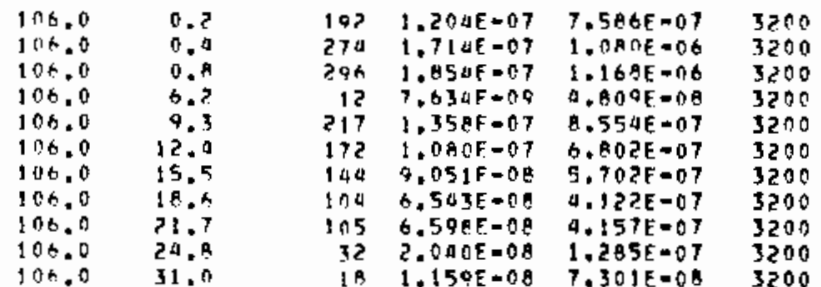

AZIMUTH HEIGHT EYPCSURE F/O DU/G DISTANCE DEGREES METEHS GM-gEC/CIIN SEC/CU.N I/SR.N METFRS $\times 10 \mathrm{E}+6$

\begin{tabular}{|c|c|c|c|c|c|}
\hline 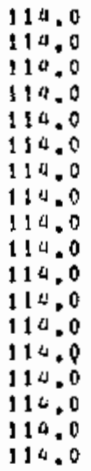 & $\begin{array}{l}0.2 \\
0.4 \\
0.9 \\
1.5 \\
3.1 \\
6.2 \\
9.3 \\
12.9 \\
15.5 \\
18.8 \\
21.7 \\
24.8 \\
31.0 \\
37.2 \\
43.4 \\
49.6 \\
55.8 \\
62.0\end{array}$ & 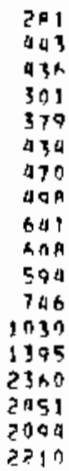 & 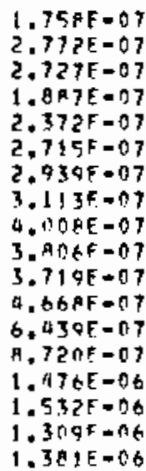 & $\begin{array}{l}1.10 A E=06 \\
1.746 E=06 \\
1.719 E=06 \\
1.189 E=06 \\
1.994 E=06 \\
1.711 E=06 \\
1.852 E=06 \\
1.961 E=06 \\
2.525 E=06 \\
2.39 B E=06 \\
2.343 E=06 \\
2.941 E=06 \\
9.057 E=06 \\
5.190 E=06 \\
9.296 E=06 \\
9.851 E=06 \\
9.24 A E=06 \\
0.702 E=06\end{array}$ & $\begin{array}{l}3200 \\
3200 \\
3200 \\
3200 \\
3200 \\
3200 \\
3200 \\
3200 \\
3200 \\
3200 \\
3200 \\
3200 \\
3200 \\
3200 \\
3200 \\
3200 \\
3200 \\
3200\end{array}$ \\
\hline
\end{tabular}

\begin{tabular}{|c|c|c|c|c|c|}
\hline $\begin{array}{l}\text { AZTNIITH } \\
\text { DETEFES }\end{array}$ & $\begin{array}{l}\text { HE IGHT } \\
\text { METERS }\end{array}$ & $\begin{array}{c}\text { EXPOSURE } \\
\text { GM }-3 E C / C U . M \\
\times 10 E+b\end{array}$ & $\begin{array}{c}E / \theta \\
\text { SEC } / \text { I. }\end{array}$ & $\begin{array}{c}E \cup / 6 \\
1 / 80 .\end{array}$ & $\begin{array}{l}\text { OISTANCE } \\
\text { ME TFRS }\end{array}$ \\
\hline $\begin{array}{l}122.0 \\
122.0 \\
122.0 \\
122.0 \\
122.0 \\
122.0 \\
122.0 \\
122.0 \\
122.0 \\
122.0 \\
122.0 \\
122.0 \\
122.0 \\
122.0 \\
122.0 \\
122.0 \\
122.0 \\
122.0\end{array}$ & $\begin{array}{l}0 . ? \\
0.9 \\
0.9 \\
1.5 \\
3.1 \\
6.2 \\
9.3 \\
12.4 \\
15.5 \\
19.6 \\
21.7 \\
29.9 \\
31.0 \\
37.2 \\
43.4 \\
49.6 \\
55.9 \\
62.0\end{array}$ & $\begin{array}{l}1711 \\
1605 \\
1769 \\
1243 \\
1651 \\
2019 \\
2659 \\
2810 \\
2701 \\
3252 \\
2826 \\
3040 \\
2940 \\
3437 \\
3472 \\
1657 \\
3769 \\
4650\end{array}$ & $\begin{array}{l}1.070 E=06 \\
1.003 F=06 \\
1.106 E=116 \\
1.770 F=01 \\
1.032 E=06 \\
1.262 E=06 \\
1.662 E=06 \\
1.757 F=06 \\
1.713 E=06 \\
2.033 E=06 \\
1.767 F=06 \\
1.900 E=06 \\
1.063 E=06 \\
2.14 F E=06 \\
2.171 E=06 \\
1.036 E=06 \\
2.355 E=06 \\
2.906 E=06\end{array}$ & $\begin{array}{l}6.739 \mathrm{E}=06 \\
6.322 \mathrm{E}=06 \\
6.96 \mathrm{E}=06 \\
4.895 \mathrm{~F}=06 \\
6.503 \mathrm{E}=06 \\
7.949 \mathrm{E}=06 \\
1.047 \mathrm{E}=05 \\
1.107 \mathrm{E}=05 \\
1.079 \mathrm{E}=05 \\
1.281 \mathrm{E}=05 \\
1.113 \mathrm{E}=05 \\
1.197 \mathrm{E}=05 \\
1.173 \mathrm{E}=05 \\
1.353 \mathrm{E}=05 \\
1.367 \mathrm{E}=05 \\
6.526 \mathrm{E}=06 \\
1.464 \mathrm{E}=05 \\
1.831 \mathrm{E}=05\end{array}$ & $\begin{array}{l}3200 \\
3200 \\
3200 \\
3200 \\
3200 \\
3200 \\
3200 \\
3200 \\
3200 \\
3200 \\
3200 \\
3200 \\
3200 \\
3200 \\
3200 \\
3200 \\
3200 \\
3200\end{array}$ \\
\hline
\end{tabular}

\begin{tabular}{|c|c|c|c|c|c|}
\hline $\begin{array}{l}\text { LIMUTM } \\
\text { DEGREES }\end{array}$ & $\begin{array}{l}\text { HE IGHT } \\
\text { ME TERS }\end{array}$ & $\begin{array}{c}\text { EXPOSUAE } \\
\text { GM-SEC/C11.M } \\
\times 10 E+6\end{array}$ & $\begin{array}{c}E / O \\
S E C / C U, N\end{array}$ & $\begin{array}{l}1 / 900 \\
1 / 90,4\end{array}$ & $\begin{array}{c}\text { OISTANCE } \\
\text { METERS }\end{array}$ \\
\hline $\begin{array}{l}130.0 \\
130.0 \\
130.0 \\
130.0 \\
130.0 \\
130.0 \\
130.0 \\
130.0 \\
130.0 \\
130.0 \\
130.0 \\
130.0 \\
130.0 \\
130.0 \\
130.0 \\
130.0 \\
130.0 \\
130.0\end{array}$ & $\begin{array}{r}0.2 \\
0.9 \\
0.8 \\
1.5 \\
3.1 \\
6.2 \\
9.3 \\
12.4 \\
15.5 \\
18.8 \\
21.7 \\
24.8 \\
31.0 \\
37.7 \\
43.9 \\
49.6 \\
55.18 \\
62.0\end{array}$ & $\begin{array}{l}502 \\
554 \\
625 \\
612 \\
145 \\
898 \\
1020 \\
1999 \\
2141 \\
2632 \\
2741 \\
2941 \\
2990 \\
2589 \\
2430 \\
1279 \\
1039 \\
653\end{array}$ & 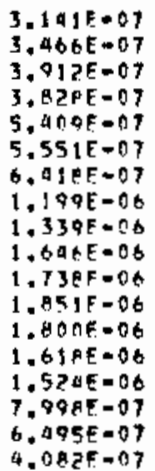 & $\begin{array}{l}1.979 E=06 \\
2.102 E=06 \\
2.465 E=00 \\
2.912 E=06 \\
3.408 E=06 \\
3.097 E=06 \\
1.043 E=06 \\
7.554 E=06 \\
6.433 E=06 \\
1.037 E=05 \\
1.095 E=05 \\
1.164 E=05 \\
1.139 E=05 \\
1.020 E=05 \\
9.600 E=06 \\
5.039 E=06 \\
1.092 E=06 \\
2.572 E=06\end{array}$ & $\begin{array}{l}3200 \\
3200 \\
3200 \\
3200 \\
3200 \\
3200 \\
3200 \\
3200 \\
3200 \\
3200 \\
3200 \\
3200 \\
3200 \\
3200 \\
3200 \\
3200 \\
3200 \\
3200\end{array}$ \\
\hline
\end{tabular}

TEST $\$ 10$

JHLY 30, 10B4 0127 10 0257 921

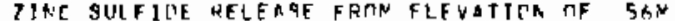
TOOON ARC SANPIER HT $1.5 \mathrm{~N}$ IIE $6.3 \mathrm{~N} / \mathrm{SFC}$ AT SBN

\begin{tabular}{|c|c|c|c|c|}
\hline $\begin{array}{l}\text { AZTRUTH } \\
\text { DEGREFG }\end{array}$ & $\begin{array}{c}\text { EXPCISUDE } \\
\text { GN=SEC/C11.M } \\
\times 1 O E+6\end{array}$ & $\begin{array}{c}F / C^{\prime} \\
S E C / C H . N\end{array}$ & $\begin{array}{l}E 11 / 6 \\
1 / 96 . N\end{array}$ & $\begin{array}{c}\text { NISTANCE } \\
\text { NETEAS }\end{array}$ \\
\hline $\begin{array}{r}98.0 \\
106.0 \\
110.0 \\
114.0 \\
118.0 \\
122.0 \\
126.0 \\
130.0\end{array}$ & $\begin{array}{r}6 \\
8 \\
390 \\
855 \\
426 \\
1032 \\
1318 \\
3\end{array}$ & $\begin{array}{l}4.246 E=09 \\
5.043 \mathrm{E}=09 \\
2.439 E-07 \\
5.347 E-07 \\
5.748 E-07 \\
1.020 \mathrm{E}=06 \\
8.244 E-07 \\
2.054 E=09\end{array}$ & $\begin{array}{l}2.706 E=08 \\
3.177 F=08 \\
1.537 E=06 \\
3.369 E=06 \\
3.646 E=06 \\
6.428 E=06 \\
5.194 E=06 \\
1.294 F=08\end{array}$ & $\begin{array}{l}7000 \\
7100 \\
7000 \\
7000 \\
7000 \\
7000 \\
1000 \\
7000\end{array}$ \\
\hline
\end{tabular}

CHOSSHINC INTEGRATELI $2.878 F-03 \quad 1.813 E-02$

IFST S10 JULY 30, 1964 0137 TO O227 PST ZINC SULFIDE RFLEASF FFCM FLEUATICH: TIF $5 \mathrm{kN}$

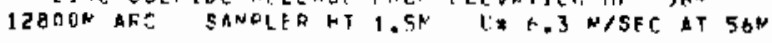

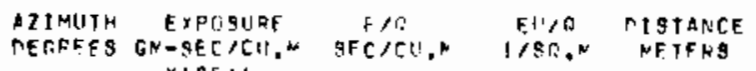
$x I \cap E+b$

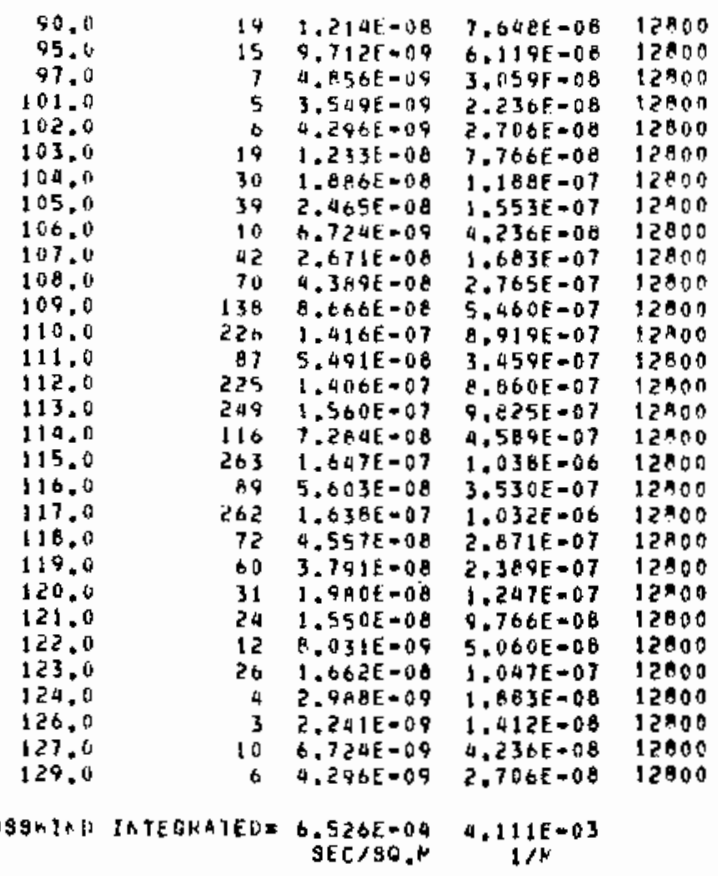


SERIES 164 EXPERIMENT: 11 ( S- 11$)$

GROUND LEYEL AND TOWER SAHFLING ON THE 1600 AND 3200 N ARCS.

GROUND LEVEL SAHPLING ONLY ON THE 7000 AND 12800 M ARCS.

SIGNIFICANT PORTION OF THE PLUHE NEYER PASSED OYER THE GRID.

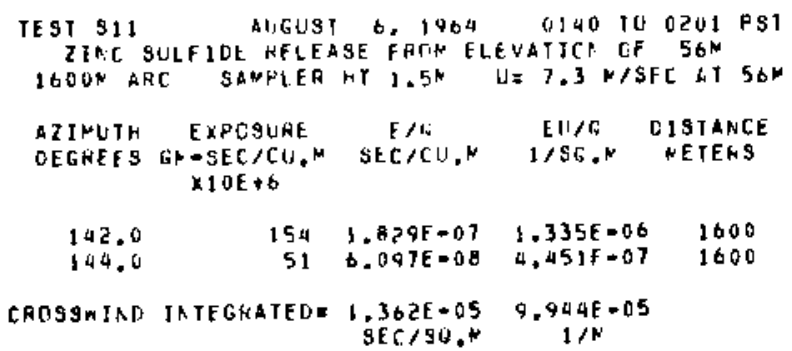

$\begin{array}{lrrrrr}130.0 & 0.2 & 160 & 2.134 E-07 & 1.550 E-06 & 1600 \\ 130.0 & 0.8 & 317 & 3.761 E-07 & 2.746 E-06 & 1600 \\ 130.0 & 0.2 & 382 & 0.532 E-07 & 3.300 E-06 & 1600 \\ 130.0 & 9.3 & 20 & 2.439 F-0.0 & 1.760 E-07 & 1600 \\ 130.0 & 12.4 & 190 & 2.256 E-07 & 1.607 E-06 & 1600\end{array}$

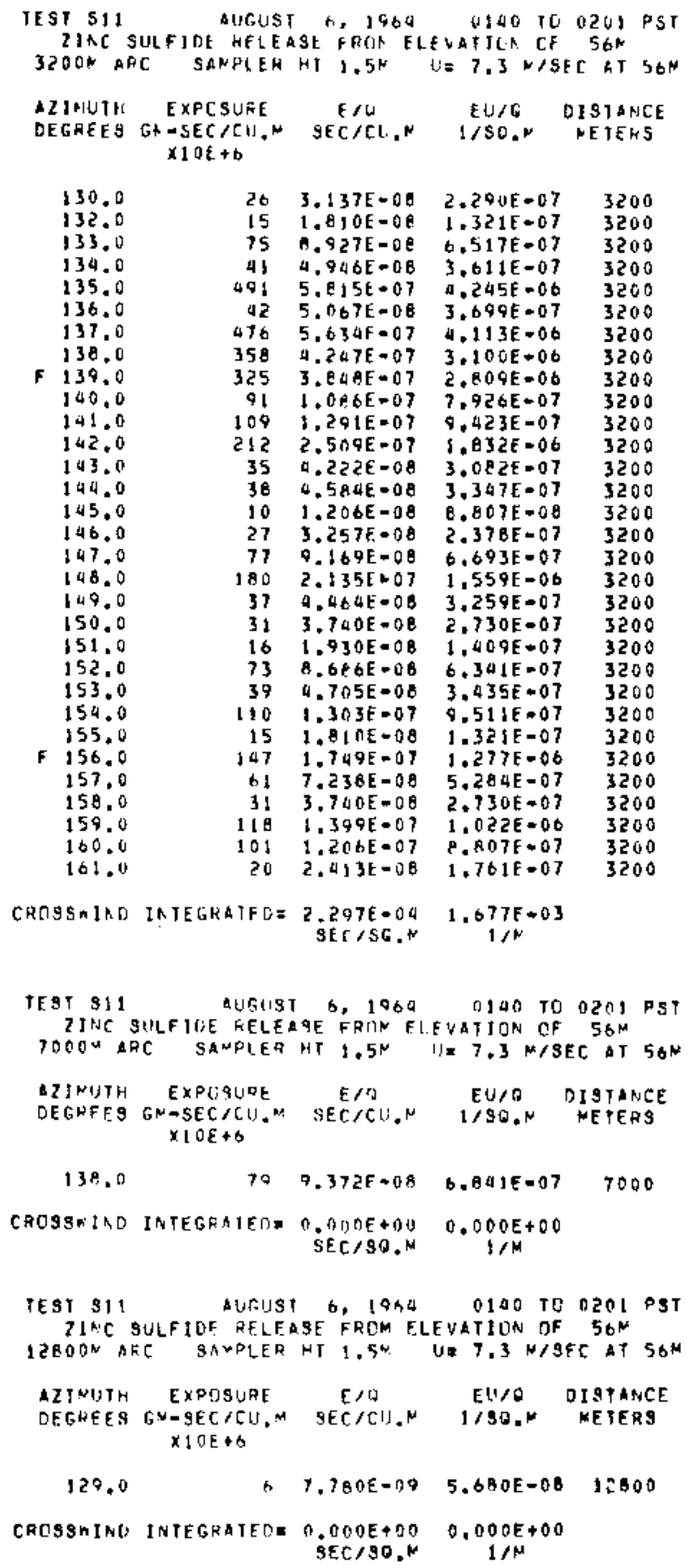


SERIES 164 EXPERIMENT: 12 (S-12)

GROUND LEYEL AND TOWER SAMPLING ON THE 1600 RND 3200 M ARCS.

GROUNDLEVEL SAMPL ING ONEY ON THE 7000 AND 12800 M ARCS.

TOMER PATA FULLTH, ...

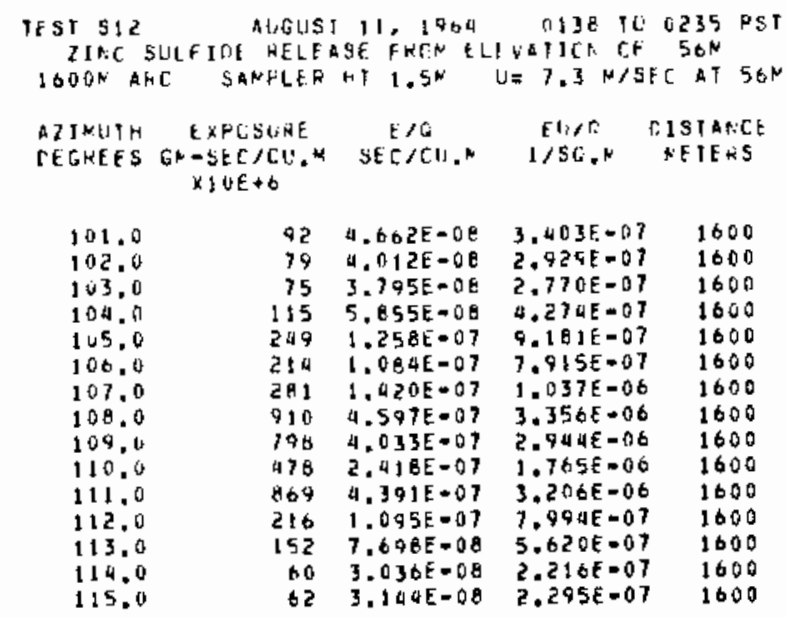
TEST \$12 AUGUST 11,1960 1139 10 0235 $95 T$ 2IRC SULFIUE KELEASE FPUN ELFVATIGN DF $56 \mathrm{M}$

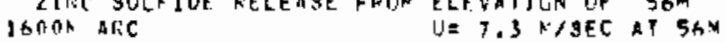

\begin{tabular}{|c|c|c|c|c|c|}
\hline $\begin{array}{l}\text { AZINUTH } \\
\text { OEGNEES }\end{array}$ & $\begin{array}{l}\text { HEIGHT } \\
\text { METEPS }\end{array}$ & 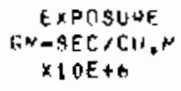 & $\begin{array}{c}E / Q \\
\text { SEr } / C \cup, N\end{array}$ & $\begin{array}{c}E U / Q \\
1 / 3 A . M\end{array}$ & $\begin{array}{l}\text { ISTAMCE } \\
\text { METERS }\end{array}$ \\
\hline $\begin{array}{l}106.0 \\
106.0 \\
106.0 \\
106.0 \\
100.0 \\
106.0 \\
106.0 \\
106.0 \\
106.0 \\
106.0 \\
106.0 \\
106.0 \\
106.0 \\
106.0 \\
106.0\end{array}$ & $\begin{array}{r}0.2 \\
0.4 \\
0.9 \\
1.5 \\
3.5 \\
6.2 \\
9.3 \\
12.4 \\
15.5 \\
18.6 \\
21.7 \\
29.8 \\
31.0 \\
37.2 \\
43.4\end{array}$ & $\begin{array}{l}112 \\
114 \\
55 \\
159 \\
179 \\
196 \\
480 \\
463 \\
437 \\
865 \\
319 \\
360 \\
162 \\
90 \\
15\end{array}$ & 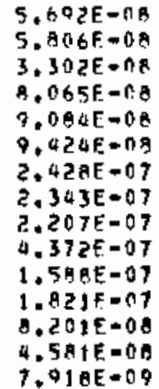 & $\begin{array}{l}4.155 E=07 \\
9.238 E-07 \\
2.010 E-07 \\
5.280 E=07 \\
6.631 E=07 \\
6.079 E=07 \\
1.772 E=06 \\
1.711 E=06 \\
1.611 E=06 \\
3.592 E=06 \\
1.159 E=06 \\
1.329 E=06 \\
5.987 E-07 \\
3.399 E=07 \\
5.760 E-01\end{array}$ & $\begin{array}{l}1600 \\
1600 \\
1600 \\
1600 \\
1600 \\
1600 \\
1600 \\
1600 \\
1600 \\
1600 \\
1600 \\
1600 \\
1600 \\
1600 \\
1600\end{array}$ \\
\hline
\end{tabular}

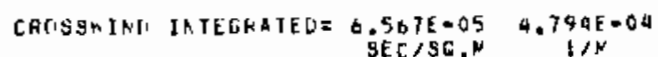

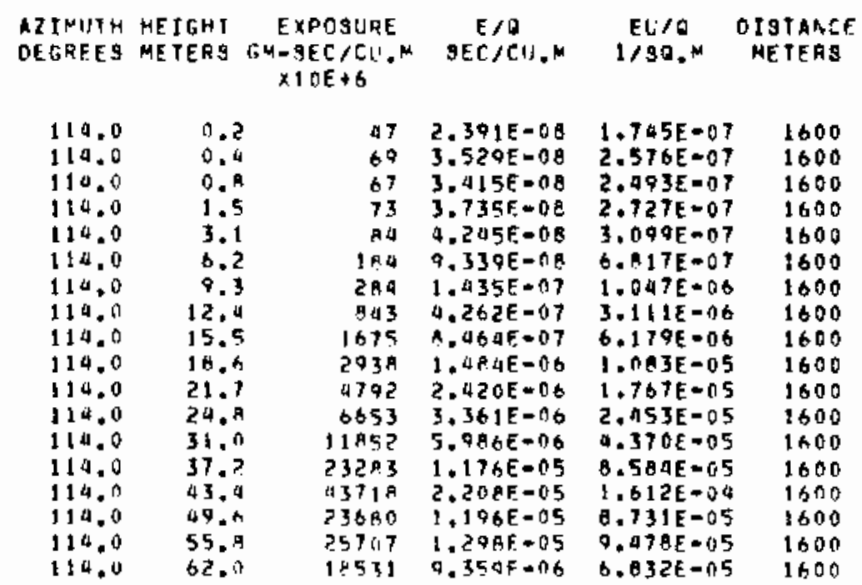

\begin{tabular}{|c|c|c|c|c|c|}
\hline $\begin{array}{l}\text { AZIMUTH } \\
\text { DEGREFS }\end{array}$ & $\begin{array}{l}\text { HEICHT } \\
\text { METEHS }\end{array}$ & 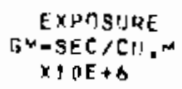 & $\begin{array}{c}5 / 6 \\
\operatorname{SEC} / \mathrm{CL} . \mathrm{N}\end{array}$ & $\begin{array}{c}E Q / 6 \\
1 / 59.4\end{array}$ & $\begin{array}{l}\text { DIST ANCE } \\
\text { METERS }\end{array}$ \\
\hline $\begin{array}{l}122.0 \\
122.0 \\
122.0 \\
122.0 \\
122.0 \\
122.0 \\
122.0 \\
122.0 \\
122.0 \\
122.0 \\
122.0\end{array}$ & $\begin{array}{r}9.3 \\
12.4 \\
15.5 \\
21.7 \\
24.8 \\
31.0 \\
37.2 \\
43.4 \\
49.6 \\
55.2 \\
62.0\end{array}$ & $\begin{array}{l}26 \\
67 \\
53 \\
16 \\
12 \\
27 \\
19 \\
19 \\
8 \\
16 \\
13\end{array}$ & $\begin{array}{l}1.35 P E=08 \\
3.396 E=08 \\
2.717 E=08 \\
8.490 E=09 \\
6.221 E=09 \\
1.4111 E=08 \\
9.615 E=09 \\
7.352 E=04 \\
4.234 E=09 \\
8.468 E=09 \\
6.820 E=09\end{array}$ & $\begin{array}{l}9.916 E=0 . \\
2.479 E=07 \\
1.983 E=07 \\
6.197 \mathrm{E}=08 \\
4.542 E=08 \\
1.032 E=07 \\
7.019 E=08 \\
5.367 E=08 \\
3.091 E=09 \\
6.182 E=08 \\
5.050 E=08\end{array}$ & $\begin{array}{l}1600 \\
1000 \\
1600 \\
1600 \\
1600 \\
1600 \\
1600 \\
1600 \\
1600 \\
160 \% \\
1600\end{array}$ \\
\hline
\end{tabular}




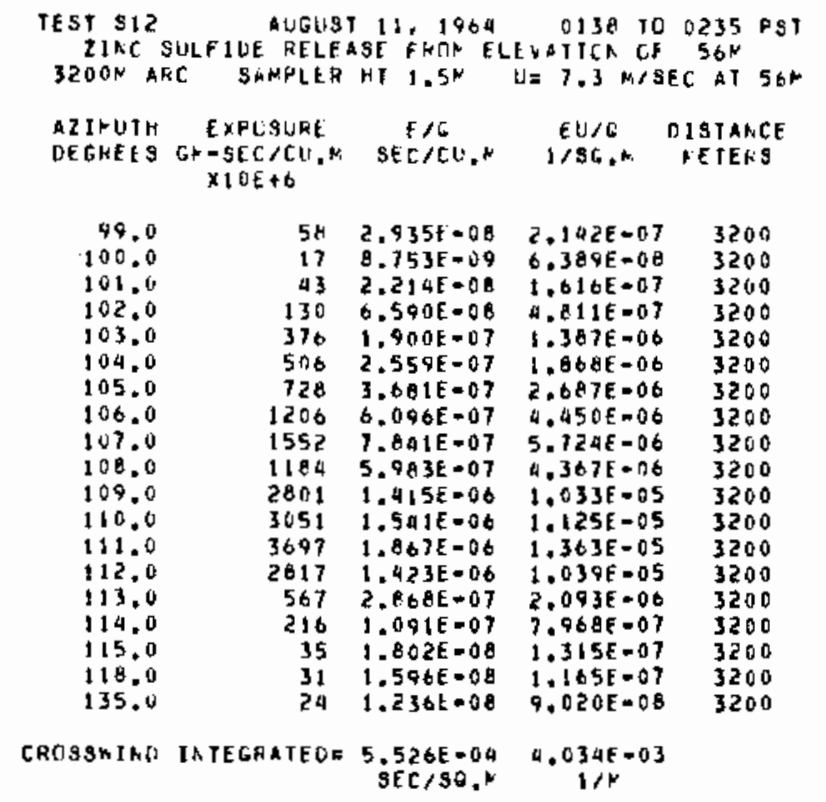

TOWER RATA FOLLINW....

\begin{tabular}{|c|c|c|c|c|c|}
\hline \multicolumn{2}{|c|}{$\begin{array}{l}\text { TEST } \$ 12 \\
\text { ZINC SULFIDE } \\
3200 \mathrm{ARC}\end{array}$} & $\begin{array}{l}\text { AUGUS' } 11 \text {, } 19 \\
\text { MELCASE FRIIA }\end{array}$ & $\begin{array}{l}9641 \text { O138 } \\
\text { FLEVATICA } \\
\text { UIX } 7.3 \mathrm{M} / \mathrm{SE}\end{array}$ & PSY & \\
\hline $\begin{array}{l}\text { AZINUPH } \\
\text { DEGREES }\end{array}$ & $\begin{array}{l}\text { HE IGH T } \\
\text { ME TERS }\end{array}$ & $\begin{array}{c}\text { EXFCSURE } \\
\text { GWO-AEC } / C U . N \\
\times 10 E+6\end{array}$ & $\begin{array}{c}E / 2 \\
S E C / C U . W\end{array}$ & $\begin{array}{c}F \cup / 6 \\
1 / 50 .\end{array}$ & $\begin{array}{l}\text { DISTANCE } \\
\text { METERg }\end{array}$ \\
\hline 106.0 & 0.2 & 918 & $0.639 E-n)$ & $3.386 E=06$ & 3200 \\
\hline 100,0 & 0.4 & $\operatorname{An} 6$ & $4.073 E-D 7$ & $2.973 \mathrm{E}$ & \\
\hline 10h.0 & 0.8 & $9 \% ?$ & $5.015 E=07$ & $3.061 E-06$ & 3200 \\
\hline 106.0 & 1,5 & 100 & $3.587 E-07$ & $2.604 E=06$ & 3200 \\
\hline 106.0 & 3.1 & Ast & $4,37 \mathrm{KE}=07$ & $3.195 E=06$ & 3200 \\
\hline 106.0 & 6.2 & A79 & 4,4 & $3.243 E=06$ & \\
\hline 106.0 & 9.3 & 522 & $2.640 \mathrm{E}-07$ & $1.927 \mathrm{E}-06$ & 3200 \\
\hline 100.0 & 12.4 & 906 & $9.57 \mathrm{BE}=07$ & $3,342 \mathrm{E}=00$ & 3200 \\
\hline $910 t .0$ & 15.5 & 595 & 3.01 & $2.1975=06$ & 3200 \\
\hline 100.0 & 18.6 & 632 & 3.19 & $2.339 \mathrm{E}-0 \mathrm{~B}$ & $\begin{array}{l}3200 \\
3300\end{array}$ \\
\hline 105.0 & 21.7 & 5 A & 2.97 & $2+1700-06$ & \\
\hline 106.0 & 24.0 & & & & \\
\hline 106.0 & 31.0 & 1 A2 & 9.22 & $6.73 \Delta E=0 T$ & 3200 \\
\hline 106.0 & 37.2 & AS & $4.339 E=00$ & $3.167 E=07$ & 3204 \\
\hline $\begin{array}{l}\text { AZIS,UTH } \\
\text { OEGHEES }\end{array}$ & $\begin{array}{l}\text { HE IGHT } \\
\text { METERS }\end{array}$ & $\begin{array}{c}\text { EXPOSUAE } \\
\text { GMEC }-3 E U . M \\
\times 1 D E+b\end{array}$ & $\begin{array}{c}E / \theta \\
S E C / C U . M\end{array}$ & $\begin{array}{l}E \cup / 0 \\
1 / 30 . M\end{array}$ & $\begin{array}{c}\text { OISTANEE } \\
\text { MEFERS }\end{array}$ \\
\hline 119.8 & 0,2 & 109 & & 1.36 & \\
\hline & & & & & \\
\hline & $n .0$ & 176 & A. 9 & 0.502 & 10 \\
\hline 1 & 1.5 & 404 & 2.0 & $1.993 E=06$ & \\
\hline & 3,1 & 3 & 1.7 & 1.3 & \\
\hline & 6. & of & $3.52 \Delta F$ & 2.57 & \\
\hline & & 13 & 6.0 & 0.05 & \\
\hline & 12 & & 1.36 & 1.01 & \\
\hline & 15.5 & $3 n 06$ & 1.867 & $1.363[-05$ & 3200 \\
\hline & & 4701 & $? .1$ & $1.7 t$ & \\
\hline & 21 & anst & $2.338 \mathrm{~F}$ & $1,700 E=05$ & 3200 \\
\hline & 24 & 7056 & 3.564 & $2.602 \mathrm{E}-05$ & \\
\hline & 31 & & 4.005 & $3.507 \mathrm{E}-05$ & \\
\hline & & 18708 & $\triangle \triangle P A E=0 B$ & $6.926 E=05$ & 3200 \\
\hline 114,0 & 45.12 & $2091)$ & $1.050 f-155$ & $7.70 \mathrm{AE}-05$ & 3509 \\
\hline & $49 . \mathrm{t}$ & 17402 & $0.264 \mathrm{E}-10$ & $a .573 E=05$ & 200 \\
\hline & & $26 n 7$ & $1.317 \mathrm{E}=116$ & $9.813 t=08$ & 3200 \\
\hline & & 1000 & $5.051 F-07$ & $3 . \Delta O 7 \mathrm{E}=06$ & 3200 \\
\hline
\end{tabular}

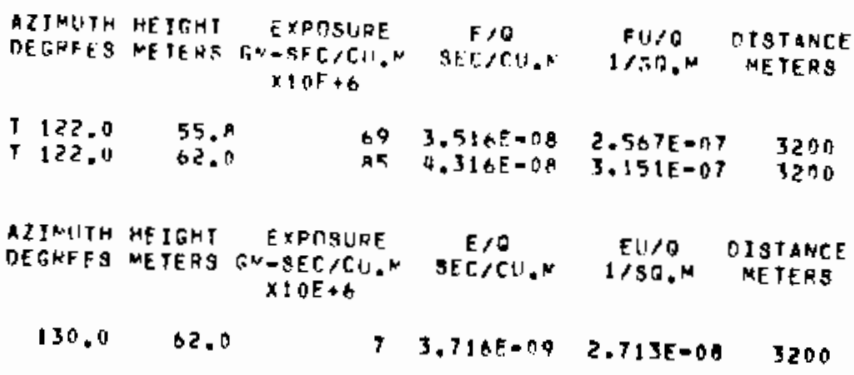


TOMFR HATA F!ULI.H: ...

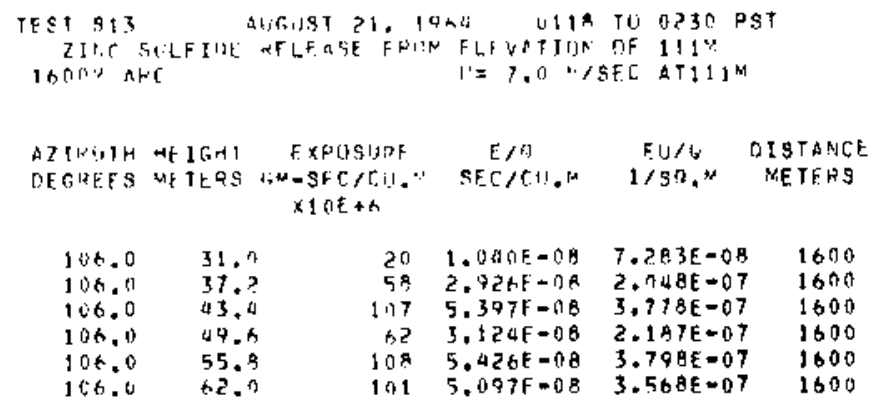

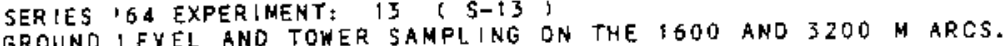

GRONND

SIGNIFICANT PORTION OF THE PLUME NEVER PASSED OVER THE GRIO. THE

TRACER WAS NOT OETECTED ON THE 1600 M ARC.

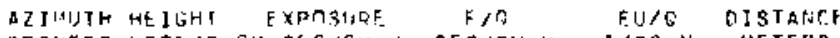

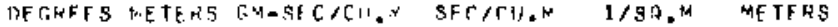
$\times 10 F+6$

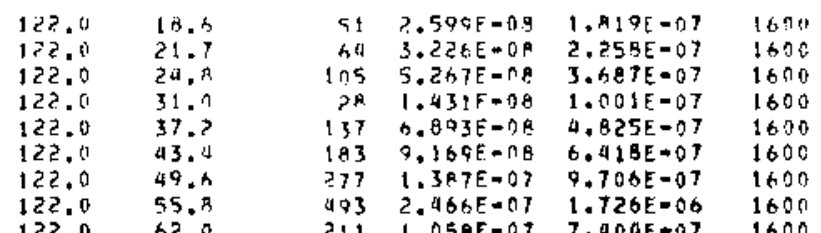

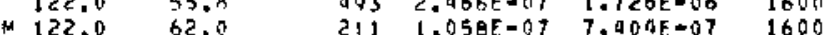

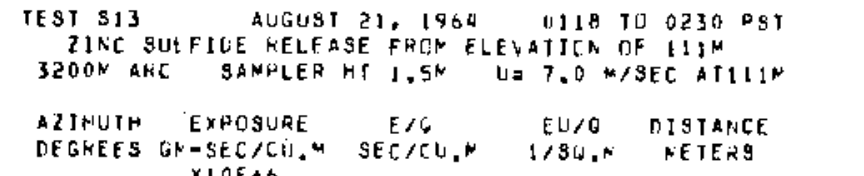

\begin{tabular}{|c|c|c|c|c|}
\hline $\begin{array}{l}71.0 \\
72.0 \\
73.0 \\
76.0 \\
77.0 \\
78.0 \\
79.0 \\
80.0 \\
81.0 \\
83.0 \\
84.0 \\
85.0 \\
66.0 \\
88.0 \\
89.0 \\
90.0 \\
92.0 \\
95.0 \\
98.0 \\
90.0 \\
100.0 \\
101.0 \\
102.0 \\
103.0 \\
104.0 \\
105.0 \\
106.0 \\
107.0 \\
108.0 \\
109.0 \\
110.0 \\
111.0 \\
112.0 \\
120.0 \\
121.0 \\
122.0 \\
123.0 \\
124.0 \\
125.0 \\
126.0\end{array}$ & $\begin{array}{l}25 \\
92 \\
15 \\
13 \\
67 \\
21 \\
11 \\
30 \\
38 \\
49 \\
10 \\
57 \\
28 \\
19 \\
12 \\
9 \\
22 \\
24 \\
66 \\
25 \\
41 \\
11 \\
62 \\
15 \\
28 \\
20 \\
19 \\
67 \\
38 \\
18 \\
47 \\
13 \\
19 \\
11 \\
8 \\
23 \\
32 \\
14 \\
110 \\
29\end{array}$ & $\begin{array}{l}1.274 E=08 \\
4.129 E=08 \\
7.646 E=09 \\
6.676 E=09 \\
3.364 E=08 \\
1.070 E=08 \\
5.607 E=09 \\
1.529 E=08 \\
1.937 E=08 \\
2.498 E=08 \\
5.097 E=09 \\
2.054 E=08 \\
1.427 E=0 B \\
9.684 E=09 \\
6.117 E=09 \\
4.587 E=09 \\
1.121 E=06 \\
1.223 E=08 \\
3.313 E=08 \\
1.274 E=08 \\
2.090 E=08 \\
5.607 E=08 \\
3.109 E=08 \\
7.646 E=09 \\
1.427 E=08 \\
1.019 E=08 \\
9.684 E=09 \\
3.364 E=08 \\
1.937 E=08 \\
9.175 E=09 \\
2.396 E=08 \\
6.626 E=09 \\
9.684 E=09 \\
5.6177 E=09 \\
4.078 E=09 \\
1.172 E=08 \\
1.631 E=06 \\
1.136 E=09 \\
5.505 E=08 \\
1.478 E=08\end{array}$ & 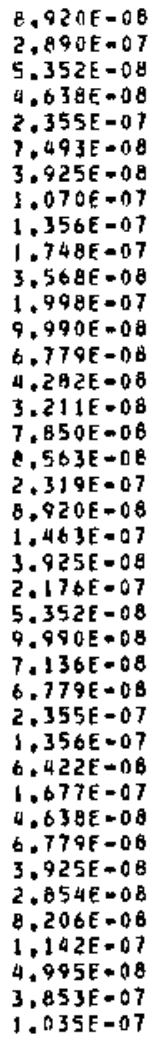 & $\begin{array}{l}3200 \\
3200 \\
3200 \\
3200 \\
3200 \\
3200 \\
3200 \\
3200 \\
3200 \\
3200 \\
3200 \\
3200 \\
3200 \\
3200 \\
3200 \\
3200 \\
3200 \\
3200 \\
3200 \\
3200 \\
3200 \\
3200 \\
3200 \\
3200 \\
3200 \\
3200 \\
3200 \\
3200 \\
3200 \\
3200 \\
3200 \\
3200 \\
3200 \\
3200 \\
3200 \\
3200 \\
3200 \\
3200 \\
3200 \\
3200\end{array}$ \\
\hline & GHA & $\begin{array}{c}4.520 E=05 \\
S E C / 90 . *\end{array}$ & $\begin{array}{c}3.1698=04 \\
1 / N\end{array}$ & \\
\hline
\end{tabular}


TOKER NATA FULLLIK....

\begin{tabular}{|c|c|c|c|c|c|}
\hline $\begin{array}{l}\text { TES S13 } \\
\text { ZINC } \\
3200 \mathrm{~N}\end{array}$ & $\begin{array}{l}\text { SULFIDE } \\
\text { RC }\end{array}$ & $\begin{array}{l}\text { AUGUST } 21 \text { P } \\
\text { HELFASE FRA }\end{array}$ & $\begin{array}{l}\text { ELEVATIOI } \\
U=7.0 \mathrm{M}\end{array}$ & $\begin{aligned} \text { TO } & 0230 \\
\text { QF } & 111 \mathrm{M} \\
\text { SEC } & 1111 \%\end{aligned}$ & PST \\
\hline $\begin{array}{l}\text { AZINELITH } \\
\text { DETSHFES }\end{array}$ & $\begin{array}{l}\text { HE ISHT } \\
\text { NETERS }\end{array}$ & $\begin{array}{c}\text { EXPOSIJRE } \\
G M-S E C / C U, M \\
\times 10 F+B\end{array}$ & $\begin{array}{c}E / 0 \\
S E C / C, 1, m\end{array}$ & $\begin{array}{l}E \cup / D \\
1 / S A, M\end{array}$ & $\begin{array}{l}\text { DTSFANE } \\
\text { METERS }\end{array}$ \\
\hline $\begin{array}{l}106.0 \\
106.0 \\
106.0 \\
106.0 \\
106.0 \\
106.0\end{array}$ & $\begin{array}{l}15.5 \\
18.8 \\
21.7 \\
24.8 \\
31.0 \\
37.2\end{array}$ & $\begin{array}{r}22 \\
69 \\
96 \\
34 \\
123 \\
14\end{array}$ & $\begin{array}{l}1.112 E=08 \\
3.475 E=08 \\
4.819 E=08 \\
1.706 E=08 \\
4.151 E=08 \\
4.206 E=08\end{array}$ & $\begin{array}{l}7.785 E-08 \\
2.033 E=07 \\
3.373 E=07 \\
1.222 E=07 \\
9.305 E=07 \\
2.994 E=07\end{array}$ & $\begin{array}{l}3200 \\
3200 \\
3200 \\
3200 \\
3200 \\
3200\end{array}$ \\
\hline $\begin{array}{l}\text { AZIMUTH } \\
\text { DEGREES }\end{array}$ & $\begin{array}{l}\text { HEIGHT } \\
\text { METERS }\end{array}$ & $\begin{array}{c}\text { EXPOSURE } \\
\text { SM- } 3 E C / C I{ }^{\prime} A \\
\times 10 E+6\end{array}$ & $\begin{array}{c}E / 2 \\
\operatorname{SEC} / C U, M\end{array}$ & $\begin{array}{l}E U / 0 \\
1 / 30, M\end{array}$ & $\begin{array}{l}\text { DI GTANC } \\
\text { METERS }\end{array}$ \\
\hline $\begin{array}{l}114.0 \\
114.0 \\
114.0 \\
114.0 \\
114.0 \\
114.0 \\
114.0\end{array}$ & $\begin{array}{l}12.0 \\
31.0 \\
37.2 \\
43.4 \\
49.6 \\
55.8 \\
62.0\end{array}$ & $\begin{array}{r}20 \\
55 \\
145 \\
234 \\
492 \\
581 \\
1000\end{array}$ & $\begin{array}{l}1.019 E=06 \\
2.778 E=08 \\
7.262 E=08 \\
1.175 E=07 \\
2.071 E=07 \\
2.906 E=07 \\
5.5026=07\end{array}$ & $\begin{array}{l}7.136 \mathrm{E}=00 \\
1.944 \mathrm{E}=07 \\
5.083 \mathrm{E}=07 \\
8.222 \mathrm{E}=07 \\
1.730 \mathrm{E}=06 \\
2.034 \mathrm{E}=06 \\
3.851 \mathrm{E}=06\end{array}$ & $\begin{array}{l}3200 \\
3200 \\
3200 \\
3200 \\
3200 \\
3200 \\
3200\end{array}$ \\
\hline $\begin{array}{l}\text { AZIMUTH } \\
\text { MESHFE } 3\end{array}$ & $\begin{array}{l}\text { HEIGHT } \\
\text { NETEHS }\end{array}$ & $\begin{array}{c}\text { EXPOSIJRF. } \\
G M=B E C>C A=14 \\
\times 10 E+b\end{array}$ & $\begin{array}{c}E / O \\
\operatorname{SEC} / \mathrm{CH} . \mathrm{N}\end{array}$ & $\begin{array}{l}E \cup / Q \\
1 / \mathrm{SO} . \mathrm{M}\end{array}$ & $\begin{array}{l}\text { DISTANC } \\
\text { METERS }\end{array}$ \\
\hline $\begin{array}{l}122.0 \\
122.0 \\
122.0 \\
122.0 \\
122.0 \\
122.0 \\
122.0 \\
122.0 \\
122.0 \\
122.0 \\
122.0 \\
122.0 \\
122.0 \\
122.0 \\
122.0 \\
122.0 \\
1122.0 \\
122.0\end{array}$ & $\begin{array}{r}0.7 \\
0.1 \\
0.8 \\
1.5 \\
3.1 \\
6.2 \\
9.3 \\
12.5 \\
15.5 \\
18.6 \\
21.7 \\
24.8 \\
31.0 \\
37.2 \\
43.8 \\
49.6 \\
55.5 \\
62.0\end{array}$ & $\begin{array}{r}141 \\
25 \\
14 \\
42 \\
31 \\
35 \\
29 \\
65 \\
79 \\
139 \\
47 \\
103 \\
153 \\
110 \\
296 \\
143 \\
311 \\
242\end{array}$ & 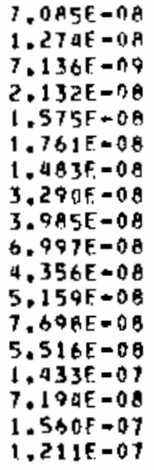 & $\begin{array}{l}4.959 E=07 \\
8.920 E=08 \\
4.995 E=08 \\
1.092 E=07 \\
1.103 E=07 \\
1.233 E=07 \\
1.036 E=07 \\
2.303 E=07 \\
2.789 E=07 \\
1.098 E=07 \\
3.049 E=07 \\
3.611 E=07 \\
5.389 E=07 \\
3.861 E=07 \\
1.003 E=06 \\
5.036 E=07 \\
1.092 E=06 \\
0.076 E=07\end{array}$ & $\begin{array}{l}3200 \\
3200 \\
3200 \\
3200 \\
3200 \\
3200 \\
3200 \\
3200 \\
3200 \\
3200 \\
3200 \\
3200 \\
3200 \\
3200 \\
3200 \\
3200 \\
3200 \\
3200\end{array}$ \\
\hline
\end{tabular}

TFST S\$3 AUFIST 21 , 1924 O11日 TO 0230 PST

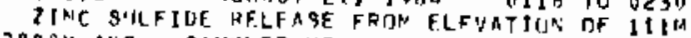

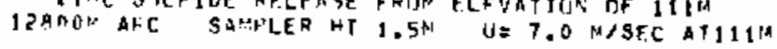

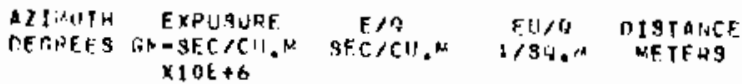

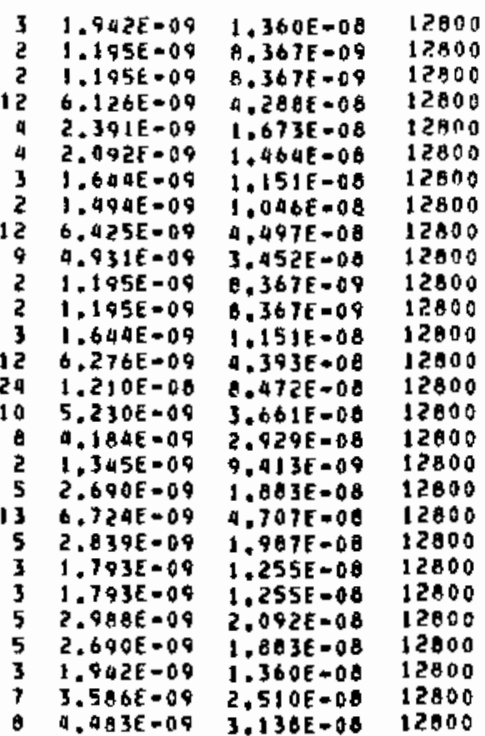

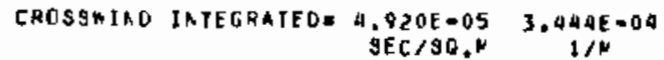

597.0

$? 98.0$

2100.0

2101.0

6102.

7103.0

4106.

3107.0

108.0

7109.0

7111.

- 112.0

6113.0

3114.0

5117.0

2116,0

2119.0

1121.0

1123.0

2124.0

426.0

2129.0

TFST $S: 3$

AZIMLIR EXPUSURE E/G EU/A DISTANCE

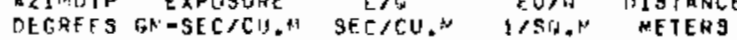
$\times 10 \mathrm{E}+6$

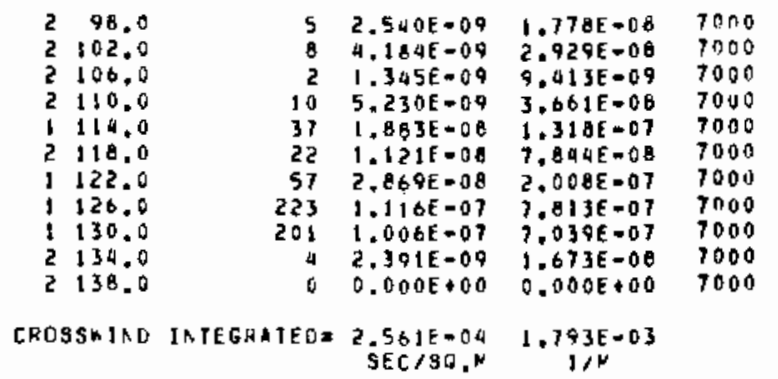


SERIES 164 EXPERIMENT: 14 ( $5-14$ )

GROUNO LEVEL SAMPLING 1600-12800 M ARCS.

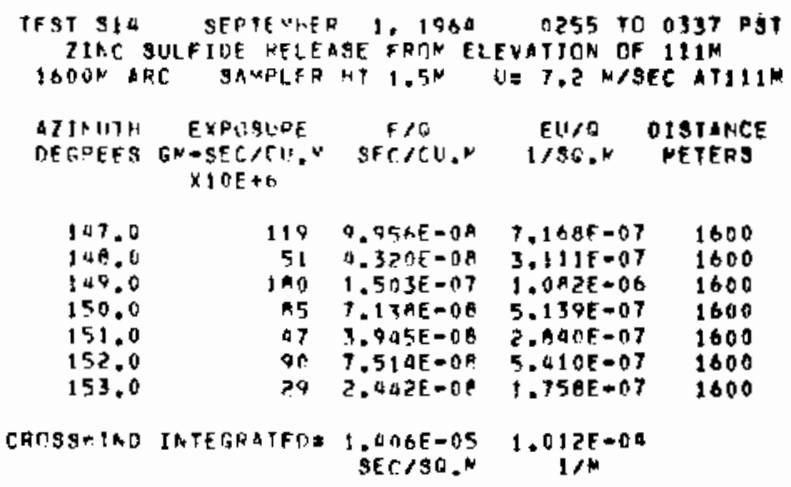

TFST 914 SEPTCHEF 1,1964 0255 TO 0337 PST 7 ISC SILFIDF HELESSE FPTH FLFVATIOA RF $11 \pm M$

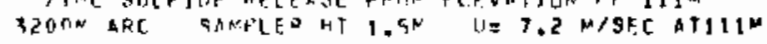

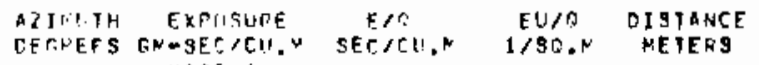
$\times 10 E+6$

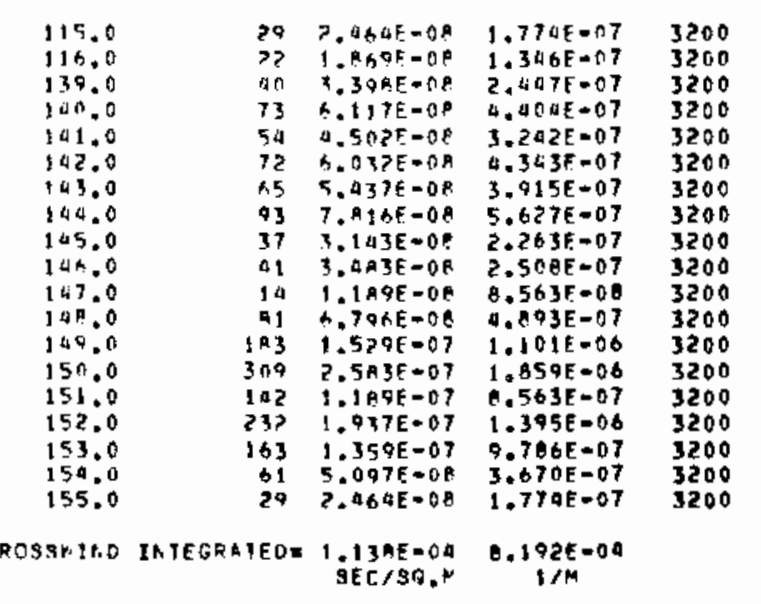

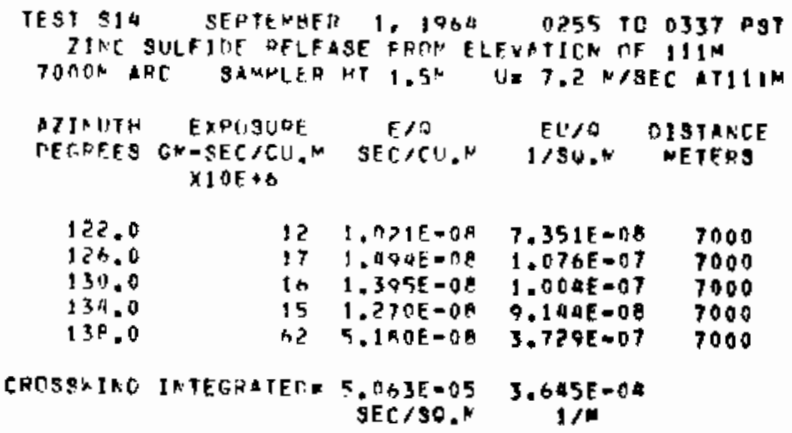




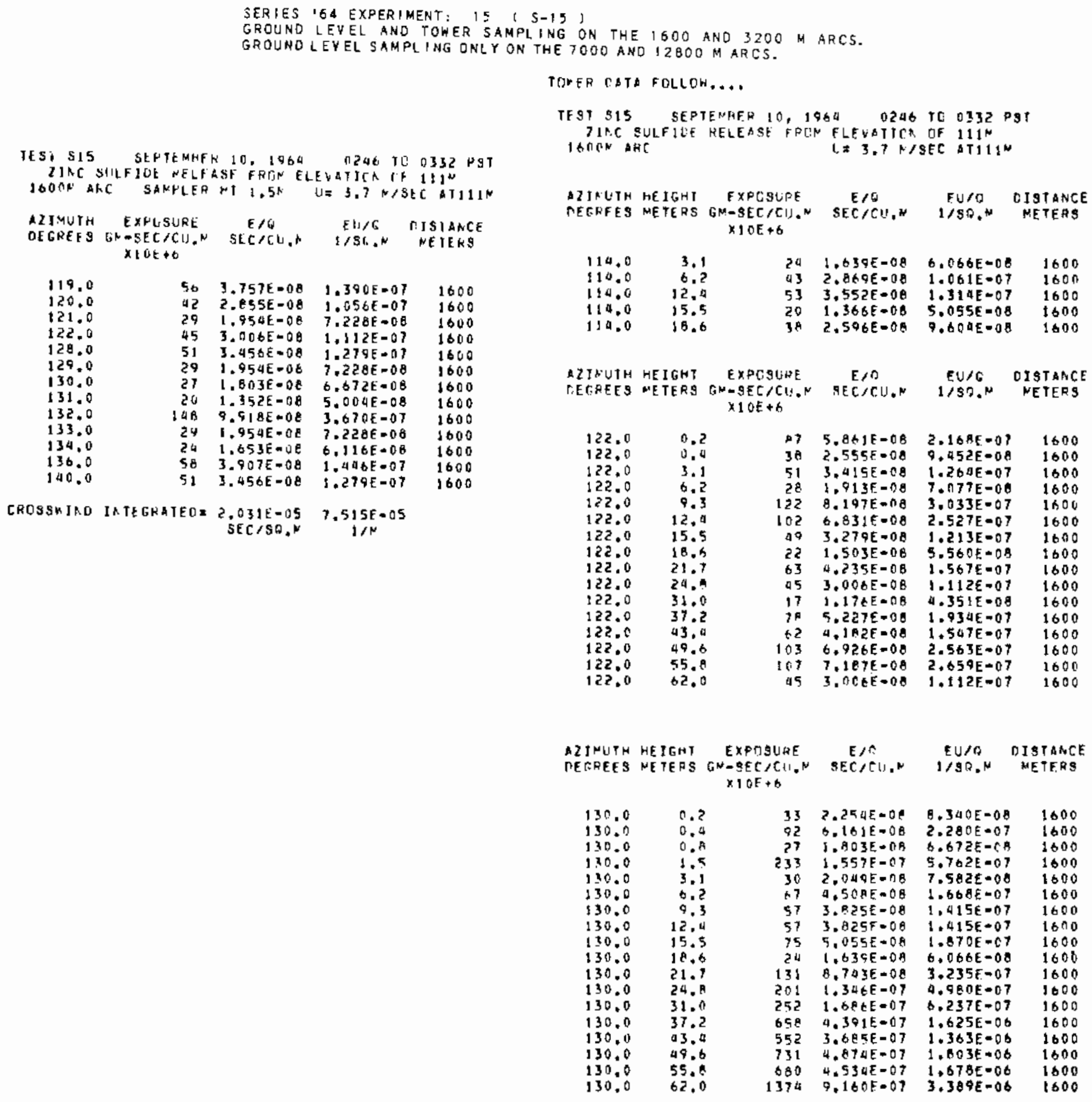




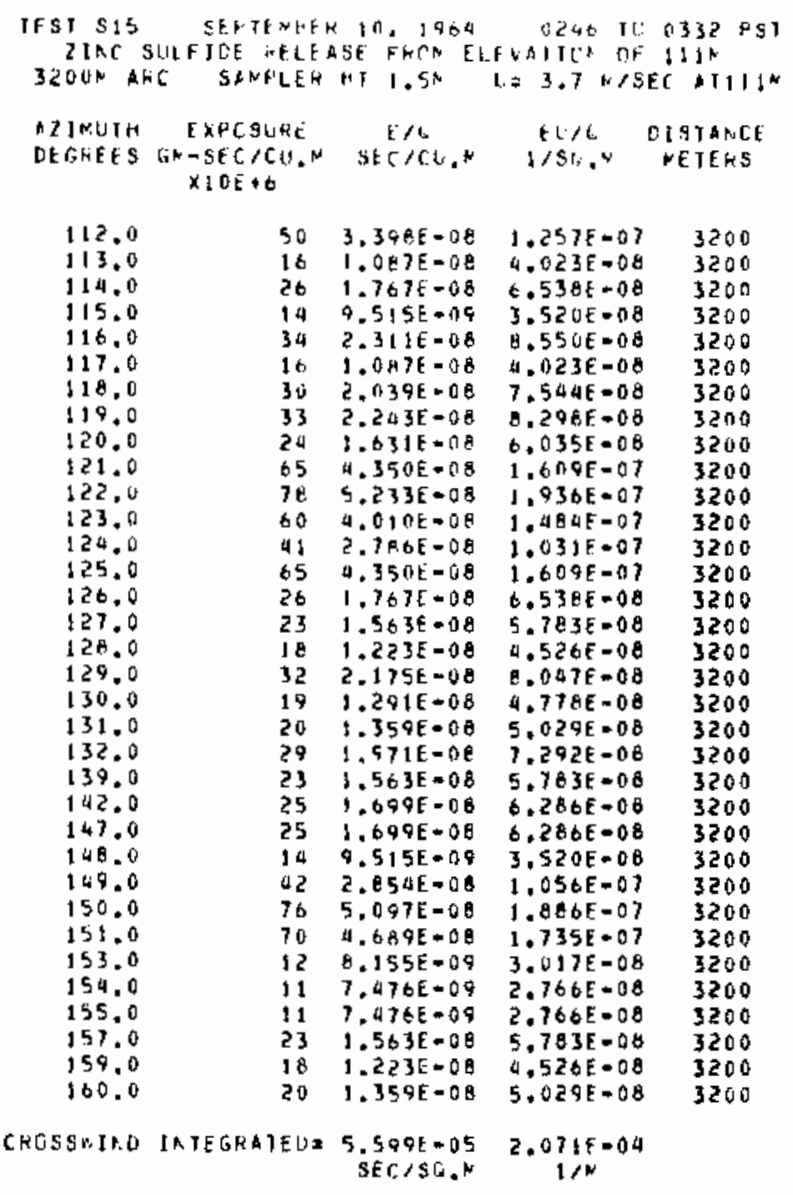

TOKFP DAFA FOLI DW....

\begin{tabular}{|c|c|c|c|c|c|}
\hline $\begin{array}{l}\text { ZJNUTH } \\
\text { EGNEES }\end{array}$ & $\begin{array}{l}\text { HE IGHT } \\
\text { METERS }\end{array}$ & $\begin{array}{c}\text { EXPCSURE } \\
G M+9 E E>C U, H \\
\times 10 E+6\end{array}$ & $\begin{array}{c}F / 0 \\
S E C / E U . N\end{array}$ & $\begin{array}{c}E \cup / 0 \\
1 / 50 . \%\end{array}$ & $\begin{array}{l}\text { DISTANCE } \\
\text { METERS }\end{array}$ \\
\hline $\begin{array}{l}122.0 \\
122.0 \\
122.0 \\
122.0 \\
122.0 \\
122.0 \\
122.0 \\
122.0 \\
122.0 \\
122.0 \\
122.0 \\
122.0 \\
122.0 \\
122.0 \\
122.0 \\
122.0 \\
122.0\end{array}$ & $\begin{array}{r}0.4 \\
0.8 \\
1.5 \\
3.1 \\
6.2 \\
9.3 \\
12.4 \\
15.5 \\
18.6 \\
21.1 \\
24.9 \\
31.0 \\
37.2 \\
43.9 \\
49.6\end{array}$ & $\begin{array}{l}36 \\
30 \\
25 \\
48 \\
52 \\
132 \\
139 \\
132 \\
100 \\
123 \\
118 \\
96 \\
04 \\
70 \\
53 \\
92 \\
64\end{array}$ & $\begin{array}{l}2.497 E=08 \\
2.039 F=00 \\
1.683 E=08 \\
3.236 E=08 \\
3.195 E=08 \\
8.803 E=08 \\
9.320 E=08 \\
6.803 E=08 \\
6.731 E=08 \\
8.220 E=08 \\
1.767 E=08 \\
5.761 E=08 \\
5.955 F=08 \\
4.980 E=08 \\
3.560 E=08 \\
5.502 E=08 \\
4.272 E=08\end{array}$ & $\begin{array}{l}9.052 E=00 \\
7.544 E=08 \\
6.227 E=00 \\
1.197 E=07 \\
1.293 E=07 \\
3.257 E=07 \\
3.449 E=07 \\
3.257 E=07 \\
2.491 \mathrm{E}=07 \\
3.041 E=07 \\
2.074 E=07 \\
2.131 E=07 \\
2.203 E=07 \\
1.044 E=07 \\
1.317 \mathrm{E}=07 \\
2.030 \mathrm{E}=07 \\
1.501 \mathrm{E}=07\end{array}$ & $\begin{array}{l}3200 \\
3200 \\
3200 \\
3200 \\
3200 \\
3200 \\
3200 \\
3200 \\
3200 \\
3200 \\
3200 \\
3200 \\
3200 \\
3200 \\
3200 \\
3200 \\
3200\end{array}$ \\
\hline
\end{tabular}

\section{AZIMUTH HEIGHT EXPOSURE E/R EU/O DISTANCE DECPEES METERS GMOSEC/CU.N SEC/CL, H I/SO.N NETERS $x, 0 E+6$}

\begin{tabular}{|c|c|c|c|c|c|}
\hline $\begin{array}{l}130.0 \\
130.0 \\
130.0 \\
130.0 \\
130.0 \\
130.0 \\
130.0 \\
130.0 \\
130.0 \\
130.0 \\
130.0 \\
130.0 \\
130.0 \\
130.0 \\
130.0 \\
130.0 \\
130.0 \\
130.0\end{array}$ & $\begin{array}{l}0.2 \\
0.1 \\
0.8 \\
1.5 \\
3.1 \\
6.2 \\
9.3 \\
12.0 \\
15.5 \\
18.6 \\
21.7 \\
24.8 \\
31.0 \\
37.2 \\
43.0 \\
09.6 \\
55.8 \\
62.0\end{array}$ & $\begin{array}{l}14 \\
11 \\
22 \\
13 \\
15 \\
54 \\
35 \\
42 \\
55 \\
139 \\
177 \\
238 \\
330 \\
295 \\
380 \\
402 \\
477 \\
270\end{array}$ & $\begin{array}{l}7.515 E=09 \\
7.476 E=09 \\
1.495 E=08 \\
9.061 E=09 \\
1.036 E=08 \\
3.625 E=08 \\
2.395 E=08 \\
2.898 E=08 \\
3.689 E=08 \\
9.320 E=08 \\
1.104 E=07 \\
1.592 E=07 \\
2.2018=07 \\
1.968 E=07 \\
2.589 E=07 \\
2.686 E=07 \\
3,184 E=07 \\
1.800 E=07\end{array}$ & $\begin{array}{l}3.520 E=08 \\
2.766 E=08 \\
5.532 E=08 \\
3.353 E=0 . \\
3.832 E=08 \\
1.341 E=07 \\
0.861 F=08 \\
1.054 E=07 \\
1.365 E=07 \\
3.449 E=07 \\
9.363 E=07 \\
5.891 E=07 \\
9.142 E=07 \\
7.280 E=07 \\
9.579 E=07 \\
9.939 E=07 \\
1.170 E=06 \\
6.682 E=07\end{array}$ & $\begin{array}{l}3200 \\
3200 \\
3200 \\
3200 \\
3200 \\
3200 \\
3200 \\
3200 \\
3200 \\
3200 \\
3200 \\
3200 \\
3200 \\
3200 \\
3200 \\
3200 \\
3200 \\
3200\end{array}$ \\
\hline
\end{tabular}

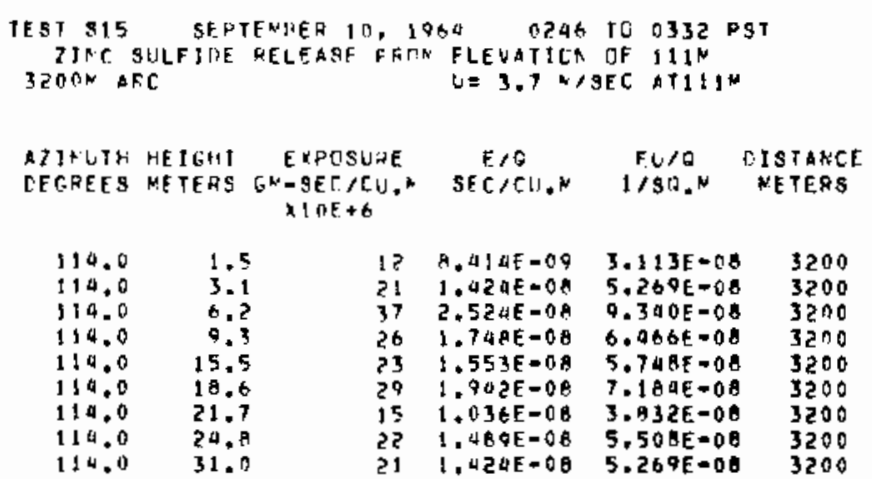




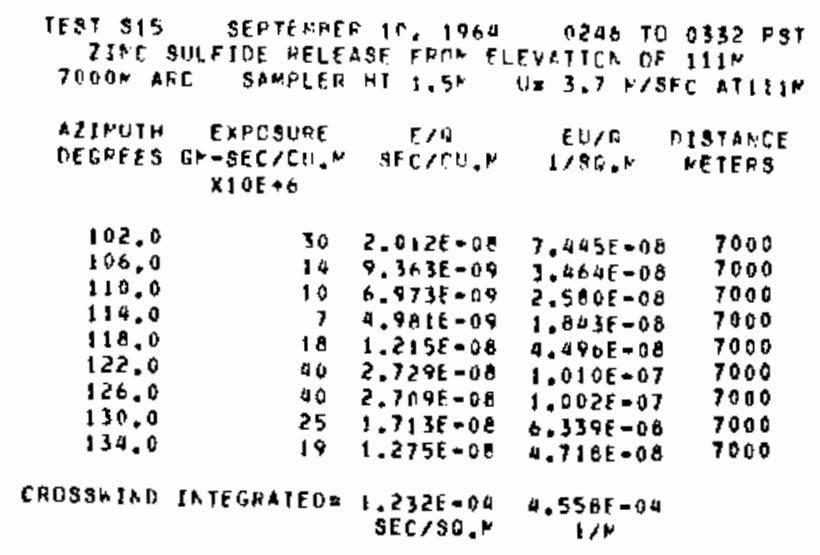

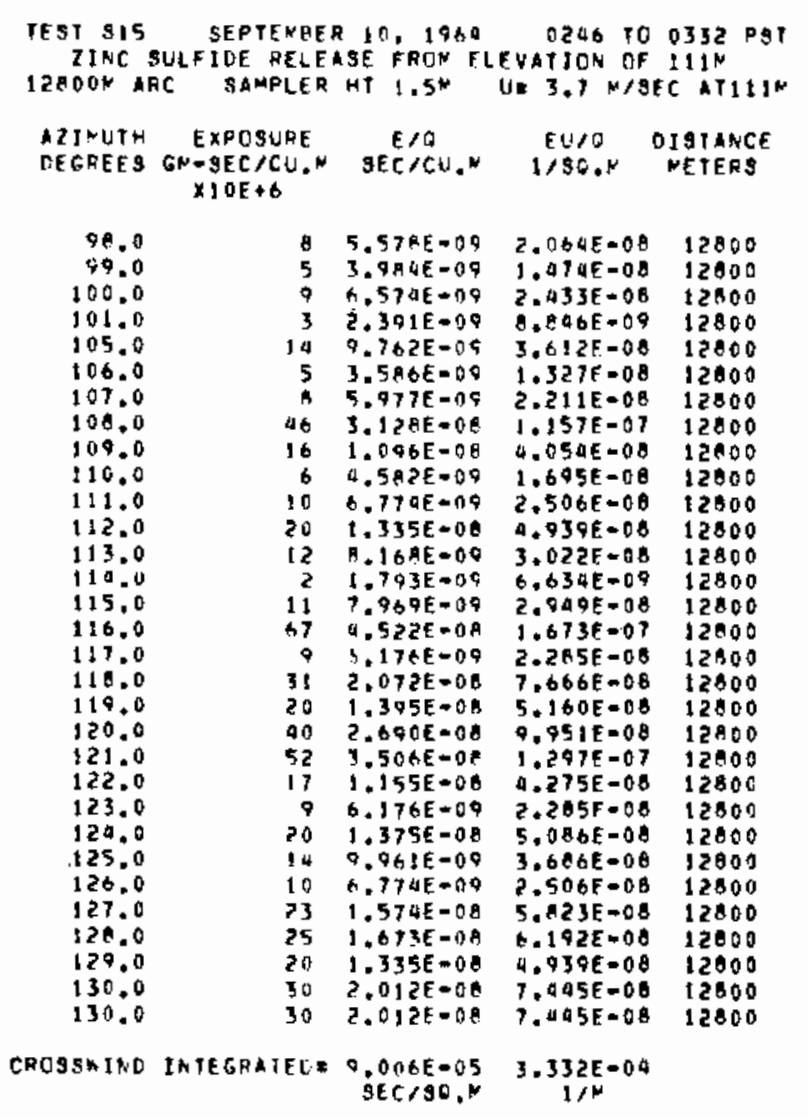


SECONDARY SERIES EXPERIHENT: Z +1

GROUND LEYEL AND TOWER SAMPLING 200-3200 M ARCS.

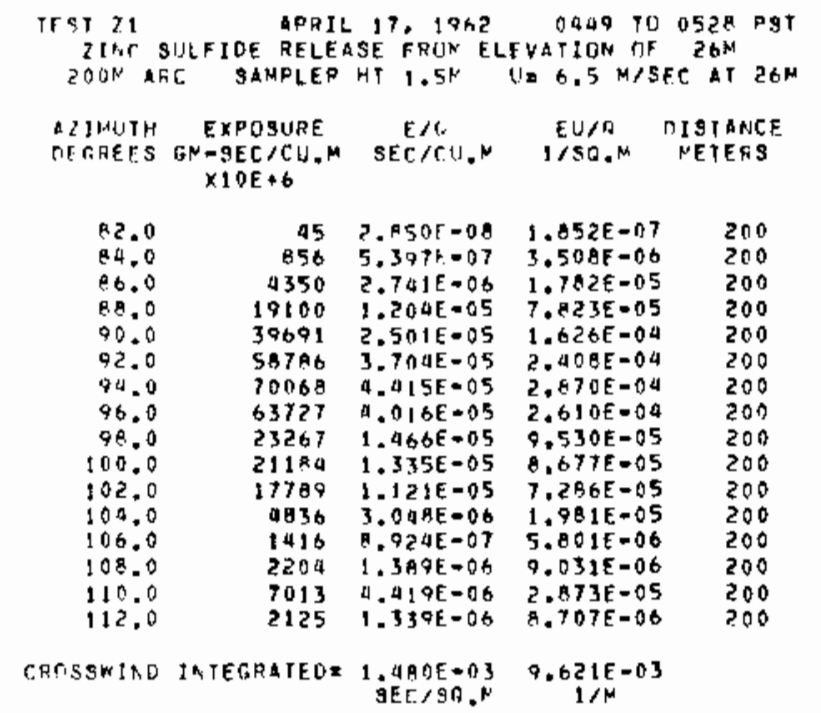

THEFI: DATA FחLITV....

\begin{tabular}{|c|c|c|c|c|c|}
\hline $\begin{aligned} \text { TESI } & 71 \\
t \mid & 1\end{aligned}$ & SILF JI: & $\begin{array}{l}\text { AFAII } 17 \text {, } 10 \\
\text { WFLFNSE FP!IH }\end{array}$ & 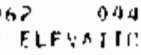 & $\begin{array}{l}\text { TL } 052 B \quad P \\
\text { HF } 2614\end{array}$ & 91 \\
\hline$\therefore$ On a & C & & $1=0.5 \%$ & $F C$ AT 264 & \\
\hline 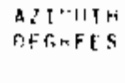 & $\begin{array}{l}\text { HE IEHOT } \\
\text { SE TE :IS: }\end{array}$ & $\begin{array}{c}\text { EXPUSUIZE } \\
{[\cdot 1-S E C / C 11, \text { AS }} \\
\times 1+F+6\end{array}$ & $\begin{array}{c}E / C^{2} \\
\text { SFC/CL, }\end{array}$ & $\begin{array}{l}E U / C \\
I / S N, M\end{array}$ & $\begin{array}{l}\text { DISTANCE } \\
\text { NETEPS }\end{array}$ \\
\hline S8. & 0.9 & 20153 & $1.52 ? F-05$ & $9.593 E=05$ & 200 \\
\hline SA. O & $n \cdot 7$ & 12659 & $7.977 \mathrm{~F}=1 \mathrm{t}$ & $5.185 E-05$ & 200 \\
\hline 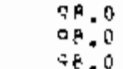 & 2.1 & $\begin{array}{l}13154 \\
21550 \\
37561\end{array}$ & $\begin{array}{l}\text { A. } 290 F=06 \\
1.35 P E=05 \\
1.28 F=05\end{array}$ & $\begin{array}{l}5.3 B A E=05 \\
8.827 E=05 \\
9.2 A>E=05\end{array}$ & $\begin{array}{l}500 \\
200 \\
200\end{array}$ \\
\hline $\begin{array}{l}=8.0 \\
48.0\end{array}$ & 4. 1 & $\begin{array}{l}32061 \\
25113\end{array}$ & $\begin{array}{l}1.028 F=05 \\
1.5429-05\end{array}$ & $\begin{array}{l}9.282 E=05 \\
1.029 E-0.4\end{array}$ & $\begin{array}{l}200 \\
200\end{array}$ \\
\hline $\begin{array}{l}9 P, 0 \\
48,0\end{array}$ & 6. & $\begin{array}{l}393 \cap 5 \\
53 \times 55\end{array}$ & $\begin{array}{l}2.477 E=05 \\
3.394 E=05\end{array}$ & $\begin{array}{l}1.510 E=04 \\
2.206 E=04\end{array}$ & $\begin{array}{l}200 \\
200\end{array}$ \\
\hline 90.0 & 9.5 & 34000 & $2.195 \%=05$ & $1.427 \mathrm{~F}=04$ & 200 \\
\hline 98.0 & 17. & 67100 & $4.2 z 8 f=05$ & $2.748 E=04$ & 200 \\
\hline 78.0 & 13.5 & $\begin{array}{r}83329 \\
175630\end{array}$ & $\begin{array}{l}5.6207=05 \\
1.107 E=01\end{array}$ & $\begin{array}{l}3.659 \mathrm{E}=04 \\
7.194 E=04\end{array}$ & $\begin{array}{l}200 \\
200\end{array}$ \\
\hline $\begin{array}{l}98.0 \\
98.0\end{array}$ & $\begin{array}{l}16.8 \\
18.9\end{array}$ & $\begin{array}{l}17563 i \\
185305\end{array}$ & $\begin{array}{l}1,107 E=04 \\
1,16 B E=0.4\end{array}$ & $\begin{array}{l}7.194 \mathrm{E} m 04 \\
7.593 \mathrm{E}=04\end{array}$ & $\begin{array}{l}200 \\
200\end{array}$ \\
\hline 88.0 & 71.6 & 270403 & $1.7045=04$ & $1.108 E-05$ & 200 \\
\hline 98.41 & 24. & $1396\} 4$ & $9.797 E-05$ & $5.718 E=04$ & 200 \\
\hline & & $12900 ?$ & $185 E=05$ & $32 \pm \varepsilon=04$ & 200 \\
\hline
\end{tabular}

\begin{tabular}{|c|c|c|c|c|c|}
\hline $\begin{array}{l}\text { A7J IUTH } \\
\text { PEGISES }\end{array}$ & $\begin{array}{l}\text { HE IGHY } \\
\text { ME TFRS }\end{array}$ & 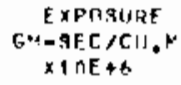 & $\begin{array}{c}E / Q \\
S E C / C H . N\end{array}$ & $\begin{array}{c}E \cup / 0 \\
1 / 39 .\end{array}$ & $\begin{array}{l}\text { DISTANCE } \\
\text { METERS }\end{array}$ \\
\hline $\begin{array}{l}106.0 \\
106.0 \\
106.0 \\
106.01 \\
104.0 \\
106.0 \\
106.0 \\
106.0 \\
106.0 \\
106.0 \\
106.0 \\
106.0 \\
106.0 \\
106.0 \\
106.0 \\
106.0\end{array}$ & $\begin{array}{r}0.3 \\
0.7 \\
1.4 \\
2.7 \\
4.1 \\
5.4 \\
6.7 \\
8.1 \\
9.4 \\
10.7 \\
13.5 \\
16.2 \\
14.0 \\
21.6 \\
24.3 \\
27.0\end{array}$ & $\begin{array}{r}1236 \\
1503 \\
997 \\
618 \\
2736 \\
1671 \\
804 \\
3039 \\
3601 \\
5506 \\
9690 \\
0539 \\
14111 \\
5653 \\
12559 \\
3079\end{array}$ & $\begin{array}{l}7.793 E=07 \\
9.073 F=07 \\
5.153 E=07 \\
3.00 C E=07 \\
1.720 E=06 \\
1.050 F=06 \\
5.700 E=07 \\
1.915 F=06 \\
7.269 E=06 \\
3.070 F=06 \\
6.106 E=06 \\
5.375 F=06 \\
9.832 E=06 \\
3.562 F=06 \\
7.913 F=06 \\
2.507 E=06\end{array}$ & $\begin{array}{l}5.065 E-06 \\
6.157 E-06 \\
3.350 E-06 \\
2.535 E-06 \\
1.121 E=05 \\
6.84 E E=06 \\
3.705 E=06 \\
1.245 E=05 \\
1.475 E=05 \\
2.255 E=05 \\
3.969 E=05 \\
3.494 E=05 \\
5.740 E-05 \\
2.316 E=05 \\
5.144 E=05 \\
1.629 E=05\end{array}$ & $\begin{array}{l}200 \\
200 \\
200 \\
200 \\
200 \\
200 \\
200 \\
200 \\
200 \\
200 \\
200 \\
200 \\
200 \\
200 \\
200 \\
200\end{array}$ \\
\hline
\end{tabular}

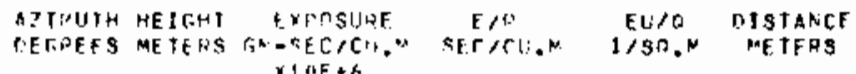
$x \perp+E+A$

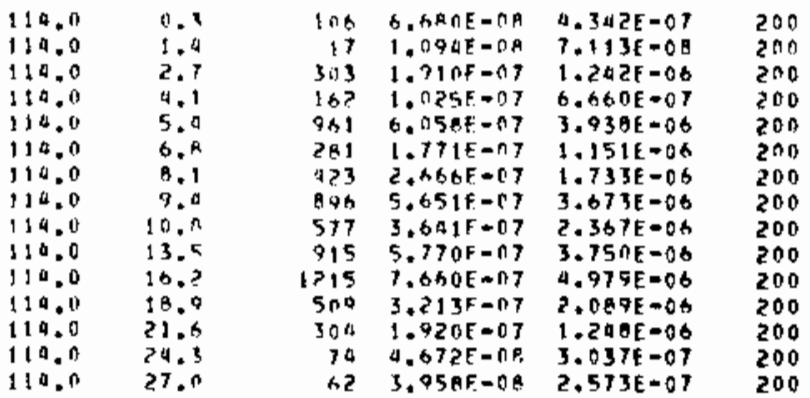

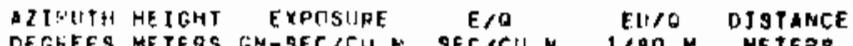

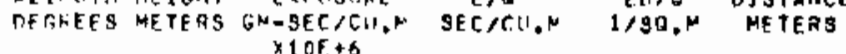

$\begin{array}{rrrrrr}122.0 & 0.2 & 221 & 1.397 F-07 & 9.07 B E-07 & 200 \\ 122.0 & 1.7 & 321 & 2.02 A E=07 & 1.316 E-06 & 200 \\ 122.0 & 1.4 & 50 & 3.193 E-08 & 2.069 E-07 & 200 \\ 122.0 & 4.1 & 25 & 1.592 E-08 & 1.035 E-07 & 200 \\ 122.0 & 10.8 & 26 & 1.6910-08 & 1.099 E-07 & 200 \\ 122.0 & 13.5 & 17 & 1.09 A E-08 & 7.113 E-08 & 200\end{array}$

DTIMUTH HEIGHI EVPISURE F/D FU/Q DISTANCE

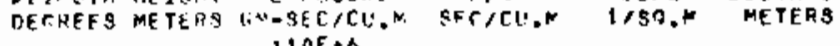

\begin{tabular}{|c|c|c|c|c|}
\hline $\begin{array}{l}130.0 \\
130.0 \\
130.0 \\
130.0 \\
130.0 \\
130.0 \\
130.0\end{array}$ & $\begin{array}{l}0.7 \\
0.7 \\
1.9 \\
2.7 \\
4.1 \\
5.0 \\
6.9\end{array}$ & $\begin{array}{r}25 \\
32 \\
9 \\
34 \\
56 \\
26 \\
20\end{array}$ & $\begin{array}{l}1.618 E=08 \\
2.024 E=08 \\
5.969 F=09 \\
2.284 E=08 \\
3.581 E=08 \\
1.691 \mathrm{E}=08 \\
1.293 \mathrm{~F}=08\end{array}$ & $\begin{array}{l}5.053 E=07 \\
1.316 E=07 \\
3.880 E=00 \\
1.487 E=07 \\
2.328 E=07 \\
1.089 E=07 \\
8.406 E=08\end{array}$ \\
\hline
\end{tabular}




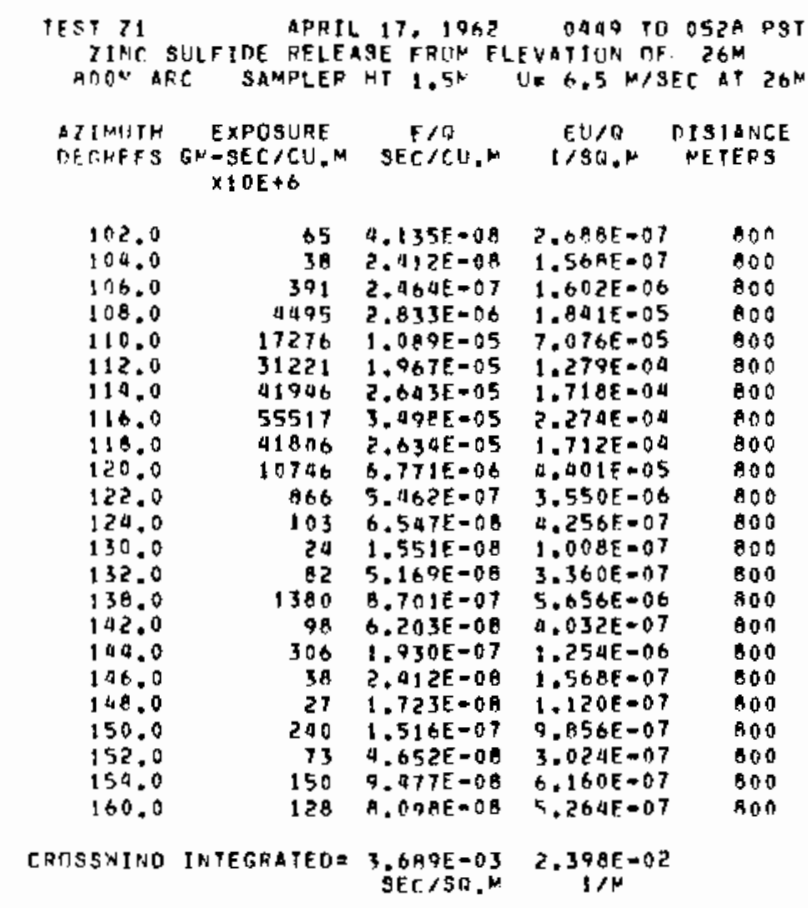

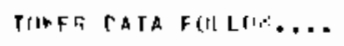

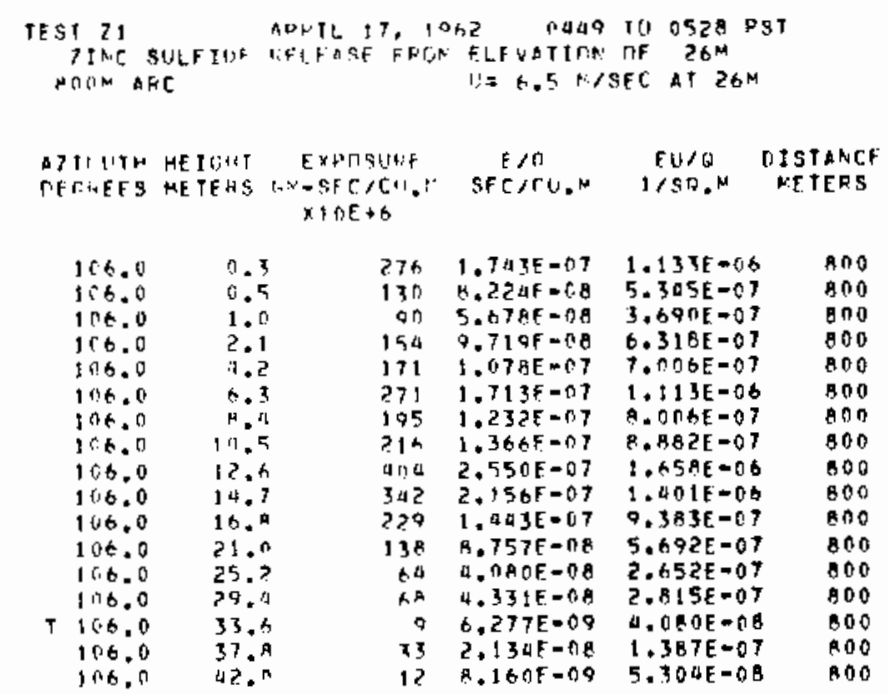

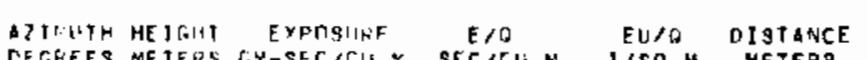

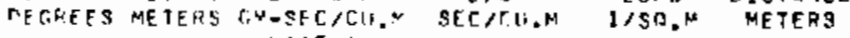
Q 1 OF $+\mathrm{B}$

\begin{tabular}{|c|c|c|c|c|c|}
\hline 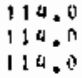 & $\begin{array}{l}0.3 \\
0.5\end{array}$ & $\begin{array}{l}10535 \\
11533 \\
231108\end{array}$ & $\begin{array}{l}1.231 F+05 \\
7.27 a F=06 \\
1.45+F=05\end{array}$ & $\begin{array}{l}A .001 E-05 \\
4.72 R E-05 \\
9.069 E-05\end{array}$ & $\begin{array}{l}800 \\
800 \\
800\end{array}$ \\
\hline 110.0 & 2.1 & ?कคA 7 & $1.69 \Delta E-05$ & $1.101 E=04$ & 800 \\
\hline 1.4 .0 & 4.? & $205 n c$ & $1.059 E-05$ & $8 E=04$ & 800 \\
\hline 114.0 & t. $x^{x}$ & $>1137$ & $1.332 F-05$ & $8.658 E-05$ & $B O O$ \\
\hline 114.0 & 4.4 & $23 \times 36$ & 1. WAOF $=05$ & $9.681 E-65$ & 900 \\
\hline 114.0 & 10.5 & ?? & $1.369 F=05$ & ค. $897 E=05$ & 000 \\
\hline 114.0 & $12 \cdot x$ & $3733^{\circ}$ & $2.3535=05$ & $1.529 E=0 A$ & 800 \\
\hline 114.0 & 14.7 & 36622 & $1.678 E=05$ & $1.090 E=04$ & 800 \\
\hline 114.0 & $10 .{ }^{2}$ & 23335 & $1.4100-115$ & $558 t=05$ & 800 \\
\hline 314.0 & 21.5 & 10425 & $917 E-05$ & $1.246 E=C 4$ & 900 \\
\hline 310.0 & 25.7 & $3+2 \geq ?$ & $=26,5=05$ & $9.021 E=05$ & $8 \cap 0$ \\
\hline 114.0 & $? 7.1$ & $493 n$ & $25 A F=06$ & $4.067 E=05$ & 90u \\
\hline 114.0 & 33.2 & 16079 & $1.113 F-05$ & $6.586 E-05$ & A00 \\
\hline 114.0 & 37.9 & 16251 & $1.02 \Delta F=05$ & $6.656 F-05$ & 200 \\
\hline 114.0 & aद. त & $12+10$ & $.9067-06$ & $5.165 E-05$ & Anก \\
\hline
\end{tabular}

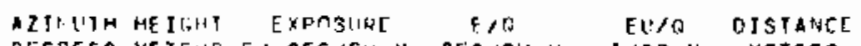

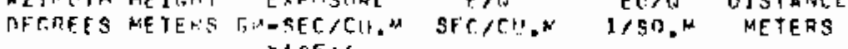
$\rightarrow 1 \cap F+x$

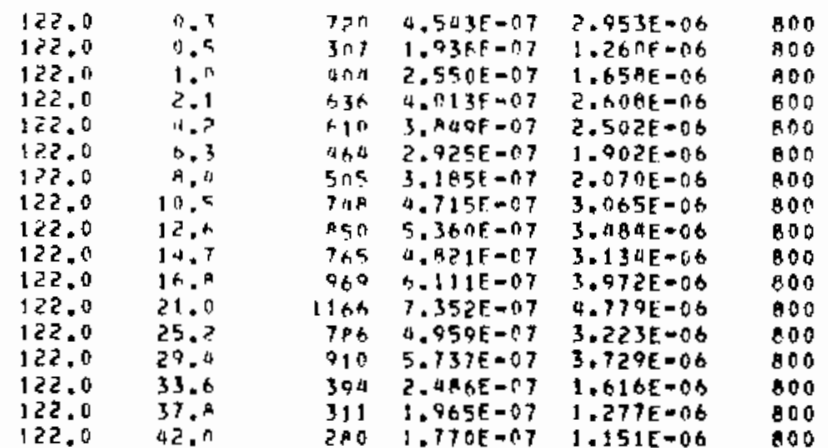

800
800
000
600
800
600
800
800
800
800
800
000
800
800
800
800
100 


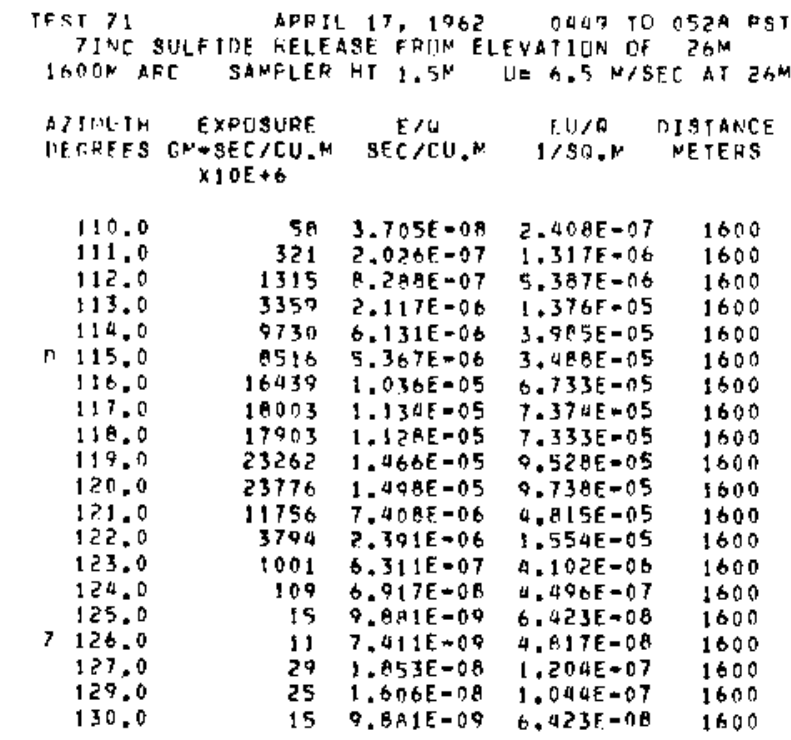

CFOSSWIND IATEGRATEDI $2.454 E-03 \quad 1.595 \mathrm{~F}=02$

SEC/ $10 . N \quad 1.595 \%$

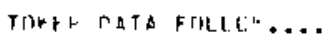

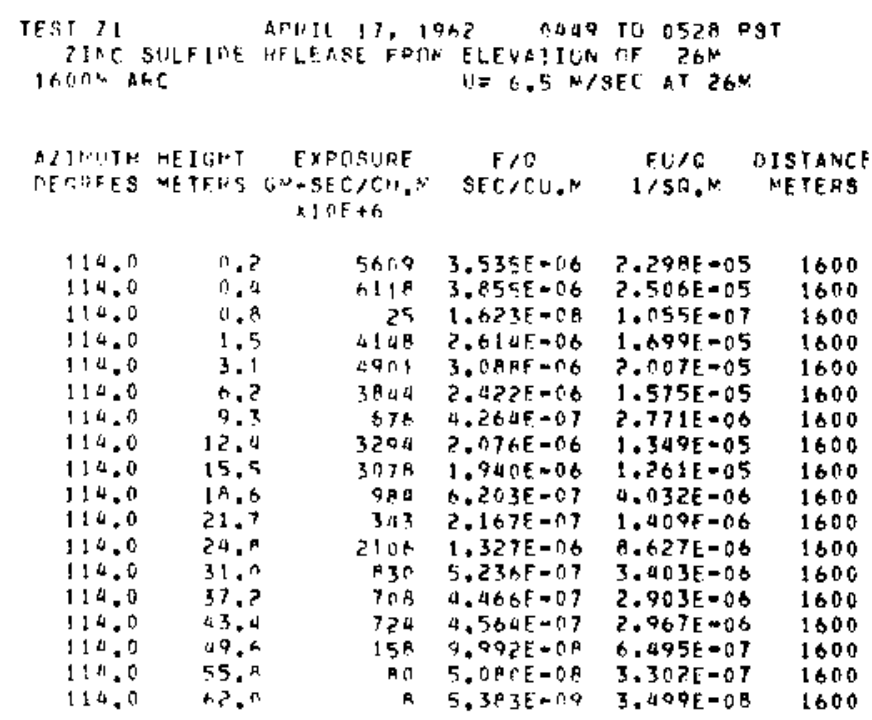

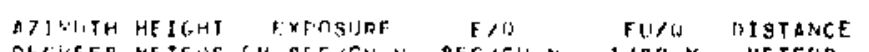

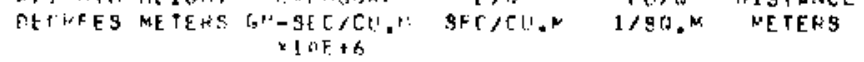

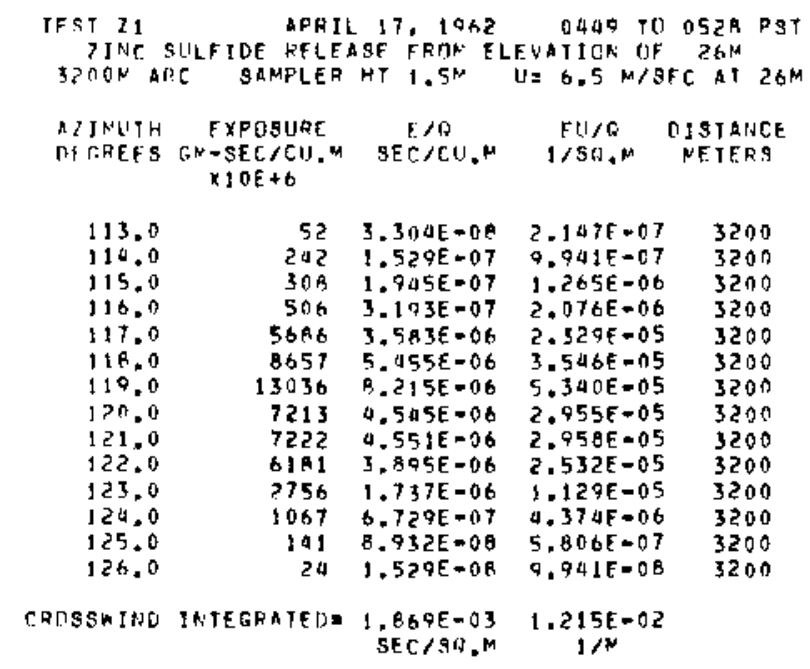

TMAFIF MATA FLHLLU. DECAEFS METEPS BMASEC/CH.M SEC/CU.M 1/90.M METERS
XIOE+G

$\begin{array}{ll}114.0 & 0.7 \\ 114.0 & 0.1 \\ 114.0 & 9.9 \\ 114.0 & 1.5 \\ 114.0 & 3.1 \\ 114.0 & 6.2 \\ 114.0 & 4.3 \\ 114.0 & 12.0 \\ 110.0 & 15.5 \\ 114.0 & 18.4 \\ 114.0 & 21.7 \\ 114.0 & 24.9 \\ 114.0 & 31.0 \\ 114.0 & 37.2 \\ 114.0 & 43.4 \\ 114.0 & 49.6\end{array}$

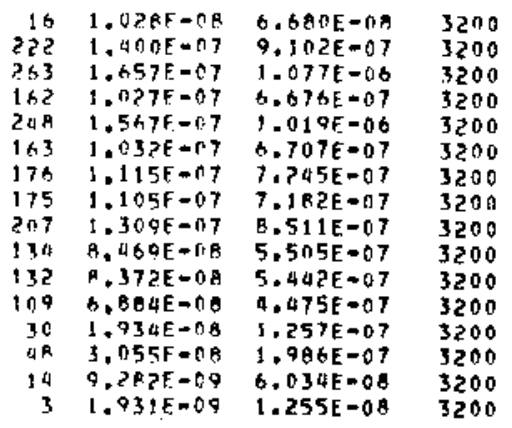

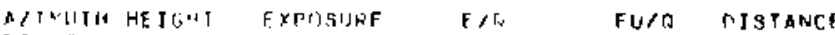

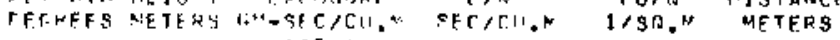

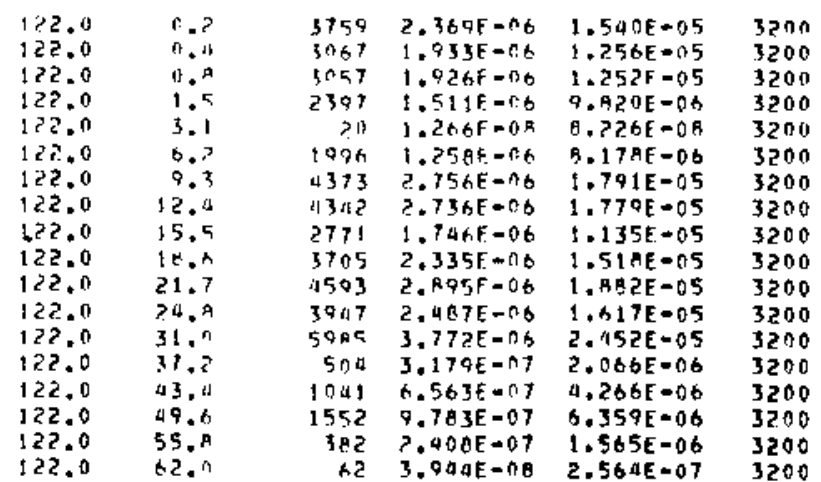




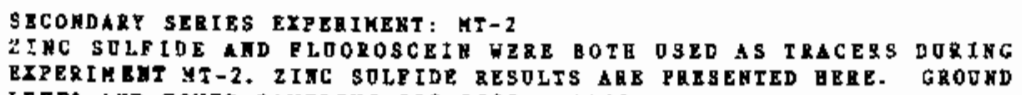
LEVEL, AHD TOUER SAMPLIHG 200-3200 HAZCS.

\begin{abstract}
TE\$T $M T 2$
7INC SULFIDE RELEA9E 19 , 1989 DQ20 TO 0440 PST $200 \mathrm{~N}$ ARC SAMPLER HF $1.5 \mathrm{M}$ U. 3.A M/SFC AF $2 \mathrm{M}$
\end{abstract}

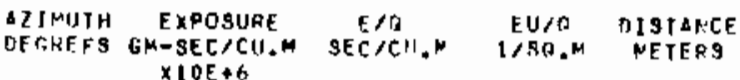
$\times 10 E+6$

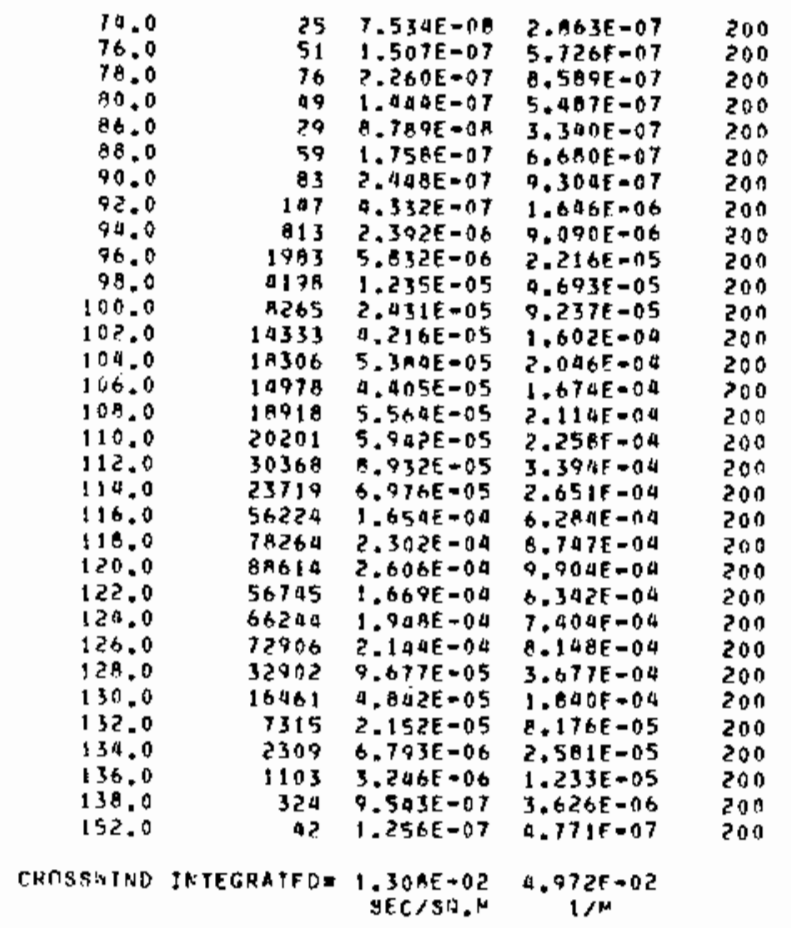

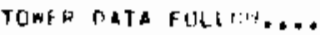

TEST HII A.AY 17, 19KA DA20 TO OA4O PQT

7 INC SULFIDE REI.FAGE FRTM ELEYATION TF $2 \mathrm{M}$

CAON ARC
}

ATINIITH HEIGHT EXPTSIIFE E/O DISTANCE

DEFHES METEFS TIN-SEC/C1I.M SEC,CU.M 1/SO,N METFRS $x$ I TE +8

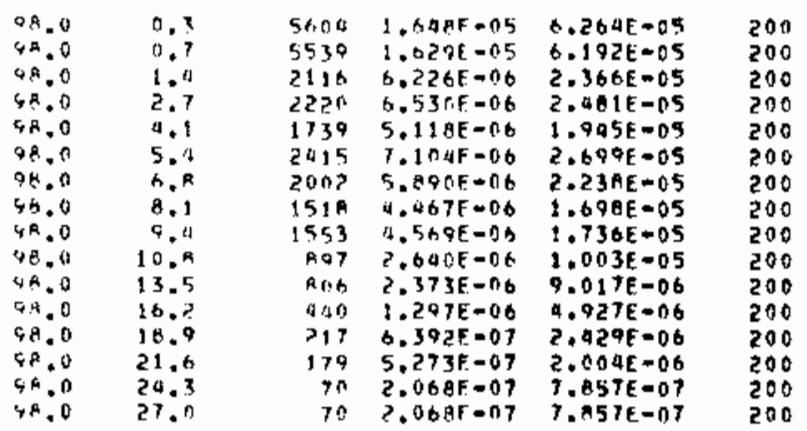

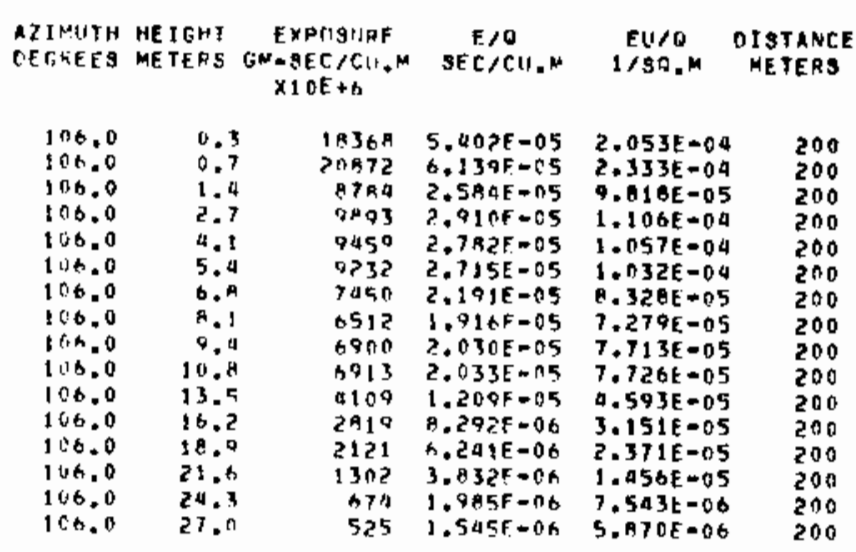

\begin{tabular}{|c|c|c|c|c|c|}
\hline $\begin{array}{l}\text { A7INITH } \\
\text { DERHFES }\end{array}$ & $\begin{array}{l}\text { HE IGHI } \\
\text { ME TE RS }\end{array}$ & 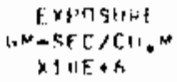 & 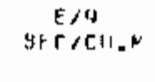 & $\begin{array}{l}\text { Eufon } \\
1 / \text { sfo.m }\end{array}$ & $\begin{array}{c}\text { OIBTANCE } \\
\text { PETERS }\end{array}$ \\
\hline 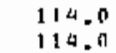 & 0.3 & $\begin{array}{l}32634 \\
3 n+11\end{array}$ & $\begin{array}{l}\Psi . \operatorname{sifF}=05 \\
1.070 F=01\end{array}$ & $\begin{array}{l}3.607 \mathrm{E}=0 \mathrm{~A} \\
0.009 \mathrm{E}=04\end{array}$ & $\begin{array}{l}200 \\
200\end{array}$ \\
\hline $\begin{array}{l}1 \\
1\end{array}$ & $\begin{array}{l}1.3 \\
3.7 \\
0.1\end{array}$ & $\begin{array}{l}15003 \\
21912 \\
20420\end{array}$ & $\begin{array}{l}0.649 E-05 \\
6.45 \text { UF -C5 } \\
6.167 t=05\end{array}$ & $\begin{array}{l}1+7 B 2 E=04 \\
2.45 P F=04 \\
2.306 E-04\end{array}$ & $\begin{array}{l}200 \\
200 \\
200\end{array}$ \\
\hline $1: 4=0$ & ." & IARTA & a. $17+F=0.5$ & $1.663 E=04$ & 200 \\
\hline 114.0 & A. $A$ & $1535 \mathrm{~h}$ & $0.5175-05$ & $1.71 \mathrm{kE}-\mathrm{nd}$ & 200 \\
\hline $\begin{array}{l}114.0 \\
110.0\end{array}$ & $\begin{array}{l}\text { A. } 1 \\
0.11\end{array}$ & $\begin{array}{l}1152 \mathrm{~h} \\
+1734\end{array}$ & $\begin{array}{l}3.300 F-05 \\
5.510 t=05\end{array}$ & $\begin{array}{l}1.288 E-04 \\
2.094 E-04\end{array}$ & $\begin{array}{l}200 \\
200\end{array}$ \\
\hline 110.0 & 10.8 & 10639 & $5.48 \geq E-0.5$ & $2.003 \mathrm{E}-0 \mathrm{a}$ & 200 \\
\hline 110.0 & 13.5 & 13755 & a. (ud d $8=0.05$ & $1.537 E=04$ & 200 \\
\hline 114,0 & 16.2 & $1>128$ & $3.567 \mathrm{E}=0.5$ & $1.355 E=09$ & 200 \\
\hline 114.0 & 18.7 & Abe 5 & 2.5 agf $=0.5$ & $9.685 E-05$ & 200 \\
\hline 114.0 & $21 . n$ & 4538 & $1.335 \mathrm{~F}-0.5$ & $5.072 E=05$ & 200 \\
\hline 11 is 0 & 24.3 & 2436 & $7.160 E=06$ & $2.723 \mathrm{E}-05$ & 200 \\
\hline , & & 14 & $0.250 E-06$ & $15 E=05$ & 200 \\
\hline
\end{tabular}

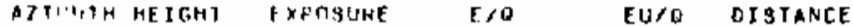

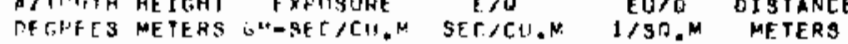

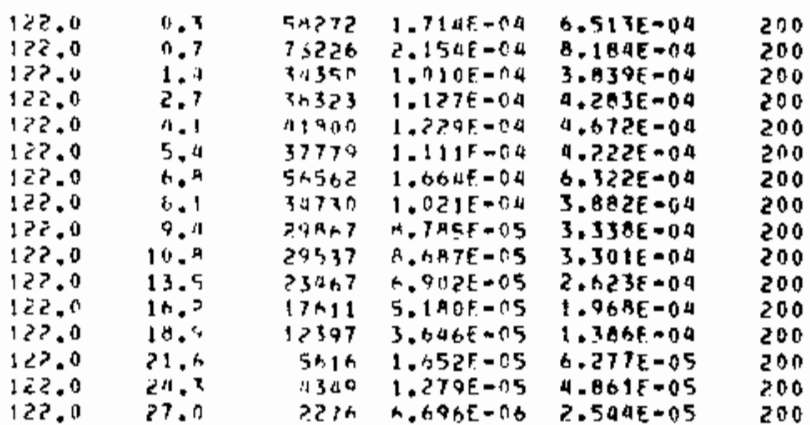




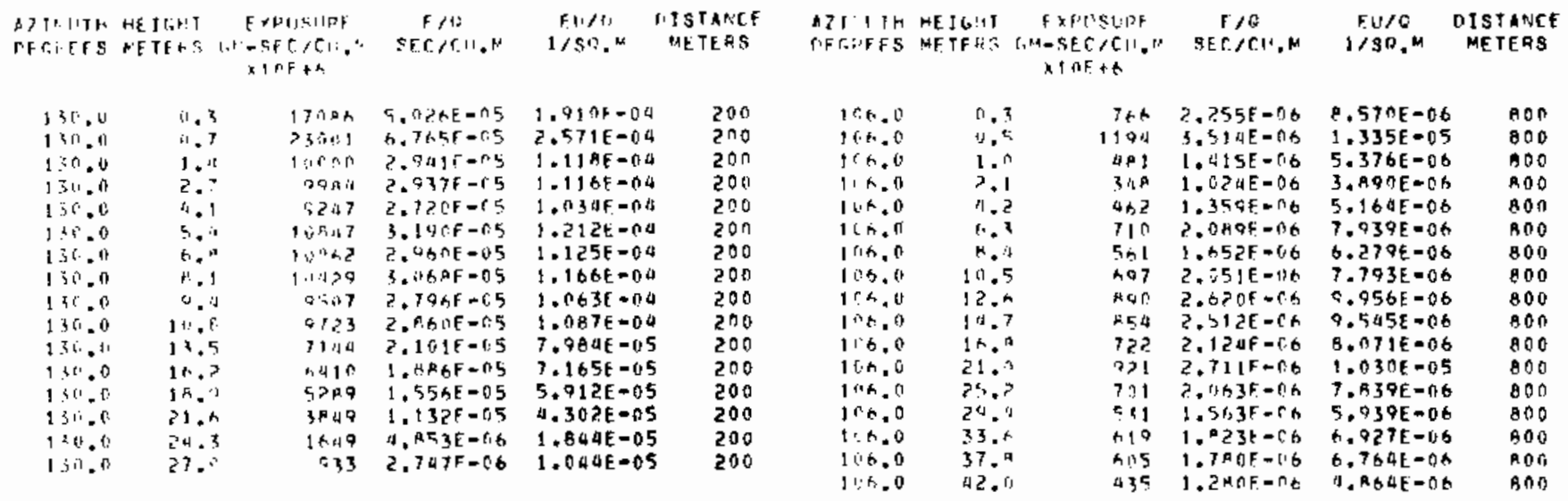

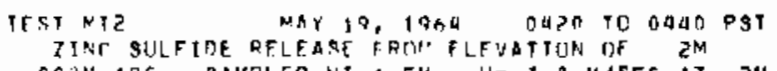
AOAN ARC GAMPIFR NT $1.5 \mathrm{~N} U=3.8 \mathrm{M} / \mathrm{SFC}$ AT $2 M$

\begin{tabular}{|c|c|c|c|c|c|}
\hline $\begin{array}{l}\text { AT } 11 \text { IH } \\
\text { MFEVEES }\end{array}$ & $\begin{array}{l}\text { HEITH'T } \\
\text { NETEKS }\end{array}$ & 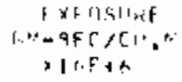 & $\begin{array}{c}\text { H } \\
\text { Serfritan }\end{array}$ & $\begin{array}{l}E \cup / 1 \\
1 / \$ 0 . M\end{array}$ & $\begin{array}{l}\text { DTSTANCE } \\
\text { METERS }\end{array}$ \\
\hline $11: 0$ & 0.3 & 7) & 1.1 H. & $4.501 t-0.5$ & सीम \\
\hline $1: 4.0$ & ri. 5 & 2975 & $0.7515=0.0$ & $3.3258-05$ & 000 \\
\hline 114.0 & $1 . n$ & 1747 & $5.1345=0.6$ & $1.053 F=05$ & 200 \\
\hline 111.0 & 2.1 & 2301 & $7.632 F-n n_{0}$ & $2.072 F=05$ & $A 00$ \\
\hline 114.0 & if.? & $32 i$ & $\nabla . \Delta T A E=r b$ & $.00 \cap F=05$ & ROA \\
\hline 114.0 & $\therefore . x$ & 33119 & $9 .+152 E=114$ & $3.744 E-05$ & $B \cap 0$ \\
\hline 114.0 & $B .0$ & 3360 & $D A A 3 E=A G$ & $3.756 \mathrm{t}=175$ & AOO \\
\hline 114,0 & 10.5 & A ח ד & $1.17 D E=\$ 5$ & $4.4805-05$ & BOO \\
\hline 114.0 & 17.4 & 4013 & $1.4: 15 E=15$ & 5.40 & 800 \\
\hline 114.0 & 11.7 & 4213 & $1 . ? 30 \mathrm{~F}=05$ & $0.700 E-05$ & $A \cap 0$ \\
\hline 114.0 & 10.9 & 3909 & $1.1506-05$ & $1.369 \mathrm{E}-05$ & 200 \\
\hline 111.0 & 21,5 & 3336 & $9.9137-06$ & $3.729 \mathrm{E}=05$ & 100 \\
\hline 114.0 & 25.2 & 2741 & $8.1635=06$ & $3.064 \mathrm{E}=05$ & 800 \\
\hline 114,0 & 29.0 & $? 167$ & 6.37 a $=0-116$ & $2.422 \mathrm{E}=05$ & 000 \\
\hline $1: 4,0$ & 33.6 & $26 \mid A$ & $7.700 E-06$ & $926 E-05$ & Bo0 \\
\hline 114.0 & & ?तon & $6.020 E=06$ & $20 A E=05$ & $B \cap 0$ \\
\hline 114.0 & 47.0 & 1505 & $14.6635=116$ & $772 \mathrm{E}-05$ & 300 \\
\hline
\end{tabular}

ATINITTH MEICWT EXPISUPF F/O FU/O DISTANCE

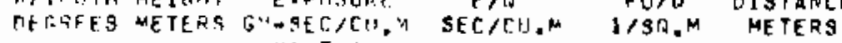

\begin{tabular}{|c|c|c|c|c|c|}
\hline $\begin{array}{l}122.0 \\
127.0 \\
122.0 \\
122.0 \\
122.0 \\
122.0 \\
122.0 \\
12100 \\
12200 \\
122.0 \\
122.0 \\
122.0 \\
127.0 \\
122.0 \\
122.0 \\
122.0 \\
122.0\end{array}$ & 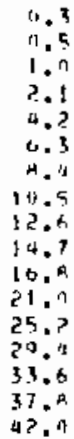 & $\begin{array}{l}3479 \\
2751 \\
1571 \\
2105 \\
2170 \\
1721 \\
2167 \\
2052 \\
2154 \\
1023 \\
2023 \\
2184 \\
2240 \\
1914 \\
1796 \\
1301 \\
977\end{array}$ & $\begin{array}{l}1.000 E=05 \\
R .034 E=06 \\
4.622 F=06 \\
6.194 E=06 \\
6.383 E=06 \\
5.062 E=106 \\
6.376 E=06 \\
7.210 E=06 \\
6.337 E=06 \\
5.363 E=06 \\
5.953 E=06 \\
6.425 E=06 \\
6.737 F=06 \\
5.347 E=06 \\
5.020 E=06 \\
3.769 E=06 \\
2.074 F=06\end{array}$ & $\begin{array}{l}3.433 E=05 \\
3.053 E=05 \\
1.756 E=05 \\
2.354 E=05 \\
2.425 E=05 \\
1.024 E=05 \\
2.025 E=05 \\
2.741 E-05 \\
2.408 E=05 \\
2.038 E=05 \\
2.262 E=05 \\
2.041 E=05 \\
2.560 E=05 \\
2.032 E=05 \\
1.908 E=05 \\
1.432 E=05 \\
1.003 E=05\end{array}$ & $\begin{array}{l}800 \\
800 \\
000 \\
000 \\
000 \\
800 \\
000 \\
000 \\
000 \\
800 \\
000 \\
000 \\
000 \\
800 \\
000 \\
000 \\
000\end{array}$ \\
\hline
\end{tabular}

\begin{tabular}{|c|c|c|c|c|c|}
\hline \multicolumn{2}{|c|}{ 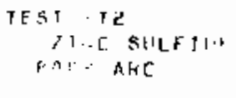 } & 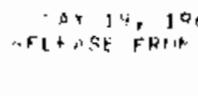 & 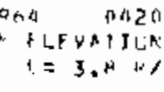 & 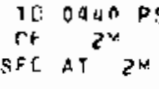 & PST \\
\hline $\begin{array}{l}\text { ATI :TH } \\
\text { MFYIIS }\end{array}$ & 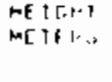 & 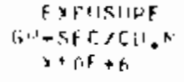 & $\begin{array}{c}t / \theta \\
S E[/ r, N, N\end{array}$ & $\begin{array}{l}F ! 1 / 6 \\
1 / 90 . \%\end{array}$ & $\begin{array}{l}\text { MISTANCE } \\
\text { METEKS }\end{array}$ \\
\hline 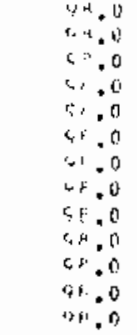 & 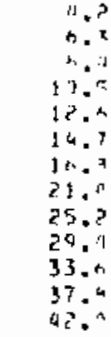 & 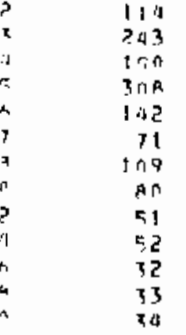 & $\begin{array}{l}3.35 t F=07 \\
7.162 F=07 \\
3.590 F=07 \\
9.083 F=07 \\
4.107 F=07 \\
2.094 E=07 \\
3.214 E=07 \\
2.376 F=07 \\
1.514 E=07 \\
1.543 E=07 \\
9.429 E=08 \\
0.714 E=08 \\
1.120 E=07\end{array}$ & $\begin{array}{l}1.774 E=06 \\
2.721 E=06 \\
2.124 E=06 \\
3.452 E=06 \\
1.593 E=06 \\
7.965 E=07 \\
1.221 E=06 \\
9.027 E=07 \\
5.754 E=07 \\
5.863 E=07 \\
3.583 E=07 \\
3.691 E=07 \\
3.909 E=07\end{array}$ & $\begin{array}{l}A 00 \\
100 \\
800 \\
800 \\
800 \\
900 \\
500 \\
000 \\
800 \\
800 \\
800 \\
800 \\
900\end{array}$ \\
\hline
\end{tabular}




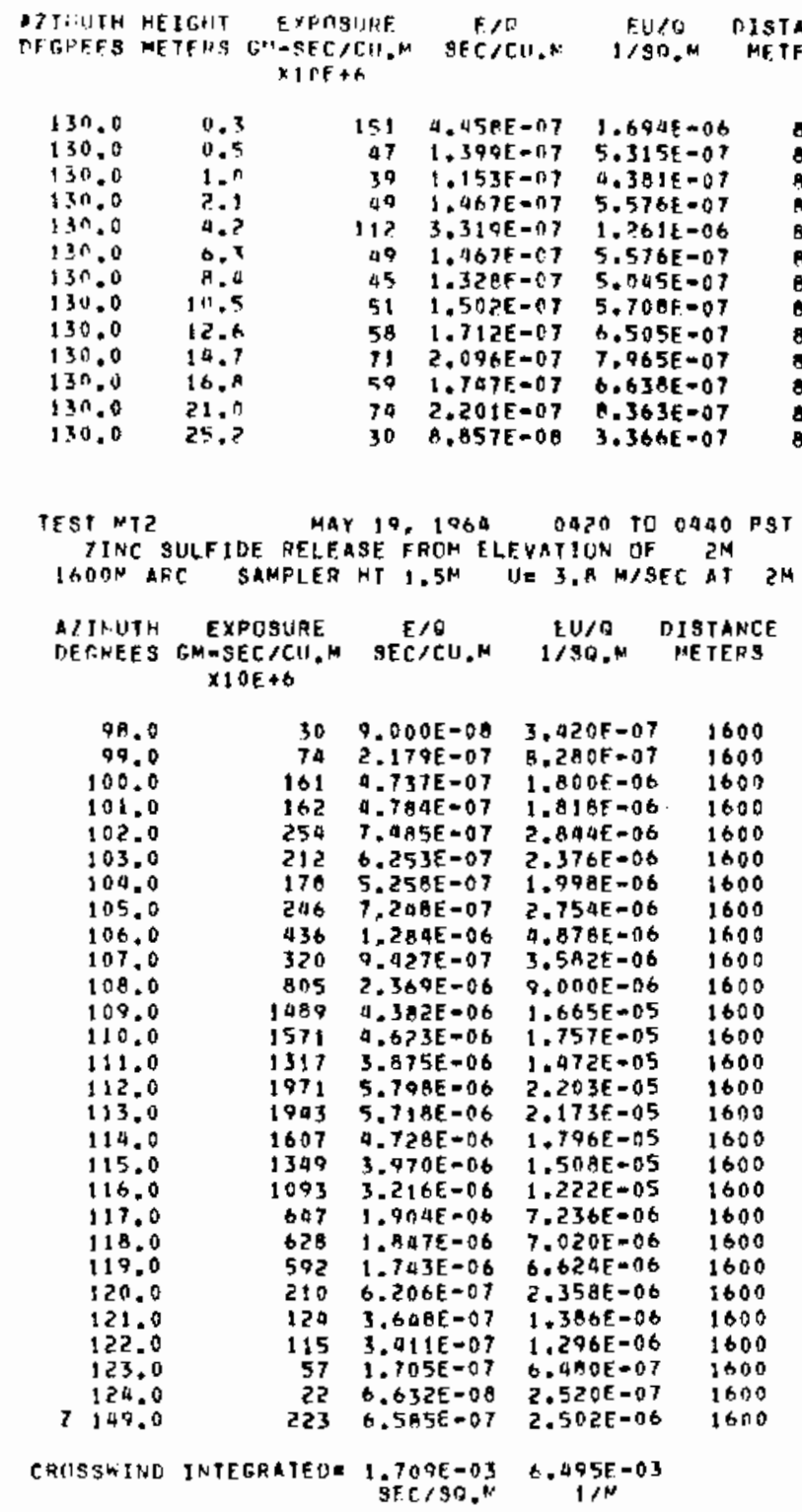

TRWFO 1:ATA FIIILL1!*....

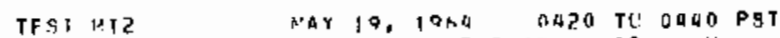

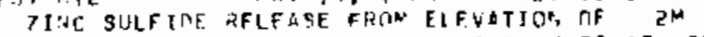
$\begin{array}{ll}1 \text { ATIO AKC } & 1=3 . R^{2} \\ & \end{array}$
\end{abstract}

ATIIHY HEIGHT FYPISUISF EAR FU/F DISTANCF

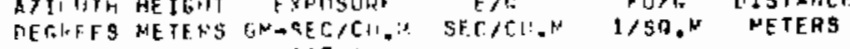
$\times 1 C E+b$

\begin{tabular}{|c|c|c|c|c|c|}
\hline 98,0 & $\mathrm{fl} . ?$ & $? 6$ & $7.9355-2 A$ & $2.9775-07$ & 1800 \\
\hline $4 \mathrm{~A}, 0$ & 6.4 & $2^{\text {月 }}$ & $A .43 A F-0 B$ & $3.206 E-07$ & 1600 \\
\hline 40.0 & 1.5 & 24 & $7.103 F-08$ & $2.699 E-07$ & 1600 \\
\hline$y^{2} \cdot 0$ & 3.1 & 19 & $5.023 E=08$ & $2.137 E-07$ & 1600 \\
\hline $9 \mathrm{M} .0$ & $0 . ?$ & $3 a$ & $1.0065-07$ & $4 \varepsilon .07$ & 1600 \\
\hline 92.0 & 12.0 & 31 & $9,175 E-08$ & $3.467 \mathrm{E}-0.7$ & 1600 \\
\hline 0 & 15.5 & 32 & $9.4715-08$ & $3.509 E-07$ & 1600 \\
\hline 0 & 18.4 & 25 & $7.695 \mathrm{E}=10 \mathrm{~B}$ & $924 E-07$ & 1600 \\
\hline 4.9 .0 & $2 \pm .7$ & 34 & $1.006 f-07$ & 3.02 AE-07 & 1600 \\
\hline $9 A .0$ & 43.4 & 18 & $5,4 B B E-O B$ & $2.085 E=07$ & 1600 \\
\hline
\end{tabular}

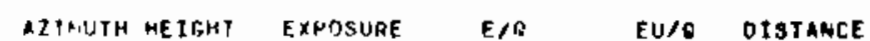
NFFHFES METEHS GM-SEC/CU.M SEC/CU.M I/SO.M METERS $\times 10 E+h$

\begin{tabular}{|c|c|c|c|c|c|}
\hline 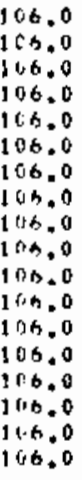 & $\begin{array}{l}0.7 \\
0.9 \\
0.4 \\
1.5 \\
3.1 \\
6.2 \\
9.3 \\
12.0 \\
15.5 \\
18.0 \\
21.7 \\
24.8 \\
31.0 \\
37.2 \\
43.0 \\
49.4 \\
55.8 \\
62.0\end{array}$ & $\begin{array}{l}132 \\
940 \\
342 \\
191 \\
716 \\
312 \\
276 \\
271 \\
924 \\
297 \\
410 \\
230 \\
190 \\
290 \\
346 \\
242 \\
212 \\
195\end{array}$ & $\begin{array}{l}5.665 E-07 \\
1.296 E-06 \\
1.007 E-06 \\
5.623 E=07 \\
6.363 E=07 \\
9.205 E-07 \\
8.139 E=07 \\
7.991 E=07 \\
1.209 E-06 \\
3.672 E=07 \\
1.208 E=06 \\
7.000 E=07 \\
5.313 E=07 \\
8.55 B E=07 \\
1.018 E-176 \\
7.128 E=07 \\
6.254 E=07 \\
5.047 E=07\end{array}$ & $\begin{array}{l}2.153 E=06 \\
4.924 E=06 \\
3.825 E=06 \\
2.137 E=06 \\
2.918 E=06 \\
3.498 E=06 \\
3.093 E=06 \\
3.037 E=06 \\
4.746 E=06 \\
3.295 E=06 \\
9.509 E=06 \\
2.664 E=06 \\
2.019 E=06 \\
3.251 E=06 \\
3.367 E=06 \\
2.709 E=06 \\
2.376 E=06 \\
2.070 F=04\end{array}$ & $\begin{array}{l}1600 \\
1600 \\
1600 \\
1600 \\
1600 \\
1600 \\
1600 \\
1600 \\
1600 \\
1600 \\
1600 \\
1600 \\
1600 \\
1600 \\
1600 \\
1600 \\
1600 \\
1600\end{array}$ \\
\hline
\end{tabular}

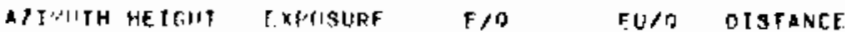

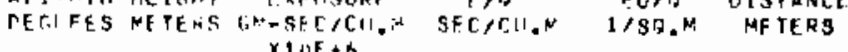
$x 1+F+b$

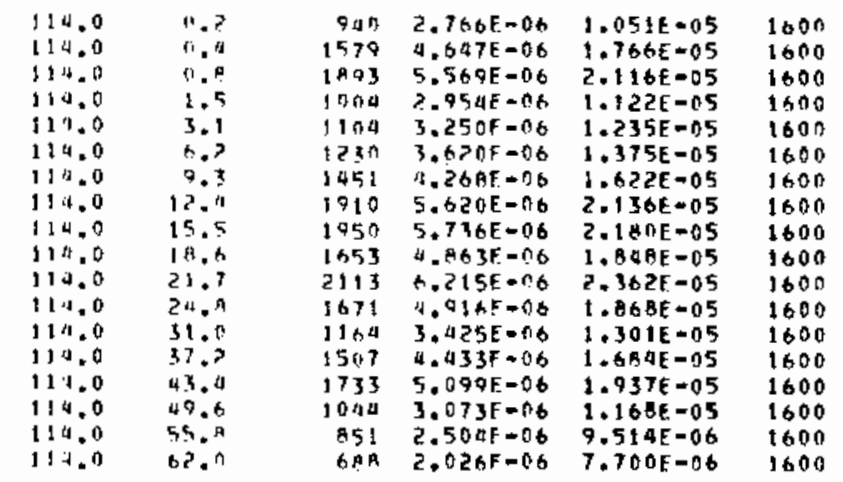

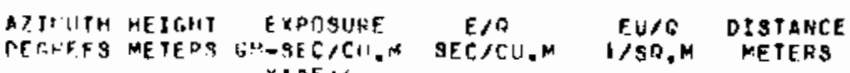

$1=3.0$

123.0

$12 \% .0$

122.0

12 ?. 0

1220

$4>2.0$

172.0

123.0

122.0

122.0

122.0

122.0

122.0

122.0

122.0

$12 ? .0$

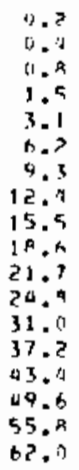
X) $0 \mathrm{E}+\mathrm{h}$

\begin{tabular}{|c|c|c|c|}
\hline $\begin{array}{l}40 \\
2 B \\
R A\end{array}$ & $\begin{array}{l}1.205 E-07 \\
8.43 \mathrm{HE}=0 \mathrm{~A} \\
7.592 \mathrm{E}-07\end{array}$ & $\begin{array}{l}4.581 E=07 \\
3.20 B E-07 \\
9.348 E-07\end{array}$ & $\begin{array}{l}1600 \\
1600 \\
1600\end{array}$ \\
\hline 117 & 3.4635 .07 & $1.316 E=06$ & 1600 \\
\hline $\begin{array}{l}\text { प्रक } \\
72\end{array}$ & $\begin{array}{l}2.545 E=07 \\
? .73 E=07\end{array}$ & $\begin{array}{l}9.672 E=07 \\
1.035 E=06\end{array}$ & $\begin{array}{l}1600 \\
1600\end{array}$ \\
\hline R5 & $2.516 \mathrm{~F}-07$ & $0 E=07$ & 1600 \\
\hline 1 tin & $1.10 E=117$ & 01 & 1600 \\
\hline 19 & $3.374 \mathrm{E}=07$ & $1.2 B 2 E=0 B$ & 1600 \\
\hline मे & $3.427 E=07$ & $22 E-07$ & 1600 \\
\hline 116 & $3.417 F=07$ & $1.2995-06$ & 1600 \\
\hline 120 & $3.542 E-07$ & $.346 \mathrm{E}-06$ & 1600 \\
\hline 55 & 1, tiat: $=07$ & $b .256 E-07$ & 1600 \\
\hline 61 & $1.821 F=97$ & $0.920 F=07$ & 1600 \\
\hline $3^{A}$ & $121 E=07$ & $259 E=07$ & 1600 \\
\hline 32 & $6397-08$ & $3.663 E-07$ & 1600 \\
\hline 41 & $33 E=07$ & $85 E \div 07$ & 1600 \\
\hline
\end{tabular}




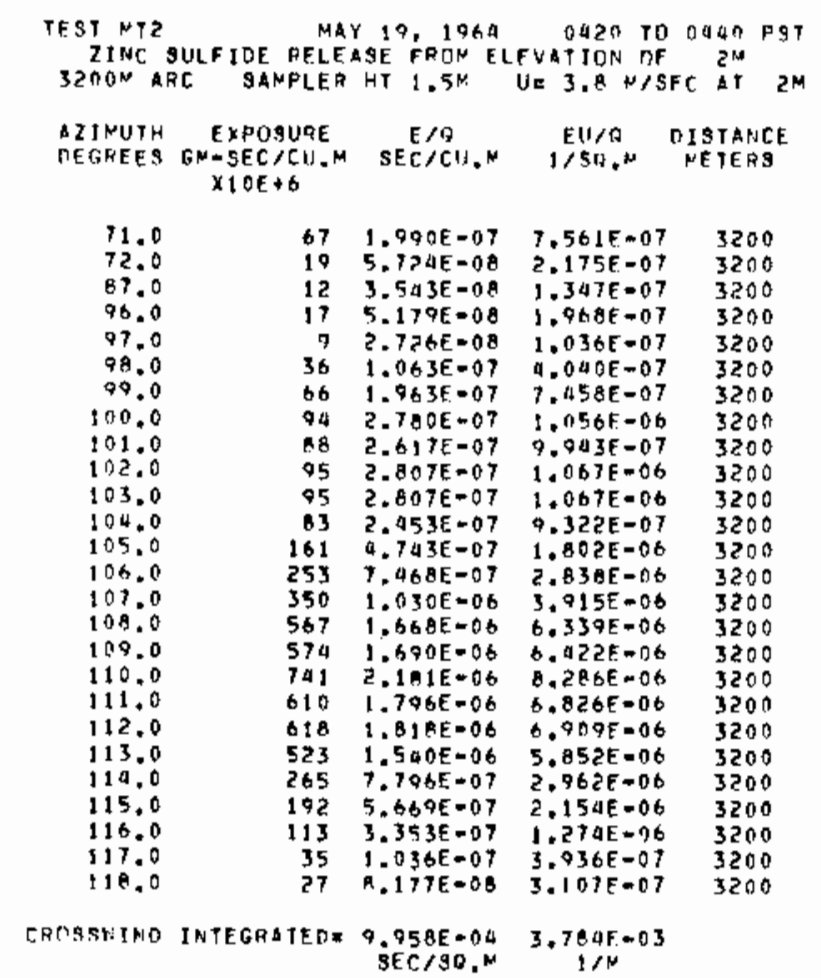

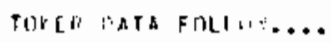

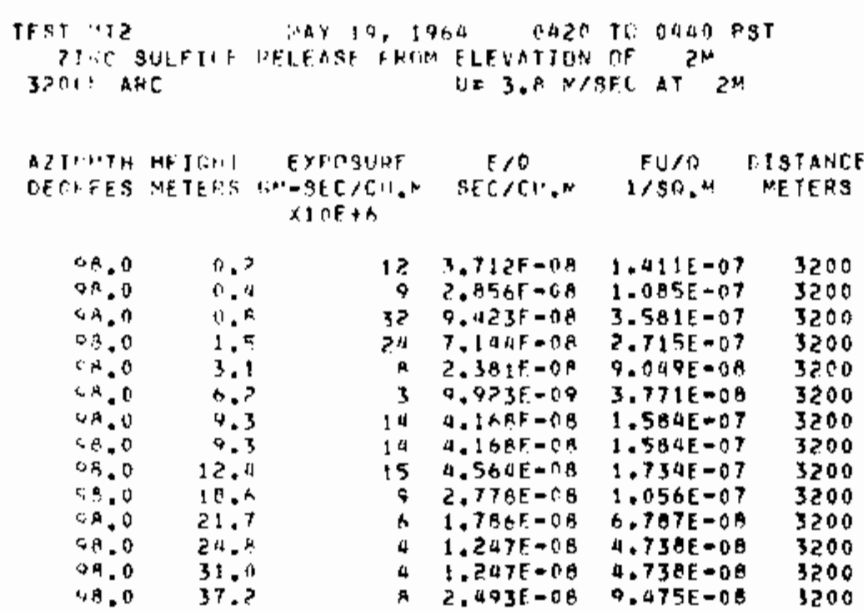

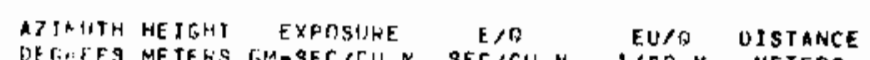
XIOE Th ON SEC/CU.N I/SO.N METERS

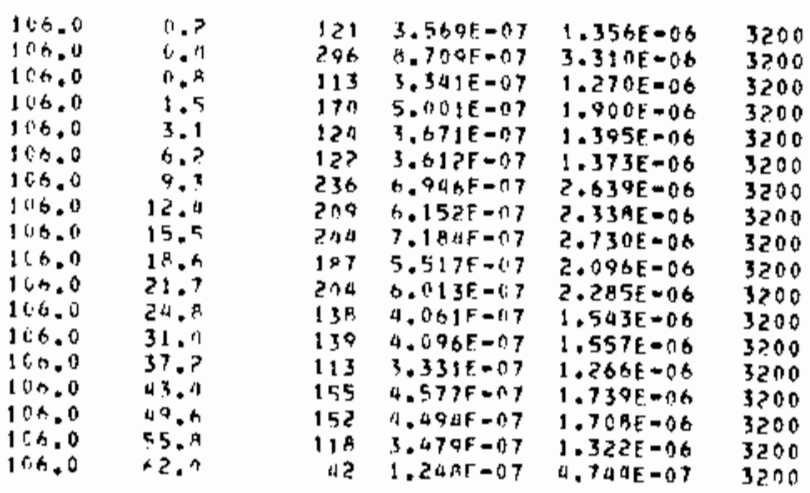

\begin{tabular}{|c|c|c|c|c|c|}
\hline $\begin{array}{l}\text { A71'1ITH } \\
\text { PETHEES }\end{array}$ & $\begin{array}{l}\text { HEIIIIT } \\
\text { MEIEUIS }\end{array}$ & 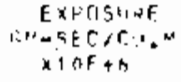 & $\begin{array}{c}E / C \\
s F t / C e, \ldots\end{array}$ & $\begin{array}{c}F u / C \\
1 / S D . M\end{array}$ & $\begin{array}{c}\text { WISTANCF } \\
\text { METERS }\end{array}$ \\
\hline $\begin{array}{lll}1 & 11 & 0 \\
1 & 10.0\end{array}$ & "1.? & $\begin{array}{l}3+2 \\
3 \geq 4\end{array}$ & $\begin{array}{l}\text { H. } 424 F=47 \\
4.033 t=07\end{array}$ & $\begin{array}{l}3 . n 49 E=n 6 \\
3.581 E=06\end{array}$ & $\begin{array}{l}3200 \\
3200\end{array}$ \\
\hline 111.0 & $\therefore$ & 107 & $5.7974-17$ & $2.203 E-06$ & $3>00$ \\
\hline 114.0 & 1. & zad & $n .3750=177$ & $3.1825-1,6$ & 3200 \\
\hline 11.0 & 3.1 & 279 & $A \cdot R I I F=07$ & $3.348 E=06$ & 3200 \\
\hline 114.0 & G. & 273 & $4.033 F=07$ & $3.2 A \cap E=06$ & 3200 \\
\hline $11+0$ & 4. & $2+?$ & $7.72 n=07$ & $2.934 E=0 \mathrm{~A}$ & 3200 \\
\hline 114.0 & 12. & 411 & $1.30 \cap 8=06$ & $0.94 \cap E=06$ & 3200 \\
\hline 114.0 & 15. & $37 \mathrm{~A}$ & $1.113 \mathrm{~F}=116$ & $4.231 \mathrm{E}=06$ & 3200 \\
\hline 1140 & 14. & 318 & $9.3678=07$ & $3,550 \mathrm{D}=06$ & 3200 \\
\hline $11 \% 0$ & $\therefore 1$. & $4>1$ & $1.23 A F=126$ & $4.706 E-06$ & 3200 \\
\hline 1140 & 24. & 304 & $1.015 t=06$ & $3.151 \mathrm{~F}=06$ & 3200 \\
\hline 114.0 & & 353 & $1.03 \mathrm{PE}-\mathrm{DO}_{6}$ & $3.946 E=06$ & 3200 \\
\hline 114.0 & & 207 & A. $745 \mathrm{~F}=07$ & $3.323 E=06$ & 3200 \\
\hline 1$\}+0$ & 43.1 & 479 & $1.263 E=06$ & $4.79 A E=06$ & 3200 \\
\hline & & $>A 1$ & A. $273 E-17$ & $3.144 E-C b$ & 3200 \\
\hline & & 273 & $0.0 \Delta 0 F=07$ & $3.055 E-06$ & 3200 \\
\hline 114.0 & 62 & $2: 3$ & 7.15 AE $=07$ & $2.720 E=06$ & 3200 \\
\hline
\end{tabular}


SECORDARY SERIES EIPERIKERT: MT-2F

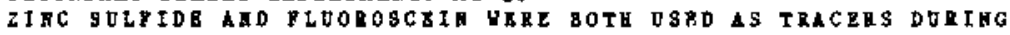

EIPERIMET KI-2. FLOORESCEIX RESOLTS ARE PRESRETBD HERE. GROOHD

LEVEL AXD TOUER SATPLIEG 200-3200 M ABCS.

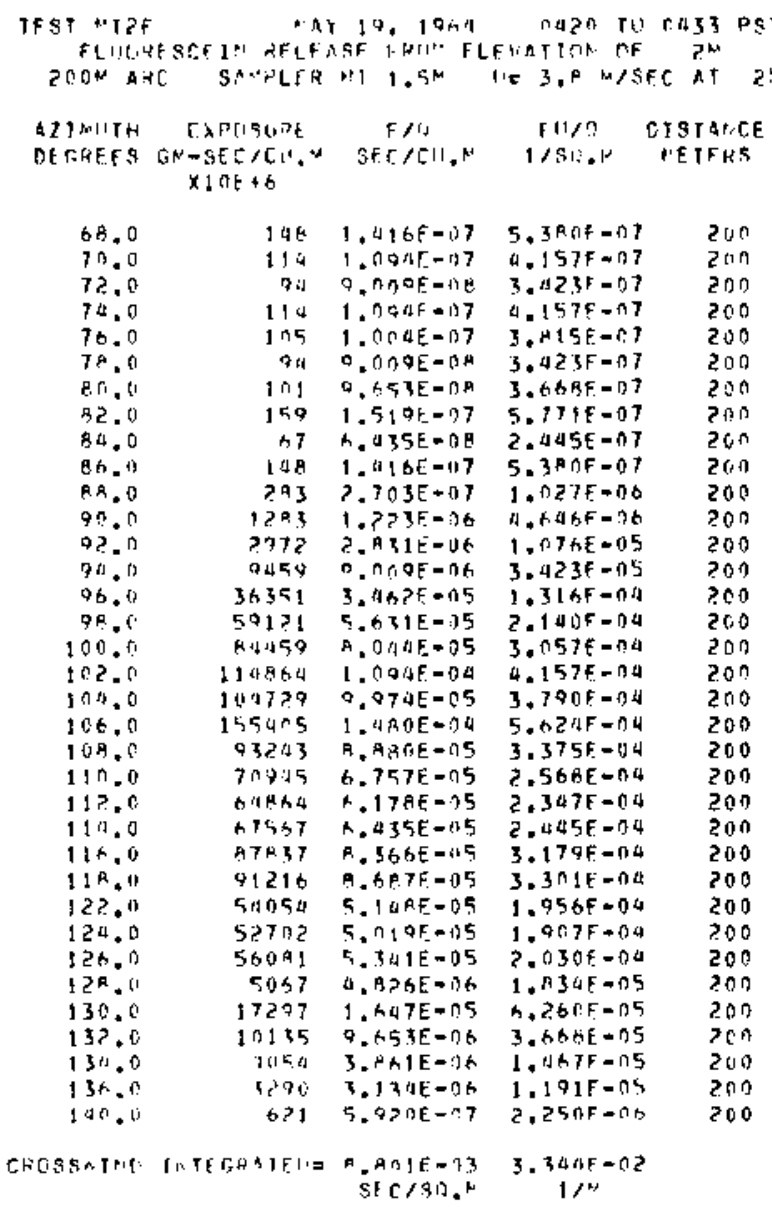

TIRER EATA FOLLCW....

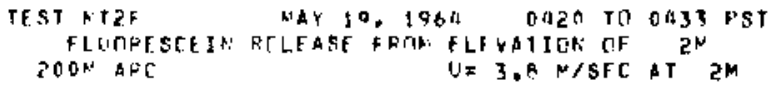

AZINHTH HEIGHF EXFISUFE. DEGHFES METEFS GU-SEC/CH.N SECACU.M 1/ST.M METEAS $\times 1 O E+6$

\begin{tabular}{|c|c|c|c|c|}
\hline $\begin{array}{l}88.0 \\
98.0 \\
98.0 \\
98.0 \\
98.0 \\
9.0 \\
99.0 \\
98.0 \\
98.0 \\
98.0 \\
98.0 \\
98.0 \\
91.0 \\
98.0 \\
98.0 \\
98.0\end{array}$ & $\begin{array}{r}0.3 \\
0.7 \\
1.4 \\
2.7 \\
4.1 \\
5.0 \\
6.8 \\
8.1 \\
9.4 \\
10.8 \\
13.5 \\
16.2 \\
18.9 \\
21.6 \\
24.3 \\
27.0\end{array}$ & $\begin{array}{l}40720 \\
54154 \\
54054 \\
54054 \\
34486 \\
43243 \\
40594 \\
33513 \\
33513 \\
28378 \\
73648 \\
7432 \\
10810 \\
6754 \\
3109 \\
1729\end{array}$ & $\begin{array}{l}0.736 E-05 \\
5.148 E=05 \\
5.14 E E-05 \\
5.148 E=05 \\
3.475 E=05 \\
4.118 F=05 \\
4.207 E=05 \\
3.192 E=05 \\
3.192 E=05 \\
2.703 E=05 \\
2.252 E-05 \\
7.070 E=06 \\
1.030 E-05 \\
6.435 E=06 \\
2.940 E=06 \\
1.0075=06\end{array}$ & $\begin{array}{l}1.800 E=114 \\
1.056 E=00 \\
1.956 E-04 \\
1.656 E=04 \\
1.320 E=04 \\
1.565 E-04 \\
1.614 E=04 \\
1.213 E=04 \\
1.213 E=04 \\
1.027 E=00 \\
0.559 E=05 \\
2.690 E=05 \\
3.912 E=05 \\
2.445 E=05 \\
1.125 E=05 \\
6.260 E=04\end{array}$ \\
\hline
\end{tabular}

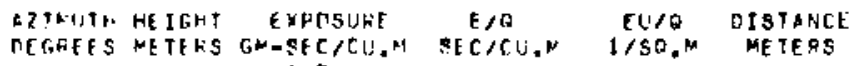
$x 1 \cap F+b$

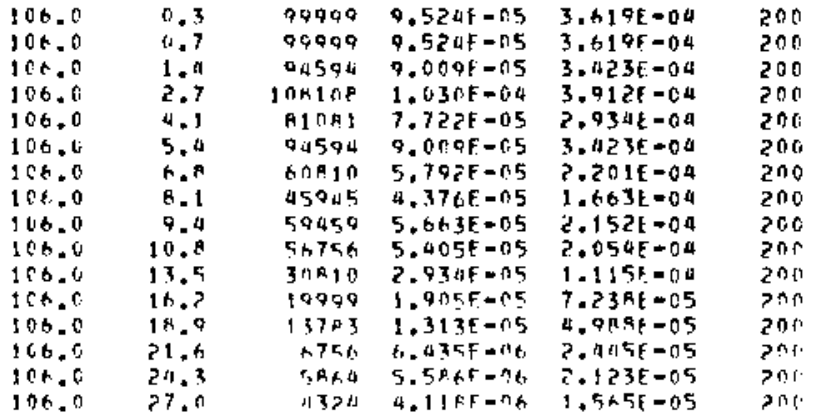

\begin{tabular}{|c|c|c|c|c|c|}
\hline $\begin{array}{l}\text { ALTUI TH } \\
\text { PERIEESS }\end{array}$ & $\begin{array}{l}\text { HE JCHT } \\
\text { RE TEP : }\end{array}$ & 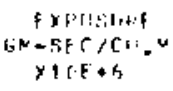 & $\begin{array}{c}E / \pi \\
\text { sec/ri:." }\end{array}$ & $\begin{array}{c}F 1 / F i \\
1 / S O . N\end{array}$ & $\begin{array}{l}\text { PISTAR:TE } \\
\text { NETFRS }\end{array}$ \\
\hline 154.0 & $r_{0} .3$ & $5,5,74$ & $5.3 n>F=0.5$ & $2.0155=04$ & $? ?_{i} \|_{1}$ \\
\hline 114.11 & 10.7 & GP1क? & $5.921,5=0.5$ & $3.25 n t-c 4$ & $p c, n$ \\
\hline 114.0 & 1.4 & 63513 & to. otict $=05$ & $2, \lambda \varnothing 9 \mathrm{~F}-04$ & 206 \\
\hline 114.0 & 2,7 & $\operatorname{si}\{5+7$ & $6.4 \times 55=05$ & 2.0 घ $5 E=00$ & $2 \cap n$ \\
\hline 114.0 & 4.1 & 66216 & $0.30+5=0.5$ & $2.3965=00$ & 300 \\
\hline 110.0 & 5.4 & i1 $? 5 \times 7$ & $0.050 .0-0.5$ & $1.5415-007$ & $20 n$ \\
\hline 114.0 & 6,8 & $\|P \in\| A$ & $4.6336=05$ & $1,761 \mathrm{E}-04$ & $7+0$ \\
\hline 114.0 & P. $t$ & 25175 & $3.34 \times=-0.5$ & $1.772 E=00$ & $p \sin$ \\
\hline $11^{4} .0$ & 9.4 & fRABA & $0.6337=05$ & $1.761 E-0.4$ & $20 n$ \\
\hline 114.0 & 10,8 & 44594 & $4.247 t=015$ & $1.614 E-04$ & 200 \\
\hline 110.0 & 13.5 & $364+6$ & $3.4755-15$ & $1.320 E-04$ & 290 \\
\hline .0 & 16.2 & $2 \geq 20 \pm$ & $2.214 f-125$ & A. $412 E-05$ & $20 n$ \\
\hline 114.0 & 19.9 & $175 \times 7$ & $1.6735=05$ & $6.35 A E-015$ & ?חn \\
\hline 110.0 & 21.1 & 8594 & $9.138 E=? 6$ & $3.07 \geqslant E-1.5$ & 200 \\
\hline & 24.3 & $75 \circ 7$ & $1.20,7=06$ & $2.739 E-0.5$ & $2 n$ \\
\hline $1 J 10$ & 27.0 & 3513 & $3.34+f=06$ & $1.2725-05$ & $2 n 0$ \\
\hline
\end{tabular}




\begin{tabular}{|c|c|c|c|c|c|}
\hline $\begin{array}{l}\text { ATINABISH } \\
\text { DEELEES }\end{array}$ & $\begin{array}{l}\text { HETEHT } \\
\text { NETEHS }\end{array}$ & 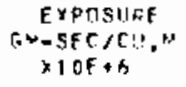 & $\begin{array}{c}E / \Pi \\
S E C / C 1, N\end{array}$ & $\begin{array}{l}F, 100 \\
1 / 50.4\end{array}$ & $\begin{array}{l}\text { CISTARCF } \\
\text { NETERS }\end{array}$ \\
\hline $\begin{array}{l}127.0 \\
122.0 \\
122.0 \\
122.0 \\
122.0 \\
122.0 \\
122.0 \\
122.0 \\
122.0 \\
122.0 \\
122.0 \\
122.0 \\
122.0 \\
122.0\end{array}$ & $\begin{array}{l}0.3 \\
0.7 \\
2.7 \\
4.1 \\
5.4 \\
5.9 \\
8.1 \\
9.9 \\
10.8 \\
13.5 \\
16.2 \\
21.6 \\
24.3 \\
27.0\end{array}$ & $\begin{array}{l}37 P 97 \\
97837 \\
49848 \\
49198 \\
43243 \\
429 \times 7 \\
41671 \\
30810 \\
37297 \\
24374 \\
21351 \\
10377 \\
7094 \\
5077\end{array}$ & $\begin{array}{l}3.552 F=0.5 \\
1.55 t F=0.5 \\
4.633 F=05 \\
9.695 E=05 \\
4.11 A E=05 \\
5.9 A 5 F=0.5 \\
3.9645=.5 \\
2.934 F=05 \\
3.552 F=05 \\
2.317 F=05 \\
7.033 F=05 \\
9.781 E=116 \\
6.757 F=06 \\
4.780 E=06\end{array}$ & 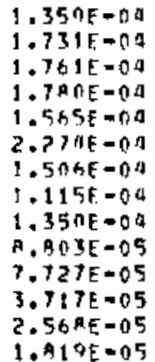 & $\begin{array}{l}200 \\
200 \\
200 \\
200 \\
200 \\
200 \\
200 \\
200 \\
200 \\
200 \\
200 \\
200 \\
200 \\
200\end{array}$ \\
\hline
\end{tabular}

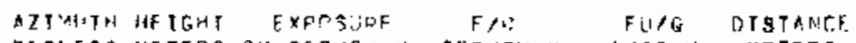

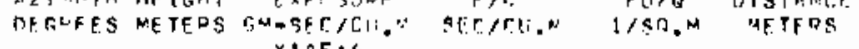

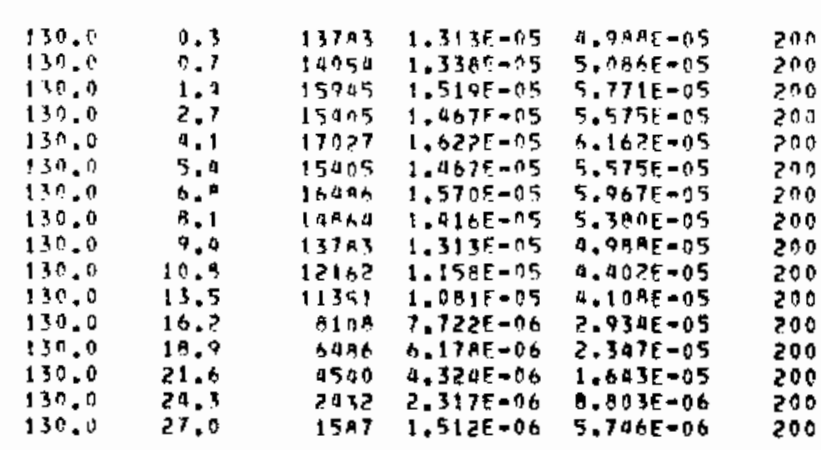

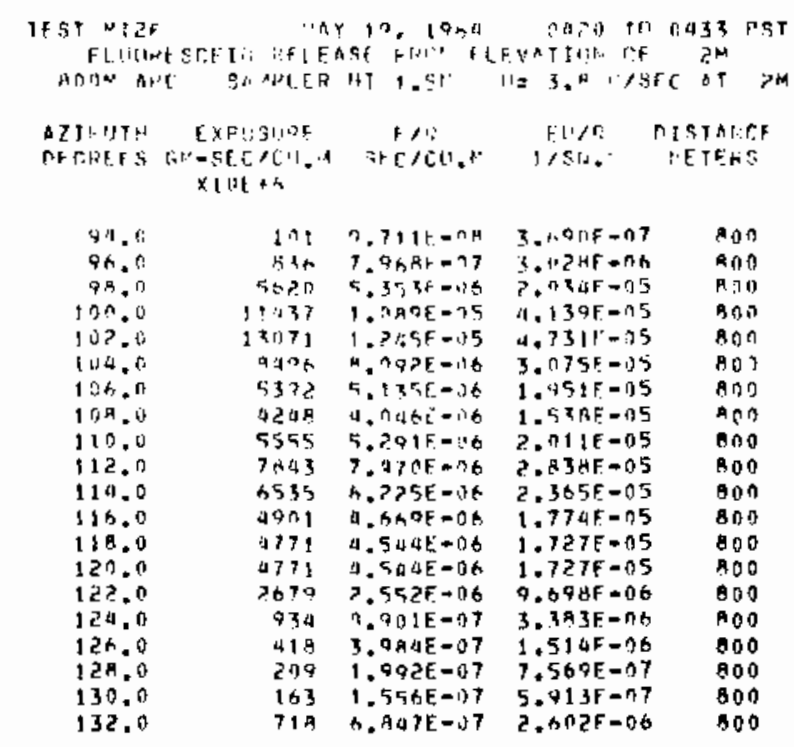

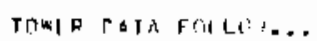

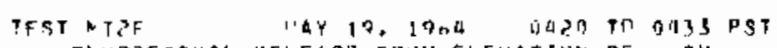

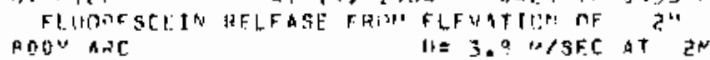

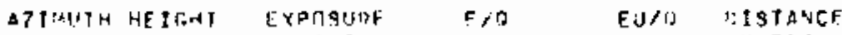

TFRREES NETFIS GM-STC/CH. 1 REC/CII.M I/SO.M METTQS $x \rightarrow 0+4$

\begin{tabular}{|c|c|c|c|c|c|}
\hline$Q R .0$ & $1 . n$ & 1073 & $1.50 \mathrm{HF}=\mathrm{nt}$ & $0.055 \mathrm{E}=0 \mathrm{~B}$ & 000 \\
\hline$Q A .0$ & 2. 1 & $1>79$ & $1,1705=16$ & $4.4 \Delta 7 E=06$ & 800 \\
\hline 94,8 & 4.7 & 5350 & $5.104 \mathrm{~F}=1 \mathrm{6}$ & $1.940 \mathrm{~A}=05$ & $A O 0$ \\
\hline 39.0 & 6.3 & 5500 & $5.32 R E=n A$ & $2.025 E-05$ & non \\
\hline 92 & A. 4 & 5439 & $5.552 F=1.6$ & 2.11 กE $\rightarrow 05$ & 100 \\
\hline $9 A .0$ & 10,5 & $5 n>1$ & 4. $93 \cap 7=06$ & $1.836 E=05$ & 800 \\
\hline 48.0 & 12.5 & 34 44 & $A, 233 \mathrm{~F}=A \mathrm{~A}$ & $1 . B O B E=05$ & 200 \\
\hline 98,0 & 14.7 & קיק & $2.116 \mathrm{~F}=\mathrm{nt}$ & $A \cdot A+2 F=06$ & 800 \\
\hline 98.0 & It. A & 3199 & $3 . O B A F=D A$ & $1.154 E-05$ & 000 \\
\hline Q. D. & 21.0 & $? 6: 9$ & $2.40 \cap E=06$ & $9.46 ? E=06$ & 900 \\
\hline QR. 0 & 25.2 & $19: \mathrm{s}$ & $1.318 E=96$ & $6.907 F=06$ & 600 \\
\hline 98.0 & 2 & 1011 & $305 \mathrm{~F}=16$ & $5.100 E-0 S$ & 900 \\
\hline 0 & 33.5 & $12 R 1$ & $220 E-06$ & $4.636 \mathrm{E}=0.6$ & ถูก \\
\hline & & $5 \mu$ S & $0 ? 5-07$ & $129 \mathrm{E}-06$ & $\sin 0$ \\
\hline & & 503 & $193 F=07$ & 1. A $21 E=06$ & 800 \\
\hline
\end{tabular}

AZJMIIT HEIGHT EXPTZIIAE E/A FUAS DISTANCF

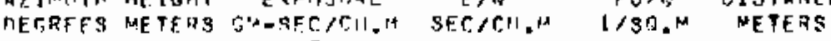

\begin{tabular}{|c|c|c|c|c|}
\hline $\begin{array}{l}106.0 \\
106.0\end{array}$ & 0.3 & $\begin{array}{l}3555 \\
39+9\end{array}$ & $\begin{array}{l}3.3 A 65=96 \\
3.385=06\end{array}$ & $\begin{array}{l}1 . Z A 7 E=C 5 \\
1.4 O O E=05\end{array}$ \\
\hline 105.12 & $1 . n$ & ज19क & $4.2 \times 3 F=n 6$ & $1.627 E+05$ \\
\hline 106.0 & 2.1 & $5 \times 10$ & $4.7 \times 1 F=00$ & 1. $917 \overline{1}=05$ \\
\hline 106.0 & $4 . ?$ & 5719 & $4.7015=16$ & $1.517 E=05$ \\
\hline $\operatorname{tnn} .0$ & 6.3 & SA4 7 & $5,37 \mathrm{HE}=0 \mathrm{~B}$ & $2.0435-05$ \\
\hline joh. 0 & P., 11 & SASA & $5.577 F=100$ & $2.119 F-05$ \\
\hline 105.0 & 111.5 & $57 \mathrm{~h}^{n}$ & $5.677 F=n 8$ & $7 E-05$ \\
\hline 106,0 & 12 & 6535 & $? 55=04$ & $E=05$ \\
\hline 106.0 & 10.7 & 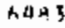 & $6.1755=100$ & - $346 \%=15$ \\
\hline $10 n .0$ & 16.7 & $.16^{\circ}$ & $5.87 \mathrm{AF}-\mathrm{n} 6$ & $.233 F=05$ \\
\hline 106.0 & $2: .9$ & A>> & $5.97 t, E=0$ S & $.271 \mathrm{~F}=05$ \\
\hline 105.0 & $25 . ?$ & $59 \mathrm{kn}$ & $5.677 F=26$ & $157 E=0 S$ \\
\hline $10 n .0$ & 3 & $5+17$ & $37 A F=16$ & 0 A ME $=05$ \\
\hline 106.0 & 3 & $5 / 137$ & $5.179 E-1.6$ & $1.96 \mathrm{RF}=115$ \\
\hline 0 & & 2193 & $\pi \Delta F=06$ & $514 F-05$ \\
\hline 06 & & 1555 & $A \times F=0 A$ & $87 F$ \\
\hline
\end{tabular}

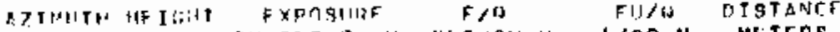

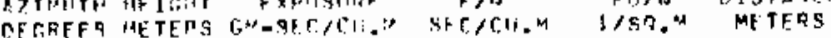

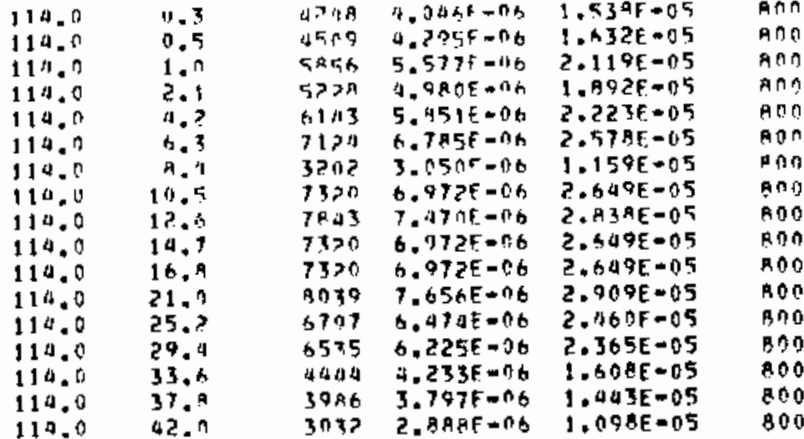

CROASWINIS INTEGFATEC= ?, 359F-1)3 R, 965F-03 


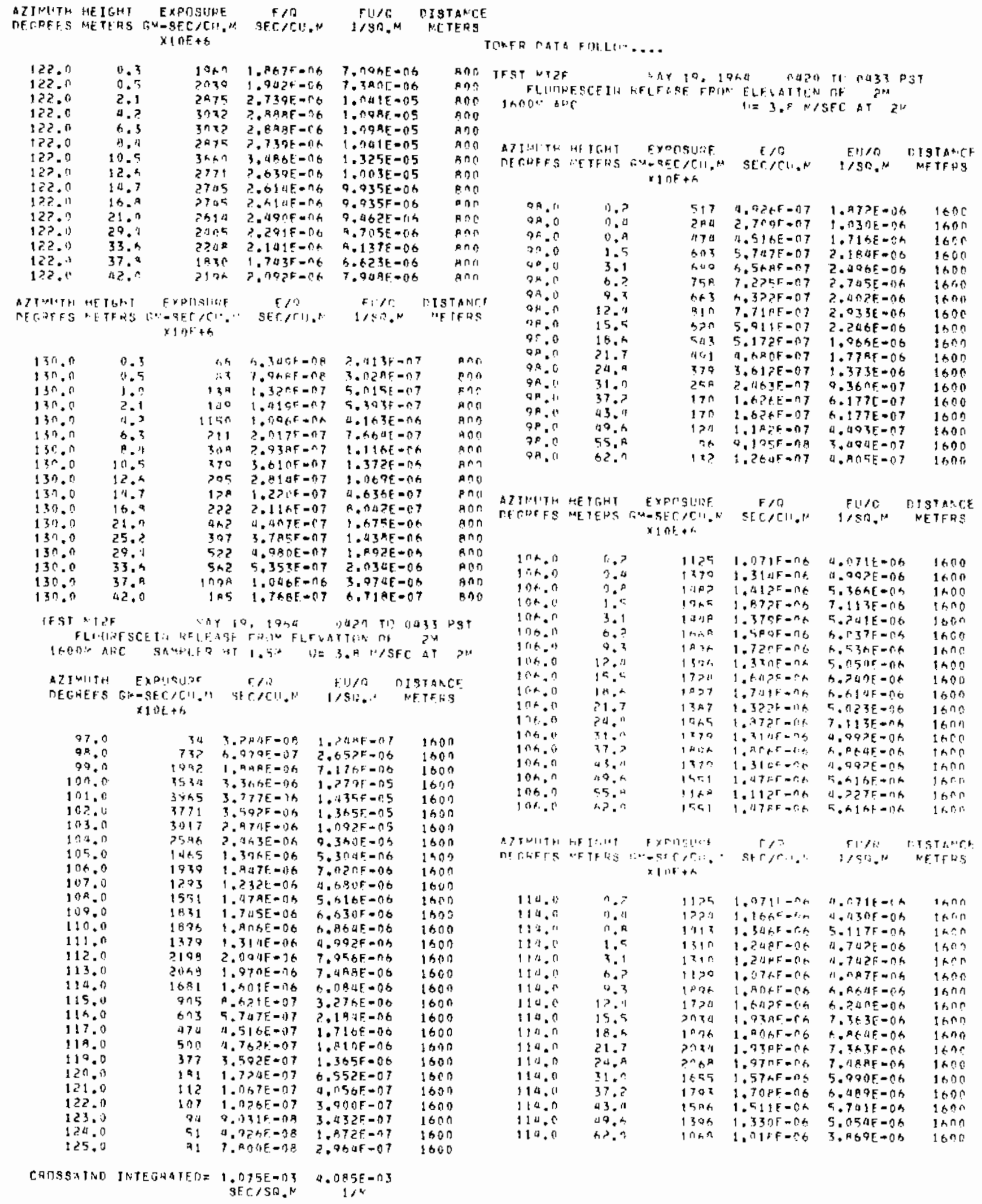




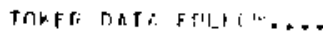

\begin{tabular}{|c|c|c|c|c|c|}
\hline 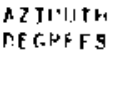 & 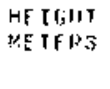 & 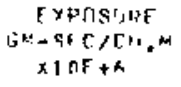 & $\begin{array}{c}f / c \\
\text { st } c / r \|, k\end{array}$ & $\begin{array}{c}E y / 0 \\
1 / 50 . N\end{array}$ & $\begin{array}{l}\text { DISTANCH } \\
\text { METERS }\end{array}$ \\
\hline $\begin{array}{l}122.0 \\
122.0 \\
122.0 \\
122.0 \\
1220.0 \\
122.0 \\
122.0 \\
12200 \\
122.0 \\
122.0 \\
122.0 \\
122.0 \\
122.0 \\
122.0 \\
122.0 \\
122.0 \\
122.0\end{array}$ & 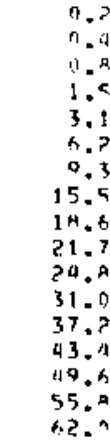 & 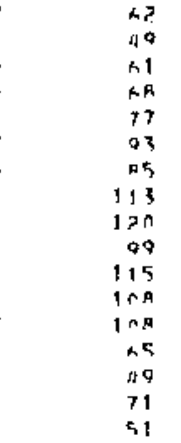 & 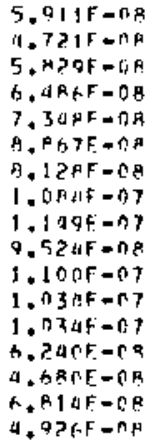 & 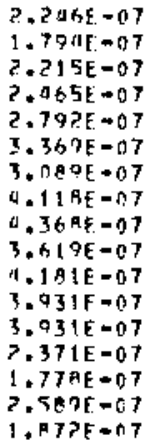 & $\begin{array}{l}1600 \\
1600 \\
1600 \\
1600 \\
1600 \\
1600 \\
1600 \\
1600 \\
1690 \\
1600 \\
1600 \\
1600 \\
1600 \\
1600 \\
1600 \\
1600 \\
1600\end{array}$ \\
\hline
\end{tabular}

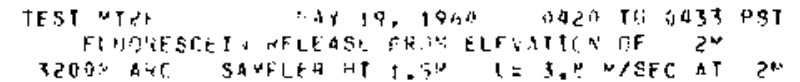

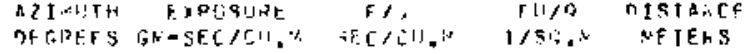
$\times 10 E+6$

\begin{tabular}{|c|c|c|c|c|}
\hline $\begin{array}{l}95.0 \\
96.0\end{array}$ & $\begin{array}{l}53 \\
95\end{array}$ & $\begin{array}{l}3.854=08 \\
0.911 k=08\end{array}$ & 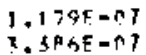 & $\begin{array}{l}3200 \\
3200\end{array}$ \\
\hline 47,0 & 211 & 2. $30 ?+-107$ & $\Leftrightarrow, 7$ व $F=07$ & 3 ? 00 \\
\hline 48.11 & $3 \div 8$ & $3.7, S E=07$ & $1.01\} F=06$ & 3200 \\
\hline 99.0 & $2 ? 5$ & $2.1545=07$ & $8.183 F=07$ & 3200 \\
\hline 06.0 & 818 & $7.7875-0 ?$ & $2.9035=0.6$ & 3200 \\
\hline 01.0 & 1354 & $3.300 f=06$ & $4.93 \times F=08$ & 3200 \\
\hline 02.0 & 857 & $9.1695=07$ & $5.104 \mathrm{~F}-06$ & 3200 \\
\hline 3.0 & 779 & $7.4>65=07$ & $2,822 E=06$ & 06 \\
\hline 0 & $4 ? 8$ & 4. $5845=07$ & $2 E-06$ & 3200 \\
\hline 0 & bh? & $6.3125=07$ & $9 F=06$ & 3200 \\
\hline .0 & 663 & $6.312 F=07$ & $397 E-06$ & 3200 \\
\hline 07.0 & 790 & $7.61 ? E=07$ & $2.902 E=06$ & 3200 \\
\hline 0 B. 0 & $b 92$ & 6. 40 तE -07 & $2.4647=06$ & 3200 \\
\hline 07.0 & $65 t$ & $6.25 B E_{-}=07$ & $2.3776-06$ & 3200 \\
\hline .0 & 623 & $5.941 E-37$ & $57 F=n 6$ & 320 \\
\hline & $6 \times 3$ & $6.03 \Delta E=07$ & $03 f-1,5$ & 3200 \\
\hline 0 & $4 \times !$ & 4. $000 E-07$ & $1, h 72 f=06$ & 3200 \\
\hline 0 & 341 & $3.249 E-07$ & $1.235 E-0.6$ & 3200 \\
\hline 0 & 253 & $3.413 E=07$ & $0.171 \mathrm{E}-07$ & 3200 \\
\hline 0 & 113 & $1.077 t-07$ & $9.002 F-07$ & 3200 \\
\hline & 69 & $6.4 Q B E=0 B$ & $3.469 f-07$ & 3200 \\
\hline & 37 & $3.5775-08$ & $3405-C 7$ & $32 n 0$ \\
\hline & 19 & $1, A, 9 E=O E$ & $0.91 \mathrm{af}=0 \mathrm{~B}$ & 3200 \\
\hline & 13 & 1. $2 A B E=O P$ & $4.707 E-08$ & $320 \mathrm{n}$ \\
\hline & 9 & $2 k=04$ & $3.52 .7 E-08$ & 3200 \\
\hline & 10 & $0.654 E-09$ & 3. $A$ GHFF $=O A$ & 3200 \\
\hline & & $\begin{array}{l}\text { 4. } 001 F=04 \\
\text { SEC }\end{array}$ & $2,2 B 0 E-113$ & \\
\hline
\end{tabular}

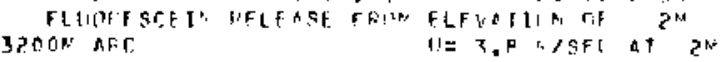

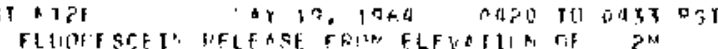

\begin{tabular}{|c|c|c|c|c|c|}
\hline $\begin{array}{l}\text { ATENITH } \\
\text { DFCHEES }\end{array}$ & $\begin{array}{l}\text { IF IEHT } \\
\text { RFTENS }\end{array}$ & 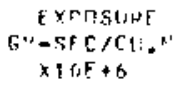 & $\begin{array}{c}E / 2 \\
\text { sf }[1<y .0\end{array}$ & $\begin{array}{l}E \forall / 1 \\
1 / 50 .\end{array}$ & $\begin{array}{l}\text { DISTOCCE } \\
\text { ME TFSS }\end{array}$ \\
\hline SP. 0 & 0.2 & $2 \div 2$ & $1.931 F=177$ & $7.337 E-0 ?$ & 3200 \\
\hline $9 A .0$ & t. .7 & $1+3$ & $1.745 F=07$ & $4.631 E-07$ & 3200 \\
\hline QP. & $0 . M$ & 257 & $2.45, F=0 ?$ & $9.312 F=07$ & 32011 \\
\hline 8.5 & 1.5 & ?an & $2.6731+107$ & $1.016 F-06$ & $32 \div n$ \\
\hline 95.6 & 3.1 & उदि & $3.3425=07$ & $1.270 E=06$ & $5=0 \pi$ \\
\hline YA. 0 & h.? & 317 & 3.0 . $55=07$ & $1.157 \mathrm{~F}-16$ & 3EAn \\
\hline 5.0 & 9.3 & रAก & $2.67 \underline{15}-07$ & $1.036 E=06$ & $3 \geq 0$ \\
\hline 80 & 12.4 & 545 & $2.673 f=07$ & $1, D I B E=06$ & 3200 \\
\hline 9. & 15.5 & PEP & $2.7 \Delta \mu F=G 7$ & $1.9 \triangle A E=O A$ & 3200 \\
\hline 98.0 & 18.4 & $?+0$ & $2.56 .3 \mathrm{~F}=\mathrm{C} 7$ & $9.755 \mathrm{E}=0.7$ & $3 \pi 00$ \\
\hline $9 ? .0$ & 21.7 & 己मn & $2.6735=17$ & $1 . A+6 F=06$ & $32 \mathrm{tr}$ \\
\hline 74.0 & 34.9 & $>27$ & $3.265 F=07$ & R. OOTE-07 & 3200 \\
\hline 30.7 & 31.0 & 206 & $\therefore .96$ AF $=07$ & 7.47 RE $=07$ & 3700 \\
\hline $9 \% . n$ & 37.2 & 171 & $1,1,3 \Delta F=n T$ & $6.20 A E=07$ & $3 z 00$ \\
\hline $9 R .0$ & 43.1 & $1 \mathrm{ad}$ & 1.37 af -07 & $5.720 E-07$ & 3200 \\
\hline$Q B .0^{\circ}$ & 69.6 & $1 \times 4$ & $1.277 F=07$ & 4. 85 पह $=07$ & 3200 \\
\hline$G A \cdot C^{\circ}$ & 55.5 & $1 \cap 1$ & $9.65 \mathrm{UF}=\mathrm{nA}$ & $3.6, E_{E}=0.7$ & 3200 \\
\hline $9 P .0$ & 62.0 & RO & $9.540 F=108$ & $3.245 E-n 7$ & 52110 \\
\hline
\end{tabular}

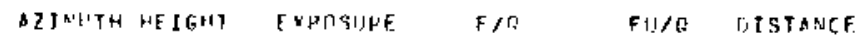

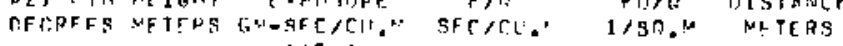
$x 1$ of $+h$

\begin{tabular}{|c|c|c|c|c|c|}
\hline int. 0 & 11.3 & 470 & $4.5675-17$ & $1.75 \% E-06$ & 520 \\
\hline 106.0 & 0.15 & $5 \times n$ & 5.05 r.F $=07$ & $1.917 F=06$ & 320 \\
\hline 106.0 & 0.4 & 497 & $4.0 .11 \pi-07$ & $1.7646=06$ & \\
\hline 106.0 & 1.5 & 400 & $4.2575=1: 7$ & 1.8 a d $=0$ th & \\
\hline 106.0 & 3.1 & 331 & $3.15,5=57$ & 1. $199 F=06$ & $3 \geq$ \\
\hline 106.0 & $6+7$ & 025 & $0.17525-0.7$ & $2.30 \cap F-n B$ & \\
\hline 104.0 & 9.3 & 412 & $5.9 .295-177$ & ?.? $15 E=06$ & 37 \\
\hline 106.0 & 12.4 & 429 & $n+0 R C F-0 ?$ & $7,31^{4}$ UE $=96$ & \\
\hline $10 \mathrm{~h} .0$ & 15.5 & that & $6.30 t k=n y$ & $? .3 O>\mathrm{E}=\mathrm{OH}$ & 32 \\
\hline $106 . n$ & 18.6 & 561 & 5.34750 .07 & $7.03 ?[0=0 \mathrm{i}$ & $2 ?$ \\
\hline 1 ith. 0 & 21.7 & 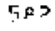 & $5.551 \mathrm{f}=07$ & $? .109 E=05$ & \\
\hline $10 x, 0$ & & $x>5$ & $A+F=n ?$ & $7.257 E-06$ & 37 \\
\hline $10 n . ?$ & $31 . n$ & $7 \times 1$ & $7.2 d n: F=n$ & $? .751 \mathrm{E}=0 \mathrm{~h}$ & 3 \\
\hline 1030 & 37.7 & $5 p$ & $5.553+=07$ & $\Rightarrow .109 E=n h$ & 3 \\
\hline 106.0 & \#3. & $5=1$ & $5,254 \mathrm{~F}=07$ & $1.99 \mathrm{hE}=0.1 \mathrm{~h}$ & 321 \\
\hline 146.0 & 49.4 & $5+1$ & $5.3+75-17$ & $?+n x ? E=06$ & 3 \\
\hline 106.0 & 45 & 426 & $5.0345=07$ & $? .295 E-n G$ & 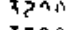 \\
\hline 1)h. .0 & 4? & 74 & $7.1 ? 0 \%=11 \%$ & ?.700E-6. & 370 \\
\hline
\end{tabular}

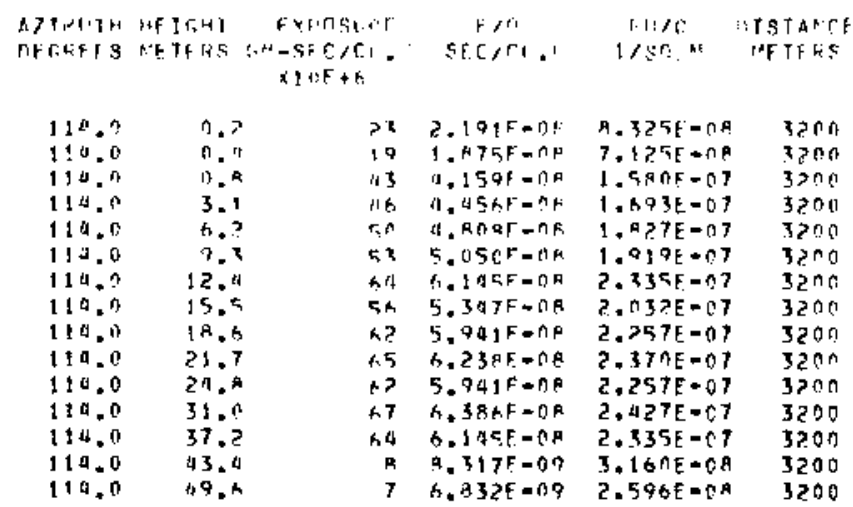


SECONDARY SERIES EXPERIHEHT: $X T-3$

ZISC SULFIDE AND FLUOAOSCEIN WERE BOTH DSED AS TRACERS DCRIAG EXPERIXETT KT-3. ZINC SULFIDE BESULTS ARE PEESEKTED BERE. GROUND LEVPL AND TOWER SAMPLIGG 200-3200 M AHCS, A SIGNTPICANT PORTION OP THE PEUHE PASSED TO TES NORTE OF THE GRID.

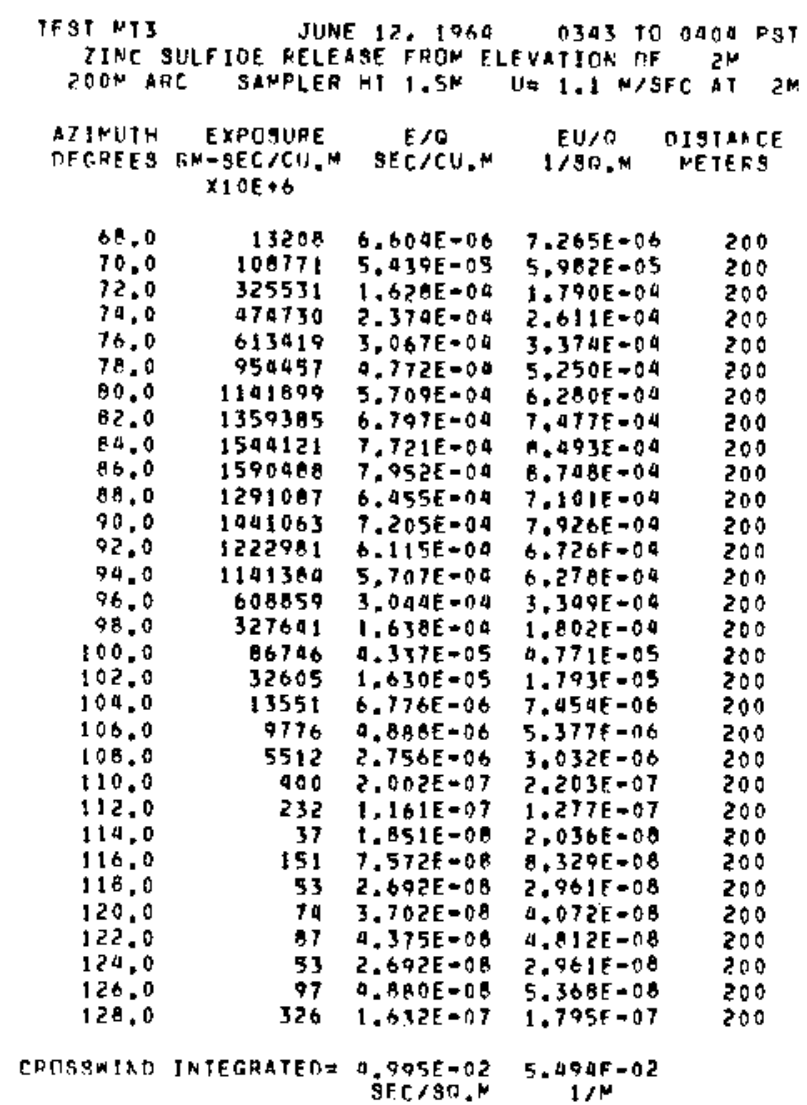

TOKEP CATA FRLLEN....

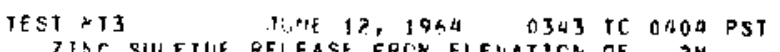

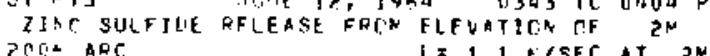

\begin{tabular}{|c|c|c|c|c|c|}
\hline $\begin{array}{l}\text { AITMU'TH } \\
\text { PEGTFES }\end{array}$ & $\begin{array}{l}\text { HE IEHT } \\
\text { METERS }\end{array}$ & 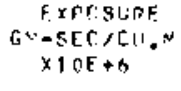 & $\begin{array}{c}E / C \\
\text { sFC'Cli.N }\end{array}$ & $\begin{array}{l}\text { Fu/k } \\
1 / \text { so.k }\end{array}$ & $\begin{array}{l}\text { OJSTANC } \\
\text { NETERS }\end{array}$ \\
\hline 9 ค. 0 & 0.3 & 156902 & $7.045 F-05$ & $0.630 E=05$ & \\
\hline 98,0 & $(1.7$ & $2 \mathrm{t}$ & .04 & 1.1 & \\
\hline$\angle \rho .0$ & 1. & 2113 & $E=04$ & 04 & 4 \\
\hline 98.0 & 2.7 & $6 ! 1$ & 4 & 04 & \\
\hline 58.0 & 4.1 & דם דמת & b. & 5. & 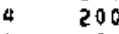 \\
\hline 9.0 & 5.4 & 1407415 & $1.037 \mathrm{E}-1.4$ & $1 E-04$ & \\
\hline 98.0 & t.. & & 7. & $\theta .31 B E=0 A$ & \\
\hline 48.0 & 4.1 & 1270 & 6. & $0.986 E=04$ & 4 \\
\hline & 9.4 & $7 ! ?$ & $61 E=04$ & A. CZTE-DO & 20 \\
\hline 59.0 & $10.0^{\circ}$ & 578 & $2.894 \mathrm{E}$ & $3.1 B<E-0 Q$ & \\
\hline $9 R .0$ & 15.5 & 190405 & & $9.767 E-05$ & \\
\hline $5 \%, 0$ & 10.2 & 72976 & $1.10 \in E=n S$ & $1.261 E=05$ & \\
\hline 98.0 & $\begin{array}{l}18 . \\
21 .\end{array}$ & 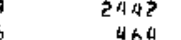 & $\begin{array}{l}1.22 ! \varepsilon=06 \\
32: E=07\end{array}$ & $1.343 \mathrm{E}-08$ & 20 \\
\hline & & & & & \\
\hline
\end{tabular}

\begin{tabular}{|c|c|c|c|c|c|}
\hline $\begin{array}{l}\text { AZTHUTH } \\
\text { DEGHEES }\end{array}$ & $\begin{array}{l}\text { MEIGHT } \\
\text { NETERS }\end{array}$ & $\begin{array}{l}\text { ExpOStiRe } \\
\text { GWEC/CH, } \\
\times 10 E+6\end{array}$ & $\begin{array}{c}E / 0 \\
\mathrm{SEC} / \mathrm{CH}, \mathrm{N}\end{array}$ & $\begin{array}{l}\text { EU/O } \\
1,90 . N\end{array}$ & $\begin{array}{l}\text { DISTANCE } \\
\text { NETERS }\end{array}$ \\
\hline $\begin{array}{l}106.0 \\
106.0\end{array}$ & 0.3 & $\begin{array}{l}3734 \\
4173\end{array}$ & $\begin{array}{l}1.8 A 7 E=06 \\
2.097 E=06\end{array}$ & $\begin{array}{l}2.054 \mathrm{E}=06 \\
2.295 \mathrm{E}=0 \mathrm{~b}\end{array}$ & 200 \\
\hline 106.0 & 1.0 & 5595 & $2.739 \mathrm{~F}=06$ & $3.07 \mathrm{AE}=06$ & $\begin{array}{l}200 \\
200\end{array}$ \\
\hline 100.0 & 2.7 & 19727 & $9,864 F=16$ & $1.005 E-05$ & $\begin{array}{l}200 \\
200\end{array}$ \\
\hline 106.0 & 4.1 & 76677 & $3.03 \cup E-05$ & $0.217 E=05$ & 200 \\
\hline 106.0 & 5.0 & 203461 & $1,017 E-64$ & $1.119 E=04$ & 200 \\
\hline 106.0 & 6.8 & anteas & $2.030 E-C 4$ & $2+243 E=04$ & 200 \\
\hline $10+.0$ & 0.1 & 601695 & $3.000 E=00$ & $3.300 \mathrm{E}=0 \mathrm{a}$ & 200 \\
\hline 100.0 & 9.0 & 605090 & 3. उटAE $=04$ & $3.661 \mathrm{E}=00$ & 200 \\
\hline 106.0 & 10.9 & +36815 & 3.1 A $\triangle E-\cap 4$ & $3.502 E=04$ & 200 \\
\hline $10 \mathrm{~A} .0$ & 13.5 & 329900 & $1.6508=00$ & $1.014 \mathrm{E}=0 \mathrm{a}$ & 200 \\
\hline 106.0 & ith.? & $1 \geqslant 044 ?$ & $6.247 E=05$ & $6.972 E=05$ & 200 \\
\hline 106.0 & 18.9 & 47165 & $2.35 B E-r 5$ & $2.594 E=05$ & 200 \\
\hline 106.0 & 21.5 & $1 \operatorname{tos} 0$ & $4,300 E=05$ & $9.130 E=05$ & 200 \\
\hline 106.0 & 24.5 & 29 ค5 & $1.493 \mathrm{~F}=0 \mathrm{t}$ & $1.642 E-06$ & 200 \\
\hline $1 \mathrm{ch} .0$ & 27.0 & 1223 & S.11GF $=07$ & $0.731 E=07$ & $2 n 0$ \\
\hline
\end{tabular}

\begin{tabular}{|c|c|c|c|c|c|}
\hline 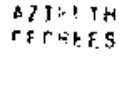 & $\begin{array}{l}\text { HEITHF } \\
\text { "ETHSS }\end{array}$ & 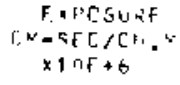 & $\begin{array}{c}F / G \\
S E[/ r 1, k\end{array}$ & $\begin{array}{l}\text { Eu/is } \\
\text { I/SR.u }\end{array}$ & $\begin{array}{l}\text { ISTAACA. } \\
\text { MFTEFS }\end{array}$ \\
\hline 114.0 & 0.3 & ay & $3.357 E-\mathrm{R} . \mathrm{A}$ & $3.693 \mathrm{E}-00$ & \\
\hline $11^{t i} \cdot 0$ & 0.7 & 11 & $1.50 n t-6 A$ & $1.749 E-C B$ & 200 \\
\hline 114.0 & 2.7 & ho & $3.475 \mathrm{~F}-18$ & $3 . A \geq 2 E=0 B$ & $2 n 0$ \\
\hline 114.0 & 4.1 & $17 \mathrm{~h}$ & $9, A 11 F-O B$ & $9.692 E-08$ & 200 \\
\hline 114.0 & 5,11 & $2 \bar{F}_{3}$ & $1,26+5=07$ & $1.3020-07$ & 200 \\
\hline 134.0 & h. & 752 & $3.7605-07$ & $4.136 \mathrm{E}=07$ & 200 \\
\hline $11: 00$ & 8.1 & 12117 & $7.2355-07$ & $1.959 \mathrm{E}=07$ & 200 \\
\hline 114.0 & 9.4 & iा? & $2.355 F=06$ & $2.591 E-06$ & 200 \\
\hline 110.0 & 10.8 & 11770 & $5.0908-06$ & $0.4795-106$ & 200 \\
\hline $11 \% .0$ & 13.5 & 15000 & $7.5405-06$ & $1.294 E-06$ & 200 \\
\hline $11^{45}=0$ & $16 . ?$ & $26 \geq 0$ & $4,310 E-0 t$ & $9.741 E-06$ & 200 \\
\hline 110.0 & 18.0 & $454:$ & $2,321 E=06$ & $2.553 E=06$ & 200 \\
\hline 1100 & 21.6 & 1035 & $5.175,5-n 7$ & $5.693 \mathrm{E}=$ & 200 \\
\hline 114.5 & 24.3 & 789 & $3.945 E=07$ & $4.340 E \div 07$ & 200 \\
\hline
\end{tabular}

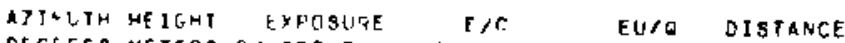

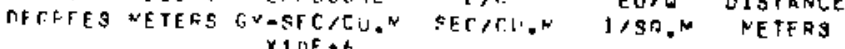

\begin{tabular}{|c|c|c|c|c|c|}
\hline $\begin{array}{l}1222.0 \\
122.0 \\
12220 \\
122.0 \\
122.0 \\
122.0 \\
12200 \\
122.0 \\
122.0\end{array}$ & $\begin{array}{l}0.3 \\
0.7 \\
1.4 \\
2.7 \\
4.1 \\
5.4 \\
6.8 \\
8.1 \\
10.9\end{array}$ & $\begin{array}{r}173 \\
53 \\
39 \\
210 \\
104 \\
119 \\
154 \\
114 \\
64\end{array}$ & $\begin{array}{l}A .057 E-08 \\
2.650 E=00 \\
1.986 E-08 \\
1.059 E-07 \\
5.212 E-08 \\
5.957 E-08 \\
7.818 E-08 \\
5.833 E=08 \\
3.227 E-08\end{array}$ & 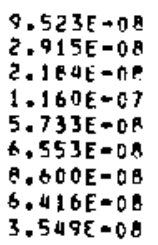 & $\begin{array}{l}200 \\
200 \\
200 \\
200 \\
200 \\
200 \\
200 \\
200 \\
200\end{array}$ \\
\hline
\end{tabular}

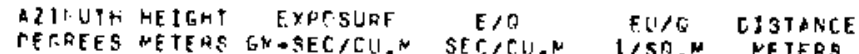

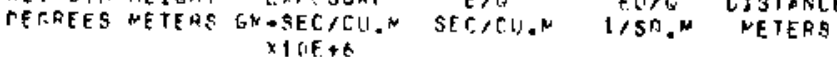

$130.0 \quad 10.4 \quad 27 \quad 1.365 F-09 \quad 1.502 E-08 \quad 200$ 


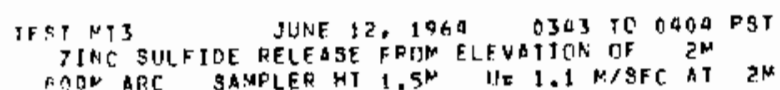

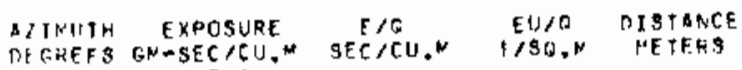
$\times 10 E+6$

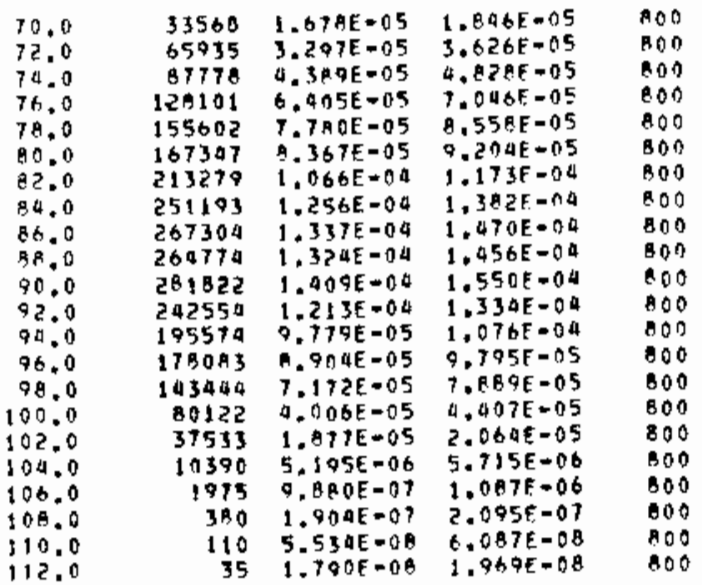

TROSENIND INTEGAATEDA 3.919E-02 $4.311 E-02$

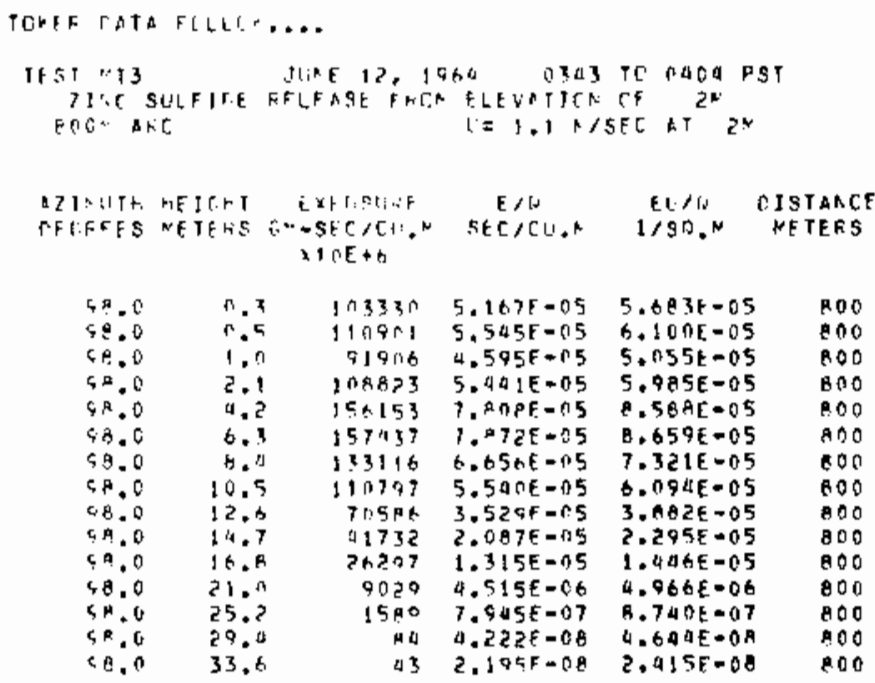

\begin{tabular}{|c|c|c|c|c|c|}
\hline $\begin{array}{l}\text { A7INWTH } \\
\text { DFTEEES }\end{array}$ & $\begin{array}{l}\text { HEICHT } \\
\text { NETEFS }\end{array}$ & $\begin{array}{c}\text { EXPCSUHE } \\
\text { GN-SEC/C11. } \\
X I U E+6\end{array}$ & $\begin{array}{c}E / 0 \\
\mathrm{SEC} / \mathrm{Cl}, \mathrm{N}\end{array}$ & $\begin{array}{l}E U / O \\
1 / 30 . m\end{array}$ & $\begin{array}{l}\text { DISTANCE } \\
\text { NETERS }\end{array}$ \\
\hline & 0.3 & & 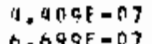 & a. $B 5 \cap E=07$ & AOO \\
\hline $\begin{array}{l}10.6 .0 \\
106.0\end{array}$ & 3.1 & $\begin{array}{r}1330 \\
5127\end{array}$ & $\begin{array}{l}0.699 E=07 \\
2.737 F=07\end{array}$ & $\begin{array}{l}7.3695=07 \\
3.011 F=07\end{array}$ & $\begin{array}{l}600 \\
000\end{array}$ \\
\hline 166.0 & 2.1 & $\{41\}$ & $7.05 S E-07$ & $51-07$ & $B O O$ \\
\hline 6.0 & 4. & 6519 & $3.255 F=06$ & $5 F=06$ & 800 \\
\hline 0,0 & 6.1 & 173 ค? & $0.691 E=06$ & $60 F=06$ & 800 \\
\hline ] & 8,4 & 34071 & $1.7108=05$ & $23 E-05$ & 800 \\
\hline .0 & 10.5 & 5.5333 & $2.7675-n 5$ & $3.043 E=05$ & AOn \\
\hline .0 & 12. & $+29 k$ & 3.1 aEF $=05$ & $063 f-05$ & BOA \\
\hline .0 & 14.7 & 407 id & $3.487 F=n 5$ & $3.836 E=05$ & 800 \\
\hline 1 & $16,0^{\circ}$ & 50 मी० & $2.903 E-115$ & $292 E=05$ & POO \\
\hline .0 & $2 ! .1$ & 32951 & $1.645 F-0,5$ & $1.814 \varepsilon=05$ & 800 \\
\hline 1 & 25.7 & 19575 & $5.263 E=06$ & $5.789 \mathrm{E}=06$ & B00 \\
\hline 1 & & 11117 & 7. $.05 c 5=06$ & $264 \mathrm{~F}=0 \mathrm{~b}$ & $a \cap 0$ \\
\hline 0 & & 1105 & $5.927 E-\cap 7$ & $52 n t-n 7$ & 800 \\
\hline & & $5 i l f_{1}$ & $.7125=07$ & $172 E-07$ & BOC \\
\hline 10 & & 1 ar & $35 F+08$ & $938 E-1,6$ & ROO \\
\hline
\end{tabular}

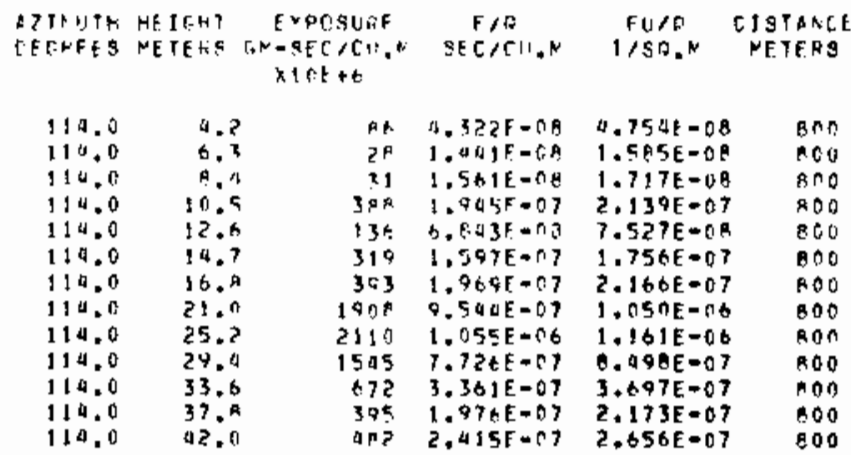

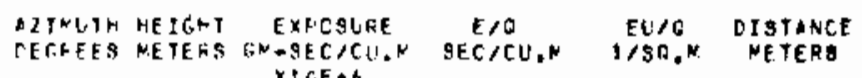

$\begin{array}{llllll}122.0 & 9.2 & 69 & 3.481 E-08 & 3.629 E-08 & 900 \\ 122.0 & 6.3 & 50 & 2.521 E-108 & 2.773 E=08 & 300\end{array}$ 


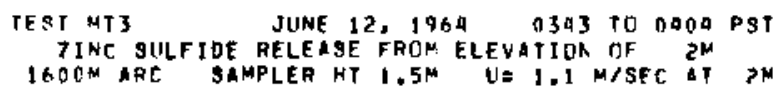

AZIMUTH EXPOSURE E/G EVIO DISTANCE DEFREES GM-SECICU.M SECICU.M T/SO.M MEZEFS $X+\triangle E+6$

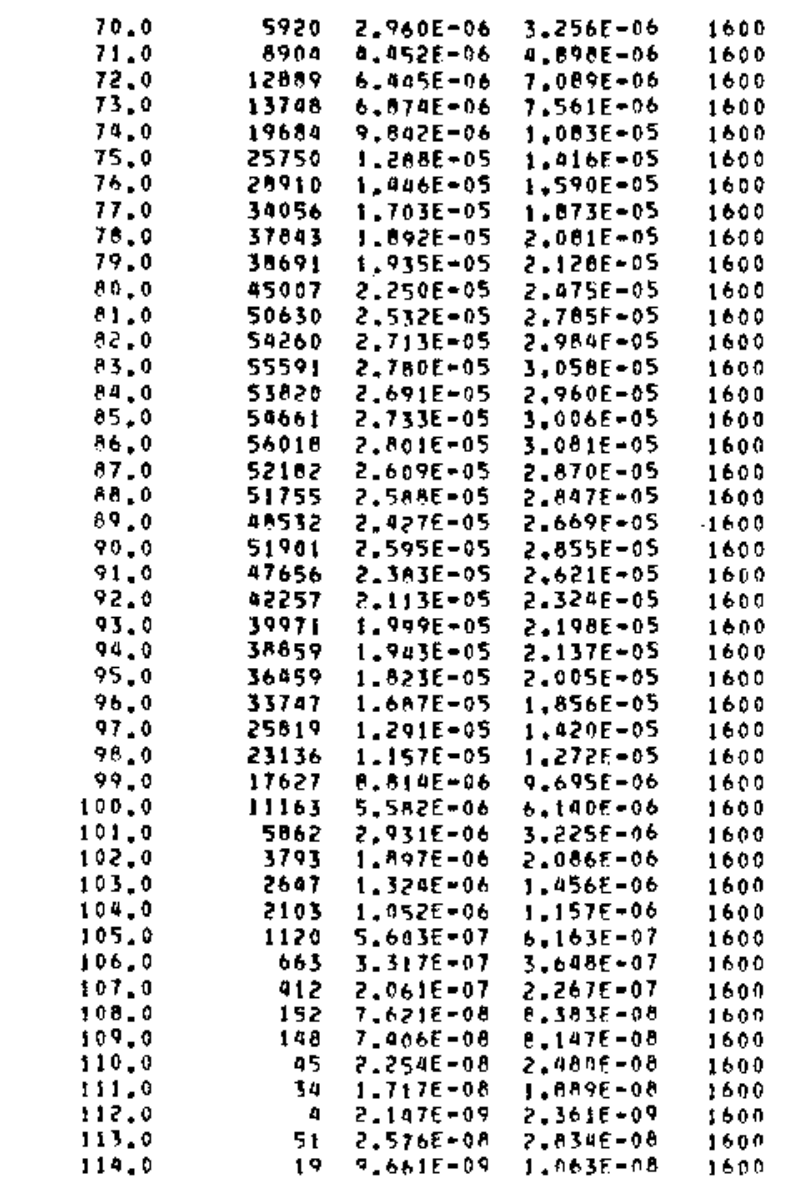

CRNSSWTHO INTEGRATEO= $1.5 \% 4 E-02 \quad 1.742 E-0 ?$

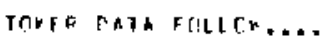

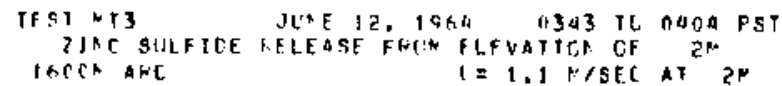

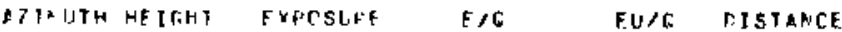

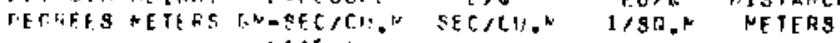
$x \in \mathbb{A B}+t$

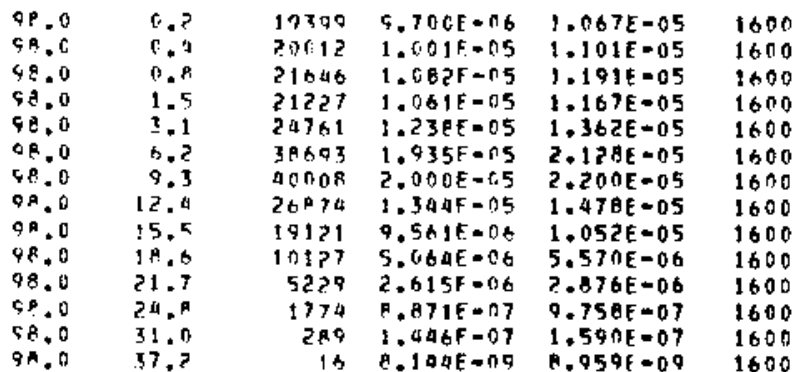

AIINITH HFIGHT EYFCSUFE F/O FU/O DISTANCE LECHEFS NETEKS GN-SFE/CL. N SEC/C11.N T/\$O,N NETERS $X \mid 0 F+6$

\begin{tabular}{|c|c|c|c|c|c|}
\hline 106.0 & $n, p$ & at 53 & $2.265 \mathrm{Fm} 07$ & $2.492 \mathrm{E}-0.7$ & 1600 \\
\hline 106.0 & 0.8 & 371 & 1. $A \in \cap C=\cap 7$ & $2.046 E=07$ & 1600 \\
\hline 156.0 & $0 . P$ & 5il & $2.558 \mathrm{E}-1.7$ & $2.81 \Delta E-07$ & 1600 \\
\hline 16.5 .0 & 1.c & $x \geqslant 4$ & $1.685 \mathrm{~F}=07$ & $1.853 \mathrm{E}-07$ & 1600 \\
\hline 106.0 & 3.1 & $I$ i $\mathrm{F}$ & $5.191 \mathrm{f}=07$ & $5.710 E=07$ & $\$ 600$ \\
\hline $1 \times 6.0$ & 0.2 & 3194 & 1. $5 \& C E-5.6$ & $1.754 \mathrm{E}=0 \mathrm{t}$ & 1600 \\
\hline $1 C B .0$ & 9.3 & $=55^{\circ}$ & $4.279 F=0.6$ & $4.707 E=06$ & 1600 \\
\hline 106.0 & 12.0 & $1151 \mathrm{~h}$ & $7.25 \mathrm{E}-n \mathrm{E}$ & $7.0 \mathrm{~B} A \mathrm{E}=06$ & 1600 \\
\hline 116.0 & 15.5 & $2113^{\circ}$ & $1.057 E-0.5$ & $1.163 t-05$ & 1600 \\
\hline 10.6 .0 & 1 A. & is;b?? & $7.61 \Delta F=n E$ & H. $595 \mathrm{E}-06$ & 1600 \\
\hline $11.6,0$ & 21.7 & 10I 10 & $9.069 E-106$ & $2.976 \varepsilon=06$ & 1600 \\
\hline 106.0 & 24.4 & A $5>0$ & 小. $262 \mathrm{~F}=06$ & 4.6 A AE $=06$ & 1600 \\
\hline 106.0 & $31 . r$ & $1 ค Q\}$ & $8 . \angle 6 Q E=07$ & $1.041 E=06$ & 1600 \\
\hline $10: 6.0$ & 37.2 & 0,77 & $3.3 A 7 E=117$ & $3.725 \mathrm{~F}-0.7$ & 1600 \\
\hline 106.0 & 43.3 & A 4 & $4.20 A E-\cap A$ & $4.629 E=0 R$ & 1600 \\
\hline
\end{tabular}

A7IMUTH HEIGHY FXPOSLPE E/E FU/G OISTANCE

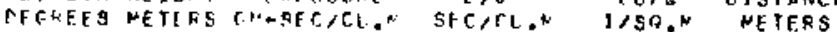
$x \in 1, F+A$

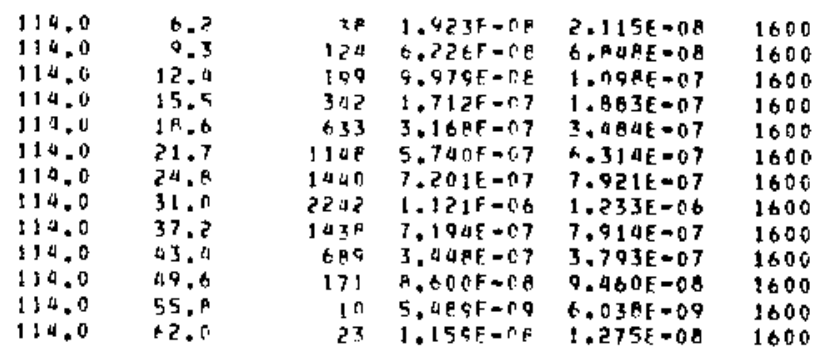

A7IVUTH HEICH1 EXPOSLILE E/G EU/C DISTANCE CFGHFES METFAS GN-SEC/CU.N SEE/CL,N 1/SQ.N FEFERS

$\begin{array}{rrrrrr}122.0 & 3.1 & 34 & 1.734 E-08 & 1.913 E-08 & 1600 \\ 122.0 & 6.2 & 34 & 1.739 E-08 & 1.913 E-00 & 1600 \\ 122.0 & 9.3 & 16 & 6.240 E-09 & 9.064 E=09 & 1600 \\ 122.0 & 15.5 & 40 & 2.014 E-08 & 2.216 E-00 & 1600\end{array}$




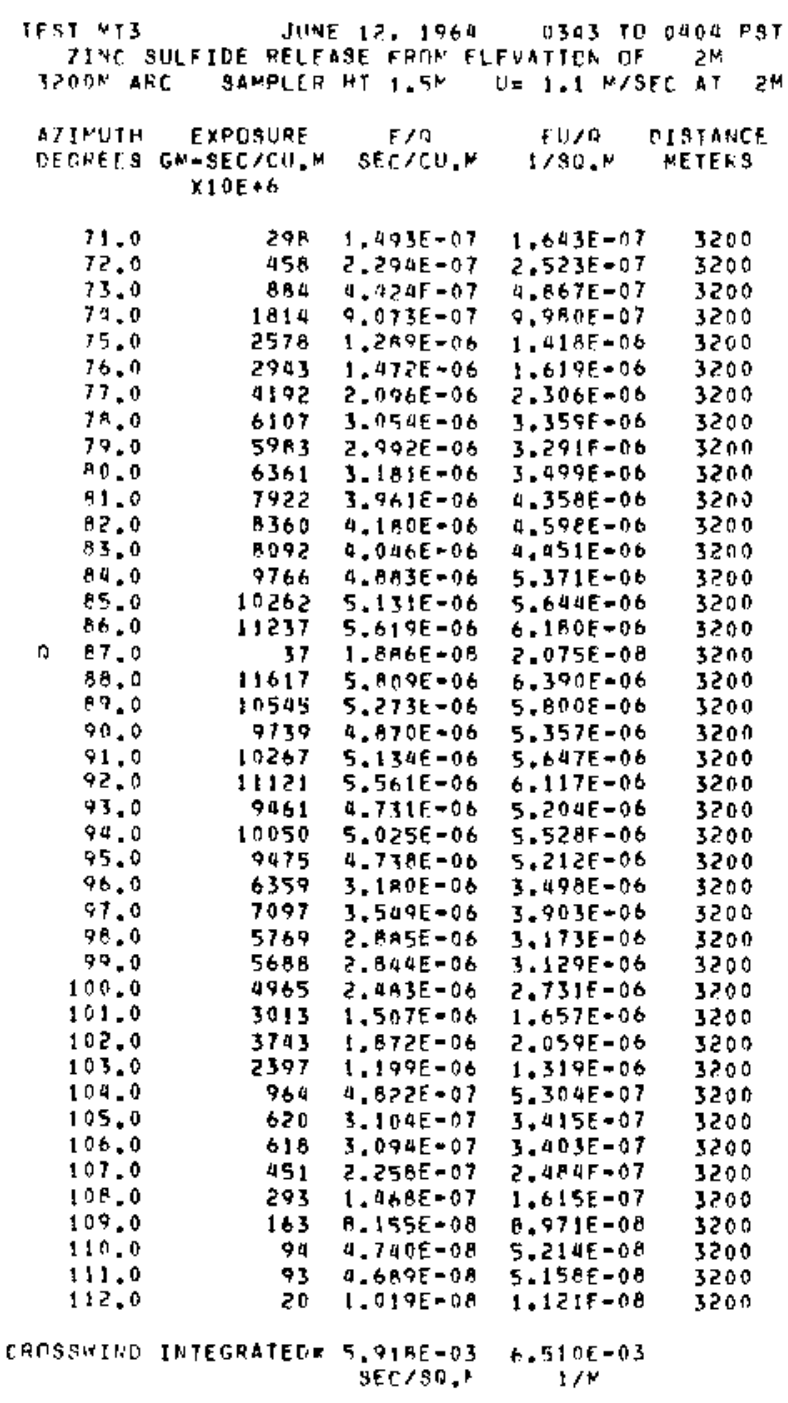

\begin{tabular}{|c|c|c|c|c|c|}
\hline \multirow{2}{*}{\multicolumn{2}{|c|}{$\begin{array}{l}\text { If } 1 \text { vis } \\
71 \times C \text { SIJLFJUE } \\
\text { 3POCN ARC }\end{array}$}} & \multirow[t]{2}{*}{ 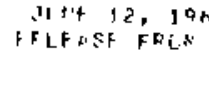 } & $\begin{array}{l}\text { OnG OB O343 } \\
\text { NELEVAIICA }\end{array}$ & $\begin{array}{l}\text { PL } \\
\text { rF }\end{array}$ & PQT \\
\hline & & & $t=1.1 \mathrm{~m}$ & SFC AT $Z^{N}$ & \\
\hline $\begin{array}{l}\text { AZINETM } \\
\text { MEREES }\end{array}$ & $\begin{array}{l}\text { HEIfRT } \\
\text { NE TFKS }\end{array}$ & 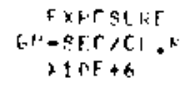 & $\begin{array}{c}E, 0 \\
s \in C, C, N\end{array}$ & $\begin{array}{c}\text { EL/C. } \\
1 / \text { SO. }\end{array}$ & $\begin{array}{l}\text { r. ISTARCE } \\
\text { NETEAS }\end{array}$ \\
\hline 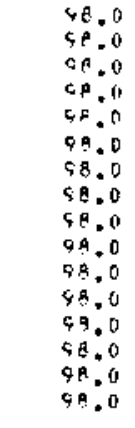 & $\begin{array}{l}3.7 \\
0.7 \\
1.5 \\
3.1 \\
6.7 \\
9.7 \\
12.4 \\
15.5 \\
18.5 \\
21.7 \\
24.8 \\
31.0 \\
37.7 \\
43.4 \\
49.6\end{array}$ & $\begin{array}{l}A(61 \\
5711 \\
7399 \\
5404 \\
7309 \\
9038 \\
8581 \\
4572 \\
6013 \\
4539 \\
3303 \\
7004 \\
807 \\
781 \\
40 \\
35\end{array}$ & 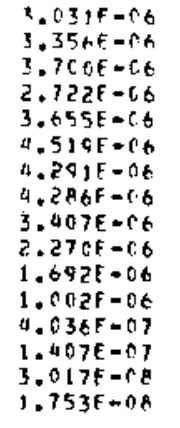 & $\begin{array}{l}3.334 E=06 \\
3.692 \mathrm{E}=06 \\
4.070 \mathrm{E}=06 \\
2.995 \mathrm{E}=06 \\
9.02 \mathrm{E}=06 \\
4.971 \mathrm{E}=06 \\
4.720 \mathrm{E}=06 \\
4.715 \mathrm{E}=06 \\
3.747 \mathrm{E}=06 \\
2.497 \mathrm{E}=06 \\
1.861 \mathrm{E}=06 \\
1.102 \mathrm{E}=06 \\
4.440 \mathrm{E}=07 \\
1.547 \mathrm{E}=07 \\
3.319 \mathrm{E}=08 \\
1.928 \mathrm{E}=08\end{array}$ & $\begin{array}{l}3200 \\
3200 \\
3200 \\
3200 \\
3200 \\
3200 \\
3200 \\
3200 \\
3200 \\
3200 \\
3200 \\
3200 \\
3200 \\
3200 \\
3200 \\
3200\end{array}$ \\
\hline $\begin{array}{l}\text { ARIAIJTH } \\
\text { TECTEES }\end{array}$ & $\begin{array}{l}\text { HE IGHT } \\
\text { NETENS }\end{array}$ & $\begin{array}{c}E+D C S U P E \\
G N=-A E C / C U . N \\
\times 1 n F+h\end{array}$ & $\begin{array}{c}E / O \\
S E[/ C L, N\end{array}$ & $\begin{array}{c}E Q / 0 \\
1 / 50 . K\end{array}$ & $\begin{array}{l}\text { DISTANCE } \\
\text { NEYERS }\end{array}$ \\
\hline $\begin{array}{l}106.0 \\
166.0 \\
160.0 \\
106.0 \\
106.0 \\
106.0 \\
106.0 \\
106.0 \\
106.0 \\
106.0 \\
106.0 \\
166.0 \\
106.0 \\
166.0 \\
106.0 \\
166.0 \\
104.0\end{array}$ & 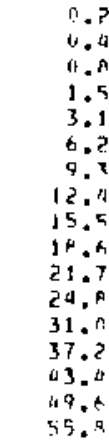 & 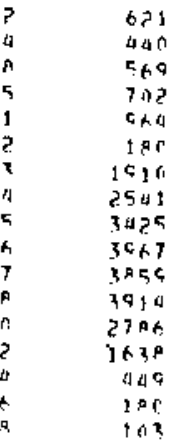 & 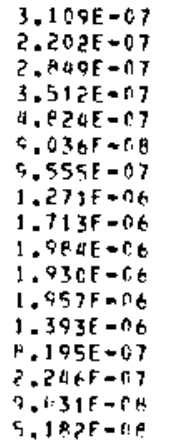 & $\begin{array}{l}3.020 E-07 \\
2.422 E-07 \\
3.134 E=07 \\
3.864 E=07 \\
5.306 E=07 \\
9.939 E=08 \\
1.051 E=06 \\
1.38 R E=06 \\
1.984 E=06 \\
2.182 E=06 \\
2.123 E=06 \\
2.153 E=06 \\
1.933 E=06 \\
4.014 E=07 \\
2.471 E=07 \\
9.934 E=0 B \\
5.700 F=08\end{array}$ & $\begin{array}{l}3200 \\
3200 \\
3200 \\
3200 \\
3200 \\
3200 \\
3200 \\
3200 \\
3200 \\
3200 \\
3200 \\
3200 \\
3200 \\
3200 \\
3200 \\
3200 \\
3200\end{array}$ \\
\hline
\end{tabular}

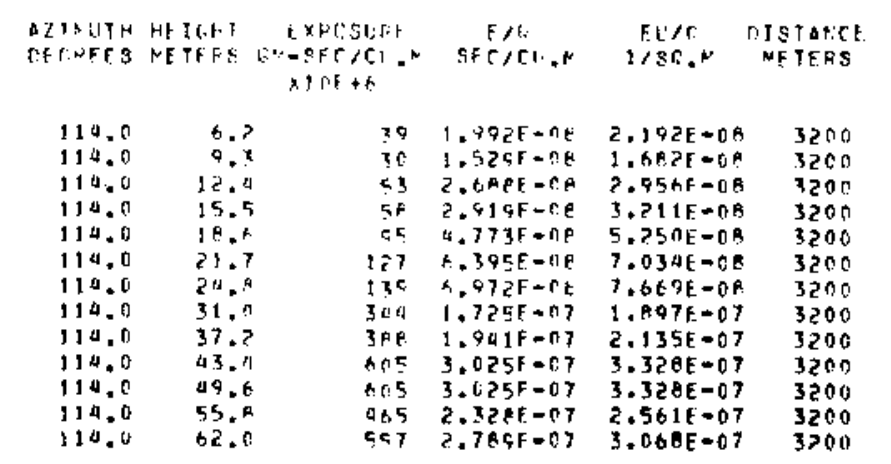


SBCOHDARY SERIES BIPEEIMENT: MT-3F

2IRC SULFIDE AND FLJOROSCEIK HERE BOTH OSED AS TRACERS DURIBG

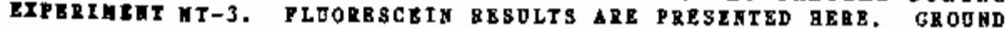
LEVEL ARD TOYBZ SAMPIIYG 200-3200 A APCS. A SIGHIFICATT PORTIOA OF TRE PLUME PASSED TO THE NORTH OF THE GIID.

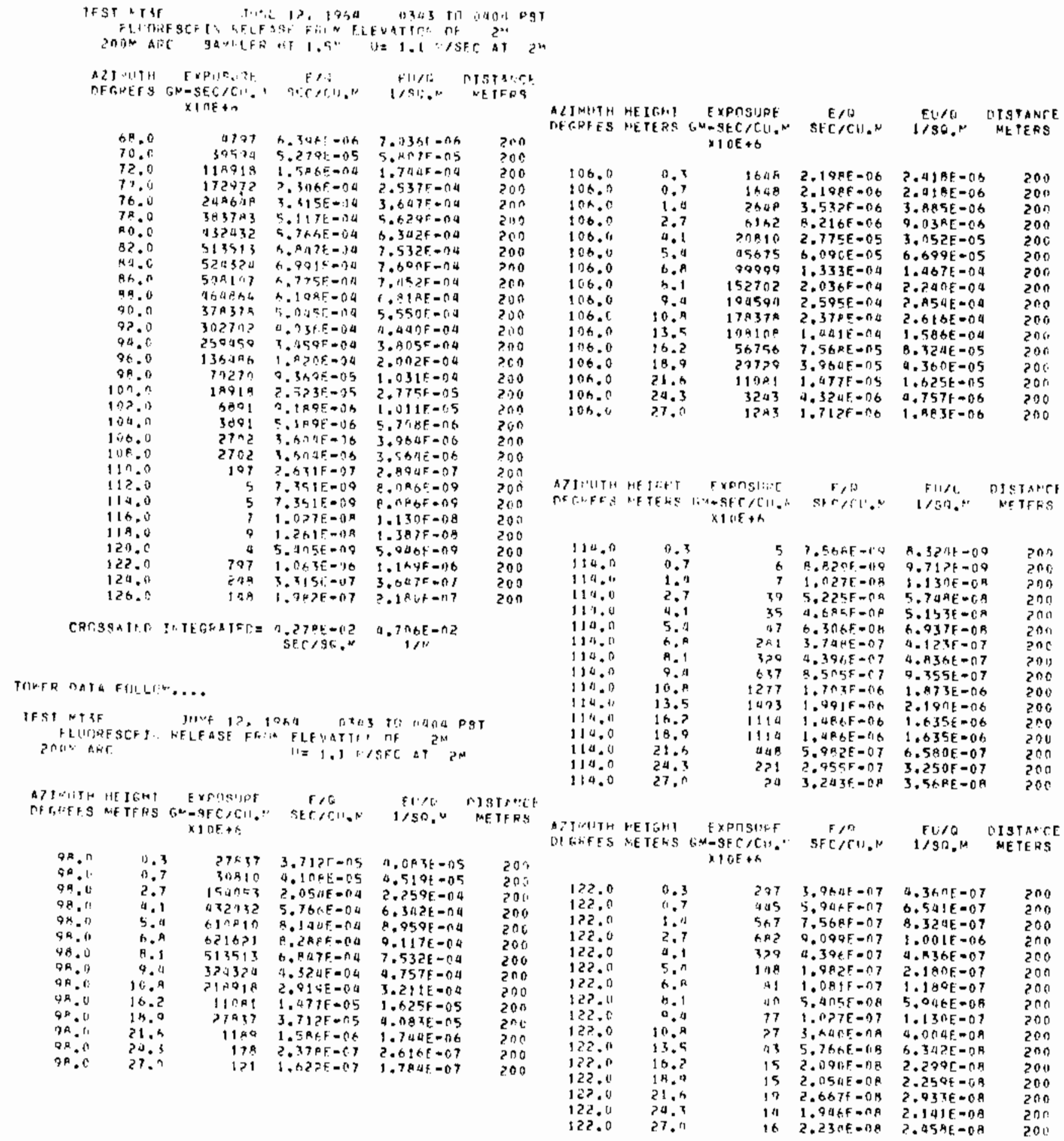




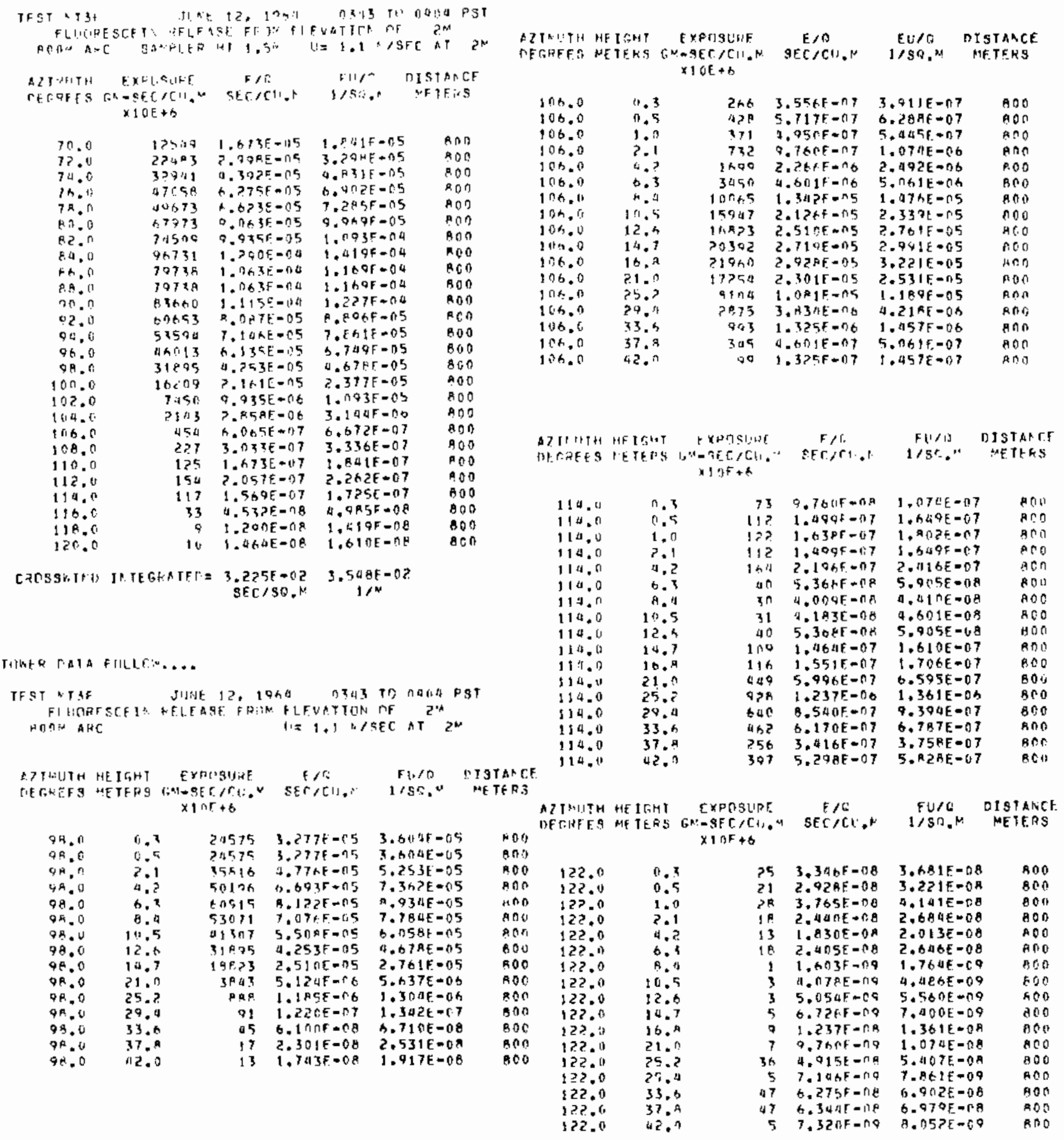




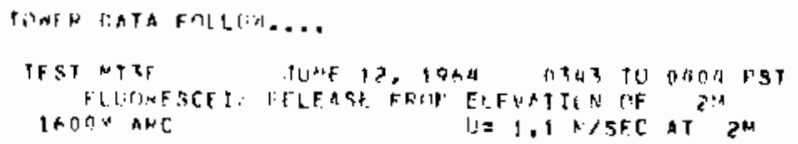

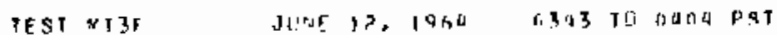

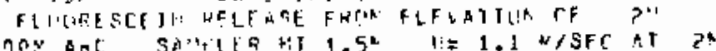

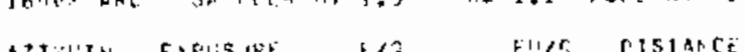

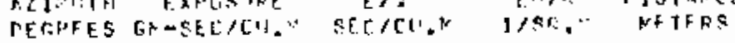
$x i$ in $+b$

\begin{tabular}{|c|c|c|c|c|}
\hline 70.0 & 7555 & $3.5+f \cdot 5=0 h$ & 3. $A O H F=06$ & 1600 \\
\hline 71.0 & 3793 & $5.057 t=06$ & $5.5035-n 6$ & 1 Ano \\
\hline 12.0 & 5615 & $7.5>9 t-66$ & $2.252 t=06$ & 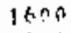 \\
\hline 73.0 & $62 P_{0}$ & $9.2765=86$ & $9.1035=106$ & 1600 \\
\hline 74.9 & R275 & $1.1 \cap 3 E-D 5$ & $1.214 F=05$ & 15012 \\
\hline 15.0 & 11734 & $1.5 \times 3 E=0.5$ & $1.730 F=n s$ & 1400 \\
\hline 76.0 & 136211 & 1. $A_{1} O E-D 5$ & $1.9985-05$ & 1600 \\
\hline 77.0 & 16206 & $2.1 \times 1 E-05$ & $2.3775-05$ & 1601 \\
\hline $7 P .0$ & $1+1 \cap 3$ & $7.414 \mathrm{E}=115$ & $7.6555-15$ & 1600 \\
\hline 79.0 & 10310 & $2.575[-15$ & $2+8.325=05$ & 1609 \\
\hline 60 & 21 kith & $3.920 E=05$ & $3.611 f-05$ & 1600 \\
\hline B1. 11 & 20055 & $3.2 A 7 E=05$ & $616 f-c 5$ & 5600 \\
\hline 32,0 & 25795 & $3.172 E-45$ & $490 E-05$ & 3601 \\
\hline $\begin{array}{r}83.0 \\
\times 4.0\end{array}$ & 253 व4 & $3.3795=05$ & $.717 \mathrm{~F}+05$ & 1600 \\
\hline $\begin{array}{l}24.0 \\
25.0\end{array}$ & 23655 & $3,7 \times 7 \bar{c}-05$ & $3.6165=05$ & 1600 \\
\hline $\begin{array}{l}25.0 \\
36.0\end{array}$ & 23655 & $7 x=05$ & $=05$ & J60? \\
\hline $\begin{array}{l}36.0 \\
97.0\end{array}$ & 22931 & 05 & 3.3 & 1607 \\
\hline 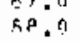 & $2 ?+13$ & $f=05$ & -05 & 1600 \\
\hline 89.6 & 203 & $\begin{array}{l}X E=15 \\
=E=05\end{array}$ & $\begin{array}{l}-185 \\
-05\end{array}$ & $\begin{array}{l}1600 \\
1000\end{array}$ \\
\hline 90.6 & $\begin{array}{l}18)=3 \\
20517\end{array}$ & $\begin{array}{l}14 E=03 \\
S B E=05\end{array}$ & $\begin{array}{l}055 F-05 \\
0.9 F-05\end{array}$ & $\begin{array}{l}1600 \\
1600\end{array}$ \\
\hline 41.4 & 175 Ah & $2.345 \mathrm{~F}=05$ & $2.570 F=0.5$ & $\begin{array}{l}1600 \\
1600\end{array}$ \\
\hline 92.0 & 13703 & $1.0 \times 9 E=05$ & $2.0,35-05$ & $\begin{array}{l}1600 \\
1600\end{array}$ \\
\hline 43.0 & $136 ? 0$ & $1.816 E=05$ & $1.906 E=1.5$ & $\begin{array}{l}1600 \\
1000\end{array}$ \\
\hline 54.6 & 12068 & $1.6 ก 9 E-05$ & $1.770 \mathrm{~F}=1 \mathrm{~s}$ & $\begin{array}{l}1600 \\
1600\end{array}$ \\
\hline 95.0 & 11034 & $1.471 E-05$ & 1, MPF $=05$ & $\begin{array}{l}1600 \\
1+00\end{array}$ \\
\hline 96.0 & 10172 & $1.350 F=05$ & $1.402 E-0.5$ & $\begin{array}{l}1400 \\
1600\end{array}$ \\
\hline 87.0 & 7758 & $1.030 E=05$ & $1.1381=05$ & $\begin{array}{l}1600 \\
1600\end{array}$ \\
\hline A. 0 & 5 झी & $7 . a_{1} \mathrm{BE}=0 \mathrm{~B}$ & $+5 O E E-N B$ & jof? \\
\hline 40.6 & $48>7$ & $6.4375=08$ & 7.rE तC =0 & joon \\
\hline$(-n .0$ & 2952 & 3. $.81+E=06$ & 4.19 คล $=06$ & jhon \\
\hline 101.1 & 2137 & ?. & $3,1 \leq \in F=n \theta$ & 1600 \\
\hline 102.0 & $13 \$ 0$ & $1.7>3 E+176$ & $1.972 \mathrm{~F}-0 \mathrm{~b}$ & 1500 \\
\hline 103.0 & 896 & $1.145 E=06$ & $1.315 \mathrm{~F}=0 \mathrm{~b}$ & $1 \sin \theta$ \\
\hline 101.0 & 551 & $7.356 E=07$ & $0.0927-0.7$ & $1+0$ \\
\hline 105.11 & 413 & $5.517 f-07$ & t.ptot=? & j6(1) \\
\hline 106.0 & $>15$ & $7.571 E-07$ & $x+1+1 k=17$ & $1+0 \bar{U}$ \\
\hline 107.0 & 159 & $1 . P+2 F-37$ & $z$. : 4 a $F=r, 7$ & 1800 \\
\hline 108.11 & Pिa & $1.103 E-n)$ & $1.2146-177$ & 1 bi: $n$ \\
\hline $\sin 9$ & 54 & $7 . F+B E=D A$ & $A .598 E=0 A$ & 1600 \\
\hline 110. & 3i. & $5.172 \mathrm{E}-1)^{2}$ & $5.6,9(45=18$ & 1600 \\
\hline-5 & $3 ?$ & $P .8 P Q E-1) F$ & $3 . P^{2} 7 F=08$ & $160 \%$ \\
\hline $1: 2,0$ & $1: 1$ & $t .379 \mathrm{~F}=.1 \mathrm{~g}$ & $1.5175=108$ & $\ln n 0$ \\
\hline 113,4 & 14 & 1.95 यf -08 & $2.149 F=f M$ & 1 thon \\
\hline 110.17 & 7 & A $A E-i s$ & $=134$ & 16in? \\
\hline & 1 & $2 . n>3 F=180$ & $>.235 F=04$ & $1+c$ \\
\hline
\end{tabular}

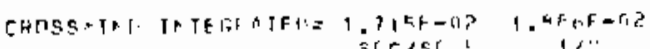

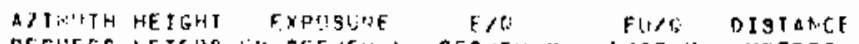

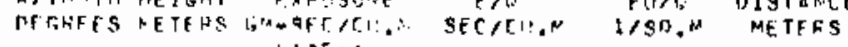
$x 10 F+C$

\begin{tabular}{|c|c|c|c|c|c|}
\hline $\begin{array}{c}9 A \\
\text { OA }\end{array}$ & 40 & JחAR & $5.475 F=11 \mathrm{~h}$ & $5.96 R E=06$ & IBt: \\
\hline QH. & if. & $5+03$ & $7.471 \mathrm{~F}=\mathrm{th}$ & R. 21 Bt $=56$ & 1000 \\
\hline 98,0 & 3.9 & 7048 & $y, 4>5 \mathrm{~F}=\mathrm{Mm}$ & $1.037 f=0.5$ & $1+00$ \\
\hline QR, $\mathrm{C}$ & 6.2 & 11551 & $1.54 C E=05$ & $1.69 .2 E=05$ & 1000 \\
\hline B. ${ }^{\prime \prime}$ & 9, & 15172 & $2.023 E=05$ & $2 ? 5 E-05$ & 16,00 \\
\hline ग 9 & 13.0 & 10172 & $3.35+F=05$ & $1,4 \$ ?[-05$ & $i \in 00$ \\
\hline QA. 6 & 15.5 & A) ค B & $1.092 F-05$ & $1.201 \mathrm{E}=05$ & isot \\
\hline 28.4 & $2 A, B$ & $42 n b$ & $5, B Q Q E=0.6$ & $3.170 t-0 t$ & 1600 \\
\hline 95. & 24.9 & $106 \%$ & $1.925 F=n_{t}$ & $1.56 \mathrm{HE}=06$ & 1000 \\
\hline & $3 ! .0$ & $10 n$ & 1,$4 ; 25 E-07$ & $5 B A E=07$ & 1600 \\
\hline 43.0 & $37 . ?$ & 14 & $50 E=08$ & $2.1+9 \mathrm{E}=0$ 月 & 1000 \\
\hline & 43.0 & $1 ?$ & $678 f-D E$ & $846 E-00$ & 1000 \\
\hline$p$ & 49 & 12 & $D \cap S E-\cap B$ & $771 E=O B$ & 1000 \\
\hline & & 7 & $1.011 \mathrm{E}-0 \mathrm{C}$ & $113 E=0 H$ & 1600 \\
\hline & & b & $080 F=00$ & 8 AE -09 & 1600 \\
\hline
\end{tabular}

A7INITH HEIGAT EXPESILE E/G FH/O OISTANCE DFGRFE METERS IFM-SEC/CII." StC/CH,N I/SC," METEHS $x+$ तE +6

\begin{tabular}{|c|c|c|c|c|c|}
\hline $106 . ?$ & 0.2 & 167 & $2.23015=117$ & $2.1153 E-117$ & $16 \cap 5$ \\
\hline 106.0 & (1. 4 & $21^{n}$ & 2. AISE-07 & $3.085 E-07$ & ח \\
\hline $\ln h .0$ & (1). A & $1 \% 2$ & $2.437 \mathrm{E}=07$ & 2, GRतE $=07$ & 1600 \\
\hline 100.0 & 1.5 & 10th & $2.62) F=117$ & Z, REBE $=$ ก 7 & $1+x \cdot n$ \\
\hline 106.0 & 3.1 & 3h? & $4.925 .5+07$ & $5.31 \cap E=07$ & 160.0 \\
\hline 106.0 & b. & TिZ & $1.310 F=r i n$ & $1.041 E=D 6$ & 1650 \\
\hline 126.0 & 9.3 & 2504 & $3.13 \times F=0$ & $3.1130 \mathrm{E}-0.6$ & $\operatorname{sen} 0$ \\
\hline 106.0 & 12.4 & $37 \div 1$ & $989 F=06$ & $5.487 t=0.6$ & $1+\pi 00$ \\
\hline 106.0 & 15 & 5250 & $7.011 \mathrm{~F}=6 \mathrm{f}$ & $7.713 E=018$ & 1600 \\
\hline 106.0 & $1 P, A$ & 44,2 & $5.9778=016$ & $i E-1, k$ & $16 n n$ \\
\hline 106.0 & $2 ! .7$ & 5258 & $0.0118+n \varepsilon$. & $7.71 \times 5=06$ & $18=0$ \\
\hline $106 . n$ & 34.7 & 3931 & $5.241 E=r .1$ & $5.7641+06$ & $\operatorname{lan} n$ \\
\hline 100.0 & 31.11 & 741 & 7. Аस्दि $=17$ & 1. $n T_{F}=E \cdot h$ & $16 n 0^{\circ}$ \\
\hline 106.0 & 37.7 & 2.41 & $3.2+115-517$ & $3.581 t=n 7$ & Jan \\
\hline 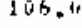 & 43.5 & 30 & $2,13+5=: 4,4$ & 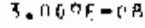 & $3+6: 11$ \\
\hline
\end{tabular}

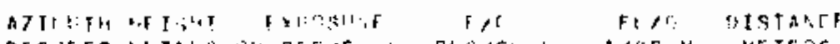

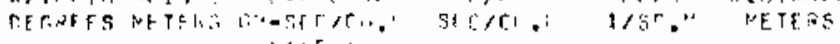

\begin{tabular}{|c|c|c|c|c|c|}
\hline 11 ik." & 3.1 & 8 & $1, j^{2}, F=n, 1$ & 1. $3 F$, VEन & $\ln x+1$. \\
\hline$\{14,1\}$ & n.? & 14 & 1. $H A 5 F=0 \hat{n}$ & $2.07 \div E=13$ & Intal \\
\hline 110.0 & 5.3 & is 4 & $5.777 \mathrm{~F}=0.0$ & $A .575 E=1,9$ & 1600 \\
\hline $114.0^{\circ}$ & 13.5 & 29 & $b .067 F=02$ & $7.33 .3 t-1 ; \mu$ & 1060 \\
\hline $114, ?$ & 19.5 & $1+1,6$ & $1.95: F-07$ & $? .1 \div 5 E-[]$. & $1 \ell r \cdot 1$ \\
\hline 114.0 & 18.5 & $15 t$ & $2,09 \mathrm{PF}=127$ & $3.30,5=07$ & 1605 \\
\hline 114.4 & 21.7 & $\{>7$ & $4.34,11=17$ & $0.215 t=0.7$ & 16 tho \\
\hline 114. & 34.4 & $5 * P$ & $7.5 ? .1-17$ & A. $x$ a $5 t=07$ & 1600 \\
\hline 114.0 & $31 . "$ & Foh & $1.105, \mathrm{~F}=\mathrm{Co}$ & $1.315 E=06$ & 1600 \\
\hline $11+4$ & 37.7 & $\operatorname{taA}$ & $3106-87$ & $1.0245=06$ & $1+n n$ \\
\hline 114.0 & 113.4 & $29 \%$ & $3, B, 2 F=07$ & 4.?? HEE -5$\}$ & 1600 \\
\hline ." & $47=n$ & $1 \times 2$ & $77(5-0) 7$ & $1.947 E-\delta 7$ & $1+06$ \\
\hline-1 & ר. & 25 & $3.401 \%=\pi p$ & $3.703 E-0 A$ & $1 \mathrm{t}^{\circ} 0$ \\
\hline 114.0 & $\operatorname{t?}, 6$ & 7 & $9.8+B \bar{c}=0 \mathrm{C}$ & $1.0976-08$ & 1600 \\
\hline
\end{tabular}

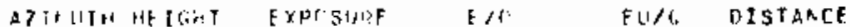

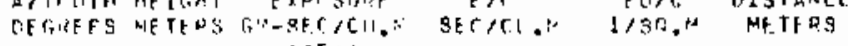
$x \in \mathbb{F}+6$

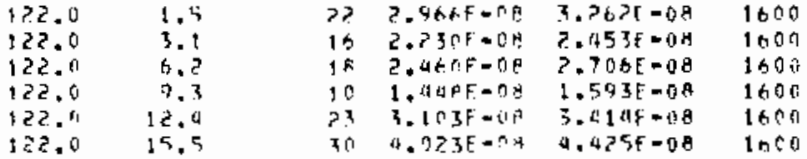




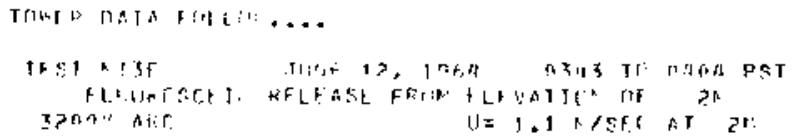

QR. U

मव.

7. 11

जh.l

जि.11

Qम.

$98.0 \quad 15.5$

9R. A $1 \mathrm{B.h}$

$9 A, 0$ it.

$98.0 \quad 37.2$

QA. 043.1

4.." 49.4

IH. 55.

SP. 6 +.?.11

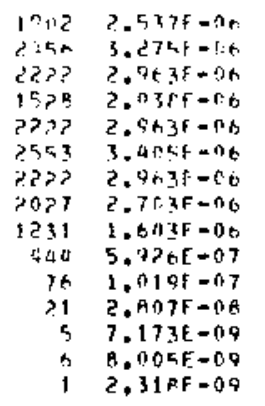

1 $2,317 F=09$

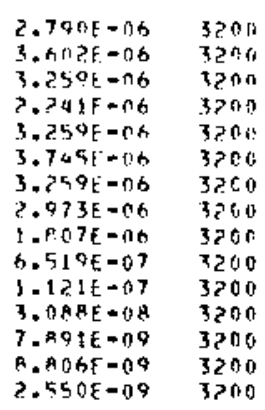

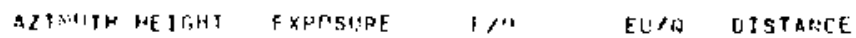

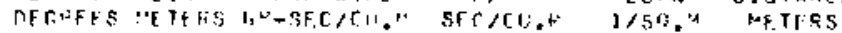
$\rightarrow 10 F+6$

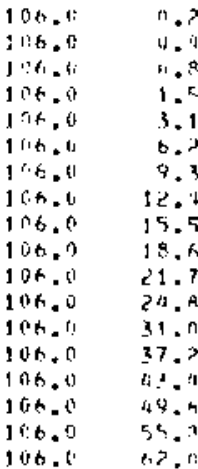

\begin{tabular}{|c|c|c|c|}
\hline $\begin{array}{l}171 \\
20 ?\end{array}$ & $\begin{array}{l}2.2 \times 7 f=01 \\
3.7035-n 7\end{array}$ & $\begin{array}{l}2.516 k=07 \\
2.073 f=07\end{array}$ & $\begin{array}{l}3200 \\
32010\end{array}$ \\
\hline $\ln x$ & $2,1$ म $] F=\cap 7$ & $3.059 \mathrm{f}-0.7$ & 3200 \\
\hline ? 10 & $2.4117 \mathrm{~F}=07$ & 3. IRRE $=07$ & 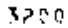 \\
\hline$? 34$ & 3. QASE - 07 & $3 . Z^{\prime A R E}=07$ & उमी \\
\hline $3 A$ & S. $125: t=n B$ & $5 .+325=6 R$ & $37 n \pi$ \\
\hline 553 & $7.3 \mu ; E=? 7$ & $B .12 B E=07$ & 3phil \\
\hline hen & A. $\cap \cap 5 E-\cap ?$ & A, fir, $f_{E}=\{1$ l & $3 ? 00$ \\
\hline 737 & $7735-07$ & $1.075 \mathrm{E}-1,6$ & $32^{40}$ \\
\hline SDR & $1.3 \times 55=0 m$ & $464 E-06$ & $3,00 n$ \\
\hline nद? & $. \Delta \cap A F=r \theta$ & $G$ A. AE. $-n h$ & 3200 \\
\hline 4 קᄀ & $1.3275-176$ & $1.34 \mathrm{OE}=\mathrm{n} \mathrm{B}$ & 3 \\
\hline 732 & $0.7736=07$ & $1.0759=06$ & 3200 \\
\hline $25 \%$ & $4.7 \mathrm{Pe}+=067$ & $5.2+1 t-0.7$ & 3 . त \\
\hline$i \neq 1$ & $?$ ?. $2 R>[+07$ & $3.51 \mathrm{CE}=0 \mathrm{C}$ & 3200 \\
\hline 72 & $9.045 \mathrm{~F}=\mathrm{nfl}$ & 1. $n t, b t=6$ ? & 3260 \\
\hline $2 r$ & $7 * 1 F-n !$ & $059 t-.9$ & לו הי \\
\hline 1 & $7 f-15$ & $1 \mathrm{nl}=6: 9$ & 3700 \\
\hline
\end{tabular}

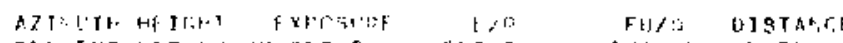

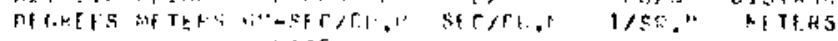
$x \rightarrow n F+\infty$

\begin{tabular}{|c|c|c|c|c|c|}
\hline 110.0 & H. & ik & $5.71,5=00$ & $6.29 n 5=00$ & 32011 \\
\hline 850.5 & 11.1 & 3 & A. $\mathrm{AnCF}=\cap \mathrm{Q}$ & $5.37)_{2 t}+1.0$ & 3200 \\
\hline סמנים מים & $\pi, 5$ & 4 & $6.030 t=10$ & $0.6355=1: 9$ & 3200 \\
\hline 114.0 & 1.5 & 4 & $5.11 \& E-n U$ & $6.790 \mathrm{E}=09$ & $32 \div 6$ \\
\hline 114,0 & 3.1 & 6 & $0.317 E-09$ & $9.11195-119$ & 3,200 \\
\hline 114. & h.? & 7 & $1 . \Omega 1 C F-\cap A$ & $1.121 \Gamma=0 R$ & 3200 \\
\hline 114.11 & ". 3 & 13 & $1, B 1 \square T=1 ; r^{3}$ & $2.0016=08$ & 3200 \\
\hline 114.0 & 12.1 & $1^{4}$ & $1.975 F=18$ & $? .173 \mathrm{f}=\mathrm{OP}$ & 3200 \\
\hline 114.0 & 15.5 & 18 & ?.वQदF = "1? & $2.745 t=0 R$ & 3700 \\
\hline 114.4 & $1 \mathrm{~h}, \mathrm{t}$ & 30 & $a .0 \Gamma 3 F=n \mu$ & $4.18,3 F-08$ & 900 \\
\hline 114,11 & 21.7 & 35 & $4.7 P C F=0 A$ & $5.2+1 E-n R$ & 3200 \\
\hline $114 . n$ & $2 \pi, n$ & 52 & $6.9025=\cap 8$ & $7.691 \mathrm{~F}-0.5$ & $3.0 \mathrm{Cu}$ \\
\hline 110,0 & $31 . n$ & 93 & $1.24, F=17$ & $1.372 \mathrm{E}-07$ & 3300 \\
\hline 114.0 & $\$ 7.2$ & 405 & $5.406, F=0.7$ & $5.947 E-07$ & 3290 \\
\hline $11 t \ldots$ & $4,3,0$ & $14 \mathrm{~h}$ & $1.955 \mathrm{r} \rightarrow 07$ & $2.150 E-07$ & $320 n$ \\
\hline 4.0 & 44.6 & $12 ?$ & $1.6435=07$ & $1.607 \mathrm{E}-07$ & $320 \mathrm{~s}^{\circ}$ \\
\hline .0 & 5 & A is & $1.071 F=0.7$ & $1.178 \mathrm{E}-07$ & 0 \\
\hline & t)? & 8.2 & $1.10 \mathrm{E}$ & $1.21 ? \mathrm{E}$ & \\
\hline
\end{tabular}


SECONDABY SBIIES ERPERIMEHT: XT-4

GRUOND LEVEL AAD TOWER SAMPIING $200-3200$ Y ARCS.

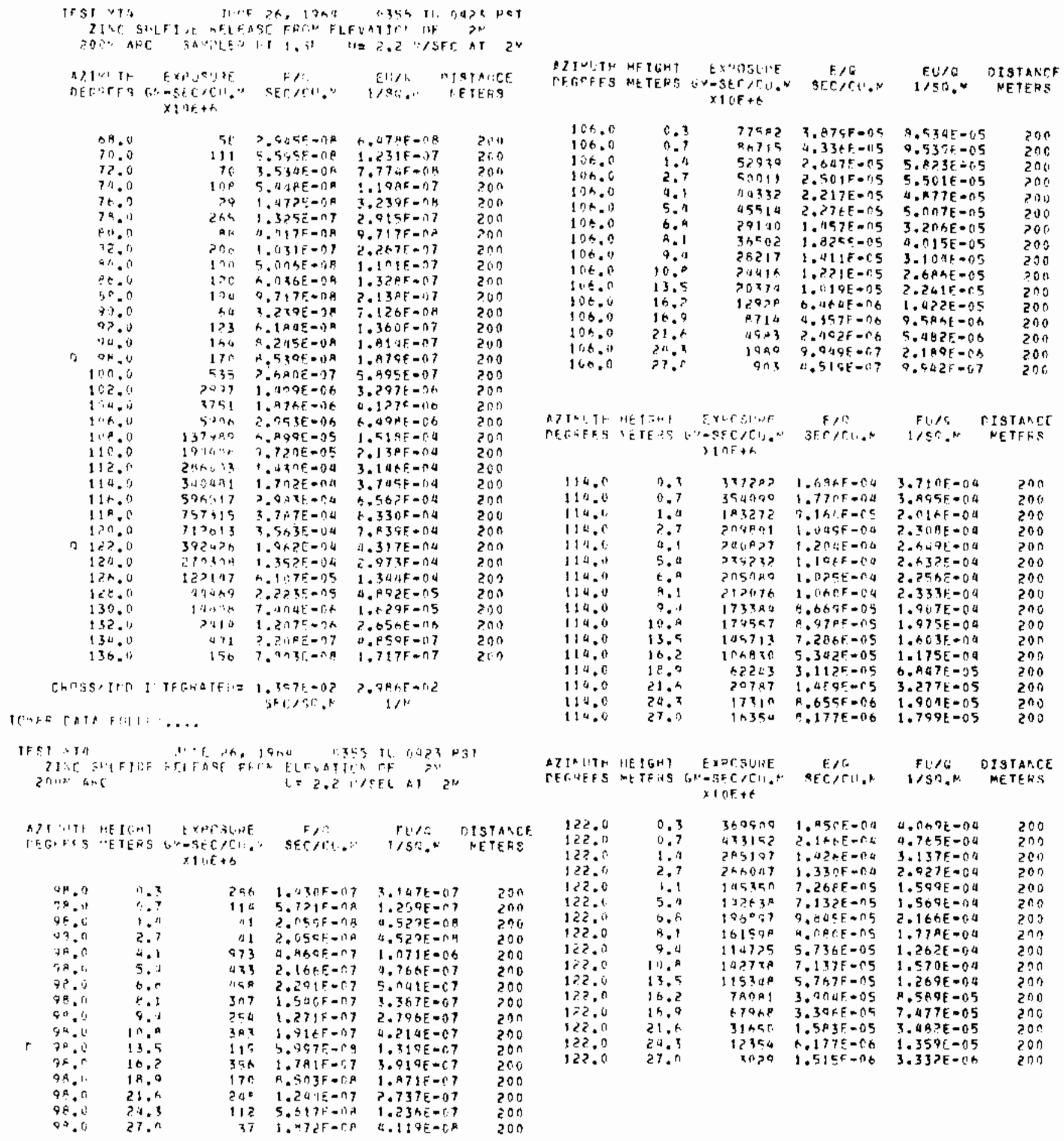




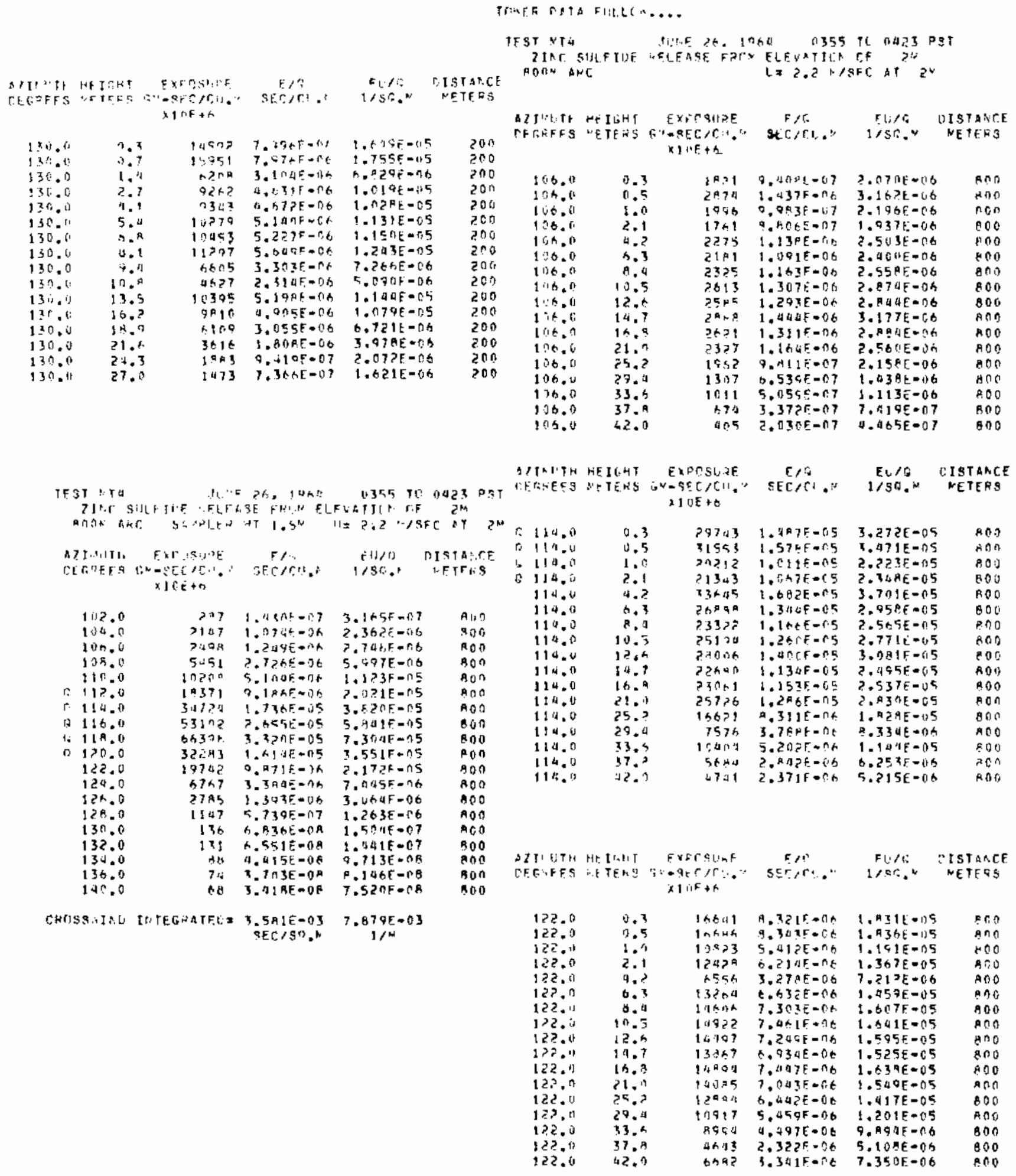




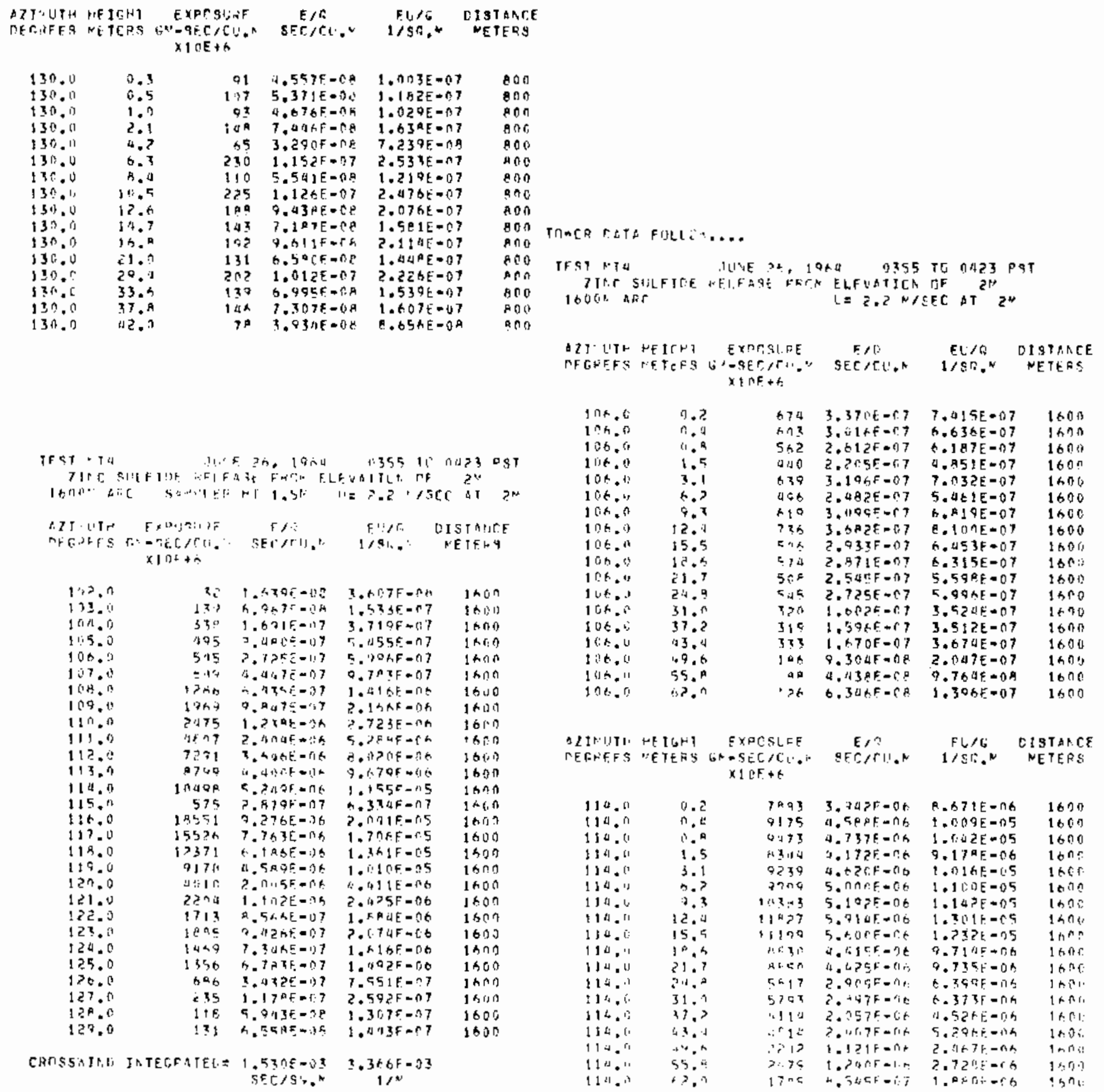




\begin{tabular}{|c|c|c|c|c|c|}
\hline 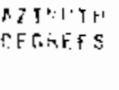 & 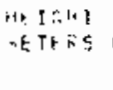 & 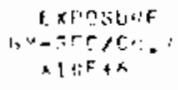 & $\begin{array}{c}f^{\prime \prime}: \\
\text { sirfri, }\end{array}$ & $\begin{array}{c}f i r r \\
1 / g r . \%\end{array}$ & $\begin{array}{l}\text { DISTAMCH } \\
\text { HETGS }\end{array}$ \\
\hline 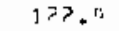 & 11.7 & $1>?$ ? & $0.1111 F-07$ & $1, x z+500$ & 1 \\
\hline $1<2$. & $\because$ & $37=$ & מ. $? 2+5=\{7$ & 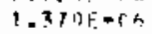 & ind \\
\hline 122. & $B=9$ & 1464 & $1,3215-57$ & E.t $11 E-n t$ & $10 r_{1}$ \\
\hline 122.11 & 1.5 & 12,3 & B.C18F- 17 & $1.32^{1} t+60$ & $160 n$ \\
\hline $1 e ? \cdot n$ & 3.1 & 1253 & $6.26 D F=\{1\}$ & $1.370 F+116$ & $\ln n: 1$ \\
\hline $12 \mathbf{z}^{2} . r$ & $\therefore$ ? & $219 n$ & $7,43 \geq E-\{1 ?$ & $1.635 E-116$ & 1600 \\
\hline $1 ? 2.6$ & 9.3 & $1+2\}$ & $5.7 n \in E-0 ?$ & $1.2555-0.5$ & 1600 \\
\hline 122.0 & 12.4 & 1773 & $5.3 n>E=n 7$ & 1.1 1E-06 & $\ln 00$ \\
\hline 122. & 15.4 & 1517 & $1.585 E \cdot 07$ & $1.669 E=0 \mathrm{~s}$ & 1400 \\
\hline 122.0 & 13.4 & $15=3$ & $7.6695-17$ & $1 . B Q 7 \bar{E}=06$ & 16001 \\
\hline $12 ? \cdot 4$ & 31.7 & 1670 & $8.387 E \cdot n T$ & $1 . \operatorname{AD} 7 E=0 B$ & 1600 \\
\hline $2 ? 0.0$ & 24.9 & 1121 & $5.0 n 9 F-n 7$ & $1.234 t-06$ & 1600 \\
\hline 122.11 & 31.0 & $127 \pi$ & $6,165 F-n 7$ & $1.35 \mathrm{ft}-12 \mathrm{~h}$ & 1600 \\
\hline $12 \bar{E} .0$ & $\$ 7.7$ & 1375 & $6.67 \mathrm{cr}-67$ & $1.513 E=00$ & $1 \neq 00$ \\
\hline 122 & 43.7 & 13.97 & $6.53 \% 5=07$ & $3.43 \mathrm{AE}-116$ & 1600 \\
\hline 122.0 & $=9.6$ & $a \neq 2$ & 4. $5635-01$ & 1.00 HE-06 & 1600 \\
\hline 172.0 & $55 . \mu$ & 1030 & $5.1 A_{2} E=\{17$ & 1.1 HOE-OS & 100. \\
\hline 122.0 & $+2 . n$ & P.gp & 4.BAIE- T? & $B . R O \cap E=O T$ & 1600 \\
\hline
\end{tabular}

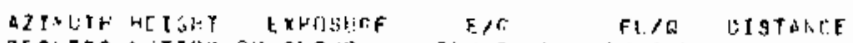

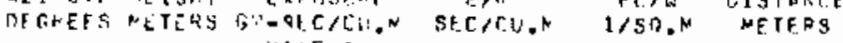

\begin{tabular}{|c|c|c|c|c|c|}
\hline $130.0^{2}$ & 0.0 & 23 & $1 .|A| F-C A$ & $2.59 \mathrm{AE}=0 \mathrm{~B}$ & 965.4 \\
\hline $13 n .0$ & $C .{ }^{A}$ & $\underline{s h}$ & I. AZKE- & $4.015 \mathrm{E}=0 \mathrm{OA}$ & 2000 \\
\hline 130. 01 & 3.1 & $i t$ & $5.547 E-(15$ & $1.220 E-05$ & 1600 \\
\hline 135.11 & t.? & 24 & $1 . \angle A C F=0 B$ & $2.7 \cup 6 E=(1 \mathrm{~A}$ & 1600 \\
\hline 130.0 & 9.3 & 12 & 0.740 .50 .5 & $1 . x>3 E-0 A$ & 1600 \\
\hline 13 ถ & 12.1 & 20 & $1.2 \Delta \Omega \mathrm{f}-\mathrm{fiA}_{\mathrm{A}}$ & $2.74 A E=58$ & jon? \\
\hline $13 \pi .0$ & 15.5 & 48 & $2.427 \mathrm{~F}=n \mathrm{~F}$ & $5.330 \mathrm{t}=0.8$ & 1000 \\
\hline 130.0 & 24.4 & 34 & $1,13 \leq E-n \in$ & $3 . P 1 ? E=A B$ & 1600 \\
\hline 130.6 & 31.5 & $? 5$ & $1.2 A B F=B A$ & $2.7735=18$ & 1600 \\
\hline 130.6 & 37.7 & 11 & $5.77 t E=019$ & $1+2 \geq 1 E=0 \theta$ & 1600 \\
\hline $13 ? .1$ & 45.1 & $>0$ & $1.20 \mathrm{FE} \cdot \mathrm{CH}$ & $2,6575-48$ & 1300 \\
\hline $13 r \cdot n$ & 49.6 & ים? & $1.012 F-08$ & $2.227 k-0.9$ & 1600 \\
\hline $13 r .9$ & $-2 . n$ & 12 & $6.220 F=05$ & $1.370 E=08$ & 16011 \\
\hline
\end{tabular}
IFET IT Th

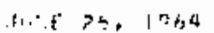

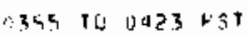

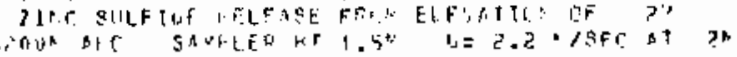

\begin{tabular}{|c|c|c|c|c|}
\hline 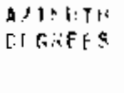 & 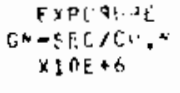 & $\begin{array}{c}\text { f/C } \\
\text { Sec/CU. }\end{array}$ & $\begin{array}{c}E, / r \\
1, s+0 .\end{array}$ & $\begin{array}{l}\text { ISTARCE } \\
\text { MEISEI: }\end{array}$ \\
\hline 102.0 & 30 & $1.505 F-\pi)$ & $1.311 F=\cap A$ & 3200 \\
\hline 108 & 111 & S. so3f-tie & $1,22 \times E-1) 7$ & 32612 \\
\hline .0 & 100 & $\because$ - WAEt $=0$ a & $2.151 \mathrm{~F}=\mathrm{n} 7$ & \\
\hline 165.0 & 277 & $1.0450+07$ & $3.260 E+07$ & 3200 \\
\hline 160.0 & 489 & $P .947 E-17$ & $5.303 F-07$ & $3 ? 00$ \\
\hline .0 & 100 & $1.951 \hat{c}=\hat{2} 7$ & $B, h=3 F=C i$ & $3>0,0$ \\
\hline 160.12 & 0.91 & $\because A c, A \in=g ?$ & $7.497 \mathrm{E}-\mathrm{n} 1$ & 3200 \\
\hline & $111^{6}$ & $5.5925-27$ & $1,>3, F=n 6$ & 3200 \\
\hline & 1195 & 5.520 & $1,21 \leqslant f=00$ & 3200 \\
\hline 10 & 1891 & $0.95+5-117$ & $2.1996-06$ & 3200 \\
\hline 0 & 7726 & $1 .{ }^{2} 6 \times t=56$ & $4.090 E-06$ & 3200 \\
\hline & 44.87 & 2.2 औt & $4.93 \mathrm{OE}=0.6$ & $3 \geqslant 00$ \\
\hline 114.0 & 4215 & $7.1 \times 8 F$ & $0.4 D I E-0 B$ & 09 \\
\hline $115,5^{1}$ & $?\{1\}$ & $1.0075=06$ & $2.215 E-7 b$ & $3 ? 00$ \\
\hline & $15 h t$ & ?. P>ne? & 1.72 U1F -06 & 3200 \\
\hline & 204 & 4.571 & $1.084 \mathrm{E}-0 \mathrm{~b}$ & 3200 \\
\hline 118.0 & 7 hot & उ. म्रवस:-67 & $4.437 F=07$ & 3200 \\
\hline 116.0 & $9 \diamond 9$ & a. $705 E-37$ & $1.0995-16$ & $3>00$ \\
\hline & 70.6 & $3.5 .5 \mathrm{E}$ & $7.711 E-07$ & 3200 \\
\hline 181.0 & 575 & $2.879 \mathrm{E}$ & $0.333 F=07$ & 3200 \\
\hline 122.0 & 452 & $2.262 \mathrm{E}$ & $4.977 \mathrm{E}=0 ?$ & 3200 \\
\hline & $33 n$ & 1.6Q4F. & $3.76 \mathrm{AF}+6 \mathrm{i}$ & 3200 \\
\hline 120,0 & 205 & $1.4>75-67$ & $3.1411 E=07$ & 3200 \\
\hline & 160 & 9.301 & $1.52+5-n 7$ & 3200 \\
\hline $12 t$ & 91 & 0.563 & $1.004 F=07$ & 3200 \\
\hline 12 & det & $2.3 \times 145-60$ & $5.126 \mathrm{E}-n 8$ & $3 \geq 00$ \\
\hline 128.0 & 11 & 5.P. $P 5 F=09$ & 1. 2 B $2 F-n g$ & 3200 \\
\hline 129.0 & 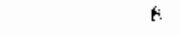 & $\because .3 n c t=09$ & $5.612 F=09$ & 3200 \\
\hline & & $\mathrm{SEC}$ & $\begin{array}{c}736 E=03 \\
110\end{array}$ & \\
\hline
\end{tabular}

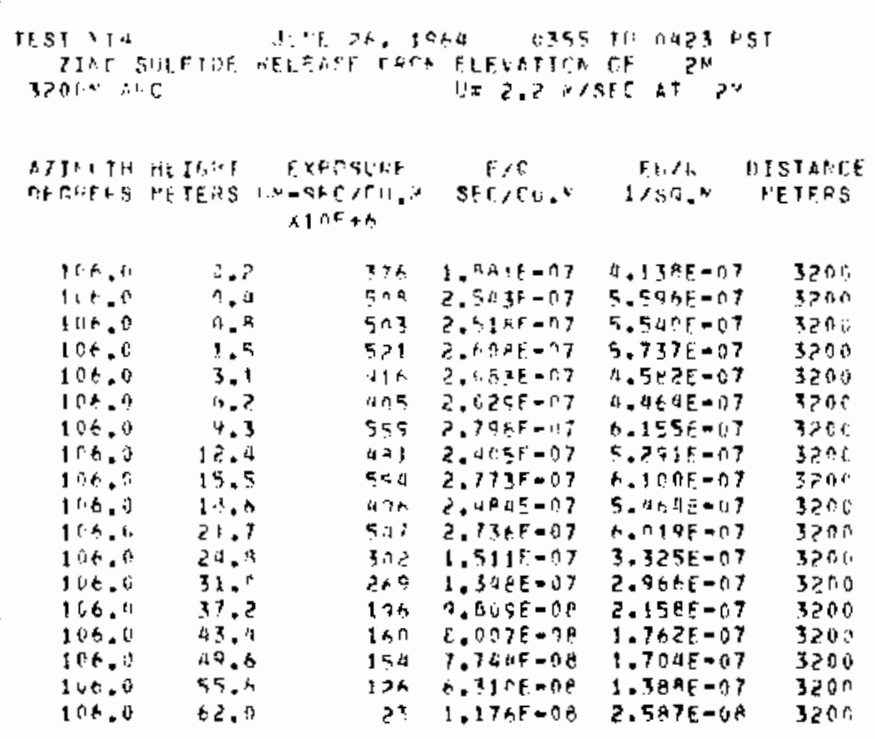

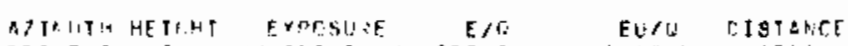

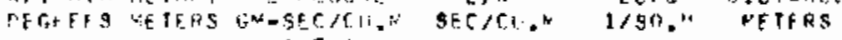
$\times 1 \cap F+h$

\begin{tabular}{|c|c|c|c|c|c|}
\hline $11^{n} \cdot n$ & 1)? & $3 n \times 3$ & $1,5375-06$ & $3.37 \mathrm{PE}-0 \mathrm{t}$ & $a$ \\
\hline 114.0 & 0.4 & Iदत & $1.05 a F=60$ & a. $? \triangle Q E=C B$ & $32 \sin$ \\
\hline $11^{4,4}$ & $A^{A}$ & 2965 & $1 . \triangle \square Z F=n h$ & $3.2 E+1 F=D A$ & $3>0$ sis \\
\hline 114.0 & 1.5 & 3537 & $1.767 F=26$ & 3. $\cap 7 \mathrm{AE}-\mathrm{CB}$ & $\div 200$ \\
\hline $114 .: 1$ & 3 & 3971 & 1.53 AE $=06$ & 1.37 A $10 \mathrm{~h}$ & $3 z^{n} ! !$ \\
\hline 114.0 & $1 . ?$ & $\{A<7$ & $1.8195-10$ & $H .2>1 F=06$ & $3 \div 00$ \\
\hline 1340 & 4.3 & $\because 433$ & E. रा $7 F+r=$ & a.a>רe-is & 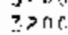 \\
\hline $1 \pm 4$ & 10. & :11? 3 & $2, d e 25-6.4$ & $A .400 E \rightarrow(16$ & $3 z^{n}$ \\
\hline 114.4 & 15.5 & $45+3$ & $2.2 \times 2 F=r i b$ & $5 . \cap 2 \cap A=\cap 6$ & 3200 \\
\hline 114.1 & 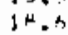 & 4857 & $2.4795=n_{6}$ & $5.4530=06$ & उ?ก! \\
\hline $111 . .1$ & ह1.7 & 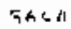 & $\therefore 947 F=06$ & $3.2 \times t 5-f_{0}$ & $3 ? 00$ \\
\hline 134.1 : & 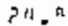 & סי.5 & 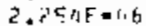 & $4.8 \in c t=n t$ & उटr. \\
\hline 114. it & 31 & acrit & $\therefore \Delta n \geq F=[1 h$ & $4 . \cos =06$ & 370. \\
\hline 1140 & & צו & $1, \ln C \mathrm{~g} F-\sin$ & I. OAYE-CG & 3206 \\
\hline 110.6 & & $x_{1} n$ & dugaf-oh & $r .>c 11-6,6$ & $x>0$ \\
\hline 110.19 & $\therefore ?$ & $z=2 t$ & $1 . i t f f=\hat{f}$ & $3.89 \cap E-1.6$ & 320 \\
\hline 314.1 & & 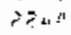 & 1. & $3.469 \div=$ th & $3>r . n$ \\
\hline $11 \% .11$ & $\therefore$. & $\because 17$ & 1. dofifolit & $2.175 \bar{p} \rightarrow 04$ & $3 e^{3}: f$ \\
\hline
\end{tabular}

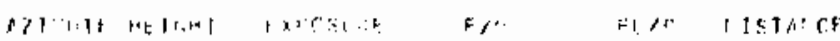

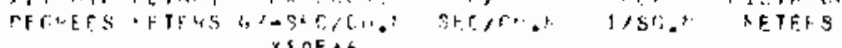

\begin{tabular}{|c|c|c|c|c|c|}
\hline $12 \bar{c}$. & . 7 & $3>1$ & $1,60 f=0.17$ & $3.537 F=07$ & $3>0 \%$ \\
\hline 127.0 & 1). 1 & 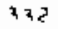 & $1 . \operatorname{Ain} p+\cos$ & $3.05+6=0 ?$ & 3द $3:$ \\
\hline $1 \geq ?$ & $?$ & 70 & $1.9715=07$ & $4.195 E-67$ & 3206 \\
\hline 122.11 & 1.3 & 341 & $1.15 \times f=67$ & 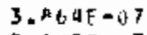 & $3 ? 031$ \\
\hline 122.0 & 3 & $4 \times 0$ & $3-32+1-04$ & $5.109 F-07$ & $3: n 0$ \\
\hline$+22.1=$ & $6 . ?$ & $4+2$ & 2,31 HF $\rightarrow C 7$ & $5.001 E=07$ & $3>0 \mathrm{O}$ \\
\hline 122.4 & 9.3 & iा 4 & $2.273 F=0.7$ & $5.000 E-07$ & $3>0$ \\
\hline $12 \hat{z} .{ }^{\prime}$ & 12." & 555 & 2.7 TE -07 & $6.10 ? E-7$ & 3000 \\
\hline 187.9 & 15.7 & $51 \%$ & $2.543[-6]$ & $5.6[2]=01)$ & $3 \varepsilon 01$ \\
\hline $1 \div 2.6$ & IE. & 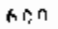 & $3 . \cap n a F+C]$ & $6.659 E-197$ & 3200 \\
\hline 172,0 & 21.7 & $47 A$ & $2,30\} E-17$ & $5.2045=17$ & $3 \geq 03$ \\
\hline 122,6 & $24 .{ }^{\circ}$ & ano & $2.3065-07$ & $5.1635=07$ & 3206 \\
\hline 122.0 & 31.0 & $4 \div 7$ & $2 . ? 19[-107$ & $4.873 E=07$ & 3200 \\
\hline 122.6 & 37.2 & $1+1$ & $1.7115 f-f: 7$ & $3.752 E=1.7$ & 3200 \\
\hline 122 & 43.4 & 1 $\div \div$ & $2.0245-07$ & $4.461 E=07$ & 3200 \\
\hline 182.4 & 49.5 & $2 ! 19$ & $1.245 E=07$ & $2.745 E-07$ & $32 \div 0$ \\
\hline $1<2$. & 55.9 & 347 & $1.735 \mathrm{E}-\mathrm{C} 7$ & $3.1^{A} E-07$ & 3200 \\
\hline 1 & $6 \% .9$ & $30 h$ & $1.9 \times \bar{L} E-0\}$ & $4.360 \varepsilon-07$ & 3200 \\
\hline
\end{tabular}

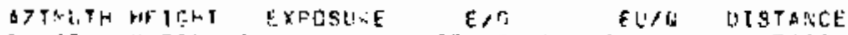

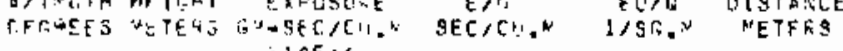
i) $\cap E+s$

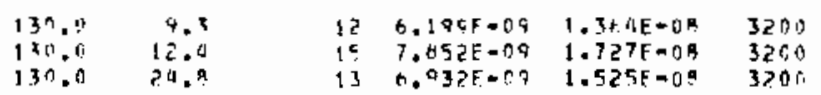


SECONDARY SERIES EXPERIMENT: $X-2$

GROUND LEVEL AND TOWER SAMPLING 600-3200 H ARCS.

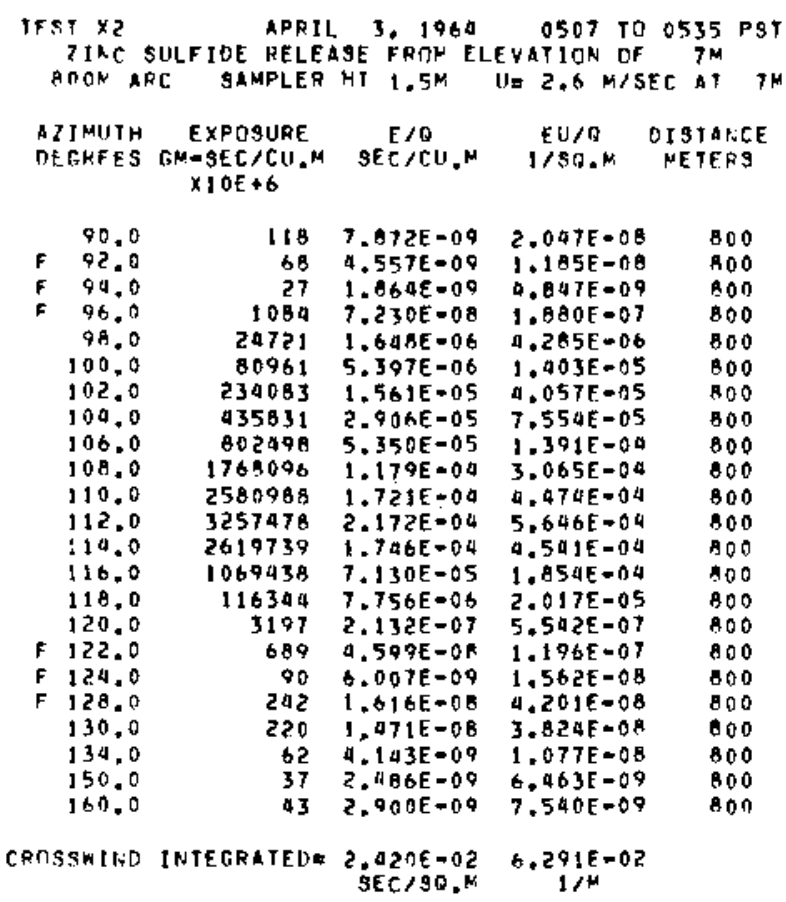

TOWEP TATA FILLOH....

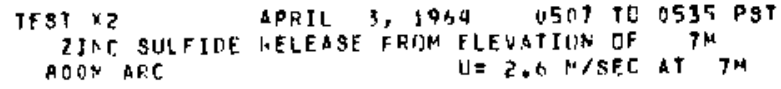

\begin{tabular}{|c|c|c|c|c|c|}
\hline $\begin{array}{l}\text { ZIRUTTH } \\
\text { EGREES }\end{array}$ & $\begin{array}{l}\text { HE IGHT } \\
\text { METERS }\end{array}$ & $\begin{array}{c}\text { EXPOSURE } \\
\text { GM-SEC } / C U . M \\
\times 10 E+6\end{array}$ & $\begin{array}{c}E / \theta \\
\operatorname{SEC} / \mathrm{Cl}, \mathrm{M}\end{array}$ & $\begin{array}{l}E \cup / 0 \\
1 / \$ Q, M\end{array}$ & $\begin{array}{l}\text { DISTANCE } \\
\text { METERS }\end{array}$ \\
\hline $\begin{array}{l}106.0 \\
106.0 \\
106.0 \\
106.0 \\
106.0 \\
106.0 \\
106.0 \\
106.0 \\
106.0 \\
106.0 \\
106.0\end{array}$ & $\begin{array}{l}0.3 \\
0.5 \\
1.0 \\
2.1 \\
4.2 \\
6.5 \\
0.4 \\
10.5 \\
12.8 \\
14.7 \\
10.4\end{array}$ & $\begin{array}{r}762505 \\
769301 \\
504054 \\
640255 \\
432494 \\
271773 \\
169003 \\
60185 \\
6903 \\
609 \\
103\end{array}$ & $\begin{array}{l}5.084 E-05 \\
5.120 E=05 \\
3.360 E-05 \\
2.935 E-05 \\
2.883 E=05 \\
1.012 E-05 \\
1.127 E=05 \\
4.012 E=06 \\
4.602 E=07 \\
4.328 E=00 \\
6.093 E=09\end{array}$ & $\begin{array}{l}1.322 E=04 \\
1.333 E=04 \\
3.737 E=05 \\
7.631 E=05 \\
7.997 E-05 \\
1.711 E=05 \\
2.929 E=03 \\
1.003 E=05 \\
1.197 E=06 \\
1.125 E=07 \\
1.792 E=08\end{array}$ & $\begin{array}{l}000 \\
000 \\
600 \\
800 \\
800 \\
000 \\
800 \\
600 \\
000 \\
000 \\
800\end{array}$ \\
\hline $\begin{array}{l}\text { DIILUTH } \\
\text { DEGREE }\end{array}$ & $\begin{array}{l}\text { HEIGHT } \\
\text { "ETERS }\end{array}$ & 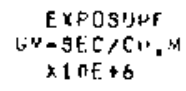 & $\begin{array}{c}F / C \\
5 E C / C U, \cdots\end{array}$ & $\begin{array}{l}E \cup / C \\
L / S O . M\end{array}$ & $\begin{array}{l}\text { DISTANCE } \\
\text { METERS }\end{array}$ \\
\hline $\begin{array}{l}114.0 \\
114.00 \\
114.0 \\
114.0 \\
114.00 \\
114.0 \\
114.0 \\
114.0 \\
114.0 \\
114.0 \\
11400 \\
119.0 \\
114.0 \\
114.0 \\
114.0\end{array}$ & $\begin{array}{r}0.3 \\
0.5 \\
1.0 \\
2.1 \\
4.2 \\
0.3 \\
6.4 \\
10.5 \\
12.6 \\
14.7 \\
16.8 \\
21.0 \\
25.2\end{array}$ & $\begin{array}{r}2243249 \\
21935199 \\
1576908 \\
1959947 \\
2198479 \\
2402265 \\
2038624 \\
1924051 \\
1305620 \\
734373 \\
375367 \\
81952 \\
12169 \\
1163 \\
55\end{array}$ & $\begin{array}{l}1.406 E-04 \\
1.402 E=04 \\
1.005 E=00 \\
1.240 E=04 \\
1.466 E=04 \\
1.602 E=04 \\
1.359 E=04 \\
1.283 E=04 \\
1.704 E=05 \\
4.696 E=05 \\
2.502 E-05 \\
5.457 E=06 \\
8.113 E=07 \\
7.755 E-08 \\
3.679 E=09\end{array}$ & $\begin{array}{l}3 . A 8 B E=04 \\
3.802 E=04 \\
2.612 E=04 \\
3.224 E=04 \\
3.811 E=04 \\
4.160 E=04 \\
3.530 E=04 \\
3.335 E=04 \\
2.263 E=04 \\
1.273 E=04 \\
6.506 E=05 \\
1.019 E-05 \\
2.109 E=06 \\
2.016 E=07 \\
9.566 E-09\end{array}$ & $\begin{array}{l}800 \\
500 \\
800 \\
600 \\
800 \\
800 \\
600 \\
800 \\
800 \\
000 \\
800 \\
000 \\
000 \\
000 \\
000\end{array}$ \\
\hline
\end{tabular}

AZTMLITH HEZGHT FXPOSURE E/O EU/O OISTANCE DEGHEFS METERS GM-SEC/CH.M SEC/CU.M 1/SO.M NETERS $X \backslash 0 E+6$

\begin{tabular}{|c|c|c|c|c|c|}
\hline $\begin{array}{l}122.0 \\
122.0 \\
122.0 \\
122.0 \\
122.0 \\
122.0 \\
122.0 \\
122.0 \\
122.0 \\
122.0 \\
122.0 \\
122.0 \\
122.0 \\
122.0 \\
122.0 \\
122.0\end{array}$ & $\begin{array}{l}0.3 \\
0.5 \\
1.0 \\
4.2 \\
6.3 \\
3.1 \\
10.5 \\
12.5 \\
19.7 \\
16.9 \\
21.0 \\
25.2 \\
29.4 \\
33.6 \\
37.8 \\
42.0\end{array}$ & $\begin{array}{r}97 \\
9 \text { A9 } \\
45 \\
225 \\
400 \\
844 \\
1560 \\
3569 \\
7807 \\
14719 \\
17963 \\
10235 \\
4380 \\
1750 \\
636 \\
257\end{array}$ & $\begin{array}{l}5.060 E=09 \\
6.597 E=08 \\
3.060 E=09 \\
1.506 F=08 \\
2.66 P E=08 \\
5.630 E=08 \\
1.040 E=07 \\
2.390 E=07 \\
5.258 E-07 \\
9.613 F=07 \\
1.198 E-06 \\
6.824 E=07 \\
3.259 E-07 \\
1.167 E-07 \\
4.241 E=08 \\
1.714 E=08\end{array}$ & $\begin{array}{l}1.523 E=06 \\
1.715 E=07 \\
7.966 E-09 \\
3.917 E=08 \\
6.937 E=08 \\
1.464 E=07 \\
2.705 E=07 \\
6.187 E=07 \\
1.367 E=06 \\
2.551 E=06 \\
3.114 E=06 \\
1.774 E=06 \\
8.473 E=07 \\
3.033 E=07 \\
1.103 E=07 \\
4.456 E=08\end{array}$ & $\begin{array}{l}000 \\
000 \\
000 \\
800 \\
000 \\
800 \\
800 \\
800 \\
800 \\
800 \\
800 \\
800 \\
000 \\
800 \\
800 \\
800\end{array}$ \\
\hline
\end{tabular}

\begin{tabular}{|c|c|c|c|c|c|}
\hline $\begin{array}{l}\text { AZINIITH } \\
\text { OE GREES }\end{array}$ & $\begin{array}{l}\text { HEIGHI } \\
\text { METERS }\end{array}$ & $\begin{array}{c}\text { EXPOSLHE } \\
\text { GU-SEC } / C \cup . \\
\times 1 O E+6\end{array}$ & $\begin{array}{c}E / \theta \\
S E C / C U . ;\end{array}$ & $\begin{array}{l}E U / 0 \\
1 / S 0 . N\end{array}$ & $\begin{array}{c}\text { DIS AAICE } \\
\text { METERS }\end{array}$ \\
\hline $\begin{array}{l}130.0 \\
130.0 \\
130.0 \\
130.0 \\
130.0 \\
130.0\end{array}$ & $\begin{array}{r}1.0 \\
4.2 \\
10.5 \\
12.6 \\
14.7 \\
42.0\end{array}$ & $\begin{array}{l}97 \\
74 \\
34 \\
91 \\
5 ! \\
33\end{array}$ & $\begin{array}{l}6.511 E=09 \\
4.979 E=09 \\
2.298 E=09 \\
6.122 E=09 \\
3.447 E=09 \\
2.2275=09\end{array}$ & $\begin{array}{l}1.603 E=06 \\
1.290 E=0 . \\
5.970 E=09 \\
1.593 E=08 \\
8.962 E=09 \\
5.790 E=09\end{array}$ & $\begin{array}{l}8,0 \\
800 \\
800 \\
800 \\
000 \\
000\end{array}$ \\
\hline
\end{tabular}




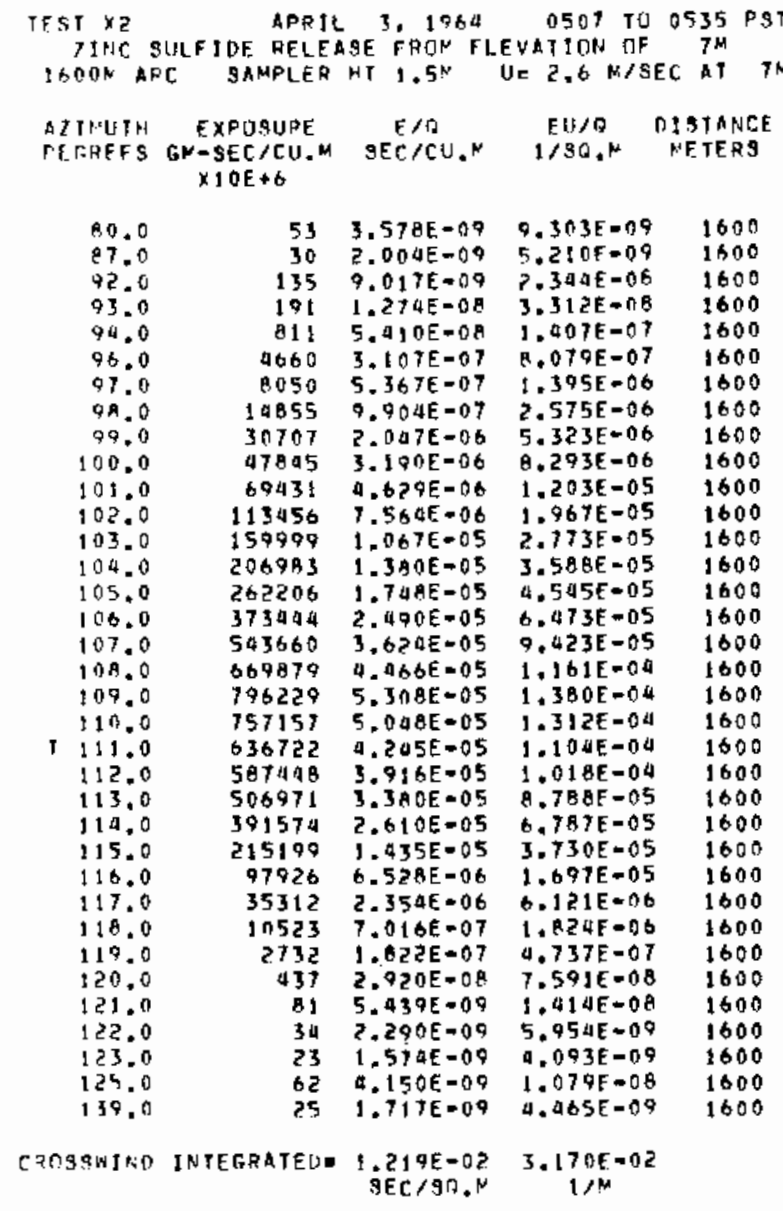

TOMER TATA F(HLLOU,...

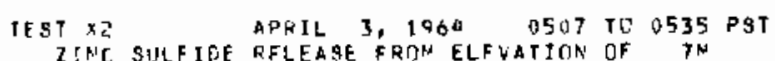

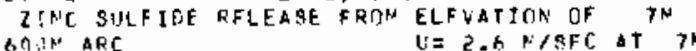

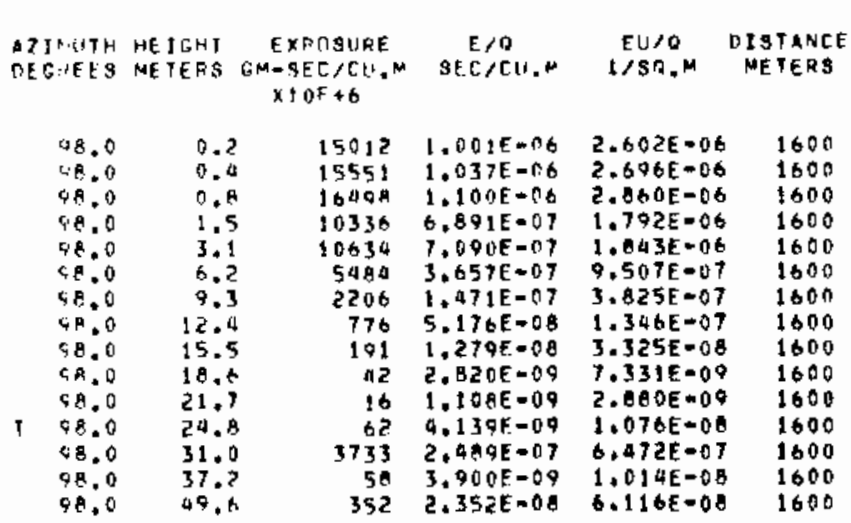

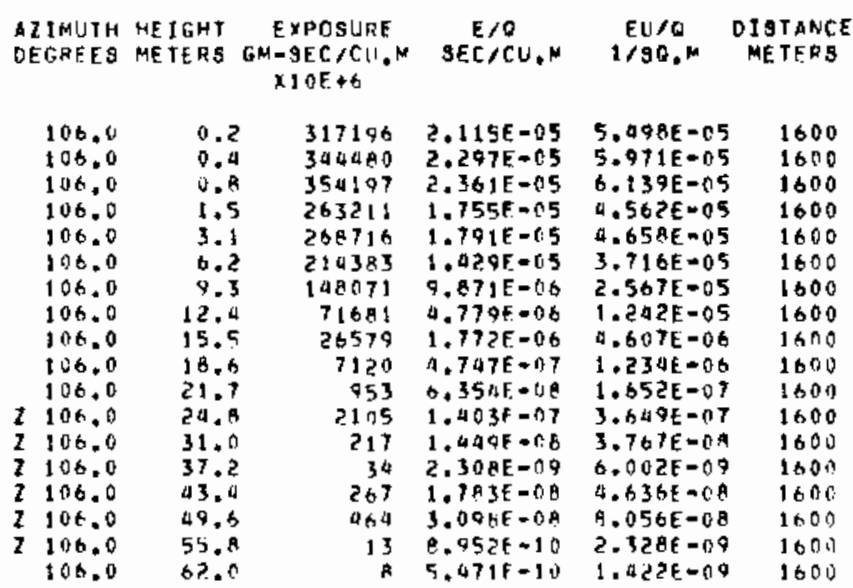

\begin{tabular}{|c|c|c|c|c|c|}
\hline $\begin{array}{l}\text { ZINUTH } \\
\text { EGHEFS }\end{array}$ & $\begin{array}{l}\text { HE IEHT } \\
\text { ME TERS }\end{array}$ & 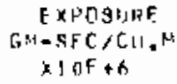 & $\begin{array}{c}E / \beta \\
S E C / r I^{\prime} . N\end{array}$ & $\begin{array}{c}C M / 0 \\
1 / S O N\end{array}$ & $\begin{array}{l}\text { PISTANCE } \\
\text { METERS }\end{array}$ \\
\hline 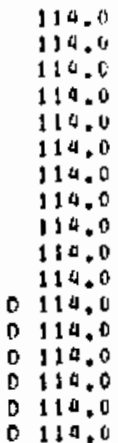 & $\begin{array}{l}0.2 \\
0.2 \\
0.8 \\
1.5 \\
3.1 \\
6.2 \\
9.1 \\
12.2 \\
15.5 \\
18.6 \\
21.7 \\
21.8 \\
31.8 \\
37.8 \\
43.5 \\
49.6\end{array}$ & $\begin{array}{r}333606 \\
344979 \\
331672 \\
202111 \\
304728 \\
474606 \\
563539 \\
512459 \\
310027 \\
110709 \\
49333 \\
922 \\
29 \\
22 \\
17 \\
20 \\
9\end{array}$ & 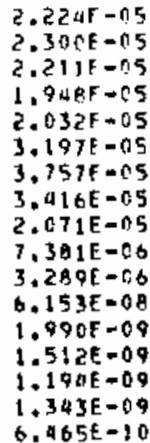 & $\begin{array}{l}5.783 E=05 \\
5.9 A 11 E=05 \\
5.749 F=05 \\
5.064 F=05 \\
5.282 E=05 \\
0.313 E=05 \\
9.76 B E=05 \\
8.083 E=05 \\
5.3 A 4 E=05 \\
1.919 E=05 \\
8.551 E=06 \\
1.600 E=07 \\
5.174 E=09 \\
3.932 E=09 \\
3.104 E=09 \\
3.491 E-09 \\
1.601 E=09\end{array}$ & $\begin{array}{l}1600 \\
1600 \\
1600 \\
1600 \\
1600 \\
1600 \\
1600 \\
1600 \\
1600 \\
1600 \\
1600 \\
1600 \\
1600 \\
1600 \\
1600 \\
1600 \\
1600\end{array}$ \\
\hline
\end{tabular}

AZIFINTH HEIGHT EXPOSURE
OEGREES METEPS GNOSEC/CU.M SEC/CH.M IJSO.M METERS $x \geq 0 E+6$

\begin{tabular}{|c|c|c|c|c|c|}
\hline $\begin{array}{l}122.0 \\
122.0 \\
122.0 \\
122.0 \\
122.0 \\
122.0 \\
122.0 \\
122.0 \\
122.0 \\
122.0 \\
122.0 \\
122.0 \\
122.0 \\
122.0 \\
122.0 \\
122.0\end{array}$ & $\begin{array}{l}0.0 \\
0.8 \\
1.5 \\
3.1 \\
9.1 \\
12.5 \\
15.5 \\
18.0 \\
21.7 \\
24.8 \\
31.1 \\
57.2 \\
43.2 \\
49.8 \\
55.8 \\
62.0\end{array}$ & $\begin{array}{r}20 \\
22 \\
15 \\
30 \\
54 \\
398 \\
2323 \\
6622 \\
10446 \\
13200 \\
13447 \\
8383 \\
2276 \\
143 \\
15 \\
23\end{array}$ & $\begin{array}{l}1.352 E=09 \\
1.503 E=09 \\
1.007 E=09 \\
2.014 E=09 \\
3.625 E=09 \\
2.659 F=08 \\
1.549 E=07 \\
9.415 E=07 \\
7.298 E=07 \\
8.864 E=07 \\
4.961 E=07 \\
5.589 E=07 \\
1.532 E=07 \\
9.599 E=09 \\
1.044 E=09 \\
1.542 E=09\end{array}$ & $\begin{array}{l}3.516 E=09 \\
3.907 E=09 \\
2.618 E=09 \\
5.237 E=09 \\
9.026 E=09 \\
6.912 E=08 \\
4.027 E=07 \\
1.198 E=06 \\
1.097 E=06 \\
2.305 E=06 \\
2.330 E=06 \\
1.053 E=06 \\
3.960 E=07 \\
2.496 E=08 \\
2.715 E=09 \\
4.009 E=00\end{array}$ & $\begin{array}{l}1600 \\
1600 \\
1600 \\
1600 \\
1600 \\
1600 \\
1600 \\
1600 \\
1600 \\
1600 \\
1600 \\
1600 \\
1600 \\
1600 \\
1600 \\
1600\end{array}$ \\
\hline $\begin{array}{l}\text { 1NUTH } \\
\text { GREES }\end{array}$ & $\begin{array}{l}\text { HE IGHS } \\
\text { NETEAS }\end{array}$ & $\begin{array}{c}\text { EXPOSURF } \\
\text { GN=SEE } \\
\times 1 \text { OF }+2\end{array}$ & $\begin{array}{c}E / \theta \\
\sec / C \|_{2} .\end{array}$ & $\begin{array}{c}F(1 / 1) \\
1 / \mathrm{SO}^{2}\end{array}$ & $\begin{array}{l}\text { CISTANCE } \\
\text { METERS }\end{array}$ \\
\hline $\begin{array}{l}130.0 \\
130.0 \\
130.0\end{array}$ & $\begin{array}{r}9.3 \\
24.9 \\
49.6\end{array}$ & $\begin{array}{r}45 \\
47 \\
14\end{array}$ & $\begin{array}{l}3.021 E=09 \\
3.184 E=09 \\
9.449 E=10\end{array}$ & $\begin{array}{l}7.655 E-09 \\
0.278 E=09 \\
2.457 E-09\end{array}$ & $\begin{array}{l}1600 \\
1600 \\
1600\end{array}$ \\
\hline
\end{tabular}




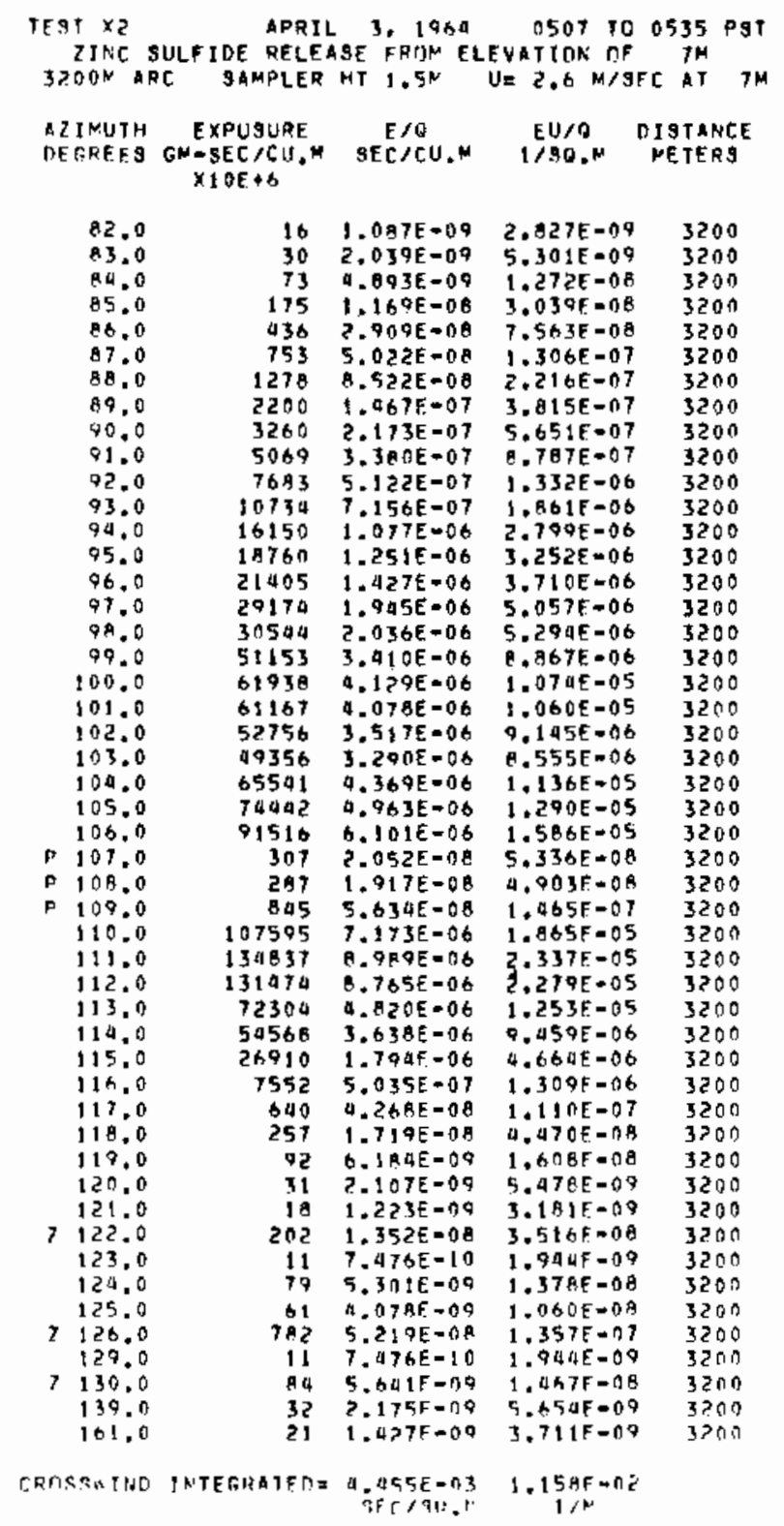

OWE DATA FOLLOW....

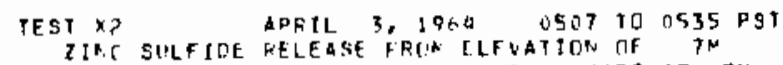

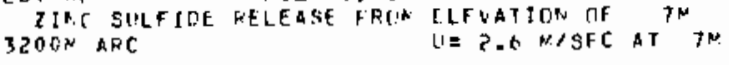

\begin{tabular}{|c|c|c|c|c|c|}
\hline $\begin{array}{l}\text { AZIFUTH } \\
\text { DEGNEES }\end{array}$ & $\begin{array}{l}\text { HEIGHT } \\
\text { METERS }\end{array}$ & $\begin{array}{c}\text { EXPOSURE } \\
G M=-S E C / C+1=M \\
\times 10 E+6\end{array}$ & $\begin{array}{c}E / C \\
s \in C / C U . F\end{array}$ & $\begin{array}{l}F u / 0 \\
\qquad / \mathrm{sog}\end{array}$ & $\begin{array}{l}\text { ISTANCE } \\
\text { METERS }\end{array}$ \\
\hline $\begin{array}{l}98.0 \\
99.0\end{array}$ & $\begin{array}{l}0.2 \\
0.4\end{array}$ & $\begin{array}{l}32879 \\
32192\end{array}$ & $\begin{array}{l}2.192 E-116 \\
2.1468-706\end{array}$ & $\begin{array}{l}5.697 E-06 \\
5.58 \cap E-06\end{array}$ & $\begin{array}{l}3250 \\
3200\end{array}$ \\
\hline Q8. 0 & 0.8 & 32113 & $2.1415-06$ & $5.566 E-06$ & 3200 \\
\hline $8 B .0$ & 1.5 & 79555 & $1.970 F-00$ & $5.123 E-06$ & 3200 \\
\hline 98.0 & 3.1 & 27701 & $1.851 \mathrm{k}=0.8$ & $4.012 \mathrm{E}=08$ & 3200 \\
\hline $90^{\circ}$ & 0.2 & 232 & $1.549[-06$ & $0.028 \varepsilon-06$ & $\$ 200$ \\
\hline 98.0 & 9.3 & 18324 & $1.227 E-06$ & $3.176 E=06$ & 3200 \\
\hline 98. & 12.9 & $377 \mathrm{~A}$ & $2.519 \mathrm{~F}-\mathrm{D}\}$ & $6.549 E-07$ & 3200 \\
\hline 98. & 15.5 & 2030 & $1.353 \mathrm{E}-07$ & $3.519 \mathrm{E}-07$ & 3200 \\
\hline 98.0 & 18.6 & 342 & 2.20 of $-n B$ & $5.943 \mathrm{E}=0 \mathrm{~B}$ & 3200 \\
\hline 98, & 21 & 95 & $0.398 E-19$ & $1.663 E-08$ & 3200 \\
\hline 98.0 & 24.8 & 14 & $9.523 t-10$ & $2.076 E-09$ & 3200 \\
\hline
\end{tabular}

\begin{tabular}{|c|c|c|c|c|c|}
\hline $\begin{array}{l}\text { DIIMUTH } \\
\text { DEGREES }\end{array}$ & $\begin{array}{l}\text { MEIGH T } \\
\text { METERS }\end{array}$ & $\begin{array}{c}\text { EXPCSUAE } \\
\text { GM-SEC } / C U . N \\
\times 10 E+6\end{array}$ & $\begin{array}{c}E / 0 \\
\text { SE.C } / \text { CU. }\end{array}$ & $\begin{array}{c}\text { EU/O } \\
1 / \$ 0 . \mathrm{H}\end{array}$ & $\begin{array}{l}\text { OISTANCE } \\
\text { METERS }\end{array}$ \\
\hline $\begin{array}{l}106.0 \\
106.0\end{array}$ & $\begin{array}{l}0.2 \\
0.4\end{array}$ & $\begin{array}{l}76321 \\
7949 ?\end{array}$ & $\begin{array}{l}5.008 E-106 \\
5.20 C F=06\end{array}$ & $\begin{array}{l}1.323 E=05 \\
1.370 E=05\end{array}$ & $\begin{array}{l}3200 \\
3200\end{array}$ \\
\hline 106.0 & $0 . \mathrm{B}$ & 77591 & $5.173 \mathrm{~F}=06$ & $1,345 E=05$ & 3200 \\
\hline 106.0 & 1.5 & 76011 & $5.067 E-06$ & $1.31 \Delta E=05$ & 3200 \\
\hline 106.0 & 3.1 & P0762 & $5,364 E=06$ & 1. $\triangle 00 E-05$ & 3200 \\
\hline 106.0 & 6.2 & 11190 & $4.746 F-06$ & $i .234 \mathrm{E}=05$ & 3200 \\
\hline 100,0 & 9,3 & 59694 & $3,980 E-06$ & $1.035 \mathrm{E}-05$ & 3200 \\
\hline 106.0 & 12,4 & 51451 & $3.430 E-06$ & $8.918 E-06$ & 3200 \\
\hline 106.0 & 15.5 & 53551 & $2,237 E-06$ & $5.016 E-06$ & 3200 \\
\hline 106.0 & 18.6 & 10577 & $1.239 E-C 6$ & $3.220 E-06$ & 3200 \\
\hline 106.0 & 21.7 & 6957 & $4.638 E-07$ & $1.206 E=06$ & 3200 \\
\hline 106. & Q4. 9 & $105 \mathrm{~F}$ & $9.72 \triangle \mathrm{E}-0 \mathrm{~g}$ & $2.52 \mathrm{BE}-07$ & 3200 \\
\hline & 31 & 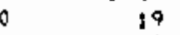 & $03 E-09$ & $388 E-09$ & 3200 \\
\hline 100.0 & 43.9 & 40 & $2.957 \mathrm{E}-09$ & $7.689 E-08$ & 3200 \\
\hline
\end{tabular}

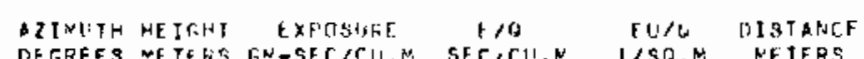
DEGRFF 3 NF TE

\begin{tabular}{|c|c|c|c|c|c|}
\hline 114.0 & 0.2 & $42+16$ & $3.2415-06$ & $A .927 E \rightarrow 0 b$ & 3200 \\
\hline 114.0 & 0.9 & 58426 & $3,805 \mathrm{E}=0 \mathrm{~b}$ & $1.013 \varepsilon-05$ & 3200 \\
\hline 119.0 & $0 . A$ & 59442 & $3.9635-06$ & $1.030 f=05$ & 3200 \\
\hline 114.0 & 1.5 & $9292 \mathrm{~A}$ & $3.520 \mathrm{E}-06$ & $9.1744-06$ & 3200 \\
\hline 114.0 & 3.1 & 52502 & $3 . \operatorname{soc} E-00$ & $9.100 E=06$ & 3206 \\
\hline 114.0 & 0. & 69934 & $0.062 E-00$ & $1.2126-05$ & 3200 \\
\hline$(34.0$ & 9.3 & 94543 & $6.303 E=106$ & $1.639 E-05$ & 3200 \\
\hline 114.0 & 12.4 & 97506 & $6.63 \mathrm{BE}-06$ & $3.726 \mathrm{E}-05$ & 3200 \\
\hline i14.0 & 15,5 & 0.5583 & $6.00 \mathrm{CF}=10$ & $1.570 E=05$ & 3206 \\
\hline 114.0 & 18.6 & $1306 t$ & $5.204 E-06$ & $1.353 t-05$ & 3200 \\
\hline 114.0 & 21.7 & 65303 & $a, 360 E-06$ & $1.133 E-05$ & 3200 \\
\hline $1: 4.0$ & 24.9 & $3 A B+2$ & $2.589 \mathrm{E}=0 \mathrm{~b}$ & $6.731 \varepsilon-06$ & $\$ 200$ \\
\hline 1140 & 31.0 & $115 n 3$ & $7,069 \mathrm{E}-07$ & $1.99 \mathrm{dE}-06$ & 3200 \\
\hline 114.0 & 37.2 & 1369 & $9.132 E-08$ & $2.374 E-07$ & 3200 \\
\hline 114.0 & 43.4 & an & $5,363 \mathrm{E}=09$ & $1.394 \mathrm{E}-0 \mathrm{O}$ & 3200 \\
\hline 114.0 & 49,6 & 6 & $4,467 \mathrm{E}=10$ & $1.101 E=09$ & 3200 \\
\hline 114.0 & $55 . \mathrm{A}$ & 24 & $1.638 E-09$ & $4.259 E-09$ & 3200 \\
\hline
\end{tabular}

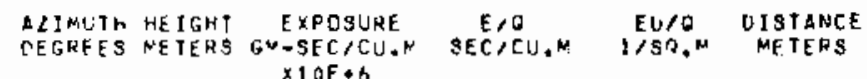

\begin{tabular}{|c|c|c|c|c|c|}
\hline 122.0 & 1.5 & 13 & $8.724 E-10$ & $2.26 B E-09$ & 3200 \\
\hline & 0.2 & 33 & & & 3200 \\
\hline 122,0 & 9.3 & 19 & $1.2800=09$ & $3.327 E=09$ & 3200 \\
\hline 122.0 & 12.4 & 187 & $1.25 \cap E=08$ & $3.251 E=08$ & 3200 \\
\hline 122.0 & 15.5 & $7>6$ & $4.845 E-08$ & $1.2608=07$ & 3200 \\
\hline 122.0 & 18. & 2357 & $1.572 E+07$ & $A .0 B 6 E=07$ & 3200 \\
\hline 122.0 & 21 & 5469 & $3,646 E-07$ & $9.979 E-07$ & 3200 \\
\hline $\begin{array}{l}122.0 \\
122.0\end{array}$ & 24 & 8944 & $5.896 E=0 ?$ & $1.5335=06$ & 3200 \\
\hline 122.0 & 31.0 & 12624 & $8.416 \mathrm{E}-07$ & $2.188 E=06$ & 3200 \\
\hline 122.0 & 37 & 8282 & $5.522 E-07$ & $1.036 \varepsilon=06$ & 3200 \\
\hline 322.0 & 03. & 2620 & $1.747 E=07$ & $4.542 E-01$ & $\$ 200$ \\
\hline 122.0 & 49.6 & 117 & $7.617 E=09$ & $2.032 \mathrm{E}-0 \mathrm{~B}$ & 3200 \\
\hline 122.0 & & 12 & $8.561 E-10$ & $2.226 E-09$ & 3200 \\
\hline
\end{tabular}

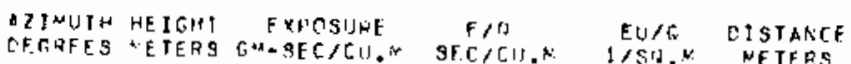
$x \perp D E+B$

$\begin{array}{llllll}130.0 & 21.7 & 20 & 1.338 E-09 & 3.478 F-09 & 3200 \\ 130.0 & 31.0 & 27 & 1.855 E-09 & 4.822 E-08 & 3200\end{array}$ 
SECONDARY SERIES EXPERIMENT: $X-4$

GROLNO LEVEL AND TOWER SAMPLING 200-3200 M ARCS.

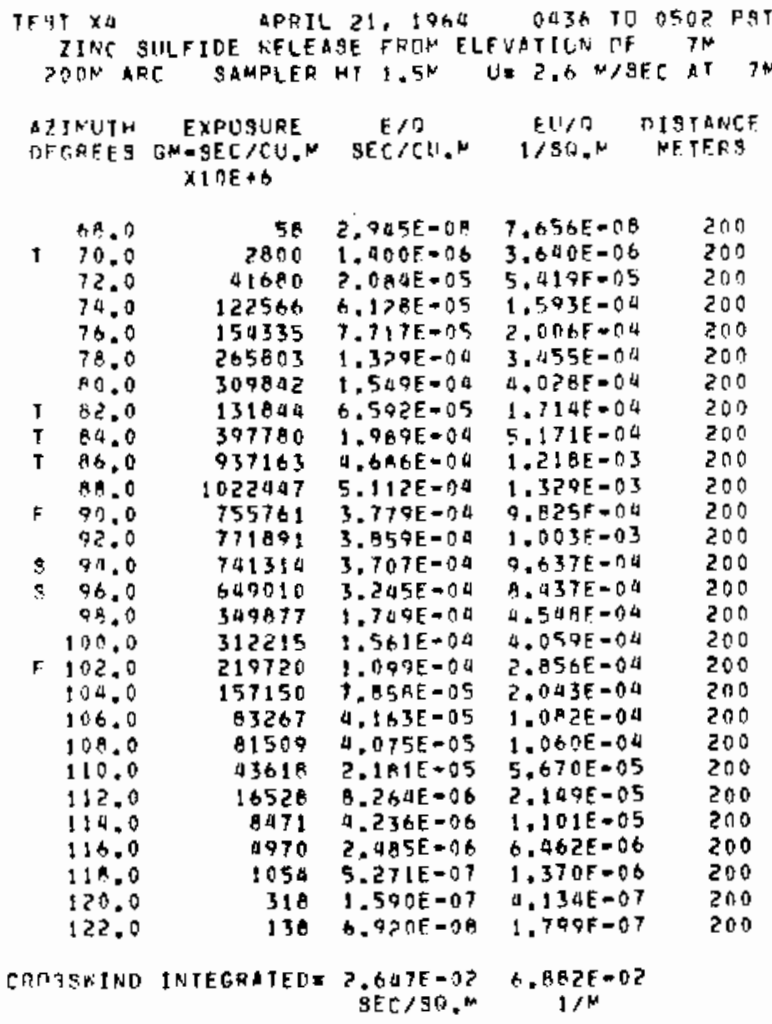

TIOAF tAIO F PLLLK....

TFST $>$ APEIL ?1, 1964 O436 TC F502 DST

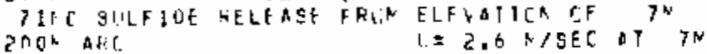

\begin{tabular}{|c|c|c|c|c|c|}
\hline $\begin{array}{l}\text { A. JYQ.TH } \\
\text { CEETEFS }\end{array}$ & $\begin{array}{l}\text { RE IGHT } \\
\text { DE TERS }\end{array}$ & 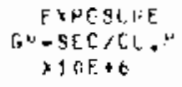 & $\begin{array}{c}E / C \\
\text { SECCL, }\end{array}$ & $\begin{array}{l}E L / 6 \\
1 / 56.2\end{array}$ & $\begin{array}{l}\text { CISTANCE } \\
\text { NEIEAS }\end{array}$ \\
\hline $\begin{array}{l}98.0 \\
98.0 \\
98.0 \\
98.0 \\
98.0 \\
98.0 \\
98.0 \\
98.0 \\
98.0 \\
98.0 \\
98.0 \\
98.0 \\
98.0 \\
98.0 \\
98.0\end{array}$ & $\begin{array}{l}0.7 \\
0.7 \\
1.4 \\
2.7 \\
4.1 \\
5.4 \\
6.8 \\
9.1 \\
10.8 \\
13.5 \\
10.2 \\
18.9 \\
21.6 \\
24.3 \\
27.0\end{array}$ & $\begin{array}{l}\text { a60401 } \\
459480 \\
359081 \\
373+53 \\
462663 \\
375597 \\
331371 \\
345178 \\
241495 \\
163021 \\
118942 \\
84916 \\
84101 \\
50721 \\
39511\end{array}$ & $\begin{array}{l}2.302 E=04 \\
2.297 E=04 \\
1.795 E=04 \\
1.065 F=04 \\
2.013 E=04 \\
1.053 E=04 \\
1.057 E=04 \\
1.726 E=04 \\
1.207 E=04 \\
8.191 E+05 \\
5.947 E=05 \\
4.246 E=05 \\
4.210 E=05 \\
2.536 E=05 \\
1.976 E-05\end{array}$ & 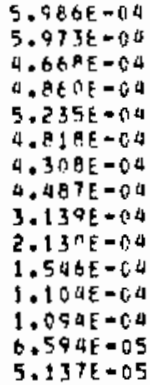 & $\begin{array}{l}200 \\
200 \\
200 \\
200 \\
200 \\
200 \\
200 \\
200 \\
200 \\
200 \\
200 \\
200 \\
200 \\
200 \\
200\end{array}$ \\
\hline
\end{tabular}

\begin{tabular}{|c|c|c|c|c|c|}
\hline $\begin{array}{l}\text { AZIMI:FH: } \\
\text { CEGHEES }\end{array}$ & $\begin{array}{l}\text { HFIGHT } \\
\text { NETEAS }\end{array}$ & $\begin{array}{c}\text { EXPCSIHE } \\
G N-S E C / C L D N \\
\times 10 F+E\end{array}$ & $\begin{array}{c}F, / C_{v} \\
S E C / C U . N\end{array}$ & $\begin{array}{l}E \cup / 4 \\
1 / 50.0\end{array}$ & $\begin{array}{l}\text { CISTANCF } \\
\text { NETERS }\end{array}$ \\
\hline $\begin{array}{l}106.0 \\
106.0\end{array}$ & 0.3 & $\begin{array}{r}95778 \\
112160\end{array}$ & $\begin{array}{l}4.789 f=05 \\
5.608 t=05\end{array}$ & $\begin{array}{l}1.245 E \sim C 4 \\
1.459 E \bullet C 4\end{array}$ & $\begin{array}{l}200 \\
200\end{array}$ \\
\hline 106.0 & 1.0 & a 3576 & $4.17 \mathrm{GE}-0.5$ & $1.086 E=04$ & 200 \\
\hline $10 t .0$ & 2.7 & 7 प 8 E 5 & $3,904 E-05$ & $1.026 E=04$ & 203 \\
\hline 106.0 & 4.1 & tQCed & $3.204 E=05$ & $8.331 E=05$ & 200 \\
\hline 106.0 & 5.4 & 402 & $3.040: E-N 5$ & $7.9041=05$ & 200 \\
\hline 100.0 & 6.1 & \pm 3157 & $5.15 \mathrm{EE}+15$ & $8.210 t+05$ & 200 \\
\hline 106.0 & 8.1 & 61570 & $5.079 f=05$ & E. $004 E=05$ & 200 \\
\hline 106.0 & $9 . i 1$ & $5 n 000$ & $2.532 E-05$ & $6.583 E-0.5$ & 200 \\
\hline $10 t .11$ & $10 . \mathrm{A}^{2}$ & 55767 & $2.785 f-05$ & $7.242 E=05$ & 200 \\
\hline 106.0 & 13.5 & $\operatorname{ls} 977$ & $2,2 s_{t}=1.5$ & $5.971 t=05$ & 200 \\
\hline 106.0 & $16 . ?$ & प1989 & $2.791 F-0.5$ & $5.43 A E-15$ & $2 \div 0$ \\
\hline .0 & 19.9 & 30096 & $1.50 \mathrm{CF}=\mathrm{r.5}$ & a. OO OAE $=05$ & 200 \\
\hline 100 & 21. & $\geq 1962$ & $1.003 E=.55$ & $2.942 t=05$ & 200 \\
\hline & & 12254 & $6.147 E-66$ & $1.58^{\circ} E-05$ & 200 \\
\hline 106.0 & $27 . n$ & 7126 & $3.3+3 F=06$ & $9,76 \times F=016$ & 206 \\
\hline
\end{tabular}

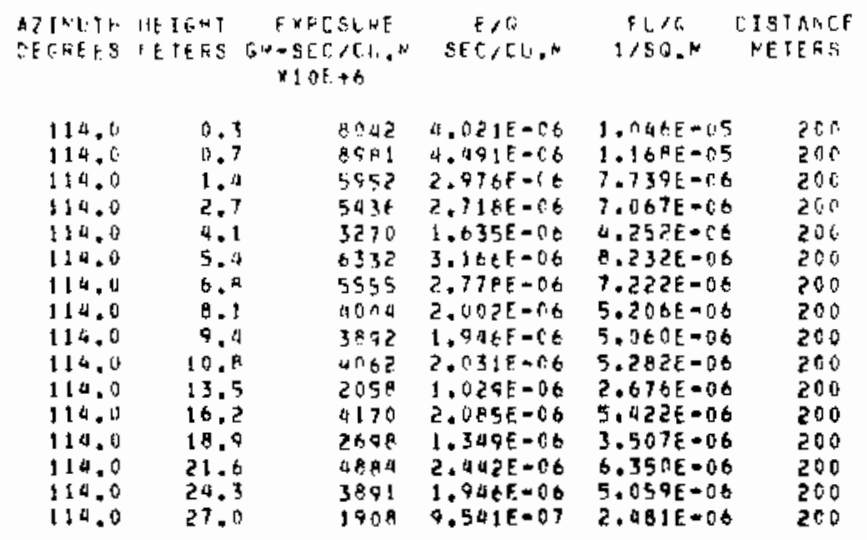

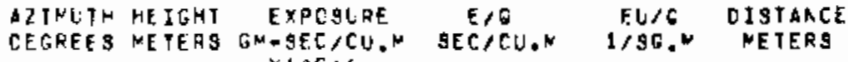
$x ! \Delta E+6$

\begin{tabular}{|c|c|c|c|c|c|}
\hline $\begin{array}{l}122.0 \\
122.0 \\
122.0 \\
122.0 \\
122.0 \\
122.0 \\
122.0 \\
122.0 \\
122.0 \\
122.0\end{array}$ & $\begin{array}{l}4.1 \\
5.4 \\
6.8 \\
8.1 \\
9.4 \\
10.8 \\
13.5 \\
16.2 \\
18.9 \\
21.9\end{array}$ & $\begin{array}{l}202 \\
444 \\
269 \\
130 \\
196 \\
215 \\
150 \\
152 \\
24 \\
31\end{array}$ & $\begin{array}{l}1.010 E-01 \\
2.222 E-07 \\
1.347 E=07 \\
6.504 E-08 \\
9.315 E-08 \\
1.077 E-07 \\
7.519 E-08 \\
7.032 E=08 \\
1.235 E-08 \\
1.511 E-08\end{array}$ & $\begin{array}{l}2.626 E=07 \\
5.778 E=07 \\
3.502 E=07 \\
1.692 E=07 \\
2.422 E-07 \\
2.001 E=07 \\
1.955 E=07 \\
1.980 E-07 \\
3.210 E=08 \\
9.085 E=08\end{array}$ & $\begin{array}{l}200 \\
200 \\
200 \\
200 \\
200 \\
200 \\
200 \\
200 \\
200 \\
200\end{array}$ \\
\hline
\end{tabular}

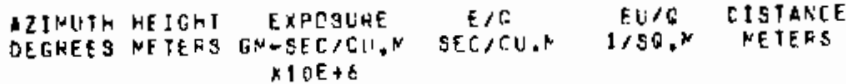

\begin{tabular}{|c|c|c|c|c|}
\hline $\begin{array}{l}130.0 \\
130.0 \\
130.0 \\
130.0 \\
130.0 \\
130.0 \\
130.0\end{array}$ & $\begin{array}{r}9.1 \\
5.4 \\
6.8 \\
8.1 \\
9.4 \\
10.8 \\
13.5\end{array}$ & $\begin{array}{l}169 \\
161 \\
105 \\
112 \\
130 \\
127 \\
96\end{array}$ & $\begin{array}{l}8.417 E=08 \\
8.081 E=08 \\
7.295 E=08 \\
5.612 E=08 \\
6.509 E=08 \\
6.397 E=08 \\
4.02 E E=08\end{array}$ & $\begin{array}{l}2.189 E=07 \\
2.101 E=07 \\
1.007 E=07 \\
1.059 E=07 \\
1.692 E=07 \\
1.083 E=07 \\
1.255 E=07\end{array}$ \\
\hline
\end{tabular}




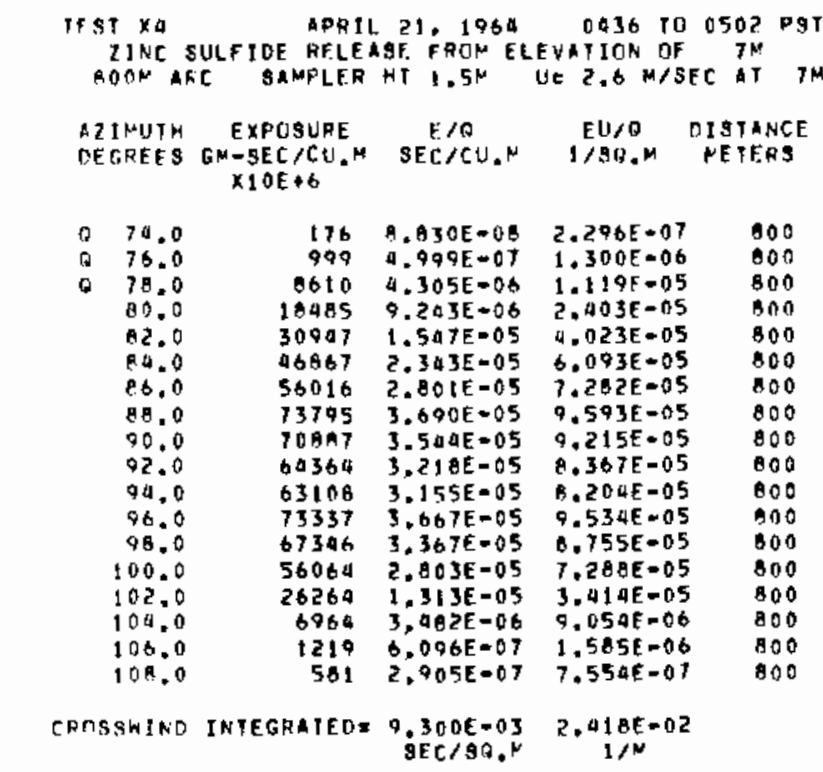

TOREF PATA FIJLLCK....

FEST X4 APKIL 21,19640436 TC 0502 PST IIAC SULFIUE RELFASE FAOT ELEVATICA OF $7 \mathrm{~N}$ AOOH ARE

AZINLTH HEIGHT EXPESLHE E/O EL/O OISTANCE DEGREFS METERS GN-9EC/CU,N SECICU.N 1/SR,N METEAS $\times 10 F+6$

\begin{tabular}{|c|c|c|c|c|c|}
\hline 98.0 & 0,3 & 53396 & $2,065 E-05$ & $0.940 E=05$ & 600 \\
\hline & 0. & $5370^{\circ}$ & $2.685 E=05$ & $6 . \triangle 82 E=05$ & 800 \\
\hline 98.0 & 1.1 & 45926 & $2.296 E-05$ & $5.970 E=05$ & 800 \\
\hline 98.0 & 2.1 & 52406 & $2.625 E=05$ & $6.825 E=05$ & 00 \\
\hline 98.0 & 4.2 & 53402 & $2.670 E=05$ & $6.042 E=05$ & 800 \\
\hline 98.0 & 0.3 & 54268 & $2.713 \mathrm{E}-0.5$ & $7.055 E-05$ & 800 \\
\hline$Q 8,0$ & B. 4 & 99297 & $2,965 \mathrm{E}-05$ & $1.709 \mathrm{E}=05$ & 000 \\
\hline 98,0 & 10.5 & 55722 & $2.789 \mathrm{E}=05$ & $7.252 E=05$ & 800 \\
\hline $9 e .0$ & 12,6 & $50 \Delta 62$ & $2.533 E=05$ & $6.586 E=05$ & 800 \\
\hline 90.0 & 14,7 & 51409 & $2.570 E=05$ & $6.6 A 3 \mathrm{E}=05$ & 000 \\
\hline 98.0 & 16.8 & 44311 & $2.216 \mathrm{E}-05$ & $5.700 E=05$ & 000 \\
\hline 98,0 & 21.0 & 37245 & $1.862 E=05$ & $4.042 \mathrm{E}=0 \mathrm{~S}$ & 000 \\
\hline 98.0 & 25.2 & 22825 & $1.241 E-05$ & $2.807 \mathrm{E}-05$ & 800 \\
\hline 98.0 & 29.4 & 16379 & $0.190 E-06$ & $2.129 \mathrm{E}-0 \mathrm{~S}$ & 000 \\
\hline 98.0 & 33. & 10241 & $8,121 E=06$ & 2.11 E-05 & 000 \\
\hline & & 13392 & $6.596 E=06$ & $1.741 E-03$ & 900 \\
\hline se. & 42.0 & 11404 & $5.702 E-06$ & 1. QE $3 E-0 S$ & 800 \\
\hline
\end{tabular}

AZIMLTH HETGMT EXPOSLTE ERO EUIG CISTANCE

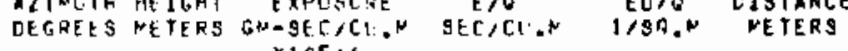

\begin{tabular}{|c|c|c|c|c|c|}
\hline 100.0 & 0.3 & 3 & $7.6178 * 07$ & $1.981 E-06$ & 800 \\
\hline 100.0 & 0.5 & $R \notin B$ & $4.4025=07$ & $1.160 E=00$ & 000 \\
\hline $10 t, 0$ & 1.0 & 779 & $3.8975-07$ & $1.013 E-0 \mathrm{~A}$ & 800 \\
\hline 100.0 & 2.1 & 859 & $4.789 E-67$ & $1.2 \Delta A \varepsilon=06$ & 800 \\
\hline 100.0 & 4.2 & 1161 & $5.808 E-C 7$ & $1.510 E-06$ & 800 \\
\hline 100.0 & 6.3 & 1424 & $1.122 E=07$ & $1 .+152 E=06$ & 600 \\
\hline 106.0 & 9.4 & 1 the 3 & $8.316 E-07$ & $2.162 \mathrm{E}=06$ & 000 \\
\hline 106.0 & 10.5 & 1719 & $8.54 P F=07$ & $2.236 E=06$ & 800 \\
\hline$j n \varepsilon .0$ & $32 . \mathrm{A}$ & 1988 & $9.91 \triangle E E-0.7$ & $2.586 \mathrm{E}=00$ & 800 \\
\hline ille. & 19.7 & 2245 & $1: 123 E=06$ & $2.019 \mathrm{E}-00$ & 800 \\
\hline 106.0 & $i 0.9$ & 2073 & $1.0 \times 1 F-06$ & $2.696 E-06$ & BOd \\
\hline 166.0 & $2 ! .0$ & 2800 & $1 . \triangle 2 \Delta E-06$ & $3.703 E=06$ & $80 \mathrm{C}$ \\
\hline$j 0 t .0$ & 25.2 & 2315 & $1,1 S E F=0 t$ & $3.010 E+0 B$ & $80 \mathrm{u}$ \\
\hline 106.0 & 29.4 & 2705 & $1,353 \mathrm{E}-n \mathrm{~B}$ & $3.517 \varepsilon-166$ & $80 \mathrm{c}$ \\
\hline 106.0 & 33.6 & 1993 & $9.9675-07$ & $2.591 k=00$ & 806 \\
\hline 106.0 & & $23 e 3$ & $1.10 \geq f-1 t$ & $3.790 \mathrm{E}-0 \mathrm{~A}$ & 900 \\
\hline 100.0 & 42,0 & 1093 & $4.4+5 F-17$ & $7.46 z t-16$ & Rnc. \\
\hline
\end{tabular}

17IRLTH HEIGHI EXPCSIJEE F,O ELIO OISTARCE CFGREF METERS GN-SFC/CL.N SFC/CU.F I/SA.N NETERS $\times 10 f+6$

\begin{tabular}{|c|c|c|c|c|c|}
\hline $\begin{array}{l}114.0 \\
114.0 \\
114.0 \\
114.0 \\
114.0 \\
114.0 \\
110.0 \\
1140.0 \\
114.0 \\
114.0 \\
114.0 \\
110.0\end{array}$ & $\begin{array}{l}9.2 \\
6.3 \\
8.4 \\
10.5 \\
22.6 \\
14.7 \\
16.8 \\
21.0 \\
25.2 \\
29.4 \\
33.4 \\
37.4\end{array}$ & $\begin{array}{l}395 \\
219 \\
136 \\
186 \\
167 \\
90 \\
136 \\
54 \\
22 \\
91 \\
32 \\
40\end{array}$ & $\begin{array}{l}1.97 \mathrm{HE}=07 \\
1.09 E E=07 \\
6.8 A(0 E=08 \\
9.336 E=08 \\
6.359 E=08 \\
4.017 E=08 \\
6.840 E=08 \\
2.714 E=08 \\
1.199 E=08 \\
4.596 E=08 \\
1.62 E E=06 \\
2.015 E=08\end{array}$ & $\begin{array}{l}5.137 E-07 \\
2.051 E-07 \\
1.77 E E-07 \\
2.027 E-07 \\
2.173 E-07 \\
1.044 E-07 \\
1.77 E E-07 \\
7.057 E-08 \\
2.927 E-08 \\
1.195 E-07 \\
4.232 E-08 \\
5.22 B E-08\end{array}$ & $\begin{array}{l}800 \\
800 \\
800 \\
000 \\
000 \\
800 \\
000 \\
000 \\
000 \\
000 \\
000 \\
000\end{array}$ \\
\hline
\end{tabular}

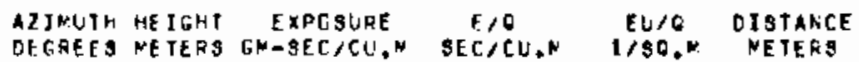
DFARES NETERS GN-3EC

$\begin{array}{rr}122.0 & 4.2 \\ 122.0 & 6.3 \\ 122.0 & 0.4 \\ 122.0 & 10.5 \\ 122.0 & 12.6 \\ 122.0 & 14.7 \\ 122.0 & 16.0 \\ 122.0 & 21.0 \\ 122.0 & 25.2 \\ 122.0 & 29.4 \\ 122.0 & 33.6 \\ 122.0 & 37.0\end{array}$

\begin{tabular}{|c|c|c|}
\hline $\begin{array}{r}293 \\
62 \\
54 \\
101 \\
136 \\
149 \\
54 \\
186 \\
22 \\
45 \\
49 \\
26\end{array}$ & $\begin{array}{l}1.466 E-07 \\
3.14 E E-08 \\
2.714 E=08 \\
7.057 E=08 \\
6.840 E=08 \\
7.491 E=08 \\
2.714 E=08 \\
9.445 E=00 \\
1.149 E-00 \\
0.787 E-08 \\
2.489 E-08 \\
1.436 E=08\end{array}$ & $\begin{array}{l}3.011 E-01 \\
6.186 E-08 \\
1.057 E=00 \\
1.835 E=07 \\
1.778 E-07 \\
1.940 E=07 \\
1.057 E=08 \\
2.456 E=07 \\
2.987 E=08 \\
1.245 E=07 \\
6.472 E=0 . \\
3.134 E=08\end{array}$ \\
\hline
\end{tabular}

800

800

800

800

000

300

800

000

000

100

800

ATIRLTM HEIEHT EXPOSLPE E/6 ELIO CISTANEE CECHEFS NETEAS GU-SEC/CU.N SECACU.N 1/SQ.N NETERS $\times 10 E+6$

$\begin{array}{rr}130.0 & 4.2 \\ 130.0 & 3.3 \\ 130.0 & 8.4 \\ 130.0 & 10.5 \\ 130.0 & 12.6 \\ 130.0 & 14.7 \\ 130.0 & 16.8 \\ 130.0 & 21.0 \\ 130.0 & 25.2 \\ 130.0 & 29.4 \\ 130.0 & 33.0\end{array}$

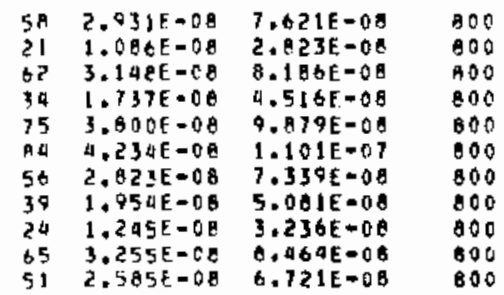




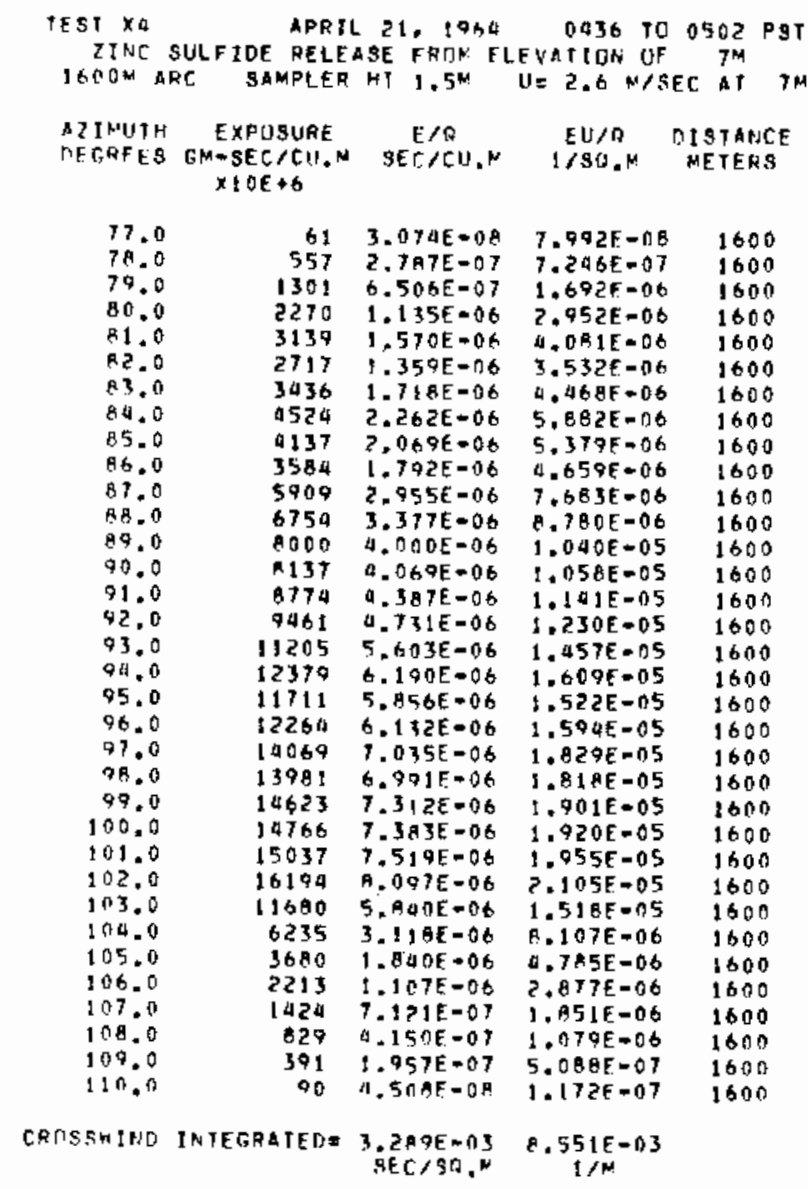

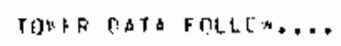

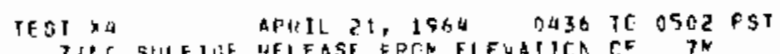
ZI: S SULFJLE IFLFASE FPEN ELEYATI

\begin{tabular}{|c|c|c|c|c|c|}
\hline 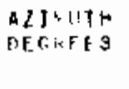 & $\begin{array}{l}\text { HEIGH I } \\
\text { NETERS }\end{array}$ & $\begin{array}{c}\text { EXPCSURE } \\
\text { GN-SEC }-C U \text { N }^{N} \\
\times 1 O E+6\end{array}$ & $\begin{array}{c}E / O \\
S E C / C L .\end{array}$ & $\begin{array}{c}E U / 6 \\
1 / S C_{*}^{*}\end{array}$ & $\begin{array}{l}\text { DISTAACE } \\
\text { NETERS }\end{array}$ \\
\hline $\begin{array}{l}4 P .0 \\
79.0 \\
79.0 \\
98.0 \\
98.0 \\
98.0 \\
98.0 \\
98.0 \\
98.0 \\
98.0 \\
98.0 \\
98.0 \\
78.0 \\
78.0 \\
98.0 \\
98.0 \\
98.0 \\
98.0\end{array}$ & $\begin{array}{l}0.2 \\
4.1 \\
0.8 \\
1.5 \\
3.1 \\
6.2 \\
9.3 \\
12.4 \\
15.5 \\
18.6 \\
21.7 \\
24.7 \\
31.0 \\
37.2 \\
45.9 \\
49.6 \\
55.9 \\
62.0\end{array}$ & $\begin{array}{l}11279 \\
12979 \\
13106 \\
13092 \\
13959 \\
12618 \\
14993 \\
15069 \\
14507 \\
9068 \\
10994 \\
11265 \\
9993 \\
6726 \\
6202 \\
2932 \\
2726 \\
2090\end{array}$ & $\begin{array}{l}5.64 C E=06 \\
0.490 E=06 \\
6.553 E=06 \\
6.541 E=06 \\
0.950 E=06 \\
6.304 E-06 \\
7.007 E=06 \\
7.915 E=06 \\
7.304 E=06 \\
4.534 E=06 \\
5.447 E=06 \\
5.633 E=06 \\
4.497 E=06 \\
3.363 E=06 \\
3.101 E=06 \\
1.460 E=06 \\
1.363 E=06 \\
1.045 E=06\end{array}$ & 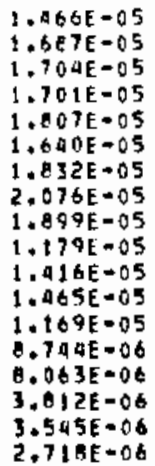 & $\begin{array}{l}1600 \\
1600 \\
1600 \\
1600 \\
1600 \\
1600 \\
1600 \\
1600 \\
1600 \\
1600 \\
1600 \\
1600 \\
1600 \\
1600 \\
1600 \\
1600 \\
1600 \\
1600\end{array}$ \\
\hline
\end{tabular}

\begin{tabular}{|c|c|c|c|c|c|}
\hline $\begin{array}{l}\text { DIFUSH } \\
\text { DECFEFS }\end{array}$ & $\begin{array}{l}\text { HETGHT } \\
\text { NETERS }\end{array}$ & $\begin{array}{l}\text { EXPOSURE } \\
\text { GNOSEC } / C U . " \\
\text { XIOE }+6\end{array}$ & $\begin{array}{c}E / \sigma \\
S E C / C U, N\end{array}$ & $\begin{array}{c}E w / 0 \\
1 / 90^{\circ}\end{array}$ & $\begin{array}{l}\text { DISTANTE } \\
\text { WETEFS }\end{array}$ \\
\hline $\begin{array}{l}106.0 \\
100.0 \\
100.0 \\
106.0 \\
106.0 \\
100.0 \\
106.0 \\
106.0 \\
106.0 \\
106.0 \\
106.0 \\
100.0 \\
100.0 \\
106.0 \\
106.0 \\
106.0 \\
100.0 \\
106.0\end{array}$ & $\begin{array}{l}0.2 \\
0.1 \\
0.8 \\
1.5 \\
3.1 \\
6.2 \\
9.2 \\
12.4 \\
15.5 \\
16.6 \\
21.7 \\
24.7 \\
31.0 \\
37.2 \\
43.0 \\
49.5 \\
55.8 \\
22.0\end{array}$ & $\begin{array}{l}1137 \\
1470 \\
1745 \\
1544 \\
2144 \\
2701 \\
1971 \\
2191 \\
2250 \\
2277 \\
2397 \\
2161 \\
1499 \\
1625 \\
1540 \\
1150 \\
897 \\
976\end{array}$ & 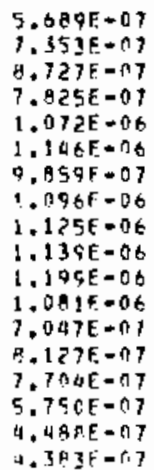 & $\begin{array}{l}1.079 E=06 \\
1.912 E=06 \\
2.269 E=06 \\
2.034 E=06 \\
3.788 E=06 \\
2.979 E=06 \\
2.563 E=06 \\
2.850 E=06 \\
2.926 E=06 \\
2.061 E=04 \\
3.116 E=06 \\
2.810 E=06 \\
1.832 E=06 \\
2.113 E=05 \\
2.003 E-06 \\
1.465 E=06 \\
1.167 E=06 \\
1.110 E=06\end{array}$ & $\begin{array}{l}1600 \\
1600 \\
1600 \\
1600 \\
1600 \\
1600 \\
1600 \\
1600 \\
1600 \\
1600 \\
1600 \\
1600 \\
1600 \\
1000 \\
1600 \\
1600 \\
1600 \\
1600\end{array}$ \\
\hline
\end{tabular}

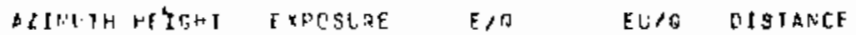
DEGREFS RETFRS GM-GEC/CI: N SEC/CU.N J/SO,N METERS
$\times 1 D E+6$

\begin{tabular}{|c|c|c|c|c|c|}
\hline 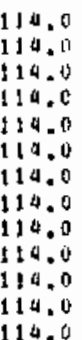 & $\begin{array}{l}3.1 \\
6.2 \\
9.3 \\
12.4 \\
15.5 \\
18.6 \\
21.7 \\
24.5 \\
31.0 \\
37.2 \\
43.9 \\
49.6 \\
55.8\end{array}$ & $\begin{array}{r}114 \\
92 \\
131 \\
89 \\
189 \\
260 \\
73 \\
13 \\
12 \\
74 \\
34 \\
10 \\
16\end{array}$ & $\begin{array}{l}6.124 E-08 \\
4.623 E=08 \\
6.55 E E-C 8 \\
4.755 E-08 \\
9.497 E-08 \\
1.303 E=07 \\
3.09 E F-08 \\
4.797 E-09 \\
6.042 E=09 \\
3.701 E-08 \\
1.737 E=08 \\
5.259 E-09 \\
0.181 E=09\end{array}$ & $\begin{array}{l}1.798 E=07 \\
1.202 E=07 \\
1.704 E=07 \\
1.152 E=07 \\
2.969 E=07 \\
3.387 E=07 \\
9.615 E=00 \\
1.767 E=08 \\
1.571 E=08 \\
9.622 E=08 \\
4.517 E=08 \\
1.367 E-08 \\
2.127 E=06\end{array}$ & $\begin{array}{l}1600 \\
1600 \\
1600 \\
1600 \\
1600 \\
1600 \\
1600 \\
1600 \\
1600 \\
1600 \\
1600 \\
1600 \\
1000\end{array}$ \\
\hline
\end{tabular}

ATINUTH HEIGHT EXPOSLIE EAR EUTG DISTARCE

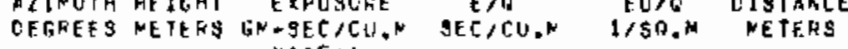
$x \perp$ OE +6

\begin{tabular}{|c|c|c|c|c|}
\hline $\begin{array}{l}122.0 \\
122.0 \\
122.0 \\
122.0 \\
122.0 \\
122.0 \\
122.0 \\
122.0 \\
122.0 \\
122.0 \\
122.0 \\
122.0 \\
122.0\end{array}$ & $\begin{array}{r}3.1 \\
6.2 \\
9.3 \\
12.9 \\
15.5 \\
18.6 \\
21.7 \\
24.4 \\
31.0 \\
37.2 \\
43.4 \\
49.6 \\
55.8\end{array}$ & $\begin{array}{l}55 \\
36 \\
53 \\
43 \\
79 \\
97 \\
26 \\
48 \\
24 \\
12 \\
34 \\
26 \\
26\end{array}$ & $\begin{array}{l}2.779 E=08 \\
1.949 E=08 \\
1.601 E=08 \\
2.185 E=08 \\
3.950 E=08 \\
4.371 E=08 \\
1.345 E=08 \\
2.917 E=08 \\
1.20 E E=08 \\
6.042 E=08 \\
1.737 E=08 \\
1.34 A E=08 \\
1.344 E=08\end{array}$ & $\begin{array}{l}7.211 E=00 \\
4.808 E=08 \\
4.371 E=08 \\
5.662 E=08 \\
1.027 E=07 \\
1.536 E=07 \\
3.496 E=08 \\
6.284 E=08 \\
3.142 E=08 \\
1.571 E=00 \\
4.517 E=00 \\
3.995 E-08 \\
3.495 E=08\end{array}$ \\
\hline
\end{tabular}

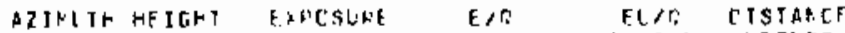

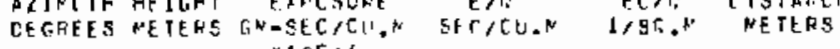

$\begin{array}{rr}130.0 & 3.1 \\ 130.0 & 6.2 \\ 130.0 & 12.0 \\ 130.0 & 15.5 \\ 130.0 & 16.6 \\ 130.0 & 21.7 \\ 130.0 & 24.0 \\ 130.0 & 31.0 \\ 130.0 & 49.6\end{array}$




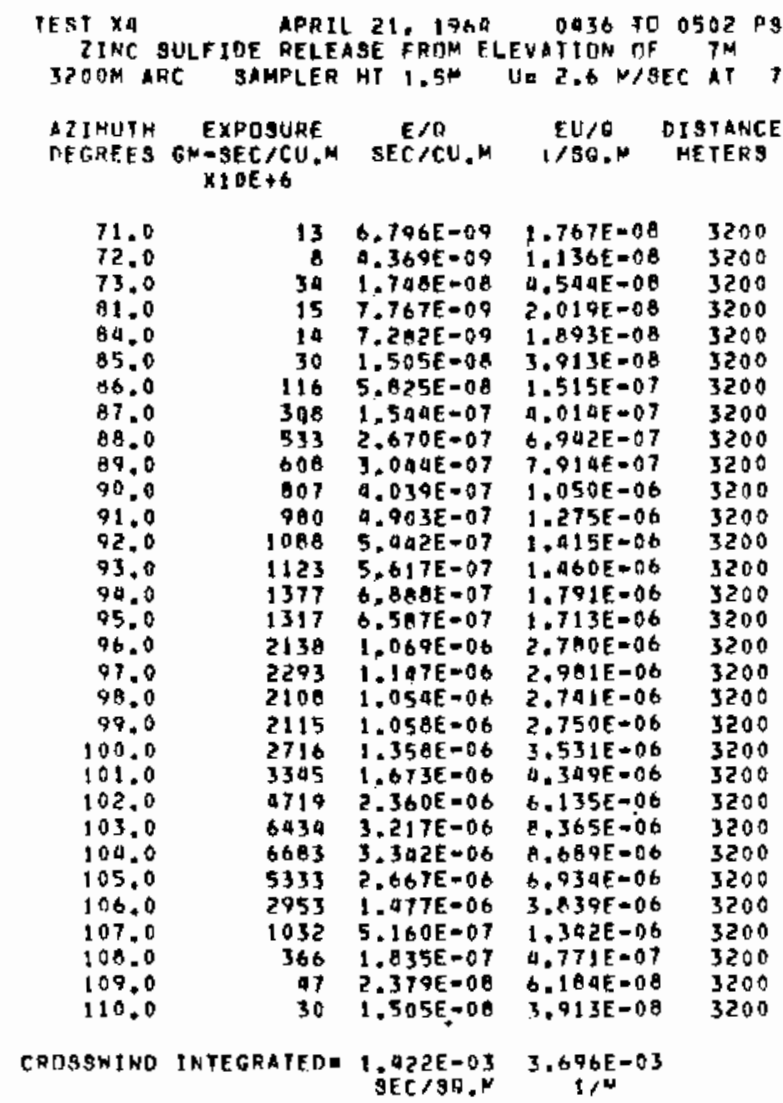

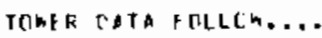

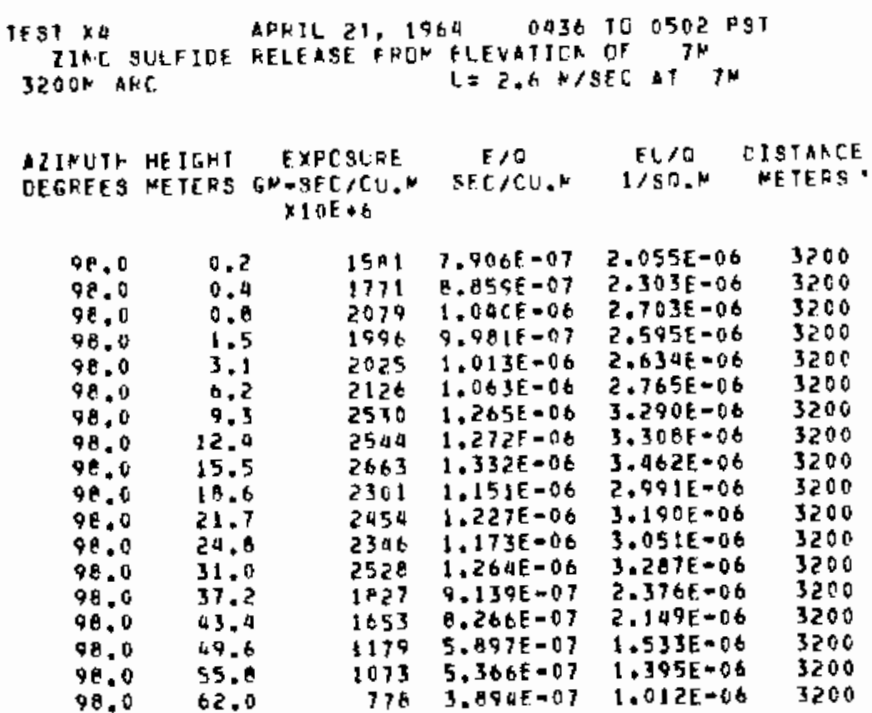

\begin{tabular}{|c|c|c|c|c|c|}
\hline $\begin{array}{l}\text { ARJKUTH } \\
\text { DEGHEES }\end{array}$ & $\begin{array}{l}\text { HEIGHI } \\
\text { METERS }\end{array}$ & $\begin{array}{c}\text { EXPOSLRE } \\
G \times-S E C / C U . N \\
\times 10 E+6\end{array}$ & $\begin{array}{c}E / 0 \\
\sec (⿻ / 2, *\end{array}$ & $\begin{array}{l}E U / 0 \\
1 / 50 .\end{array}$ & $\begin{array}{l}\text { DISTARCE } \\
\text { METERS }\end{array}$ \\
\hline $\begin{array}{l}106.0 \\
106.0 \\
106.0 \\
106.0 \\
106.0 \\
106.0 \\
106.0 \\
106.0 \\
106.0 \\
106.0 \\
106.0 \\
106.0 \\
106.0 \\
106.0 \\
106.0 \\
100.0 \\
106.0 \\
106.0\end{array}$ & $\begin{array}{l}0.2 \\
0.0 \\
0.6 \\
1.5 \\
3.1 \\
6.2 \\
9.3 \\
12.0 \\
15.5 \\
18.6 \\
21.7 \\
29.8 \\
31.0 \\
37.2 \\
43.1 \\
49.6 \\
55.8 \\
62.0\end{array}$ & $\begin{array}{l}2343 \\
3370 \\
3015 \\
2402 \\
3076 \\
3495 \\
4156 \\
4650 \\
4635 \\
4820 \\
5115 \\
4156 \\
4920 \\
4710 \\
3478 \\
3547 \\
2828 \\
674\end{array}$ & $\begin{array}{l}1.172 E=06 \\
1.685 E=06 \\
1.508 E=06 \\
1.341 E=06 \\
1.537 E=06 \\
1.741 E=06 \\
2.078 E=06 \\
2.325 E=06 \\
2.318 E=06 \\
2.410 E=06 \\
2.558 E=06 \\
2.078 E=06 \\
2.46 C E=06 \\
2.005 E=06 \\
1.739 E=06 \\
1.774 E=06 \\
1.449 E=06 \\
3.3714=07\end{array}$ & $\begin{array}{l}3.047 E=06 \\
4.381 E=06 \\
3.920 E=06 \\
3.488 E=06 \\
3.997 E=06 \\
4.531 E-06 \\
5.403 E=06 \\
6.04 A E=06 \\
6.026 E=06 \\
6.267 E=06 \\
6.650 E=06 \\
5.403 E=06 \\
6.397 E=06 \\
5.214 E=06 \\
4.522 E=06 \\
4.612 E-06 \\
3.755 E=06 \\
4.745 f=07\end{array}$ & $\begin{array}{l}3200 \\
3200 \\
3200 \\
3200 \\
3200 \\
3200 \\
3200 \\
3200 \\
3200 \\
3200 \\
3200 \\
3200 \\
3200 \\
3200 \\
3200 \\
3200 \\
3200 \\
3200\end{array}$ \\
\hline $\begin{array}{l}\text { ZINLIH } \\
\text { EGREES }\end{array}$ & $\begin{array}{l}\text { IIE IGHT } \\
\text { SIETERS }\end{array}$ & $\begin{array}{l}\text { EXPCSUAE } \\
\text { ON }=\text { SEC } / C L . K \\
\times 10 E+4\end{array}$ & $\begin{array}{c}E / G \\
S E C / C(1, N\end{array}$ & $\begin{array}{c}50 / 0 \\
1 / 50 .\end{array}$ & $\begin{array}{l}\text { DI STANCF } \\
\text { NETEFS }\end{array}$ \\
\hline $\begin{array}{l}114.0 \\
1140.0 \\
1110.0 \\
114.0 \\
114.0 \\
114.0 \\
114.0\end{array}$ & $\begin{array}{r}0.2 \\
0.4 \\
1.5 \\
1.9 \\
21.7 \\
37.2 \\
62.0\end{array}$ & $\begin{array}{l}4 \\
92 \\
18 \\
20 \\
23 \\
22 \\
26\end{array}$ & $\begin{array}{l}4.547 E=09 \\
4.027 E=08 \\
9.267 E=00 \\
1.019 E=08 \\
1.15 E E=06 \\
1.134 E=08 \\
1.335 E=08\end{array}$ & $\begin{array}{l}1.193 E=08 \\
1.047 E=07 \\
2.010 E=00 \\
2.050 E=08 \\
3.012 E=08 \\
2.944 E=00 \\
3.071 E=00\end{array}$ & $\begin{array}{l}3200 \\
3200 \\
3200 \\
3200 \\
3200 \\
3200 \\
3200\end{array}$ \\
\hline
\end{tabular}

AZINUTH HELGH EXPOSURE EFO ELIO DISTANCE

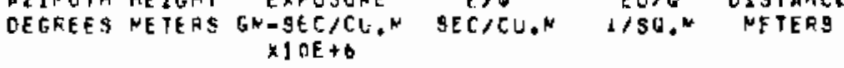

\begin{tabular}{|c|c|c|c|c|}
\hline $\begin{array}{l}122.0 \\
122.0 \\
122.0 \\
122.0 \\
122.0 \\
122.0 \\
122.0 \\
122.0 \\
122.0 \\
122.0 \\
122.0 \\
122.0 \\
122.0\end{array}$ & $\begin{array}{l}3.1 \\
6.2 \\
9.3 \\
12.0 \\
15.5 \\
18.6 \\
21.7 \\
24.8 \\
31.0 \\
37.2 \\
43.4 \\
55.8 \\
62.0\end{array}$ & $\begin{array}{l}23 \\
14 \\
18 \\
13 \\
18 \\
19 \\
13 \\
06 \\
20 \\
8 \\
14 \\
17 \\
28\end{array}$ & $\begin{array}{l}1.152 E=08 \\
7.414 E=09 \\
9,267 E=00 \\
1.66 F E=08 \\
9.267 E=09 \\
9.731 E=09 \\
6.951 E=08 \\
2.312 E=08 \\
1.047 E=08 \\
4.362 E=09 \\
7.416 E=09 \\
9.909 E=09 \\
1.409 E=08\end{array}$ & $\begin{array}{l}3.012 E=00 \\
1.92 B E=08 \\
2.410 E=08 \\
4.337 E=08 \\
2.410 E=00 \\
2.530 E=08 \\
1.007 E=08 \\
6.011 E=08 \\
2.722 E=08 \\
1.134 E=08 \\
1.928 E=08 \\
2.314 E=08 \\
3.664 E=08\end{array}$ \\
\hline
\end{tabular}


SECONDARY SERIES EXPERIMENT: XE-1

GROUNO LEYEL ANO TOMER SAMPLING 200-3200 ARCS. A SIGRIFICANT PORTION OF THE PLUHE NEYER PASSEO OVER THE GRID.

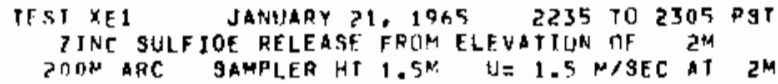

\begin{tabular}{|c|c|c|c|c|}
\hline $\begin{array}{l}\text { AZ JMUTH } \\
\text { DE ViHEFS }\end{array}$ & $\begin{array}{l}\text { EXPUSURE } \\
\text { GN-SEC } / C U . M \\
\times 1 O E+6\end{array}$ & $\begin{array}{c}E / Q \\
3 E C / C U . \mu\end{array}$ & 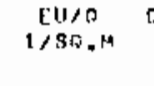 & $\begin{array}{l}\text { DISTANCE } \\
\text { NETER3 }\end{array}$ \\
\hline $\begin{array}{l}68.0 \\
70.0 \\
72.0 \\
79.0 \\
76.0 \\
72.0 \\
90.0 \\
82.0 \\
90.0 \\
86.0 \\
89.0 \\
90.0 \\
92.0 \\
94.0 \\
96.0 \\
98.0 \\
100.0 \\
102.0 \\
104.0 \\
106.0 \\
108.0 \\
110.0 \\
112.0 \\
114.0 \\
116.0\end{array}$ & $\begin{array}{l}196171 \\
618394 \\
701085 \\
538505 \\
345183 \\
190164 \\
135899 \\
85813 \\
49372 \\
43346 \\
48492 \\
43915 \\
52993 \\
59520 \\
37994 \\
16302 \\
94203 \\
117740 \\
120146 \\
101453 \\
107290 \\
74393 \\
35396 \\
15836 \\
1406\end{array}$ & $\begin{array}{l}1.691 E=00 \\
5.331 E=04 \\
6.094 E=04 \\
4.6 A 2 E=04 \\
2.976 E=04 \\
1.639 E=04 \\
1.172 E=04 \\
7.398 E=05 \\
4.256 E=05 \\
3.737 E=05 \\
9.120 E=05 \\
3.70 B E=05 \\
0.56 A E=05 \\
5.131 E=05 \\
3.275 E=05 \\
1.905 E=05 \\
8.171 E=05 \\
1.015 E=04 \\
1.036 E=04 \\
9.746 E=05 \\
9.249 E=05 \\
6.413 E=05 \\
3.051 E=05 \\
1.365 E=05 \\
1.290 E=06\end{array}$ & $\begin{array}{l}2.537 E=04 \\
7.996 E=04 \\
9.066 E=04 \\
6.963 E=04 \\
4.064 E=04 \\
2.459 E=04 \\
1.757 E=04 \\
1.110 E=04 \\
6.384 E=05 \\
5.605 E=05 \\
6.271 E=05 \\
5.562 F=05 \\
6.953 E=05 \\
7.697 E=05 \\
4.913 E=05 \\
2.108 E=05 \\
1.210 E=04 \\
1.523 E=04 \\
1.554 E=04 \\
1.312 E=04 \\
1.387 E=04 \\
9.620 E=05 \\
4.577 E=05 \\
2.048 E=05 \\
1.936 E=06\end{array}$ & $\begin{array}{l}200 \\
200 \\
200 \\
200 \\
200 \\
200 \\
200 \\
200 \\
200 \\
200 \\
200 \\
200 \\
200 \\
200 \\
200 \\
200 \\
200 \\
200 \\
200 \\
200 \\
200 \\
200 \\
200 \\
200 \\
200\end{array}$ \\
\hline
\end{tabular}

CROSEHIHO INTEGRATEDE $2.305 E-02 \quad 3.458 E-02$

\begin{tabular}{|c|c|c|c|c|c|}
\hline $\begin{array}{l}\text { A } 7 \text { INLITH } \\
\text { DEGREES }\end{array}$ & $\begin{array}{l}\text { KE IGHT } \\
\text { METERS }\end{array}$ & $\begin{array}{c}\text { EXPOSUAE } \\
\text { GIA-SEC CU,M } \\
\times 1 O E+6\end{array}$ & $\begin{array}{c}E / 0 \\
\sec \varepsilon / C U_{*} m\end{array}$ & $\begin{array}{c}E U / O \\
1 / S N . M\end{array}$ & $\begin{array}{l}\text { DISTANC } \\
\text { METERS }\end{array}$ \\
\hline $\begin{array}{l}106.0 \\
106.0 \\
106.0 \\
106.0 \\
106.0 \\
106.0 \\
106.0 \\
106.0 \\
106.0 \\
106.0 \\
106.0 \\
100.0 \\
106.0\end{array}$ & $\begin{array}{r}0.3 \\
0.7 \\
1.9 \\
2.7 \\
4.7 \\
5.4 \\
6.9 \\
1.1 \\
4.7 \\
10.8 \\
13.5 \\
16.2 \\
16.9\end{array}$ & $\begin{array}{r}80 R 96 \\
63235 \\
104034 \\
117095 \\
115391 \\
117470 \\
134154 \\
109707 \\
03953 \\
51058 \\
10166 \\
696 \\
158\end{array}$ & $\begin{array}{l}7.319 E-05 \\
5.451 E=05 \\
8.969 E=05 \\
1.004 E=00 \\
9.948 E=05 \\
1.016 F=04 \\
1.070 F=04 \\
9.406 F=05 \\
7.237 E=05 \\
9.402 E=05 \\
8.676 E=06 \\
6.002 E=07 \\
1.196 F=07\end{array}$ & $\begin{array}{l}1.098 E=04 \\
8.177 E-05 \\
1.345 E-04 \\
1.514 E=04 \\
1.492 E=04 \\
1.524 E=04 \\
1.605 E=04 \\
1.411 E=04 \\
1.066 E-04 \\
6.602 E=05 \\
1.302 E-05 \\
9.003 E=07 \\
1.79 a E=07\end{array}$ & $\begin{array}{l}200 \\
200 \\
200 \\
200 \\
200 \\
200 \\
200 \\
200 \\
200 \\
200 \\
200 \\
200 \\
200\end{array}$ \\
\hline
\end{tabular}

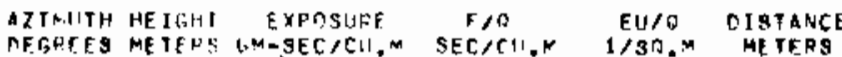
$\times 1 \cap F+h$

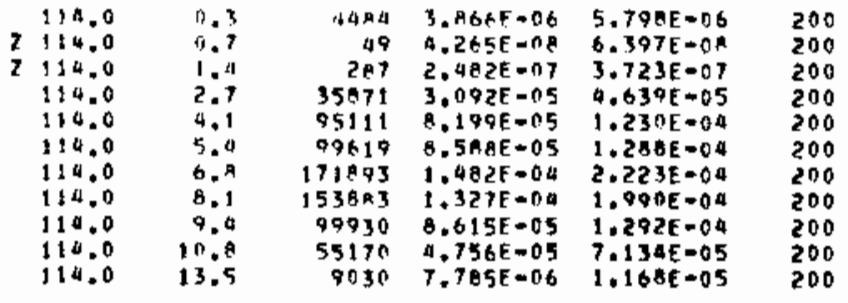

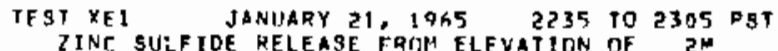
AOON ARC SAMPLER HT $1.5 \mathrm{~S}$ JE $1.5 \mathrm{M} / \mathrm{SEC}$ AT $2 \mathrm{M}$

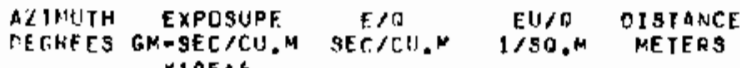
$\times 10 \mathrm{E}+6$

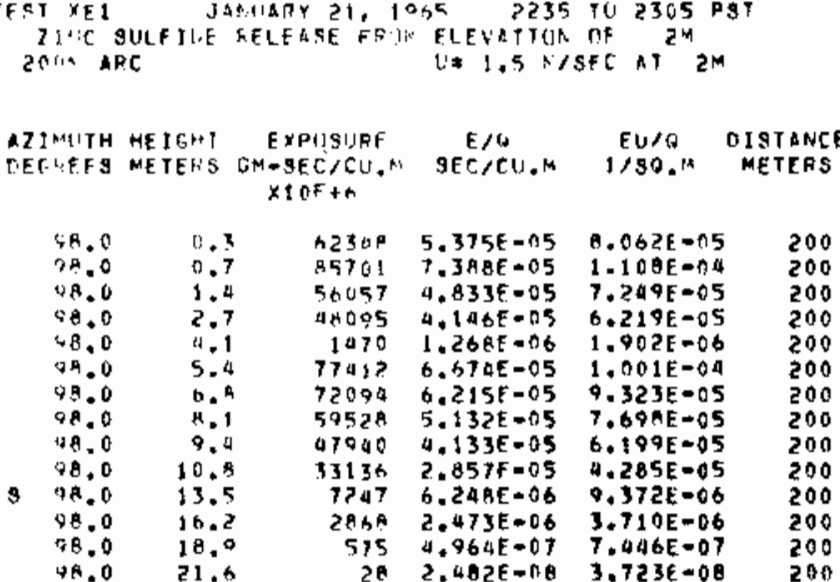

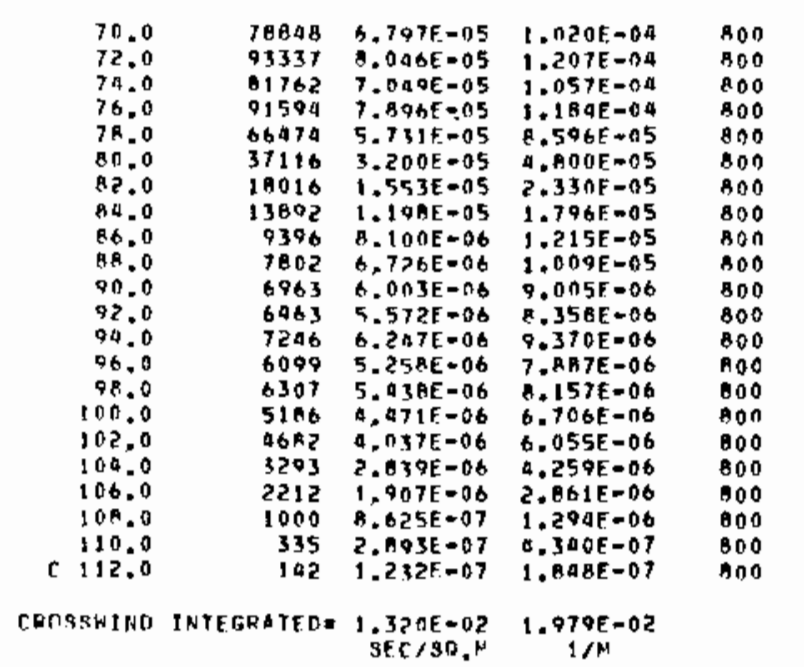


TOWEF TATAFOLLUW....

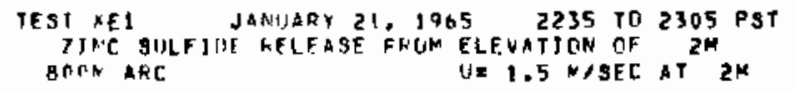

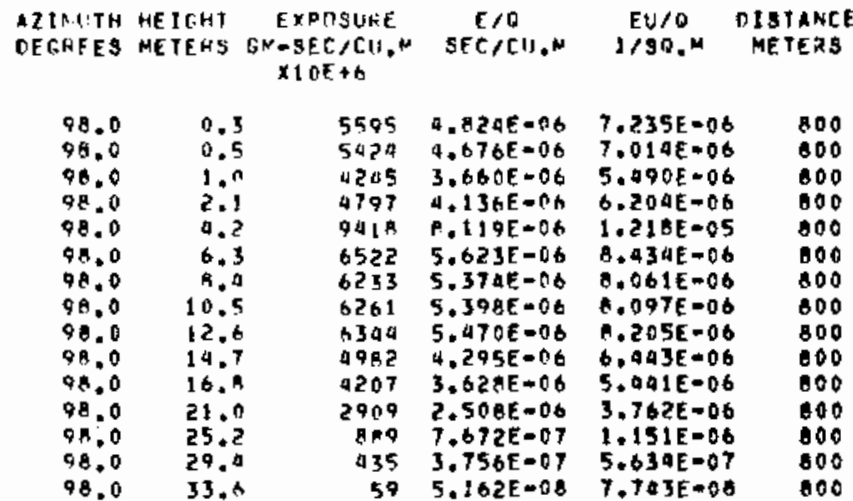

\begin{tabular}{|c|c|c|c|c|c|}
\hline $\begin{array}{l}\text { ALIMIITH } \\
\text { DEFREES }\end{array}$ & $\begin{array}{l}\text { HE IGH T } \\
\text { ME TERS }\end{array}$ & $\begin{array}{l}\text { EXPQSURE } \\
\text { GM-SEC } / C U, M \\
\text { XIOE } \$ O\end{array}$ & $\begin{array}{c}E / 0 \\
\mathrm{SEC} / \mathrm{CU}, \mathrm{M}\end{array}$ & $\begin{array}{c}E U / 0 \\
1 / \$ 0 . M\end{array}$ & $\begin{array}{l}\text { OISTRNCE } \\
\text { WFTERS }\end{array}$ \\
\hline $\begin{array}{l}106.0 \\
106.0 \\
100.0 \\
106.0 \\
106.0 \\
106.0 \\
164.0 \\
106.0 \\
106.0 \\
106.0 \\
106.0 \\
106.0 \\
106.0 \\
106.0 \\
106.0\end{array}$ & $\begin{array}{l}0.3 \\
0.5 \\
1.0 \\
2.1 \\
4.2 \\
6.3 \\
8.4 \\
10.5 \\
12.8 \\
10.7 \\
16.8 \\
21.0 \\
25.2 \\
29.4 \\
33.6\end{array}$ & $\begin{array}{r}309 \\
987 \\
1048 \\
1000 \\
3731 \\
5592 \\
8305 \\
6037 \\
0109 \\
5023 \\
4001 \\
000 \\
262 \\
152 \\
66\end{array}$ & $\begin{array}{l}7.750 E-07 \\
8.516 E-07 \\
9.036 E=07 \\
1.552 E=06 \\
3.217 E=06 \\
4.821 F=06 \\
7.238 E-06 \\
5.980 E=06 \\
6.991 E=06 \\
4.330 E-06 \\
3.527 E-06 \\
6.897 E-07 \\
2.201 E=07 \\
1.317 E=07 \\
5.696 E-08\end{array}$ & $\begin{array}{l}1.162 E=06 \\
1.277 E=06 \\
1.355 E=06 \\
2.328 E-06 \\
4.020 E=06 \\
7.232 E=06 \\
1.086 E-05 \\
8.071 E=06 \\
1.049 E=05 \\
6.096 E=06 \\
5.291 E=06 \\
1.035 E=06 \\
3.391 E=07 \\
1.976 E=07 \\
6.544 E=08\end{array}$ & $\begin{array}{l}000 \\
000 \\
800 \\
800 \\
000 \\
000 \\
800 \\
000 \\
000 \\
000 \\
000 \\
800 \\
800 \\
800 \\
800\end{array}$ \\
\hline
\end{tabular}

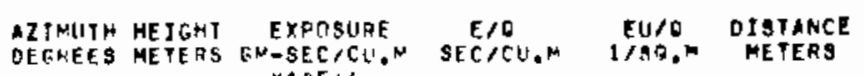

\begin{tabular}{|c|c|c|c|c|}
\hline $\begin{array}{l}114.0 \\
114.0 \\
114.00 \\
114.0 \\
114.0 \\
114.0 \\
114.0 \\
114.0 \\
114.0\end{array}$ & $\begin{array}{l}2.1 \\
4.2 \\
6.3 \\
0.8 \\
10.5 \\
12.6 \\
214.7 \\
16.8 \\
21.0\end{array}$ & $\begin{array}{r}37 \\
392 \\
724 \\
2220 \\
4811 \\
7289 \\
7119 \\
4582 \\
1010\end{array}$ & $\begin{array}{l}3.274 F=00 \\
3.383 E=07 \\
6.242 E=07 \\
1.914 E=06 \\
4.208 E=06 \\
6.284 E=06 \\
6.138 E=06 \\
3.933 E=06 \\
6.709 E=07\end{array}$ & $\begin{array}{l}4.911 E=06 \\
5.075 E=07 \\
9.364 E=07 \\
2.871 E=06 \\
6.312 E=06 \\
9.026 E=06 \\
9.206 E=06 \\
5.900 E=06 \\
1.306 E=06\end{array}$ \\
\hline
\end{tabular}

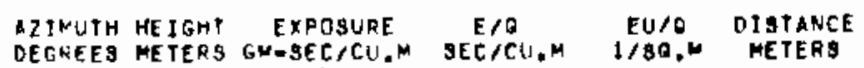
$10 E+6$

\begin{tabular}{|c|c|c|c|c|c|}
\hline $\begin{array}{l}122.0 \\
122.0 \\
122.0 \\
122.0 \\
122.0 \\
122.0\end{array}$ & $\begin{array}{r}0.3 \\
1.0 \\
2.1 \\
4.2 \\
6.3 \\
0.4 \\
20.4\end{array}$ & $\begin{array}{l}181 \\
17 \\
27 \\
53 \\
90 \\
17 \\
24\end{array}$ & $\begin{array}{l}1.562 E=07 \\
1.52 B E=08 \\
2.401 E=08 \\
0.584 E=08 \\
8.731 E=09 \\
1.52 E E=08 \\
2.136 E=08\end{array}$ & $\begin{array}{l}2.343 E=07 \\
2.292 E-08 \\
1.601 E=08 \\
6.075 E=08 \\
1.310 E=08 \\
7.292 E=08 \\
3.204 E=08\end{array}$ & $\begin{array}{l}000 \\
800 \\
000 \\
000 \\
000 \\
000 \\
000\end{array}$ \\
\hline
\end{tabular}

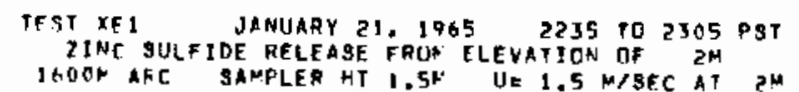
DIFUTH EXPOSURE
DECHEFS GNOSECICU.M SEC/CU.M I/SO.M NISTANEE $X \mathbb{O O E} \rightarrow b$

10.0

71.0

$P 73.0$

p 70.0

P 75.0

76.0

77.0

$7 \mathrm{R} .0$

79.0

00.0

81.0

83,0

84.0

85.0

B. 0

8. 0

36.0

89.0

P 9.0

9.0

95.0

96.0

97.0

9.0

99.0

100,0

101.0

102.0

103.0

100.0

105.0

106,0

101.0

108.0

108.0
109.0

110.0

111.0

112.0

$1: 3.0$

119.0

115.0

117.0

20,

121.0

122.0
130.0

2

$017.033 E-00 \quad 1.055 E=07 \quad 1600$

$225 \quad 1.943 E-07 \quad 2.915 E-07$

$55 \quad 9.612 E=08 \quad 7.218 F-08$

47 A.DTPE=0B 6.107E-08 1600

$156 \quad 1.351 \mathrm{E}-07 \quad 2.027 \mathrm{~F}-07 \quad 1600$

103 O.ABE-OB $1.332 E-07$
11310000

$104420.0025-06 \quad 1.3505-05$

$10055 \quad 0.669 E-16 \quad 1.300 E-05 \quad 1600$

711 6.648E-OQ $9.912 E-06 \quad 1600$

$3140 \quad 6.155 E=06 \quad 9.233 E=06 \quad 1600$

$6925 \quad 5.970 E-06 \quad 8.955 E-06 \quad 1600$

$5545 \quad 4.7 \mathrm{AOE}=06 \quad 7.171 \mathrm{EE}=06 \quad 1600$

54as $0.697 \mathrm{E}-06 \quad 7.096 \mathrm{E}-06 \quad 1600$

$3677 \quad 3.342 E-06 \quad 5.014 E=06 \quad 1600$

$3740 \quad 3,228 E-06 \quad 4.841 E=06 \quad 1600$

2756 2,376E-06 3,564E-06 1600

$2535 \quad 2.186 E=06 \quad 3.279 E=06 \quad 1600$

$2460 \quad 2.121 E=06 \quad 3.1$ A1E $=06 \quad 1600$

1957 1.6BAE-06 2.532E-06 1600

$21 \quad 1.851 \mathrm{E}-0 \mathrm{~B} \quad 2.776 \mathrm{E}=08 \quad 1600$

$197 \quad 7,736 \mathrm{E}-07 \quad 1.160 \mathrm{E}-06 \quad 1600$

$1021 \quad 0,809 E-07 \quad 1,321 E-06 \quad 1600$

991 . $550 E-07 \quad 1.283 E-06 \quad 1600$

$1017 \quad 0.772 E-07 \quad 1.3165-06 \quad 1600$

$6976.015 E=07$ 9.022E-07 1600

$903 \quad 7.791 \mathrm{E}-07 \quad 1.169 \mathrm{E}-06 \quad 1600$

$837 \quad 7.21 A E-07 \quad 1.083 E=06 \quad 1600$

6 5.5HQE-07 H.3A4E-07 1600

661 5,700E-07 $0.550 E=07 \quad 1600$

562 H. $949 E-07$ T.273E-07 1600

$652 \quad 5.626 E-07$ B. $039 E-07 \quad 1600$

$751 \quad 6.477 E-07 \quad 9.716 E-07 \quad 1600$

699 $5.941 E=07 \quad 8.911 E=07 \quad 1600$

$5474.719 E-07 \quad 7.079 E-07 \quad 1600$

485 4.1A3E-07 6.27AE-07 1600

392 3.3A7E-07 5.080E-17 1000

$212 \quad 2.350 E=07 \quad 3.526 E-07 \quad 1000$

$339 \quad 7.924 E=07 \quad 0.386 E-07 \quad 1600$

161 1. 3RAE-07 2.082E-07 1500

B5 7.AतSE=0 $1.110 E=07$ 1600

$55 \quad 0.012 \mathrm{E}=08 \quad 7.218 \mathrm{~F}=08 \quad 1600$

$45 \quad 3.8 B 6 F-0 R \quad 5.830 E-18 \quad 1600$

$64 \quad 5.552 E-08 \quad 6.326 E=08 \quad 1600$

27 ?. $006 E-0$ B $3.6095-08 \quad 1600$

$30>.501 E-08$ 3.AAGE-0E 1500

$23 \quad 2.036 \mathrm{E}-98 \quad 3.054 \mathrm{AE}-08 \quad 1600$

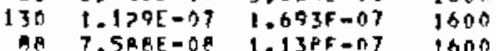

CRISSMIAO INTEGAATED= P.397E-133 3.596E=03 
TOWFR DATA FOLILW, .

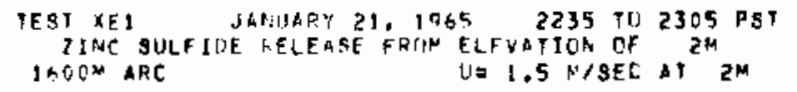

\begin{tabular}{|c|c|c|c|c|c|}
\hline $\begin{array}{l}2 T^{\circ} \text { UUTH } \\
\text { ESPEES }\end{array}$ & $\begin{array}{l}\text { HE IGIT } \\
\text { METE FS }\end{array}$ & $\begin{array}{c}\text { EXPQSLRE } \\
64=9 E C / C 11 . M \\
\times 10 E+6\end{array}$ & $\begin{array}{c}F / B \\
S E C / C, N, N\end{array}$ & $\begin{array}{c}E U / O \\
1 / 30, M\end{array}$ & $\begin{array}{l}\text { STANCE } \\
\text { ETERS }\end{array}$ \\
\hline $\begin{array}{l}48.0 \\
98.0 \\
98.0 \\
48.0 \\
98.0 \\
98.0 \\
98.0 \\
98.0 \\
98.0 \\
48.0 \\
98.0 \\
98.0 \\
08.0 \\
98.0 \\
98.0 \\
98.0\end{array}$ & $\begin{array}{l}0.2 \\
0.4 \\
0.9 \\
1.5 \\
3.1 \\
6.2 \\
4.3 \\
12.4 \\
15.5 \\
18.6 \\
21.7 \\
24.8 \\
31.0 \\
37.2 \\
43.4 \\
47.6\end{array}$ & $\begin{array}{l}703 \\
834 \\
750 \\
507 \\
766 \\
1300 \\
1205 \\
2152 \\
1610 \\
1629 \\
1593 \\
966 \\
363 \\
227 \\
51 \\
33\end{array}$ & $\begin{array}{l}6.063 E=07 \\
7.190 E=07 \\
6.607 E=07 \\
5.154 E=07 \\
6.604 E=07 \\
1.171 E=06 \\
1.030 E=06 \\
1.856 E=06 \\
1.398 E=06 \\
1.405 E=06 \\
1.373 E=08 \\
8.331 E=07 \\
3.13 B E=07 \\
1.958 E=07 \\
4.443 E=08 \\
2.915 E=08\end{array}$ & $\begin{array}{l}9.094 E=07 \\
1.07 B E+06 \\
9.910 E=07 \\
7.732 E=07 \\
9.906 E=07 \\
1.682 E=06 \\
1.559 E=06 \\
2.784 E=06 \\
2.082 E=06 \\
2.107 E=06 \\
2.060 E=06 \\
1.250 E=06 \\
9.707 E-07 \\
2.937 E=07 \\
6.665 E-06 \\
0.373 E-08\end{array}$ & $\begin{array}{l}1600 \\
1600 \\
1600 \\
1600 \\
1600 \\
1600 \\
1600 \\
1600 \\
1600 \\
1600 \\
1600 \\
1600 \\
1600 \\
1600 \\
1600 \\
1600\end{array}$ \\
\hline
\end{tabular}

DZTHITH HEIGHT EXPOSURE E/O EU/R DIBTANCE DEFPFES METERS GMASEC/CI1.4 SEC/CU,M 1/SR.M METERS

\begin{tabular}{|c|c|c|c|c|c|}
\hline $\begin{array}{l}160.0 \\
106.0 \\
106.0 \\
106.0 \\
106.0 \\
106.0 \\
106.0 \\
106.0 \\
106.0 \\
106.0 \\
106.0 \\
106.0 \\
106.0 \\
106.0 \\
100.0\end{array}$ & $\begin{array}{l}0.2 \\
0.4 \\
0.7 \\
1.5 \\
3.1 \\
6.7 \\
9.3 \\
12.4 \\
15.5 \\
18.0 \\
21.7 \\
24.8 \\
31.0 \\
37.2 \\
43.2\end{array}$ & $\begin{array}{r}739 \\
547 \\
519 \\
322 \\
529 \\
657 \\
1151 \\
1289 \\
741 \\
653 \\
617 \\
563 \\
252 \\
98 \\
49\end{array}$ & $\begin{array}{l}6.374 E-07 \\
4.722 E=07 \\
4.35 \% E=07 \\
2.7 A 3 E=07 \\
4.562 E=07 \\
5.665 E-07 \\
9.930 E-07 \\
1.112 E=06 \\
6.390 E-07 \\
5.632 F=07 \\
5.319 E-07 \\
4.860 E=07 \\
2.130 E-07 \\
8.470 E-0 E \\
4.165 F=08\end{array}$ & 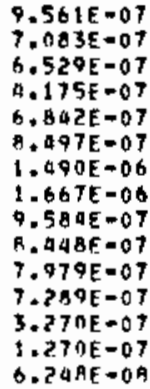 & $\begin{array}{l}1600 \\
1600 \\
1600 \\
1600 \\
1600 \\
1600 \\
1600 \\
1600 \\
1600 \\
1600 \\
1600 \\
1600 \\
1600 \\
1600 \\
1000\end{array}$ \\
\hline
\end{tabular}

MIIRE:TH HEIGHT EXPNSUAE E/Q EU/O OISTANCE DETHEES METERS GM-SEC/CU.M SEC/Cl., I/GR,M HETERS $\times 10 E+6$

$\begin{array}{ll}114.0 & 0.2 \\ 114.0 & 0.0 \\ 114.0 & 0.7 \\ 114.0 & 1.5 \\ 114.0 & 3.1 \\ 114.0 & 6.7 \\ 110.0 & 9.3 \\ 114.0 & 12.4 \\ 114.0 & 15.5 \\ 114.0 & 14.6 \\ 114.0 & 21.7 \\ 119.0 & 24.8 \\ 114.0 & 31.0 \\ 114.0 & 37.2\end{array}$

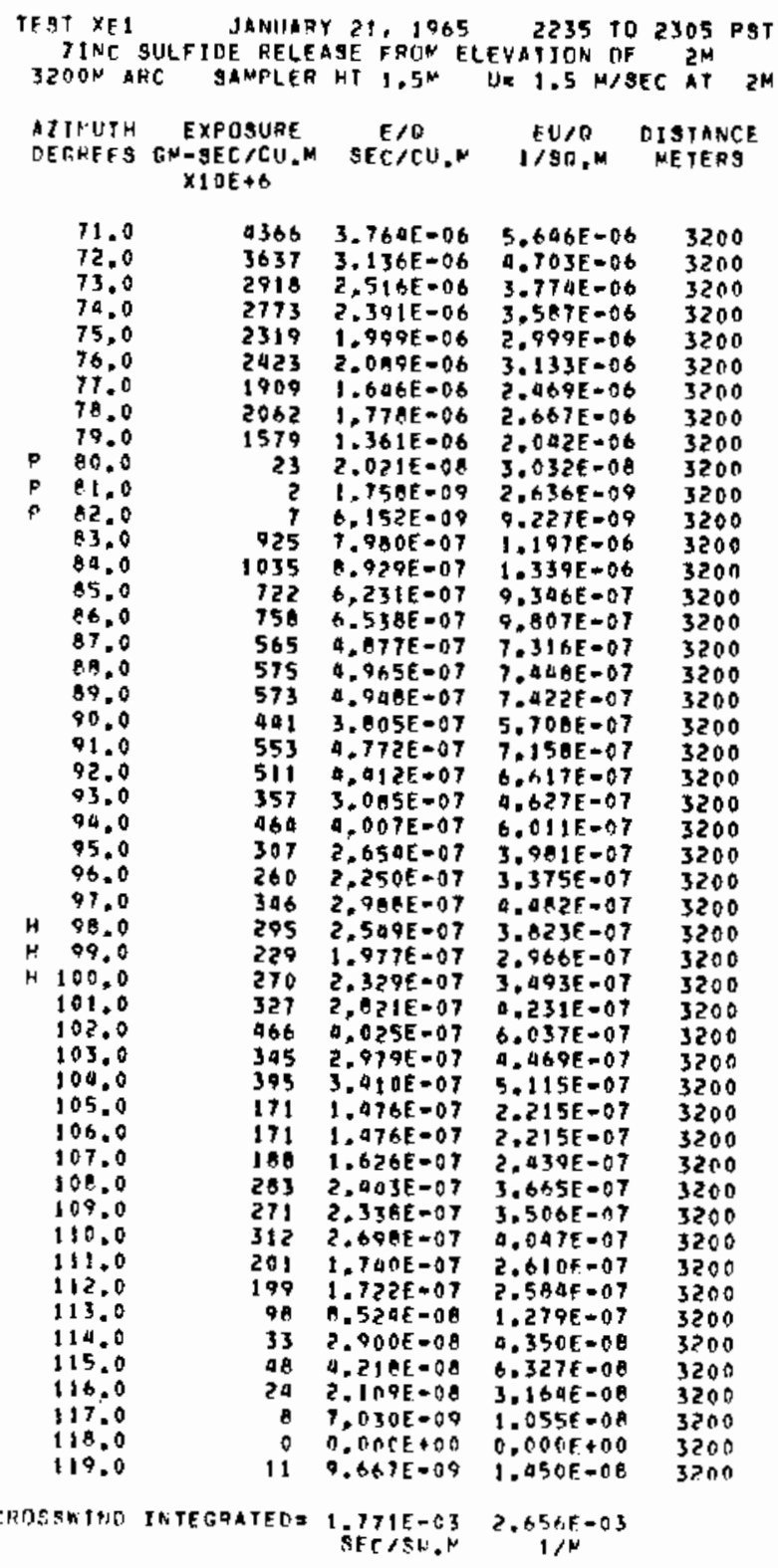

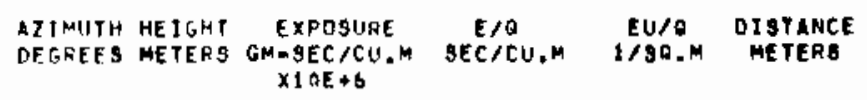

$\begin{array}{rrrrrr}122.0 & 0.2 & 39 & 3.303 E-00 & 4.955 E-08 & 1600 \\ 122.0 & 0.9 & 74 & 6.913 E=08 & 9.619 E=08 & 1600 \\ 122.0 & 9.3 & 19 & 1.647 E-08 & 2.470 E-08 & 1600 \\ 122.0 & 12.4 & 19 & 1.667 E-00 & 2.470 E-00 & 1600\end{array}$


TOMER DATA FOLLOH....

\begin{tabular}{|c|c|c|c|c|c|}
\hline $\begin{array}{l}\text { TESI XEL } \\
\text { TI NAE } \\
\text { 3200N" }\end{array}$ & Sth & $\begin{array}{l}\text { NUARY } 21 \text {. } \\
\text { RFLEASE FR }\end{array}$ & $\begin{array}{l}65 \text { ELEVATION } \\
\text { U. } 1.5 \text { W }\end{array}$ & 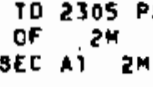 & \\
\hline $\begin{array}{l}\text { AZIMUTH } \\
\text { DEGREES }\end{array}$ & $\begin{array}{l}\text { HE IGNT } \\
\text { METERS }\end{array}$ & $\begin{array}{c}\text { EXP NSURE } \\
\text { GH-SEC } / C U . M \\
\text { XODE }+6\end{array}$ & $\begin{array}{c}E>0 \\
S E C / C L, H\end{array}$ & $\begin{array}{l}F U / 0 \\
1 / 90 . M\end{array}$ & $\begin{array}{l}\text { DISTANCE } \\
\text { METERS }\end{array}$ \\
\hline $\begin{array}{l}9.00 \\
98.0 \\
90.0 \\
98.0 \\
98.0 \\
98.0 \\
90.0 \\
98.0 \\
90.0 \\
98.0 \\
98.0 \\
48.0 \\
98.0 \\
98.0 \\
98.0 \\
98.0 \\
98.0 \\
98.0\end{array}$ & $\begin{array}{l}0.2 \\
0.0 \\
0.0 \\
1.5 \\
3.1 \\
6.2 \\
9.0 \\
12.5 \\
15.5 \\
18.6 \\
21.7 \\
24.0 \\
31.0 \\
37.2 \\
43.0 \\
49.8 \\
55.2 \\
62.0\end{array}$ & $\begin{array}{l}211 \\
231 \\
164 \\
281 \\
337 \\
523 \\
666 \\
726 \\
973 \\
961 \\
607 \\
603 \\
676 \\
559 \\
454 \\
162 \\
100 \\
101\end{array}$ & $\begin{array}{l}1.821 E=07 \\
1.995 E=07 \\
1.415 E-07 \\
2.427 E=07 \\
2.913 E-07 \\
4.511 E=07 \\
5.742 E=07 \\
6.260 E=07 \\
7.533 E=07 \\
0.266 E=07 \\
6.964 E=07 \\
5.896 E-07 \\
5.032 E-07 \\
4.025 E=07 \\
3.955 F=07 \\
1.403 E=07 \\
6.910 E=08 \\
8.757 F=08\end{array}$ & $\begin{array}{l}2.821 E=07 \\
2.992 E=07 \\
2.122 E=07 \\
3.641 E=07 \\
4.369 E=07 \\
6.767 E=07 \\
0.612 E=07 \\
9.391 E=07 \\
1.130 E=06 \\
1.243 E=06 \\
1.045 E=06 \\
1.044 E=07 \\
0.748 E=07 \\
7.238 E-07 \\
5.932 E=07 \\
2.109 E=07 \\
1.037 E=07 \\
1.319 E=07\end{array}$ & $\begin{array}{l}3200 \\
3200 \\
3200 \\
3200 \\
3200 \\
3200 \\
3200 \\
3200 \\
3200 \\
3200 \\
3200 \\
3200 \\
3200 \\
3200 \\
3200 \\
3200 \\
3200 \\
3200\end{array}$ \\
\hline $\begin{array}{l}\text { AZIMUTH } \\
\text { DEGHEE S }\end{array}$ & $\begin{array}{l}\text { HE IGHT } \\
\text { METERS }\end{array}$ & $\begin{array}{c}\text { EXPRSUPE } \\
G M=-S E C / C \cup . N \\
\times 1 O E+b\end{array}$ & $\begin{array}{c}E / \sigma \\
\text { gEC/RU, }\end{array}$ & $\underset{1 / S O, M}{E U / O}$ & $\begin{array}{c}\text { DISTANEE } \\
\text { METERS }\end{array}$ \\
\hline $\begin{array}{l}106.0 \\
106.0 \\
106.0 \\
106.0 \\
106.0 \\
106.0 \\
106.0 \\
106.0 \\
106.0 \\
106.0 \\
106.0 \\
0106.0 \\
Q 106.0 \\
0108.0\end{array}$ & $\begin{array}{l}3.1 \\
6.2 \\
9.3 \\
12.5 \\
15.5 \\
18.6 \\
21.7 \\
24.8 \\
31.0 \\
37.2 \\
43.8 \\
49.8 \\
55.9 \\
62.0\end{array}$ & $\begin{array}{r}99 \\
214 \\
553 \\
519 \\
347 \\
502 \\
46 \\
538 \\
569 \\
527 \\
04 \\
166 \\
61 \\
30\end{array}$ & $\begin{array}{l}8.621 E=08 \\
1.850 E=07 \\
4.771 E=07 \\
4.478 E-07 \\
2.996 E=07 \\
4.335 E=07 \\
1.449 E-08 \\
4.642 E-07 \\
4.905 E=07 \\
4.546 E=07 \\
3.635 E=08 \\
1.437 E-07 \\
5.26 E F=08 \\
2.600 E=08\end{array}$ & $\begin{array}{l}1.293 E-07 \\
2.775 E=07 \\
7.156 E=07 \\
6.717 E=07 \\
0.494 E=07 \\
6.503 E=07 \\
1.117 E=07 \\
6.963 E=07 \\
7.35 E E=07 \\
6.019 E=07 \\
5.752 E=00 \\
2.155 E=07 \\
7.902 E=0 . \\
3.900 E=08\end{array}$ & $\begin{array}{l}3200 \\
3200 \\
3200 \\
3200 \\
3200 \\
3200 \\
3200 \\
3200 \\
3200 \\
3200 \\
3200 \\
3200 \\
3200 \\
3200\end{array}$ \\
\hline
\end{tabular}

DZINUTH HEIGHT EXPOSURE E/Q EUTO OISTANCE DECREES METEFS GM-SEC/CI1.M SEC/CII.M I/SO.M METERS तi 1 if $\rightarrow$ A

\begin{tabular}{|c|c|c|c|c|c|}
\hline $\begin{array}{l}114.0 \\
110.0 \\
114.0 \\
114.0 \\
114.0 \\
114.0 \\
114.0 \\
110.0 \\
114.0 \\
114.0 \\
114.0 \\
1124.0 \\
119.0 \\
114.0 \\
110.0 \\
114.0 \\
114.0 \\
119.0\end{array}$ & $\begin{array}{r}0.7 \\
0.7 \\
0.0 \\
1.5 \\
3.1 \\
6.2 \\
9.3 \\
12.0 \\
15.5 \\
18.6 \\
21.7 \\
24.5 \\
31.0 \\
37.2 \\
93.4 \\
09.6 \\
55.8 \\
62.0\end{array}$ & $\begin{array}{l}54 \\
63 \\
39 \\
63 \\
62 \\
85 \\
191 \\
162 \\
234 \\
159 \\
13 \\
85 \\
47 \\
48 \\
105 \\
81 \\
23 \\
20\end{array}$ & $\begin{array}{l}4.65 A E=0 A \\
5.449 E=08 \\
3.427 F=08 \\
5.440 E=0 B \\
5.357 E=08 \\
7.365 E=08 \\
1.214 E=07 \\
1.398 E=07 \\
2.050 E-07 \\
1.373 E=07 \\
1.172 E=08 \\
7.590 E=08 \\
4.074 E=08 \\
4.154 E=08 \\
4.100 E=08 \\
7.047 E=08 \\
2.052 E=08 \\
1.779 E=08\end{array}$ & $\begin{array}{l}6.9 B 7 E=0 B \\
R .173 E=08 \\
5.141 E=08 \\
8.160 E=08 \\
8.035 E=08 \\
1.105 E=07 \\
1.820 E=07 \\
2.097 E=07 \\
3.08 B E=07 \\
2.059 E=07 \\
1.758 E=08 \\
1.13 B E=07 \\
6.112 E=08 \\
6.232 E=00 \\
1.366 E=07 \\
1.057 E=07 \\
3.079 E=0 B \\
2.66 B E-0 B\end{array}$ & $\begin{array}{l}3200 \\
3200 \\
3200 \\
3200 \\
3200 \\
3200 \\
3200 \\
3200 \\
3200 \\
3200 \\
3200 \\
3200 \\
3200 \\
3200 \\
3200 \\
3200 \\
3200 \\
3200\end{array}$ \\
\hline
\end{tabular}


SECONDARY SERIES EXPERIMENT: $X E-2$

GROUND LEYEL SAMPLING 200-3200 M ARCS.

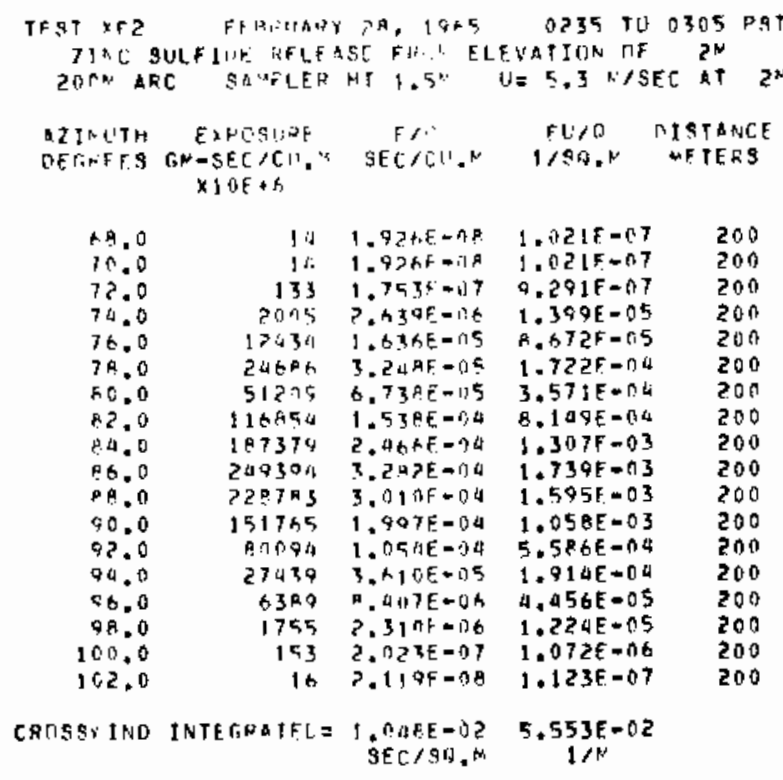

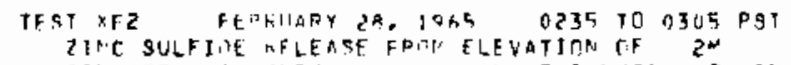

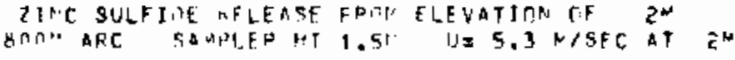

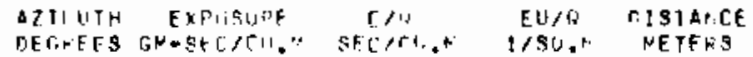

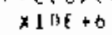

\begin{tabular}{|c|c|c|c|c|}
\hline 74.0 & c & C. D ir $6 E+19$ ) & $0.0006+00$ & 900 \\
\hline 76.0 & 56 & $7.45 \mathrm{AF}=.7 \mathrm{H}$ & $3.551 \%-0.7$ & 100 \\
\hline $7 \pi .0$ & 1151 & $1.515 F=16$ & $8.030 F=06$ & BeO \\
\hline 20.0 & $35 ? 8$ & $4.65+6 F=10 A$ & $2.461 \mathrm{~F}=05$ & 800 \\
\hline 82.0 & $9 \times 53$ & $.270 E=05$ & $0.732 F-0.5$ & 800 \\
\hline 50.0 & 25994 & $3.157 F=05$ & $1.673 E=04$ & 000 \\
\hline 16.0 & 23599 & 3.1 a $\triangle E=05$ & $1.660 E-04$ & 600 \\
\hline AP.O & 10507 & $1.3 \times 3 E=0.5$ & $327 E-05$ & $A_{00}$ \\
\hline .0 & $46 ? 1$ & $O A 1 E=06$ & $25 E-05$ & 800 \\
\hline$\$ 2,0$ & 1065 & $1.491 E-1.6$ & $7.427 E-06$ & 800 \\
\hline & 273 & $? .9 a d E=07$ & $1.56 \pm E=00$ & 800 \\
\hline
\end{tabular}

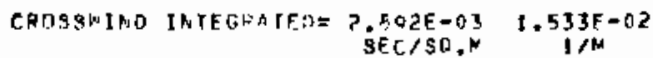

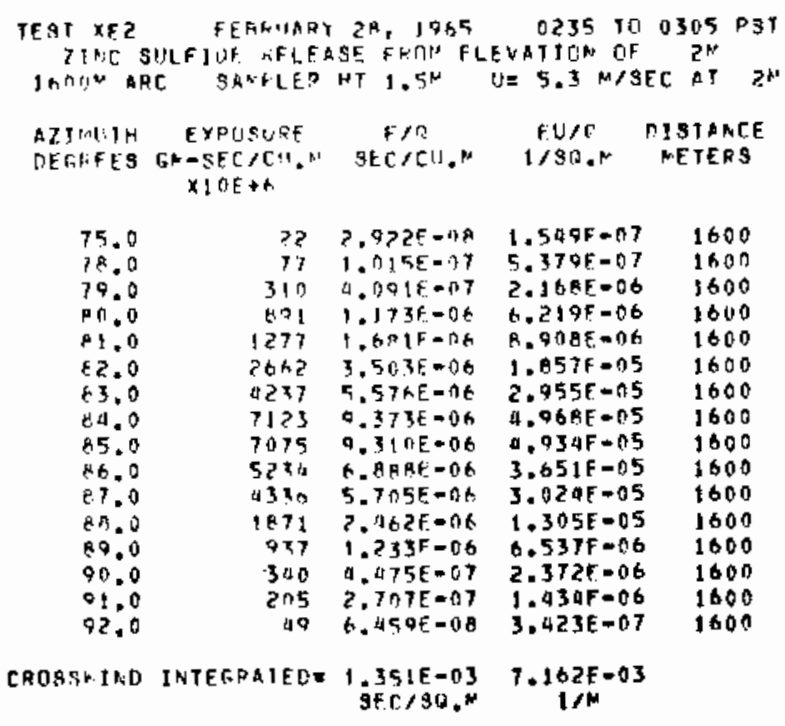

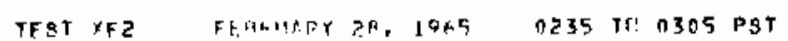

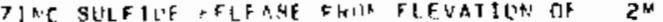

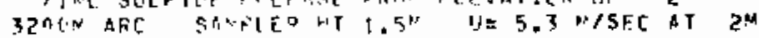

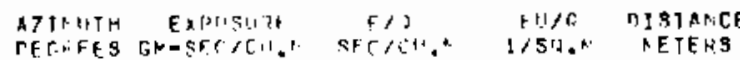
XIAE \&

\begin{tabular}{|c|c|c|c|c|}
\hline $\begin{array}{l}0.0 \\
0.0\end{array}$ & $\begin{array}{ll}1 & 3 t . \\
0 & 105\end{array}$ & $\begin{array}{l}\text { 1. } 78 \mathrm{AH}=01 \% \\
1.9725=0 \mathrm{~A}\end{array}$ & $\begin{array}{l}0.59 B F=07 \\
5.6 A O F=06\end{array}$ & $\begin{array}{l}350,19 \\
3>00\end{array}$ \\
\hline$P 2.0$ & ?4दी & $3.311 E=00$ & $1.702 F=0.5$ & 3200 \\
\hline 5.0 & $459 i_{11}$ & $\therefore$. U a $5 F=0 t$ & $\begin{array}{l}04 E-05 \\
84 F-05\end{array}$ & $\begin{array}{l}3200 \\
3200\end{array}$ \\
\hline 85.0 & 36 t. & औ. คว & $556 E-65$ & 3200 \\
\hline 56,0 & $3 i \cap 3$ & 2.63 the $=16$ & $97 E=05$ & 3200 \\
\hline 2.7 .0 & 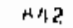 & $1.1 \times 9[-n 6$ & $976 E-06$ & 3200 \\
\hline 98.0 & 3 ก5 & $4.071 \varepsilon=07$ & $1315-06$ & 3200 \\
\hline 89.0 & a? & $023 E-n 7$ & $2 E=0 ?$ & 0 \\
\hline & र९ & $173 F-39$ & $+2 E=07$ & 20 \\
\hline
\end{tabular}

CRESS: TOO INTEGRAIELI = $1.475 F-037.9165-03$ 


\begin{tabular}{ll} 
Xenon Data for Xe-2 \\
Position* & $\frac{E / Q}{}$ \\
\hline $1-080$ & $7.78-5$ \\
$1-090$ & $1.95-4$ \\
$1-100$ & $3.02-7$ \\
$3-070$ & $2.33-8$ \\
$3-080$ & $2.19-5$ \\
$3-090$ & $7.67-7$
\end{tabular}

*Stable grid 
SBCONDARY SERIES EXPERIHENT: XR-3

ZIRC SULFIDE AMD FLJOROSCEIN WERE BOTE OSRD AS TRACBRS DUEIKO EXPBPIGBAT: IE-3. ZIRC PLUORIDE RESOLTS ABE PRESERTED HERE. GROOUD LEVEL AMD TOUER 3AHPLING 200-1600 M ARCS.

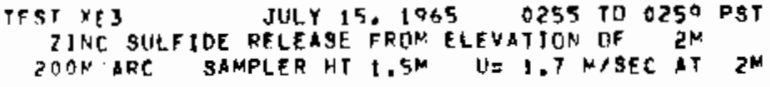

200

CPISOWIND INTEGRATFDE 1.81 aE -02 $3,084 F-02$
SEC $190 . \mathrm{M}$

TURFF BATA FrILLIIT....

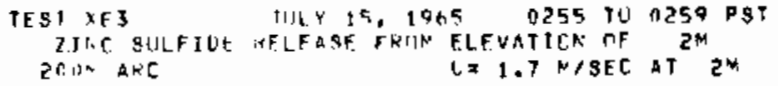

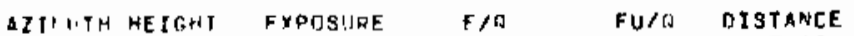

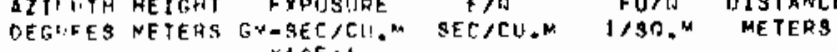
$\times 10 \mathrm{E}+6$

\begin{tabular}{|c|c|c|c|c|}
\hline $\begin{array}{l}98.0 \\
9.0 \\
48.0 \\
98.0 \\
9.00 \\
98.0 \\
98.0 \\
98.0 \\
98.0 \\
98.0 \\
48.0 \\
98.0 \\
9 R .0 \\
9 R .0\end{array}$ & $\begin{array}{l}0.3 \\
0.7 \\
1.4 \\
2.7 \\
4.1 \\
5.7 \\
6.9 \\
13.1 \\
9.4 \\
10.9 \\
13.5 \\
16.2 \\
15.9 \\
21.6\end{array}$ & $\begin{array}{r}121 \\
141 \\
23 \\
61 \\
33 \\
20 \\
31 \\
29 \\
23 \\
11 \\
08 \\
17 \\
19 \\
6\end{array}$ & $\begin{array}{l}5.968 E=07 \\
7.957 E=07 \\
1.174 E=07 \\
3.010 E=07 \\
1.652 E=07 \\
1.028 E=07 \\
1.541 E=07 \\
1.431 E=07 \\
1.17 a E=07 \\
5.505 E=08 \\
2.386 E=07 \\
8.808 E=08 \\
6.973 E=08 \\
3.303 E=08\end{array}$ & $\begin{array}{l}1.015 E=06 \\
1.353 E=06 \\
1.997 E=07 \\
5.116 E=07 \\
2.008 E=07 \\
1.747 E=07 \\
2.620 E=07 \\
2.435 E=07 \\
1.997 E=07 \\
9.359 E=08 \\
1.056 E=07 \\
1.497 E=07 \\
1.155 E=07 \\
5.615 E=08\end{array}$ \\
\hline
\end{tabular}

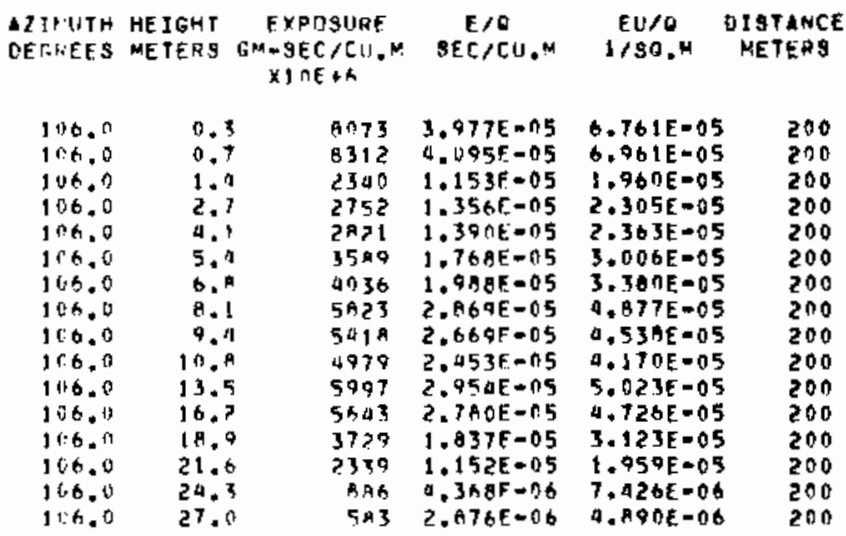

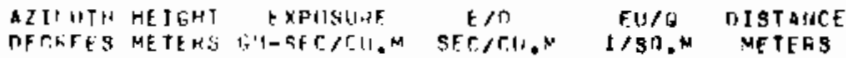
11 fif $+h$

\begin{tabular}{|c|c|c|c|c|c|}
\hline $\begin{array}{l}114.0 \\
114.6\end{array}$ & $? ?$ & $\begin{array}{l}1301>0 \\
12 \text { समतS }\end{array}$ & $\begin{array}{l}3.410 E-04 \\
5.349 T=04\end{array}$ & $\begin{array}{l}1.091 E-03 \\
1.079 E=03\end{array}$ & $\begin{array}{l}200 \\
200\end{array}$ \\
\hline $\begin{array}{l}114.0 \\
114.0\end{array}$ & $\begin{array}{l}1.4 \\
2.7\end{array}$ & $\begin{array}{l}\text { Putho } \\
\text { s5A18 }\end{array}$ & $\begin{array}{l}1.412 E=04 \\
1.725 E=04\end{array}$ & $\begin{array}{l}2.001 E=04 \\
2.052 E=04\end{array}$ & $\begin{array}{l}200 \\
200\end{array}$ \\
\hline 114.0 & 1.1 & 30139 & $1.5227=04$ & 5 BAE -0 O & 200 \\
\hline $\begin{array}{l}114.0 \\
114.0 \\
114.0\end{array}$ & $\begin{array}{l}5.11 \\
6.9 \\
0.1\end{array}$ & $\begin{array}{l}33133 \\
271249 \\
76163\end{array}$ & $\begin{array}{l}1.832 E=00 \\
1.337 E=04 \\
1.2 \text { A } 4 E-04\end{array}$ & $\begin{array}{l}2.775 E=04 \\
2.273 E=04 \\
2.1 B 3 E=04\end{array}$ & $\begin{array}{l}200 \\
200 \\
200\end{array}$ \\
\hline $\begin{array}{l}119.0 \\
114.0\end{array}$ & $\begin{array}{r}9.8 \\
10.9\end{array}$ & $\begin{array}{l}75345 \\
70047\end{array}$ & $\begin{array}{l}1.2505-04 \\
1.027 F-00\end{array}$ & $\begin{array}{l}2.126 E=04 \\
1.746 E=04\end{array}$ & $\begin{array}{l}200 \\
200\end{array}$ \\
\hline 114.0 & 13.5 & 14659 & $7.226 E-05$ & $1.228 E=0 a$ & 200 \\
\hline $\begin{array}{l}114.0 \\
114.0\end{array}$ & $16, \bar{c}$ & $\begin{array}{l}\text { A193 } \\
\text { and }\end{array}$ & $4.036 E-05$ & $6.861 E-05$ & 200 \\
\hline 110.0 & 21.5 & $\cos 8$ & $1.014 \mathrm{E}=05$ & $4 E=05$ & 200 \\
\hline $\begin{array}{l}114.0 \\
11 \\
114.0\end{array}$ & $\begin{array}{l}24.3 \\
27 . n\end{array}$ & $\begin{array}{r}1 \pm 15 \\
450\end{array}$ & $\begin{array}{l}5,490 E=06 \\
7.220 E=06\end{array}$ & $\begin{array}{l}9.340 E=06 \\
3.774 E=06\end{array}$ & $\begin{array}{l}200 \\
200\end{array}$ \\
\hline
\end{tabular}

A7TIUTH HEIGHT EXPOSURE E/D EU/O DISTANCE DEGHEFS METERY GM-SEC/CU.M SEC/CE.M 1/90,M METERS $x \cap \cap E+4$

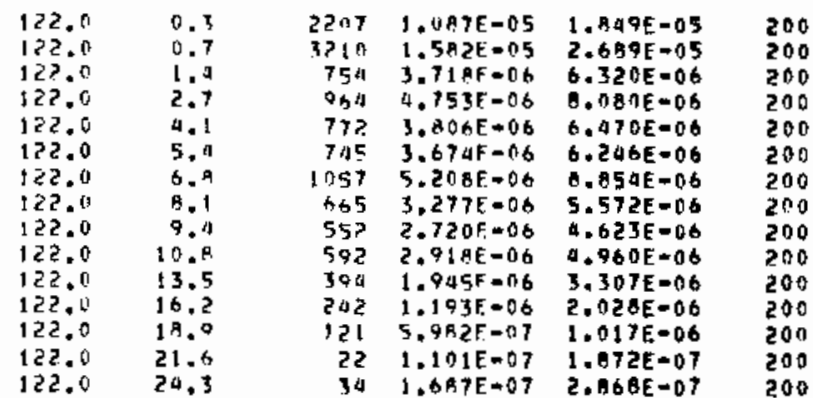




\begin{tabular}{|c|c|c|c|c|c|}
\hline $\begin{array}{l}\text { AZTPUUTH } \\
\text { OEREEES }\end{array}$ & $\begin{array}{l}\text { ME IGHI } \\
\text { METERS }\end{array}$ & $\begin{array}{c}\text { EXPOSUHF } \\
6+4-S E C / C 11 . \\
\times \square 0 E+6\end{array}$ & 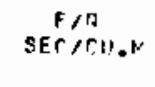 & $\begin{array}{c}E U / C \\
1 / \mathrm{SO}, M\end{array}$ & $\begin{array}{l}\text { DISTAND } \\
\text { METERS }\end{array}$ \\
\hline $\begin{array}{l}130.0 \\
130.0 \\
130.0 \\
130.0 \\
130.0 \\
130.0 \\
130.0\end{array}$ & $\begin{array}{r}1.11 \\
2.7 \\
4.1 \\
5.9 \\
6.8 \\
18.9 \\
21.4\end{array}$ & $\begin{array}{r}10 \\
17 \\
582 \\
35 \\
61 \\
65 \\
17\end{array}$ & $\begin{array}{l}5.138 E=08 \\
3.238 E=08 \\
2.870 E=06 \\
1.762 F=07 \\
3.010 E=07 \\
3.266 E=07 \\
8.806 E=08\end{array}$ & $\begin{array}{l}0.735 E=08 \\
1.061 E=07 \\
8.079 E-06 \\
2.995 E=07 \\
5.116 E=07 \\
5.553 E=07 \\
1.497 E=07\end{array}$ & $\begin{array}{l}200 \\
200 \\
200 \\
200 \\
200 \\
200 \\
200\end{array}$ \\
\hline
\end{tabular}

TEST XE3 JULY 15. 1965 O5 0255 TO 0259 PST ZINC SULFIDE RELEASE FFOM ELEVATICN OF $2 \mathrm{M}$ TOM

AZJMUTH EXPOSURE E/L ELI/O DISTARCE DEFFEFS GM-SEC/CU.M SECICU.N 1/SOAM METERS $X 10 E+6$

\begin{tabular}{|c|c|c|c|c|}
\hline $\begin{array}{r}01.0 \\
90.0 \\
94.0 \\
96.0 \\
98.0 \\
100.0 \\
102.0 \\
C 109.0 \\
1110.0 \\
112.0 \\
119.0 \\
116.0 \\
119.0 \\
120.0 \\
120.0 \\
122.0\end{array}$ & $\begin{array}{r}32 \\
56 \\
35 \\
70 \\
112 \\
529 \\
157 \\
35 \\
136 \\
15702 \\
22160 \\
12162 \\
3572 \\
157 \\
157 \\
35\end{array}$ & 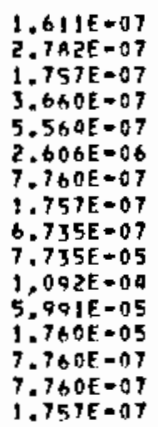 & $\begin{array}{l}2.73 R E=07 \\
0.729 E=07 \\
2.987 E=07 \\
6.223 E=07 \\
9.458 E=07 \\
4.931 E=06 \\
1.319 E=06 \\
2.987 E=07 \\
1.145 E=06 \\
1.315 E=04 \\
1.856 E=00 \\
1.019 E=04 \\
2.992 E=05 \\
1.319 E=06 \\
1.319 E=06 \\
2.987 E=07\end{array}$ & $\begin{array}{l}000 \\
000 \\
000 \\
800 \\
800 \\
800 \\
800 \\
100 \\
800 \\
000 \\
800 \\
000 \\
000 \\
000 \\
800 \\
800\end{array}$ \\
\hline $89 \mathrm{WI}$ & At & $\begin{array}{l}7.5 A B E=03 \\
\text { SEC / SD.M }\end{array}$ & $1.2905=02$ & \\
\hline
\end{tabular}

FOHFR MBTA FULLL":...

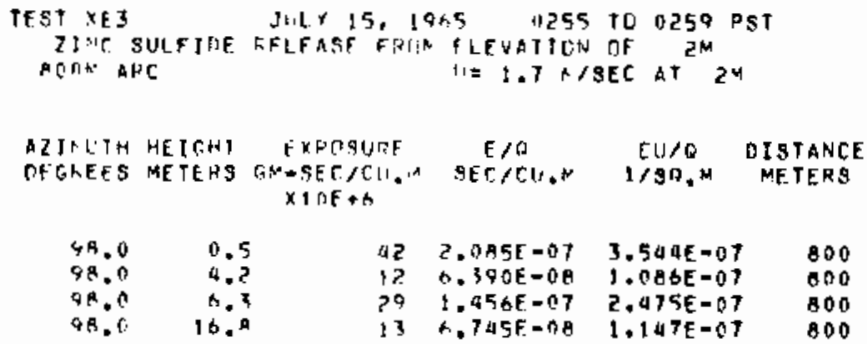

L2JMIITH HEIGH'T EXPOSUAE SE/O EU/O DISTANCE DEGLEES METERS GN-SEC/CU.N SEC/CU.M IISO.M METERS
XIOE \&

\begin{tabular}{|c|c|c|c|c|c|}
\hline 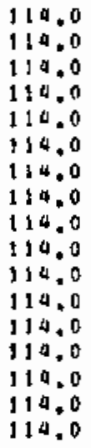 & $\begin{array}{l}0.5 \\
0.5 \\
1.0 \\
2.5 \\
4.7 \\
6.3 \\
0.4 \\
10.5 \\
12.5 \\
19.7 \\
16.7 \\
21.0 \\
25.2 \\
29.4 \\
33.8 \\
37.4 \\
42.0\end{array}$ & $\begin{array}{l}16973 \\
17454 \\
4938 \\
4897 \\
5349 \\
5584 \\
3739 \\
5473 \\
4856 \\
4350 \\
2978 \\
2426 \\
1178 \\
598 \\
392 \\
291 \\
165\end{array}$ & $\begin{array}{l}8.361 E-05 \\
2.403 E-05 \\
2.433 E=05 \\
2.408 E=05 \\
2.635 E=05 \\
2.752 E=05 \\
1.042 E=05 \\
2.696 E=05 \\
2.392 E=05 \\
2.143 E=05 \\
1.467 E-05 \\
1.195 E=05 \\
5.00 A E=06 \\
2.948 E-06 \\
1.933 E=06 \\
1.436 E=06 \\
8.161 E-07\end{array}$ & $\begin{array}{l}1.021 E=04 \\
1.063 E=04 \\
4.135 E=05 \\
0.093 E=05 \\
4.480 E=05 \\
4.678 E=05 \\
3.132 E=05 \\
9.584 E=05 \\
4.067 E=05 \\
3.044 E=05 \\
2.090 E=05 \\
2.032 E=05 \\
9.867 E=06 \\
5.012 E=06 \\
3.206 E=06 \\
2.441 E=06 \\
1.307 E=06\end{array}$ & $\begin{array}{l}300 \\
800 \\
800 \\
000 \\
800 \\
800 \\
800 \\
000 \\
800 \\
800 \\
800 \\
800 \\
100 \\
000 \\
000 \\
100 \\
800\end{array}$ \\
\hline
\end{tabular}

DZJTUTH HEIFHT EXPOSUAF F/O DE EUTO DISTANCE PEGHEES METERS GW-QEC/CU,M SEC/CU,M I/9O.M METERS $x] \cap E+6$

\begin{tabular}{|c|c|c|c|c|c|}
\hline $\begin{array}{l}122.0 \\
122.0 \\
122.0 \\
122.0 \\
122.0 \\
122.0 \\
122.0 \\
122.0\end{array}$ & $\begin{array}{r}0.5 \\
2.1 \\
10.5 \\
14.7 \\
25.2 \\
29.9 \\
33.6 \\
37.8\end{array}$ & $\begin{array}{r}100 \\
21 \\
20 \\
10 \\
15 \\
16 \\
12\end{array}$ & $\begin{array}{l}0.971 E=07 \\
1.065 E=07 \\
9.941 F=08 \\
9.231 F=08 \\
7.396 E=08 \\
7.906 E=08 \\
6.375 E=08 \\
4.335 E=08\end{array}$ & $\begin{array}{l}1.051 E=07 \\
1.011 E=07 \\
1.600 E=07 \\
1.569 E=07 \\
1.257 E=07 \\
1.340 E=07 \\
1.0045=07 \\
1.370 E=04\end{array}$ & $\begin{array}{l}000 \\
800 \\
000 \\
000 \\
000 \\
000 \\
000 \\
000\end{array}$ \\
\hline
\end{tabular}

\begin{tabular}{|c|c|c|c|c|c|}
\hline $\begin{array}{l}2 J 11 \text { TH } \\
\text { ESREES }\end{array}$ & $\begin{array}{l}\text { HE IGHT } \\
\text { METERS }\end{array}$ & $\begin{array}{c}\text { EXPCGHRE } \\
\text { GN=-SEC/CU. } \\
\times 10 E+b\end{array}$ & $\begin{array}{c}E / C) \\
\text { SE } t, / C 1 ! N\end{array}$ & $\begin{array}{c}f, 0 / 6 \\
1 / 30 .\end{array}$ & $\begin{array}{l}\text { DI ITANCE } \\
\text { METERS }\end{array}$ \\
\hline $\begin{array}{l}10 h .0 \\
104.0 \\
106.0 \\
117.0 \\
106.0 \\
116.0 \\
106.0 \\
106.0 \\
106.0 \\
106.0 \\
106.0 \\
106.0 \\
106.0\end{array}$ & $\begin{array}{l}1.7 \\
6.3 \\
8.4 \\
10.5 \\
12.4 \\
14.7 \\
16.9 \\
21.7 \\
25.7 \\
29.1 \\
33.6 \\
37.9 \\
42.1\end{array}$ & $\begin{array}{l}13 \\
33 \\
51 \\
76 \\
47 \\
162 \\
152 \\
134 \\
102 \\
134 \\
136 \\
40 \\
79\end{array}$ & $\begin{array}{l}6.745 E=00 \\
1.569 F=07 \\
2.556 F=07 \\
3.753 E=07 \\
2.343 E=07 \\
3.024 E=07 \\
7.401 E=07 \\
6.6395=07 \\
7.2915=07 \\
6.630 E=07 \\
6.707 F=07 \\
5.753 E=07 \\
3.927 E=07\end{array}$ & $\begin{array}{l}1.147 E=07 \\
2.137 E=07 \\
4.345 E=07 \\
6.349 E=07 \\
3.983 E=07 \\
1.364 E=06 \\
1.273 E=06 \\
1.129 E=06 \\
1.240 E=06 \\
1.127 E=06 \\
1.140 E=06 \\
6.720 E=07 \\
6.676 E=07\end{array}$ & $\begin{array}{l}800 \\
000 \\
000 \\
800 \\
800 \\
000 \\
000 \\
800 \\
800 \\
800 \\
000 \\
000 \\
000\end{array}$ \\
\hline
\end{tabular}




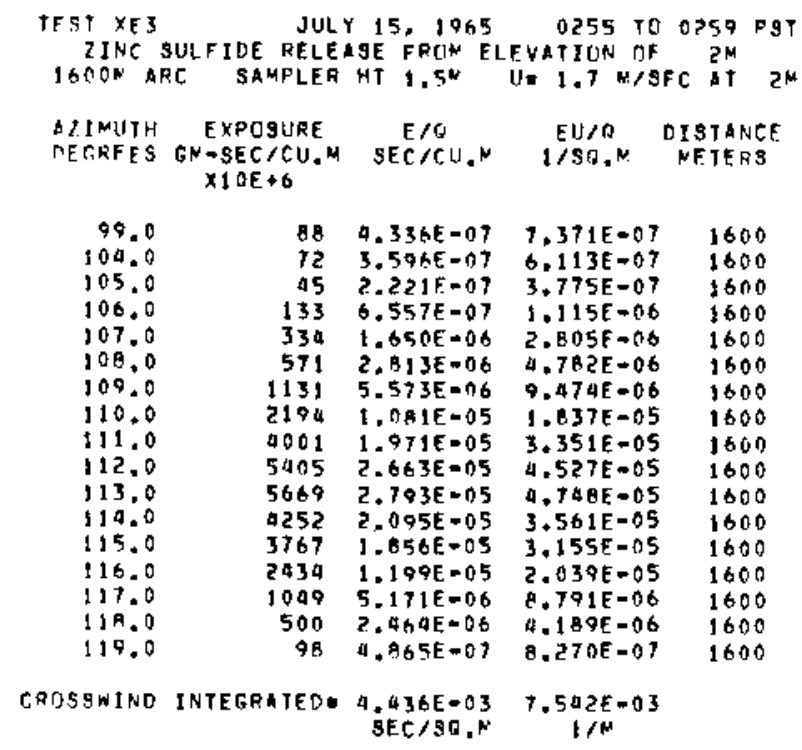

TOH.F NATA FILLPי,...

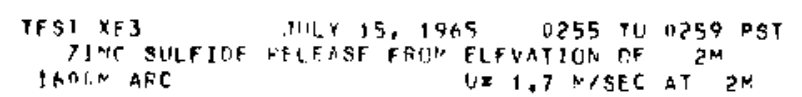

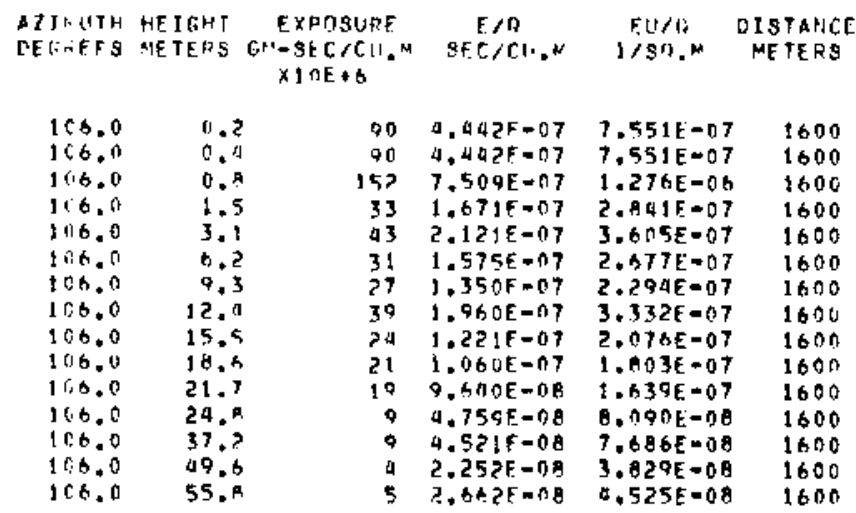

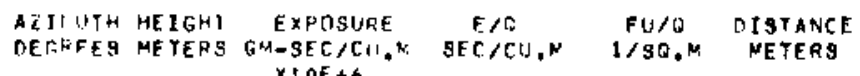
$x \perp n+6$

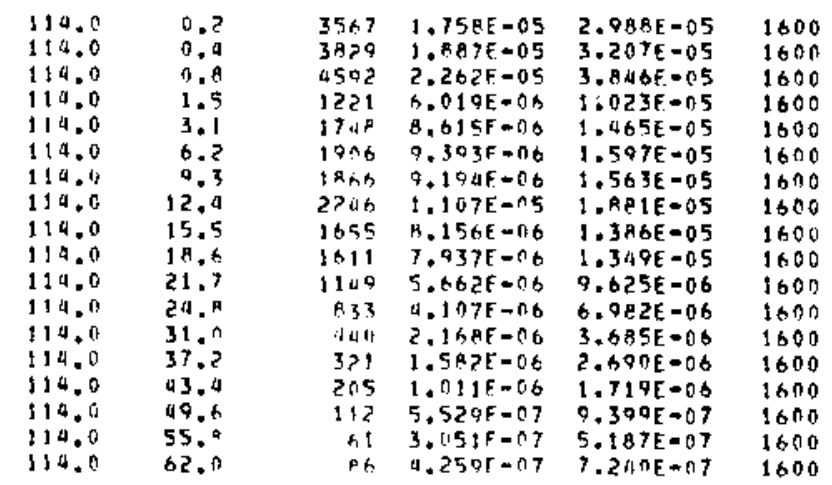


SECOHDARY SERIBS EXPERTERAT: XZ-3P

$2 I$ YC SULIDE AOD PLOOROSCBIH HERE BOTH USED AS TRACERS DURIHG

RIPERIXEET IE-3, FLDORESCEIS TZSOLIS ARE PRESBHTED HBRE. CROOHD

LEVEL AND TONER SAMPLIGG 200-1600 H ARCS.

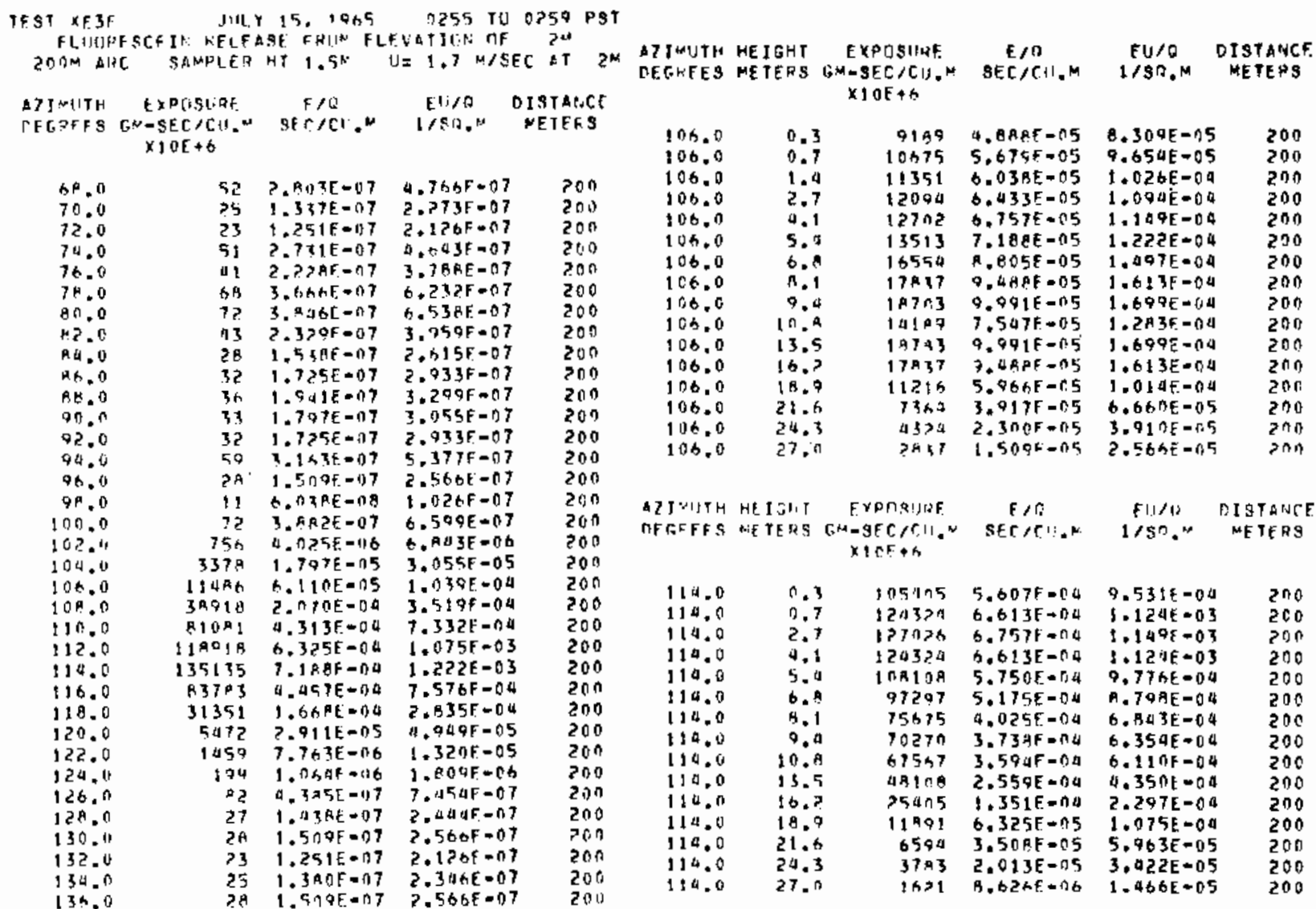

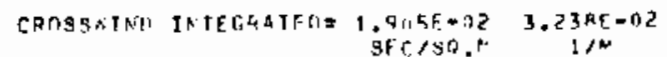

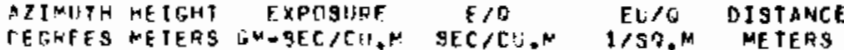

TrJiE.E TATA FIULLIJ!E.... $\times 10 \mathrm{E}+\mathrm{A}$

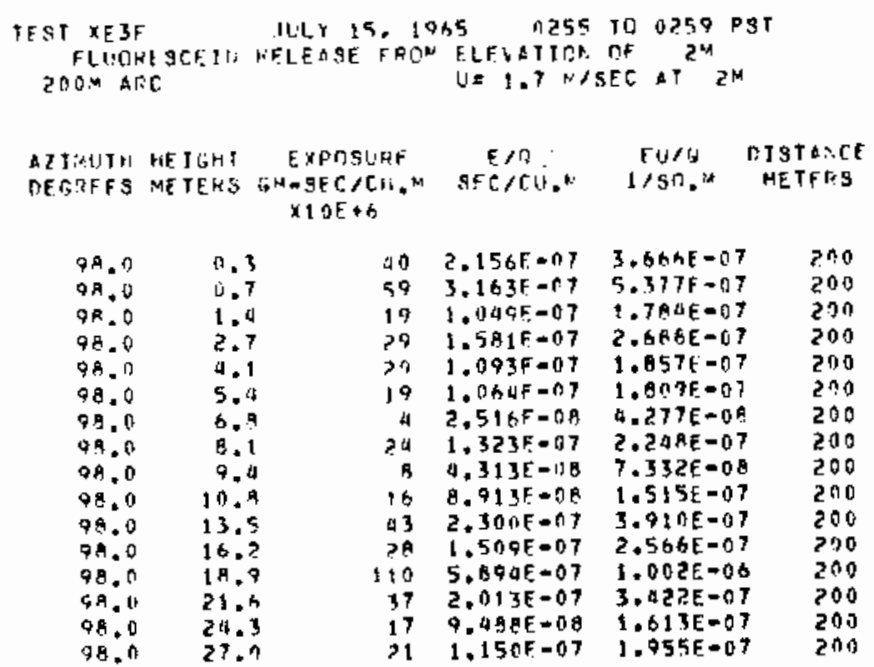

\begin{tabular}{|c|c|c|c|c|c|}
\hline $\begin{array}{l}122.0 \\
122.0 \\
122.0 \\
122.0 \\
122.0 \\
122.0 \\
122.0 \\
122.0 \\
122.0 \\
122.0 \\
122.0 \\
122.0 \\
122.0 \\
122.0 \\
122.0 \\
122.01\end{array}$ & $\begin{array}{l}0.3 \\
0.7 \\
1.0 \\
2.7 \\
4.1 \\
5.7 \\
6.8 \\
4.1 \\
9.4 \\
10.4 \\
13.5 \\
16.2 \\
18.9 \\
25.8 \\
24.3 \\
27.0\end{array}$ & $\begin{array}{l}\text { A10 } \\
1010 \\
1077 \\
1162 \\
986 \\
9196 \\
1310 \\
797 \\
7 ? 9 \\
801 \\
? 09 \\
308 \\
90 \\
91 \\
74 \\
15\end{array}$ & 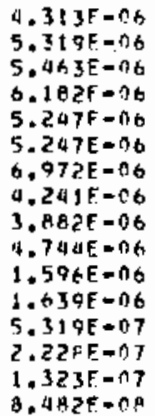 & 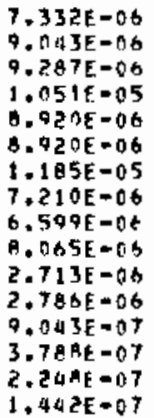 & $\begin{array}{l}200 \\
200 \\
200 \\
200 \\
200 \\
200 \\
200 \\
200 \\
200 \\
200 \\
200 \\
200 \\
200 \\
200 \\
200 \\
200\end{array}$ \\
\hline
\end{tabular}




\begin{tabular}{|c|c|c|c|c|c|c|c|c|c|c|c|}
\hline $\begin{array}{l}\text { ATIAH YH } \\
\text { IFCIFES }\end{array}$ & $\begin{array}{l}\text { MEIGHT } \\
\text { ME Tr.RS }\end{array}$ & 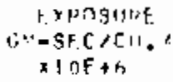 & 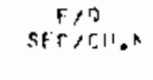 & $\begin{array}{l}F \cup / n \\
1 / S 0 . *\end{array}$ & $\begin{array}{l}\text { ISTANIT } \\
\text { F TERS }\end{array}$ & & & & & & \\
\hline & & & & & & $\begin{array}{l}\text { AZINITH } \\
\text { PERTEFS }\end{array}$ & $\begin{array}{l}\text { HE JGHI } \\
\text { METEHS }\end{array}$ & $\begin{array}{c}\text { EXPCSUPE } \\
G=S F C / C I: .\end{array}$ & 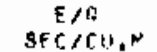 & $\begin{array}{c}E U / 1 \\
1 / 50.4\end{array}$ & $\begin{array}{l}\text { DISTANCE } \\
\text { METERS }\end{array}$ \\
\hline $\begin{array}{l}130.0 \\
13 n .0\end{array}$ & 0.3 & $\begin{array}{l}12 \\
17\end{array}$ & 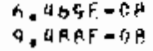 & $\begin{array}{l}1.100 E-07 \\
1.613 F=07\end{array}$ & $\begin{array}{l}500 \\
500 \\
500\end{array}$ & & & I $10 F+A$ & & & \\
\hline 1311.0 & 1.1 & 12 & $6.7575=0 \mathrm{~A}$ & $1.1 \pm 9 E=07$ & 200 & & & & & & \\
\hline $13 n .6$ & 2.7 & $1 ?$ & $5.757 F=0.0$ & $1.1 \Delta 9 E * 07$ & $2 \mathrm{A!} !$ & 116.0 & 0.3 & 15 & R. 25 \& $-0 \mathrm{H}$ & $1.403 \xi-6.7$ & $\mathrm{ACO}$ \\
\hline 130.0 & 4.1 & $>0$ & 1.0 ดी $=$ กी & $1.815 t-07$ & 200 & 106.0 & C. 5 & 16 & $5.575 f-02$ & $1.458 E=0.7$ & $\mathrm{BOO}$ \\
\hline 130.0 & 5.4 & 30 & $1.0715=07$ & $1.921 E=07$ & 200 & 105.0 & 1.1 & 11 & $7.612 f-0$. & $1.2405=07$ & 800 \\
\hline 130.6 & 0.9 & 7 & 5. हR? $=08$ & $6.599\{-08$ & 200 & $1^{1 \cdot 4}, 0$ & 2.1 & 3 & $? .01 E E=08$ & $3.03 \cap E=c B$ & 400 \\
\hline 1.30 .6 & N. 1 & 1? & $0.460 F=08$ & $1.10 n E=07$ & 2 no & 156.0 & 4.? & 13 & $0.97 \cap F=08$ & $1.185 E-67$ & 800 \\
\hline 130.0 & 9.1 & 22 & $1.178 F-07$ & $2.0011 E-07$ & 200 & $\begin{array}{l}106.0 \\
11000\end{array}$ & $\begin{array}{l}\text { A. } \\
\text { A. }\end{array}$ & $\begin{array}{l}14 \% \\
148\end{array}$ & $\begin{array}{l}7.70 \text { HE }=07 \\
7.8 B T F=07\end{array}$ & $\begin{array}{l}1.310 \mathrm{E}=06 \\
1.341 \mathrm{E}=06\end{array}$ & $\begin{array}{l}300 \\
\text { मी० }\end{array}$ \\
\hline 130.0 & 10.9 & 9 & $\triangle, B R A F=0 A$ & $A .309 E-O A$ & 200 & $\begin{array}{l}1136.0 \\
106.0\end{array}$ & 10.5 & 179 & $\begin{array}{l}7.987 F=197 \\
9.53 \times F=67\end{array}$ & $1.621 \mathrm{~F}=06$ & $\begin{array}{l}800 \\
800\end{array}$ \\
\hline $\begin{array}{l}130.0 \\
134.0\end{array}$ & $\begin{array}{l}13.5 \\
10.7\end{array}$ & $\begin{array}{r}17 \\
8\end{array}$ & $\begin{array}{l}9.500 F=08 \\
4.672 E=04\end{array}$ & $\begin{array}{l}1.590 E=07 \\
7.943 E=(189\end{array}$ & $\begin{array}{l}200 \\
200\end{array}$ & 106.0 & $12 . \mathrm{h}$ & in 1 & $7,520 t=0.7$ & $1 . ? 78 E=0 A$ & POO \\
\hline 130.0 & $1 \times .8$ & 10 & $5.3915=08$ & $0.165 E-08$ & 200 & 106.0 & 14.7 & 543 & $7.3125-(1)$ & $1.294 \mathrm{E}=06$ & arc \\
\hline $13 \cap .0$ & 21.4 & 6 & $3.522 F=0 p$ & $5.988 E-08$ & 200 & $10 b .0$ & $16 n^{2}$ & 255 & $1.357 F=0.6$ & $2.307 E-06$ & $\begin{array}{l}A \cap 0 \\
B O 0\end{array}$ \\
\hline 130.0 & 24.3 & A & $4.4575=08$ & $7.576 E=0 \mathrm{~A}$ & 200 & 106.0 & $\begin{array}{l}21.7 \\
25 . ?\end{array}$ & $\begin{array}{l}209 \\
342\end{array}$ & $\begin{array}{l}1.5975=06 \\
1.92+E=16\end{array}$ & $\begin{array}{l}2.697 E-06 \\
3.27 \Delta E-06\end{array}$ & $\begin{array}{l}800 \\
800\end{array}$ \\
\hline 150.0 & 27.0 & A & $4.67 ? 5=0 A$ & $7.943 E=09$ & 200 & $\begin{array}{l}106.0 \\
500.0\end{array}$ & 24.1 & $\begin{array}{l}3 \times 2 \\
3 \times 2\end{array}$ & 1.50 \& -06 & $3.557 \mathrm{E}-06$ & 800 \\
\hline & & & & & & $1) 6$. & & 322 & $1.715 \mathrm{~F}=\mathrm{no}$ & $915 E-06$ & Bno \\
\hline & & MilY Y I & 10,5 & 55 TC $025 ?$ & PST & 106.0 & 37.2 & $2+1$ & $1.2 R \& E-C A$ & $1 \mathrm{ABE}-0 \mathrm{~S}$ & AOO \\
\hline & $F<C$ & WELFASE & Hath & {$[1: A, \quad n F$} & & $13+2$ & 42.3 & ? अ & $1.102 F=1,2$ & $2.0275=\mathrm{Ch}$ & her \\
\hline
\end{tabular}

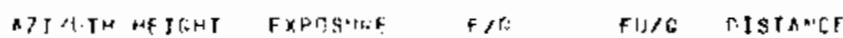

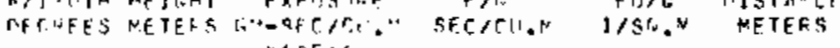
$x 10 \mathrm{f}+\mathrm{h}$

\begin{tabular}{|c|c|c|c|c|}
\hline sh. n & $1 n$ & $5.71) 25-117$ & $9.693 E-17$ & $A(0)$ \\
\hline P. E. 0 & 94 & a. $5>n E-47$ & $7.6935-197$ & 900 \\
\hline 90.16 & $5 ?$ & $? .7 \circ 1[-17$ & $4.7>A F-07$ & 85,0 \\
\hline ; 0 & 96 & $5.145 E-1.7$ & $9.747 f=07$ & 800 \\
\hline 94.0 & ${ }^{A} ?$ & a. 3 हnE-ก7 & $7.4078-07$ & 800 \\
\hline $9 n .0$ & 9 & $4.967 F=0 A$ & $P .274 F=n R$ & $R 00$ \\
\hline $4 R \cdot ?$ & 35 & 1. $977 \mathrm{~F}-07$ & $3.1915=07$ & 800 \\
\hline 100.0 & $5 \mathrm{H}$ & $074 E-07$ & $005=0.7$ & 800 \\
\hline $1: 2.0$ & 128 & h. $R, 4 E=07$ & $1,158 \mathrm{~F}-06$ & An? \\
\hline 104.0 & 15 & $R .2 \cap 5 E-3 R$ & $1,3 \div 5, E-07$ & 900 \\
\hline 106.0 & 14 & $7.027 E=00$ & $1.3+8 \mathrm{f}=07$ & 800 \\
\hline 109.0 & 15 & $A . \cap A B E=90$ & $1.3715-07$ & 800 \\
\hline 110.0 & $\geqslant 4$ & $1.2 \$ 3 E-n\rangle$ & $199 F-07$ & Pon \\
\hline 112.0 & $\begin{cases}300 \\
0\end{cases}$ & $7.7345=05$ & $655 \mathrm{~F}-14$ & RoO 0 \\
\hline 119.0 & 23006 & $1.2 ?$ at $=00$ & QAOE-0A & A 00 \\
\hline 116.0 & $1332 A$ & $7.127 E=05$ & 1. $212 F=04$ & A0O \\
\hline 118.0 & 3930 & $2.037 f-05$ & $3.4635-05$ & $A 00$ \\
\hline 120.0 & 156 & A. $3 a A F=07$ & 1.41 HE -06 & 900 \\
\hline$: 27.0$ & 24 & $1,3 ? 15-107$ & $.2465=07$ & AOn \\
\hline 124,1 & 35 & $1 .{ }^{A} 77 E-6.7$ & $191 F-07$ & $F 00$ \\
\hline & a)! & $.325 E-07$ & $7 \times 3 F-0.7$ & 800 \\
\hline 12 & 83 & $4.4508-107$ & $5 \times 5 F=07$ & Aon \\
\hline$\vdots 3$ & 49 & $2.5735-07$ & $374 E-0.7$ & 900 \\
\hline
\end{tabular}

114.0
114.0
114.0
114.0
114.0
114.0
114.0
1114.0
114.0
114.0
114.0
114.0
114.0
114.0
119.0
114.0

0.3
0.5
2.1
4.2
6.3
6.4
10.5
12.4
14.7
16.4
21.0
25.2
29.4
33.6
37.8
42.4

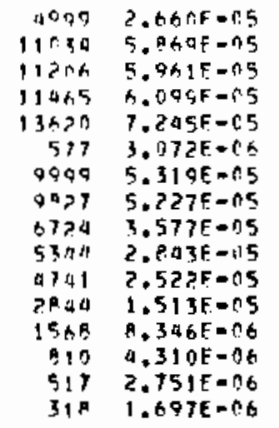

a. $521 E \cdot 05$

Q. STAE-AS

$1.0: 3 t=10$

$1.037 \mathrm{E}=\mathrm{AB}$

$1.232 E-04$

$5.223 E=06$

$9.043 E=05$

A. $807 E=05$

6. DAnE-05

$4.833 E-05$

$4.2 A 7 E-05$

$2.572 E=05$

1. $419 E=05$

$7.328 E=06$

$4.677 E-06$

2. BADE-D6

900

คก

\&ดก

8no

A0
B०

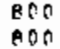

BOD

PSO

BOA

ROO

800
800

00

AOO

AZIMIJTH HEIGHT EXPOSURE E/A EHIQ DISTANCE

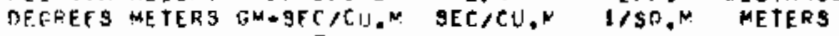
$\times 10 E+6$

\begin{tabular}{|c|c|c|c|c|c|}
\hline $\begin{array}{l}122.0 \\
12200 \\
122.0 \\
12200 \\
122.0 \\
12200 \\
122.0 \\
12200 \\
122.0 \\
122.0 \\
122.0 \\
12200 \\
12200 \\
122.0 \\
12200 \\
12200 \\
127.0\end{array}$ & $\begin{array}{l}0.3 \\
0.5 \\
1.0 \\
2.1 \\
4.2 \\
6.3 \\
8.0 \\
10.5 \\
12.5 \\
19.7 \\
16.9 \\
21.0 \\
25.2 \\
29.4 \\
33.4 \\
37.9 \\
42.0\end{array}$ & $\begin{array}{l}12 \\
14 \\
20 \\
16 \\
15 \\
90 \\
18 \\
16 \\
18 \\
9 \\
18 \\
9 \\
79 \\
31 \\
16 \\
75 \\
73\end{array}$ & $\begin{array}{l}6.695 E=0.9 \\
7.612 F=08 \\
1.042 F=07 \\
9.621 E=08 \\
9.629 E=08 \\
0.264 E=07 \\
9.813 E=08 \\
8.621 E=08 \\
9.721 E=08 \\
5.136 E=08 \\
4.585 E=08 \\
5.136 E=08 \\
1.559 E=07 \\
1.697 F=07 \\
8.621 E=08 \\
1.376 E=07 \\
1.23 P F=07\end{array}$ & 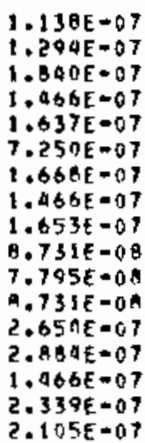 & $\begin{array}{l}800 \\
800 \\
800 \\
800 \\
800 \\
800 \\
500 \\
0100 \\
800 \\
800 \\
000 \\
100 \\
800 \\
000 \\
000 \\
000 \\
000\end{array}$ \\
\hline
\end{tabular}

\begin{tabular}{|c|c|c|c|c|c|c|c|}
\hline 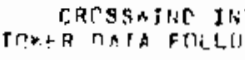 & $\begin{array}{l}\text { EGNATEDA } \\
\therefore\end{array}$ & ค. An & $\begin{array}{l}9 E-.93 \\
130 . M\end{array}$ & & $\begin{array}{l}066^{\circ} \\
1 / 4\end{array}$ & 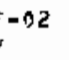 & \\
\hline $\begin{array}{l}F \leqslant I \quad X E 3 F \\
\text { FLLiifKE } \$ C\end{array}$ & $\begin{array}{l}\text { WUY } 15 \\
\text { PELFกSE }\end{array}$ & : & FLEVA & $\begin{array}{l}0259 \\
\text { T16: }\end{array}$ & $\begin{array}{l}\text { IU } \\
\text { nf }\end{array}$ & $\begin{array}{r}0259 \\
2 \%\end{array}$ & $9 \mathrm{Ps}$ \\
\hline Roov dic & & & $\eta=1$. & $7: 18$ & $\$ F C$ & AT & $2 \times$ \\
\hline
\end{tabular}

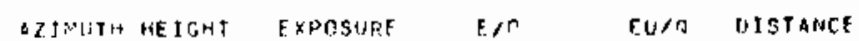

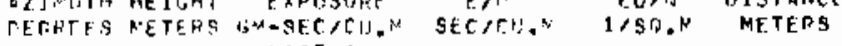
$\times 10 \mathrm{fF}+6$

\begin{tabular}{|c|c|c|c|c|c|}
\hline 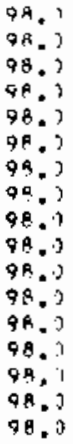 & $\begin{array}{l}5.3 \\
0.5 \\
1.9 \\
? .1 \\
7.3 \\
0.3 \\
9.4 \\
10.5 \\
12.5 \\
14.7 \\
16.0 \\
21.0 \\
25.2 \\
29.9 \\
33.0 \\
37.8 \\
42.0\end{array}$ & $\begin{array}{r}0 \\
7 \\
4 \\
18 \\
42 \\
34 \\
35 \\
37 \\
20 \\
10 \\
10 \\
10 \\
15 \\
9 \\
49 \\
94 \\
53\end{array}$ & 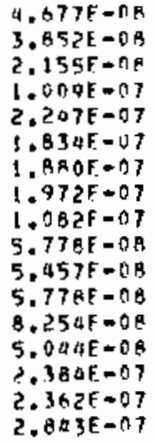 & 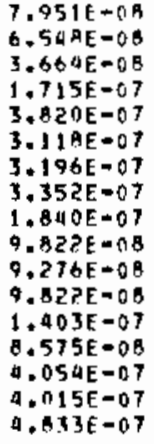 & $\begin{array}{l}800 \\
800 \\
800 \\
800 \\
800 \\
800 \\
800 \\
800 \\
800 \\
000 \\
800 \\
800 \\
800 \\
800 \\
800 \\
100 \\
800\end{array}$ \\
\hline
\end{tabular}




\begin{tabular}{|c|c|c|c|c|c|}
\hline $\begin{array}{l}\text { SZ I TH TH } \\
\text { SETKEES }\end{array}$ & $\begin{array}{l}\text { HEIGHI } \\
\text { METEKS }\end{array}$ & $\begin{array}{c}F x=n S 1, R F \\
6 \times-9 E C / C+1.2 \\
\times 10 E+6\end{array}$ & $\begin{array}{c}F / 6 \\
\text { SEt: } / C^{2}:{ }^{2}\end{array}$ & $\begin{array}{c}F \cup / 0 \\
1 / 50.4\end{array}$ & $\begin{array}{l}\text { OISTA'OCE } \\
\text { METERS }\end{array}$ \\
\hline $\begin{array}{l}130.0 \\
130.0 \\
130.0 \\
130.0 \\
130.0 \\
1370\end{array}$ & $\begin{array}{l}0.3 \\
0.5 \\
1.5 \\
2.1 \\
4.2 \\
3.3\end{array}$ & $\begin{array}{l}18 \\
23 \\
15 \\
30 \\
19 \\
98\end{array}$ & $\begin{array}{l}9.6795=0 F \\
1.238 F=07 \\
9.162 F=09 \\
1.605 E=07 \\
1.036 E=07 \\
3.119 E=07\end{array}$ & $\begin{array}{l}1.637 E-07 \\
2.105 E=07 \\
1.36 A E=07 \\
2.72 P E-07 \\
1.762 E-07 \\
5.301 E=07\end{array}$ & $\begin{array}{l}800 \\
800 \\
100 \\
800 \\
800 \\
900\end{array}$ \\
\hline
\end{tabular}

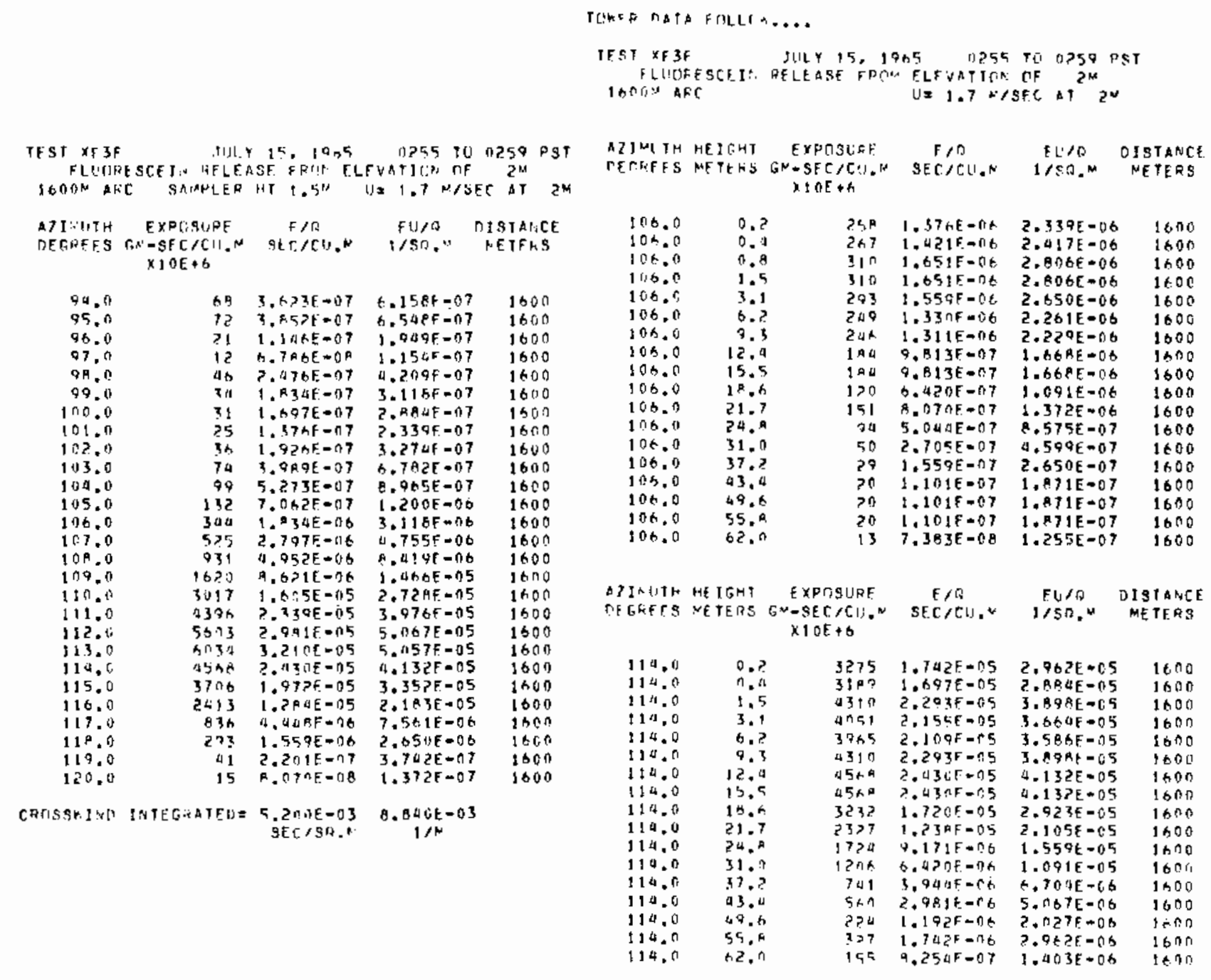




\begin{tabular}{|c|c|}
\hline Position* & $E / Q$ \\
\hline $1-096$ & $1.65-6$ \\
\hline $1-100$ & $4.53-6$ \\
\hline $1-108$ & $1.68-4$ \\
\hline $1-112$ & $7.09-4$ \\
\hline $2-096$ & $1.44-6$ \\
\hline $2-100$ & $2.67-6$ \\
\hline $2-112$ & $1.10-4$ \\
\hline $2-116$ & $9.80-5$ \\
\hline $3-096$ & $8.97-7$ \\
\hline $3-108$ & $1.16-5$ \\
\hline $3-111$ & $3.92-5$ \\
\hline $3-114$ & $4.18-5$ \\
\hline $3-117$ & $1.12-5$ \\
\hline
\end{tabular}

*Stable grid 


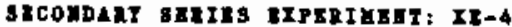

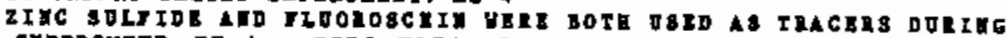

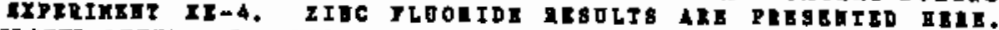 GROTID LEVRL AHD TOUKR SAKPLIGG $200-1600$ M ABCS.}

\begin{tabular}{|c|c|c|c|c|}
\hline $\begin{array}{l}\text { ST } X E-\pi \\
\text { ZIRC } 5 \\
\text { 20OH AK }\end{array}$ & $\begin{array}{l}\text { BUGLS } \\
\text { ILFIIIF PFLF } \\
\text { SAHFL.EF }\end{array}$ & 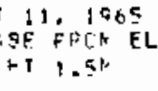 & 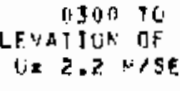 & $\begin{array}{rl}030) & P 5 T \\
21 & \\
6 & 41\end{array}$ \\
\hline $\begin{array}{l}\text { AZTRETH } \\
\text { DETGRFE } 3\end{array}$ & 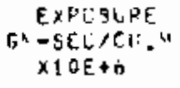 & 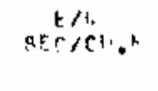 & $\begin{array}{c}E 1,10 \\
1 / \operatorname{san}^{2}:\end{array}$ & $\begin{array}{l}\text { CISTARCE } \\
\text { NETEFS }\end{array}$ \\
\hline 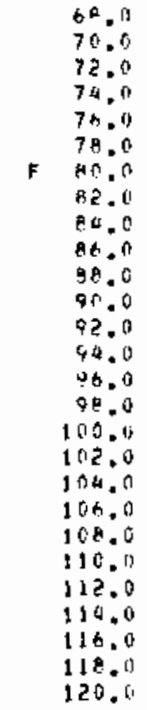 & 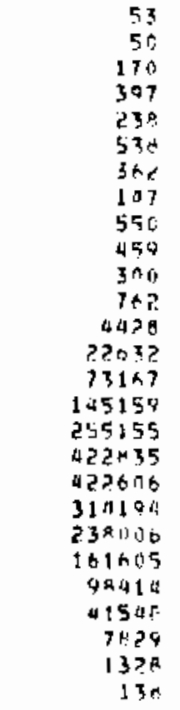 & 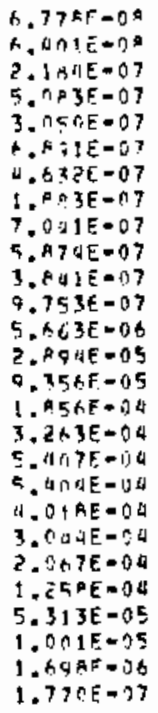 & 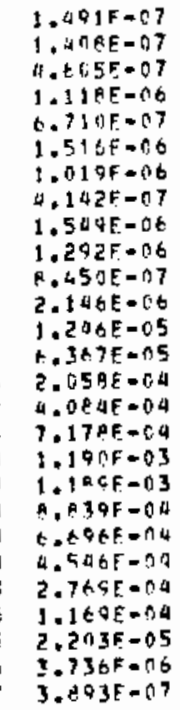 & $\begin{array}{l}200 \\
200 \\
200 \\
200 \\
200 \\
200 \\
200 \\
200 \\
200 \\
200 \\
200 \\
200 \\
200 \\
200 \\
200 \\
200 \\
200 \\
200 \\
200 \\
200 \\
200 \\
200 \\
200 \\
200 \\
200 \\
200 \\
200\end{array}$ \\
\hline $15.12>0$ & 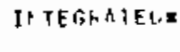 & $\begin{array}{l}1.978 \\
3001\end{array}$ & $1 / 4$ & \\
\hline
\end{tabular}

TOKE TATH FILLLLE....

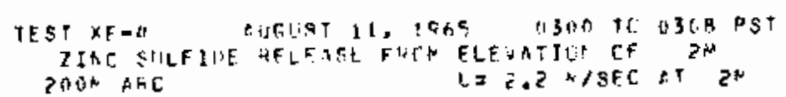

\begin{tabular}{|c|c|c|c|c|c|}
\hline $\begin{array}{l}\text { AZINLIIH } \\
\text { TEGREES }\end{array}$ & $\begin{array}{l}\text { HEIGHT } \\
\text { NEYEFS }\end{array}$ & 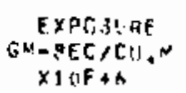 & $\begin{array}{c}E / \theta \\
\text { SEt/Cu,N }\end{array}$ & $\begin{array}{l}E L / A \\
1 / S R . M\end{array}$ & $\begin{array}{l}\text { DT } 9 T A N C E \\
\text { NETEAS }\end{array}$ \\
\hline $10 t .0$ & 0.3 & SAETP & $3.693 E-04$ & $8.124 \mathrm{E}=0 \mathrm{~g}$ & 2 \\
\hline 106.0 & 0.7 & 581364 & $4.877 \mathrm{E}=04$ & $1+073 E-03$ & 200 \\
\hline 100.0 & 3.1 & 297070 & $3.810 E-00$ & $A .303 \mathrm{E}=0 \mathrm{Q}$ & $2 n 0$ \\
\hline 106.0 & 2.7 & 255857 & $3.272 F=04$ & $7.199_{E}=04$ & 200 \\
\hline $10 b .0$ & 4.1 & 294333 & $3.6364=04$ & $7.999 E-04$ & 200 \\
\hline 106.0 & 5.4 & $\ln \theta \sin$ & $3,949 E=\cap 4$ & A.BO5E=04 & 200 \\
\hline 100.0 & $B . A$ & en?hQ9 & $2.5900-00$ & $5.697 E=00$ & 200 \\
\hline 106.0 & A. 1 & $23209 \mathrm{~A}$ & $2.975 E=14$ & $0.555 \mathrm{E}=0 \mathrm{~A}$ & 200 \\
\hline 105.0 & $\$ .1$ & 222679 & $2.54 A F=04$ & $4.265 E=04$ & 200 \\
\hline 106.0 & 10.6 & lofic 42 & $2.05 ?+=04$ & $1.513 \epsilon=04$ & 2 no \\
\hline 106.8 & 13.5 & $11+543$ & $1.49 G F-A 4$ & $3.279 E-04$ & $20 n$ \\
\hline 100.0 & 16.2 & $4450 A$ & R. $305 F=n 5$ & $\therefore$ - $27 \mathrm{E}=04$ & 200 \\
\hline $1 \cap 6, n$ & 18.9 & $443 \cap A$ & $0.305 E=n 5$ & $1.387 \mathrm{E}-04$ & $20 n$ \\
\hline 106.0 & al.n & $2 x+3$ & $3.359 \mathrm{E}-n 5$ & $7.389 \mathrm{~B}-\mathrm{CS}$ & 200 \\
\hline 106.0 & 24.3 & $|.151|$ & $1.3 \triangle A E-C S$ & $2.957 E=r 5$ & 200 \\
\hline 106.0 & 27.0 & wa>n & $5.6535-06$ & $1.244 E=05$ & 2011 \\
\hline D2 INUITH. & HEICIHT & GXPRSLEF & $r, \pi$ & E1! $/ r_{0}$ & PISTANCF \\
\hline "Eringers & P'ETEPS & 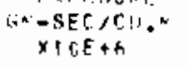 & r & isnon & 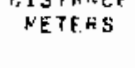 \\
\hline 110.0 & 0.3 & $x>9>?$ & 4.216 & $9.262 E=05$ & 200 \\
\hline 1100 & 0.7 & $4+975$ & sonif-rs & $1.320 \mathrm{~N}=1 \mathrm{a}$ & 200 \\
\hline 114,8 & 1.4 & 32271 & $4.1275-t 5$ & $9.379 E-05$ & 200 \\
\hline 114.0 & 2.7 & 15 21 & $4.5 e 1 F=05$ & $1.00 \mathrm{AE}=04$ & 200 \\
\hline$t 12.0$ & 4.1 & 34799 & $5 . M Q \subseteq F .05$ & $1.1 \geq 0 \mathrm{E}=04$ & 200 \\
\hline 110.6 & 5.4 & 43737 & 5.50 af & $1.211 \mathrm{E}=04$ & $20 \mathrm{c}$ \\
\hline 114.0 & $6, A$ & 34710 & $3.70 Q E-15$ & Q.359E=05 & 200 \\
\hline 114.0 & A. 1 & X5an? & $4.665 E-05$ & $1.026 E=04$ & 200 \\
\hline 114.0 & 9.1 & 34403 & 4. उ9GE. & $0.679 E=05$ & 200 \\
\hline 114.0 & 10.8 & 34471 & $3.4 R Q F-05$ & $9.6 \triangle A E=05$ & 200 \\
\hline 110.0 & 13.5 & 24071 & $3.193 E-05$ & $7.025 E-05$ & 200 \\
\hline 114.0 & $16 . ?$ & 16309 & $2.096 E-05$ & U.t1IE-05 & 200 \\
\hline 154.0 & 18.0 & 7900 & $1.010 t-05$ & $2.223 E=05$ & 200 \\
\hline 114.0 & $21, x$ & 3646 & $4 . B C 9 E-06$ & $1.032 E-05$ & 200 \\
\hline 114,6 & 24.3 & 2372 & $3.0345-0.6$ & $6.676 E=06$ & 200 \\
\hline
\end{tabular}

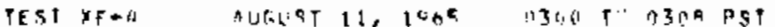

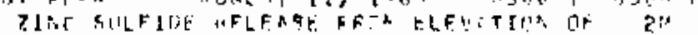

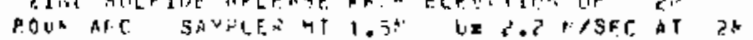




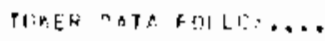

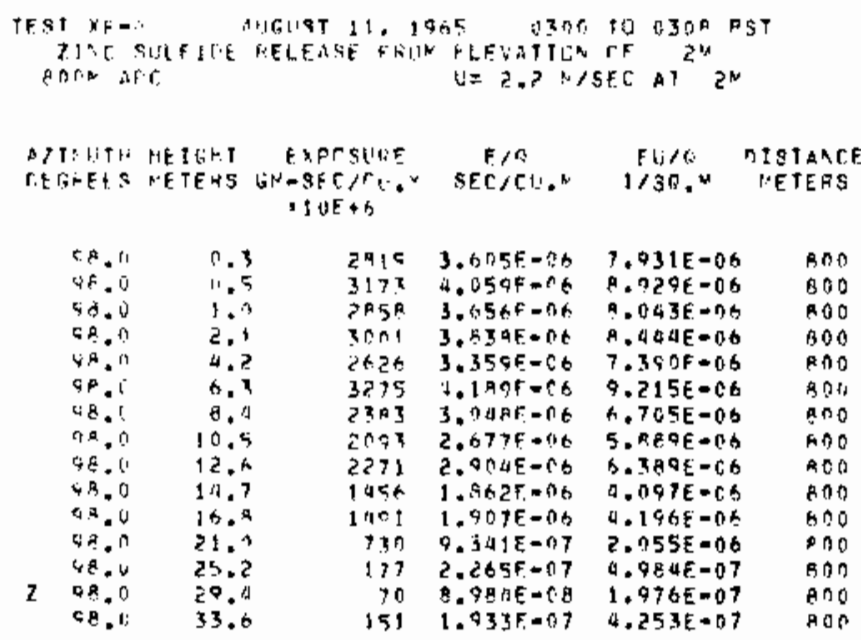

\begin{tabular}{|c|c|c|c|c|c|}
\hline $\begin{array}{l}\text { AZIH:TH } \\
\text { CEGREA }\end{array}$ & $\begin{array}{l}\text { NE IGHT } \\
\text { METERS }\end{array}$ & $\begin{array}{c}\text { EXPOSUDE } \\
G+S E C / C+i * \\
\times 1 O E+h\end{array}$ & $\begin{array}{c}\text { ES } \\
\text { SEC }\end{array}$ & $\begin{array}{l}5 L / 0 \\
1 / S N_{*}\end{array}$ & $\begin{array}{l}\text { CISTANCE } \\
\text { NETERS }\end{array}$ \\
\hline $100 . t$ & 17.2 & $3 \geq ? 45$ & $4.099 F=05$ & 9. ก15E $=05$ & 800 \\
\hline 1120.0 & 4.5 & $3 \mathrm{HO}+2$ & 4. senE-0.5 & $1.096 E-0 \mathrm{a}$ & Ann \\
\hline$F 1(x, 4)$ & $i . n$ & $31 \ln 4$ & U. DETE- AS & $9.94 R E=0.5$ & 800 \\
\hline $106 . n$ & 2.1 & $3 \div 7+1$ & $4, Y 55 E-C 5$ & $1.791 E=04$ & 800 \\
\hline $1: 6.6$ & 4.2 & $5 \times 5.5$ & $7.1085=15$ & $1.56^{4} \mathrm{E}=04$ & 800 \\
\hline 10.4 .0 & 6.3 & $4098+6$ & $5.226 F=015$ & $1 . \$ 50 E-04$ & AOO \\
\hline 100.0 & $*$ & 42910 & $6.255 \mathrm{~F}=0.5$ & 1.37 SE $=04$ & 800 \\
\hline $10 t=0$ & 10.5 & 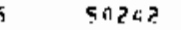 & $6.925 E=05$ & $1.413 E=04$ & 890 \\
\hline $1: 6.0$ & 12.4 & $43 \times 63$ & 5.6025 .05 & $1.232 E=04$ & POO \\
\hline $1: 6.0$ & 14.7 & 106372 & $5.530 \mathrm{E}=1 \mathrm{AS}$ & $1 . \operatorname{s} \cap 5 E-04$ & 800 \\
\hline 108.0 & 10.8 & $447: 7$ & $5.727 F=115$ & $1.259 E-00$ & प्र०0 \\
\hline 106.0 & $21.1)$ & 2.064 & 3.7 ก $F=05$ & A. 27 ZE -05 & 800 \\
\hline 10.0 & 25.2 & PABA & 1.13 t $F=115$ & $2.095 E=05$ & A०O \\
\hline 1 & & 0332 & $1.193 E=05$ & $2.62 h E=05$ & BOo \\
\hline 0 & 33 & 4783 & $6.117 \mathrm{~F}=18$ & $1.3146 E-05$ & ADO \\
\hline & 37. & 2703 & 3 , ito 5F.0.6 & $7.622 E=06$ & $8 \cap n$ \\
\hline & 42,0 & $13 \times 2$ & $B 7 E=\wedge s$ & IEBF $=06$ & $\mathrm{HOC}$ \\
\hline
\end{tabular}

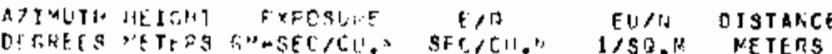
× $10+4$

\begin{tabular}{|c|c|c|c|c|}
\hline 154.0 & 1.3 & $A 5$ & 3. $3265=$ r. & $1 . \times 3 ?, E-07$ \\
\hline 114,0 & 1.5 & 37 & $4.70 D E-D B$ & $1.054 \varepsilon=07$ \\
\hline $1: 4.0$ & 2.1 & 10 & $2.3955-08$ & $9 E-08$ \\
\hline 114.0 & d.? & $1 \mathrm{nz}$ & $1.377 \mathrm{E}=0.7$ & $03 \cap E=07$ \\
\hline $1 ! 4,6$ & $x .3$ & Ab a & GRQE-08 & $251 E=07$ \\
\hline 114,0 & 8.4 & 10 & $096 F=0 \mathrm{~A}$ & $11 E=0 \mathrm{~A}$ \\
\hline 114.0 & 10.5 & 121 & $1.557 F-07$ & $i E=07$ \\
\hline 114.0 & 12.6 & 150 & $E F=07$ & $E=0 t$ \\
\hline 114.6 & 19,7 & 2.34 & $9945-07$ & $6.587 E=07$ \\
\hline 134.0 & 16.3 & $? .92$ & 3.7 A $3 E-0 ?$ & $234 E-07$ \\
\hline $\begin{array}{l}114.0 \\
114.0\end{array}$ & 21.7 & $\begin{array}{l}\text { a } 66 \\
140\end{array}$ & $\begin{array}{l}5.444 E=07 \\
1.797 E=07\end{array}$ & $\begin{array}{l}1.190 E=06 \\
3.953 E=07\end{array}$ \\
\hline 114.0 & 29,0 & 169 & 2.1 ABE-OT & $4.769 E-07$ \\
\hline 114.0 & 33.6 & 305 & $a .414 E=07$ & $10 E-07$ \\
\hline 114.0 & 37.9 & 160 & $2.051 E-07$ & $311 E=07$ \\
\hline 134.0 & & 150 & $2,051 E=07$ & $4.46 B E=07$ \\
\hline
\end{tabular}

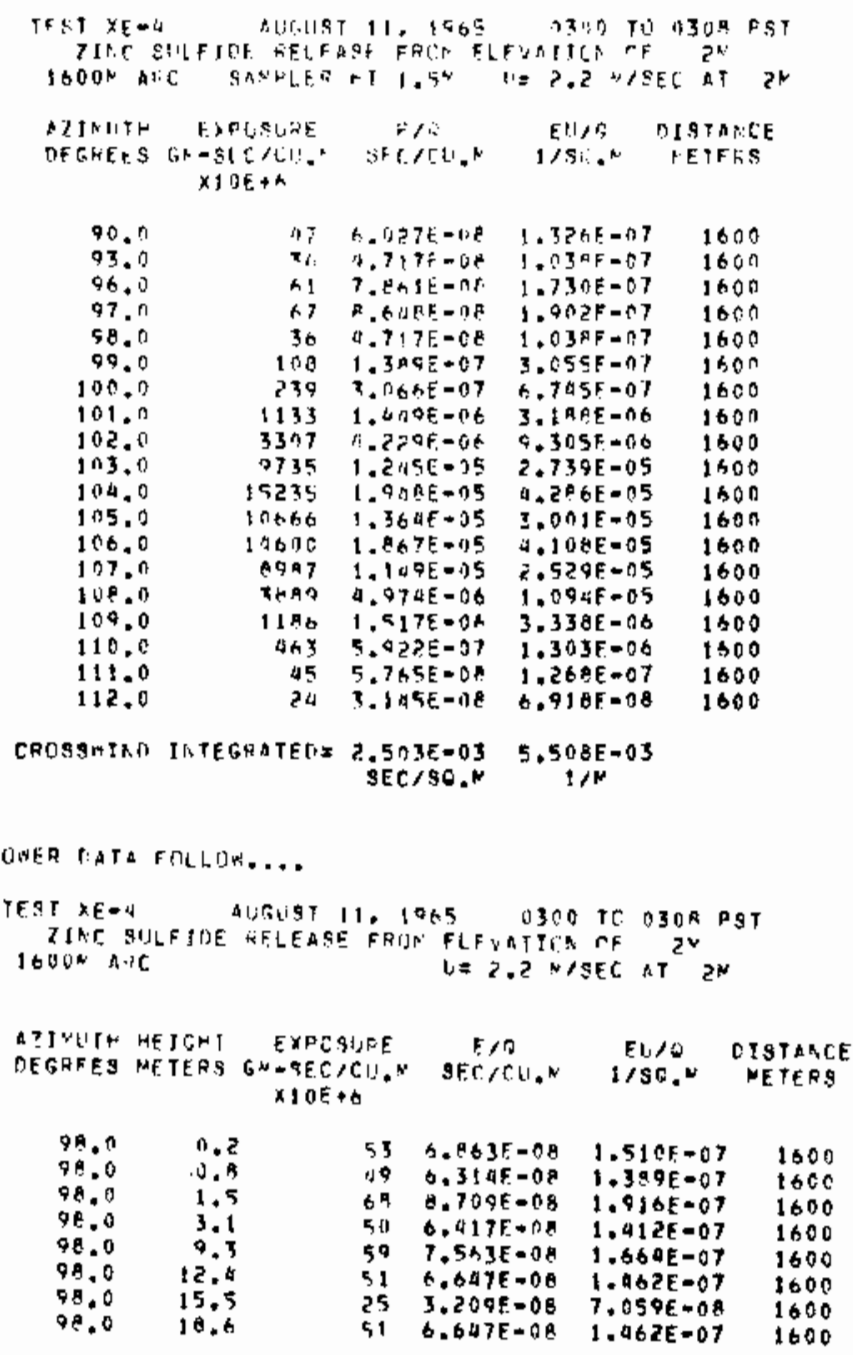

AZINUTH HEIGHT EYPDSURE

E 10 EU/C DISTANCE $\times 10 \mathrm{E}+6$

$1 / \$ 0 . N$ NETERS

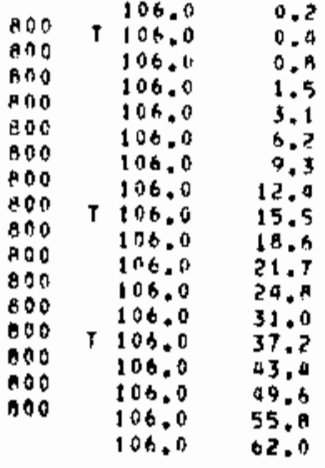

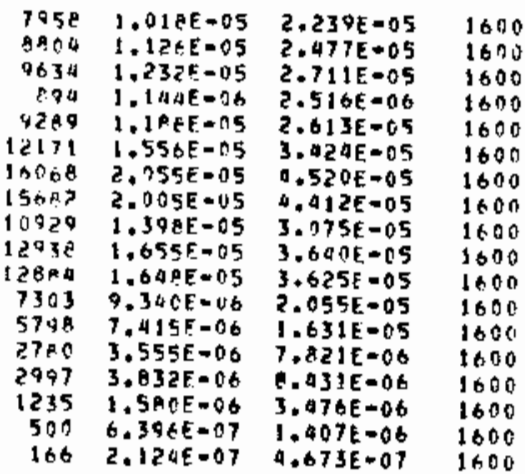


SECOADART SBRIES CXPSPIXRMT: IB-4F

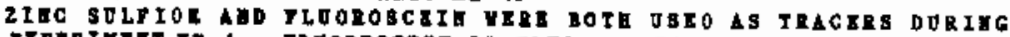

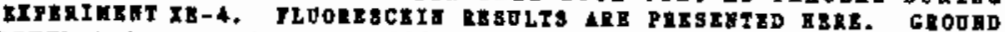

LETEL AEO TOUED BAXPLTEG $200-1600$ K ARGB.

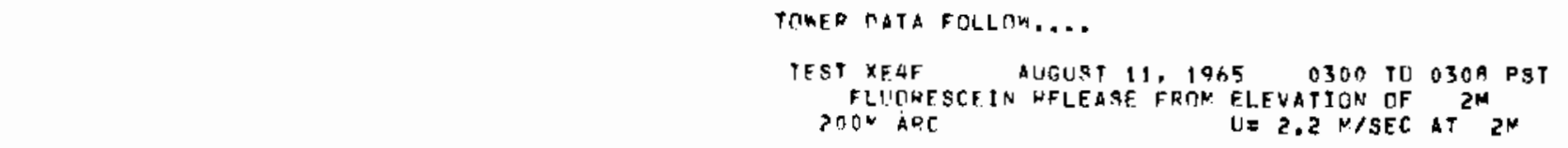

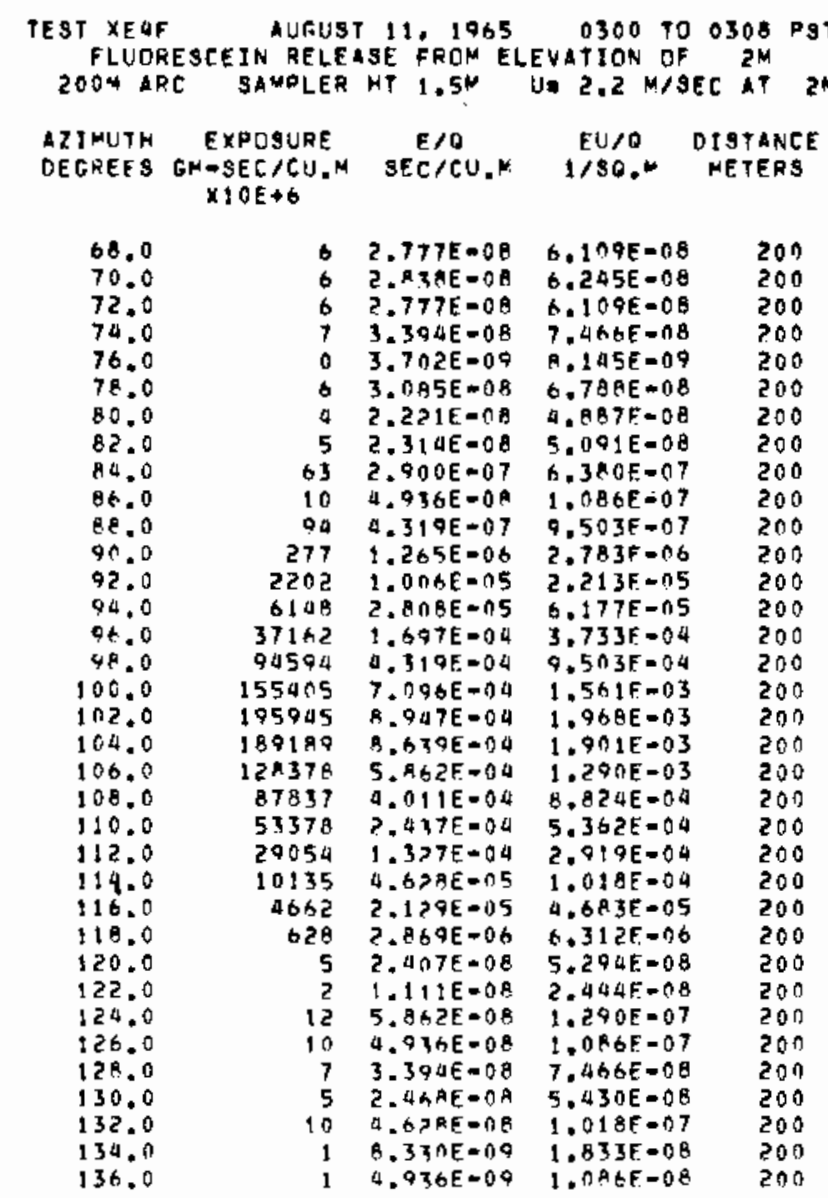

LIJMITH HE ISHT

EXPDSURE

$E / 0$

EUTO OISTANCE M DEGHEES MEYERS GM-SEC/CI." SEC/CU.M I/SH.M HETERS $\times 10 E+6$

\begin{tabular}{|c|c|c|c|c|c|}
\hline $\begin{array}{l}9 A .0 \\
98.0 \\
98.0 \\
98.0 \\
98.0 \\
98.0 \\
98.0 \\
98.0 \\
98.0 \\
98.0 \\
98.0 \\
98.0 \\
98.0 \\
98.0 \\
98.0 \\
9 A .0\end{array}$ & $\begin{array}{l}0.3 \\
0.7 \\
1.4 \\
2.7 \\
4.1 \\
5.9 \\
6.1 \\
9.1 \\
9.4 \\
10.8 \\
13.5 \\
16.2 \\
18.9 \\
21.6 \\
24.3 \\
27.0\end{array}$ & 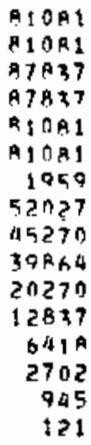 & $\begin{array}{l}3.702 E=04 \\
3.702 E=04 \\
4.011 E=04 \\
4.011 E=04 \\
3.702 E=04 \\
3.702 E=04 \\
9.947 F=06 \\
2.37+E=04 \\
2.067 E=04 \\
1.820 E=04 \\
9.256 E=05 \\
5.862 E=05 \\
2.031 E=05 \\
1.234 E=05 \\
9.319 E=06 \\
5.553 E=07\end{array}$ & $\begin{array}{l}A .145 E=04 \\
B .145 E=04 \\
8.824 E=04 \\
8.824 E=04 \\
8.145 E=04 \\
8.145 E=04 \\
1.968 E=05 \\
5.226 E=04 \\
9.54 A E=04 \\
4.005 E=04 \\
2.036 E=04 \\
1.200 E=04 \\
6.448 E=05 \\
2.715 E=05 \\
9.503 E=06 \\
1.222 E=06\end{array}$ & $\begin{array}{l}200 \\
200 \\
200 \\
200 \\
200 \\
200 \\
200 \\
200 \\
200 \\
200 \\
200 \\
200 \\
200 \\
200 \\
200 \\
200\end{array}$ \\
\hline
\end{tabular}

\begin{tabular}{|c|c|c|c|c|c|}
\hline $\begin{array}{l}\text { DIMUTH } \\
\text { OEGREES }\end{array}$ & $\begin{array}{l}\text { HEIGHT } \\
\text { METERS }\end{array}$ & $\begin{array}{l}\text { EXPOSURE } \\
\text { GM-SEC } / \text { CII.M } \\
\times 1 O E+6\end{array}$ & $\begin{array}{c}E / Q \\
S E E / C U_{*} N\end{array}$ & $\begin{array}{c}E U / O \\
1 / S Q . N\end{array}$ & $\begin{array}{c}\text { DIST ANCE } \\
\text { METERS }\end{array}$ \\
\hline $\begin{array}{l}106.0 \\
106.0 \\
106.0 \\
106.0 \\
106.0 \\
106.0 \\
106.0 \\
106.0 \\
106.0 \\
106.0 \\
106.0 \\
106.0 \\
106.0 \\
106.0 \\
106.0\end{array}$ & $\begin{array}{r}0.3 \\
0.7 \\
1.4 \\
4.1 \\
5.4 \\
6.8 \\
8.1 \\
9.4 \\
10.9 \\
13.5 \\
16.2 \\
18.9 \\
21.6 \\
24.3 \\
27.0\end{array}$ & $\begin{array}{l}114964 \\
128378 \\
135135 \\
121621 \\
110864 \\
101351 \\
87437 \\
74374 \\
52702 \\
41891 \\
48649 \\
18243 \\
8783 \\
4739 \\
2432\end{array}$ & $\begin{array}{l}5.245 E=04 \\
5.862 E=04 \\
6.171 E=04 \\
5.553 E=04 \\
5.245 E=04 \\
4.628 E=04 \\
4.011 E=04 \\
3.394 E=04 \\
2.407 E=04 \\
1.813 E=04 \\
2.221 E=04 \\
8.330 E=05 \\
4.011 E=05 \\
2.160 E=05 \\
1.111 E=05\end{array}$ & $\begin{array}{l}1.154 E=03 \\
1.240 E=03 \\
1.358 E=03 \\
1.222 E=03 \\
1.154 E=03 \\
1.018 E=03 \\
8.82 A E=04 \\
7.466 E=04 \\
5.294 E=04 \\
4.208 E=04 \\
4.887 E=04 \\
1.833 E=04 \\
8.824 E=05 \\
4.751 E=05 \\
2.444 E=05\end{array}$ & $\begin{array}{l}200 \\
200 \\
200 \\
200 \\
200 \\
200 \\
200 \\
200 \\
200 \\
200 \\
200 \\
200 \\
200 \\
200 \\
200\end{array}$ \\
\hline
\end{tabular}

DZJMITH HEIGMT EXPTSLIPE E/O EU/G DISTANTE DEGHEES RETERS GM-SEC/CIIM SEC/CU.N 1/SO.M METERS $\times 10 E+6$

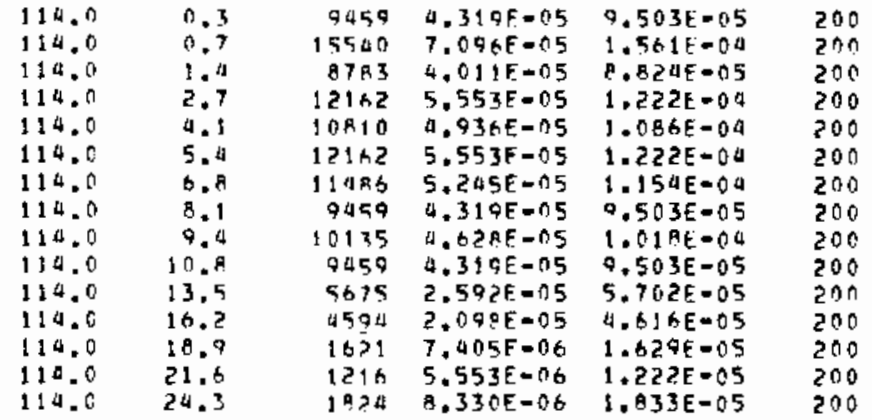




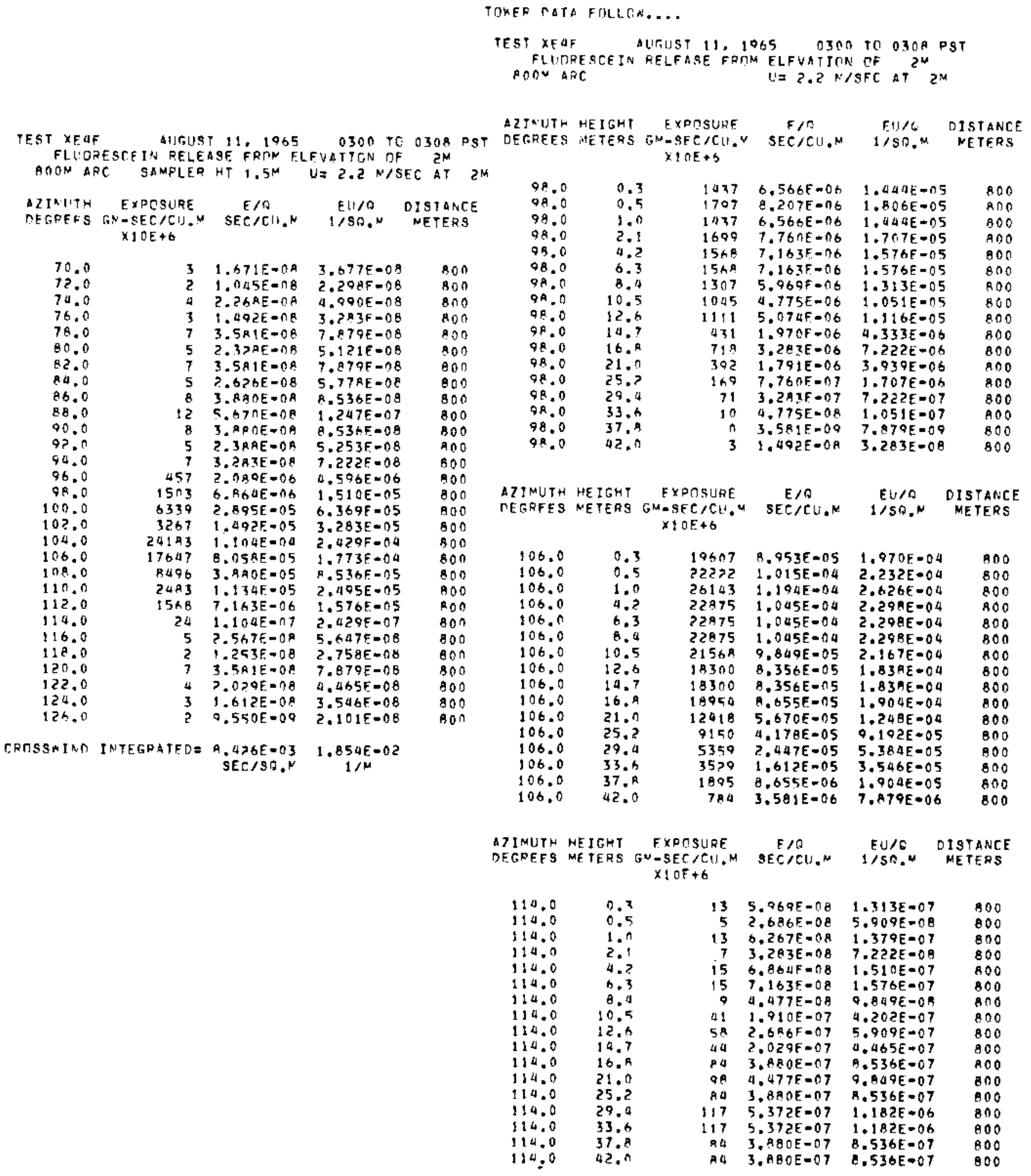




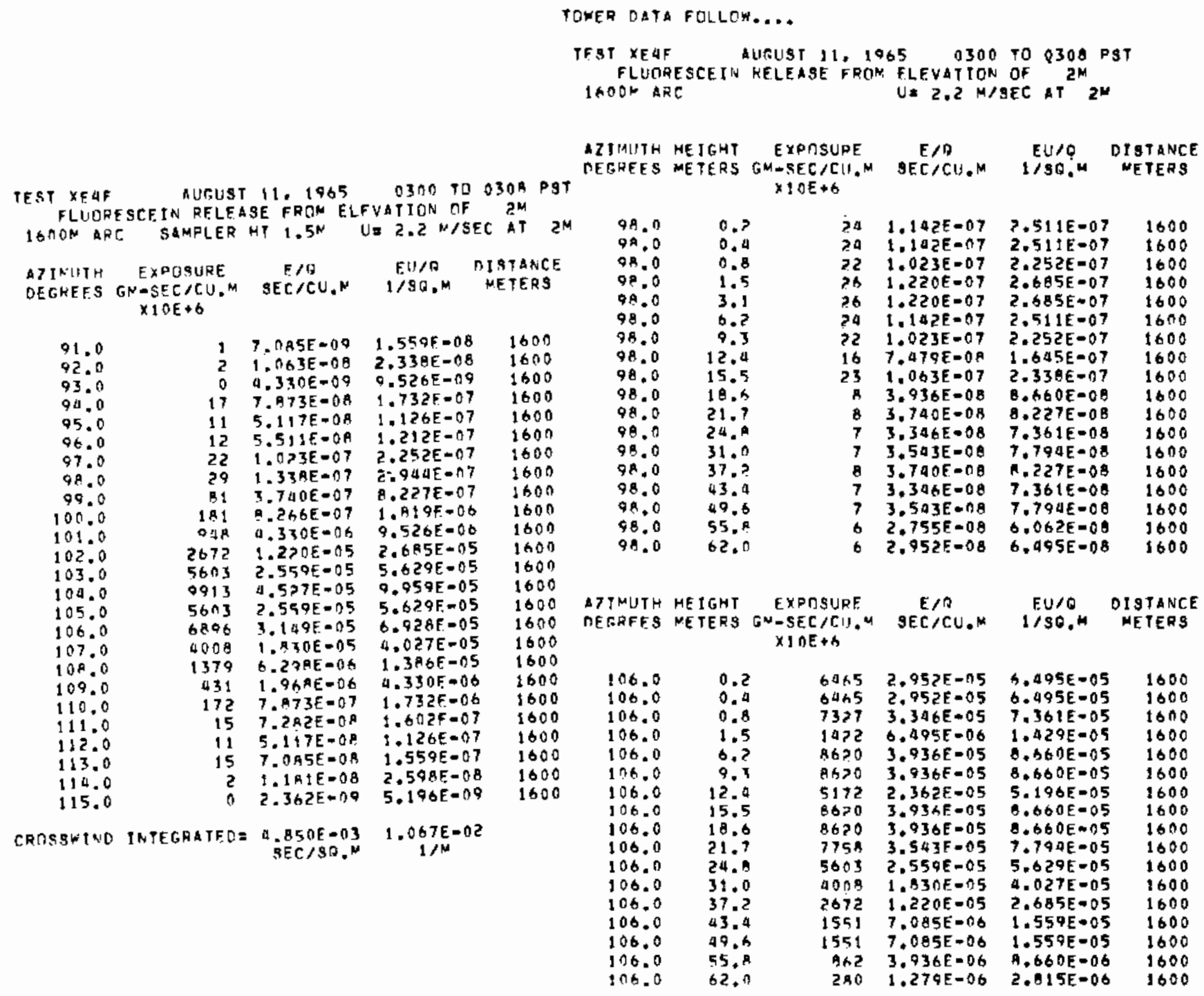




\begin{tabular}{lll} 
Xenon Data for Xe-4 & \\
Position* & & E/ ? \\
\hline $1-094$ & & $3.66-5$ \\
$1-102$ & & $7.89-4$ \\
$1-106$ & & $5.38-4$ \\
$1-110$ & & $3.01-4$ \\
$1-114$ & & $7.47-5$ \\
& & \\
$2-098$ & & $5.94-6$ \\
$2-102$ & & $2.09-5$ \\
$2-106$ & & $1.07-4$ \\
$2-110$ & & $1.32-5$ \\
& \\
$3-100$ & & $1.80-6$ \\
$3-103$ & $3.31-5$ \\
$3-106$ & $4.23-5$ \\
$3-109$ & $3.58-6$ \\
$3-112$ & $3.38-7$
\end{tabular}

*Stable grid 
SEGOHDARY BERIES EXPBHIMENT: XE-5

ZINC SULFIDB AND FLUOROSCEIN WERE BOTA DSED AS TAACERS DURING

ZXPBRINETT XE-5. ZINC FLTORIDE RESULTS AEE PRESEHTED HERE.

GROUHD LEVEL AND TOKER SAMPLING 200-1500 M ARCS.

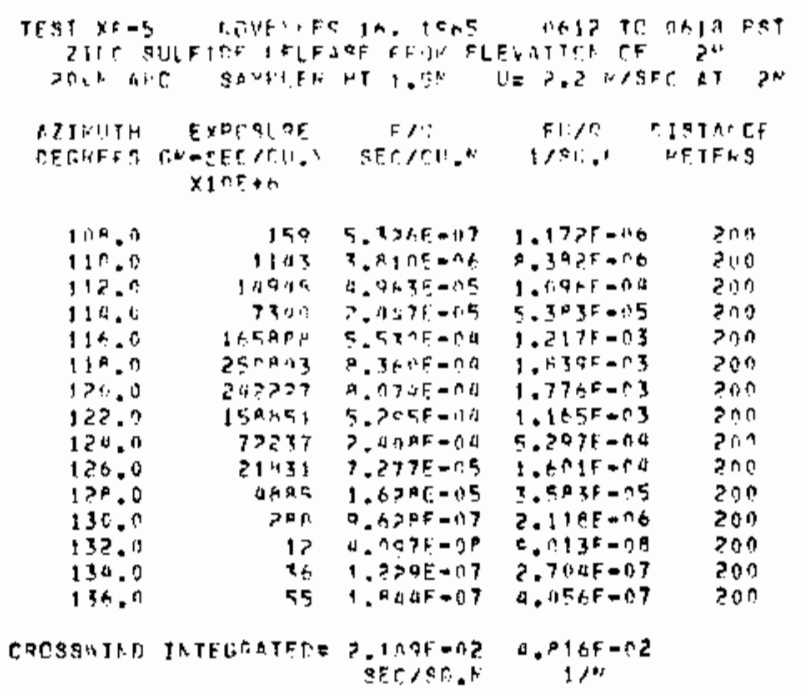

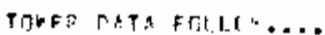

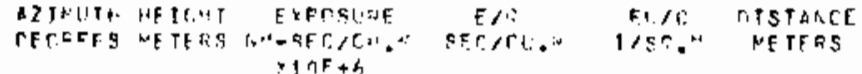

\begin{tabular}{|c|c|c|c|c|}
\hline 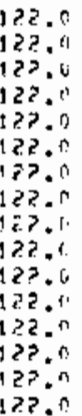 & $\begin{array}{l}0.3 \\
0.7 \\
1.3 \\
2.7 \\
1.1 \\
5.4 \\
5.4 \\
8.1 \\
9.8 \\
10.9 \\
13.5 \\
16.3 \\
18.9 \\
71.4 \\
54.3 \\
? 7.0\end{array}$ & 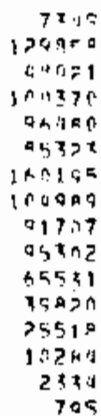 & 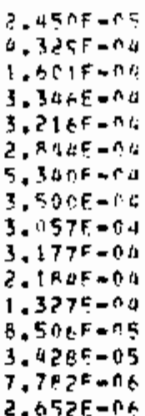 & $\begin{array}{l}5.390 E=05 \\
9.523 E=04 \\
3.522 E=04 \\
7.360 E=14 \\
7.075 E=04 \\
6.257 E=01 \\
1.175 E=03 \\
7.657 E=04 \\
6.725 E=04 \\
6.909 E=04 \\
1.906 E=04 \\
2.920 E=00 \\
1.871 E=04 \\
7.542 E=05 \\
1.713 E=05 \\
5.835 E=00\end{array}$ \\
\hline
\end{tabular}

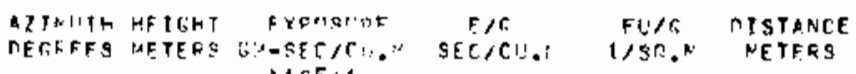
A I G F t h

\begin{tabular}{|c|c|c|c|c|c|}
\hline 131.0 & 0.3 & $4 \downarrow T$ & $1.492 E=\mathrm{nA}$ & $3,2 B 2[-00$ & 200 \\
\hline $13 r .0$ & 0.7 & 494 & $1.015 E=n t$ & $X .550 \mathrm{~F}=0 \mathrm{~A}$ & 200 \\
\hline $13 n .0$ & 1. a & 0,3 & $3.2457=80$ & $7.13 A E=06$ & 200 \\
\hline 132.11 & 2.7 & 72 & $1.067 F-07$ & $2.3 \cup A E-67$ & $20 n$ \\
\hline $13 n, 0$ & 4.1 & 204 & 9. R1 1 SE $=07$ & $2.160 E-0 B$ & $2 \times 0$ \\
\hline $13 n . n$ & 5.4 & $3 \cap r$ & $1 . \ln \geq 5=n 6$ & $z, 707 E=.56$ & 200 \\
\hline 130.0 & 6. 0 & $47 x$ & 1.58 त $5=06$ & T. $.755=06$ & 200 \\
\hline $13 n .0$ & 8.1 & ¿1 & $1.7 C F E=n 7$ & $3.757 \mathrm{~F}-0.7$ & 200 \\
\hline 13 & 8. . 1 & $1 \pi ?$ & $\because 41 \mid F-n 7$ & $0.7055=017$ & 200 \\
\hline $13 f$ & 10.8 & $<1$ & $1.7500-27$ & $3.757 f=07$ & $20 n$ \\
\hline 13 & 13.5 & $1 \mathrm{~A} a$ & $5.55 \cap .5-\cap 7$ & $1.221 E=06$ & 200 \\
\hline 130.0 & 16.7 & $n !$ & $3.0 \leftrightarrow \cap f=07$ & $6.731 E-67$ & 200 \\
\hline $13 n . n$ & 1ค.? & रे & $1, n \in ? F=n]$ & $2.34 A E-07$ & 200 \\
\hline
\end{tabular}

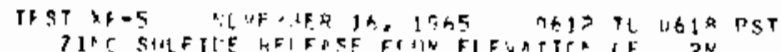

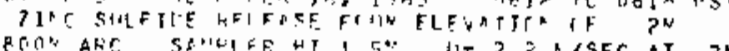

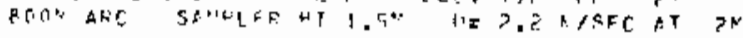

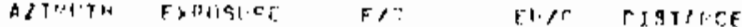

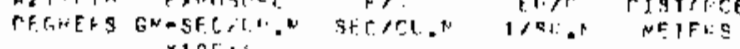
$\times 1 \cap[+\varepsilon$

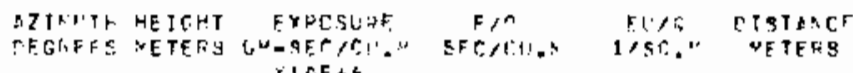
$x I P: F+h$

$.590 F=04 \quad 200$

$111 . n$

110.1

110.6

$114 . \hat{0}$

$F$ is.

114.0

110.0

114.8

114.0

110.0

114.0

10.0

10.0

$110.0 \quad 77.4$

\begin{tabular}{|c|c|c|c|c|}
\hline $114 . ?$ & $2+1$ & $\Rightarrow 1 \wedge \Gamma-07$ & $1 . S 1 H F=n_{n}$ & A 0 n \\
\hline $11+.11$ & $115 \%$ & A. FA:IIF - nt & $5.501 F=16$ & ann \\
\hline 118.0 & 111 月1 & I.71K5-115 & $\because .176, F=05$ & Acon \\
\hline $1 \hat{c}^{n}=0$ & 320,110 & $1 . " \therefore A=-n$ & $2.350 .5-f 4$ & A00 \\
\hline 122.11 & $x>x+$ & $1 . ? 5 \mathrm{a} A \mathrm{E}=0 \Delta$ & $2.760 .5=04$ & AOก \\
\hline 124.0 & 16977 & $5.0 .09-05$ & $1.205 F-0,4$ & Bกn \\
\hline 126.0 & 3542 & $1.1 \times\{=-75$ & $3.598 \mathrm{~F}+155$ & AnO \\
\hline 12 & $4 \cap \Delta$ & $1.3+7 F=10$ & $2.0441=06$ & 800 \\
\hline $13[0,0$ & 1 if & H.QC IF $=$ ne & $1.000=07$ & $A \cap C$ \\
\hline
\end{tabular}

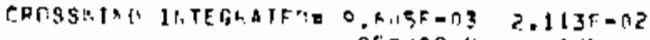




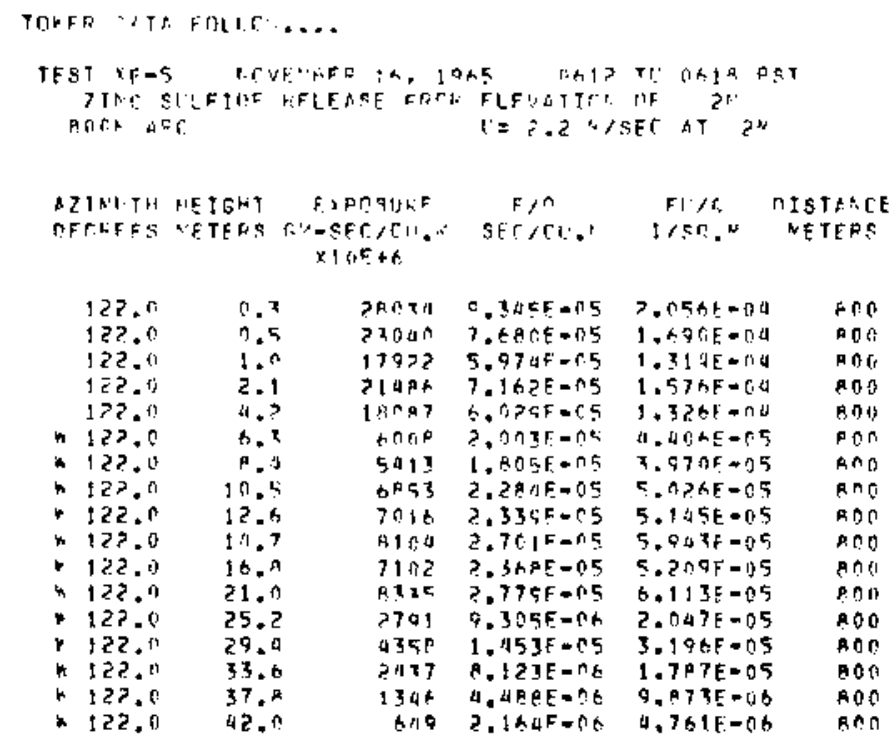

THEF IIATS FOLLCH....

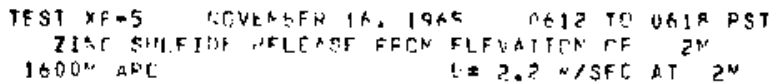

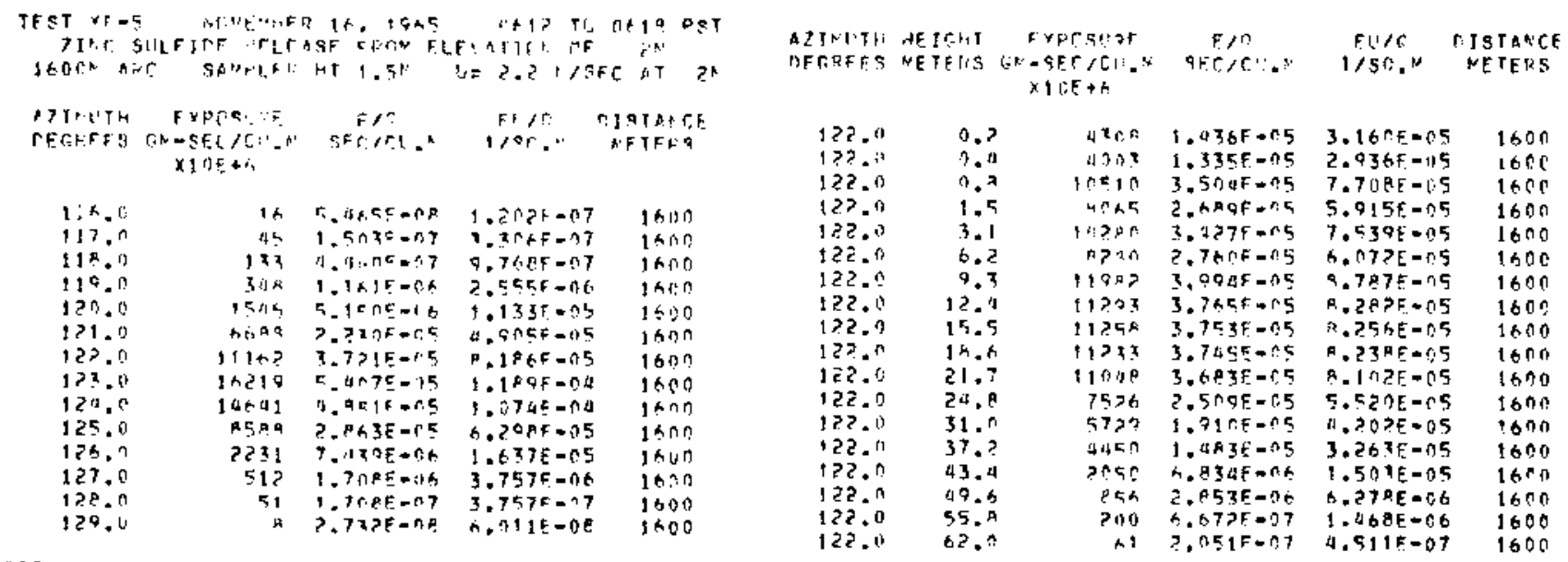

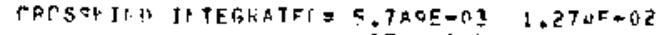

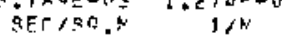


SECOFDARY SERIES BXPERIMZHT: XE-5F

ZINC SULYIDE AHD FLOOROSCEIN WERE BOTH USED AS TRACQRS DORING EXPEQIHSTT KE-5. FLDORESCEIN RESDLTS ARE PRESEHTED HERE, GROUND LBVEL AND TOWER SAMPLING 200-1600 B ARCS.

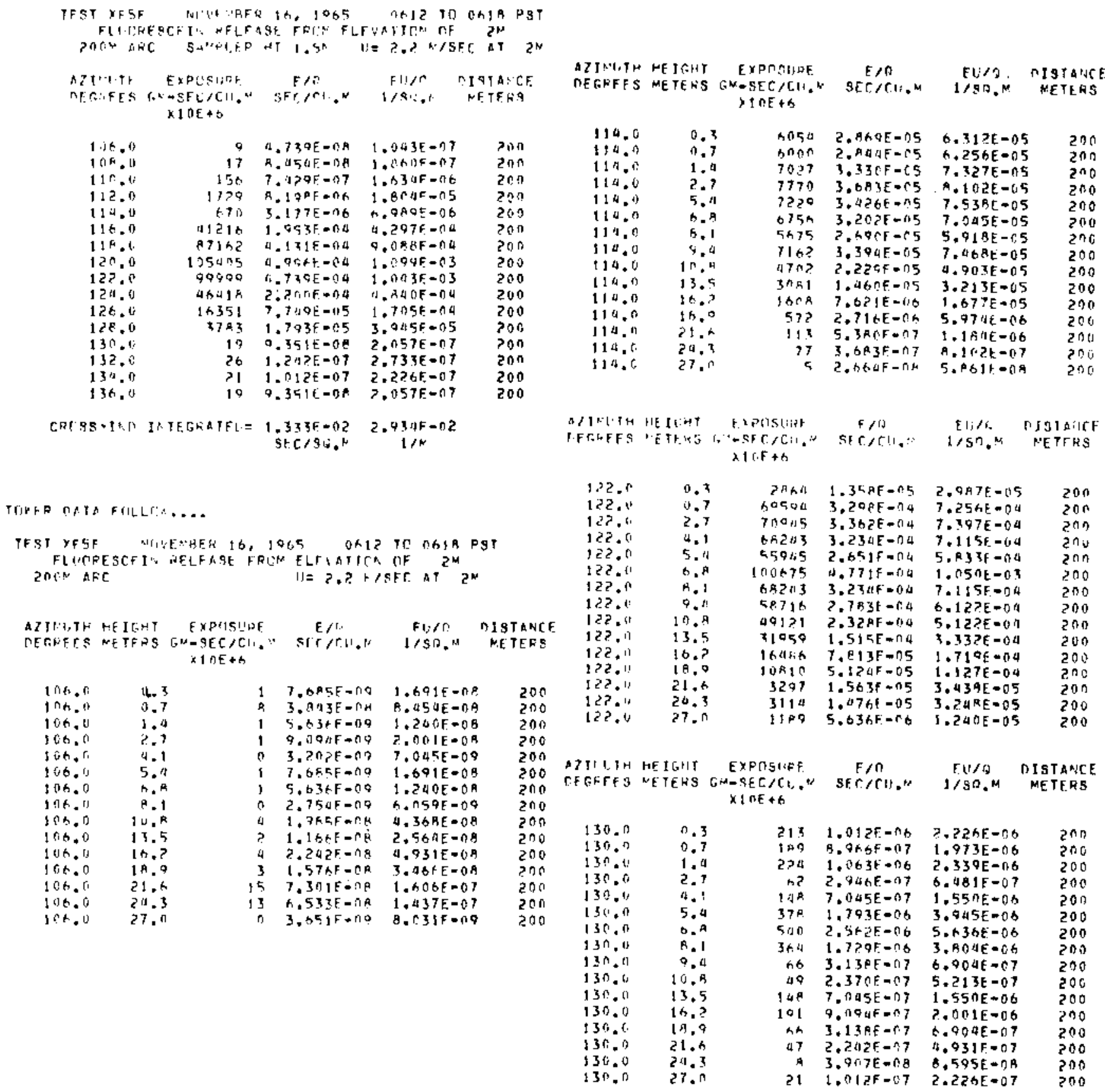




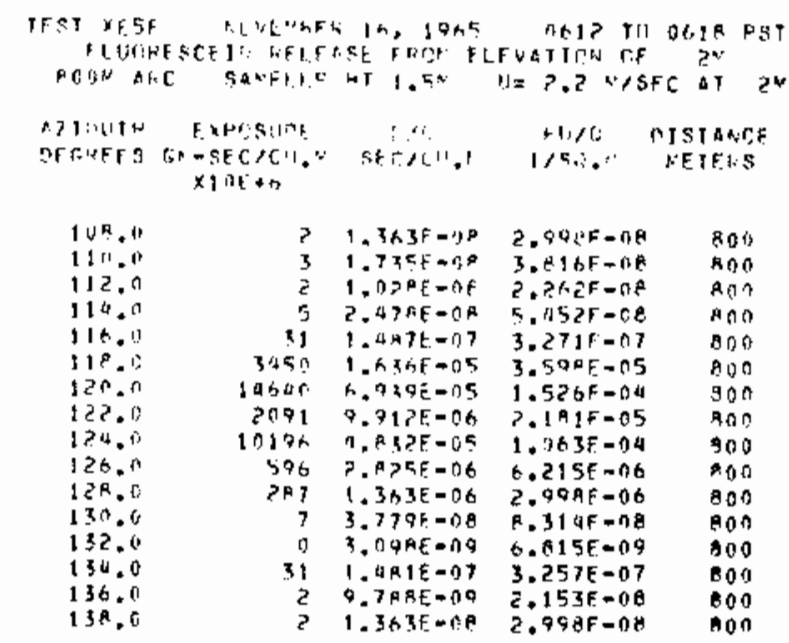

CENSSRIAD IATERHATETS

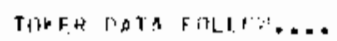

AZINIITH HEIGHT EXPDSUNE E/R EIJO DISTANCE

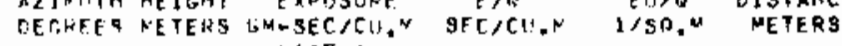

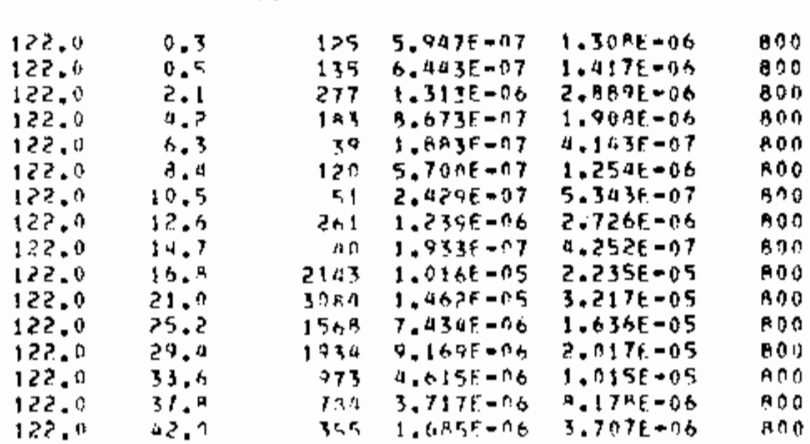

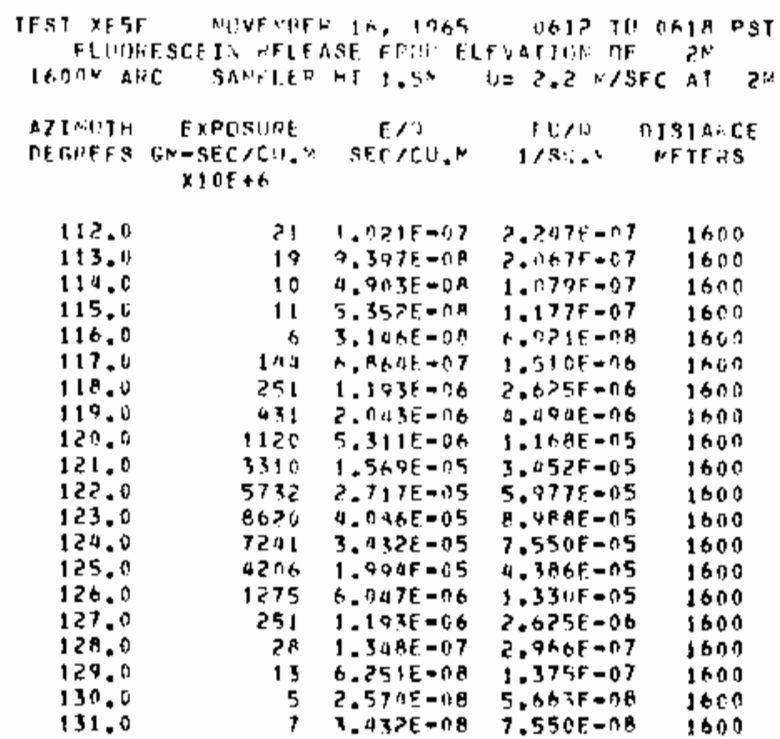

CFOSSXIFD INTEGRATEIY= 4.3

$$
\text { SEC.SOA R }
$$

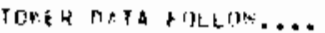

IFST XF5F NUVEPEP IS, 1965 OBD TO 0618 PST

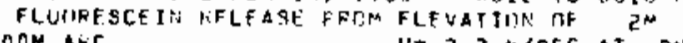

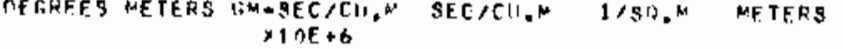

\begin{tabular}{|c|c|c|c|c|c|}
\hline 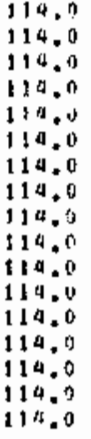 & $\begin{array}{l}0.7 \\
0.1 \\
0.9 \\
1.5 \\
3.1 \\
6.2 \\
9.3 \\
12.4 \\
15.5 \\
18.6 \\
21.7 \\
24.8 \\
31.0 \\
37.2 \\
43.4 \\
55.8 \\
62.0\end{array}$ & $\begin{array}{r}4 \\
1 \\
n \\
1 \\
1 \\
7 \\
20 \\
15 \\
6 \\
7 \\
6 \\
11 \\
20 \\
18 \\
2 \\
1 \\
10\end{array}$ & 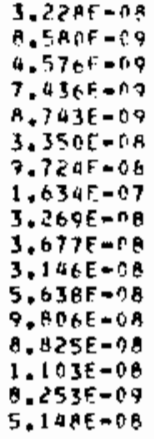 & $\begin{array}{l}7.101 E=0 A \\
1.08 B E=0 A \\
1.007 E=0 B \\
1.036 E=0 R \\
1.924 E=0 B \\
7.370 E=0 A \\
2.139 E=07 \\
3.595 E=07 \\
7.191 E=0 B \\
8.090 E=0 B \\
6.921 E=03 \\
1.240 E=07 \\
2.157 E=07 \\
1.941 E=07 \\
2.427 E=0 B \\
1.916 E=0 B \\
1.133 E=07\end{array}$ & $\begin{array}{l}1600 \\
1600 \\
1600 \\
1600 \\
1600 \\
1600 \\
1600 \\
1600 \\
1600 \\
1600 \\
1600 \\
1600 \\
1600 \\
1600 \\
1600 \\
1600 \\
1600\end{array}$ \\
\hline
\end{tabular}




\begin{tabular}{|c|c|c|c|c|c|}
\hline 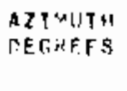 & $\begin{array}{l}\text { WEITHT } \\
\text { NETERS }\end{array}$ & $\begin{array}{c}E x P D S I N E \\
G x=S E C>C=.4 \\
x+0 E+A\end{array}$ & $\begin{array}{c}E / r \\
\text { SEC } / \mathrm{CH}, \mathrm{M}\end{array}$ & $\begin{array}{l}511 / 0 \\
1 / 5,2.02\end{array}$ & $\begin{array}{l}\text { DISTANEE } \\
\text { NEYERS }\end{array}$ \\
\hline $\begin{array}{l}122.0 \\
122.0 \\
122.0 \\
122.0 \\
122.0 \\
122.0 \\
122.0 \\
122.0 \\
122.0 \\
122.0 \\
122.0 \\
122.0 \\
122.0 \\
122.0 \\
122.0 \\
122.0 \\
122.0\end{array}$ & $\begin{array}{l}0 . ? \\
0.4 \\
1.5 \\
3.1 \\
6.2 \\
7.5 \\
12.7 \\
15.5 \\
18.5 \\
21.7 \\
24.8 \\
51.0 \\
37.2 \\
43.4 \\
48.5 \\
55.8 \\
52.0\end{array}$ & 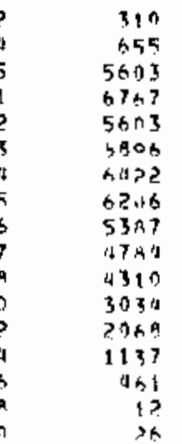 & 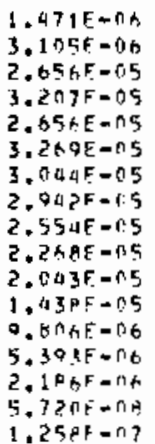 & 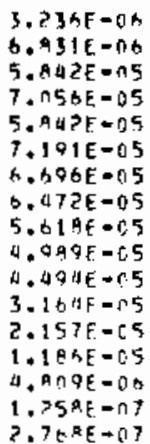 & $\begin{array}{l}16019 \\
1600 \\
1600 \\
1690 \\
1600 \\
1600 \\
1600 \\
1000 \\
1600 \\
1600 \\
1600 \\
1600 \\
1600 \\
1600 \\
1600 \\
1600 \\
16011\end{array}$ \\
\hline
\end{tabular}


Xenon Data for $\mathrm{Xe}-5$

$\begin{array}{lll}\text { Position } & & E / Q \\ 1-108 & & 5.15-6 \\ 1-114 & & 2.16-4 \\ 1-120 & & 8.85-4 \\ 1-126 & & 1.05-4 \\ 1-130 & & 3.12-6 \\ & & \\ 2-114 & & 1.67-7 \\ 2-120 & & 1.72-4 \\ 2-126 & & 5.96-5 \\ 2-130 & & 2.12-5 \\ & & \\ 3-118 & & 4.48-6 \\ 3-122 & & 8.15-5 \\ 3-126 & & 1.02-5\end{array}$

*Stable grid 


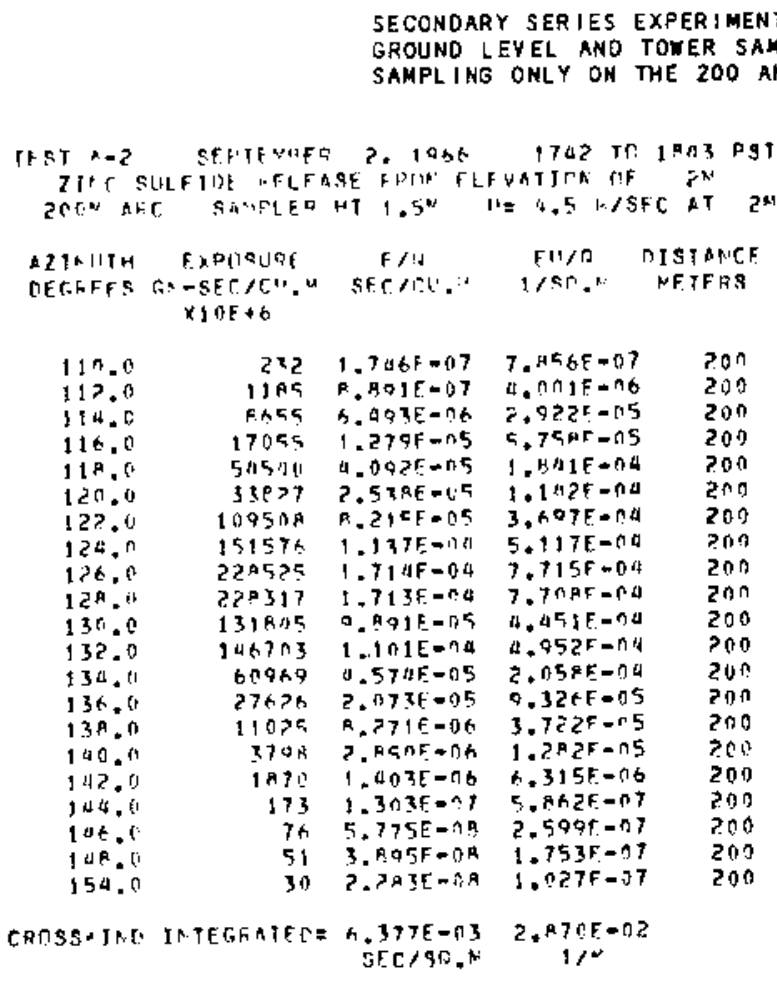

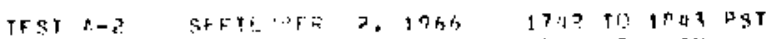

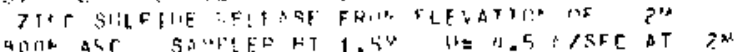

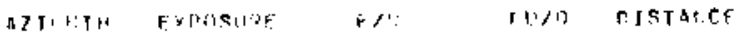

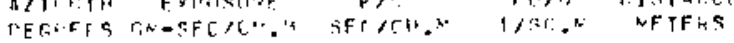
$x i n t+1$

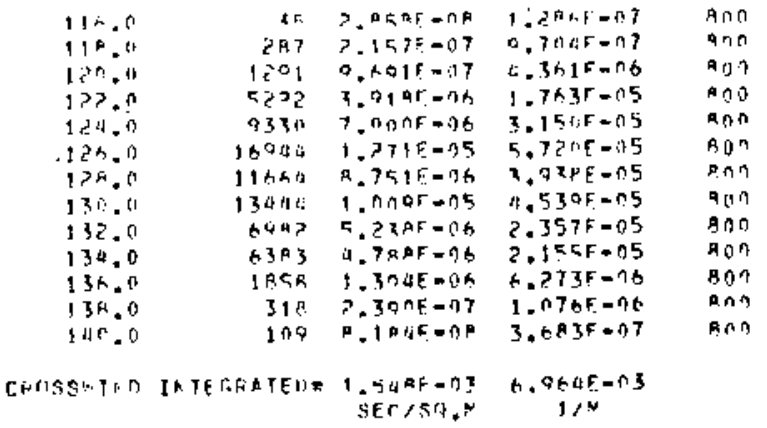

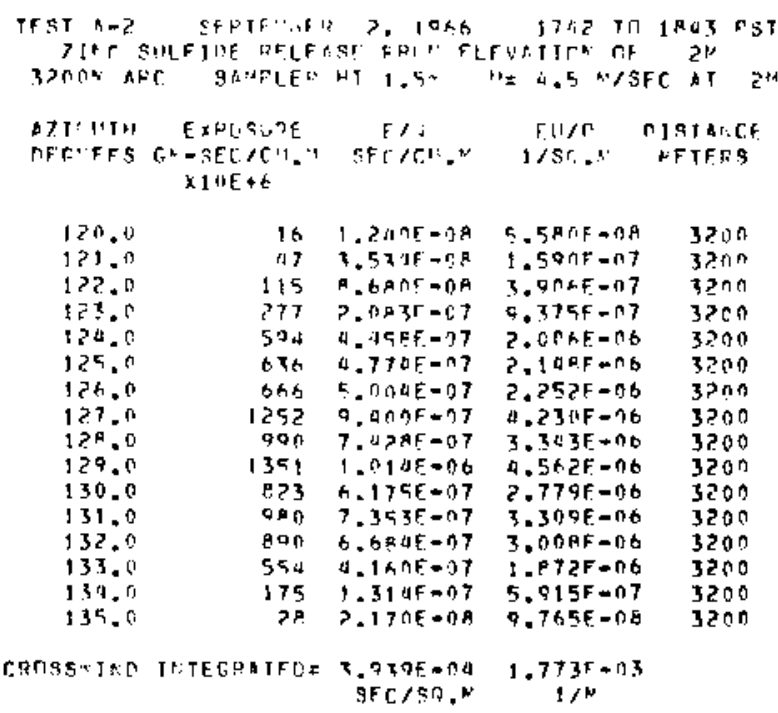




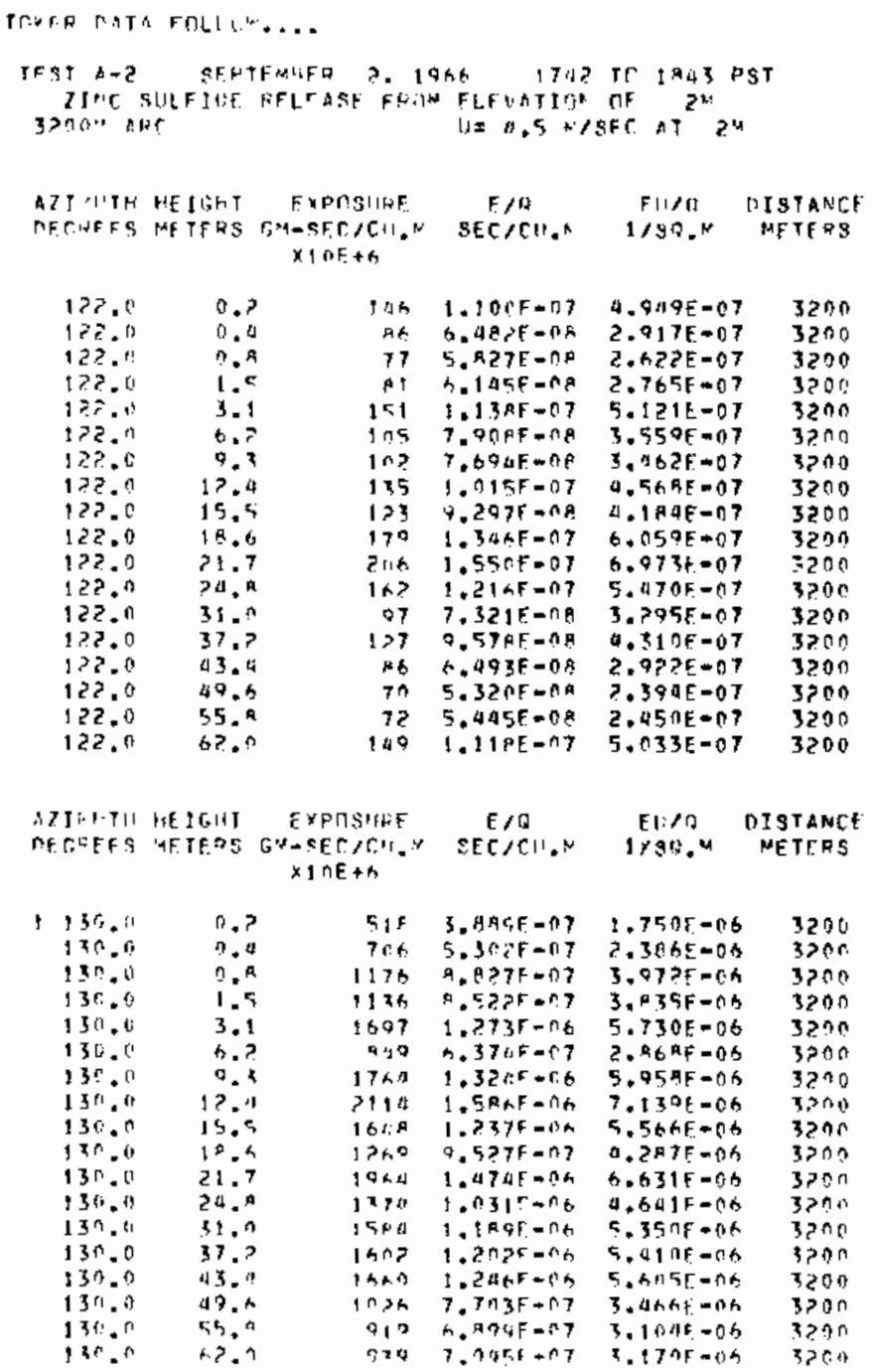


SECONDARY SERIES EXPERIMENT: $A-5$

GROUNO LEVEL AND TOHER SAMPLING ON THE 3200 M ARC. GROUND LEYEL SAMPLING ONLY ON THE BOO M ARC.

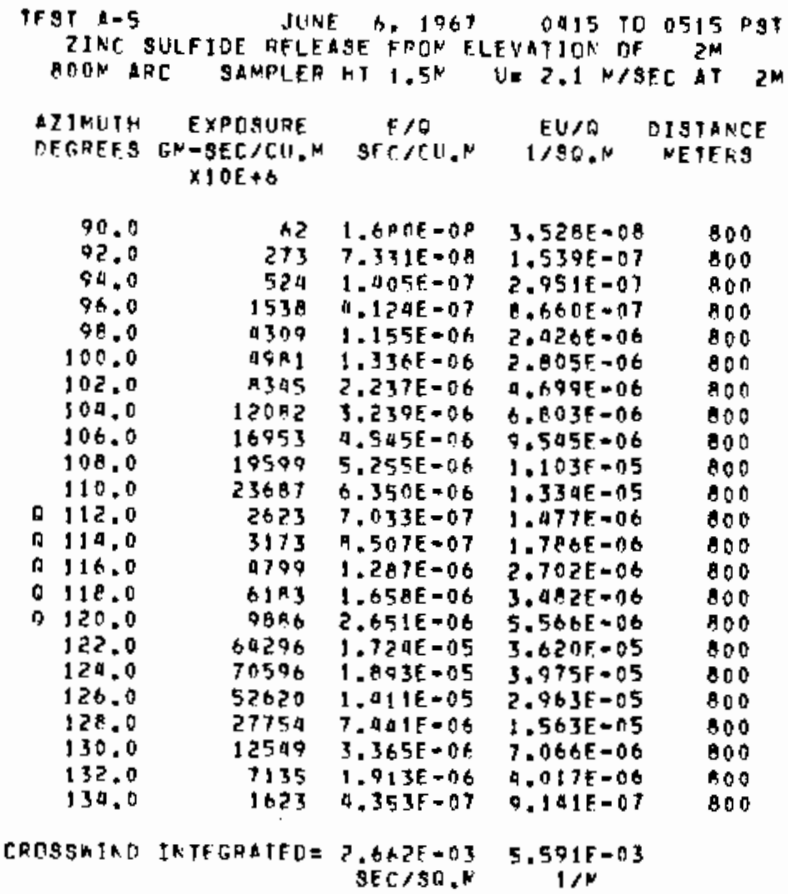

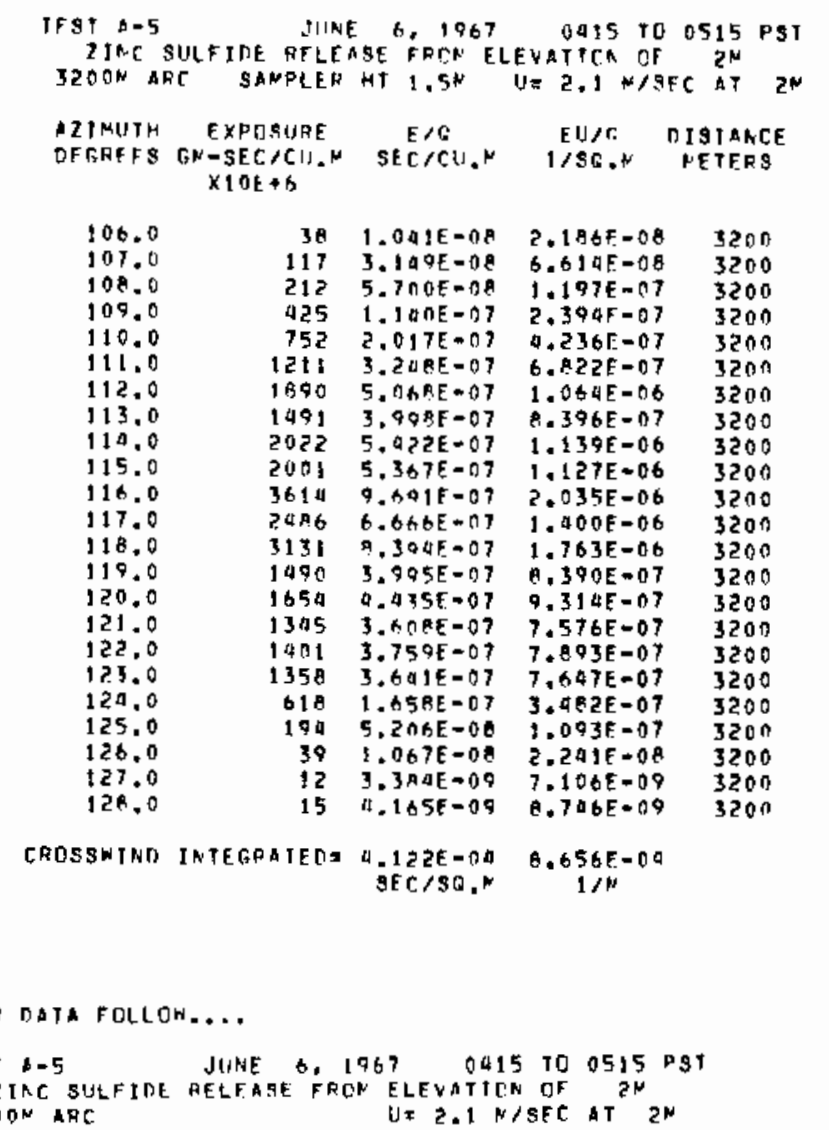

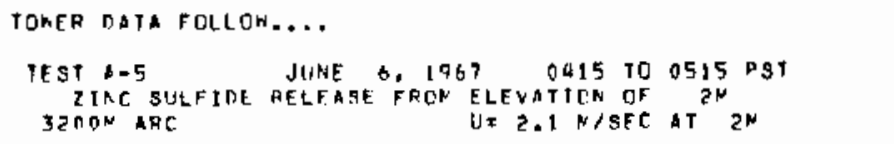
AZJNUTH HETGHT EXPOSURE F/O EU/Q DISTANCE DETREES METERS GM-SECICH.N SECICU.N IISA.M NETFRS $10 E+6$

\begin{tabular}{|c|c|c|c|c|c|}
\hline $\begin{array}{l}506.0 \\
108.0 \\
106.0 \\
106.0 \\
106.0 \\
106.0 \\
106.0 \\
106.0 \\
106.0 \\
106.0 \\
106.0 \\
106.0 \\
106.0 \\
106.0 \\
100.0 \\
106.0 \\
106.0 \\
106.0\end{array}$ & $\begin{array}{l}0.2 \\
0.4 \\
0.8 \\
1.5 \\
3.1 \\
6.2 \\
9.3 \\
12.1 \\
15.5 \\
18.6 \\
21.7 \\
24.8 \\
11.0 \\
37.2 \\
43.4 \\
49.6 \\
55.8 \\
62.0\end{array}$ & $\begin{array}{l}110 \\
142 \\
130 \\
182 \\
219 \\
279 \\
287 \\
275 \\
215 \\
170 \\
179 \\
197 \\
293 \\
319 \\
392 \\
454 \\
509 \\
96\end{array}$ & 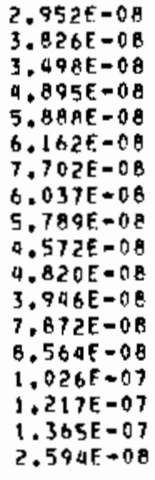 & $\begin{array}{l}6.198 E=0 R \\
8.035 E=08 \\
7.346 E=0 A \\
1.028 E=07 \\
1.237 E=07 \\
1.294 E=07 \\
1.617 E=07 \\
1.26 A E=07 \\
1.216 E=07 \\
9.600 E=08 \\
1.012 E=07 \\
8.287 E=06 \\
1.653 E=07 \\
1.199 E-07 \\
2.156 E=07 \\
2.557 E=07 \\
2.066 E=07 \\
5.047 E=0 A\end{array}$ & $\begin{array}{l}3200 \\
3200 \\
3200 \\
3200 \\
3200 \\
3200 \\
3200 \\
3200 \\
3200 \\
3200 \\
3200 \\
3200 \\
3200 \\
3200 \\
3200 \\
3200 \\
3200 \\
3200\end{array}$ \\
\hline
\end{tabular}




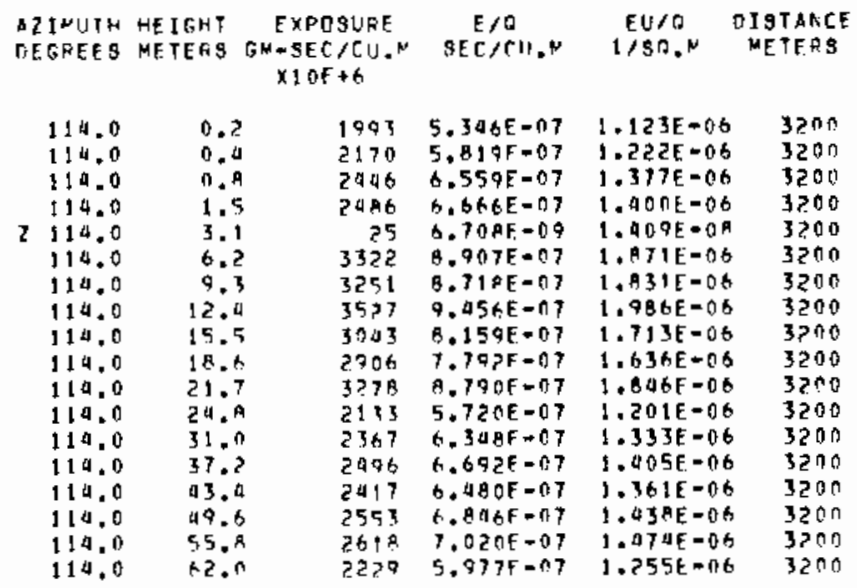

\begin{tabular}{|c|c|c|c|c|c|}
\hline $\begin{array}{l}\text { AZINLTH } \\
\text { DETEFES }\end{array}$ & $\begin{array}{l}\text { ME IEHT } \\
\text { ME TERS }\end{array}$ & 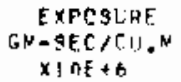 & $\begin{array}{c}E / Q \\
S E E / C U, N\end{array}$ & $\begin{array}{l}F \cup / 0 \\
1 / S O . M\end{array}$ & $\begin{array}{l}\text { DJGTANCE } \\
\text { WETEAS }\end{array}$ \\
\hline $\begin{array}{l}122.0 \\
122.0 \\
122.0 \\
122.0 \\
122.0 \\
122.0 \\
122.0 \\
122.0 \\
122.0 \\
122.0 \\
122.0 \\
122.0 \\
122.0 \\
122.0 \\
122.0 \\
122.0 \\
122.0 \\
122.0\end{array}$ & $\begin{array}{r}0.2 \\
0.4 \\
0.8 \\
1.5 \\
3.1 \\
6.2 \\
9.3 \\
12.4 \\
15.5 \\
18.0 \\
21.7 \\
24.8 \\
31.0 \\
37.2 \\
43.9 \\
49.6 \\
55.8 \\
62.0\end{array}$ & $\begin{array}{l}1644 \\
1636 \\
1537 \\
1546 \\
1352 \\
1990 \\
2394 \\
2028 \\
2798 \\
2232 \\
2770 \\
2153 \\
2029 \\
1472 \\
1793 \\
1718 \\
1711 \\
2077\end{array}$ & 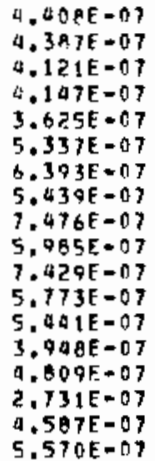 & $\begin{array}{l}9.250 E=07 \\
9.212 E=07 \\
8.655 E=07 \\
8.708 E=07 \\
7.612 E=07 \\
1.121 E=06 \\
1.342 E=06 \\
1.142 E=06 \\
1.570 E=06 \\
1.257 E=06 \\
1.560 E=06 \\
1.212 E-06 \\
1.103 E=06 \\
6.292 E=07 \\
1.010 E=06 \\
5.735 E=07 \\
9.633 E=07 \\
1.170 E=06\end{array}$ & $\begin{array}{l}3200 \\
3200 \\
3200 \\
3200 \\
3200 \\
3200 \\
3200 \\
3200 \\
3200 \\
3200 \\
3200 \\
3200 \\
3200 \\
3200 \\
3200 \\
3200 \\
3200 \\
3200\end{array}$ \\
\hline
\end{tabular}


SECONDARY SER IES EXPERIMENT; $A=6$

GROUNO LEYEL AND TOWER SAMPLING ON THE 3200 M ARC. GROUND LEVEL

SAMPI ING DNLY ON THE BDO H ARC. A SIGNIFICANT PORTION OF THE

PL, UME NEVER PASSEO OVER THE GRIO.

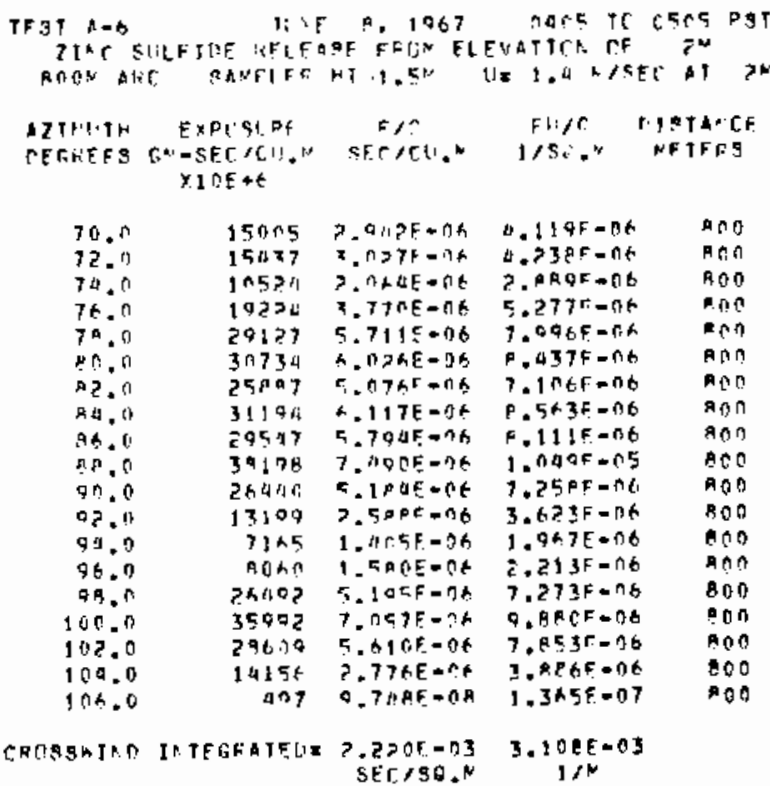

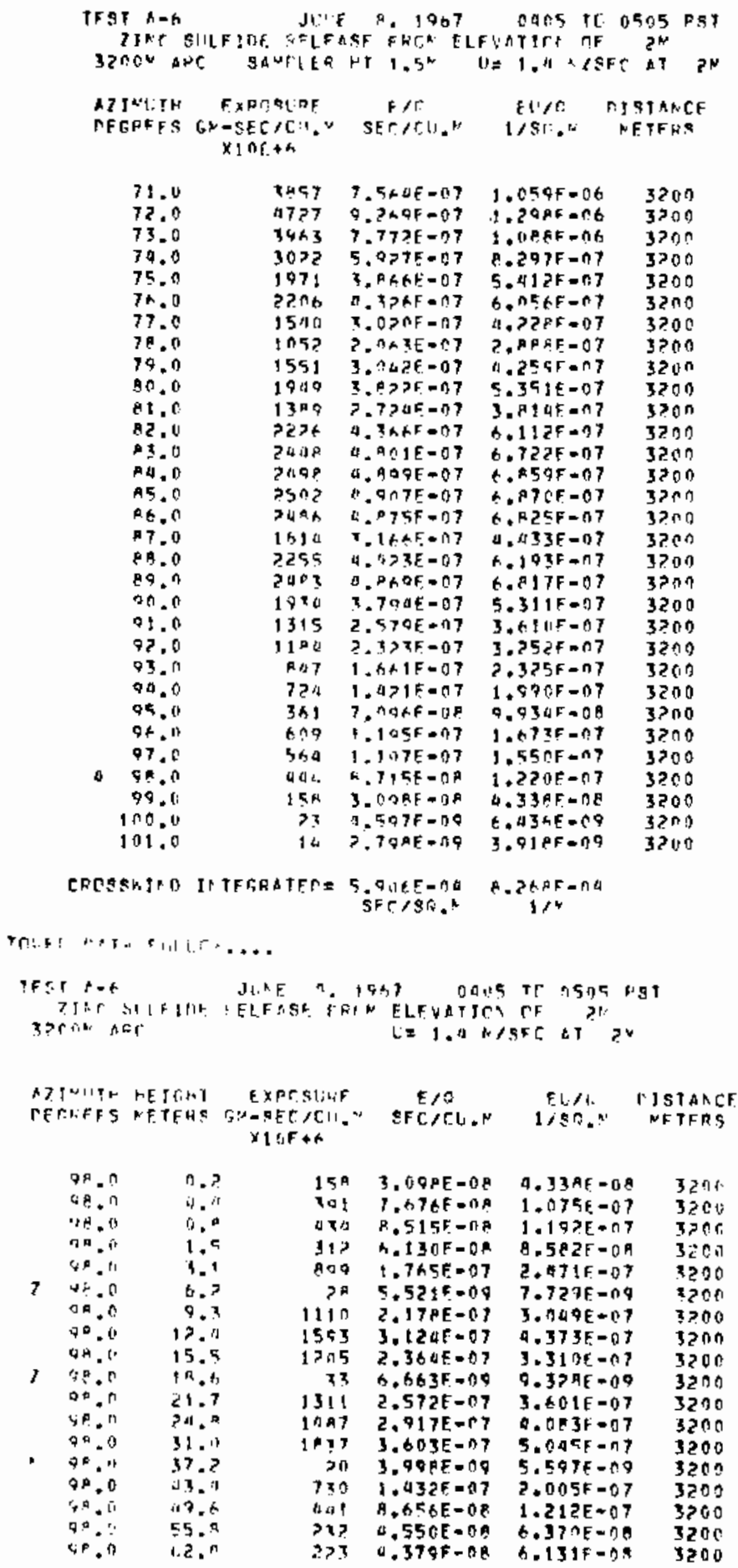


SECONDARY SERIES EXPERIHENT: A-7

GROUND LEYEL ANO TOWER SAMPLING ON THE 3200 M ARC. GROUND LEVEL SAMPLING ONLY ON THE 800 M ARC. A SIGNIFICANT PORTION OF THE PLUIE NEVER PASSED OVER THE GRID.

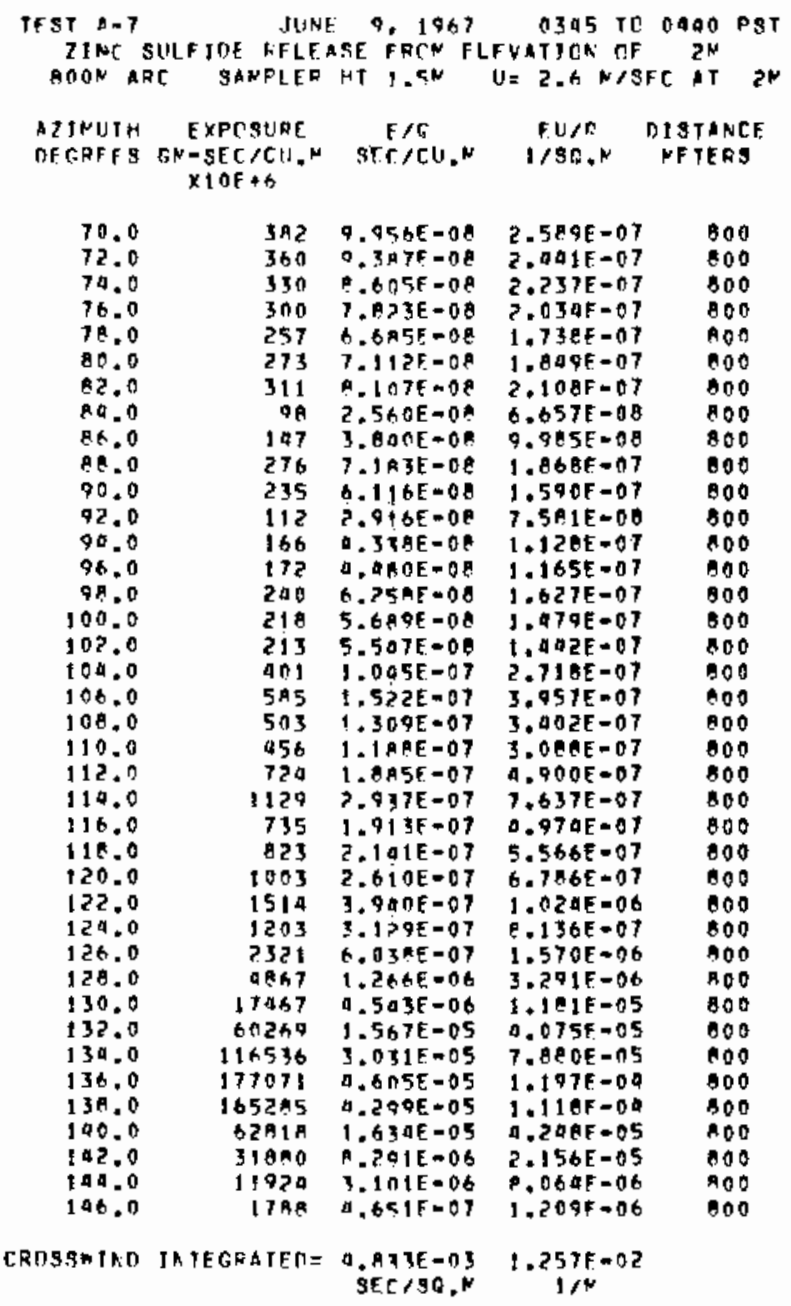




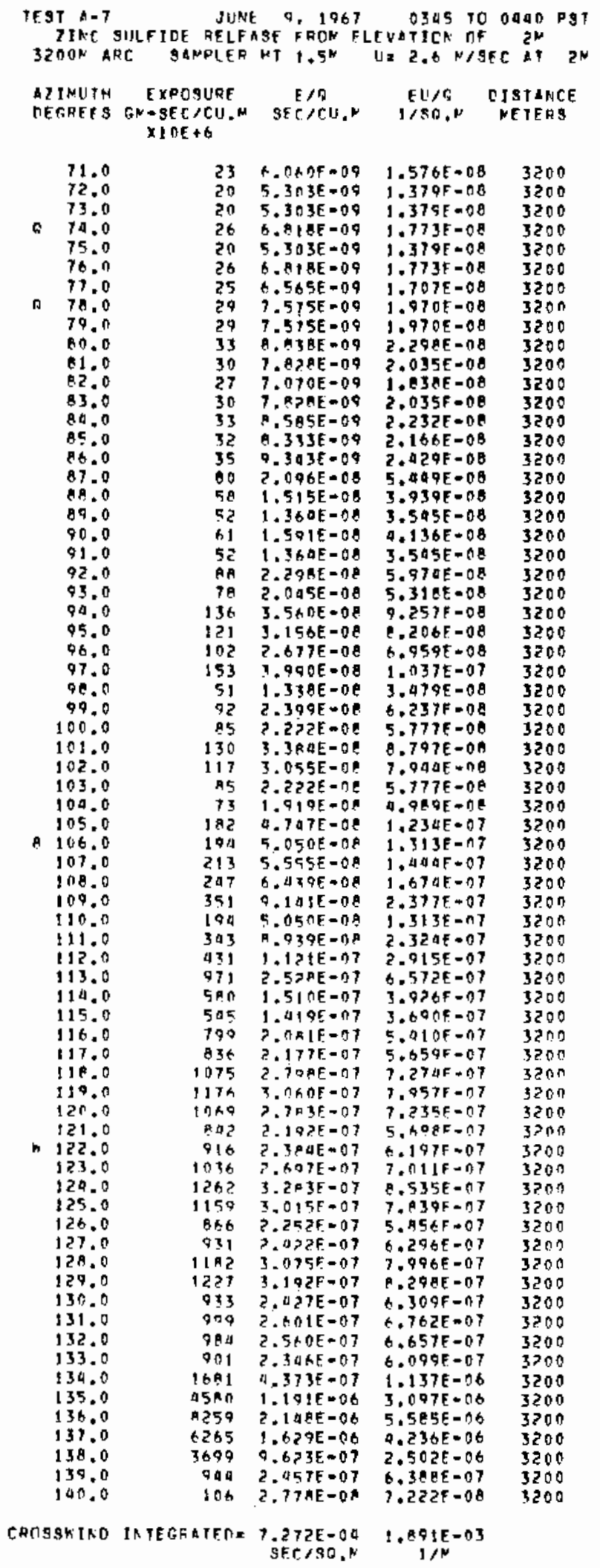

TOKER TATA FOLLOL....

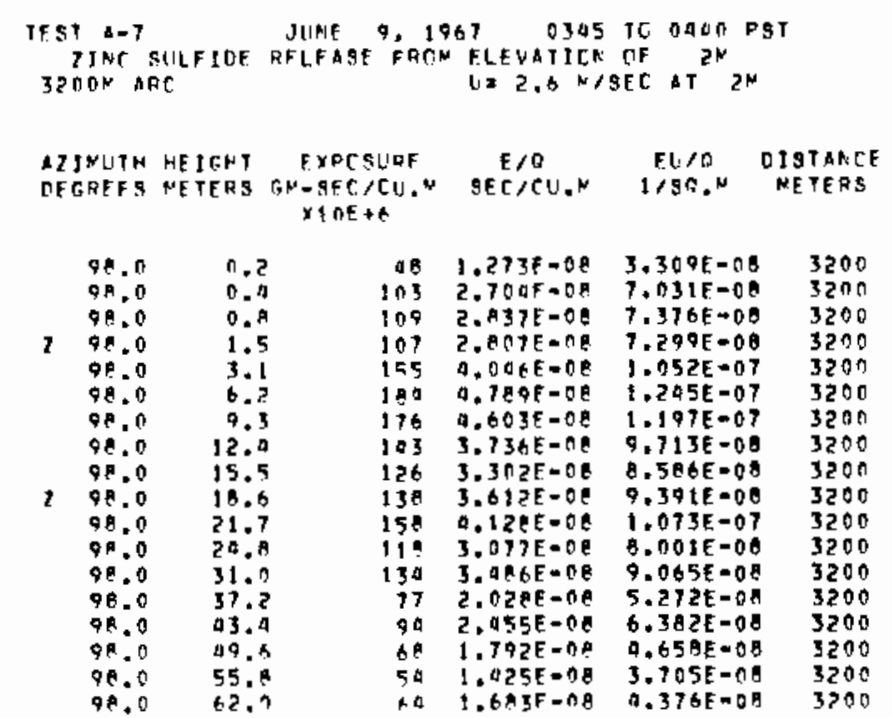
ATIKLTH HEIGHT EXPCSURE E/D EUTG DISTANCE TFERFES NETERS GN-SEC/CI!N SEC/TU.N J/SR.N NETERS $\times 10 E+6$

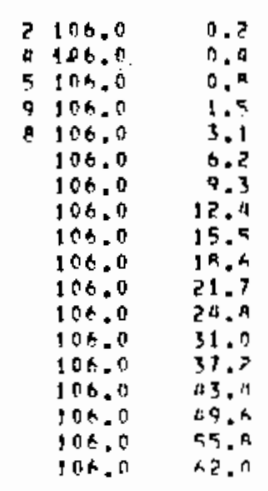
DZJKITH HEIEMT FXPCSURE F/G FU/G DISTARCF. TEGPFES NETERS GM-SEC/CII." SFCICH.N 1/3A.N NFTEFS

\begin{tabular}{|c|c|c|c|c|c|}
\hline 114.0 & 0.2 & A07 & $2.333 f=07$ & $\begin{array}{l}6.066 E=07 \\
5.34 Z E=07\end{array}$ & $3 ? 0 n$ \\
\hline 114.0 & 0.4 & $70 \%$ & $5 E=17$ & & $32 \pi n$ \\
\hline $\begin{array}{l}110.0 \\
119.0\end{array}$ & 0.9 & 653 & $A E+07$ & A. 01 SE -07 & $320 n$ \\
\hline $\begin{array}{l}119.0 \\
110.0\end{array}$ & 1.5 & 593 & $5 \Delta U E=07$ & $0.0148-0.7$ & $32 n 0$ \\
\hline $\begin{array}{l}110.0 \\
114.0\end{array}$ & 3. & $7>1$ & $1.876 .5=0.7$ & A.A7RF $=0\}$ & 3200 \\
\hline $\begin{array}{l}114,0 \\
119,0\end{array}$ & $6 . ?$ & $0>2$ & $P A E-57$ & $6.236 E=07$ & $32 \cap 0$ \\
\hline 119,0 & 9.3 & 077 & $503 E=07$ & $6.6125=07$ & 3200 \\
\hline $\begin{array}{l}1 ! 4.0 \\
1 ! 1,0\end{array}$ & 12.4 & 425 & $1075=07$ & $7 E=07$ & 3200 \\
\hline 1111.0 & 15.5 & 802 & ZE=n & $0.037 E=07$ & 3200 \\
\hline 114.0 & I 月 & 1043 & 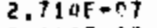 & $7.057 E-07$ & 3200 \\
\hline $\begin{array}{l}110.0 \\
113.0\end{array}$ & 21.7 & $7 \times 7$ & प $A F-07$ & 5.32 UE -07 & 3200 \\
\hline $\begin{array}{l}114,0 \\
1140\end{array}$ & $24 . A$ & 759 & $1.9765-07$ & $5.138 E-07$ & 3200 \\
\hline $\begin{array}{l}114.0 \\
114.0\end{array}$ & 31.0 & 713 & $1.907 \mathrm{E}+1 \mathrm{q}$ ? & $0.958 E-07$ & 3200 \\
\hline $\begin{array}{l}114=0 \\
110.0\end{array}$ & 37.7 & poz & $2.086 E-07$ & $5.025 t-07$ & 3200 \\
\hline $\begin{array}{l}110.0 \\
110.0\end{array}$ & t3.t & क्य & $1.7 P G E=07$ & $0.652 E-07$ & 3200 \\
\hline $\begin{array}{l}114.0 \\
114.0\end{array}$ & 49.6 & 550 & $1.4555=07$ & $3.783 E-07$ & 200 \\
\hline 110.0 & 45.9 & $\begin{array}{l}509 \\
\text { aी }\end{array}$ & $\begin{array}{r}1.3206=07 \\
1668=07\end{array}$ & $3.031 E=0.7$ & 3200 \\
\hline & & $40 \%$ & & & \\
\hline
\end{tabular}




\begin{tabular}{|c|c|c|c|c|c|}
\hline $\begin{array}{l}\text { AZIKUTTH } \\
\text { DECREES }\end{array}$ & $\begin{array}{l}\text { HE IGHT } \\
\text { ME TERS }\end{array}$ & $\begin{array}{c}\text { EXPCBURF } \\
G \omega-\leq E C>C L, N \\
\times 10 F+a\end{array}$ & $\begin{array}{c}E / \theta \\
S E C / C U . K\end{array}$ & $\begin{array}{l}F \cup / 0 \\
1 / S A, M\end{array}$ & $\begin{array}{l}\text { DISIANCE } \\
\text { NETERS }\end{array}$ \\
\hline $\begin{array}{r}122.0 \\
122.0 \\
122.0 \\
122.0 \\
122.0 \\
122.0 \\
122.0 \\
122.0 \\
122.0 \\
122.0 \\
122.0 \\
122.0 \\
122.0 \\
122.0 \\
122.0 \\
122.0 \\
122.0 \\
122.0\end{array}$ & $\begin{array}{l}0.2 \\
0.21 \\
0.2 \\
1.5 \\
3.1 \\
6.2 \\
9.3 \\
12.4 \\
15.5 \\
18.6 \\
25.7 \\
29.8 \\
31.0 \\
37.2 \\
03.4 \\
49.8 \\
55.4 \\
62.0\end{array}$ & $\begin{array}{l}11 \times 5 \\
1176 \\
1196 \\
997 \\
978 \\
1232 \\
1112 \\
1328 \\
1234 \\
1134 \\
1198 \\
1006 \\
1071 \\
943 \\
679 \\
620 \\
6 \times 7 \\
591\end{array}$ & 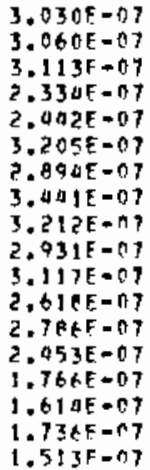 & 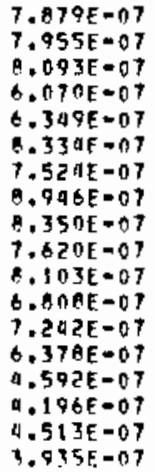 & $\begin{array}{l}3200 \\
3200 \\
3200 \\
3200 \\
3200 \\
3200 \\
3200 \\
3200 \\
3200 \\
3200 \\
3200 \\
3200 \\
3200 \\
3200 \\
3200 \\
3200 \\
3200 \\
3290\end{array}$ \\
\hline
\end{tabular}

\begin{tabular}{|c|c|c|c|c|c|}
\hline $\begin{array}{l}\text { MIJMETH } \\
\text { rEGREES }\end{array}$ & $\begin{array}{l}\text { HE ICHT } \\
\text { NETEAS }\end{array}$ & $\begin{array}{c}\text { ExFCSUEF } \\
\text { GN }-S F C>C U=N \\
\times 1 \cap F+*\end{array}$ & $\begin{array}{c}E>0 \\
S F t / C U, N\end{array}$ & $\begin{array}{c}F U / C \\
1 / \text { SO. }\end{array}$ & $\begin{array}{l}\text { DISTANCE } \\
\text { METFRS }\end{array}$ \\
\hline $\begin{array}{l}130.0 \\
130.0 \\
130.0 \\
130.0 \\
130.0 \\
130.0 \\
130.0 \\
130.0 \\
130.0 \\
130.0 \\
130.0 \\
130.0 \\
130.0 \\
130.0 \\
130.0 \\
130.0 \\
130.0 \\
130.0\end{array}$ & 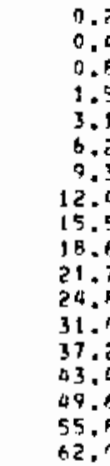 & $\begin{array}{l}1590 \\
1470 \\
1492 \\
1157 \\
1077 \\
1074 \\
1064 \\
1272 \\
1011 \\
1545 \\
1745 \\
1573 \\
1501 \\
1438 \\
1205 \\
921 \\
900 \\
1009\end{array}$ & 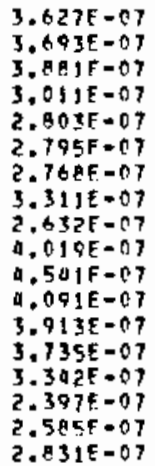 & 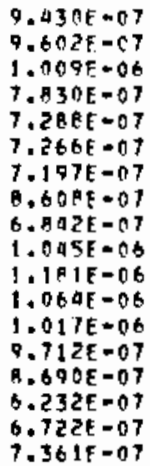 & $\begin{array}{l}1200 \\
3200 \\
3200 \\
3200 \\
3200 \\
3200 \\
3200 \\
3200 \\
3200 \\
3200 \\
3200 \\
3200 \\
3200 \\
3200 \\
3200 \\
3200 \\
3200 \\
3700\end{array}$ \\
\hline
\end{tabular}


SECONDARY SERIES EXPERI HENT; $A-8$

GROUND LEVEL AND YOHER SAMPLING ON THE 3200 M ARC. GROUND LEVEL SAMPLING ONLY ON THE 800 M ARC.

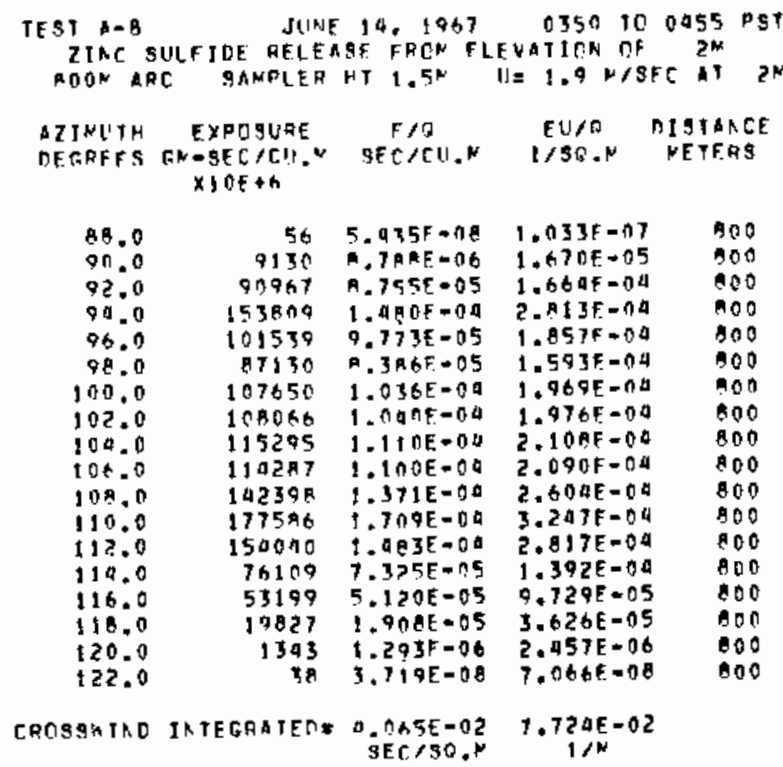

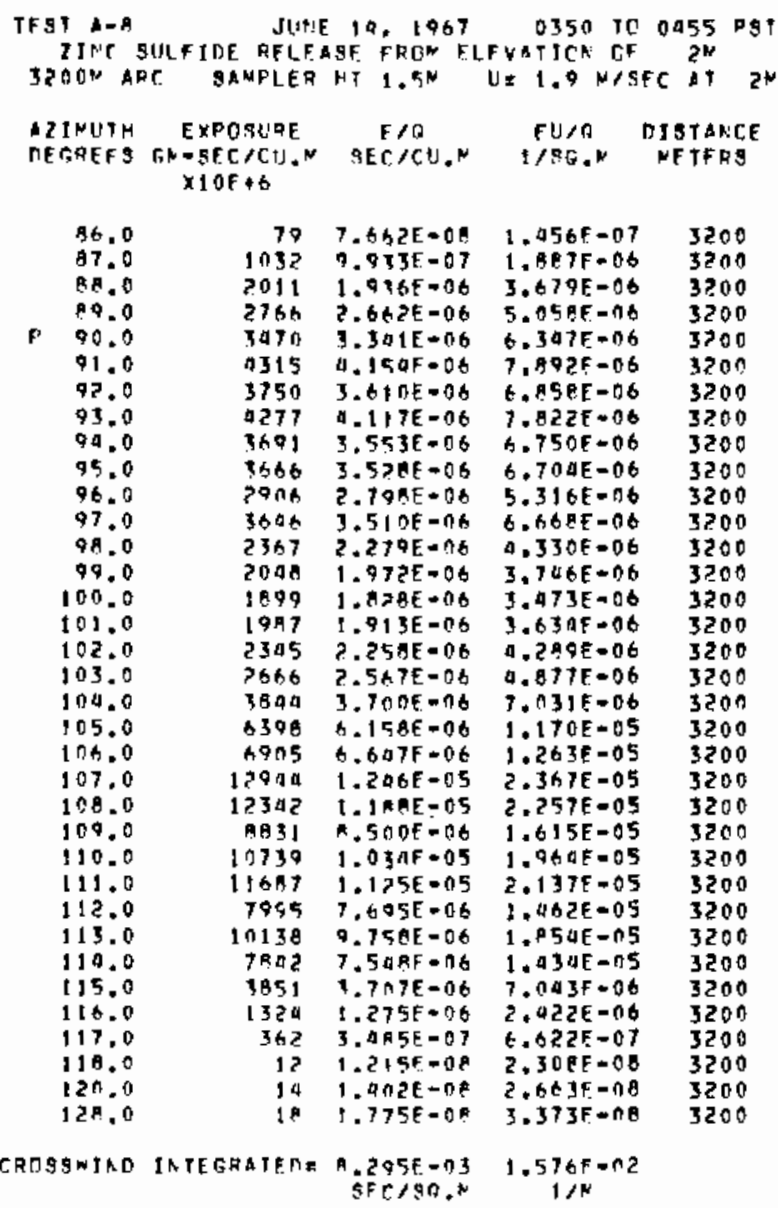


TOKEEP NATA FOLLUW...

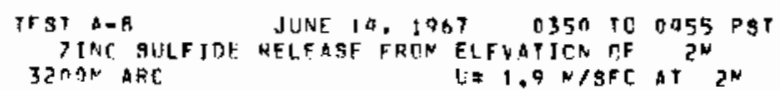

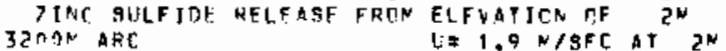

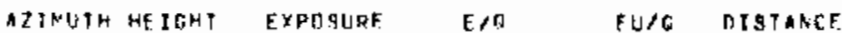

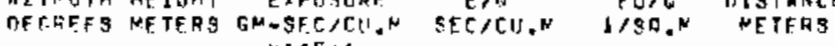
$\times 10 \mathrm{~F}+\mathrm{A}$

\begin{tabular}{|c|c|c|c|c|}
\hline $\begin{array}{l}9 A .0 \\
98.0 \\
98.0 \\
9 A .0 \\
9 A .0 \\
98.0 \\
9 A .0 \\
98.0 \\
98.0 \\
98.0 \\
98.0 \\
98.0 \\
98.0 \\
9 A .0\end{array}$ & $\begin{array}{l}0 . ? \\
0.9 \\
0.8 \\
1.5 \\
3.1 \\
6.2 \\
9.3 \\
12.0 \\
15.5 \\
18.6 \\
21.7 \\
24.8 \\
31.0 \\
37.7\end{array}$ & $\begin{array}{l}1217 \\
2511 \\
2451 \\
3018 \\
3371 \\
4019 \\
5079 \\
5946 \\
5197 \\
3555 \\
2627 \\
1012 \\
141 \\
79\end{array}$ & $\begin{array}{l}1.171 E=06 \\
2.919 F=06 \\
2.562 E=06 \\
2.892 E=06 \\
3.295 F=06 \\
3.869 E-06 \\
4.922 F=06 \\
5.743 E=06 \\
4.993 F=06 \\
3.422 F=06 \\
2.529 F=06 \\
9.740 E=07 \\
1.359 F=0.7 \\
2.319 F=09\end{array}$ & $\begin{array}{l}2.226 E=06 \\
4.593 E=06 \\
5.867 E=06 \\
5.502 E=06 \\
6.166 E=06 \\
7.350 E=06 \\
9.162 E=06 \\
1.091 E=05 \\
9.466 E=06 \\
6.502 E=06 \\
4.809 E=06 \\
1.951 E=06 \\
2.592 E=07 \\
4.494 E=08\end{array}$ \\
\hline
\end{tabular}

A7IPLTH HEIGHT EXPOSUHE ERO EUSO CISTAMCE DFTREFS METERS GN-SEC/CII.N SEC/CL.N 1/SO.N NETERS $\times 10 E+B$

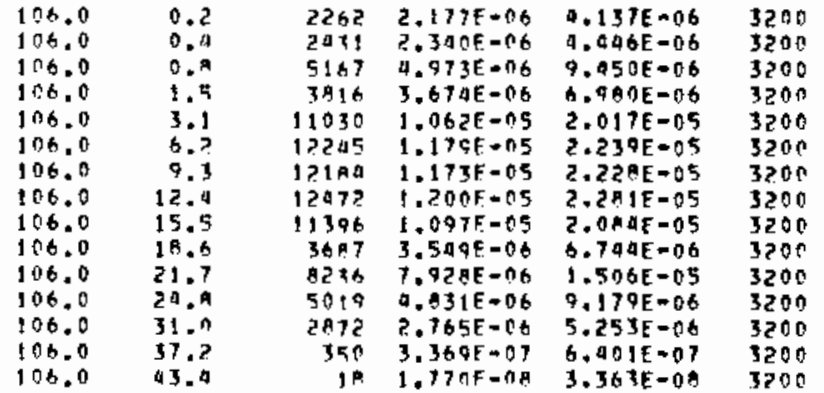

\begin{tabular}{|c|c|c|c|c|c|}
\hline $\begin{array}{l}\text { ZINUTH } \\
\text { F GPFES }\end{array}$ & $\begin{array}{l}\text { HE IGHT } \\
\text { HETERS }\end{array}$ & $\begin{array}{c}\text { EXPCSERF } \\
\text { GN-SEC,CU.N } \\
\times 1 \cap E+6\end{array}$ & $\begin{array}{c}E / n \\
S E C / C U, N\end{array}$ & $\begin{array}{c}F \| / 6 \\
1 / 30 . N\end{array}$ & $\begin{array}{l}\text { DISTANCE } \\
\text { MEYEAS }\end{array}$ \\
\hline 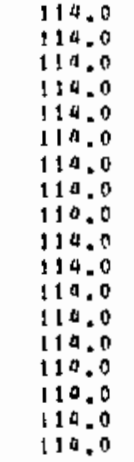 & $\begin{array}{l}0.2 \\
0.7 \\
0.8 \\
1.5 \\
3.1 \\
6.2 \\
9.3 \\
12.4 \\
15.5 \\
10.6 \\
21.7 \\
24.8 \\
31.0 \\
37.2 \\
43.9 \\
49.5 \\
55.4 \\
62.0\end{array}$ & $\begin{array}{l}6975 \\
6160 \\
7173 \\
6901 \\
7757 \\
7969 \\
9395 \\
8900 \\
7667 \\
9591 \\
9751 \\
6396 \\
3177 \\
1090 \\
279 \\
122 \\
79 \\
03\end{array}$ & 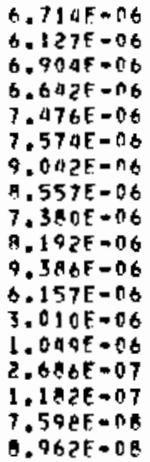 & $\begin{array}{l}1.276 E=05 \\
1.160 E=05 \\
1.312 E=05 \\
1.262 E-05 \\
1.020 E=05 \\
1.439 E=05 \\
1.718 E=05 \\
1.426 E=05 \\
1.402 E=05 \\
1.557 E=05 \\
1.703 E=05 \\
1.170 E=05 \\
5.719 E=06 \\
1.99 A E=06 \\
5.100 E=07 \\
2.206 E=07 \\
1.000 E=07 \\
1.703 E=07\end{array}$ & $\begin{array}{l}3200 \\
3200 \\
3200 \\
3200 \\
3200 \\
3200 \\
3200 \\
3200 \\
3200 \\
3200 \\
3200 \\
3200 \\
3200 \\
3200 \\
3200 \\
3200 \\
3200 \\
3200\end{array}$ \\
\hline
\end{tabular}

\begin{tabular}{|c|c|c|c|c|c|}
\hline $\begin{array}{l}\text { AZTMUITH } \\
\text { OEGREES }\end{array}$ & $\begin{array}{l}\text { HE IGH I } \\
\text { ME TERS }\end{array}$ & $\begin{array}{l}\text { EXPCSURE } \\
\text { GN-SEC }>C ! !, N \\
\times 10 E+6\end{array}$ & $\begin{array}{c}E / 0 \\
S E C / C U . *\end{array}$ & $\begin{array}{l}E \cup / 0 \\
1 / 90, N\end{array}$ & $\begin{array}{l}\text { DISTANCE } \\
\text { METERS }\end{array}$ \\
\hline $\begin{array}{l}122.0 \\
122.0 \\
122.0 \\
122.0 \\
122.0 \\
122.0 \\
122.0 \\
122.0 \\
122.0 \\
122.0 \\
122.0 \\
122.0 \\
122.0\end{array}$ & $\begin{array}{l}3.1 \\
9.3 \\
12.0 \\
15.5 \\
10.6 \\
21.7 \\
20.9 \\
31.0 \\
37.2 \\
43.4 \\
49.4 \\
55.9 \\
62.0\end{array}$ & $\begin{array}{l}40 \\
07 \\
22 \\
38 \\
33 \\
90 \\
21 \\
13 \\
35 \\
19 \\
29 \\
41 \\
98\end{array}$ & $\begin{array}{l}3.923 E=08 \\
4.577 E=08 \\
2.207 F=08 \\
3.67 A E=08 \\
3.197 E=08 \\
3.923 E=08 \\
2.006 F=08 \\
1.264 E=08 \\
3.913 E=08 \\
1.833 E=08 \\
2.857 F=08 \\
3.961 E=08 \\
5.650 E=0 A\end{array}$ & 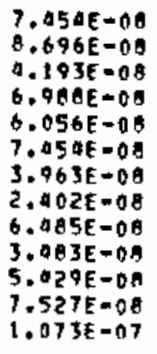 & $\begin{array}{l}3200 \\
3200 \\
3200 \\
3200 \\
3200 \\
3200 \\
3200 \\
3200 \\
3200 \\
3200 \\
3200 \\
3200 \\
3200\end{array}$ \\
\hline $\begin{array}{l}\text { DIPUTH } \\
\text { DE EREFS }\end{array}$ & $\begin{array}{l}\text { HE ICHT } \\
\text { METERS }\end{array}$ & $\begin{array}{c}\text { FXPCSUFF } \\
\text { GN-SFC }>\text { CI: } \\
\times 1 \cap F+6\end{array}$ & $\begin{array}{c}E / 0 \\
\text { ser }\end{array}$ & $\begin{array}{c}F(1 / 0 \\
1 / 50 .\end{array}$ & $\begin{array}{l}\text { CISTANCF } \\
\text { NFTERS }\end{array}$ \\
\hline $\begin{array}{l}130.0 \\
130.0 \\
130.0 \\
130.0 \\
130.0 \\
130.0 \\
130.0 \\
130.0 \\
130.0 \\
130.0 \\
130.0\end{array}$ & $\begin{array}{l}6.7 \\
9.3 \\
12.9 \\
15.5 \\
18.6 \\
21.7 \\
24.0 \\
11.0 \\
49.6 \\
55.9 \\
62.0\end{array}$ & $\begin{array}{l}11 \\
30 \\
91 \\
79 \\
33 \\
75 \\
39 \\
75 \\
7 \\
13 \\
32\end{array}$ & $\begin{array}{l}1.062 F=08 \\
2.942 F=08 \\
8.827 E-08 \\
2.860 E=08 \\
3.265 F=08 \\
2.052 E=08 \\
3.793 F=09 \\
2.465 E=08 \\
7.144 E-09 \\
1.294 E=08 \\
3.117 E=08\end{array}$ & 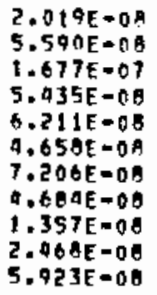 & $\begin{array}{l}3200 \\
3200 \\
3200 \\
3200 \\
3200 \\
3200 \\
3200 \\
3200 \\
3200 \\
3200 \\
3200\end{array}$ \\
\hline
\end{tabular}

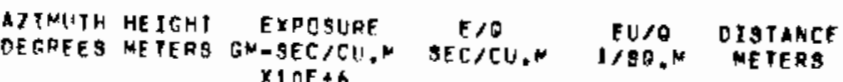

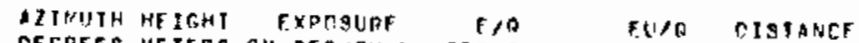


SECONDARY SERIES EXPERIMENT: $1-9$

GROUND LEVEL AND TOWER SAMPLING ON THE 3200 M ARC. GROUND LEYEL SAMPLING ONLY ON THE BOO M ARC. A SIGNIFICANT PORTION OF THE PLUME NEYER PASSEO OVER THE GRIO.

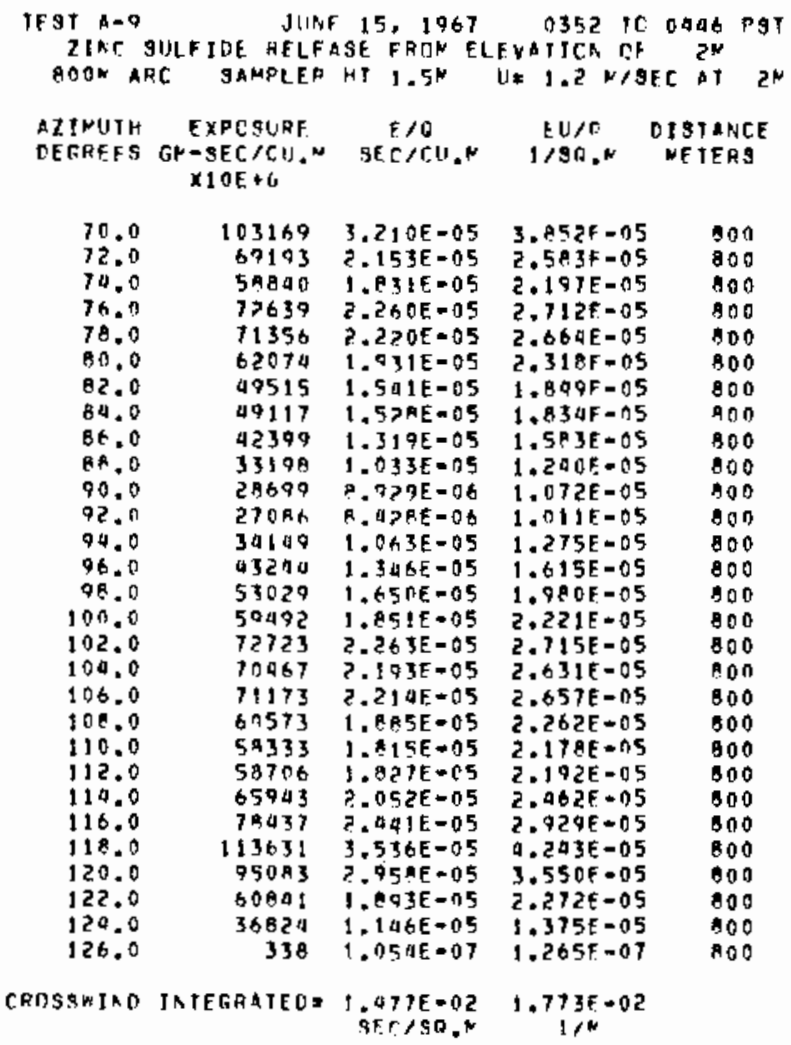

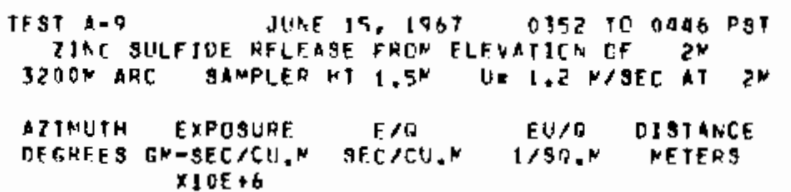

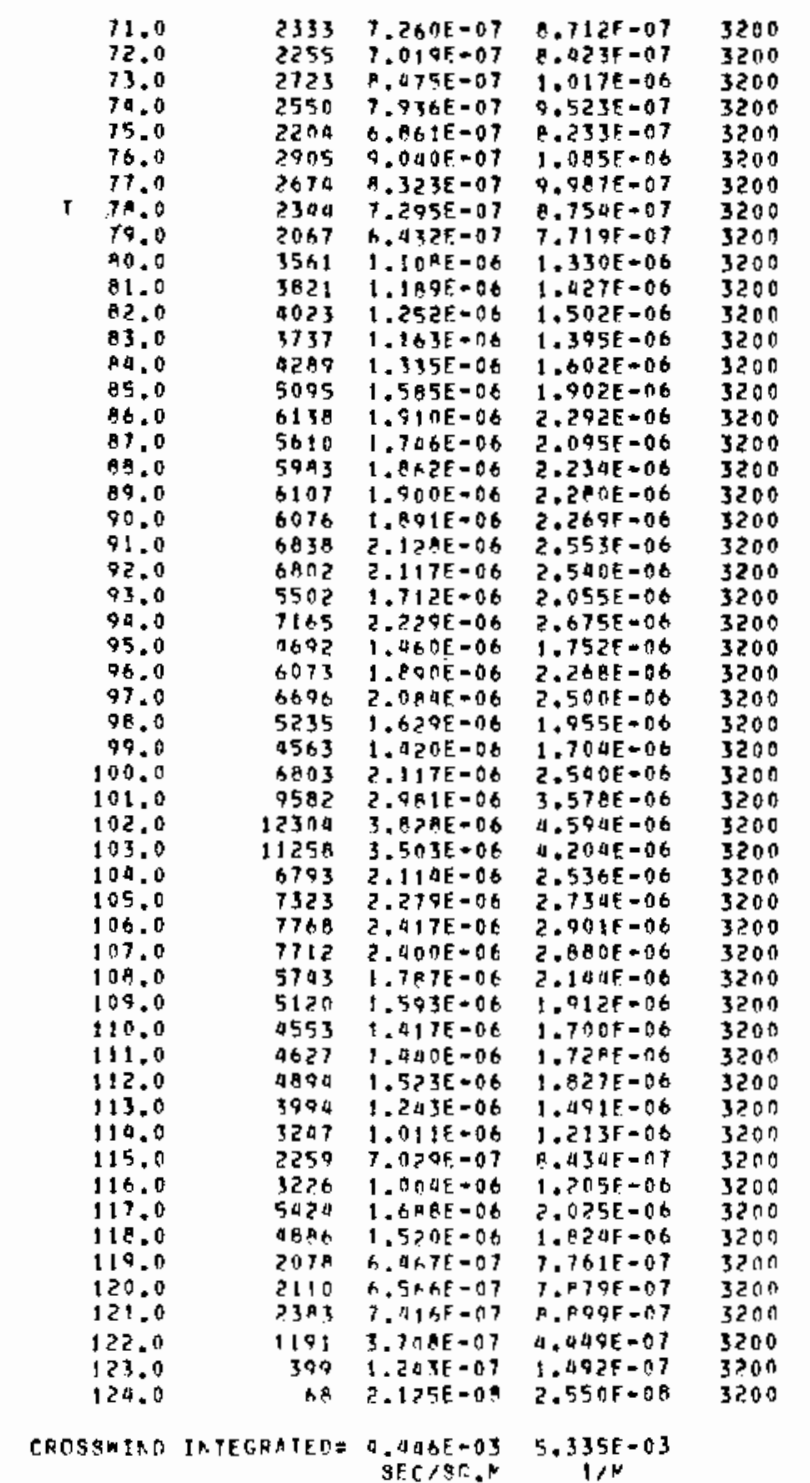




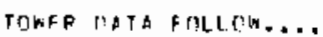

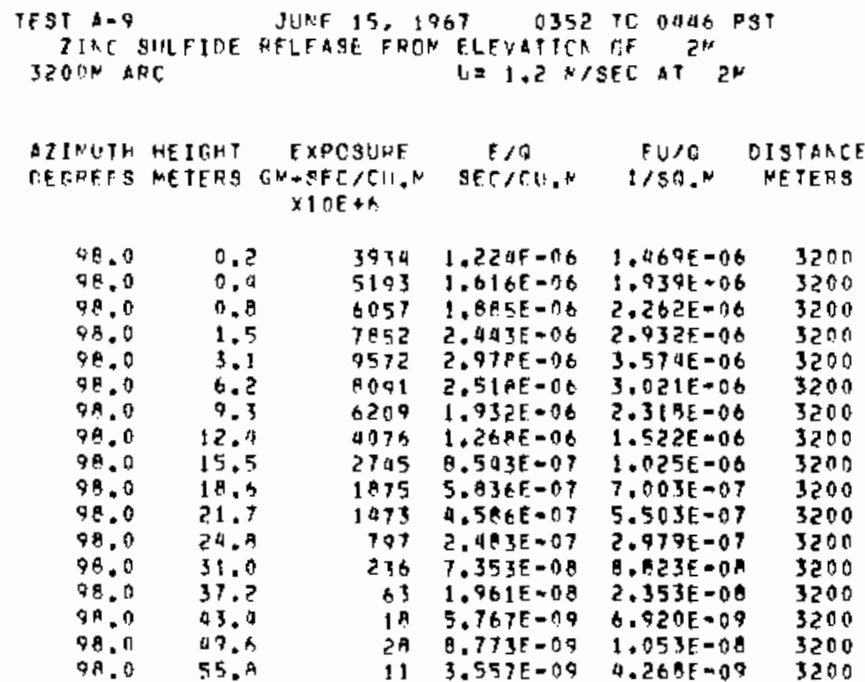

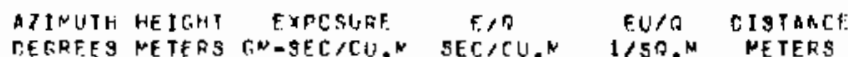
$X \perp 0 E+h$

\begin{tabular}{|c|c|c|c|c|c|}
\hline $\begin{array}{l}106.0 \\
106.0 \\
106.0 \\
106.0 \\
106.0 \\
106.0 \\
106.0 \\
106.0 \\
106.0 \\
106.0 \\
104.0 \\
106.0 \\
106.0 \\
106.0 \\
106.0 \\
106.0 \\
104.0\end{array}$ & $\begin{array}{l}0.2 \\
0.4 \\
0.8 \\
1.5 \\
3.5 \\
6.2 \\
9.3 \\
12.9 \\
15.5 \\
18.6 \\
21.7 \\
24.4 \\
31.0 \\
37.2 \\
43.1 \\
49.6 \\
55.9\end{array}$ & $\begin{array}{l}6505 \\
7062 \\
4191 \\
7102 \\
6853 \\
4225 \\
2610 \\
1609 \\
0870 \\
7612 \\
4933 \\
3059 \\
1691 \\
774 \\
363 \\
A 9 \\
77\end{array}$ & $\begin{array}{l}2.024 F=06 \\
2.137 F=06 \\
2.520 F=06 \\
2.210 E=06 \\
2.132 F=06 \\
1.315 E=06 \\
8.135 E=07 \\
5.009 E=07 \\
2.760 F=06 \\
2.369 E=08 \\
1.500 E=06 \\
9.515 E=07 \\
5.262 F=07 \\
2.111 E=07 \\
1.110 E-0 ? \\
2.490 F=08 \\
8.536 F=09\end{array}$ & $\begin{array}{l}2.427 E=06 \\
2.637 E=06 \\
3.029 E=06 \\
2.652 E=06 \\
2.559 E=06 \\
1.579 E=06 \\
0.162 E=07 \\
6.010 E=07 \\
3.312 E=06 \\
2.842 E=06 \\
1.805 E=06 \\
1.142 E=06 \\
6.315 E=07 \\
2.893 E=07 \\
1.356 F=07 \\
2.989 E=08 \\
1.924 E=0 A\end{array}$ & $\begin{array}{l}3200 \\
3200 \\
3200 \\
3200 \\
3200 \\
3200 \\
3200 \\
3200 \\
3200 \\
3200 \\
3200 \\
3200 \\
3200 \\
3200 \\
3200 \\
3200 \\
3200\end{array}$ \\
\hline
\end{tabular}



APPENDIX B

DIFFUSION SUMMARIES

B-1 



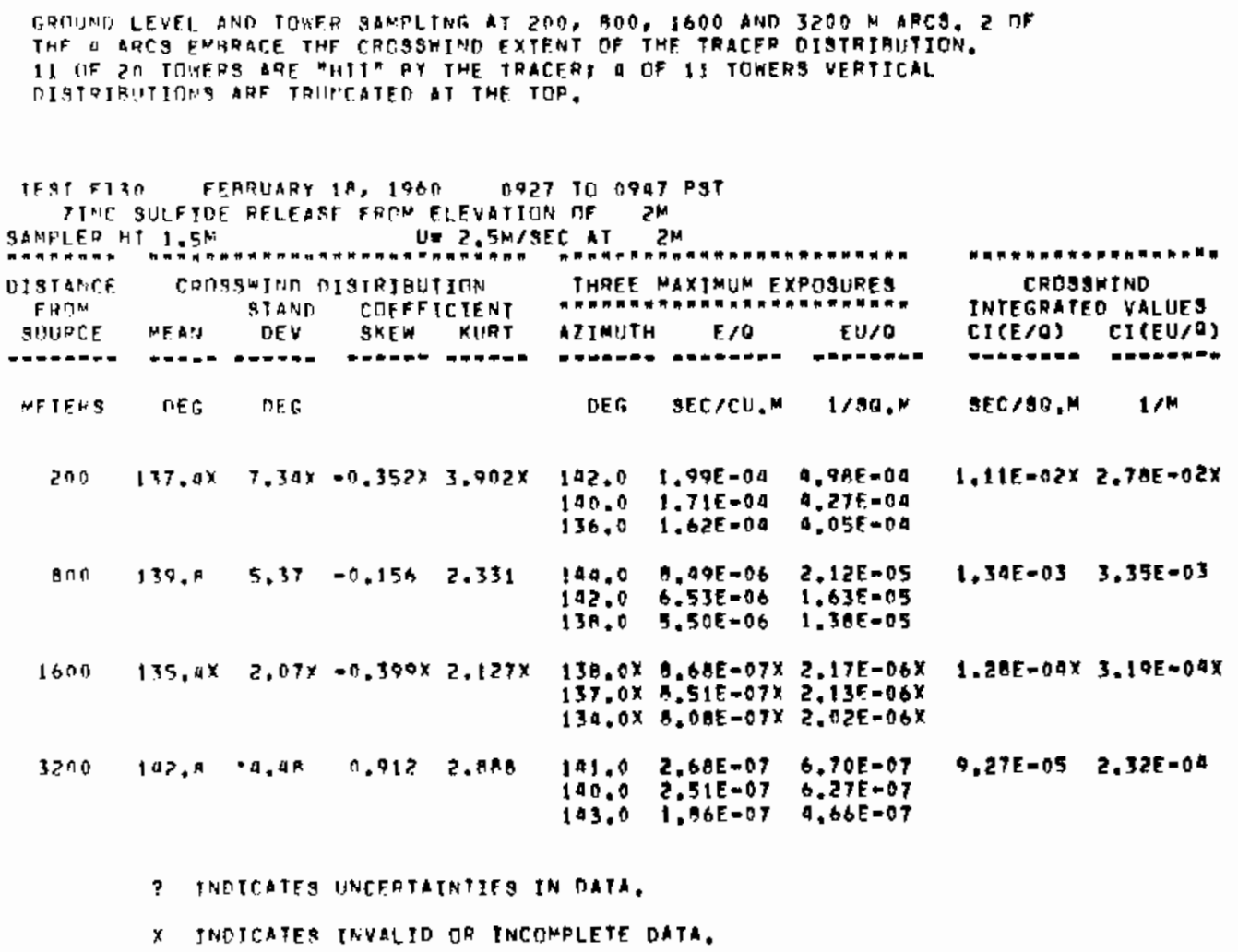




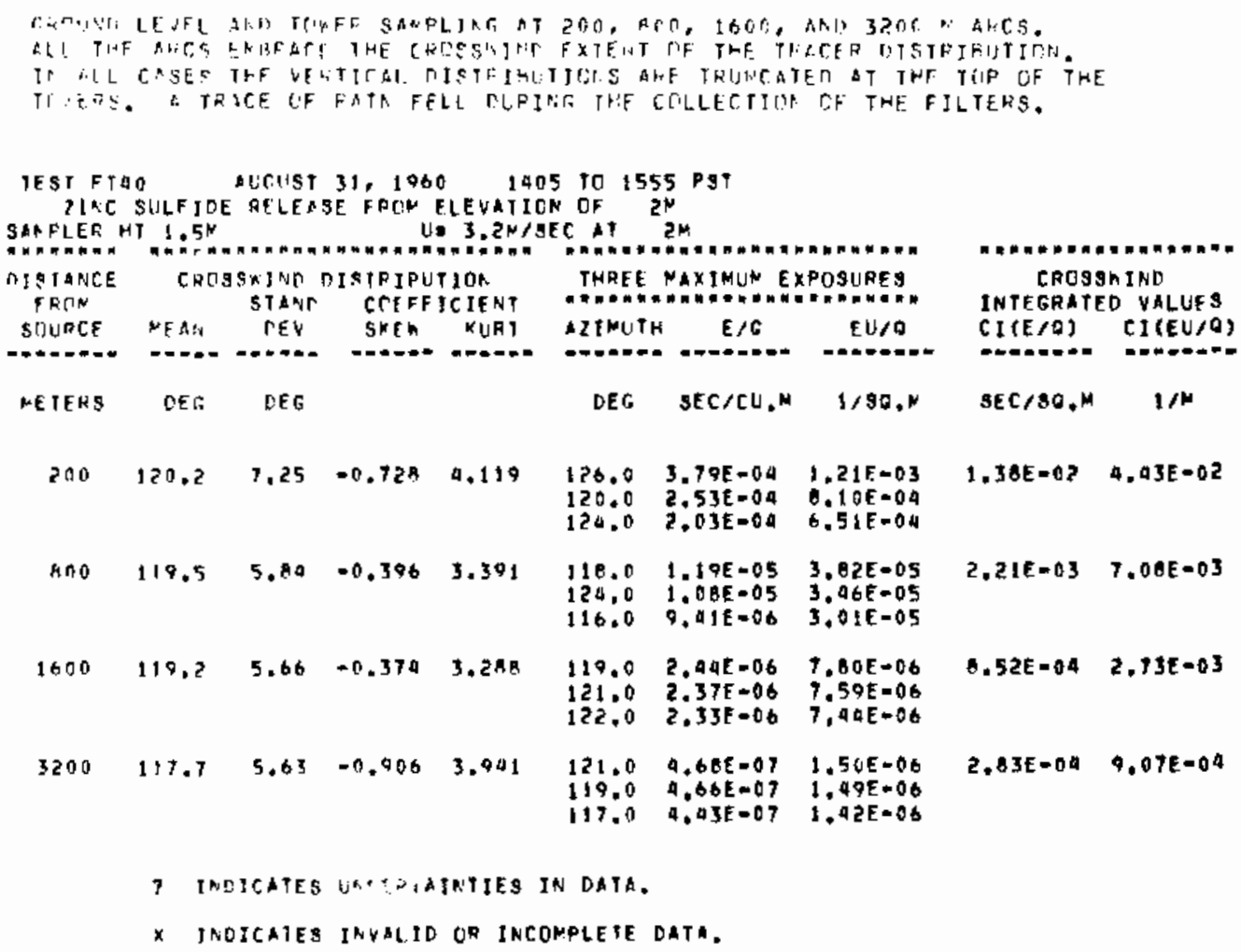




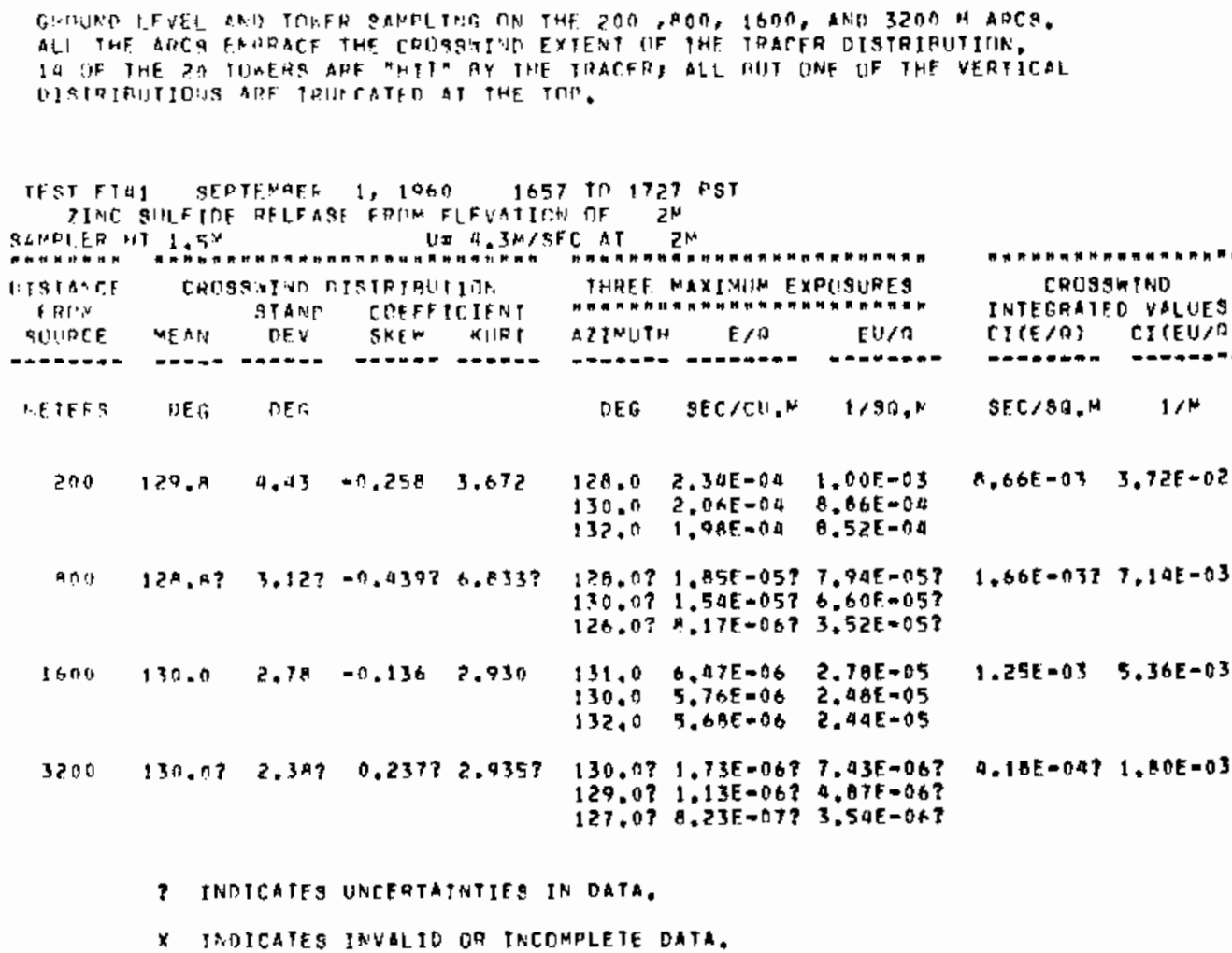




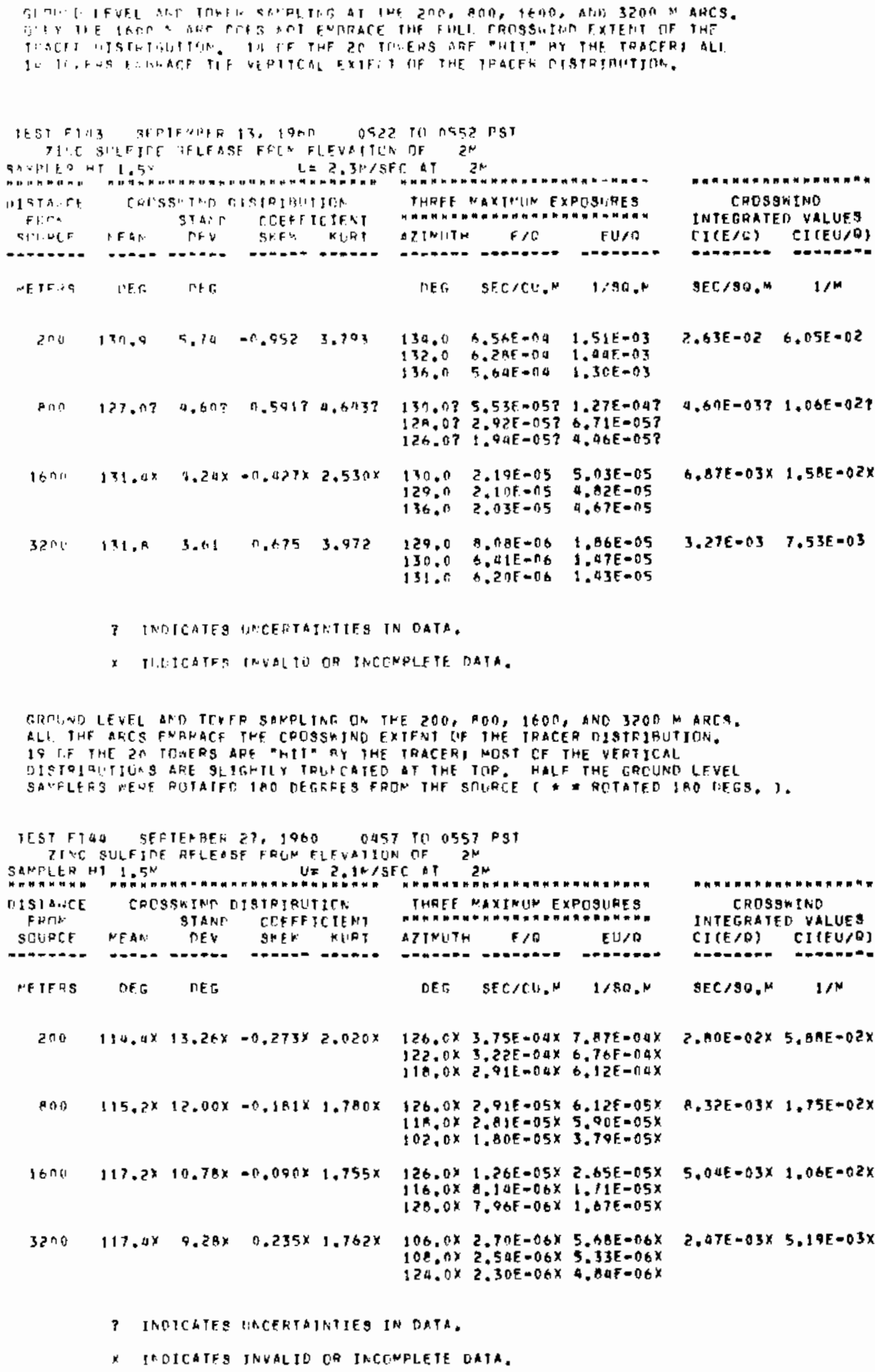

? IMOICATER HACERTAINTIES IN DATA.

* Itodcates jnvalio or incenplete onta. 


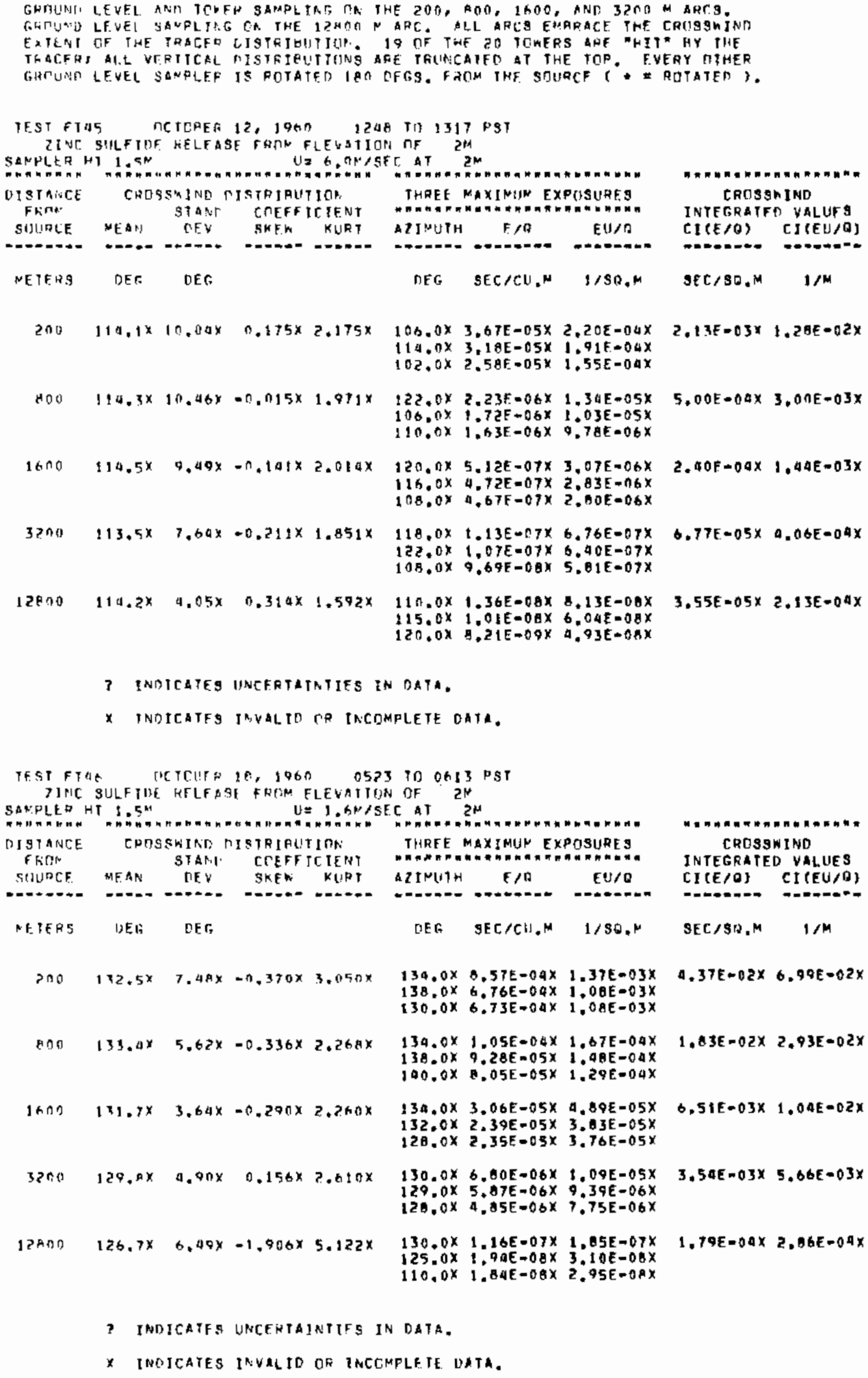




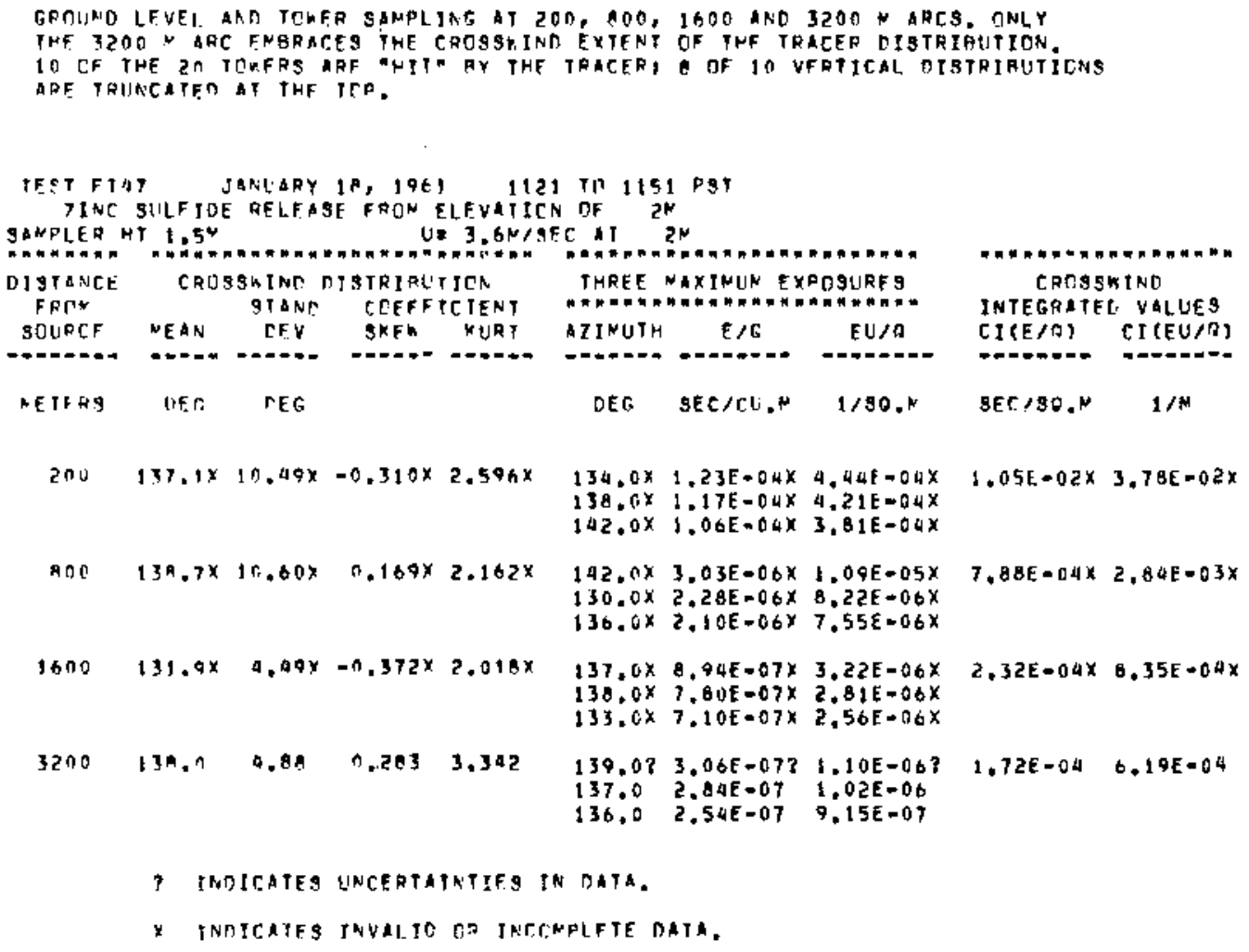

? THDICATES URCERTATATIES IN

$x$ TNOICATES IRUALIT CP JNCENPLETE DATA. 


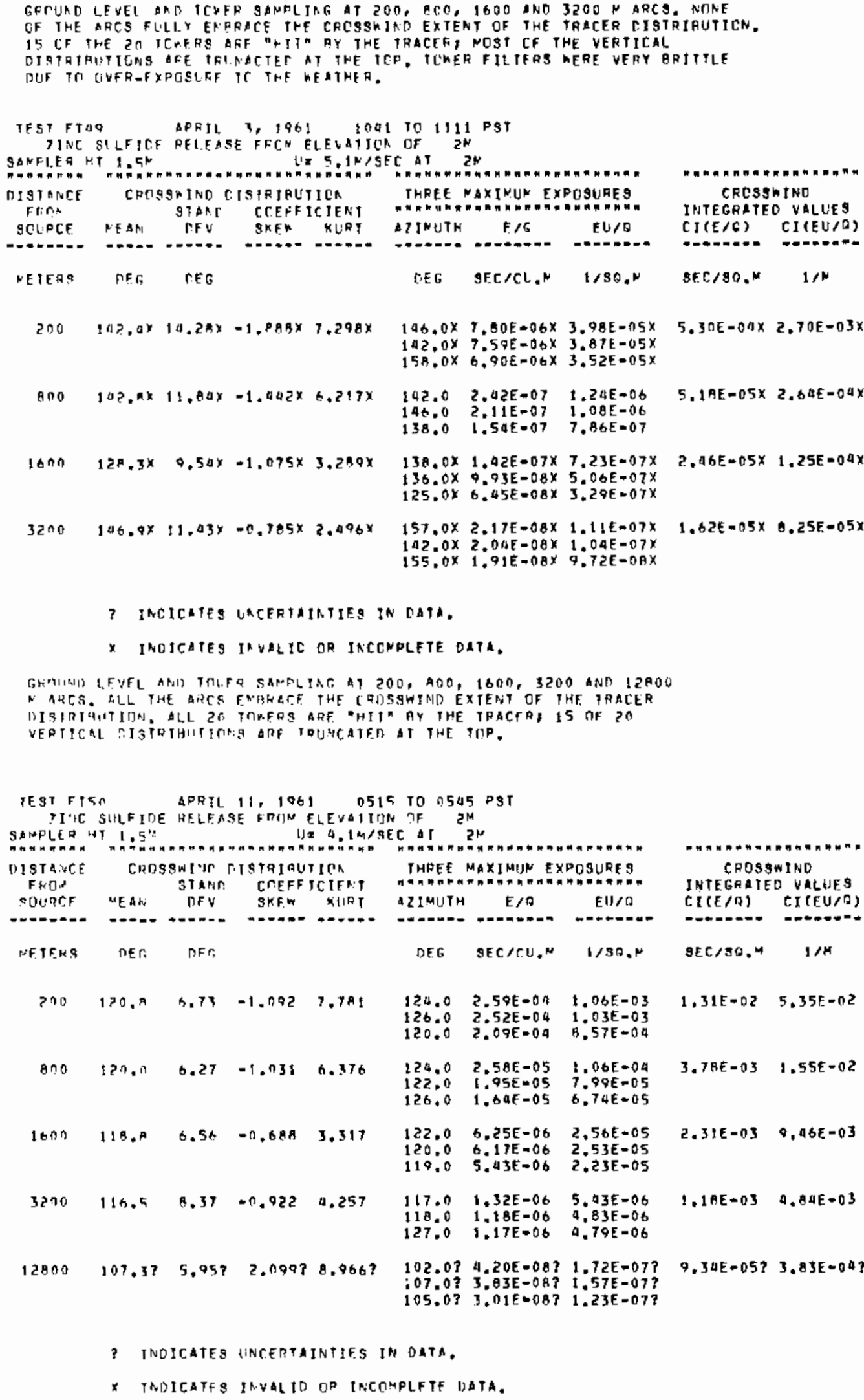




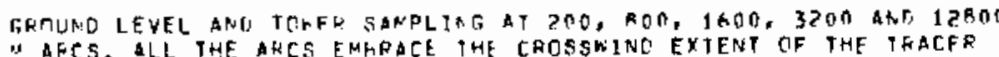
DISTRIBUTION, IS OF 20 TC, EHS AFE "HIT" BT THE TRACER

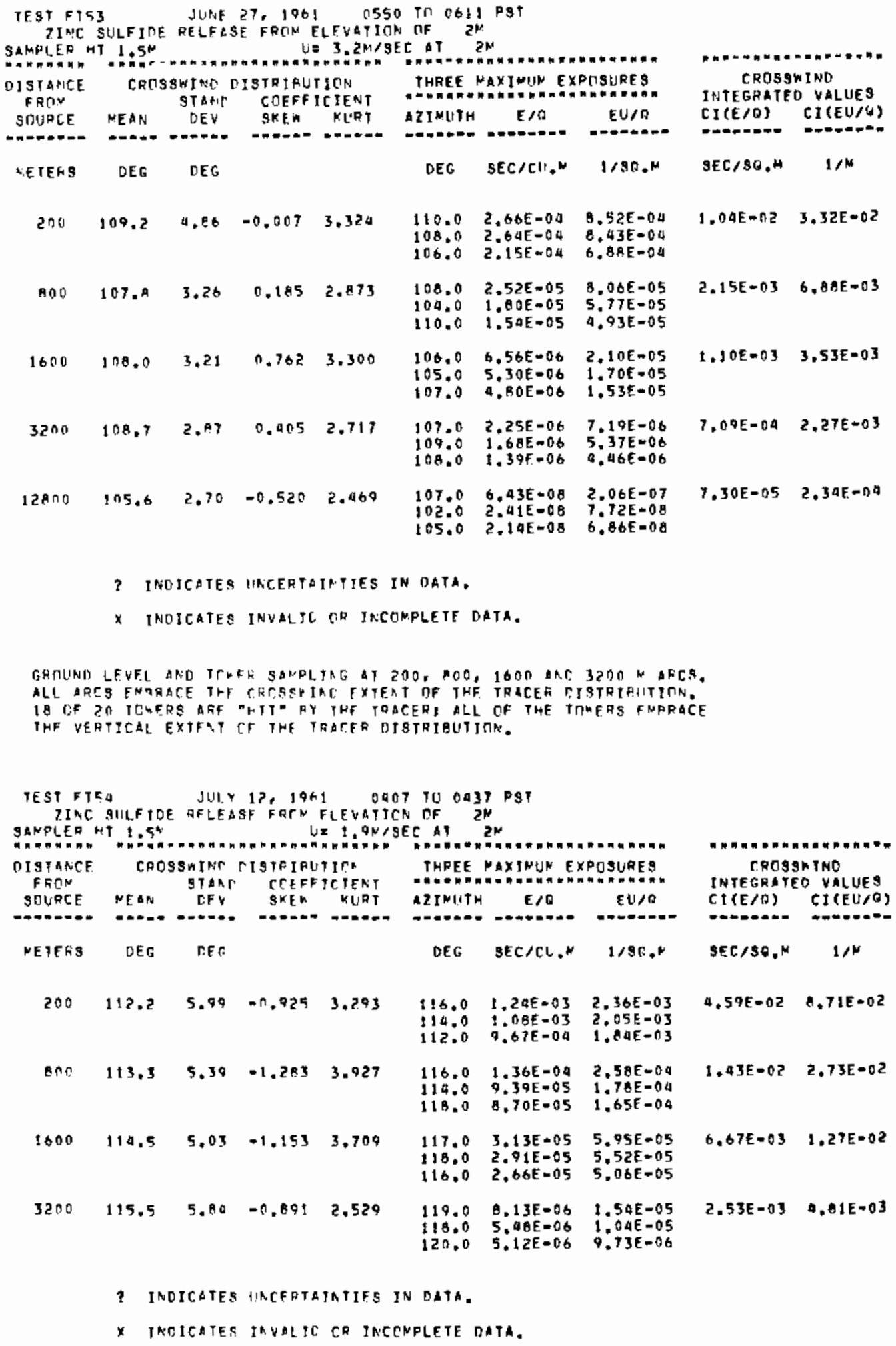

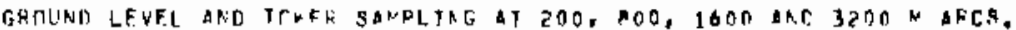

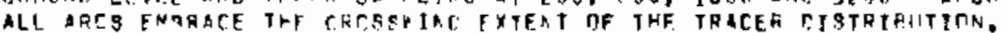
18 OF ZO ICWERS AGF "HTT" PY THF TOACERI ALL OF THE THMERS FMPRACE IHF VERTICAL EXTFVT CF THF TADTEA DISTRIBUTIUR. 
GAOUND LEVEL ARD TOMFR SARPLJAG AT 200, MO0, 1600 AME 3200 ARCS. LLL ARCS ENAAACE THE CRCSSUIAC EYTEAT OF THE TRACEF DISTRTOUTIDN, 18 OF 20 TOMERS ARE "HIT." EY THE TRACER.

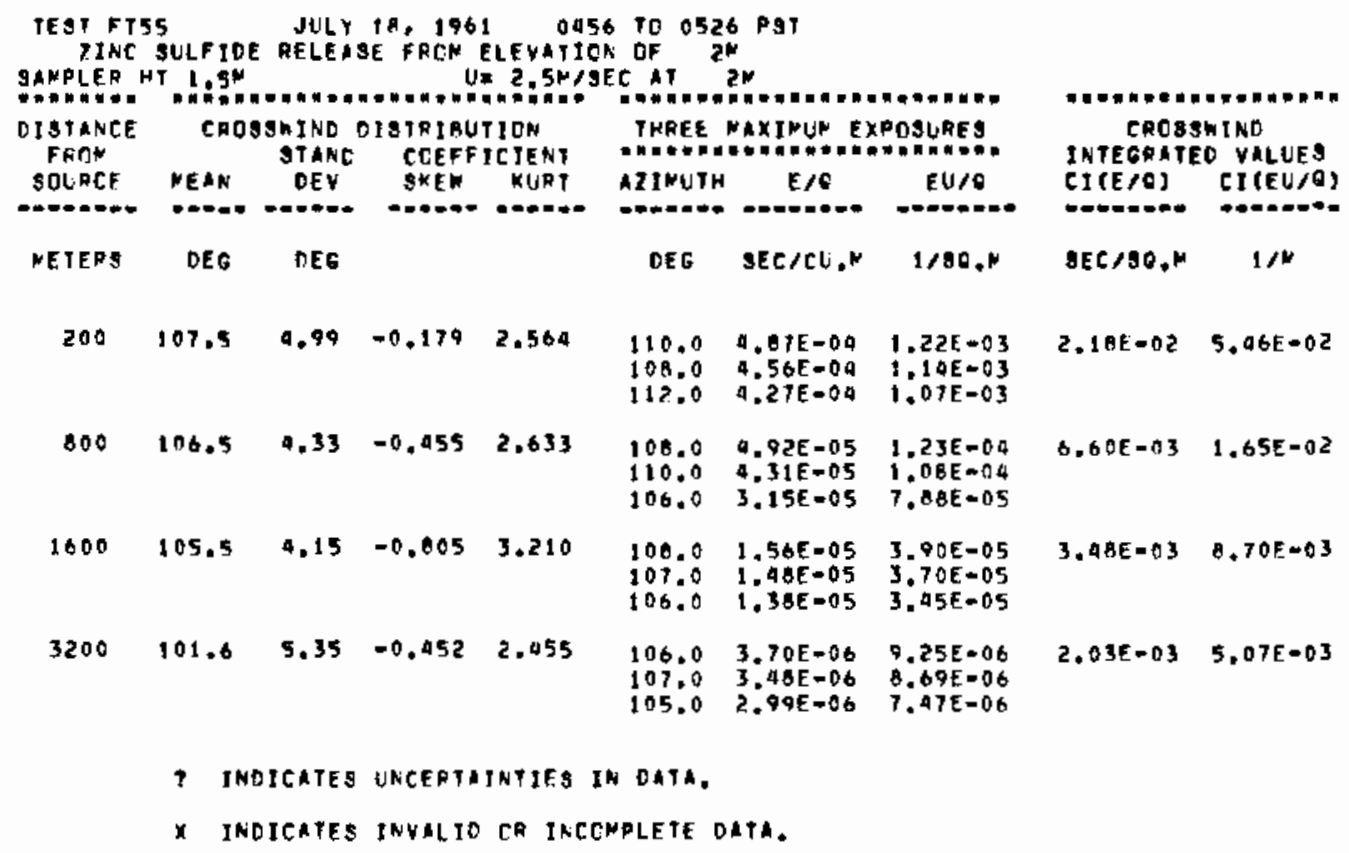

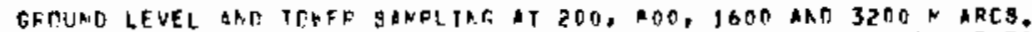
ALL THE RRCS ENAFACF THF COCSSTIND FXIENT DF THF TRACFR OISTRTOUTION. ALL THF TOKERS APF."HIT. EY THE TRACEA INR ENBRACE THE VFRTICAL DISTRIAUTIOAS.

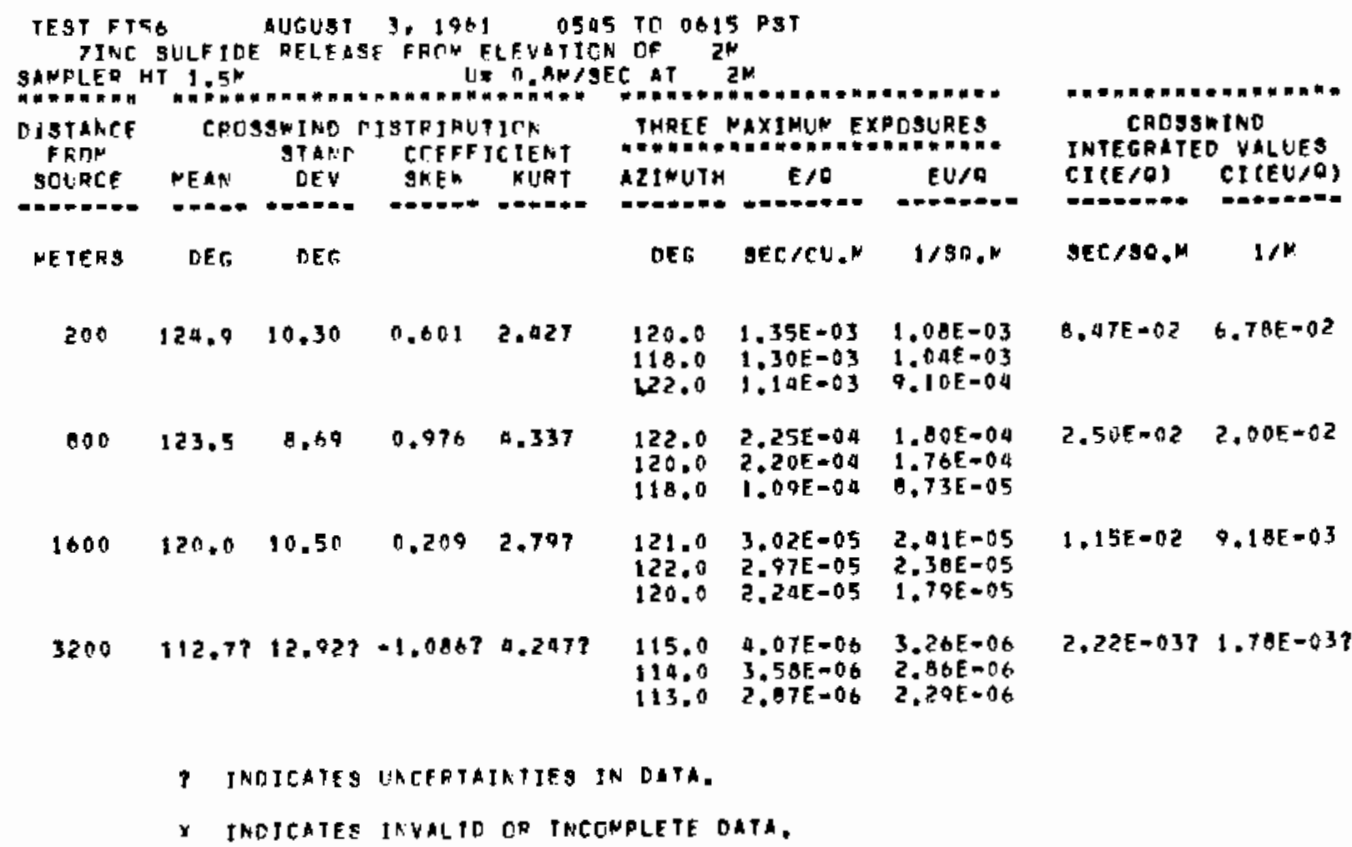


GRTUND LEVEL AND TOKER SAMPLING AT $200,500,1600$ AND $3200 \mathrm{M}$ ARCA.

DNLY THE $200 \mathrm{M}$ ARC DID NOT EMRRACE THE CROSSIIND EXTENT OF THE TRACER

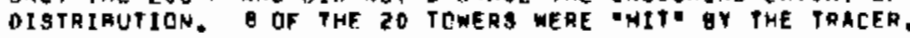

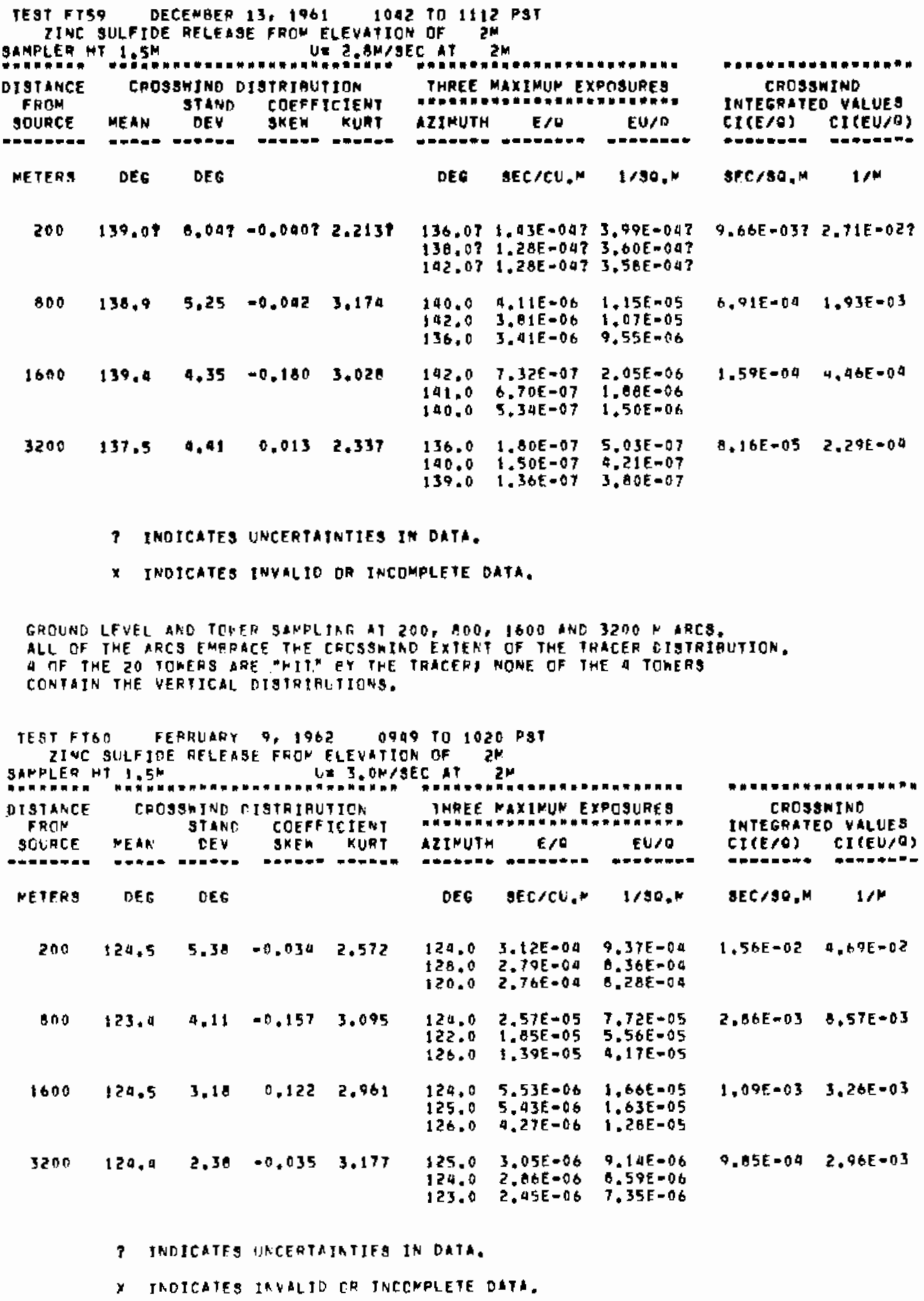


GROUAD LEVEL AND TOMEA SAKPLJAS AT 200, 800, 1600 ERO 3200 M ARCS.

ALL CF THE ARCS FWARACT THE CRISSATMD EXTENT DF THE TRACEF DISTRIBUTHCN.

IS OF 70 TOREAS ANE MHIT" GY THE TRACER

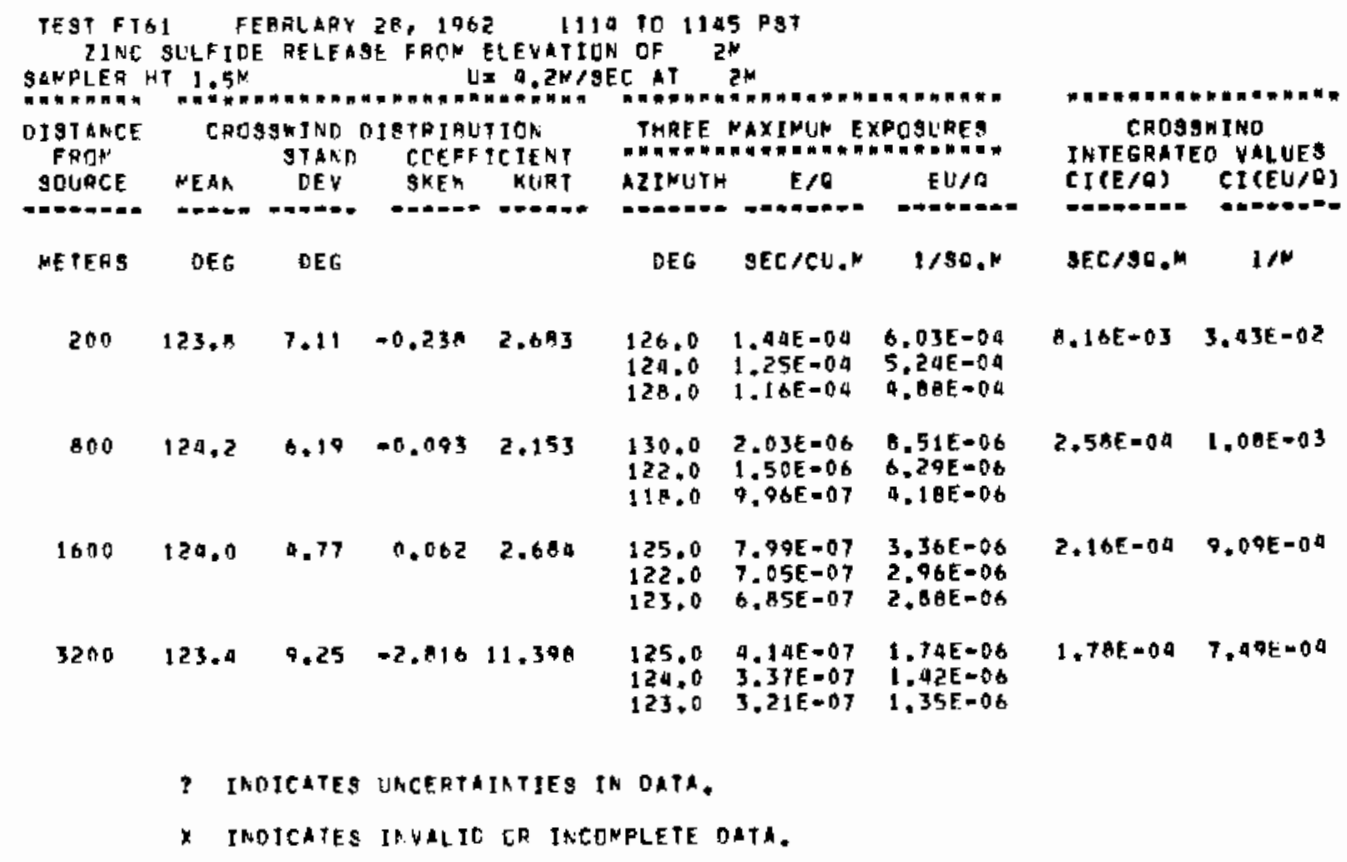

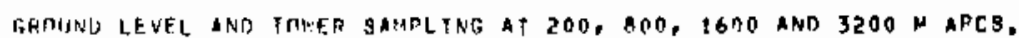
NUNE IF THF ARCS ENPAACE THE CODSBNIND EXIENT OF THE TRACER DISIRIAUTIDN. 11 UF 2 D TONFHS ARE "HIT" AY THE TRACER

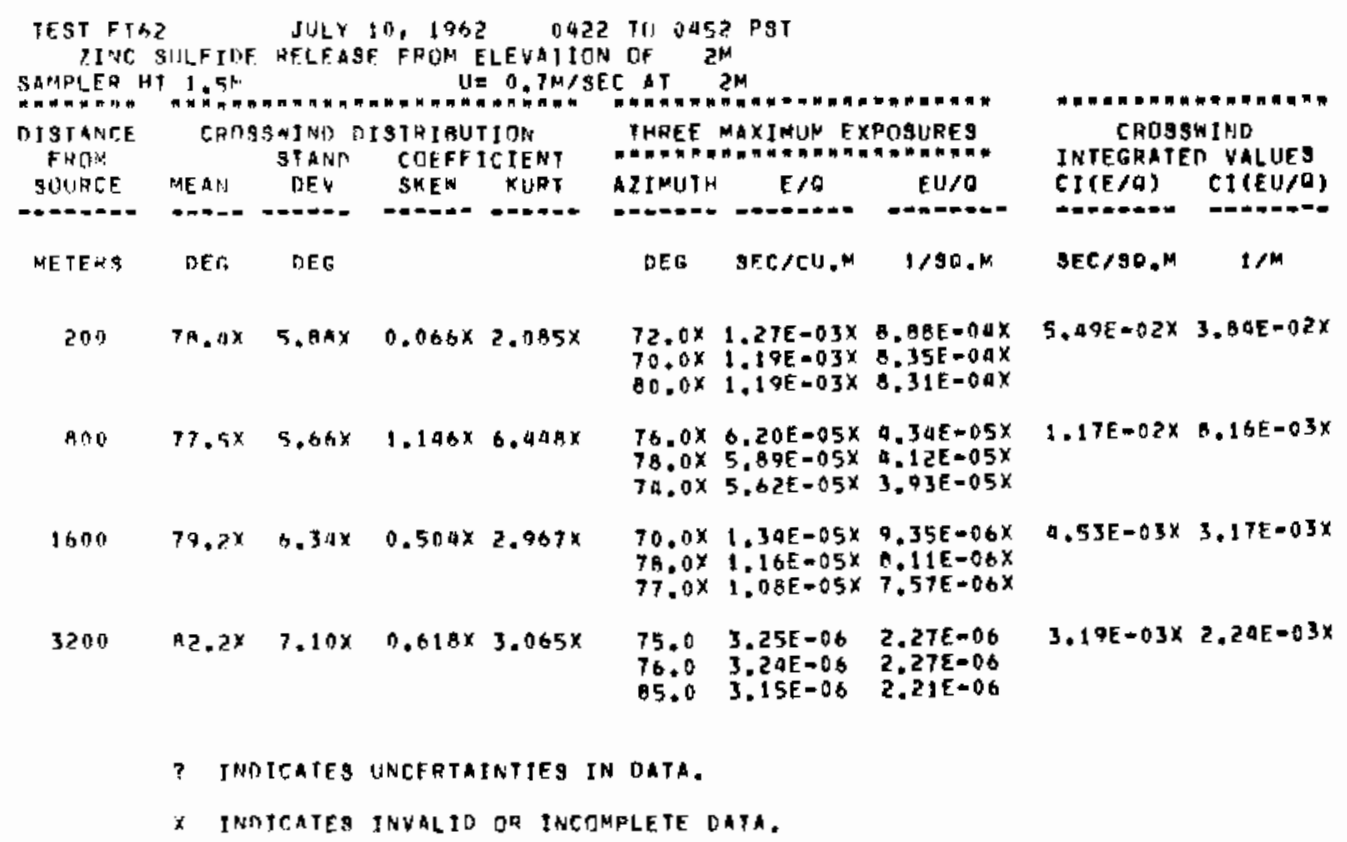




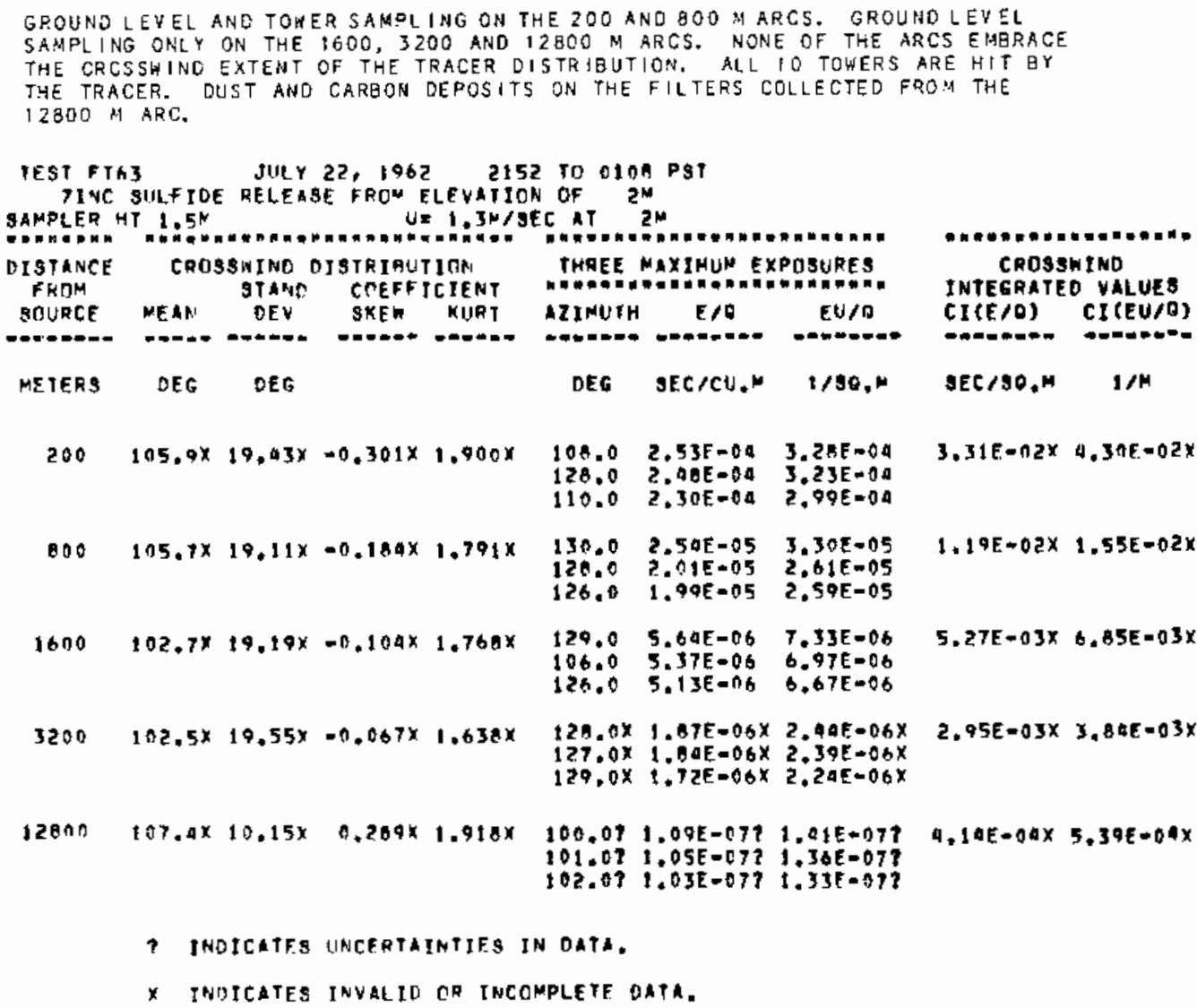

GROUNO LEVEL AND TOWER SAMPL ING ON THE 200 ANO $O 0 O$ M ARCS. GROUNOLEVEL SAMPL ING ONLY ON THE 1600 . ANO 3200 M ARCS. RLL OF THE AACS EMGRACE THE CROSSVINO EXTENT OF THE TRRCER DISTRIBUTION, ALL IO TOLERS ARE HIT BY THE TRACCR.

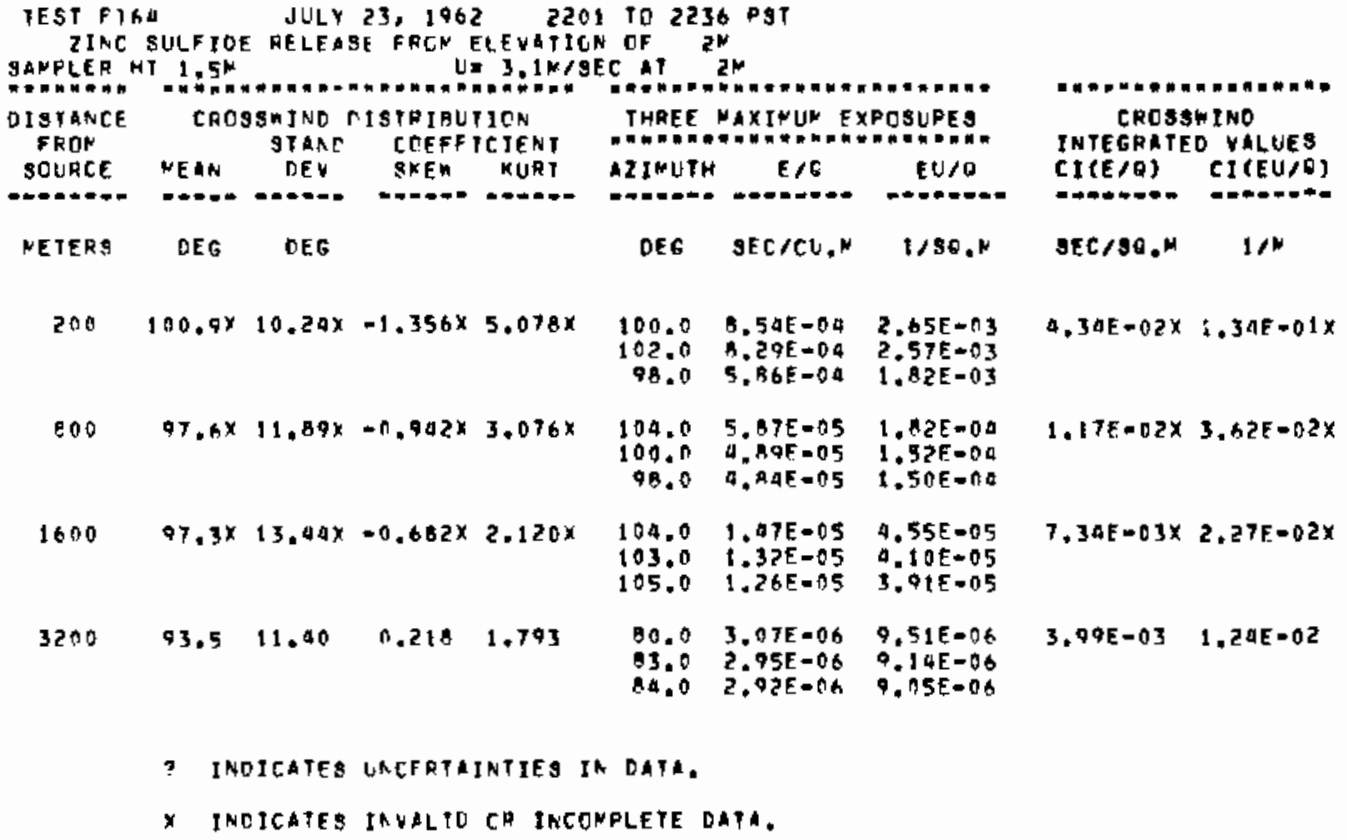


GROUND LEVEL SAMFLIAG AT 200 , ROR, 1600,3200 ANA 12800 N ARCSE TCMER

SAMPLING AT 200 NND BOO AFCS. ALL THE ARCS ENBRACE THE CROSSHINO

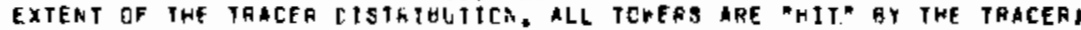

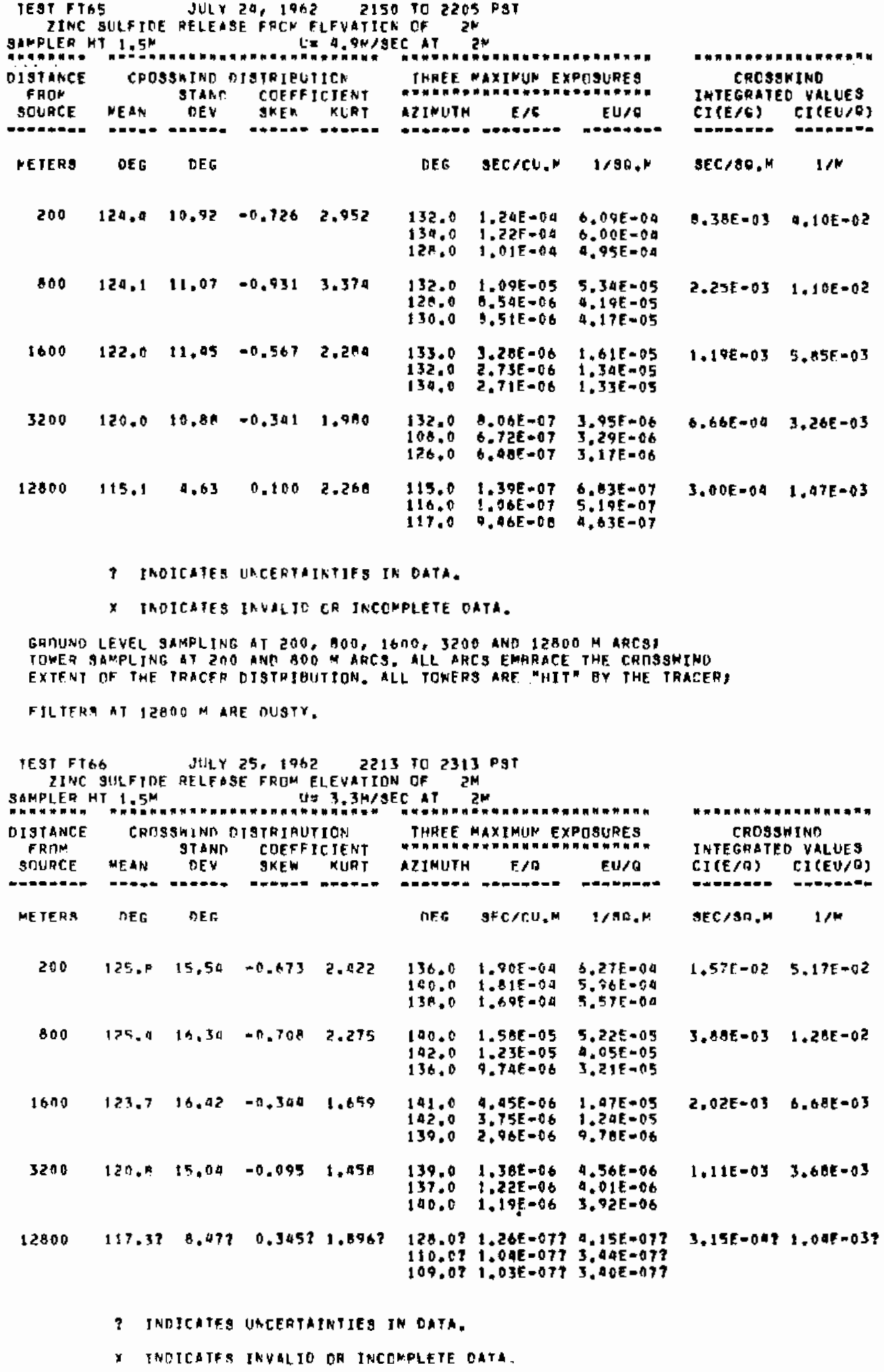

? INDICATES UACERTATNIIES IN OATA.

$x$ INOICATES INVALIO DR INCEMPLETE OATA. 


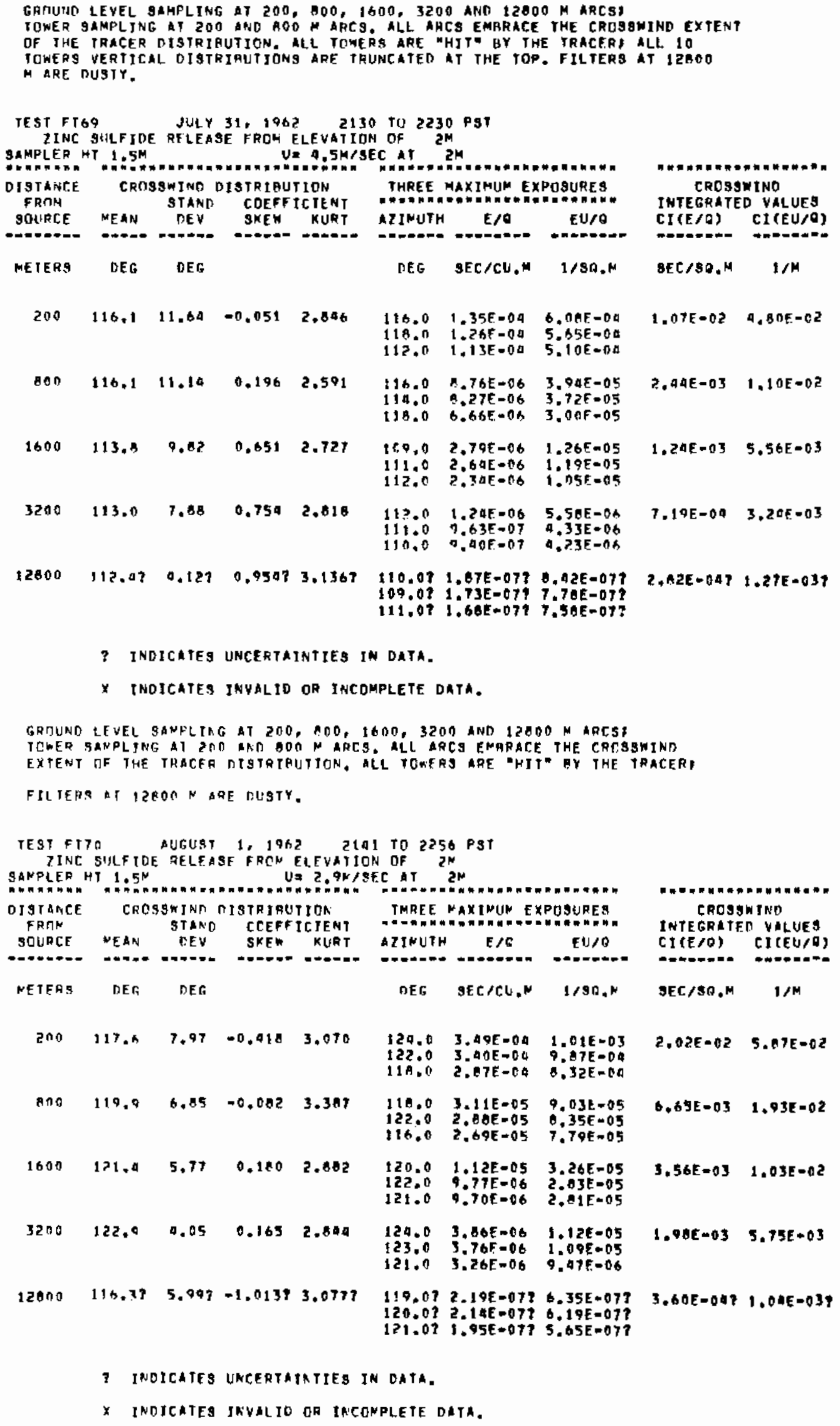

NETEAS DEF DEG DEG SEC/CU.N 1/SB,N SEC/SO,M 1/M

200 117.5 7.97 $=0.418 \quad 3.070$ 120.0 3.49E-04 1.01E-03 2.02E-02 5.07E-02

\section{CROSSTIND}

INTEGRATED VALUES

CI $(E / O)$ CI $(E \cup / Q)$

$6.69 E=03 \quad 1.93 E-02$

$3.56 E=03 \quad 1.03 E-02$

$1.90 E-03 \quad 5.75 E+03$

$3.60 E-0421.046-037$

7 INOICATES UNCERTATATES IN DATA.

X INDICATES JNVHLIO OR TREONPLETE DATA. 


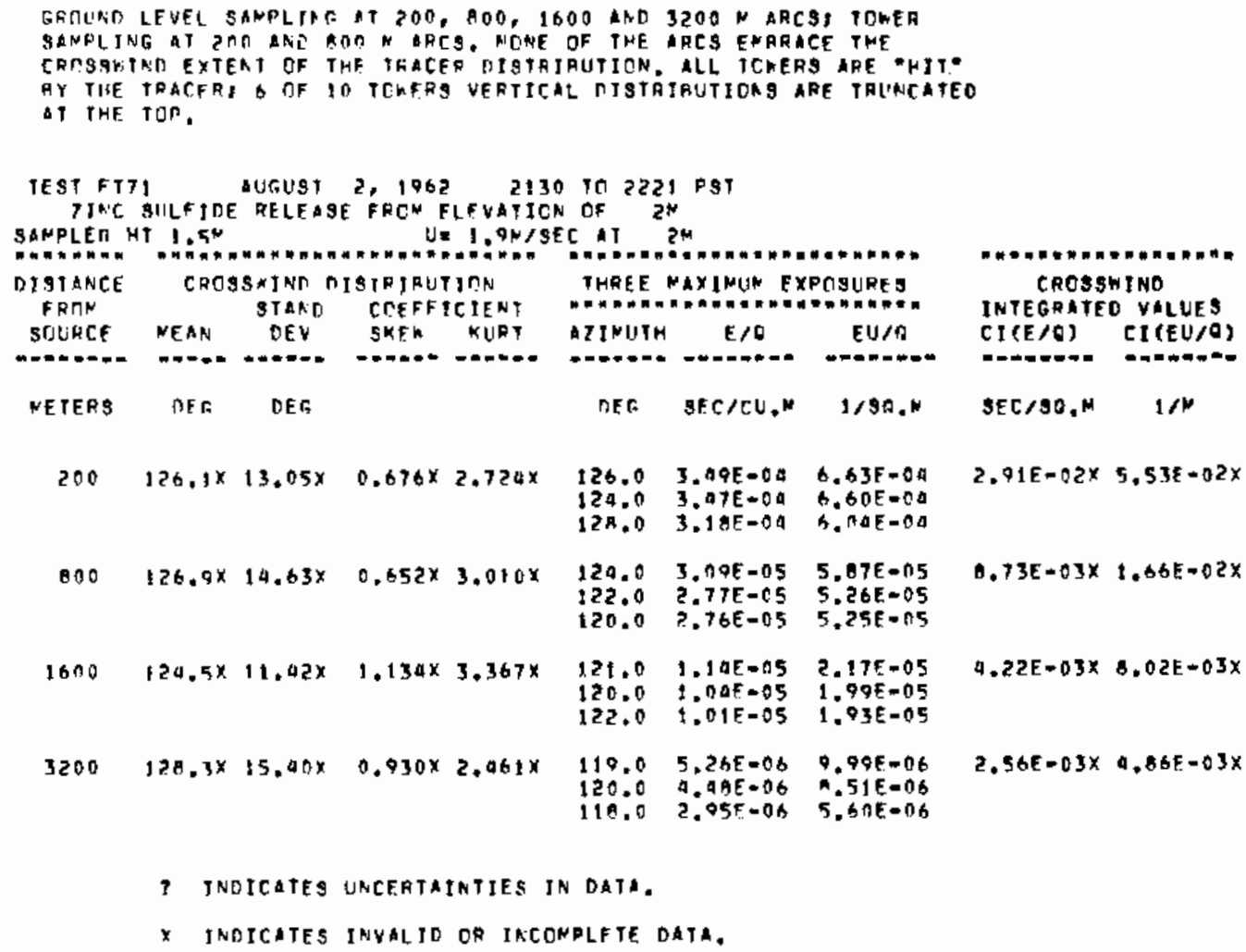




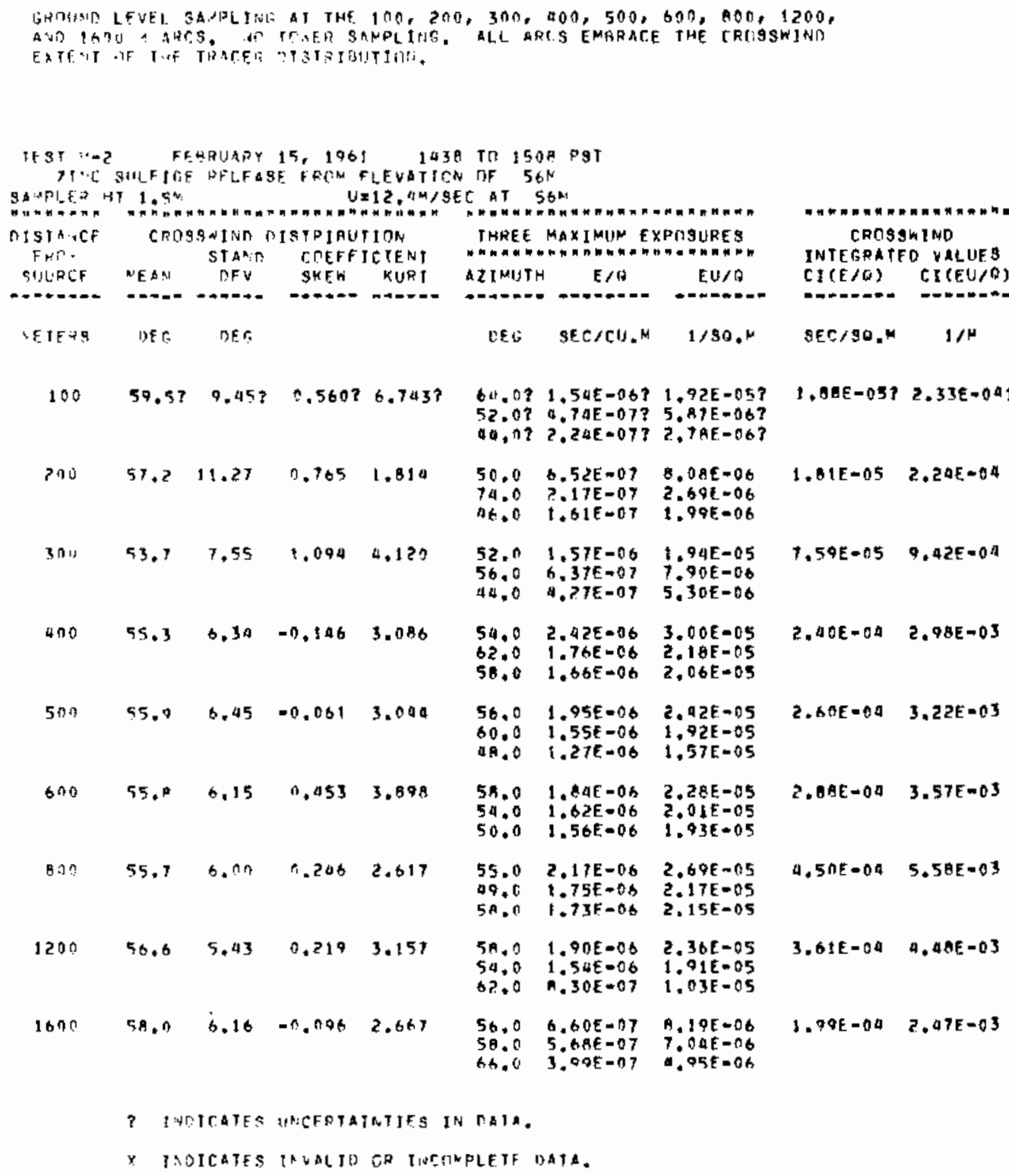


GROUND LEYEL SAMPL ING AT $100,200,300,400,500,500,800,1200$, AND I600 H ARCS. NO TOWER SAMPLING. THE TRACER WAS NOT DETECTED ON THE 100 M ARC;

ALL THE OTHER ARCS EMBRACE THE FULL CROSSWINO EXTENT OF THE TRACER

OISTRIBUTION.

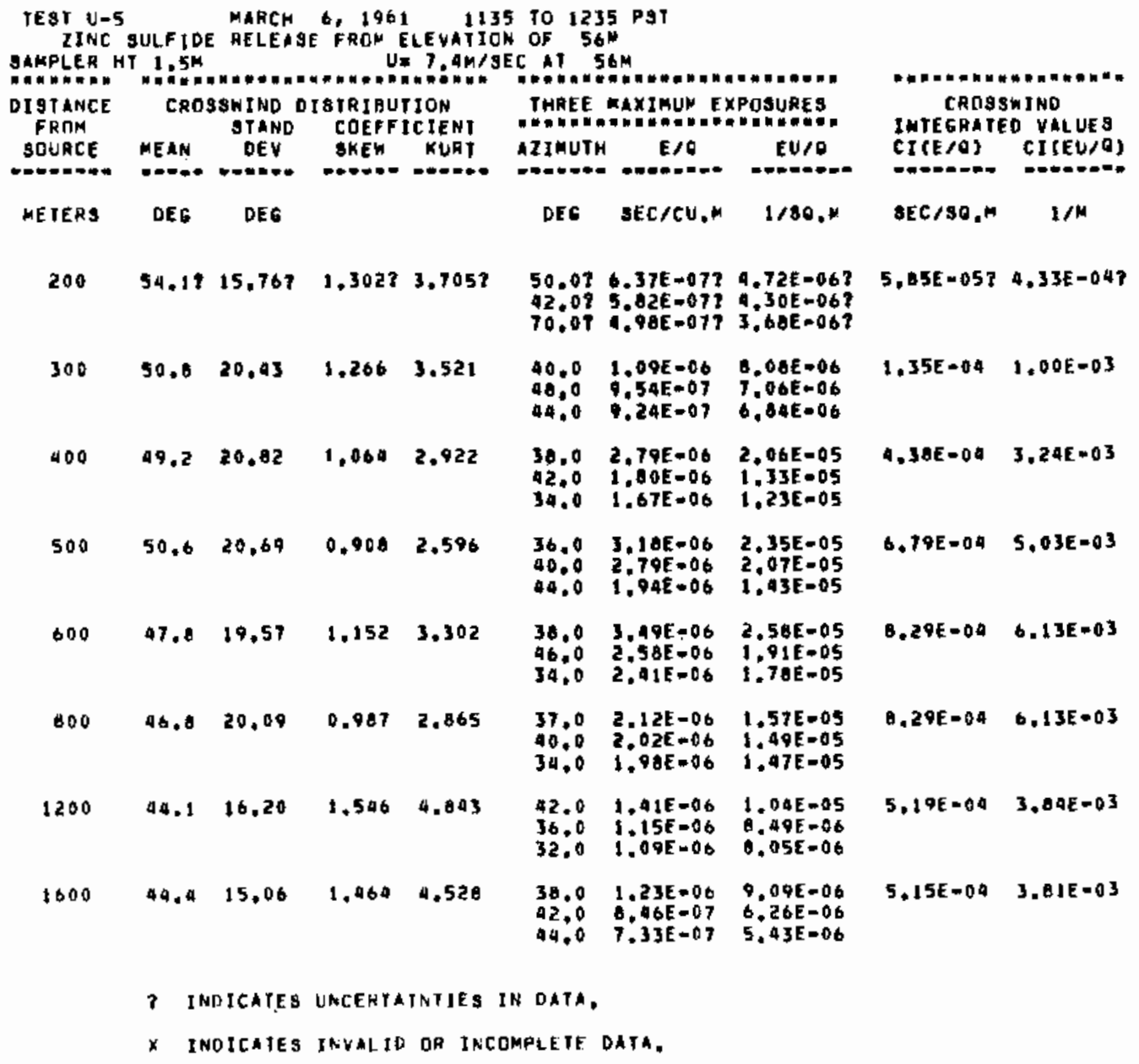




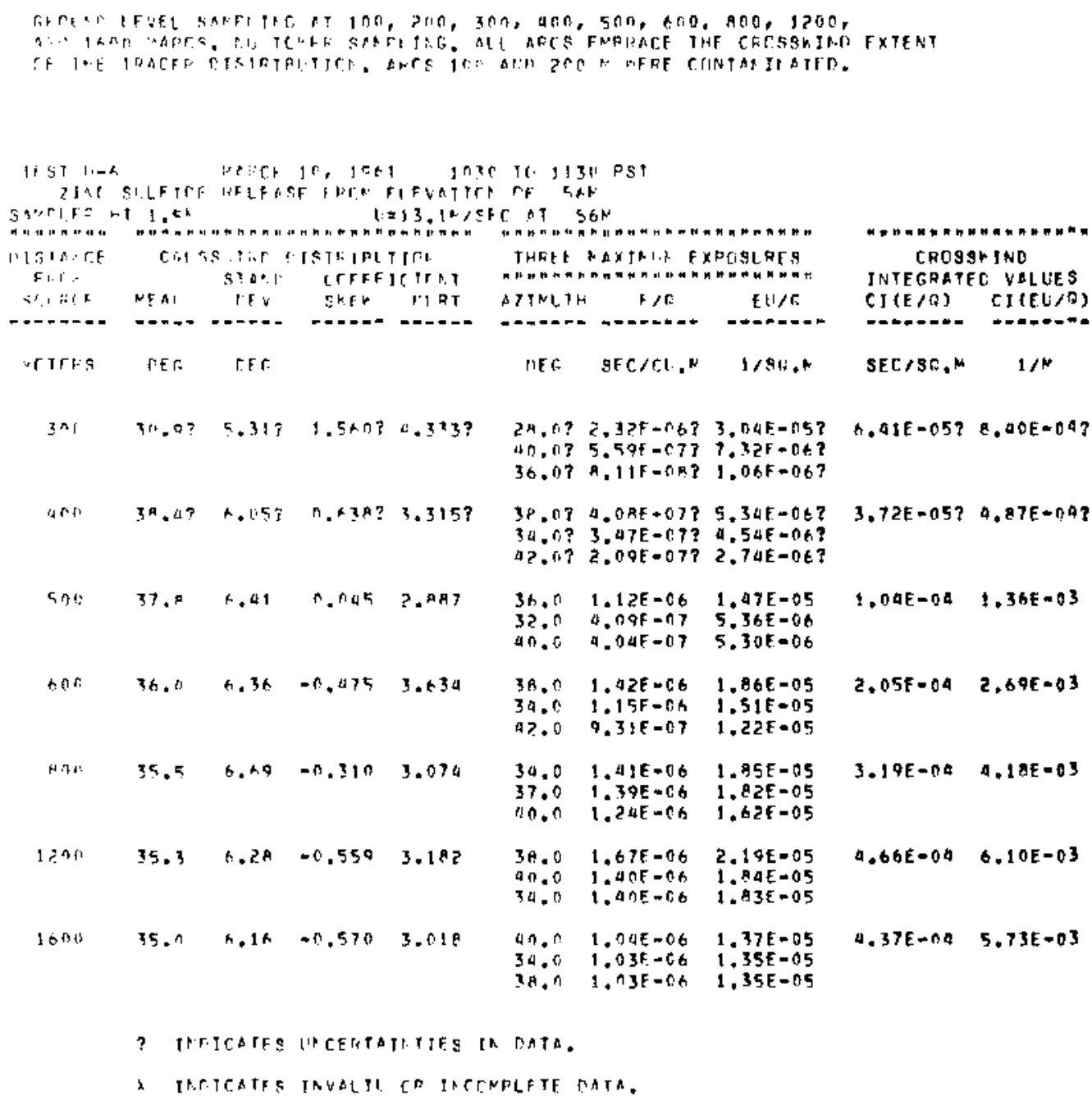




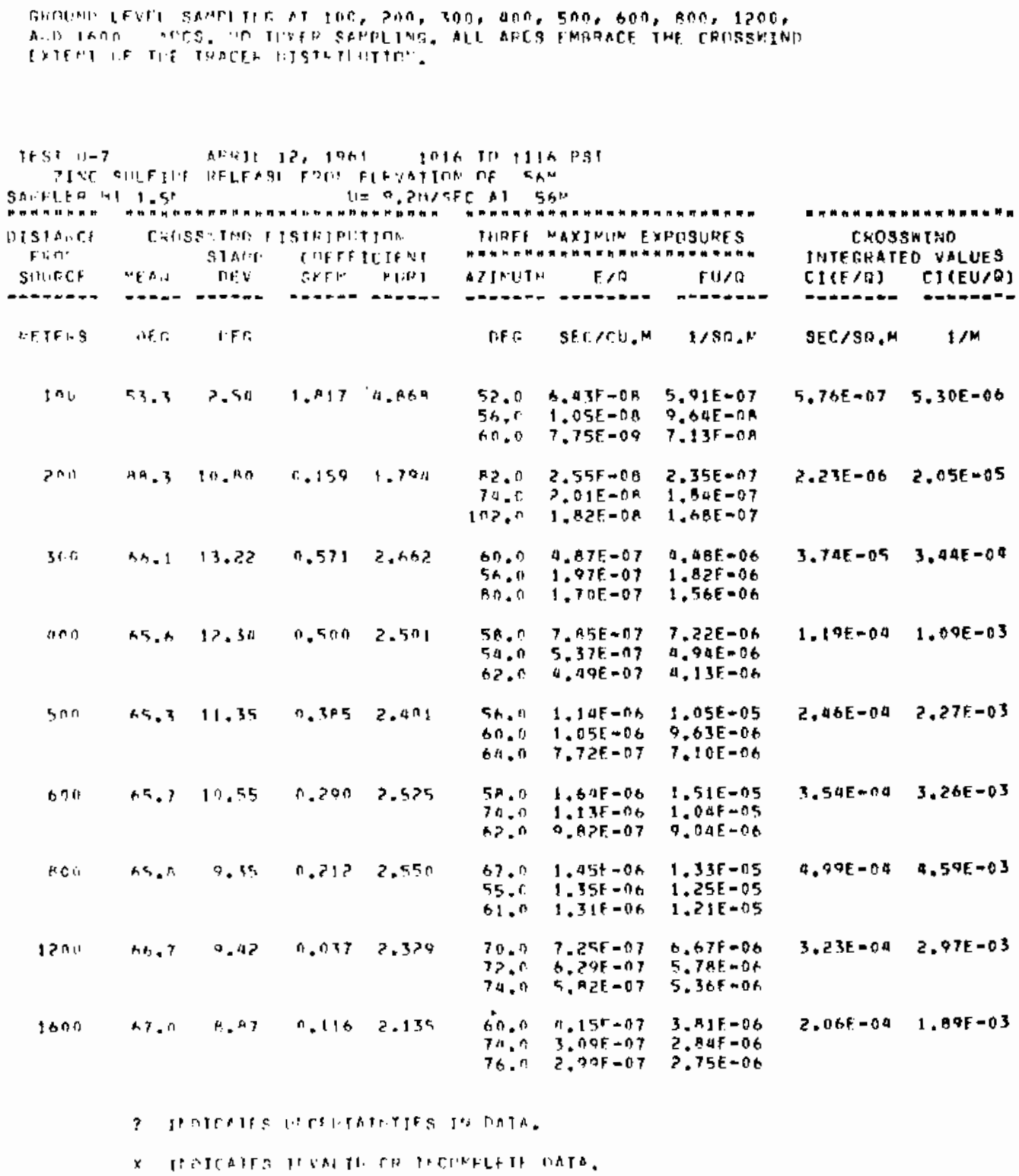




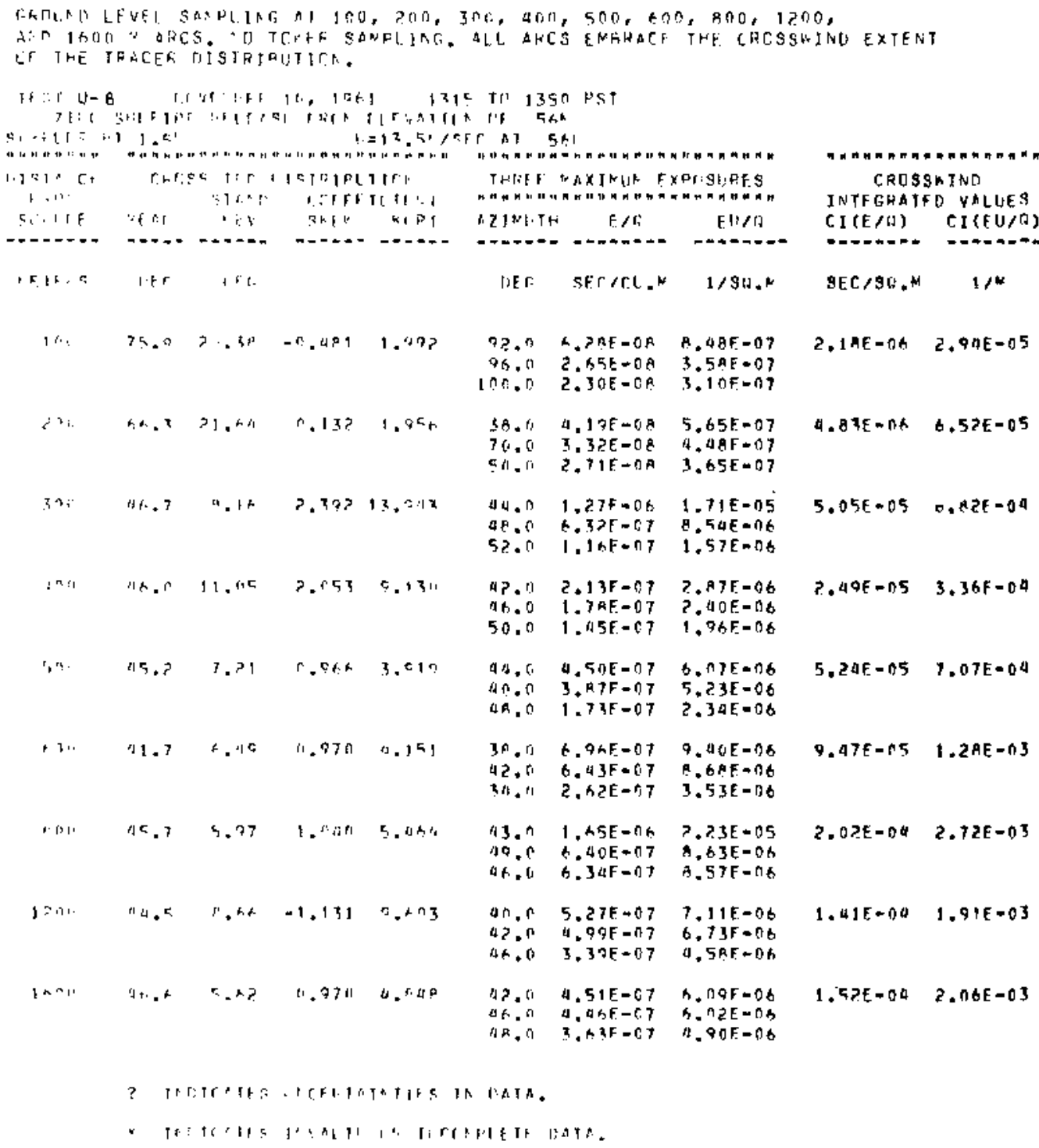




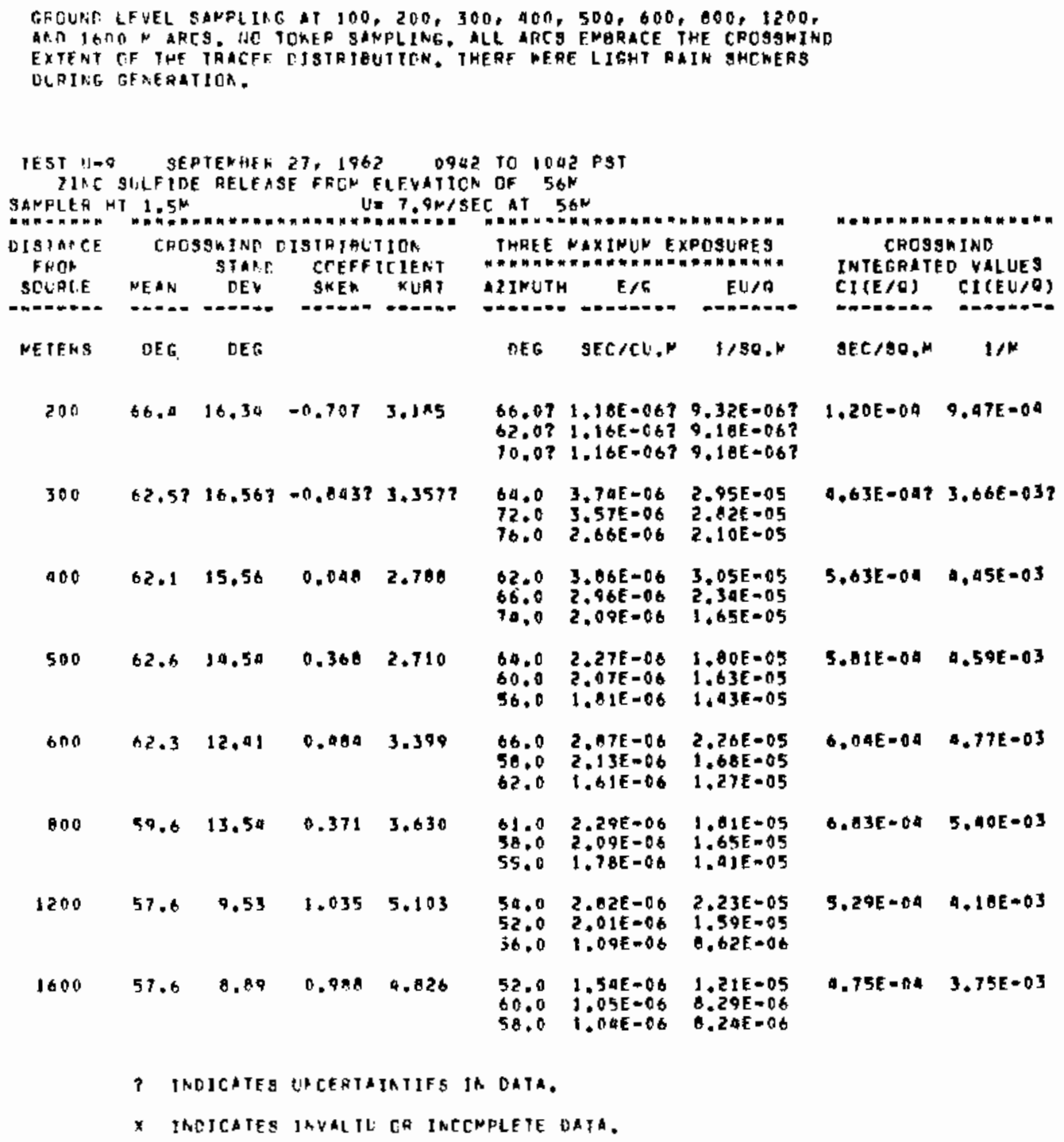




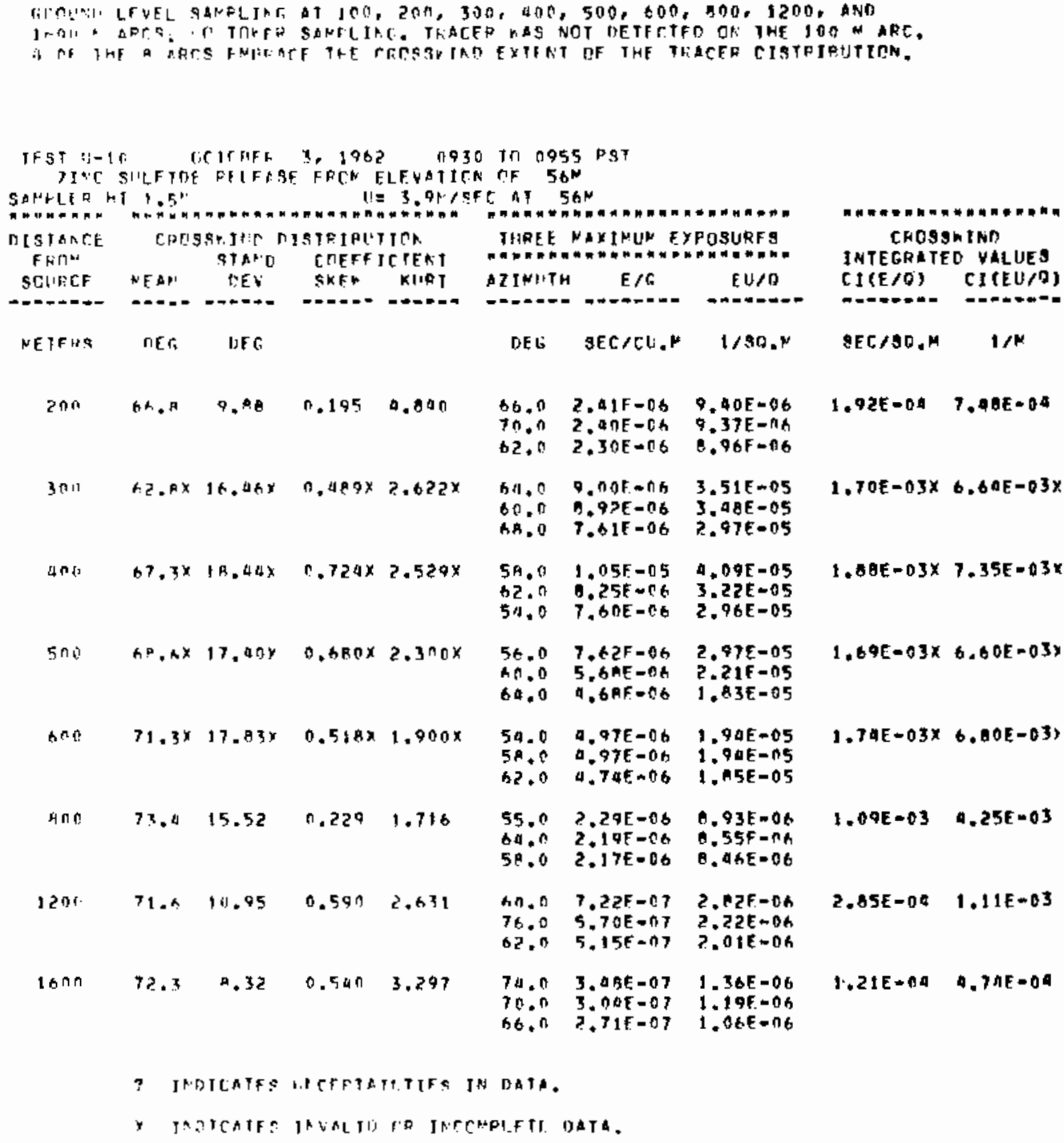

CHOSOMtNin INTEGRATED YALUES $C I(E / O)$ CI $(E U / D)$ SEC $100 . M \quad 1 / \mathrm{K}$ 


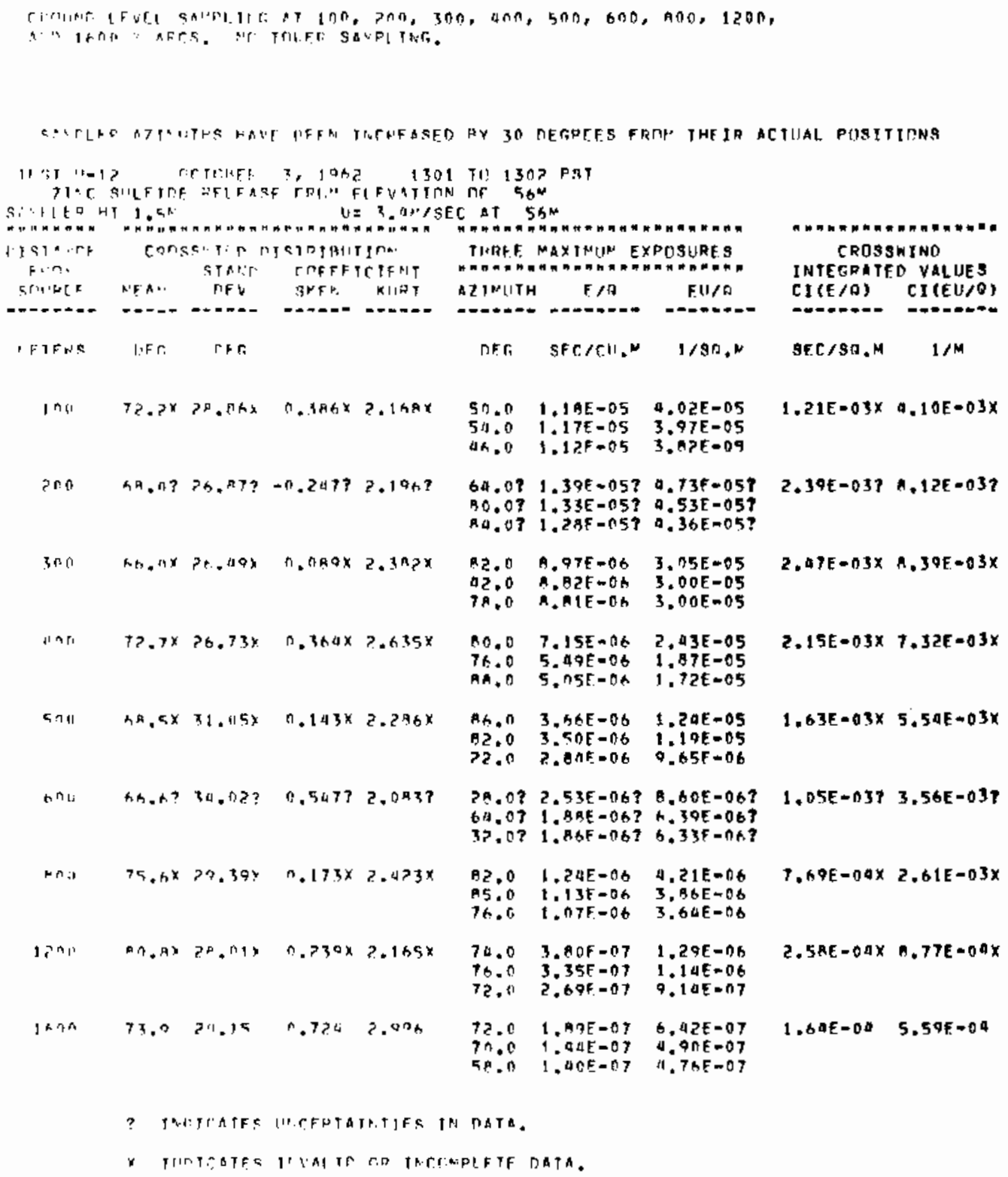




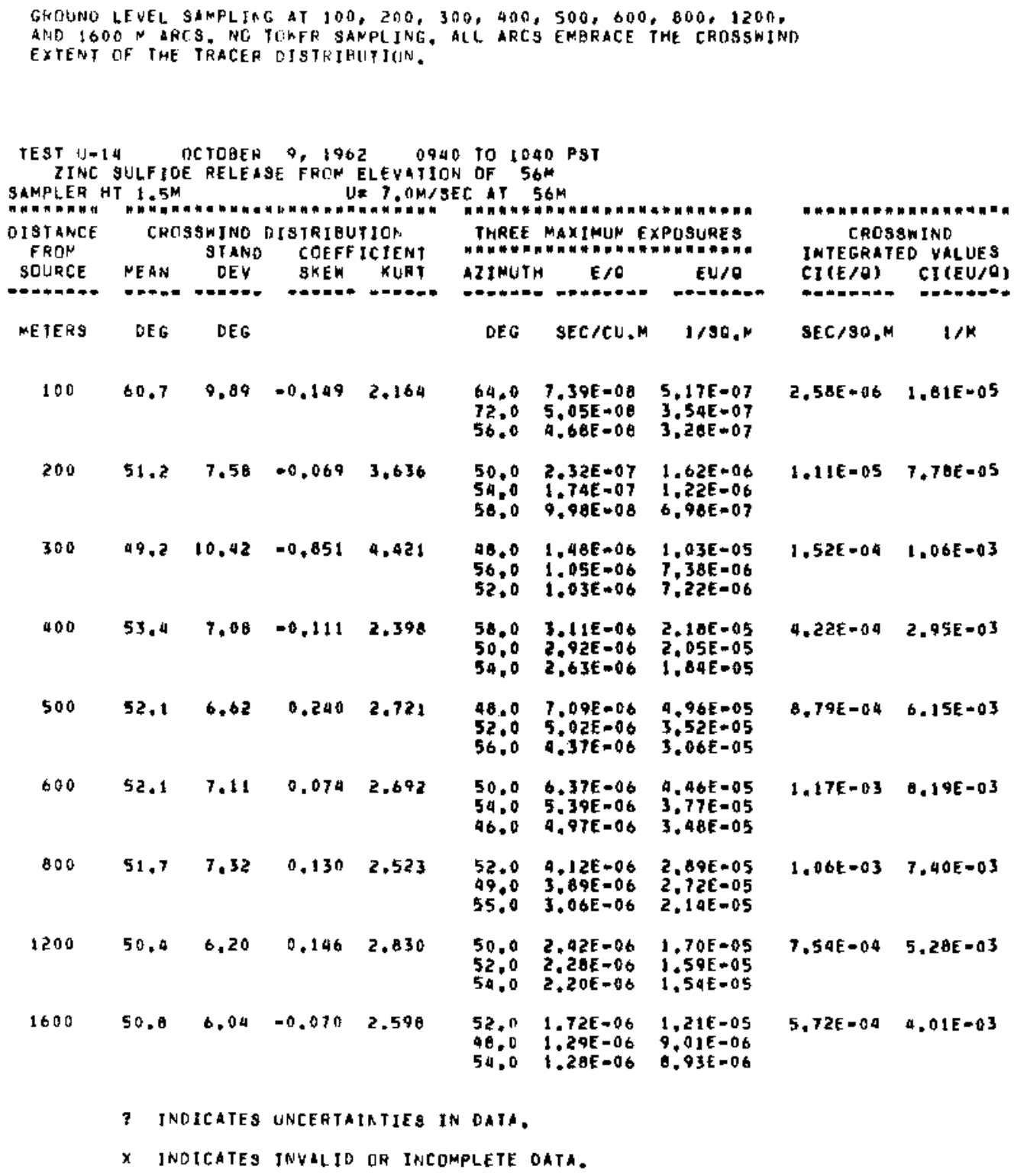


GROUND LEVEL SAMPLIRT, AT $100,200,300,400,500,600,000,1200$, AHS $1600 \mathrm{Y}$ APCS. NO TOWFR SANFLTNF. 7 OF THE 9 ARCS EHRFACE THE COMSARIND EXTENT RF THF TFACFK DISTRIRLITION.

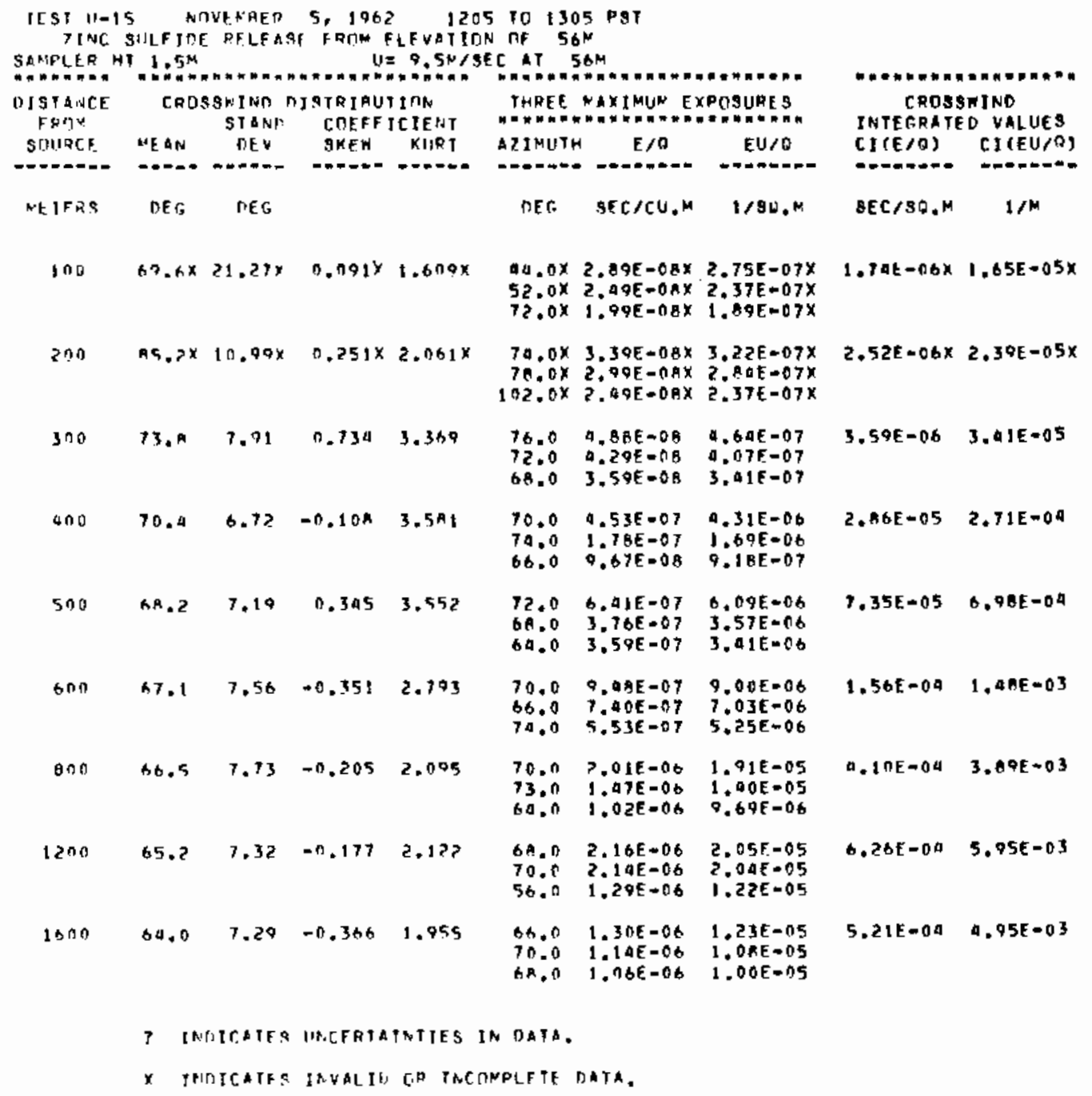




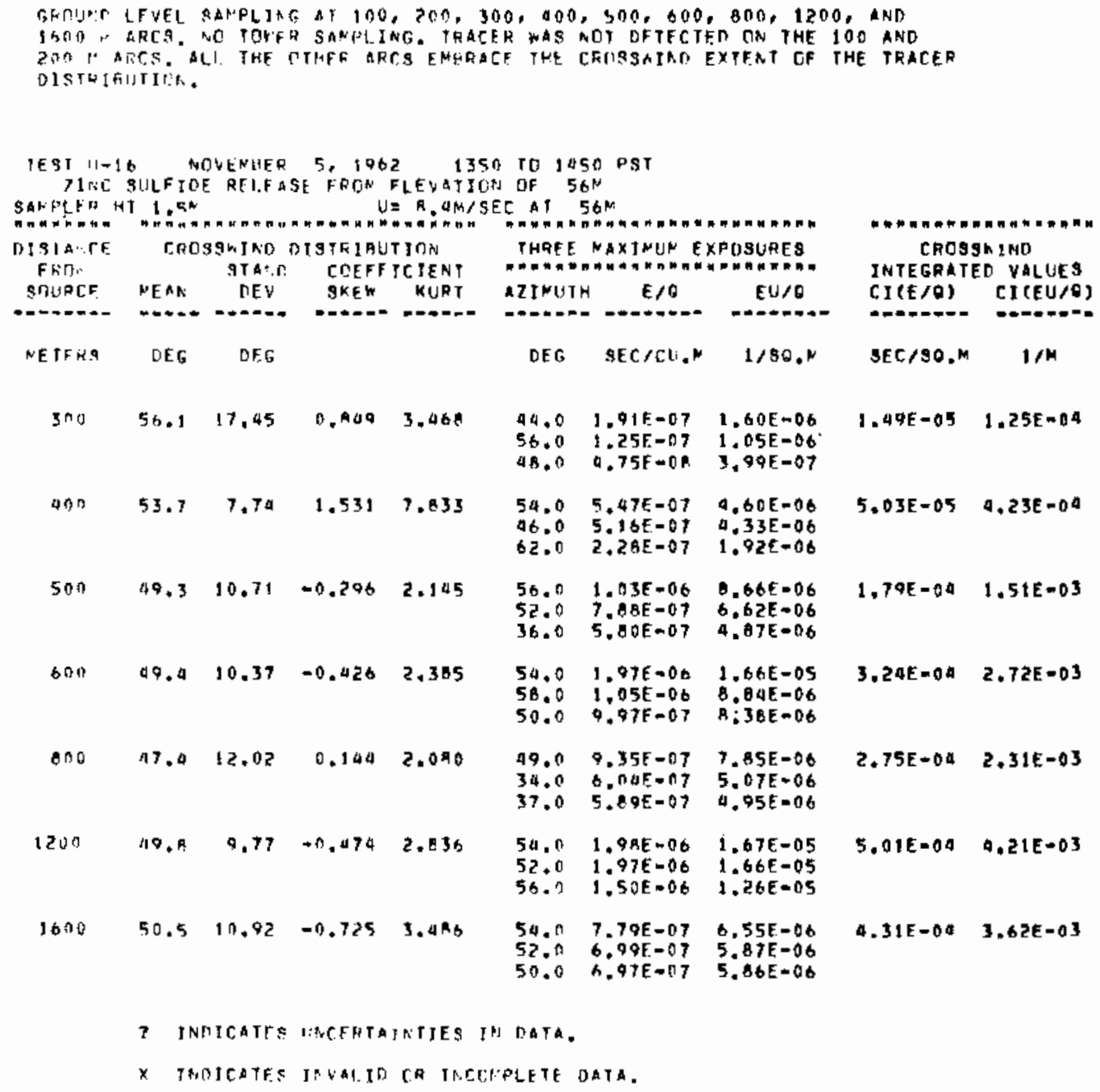




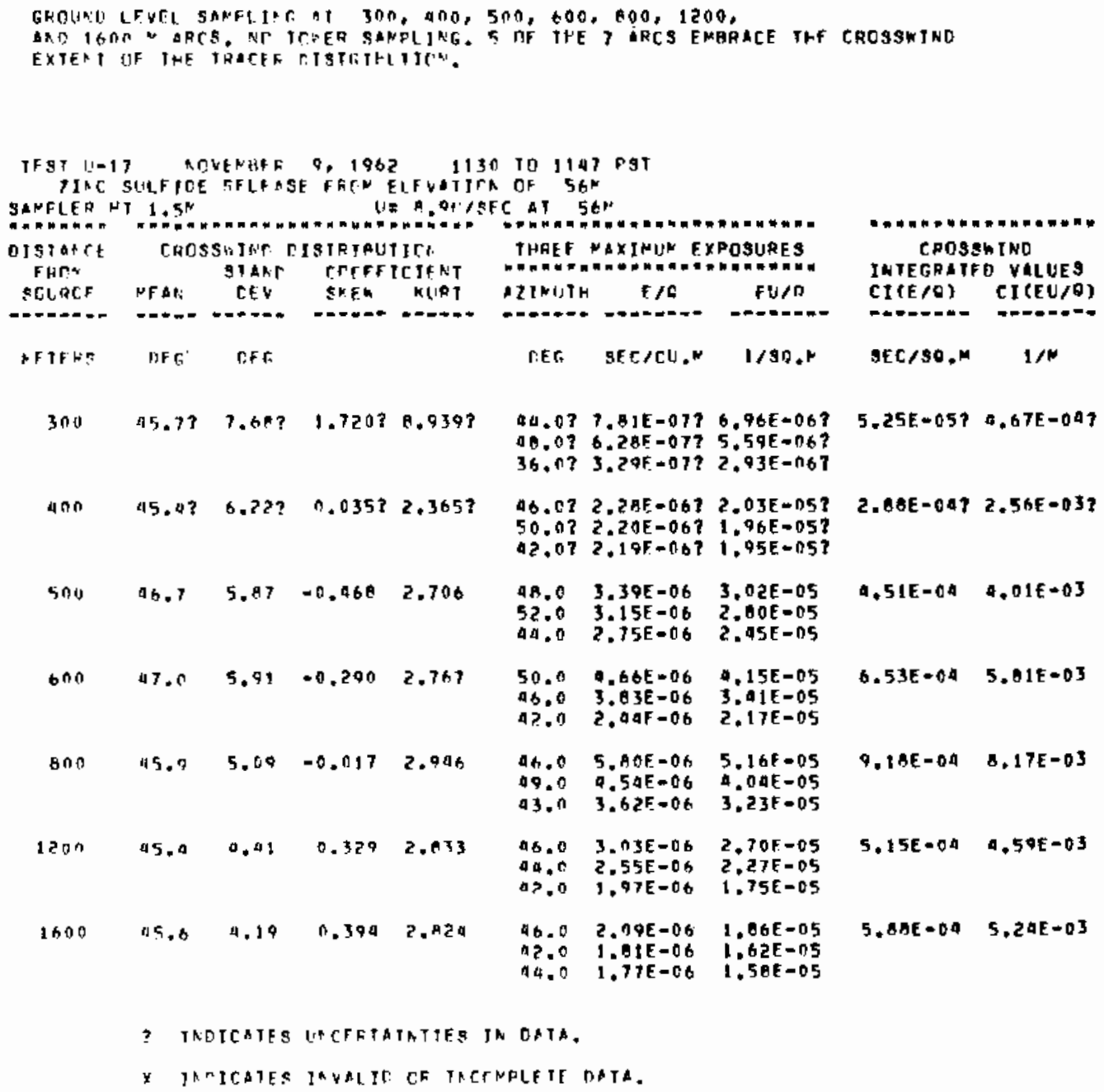




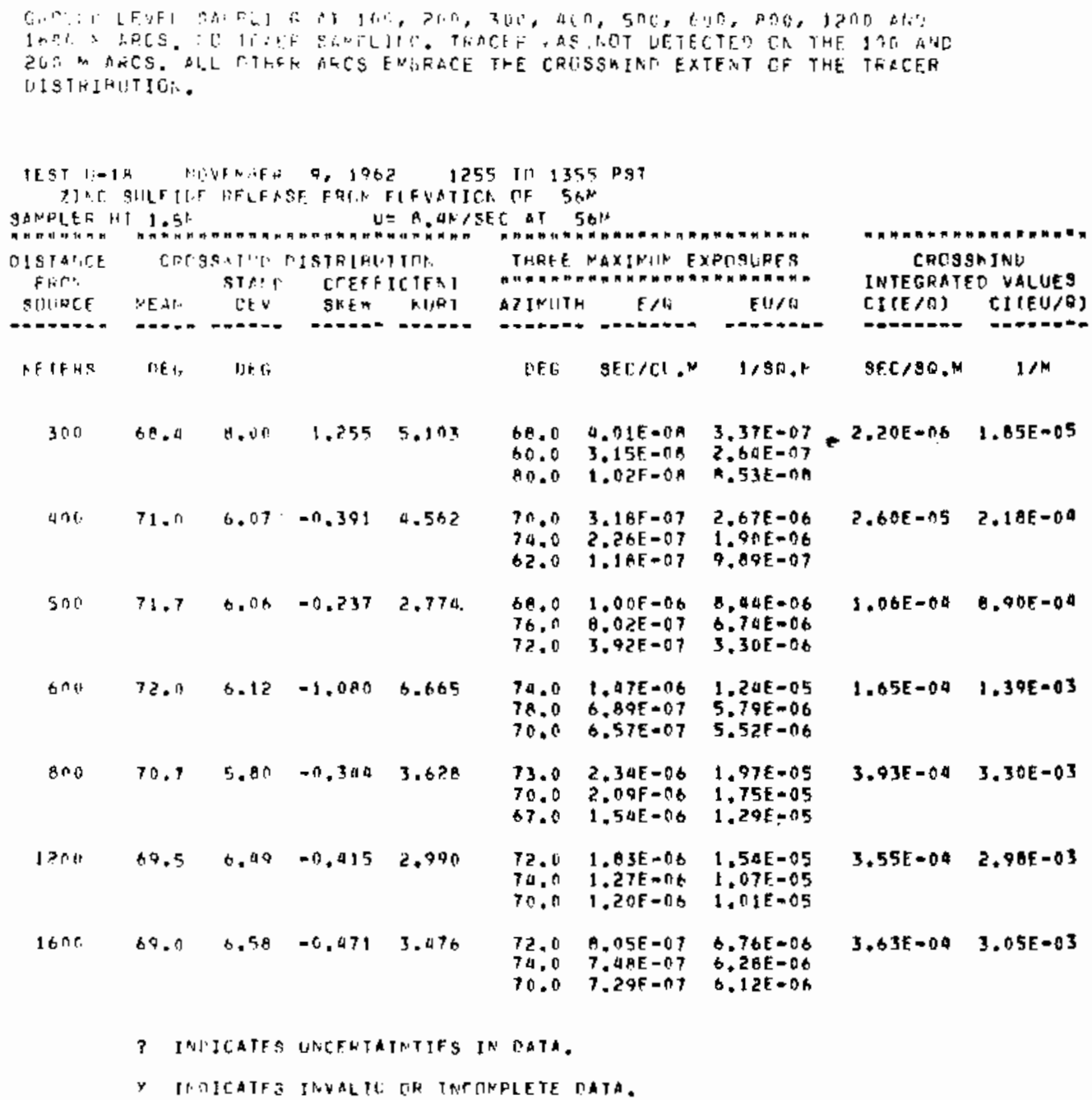




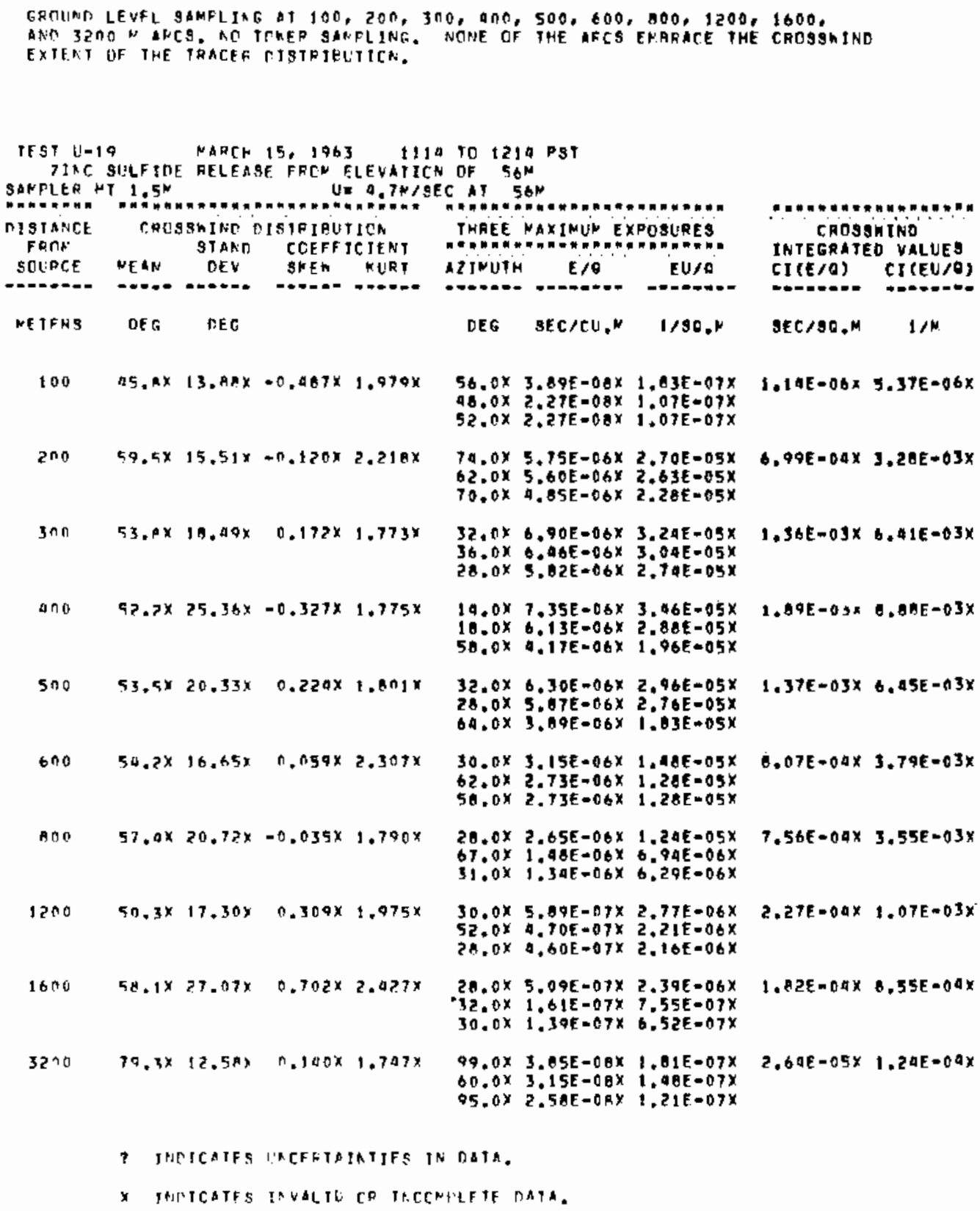




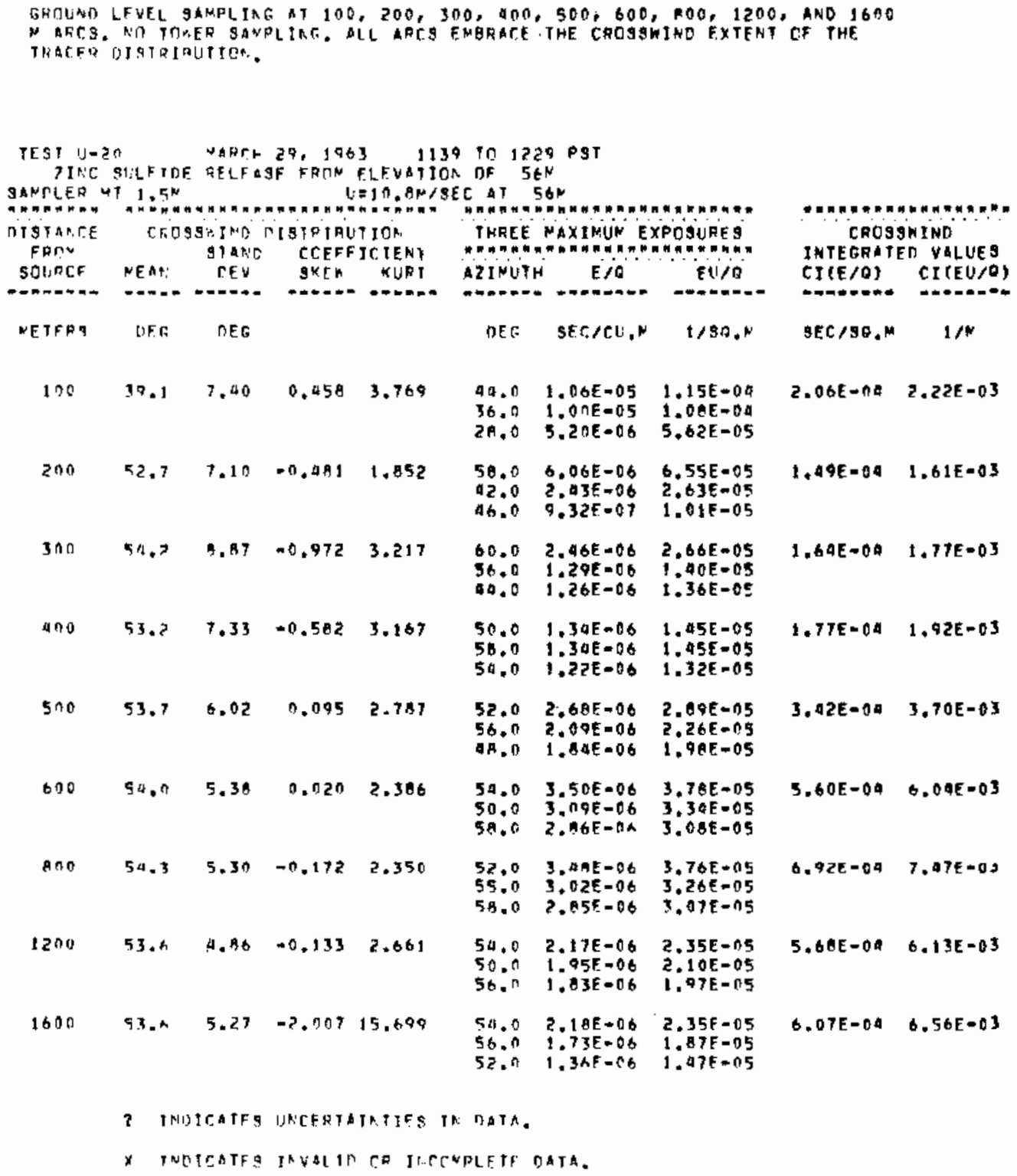




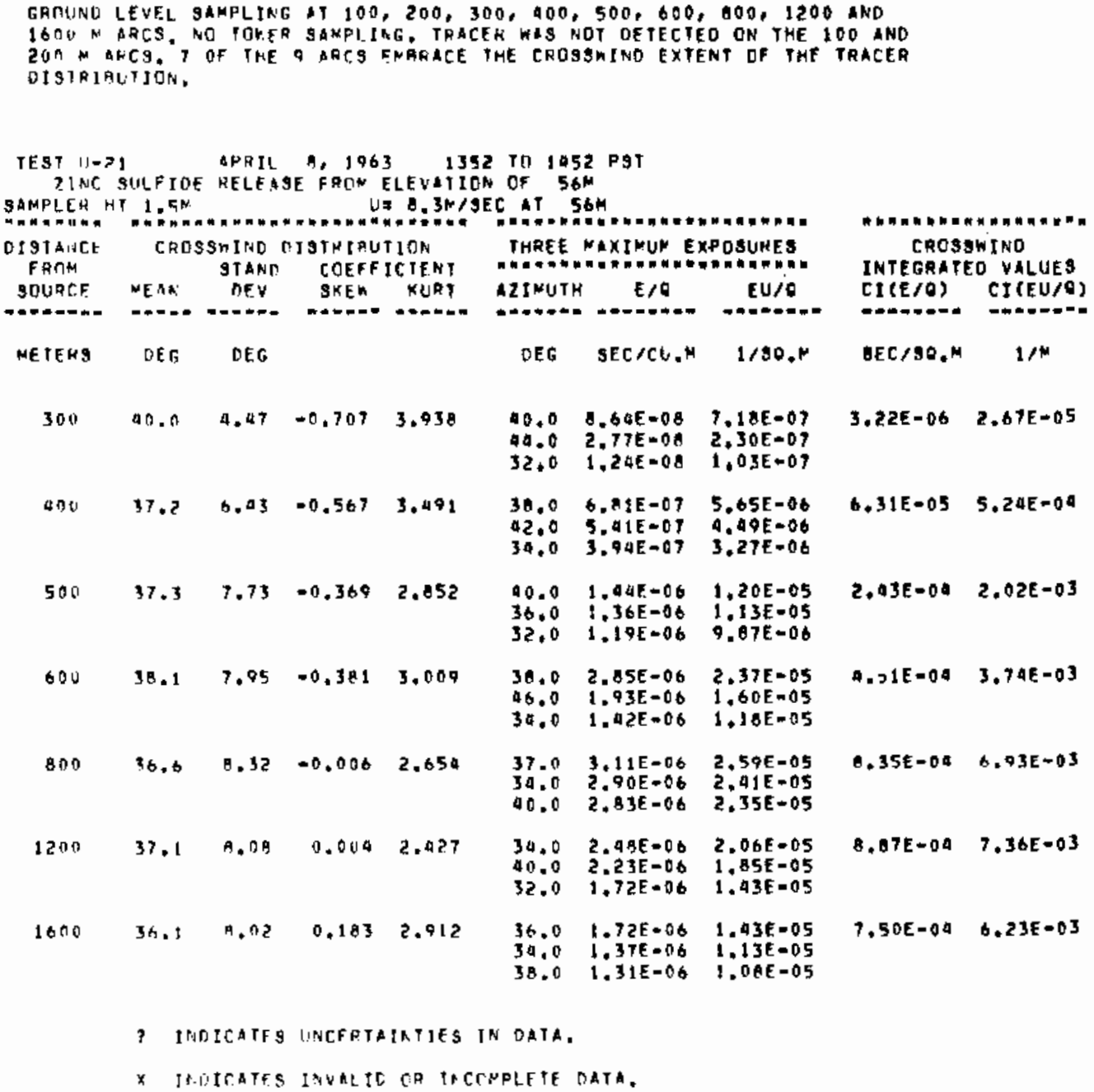




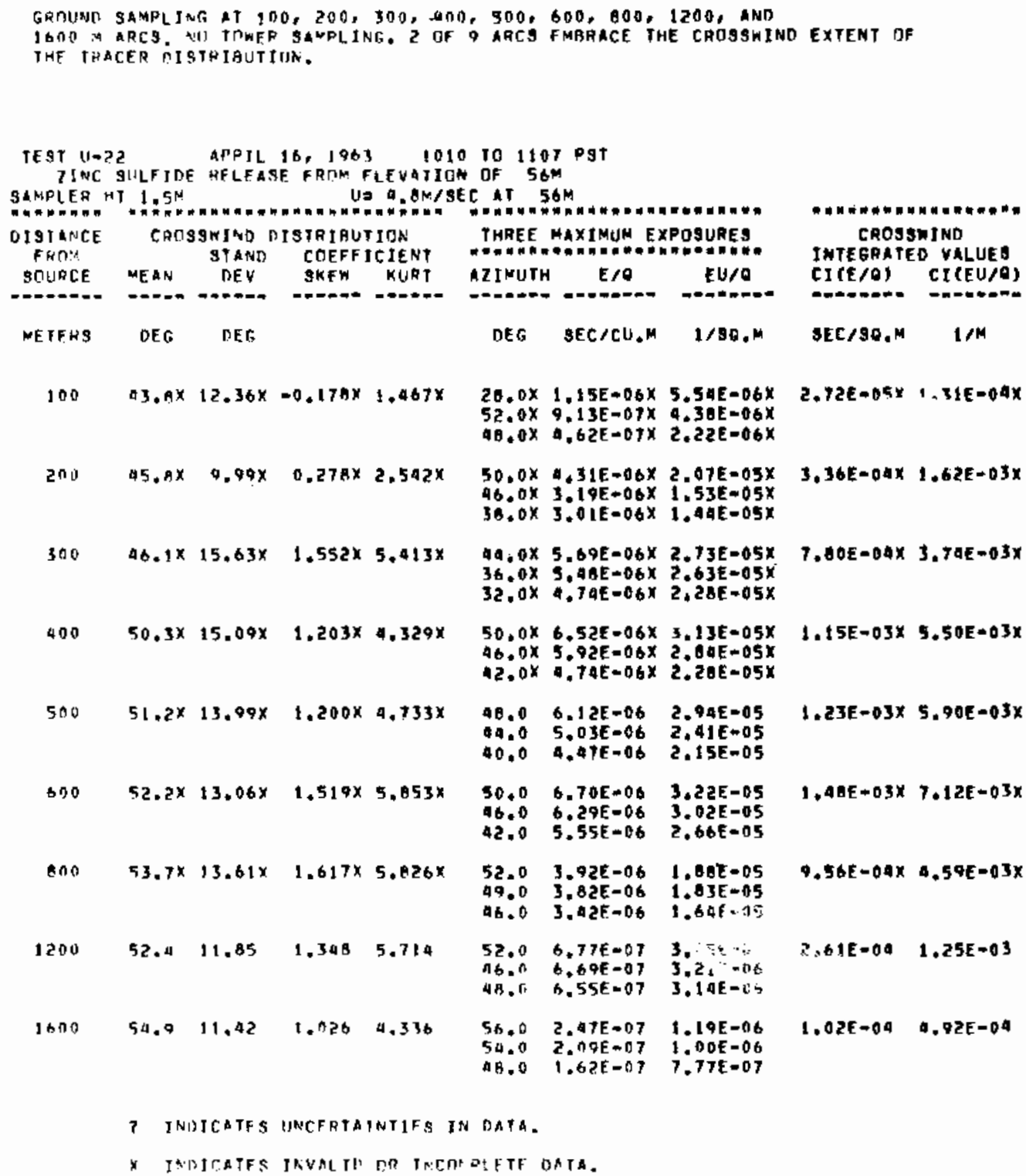




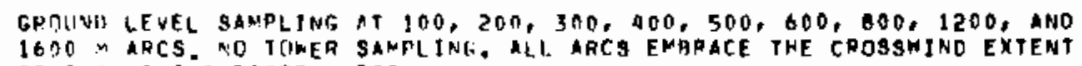
DF THE TRACFR DESTPIRLTITH.

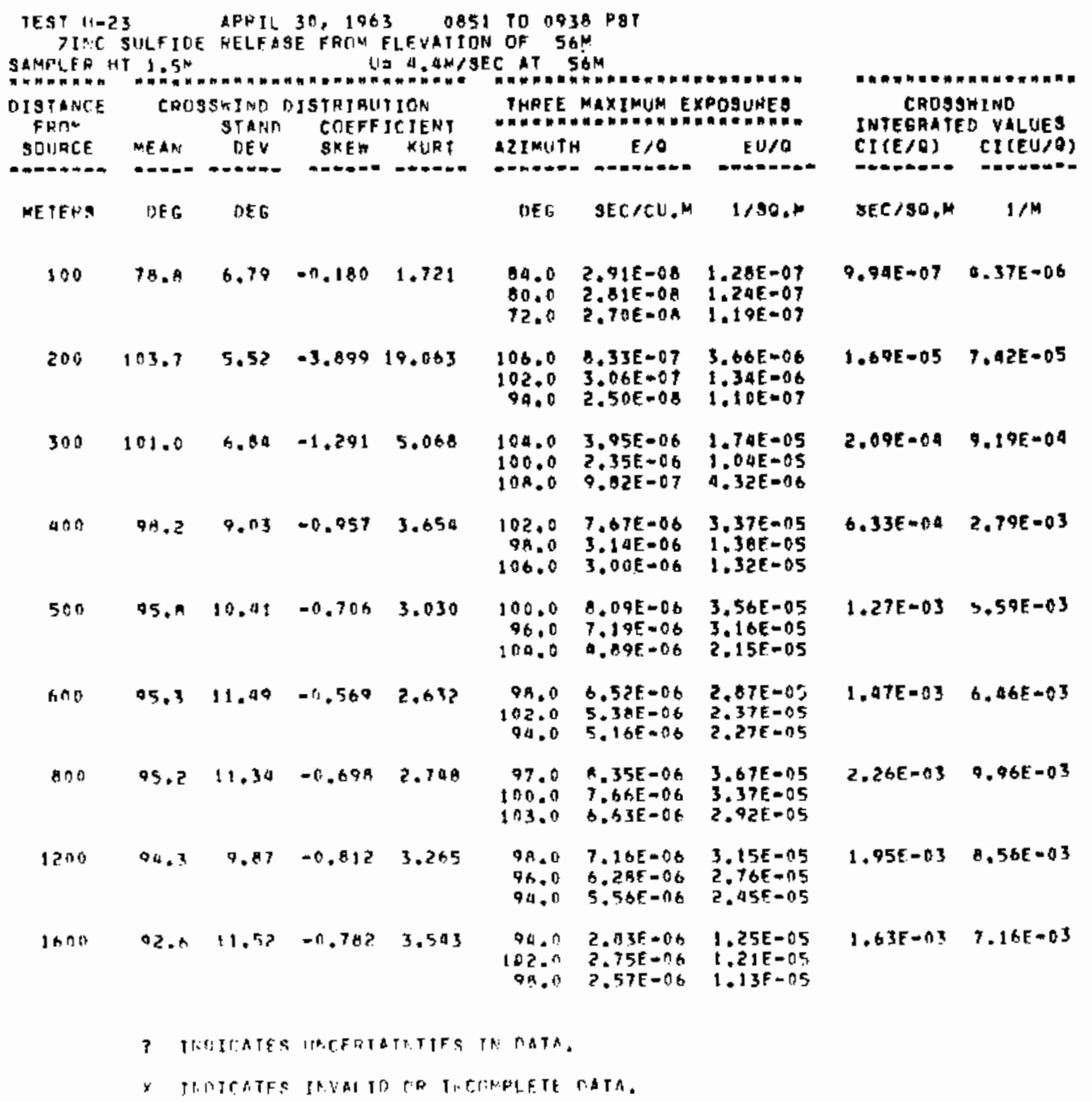




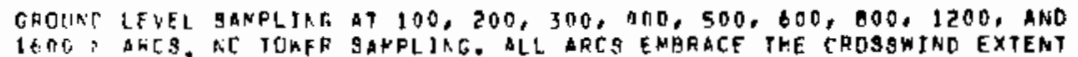
OFF THE TFACF CISTRIAUTICK.

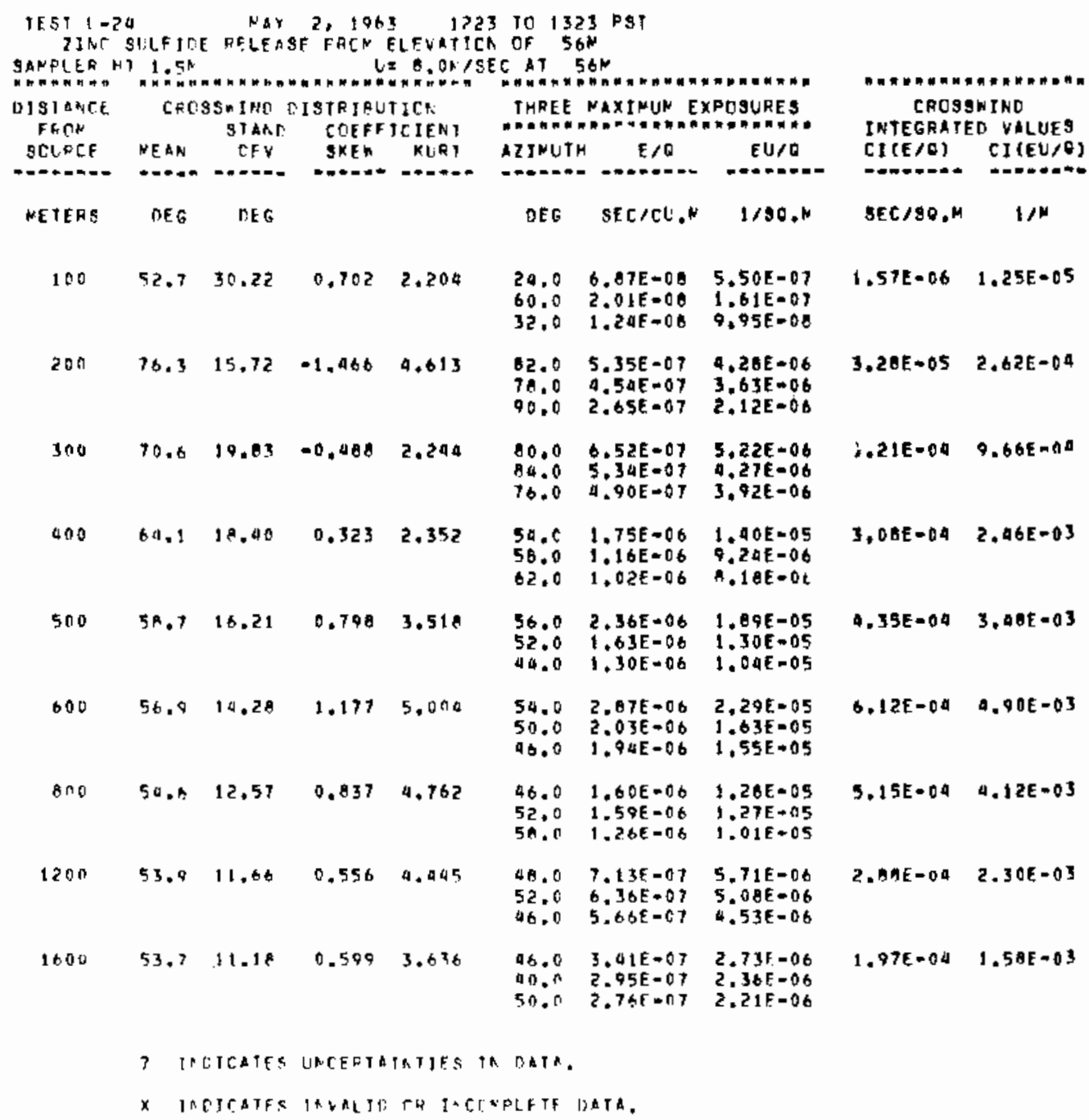




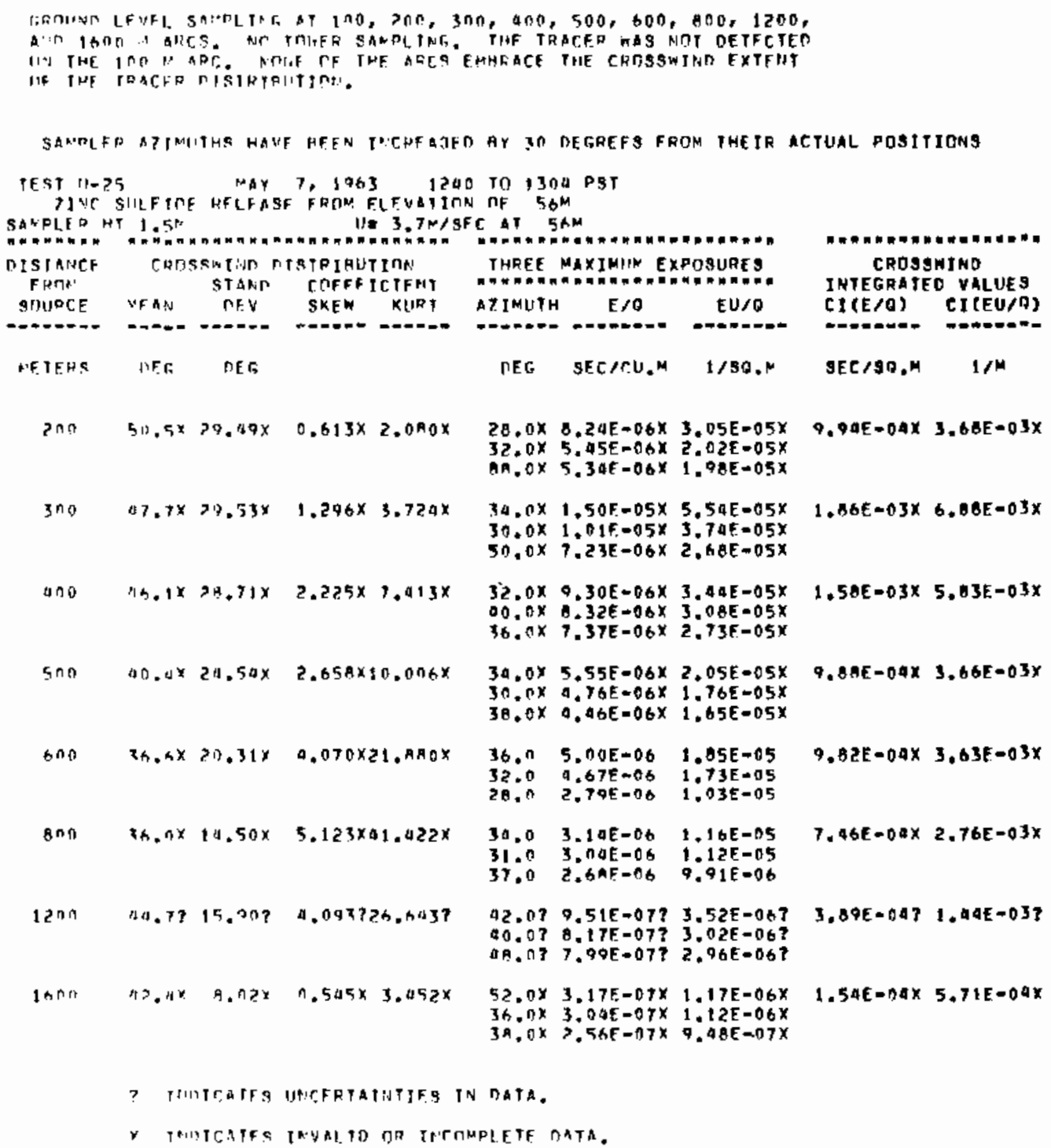




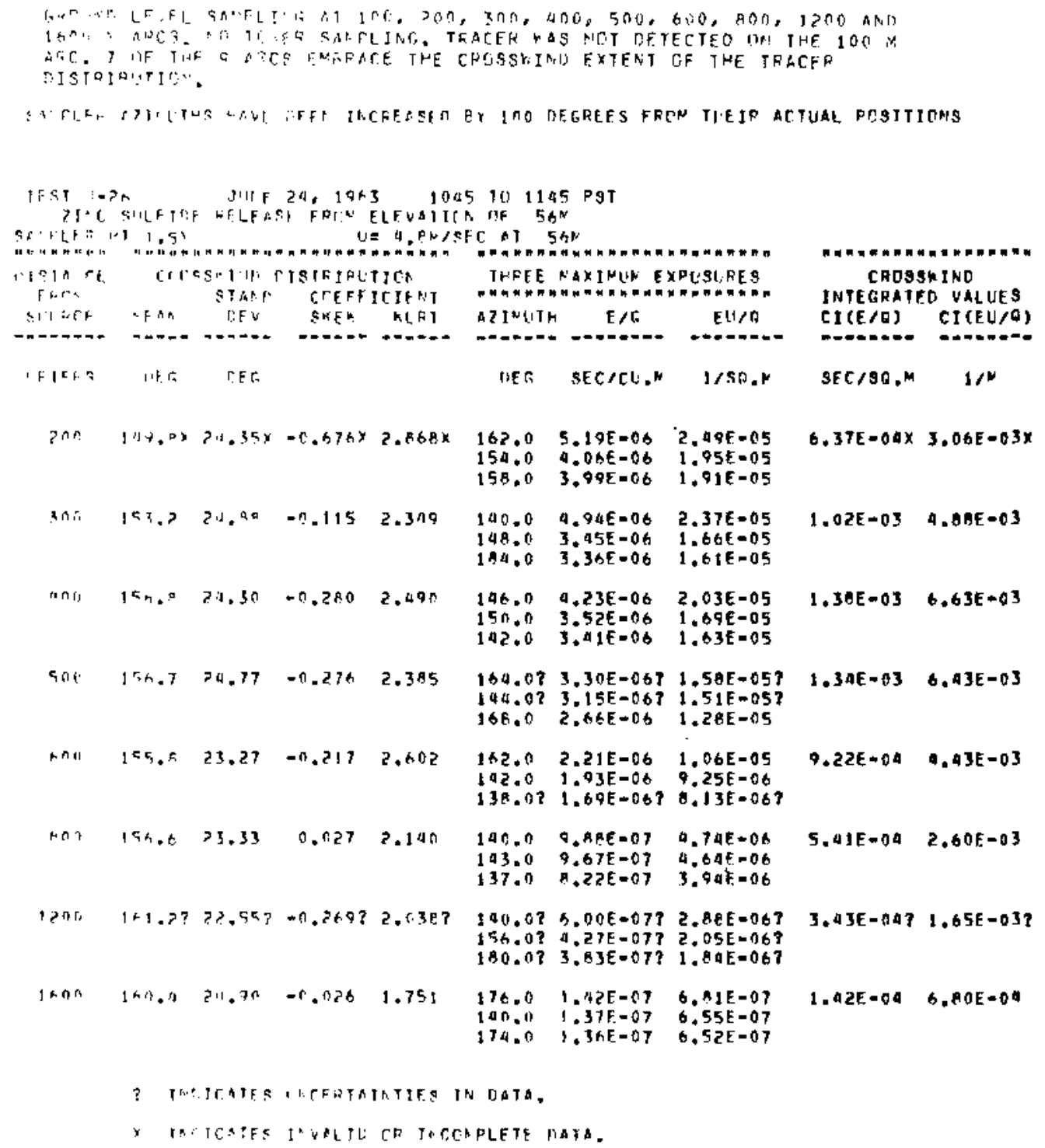




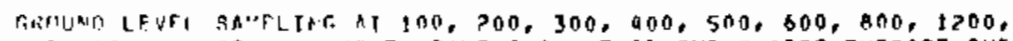

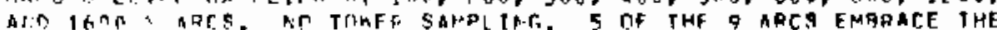
THF CFOSSUIPI: EXTERT OF THF TPACEE CISTRIRUTION.

\begin{tabular}{|c|c|c|c|c|c|c|c|c|c|}
\hline $5 n_{1}, P\lfloor F$ & 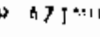 & ITHS HAV & If $\quad P^{2} F F^{N} I$ & INTHFASED & AY 30 & DEGAEES FF & FROM THE TR & \multicolumn{2}{|c|}{ METUAL PQSTTJONS } \\
\hline 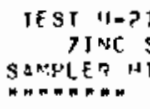 & 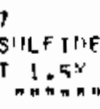 & 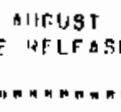 & 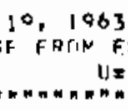 & $\begin{array}{l}1258 \\
\text { FFUATICR } \\
=3.3 \mathrm{~N} / 3 \mathrm{C} C\end{array}$ & $\begin{array}{rr}T 0 & 135 \\
0 F & 5 b \\
C \text { NT } & 5\end{array}$ & 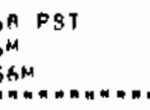 & & 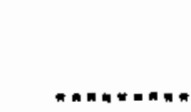 & 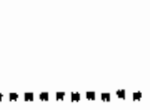 \\
\hline $\begin{array}{l}\text { DISTANCE } \\
\text { FRMA }\end{array}$ & CRris & 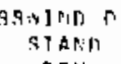 & $\begin{array}{l}\text { ISTRIAUT } \\
\text { CAFFFT }\end{array}$ & $\begin{array}{l}\text { TIRR. } \\
\text { TCTENT }\end{array}$ & 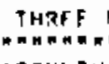 & $\operatorname{Max}_{\operatorname{mat}}$ & XPOSURES & $\begin{array}{r}\text { CROSS } \\
\text { INTEGRATE }\end{array}$ & WWINI \\
\hline SCIIIRCF & NEAH & NF. & SKFW & KUQT & AZIMUTH & $E / D$ & EU/G & $C I(E / \pi)$ & $\operatorname{CI}(E U / \theta)$ \\
\hline$=-0-0=0$ & $-0=0$ & $--\infty-n$ & $-\infty \rightarrow-\infty-\infty$ & $-=-=-0$ & $-\infty+-+\infty$ & $-\infty+\infty+\infty=$ & $-0-\infty-2-0$ & $=-\infty-\infty--\infty$ & $=-\infty-0--\infty$ \\
\hline NEFF" & ner. & MER. & & & DE G & SEC/CU, & $1 / \sin . N$ & SEC/SO.M & $1 / 4$ \\
\hline 100 & $59.0 x$ & 34.674 & $0.336 x$ & $2,111 x$ & $\begin{array}{l}10.0 x \\
10.0 x \\
10.0 x\end{array}$ & $\begin{array}{l}2.02 f=05 x \\
2.60 E=05 x \\
2.23 E=05 x\end{array}$ & $\begin{array}{l}9.31 E-05 x \\
0.84 E=05 x \\
7.35 \xi-05 x\end{array}$ & $2,26 E-0.3 x$ & 7. QAE $-03 x$ \\
\hline $2 n 0$ & $77.5 x$ & $35.92 x$ & $0.140 x$ & $1.930 \times$ & $\begin{array}{l}68.0 \\
94.0 \\
72.0\end{array}$ & $\begin{array}{l}6.01 E-06 \\
5.97 E-06 \\
5.31 E-06\end{array}$ & $\begin{array}{l}1.98 E=05 \\
1.97 F=05 \\
1.75 E-05\end{array}$ & $1.68 E-133 x$ & $5.53 E=03 x$ \\
\hline 300 & $79.6 x$ & $29.68 X$ & $n . n 94 x$ & $2,300 x$ & $\begin{array}{l}54.0 \\
94.0 \\
5 \% .0\end{array}$ & $\begin{array}{l}6.48 E=06 \\
h .16 E=06 \\
4.20 E=06\end{array}$ & $\begin{array}{l}2.1 \text { aE }-05 \\
2.03 E-05 \\
1.30 F=05\end{array}$ & $1.60 \varepsilon-05 x$ & 5. $2 A E-03 x$ \\
\hline 400 & $A \cap . A X$ & $29.75 x$ & $0.101 x$ & $2.301 x$ & $\begin{array}{l}96.0 \\
92.0 \\
9.0\end{array}$ & $\begin{array}{l}4.02 E=06 \\
9.44 E-06 \\
3.96 E-06\end{array}$ & $\begin{array}{l}1.59 E=05 \\
1.47 E=05 \\
1.31 E=05\end{array}$ & $1.61 E=03 x$ & $5,31 E-0.5 x$ \\
\hline 5 ก0 & A 3.3 & 26.90 & $0.27 \%$ & 2.413 & $\begin{array}{l}90.0 \\
90.07 \\
70.0\end{array}$ & $\begin{array}{l}3.34 E=06 \\
2.73 F=06 \\
2.20 E=06\end{array}$ & $\begin{array}{l}1.10 E=05 \\
9.03 E=06 \\
7.26 E=06\end{array}$ & $1.13 E-03$ & $3.73 E-03$ \\
\hline att & A2. 1 & $? 1.90$ & 0.277 & 2.697 & $\begin{array}{l}88.0 \\
92.0 \\
76.0\end{array}$ & $\begin{array}{l}1.99 E=06 \\
1.51 E=A 6 \\
1.34 E=06\end{array}$ & $\begin{array}{l}5.56 E=06 \\
5.00 F=06 \\
0.03 E=06\end{array}$ & 7. $\mathrm{x}$ aE $=\cap a$ & $2.52 E=03$ \\
\hline $\operatorname{AnO}$ & $a_{n}, 6$ & $24.7 \mathrm{R}$ & 0.122 & 2.609 & $\begin{array}{l}73.0 \\
91.0 \\
01100\end{array}$ & $\begin{array}{l}7.07 E=07 \\
5.96 E=07 \\
5.71 F=07\end{array}$ & $\begin{array}{l}2.33 F=06 \\
1.97 E=06 \\
1.98 F-06\end{array}$ & $4.11 E-1.4$ & $1.36 E-03$ \\
\hline $1 p^{n i 1}$ & A. 5 & 18.61 & $n .71 n$ & 3.297 & $\begin{array}{l}9.00 \\
30.0 \\
76.0\end{array}$ & $\begin{array}{l}2.95 E=07 \\
? .77 E=07 \\
2.29 E=07\end{array}$ & $\begin{array}{l}0.73 E=07 \\
9.15 E=07 \\
7.55 E=07\end{array}$ & $1.83 \mathrm{t}=0 \mathrm{a}$ & $0.02 E=0$. \\
\hline $1+n !$ & $n n \cdot a$ & 17.07 & $n .0 \times 3$ & 2.325 & $\begin{array}{l}90.0 \\
72.0 \\
8 \% .0\end{array}$ & $\begin{array}{l}1.6 P E=07 \\
1.25 F=07 \\
1.0 ? E=07\end{array}$ & $\begin{array}{l}5.55 E-07 \\
a .12 F=07 \\
3.36 E=07\end{array}$ & $5.0>E=04$ & $3.38 \mathrm{E}=00$ \\
\hline & $?$ & ITHFS & INCEATAT & INTJFS IN & TATA. & & & & \\
\hline & $x^{x}$ & s & VATII: & CR TRC & (E. & m & & & \\
\hline
\end{tabular}




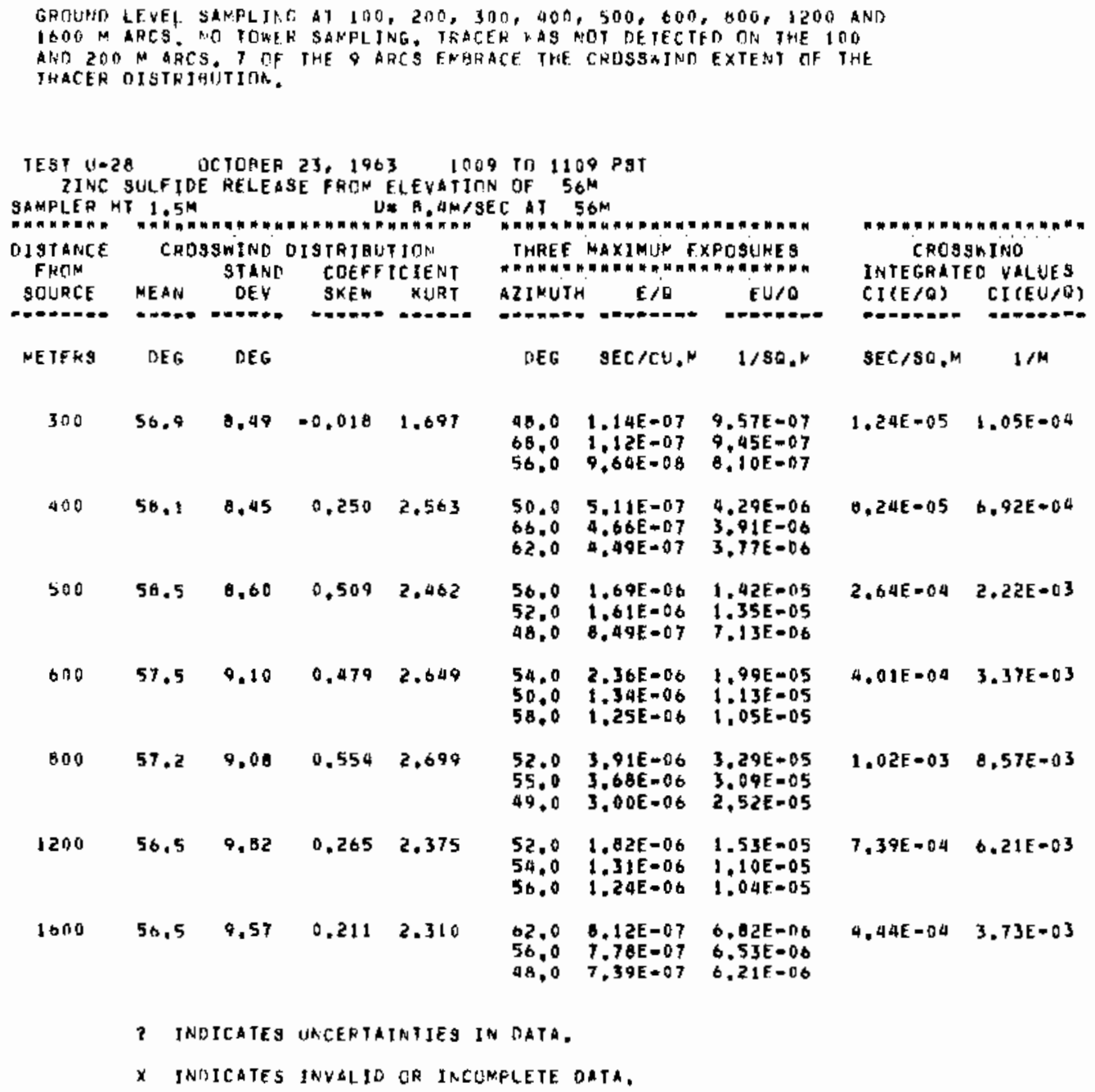




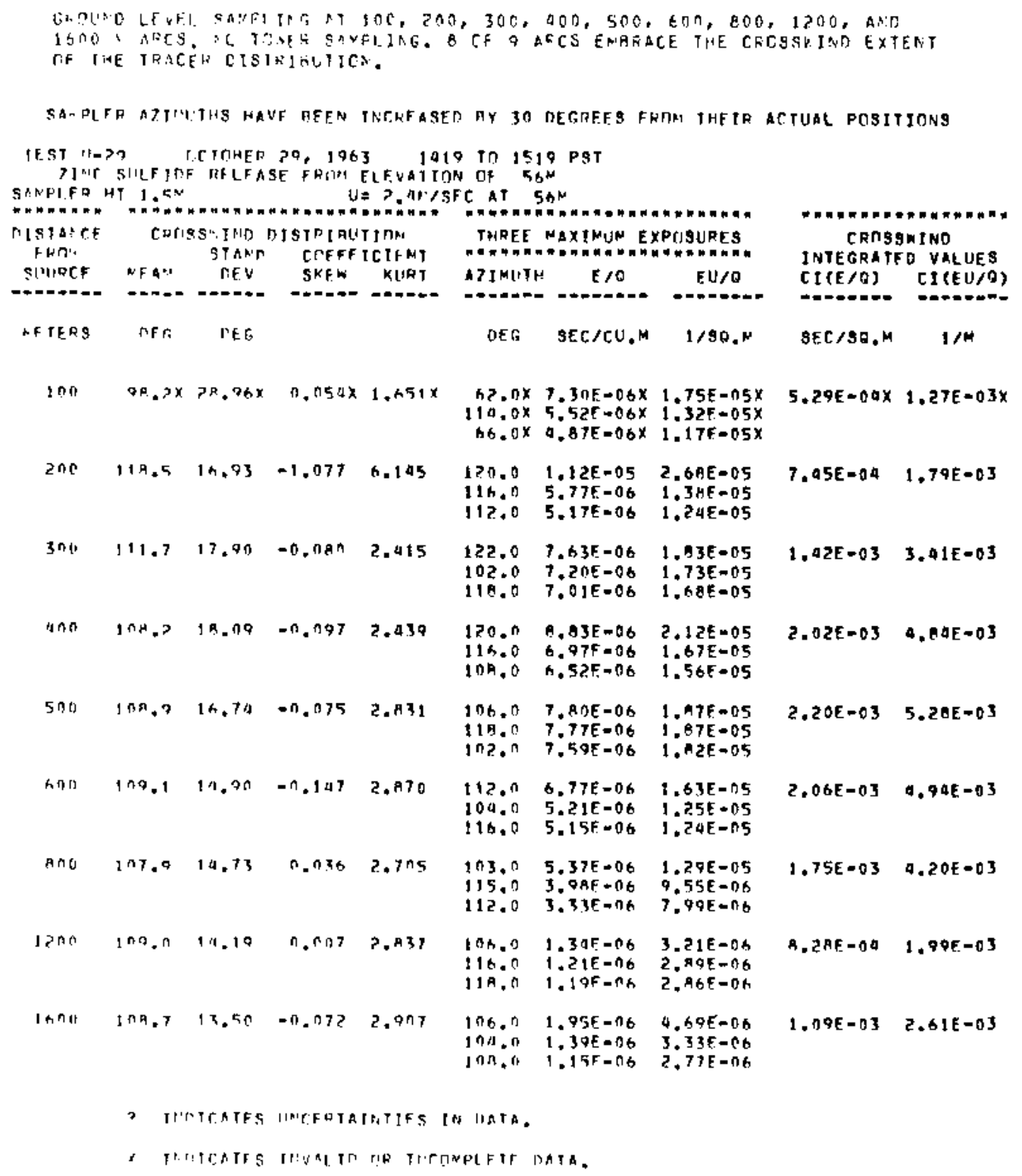




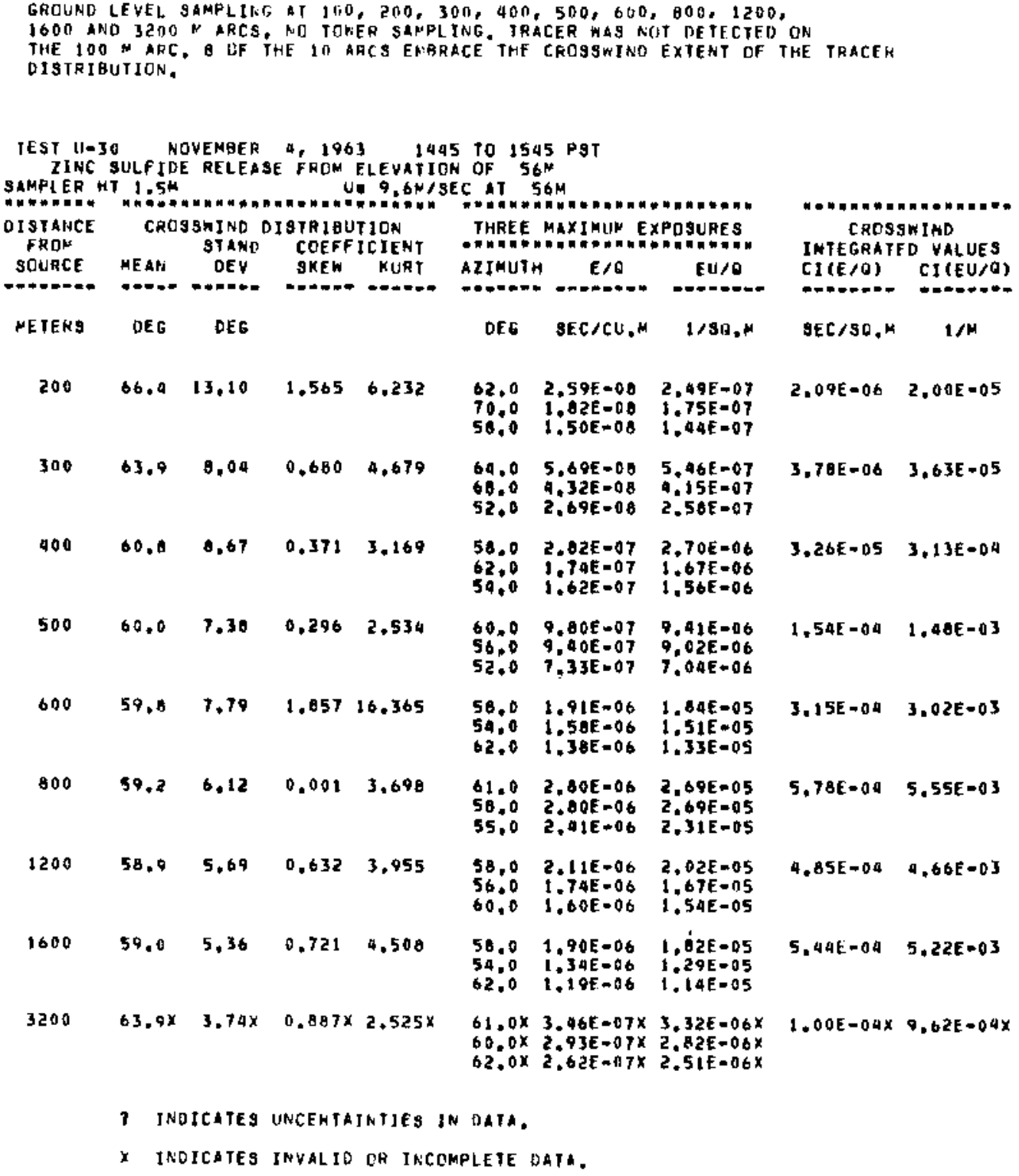




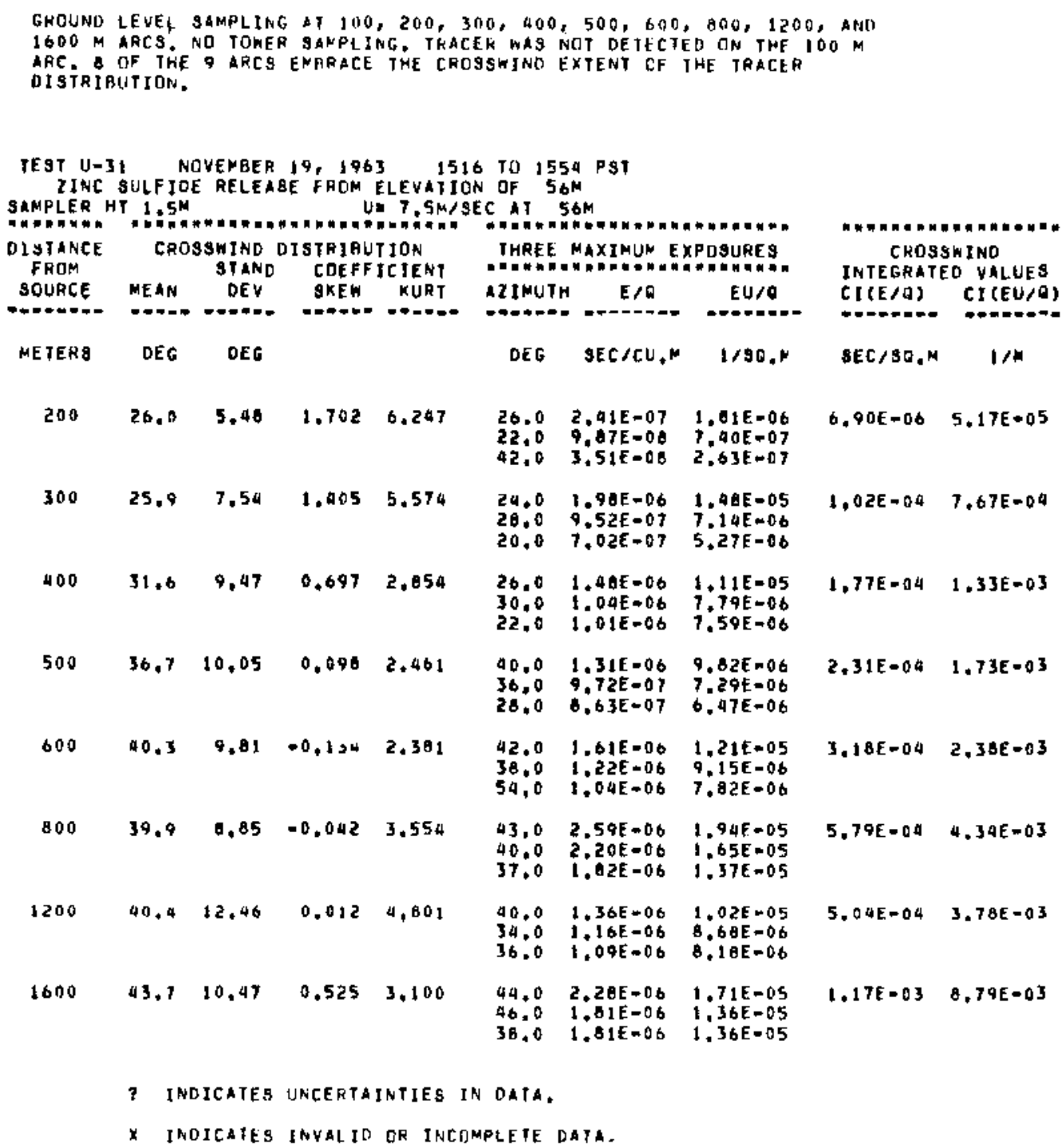




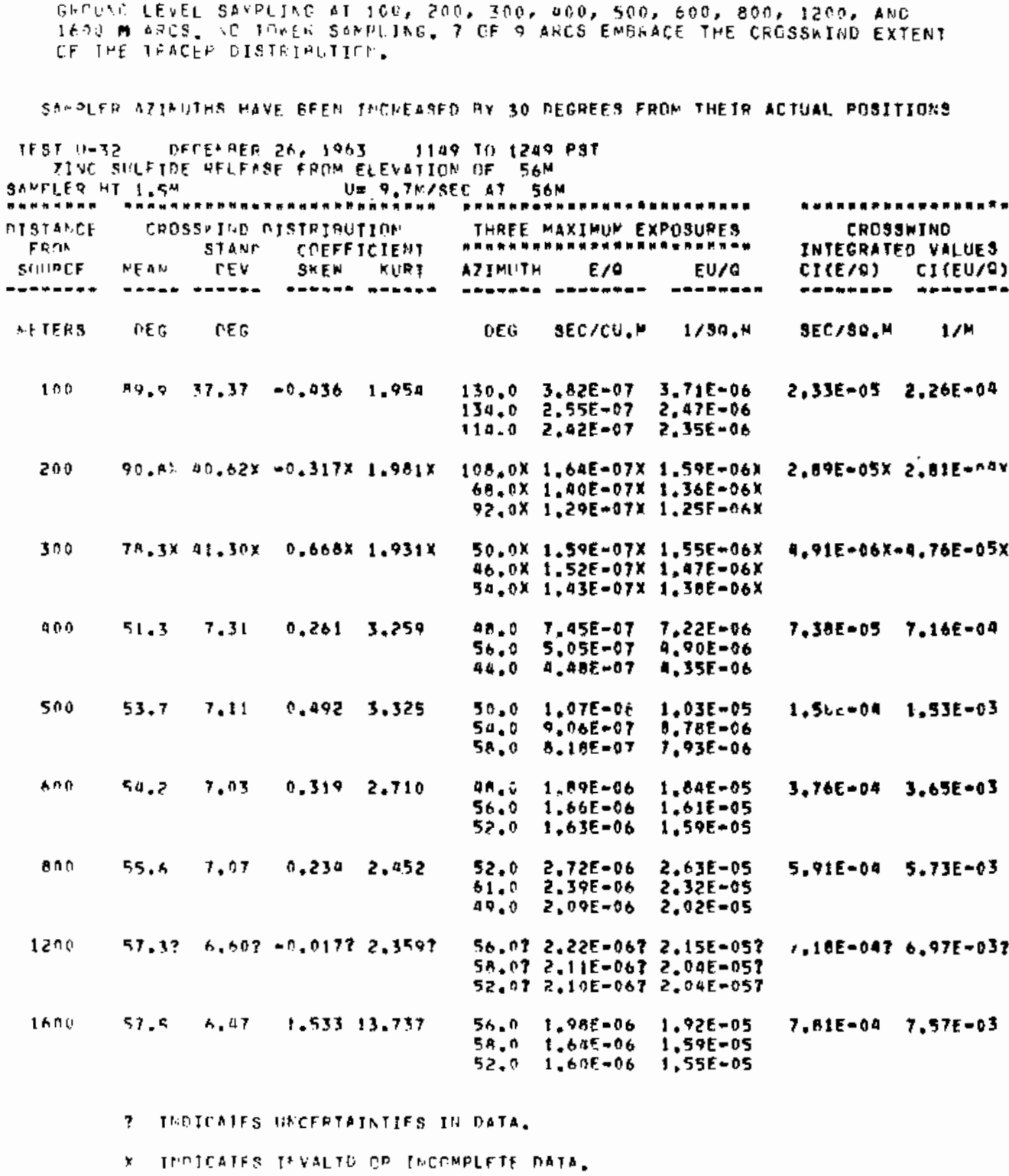




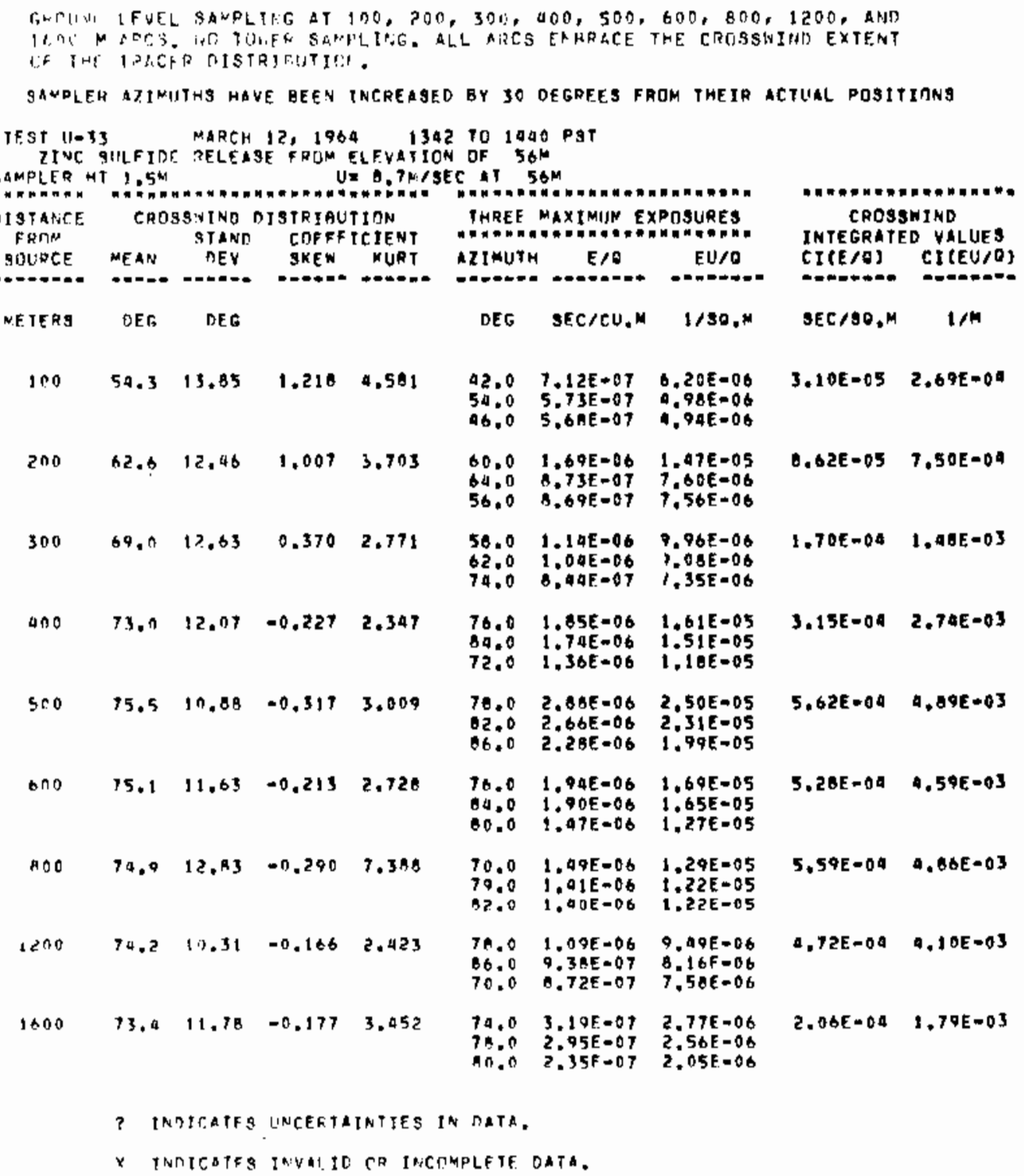




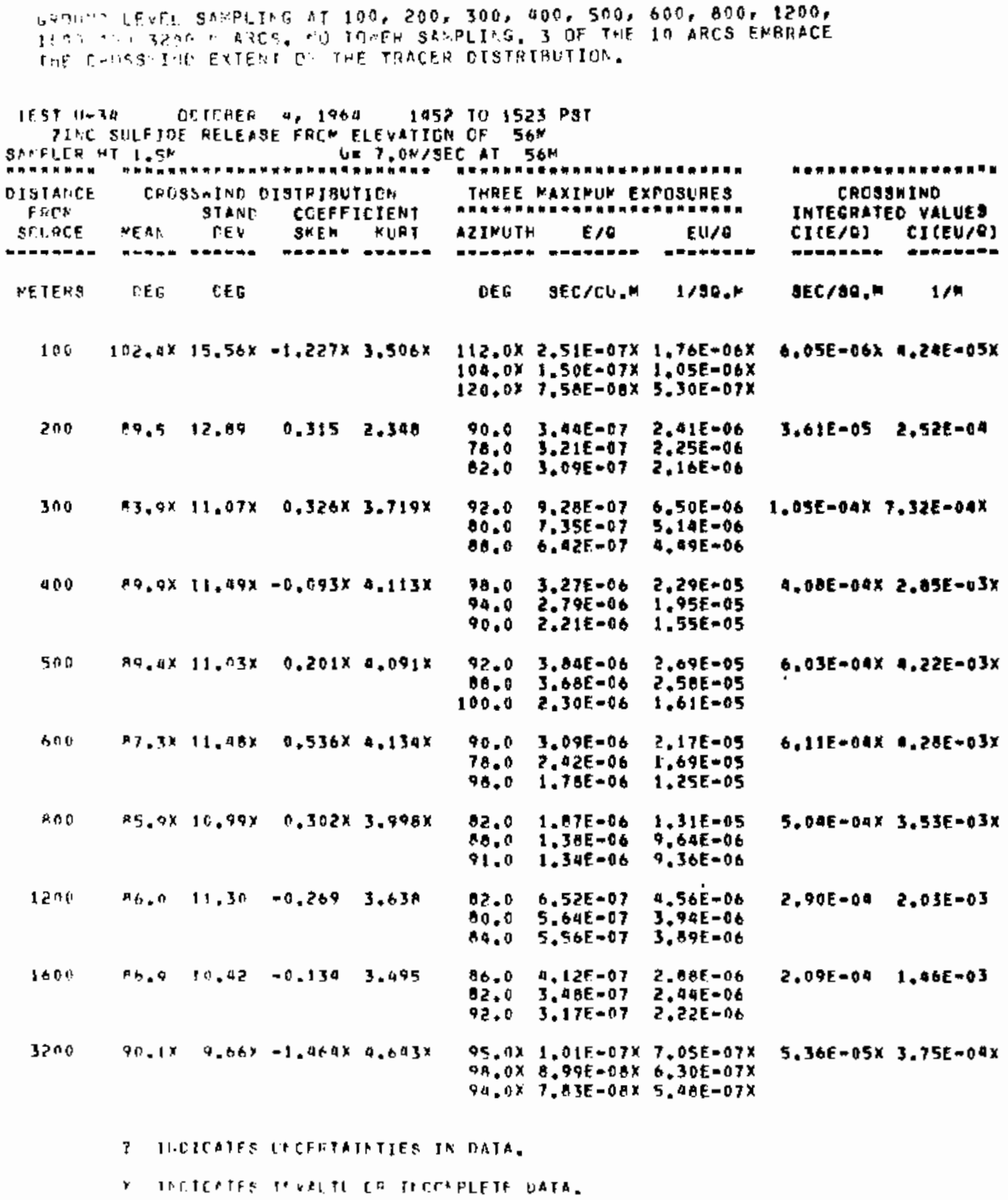

? MICICAIFS LRCFITALTIES IN DATA.

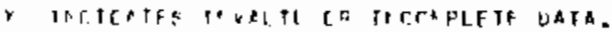




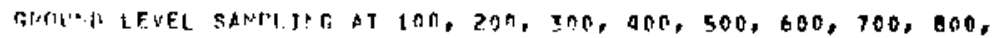

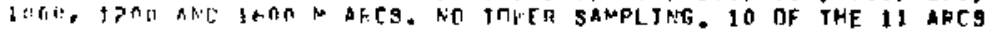

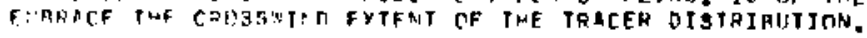

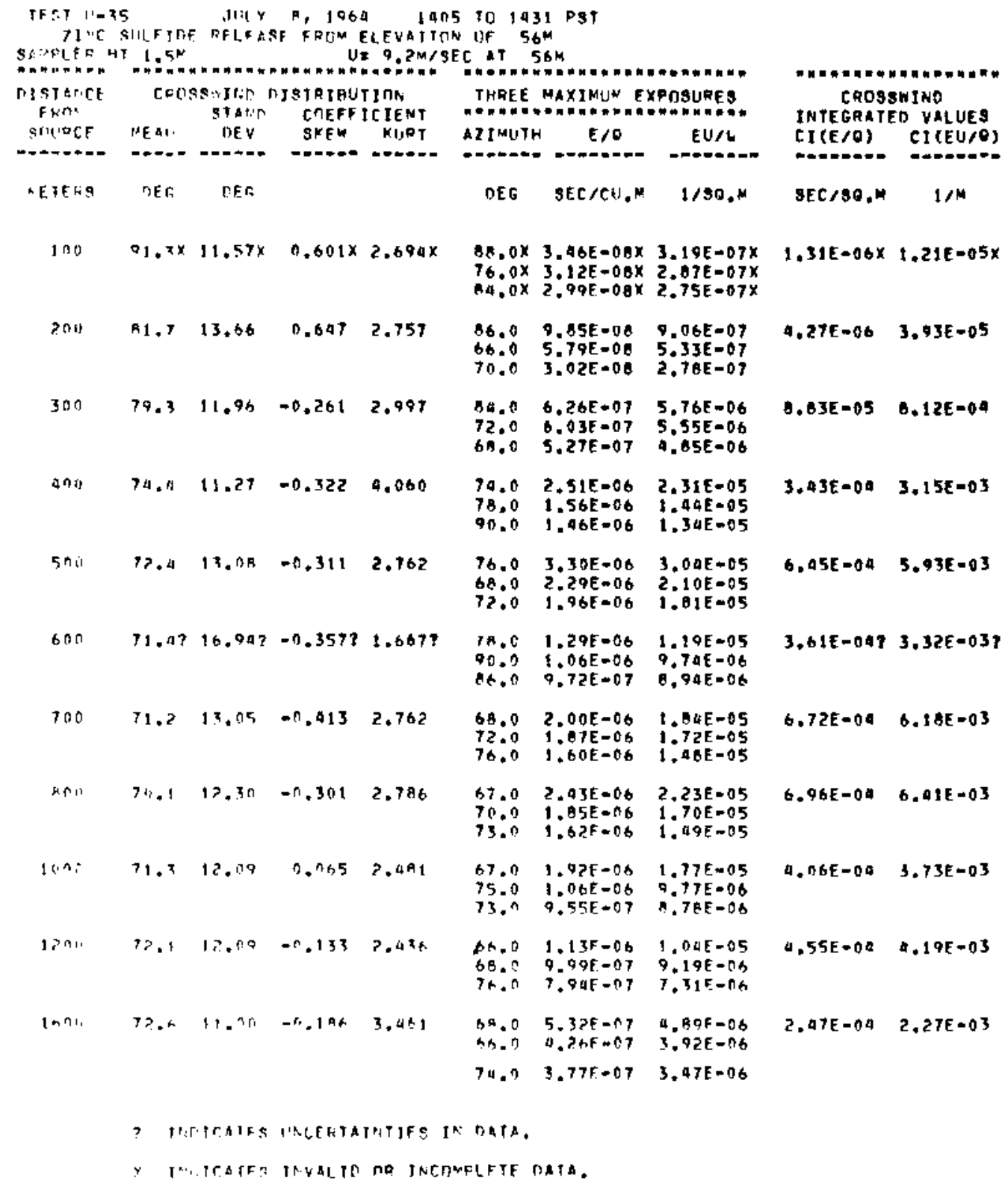




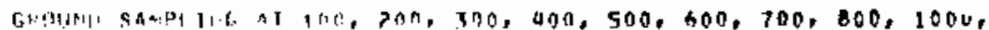

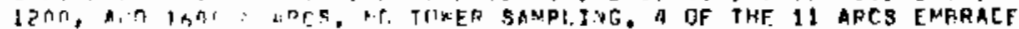

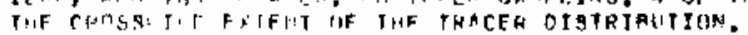

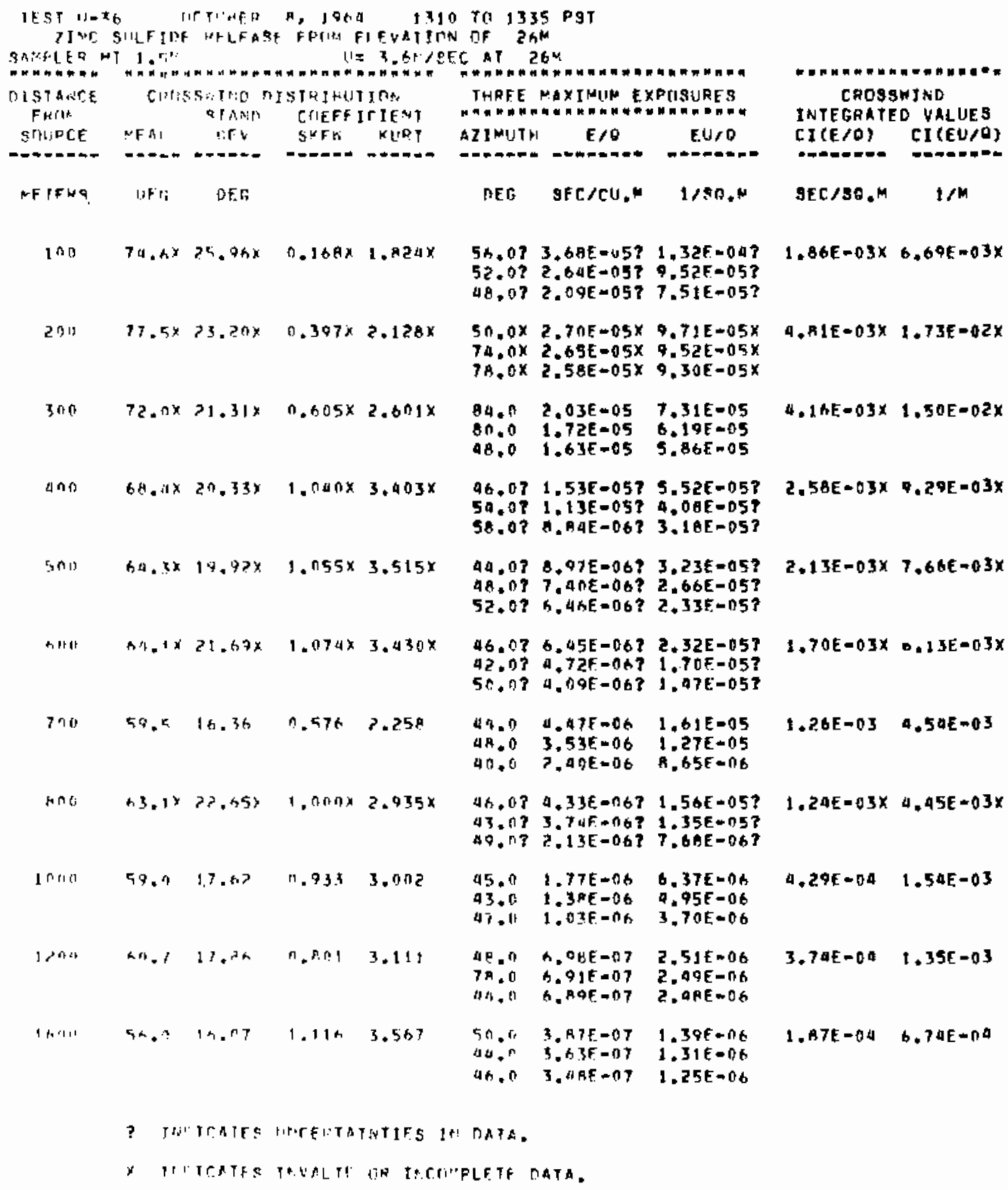




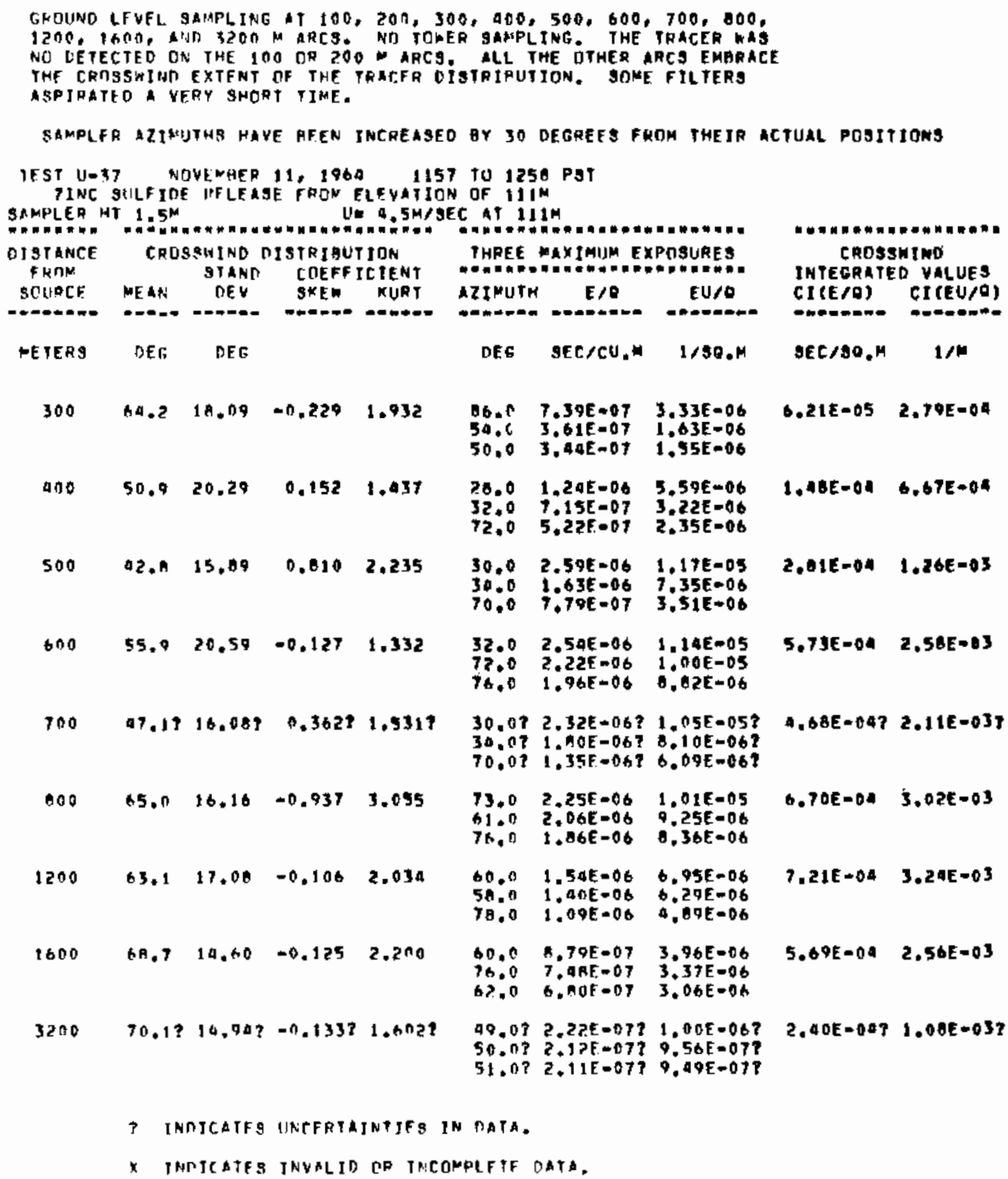




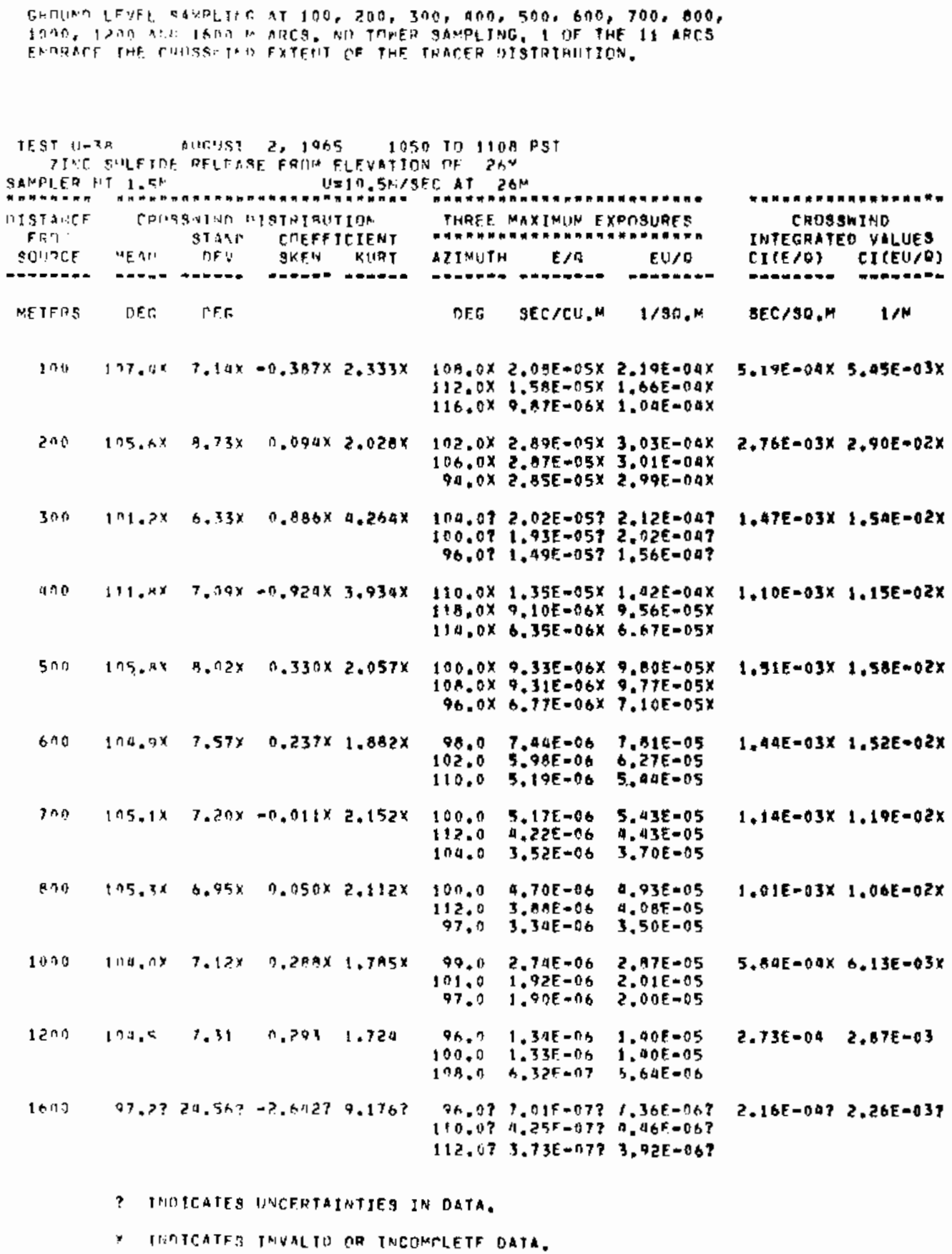




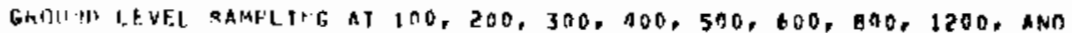

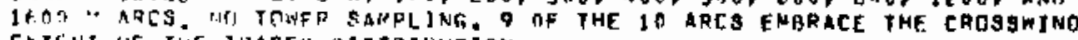

ExiENT th THF IHACE! OISTRTHITION.

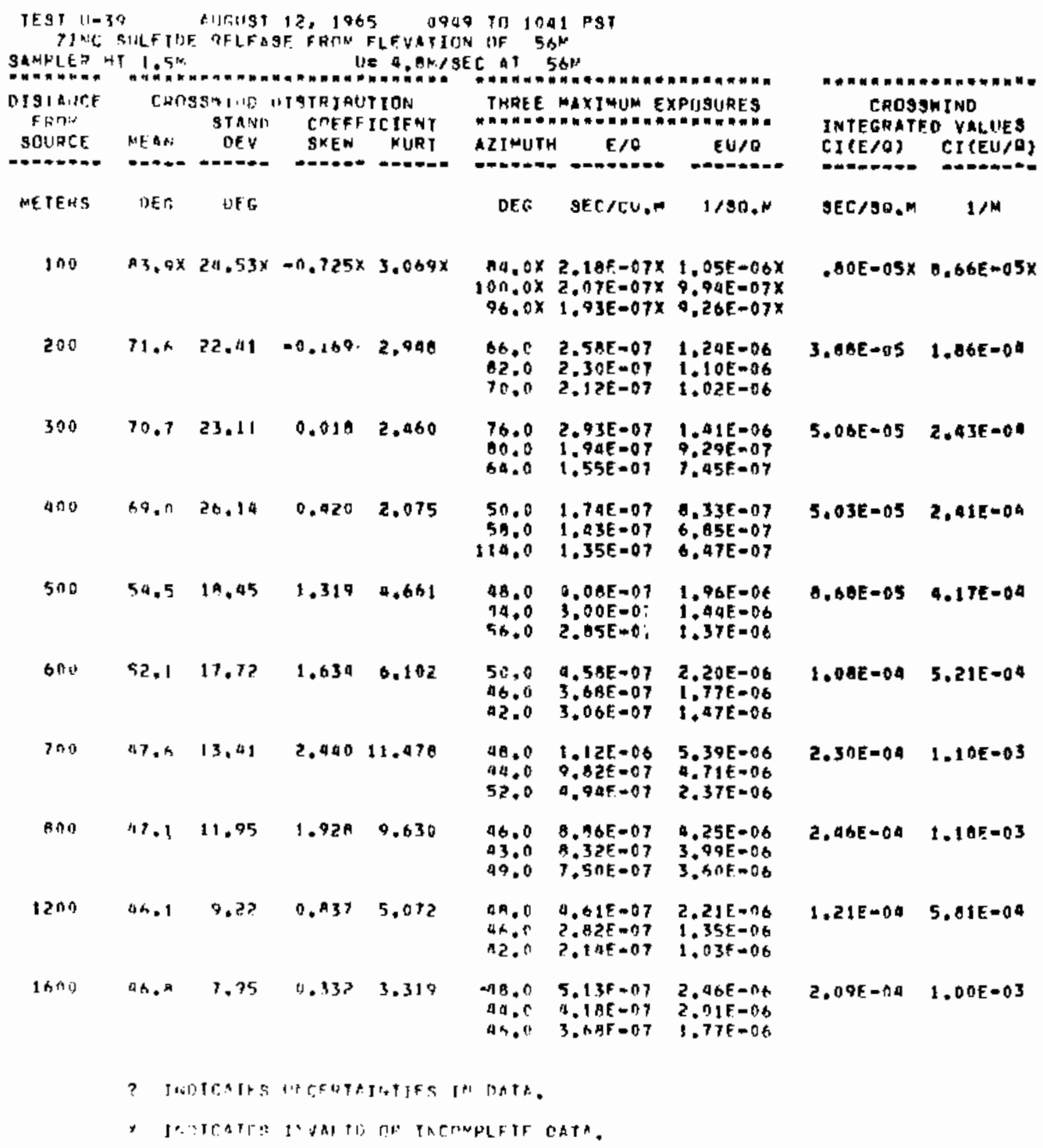




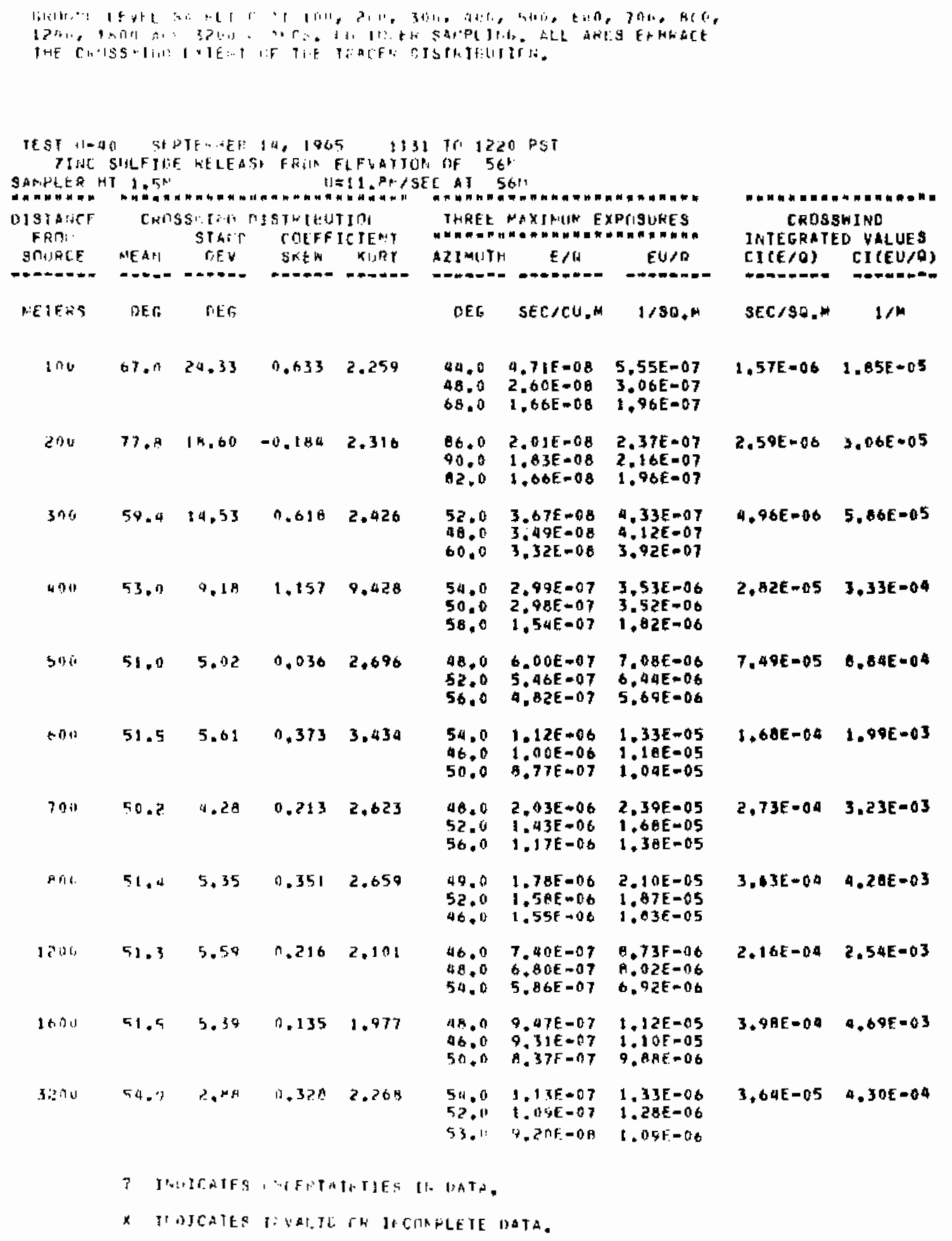




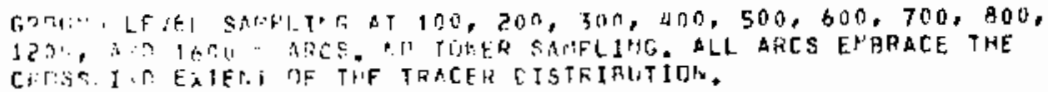

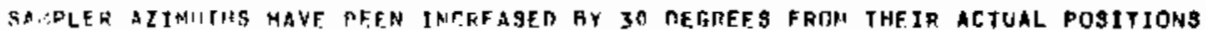

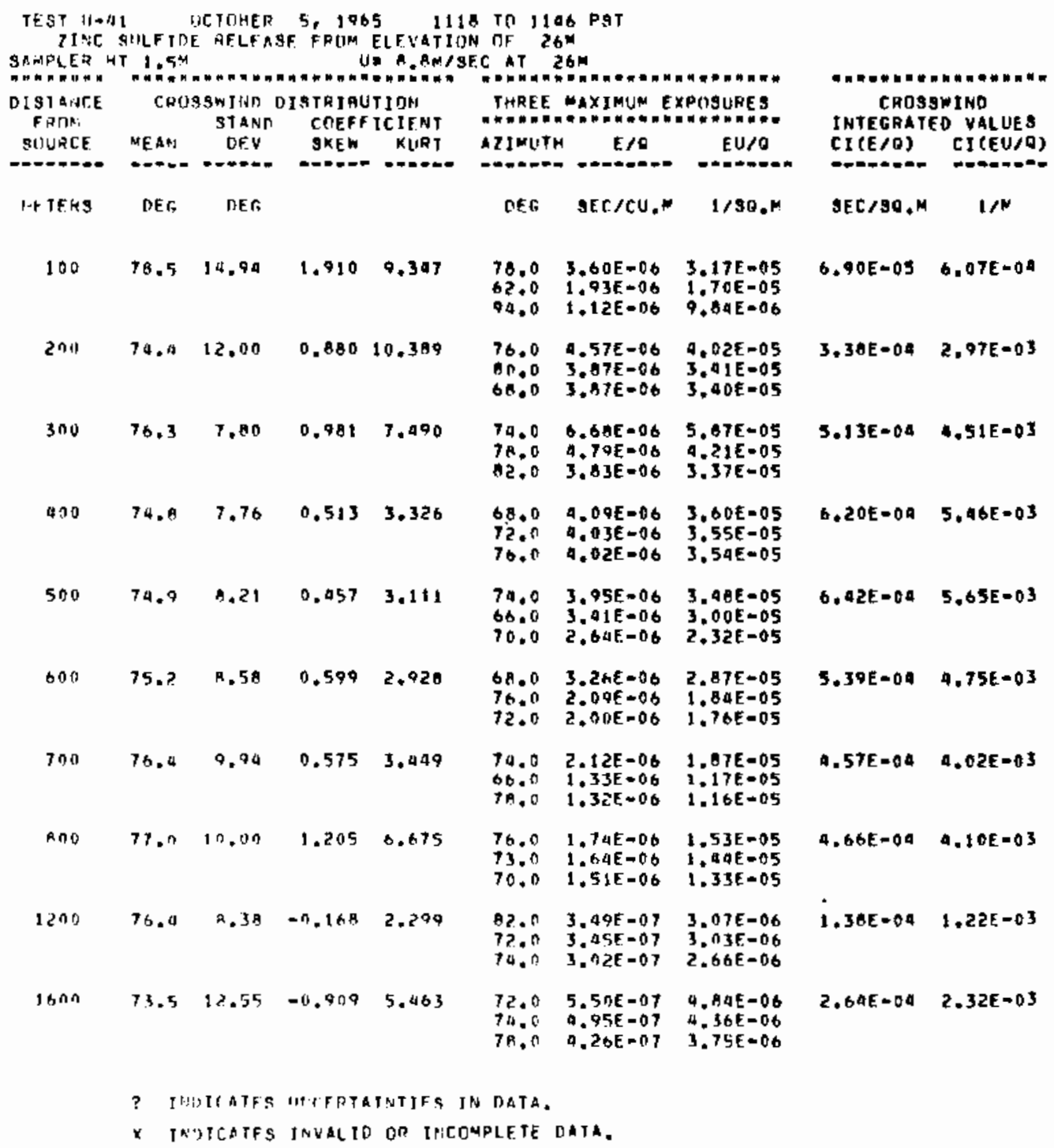




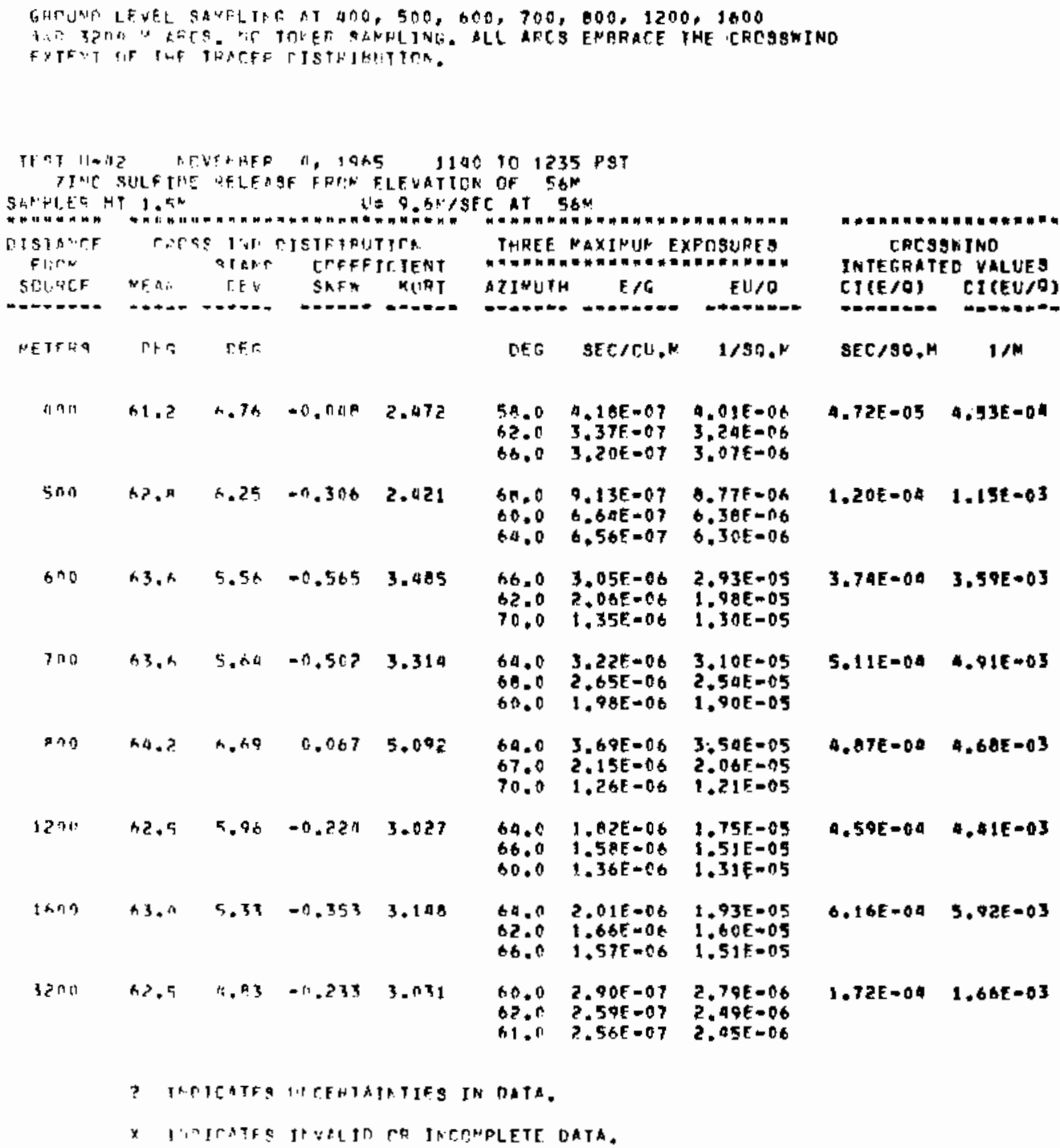




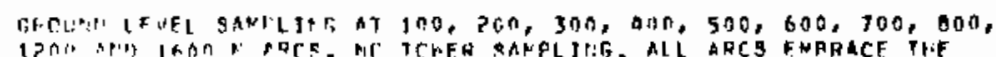

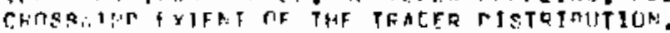

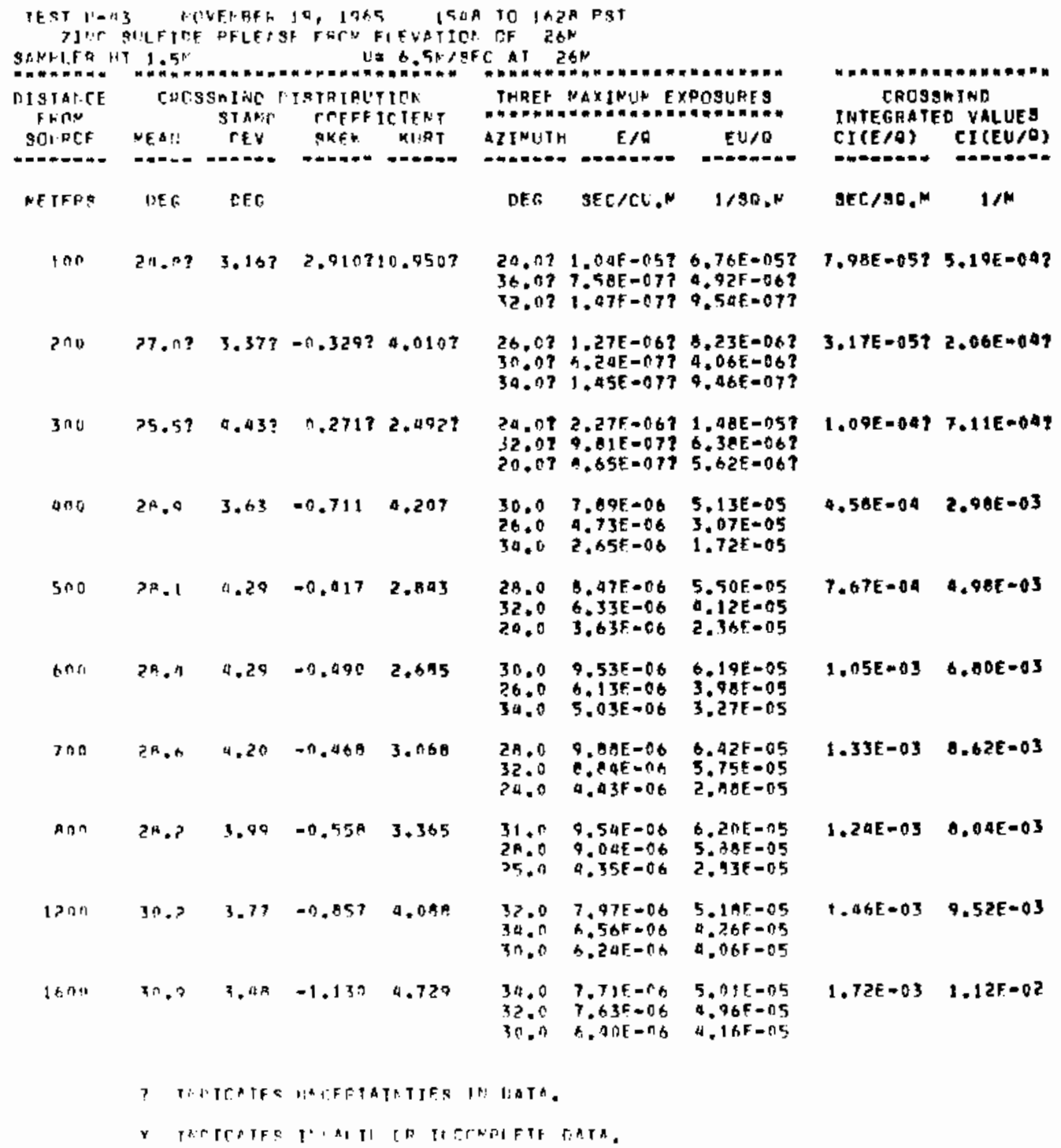




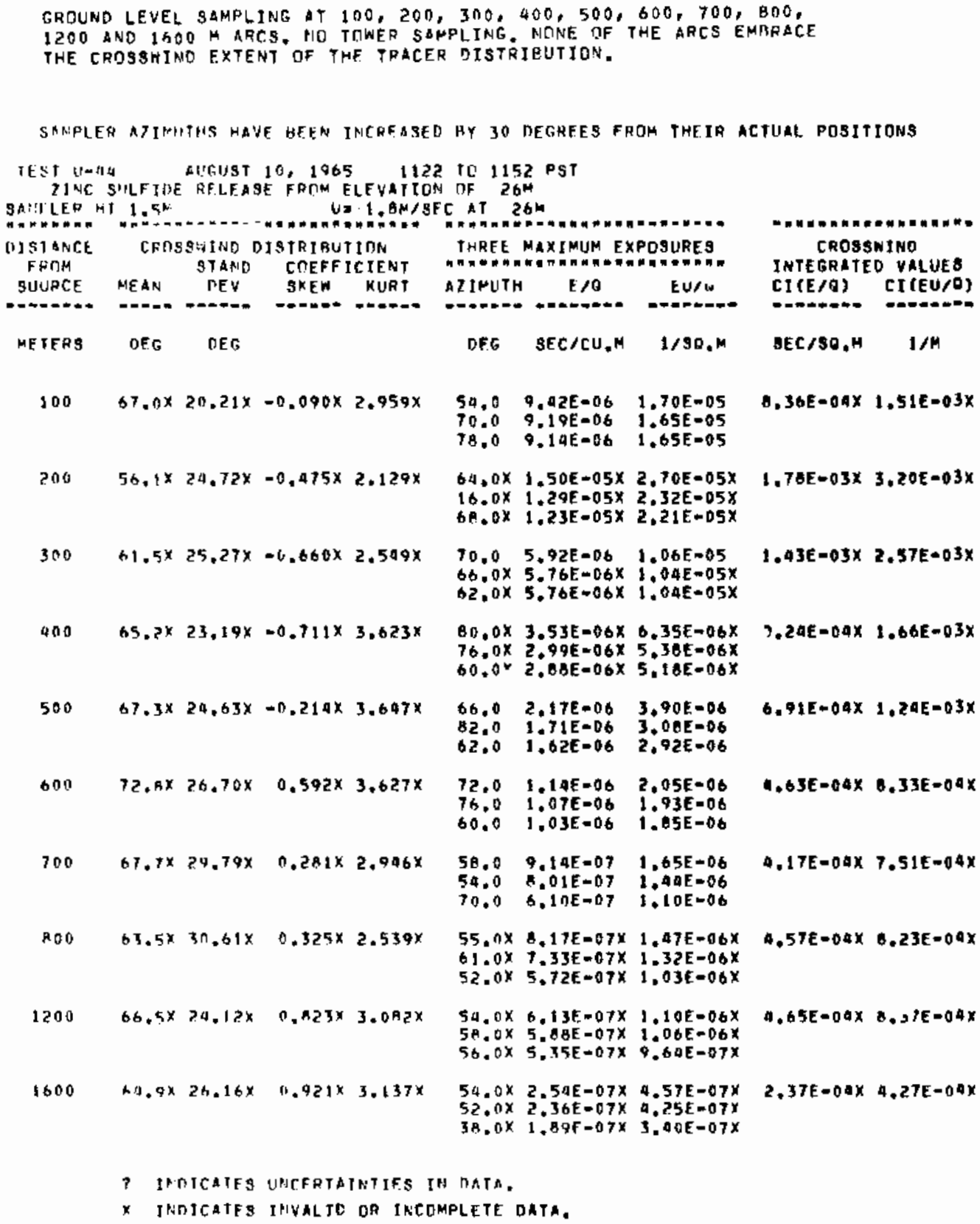


IINC BHLFIOE AFIFASE, RHOUHD LEVEL SAMPLING AT 200, DOO, 600 ,

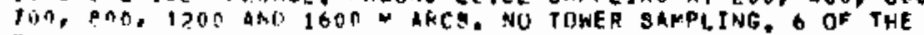

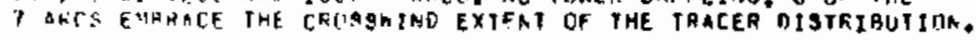

\begin{tabular}{|c|c|c|c|c|c|c|c|c|c|}
\hline $\begin{array}{l}\text { OISTANCE } \\
\text { FROM }\end{array}$ & & $\begin{array}{l}\text { SSWIND D } \\
\text { STAND }\end{array}$ & $\begin{array}{l}\text { DISTRIRUT } \\
\text { COEFF? }\end{array}$ & $\begin{array}{l}\text { TIDN } \\
\text { ICIENT }\end{array}$ & THREE $_{n \rightarrow \infty}$ & 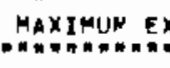 & $\begin{array}{l}\text { XPOSURES } \\
\text { nam }\end{array}$ & $\begin{array}{r}\text { CROSS } \\
\text { INTEGRATE }\end{array}$ & $\begin{array}{l}\text { AWTNO } \\
\text { ED VALUES }\end{array}$ \\
\hline SOURCE & MEAN & DEV & SKEN & KURT & AZIMUTH & $E / 6$ & EU/O & $C I(E / Q)$ & $\operatorname{CI}(E) / \theta)$ \\
\hline 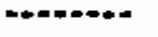 & $-\infty-\infty$ & $\cdots \cdots$ & \#... & -1-n- & - & " - - & 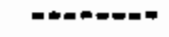 & 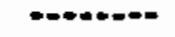 & 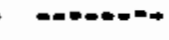 \\
\hline METERS & DEG & DEG & & & DEG & $\mathrm{SEC} / \mathrm{CU}, \mathrm{m}$ & $1 / 30 . N$ & SEC/SO.M & $1 / \mu$ \\
\hline 200 & 38,3 & 5.77 & -0.496 & 4.139 & $\begin{array}{l}40.0 \\
36.0 \\
44.0\end{array}$ & $\begin{array}{l}2.68 E-09 \\
2.22 E-09 \\
1.68 E-04\end{array}$ & $\begin{array}{l}1.82 E-03 \\
1.51 E-03 \\
1.14 E-03\end{array}$ & $1.20 E-02$ & $8.18 \mathrm{E}-02$ \\
\hline 400 & 18.7 & 5.48 & -0.050 & 3.254 & $\begin{array}{l}18.0 \\
22.0 \\
14.0\end{array}$ & $\begin{array}{l}7.26 E-05 \\
6.40 E=05 \\
4.86 E=05\end{array}$ & $\begin{array}{l}4.94 E=04 \\
4.41 E=04 \\
3.31 E=04\end{array}$ & $6.93 \mathrm{E}=03$ & $4.71 E-02$ \\
\hline 600 & 18.7 & 4.69 & -0.287 & 3,057 & $\begin{array}{r}18.0 \\
-22.0 \\
14.0\end{array}$ & $\begin{array}{l}3.09 E-05 \\
3.09 E-05 \\
1.55 E-05\end{array}$ & $\begin{array}{l}2.38 E-04 \\
2.10 E=04 \\
1.05 E-04\end{array}$ & $4.25 E=03$ & $2.69 E=02$ \\
\hline 700 & 19.0 & 5,03 & -0.290 & 2,549 & $\begin{array}{l}20.0 \\
24.0 \\
16.0\end{array}$ & $\begin{array}{l}2.02 E=05 \\
1.94 E-05 \\
1.70 E=05\end{array}$ & $\begin{array}{l}1.37 E=04 \\
1.32 E=04 \\
1.16 E=04\end{array}$ & $3,58 E-03$ & $2.44 E-02$ \\
\hline$\theta 00$ & 19.0 & 0.65 & -0.207 & 2.509 & $\begin{array}{l}22.0 \\
19.0 \\
16.0\end{array}$ & $\begin{array}{l}1.95 E-05 \\
1.71 E=05 \\
1.37 E=05\end{array}$ & $\begin{array}{l}1.32 E=04 \\
1.16 E=04 \\
9,29 E=05\end{array}$ & $3.28 E-03$ & $2.23 E+02$ \\
\hline 1200 & 18.9 & 3.87 & -0.128 & 3.138 & $\begin{array}{l}18.0 \\
20.0 \\
22.0\end{array}$ & $\begin{array}{l}9.32 E=06 \\
9.12 E-06 \\
6.18 E=06\end{array}$ & $\begin{array}{l}6.34 E=05 \\
6.20 E=05 \\
4.21 E=05\end{array}$ & $1.76 E=03$ & $1,20 E=02$ \\
\hline 1600 & $27.3 x$ & $24.76 x$ & $2.646 x$ & $8.824 x$ & $\begin{array}{l}18.0 x \\
20.0 x \\
16.0 x\end{array}$ & $\begin{array}{l}3.54 E=06 x \\
3.09 E=06 x \\
2.59 E=06 x\end{array}$ & $\begin{array}{l}2.41 E=05 x \\
2.10 E=05 x \\
1.76 E=05 x\end{array}$ & $9.65 E-04 x$ & $6.56 E=03 x$ \\
\hline & INO & ES & TAI & INTIES & DATA. & & & & \\
\hline & $\pi$ & CATES & & OR IN & LETE & $T A$. & & & \\
\hline
\end{tabular}


FLUOHOSCEIR RELEASE, GLOUHD LEVEL SAKPLISG AT 200,400,600,

$700, B 00,1200$ AHD 1600 M ABCE. SO TOUER SAMPEIXG, ALI OP THE ARCS

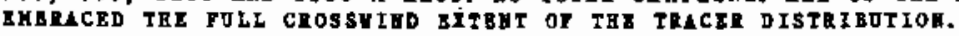

\begin{tabular}{|c|c|c|c|c|c|c|c|c|c|}
\hline \multirow{2}{*}{ 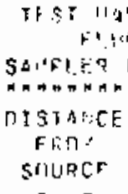 } & \multicolumn{2}{|c|}{$\begin{array}{c}\text { CRISSTIVD } \\
\text { STANOB }\end{array}$} & \multicolumn{2}{|c|}{$\begin{array}{l}\text { DISTRIRHTINN } \\
\text { COEFFTCIENT }\end{array}$} & \multicolumn{3}{|c|}{ 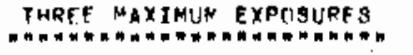 } & \multicolumn{2}{|c|}{$\begin{array}{l}\text { CROSSHIND } \\
\text { INTEGRATED VALUES }\end{array}$} \\
\hline & NFAR & DEV & SKE.W & KURT & AZ JMUTH & $E / O$ & $E U / \sigma$ & $C I(E / O)$ & CI $(E(L / D)$ \\
\hline - - & $\cdots-$ & $\cdots$ & -.... & $--\infty-\infty$ & $=-\infty$ & - & - - - & $-\infty n+\infty)$ & 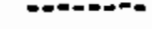 \\
\hline NETFQS & $r, f_{i}$ & $D E C_{0}$ & & & DEG & SEC/CU,M & $1 / \mathrm{so.m}$ & sEC $/ 3 ه . M$ & $1 / m$ \\
\hline $2 n t$ & $17.1 ?$ & $9.95 ?$ & $9.774 ?$ & 7.5912 & $\begin{array}{l}36.0 \\
40.0 \\
32.0\end{array}$ & $\begin{array}{l}2.26 E=06 \\
1.93 E=06 \\
1.50 F=06\end{array}$ & $\begin{array}{l}2.4 a E-05 \\
2.0 A E-05 \\
1.62 E-05\end{array}$ & $1.20 E-043$ & $1.305-031$ \\
\hline 400 & at.n? & 6.278 & n. naE? & 4.0522 & $\begin{array}{l}24.0 \\
20.0 \\
20.0\end{array}$ & $\begin{array}{l}M .01 E-0 t \\
8.33 E-06 \\
.00 E=06\end{array}$ & $\begin{array}{l}9.52 E-05 \\
9.0 N E-05 \\
9.97 E-05\end{array}$ & $9.08 E-04 ?$ & $9.00 E=03 ?$ \\
\hline $6(1)$ & 31.7 & 5.15 & 0.586 & 6.637 & $\begin{array}{l}24.0 \\
20.0 \\
16.0\end{array}$ & $\begin{array}{l}9.39 F-06 \\
.81 E=06 \\
5.01 E=06\end{array}$ & $\begin{array}{l}1.01 E-06 \\
9.52 E-05 \\
5.41 E-05\end{array}$ & $1.31 E=03$ & $1.41 E-02$ \\
\hline 700 & 39.9 & 5.19 & -19.371 & 3.349 & $\begin{array}{l}20.0 \\
20.0 \\
16.0\end{array}$ & $\begin{array}{l}0.53 E-06 \\
6.31 E=06 \\
0.1 B E-06\end{array}$ & $\begin{array}{l}9.22 E=05 \\
6.81 E-05 \\
6.67 E=05\end{array}$ & $1+31 E-03$ & $1.41 E-02$ \\
\hline Bno & 19.7 & 5.02 & $=0.0 R \theta$ & 3.433 & $\begin{array}{l}19.0 \\
22.0 \\
16.0\end{array}$ & $\begin{array}{l}7.05 E-06 \\
7.42 E=06 \\
5.34 E=06\end{array}$ & $\begin{array}{l}A, 4 B E-05 \\
8.01 E-05 \\
5.77 E-05\end{array}$ & $\begin{array}{c}1.37 E-03 \\
.\end{array}$ & $1.4 A E=02$ \\
\hline 1200 & $18 . A ?$ & $4.31\}$ & -0.2617 & 3.0047 & $\begin{array}{l}18.0 ? \\
20.0 ? \\
20.0 ?\end{array}$ & $\begin{array}{l}0.92 E-06 ? \\
4.50 E-067 \\
3.37 \mathrm{E}=06 ?\end{array}$ & $\begin{array}{l}5.31 F-05 ? \\
4.86 E-05 ? \\
3,64 E-05 ?\end{array}$ & $9.63 \mathrm{E}-047$ & $1.04 E-02 ?$ \\
\hline $16 n n$ & 19.7 & 4.34 & 1.253 & 11.638 & $\begin{array}{l}1 A .0 \\
20.0 \\
22.0\end{array}$ & $\begin{array}{l}2.90 E=06 \\
2.50 E=06 \\
2.08 E=06\end{array}$ & $\begin{array}{l}3.22 E-05 \\
2.70 E-05 \\
2.25 E=05\end{array}$ & $6.7 \mathrm{AE}-0 \mathrm{~d}$ & $7.28 E-03$ \\
\hline & $?$ & MCATE 8 & $\begin{array}{l}\text { IJNCFAT } \\
\text { TWVRLT }\end{array}$ & {$[n+1 F S$} & $\begin{array}{l}\text { DATA. } \\
\text { DETEO }\end{array}$ & OATA. & & & \\
\hline
\end{tabular}




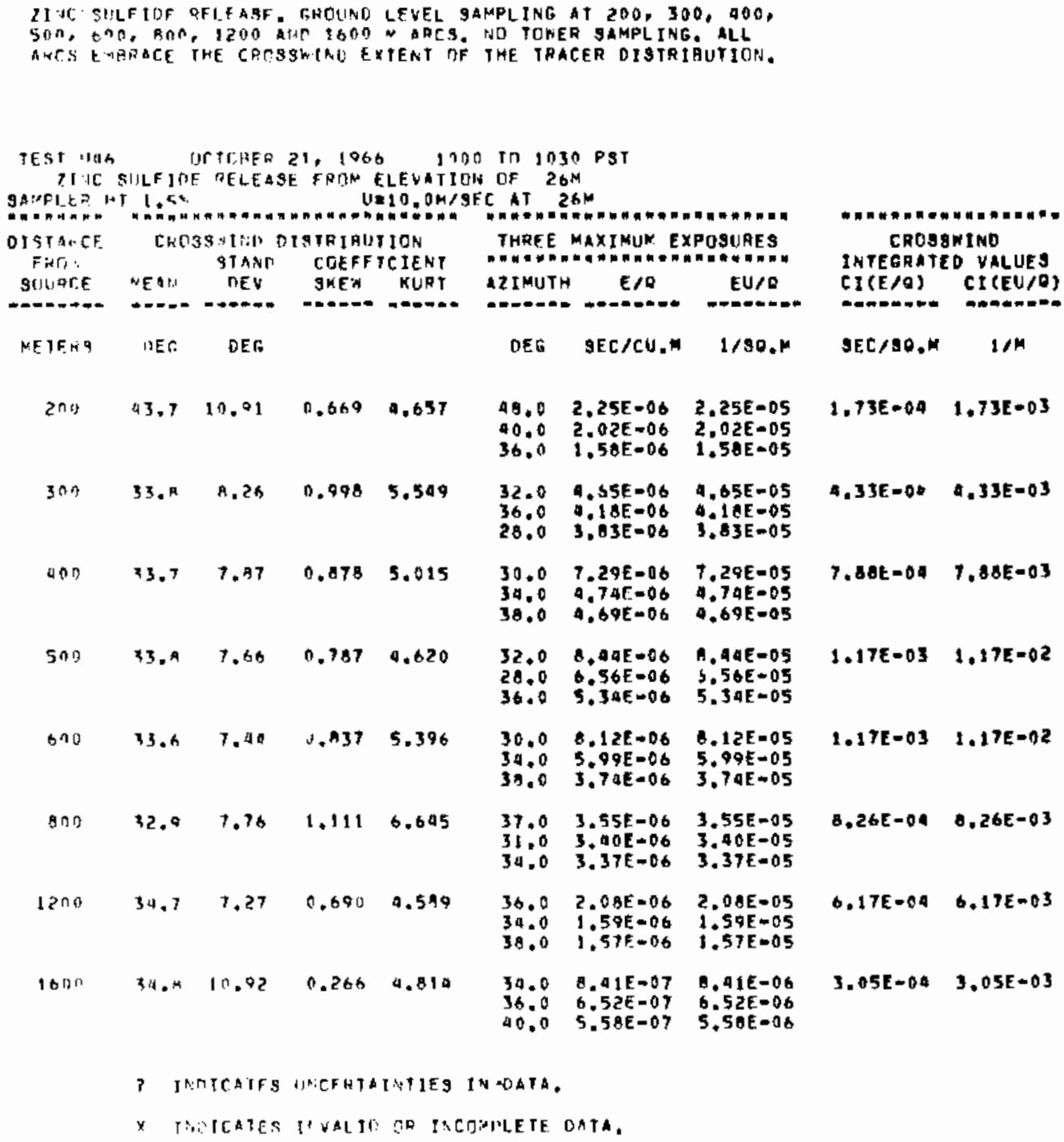




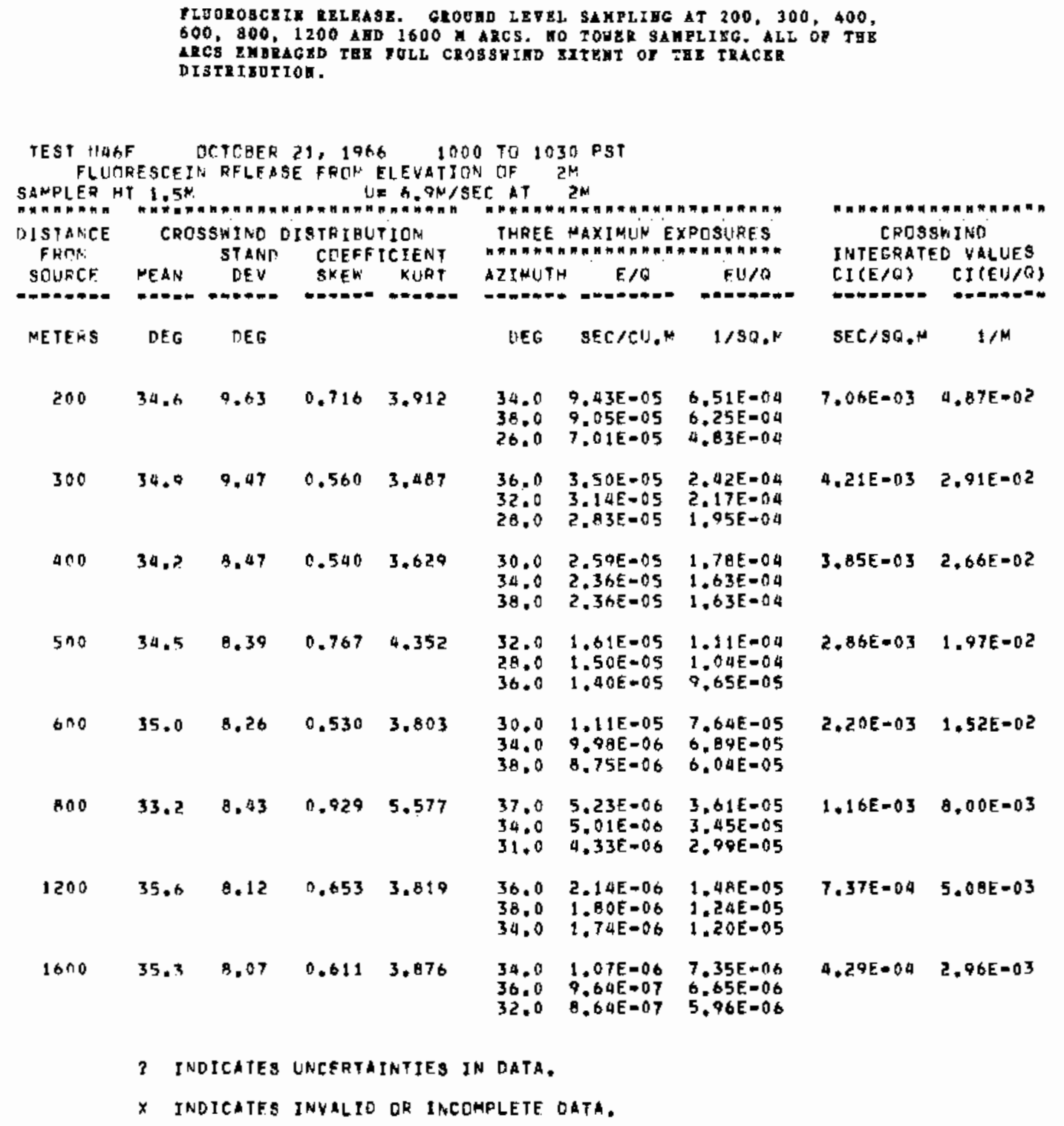


ZIIC 3:ILFTDE RELEASF. GROUND LEVEL SAMPLING AT 100, 200, 300, 400, $50 \%, 60 \%, 70 \%, 000,1200$ AND 1600 HRC 3 , NO TOWER SAMPLING, ALL ANCS FNHHACF: THE CACSGMIND EXTENT OF THE TRACER OISTRIBUTION.

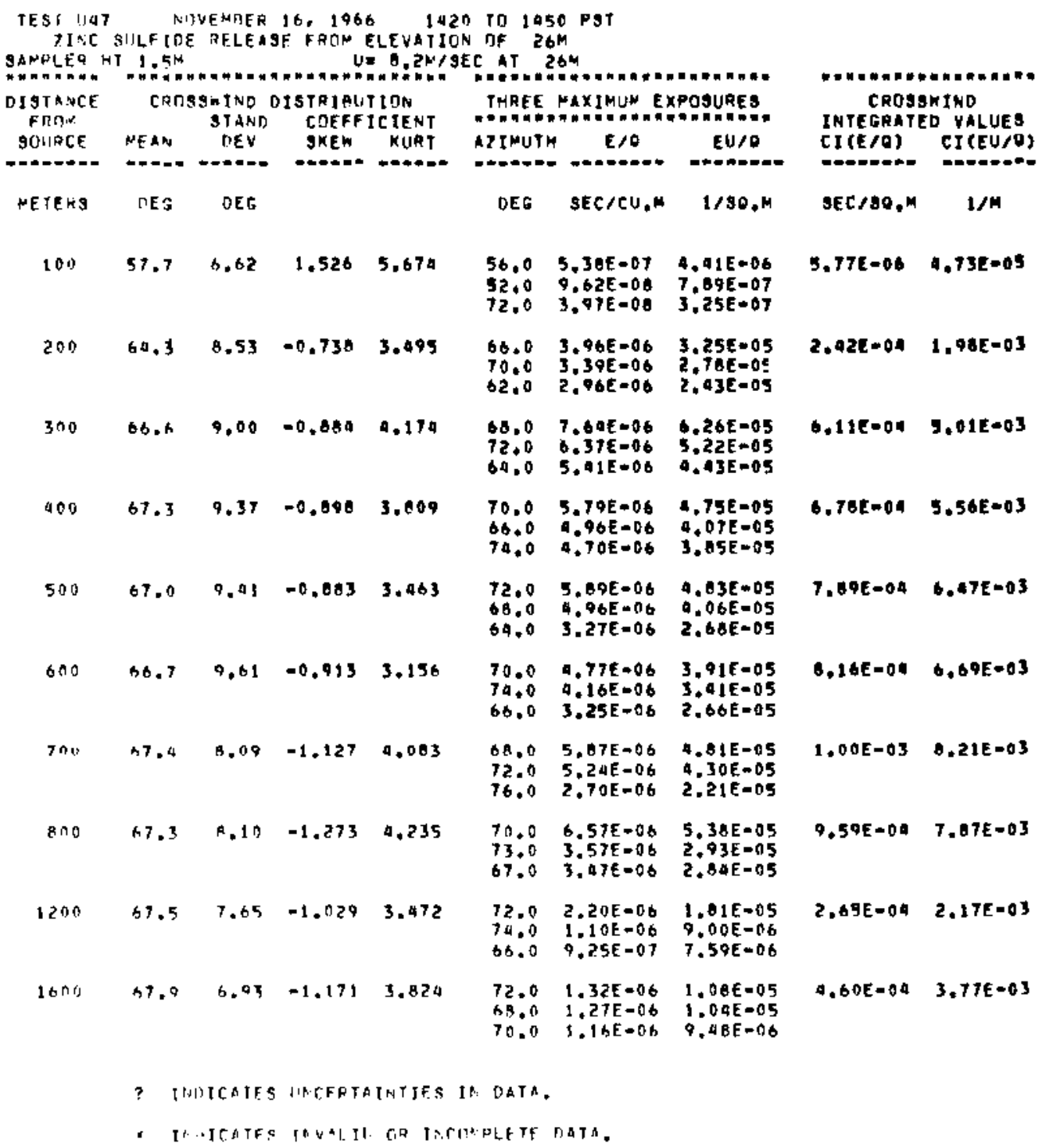




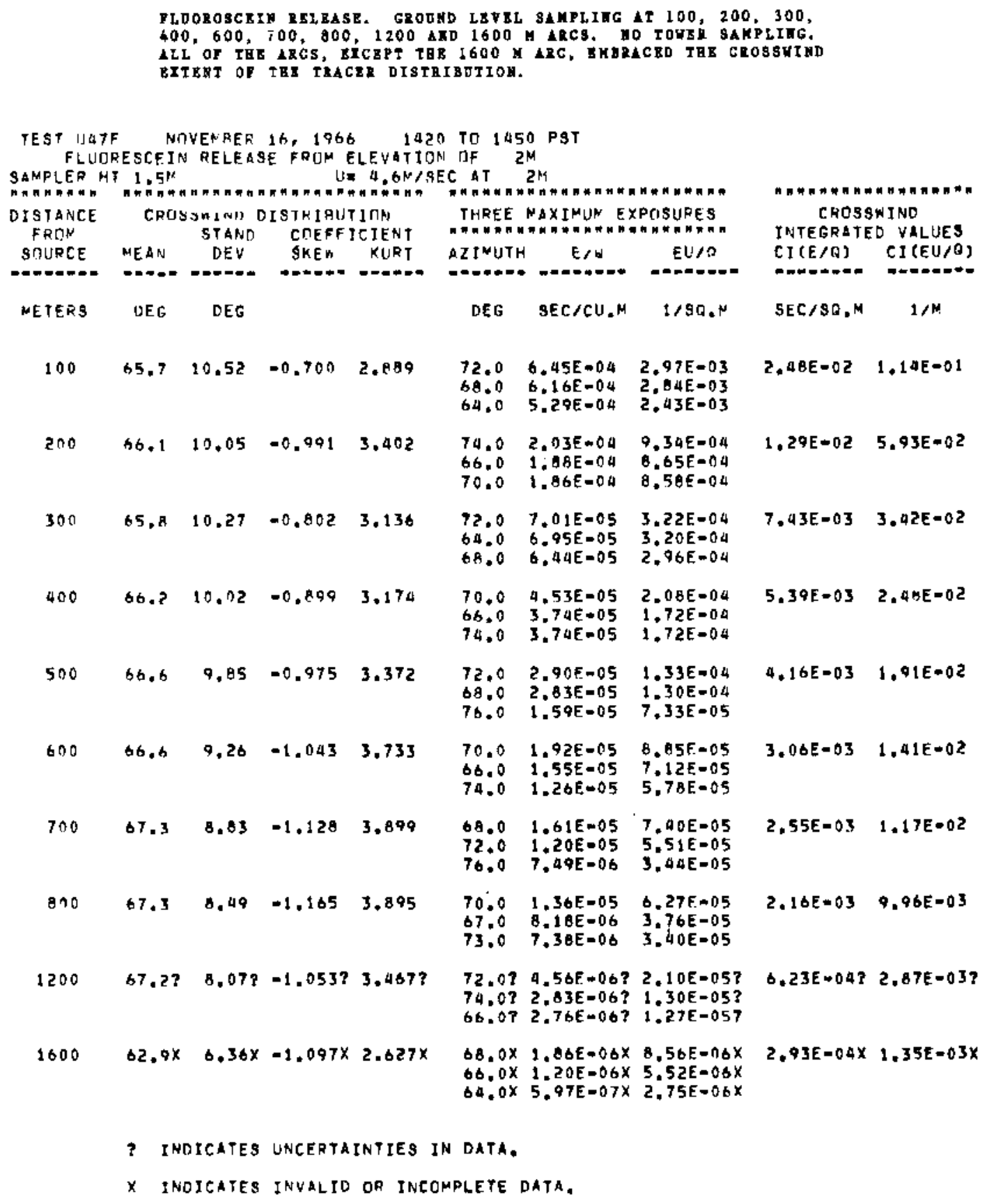




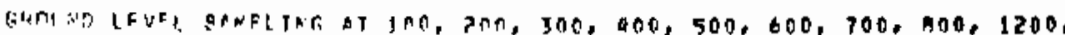

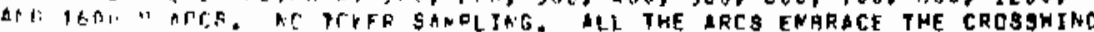

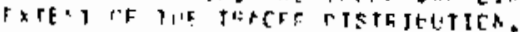

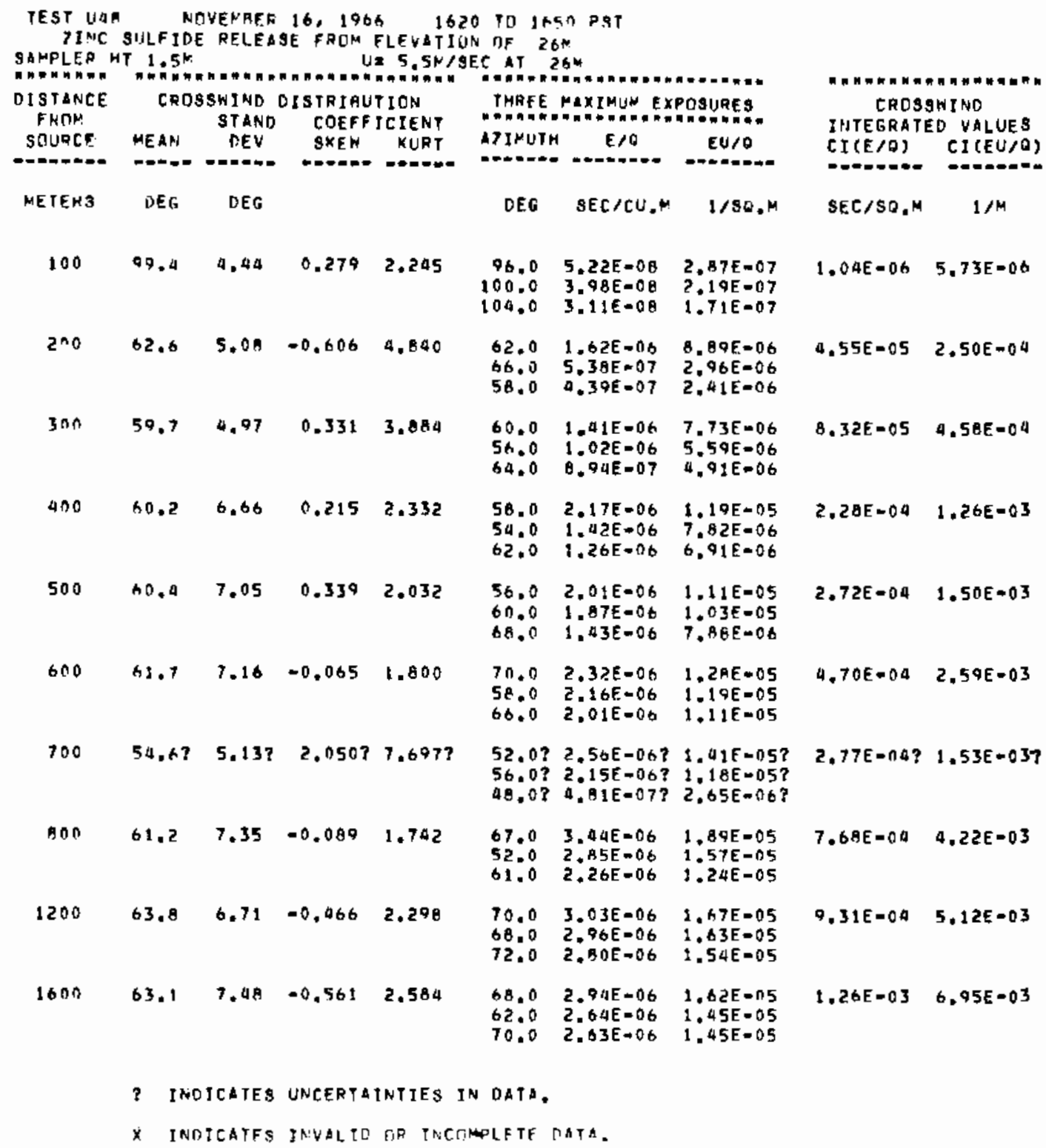




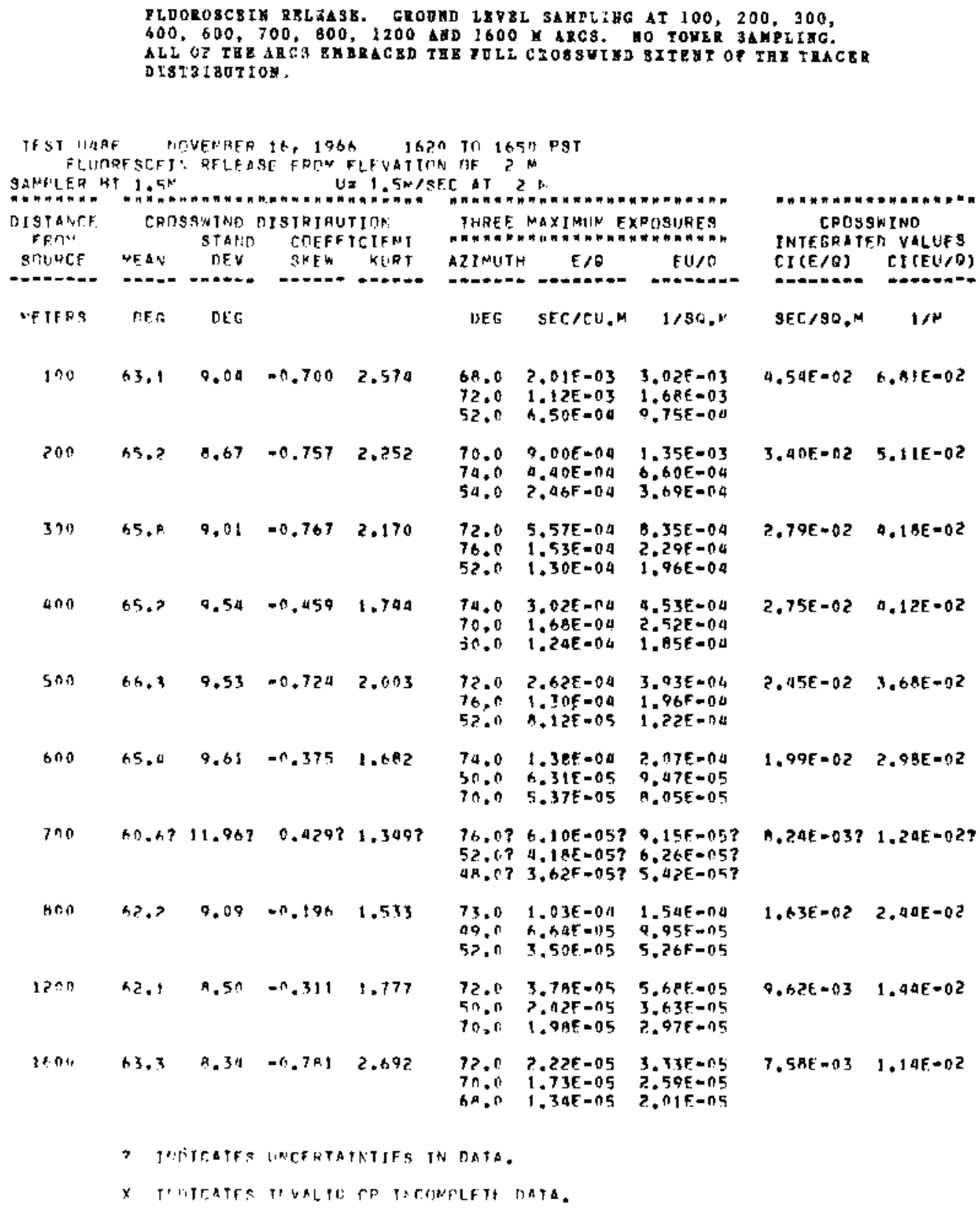




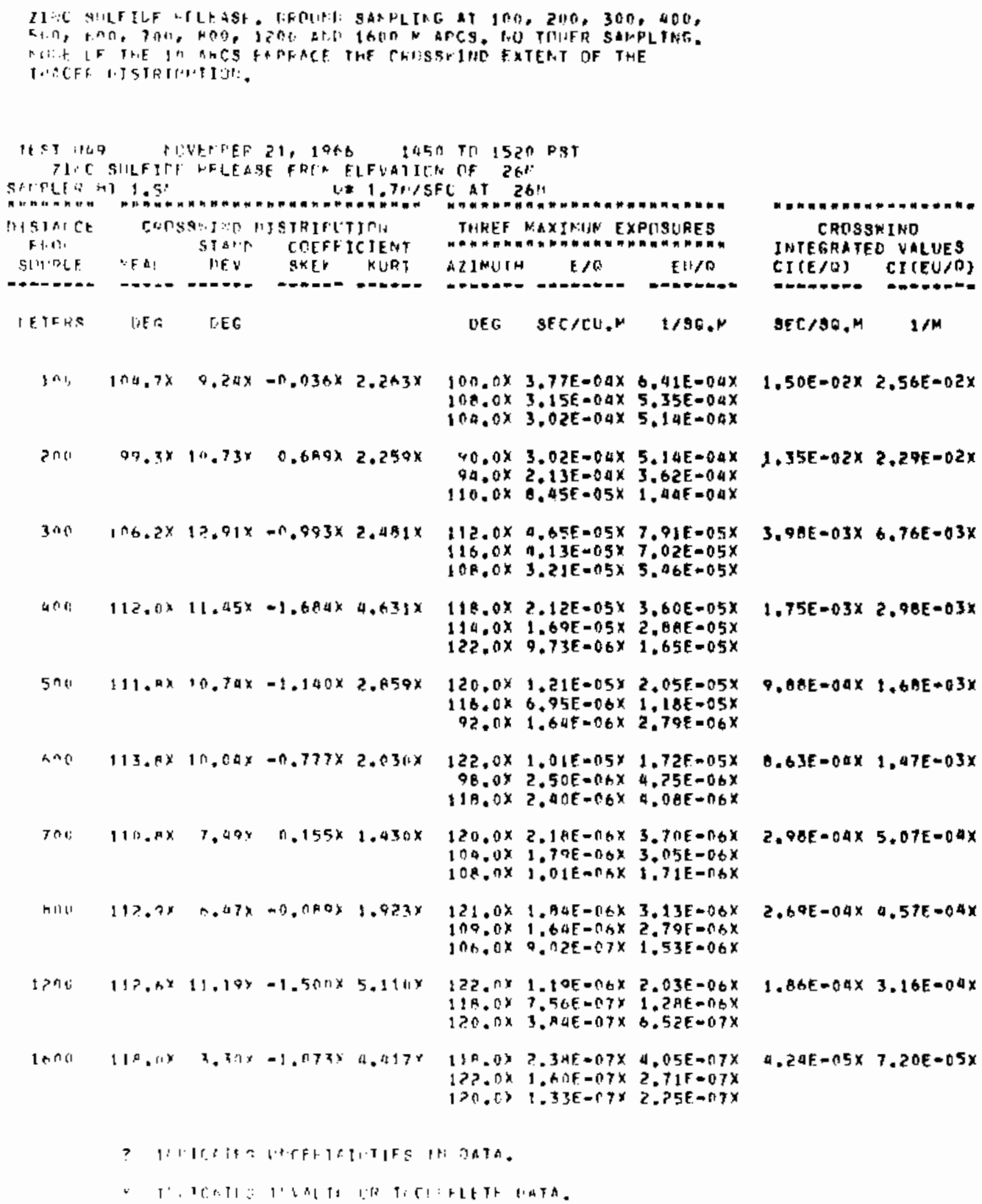




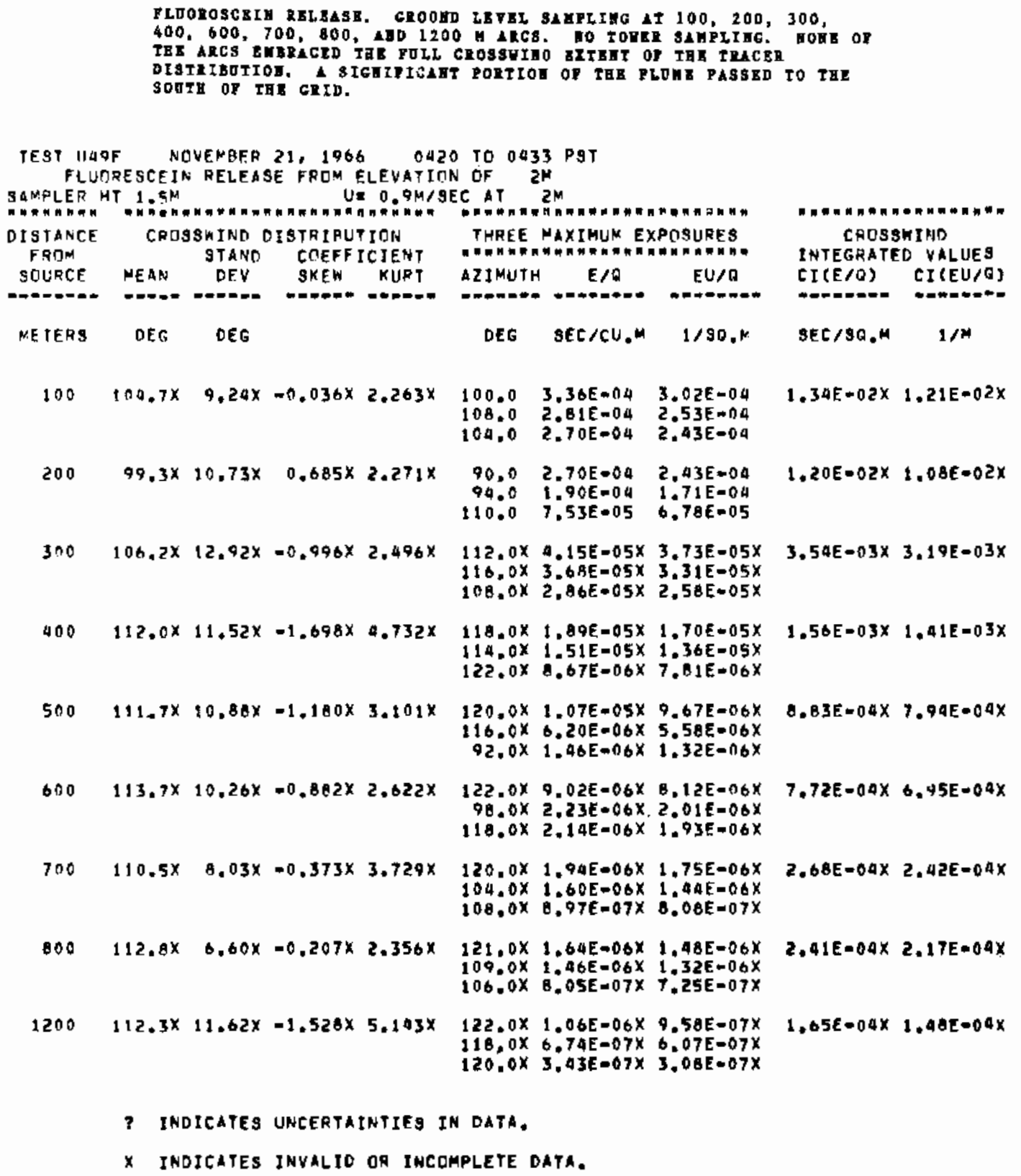




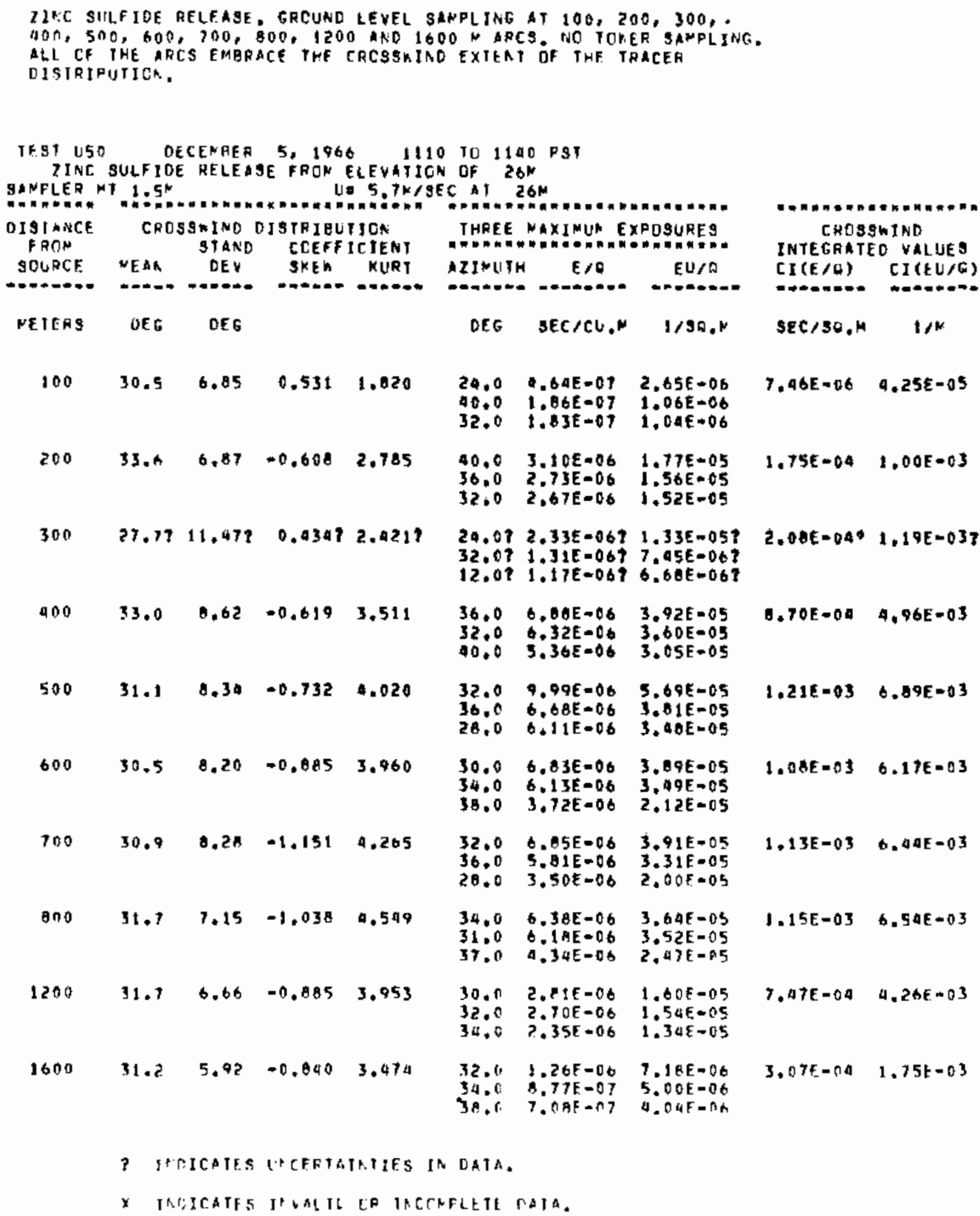




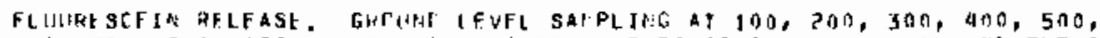

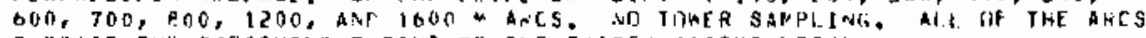

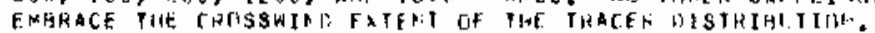

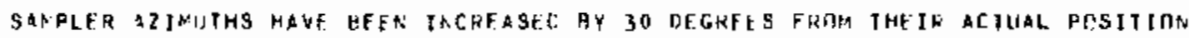

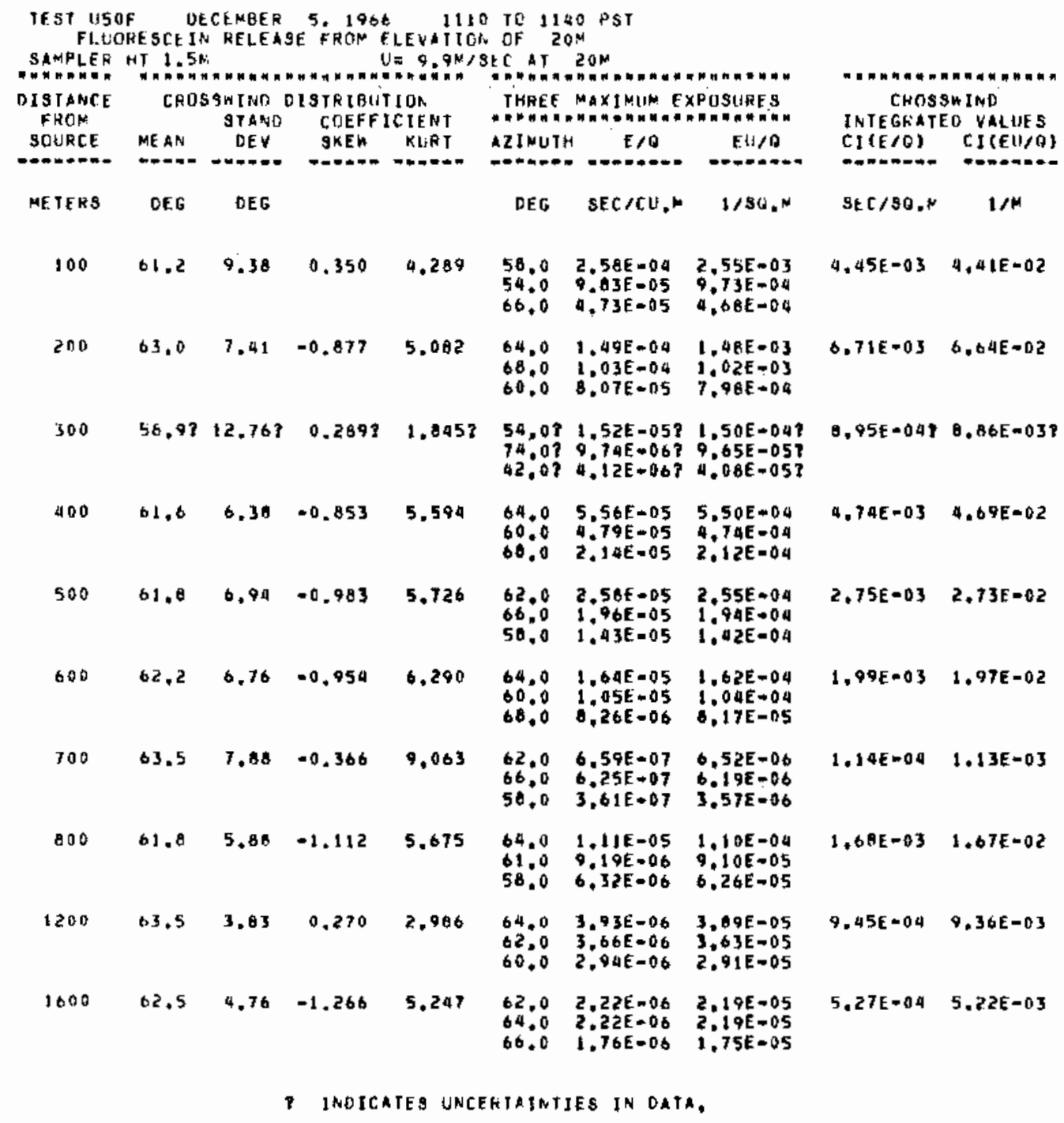

X INOICAJES INVALJO OR INCOMPLETE DATA. 


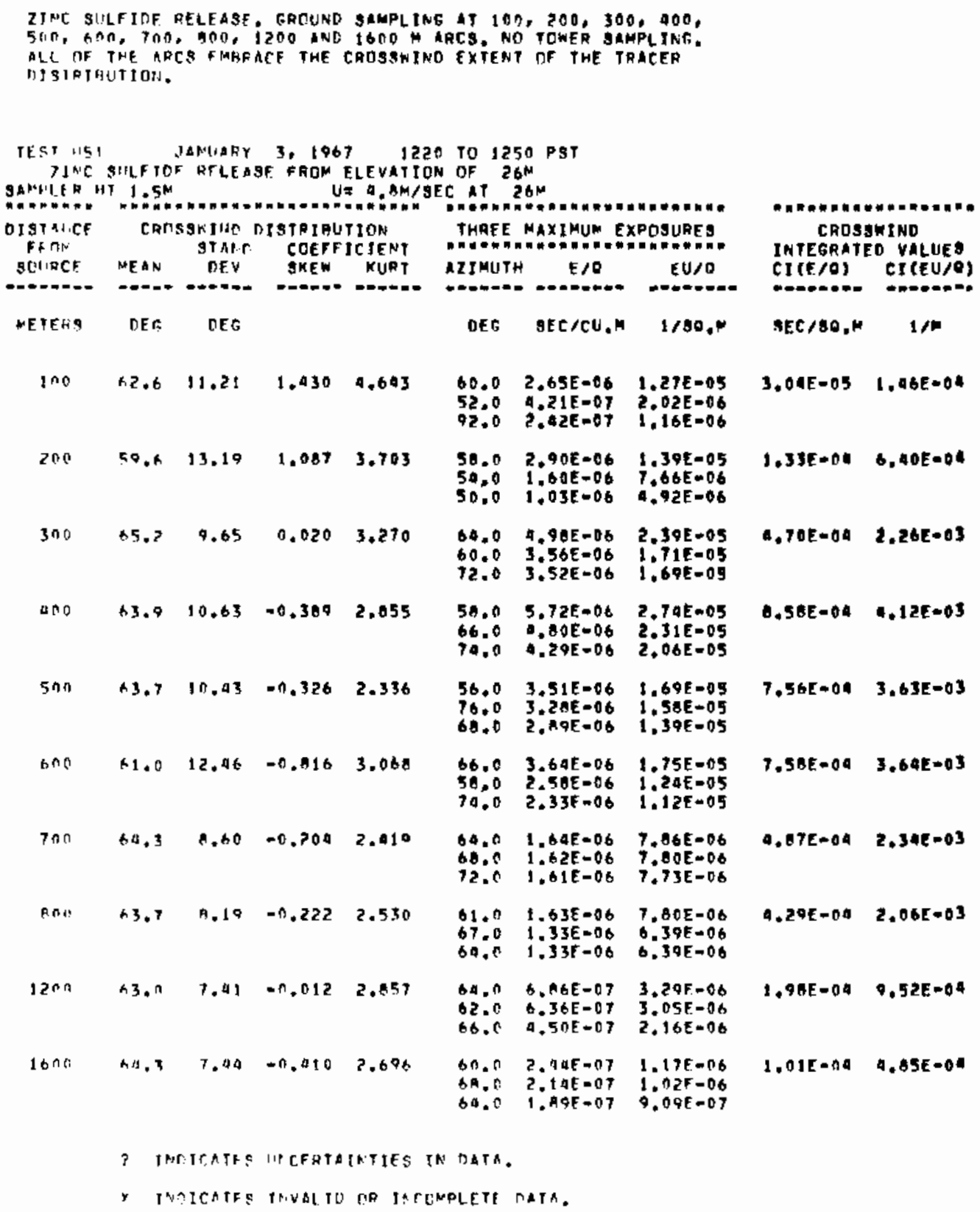


FLDURESCEIN RELEASE, GROUND LEVEL SAMPLING ONLY AT THE 1 BOO M ARC, THF ARC FMARACEO THE CROSSMIND EXTENT OF THE TRACER DISTRIAUTION.

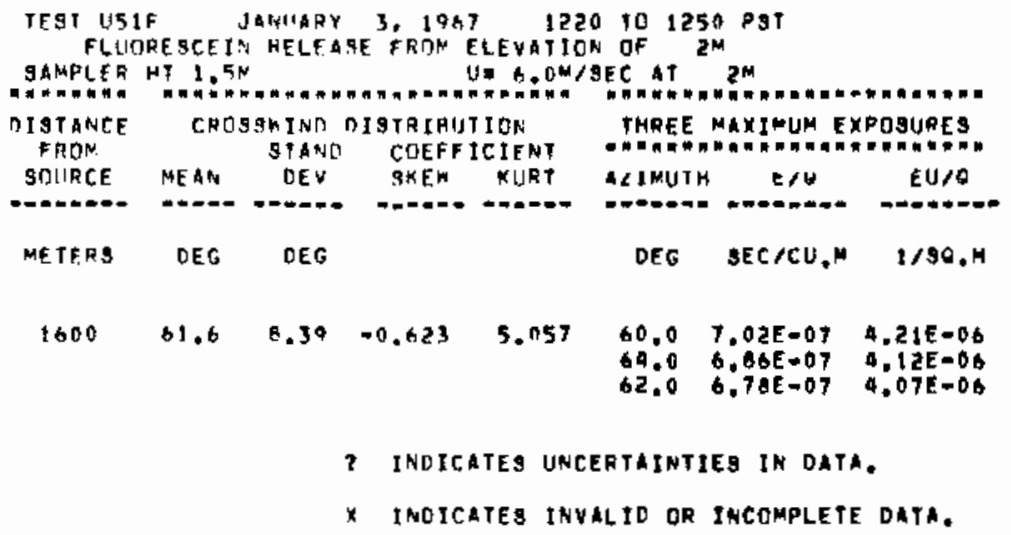




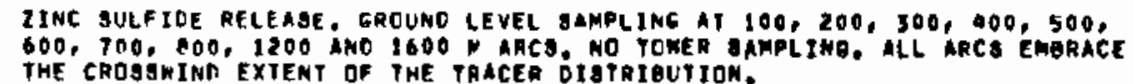

$600,700,100,1200$ ANO 1600 AACS. NO TOWER JAMPLINO. ALL ARC EMGRACE THE CROSGWINA EXTENT DF THE TAACE DIATRIOUTION.

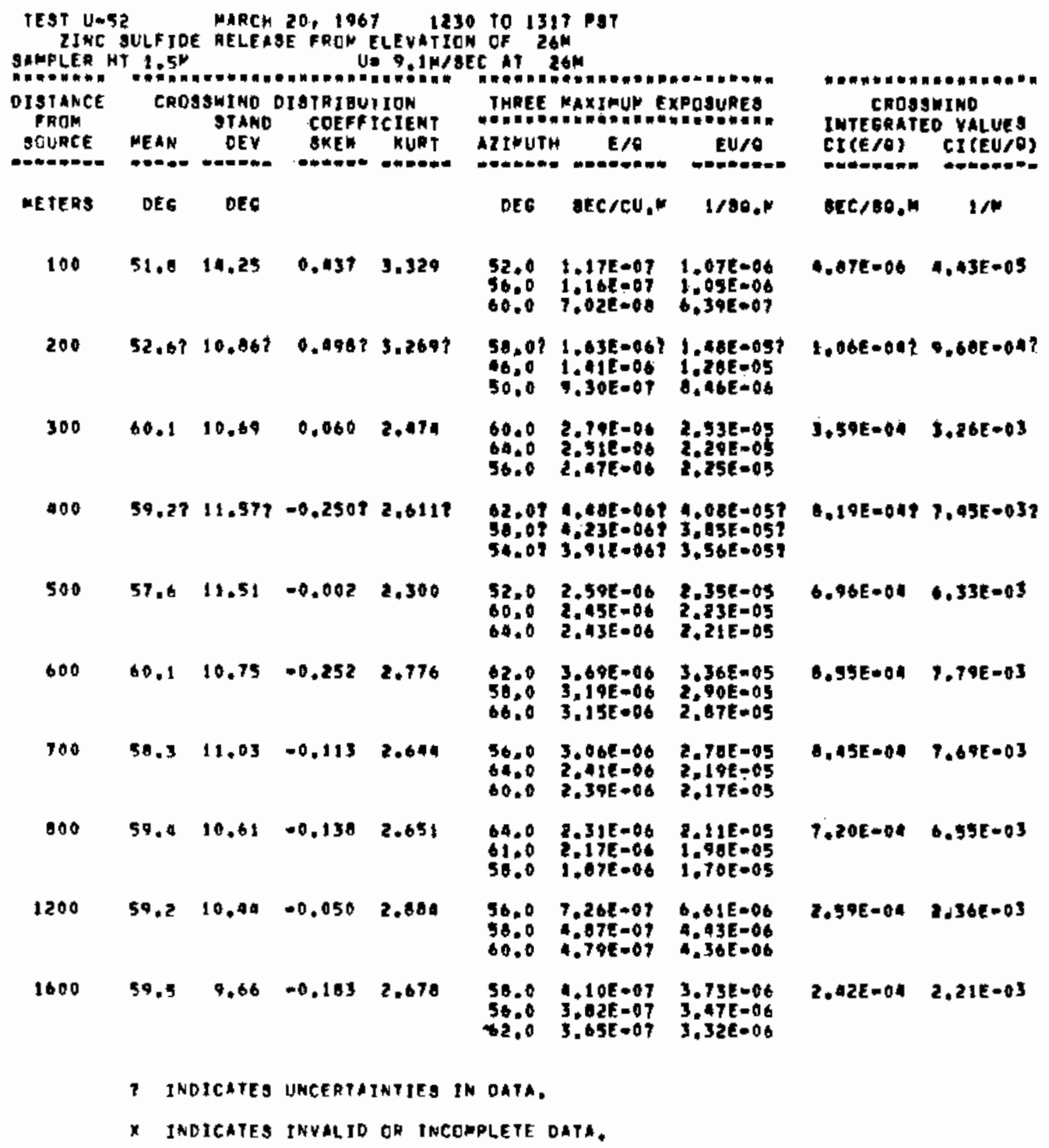




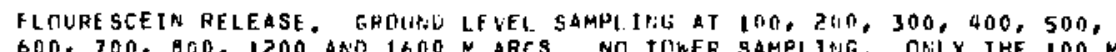
600,700 , 600, 1200 ANO $1600 \mathrm{~N}$ ARES. NO TOMER SAMPLJNG. ORIY THE 100 AAC DOES ROT ENHRACE THE CROSSWIAN EXTENT OF THE TRACFR OISTRIRUTION.

SAMPLER AZIMUTHS HAVE GFEN INTREAGE OY 30 DEGREES FRON THEIR ACTUAL POSITION

\begin{tabular}{|c|c|c|c|c|c|c|c|c|c|}
\hline $\begin{array}{r}\text { IEST USZ } \\
\text { FLUSO } \\
\text { SANPLER } \\
\text { W WW WHE }\end{array}$ & $\begin{array}{l}F \\
\text { RE SCE IN } \\
\text { HT } 1.5 \mathrm{~F}\end{array}$ & $\begin{array}{r}\text { MARCH } \\
\text { RELEA } \\
\text { R. }\end{array}$ & 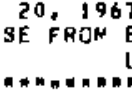 & 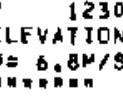 & $\begin{array}{l}\text { TO } 131 \\
\text { OF } \\
\text { OF } \\
\text { OE AT }\end{array}$ & 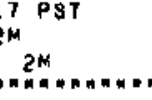 & 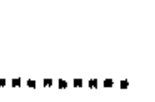 & \multicolumn{2}{|c|}{ 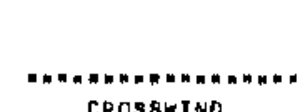 } \\
\hline & & $\begin{array}{l}\text { SSKIND } \\
\text { STAND }\end{array}$ & $\begin{array}{l}\text { A I S TRIBUT } \\
\text { CDEFF }\end{array}$ & $\begin{array}{l}\text { ION } \\
\text { CIENT }\end{array}$ & 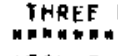 & $\begin{array}{l}M A X I N U M \\
M Y X Y M\end{array}$ & 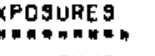 & $\begin{array}{r}\text { CROSE } \\
\text { INTEGRATE }\end{array}$ & $\begin{array}{l}\text { I IND } \\
\text { D VALUES }\end{array}$ \\
\hline SOURCE & ME AN & DEV & SKEW & KURT & AZINUTH & $E / Q$ & $E \cup / Q$ & $\operatorname{CJ}(t / 0)$ & $C I(E I) / 0)$ \\
\hline 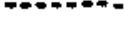 & - & $--0+\infty$ & $=-\infty+\infty=$ & 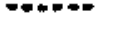 & $=0-\infty+\infty$ & $+\infty+\infty+\infty+\infty$ & 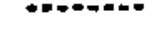 & O- & (-) \\
\hline ME TE R 3 & OEG & DEG & & & OE 6 & set/CU.H & $1 / 80,14$ & $S E C / 90, N$ & $1 / m$ \\
\hline 100 & BE, : $x$ & $14.74 x$ & $-0.093 x$ & $2.005 x$ & $\begin{array}{r}106.0 \\
82.0 \\
94.0\end{array}$ & $\begin{array}{l}1.58 E=04 \\
1.56 E=09 \\
1.56 E=04\end{array}$ & $\begin{array}{l}1.07 E=03 \\
1.06 E=03 \\
1.06 E=03\end{array}$ & $9.12 \mathrm{E}=03 \mathrm{x}$ & $6.20 E-02 x$ \\
\hline 200 & $63.2 ?$ & 14.117 & 0.5007 & $2.466 ?$ & $\begin{array}{r}88.07 \\
108.0 ? \\
84.01\end{array}$ & $\begin{array}{l}4.05 E=057 \\
3.99 E=05 ? \\
3.93 E=05 ?\end{array}$ & $\begin{array}{l}3,30 E=047 \\
2,71 E=047 \\
2.68 E=047\end{array}$ & $3.49 E-037$ & $2,34 E=02 ?$ \\
\hline 300 & 91.4 & 10.48 & 0.037 & 2.930 & $\begin{array}{l}90.0 \\
82.0 \\
86.0\end{array}$ & $\begin{array}{l}2.07 E-05 \\
2.77 E-05 \\
2.50 E=05\end{array}$ & $\begin{array}{l}1.95 E-04 \\
1.08 E-04 \\
1.75 E-04\end{array}$ & $4,06 E-03$ & $2.76 E-02$ \\
\hline 400 & 90.1 & 12.49 & -0.350 & 2.466 & $\begin{array}{l}98,0 \\
92,0 \\
96,0\end{array}$ & $\begin{array}{l}1.49 E-05 \\
1.46 E=05 \\
1.20 E-05\end{array}$ & $\begin{array}{l}1.01 E=04 \\
9.94 E-05 \\
0.18 E-05\end{array}$ & $5,13 E-03$ & $2.13 E=02$ \\
\hline 500 & 88.5 & 12.49 & $=0.230$ & 2.414 & $\begin{array}{l}90.0 \\
86.0 \\
94.0\end{array}$ & $\begin{array}{l}1.00 E=05 \\
7.64 E=06 \\
6.59 E=06\end{array}$ & $\begin{array}{l}6.02 E=05 \\
5.20 E-05 \\
4.40 E+05\end{array}$ & $2,26 E-03$ & $1.5 \mathrm{AE}=02$ \\
\hline 600 & 89.6 & 12.16 & -0.290 & 2.512 & $\begin{array}{l}92,0 \\
88,0 \\
96,0\end{array}$ & $\begin{array}{l}7.07 E=06 \\
6,30 E=06 \\
4,07 E=06\end{array}$ & $\begin{array}{l}4.11 E-05 \\
4.29 E-05 \\
3.31 E-05\end{array}$ & $1.88 \mathrm{E}=03$ & $1,28 E=02$ \\
\hline 700 & 89.7 & 12.20 & -0.187 & $2 . \$ 47$ & $\begin{array}{l}86,0 \\
90,0 \\
94,0\end{array}$ & $\begin{array}{l}3.01 E-06 \\
3.81 E=06 \\
3.81 E=06\end{array}$ & $\begin{array}{l}2.59 E-05 \\
2.59 E-05 \\
2.50 E-05\end{array}$ & $1.50 E=03$ & $1.02 \mathrm{E}-02$ \\
\hline 800 & 90,2 & 11.98 & -0.218 & 2.400 & $\begin{array}{l}91,0 \\
94,0 \\
08,0\end{array}$ & $\begin{array}{l}3.40 E-06 \\
3.28 E=06 \\
2.68 E-06\end{array}$ & $\begin{array}{l}2.31 E=05 \\
2.23 E=05 \\
1.82 t=05\end{array}$ & $1.25 E-03$ & $8.50 E \div 03$ \\
\hline $1=00$ & 91,0 & 11.35 & $=0.363$ & 2.597 & $\begin{array}{l}94.0 \\
90.0 \\
92.0\end{array}$ & $\begin{array}{l}1.05 E=06 \\
9.19 E=07 \\
9.08 E-07\end{array}$ & $\begin{array}{l}7.15 E-106 \\
6.25 E=06 \\
0.17 E=06\end{array}$ & $4.93 E=0 a$ & $3.35 E=03$ \\
\hline
\end{tabular}

? INDTCATES UNCERTAINTIES JN DATA.

x INDICATES INVALID OR INCONPLETE DATA. 


\begin{tabular}{|c|c|c|c|c|c|c|c|c|c|}
\hline $\begin{array}{l}\text { TEST U-S } \\
\text { ZINC. } \\
\text { SAMPLER H } \\
\text { A }\end{array}$ & $\begin{array}{l}3 \\
\text { SULF IDE } \\
1\end{array}$ & $\begin{aligned} & \text { MARCH } \\
\text { RELEAS } & \text { RE } \\
& \text { NAOHNA }\end{aligned}$ & $\begin{array}{l}23,1967 \\
\text { rROM E }\end{array}$ & 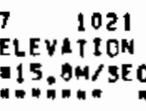 & $\begin{array}{ll}T 0 & 112 \\
0 F & 26 \\
C & ?\end{array}$ & 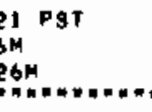 & 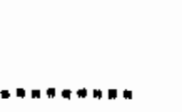 & 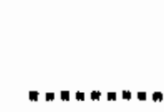 & 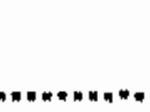 \\
\hline $\begin{array}{l}\text { DIST ANE E } \\
\text { FROM }\end{array}$ & CaOs & $\begin{array}{l}\text { SBWTNDD } \\
\text { STAND }\end{array}$ & $\begin{array}{r}\text { ISTRIAUT } \\
\text { COEFFY }\end{array}$ & $\begin{array}{l}\text { TION } \\
\text { ICIENT }\end{array}$ & THREE & MAXXIHUN E & XPOSUAES & $\begin{array}{r}\text { CRUSS } \\
\text { IMTEGRATE }\end{array}$ & $\begin{array}{l}\text { SWINO } \\
\text { SO VAL UES }\end{array}$ \\
\hline $\begin{array}{l}\text { SOURCE } \\
\text { SOL }\end{array}$ & MEAN & DEV & SKEW & KURT & AZIMUIN & $\begin{array}{c}E / 0 \\
-\infty\end{array}$ & EU/A & CI $(E / 0)$ & $C I(E \cup / Q\}$ \\
\hline METERS & DEG & OEG & & & DEG & $\mathrm{sec} / \mathrm{CU}_{\mathbf{N}} \mathrm{M}$ & $1 / 86$. & $s \in C / 90, \mu$ & $1 / M$ \\
\hline 100 & 53.5 & 15.20 & 0.334 & 2.163 & $\begin{array}{l}98.0 \\
56.0 \\
90.0\end{array}$ & $\begin{array}{l}1.56 E=08 \\
9.96 E=09 \\
9.15 E=09\end{array}$ & $\begin{array}{l}2.47 E-07 \\
1.57 E-07 \\
1.45 E-07\end{array}$ & $7.7 B E-07$ & $1.23 E-05$ \\
\hline 200 & 39.0 & 10.18 & 0.618 & 2.A85 & $\begin{array}{l}36.0 \\
30.0 \\
30.0\end{array}$ & $\begin{array}{l}1.05 E=07 \\
6.09 E=0 D \\
6.70 E=08\end{array}$ & $\begin{array}{l}1.62 E-06 \\
1.09 E=06 \\
1.06 E-06\end{array}$ & $7.97 \mathrm{E}-06$ & $1,26 E=04$ \\
\hline 300 & 36.97 & 12.797 & $1.04 \cap ?$ & 4.1157 & $\begin{array}{l}32.07 \\
28.0 ? \\
40.01\end{array}$ & $\begin{array}{l}1.77 E-077 \\
1.56 E=077 \\
1.52 E-077\end{array}$ & $\begin{array}{l}2.00 E=067 \\
2.06 E=067 \\
2.40 E=067\end{array}$ & $2.15 E=051$ & $3,4 \mathrm{AE}-04\}$ \\
\hline $4 \cap 0$ & 39.7 & 12.55 & 0.000 & 3.660 & $\begin{array}{l}38.0 \\
34.0 \\
30.0\end{array}$ & $\begin{array}{l}3.30 E-07 \\
1.51 E=07 \\
1.94 E=07\end{array}$ & $\begin{array}{l}5.22 E=06 \\
2.36 E-06 \\
2.27 E-06\end{array}$ & $3.90 E-05$ & $6.17 \mathrm{E}=\mathrm{O}^{4}$ \\
\hline 500 & 37.1 & 10.26 & 0.776 & 3.672 & $\begin{array}{l}32.0 \\
40.0 \\
36.0\end{array}$ & $\begin{array}{l}5.39 E-07 \\
.32 E=07 \\
4.30 E=07\end{array}$ & $\begin{array}{l}6.51 E=06 \\
7.62 E=06 \\
6.93 E=06\end{array}$ & 9. DTE- O5 & $1,56 E-03$ \\
\hline 600 & 36.3 & 9.95 & 0.944 & 3.664 & $\begin{array}{l}34.0 \\
30.0 \\
30.0\end{array}$ & $\begin{array}{l}6.03 E=07 \\
5.26 E=07 \\
4.20 E=07\end{array}$ & $\begin{array}{l}9.3 x E=06 \\
6.32 E=06 \\
6.60 E=06\end{array}$ & $1,18 E-04$ & $1.07 \varepsilon-03$ \\
\hline 700 & 35.5 & 9.40 & 0.069 & 3.666 & $\begin{array}{l}32.0 \\
36.0 \\
28.0\end{array}$ & $\begin{array}{l}0.26 E=07 \\
4.73 E=07 \\
0.64 E=07\end{array}$ & $\begin{array}{l}1.31 E=05 \\
7.476=06 \\
7.33 E=06\end{array}$ & $1.59 E-09$ & $2.52 E-03$ \\
\hline Bon & 36,3 & 9.30 & ก.А & 3.591 & $\begin{array}{l}31.0 \\
37.0 \\
34.0\end{array}$ & $\begin{array}{l}3.785-07 \\
3.76 E=07 \\
3.37 E=07\end{array}$ & $\begin{array}{l}5.98 E-06 \\
5.90 E-06 \\
5.33 F=06\end{array}$ & $1.03 E-04$ & I. . $3 E-0.3$ \\
\hline 1200 & 35.1 & 0.08 & 0.689 & 3.640 & $\begin{array}{l}30.0 \\
32.0 \\
34.0\end{array}$ & $\begin{array}{l}3.97 E=07 \\
3.66 E=07 \\
3.57 E=07\end{array}$ & $\begin{array}{l}6.27 E=06 \\
5.79 E=06 \\
5.64 E=06\end{array}$ & $1.3 n E=04$ & $2.0 B E-03$ \\
\hline \multirow[t]{3}{*}{1600} & $37 . ?$ & 9.87 & 0.753 & 2.747 & $\begin{array}{l}32.0 \\
28.0 \\
39.0\end{array}$ & $\begin{array}{l}1.73 E-07 \\
1.39 E=07 \\
1.24 E=1.7\end{array}$ & $\begin{array}{l}2.74 E=06 \\
2.20 E=06 \\
1.96 E=06\end{array}$ & $6.53 E-05$ & $1.035=05$ \\
\hline & $I^{* 1+}$ & JCATFs & lince & TNTIEA IN & DATA, & & & & \\
\hline & INDI & ICATES & INVAL ID & OR INCERP & PLETE DA & 2. & & & \\
\hline
\end{tabular}


FLOURESCEIN RELFASF. GRTUND LEVFL SAMPLJAG AT $100,200,300,400,500$, $000,700,800,1200$, AND 1600 M ARCS. NO TOWER SAMPLING.

SAMPLER LZIMUTHS HAVE BEEN INCGEASED GY 30 DEGKEES FROM THEIF ACTUAL POSITIOA

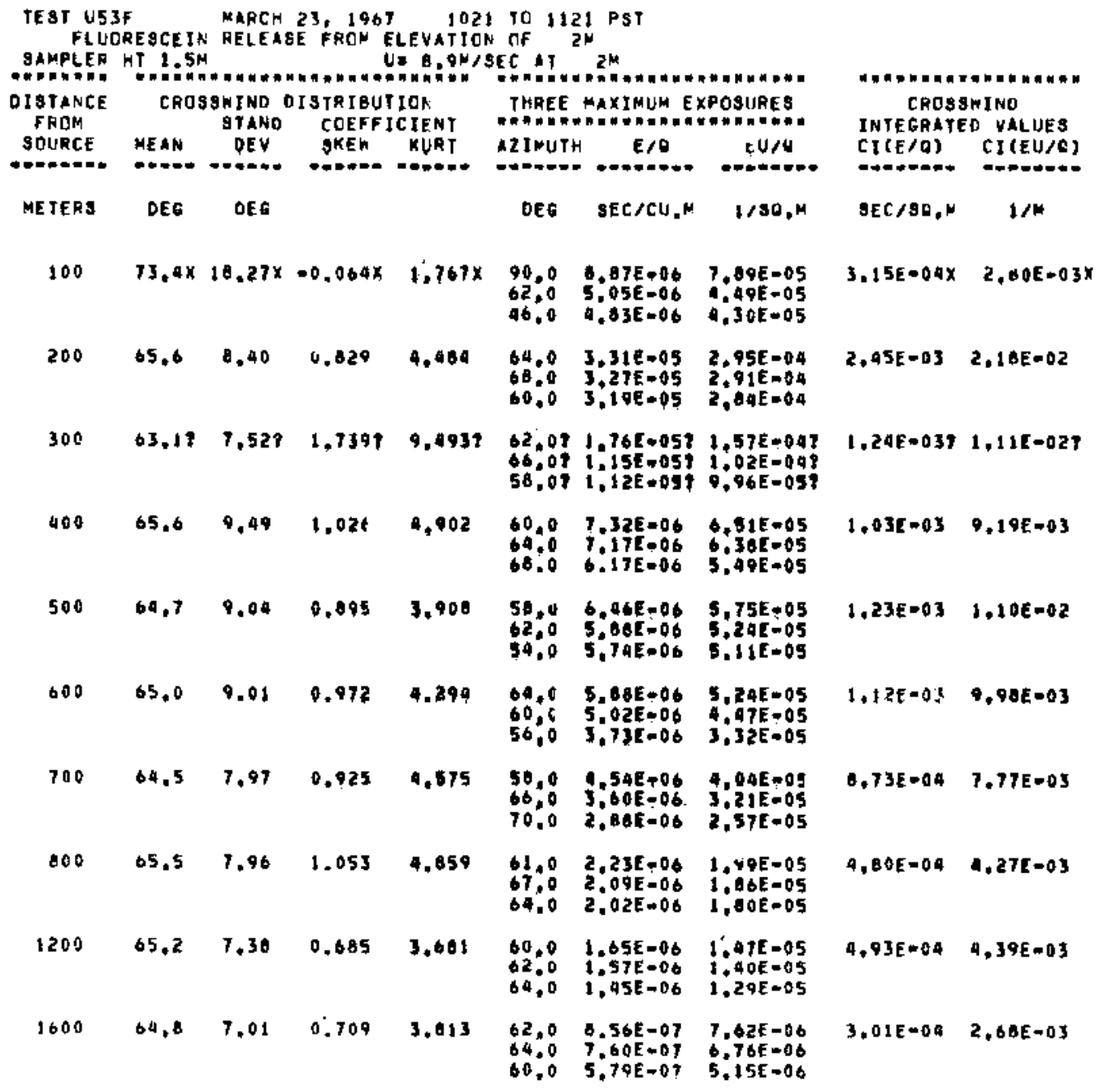

? INDICATES UNCERTAINTIES IN DATA,

X INDICATES INVALIO OR INCOMPLETE DATA. 
ZIMC SULFIOE RELEAGE. GROUND LEYEL SAMPLIMS AT $100,200,300,400,500$, $600,700,000,1200$ iNO 1600 ARCS, NO TOWER OAMPLINO, ALL AFCS EMBRACE THE CROSBMIND EXTENT DF THE TARCER DISTRIOUTION.

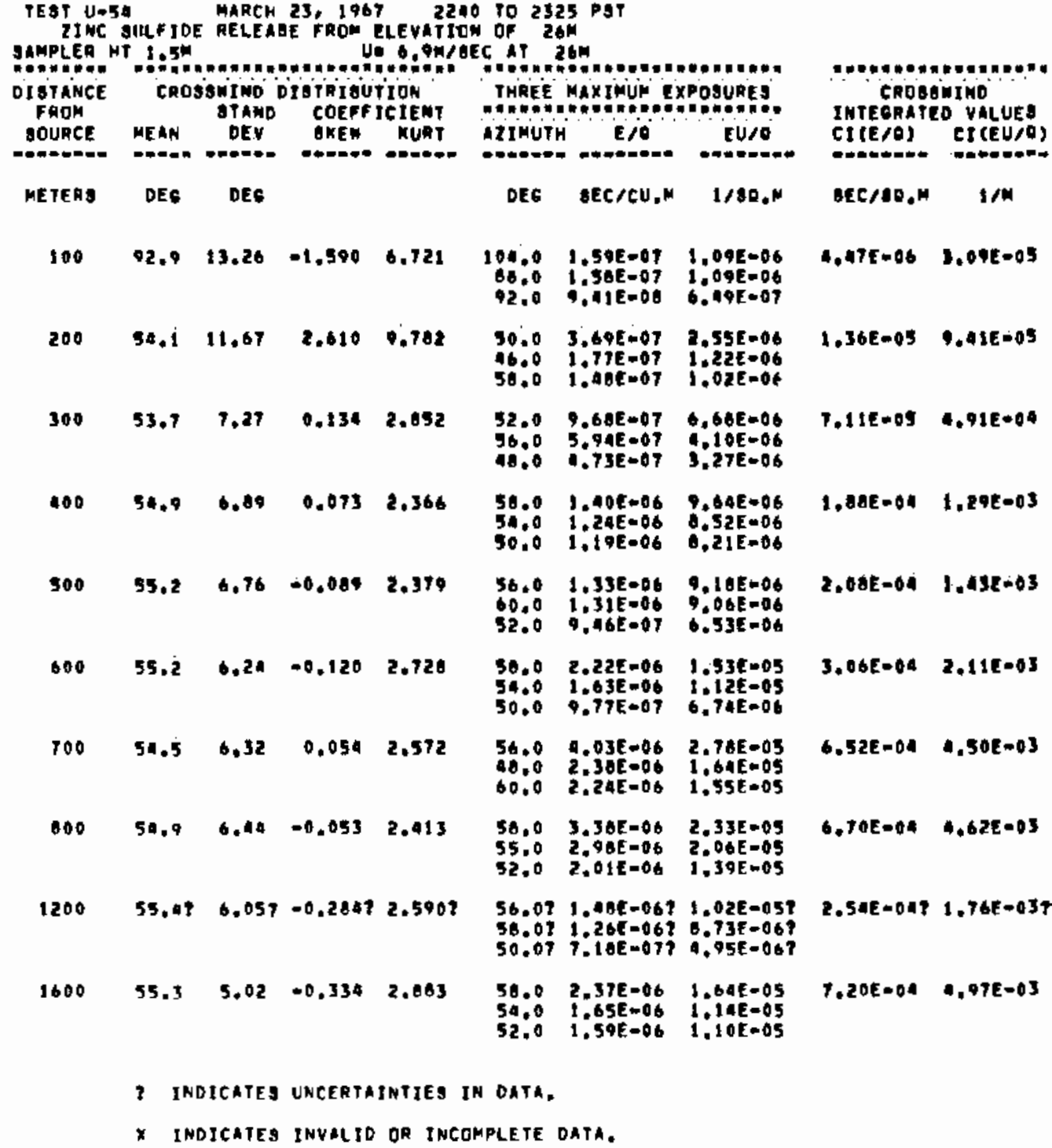

$?$ INDICATES UNCERTAINTES IH OATA.

X INDICATES INVALIO OR INCOMPLETE DATA. 
FLOUPESCEIN PELEASE. GROUUND LEVEL SAMPLING AT 100, 200, 300, 000,500, $600,70 \mathrm{n}, A 00,1200$ ANO $1600 \mathrm{~N}$ ARCS. NO TOMER SAMPLING. ALL ARCS FNAGACE THE CRCSSWIRT EXTENT DF THE TRACER DISTRIRUTION.

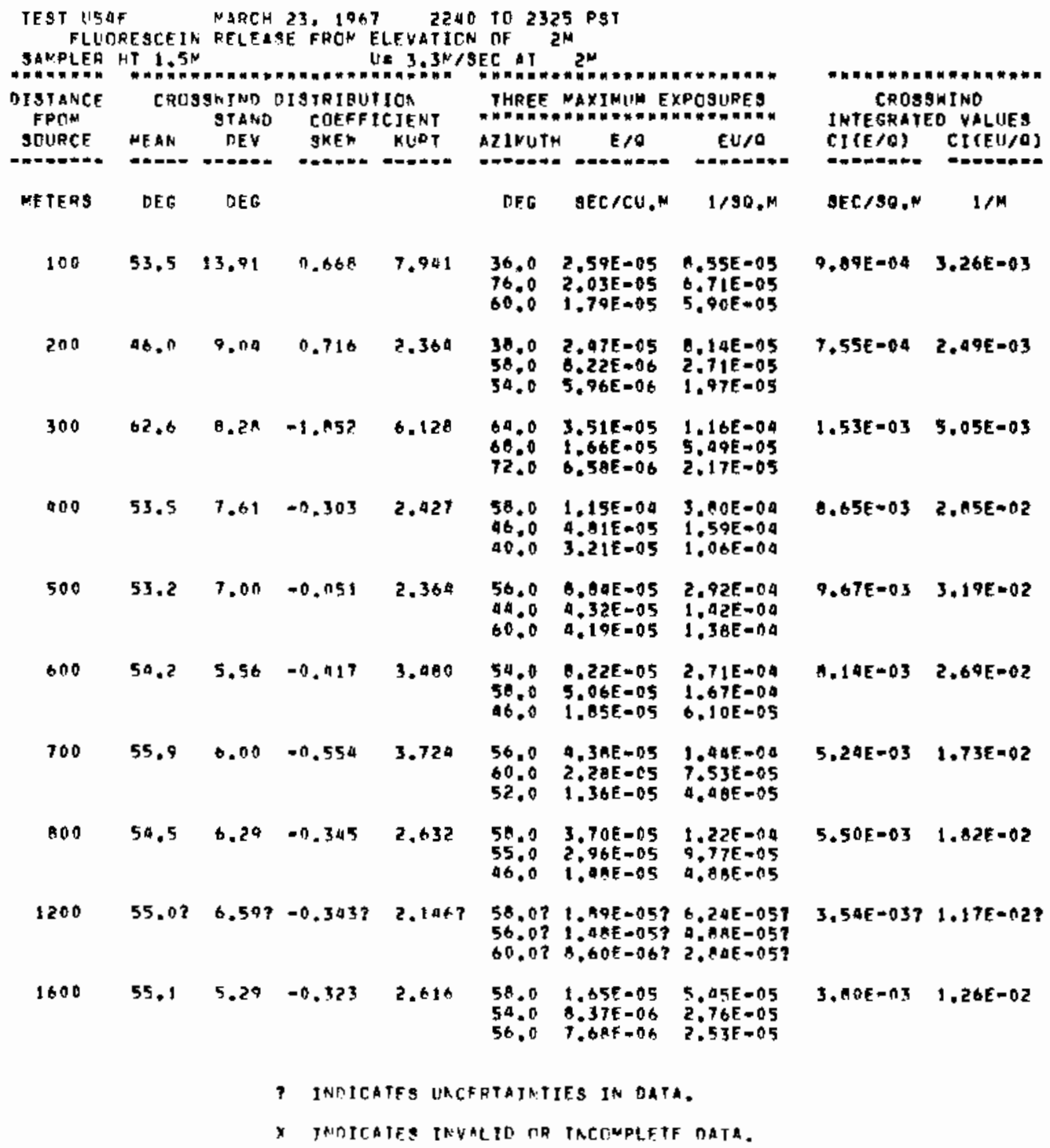


GROUKD LEVEL SANFLIAG $1100,200,300,000,500,600,700,000$,
1200, ANO 1600 ARCS. RO TCKER SANPLING. ALL ARC E EMBRACE THE CAOSGRIND EXIENT OF THE TRICER DISTFIBUTICN.

SANPLER AZINUTHS HAVE BEEA INCREASE BY 30 DEGREE FROM IHEIF ACTUAL PUSITIGKS

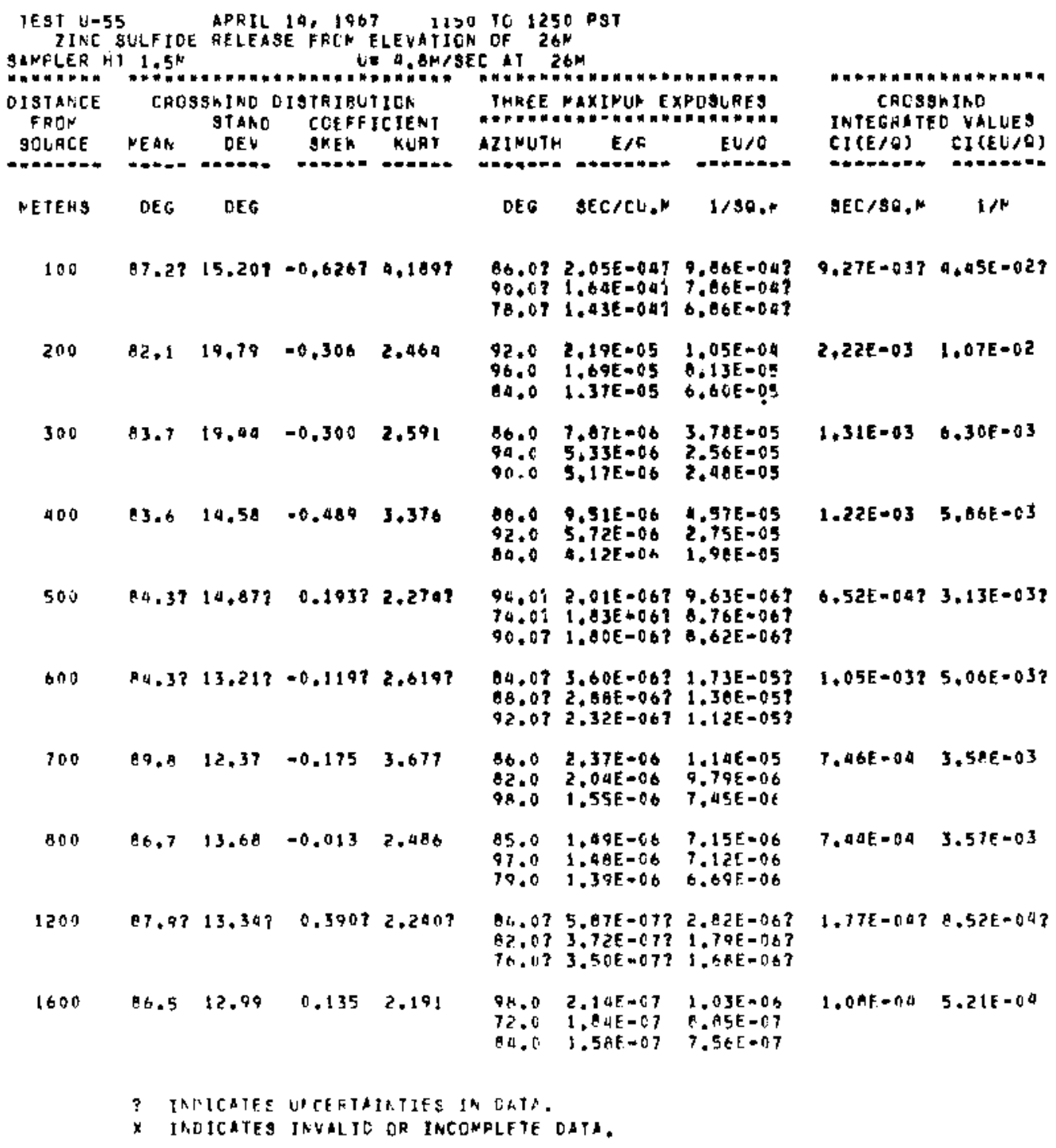




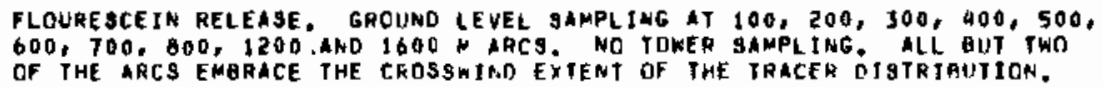
OF THE ARCS EMARACE THE tROSSMIAD EXTENT OF THE TRACER DISTRIAUTION.

SAMPLER AZIMUTHS HAVE BEEN JKCREASED GY JO DEgREES FRDM THEIG ACTUAL POSITION

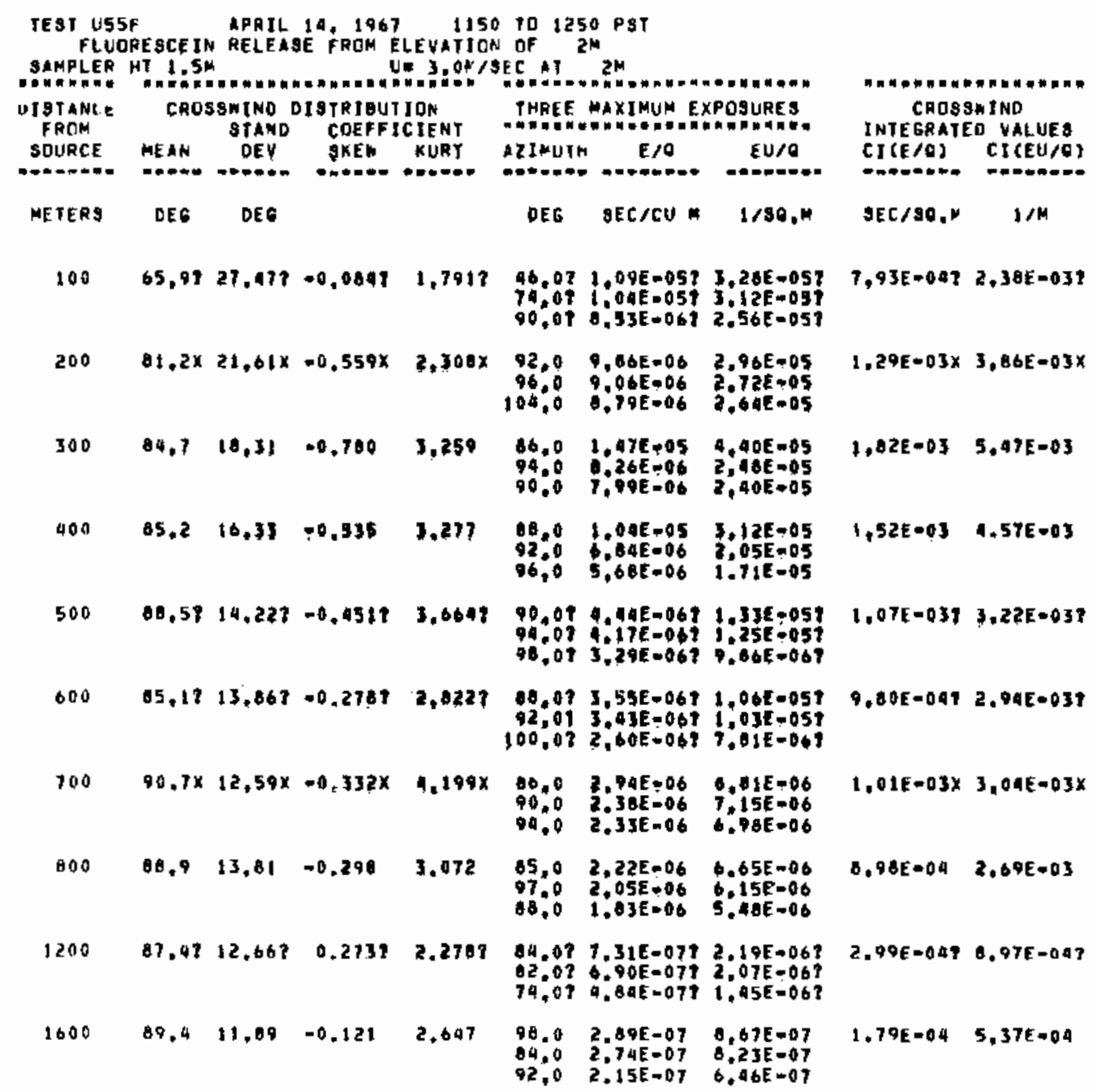

$?$ INDICATES UNCEATAINTIES IN DATA,

X INOICATE INVALIO OR INCOMPLETE DATA. 


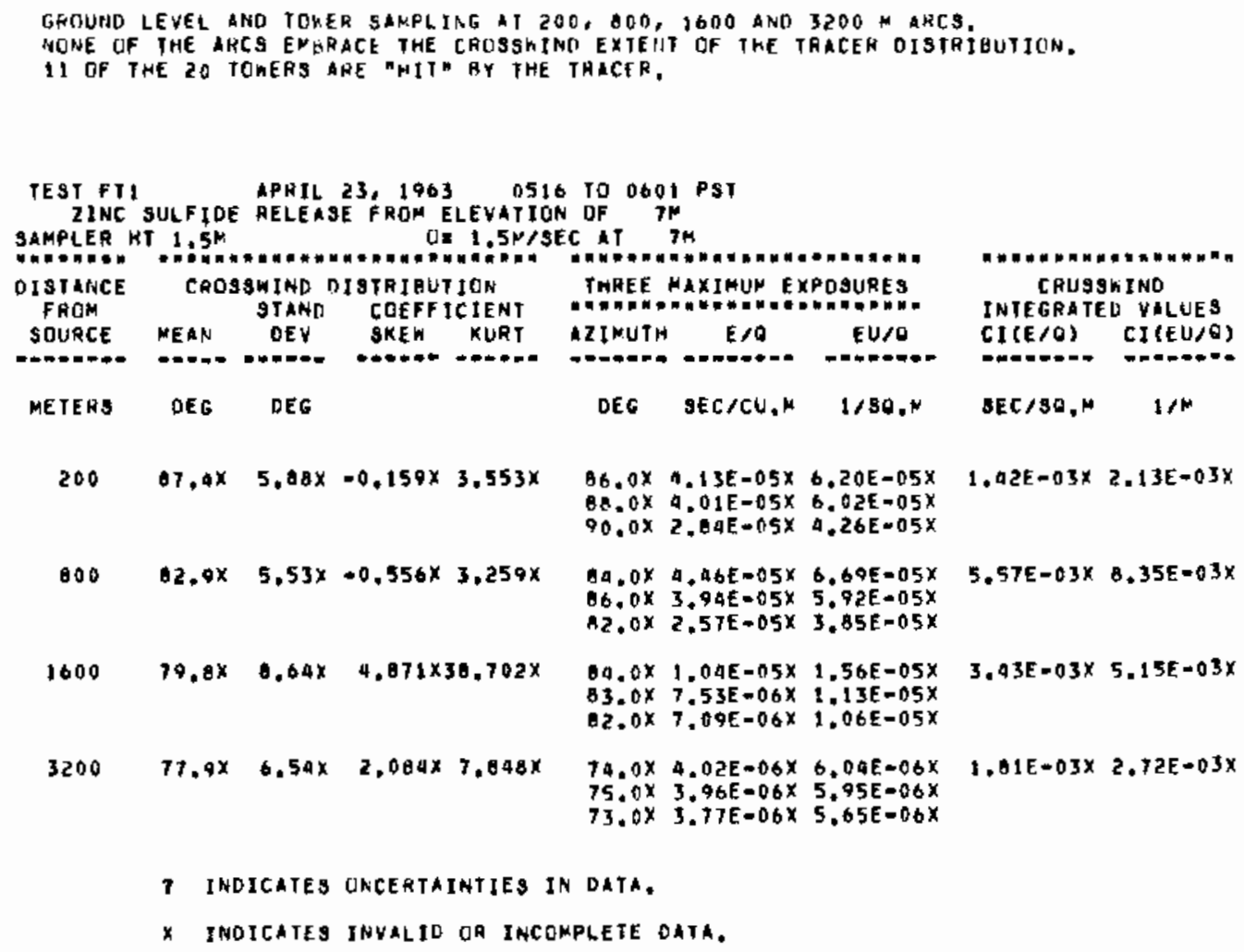$$
\Delta a .0 X \quad 4.46 E=05 x \quad 6.69 E=05 X
$$$$
06.0 \times 3.94 E-05 \times 5.92 E=05 X
$$$$
82.0 \times 2.57 \mathrm{E}=05 \times \quad 3.85 \mathrm{E}=05 \mathrm{X}
$$

$5.57 E-03 x \quad 8.35 E=03 x$ $22.0 \times 2.57 \mathrm{E}=05 \mathrm{X} \quad 3.85 \mathrm{E}-05 \mathrm{X}$

7 INDICATES UNCERTAINTIES IN DATA,

X INOICATES INVALID OA INCOMPLETE DATA. 


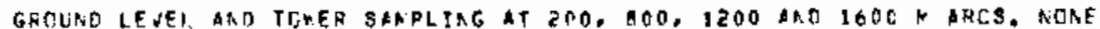
CF THE LACS EUAPACF THE CACBSKIND EXTENT (IF THE TRACER DISTRIPUTICN. ALL CF THE TTWERS WERE NHIT," PY THE TRACER,

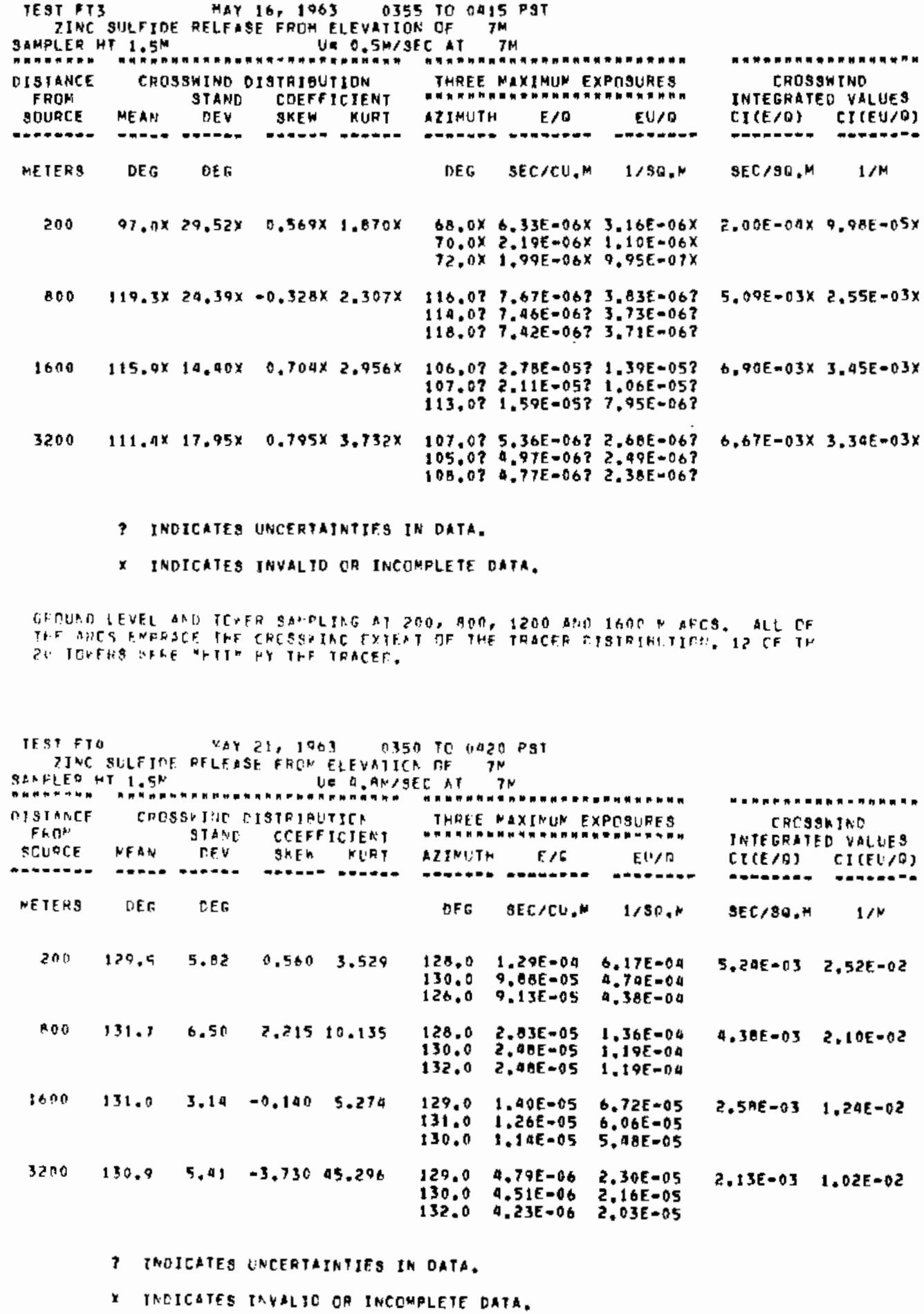


GRDUND LEVEL AND TOMER SAMPLING IT $200,800,1600$ AND 3200 P RCS.

ALL DF THE ARCS EMgRACF. THE CROSSWIND EXTENT DF THE TRACER DISTRIAUTION.

11 DF THE 20 TOWERS TEPE "MIT" BY THE TRACER

\begin{tabular}{|c|c|c|c|c|c|c|c|c|c|}
\hline $\begin{array}{l}\text { DISTANCE } \\
\text { FROM }\end{array}$ & & $\begin{array}{l}\text { IOMIND O } \\
\text { STAND }\end{array}$ & $\begin{array}{l}\text { ISTAIAU } \\
\text { CDEFF }\end{array}$ & $\begin{array}{l}\text { TION } \\
\text { TCIENT }\end{array}$ & THREE & 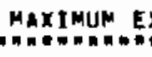 & Xe08UREs & $\begin{array}{l}\text { CROSS } \\
\text { INTEGRATE }\end{array}$ & $\begin{array}{l}\text { OWIND } \\
\text { EO YALUES }\end{array}$ \\
\hline SOURCE & ME AN & DEV & SKEW & KURT & AIIMUTH & $E / C$ & EU/O & Cl(E/e) & CI $(E \cup / O)$ \\
\hline$+\infty+\infty$ & 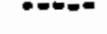 & n-mene & $x=-$ & - & 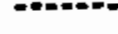 & r & - & 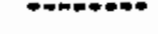 & $x+\operatorname{cosec}$ \\
\hline ME TERS & DEG & DEG & & & DEF & $S E C / C U, M$ & $1 / 80, N$ & SEC/80,M & $1 / M$ \\
\hline 200 & 129.4 & 4.42 & $-0,200$ & 2.907 & $\begin{array}{l}130.0 \\
132.0 \\
128.0\end{array}$ & $\begin{array}{l}2.30 E-0 a \\
1.78 E+0 a \\
1.74 E-04\end{array}$ & $\begin{array}{l}9.68 E=04 \\
7.4 B E=04 \\
7.30 E=04\end{array}$ & $7.94 E=03$ & $3,33 E=02$ \\
\hline 800 & 129.5 & 3.27 & $-0,216$ & 2.620 & $\begin{array}{l}132.0 \\
130.0 \\
128.0\end{array}$ & $\begin{array}{l}6.29 E=05 \\
5.48 E=05 \\
5.37 E=05\end{array}$ & $\begin{array}{l}2.64 E-04 \\
2.30 E=02 \\
2.26 E-04\end{array}$ & $7.96 E-03$ & $3.13 E=02$ \\
\hline 1600 & 129.6 & 2.66 & -0.194 & 2.540 & $\begin{array}{l}131.0 \\
129.0 \\
130.0\end{array}$ & $\begin{array}{l}2.03 E-05 \\
2.36 E=05 \\
2.21 E=05\end{array}$ & $\begin{array}{l}1.02 E=04 \\
9.92 E=05 \\
9.29 E=05\end{array}$ & $4,82 E-03$ & $2.03 E-02$ \\
\hline 3200 & 131.7 & 2.32 & -0.337 & 3.336 & $\begin{array}{l}130.0 \\
131.0 \\
132.0\end{array}$ & $\begin{array}{l}9.97 E=06 \\
7.99 E-06 \\
6,76 E=06\end{array}$ & $\begin{array}{l}3.9 \theta E=05 \\
3.15 E=05 \\
2.04 E=05\end{array}$ & $2.96 E=03$ & $1.24 \mathrm{E}-02$ \\
\hline & $?$ & ICATES & UNCEATA & INTIES & DRTA. & DHTA & & & \\
\hline
\end{tabular}

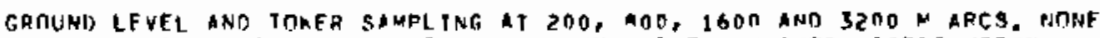
OF IHE ARCS EMPRACE IHF CROBSVITNR EXTEHT OF THE TRACER DISTRBPUIIDN. 3 NF THF. 20 TOWERS WFAF "HIT" AY THE TKACER

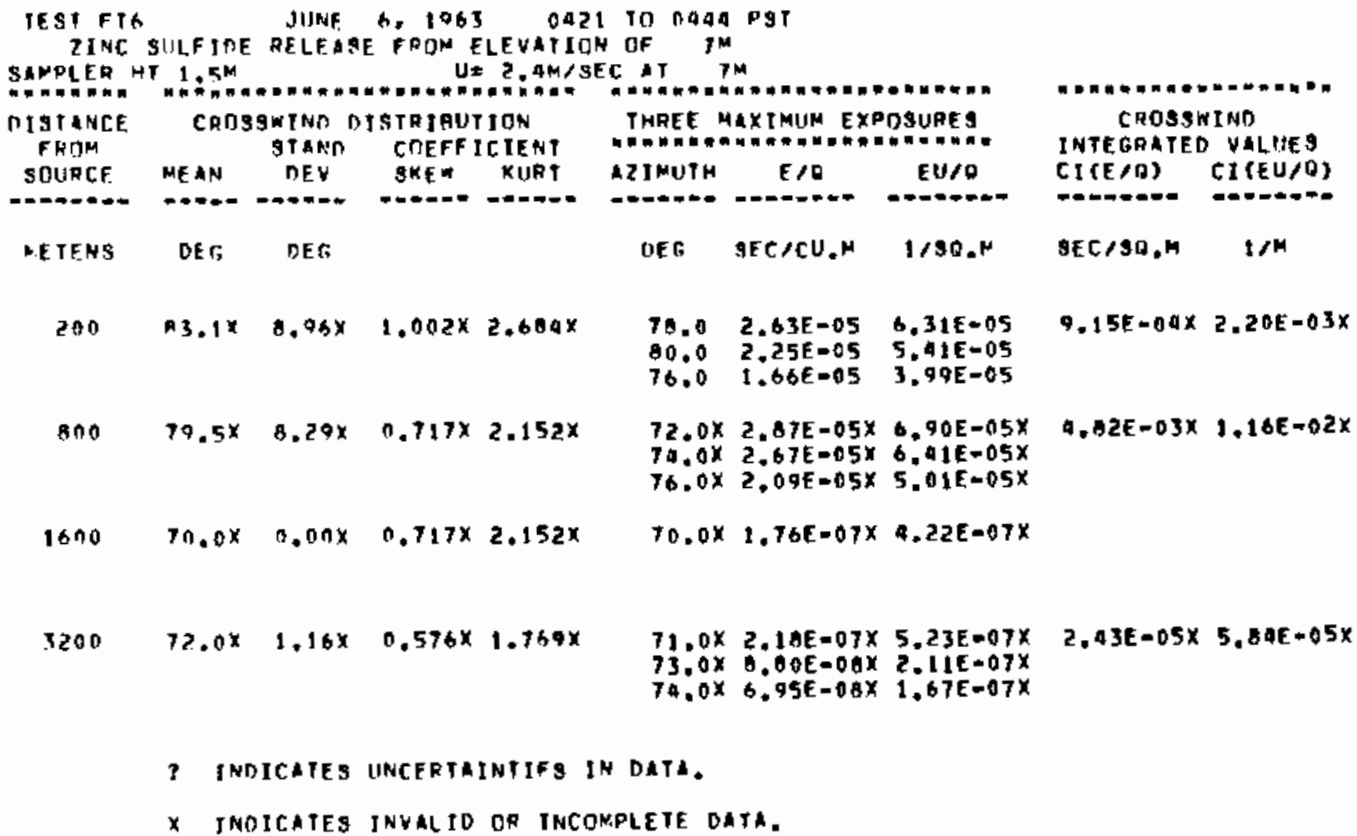




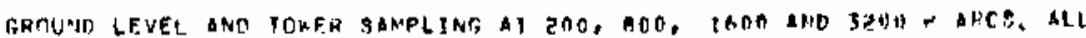
DF THE APCS EMRRACF THE CROSSWIND EXIENT [HF THE TRACFR OISTRIAUTION. IA UF THE 2 T! TDWERS WERE "HIT" AY THE TRACER

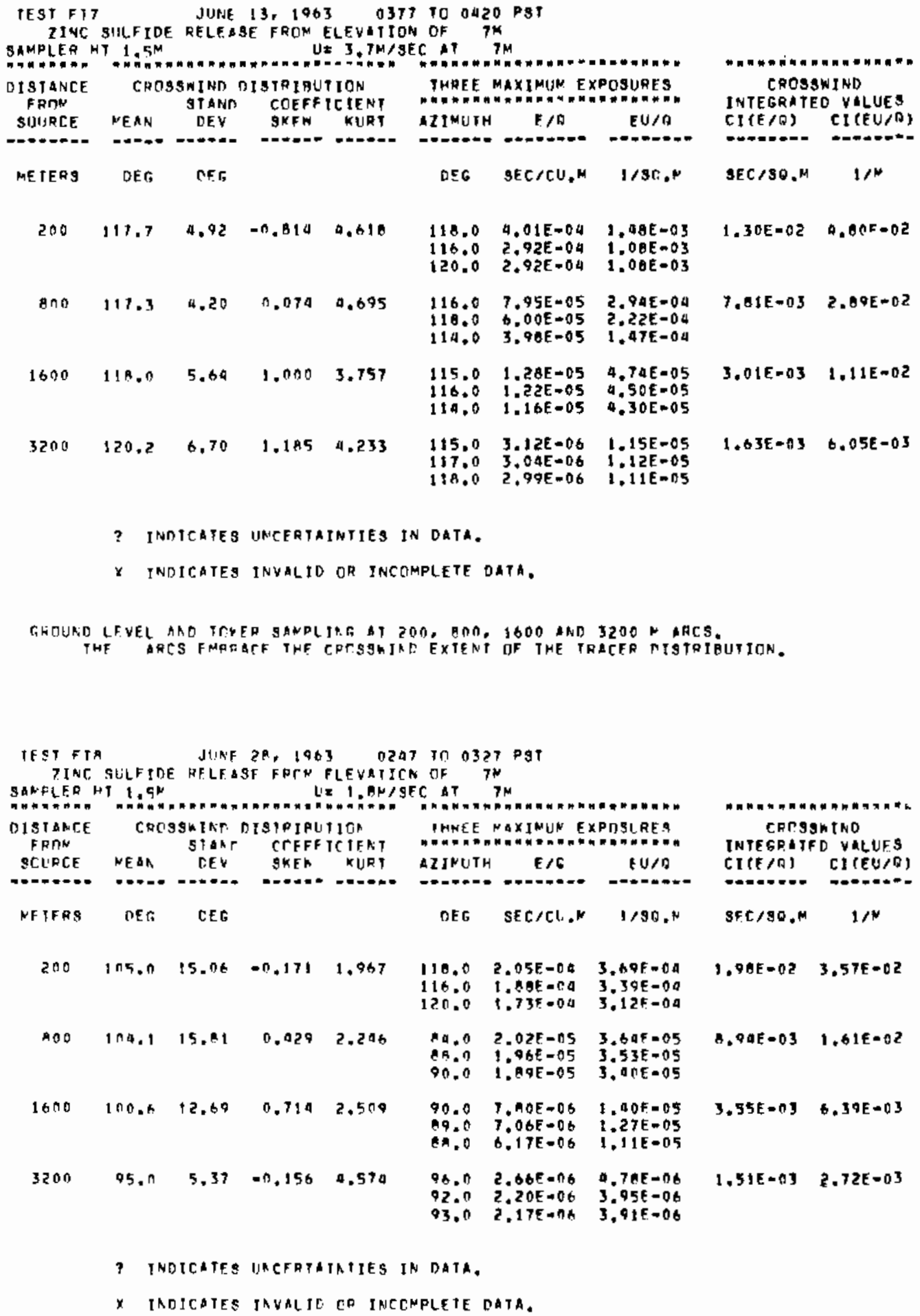




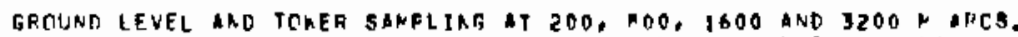

ALI CF THE IRCS EMANACE THF CROBSRIND EXTENT OF THF TRACER DISTHIEUTIOH.

19 TF THE 2O TCAFAS KERE *HTH" EY THE TRACER

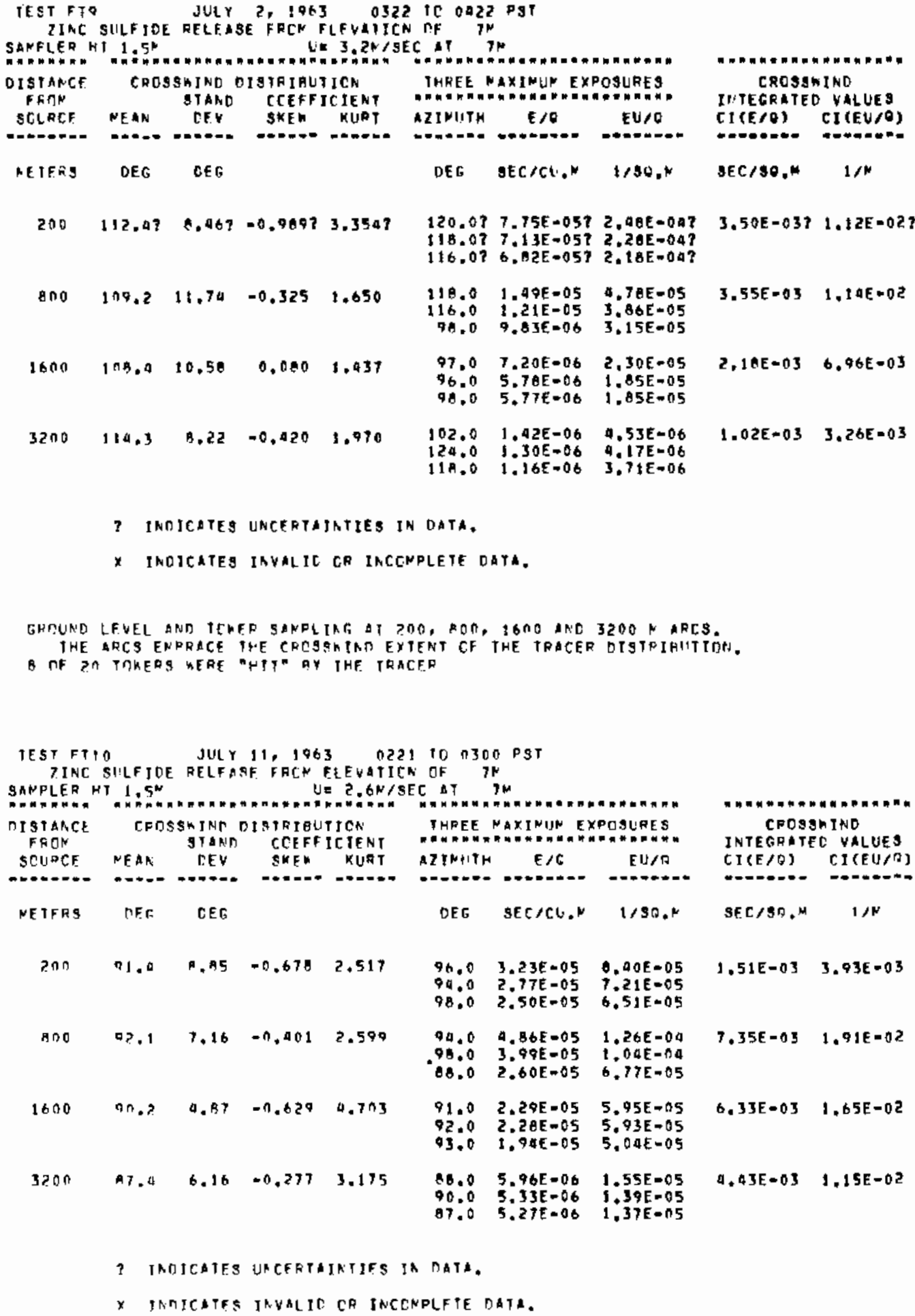




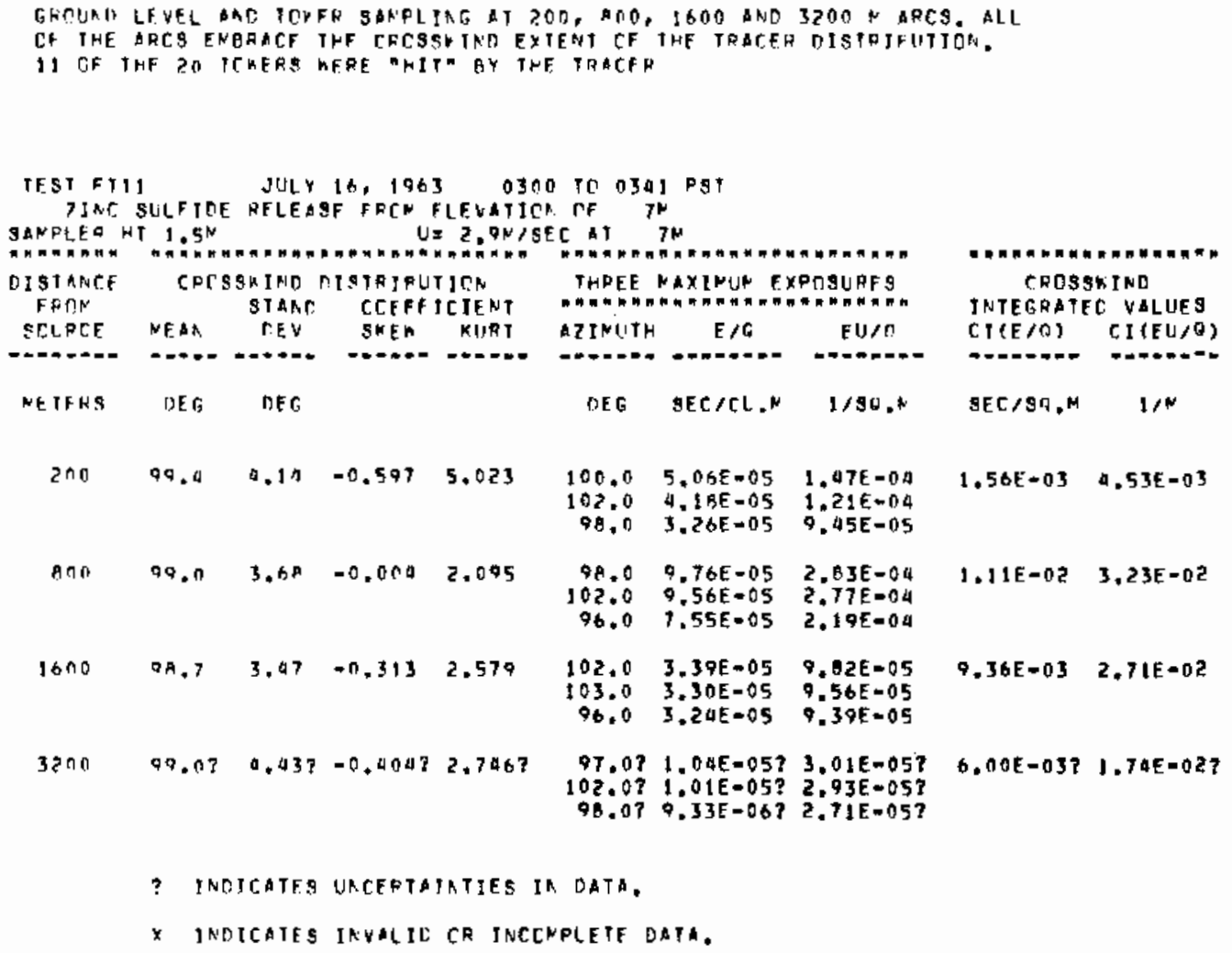

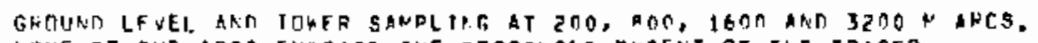
MLNE OF THE ARTS ENPAACE THE CRCSBMTKD EXTENT OF THE TRACFA

CISIRIAUTIOR. D TF THE 2 O TCKERS MEAF "HJT." PY THE TRACER

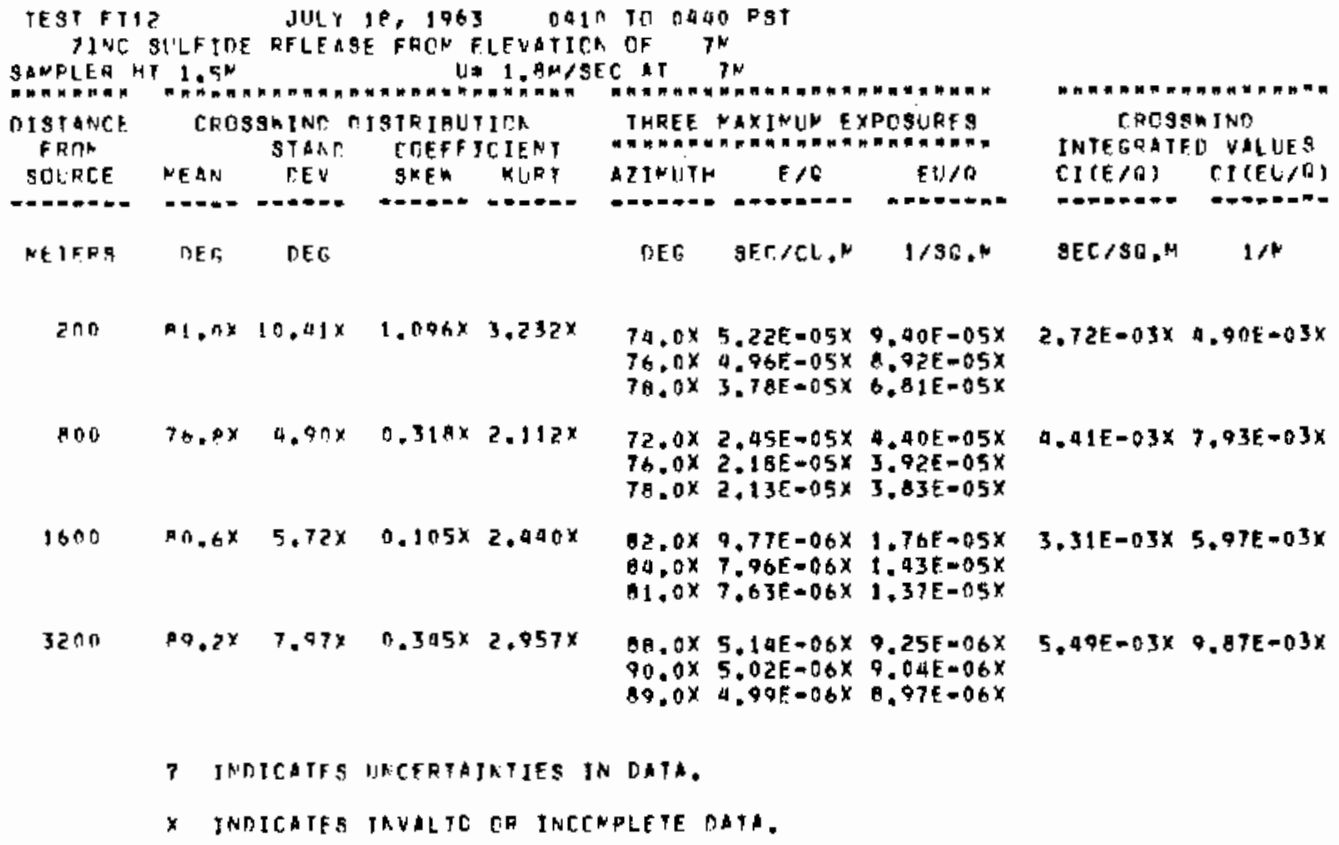




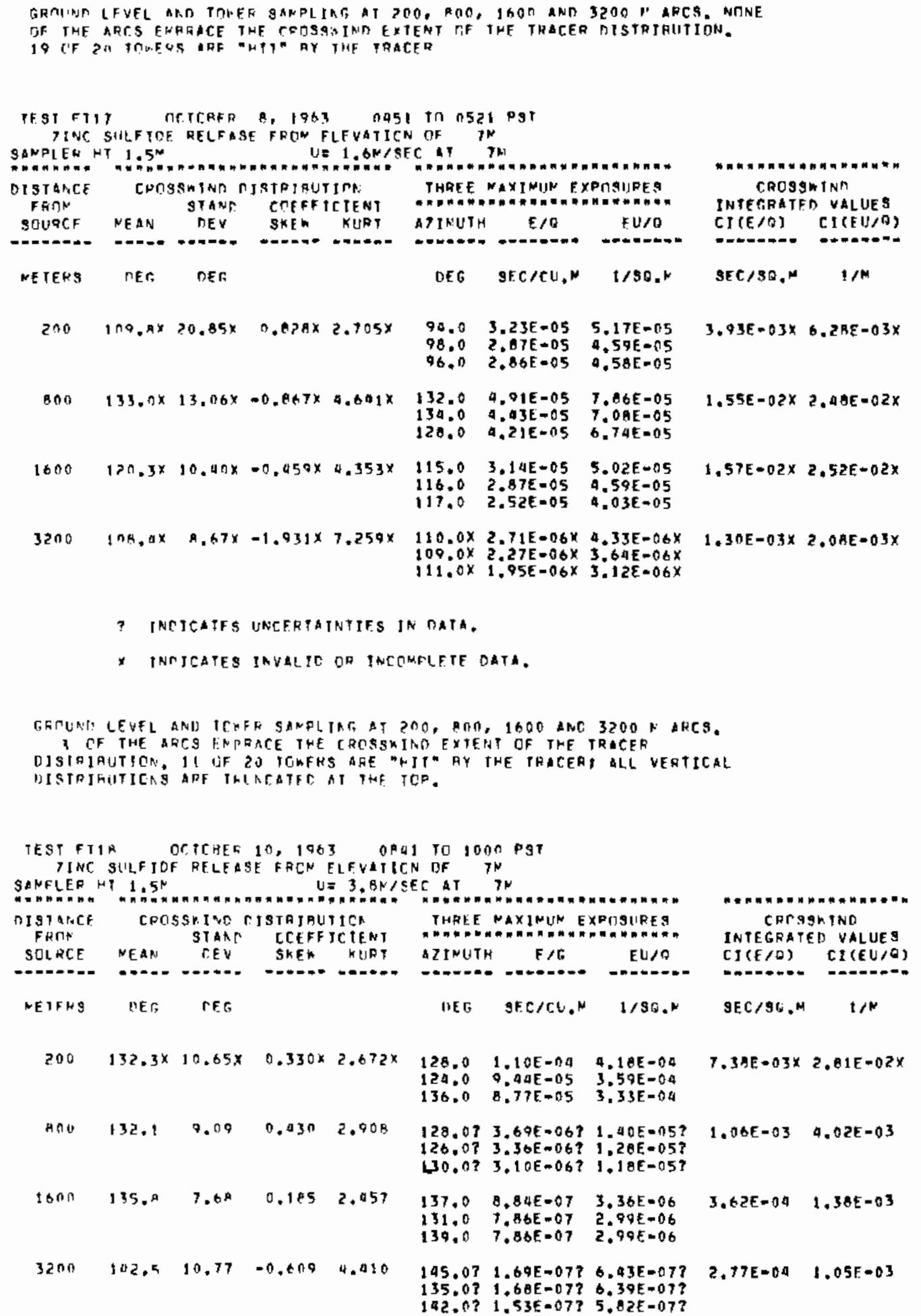


GFOUND IFVEL ANH IOLFR SAMPLIFG AT $200,000,1600$ HND 3200 M ARCSI

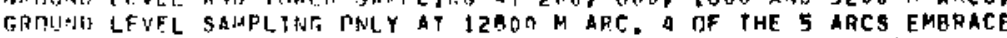
THE ChOSgMIODO EXTENT DF THE TRACER OISTRIAUTION. IR OF 20 TOWERS

AHE "HIT" AY THE TAACER

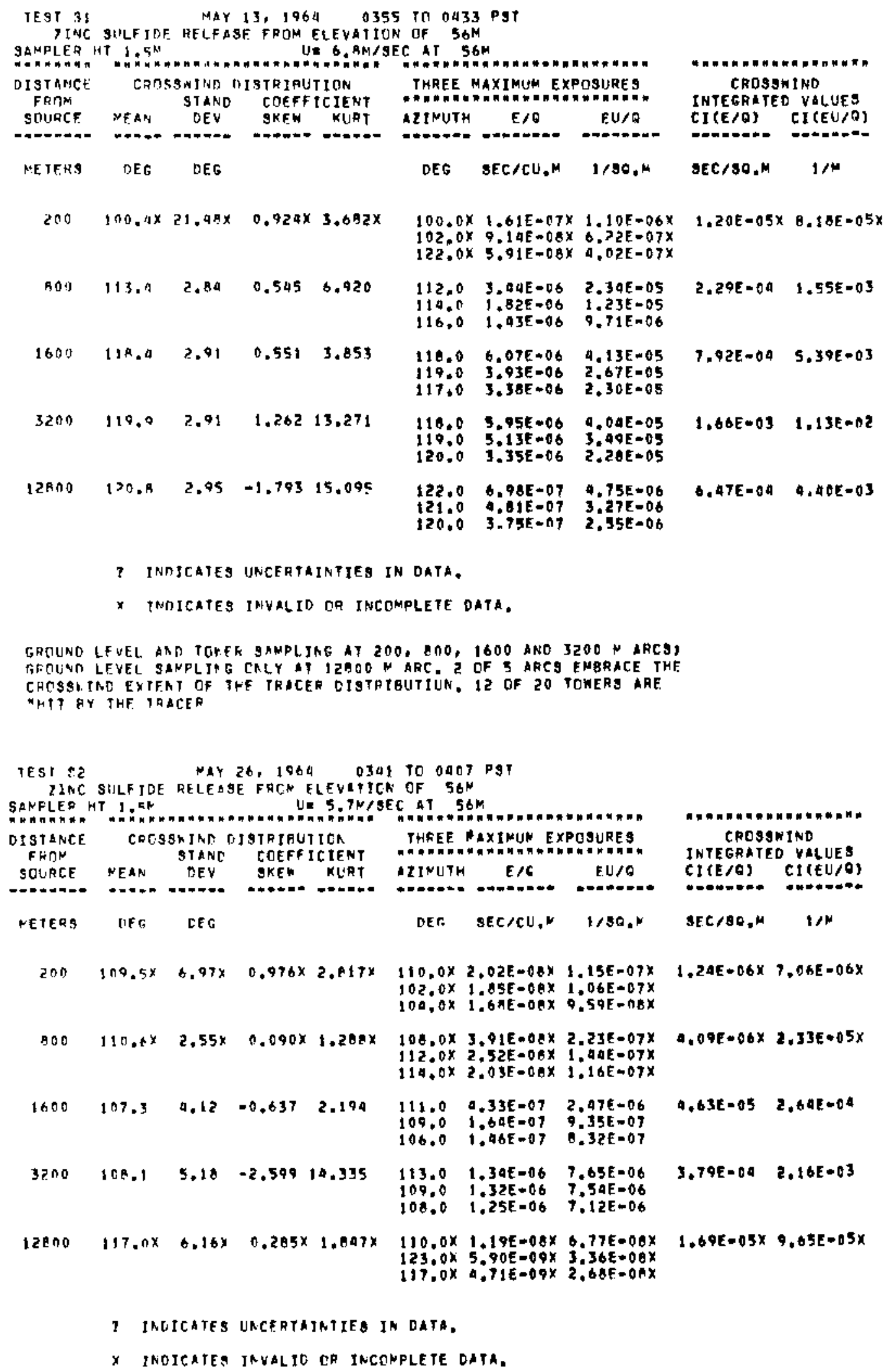

7 INUICATES URCFRTHINTIES IN DATA.

x INOICATES JAVAlio CR INCONPLETE DATA. 


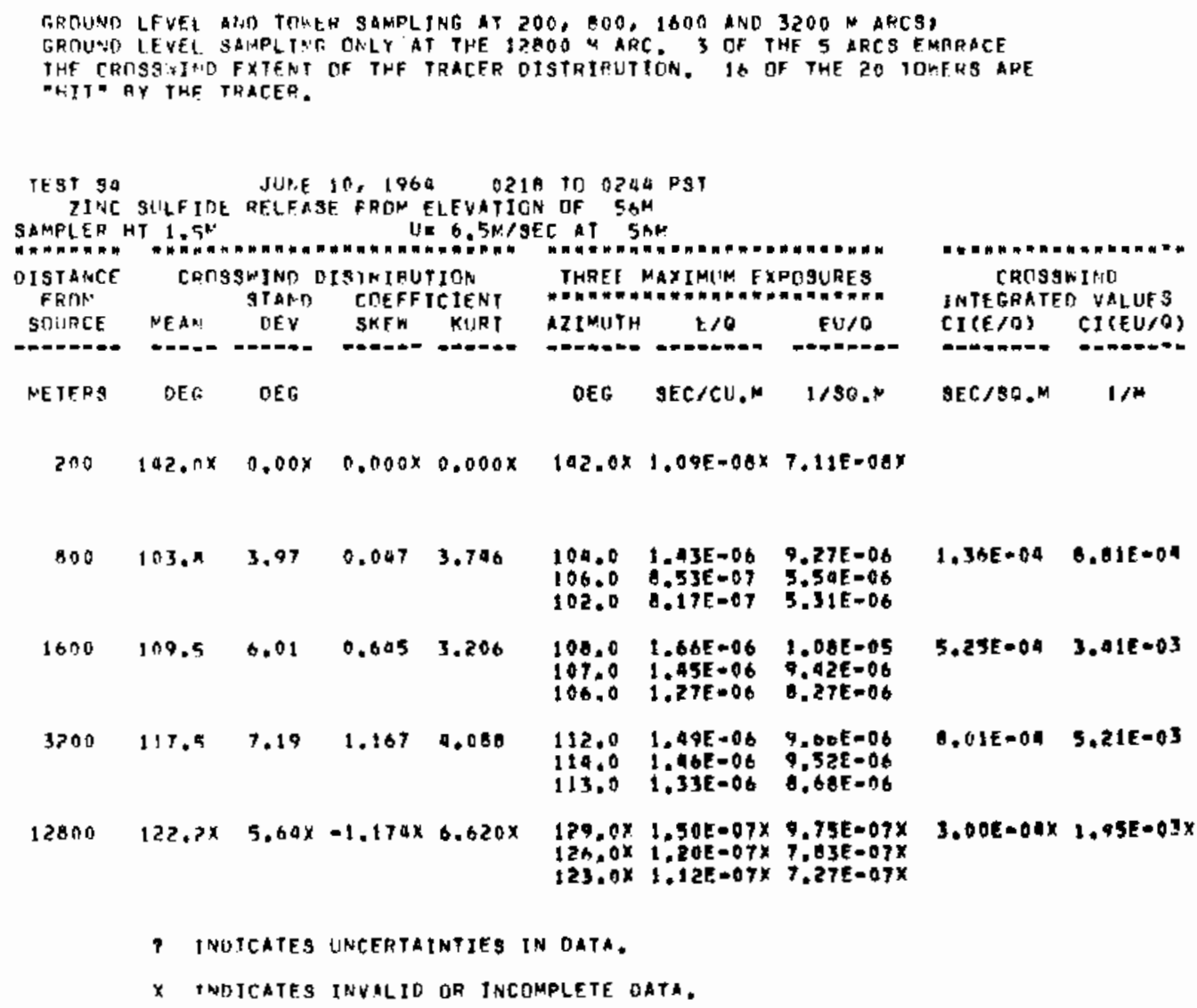


GRMIIND LEVEL AND TOKFR SANPLING AT 200, DOO, 1600 AND $3200 \mathrm{M}$ ARCS ALI. ARES EMARACE THE CRTSSWIND EXTENT OF THE TRACER DISTRIRUTION. 17 DE THF ? TOUERS AHE MTI" AY THE TRACER

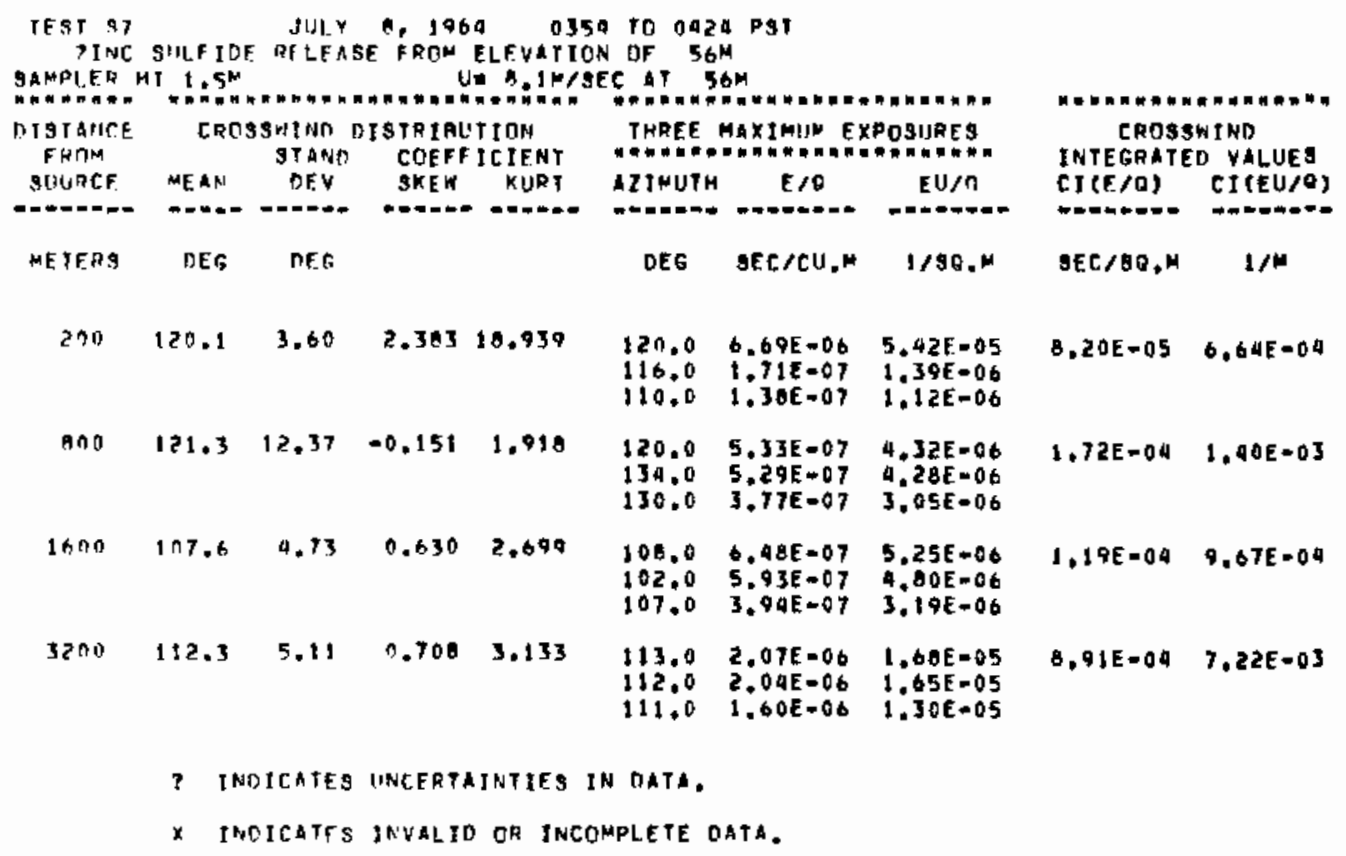

GAIUND LEVEL ANI) TOHER SAMPLINF AT 1600 APO 3200 M ARCSI GROUAO LEVEL SAMPLING UNLY AT TOND AND $12 B 00$ M ARCS, 2 OF THE A ARCS EVBRACE THE CHOSSHIHA EXTEIST OF THE THACER DISTRIBUTIUHY. 7 DF 10 TOMFRS ARE "MIT" BY THF TRACER.

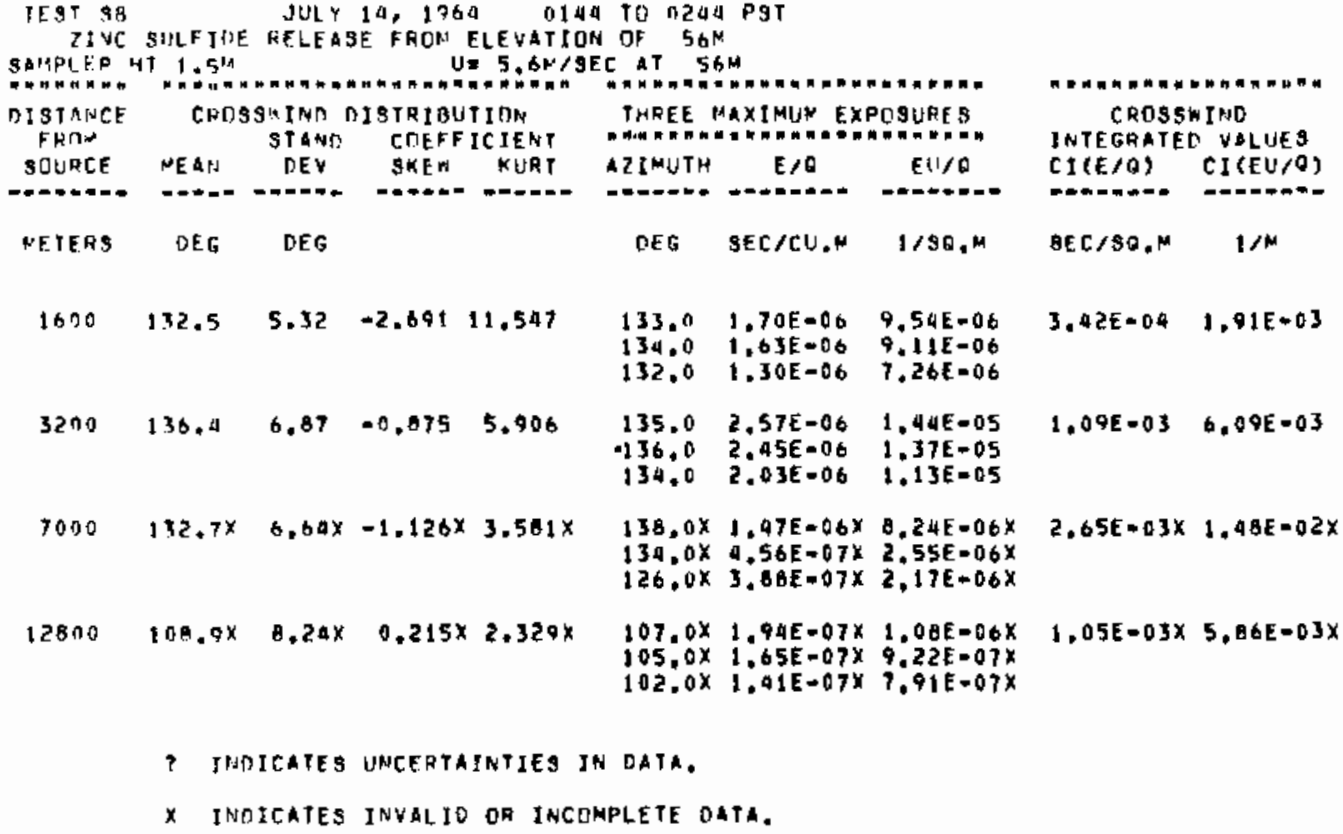




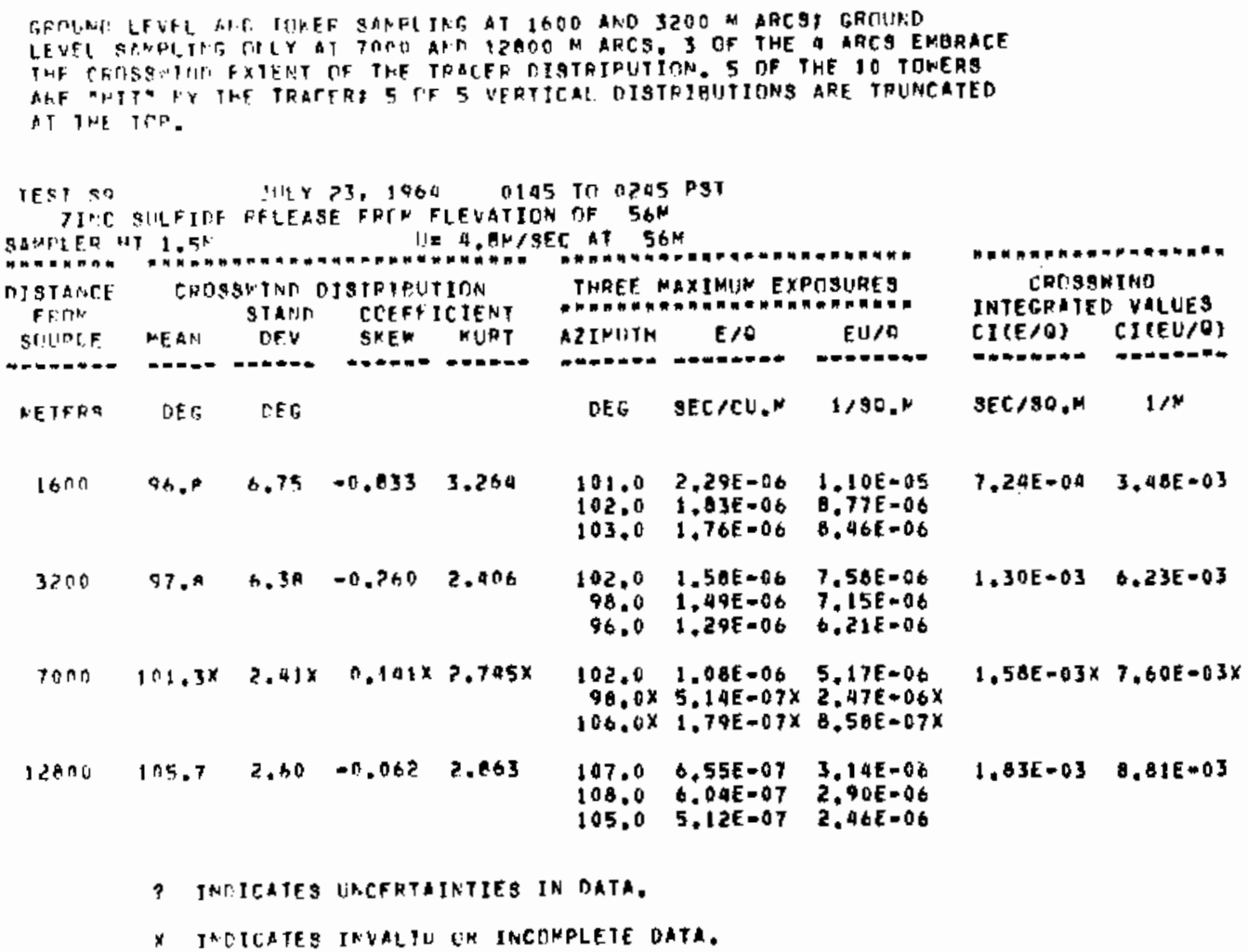

GROUNT TEVEL AND TCRER SAMPLING AT 1600 MND $3200 \mathrm{M}$ ARCS. GROUND LEVEL SANPLING ONIY AT 1000 AND 12000 ARCS. ALL ARCS ENBRACE THE CRCSSKINO EXTENT OF THE TRACER DISTRIBITJOA. B OF THE 10 TONERS ARE "HIT" OY THE TRACER

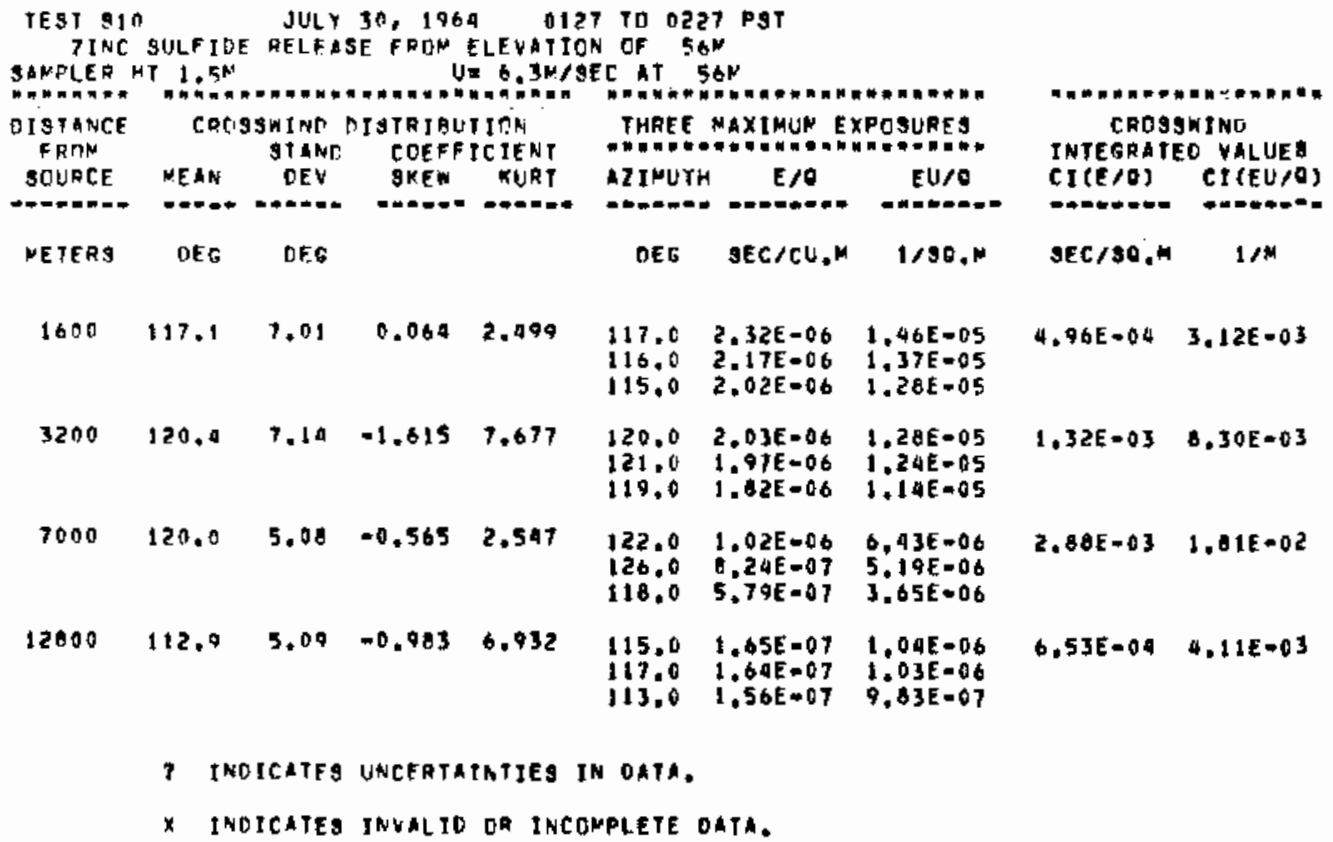




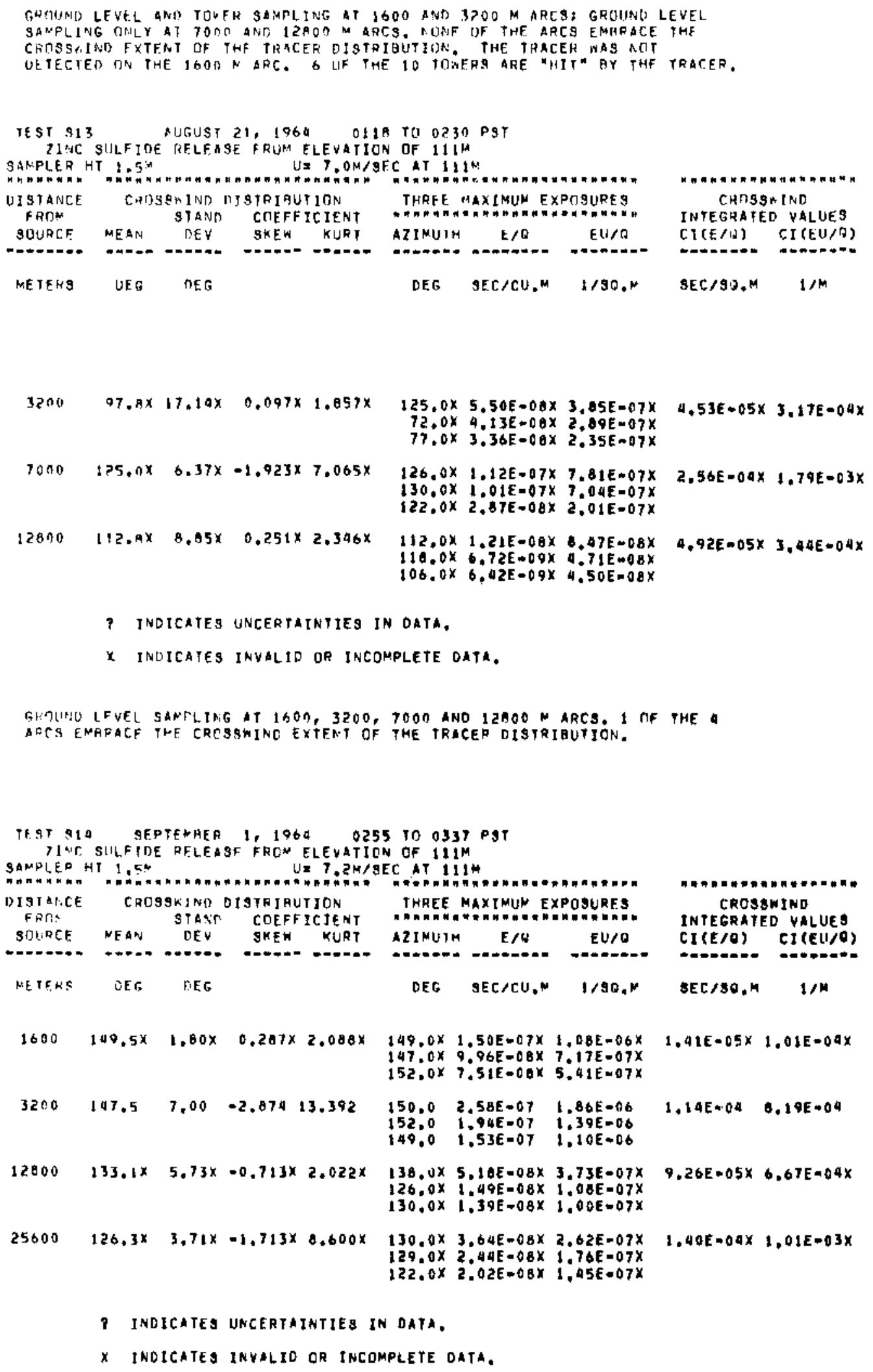

P INDICATES UNCERTAINTIES IN DATA.

$X$ INDICATES INYLLIO QR TNCOMPLETE DATA. 


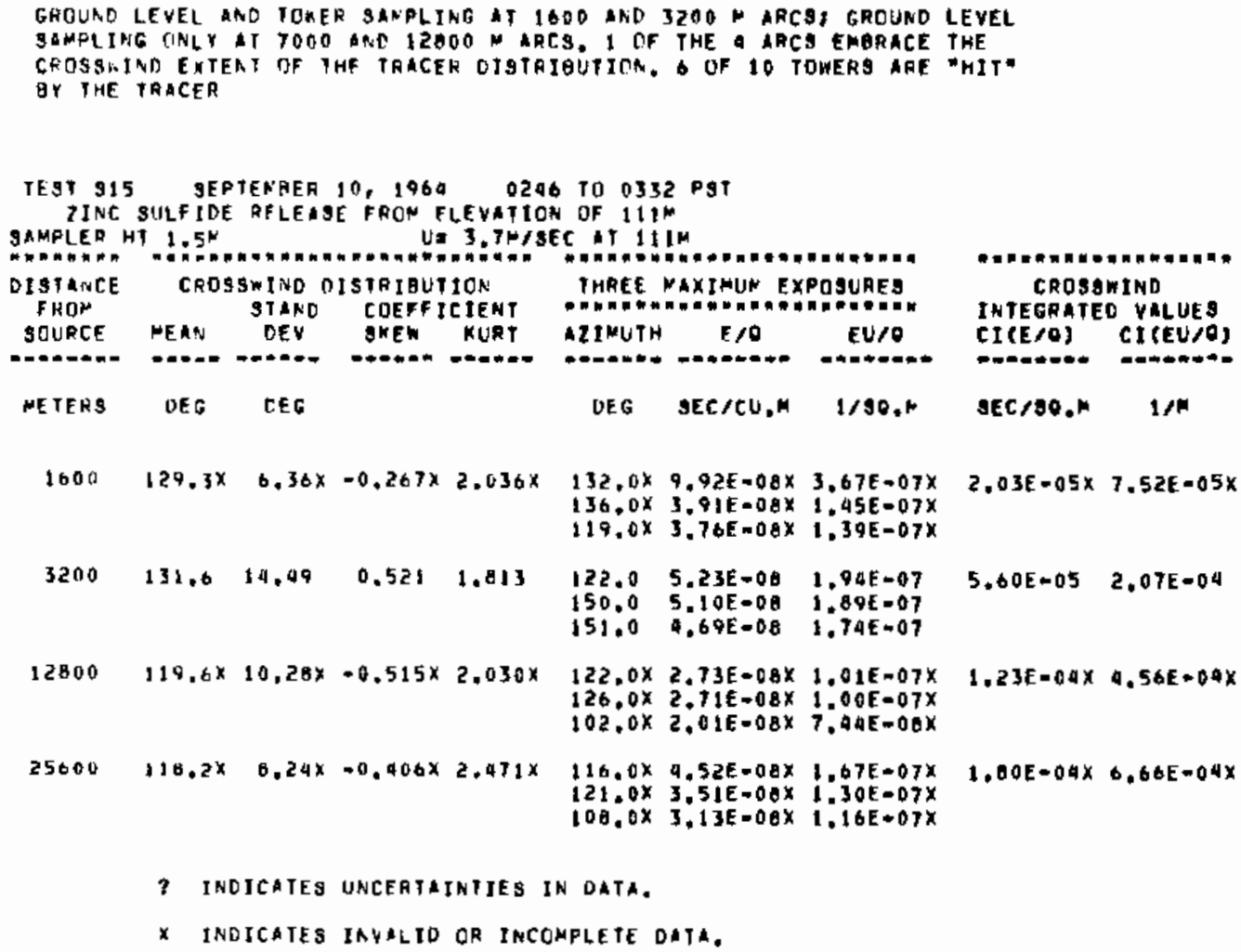




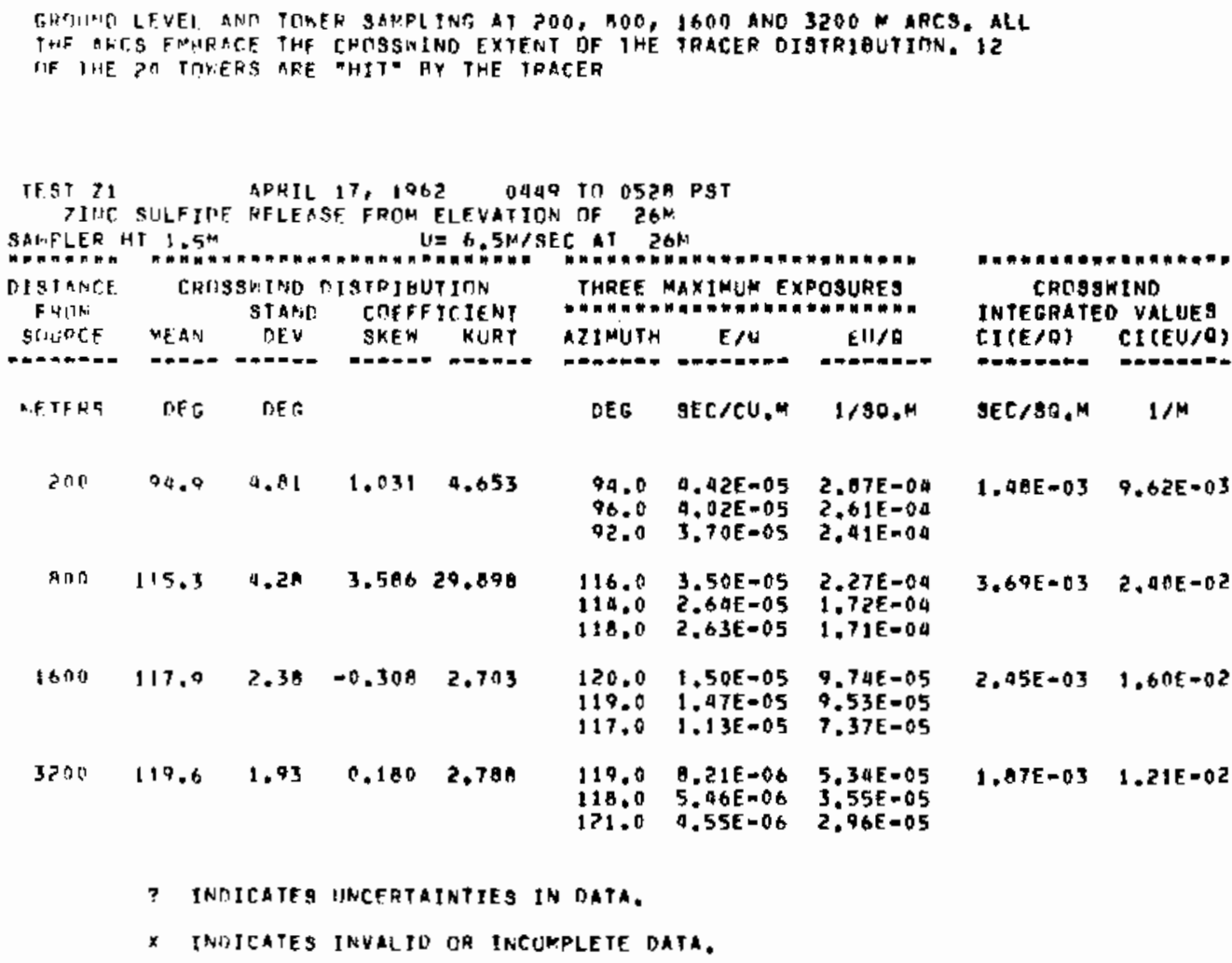

? INDICATEG IJNCERTAINTIES IN DATA.

x INDJCATES INVALIO OR INCUMPLETE DATA. 
ZIMC SULFIDE RELEASE, GROOHD LZVEL AFD TOUER SAMPLIKG AT 200,

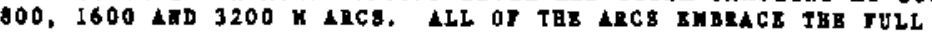

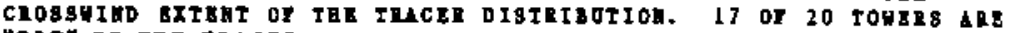

"RIT" BI THE TRACBR.

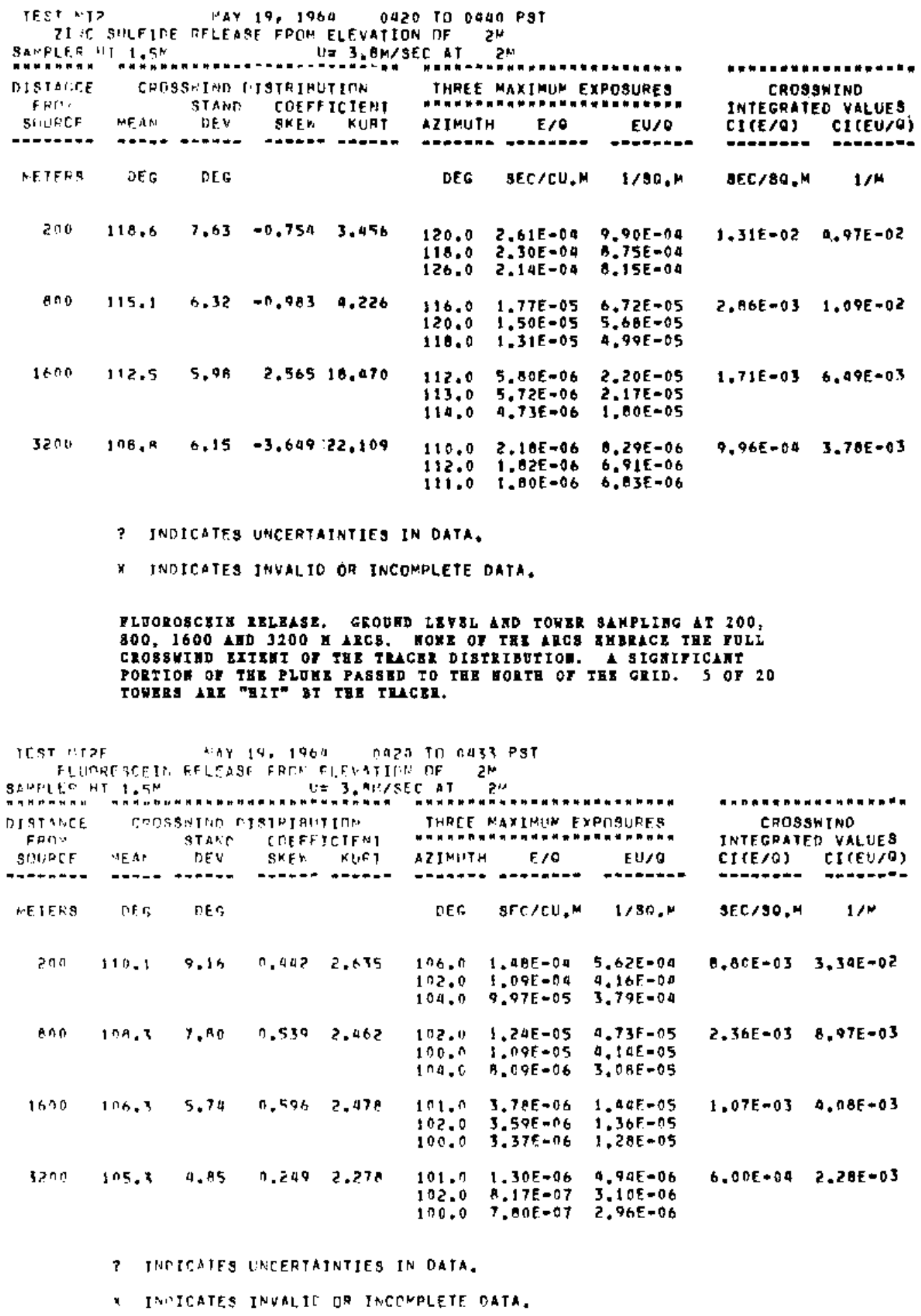




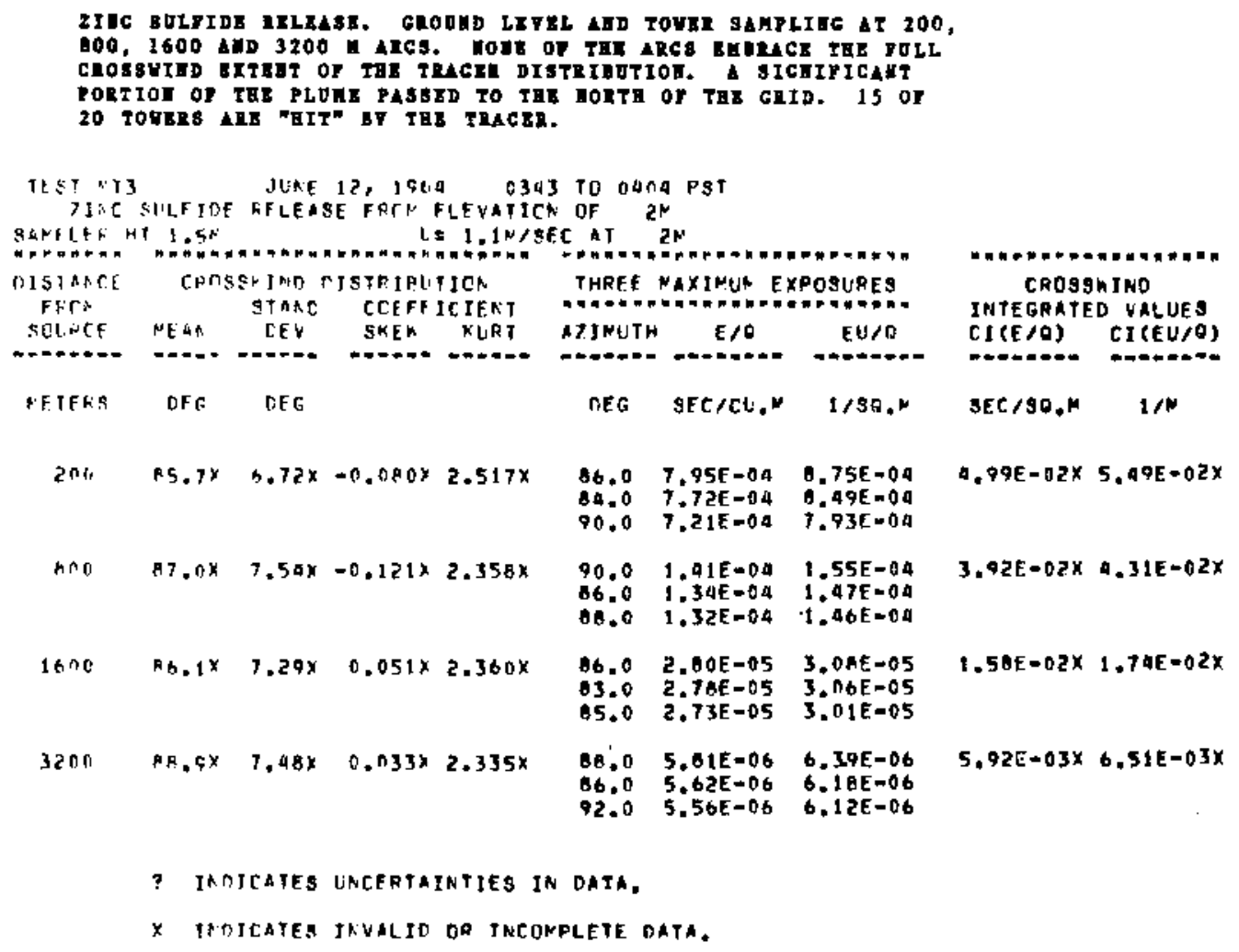




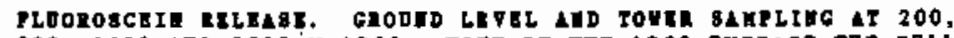

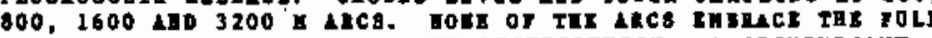
CRos

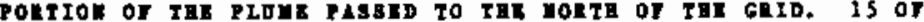
20 rOgER AIE "BIT" UT TEE THCEL.

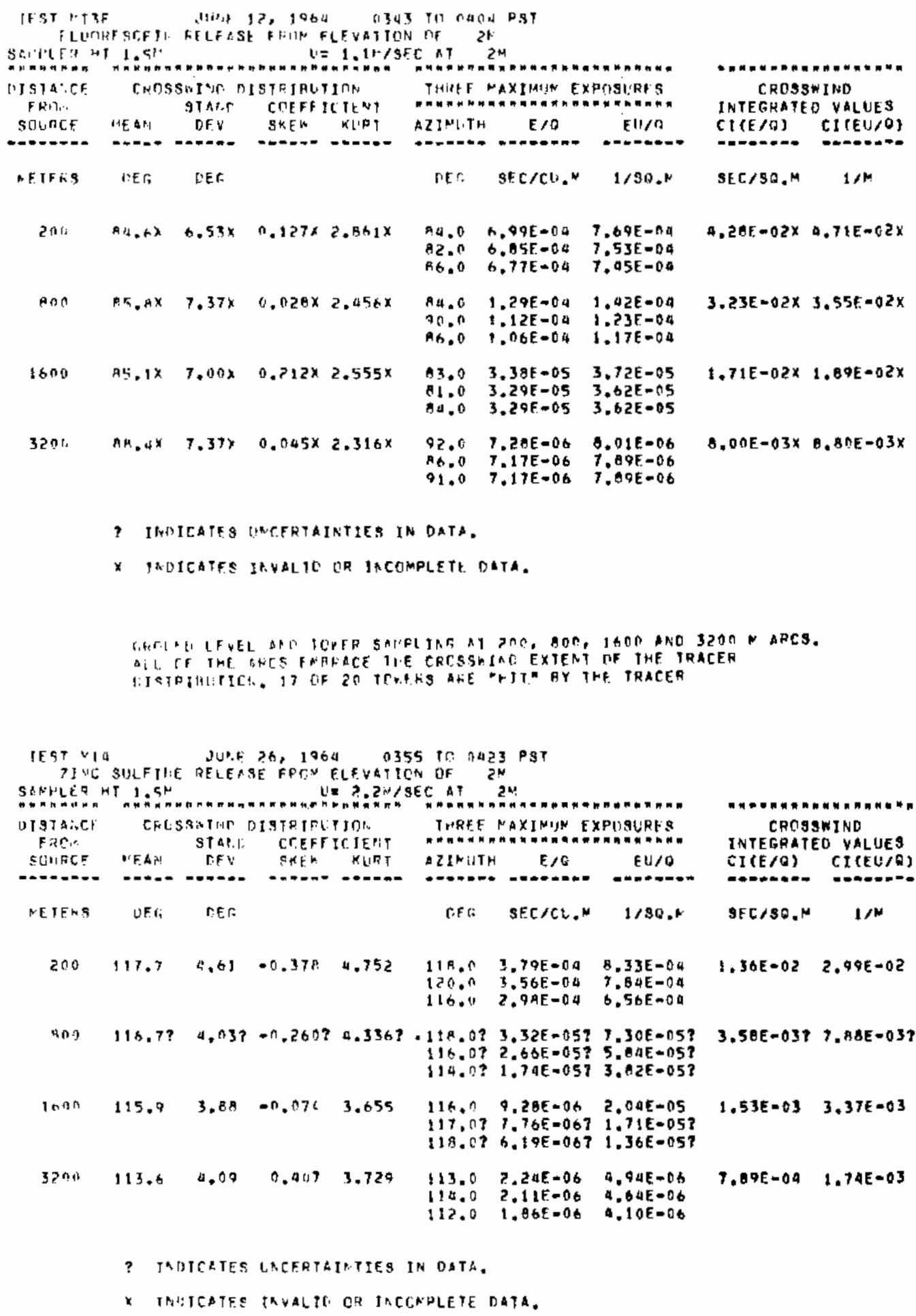

$1.36 E-02 \quad 2.49 E-02$

$3.58 E-0377 . A 8 E-037$

$1.53 E-03 \quad 3,37 E=03$

$7.09 E=09 \quad 1.74 E-03$

? TABICATES LNEFRTAIRTIES IN OSTA.

r thiticates thyalfo or tacchplete data. 
GEMUND LEVEL AMN TORER SANPLTHA AT 900,9600 ANO 3200 M ARCS. ALL GF THE ARCS FNBRACE THE CROSSAIND EXTFNT CIF THE TRACER DPSTRIAUTICH. ALL 15 TOWERS AIE. "HIT" BY THE TPACFA.

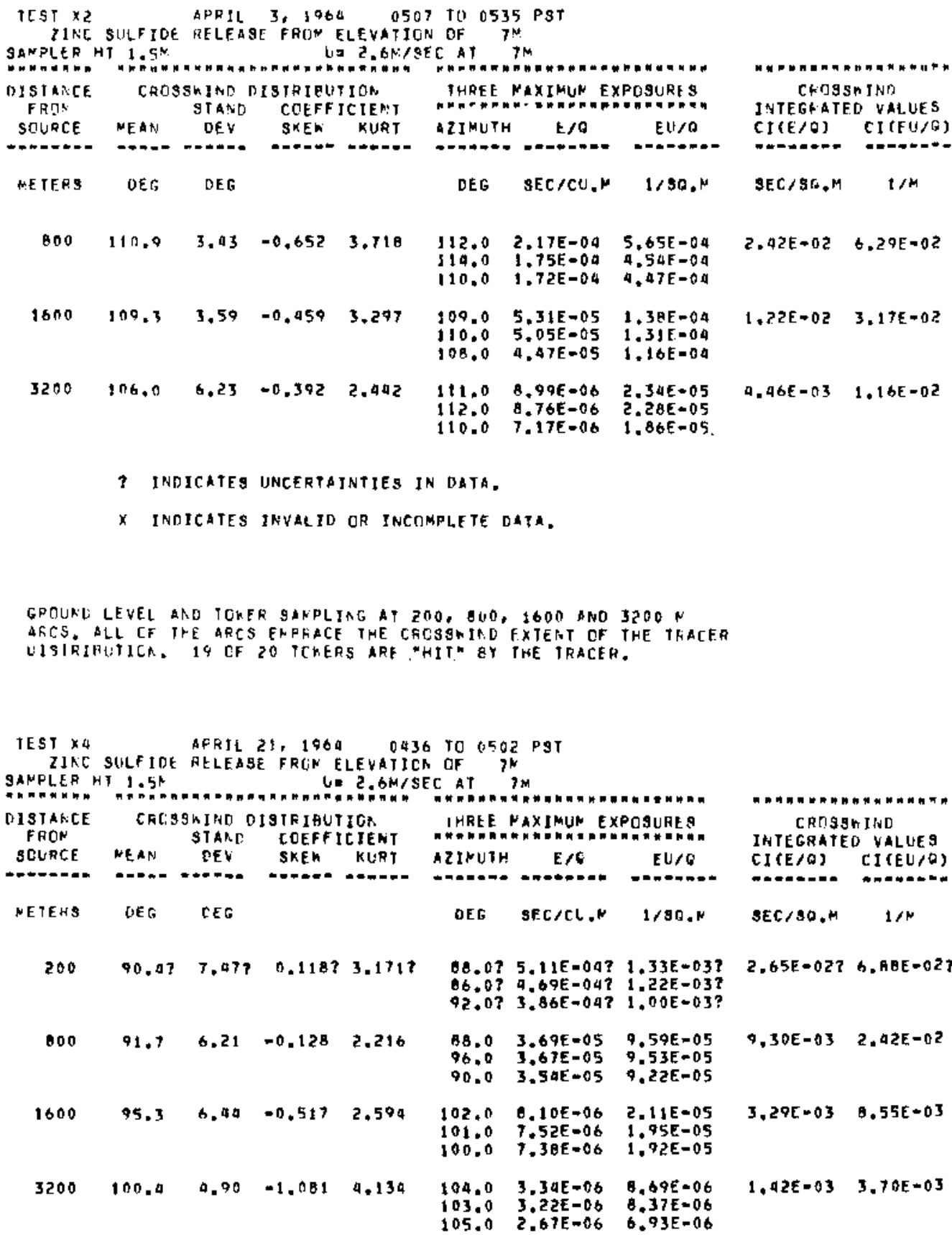


GKMUNO LEVEL ANO TOMER SHMPLING NT $200,800,1600$ ANO 3200 M ARC HC'CE OF THE ARCS ENAPACE TME CROSSHIMD EXIENT OF THE TRACER OIBTRIBUTION. 14 CF THE 20 TOYESE ARE "KITP BY THE TRACER

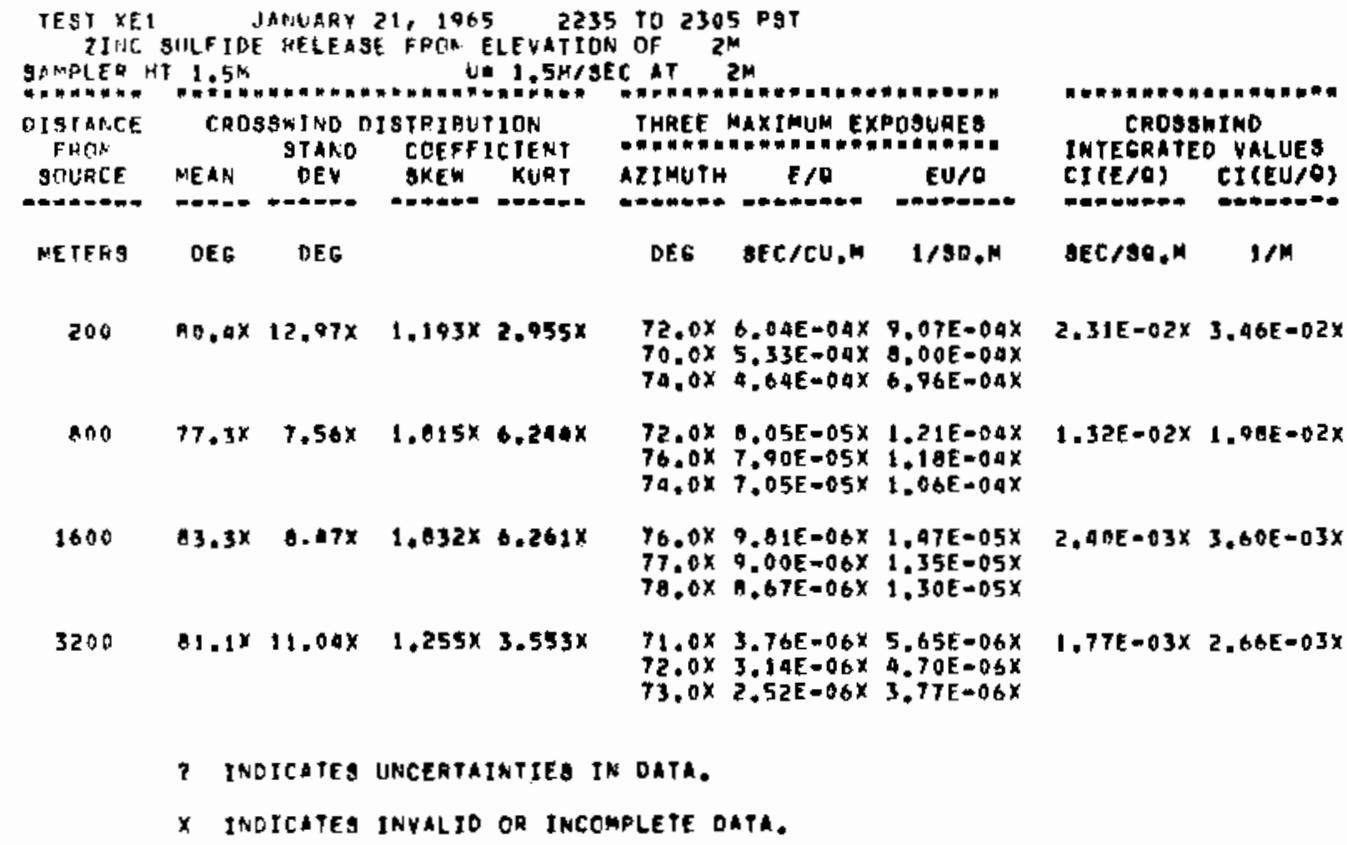

GROUNE LEVEL SAMPLIAG AT $200,000,1600$ AND 3200 ARTS. ALL DF THE AHCS EMREACE THE TROSSNINR EXTENT OF THE TRACER DISTRIBUTION.

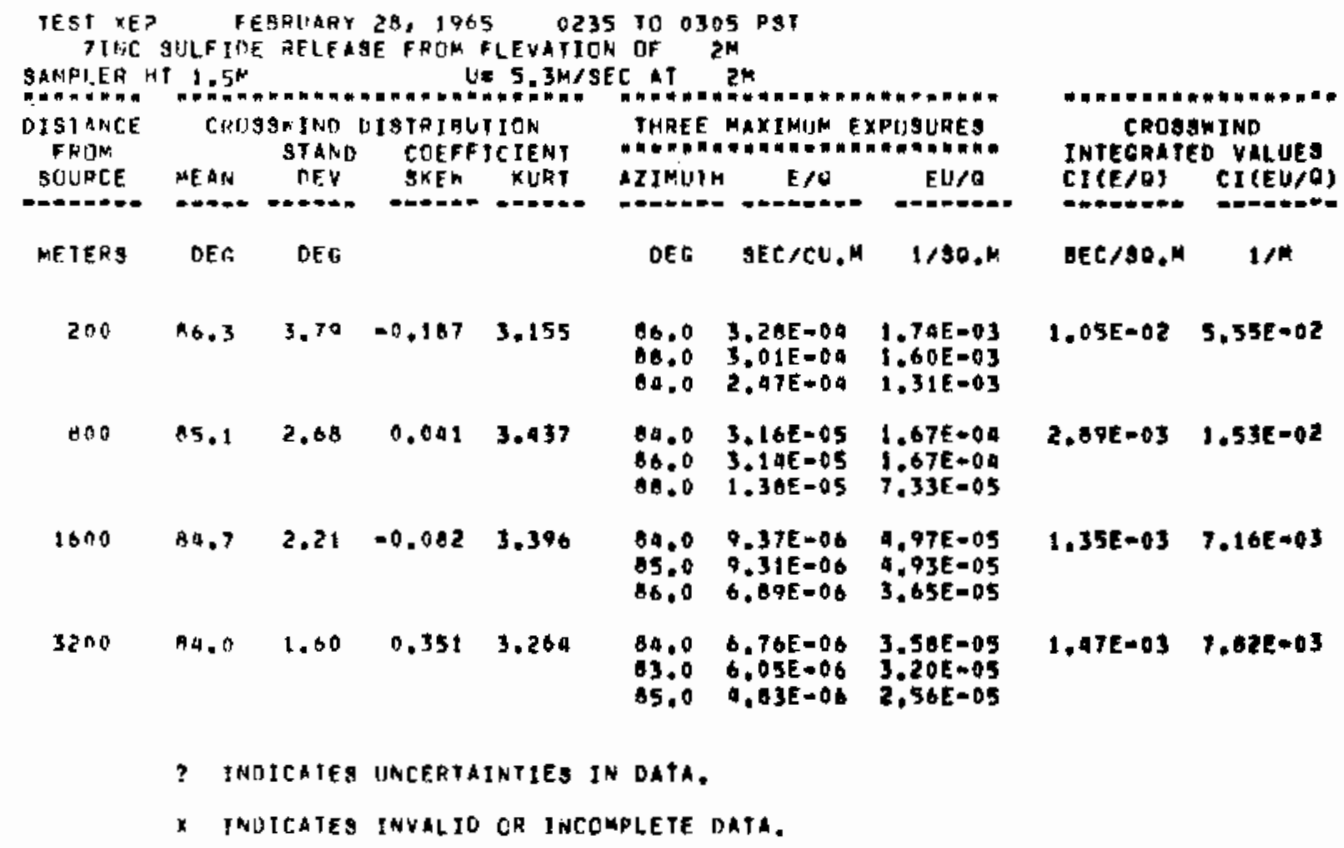




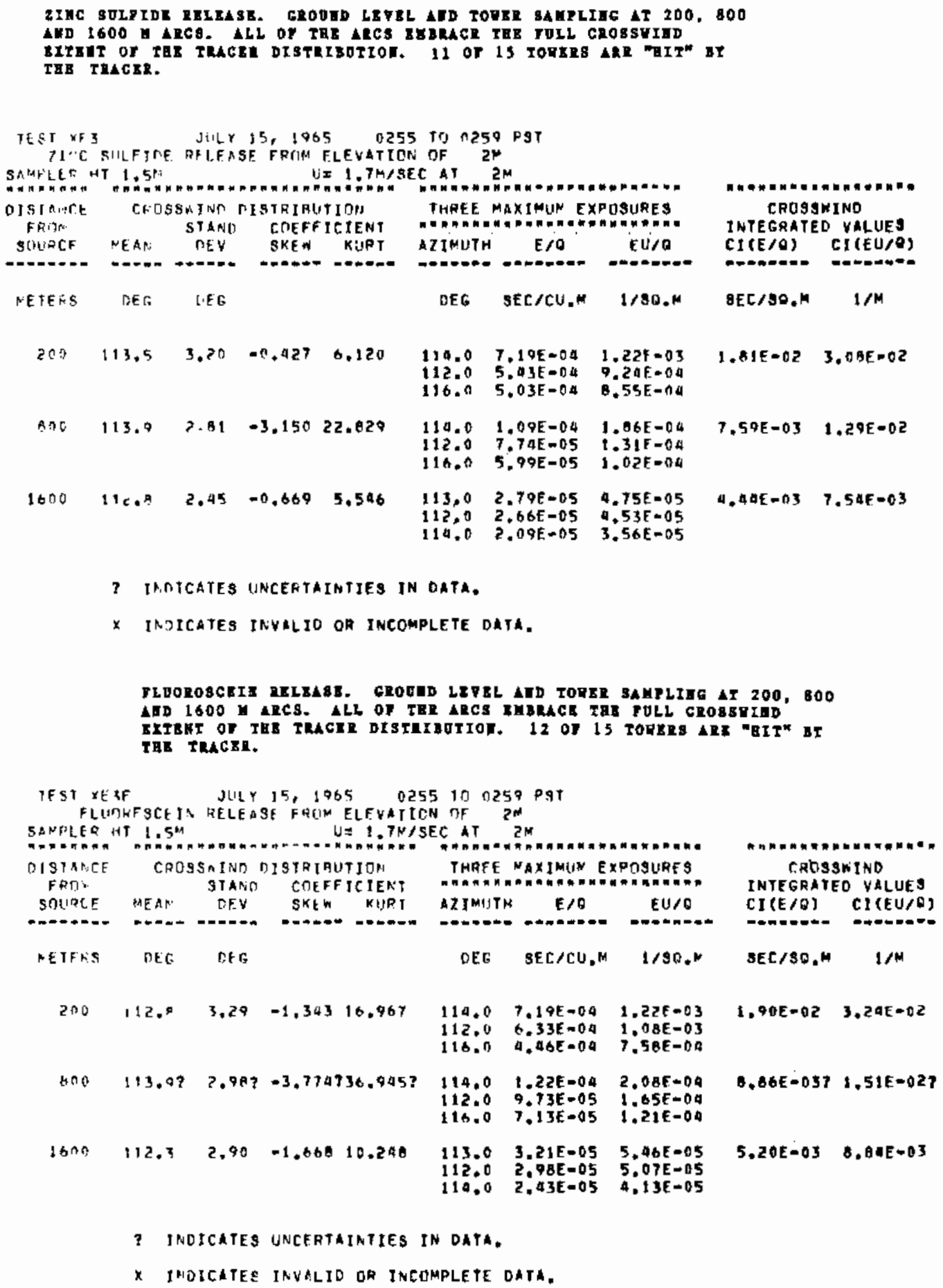




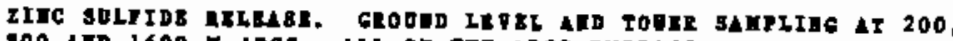

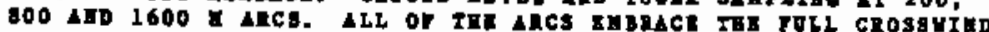

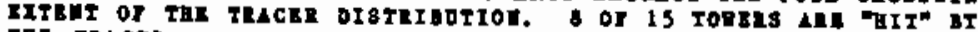

TER TACEL.

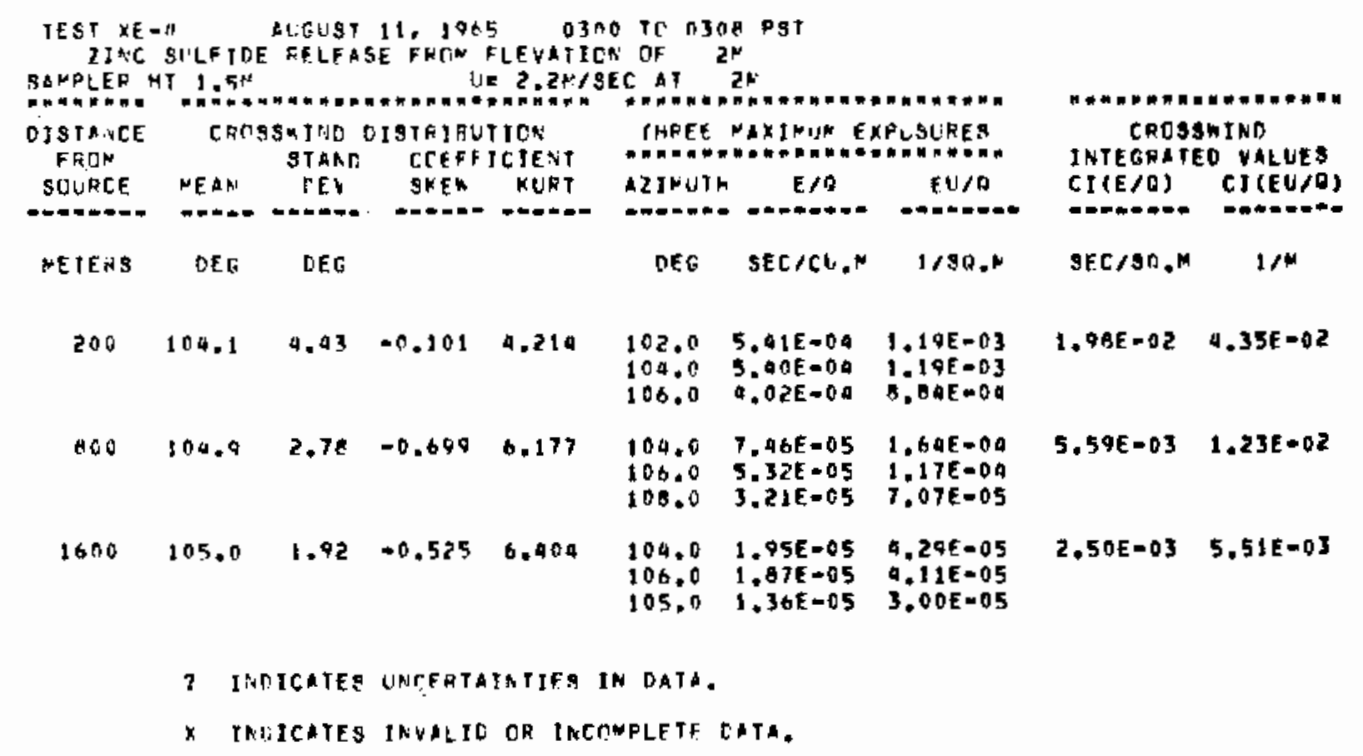

FLDOROSGEIF RELSASE. GRODUD LEFRL AID TORER SAMPLITE AT 200, 800

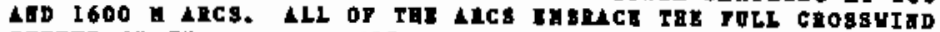

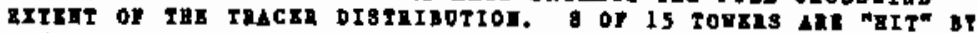
TEE TMACE.

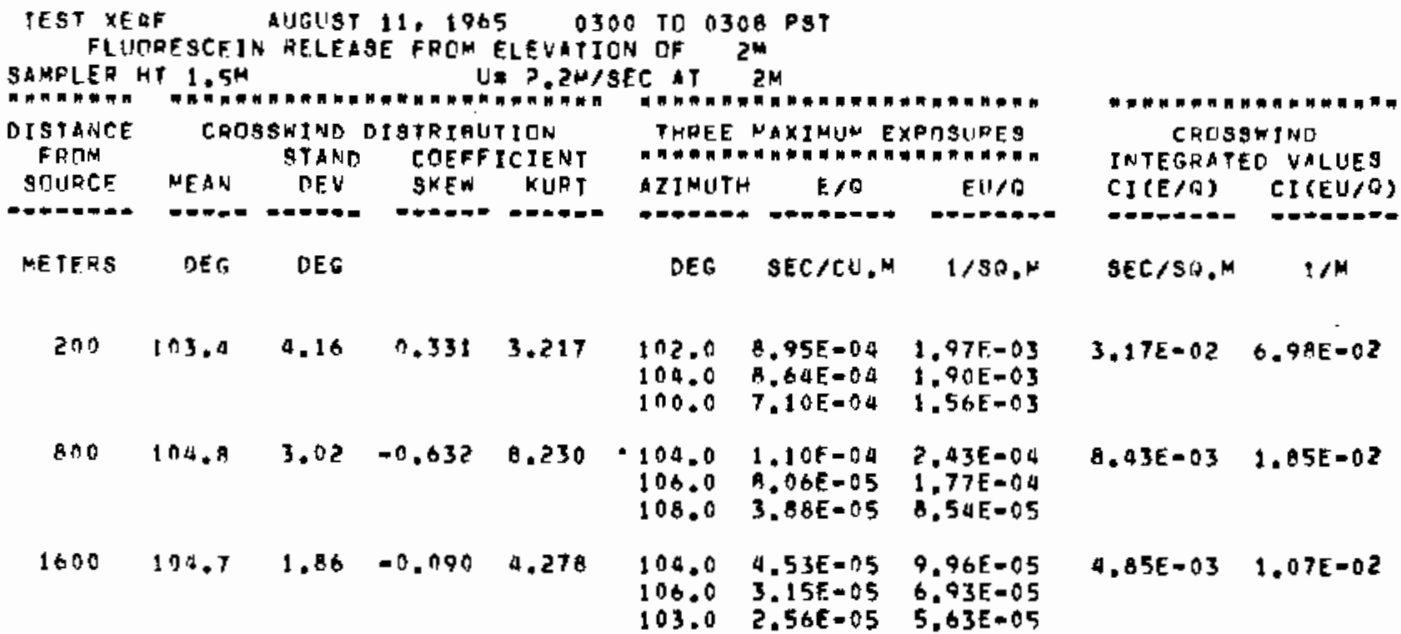

? INOICATES UNCEFTAINTIES JN DATA.

X INDICATES INVALID OR INCOMPLFTE OATA. 


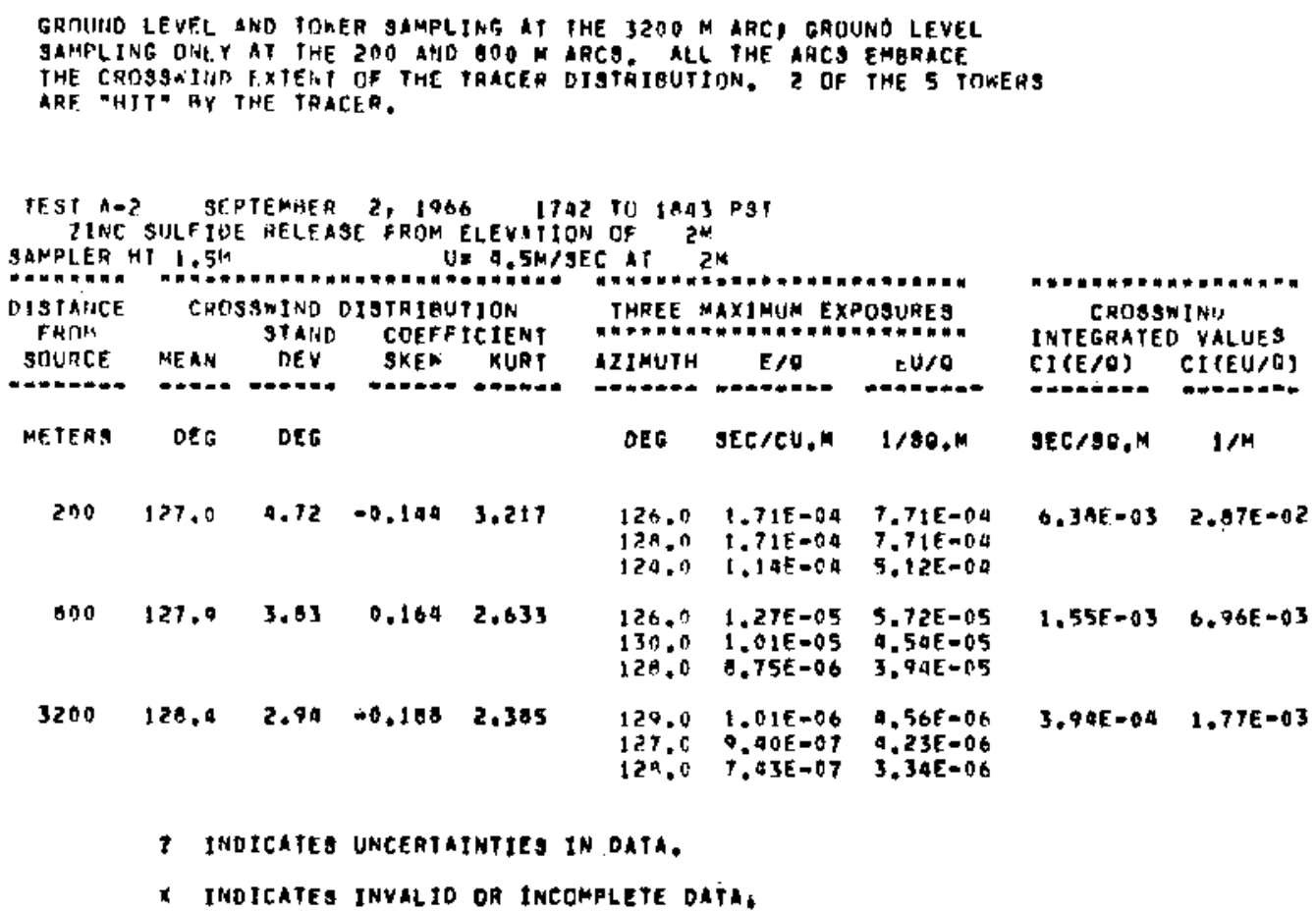

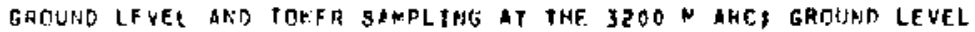
SANPLI ULG (NLY IT THE BOO ARC. ROTH ARCS EMURACE THE CROSSWIND EXTENT OF THE THACER DJGTEIBUTIUN, J TF THE 5 TOKEHS ARE "HIT" BY THE IRACFA.

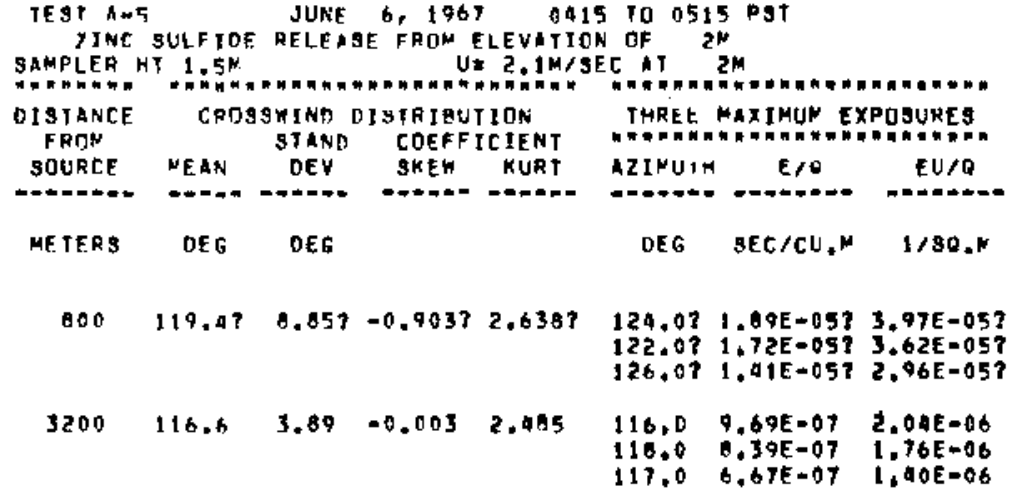


GKOUND LEVET ANO TMAER SANPLIAT AF THE 3200 NAHC; GNIIJND LEVEL SAMPLING ONLY AT THE HOD $\mathrm{K}$ ARC, MEITHF ARC FMRRACES THE CROSSWIND EXTEHT UF THE TRACER DISTRIBUTION, I TF THE 5 FORERS ARE. "HIT" GY THE THACER.

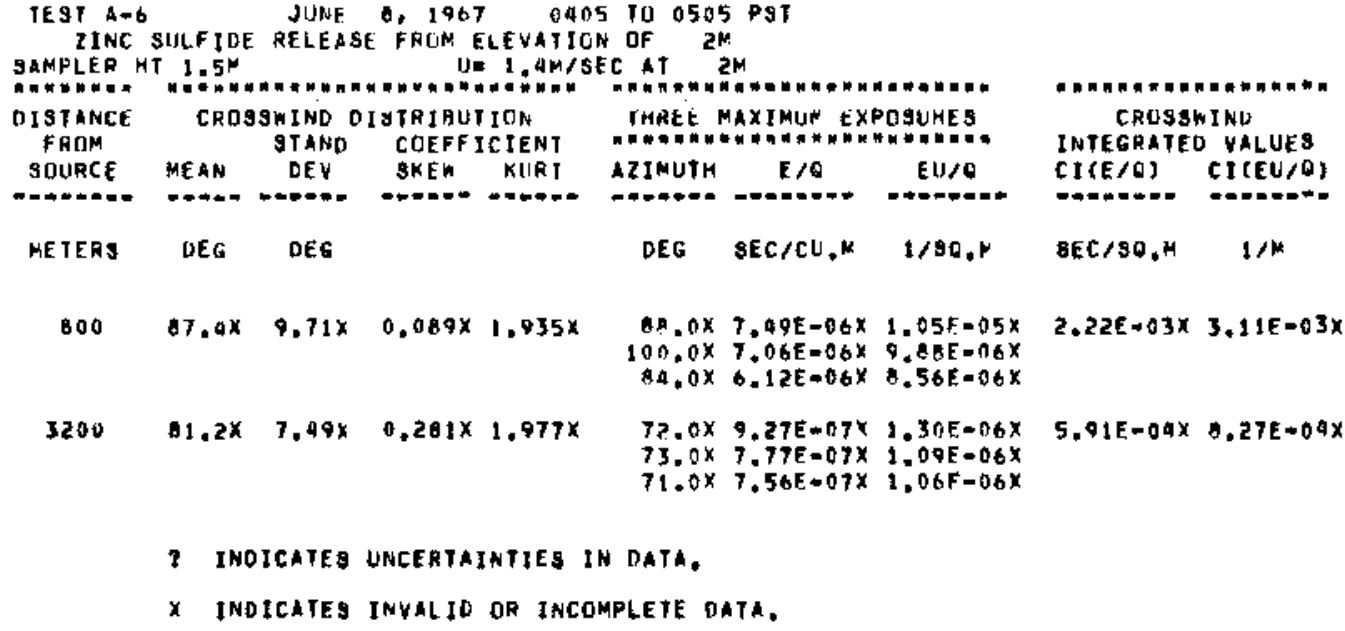

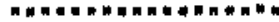
CROSBHIND INTEGRATED VALUES CI $(E / Q)$ CJ $(E U / O)$ SEC/30,H I/H

$0.03 E-03 x \quad 1.26 E-02 x$

$7.27 E-04 \times 1.096-01 x$

7 INOICATES UNCERTAINTIES IN DATA.

$X$ INDICATES INVALID DR INCOMPLETE OATA. 
GRIIP'D LEVEL AND TOWE, SAMPLING AT THE $3200 \mathrm{M}$ ARC, GROUND LEVEL

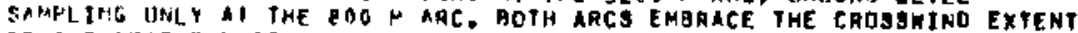

(IF IHE THACER ISISTRIMLITIN. ALL 5 TOWERS ARE OHIT GY THE TRACEA

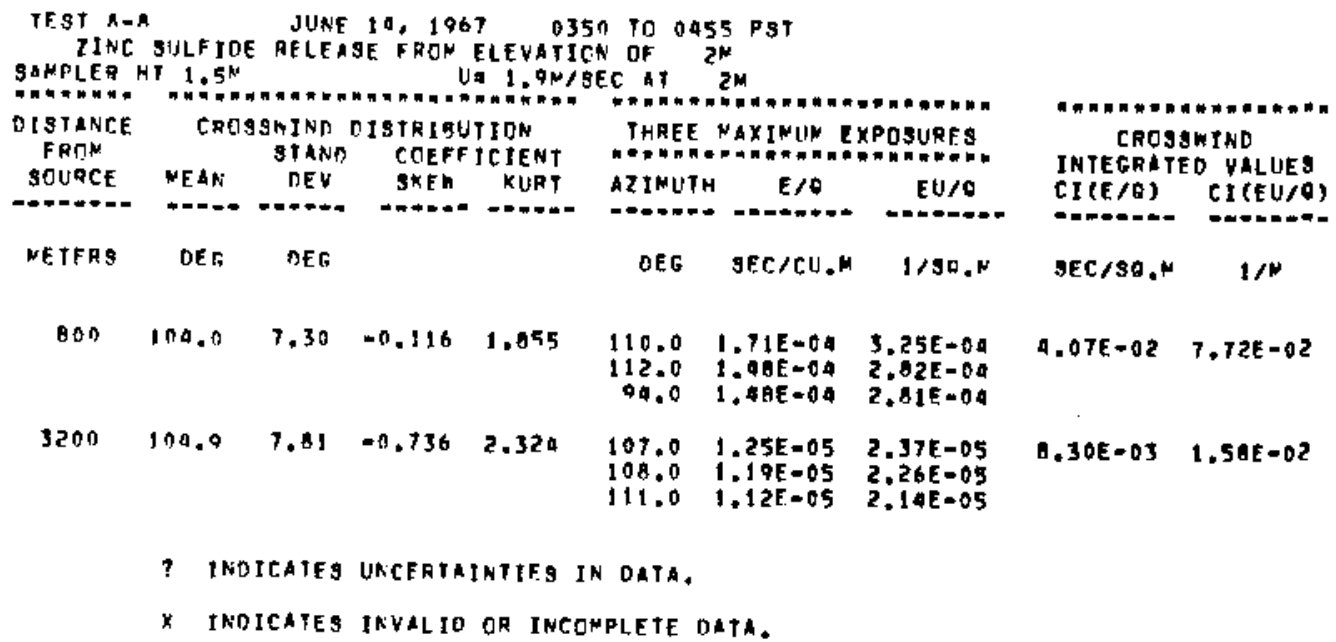


APPENDIX C

METEOROLOGICAL DATA

C-1 

APPENDIX C - METEOROLOEICAL DATA

30 SERIES

\begin{tabular}{|c|c|c|c|c|c|c|c|c|c|c|}
\hline & & $400 \mathrm{ET}$ & TOUER & & & & PORT & BEE MI & ST & \\
\hline & $\begin{array}{c}\text { HEICHT } \\
(\mathrm{ft})\end{array}$ & $\begin{array}{c}v \\
(n / s)\end{array}$ & $\left(\begin{array}{c}\theta \\
(d e g)\end{array}\right.$ & $\sigma_{\theta}(d \in q)$ & $(\circ \mathrm{F})$ & $\begin{array}{c}\text { HEICHT } \\
(f t)\end{array}$ & 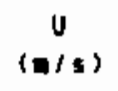 & $\stackrel{\theta}{(d \mathbf{e g})}$ & $\begin{array}{c}\sigma_{\theta} \\
(d e q)\end{array}$ & $\begin{array}{c}T \\
(0 \mathrm{E})\end{array}$ \\
\hline$F T-30$ & 7 & 2.5 & 320 & 11.0 & 39.4 & 2.5 & 2.0 & 339 & & 39.7 \\
\hline & 50 & 3.4 & 327 & 10.9 & 38.3 & 5 & 3.5 & $\mathbf{M}$ & $\mathbf{M}$ & 39.2 \\
\hline & 100 & 3.5 & 327 & 10.4 & 37.6 & 10 & 3.0 & 348 & & 38.8 \\
\hline & 150 & 3. 4 & 328 & 10.7 & 37.3 & 20 & 3.4 & $\mathbf{M}$ & $\mathbf{M}$ & 38.4 \\
\hline & 200 & 3.9 & 33 & 9.1 & 37.3 & 40 & $\mathbf{M}$ & 336 & & 38.2 \\
\hline & 250 & 1.0 & 326 & 8.5 & 36.9 & 80 & $M$ & 342 & & 39.0 \\
\hline & 300 & $\mathbf{M}$ & 329 & 8. 0 & 36.6 & & & & & \\
\hline & 100 & 3.6 & 328 & 7.3 & 36.4 & & & & & \\
\hline ET -31 & 7 & 3.4 & 307 & D. $B$ & 39.4 & 2.5 & 1.7 & 327 & H & H \\
\hline & 50 & 4. 2 & 307 & 1.2 & 3 B. 2 & 5 & 2.9 & M & $\mathbf{r}$ & $\mathbf{n}$ \\
\hline & 100 & 4.6 & 307 & 5.4 & 37.5 & 10 & $M$ & 318 & $\mathbf{H}$ & $\mathbf{M}$ \\
\hline & 130 & 4. 9 & 309 & 4.6 & 37.2 & 20 & 3.2 & M & M & $M$ \\
\hline & 200 & 5.0 & 313 & 5.0 & 37.2 & 40 & $n$ & 320 & M & H \\
\hline & 250 & 54 & 308 & 1.8 & 36.7 & 80 & $M$ & 322 & H & $\mathbf{M}$ \\
\hline & 300 & 4. 9 & 313 & 4.9 & 36.7 & & & & & \\
\hline & 100 & 5.1 & 316 & 5.5 & 36.9 & & & & & \\
\hline FT- 40 & 7 & 4. 1 & 296 & 10.3 & 69.3 & 2.5 & 3.2 & $\mathbf{M}$ & n & 68.7 \\
\hline & 50 & 5.7 & 301 & 9.7 & 67.2 & 5 & 3.1 & $\mathbf{x}$ & $M$ & 68.1 \\
\hline & 140 & 6.1 & 301 & B. 8 & 66.3 & 10 & 4.5 & 314 & 6.1 & $\mathbf{A}$ \\
\hline & 150 & 6.6 & 295 & 8.4 & 66.0 & 20 & 4.9 & M & $M$ & 67.1 \\
\hline & 200 & 6.6 & 298 & 7.6 & 66.2 & 40 & 5.5 & 310 & 3.5 & 66.8 \\
\hline & 250 & 6.8 & 299 & 7.1 & 65.6 & BO & 5.6 & 318 & 3.8 & 66.1 \\
\hline & 300 & 6.7 & 298 & 6.7 & 65.6 & & & & & \\
\hline & 100 & 6.9 & 295 & 6.1 & 65.3 & & & & & \\
\hline FT- 41 & 7 & 6.7 & $30 B$ & 4. 6 & 68.5 & 2.5 & 4.3 & $y$ & $\mathbf{M}$ & 66.6 \\
\hline & 50 & 9.9 & 316 & 9. 0 & 67.7 & 5 & $\mathbf{M}$ & $\mathbf{M}$ & $M$ & 66.4 \\
\hline & 100 & 10.5 & 312 & 3.5 & 67.0 & 10 & 5.7 & 326 & 3.4 & 66.3 \\
\hline & 150 & 11.6 & 305 & 3.3 & 66.6 & 20 & 7.1 & $\mathbf{M}$ & $M$ & 66.2 \\
\hline & 240 & 11.8 & 309 & 3.2 & 67.0 & 40 & $n$ & 323 & 4. 7 & 63.9 \\
\hline & 250 & 11.8 & 304 & 3. 2 & 66.0 & 80 & 9.4 & 336 & 5.5 & 65.7 \\
\hline & 300 & 12.6 & 310 & 2.0 & 65.9 & & & & & \\
\hline & 400 & 13.8 & 306 & 2.8 & 65.7 & & & & & \\
\hline $5 T-42$ & 7 & 2.2 & 297 & 9.6 & 53.1 & 2.5 & H & $\mathbf{n}$ & $x$ & 52.6 \\
\hline & 50 & 5.0 & 284 & 11.2 & 56.1 & 5 & 3.5 & M & $\mathbf{H}$ & 53.0 \\
\hline & 100 & 5.9 & 304 & 8.7 & 57.2 & 10 & 4. $B$ & $x$ & $\mathbf{M}$ & 33.6 \\
\hline & 150 & 6.5 & 307 & 0.2 & 57.8 & 20 & $M$ & n & $\mathbf{M}$ & 54.3 \\
\hline & 200 & 6.5 & 317 & 5.9 & 58.2 & 40 & 1. 7 & 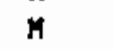 & M & $55 . \mathrm{s}$ \\
\hline & 250 & 6.2 & $3: ?$ & i. 3 & 58.2 & 80 & $\mathbf{M}$ & $\mathbf{M}$ & $\mathbf{M}$ & 56.5 \\
\hline & 300 & $M$ & 327 & 4.5 & 58.3 & & & & & \\
\hline & 400 & 6.8 & 325 & 5.1 & 58.1 & & & & & \\
\hline
\end{tabular}




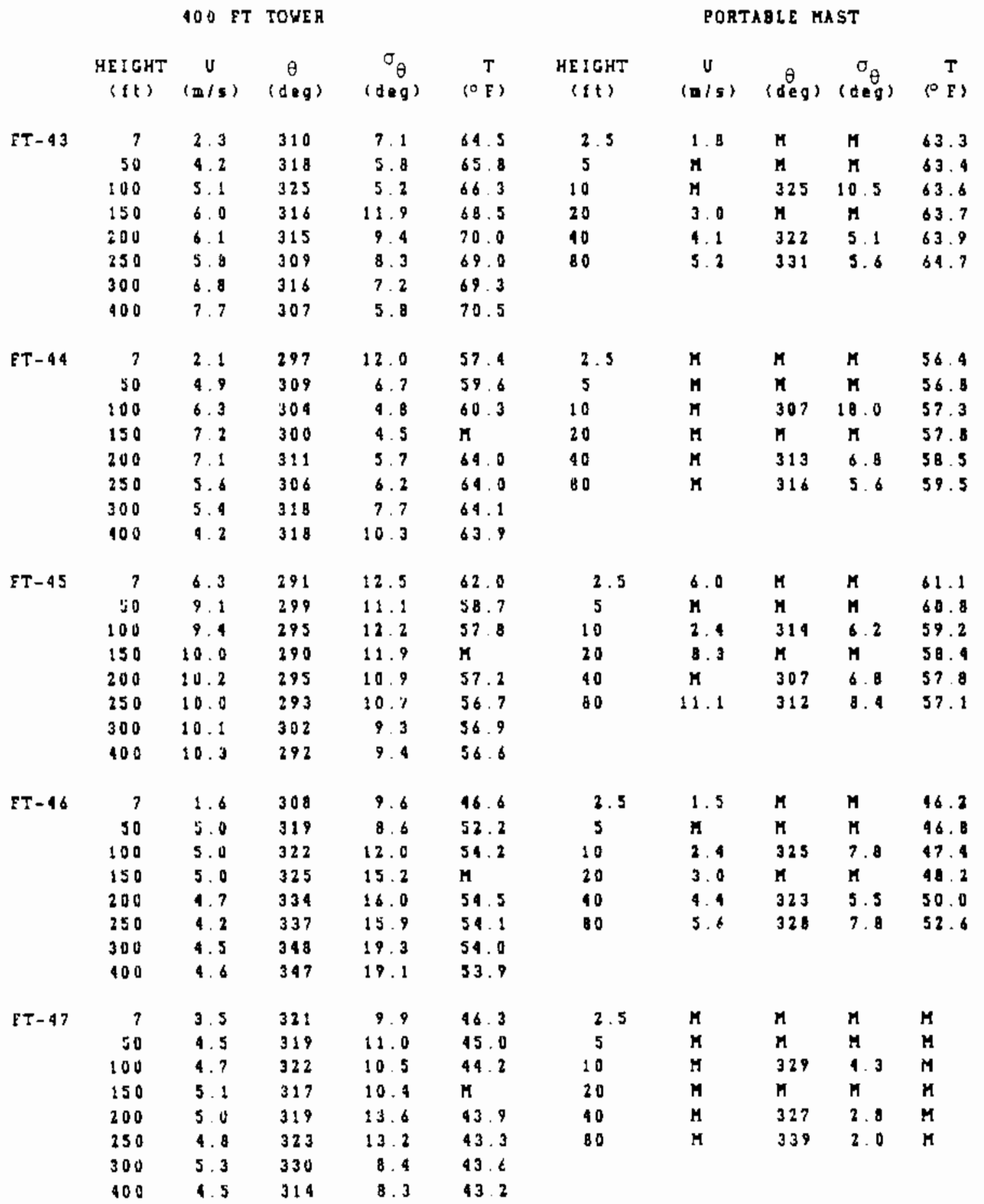


100 TT TOUER

PORTABLE MAST

\begin{tabular}{|c|c|c|c|c|c|c|c|c|c|c|}
\hline & $\begin{array}{l}\text { HEICHT } \\
(\mathrm{ft})\end{array}$ & $\begin{array}{c}U \\
(\mathrm{n} / \mathrm{s})\end{array}$ & $\left(\begin{array}{c}\theta \\
(\operatorname{deq}\rangle)\end{array}\right.$ & $\underset{\theta}{\sigma} \theta$ & $\left(\begin{array}{c}T \\
(0)\end{array}\right.$ & $\begin{array}{l}\text { HEIGHT } \\
(f t)\end{array}$ & $\begin{array}{c}U \\
(m / 5)\end{array}$ & $\left(\begin{array}{c}\theta \\
(d e g)\end{array}\right.$ & $\begin{array}{c}\sigma_{\theta} \\
(\operatorname{deg})\end{array}$ & $\begin{array}{c}T \\
\text { to } F \text { ) }\end{array}$ \\
\hline \multirow[t]{8}{*}{$8 T-48$} & 7 & 2.9 & 308 & 16.4 & 47.9 & 2.5 & M & $M$ & M & 17.8 \\
\hline & 50 & 3.7 & 317 & 15.6 & 46.6 & 5 & $\mathbf{K}$ & H & $\mathbf{H}$ & 47.7 \\
\hline & 100 & 3.8 & 314 & 12.5 & 45.7 & 10 & M & 335 & 17.2 & 46.0 \\
\hline & 150 & 4. 2 & $3 \pm 1$ & 12.3 & $\mathbf{H}$ & 20 & 4. 1 & I & $M$ & 45.4 \\
\hline & 200 & 4.1 & 316 & 13.5 & 45.3 & 90 & 4.5 & 331 & 17.0 & 45.2 \\
\hline & 250 & 4. 1 & 312 & 13.6 & 94.6 & 80 & $n$ & 336 & 19.1 & 42.5 \\
\hline & 300 & 4. 1 & 325 & 12.8 & 49.8 & & & & & \\
\hline & 400 & 4. $t$ & 300 & 11.6 & 44.8 & & & & & \\
\hline \multirow[t]{8}{*}{$E T-49$} & $?$ & 5,1 & 322 & 19.3 & 69.9 & 2.5 & n & 343 & g. 5 & 64.4 \\
\hline & 50 & 6.9 & 325 & 20.5 & 61.9 & 5 & H & $\mathbf{M}$ & $\mathbf{H}$ & 63.8 \\
\hline & 100 & 7.6 & 326 & 19.5 & 60.1 & 10 & H & 339 & 8.1 & $\$ 2.0$ \\
\hline & 150 & 8. 0 & 323 & 18.1 & $\mathbf{H}$ & 20 & $\mathbf{M}$ & H & $\mathbf{M}$ & 61.1 \\
\hline & 200 & 7.9 & 326 & 17.8 & 59.1 & 40 & $\mathbf{M}$ & 337 & 12.5 & 60.9 \\
\hline & 250 & 8.0 & 321 & 18.1 & $\mathbf{M}$ & 80 & $y$ & 327 & 12.1 & 59.6 \\
\hline & 300 & 8.1 & 336 & 16.5 & 53.5 & & & & & \\
\hline & 100 & g. 1 & 321 & 17.2 & 53.6 & & & & & \\
\hline \multirow[t]{8}{*}{ FT-SU } & 7 & 4.1 & 298 & 8.0 & 51.6 & 2.5 & $M$ & 317 & 7.0 & 50.7 \\
\hline & 50 & 6.5 & 304 & 6.9 & 52.5 & 5 & $\mathbf{M}$ & $M$ & M & 50.9 \\
\hline & 100 & 7.3 & $30:$ & 7.0 & 52.3 & 10 & $\mathbf{M}$ & 311 & 6.0 & 51.3 \\
\hline & 150 & a. 3 & 296 & 6.3 & M & 20 & $\mathbf{M}$ & H & $M$ & 51.5 \\
\hline & 200 & 8.8 & 302 & 5.6 & $52 . B$ & 90 & 6.5 & 326 & 6.1 & 51.6 \\
\hline & 250 & K & 296 & 4. 8 & 52.8 & 90 & $\mathbf{M}$ & 313 & 6.5 & 51.9 \\
\hline & 300 & 9.0 & 307 & 4.4 & 52.9 & & & & & \\
\hline & 400 & 6. 8 & 293 & 3.9 & 53.2 & & & & & \\
\hline \multirow[t]{8}{*}{$\varepsilon T-51$} & 7 & 4. 8 & 299 & 11.3 & 65.4 & 2.5 & $\mathbf{M}$ & 314 & g. 9 & 65.4 \\
\hline & 50 & 6.2 & 303 & 10.0 & 62.5 & 5 & M & $M$ & M & 63.8 \\
\hline & 100 & 6.5 & 300 & 9.5 & $6: .6$ & 10 & $M$ & 316 & 6.9 & 63.9 \\
\hline & 150 & 6.8 & 296 & g. 8 & $\mathbf{M}$ & 20 & $\mathbf{H}$ & $x$ & $M$ & 62.2 \\
\hline & 200 & 6.8 & 301 & 8. 0 & 60.7 & 40 & $M$ & 316 & 6.6 & 61.1 \\
\hline & 250 & 7.0 & 298 & 7.3 & 60.1 & 80 & $M$ & 310 & 1. 9 & 60.8 \\
\hline & 300 & 6.9 & 321 & 6.4 & 60.3 & & & & & \\
\hline & 100 & 6.8 & 298 & 6.9 & 60.1 & & & & & \\
\hline \multirow[t]{8}{*}{ FT -52} & 7 & 3. 8 & 286 & 9.9 & 53.8 & 2.5 & 2.7 & 319 & 6.1 & 53.6 \\
\hline & 50 & 'j. 3 & 294 & g. 9 & 53.0 & 5 & $\mathbf{M}$ & $\mathbf{M}$ & $\mathbf{M}$ & 53.1 \\
\hline & 100 & 5.7 & 291 & 10.6 & 52.5 & 10 & $M$ & 314 & 11.6 & 53.2 \\
\hline & 150 & 6. 3 & 285 & 10.8 & 52.2 & 20 & 4.6 & $n$ & $M$ & 53.1 \\
\hline & 200 & 6.6 & 292 & 10.4 & $\$ 2.7$ & 40 & $M$ & 322 & 19.7 & 53.0 \\
\hline & 250 & 7.2 & 289 & 10.5 & 52.2 & 80 & $H$ & 309 & 7.9 & 52.8 \\
\hline & 300 & 7.9 & 304 & 9.7 & 52.1 & & & & & \\
\hline & 400 & 8.3 & 292 & 9.4 & 52.0 & & & & & \\
\hline
\end{tabular}


\$0O FT TOWER

PORTABLE MAST

\begin{tabular}{|c|c|c|c|c|c|c|c|c|c|c|}
\hline & $\begin{array}{c}\text { HEICHT } \\
(\mathrm{ft})\end{array}$ & $\begin{array}{c}U \\
(m / 5)\end{array}$ & $\begin{array}{c}\theta \\
(\operatorname{tg} g)\end{array}$ & $\begin{array}{c}\sigma_{\theta} \\
(d e g)\end{array}$ & $\begin{array}{c}\mathrm{T} \\
\left({ }^{\circ} \mathrm{F}\right)\end{array}$ & $\begin{array}{c}\text { HEICHT } \\
(s t)\end{array}$ & $\begin{array}{c}U \\
(x / 5)\end{array}$ & $\begin{array}{c}\theta \\
(d \in q)\end{array}$ & $\begin{array}{c}\sigma_{\theta} \\
(\text { ded })\end{array}$ & $\begin{array}{c}T \\
\langle\sigma F\rangle\end{array}$ \\
\hline \multirow[t]{8}{*}{$5 T-53$} & 7 & 3.2 & 294 & 8.2 & 58.2 & & & & & \\
\hline & 50 & 4. 5 & 295 & 5.3 & 58.0 & & & & & \\
\hline & 100 & 5.4 & 296 & 5.4 & 57.2 & & & & & \\
\hline & 150 & 6.2 & 292 & 5.9 & 57.2 & & & & & \\
\hline & 200 & 6.9 & 303 & 3.6 & 57.6 & & & & & \\
\hline & 250 & 7.4 & 295 & 4.0 & 57.1 & & & & & \\
\hline & 300 & 7.7 & 300 & 3.9 & 56.8 & & & & & \\
\hline & 100 & 8.9 & 296 & 2.9 & 56.9 & & & & & \\
\hline \multirow[t]{8}{*}{$F ' \Gamma-54$} & 7 & 1.9 & 295 & 10.0 & 73.5 & & & & & \\
\hline & 50 & 4.6 & 304 & 5.6 & 77.6 & & & & & \\
\hline & 100 & 6.5 & 307 & 3. 9 & 79.2 & & & & & \\
\hline & 150 & 7.9 & 307 & 2.8 & 80.3 & & & & & \\
\hline & 200 & 7.6 & 312 & 3. 1 & 82. 6 & & & & & \\
\hline & 250 & 7.1 & 310 & 3.0 & 92.6 & & & & & \\
\hline & 300 & 6.6 & 348 & 3.3 & B2. 3 & & & & & \\
\hline & 400 & 6.2 & 320 & 2.0 & 83.0 & & & & & \\
\hline \multirow[t]{8}{*}{$E T-55$} & 7 & 2.5 & 287 & 3.4 & 66.7 & & & & & \\
\hline & 50 & 4. 5 & 292 & 3.7 & 68.1 & & & & & \\
\hline & 100 & 5.7 & 291 & 3.4 & 68.1 & & & & & \\
\hline & 150 & 6.9 & 289 & 2.9 & 68.2 & & & & & \\
\hline & 200 & 7.6 & 298 & 2.8 & 68.6 & & & & & \\
\hline & 250 & 8. 0 & 293 & 2.7 & 68.6 & & & & & \\
\hline & 300 & 8.9 & 330 & 0.7 & 69.4 & & & & & \\
\hline & 100 & 9.6 & 300 & $3: 6$ & 70.5 & & & & & \\
\hline \multirow[t]{8}{*}{ FT-56 } & 7 & 0.8 & 308 & 10.6 & 75.4 & 2.5 & 0.8 & 328 & 10.1 & 74.7 \\
\hline & $\$ 0$ & 3.3 & 310 & 7.0 & 78.4 & 5 & $\mathbf{M}$ & $\mathbf{M}$ & $\mathbf{H}$ & 74.9 \\
\hline & 100 & 4.5 & 305 & 5. $\theta$ & 78.8 & 10 & $\mathbf{M}$ & 323 & 9.0 & 76.2 \\
\hline & 150 & 5.6 & 300 & 6.5 & 79.1 & 20 & 11.7 & $n$ & $\mathbf{M}$ & 77.1 \\
\hline & 200 & 6. 3 & 3० 4 & 4.2 & 79.9 & 40 & $M$ & 329 & B. 4 & 78.4 \\
\hline & 350 & 6.9 & 295 & 4.5 & 79.9 & 80 & $\mathbf{H}$ & 324 & 6.7 & 780 \\
\hline & 300 & 1.9 & $M$ & $n$ & 80.1 & & & & & \\
\hline & 100 & 8.6 & 295 & 4.2 & 80.4 & & & & & \\
\hline \multirow[t]{8}{*}{$5 T-57$} & 7 & 2.6 & 290 & 4.4 & 69.7 & 2.5 & M & 306 & 1.5 & 67.6 \\
\hline & 50 & 4.5 & 291 & 3.0 & 710 & 5 & M & I & M & $67 . B$ \\
\hline & 100 & 6.0 & 293 & 2.9 & 71.2 & 10 & $n$ & 295 & 3.0 & 68.8 \\
\hline & 150 & 7.4 & 291 & 2.3 & 31.6 & 20 & 3.1 & $\boldsymbol{M}$ & I & 69.3 \\
\hline & 200 & 8.1 & 297 & 3.2 & 72.0 & 40 & $n$ & 303 & 1.9 & M \\
\hline & 250 & 9.2 & 295 & 3. 4 & 72.4 & 80 & $n$ & 298 & 2.2 & 69.4 \\
\hline & 300 & B. $B$ & $2 \mathrm{BO}$ & 2.5 & 73.0 & & & & & \\
\hline & 100 & 8.8 & 302 & 3.3 & 73.6 & & & & & \\
\hline
\end{tabular}




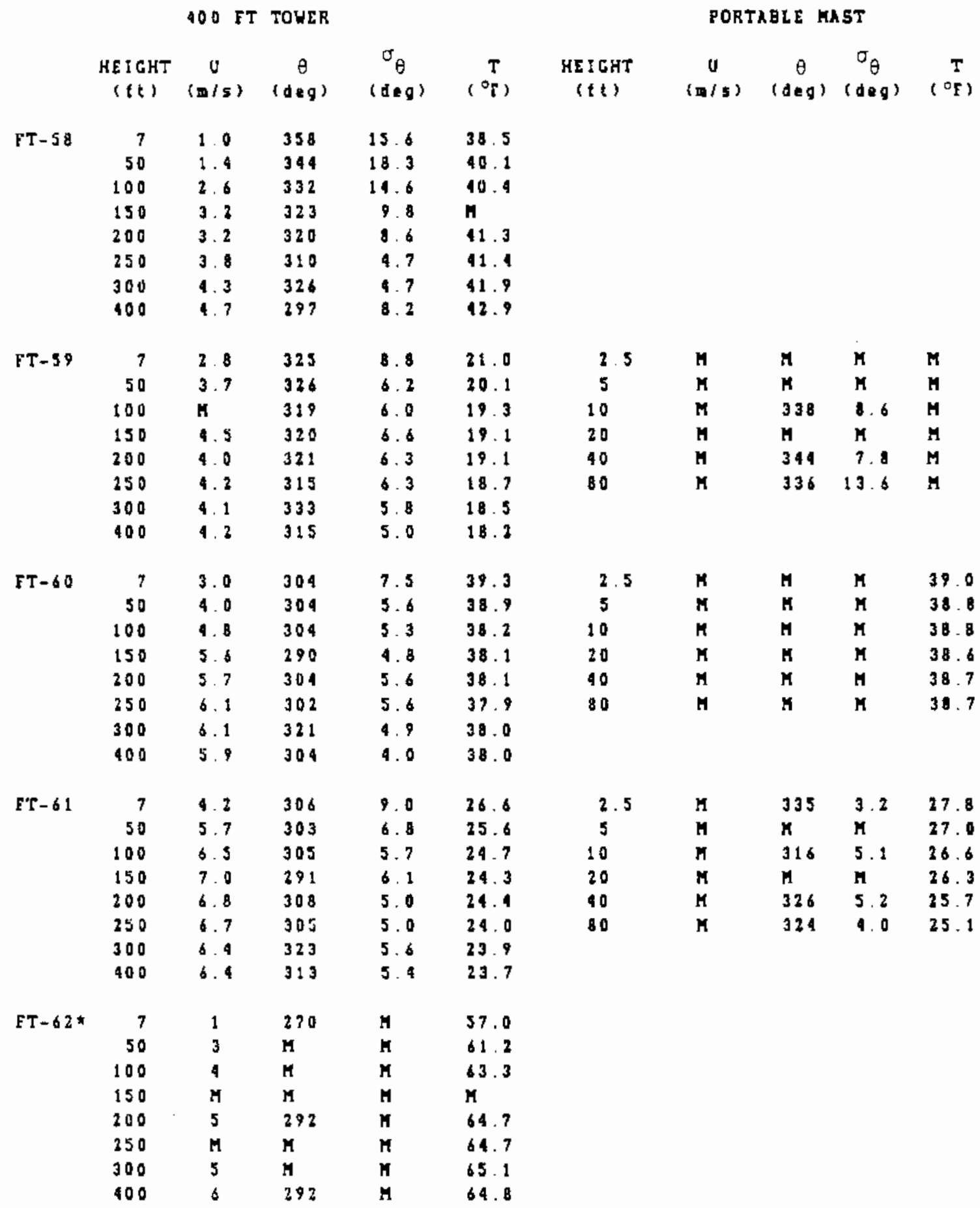

- Data from nearest hourly observation 
QDO FT TOUER HEIGHT
(it) (n) $F T-63$

7
50
100
150
200
210
300
400

$5 T-64$

7
50
100
150
200
250
300
400

1. 3

3. 3

4.7

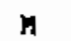

6.9

II

7. 2

0.9

$\theta$
(deg)

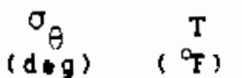

HEIGHT

(ft)

$\begin{array}{cccc}U & \theta & \sigma_{\theta} & T \\ (\mathbb{D} / s) & (\operatorname{deg})\left(\operatorname{dez}^{\prime}\right) & (\mathrm{T})\end{array}$

\section{PORTAGLE MAST}

$\begin{array}{lcl}274 & 29.7 & 79.0 \\ 294 & 16.6 & 85.2 \\ 294 & 13.2 & 85.8 \\ \text { H } & \text { M } & 86.3 \\ 301 & 10.7 & 97.2 \\ \text { H } & \text { M } & 87.1 \\ \text { M } & \text { H } & 87.8 \\ \text { M } & M & 98.6\end{array}$

$278 \quad 12.2 \quad 02.8$

$294 \quad 11.8 \quad 86.6$

293 d.3 B7.3

$\begin{array}{lll}4 & \text { B } & \text { B. }\end{array}$

$297 \quad 3.7 \quad 87.8$

$M \quad M \quad 89.2$

M $M \quad 90.5$

292 4 91.9

$\begin{array}{llllll}\text { FT-65 } & 7 & 4.9 & 310 & 9.5 & 6.6\end{array}$

$\begin{array}{lllll}50 & 6.7 & 305 & 9.7 & 87.1\end{array}$

$\begin{array}{lllll}100 & 8.7 & 306 & 8.0 & 87.0\end{array}$

$150 \quad 10.0 \quad 290 \quad 6.3 \quad 86.9$

$\begin{array}{llllll}300 & 10.4 & 304 & 57 & 87.4\end{array}$

$250 \quad 11.6 \quad 301 \quad 4.7 \quad 87.5$

$\begin{array}{lllll}300 & 12.0 & 272 & 3.9 & 88.3\end{array}$

100 M $M$ M M 89.0

$\begin{array}{llllll}\text { ET-65 } & 7 & 3.3 & 300 & 15.9 & 84.1\end{array}$

$\begin{array}{lllll}50 & 5.3 & 308 & 14.3 & \$ 5.2\end{array}$

$100 \quad 7.0 \quad 308 \quad 12.8 \quad 85.2$

$150 \quad 8.0 \quad 290 \quad 11.8 \quad 85.1$

$200 \quad$ B. $\quad 306 \quad 10.3 \quad 05.6$

$\begin{array}{lllll}250 & 9.5 & 299 & 0.6 & 85.5\end{array}$

$\begin{array}{lllll}300 & 10.0 & 269 & 7.3 & 85.8\end{array}$

400 I I I 86.4

$\begin{array}{rrrrrr}\text { FT-67 } & 7 & 1.7 & 280 & 1.0 & 79.9 \\ 50 & 3.4 & 286 & 6.7 & 02.5 \\ 100 & 1.9 & 308 & 4.7 & 82.7 \\ 150 & 5.8 & 281 & 3.0 & 83.1 \\ 200 & 7.3 & 302 & 2.8 & 14.5 \\ 250 & \text { M } & 196 & 2.8 & 15.2 \\ 300 & 9.1 & 270 & 1.5 & 36.3 \\ 400 & \text { M } & \text { M } & \text { M } & 88.1\end{array}$




\begin{tabular}{|c|c|c|c|c|c|c|c|c|c|c|}
\hline & $\begin{array}{c}\text { HE I GHT } \\
(\mathrm{ft})\end{array}$ & $\begin{array}{c}U \\
(\mathbf{a} / \mathbf{s})\end{array}$ & $\left(d^{\theta} \theta g\right)$ & $\begin{array}{c}\sigma_{\theta} \\
\left(d_{e}\right)\end{array}$ & $\begin{array}{c}T \\
(0 F)\end{array}$ & $\begin{array}{c}\text { HEIGHT } \\
(t)\end{array}$ & $\begin{array}{c}V \\
(\mathrm{~m} / \mathrm{s})\end{array}$ & $\begin{array}{c}\theta \\
(d \in q)\end{array}$ & $\begin{array}{c}\sigma_{\theta} \\
(\mathrm{deg})\end{array}$ & $\begin{array}{c}T \\
(\circ F)\end{array}$ \\
\hline \multirow[t]{8}{*}{$F T-68$} & 7 & 3.8 & 310 & S. 5 & 87.2 & & & & & \\
\hline & 50 & 6.2 & 311 & 4. 2 & 68.2 & & & & & \\
\hline & 100 & 7.9 & 308 & 4.0 & 08.3 & & & & & \\
\hline & 150 & 9.3 & 291 & 3.6 & 88.3 & & & & & \\
\hline & 200 & 10.0 & 308 & 3.0 & 68.7 & & & & & \\
\hline & 250 & 10.7 & 299 & 2.1 & 08.6 & & & & & \\
\hline & 300 & 11.3 & 273 & 3 & 58.8 & & & & & \\
\hline & 900 & $x$ & $\mathbf{H}$ & $\mathbf{M}$ & B B, 4 & & & & & \\
\hline \multirow[t]{8}{*}{ FT-69 } & 7 & 4.5 & 300 & 13.6 & 83.7 & & & & & \\
\hline & 50 & 6.6 & 298 & 13.2 & 84.9 & & & & & \\
\hline & 100 & 8. 0 & 297 & 12.1 & 89.3 & & & & & \\
\hline & 150 & 9.0 & 379 & 11.3 & 89. 1 & & & & & \\
\hline & 200 & 9.2 & 293 & 11.1 & 04.4 & & & & & \\
\hline & 250 & 9.7 & 288 & 10.1 & 94.0 & & & & & \\
\hline & 300 & 10.3 & 261 & 1. 6 & 84.2 & & & & & \\
\hline & 100 & $n$ & M & $M$ & \$4. 1 & & & & & \\
\hline \multirow[t]{8}{*}{$F T=70$} & 7 & 2. 9 & 298 & 9.0 & $\$ 1.0$ & & & & & \\
\hline & 50 & 4. 6 & 294 & 9. 7 & 01.7 & & & & & \\
\hline & 100 & 5.9 & 295 & 7.6 & 81.3 & & & & & \\
\hline & 150 & 6.4 & 276 & 7.9 & 81.1 & & & & & \\
\hline & 200 & 6.4 & 291 & 7.2 & 01.3 & & & & & \\
\hline & 250 & 6.9 & 289 & 6.6 & 81.1 & & & & & \\
\hline & 300 & 7.2 & 259 & 6.1 & 81.0 & & & & & \\
\hline & 100 & $n$ & M & I & 91.0 & & & & & \\
\hline \multirow[t]{8}{*}{ FT-71 } & 7 & 1.9 & 315 & 21.2 & 74.4 & & & & & \\
\hline & 50 & 3.1 & 310 & 18.2 & 74.7 & & & & & \\
\hline & 100 & 4. 3 & 309 & 16.5 & 74.3 & & & & & \\
\hline & 150 & 5.2 & 292 & 19.6 & 74.2 & & & & & \\
\hline & 200 & 5.0 & 306 & 11.8 & 75.0 & & & & & \\
\hline & 250 & 4. 8 & 294 & 11.3 & 75.2 & & & & & \\
\hline & 300 & 4.7 & 265 & 15.9 & 75.6 & & & & & \\
\hline & 900 & $n$ & $M$ & M & 75.7 & & & & & \\
\hline
\end{tabular}

U SERIES

\begin{tabular}{|c|c|c|c|c|c|c|c|c|c|c|}
\hline$v-2$ & 7 & 7.7 & 231 & 8.3 & 56.0 & 2.5 & H & $\mathbf{n}$ & $\mathbf{H}$ & $5 B .6$ \\
\hline & 50 & 10.2 & 236 & 73 & 54.9 & 5 & 5.9 & $M$ & $\mathbf{H}$ & 58.7 \\
\hline & 100 & 11.3 & 232 & 6.8 & 59.3 & 10 & $\mathbf{M}$ & H & I & 58.2 \\
\hline & 150 & 11.9 & 230 & 6.5 & $\mathbf{A}$ & 20 & B. 1 & H & $M$ & 58.0 \\
\hline & 300 & 12.4 & 229 & 6. 3 & 54.1 & 90 & $M$ & 243 & 4.0 & 57.4 \\
\hline & 250 & 12.6 & 227 & 6.5 & 53.1 & 80 & M & 245 & 5.6 & 56.3 \\
\hline & 300 & 12.9 & 252 & 5.6 & 53.0 & & & & & \\
\hline & 40.0 & 12.8 & 238 & 56 & 525 & & & & & \\
\hline
\end{tabular}


100 FT TOWEA

PORTAGLE MAST

\begin{tabular}{|c|c|c|c|c|c|c|c|c|c|c|}
\hline & $\begin{array}{c}\text { HEICHT } \\
(t \mathrm{t})\end{array}$ & $\begin{array}{c}U \\
(m / s)\end{array}$ & $\left(\begin{array}{c}\theta \\
(d \in q)\end{array}\right.$ & (deg) & $\begin{array}{c}T \\
\left({ }^{\circ} \mathrm{F}\right)\end{array}$ & $\begin{array}{c}\text { HEICHT } \\
(\mathrm{ft})\end{array}$ & $\begin{array}{c}U \\
(\mathrm{~d} / 5)\end{array}$ & $\begin{array}{c}\theta \\
\{d: g\rangle\end{array}$ & $\begin{array}{c}\sigma_{\theta} \\
(d e g)\end{array}$ & $\begin{array}{c}T \\
\left({ }^{\circ} \mathrm{F}\right)\end{array}$ \\
\hline \multirow[t]{8}{*}{$\mathrm{J}=5$} & 7 & 4. 6 & 220 & 17.3 & 43.2 & 2.5 & $n$ & $\mathbf{M}$ & $M$ & 50.0 \\
\hline & 50 & 6.8 & 224 & 16.9 & 47.0 & 5 & 4. 7 & $M$ & H & 49.6 \\
\hline & 100 & 6.8 & 223 & 15.0 & 46.2 & 10 & $M$ & 230 & 13.4 & 49.5 \\
\hline & 150 & 7.4 & 216 & 16.2 & 45.8 & 20 & 5. 7 & H & $\mathbf{M}$ & 49.0 \\
\hline & 200 & 7.4 & 218 & 15.3 & 46.2 & 40 & 6.3 & 233 & 15.9 & 48.9 \\
\hline & 250 & 7.5 & 217 & 14.5 & 45.3 & 10 & $\mathbf{M}$ & 230 & 10.1 & 49.9 \\
\hline & 300 & 7.9 & 244 & $13 \cdot 4$ & 45.3 & & & & & \\
\hline & 400 & 7.8 & 219 & 14.1 & 44.9 & & & & & \\
\hline \multirow[t]{8}{*}{$v-6$} & 7 & 7.9 & 210 & 7.6 & 48.4 & 2.5 & $x$ & n & $\boldsymbol{M}$ & 51.9 \\
\hline & so & 11.0 & 216 & 6.9 & 45.9 & 5 & 6.8 & H & $M$ & 50.9 \\
\hline & 100 & 11.9 & 213 & 6.4 & 45.1 & 10 & H & 222 & 7.1 & 50.5 \\
\hline & 150 & 12.7 & 205 & క. 7 & $M$ & 20 & 9.4 & $\mathbf{r}$ & $M$ & 49.7 \\
\hline & 200 & 13.1 & 209 & 6.1 & 94.6 & 40 & 11.0 & 215 & 4.2 & 49.0 \\
\hline & 250 & 13.4 & 210 & 5.6 & 44.0 & 00 & $n$ & $2: 9$ & 3.5 & 47.7 \\
\hline & 300 & $M$ & $M$ & $n$ & 43.8 & & & & & \\
\hline & 100 & 13.6 & $2: 0$ & 5.6 & 43.5 & & & & & \\
\hline \multirow{8}{*}{$\mathrm{y}-7$} & 7 & 6.5 & 292 & 13.2 & 58.7 & 2.5 & $\mathbf{M}$ & 267 & 52.0 & 61.4 \\
\hline & 50 & 8.3 & 246 & 11.1 & 55.5 & 5 & $M$ & $\mathbf{M}$ & H & 61.0 \\
\hline & 100 & 9.0 & 244 & 10.8 & 54.7 & 10 & $\mathbf{M}$ & 252 & 5.0 & 59.2 \\
\hline & 150 & 9.3 & 235 & 12.0 & 53.9 & 20 & $\mathbf{H}$ & $\mathbf{M}$ & $n$ & 58.2 \\
\hline & 200 & 9.2 & 239 & 10.8 & 53.7 & 40 & $M$ & 261 & 10.4 & 57.6 \\
\hline & 250 & 9.5 & 340 & 10.5 & 53.7 & 60 & $M$ & 258 & 9.4 & 56.0 \\
\hline & 300 & 9.5 & 254 & 10.0 & 53.5 & & & & & \\
\hline & 400 & 9.7 & 239 & 10.1 & 53.2 & & & & & \\
\hline \multirow[t]{8}{*}{ U-8 } & 7 & 7.5 & 225 & 8.5 & 56.8 & 2.5 & $M$ & 253 & 12.3 & 59.8 \\
\hline & 50 & 10.8 & 223 & 6.7 & 56.2 & 5 & $H$ & $\mathbf{H}$ & $\mathbf{M}$ & 58.4 \\
\hline & 100 & 12.0 & 219 & 7.0 & 55.7 & 10 & $\mathbf{H}$ & 235 & 6.8 & 59.1 \\
\hline & 150 & 13.1 & 216 & 6.9 & $M$ & 20 & $n$ & $\mathbf{M}$ & $\mathbf{H}$ & 59.0 \\
\hline & 200 & 13.5 & 219 & 5.3 & 55.2 & 40 & $\mathbf{M}$ & 243 & 5.4 & 58.6 \\
\hline & 250 & 14.0 & 218 & 6.6 & 54.8 & 80 & $M$ & 242 & 5.2 & 58.1 \\
\hline & 300 & 14.4 & 236 & 6.1 & 54.6 & & & & & \\
\hline & 100 & 14.6 & 219 & 6.2 & 54.3 & & & & & \\
\hline \multirow[t]{8}{*}{ U-9 } & 7 & 5.0 & 236 & 16.8 & 68.0 & 2.5 & M & 253 & 12.9 & 67.3 \\
\hline & 50 & 6.5 & 234 & 15.6 & 67.0 & 5 & H & H & I & 66.9 \\
\hline & 100 & 7.5 & 236 & 14.6 & 66.3 & 10 & $\mathbf{M}$ & 247 & 13.9 & 66.5 \\
\hline & 150 & \&. 1 & 227 & 15.3 & 65.7 & 20 & $M$ & $\boldsymbol{M}$ & $M$ & 66.2 \\
\hline & 200 & 7.9 & 235 & 14.5 & 65.6 & 40 & $M$ & 253 & 13.5 & 65.7 \\
\hline & 250 & 8. 3 & 237 & 14.1 & 65.4 & 0 & M & 254 & 12.9 & 65.4 \\
\hline & 300 & 7.6 & 205 & 14.3 & 65.3 & & & & & \\
\hline & 400 & 6. 1 & 236 & 14.4 & 64.6 & & & & & \\
\hline
\end{tabular}




\begin{tabular}{|c|c|c|c|c|c|c|c|c|c|c|}
\hline \multicolumn{7}{|c|}{ 400 FT TOUER } & \multicolumn{4}{|c|}{ PORTAELE MAST } \\
\hline & $\begin{array}{c}\text { HE ICHT } \\
\text { (ft) }\end{array}$ & $\begin{array}{c}U \\
(m / 5)\end{array}$ & $\begin{array}{c}\theta \\
(d * 9)\end{array}$ & $\begin{array}{c}\sigma_{\theta} \\
(d \neq g)\end{array}$ & $\left({ }^{\circ} \mathrm{F}\right)$ & $\begin{array}{c}\text { HE ICHT } \\
(t t)\end{array}$ & $\begin{array}{c}\mathrm{U} \\
(\mathrm{m} / \mathrm{s})\end{array}$ & $\begin{array}{c}\theta \\
(d \times g)\end{array}$ & $\begin{array}{c}\sigma_{\theta} \\
(d=g)\end{array}$ & $\left(\begin{array}{c}T \\
\left({ }^{\circ}\right)\end{array}\right.$ \\
\hline \multirow[t]{7}{*}{$U=10$} & 7 & 2.7 & 249 & 23.5 & 62.5 & 2.5 & $M$ & 273 & 14.0 & 62.4 \\
\hline & 50 & 3.3 & 247 & 23.2 & 60.3 & 5 & $H$ & H & $\boldsymbol{M}$ & 61.2 \\
\hline & 100 & 3.9 & 245 & 21.2 & 59.7 & 10 & $\mathbf{n}$ & 268 & 14.5 & 60.7 \\
\hline & 150 & 4. 4 & 238 & 21.6 & 59.0 & 20 & I & $n$ & $\mathbf{M}$ & 60.5 \\
\hline & 200 & 3.9 & 245 & 21.1 & 58.8 & 40 & $M$ & 270 & 10.8 & 60.0 \\
\hline & 250 & 4. 1 & 246 & 18.4 & 58.9 & 80 & $M$ & 274 & 12.4 & 59.1 \\
\hline & $\begin{array}{l}300 \\
100\end{array}$ & $\begin{array}{l}3.9 \\
3.8\end{array}$ & $\begin{array}{l}217 \\
247\end{array}$ & $\begin{array}{l}17.0 \\
26.1\end{array}$ & $\begin{array}{l}59.0 \\
58.5\end{array}$ & & & & & \\
\hline \multirow[t]{8}{*}{ V-11 } & 7 & 1.8 & 260 & 26.0 & 63.9 & 2.5 & $n$ & 279 & 19.6 & 64.0 \\
\hline & 50 & 2.2 & 257 & 20.5 & 62.6 & 5 & $\mathbf{H}$ & $H$ & $\mathbf{M}$ & 63.0 \\
\hline & 100 & 2.6 & 254 & 20.2 & 62.0 & 10 & $M$ & 275 & 17.0 & 62.7 \\
\hline & 150 & 3.0 & 242 & 21.0 & 61.2 & 20 & $\mathbf{H}$ & $\mathbf{H}$ & $\mathbf{H}$ & 63.2 \\
\hline & 200 & 2.5 & 252 & 18.7 & 61.2 & 40 & $\mathbf{H}$ & 277 & 17.0 & 61.5 \\
\hline & 250 & 3. 1 & 254 & 17.3 & 61.0 & 80 & $\mathbf{M}$ & 280 & 19.6 & 61.0 \\
\hline & 300 & 2.7 & 223 & 15.2 & 60.9 & & & & & \\
\hline & 100 & 2. 7 & 251 & 16.8 & 60.6 & & & & & \\
\hline \multirow[t]{8}{*}{$v-12$} & 7 & 2.0 & 220 & 49.8 & 66.9 & 2.5 & $\mathbf{M}$ & 244 & 33.8 & 60.1 \\
\hline & 50 & 2.9 & 220 & 37.4 & 65.5 & 5 & $\mathbf{H}$ & I & $\mathbf{M}$ & 64.6 \\
\hline & 100 & 3.2 & 220 & 34.4 & 64.5 & 10 & K & 242 & 32.0 & 65.8 \\
\hline & 150 & 3.8 & 211 & 35.0 & 64.0 & 20 & $n$ & M & $\mathbf{M}$ & 65.3 \\
\hline & 200 & 3.4 & 217 & 32.9 & 64.0 & 40 & M & 250 & 28.8 & 64.8 \\
\hline & 250 & 3.9 & 218 & 30.0 & 63.5 & 80 & M & 248 & 30.8 & 64.0 \\
\hline & 300 & 3.6 & 187 & 29.1 & 63.5 & & & & & \\
\hline & 400 & 3.6 & 224 & 37.5 & 63.4 & & & & & \\
\hline \multirow[t]{8}{*}{$\mathrm{U}-13$} & 7 & 7.2 & 211 & 16.1 & 60.4 & 2.5 & $\mathbf{H}$ & 232 & 7.2 & 61.2 \\
\hline & $\$ 0$ & 10.9 & 210 & 34.4 & 58.4 & 5 & $M$ & H & $\mathbf{M}$ & 60.5 \\
\hline & 100 & 12.0 & 208 & 26.9 & 57.7 & 10 & $\mathbf{M}$ & 230 & 5.8 & 60.0 \\
\hline & 150 & 13.0 & 199 & 39.0 & 57.0 & 20 & $\mathbf{M}$ & $\mathbf{H}$ & $M$ & 59.5 \\
\hline & 200 & 12.9 & 204 & 28.8 & 57.0 & 40 & $\mathbf{H}$ & 240 & 5.3 & 56.9 \\
\hline & 250 & 13.1 & 206 & 29.3 & 56.4 & 80 & $\mathbf{M}$ & 240 & 5. 3 & 58.0 \\
\hline & 300 & 12.2 & 174 & 27.3 & 56.6 & & & & & \\
\hline & 400 & 12.7 & 205 & 28.5 & 56.4 & & & & & \\
\hline \multirow[t]{8}{*}{$U-14$} & 7 & 9. 2 & 231 & 9.7 & 58.5 & 2.5 & $\mathbf{M}$ & 249 & 10.4 & 58.8 \\
\hline & 50 & 5.9 & 231 & 9.2 & $\$ 7.9$ & 5 & $\mathbf{M}$ & $H$ & M & 58.3 \\
\hline & 100 & 6.9 & 227 & 8.6 & 57.2 & 10 & M & 247 & 8.5 & 58.1 \\
\hline & 150 & 1.0 & 218 & 8.3 & 56.7 & 20 & $M$ & $\mathbf{M}$ & $\mathbf{M}$ & 57.3 \\
\hline & 200 & 7.0 & 225 & 6. 8 & 36.9 & 40 & $\mathbf{y}$ & 253 & 9. 9 & 57.4 \\
\hline & 350 & 7.1 & 223 & 7.9 & 56.4 & 80 & $\mathbf{M}$ & 271 & 7.0 & 53.1 \\
\hline & 300 & 6.7 & 214 & B. 6 & 56.4 & & & & & \\
\hline & 400 & 7.4 & 223 & B. 1 & 55.9 & & & & & \\
\hline
\end{tabular}


100 FT TOUER

PORTAGLE MAST

\begin{tabular}{|c|c|c|c|c|c|c|c|c|c|c|}
\hline & $\begin{array}{c}\text { HE I GHT } \\
\text { (tt) }\end{array}$ & $\begin{array}{c}U \\
(\mathbf{a} / \mathbf{s})\end{array}$ & $\langle\stackrel{\theta}{\langle\in g}\rangle$ & $\begin{array}{c}\sigma_{\theta} \\
\text { (deg) }\end{array}$ & $\begin{array}{c}T \\
(\circ F)\end{array}$ & $\begin{array}{c}\text { HEICHT } \\
(t+)\end{array}$ & $\begin{array}{c}U \\
(m / 5)\end{array}$ & $\begin{array}{c}\theta \\
(d e g)\end{array}$ & $\begin{array}{c}\sigma_{\theta} \\
\text { (deq) }\end{array}$ & $\begin{array}{c}T \\
(0 E)\end{array}$ \\
\hline \multirow[t]{8}{*}{$U-15$} & 7 & 5.5 & 243 & 9.4 & 56.1 & 2.5 & $\mathbf{M}$ & 269 & 8.7 & 54.0 \\
\hline & 50 & 7.7 & 243 & 8.8 & 56.0 & 5 & $x$ & M & $n$ & 53.8 \\
\hline & 100 & 8. 9 & 243 & a. 0 & 55.2 & 10 & m & 263 & B. 0 & 53.7 \\
\hline & 150 & 9.7 & 232 & 7.6 & 55.0 & 20 & $n$ & M & M & 53.6 \\
\hline & 200 & 9.5 & 238 & 7.5 & 53.2 & 40 & $\mathbf{M}$ & 274 & 8.5 & 53.5 \\
\hline & 250 & 9.6 & 235 & 6.6 & 54. & 80 & $M$ & 274 & 7.0 & 53.1 \\
\hline & 300 & 9.3 & 227 & 6.8 & 59.7 & & & & & \\
\hline & 400 & 10.3 & 240 & 6.8 & 54.2 & & & & & \\
\hline \multirow[t]{8}{*}{$U-16$} & 7 & 4. 7 & 232 & 10.2 & 58.0 & 2.5 & $\mathbf{M}$ & 258 & 9.2 & 55.9 \\
\hline & 50 & 6.8 & 229 & 10.6 & 58.0 & 5 & $x$ & 月 & $\mathbf{H}$ & 55.6 \\
\hline & 100 & 7.6 & 223 & 10.0 & 57.3 & 10 & $n$ & 254 & 10.2 & $\$ 5.5$ \\
\hline & 150 & 6.3 & 217 & 10.1 & 57.0 & 20 & $M$ & I & ! & 53.5 \\
\hline & 300 & 8. 4 & 225 & 10.2 & 57.2 & 40 & $n$ & 262 & 8.1 & 55.3 \\
\hline & 250 & 8.8 & 225 & 10.1 & 56.7 & 10 & $y$ & 262 & 9.6 & 54.9 \\
\hline & 300 & 8.3 & 214 & 10.0 & 56.7 & & & & & \\
\hline & 400 & 9.3 & 726 & 9.5 & 56.2 & & & & & \\
\hline \multirow[t]{8}{*}{$U=! 7$} & 7 & 5.6 & 223 & 7.7 & 60.5 & 2.5 & 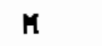 & 239 & 2.0 & 60.0 \\
\hline & 50 & 7.9 & 223 & 6.6 & 59.3 & 5 & $\mathbf{H}$ & M & $\mathbf{x}$ & 59.1 \\
\hline & 100 & 6.3 & 220 & 6.0 & 50.6 & 10 & $M$ & 237 & 0.6 & 58,1 \\
\hline & 150 & 9.0 & 213 & 5.4 & 58.1 & 20 & $\mathbf{n}$ & M & M & 58.8 \\
\hline & 200 & 8. 9 & 220 & 5.9 & 58.5 & 10 & $M$ & 243 & 2.0 & 58.8 \\
\hline & 250 & 9.1 & 219 & 6.1 & 57.7 & 80 & $\mathbf{M}$ & 346 & 4.9 & $n$ \\
\hline & 300 & B. 5 & 209 & 6. 3 & 57.8 & & & & & \\
\hline & 400 & 9.1 & 322 & 5.2 & 57.5 & & & & & \\
\hline \multirow[t]{8}{*}{$U-18$} & 7 & 5.6 & 248 & 8.9 & 61.1 & 2.5 & $M$ & 268 & 8,5 & 60.2 \\
\hline & 50 & 7.4 & 248 & 8.5 & 60.2 & 5 & $n$ & $\mathbf{H}$ & $\mathbf{M}$ & 59.2 \\
\hline & 100 & s. 3 & 248 & 7.5 & 59.5 & 10 & $\mathbf{H}$ & 260 & 6.6 & 59.1 \\
\hline & 150 & B. 7 & 235 & 7.5 & 58.9 & 20 & $n$ & $n$ & M & 58.9 \\
\hline & 200 & B. 4 & 240 & 7.4 & 59.2 & 40 & H & 264 & 5.7 & $5 B .4$ \\
\hline & 250 & 0.6 & 241 & 6.6 & 58.5 & 80 & $x$ & 269 & 5.0 & $n$ \\
\hline & 300 & 7.9 & 229 & 6.4 & 58.5 & & & & & \\
\hline & 400 & 9.0 & 245 & 6.2 & 58.4 & & & & & \\
\hline \multirow[t]{8}{*}{$U-19$} & 7 & 3.1 & 225 & 24.4 & 50.1 & & & & & \\
\hline & 50 & 4.2 & 228 & 22.9 & 48.0 & & & & & \\
\hline & 100 & 4. 4 & 223 & 21.9 & 47.3 & & & & & \\
\hline & 150 & 4.1 & 212 & 21.9 & 96.6 & & & & & \\
\hline & 200 & 4.7 & 219 & 31.5 & 16.6 & & & & & \\
\hline & 250 & 4.6 & 319 & 19.6 & 46.2 & & & & & \\
\hline & 300 & 9. 3 & 213 & 19.2 & 46.2 & & & & & \\
\hline & 400 & \$. 1 & 222 & 17.6 & 16.0 & & & & & \\
\hline
\end{tabular}


100 FT TOWE

PORTABLE MAST

\begin{tabular}{|c|c|c|c|c|c|c|c|c|c|}
\hline $\begin{array}{l}\text { HEICHT } \\
\text { (ft) }\end{array}$ & $\begin{array}{c}U \\
(m / 5)\end{array}$ & $\begin{array}{c}\theta \\
(d \notin g)\end{array}$ & $\begin{array}{c}\sigma_{\theta} \\
(\phi \in g)\end{array}$ & $\left(\begin{array}{c}T \\
\left(0^{2}\right)\end{array}\right.$ & $\begin{array}{c}\text { HEICHT } \\
(\mathrm{ft}\rangle\end{array}$ & $\begin{array}{c}U \\
(\mathrm{n} / \mathrm{s})\end{array}$ & $\begin{array}{c}\theta \\
(\operatorname{deg})\end{array}$ & $\sigma_{\theta}$ & $\left(\begin{array}{c}T \\
\left({ }^{2}\right)\end{array}\right.$ \\
\hline
\end{tabular}

$\begin{array}{llllll}0-20 & 7 & 7.0 & 233 & 7.6 & 50.1\end{array}$

$\begin{array}{rrrrr}50 & 9.1 & 232 & 6.0 & 49.3 \\ 100 & 10.1 & 228 & 5.6 & 48.7 \\ 150 & 10.0 & 216 & 6.0 & 48.2 \\ 200 & 10.8 & 223 & 5.9 & 47.7 \\ 250 & 11.4 & 224 & 5.7 & 48.0 \\ 300 & 10.9 & 217 & 5.8 & 48.0 \\ 100 & 11.9 & 224 & 5.4 & 47.6\end{array}$

$\begin{array}{llllll}\mathrm{U}-21 & 7 & 5.2 & 217 & 7.7 & 55.5\end{array}$

$\begin{array}{lllll}50 & 6.9 & 215 & 7.7 & 54.5\end{array}$

$\begin{array}{lllll}100 & 7.7 & 210 & 7.1 & 53.7\end{array}$

$150 \quad 7.6 \quad 199 \quad 5.6 \quad 53.2$

$200 \quad 8.3 \quad 206 \quad 7.3 \quad 53.6$

$250 \quad 8.4 \quad 206 \quad 6.6 \quad 52.9$

$300 \quad 8.0 \quad 216 \quad 5.1252 .8$

$400 \quad 8.4 \quad 207 \quad 5.4 \quad 52.3$

$\begin{array}{llllll}\text { U-22 } & 7 & 3.5 & 233 & 24.3 & 49.9\end{array}$

$\begin{array}{lllll}50 & 4.3 & 232 & 18.8 & 48.0\end{array}$

$\begin{array}{lllll}100 & 9.6 & 229 & 17.7 & 47.0\end{array}$

$\begin{array}{lllll}150 & 4.2 & 219 & 16.2 & 46.5\end{array}$

$200 \quad 8 \quad 227 \quad 15.7 \quad 46.2$

$\begin{array}{lllll}250 & 4.8 & 227 & 14.6 & 46.2\end{array}$

$\begin{array}{lllll}300 & 4.6 & 232 & 14.0 & 46.1\end{array}$

$400 \quad 4.9230 \quad 13.2 \quad 45.7$

$\begin{array}{llllll}\text { U-23 } & 7 & 3.1 & 273 & 13.3 & 57.7\end{array}$

$50 \quad 3.8 \quad 275 \quad 12.1 \quad 56.8$

$\begin{array}{lllll}100 & 4.2 & 270 & 12.4 & 56.1\end{array}$

$150 \quad 3.8261 \quad 12.455 .6$

$200 \quad 9.4 \quad 269 \quad 11.8 \quad 56.0$

$\begin{array}{lllll}250 & 4.6 & 268 & 11.0 & 53.3\end{array}$

$\begin{array}{lllll}300 & 3.7 & 272 & 10.7 & 55.2\end{array}$

$\begin{array}{lllll}600 & 4.4 & 267 & 10.7 & 54.8\end{array}$

$\begin{array}{rrrrrr}\text { J-24 } & 7 & 5.4 & 238 & 22.9 & 61.2 \\ 50 & 6.8 & 238 & 21.8 & 57.2 \\ 100 & 7.5 & 235 & 19.4 & 56.2 \\ 150 & 7.2 & 223 & 18.7 & 55.5 \\ 200 & 8.0 & 231 & 19.0 & 55.4 \\ 250 & \text { B.3 } & 232 & 17.6 & 54.9 \\ 300 & 7.5 & 236 & 16.5 & 54.9 \\ 100 & 8.1 & 231 & 16.6 & 54.6\end{array}$


400 FT TOUER

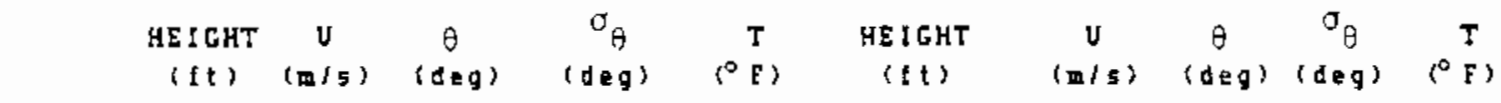

$\begin{array}{rllll}7 & 1.9 & 188 & 47.8 & 56.5 \\ 50 & 3.1 & 201 & 39.8 & 55.2 \\ 100 & 3.4 & 194 & 35.8 & 54.6 \\ 150 & 3.0 & 184 & 39.2 & 54.1 \\ 200 & 3.7 & 19 . & 34.2 & 54.2 \\ 250 & 3.8 & 192 & 30.7 & 53.4 \\ 300 & 3.5 & 200 & 30.1 & 53.5 \\ 100 & 3.4 & 195 & 29.8 & 53.2\end{array}$

\begin{tabular}{|c|c|c|c|c|c|}
\hline \multirow[t]{8}{*}{$U-26$} & 7 & 3.5 & 229 & 33.9 & 74.8 \\
\hline & 50 & 4.4 & 237 & 29.5 & 72.9 \\
\hline & 100 & 9.9 & 232 & 27.2 & 71.2 \\
\hline & 150 & 4. 8 & 229 & 25.8 & 70.6 \\
\hline & 200 & 4.8 & 233 & 24.7 & 70.6 \\
\hline & 250 & S. 2 & 233 & 22.6 & 70.0 \\
\hline & 300 & 4.6 & 235 & 22.3 & 70.0 \\
\hline & 400 & 5.1 & 233 & 20.2 & 70.0 \\
\hline \multirow[t]{8}{*}{ U- 27} & 7 & 2.2 & 228 & $42 \cdot 1$ & 76.6 \\
\hline & 50 & 2.7 & 221 & 36.0 & 74.6 \\
\hline & 100 & 2.9 & $23 \mathrm{t}$ & 29.7 & 73.4 \\
\hline & 150 & 3. 1 & 225 & 31.7 & 72.9 \\
\hline & 200 & 3.3 & 230 & 2. B & 72.9 \\
\hline & 250 & 3.1 & 234 & 27.5 & 72.7 \\
\hline & 300 & 3.4 & 233 & 25.9 & 72.9 \\
\hline & 400 & 3.2 & 231 & 23.9 & 72.2 \\
\hline \multirow[t]{8}{*}{$U-28$} & 7 & 5.5 & 241 & 18.6 & 59.2 \\
\hline & 50 & 7.4 & 226 & 11.1 & 56.9 \\
\hline & 100 & 7.6 & 236 & 10.4 & 56.1 \\
\hline & 150 & B. 1 & 233 & 9.8 & 55.0 \\
\hline & 200 & 8.1 & 240 & 9.5 & 55.2 \\
\hline & 250 & 3.5 & 233 & 9.2 & 54.0 \\
\hline & 300 & 8. 3 & 229 & 9.5 & 54.9 \\
\hline & 400 & d. 7 & 232 & 9.0 & 54.7 \\
\hline
\end{tabular}

$\begin{array}{rrrrrr}U-29 & 7 & 1.7 & 269 & 26.7 & 60.1 \\ & 50 & 2.2 & 254 & 19.8 & 59.2 \\ 100 & 2.4 & 264 & 17.0 & 58.0 \\ 150 & 2.4 & 259 & 16.0 & 57.6 \\ 200 & 2.4 & 262 & 15.4 & 57.7 \\ 250 & 2.6 & 257 & 14.9 & 57.6 \\ 300 & 2.8 & 259 & 14.5 & 57.4 \\ 100 & 2.8 & 254 & 14.6 & 57.3\end{array}$




\begin{tabular}{|c|c|c|c|c|c|c|c|c|c|c|}
\hline & & $100 \mathrm{~F}$ & TOUER & & & & PORT & ALE KA & Ast & \\
\hline & $\begin{array}{c}\text { HEICHT } \\
(t:)\end{array}$ & $\begin{array}{c}U \\
(\mathrm{~m} / \mathrm{s})\end{array}$ & $\begin{array}{c}\theta \\
(d \in q)\end{array}$ & $\begin{array}{c}\sigma_{\theta} \\
(d \notin g)\end{array}$ & $\left(\begin{array}{c}T \\
(0)\end{array}\right.$ & $\begin{array}{c}\text { HEIGHT } \\
\text { (Et) }\end{array}$ & $\begin{array}{c}U \\
(a / 5)\end{array}$ & $\begin{array}{c}\theta \\
(d \in q)\end{array}$ & $\begin{array}{c}\sigma_{\theta} \\
(d e g)\end{array}$ & $\left(\begin{array}{l}\mathrm{T} \\
\mathrm{F})\end{array}\right.$ \\
\hline $0-30$ & 7 & 6.0 & 249 & B. 2 & 57.5 & & & & & \\
\hline & 50 & B. & 236 & 7.7 & 56.6 & & & & & \\
\hline & 100 & 9.0 & 249 & 7.7 & 55.7 & & & & & \\
\hline & $\$ 50$ & ค. 4 & 237 & 7.3 & 54.9 & & & & & \\
\hline & 200 & 9.6 & 243 & 7.0 & 55.2 & & & & & \\
\hline & 250 & 9.6 & 235 & 6.4 & 53.0 & & & & & \\
\hline & 300 & 9.4 & 231 & 6.0 & 34.8 & & & & & \\
\hline & 400 & 10.3 & 239 & 5.7 & 54.6 & & & & & \\
\hline$U-31$ & 7 & 3.9 & 233 & 10.7 & 55.8 & & & & & \\
\hline & 50 & 5.6 & 215 & 10.2 & 56.4 & & & & & \\
\hline & 100 & 6.9 & 222 & a. 7 & 53.9 & & & & & \\
\hline & 150 & 7.0 & 218 & 7.8 & 55.7 & & & & & \\
\hline & 200 & 7.5 & 223 & 7.1 & 55.8 & & & & & \\
\hline & 250 & 8. 0 & 216 & 7.6 & 55.6 & & & & & \\
\hline & 300 & 7.6 & 212 & 7.8 & $\$ 5.4$ & & & & & \\
\hline & 400 & b. 3 & 215 & 7.7 & 55.1 & & & & & \\
\hline $4-32$ & 7 & 4.5 & 218 & 8.0 & 60.1 & & & & & \\
\hline & 50 & 7.6 & 199 & 8.1 & 60.4 & & & & & \\
\hline & 100 & 8. 7 & 210 & 7.7 & 59.8 & & & & & \\
\hline & 150 & 9.2 & 204 & 7.2 & 59.6 & & & & & \\
\hline & 200 & 9.7 & 207 & 7.7 & 59.7 & & & & & \\
\hline & 250 & 10.4 & 202 & 7.4 & 59.5 & & & & & \\
\hline & 300 & 10.1 & 199 & 7.1 & 59.3 & & & & & \\
\hline & 400 & 10.9 & 205 & 7.2 & 59.0 & & & & & \\
\hline V-33 & 7 & 6.0 & 233 & 13.0 & 48.5 & & & & & \\
\hline & 30 & 7.6 & 213 & 12.1 & 96.1 & & & & & \\
\hline & 100 & 8.0 & 228 & 11.4 & 44.8 & & & & & \\
\hline & 150 & 7.3 & 224 & 11.4 & 4 4.9 & & & & & \\
\hline & 200 & 8.0 & 225 & 11.3 & 94.0 & & & & & \\
\hline & 250 & 1.9 & 224 & 11.3 & 43.0 & & & & & \\
\hline & 300 & 8. 3 & 220 & 11.6 & 43.8 & & & & & \\
\hline & 900 & B. 6 & 222 & 10.8 & 43.5 & & & & & \\
\hline $\mathrm{J}-3 \mathrm{~A}$ & 1 & 5. 0 & 269 & 16.1 & 63.6 & & & & & \\
\hline & 50 & 6.3 & 261 & 16.2 & 60.8 & & & & & \\
\hline & 100 & 6.5 & 269 & 15.5 & 60.0 & & & & & \\
\hline & 150 & 6. 8 & 263 & 15.0 & 59.1 & & & & & \\
\hline & 206 & 7.0 & 259 & 14.6 & 59.0 & & & & & \\
\hline & 250 & 7. 2 & 262 & 14.5 & 58.7 & & & & & \\
\hline & 300 & 6.8 & 257 & 14.8 & 38.8 & & & & & \\
\hline & 100 & 7.3 & 257 & 19.8 & 58.5 & & & & & \\
\hline
\end{tabular}


100 FT TOYER

PORTABLE MAST

\begin{tabular}{|c|c|c|c|c|c|c|c|c|c|c|}
\hline & $\begin{array}{c}\mathrm{HE} ! \mathrm{GHT} \\
(\mathrm{t} b)\end{array}$ & $\begin{array}{c}U \\
(\pi / 5)\end{array}$ & $\begin{array}{c}\theta \\
(d \times g)\end{array}$ & $\begin{array}{c}\sigma_{\theta} \\
(d e g)\end{array}$ & $\left(\begin{array}{c}T \\
0\end{array}\right.$ & $\begin{array}{c}\text { HE IGHT } \\
(b \mathrm{t})\end{array}$ & $\begin{array}{c}4 \\
(m / s)\end{array}$ & $\begin{array}{c}\theta \\
(d \in g)\end{array}$ & $\begin{array}{c}\sigma_{\theta} \\
\text { (deg) }\end{array}$ & $\begin{array}{c}T \\
\left({ }^{\circ} \mathrm{F}\right)\end{array}$ \\
\hline \multirow[t]{8}{*}{ U-35 } & 1 & 6.6 & 257 & 12,5 & 97.0 & & & & & \\
\hline & 50 & 8.0 & 249 & 12.2 & 92.0 & & & & & \\
\hline & 100 & 8. 9 & 261 & 12.1 & 90.0 & & & & & \\
\hline & 150 & 9.1 & 357 & 11.3 & 89.9 & & & & & \\
\hline & 200 & 9.2 & 252 & 11.3 & 89.7 & & & & & \\
\hline & 250 & 9.1 & 250 & 10.9 & B9. 4 & & & & & \\
\hline & 300 & 9.0 & 255 & 10. & 89.3 & & & & & \\
\hline & 400 & 9.4 & 296 & $10 . B$ & 89.1 & & & & & \\
\hline \multirow[t]{8}{*}{$U-36$} & 7 & 3.0 & 230 & 34.8 & 75.1 & 2.5 & 2.4 & 270 & 36.5 & 76.5 \\
\hline & 50 & 3.3 & 253 & 33.3 & 72.1 & 5 & 2.9 & 270 & 37.7 & 74.1 \\
\hline & 100 & 3.6 & 261 & $33 \cdot 3$ & 71.2 & 10 & 3.3 & 268 & 33.4 & 73.1 \\
\hline & 150 & 3.6 & 361 & 31.6 & 70.2 & 20 & 3.6 & 266 & 31.2 & 72.6 \\
\hline & 200 & 3.7 & 262 & 33.4 & 70.5 & 40 & 3.8 & 269 & 32.0 & 71.9 \\
\hline & 250 & 4. 2 & 364 & 29.1 & 69.9 & 80 & 4. I & 268 & 29.3 & 71.5 \\
\hline & 300 & 3.9 & 260 & 27.8 & 69.9 & & & & & \\
\hline & 400 & 4. 2 & 262 & 26.1 & 69.8 & & & & & \\
\hline \multirow[t]{8}{*}{ U- 37} & 7 & 2.5 & 185 & 28.7 & 49.6 & & & & & \\
\hline & 50 & 3.9 & 209 & 21.5 & 18.5 & & & & & \\
\hline & 100 & 4. 1 & 213 & 19.9 & 42.9 & & & & & \\
\hline & 150 & 1.2 & 211 & 19.2 & 47.2 & & & & & \\
\hline & 200 & 4. 3 & 213 & 19.3 & 47.3 & & & & & \\
\hline & 250 & 4. 7 & 216 & 17.9 & 16.8 & & & & & \\
\hline & 300 & 4. 2 & 210 & 17.5 & 16.9 & & & & & \\
\hline & 100 & 4.5 & 211 & 16.5 & 47.0 & & & & & \\
\hline \multirow[t]{8}{*}{$U-38$} & 7 & 3.0 & 206 & 12.2 & 83.2 & 2.5 & 2.7 & 289 & 14.0 & 85.8 \\
\hline & 50 & 4.5 & 283 & 9.0 & 00.5 & 5 & 3,4 & 289 & 12.8 & 84.4 \\
\hline & 100 & 4.3 & 284 & 8. 4 & 79.7 & 10 & 3.9 & 288 & 12.0 & d. 2 \\
\hline & 150 & M & $n$ & H & 78.9 & 20 & 4. $z$ & 287 & 13.2 & 82. 7 \\
\hline & 200 & 4. 8 & 287 & 7.9 & 79.1 & 40 & 1. 1 & 292 & 10.7 & 81.6 \\
\hline & 250 & 5.0 & 285 & 6.7 & 78.4 & 60 & 4. 8 & 288 & 9.1 & 81.2 \\
\hline & 300 & 3. 1 & 292 & 6.3 & 78.4 & & & & & \\
\hline & 400 & 5.3 & 281 & 6.0 & 78.2 & & & & & \\
\hline \multirow[t]{8}{*}{$V=39$} & 7 & B. 0 & 226 & 10.3 & 79.2 & 2.5 & 5.4 & 131 & 12.8 & 9 1.2 \\
\hline & 50 & 10.5 & 217 & 10.0 & 75.8 & 5 & 6.6 & 323 & 13.4 & 80.1 \\
\hline & 100 & 11.0 & 223 & 9.B & 74.6 & 10 & 7.8 & 231 & 12.3 & 79.2 \\
\hline & 150 & $n$ & $n$ & $x$ & 73.7 & 20 & 9.1 & 227 & 13.5 & 7 B. 1 \\
\hline & 200 & 12.3 & 224 & 9.1 & 73.7 & 40 & 10.5 & 231 & 11.3 & 77.3 \\
\hline & 250 & 12.9 & 225 & B. 7 & 73.1 & 80 & 11.6 & 228 & 10.5 & 76.5 \\
\hline & 300 & 12.3 & 229 & 8.6 & 72.8 & & & & & \\
\hline & 100 & 12.9 & 225 & 7.4 & 72.3 & & & & & \\
\hline
\end{tabular}


\$OO ET TOWER

PORTAELE MAST

\begin{tabular}{|c|c|c|c|c|c|c|c|c|c|c|}
\hline & $\begin{array}{c}\text { HEICHT } \\
(t t)\end{array}$ & $\begin{array}{c}U \\
(m / s)\end{array}$ & $\begin{array}{c}\theta \\
(d e g)\end{array}$ & $\begin{array}{c}\sigma_{\theta} \\
(d \theta g)\end{array}$ & $\left(\begin{array}{c}T \\
\left.{ }^{\circ} \mathrm{F}\right)\end{array}\right.$ & $\begin{array}{c}\text { HEICHT } \\
(\mathrm{Et})\end{array}$ & $\begin{array}{c}U \\
(m / \equiv)\end{array}$ & $\begin{array}{c}\theta \\
(\text { deg })\end{array}$ & $\begin{array}{c}\sigma_{\theta} \\
(d e q)\end{array}$ & $\left(\begin{array}{c}T \\
\left({ }^{\circ}\right)\end{array}\right.$ \\
\hline \multirow{8}{*}{$\mathrm{U}-40$} & 7 & 7.5 & 234 & 8.3 & 73.5 & 3.5 & 9. 7 & 239 & 9.2 & 73.9 \\
\hline & 50 & 9.5 & 228 & 7.1 & 72.0 & 5 & 5.9 & 231 & 9.6 & 73.5 \\
\hline & 100 & 10.5 & 229 & 6.9 & 71.1 & 10 & 7.0 & 238 & 8.7 & 73.0 \\
\hline & 150 & $K$ & I & M & 70.5 & 20 & 1. 2 & $n$ & $\mathbf{H}$ & 72.5 \\
\hline & 200 & 11.8 & 230 & 6.6 & 70.5 & 90 & 9.5 & 238 & 7.9 & 71.8 \\
\hline & 250 & 11.9 & 232 & 5.9 & 70.0 & 80 & 10.6 & 235 & 7.1 & 71.4 \\
\hline & 300 & 11.8 & 238 & 5.8 & 69.8 & & & & & \\
\hline & 100 & 12.4 & 232 & 5.1 & 69.4 & & & & & \\
\hline \multirow[t]{8}{*}{$U-91$} & 7 & 6.2 & 223 & 11.3 & 71.2 & & & & & \\
\hline & 50 & Q. 0 & 119 & 8. 7 & 68.3 & & & & & \\
\hline & 100 & B. B & 221 & 7.8 & 67.4 & & & & & \\
\hline & 150 & H & H & $x$ & 66.4 & & & & & \\
\hline & 200 & 9.2 & 226 & 6.9 & 66.9 & & & & & \\
\hline & 250 & 9.8 & 225 & 6.6 & 66.1 & & & & & \\
\hline & 300 & 9.3 & 232 & 6.7 & 65.9 & & & & & \\
\hline & 100 & 9.5 & 222 & 6.5 & 65.7 & & & & & \\
\hline \multirow[t]{8}{*}{$\mathrm{v}-42$} & 7 & 6.3 & 242 & 9.1 & 61.9 & 2.5 & 4. 1 & 248 & 10.3 & $\mathbf{H}$ \\
\hline & 50 & 8. 1 & 237 & 8.0 & 60.3 & 5 & 5.1 & 249 & 10.0 & 61.2 \\
\hline & 100 & 9.1 & 239 & 7.6 & 59.7 & 10 & 6.1 & 247 & 9.1 & 60.9 \\
\hline & 150 & 9.5 & 346 & 7.5 & 58.2 & 20 & 7.1 & 292 & 9.9 & 60.7 \\
\hline & 200 & 9.6 & 237 & 6.6 & 58.7 & 40 & 6.1 & 249 & B. 8 & 59.8 \\
\hline & 250 & 10.3 & 243 & 6.6 & 58.6 & 80 & 9.0 & 249 & 7. 4 & 59.6 \\
\hline & 300 & 10.0 & 250 & 6.4 & 58.5 & & & & & \\
\hline & 100 & 10.3 & 239 & 6.7 & 50.4 & & & & & \\
\hline \multirow[t]{8}{*}{$U-13$} & 7 & 2.2 & 209 & 8.5 & 52.2 & 2. 5 & 2. 3 & 212 & 8. 9 & $M$ \\
\hline & 50 & 5.3 & 201 & 4. 2 & 33.7 & 5 & 2.8 & 212 & 5.9 & 51. \\
\hline & 100 & 6.5 & 204 & 3.7 & 54.1 & 10 & 3.3 & 210 & 5.7 & 51.6 \\
\hline & 150 & $M$ & 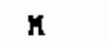 & M & $\mathbf{M}$ & 20 & 4.0 & 201 & 5.6 & 52.0 \\
\hline & 300 & 7.5 & 202 & 3. 3 & 54.2 & 90 & 5.1 & 209 & 5.1 & 52.4 \\
\hline & 250 & 3.0 & 208 & 3.8 & 53.9 & 80 & 6.2 & 207 & 4. 4 & 53.0 \\
\hline & 300 & 8.0 & 217 & 3.7 & 53.8 & & & & & \\
\hline & 100 & b. $?$ & 309 & 4. 0 & 53.5 & & & & & \\
\hline \multirow[t]{8}{*}{$\mathrm{V}-44$} & 1 & 1.6 & 156 & 60.8 & 80.9 & 2.5 & 1.7 & 215 & 73.9 & $\mathbf{M}$ \\
\hline & 50 & 2.4 & 161 & 56.9 & 78.7 & 5 & 1.9 & 216 & 73.2 & B Z, 1 \\
\hline & 100 & 1.8 & 166 & 55.2 & 78.2 & 10 & 2.2 & 208 & 73,3 & 81.3 \\
\hline & 150 & 1.7 & 165 & 54.0 & 77.5 & 20 & 2.3 & 198 & 59.8 & 80.7 \\
\hline & 200 & 2.0 & 170 & 46.9 & 77.6 & 40 & 2. 4 & 195 & 65.6 & 80.4 \\
\hline & 250 & 2.1 & 182 & 45.5 & 76.9 & 80 & 2.6 & 199 & 63.2 & 80.0 \\
\hline & 300 & 2.0 & 200 & 45.9 & 76.6 & & & & & \\
\hline & 400 & 1.9 & 187 & 35.9 & 76.7 & & & & & \\
\hline
\end{tabular}


4QO FT TOUER

PORTABLE MAST

\begin{tabular}{|c|c|c|c|c|c|c|c|c|c|c|}
\hline & $\begin{array}{c}\text { HEIGHT } \\
(t \mathrm{t})\end{array}$ & $\begin{array}{c}U \\
(m / s)\end{array}$ & $\left(\begin{array}{c}\theta \\
(d e g\end{array}\right)$ & $\begin{array}{c}\sigma_{\theta} \\
(\operatorname{deg})\end{array}$ & $\begin{array}{c}T \\
\left({ }^{\circ} \mathbf{F}\right)\end{array}$ & $\begin{array}{c}\text { HEICHT } \\
(t(t)\end{array}$ & $\begin{array}{c}U \\
(\mathrm{~m} / \mathrm{s})\end{array}$ & $\begin{array}{c}\theta \\
(d \in g)\end{array}$ & $\begin{array}{c}\sigma_{\theta} \\
(d e g)\end{array}$ & $\begin{array}{c}T \\
\left({ }^{\circ} \mathrm{F}\right)\end{array}$ \\
\hline \multirow[t]{8}{*}{$u-45$} & 7 & 6.8 & 183 & 6.0 & 67.6 & 2.5 & 4.7 & 200 & B. 3 & $x$ \\
\hline & 50 & 9.7 & 197 & 7.1 & 67.1 & 5 & 6.0 & 199 & 7.3 & 66.9 \\
\hline & 100 & 10.8 & 196 & 5. 4 & 67.0 & 10 & 7.0 & 199 & 7.2 & 66.9 \\
\hline & 150 & B. 9 & 195 & 5.2 & 66.5 & 20 & 8. 0 & 191 & 7.0 & 66.6 \\
\hline & 200 & 11.5 & 195 & 5.2 & 66.9 & 40 & 9.3 & 200 & 6.5 & 66.7 \\
\hline & 250 & $\$ 1 . \$$ & 197 & 5.0 & 66.7 & 80 & 10.4 & 200 & 6.2 & 66.3 \\
\hline & 300 & 11.5 & 218 & 5.0 & 66.1 & & & & & \\
\hline & 100 & 12.2 & 202 & 4.7 & 66.1 & & & & & \\
\hline
\end{tabular}

$\begin{array}{rrrrrrrrrrr}\text { U-46 } & 7 & 6.9 & 213 & 12.4 & 52.2 & 2.5 & 1.9 & 216 & 11.6 & M \\ & 50 & 9.4 & 197 & 10.9 & 49.7 & 5 & 6.0 & 216 & 10.5 & M \\ 100 & 10.0 & 209 & 10.0 & 49.3 & 10 & 7.1 & 215 & 10.9 & M \\ 150 & 10.1 & 209 & 9.3 & 48.6 & 20 & 8.1 & 208 & 11.7 & 11 \\ 200 & 10.2 & 209 & 9.3 & 48.7 & 40 & 9.1 & 216 & 11.1 & M \\ 250 & 10.6 & 213 & 9.1 & 48.2 & 80 & 9.5 & 215 & 10.2 & M \\ & 300 & 10.9 & 233 & 8.8 & 48.0 & & & & & \\ 100 & 11.0 & 217 & 0.9 & 47.8 & & & & \end{array}$

\begin{tabular}{|c|c|c|c|c|c|c|c|c|c|c|}
\hline \multirow[t]{8}{*}{$\mathrm{U}=47$} & 7 & 4.6 & 256 & 9.2 & 57.1 & 2.5 & $\mathbf{M}$ & M & M & $M$ \\
\hline & 50 & 6.3 & 231 & 9.6 & 56.1 & 5 & M & $\mathbf{M}$ & M & $M$ \\
\hline & 100 & 8. 2 & 228 & 9.2 & 35.5 & 10 & 4.9 & 249 & 10.0 & $M$ \\
\hline & 150 & 7.7 & 239 & 9.6 & 55.2 & 20 & 5.6 & 294 & 10.8 & 56.1 \\
\hline & 200 & 7.4 & 241 & 10.0 & 55.1 & 40 & 6.4 & 250 & 11.1 & 57.2 \\
\hline & 250 & 7.7 & 260 & 0. 9 & 54.6 & 80 & 7.1 & 292 & 9.7 & 53.9 \\
\hline & 300 & 7.6 & 258 & 9.8 & 54.5 & & & & & \\
\hline & 100 & 7.9 & 246 & B. 8 & 59.6 & & & & & \\
\hline \multirow[t]{8}{*}{$U-48$} & 7 & 1.3 & 248 & 10.8 & 50.5 & 2.5 & H & $M$ & I & $M$ \\
\hline & 50 & 3. & 226 & B. 2 & 52.6 & 5 & $\mathbf{M}$ & ท & $\mathbf{H}$ & $M$ \\
\hline & 100 & 5.5 & 223 & 7.1 & 52.7 & 10 & 2.2 & 246 & 11.7 & M \\
\hline & 150 & 5.5 & 233 & 6.3 & 52.9 & 20 & 2.6 & 241 & 10.7 & 51.3 \\
\hline & 200 & $M$ & 237 & 5. 7 & 53.0 & 40 & 3.6 & 246 & 8.4 & 53.2 \\
\hline & 250 & 5.1 & 253 & 7.0 & 52.5 & 00 & 4.2 & 241 & 6.9 & 50.9 \\
\hline & 300 & 5.5 & $\mathbf{n}$ & $M$ & 52.4 & & & & & \\
\hline & 100 & 5.9 & 240 & 4. 3 & 52.5 & & & & & \\
\hline
\end{tabular}

\begin{tabular}{|c|c|c|c|c|c|}
\hline \multirow[t]{2}{*}{$U-49$} & 7 & 0.9 & 326 & 24.0 & 50.8 \\
\hline & 50 & 1.1 & 298 & 25.9 & 50.5 \\
\hline & 100 & 1. 7 & $2 B 9$ & 25.9 & 50.1 \\
\hline & 150 & 1.4 & 296 & 24.0 & 49.9 \\
\hline & 200 & 0.9 & 289 & 21,3 & 19.9 \\
\hline & 250 & 0.9 & 301 & 23.4 & 49.3 \\
\hline & 300 & 1.1 & 296 & 23.7 & 49.2 \\
\hline & 100 & 1.0 & 271 & 25.? & 49 \\
\hline
\end{tabular}


100 FT TOWER

PORTABLE MAST

\begin{tabular}{|c|c|c|c|c|c|c|c|c|c|c|}
\hline & $\begin{array}{c}\text { HE IGHT } \\
(t())\end{array}$ & $\begin{array}{c}U \\
(m / s)\end{array}$ & $\stackrel{\theta}{(d \bullet g)}$ & $(\stackrel{\sigma}{\theta})$ & $\begin{array}{c}T \\
(\circ \mathrm{F})\end{array}$ & $\begin{array}{c}\text { HEIGHT } \\
(t))^{2}\end{array}$ & $\begin{array}{c}U \\
(a / s)\end{array}$ & $\begin{array}{c}\theta \\
(d e g)\end{array}$ & $\begin{array}{c}\sigma_{\theta} \\
(d \in q)\end{array}$ & $\begin{array}{c}T \\
(\circ E)\end{array}$ \\
\hline \multirow[t]{8}{*}{$\mathrm{U}-50$} & 7 & 6.8 & 214 & 9.7 & 49.3 & 2. 5 & 4.7 & 213 & 10.5 & H \\
\hline & 30 & 9.1 & 197 & B. 2 & 47.9 & 5 & 5.7 & 214 & 9. B & $M$ \\
\hline & 100 & 9.9 & 192 & 7.8 & 47.5 & 10 & 6.6 & 212 & 10.0 & $\mathbf{n}$ \\
\hline & 150 & 8.0 & 204 & 6.8 & 47.0 & 20 & 7.5 & 203 & 10.6 & $M$ \\
\hline & 200 & 10.8 & 210 & 7.1 & 46.7 & 10 & 8.6 & 214 & 9.8 & $M$ \\
\hline & 250 & 10.6 & 228 & 6.8 & 47.0 & 80 & 9.3 & 210 & 8.3 & n \\
\hline & 300 & $\mathbf{H}$ & 210 & 6.0 & 46.2 & & & & & \\
\hline & 100 & 11.2 & 214 & 6.3 & 46.3 & & & & & \\
\hline \multirow[t]{8}{*}{$u-5 t$} & 7 & 4.0 & 250 & 14.4 & 54.5 & 2.5 & 3.0 & 212 & 14.1 & $\mathbf{M}$ \\
\hline & 50 & 5.7 & 237 & 12.1 & 52.9 & 5 & $n$ & 238 & 13.9 & 53.5 \\
\hline & 100 & 6.0 & 222 & 10.2 & 52.2 & 10 & 1. 1 & 242 & 13.6 & 53.4 \\
\hline & 150 & 4.9 & 235 & 9.9 & 51.8 & 20 & 4.7 & 237 & 19.3 & 52.7 \\
\hline & 200 & 6.3 & 240 & 9.7 & 51.7 & 40 & 5. 4 & 243 & 12.5 & 52,1 \\
\hline & 250 & 6.3 & 360 & 9.6 & 51.2 & 80 & 5.8 & 240 & 10.5 & 51.3 \\
\hline & 300 & 6.3 & 240 & 9.8 & 51.0 & & & & & \\
\hline & 400 & 6.6 & 244 & 0.6 & 51.0 & & & & & \\
\hline \multirow[t]{8}{*}{$U-52$} & 7 & 6.8 & 242 & 14.7 & 61.3 & 2.5 & 4. 7 & 243 & 6. 3 & M \\
\hline & 50 & a. 4 & 223 & 13.0 & 58.1 & 5 & 5.6 & $\mathbf{M}$ & $\mathbf{H}$ & 56.5 \\
\hline & 100 & 9.1 & $22 t$ & 12.0 & 57.6 & 10 & 6.5 & 243 & 8. 3 & 56.2 \\
\hline & 150 & $n$ & H & $\mathbf{F}$ & 56.9 & 30 & 7.5 & 237 & 9.0 & 55.1 \\
\hline & 200 & 9.6 & 236 & 11.6 & 56.5 & 40 & 8. 3 & 243 & 0.0 & 59.4 \\
\hline & 250 & $n$ & $\mathbf{M}$ & $\mathbf{M}$ & 56.2 & $B O$ & 9.0 & 238 & 7.3 & 53.3 \\
\hline & 300 & $\mathbf{M}$ & $\mathbf{H}$ & H & 56.0 & & & & & \\
\hline & 100 & H & M & $x$ & 55.9 & & & & & \\
\hline \multirow[t]{8}{*}{$U-53$} & 7 & 10.9 & $2: 9$ & 9.8 & 55.9 & 2.5 & 7.6 & 219 & 10.4 & 53.0 \\
\hline & 50 & 13.7 & 199 & 8.2 & 55.8 & 5 & 8. 9 & 216 & 9.8 & 51.6 \\
\hline & 100 & 15.0 & 198 & 7.9 & 53.5 & 10 & 10.0 & 217 & 10.2 & 51.1 \\
\hline & 150 & 15.5 & 207 & 7.8 & 54.7 & 20 & 11.5 & 210 & 10.5 & $\boldsymbol{M}$ \\
\hline & 200 & 16.0 & 211 & 7.4 & 54.7 & 90 & 13.0 & 218 & 9.6 & 50.0 \\
\hline & 250 & 15.8 & 233 & 7.4 & 54.1 & 80 & 14.3 & 219 & 9. 7 & 48.8 \\
\hline & 300 & $\mathbf{K}$ & $\mathbf{H}$ & $n$ & 53.0 & & & & & \\
\hline & 400 & 16.5 & 221 & 7.1 & 53.8 & & & & & \\
\hline \multirow[t]{8}{*}{ U-51 } & 7 & 3.3 & 232 & d. 7 & 10.5 & 2.5 & 2.9 & 240 & 10.4 & 38.4 \\
\hline & 50 & 5.6 & 222 & 6.8 & 42,3 & 5 & 2,9 & 239 & 10.3 & 38. \\
\hline & 100 & 6.9 & 215 & 5.9 & 42.1 & 10 & 3.4 & 240 & 10.6 & 38.9 \\
\hline & 150 & $x$ & $M$ & $n$ & 42.5 & 20 & 4. 1 & 234 & 10.7 & 39.5 \\
\hline & 200 & B. 4 & 234 & 4. 4 & 42.9 & 40 & 5.0 & 241 & 8.8 & 39.8 \\
\hline & 250 & $n$ & $n$ & $\mathbf{n}$ & 92.8 & 80 & 6.0 & 235 & 6.8 & 39.9 \\
\hline & 300 & $\mathbf{H}$ & 11 & H & 42.9 & & & & & \\
\hline & 100 & $x$ & $\mathbf{n}$ & $\mathbf{n}$ & 43. 4 & & & & & \\
\hline
\end{tabular}




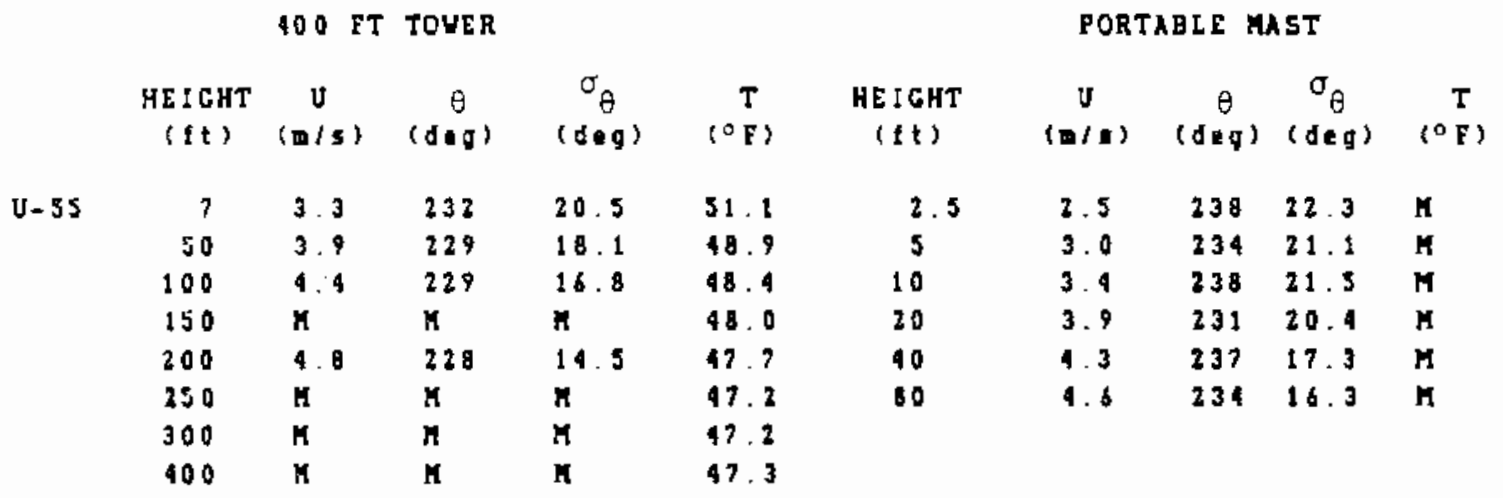

63 SERIES

\begin{tabular}{|c|c|c|c|c|c|}
\hline \multirow[t]{8}{*}{ FT $-1=63$} & $\gamma$ & 0.6 & 222 & 13.6 & 12.: \\
\hline & 50 & 2.1 & 256 & 19.9 & 14.1 \\
\hline & 100 & 3.5 & 256 & 12.6 & 45.1 \\
\hline & 130 & 4. 1 & 248 & 12.2 & 16.2 \\
\hline & 200 & 5.5 & 258 & 9.4 & 17.2 \\
\hline & 230 & 5.7 & 254 & 8.5 & 47.7 \\
\hline & 300 & 5.3 & 261 & 9.0 & 47.6 \\
\hline & 400 & 5.4 & 261 & 9.1 & 17.6 \\
\hline \multirow{8}{*}{$F T-3-63$} & 7 & 0.1 & 011 & 66.1 & 42.0 \\
\hline & 50 & 0.5 & 288 & 48.0 & 47.7 \\
\hline & 100 & 1.2 & 297 & 14.5 & 18.2 \\
\hline & 150 & 2. 1 & 293 & 11.2 & 18.6 \\
\hline & 200 & 2.8 & 300 & 6.3 & 49.5 \\
\hline & 250 & 3.7 & 298 & 5.4 & 19.8 \\
\hline & 300 & 3.9 & 300 & 3.2 & 50.3 \\
\hline & 400 & 4.7 & 291 & 3.6 & 50.7 \\
\hline \multirow[t]{8}{*}{ FT $-4-63$} & 7 & 3.4 & 311 & 8. 0 & 61.3 \\
\hline & 50 & 6.2 & 314 & $\$ .5$ & 63.0 \\
\hline & 100 & 7.7 & 308 & 4.0 & 63.6 \\
\hline & 150 & 9. 8 & 300 & 2.9 & 64.7 \\
\hline & 200 & 9.6 & 311 & 2.9 & 66.7 \\
\hline & 250 & 8.6 & 310 & 3.4 & 67.8 \\
\hline & 300 & 0. 4 & 317 & 3.9 & 68.2 \\
\hline & 100 & 2.1 & 318 & 5. $t$ & 68.4 \\
\hline \multirow[t]{8}{*}{$F T-5-63$} & 7 & 2.7 & 309 & 4. 7 & 61.8 \\
\hline & 50 & 5.8 & $3: 2$ & 3.8 & 64.0 \\
\hline & 100 & 1. 2 & 310 & 3.1 & 66.4 \\
\hline & 150 & 8.5 & 308 & 3. 8 & 69.5 \\
\hline & 200 & d. 2 & 320 & 2.7 & 70.0 \\
\hline & 250 & 7.3 & 320 & 3.7 & 70.0 \\
\hline & 300 & 7.2 & 327 & 3.7 & 69.9 \\
\hline & 400 & 6.5 & 330 & 5.1 & 69.8 \\
\hline
\end{tabular}


100 ET TOWER

PORTAgLE MAST

\begin{tabular}{|c|c|c|c|c|c|c|c|c|c|c|}
\hline & $\begin{array}{l}\text { EIG TTT } \\
(t t)\end{array}$ & $\begin{array}{c}U \\
(m / 5)\end{array}$ & $\begin{array}{c}\theta \\
(d \in g)\end{array}$ & $\begin{array}{c}\sigma_{\theta} \\
(d \bullet g)\end{array}$ & $\begin{array}{c}T \\
(O E)\end{array}$ & $\begin{array}{c}\text { HEIGHT } \\
(t \mathrm{t})\end{array}$ & $\begin{array}{c}v \\
(n / s)\end{array}$ & $\left(\begin{array}{c}\theta \\
(d \in g)\end{array}\right.$ & $\begin{array}{c}\sigma_{\theta} \\
(d \in g)\end{array}$ & $\begin{array}{c}T \\
(\circ F)\end{array}$ \\
\hline \multirow[t]{8}{*}{$F T-6-63$} & 7 & 1.3 & 246 & 20.6 & 55.7 & & & & & \\
\hline & 50 & 3.4 & 259 & 16.8 & 57.4 & & & & & \\
\hline & 100 & 3.6 & 252 & 14.0 & 57.6 & & & & & \\
\hline & 150 & 3.5 & 242 & 10.9 & 57.7 & & & & & \\
\hline & 200 & 4.2 & 252 & 10.9 & 58.1 & & & & & \\
\hline & 250 & 3.5 & 253 & 9.7 & 57.9 & & & & & \\
\hline & 300 & 4. 5 & 266 & 9.9 & 57.7 & & & & & \\
\hline & 400 & 4.4 & 264 & 9.0 & 57.3 & & & & & \\
\hline \multirow[t]{8}{*}{ FT $-7-63$} & 7 & 2.7 & 293 & 7.0 & 62.7 & & & & & \\
\hline & 50 & 4.6 & 294 & 5.8 & 63.3 & & & & & \\
\hline & 100 & 3.9 & 292 & 6.1 & 63.2 & & & & & \\
\hline & 150 & 5.6 & 291 & 5.7 & 63.1 & & & & & \\
\hline & 200 & 7.1 & 291 & 5.9 & 63.5 & & & & & \\
\hline & 250 & 4.0 & 300 & 4.2 & 63.2 & & & & & \\
\hline & 300 & 7.6 & $30 \pm$ & 4. 7 & 63.3 & & & & & \\
\hline & 400 & 9.1 & 297 & 4. 1 & 63.4 & & & & & \\
\hline \multirow[t]{8}{*}{ FT $-8-63$} & 7 & 1.2 & 278 & 21.1 & 59.1 & & & & & \\
\hline & 50 & 2.3 & 277 & 19.0 & 59.4 & & & & & \\
\hline & 100 & 3.2 & 283 & 16.9 & 59.0 & & & & & \\
\hline & 150 & 2.6 & 278 & 17.0 & 58.7 & & & & & \\
\hline & 200 & 3.6 & 202 & 16.8 & 58.9 & & & & & \\
\hline & 250 & 4.5 & 280 & 17.5 & 58.7 & & & & & \\
\hline & 300 & 4.0 & 291 & 15.8 & $5 B .6$ & & & & & \\
\hline & 900 & 4. 7 & 278 & 17.9 & 58.4 & & & & & \\
\hline \multirow[t]{8}{*}{ FT $-9-63$} & 7 & 2.2 & 289 & 10.8 & 67.8 & & & & & \\
\hline & 50 & 4. 3 & 287 & 11.7 & 70.4 & & & & & \\
\hline & 100 & 5.3 & 298 & 10.5 & 70.0 & & & & & \\
\hline & 150 & 5.4 & 297 & 9.9 & 69.9 & & & & & \\
\hline & 200 & 7.5 & 302 & 1. 6 & 70.5 & & & & & \\
\hline & 250 & 6. 2 & 304 & 7.3 & 72.2 & & & & & \\
\hline & 300 & D. 2 & 304 & 6.8 & 70.7 & & & & & \\
\hline & 900 & 9.4 & 300 & 5.1 & 71.3 & & & & & \\
\hline \multirow[t]{8}{*}{$F T-10-63$} & 7 & 1. 7 & 274 & 7.2 & 58.4 & & & & & \\
\hline & 50 & 3.6 & 269 & 6.2 & 62.3 & & & & & \\
\hline & 100 & 3.8 & 280 & 6.1 & 61.6 & & & & & \\
\hline & 150 & 3.6 & 279 & 6.8 & 62.4 & & & & & \\
\hline & 200 & 4. 8 & 284 & 6.2 & 61.7 & & & & & \\
\hline & 250 & 4. 5 & 283 & 5.6 & 61.4 & & & & & \\
\hline & 300 & 4. 4 & 282 & 5.5 & 61.3 & & & & & \\
\hline & 400 & 5.1 & 282 & 5.6 & 60.8 & & & & & \\
\hline
\end{tabular}


40O FT TOWER

PORTARLE MAST

\begin{tabular}{|c|c|c|c|c|c|c|c|c|c|}
\hline $\begin{array}{c}\text { HE I GHT } \\
(t t)\end{array}$ & $\begin{array}{c}6 \\
(m / 5)\end{array}$ & $\begin{array}{c}\theta \\
(d \in g)\end{array}$ & $\begin{array}{c}\sigma_{\theta} \\
(\boldsymbol{d} \mathbf{e})\end{array}$ & $\begin{array}{c}T \\
\left({ }^{\circ} \mathrm{F}\right)\end{array}$ & $\begin{array}{c}\text { HEIGHT } \\
(\mathrm{t} t)\end{array}$ & $\begin{array}{c}U \\
( \pm / 5)\end{array}$ & $\begin{array}{c}\theta \\
(d e g)\end{array}$ & $\begin{array}{c}\sigma_{\theta} \\
(d \in g)\end{array}$ & $\begin{array}{c}\mathrm{T} \\
\left({ }^{\circ} \mathrm{F}\right)\end{array}$ \\
\hline$E \tau-11-63 \quad 7$ & 1.6 & 272 & 6.2 & 57.6 & & & & & \\
\hline 50 & 4.2 & 277 & 4.0 & 61.6 & & & & & \\
\hline 100 & 9. 9 & 289 & 3.2 & 62.0 & & & & & \\
\hline 150 & 4. 7 & 286 & 4.0 & 62.0 & & & & & \\
\hline 200 & 6.2 & 291 & 3. 5 & 62.3 & & & & & \\
\hline 250 & 5.9 & 288 & 3.0 & 63.0 & & & & & \\
\hline 300 & 5.9 & 289 & 3.0 & 62.0 & & & & & \\
\hline 400 & 6.7 & 290 & 3.9 & 62.0 & & & & & \\
\hline ET- $12-63 \quad 7$ & 1. 1 & 245 & 20.2 & 60.1 & & & & & \\
\hline 50 & 2.5 & 253 & 21.9 & 62.7 & & & & & \\
\hline 100 & 3.0 & 261 & 23.2 & 62.7 & & & & & \\
\hline 150 & 3.2 & 261 & 19.4 & 63.2 & & & & & \\
\hline 200 & 4. 1 & 268 & 16.0 & 64.5 & & & & & \\
\hline 250 & 4. 6 & 268 & 14.0 & 69.6 & & & & & \\
\hline 300 & \$. 3 & 268 & $1: 5$ & 64.9 & & & & & \\
\hline 100 & 6.2 & $27 \%$ & 8.6 & 65.2 & & & & & \\
\hline FT-13-63 7 & 0.5 & 286 & 6.8 & 68.2 & & & & & \\
\hline 50 & 2.1 & 288 & 7.5 & 72.0 & & & & & \\
\hline 100 & H & 293 & 7.8 & 72.7 & & & & & \\
\hline 150 & 2. 8 & 290 & 5,0 & 72.9 & & & & & \\
\hline 200 & 3.7 & 292 & 4. 3 & 73.7 & & & & & \\
\hline 250 & 4.2 & 296 & 4. 0 & 73.3 & & & & & \\
\hline 300 & 4. 3 & 287 & 1. 0 & 73.3 & & & & & \\
\hline 400 & 4.9 & 286 & 4.3 & 73.3 & & & & & \\
\hline $57-14-637$ & 2.0 & 299 & 7.9 & 64.6 & & & & & \\
\hline 50 & 3.5 & 297 & 6.0 & 65.2 & & & & & \\
\hline 100 & 4.4 & 307 & 4.3 & 65.9 & & & & & \\
\hline 150 & 9.6 & 307 & 3.6 & 65.0 & & & & & \\
\hline 200 & 3. 8 & 310 & 3.2 & 65.4 & & & & & \\
\hline 250 & 6.6 & 312 & 3.3 & 65.2 & & & & & \\
\hline 300 & 6.9 & 305 & 2. 8 & 65.1 & & & & & \\
\hline 100 & 5. 0 & 306 & 2.7 & 65.2 & & & & & \\
\hline ET-15-63 7 & 0.8 & 297 & 14.4 & 58.8 & & & & & \\
\hline 50 & 3.5 & 304 & 5.1 & 63.2 & & & & & \\
\hline 100 & 4.6 & 310 & 2.0 & 63.7 & & & & & \\
\hline 150 & 5.0 & 309 & 2.4 & 63.4 & & & & & \\
\hline 200 & 6.4 & 313 & 2.5 & 64.2 & & & & & \\
\hline 250 & 6.9 & 312 & 2.5 & 64.0 & & & & & \\
\hline 300 & 6.9 & $3 \div 0$ & 3.7 & 64.0 & & & & & \\
\hline 100 & 6. 1 & 309 & 2.2 & 64.2 & & & & & \\
\hline
\end{tabular}


100 FT TOUER

PORTABLE MAST

\begin{tabular}{|c|c|c|c|c|c|c|c|c|c|}
\hline $\begin{array}{c}\text { HE I CHT } \\
(t) \text { (t) }\end{array}$ & $\begin{array}{c}U \\
(m / s)\end{array}$ & $\begin{array}{c}\theta \\
(d \in \theta)\end{array}$ & $\begin{array}{c}\sigma_{\theta} \\
(d \bullet q)\end{array}$ & $\left(0^{\circ} \mathrm{F}\right)$ & $\begin{array}{c}\text { HEICHT } \\
(t t)\end{array}$ & $\begin{array}{c}\mathrm{U} \\
\langle\mathrm{w} / \mathrm{s}\rangle\end{array}$ & $\begin{array}{c}\theta \\
(d \pm g)\end{array}$ & $\begin{array}{c}\sigma_{\theta} \\
(d \in \boldsymbol{q})\end{array}$ & $\begin{array}{c}\mathrm{T} \\
\left({ }^{\circ} \mathrm{F}\right)\end{array}$ \\
\hline ET-16-837 & 0.4 & 291 & 29.4 & 61.9 & & & & & \\
\hline 50 & 2.4 & 312 & 17.4 & 69.2 & & & & & \\
\hline 100 & 3.1 & 313 & 15. 1 & 69.8 & & & & & \\
\hline 150 & 3.9 & 323 & 11.7 & 70.2 & & & & & \\
\hline 200 & 4.2 & 302 & 9.4 & 71.0 & & & & & \\
\hline 250 & 4.7 & 199 & 9. 6 & 71.0 & & & & & \\
\hline 301 & 1.9 & 296 & 7. 7 & 71.0 & & & & & \\
\hline 100 & 5.7 & 193 & 6.4 & 71.0 & & & & & \\
\hline ET-17-63 7 & 0.6 & 305 & 26.4 & 51.6 & & & & & \\
\hline 50 & 3.4 & 291 & 14.1 & 53.7 & & & & & \\
\hline 100 & 3.5 & 296 & 3.9 & 54.1 & & & & & \\
\hline 150 & 4. 2 & 295 & 3.4 & 54.0 & & & & & \\
\hline 200 & 5.1 & 300 & 4.2 & 55.3 & & & & & \\
\hline 250 & 6.7 & 302 & 5.4 & 55. B & & & & & \\
\hline 300 & 6.7 & 299 & 5.6 & 56.3 & & & & & \\
\hline 100 & 7.7 & 307 & 5.3 & 56.9 & & & & & \\
\hline FT $-18-63 ?$ & 3.3 & 323 & 26.4 & 66.1 & & & & & \\
\hline 50 & $\mathbf{4}: 3$ & 309 & 13.4 & 64.3 & & & & & \\
\hline 100 & 4. 4 & 321 & 12.2 & 63.3 & & & & & \\
\hline 150 & 1. 2 & 320 & 11.0 & 62.7 & & & & & \\
\hline 200 & 4. 3 & 324 & 10.9 & 62.9 & & & & & \\
\hline 250 & 5.0 & 323 & 10.5 & 62.7 & & & & & \\
\hline 300 & 4.6 & 316 & 10.6 & 62.6 & & & & & \\
\hline 100 & 1. 6 & 319 & 11.2 & 62.0 & & & & & \\
\hline
\end{tabular}

164 SERIES

$\begin{array}{cccccc}5-64-1 & 7 & 1.9 & 304 & 6.6 & 39.8 \\ & 50 & 4.2 & 297 & 5.5 & 11.0 \\ 100 & 5.5 & 311 & 5.3 & 11.9 \\ & 150 & 5.7 & 308 & 5.2 & 41.6 \\ & 200 & 6.8 & 300 & 5.7 & 42.1 \\ & 250 & 7.7 & 304 & 5.3 & 41.9 \\ & 300 & 7.6 & 305 & 6.5 & 41.9 \\ & 400 & 8.5 & 302 & 5.4 & 12.0 \\ & & & & & \\ 5-64-2 & 7 & 1.3 & 279 & 7.2 & 51.0 \\ & 50 & 3.5 & 282 & 4.3 & 54.2 \\ & 100 & 4.5 & 302 & 3.8 & 54.6 \\ & 150 & 1.6 & 100 & 3.4 & 54.9 \\ & 200 & 5.7 & 294 & 3.3 & 55.6 \\ & 250 & 6.6 & 296 & 3.7 & 55.8 \\ & 300 & 6.8 & 300 & 2.8 & 56.3 \\ 100 & 7.1 & 305 & 5.3 & 57.1\end{array}$


$100 \mathrm{FT}$ TOWER PORTABLE MAST

\begin{tabular}{|c|c|c|c|c|c|c|c|c|c|c|}
\hline & $\begin{array}{l}\text { HEIEHT } \\
(f t)\end{array}$ & $\begin{array}{c}U \\
(x / 5)\end{array}$ & $\begin{array}{c}\theta \\
(d e g)\end{array}$ & $\begin{array}{c}\sigma_{\theta} \\
(d \in g)\end{array}$ & $\begin{array}{c}T \\
\left({ }^{\circ} \mathrm{E}\right)\end{array}$ & $\begin{array}{l}\text { HEICHT } \\
(f t)\end{array}$ & $\begin{array}{c}U \\
(m / 5)\end{array}$ & $\begin{array}{c}\theta \\
(\theta \in g)\end{array}$ & $\begin{array}{c}\sigma_{\theta} \\
(\text { deg })\end{array}$ & $\begin{array}{c}T \\
(\circ \mathrm{F})\end{array}$ \\
\hline \multirow[t]{8}{*}{$5-64-4$} & 7 & 1.0 & 290 & 14.3 & $3 B .3$ & & & & & \\
\hline & 50 & 3.0 & 291 & 9.3 & 81.7 & & & & & \\
\hline & 100 & 4. 1 & 308 & 5.7 & 62.0 & & & & & \\
\hline & 150 & 4. 5 & 309 & 5.0 & 62.4 & & & & & \\
\hline & 200 & 6.5 & 308 & 4. 3 & 63.4 & & & & & \\
\hline & 250 & 7.9 & 307 & 2.5 & 64.5 & & & & & \\
\hline & 300 & B. 0 & 312 & 3.1 & 65.7 & & & & & \\
\hline & 400 & 1.0 & 309 & 1.7 & 67.2 & & & & & \\
\hline \multirow[t]{8}{*}{$5-64-5$} & 7 & 1.9 & 335 & 20.6 & 39.5 & & & & & \\
\hline & 50 & 4. 2 & 319 & 17.5 & 63.8 & & & & & \\
\hline & 100 & 4. 9 & 326 & 14.1 & 64.6 & & & & & \\
\hline & 150 & 4. 8 & 314 & 12.1 & 64.8 & & & & & \\
\hline & 200 & 6.0 & 301 & 10.1 & 65.2 & & & & & \\
\hline & 250 & 6.7 & 296 & 7.9 & 65.4 & & & & & \\
\hline & 300 & 6.7 & 300 & 6.6 & 65.6 & & & & & \\
\hline & 400 & B. 0 & 290 & 5. 2 & 66.2 & & & & & \\
\hline \multirow[t]{8}{*}{$S-64-6$} & 3 & 1.0 & 328 & 9.7 & 63.3 & & & & & \\
\hline & 50 & K & 4 & Y & 69.6 & & & & & \\
\hline & 100 & 3.1 & 341 & 8. 9 & 69.8 & & & & & \\
\hline & 150 & 2.9 & 337 & 7.1 & 69.7 & & & & & \\
\hline & 200 & 3.2 & 330 & 7.6 & 69.9 & & & & & \\
\hline & 250 & 3.5 & 328 & 8.2 & 69.7 & & & & & \\
\hline & 300 & 3. 5 & 334 & 7.5 & 69.6 & & & & & \\
\hline & 400 & 4. 5 & 326 & 10.0 & 69.3 & & & & & \\
\hline \multirow[t]{8}{*}{$S-64-7$} & 7 & 2.8 & 29: & d. 2 & 70.4 & & & & & \\
\hline & 50 & S. 4 & 286 & 6.7 & 73.8 & & & & & \\
\hline & 100 & 6.8 & 303 & 6.7 & 73.8 & & & & & \\
\hline & 150 & 6.7 & 903 & 6.5 & 74.3 & & & & & \\
\hline & 200 & 8. 1 & 300 & 6.8 & 75.2 & & & & & \\
\hline & 250 & 8.6 & 300 & 6.5 & 75.4 & & & & & \\
\hline & 300 & 8.0 & 304 & 7.1 & 75.6 & & & & & \\
\hline & 400 & 8. 6 & 305 & 5.5 & 76.6 & & & & & \\
\hline \multirow[t]{8}{*}{$5-64-8$} & 7 & 0.8 & 319 & 15.8 & 68.0 & & & & & \\
\hline & 50 & 3.6 & 310 & 12.3 & 70.7 & & & & & \\
\hline & 100 & 4.6 & 323 & 10.1 & 71.1 & & & & & \\
\hline & 150 & 1. 4 & 319 & 9.2 & 71.3 & & & & & \\
\hline & 200 & 5.6 & 311 & B. 0 & 71.8 & & & & & \\
\hline & 250 & 5.6 & 304 & 7.5 & 72.0 & & & & & \\
\hline & 300 & 5.5 & 312 & B. 2 & 72.3 & & & & & \\
\hline & 400 & 5.8 & 396 & 9.3 & 72.8 & & & & & \\
\hline
\end{tabular}




\begin{tabular}{|c|c|c|c|c|c|c|c|c|c|c|}
\hline & $\begin{array}{c}\text { HEI GHT } \\
(t \mathrm{t})\end{array}$ & $\begin{array}{c}4 \\
(m / 5)\end{array}$ & $\begin{array}{c}\theta \\
(d \in q)\end{array}$ & $\begin{array}{c}\sigma_{\theta} \\
(d \bullet g)\end{array}$ & $\left(\begin{array}{c}T \\
\left({ }^{\circ}\right)\end{array}\right.$ & $\begin{array}{c}\text { HEICHT } \\
\text { (It) }\end{array}$ & $\begin{array}{c}U \\
(n / g)\end{array}$ & $\left(\begin{array}{c}\theta \\
(d e g)\end{array}\right.$ & $\begin{array}{c}\sigma_{\theta} \\
\left(\operatorname{deq}^{\prime}\right)\end{array}$ & $\begin{array}{c}T \\
\left({ }^{\circ} E\right)\end{array}$ \\
\hline \multirow[t]{8}{*}{$5-69-9$} & 7 & 1.4 & 274 & 14.4 & 36.2 & 2.5 & 1. 4 & 267 & M & 57.1 \\
\hline & 50 & 3.1 & 271 & 13.4 & 59.7 & 5 & 1.6 & 269 & H & 58.5 \\
\hline & 100 & 4.0 & 289 & 10.5 & 60.0 & 10 & 2.2 & 271 & $\mathbf{M}$ & 59.6 \\
\hline & 150 & 3. 7 & 287 & 9.1 & 59.7 & 20 & 2.0 & 260 & $\mathbf{M}$ & 60.6 \\
\hline & 200 & 4.8 & 286 & 7.5 & 59.6 & 40 & 3.4 & 276 & $\mathbf{H}$ & 61.4 \\
\hline & 250 & 5.6 & 280 & 6.6 & 59.6 & 80 & 4.6 & 273 & $\mathbf{M}$ & 61.7 \\
\hline & 300 & 5. 7 & 296 & 5.9 & 59.6 & & & & & \\
\hline & 400 & 1.0 & 289 & 5.0 & 59.6 & & & & & \\
\hline \multirow[t]{8}{*}{$5-69-10$} & 7 & 2.0 & 305 & 14.2 & 66.0 & & & & & \\
\hline & 50 & 4. 1 & 298 & B. 7 & 67.7 & & & & & \\
\hline & 100 & 5.3 & $3: 3$ & 6.5 & 68.0 & & & & & \\
\hline & 150 & 5.2 & 311 & 5.8 & 68.0 & & & & & \\
\hline & 200 & 6.3 & 305 & 3.0 & 68.4 & & & & & \\
\hline & 250 & $n$ & Y & H & 68.3 & & & & & \\
\hline & 300 & 6.7 & 313 & 5.3 & 68.4 & & & & & \\
\hline & 400 & 1. 4 & 300 & 3.9 & 68.6 & & & & & \\
\hline \multirow[t]{8}{*}{$5-64-11$} & 7 & 0.8 & 306 & 3.6 & 56.8 & 3.5 & 0.9 & 307 & 8. 0 & 56.8 \\
\hline & 50 & 3.5 & 312 & 4. 0 & 64.9 & 5 & 1.2 & 305 & 4.7 & 58.4 \\
\hline & 100 & 3.7 & 341 & 8.5 & 68.1 & 10 & 1.7 & 304 & 2.8 & 59.9 \\
\hline & 150 & 2. $B$ & 349 & 11.6 & 68.2 & 20 & 2.6 & $\mathbf{M}$ & $M$ & 62.4 \\
\hline & 200 & 2.8 & 349 & 11.8 & 67.7 & 40 & 3.9 & 313 & 2.7 & 64.6 \\
\hline & 250 & 2.6 & 013 & 12.0 & 68.2 & 80 & 4.8 & 317 & 6.2 & 67.7 \\
\hline & 300 & 2.3 & 005 & 12.1 & 68.0 & & & & & \\
\hline & 100 & 2.4 & 354 & 11.1 & 67.6 & & & & & \\
\hline \multirow[t]{8}{*}{$5-64-12$} & 7 & 1.5 & 286 & 12.0 & 67.6 & & & & & \\
\hline & 50 & 3.6 & 288 & 7.0 & 71.0 & & & & & \\
\hline & 100 & 5.5 & 307 & 3. 4 & 72.8 & & & & & \\
\hline & 150 & 5.5 & 305 & 3.4 & 73.5 & & & & & \\
\hline & 200 & 7.3 & 302 & 3.1 & 74.7 & & & & & \\
\hline & 250 & 7.2 & 330 & 3.0 & 75.4 & & & & & \\
\hline & 300 & 5.8 & 315 & 4.2 & 75.0 & & & & & \\
\hline & 100 & 1.9 & 305 & 6.1 & 76.2 & & & & & \\
\hline \multirow[t]{8}{*}{$5-64-13$} & 7 & 1.1 & 282 & 30.7 & 61.3 & & & & & \\
\hline & 50 & 3.3 & 288 & 20.5 & 67.1 & & & & & \\
\hline & 100 & 4.0 & 304 & 16.3 & 67.9 & & & & & \\
\hline & 150 & 1. 5 & 306 & 14.8 & 68.0 & & & & & \\
\hline & 200 & 5.6 & 302 & 12.1 & 68.7 & & & & & \\
\hline & 250 & 6.4 & 328 & 9.4 & 68.6 & & & & & \\
\hline & 300 & 6.2 & 318 & B. 1 & 68.7 & & & & & \\
\hline & 400 & 7.6 & 302 & 5.5 & 69.8 & & & & & \\
\hline
\end{tabular}


100 FT TOYER

PORTAgLE MAST

\begin{tabular}{|c|c|c|c|c|c|c|c|c|c|c|}
\hline & $\begin{array}{c}\text { HEI GHT } \\
\text { (Et) }\end{array}$ & $\begin{array}{c}\mathrm{U} \\
(\mathrm{m} / \mathrm{s})\end{array}$ & $\begin{array}{c}\theta \\
(d \in q)\end{array}$ & $\begin{array}{c}\sigma_{\theta} \\
(d \in q)\end{array}$ & $\begin{array}{c}T \\
(O E)\end{array}$ & $\begin{array}{c}\text { HE I GHT } \\
\text { (E: })\end{array}$ & $\begin{array}{c}U \\
(m / 5)\end{array}$ & $\begin{array}{c}\theta \\
(d \in g)\end{array}$ & $\begin{array}{c}\sigma_{\theta} \\
(d \in g)\end{array}$ & $\begin{array}{c}T \\
(\circ \mathrm{F})\end{array}$ \\
\hline \multirow[t]{8}{*}{$5-64-14$} & 7 & 2.2 & 331 & 6.2 & 58.6 & & & & & \\
\hline & 50 & 3.9 & 331 & 4. 9 & 59.5 & & & & & \\
\hline & 100 & 5.0 & 337 & 4.2 & 59.6 & & & & & \\
\hline & 150 & 5.2 & 340 & 3.6 & 59.3 & & & & & \\
\hline & 200 & 6.3 & 334 & 1.0 & 59.6 & & & & & \\
\hline & 250 & 7.2 & 331 & 3.3 & 59.3 & & & & & \\
\hline & 300 & 6.8 & 341 & 3.9 & 59.2 & & & & & \\
\hline & 100 & 7.7 & 336 & 3.6 & 60.1 & & & & & \\
\hline \multirow[t]{8}{*}{$s-64-15$} & 7 & 0.6 & 264 & 23.0 & 19.6 & 2.5 & 0.8 & 248 & 35.1 & 48.2 \\
\hline & 50 & 2.2 & 287 & 23.8 & 36.3 & 5 & 1.2 & 352 & 25.2 & $50 .:$ \\
\hline & 100 & 2.4 & 312 & 24.0 & 57.1 & 10 & 1.7 & 259 & 25.9 & 51.7 \\
\hline & 150 & 2.1 & 328 & 23.9 & 57.3 & 20 & 2.2 & 261 & 26.1 & 54.4 \\
\hline & 200 & 3.3 & 331 & 23.9 & 58.0 & 40 & $\mathbf{H}$ & 285 & 28.5 & 56.5 \\
\hline & 250 & 4. 1 & 337 & 24.3 & 58.0 & 80 & 4. 1 & 295 & 29.5 & 57.3 \\
\hline & 300 & 3.4 & 350 & 21.9 & 50.1 & & & & & \\
\hline & 100 & 1.0 & 350 & 21.6 & 58.9 & & & & & \\
\hline
\end{tabular}

7 SERIES

\begin{tabular}{|c|c|c|c|c|c|c|c|c|c|c|}
\hline \multirow[t]{8}{*}{$2-1$} & 7 & 2.5 & 300 & 7.6 & 49.6 & 2.5 & $\mathbf{H}$ & $\mathbf{H}$ & $\mathbf{M}$ & $\mathbf{M}$ \\
\hline & 50 & 5.0 & 305 & 1.0 & 30.8 & 5 & 11 & $M$ & H & M \\
\hline & 100 & 6.7 & 302 & 3.2 & 51.5 & 10 & $\mathbf{M}$ & 319 & 4. 1 & 49.0 \\
\hline & 150 & $\mathbf{H}$ & M & $M$ & 52.0 & 20 & $n$ & $\mathbf{M}$ & H & 49.3 \\
\hline & 300 & 9.2 & 303 & 3.2 & 53.8 & 40 & $\boldsymbol{K}$ & 325 & 2.5 & 50.0 \\
\hline & 250 & 9.3 & 301 & 2.4 & 54.8 & 80 & $\mathbf{H}$ & 318 & 10.8 & 50.7 \\
\hline & 300 & 9. 1 & 341 & 3.0 & 55.6 & & & & & \\
\hline & 100 & 8.3 & 309 & 2.3 & 56.0 & & & & & \\
\hline
\end{tabular}

ITT SERIES

\begin{tabular}{|c|c|c|c|c|c|}
\hline \multirow[t]{8}{*}{$\pi T-2$} & 7 & 3.6 & 295 & 7.2 & 60.1 \\
\hline & 50 & 6.0 & 288 & 7.6 & 61.2 \\
\hline & 100 & I & n & $n$ & 61.0 \\
\hline & 150 & 6.9 & 297 & 6.6 & 60.7 \\
\hline & 200 & I & I & $x$ & 61.0 \\
\hline & 250 & n & $\mathbf{M}$ & M & 60.7 \\
\hline & 300 & $M$ & H & H & 60.6 \\
\hline & 100 & 10.1 & 294 & 3.6 & 60.4 \\
\hline \multirow[t]{8}{*}{$\mathrm{MT}-3$} & 7 & 1.1 & 291 & 7.5 & 53.8 \\
\hline & 50 & 3. 2 & 289 & 1.0 & 57.5 \\
\hline & 100 & 4.6 & 313 & 4.8 & 57.5 \\
\hline & 150 & 3.8 & 309 & 3.5 & 58.8 \\
\hline & 200 & 5.1 & 303 & 4. 3 & 59.4 \\
\hline & 250 & 5.3 & 302 & 4.1 & 59.2 \\
\hline & 300 & 5.1 & 305 & 4. 7 & 59.2 \\
\hline & 400 & 5.4 & 298 & 1. 6 & 59.1 \\
\hline
\end{tabular}




\begin{tabular}{|c|c|c|c|c|c|c|c|c|c|c|}
\hline & $\begin{array}{c}\text { HEIGHT } \\
(f t)\end{array}$ & $\begin{array}{c}0 \\
(m / 5)\end{array}$ & $\left(\begin{array}{c}\theta \\
(1 \in 9\end{array}\right)$ & $\begin{array}{c}\sigma_{\theta} \\
(\boldsymbol{d} \in \boldsymbol{q})\end{array}$ & $\begin{array}{c}T \\
(0 F)\end{array}$ & $\begin{array}{c}\text { HE IGHT } \\
\text { (ft) }\end{array}$ & $\begin{array}{c}U \\
(\mathrm{a} / \mathrm{s})\end{array}$ & $\begin{array}{c}\theta \\
(\operatorname{deg})\end{array}$ & $\begin{array}{c}\sigma_{\theta} \\
(d \in g)\end{array}$ & $\begin{array}{c}T \\
(\circ F)\end{array}$ \\
\hline \multirow[t]{8}{*}{$M T-4 \hbar$} & 7 & 2.2 & $\mathbf{H}$ & $\mathbf{M}$ & 72.8 & & & & & \\
\hline & 50 & 4. 5 & 292 & $\mathbf{M}$ & 73.9 & & & & & \\
\hline & 100 & 5.8 & H & M & 73.8 & & & & & \\
\hline & 150 & H & K & $\mathbf{M}$ & $n$ & & & & & \\
\hline & 200 & 7.2 & 292 & $\mathbf{M}$ & 74.3 & & & & & \\
\hline & 250 & H & $\mathbf{M}$ & $\mathbf{H}$ & 74.2 & & & & & \\
\hline & 300 & 6.7 & $\mathbf{K}$ & $\mathbf{M}$ & 74.6 & & & & & \\
\hline & 100 & 6.7 & 315 & $\mathbf{H}$ & 76.2 & & & & & \\
\hline \multicolumn{11}{|c|}{ I SERIES } \\
\hline \multirow[t]{8}{*}{$x-2$} & 7 & 1.2 & 298 & 5.0 & 35.0 & & & & & \\
\hline & 50 & 4.1 & 301 & 2. 7 & 39.3 & & & & & \\
\hline & 100 & 5.2 & 319 & 2.6 & 40.1 & & & & & \\
\hline & 150 & 5.8 & 317 & 3.1 & 11.0 & & & & & \\
\hline & 200 & 1.4 & $3: 4$ & 4. 4 & 43. 0 & & & & & \\
\hline & 250 & 7.7 & 325 & 5.2 & 49.4 & & & & & \\
\hline & 300 & 6.9 & 326 & 4. 0 & 45.0 & & & & & \\
\hline & 400 & 6.5 & 332 & 3.7 & 45.1 & & & & & \\
\hline \multirow[t]{8}{*}{$x-4$} & 7 & 2.1 & 272 & 9.8 & 13.9 & & & & & \\
\hline & 50 & 3.6 & 265 & 9.4 & 46.0 & & & & & \\
\hline & 100 & 4.1 & 283 & 7.1 & 15.9 & & & & & \\
\hline & 150 & 4. 1 & 282 & 7.5 & 45.8 & & & & & \\
\hline & 200 & 5.3 & 282 & 6.8 & 46.3 & & & & & \\
\hline & 250 & 6.2 & 288 & 6.0 & 46.1 & & & & & \\
\hline & 300 & 6.6 & 286 & 5.2 & 46.2 & & & & & \\
\hline & 400 & 8.0 & 290 & 3.5 & 16.2 & & & & & \\
\hline
\end{tabular}

I. SERIES

$\begin{array}{rrrrrrrrrrr}14-1 & 7 & 1.0 & 249 & 22.3 & 29.0 & 2.5 & 1.1 & 253 & 23.1 & 29.3 \\ & 50 & 2.9 & 272 & 16.4 & 34.6 & 5 & 1.5 & 245 & 21.5 & 29.6 \\ 100 & 3.8 & 297 & 16.2 & 36.4 & 10 & 2.0 & 254 & 20.6 & 30.8 \\ 150 & 4.1 & 310 & 13.7 & 14 & 20 & 2.6 & 242 & 32.5 & 32.2 \\ & 200 & 1.8 & 312 & 7.3 & 38.6 & 40 & 2.9 & 1 & 11 & 34.6 \\ 250 & 5.2 & 313 & 2.4 & 38.8 & 80 & 1.1 & 262 & 11.6 & 35.1 \\ 300 & 4.9 & 310 & 2.1 & 39.2 & & & & & \\ 400 & 5.5 & 315 & 4.0 & 39.6 & & & & & \end{array}$

- Jaba from nearest houriy observation 


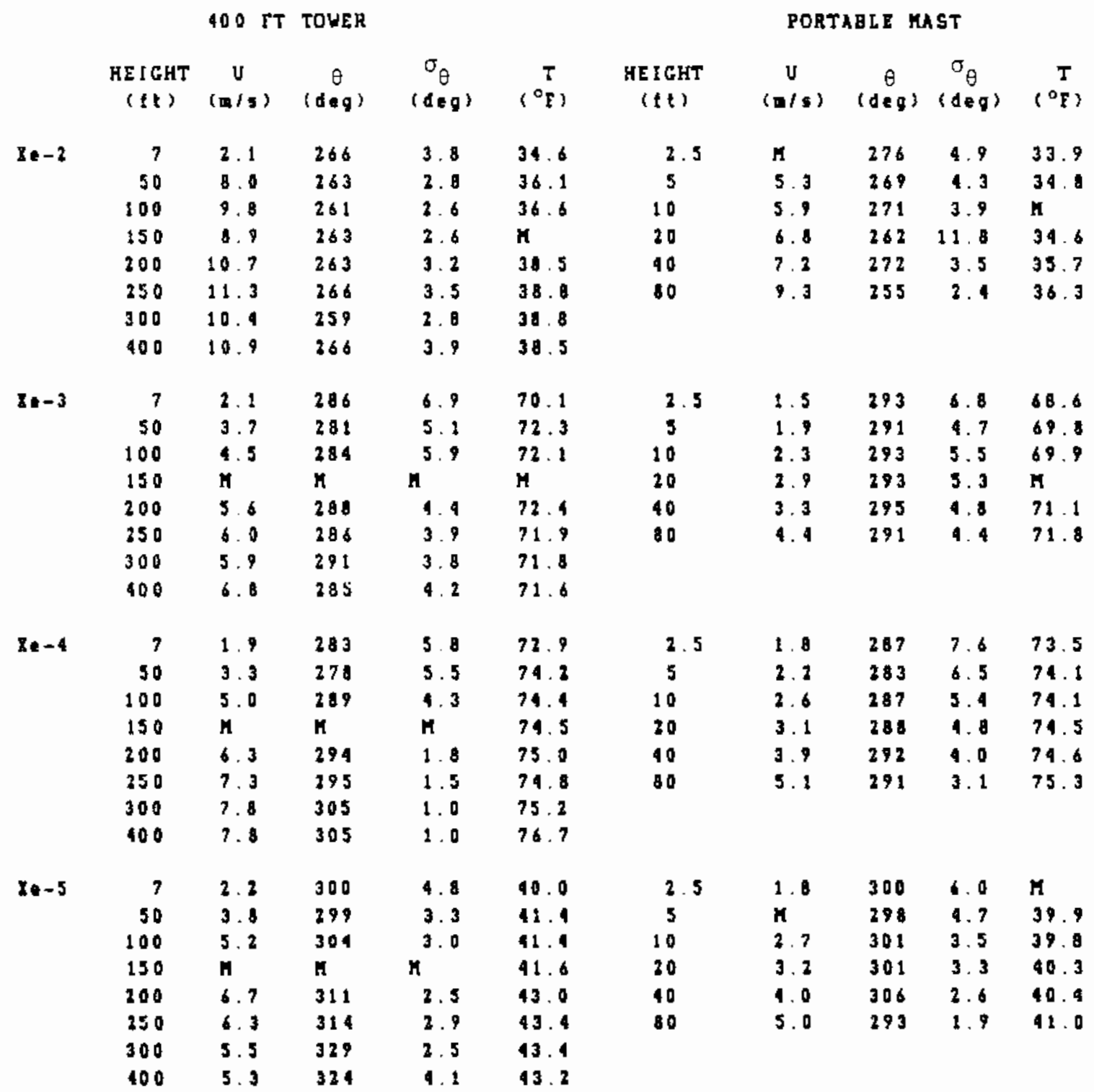

A SERIES

\begin{tabular}{|c|c|c|c|c|c|}
\hline \multirow[t]{8}{*}{$\lambda-2$} & 7 & 4.5 & 310 & 5. 9 & 00.5 \\
\hline & 50 & $5.6 *$ & $292 *$ & $H$ & 0.2 \\
\hline & 100 & $6.7 *$ & n & $\mathbf{M}$ & 80.6 \\
\hline & 150 & H & $\mathbf{M}$ & $n$ & $n$ \\
\hline & 200 & $7.4 \div$ & $304 \pi$ & $\pi$ & 79.6 \\
\hline & 350 & $\mathbf{M}$ & H & $\mathbf{M}$ & 78.8 \\
\hline & 300 & 6. $0 *$ & $n$ & $n$ & 78.8 \\
\hline & 900 & B. 9* & $315 \%$ & $x$ & 787 \\
\hline
\end{tabular}

Data from nearest hourly obsetvation 
400 ET TOUER

PORTABLE MAST

\begin{tabular}{|c|c|c|c|c|c|c|c|c|c|c|}
\hline & $\begin{array}{c}\text { HEICHT } \\
(f t)\end{array}$ & $\begin{array}{c}U \\
(\pi / s)\end{array}$ & $\begin{array}{c}\theta \\
(d \in g)\end{array}$ & $\begin{array}{c}\sigma_{\theta} \\
(d e g)\end{array}$ & $\left(\begin{array}{c}\mathrm{T} \\
\left.{ }^{\circ} \mathrm{F}\right)\end{array}\right.$ & $\begin{array}{c}\text { HEICHT } \\
(f t)\end{array}$ & $\begin{array}{c}U \\
(n / s)\end{array}$ & $\begin{array}{c}\theta \\
\langle d \in g\end{array}$ & $\begin{array}{c}\sigma_{\theta} \\
(d e g)\end{array}$ & $\left(\begin{array}{c}T \\
\left({ }^{\circ} \mathrm{F}\right)\end{array}\right.$ \\
\hline \multirow[t]{8}{*}{$\lambda-5$} & 7 & 3.1 & 302 & 14.9 & 67.6 & 2.5 & 2.1 & 299 & 18.8 & 67.2 \\
\hline & 50 & 4. 8 & 296 & 14.2 & 68.6 & 5 & 2.7 & 301 & 16.7 & 68.0 \\
\hline & 100 & 4.9 & 294 & 14.8 & 68.4 & 10 & 3.2 & 302 & 15.8 & 68.1 \\
\hline & 150 & $\boldsymbol{H}$ & $n$ & M & 68.4 & 20 & 3.9 & 298 & 15.8 & 68.6 \\
\hline & 300 & 5.5 & 289 & 12.4 & $6 B .6$ & 90 & 4.5 & 300 & 15.2 & 68.9 \\
\hline & 250 & Y & I & $M$ & 68,0 & 80 & 5.1 & 396 & 14.1 & 68.9 \\
\hline & 300 & 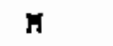 & $\mathbf{H}$ & H & 68.0 & & & & & \\
\hline & 900 & 6.0 & 285 & 9.1 & 68.0 & & & & & \\
\hline \multirow[t]{8}{*}{$x-6$} & 7 & 1.5 & 254 & 26.0 & 54.5 & 2.5 & 1.1 & 251 & 22.7 & 58.2 \\
\hline & 50 & 2.9 & 240 & 18.0 & 55.2 & 5 & 1.4 & $\mathbf{H}$ & $\mathbf{H}$ & 56.8 \\
\hline & 100 & 3.7 & 239 & 17.3 & $5 \mathrm{~J} .5$ & 10 & 1. 8 & 249 & 21.0 & 58.5 \\
\hline & 150 & $\mathbf{M}$ & M & $n$ & 53.0 & 20 & 2.2 & 243 & 20.5 & 58.9 \\
\hline & 200 & 4. 1 & 243 & 13.5 & 57.3 & 40 & 2.9 & 245 & 19.2 & 59.3 \\
\hline & 250 & 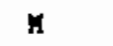 & $\boldsymbol{H}$ & $n$ & 56.6 & 80 & 3.6 & 240 & 18.0 & 59.6 \\
\hline & 300 & $\mathbf{H}$ & $x$ & A & 56.9 & & & & & \\
\hline & 400 & 4.6 & 261 & 11.0 & 57.4 & & & & & \\
\hline \multirow[t]{8}{*}{$\lambda-?$} & 7 & 2.8 & 320 & 5.1 & 57.5 & 2.5 & 2.1 & 322 & 7.8 & 62.3 \\
\hline & 50 & 4.8 & 312 & 4. 1 & 57.9 & 5 & 2.6 & $\mathbf{M}$ & $\pi$ & 62.8 \\
\hline & 100 & 5.9 & 315 & 3.2 & 58. 1 & 10 & 3.3 & 320 & 4.9 & 62.7 \\
\hline & 150 & $\mathbf{M}$ & $\mathbf{M}$ & 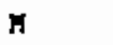 & 58.4 & 20 & 3.9 & 321 & 4. 4 & 62.5 \\
\hline & 200 & 7.9 & 310 & 1. 4 & 59.0 & 90 & 1. 8 & 322 & 3.9 & 63.1 \\
\hline & 250 & $\mathbf{n}$ & I & $n$ & 59.3 & 80 & 5.8 & 317 & 4. 0 & 63.1 \\
\hline & 300 & $K$ & $\mathbf{M}$ & $\mathbf{M}$ & 59.7 & & & & & \\
\hline & 400 & 7.6 & 312 & 3.3 & 60.4 & & & & & \\
\hline \multirow[t]{8}{*}{$A-8$} & 7 & 1.8 & $28 ?$ & 7.5 & 61.0 & 2.3 & 1.7 & 289 & 9.1 & 66.0 \\
\hline & 50 & 1. 3 & 285 & 4. 2 & 64.7 & 5 & 1.9 & 290 & 8. 3 & 66.0 \\
\hline & 100 & 6.2 & 297 & 3.2 & 65.9 & 10 & 2.4 & 289 & 7.5 & 66.9 \\
\hline & 150 & и & $\mathbf{H}$ & $\mathbf{H}$ & 66.9 & 20 & 3.1 & 289 & 6.6 & 67.0 \\
\hline & 200 & 6.0 & 299 & 2.2 & 67.2 & 40 & 4.2 & 296 & 9. 6 & 68.8 \\
\hline & 250 & $\mathbf{H}$ & $\mathbf{M}$ & $\mathbf{M}$ & 67.0 & 80 & 5.6 & 297 & 2. 6 & 69.8 \\
\hline & 300 & $n$ & M & M & 67.6 & & & & & \\
\hline & 400 & 5.0 & 314 & 5. 1 & 68.3 & & & & & \\
\hline \multirow[t]{8}{*}{$\lambda-9$} & 7 & 0.8 & 260 & 37.2 & 62.6 & 2.5 & 0.8 & 251 & 41.2 & 65.9 \\
\hline & 50 & 2.7 & 285 & 6.8 & 68.1 & 5 & 1.2 & 259 & 37.9 & 67.8 \\
\hline & 100 & 3.7 & 296 & 5.5 & 68.2 & 10 & 1.5 & 265 & 31.8 & 69.5 \\
\hline & 150 & $\mathbf{H}$ & $\mathbf{n}$ & $x$ & 68.4 & 20 & 2.0 & 276 & 23.0 & 71.0 \\
\hline & 200 & 5.1 & 299 & 3.0 & 68.9 & 40 & 2.8 & 291 & 10.9 & 12.5 \\
\hline & 250 & M & M & $M$ & 68.7 & 80 & 3.5 & 396 & $5 .:$ & 72. \\
\hline & 300 & $\mathbf{M}$ & $n$ & $M$ & 69.3 & & & & & \\
\hline & 100 & 7.1 & 299 & 1.7 & 70.7 & & & & & \\
\hline
\end{tabular}





\begin{tabular}{|c|c|c|c|}
\hline \multicolumn{2}{|c|}{$\begin{array}{l}\text { NRC FOAM 335 } \\
\text { U.S. NUCLEAR AEGULATOAY COMMISSION } \\
\text { BIBLIOGRAPHIC DATA SHEET }\end{array}$} & \multicolumn{2}{|c|}{$\begin{array}{l}\text { 1. REPORT NUMEEA (A Is gned oy ODC) } \\
\text { NUREG/CR-3456 } \\
\text { PNL-4814 }\end{array}$} \\
\hline \multirow{2}{*}{\multicolumn{2}{|c|}{ 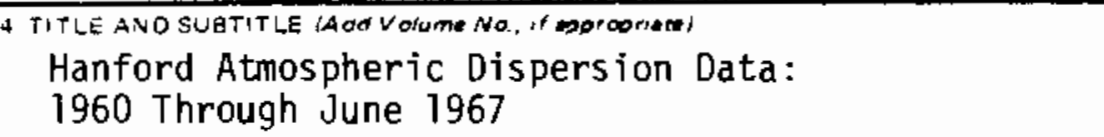 }} & \multicolumn{2}{|l|}{ 2. (Lewe Dism $x)$} \\
\hline & & \multicolumn{2}{|c|}{ 3. RECIPIENT'S ACCESSION NO. } \\
\hline \multicolumn{2}{|c|}{ PW Nickola, JV Ramsdel1, CS Glantz, RE Kerns } & \multicolumn{2}{|c|}{ 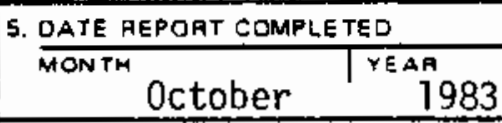 } \\
\hline \multirow{2}{*}{\multicolumn{2}{|c|}{$\begin{array}{l}9 \text { PEAFOAMING OAGANIZATION NAME ANO MAILING ADORESS Hnetud Zip COAE } \\
\text { GeOSCiences R\&E Department } \\
\text { Pacific Northwest Laboratory } \\
\text { Richland, Washington } 99352\end{array}$}} & \multicolumn{2}{|c|}{\begin{tabular}{|l|l|} 
DATE REPORT ISSUEO & \\
MONTH NOvember & 1983 \\
\end{tabular}} \\
\hline & & \multicolumn{2}{|l|}{6 (Latue doenk) } \\
\hline & & \multicolumn{2}{|l|}{ 8. (Leare $\operatorname{dan} k$ ) } \\
\hline \multirow{2}{*}{\multicolumn{2}{|c|}{$\begin{array}{l}\text { 12. SPONSORING OAGANIZATION NAME AND MAILING AODRESS anciud Zio Code' } \\
\text { Division of Health, Siting and Waste Management } \\
\text { Office of Nuclear Regulatory Research } \\
\text { U.S. Nuclear Regulatory Comission } \\
\text { Washington, DC } 20555\end{array}$}} & \multicolumn{2}{|c|}{ 10. PQOJECT, TASK/WORK UNIT NO. } \\
\hline & & \multicolumn{2}{|l|}{$\begin{array}{l}\text { 11. FIN NO. } \\
\text { FIN B2384 }\end{array}$} \\
\hline \multicolumn{4}{|l|}{$\begin{array}{l}\text { 13. TYPE OF REPORT } \\
\text { Technical Report }\end{array}$} \\
\hline \multicolumn{2}{|l|}{ 15. SUPPLEMENTARY NOTES } & \multicolumn{2}{|l|}{ 14. (Leave aromit) } \\
\hline \multicolumn{4}{|c|}{$\begin{array}{l}\text { 15. ABSTRACT } 200 \text { words or lessi } \\
\text { This volume presents dispersion and supporting meteorological data from } \\
\text { experiments conducted over relatively flat terrain at Hanford, Washington } \\
\text { from January } 1960 \text { through June } 1967 \text {. The nature of the experiments, the } \\
\text { sampling grids, and the tracer techniques used are described in the } \\
\text { narrative portion of the document. Appendices contain the time-integrated } \\
\text { concentrations for samplers within the plumes, summaries of the concen- } \\
\text { tration distributions across the plumes, and wind and temperature profile } \\
\text { data for each release period. }\end{array}$} \\
\hline \multicolumn{4}{|c|}{$\begin{array}{l}\text { Atmospheric Dispersion } \\
\text { Tracer Dispersion Experiments }\end{array}$} \\
\hline \multicolumn{4}{|c|}{ 17D. IDENTIFIERS, GPEN.ENDED TERUIS } \\
\hline \multirow[t]{2}{*}{$\begin{array}{l}\text { 1G. AVALABLLITY STATEMENT } \\
\text { Unlimited }\end{array}$} & \multirow{2}{*}{\multicolumn{2}{|c|}{ 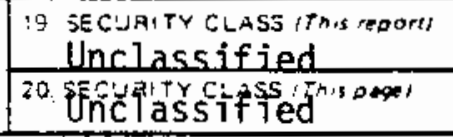 }} & 2: YO OFPAUES \\
\hline & & & $\mid \begin{array}{l}22 \text { ORICE } \\
5\end{array}$ \\
\hline
\end{tabular}


